bootstrap 1

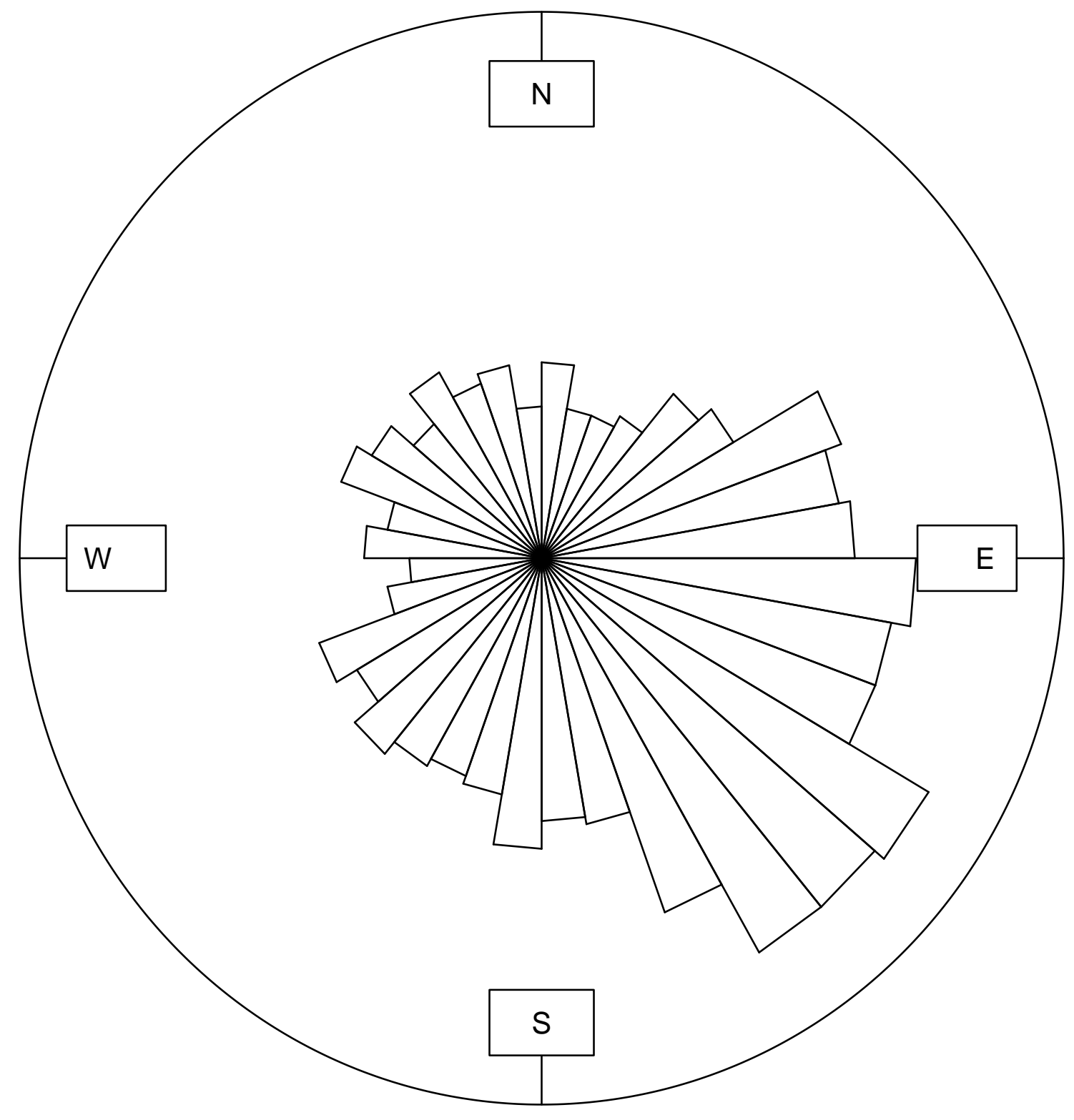


bootstrap 2

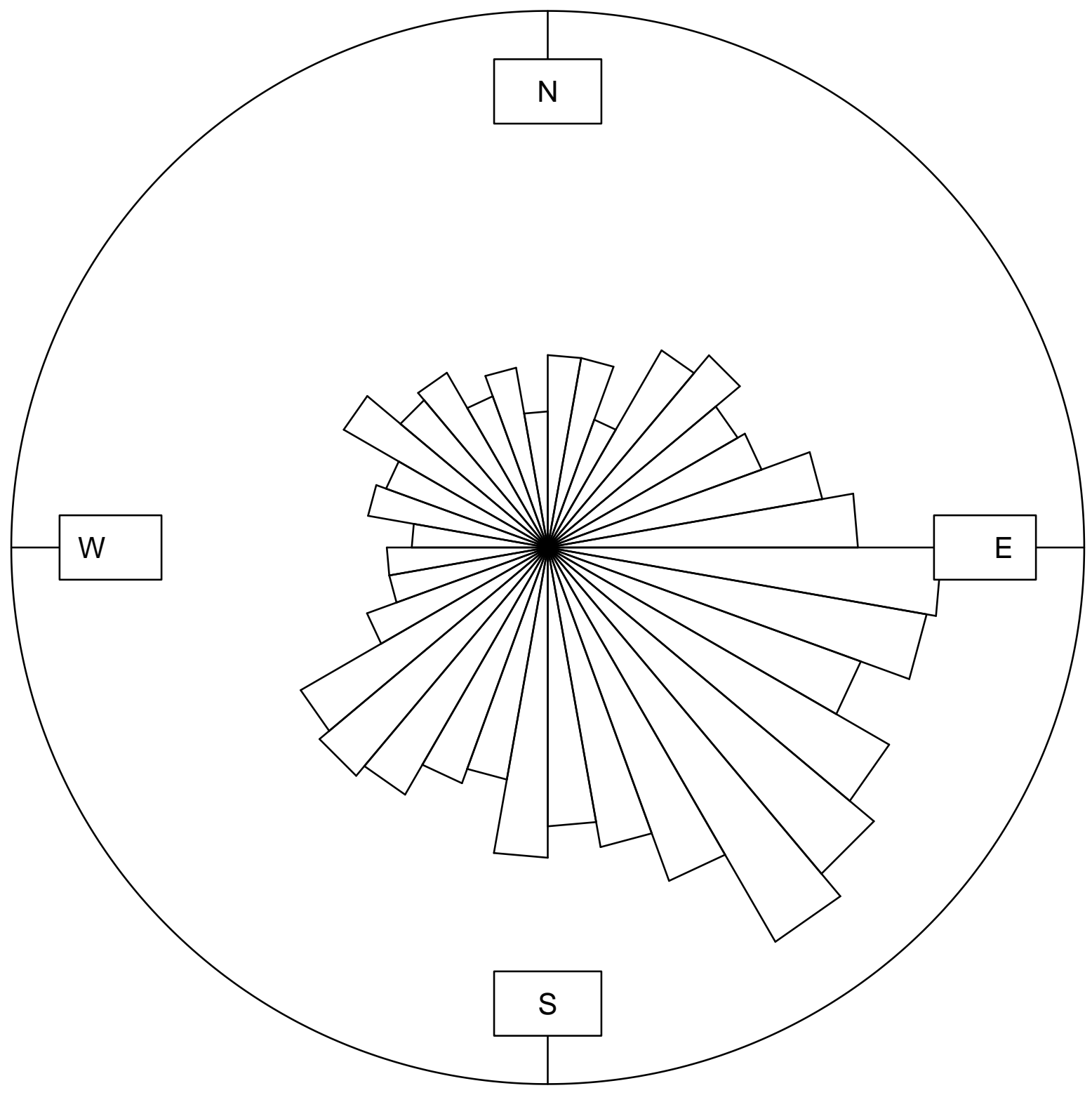


bootstrap 3

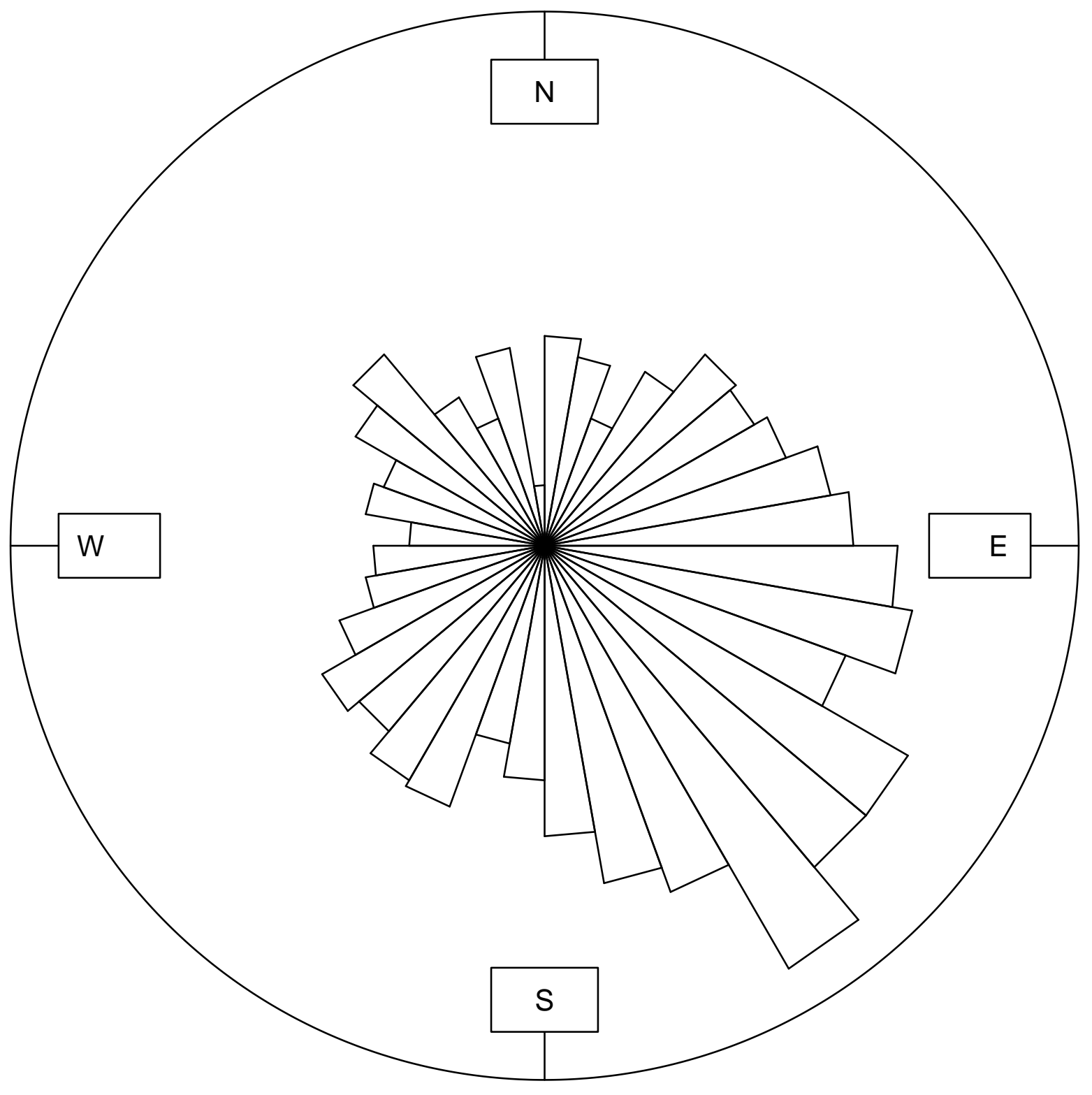


bootstrap 4

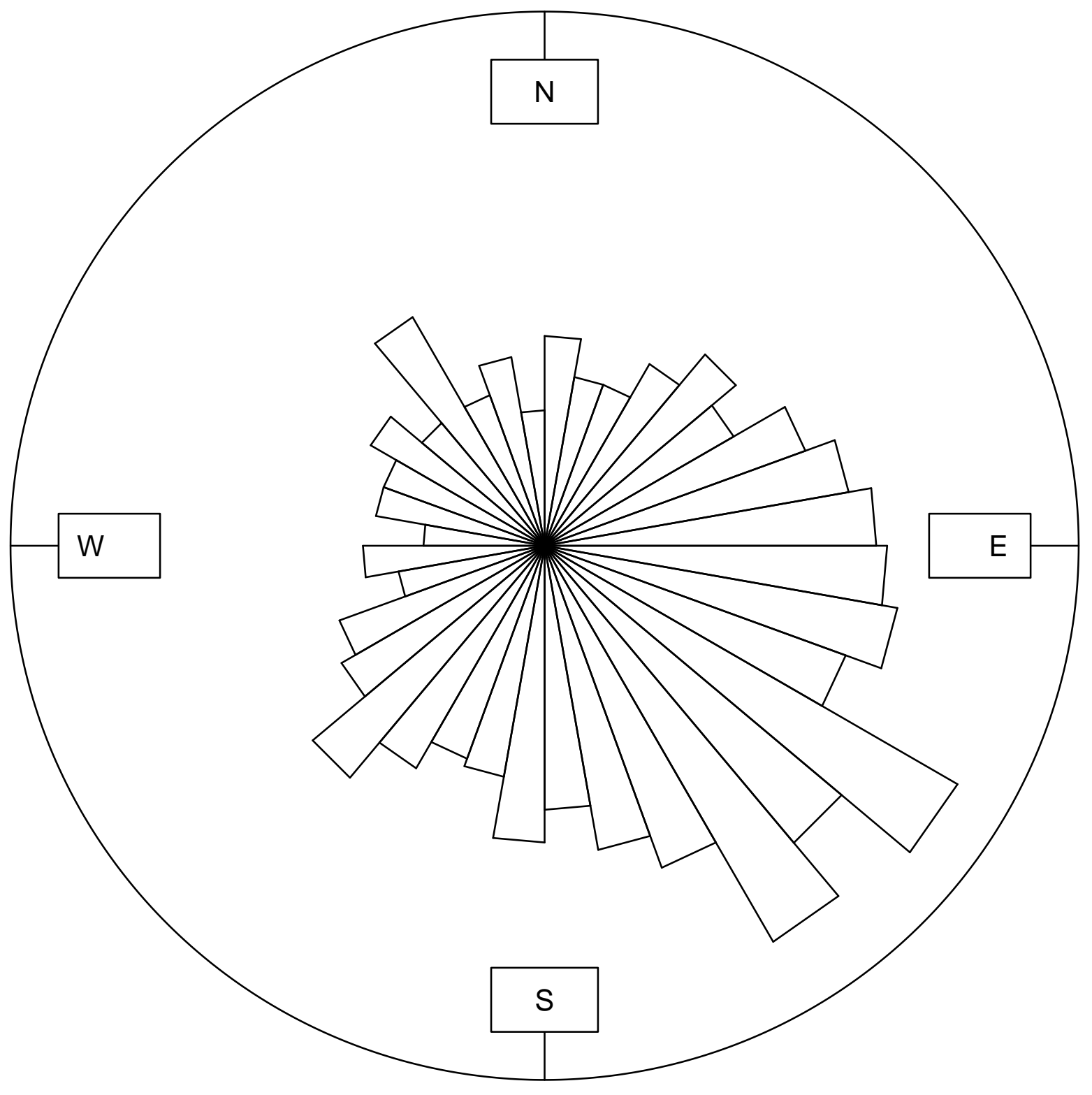


bootstrap 5

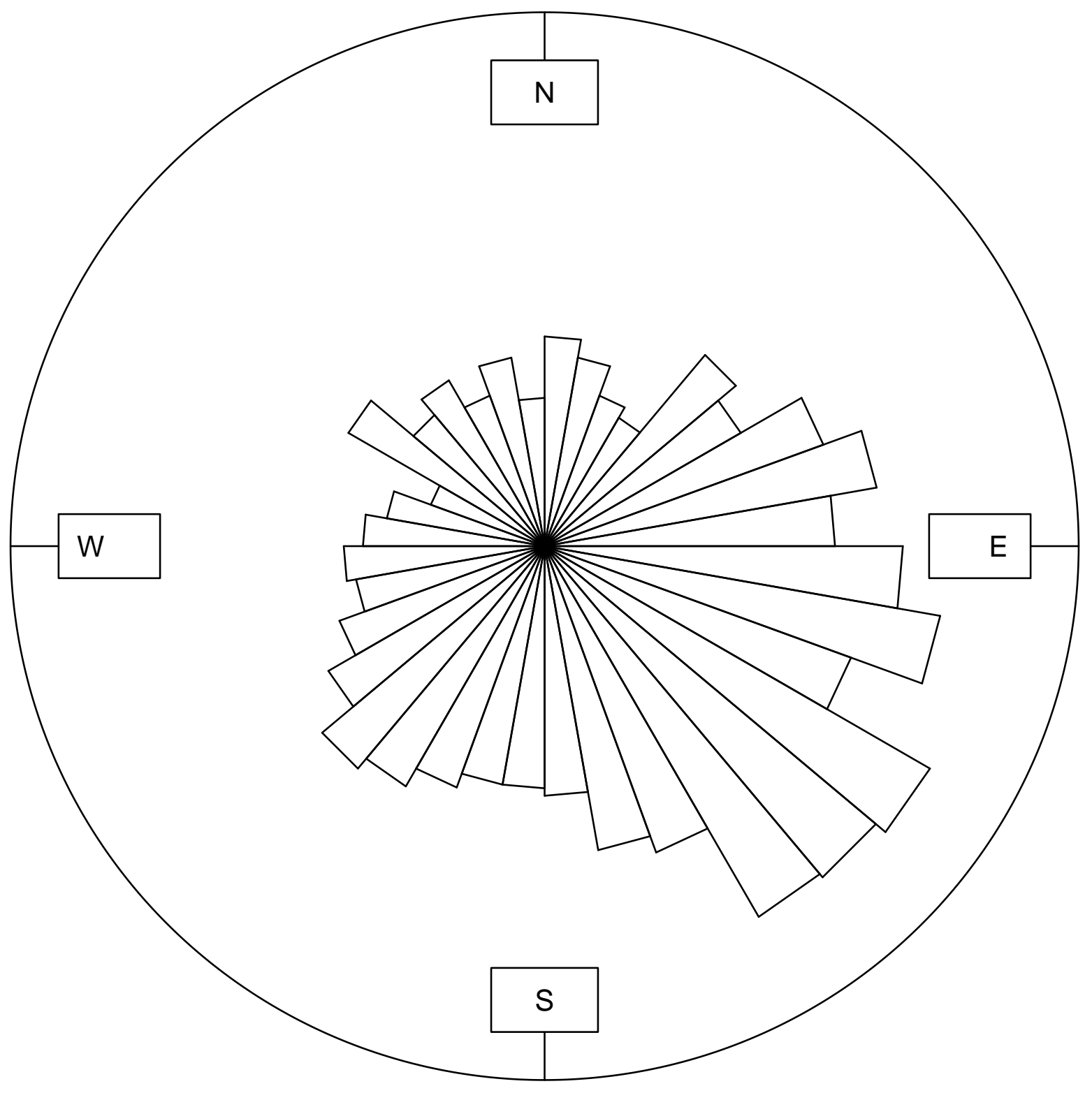


bootstrap 6

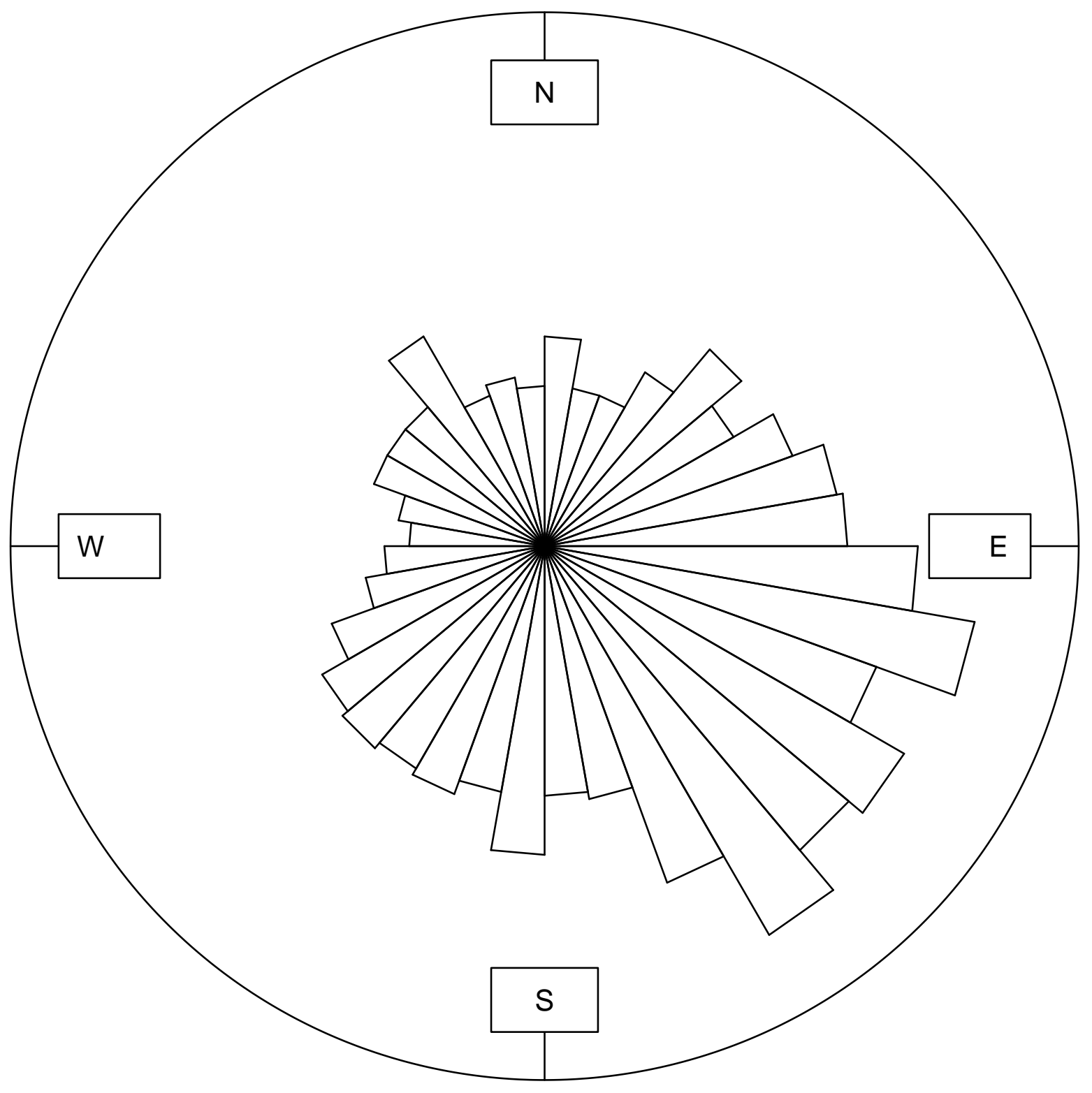


bootstrap 7

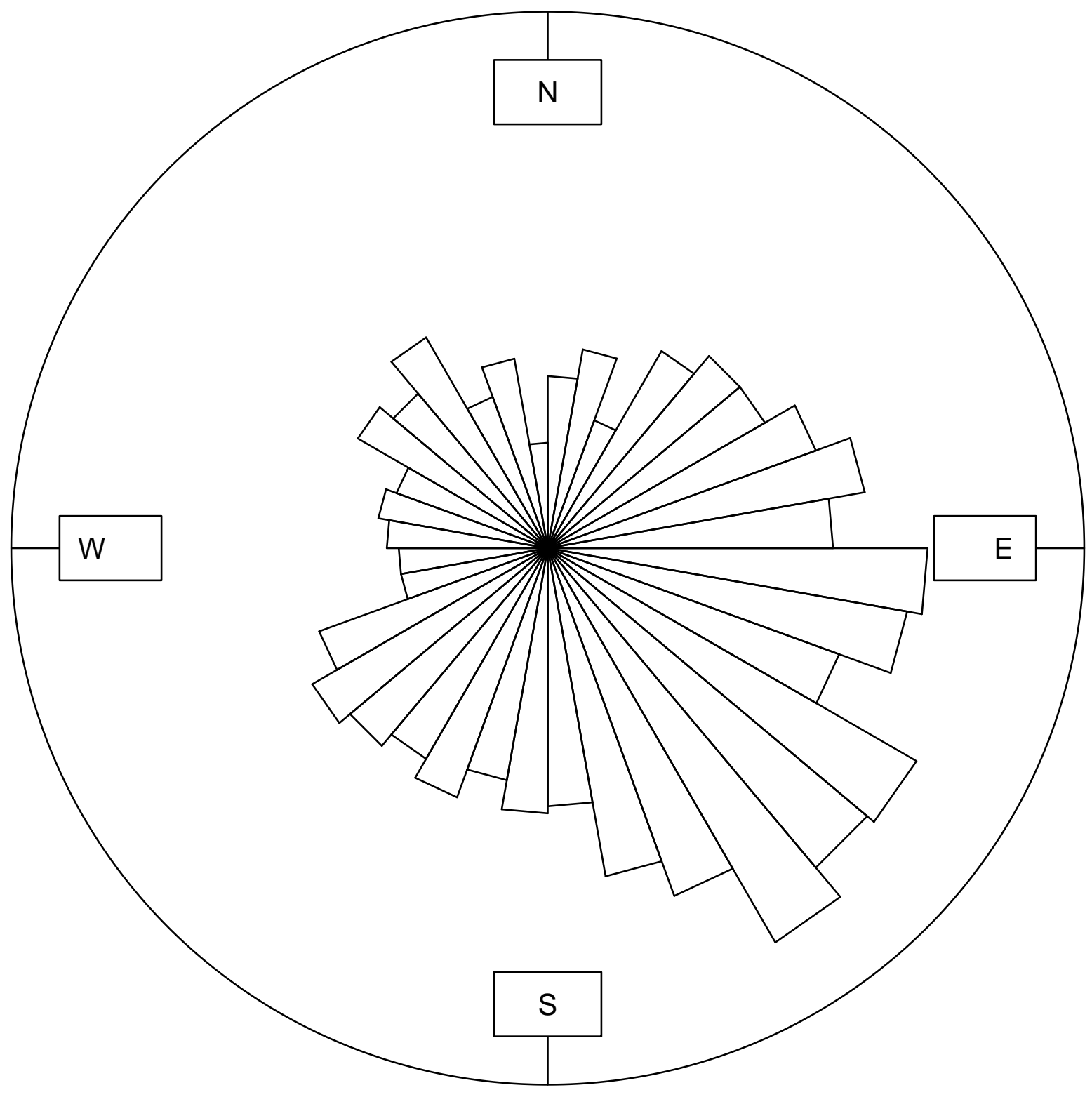


bootstrap 8

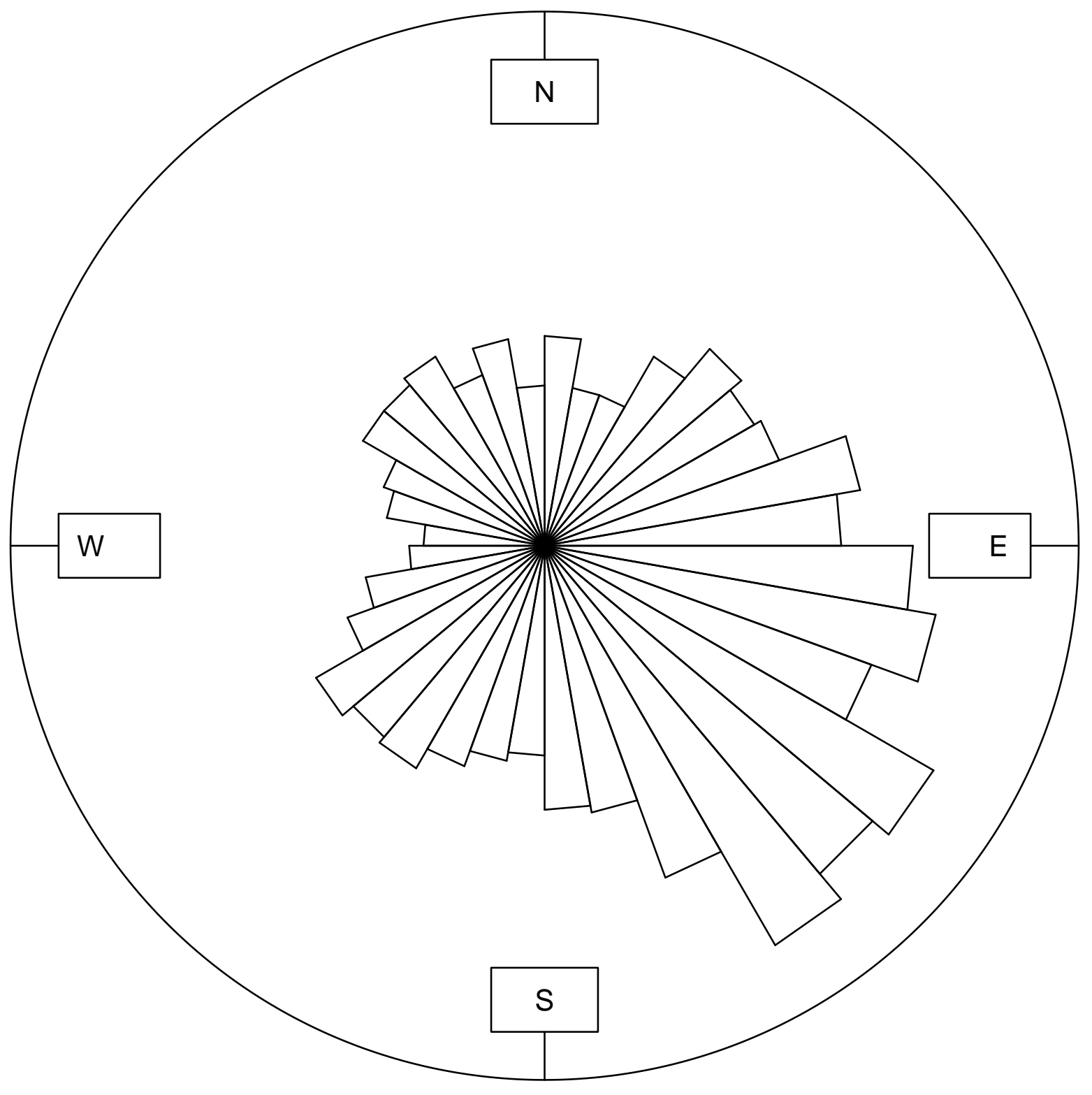


bootstrap 9

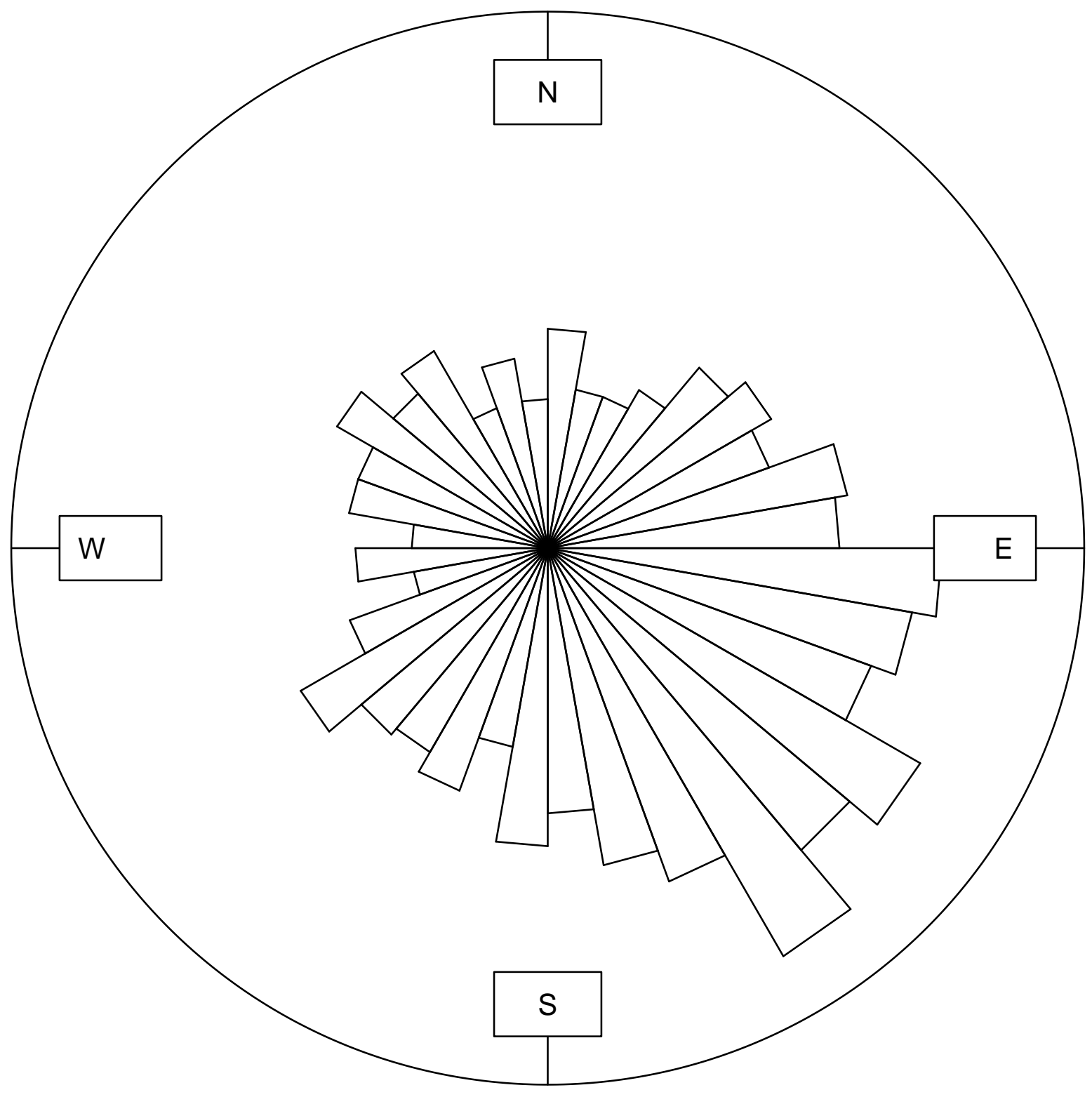


bootstrap 10

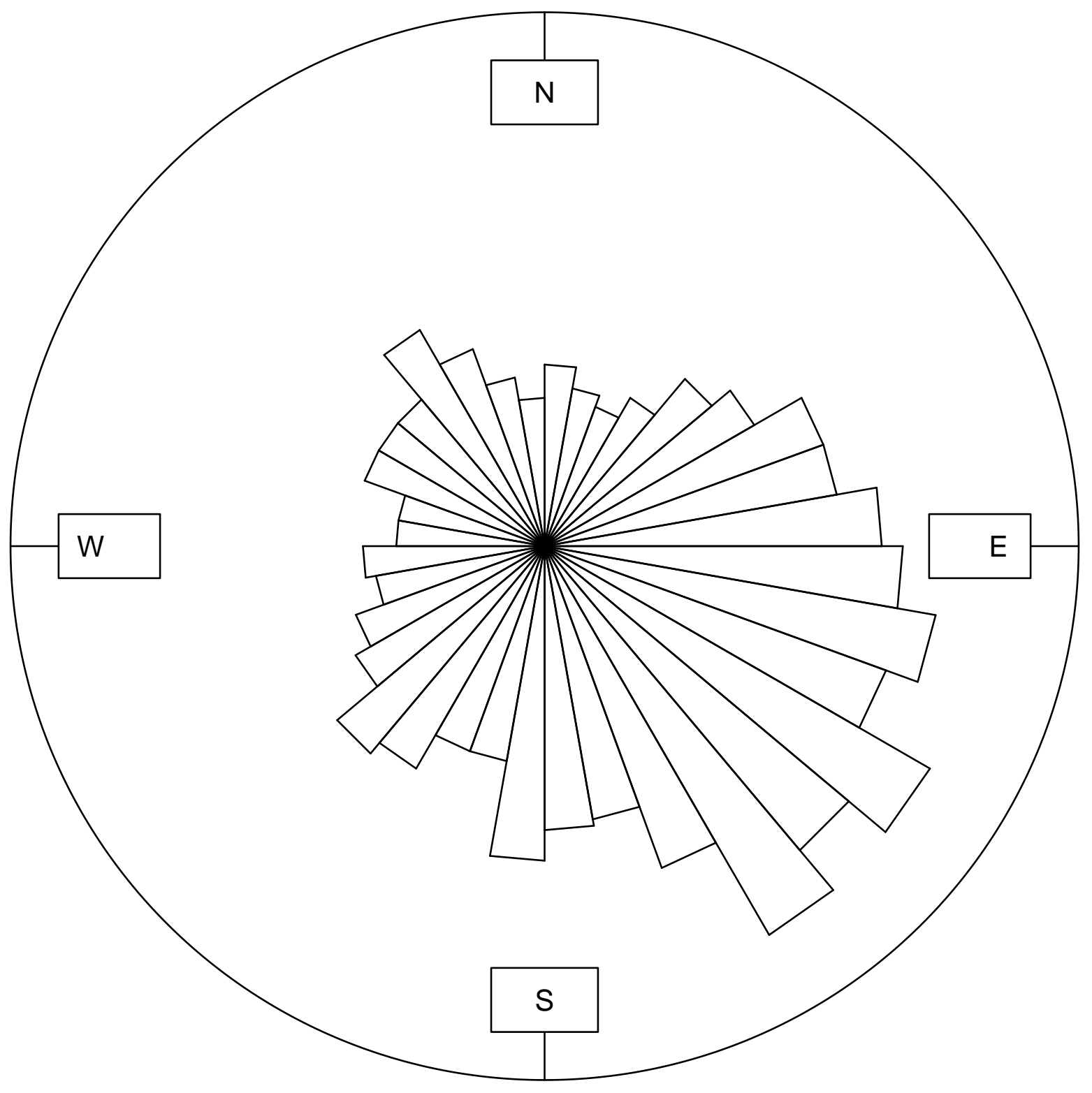




\section{bootstrap 11}

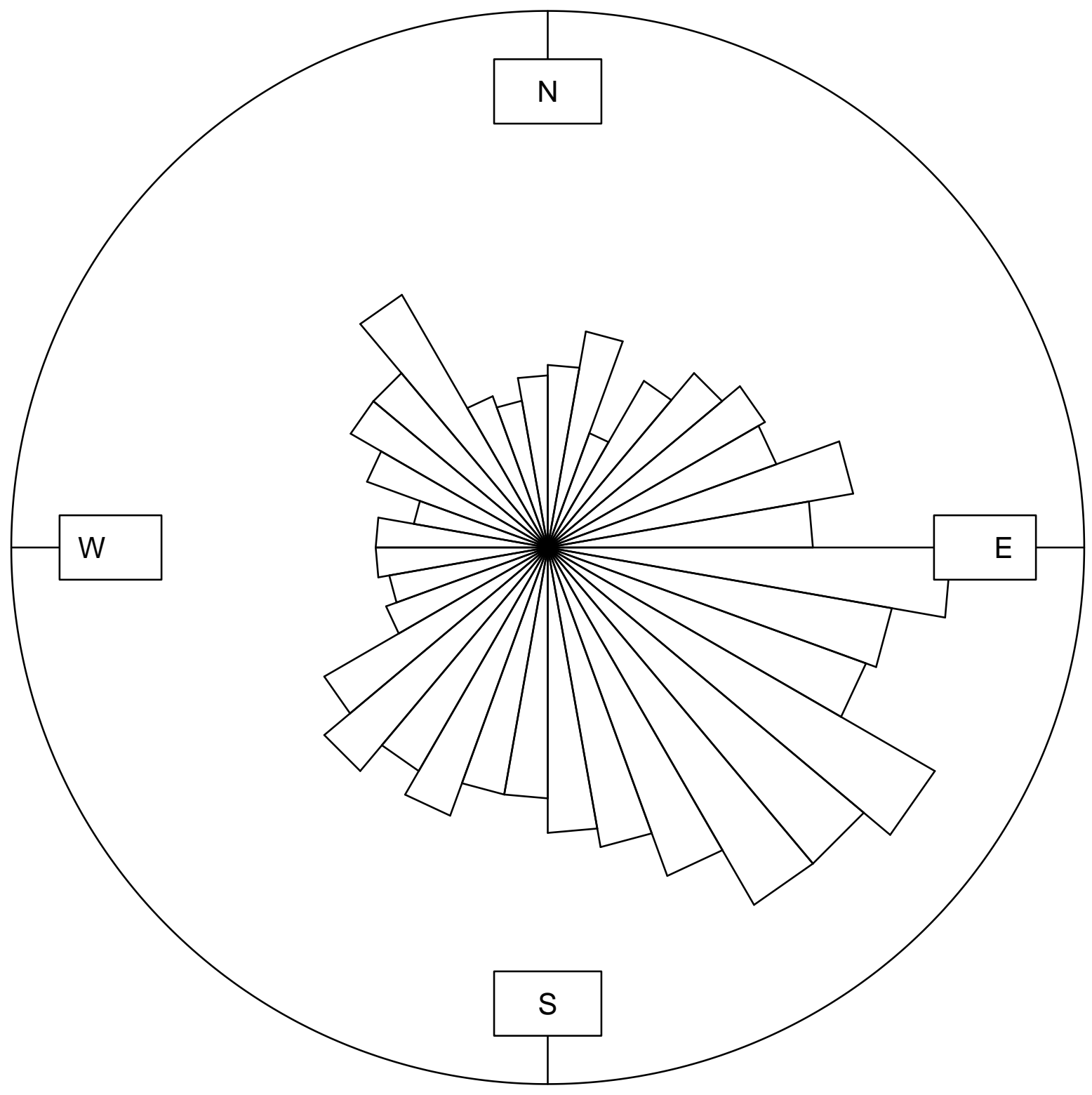


bootstrap 12

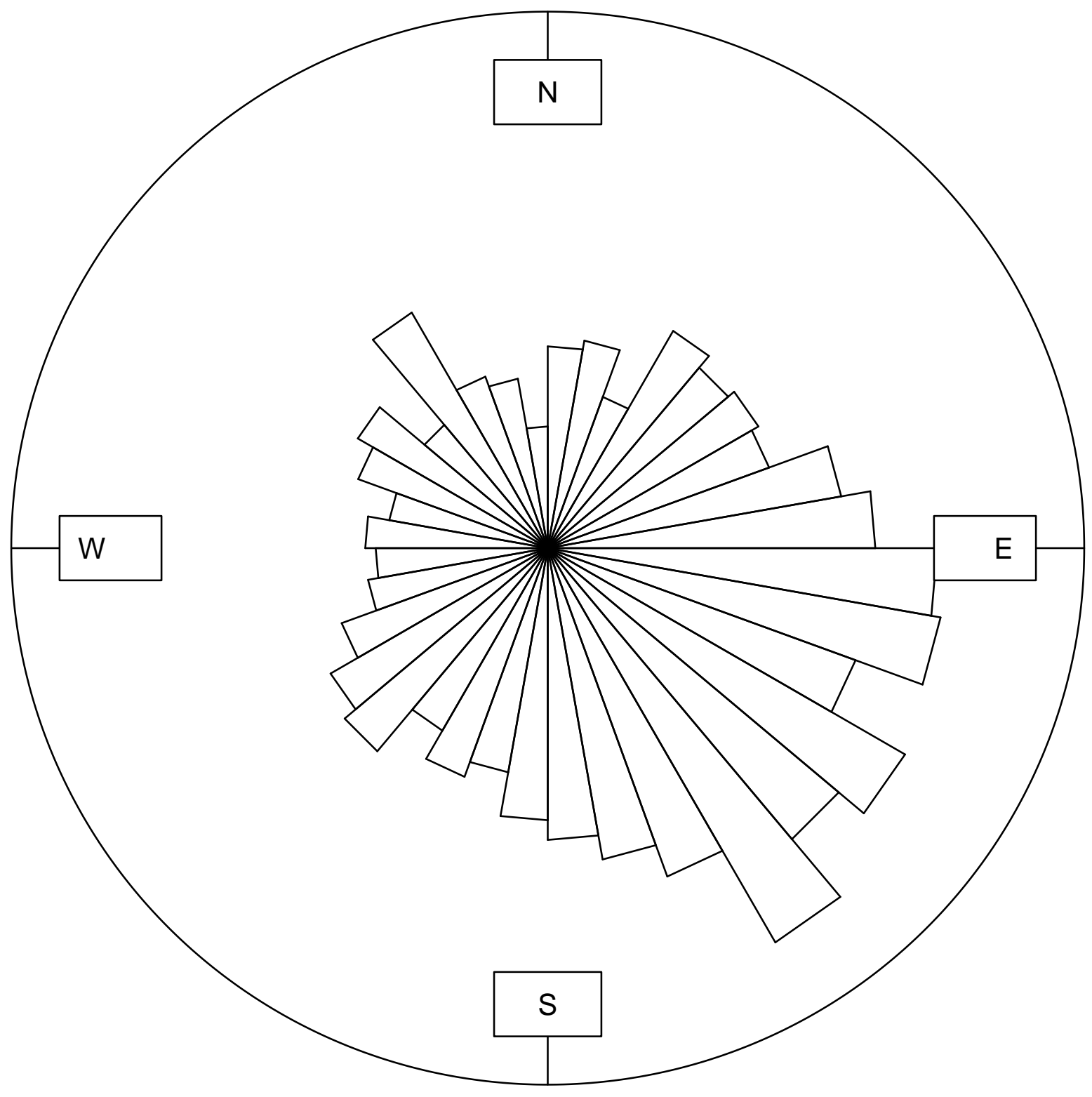




\section{bootstrap 13}

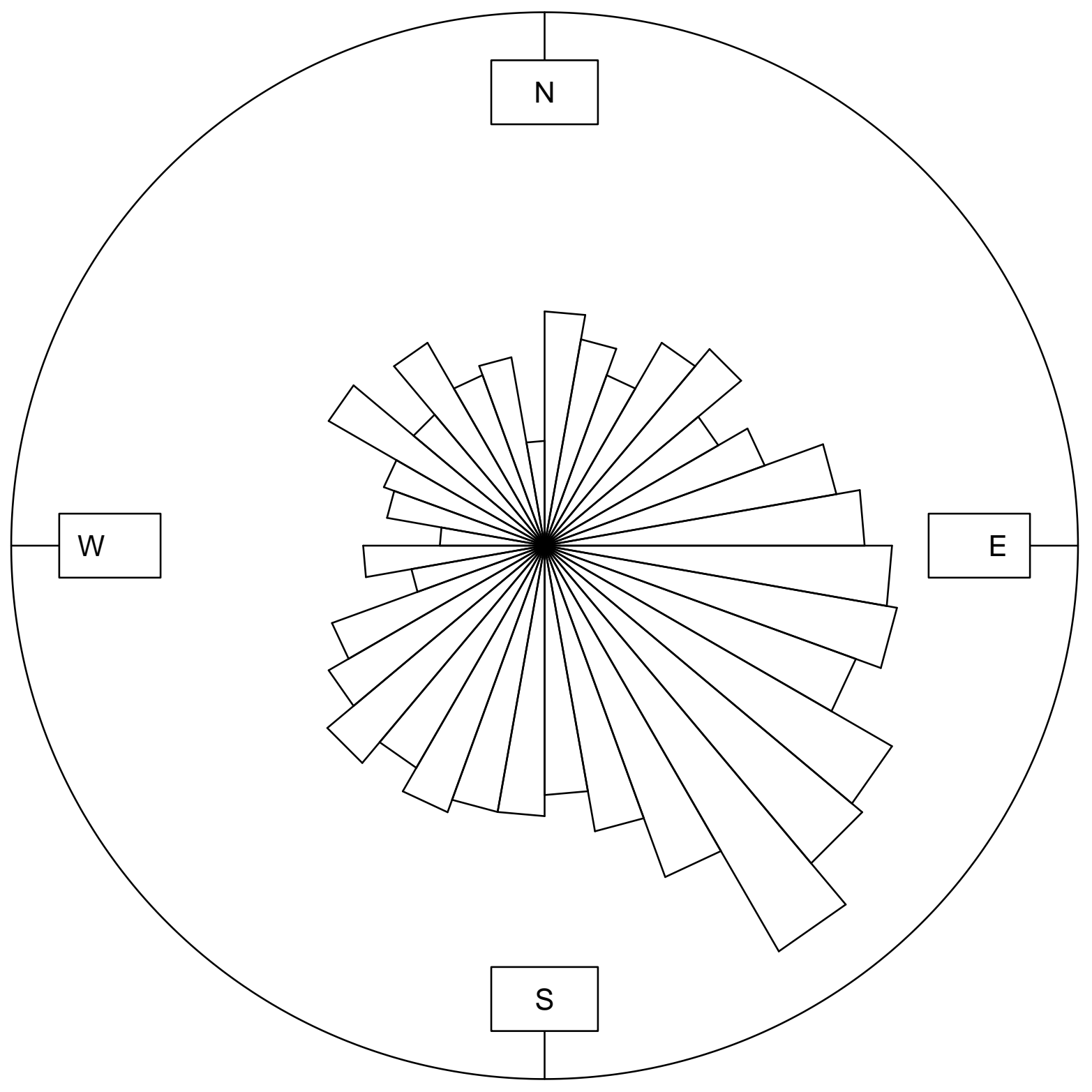




\section{bootstrap 14}

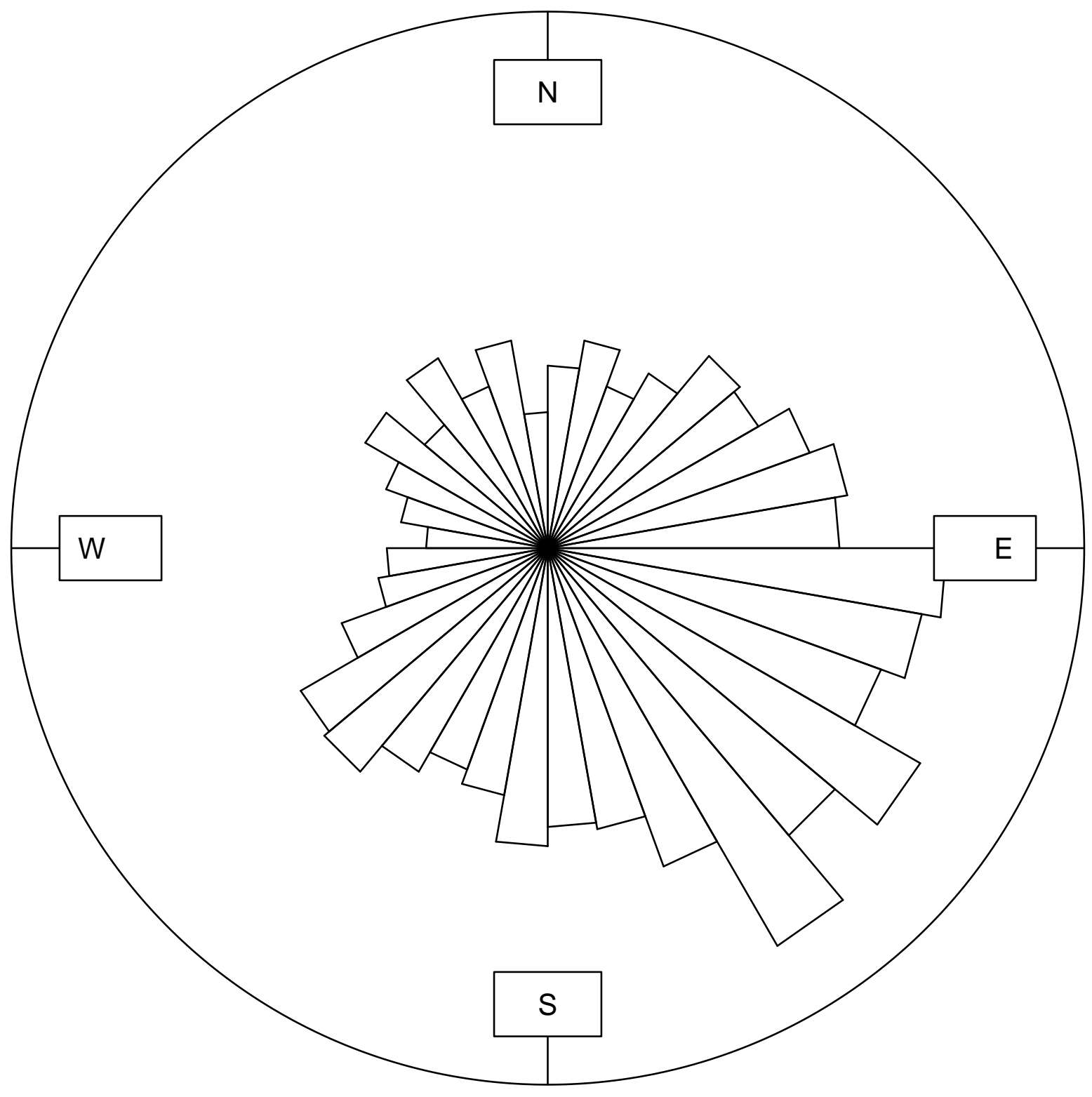


bootstrap 15

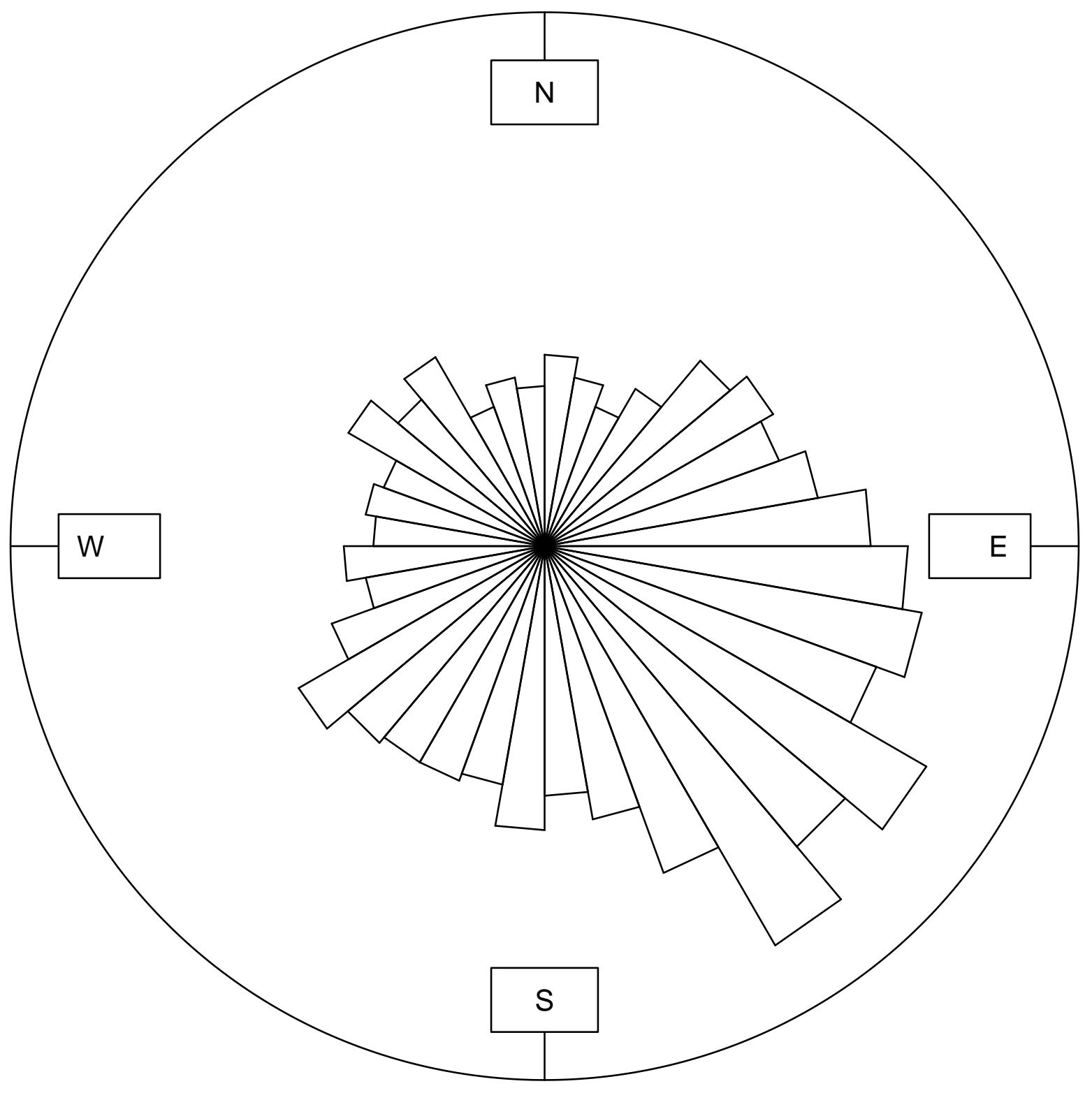


bootstrap 16

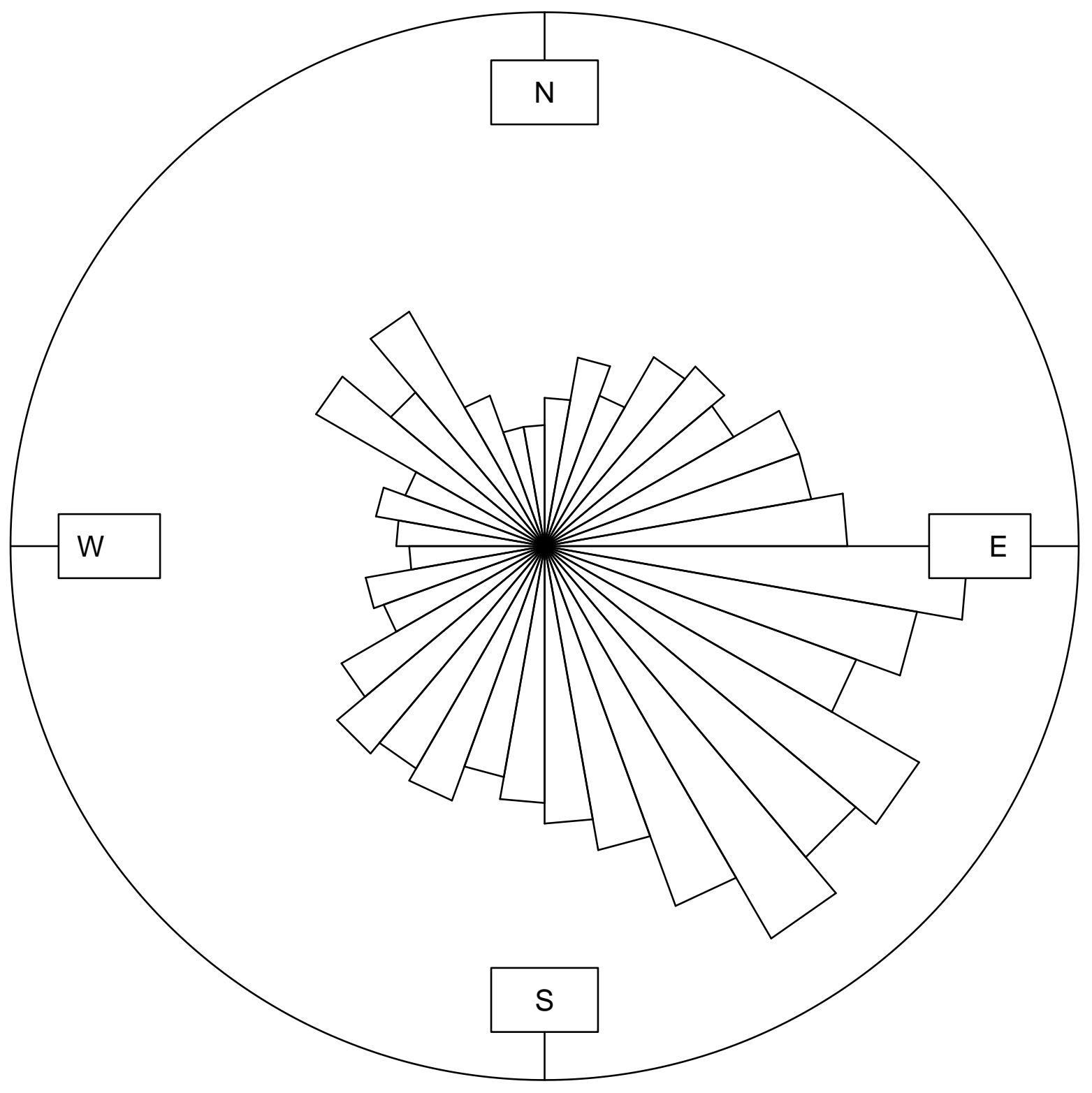


bootstrap 17

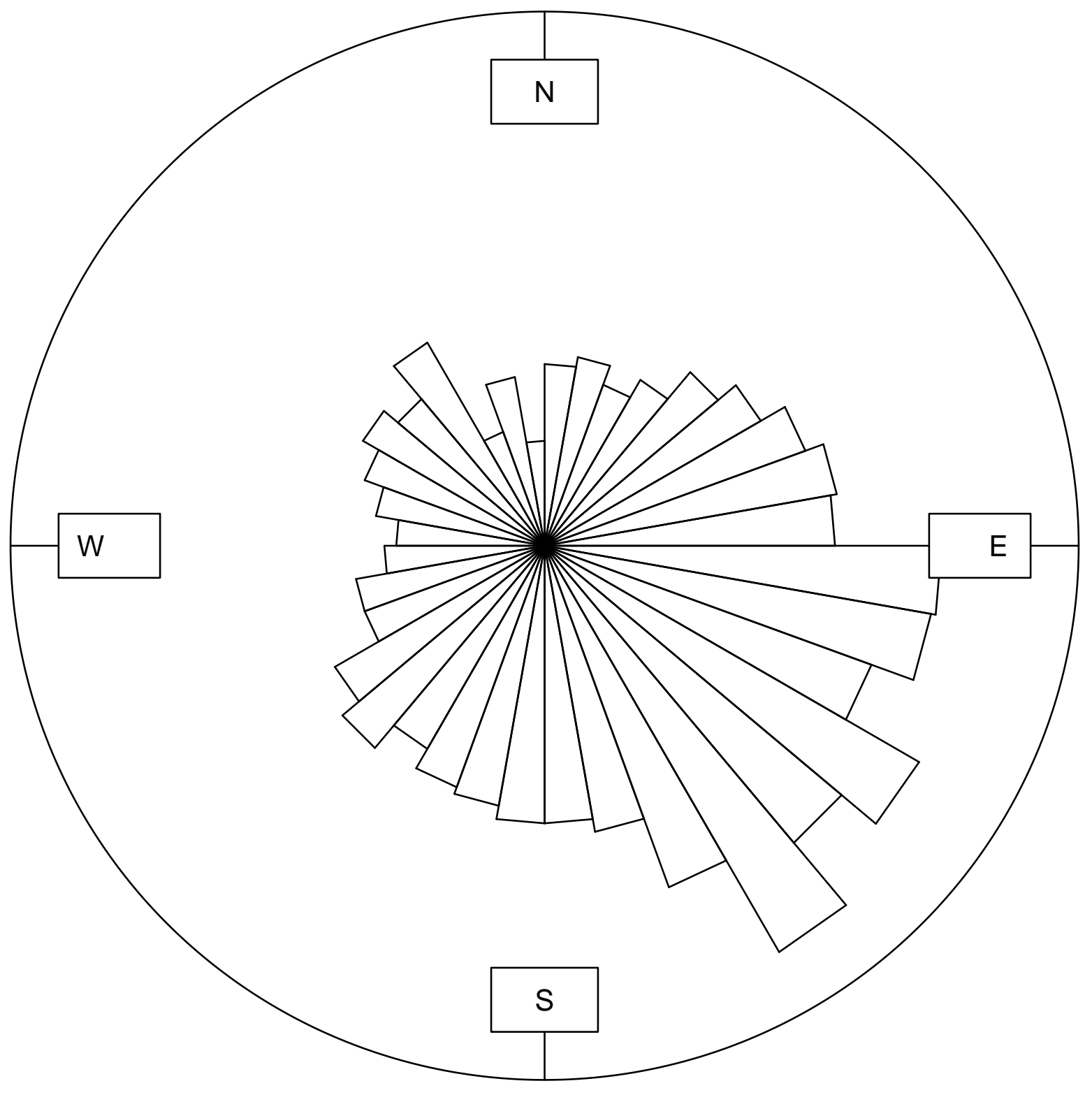




\section{bootstrap 18}

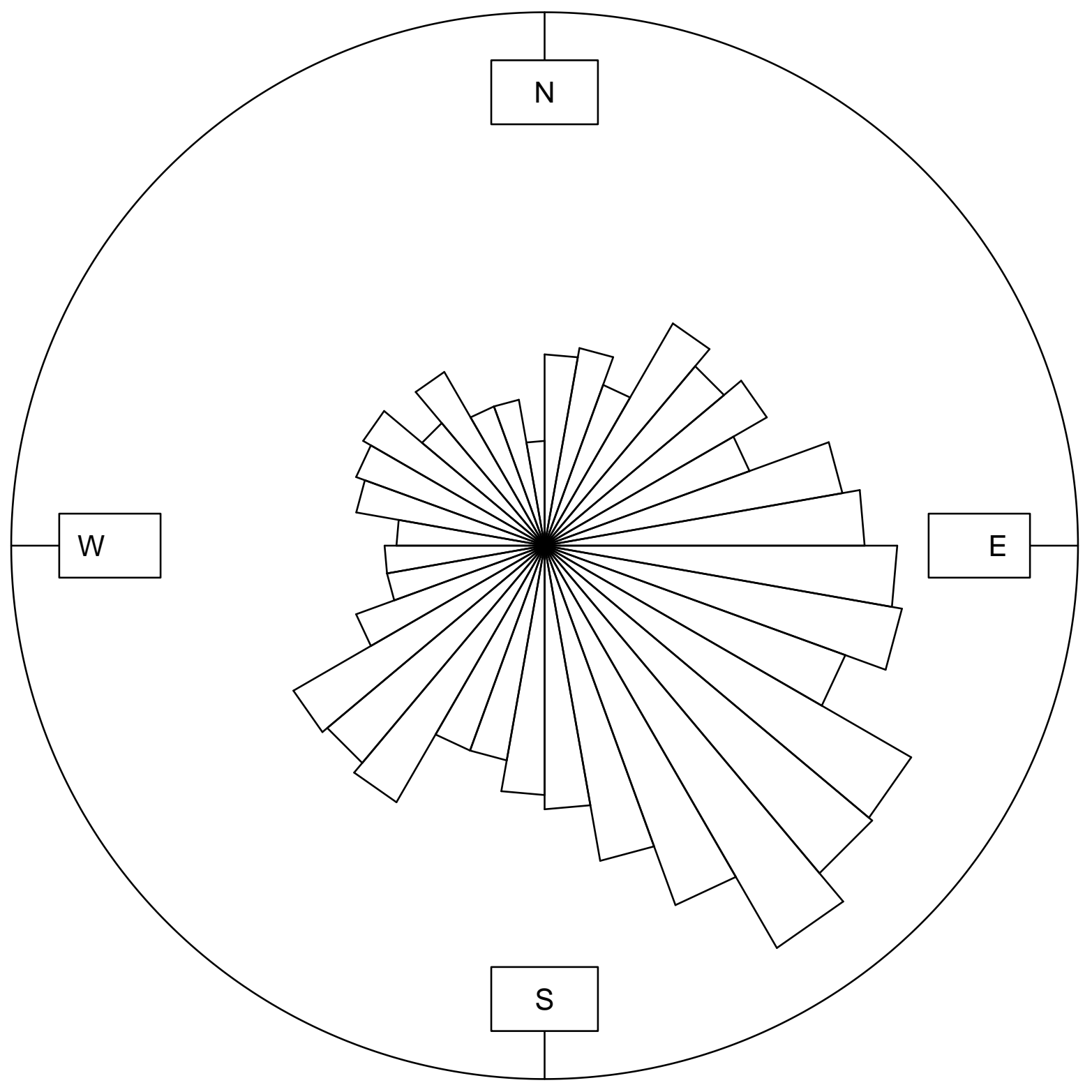


bootstrap 19

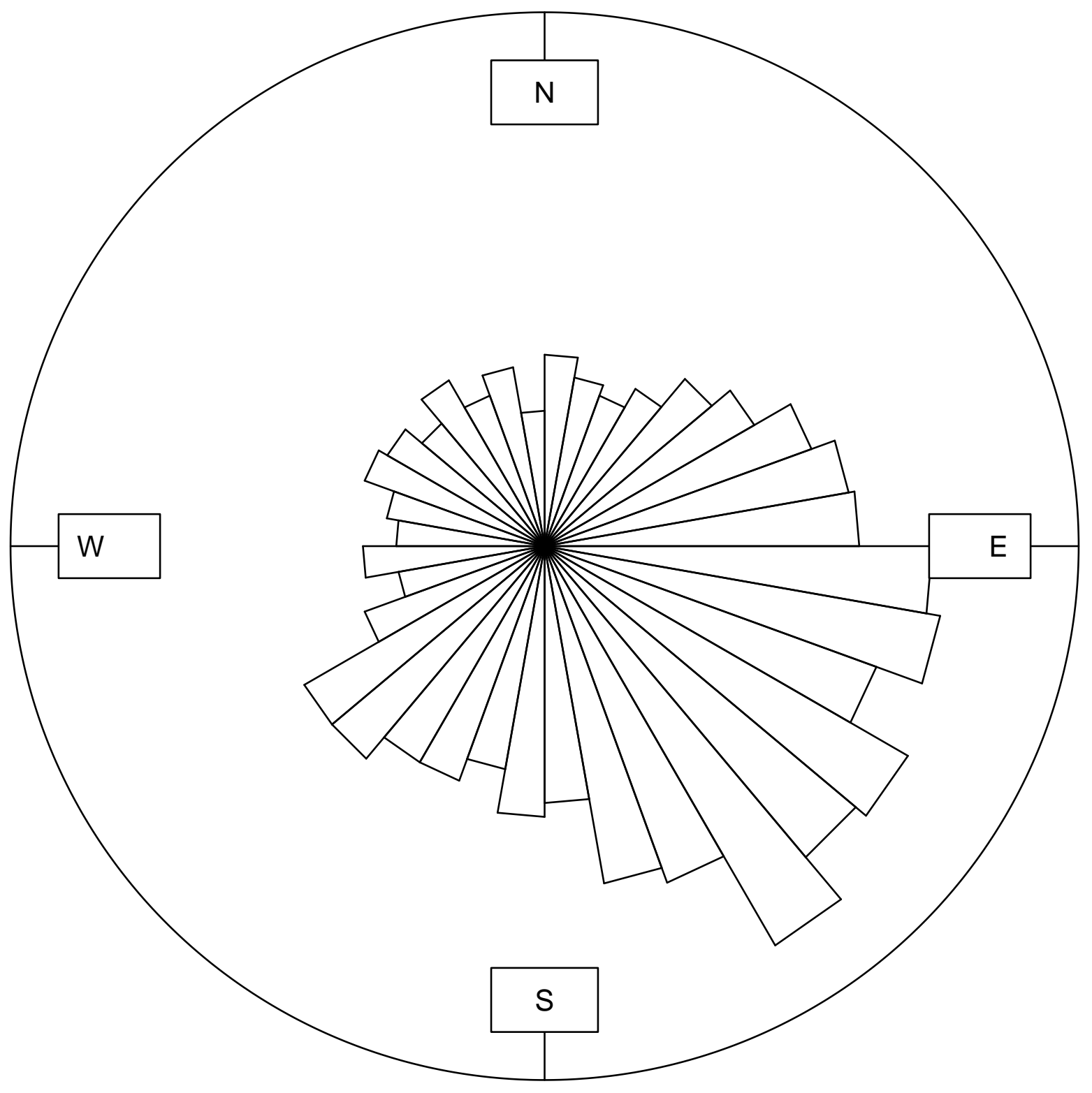


bootstrap 20

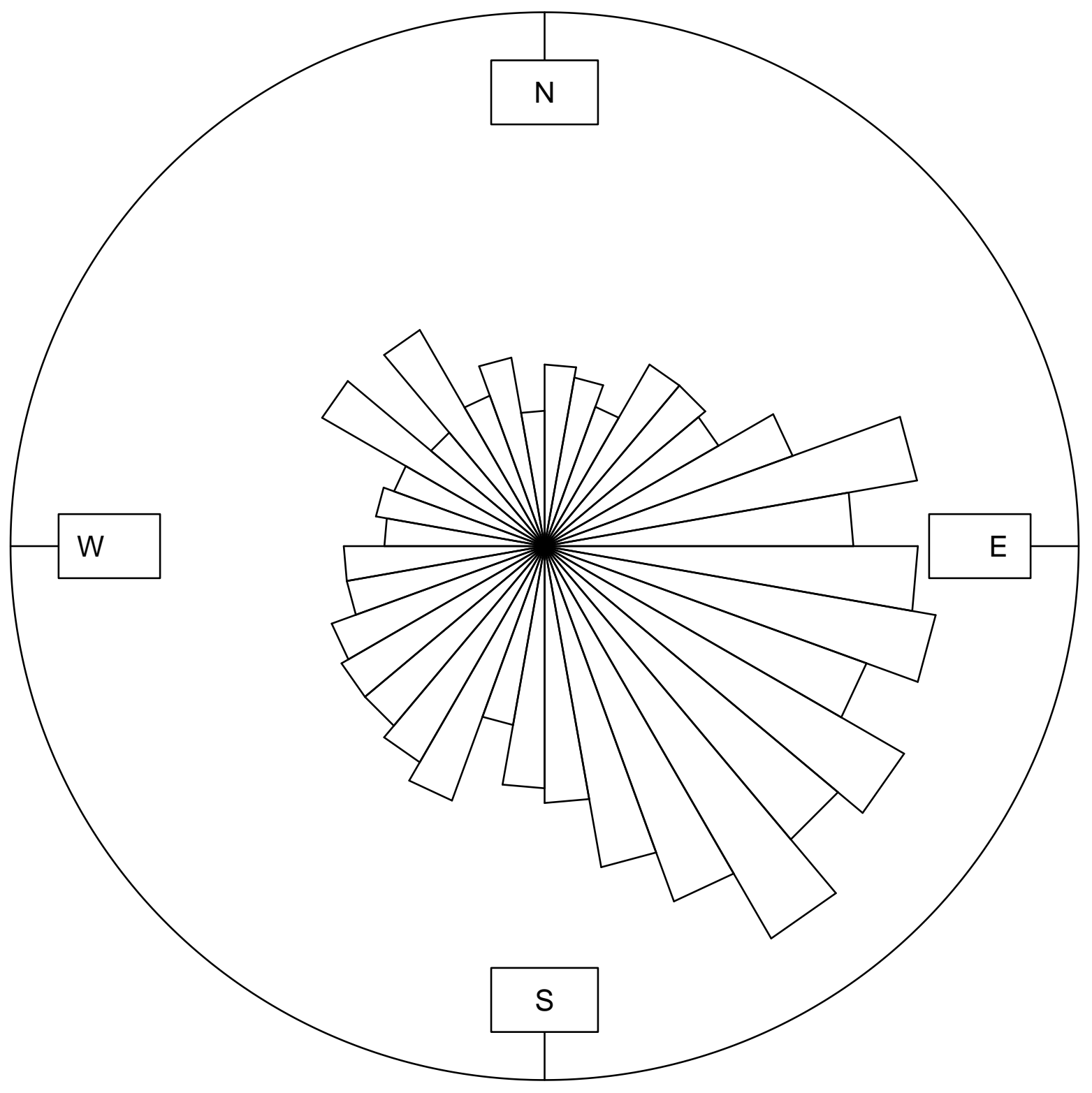




\section{bootstrap 21}

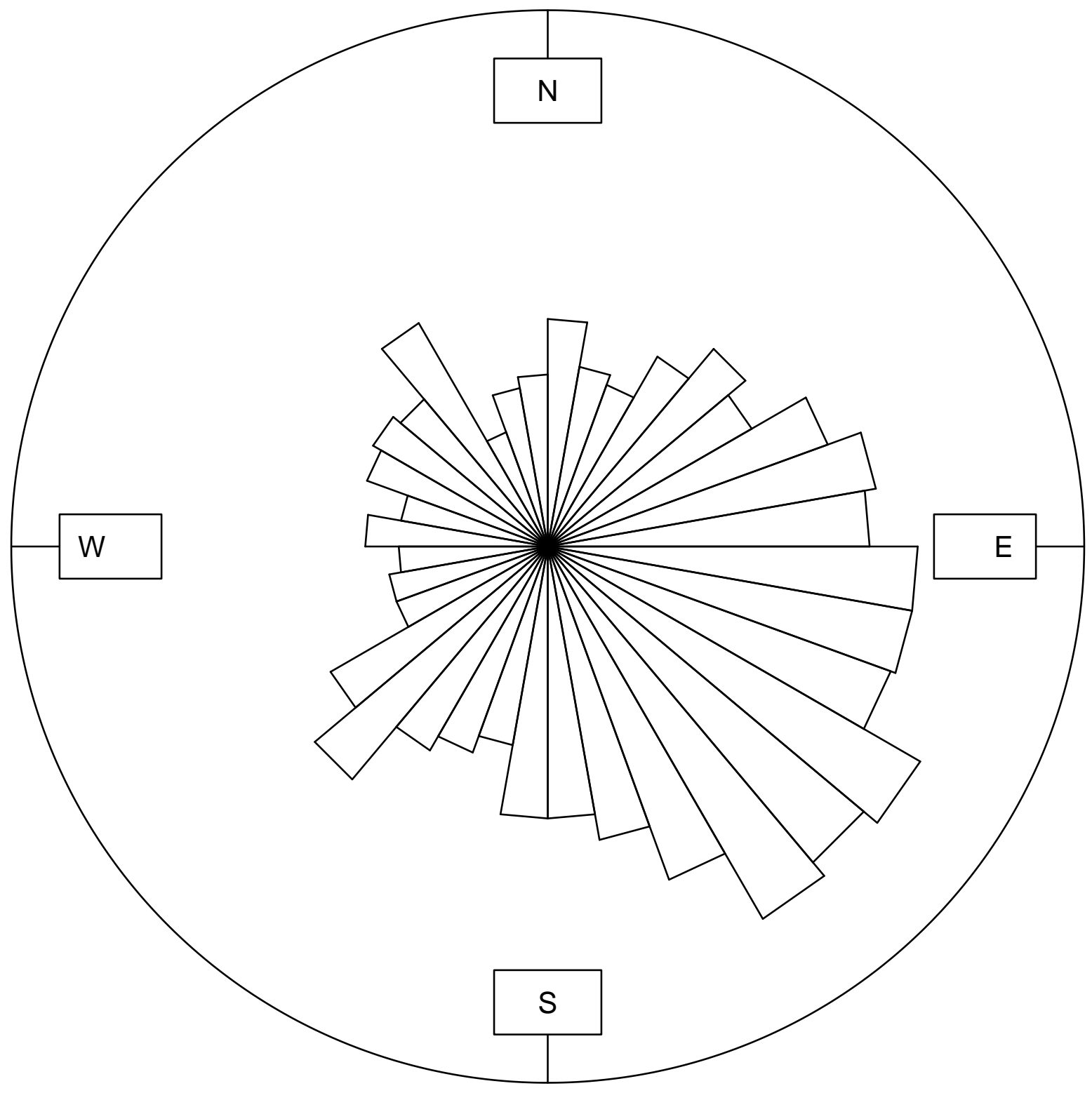


bootstrap 22

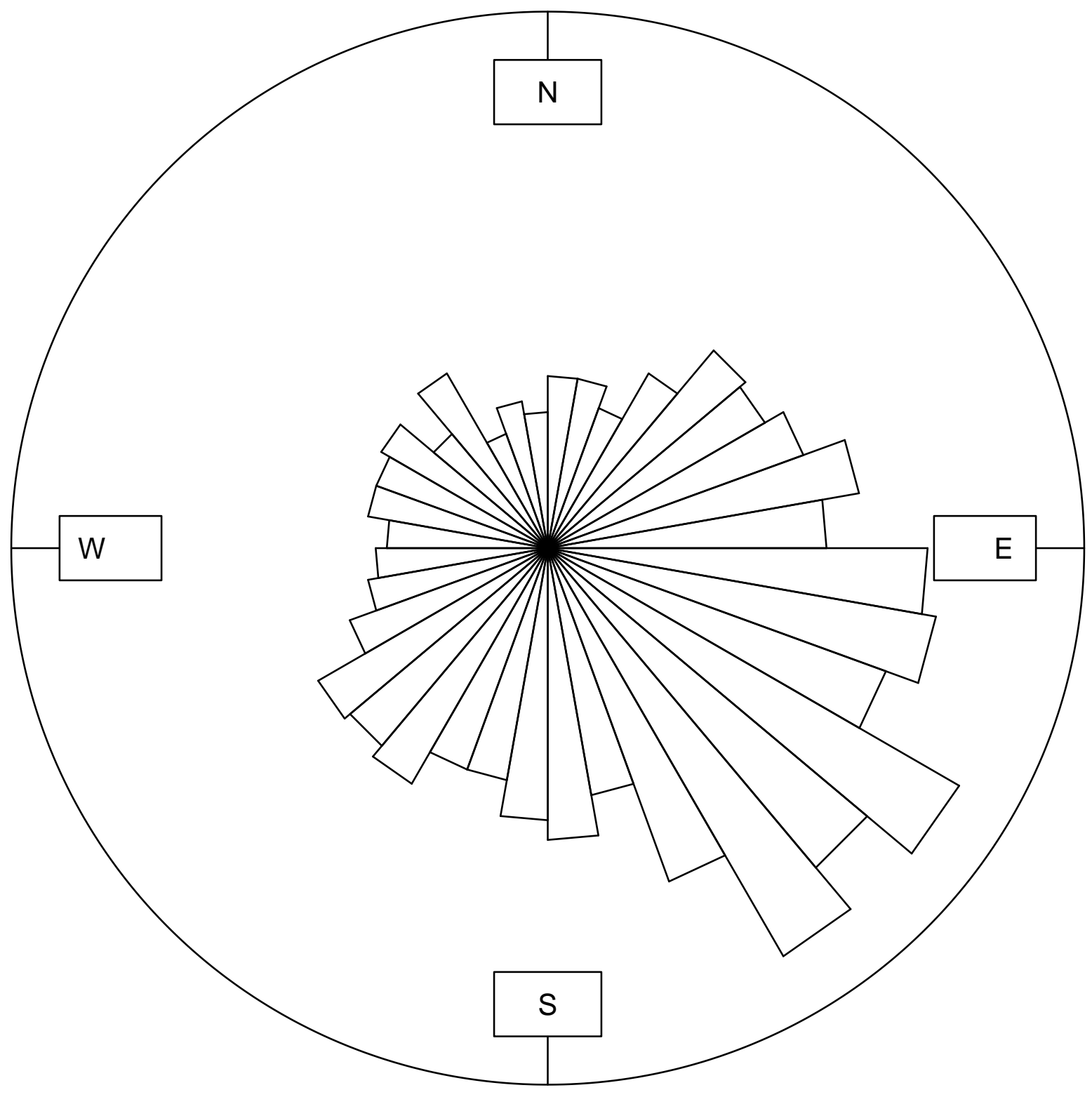


bootstrap 23

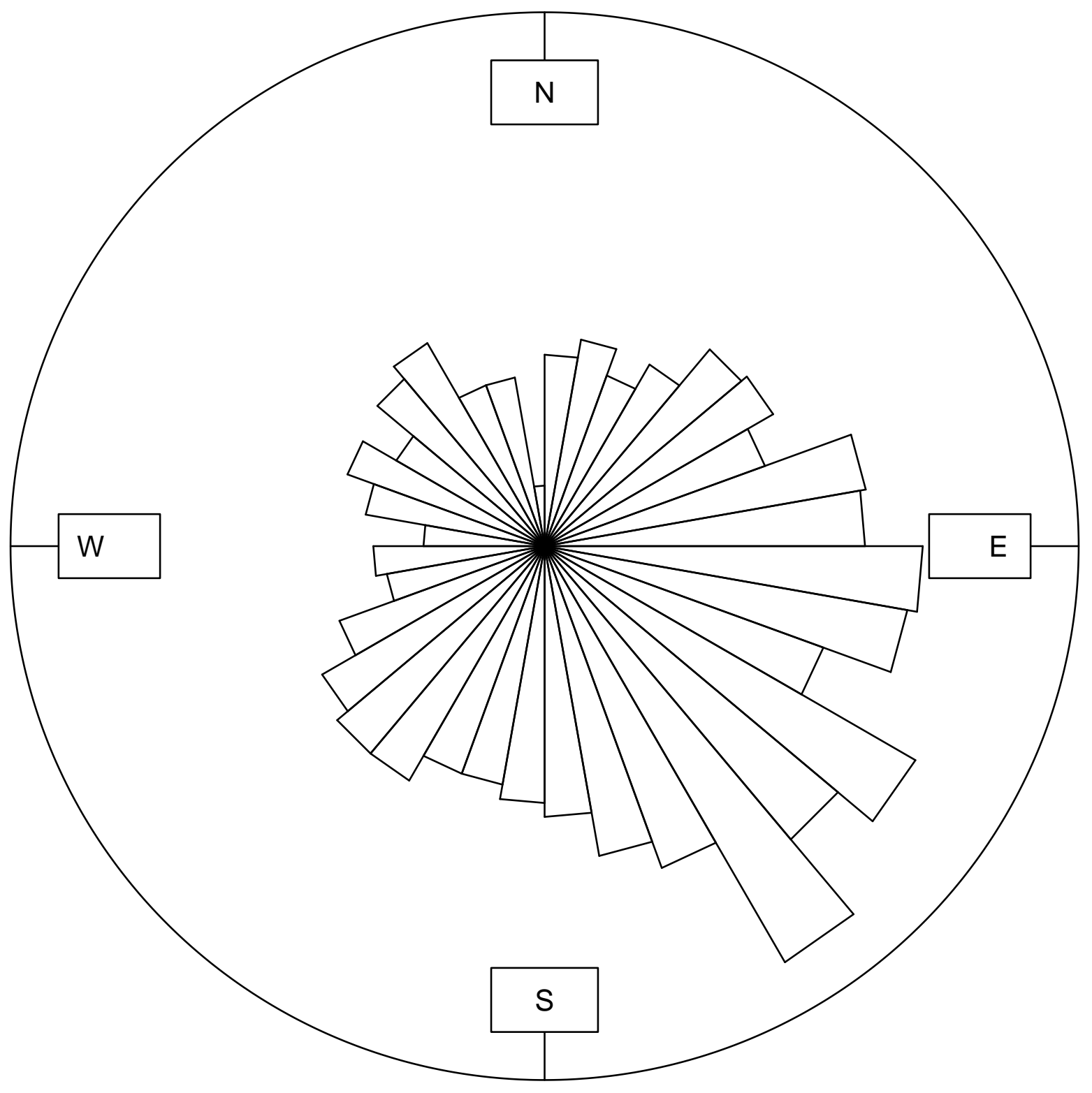




\section{bootstrap 24}

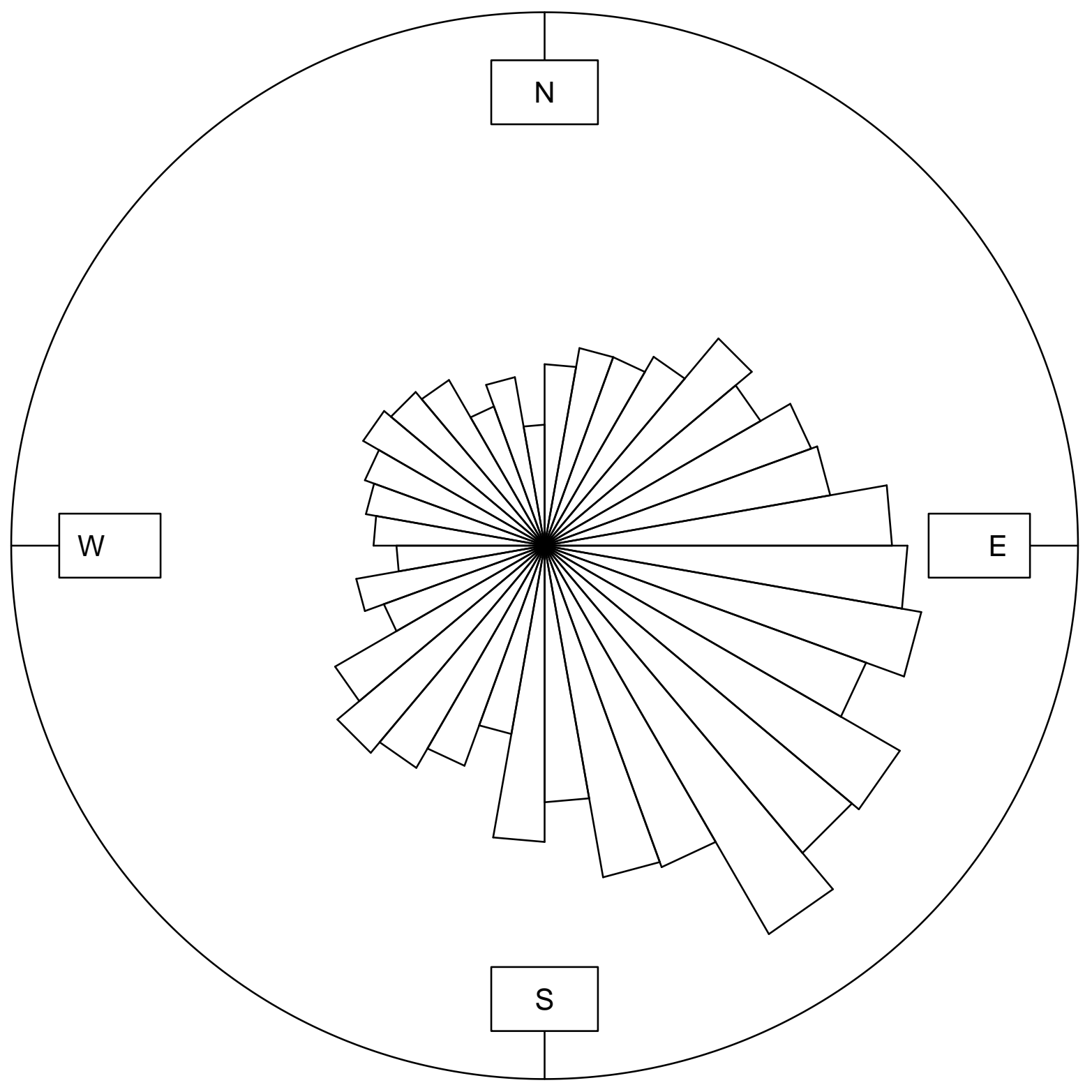


bootstrap 25

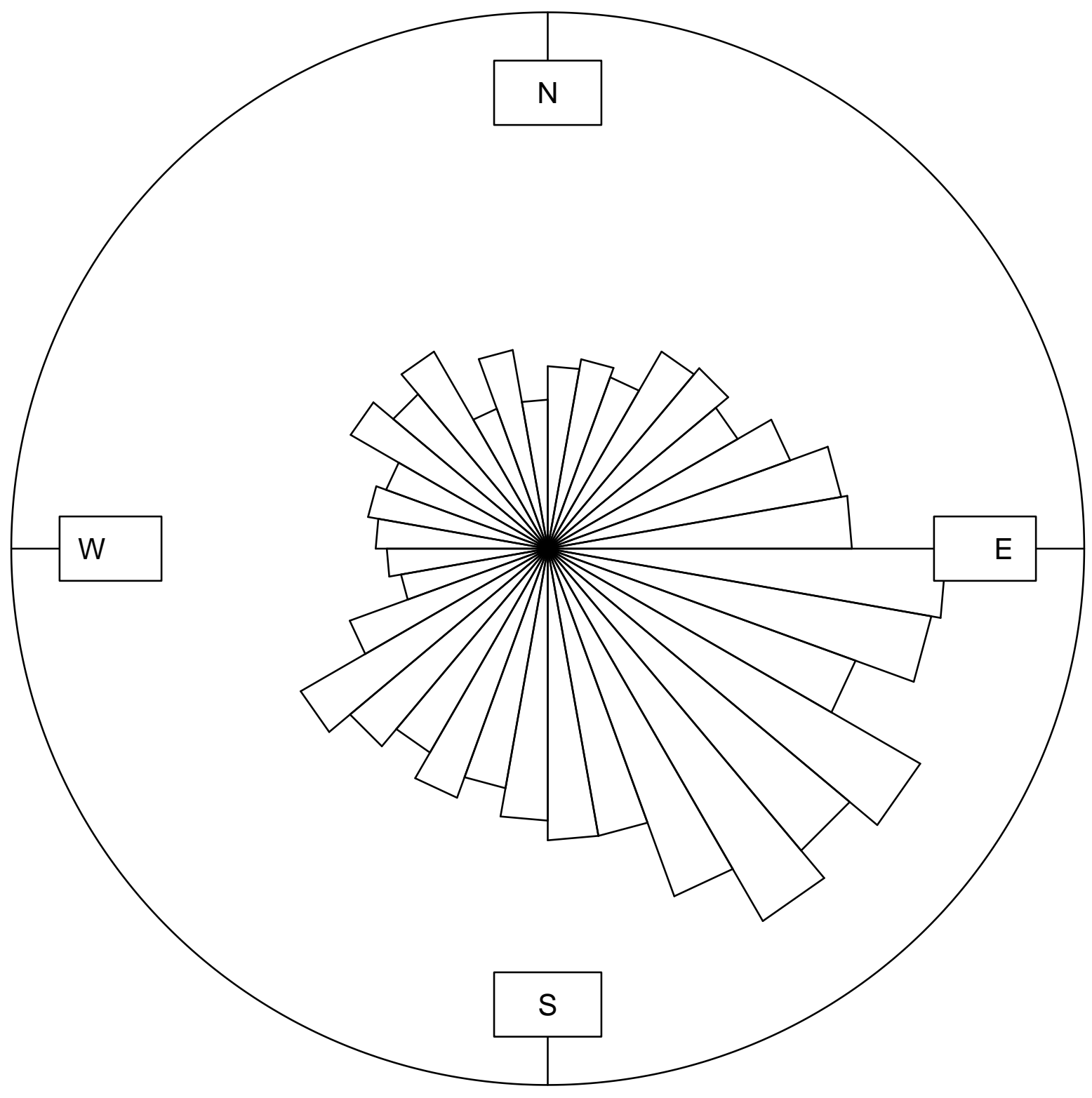


bootstrap 26

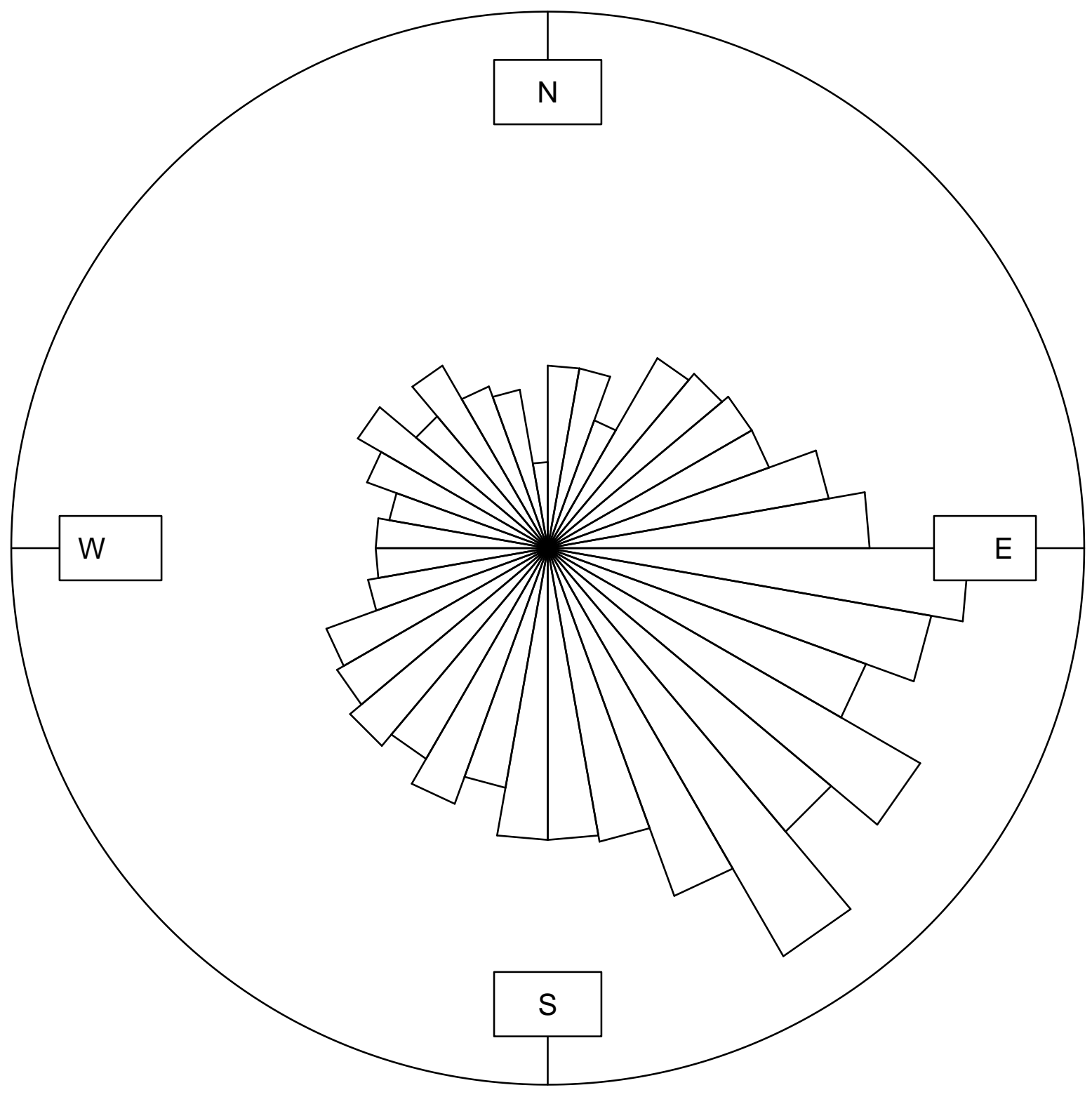


bootstrap 27

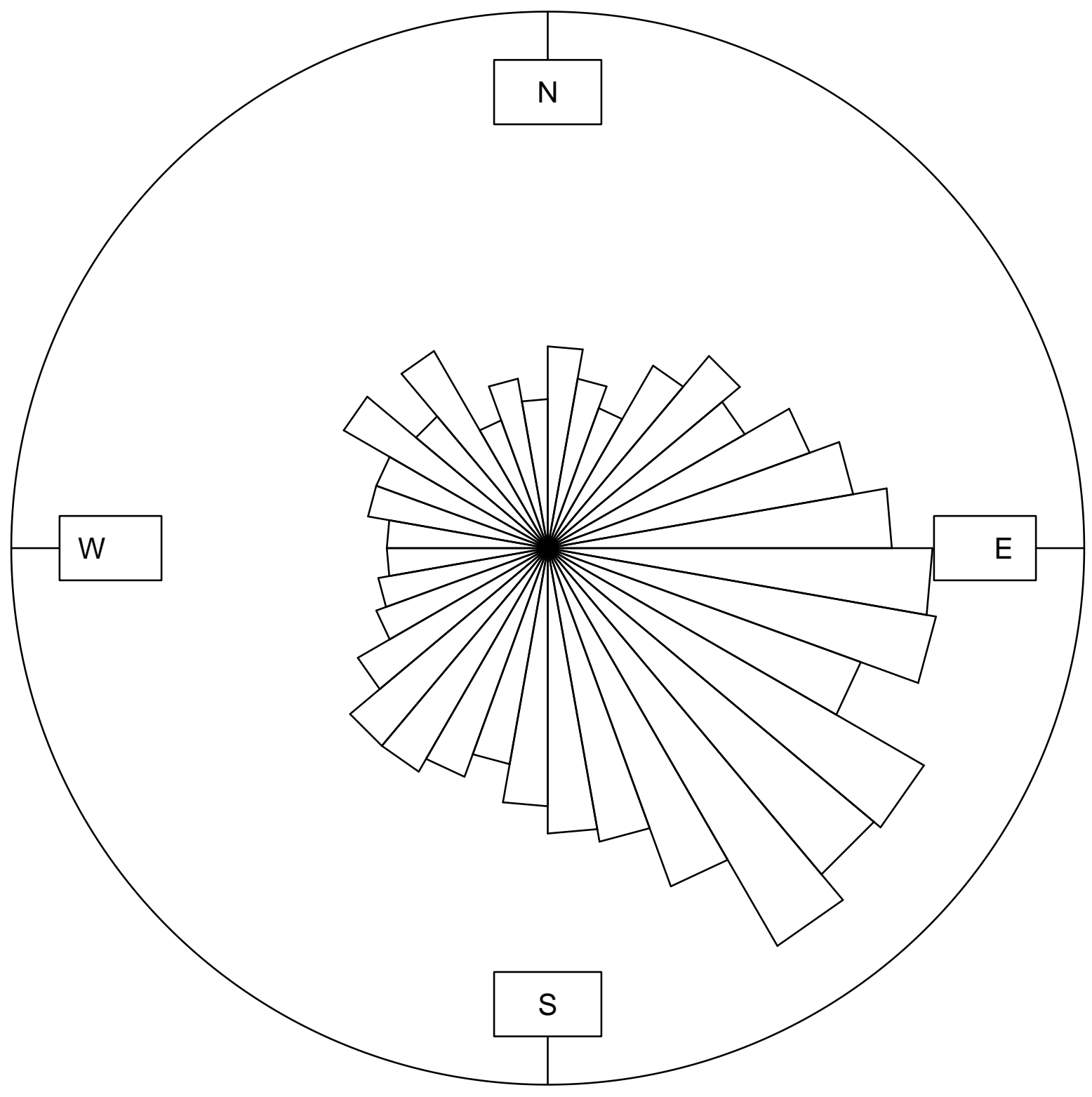


bootstrap 28

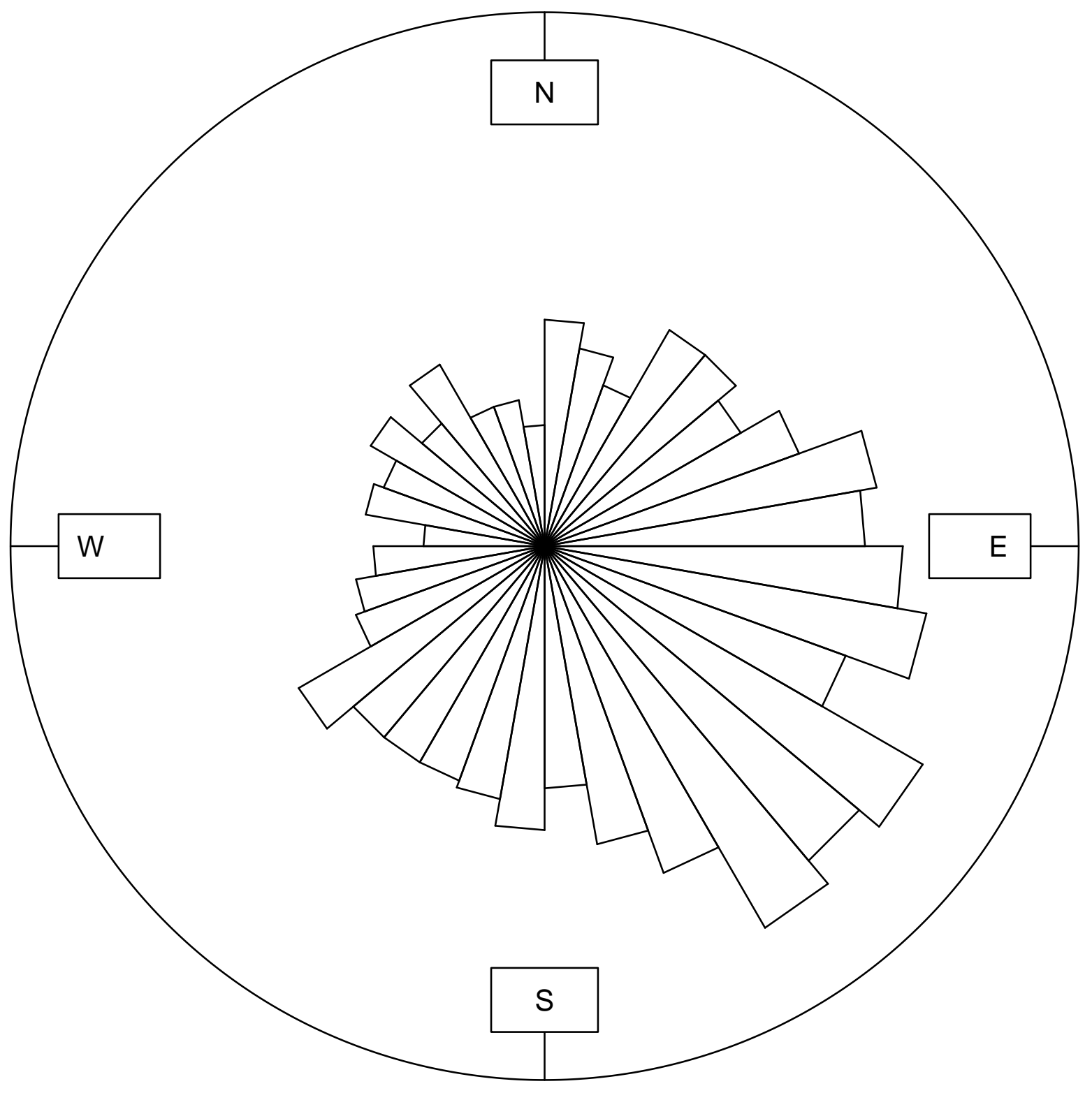


bootstrap 29

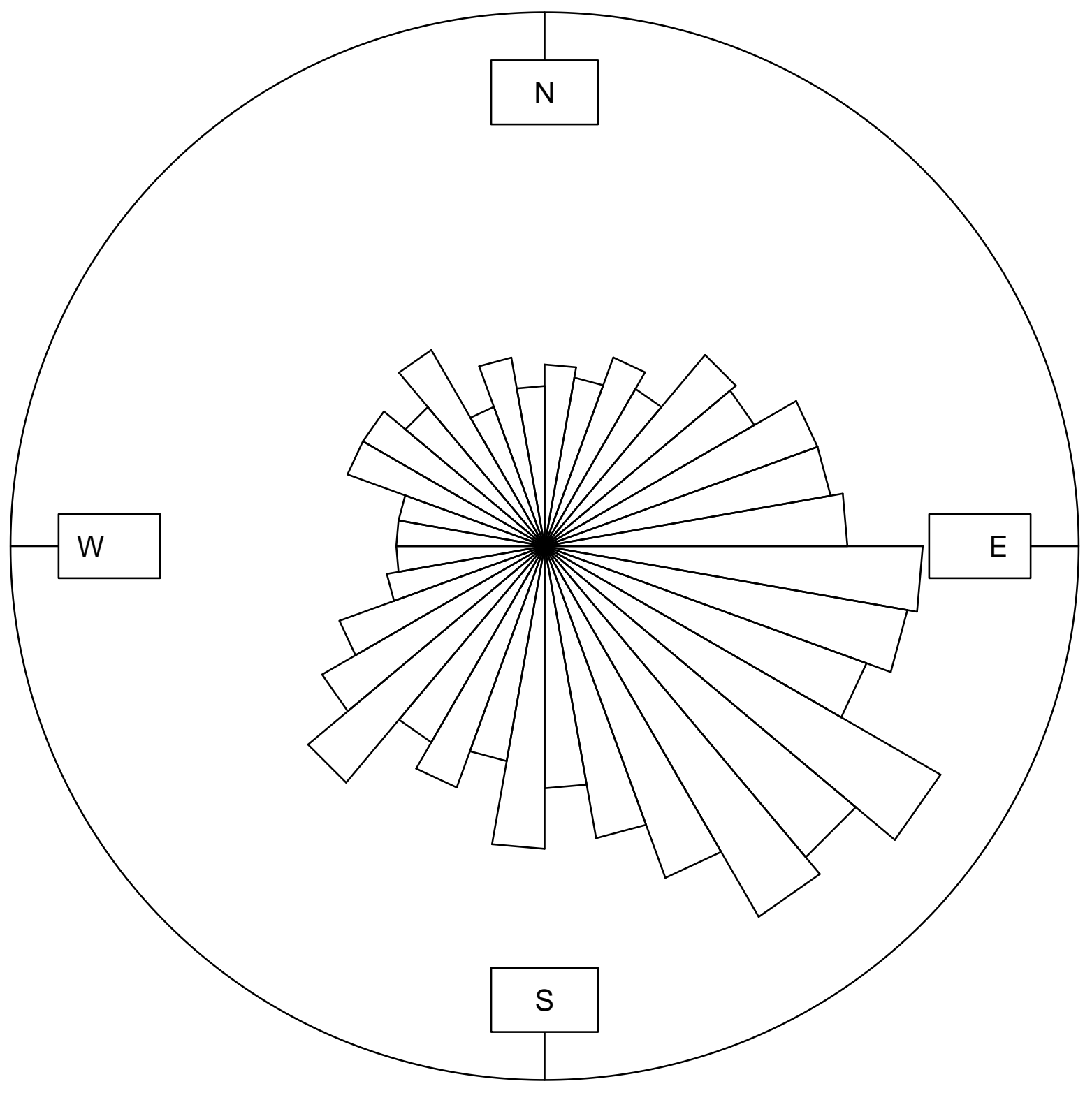


bootstrap 30

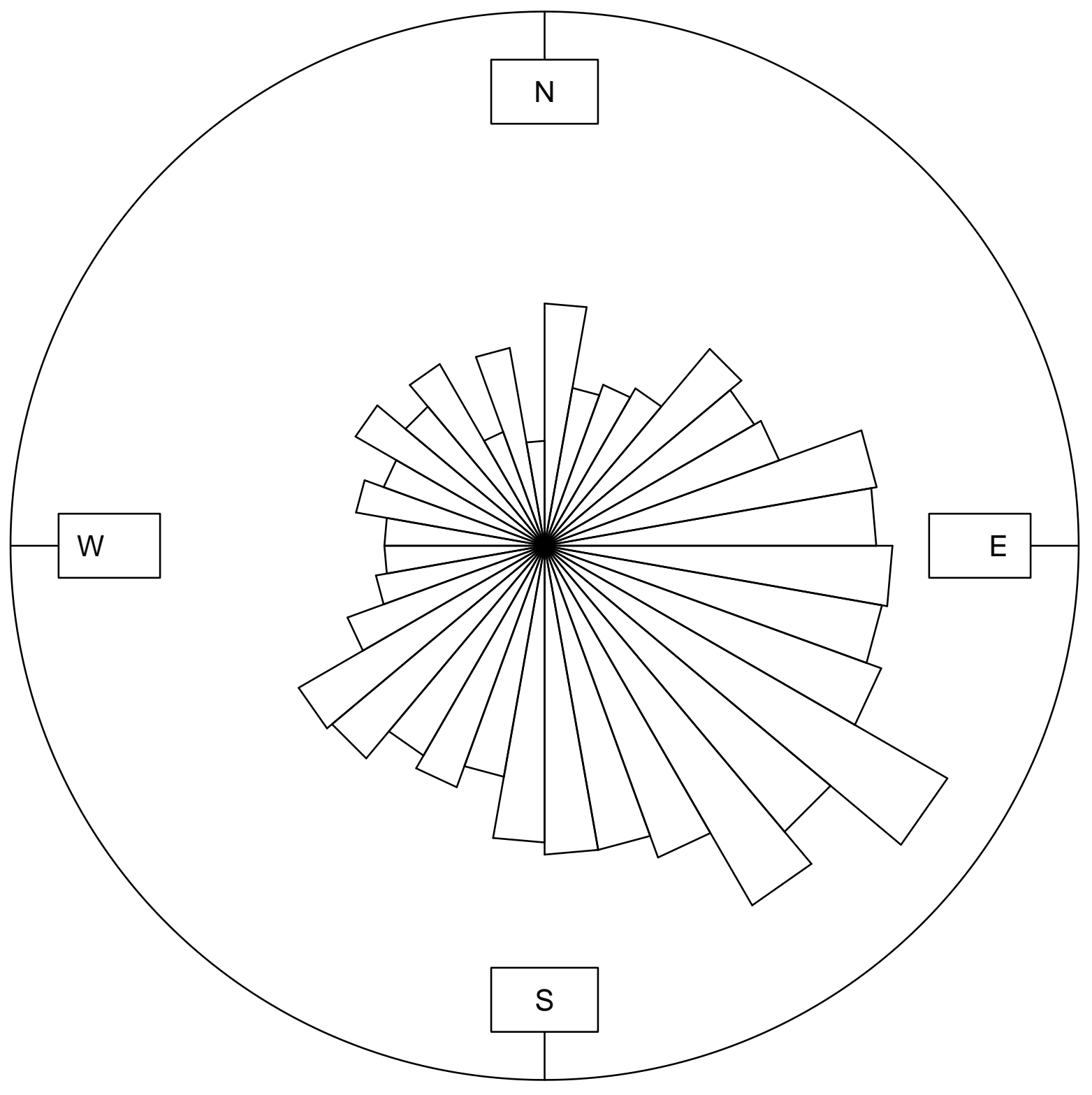




\section{bootstrap 31}

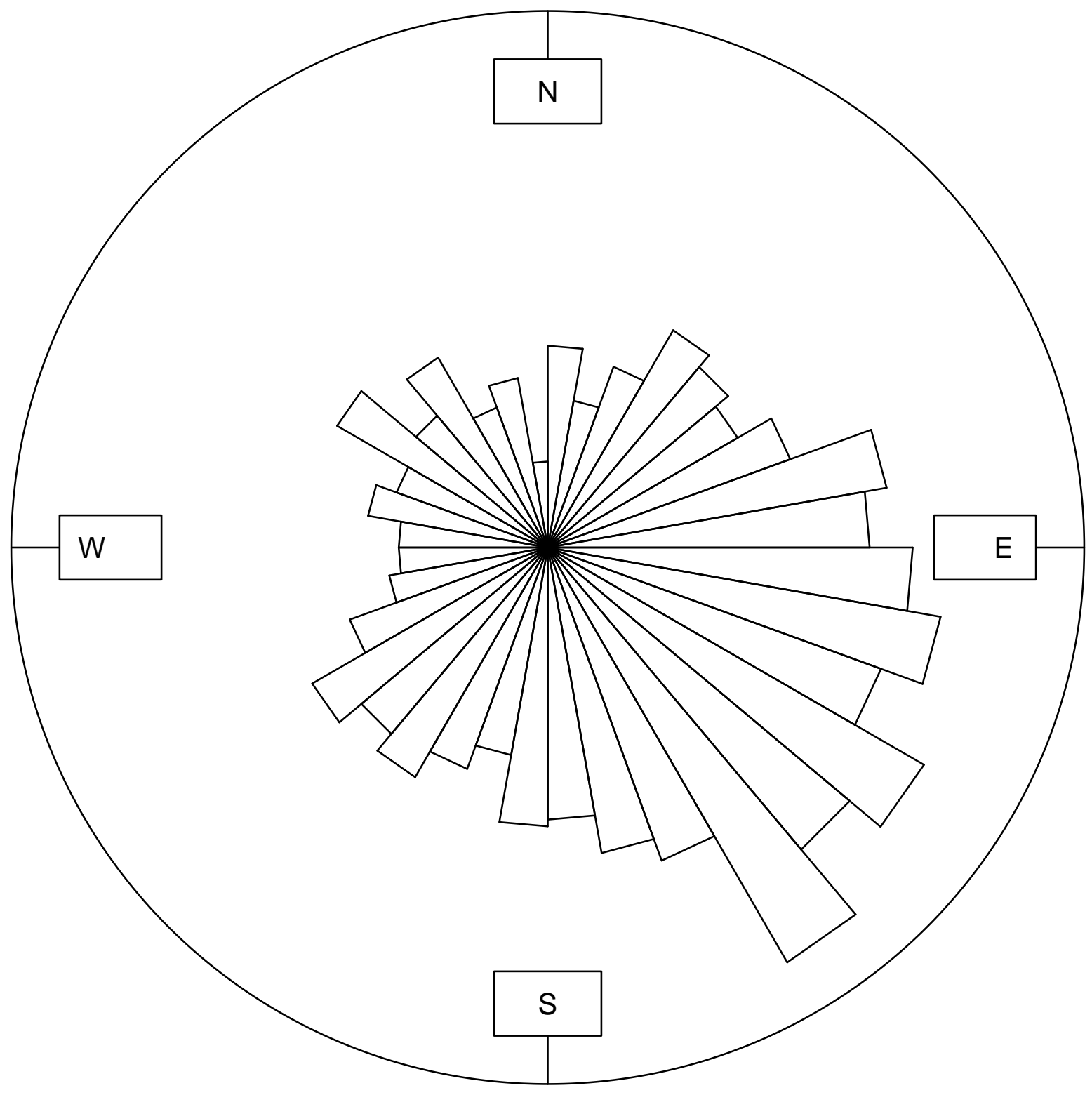


bootstrap 32

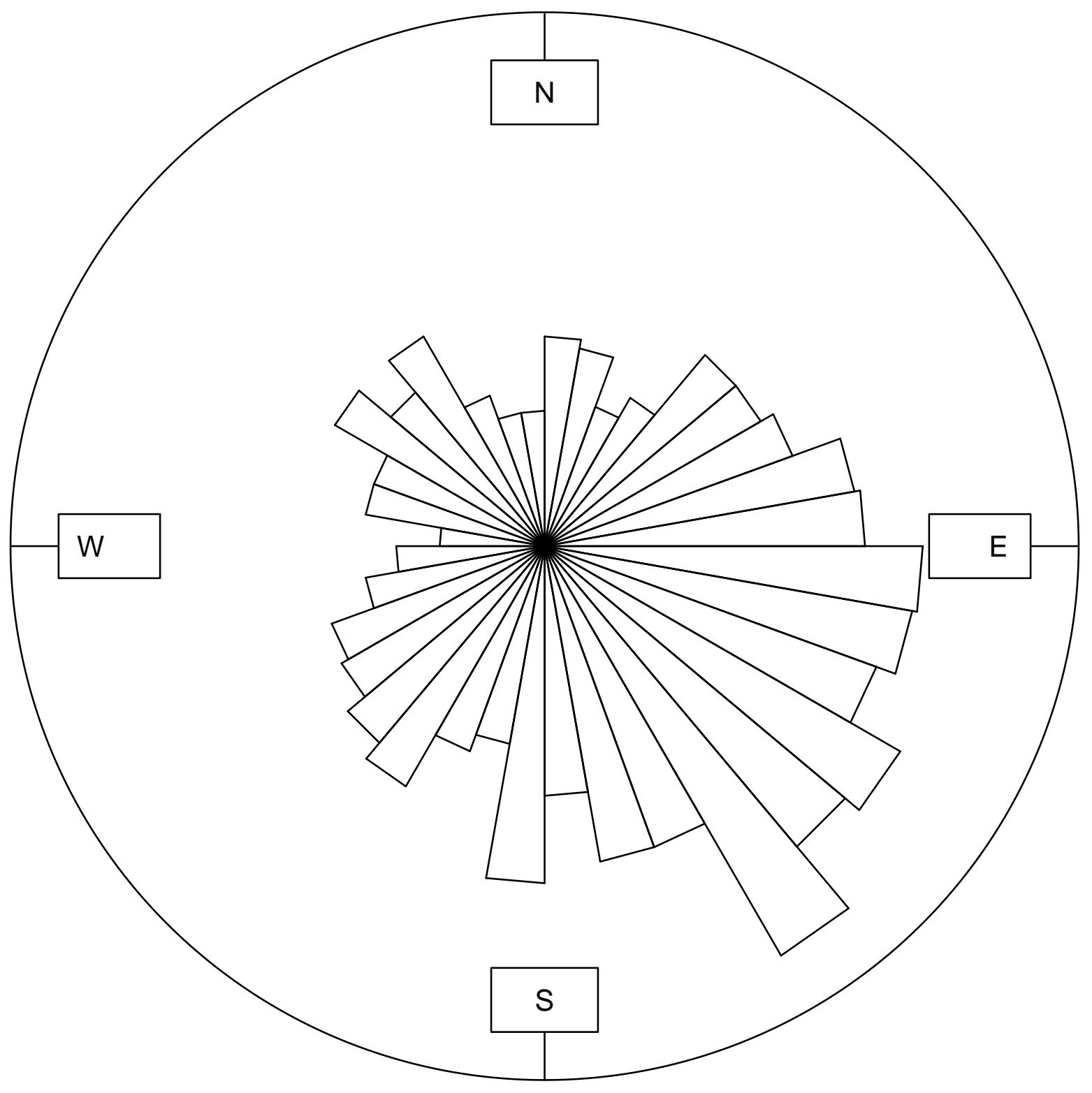




\section{bootstrap 33}

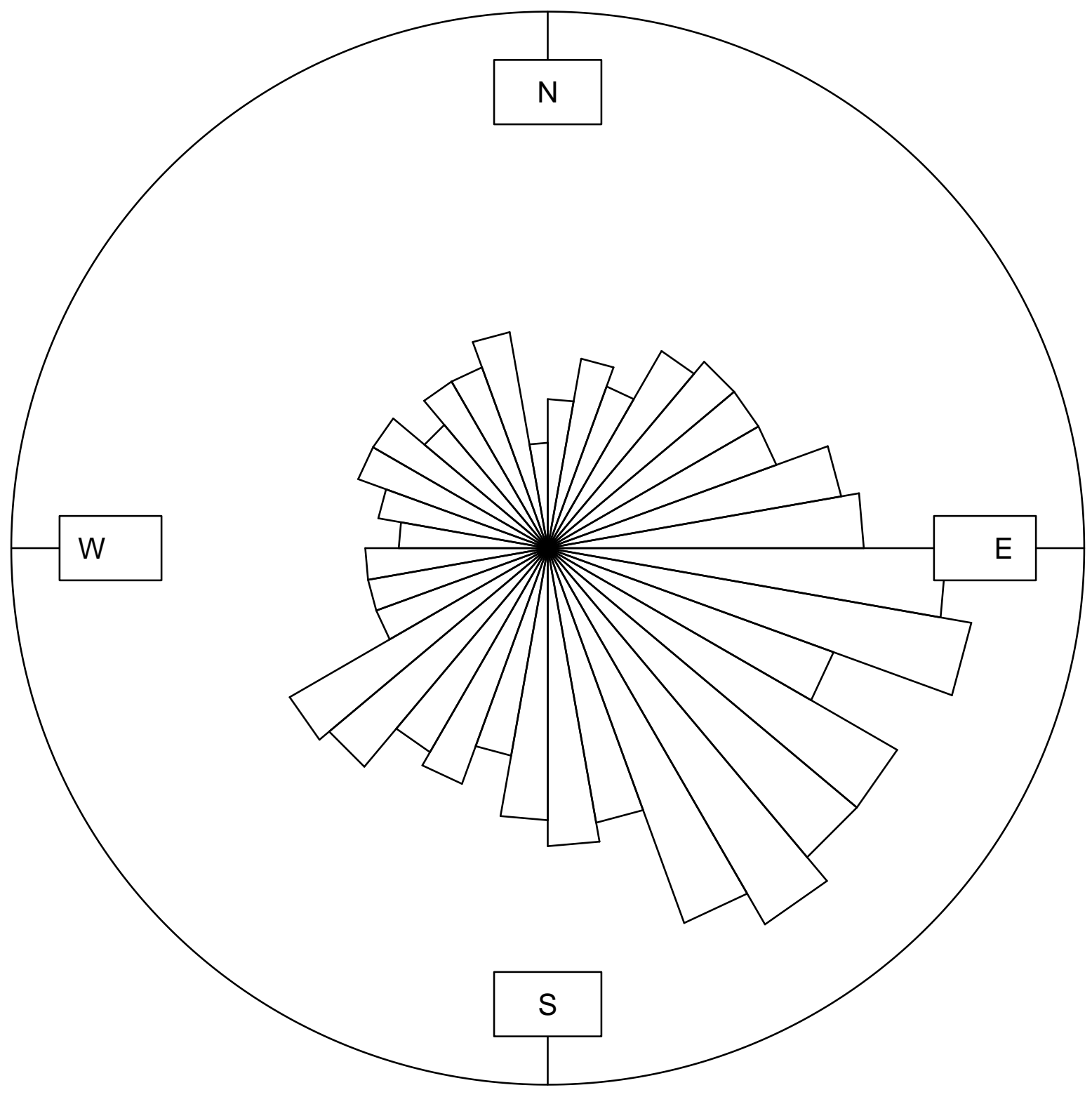




\section{bootstrap 34}

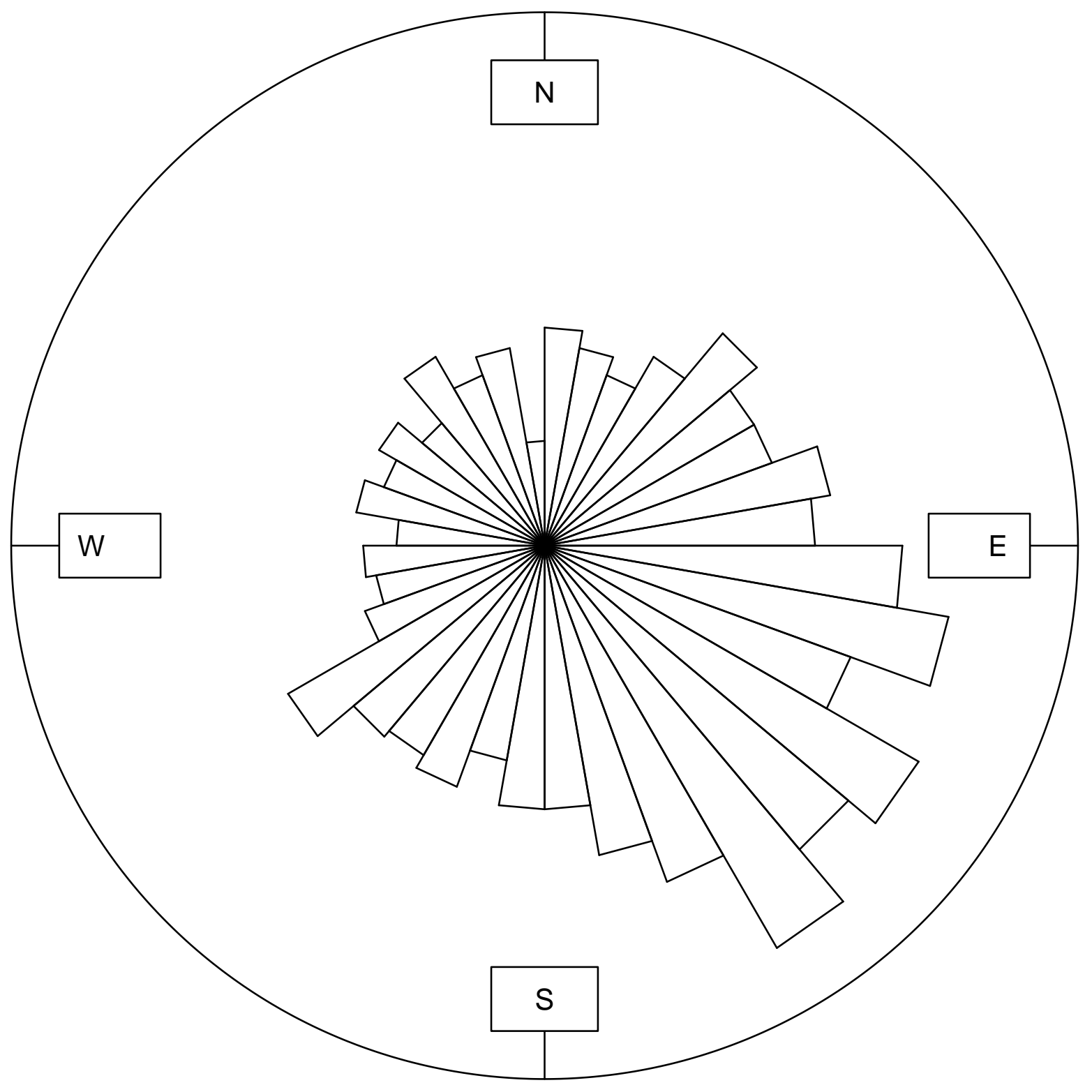


bootstrap 35

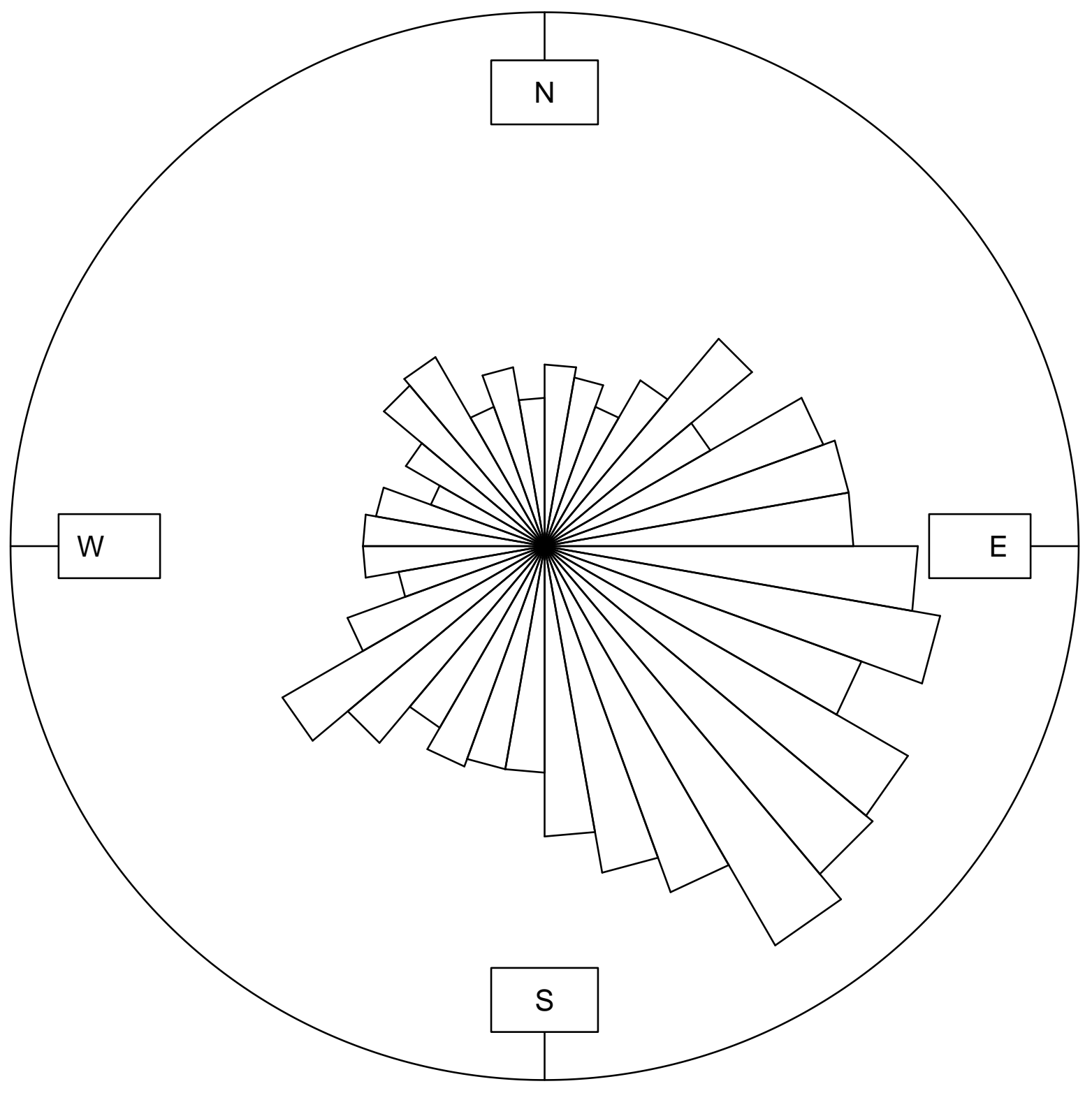


bootstrap 36

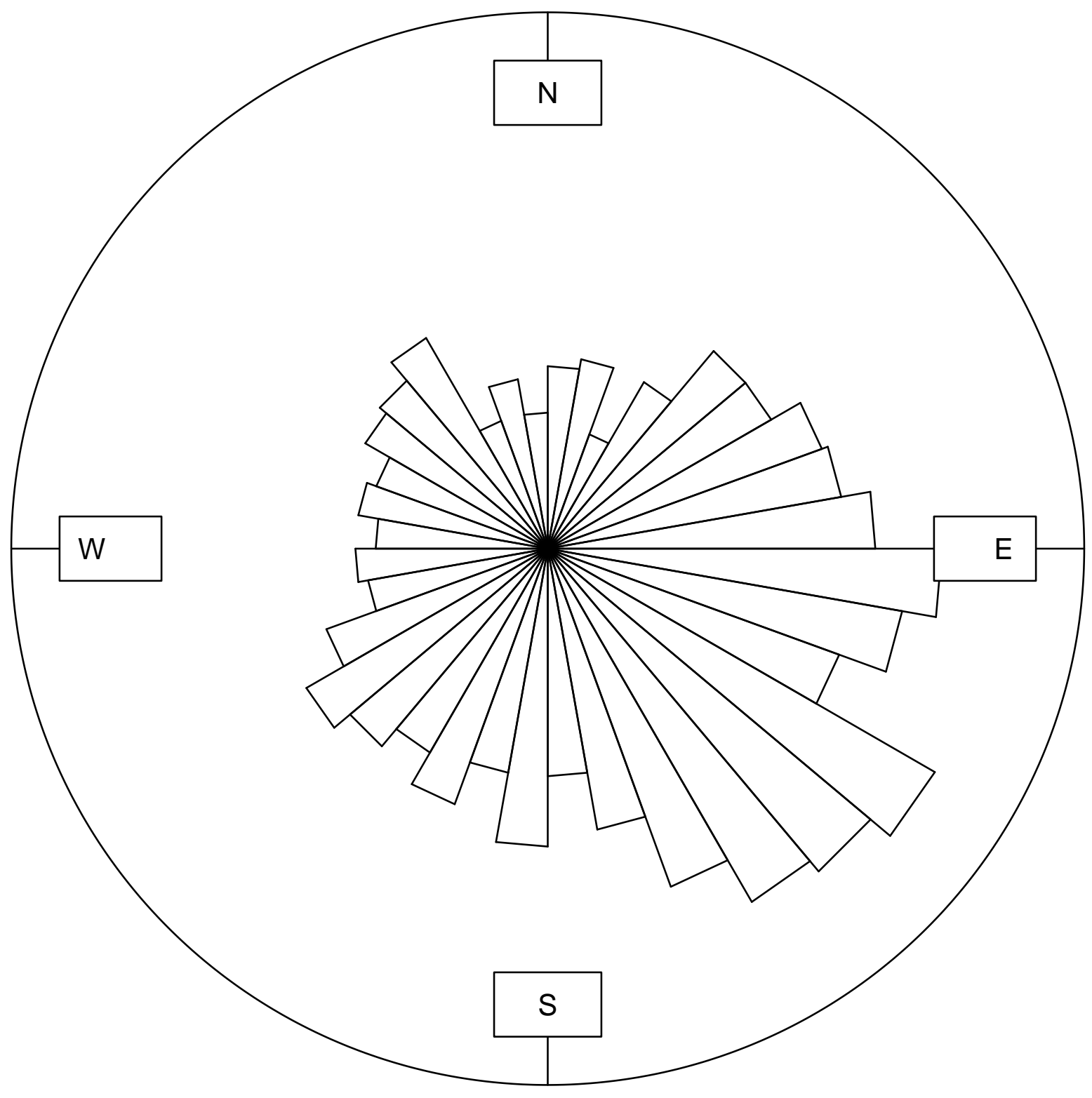


bootstrap 37

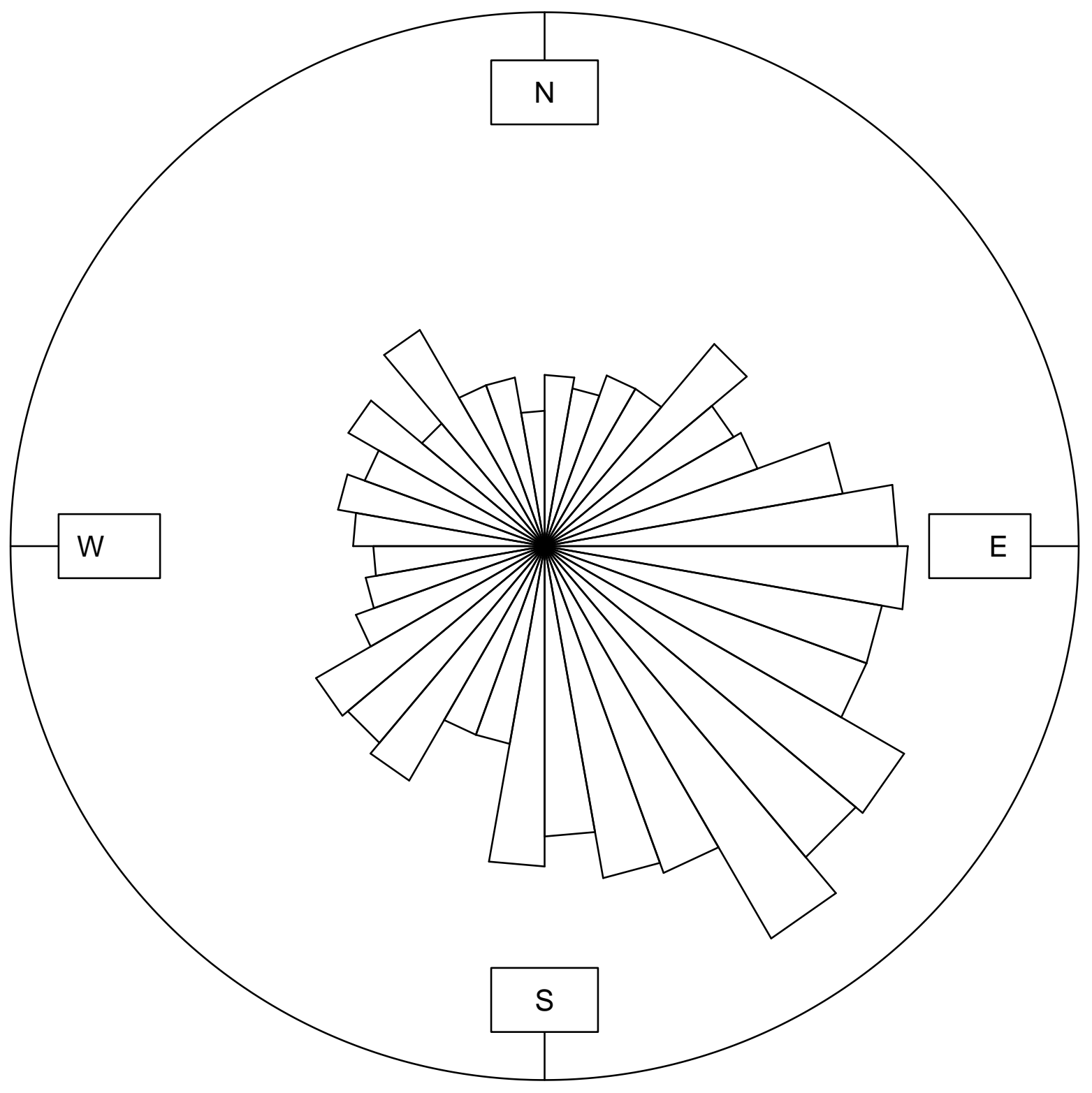


bootstrap 38

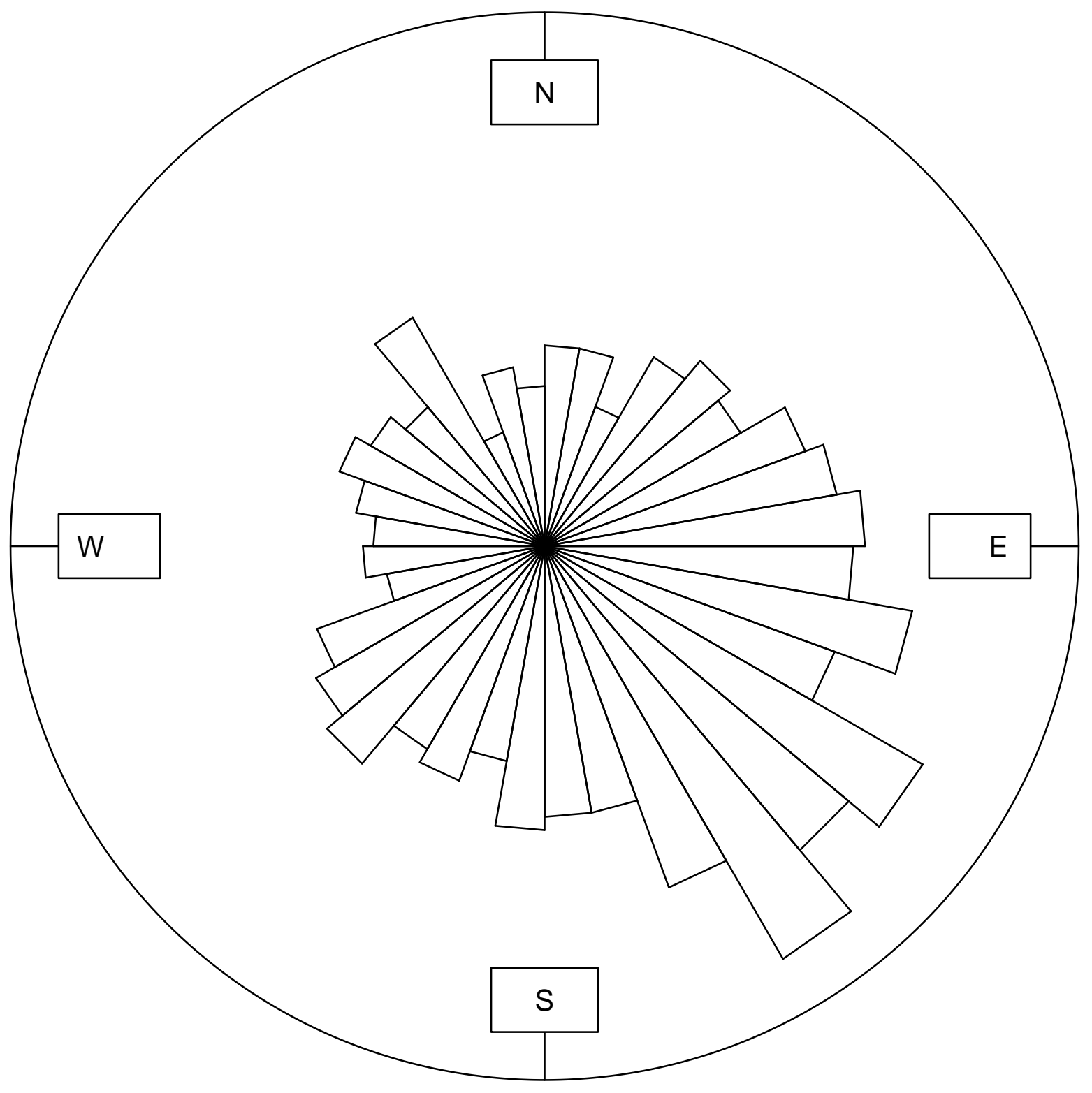


bootstrap 39

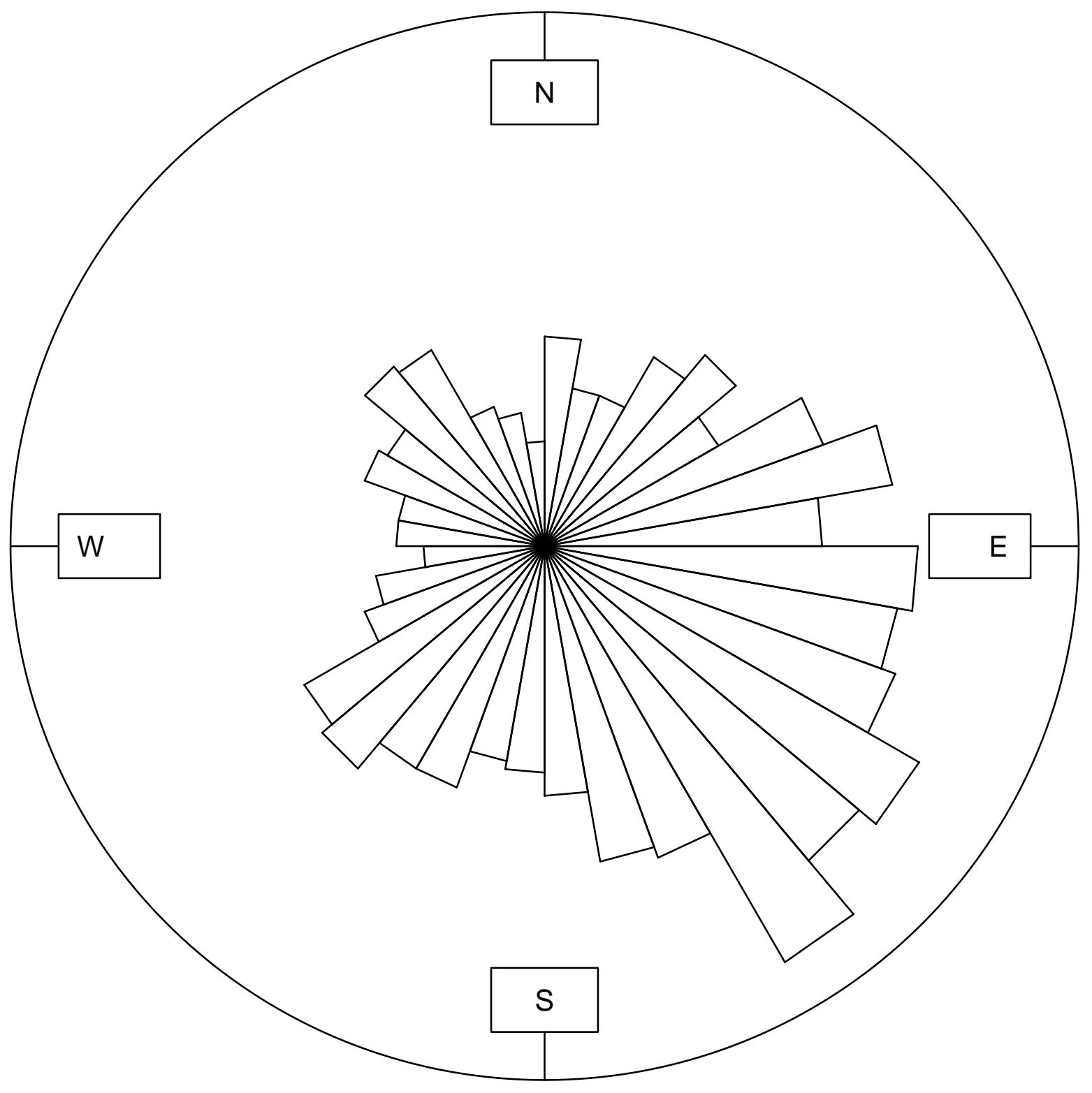


bootstrap 40

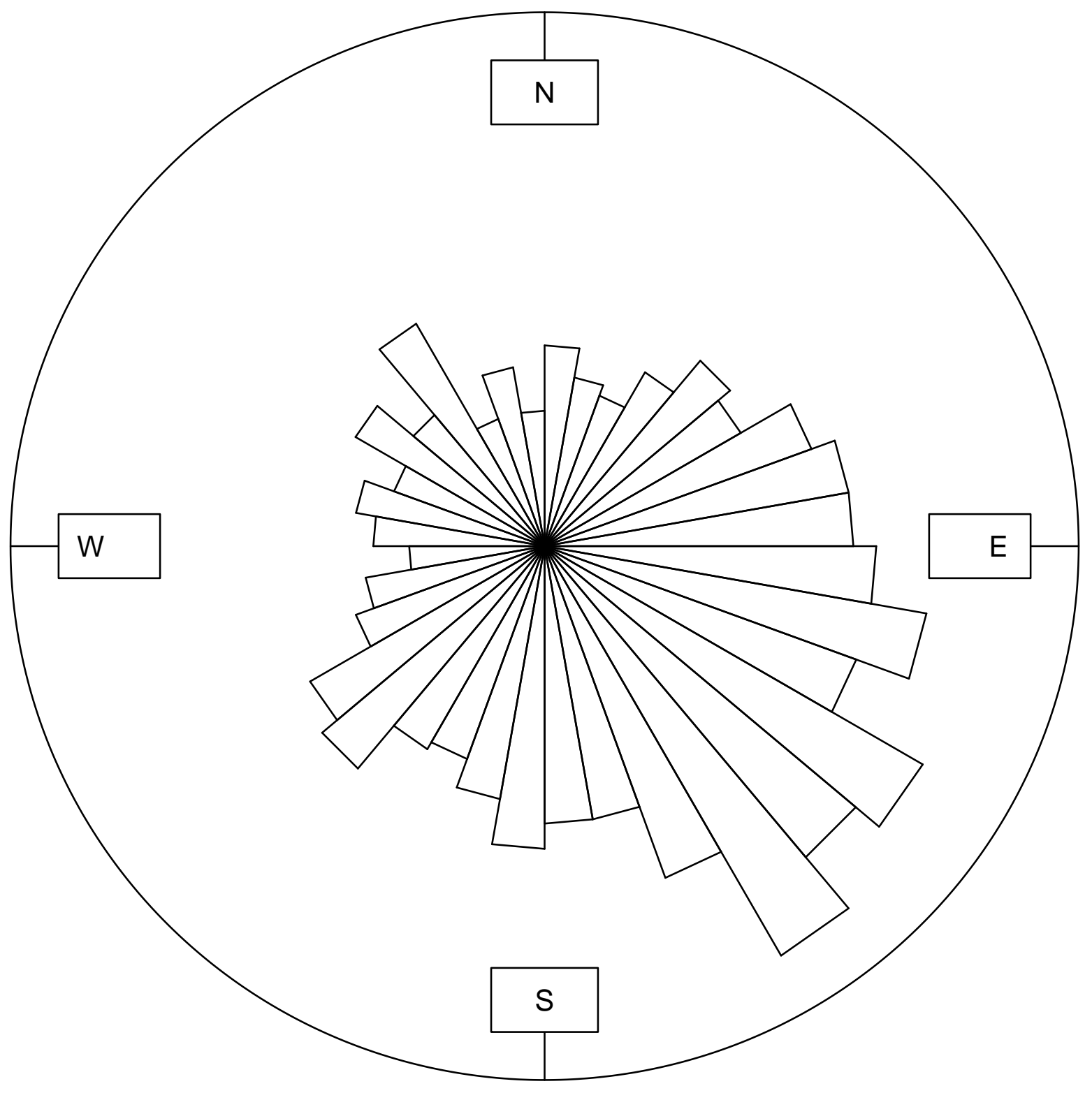




\section{bootstrap 41}

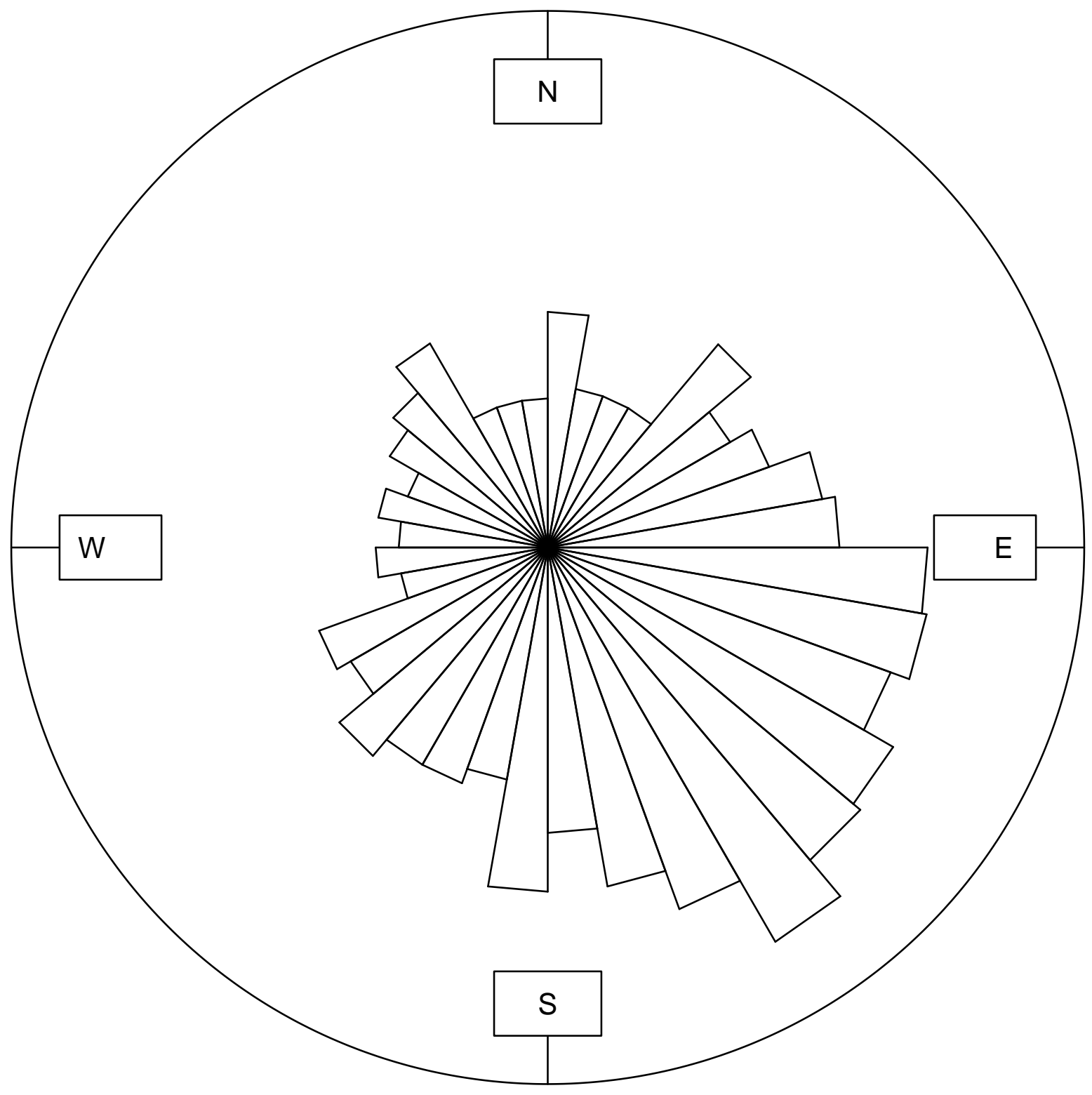


bootstrap 42

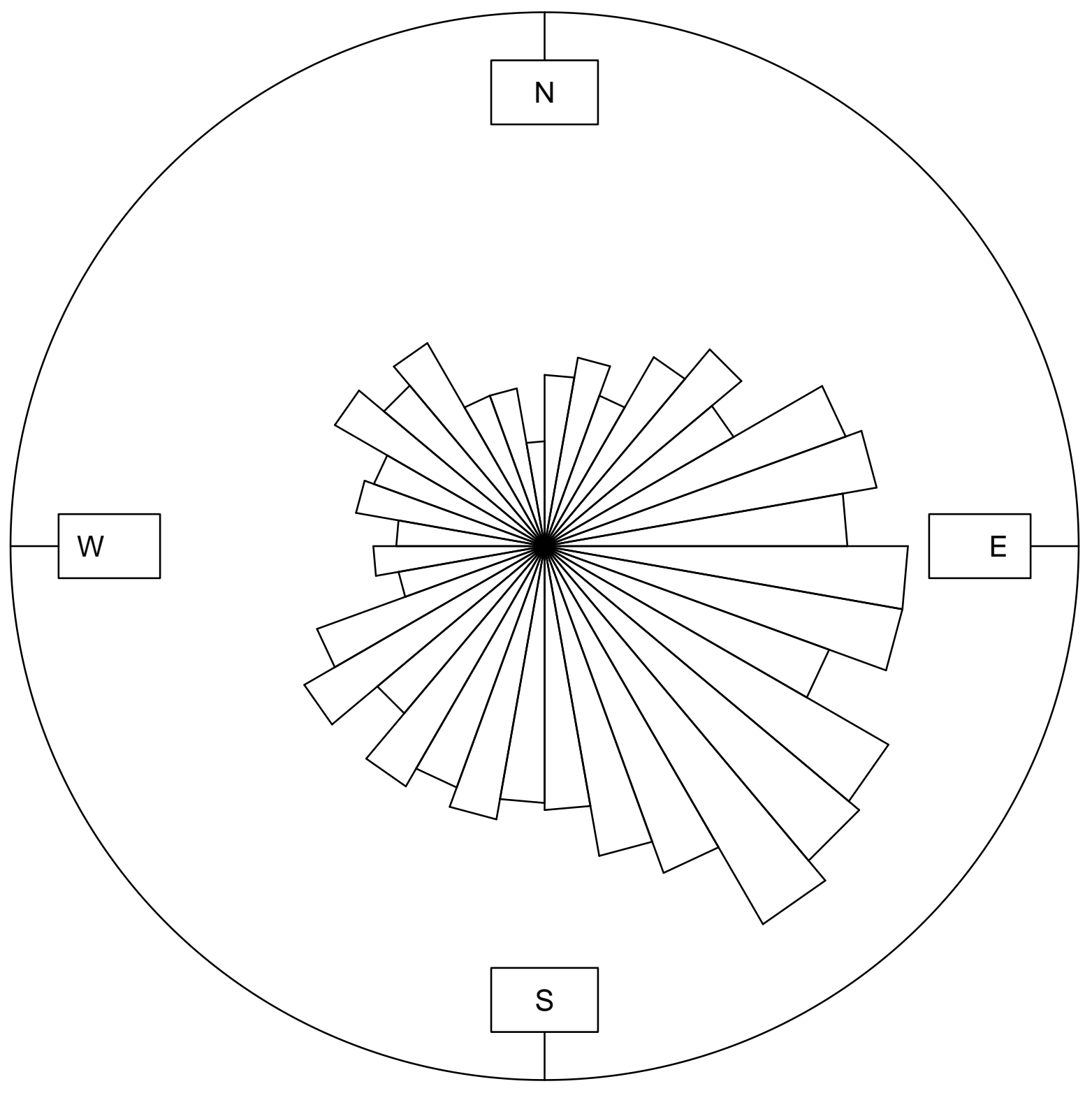




\section{bootstrap 43}

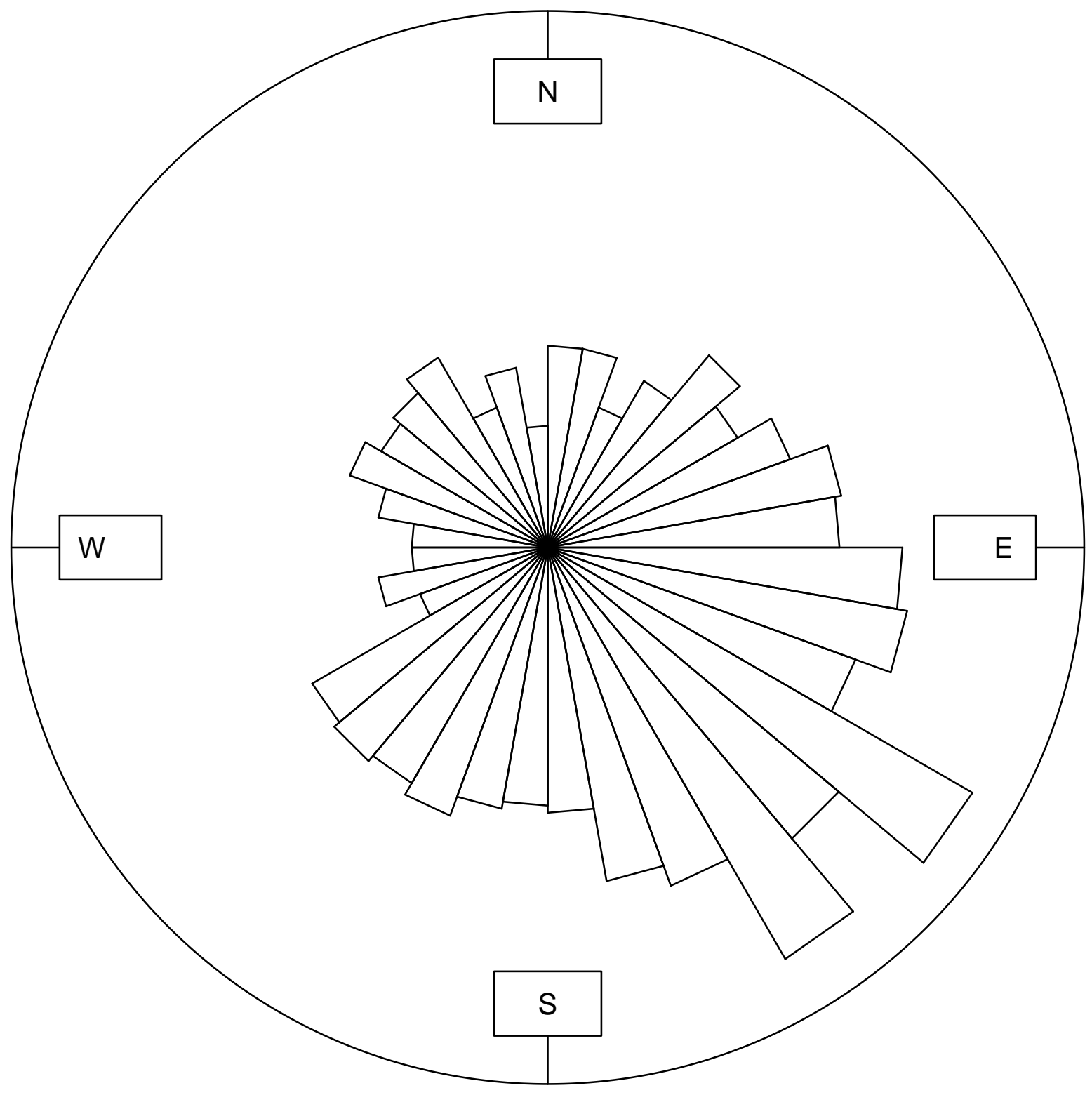




\section{bootstrap 44}

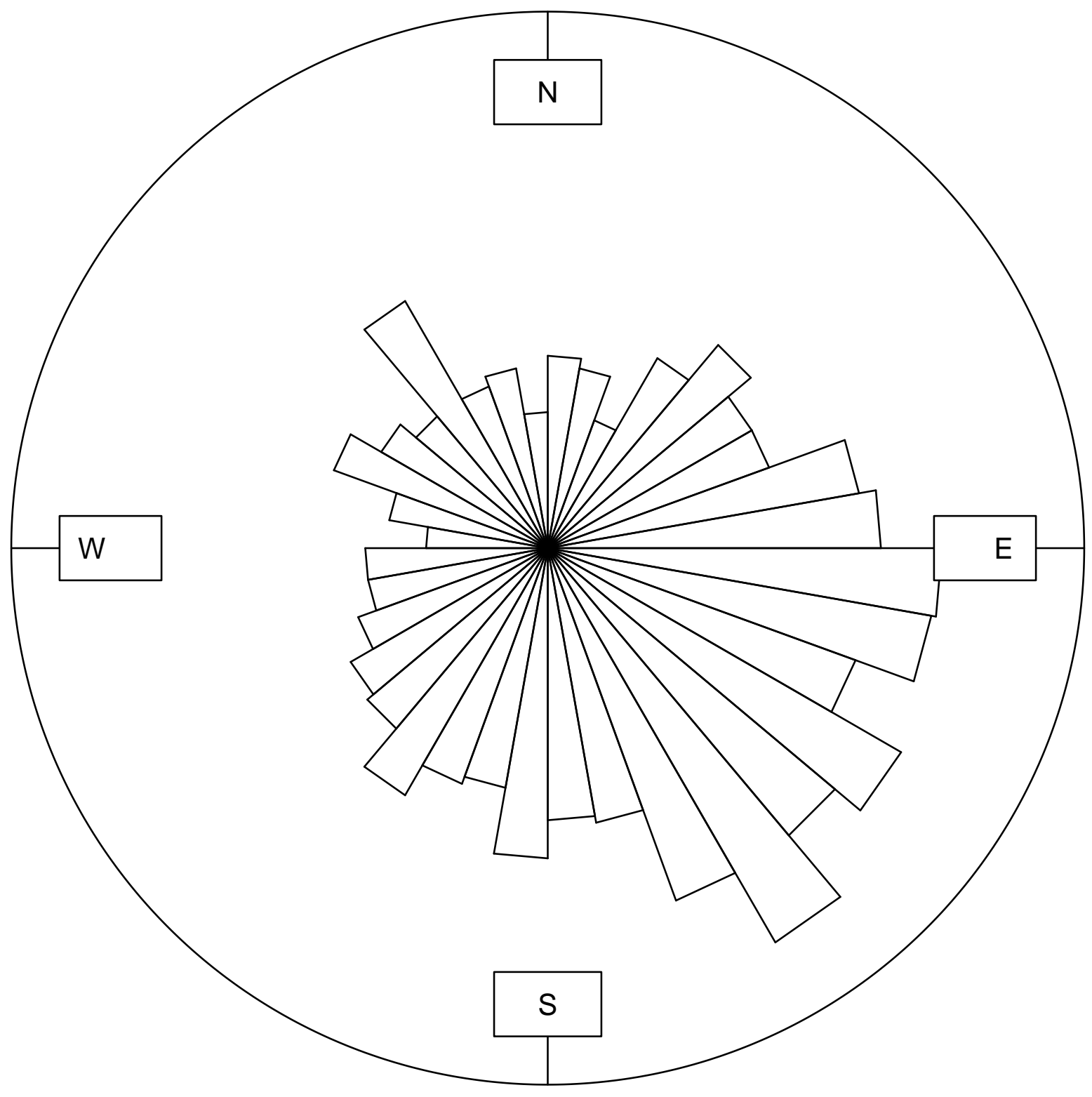


bootstrap 45

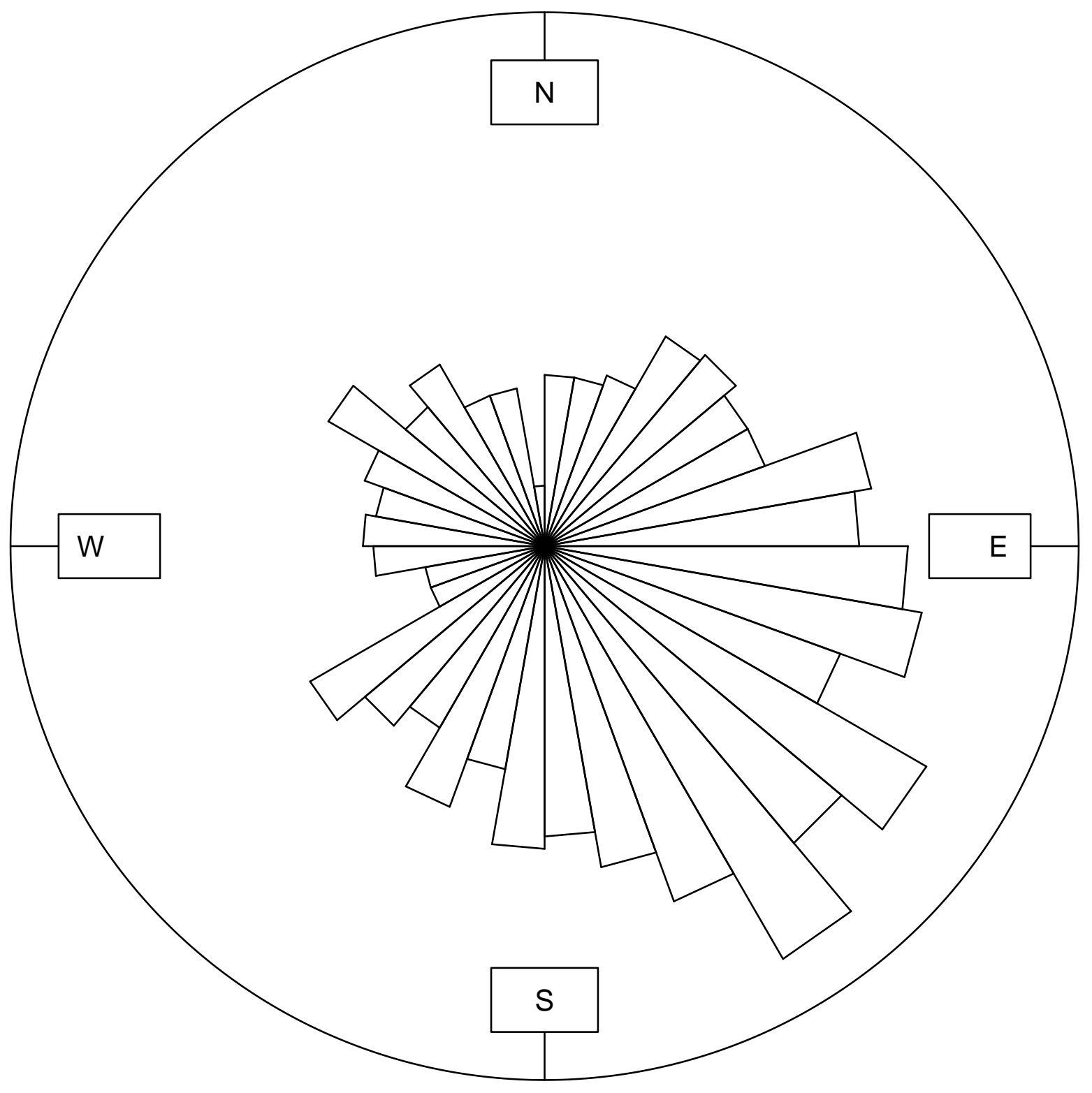


bootstrap 46

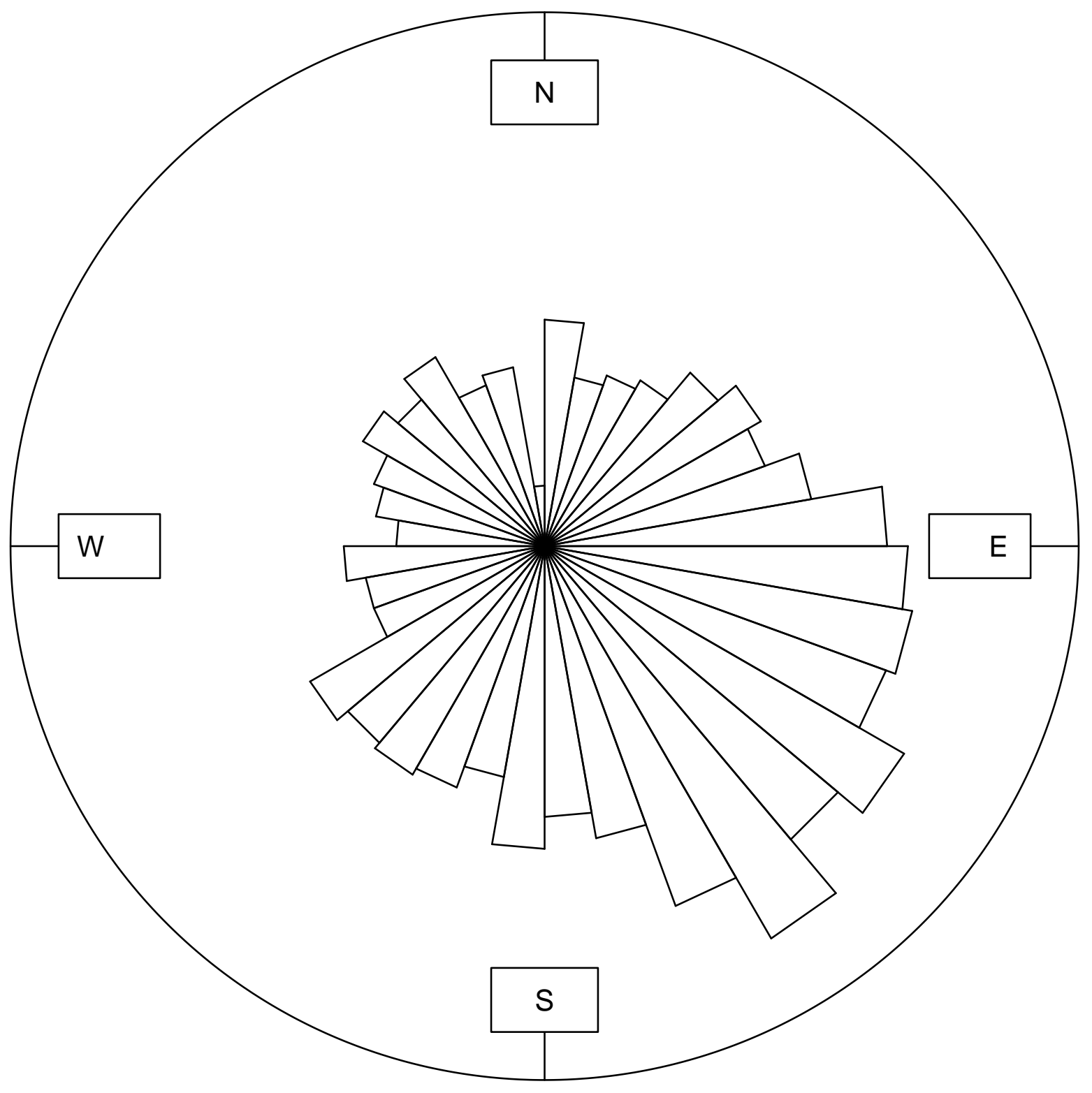


bootstrap 47

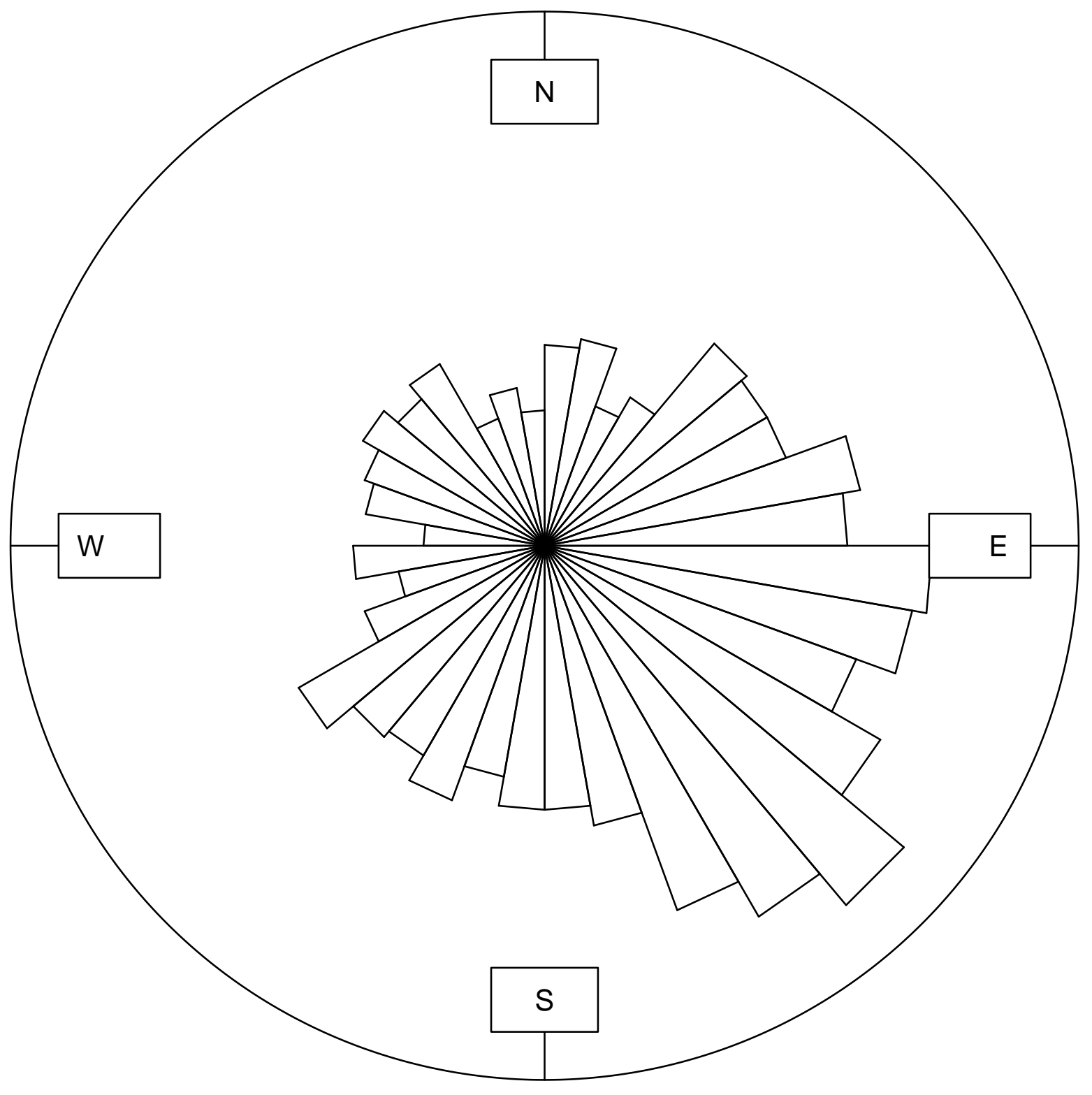


bootstrap 48

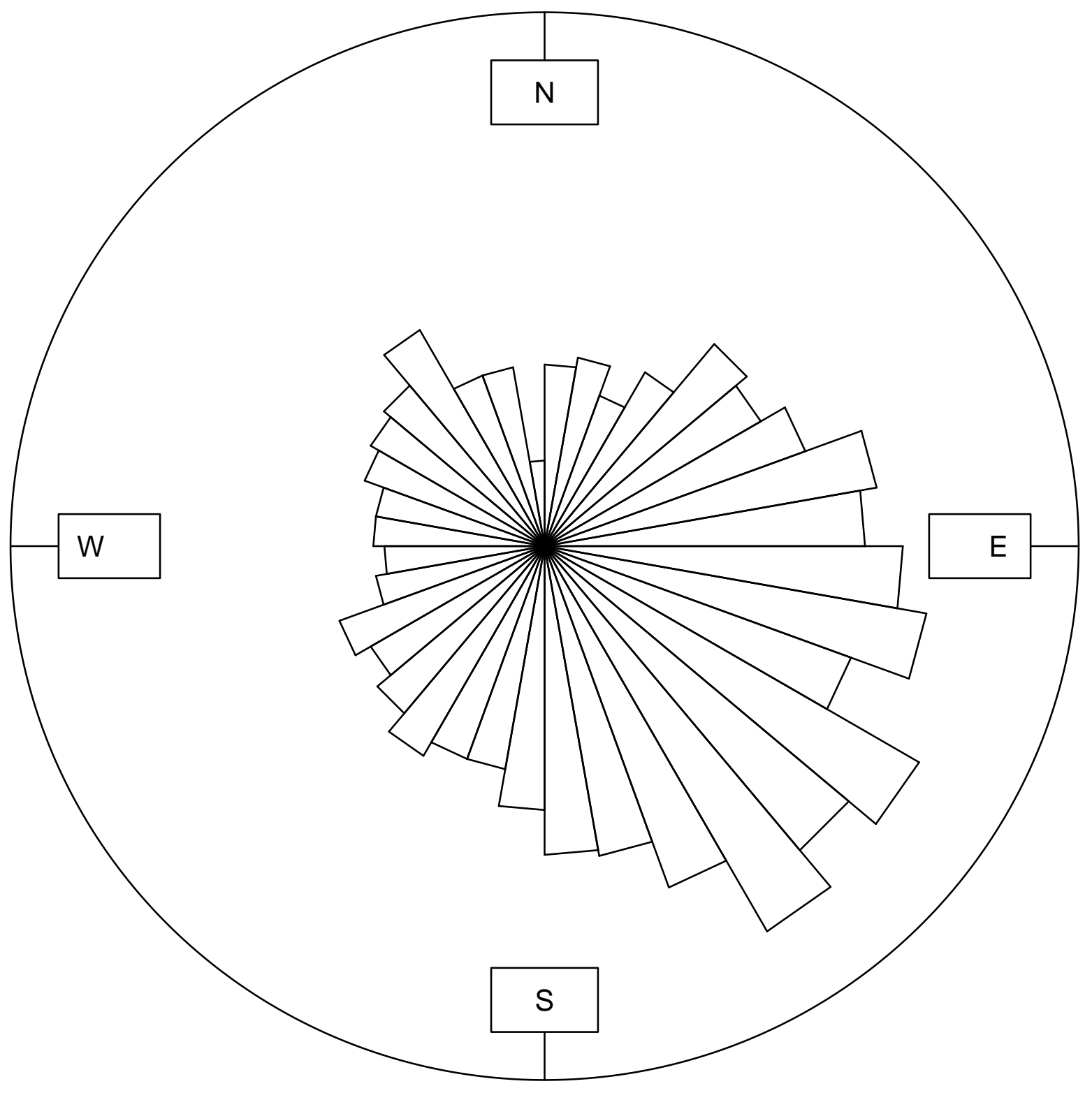


bootstrap 49

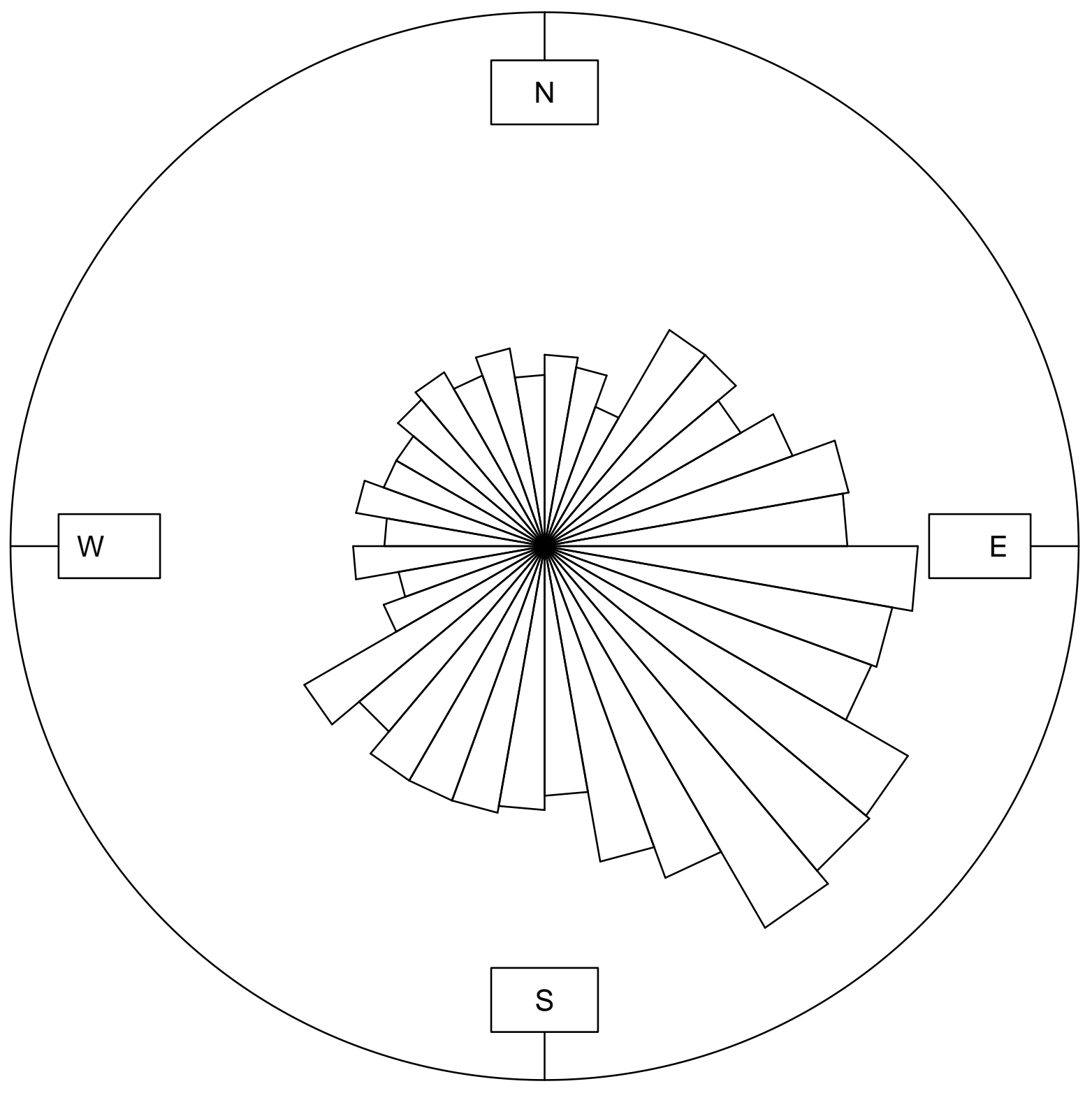


bootstrap 50

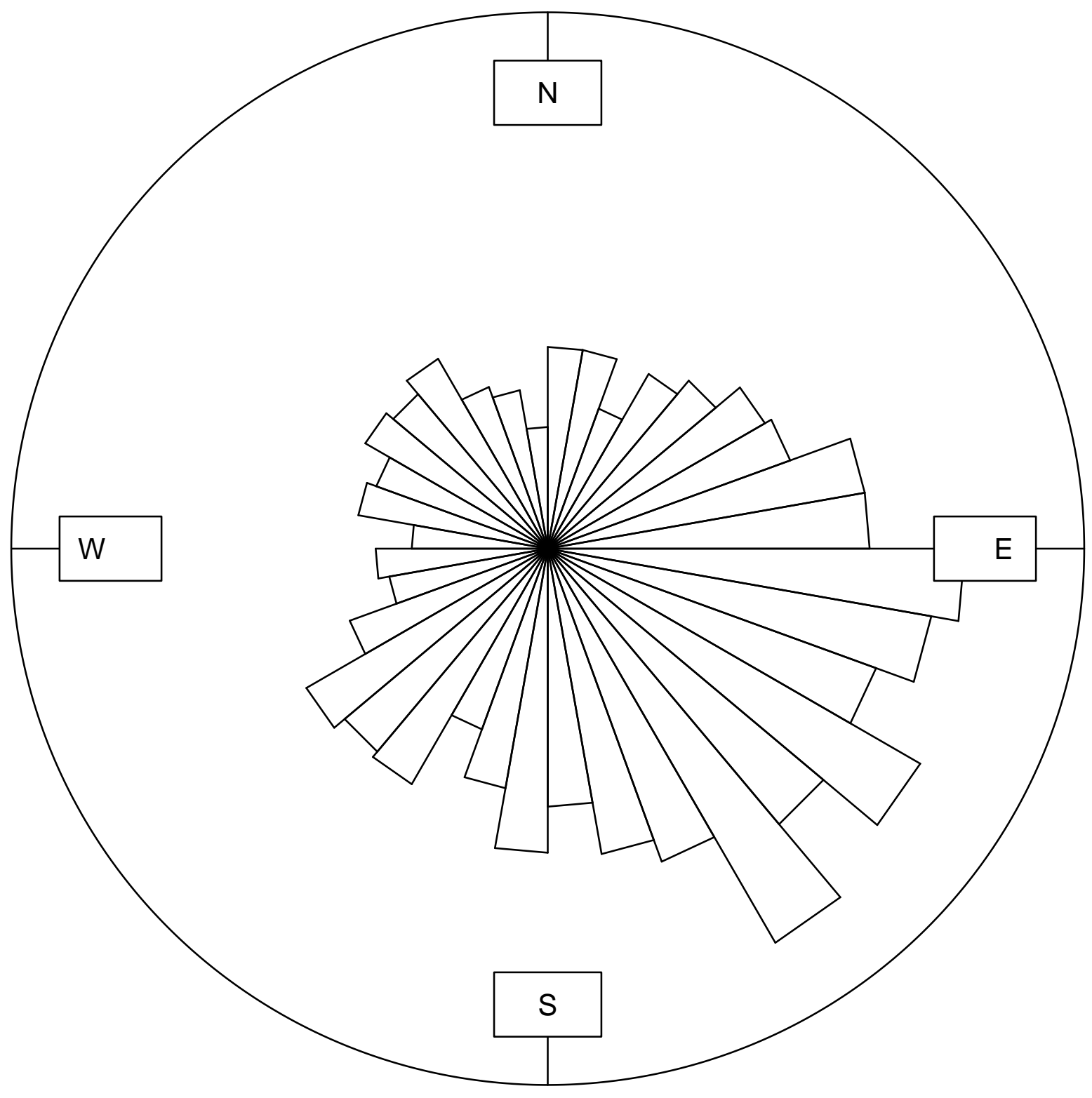




\section{bootstrap 51}

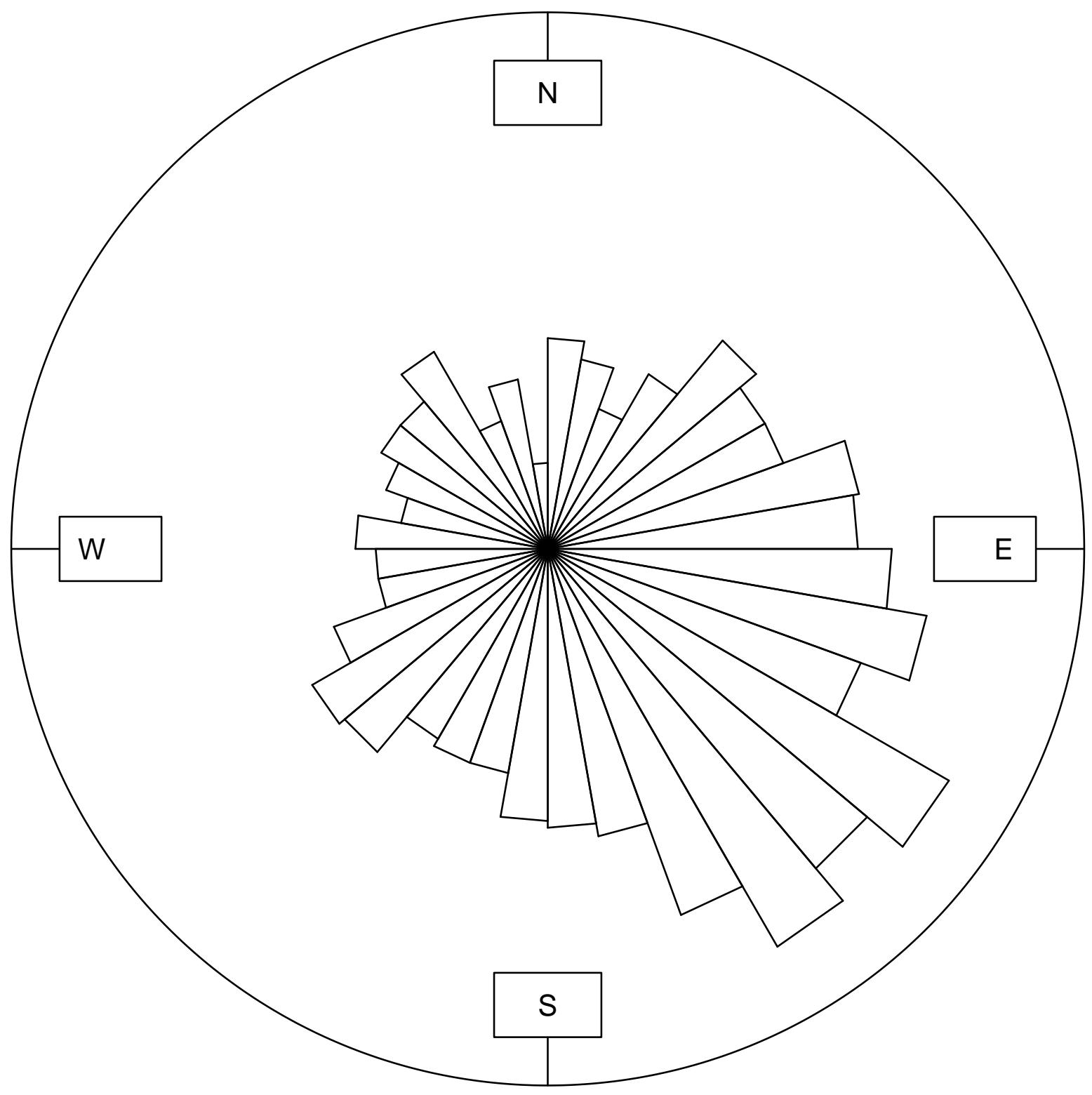


bootstrap 52

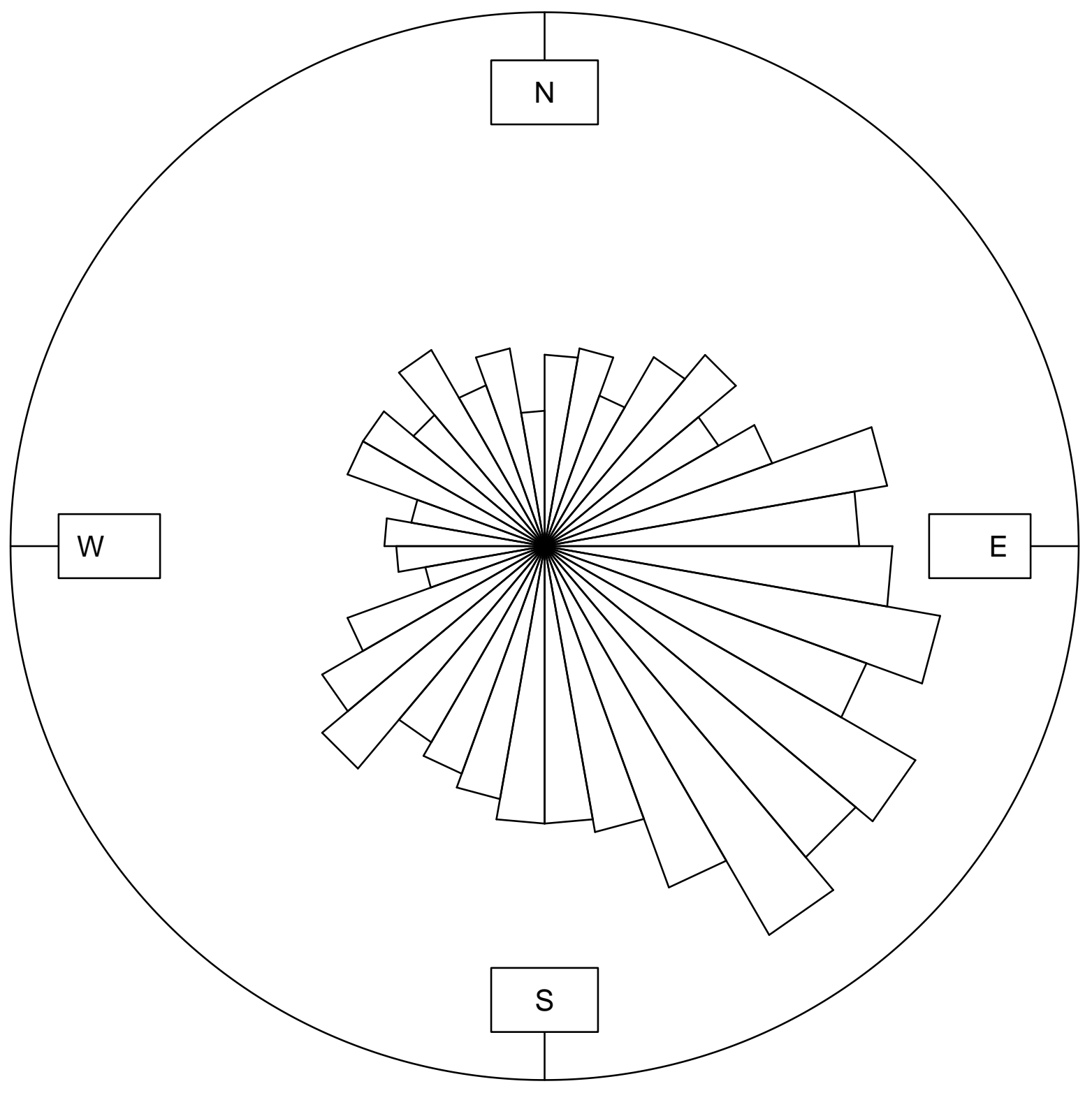




\section{bootstrap 53}

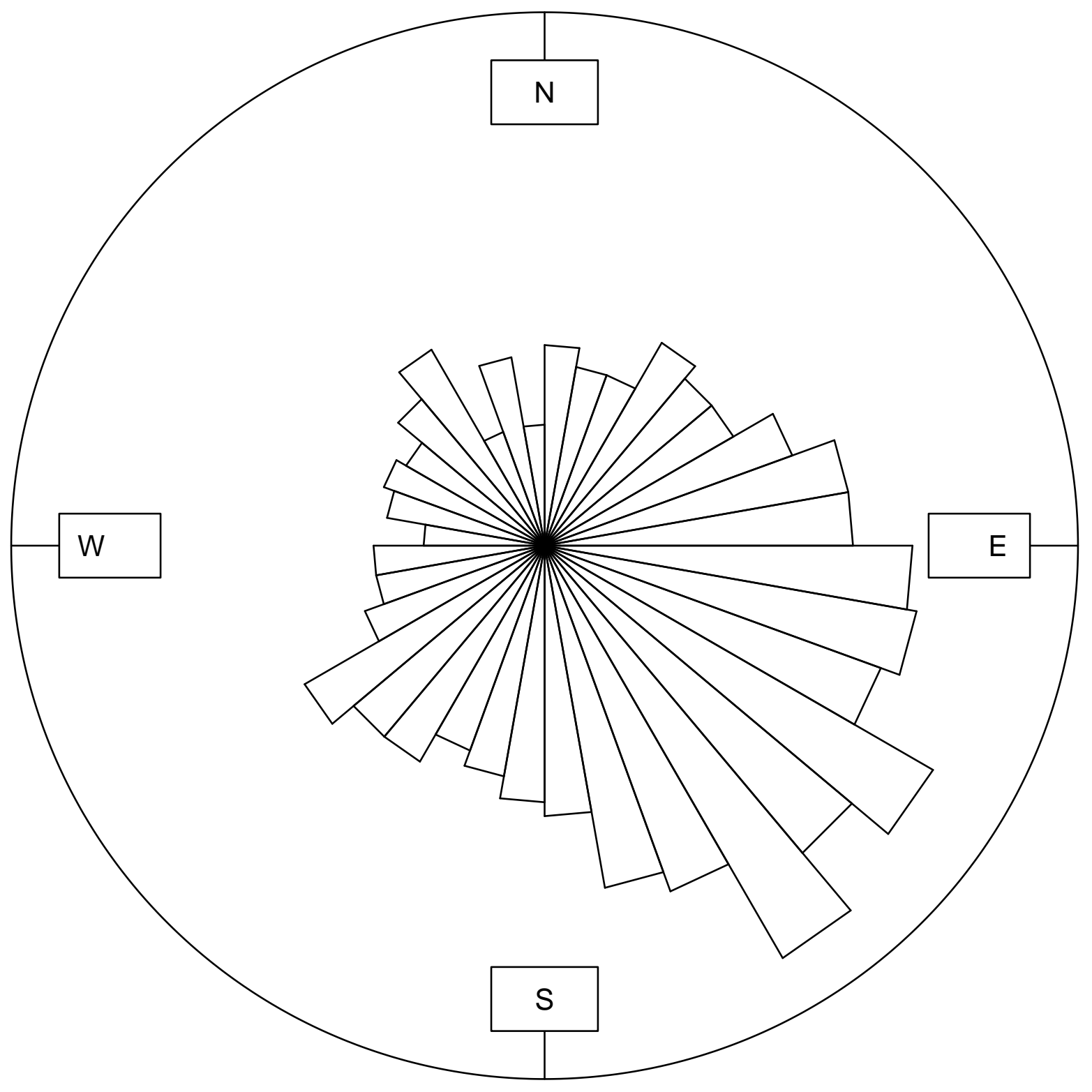




\section{bootstrap 54}

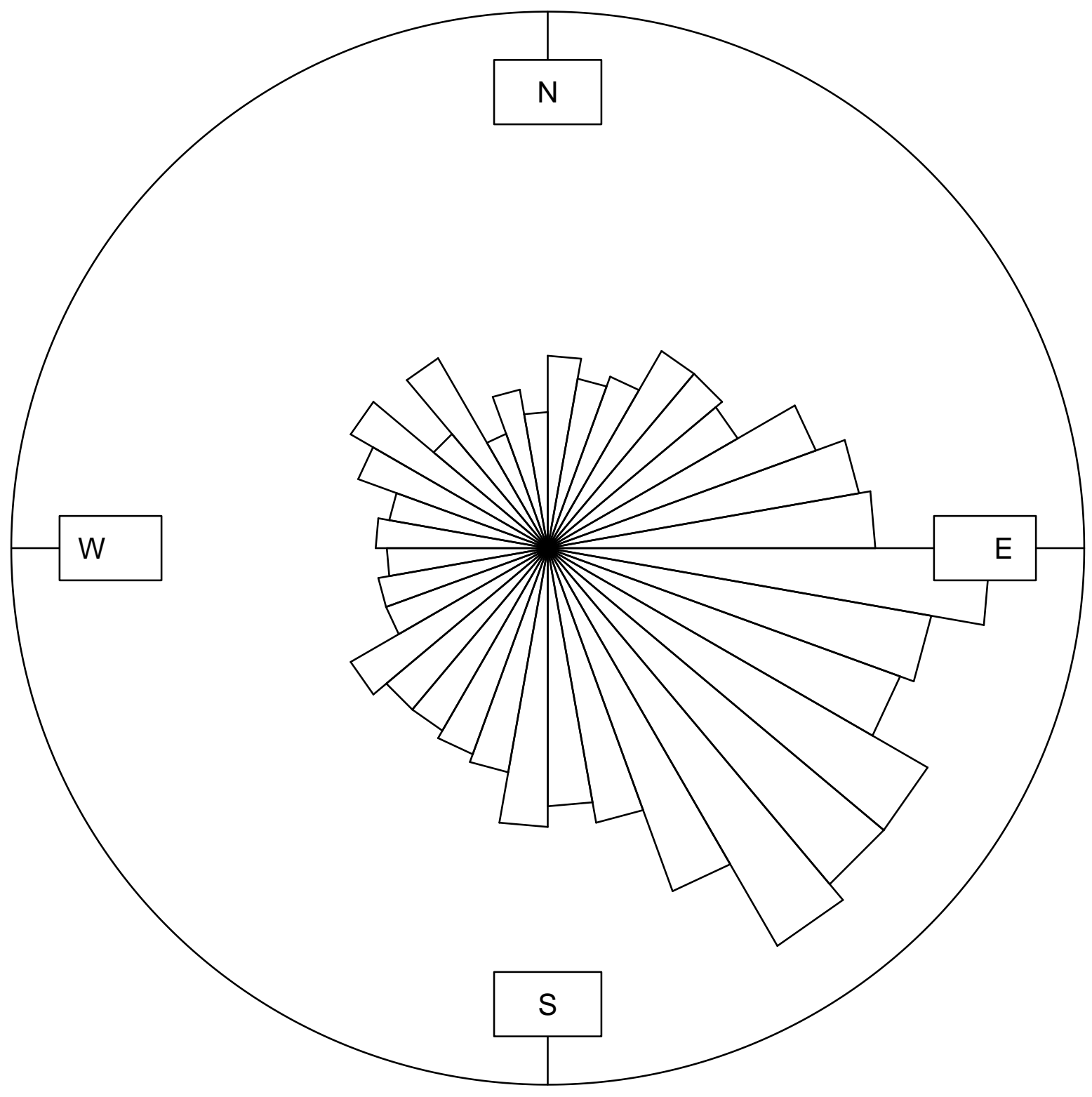




\section{bootstrap 55}

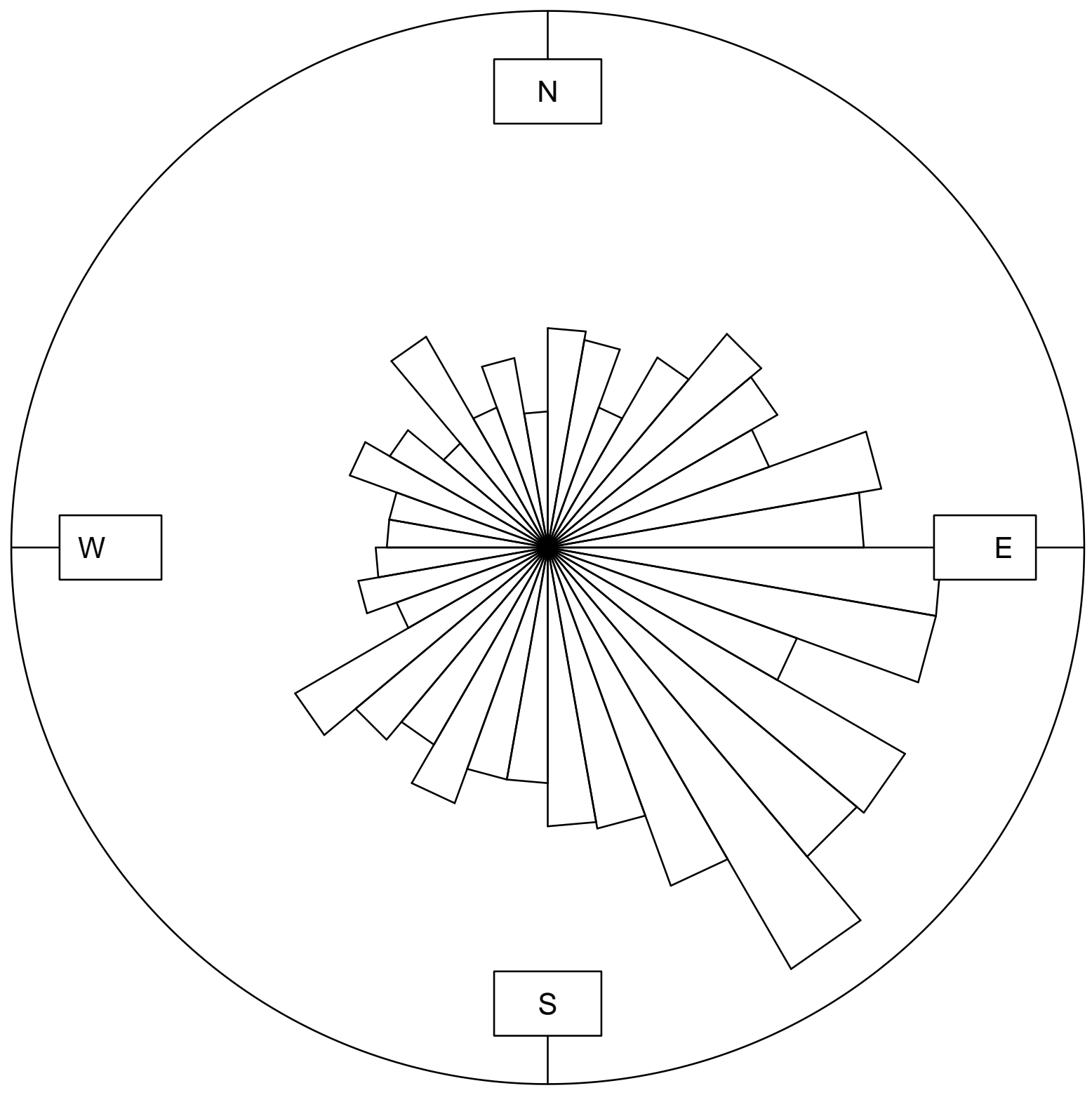




\section{bootstrap 56}

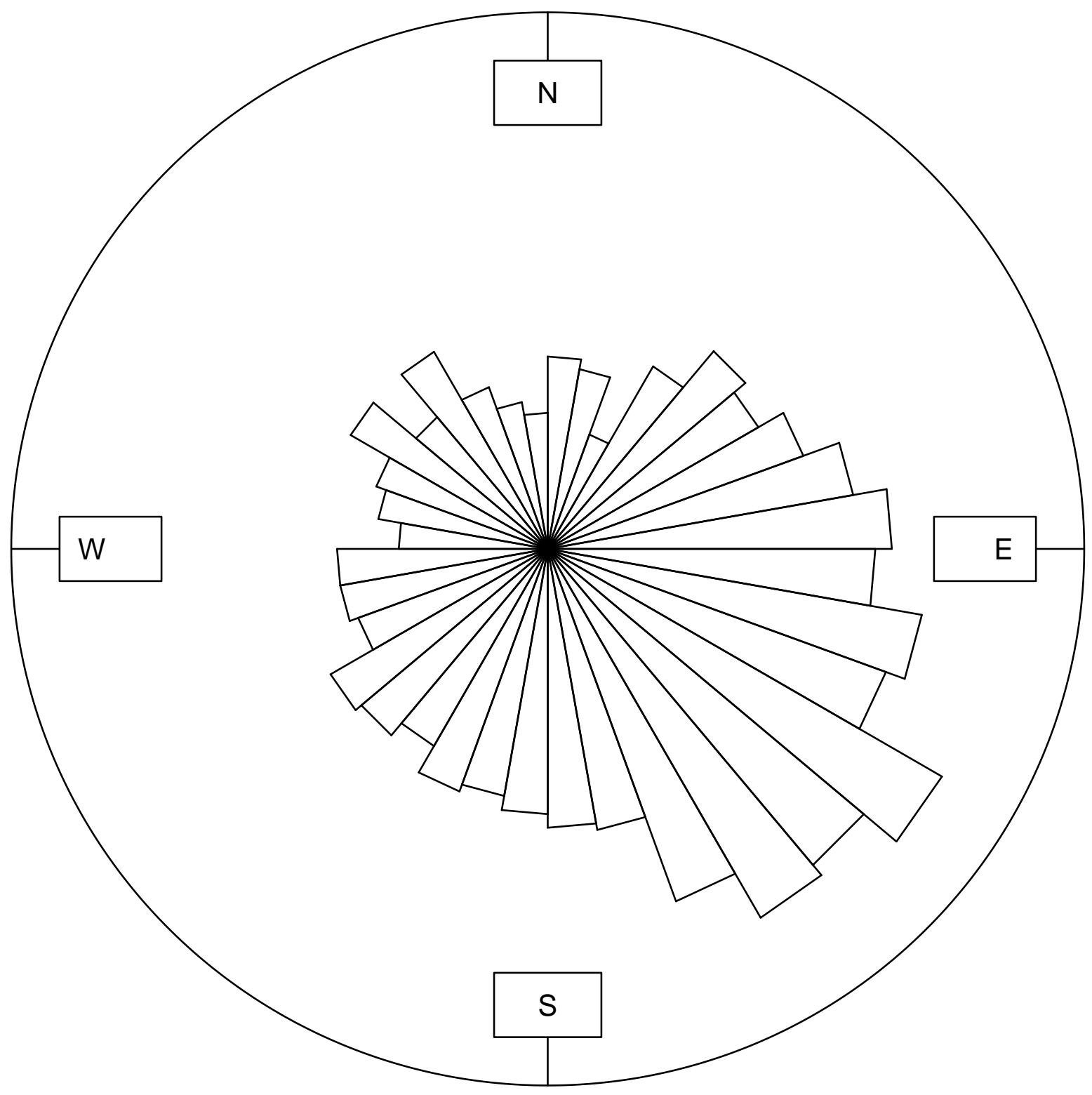


bootstrap 57

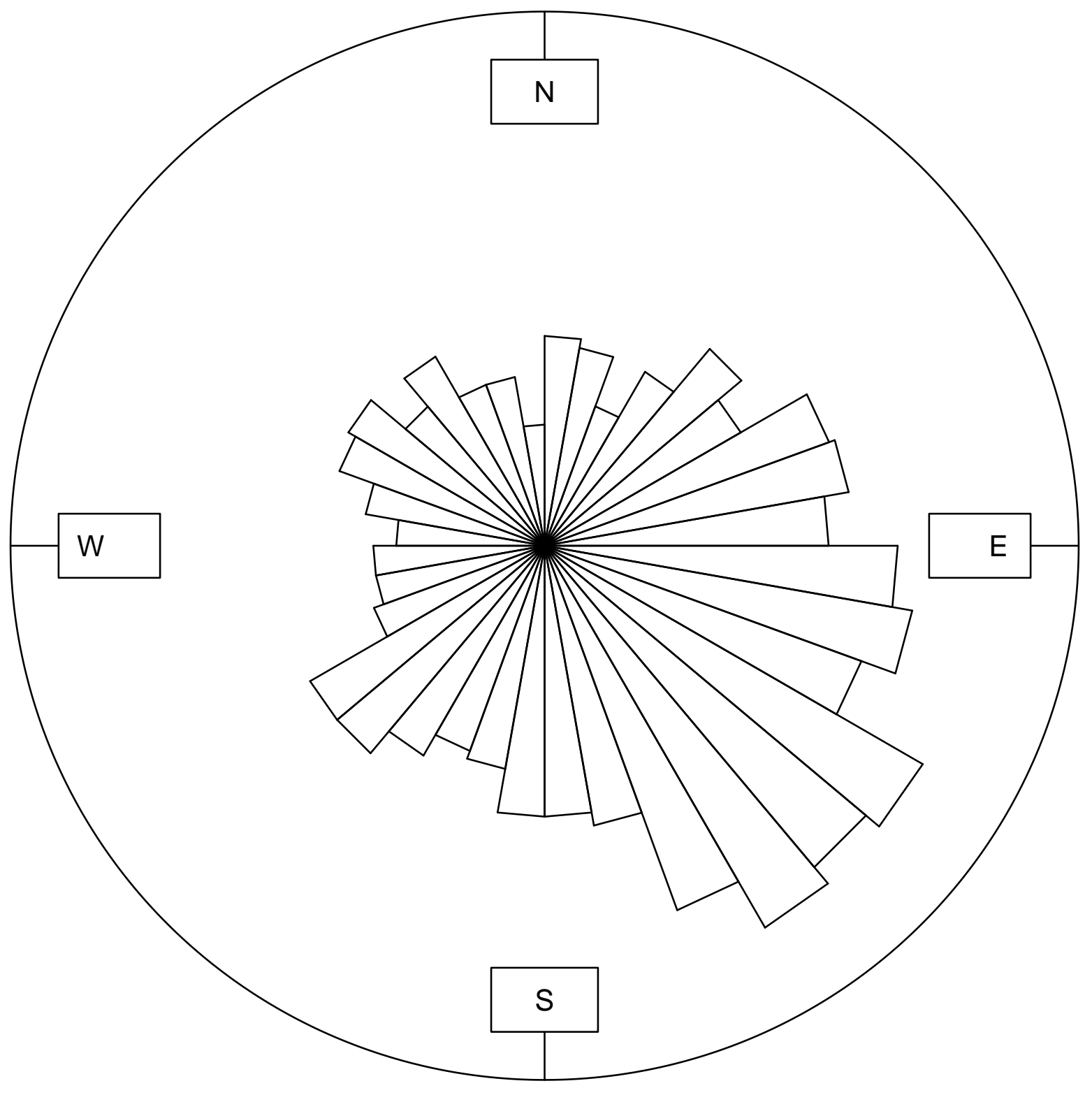




\section{bootstrap 58}

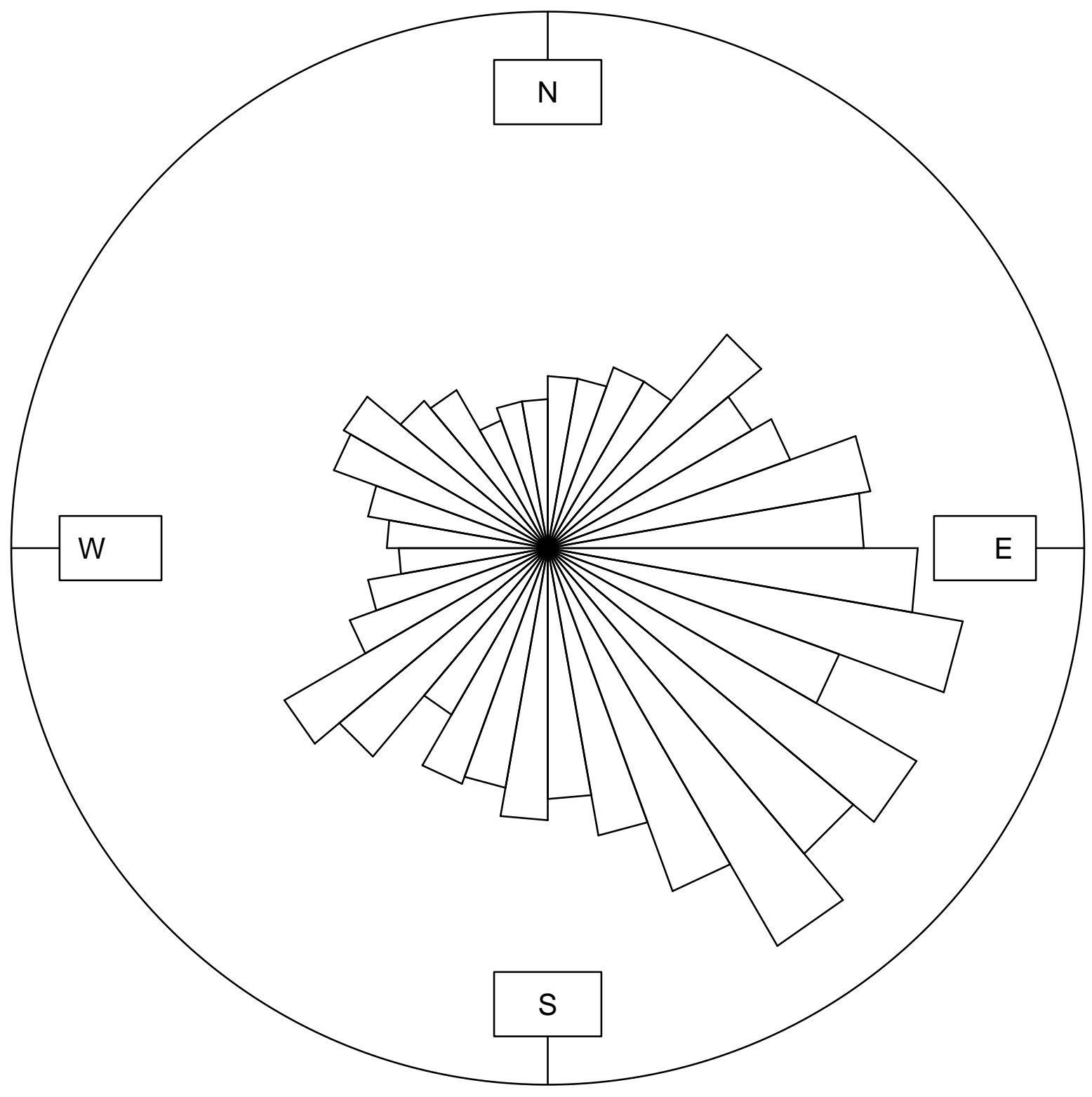


bootstrap 59

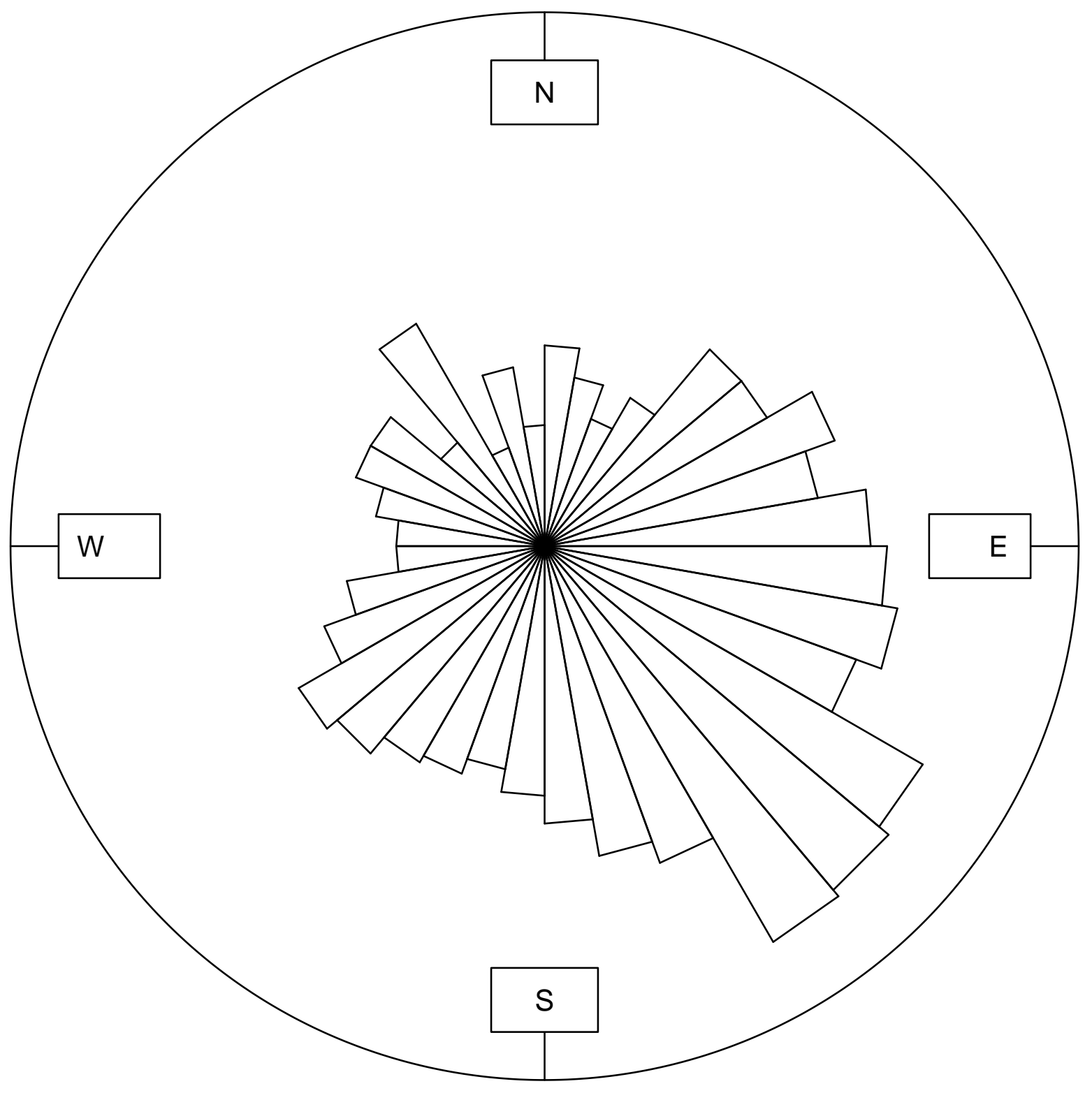


bootstrap 60

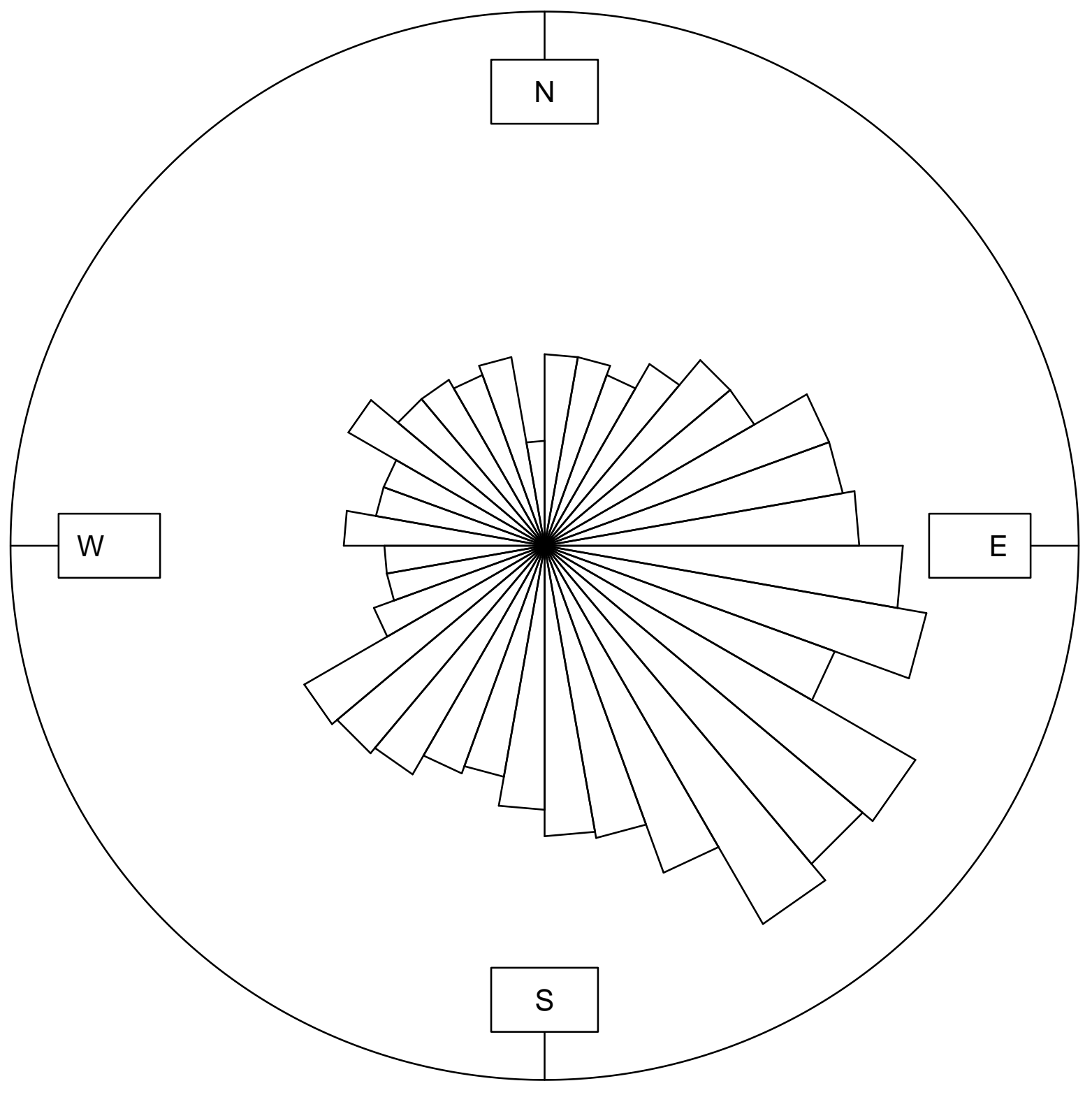


bootstrap 61

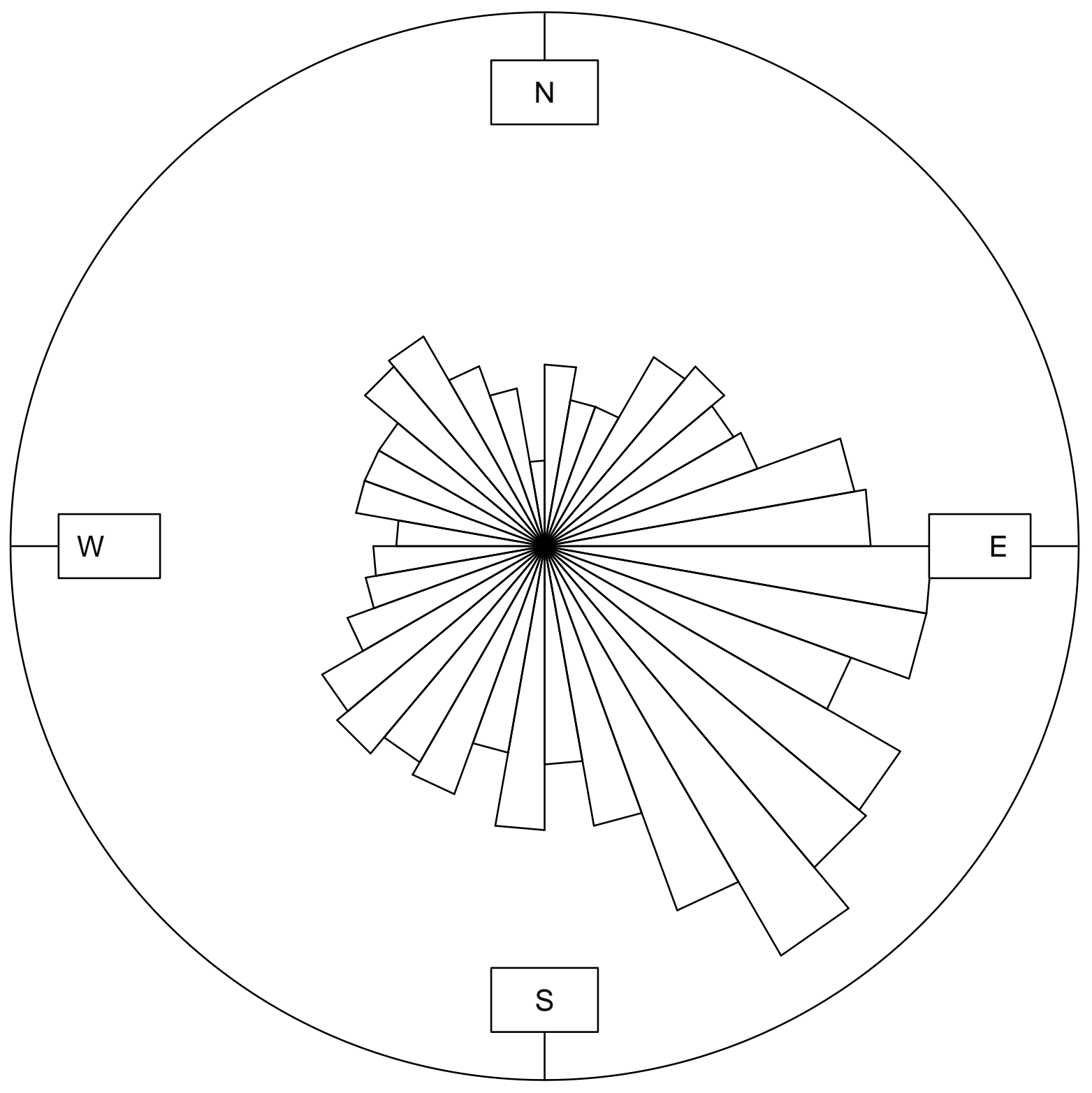


bootstrap 62

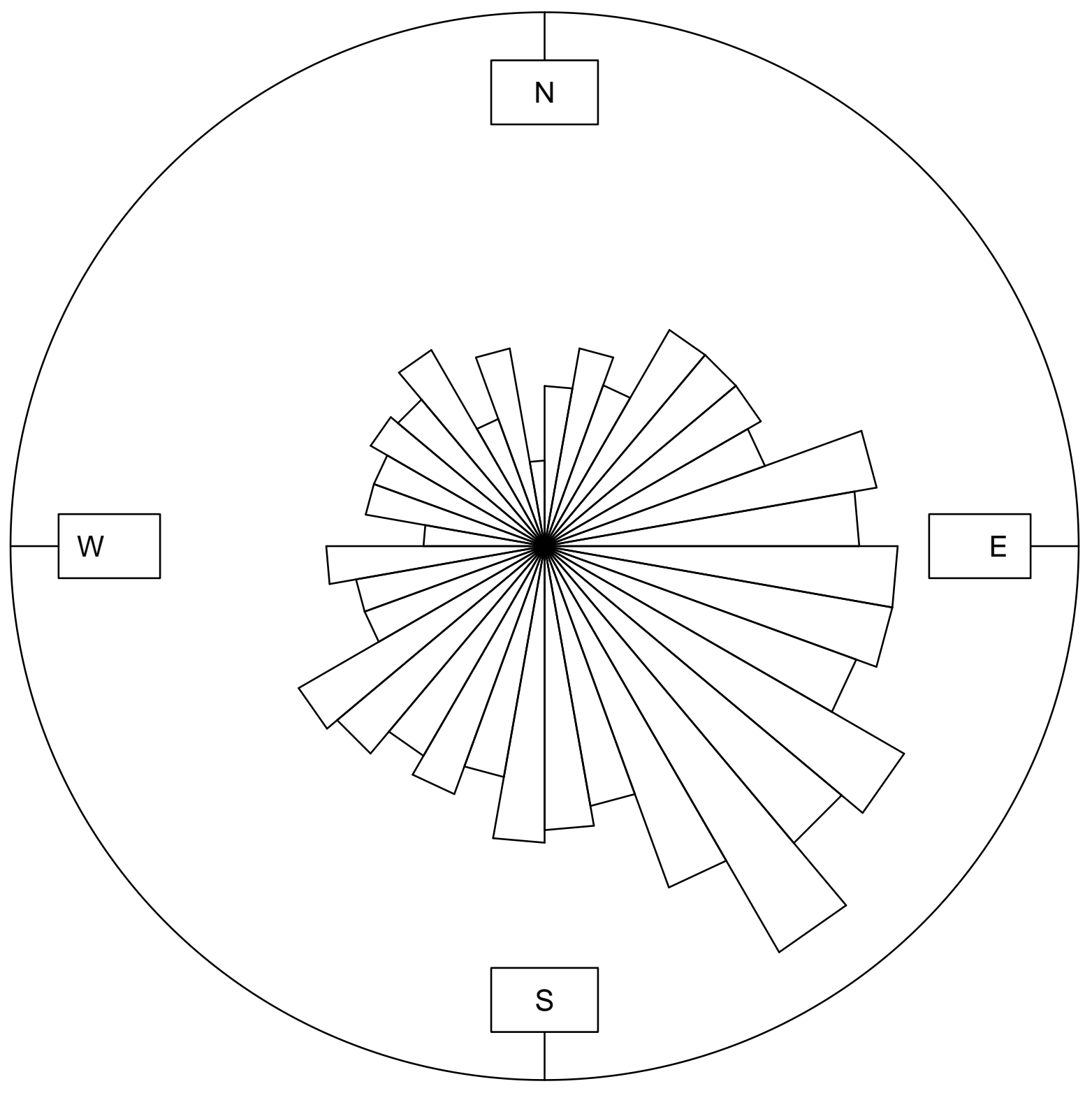




\section{bootstrap 63}

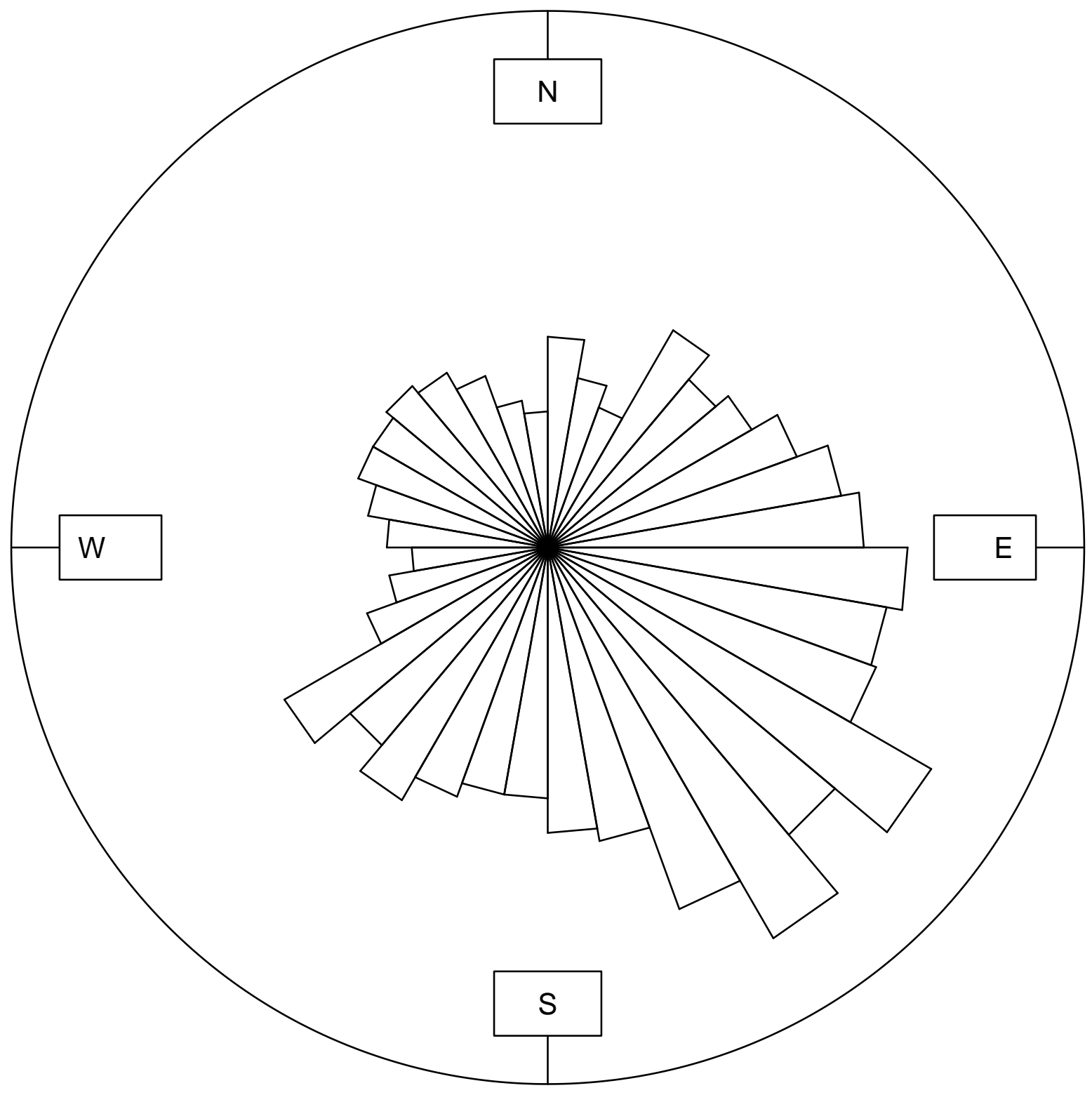




\section{bootstrap 64}

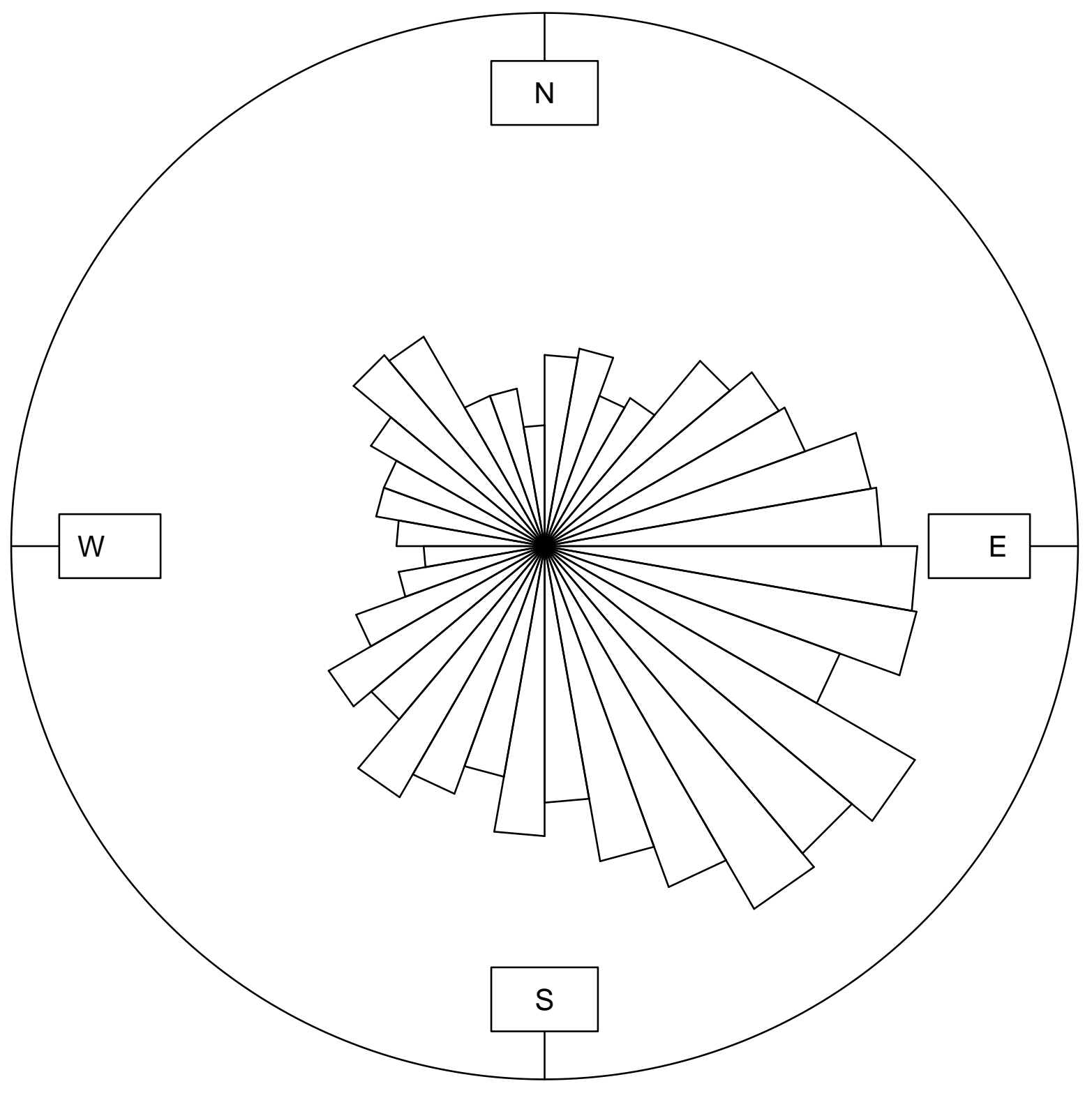




\section{bootstrap 65}

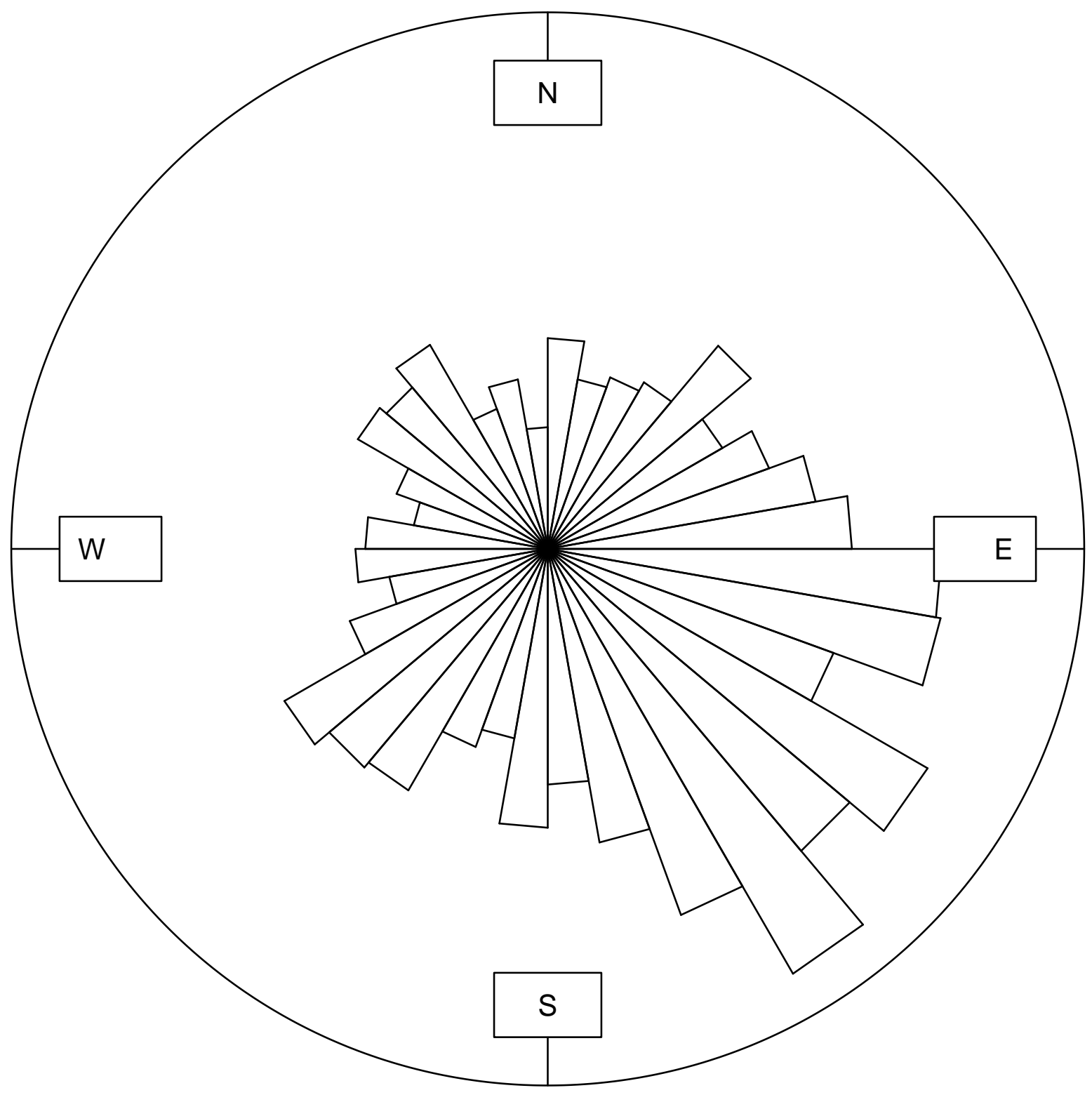




\section{bootstrap 66}

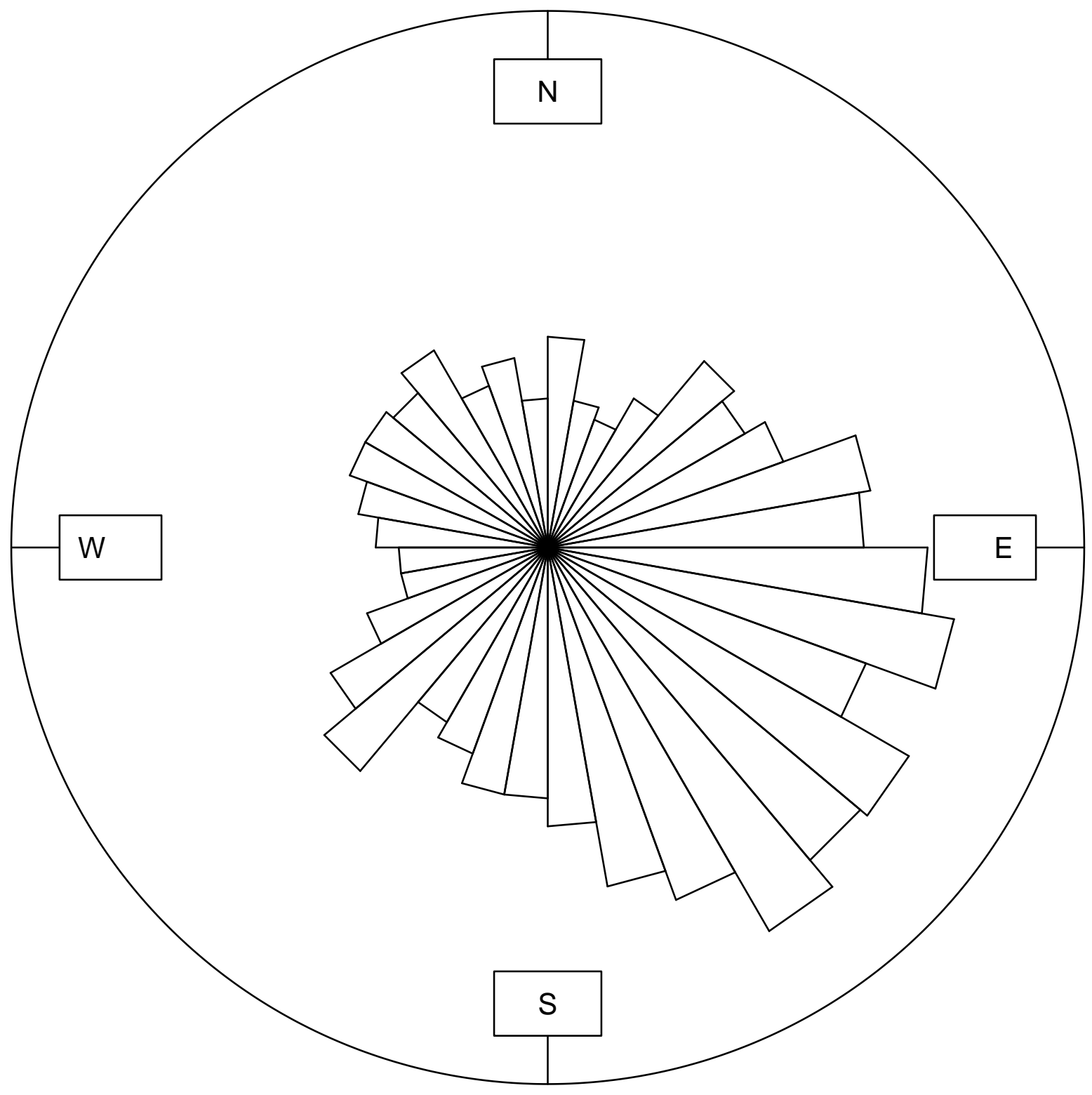


bootstrap 67

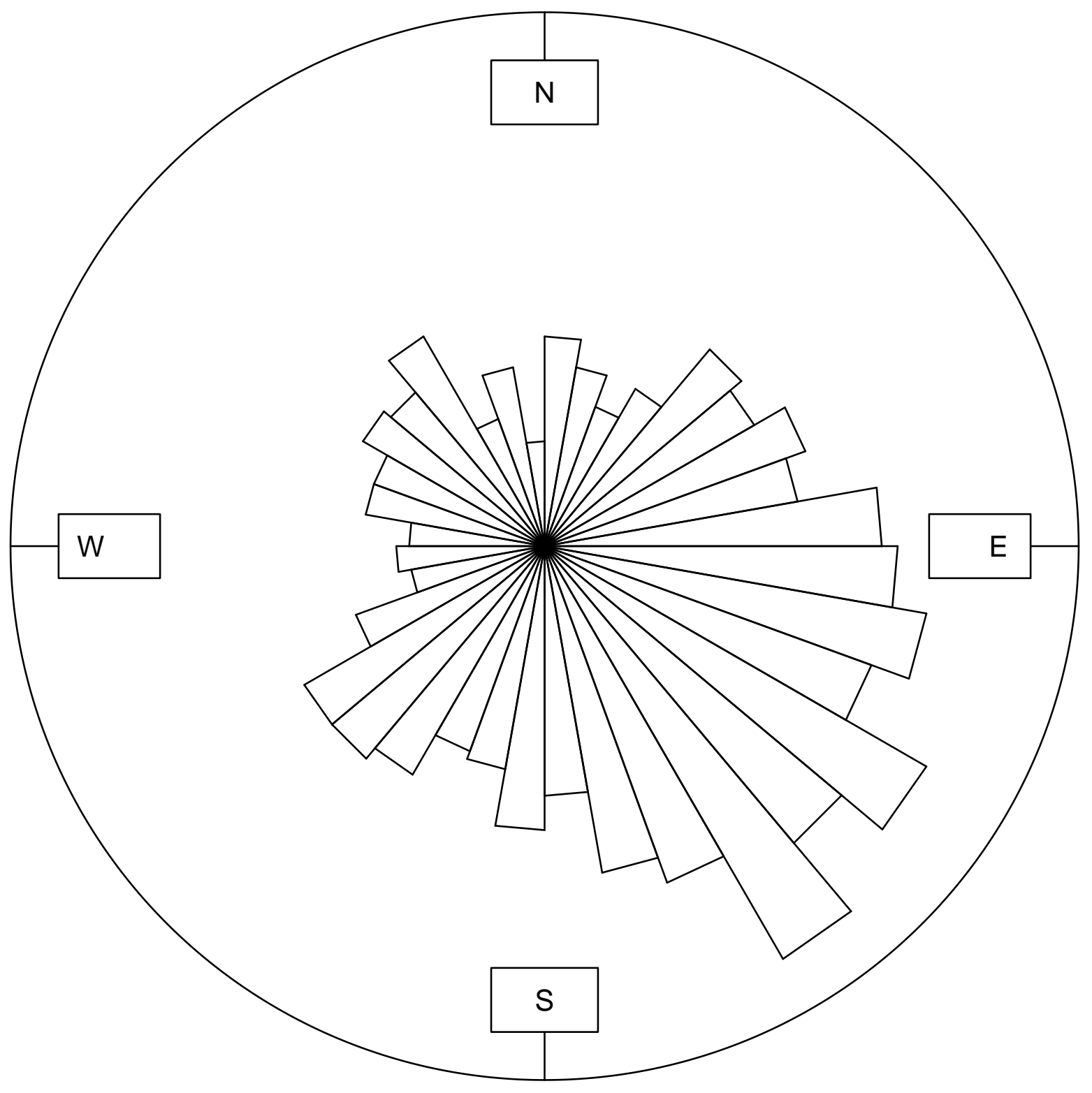




\section{bootstrap 68}

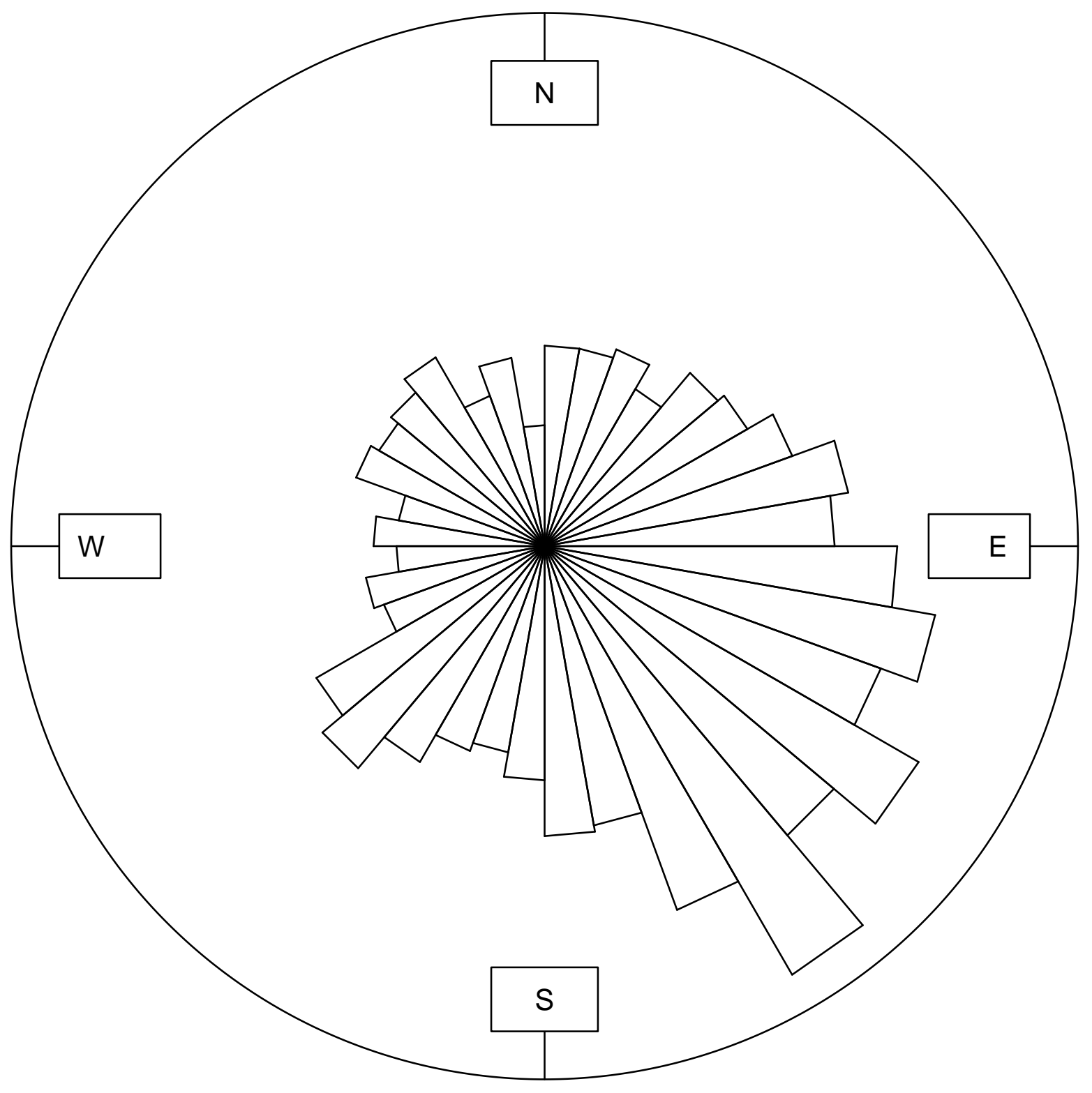




\section{bootstrap 69}

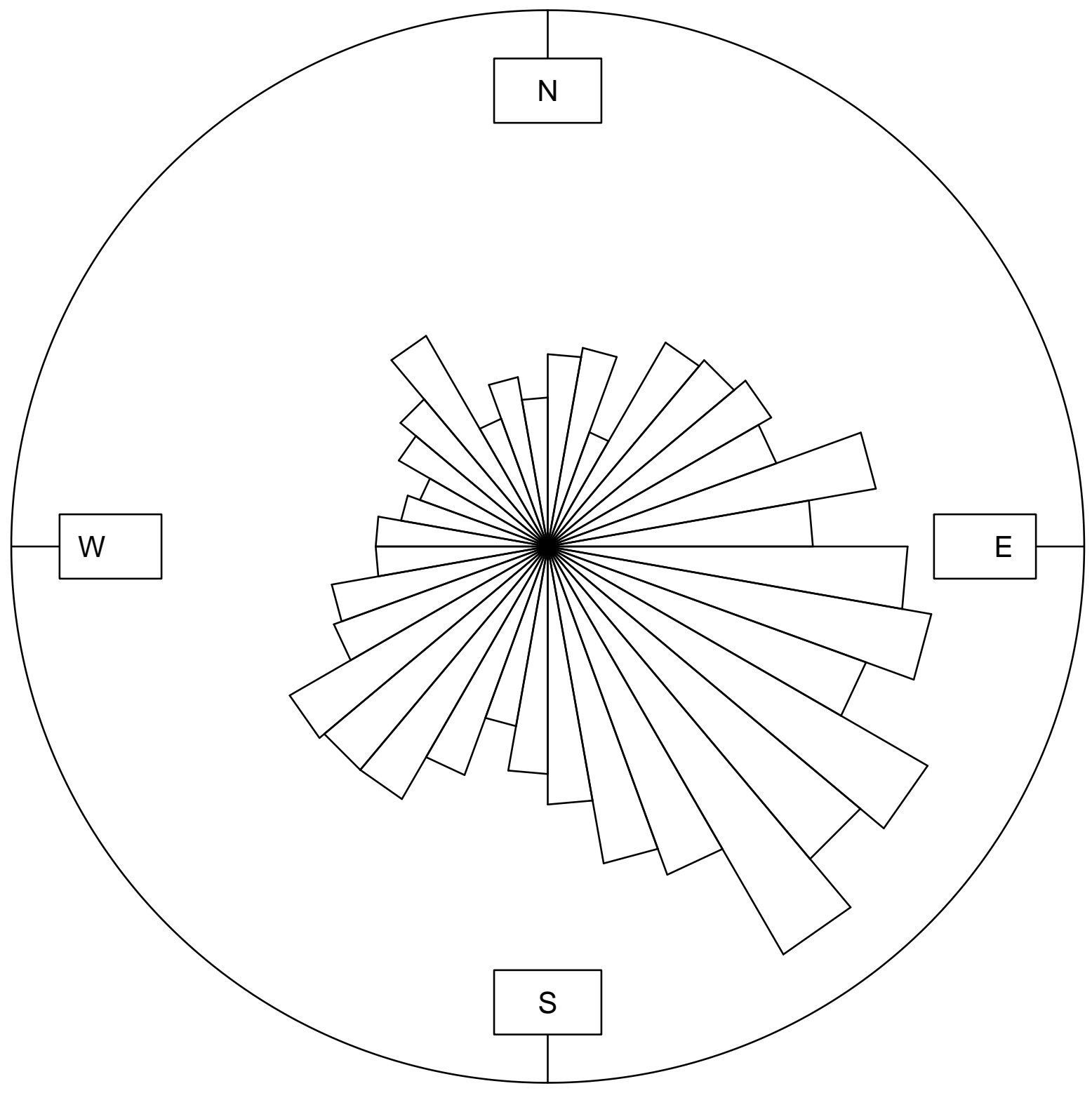


bootstrap 70

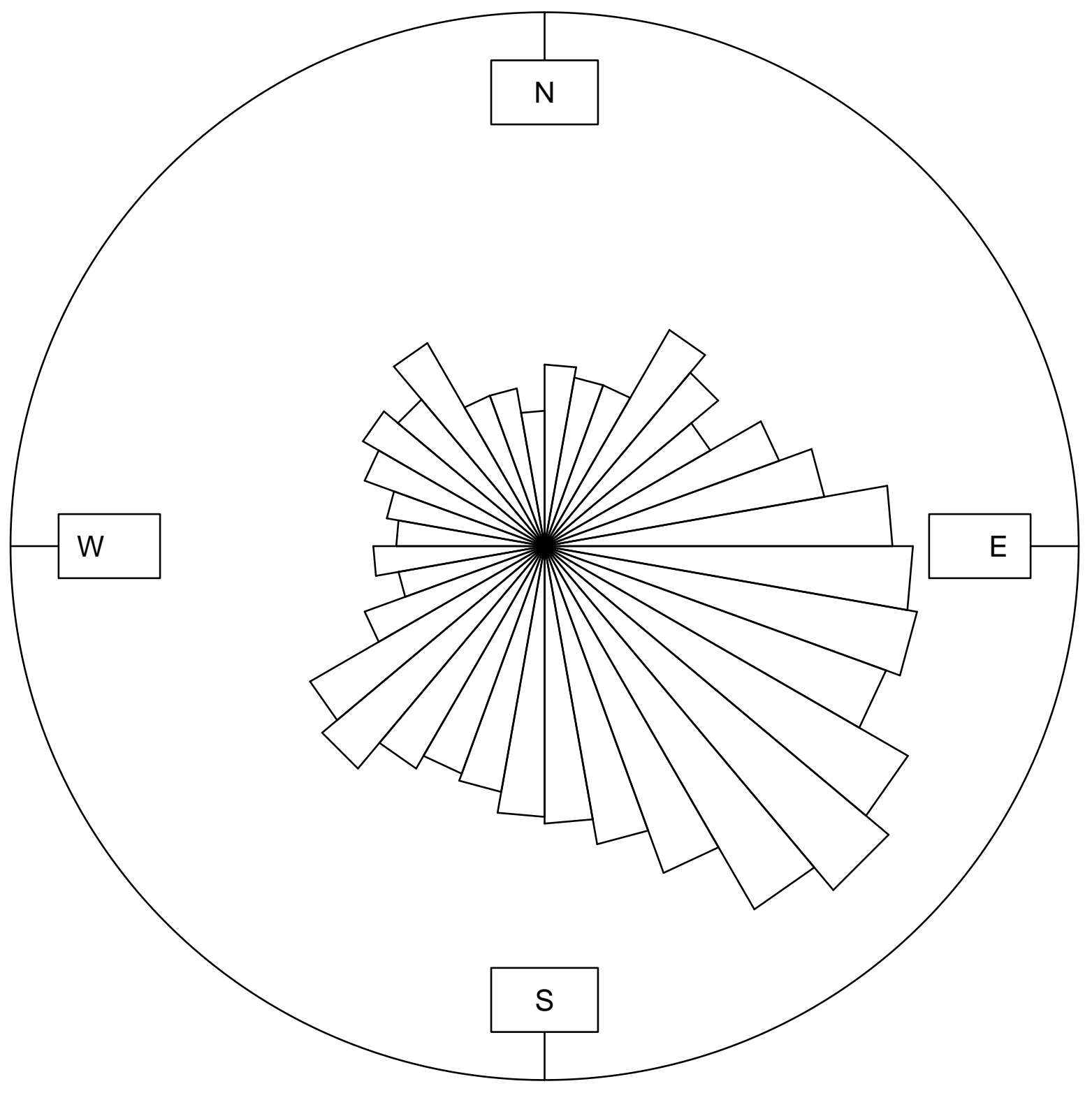




\section{bootstrap 71}

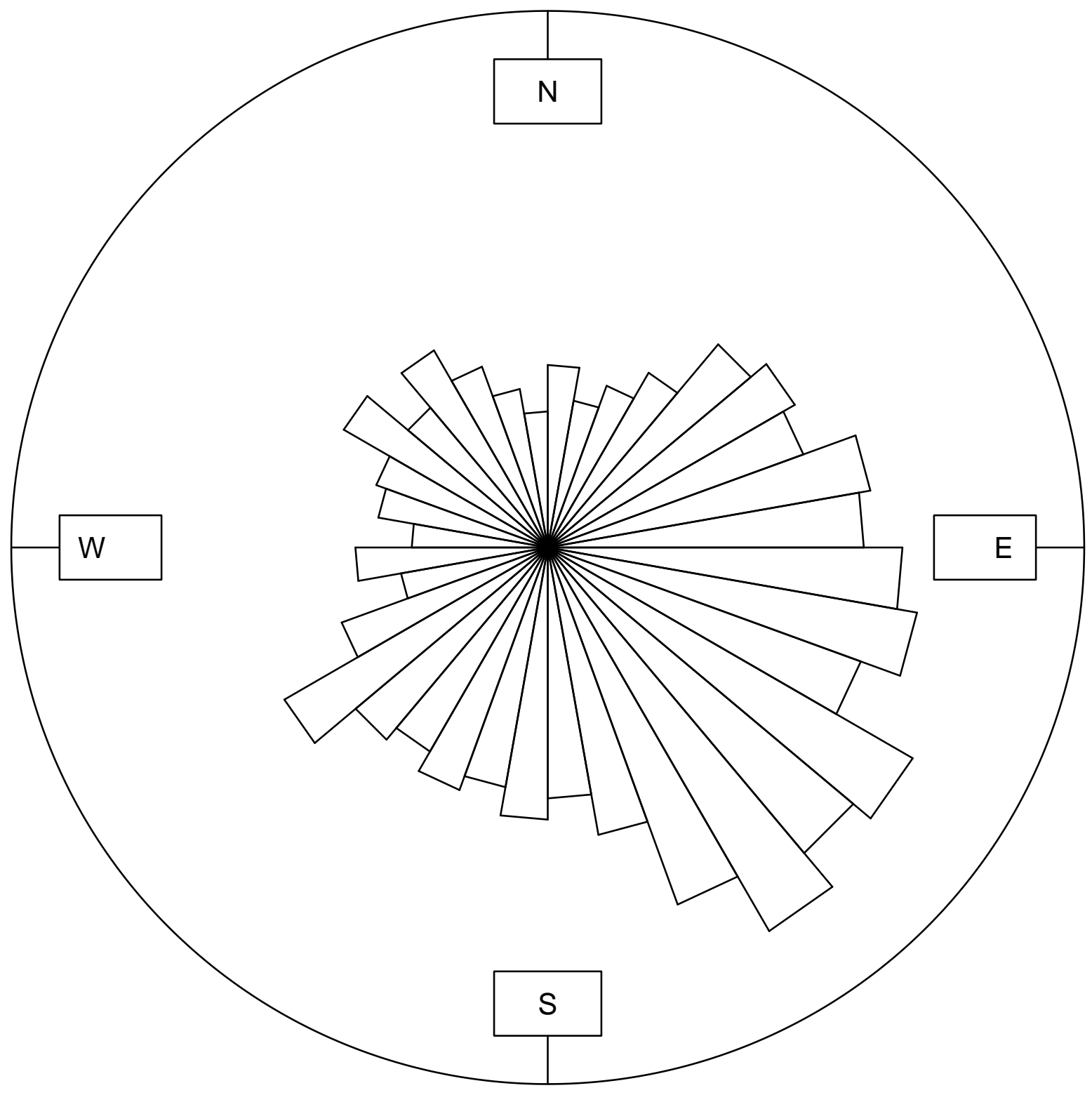




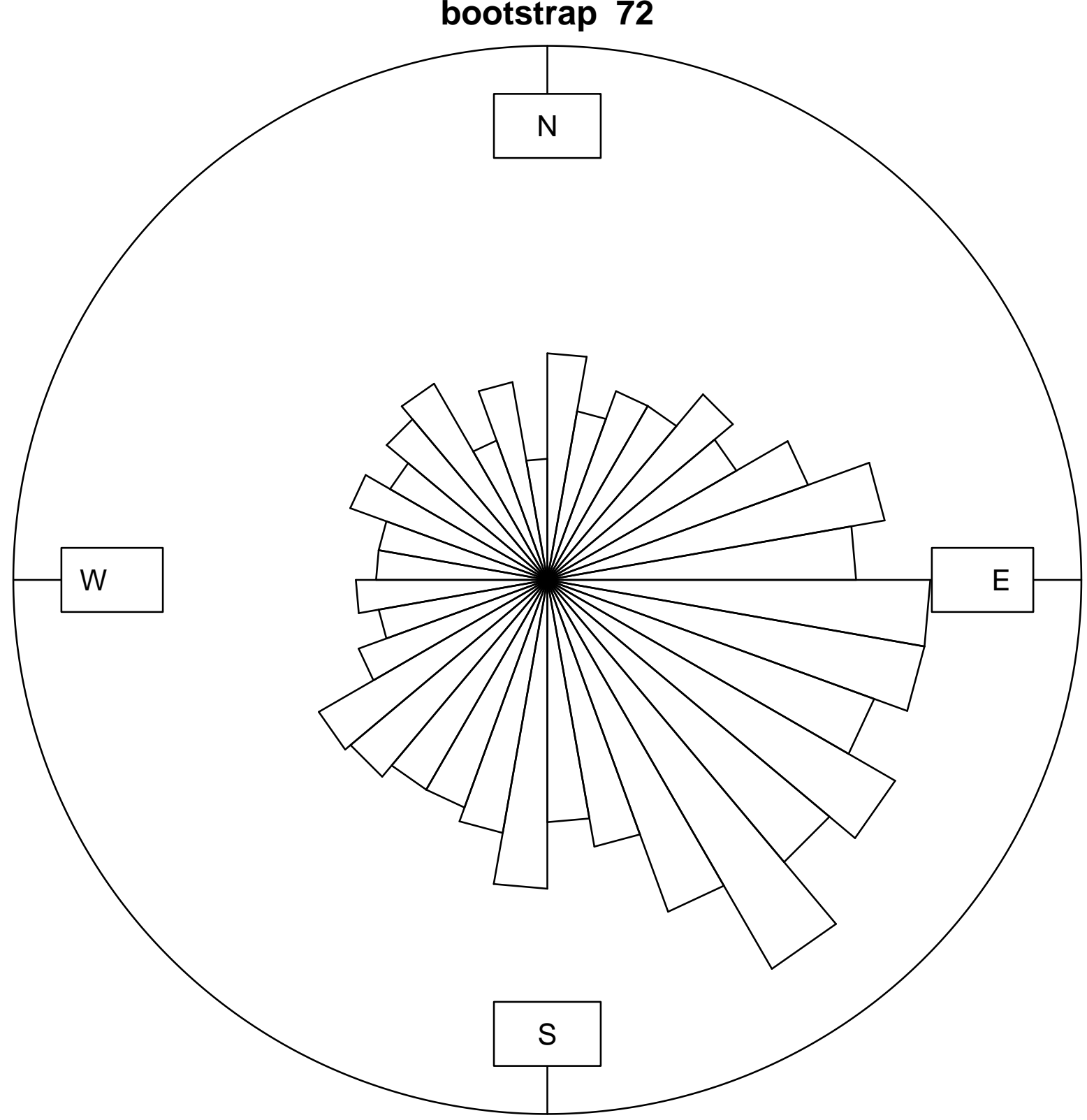


bootstrap 73

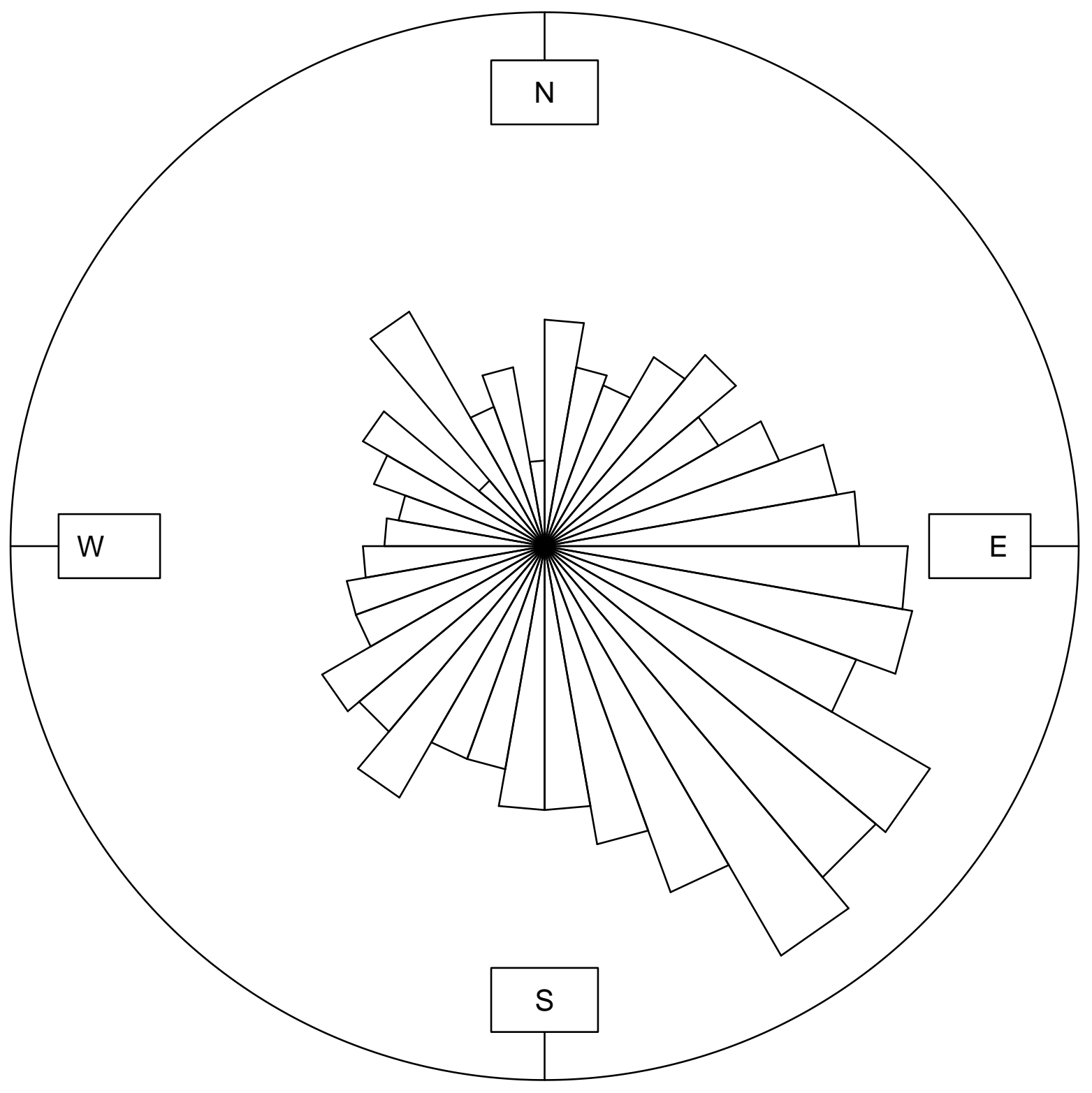




\section{bootstrap 74}

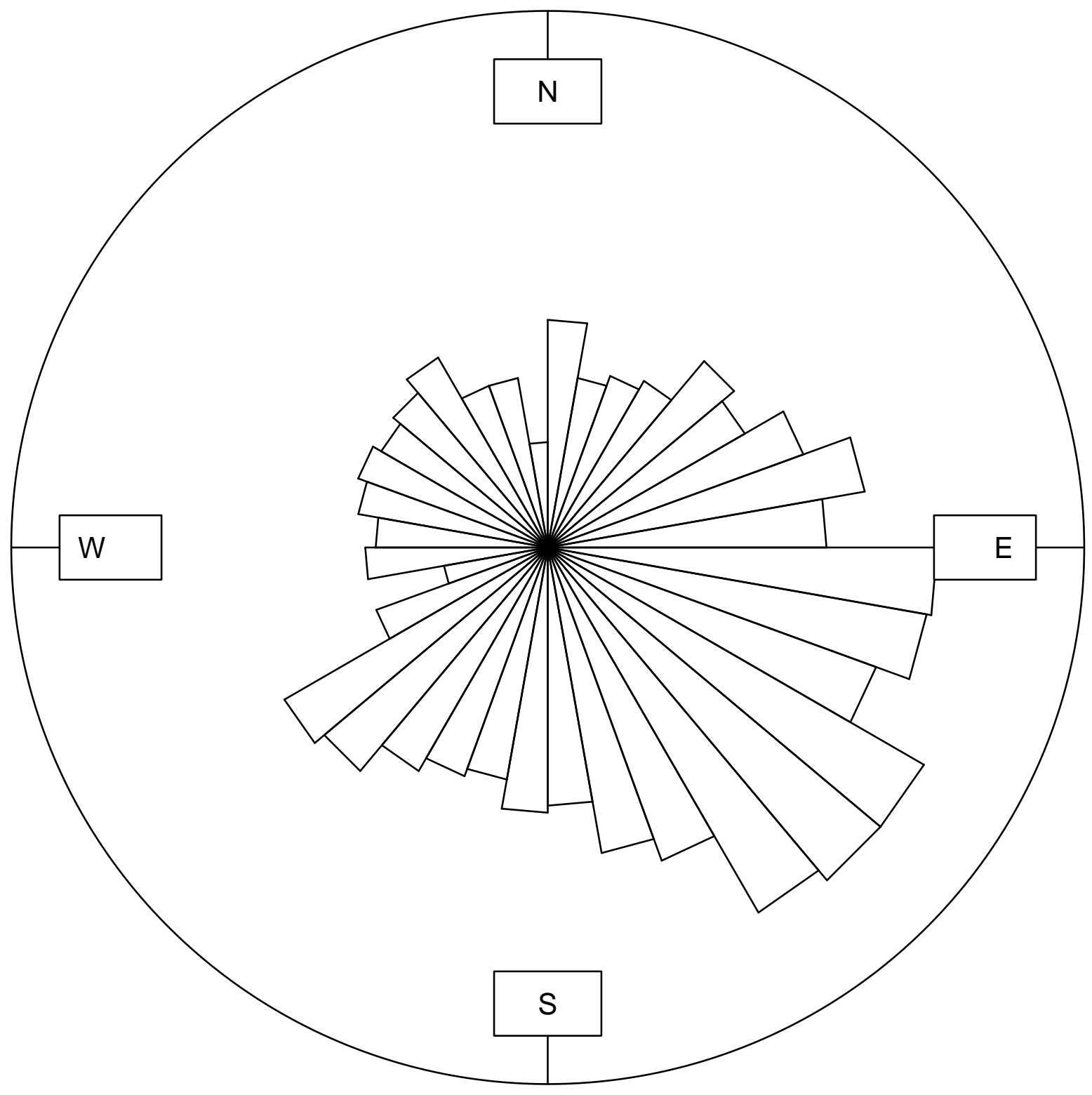


bootstrap 75

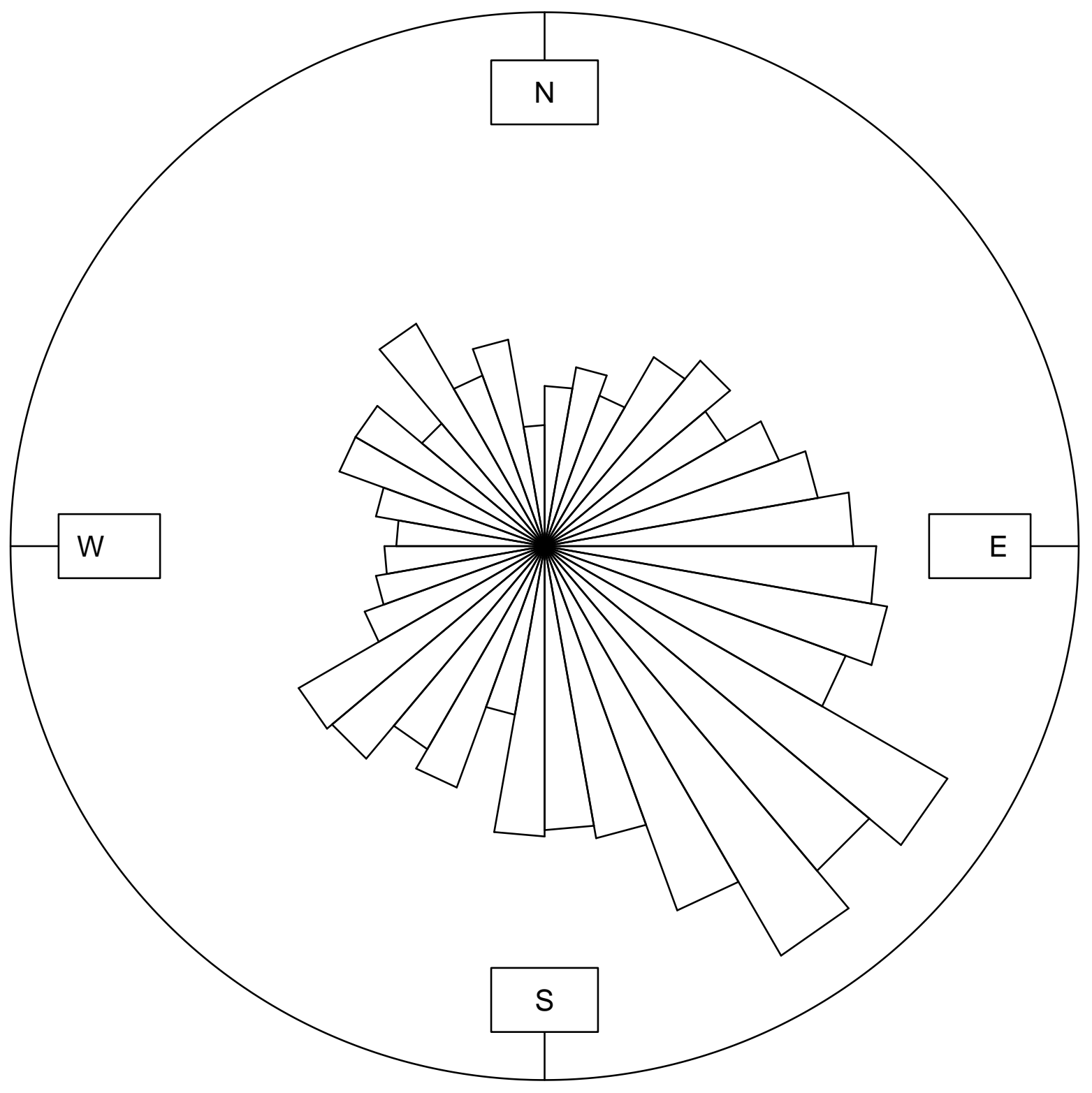


bootstrap 76

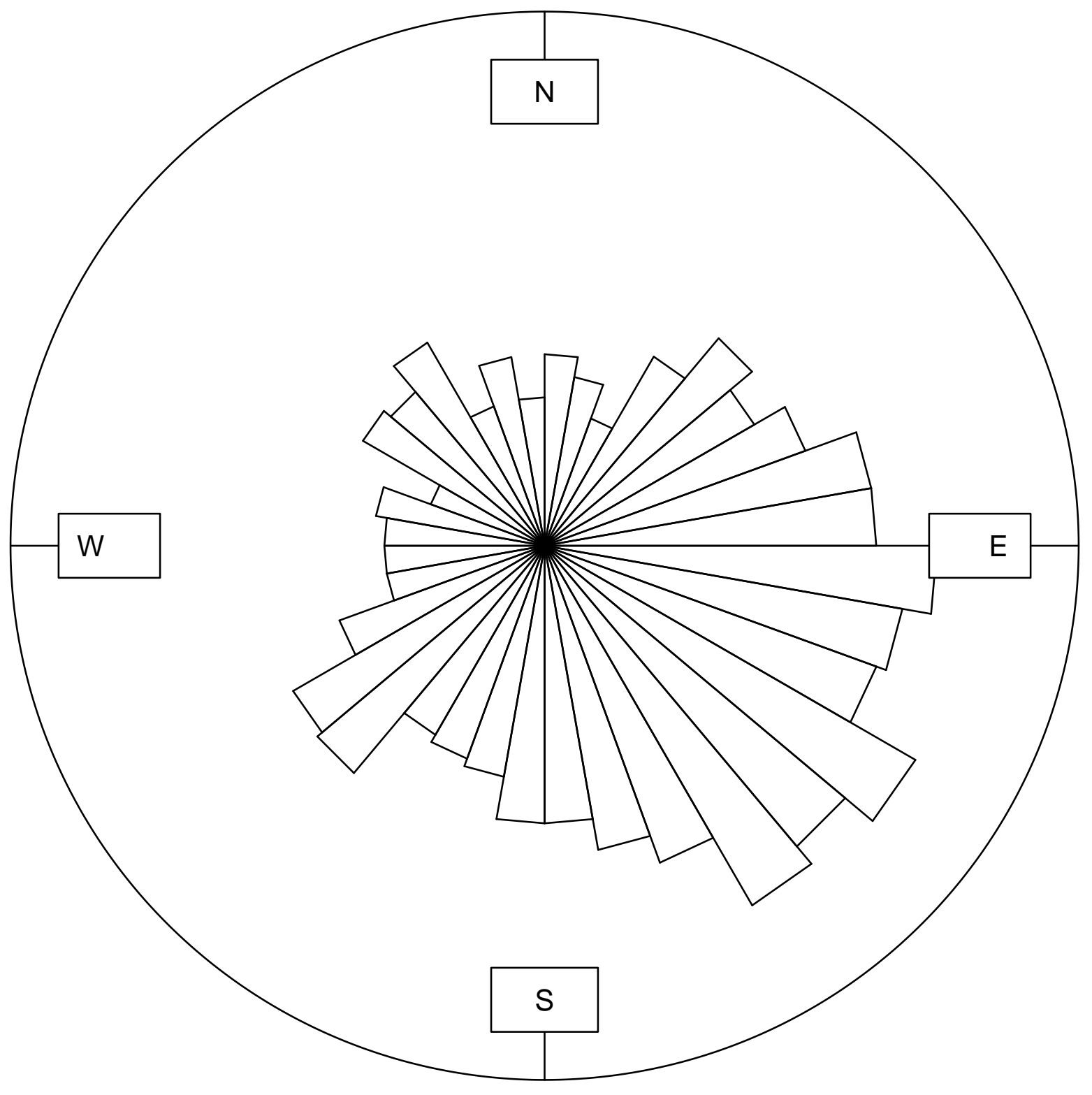


bootstrap 77

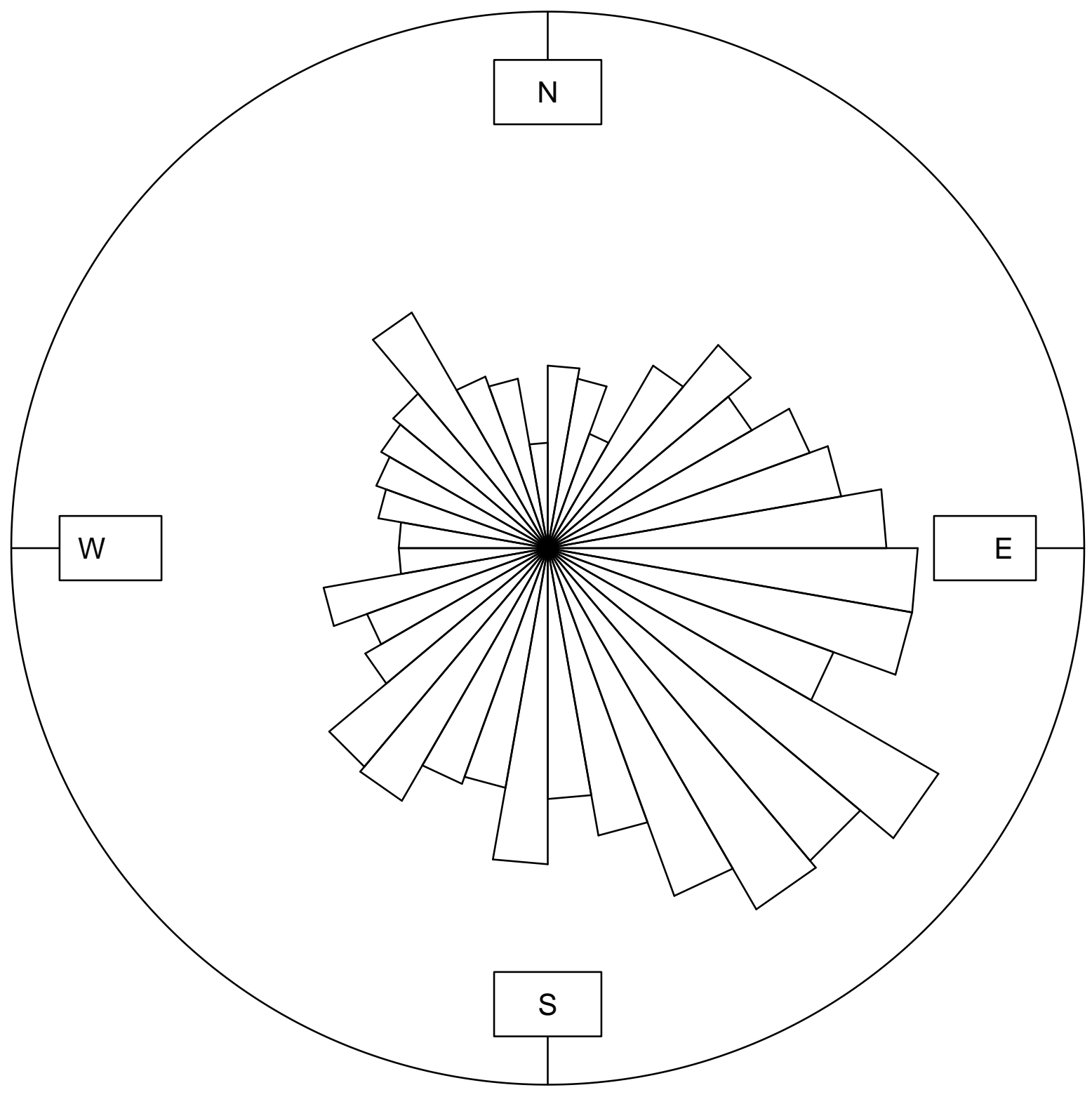


bootstrap 78

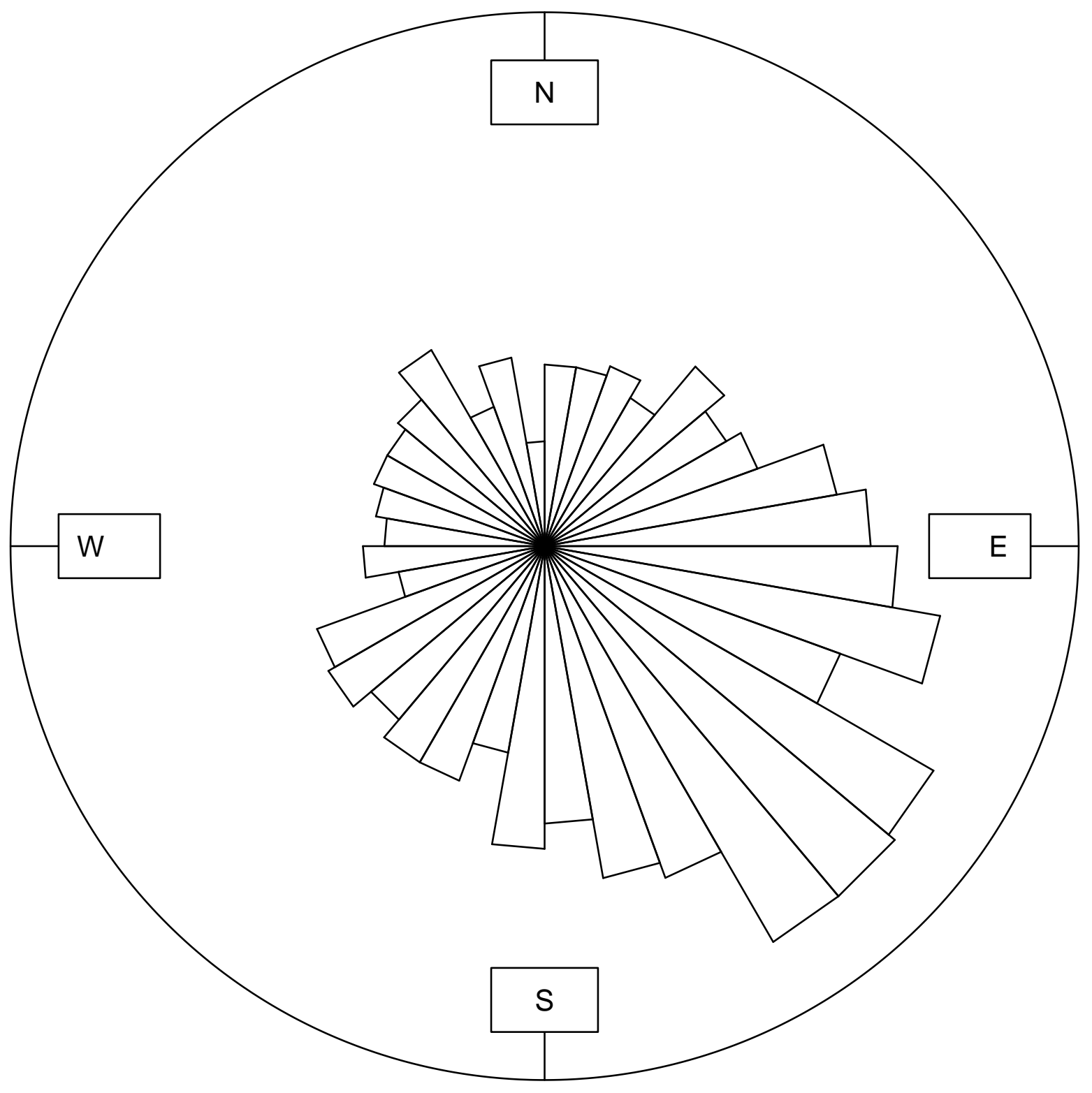


bootstrap 79

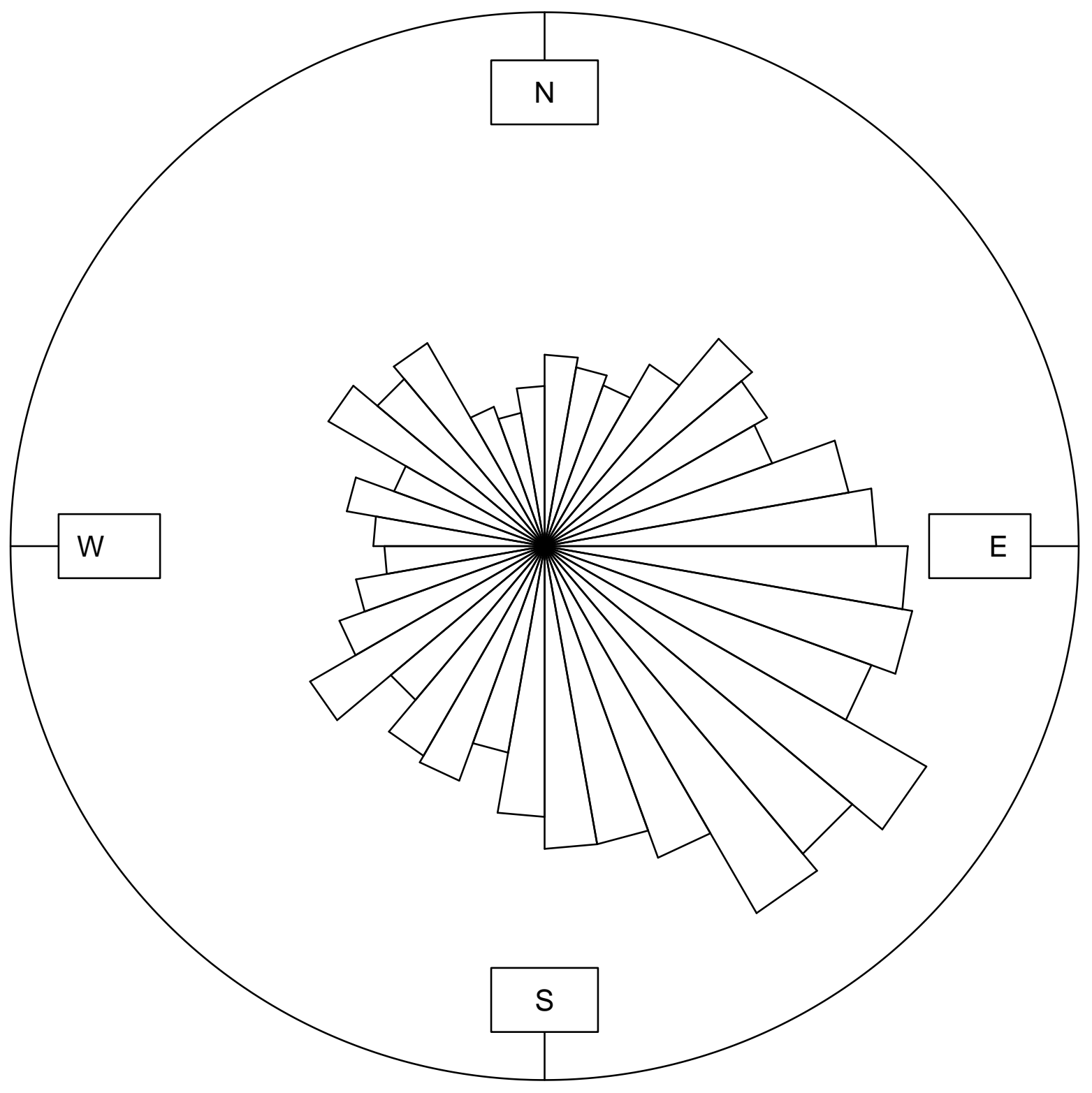


bootstrap 80

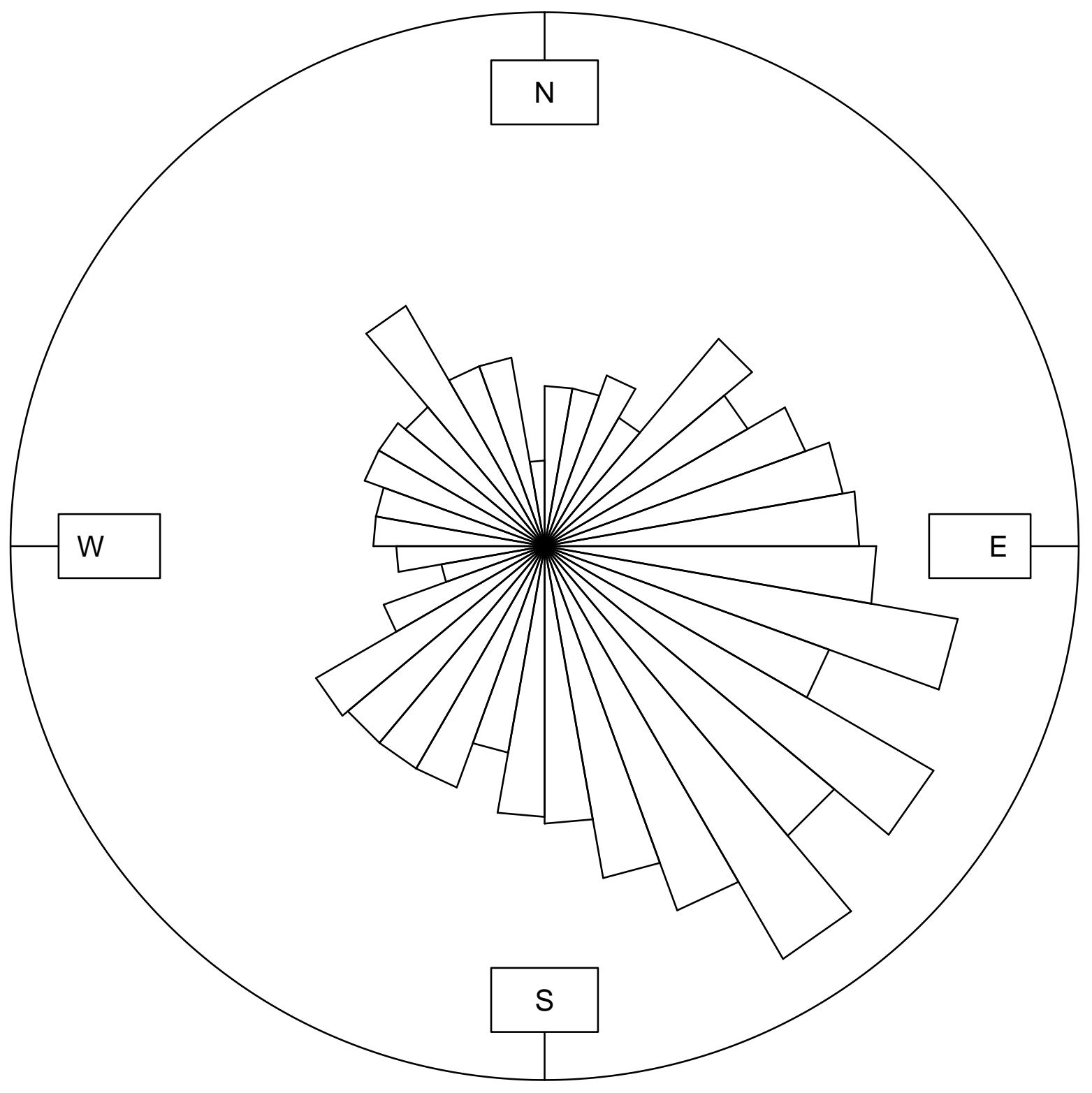




\section{bootstrap 81}

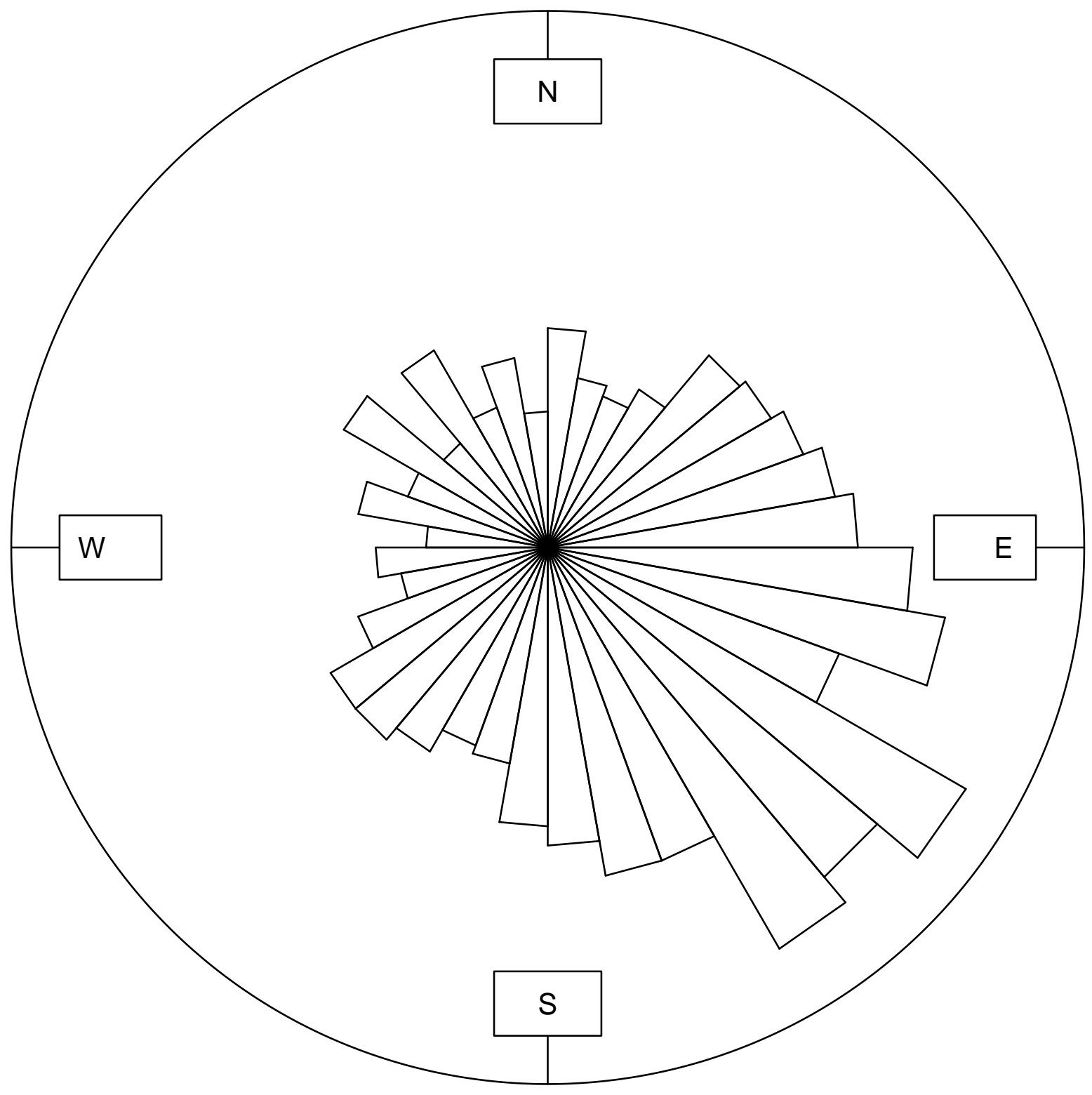


bootstrap 82

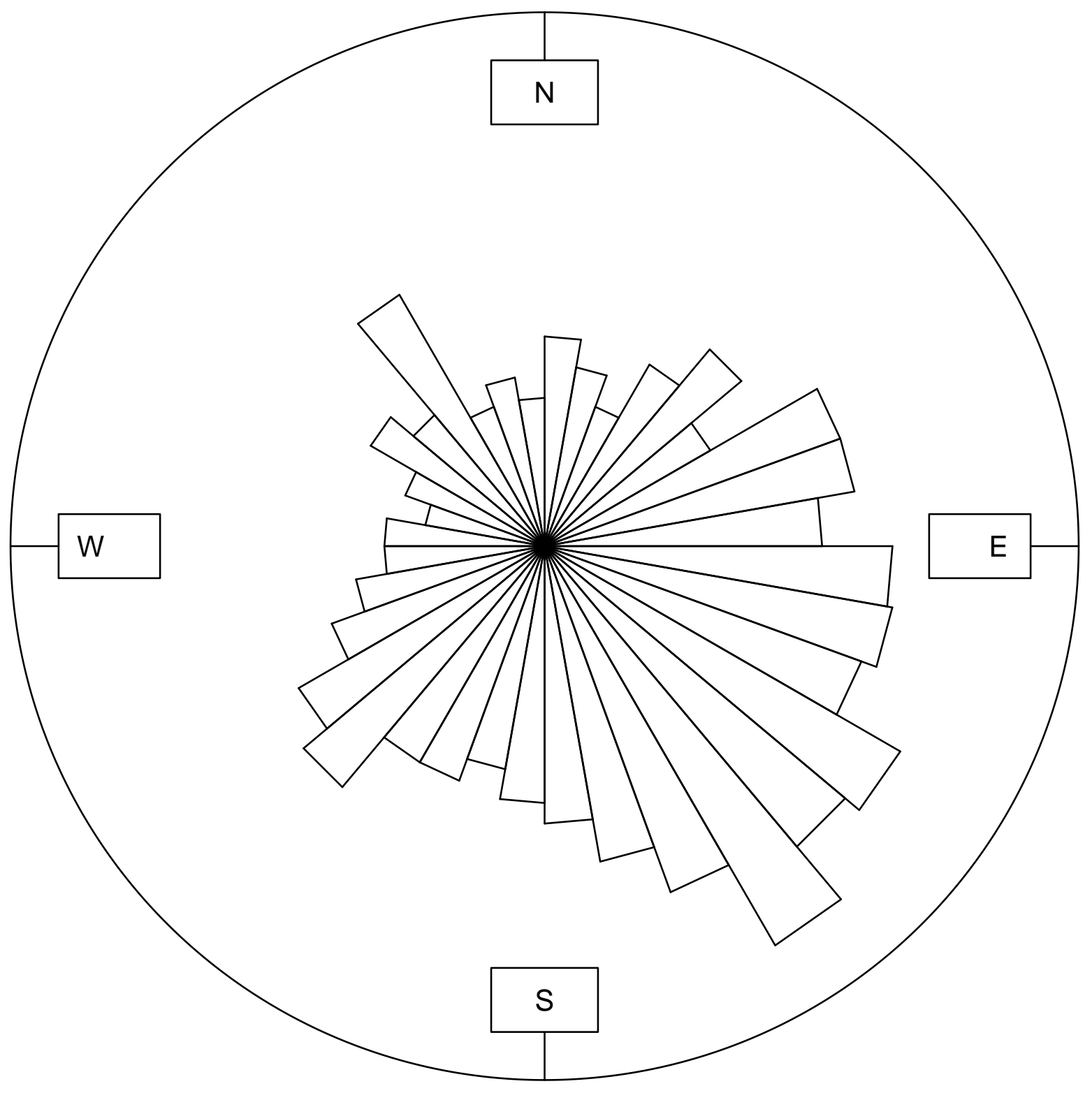




\section{bootstrap 83}

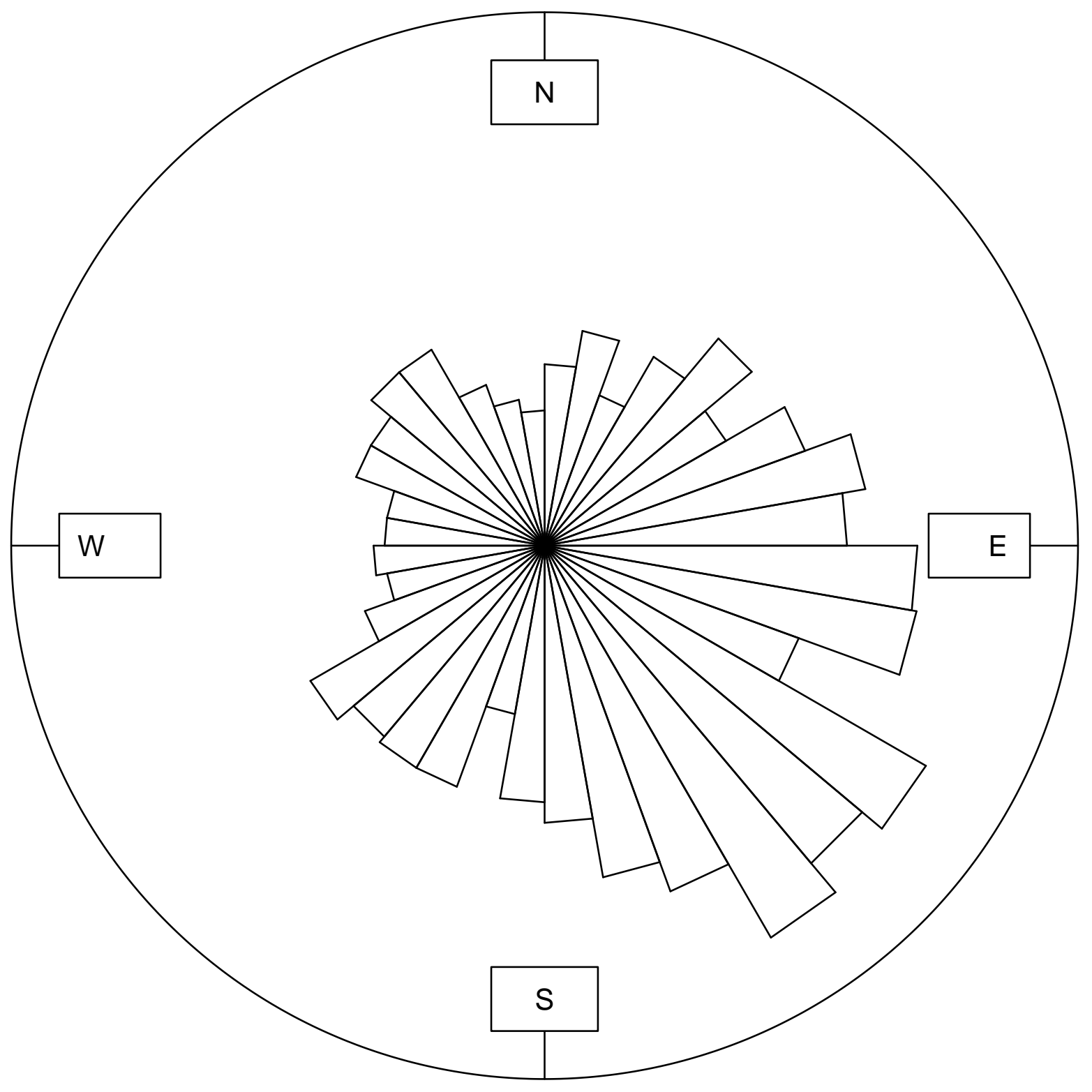




\section{bootstrap 84}

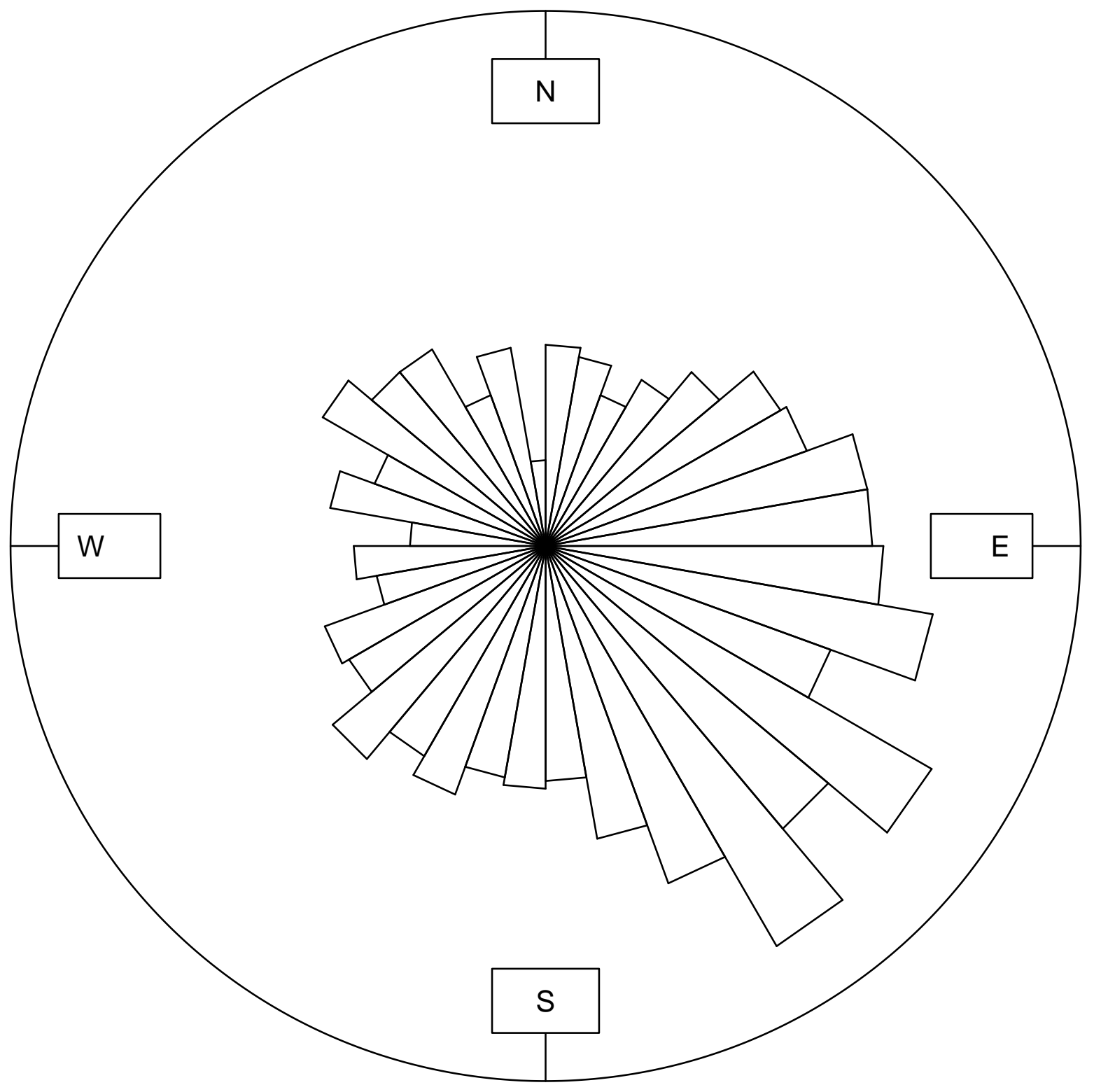




\section{bootstrap 85}

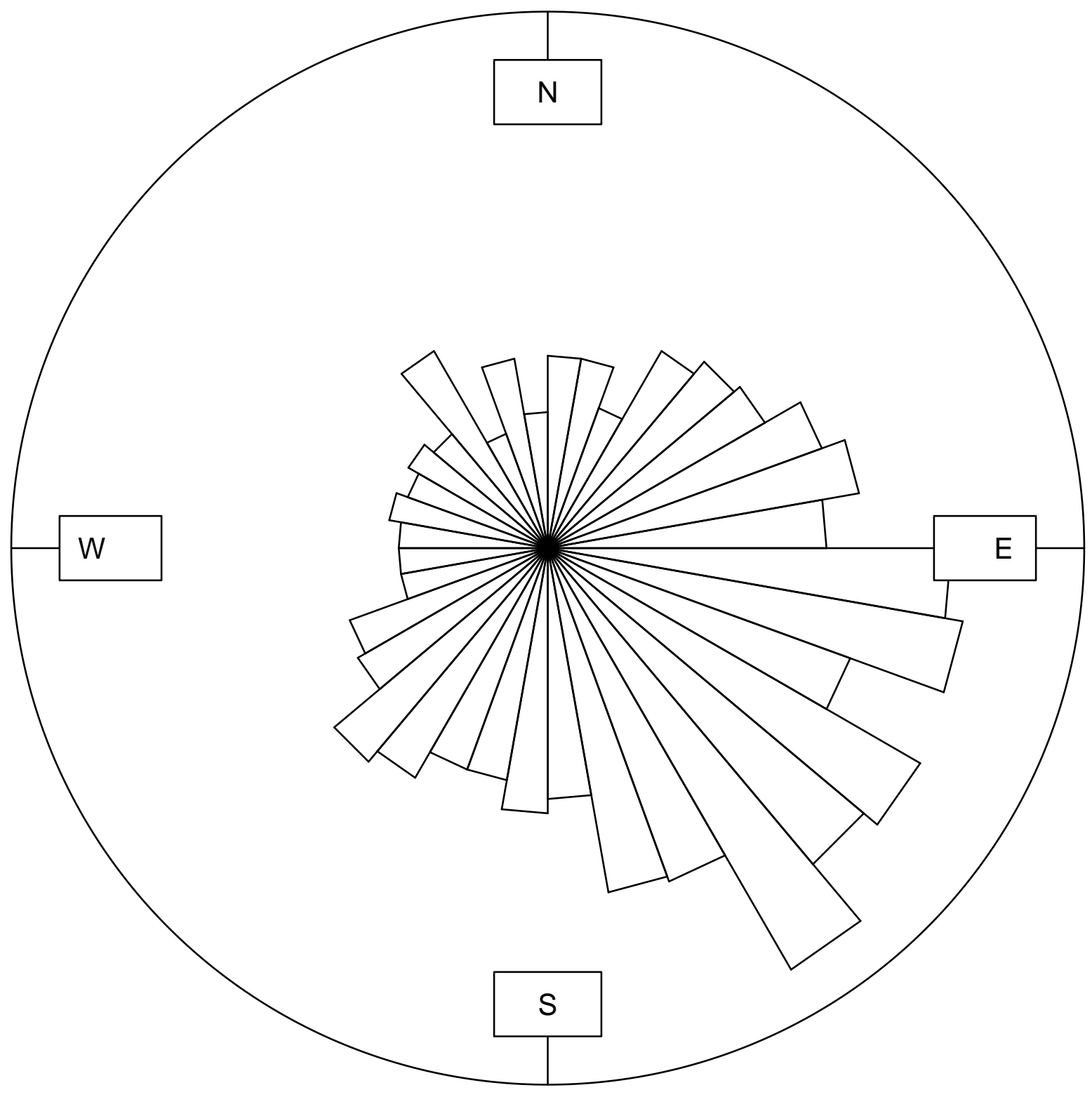




\section{bootstrap 86}

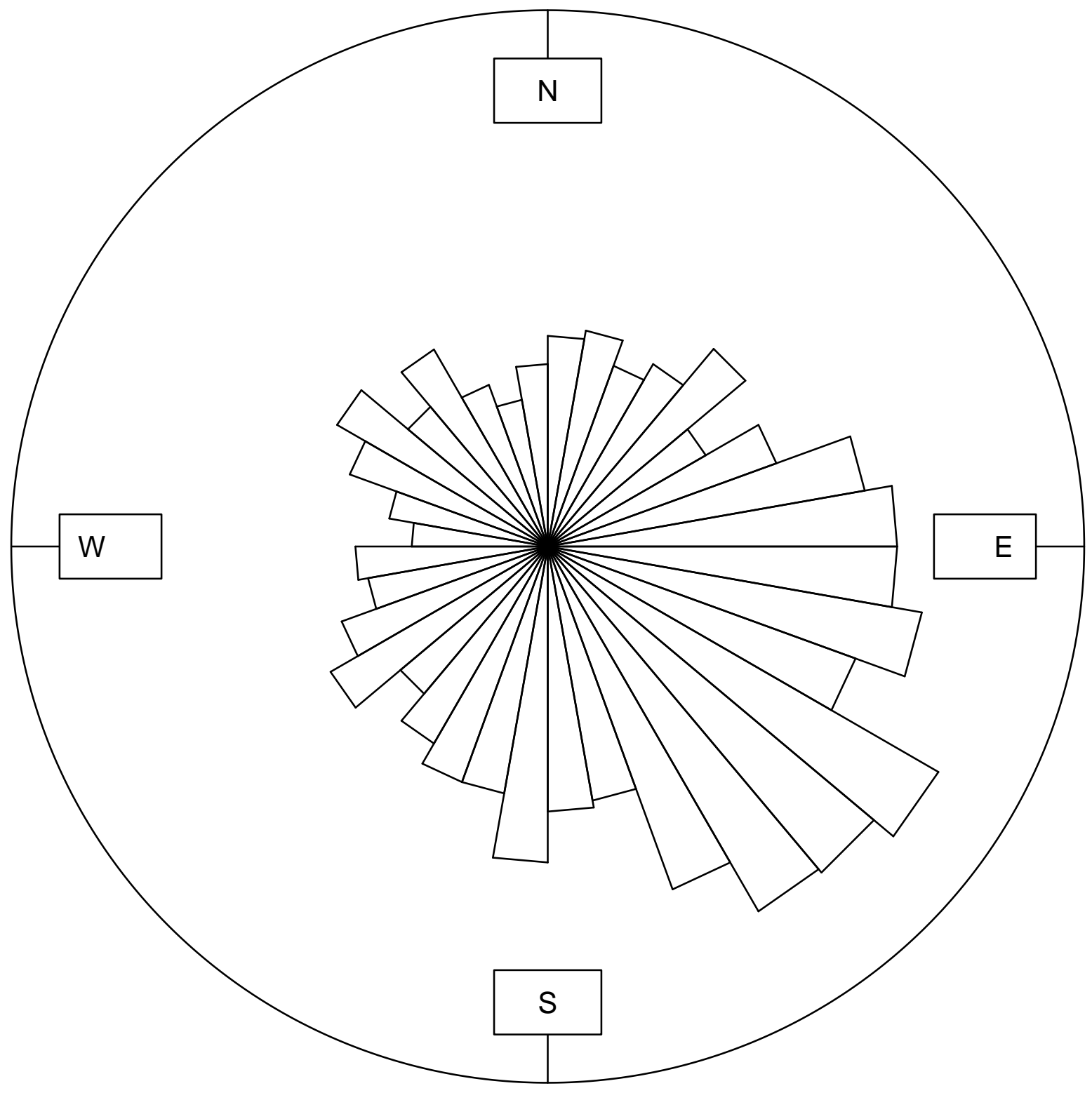


bootstrap 87

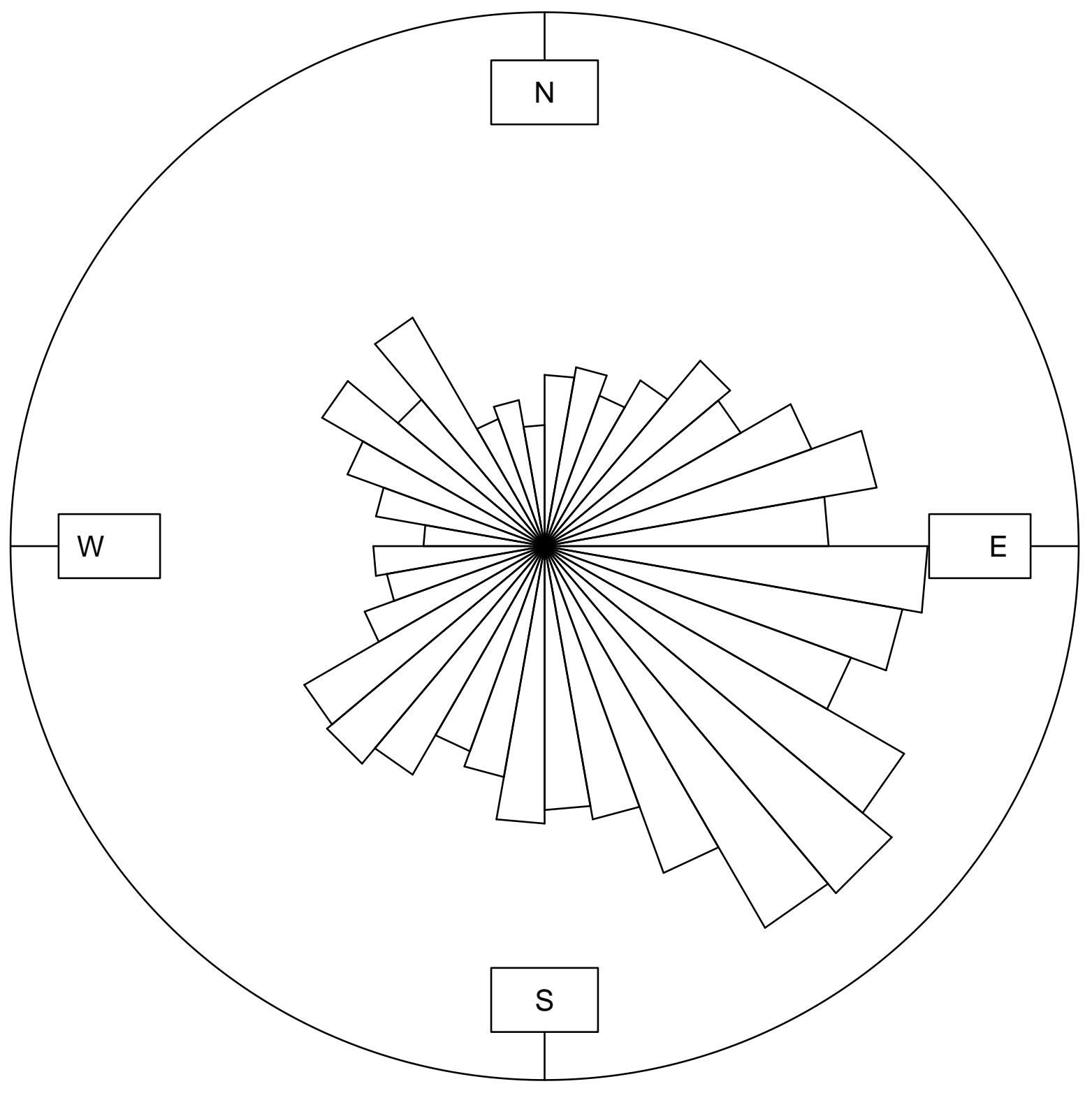




\section{bootstrap 88}

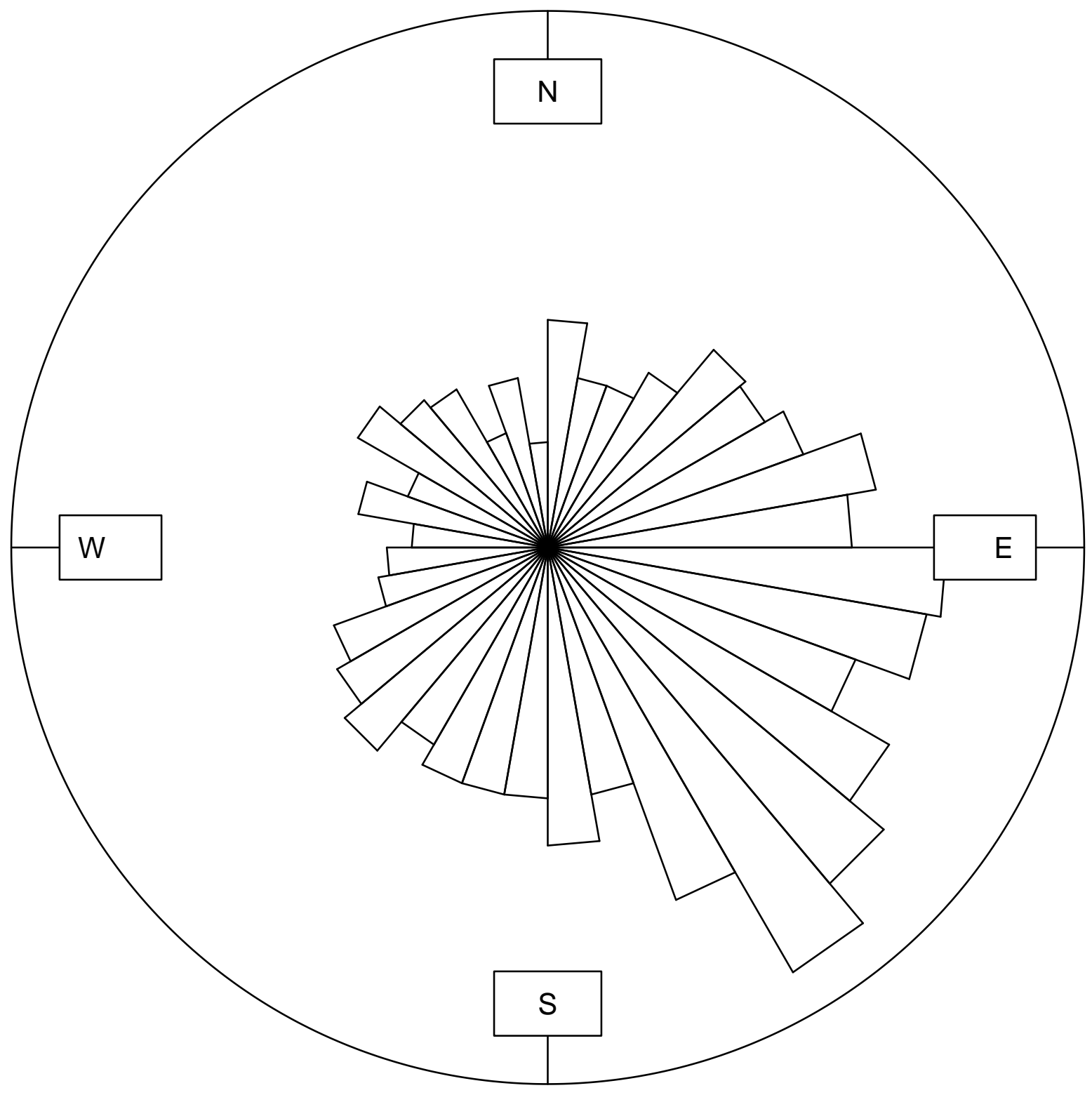


bootstrap 89

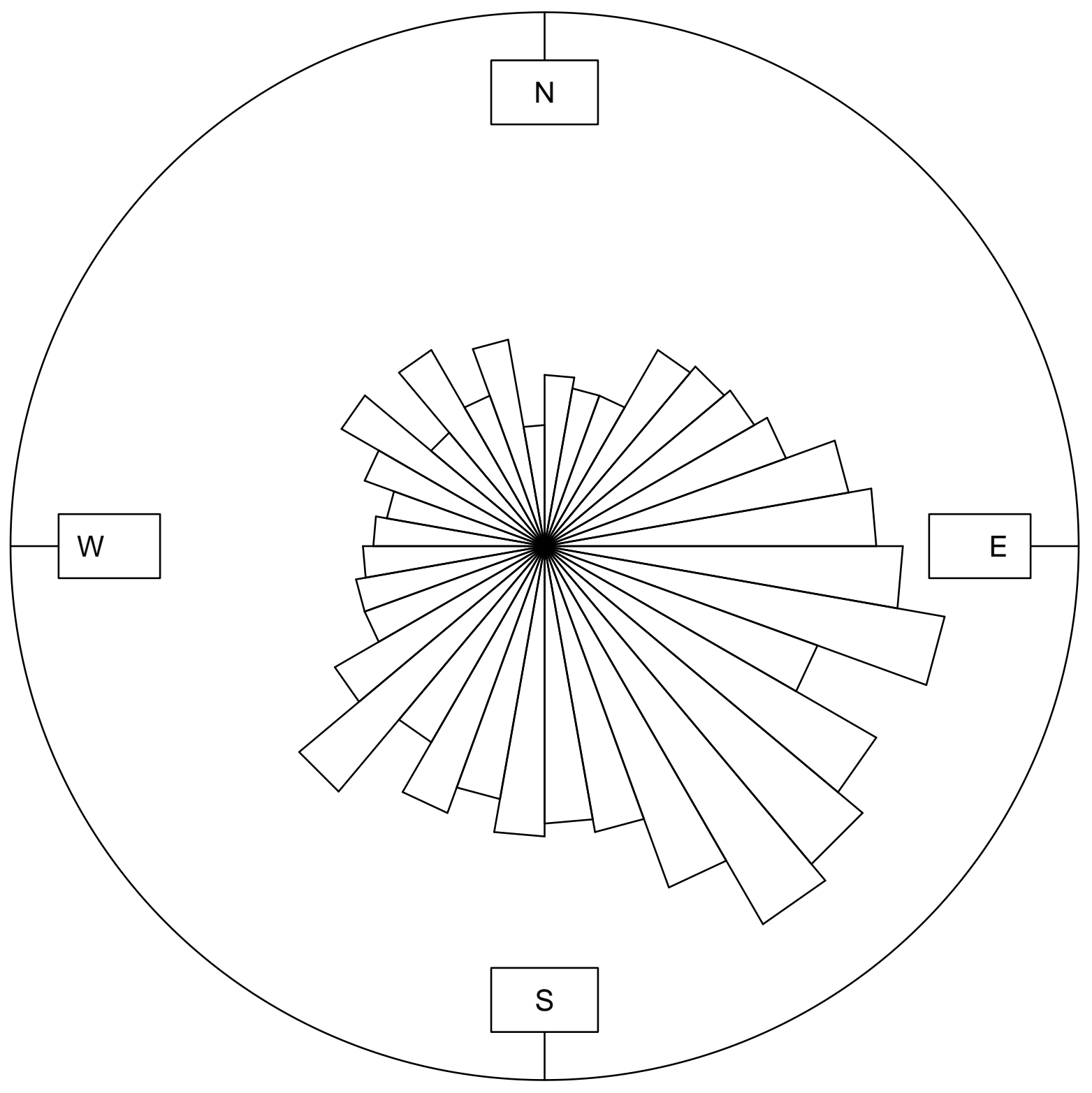


bootstrap 90

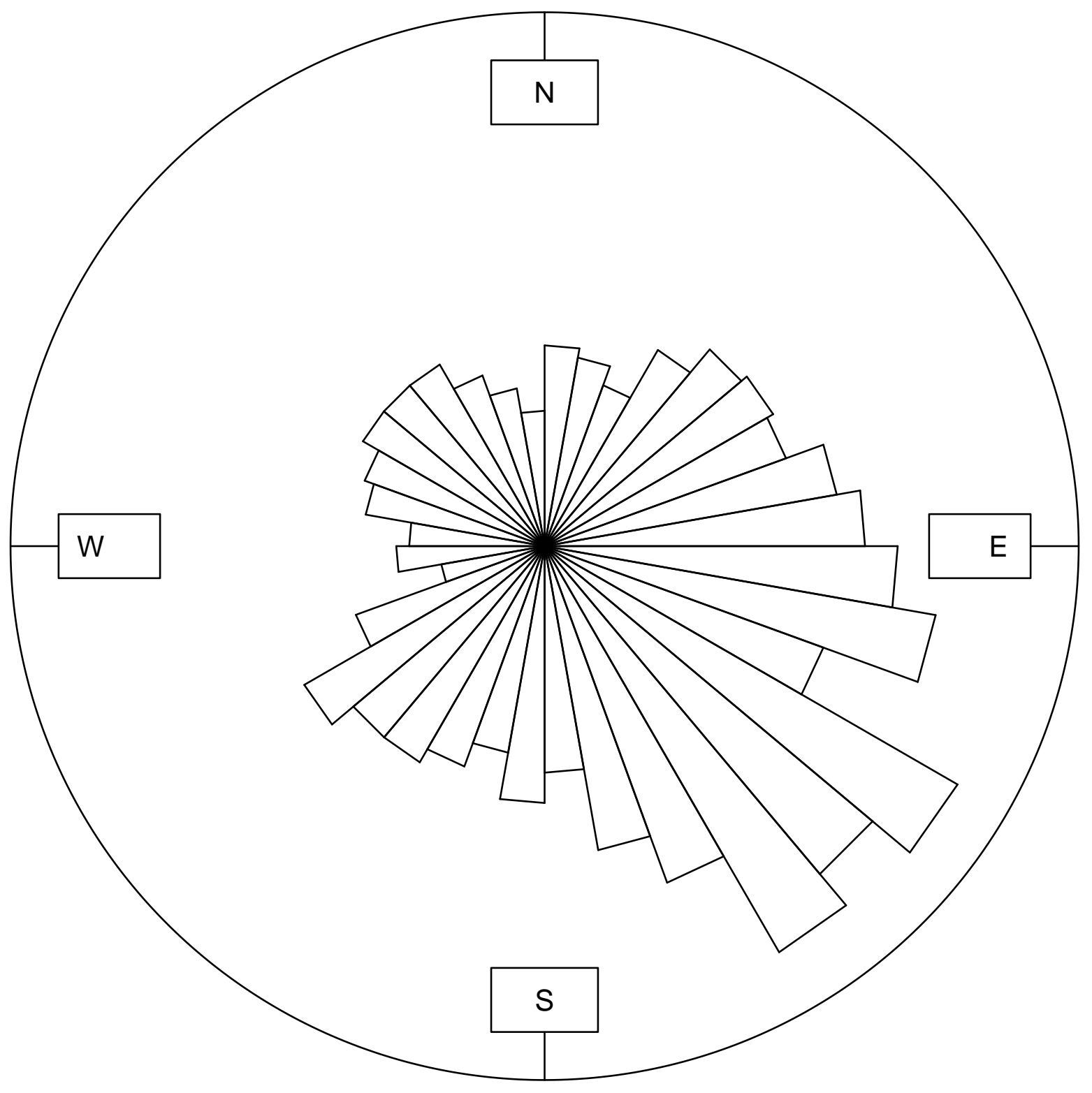




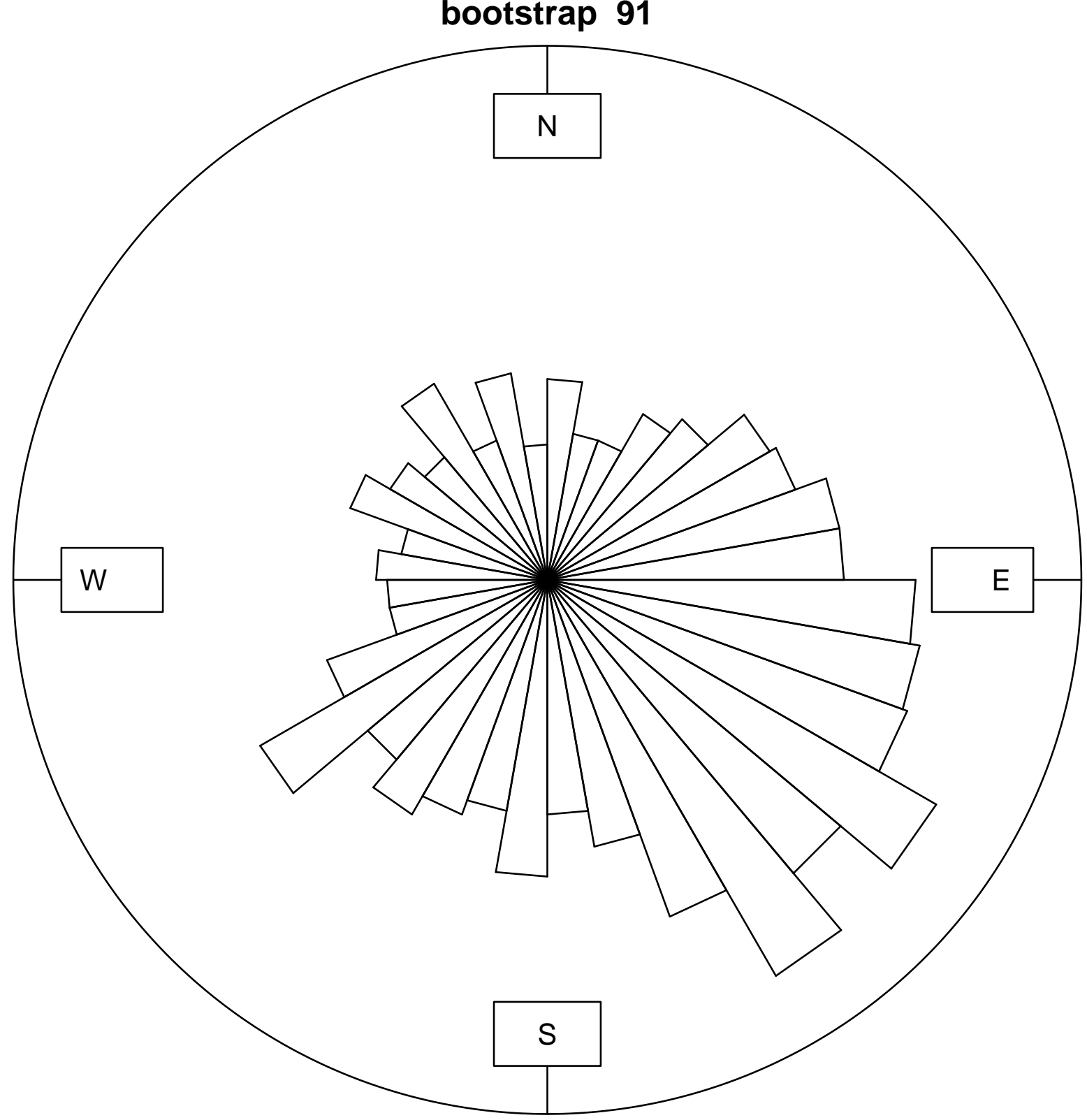




\section{bootstrap 92}

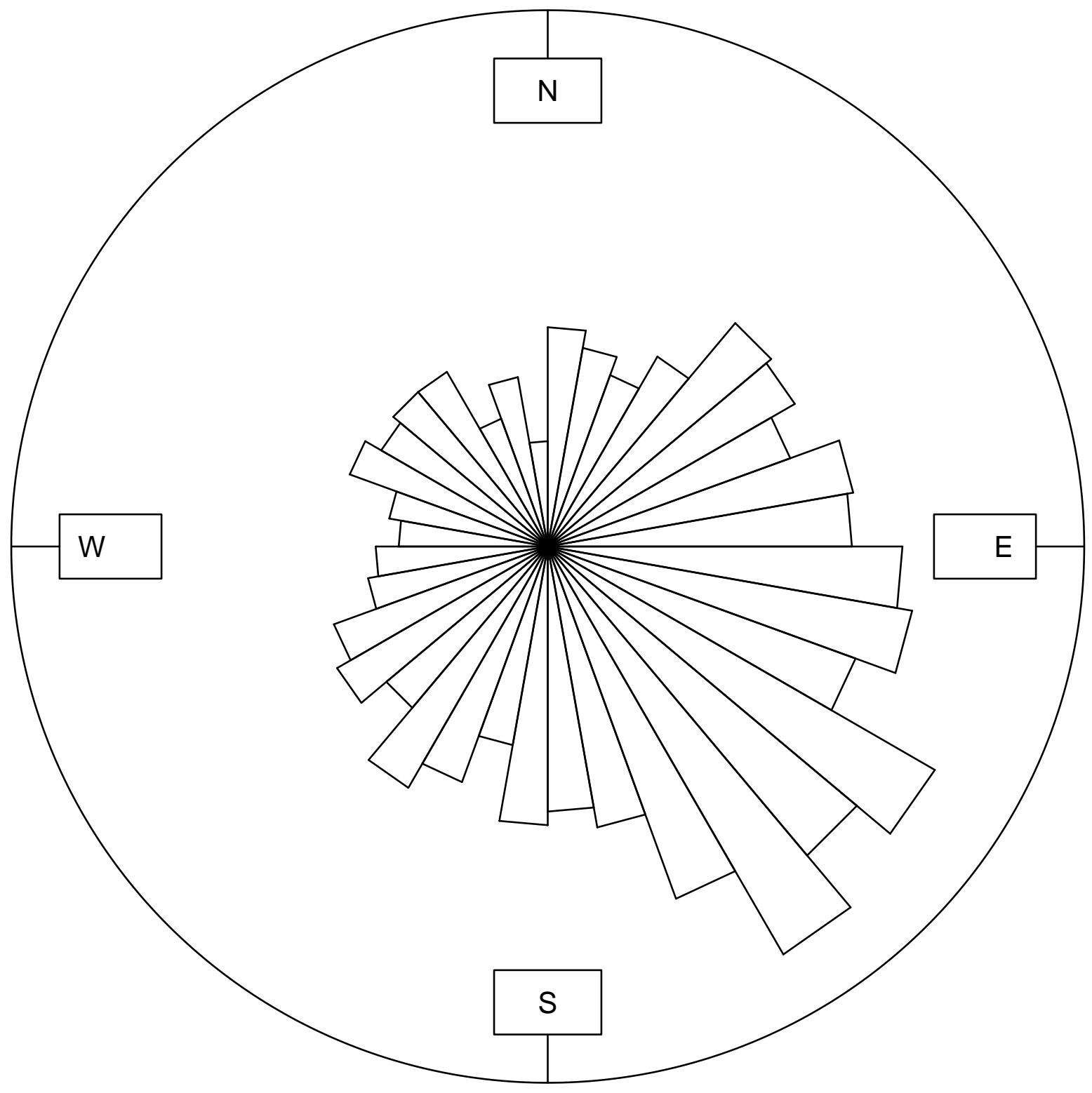


bootstrap 93

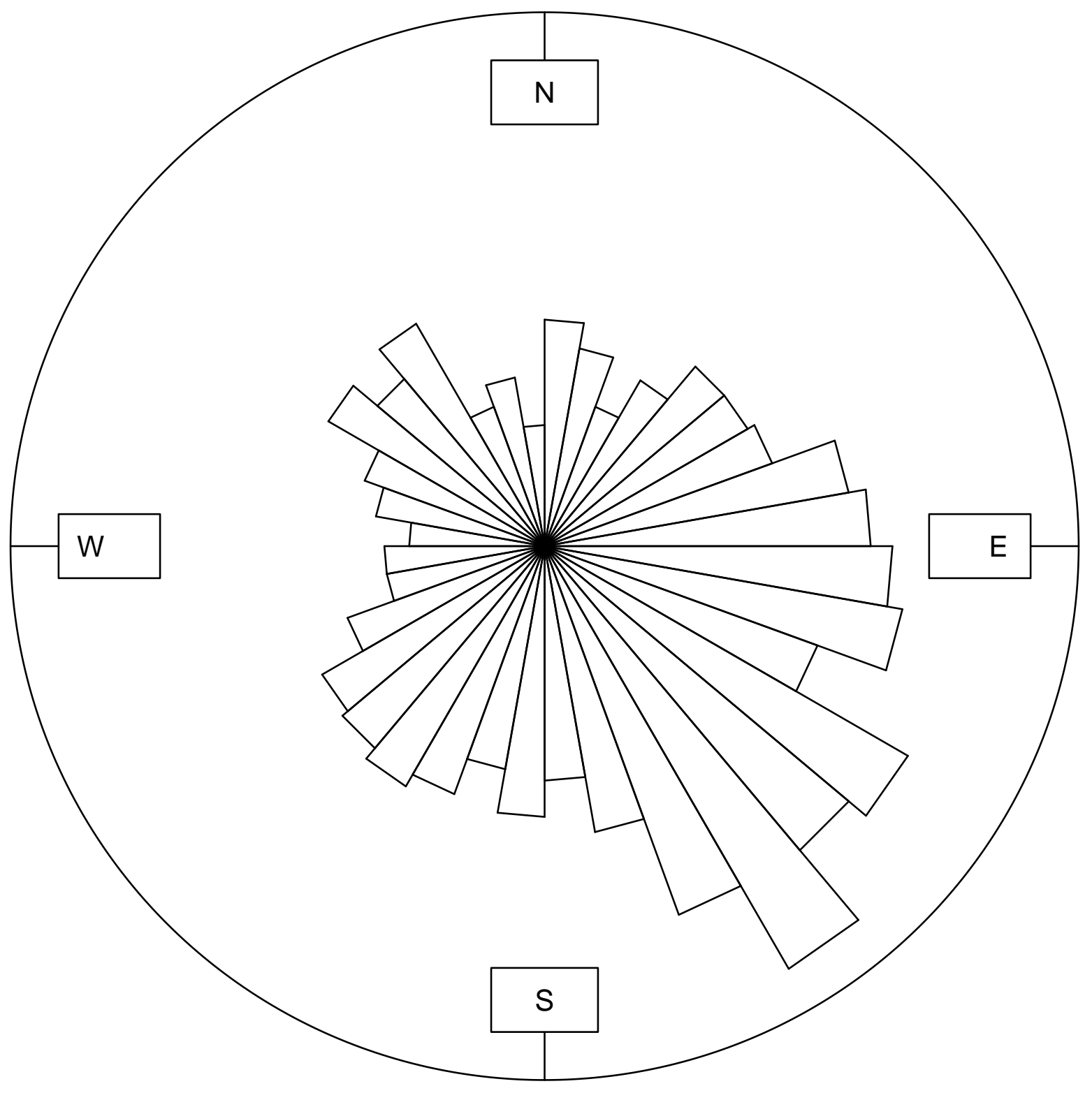




\section{bootstrap 94}

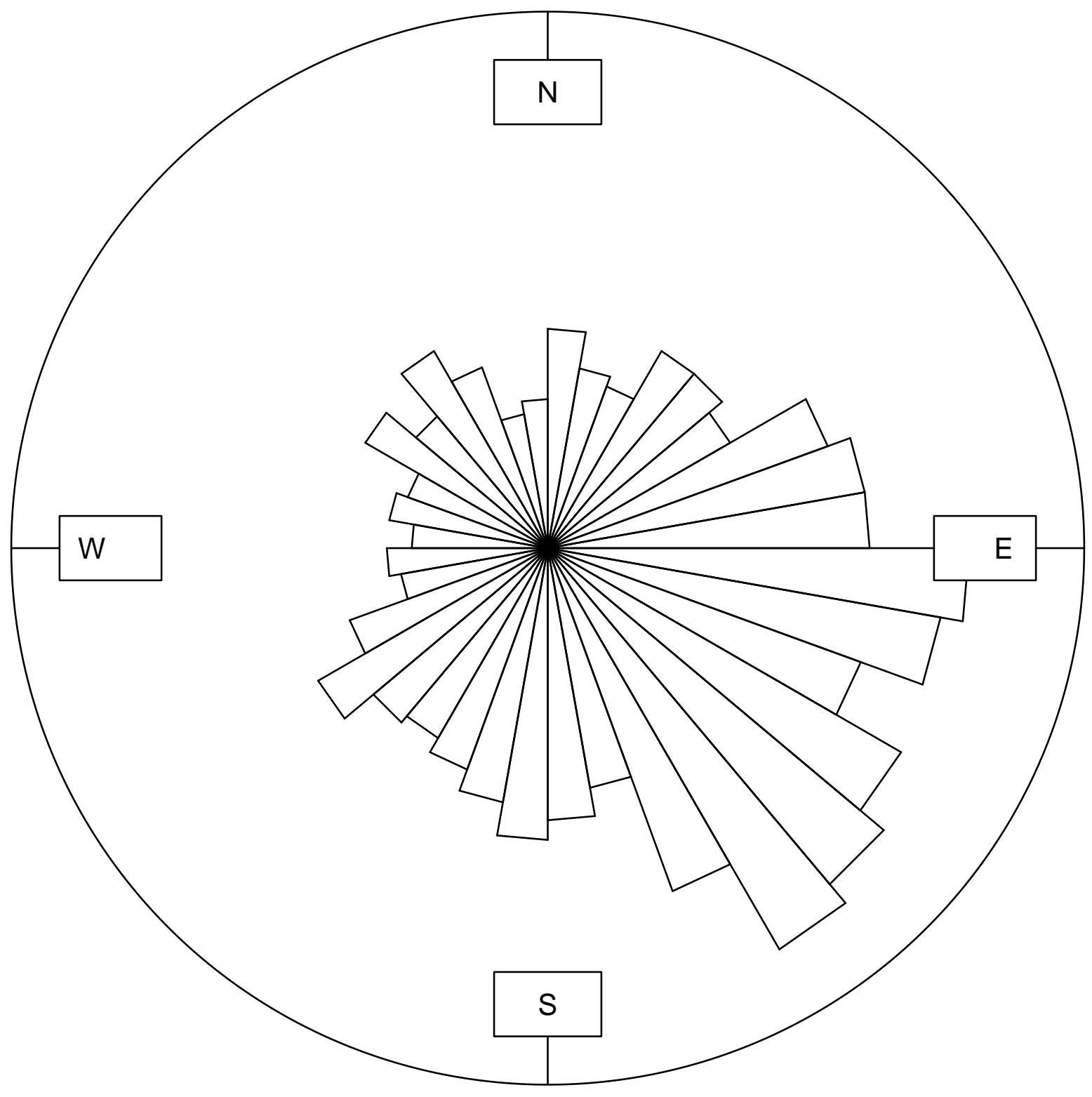


bootstrap 95

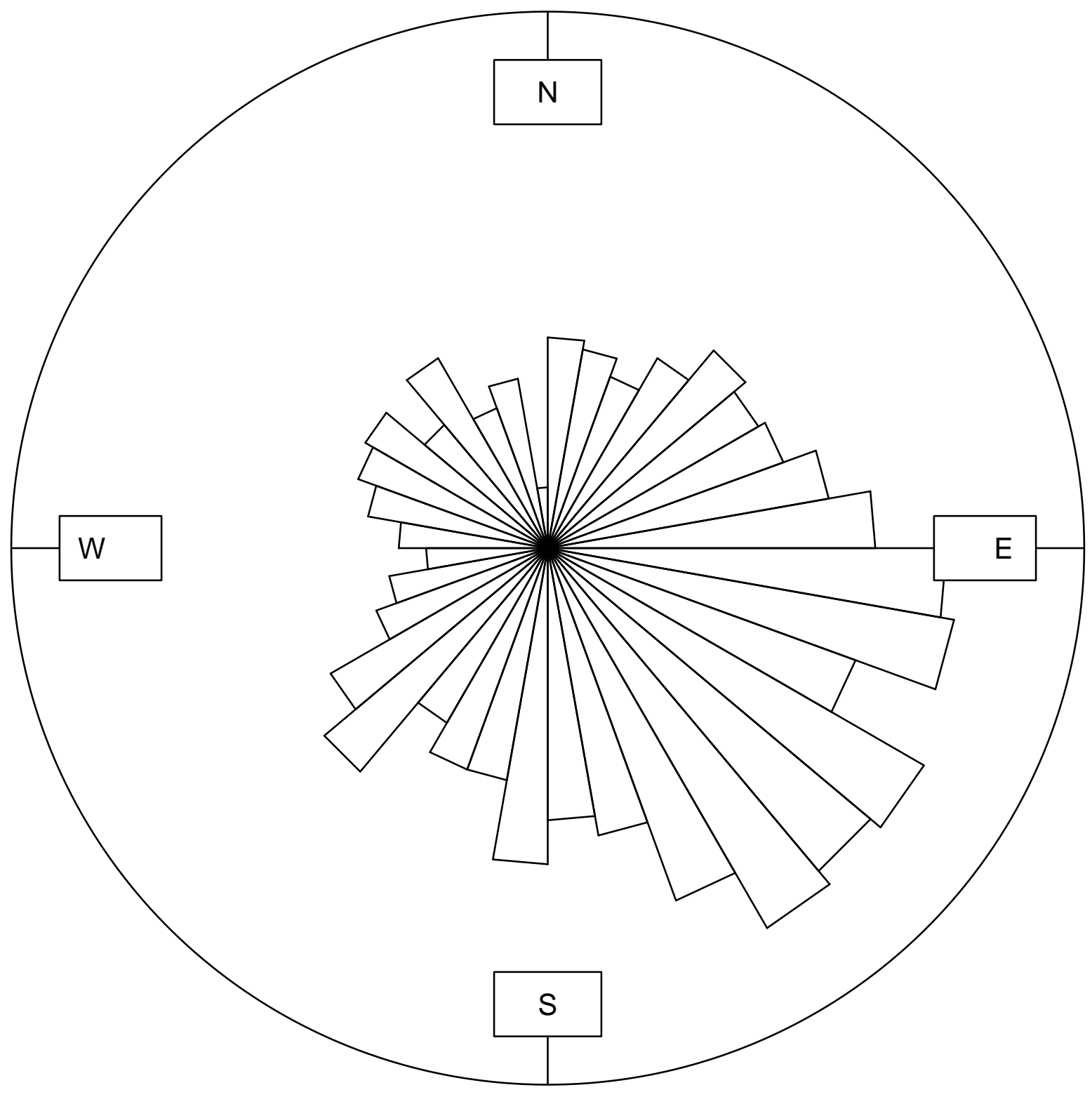


bootstrap 96

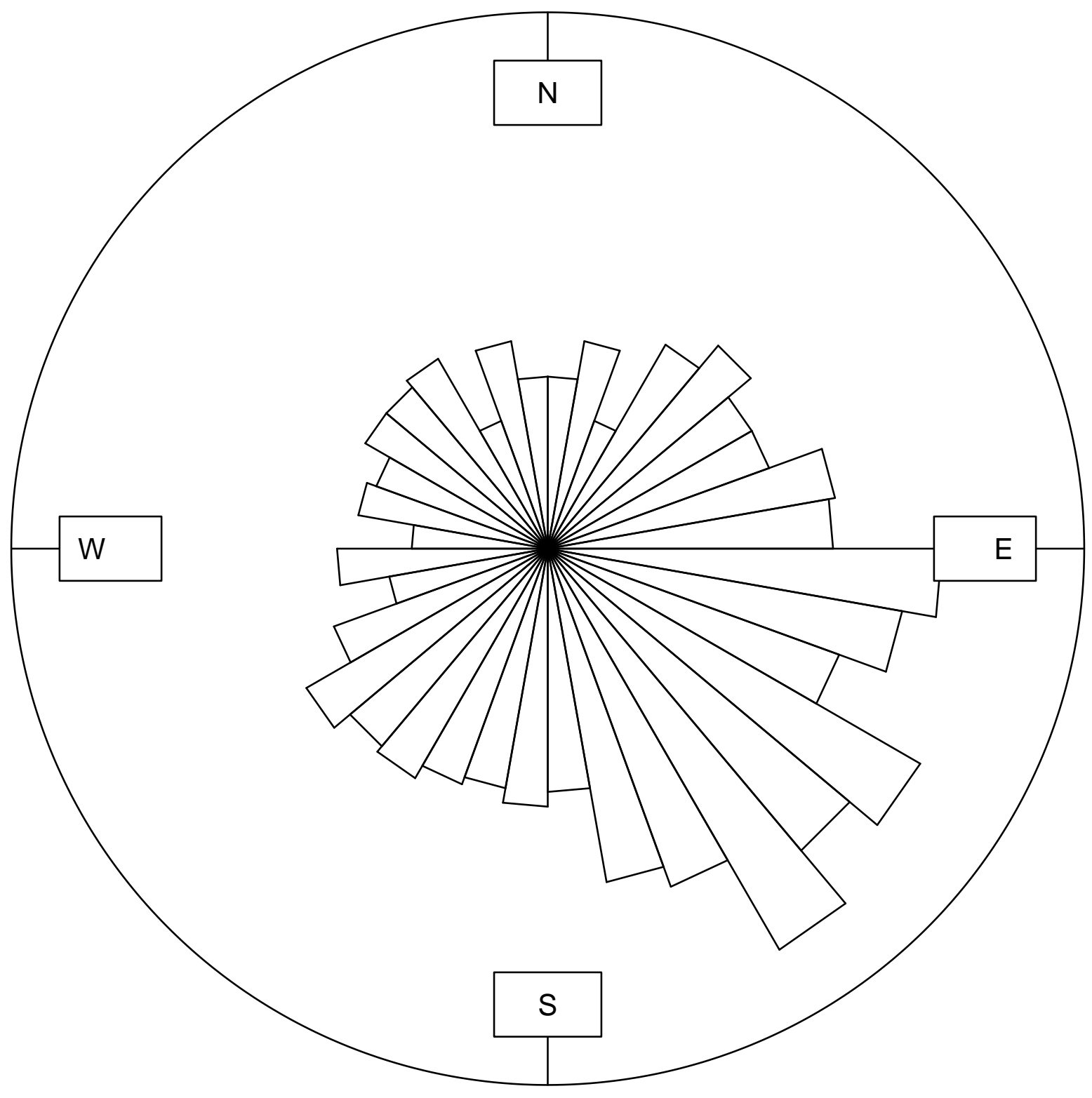


bootstrap 97

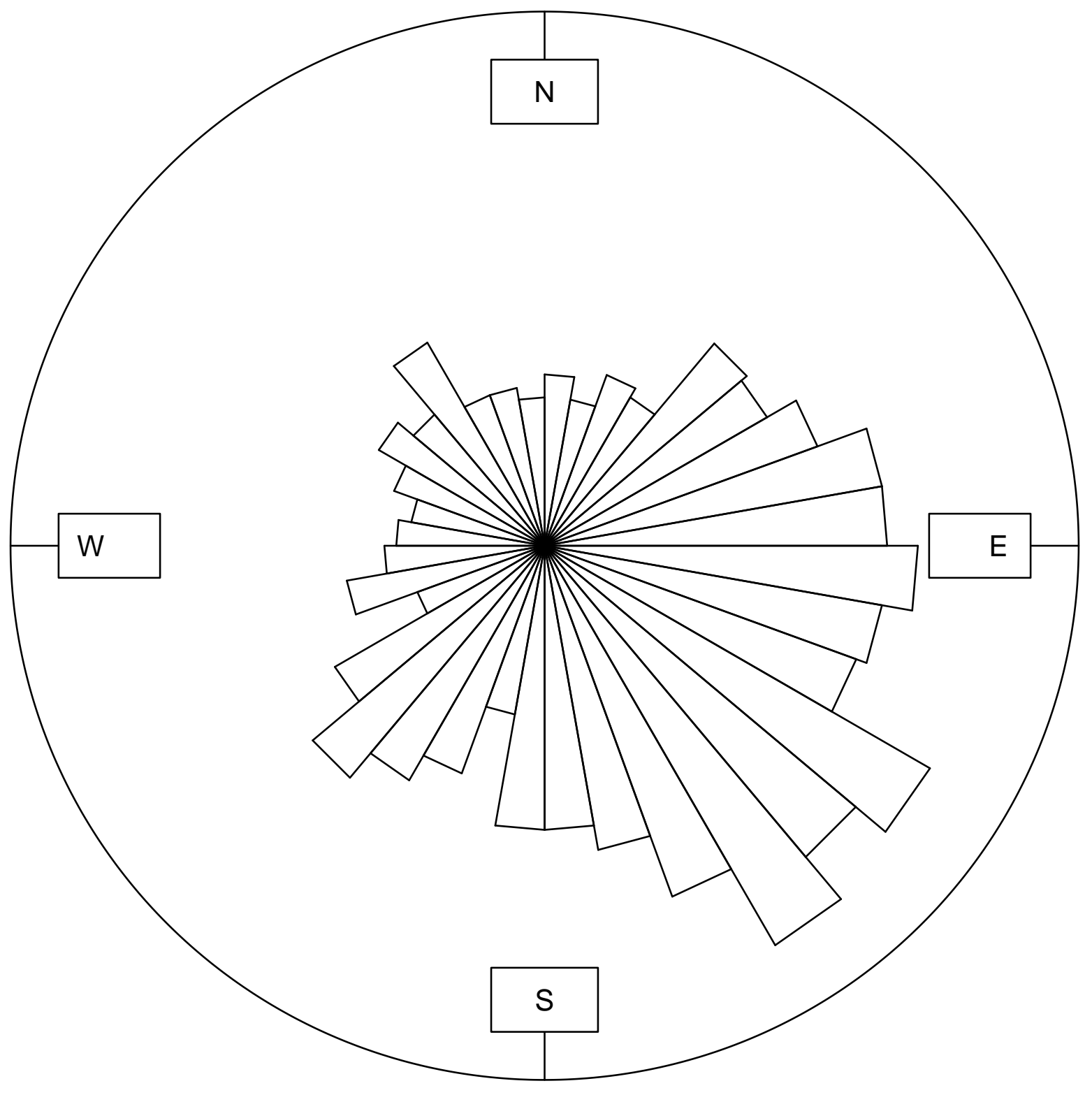




\section{bootstrap 98}

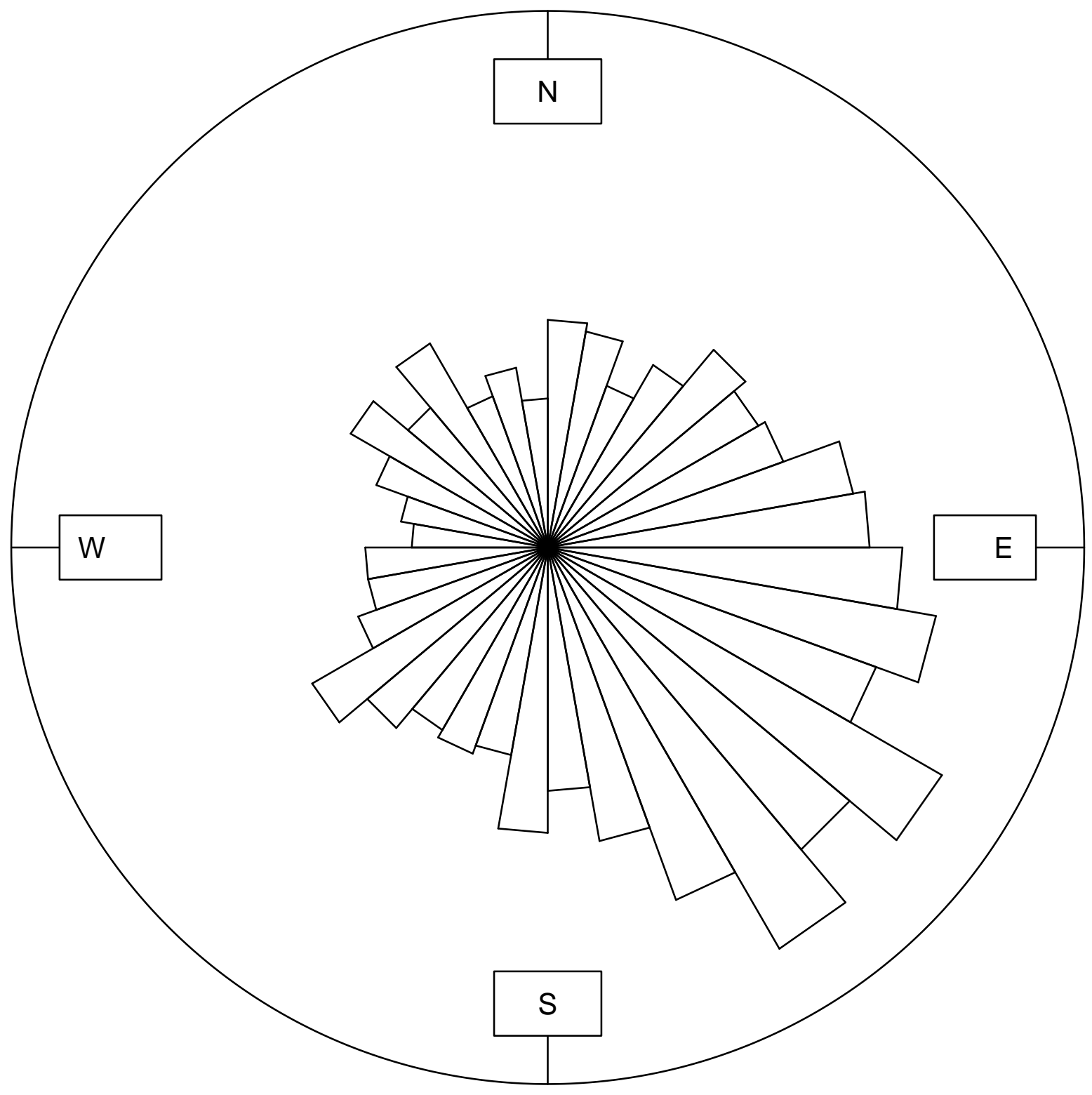


bootstrap 99

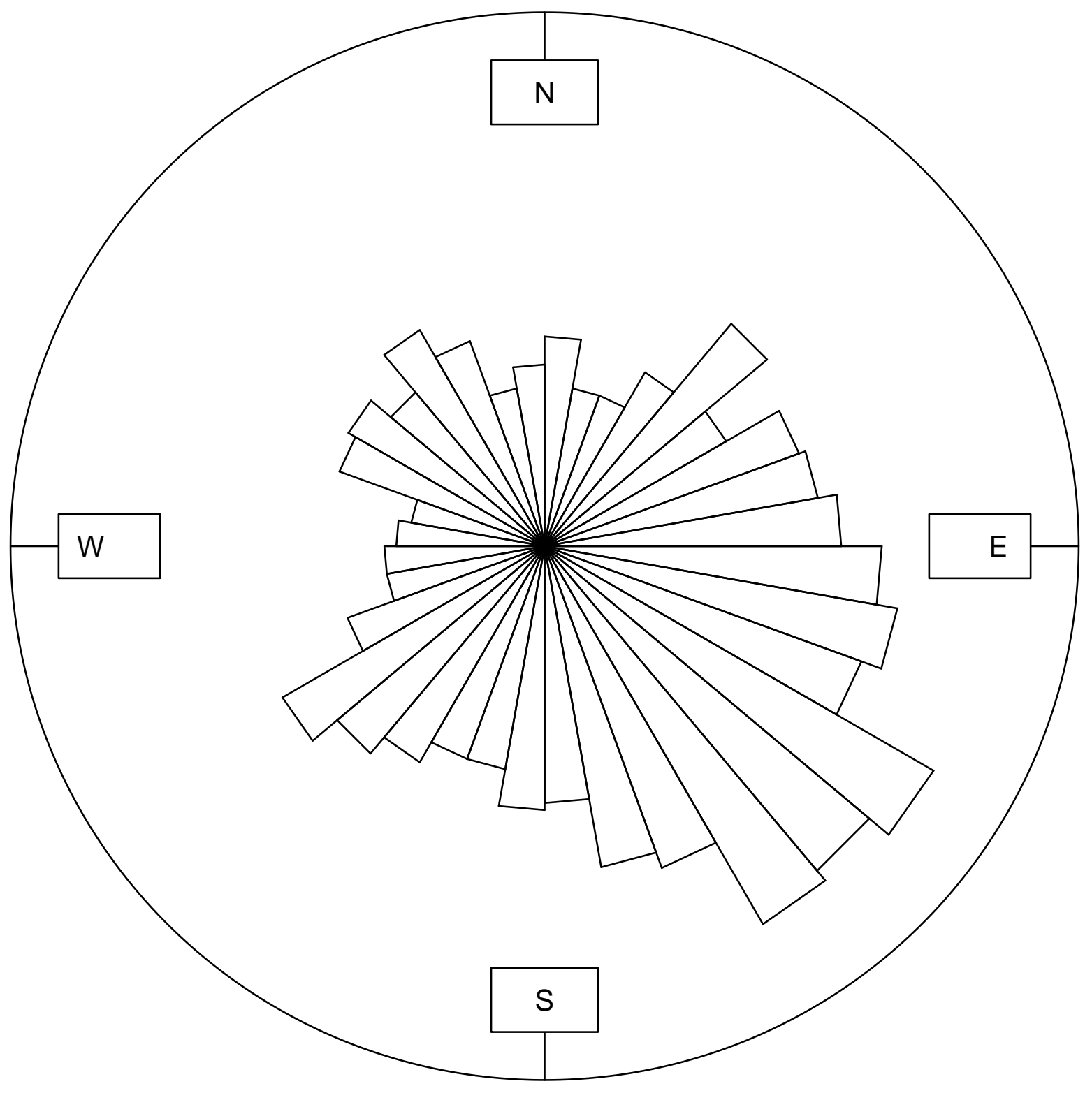


bootstrap 100

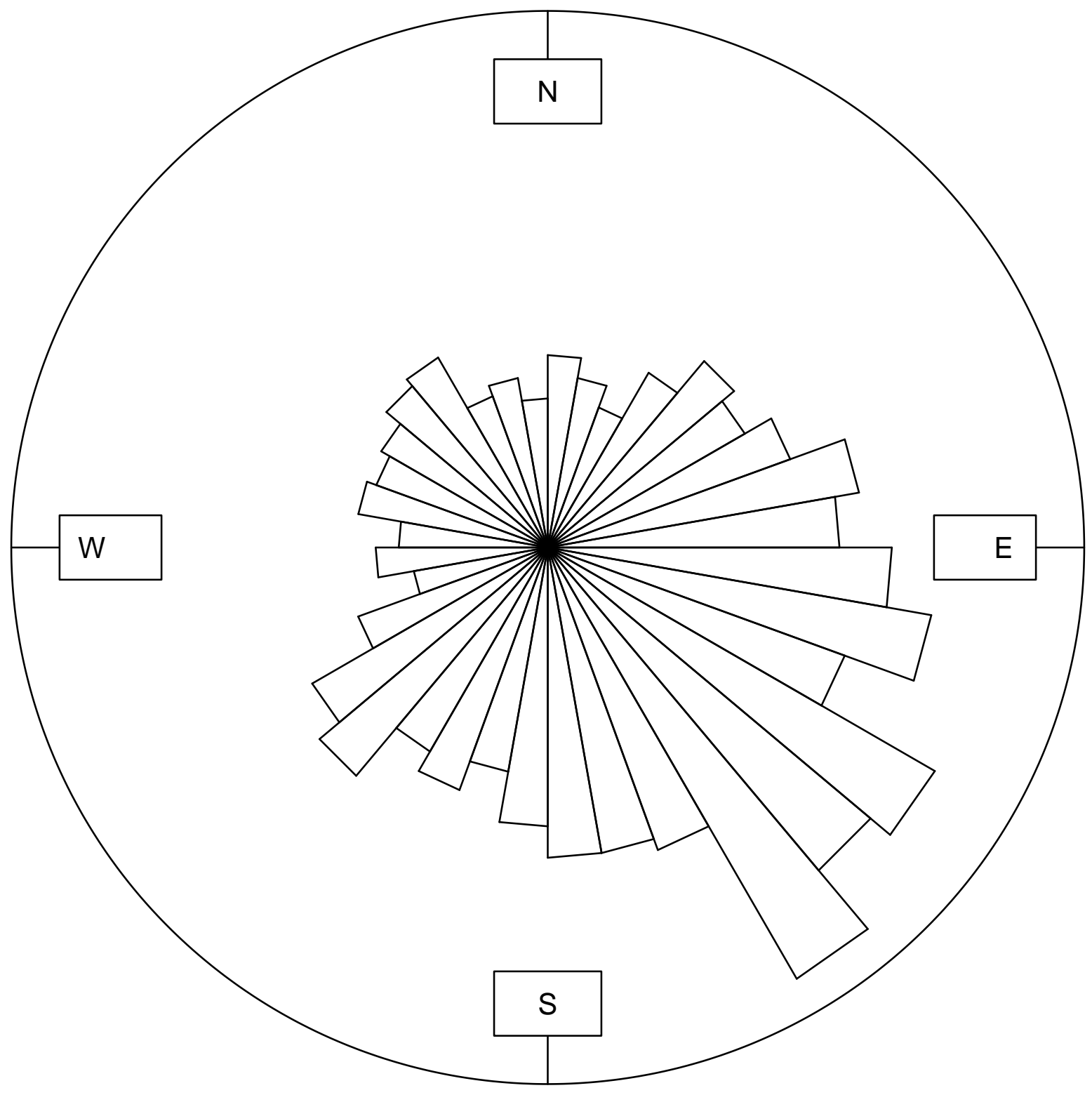


bootstrap 101

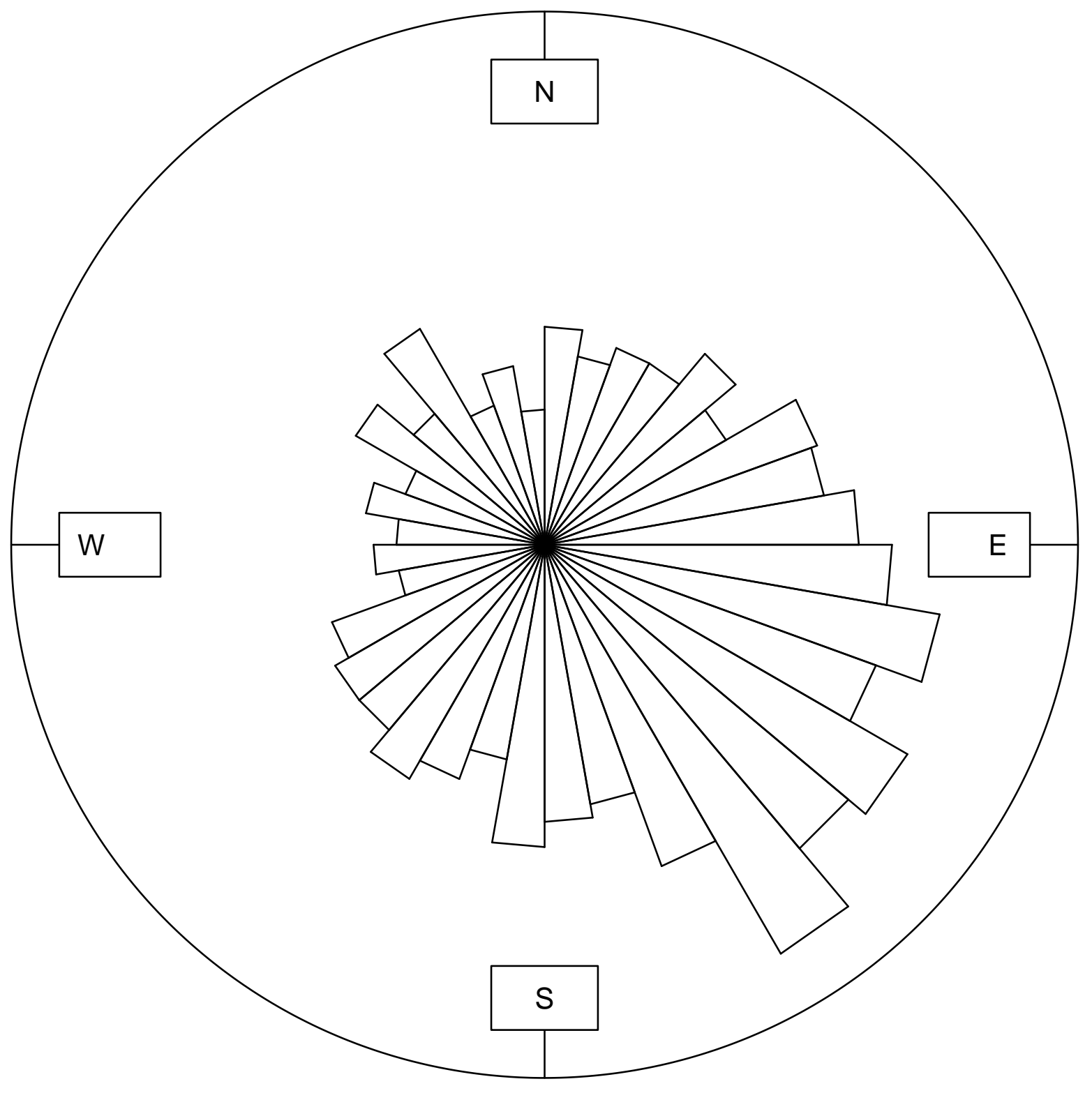


bootstrap 102

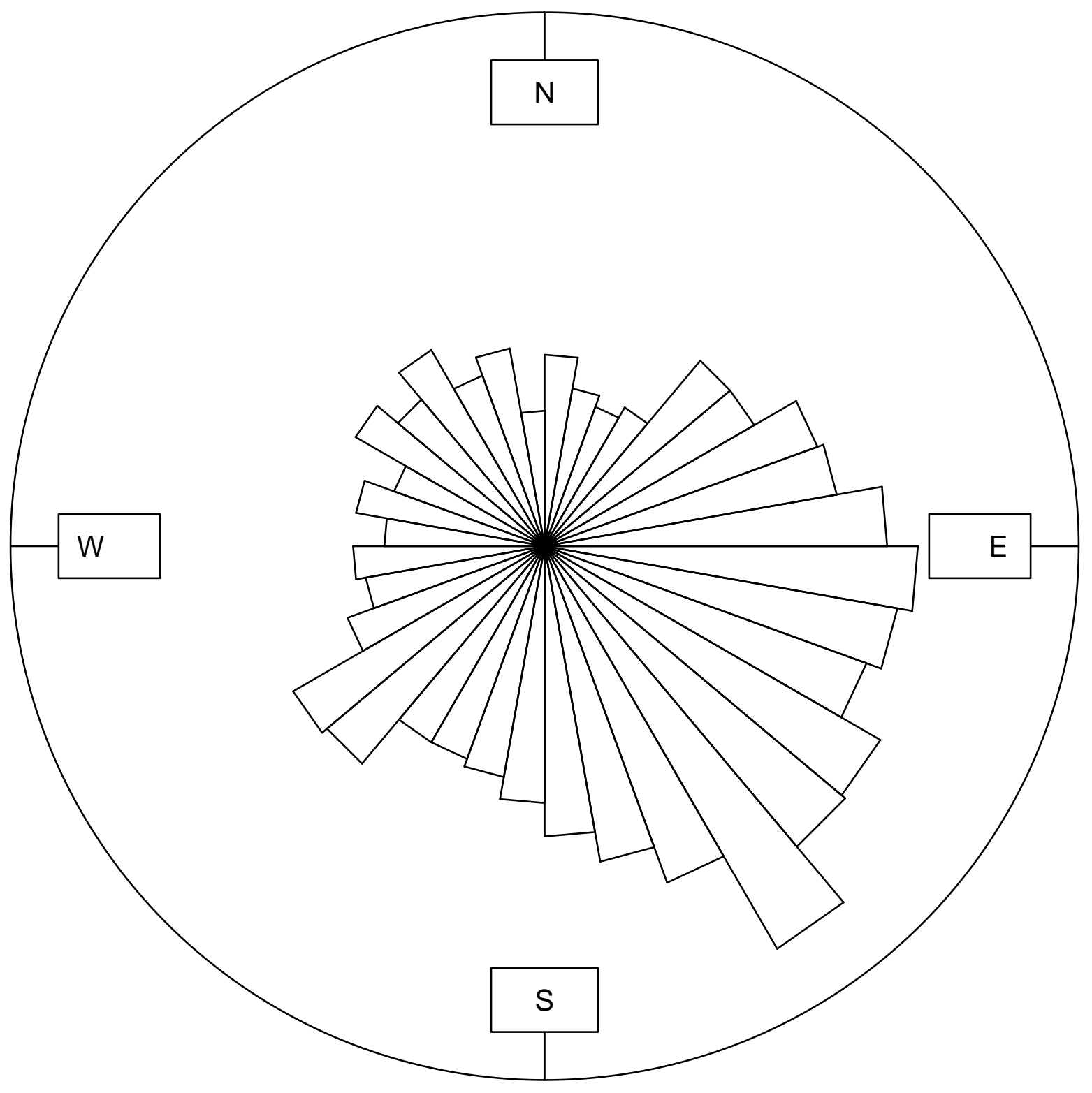


bootstrap 103

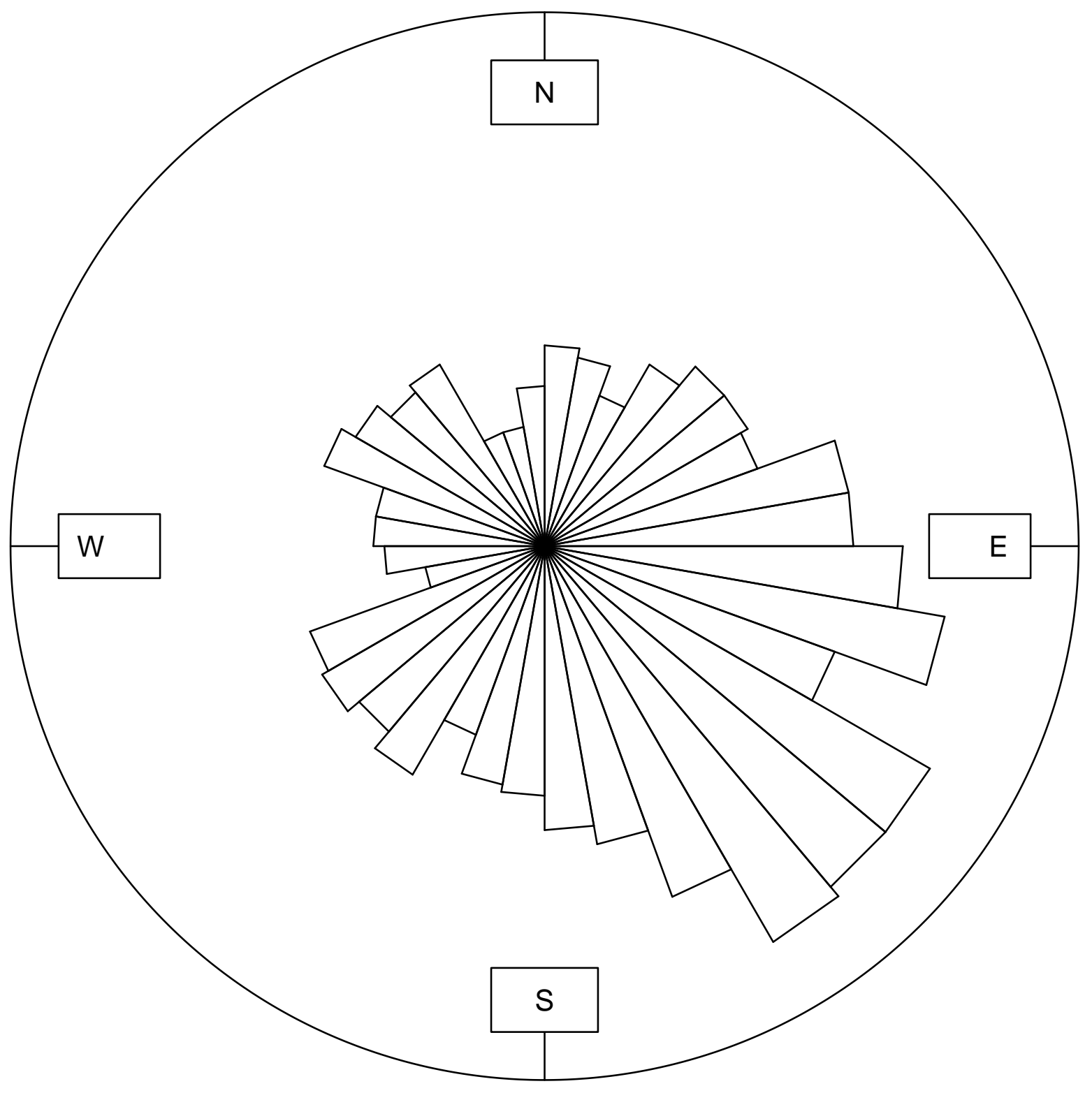


bootstrap 104

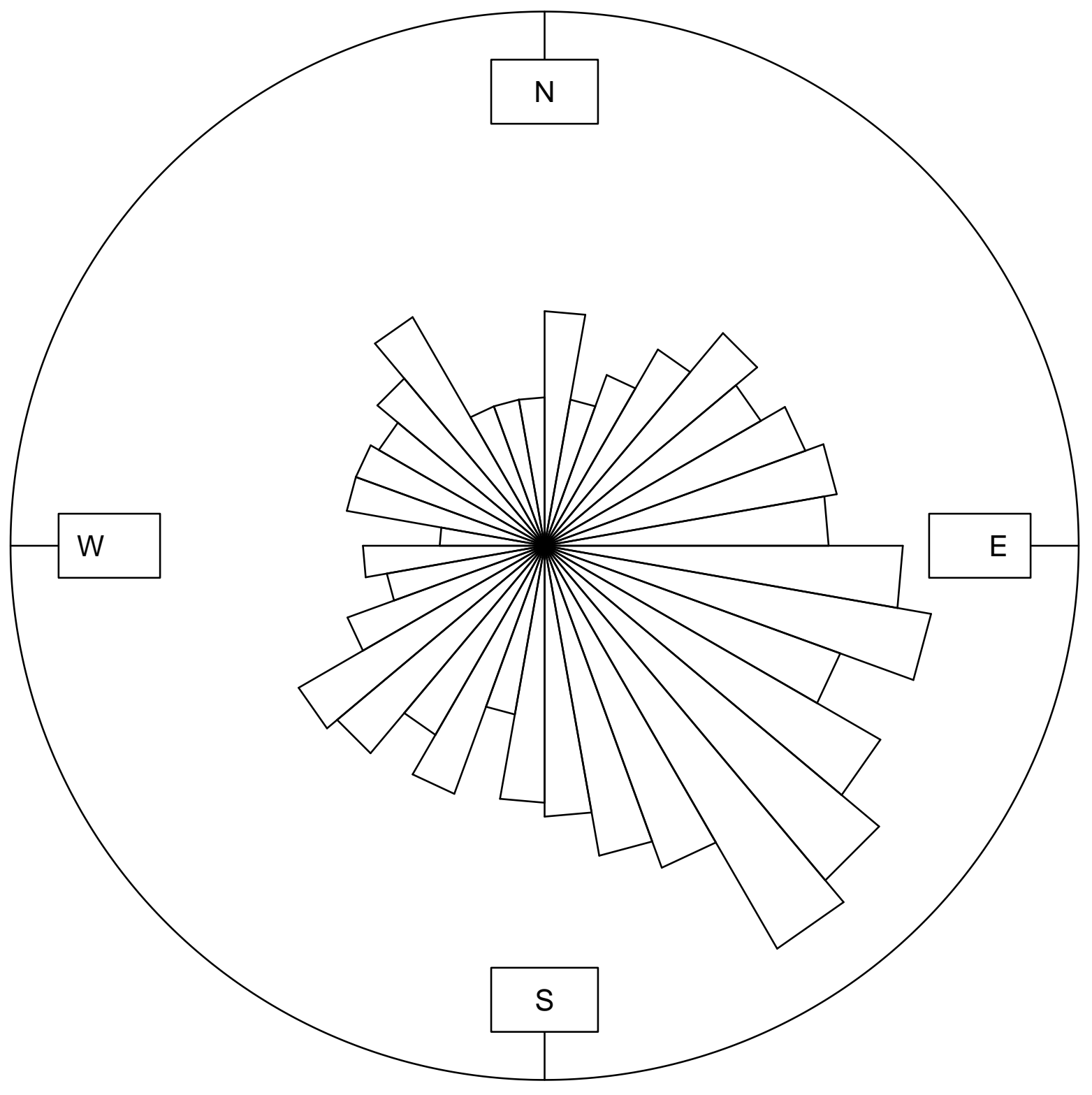


bootstrap 105

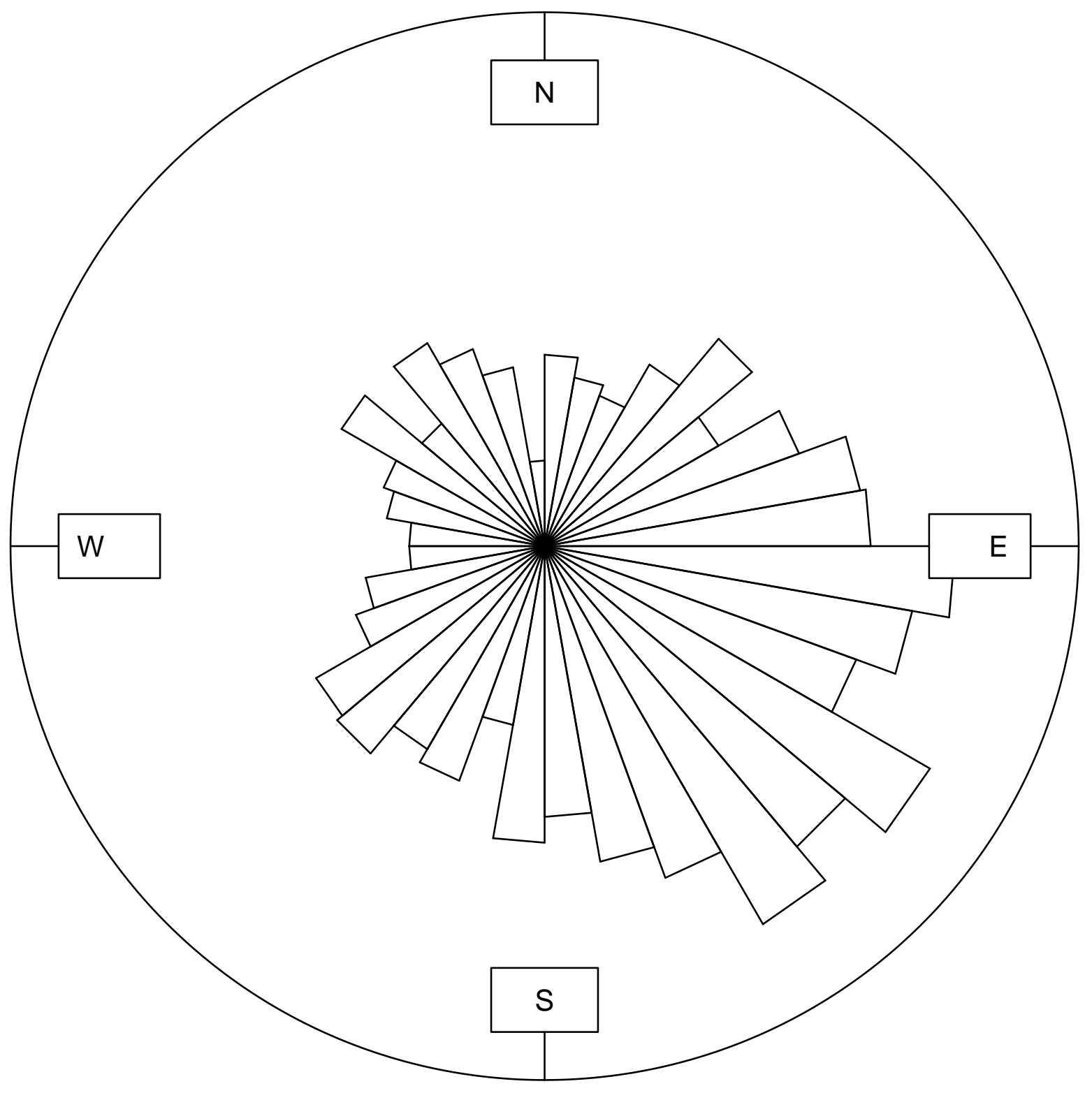


bootstrap 106

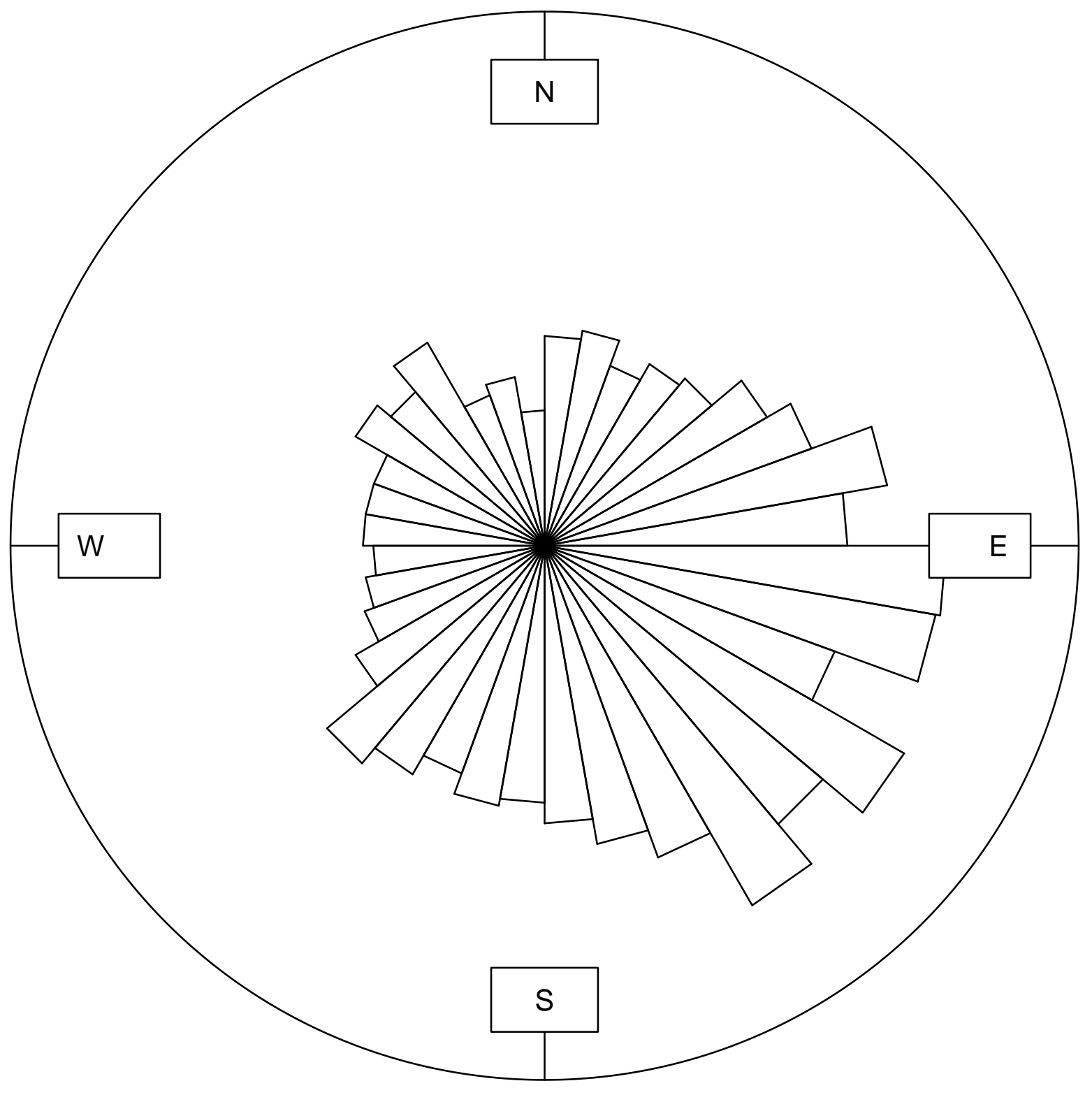


bootstrap 107

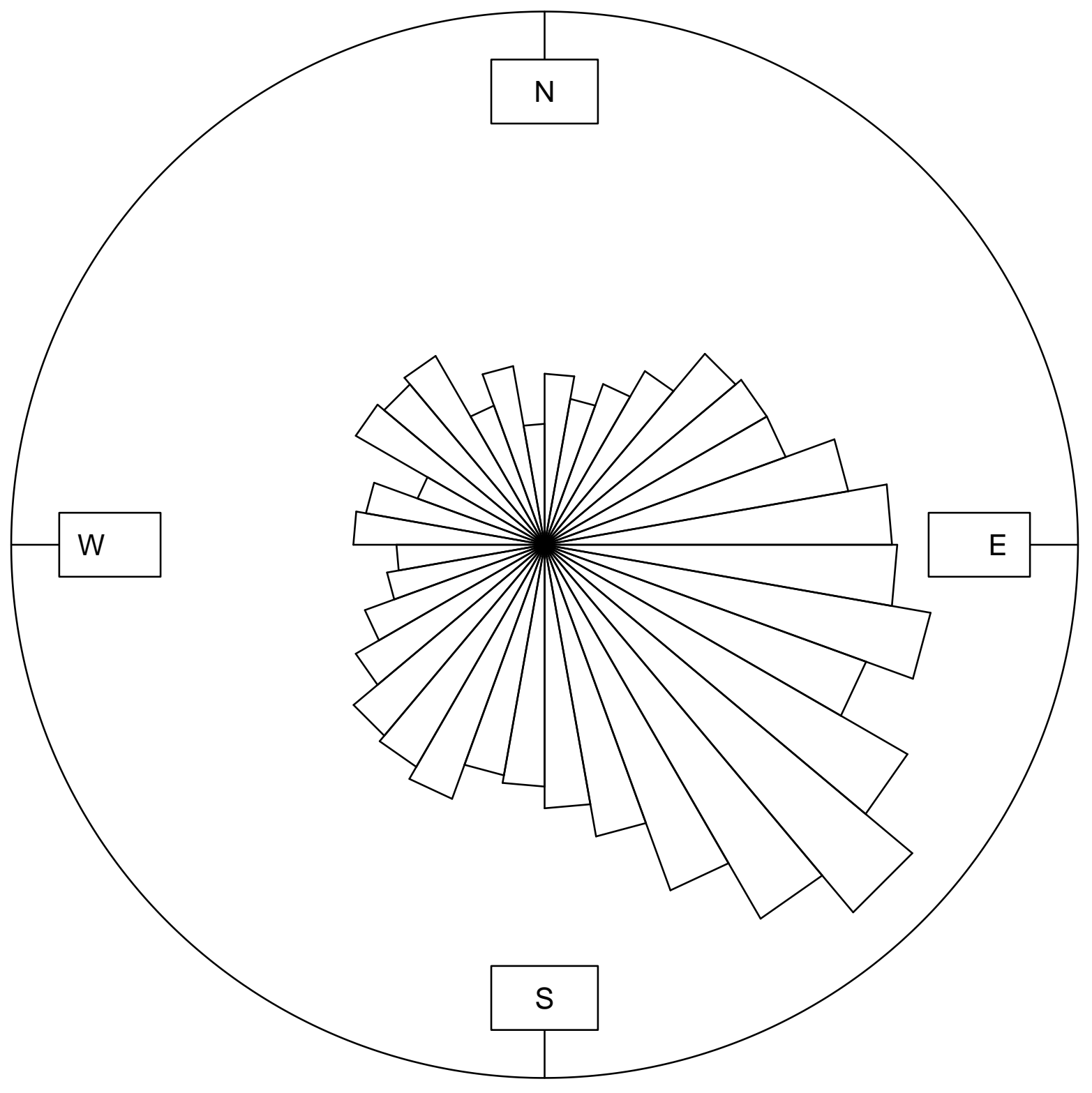


bootstrap 108

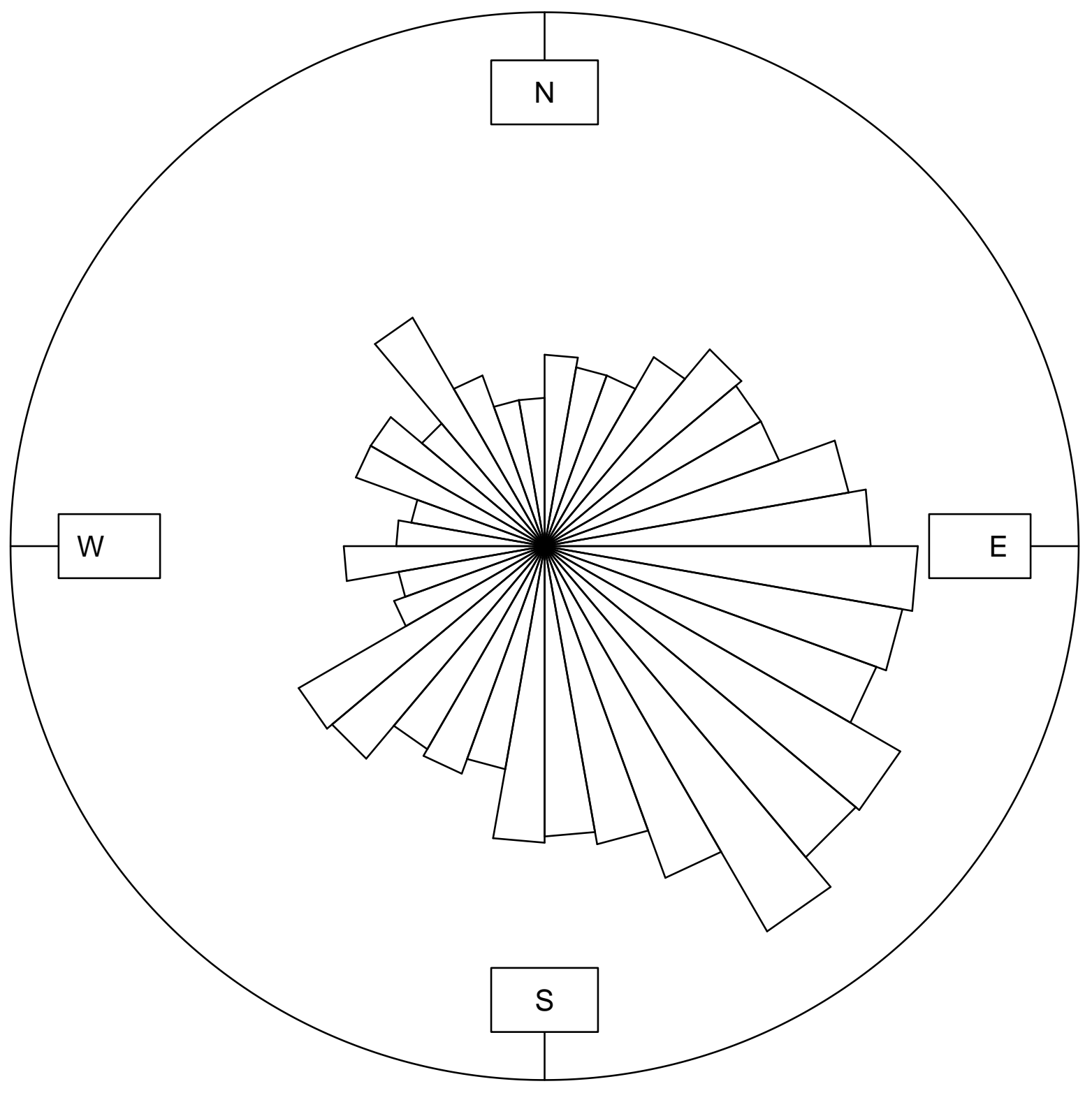


bootstrap 109

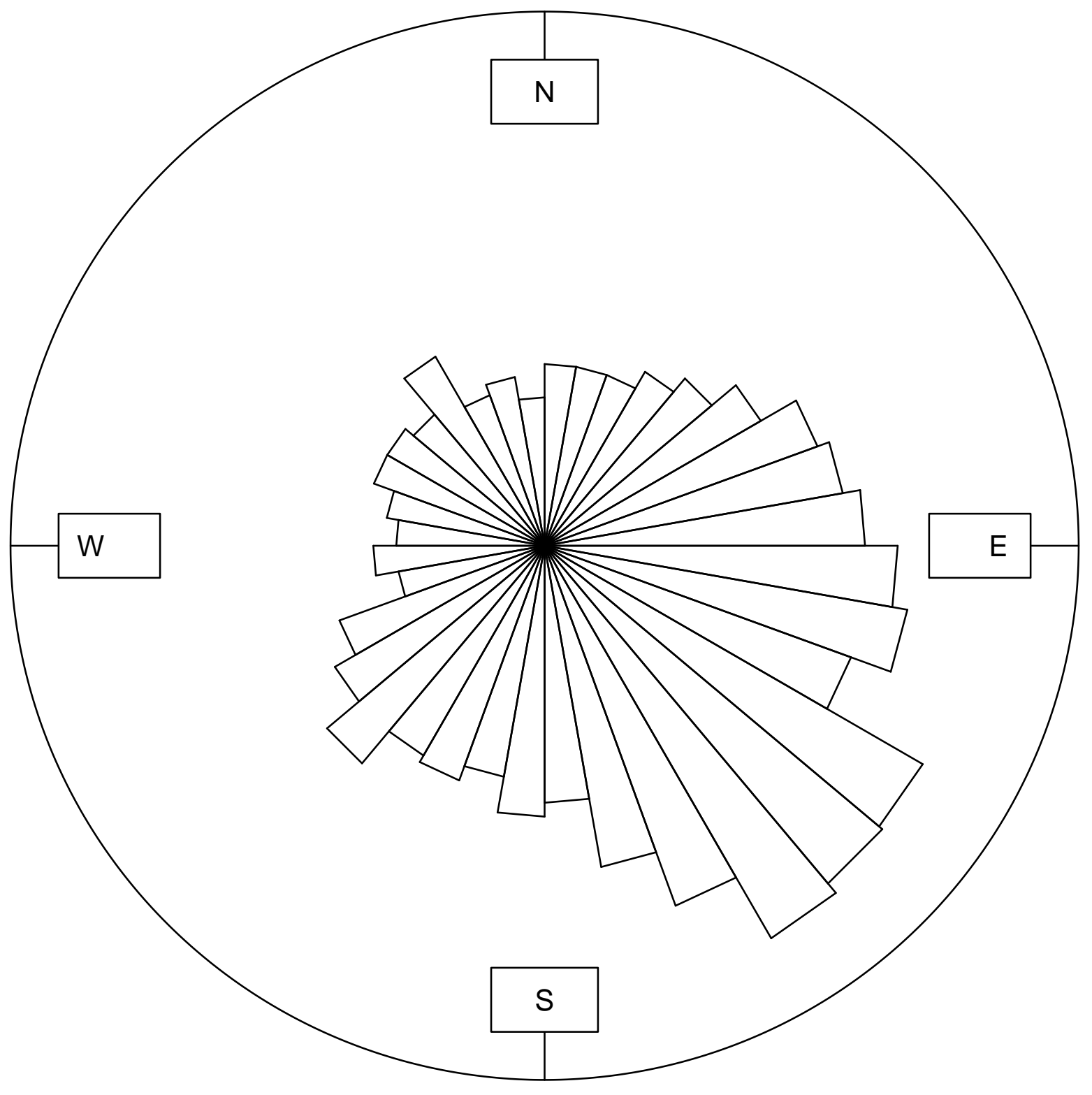


bootstrap 111

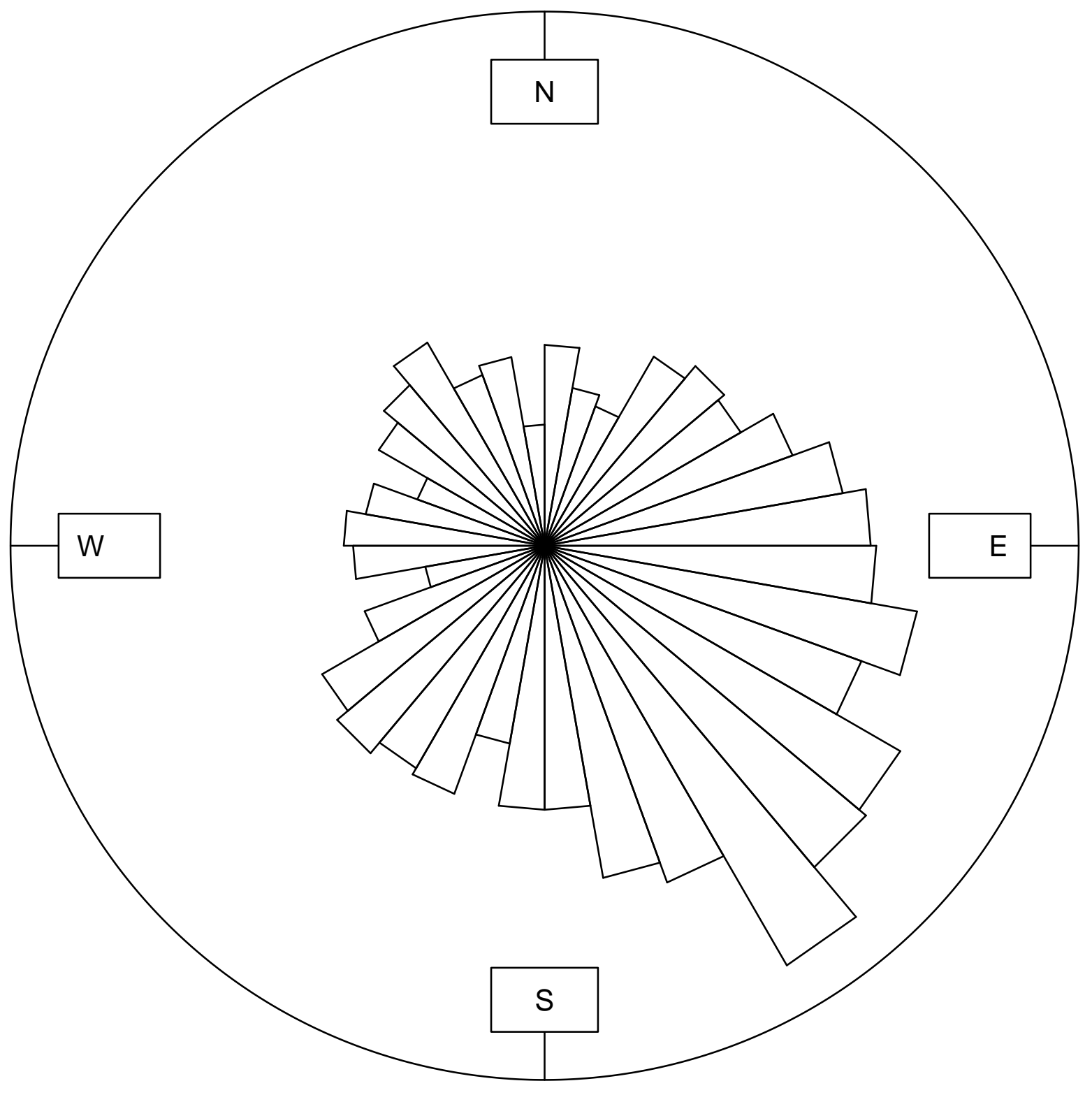


bootstrap 112

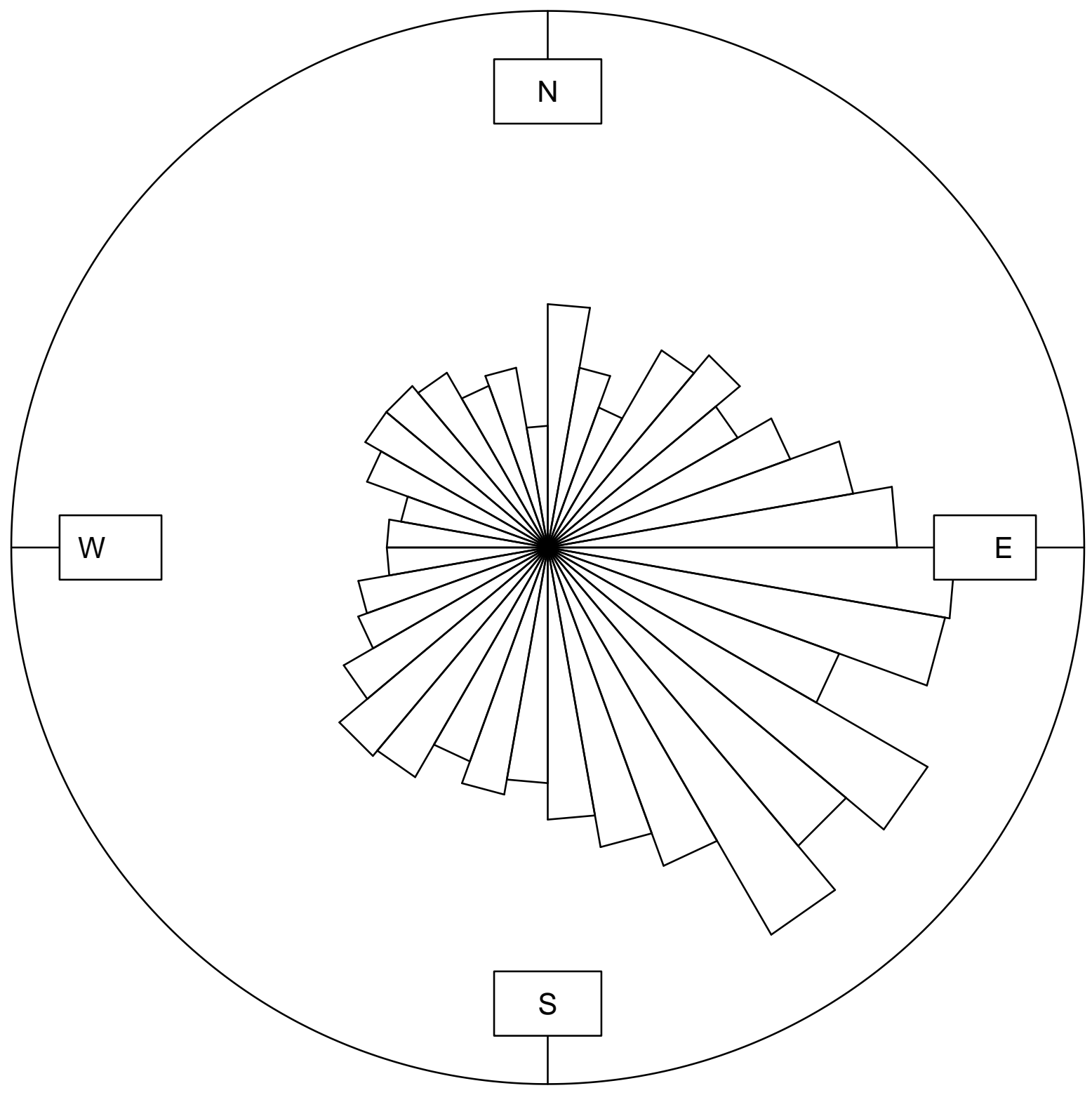


bootstrap 113

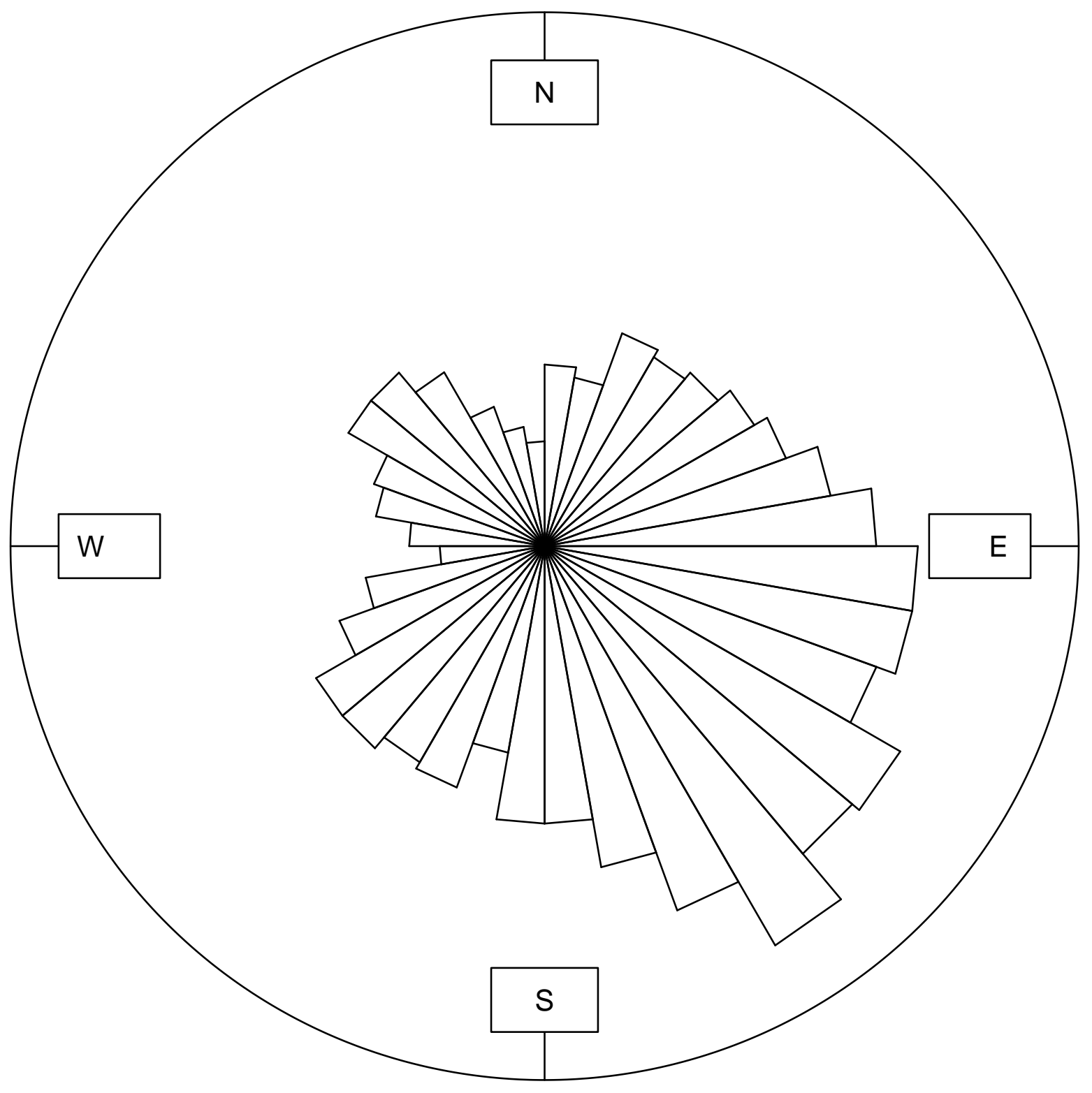


bootstrap 114

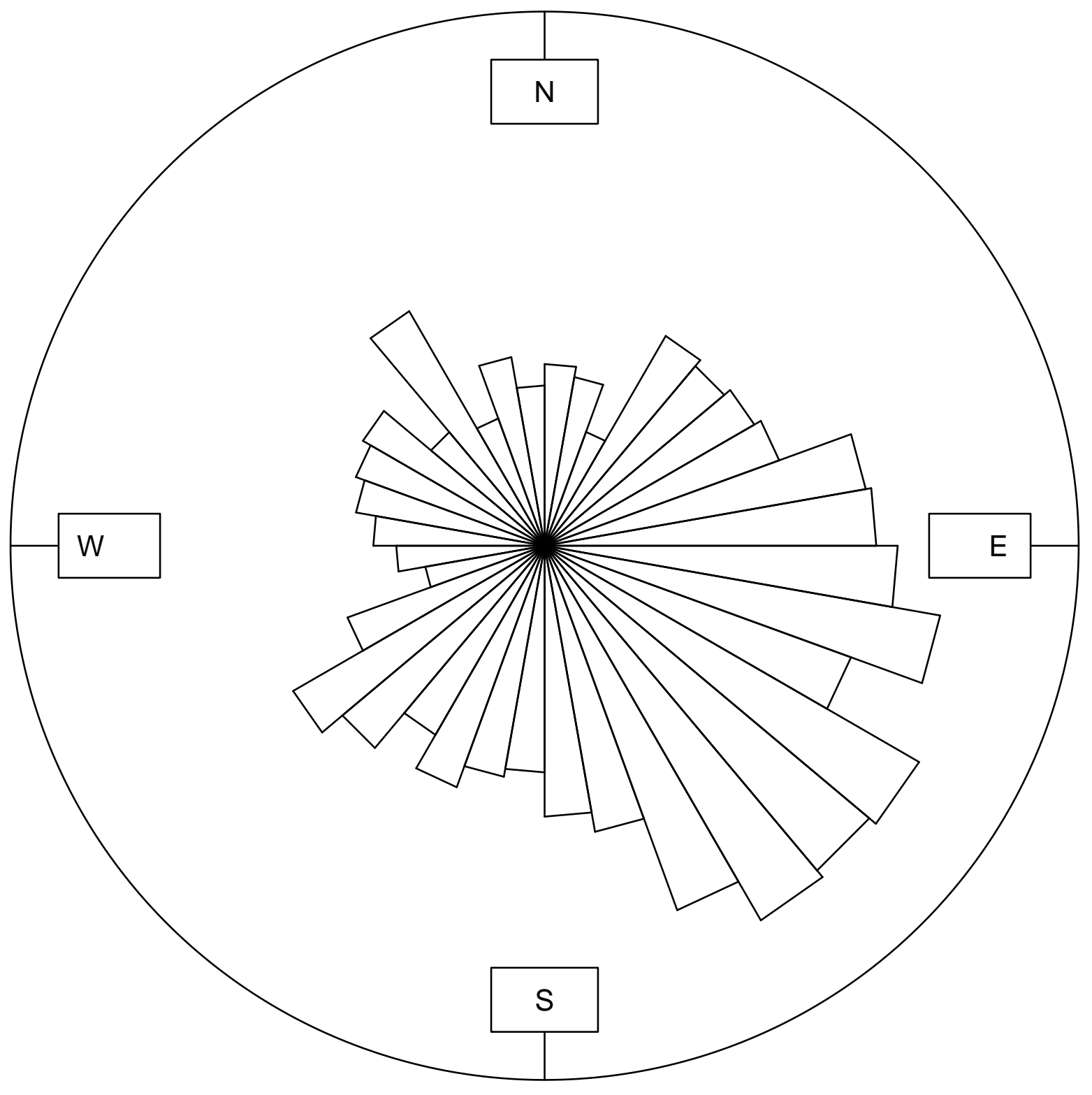




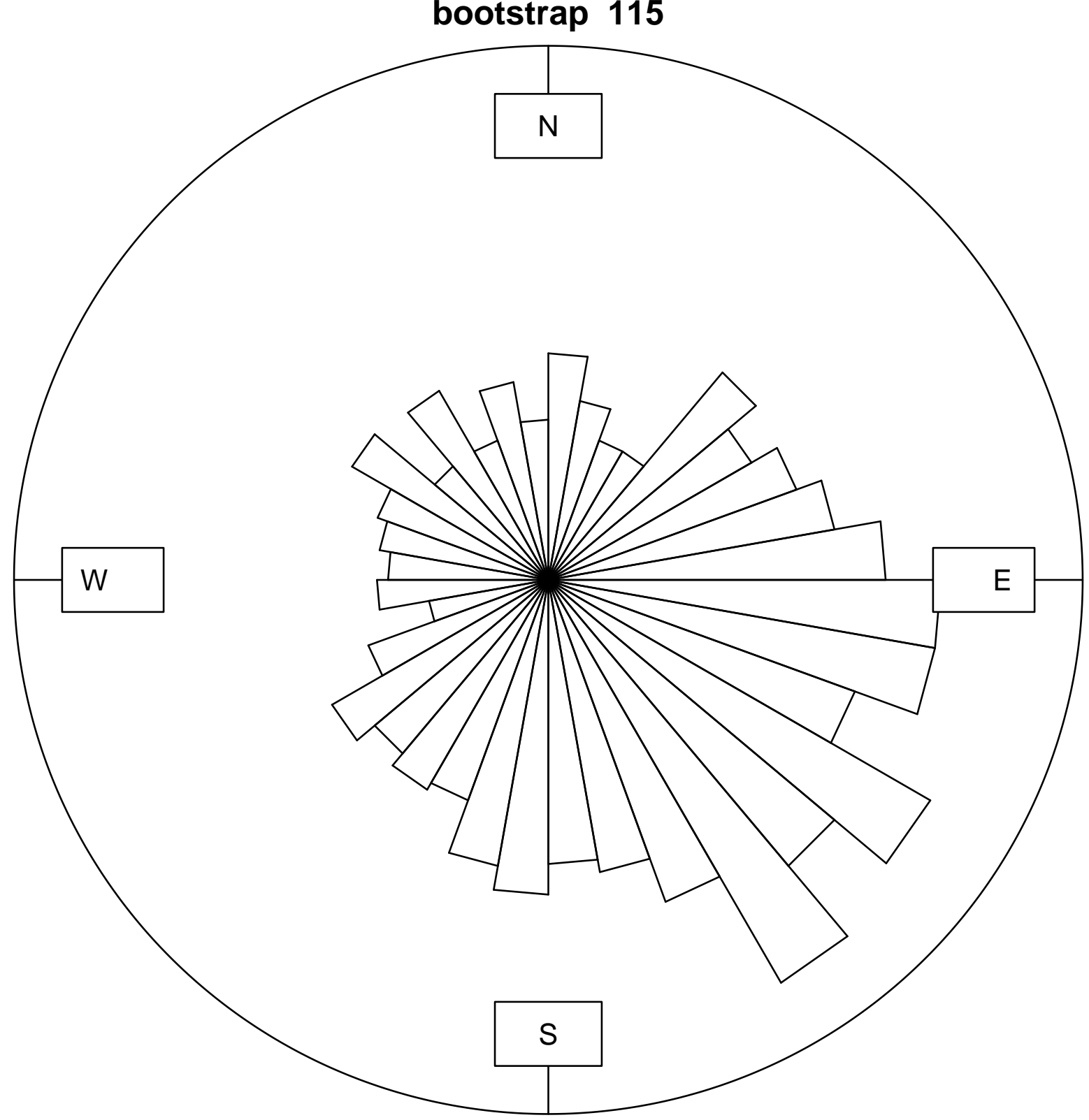


bootstrap 116

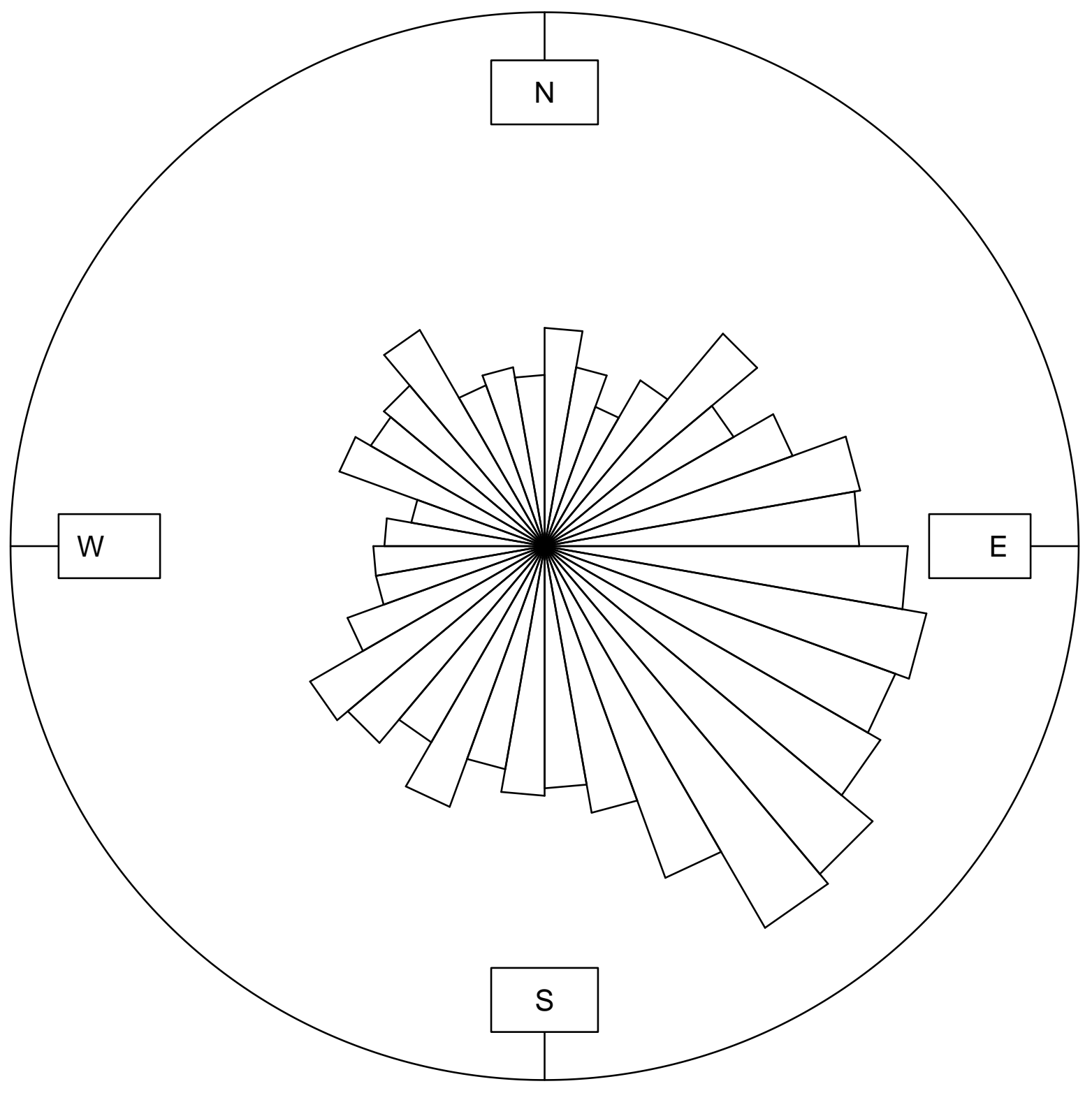


bootstrap 117

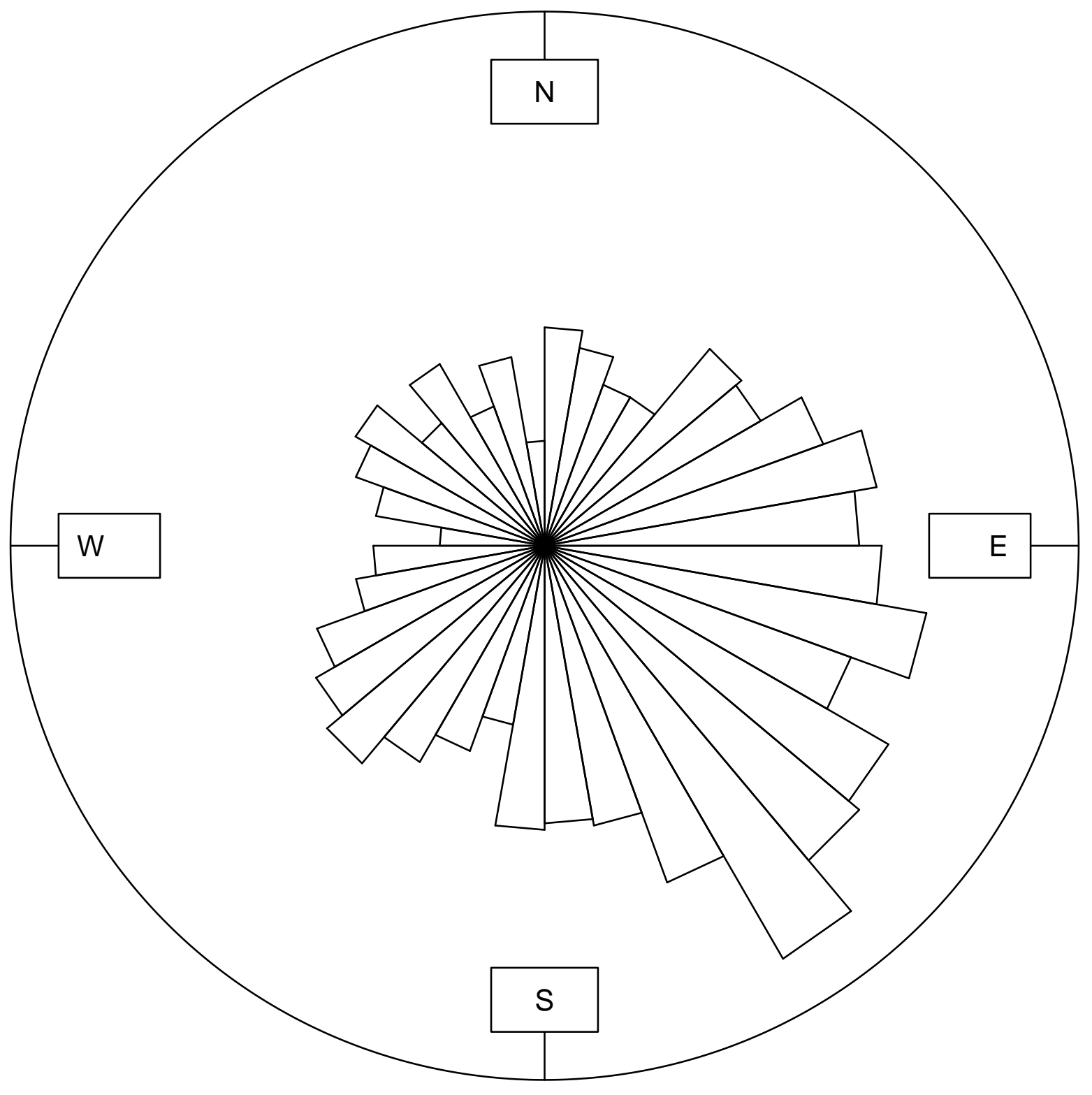


bootstrap 118

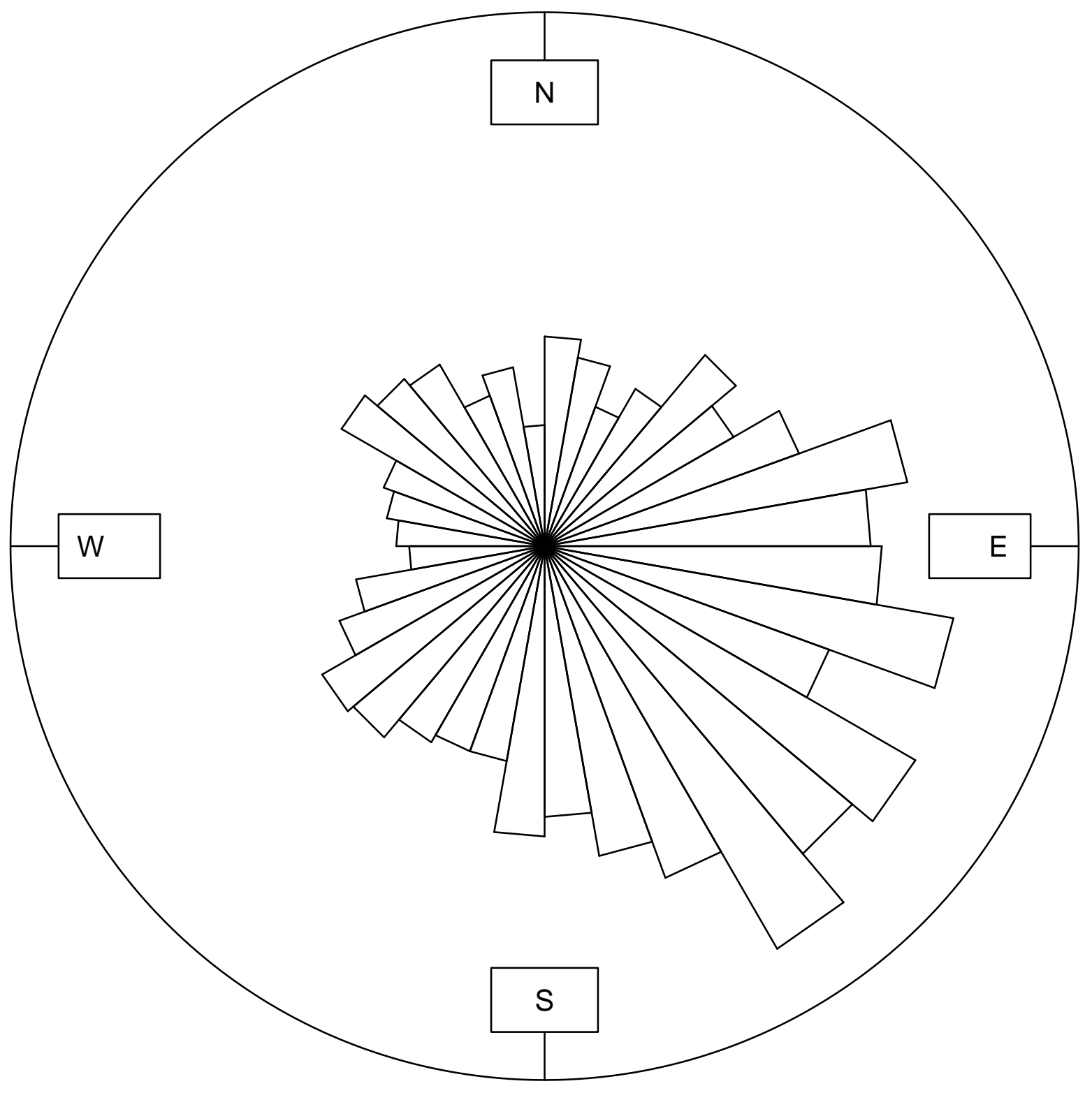


bootstrap 119

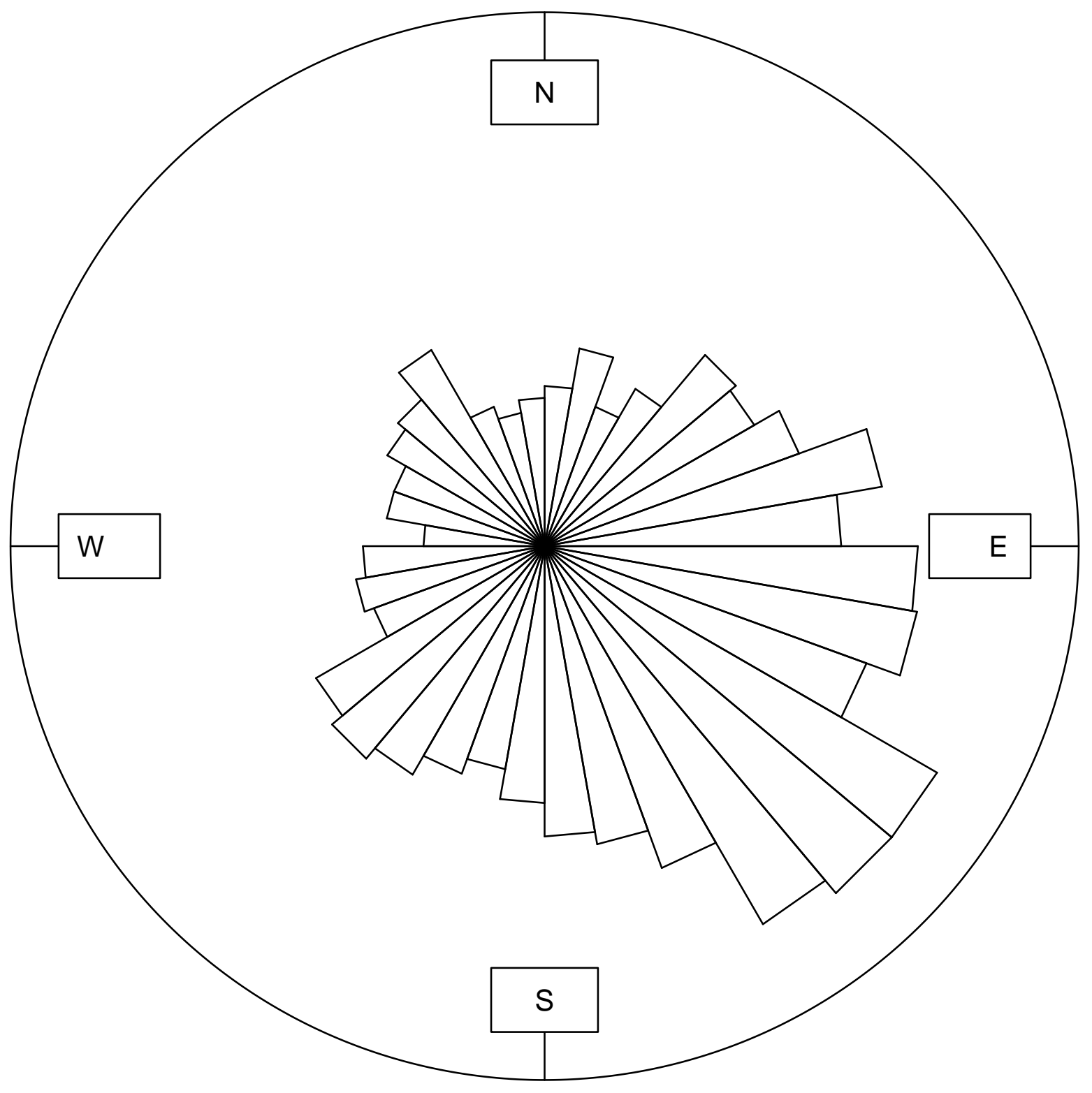


bootstrap 120

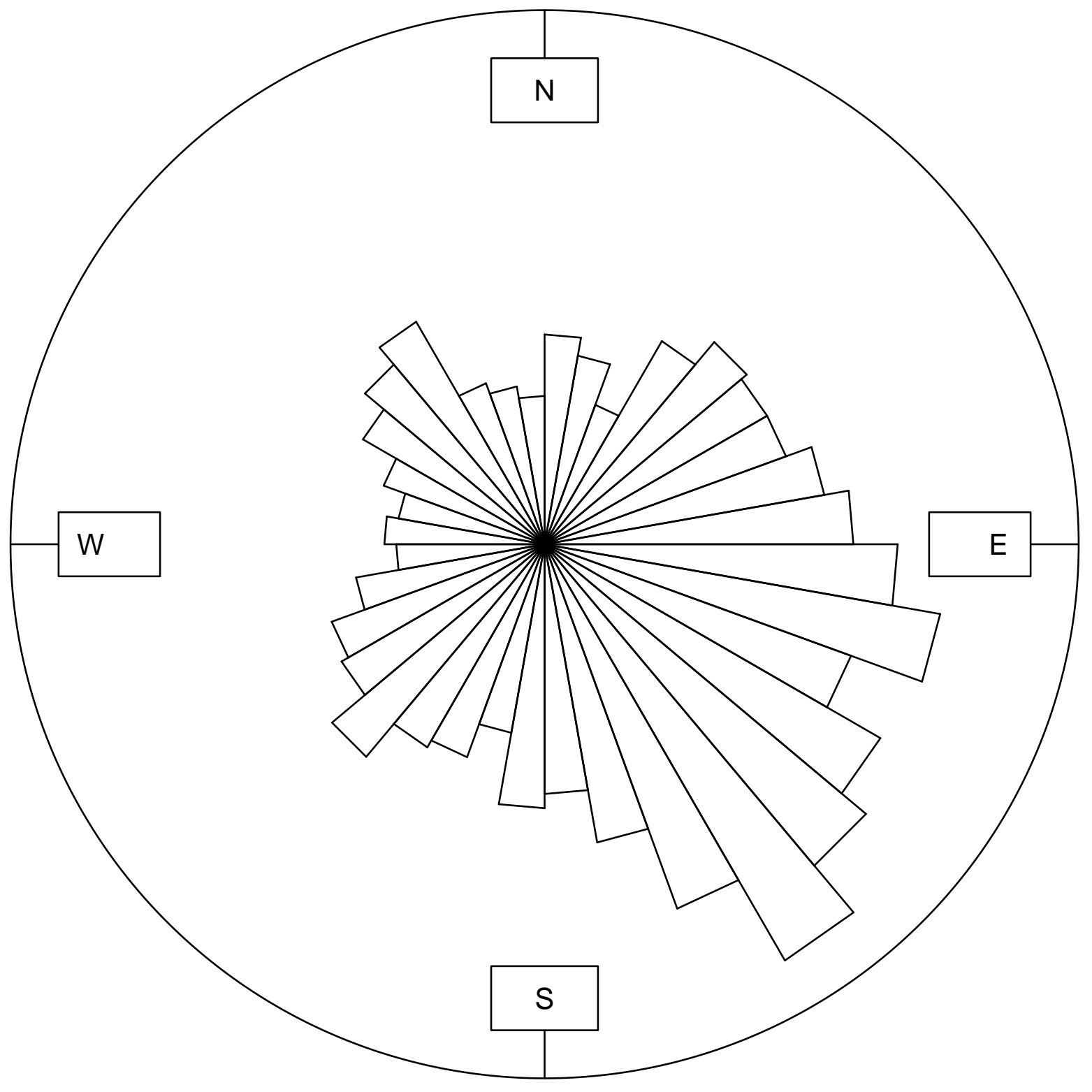


bootstrap 121

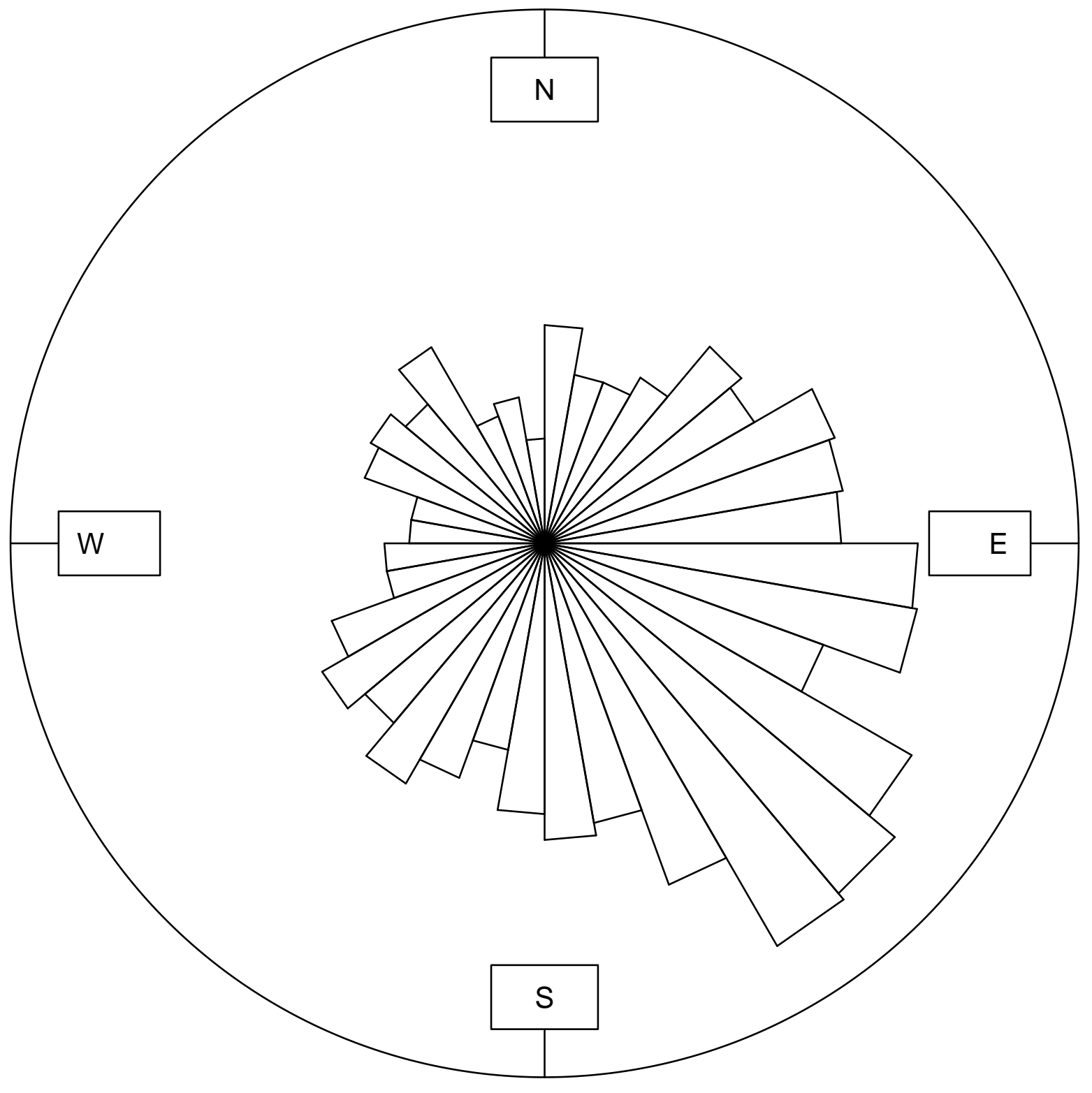


bootstrap 122

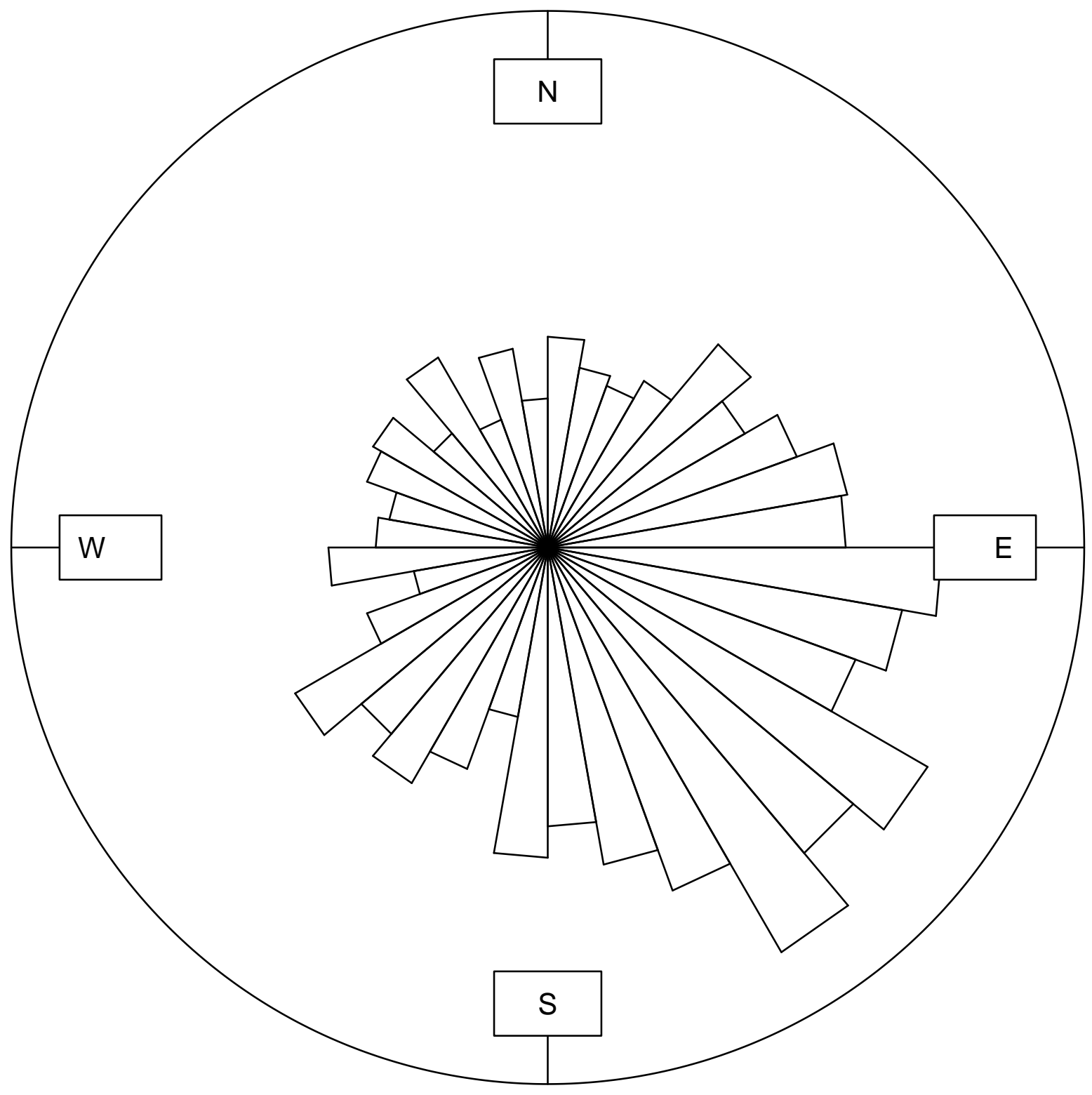


bootstrap 123

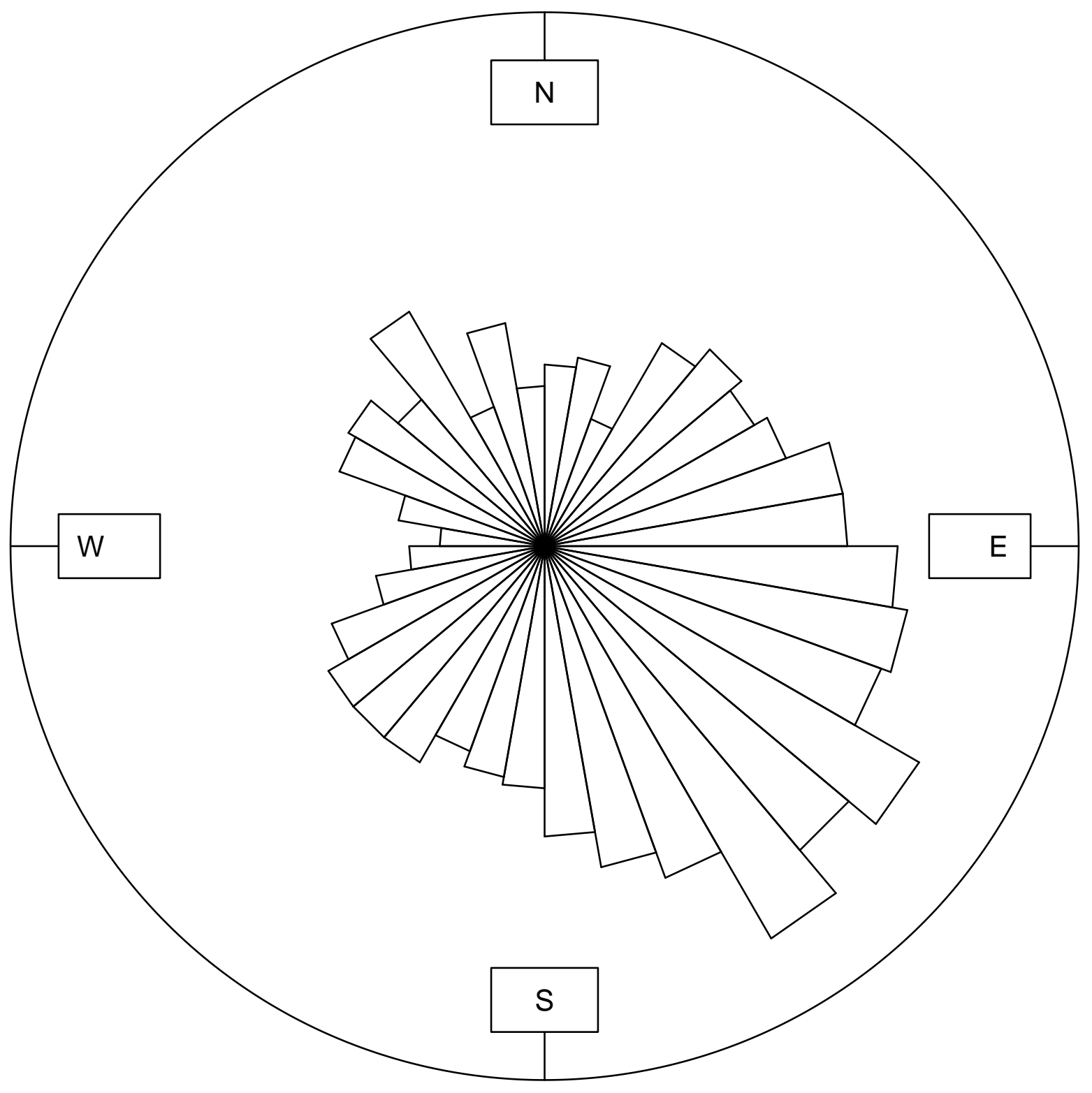




\section{bootstrap 124}

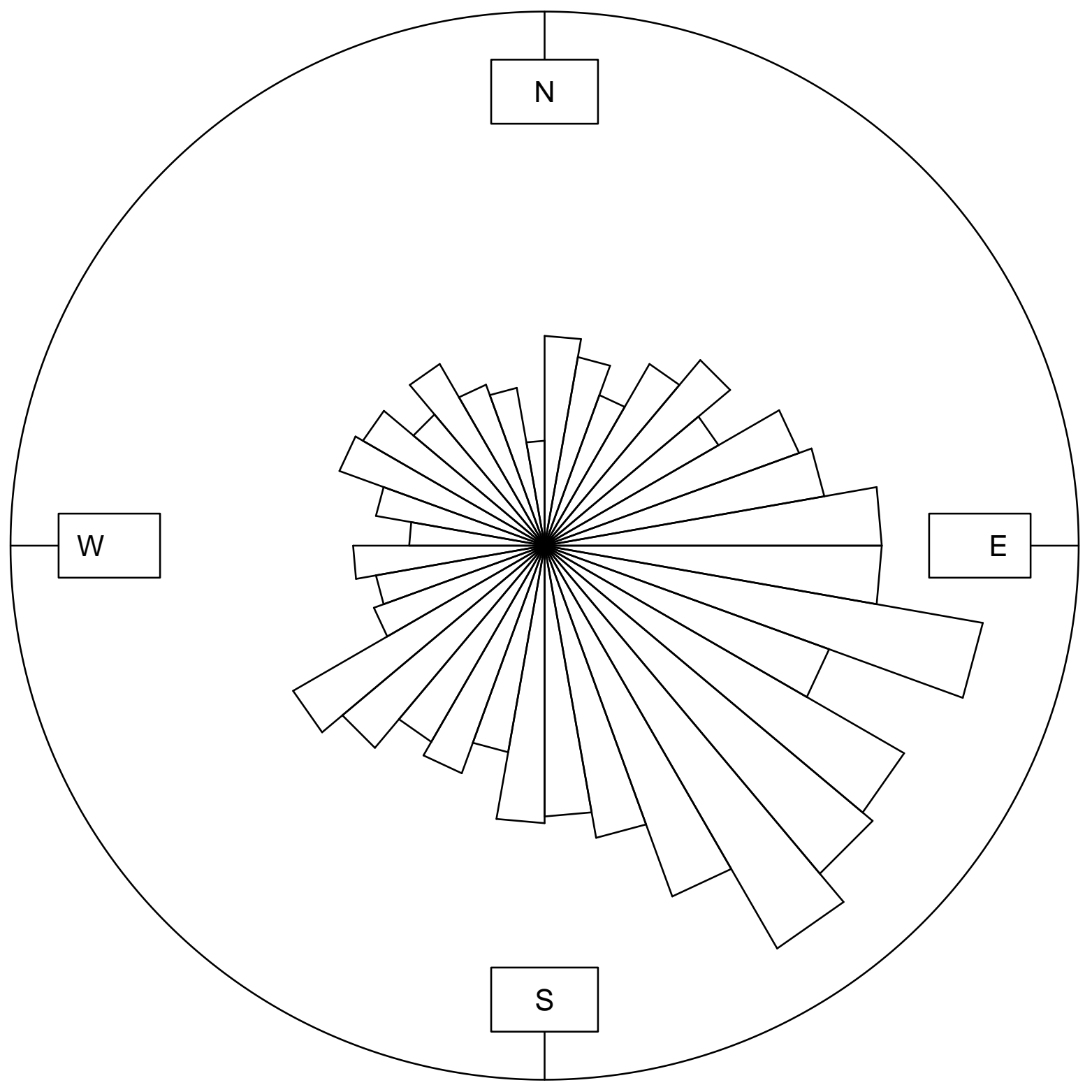




\section{bootstrap 125}

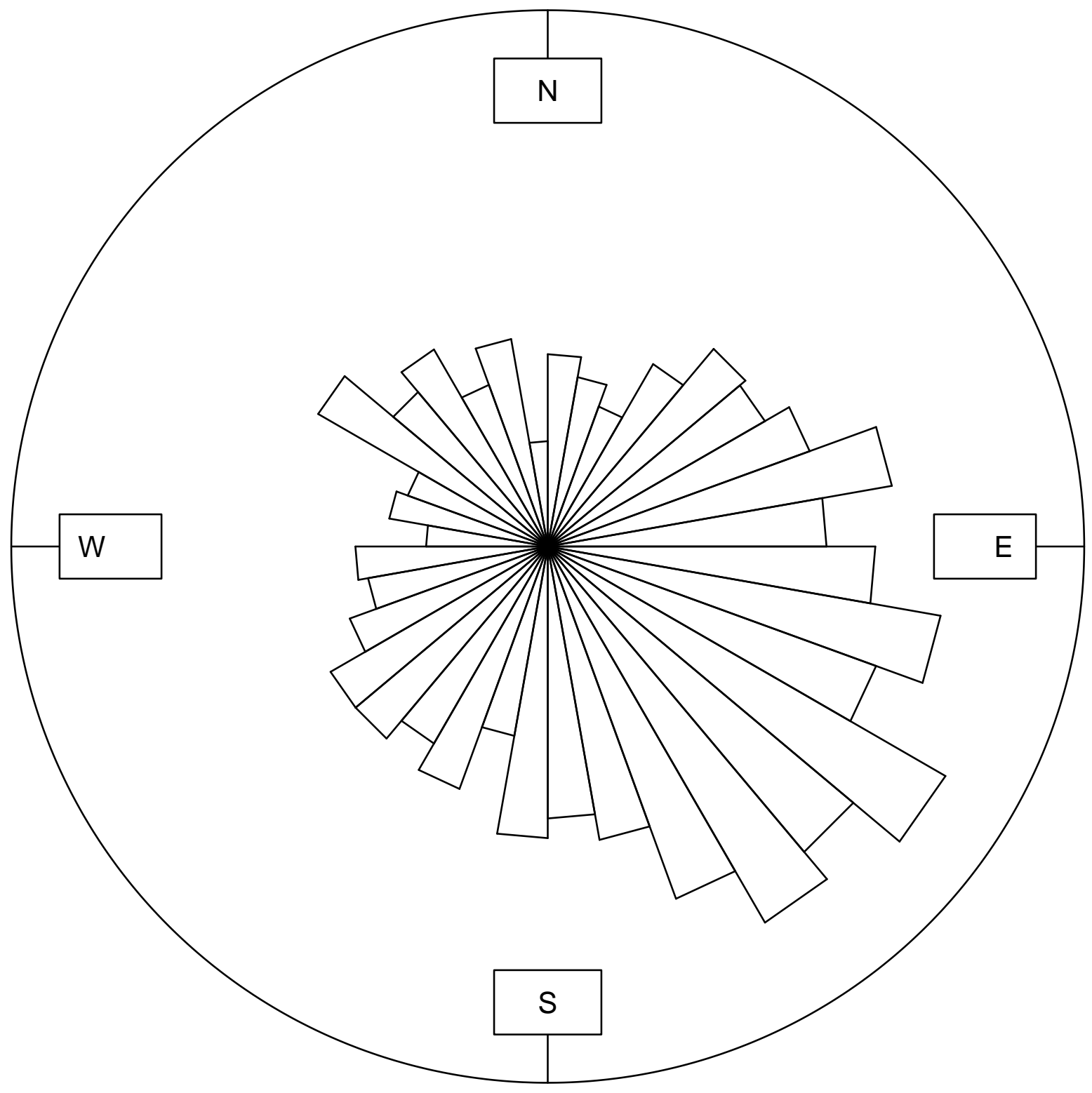


bootstrap 126

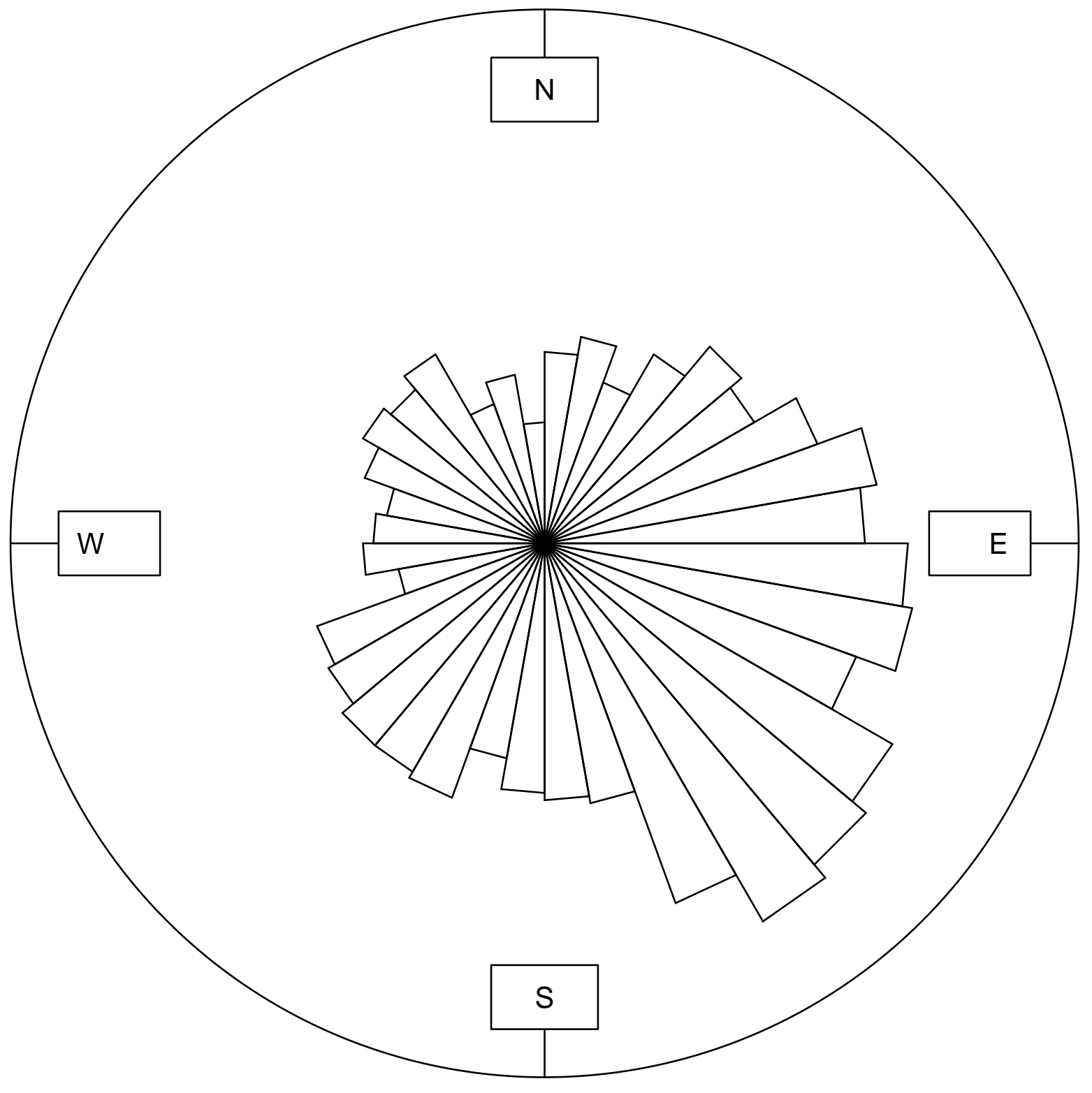


bootstrap 127

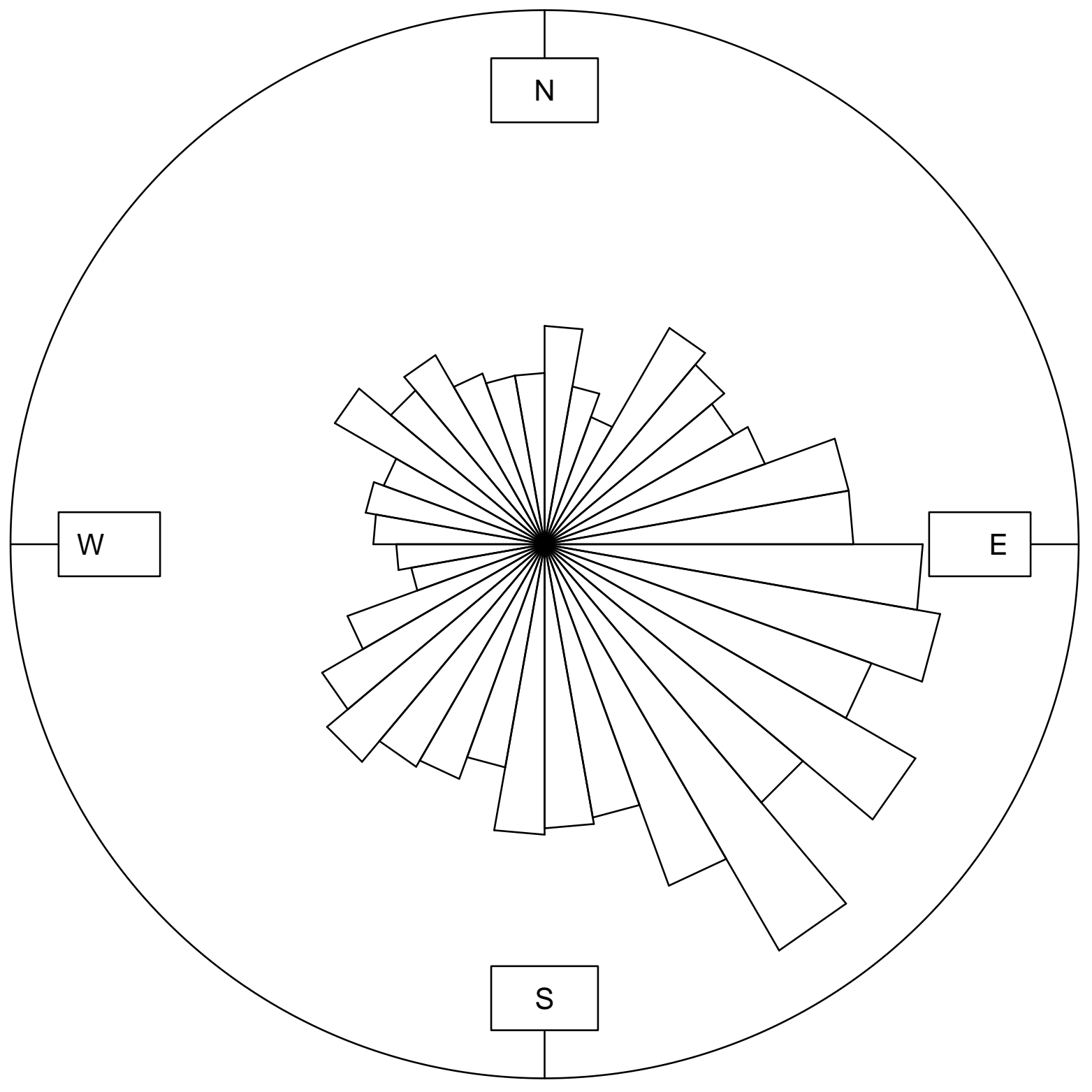


bootstrap 128

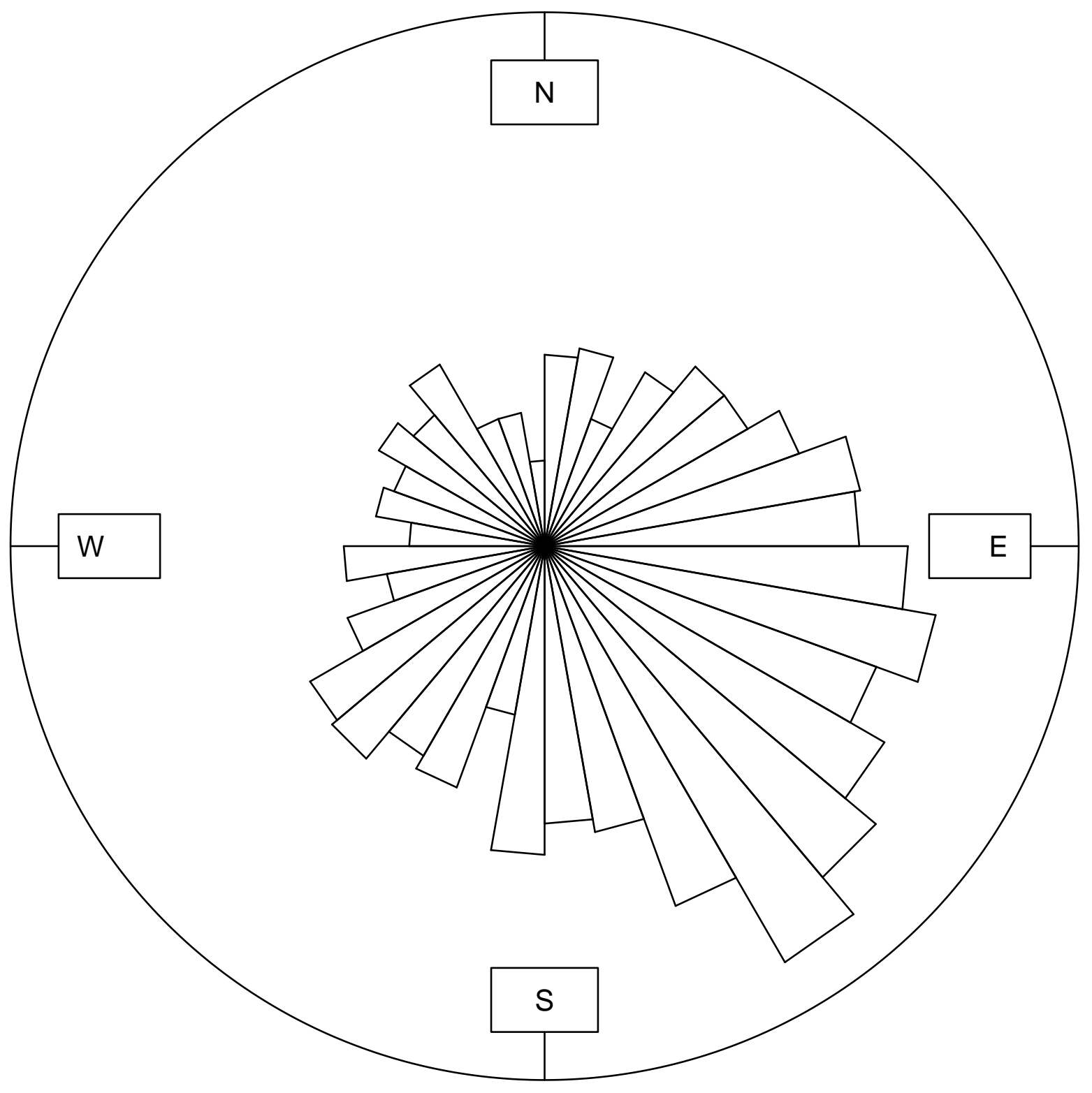


bootstrap 129

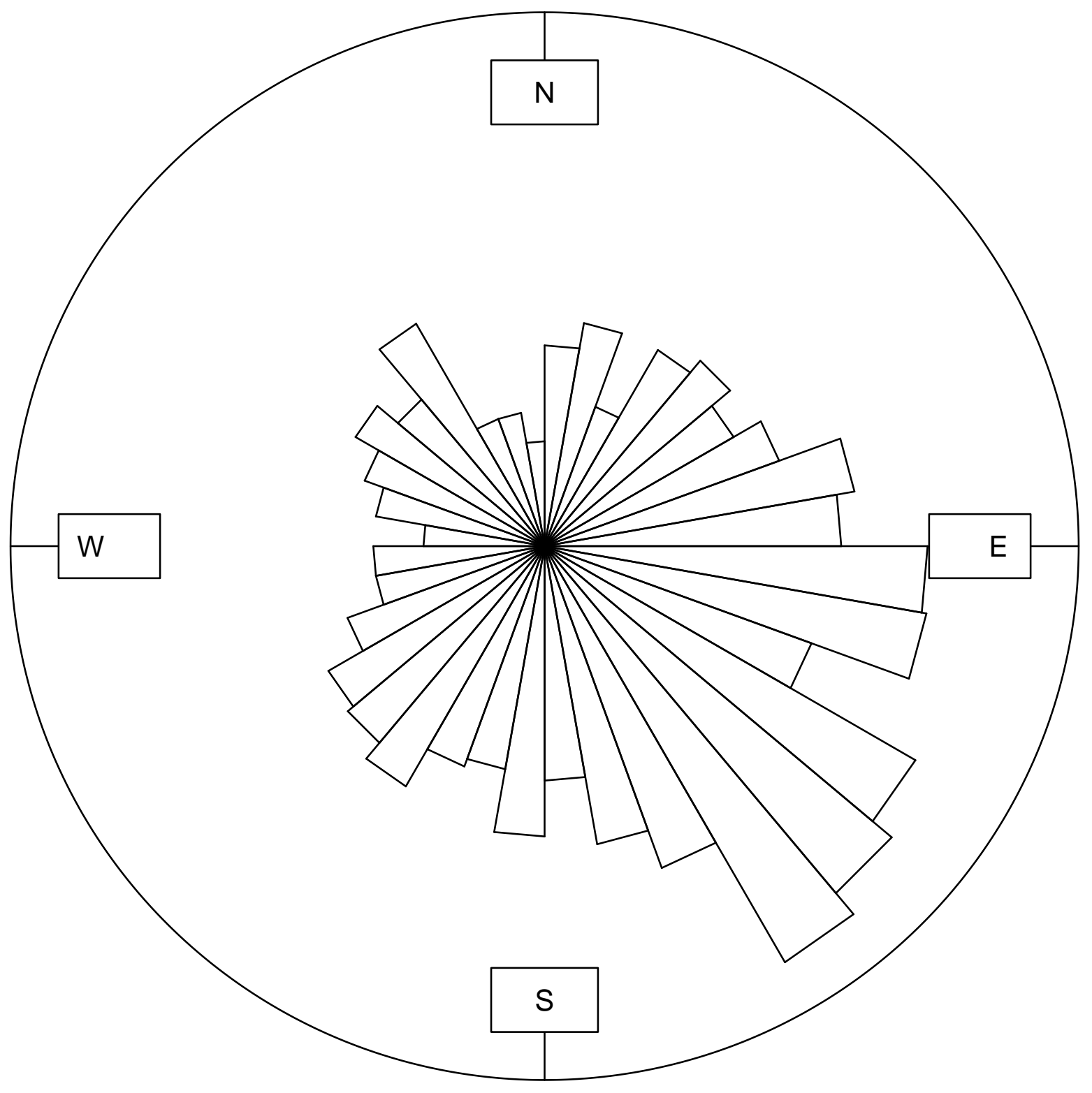


bootstrap 130

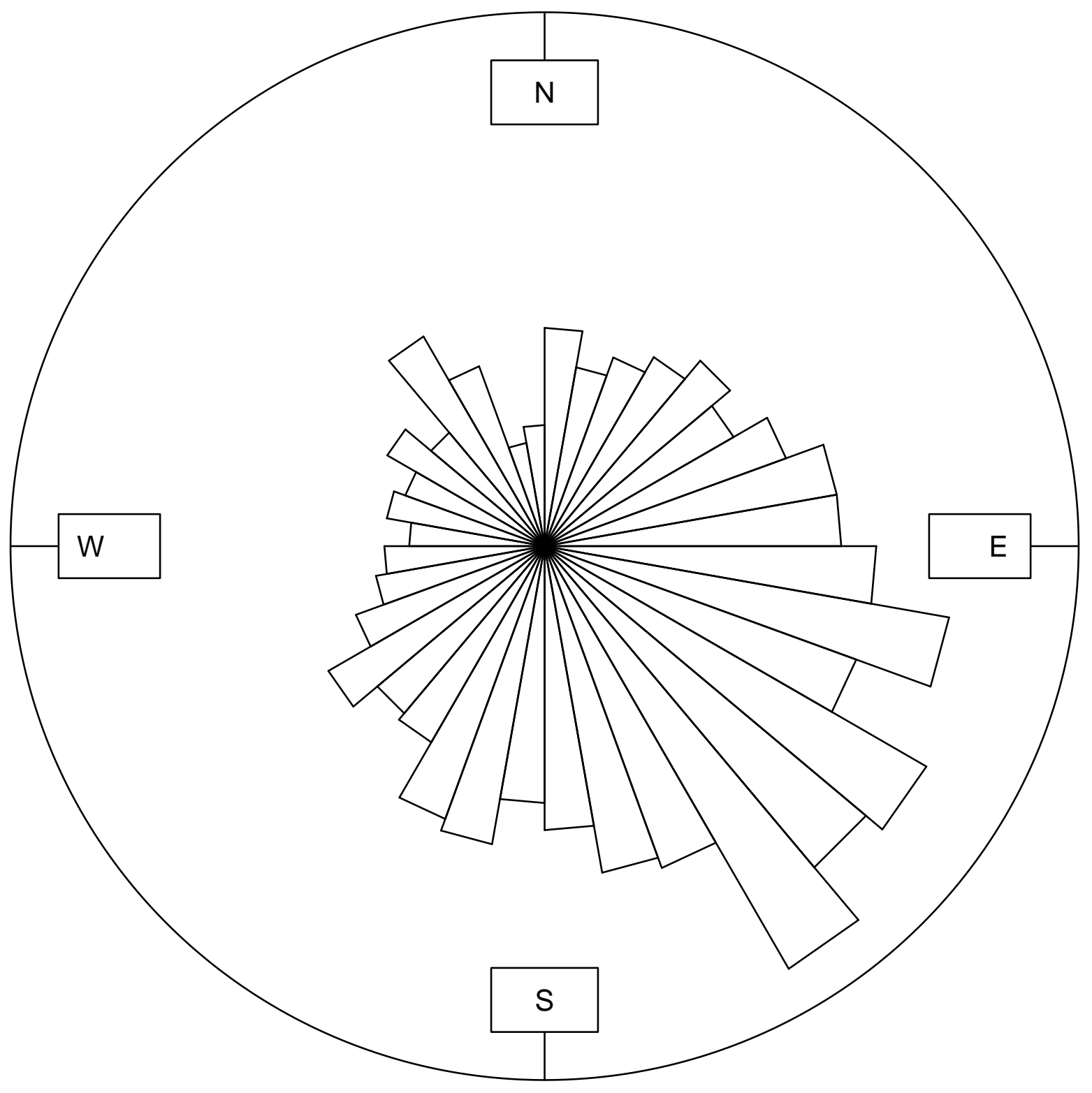


bootstrap 131

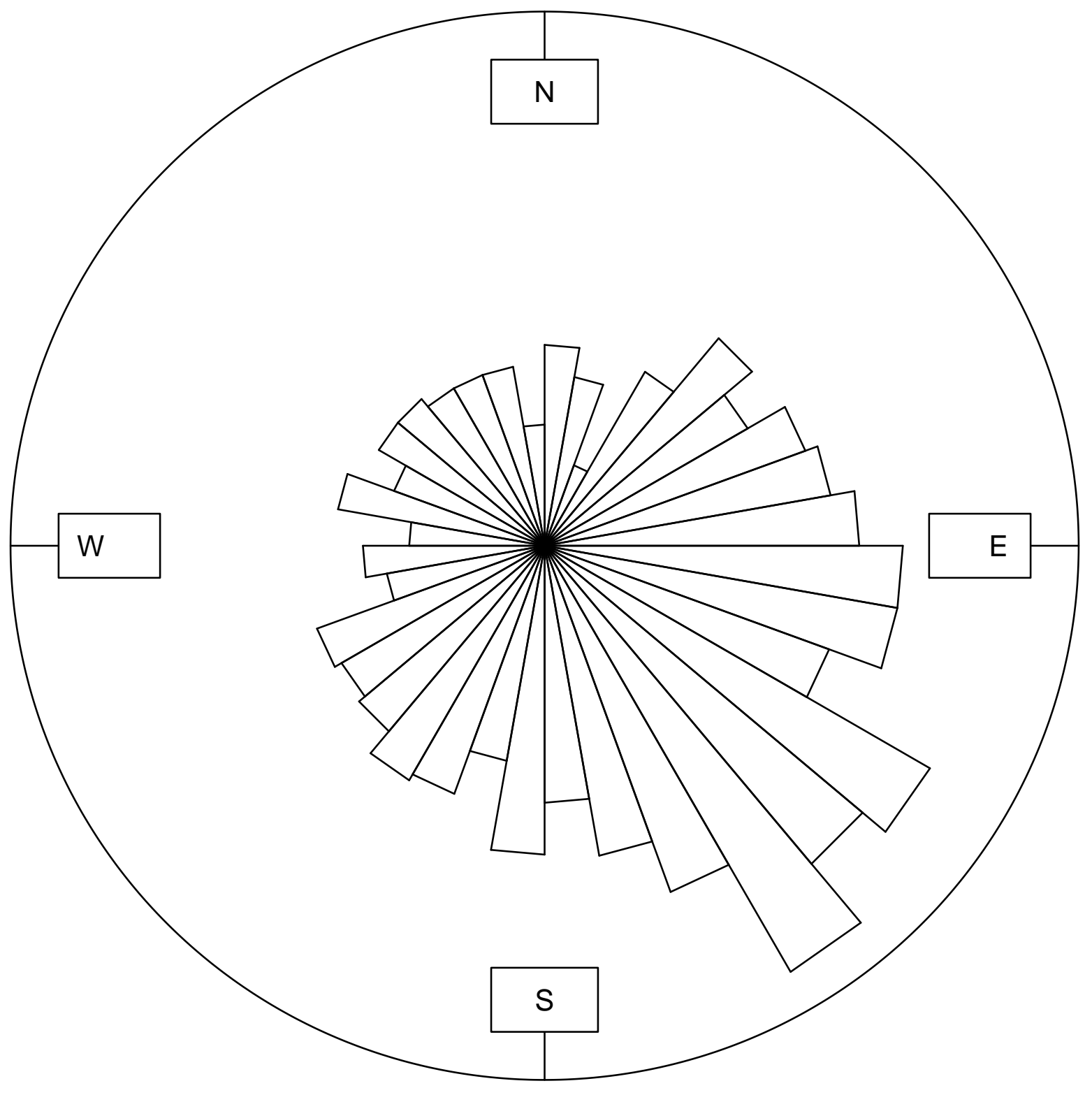


bootstrap 132

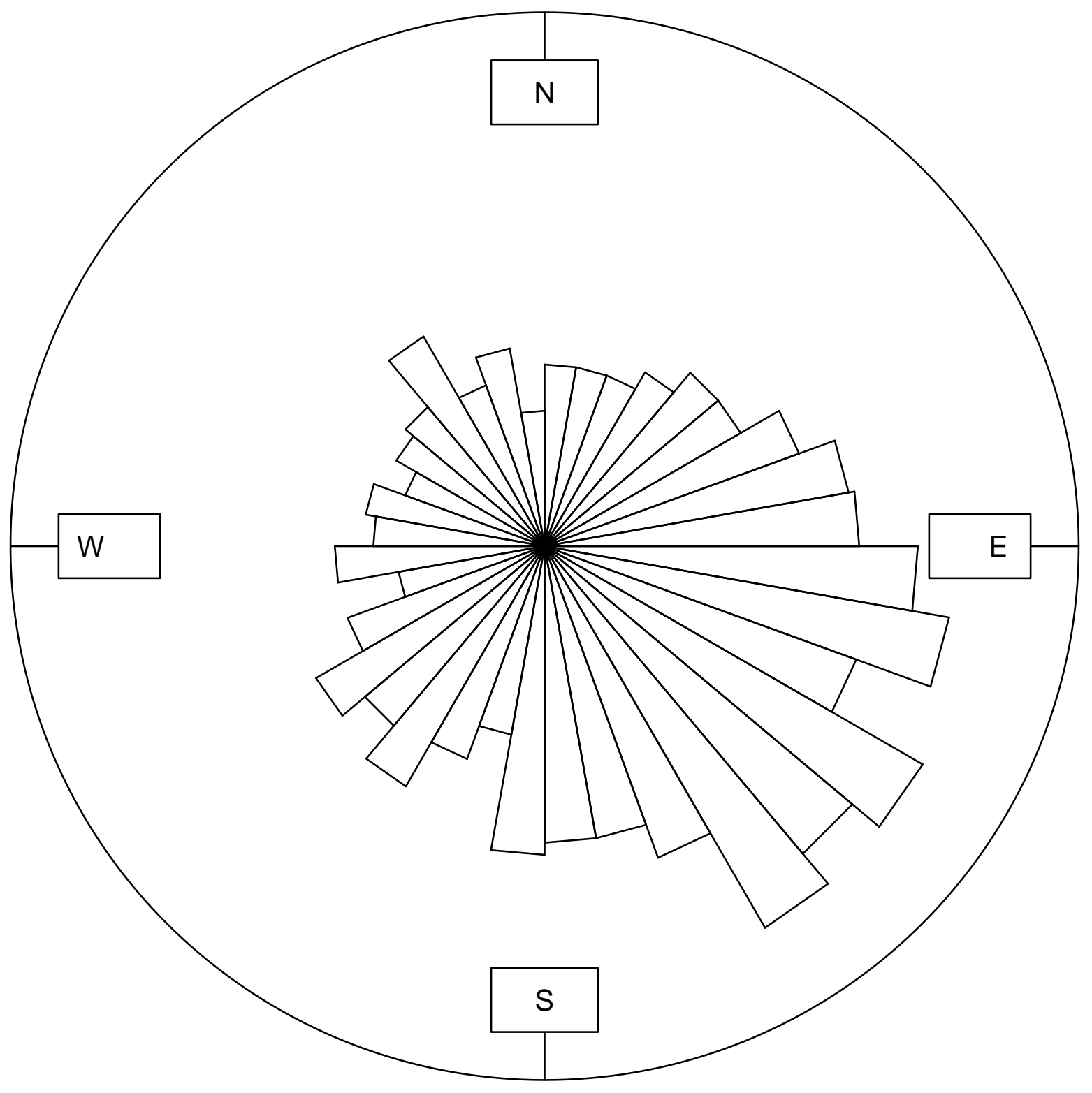




\section{bootstrap 133}

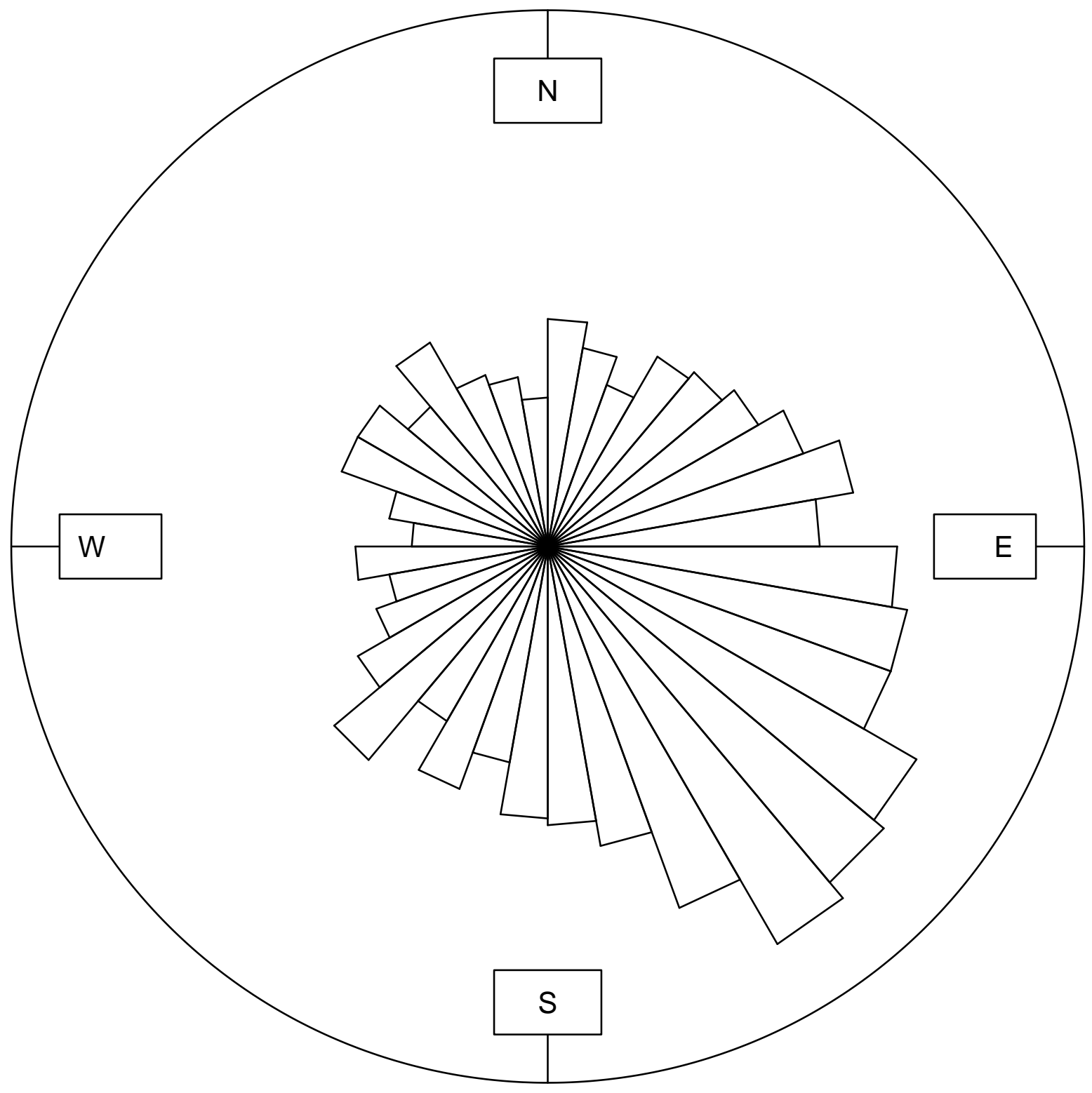


bootstrap 134

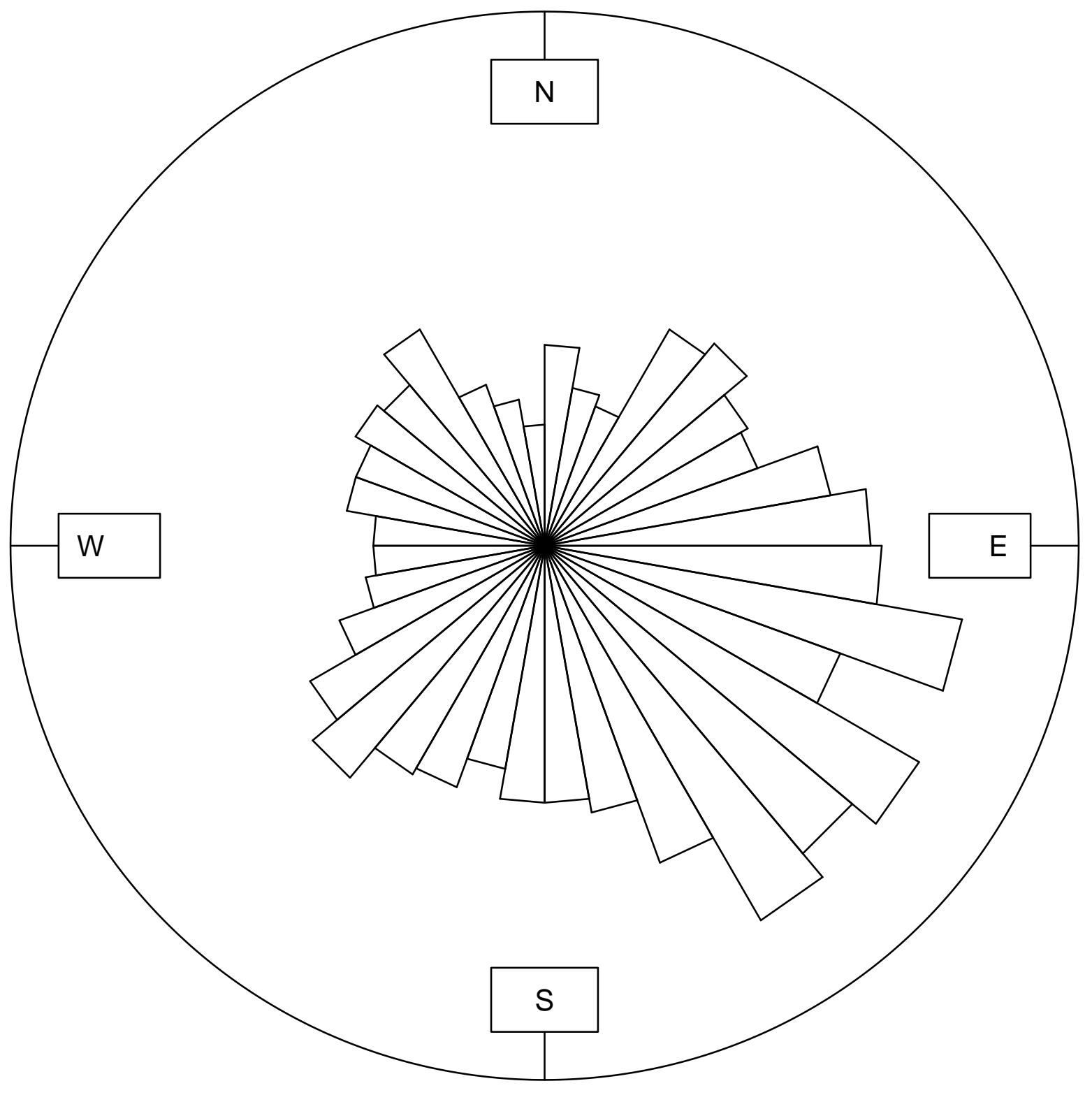




\section{bootstrap 135}

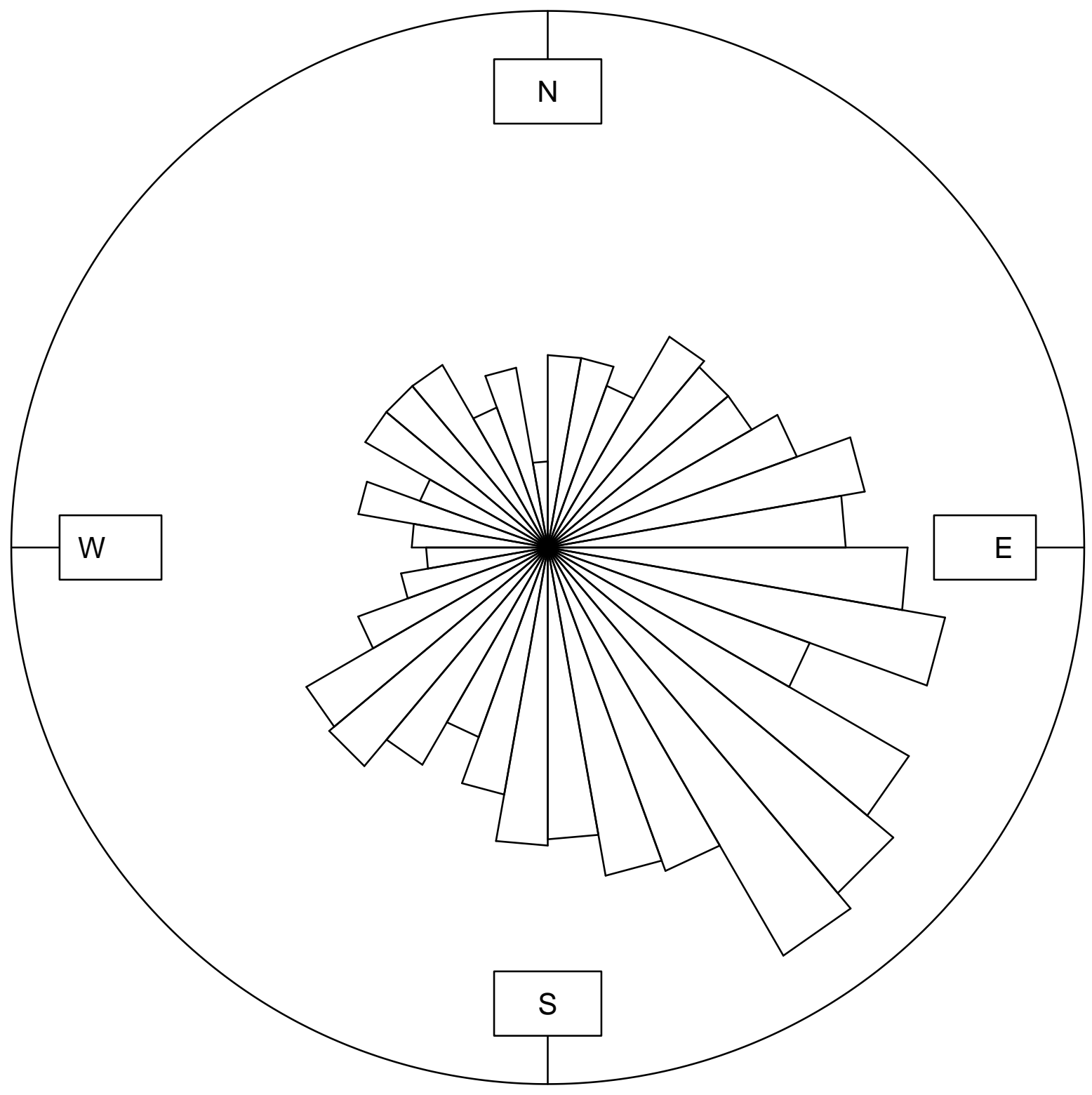




\section{bootstrap 136}

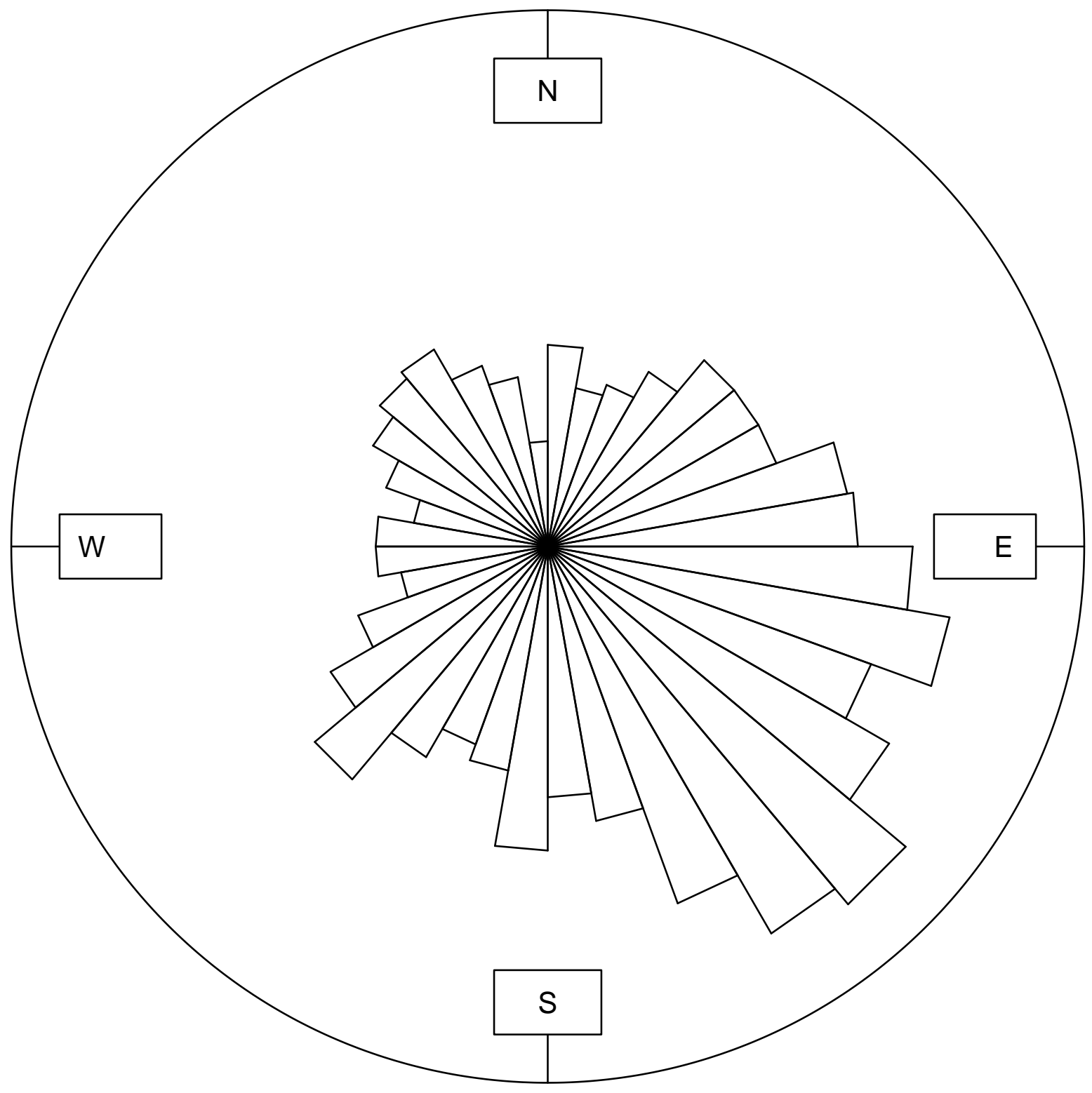


bootstrap 137

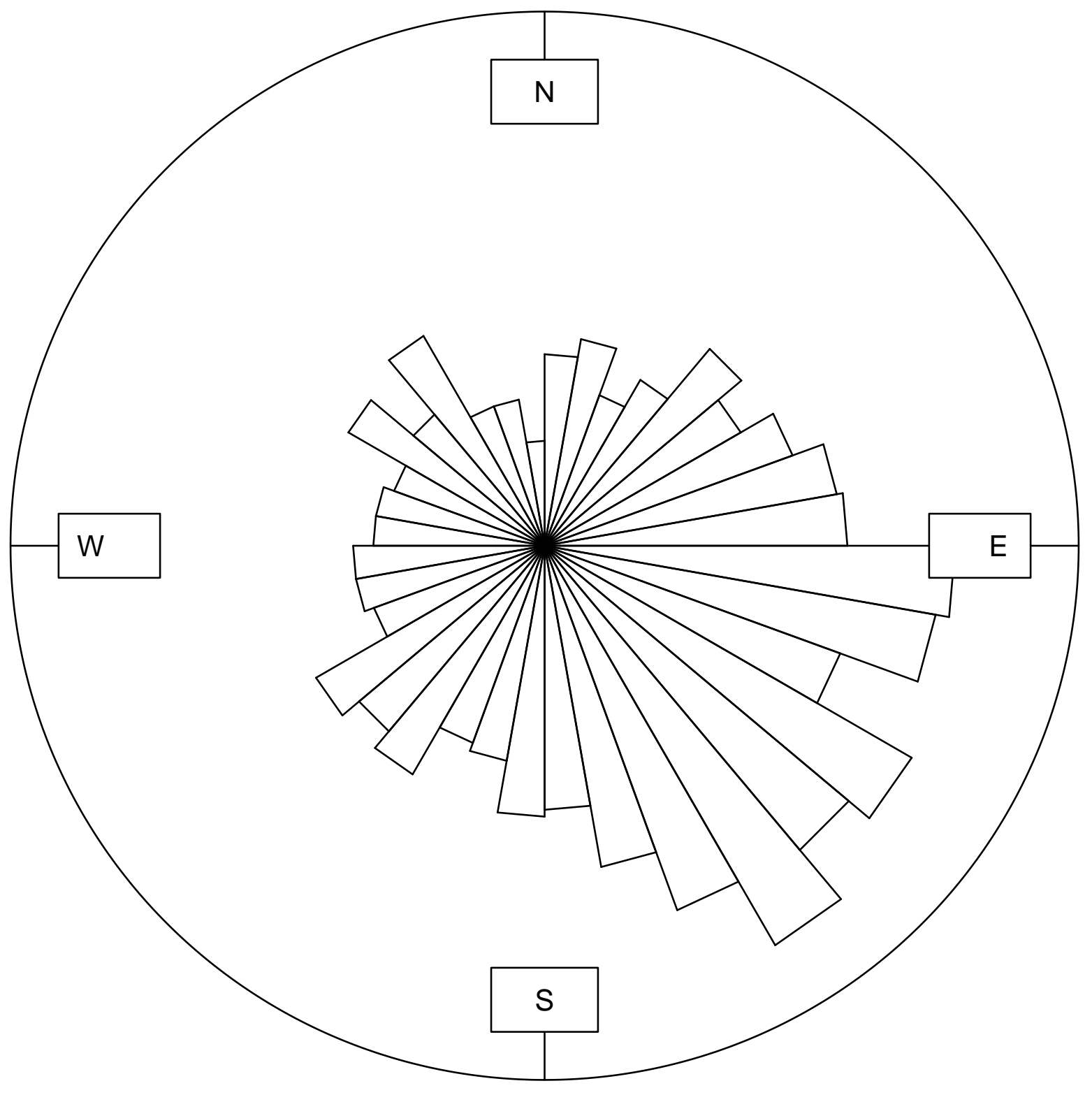


bootstrap 138

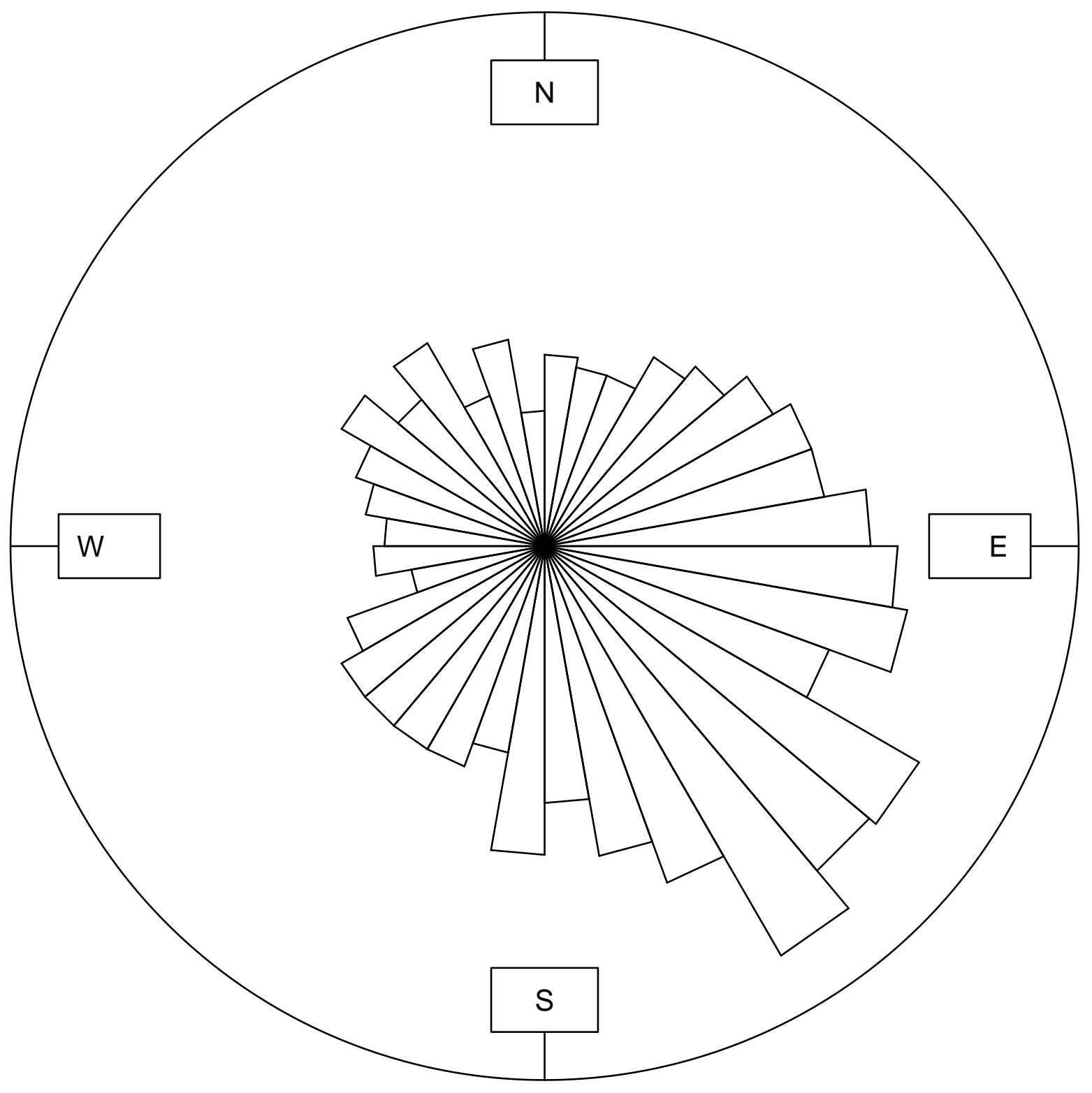


bootstrap 139

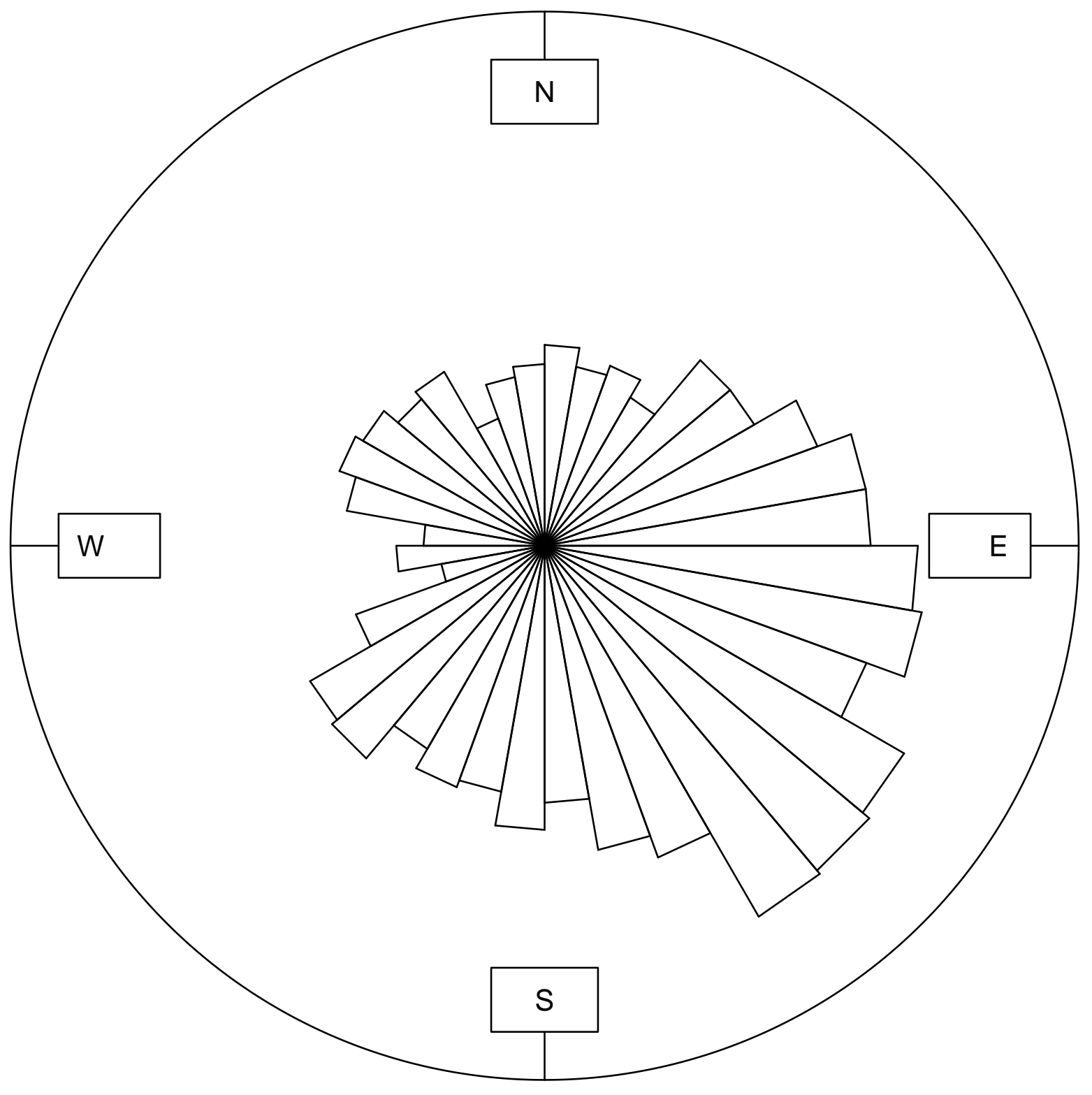


bootstrap 140

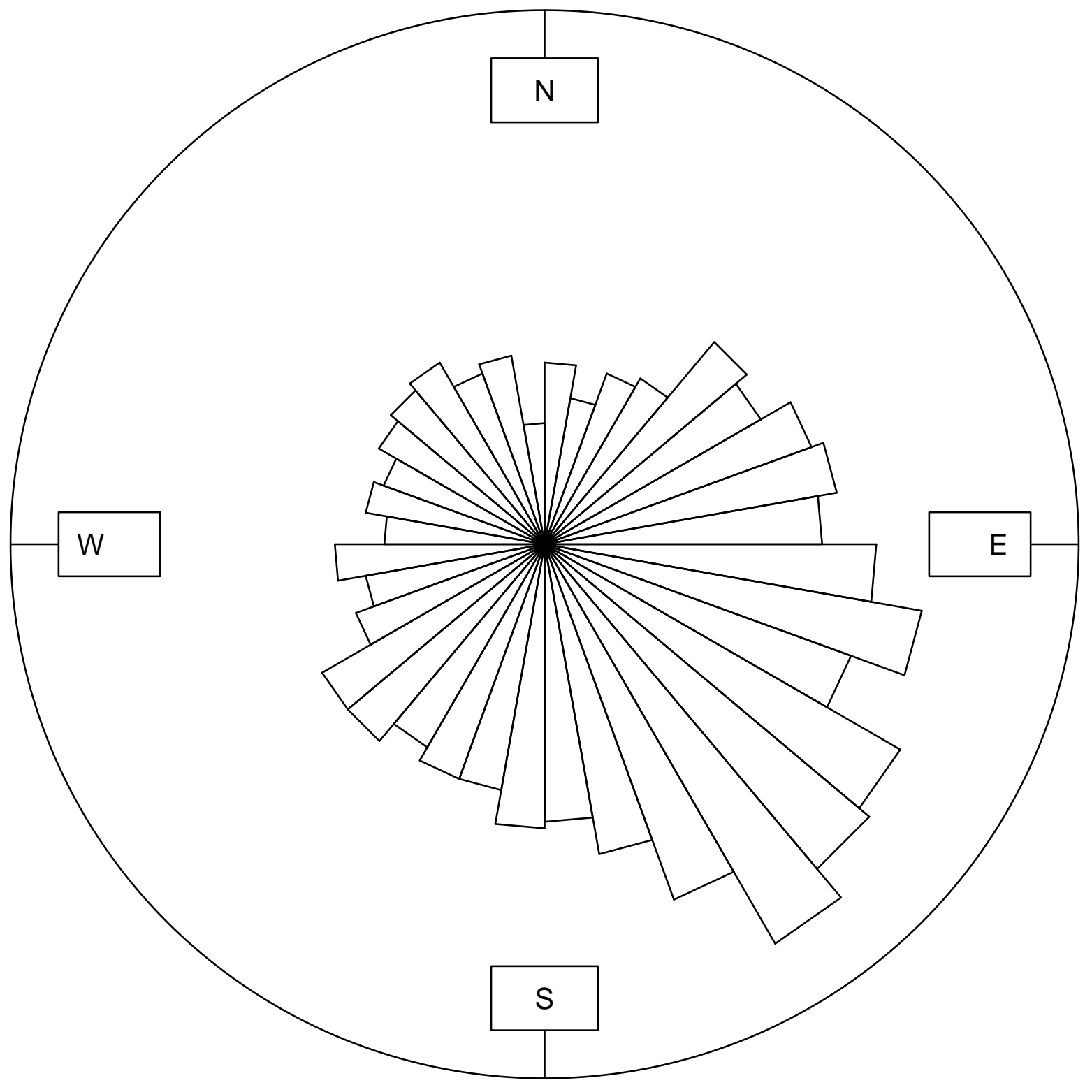


bootstrap 141

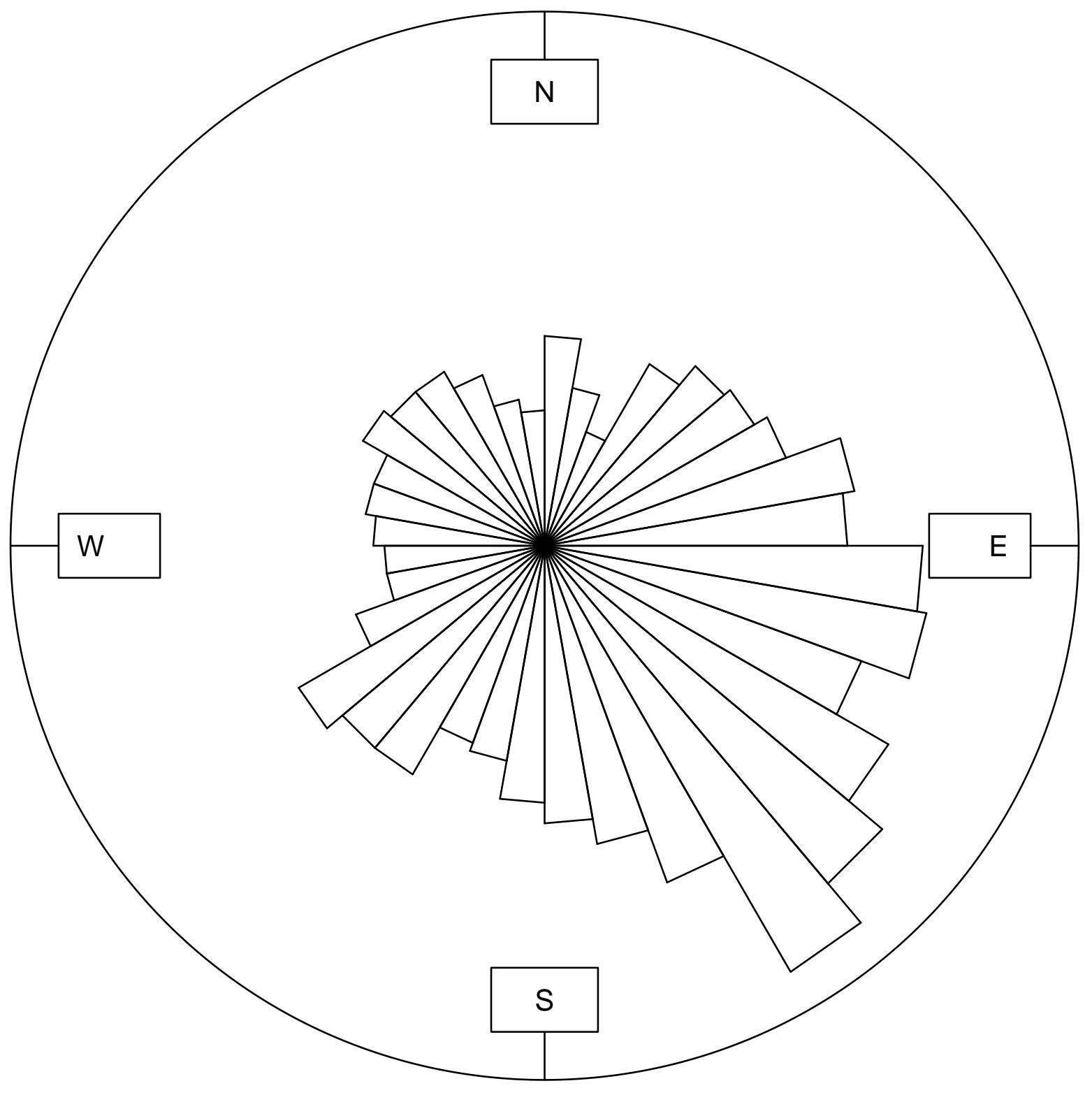


bootstrap 142

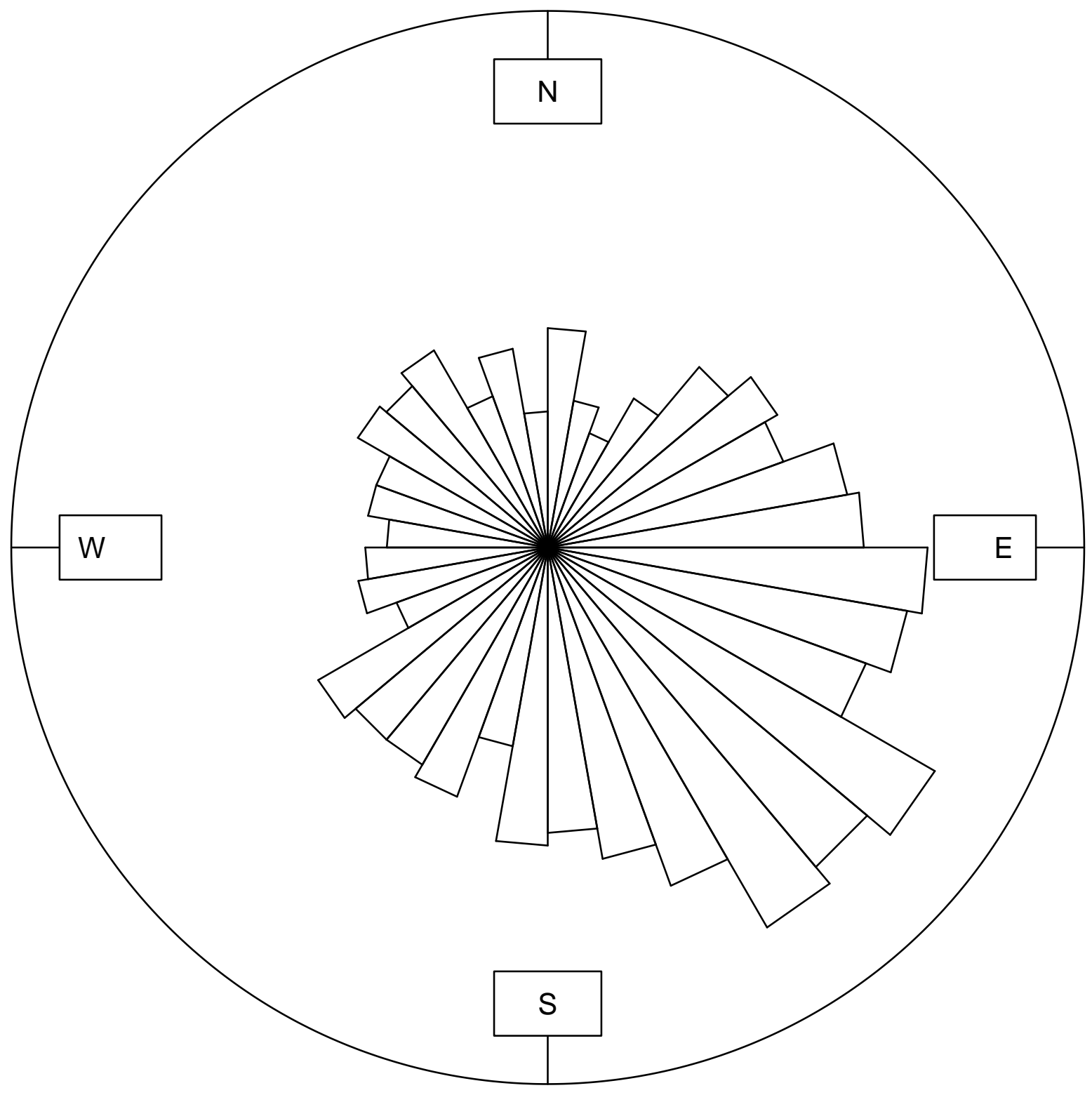




\section{bootstrap 143}

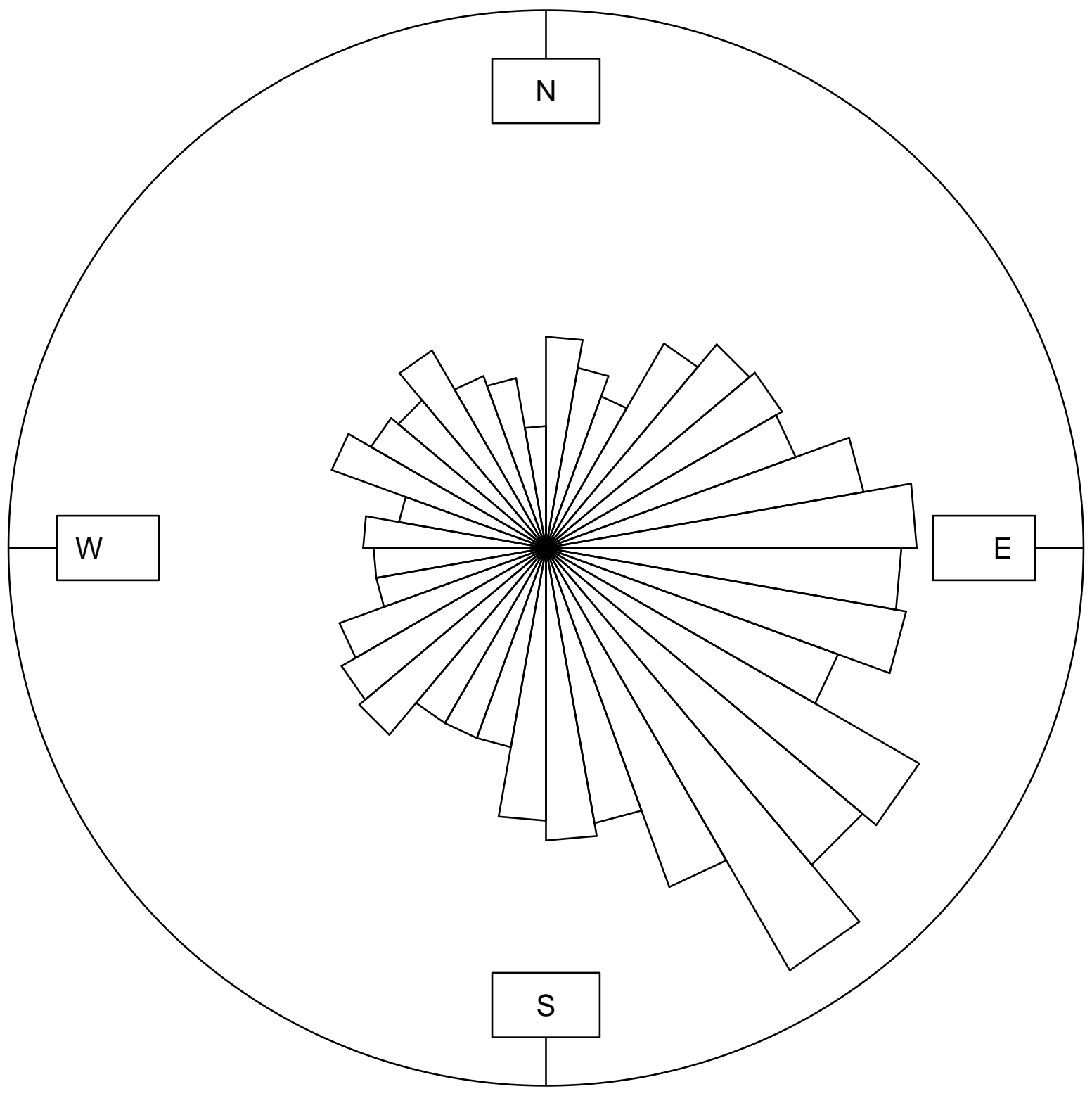


bootstrap 144

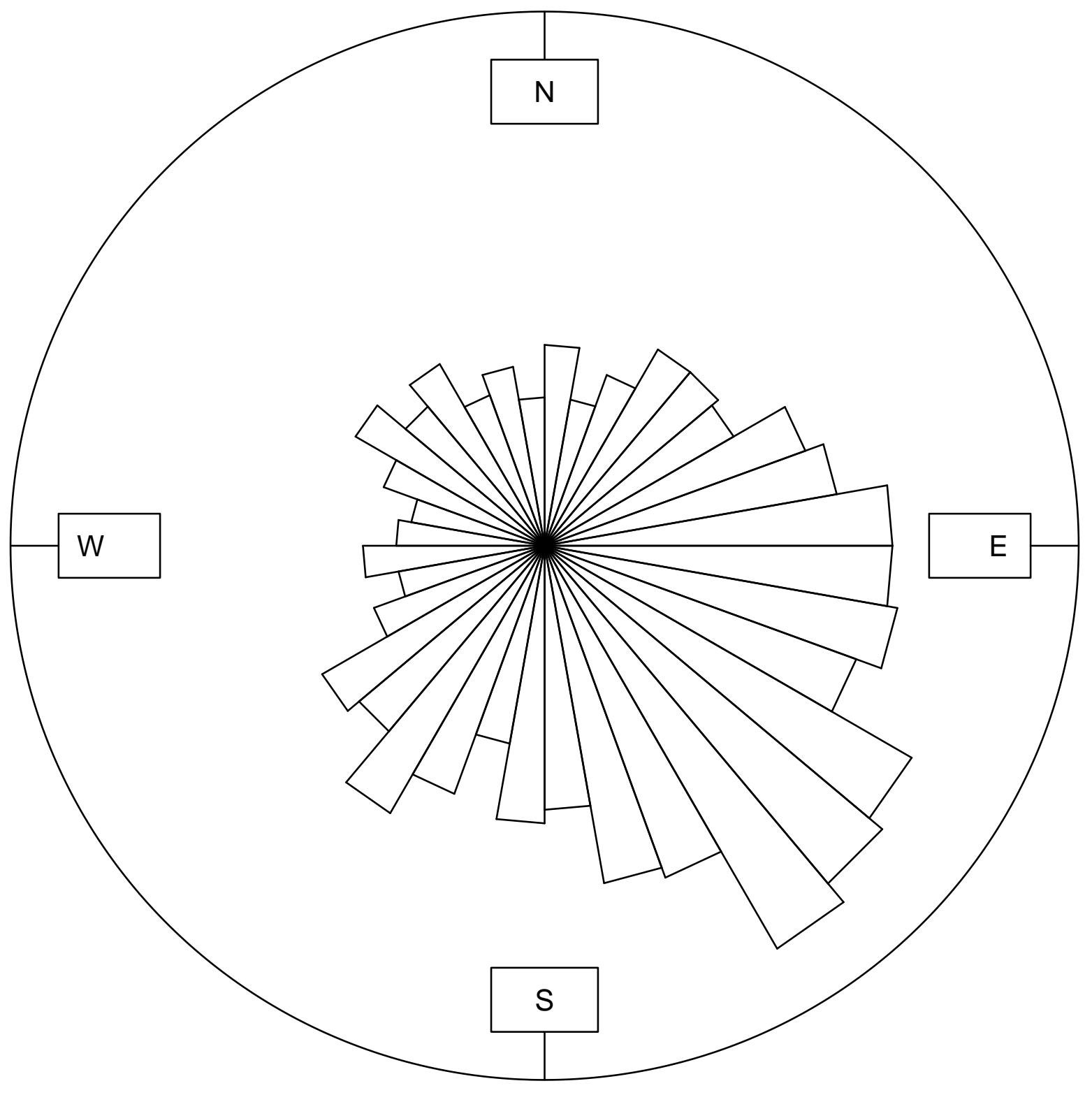


bootstrap 145

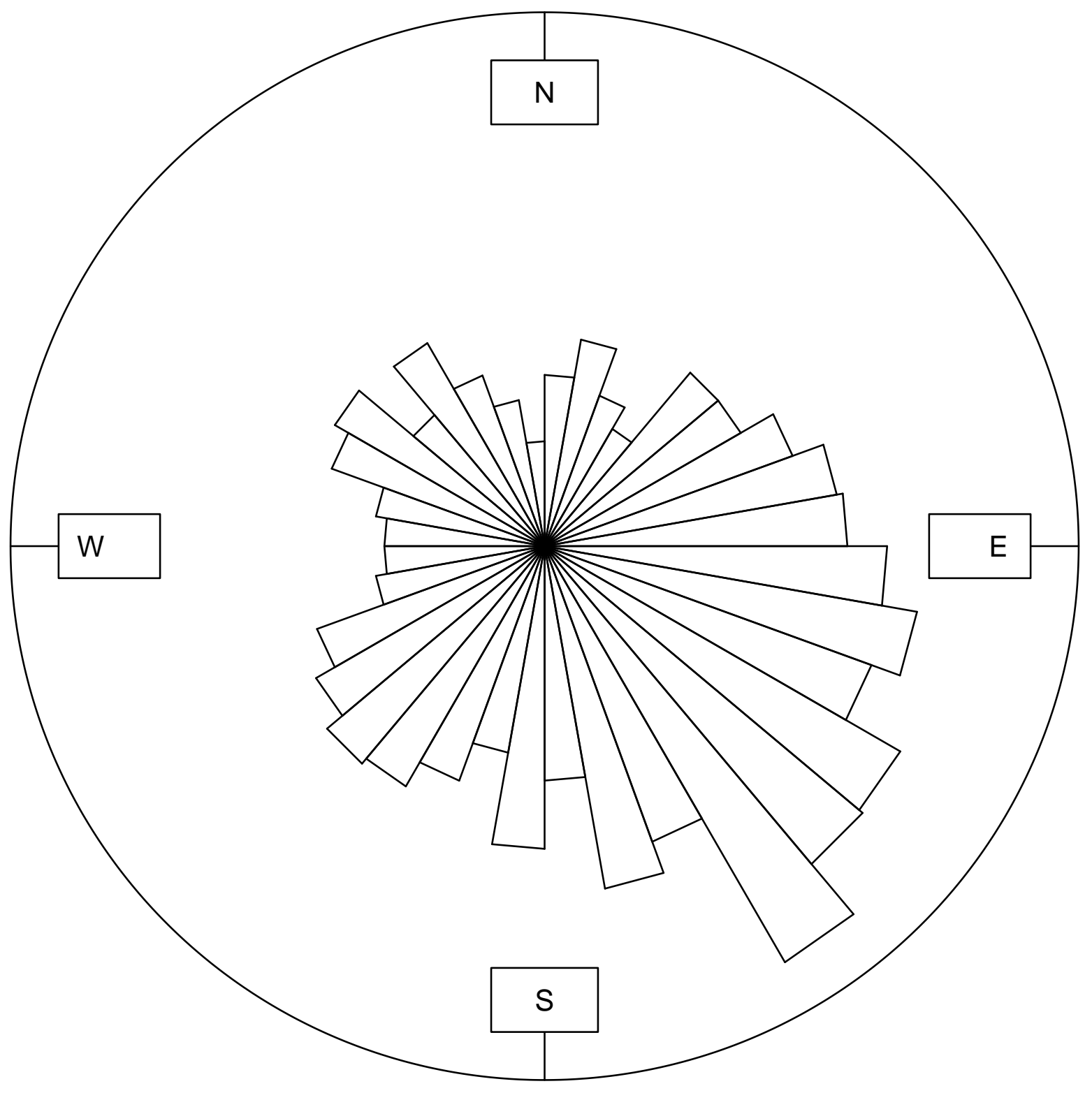


bootstrap 146

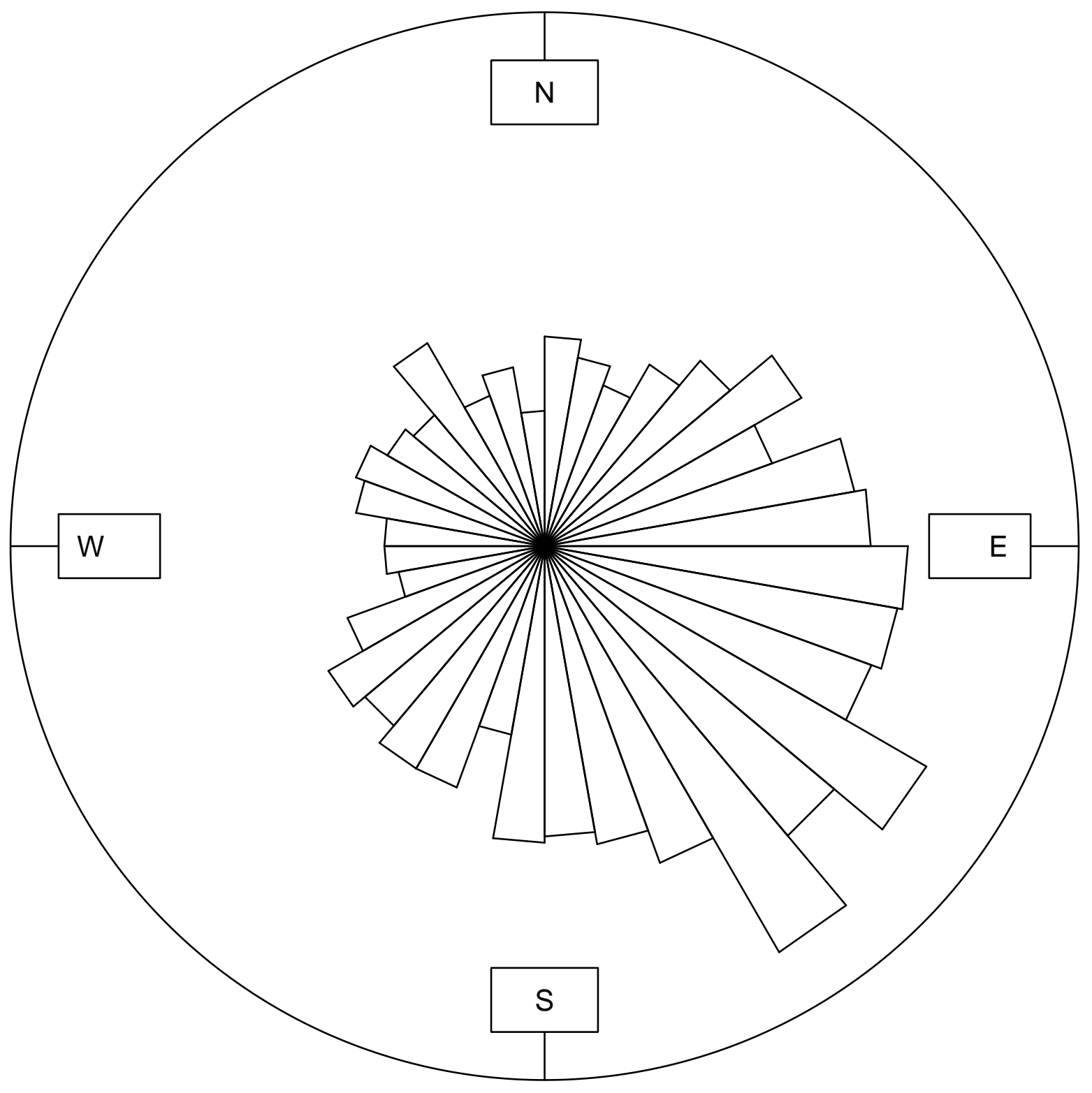


bootstrap 147

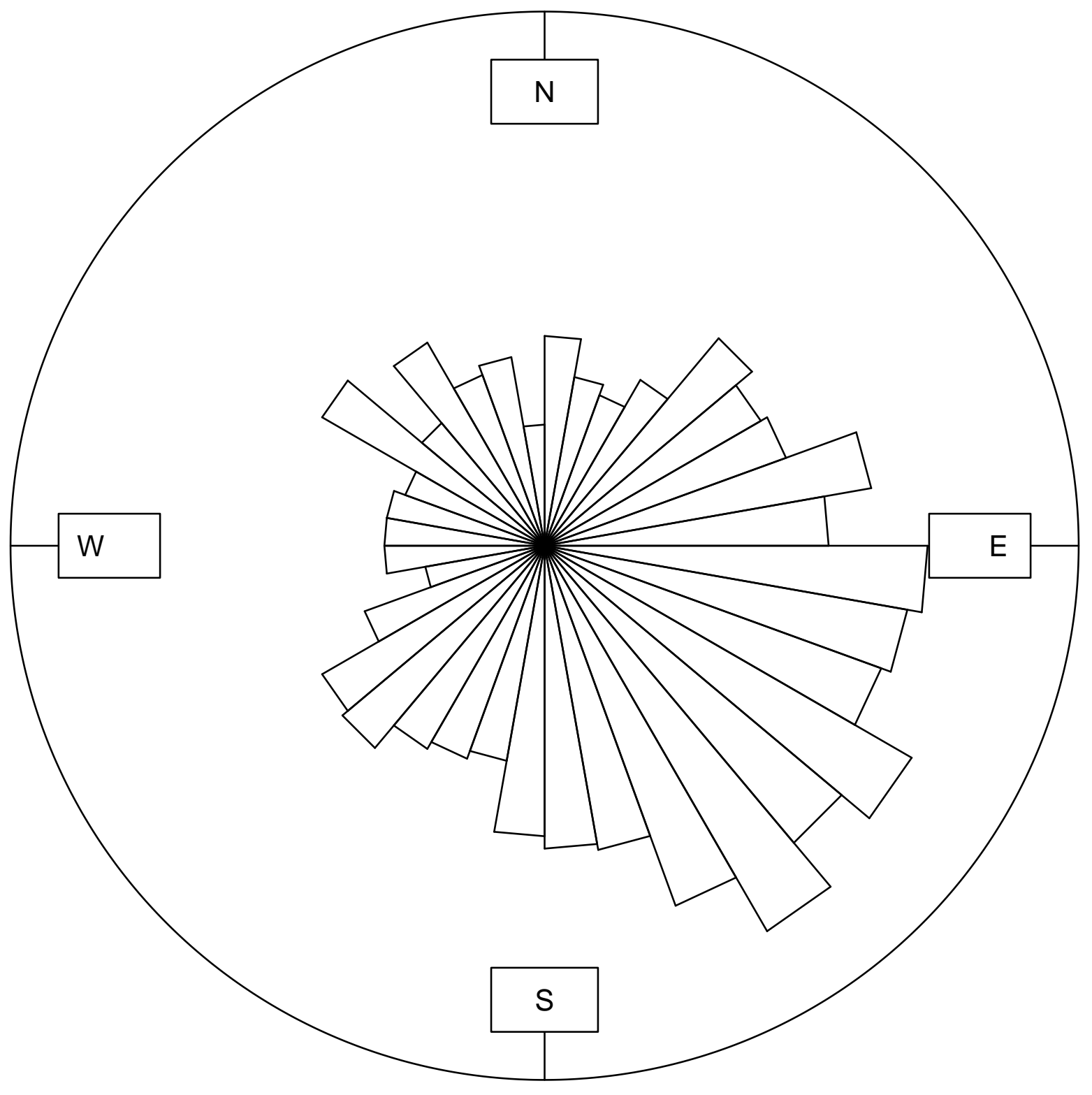


bootstrap 148

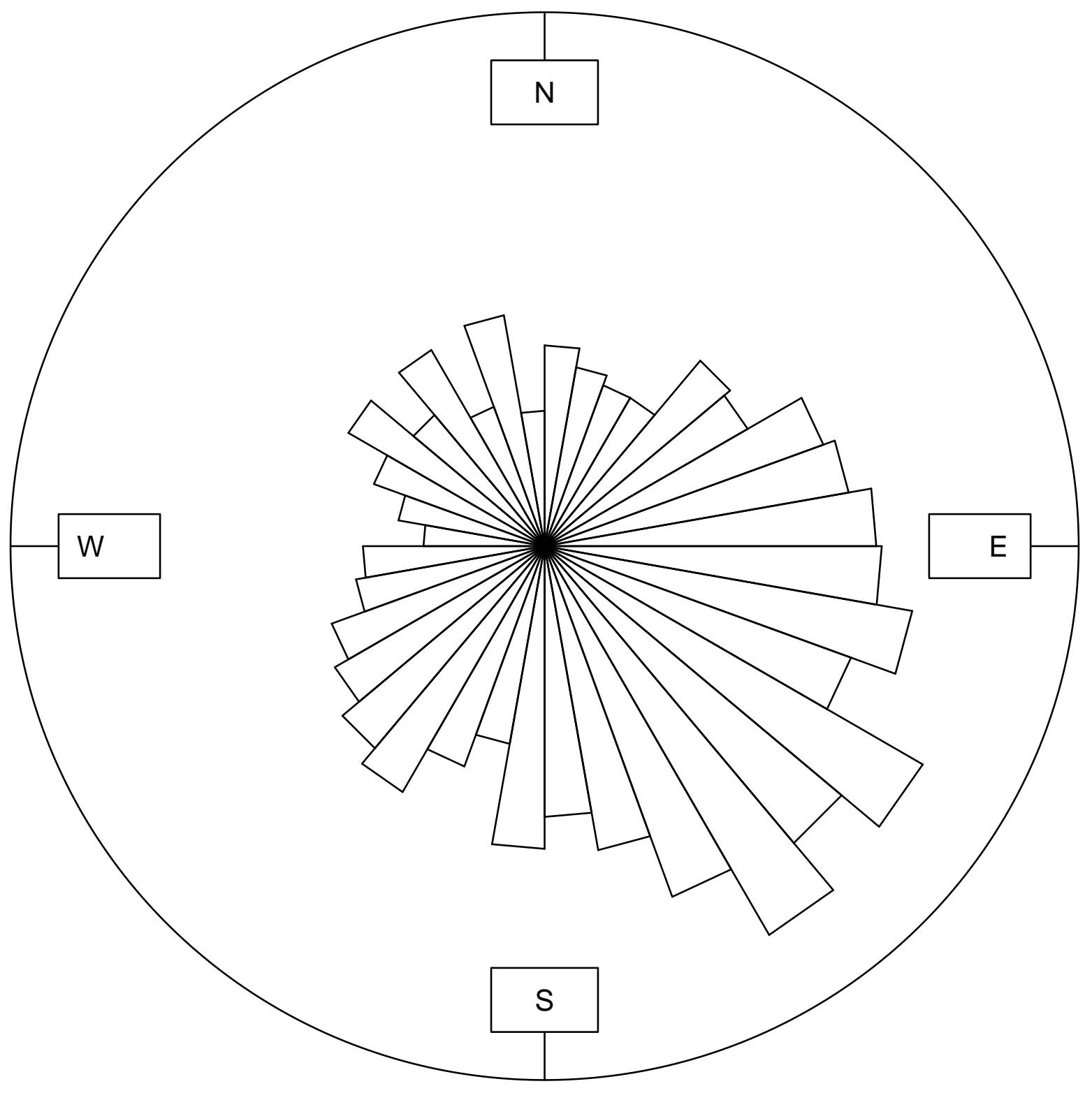


bootstrap 149

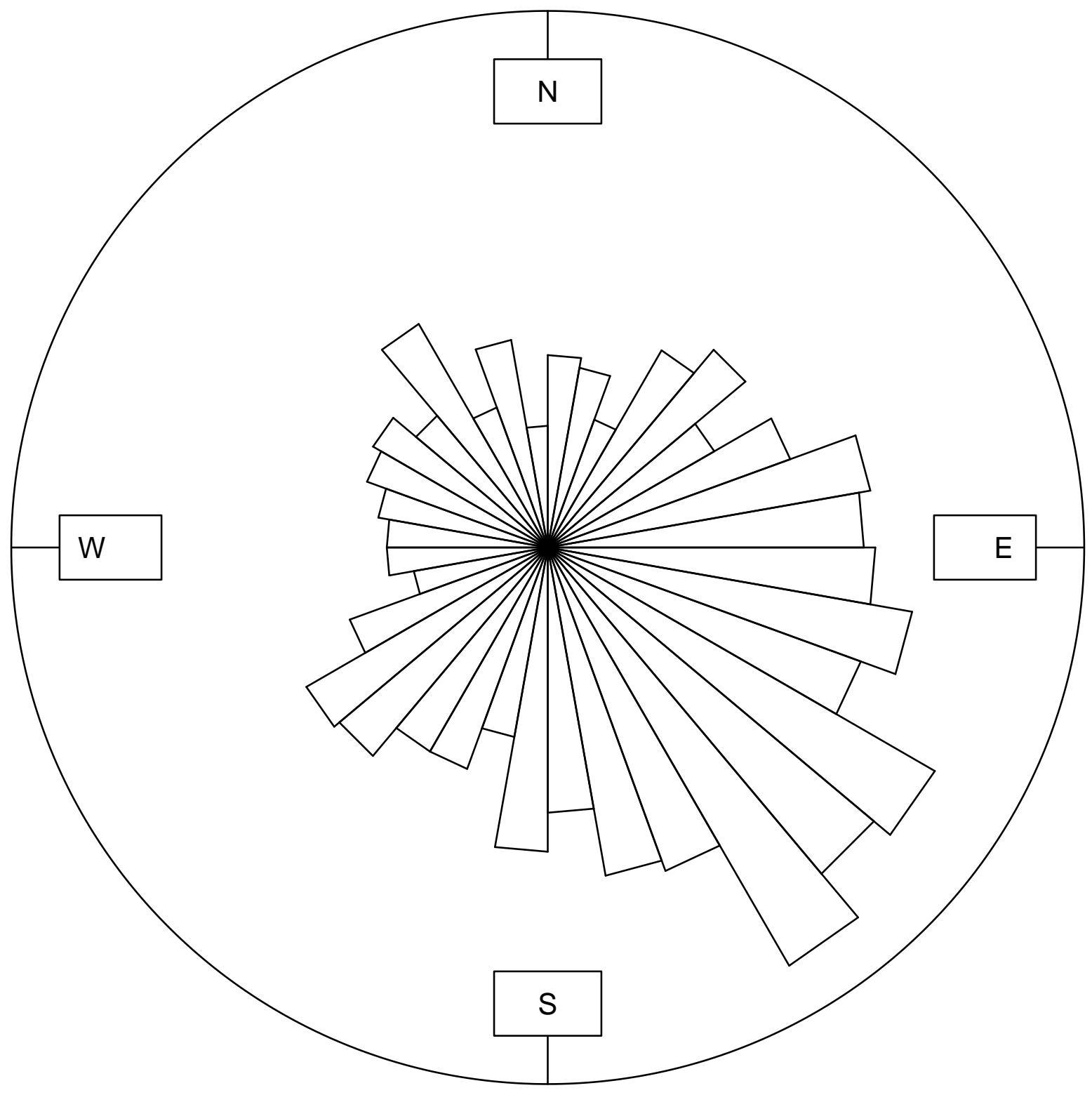


bootstrap 150

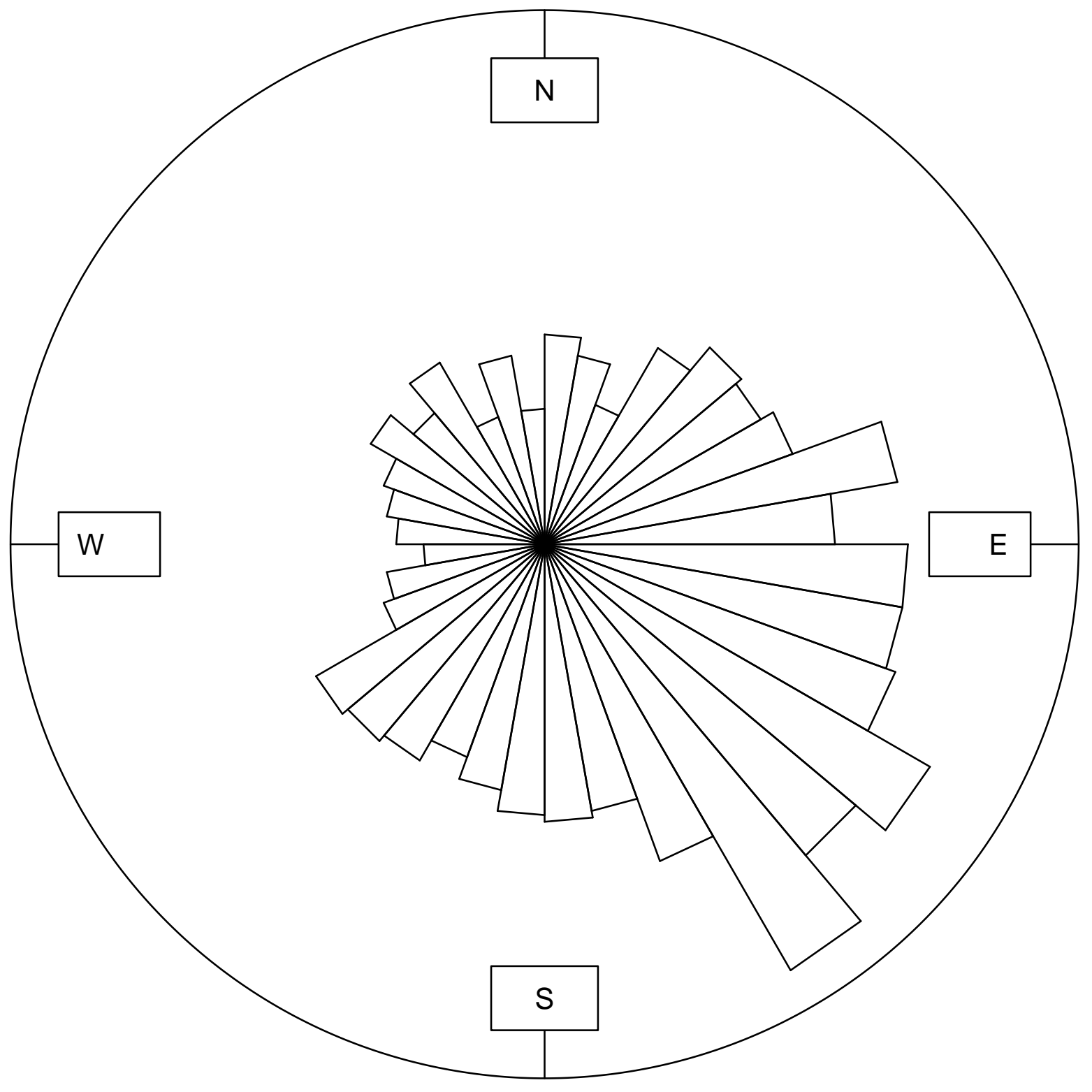


bootstrap 151

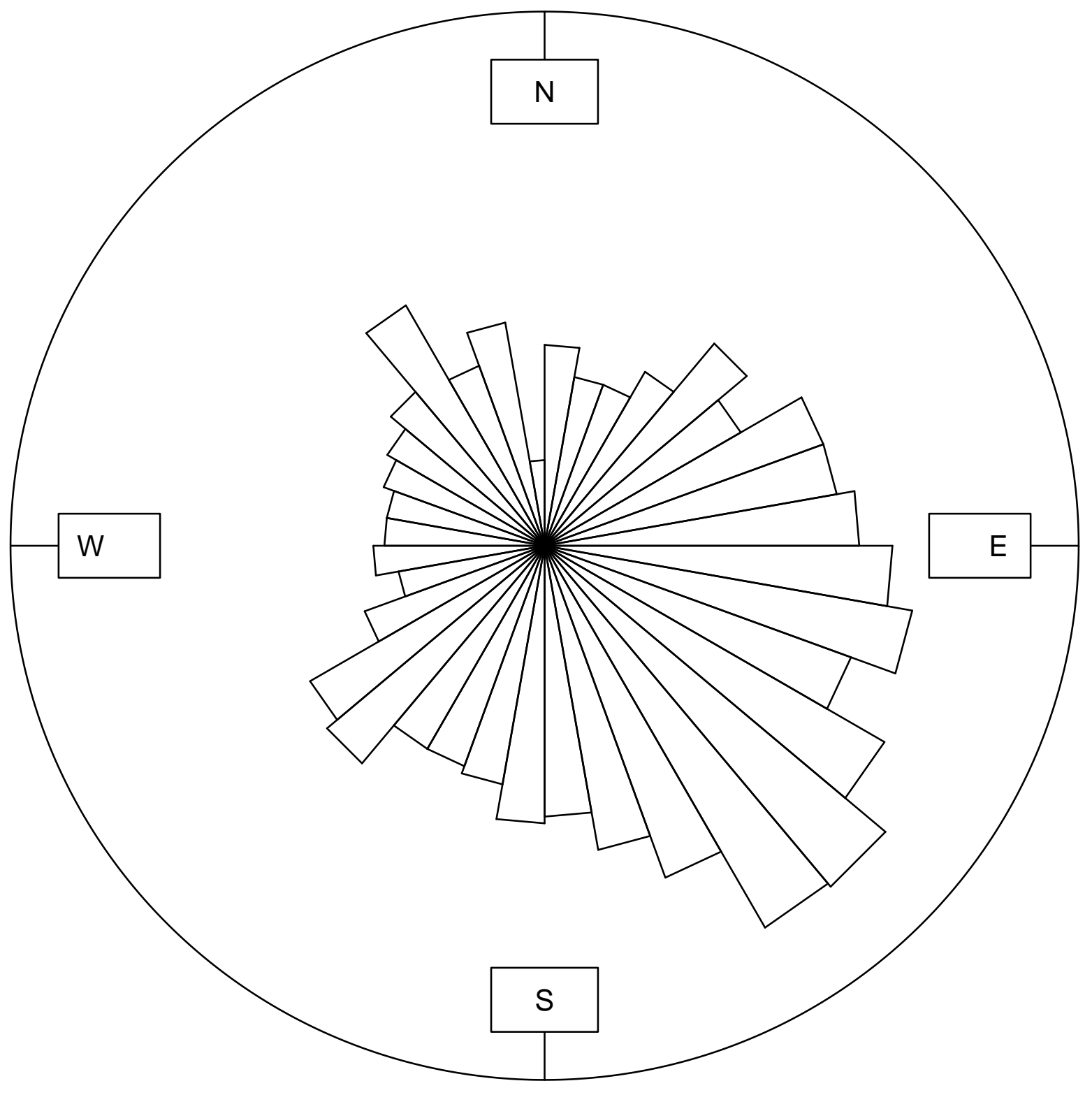


bootstrap 152

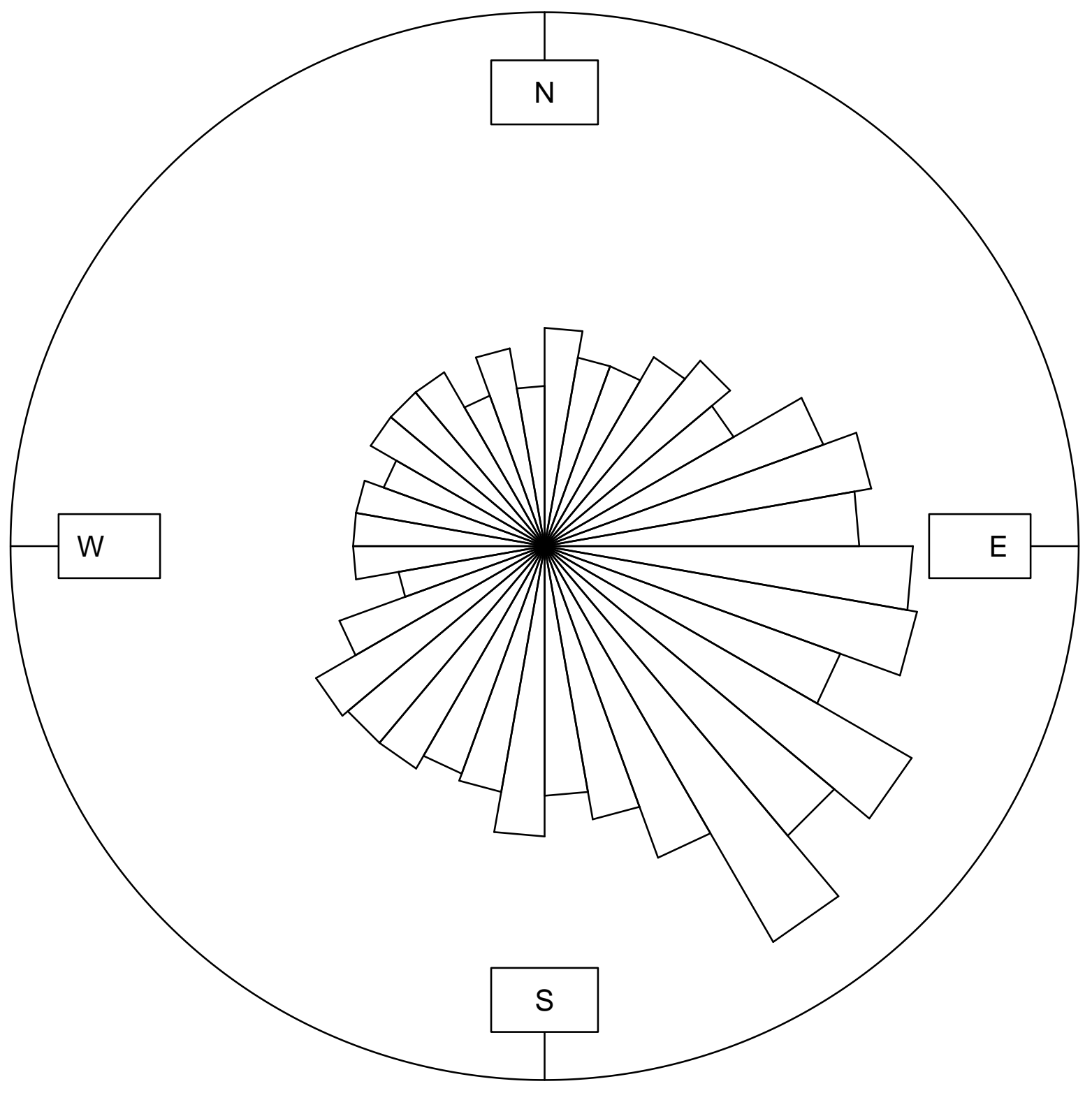




\section{bootstrap 153}

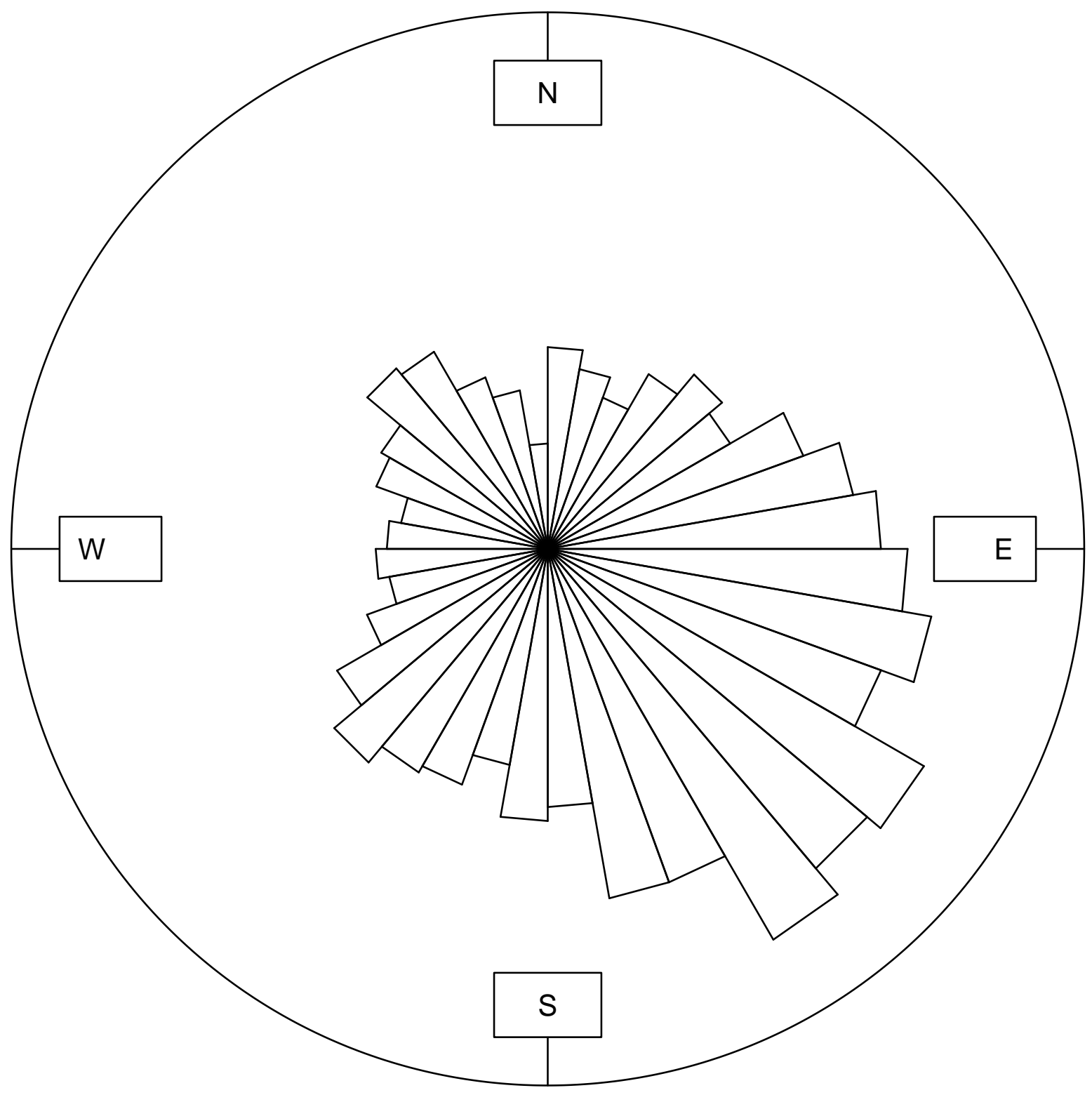




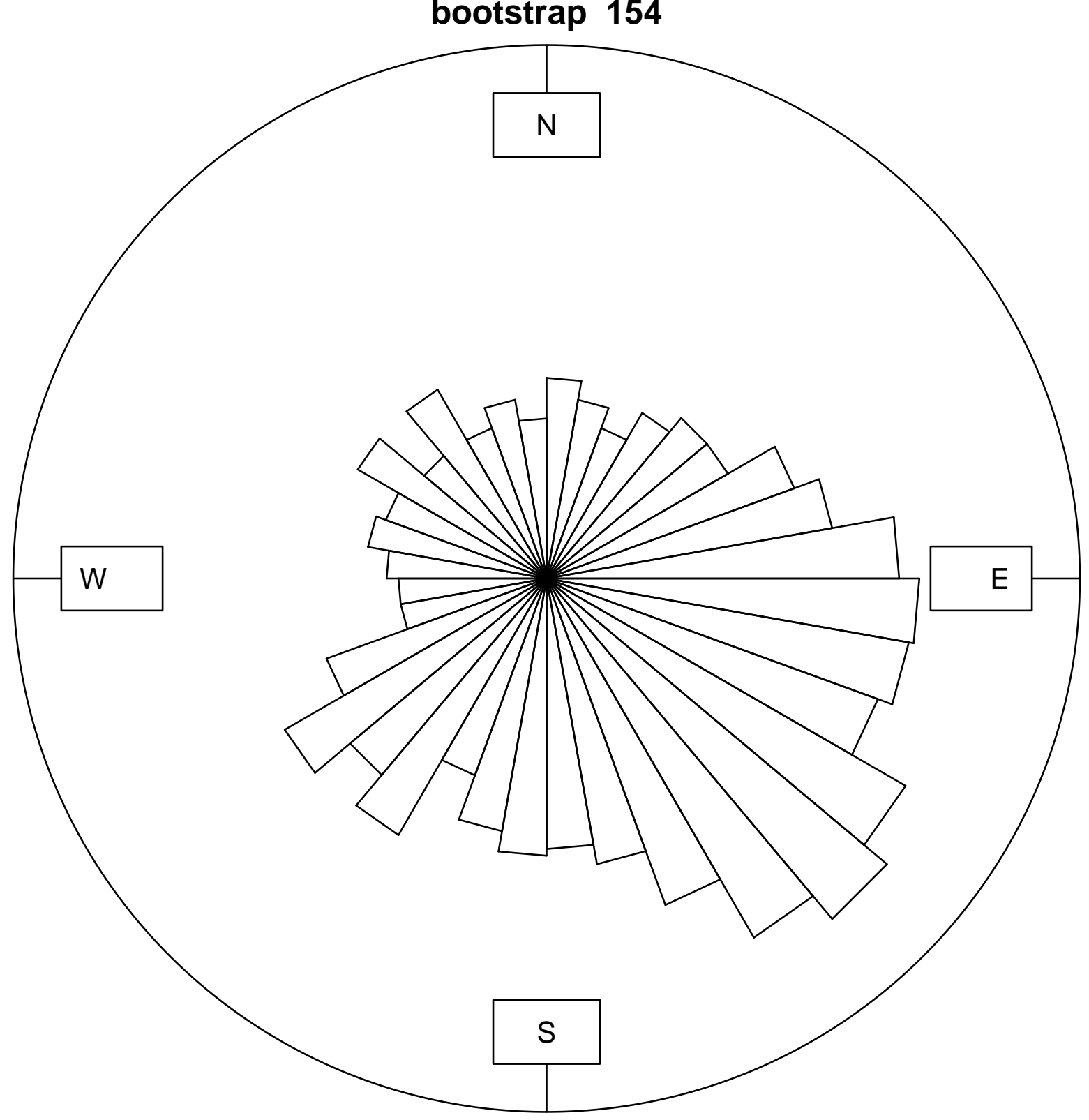




\section{bootstrap 155}

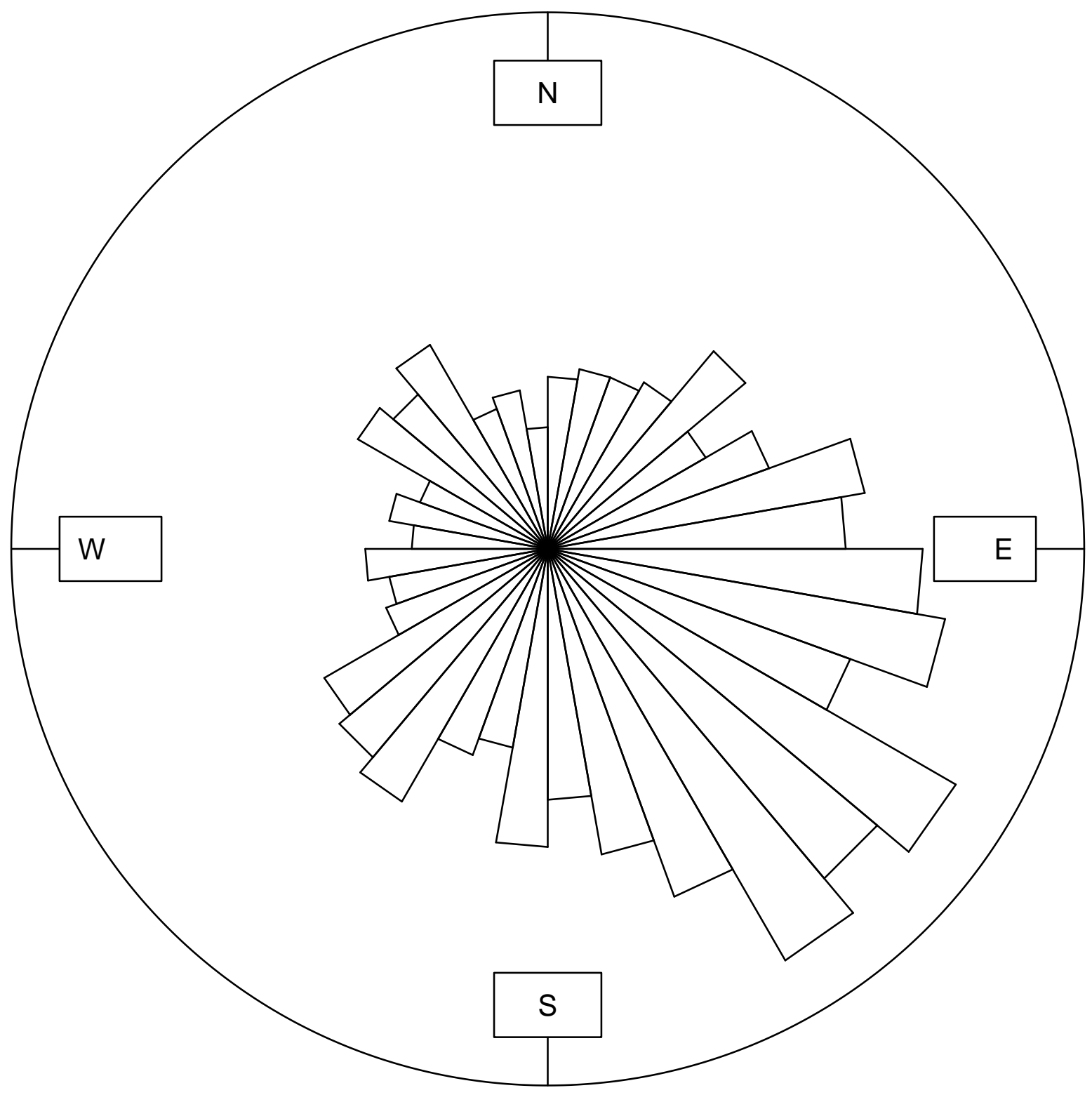




\section{bootstrap 156}

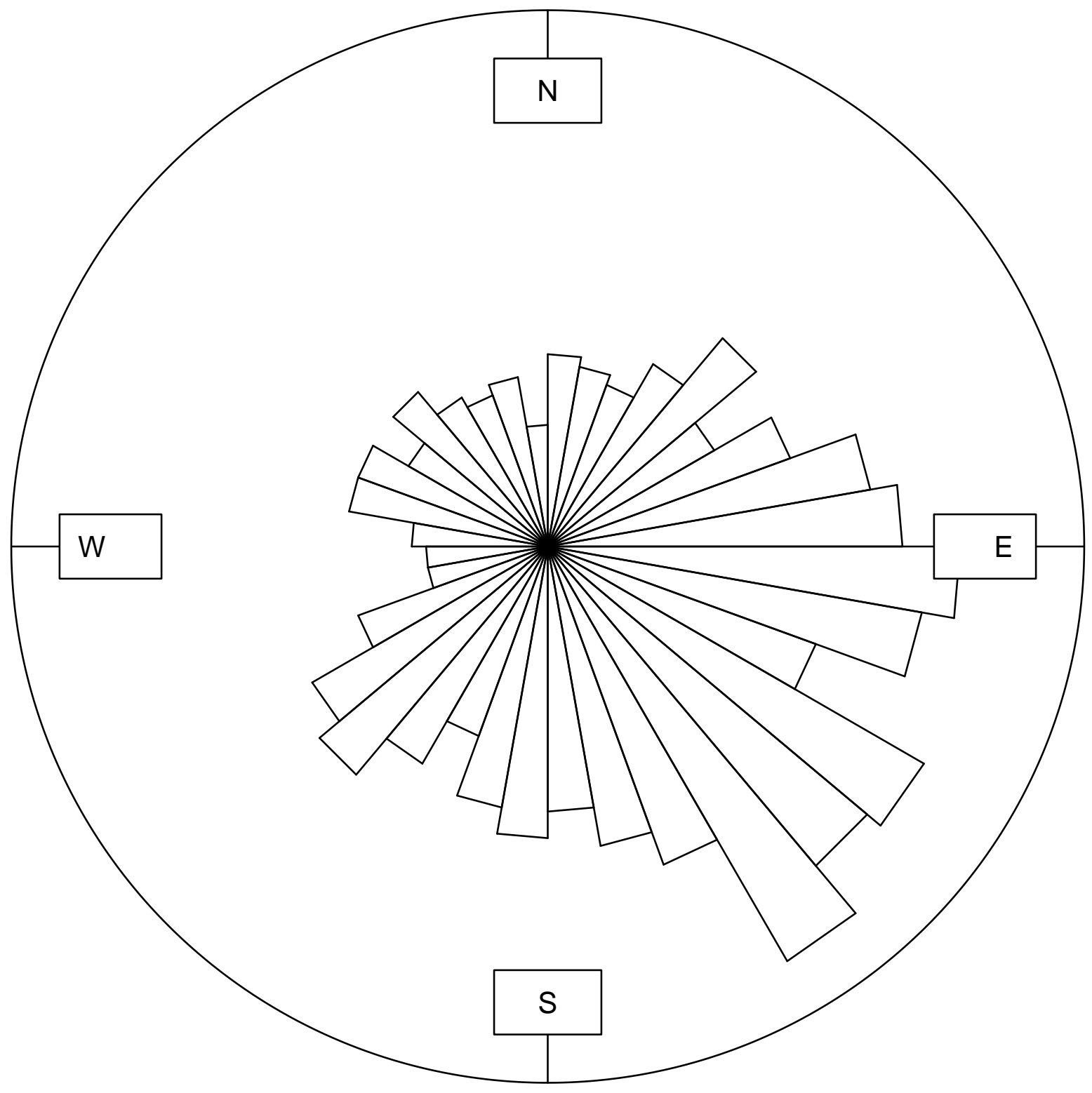


bootstrap 157

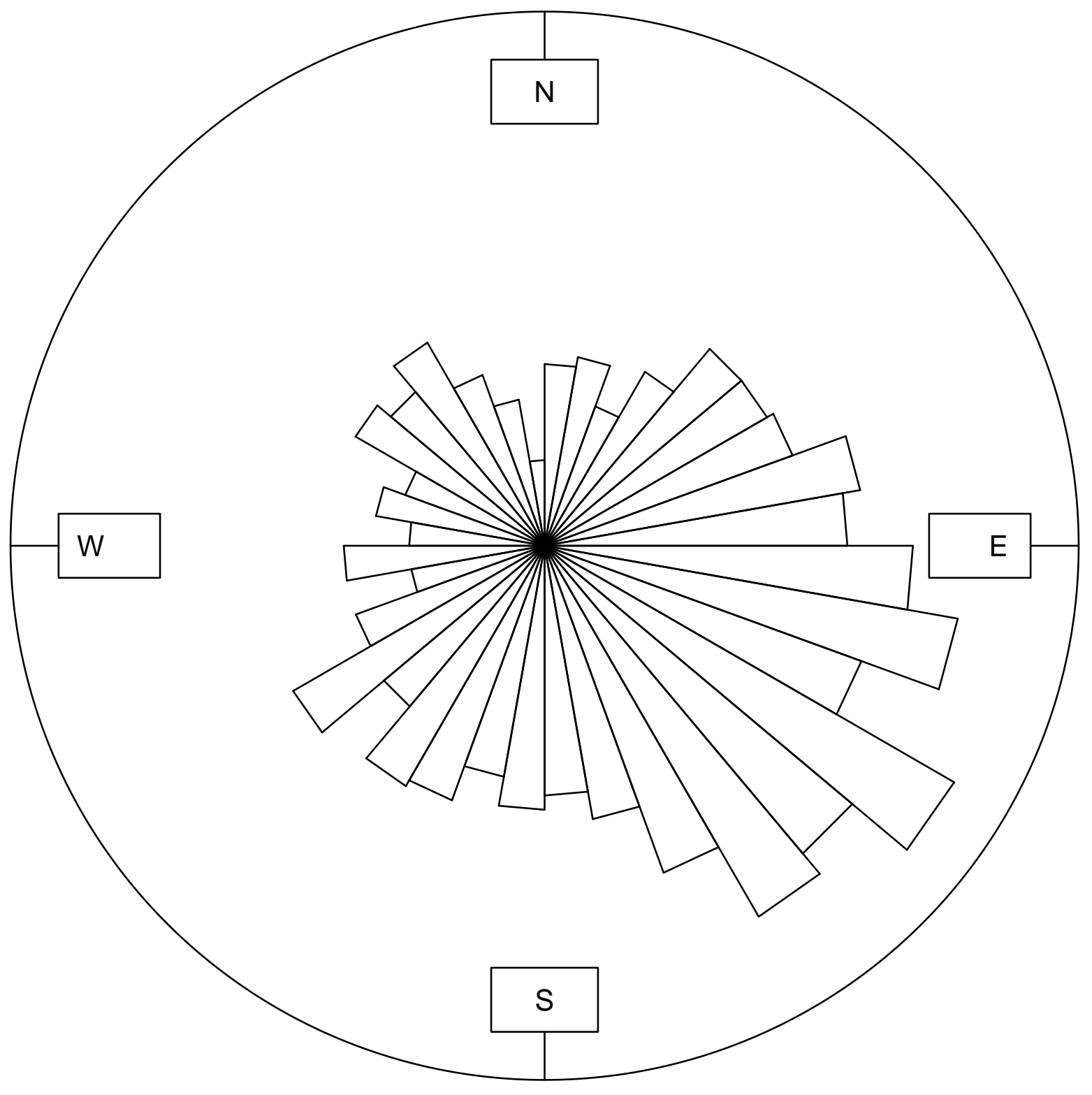


bootstrap 158

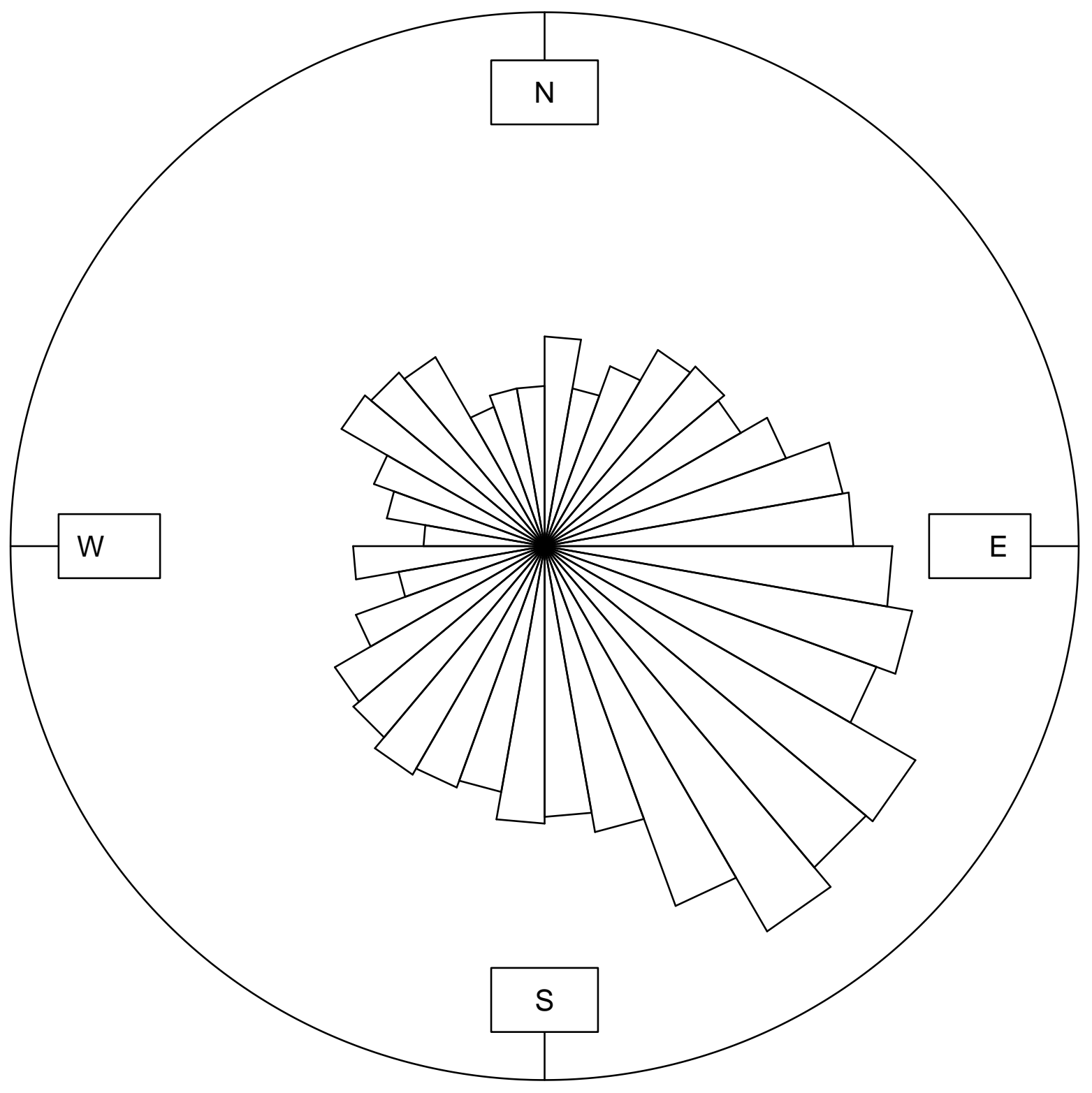


bootstrap 159

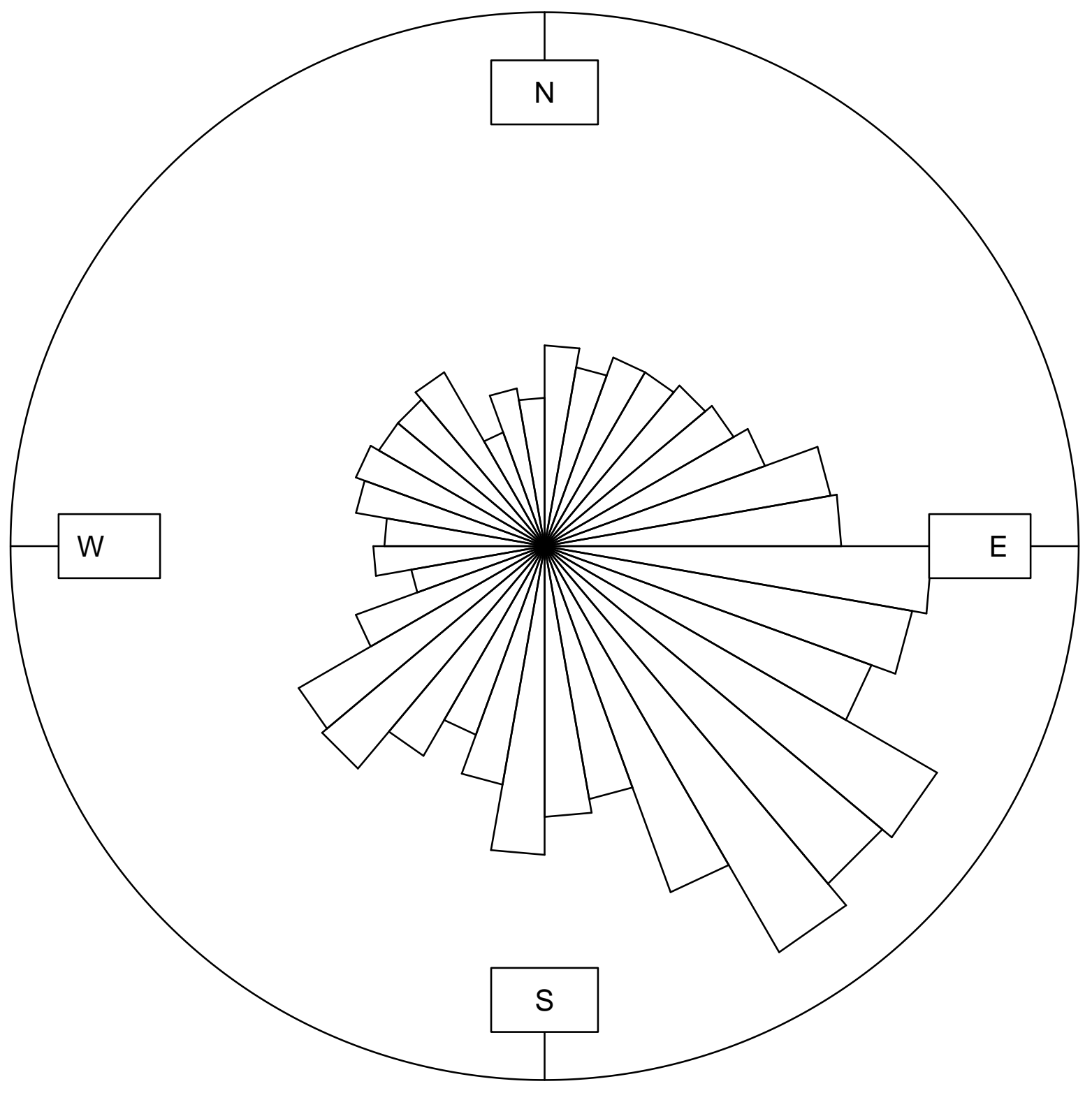


bootstrap 160

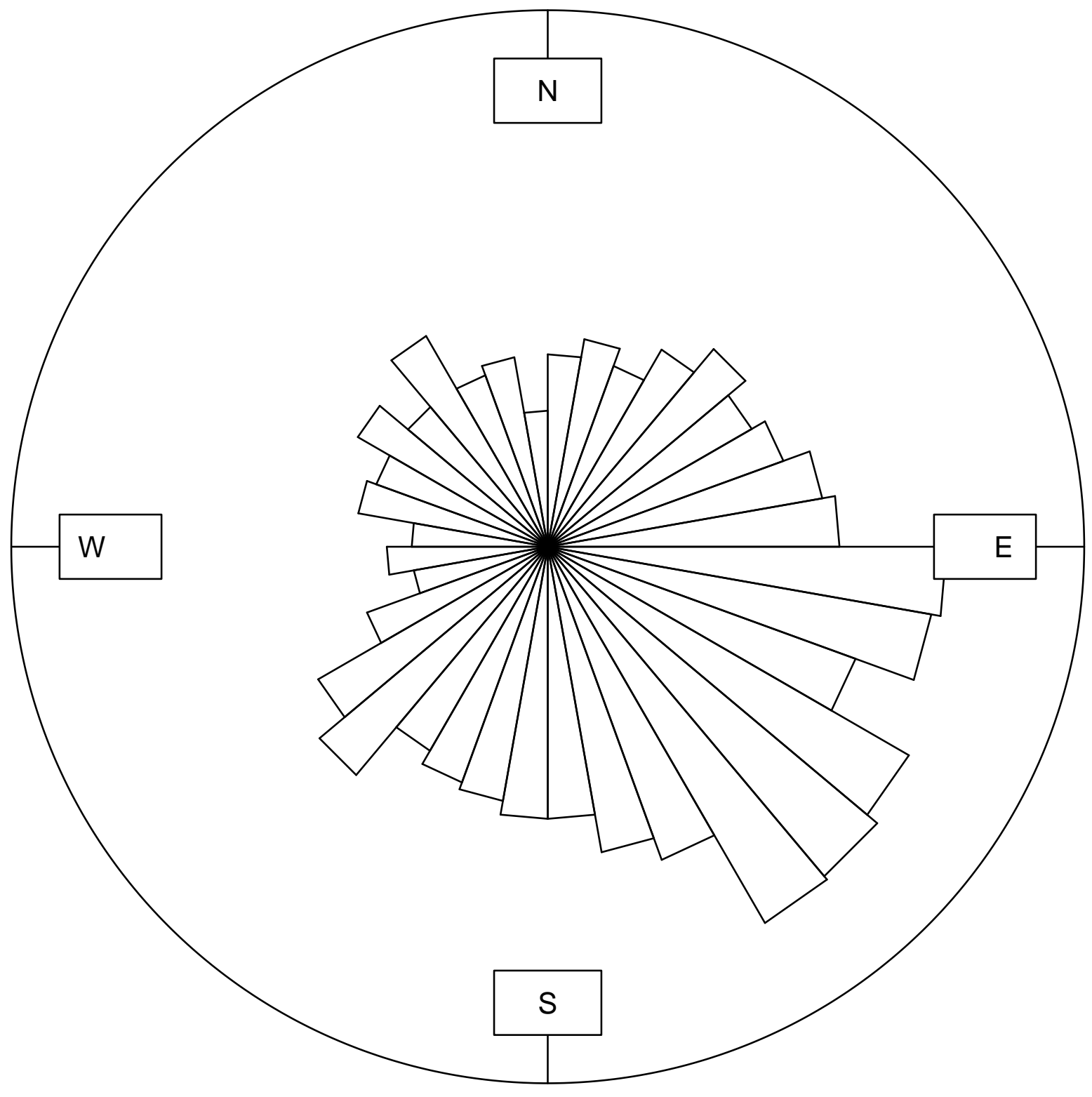


bootstrap 161

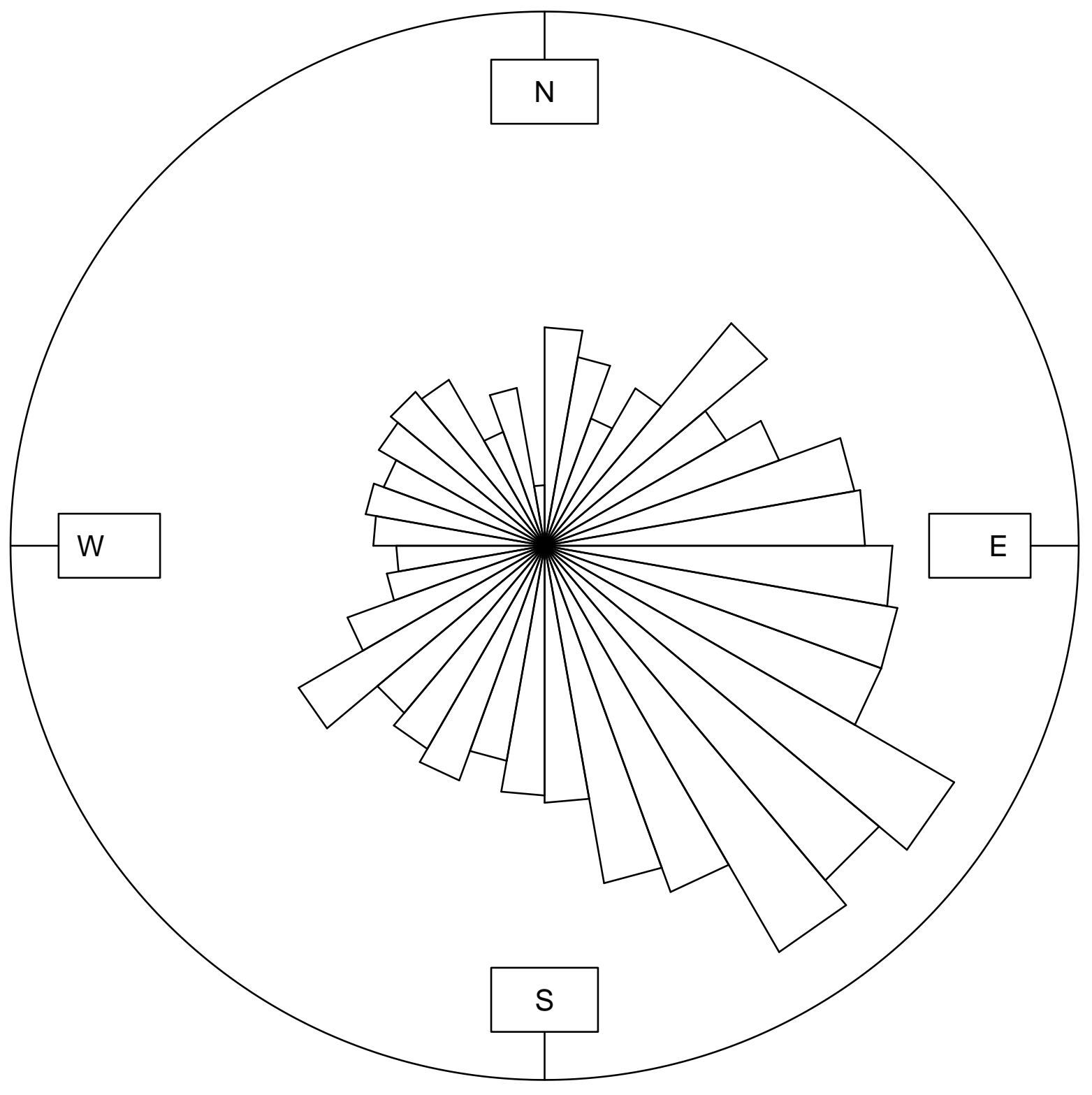


bootstrap 162

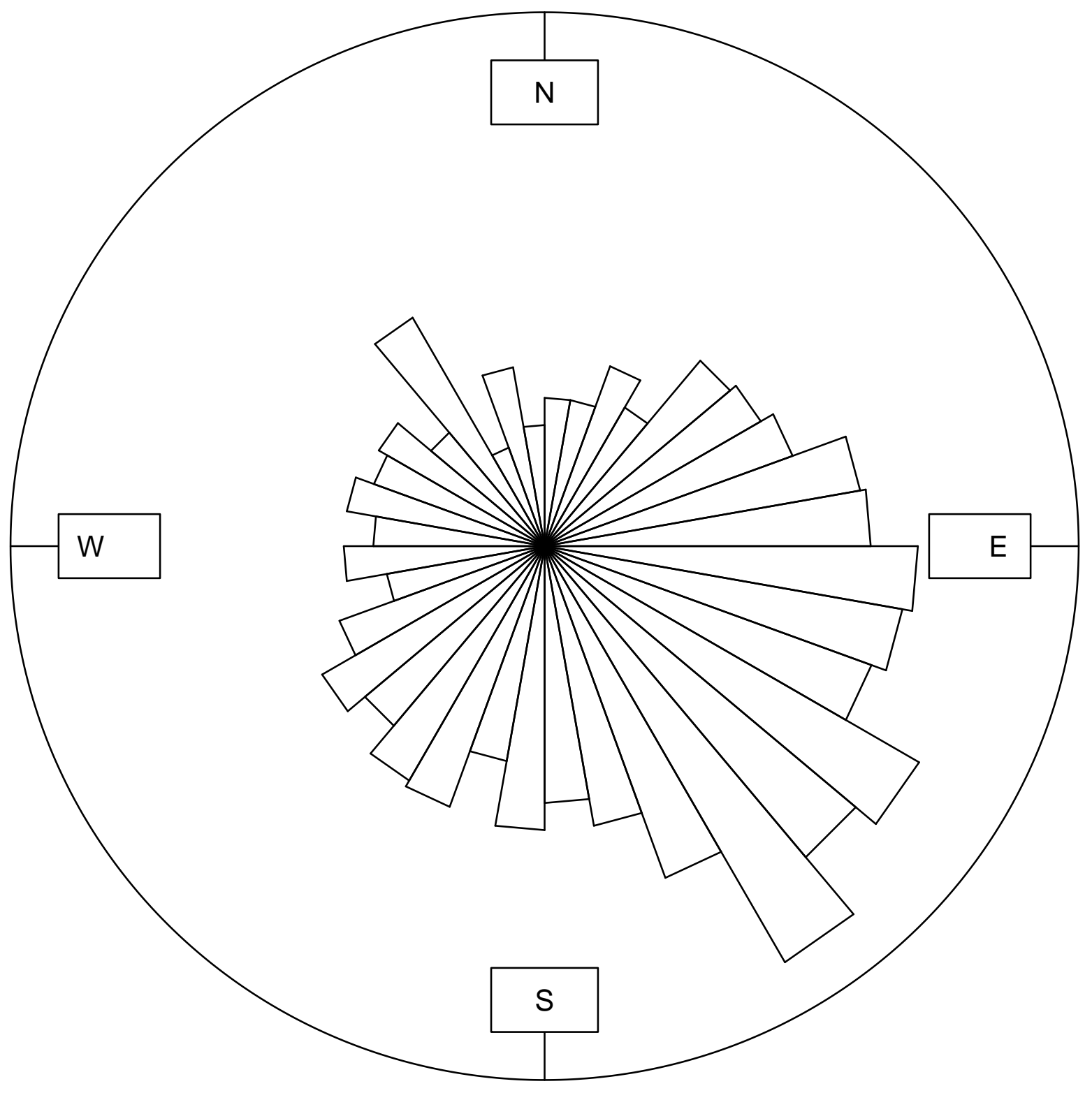


bootstrap 163

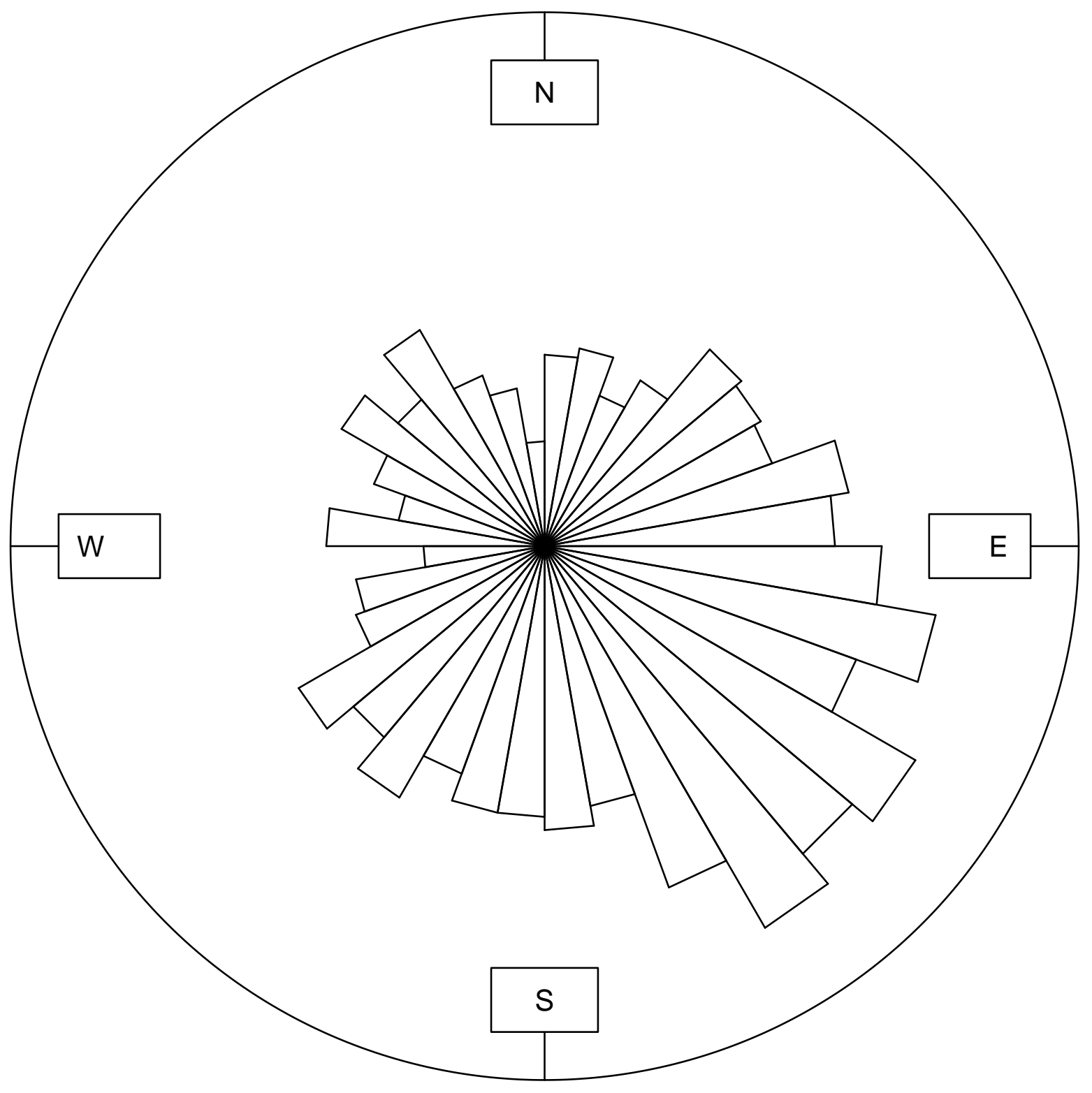




\section{bootstrap 164}

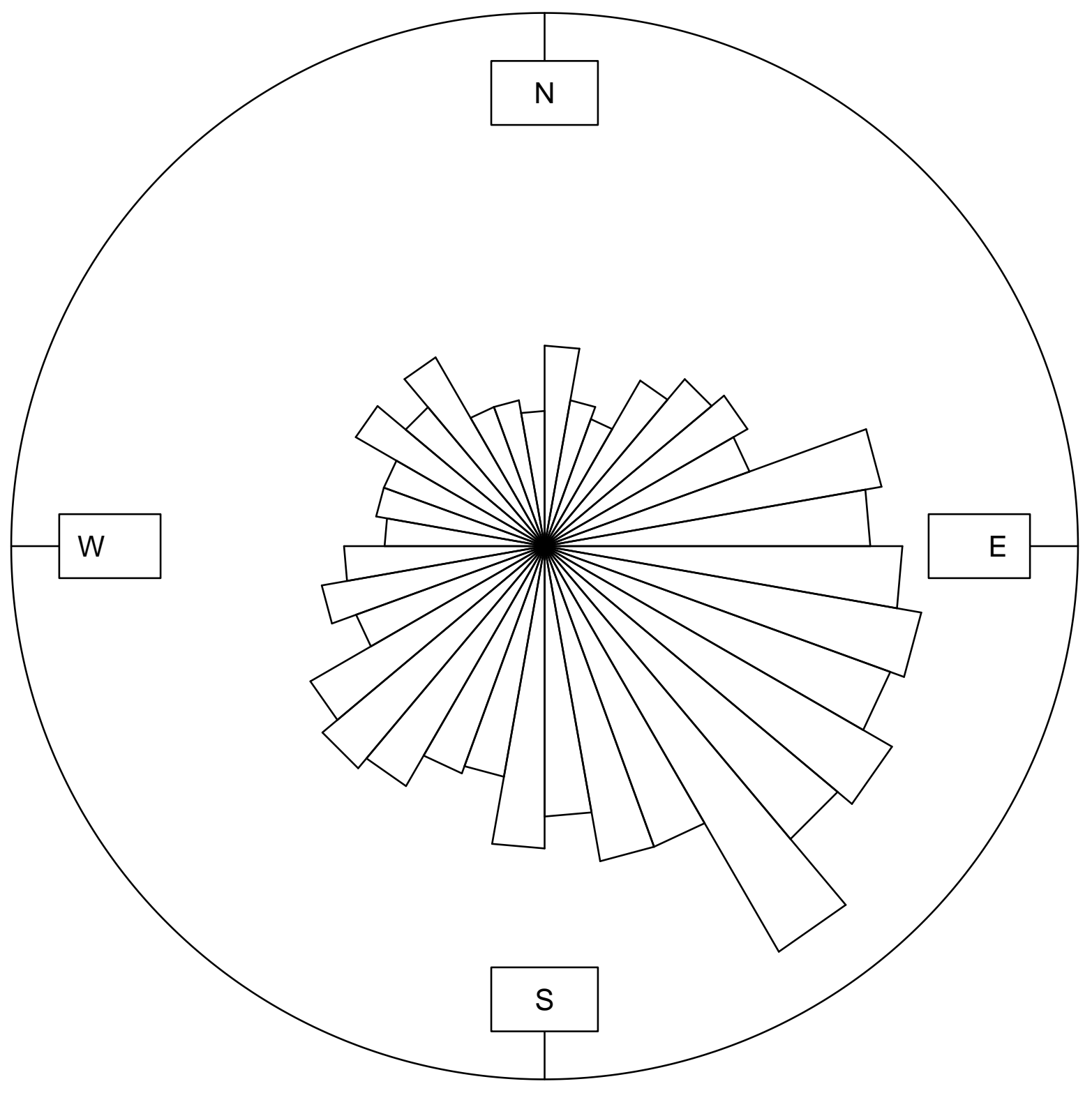


bootstrap 165

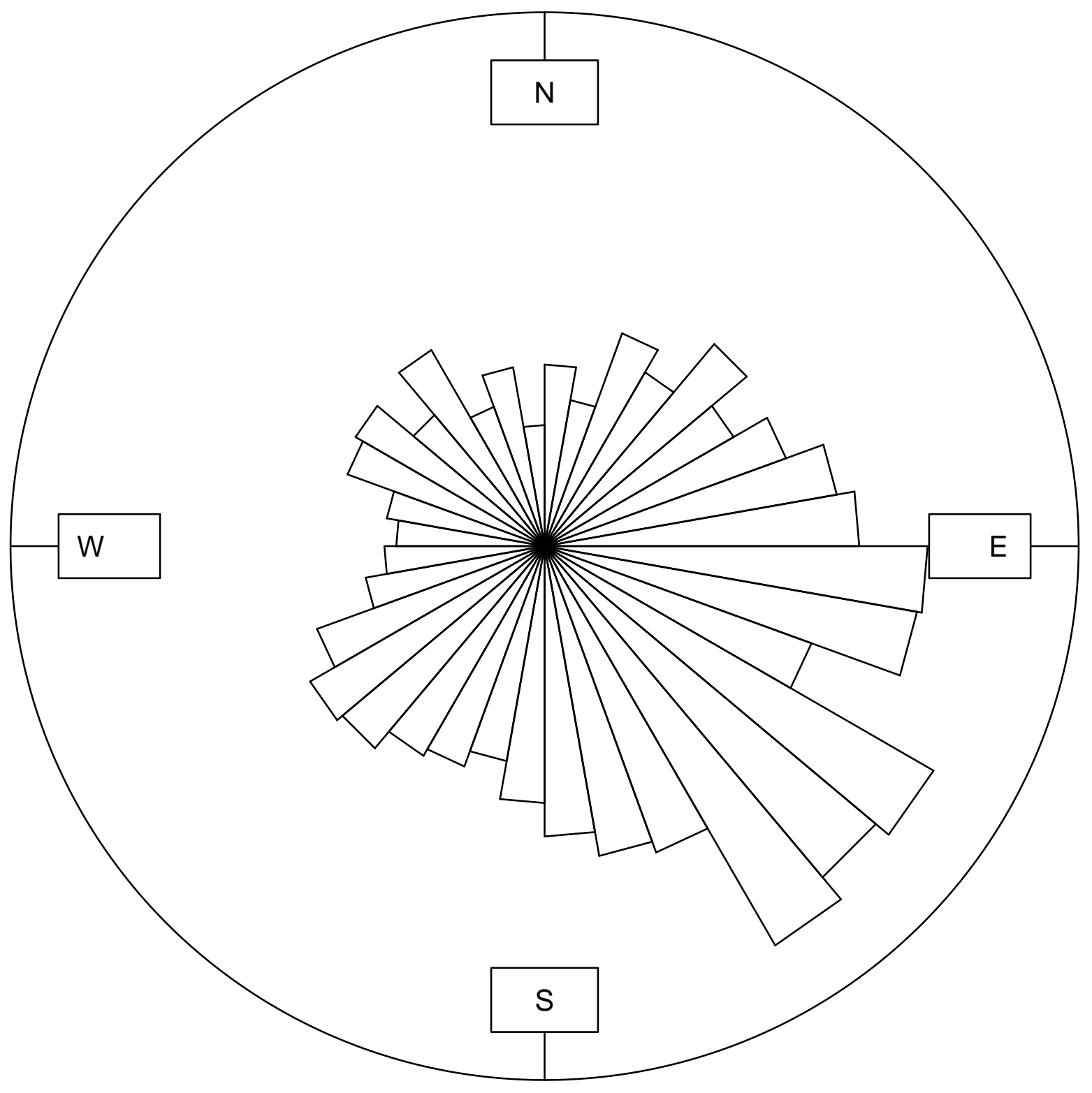


bootstrap 166

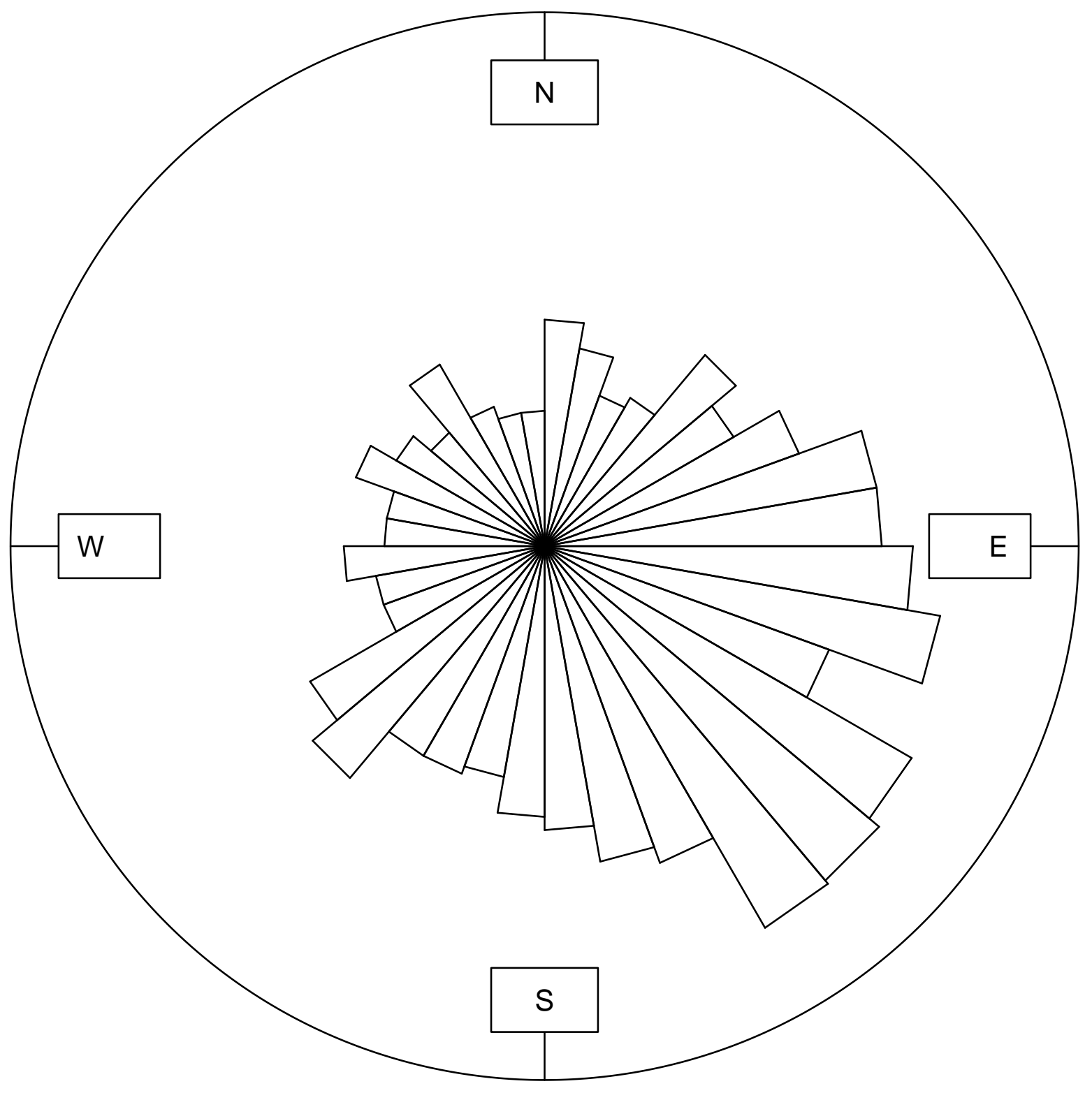


bootstrap 167

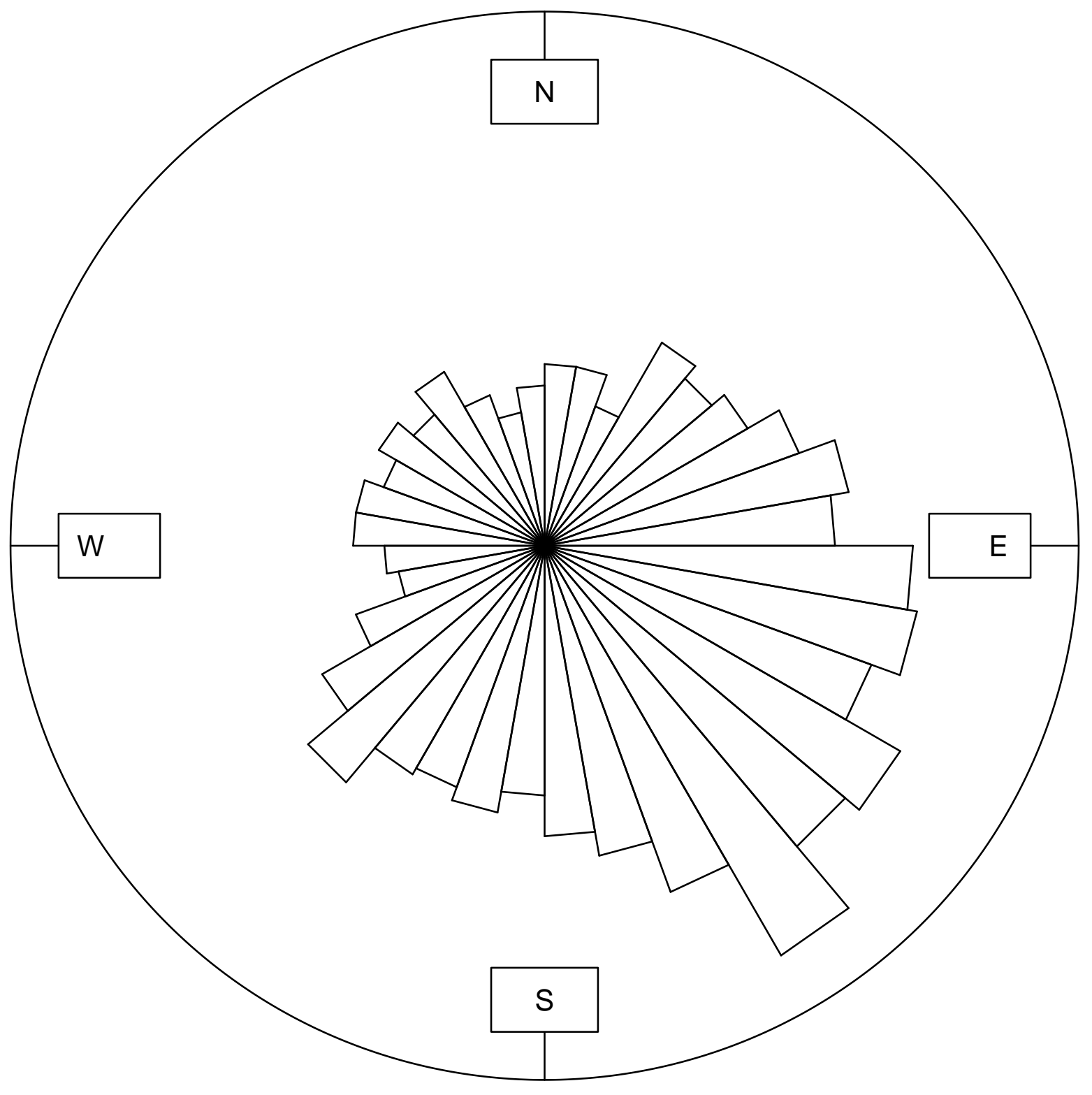


bootstrap 168

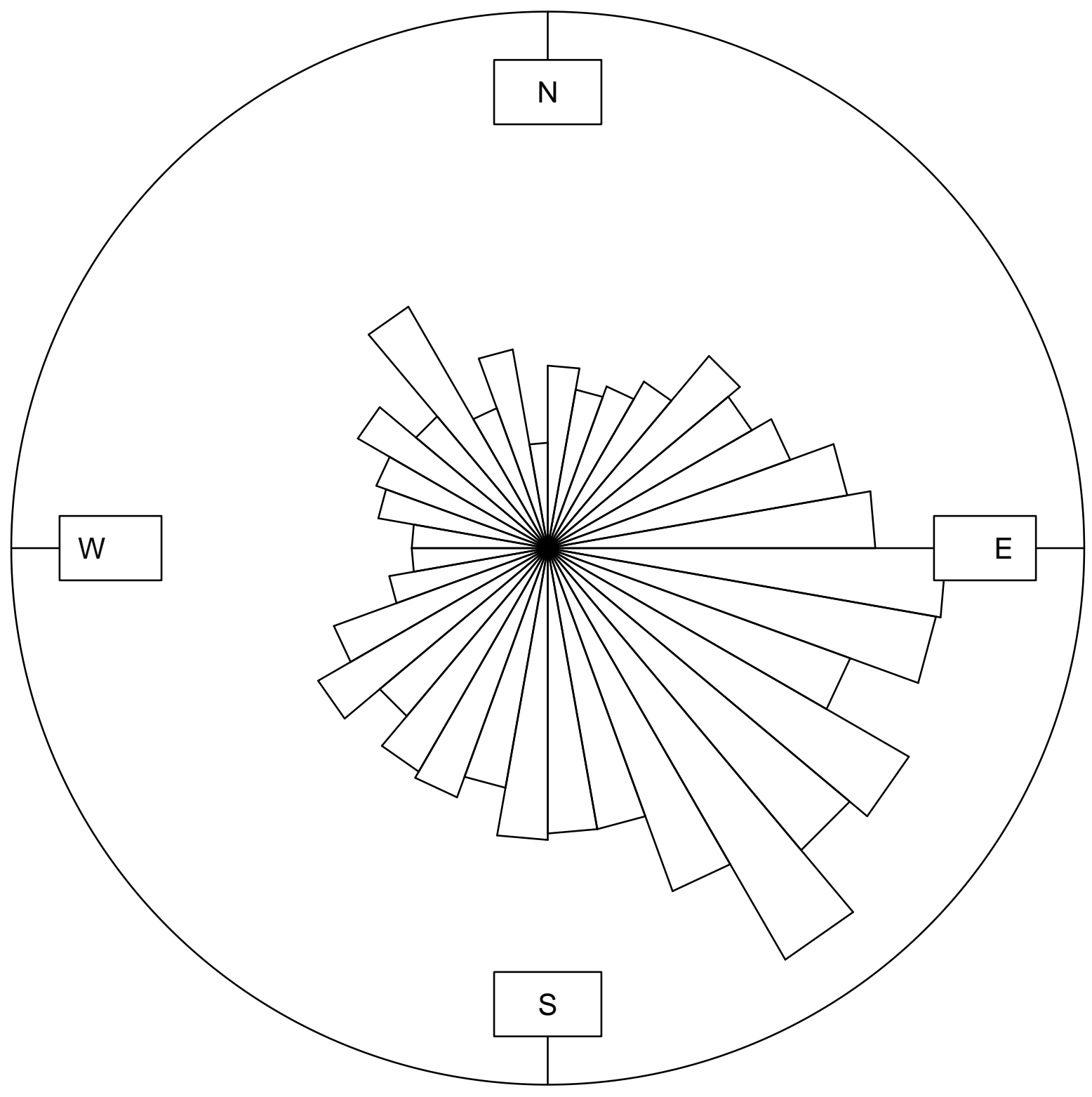


bootstrap 169

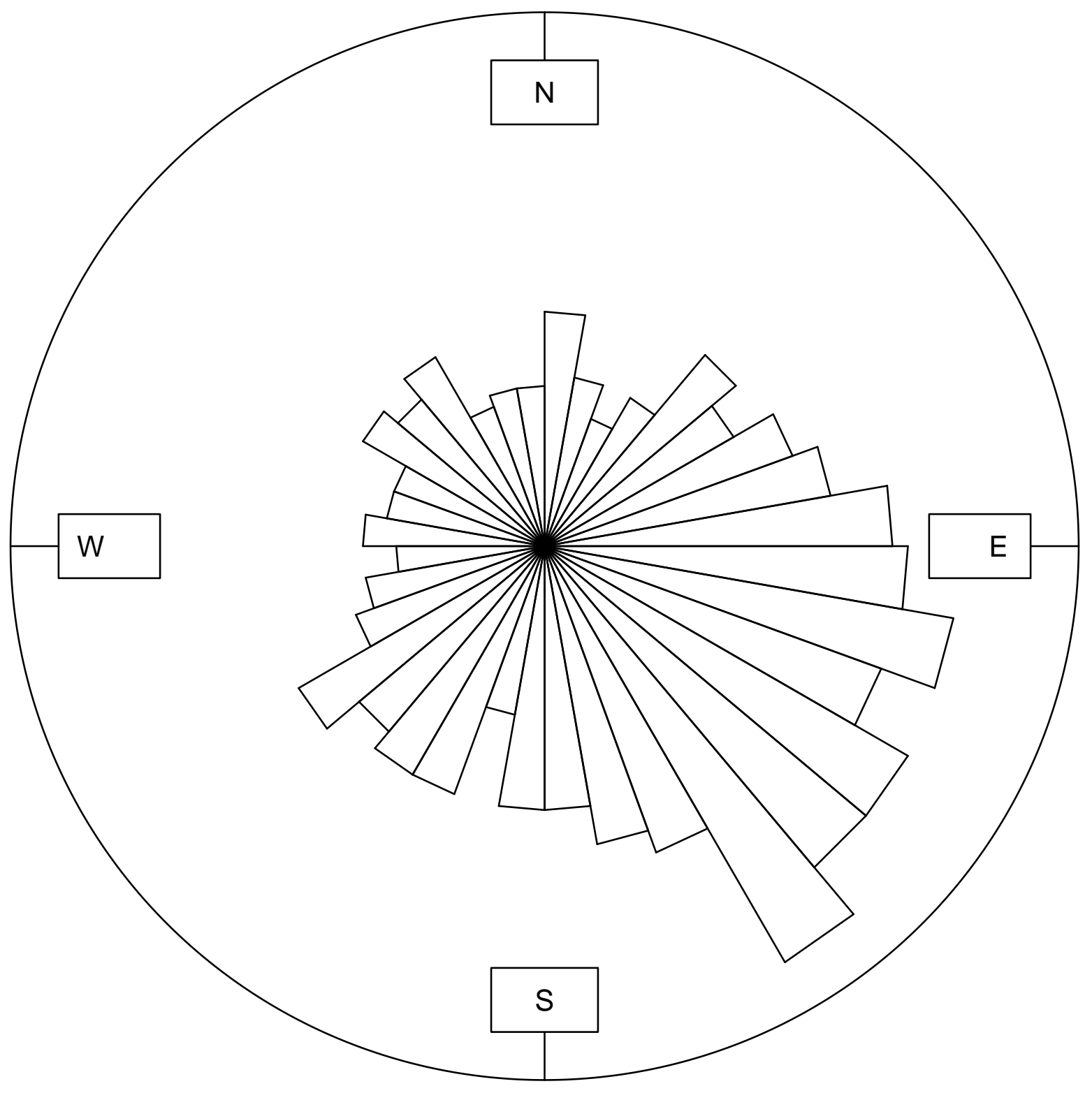


bootstrap 170

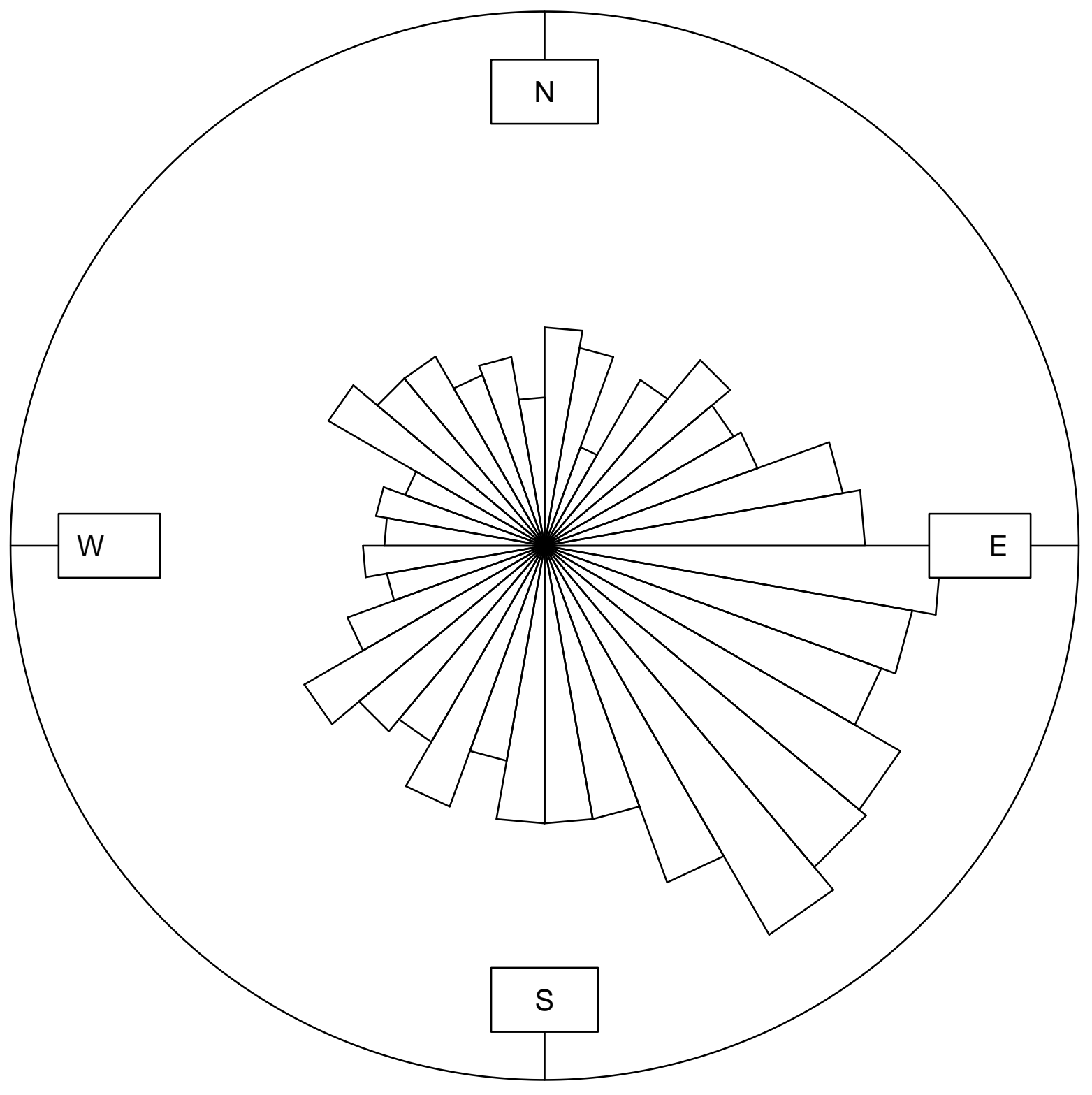


bootstrap 171

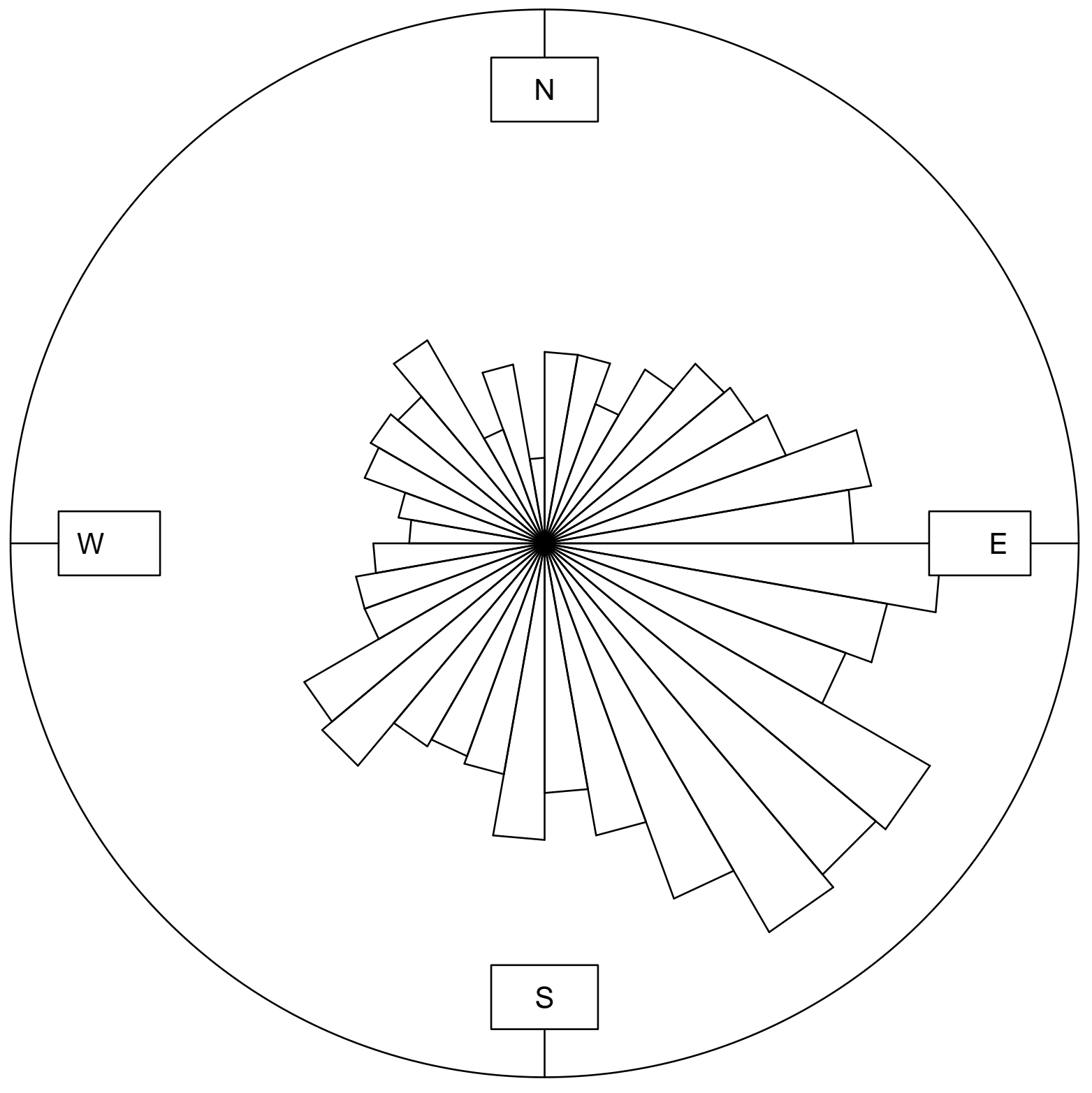


bootstrap 172

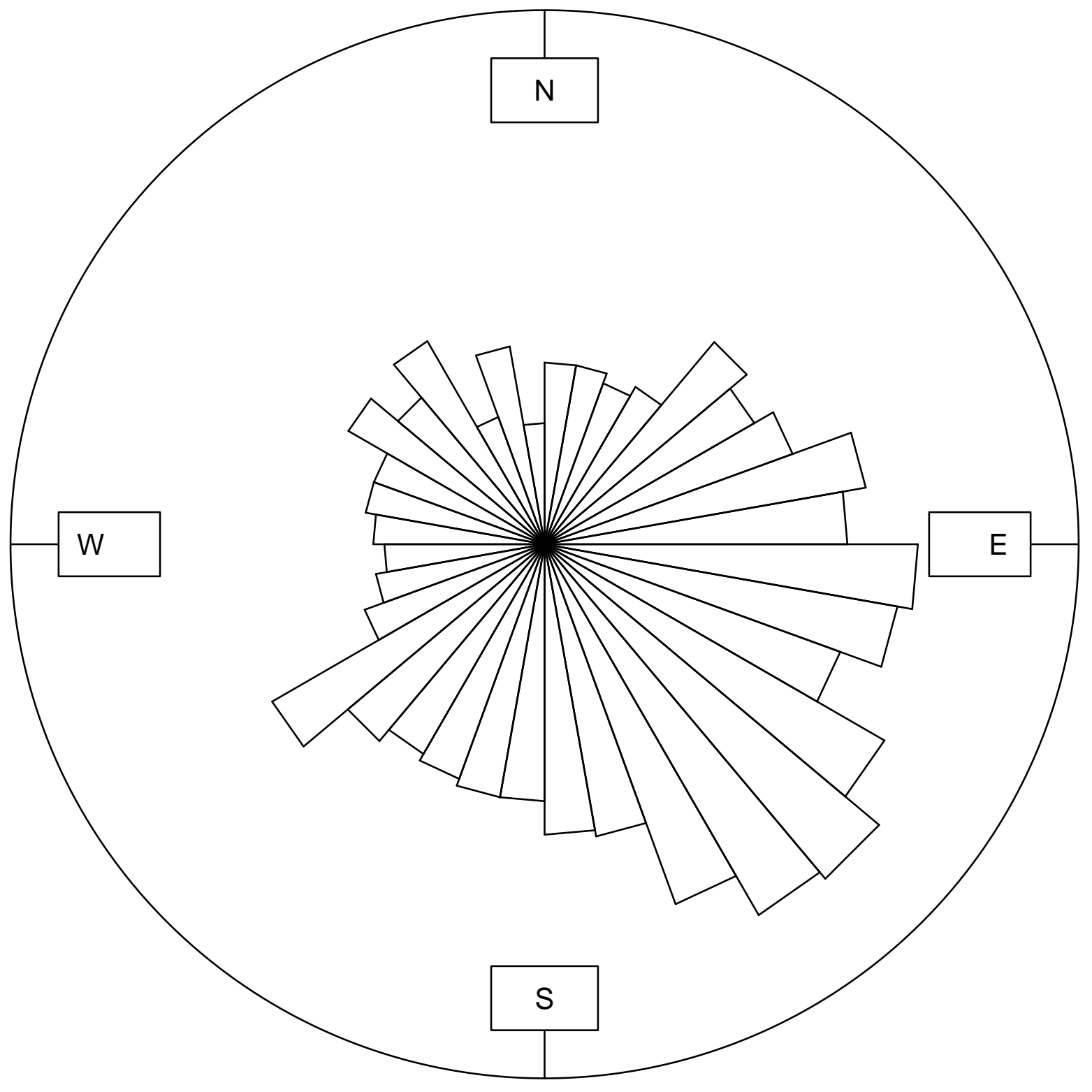


bootstrap 173

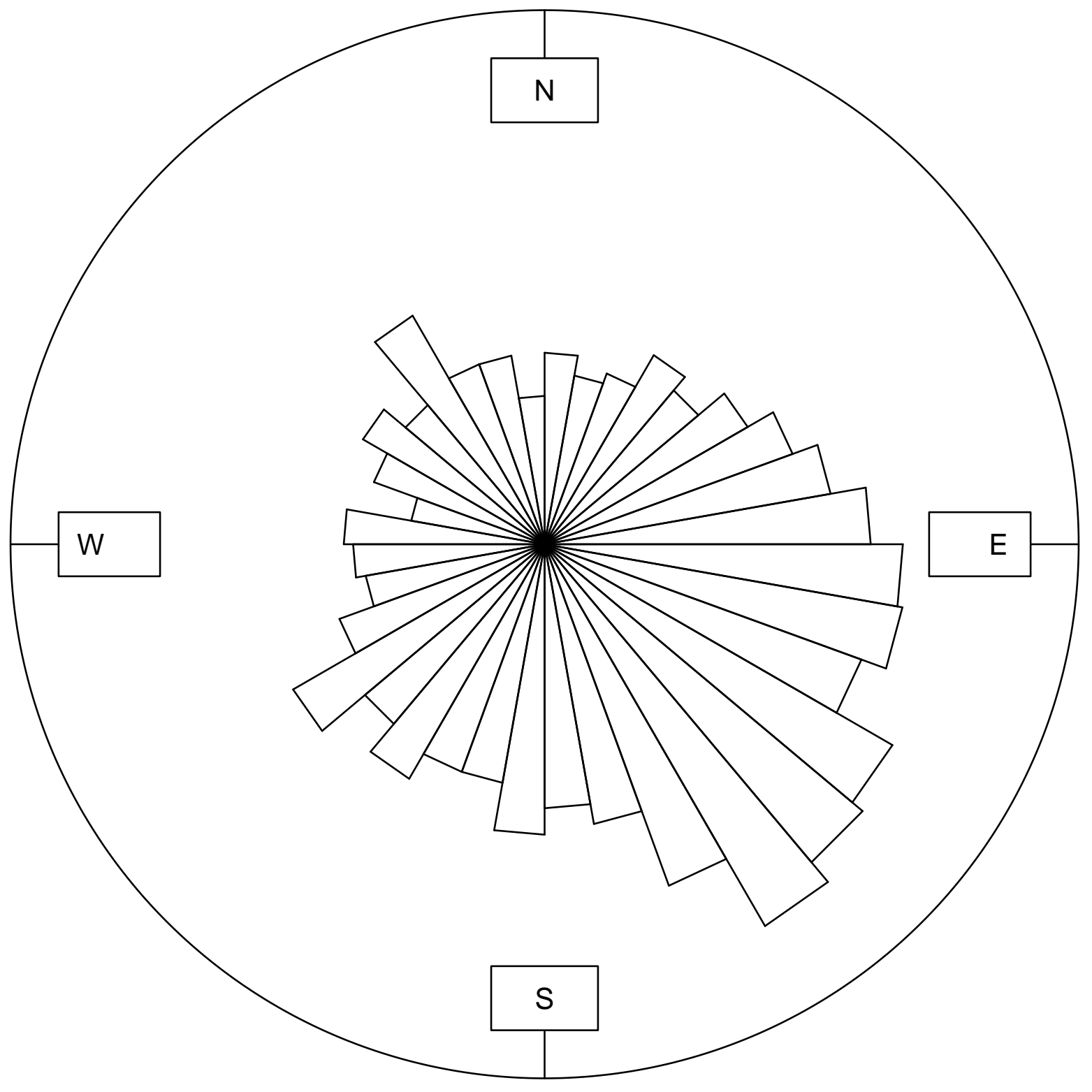


bootstrap 174

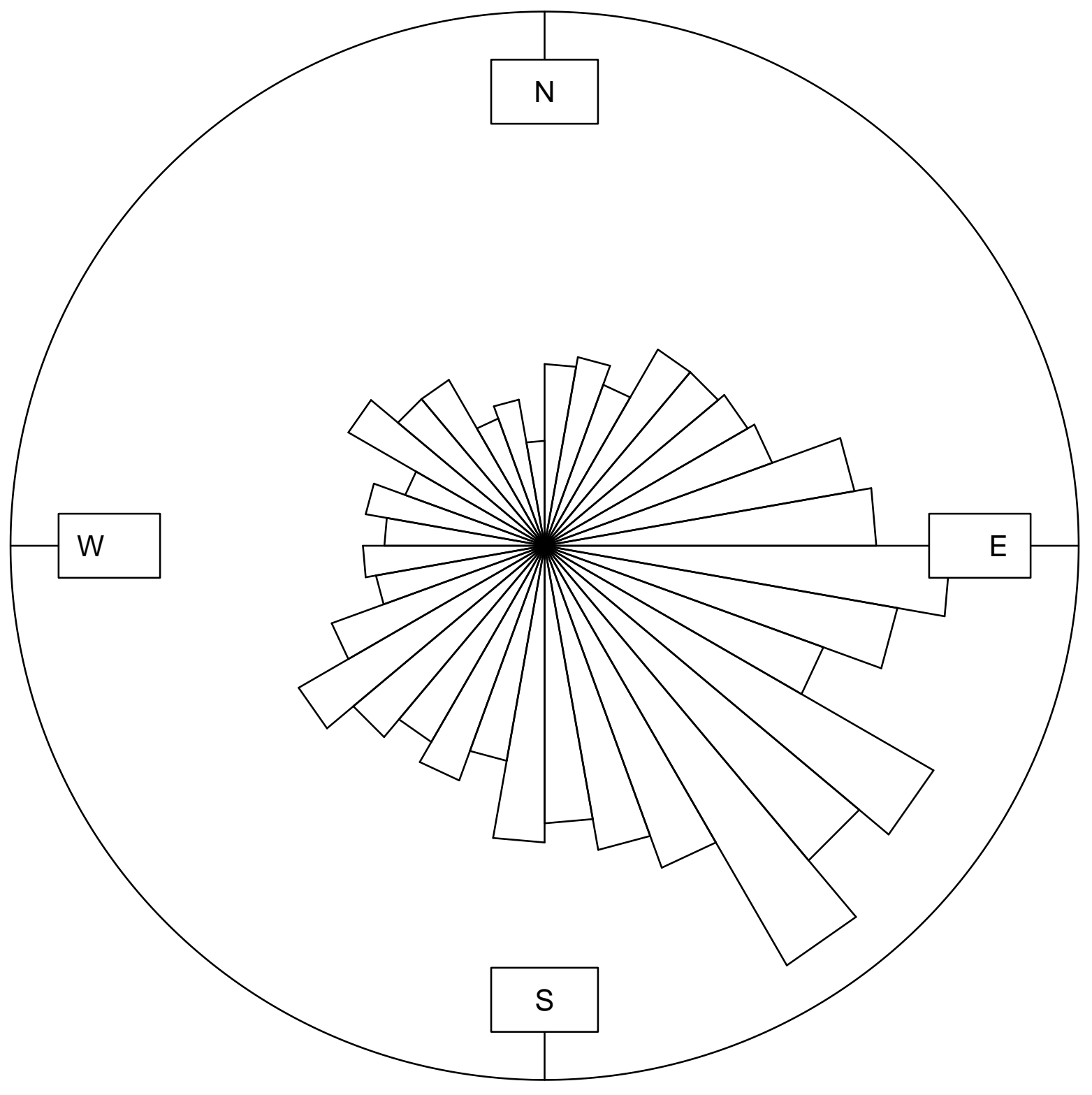


bootstrap 175

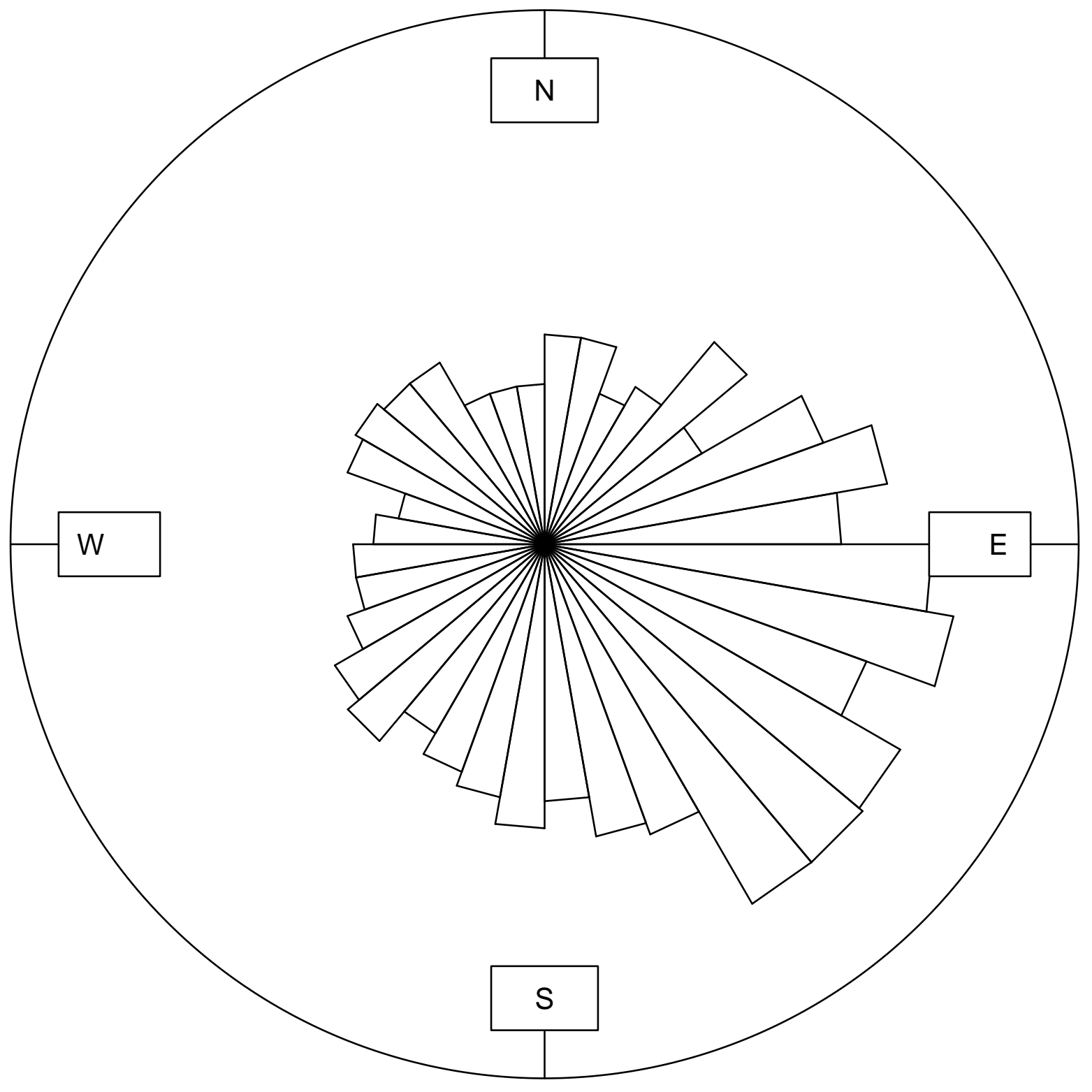


bootstrap 176

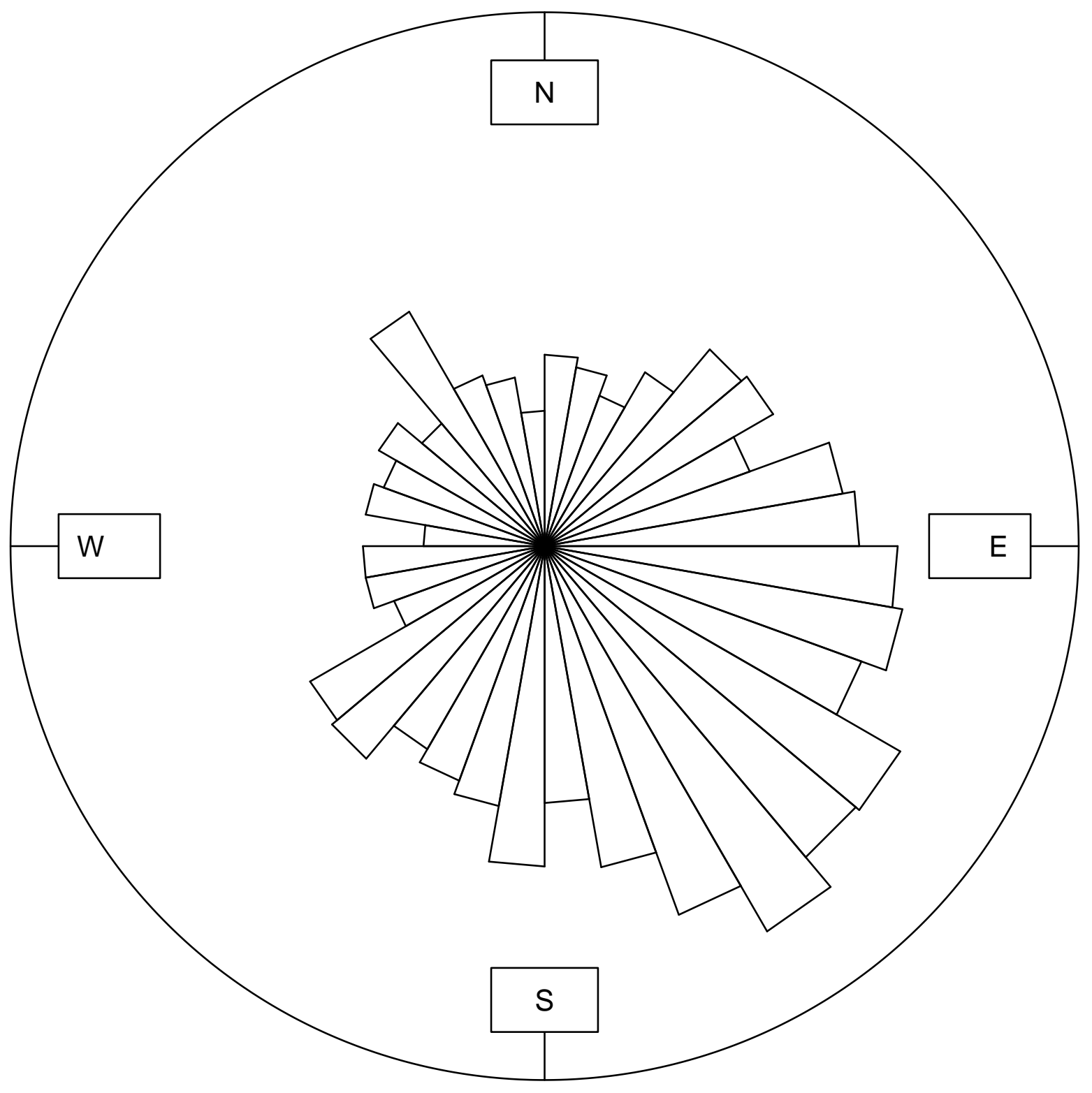


bootstrap 177

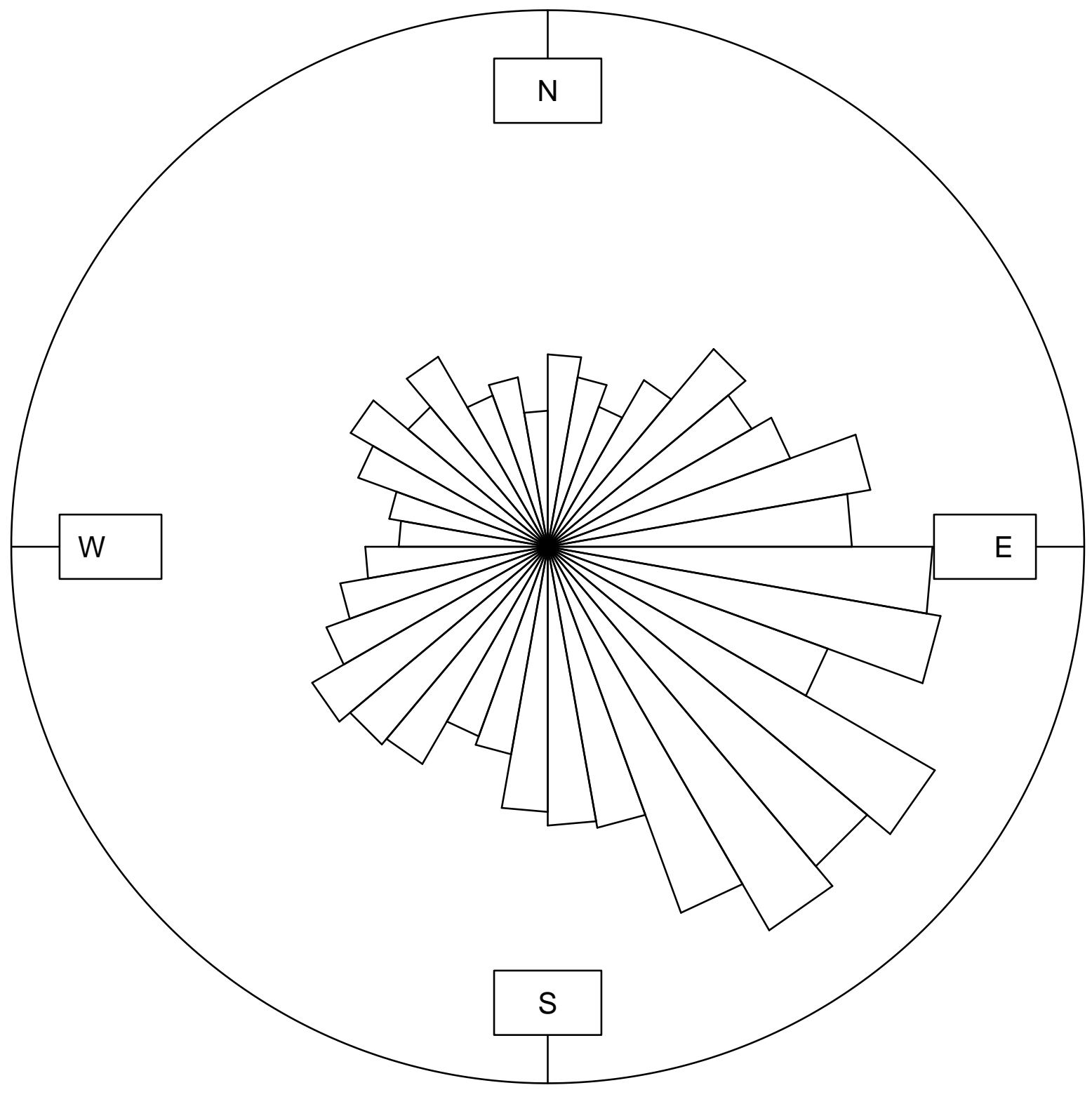


bootstrap 178

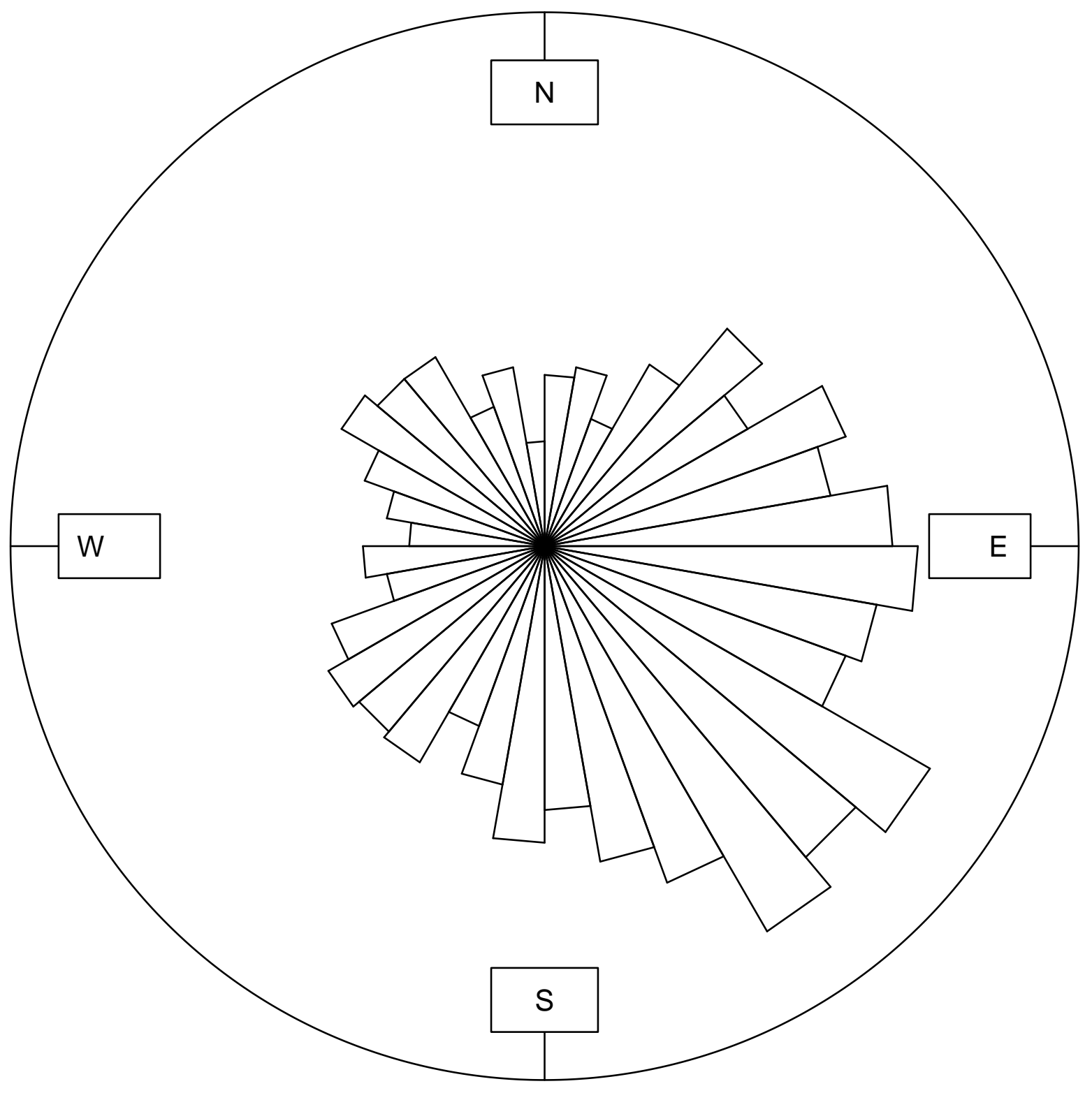


bootstrap 179

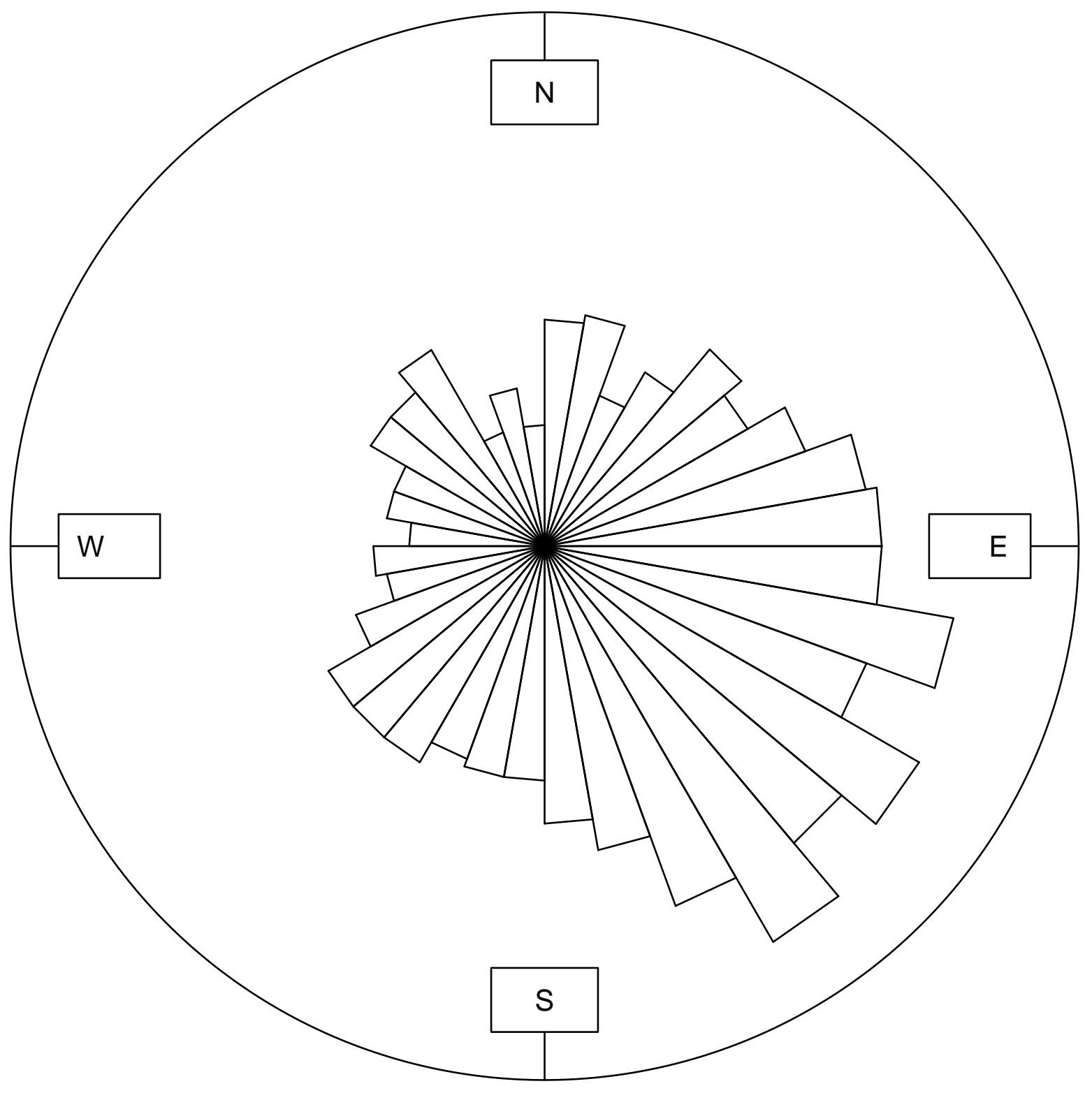


bootstrap 180

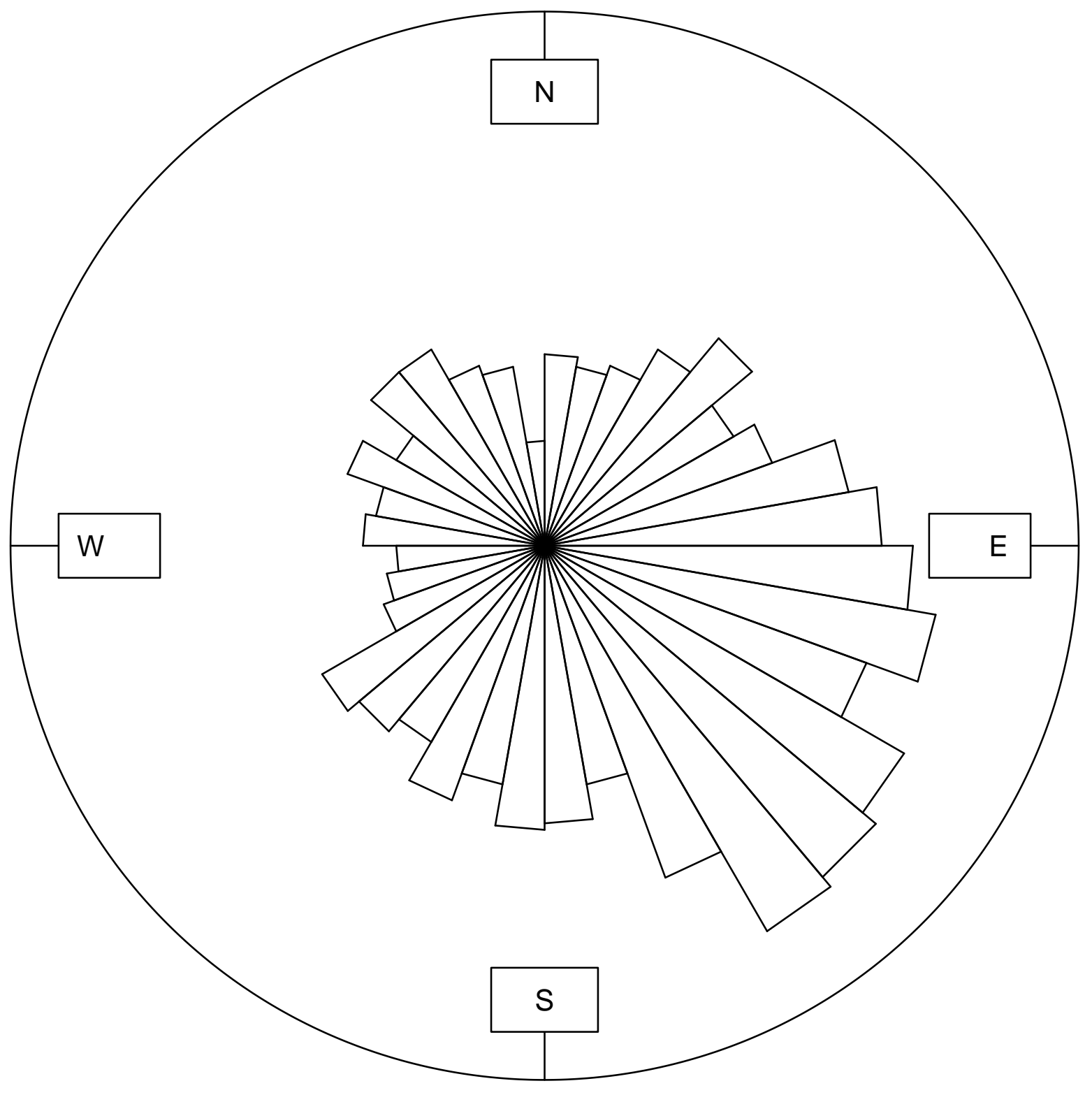




\section{bootstrap 181}

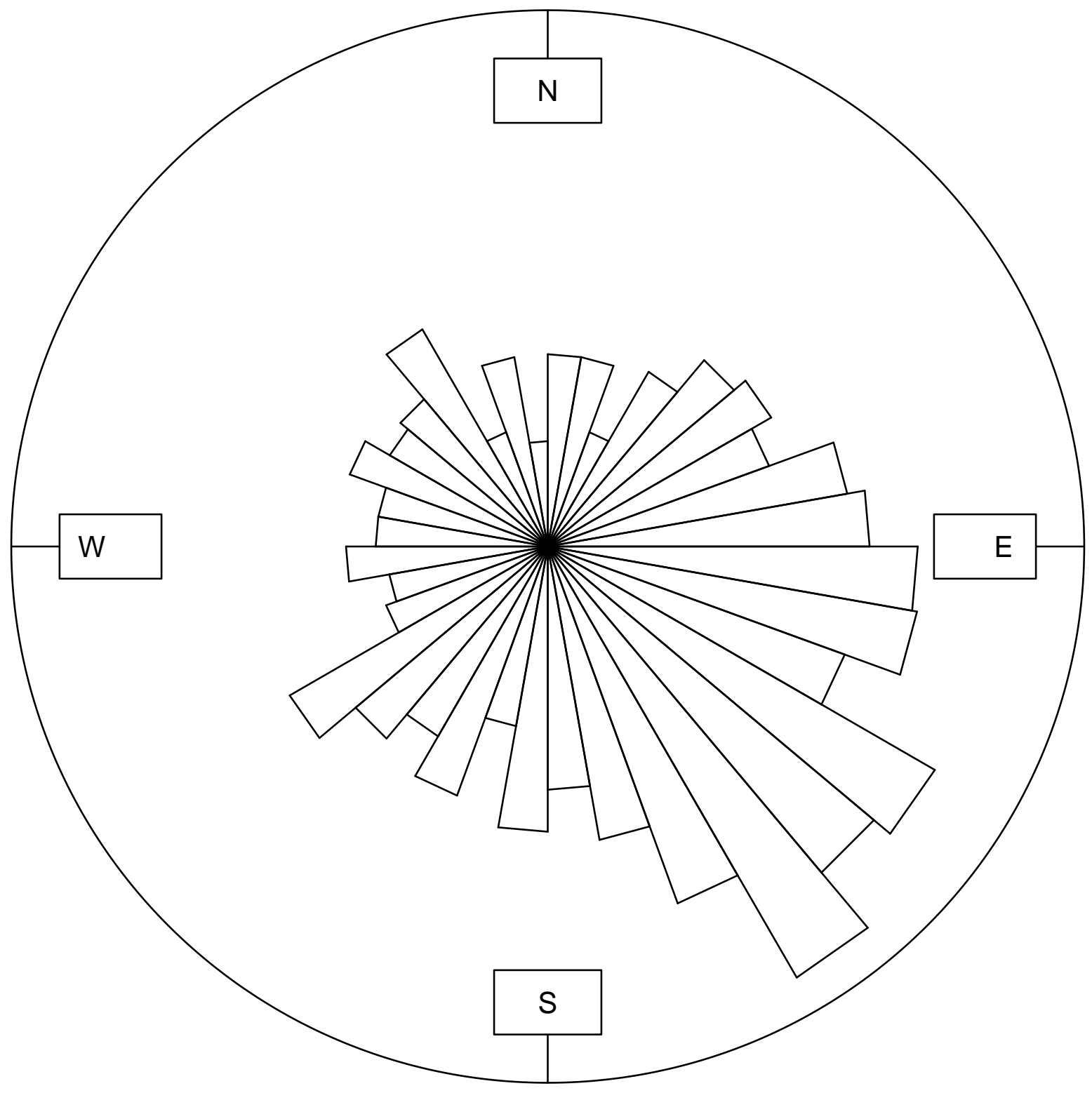


bootstrap 182

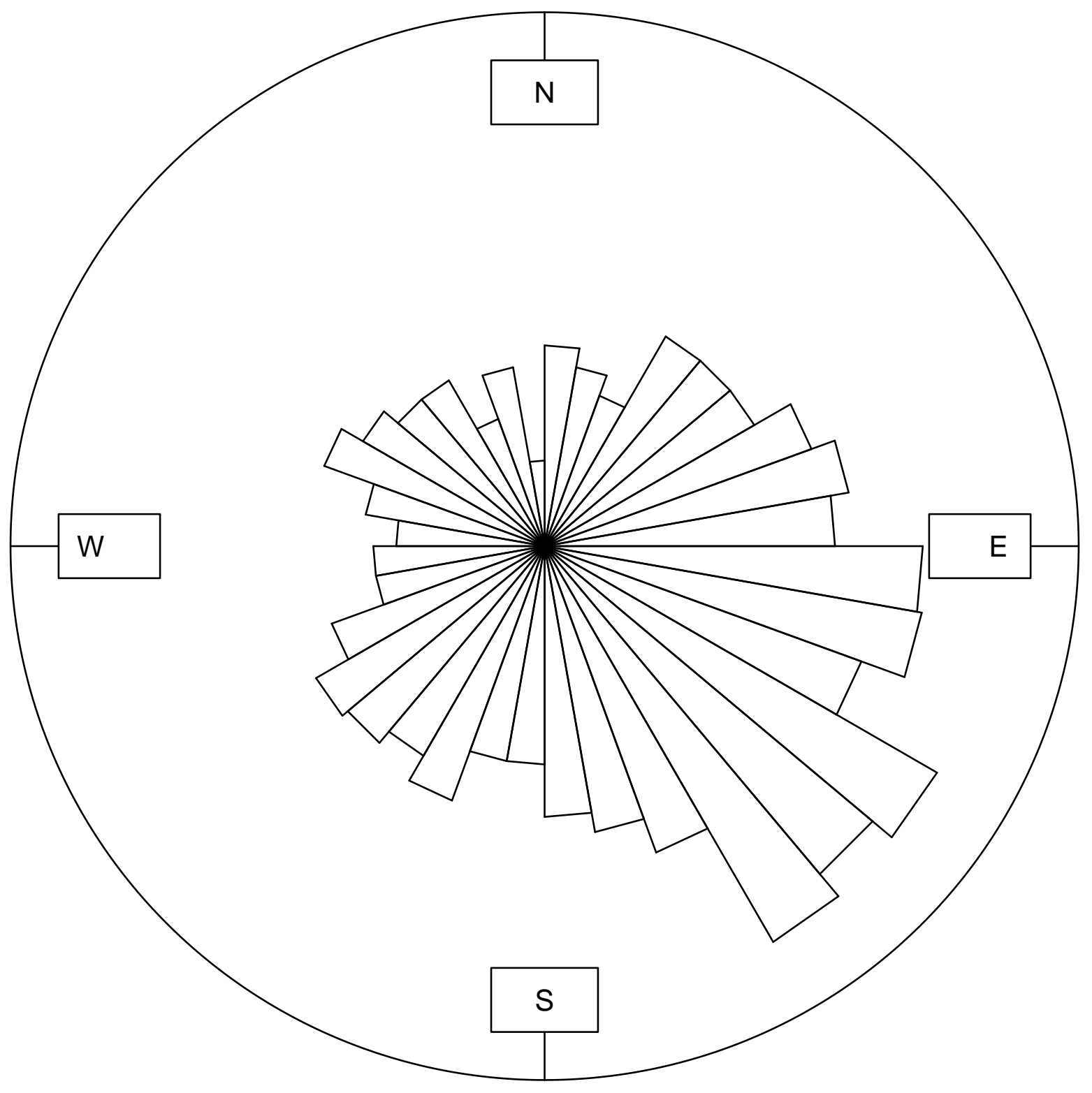


bootstrap 183

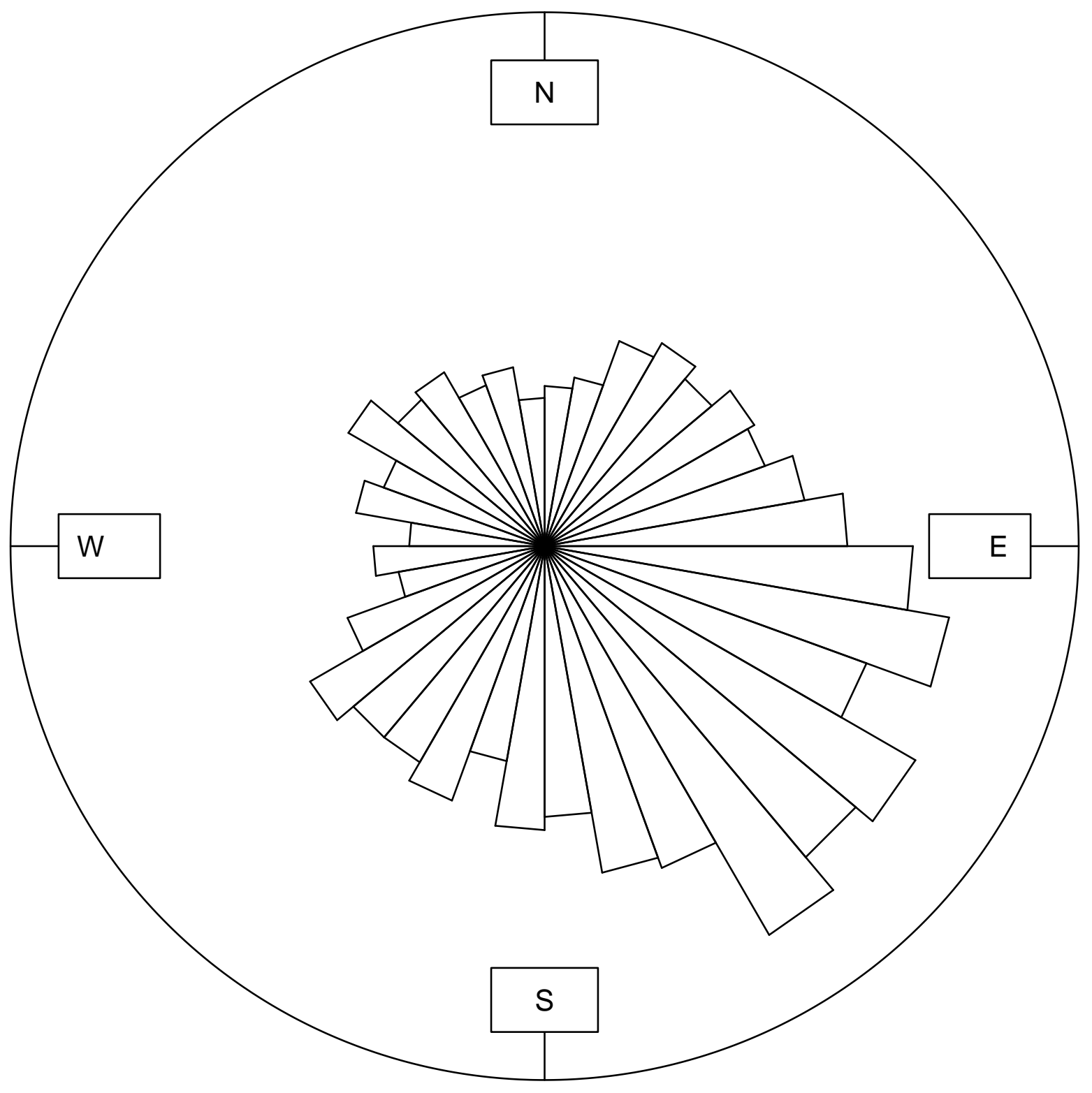


bootstrap 184

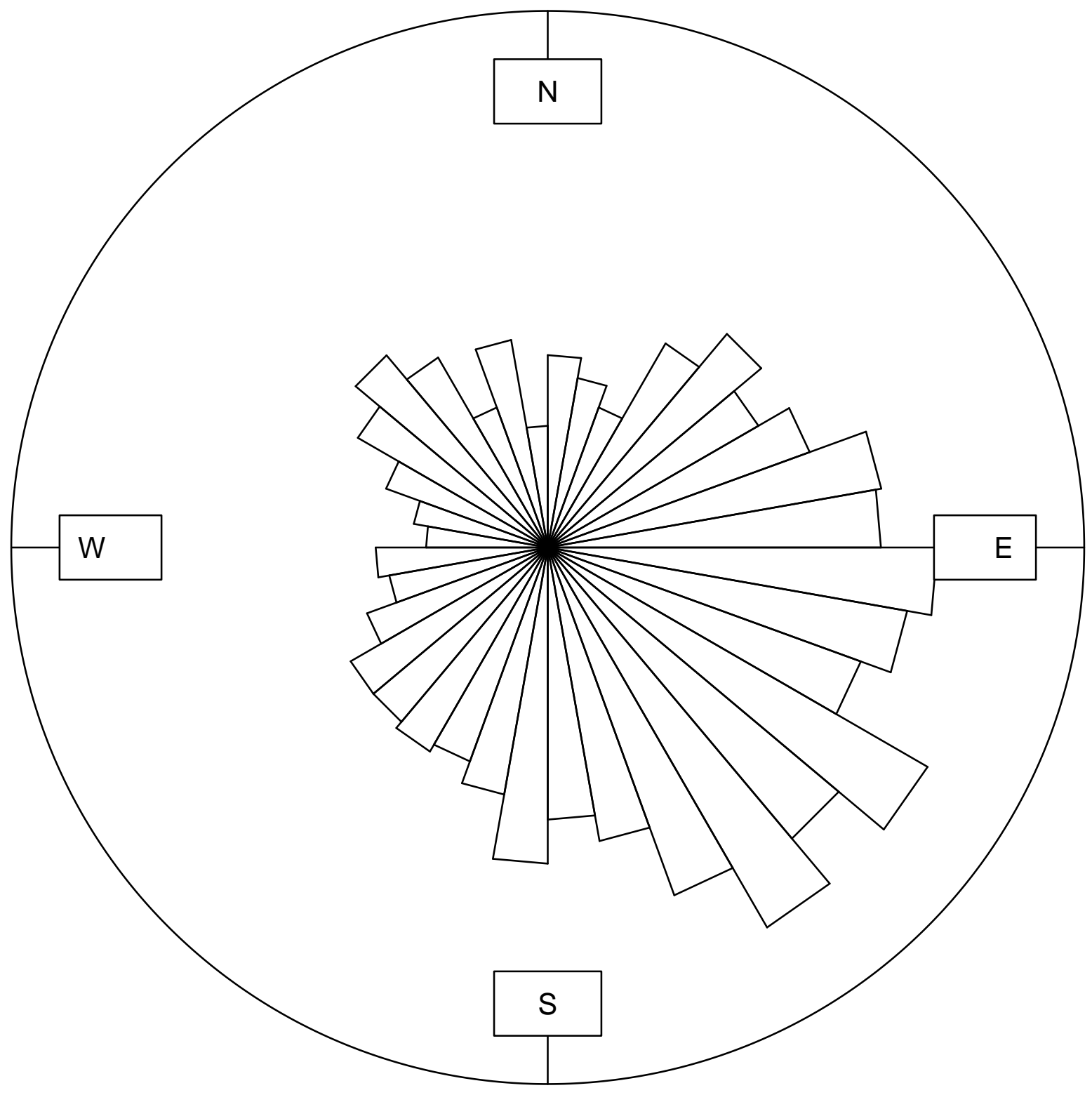




\section{bootstrap 185}

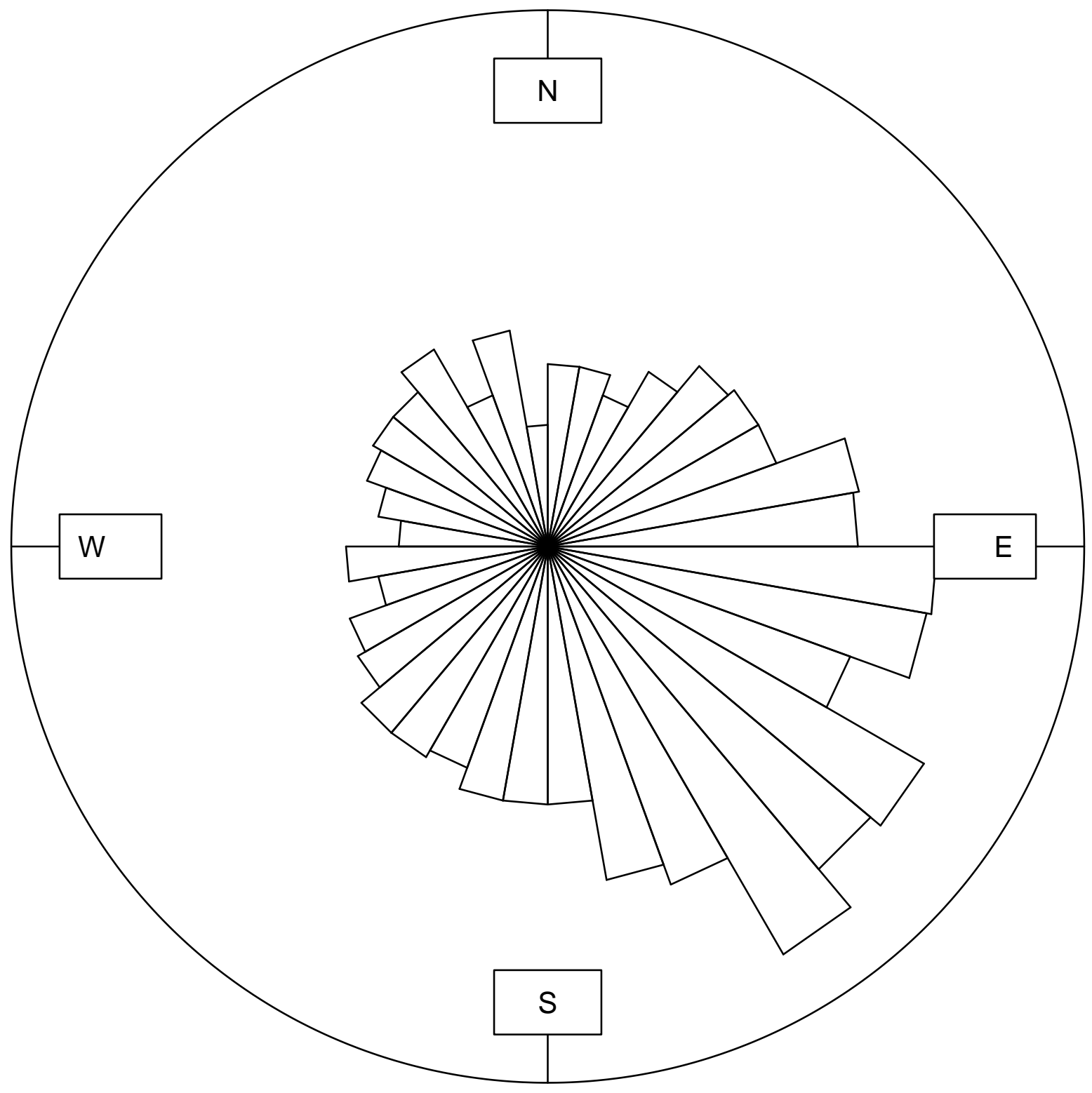


bootstrap 186

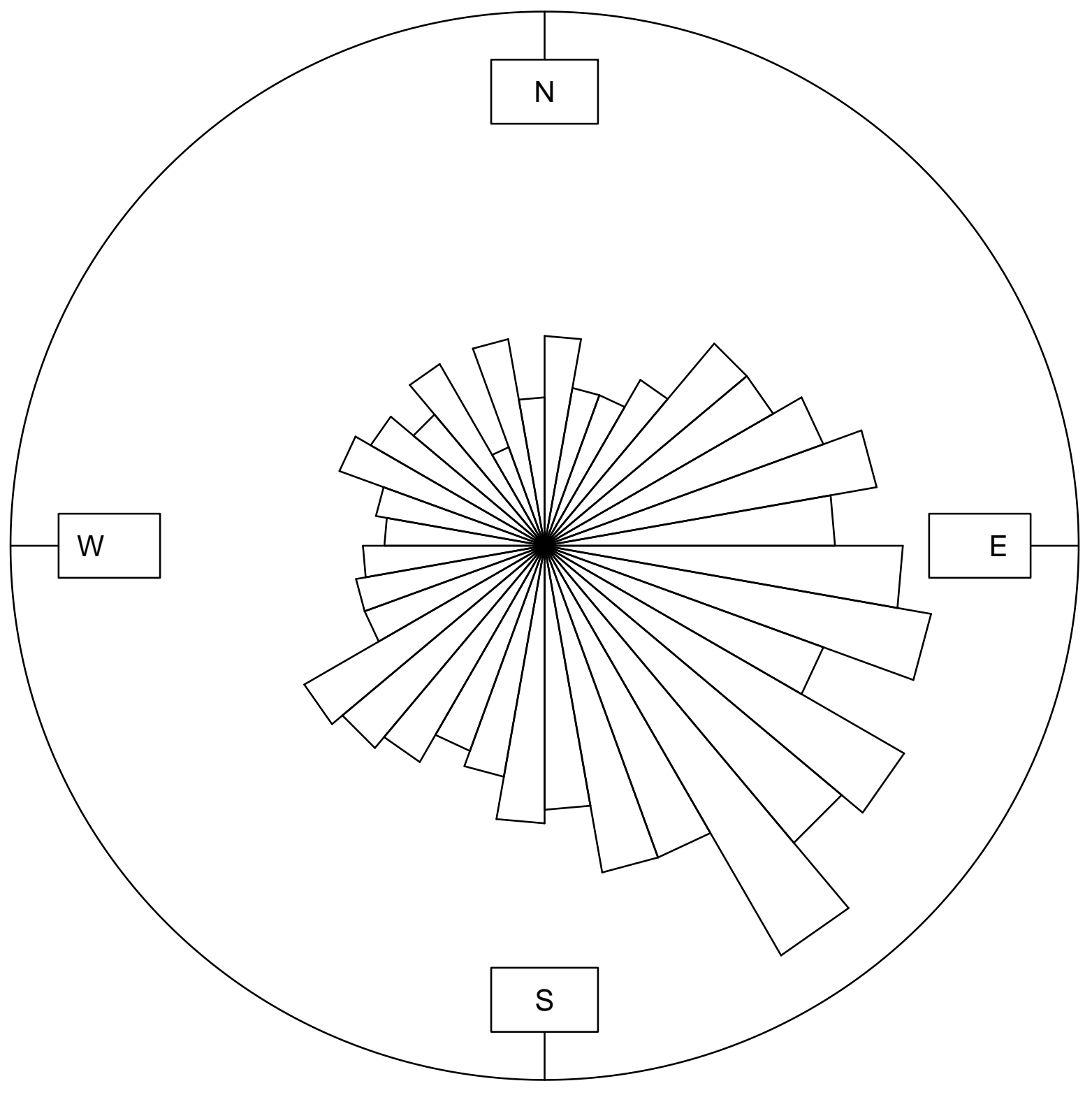


bootstrap 187

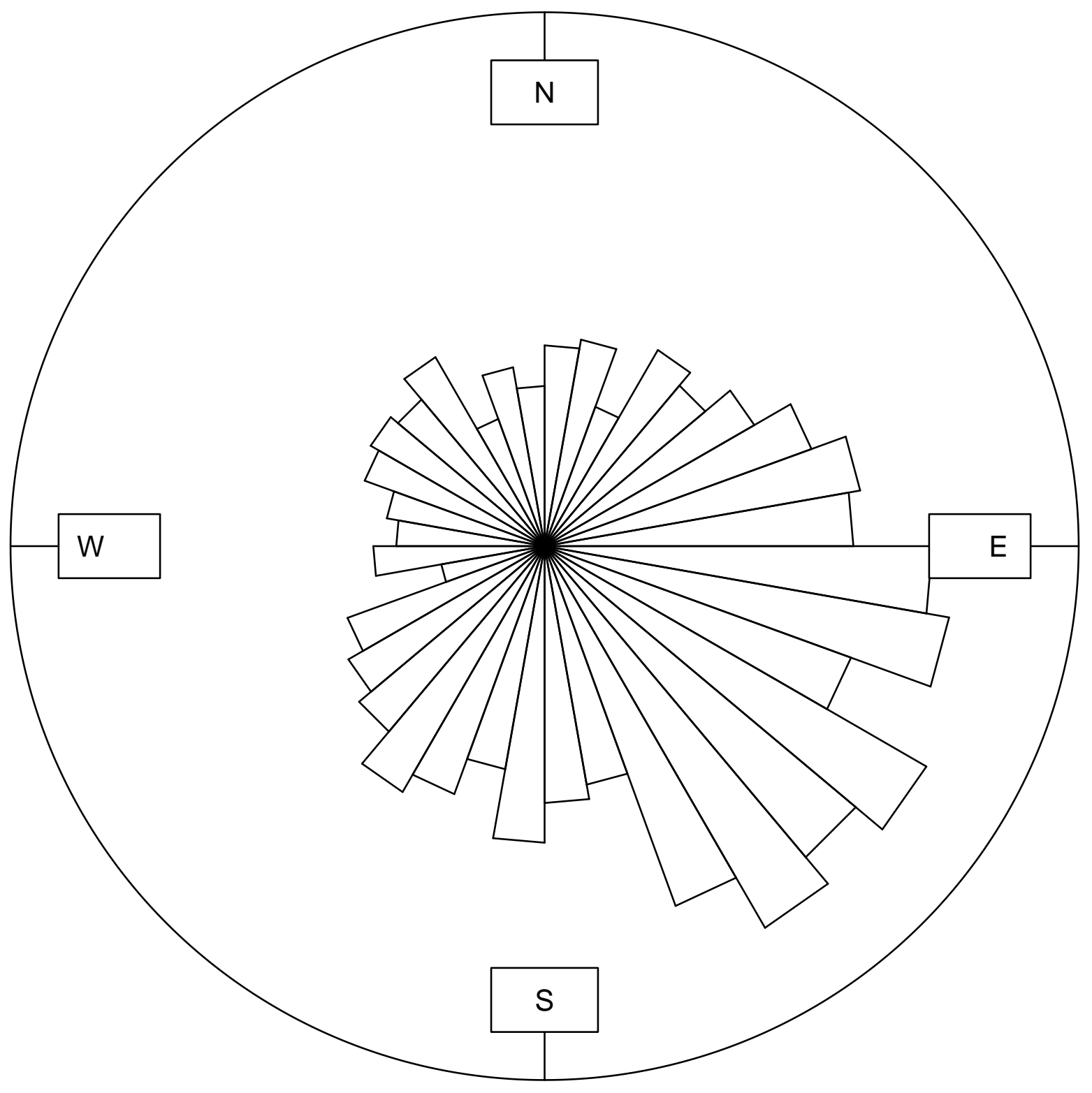


bootstrap 188

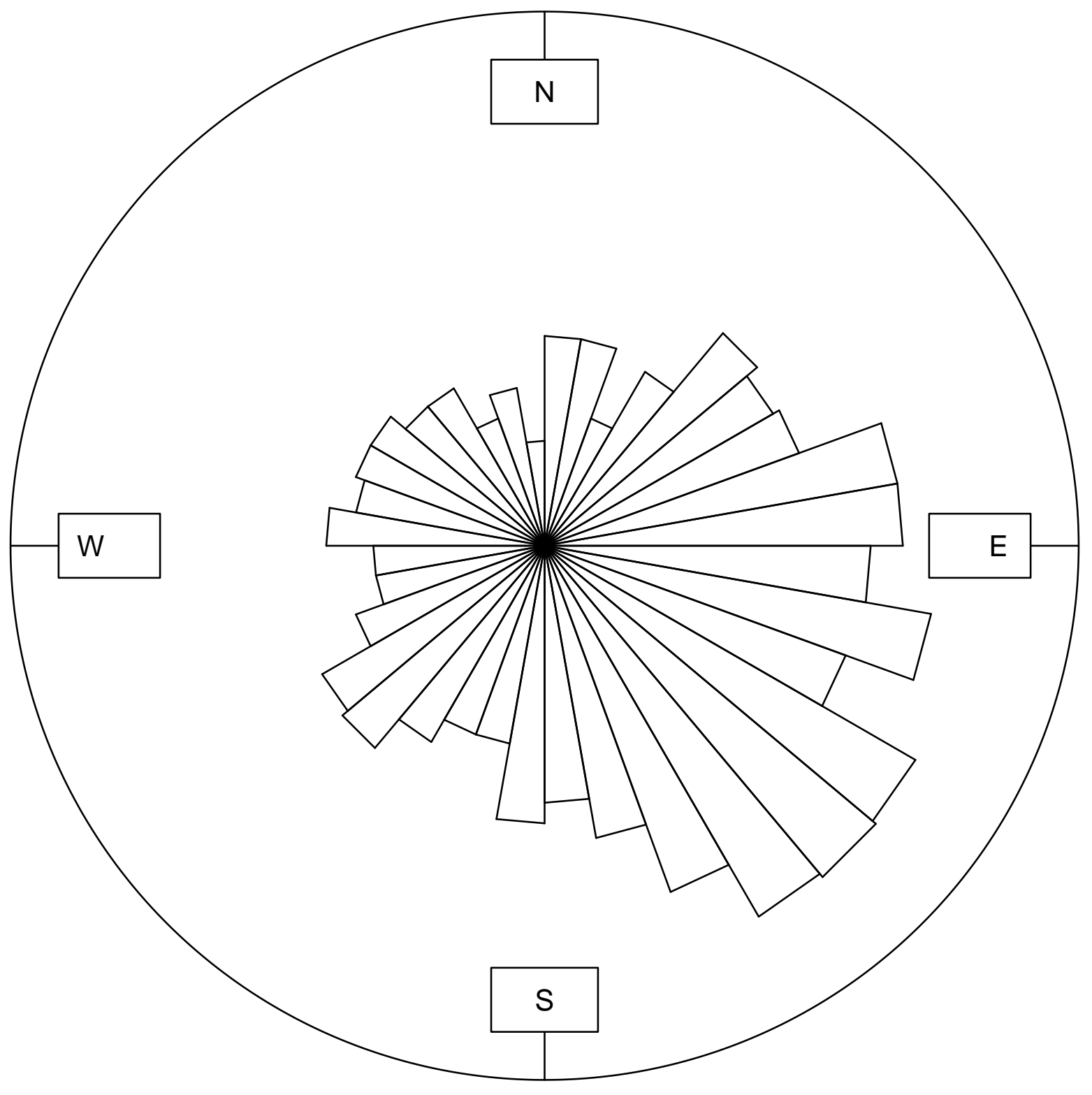


bootstrap 189

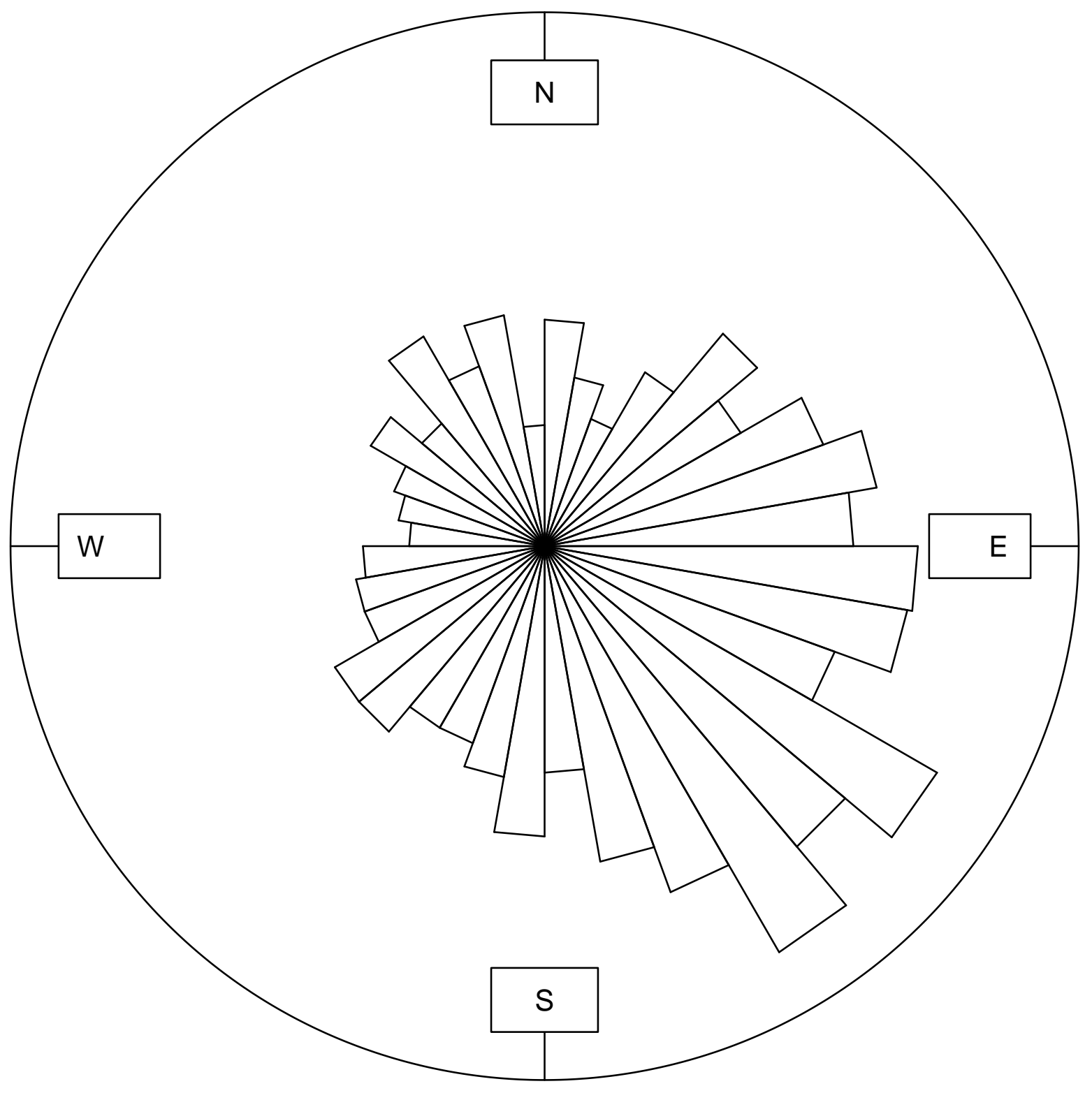


bootstrap 190

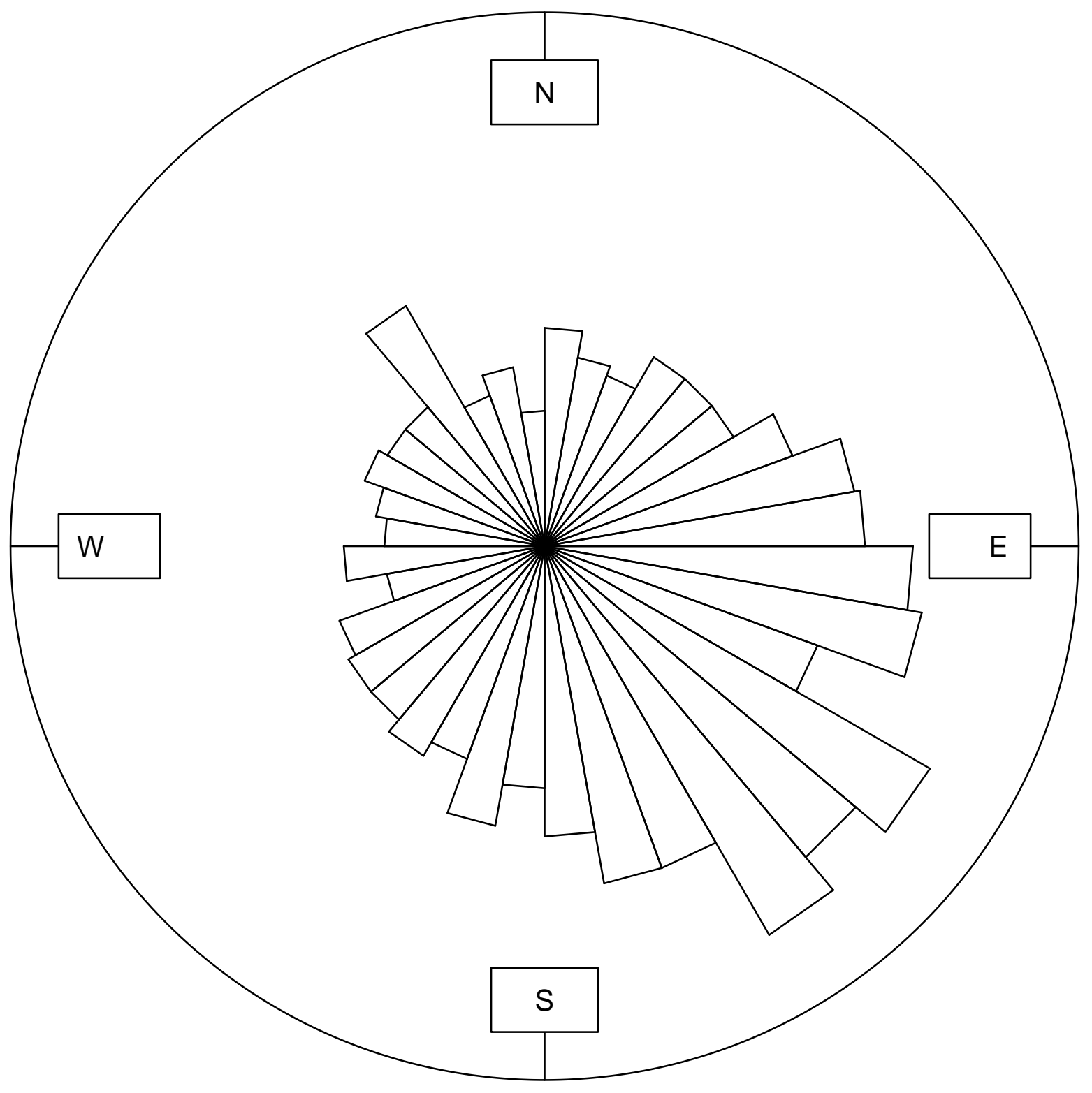




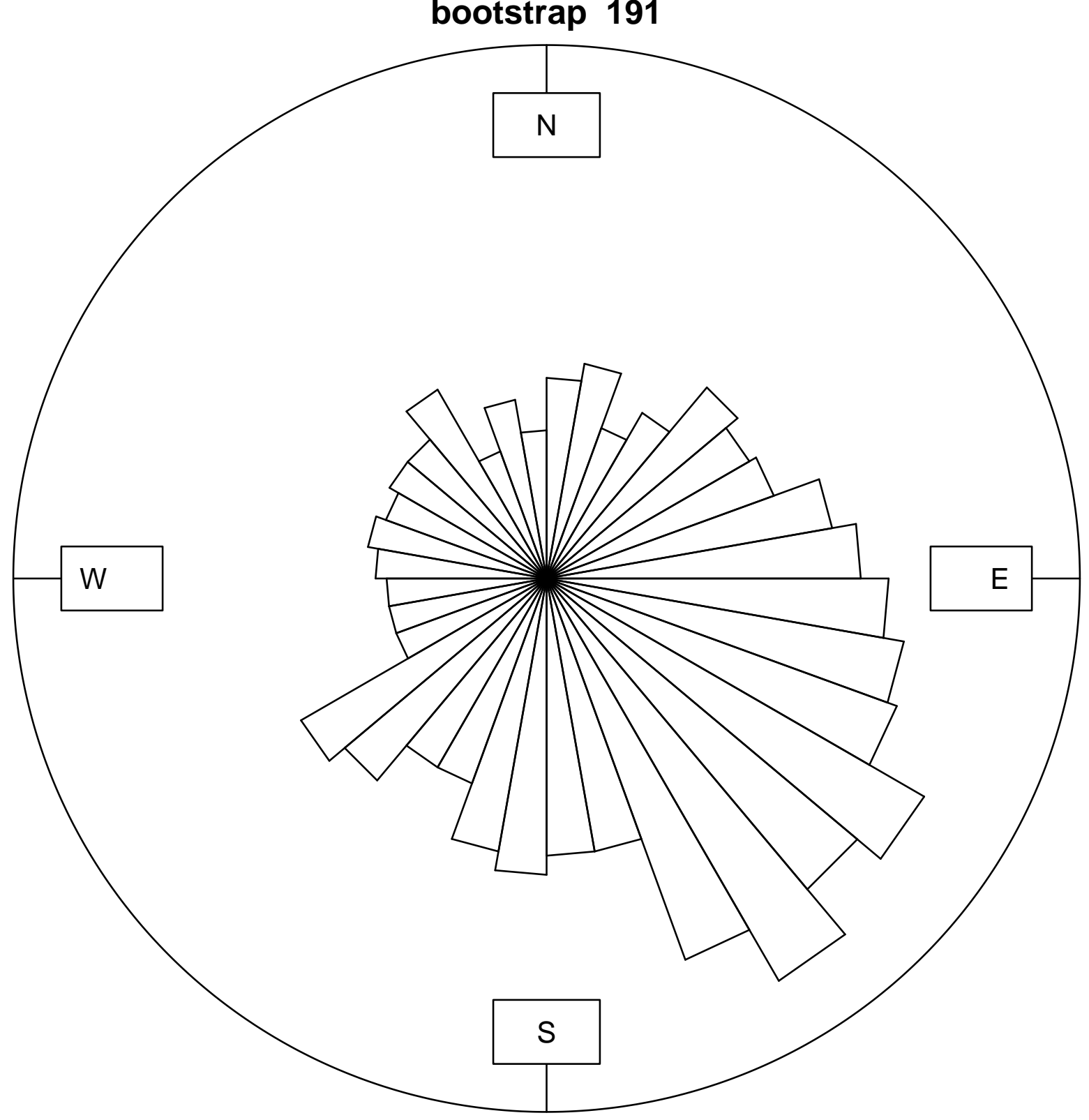


bootstrap 192

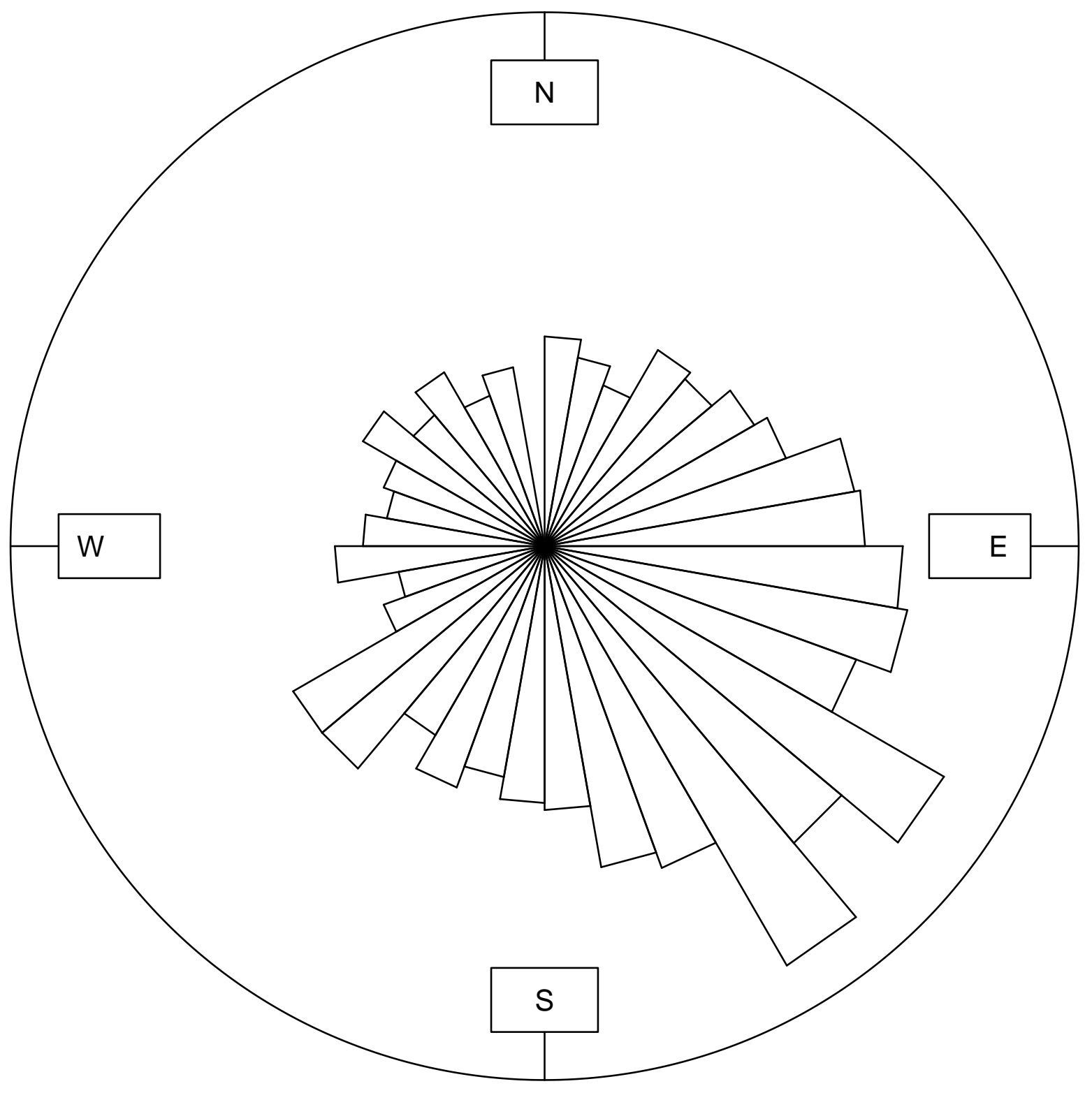




\section{bootstrap 193}

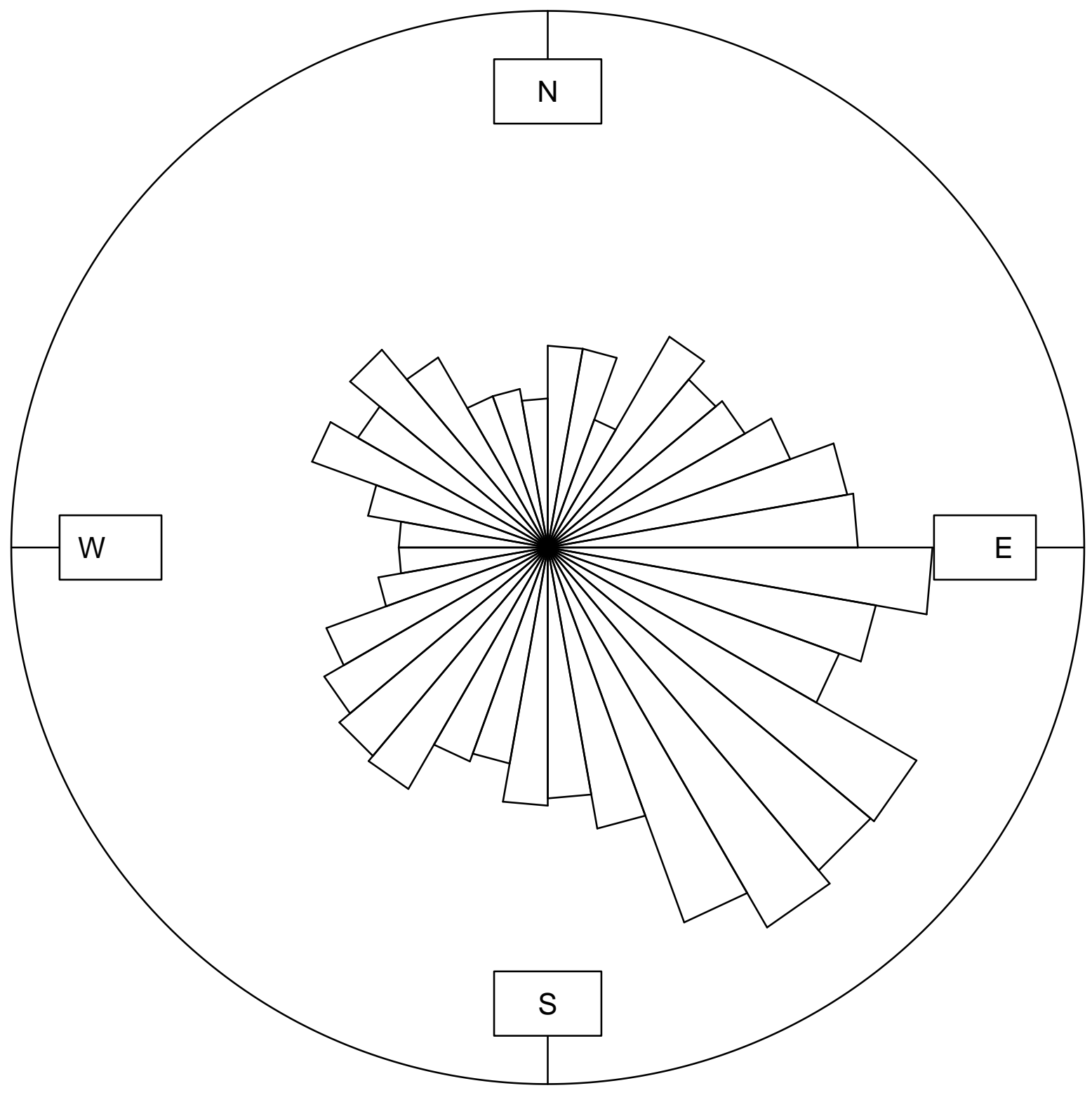




\section{bootstrap 194}

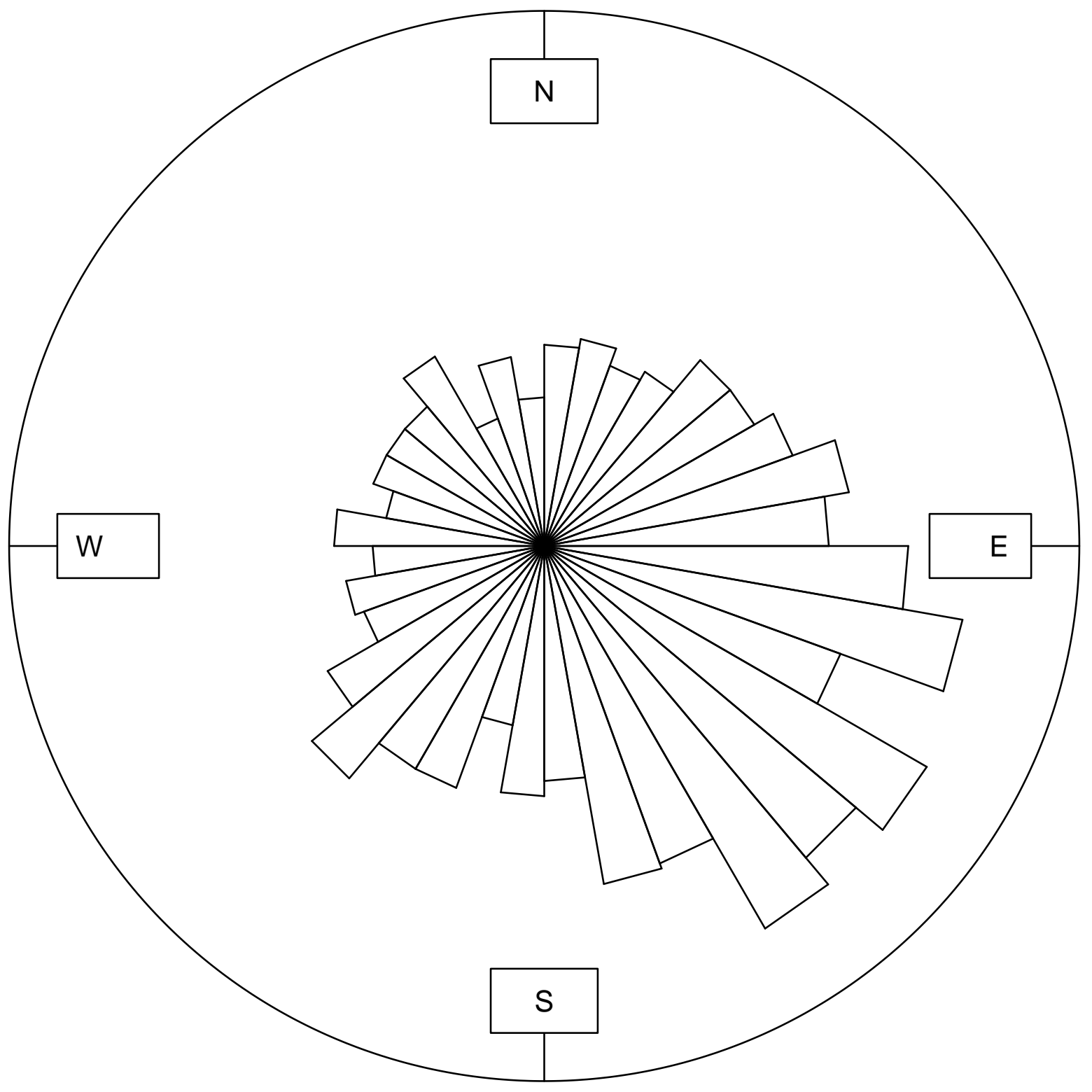


bootstrap 195

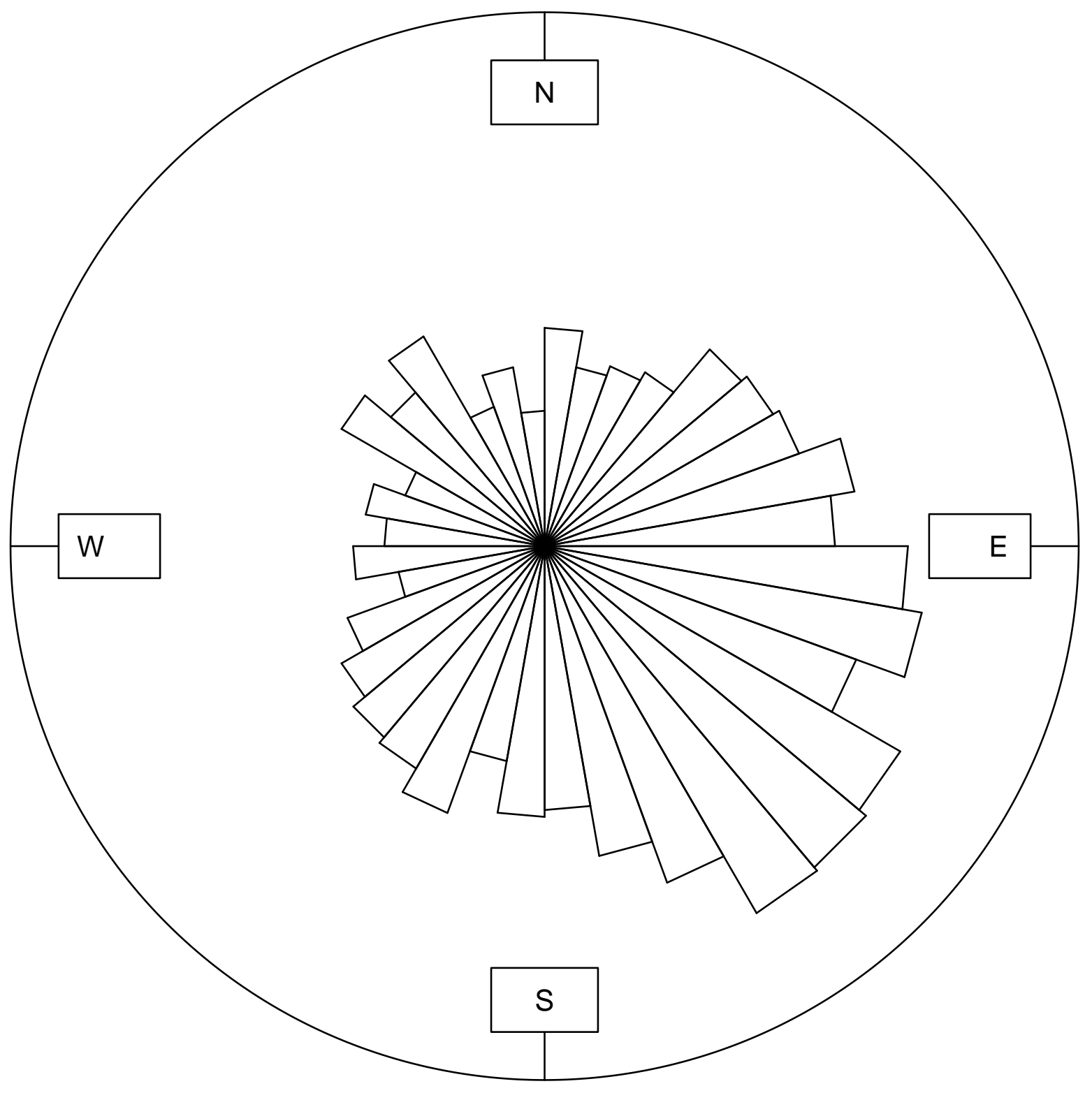


bootstrap 196

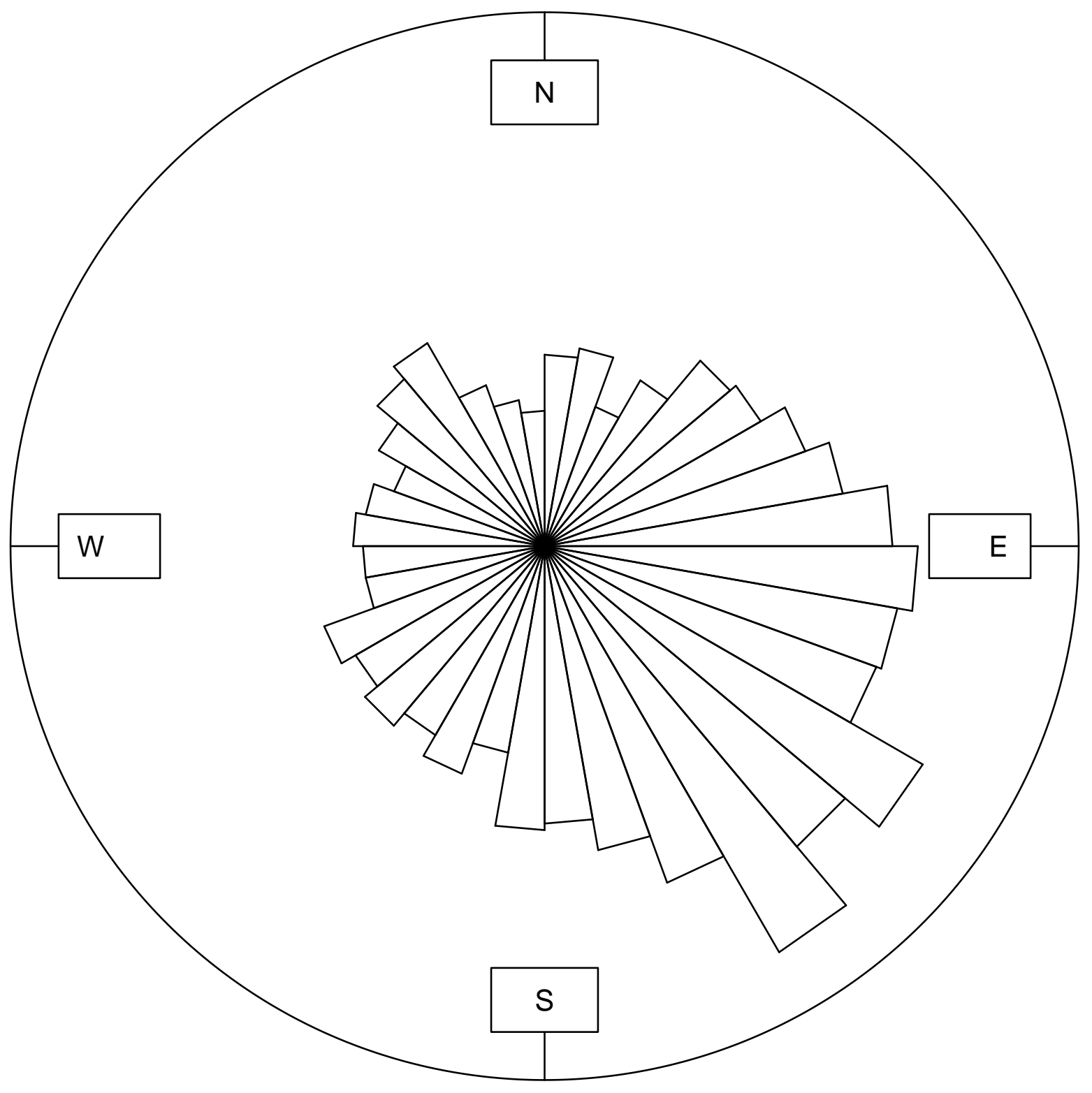


bootstrap 197

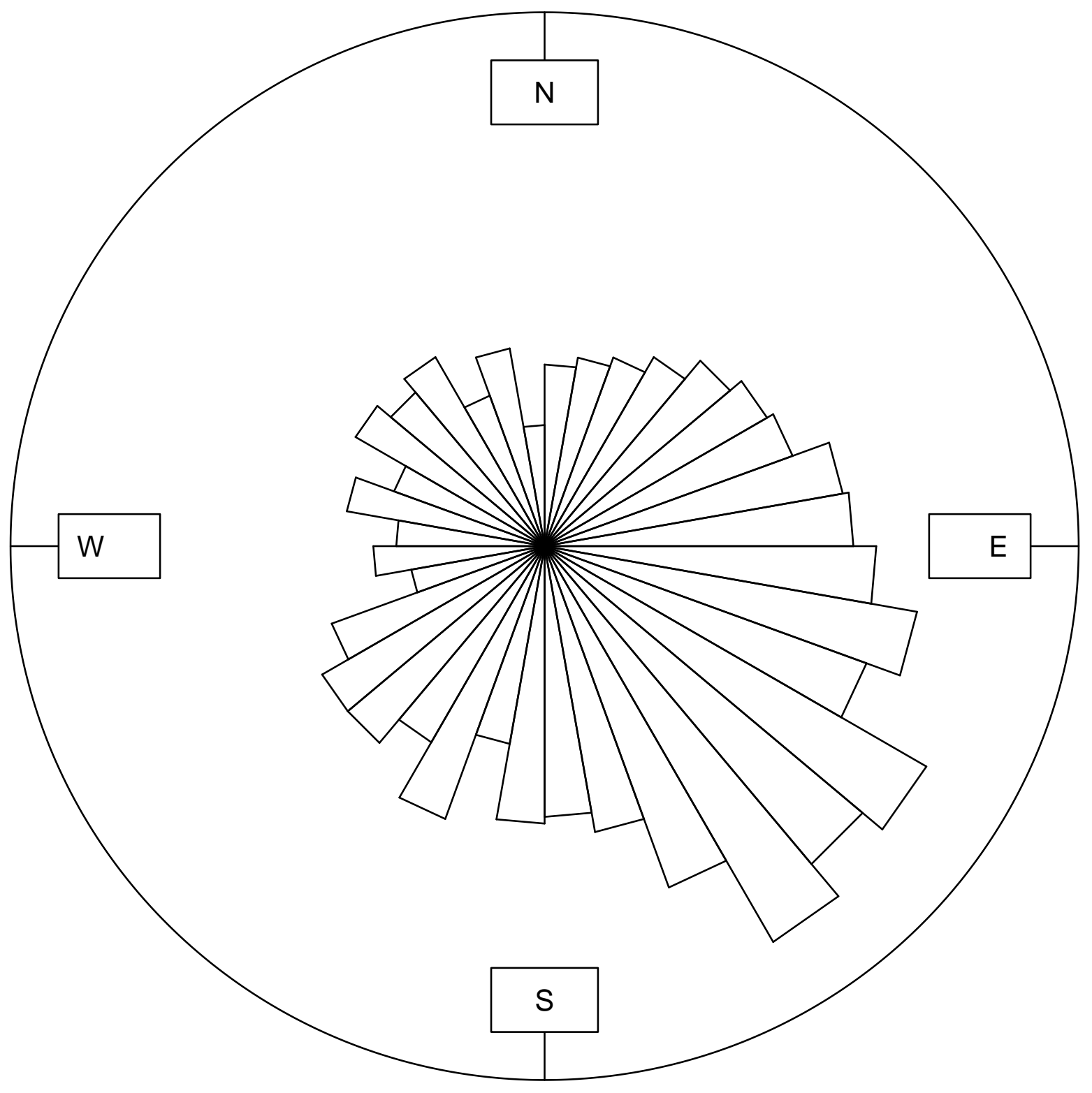


bootstrap 198

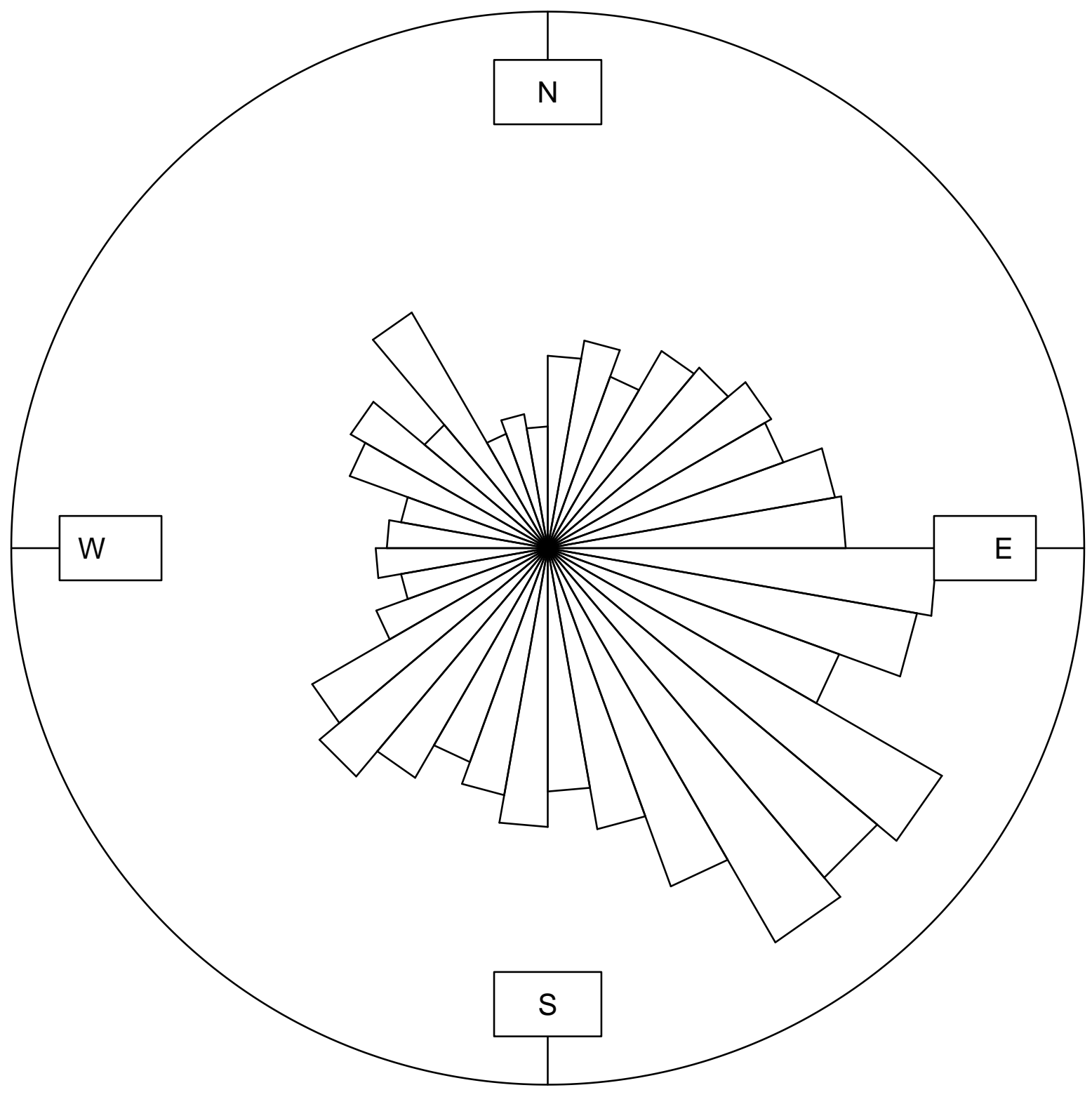


bootstrap 199

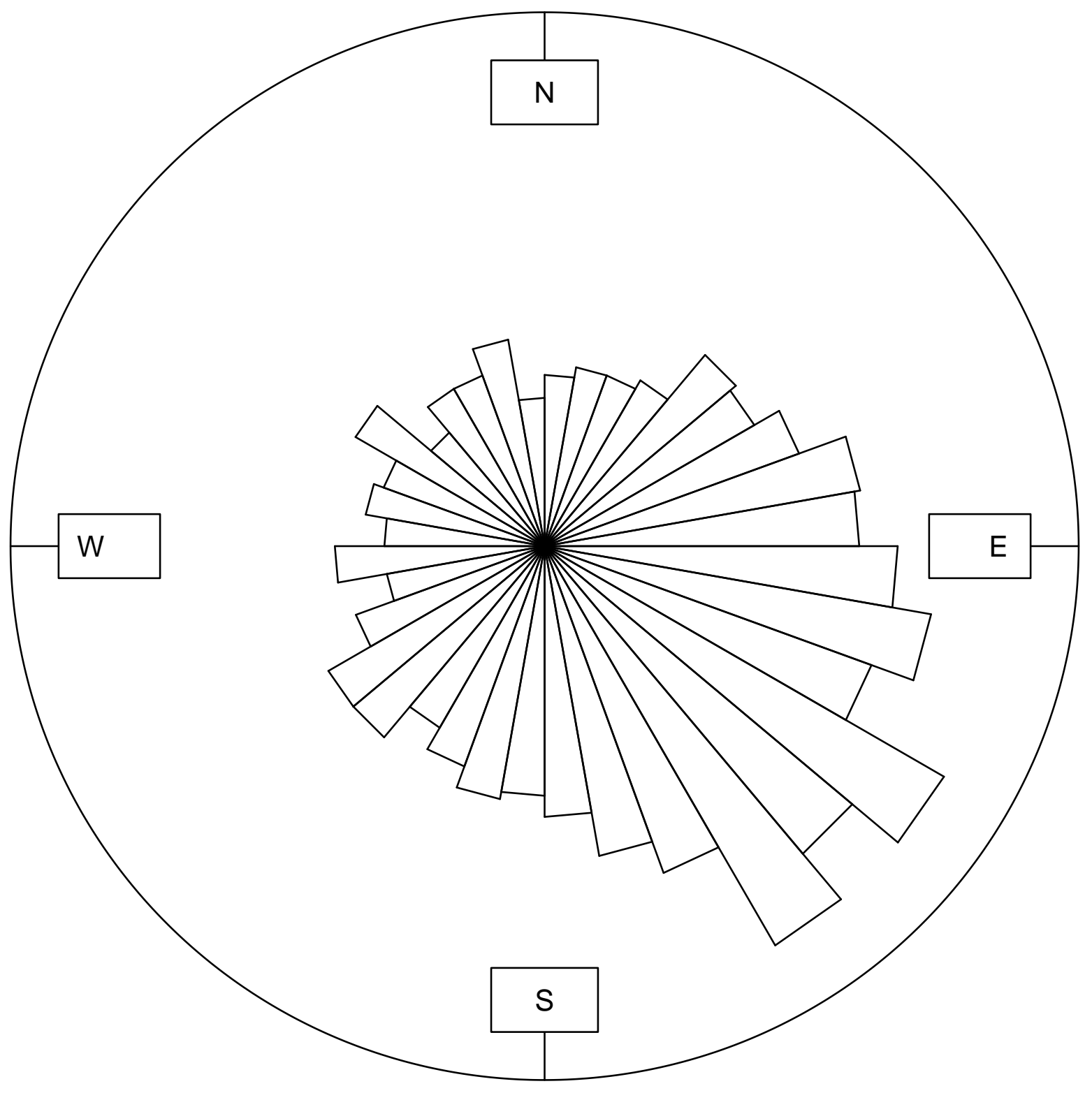


bootstrap 200

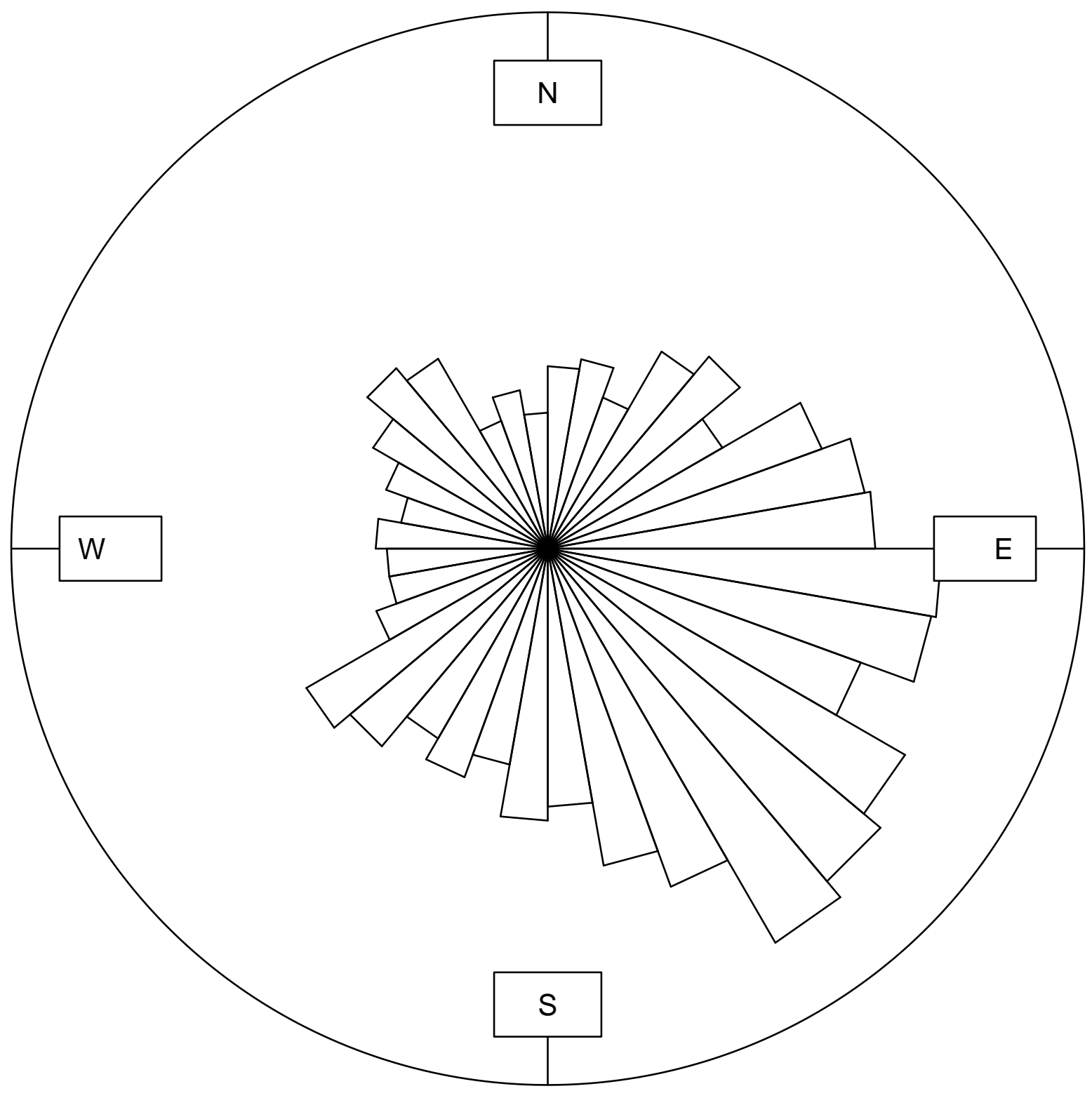


bootstrap 201

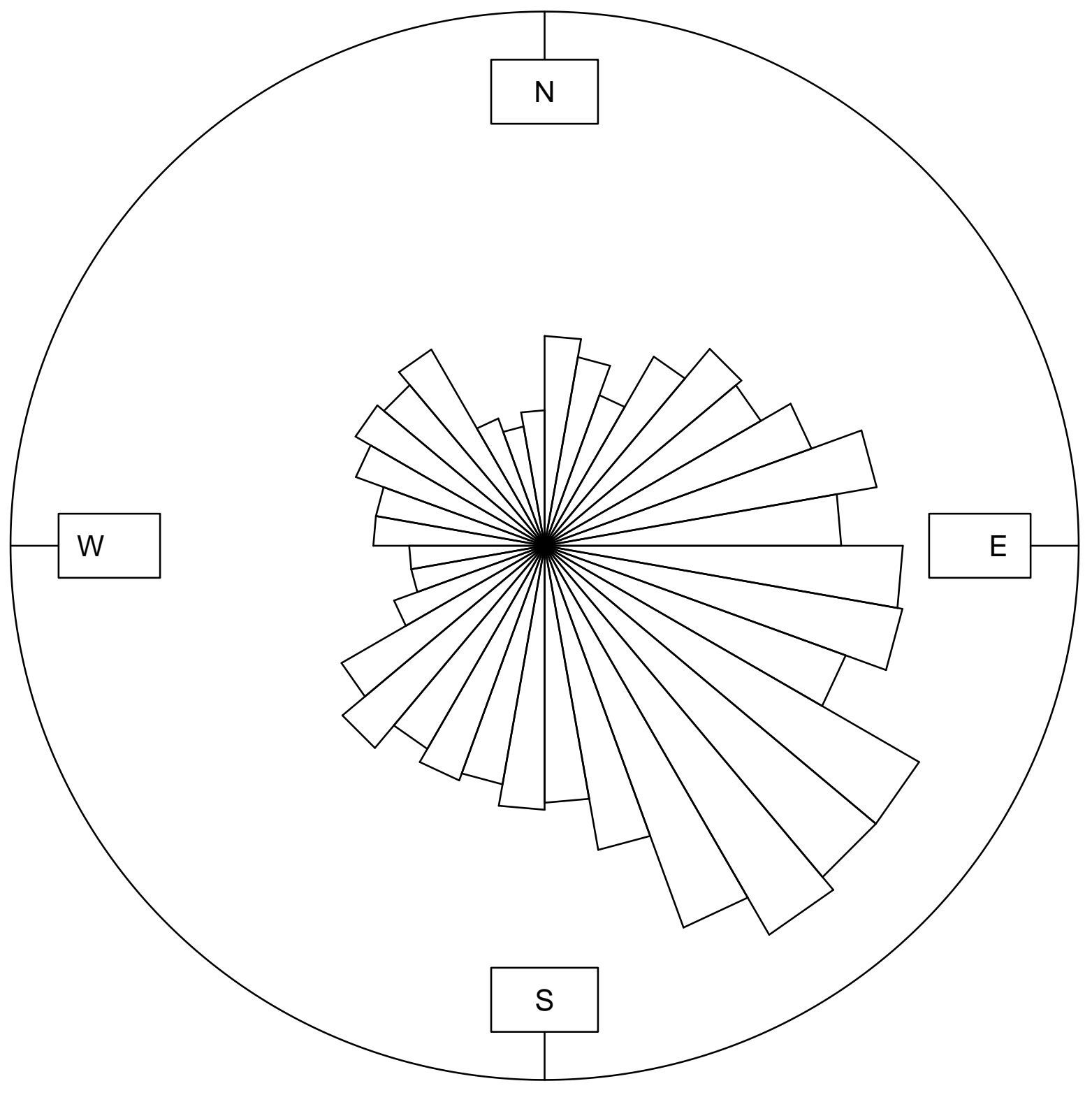


bootstrap 202

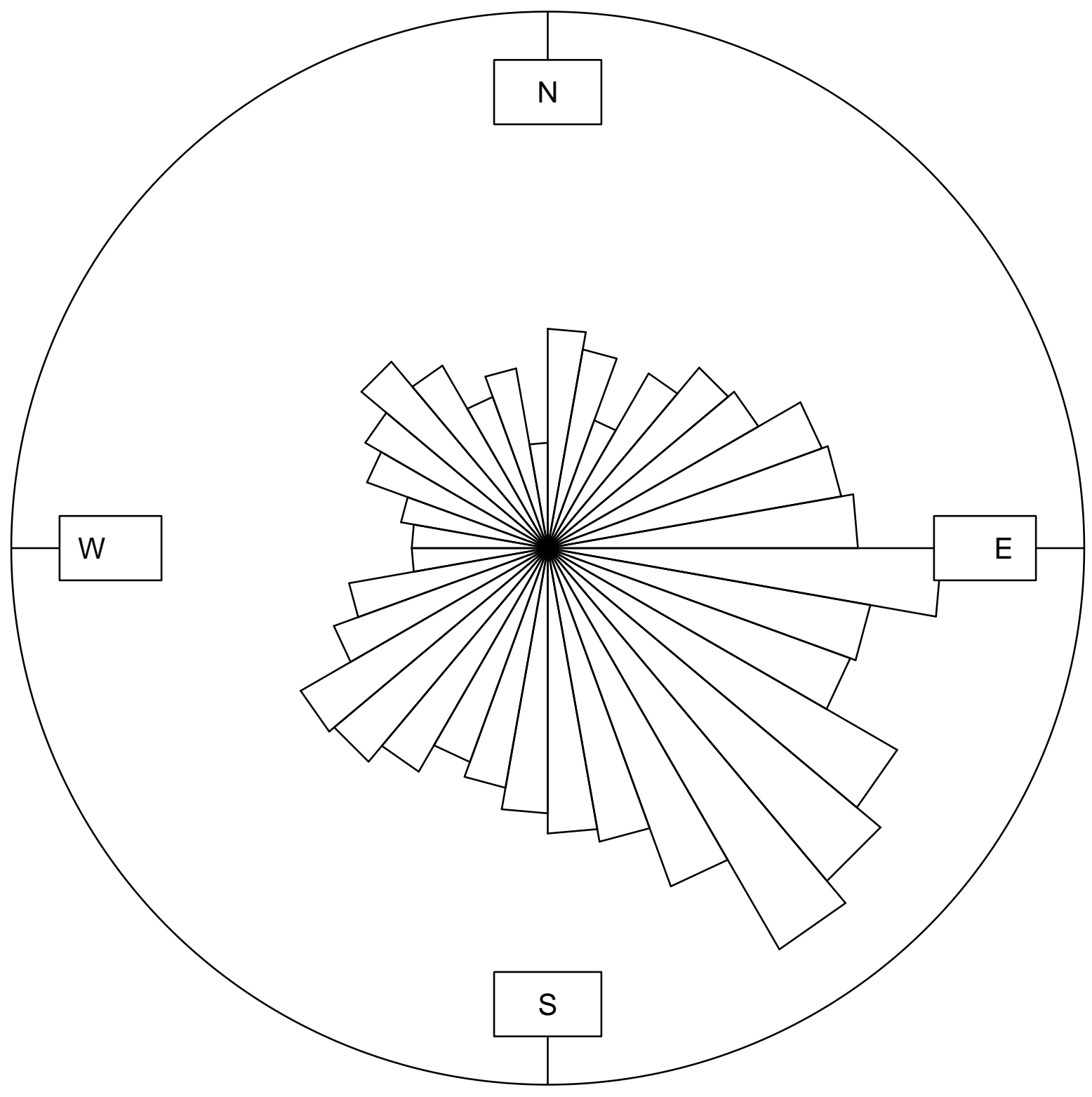




\section{bootstrap 203}

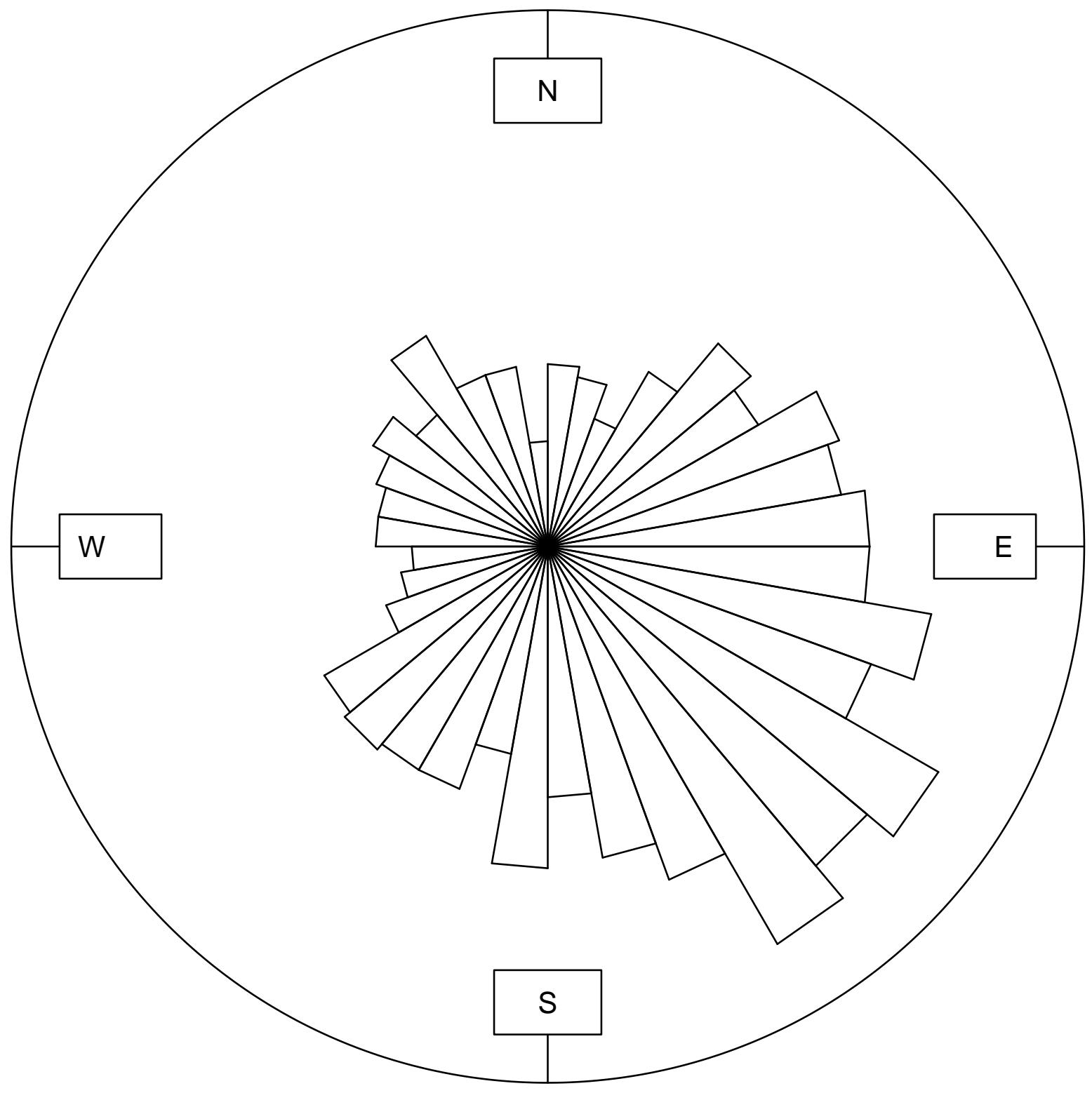


bootstrap 204

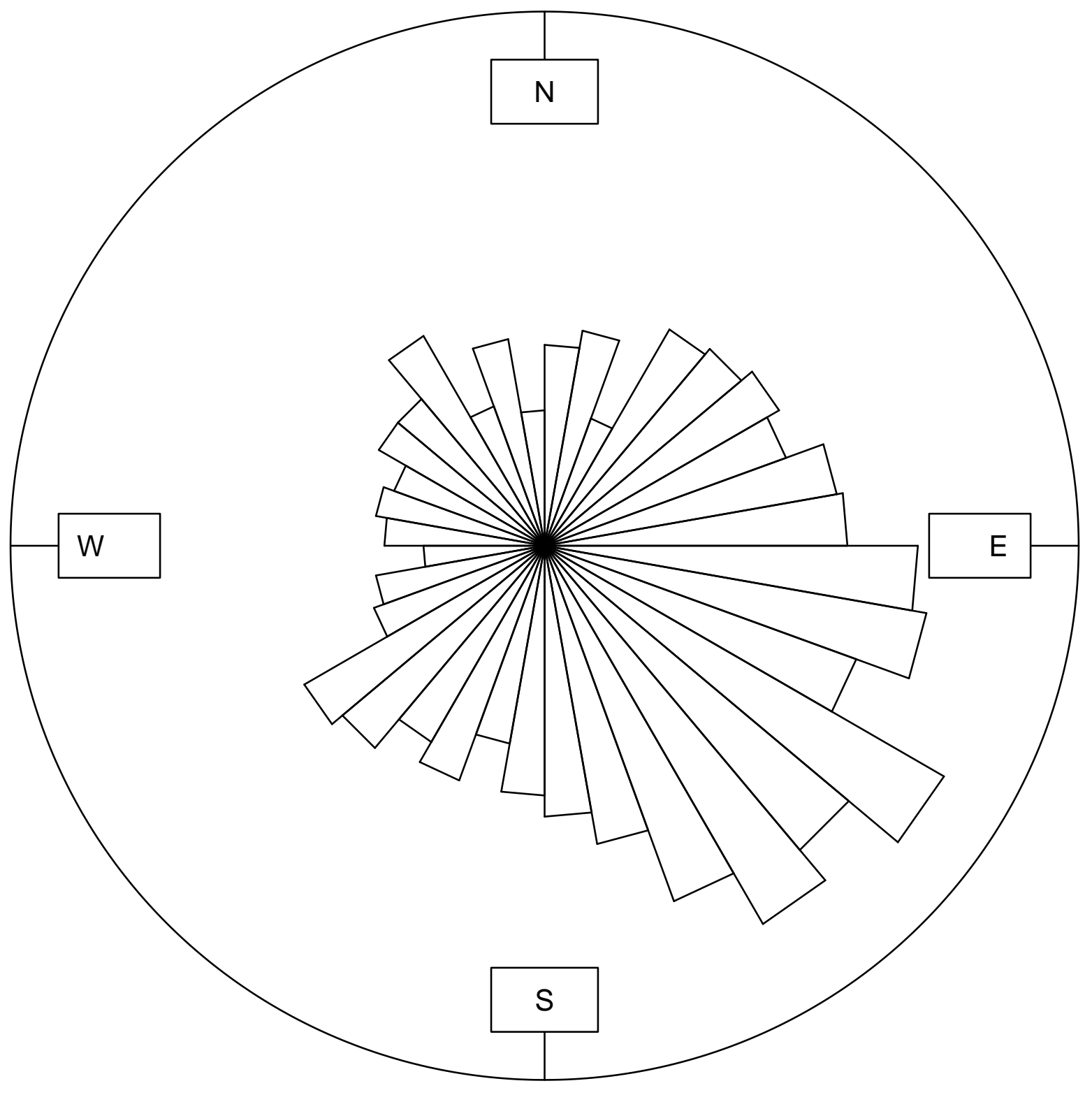


bootstrap 205

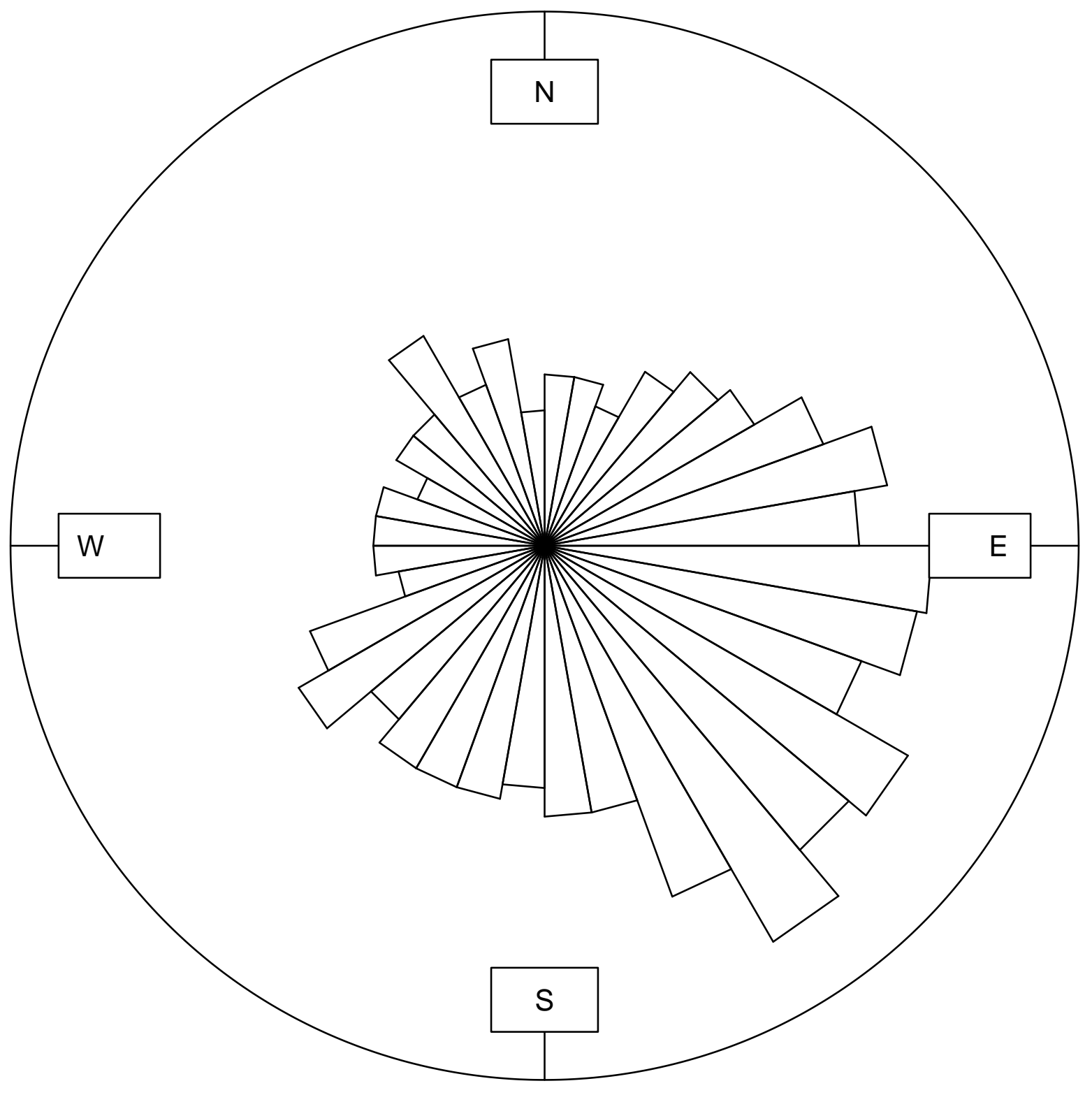


bootstrap 206

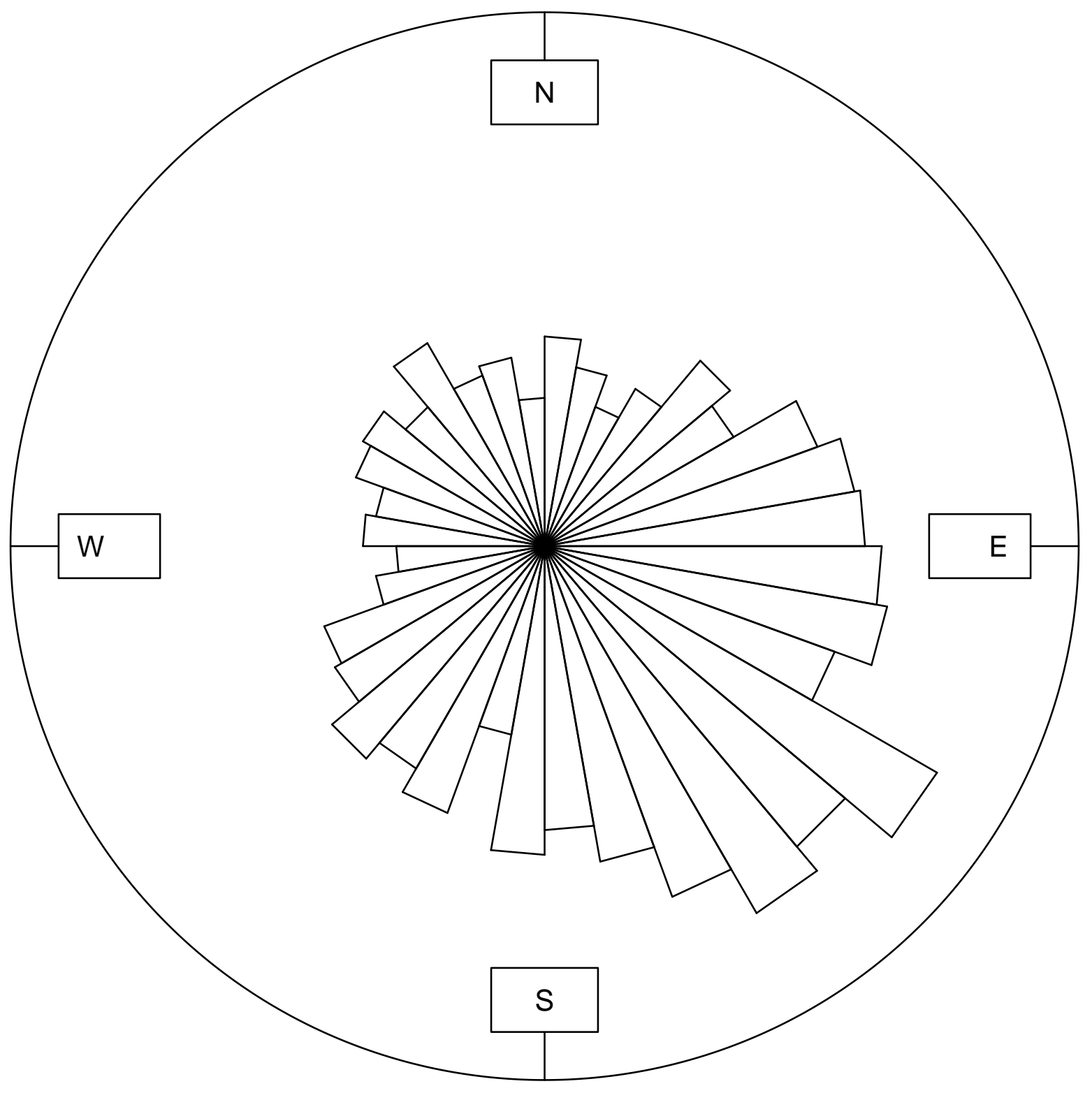


bootstrap 207

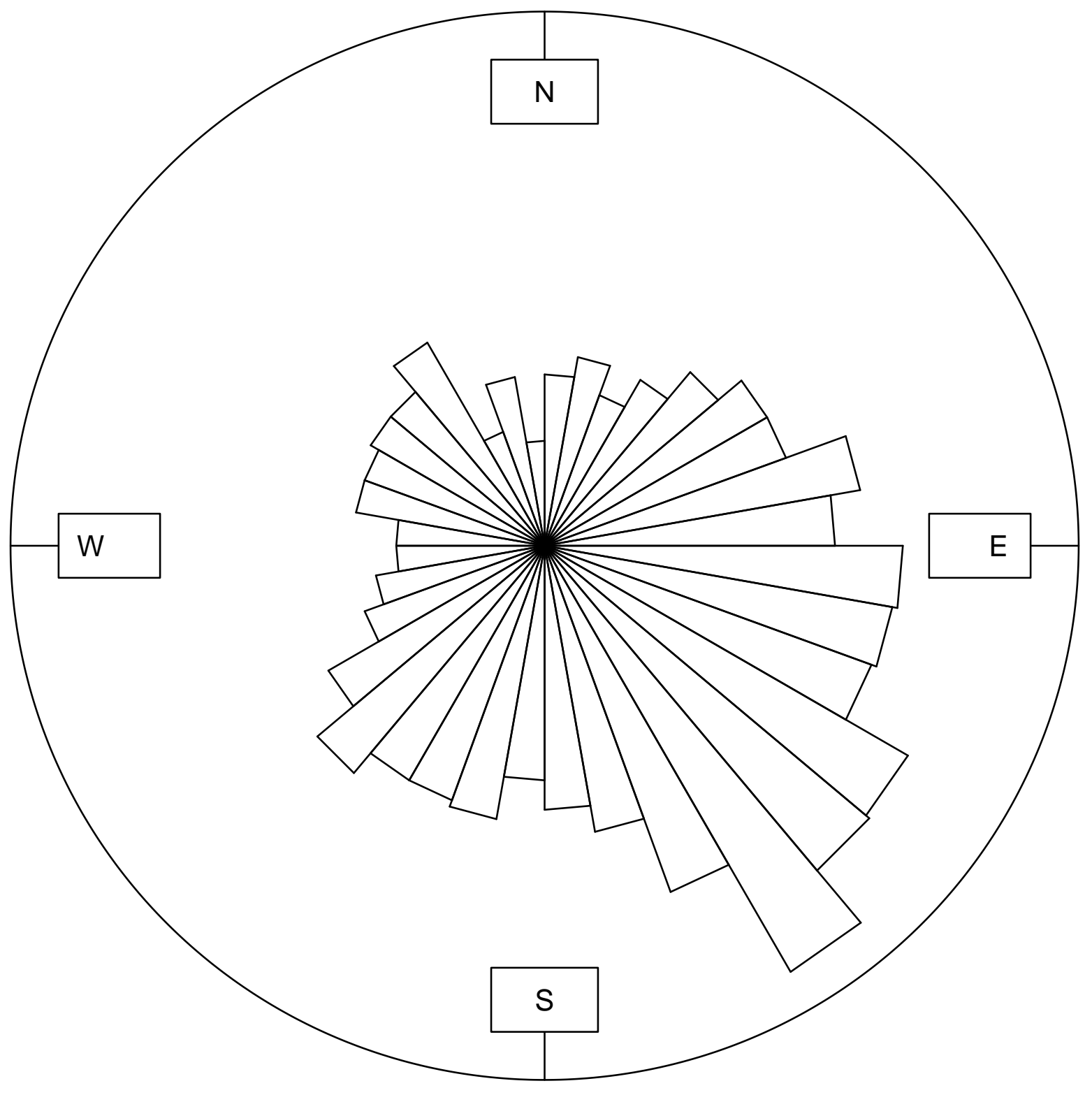


bootstrap 208

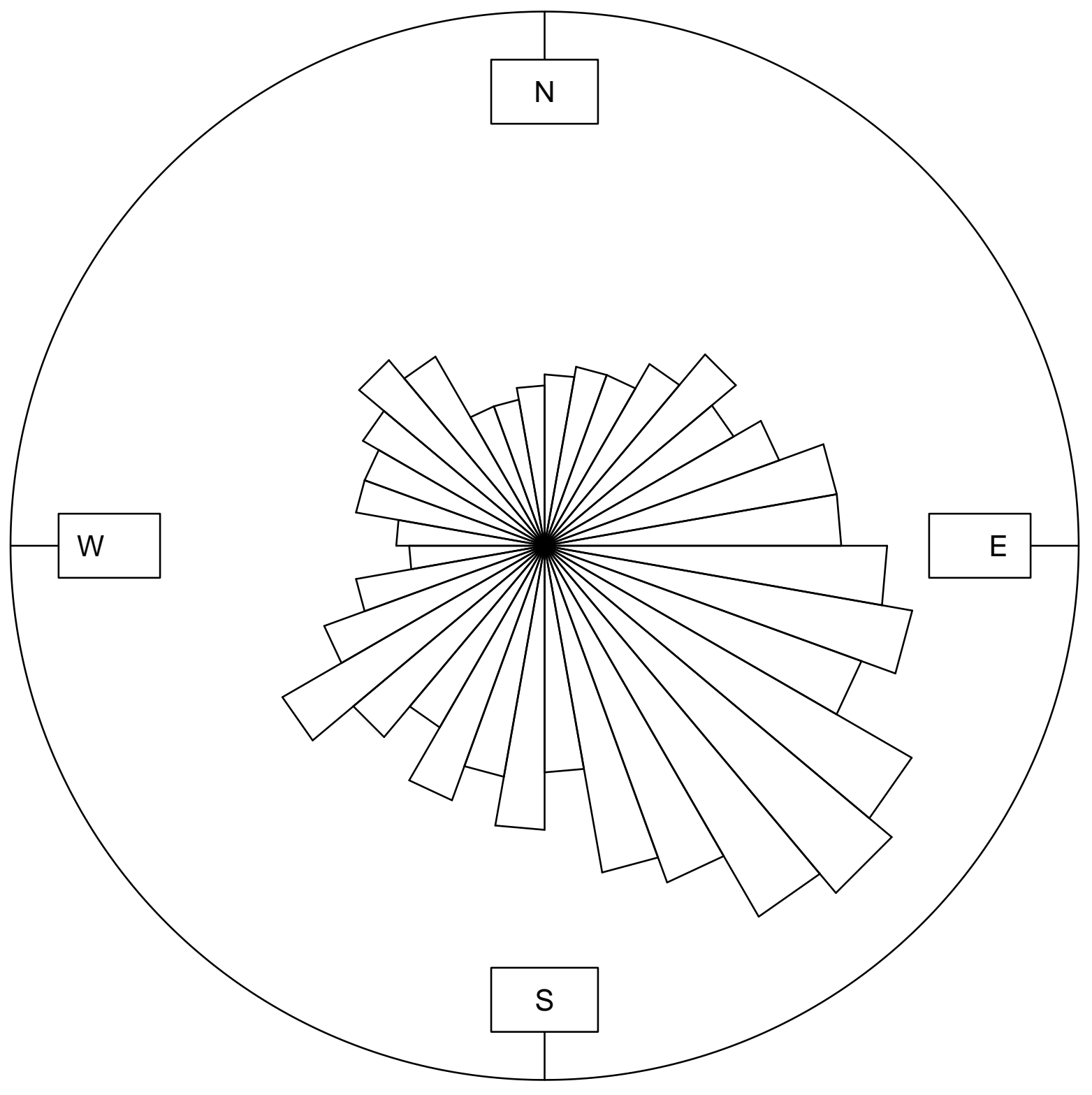


bootstrap 209

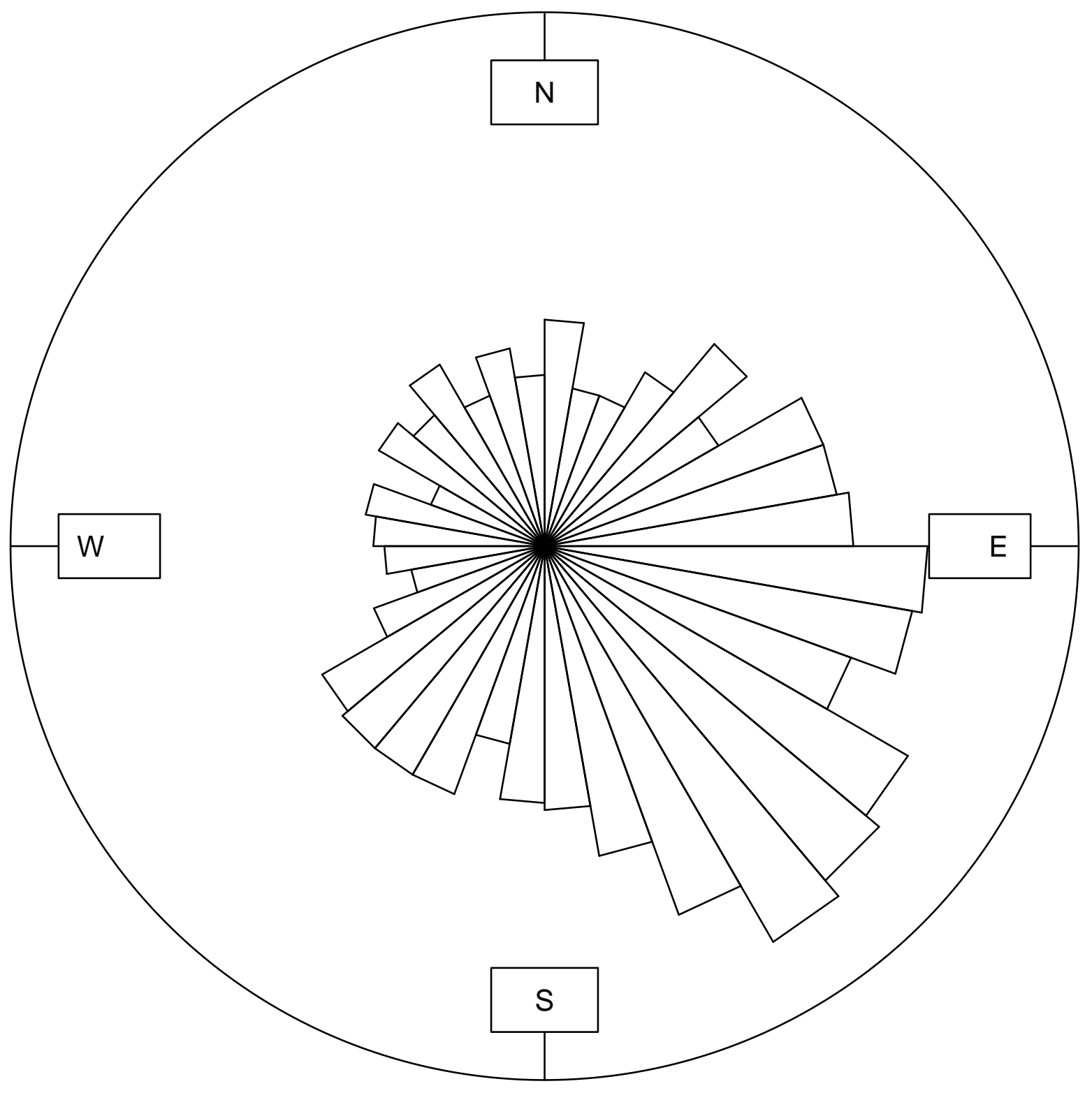


bootstrap 210

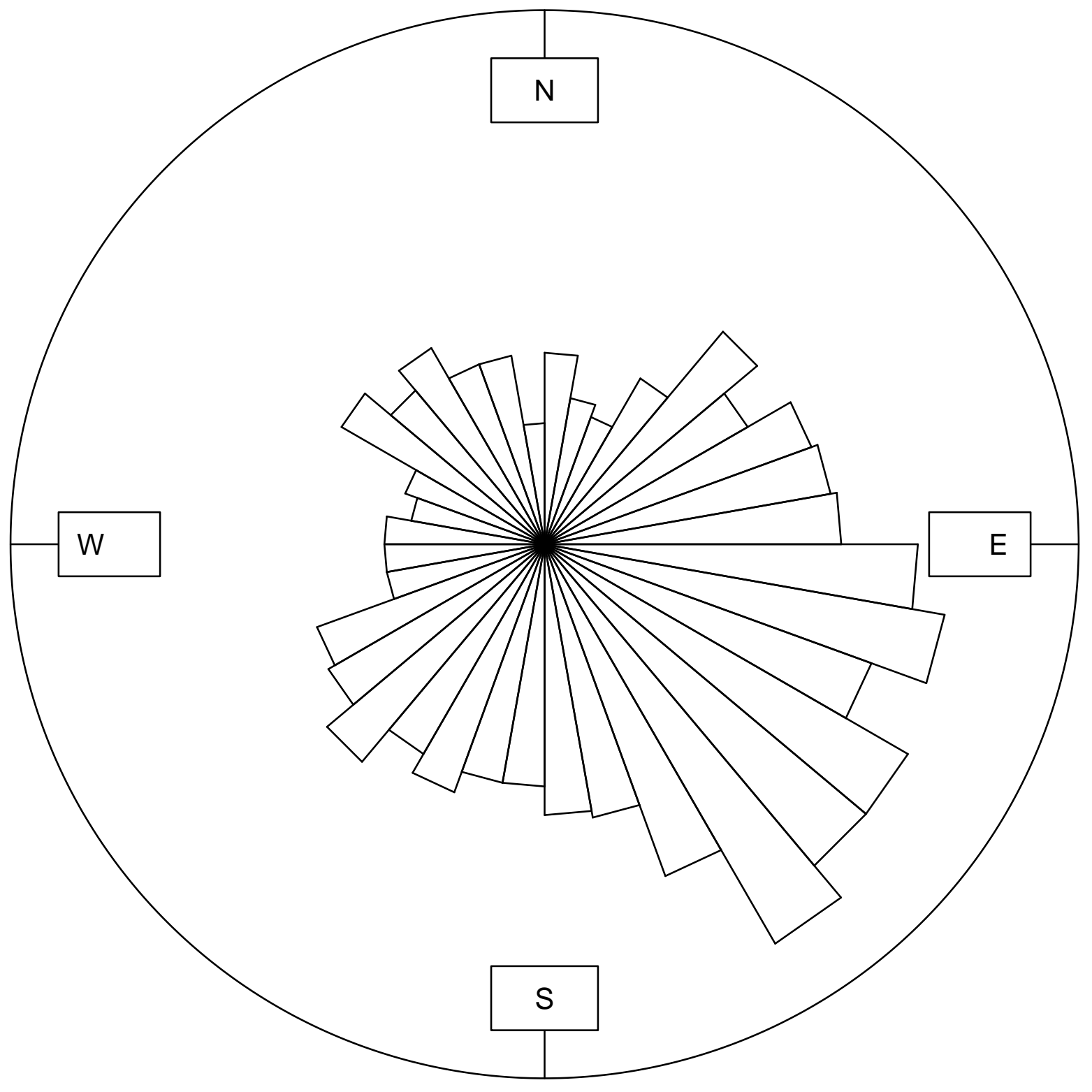


bootstrap 211

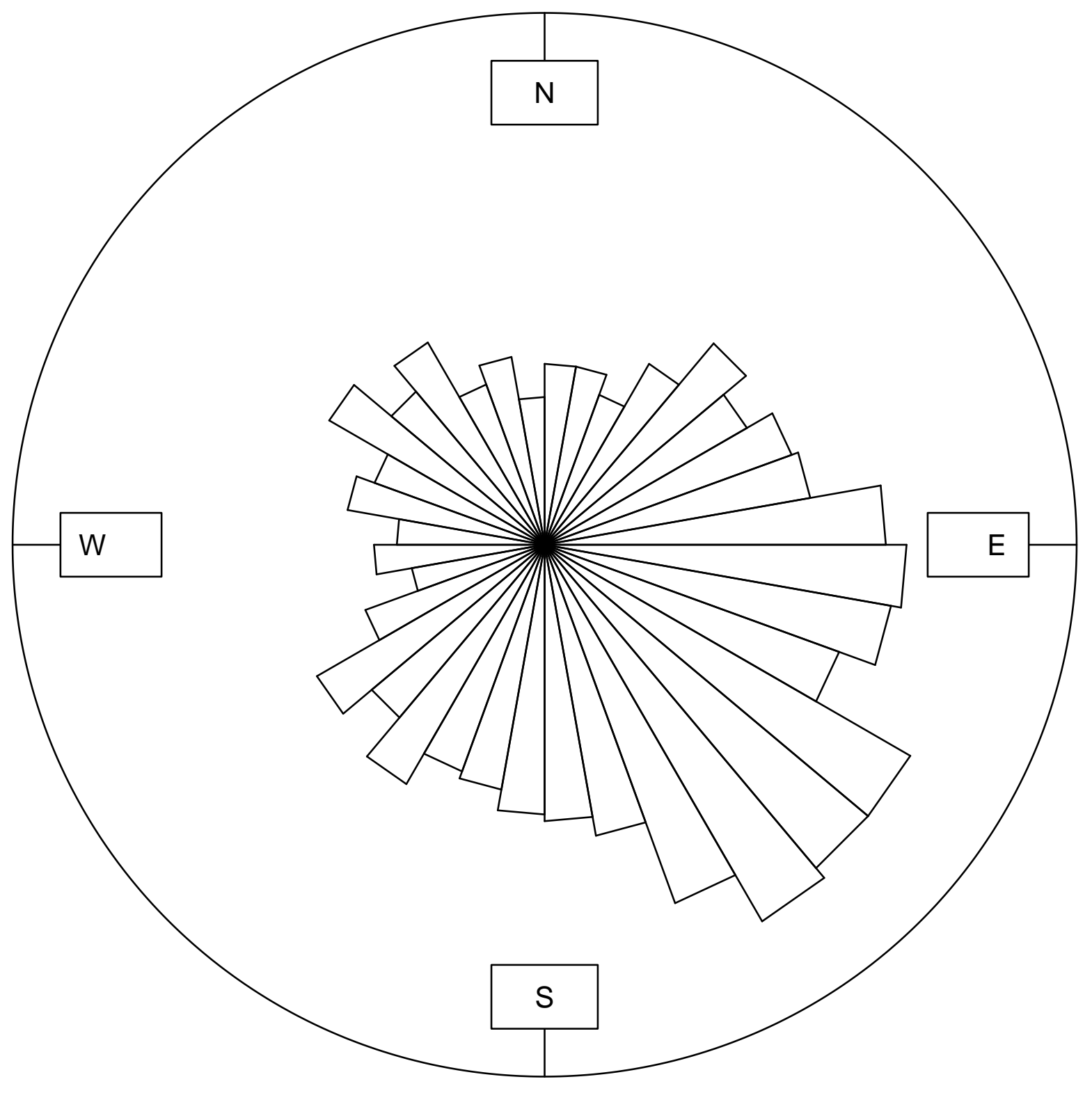


bootstrap 212

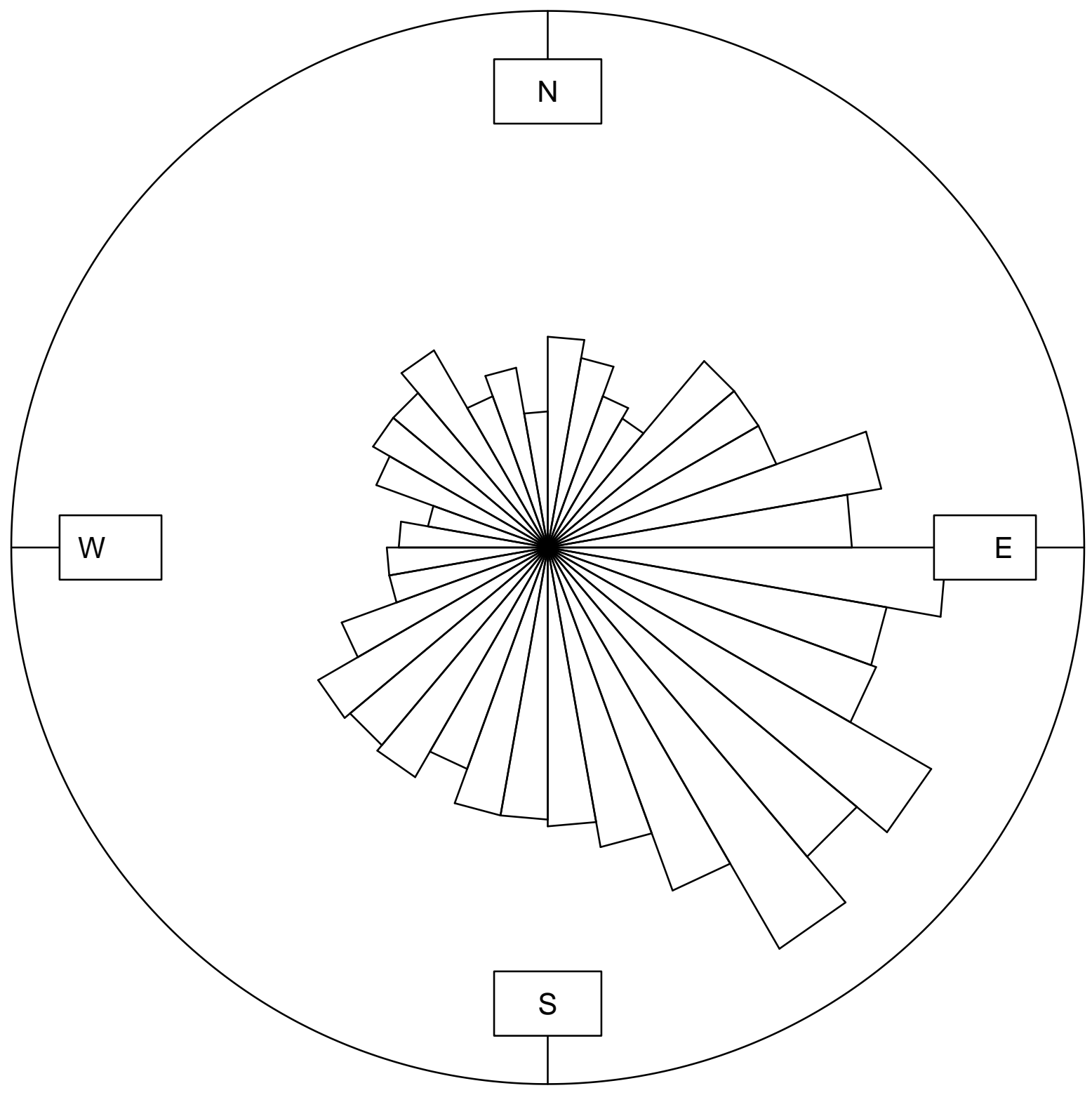


bootstrap 213

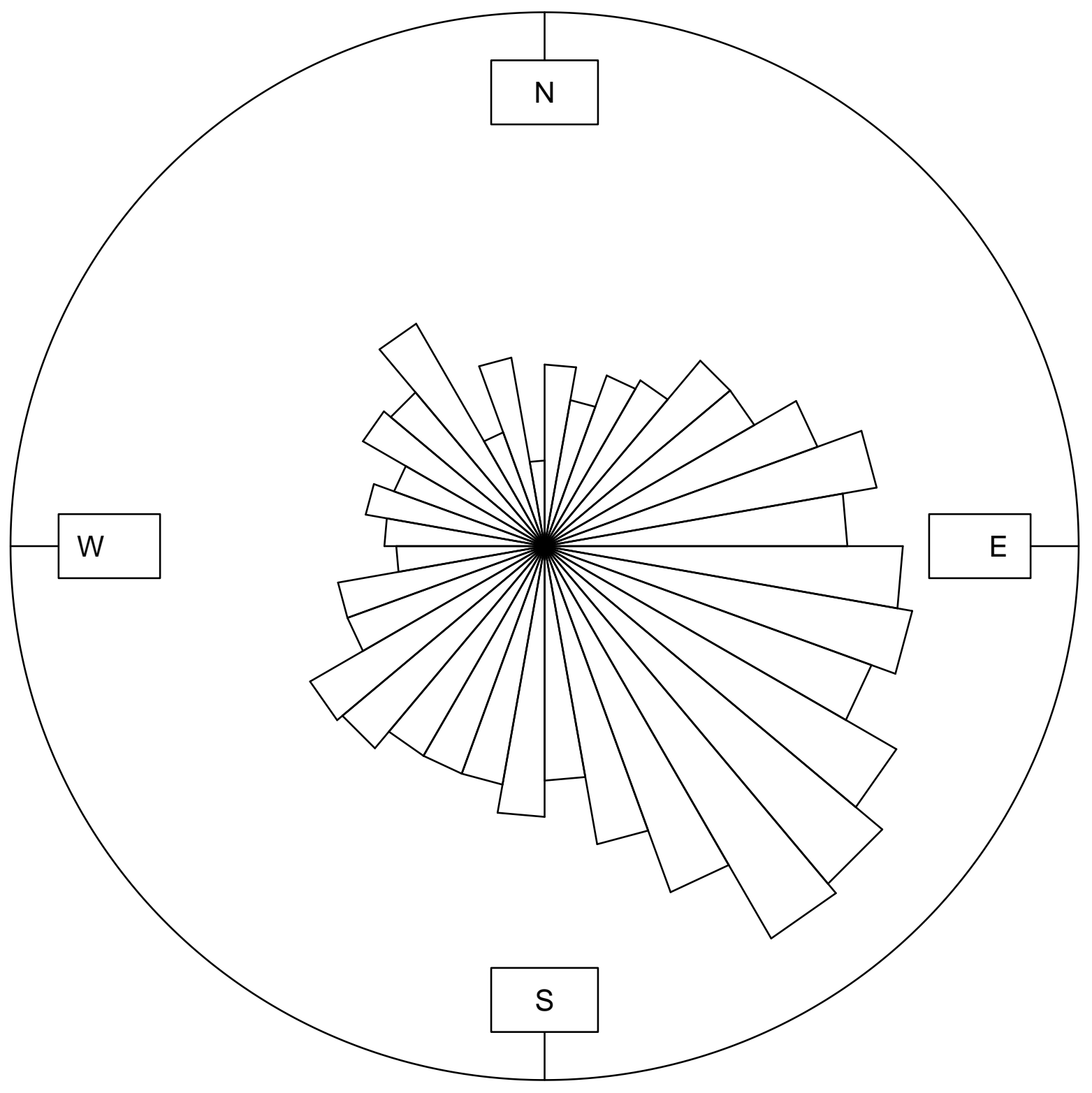


bootstrap 214

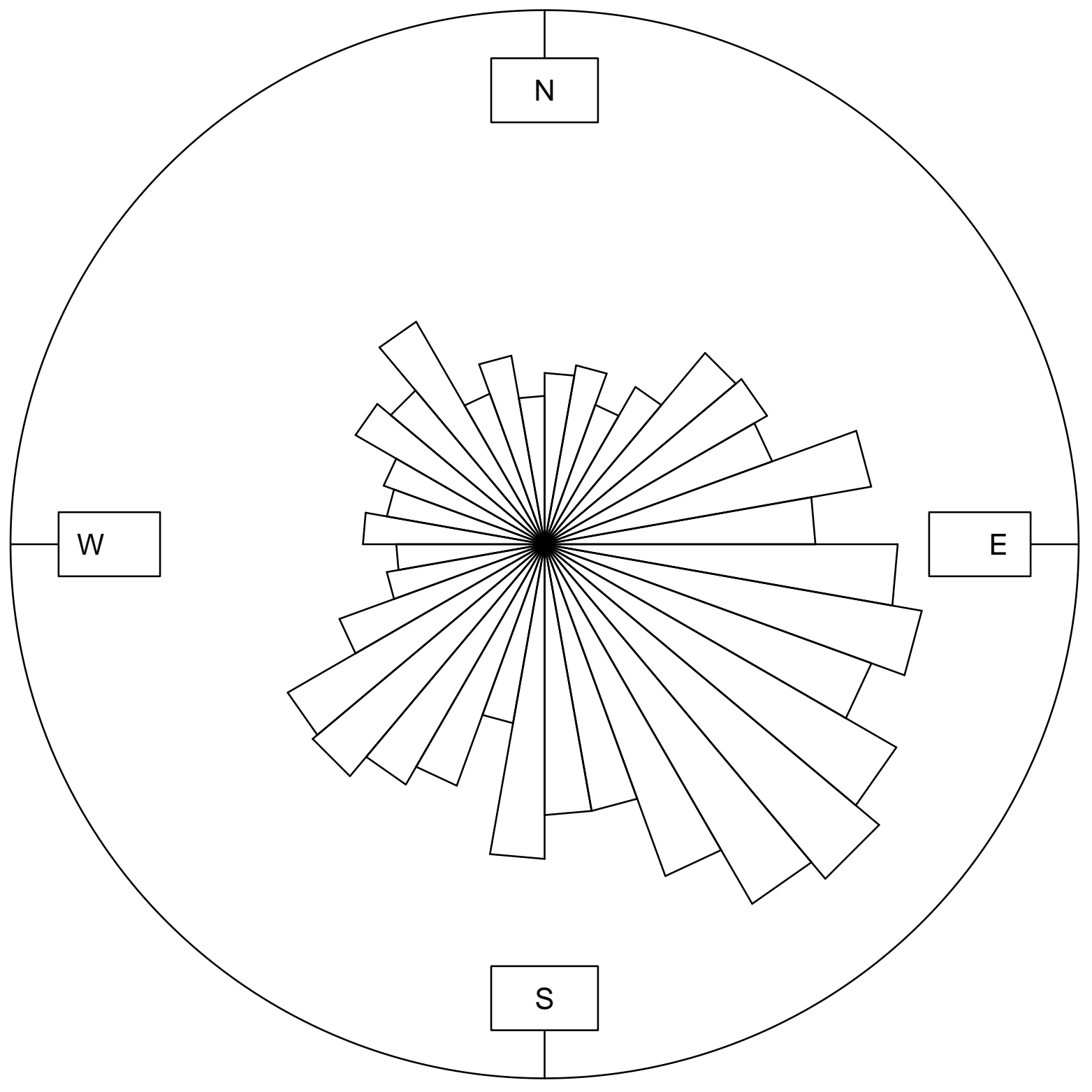


bootstrap 215

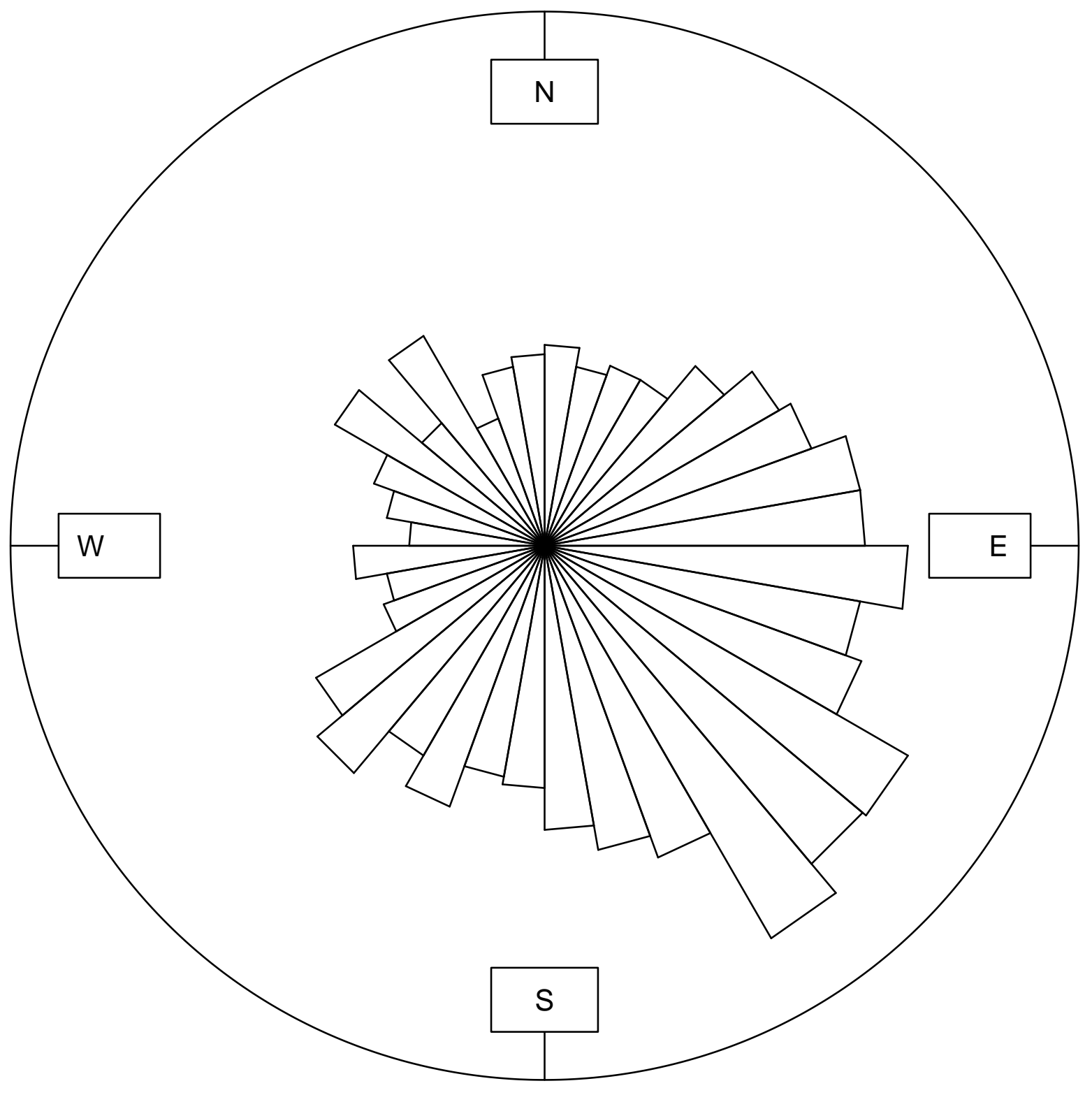


bootstrap 216

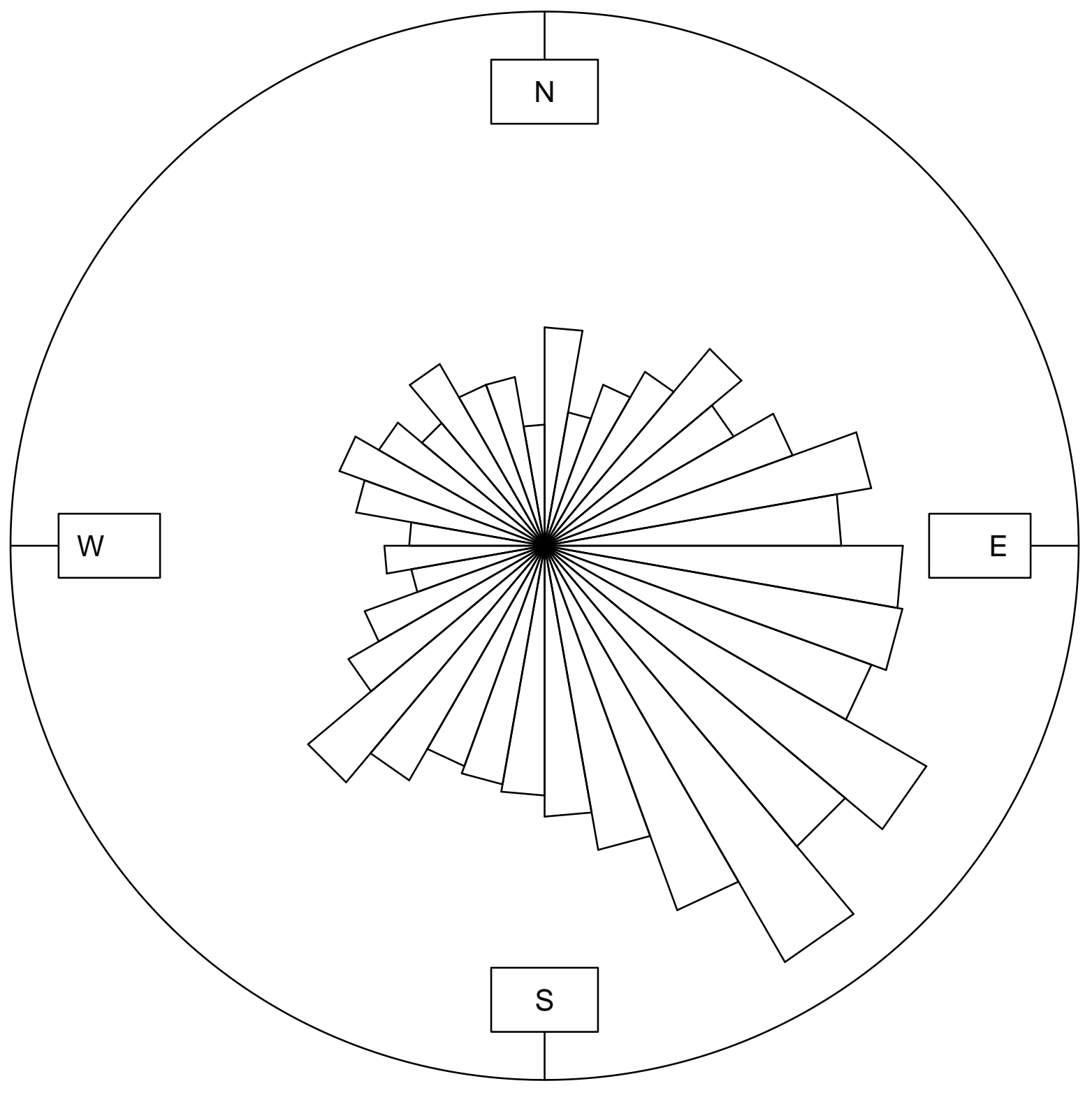


bootstrap 217

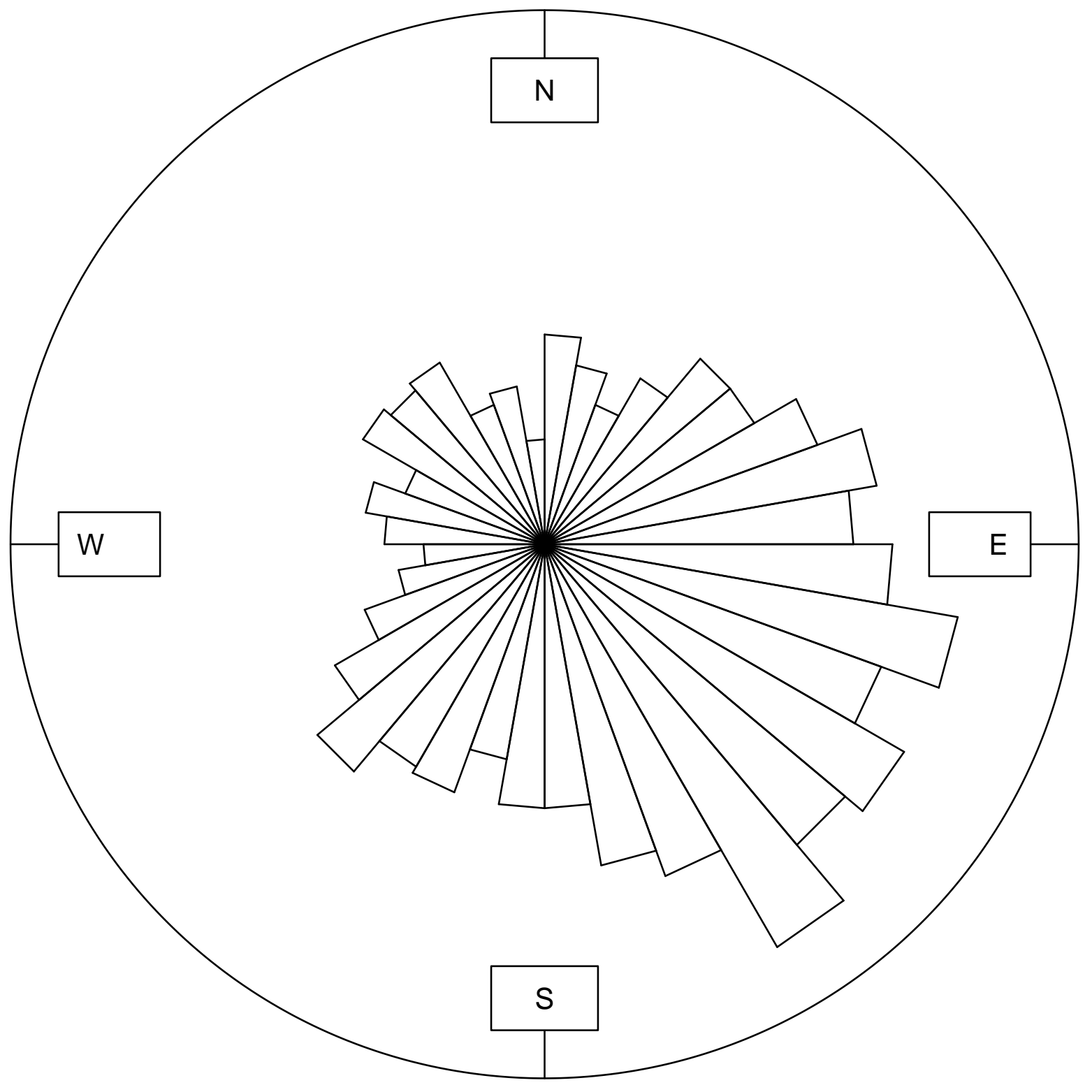


bootstrap 218

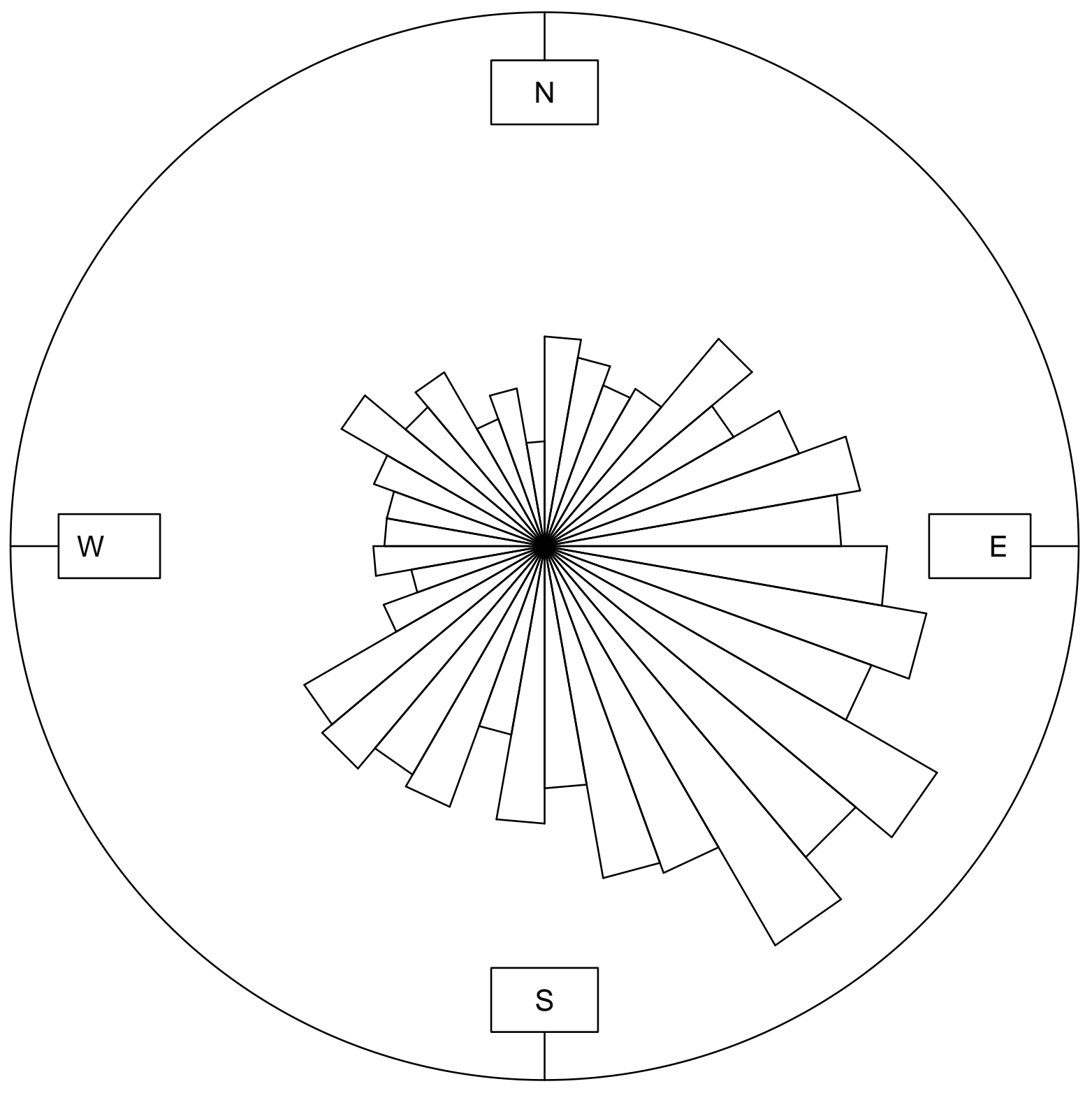


bootstrap 219

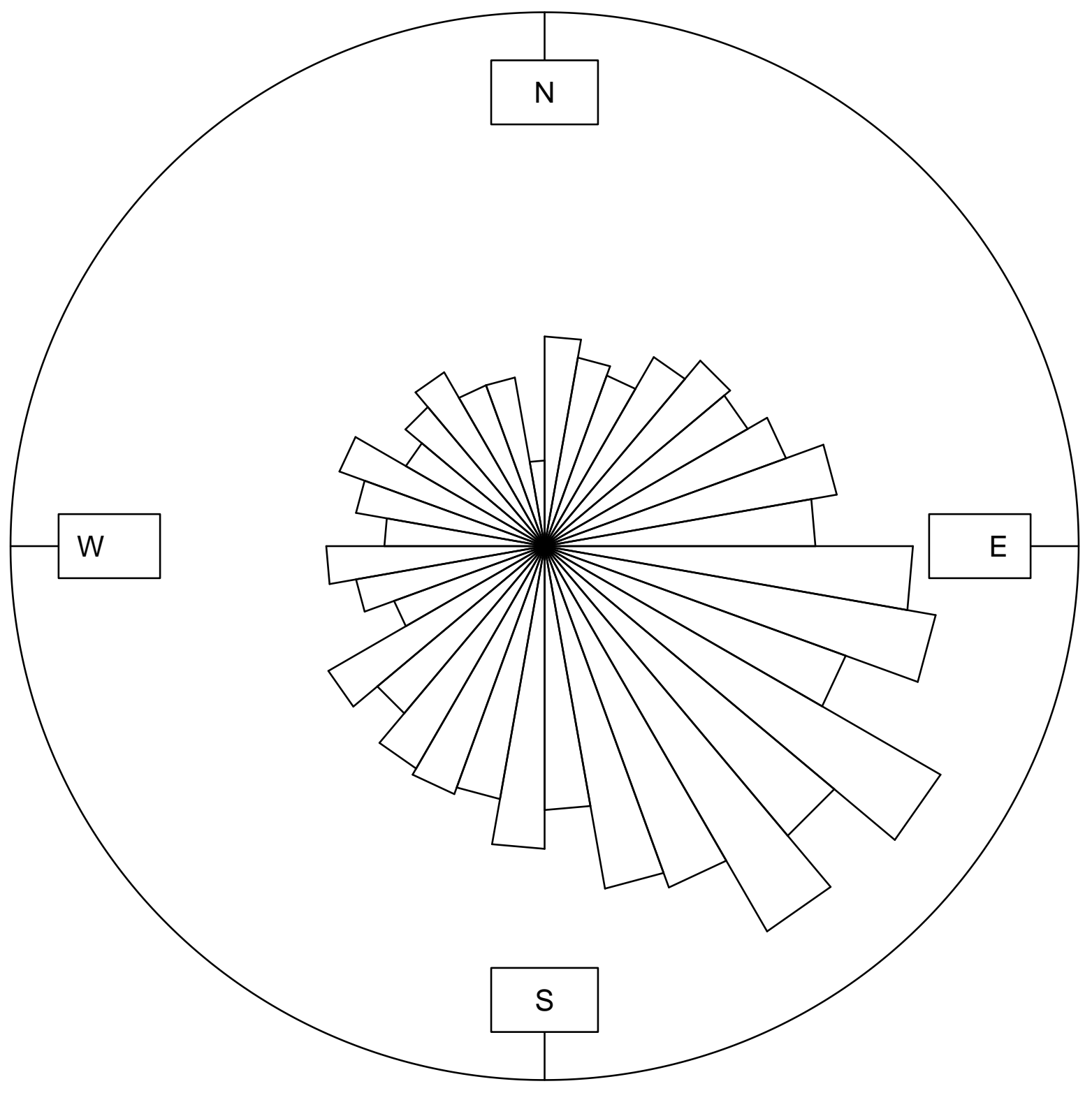


bootstrap 220

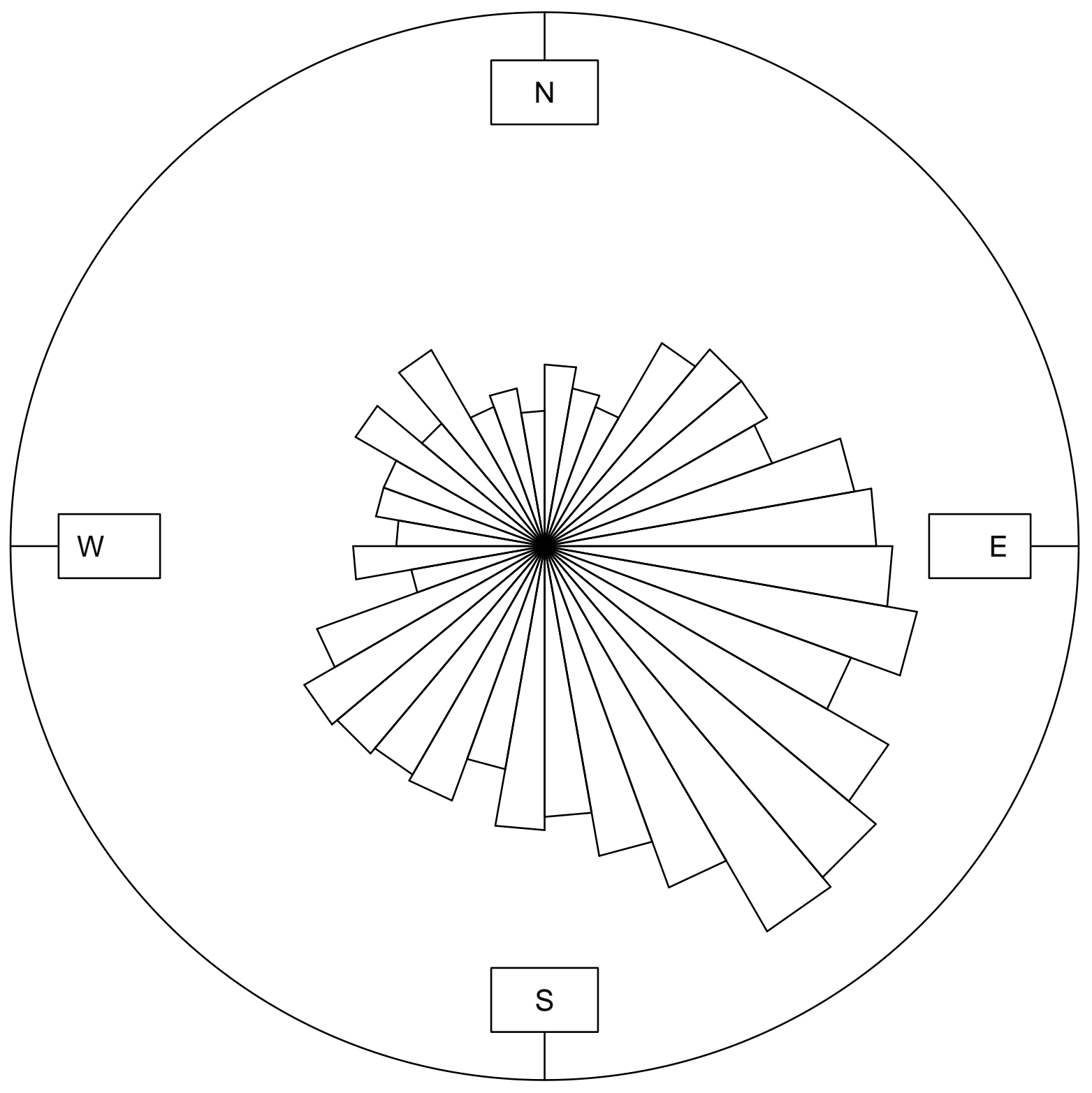


bootstrap 221

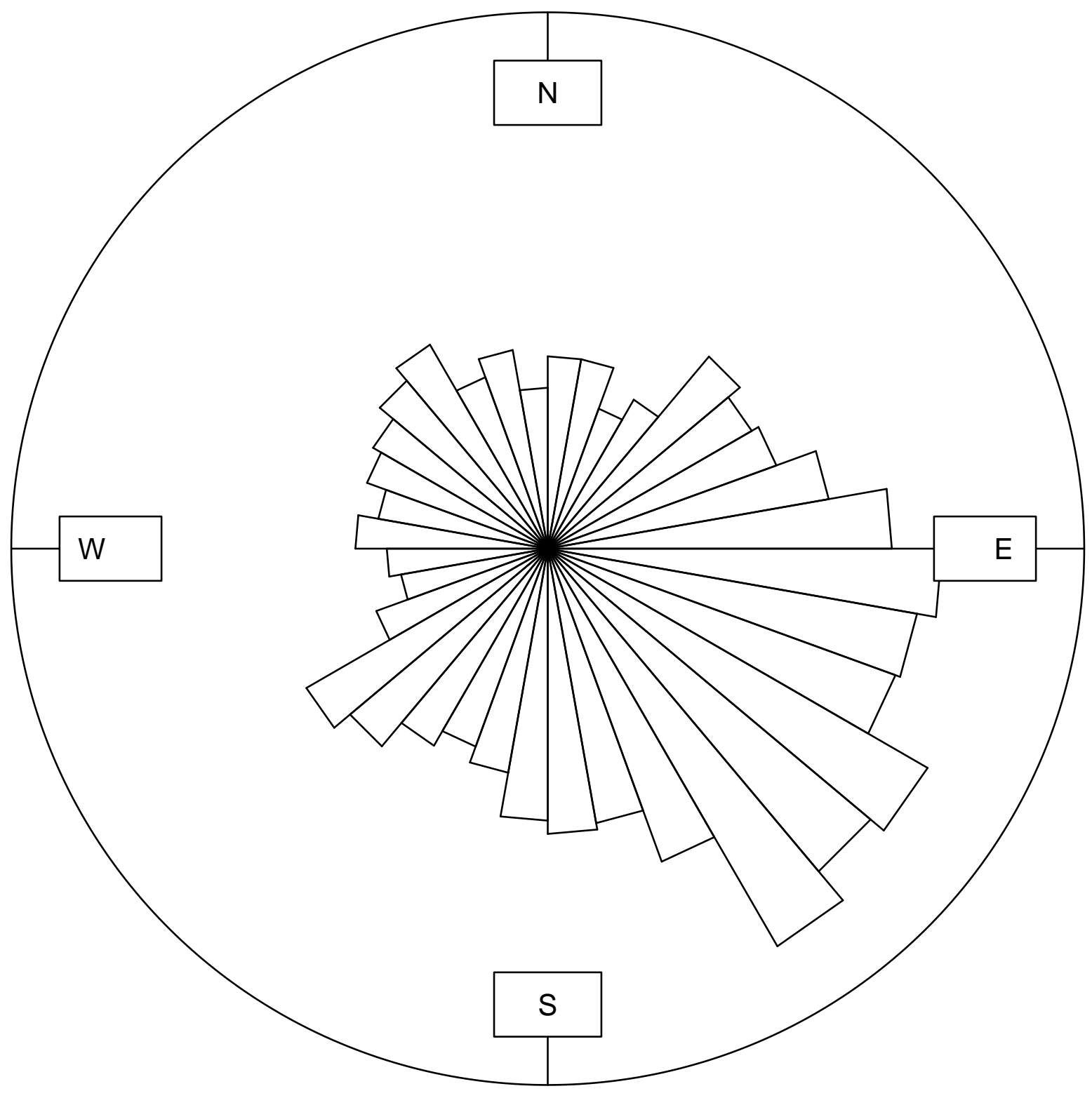


bootstrap 222

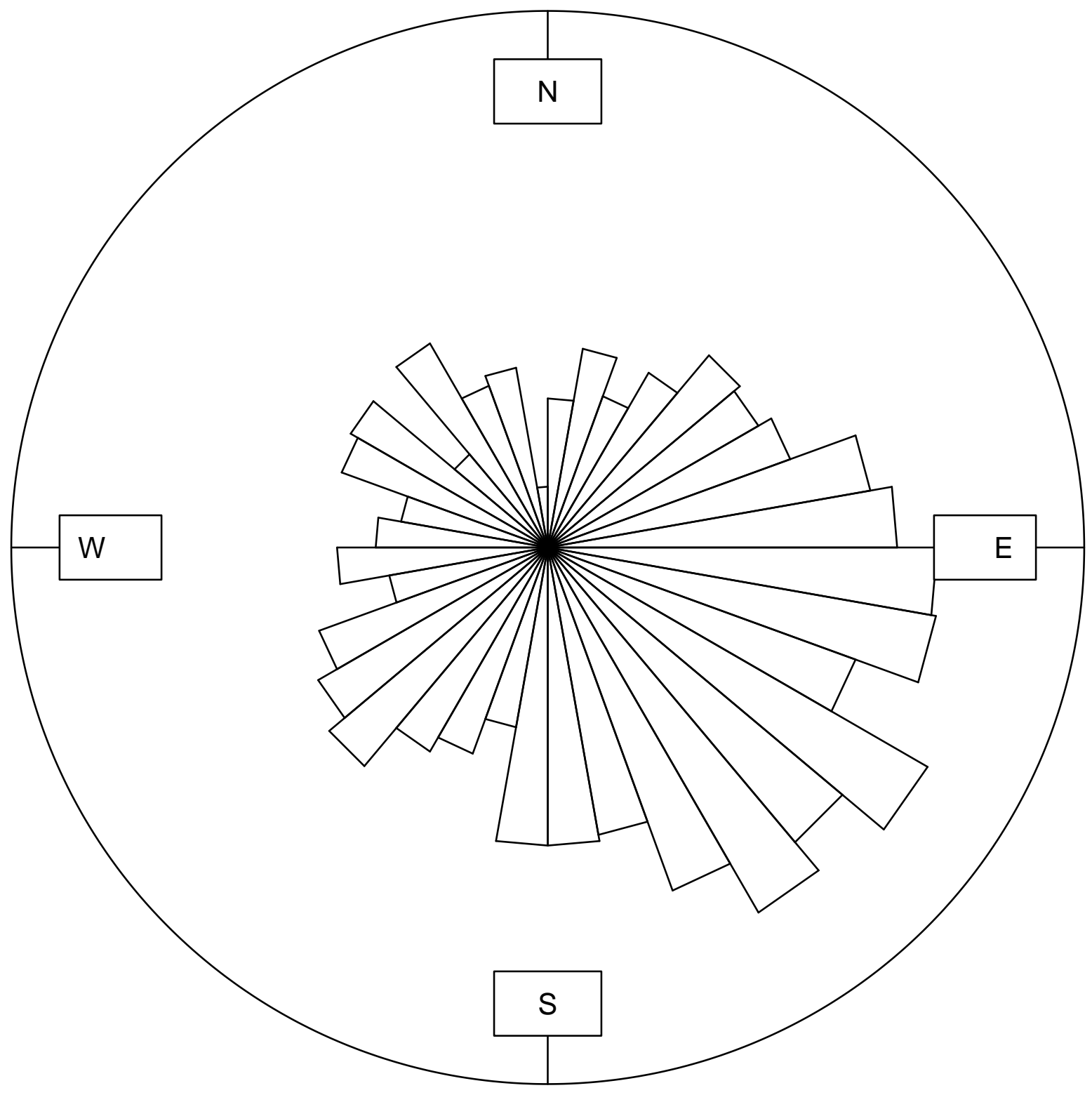


bootstrap 223

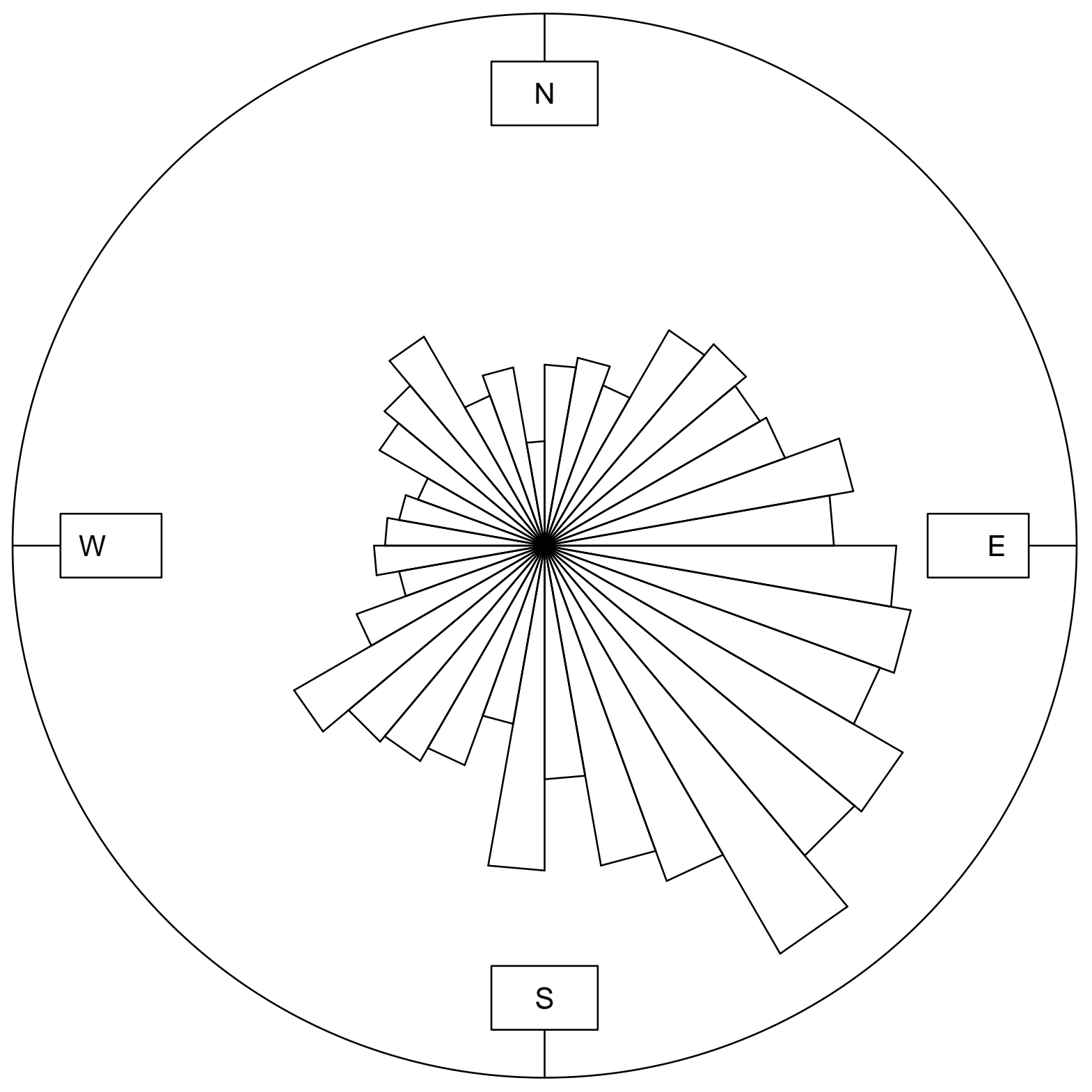


bootstrap 224

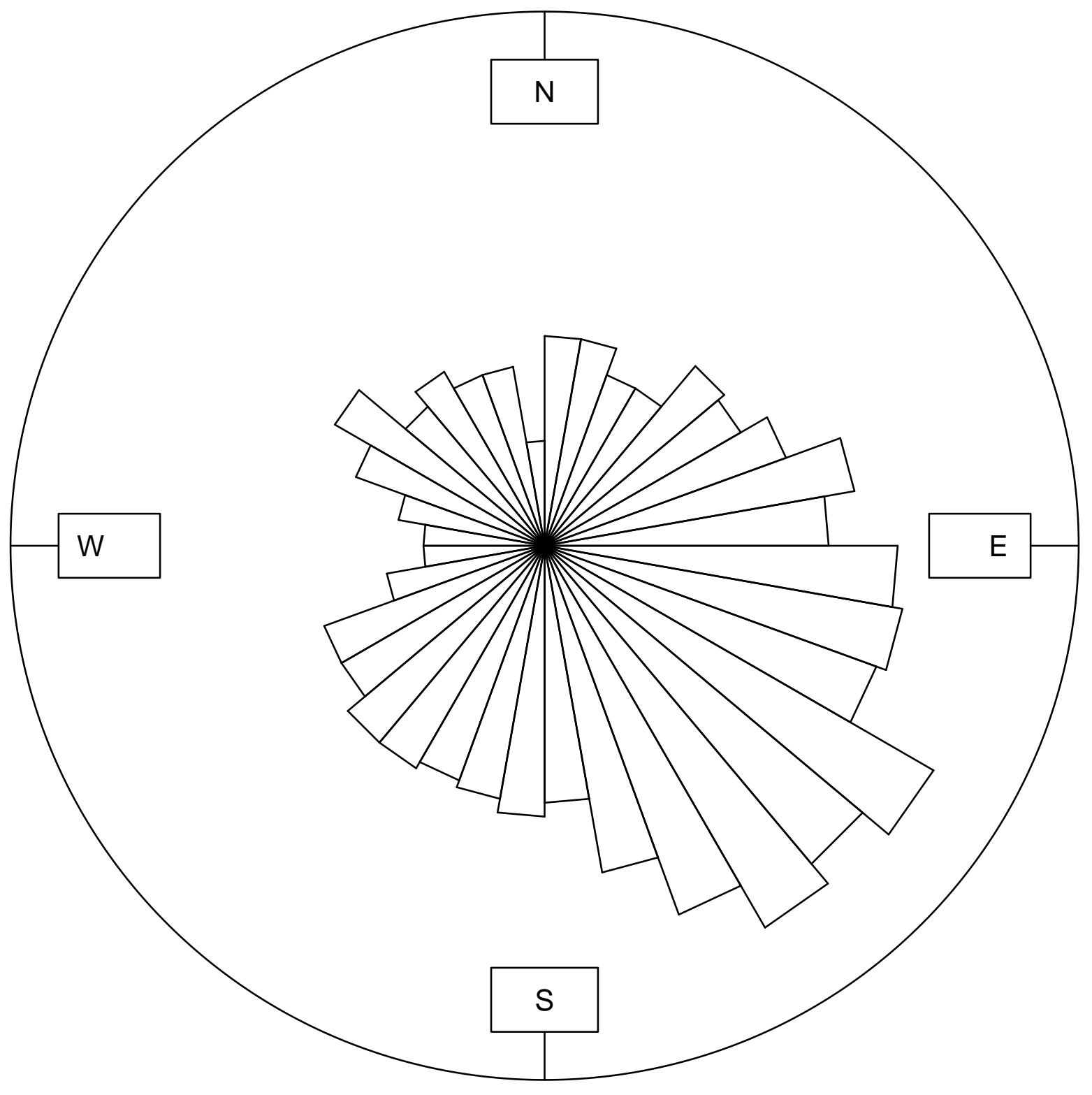


bootstrap 225

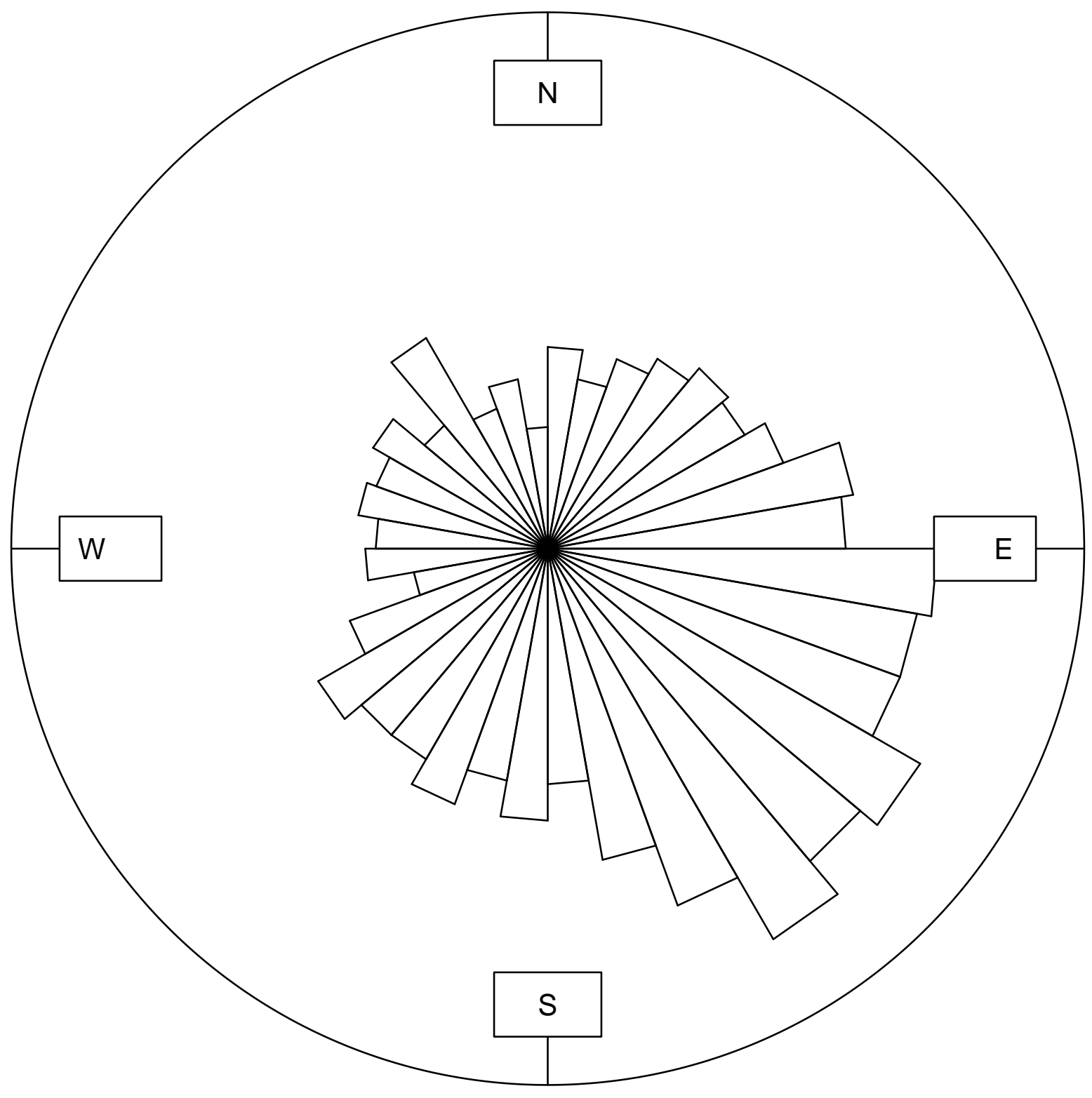


bootstrap 226

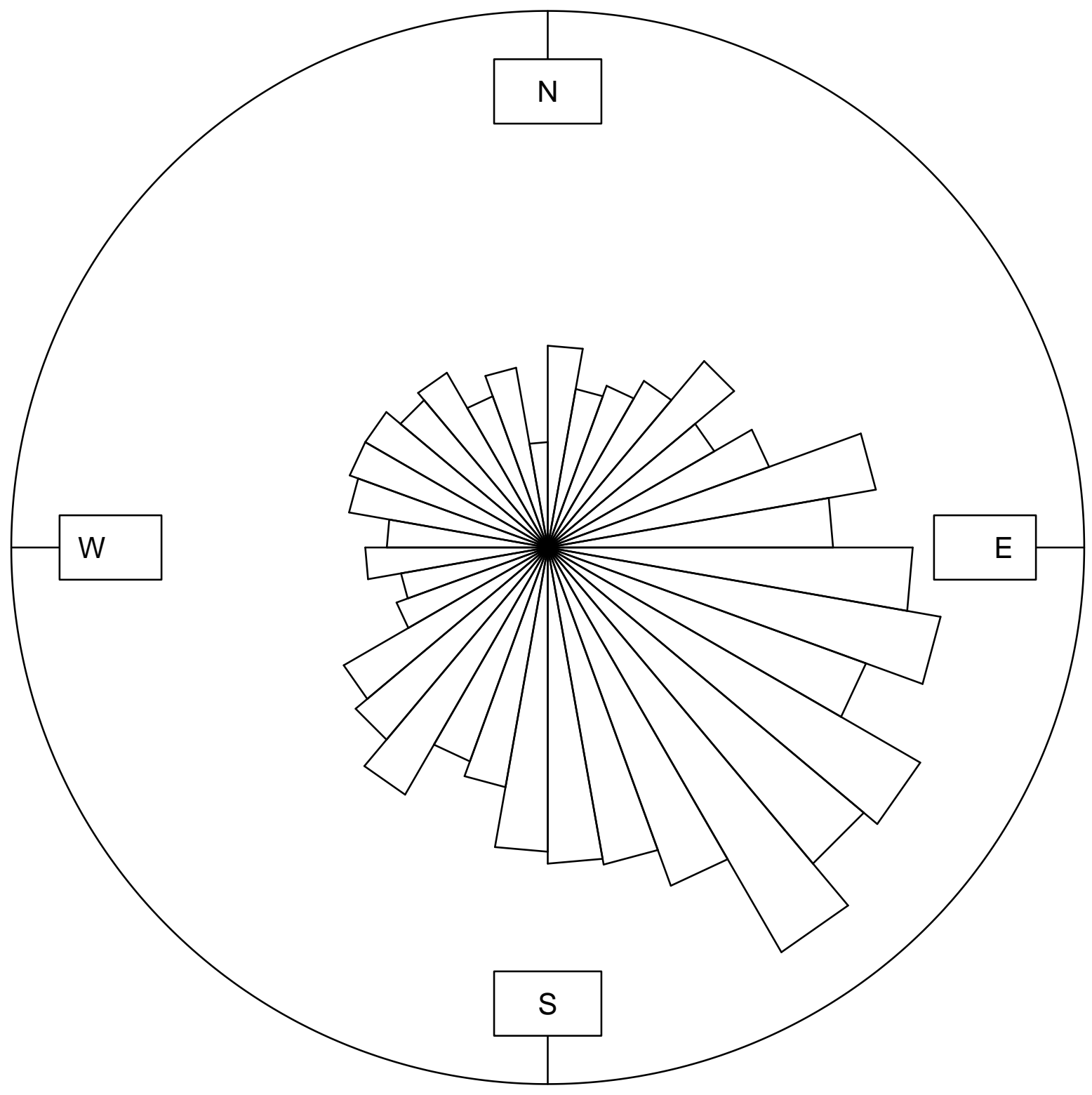


bootstrap 227

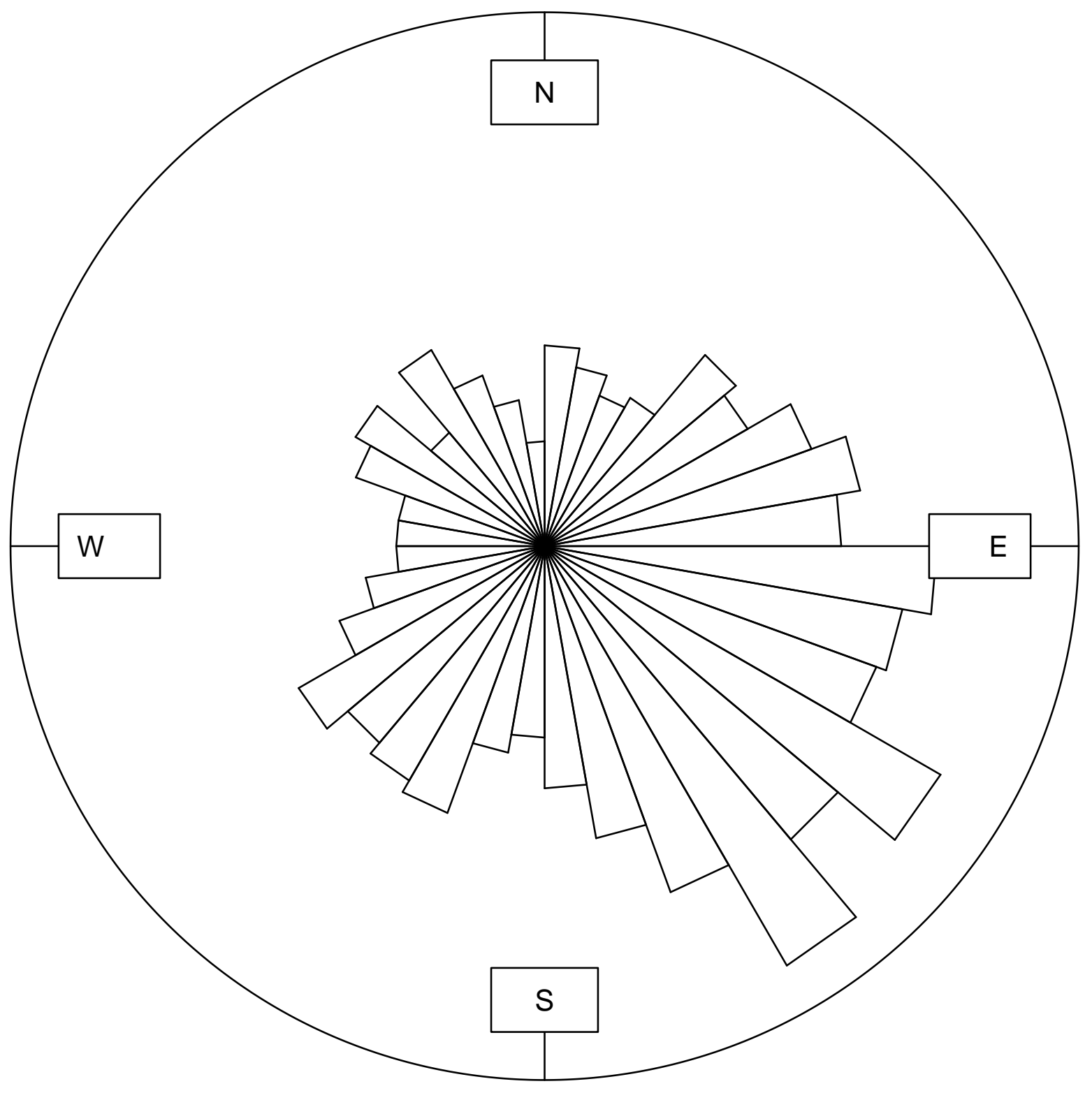


bootstrap 228

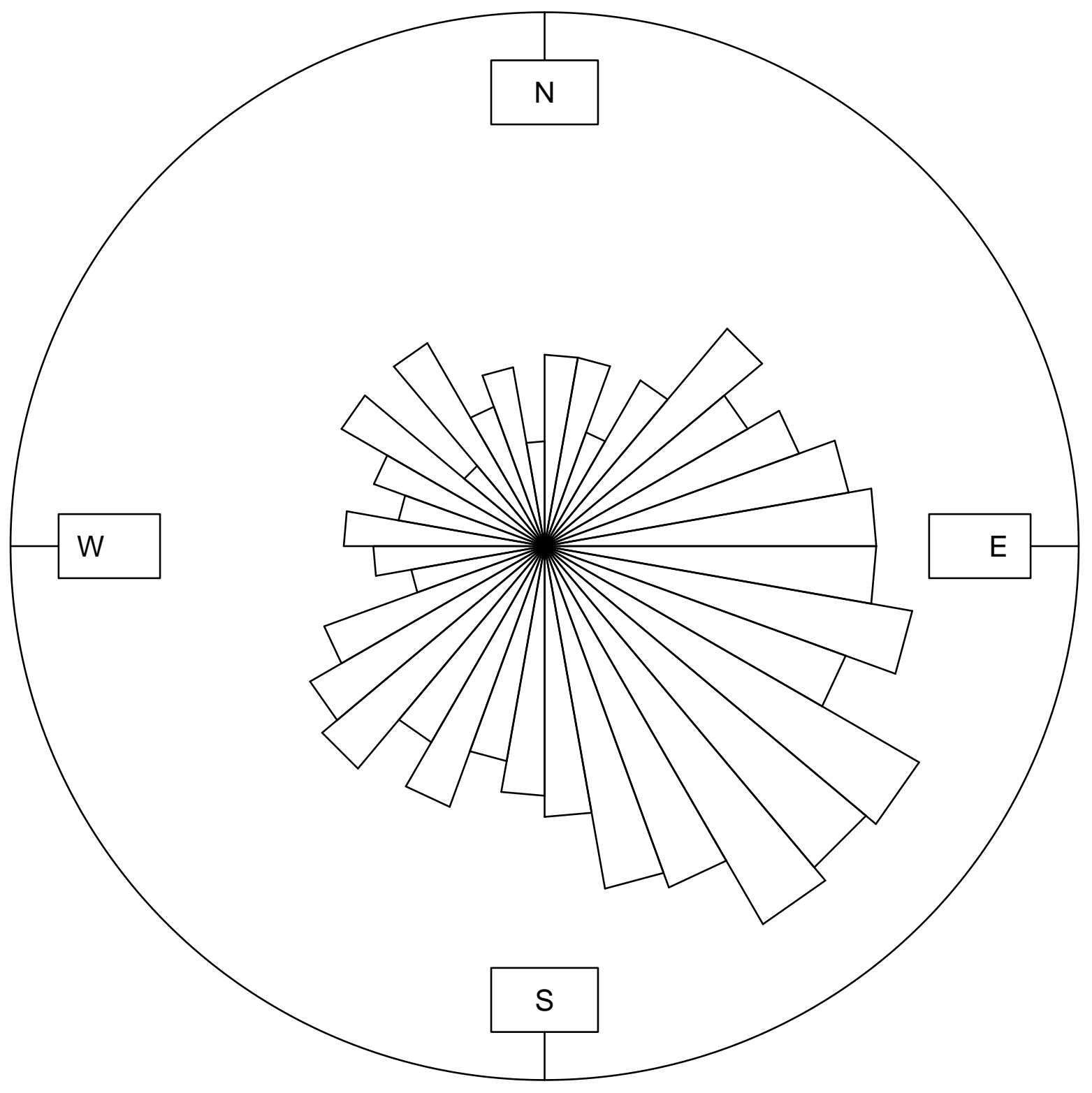


bootstrap 229

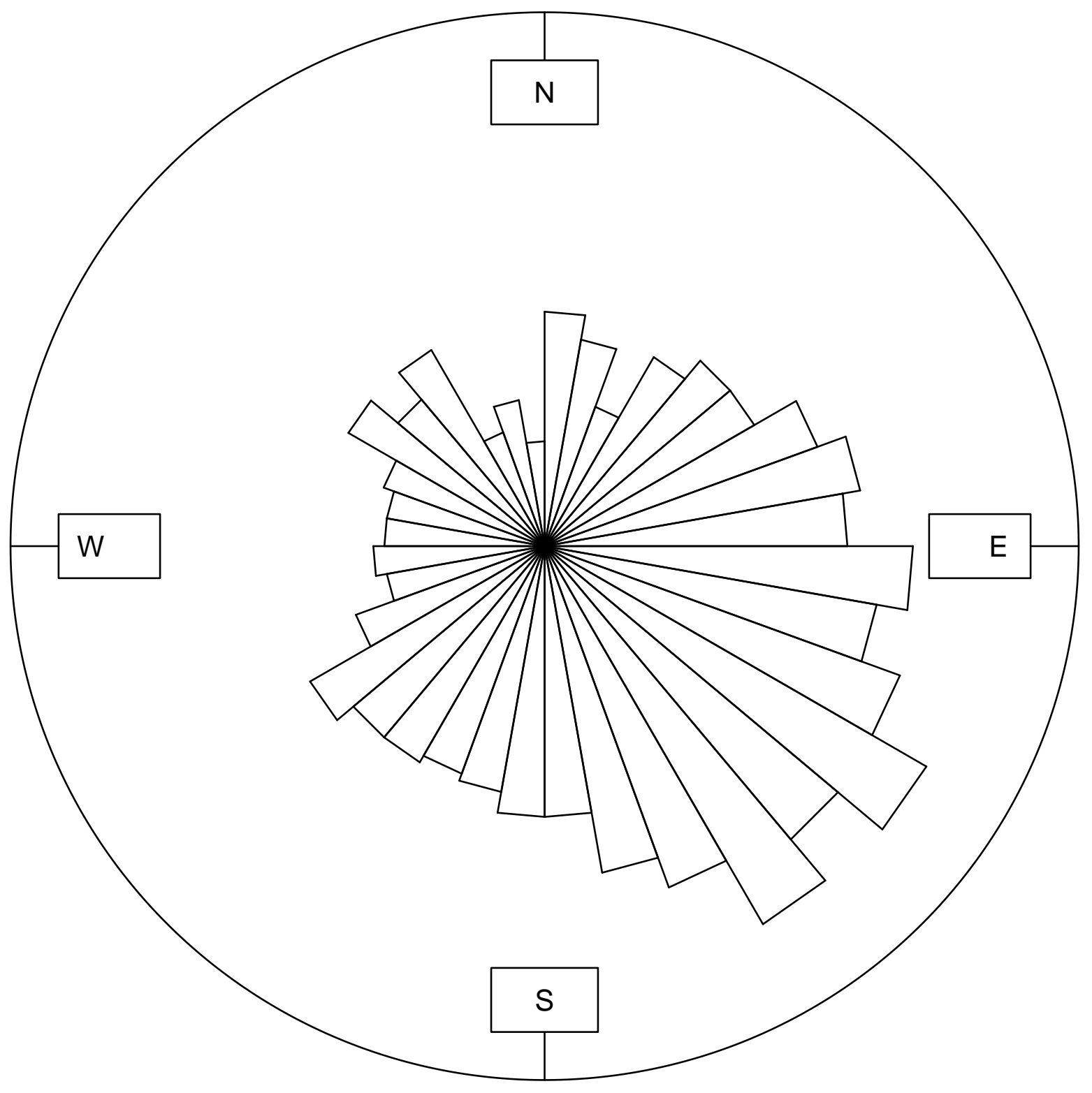


bootstrap 230

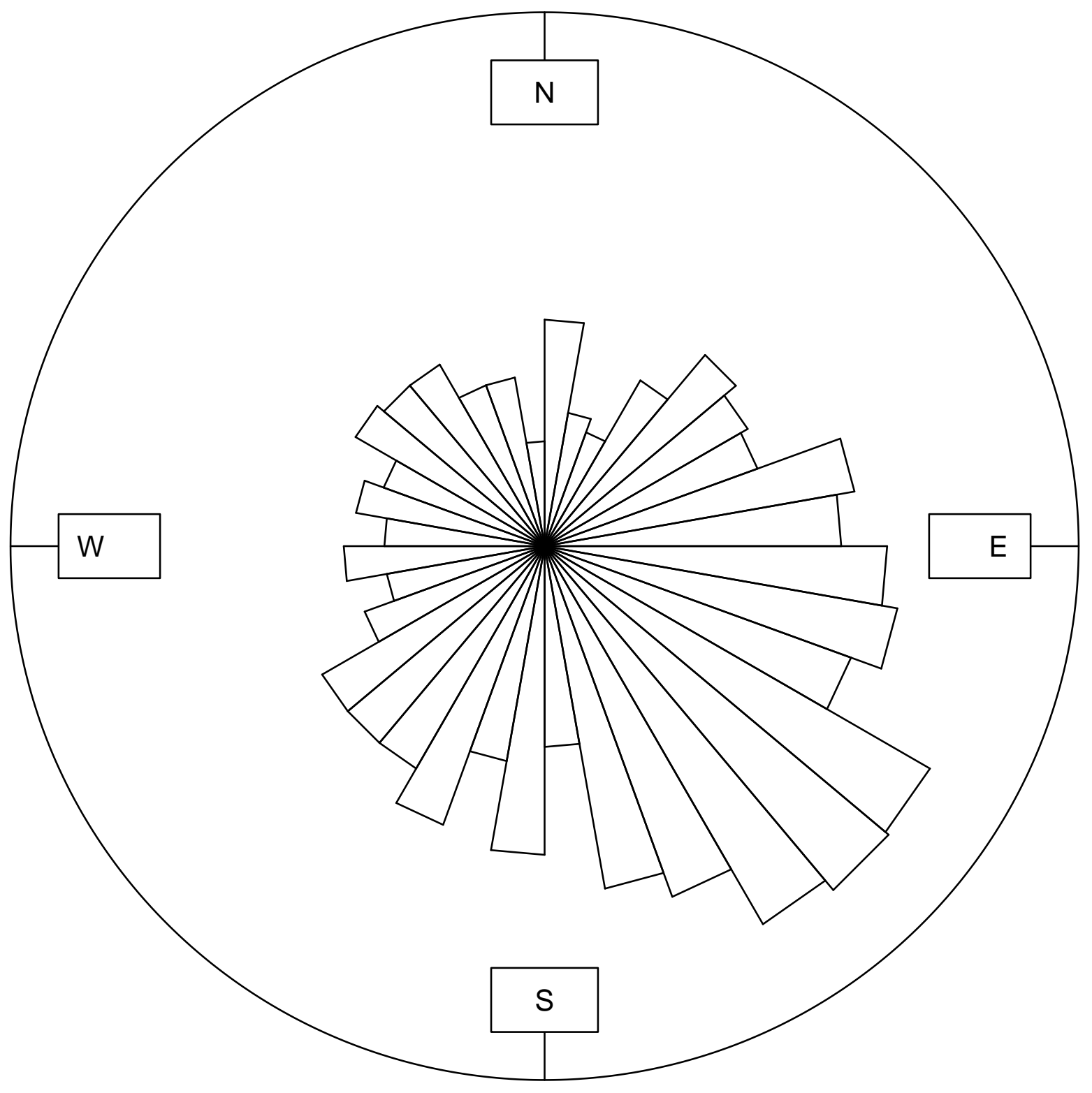


bootstrap 231

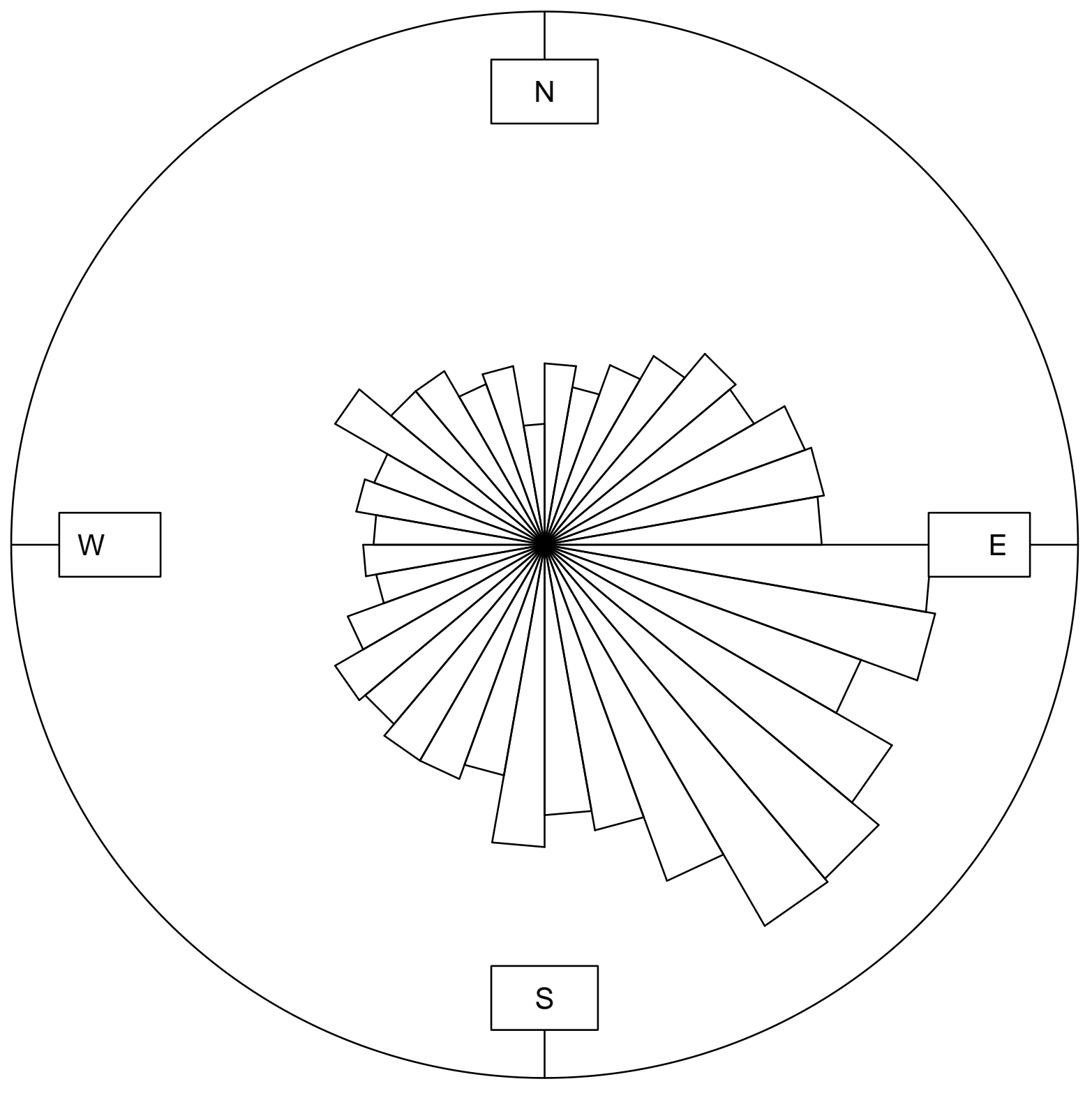


bootstrap 232

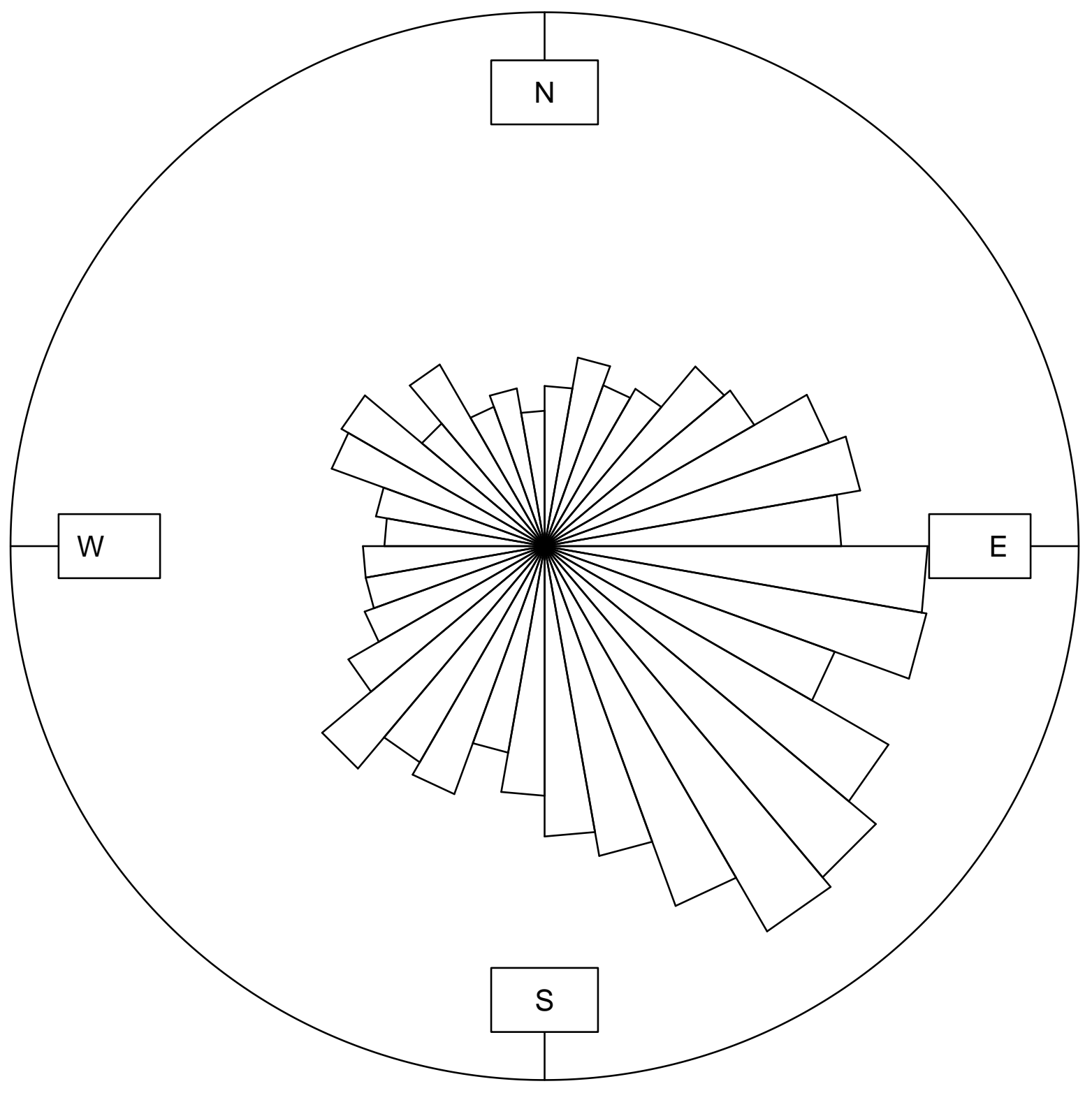




\section{bootstrap 233}

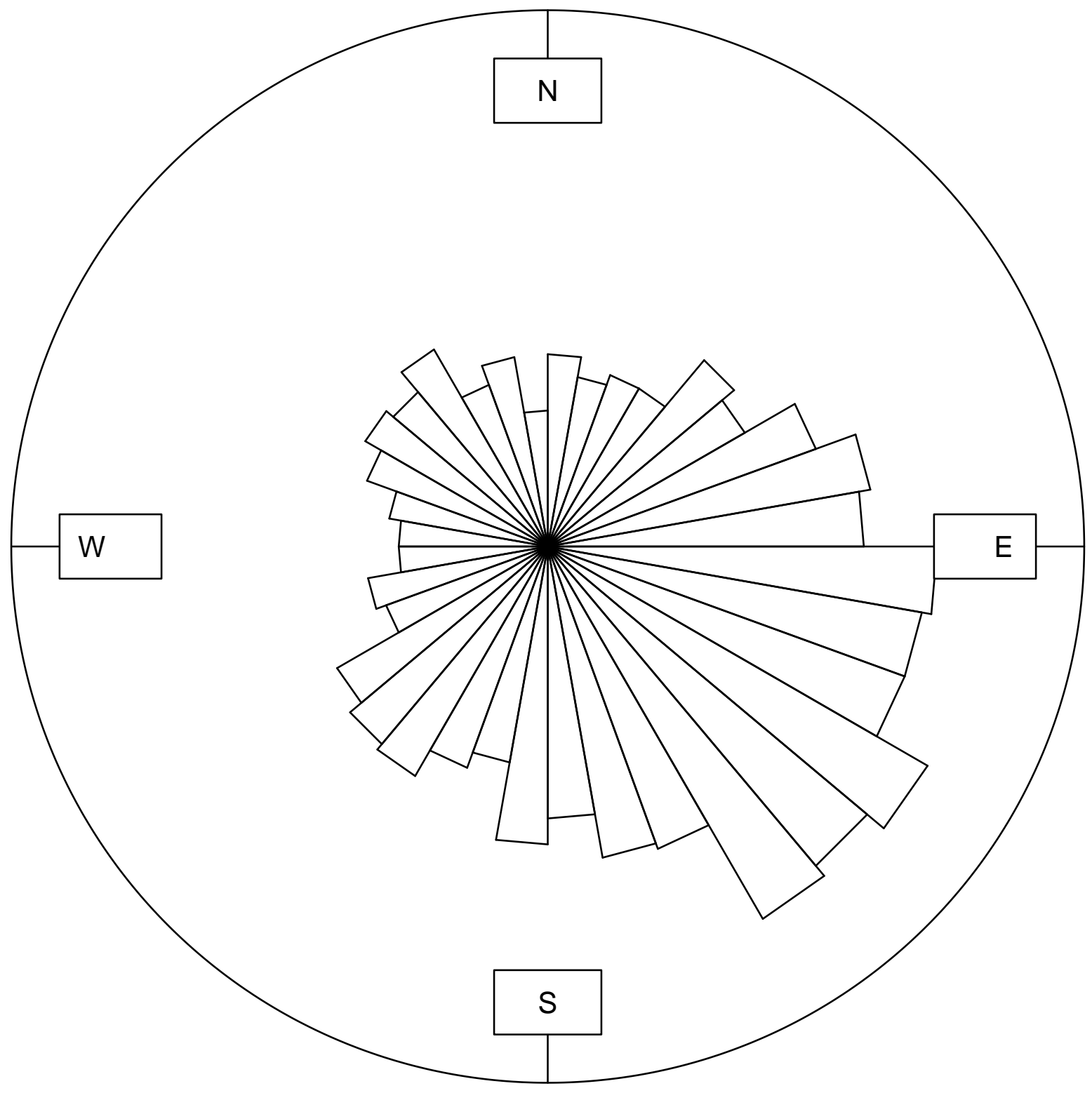


bootstrap 234

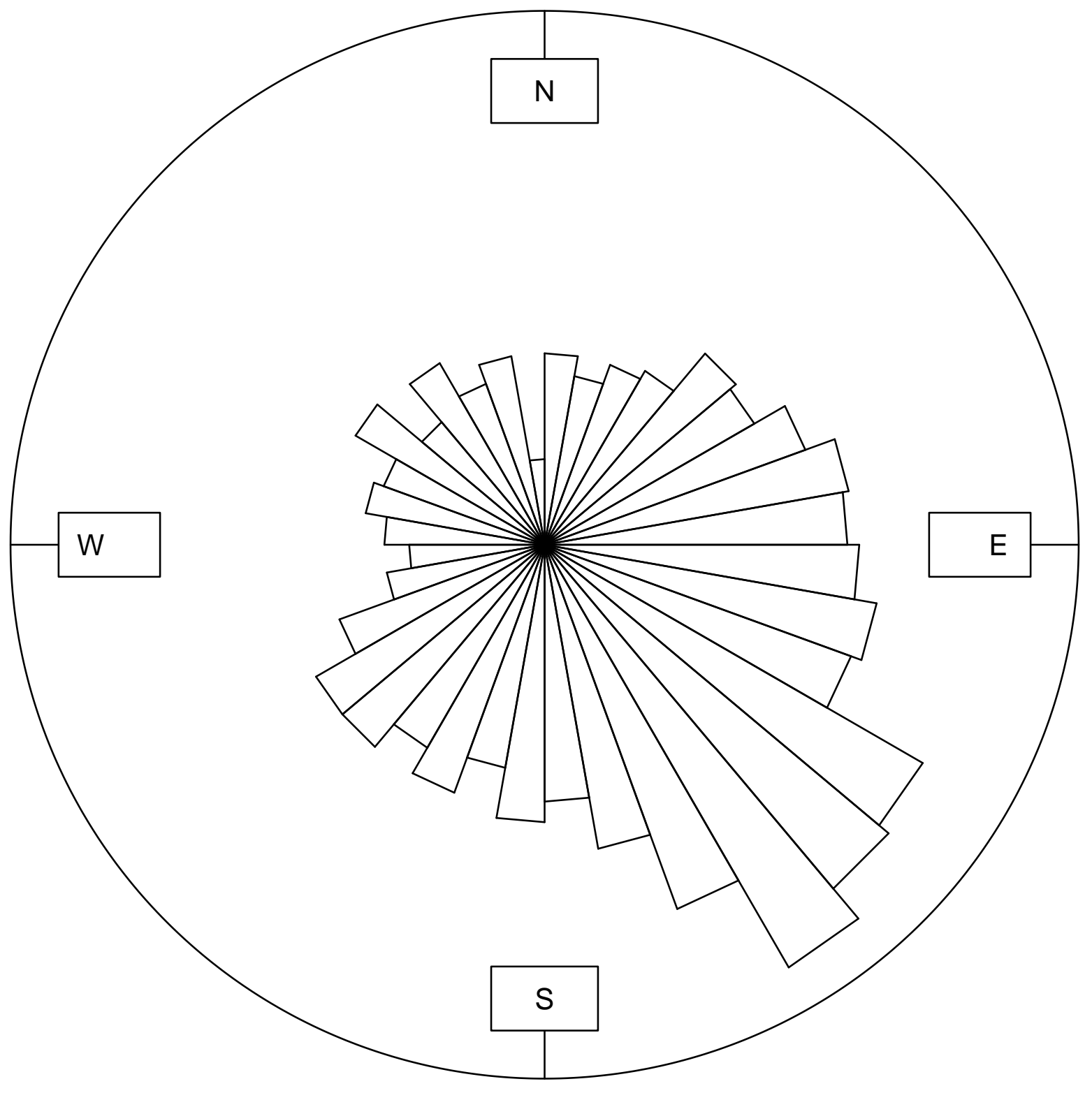




\section{bootstrap 235}

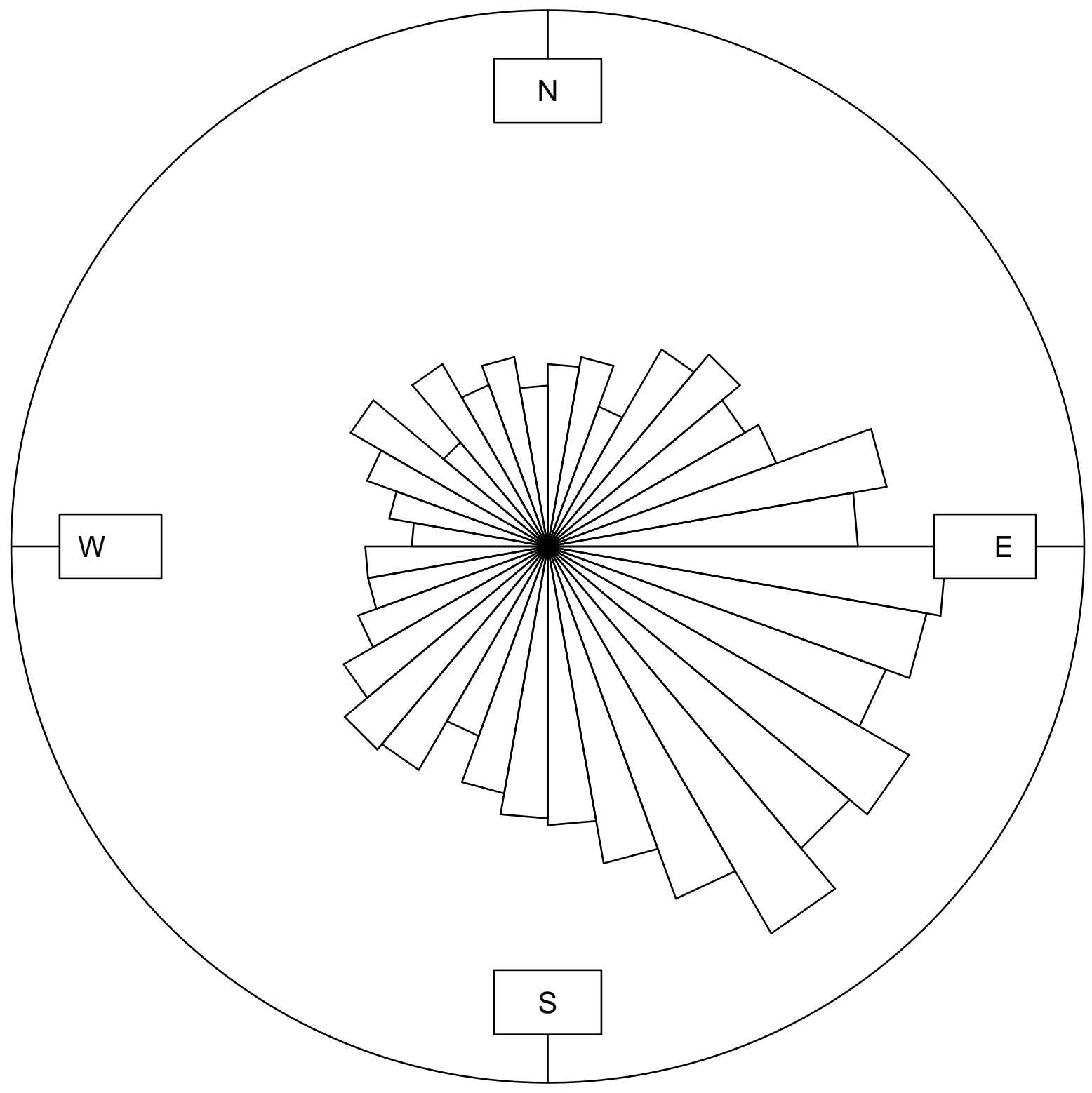


bootstrap 236

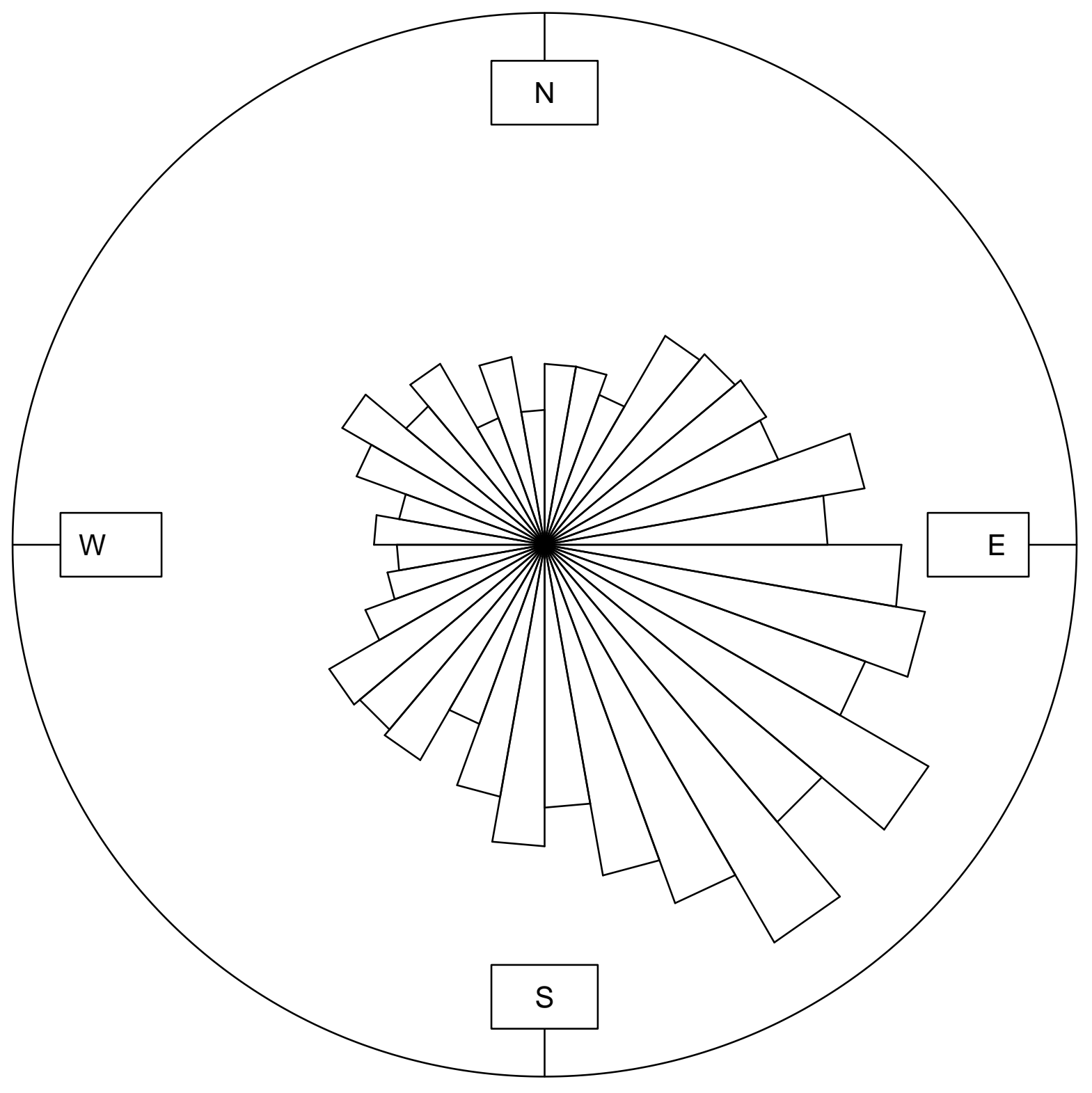


bootstrap 237

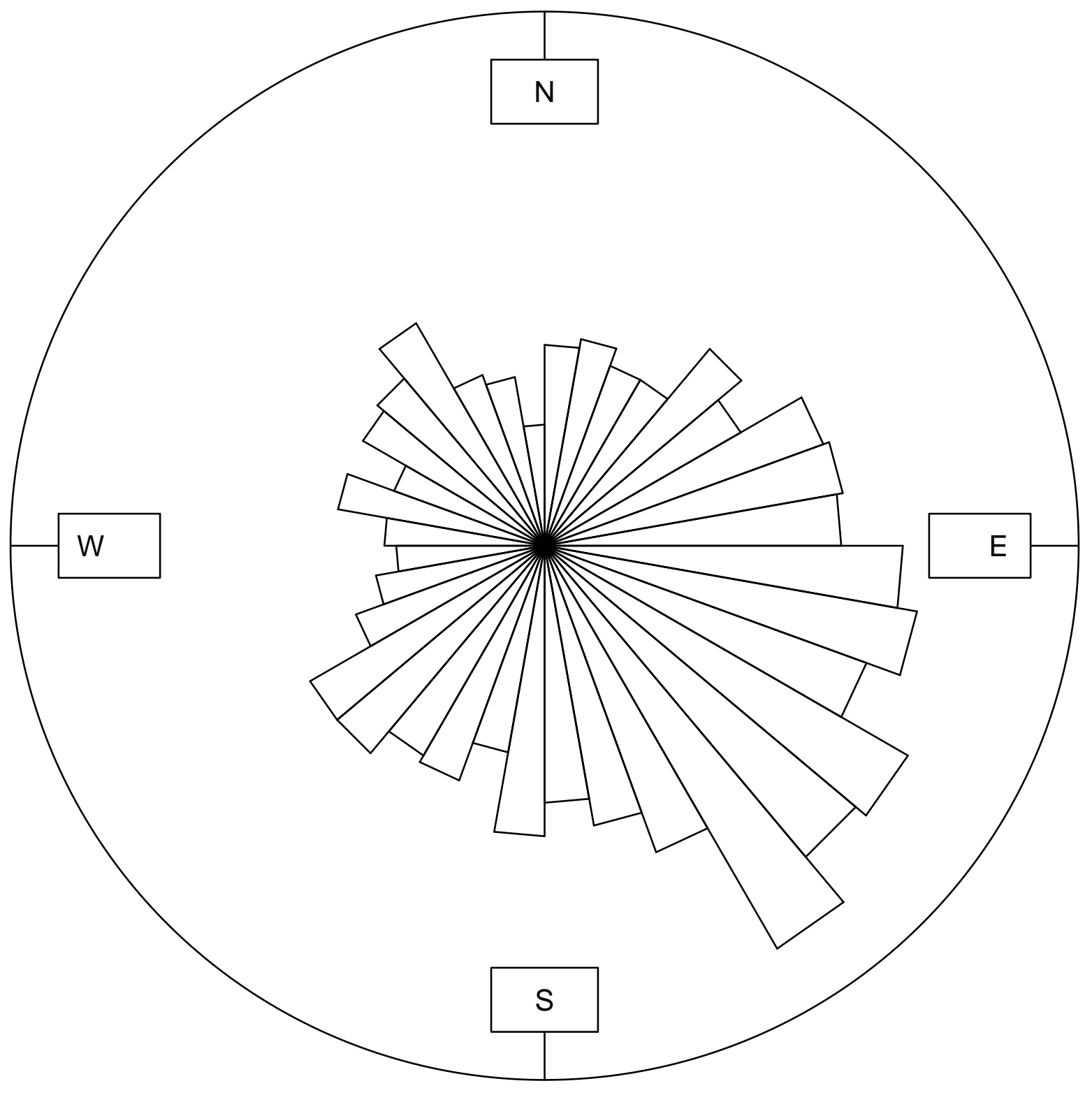


bootstrap 238

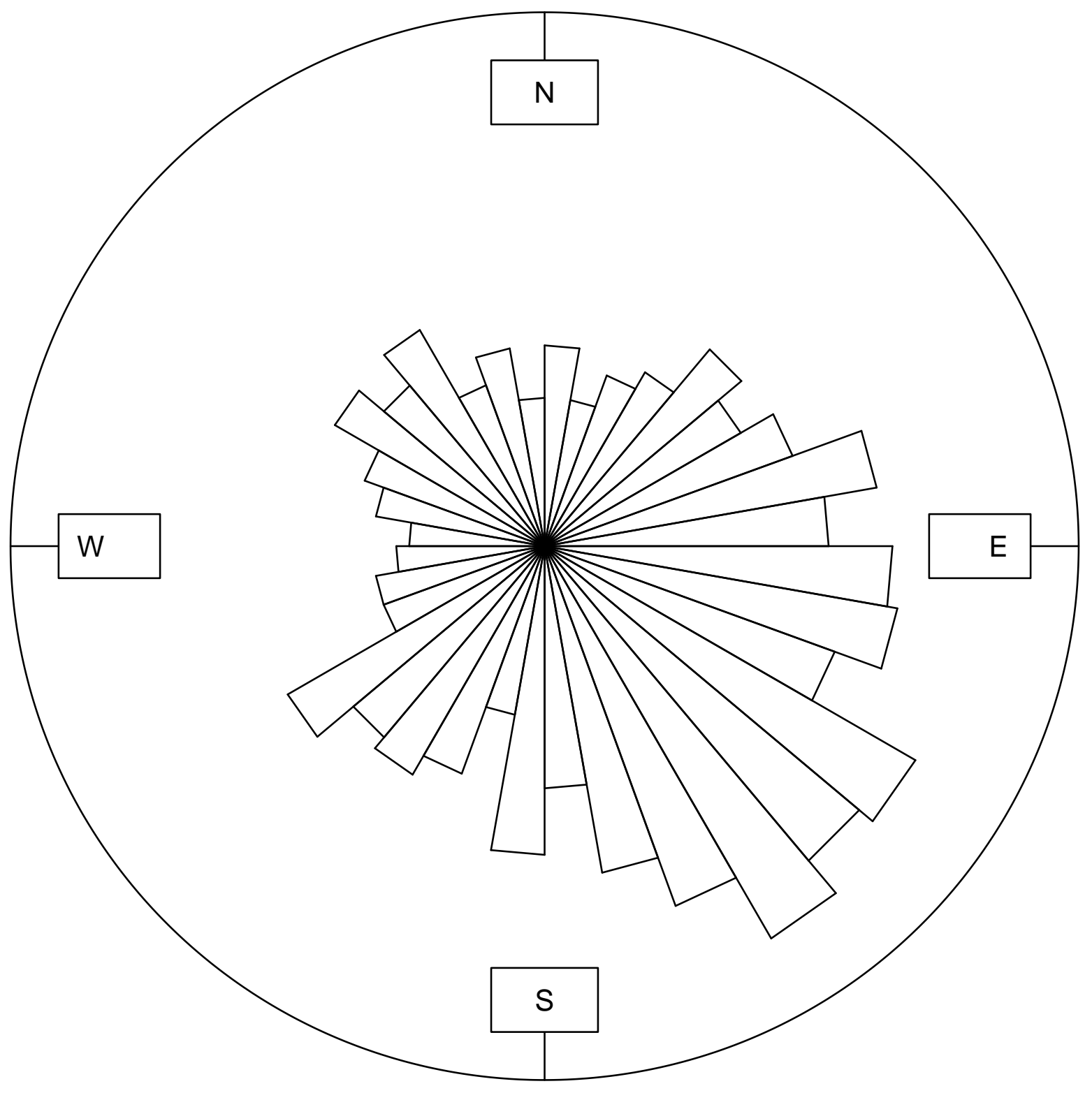


bootstrap 239

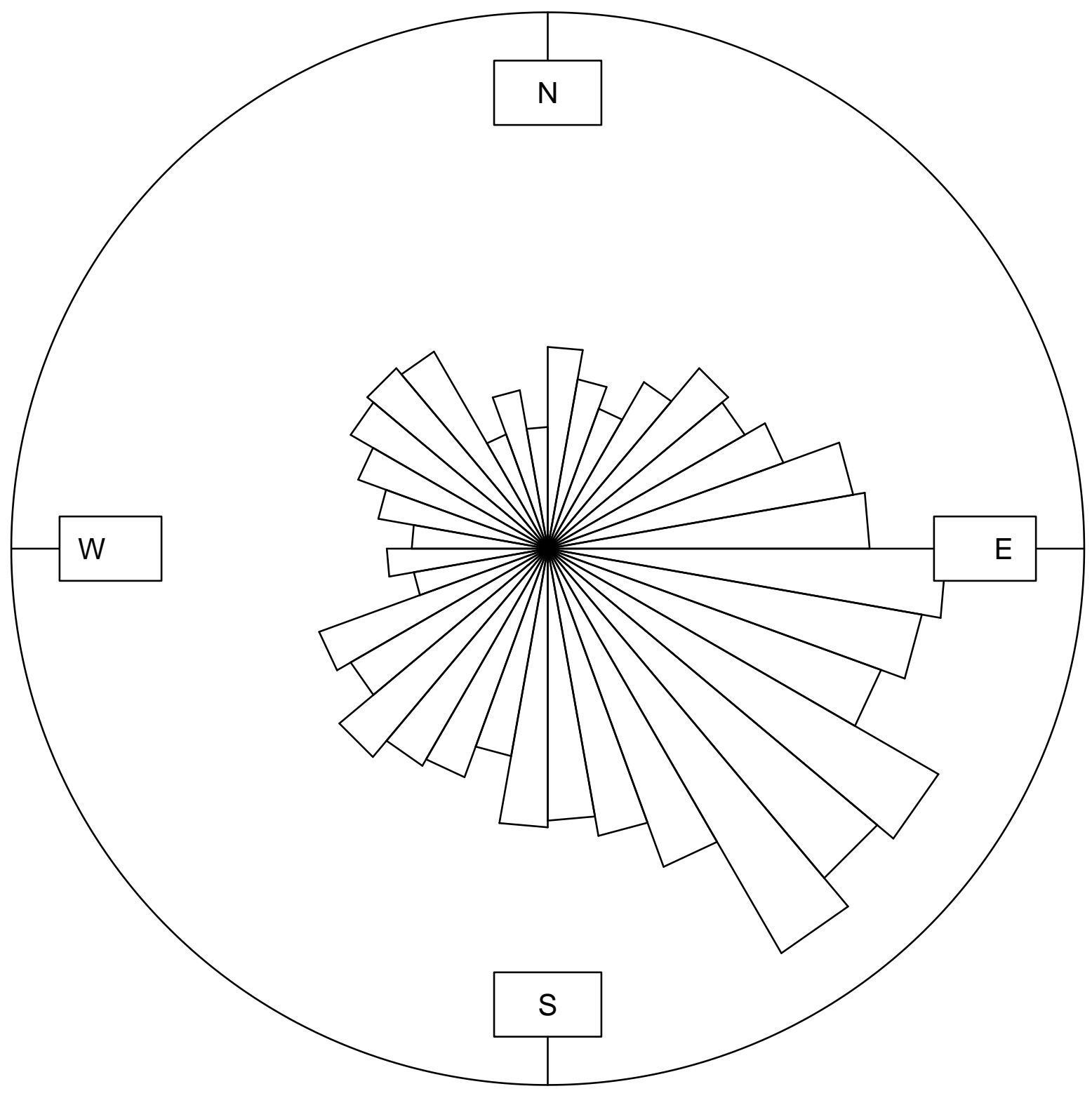


bootstrap 240

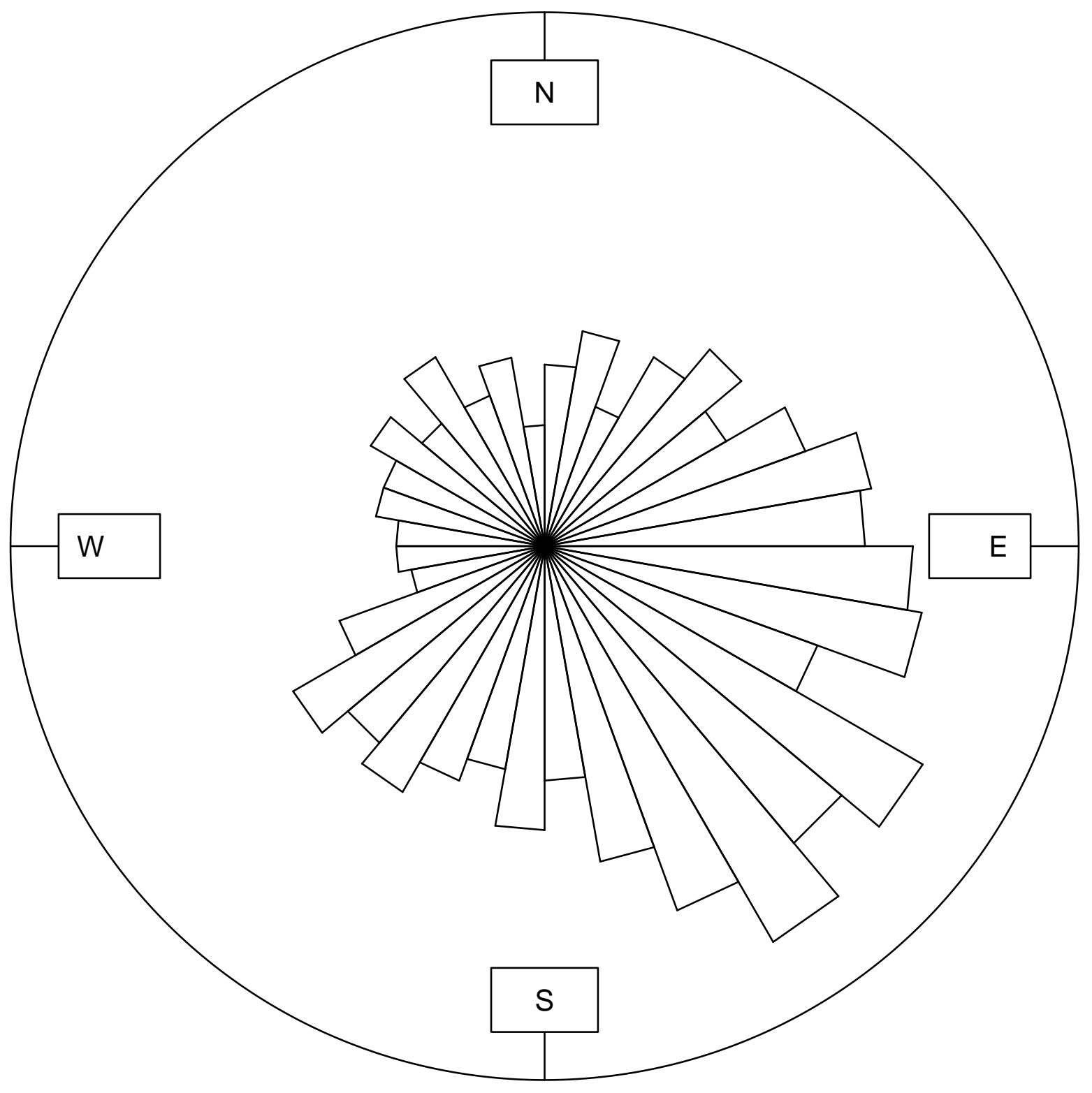


bootstrap 241

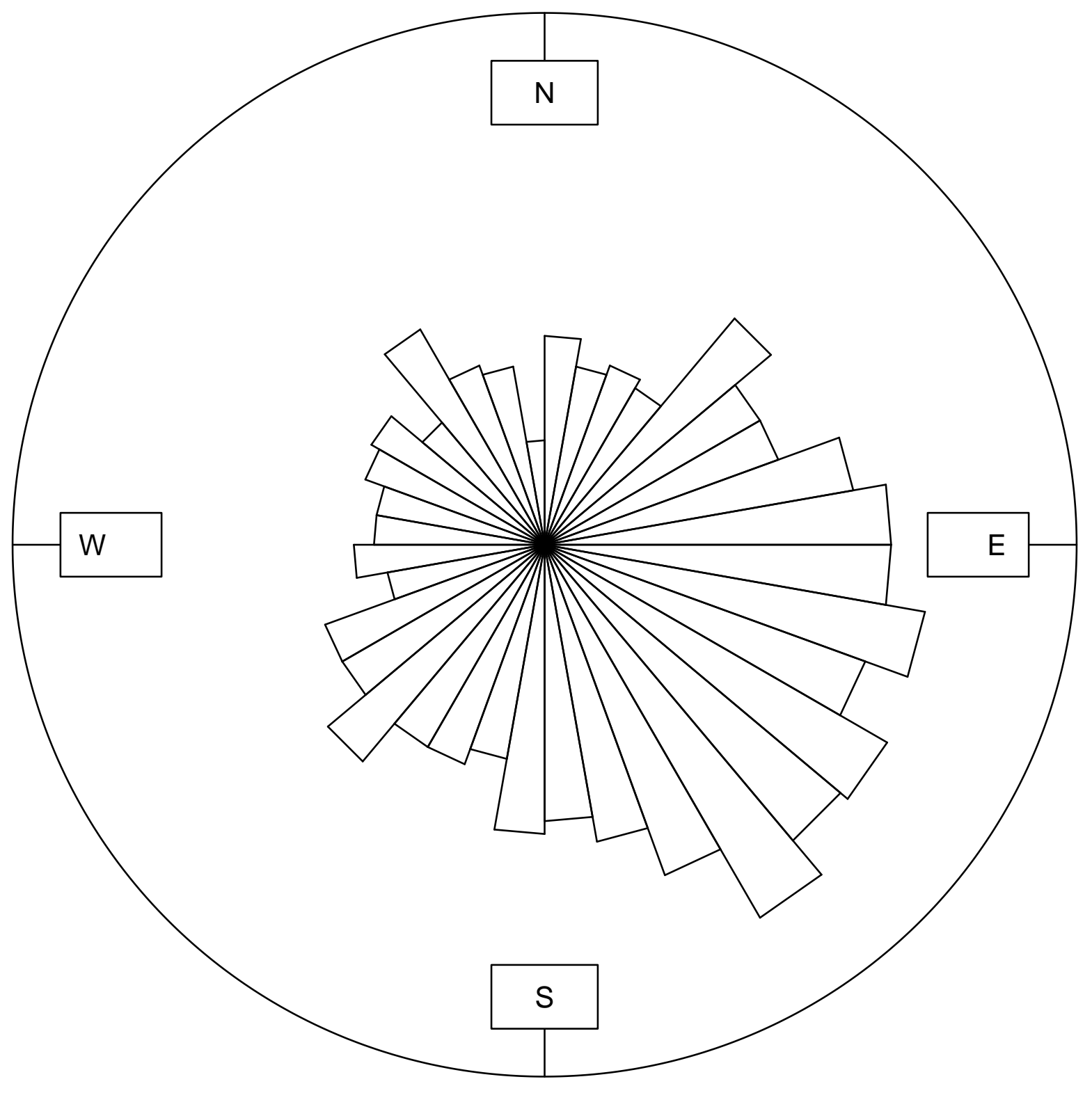


bootstrap 242

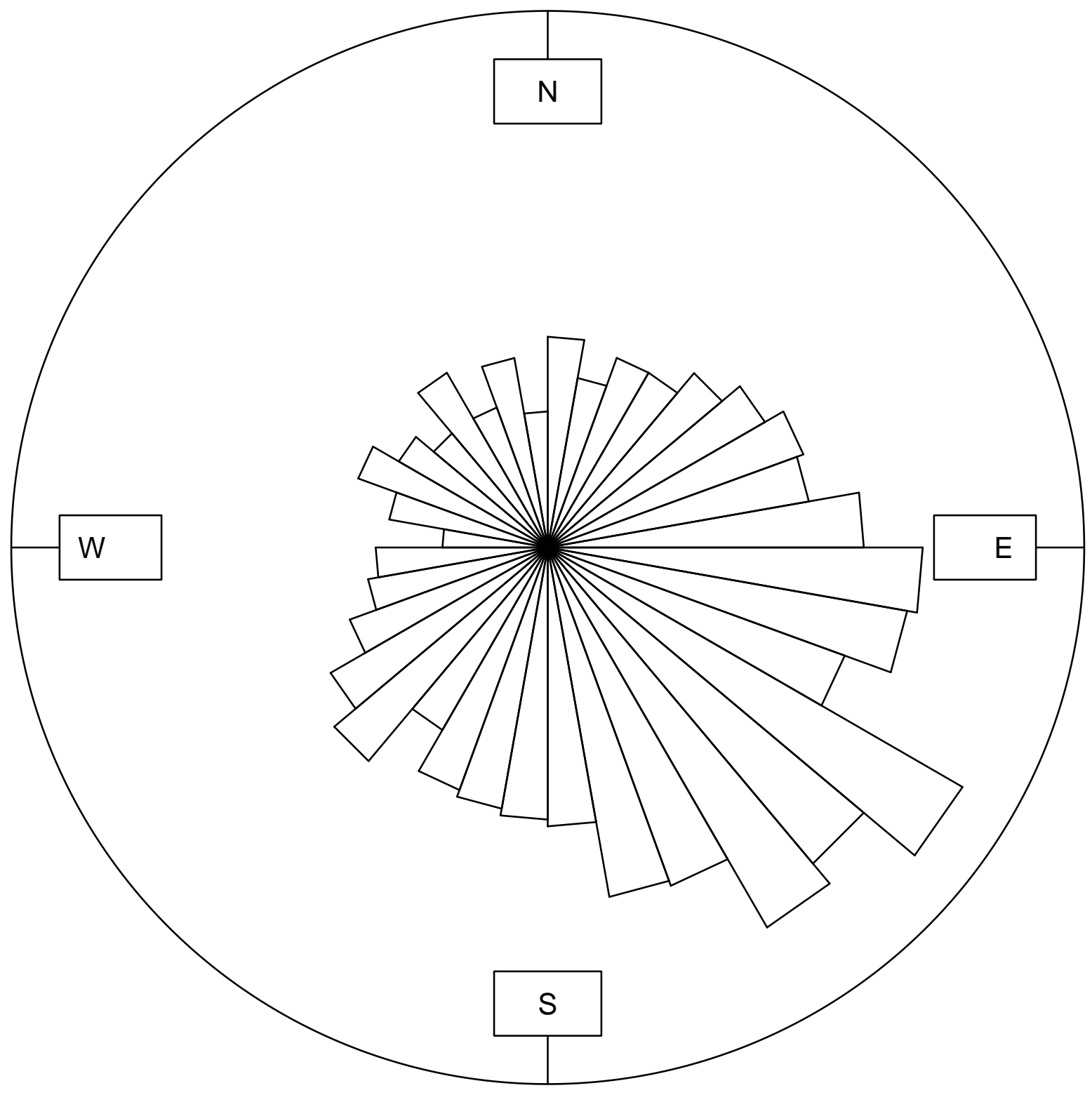




\section{bootstrap 243}

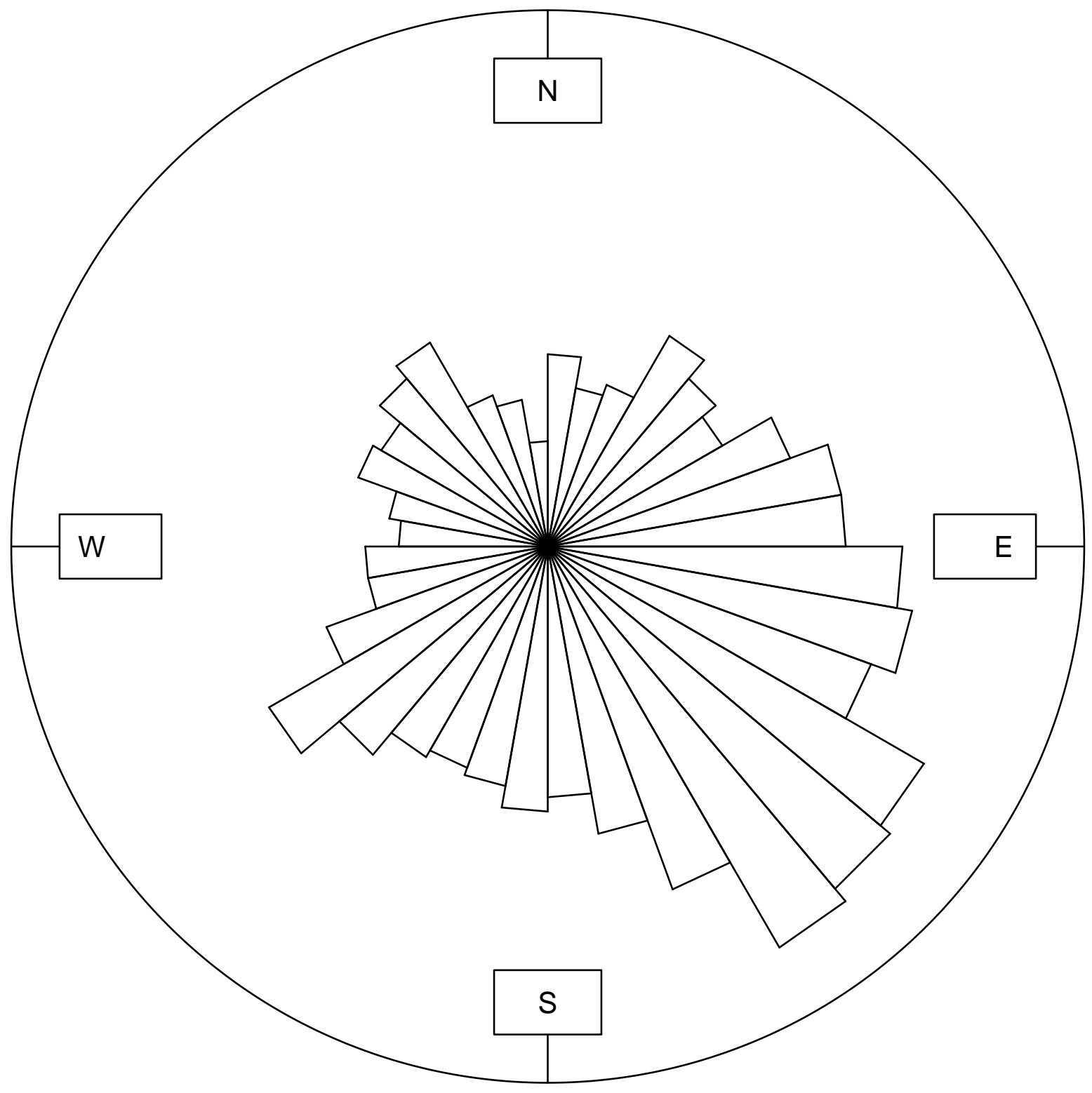


bootstrap 244

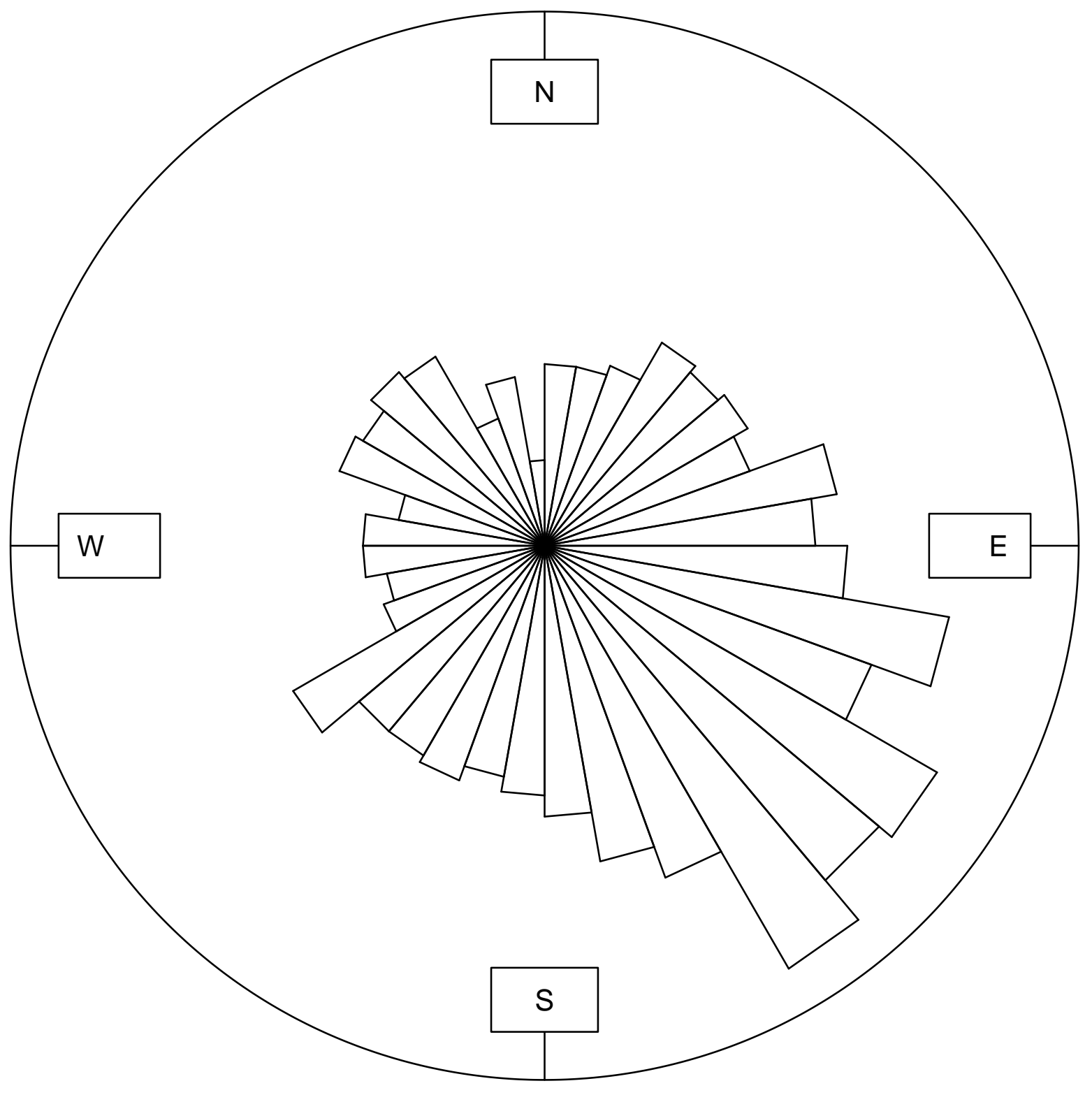


bootstrap 245

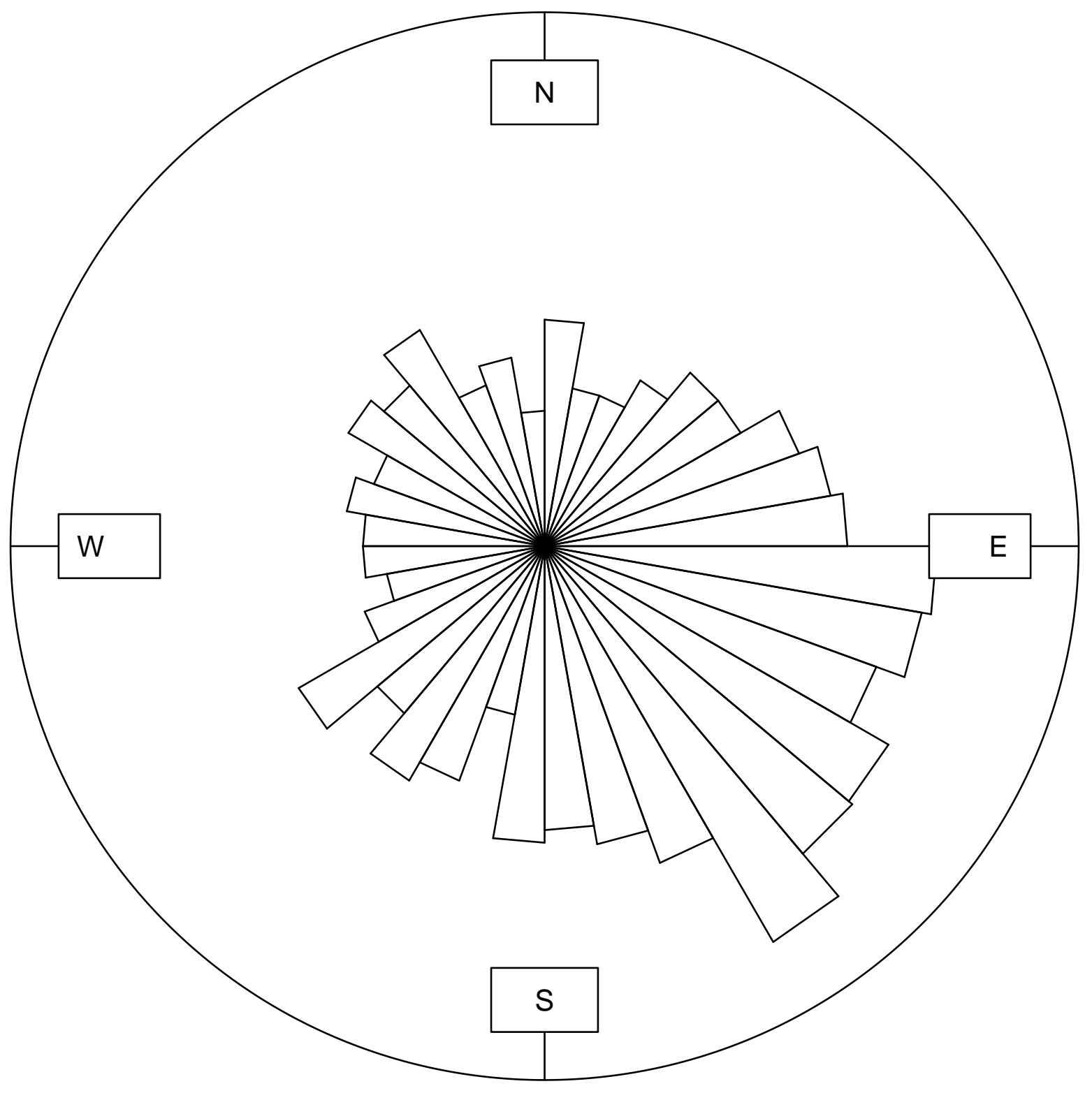


bootstrap 246

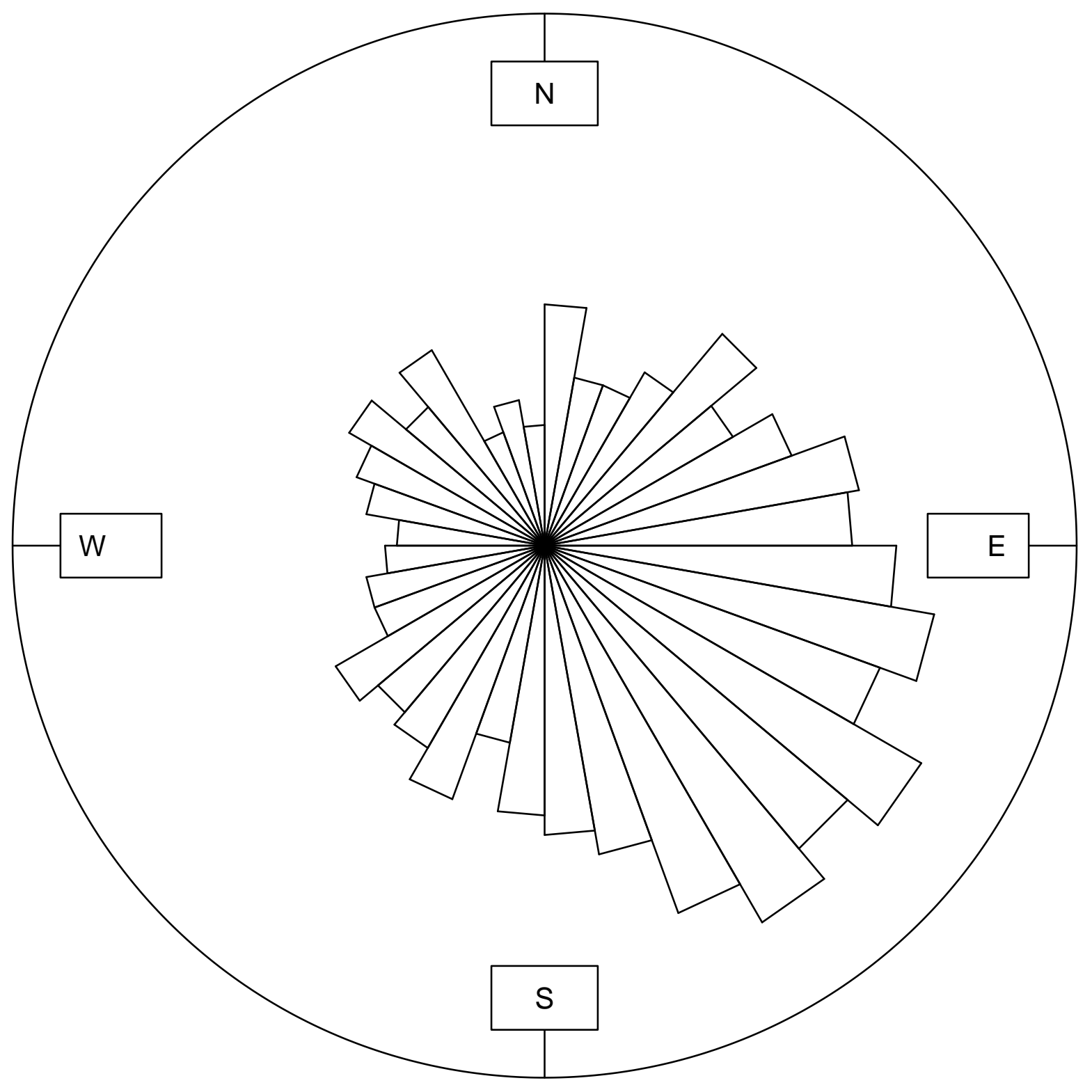


bootstrap 247

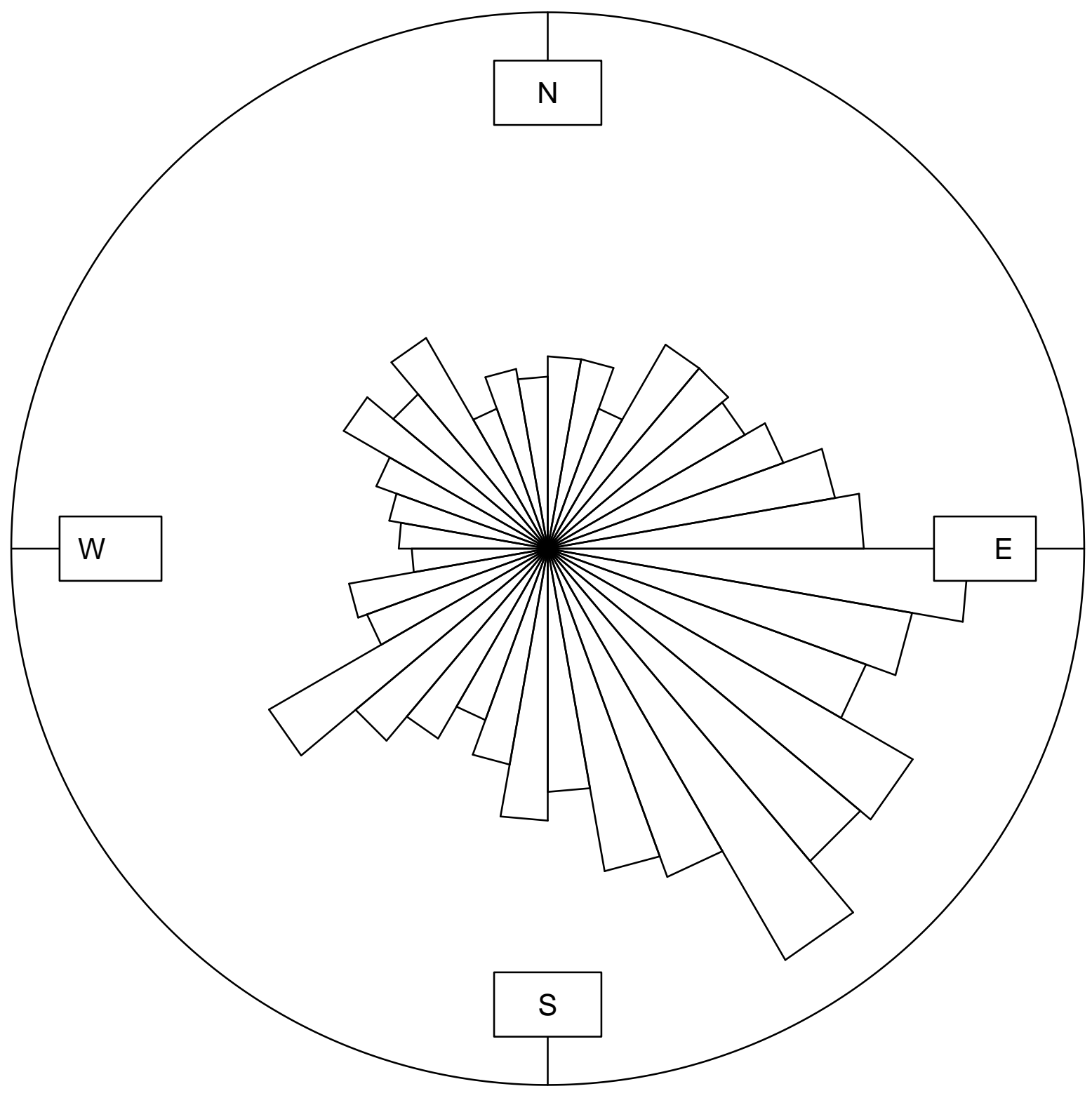


bootstrap 248

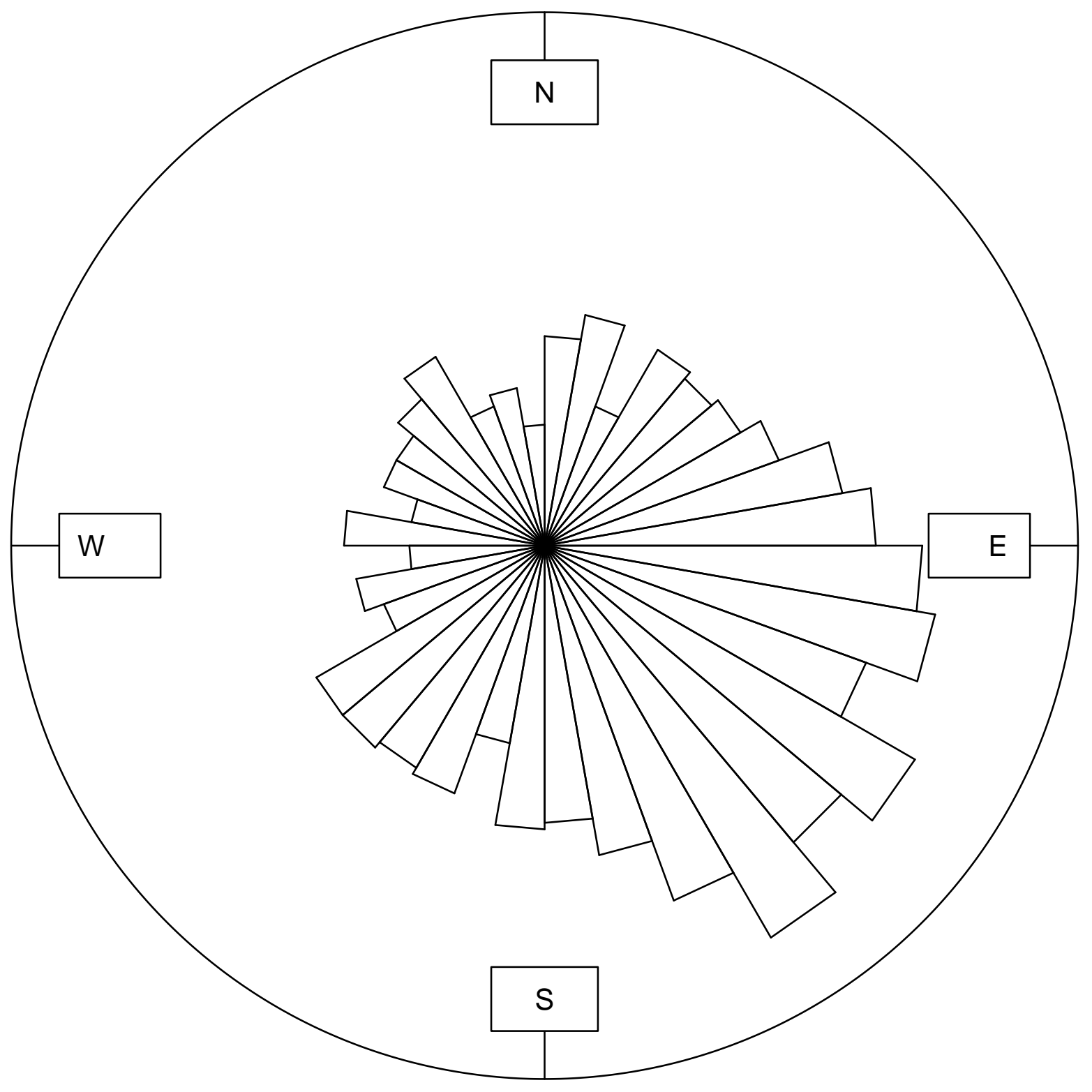


bootstrap 249

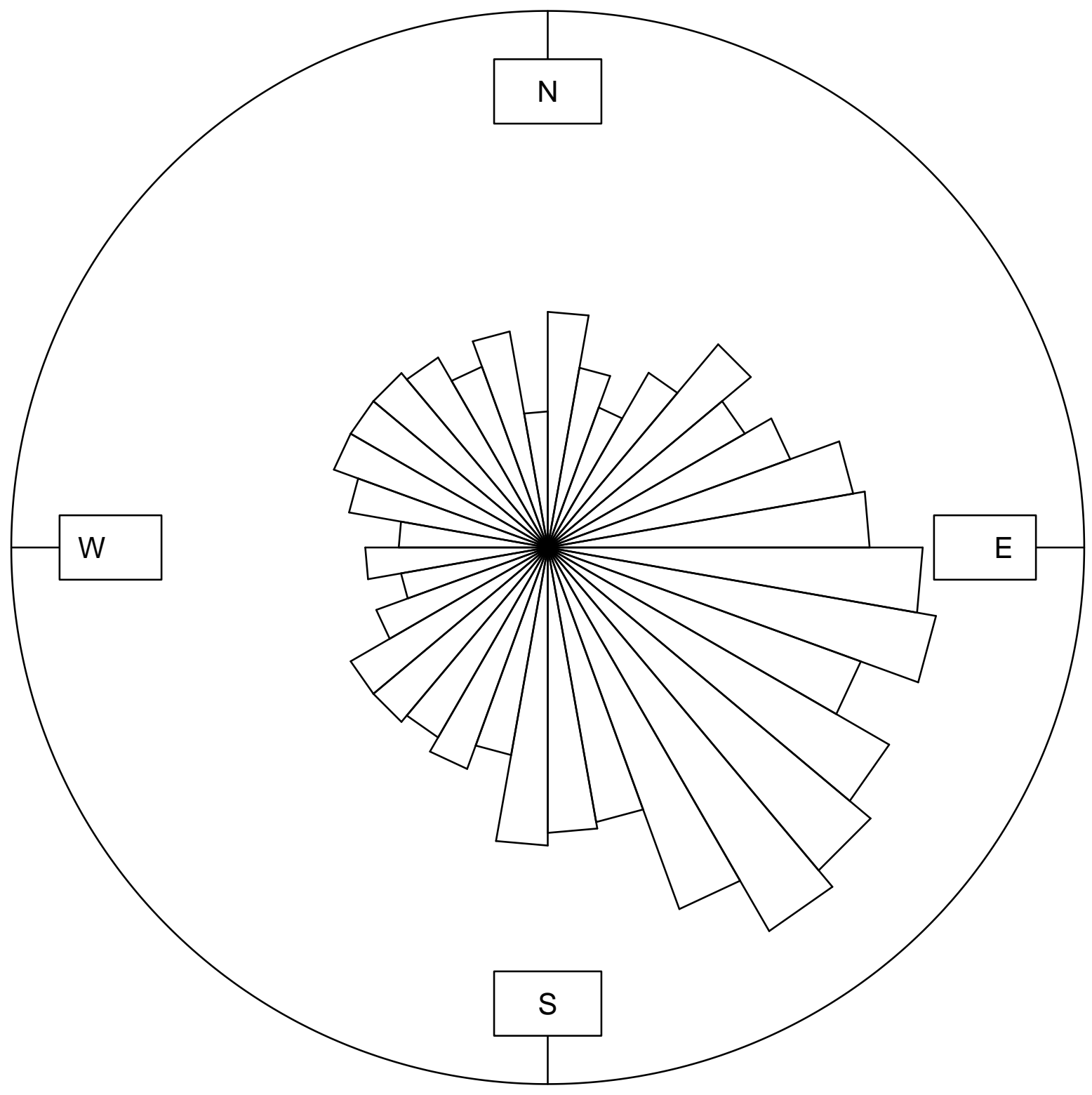


bootstrap 250

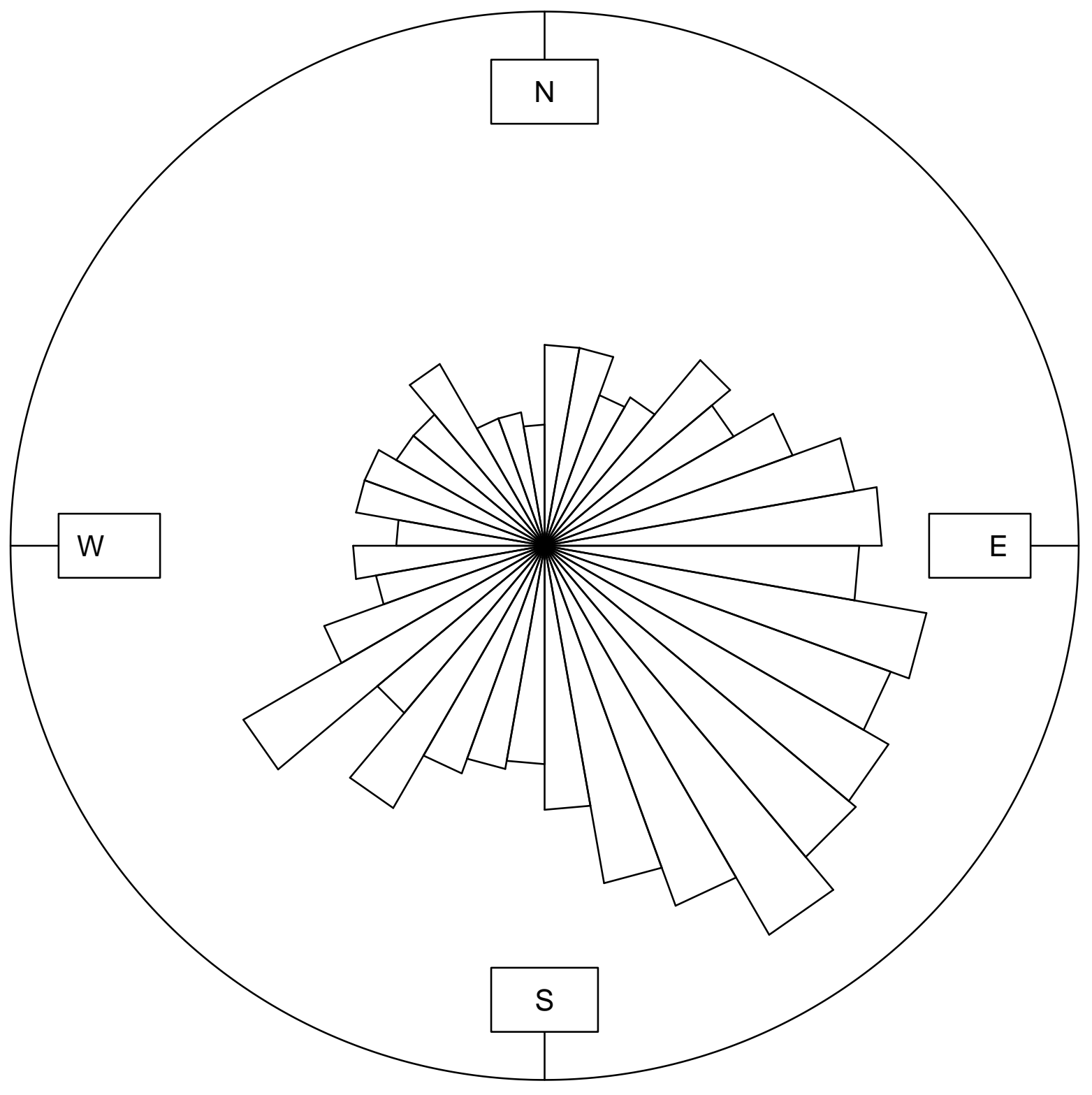


bootstrap 251

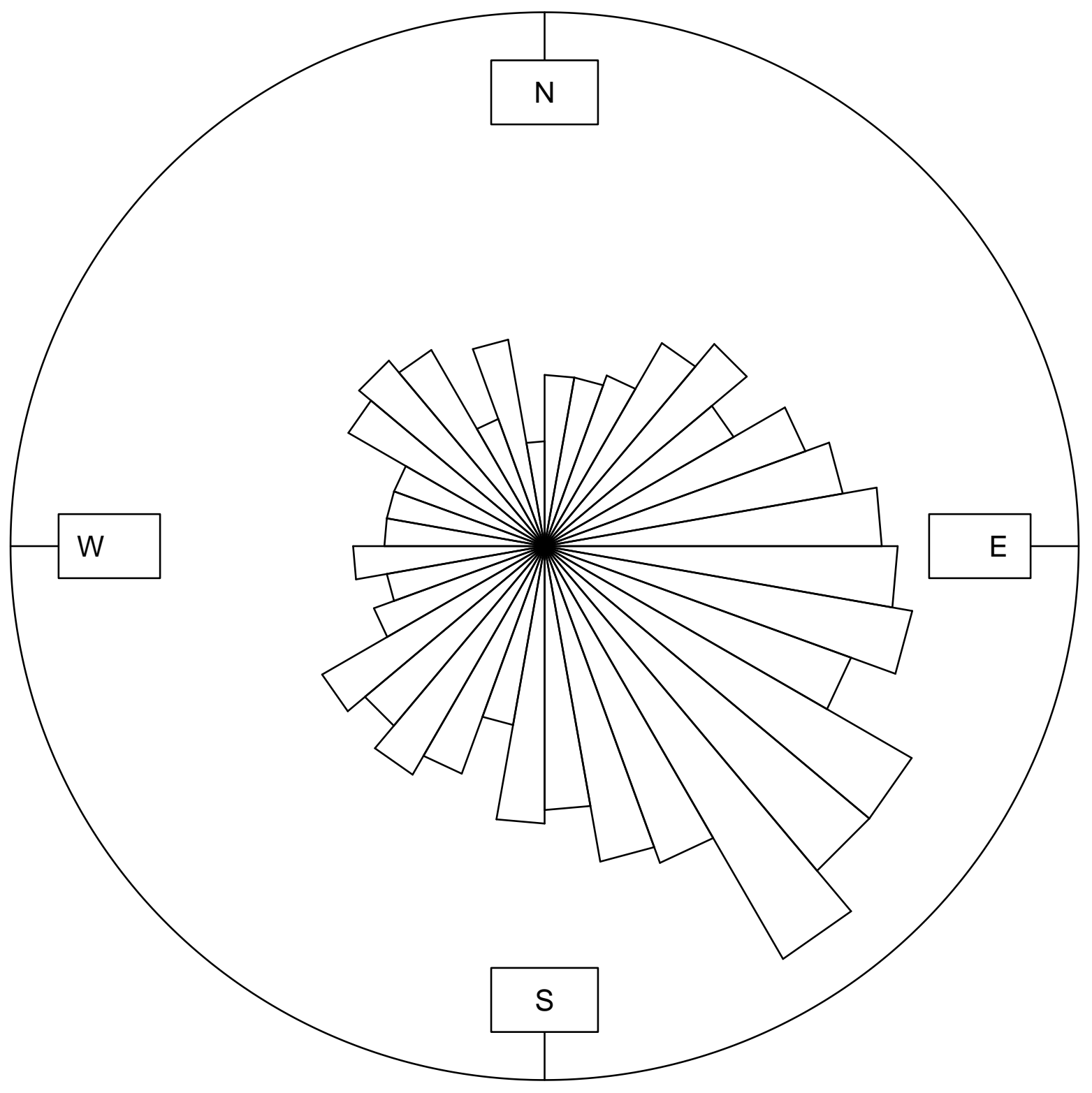


bootstrap 252

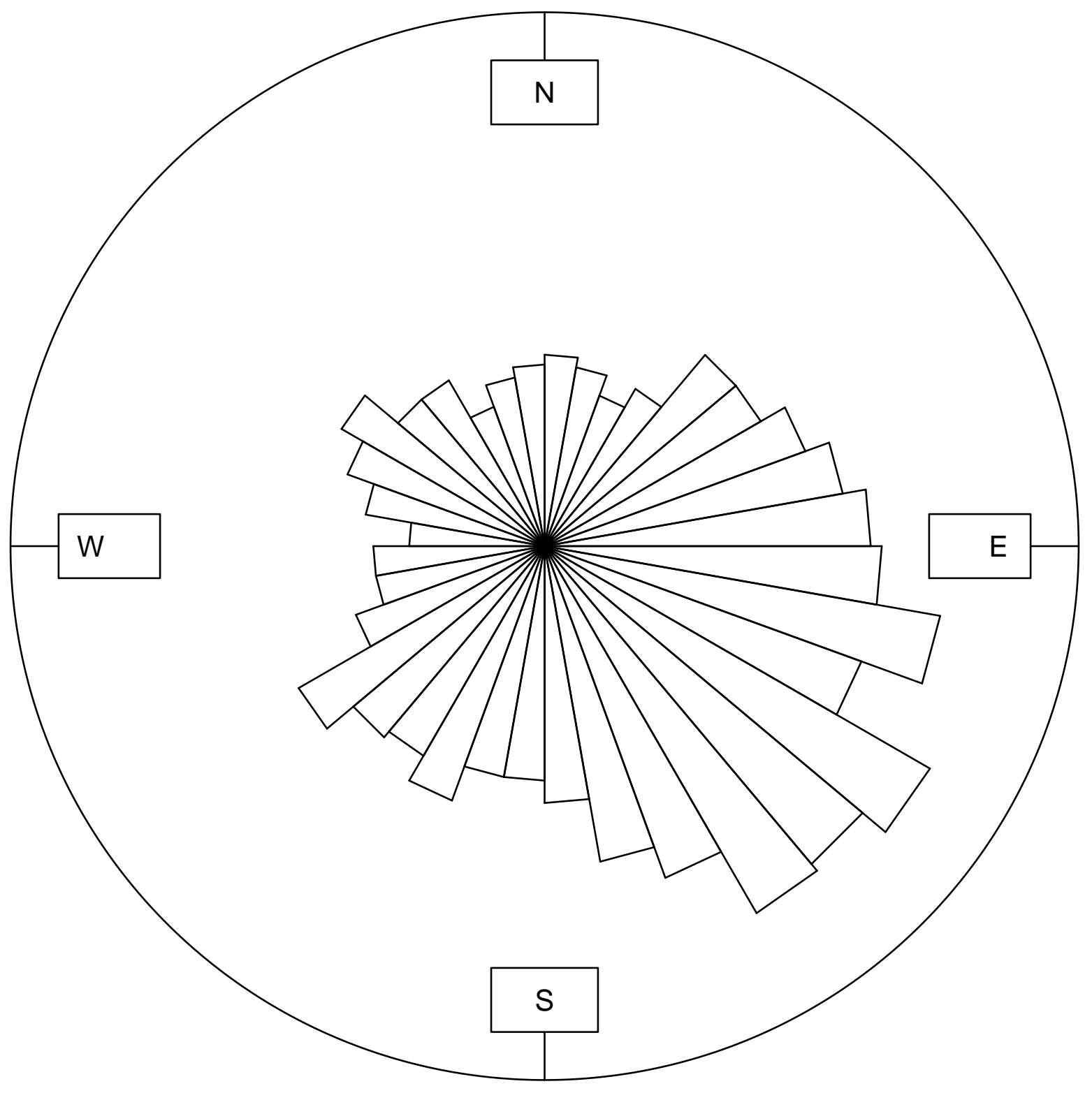




\section{bootstrap 253}

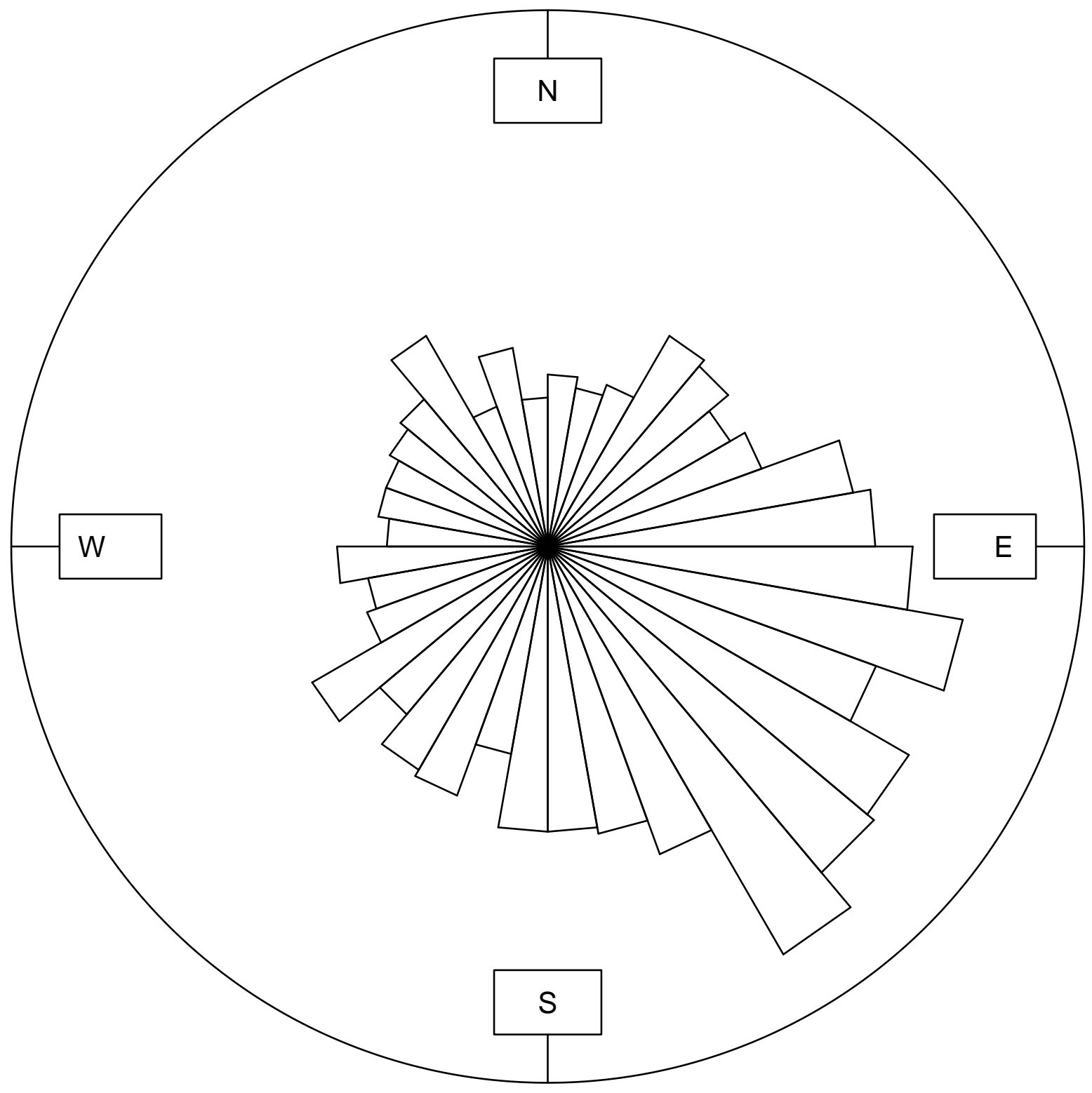


bootstrap 254

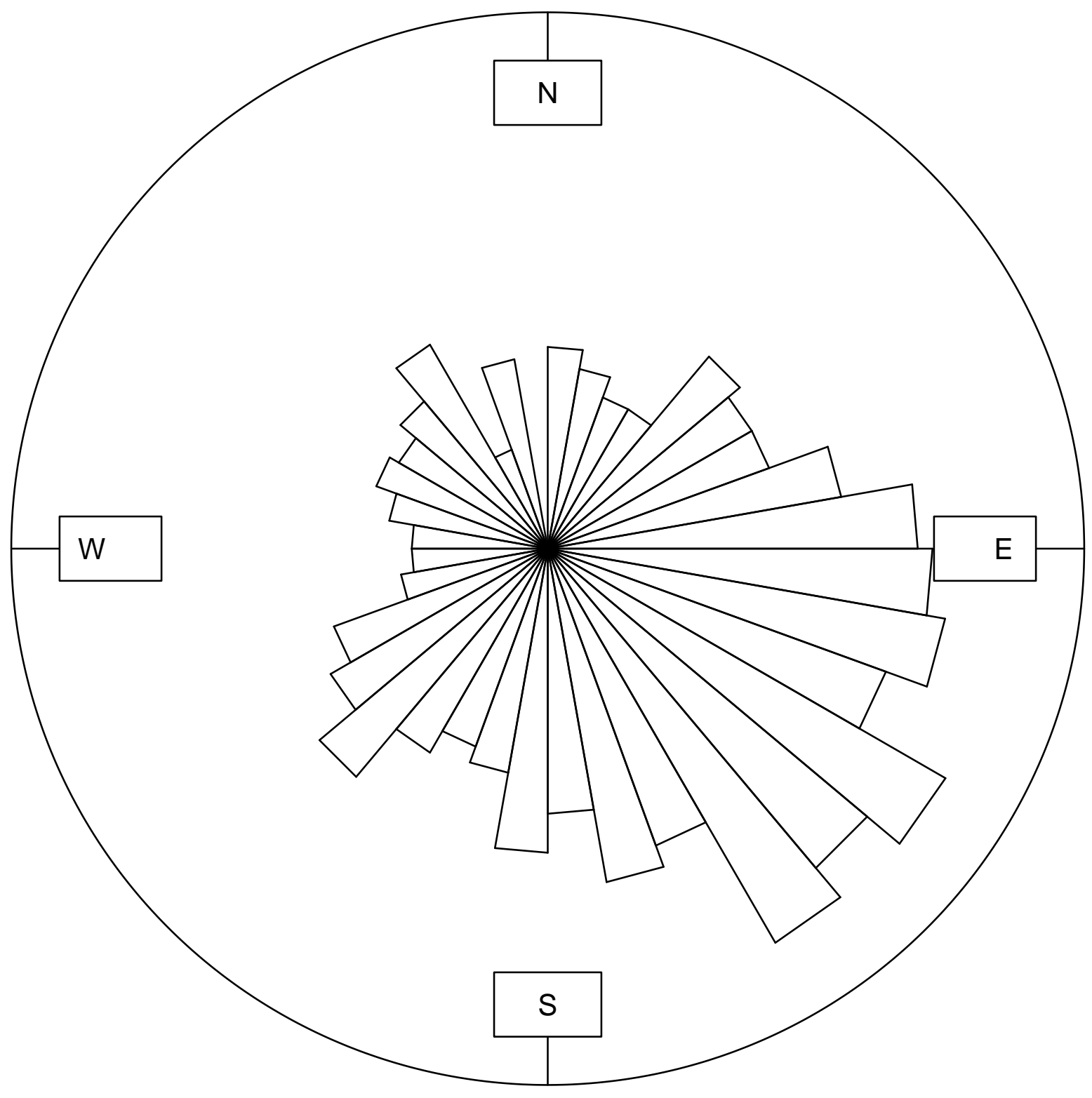




\section{bootstrap 255}

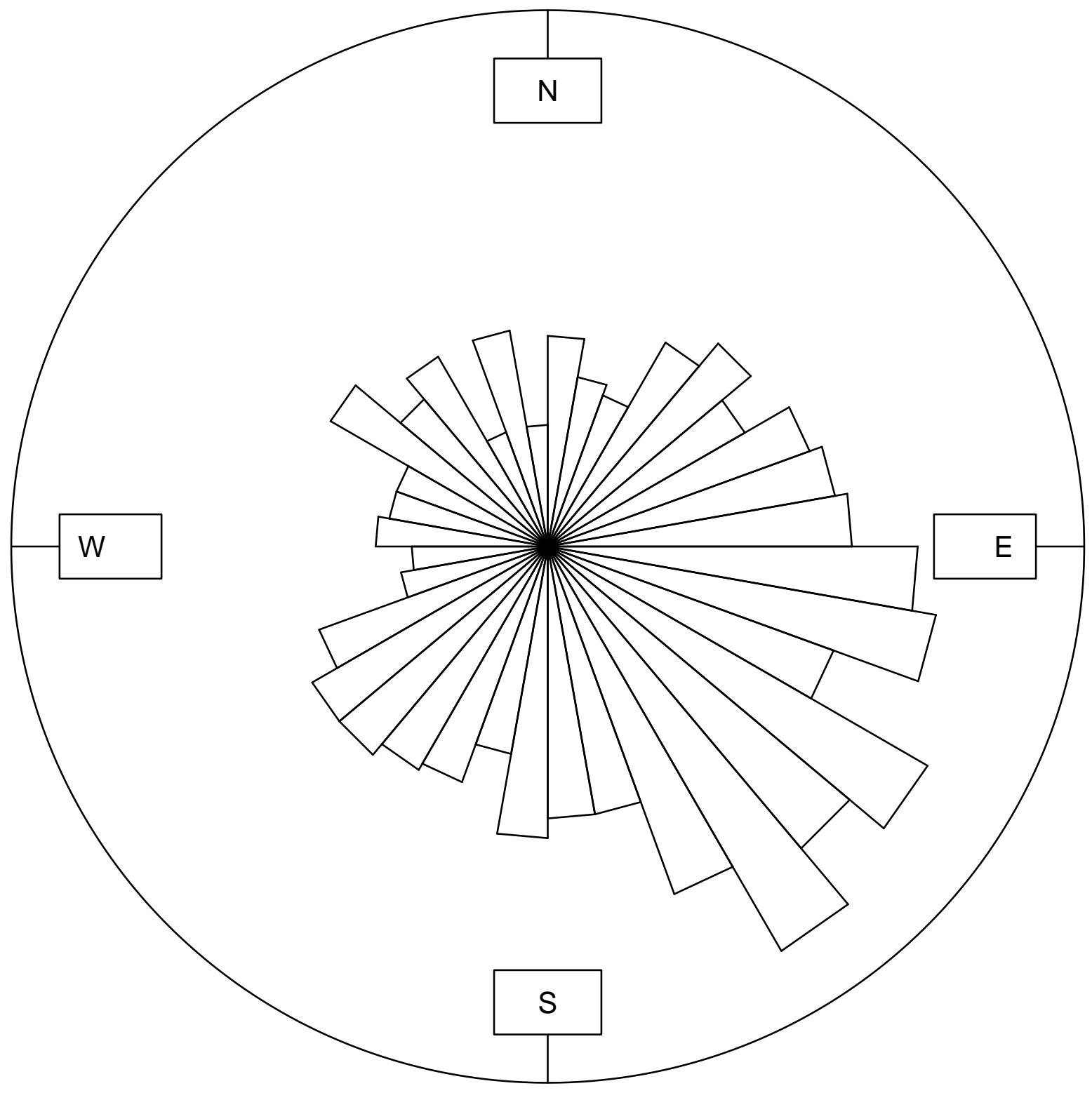


bootstrap 256

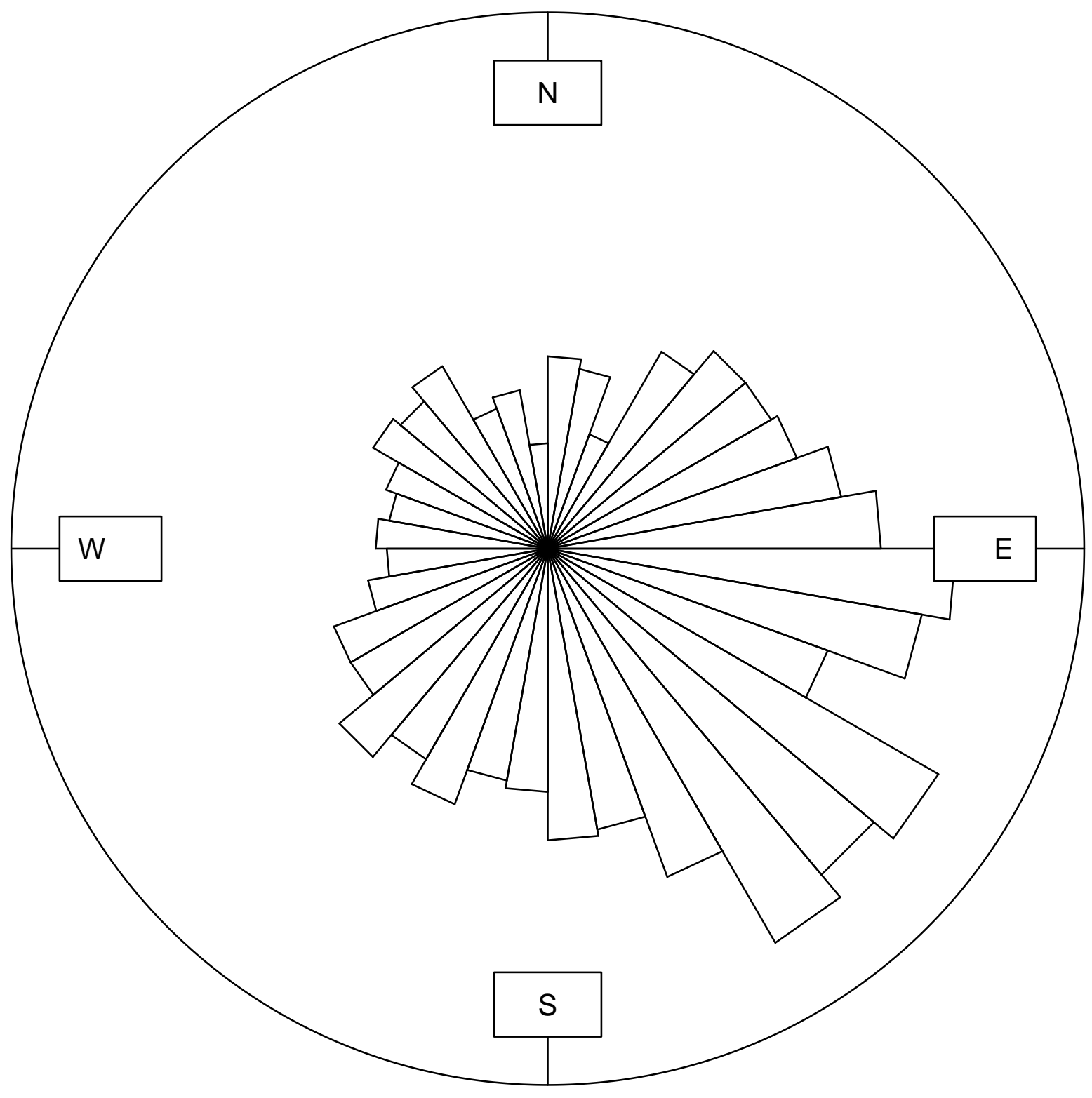


bootstrap 257

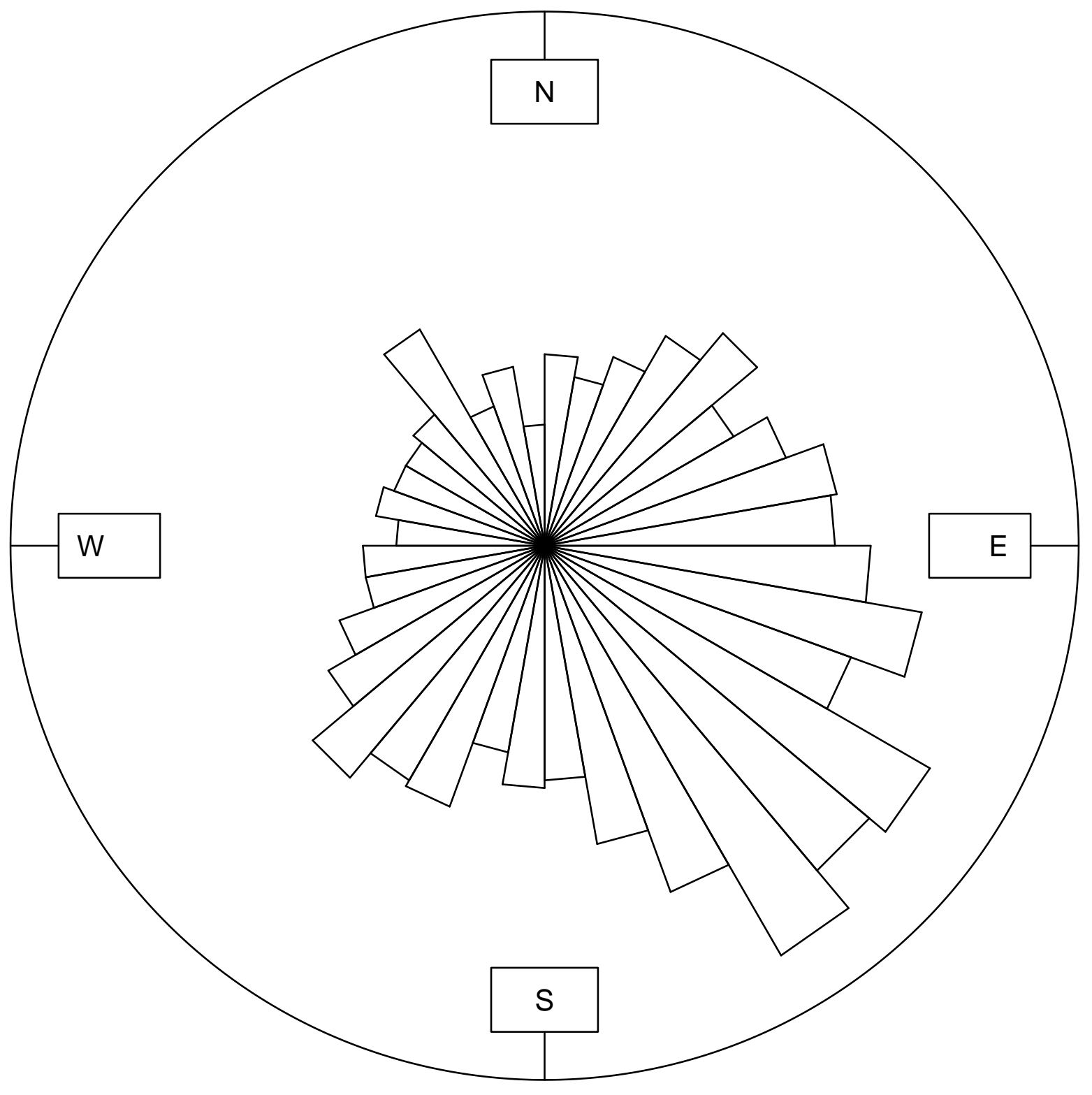


bootstrap 258

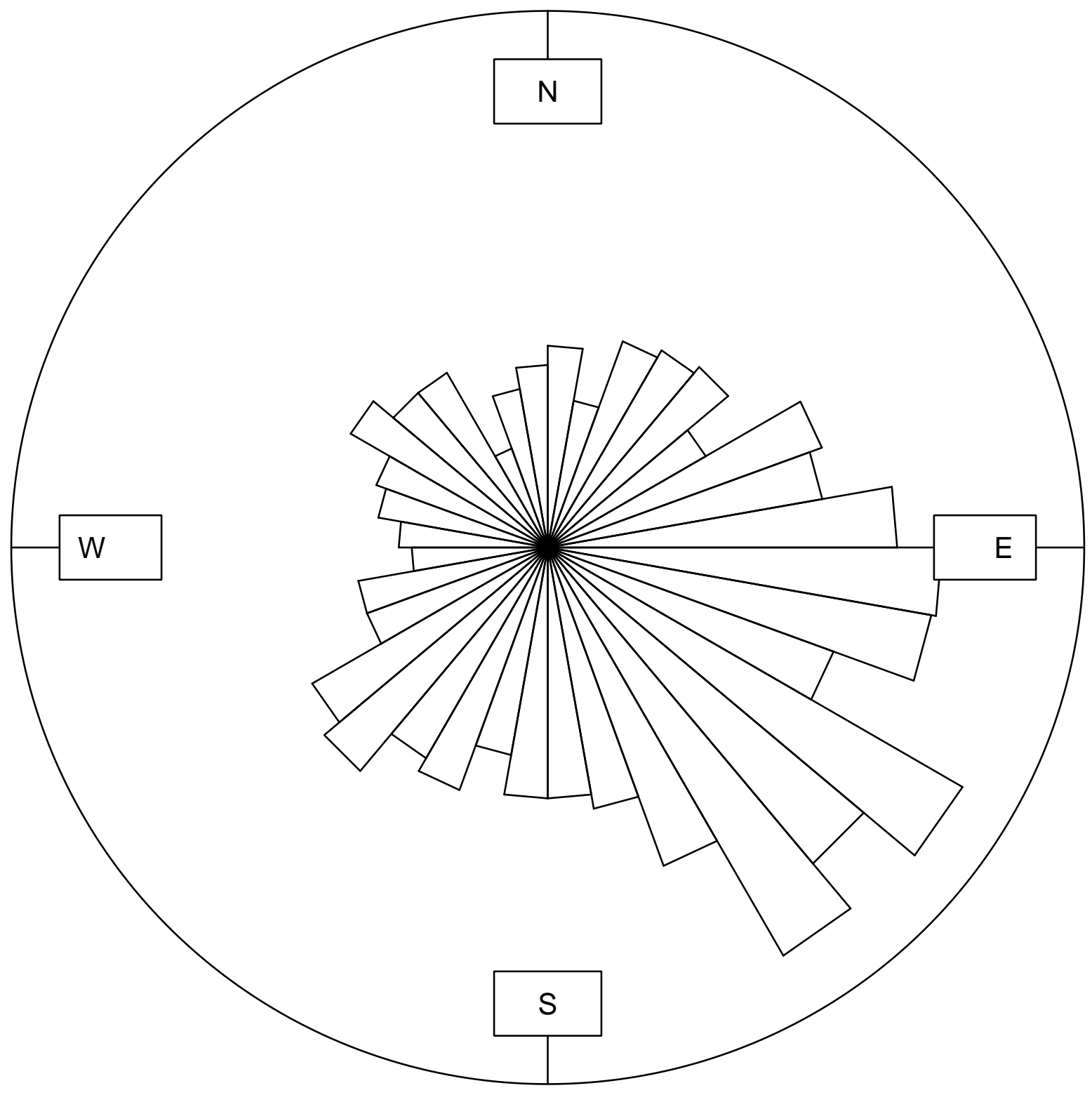


bootstrap 259

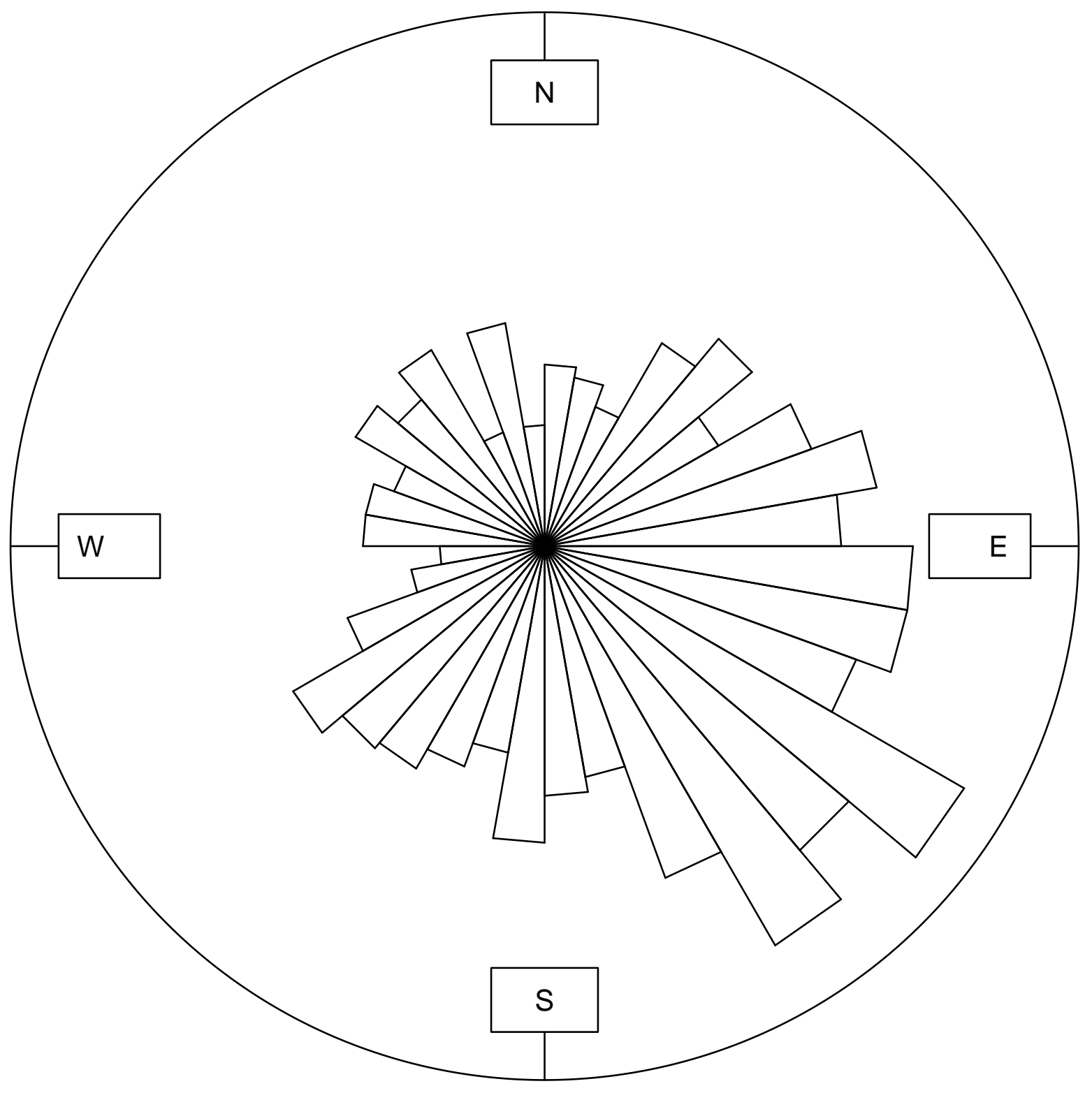


bootstrap 260

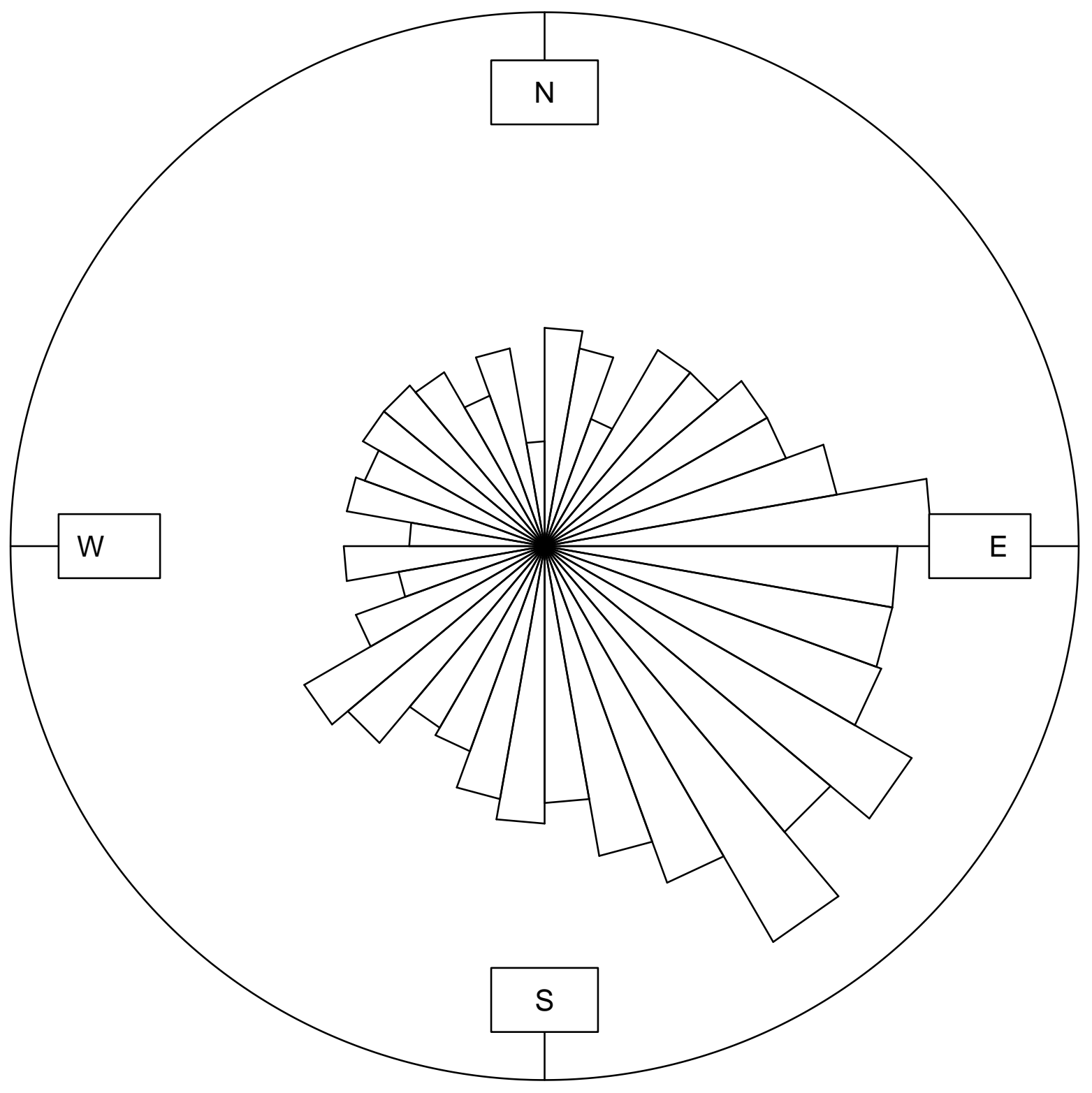


bootstrap 261

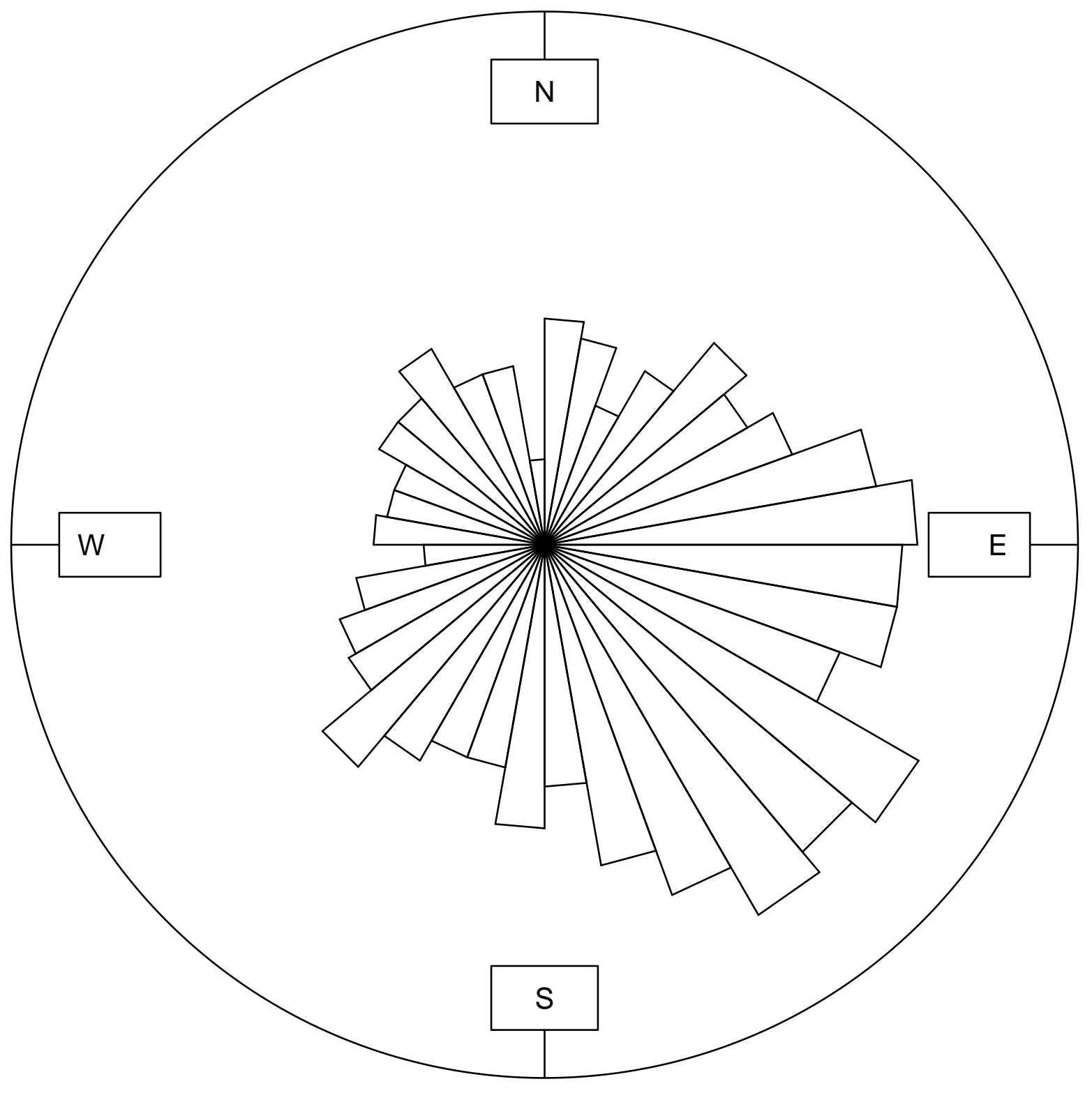


bootstrap 262

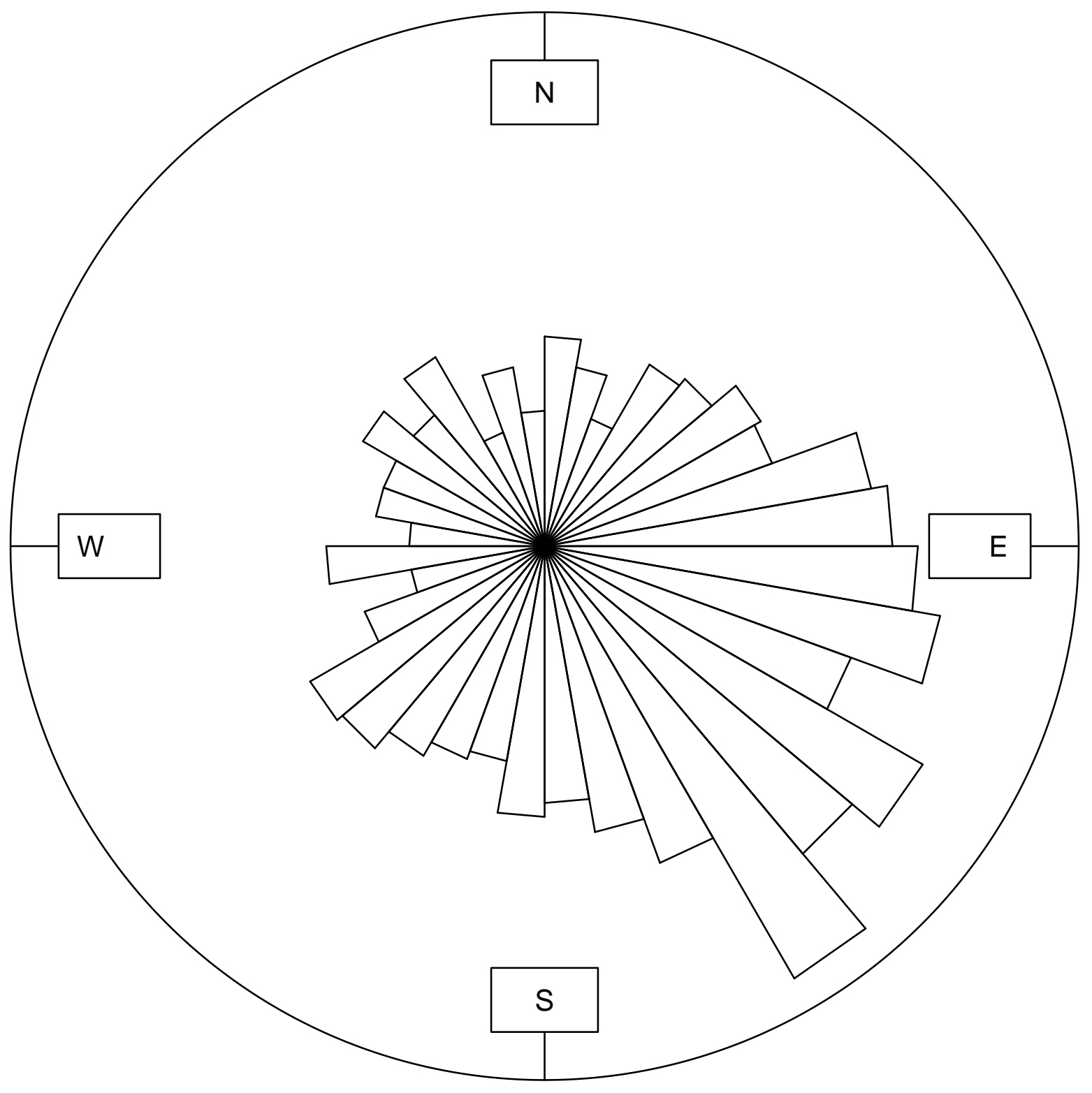




\section{bootstrap 263}

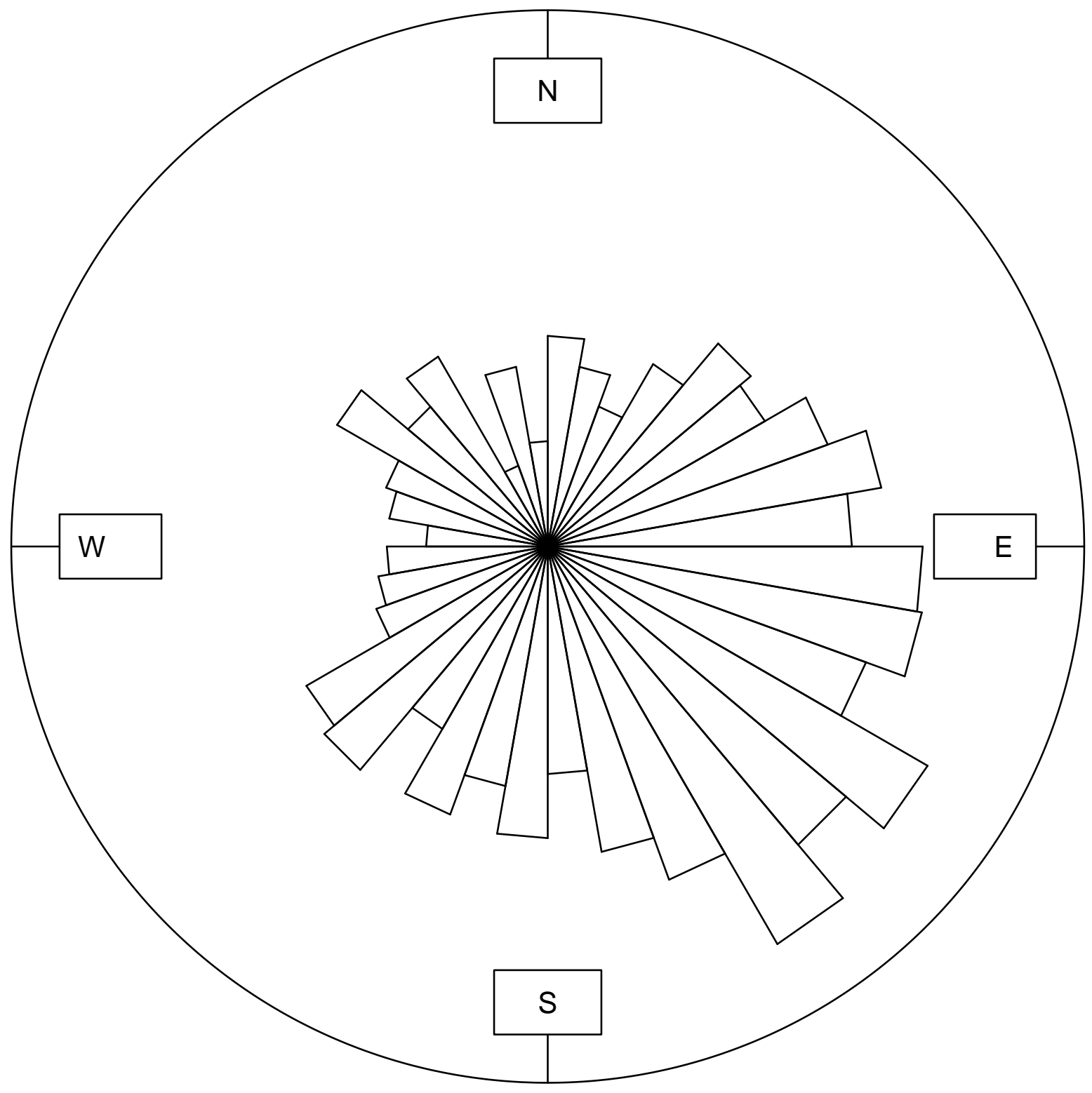




\section{bootstrap 264}

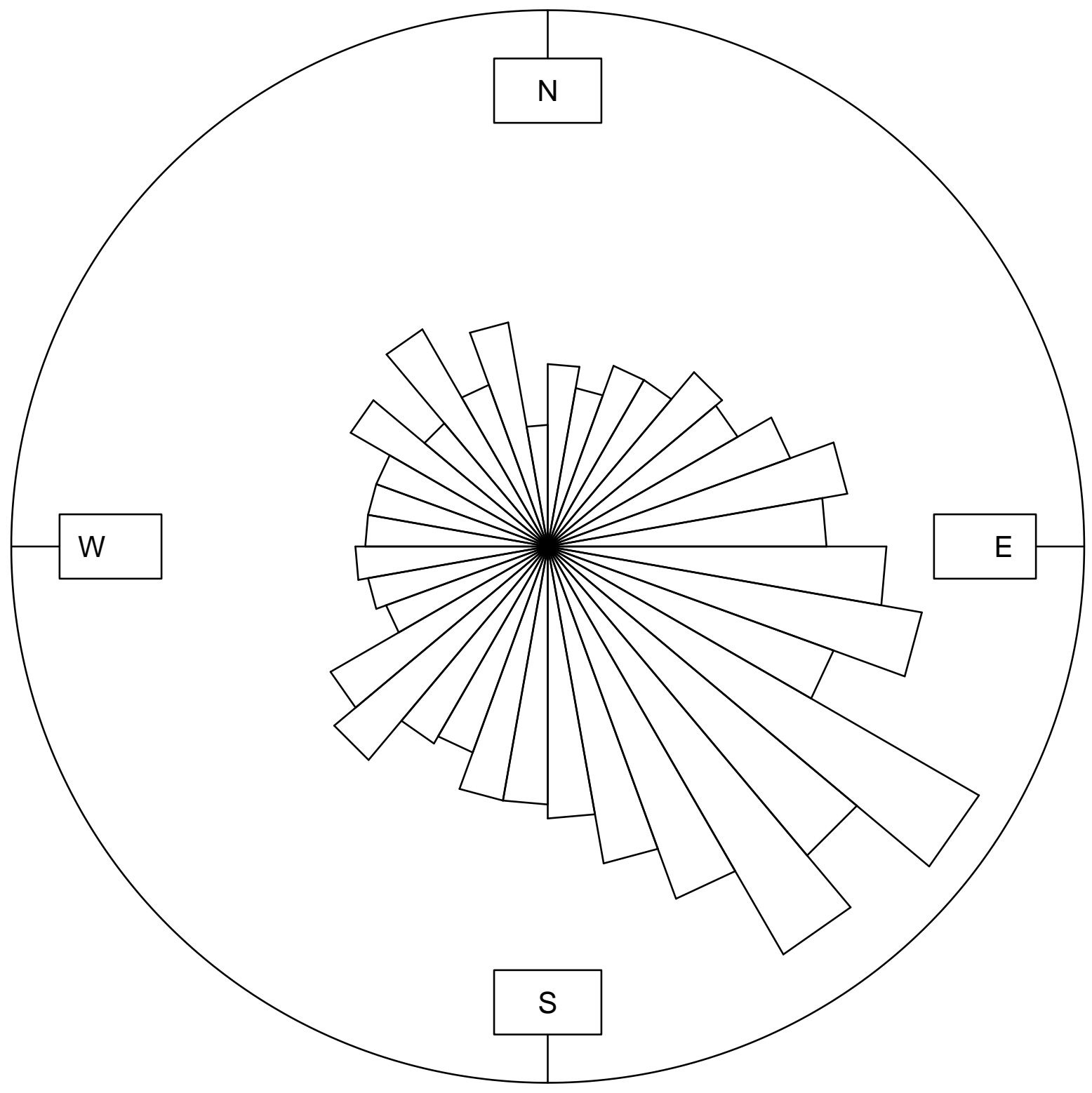


bootstrap 265

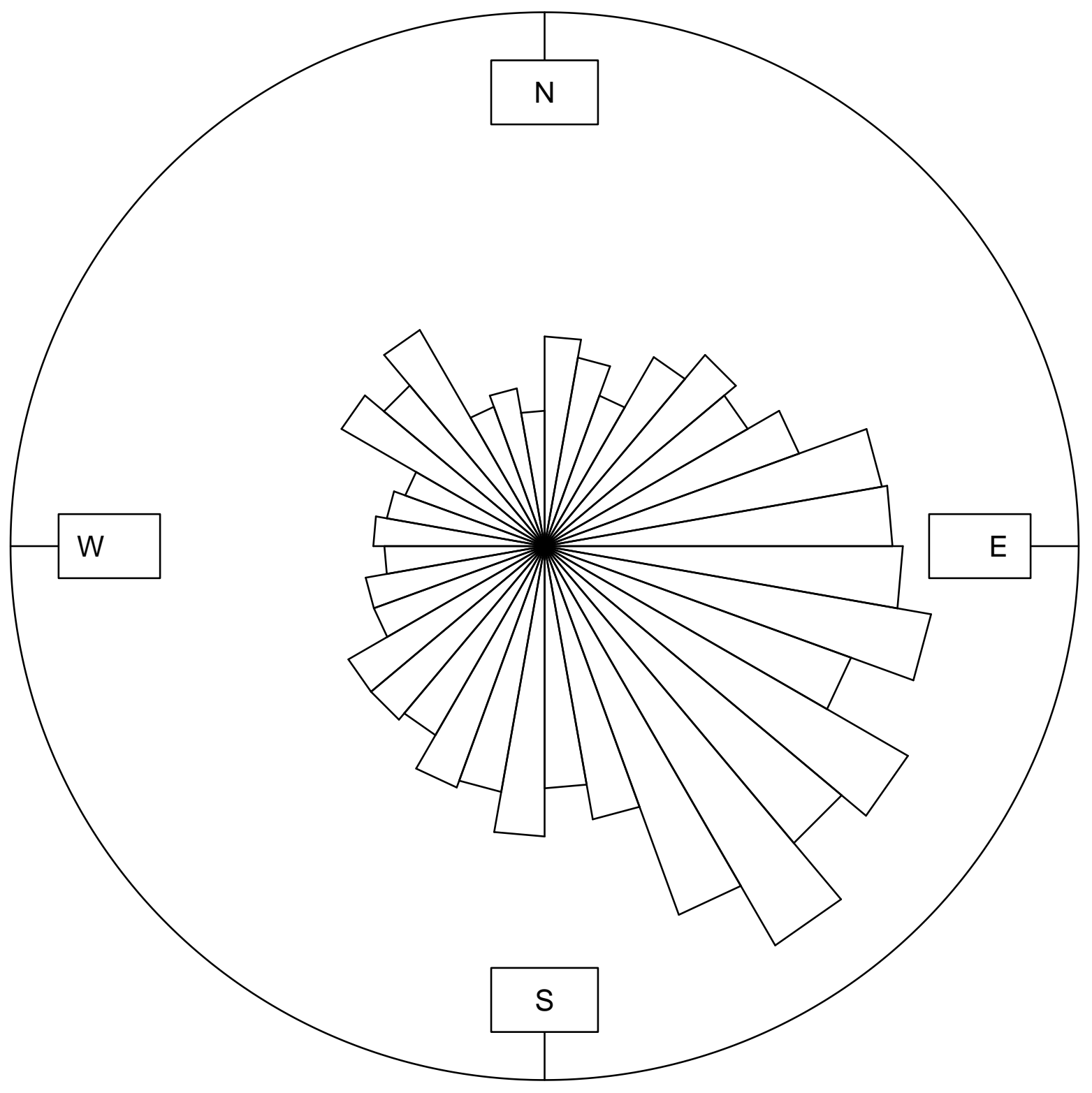




\section{bootstrap 266}

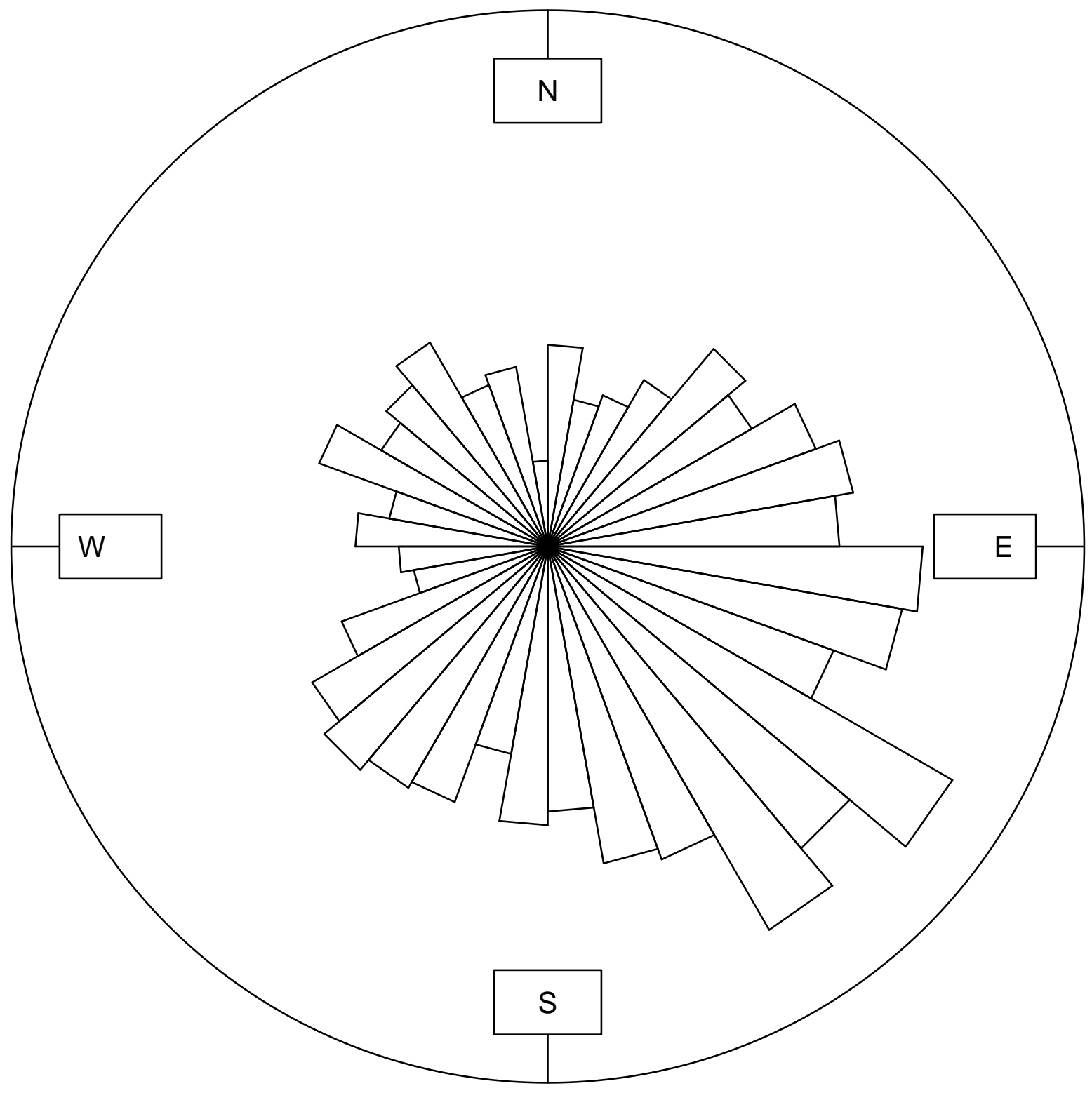


bootstrap 267

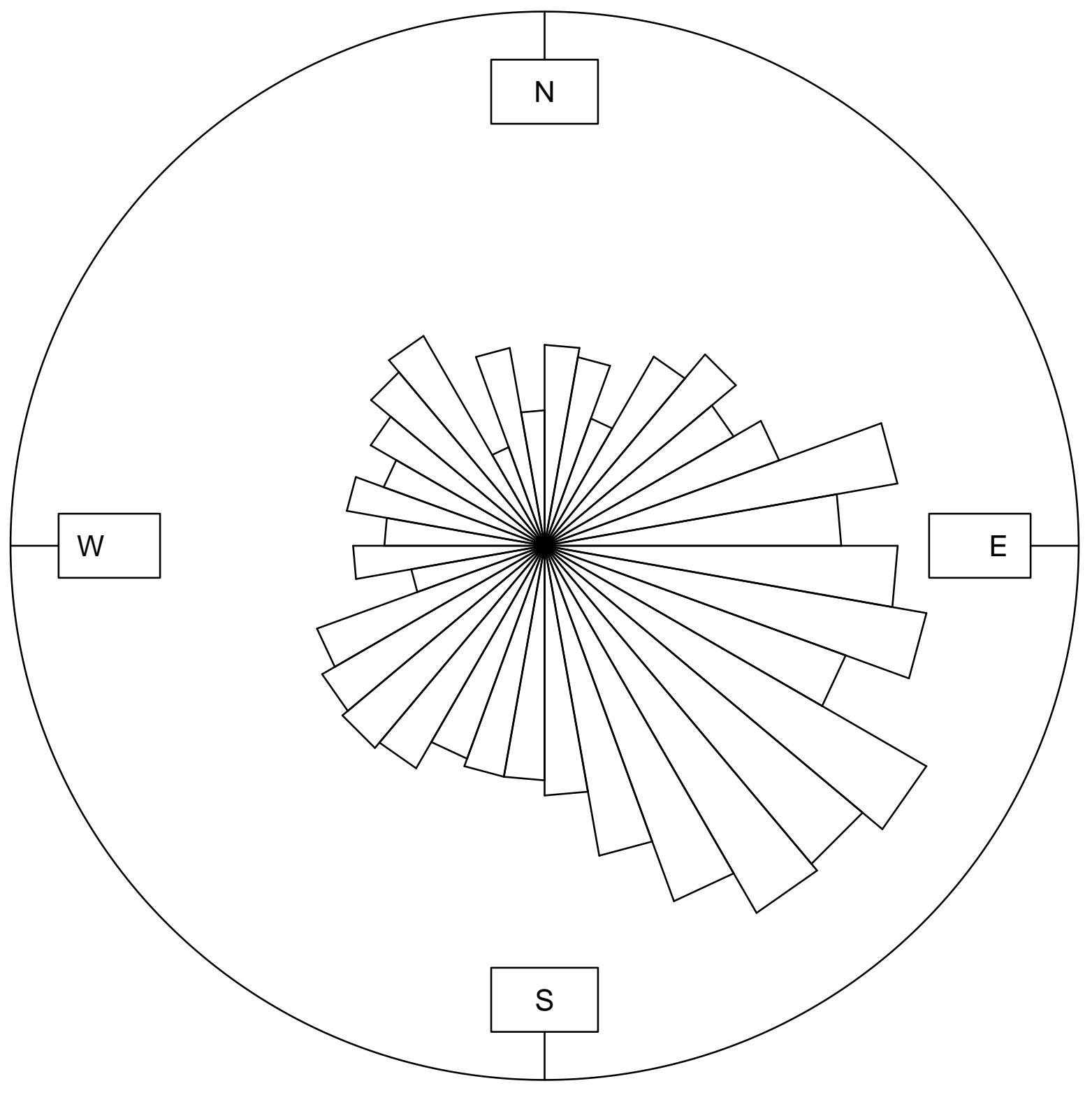


bootstrap 268

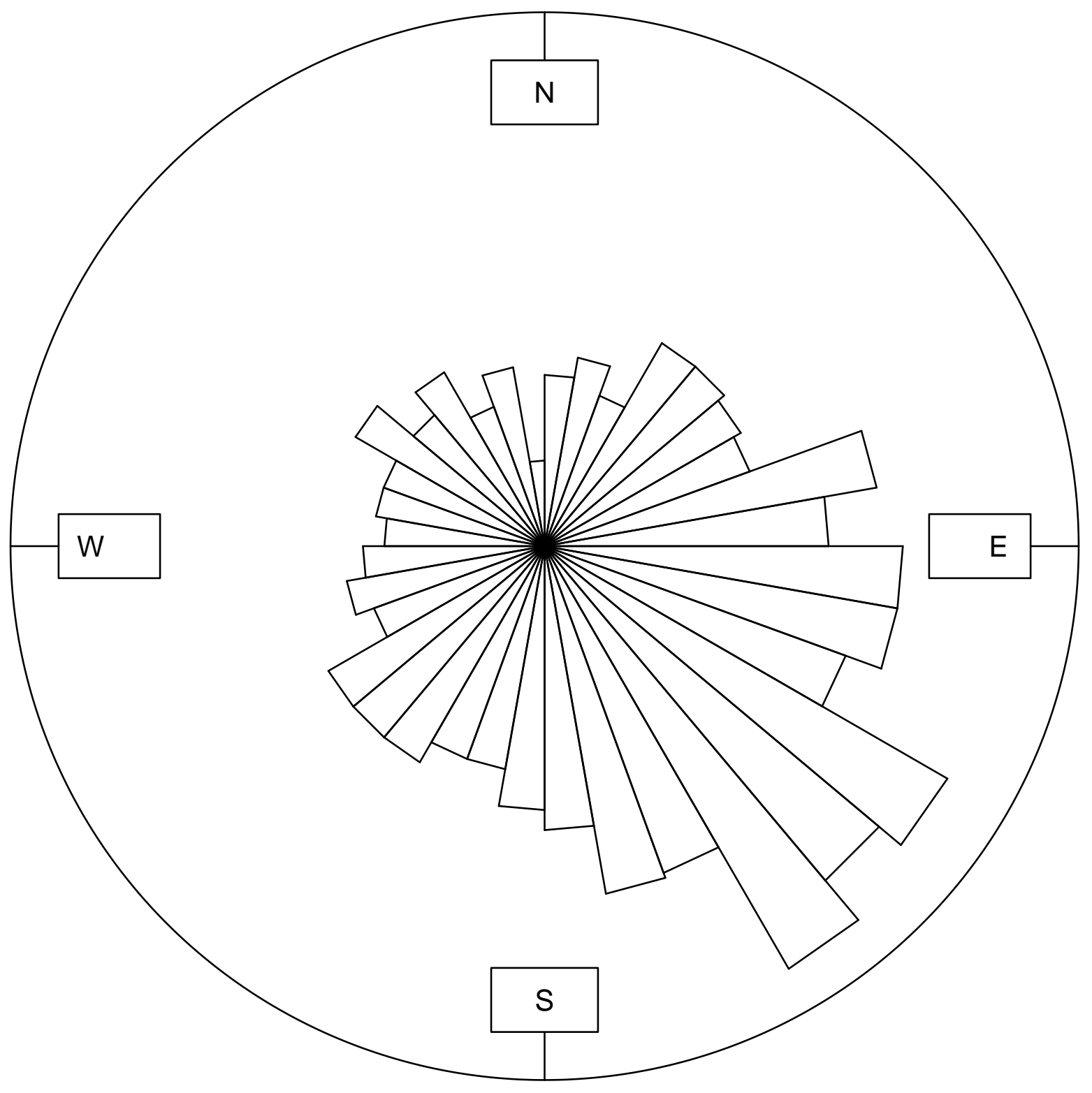


bootstrap 269

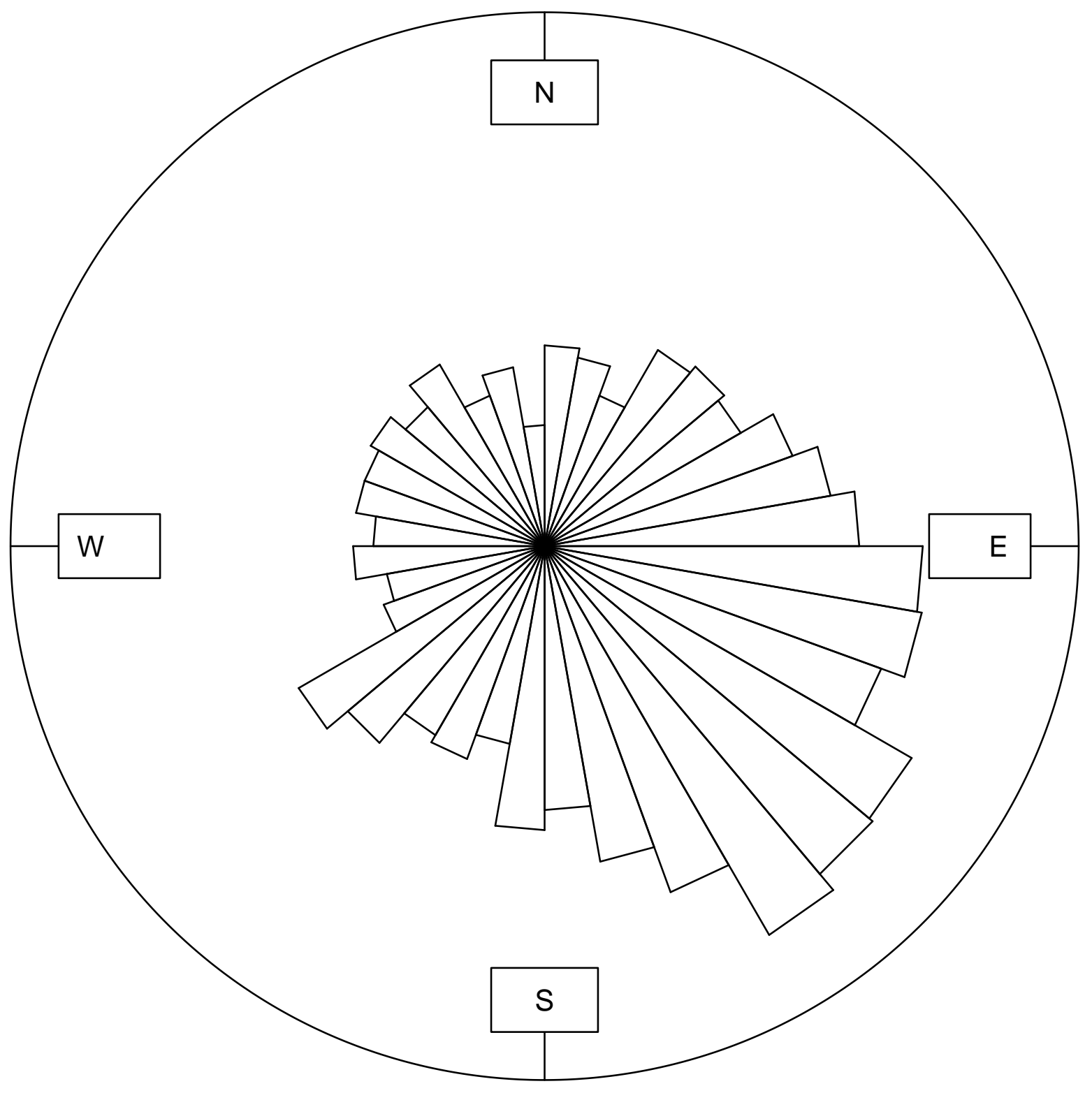


bootstrap 270

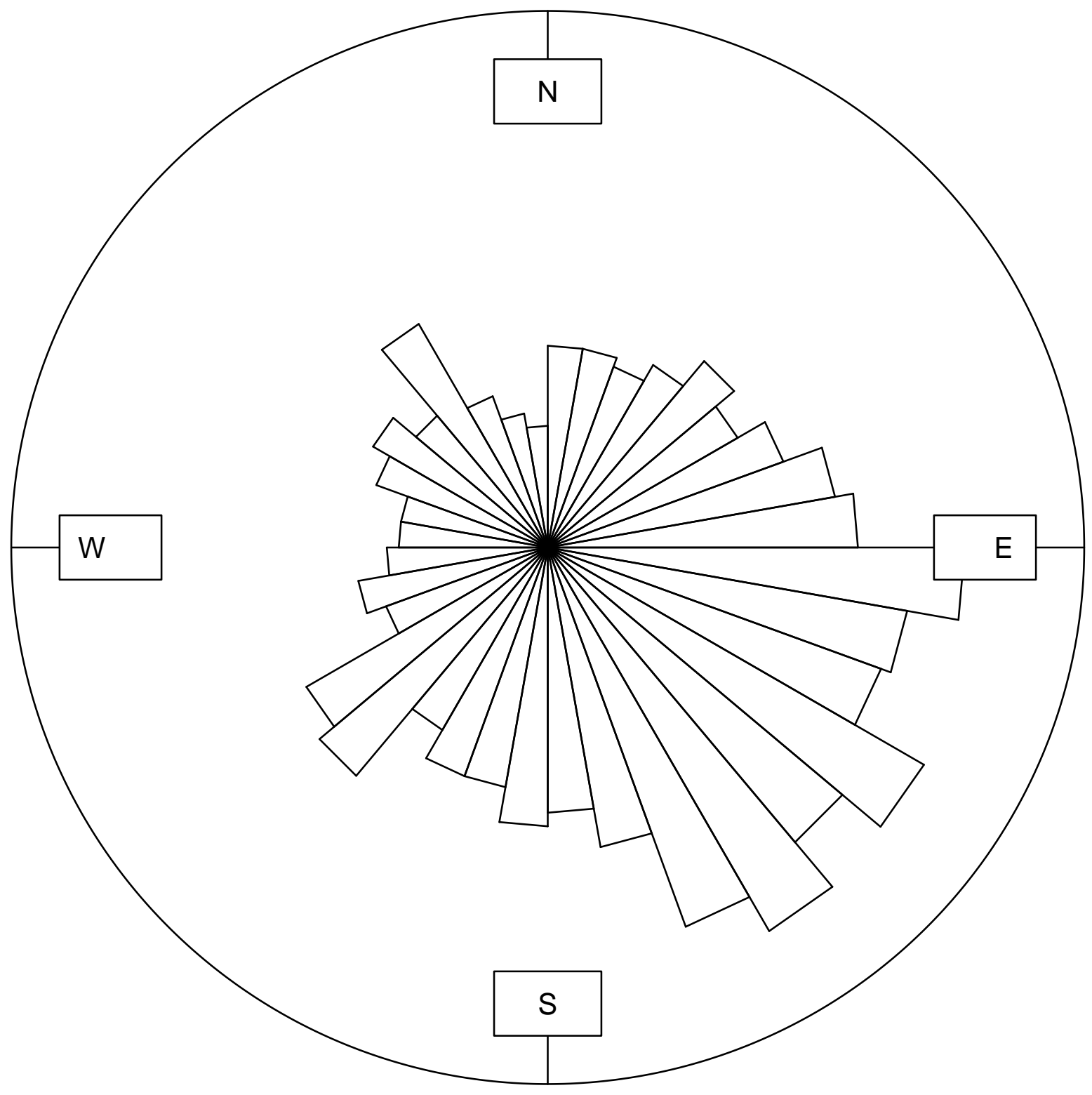


bootstrap 271

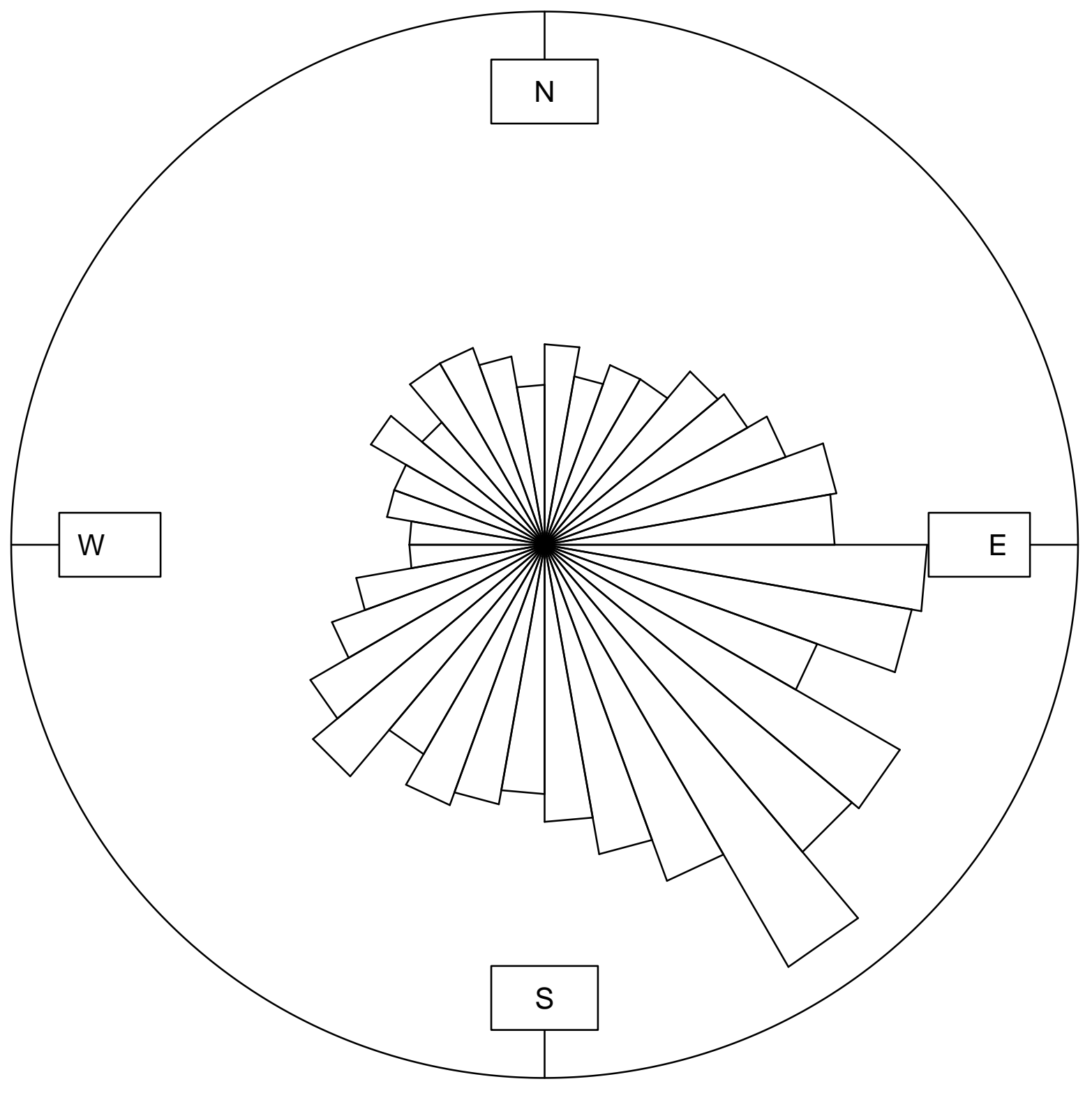


bootstrap 272

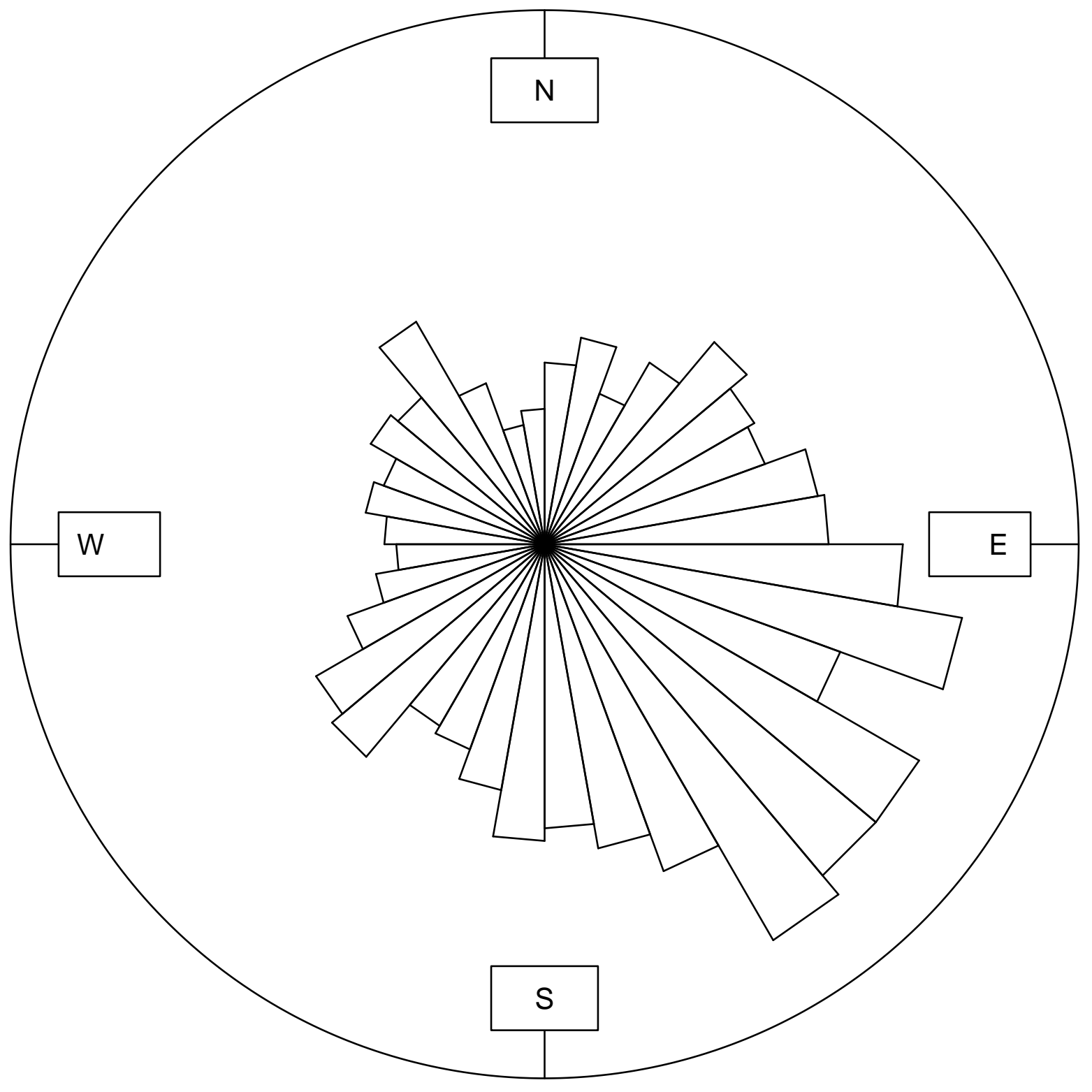


bootstrap 273

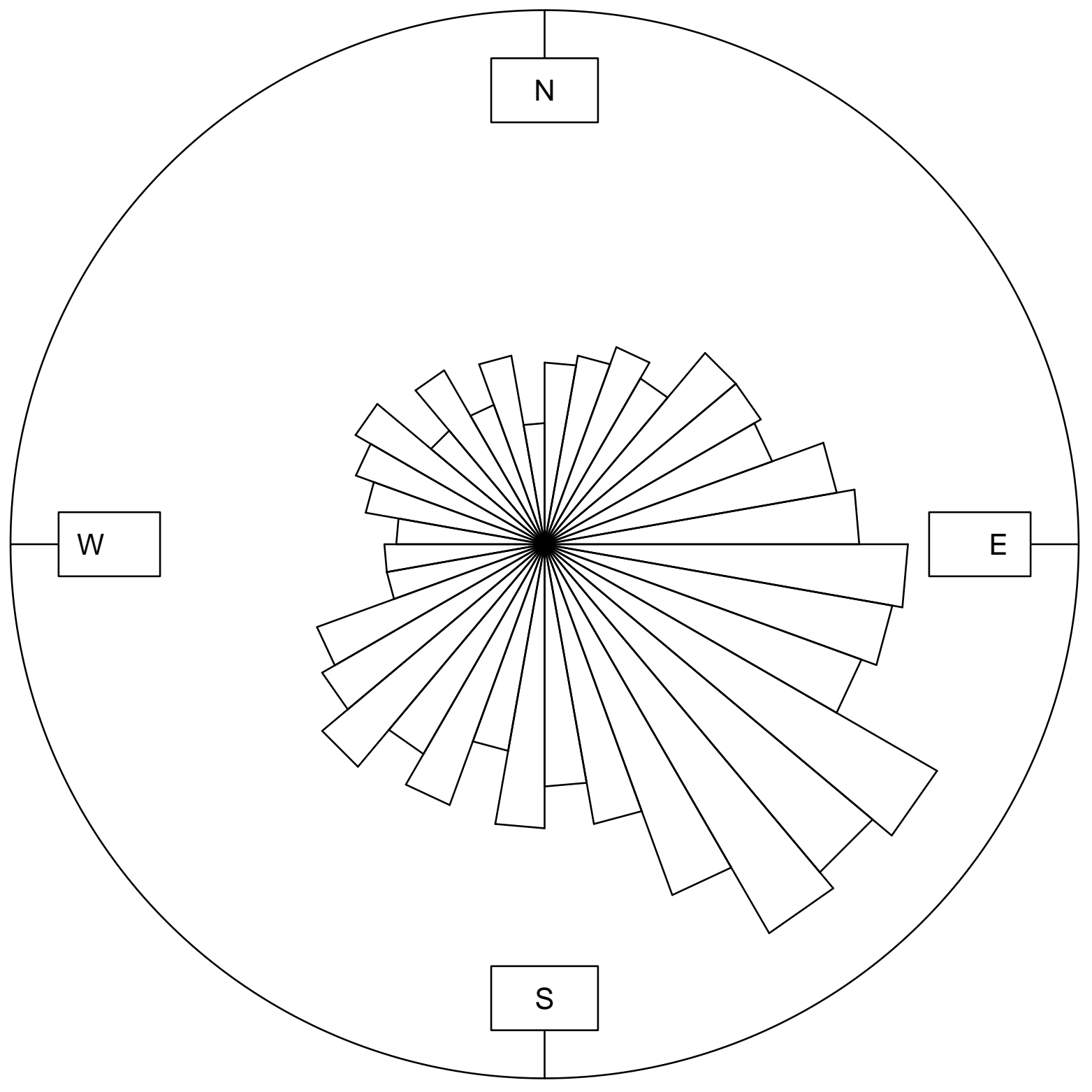


bootstrap 274

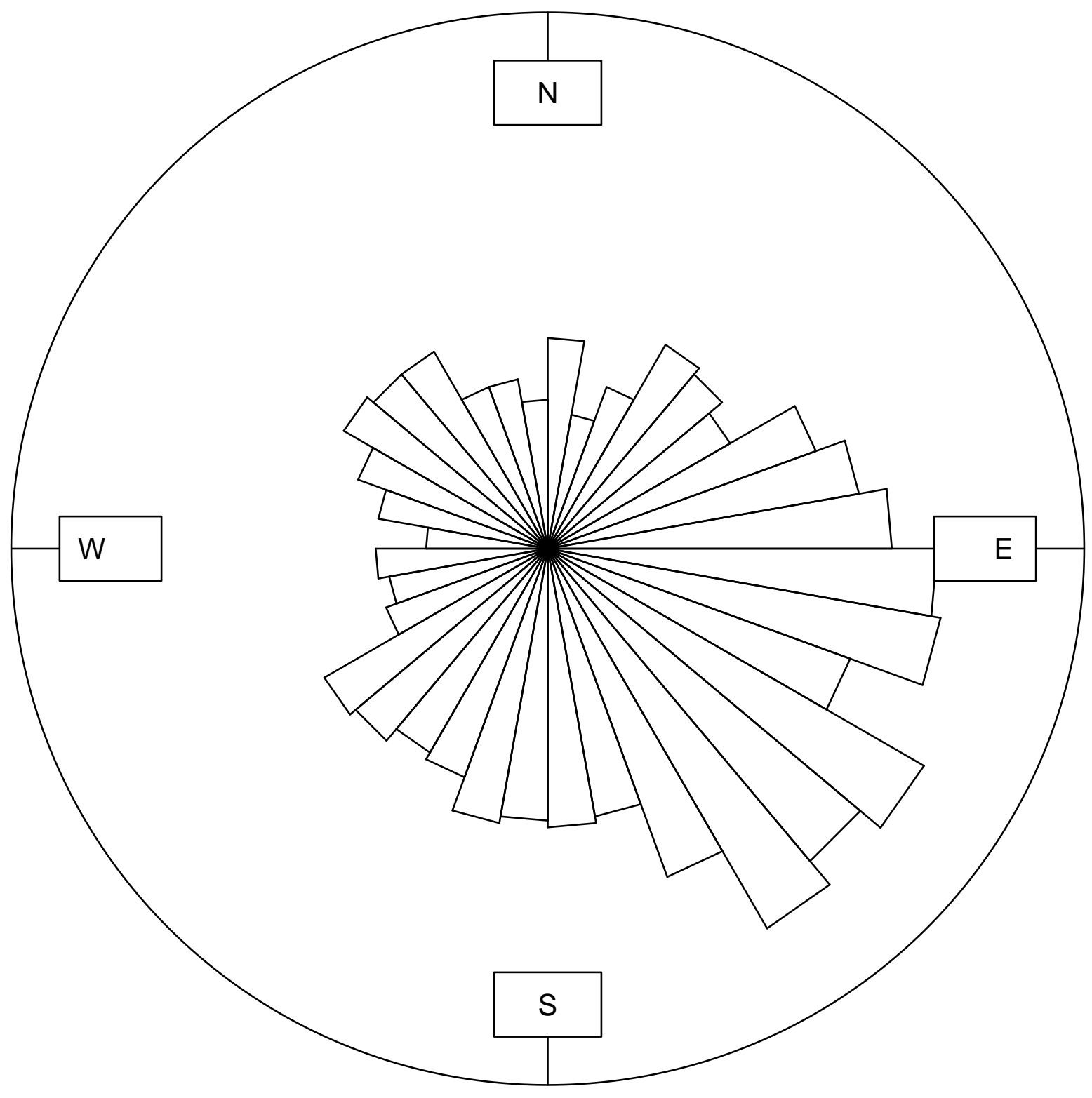


bootstrap 275

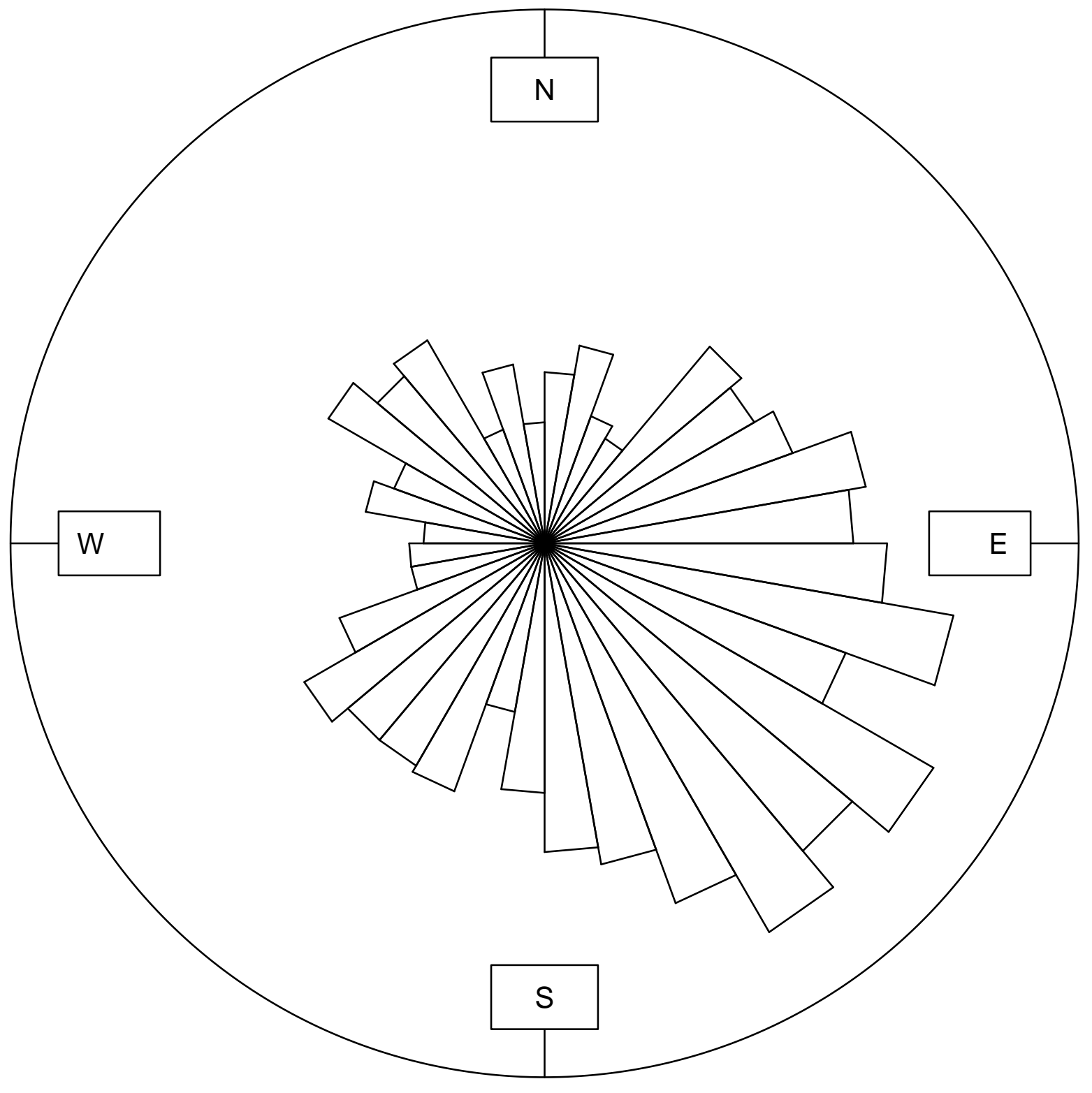


bootstrap 276

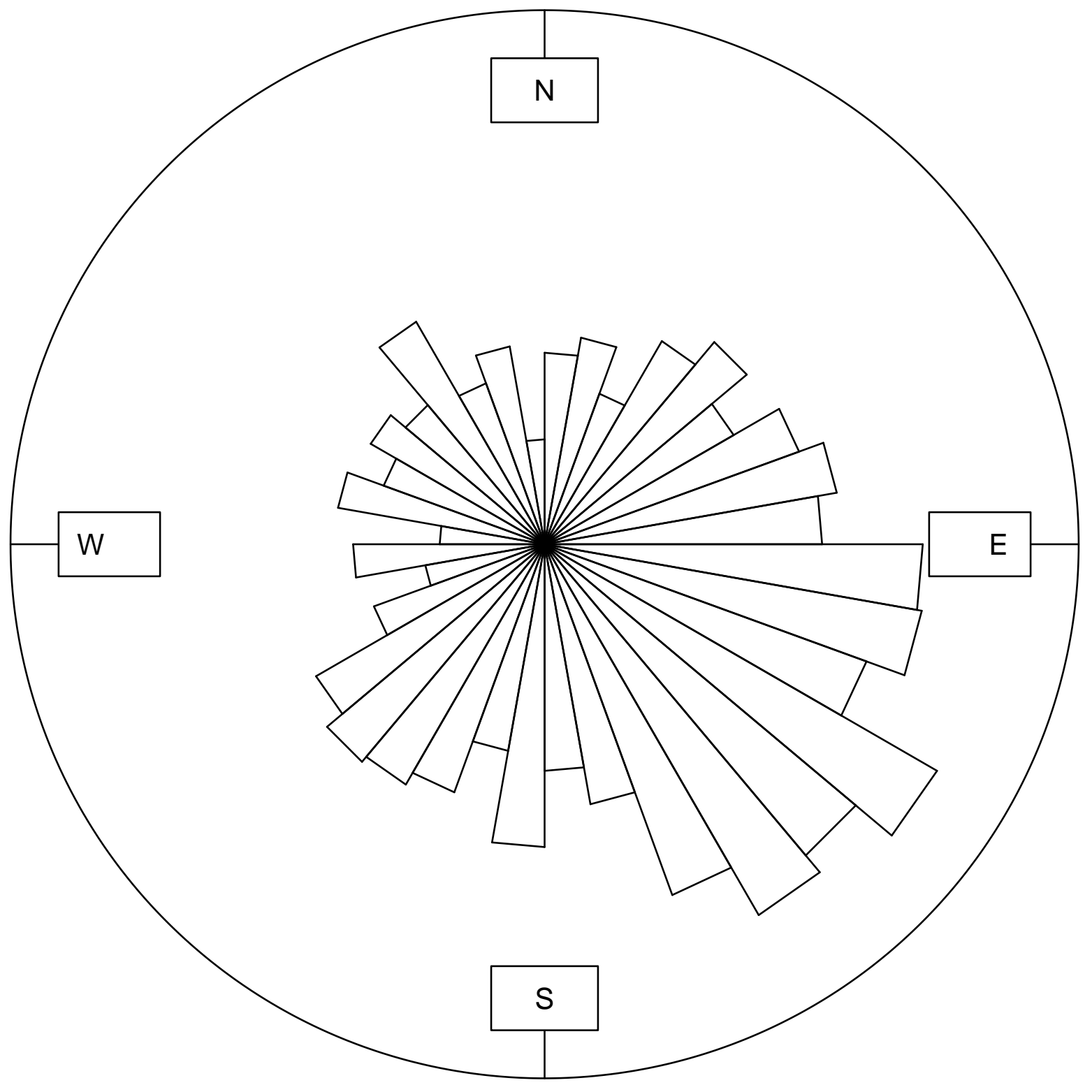


bootstrap 277

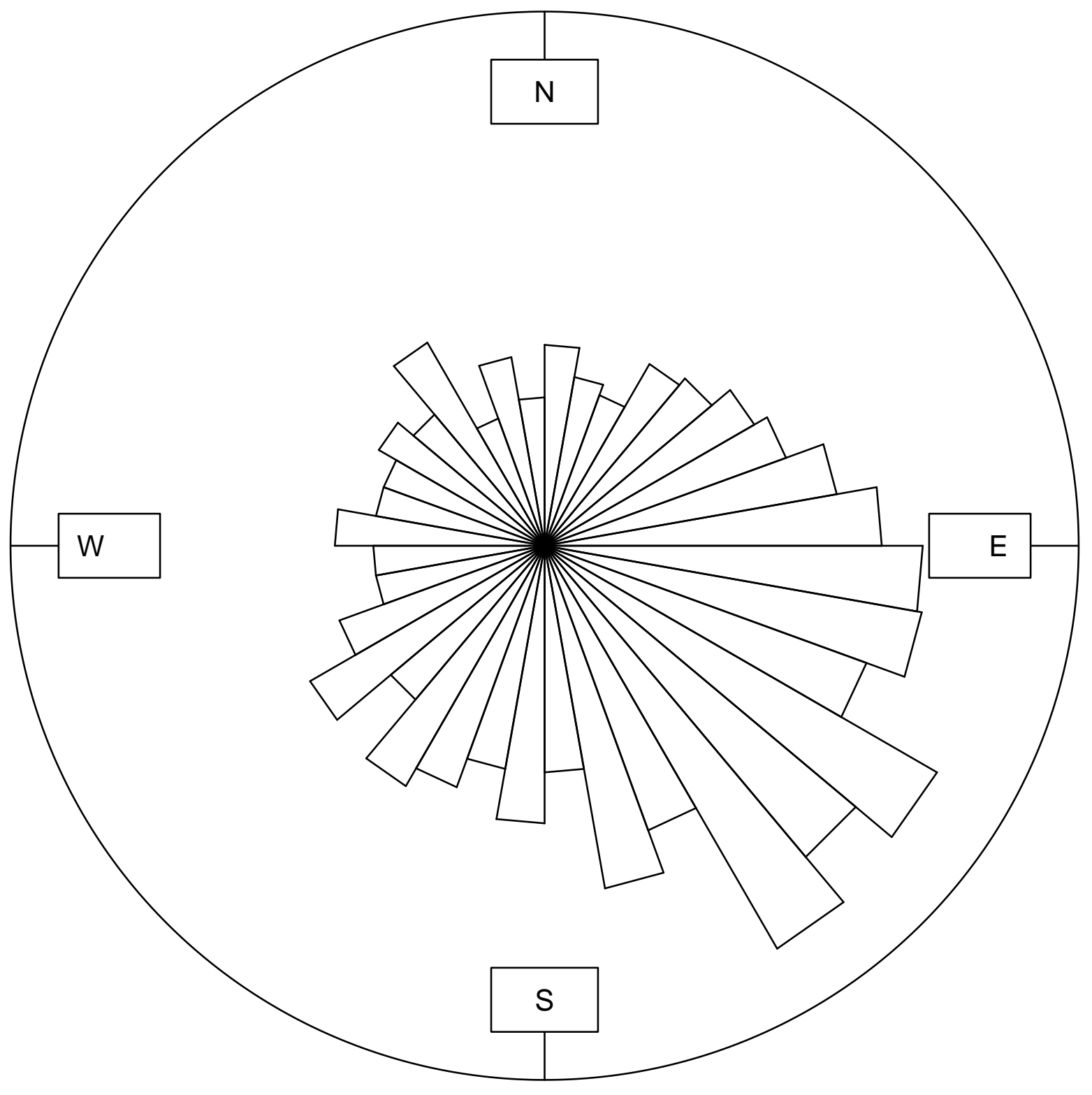


bootstrap 278

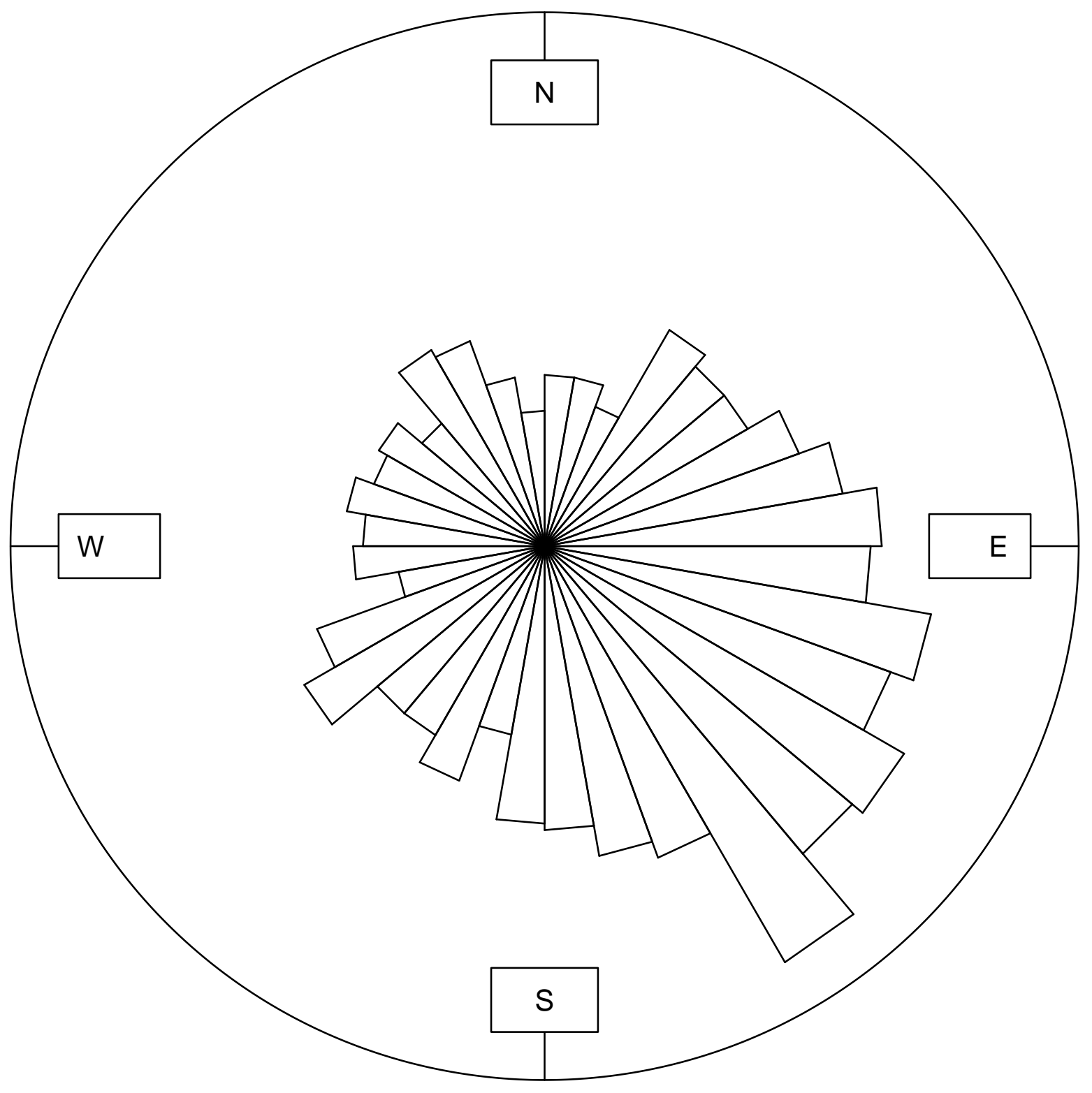


bootstrap 279

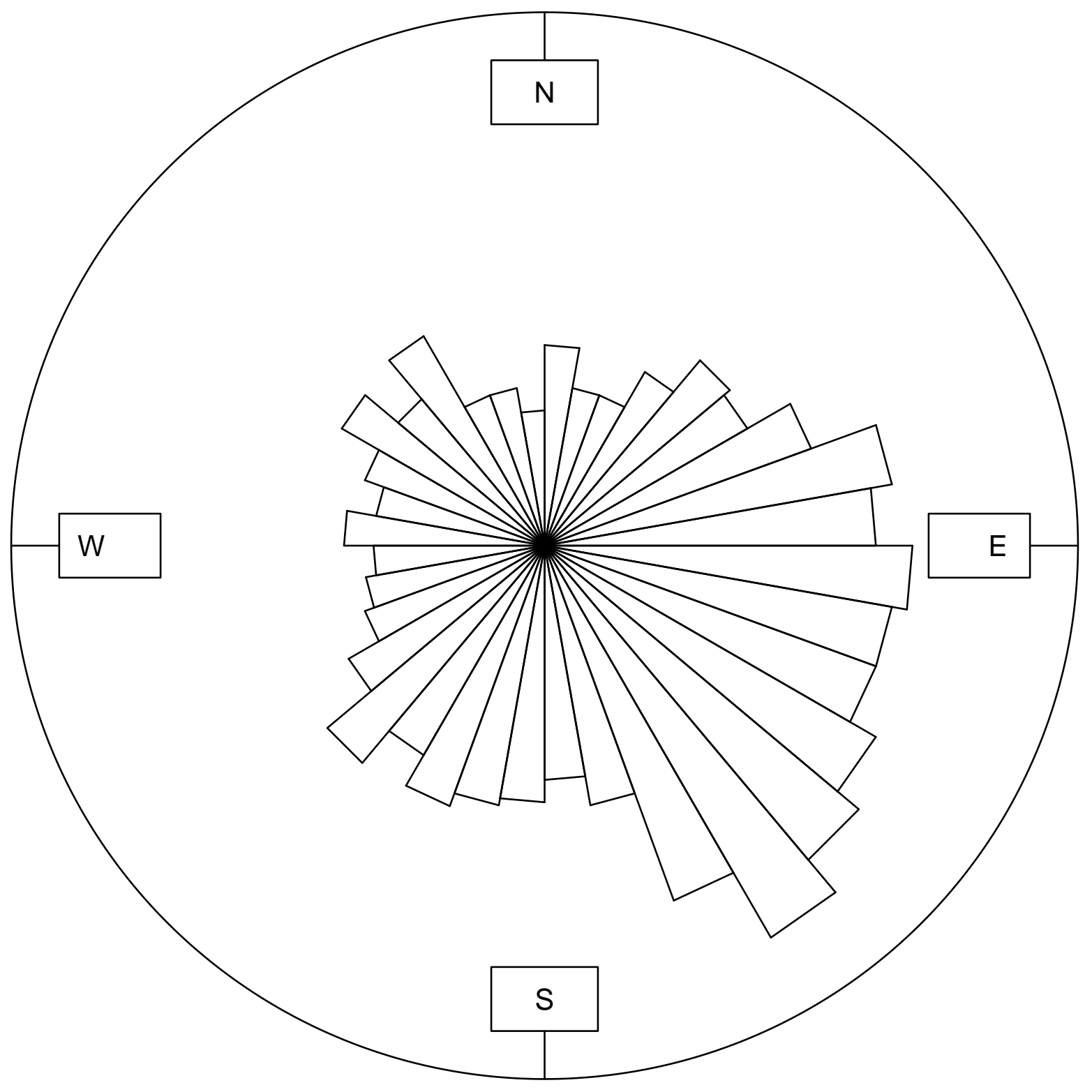


bootstrap 280

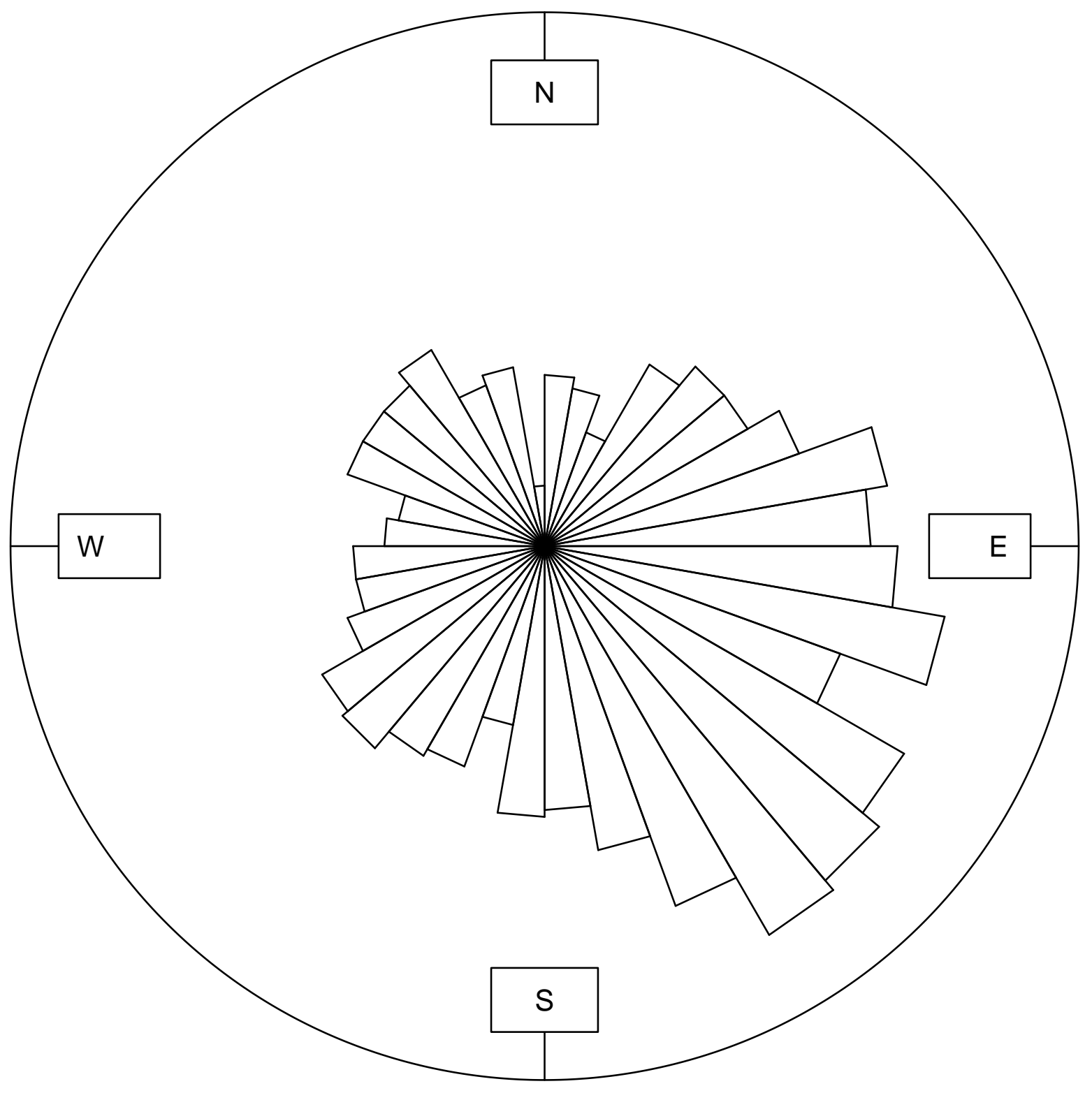


bootstrap 281

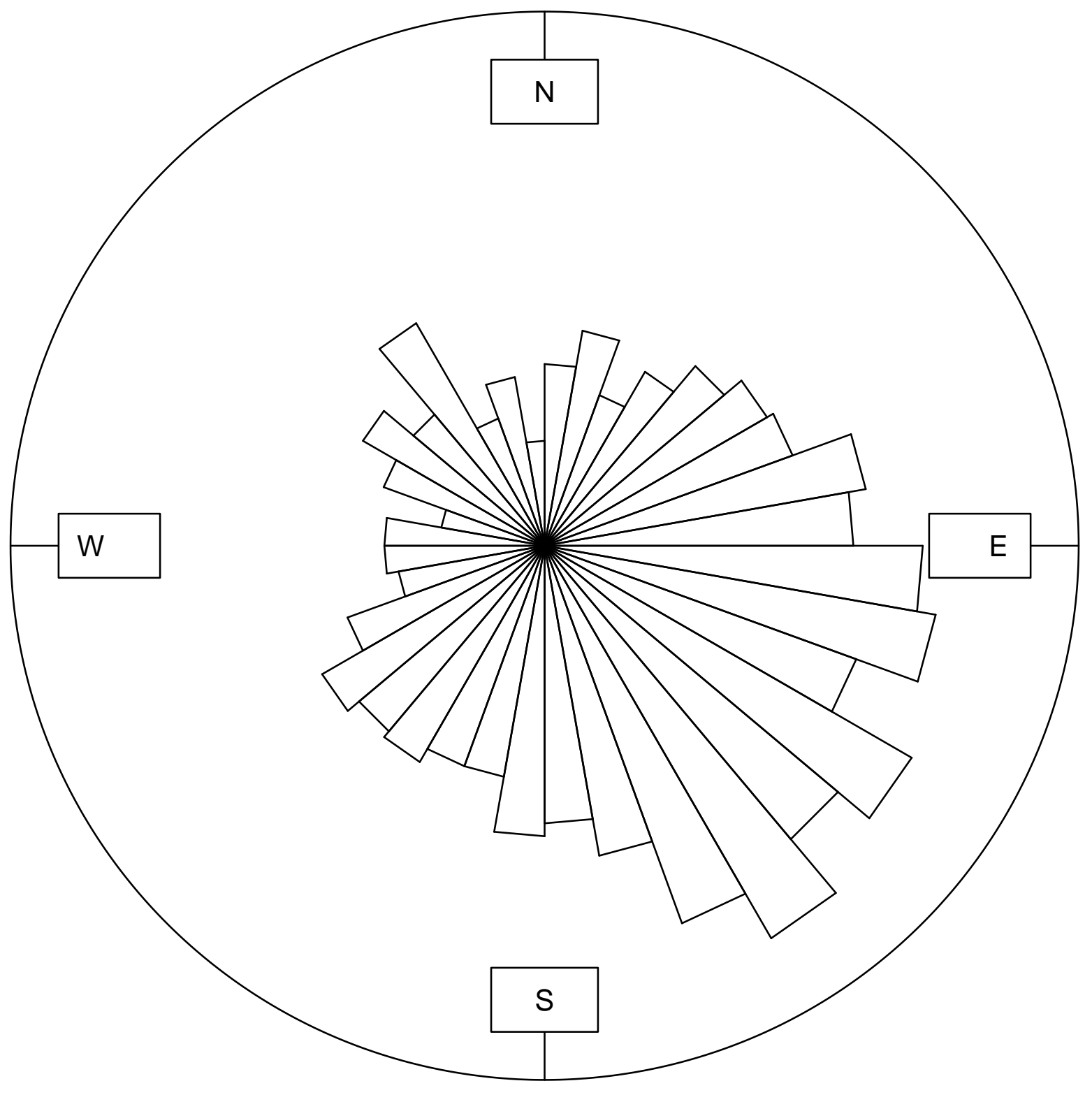


bootstrap 282

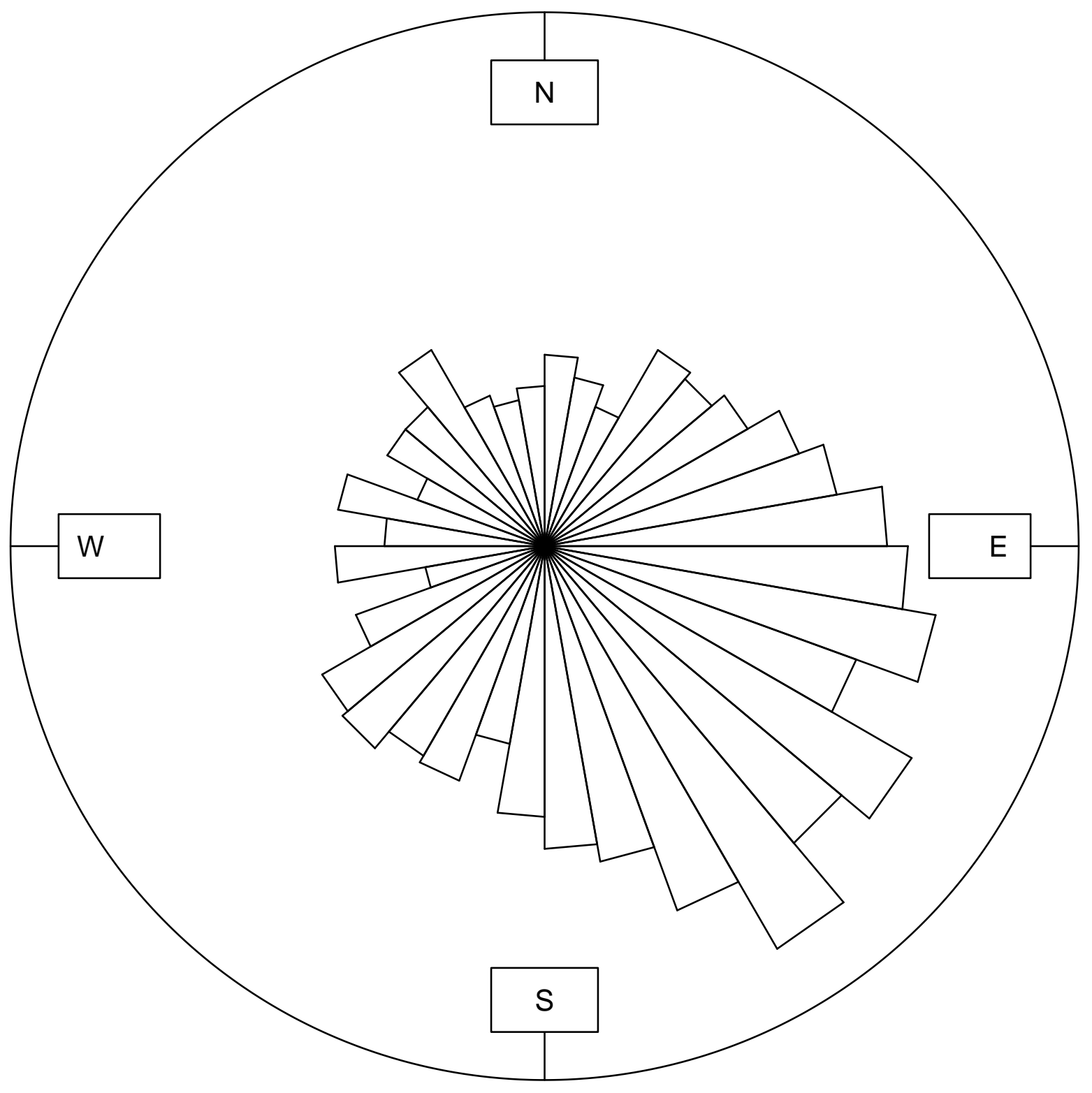


bootstrap 283

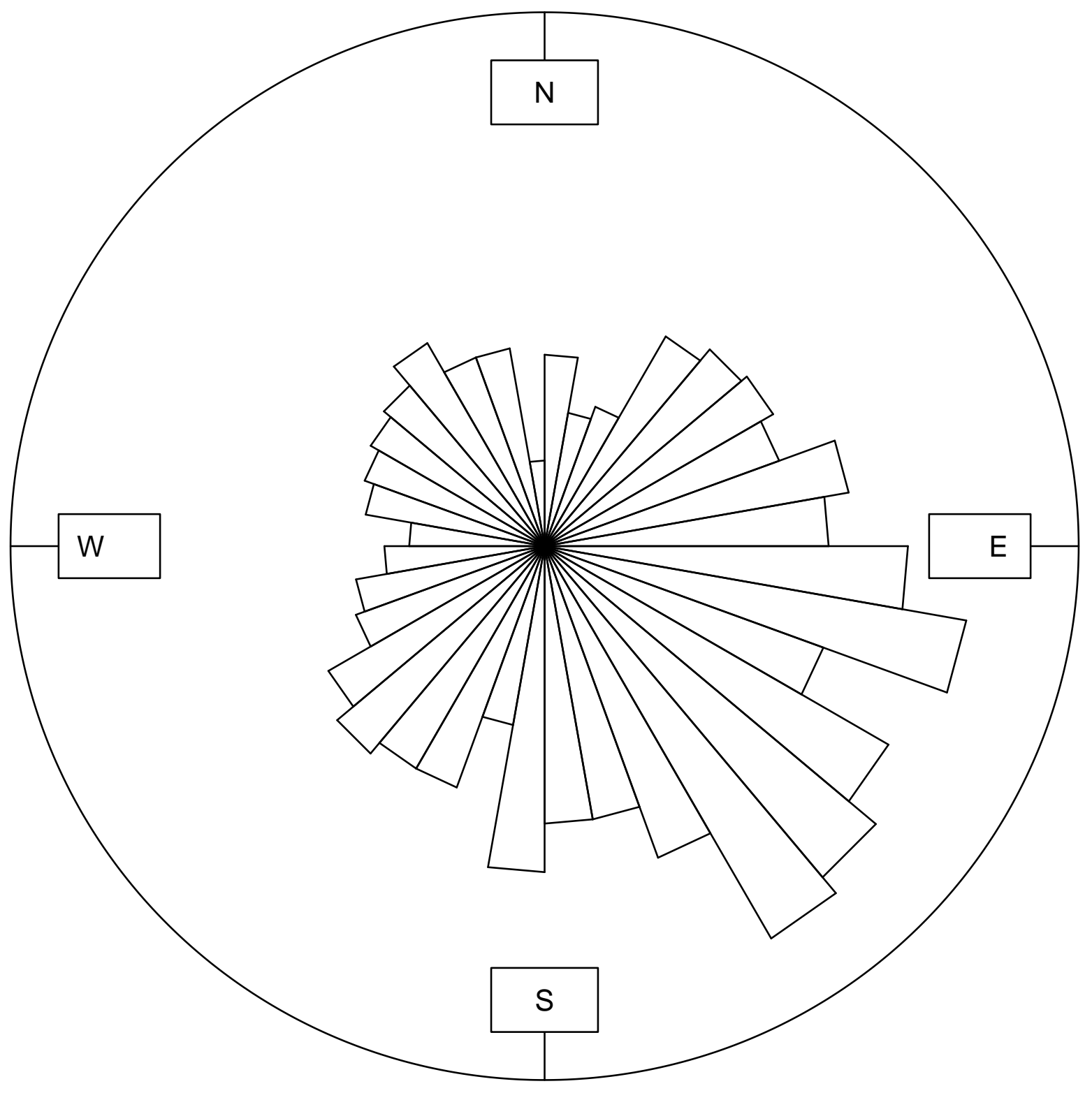


bootstrap 284

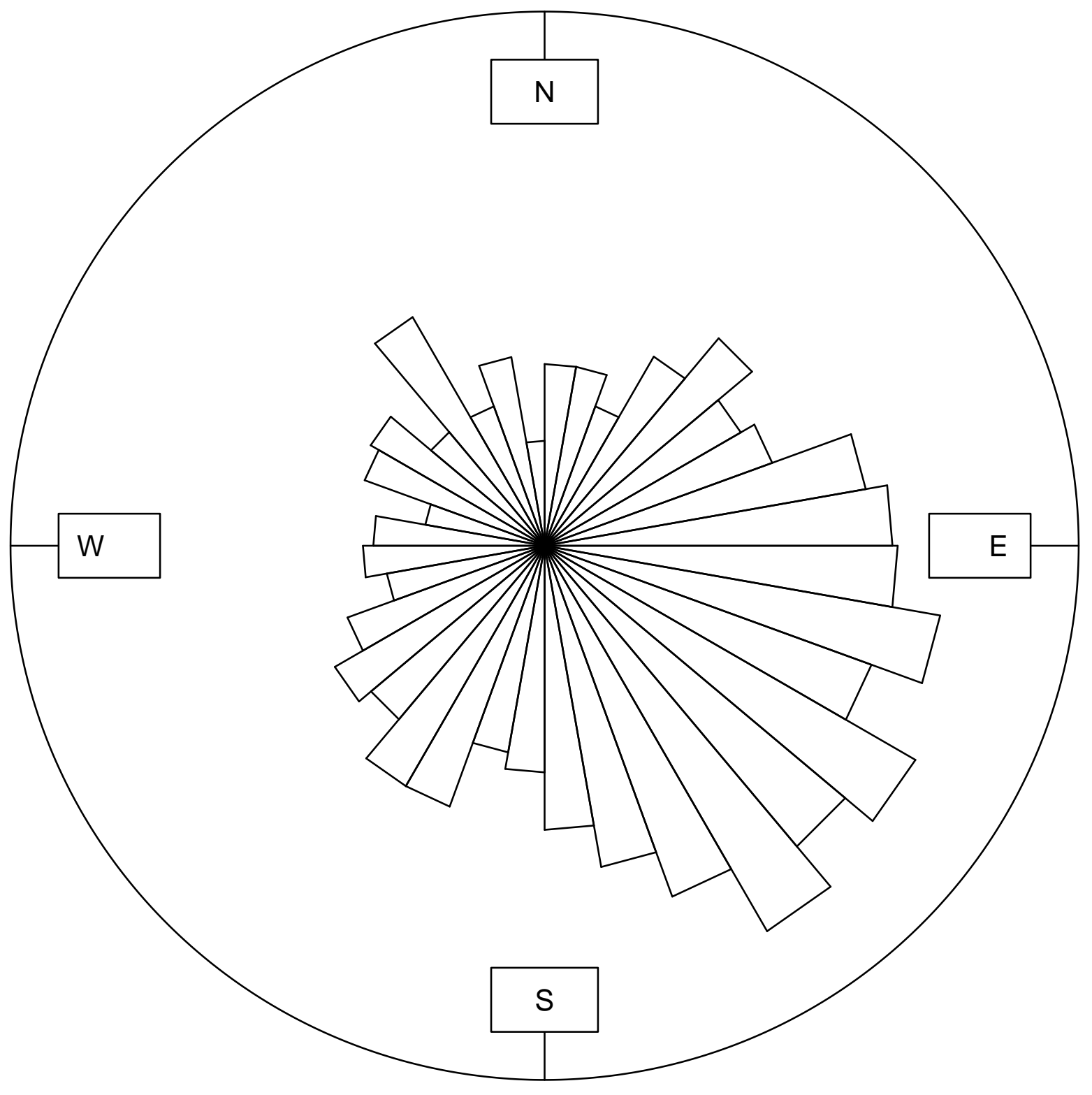


bootstrap 285

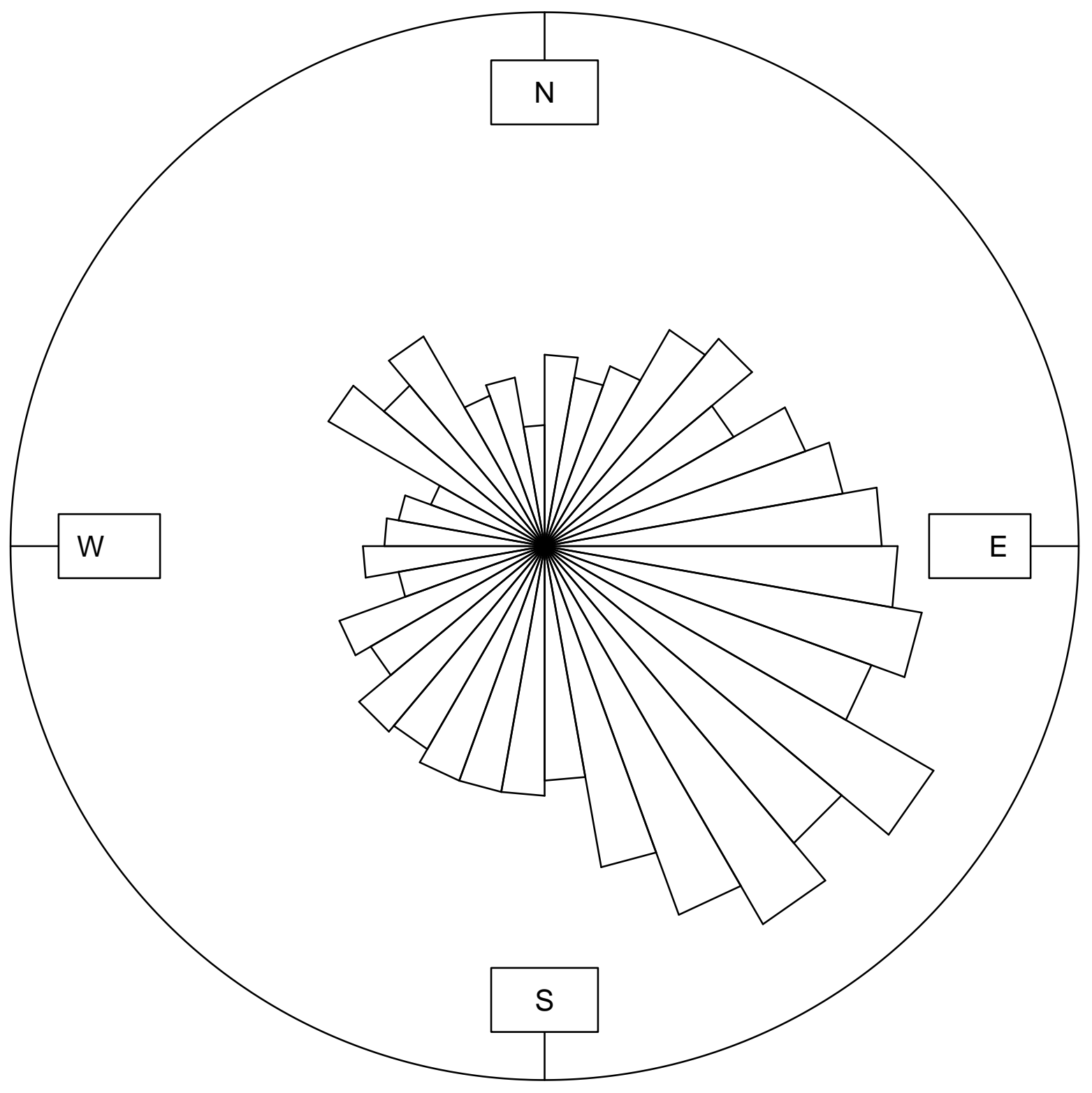


bootstrap 286

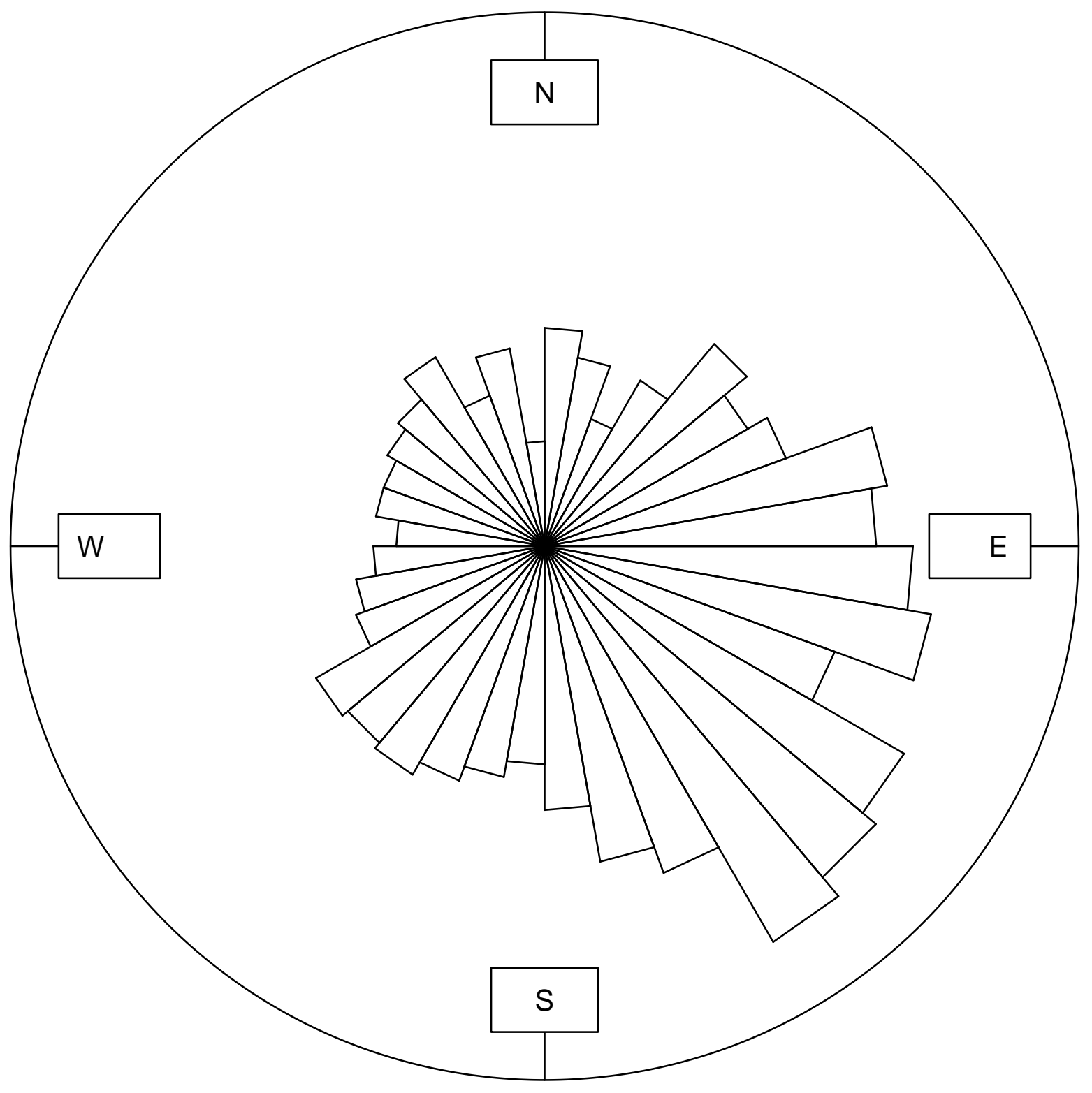


bootstrap 287

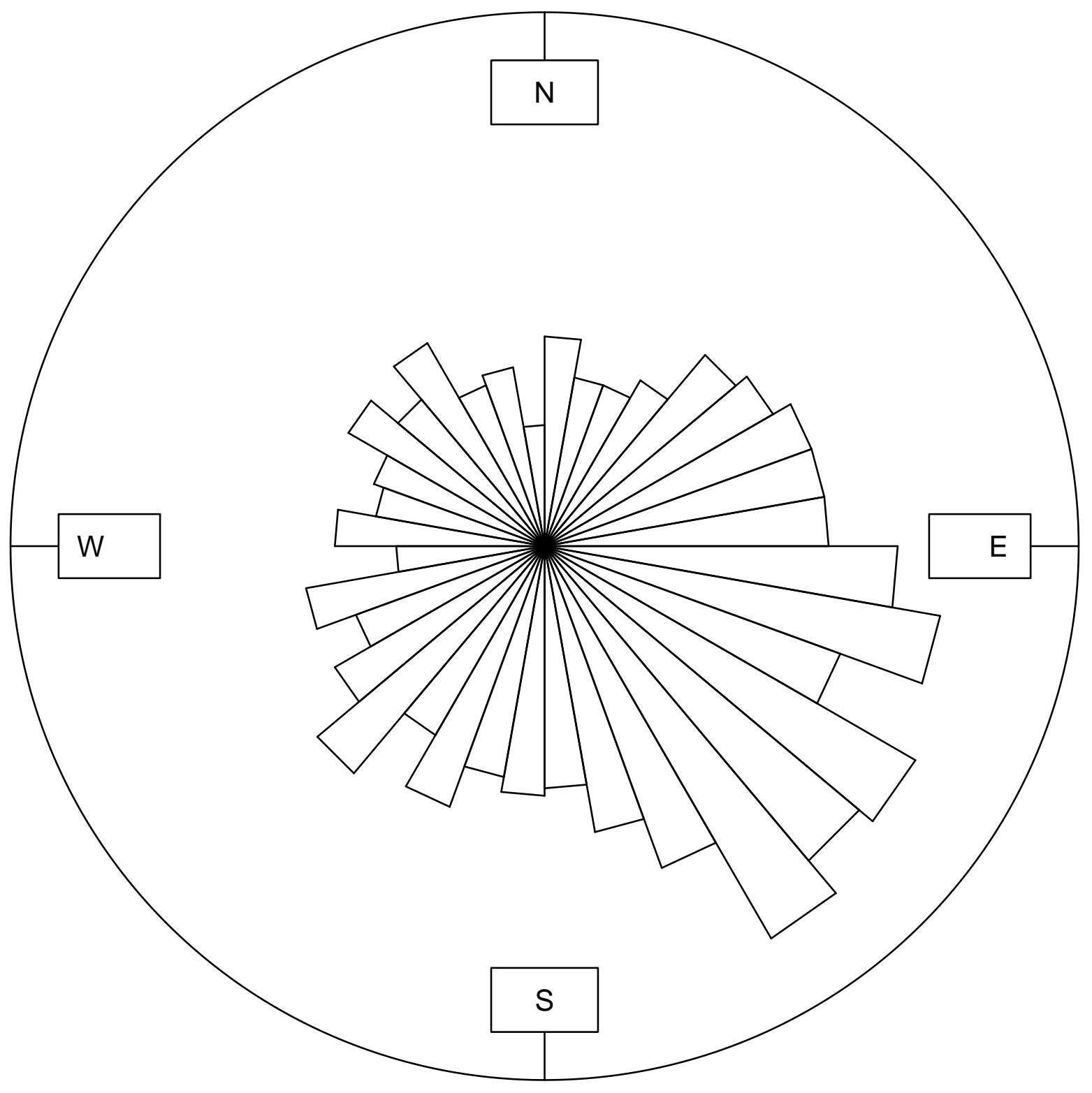


bootstrap 288

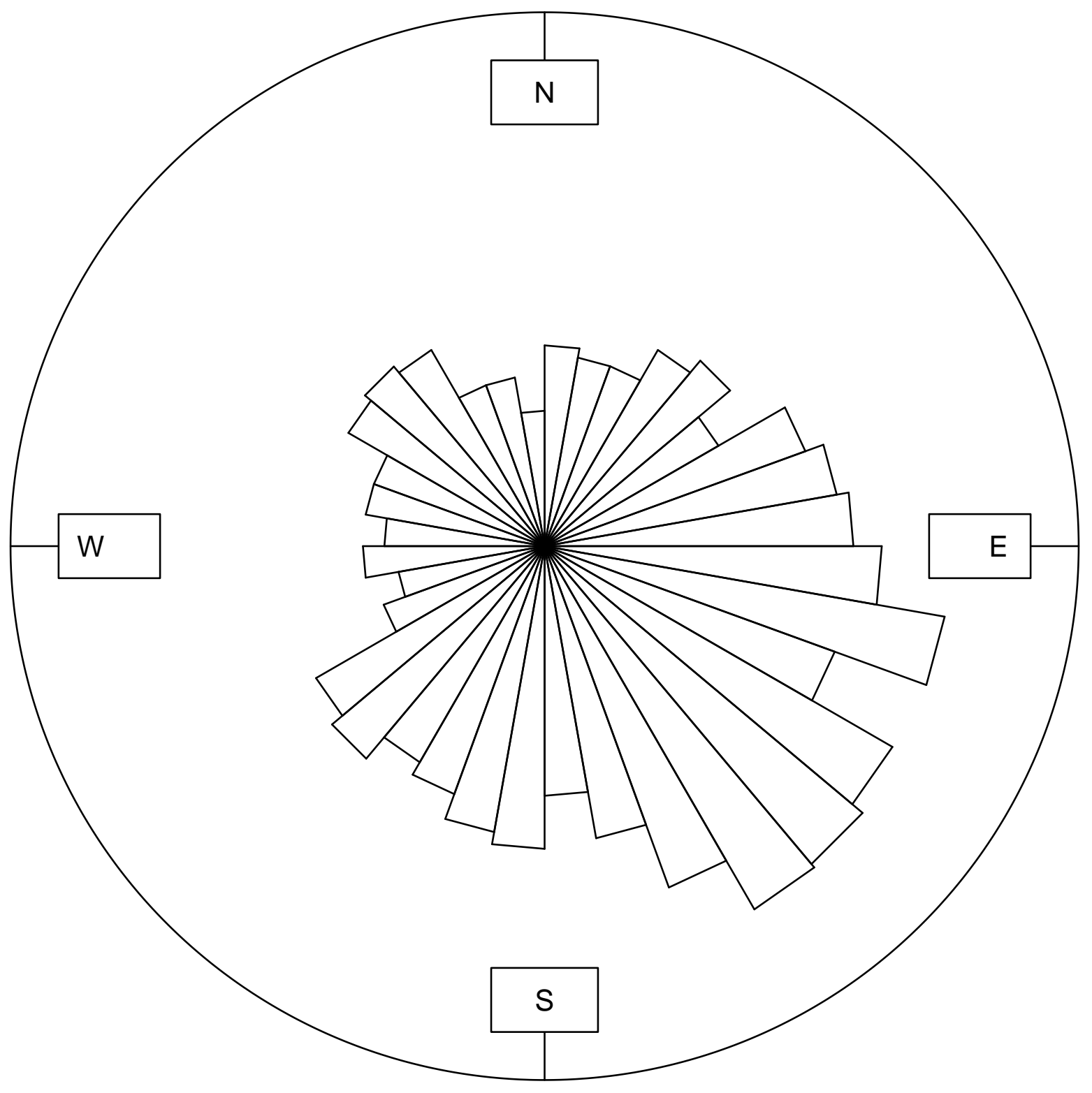


bootstrap 289

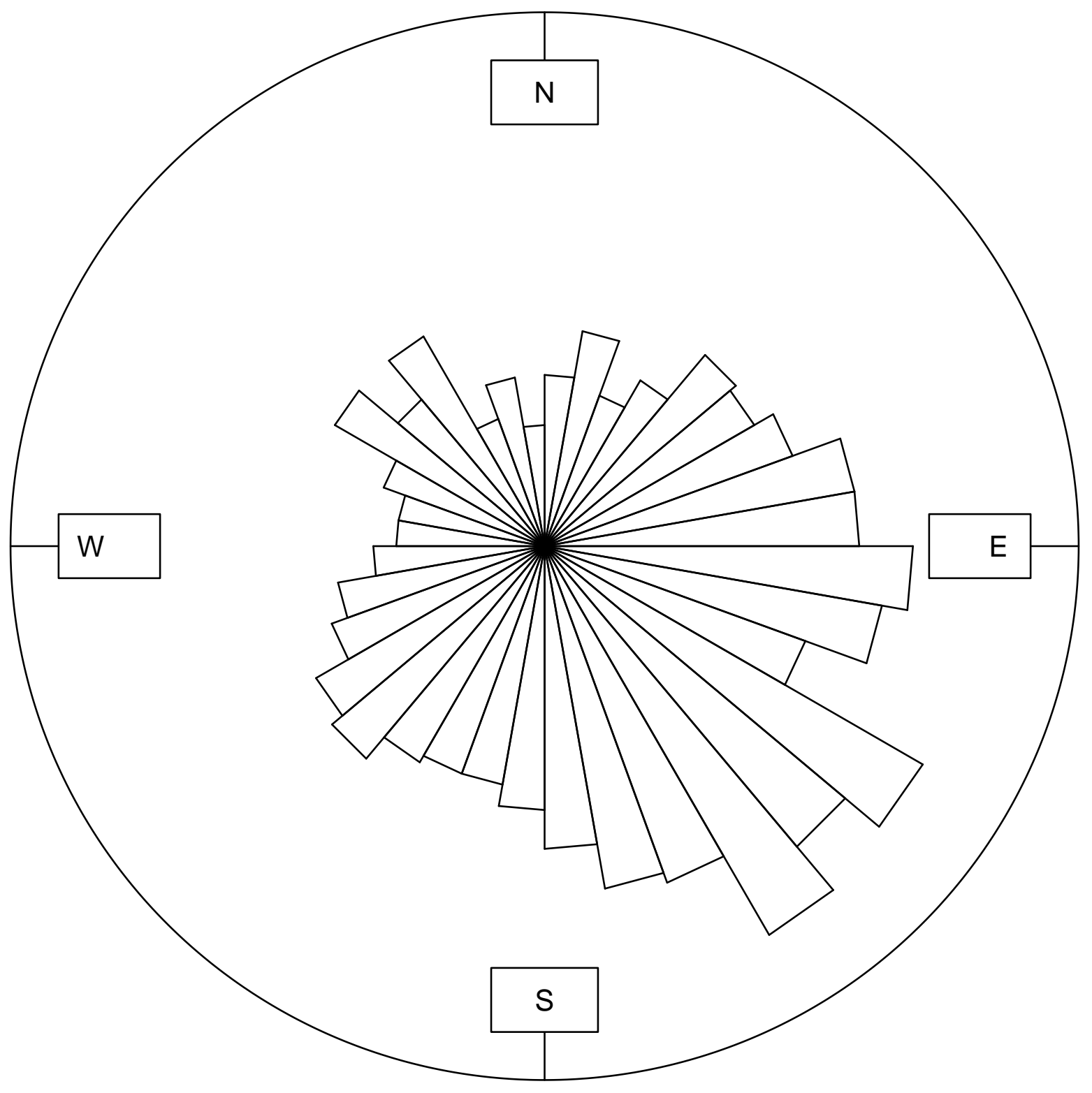


bootstrap 290

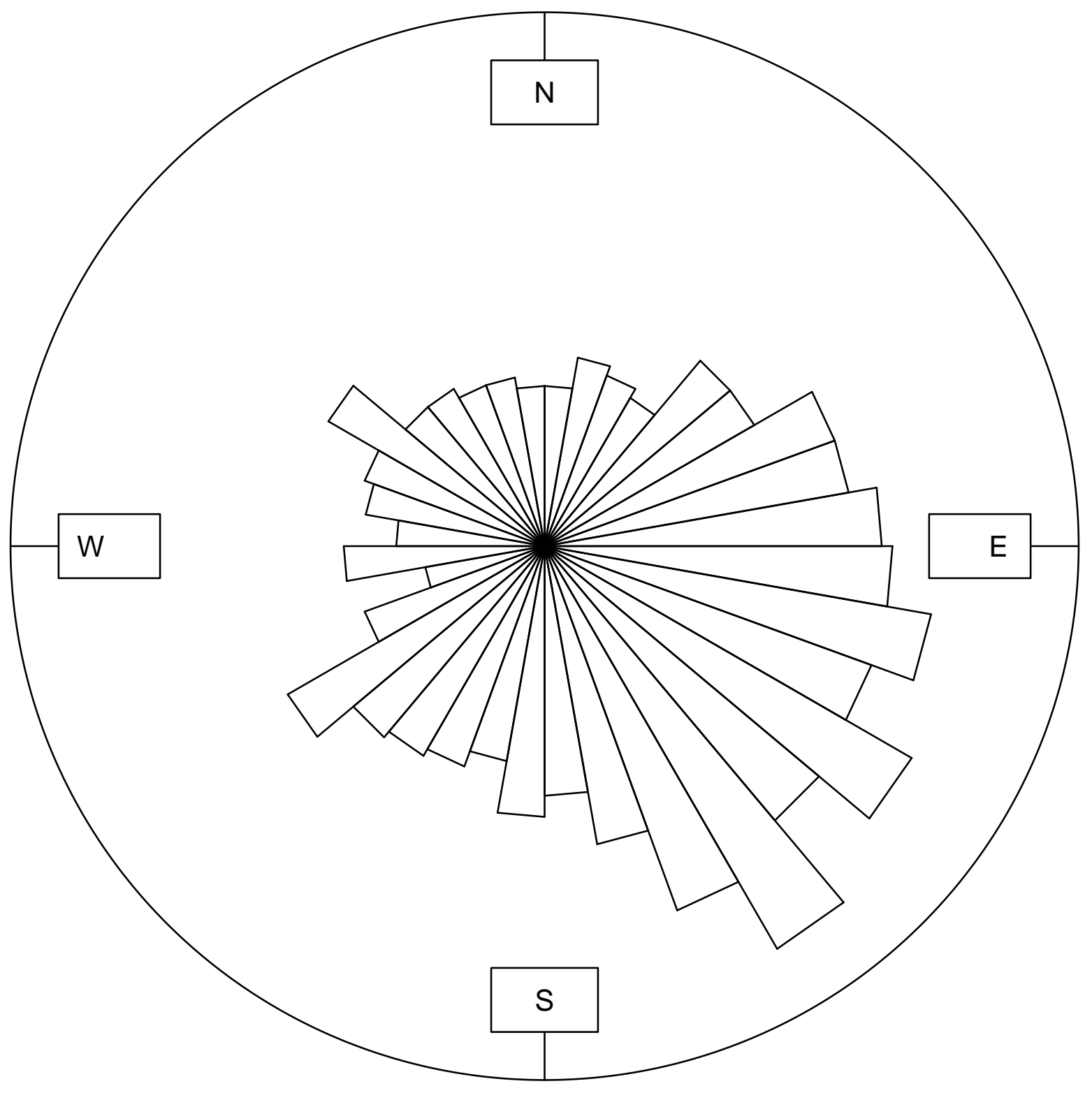


bootstrap 291

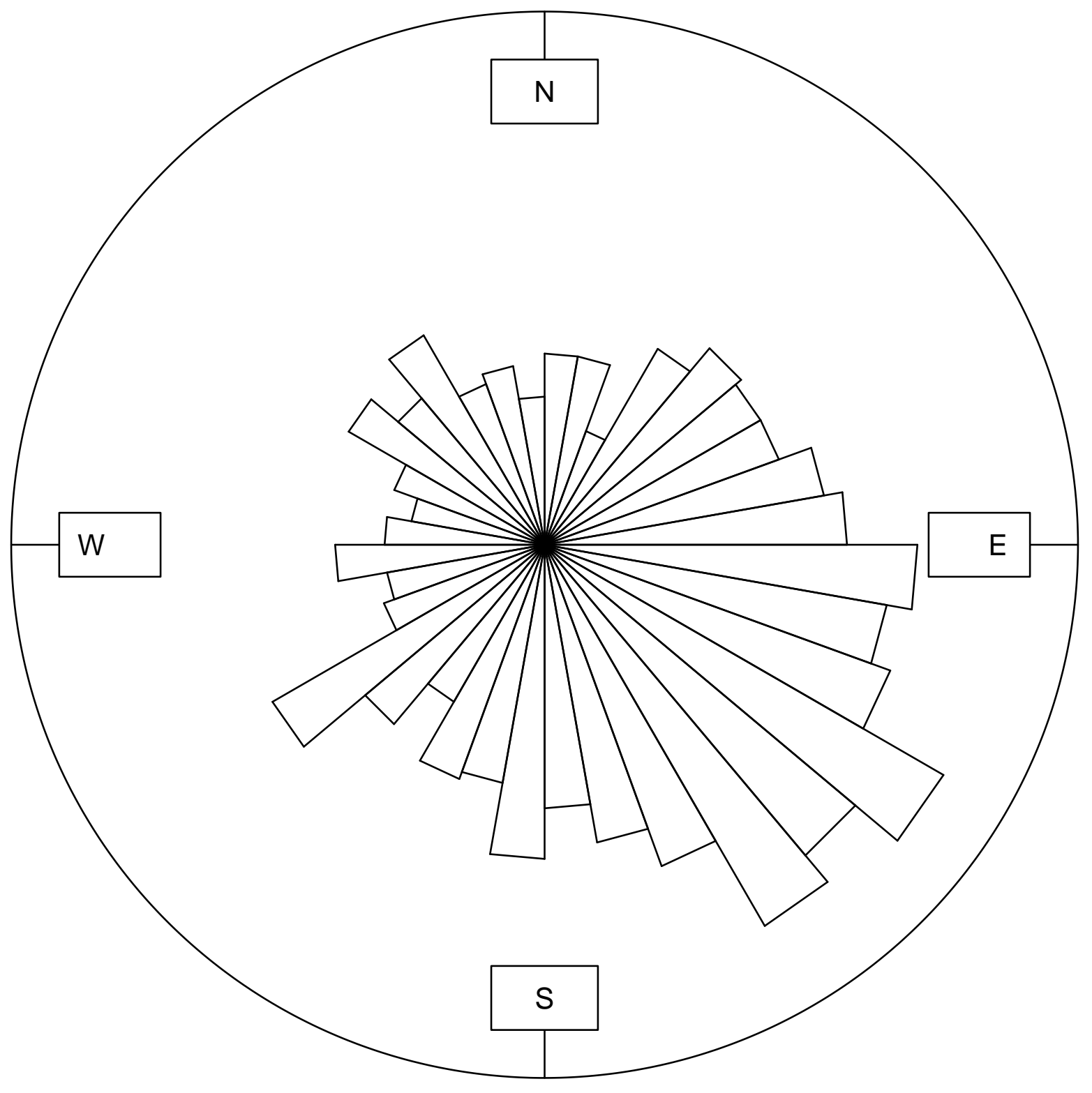


bootstrap 292

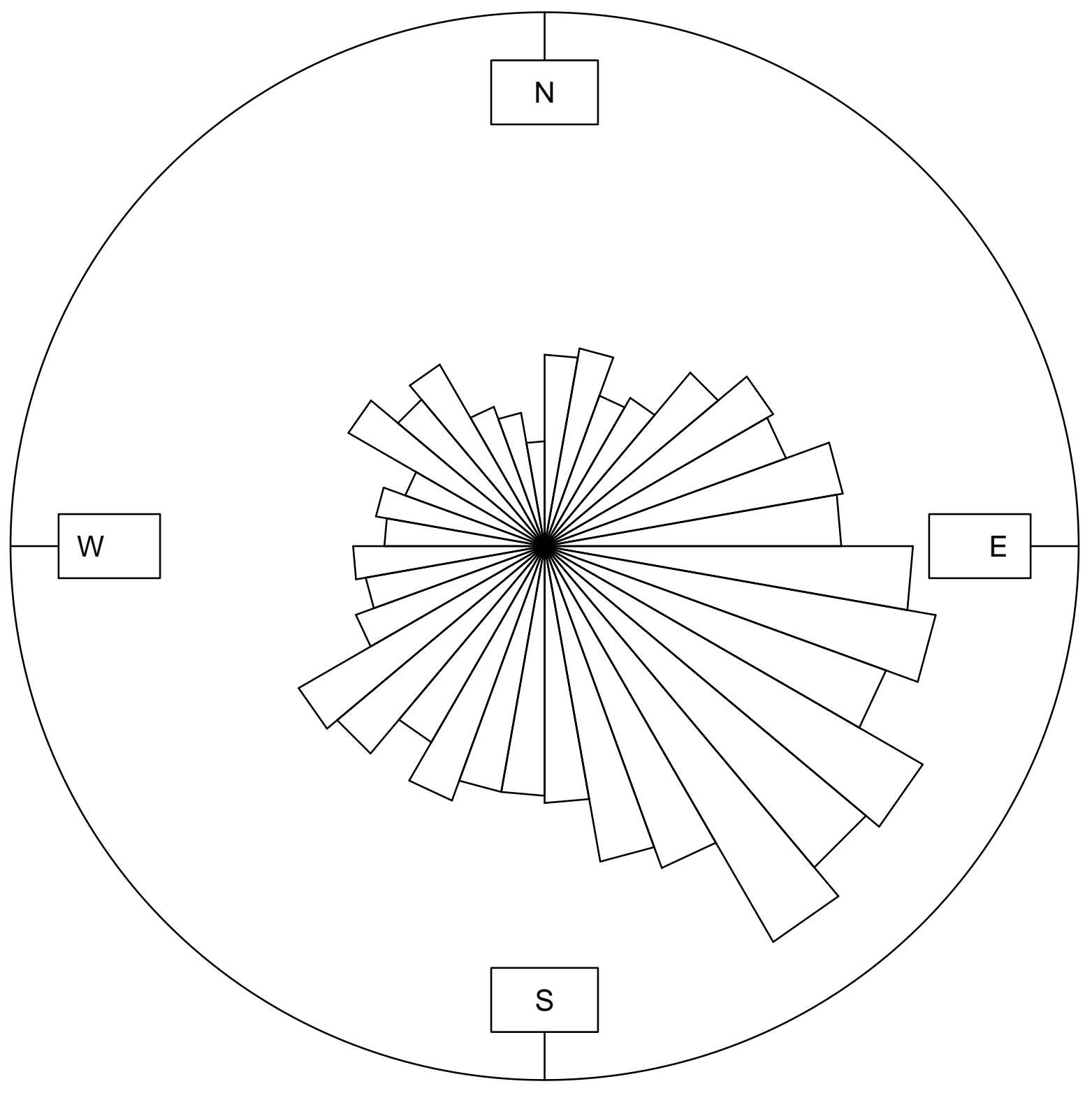




\section{bootstrap 293}

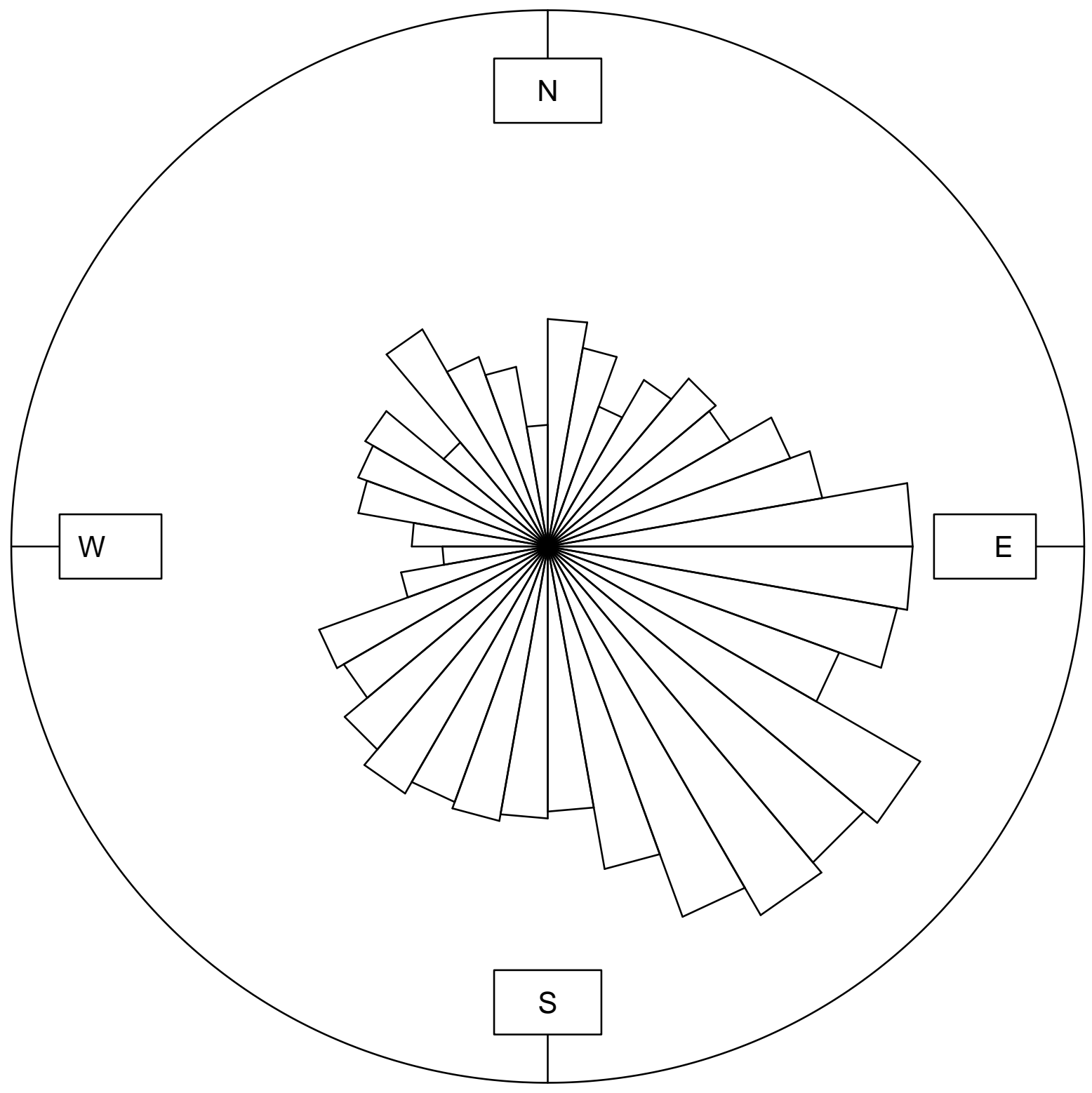


bootstrap 294

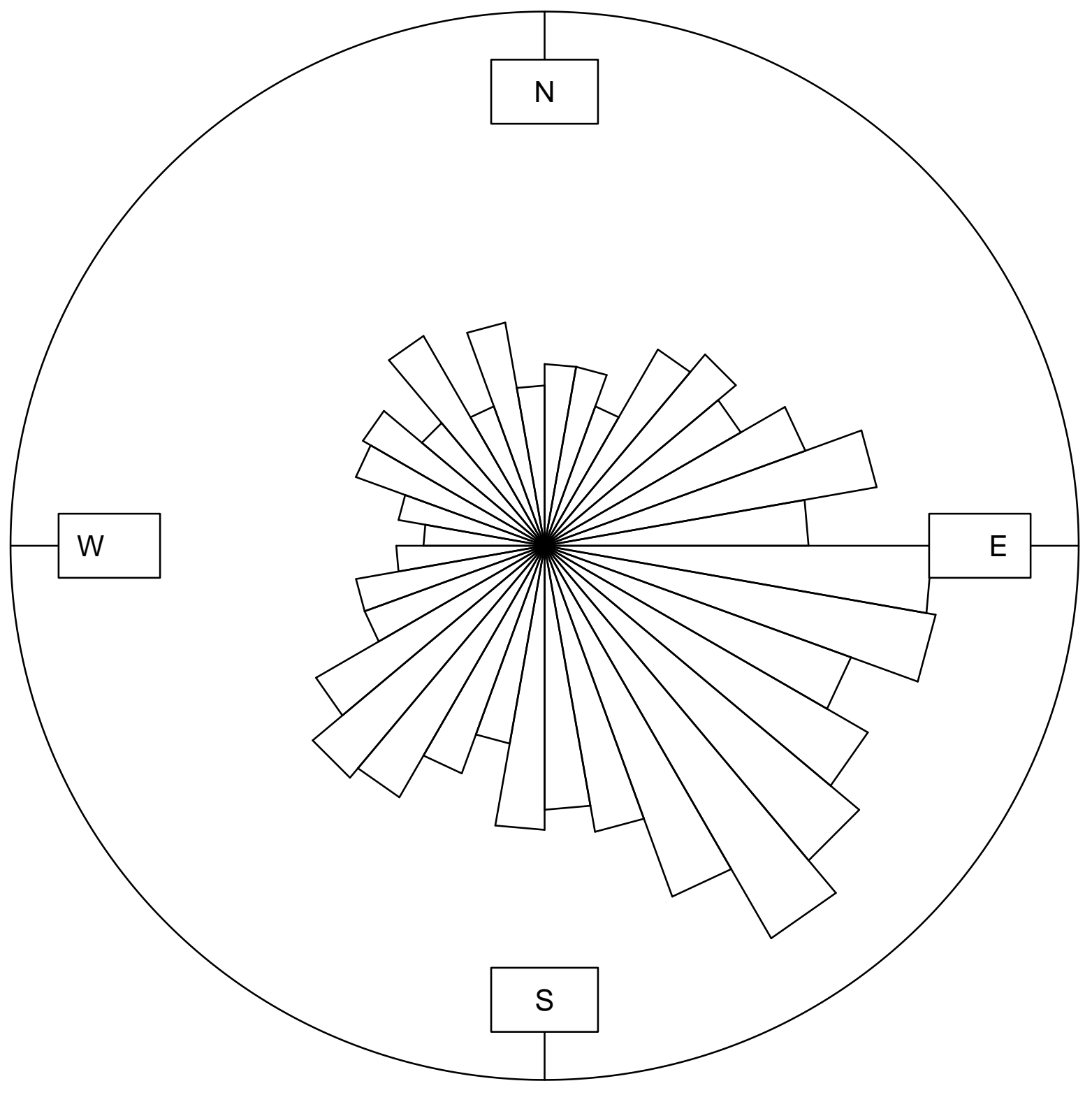


bootstrap 295

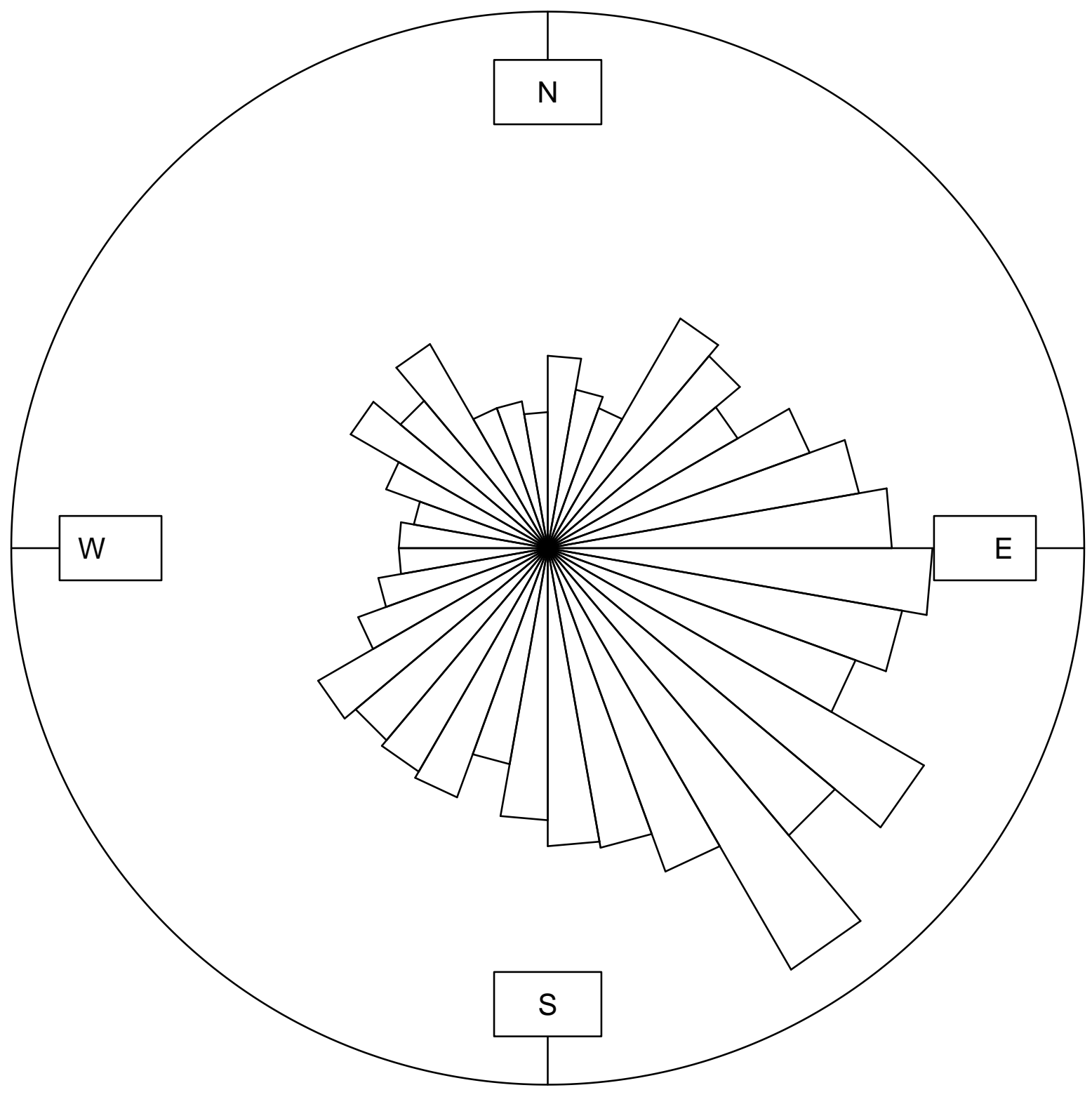


bootstrap 296

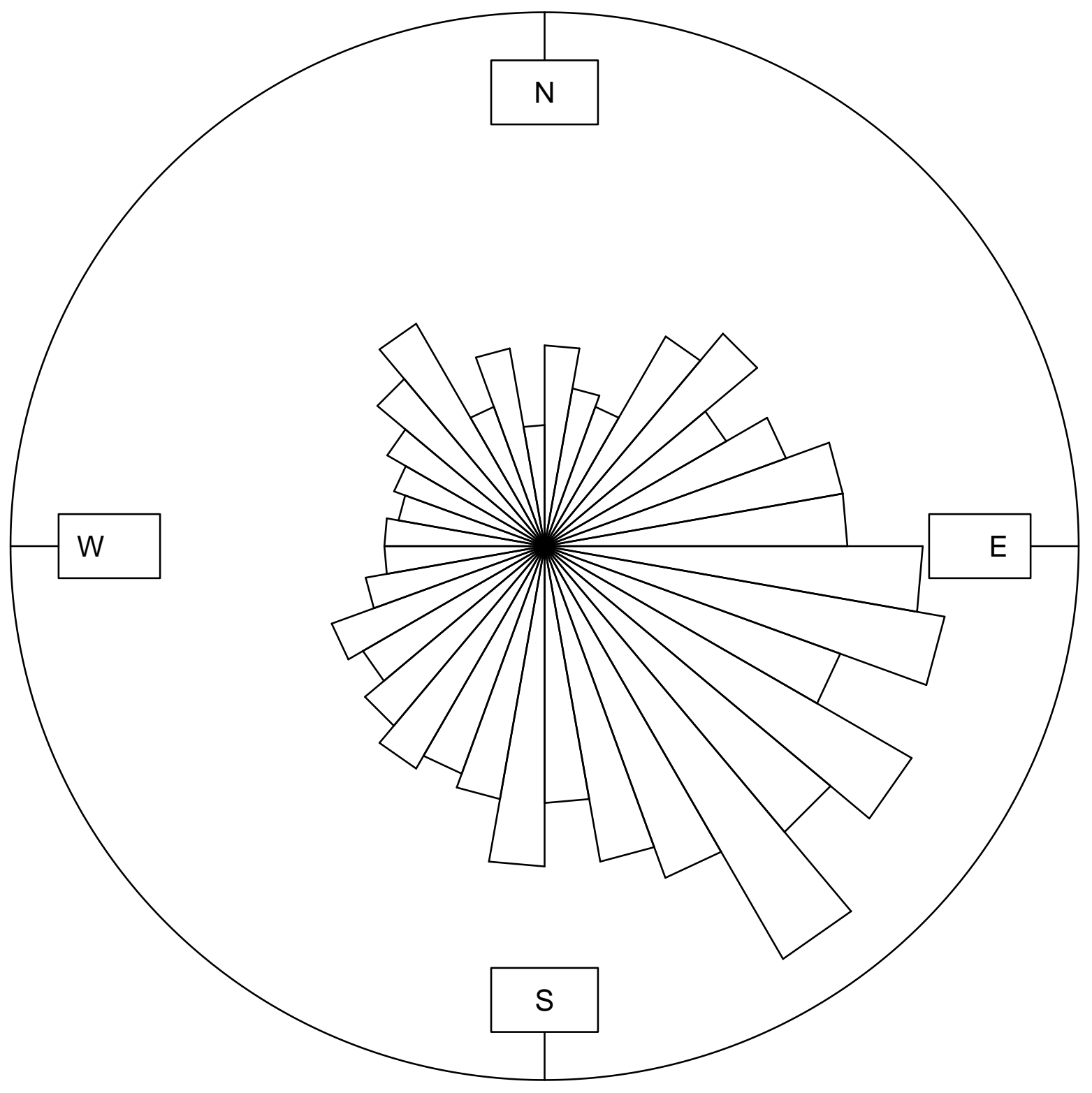


bootstrap 297

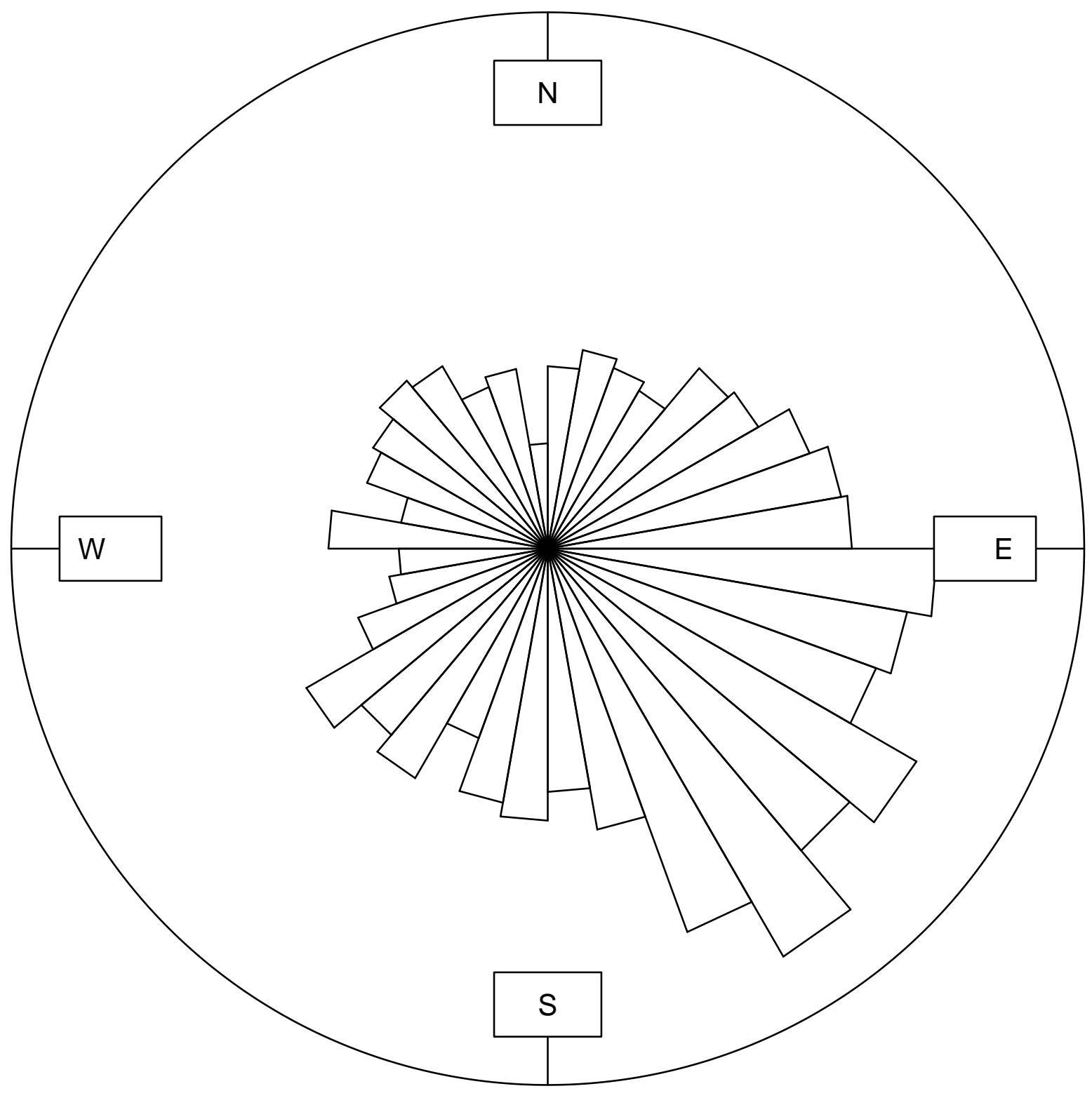


bootstrap 298

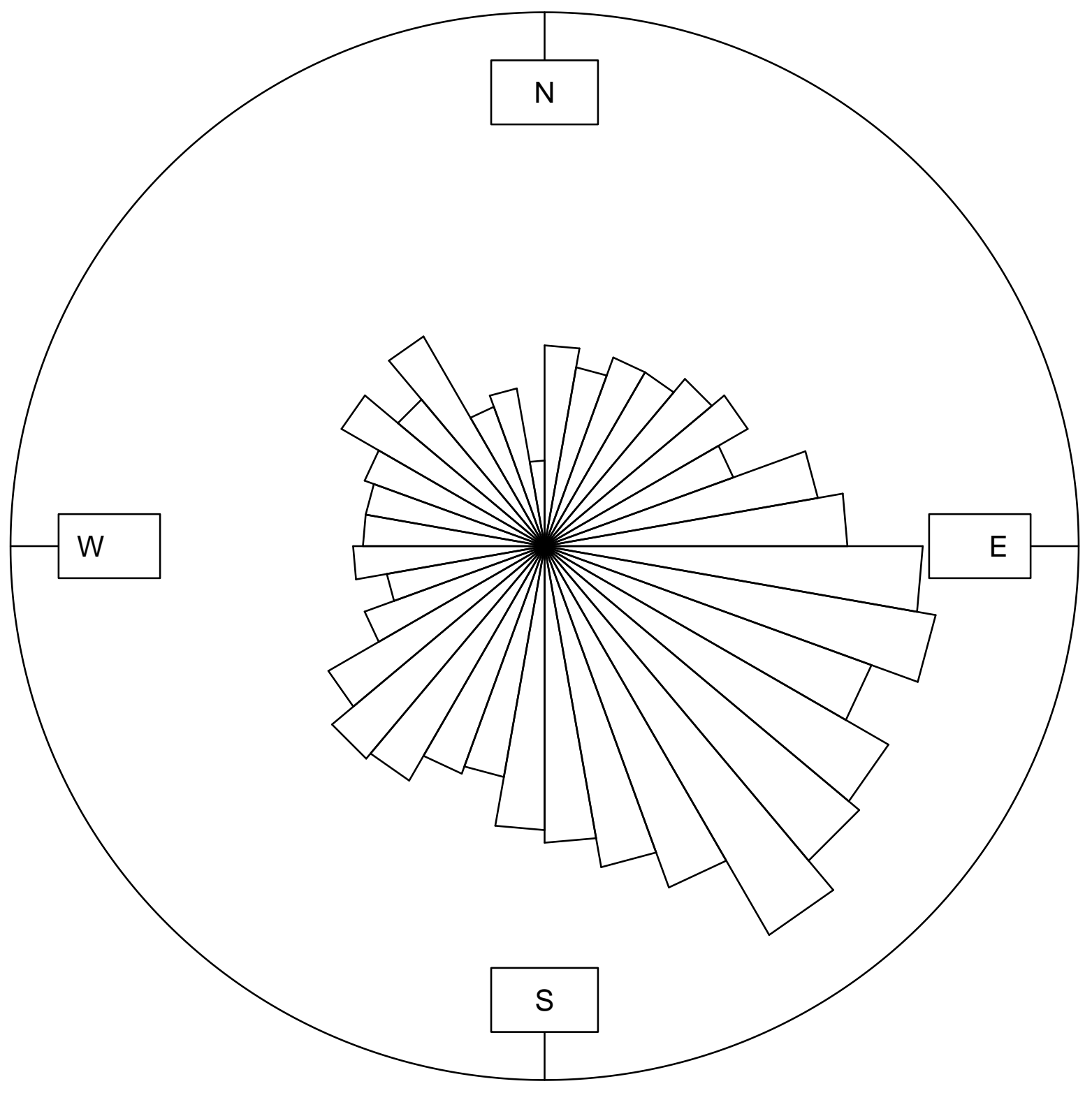


bootstrap 299

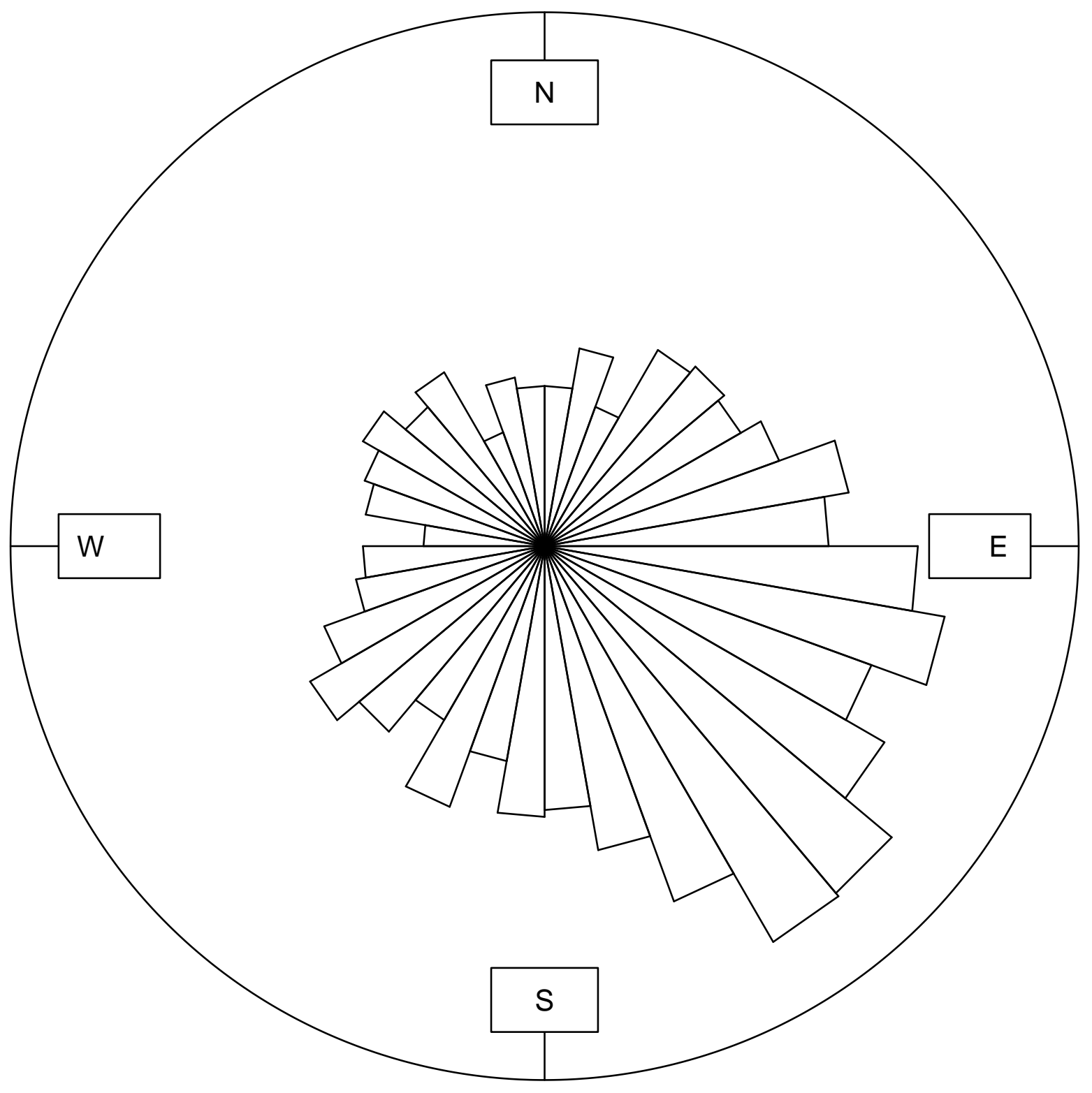


bootstrap 300

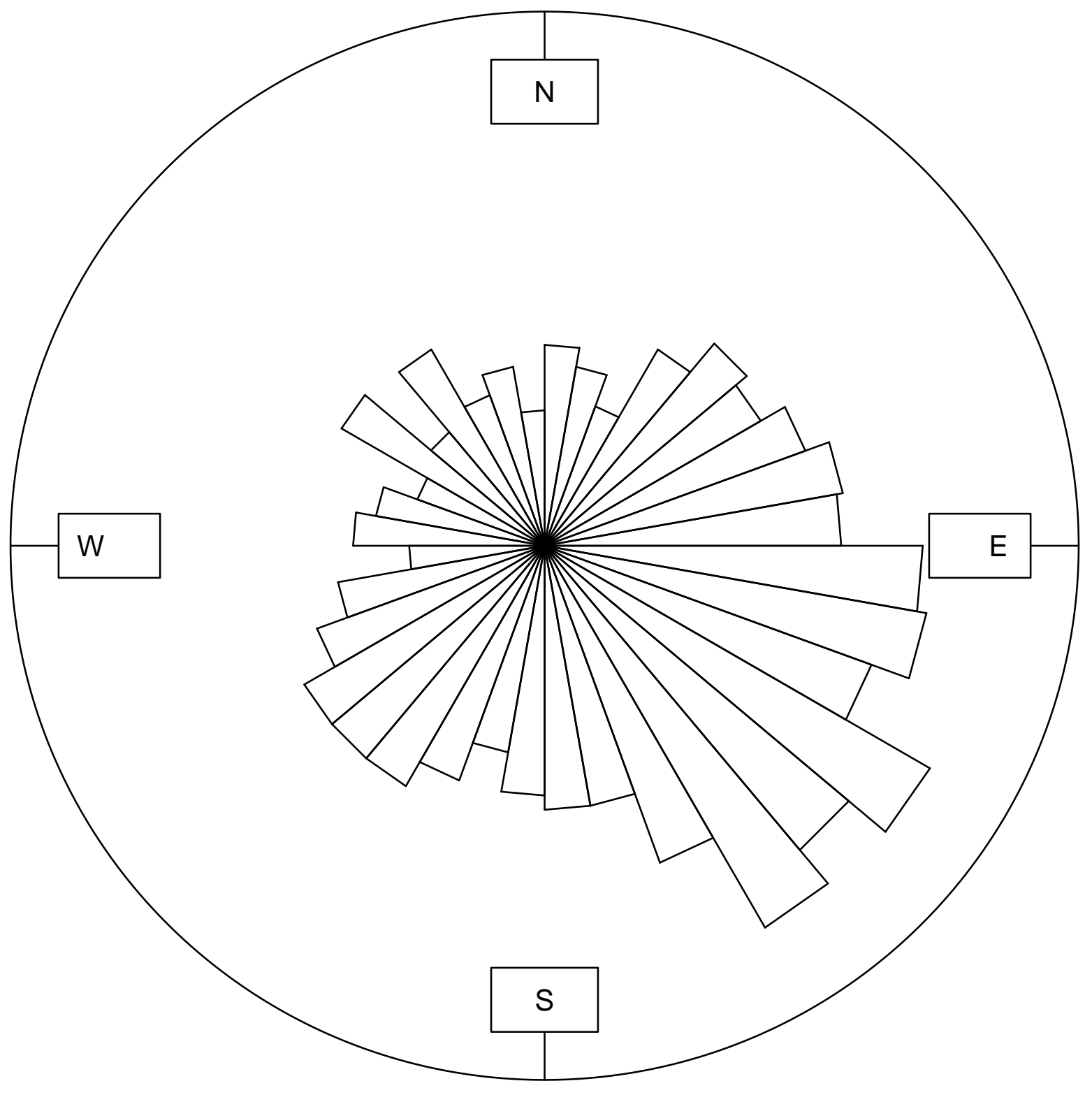


bootstrap 301

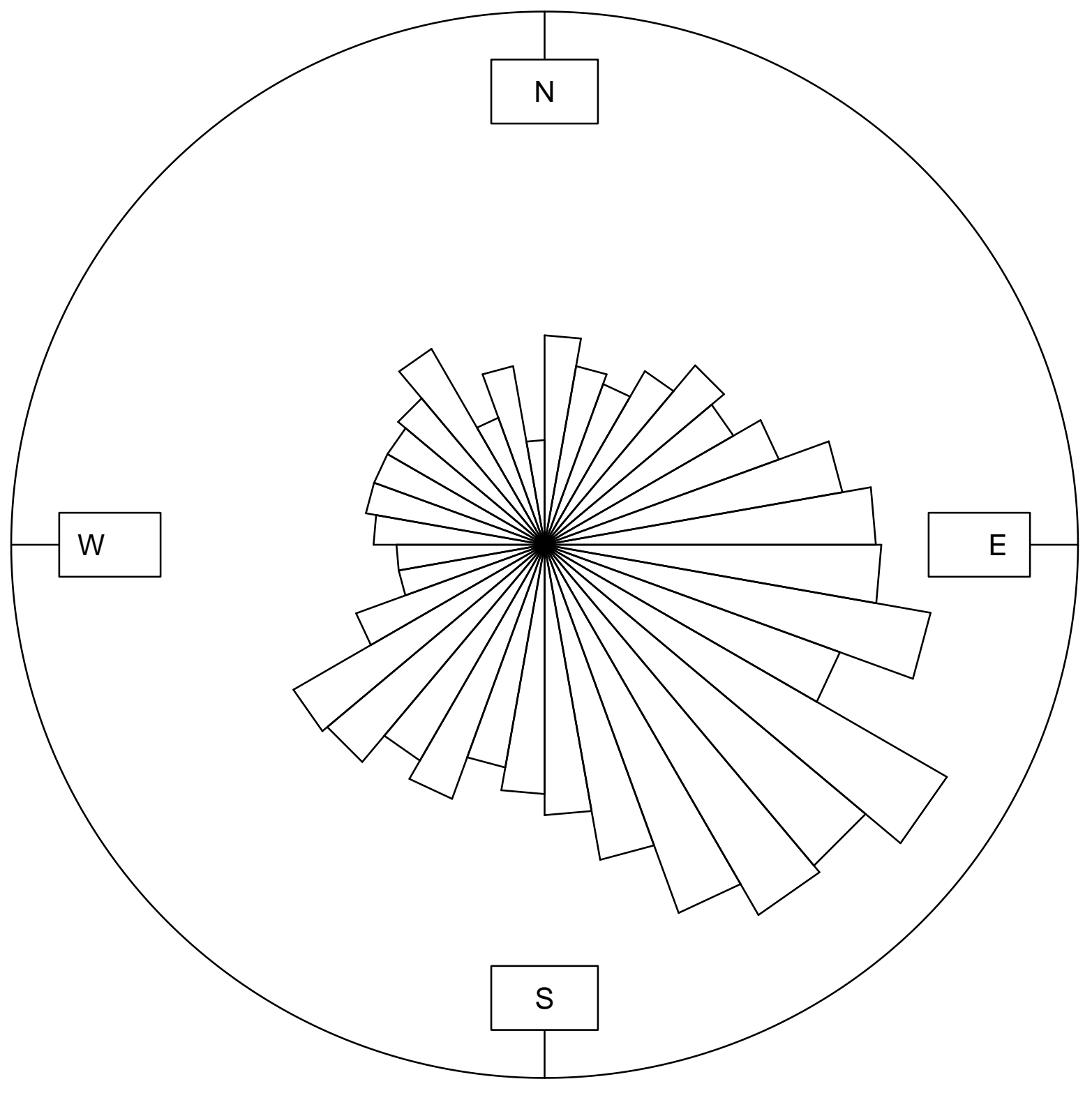


bootstrap 302

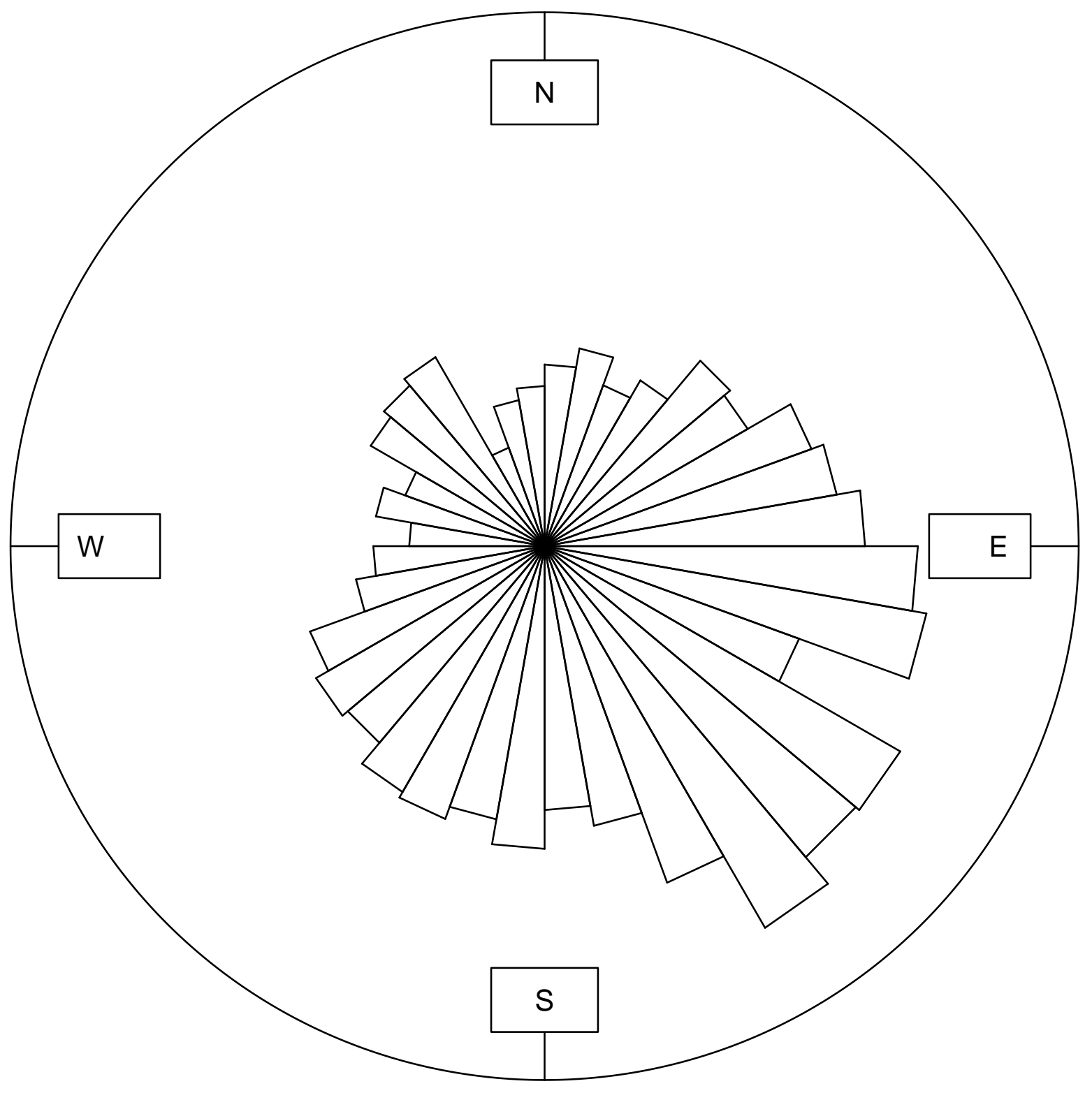


bootstrap 303

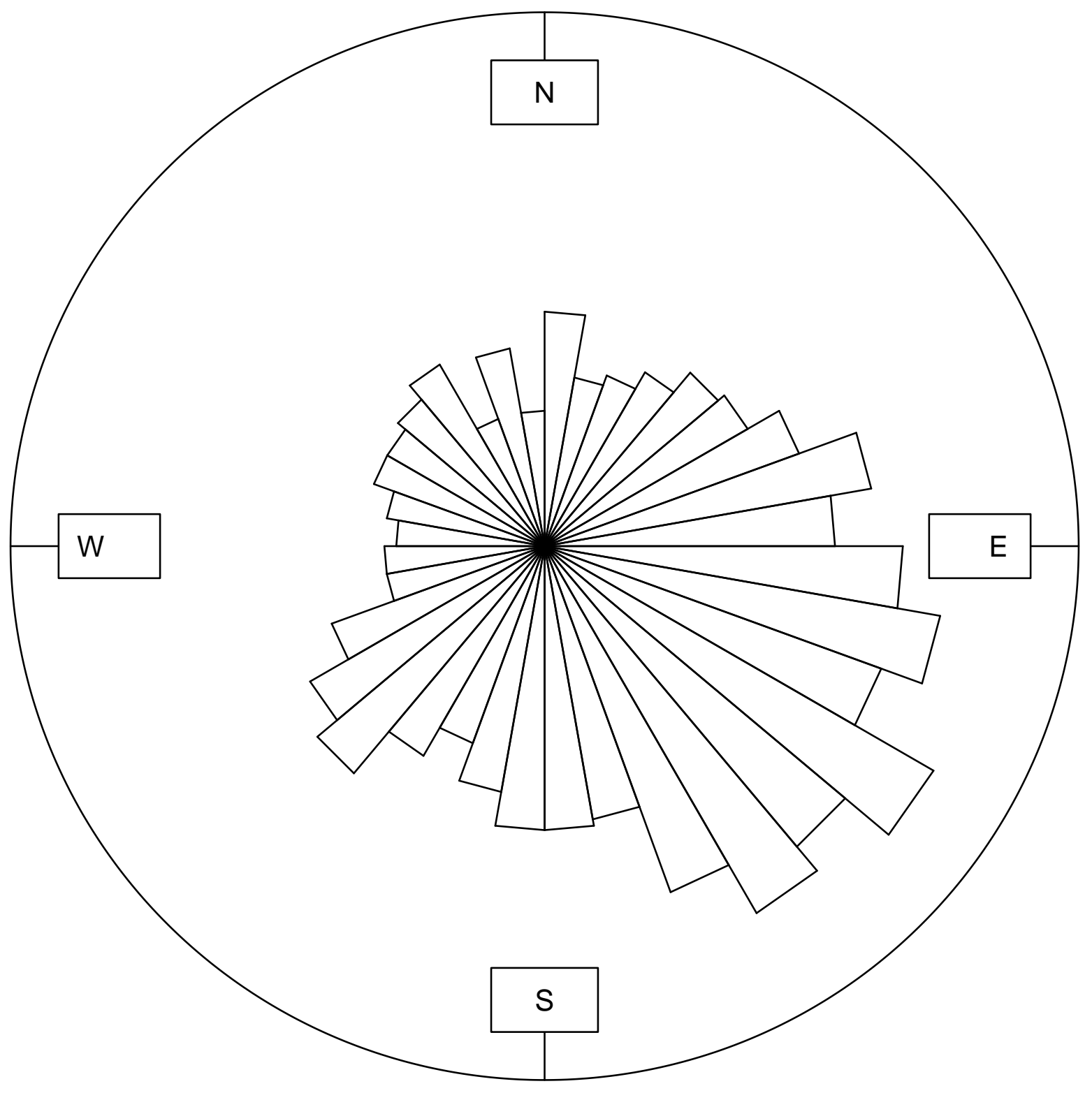


bootstrap 305

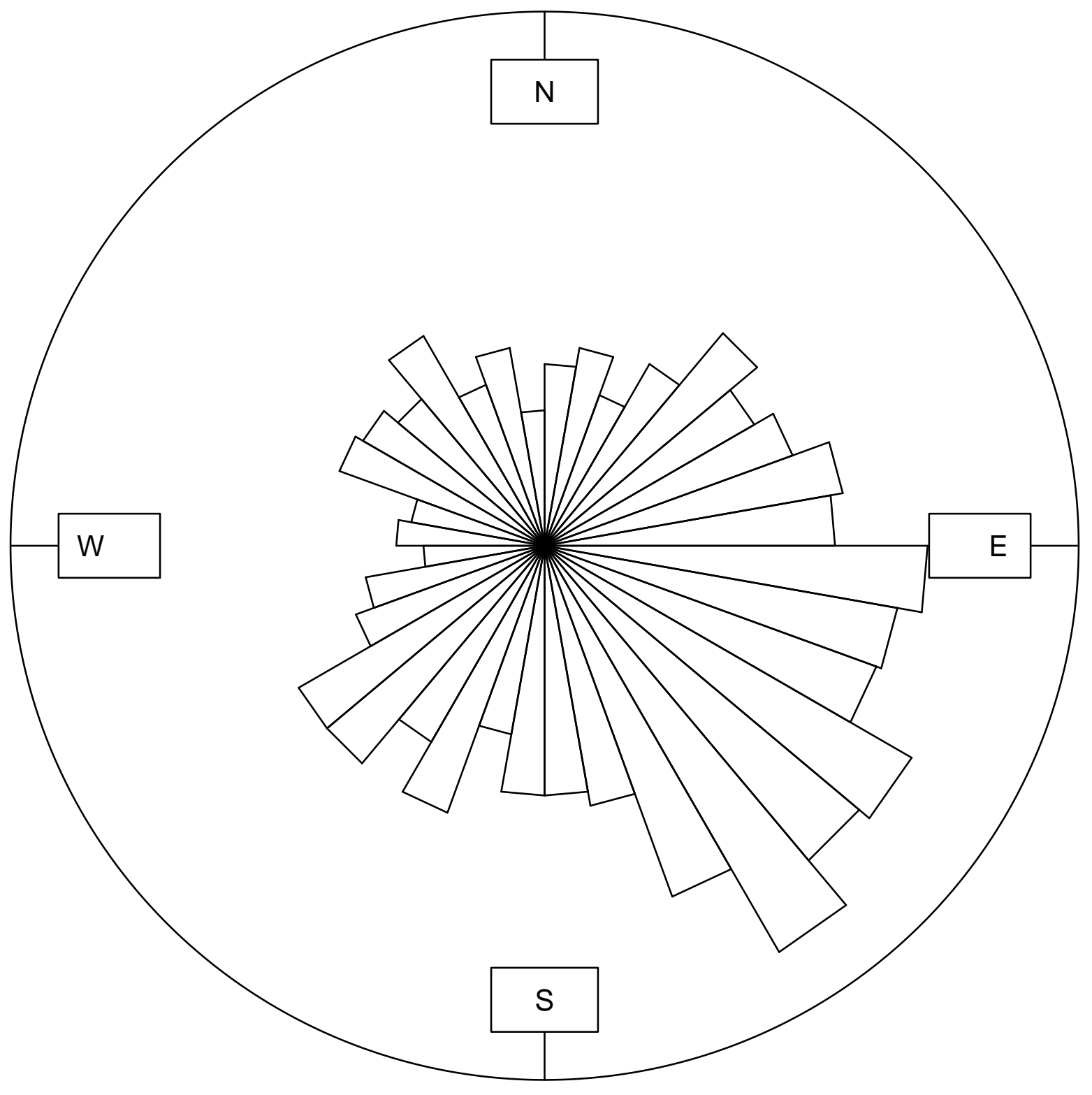


bootstrap 306

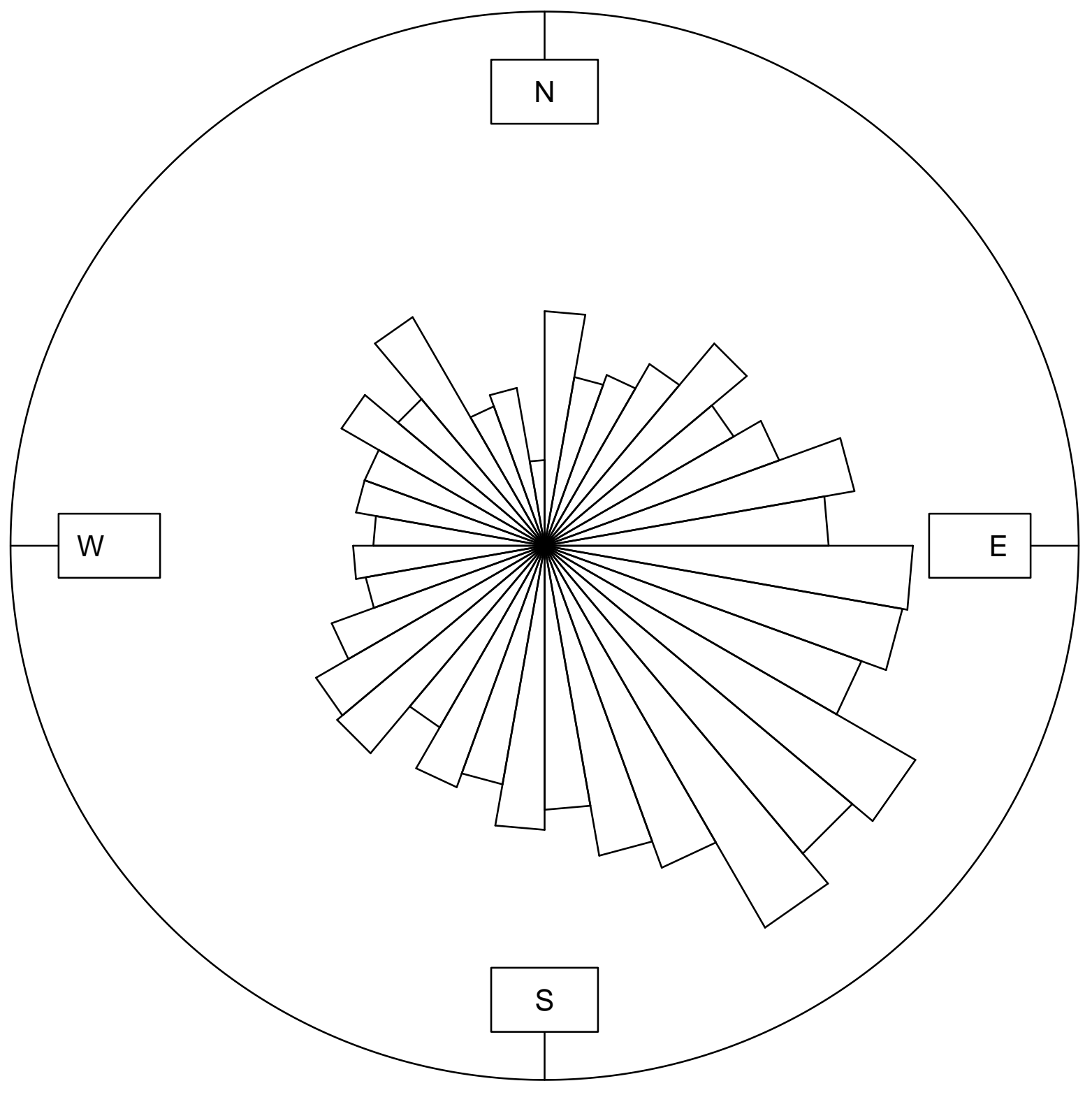


bootstrap 307

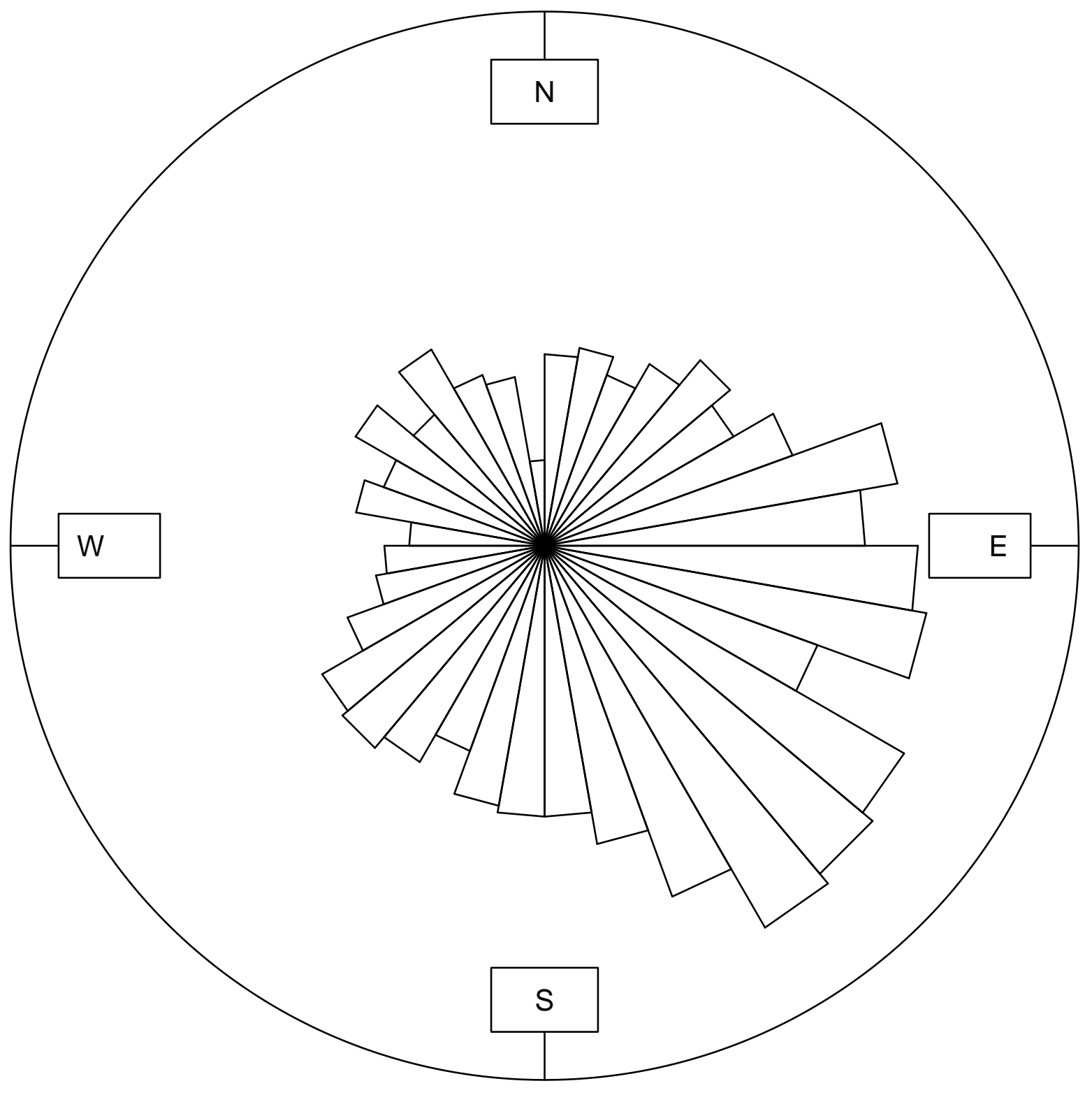


bootstrap 308

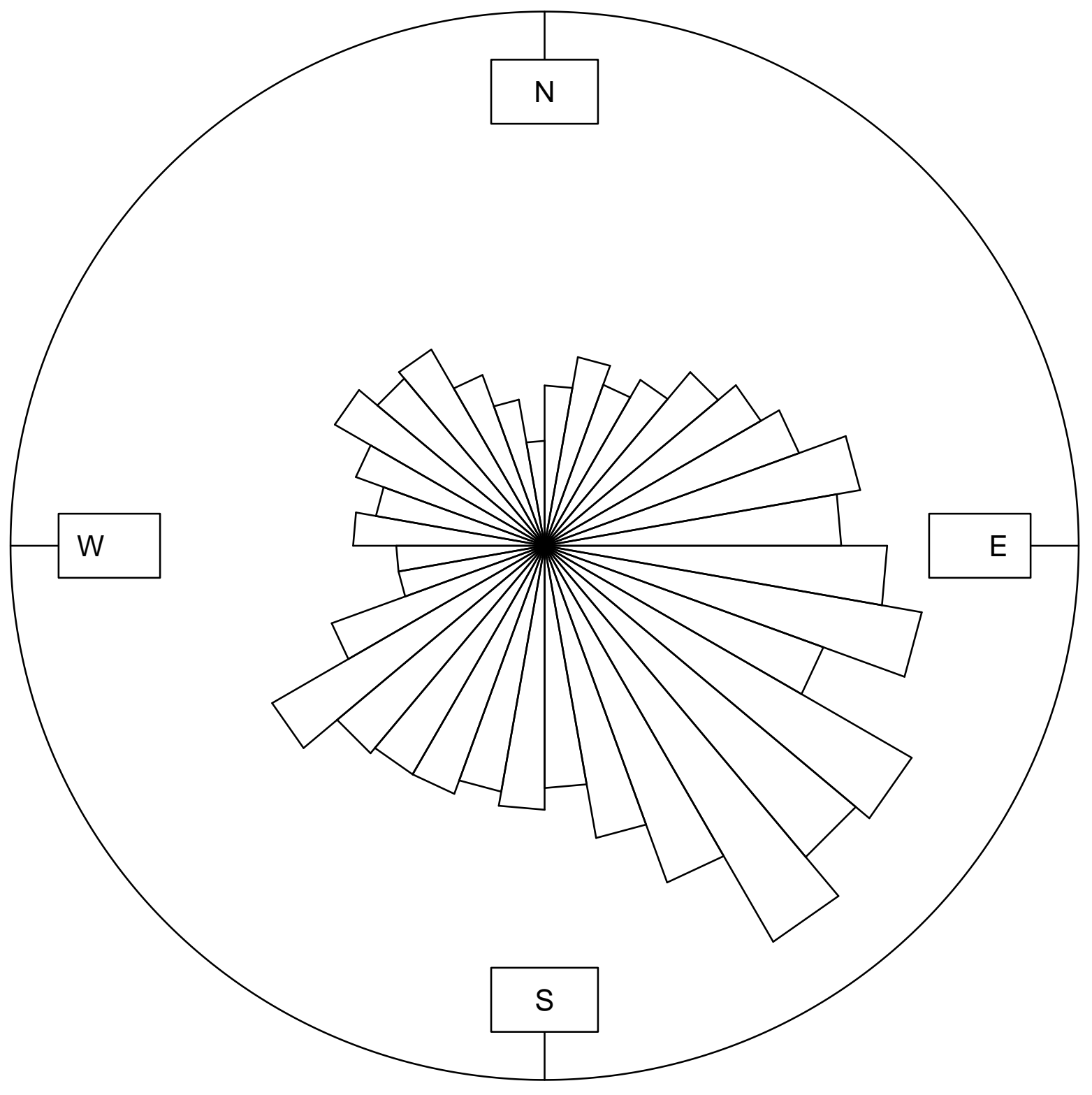


bootstrap 309

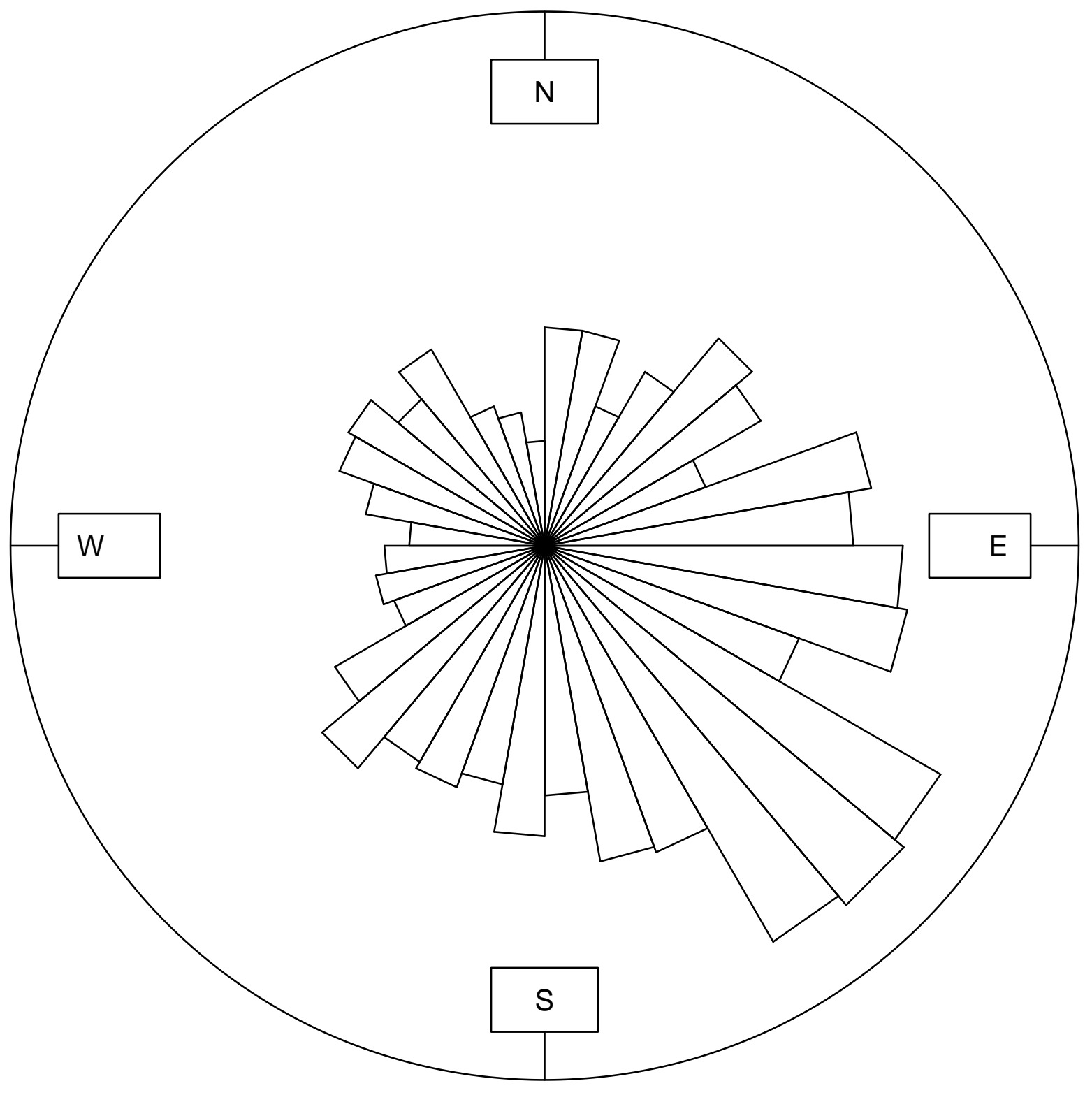


bootstrap 310

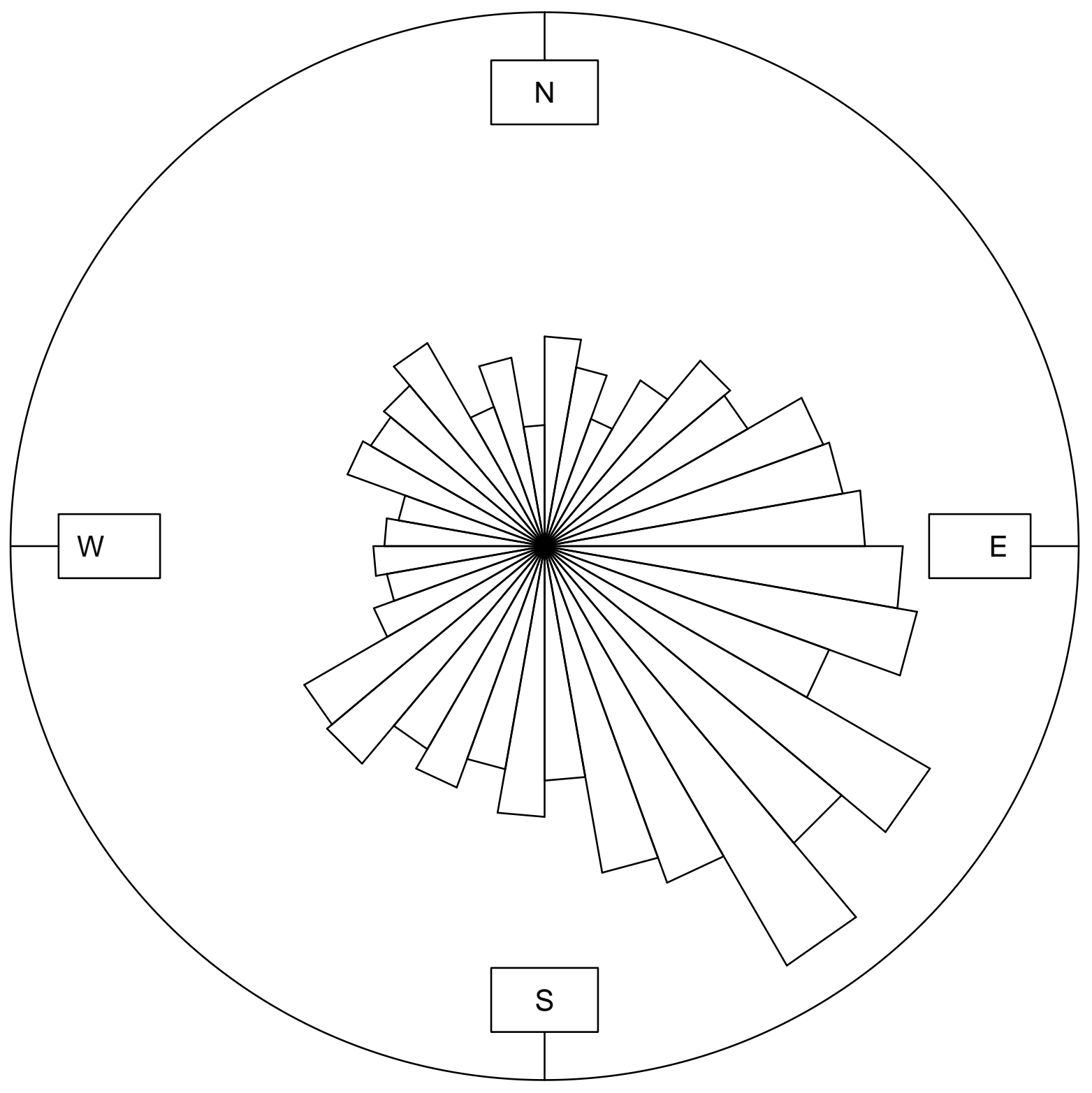




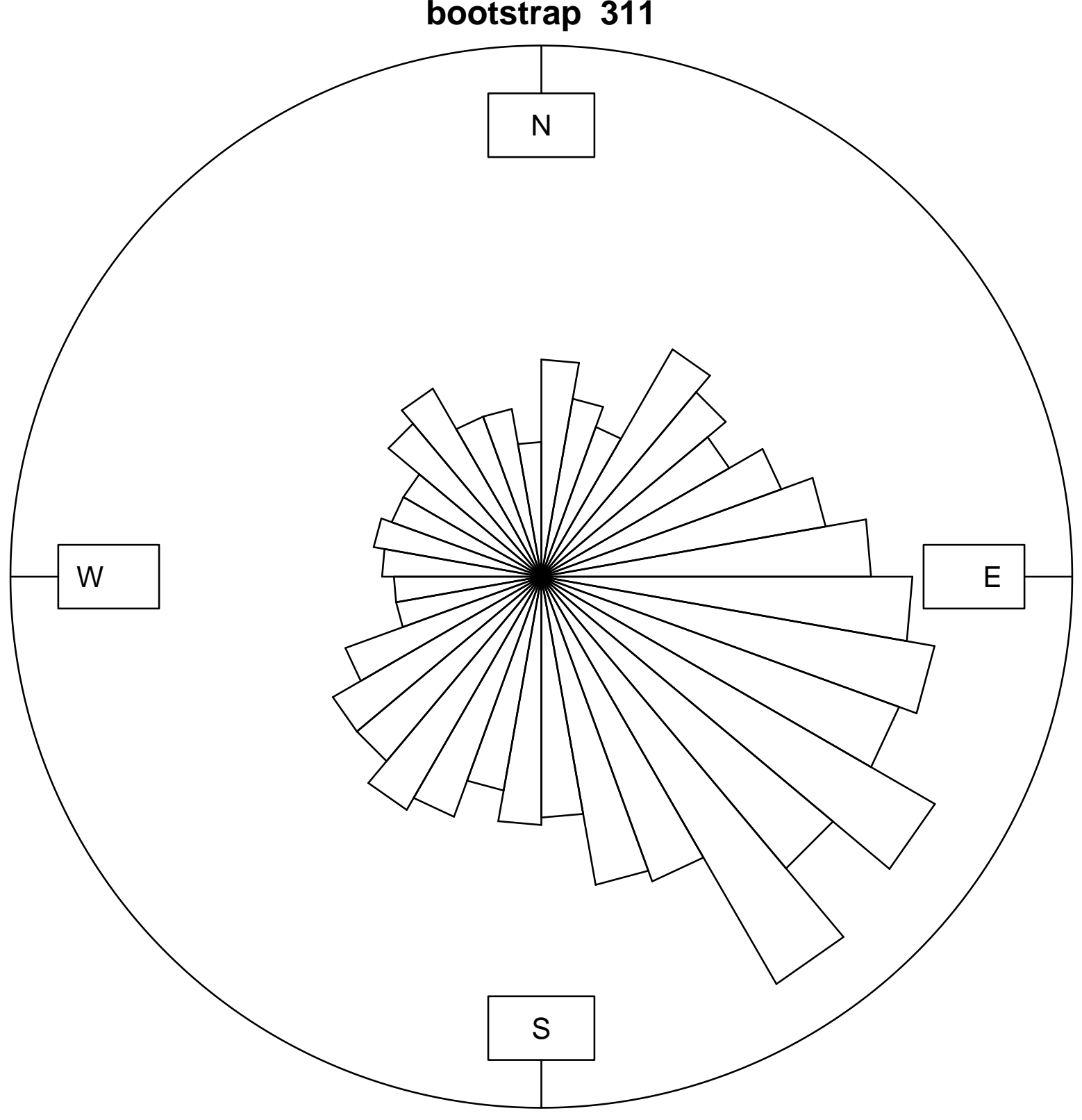


bootstrap 312

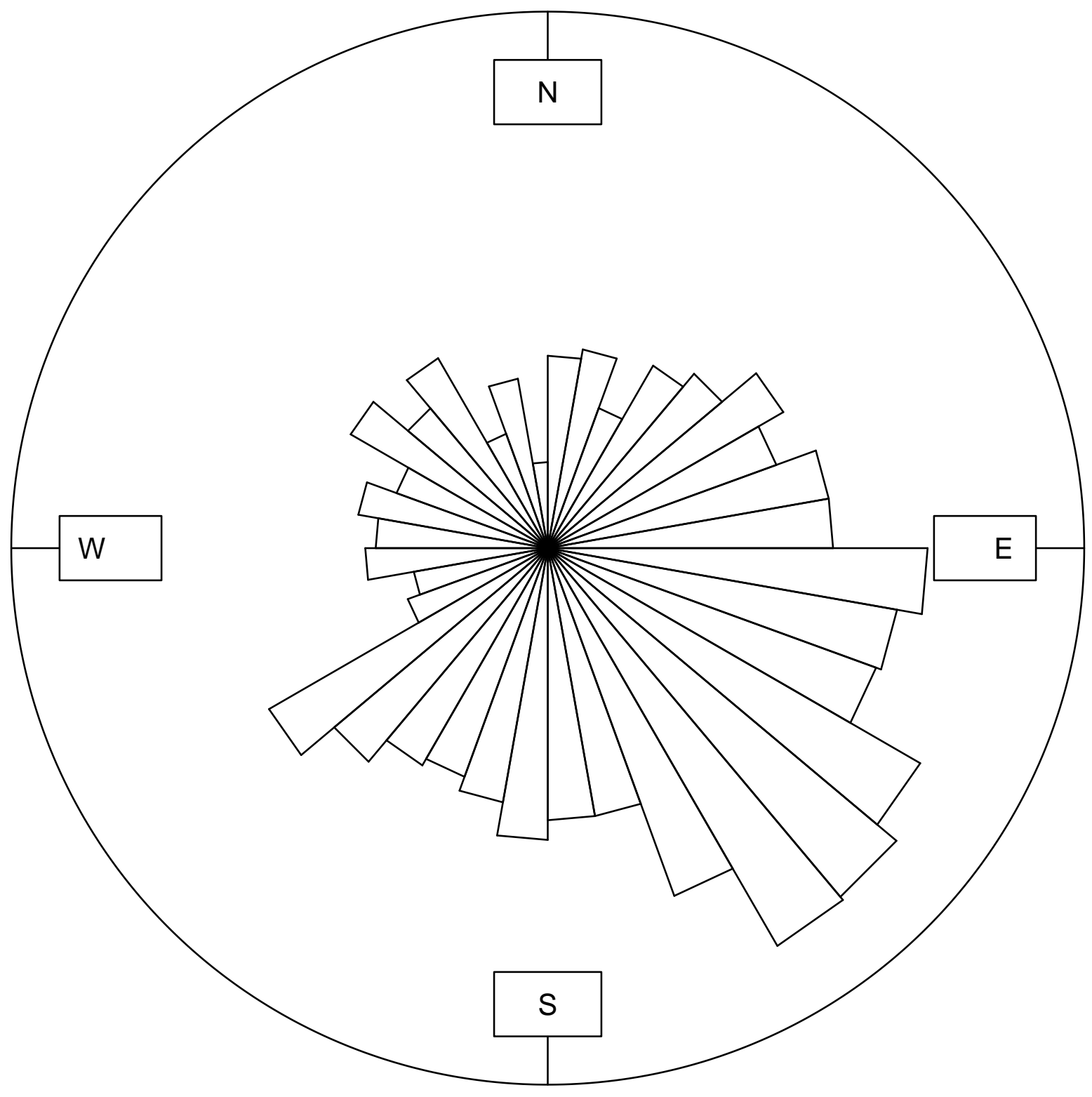


bootstrap 313

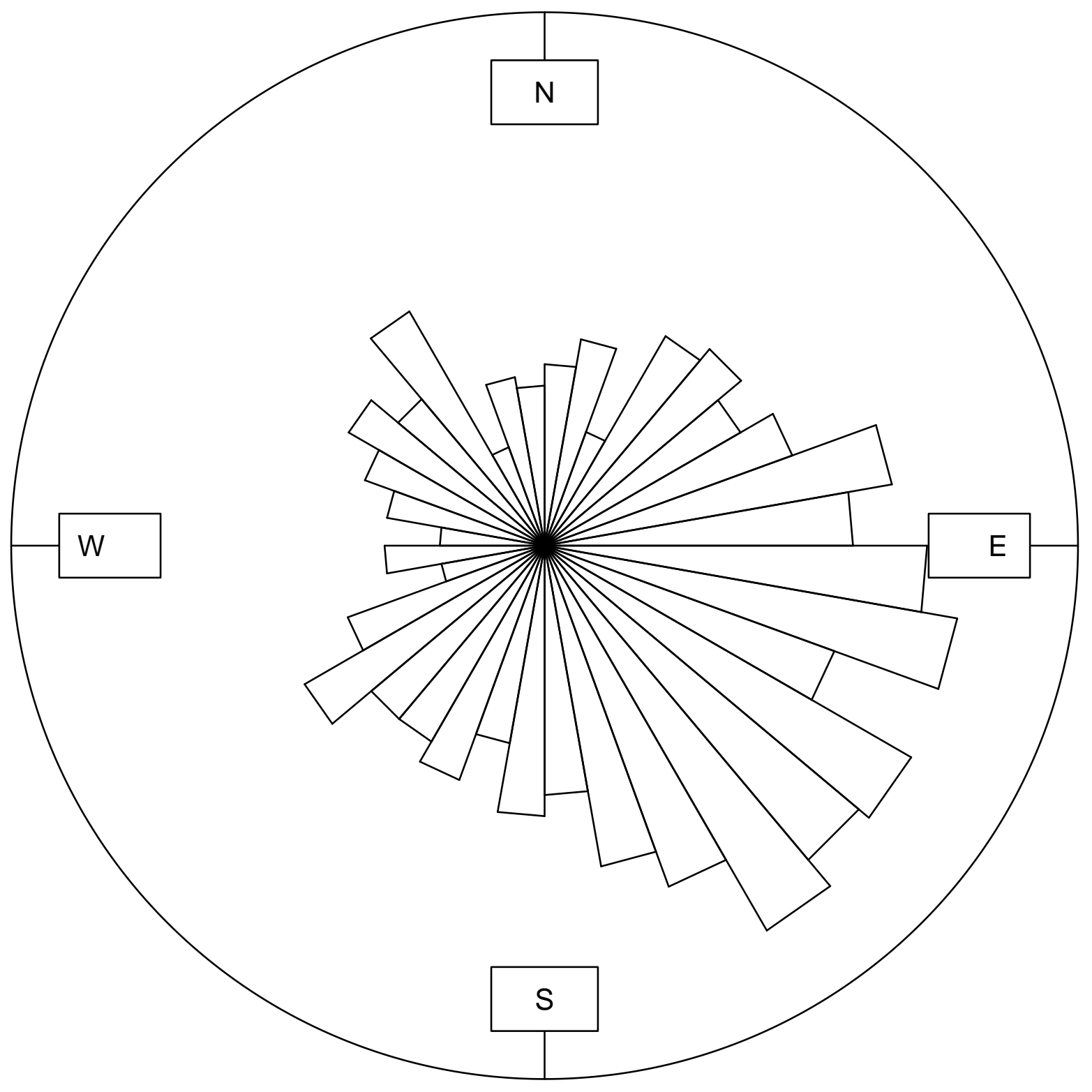


bootstrap 314

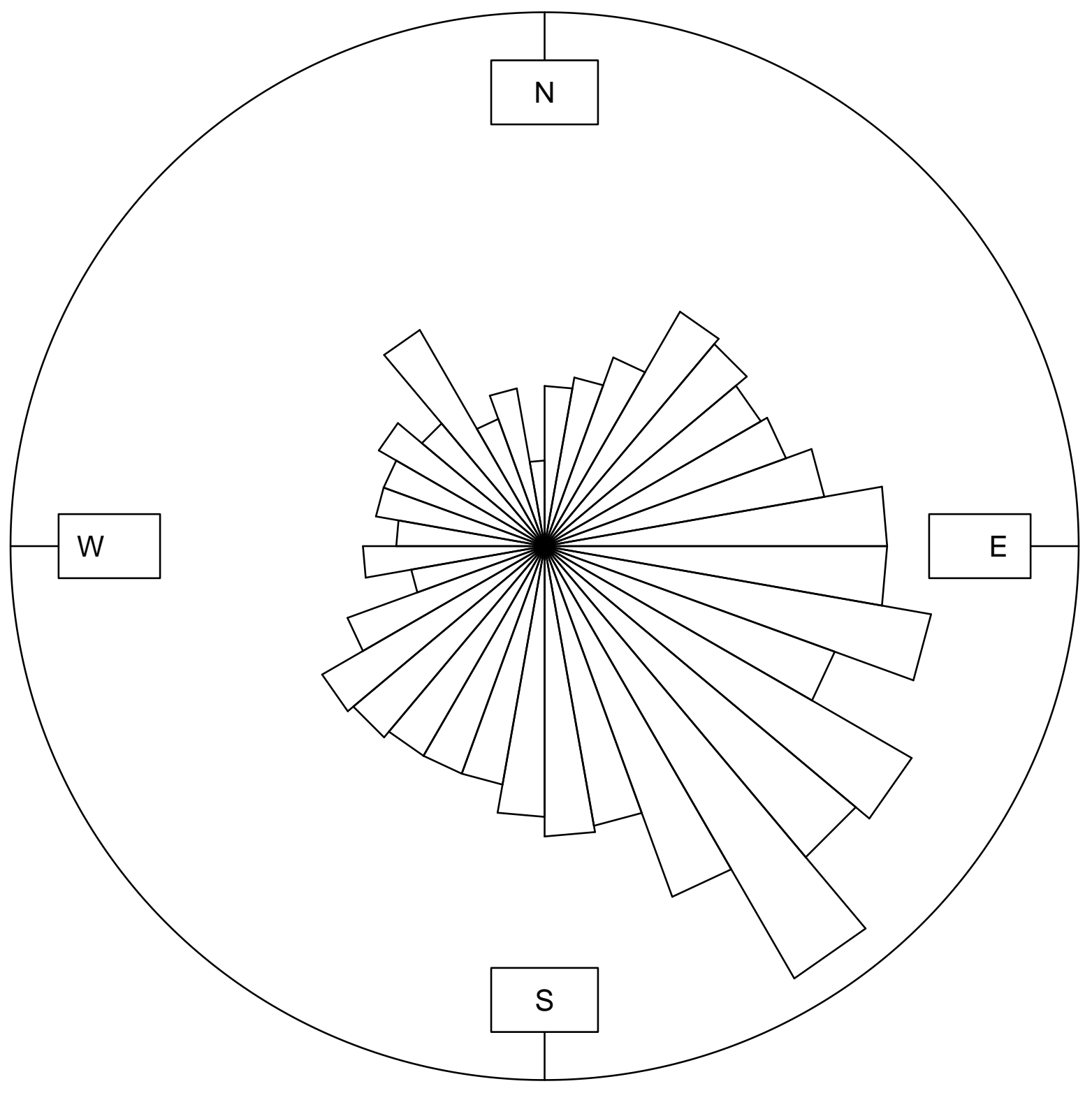


bootstrap 315

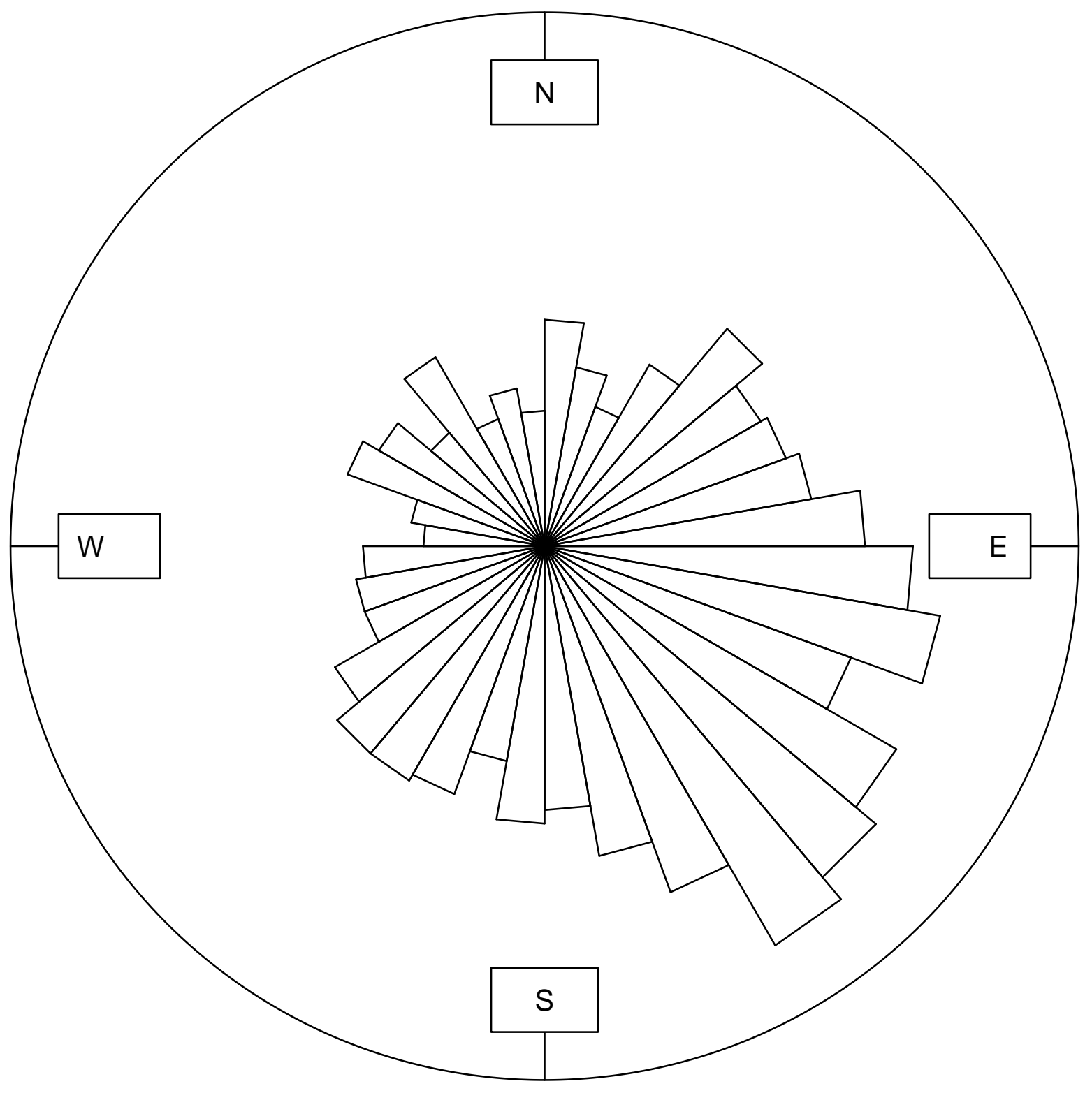


bootstrap 317

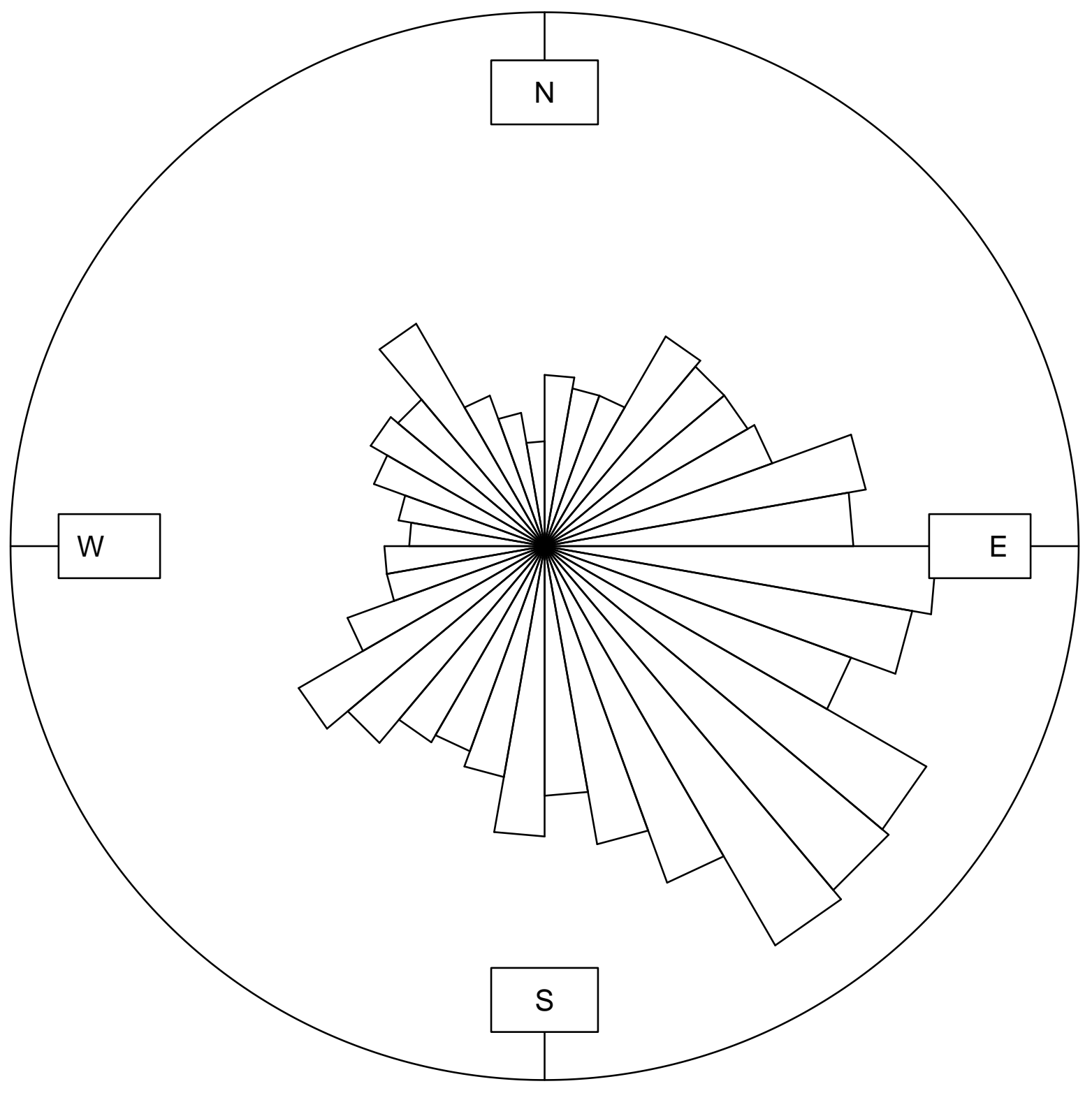


bootstrap 318

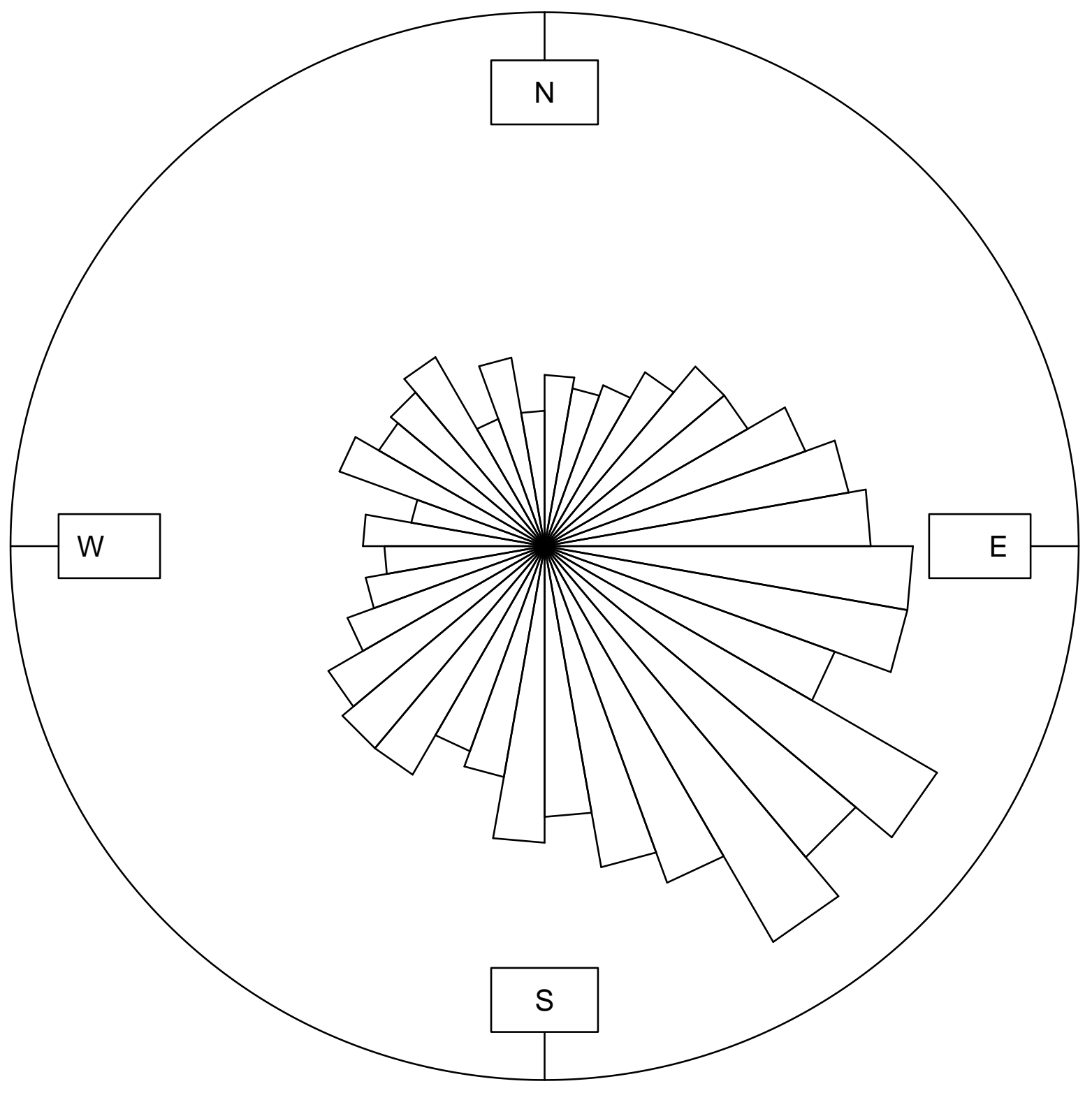


bootstrap 319

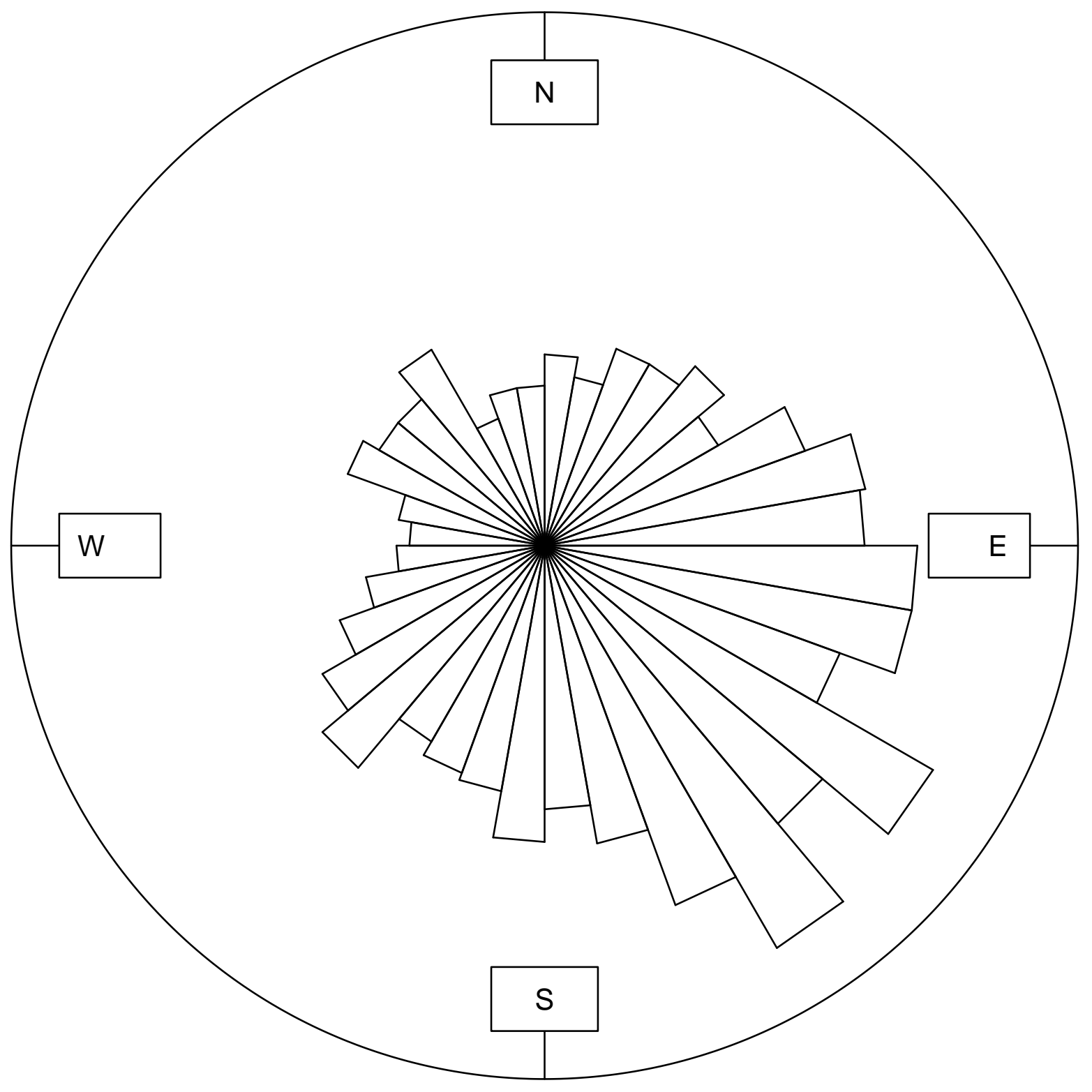


bootstrap 320

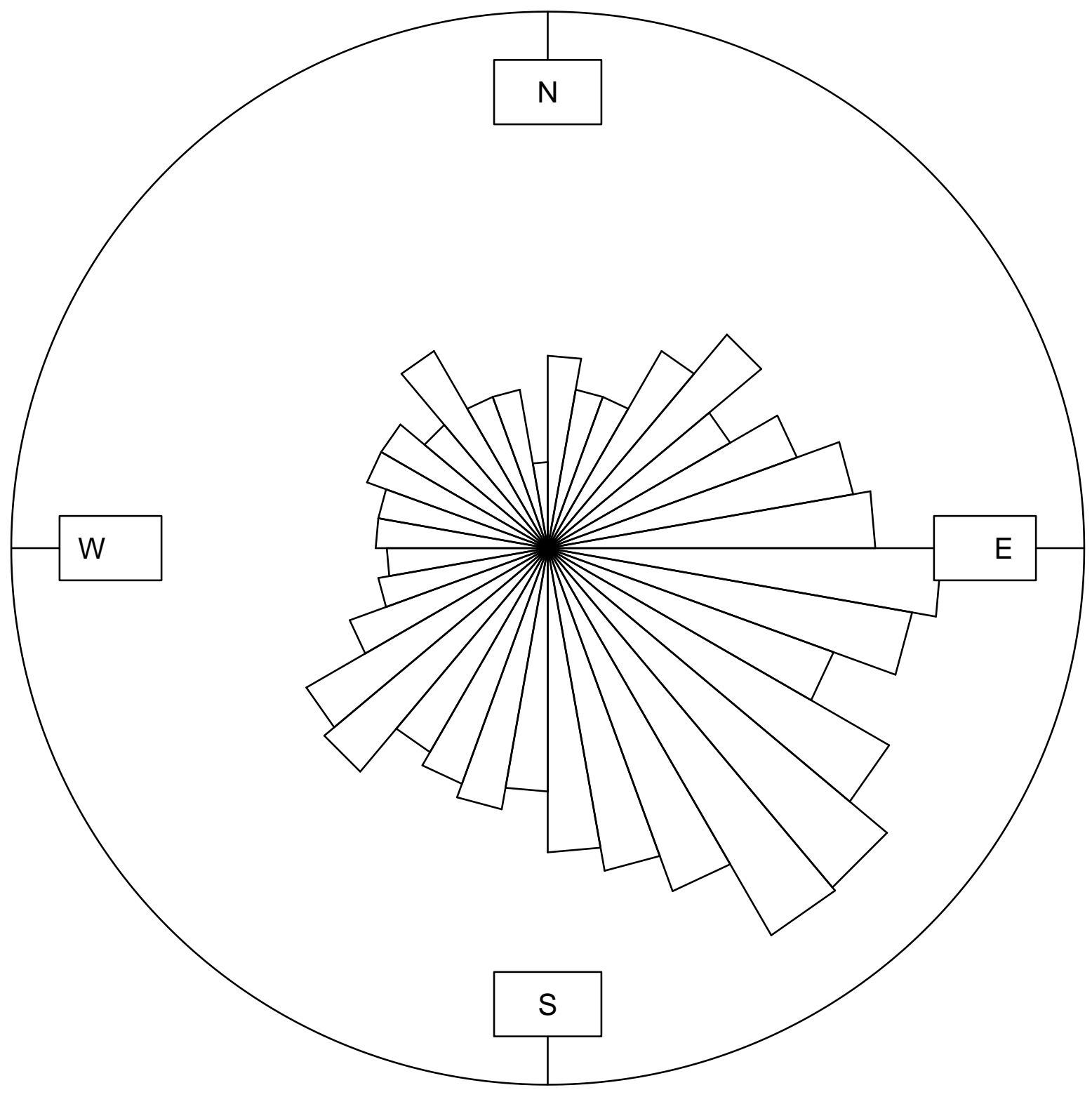


bootstrap 321

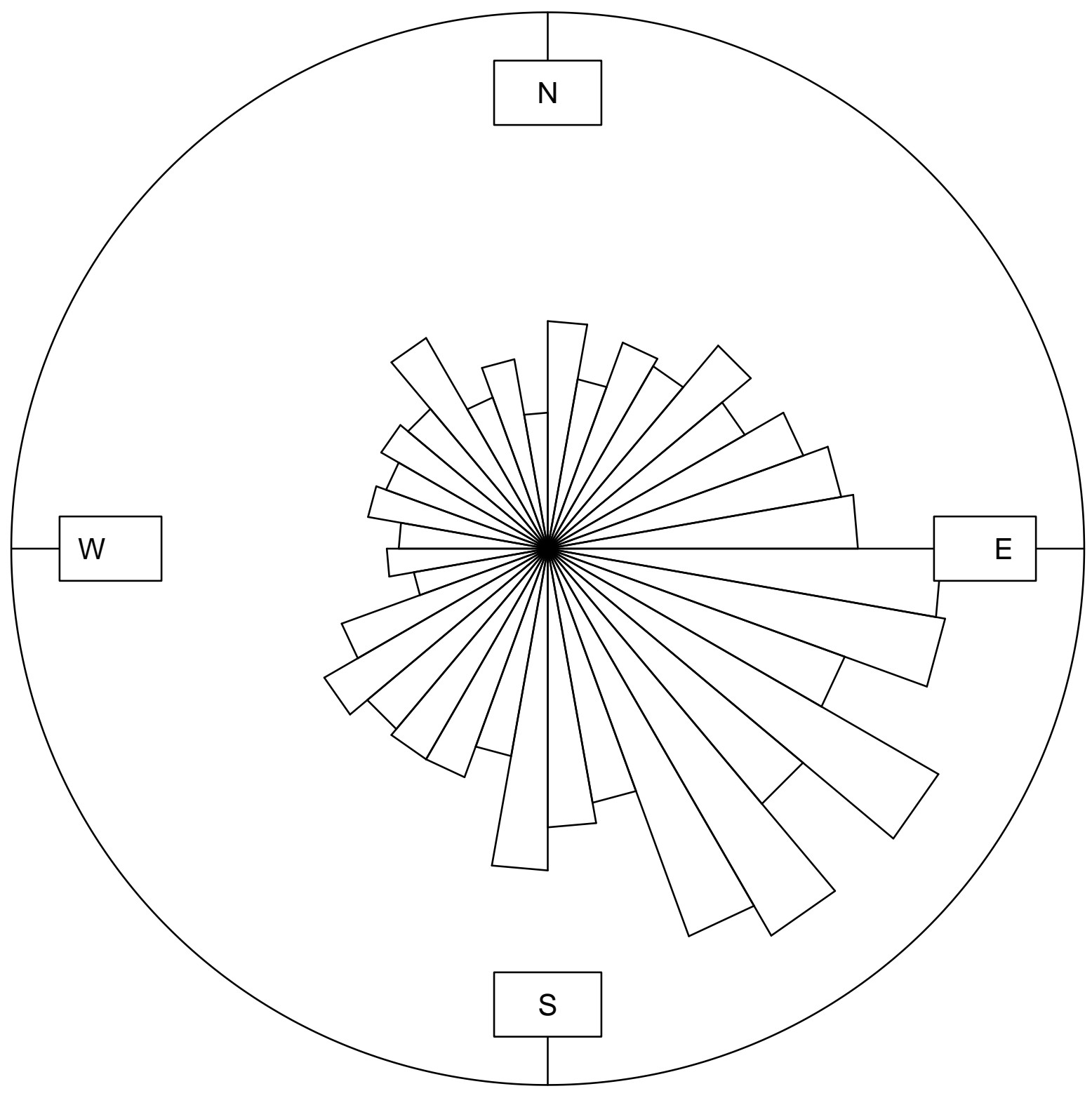


bootstrap 322

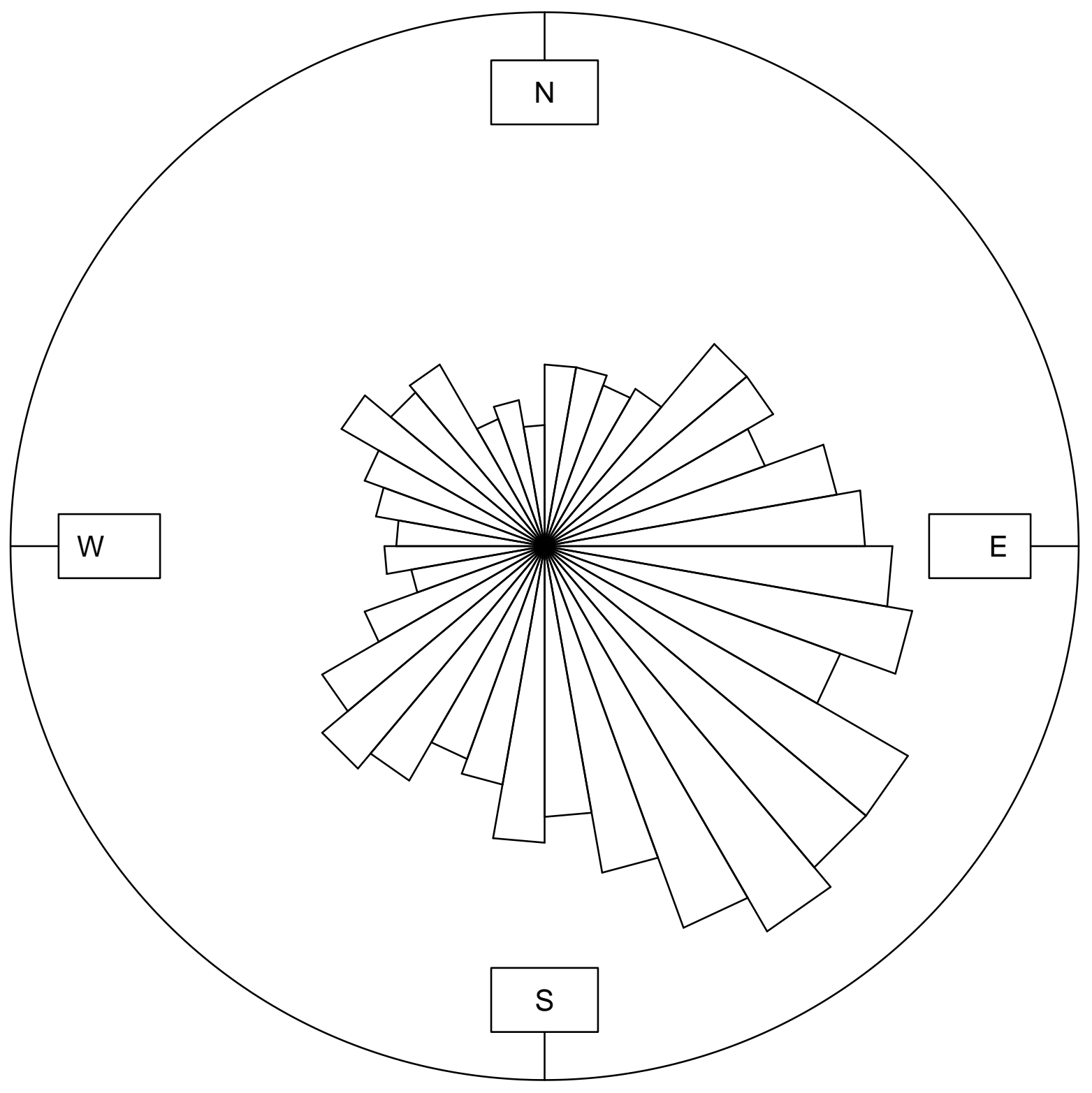


bootstrap 323

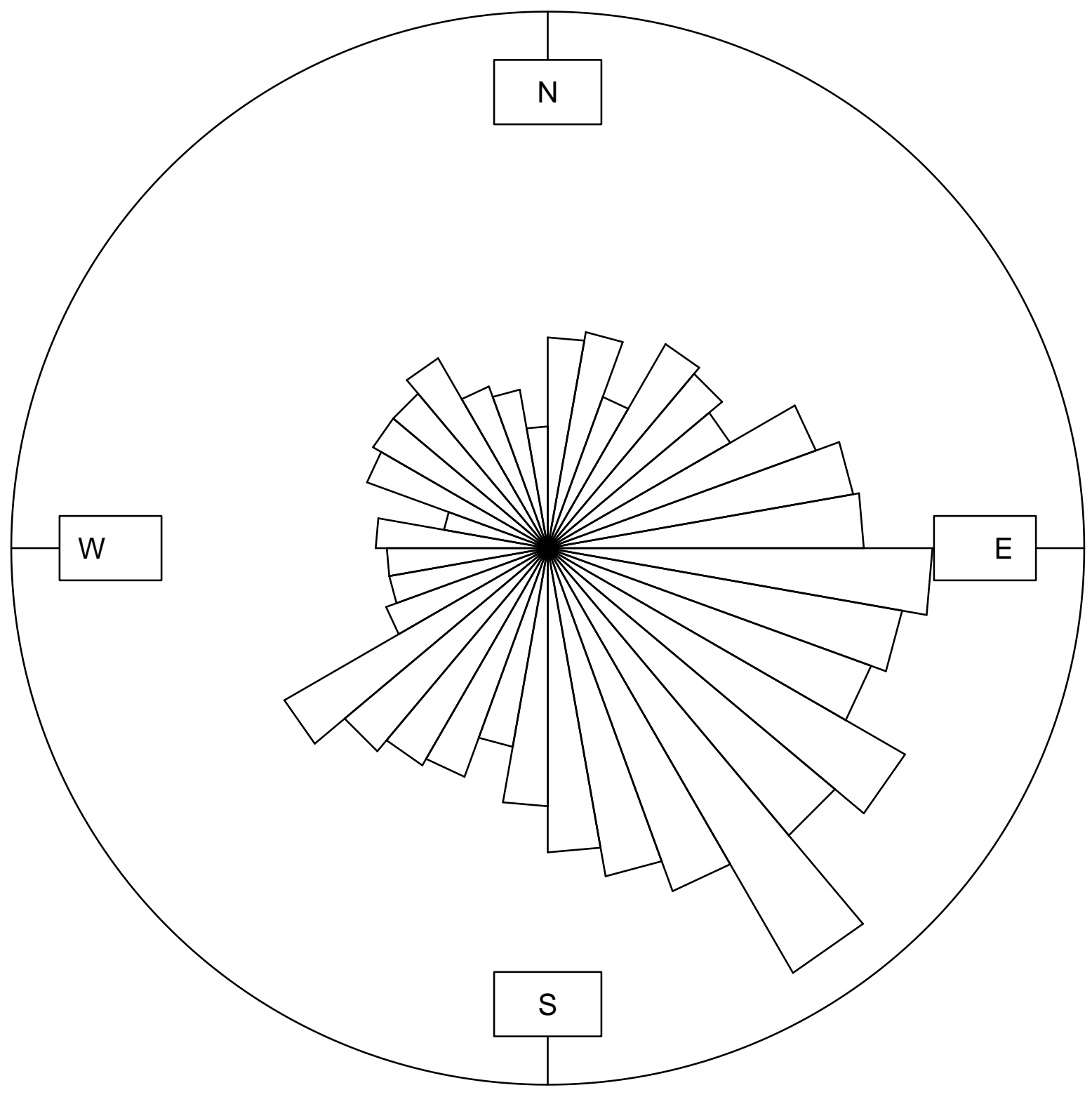


bootstrap 324

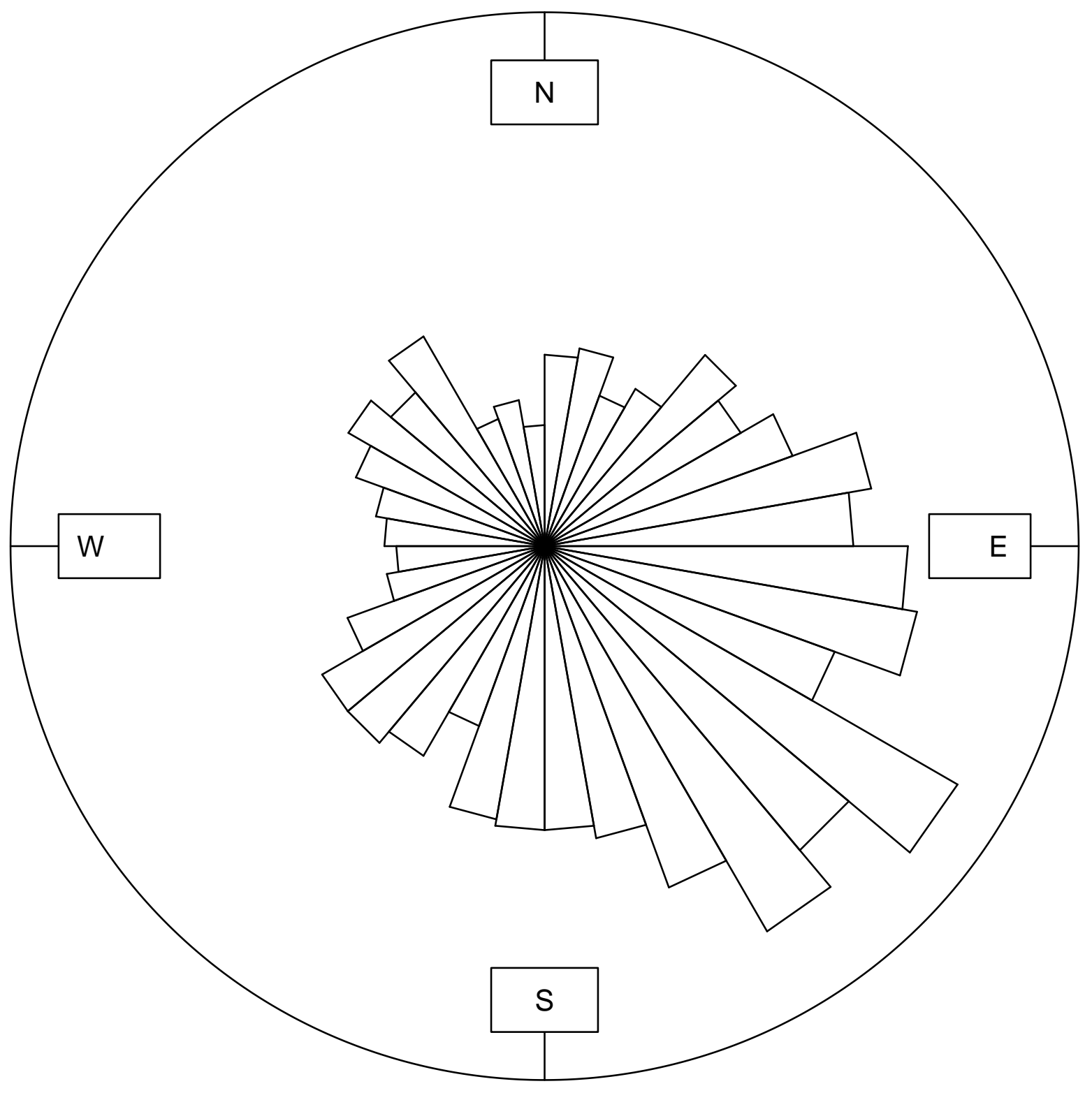


bootstrap 325

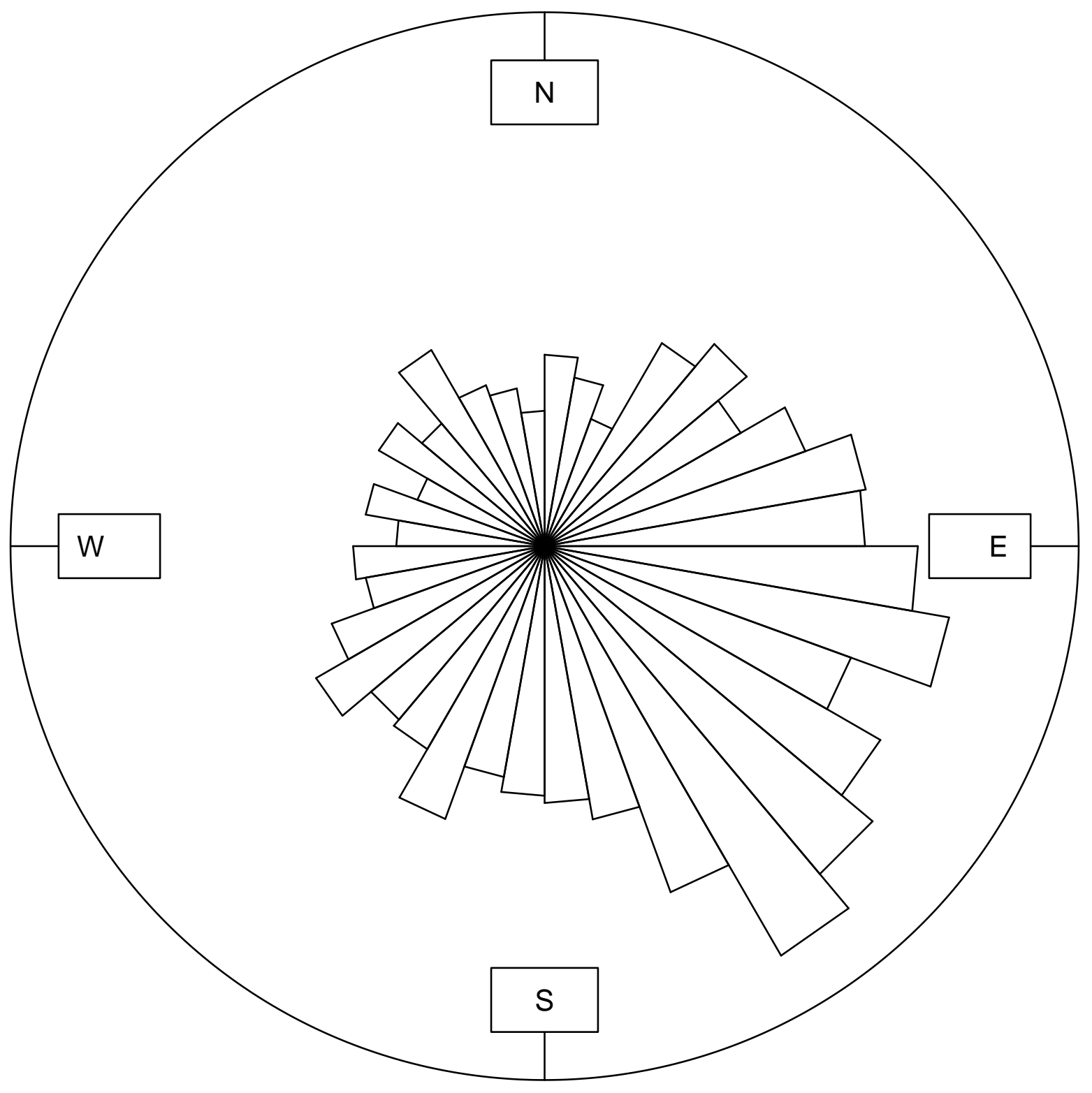


bootstrap 326

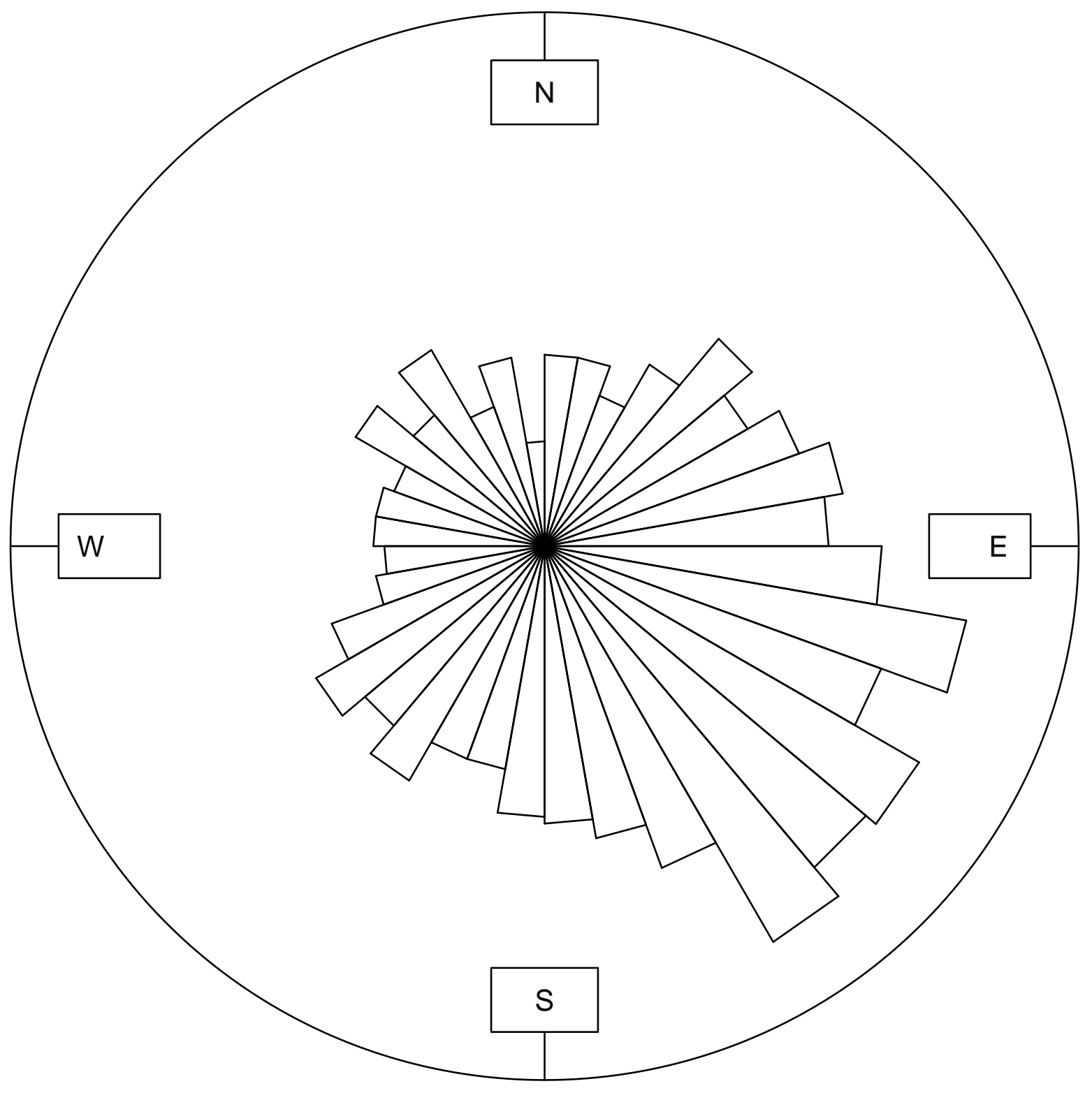


bootstrap 328

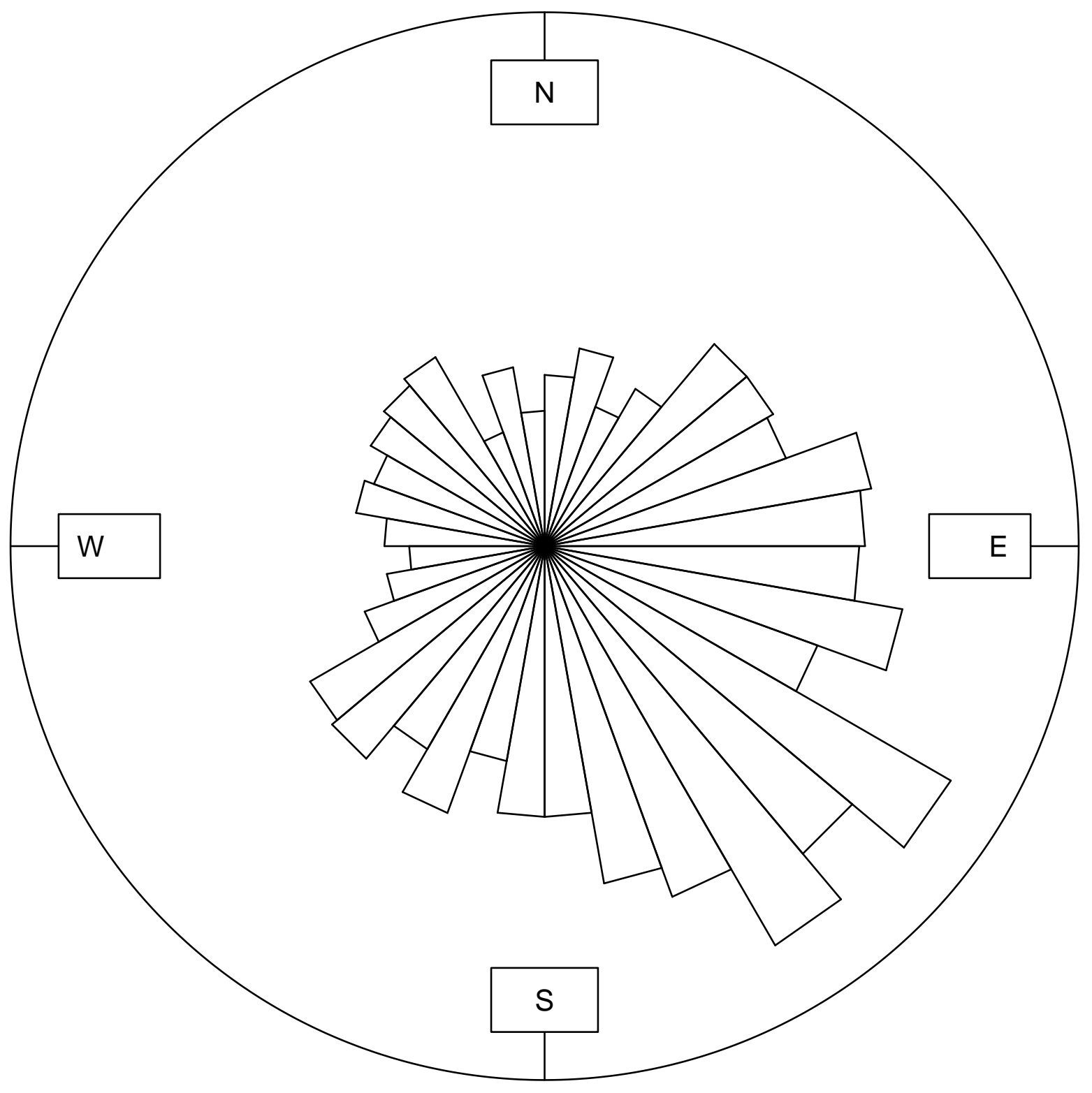


bootstrap 329

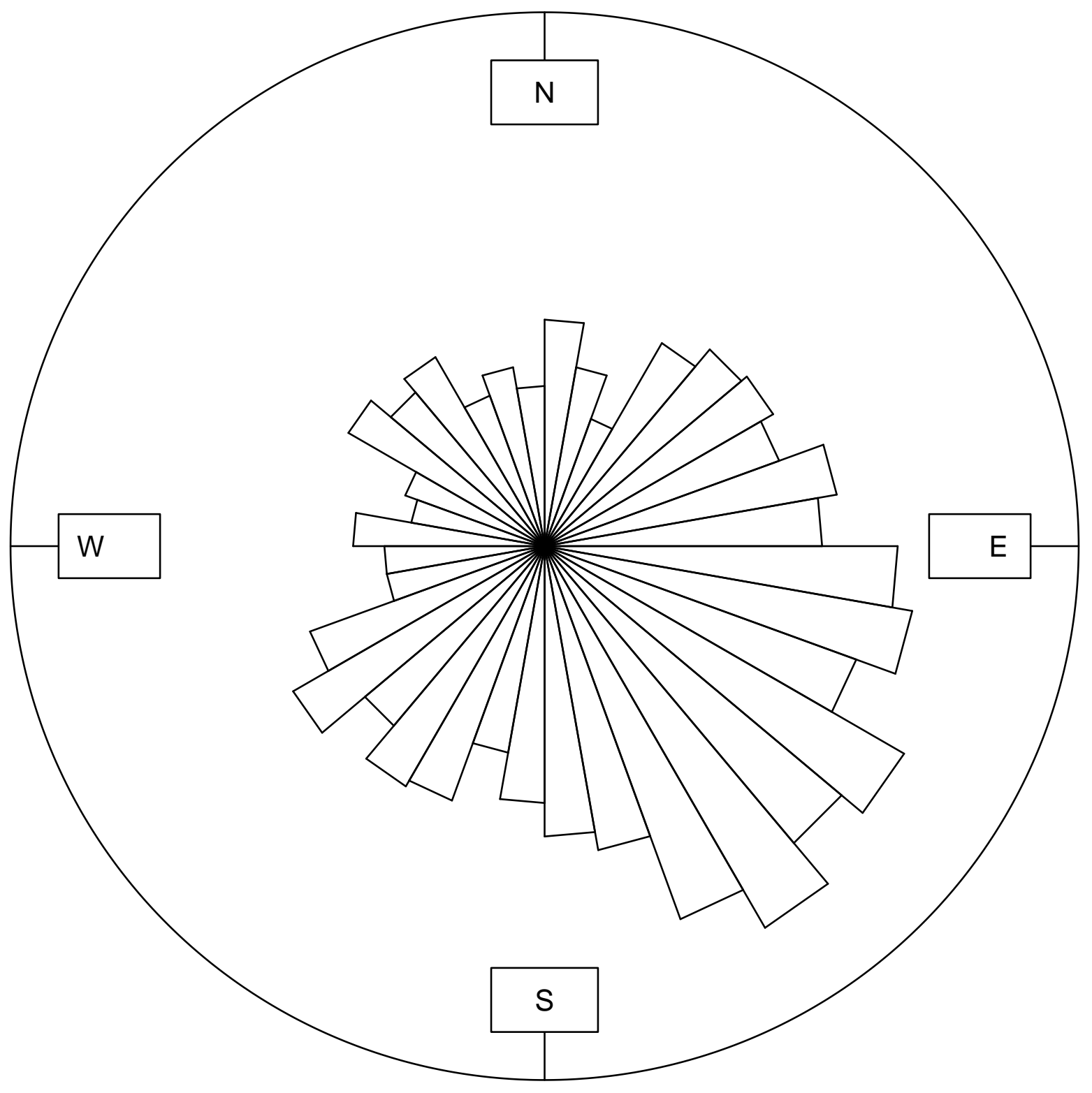


bootstrap 330

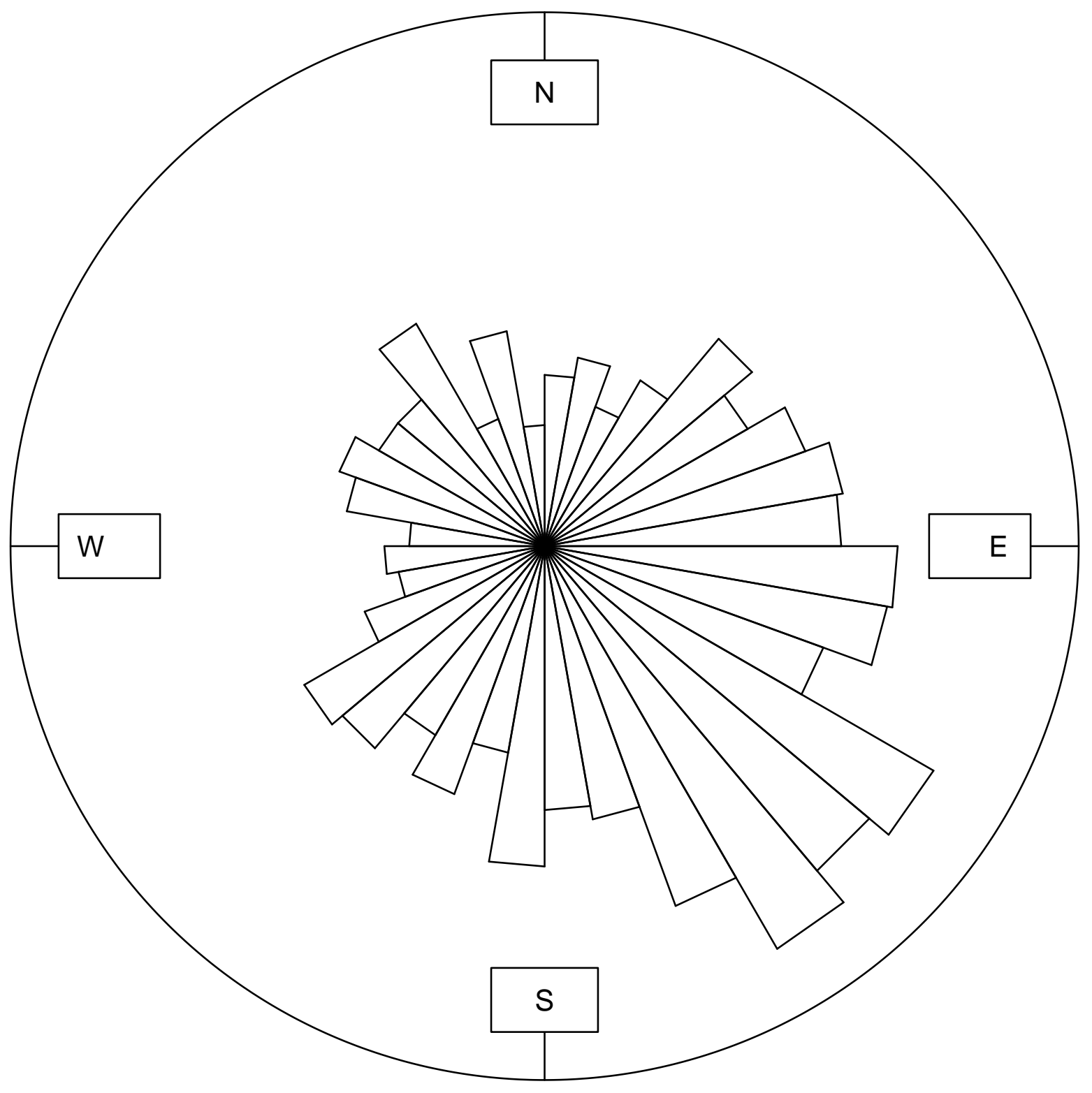


bootstrap 331

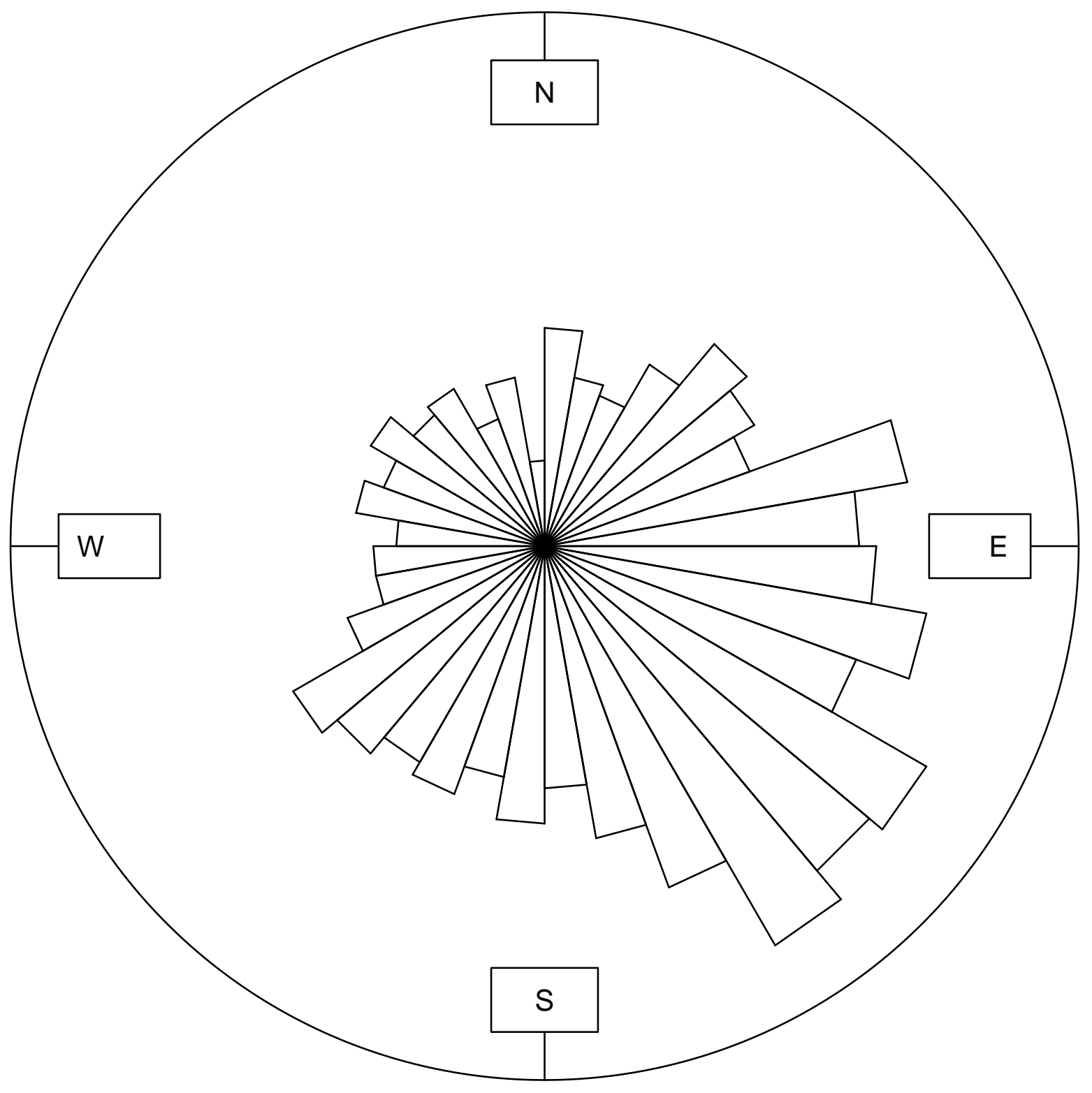


bootstrap 332

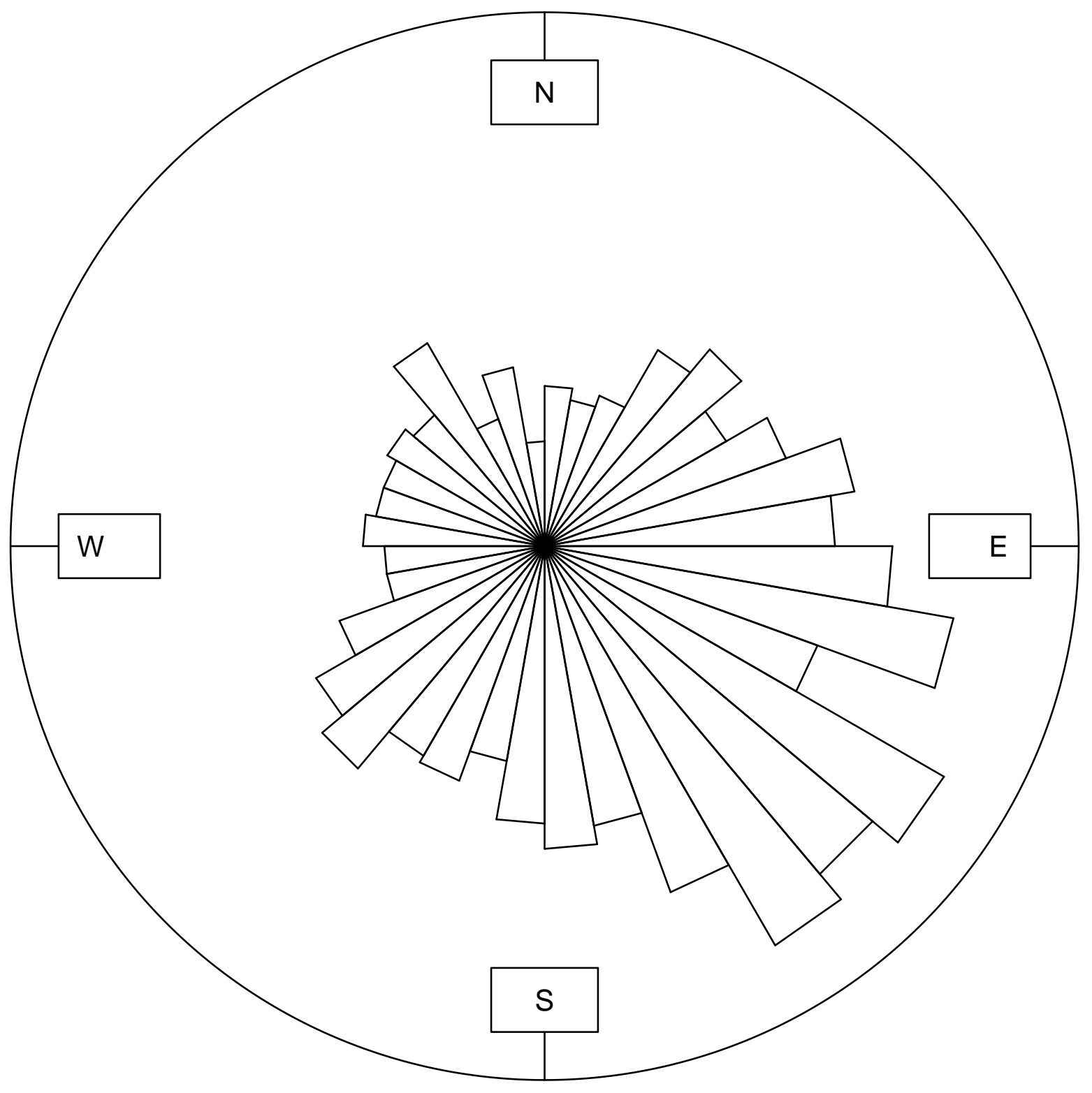




\section{bootstrap 333}

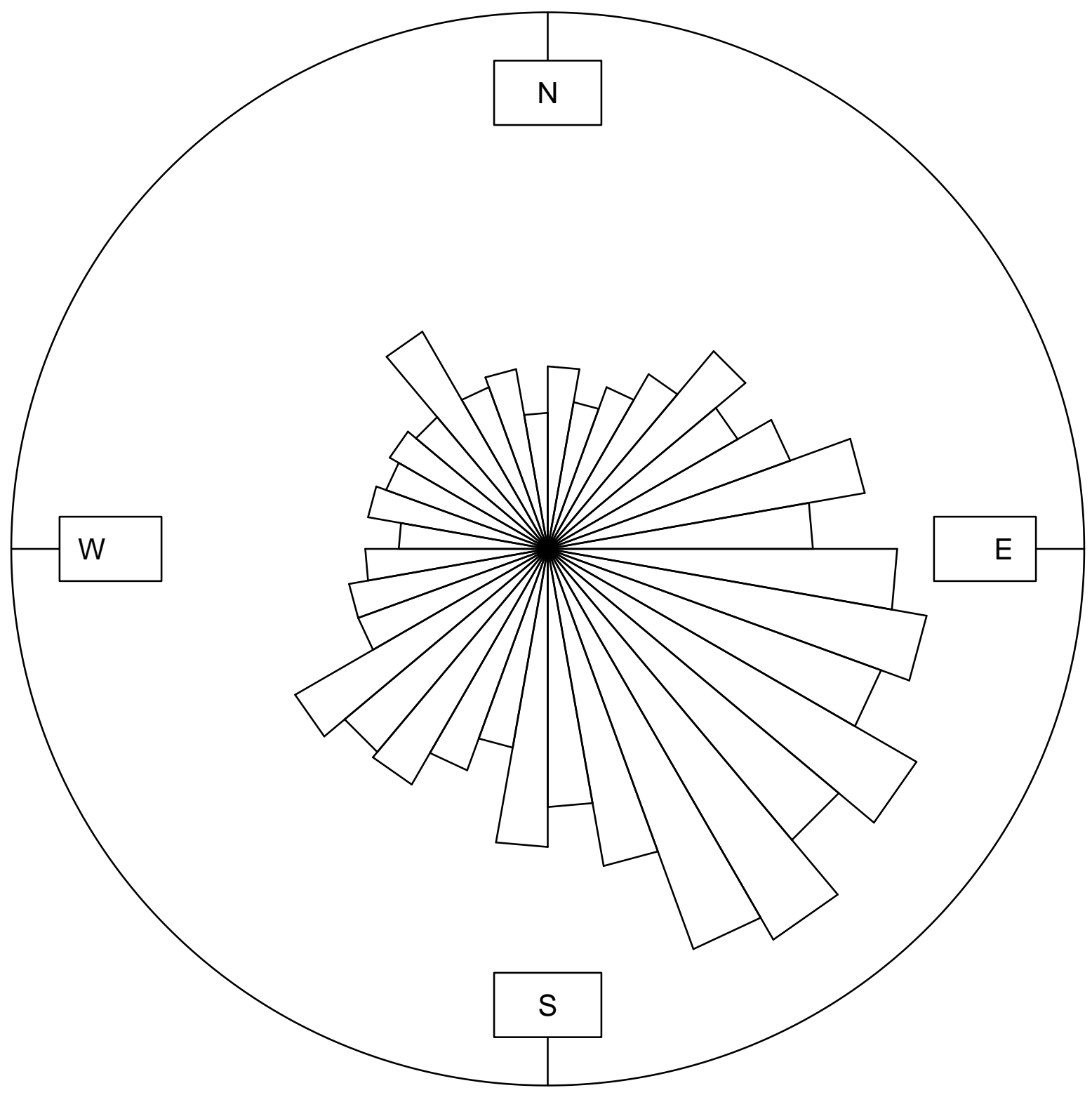




\section{bootstrap 334}

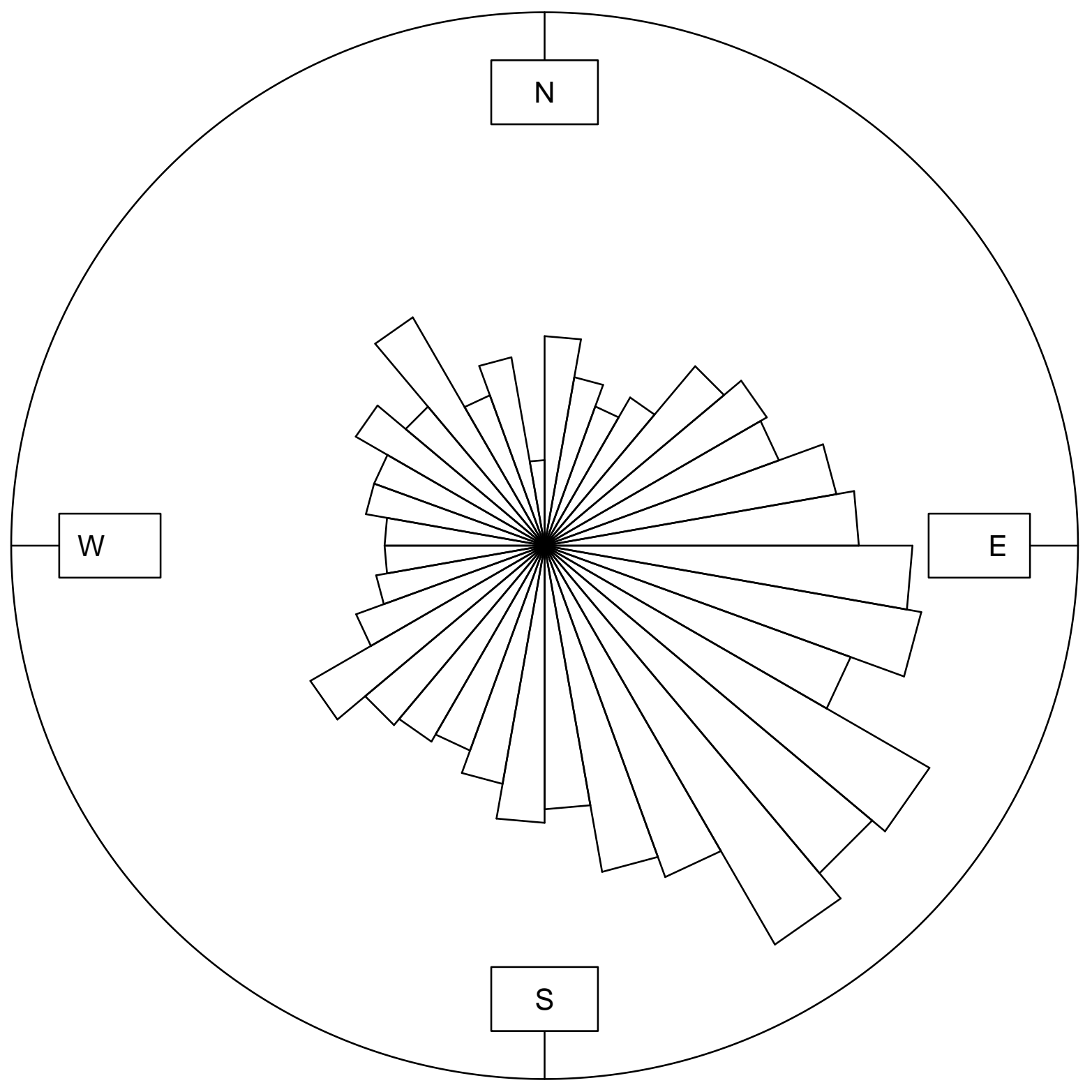




\section{bootstrap 335}

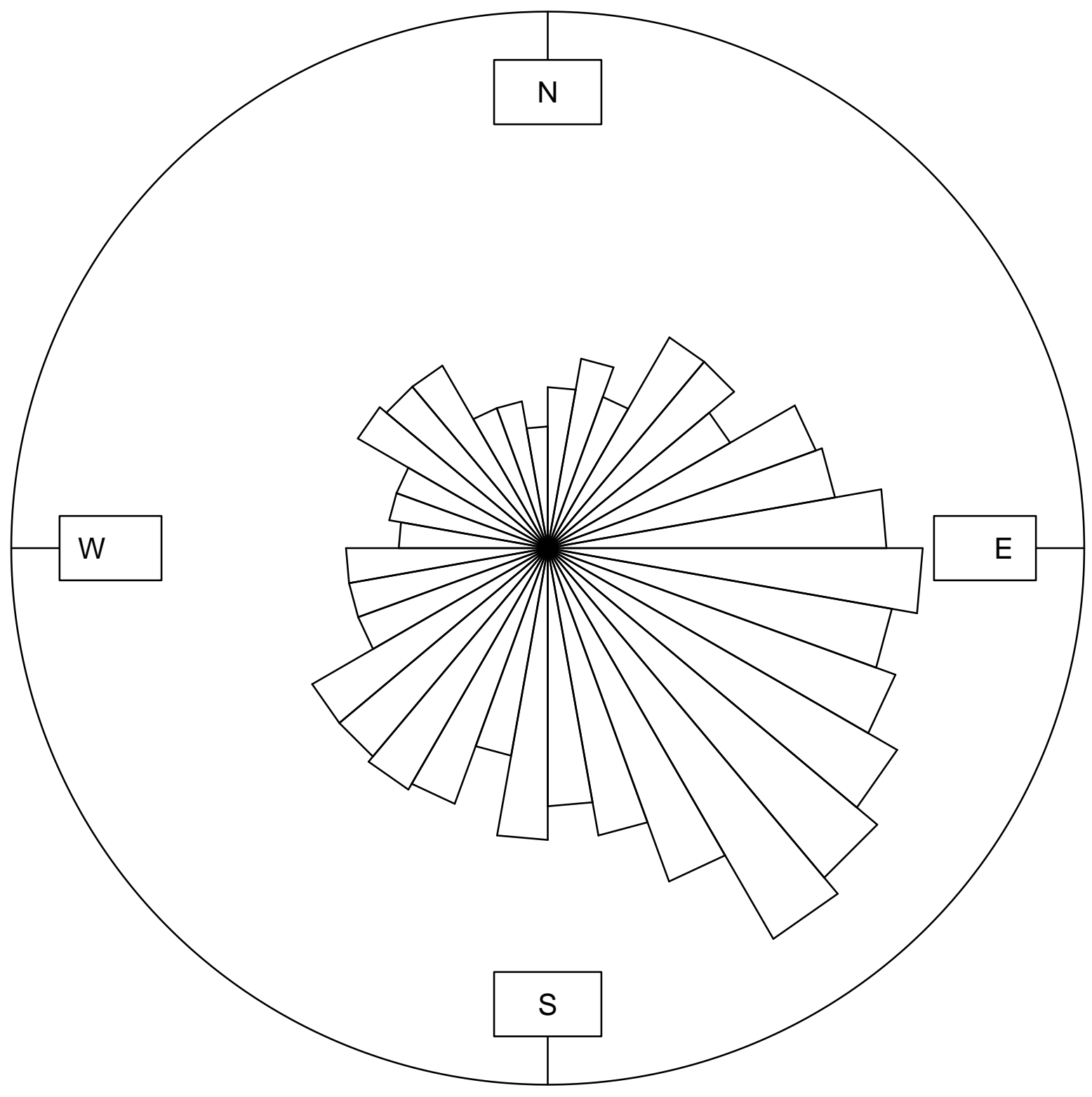




\section{bootstrap 336}

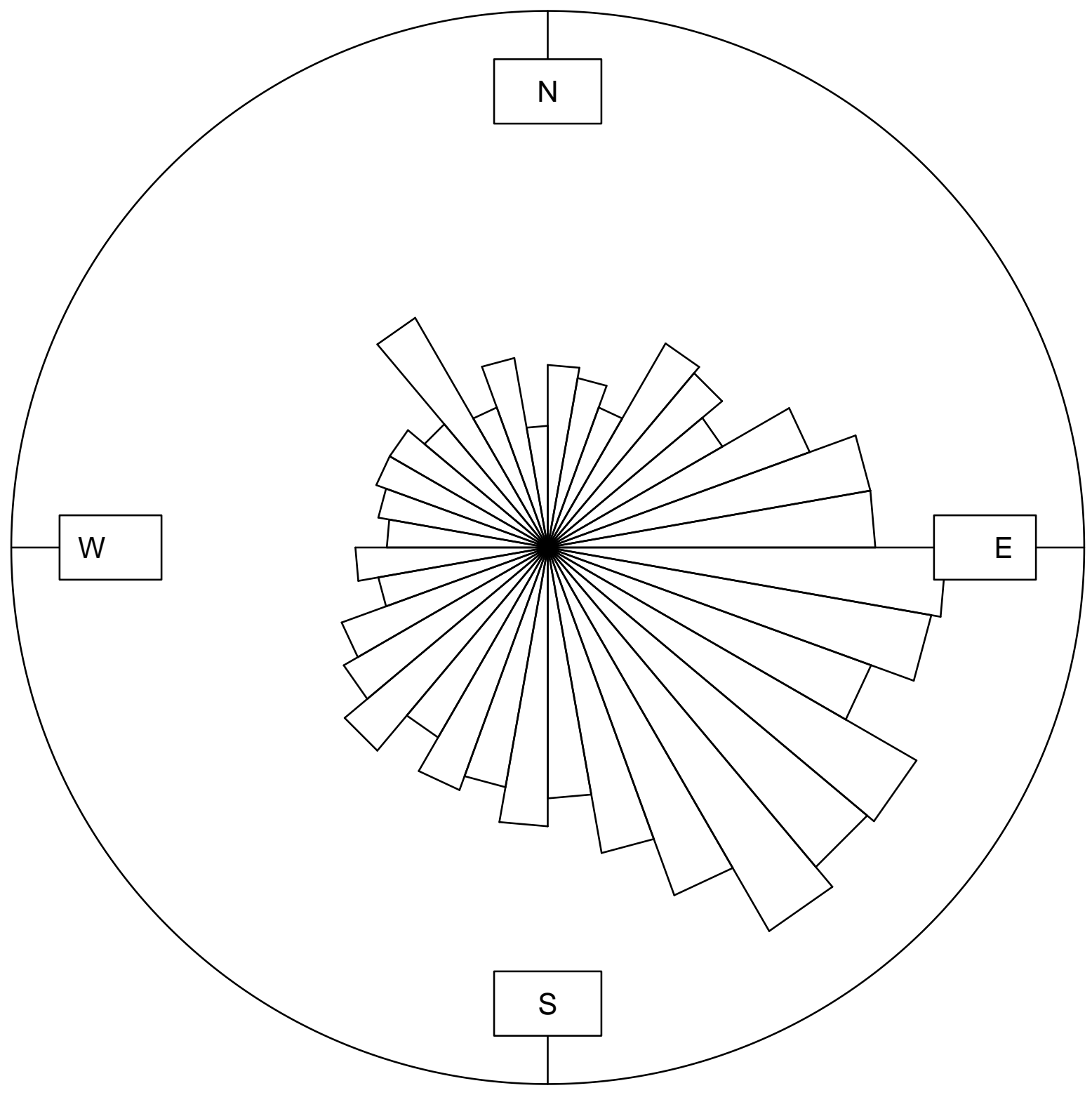


bootstrap 337

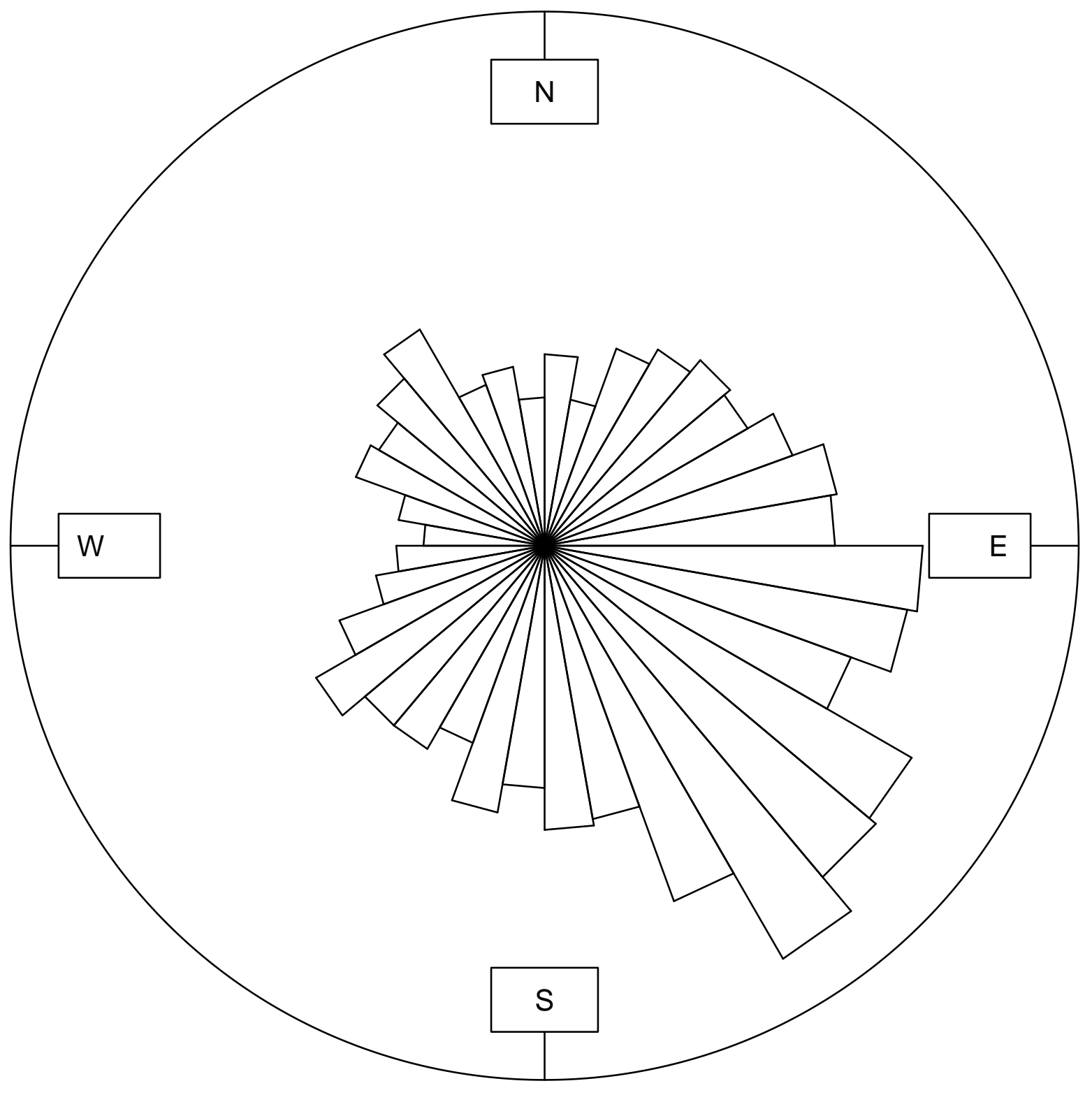


bootstrap 338

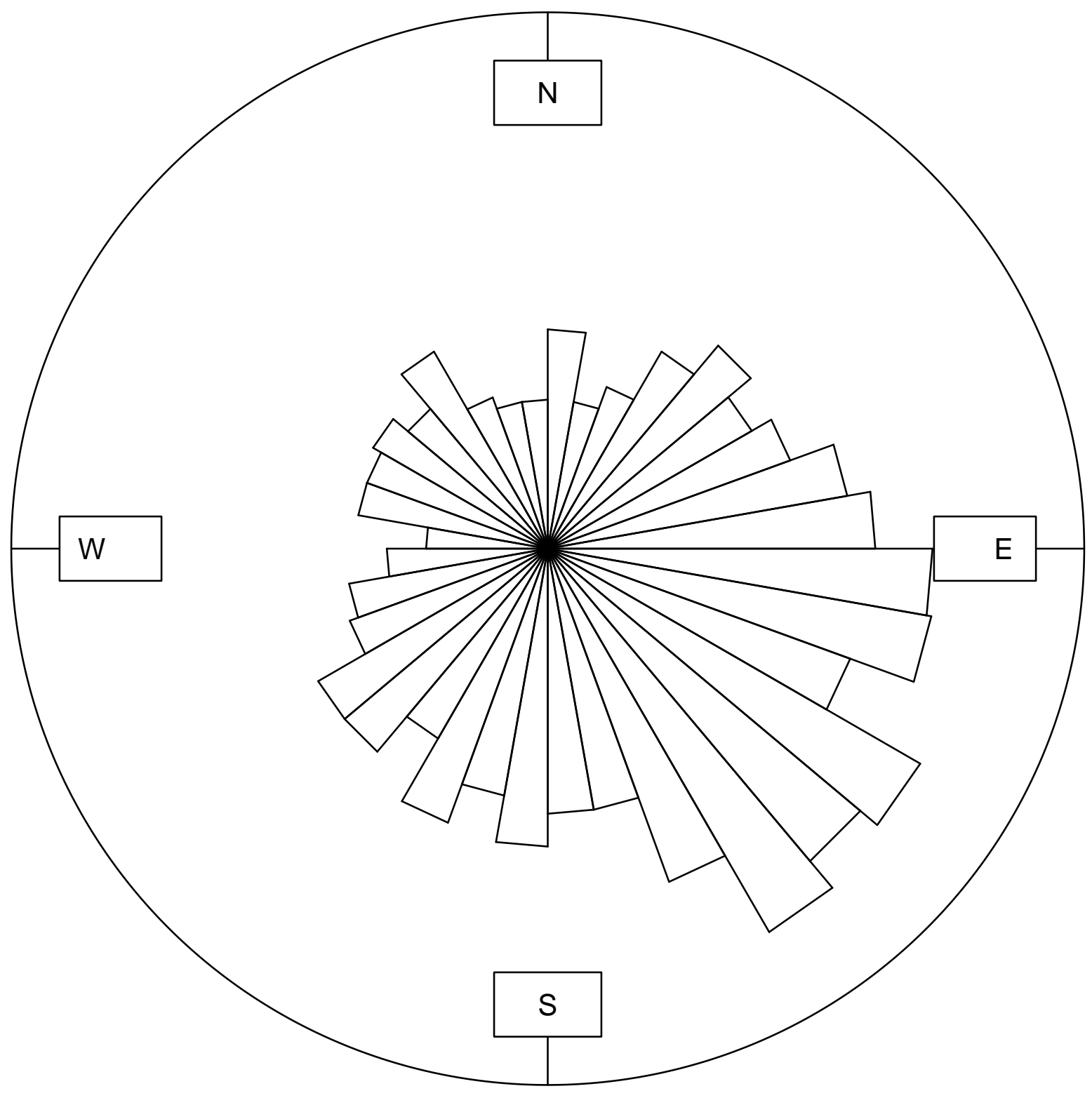


bootstrap 340

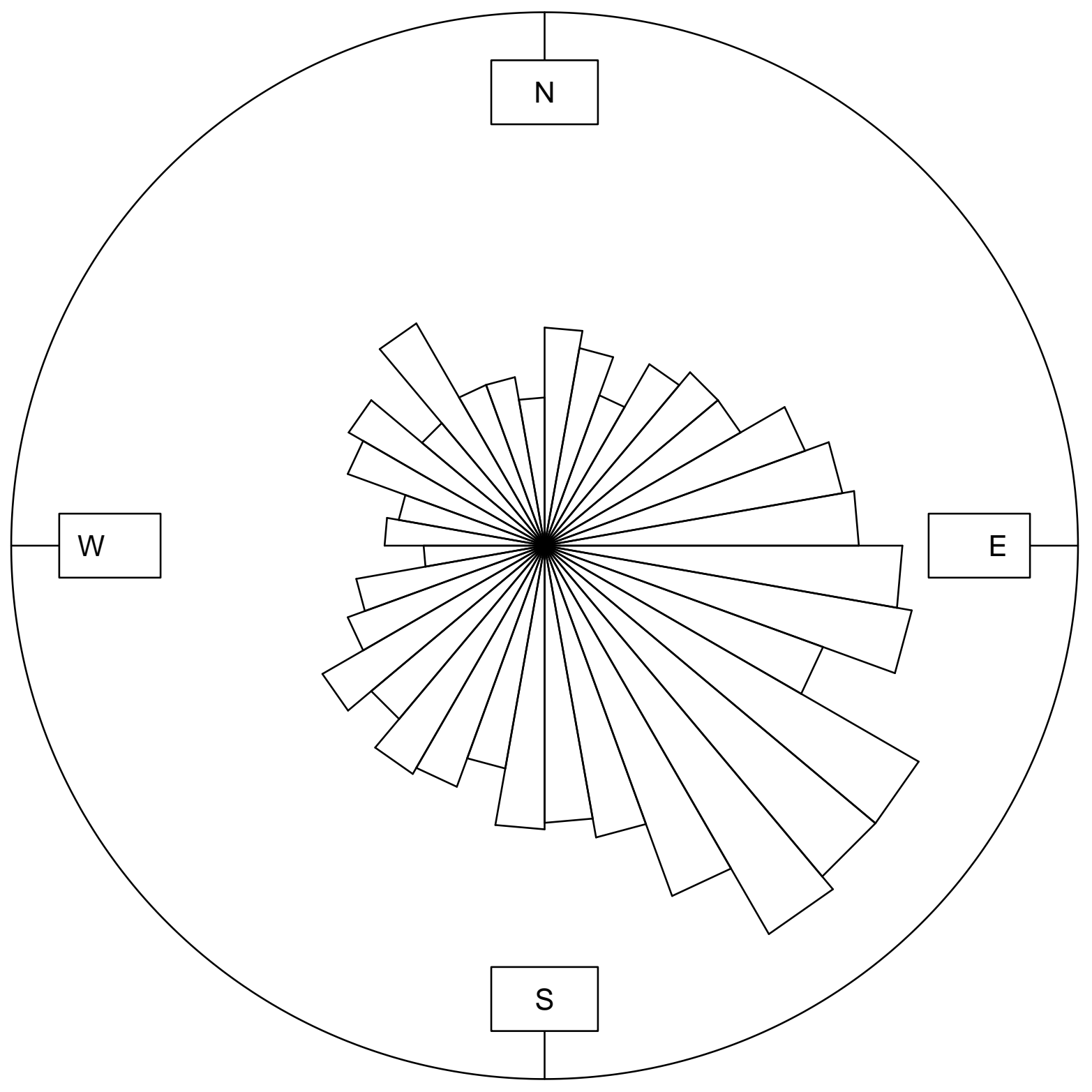


bootstrap 341

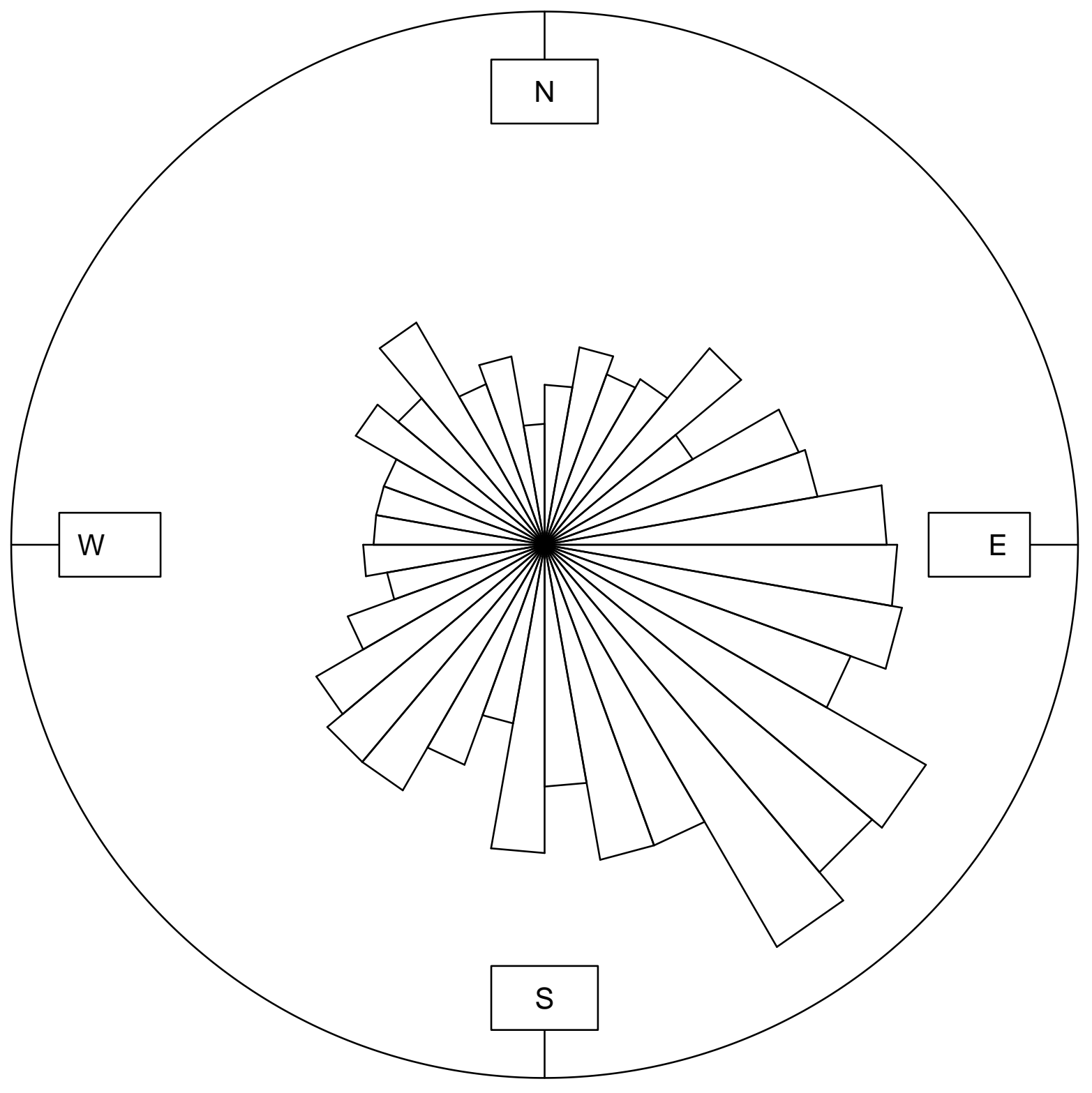


bootstrap 342

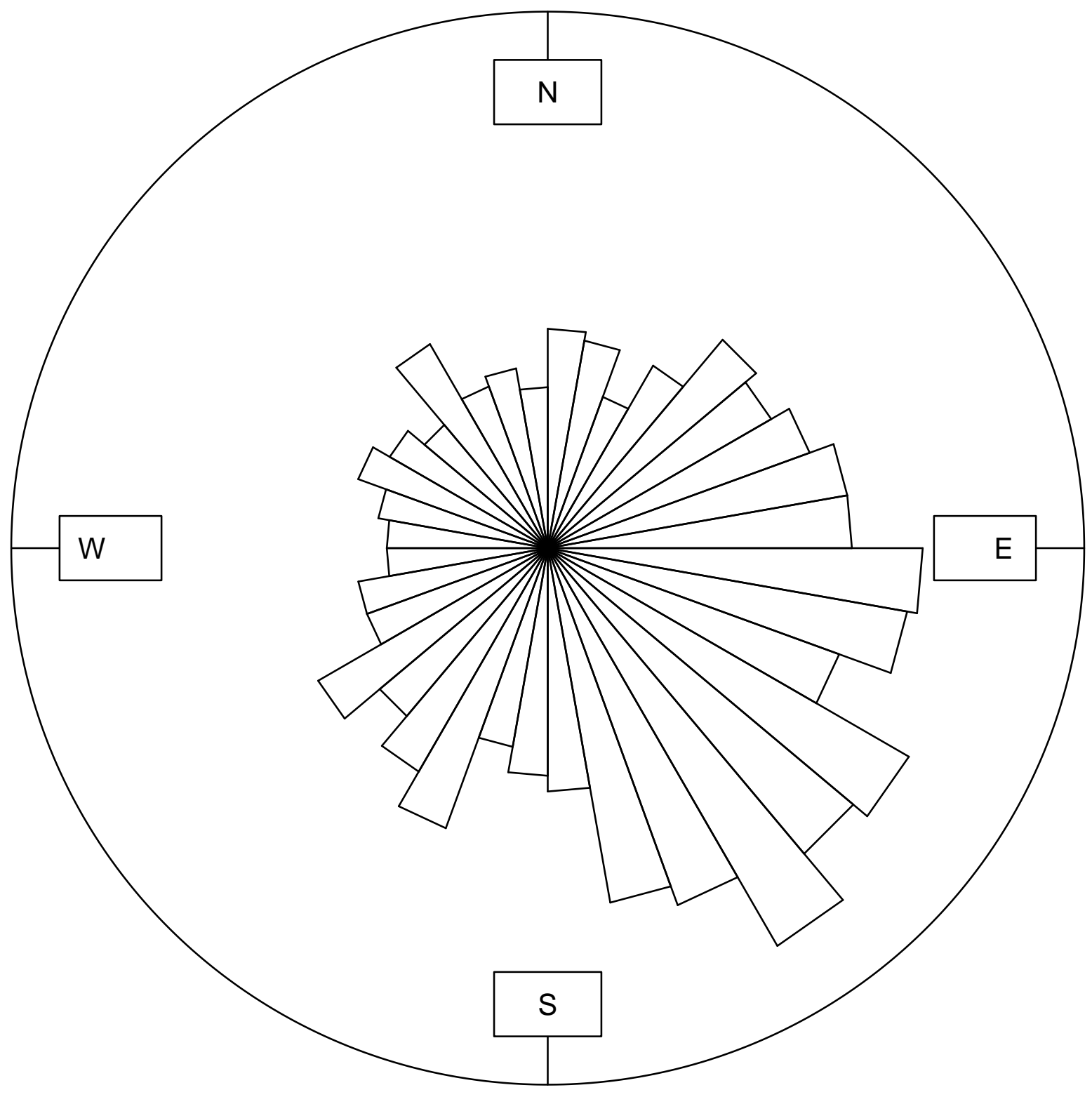




\section{bootstrap 343}

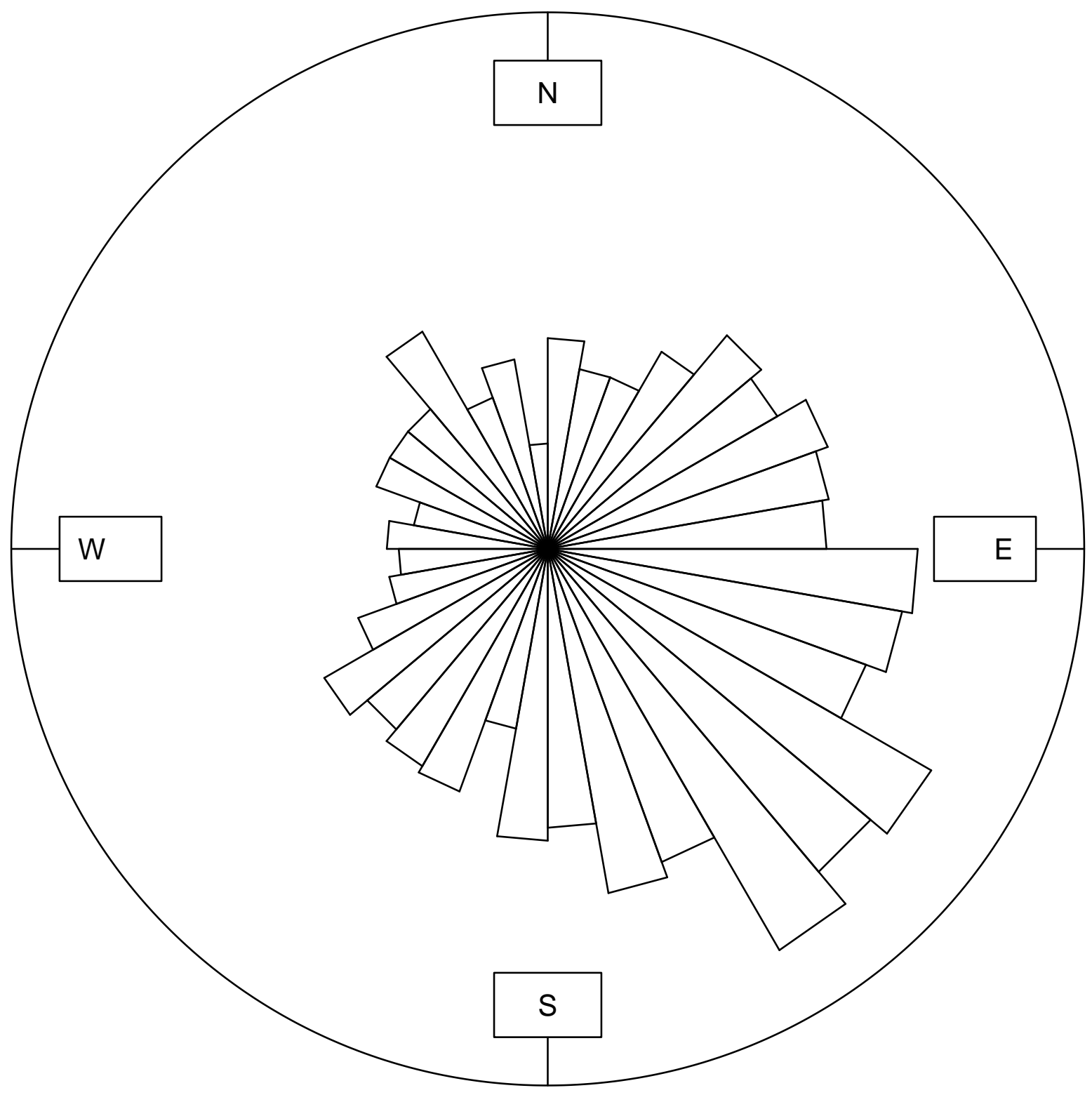


bootstrap 344

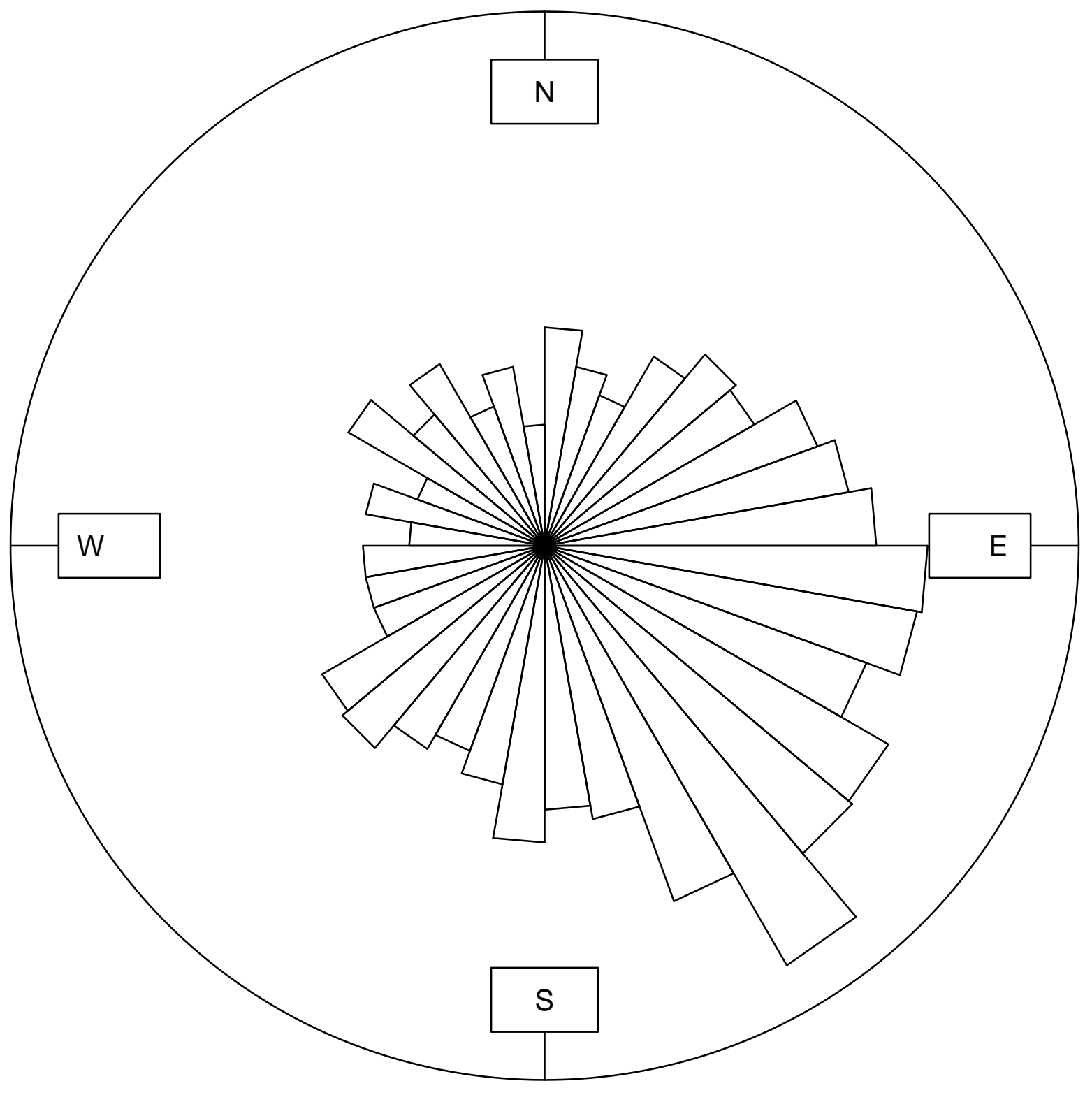


bootstrap 345

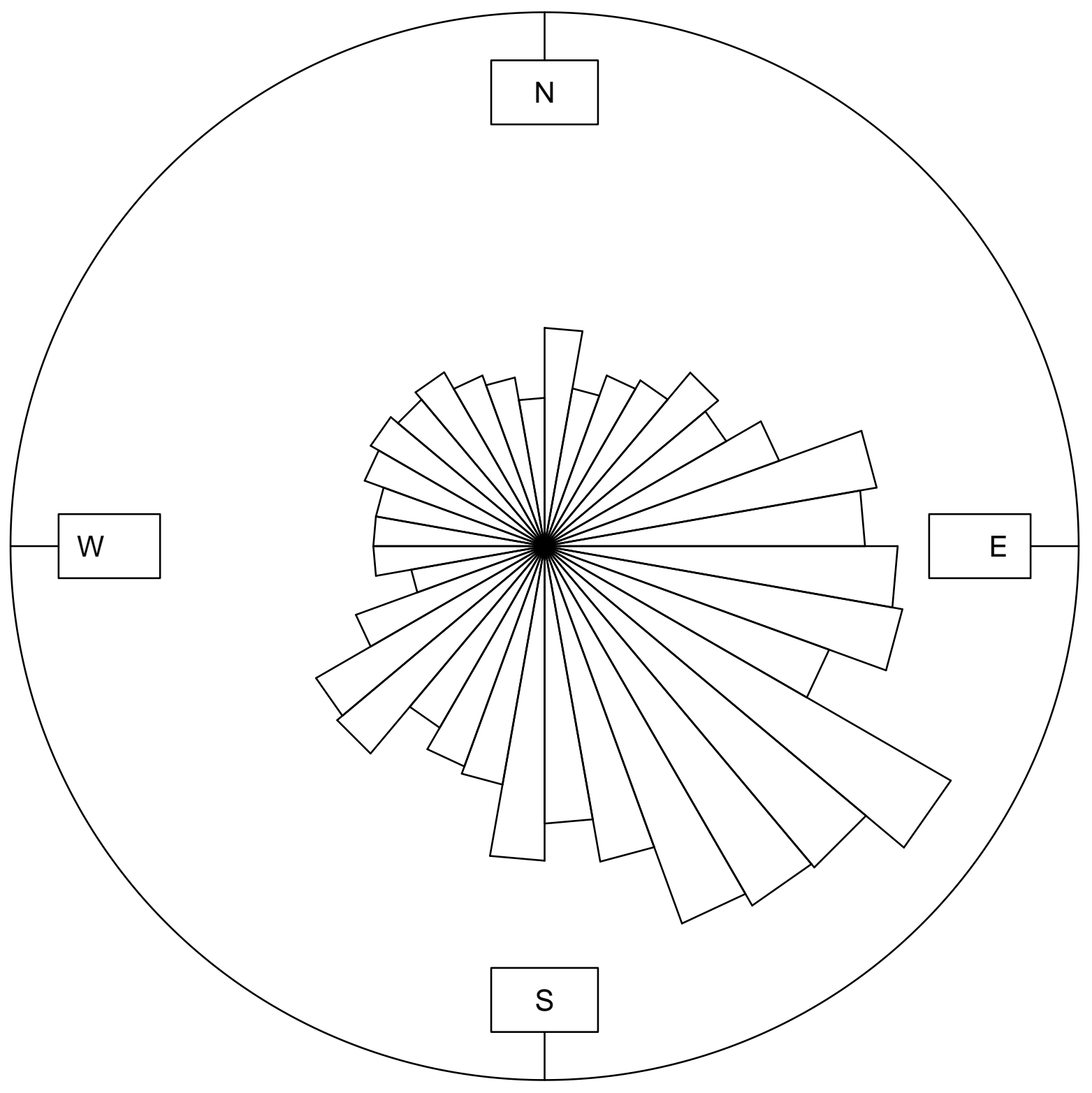


bootstrap 346

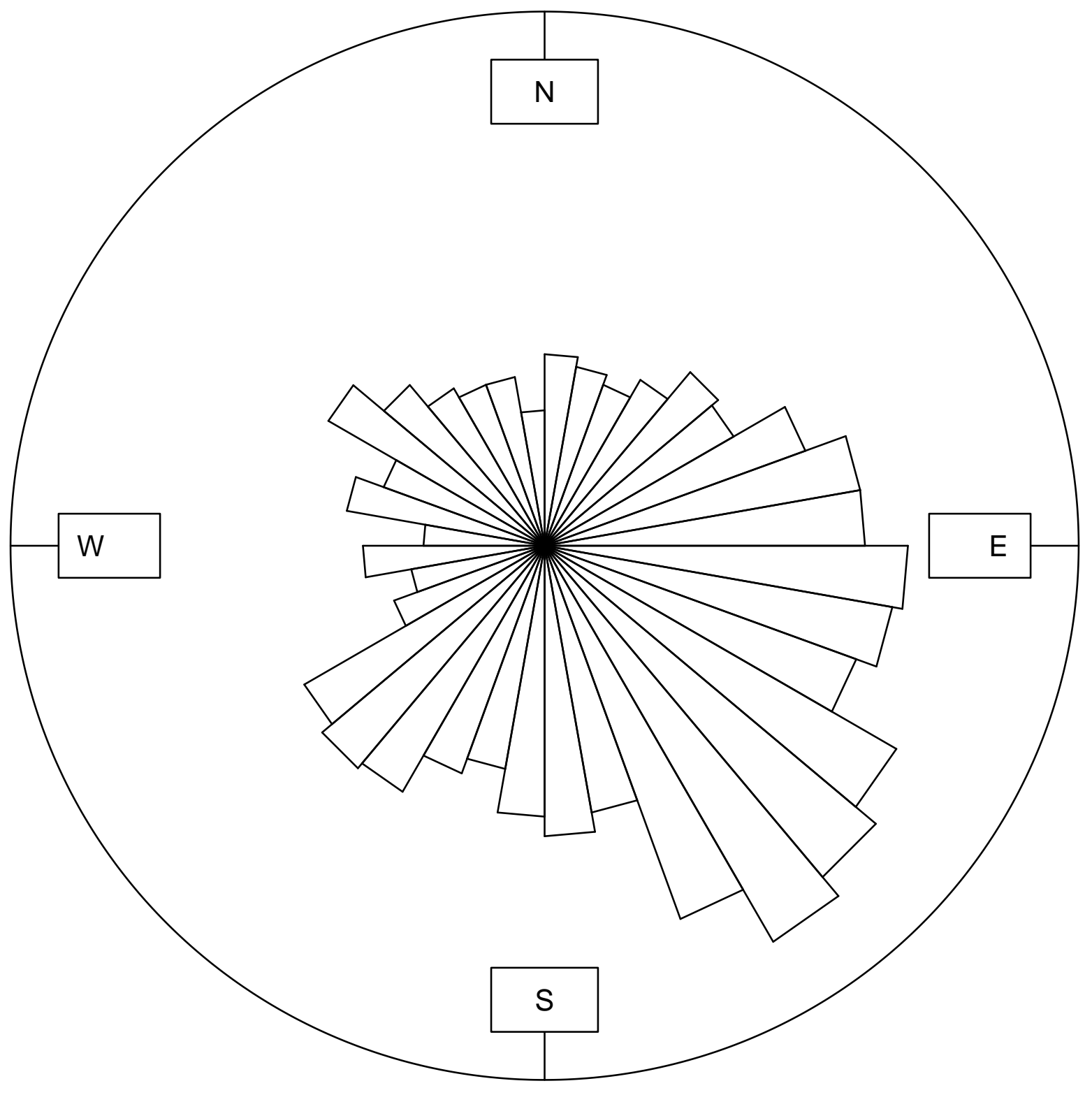


bootstrap 347

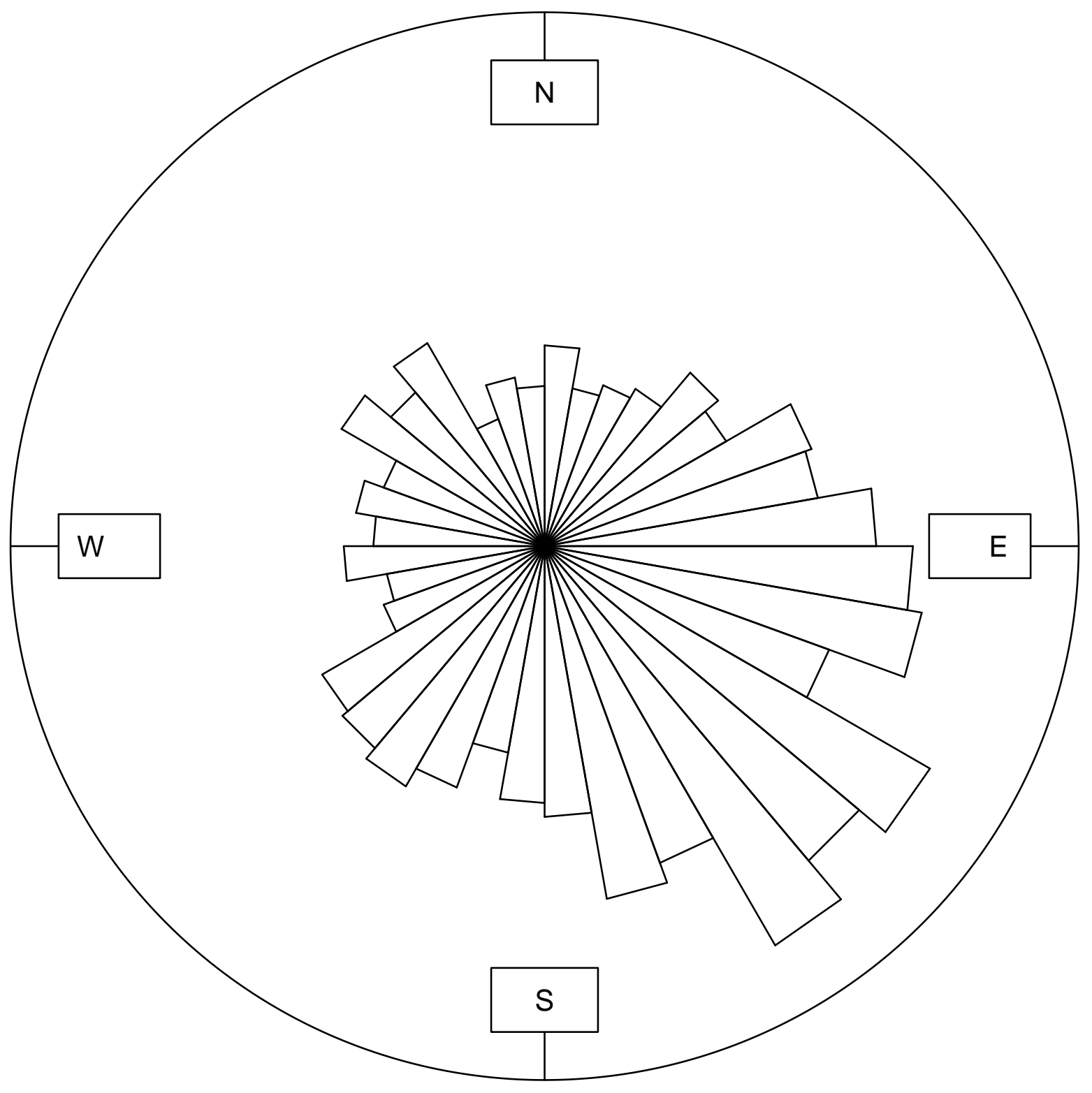


bootstrap 348

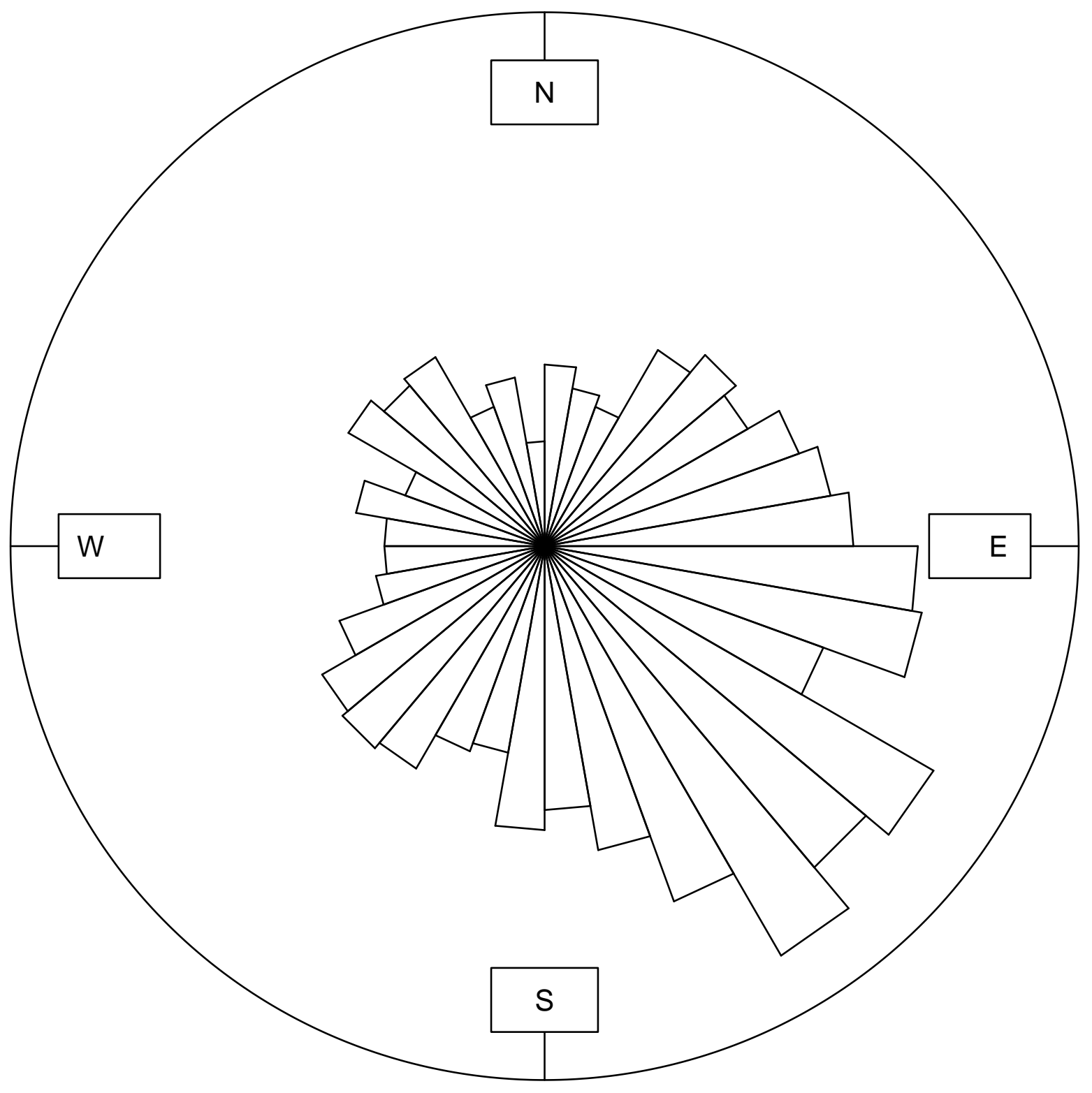


bootstrap 349

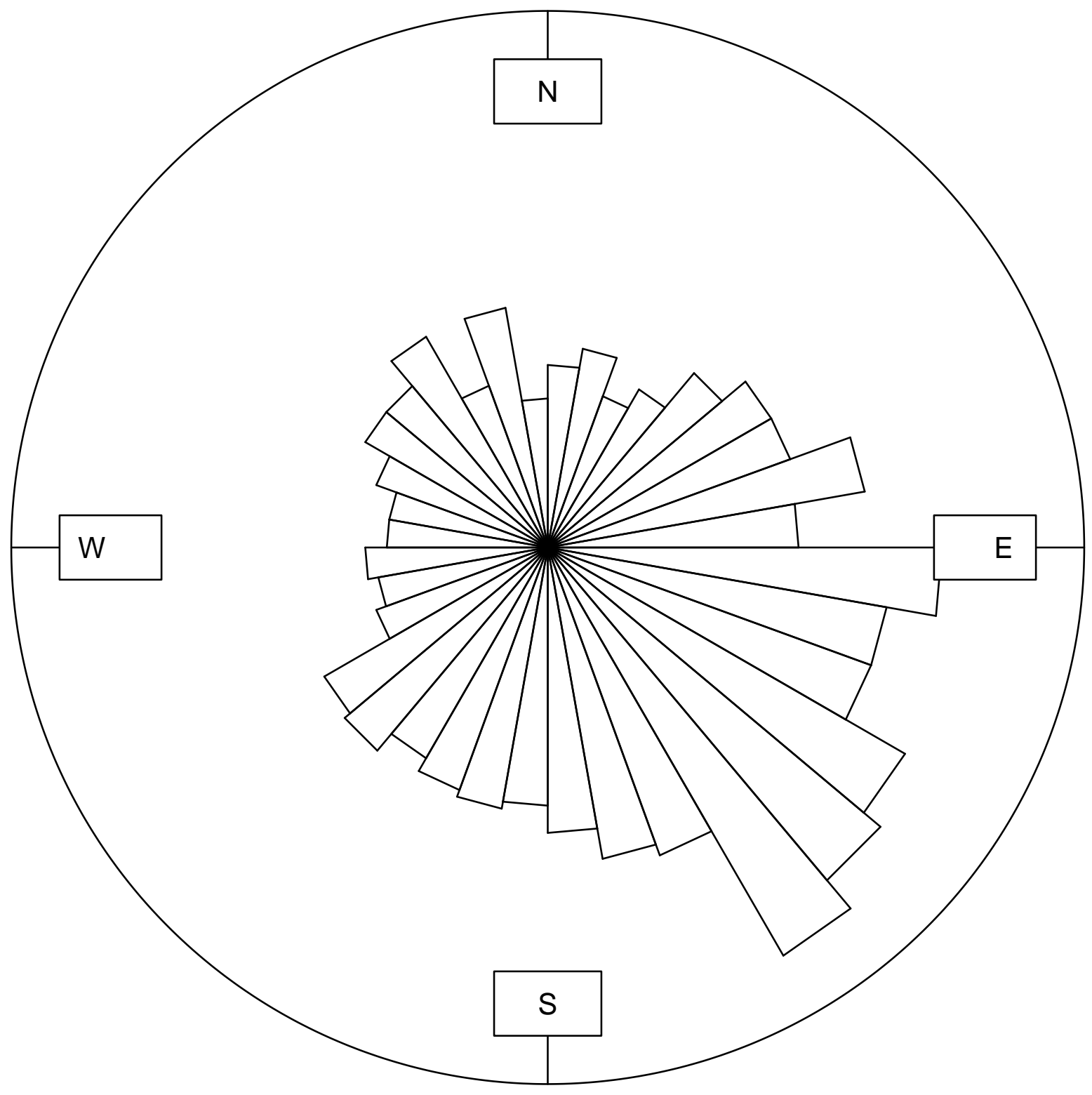


bootstrap 350

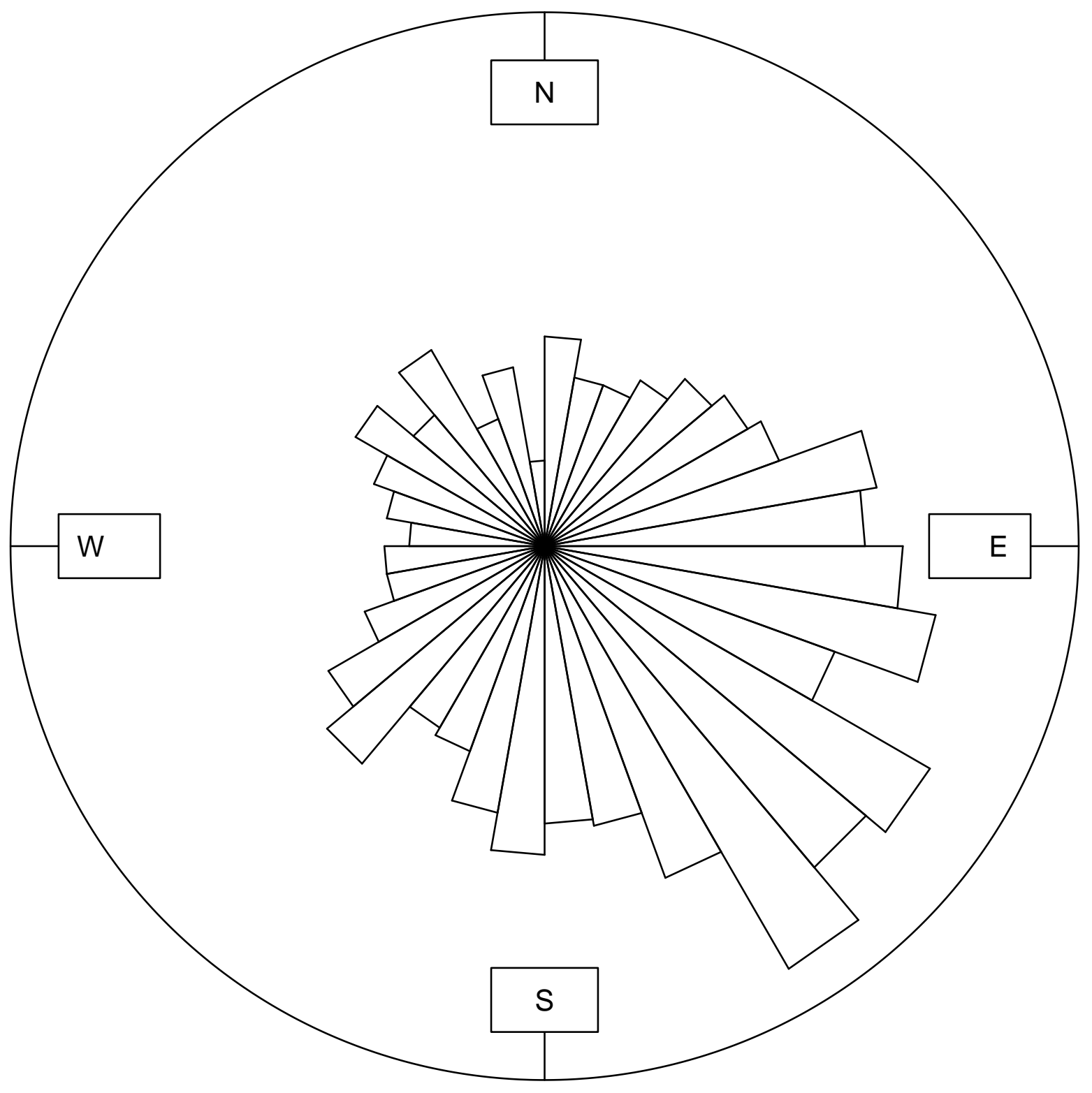




\section{bootstrap 351}

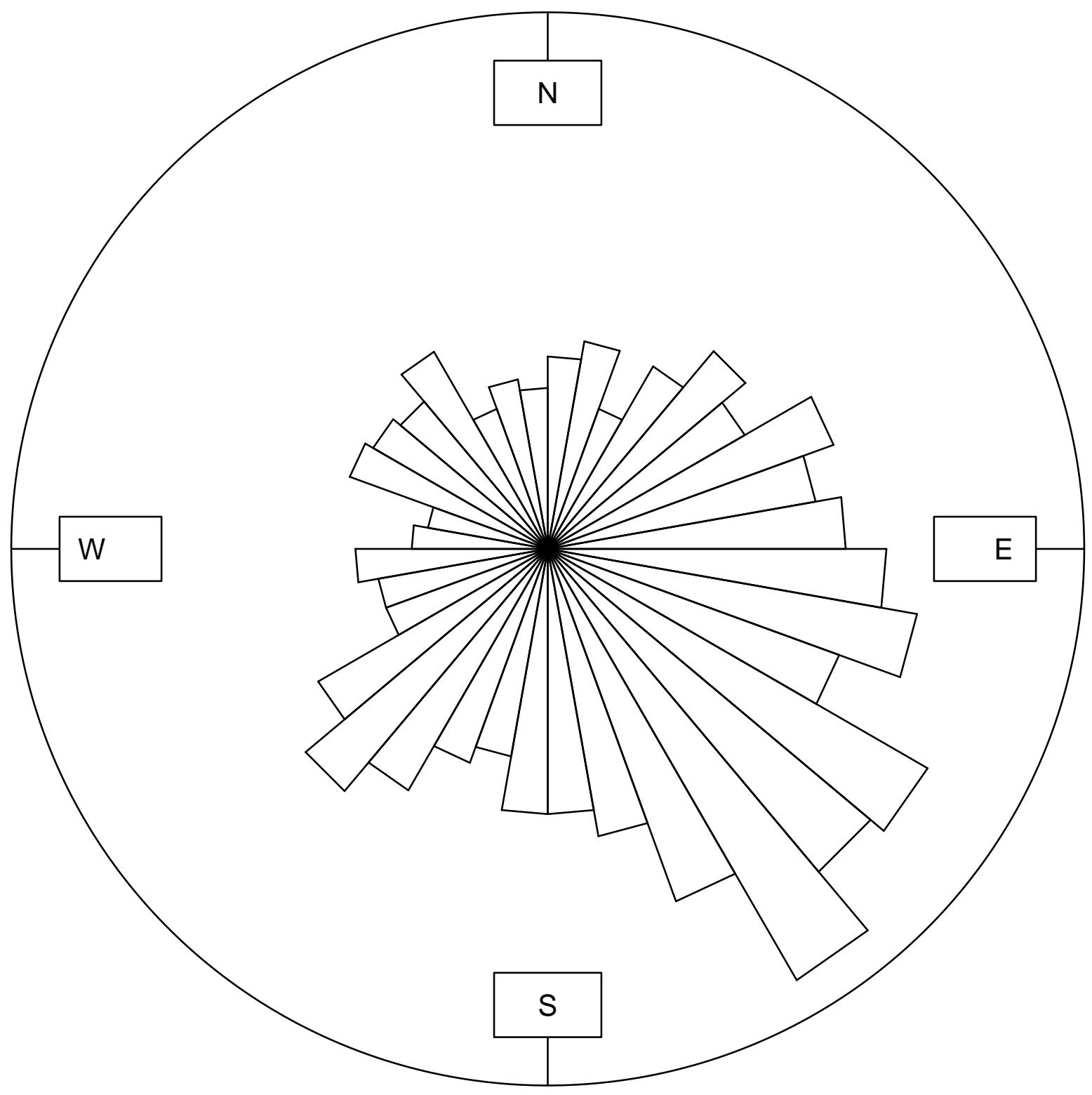


bootstrap 352

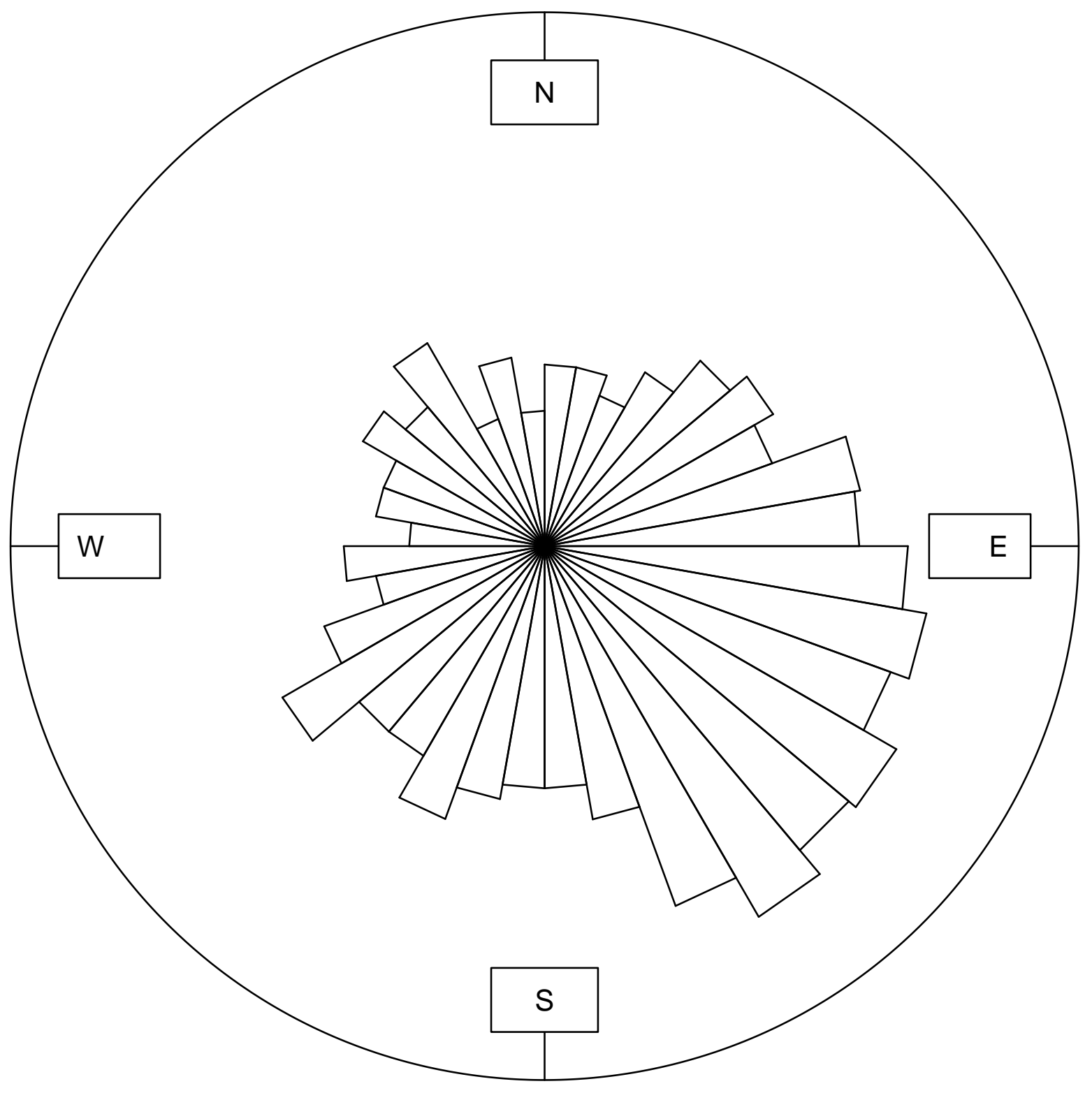




\section{bootstrap 353}

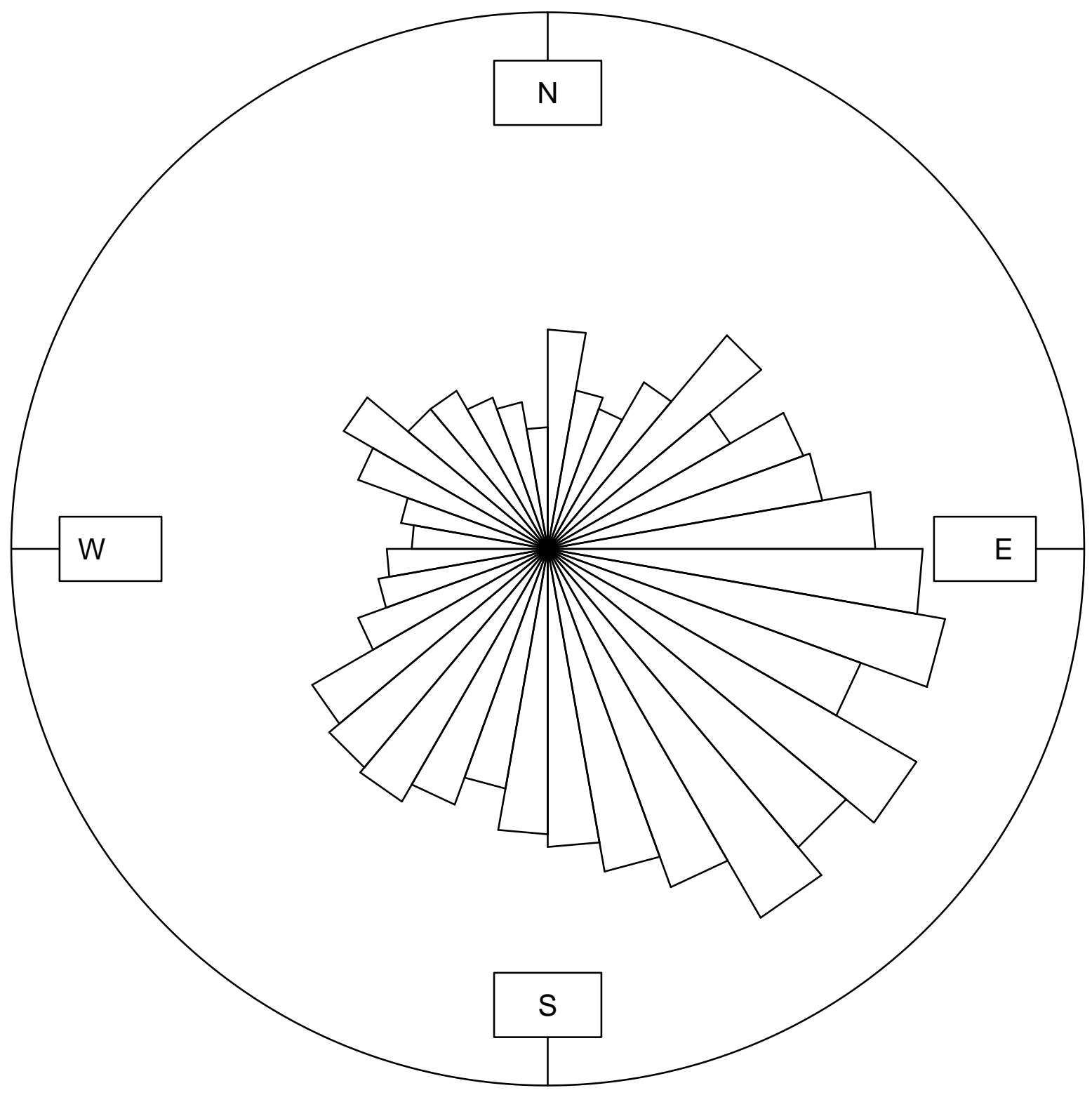


bootstrap 354

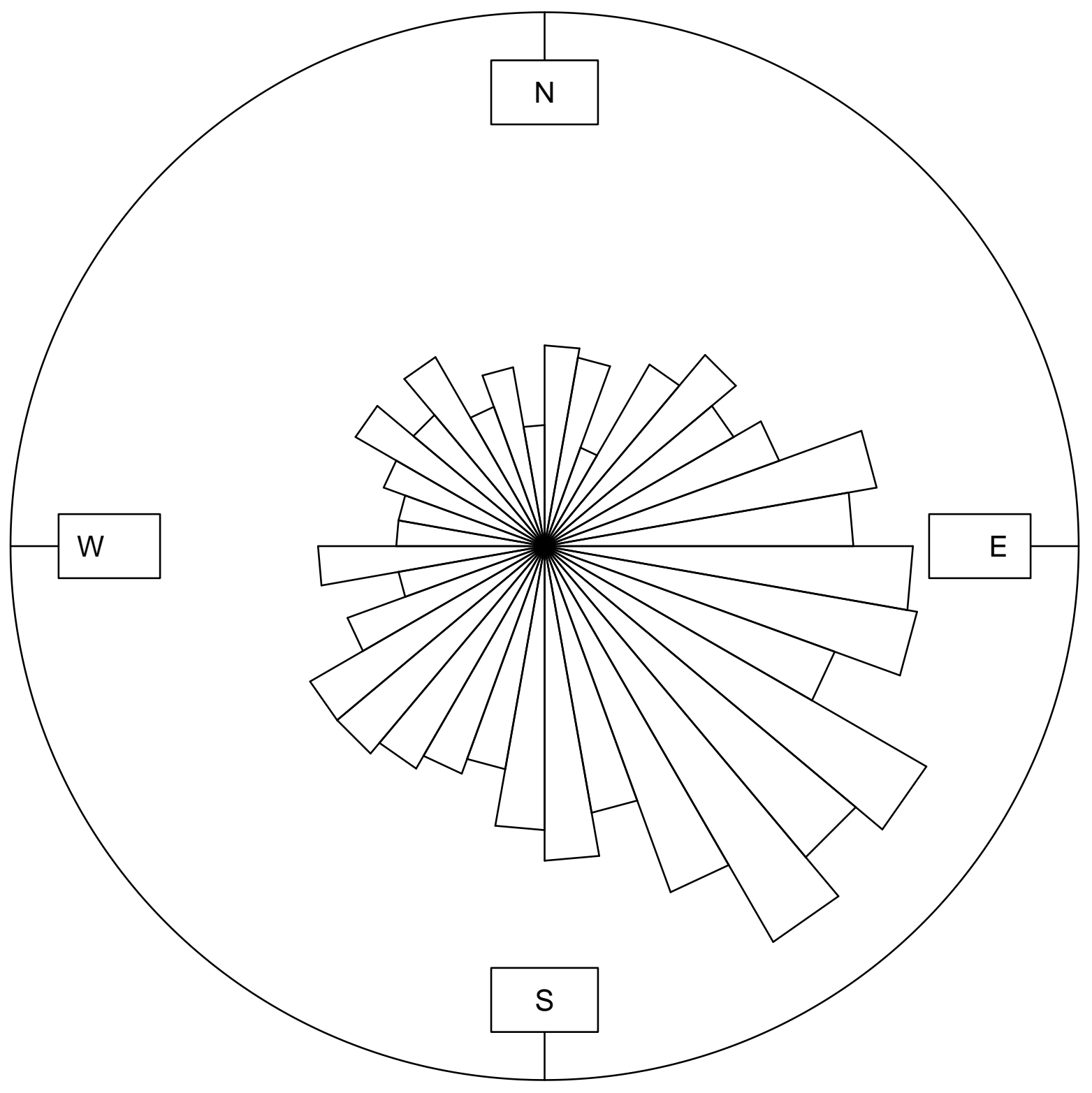




\section{bootstrap 355}

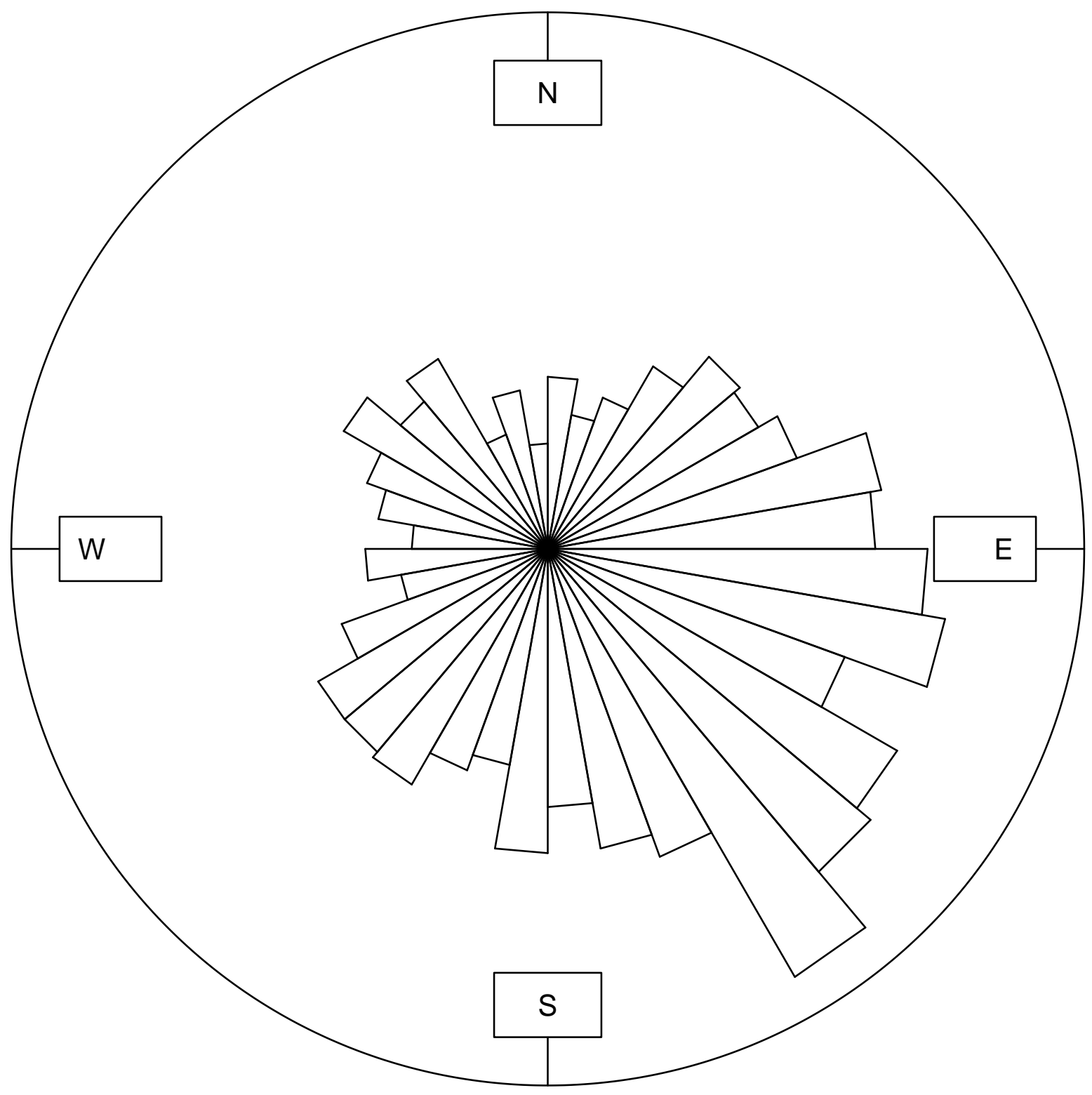




\section{bootstrap 356}

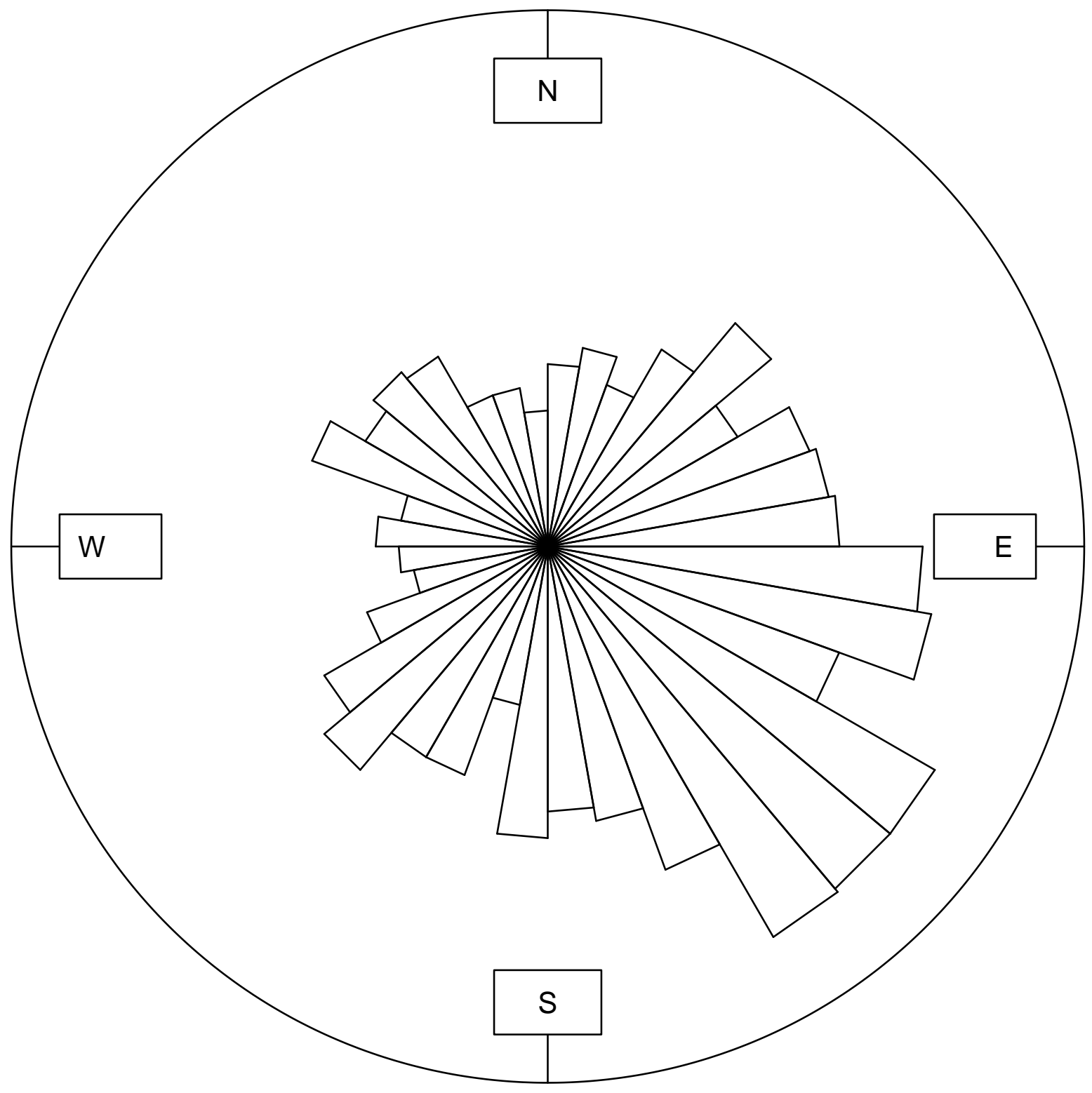


bootstrap 357

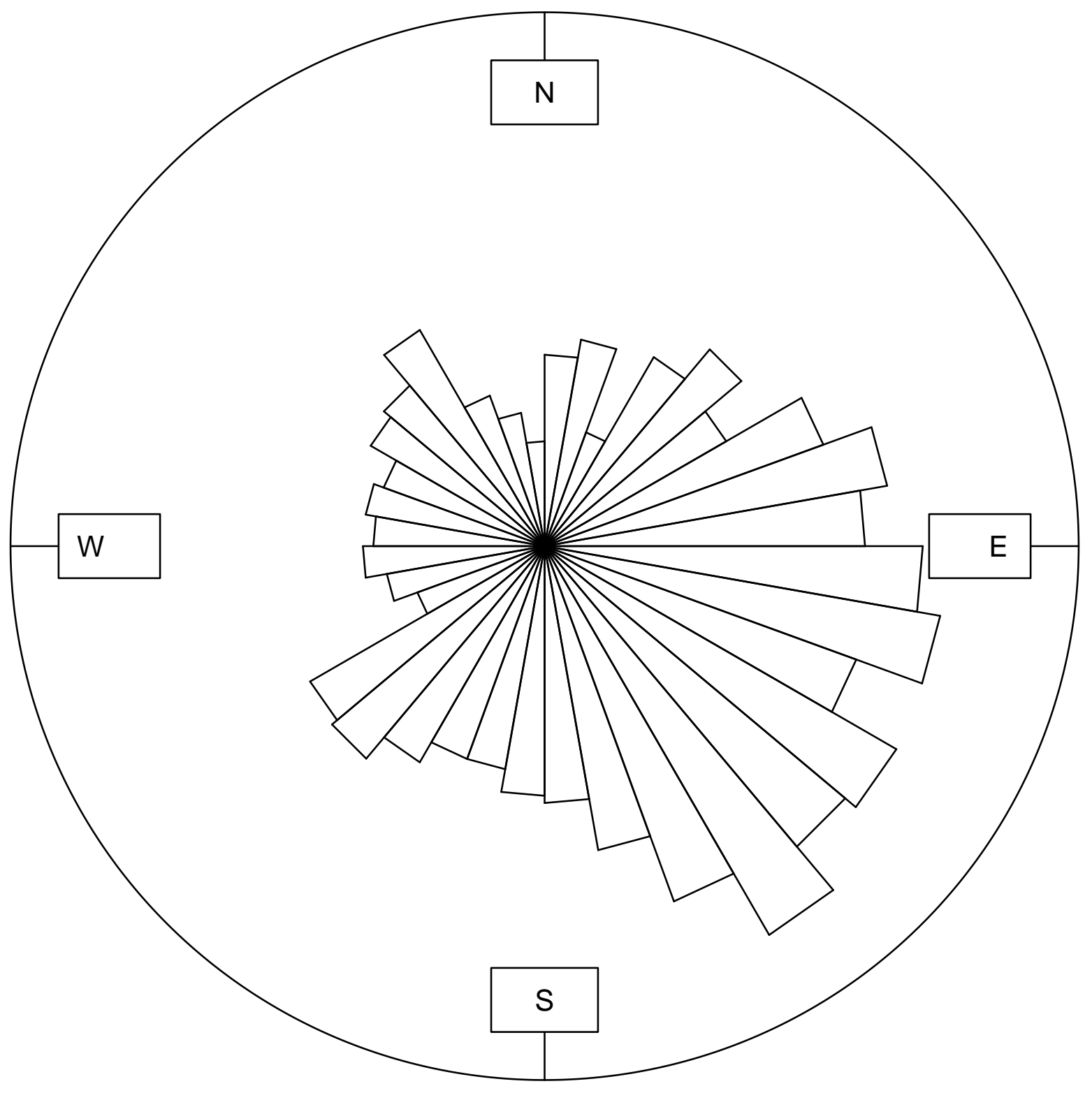


bootstrap 358

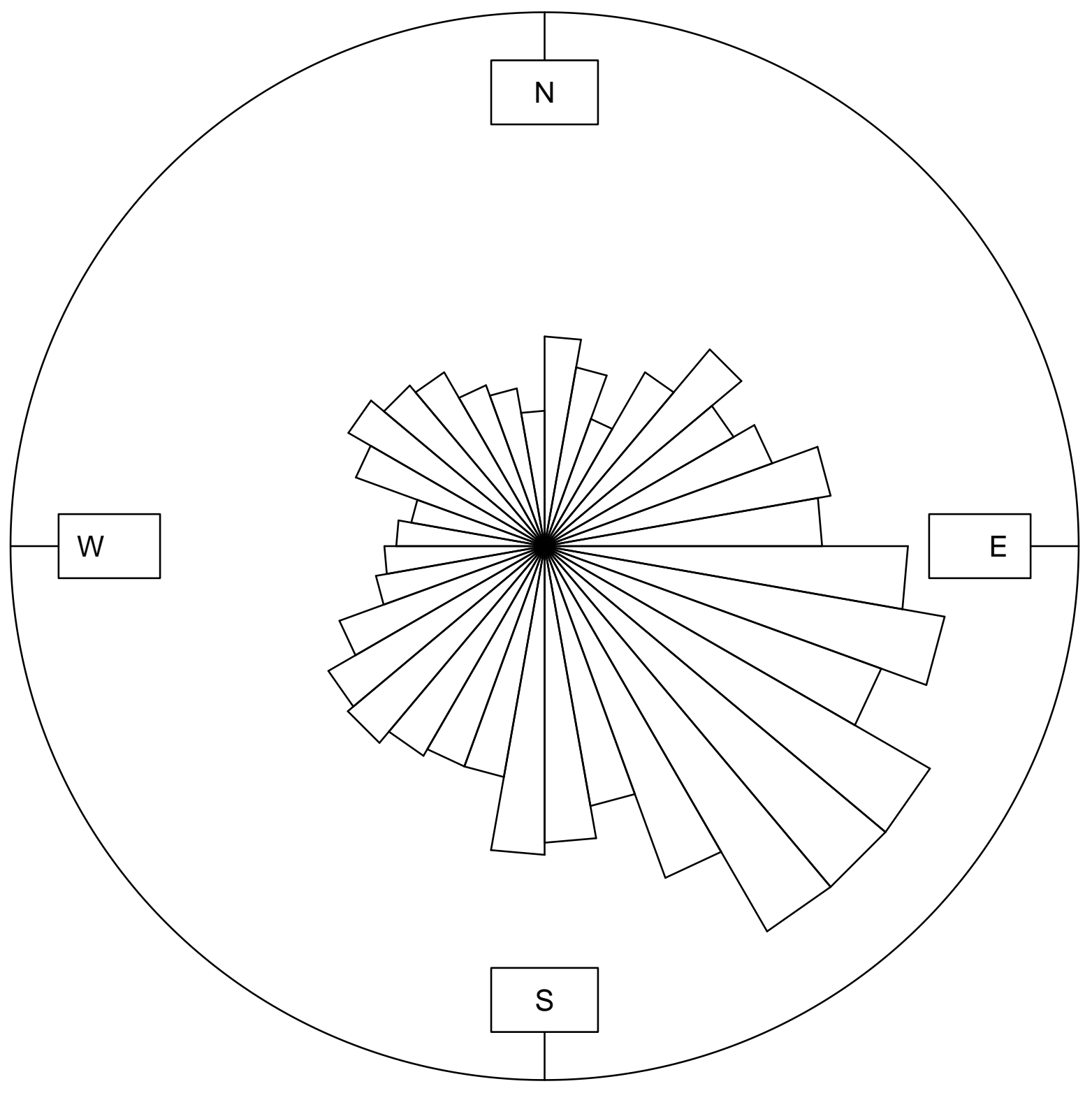


bootstrap 359

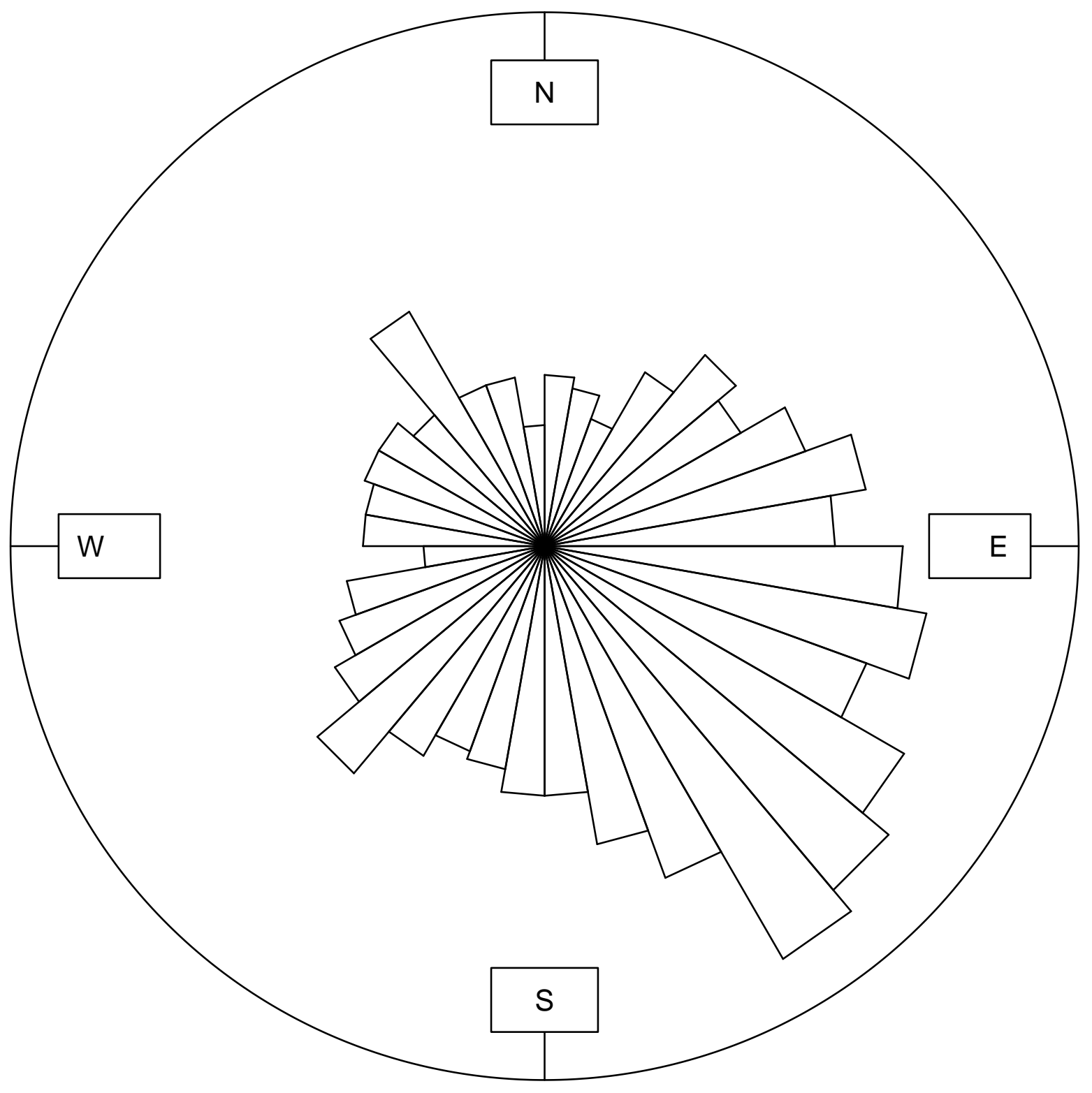


bootstrap 360

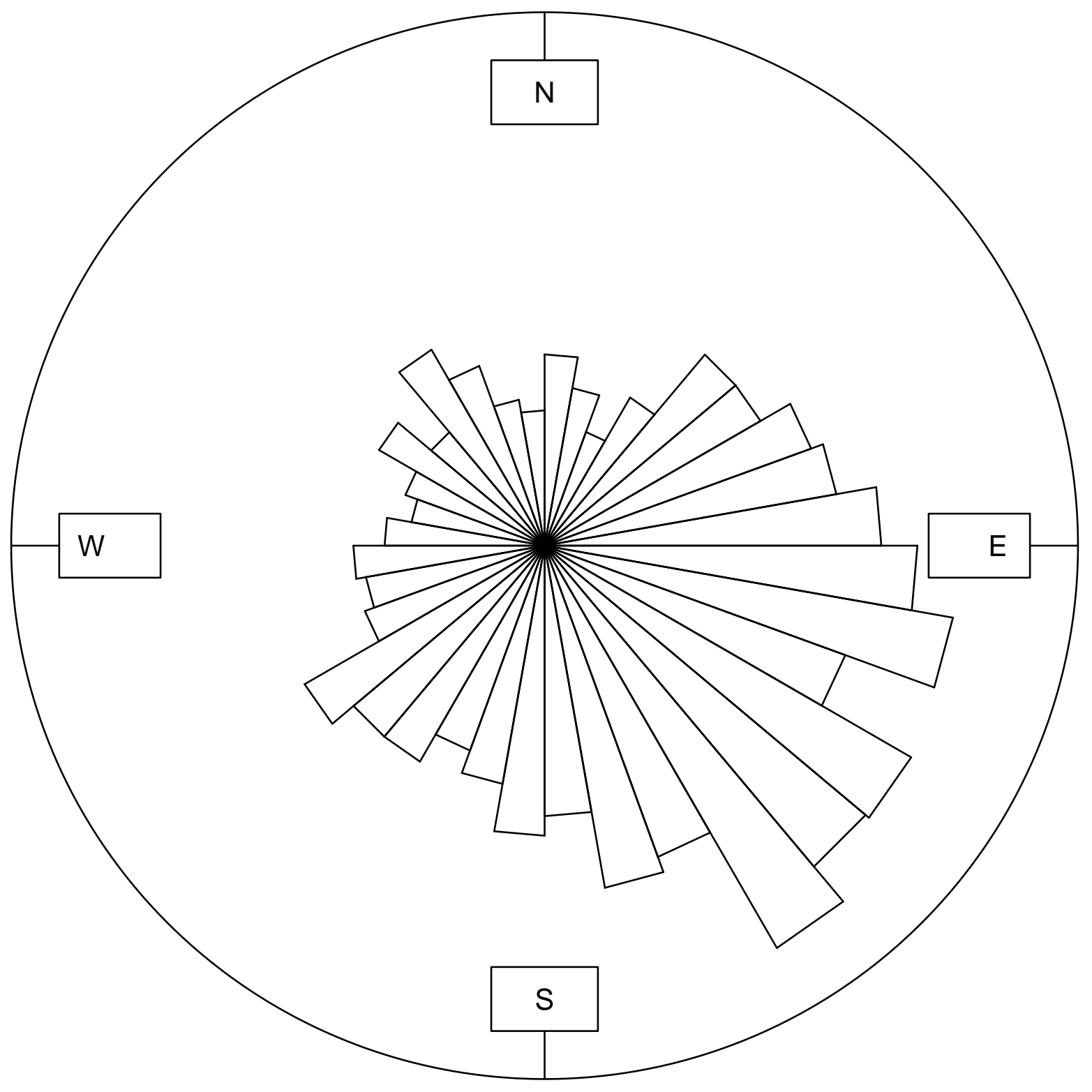


bootstrap 361

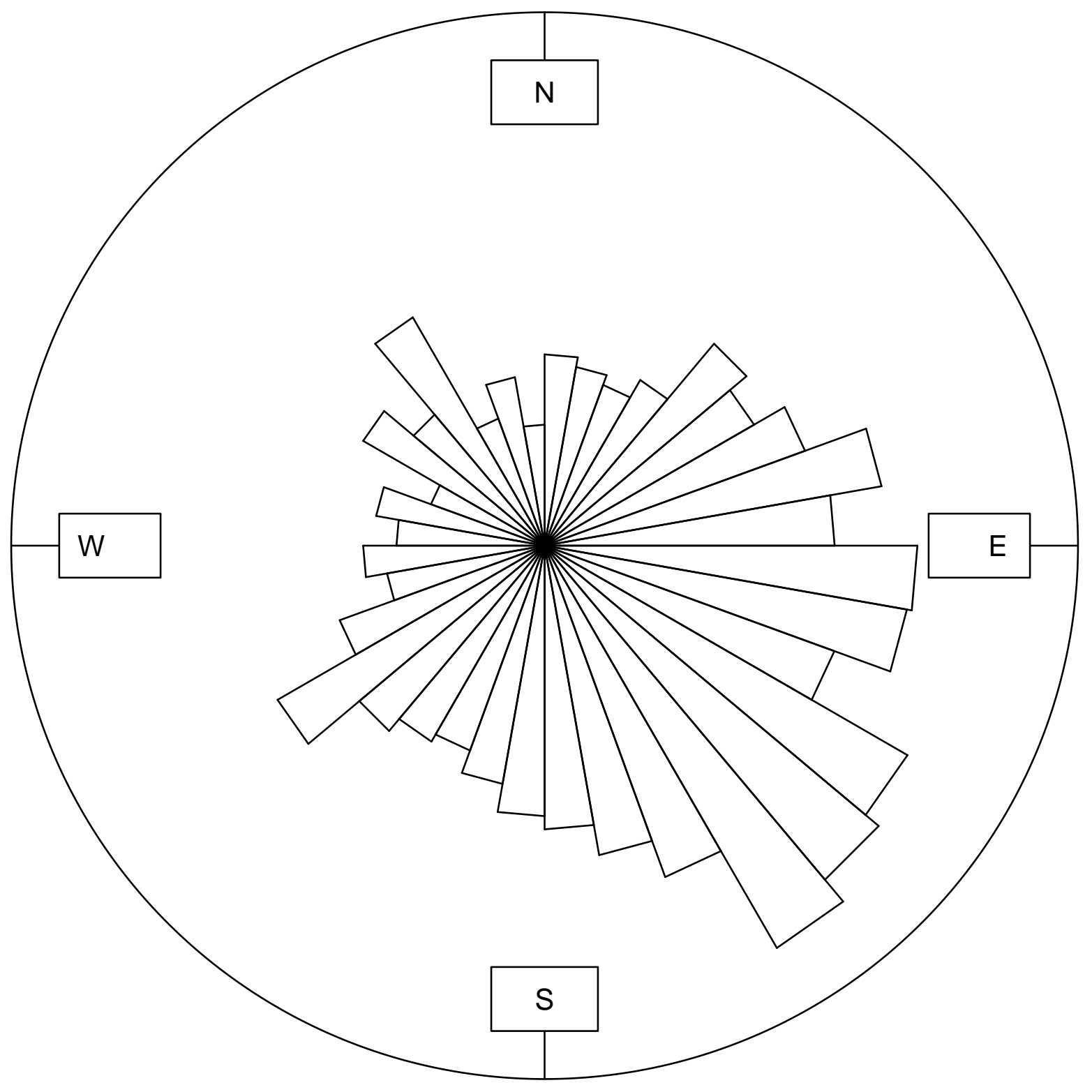


bootstrap 362

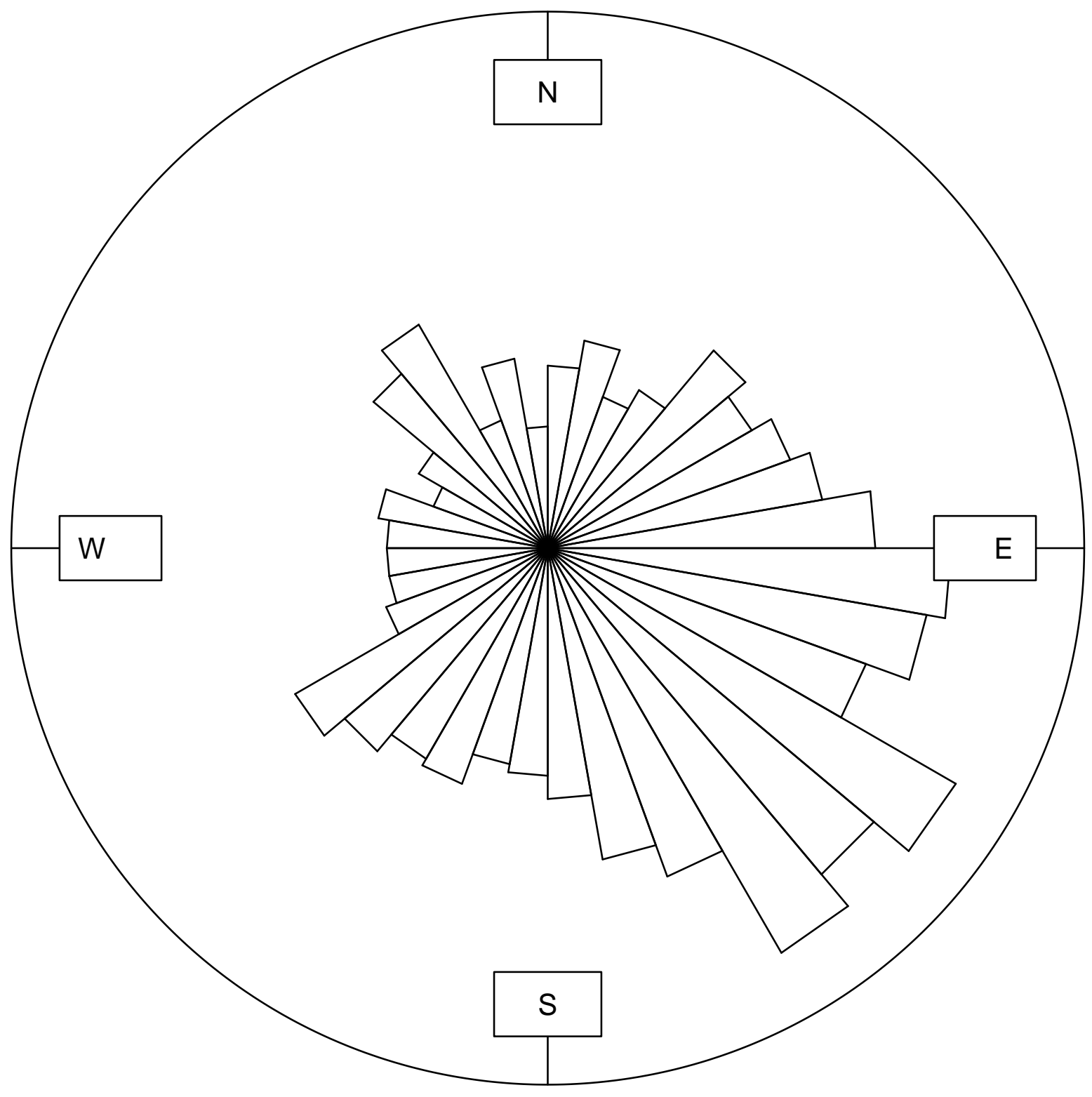


bootstrap 363

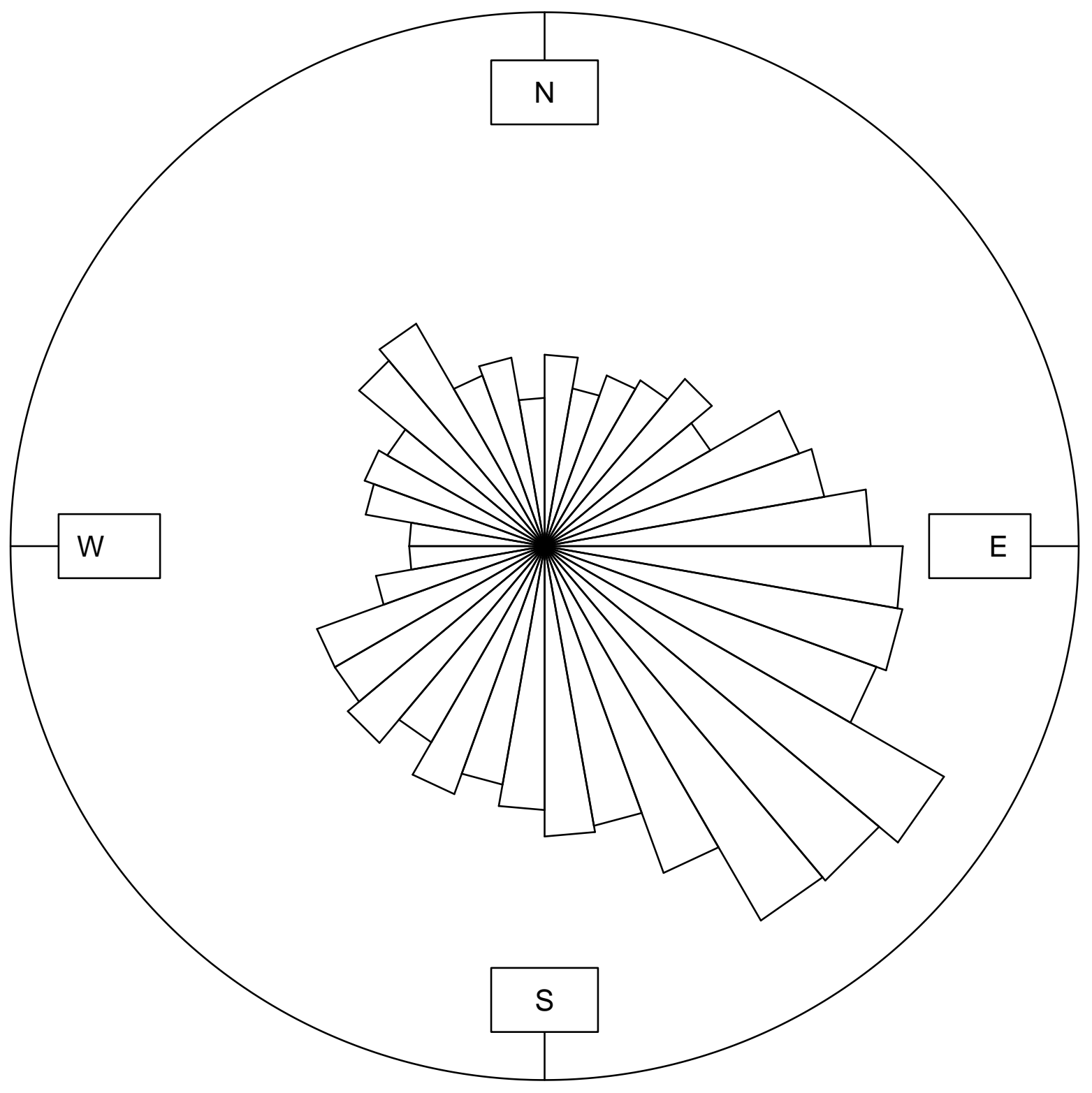


bootstrap 364

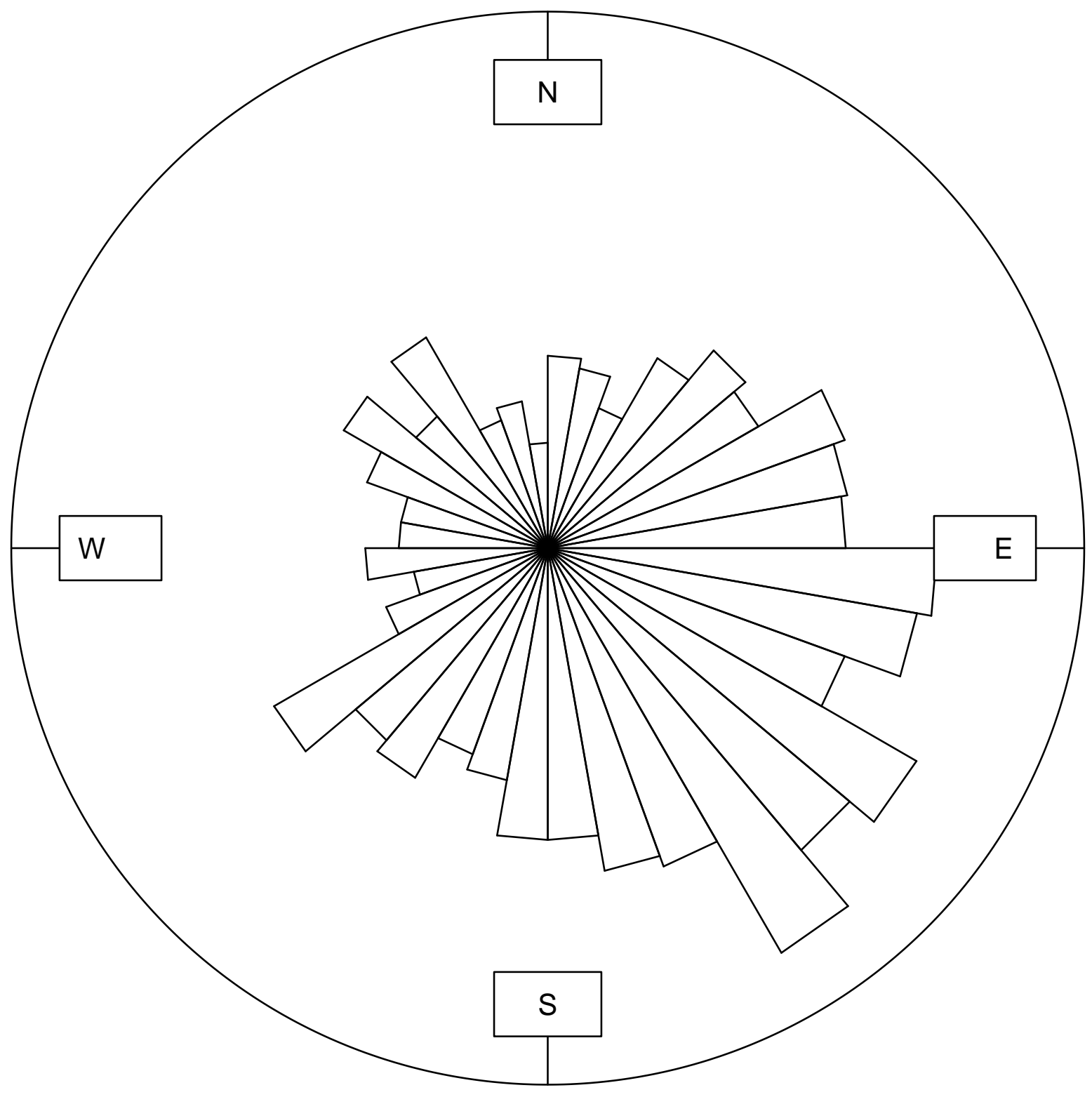


bootstrap 365

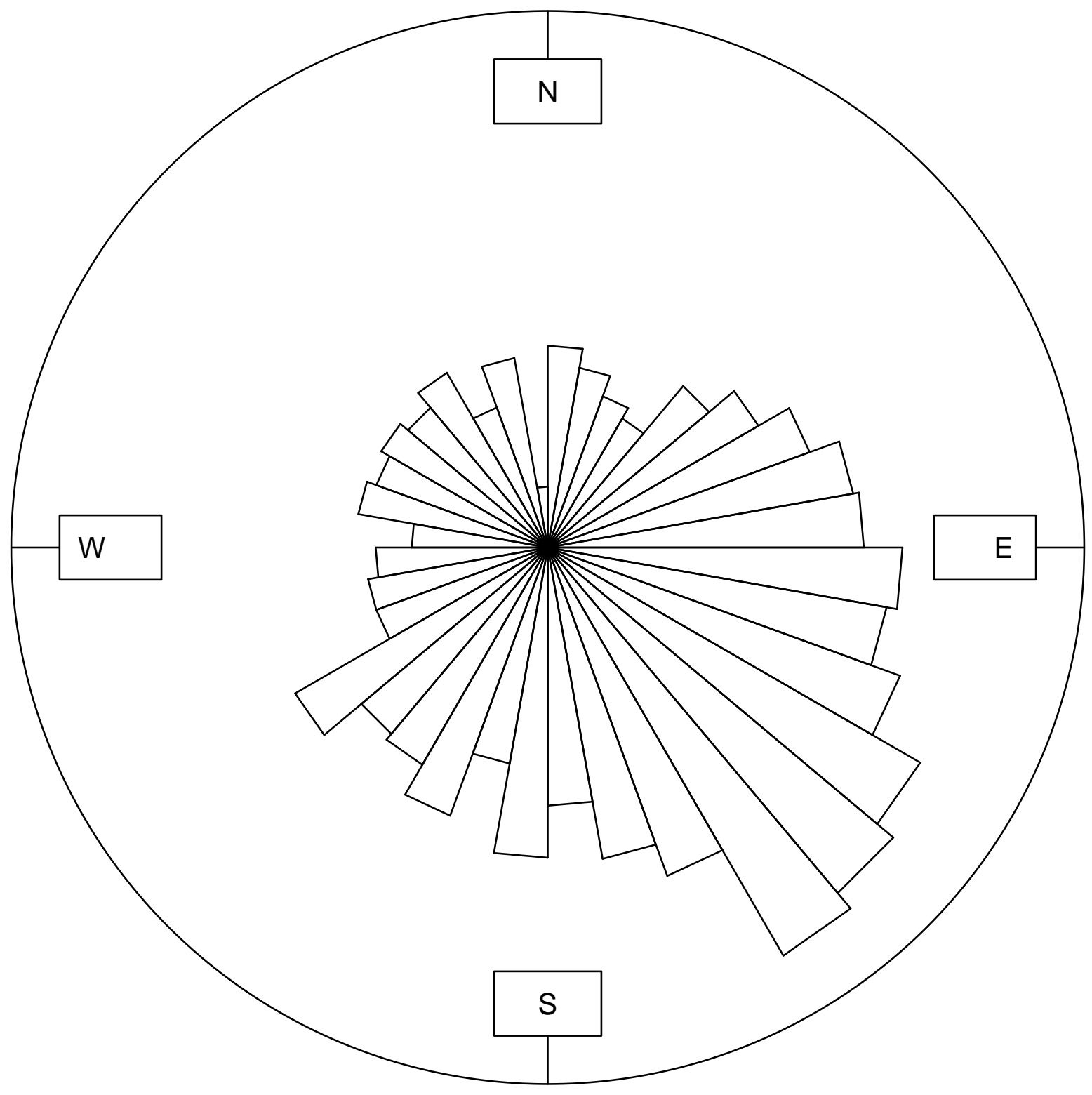


bootstrap 366

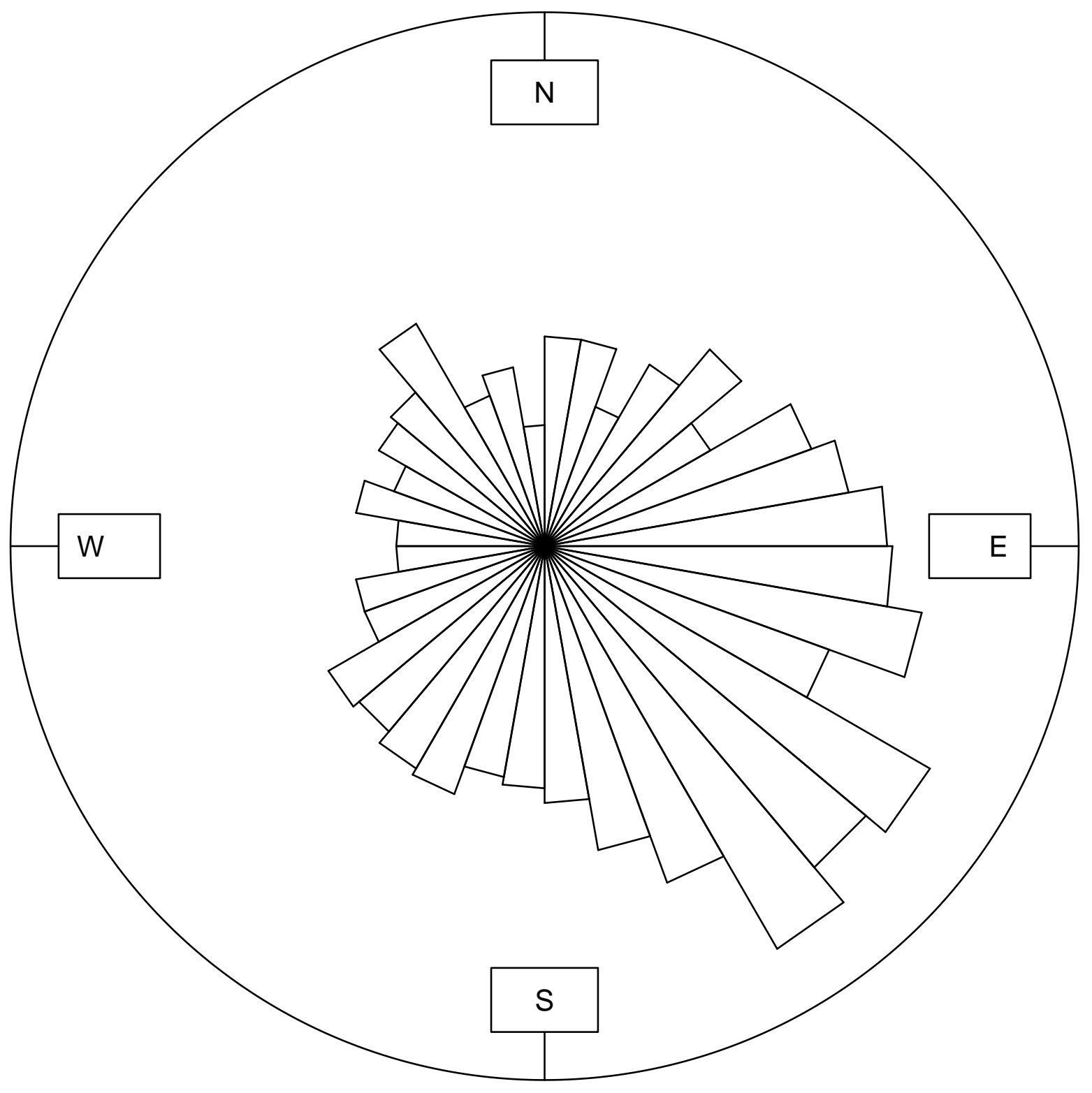


bootstrap 367

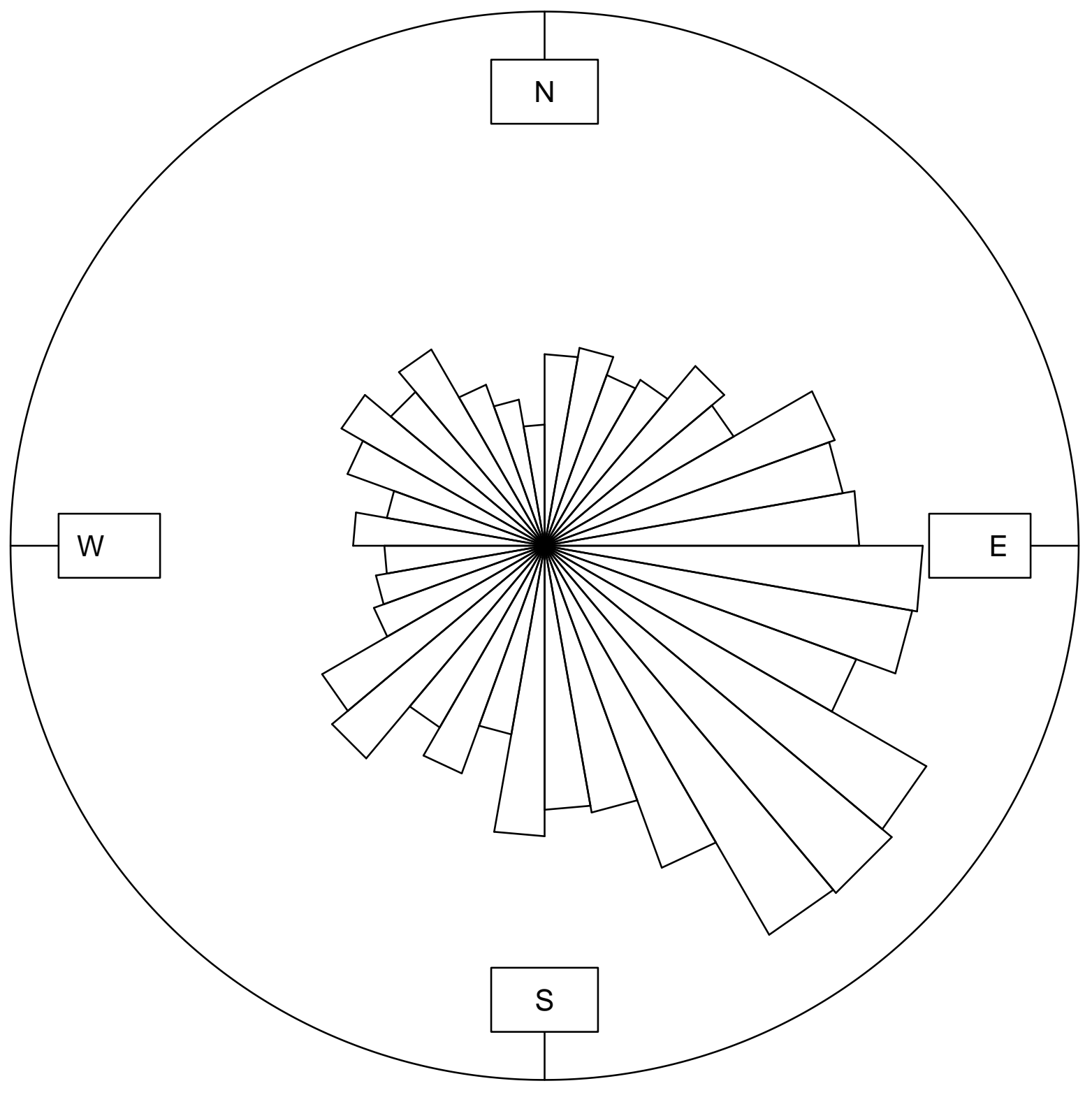


bootstrap 368

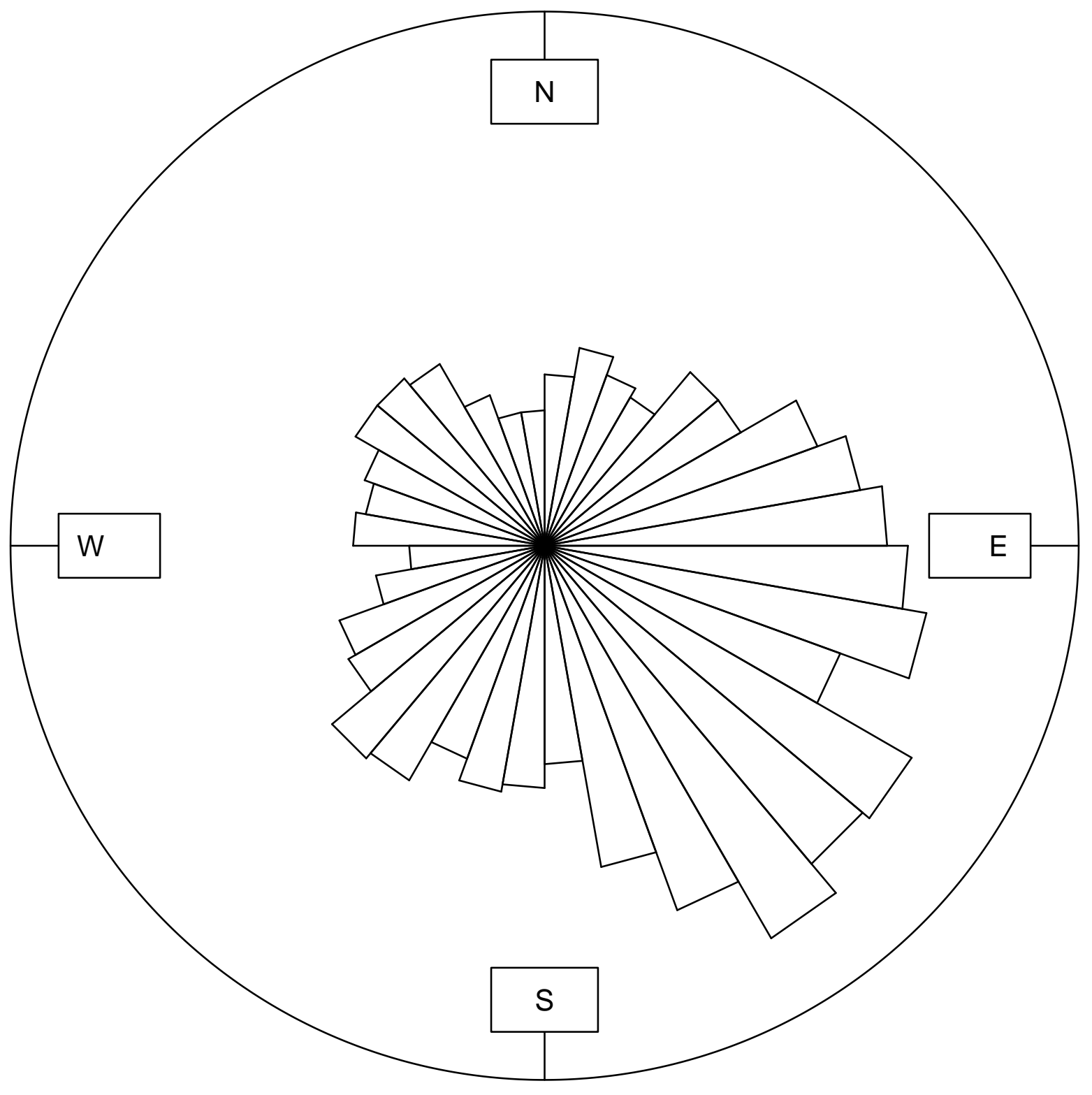


bootstrap 369

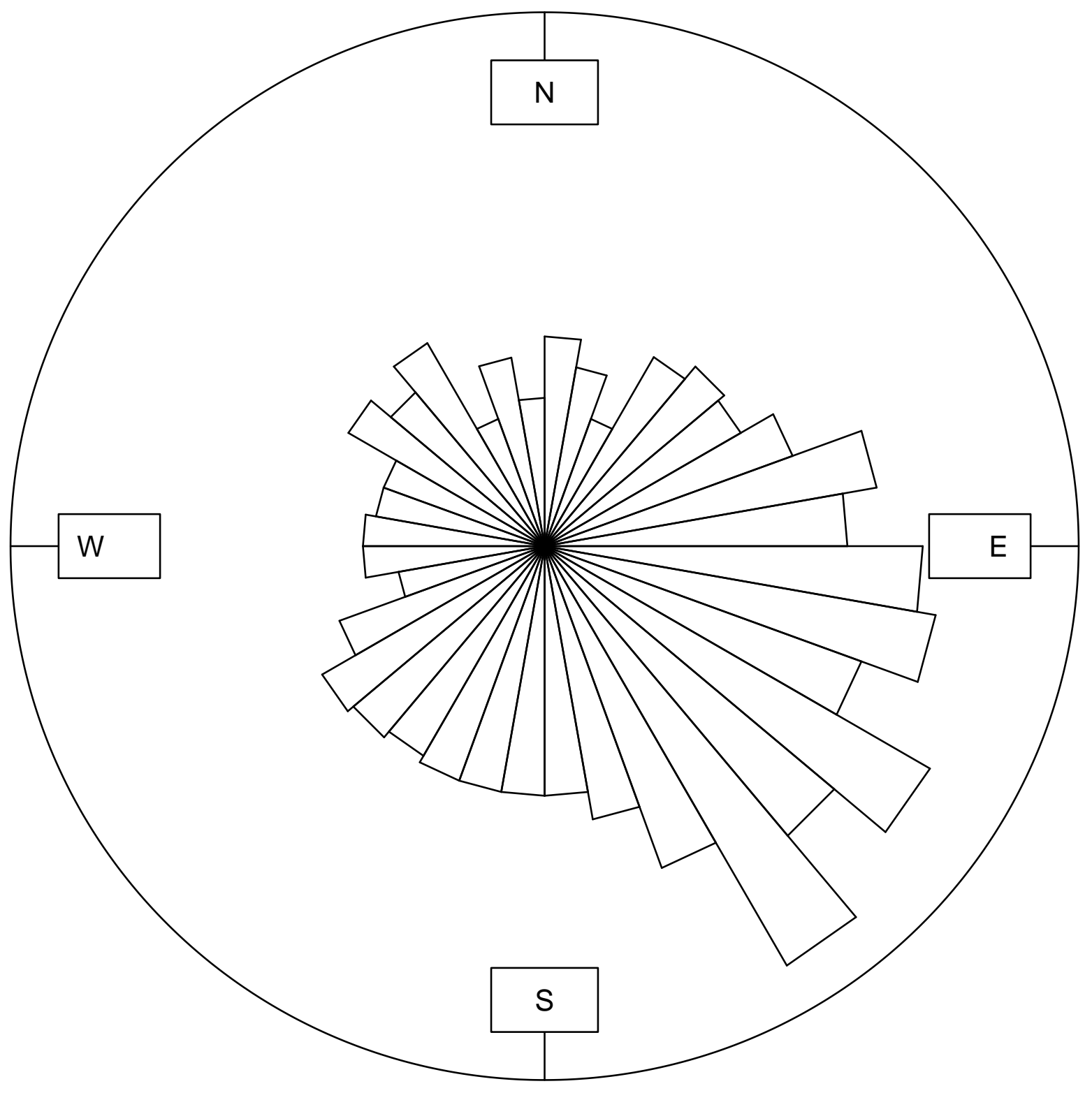


bootstrap 370

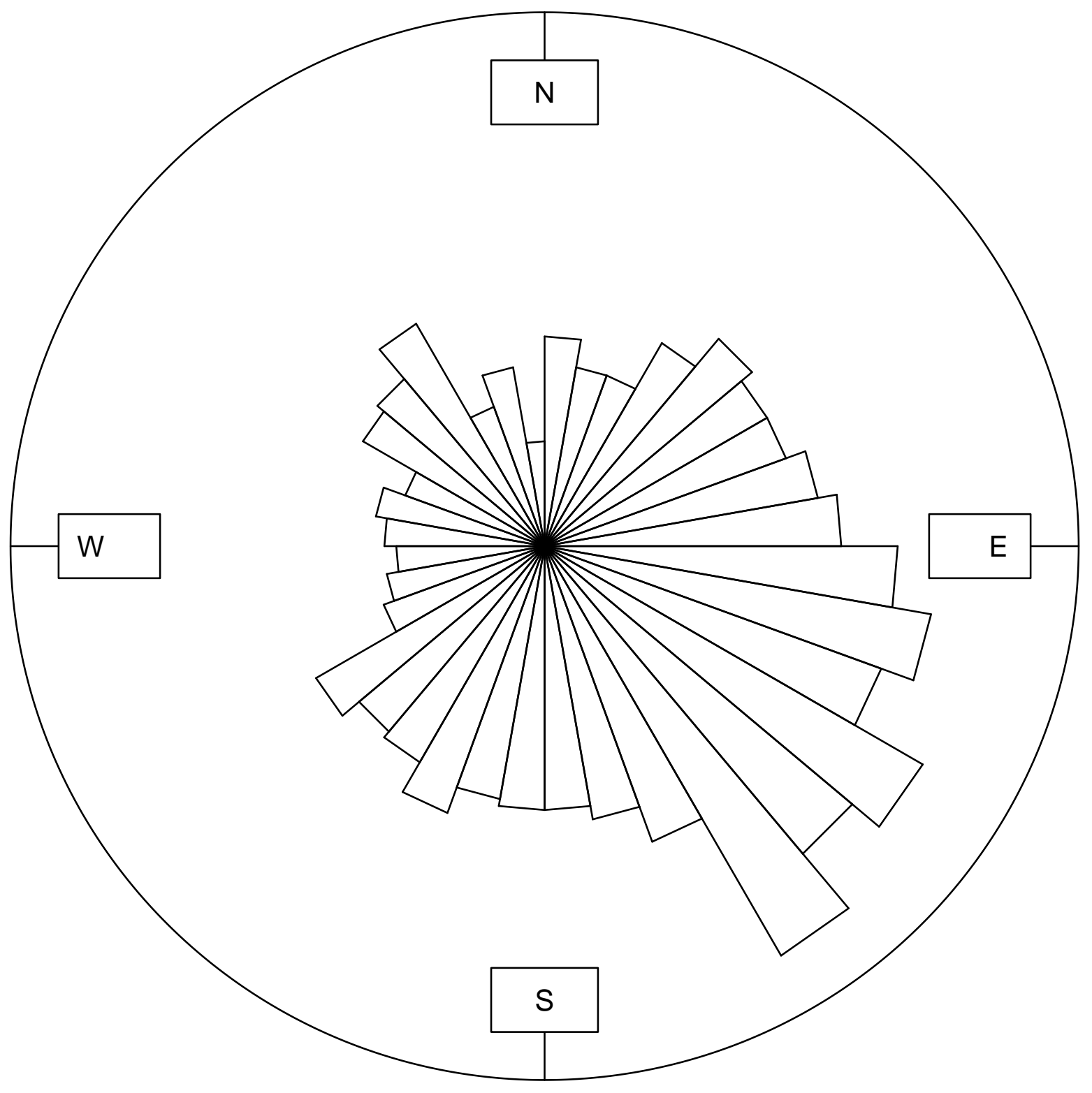


bootstrap 371

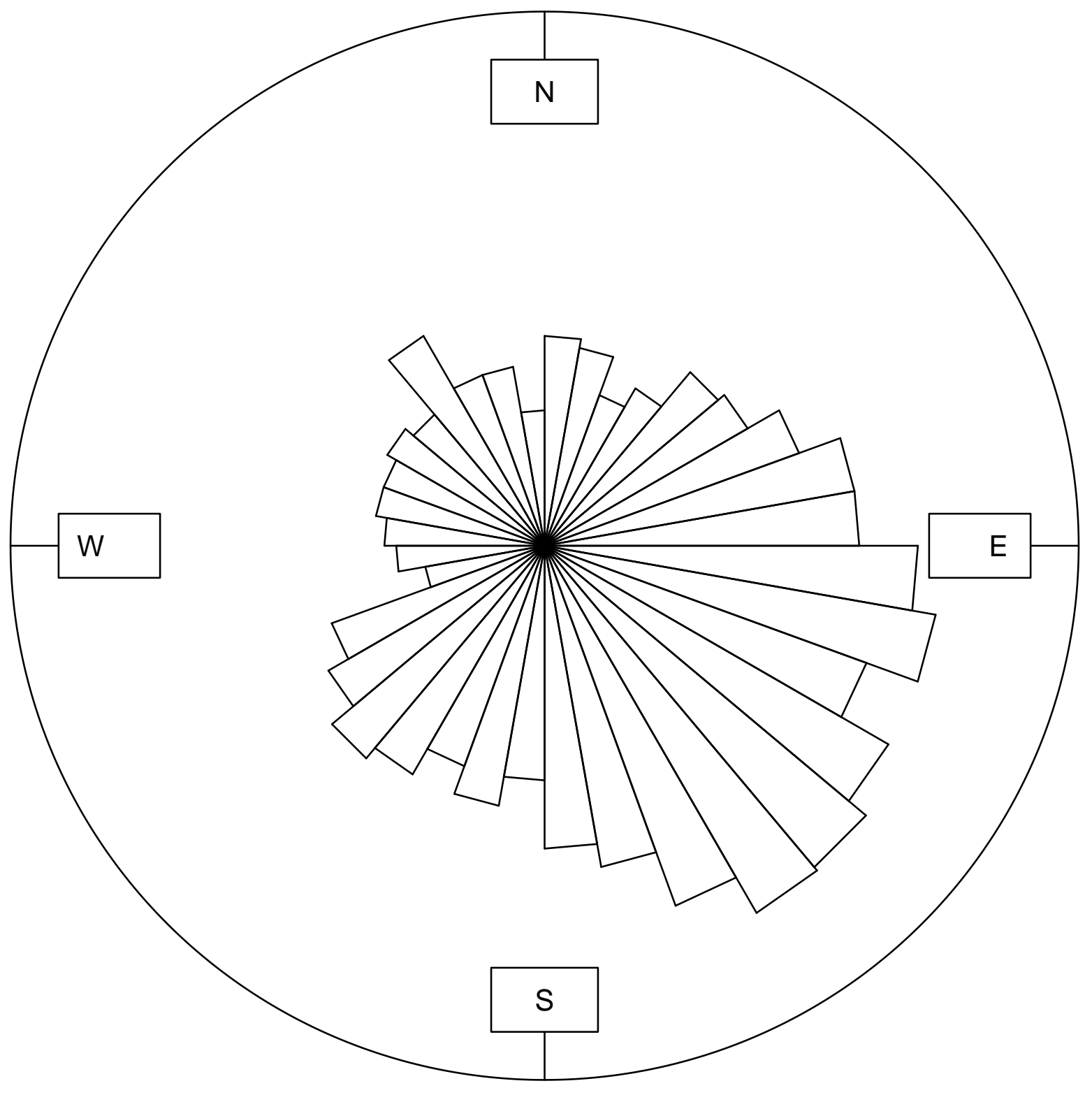


bootstrap 372

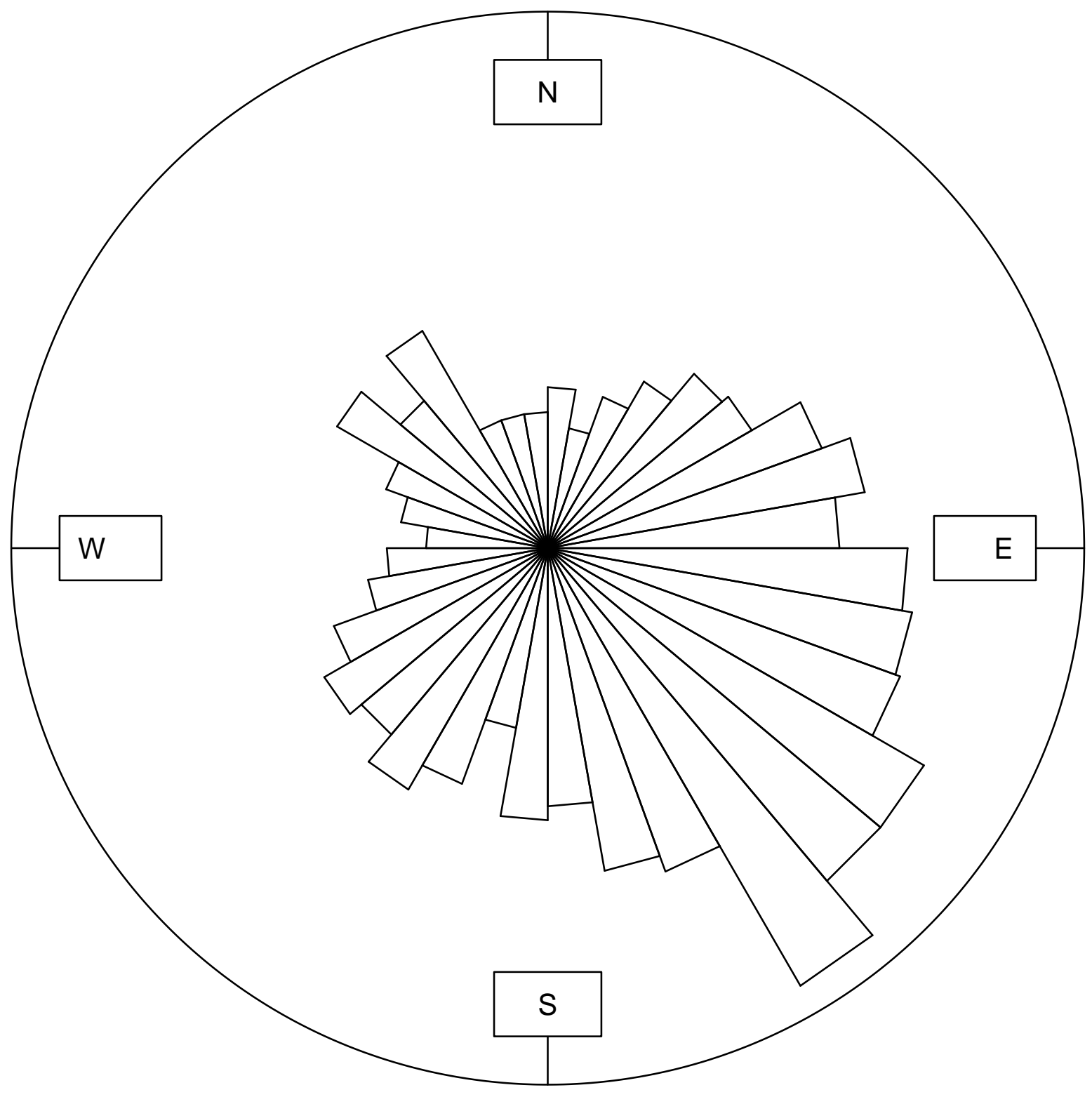


bootstrap 373

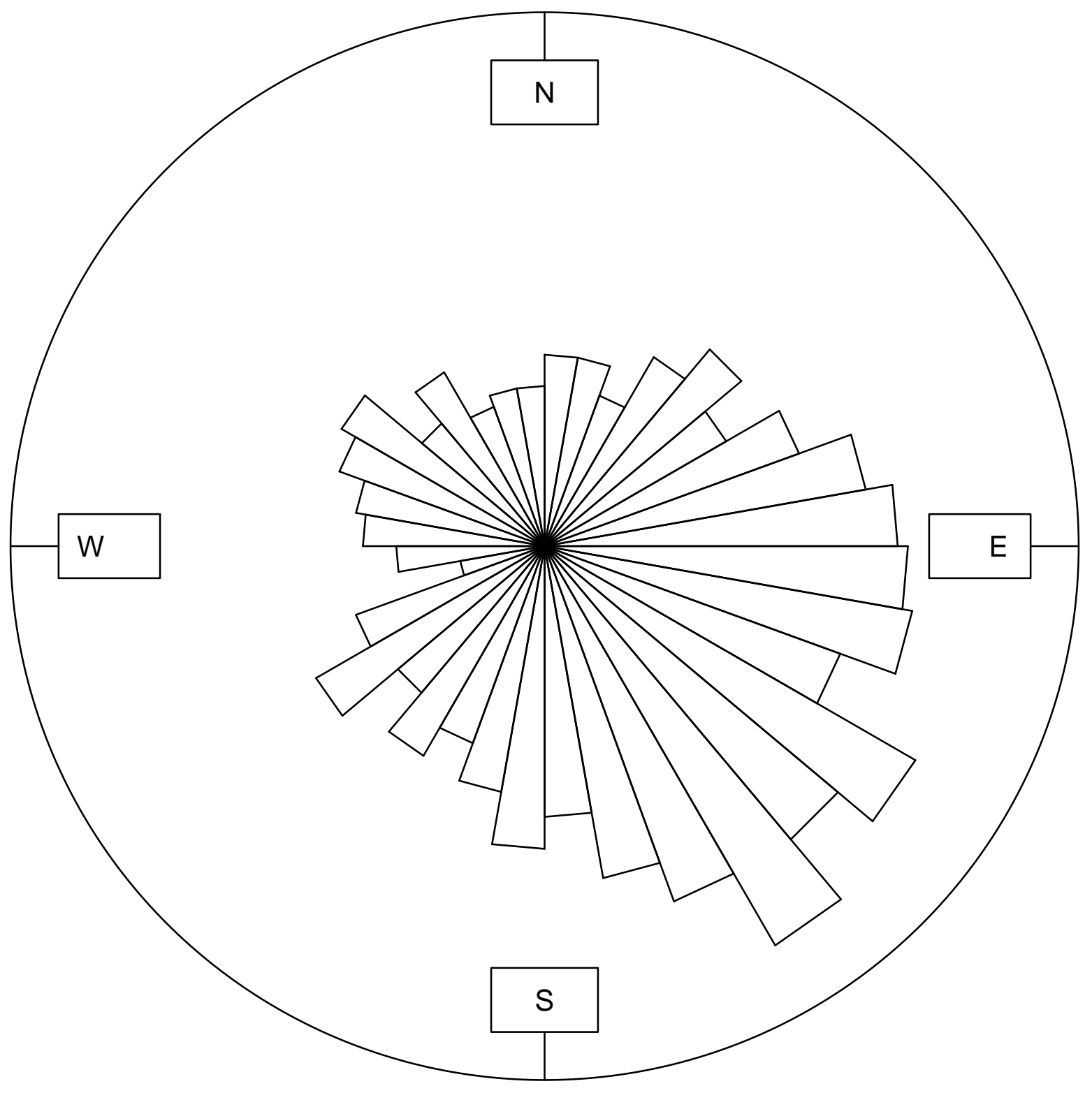


bootstrap 374

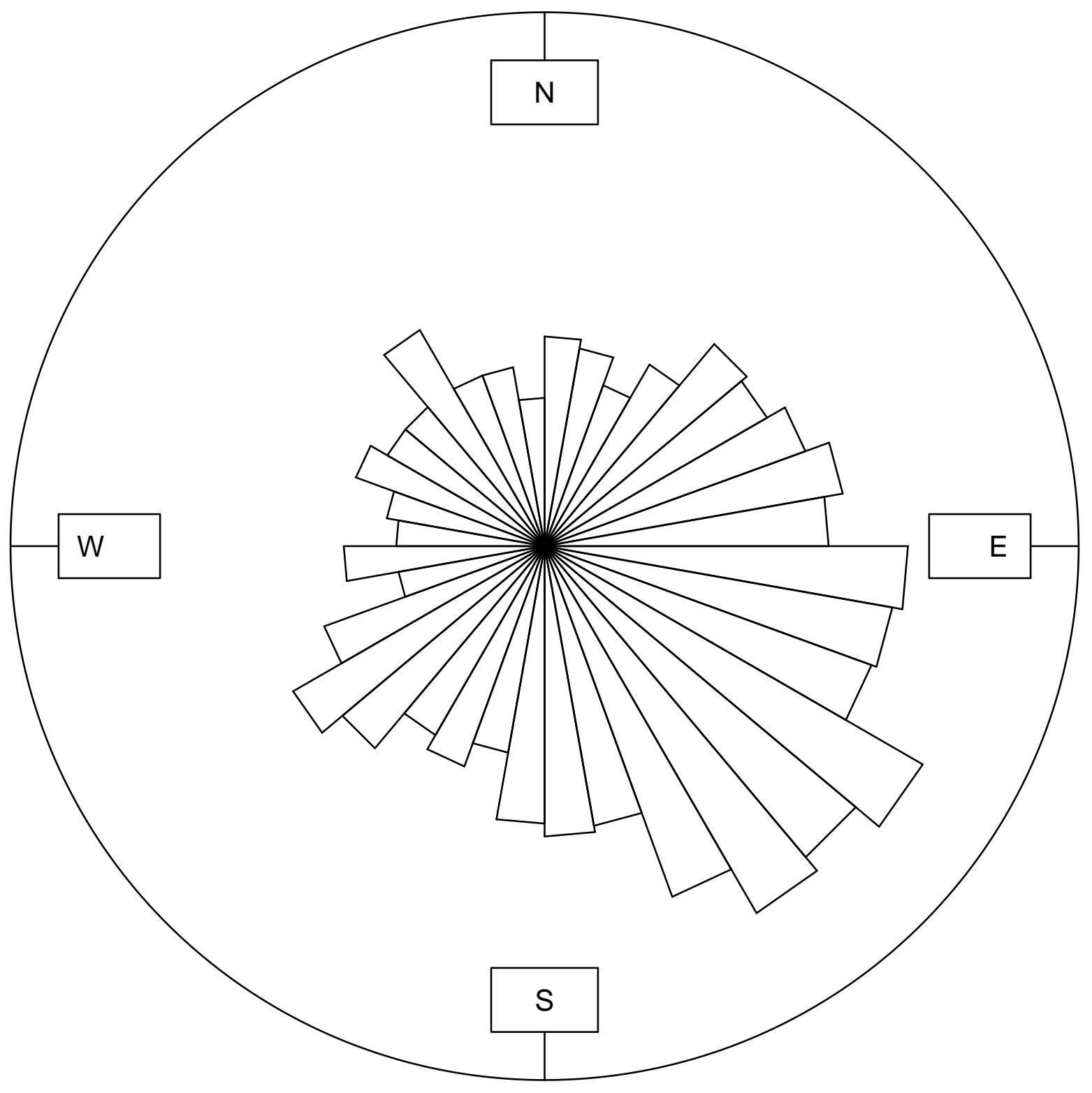


bootstrap 375

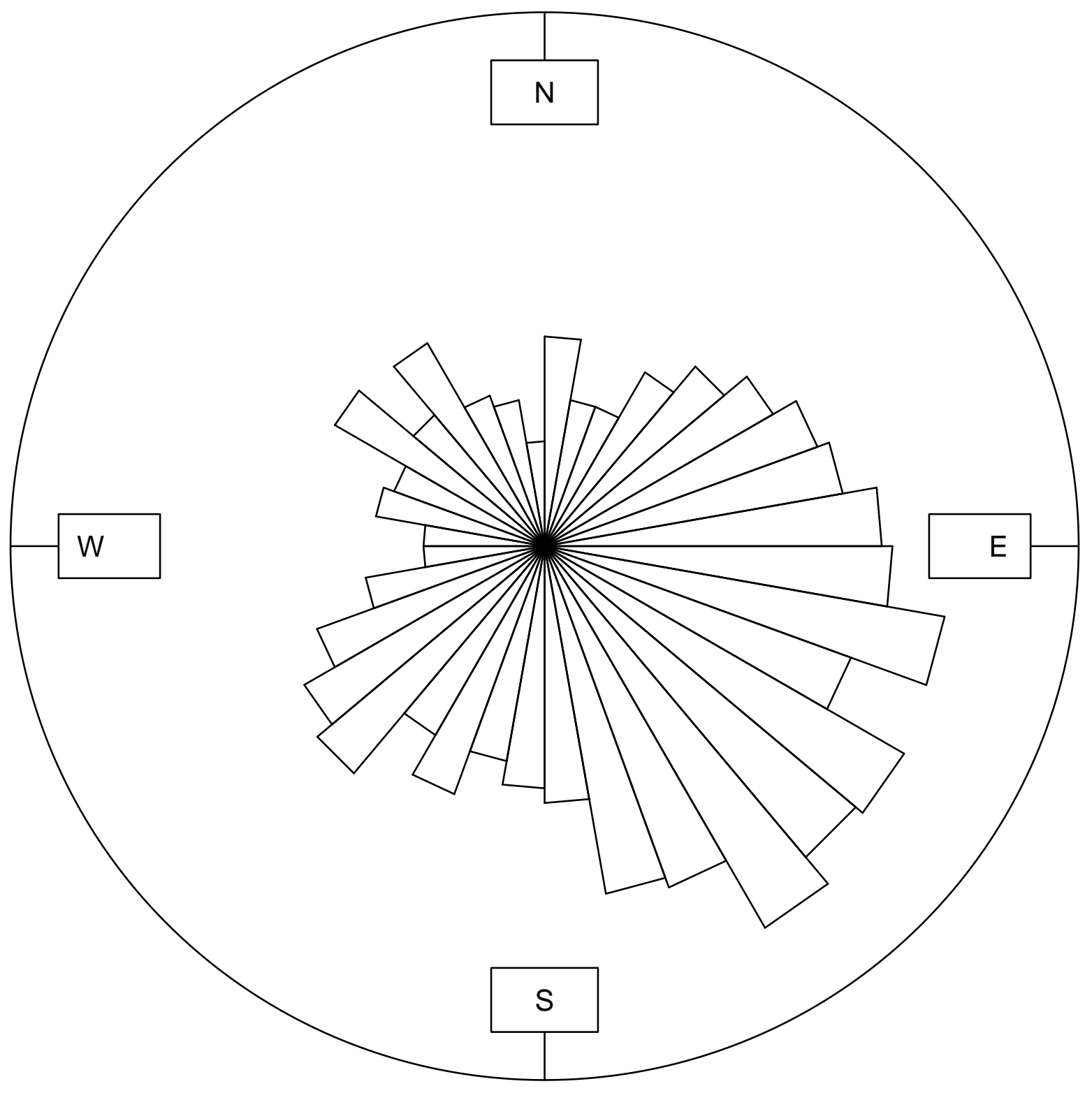


bootstrap 376

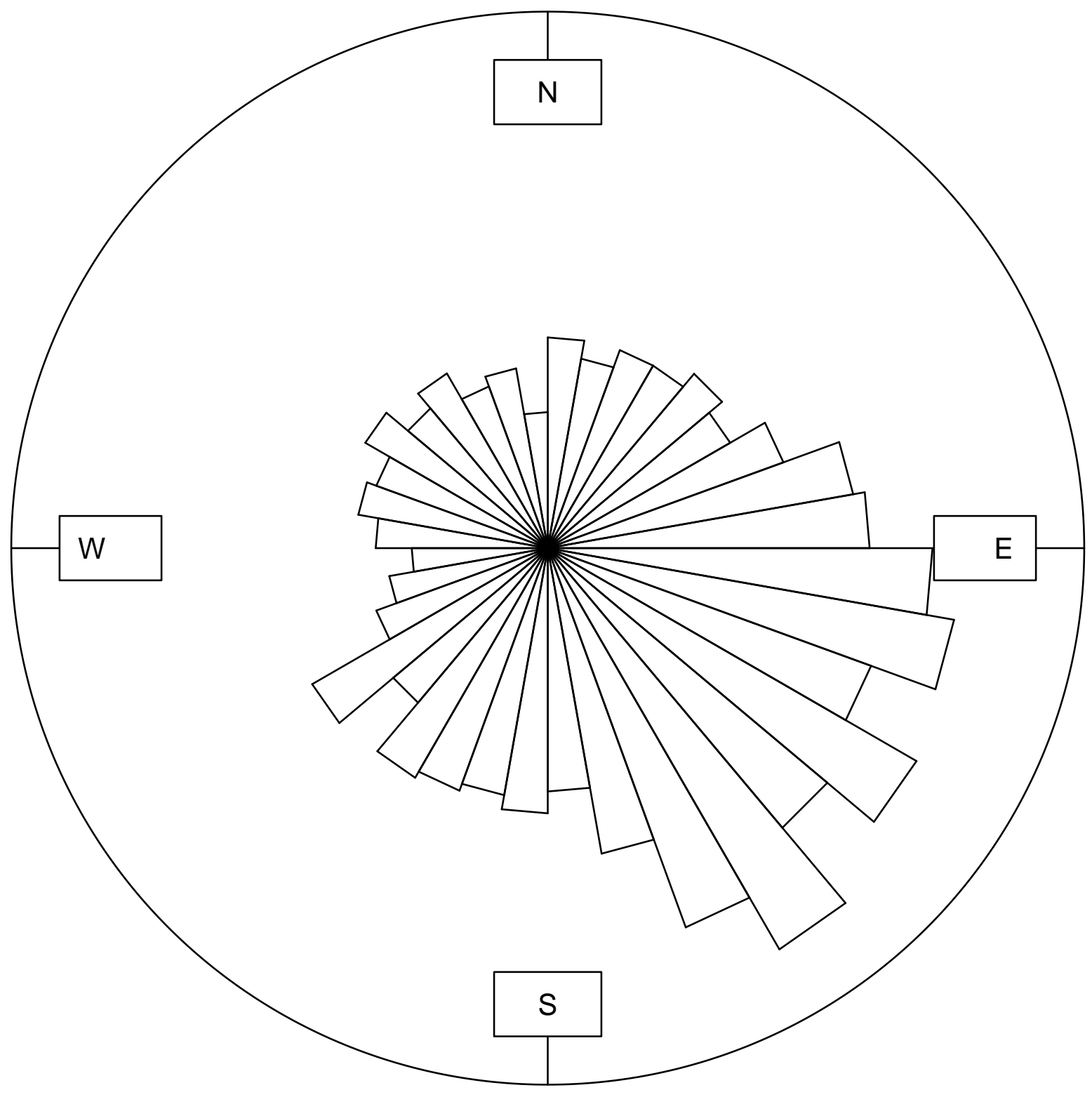




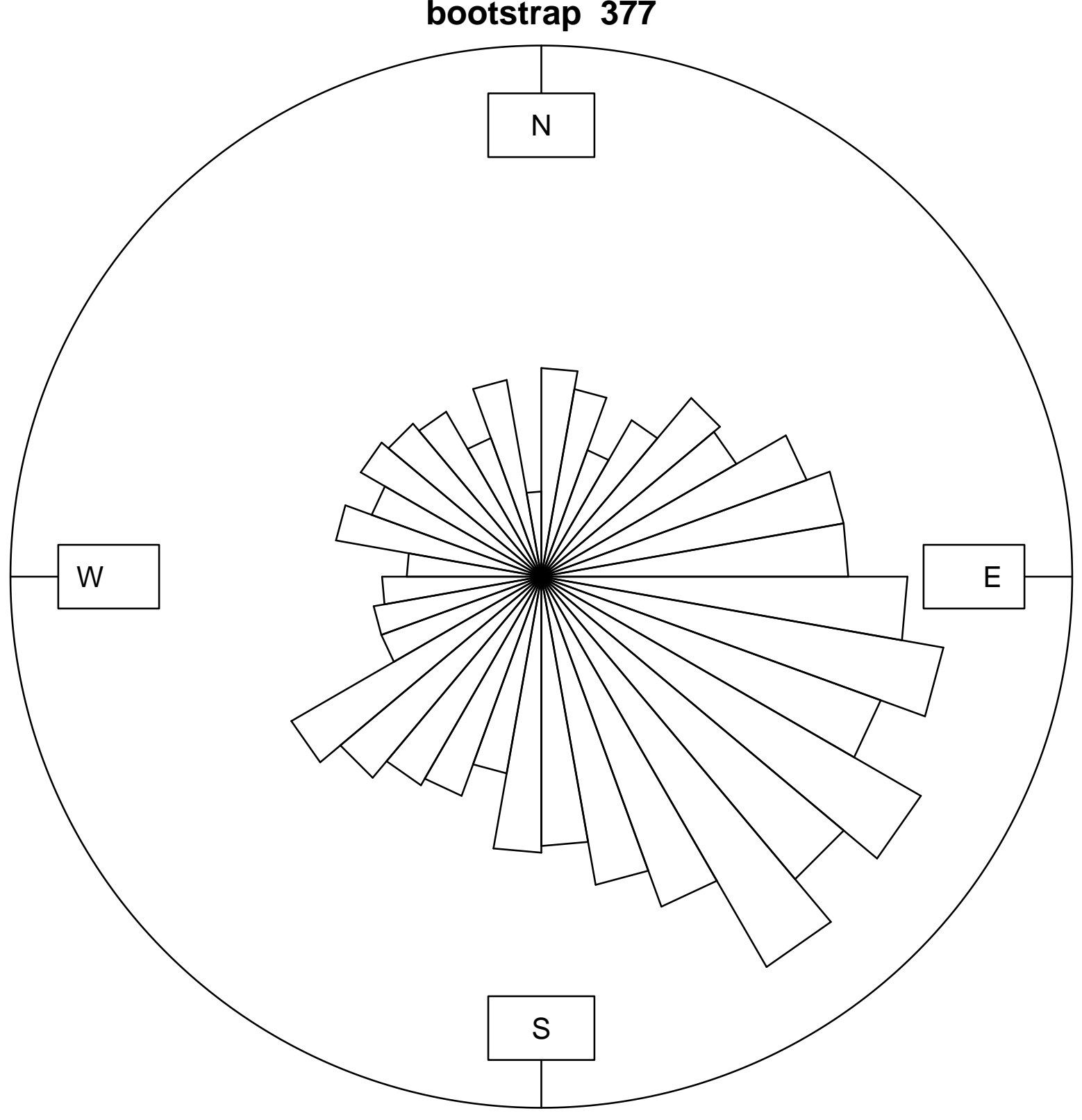


bootstrap 378

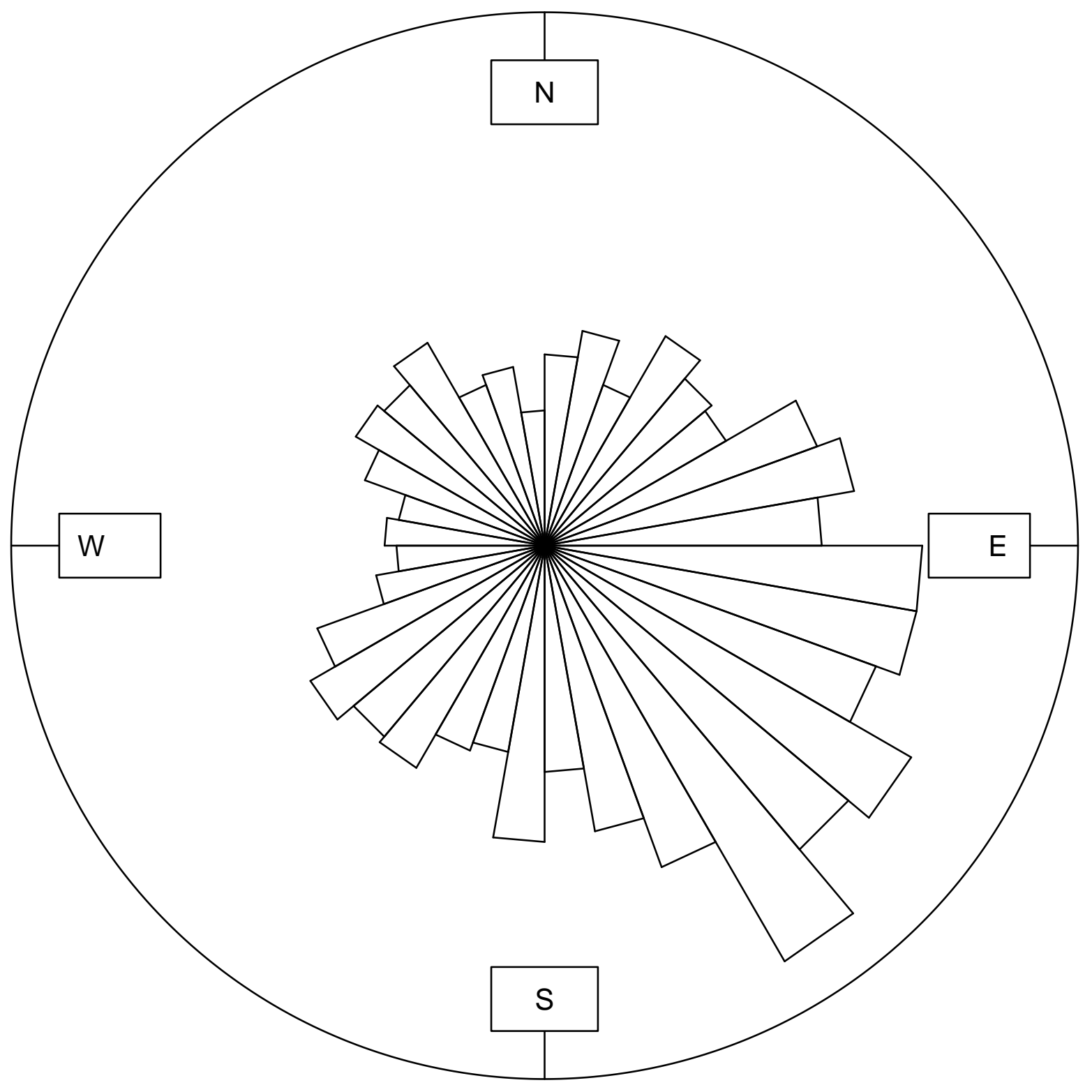


bootstrap 379

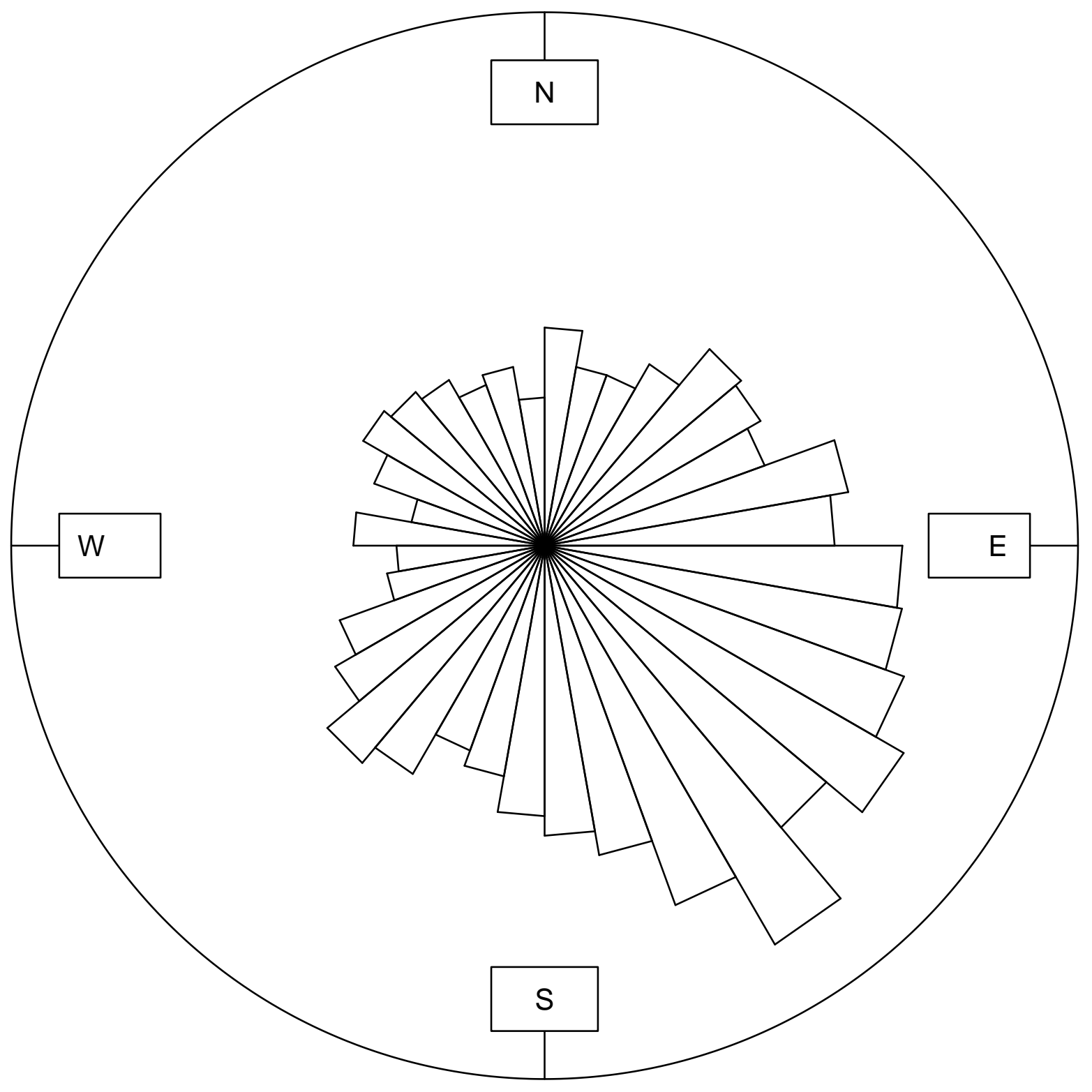


bootstrap 380

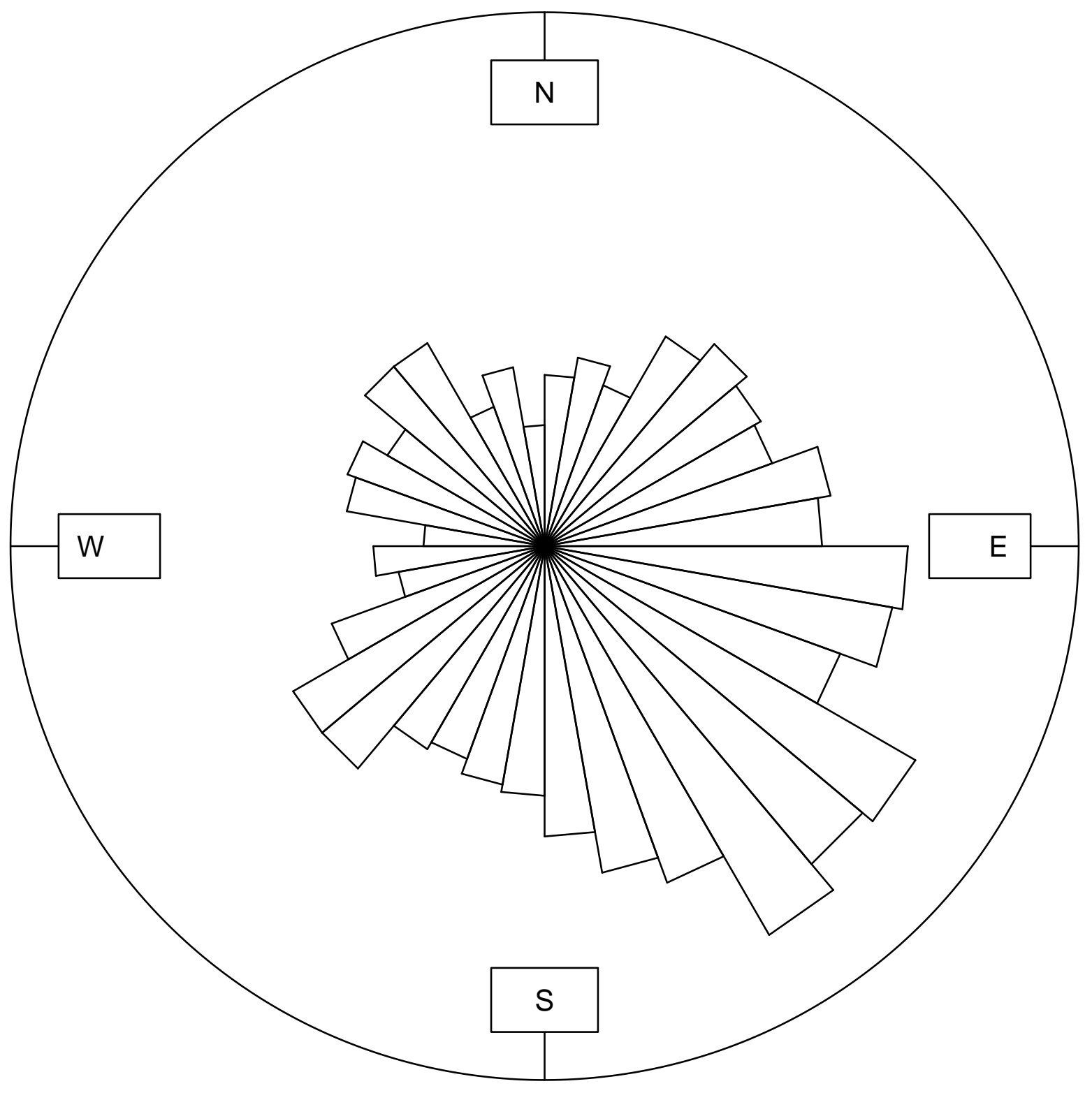




\section{bootstrap 381}

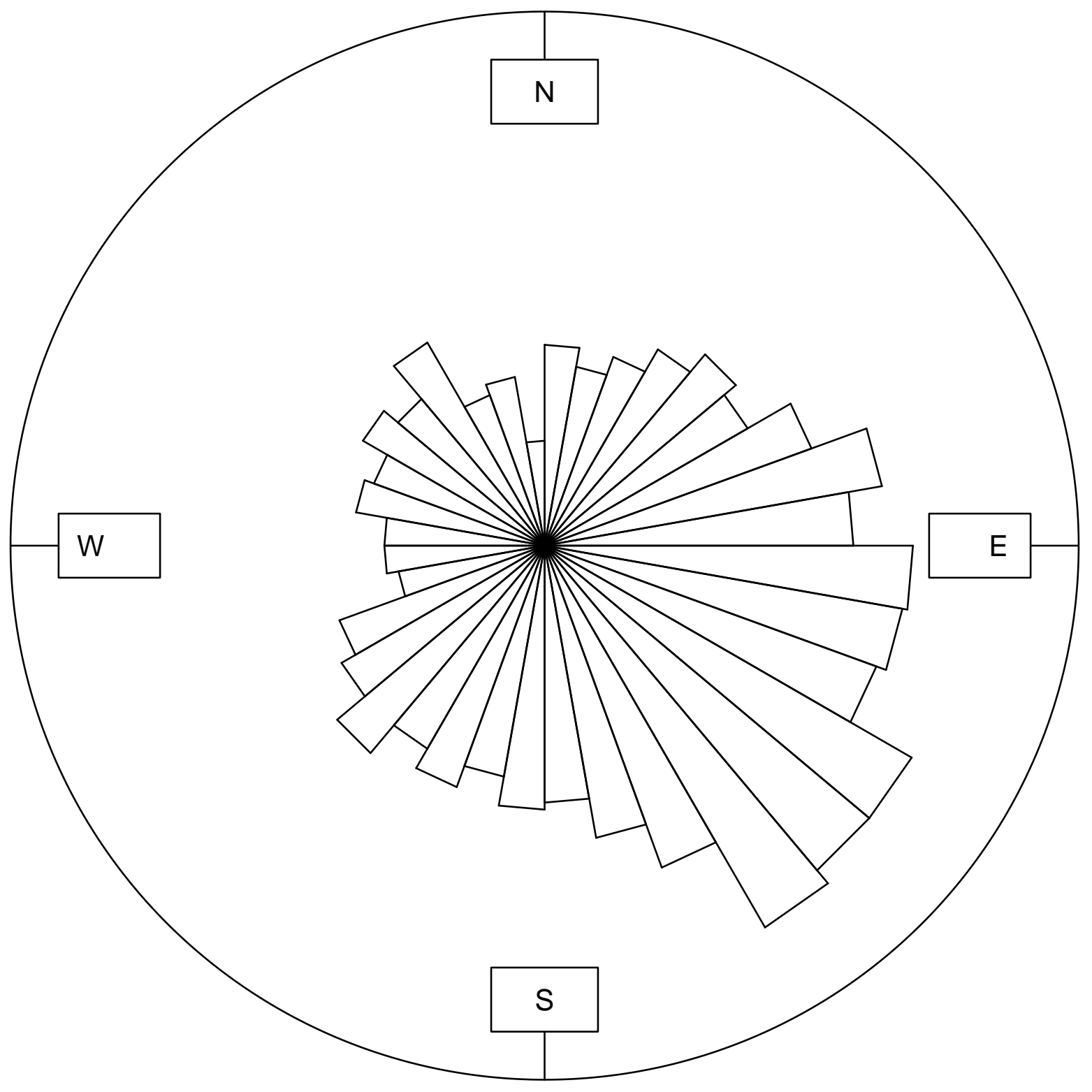


bootstrap 382

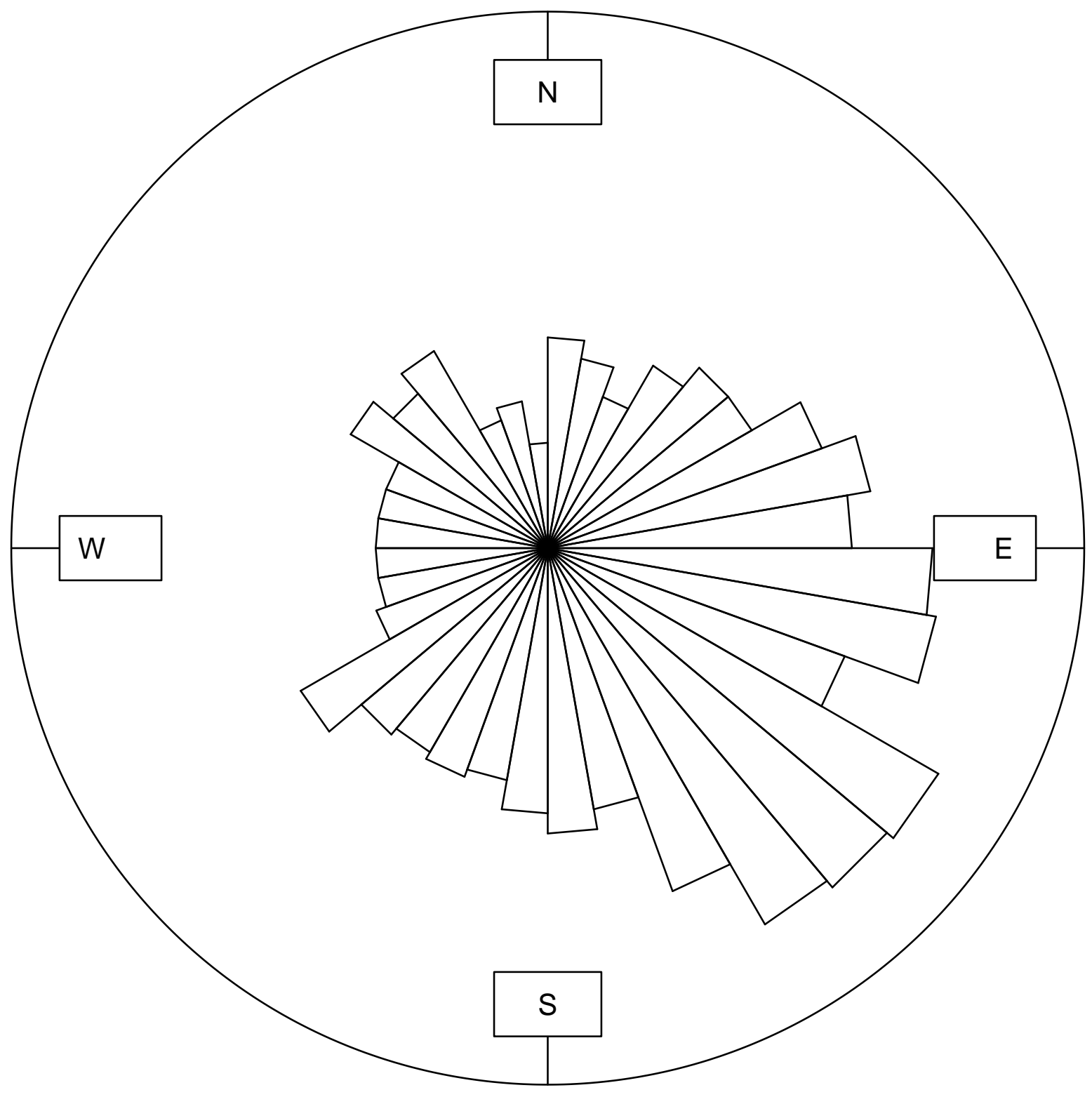




\section{bootstrap 383}

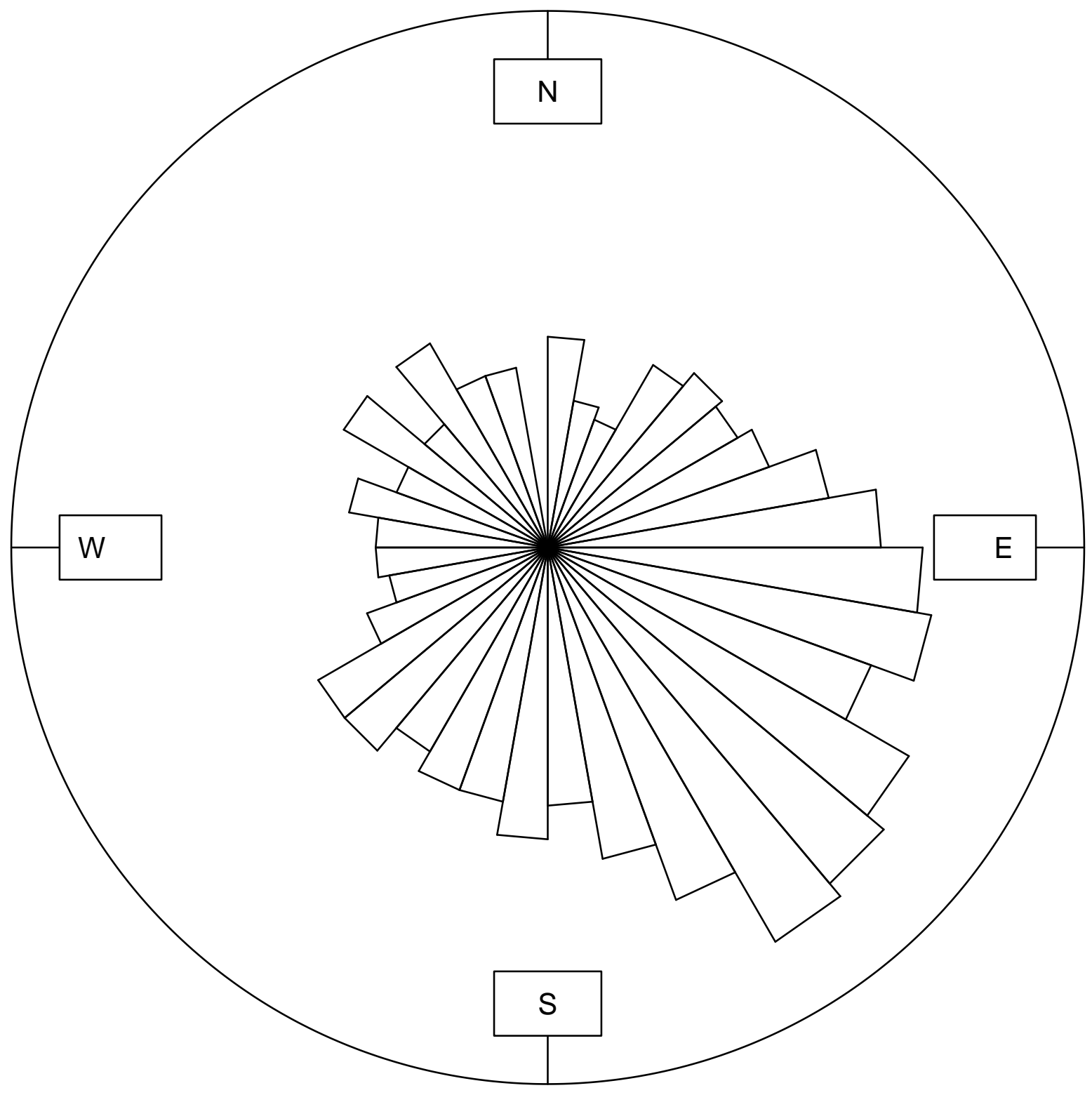


bootstrap 384

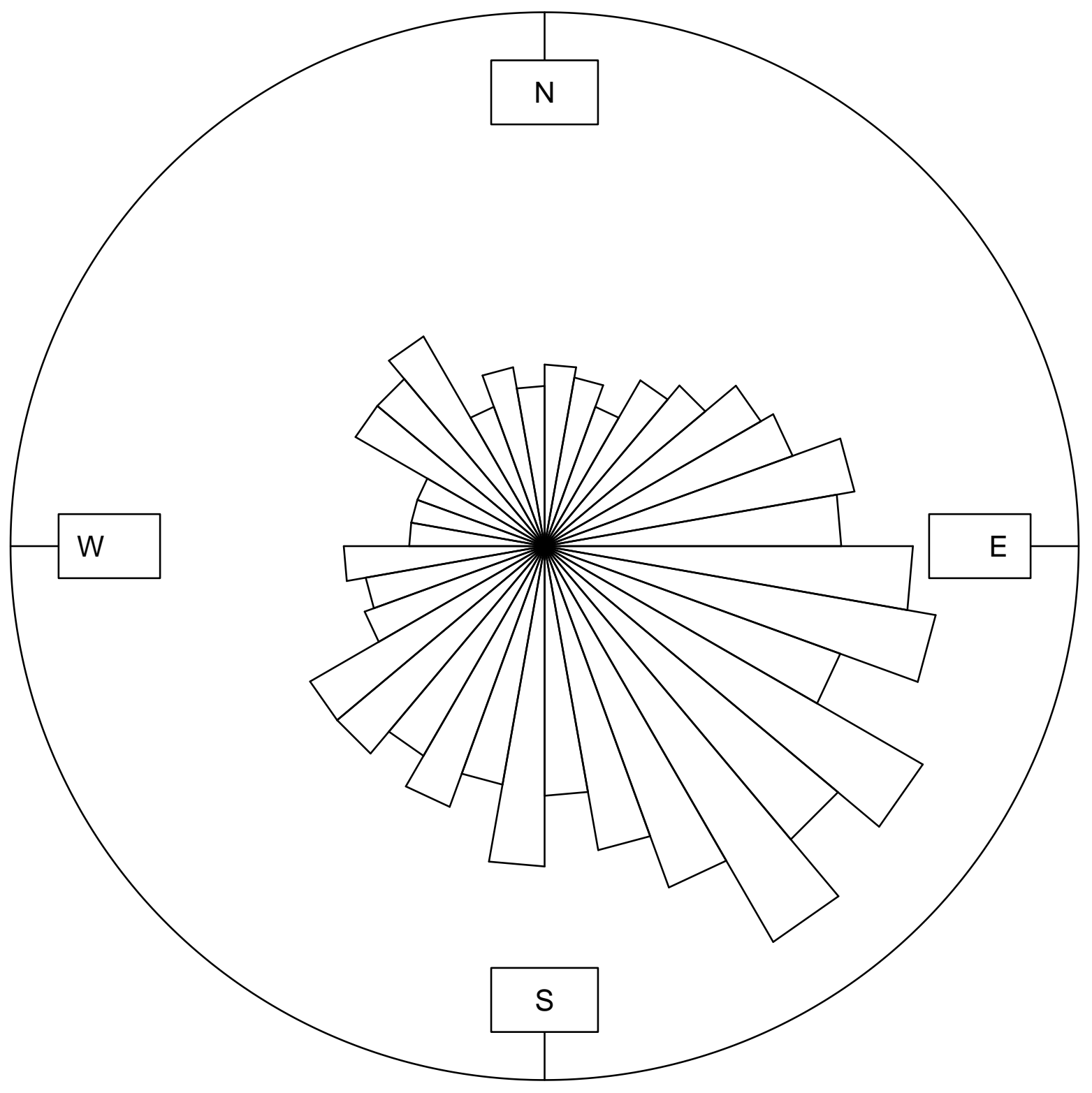




\section{bootstrap 385}

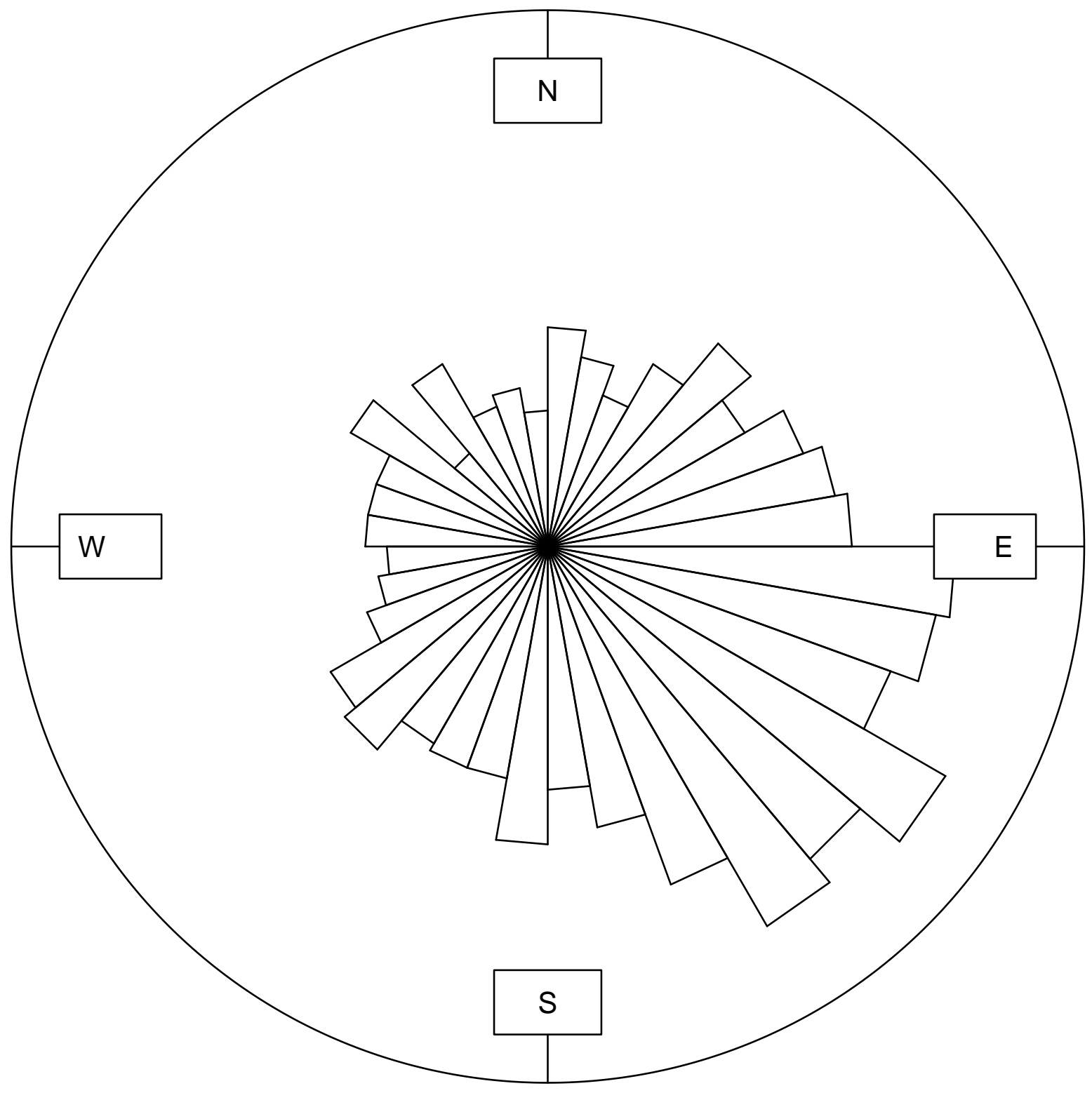


bootstrap 386

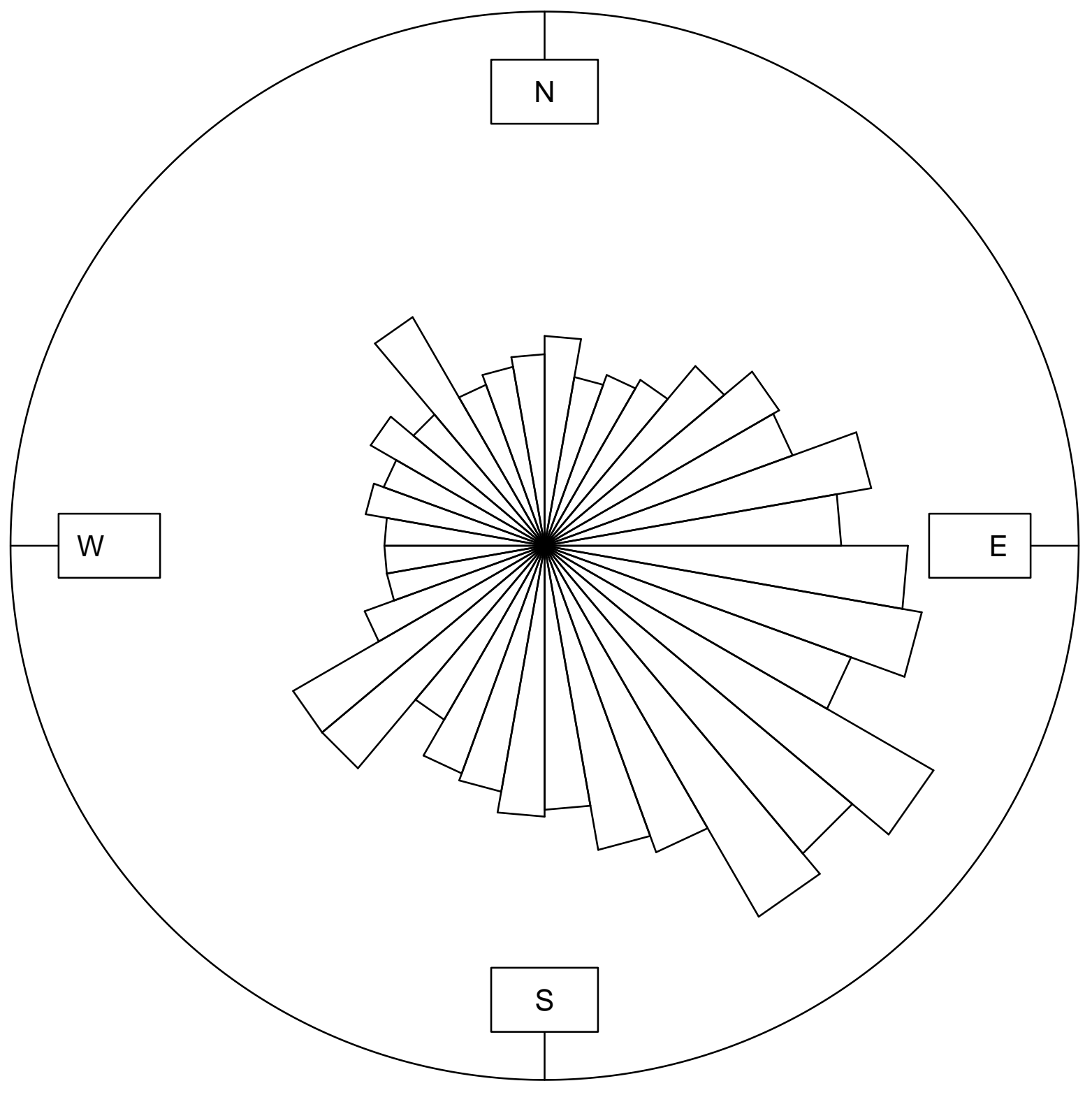


bootstrap 387

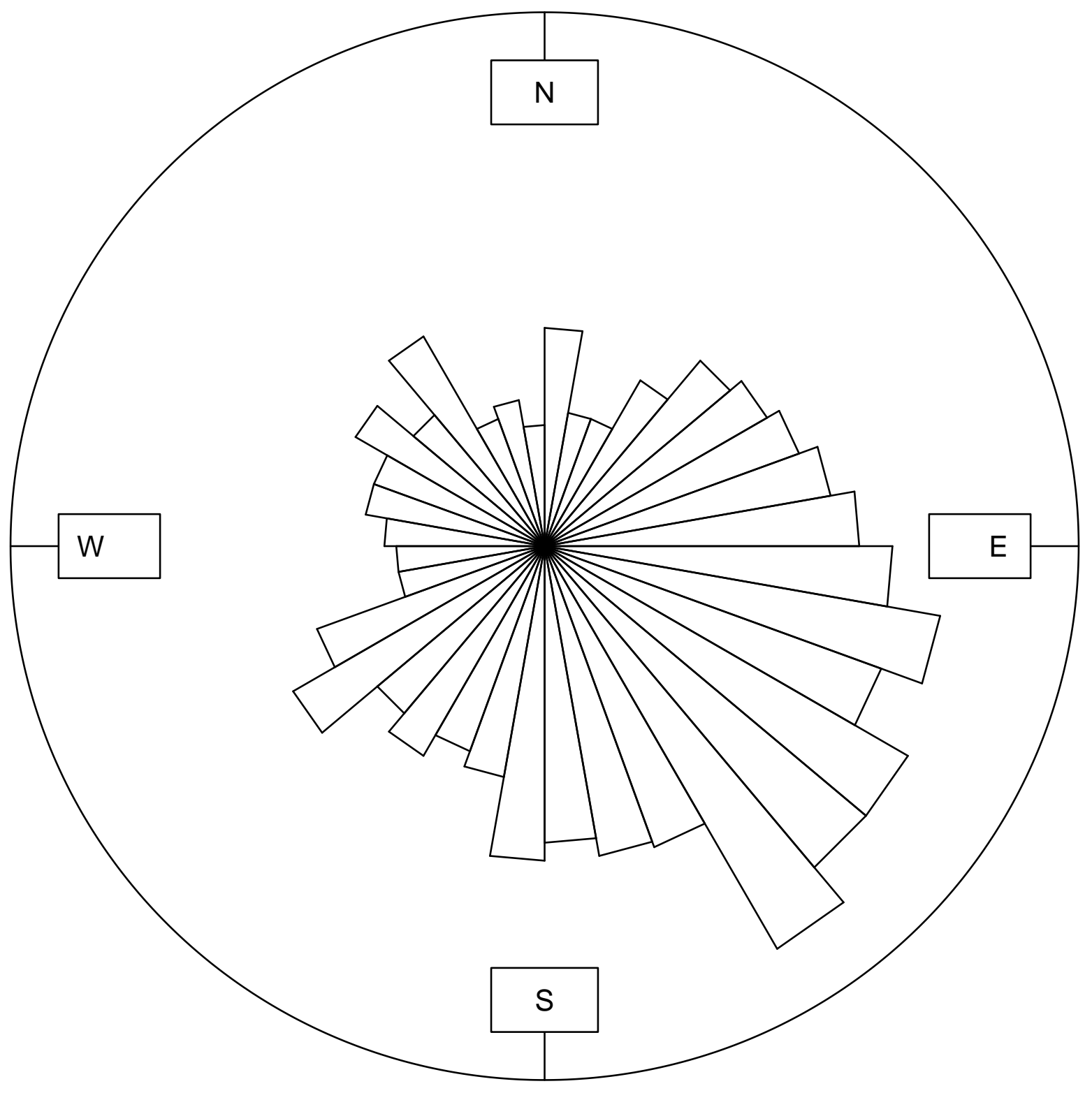


bootstrap 388

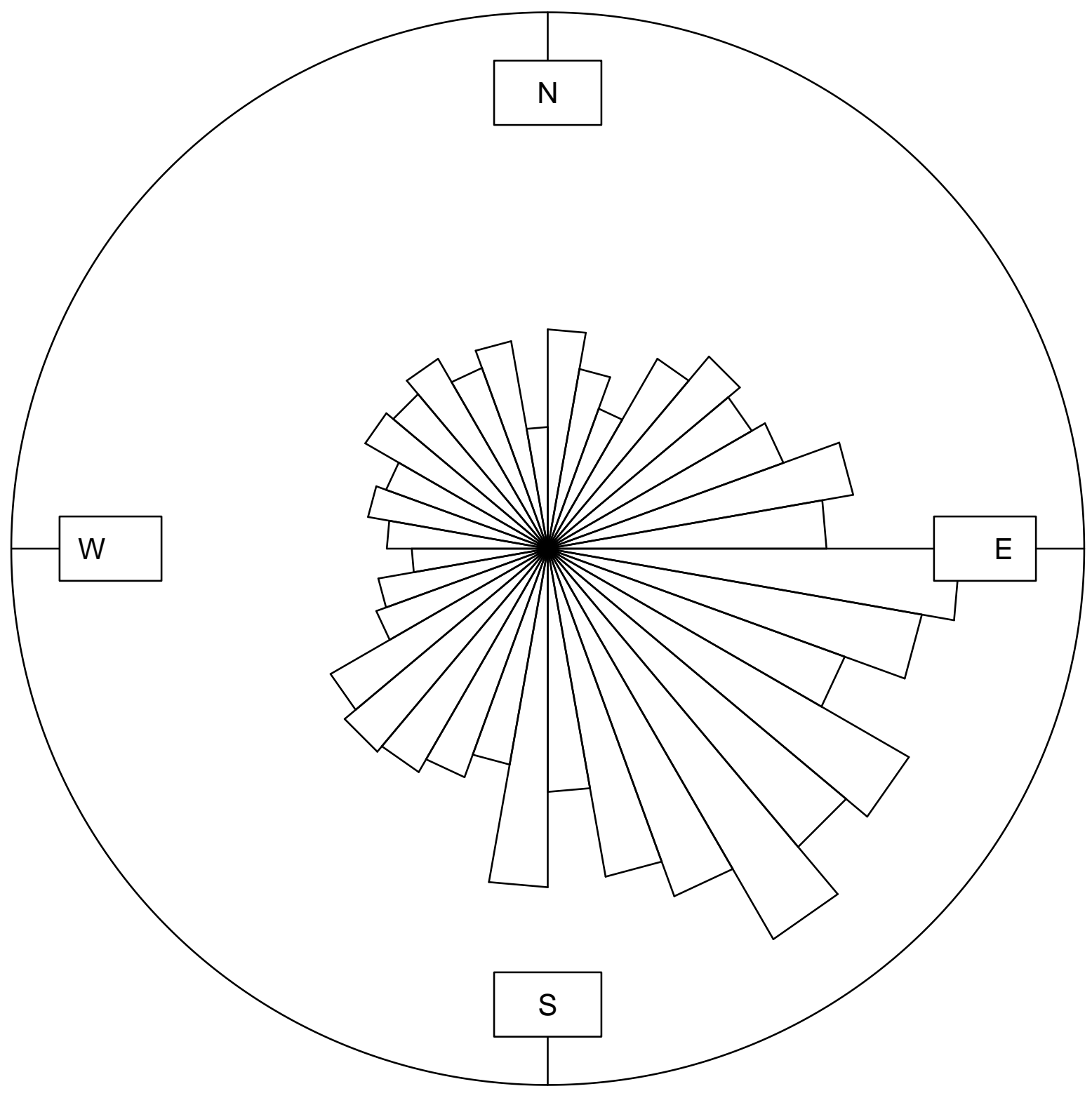


bootstrap 390

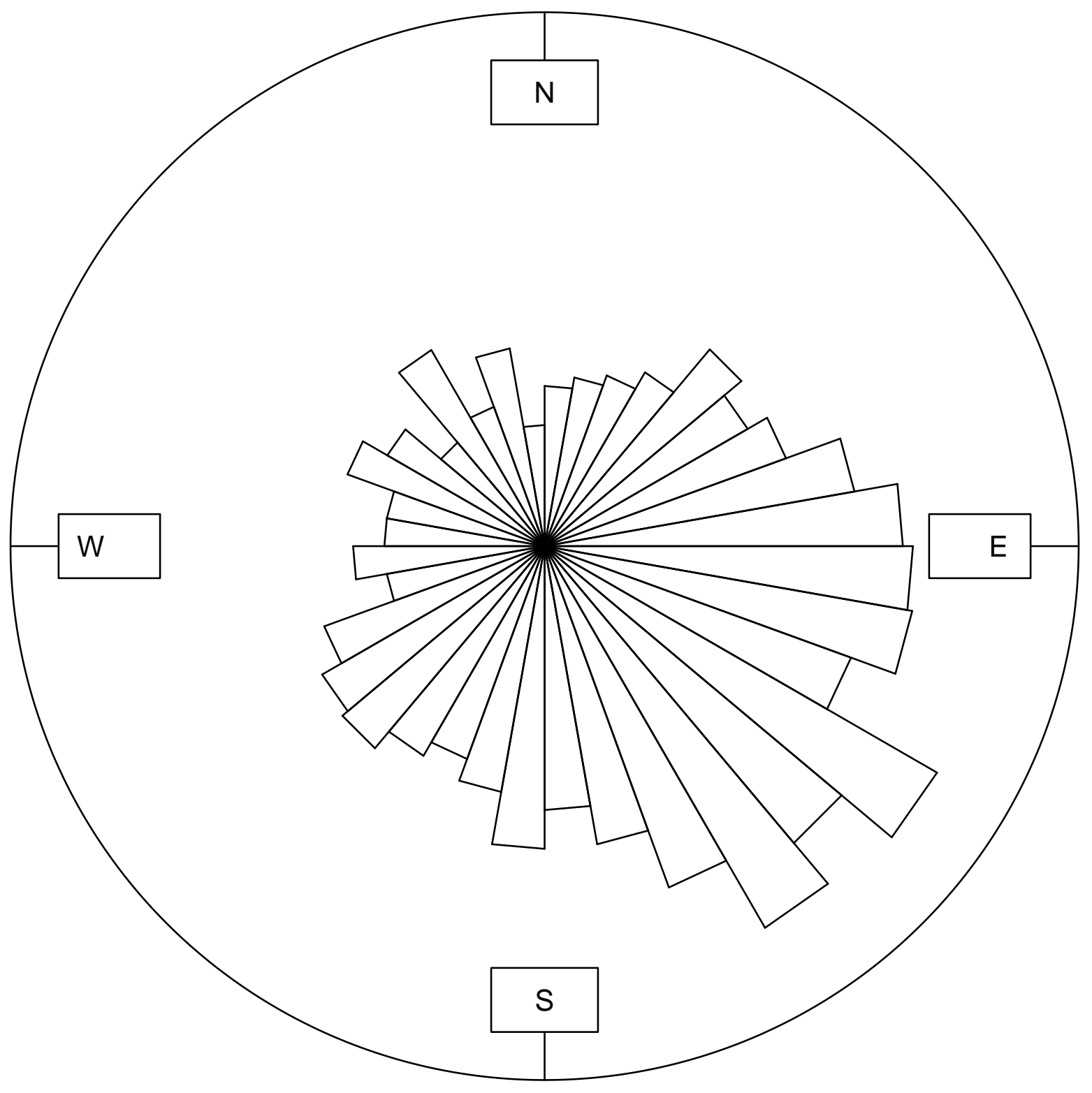


bootstrap 391

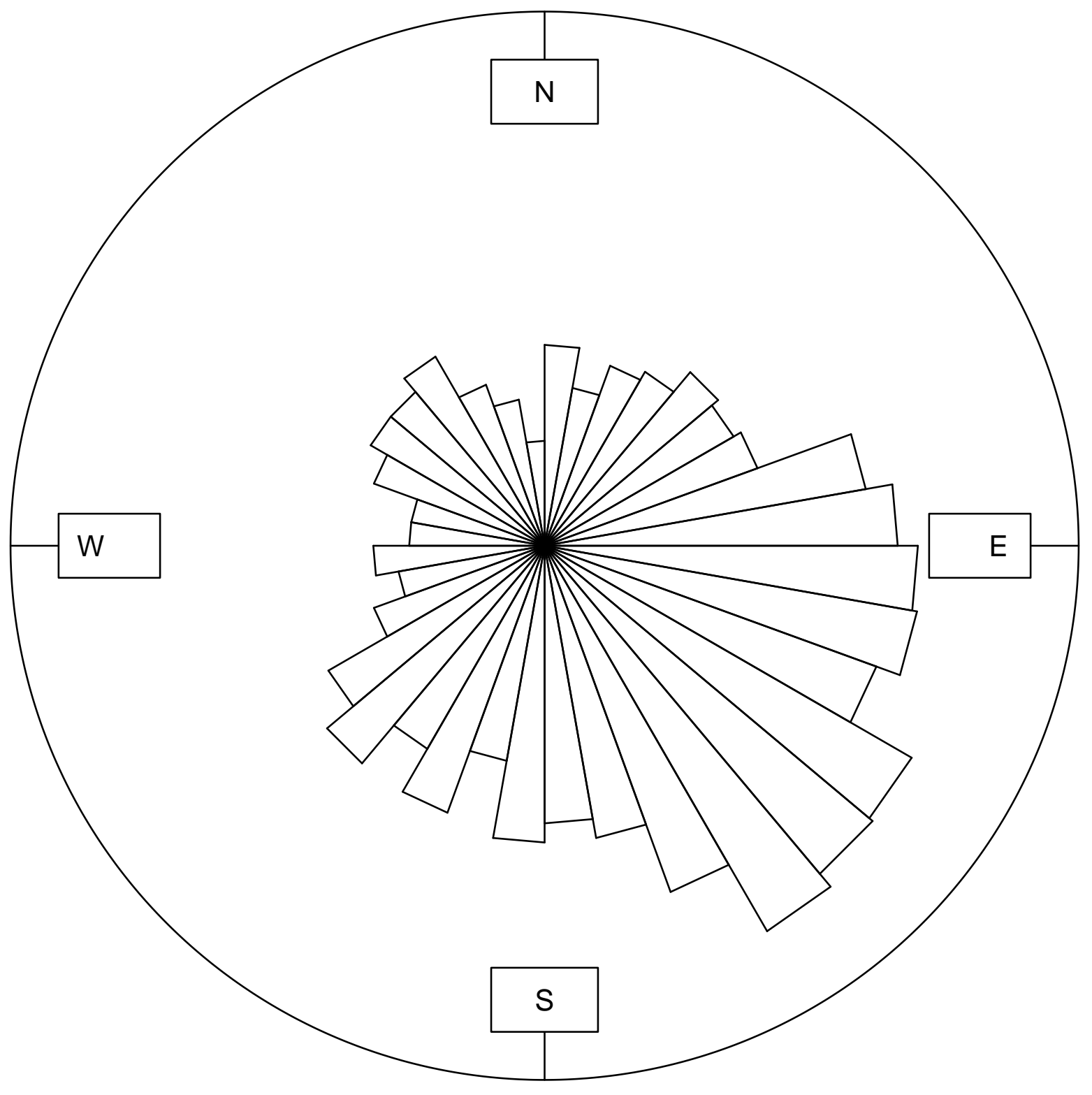


bootstrap 392

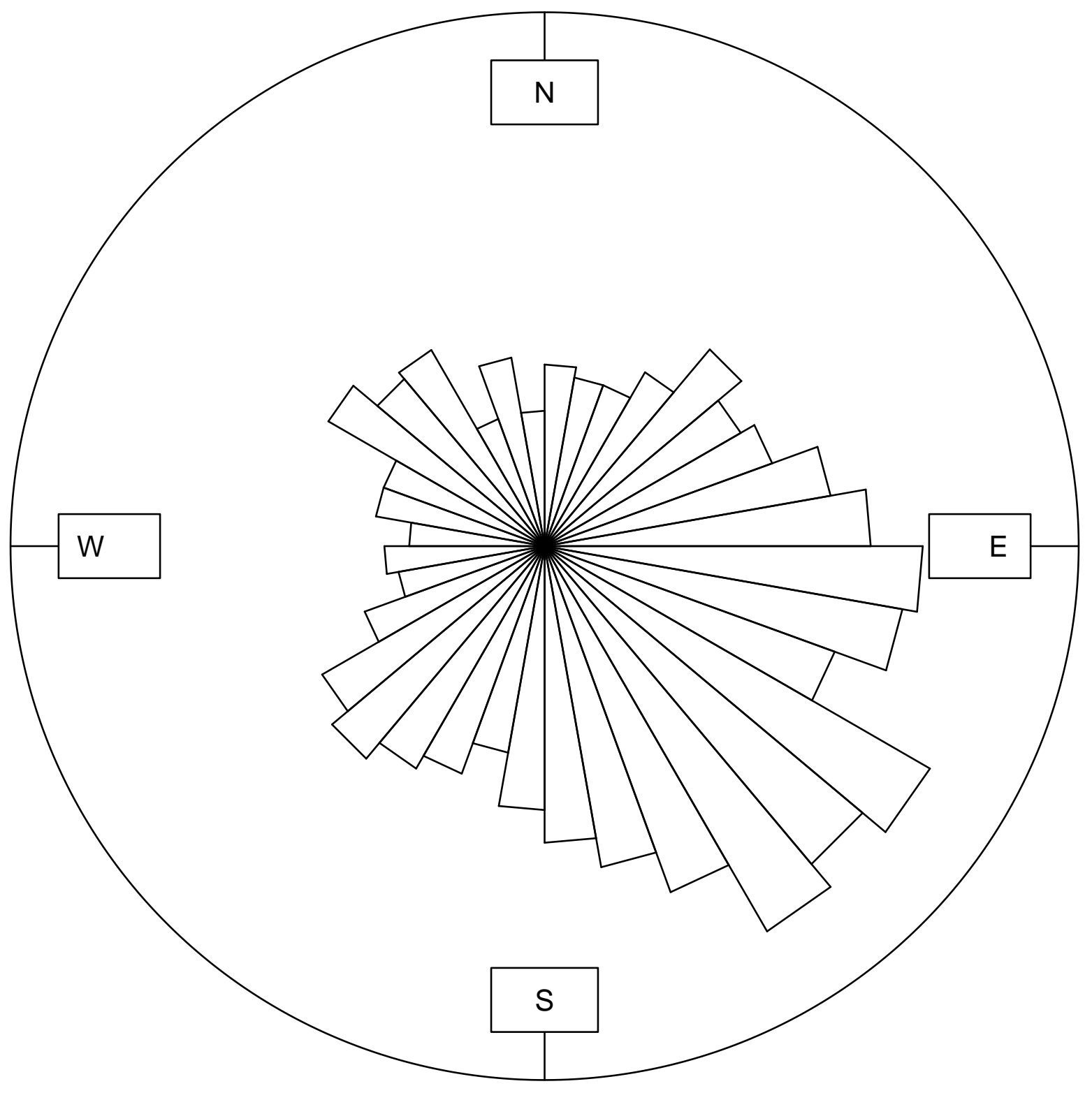




\section{bootstrap 393}

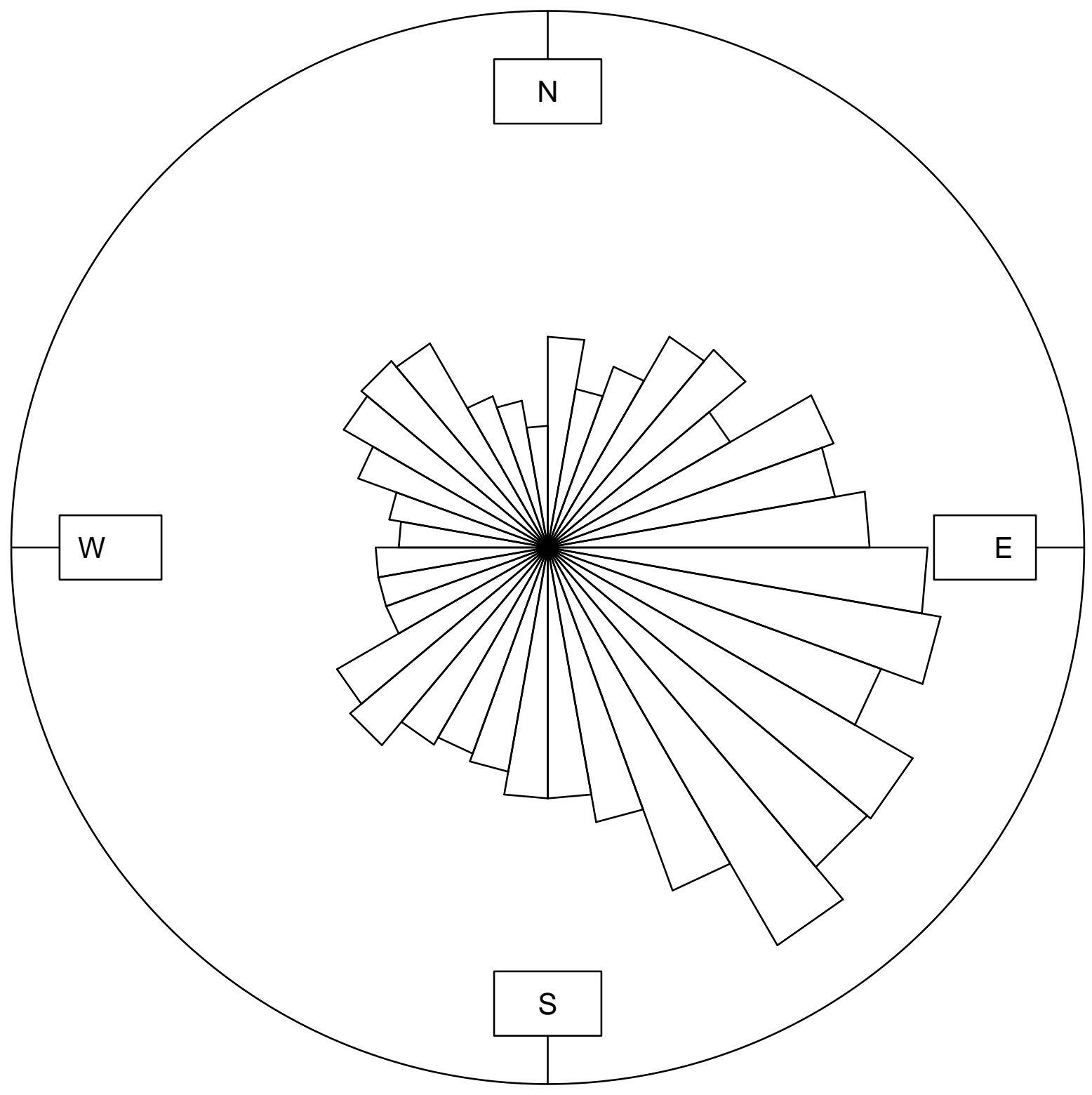


bootstrap 394

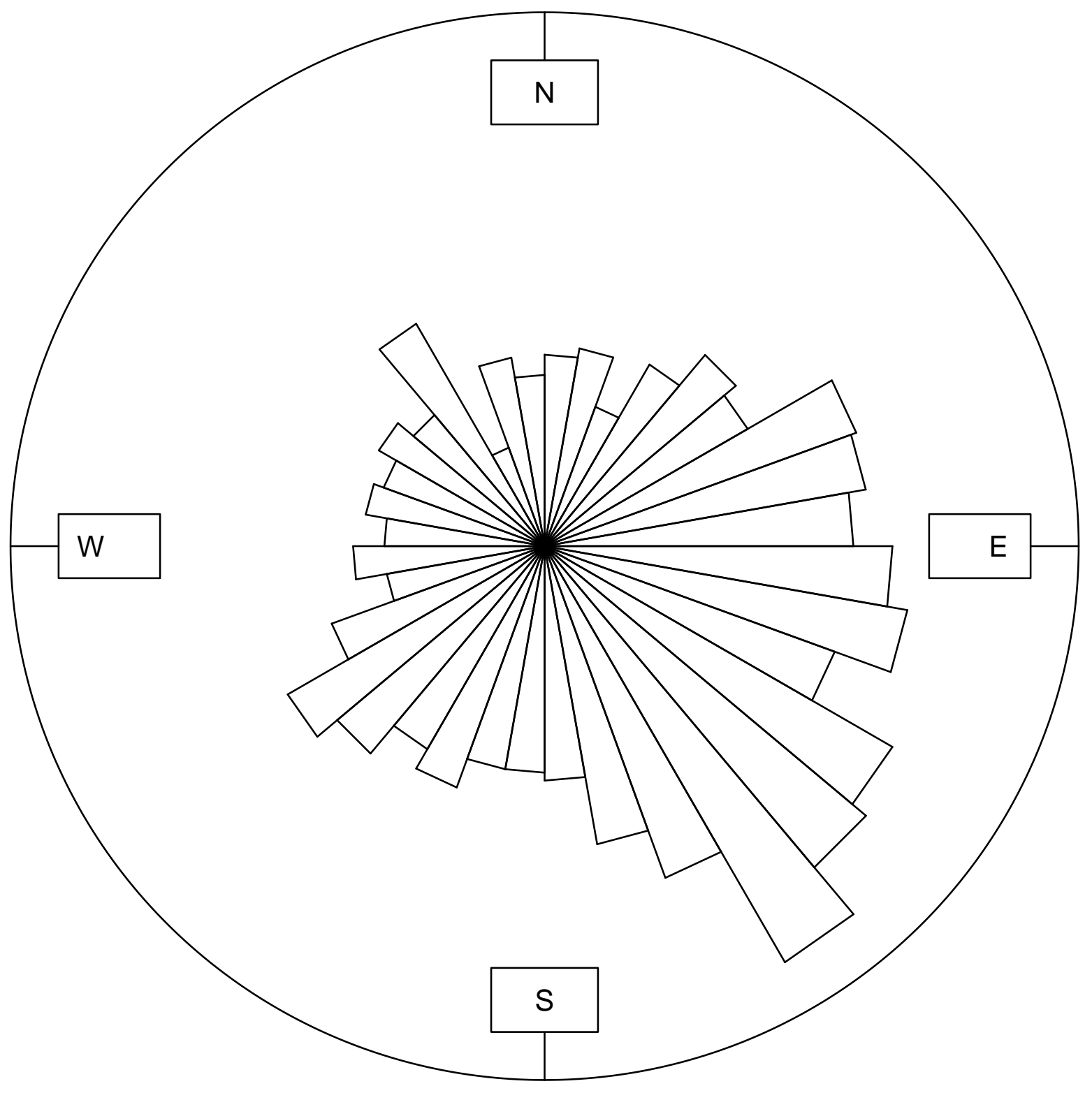


bootstrap 395

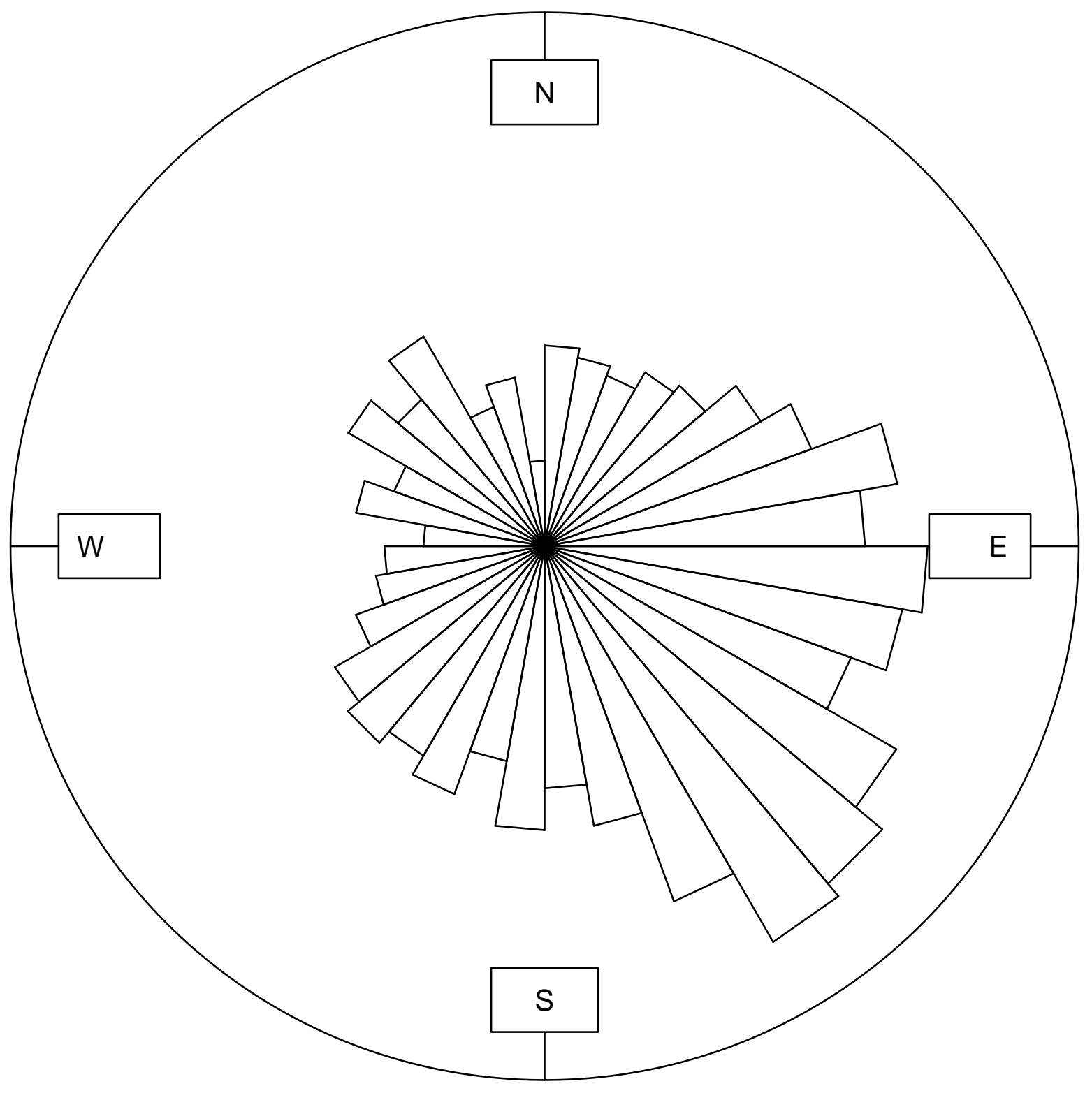


bootstrap 396

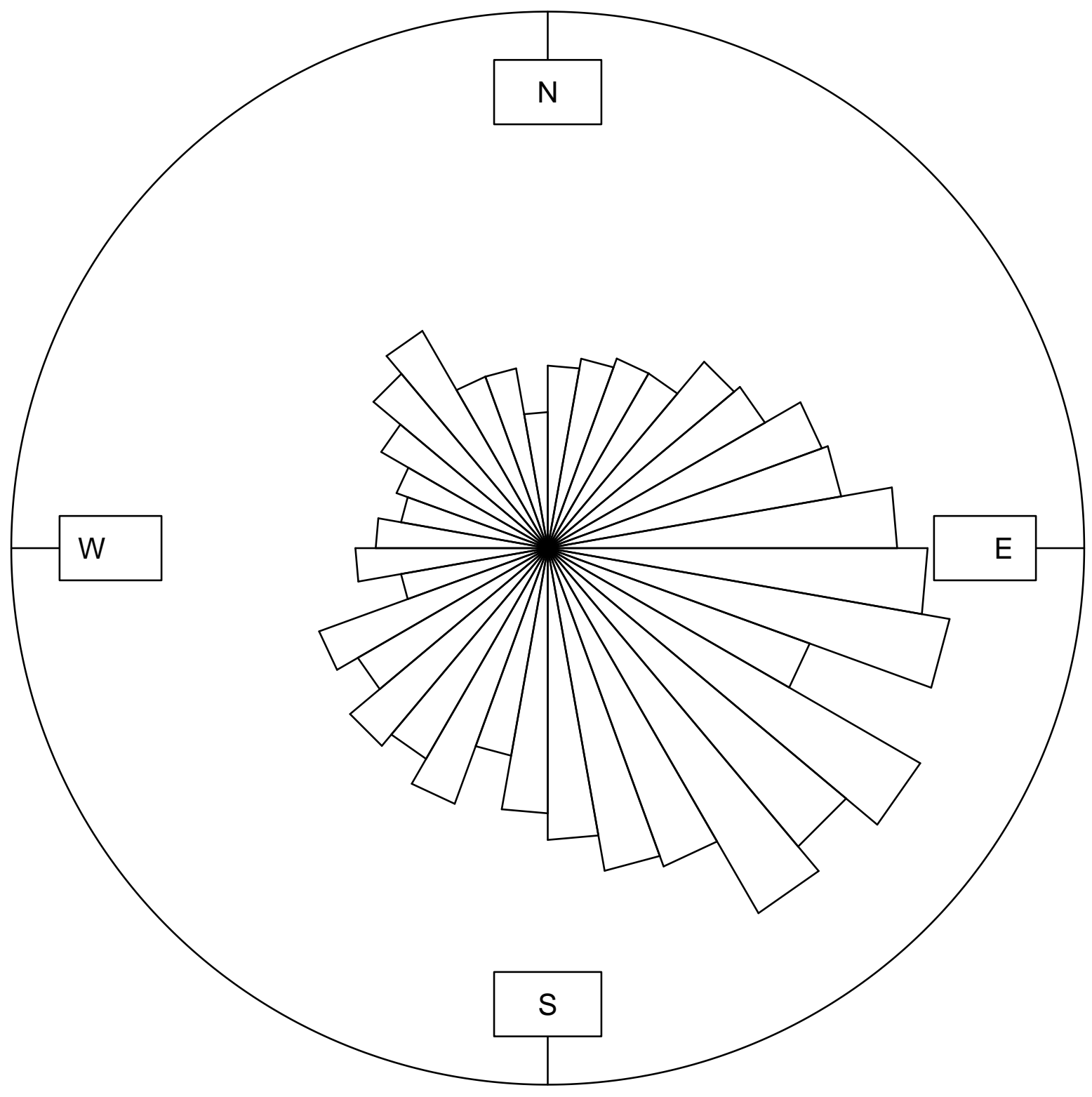


bootstrap 397

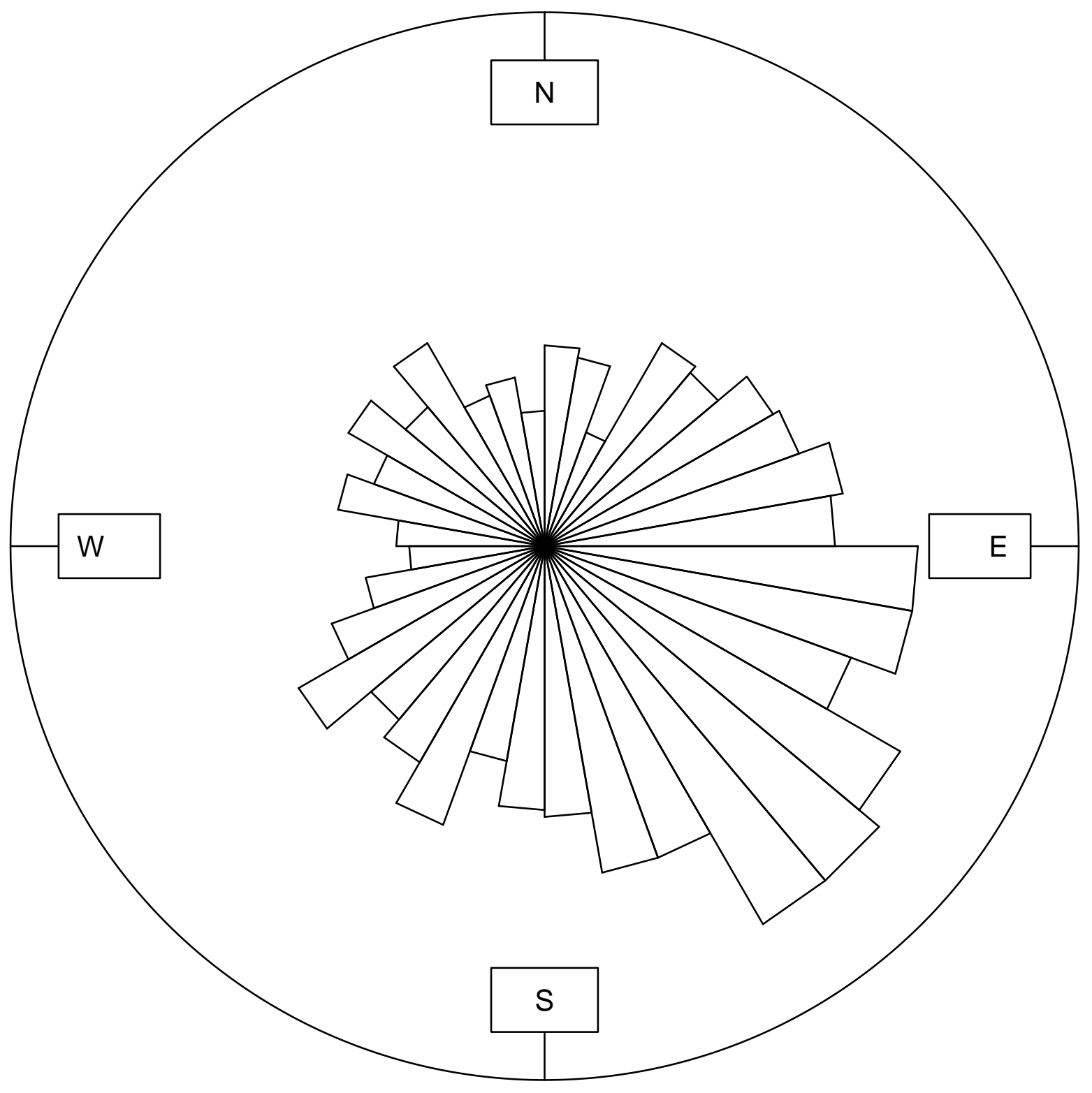


bootstrap 398

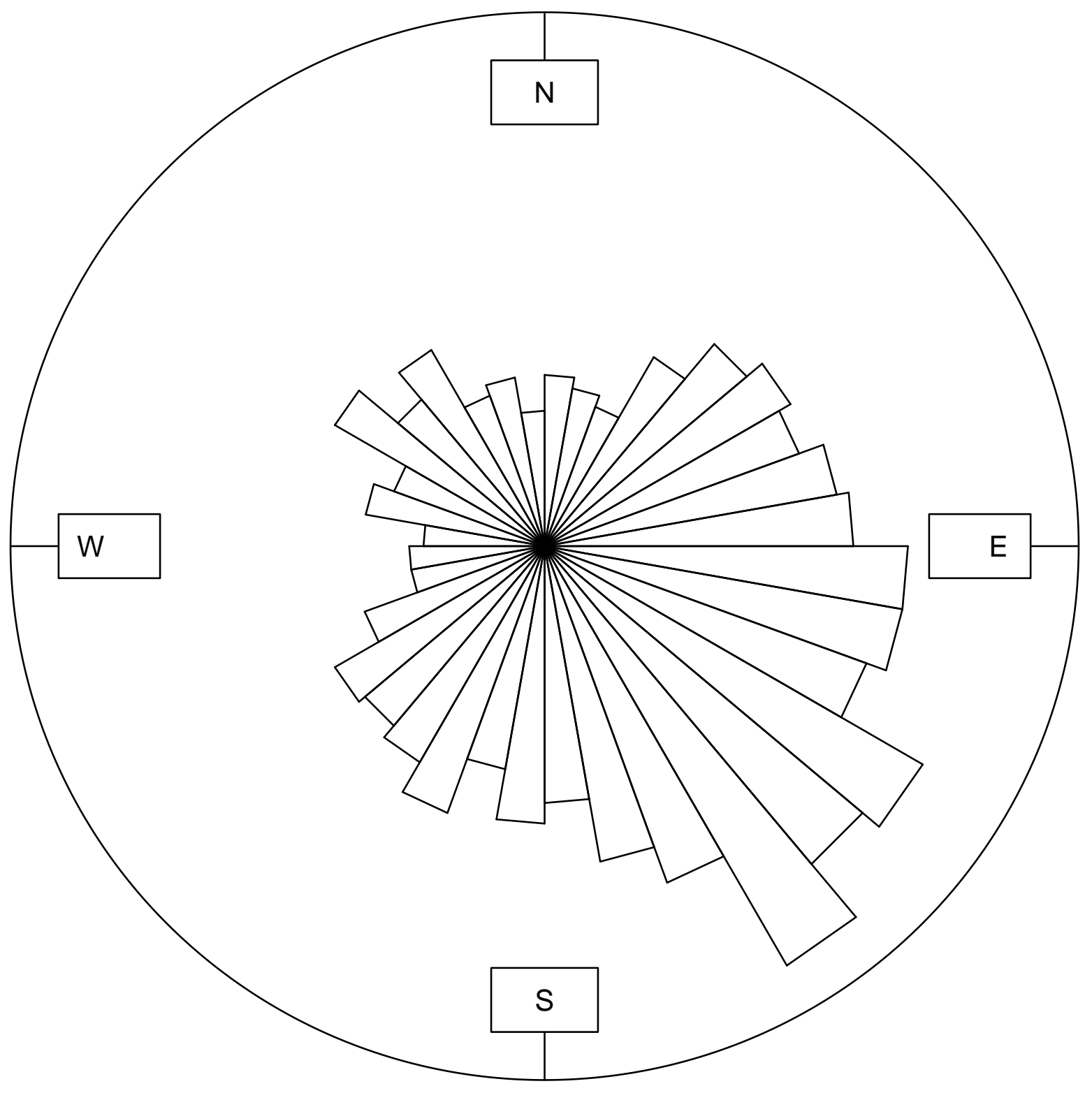




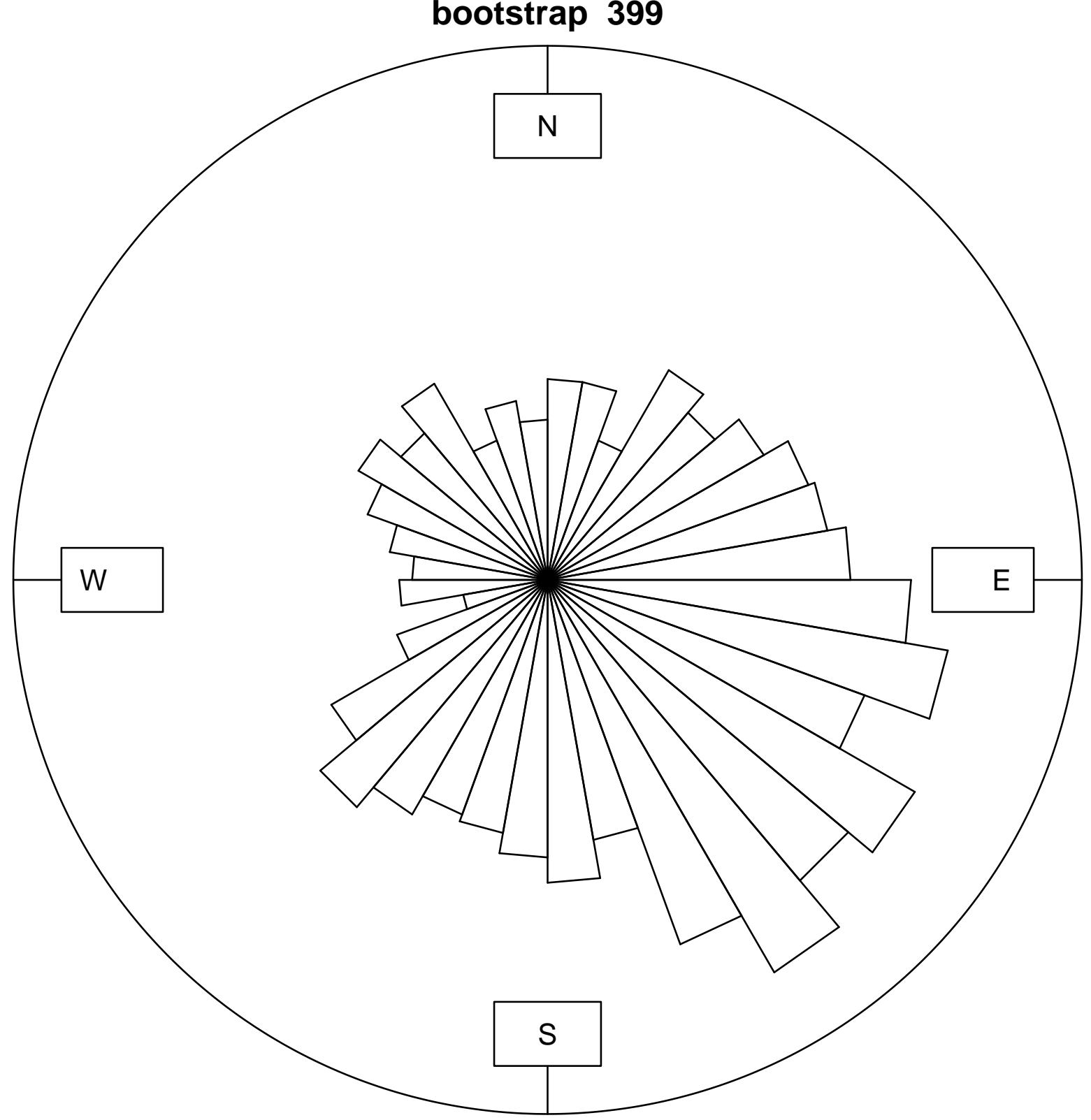


bootstrap 400

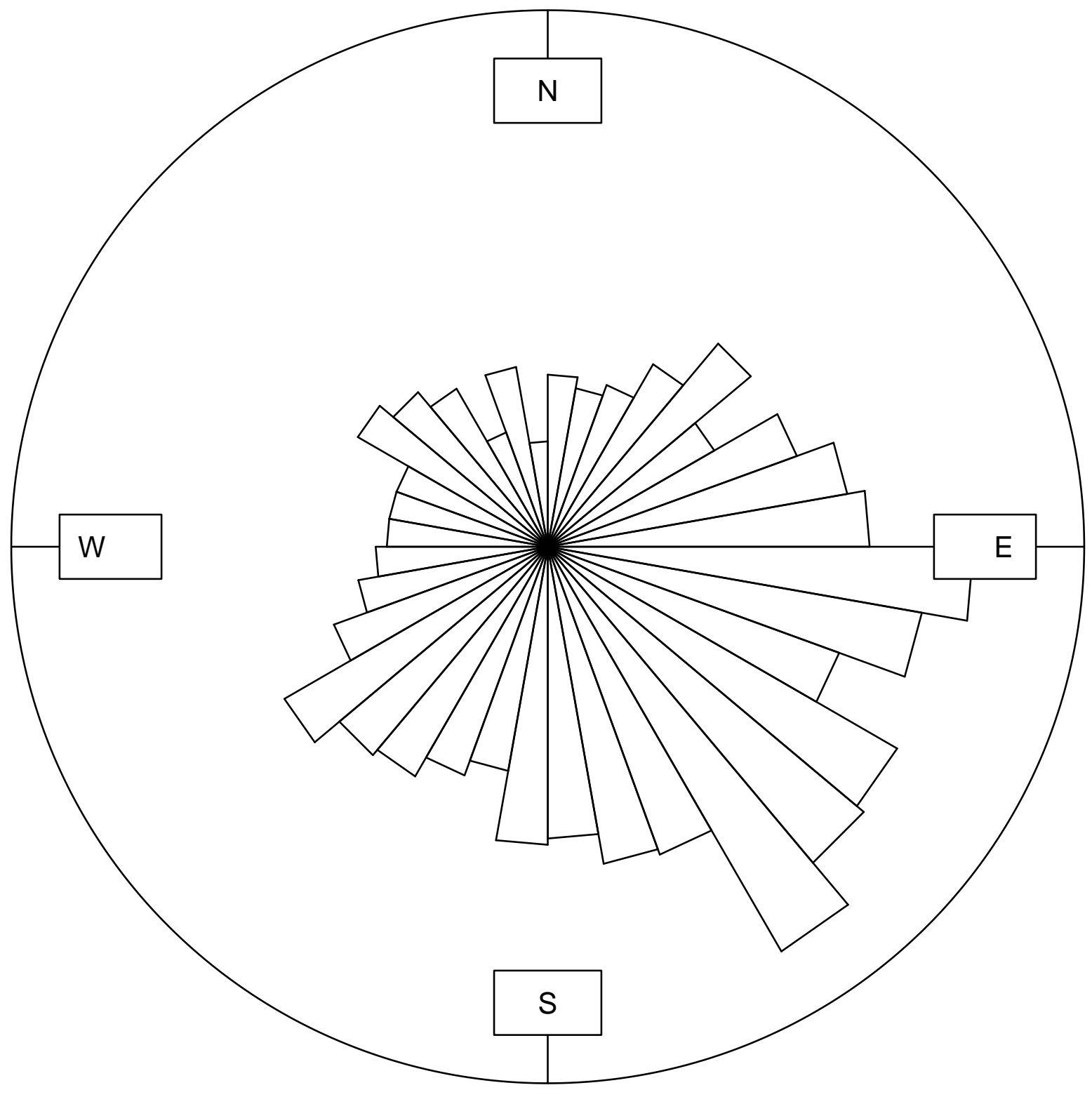


bootstrap 401

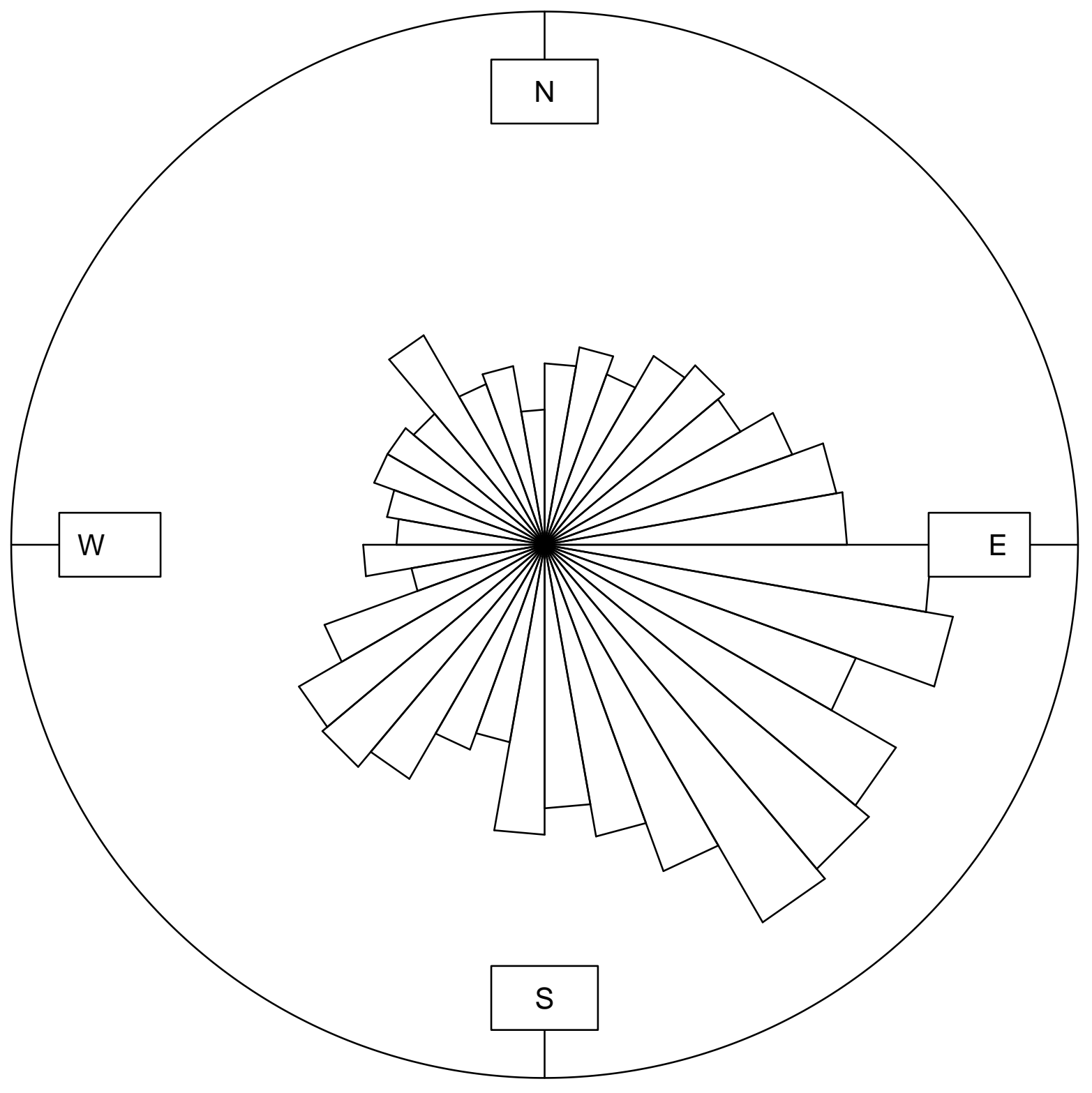


bootstrap 402

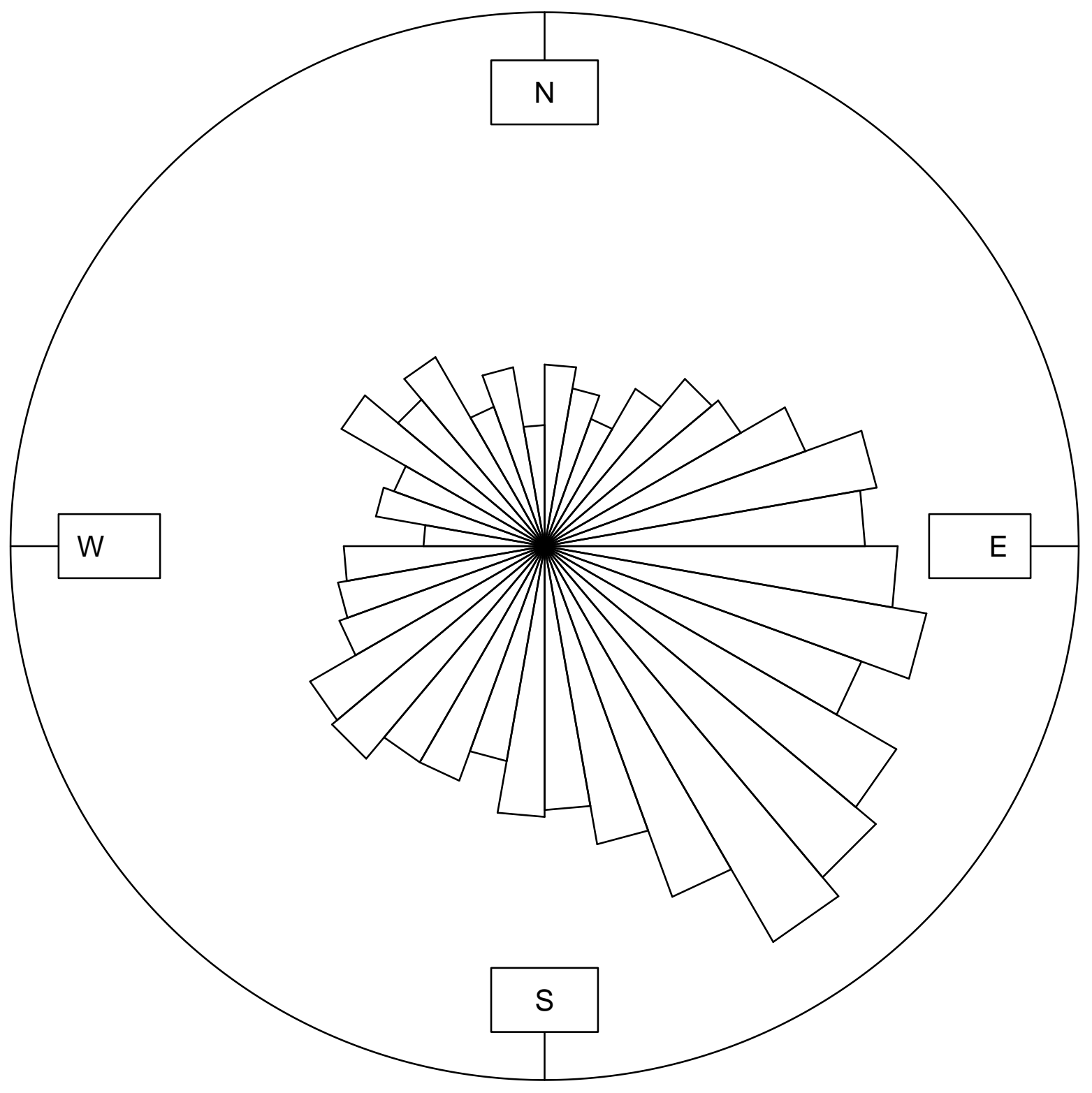




\section{bootstrap 403}

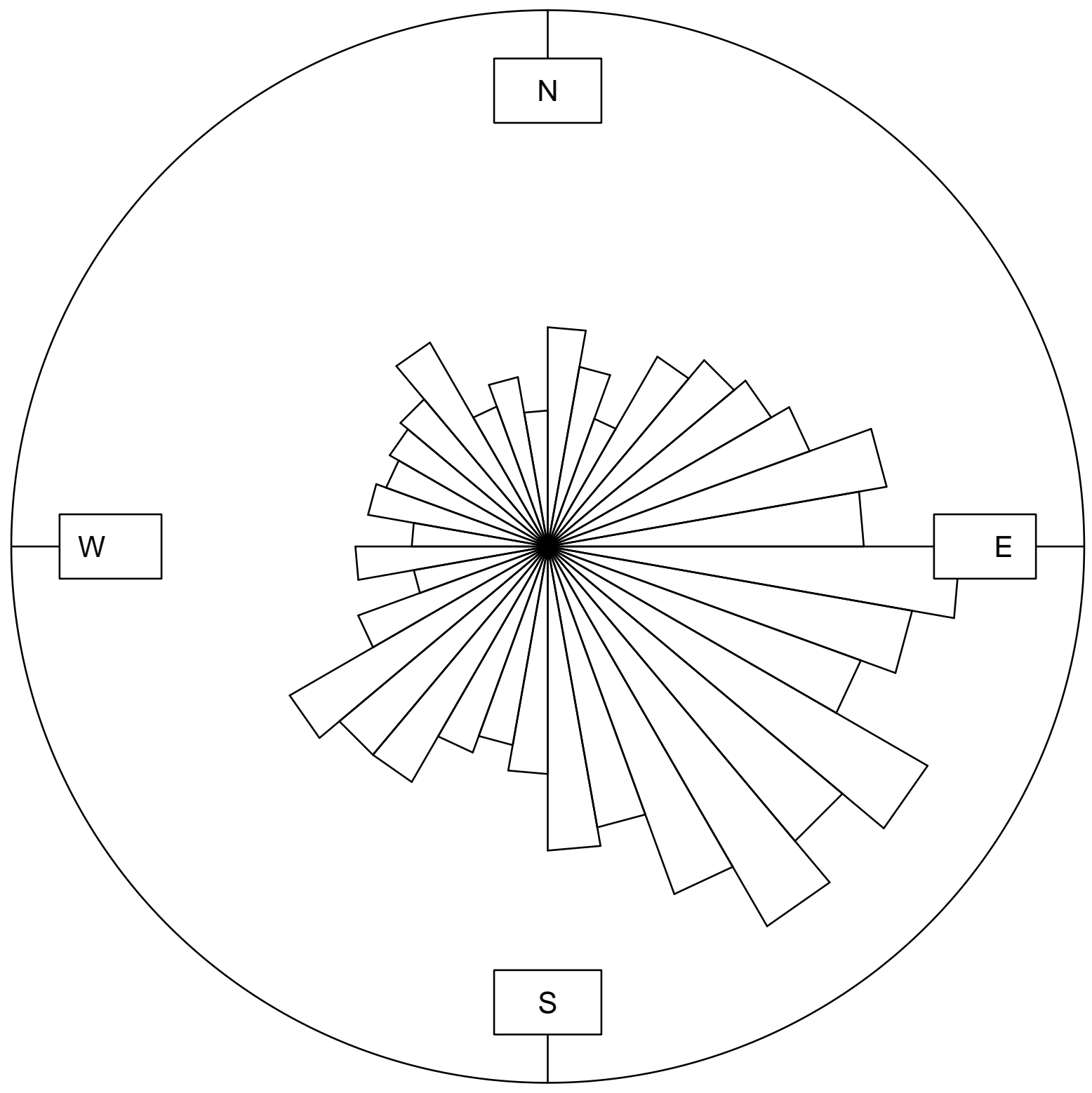


bootstrap 404

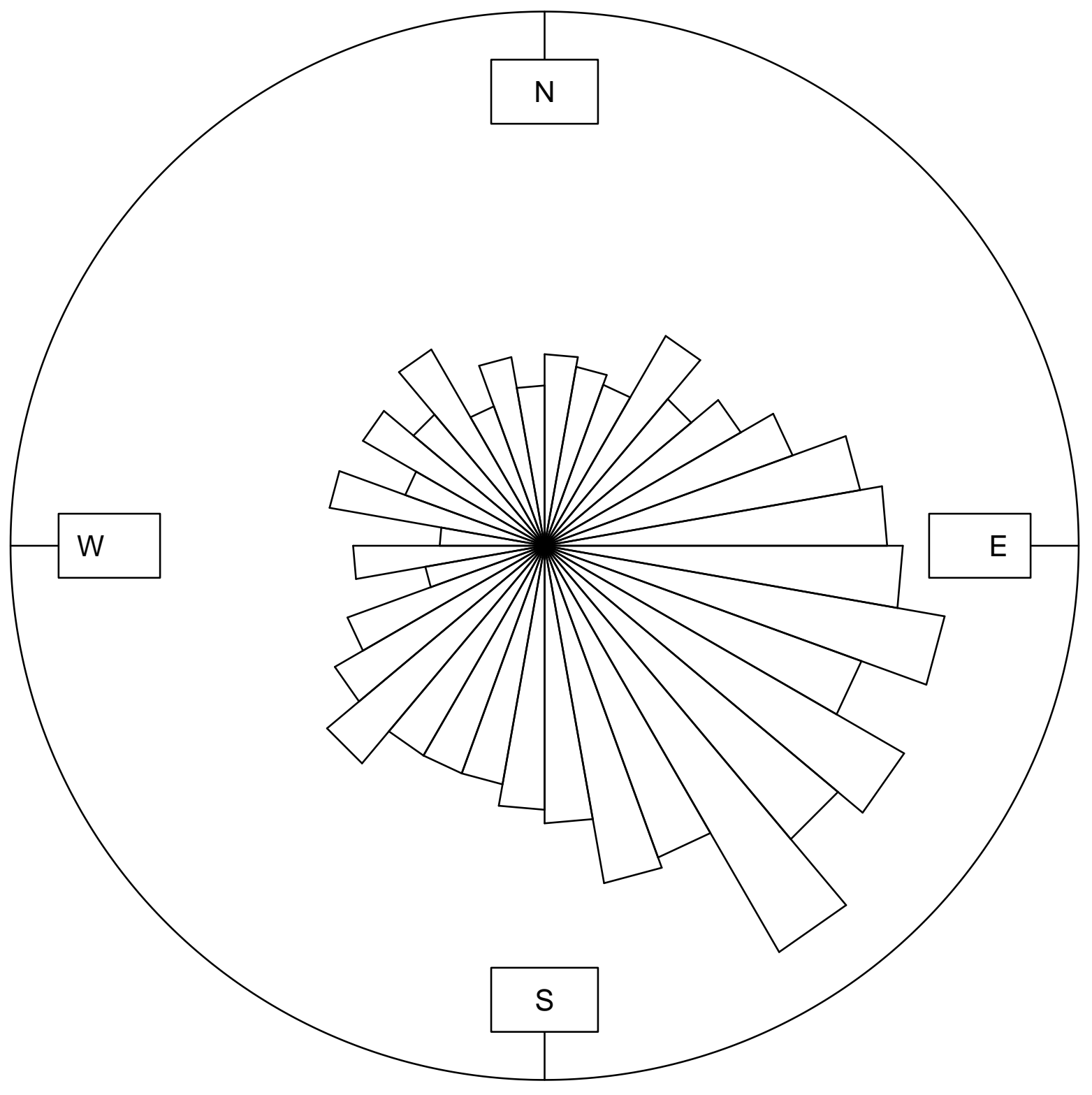




\section{bootstrap 405}

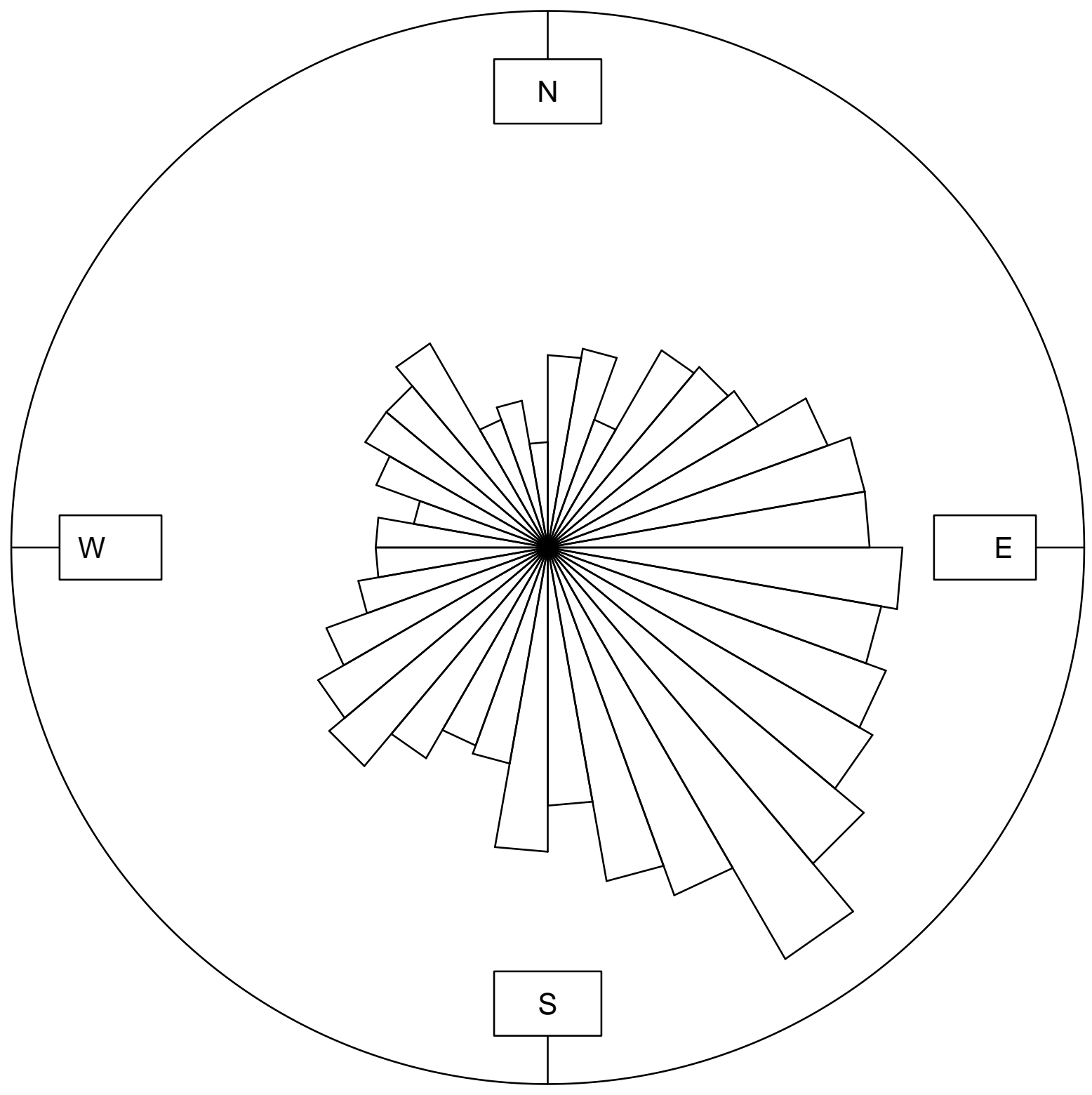


bootstrap 406

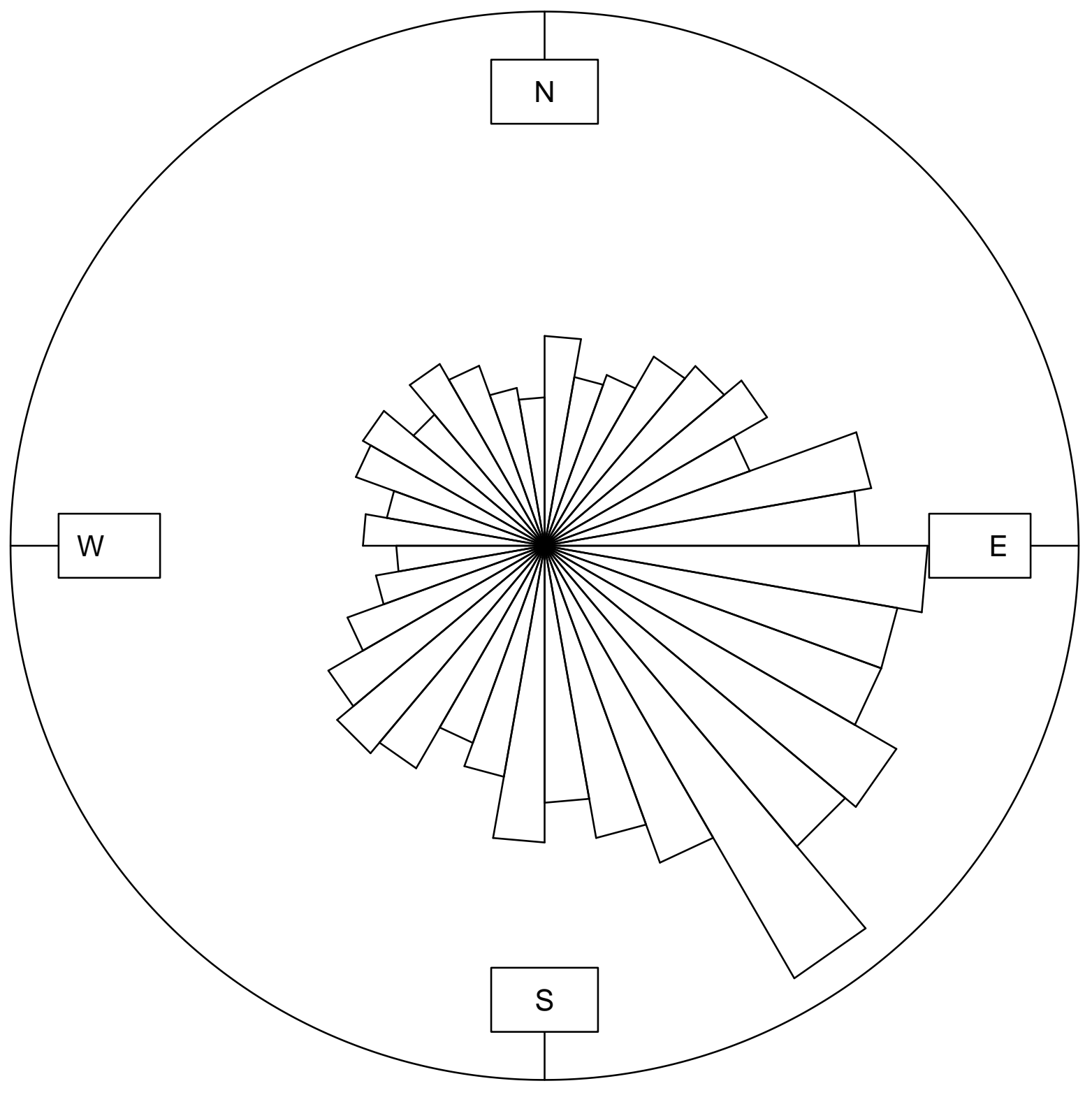


bootstrap 407

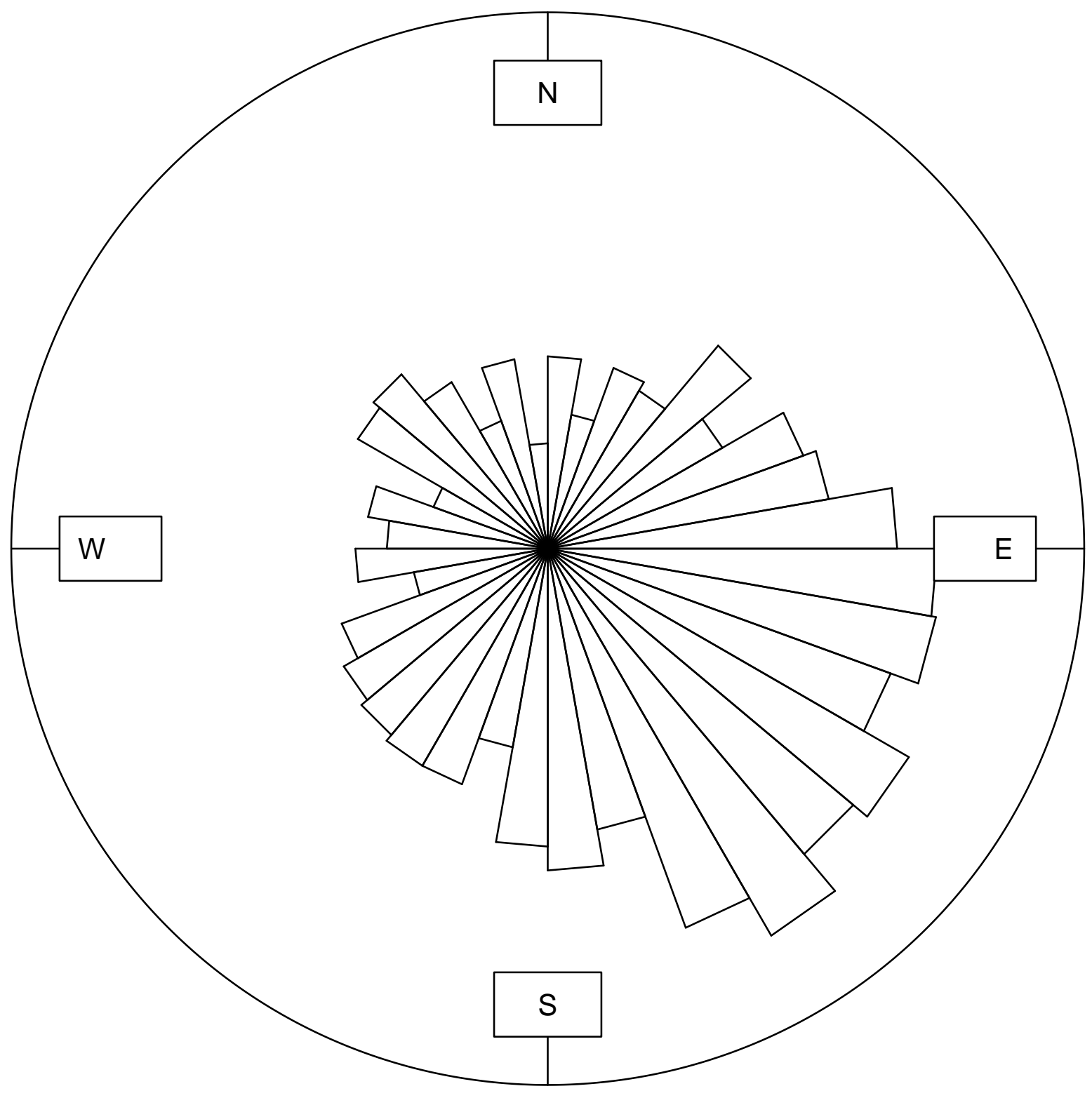


bootstrap 408

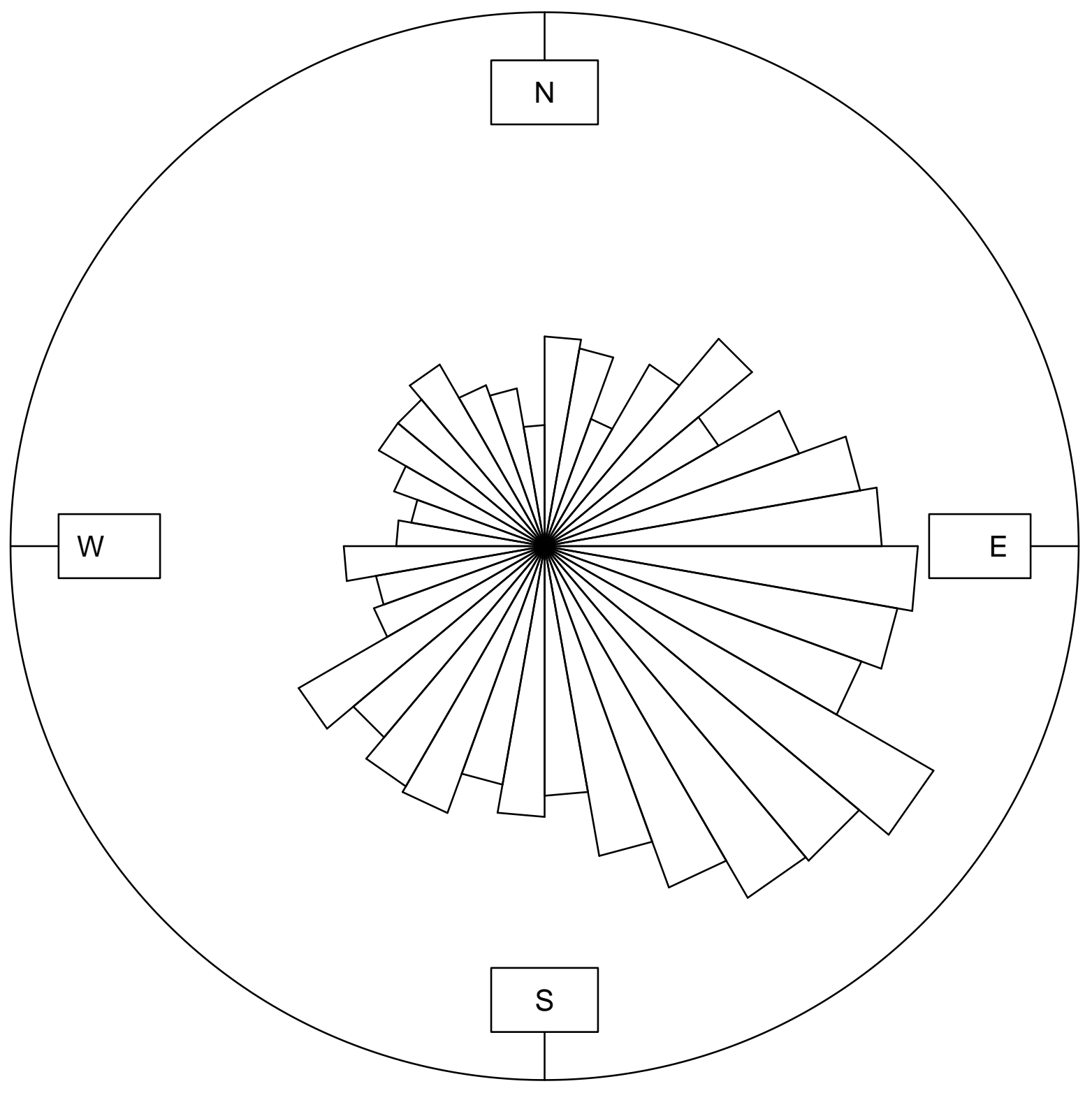


bootstrap 409

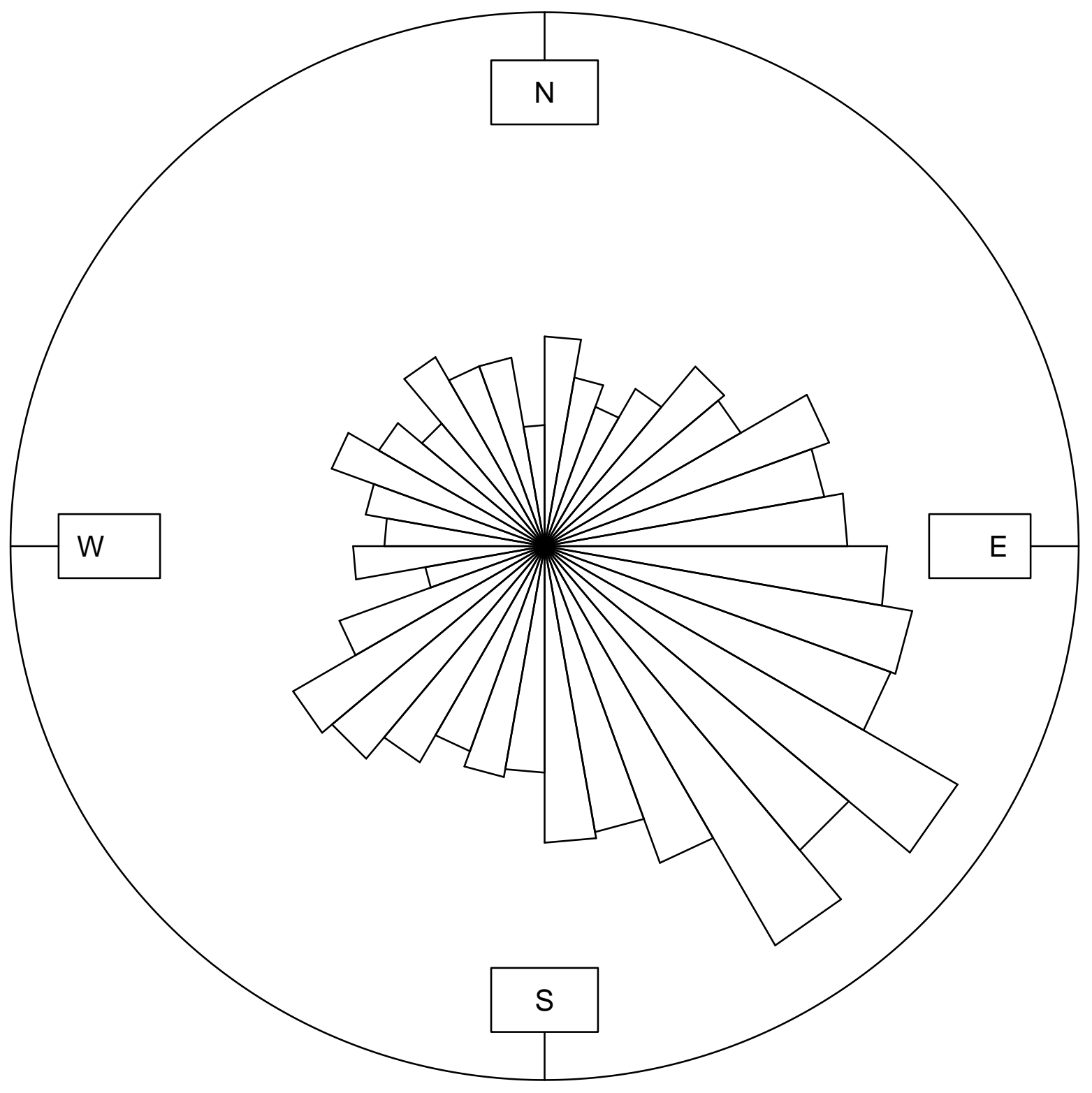


bootstrap 410

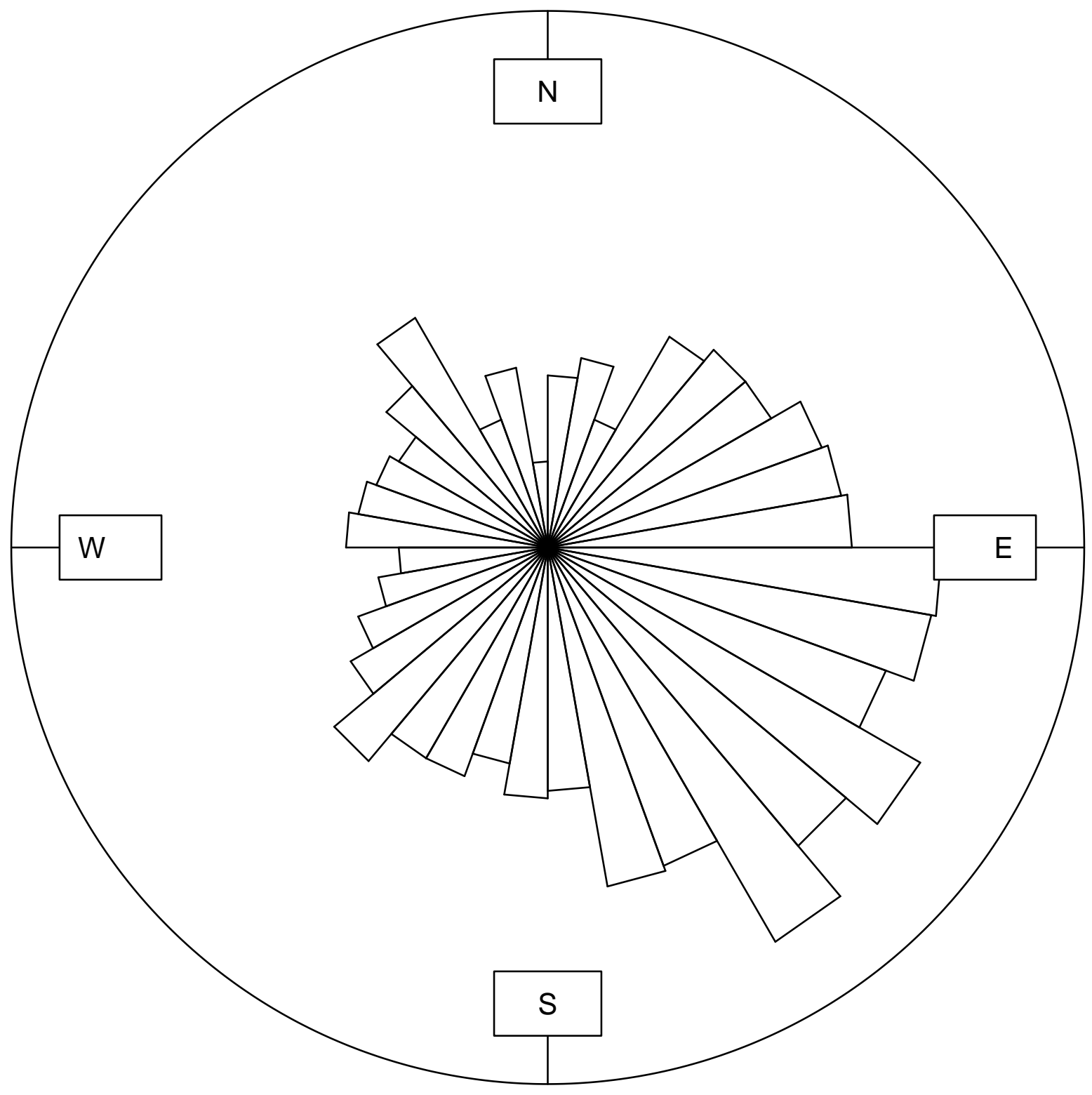


bootstrap 411

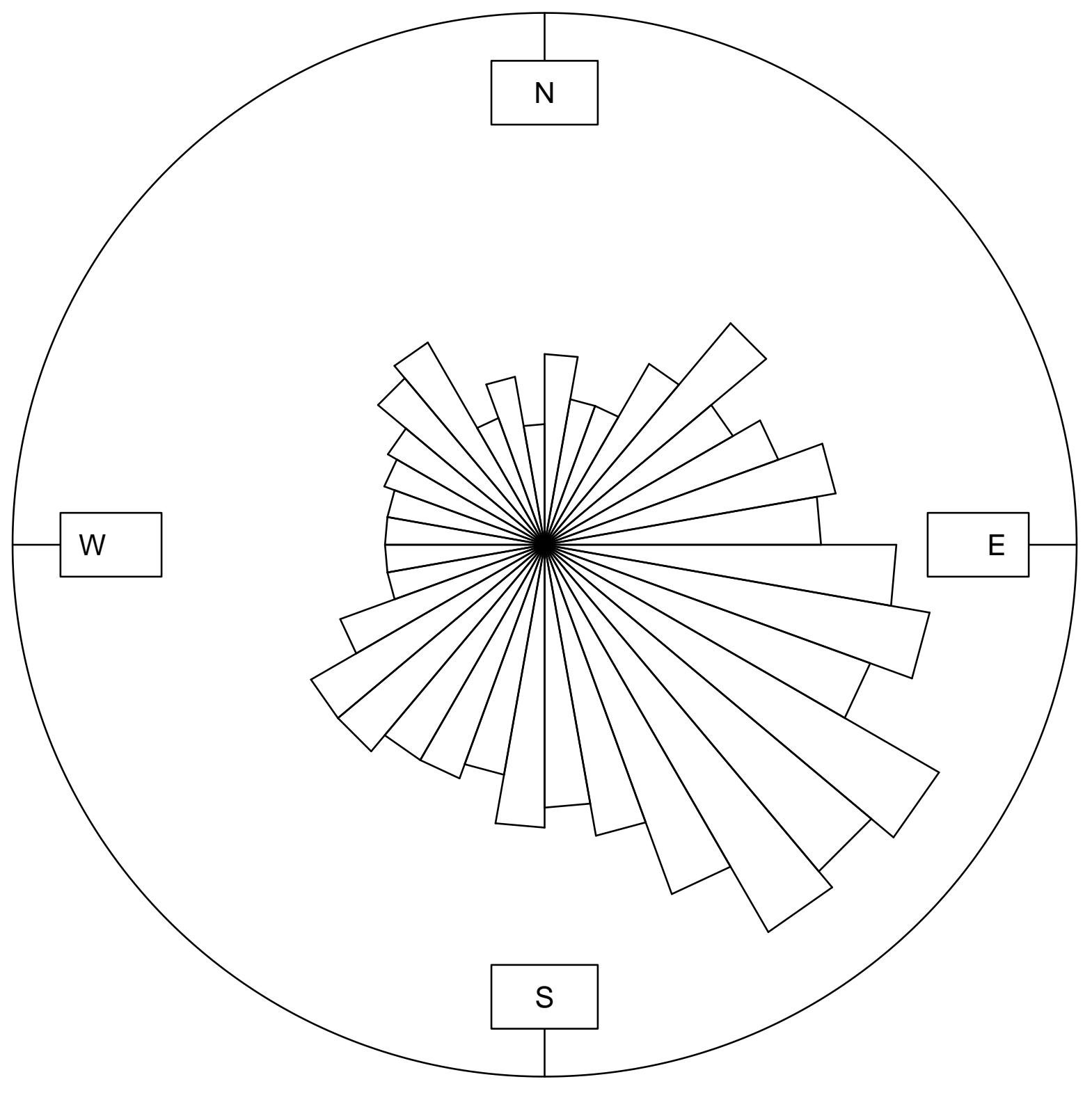


bootstrap 412

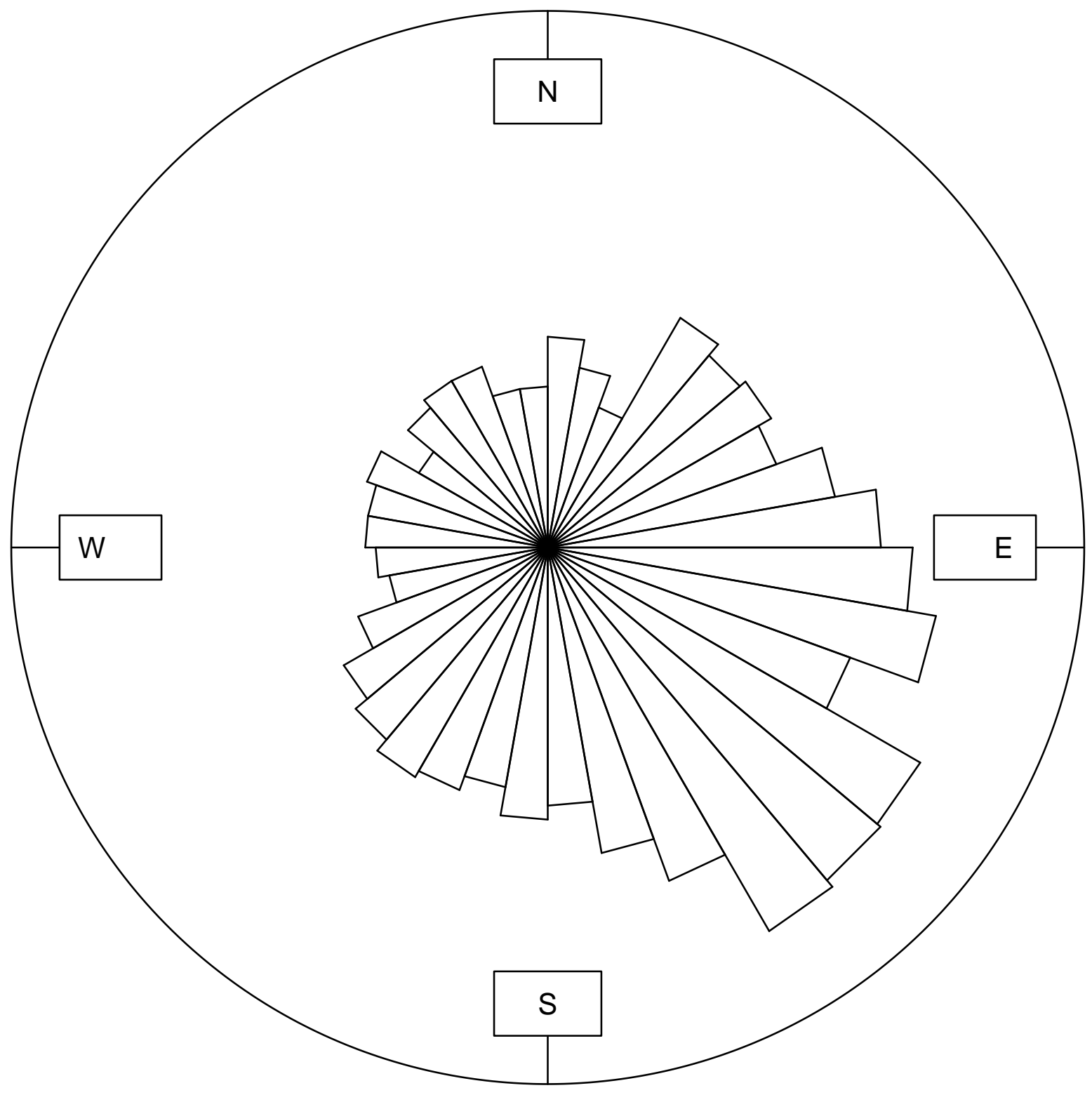


bootstrap 413

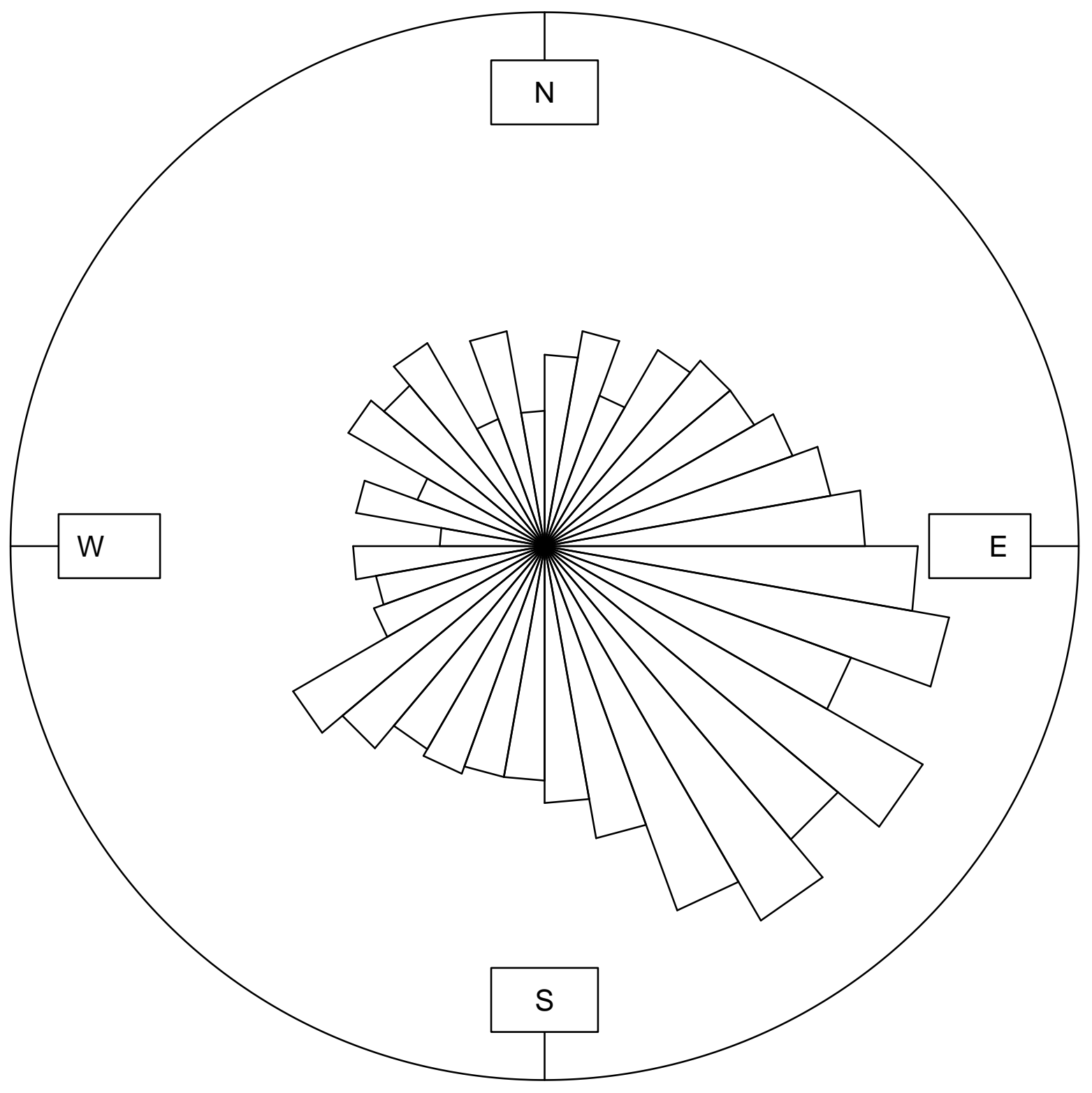


bootstrap 414

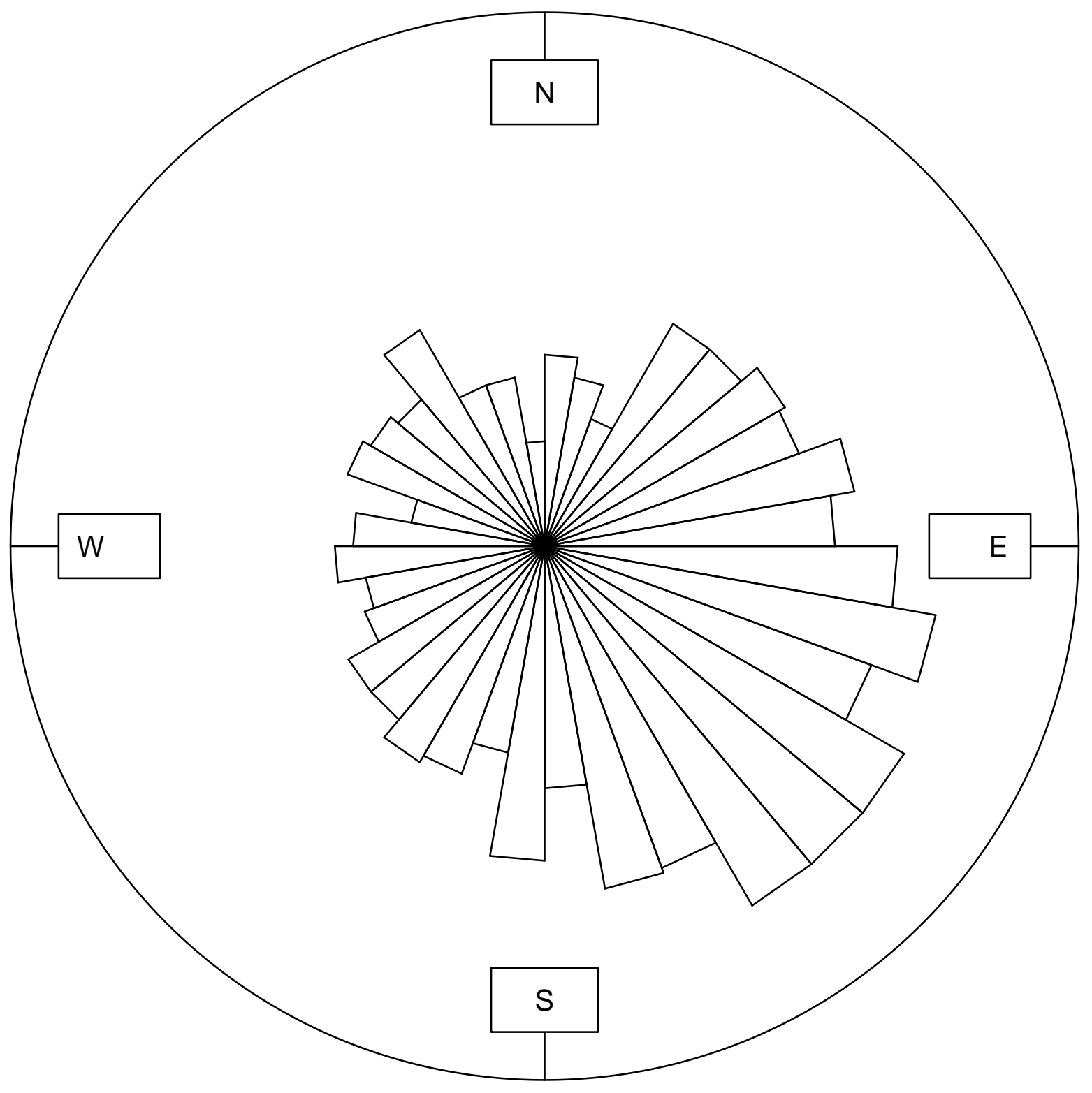




\section{bootstrap 415}

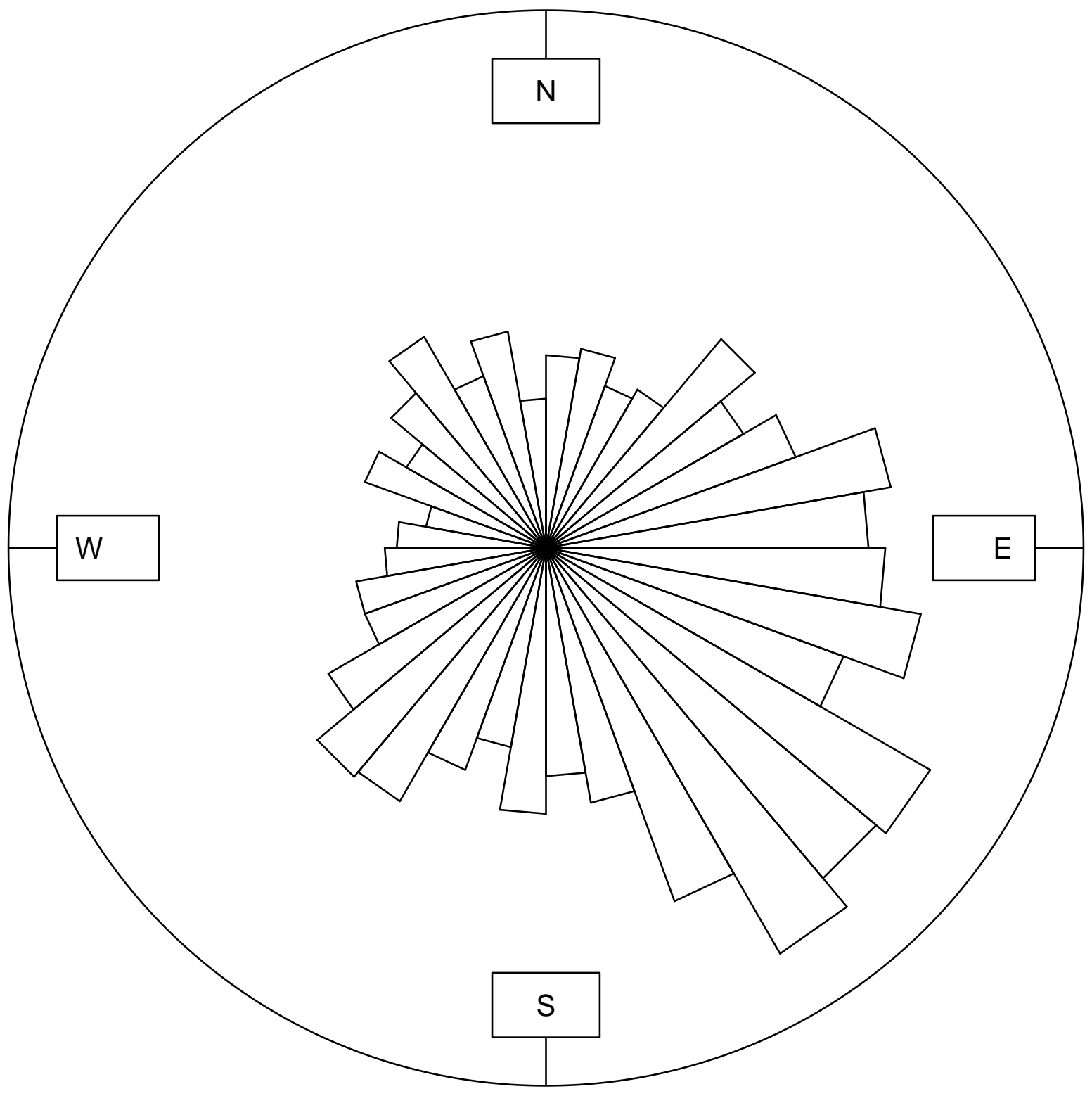




\section{bootstrap 416}

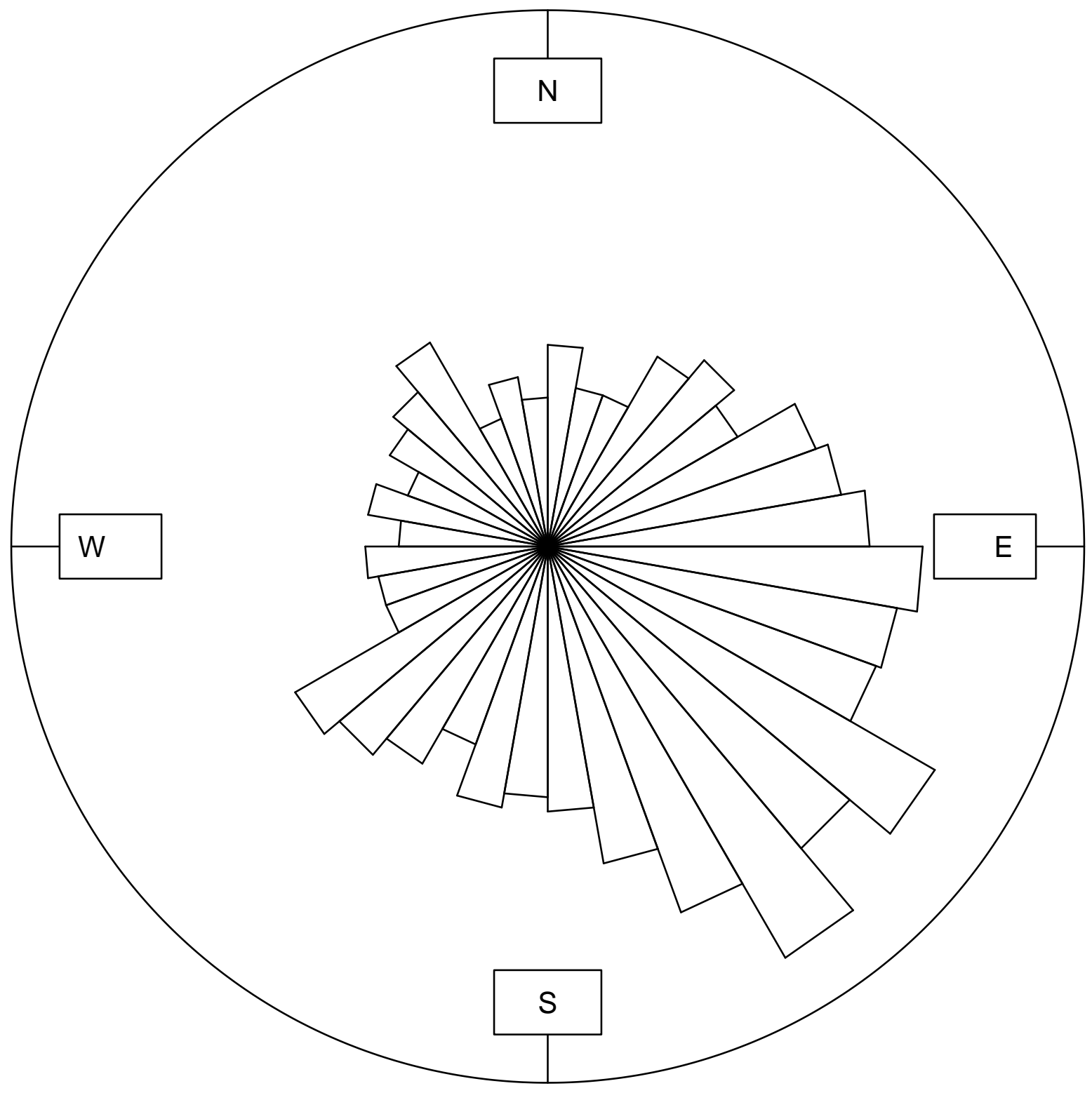


bootstrap 417

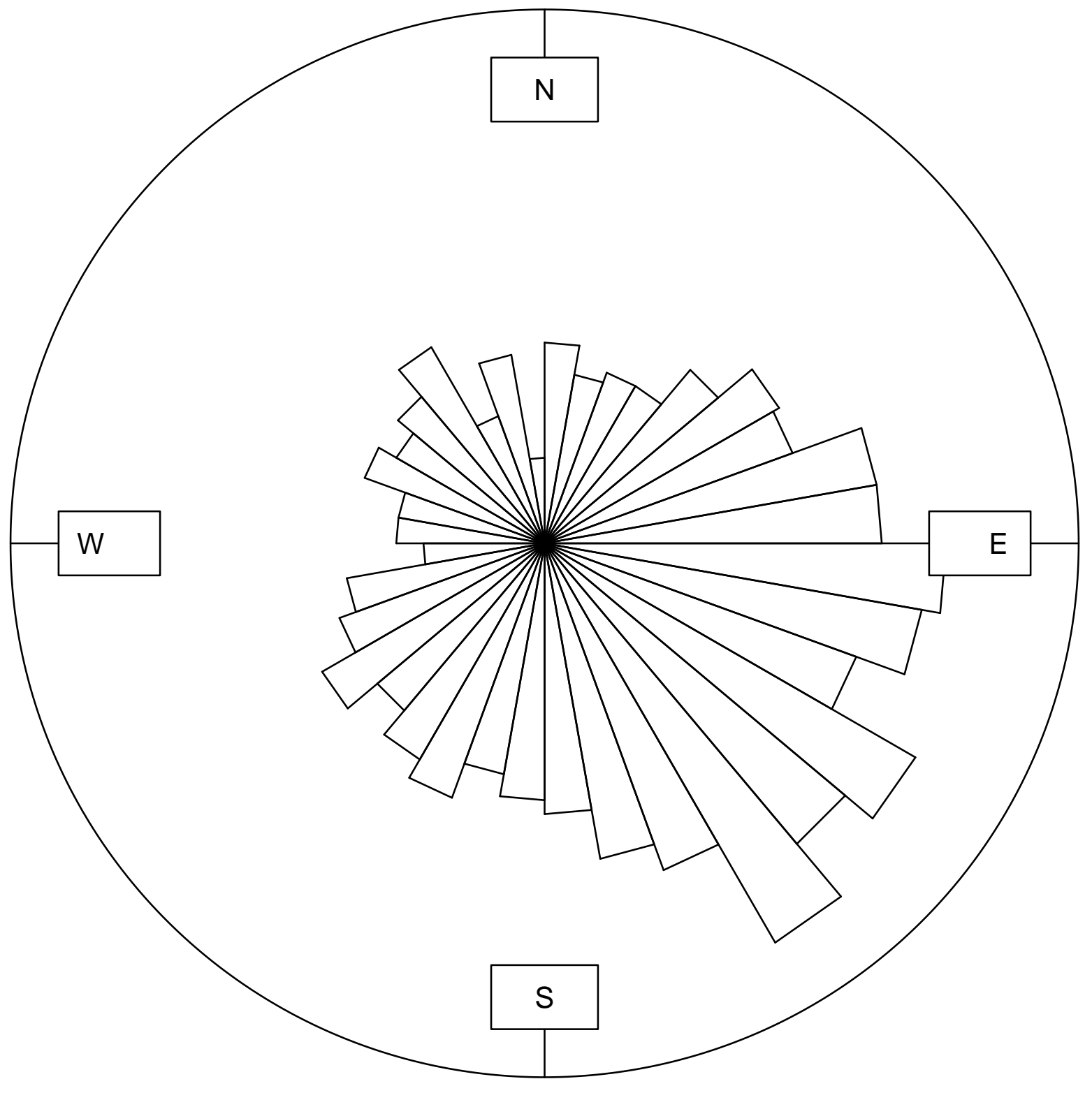


bootstrap 418

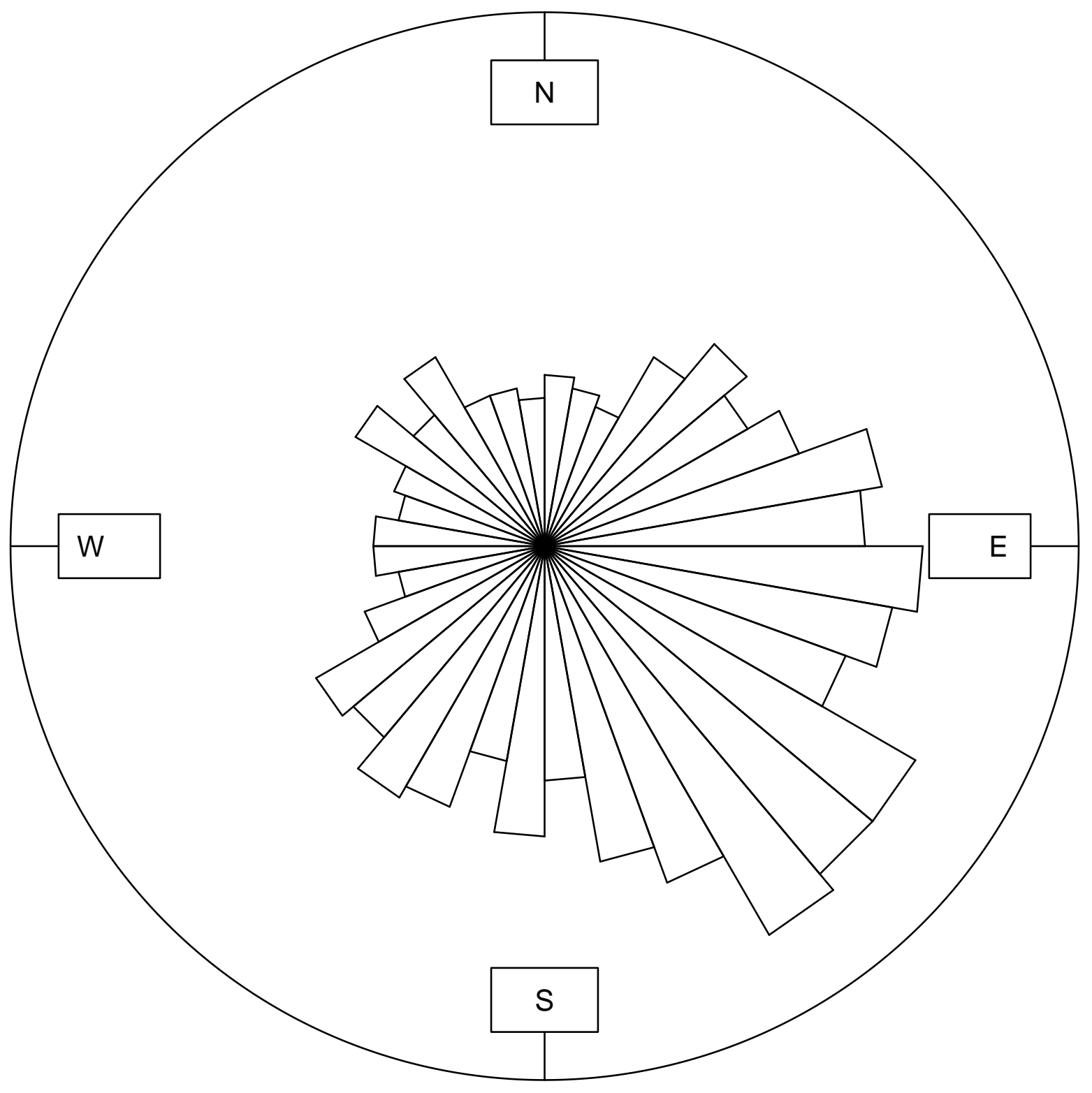


bootstrap 419

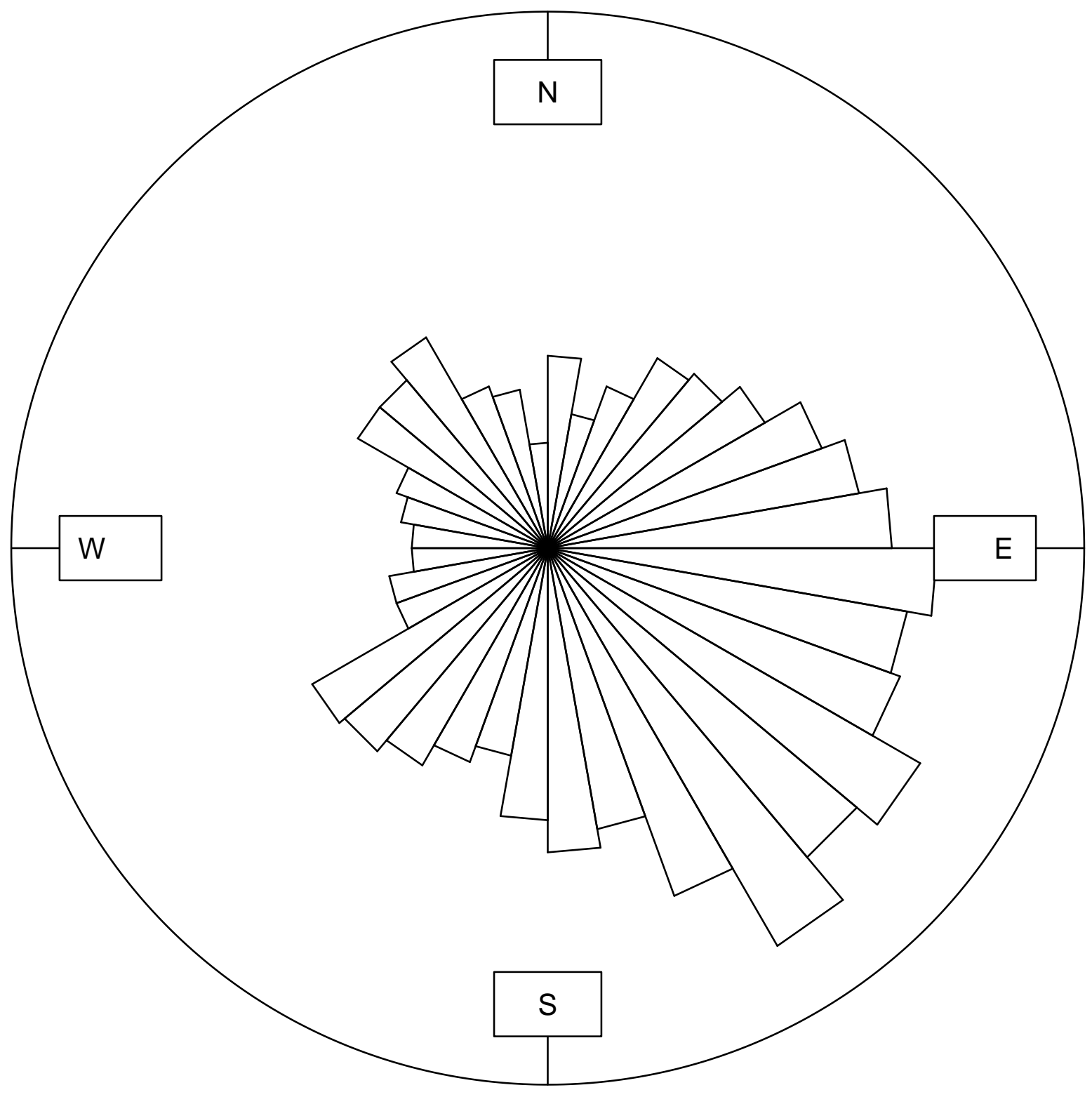


bootstrap 420

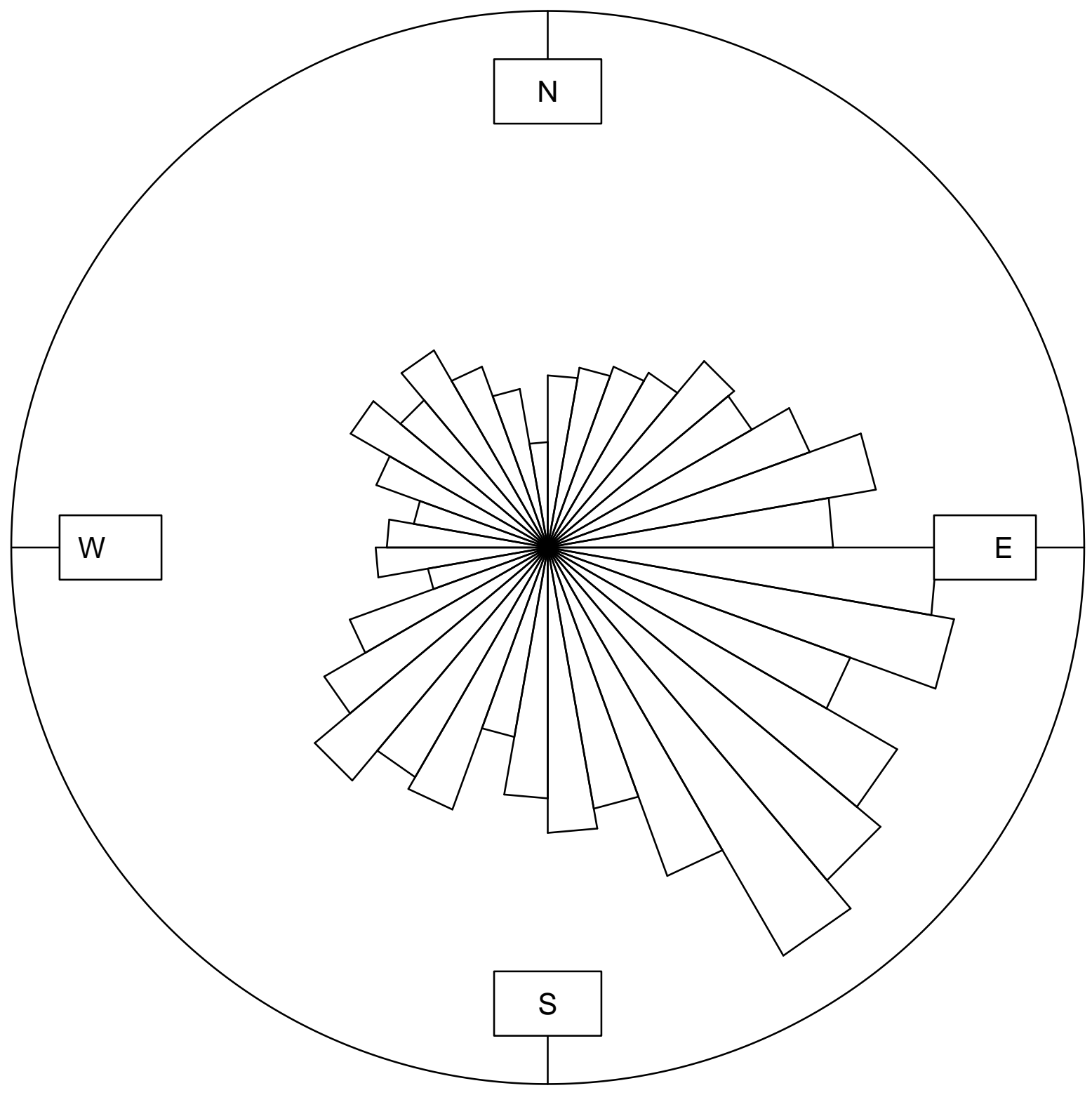


bootstrap 421

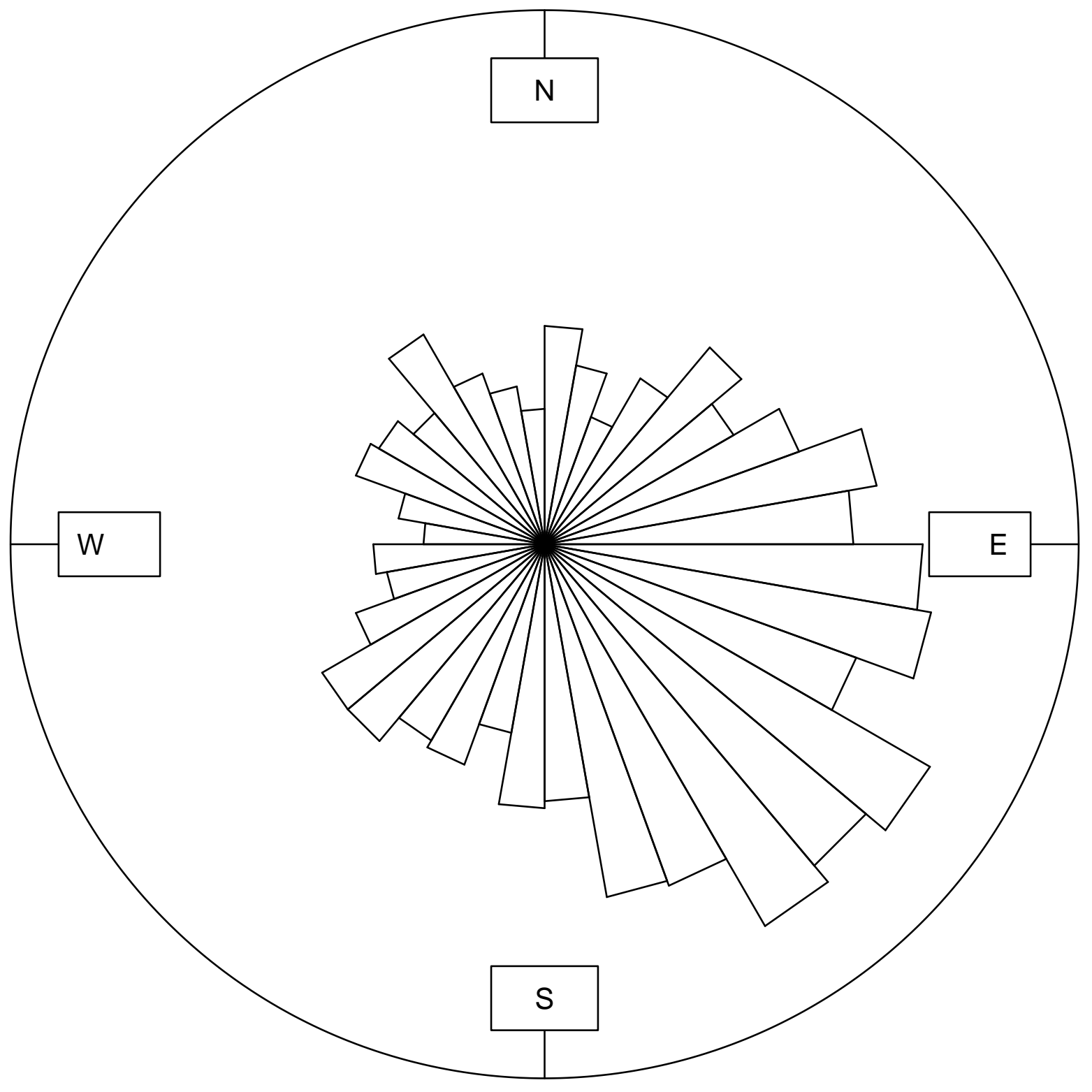


bootstrap 422

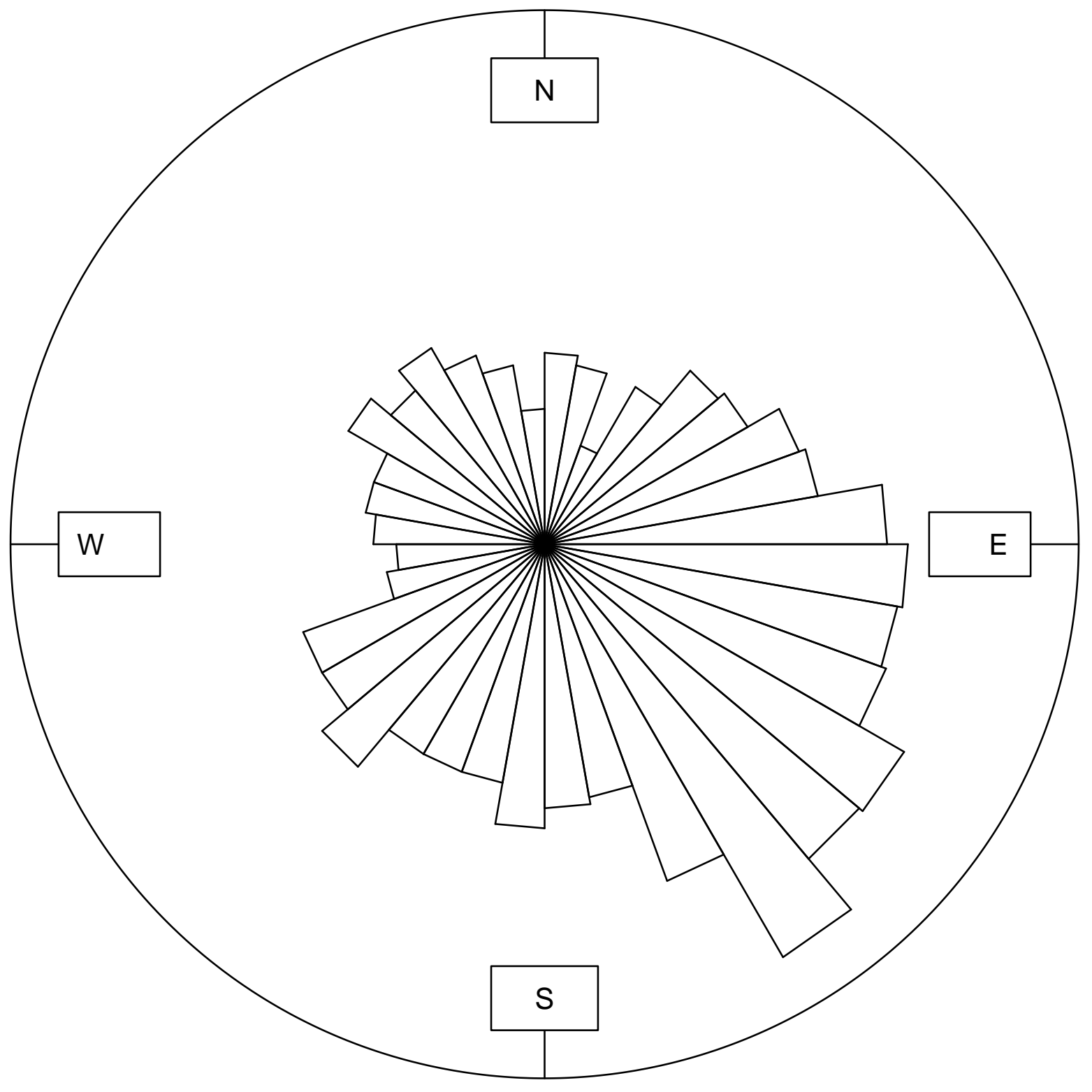


bootstrap 423

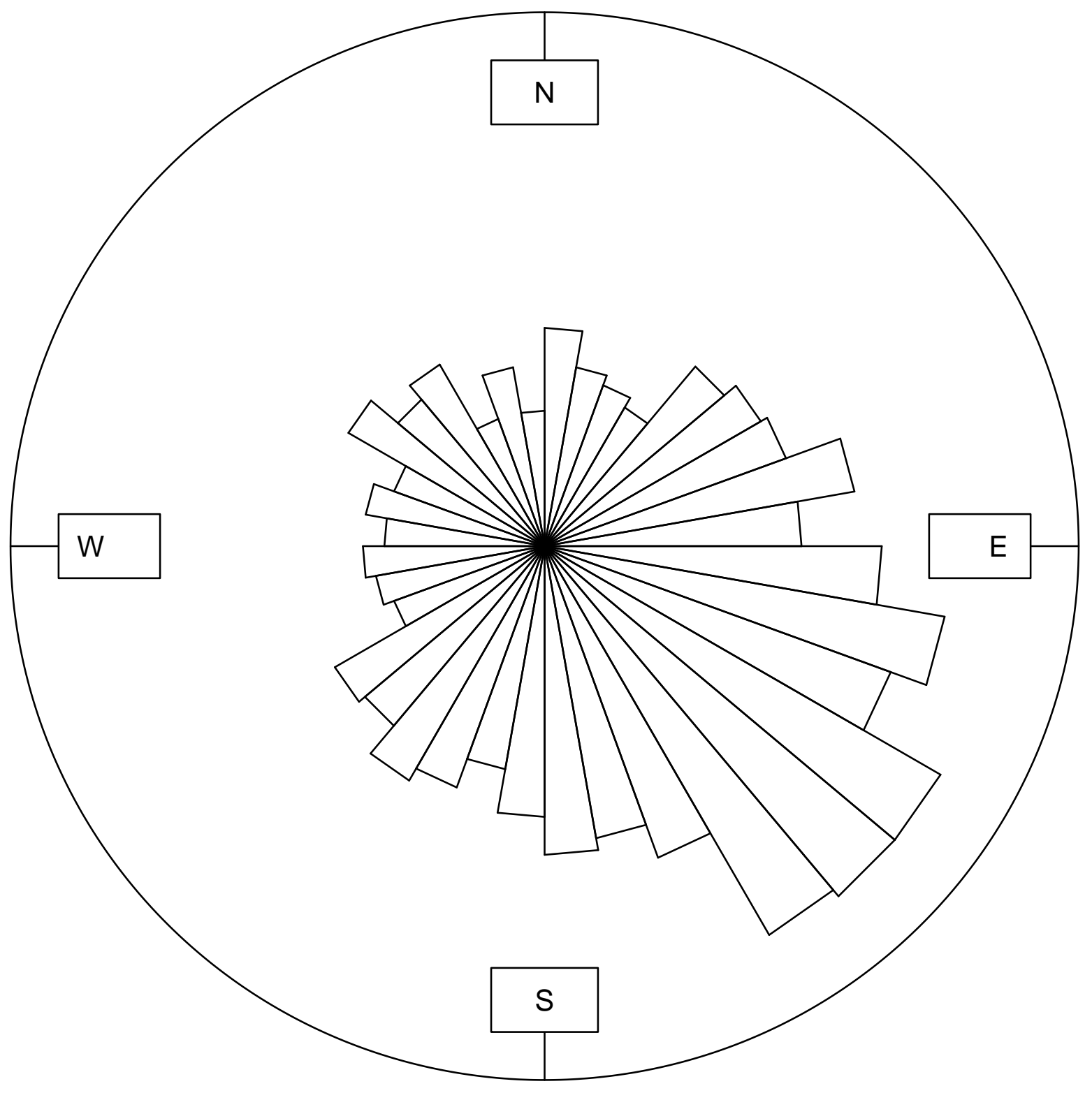


bootstrap 424

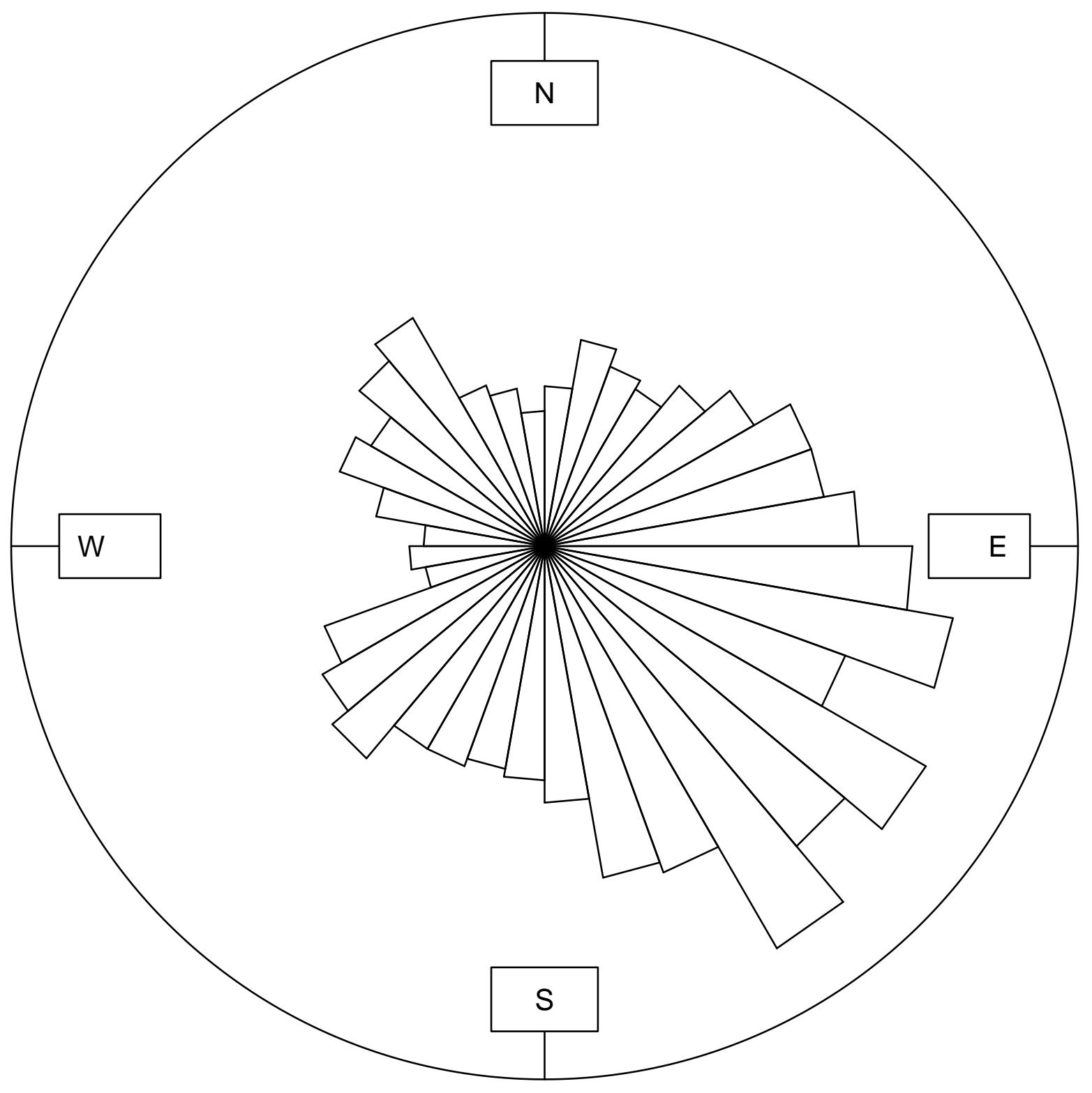


bootstrap 425

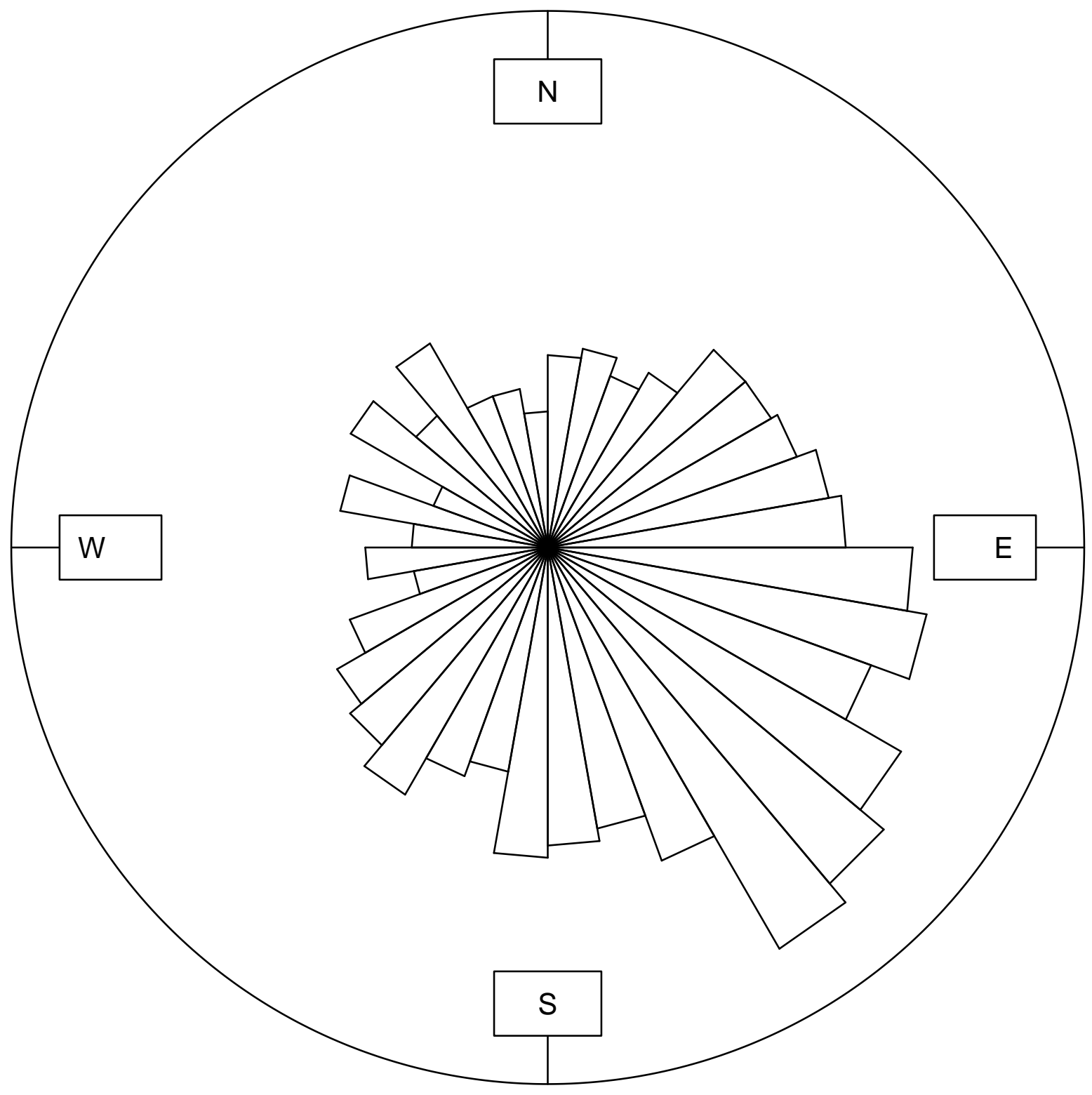




\section{bootstrap 426}

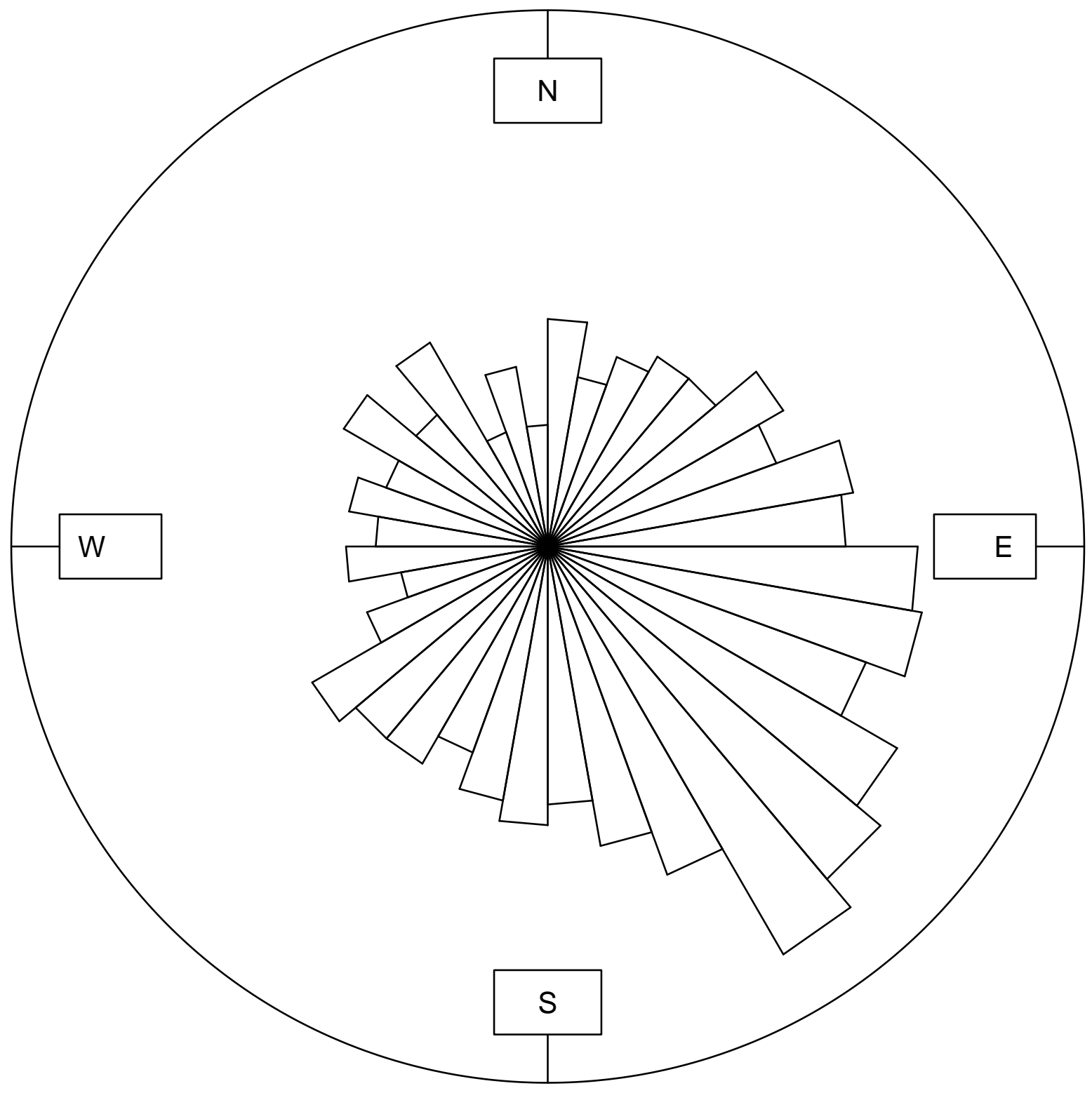


bootstrap 427

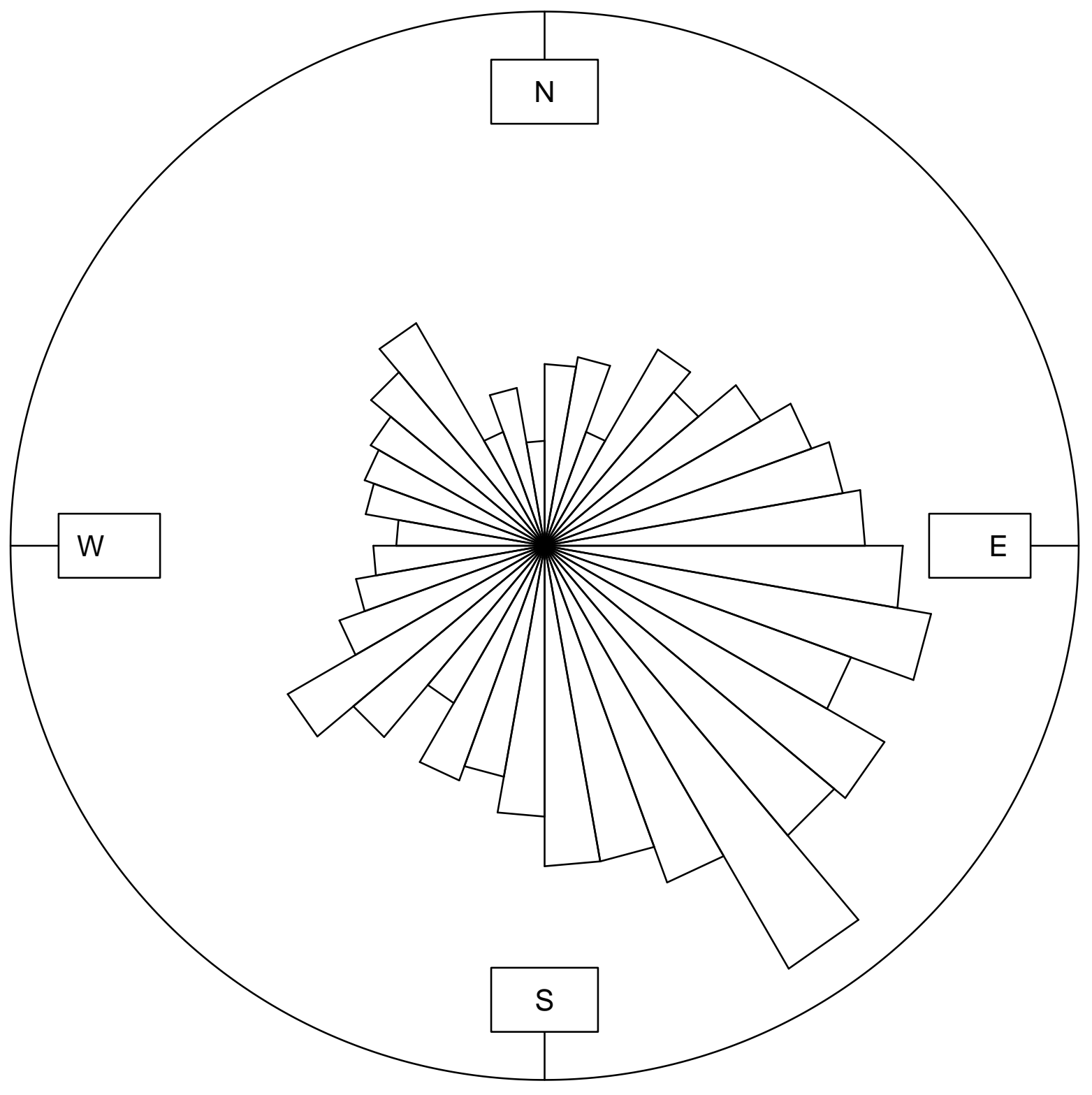


bootstrap 428

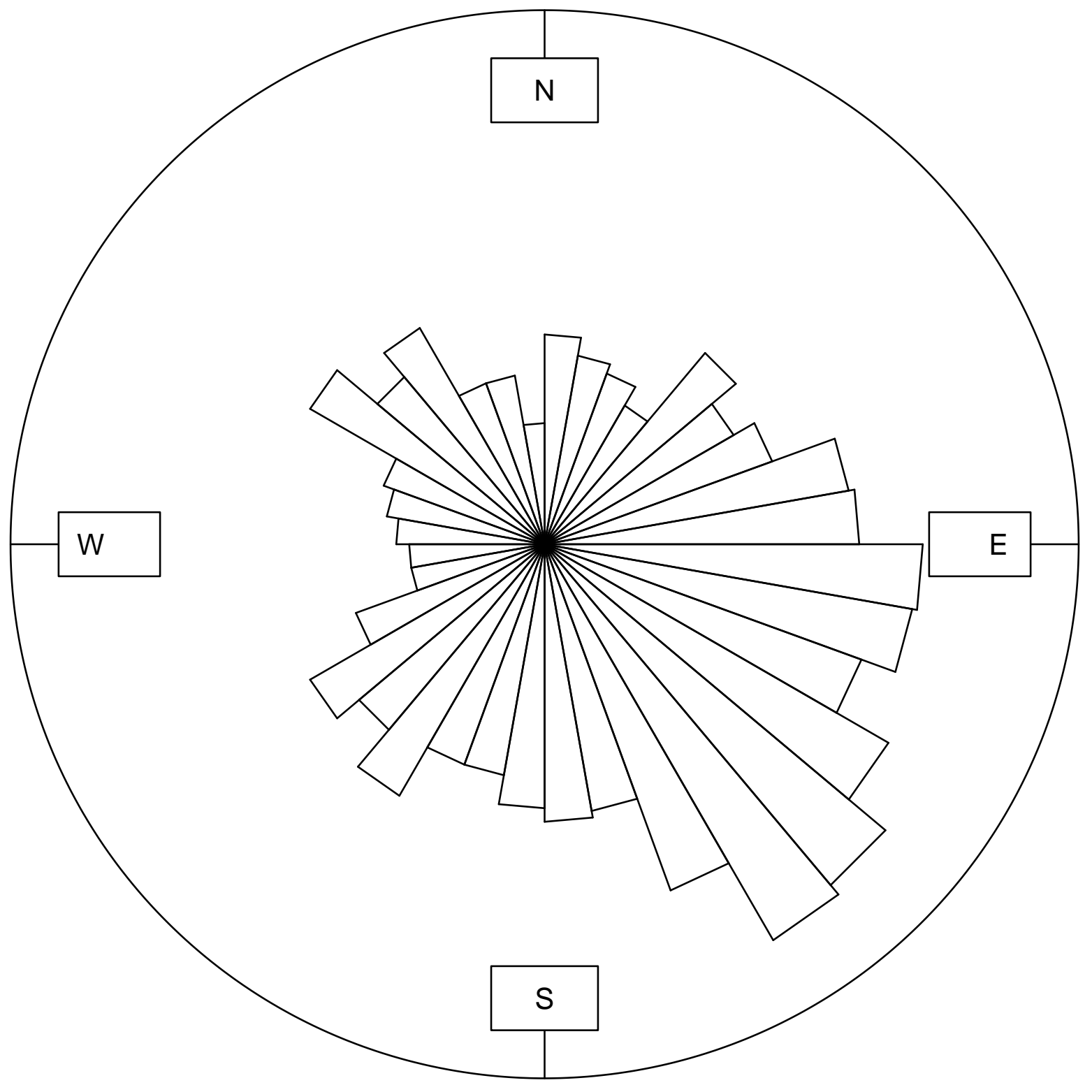


bootstrap 429

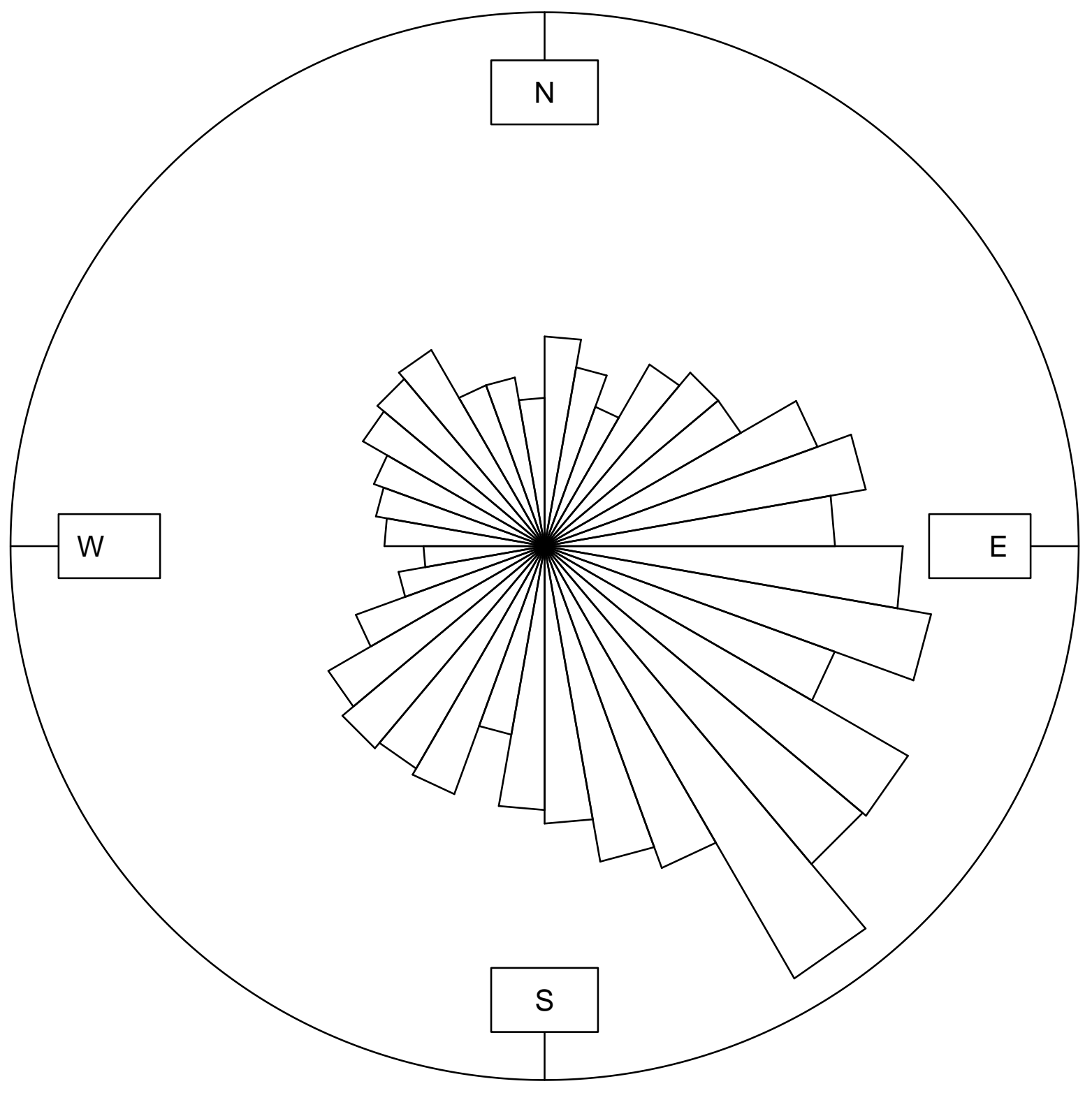


bootstrap 430

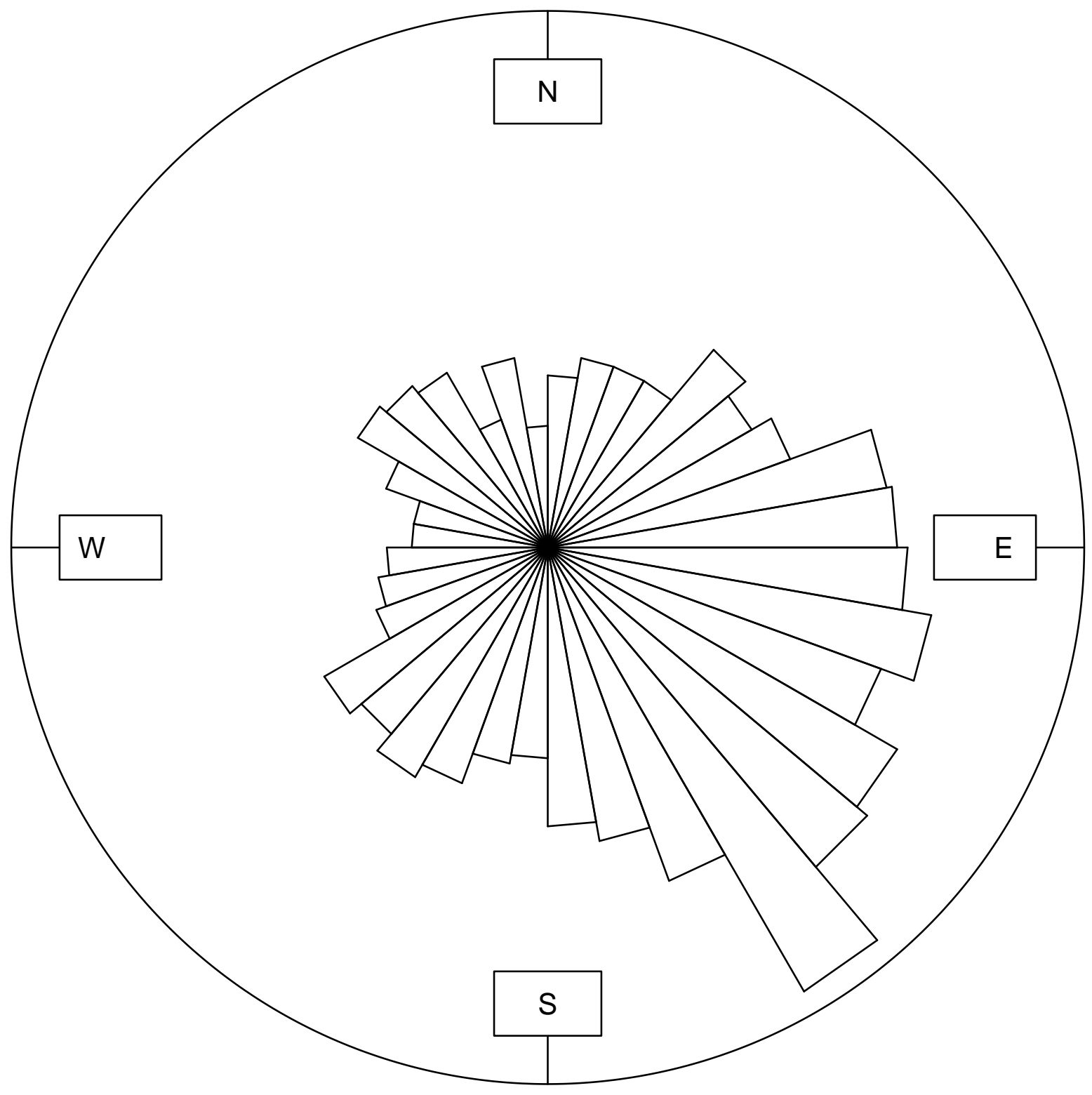


bootstrap 431

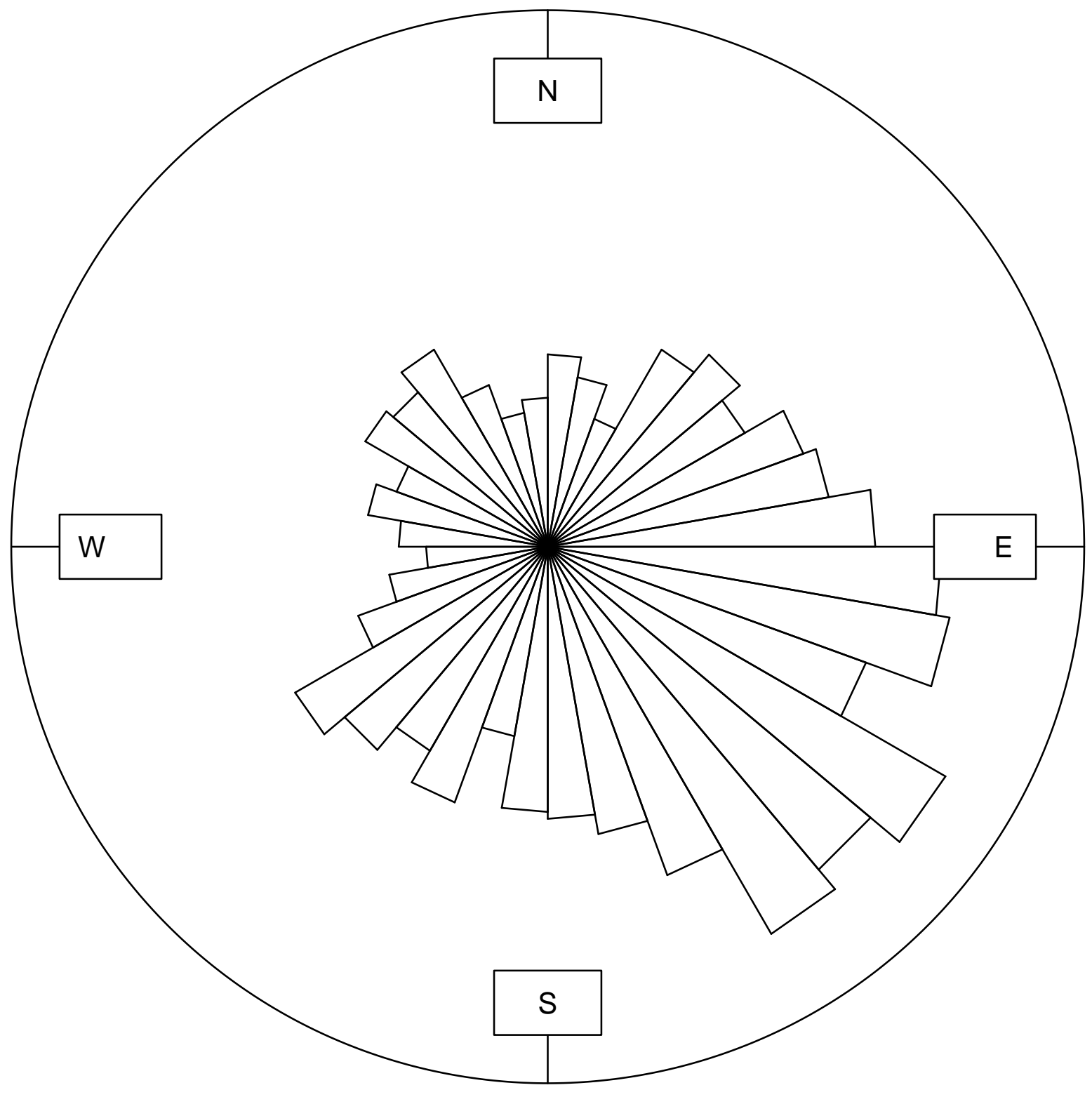


bootstrap 432

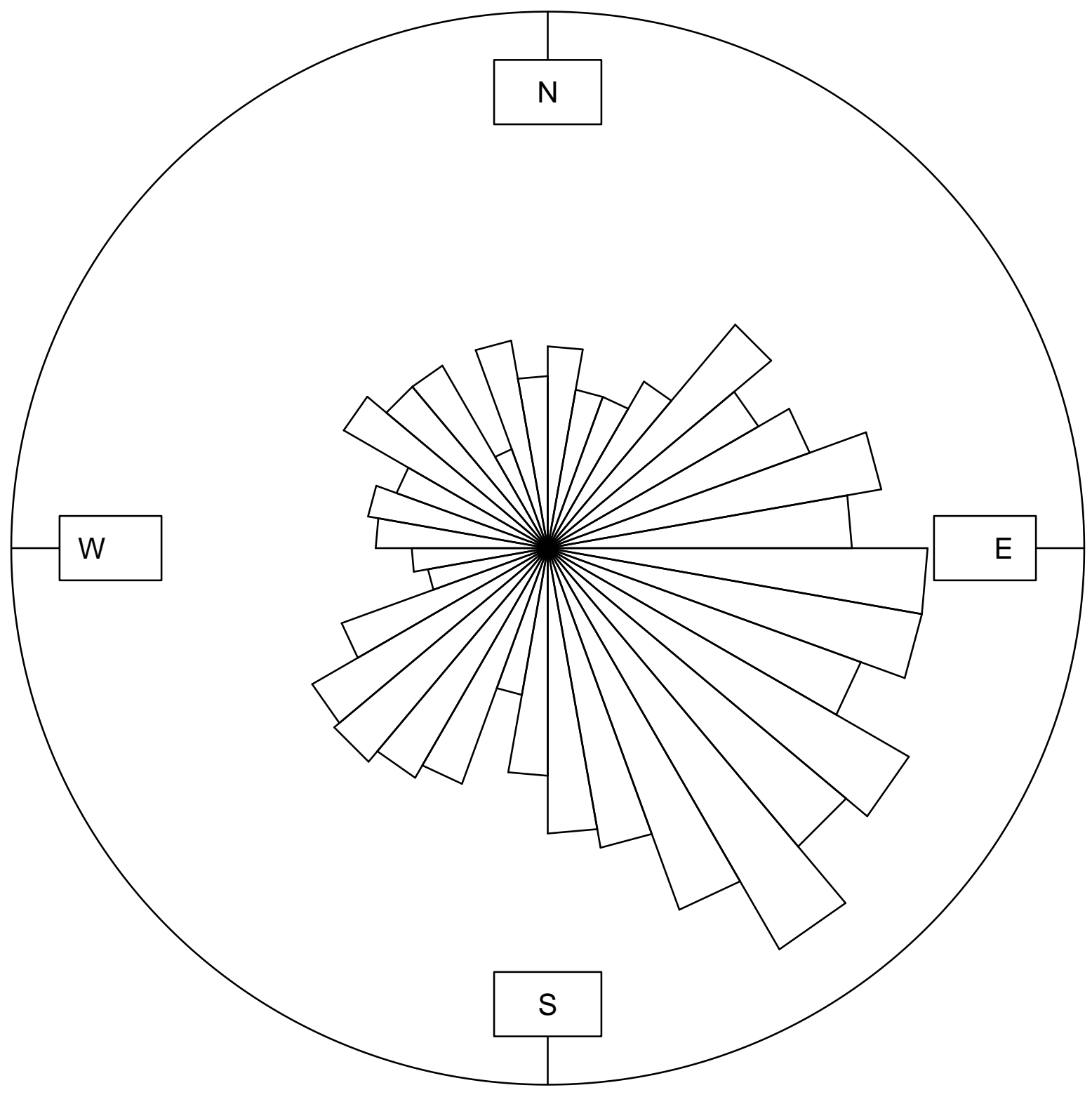




\section{bootstrap 433}

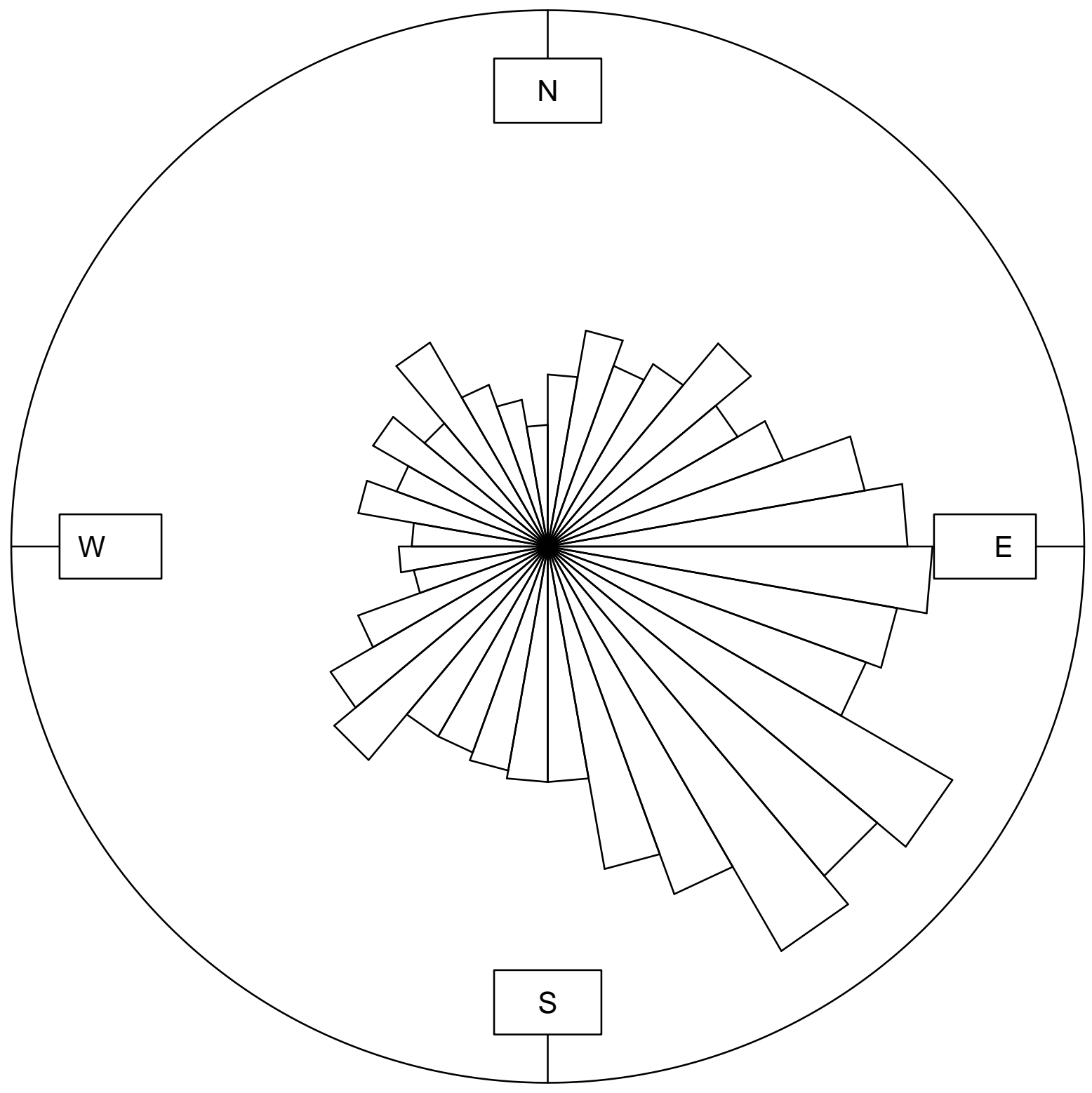


bootstrap 434

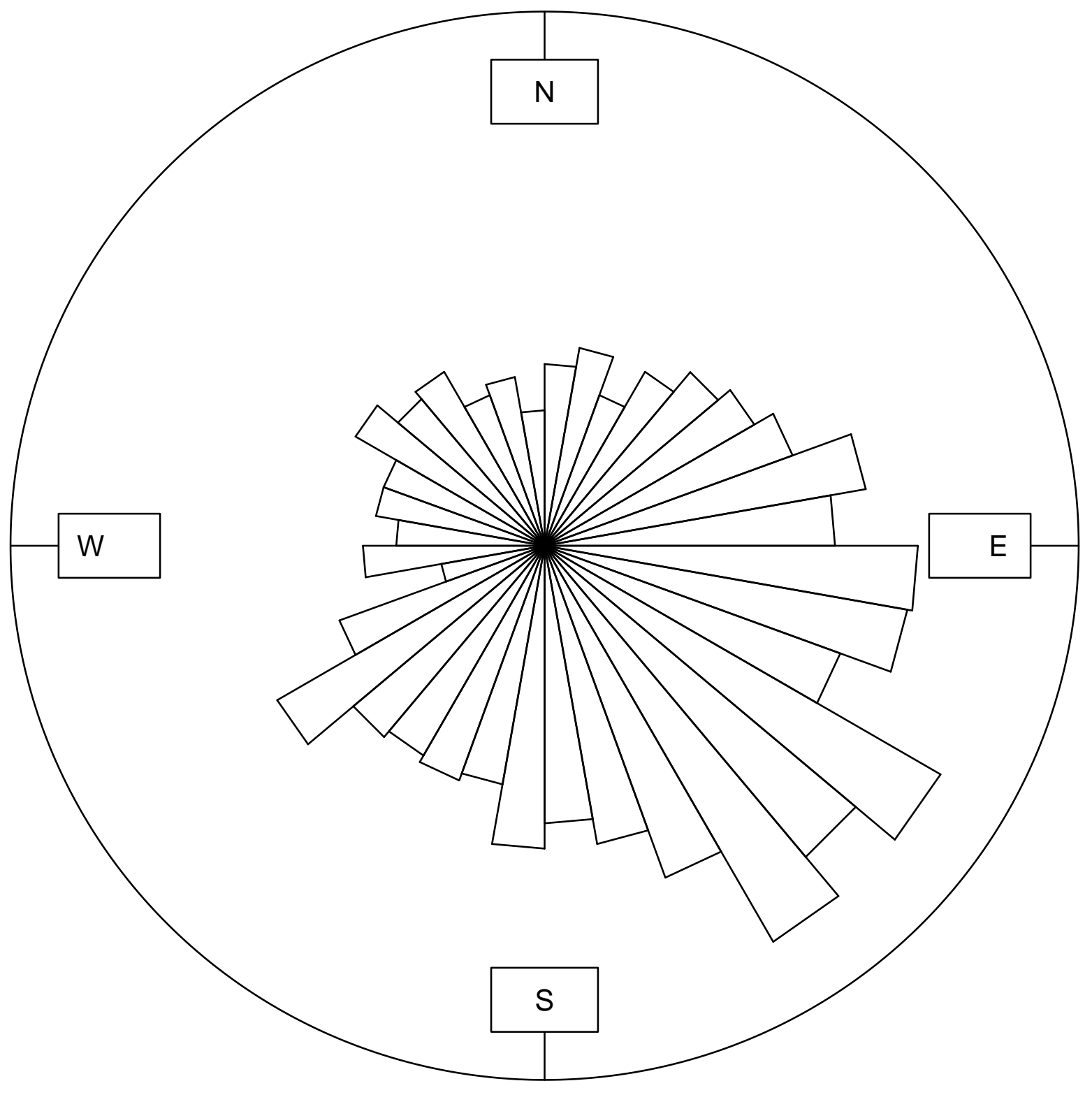




\section{bootstrap 435}

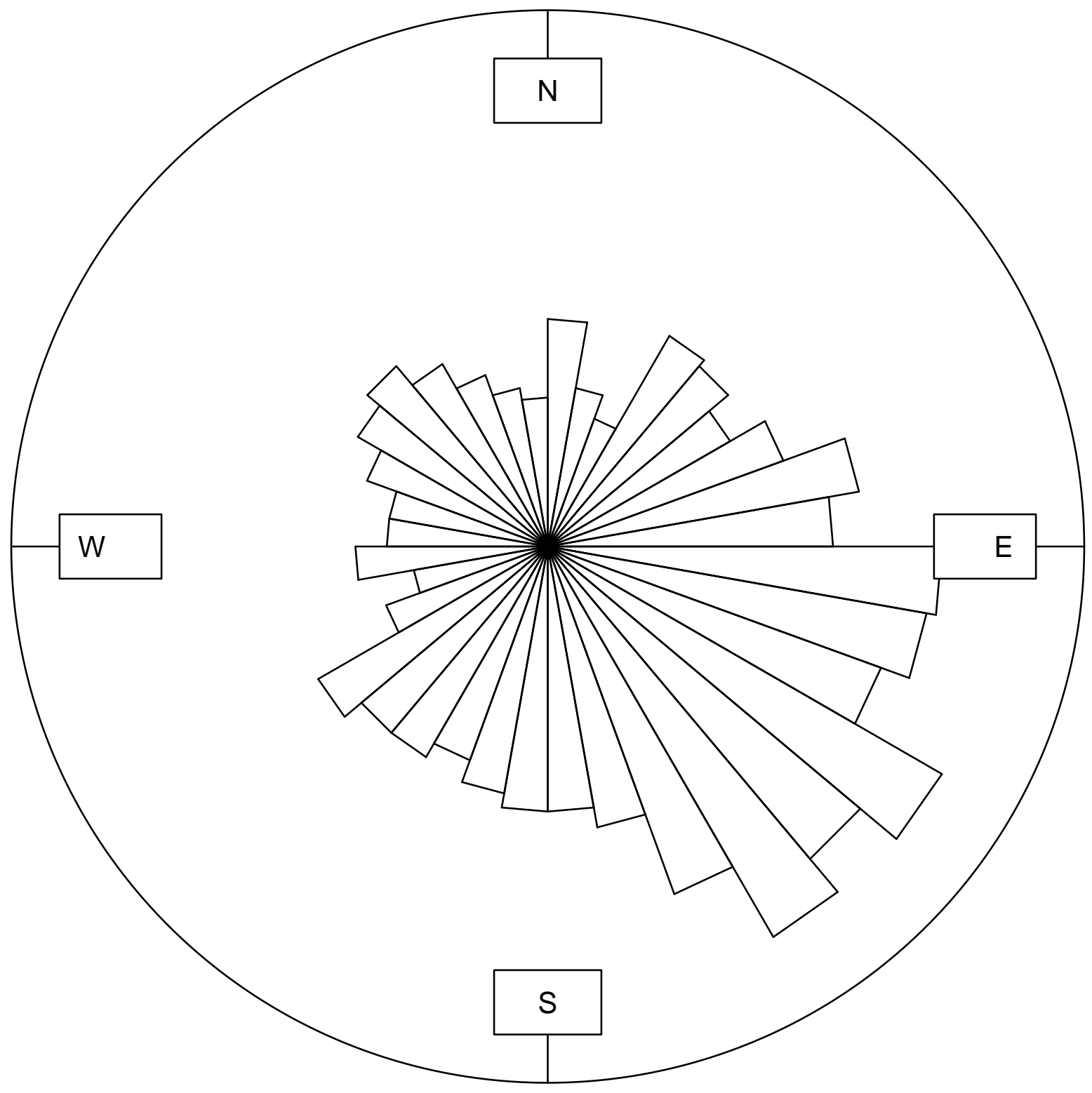




\section{bootstrap 436}

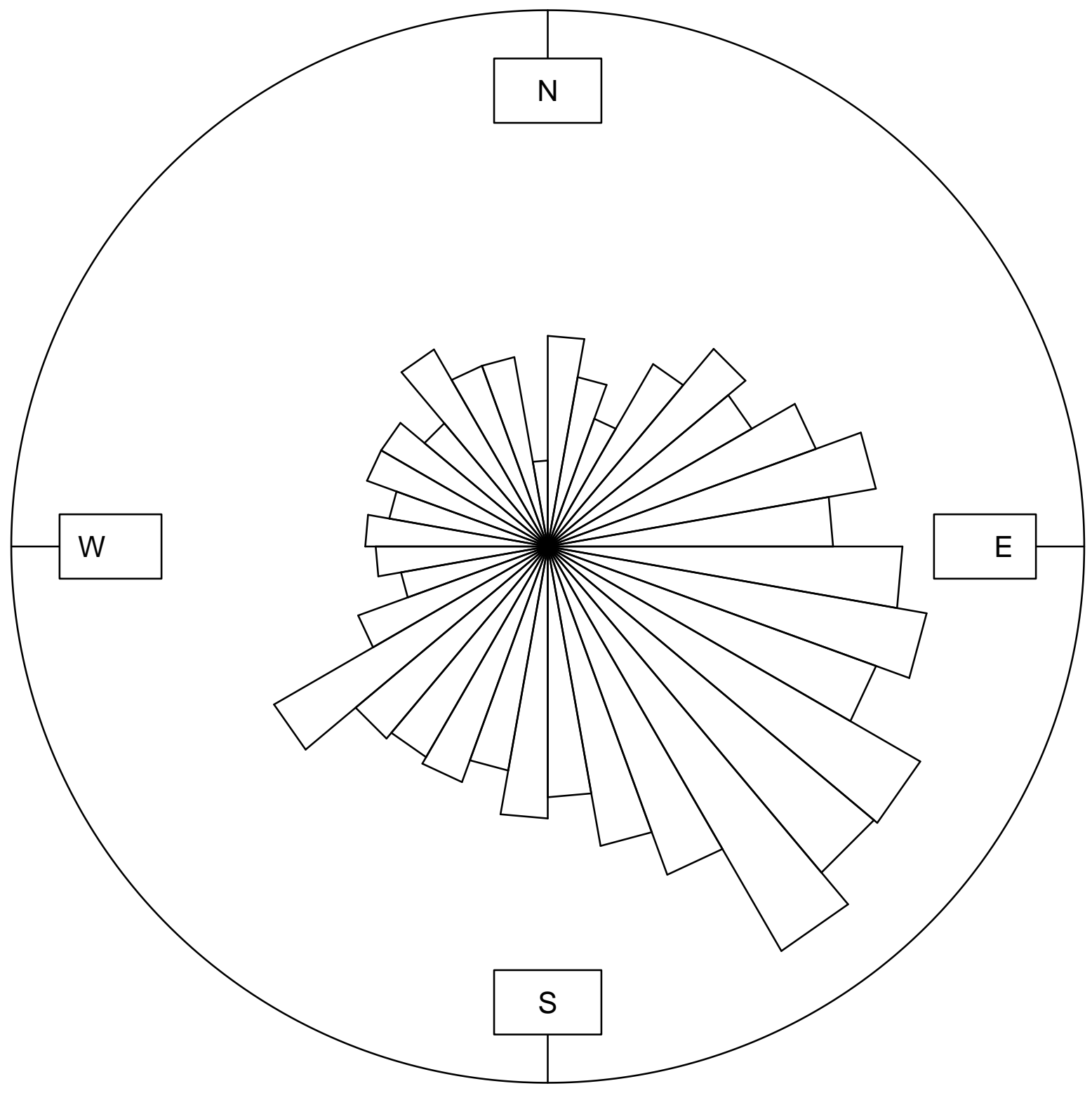


bootstrap 437

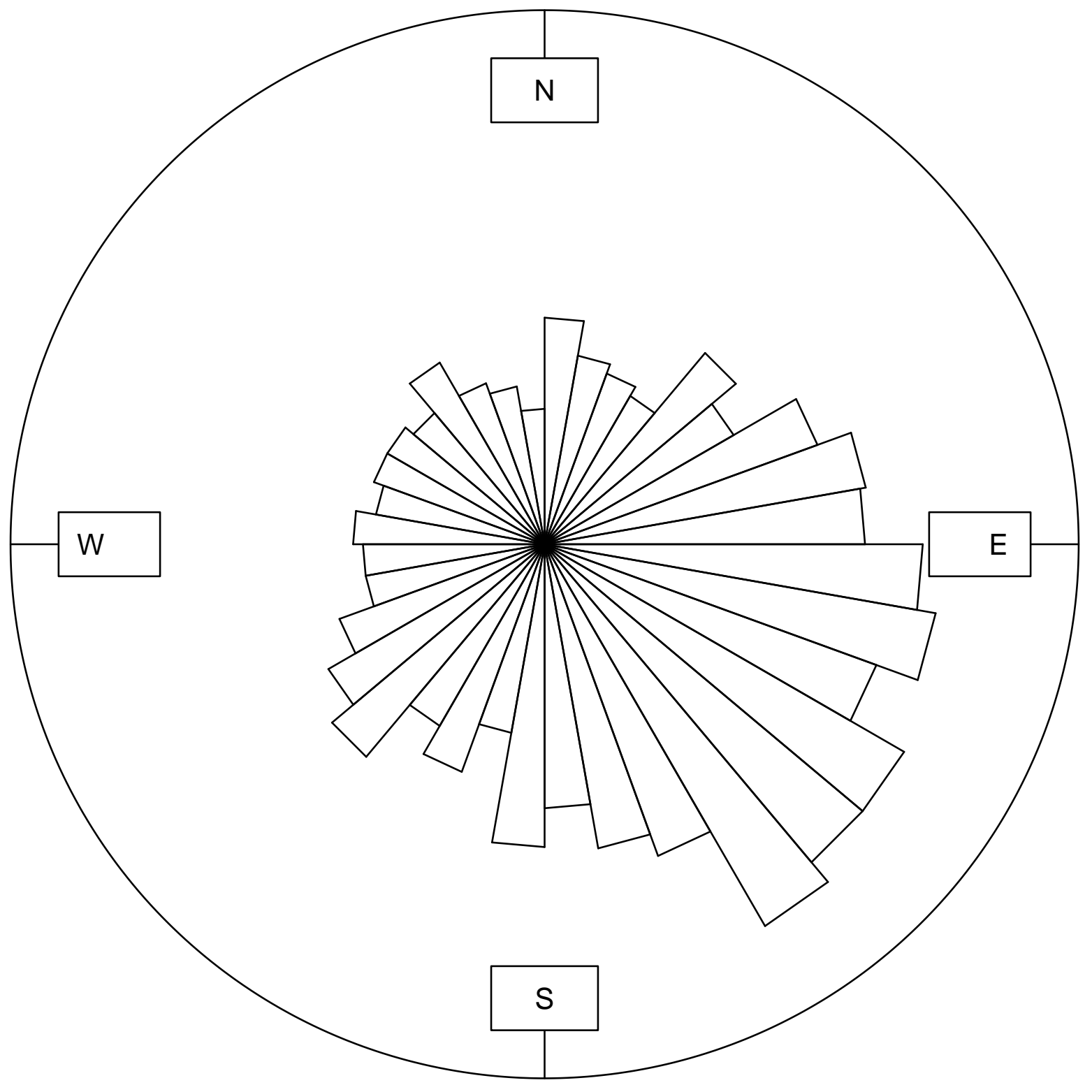




\section{bootstrap 438}

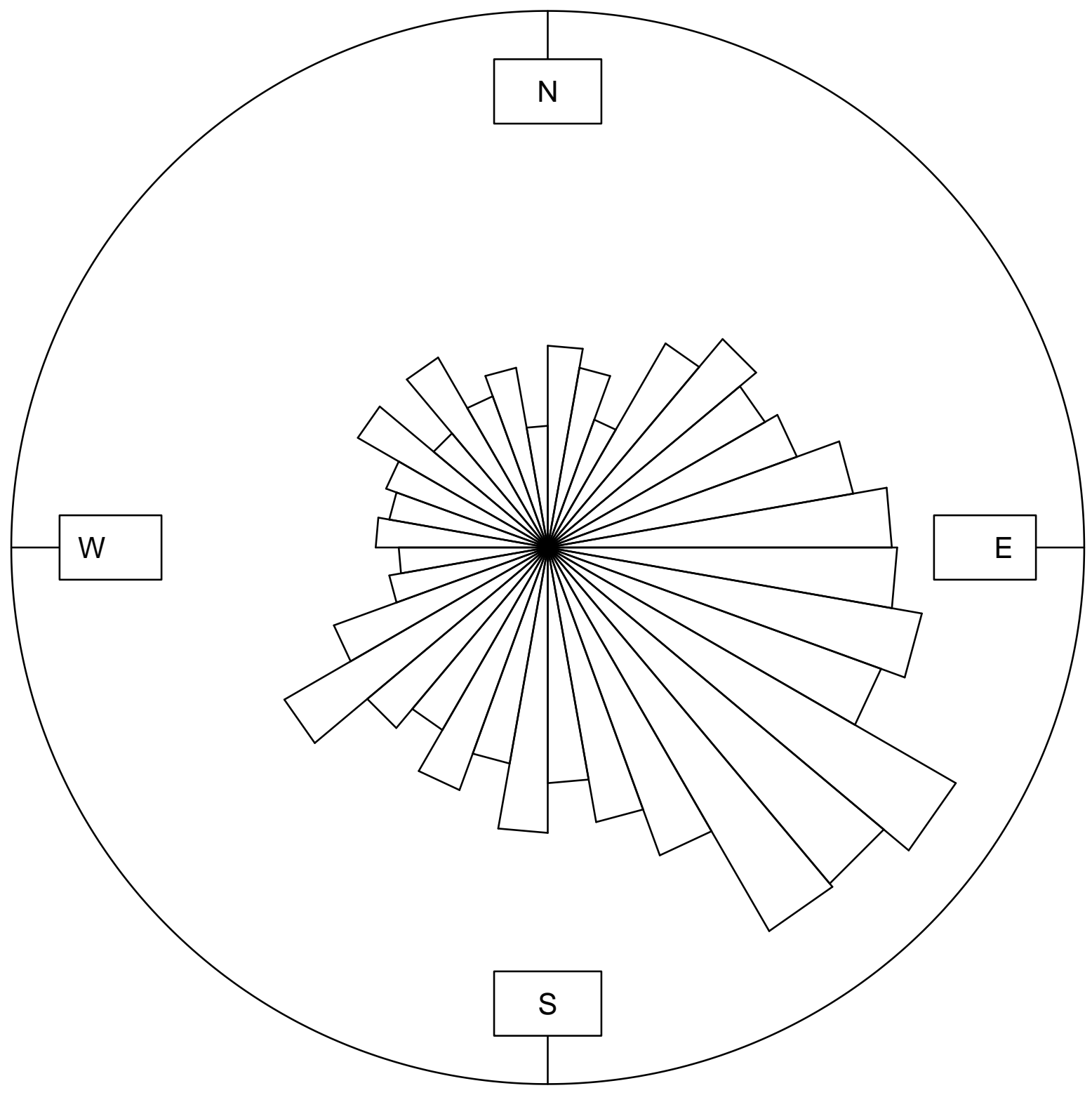


bootstrap 439

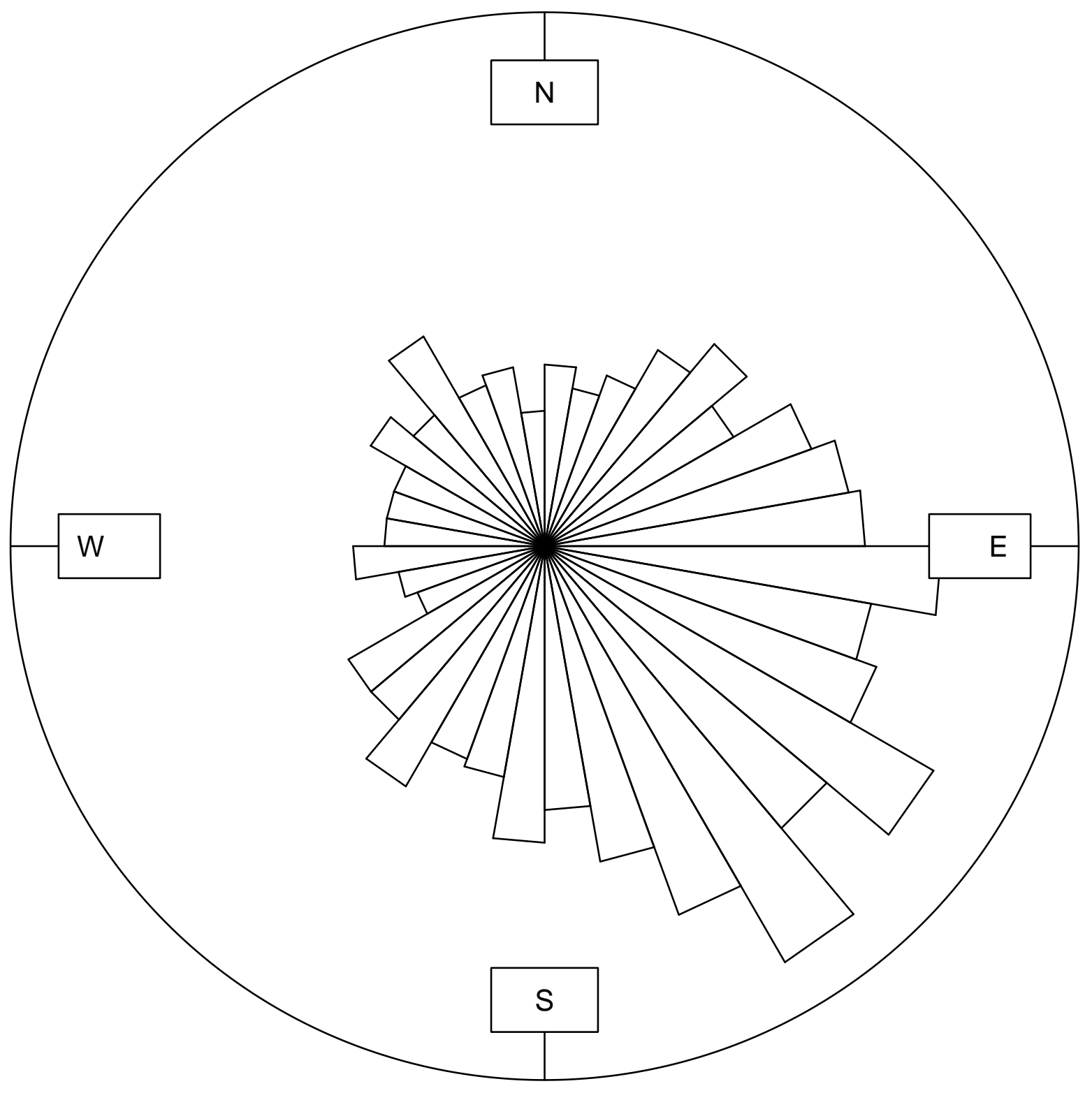


bootstrap 440

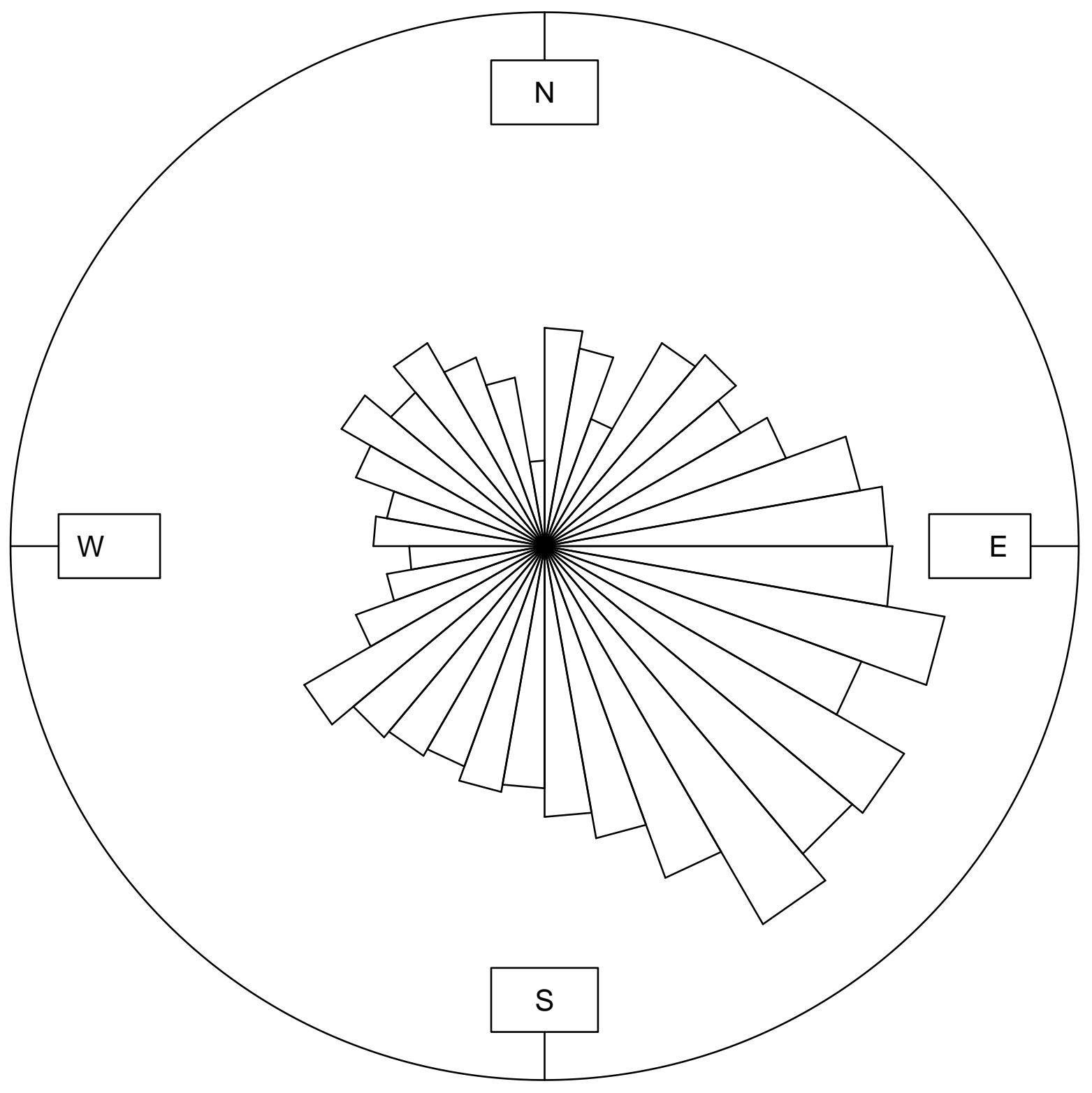


bootstrap 441

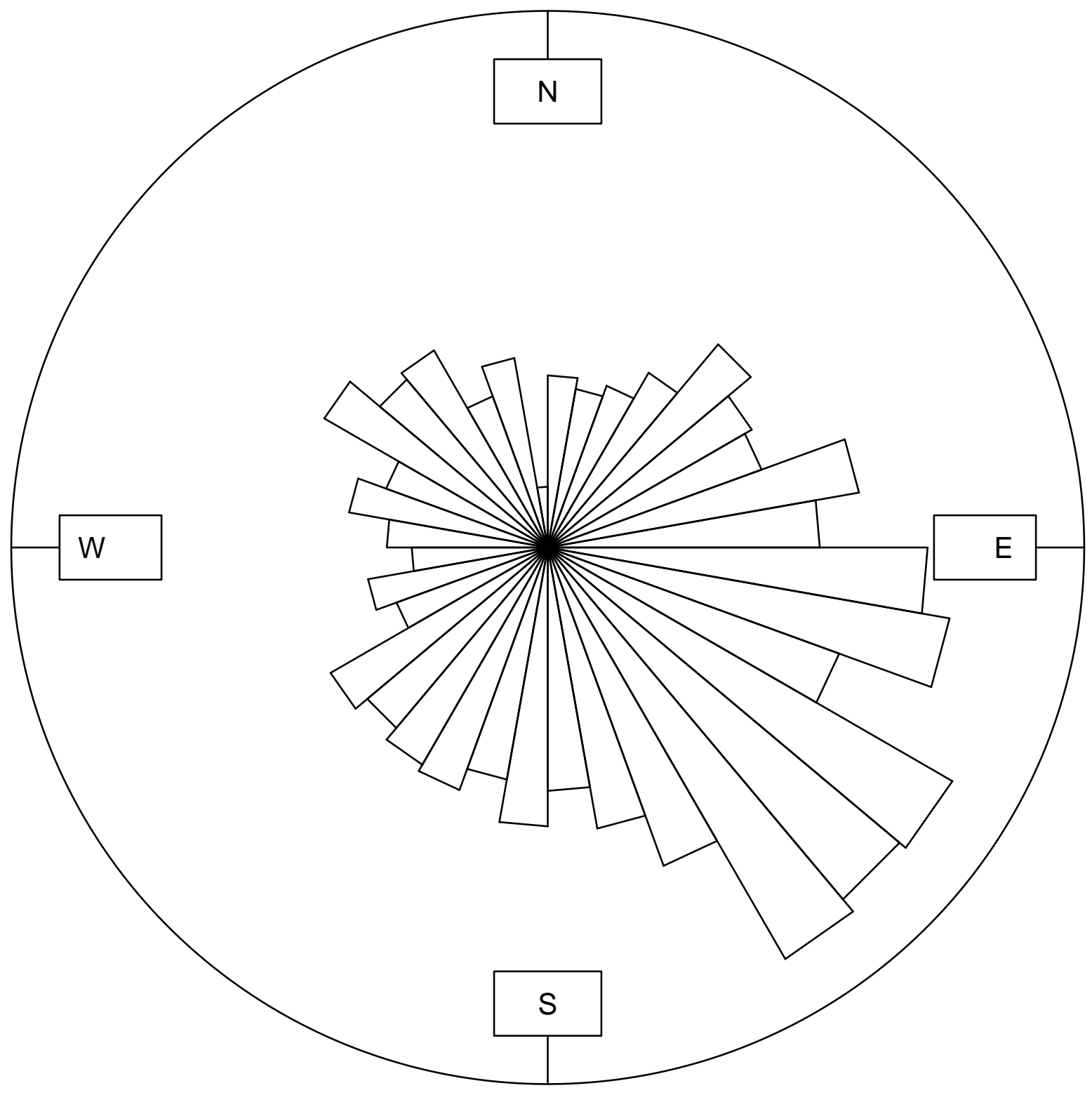


bootstrap 442

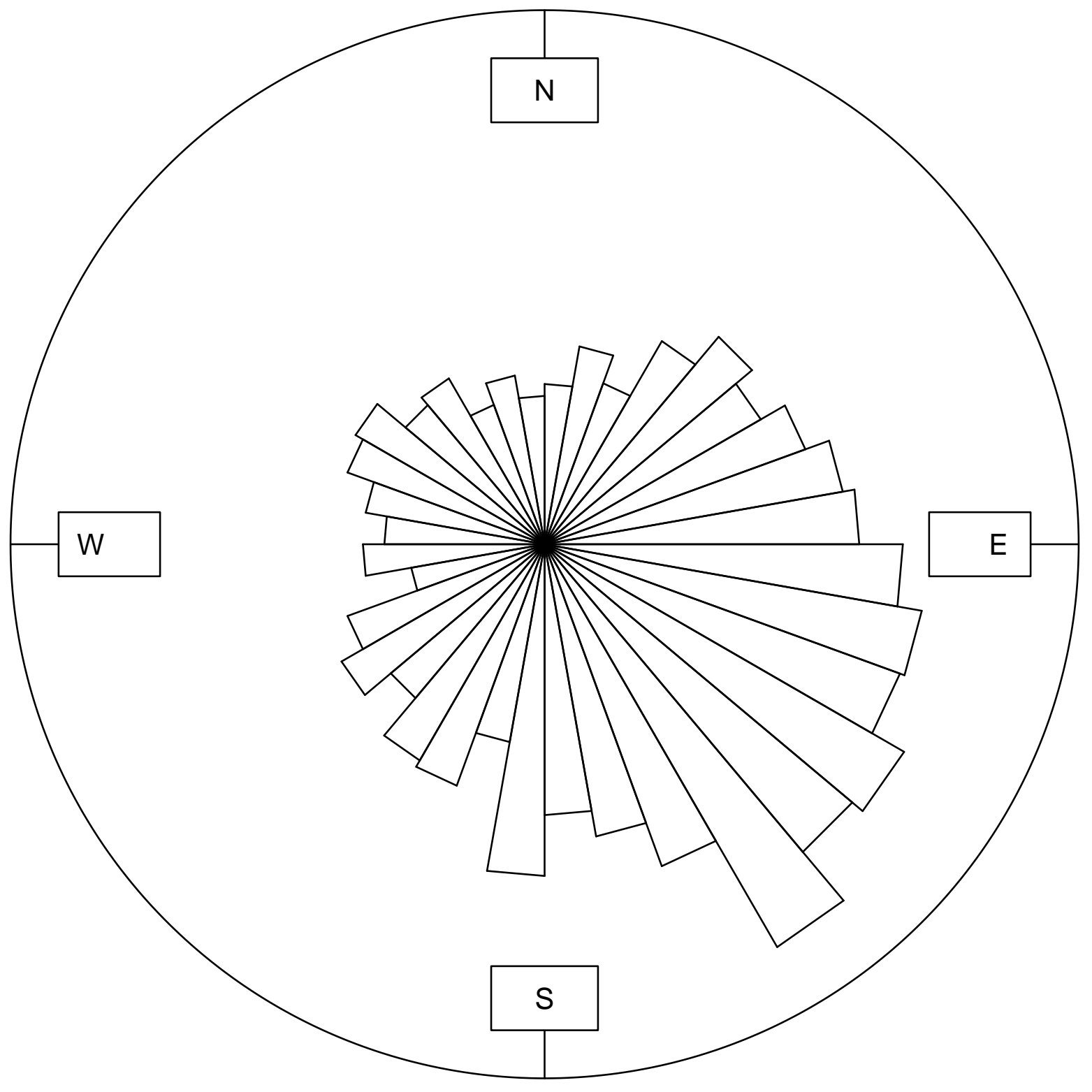


bootstrap 443

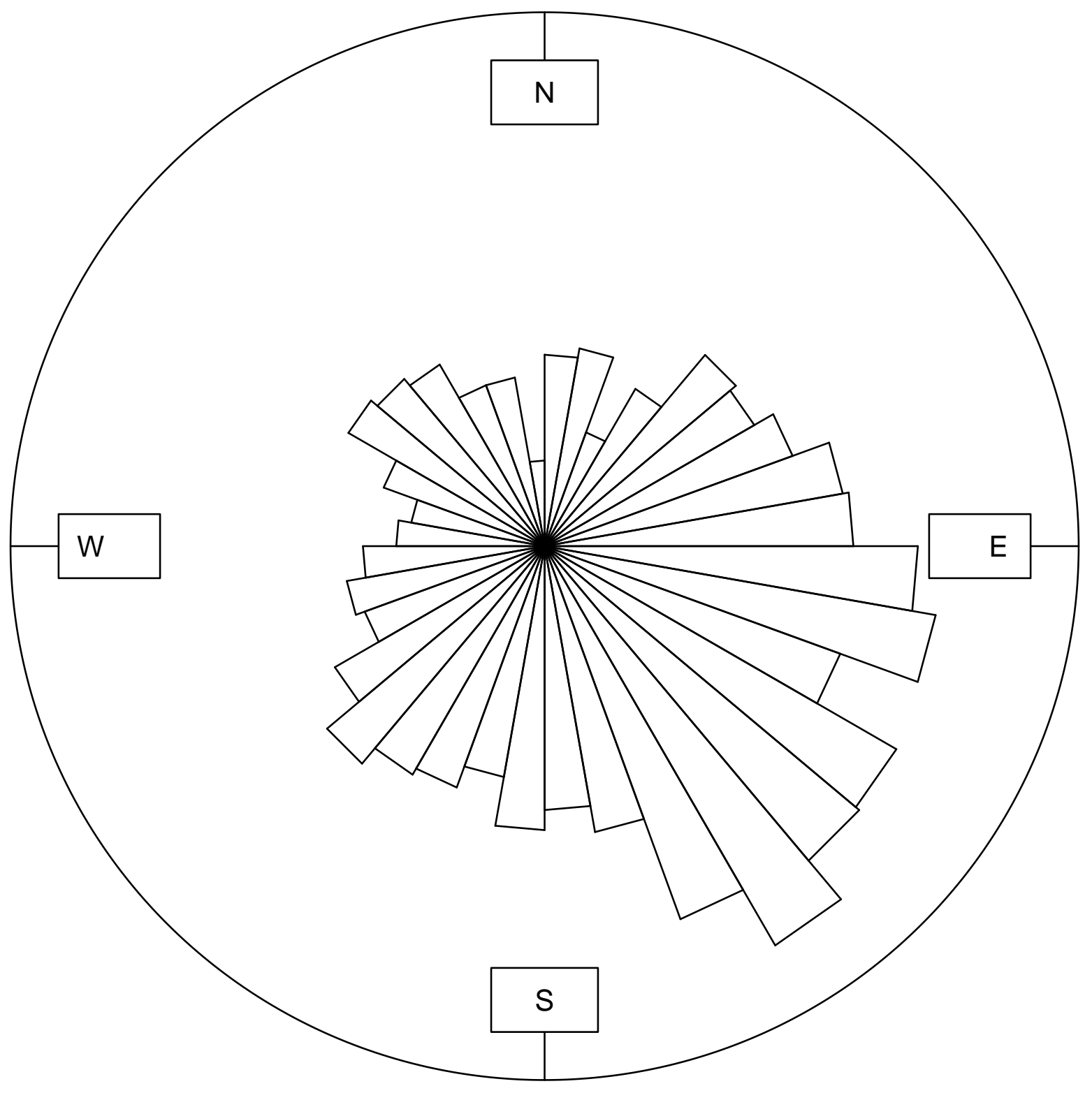


bootstrap 444

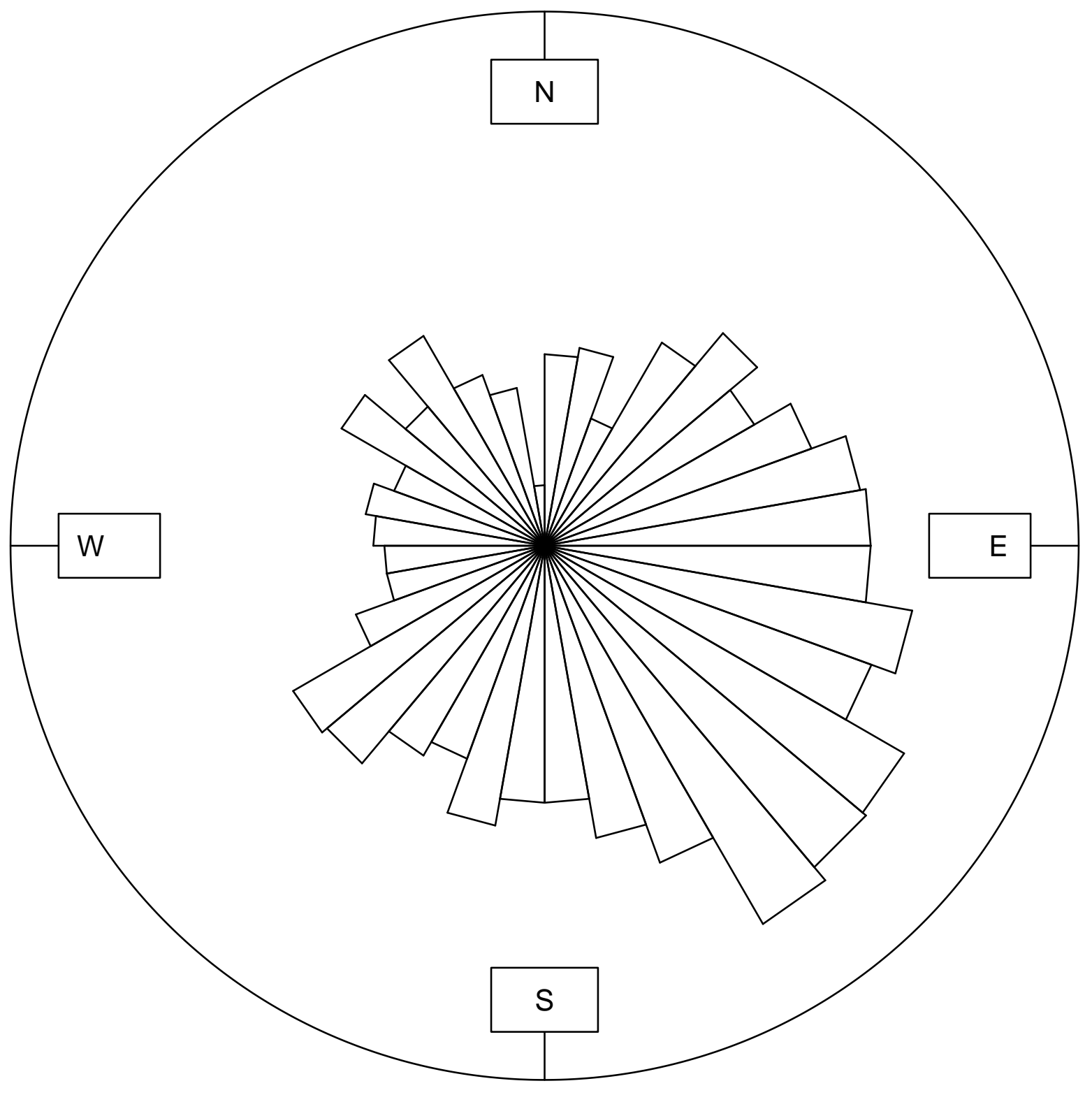




\section{bootstrap 445}

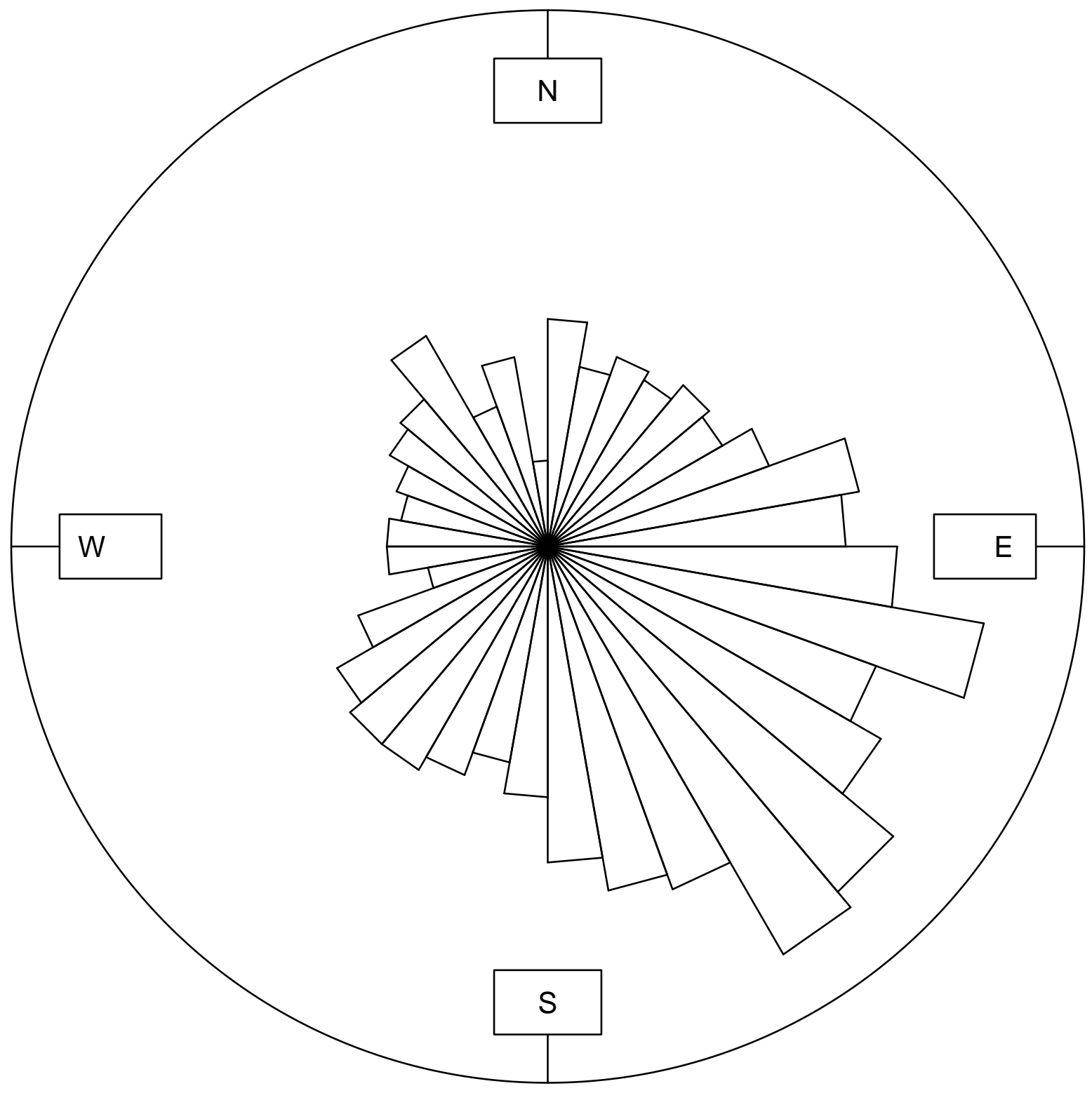


bootstrap 446

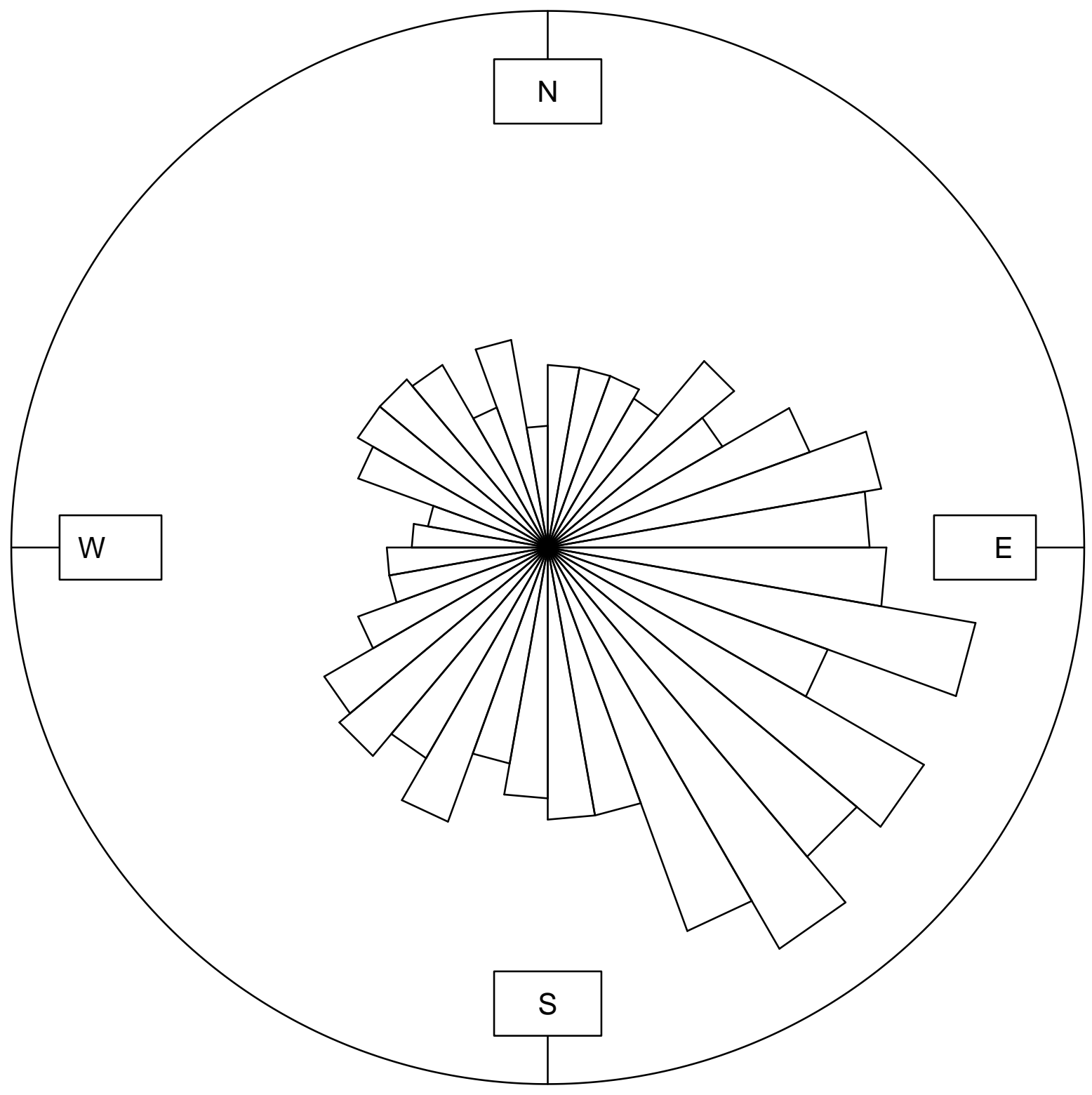


bootstrap 447

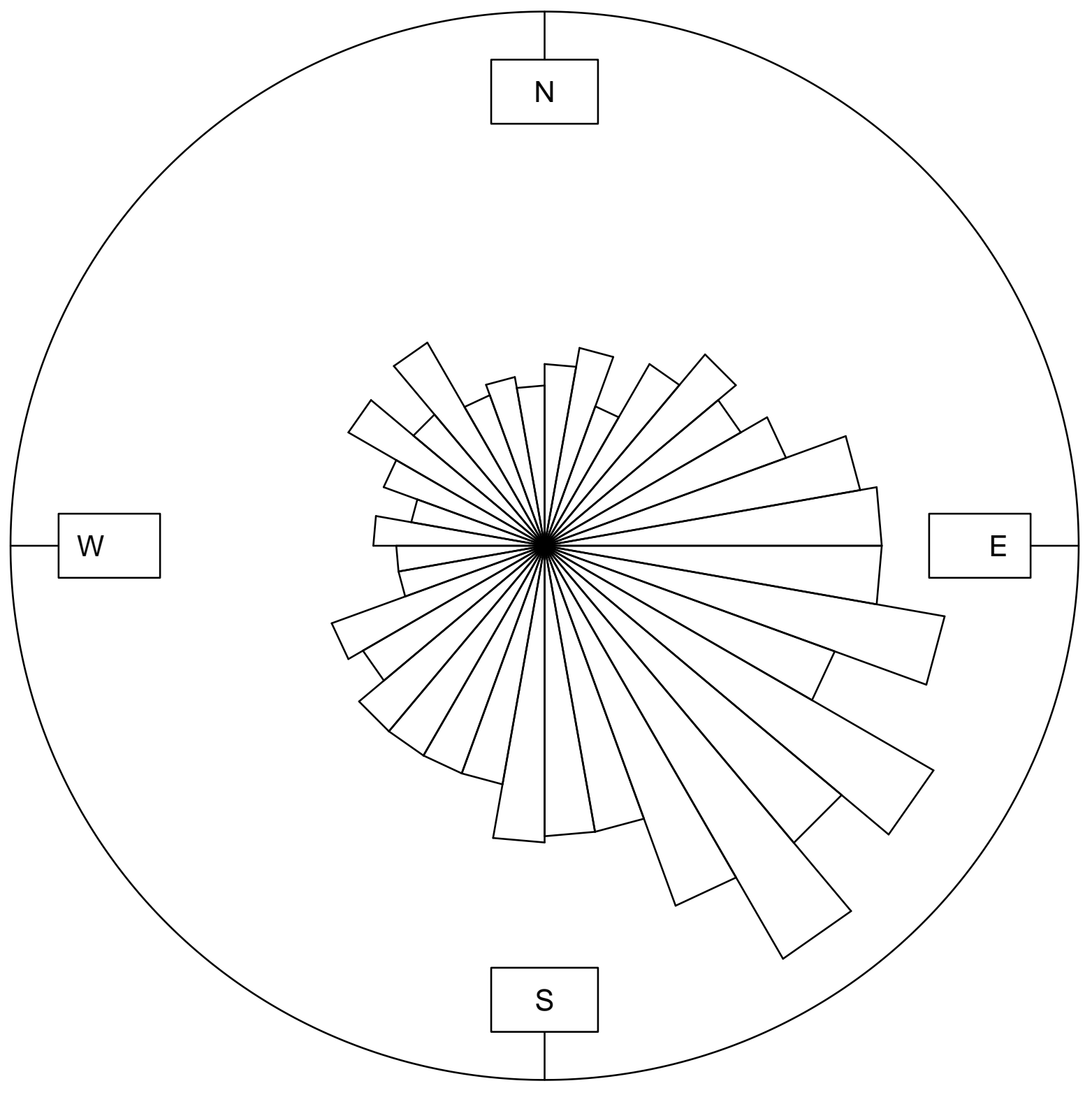


bootstrap 448

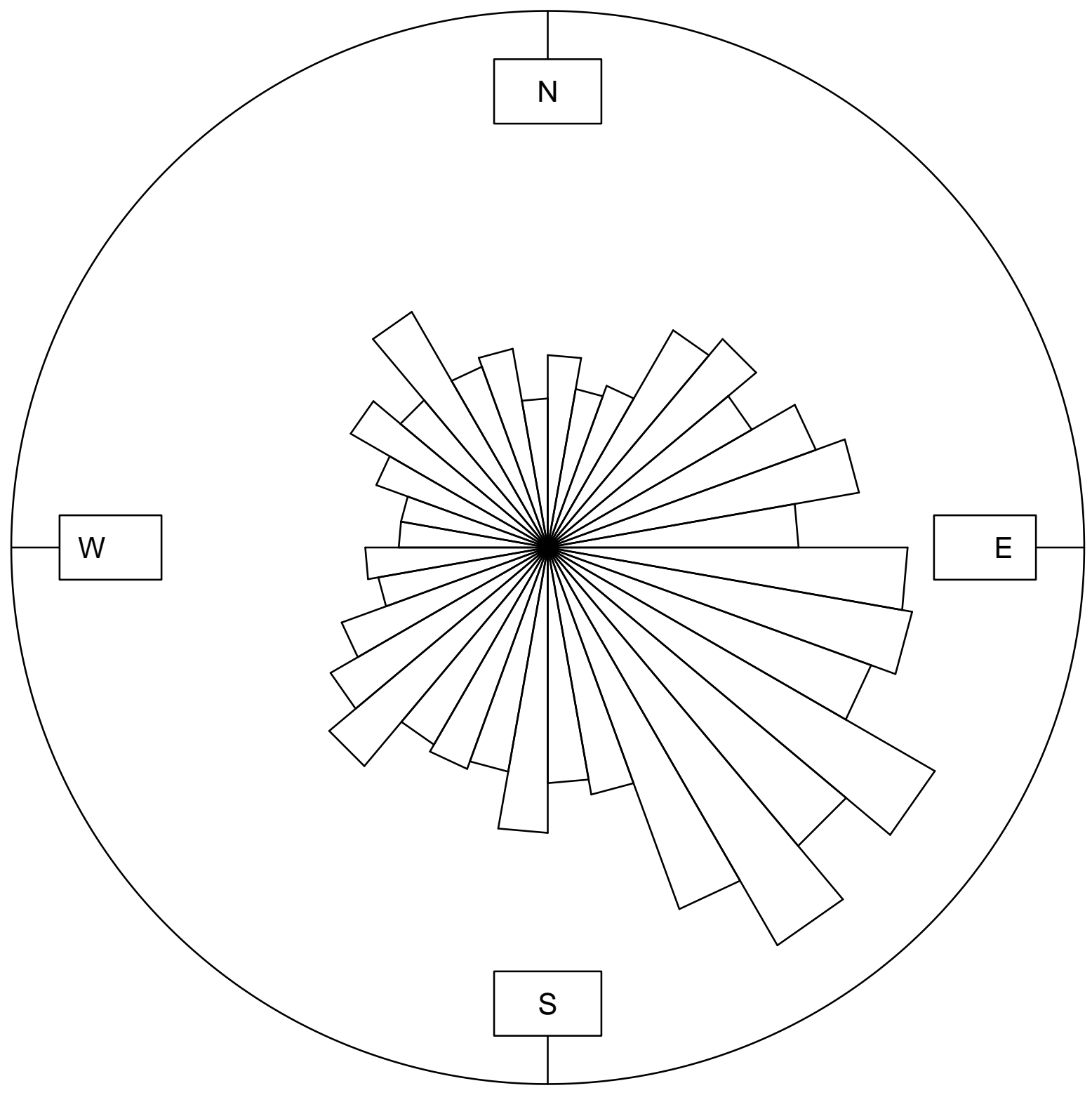


bootstrap 449

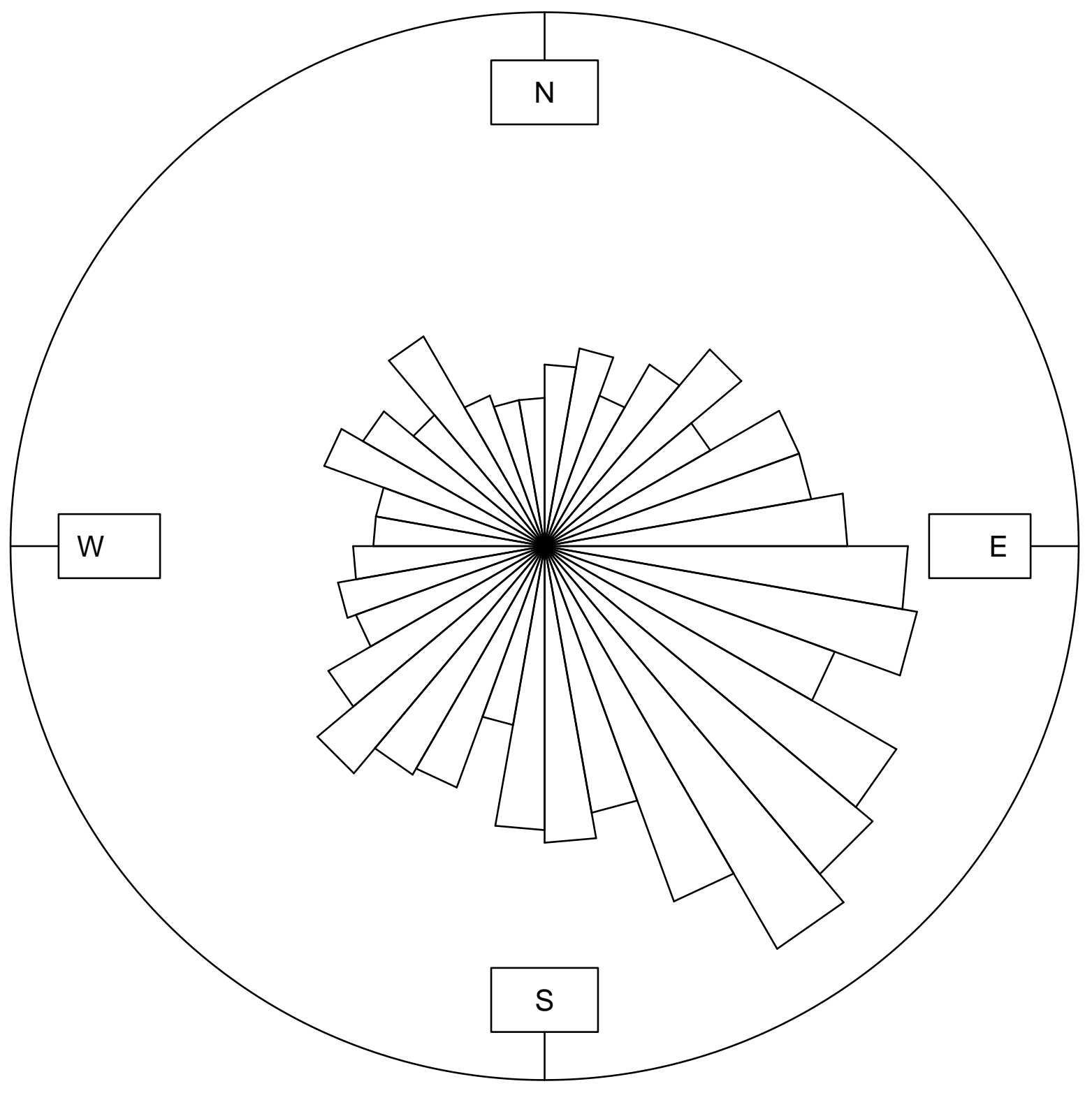


bootstrap 450

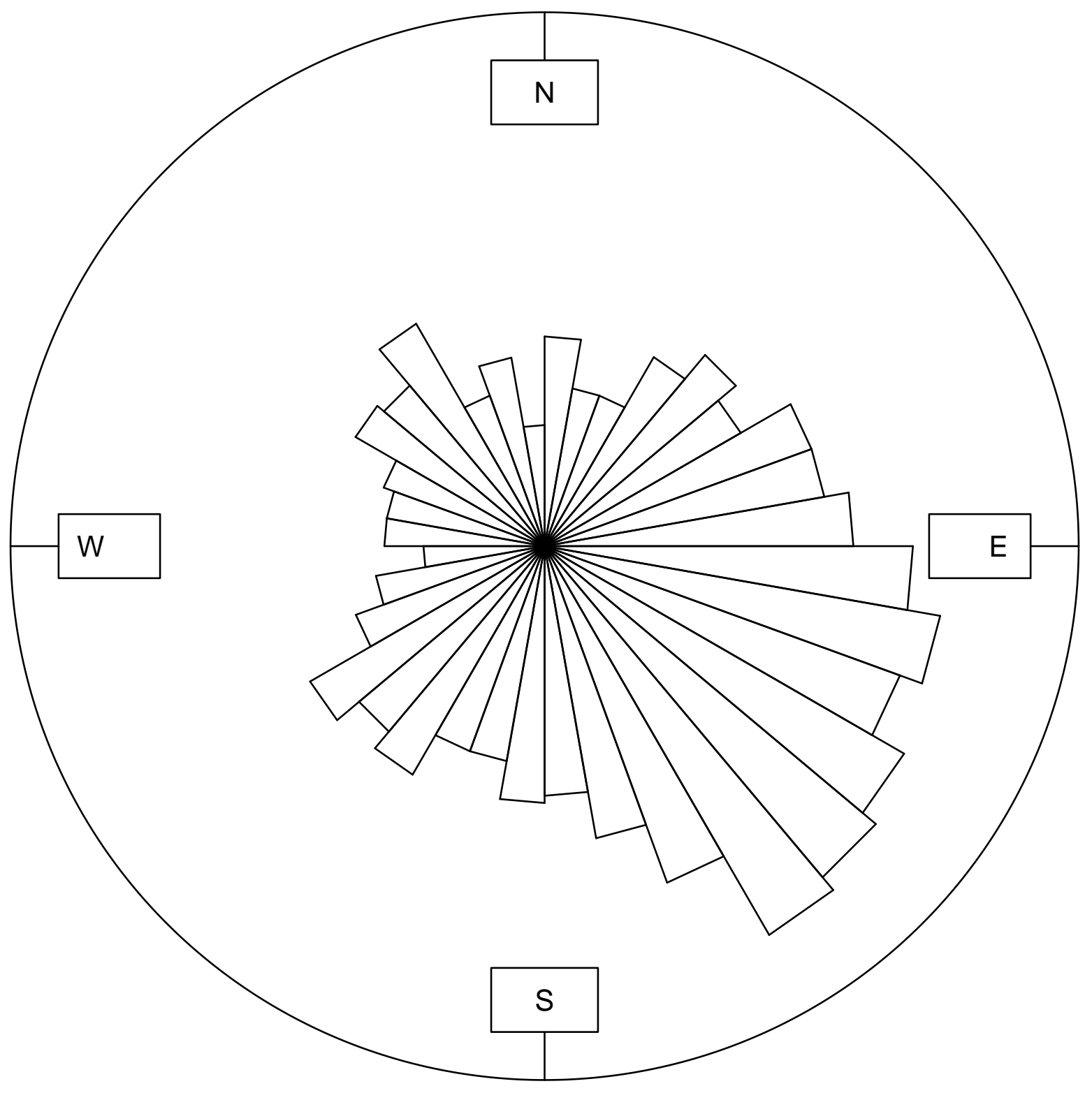


bootstrap 451

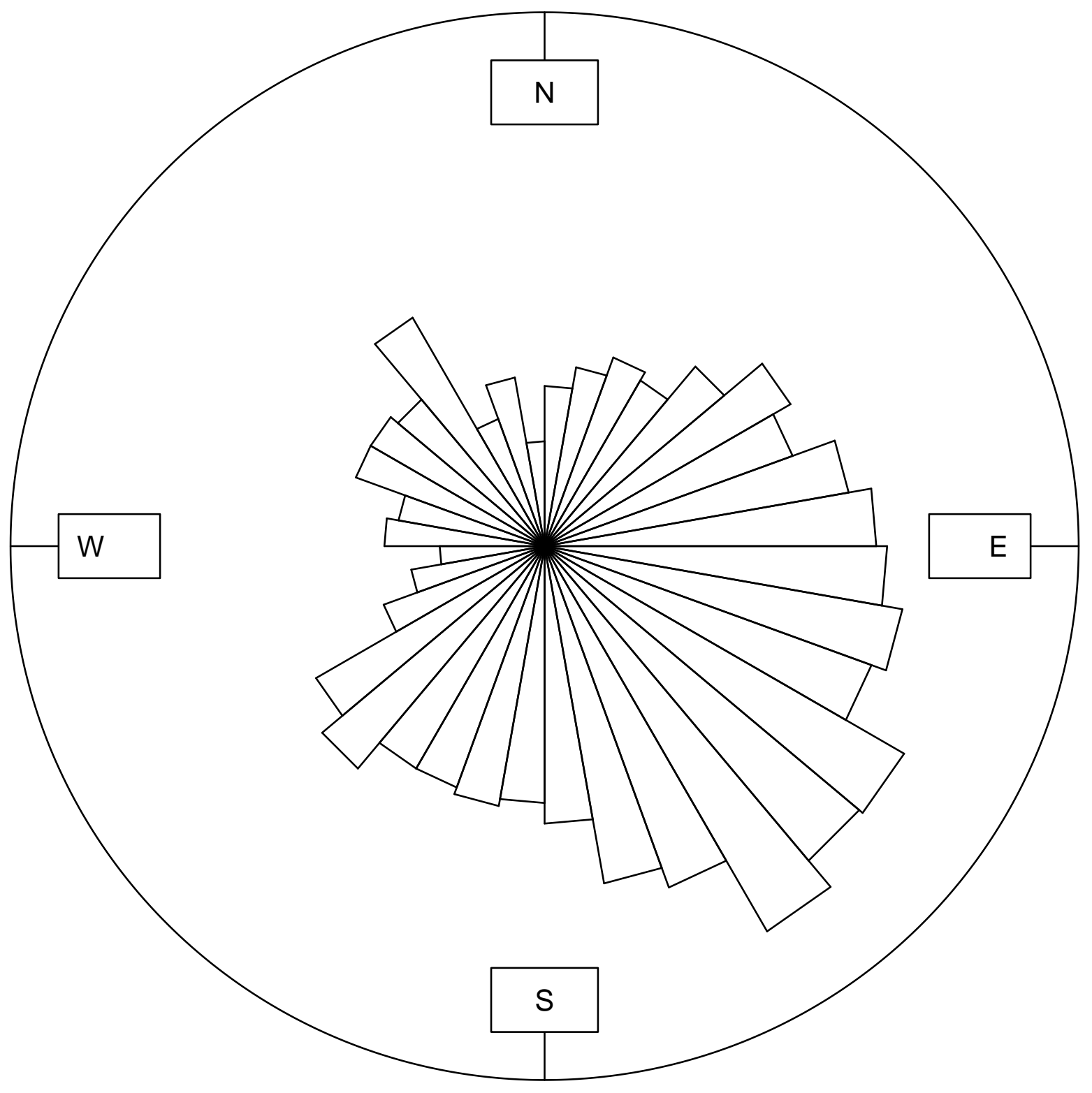


bootstrap 452

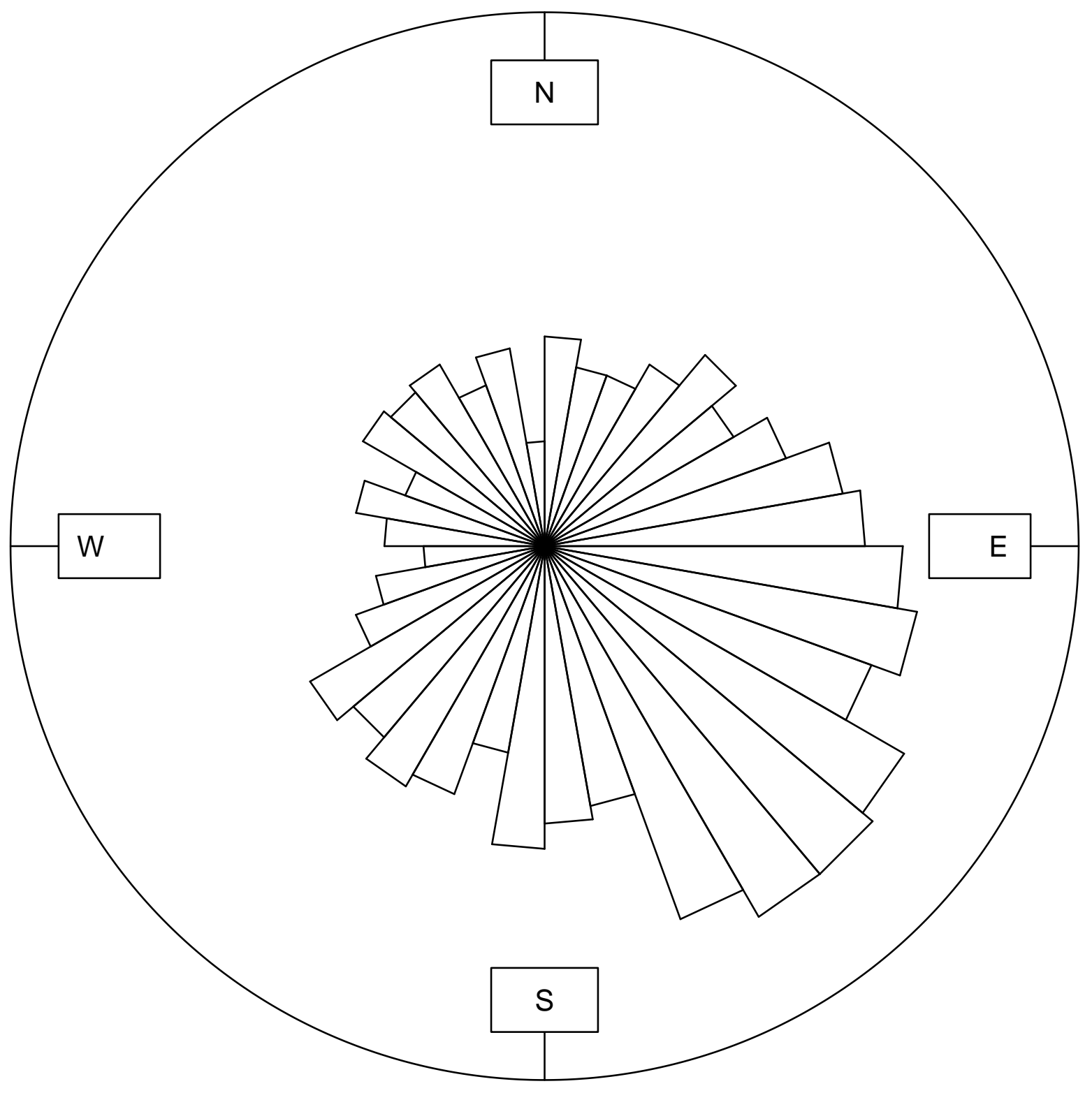




\section{bootstrap 453}

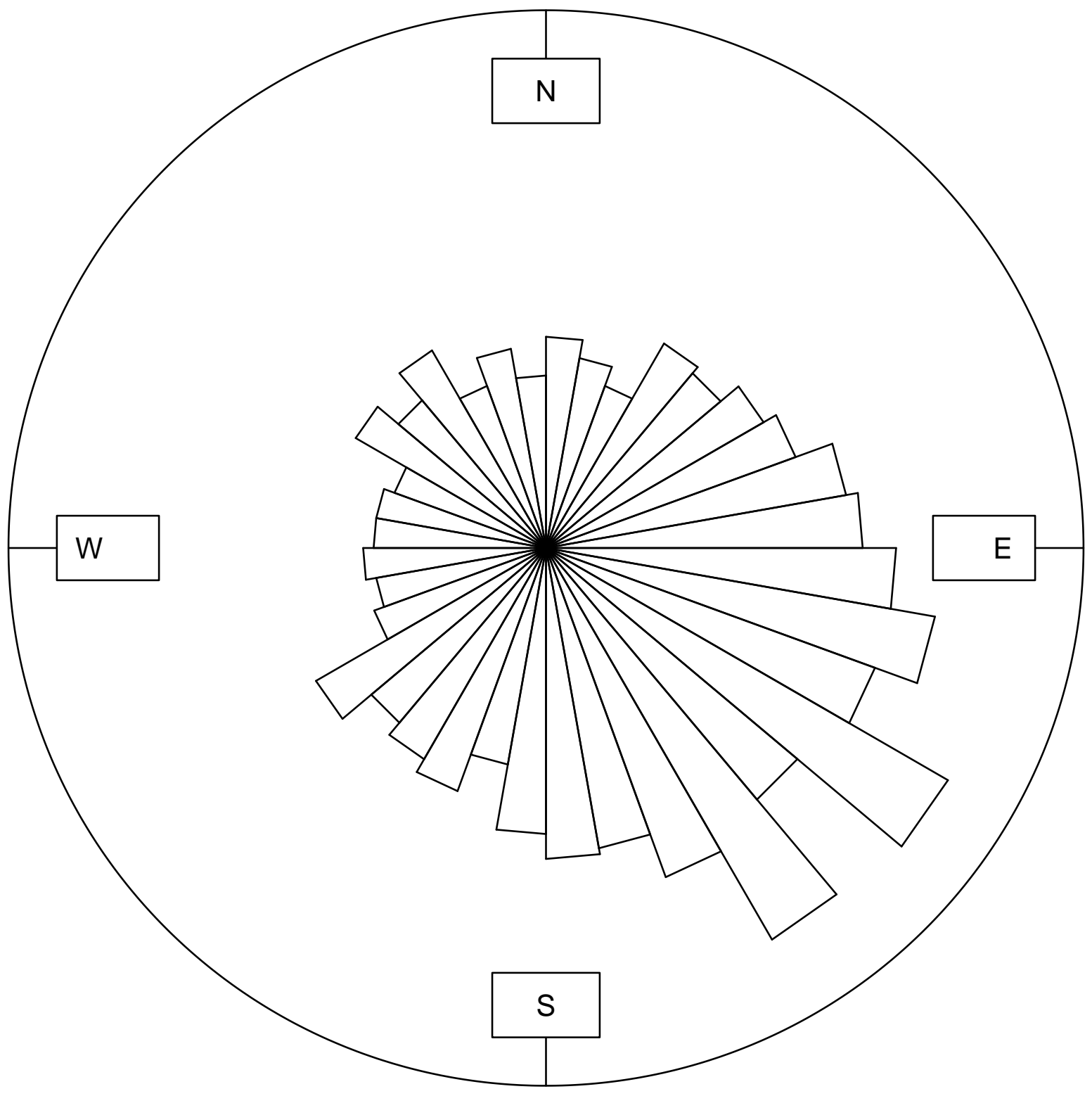




\section{bootstrap 454}

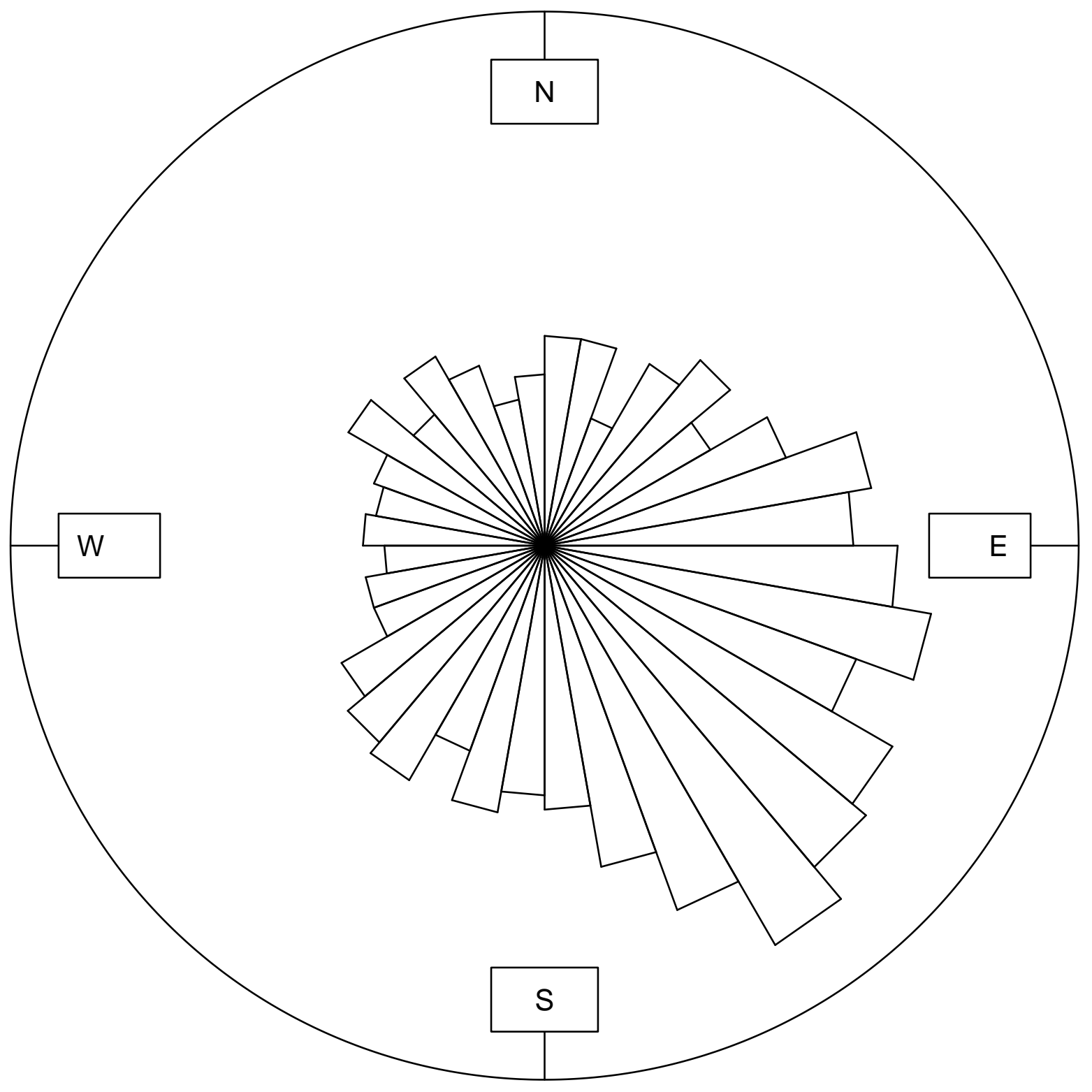




\section{bootstrap 455}

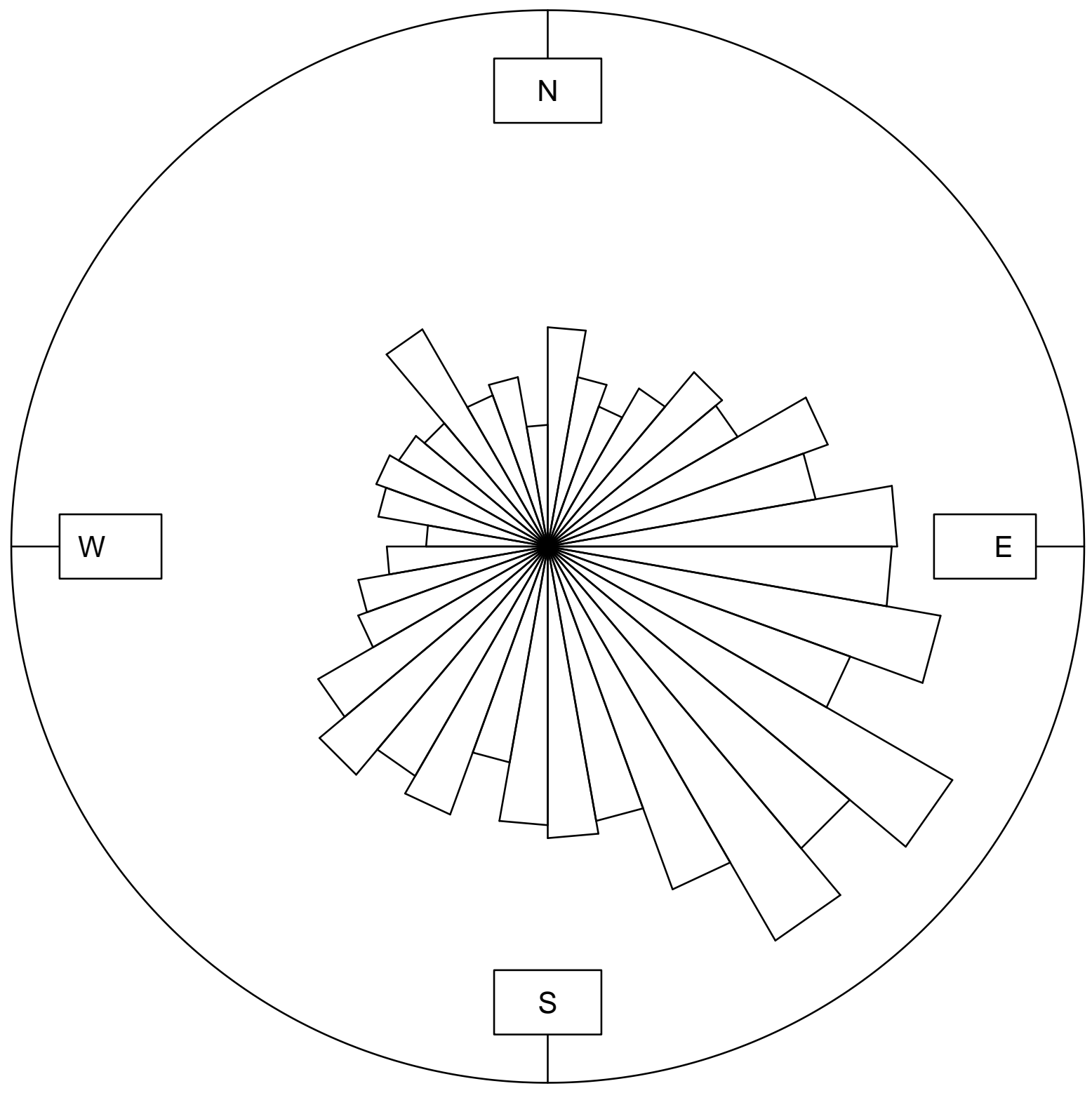




\section{bootstrap 456}

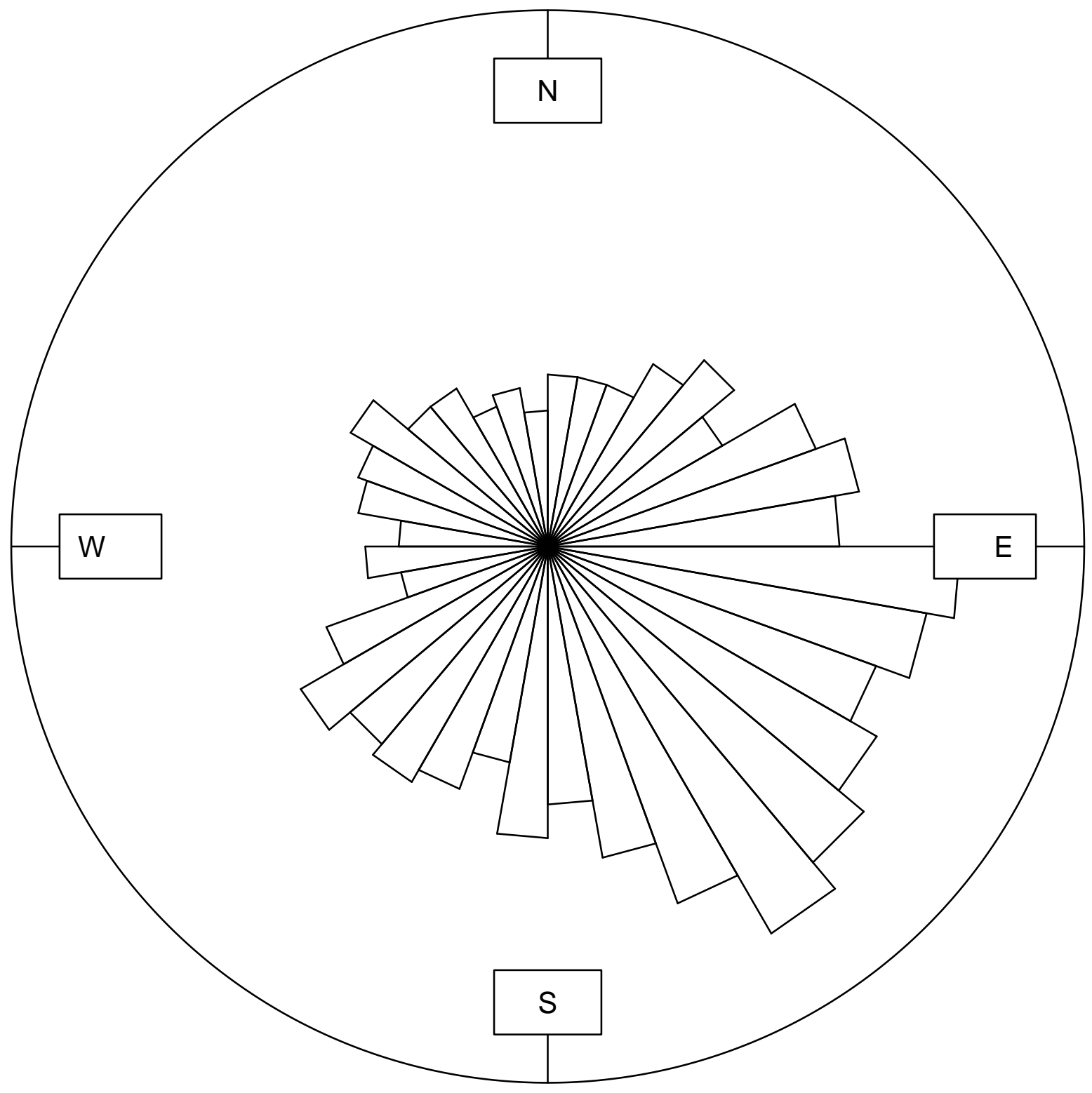


bootstrap 457

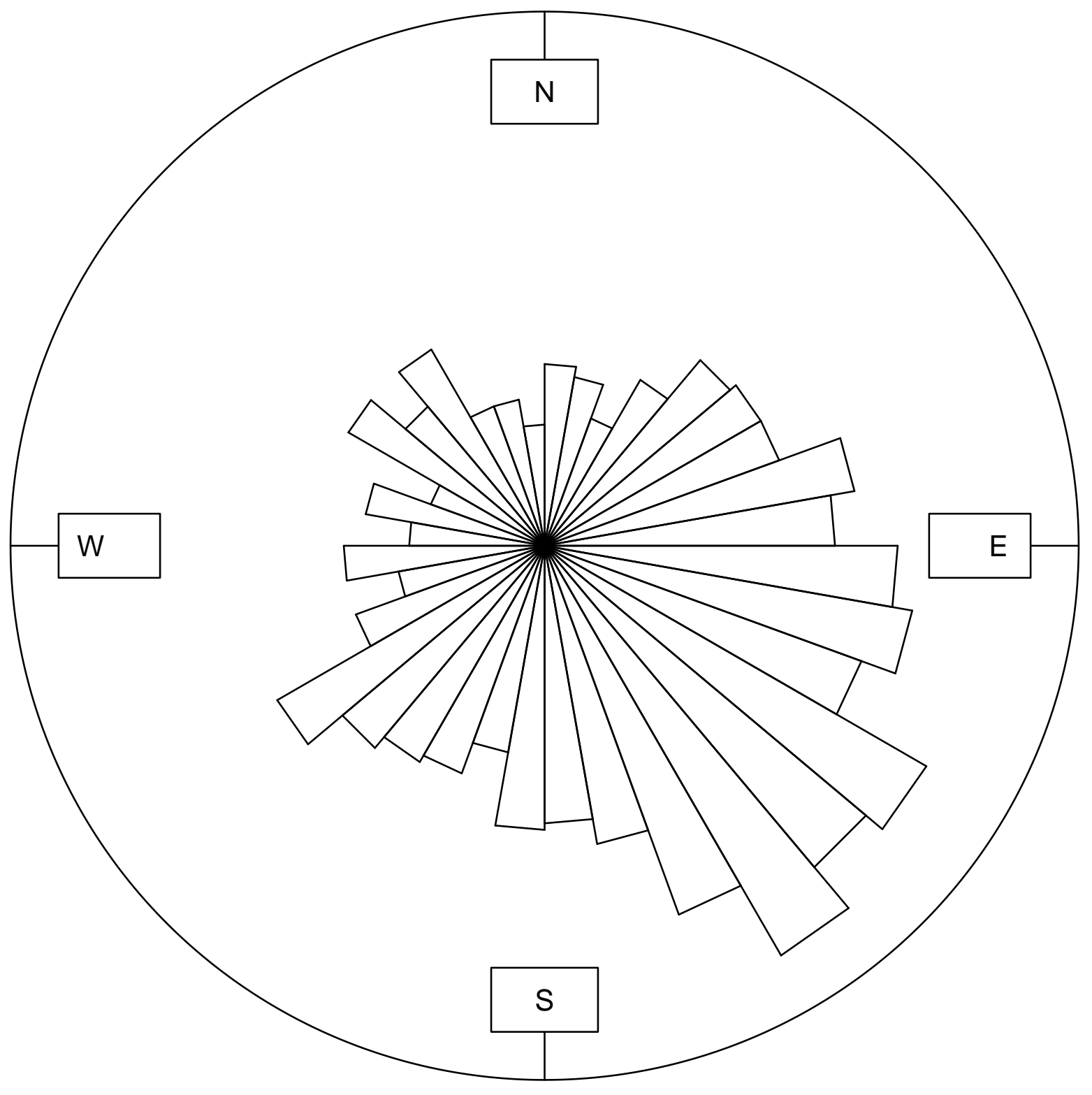


bootstrap 458

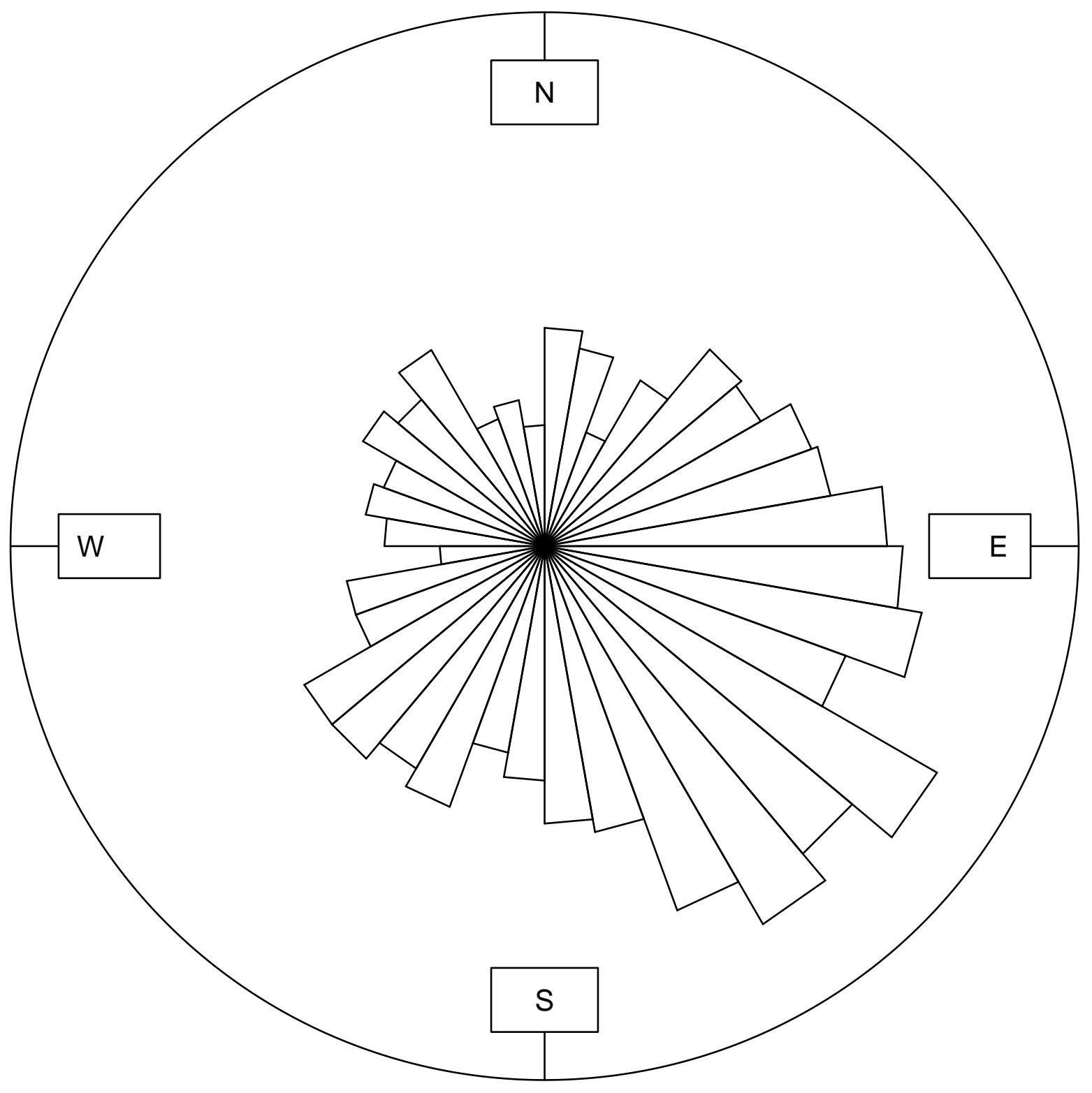


bootstrap 459

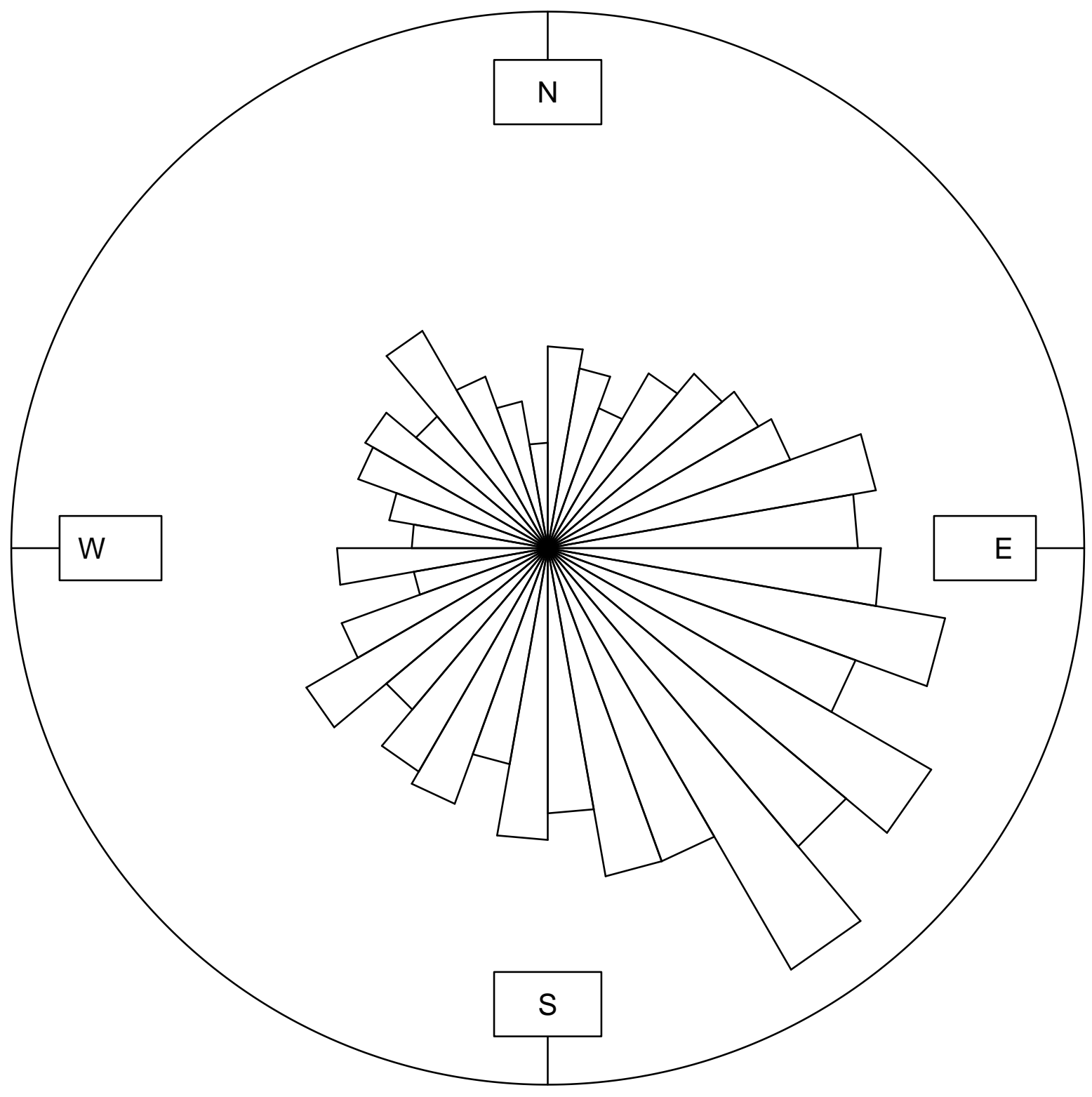


bootstrap 460

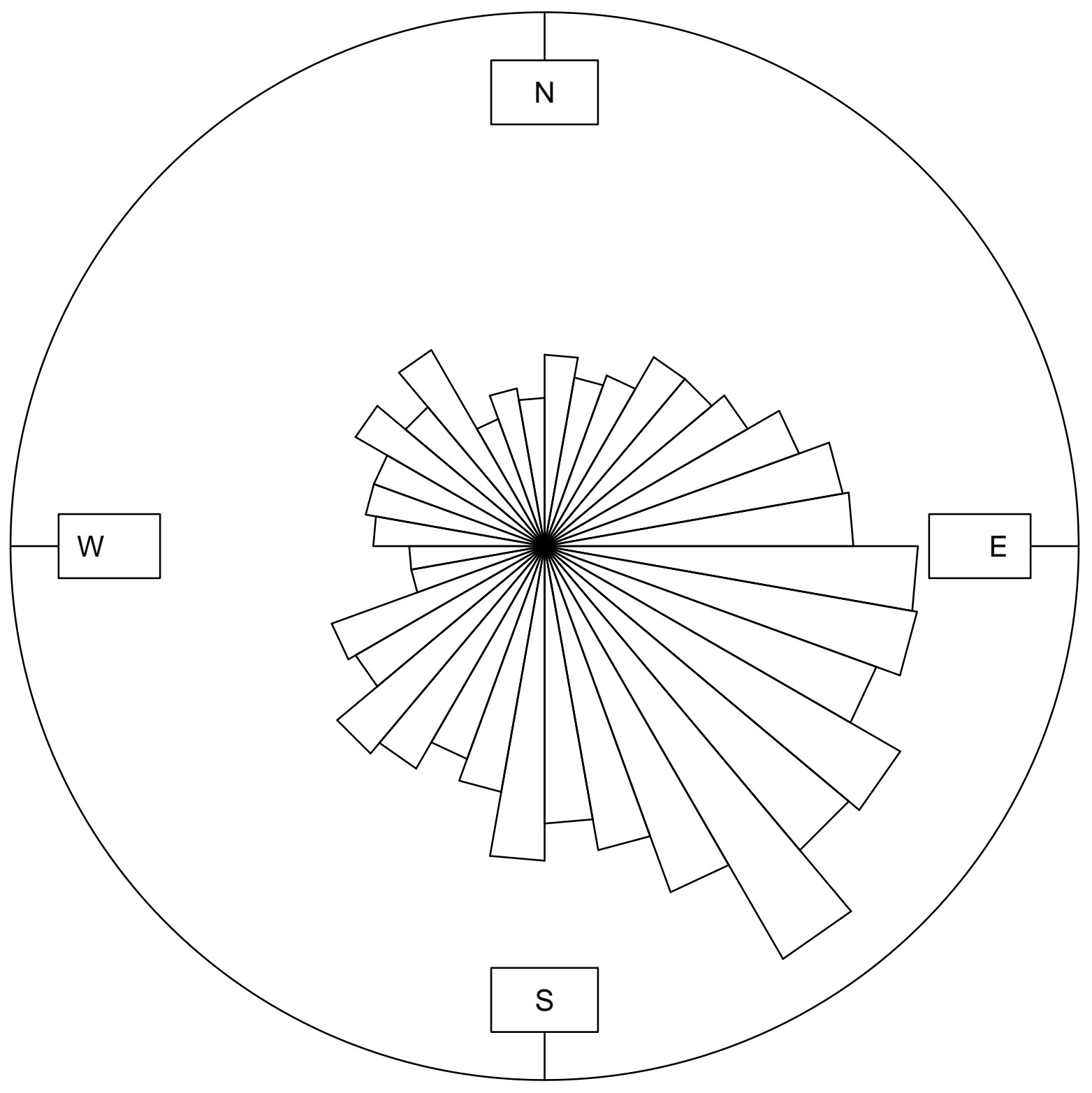


bootstrap 461

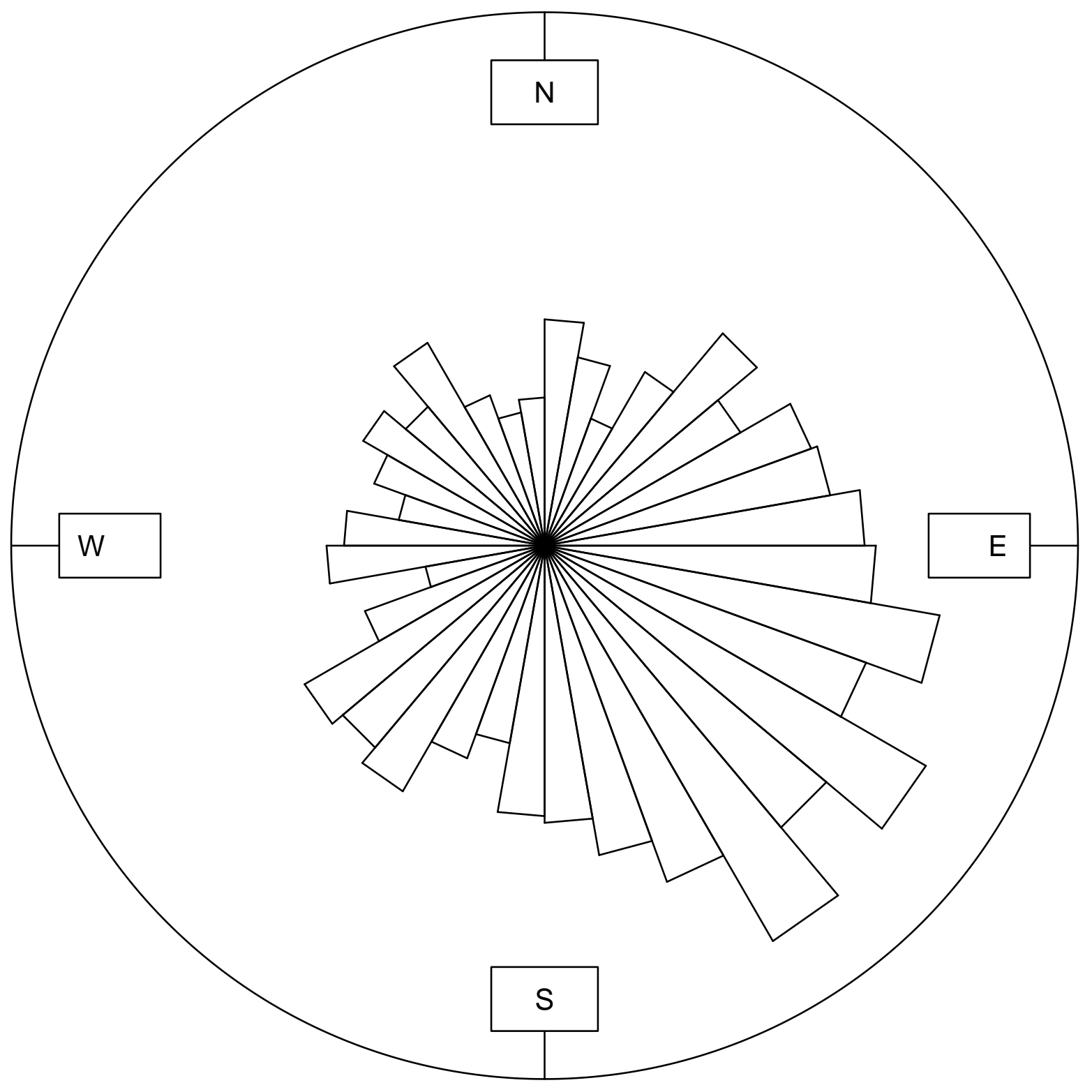


bootstrap 462

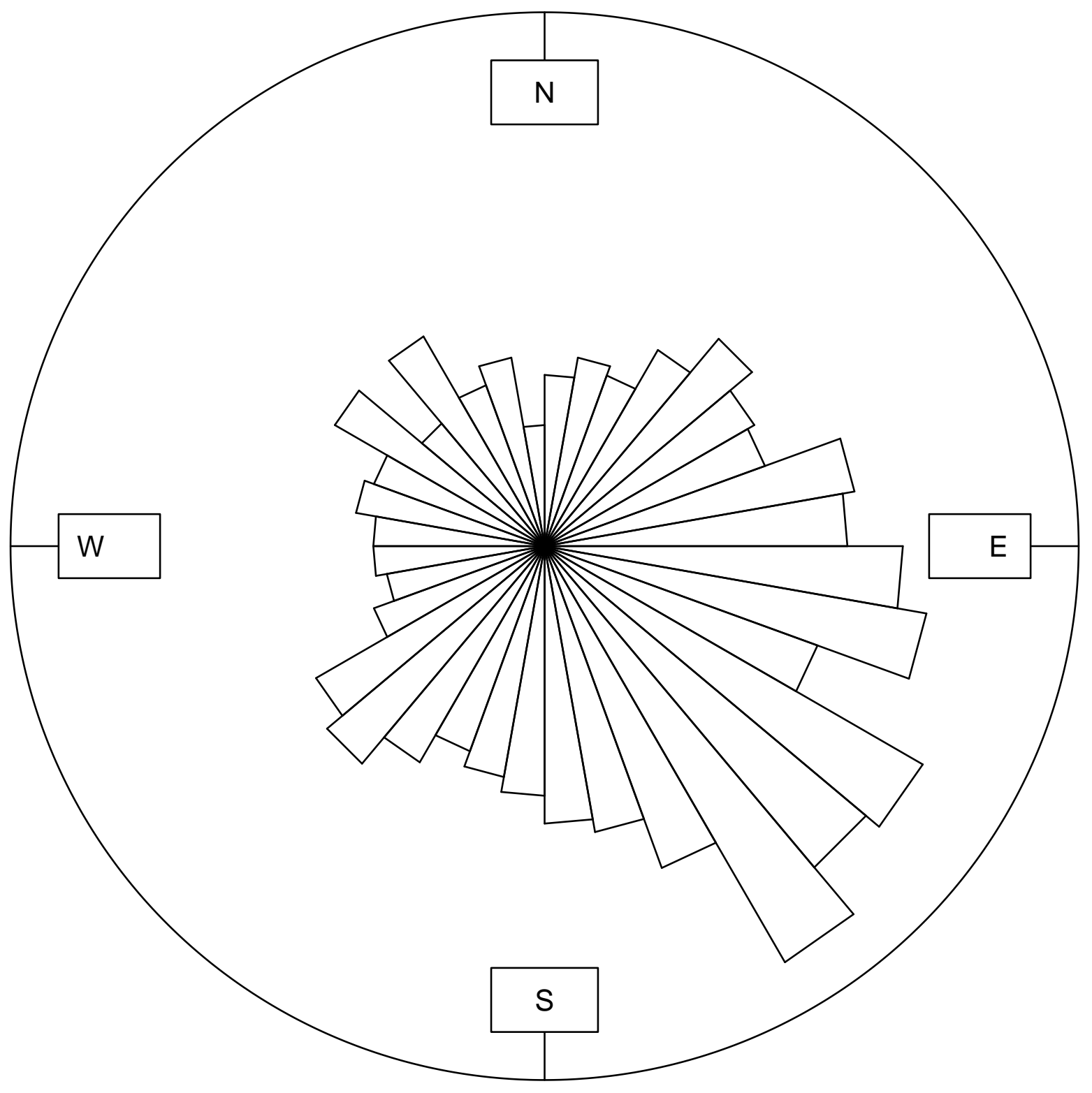




\section{bootstrap 463}

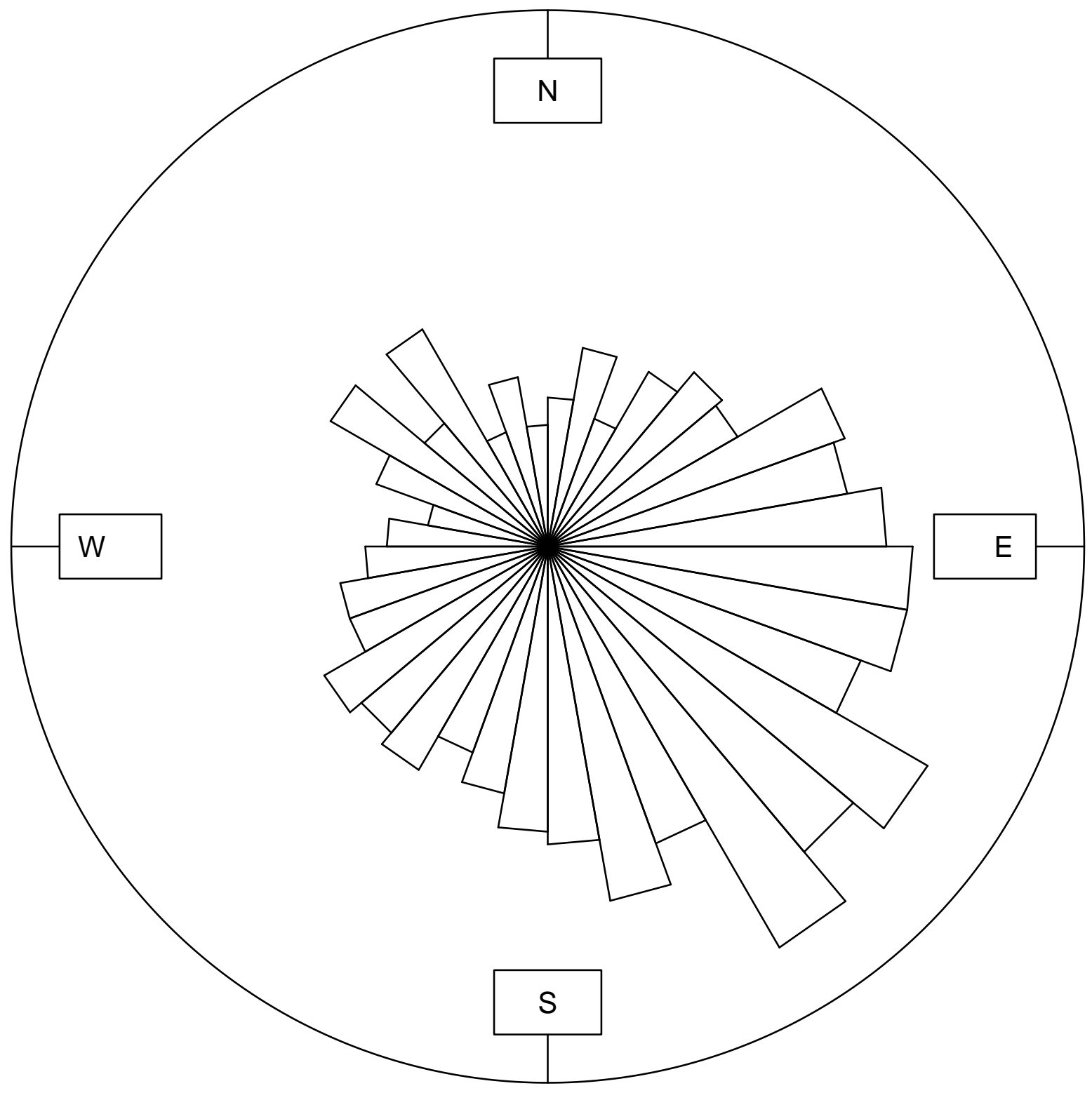


bootstrap 464

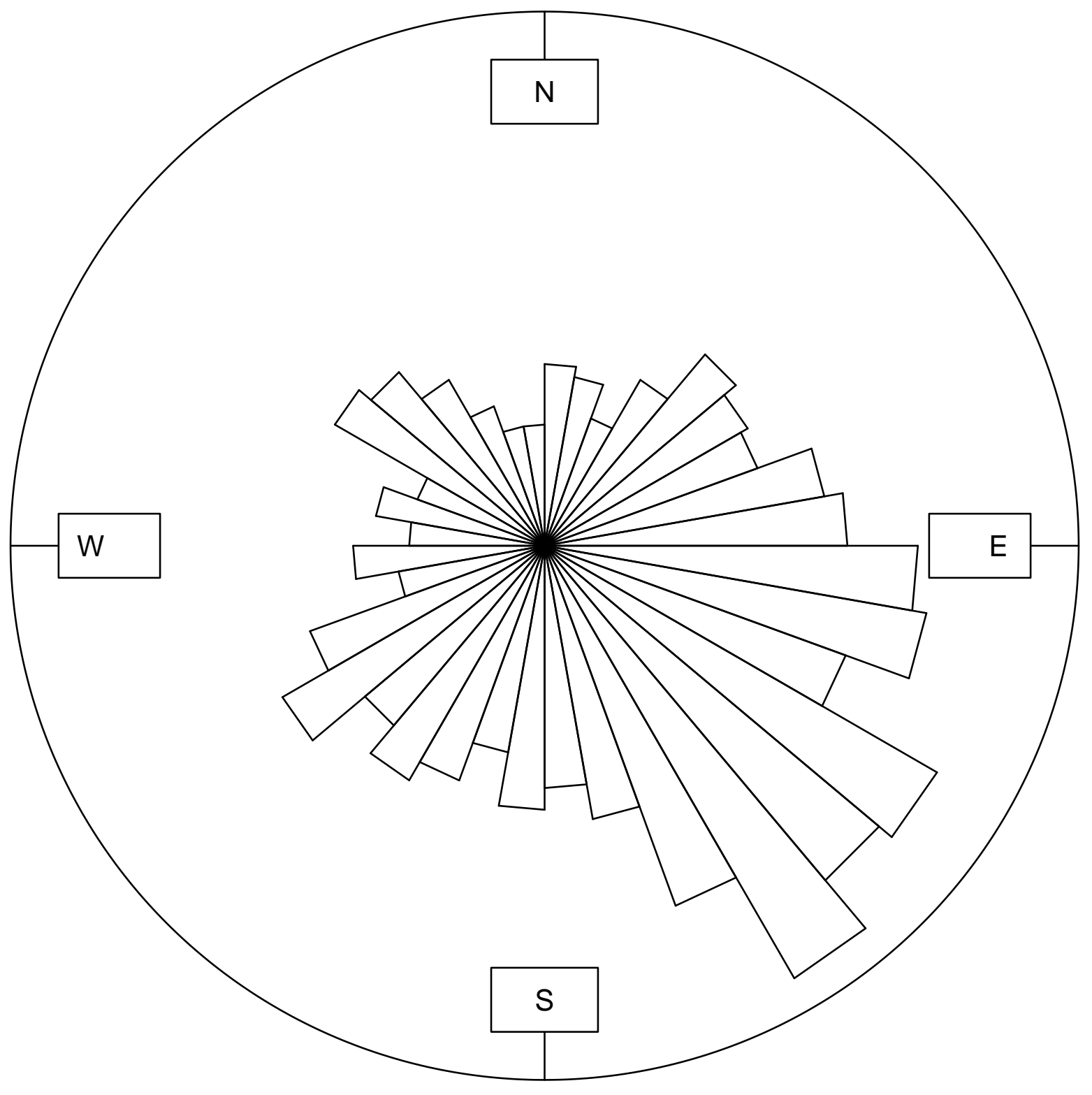


bootstrap 465

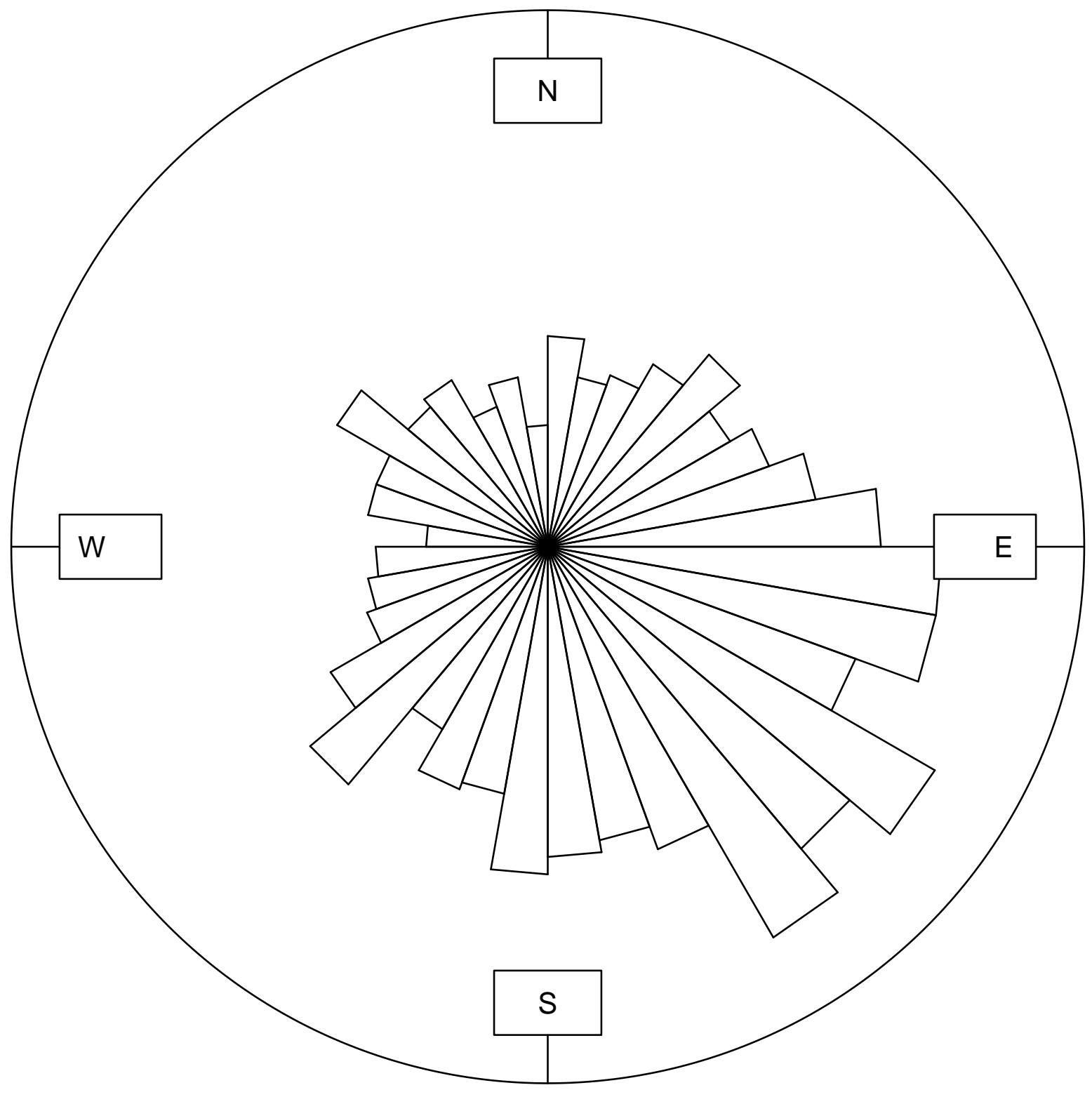




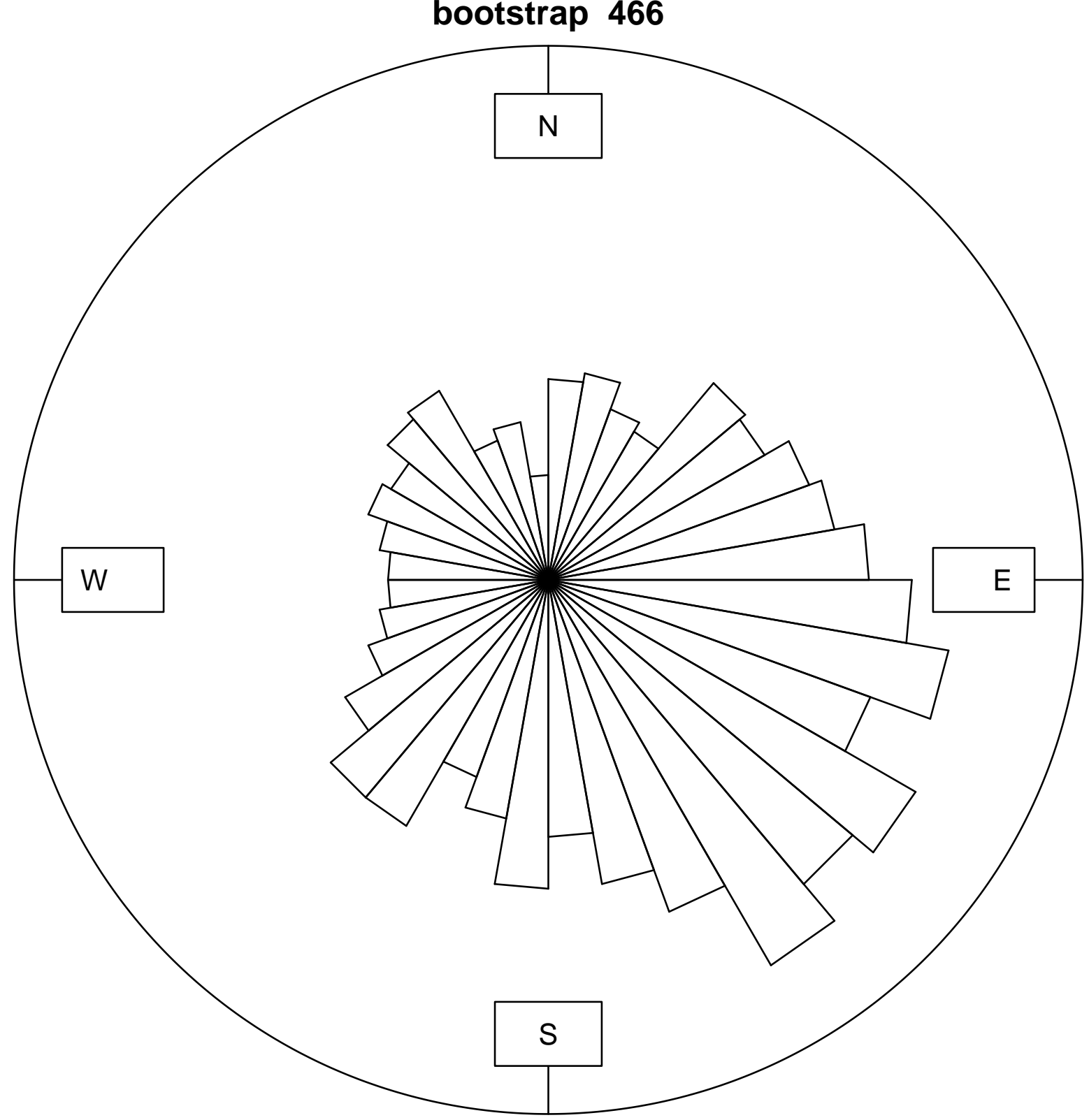


bootstrap 467

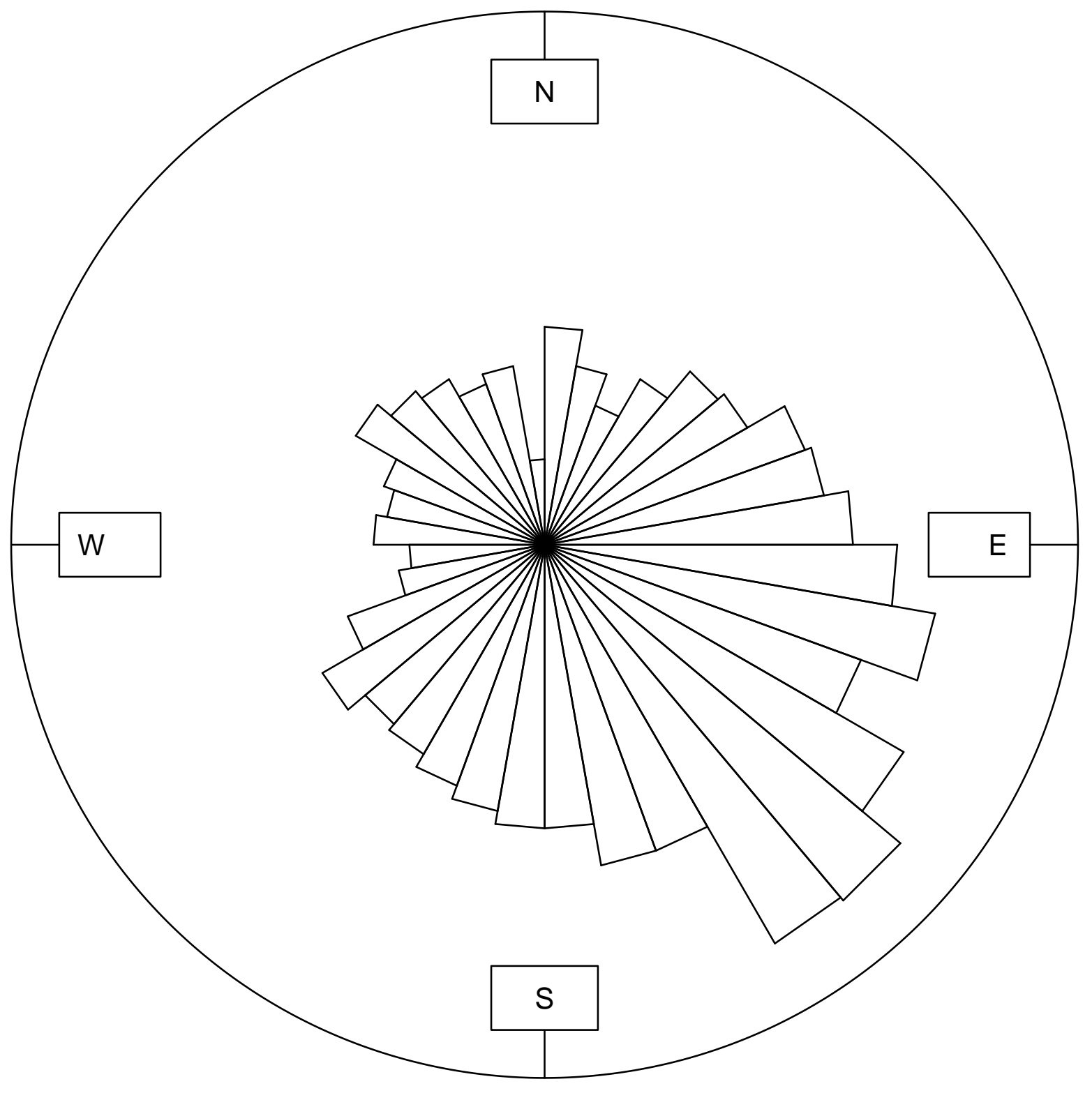


bootstrap 468

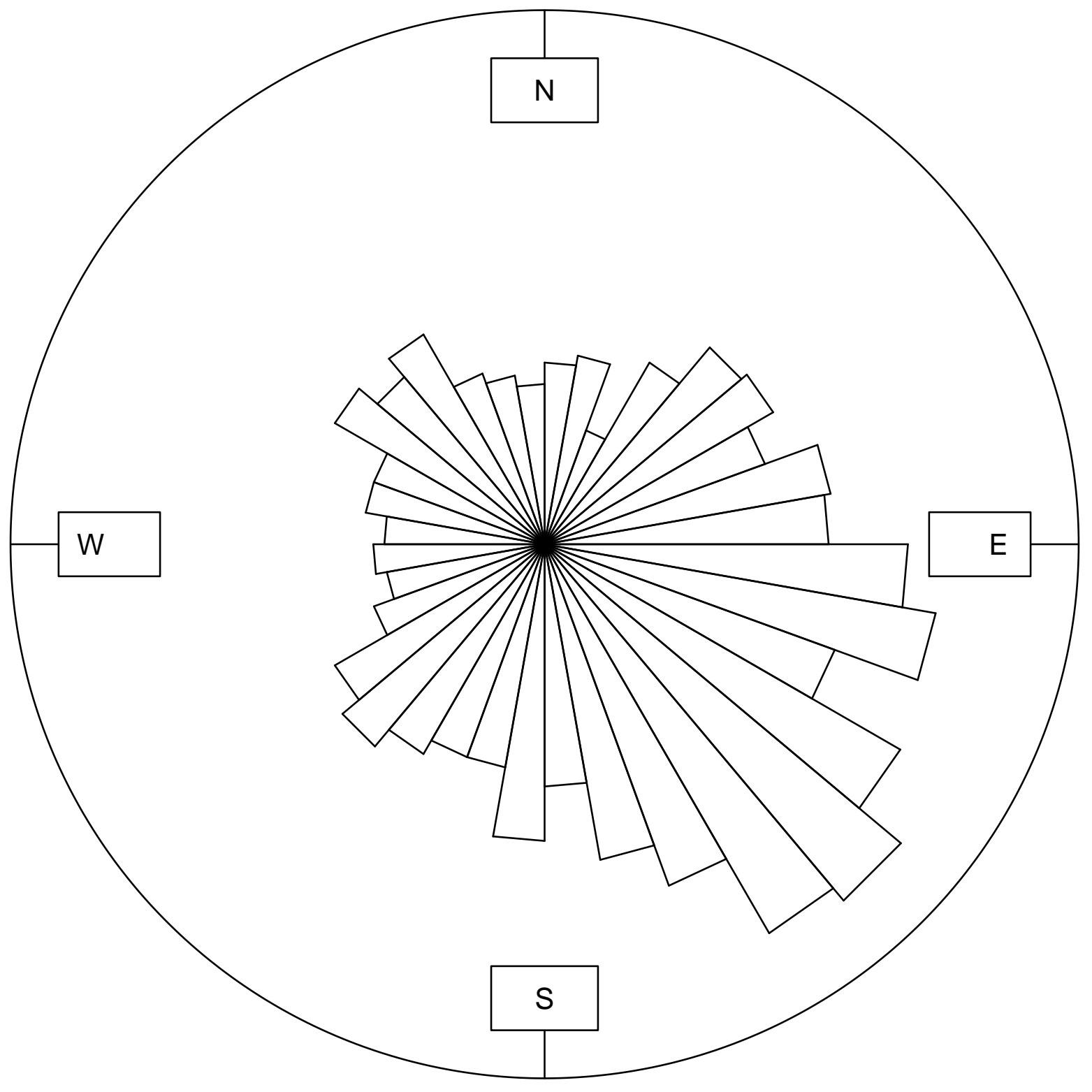


bootstrap 469

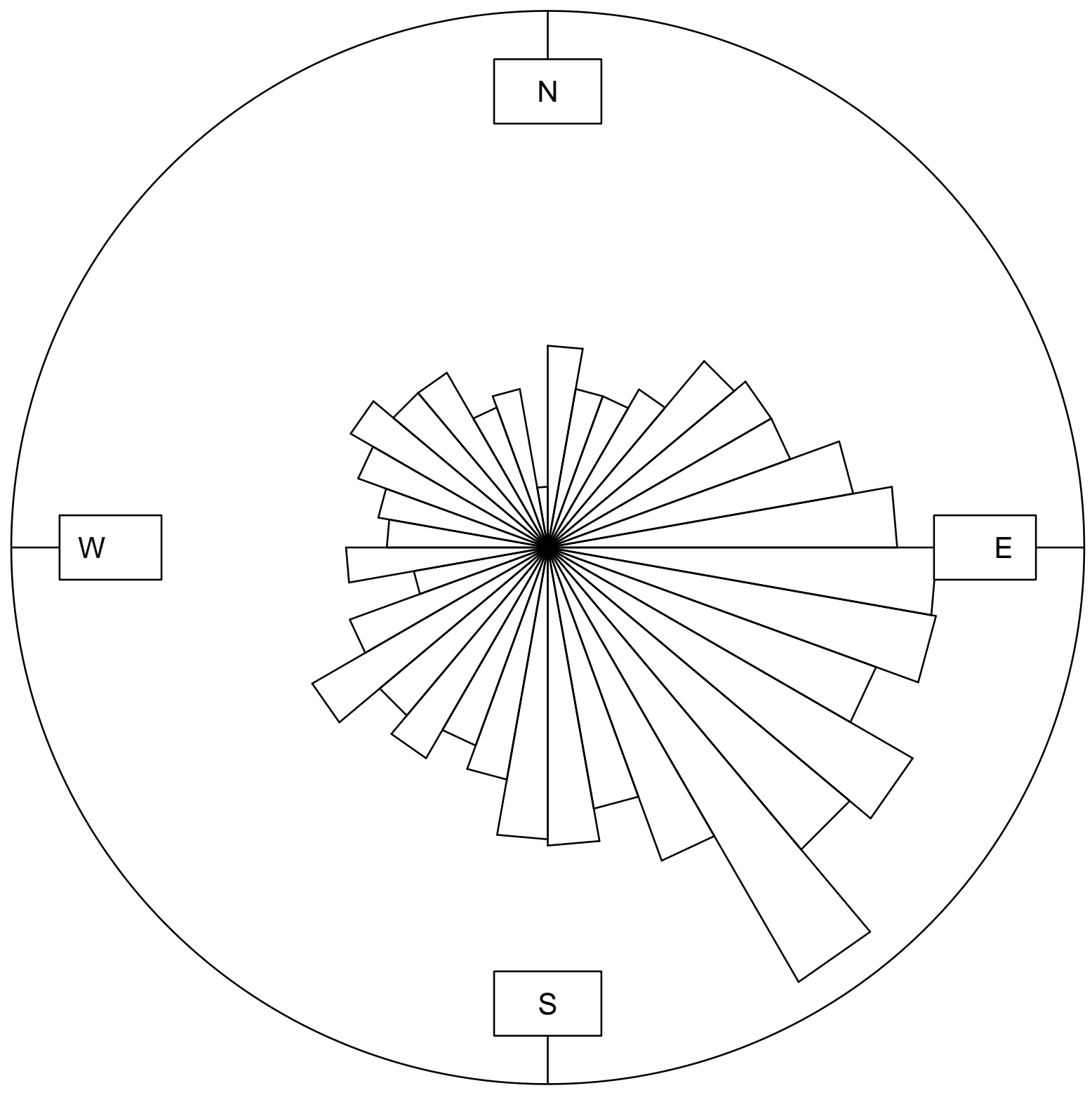


bootstrap 470

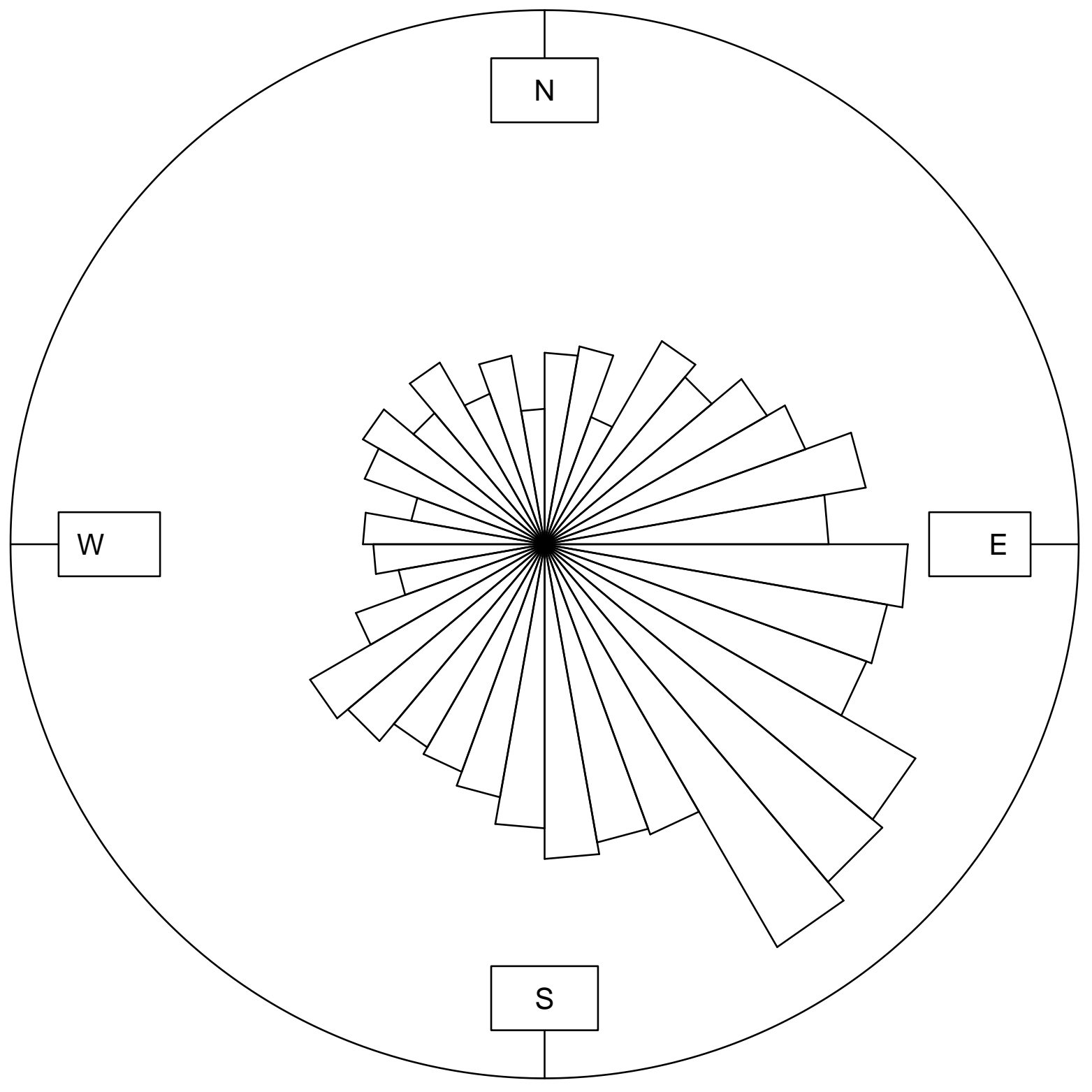


bootstrap 471

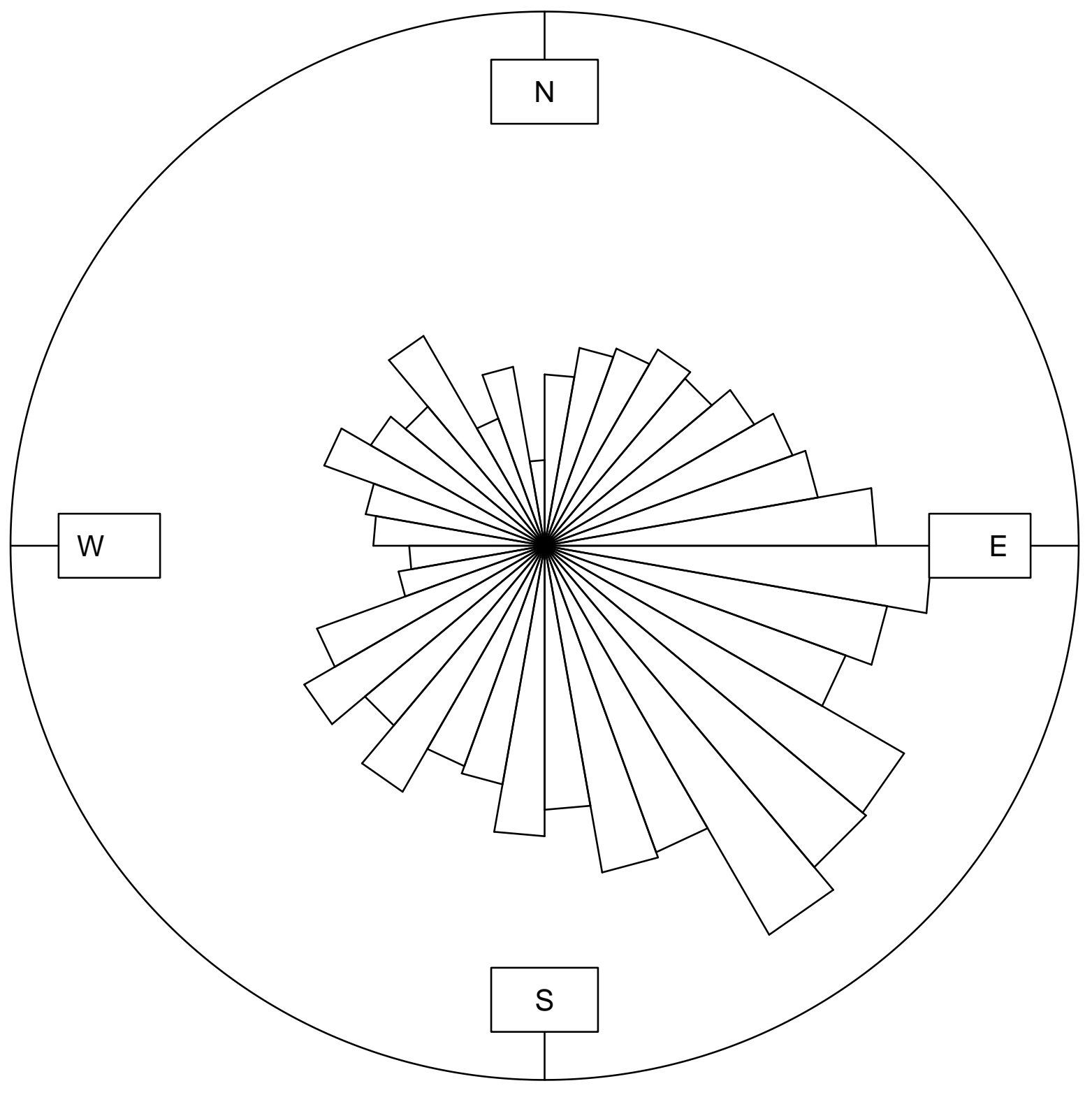


bootstrap 472

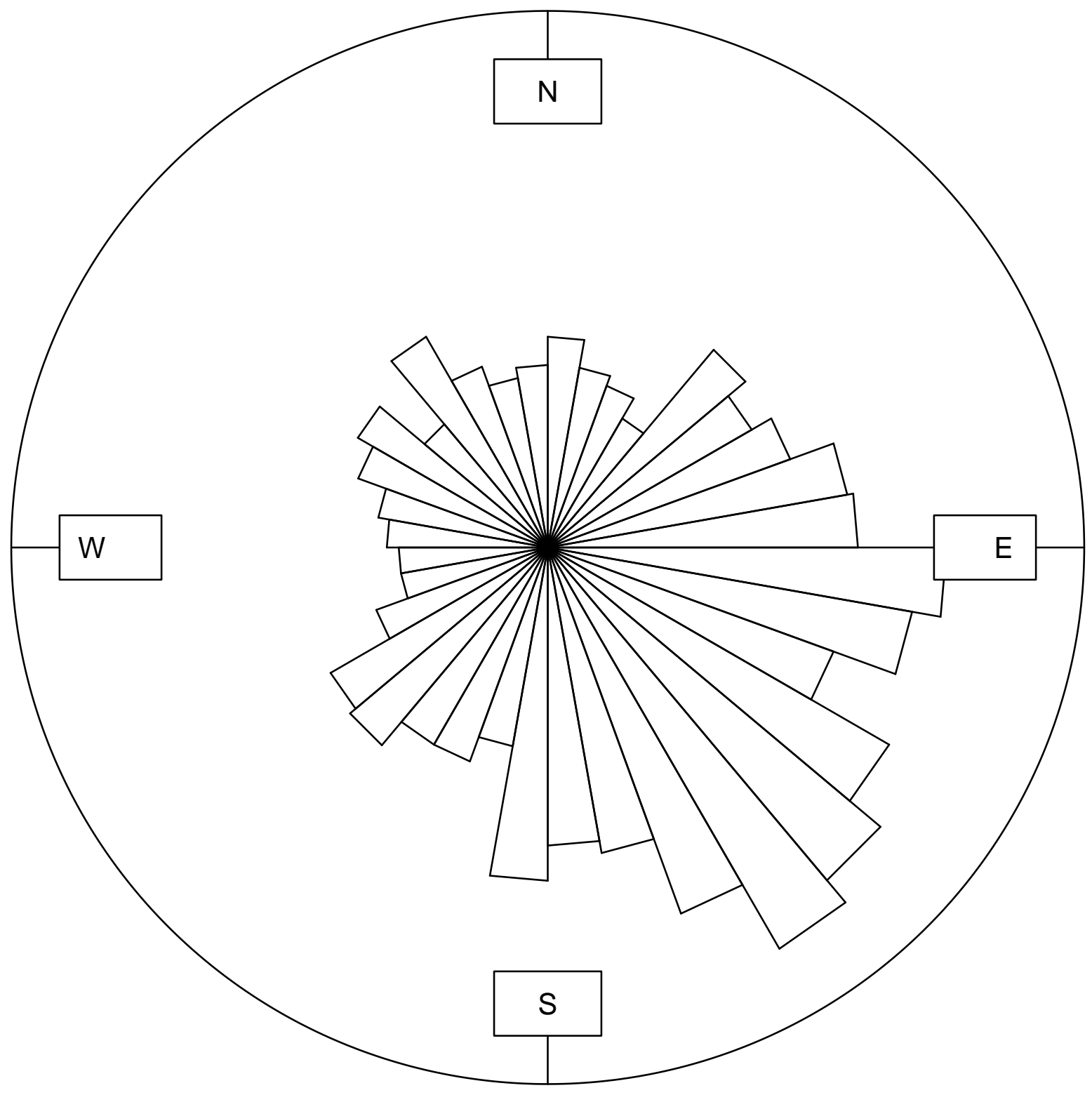


bootstrap 473

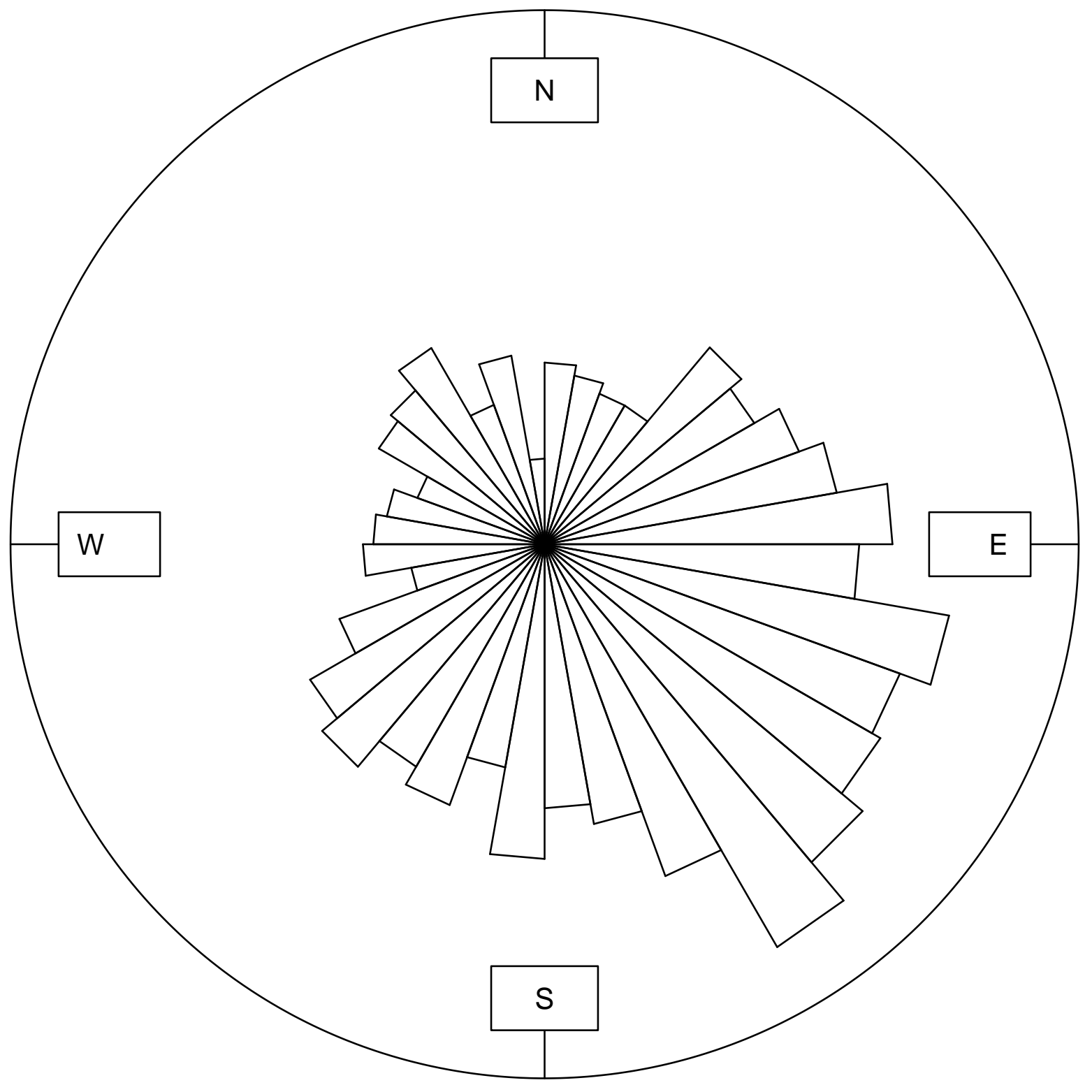


bootstrap 474

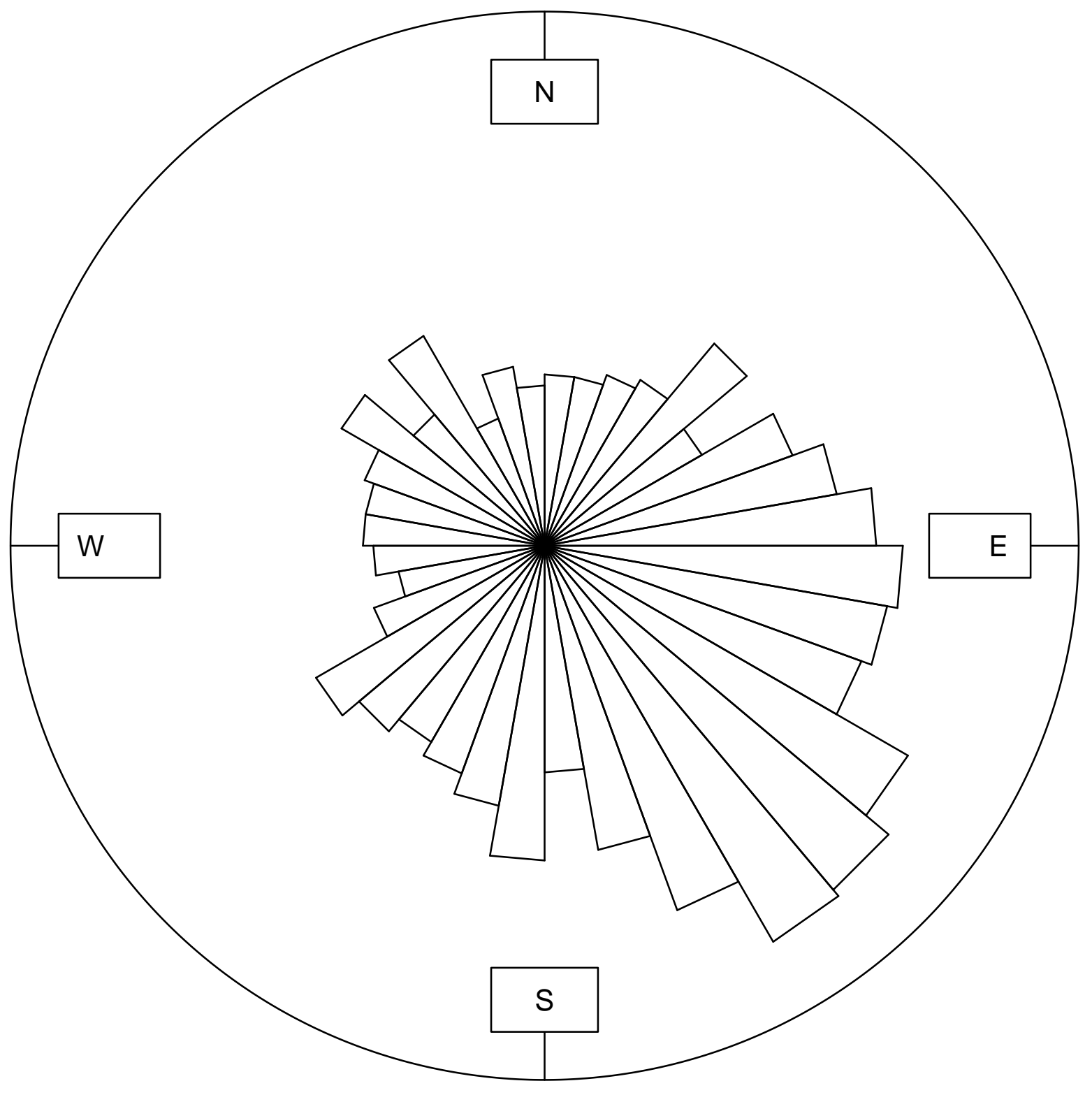


bootstrap 475

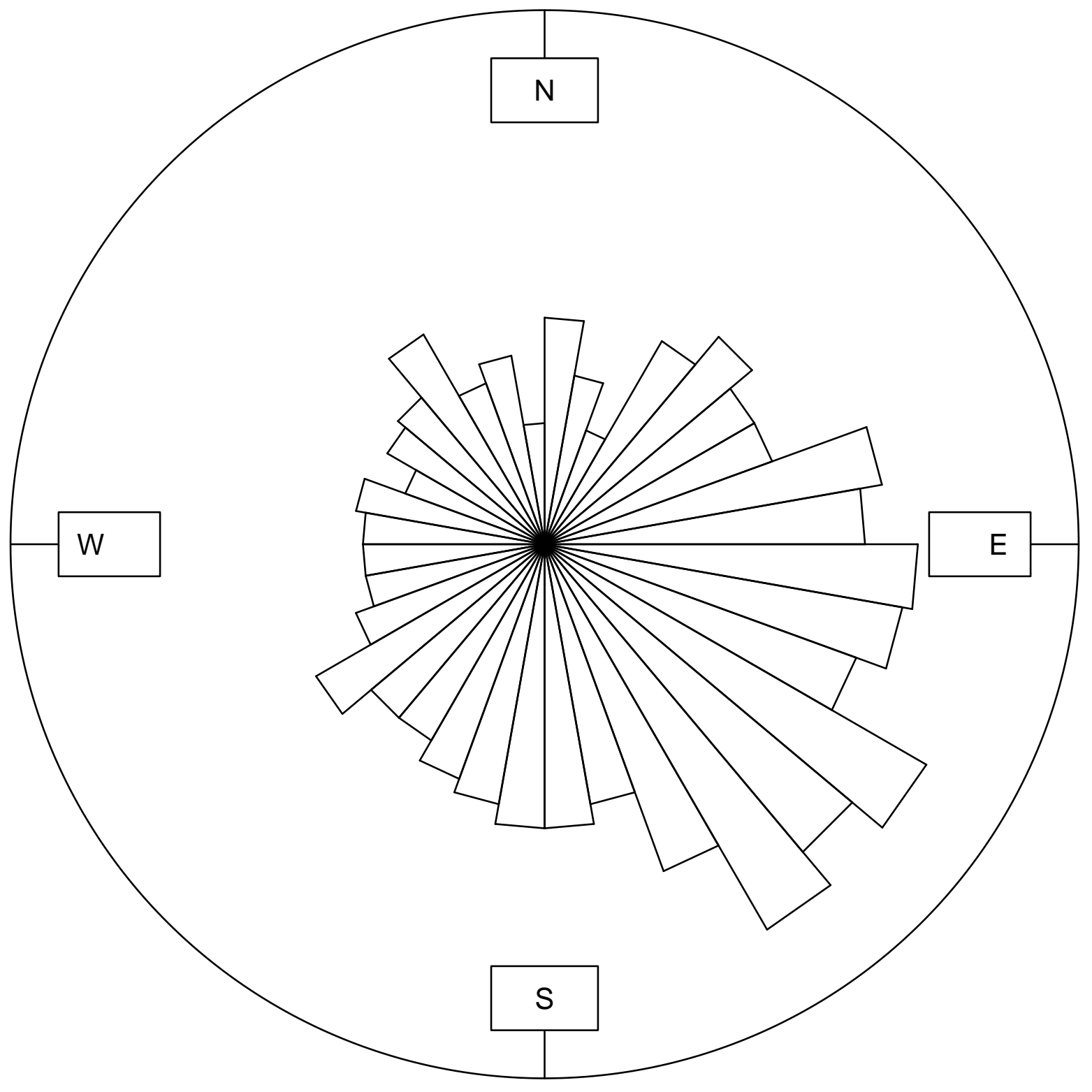


bootstrap 476

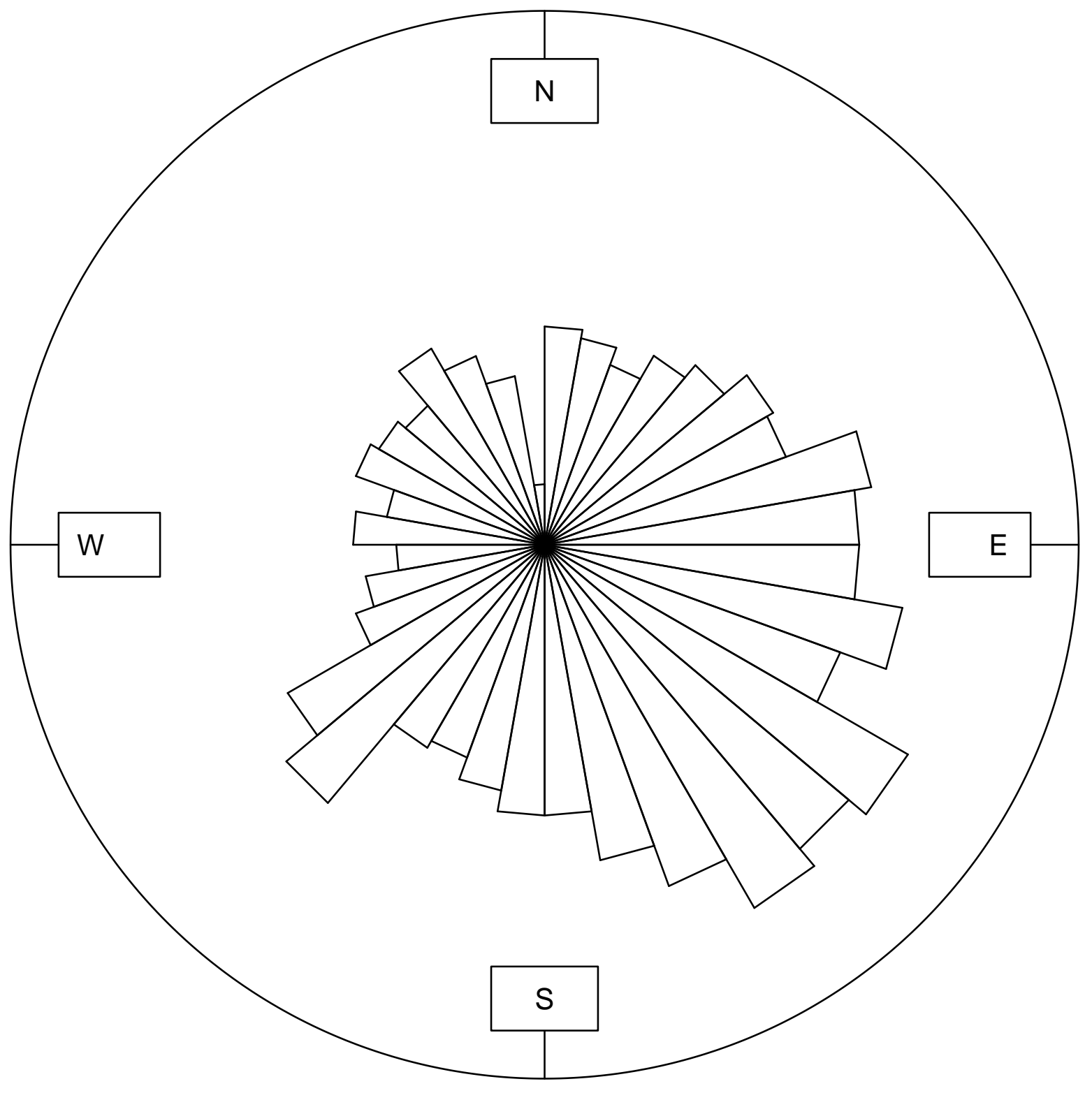


bootstrap 477

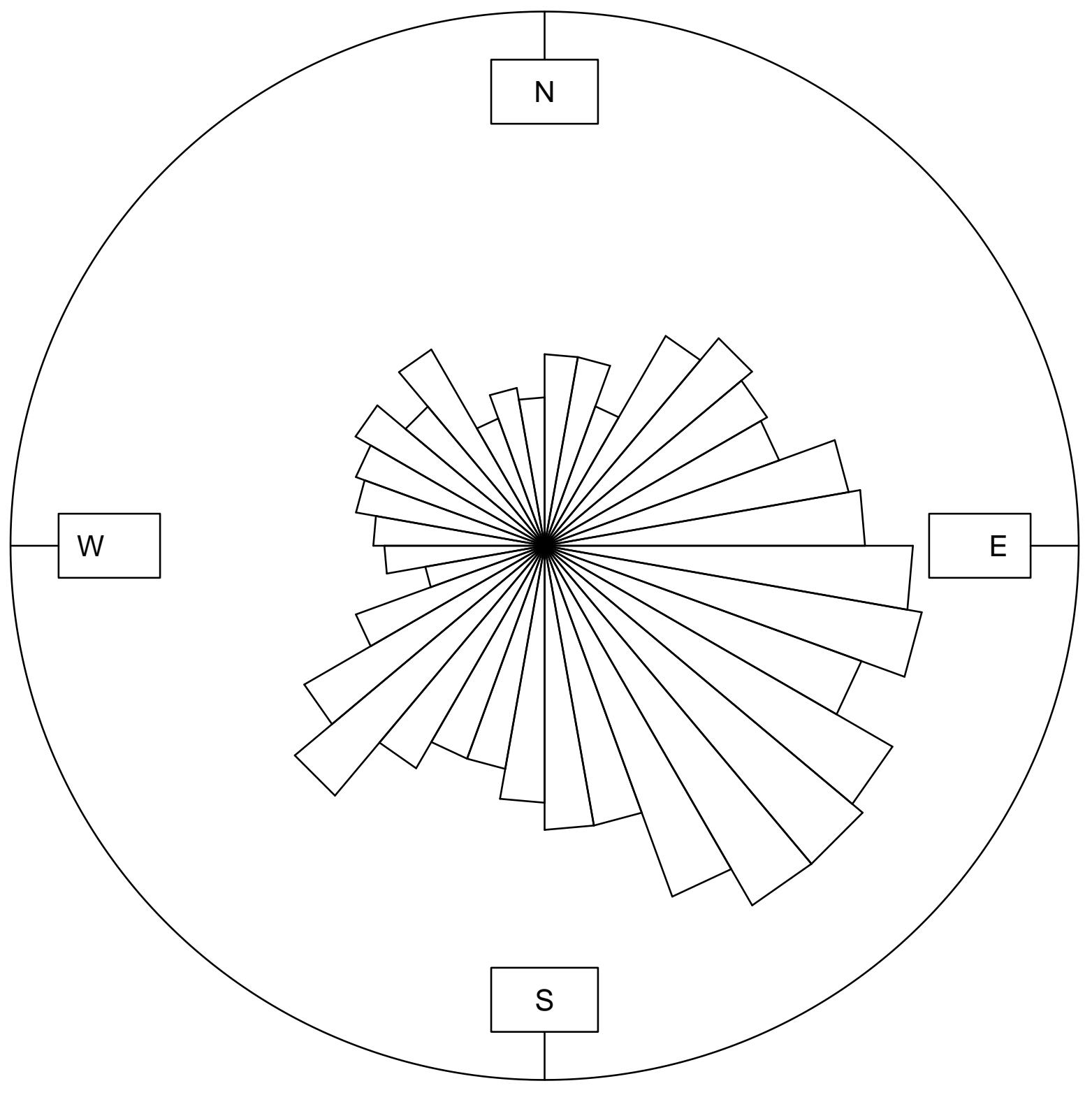


bootstrap 478

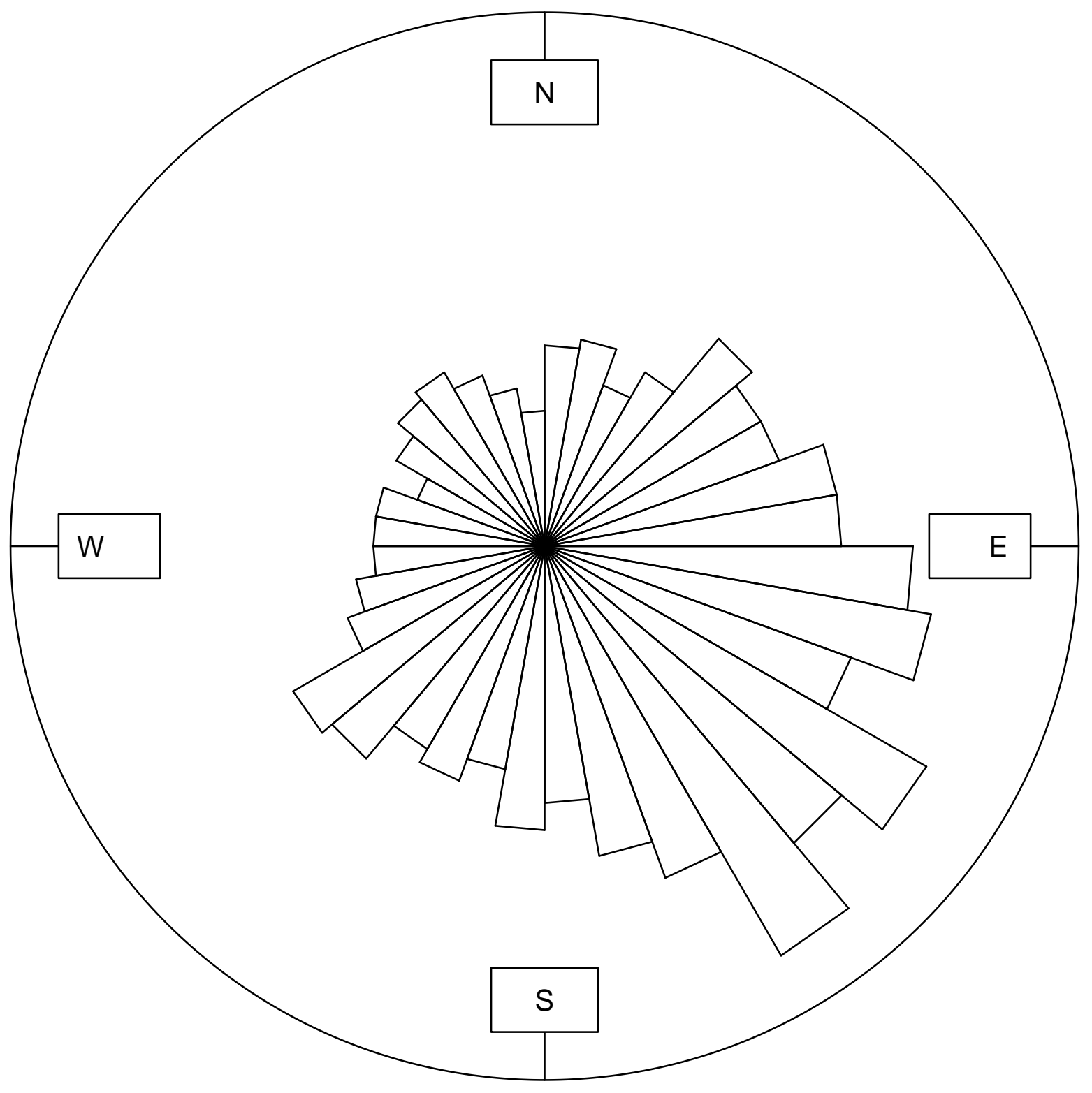


bootstrap 479

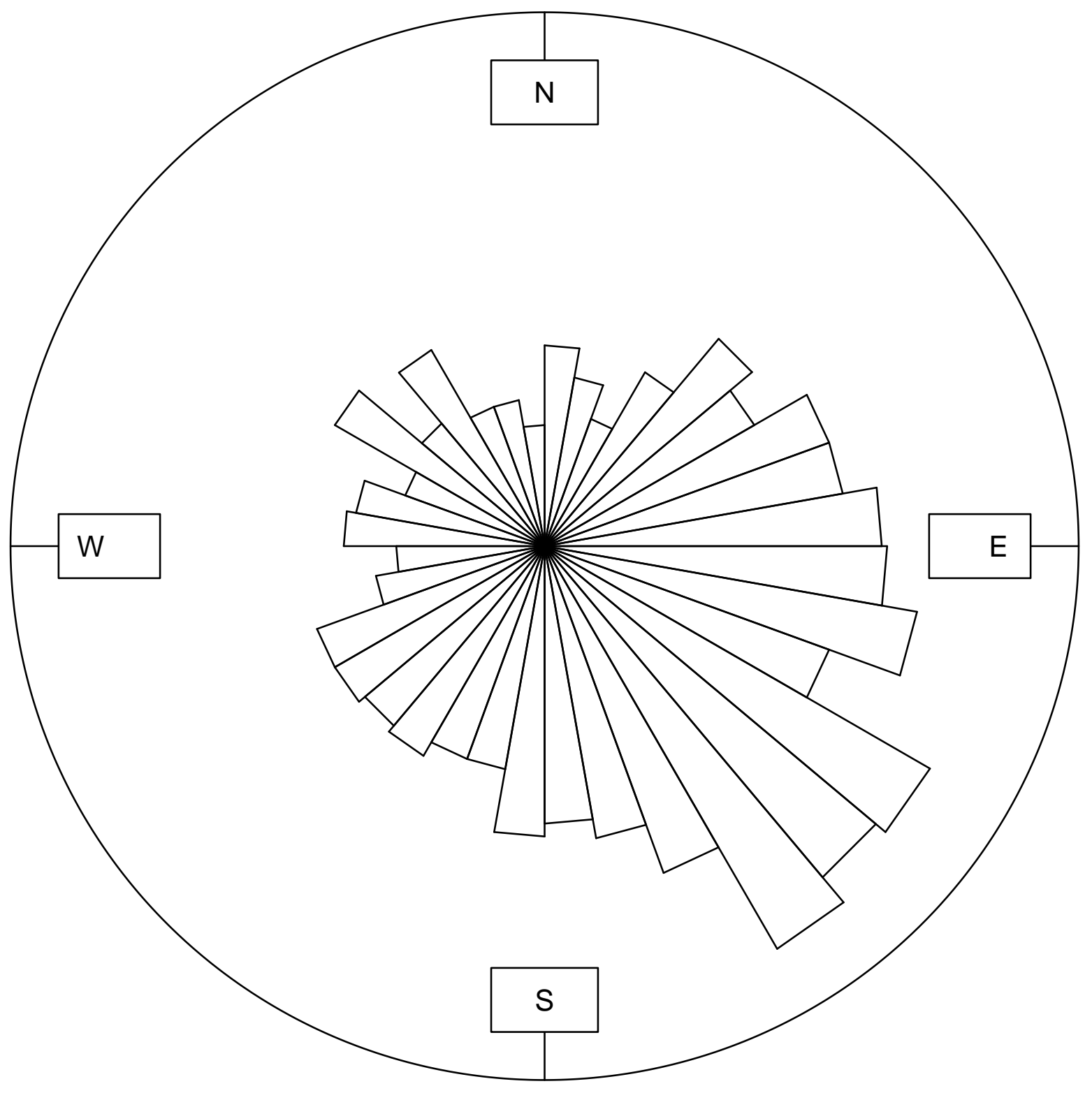


bootstrap 480

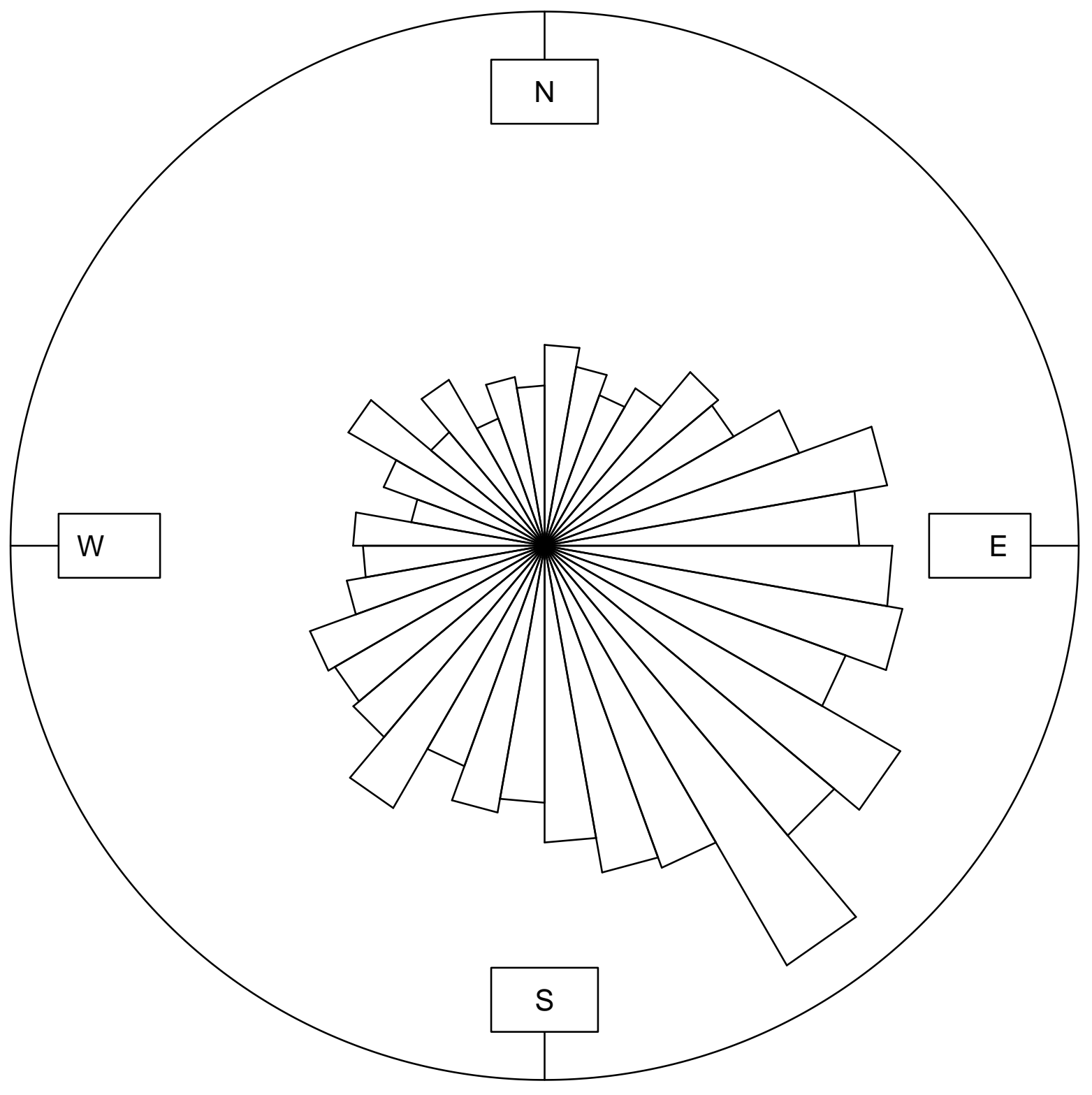


bootstrap 481

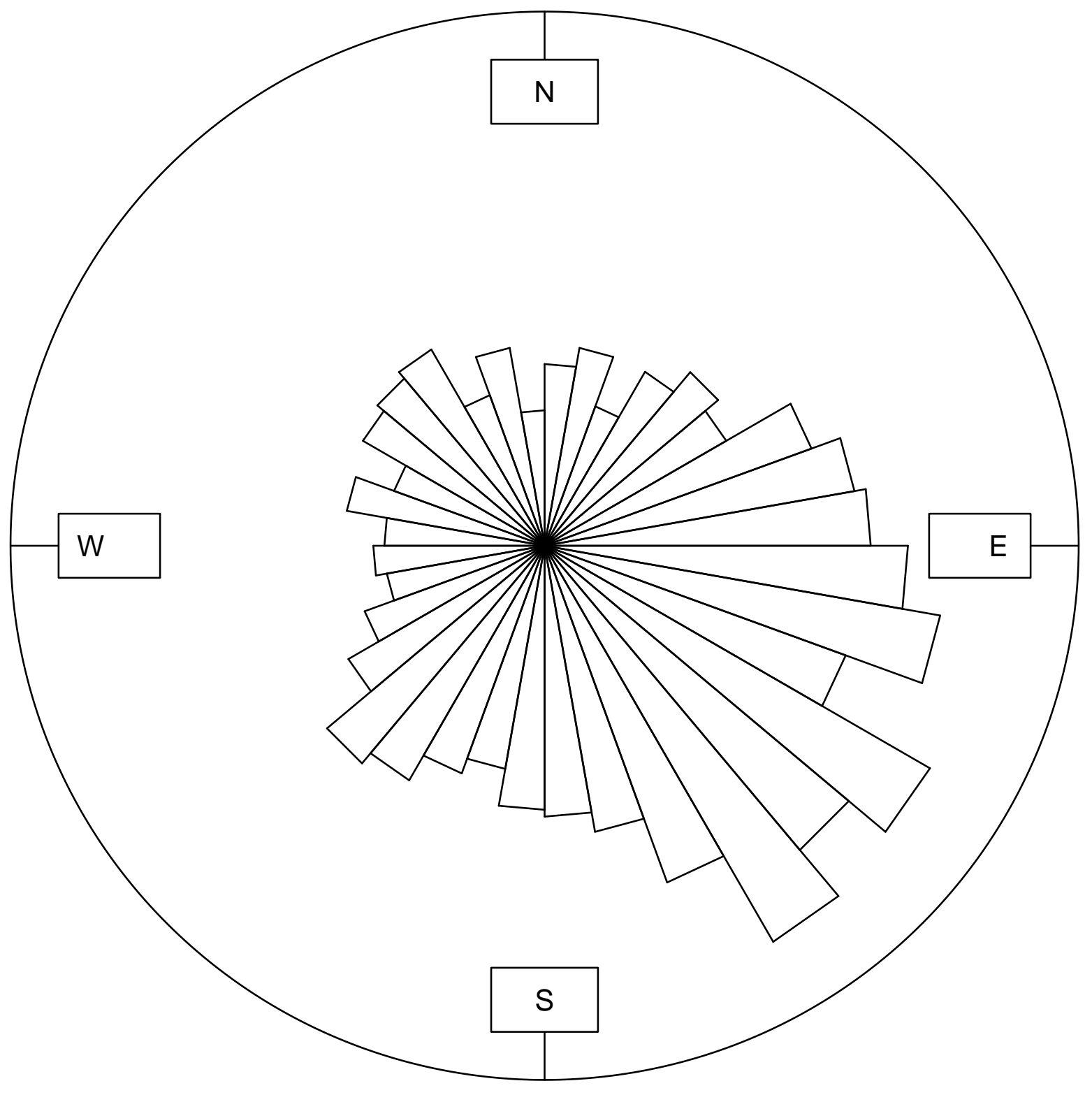


bootstrap 482

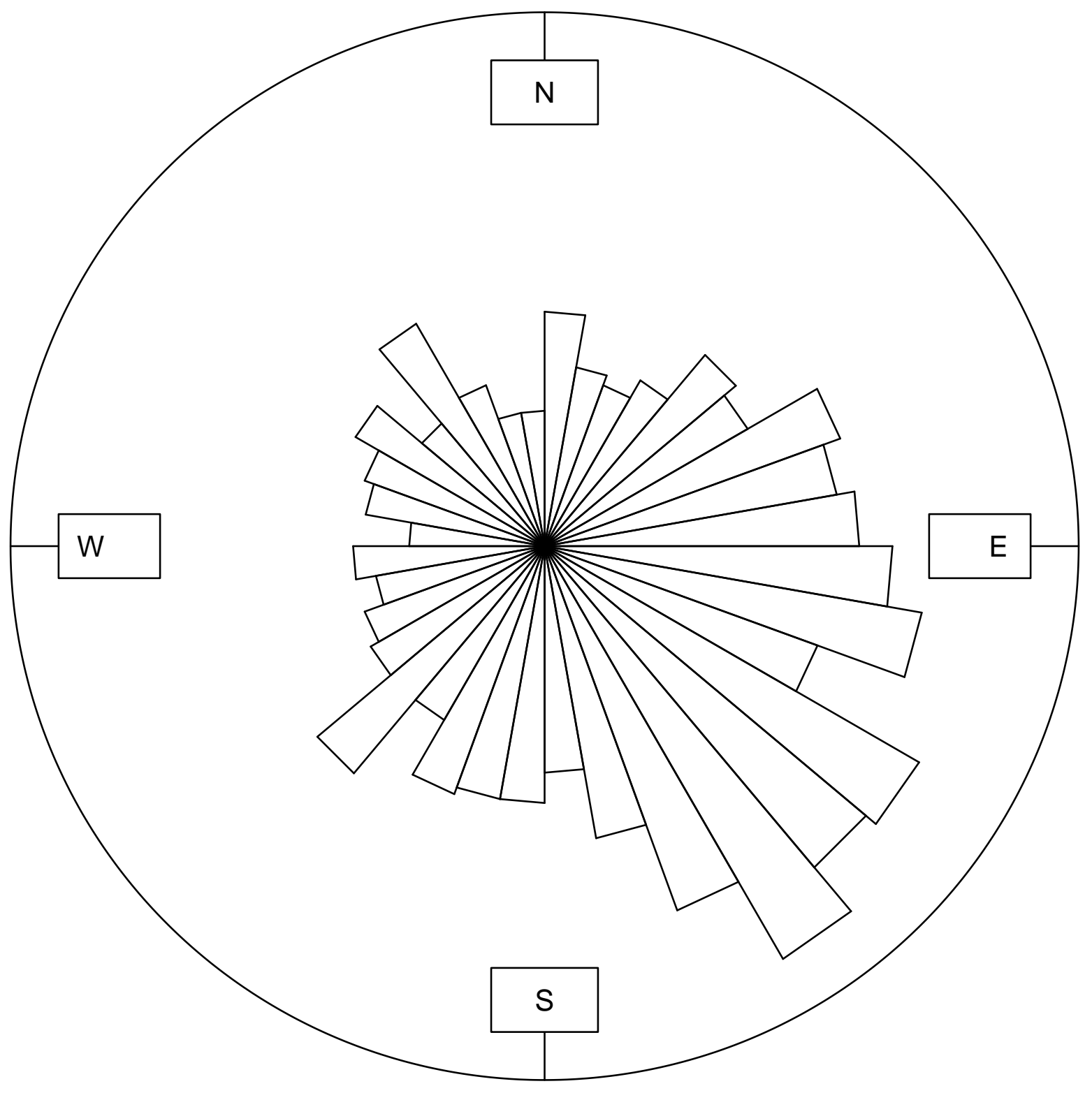




\section{bootstrap 483}

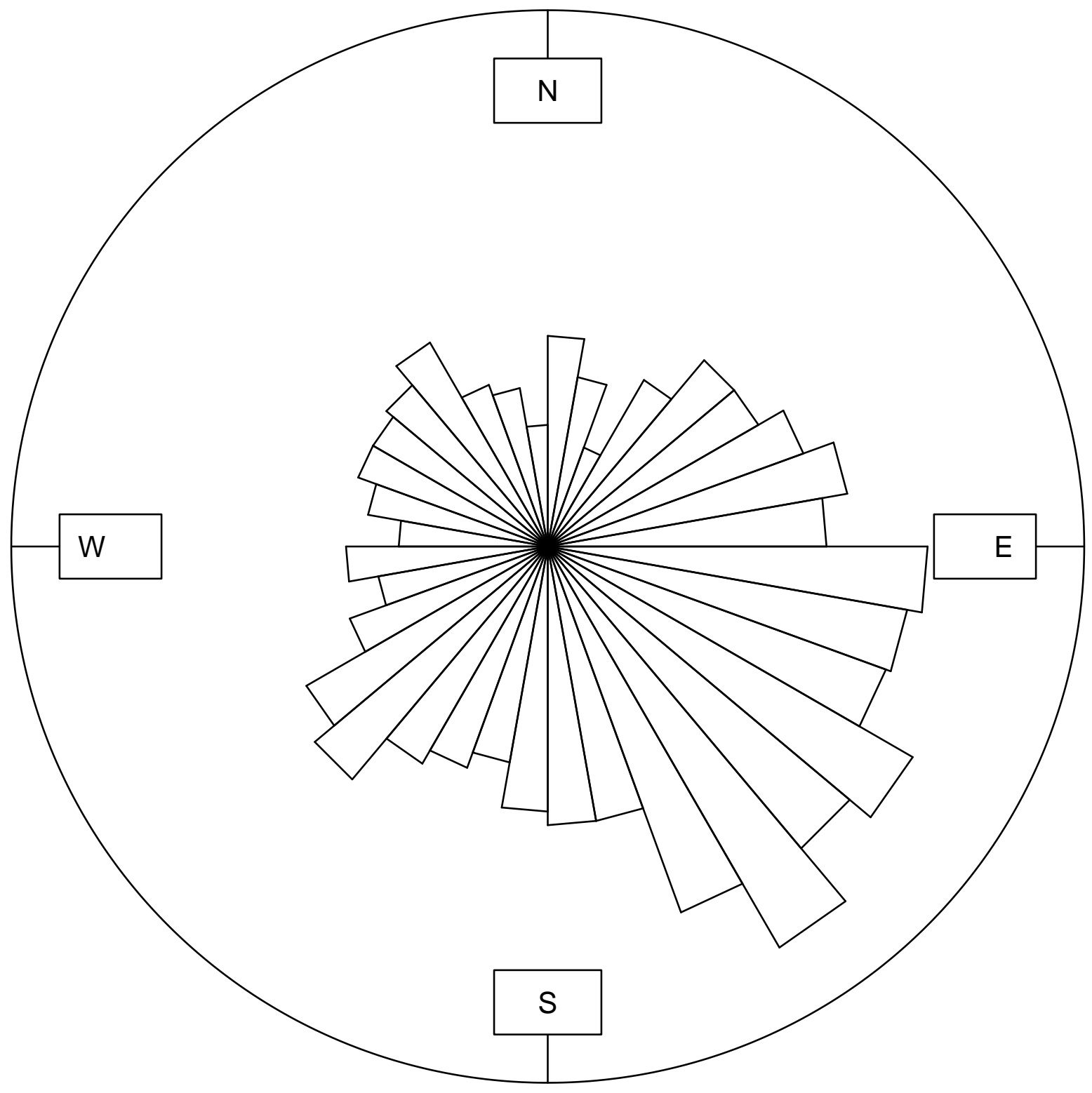


bootstrap 484

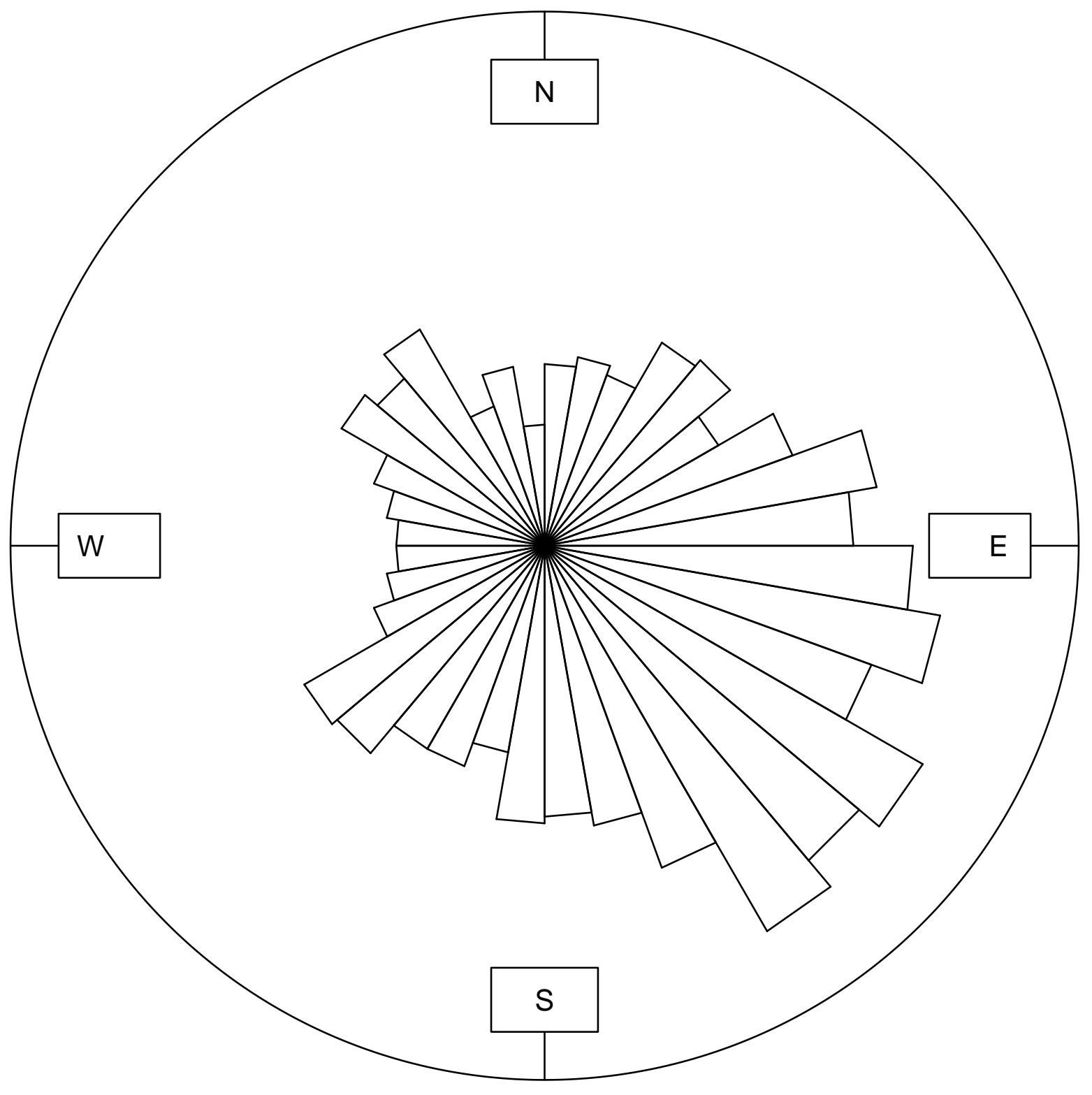




\section{bootstrap 485}

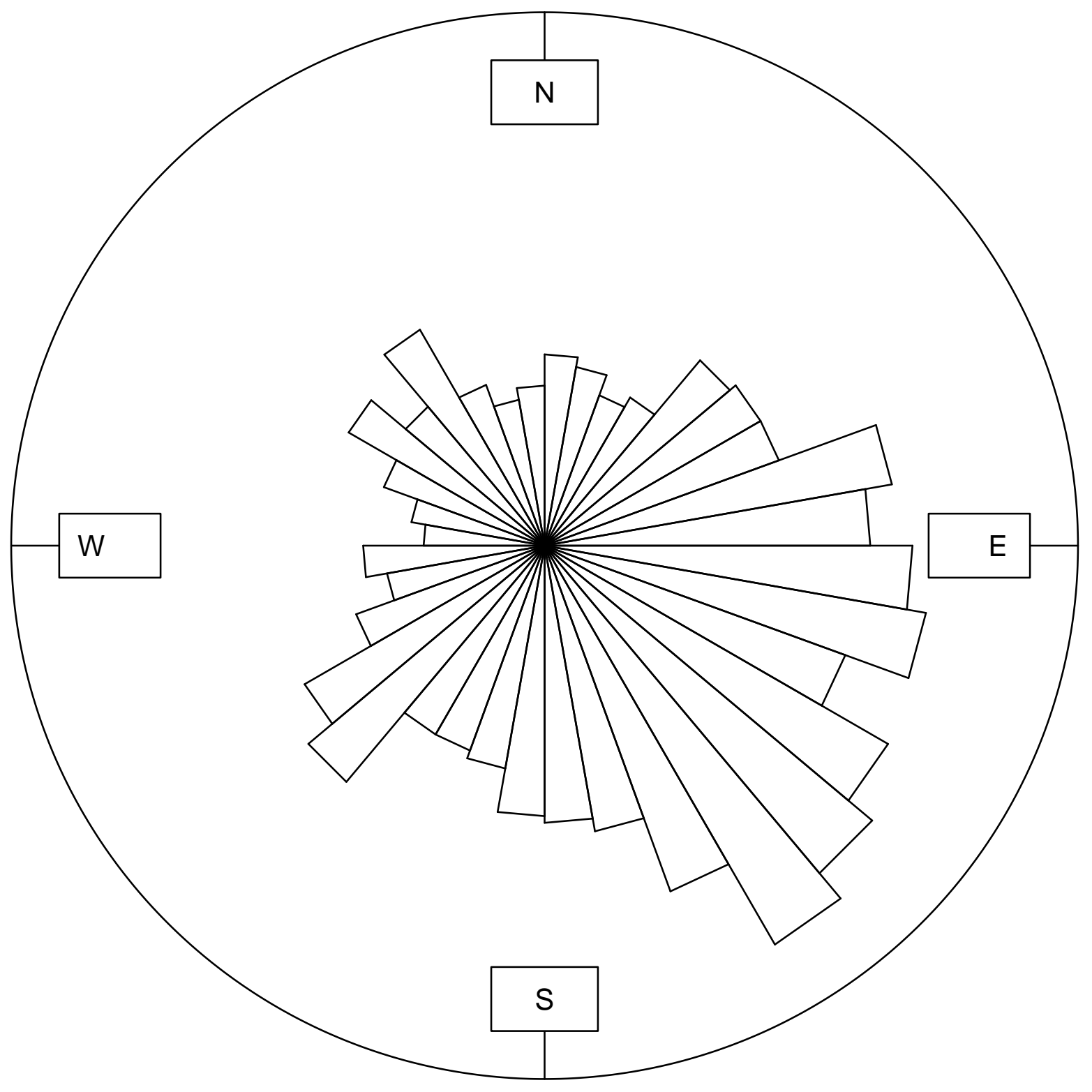


bootstrap 486

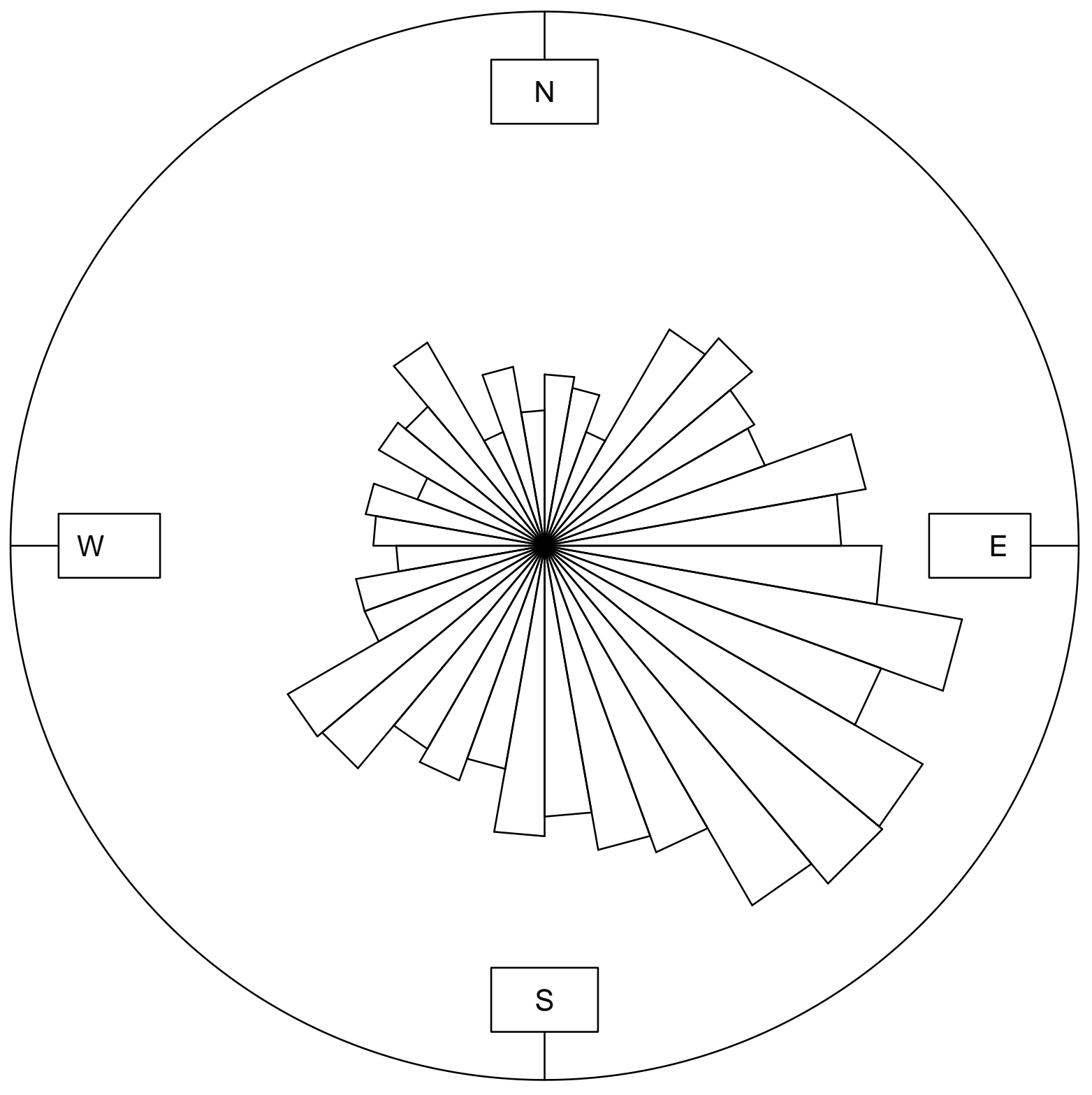


bootstrap 487

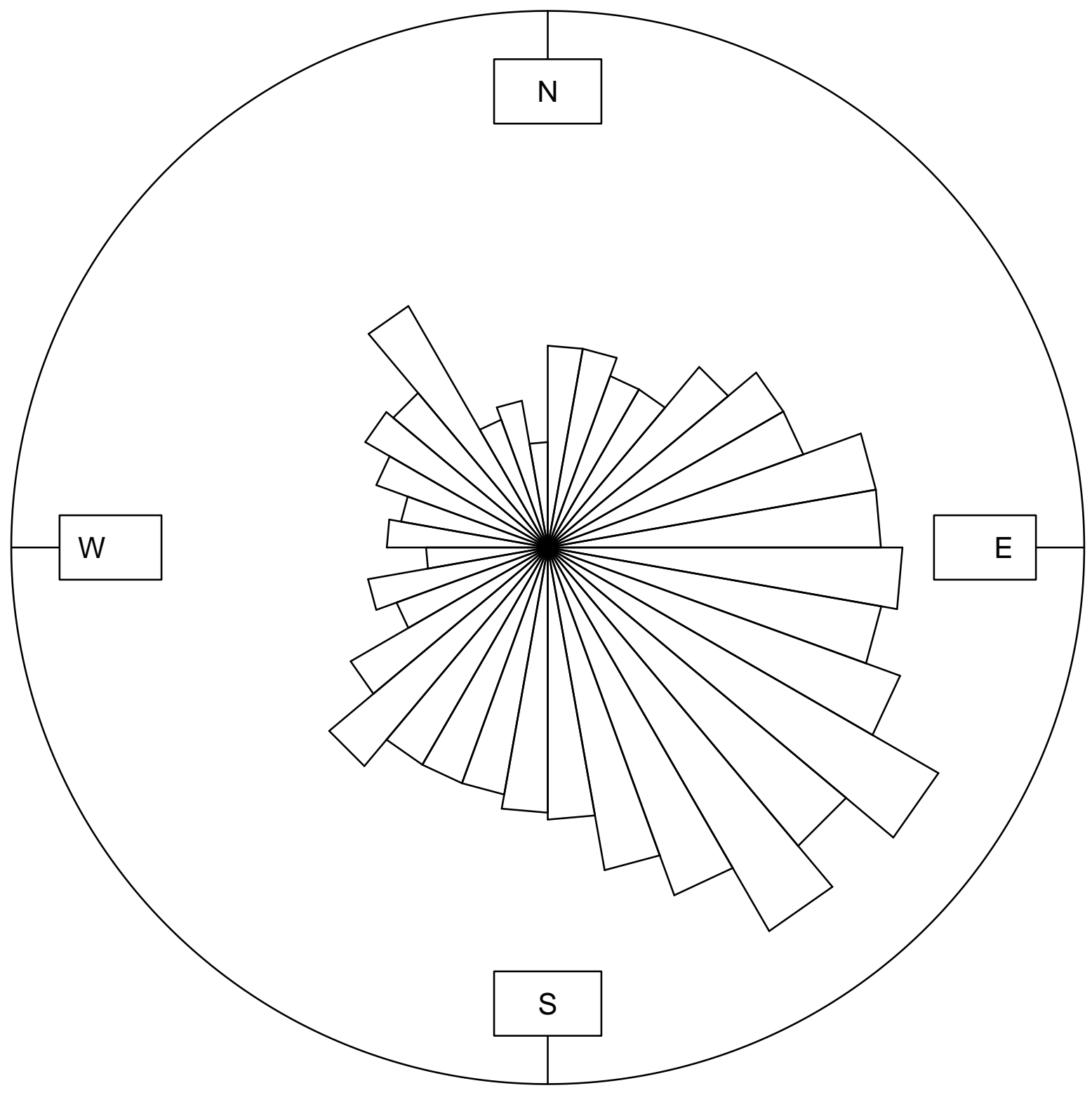


bootstrap 488

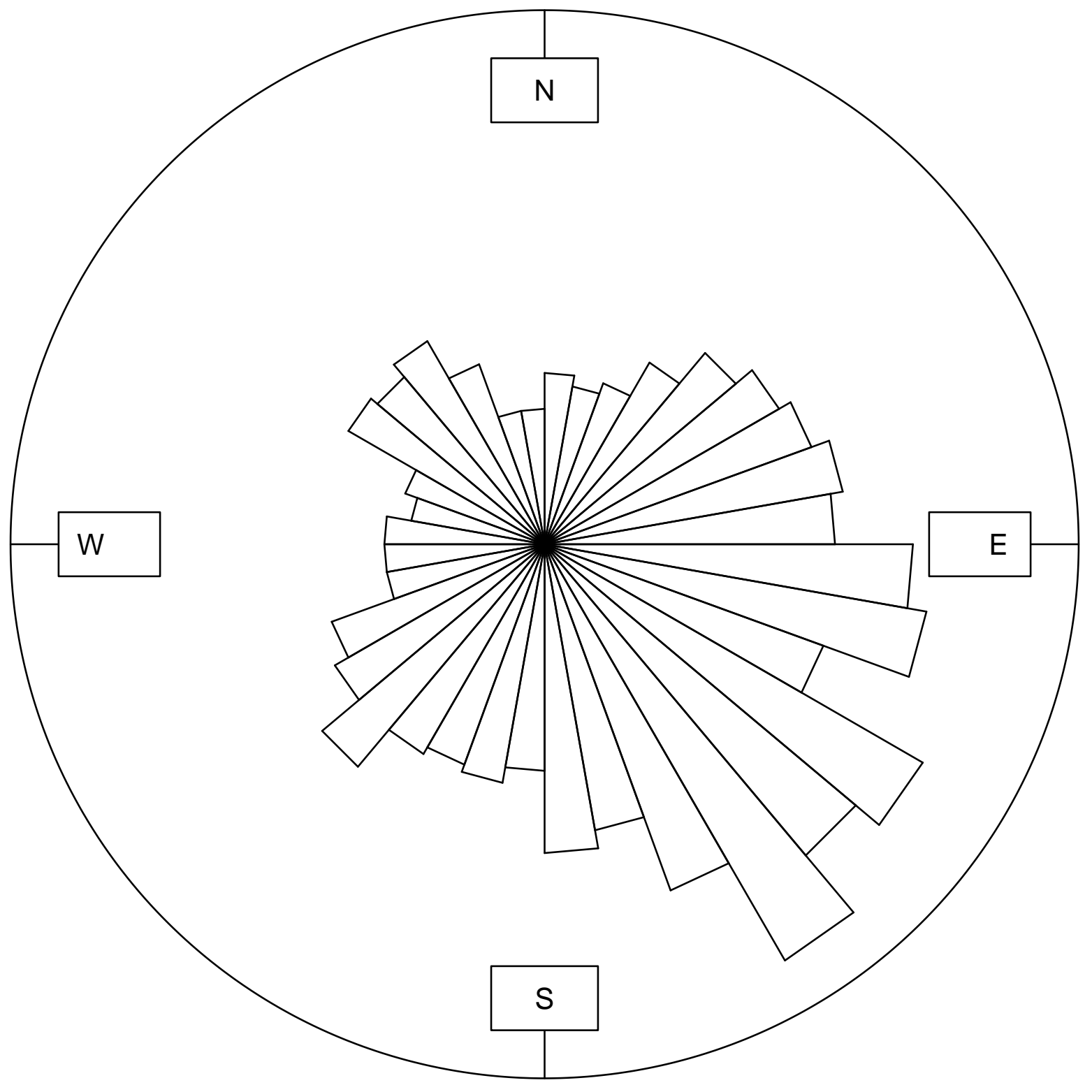


bootstrap 489

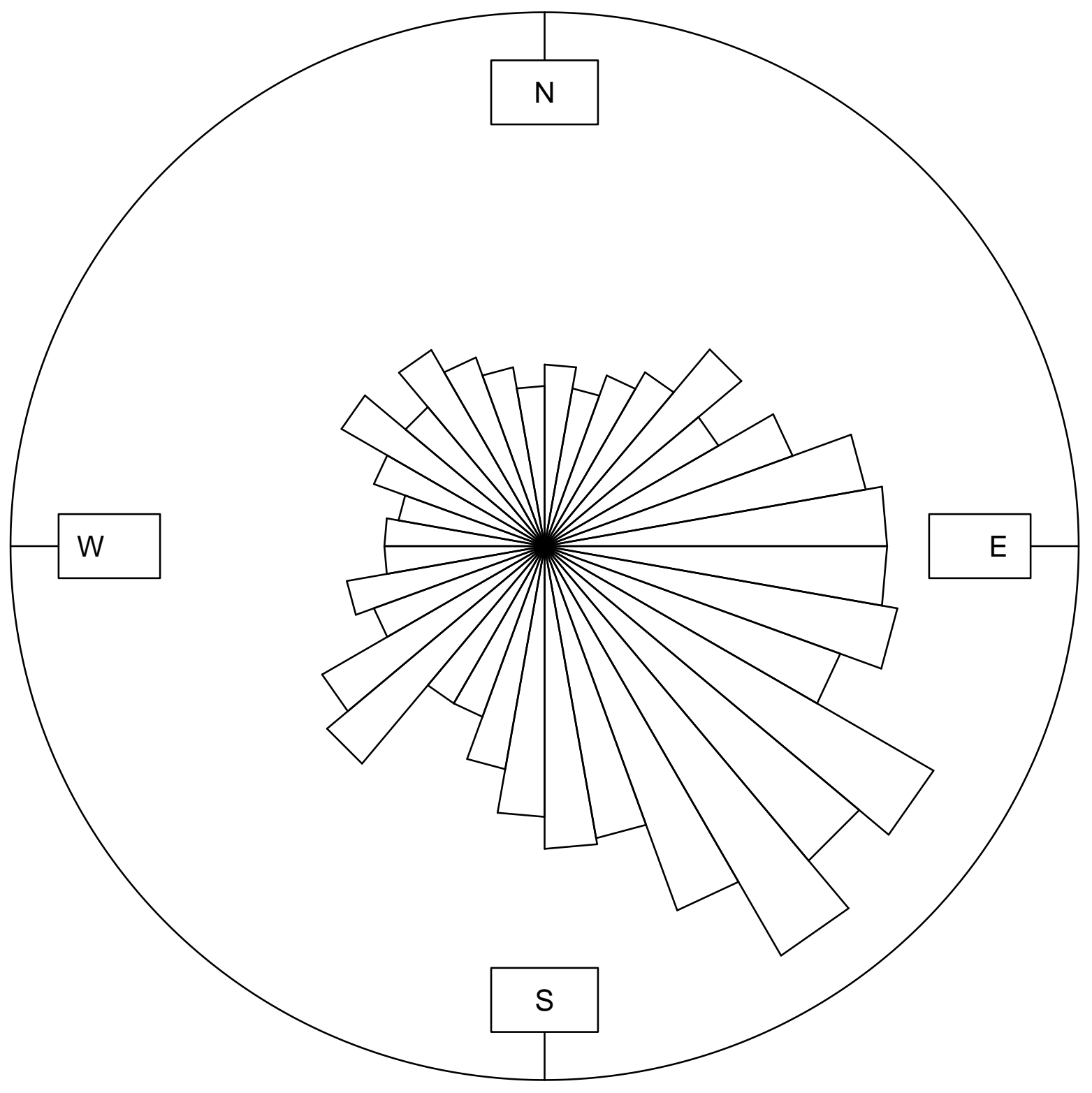


bootstrap 490

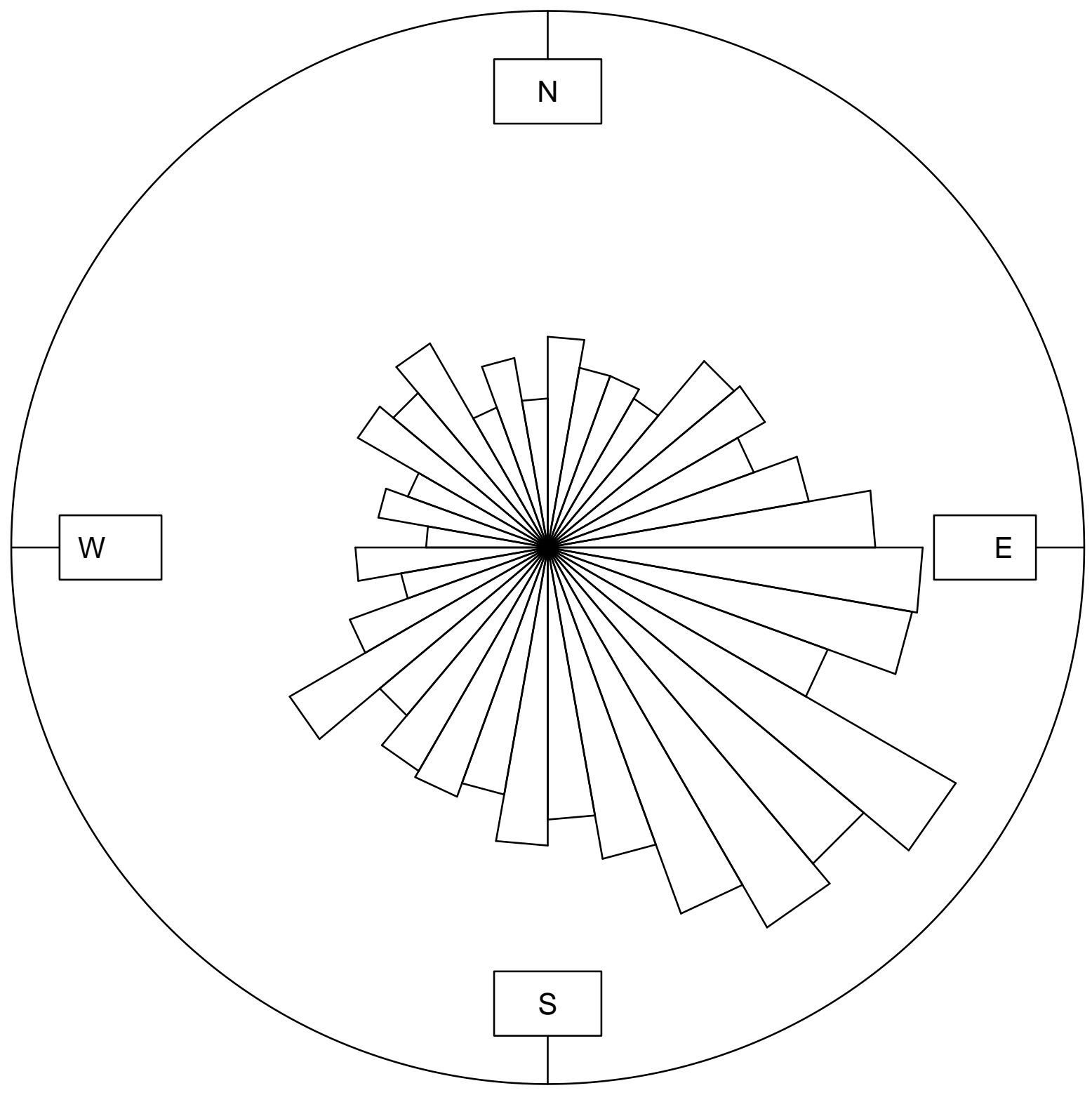


bootstrap 491

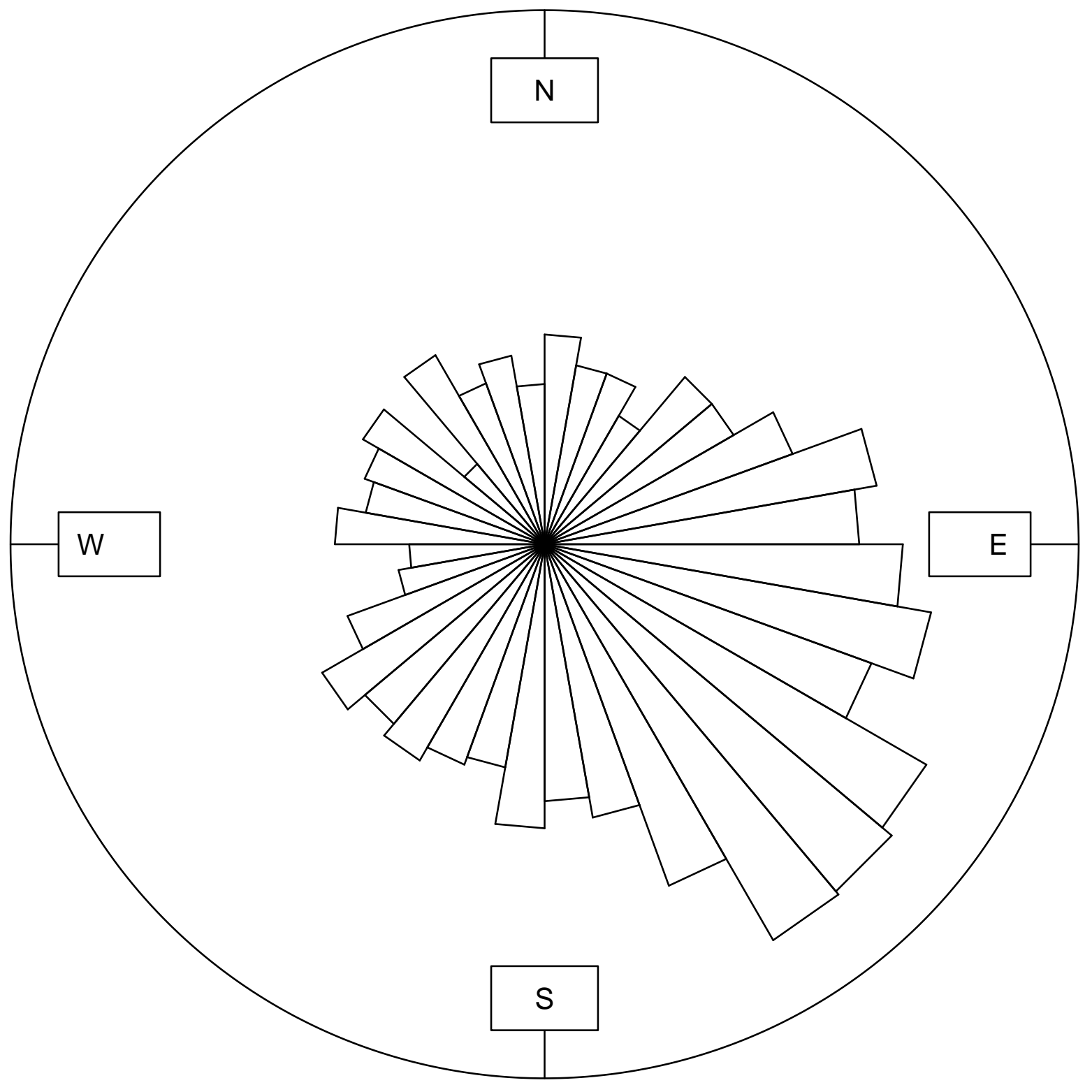


bootstrap 492

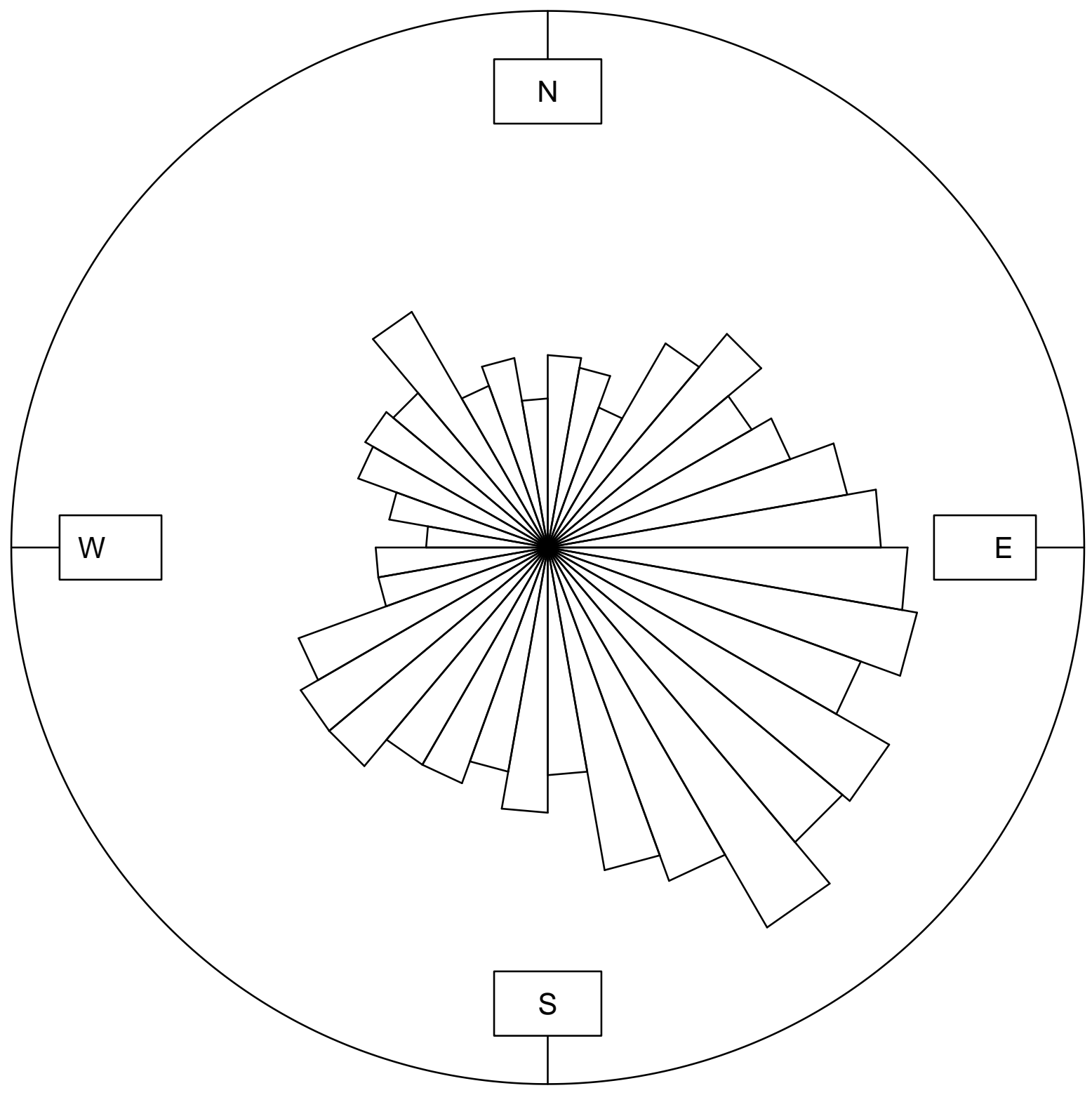




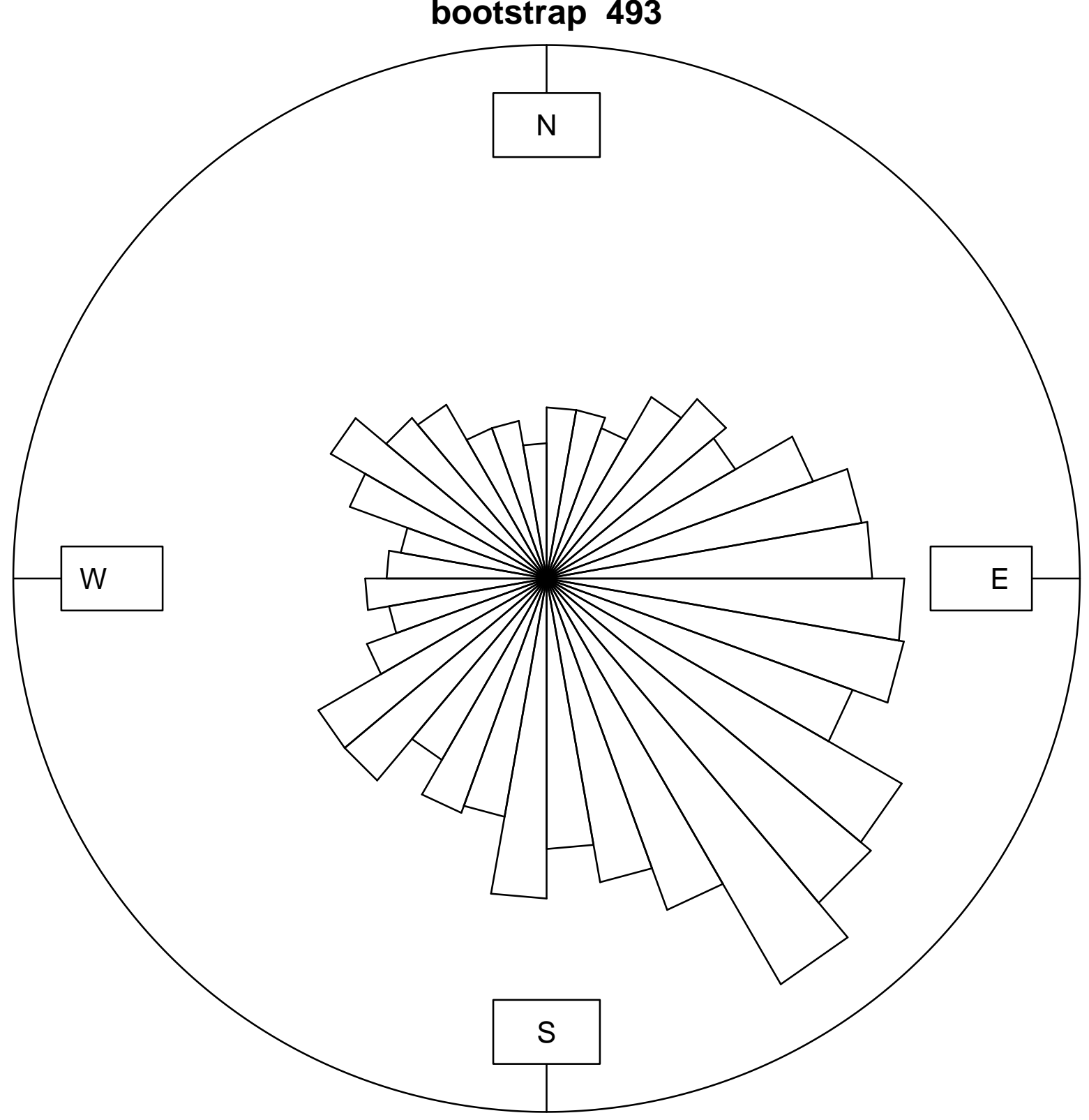


bootstrap 494

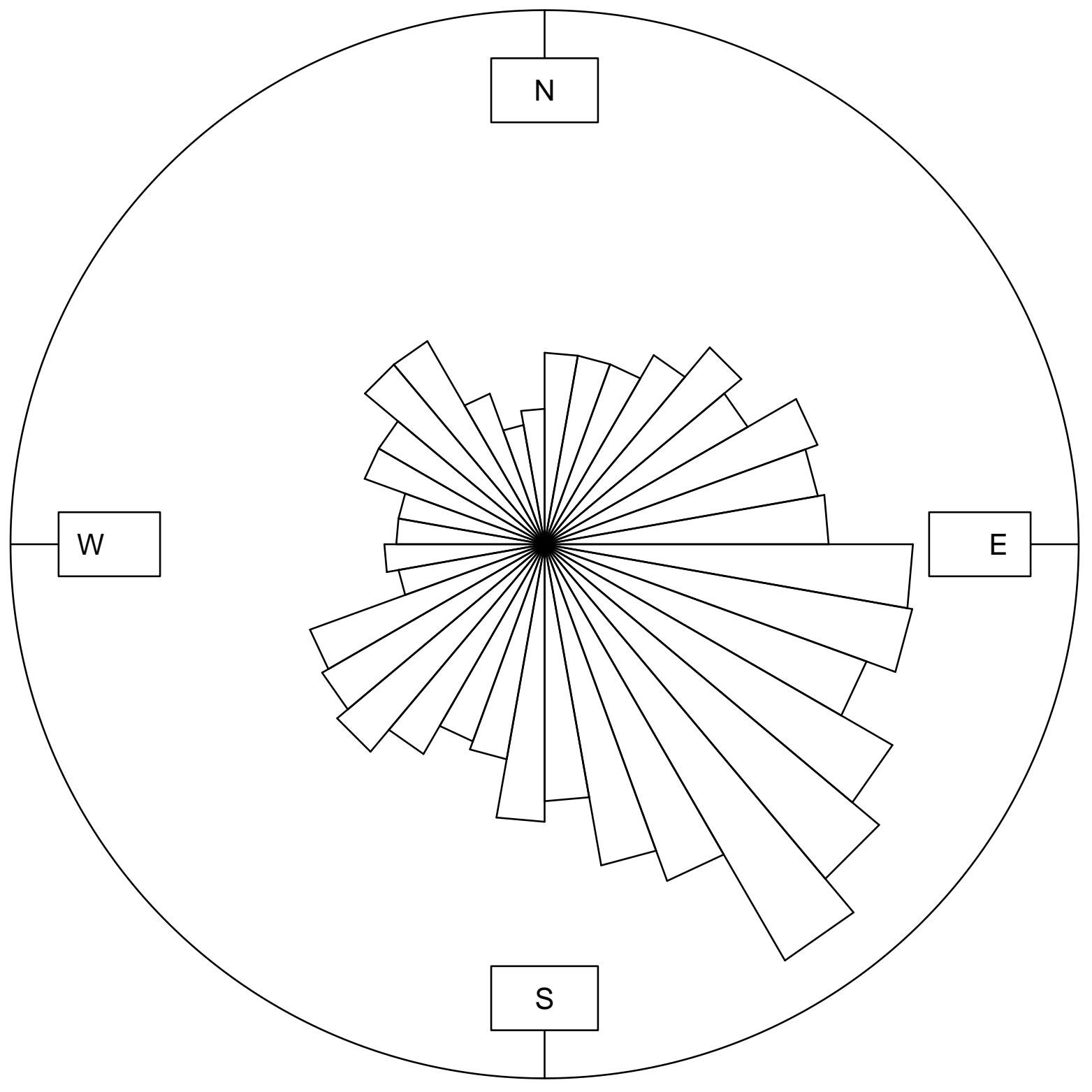


bootstrap 495

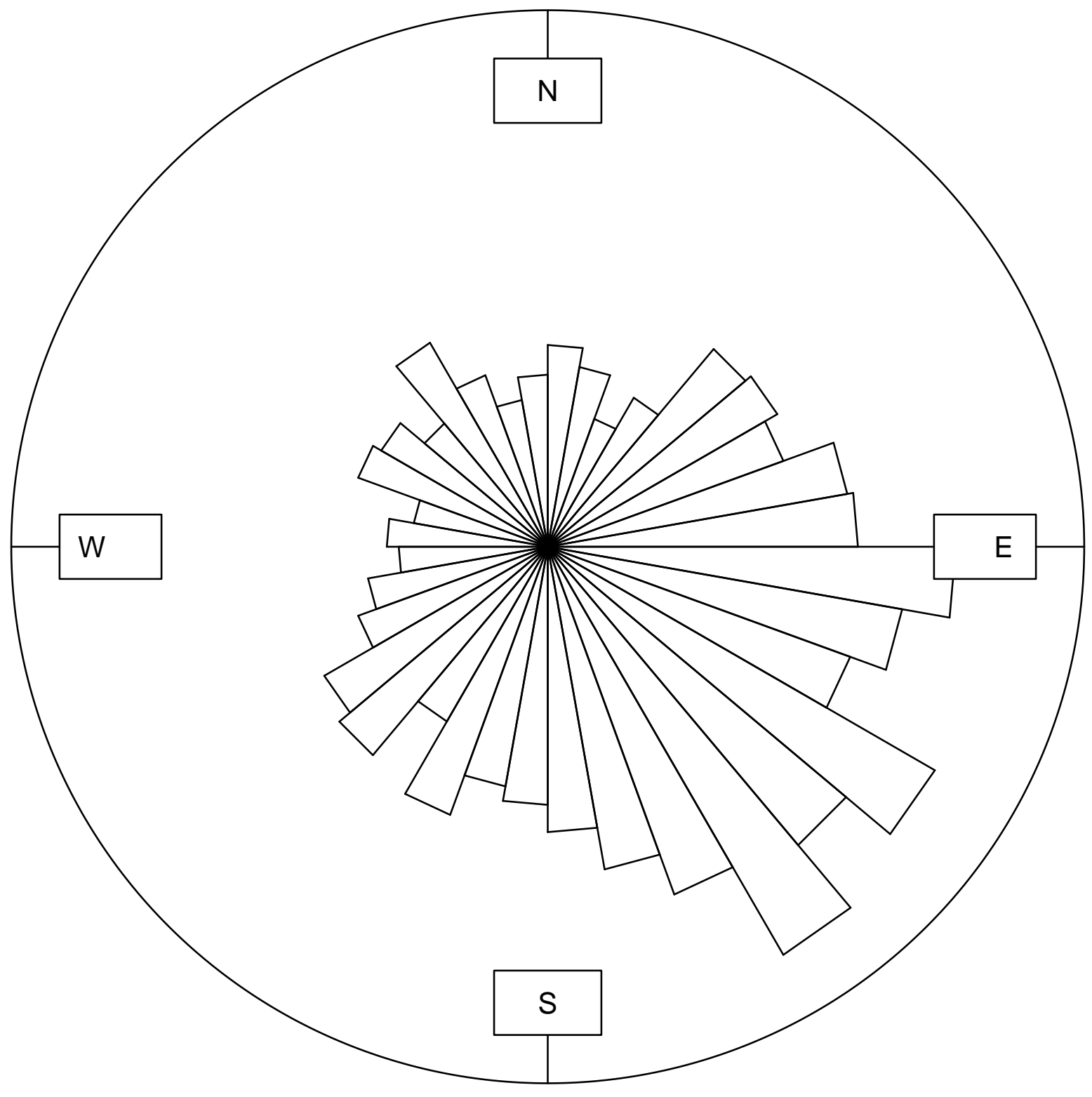




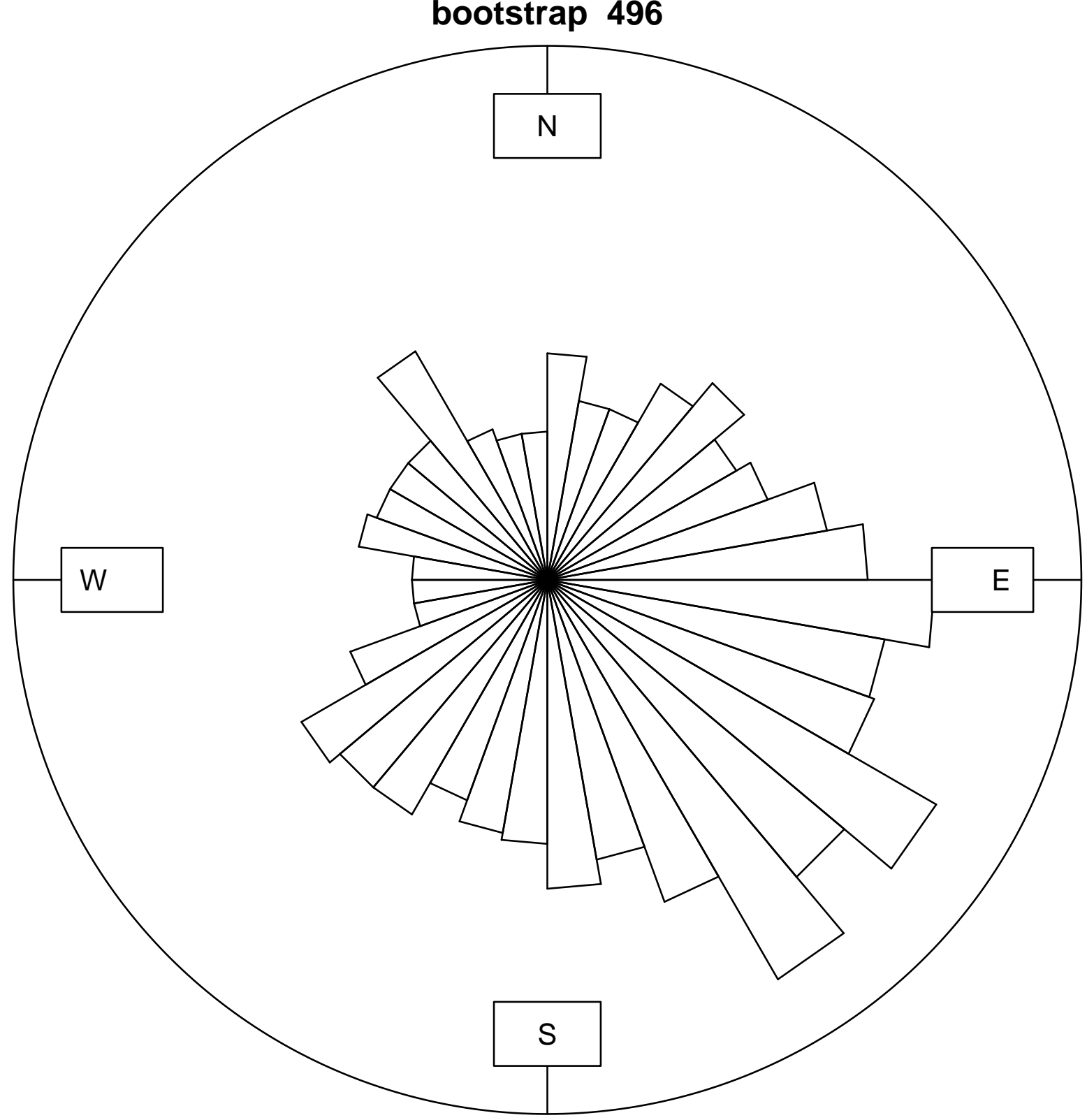


bootstrap 497

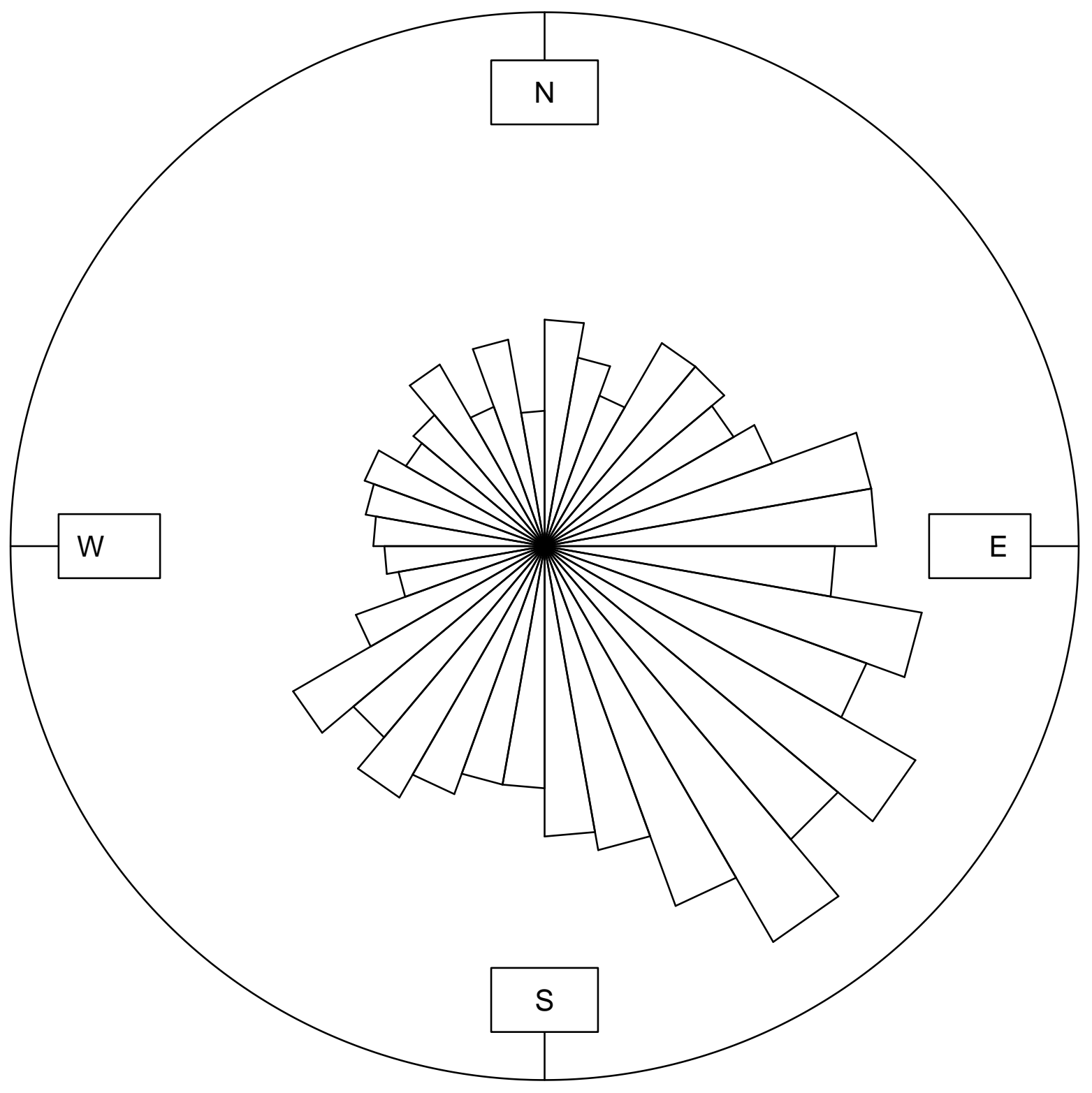


bootstrap 498

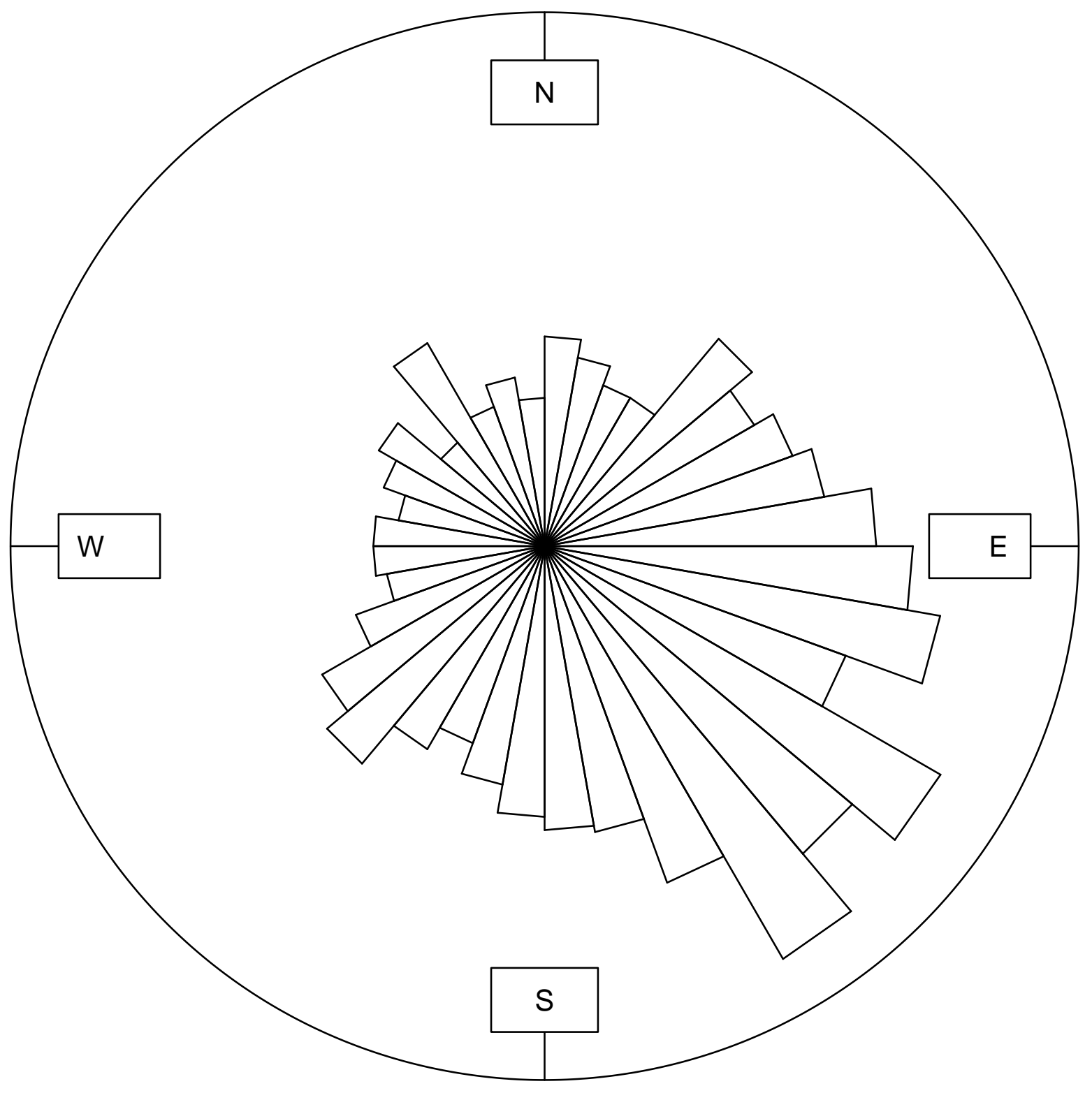


bootstrap 499

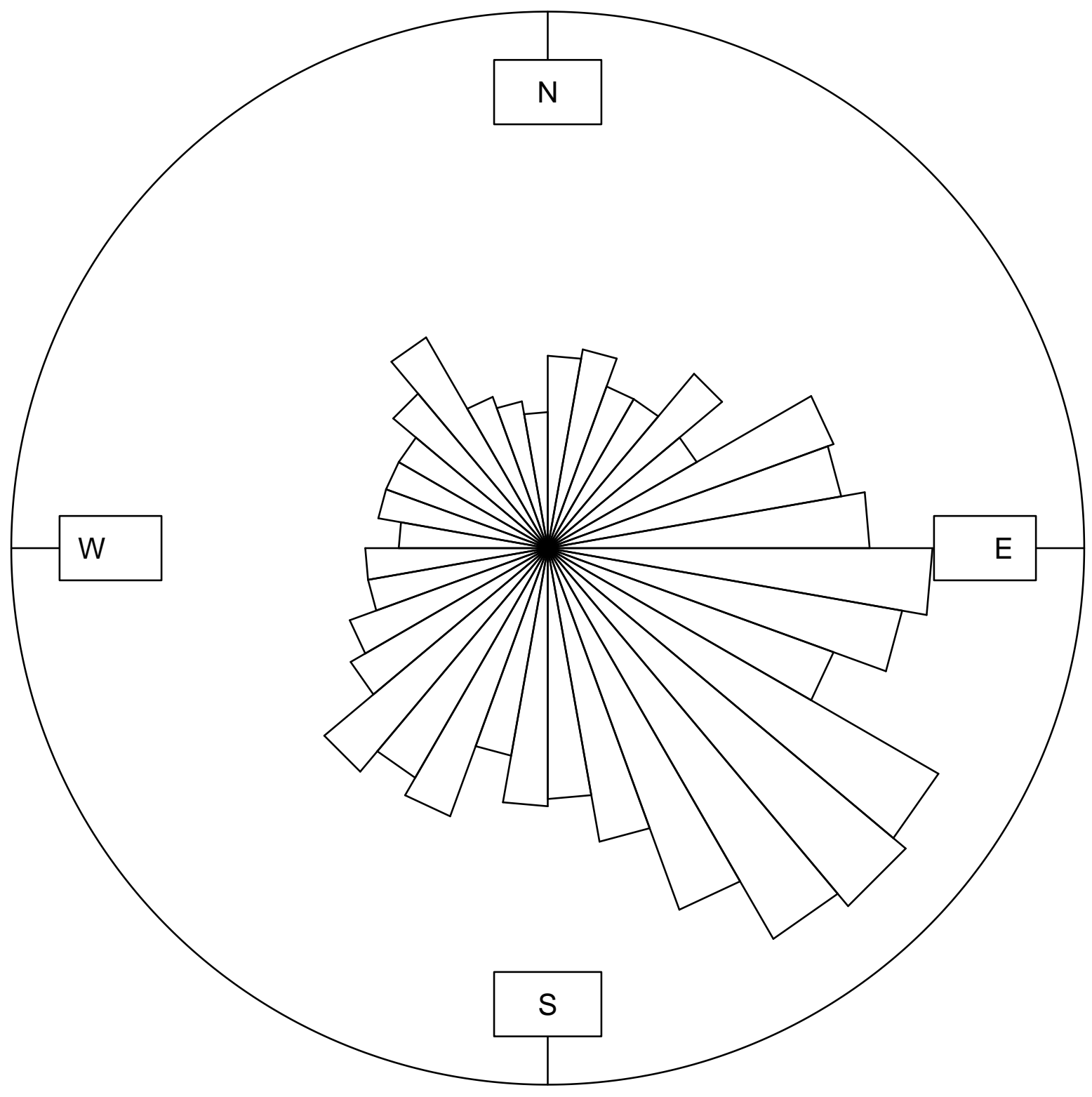




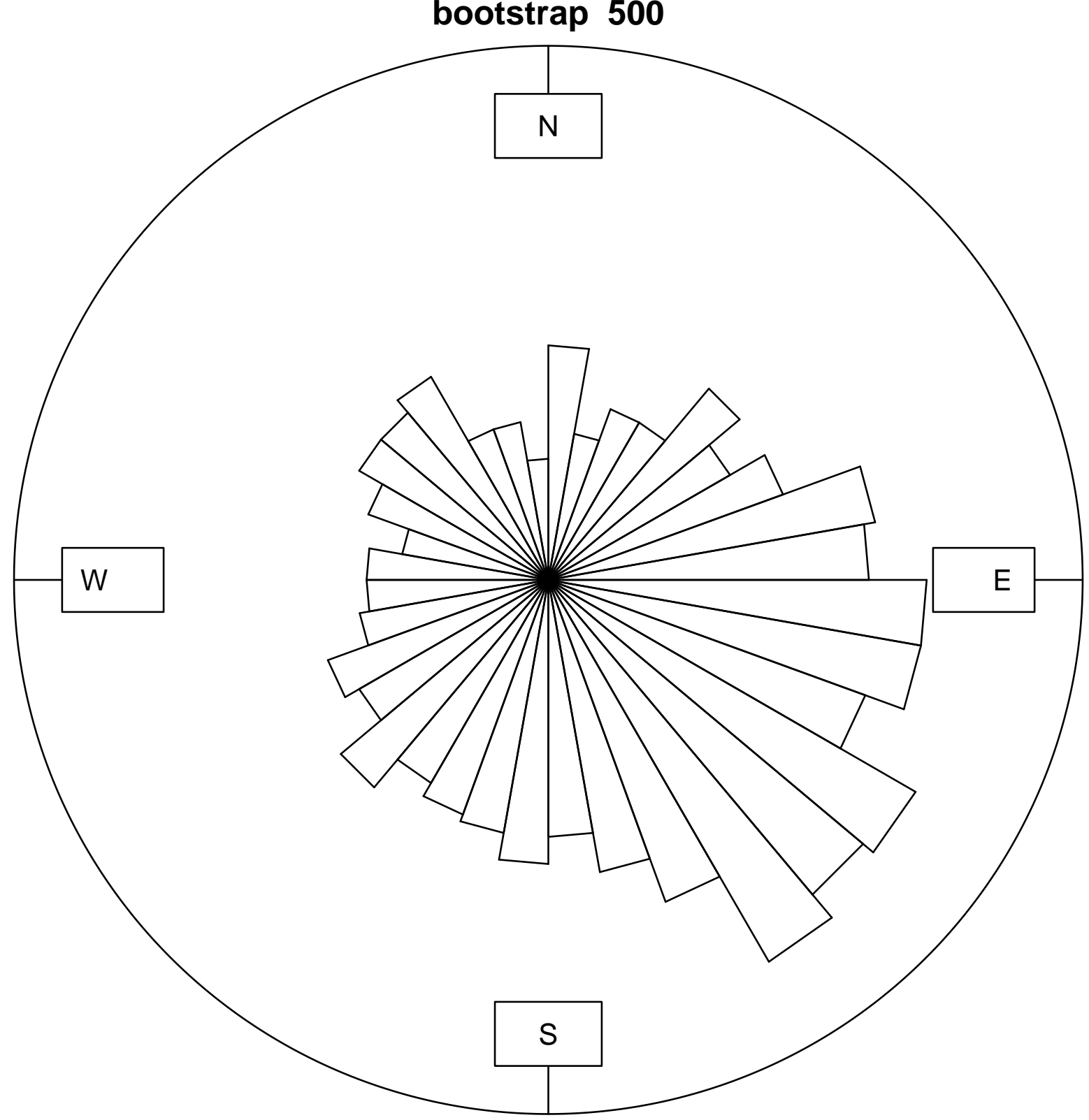


bootstrap 501

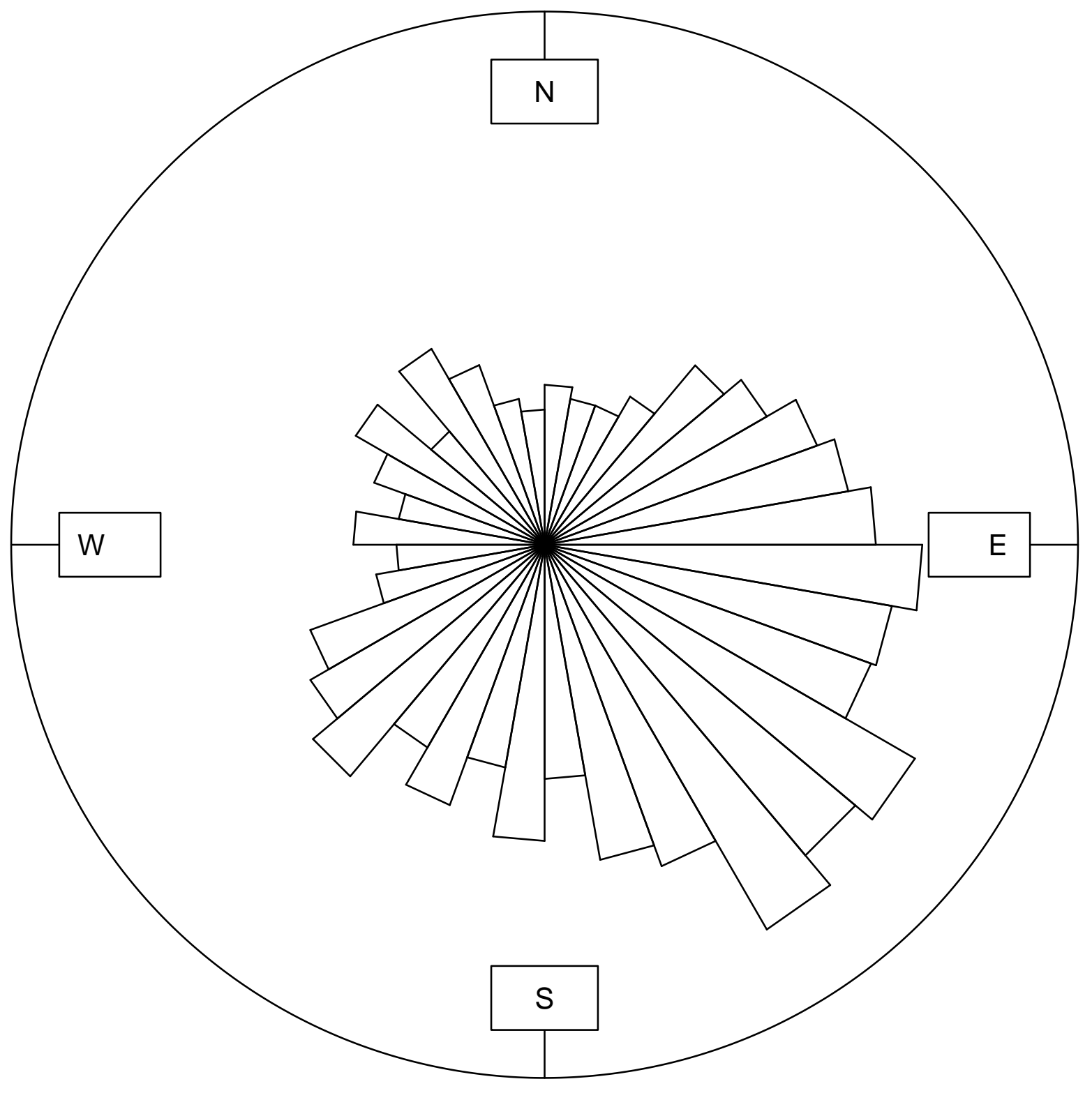


bootstrap 502

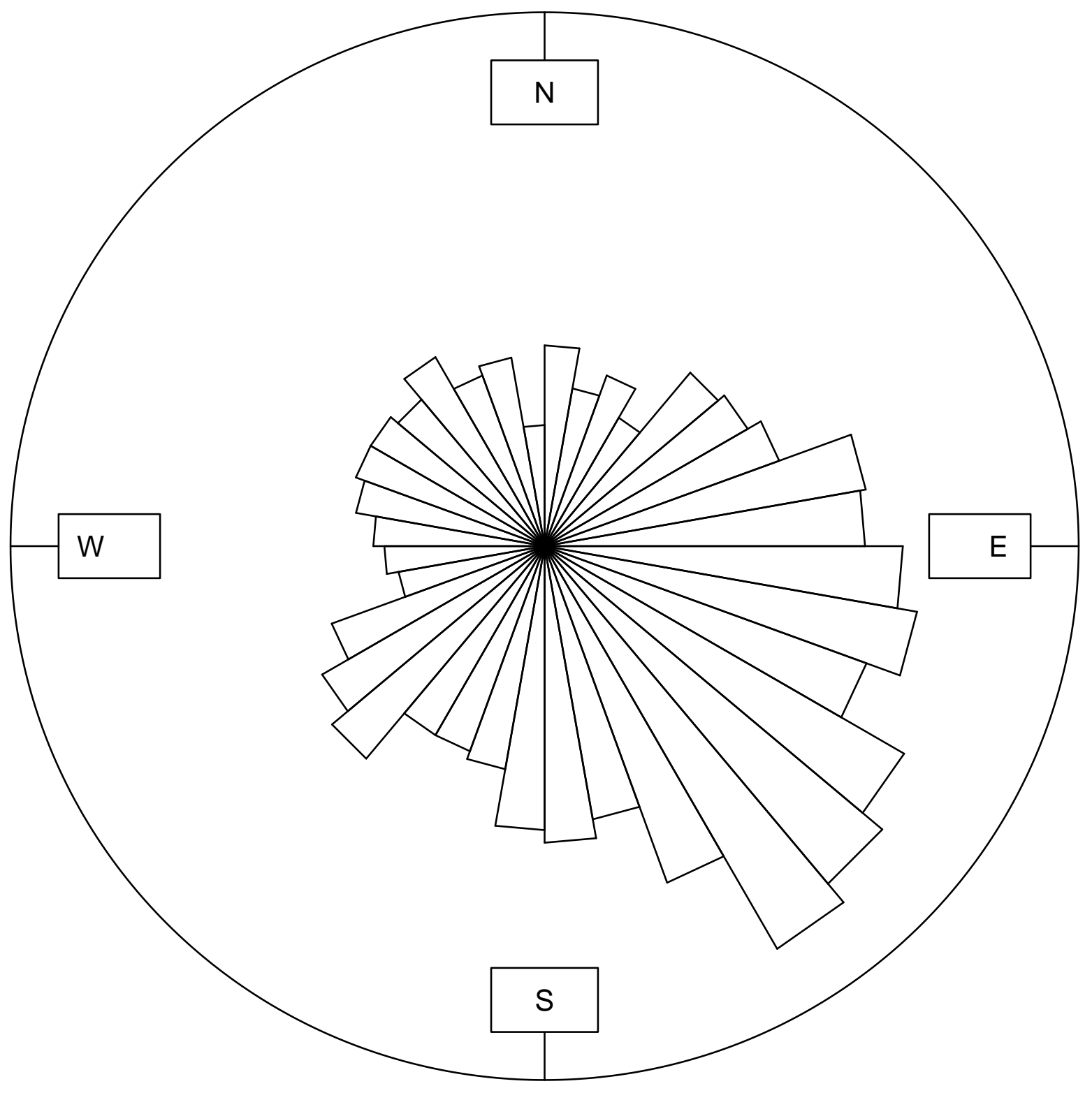




\section{bootstrap 503}

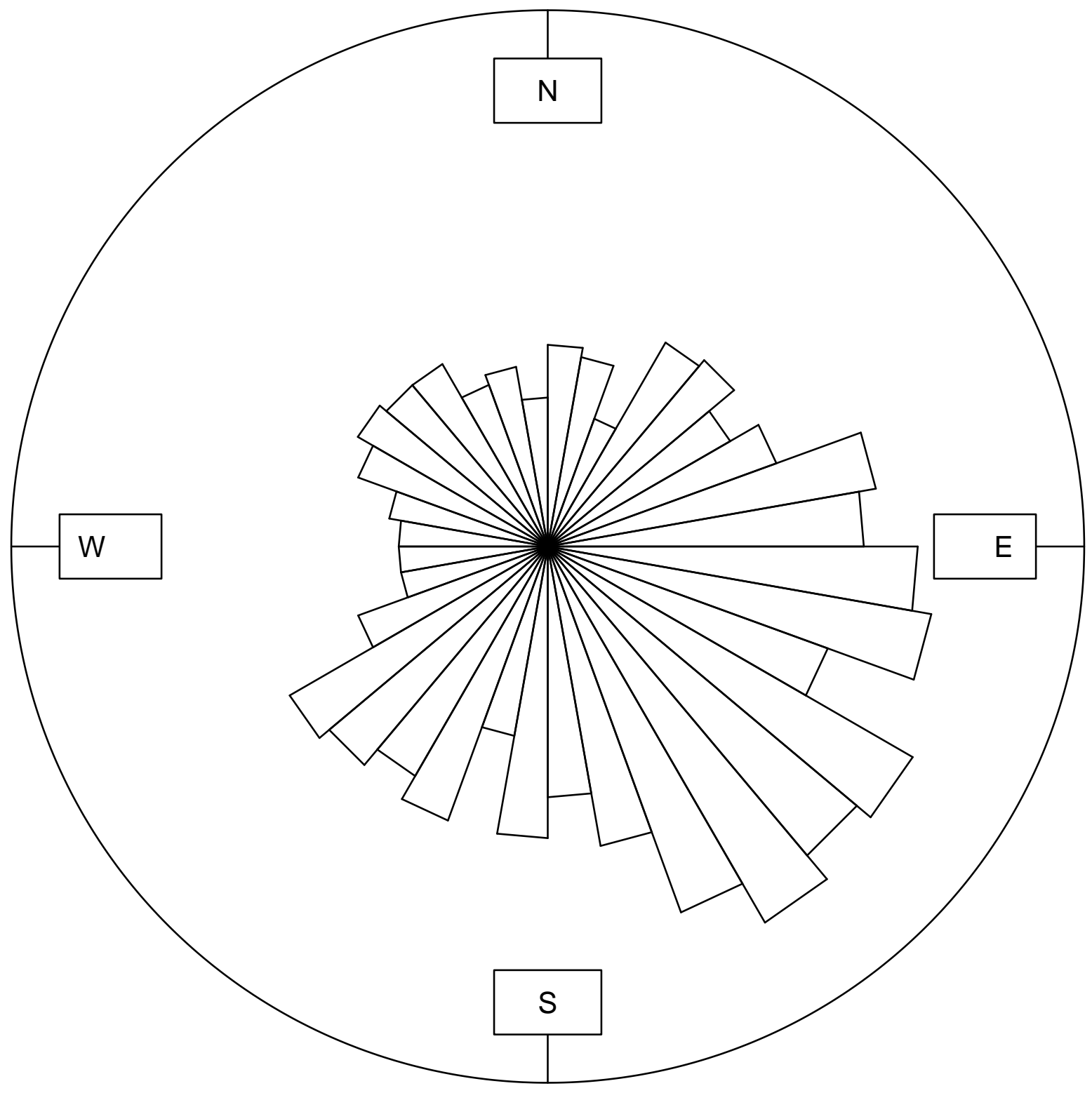


bootstrap 504

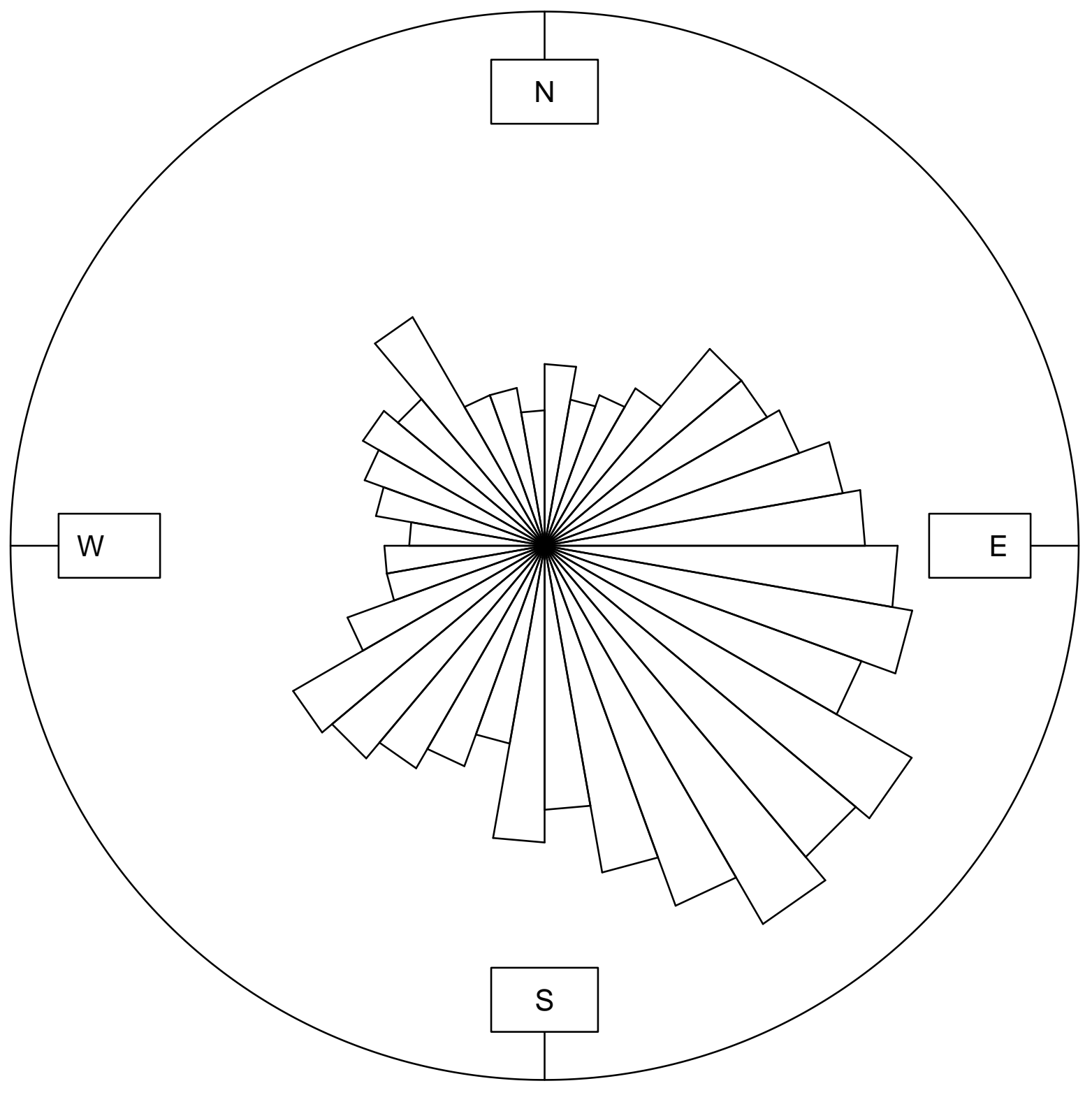


bootstrap 505

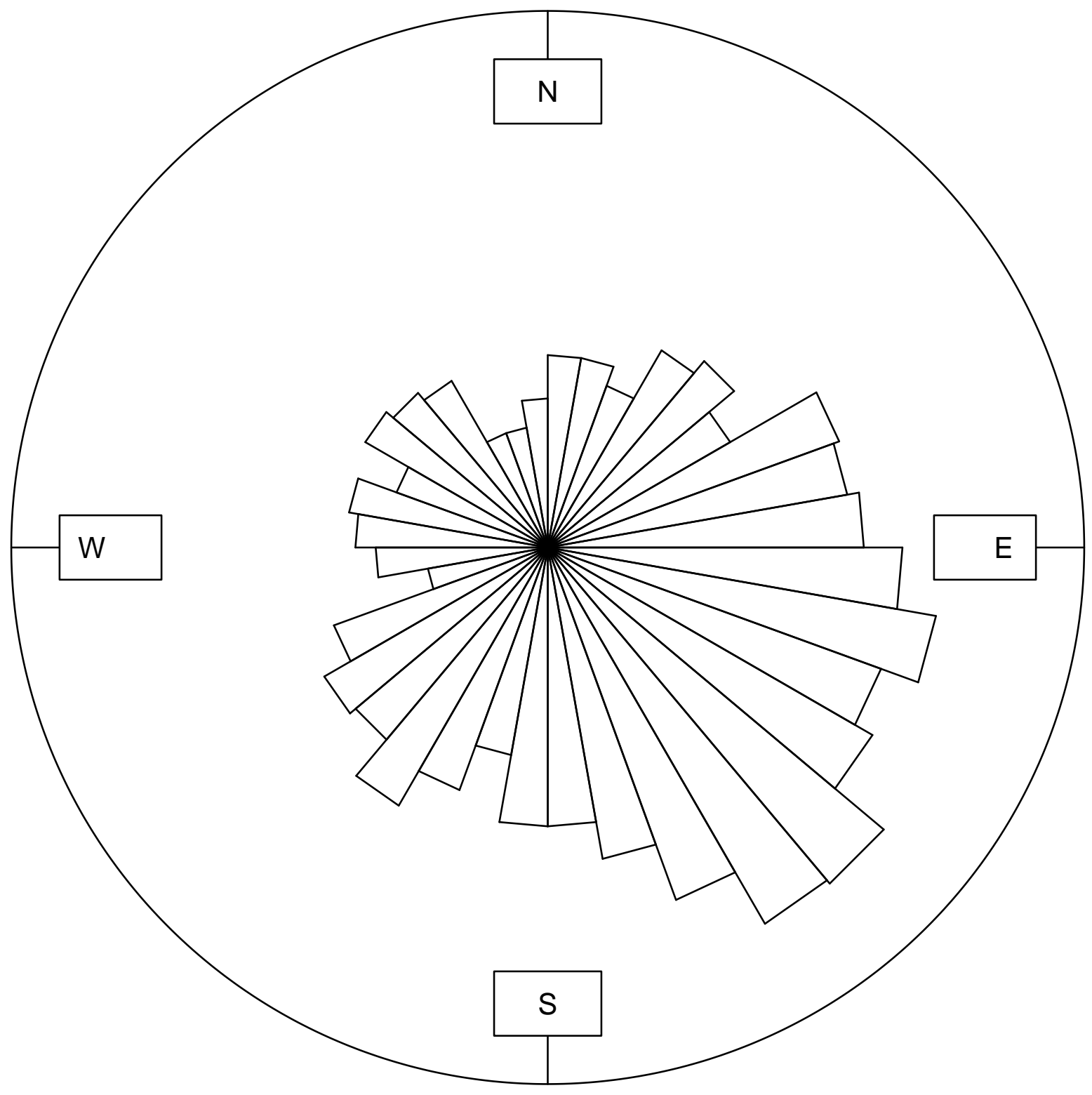


bootstrap 506

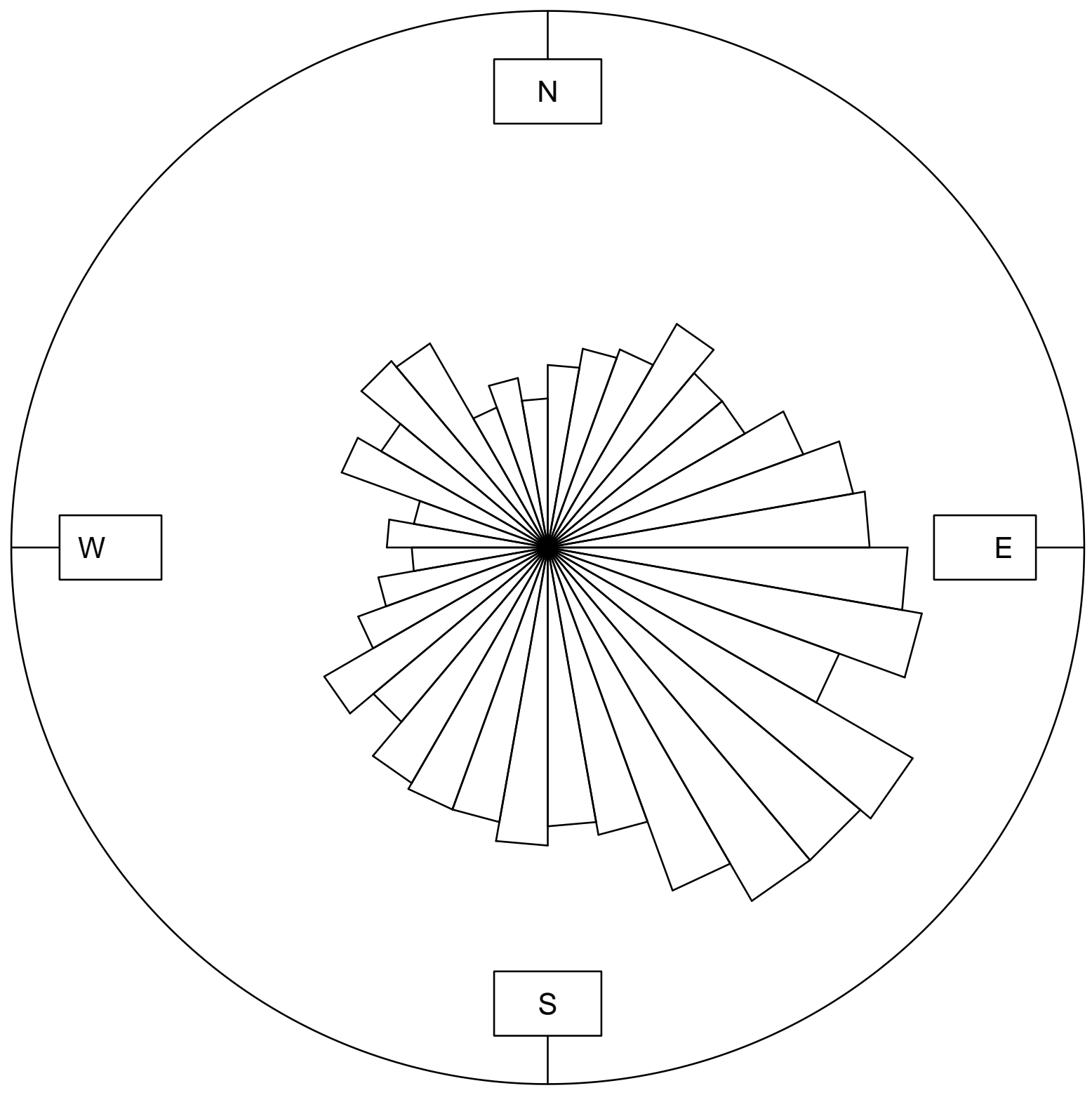


bootstrap 507

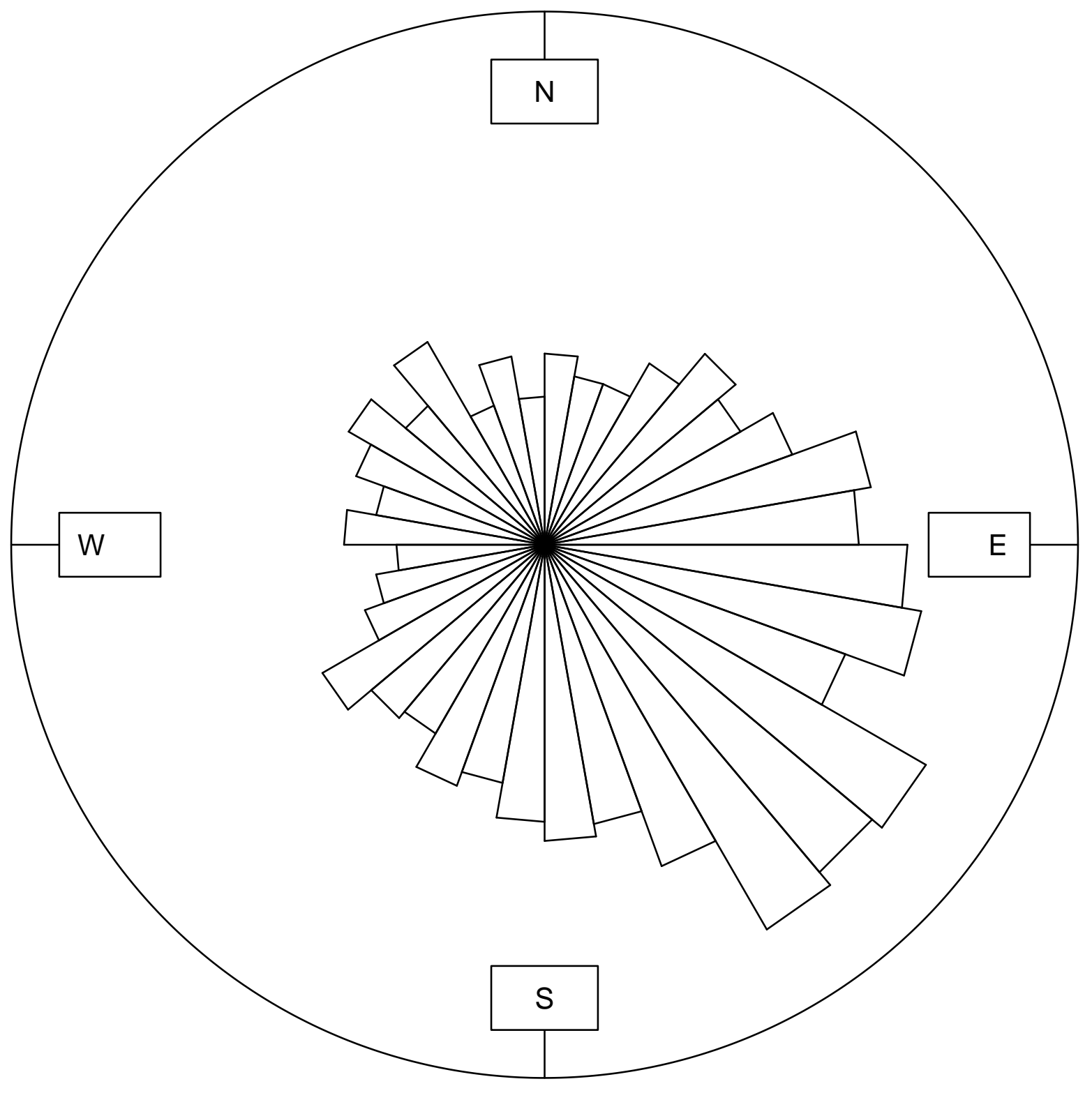


bootstrap 508

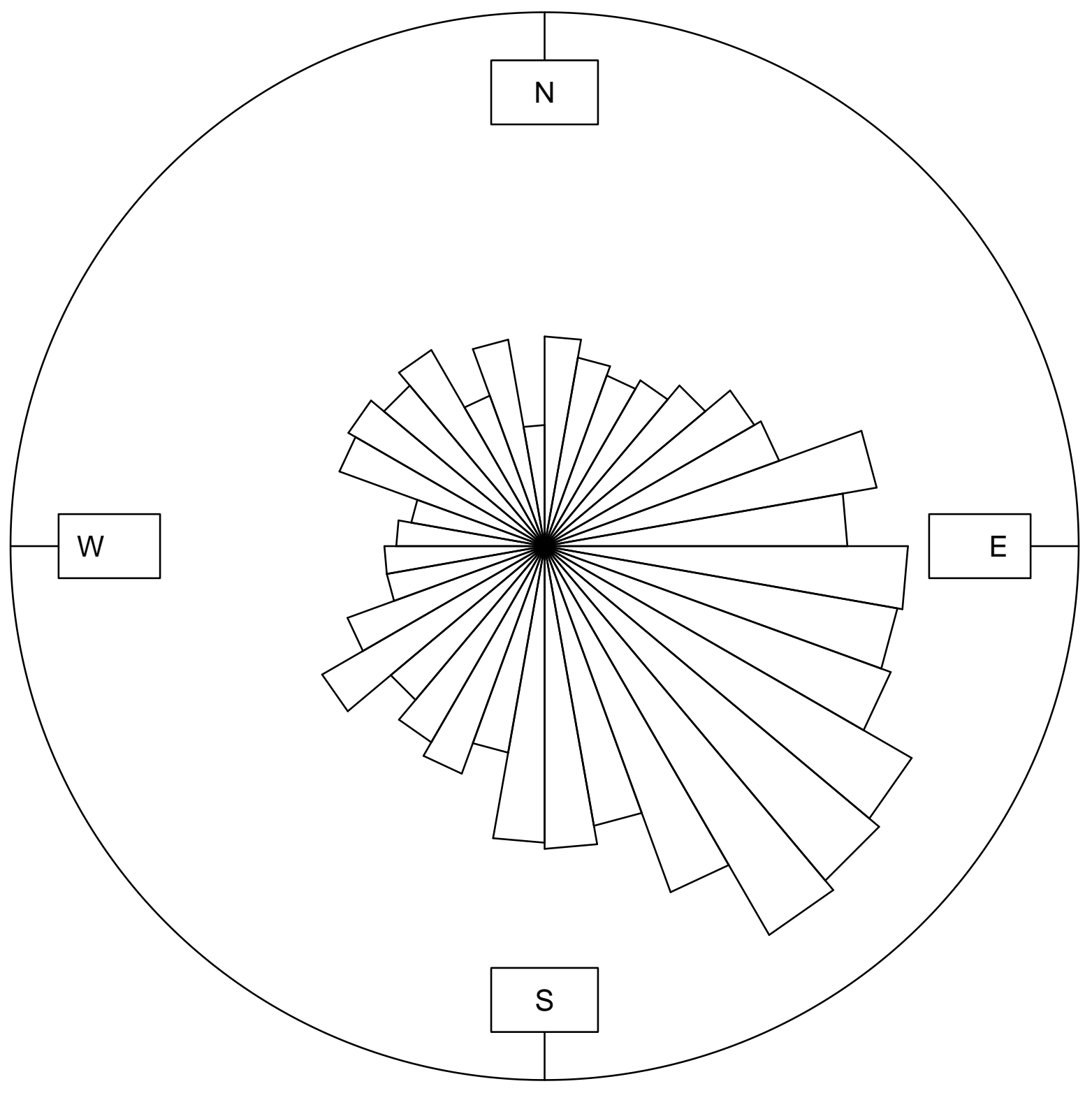


bootstrap 509

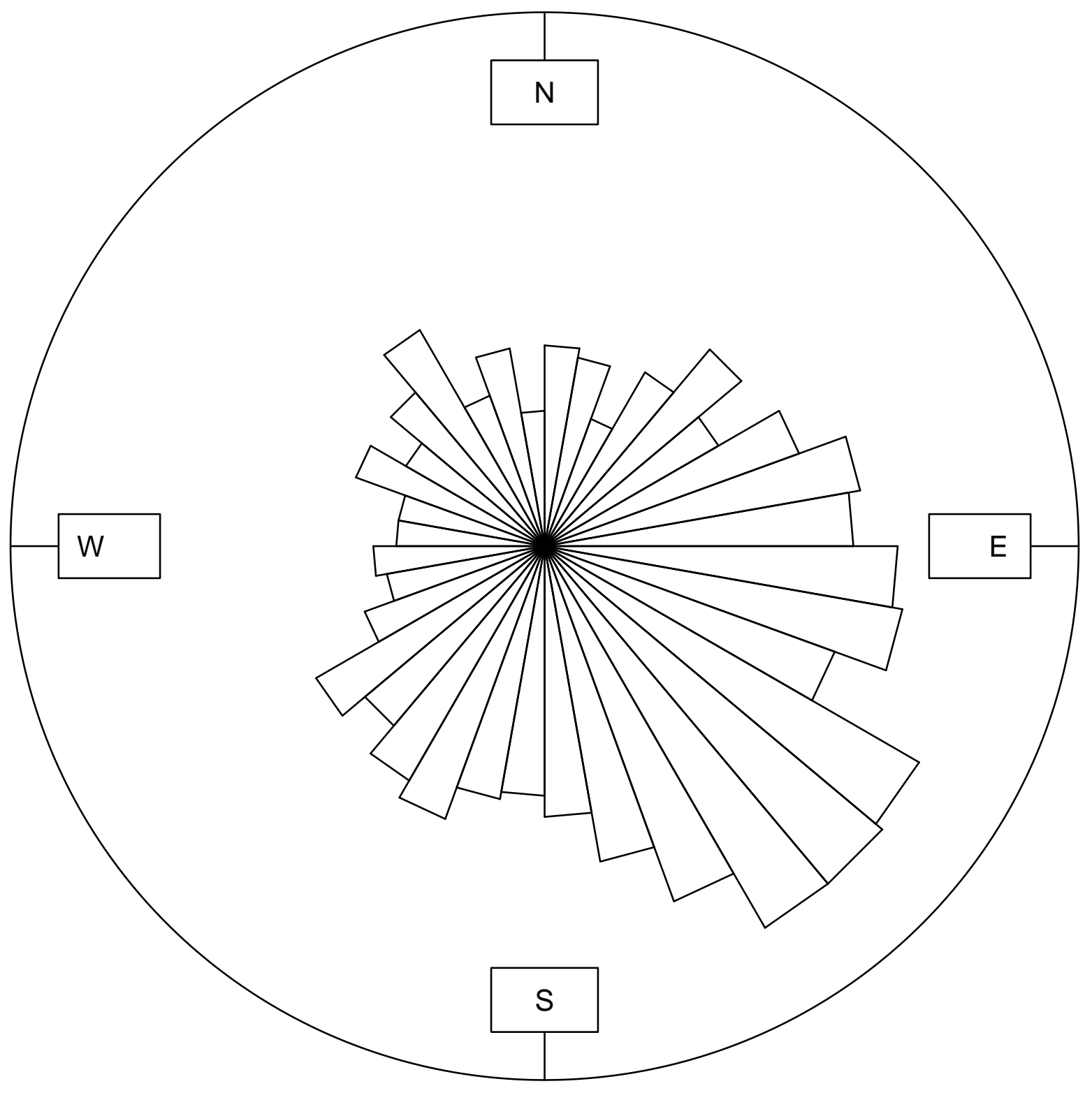


bootstrap 510

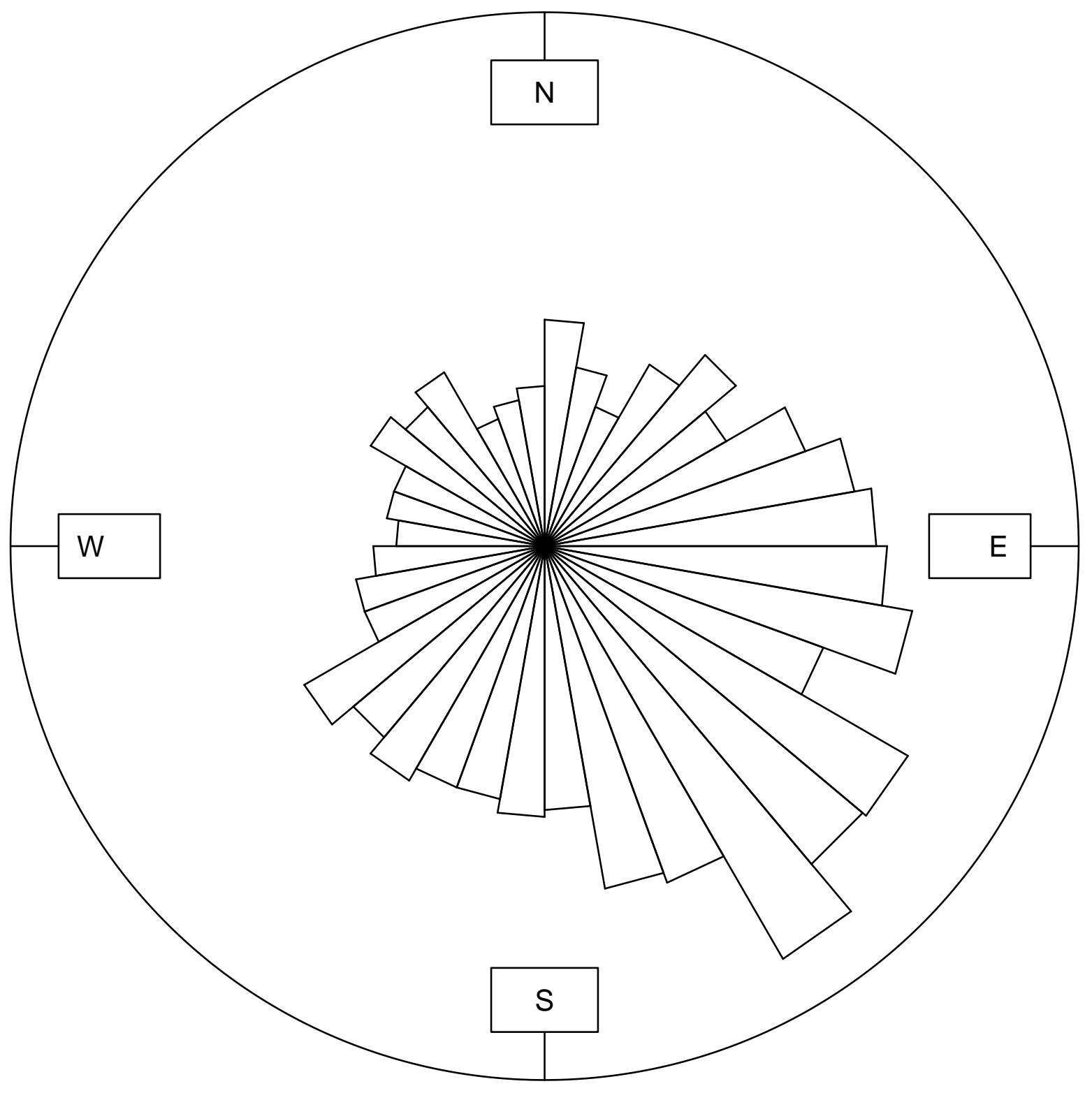


bootstrap 511

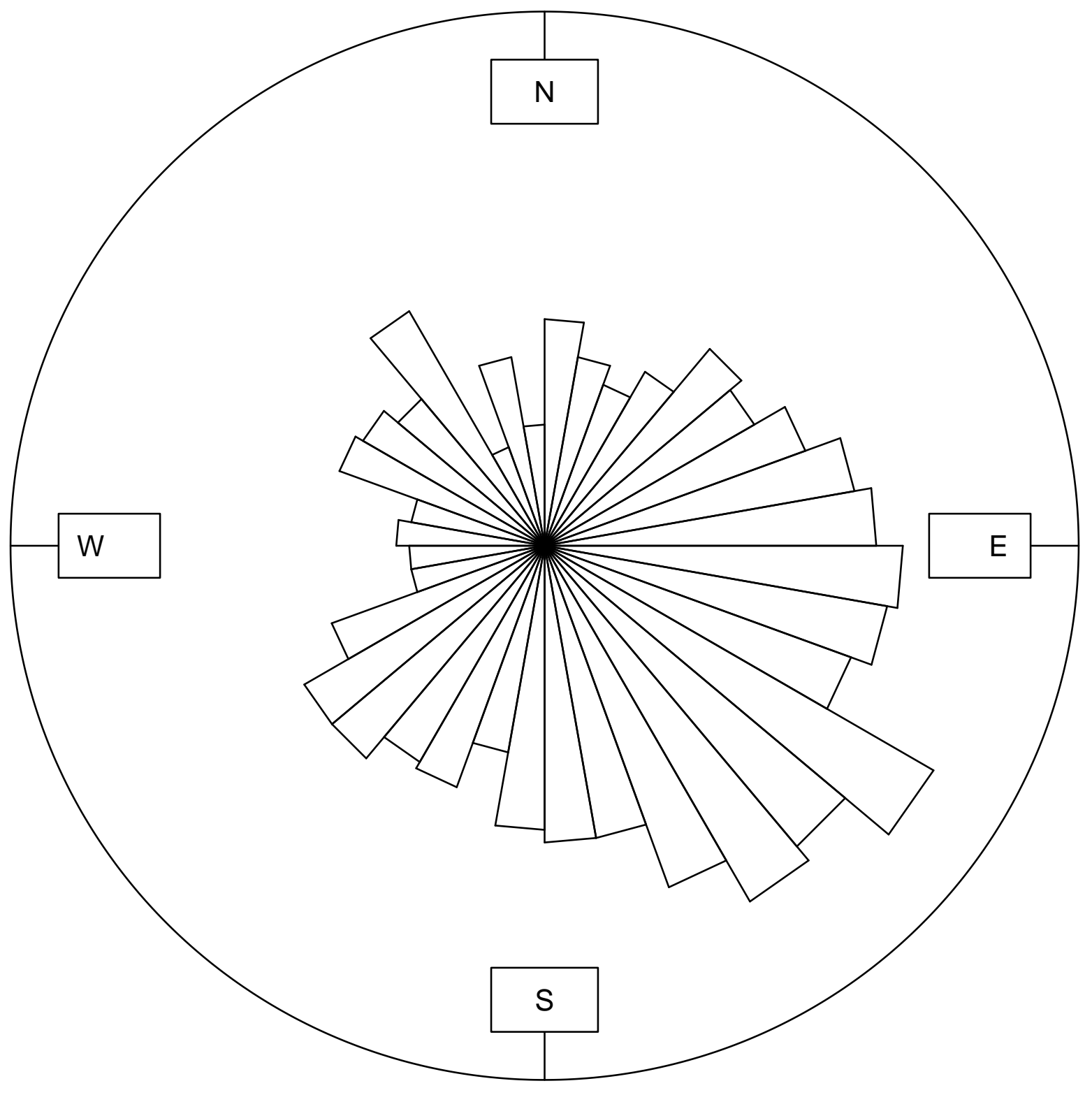


bootstrap 512

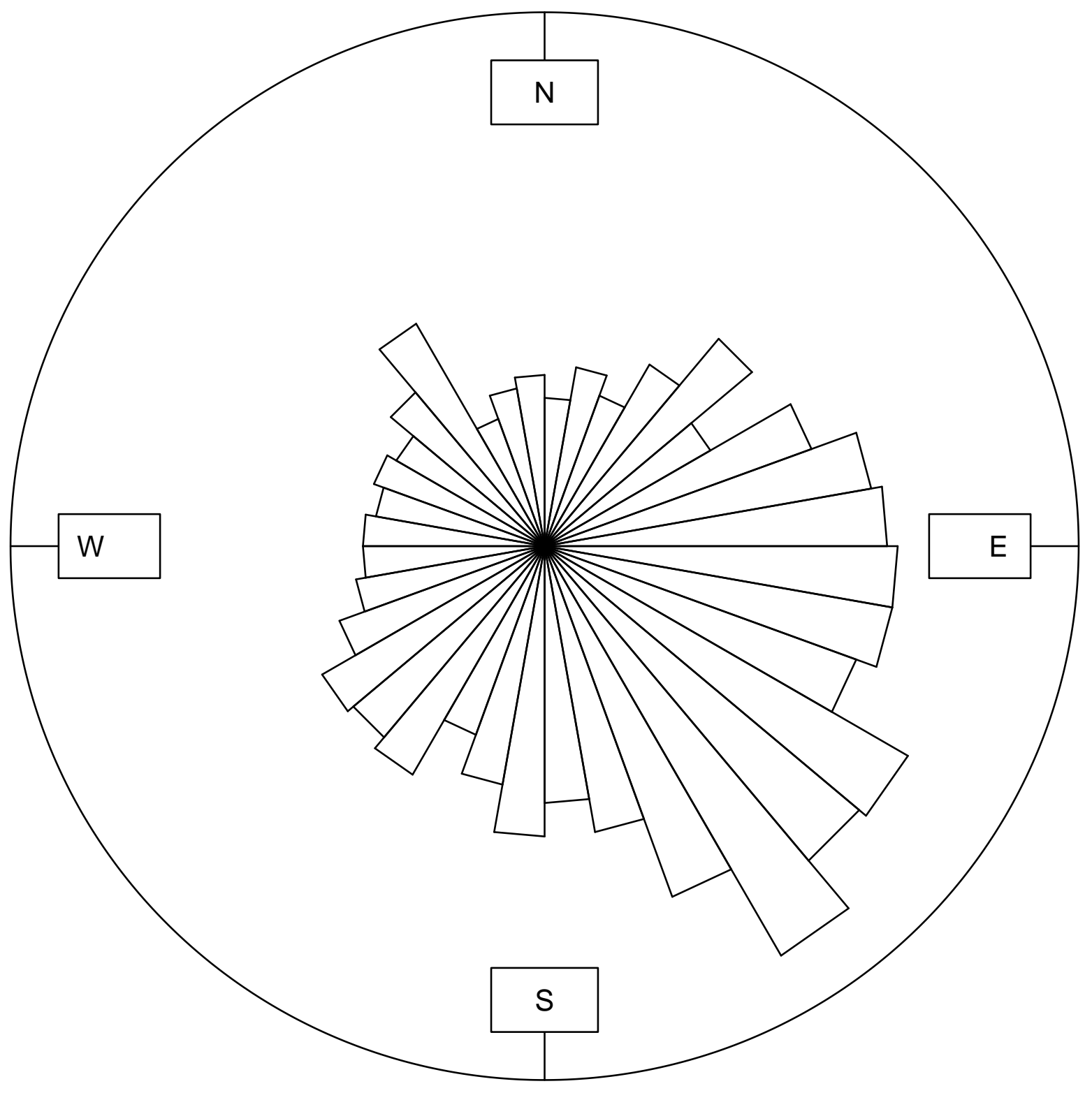


bootstrap 513

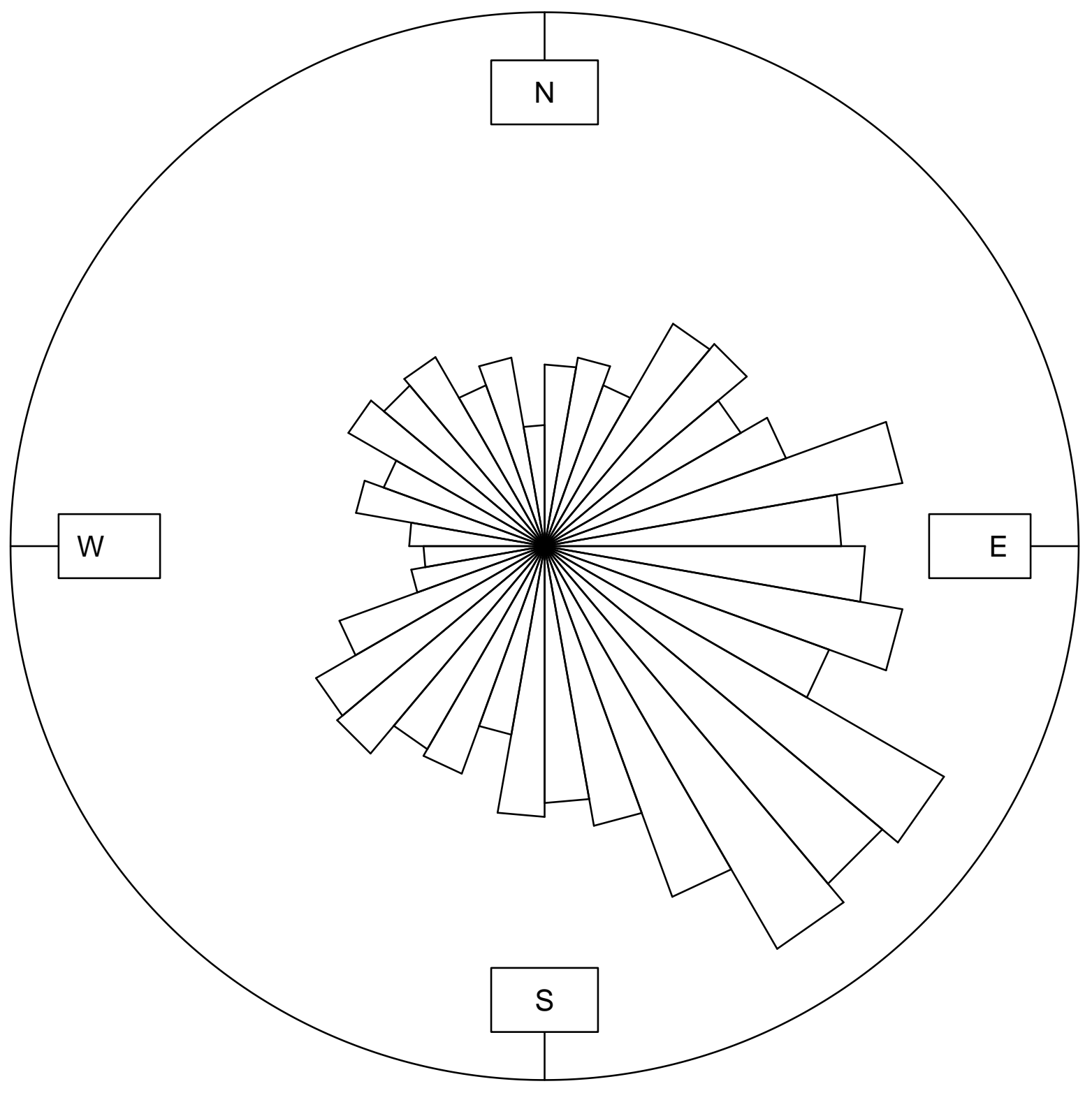


bootstrap 514

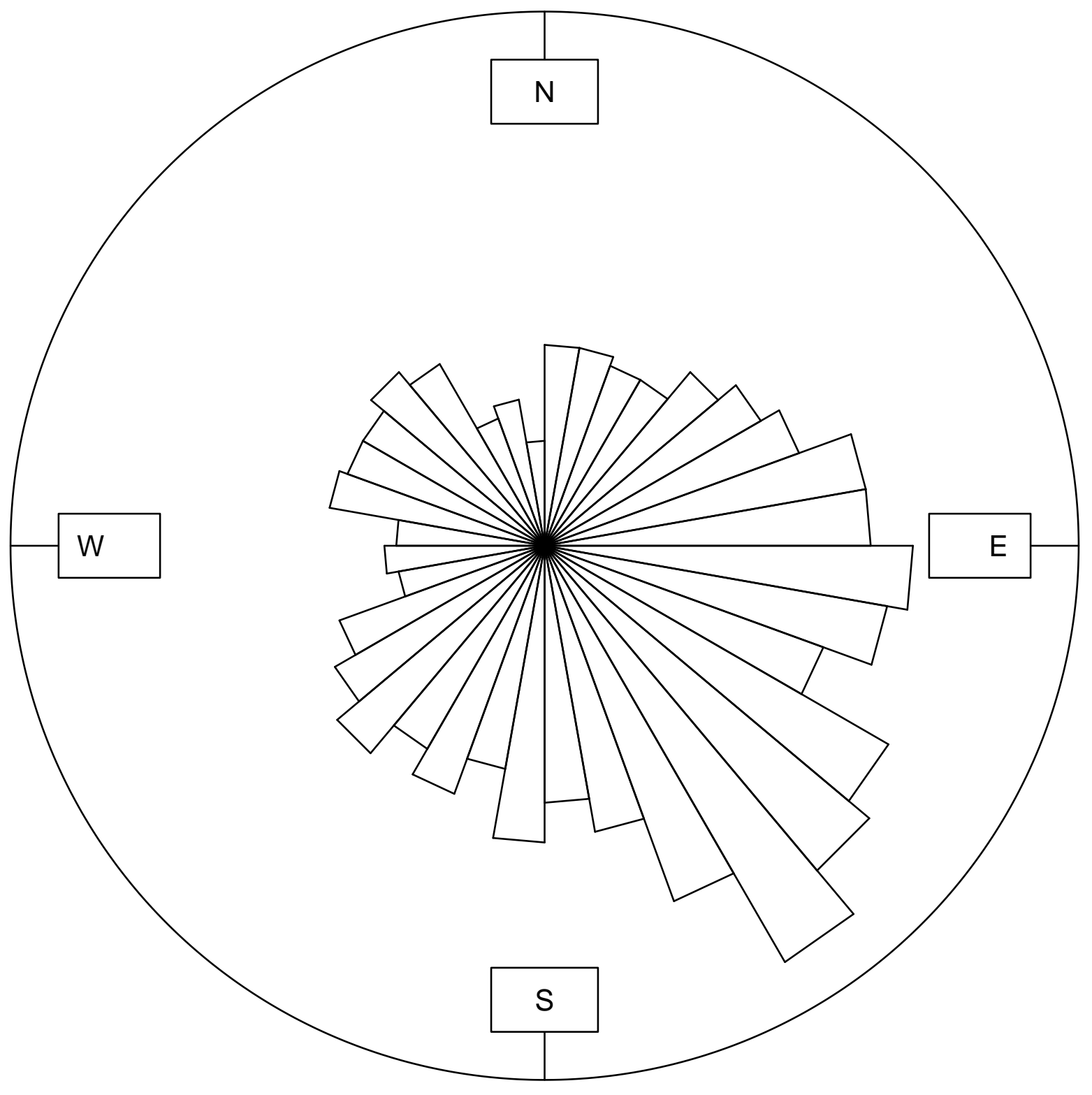




\section{bootstrap 515}

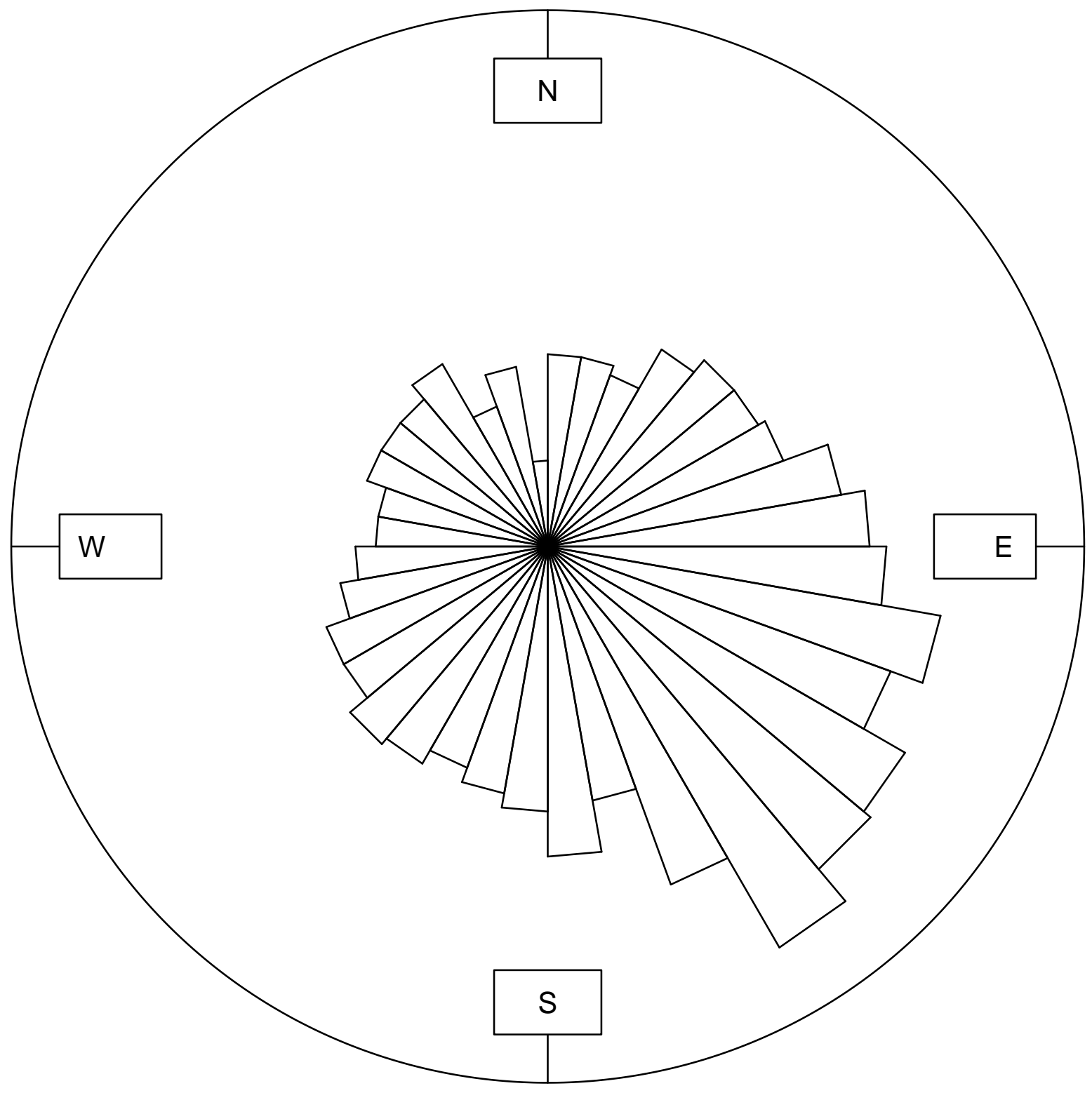


bootstrap 516

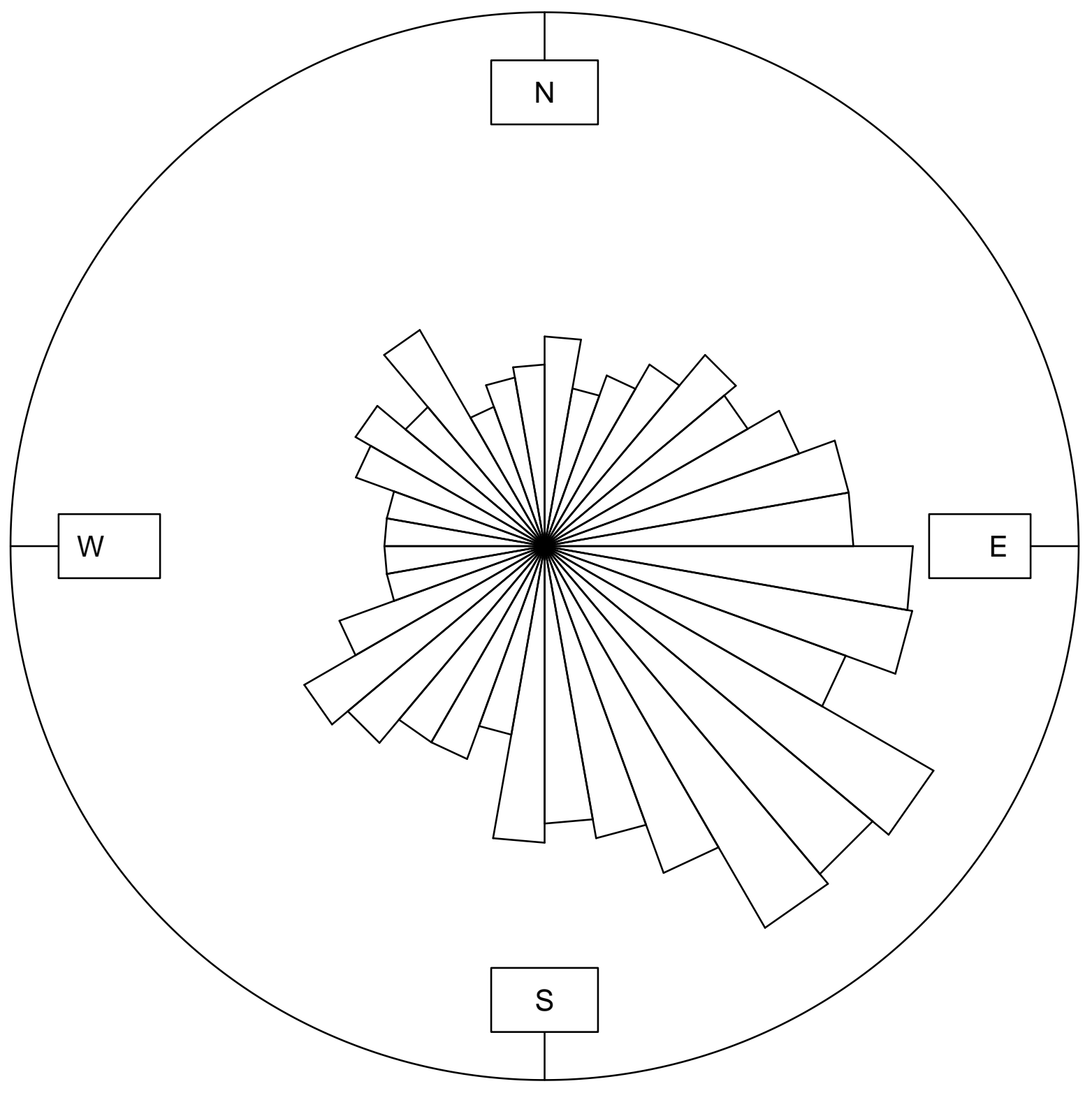


bootstrap 517

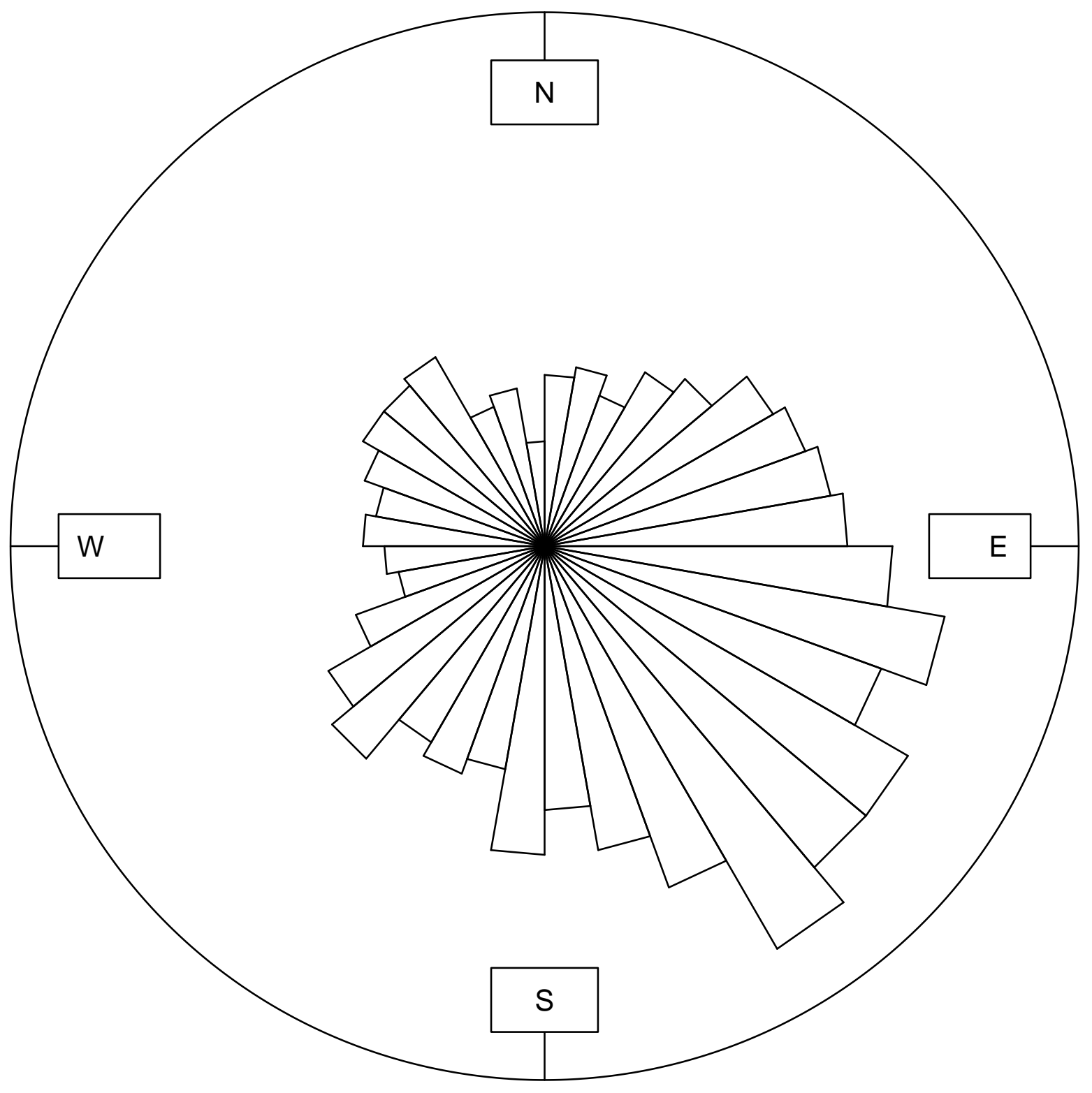


bootstrap 518

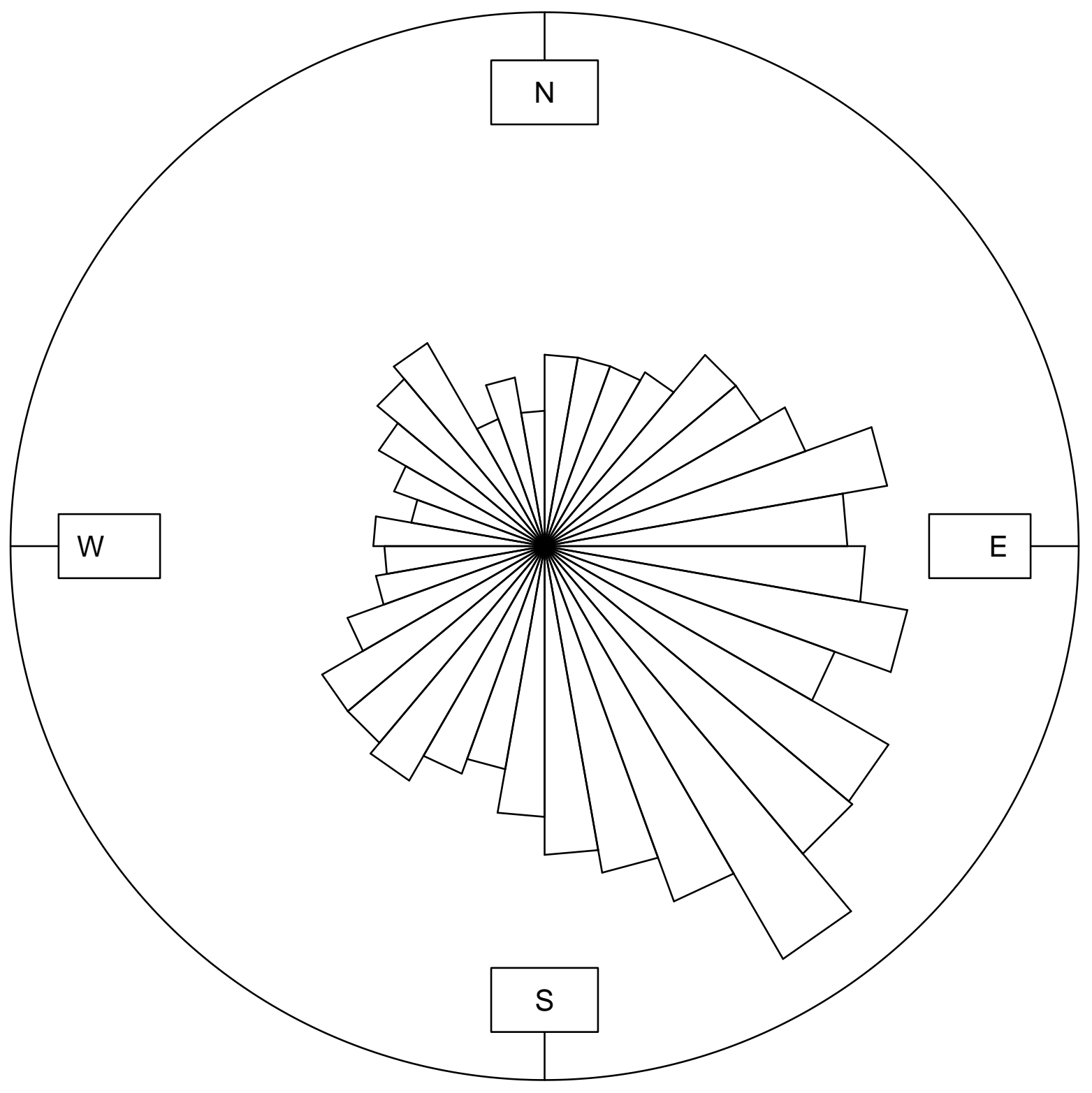


bootstrap 519

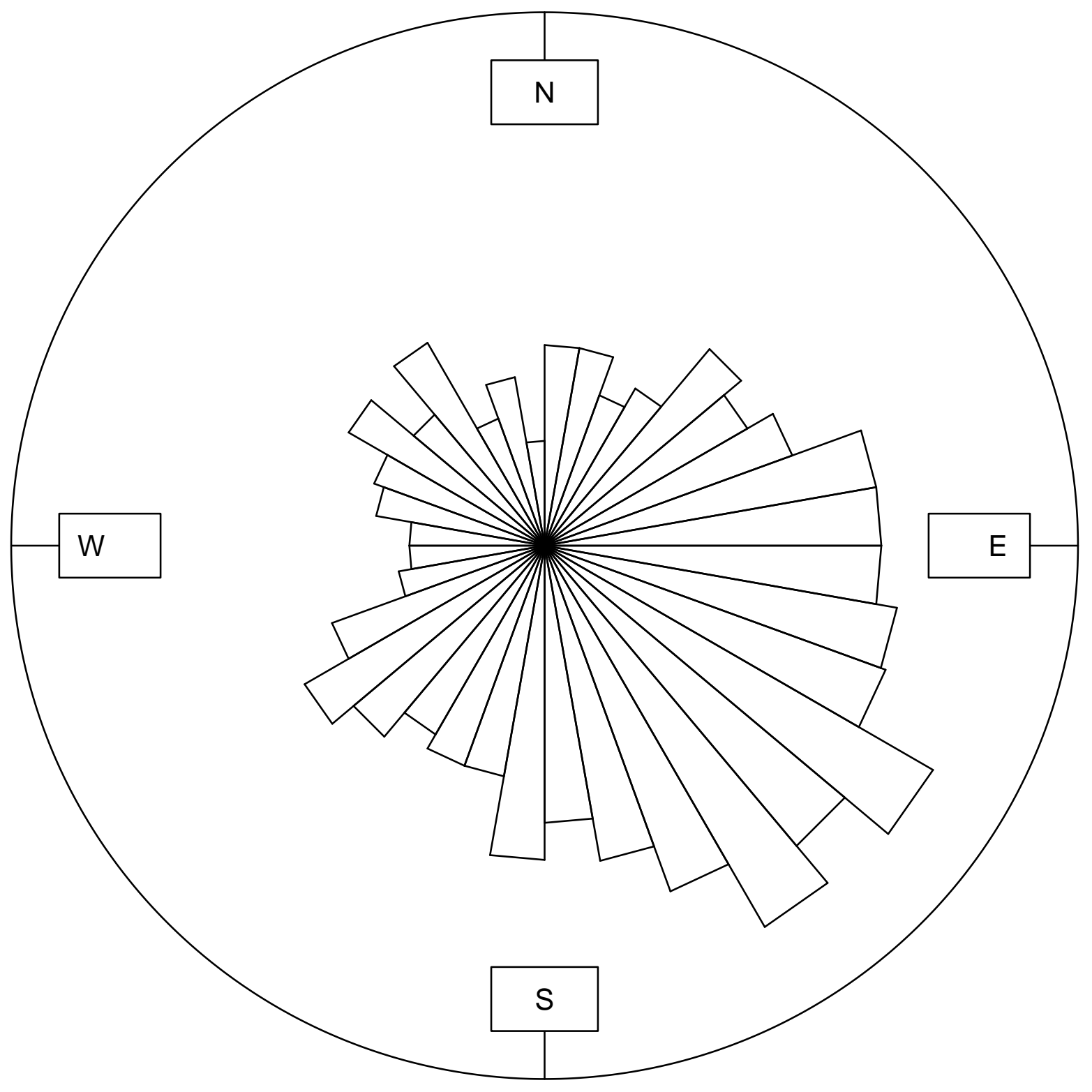


bootstrap 520

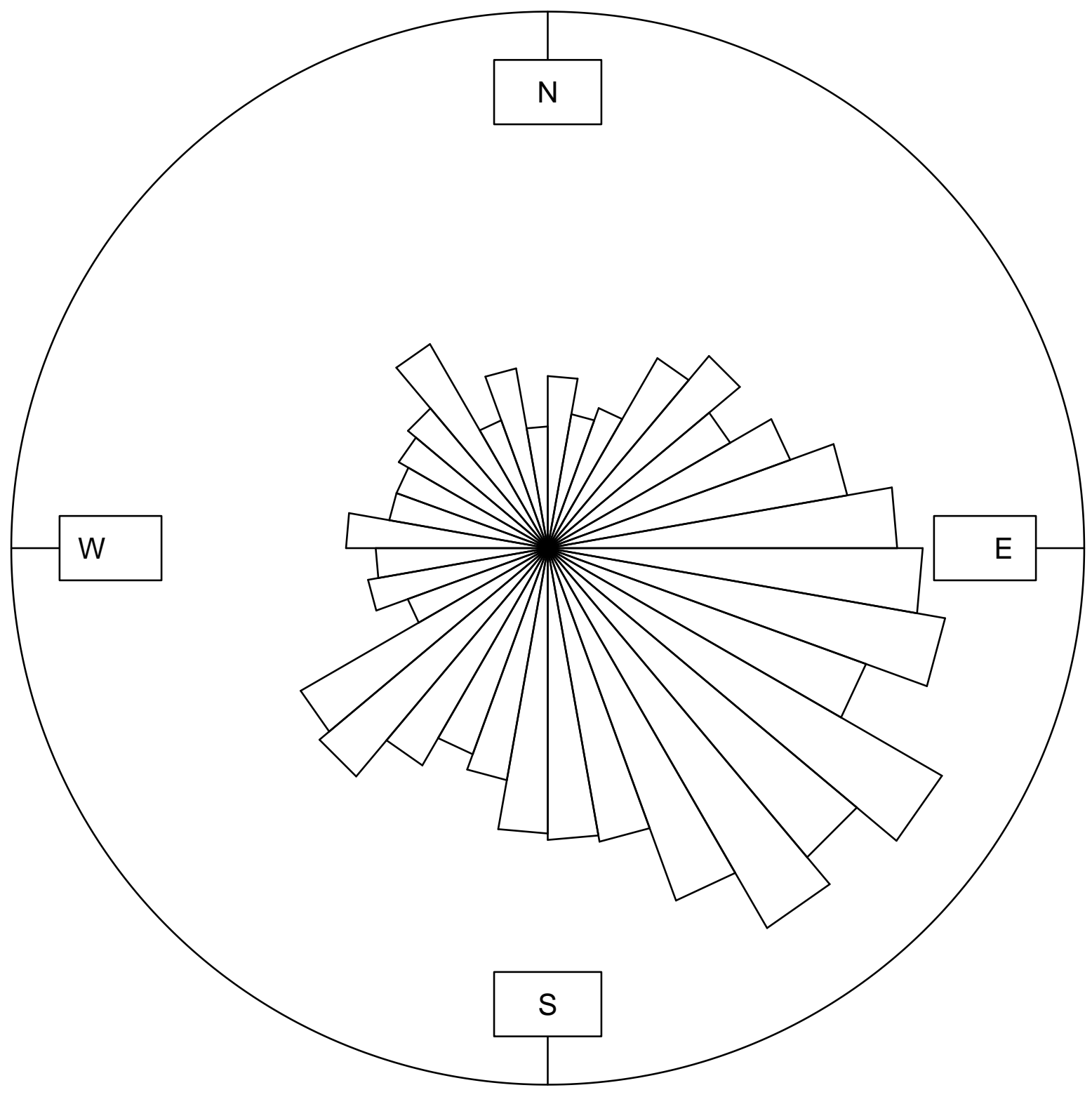


bootstrap 521

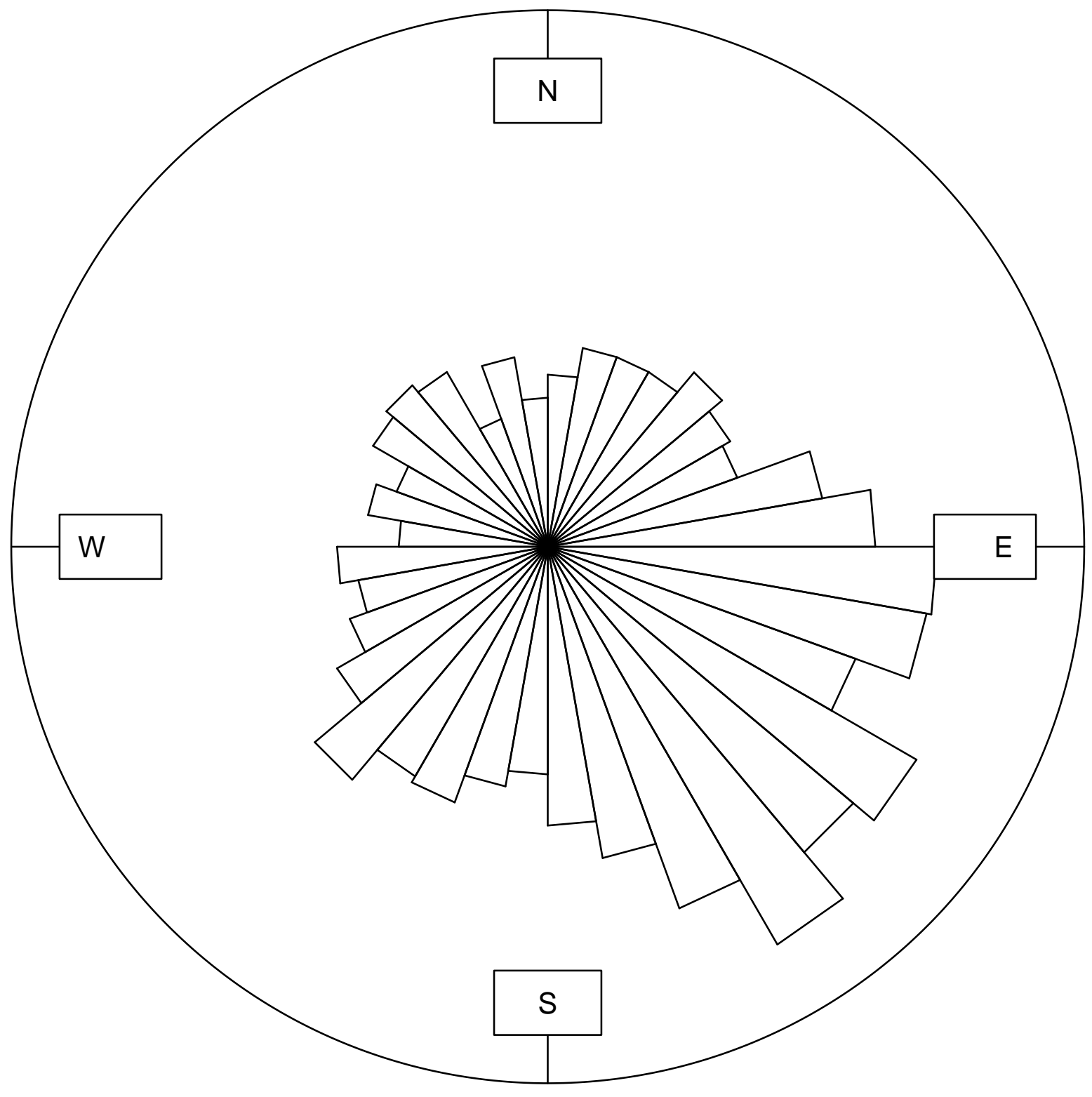


bootstrap 522

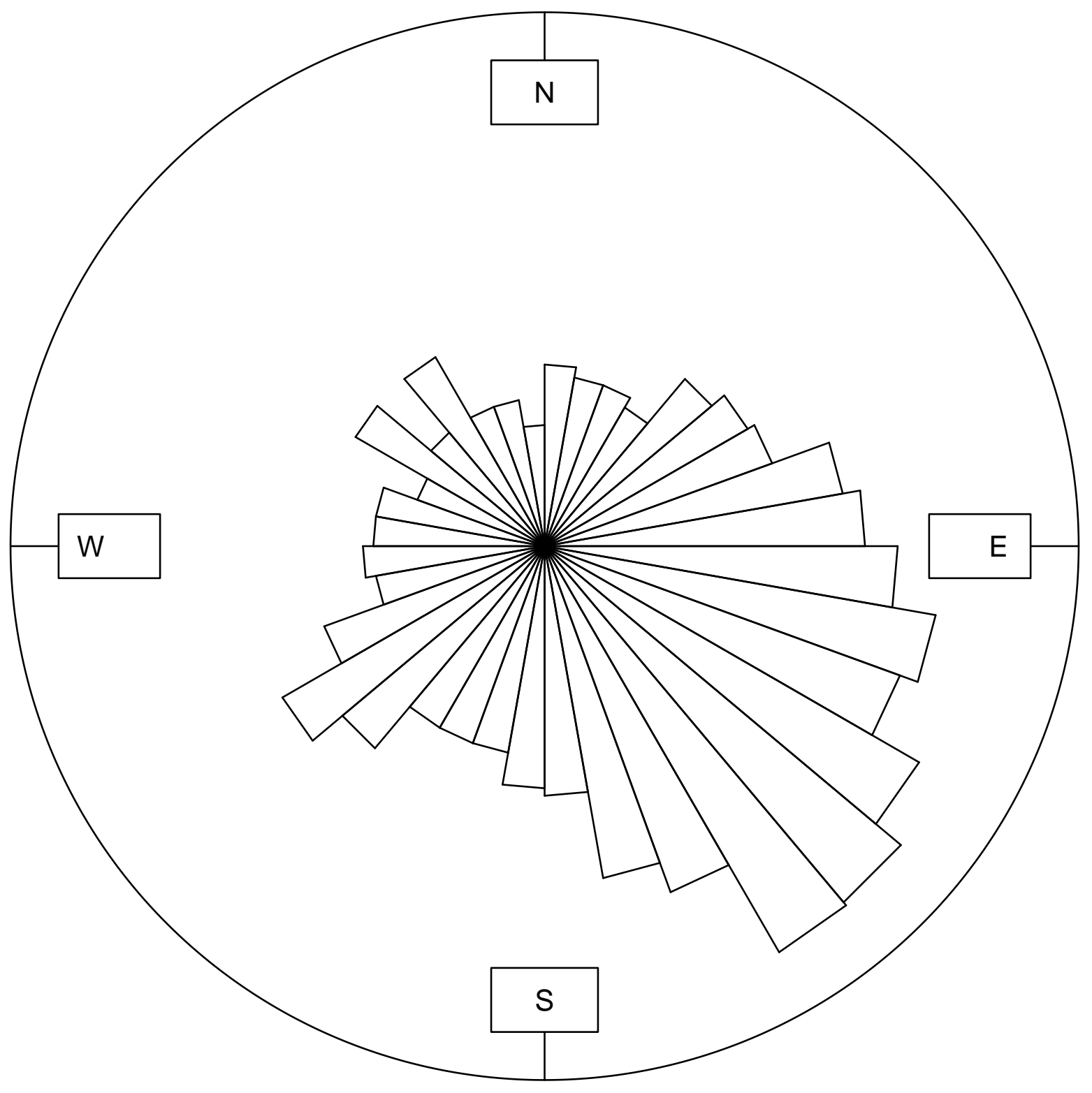


bootstrap 523

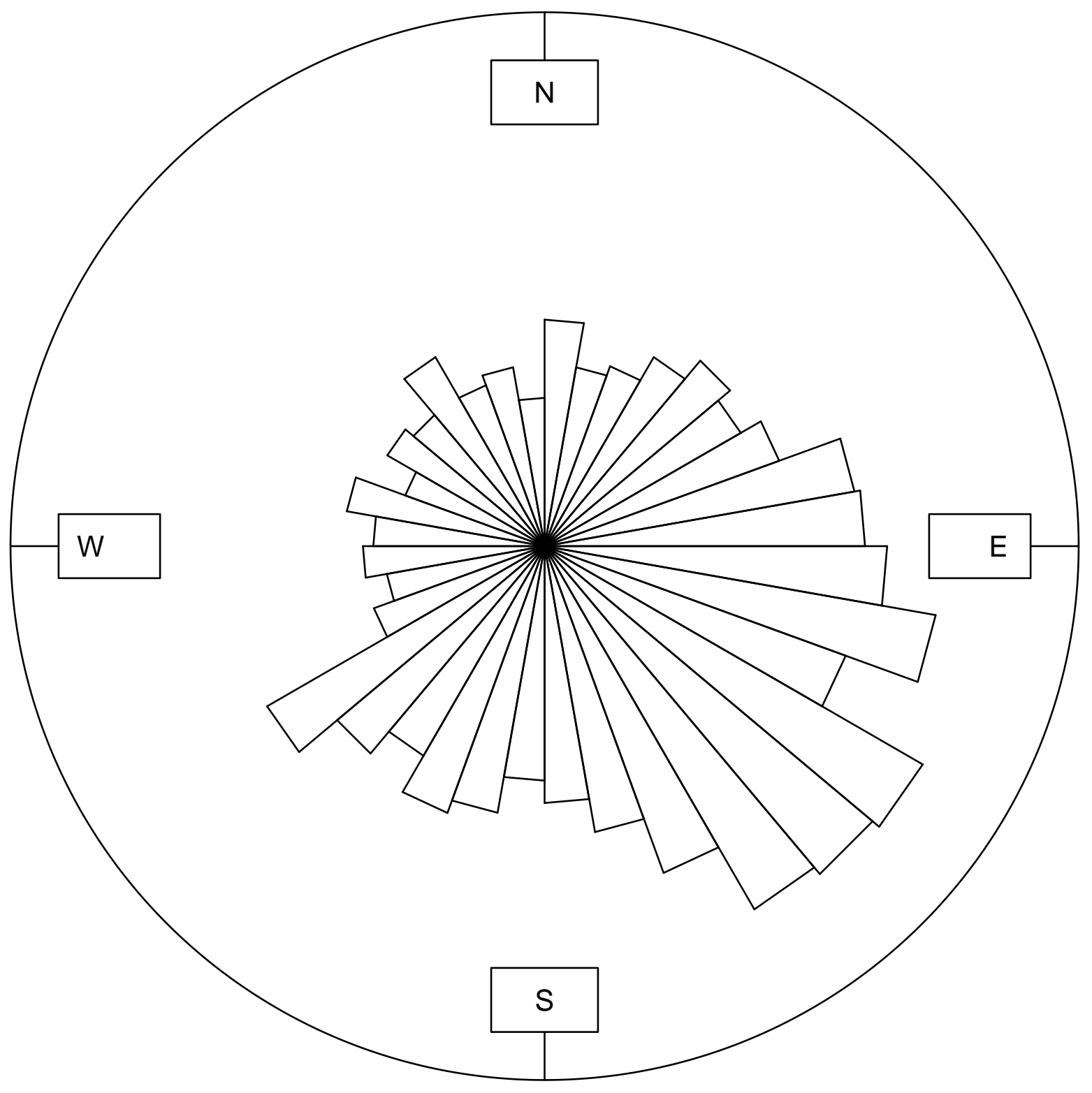


bootstrap 524

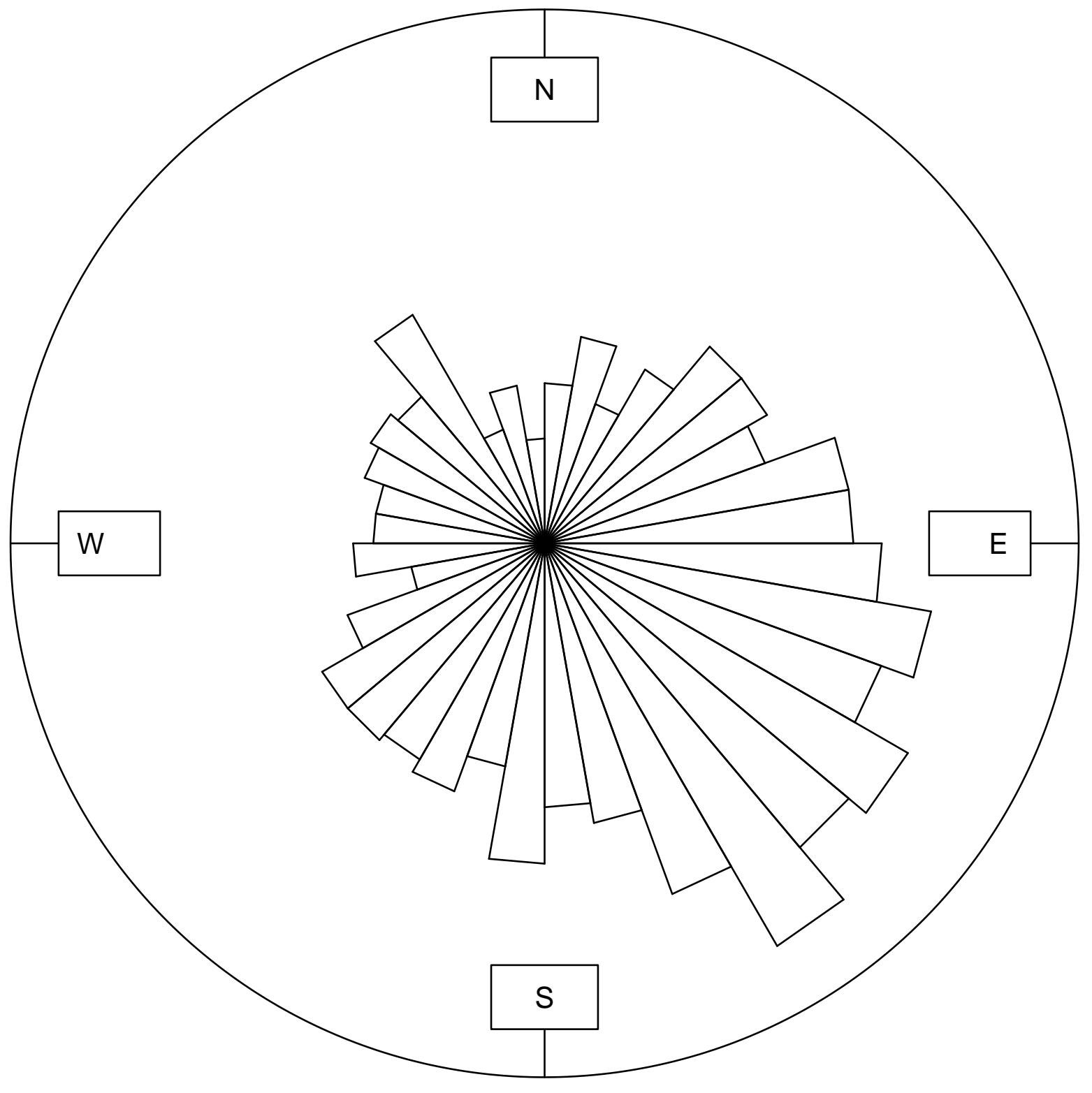




\section{bootstrap 525}

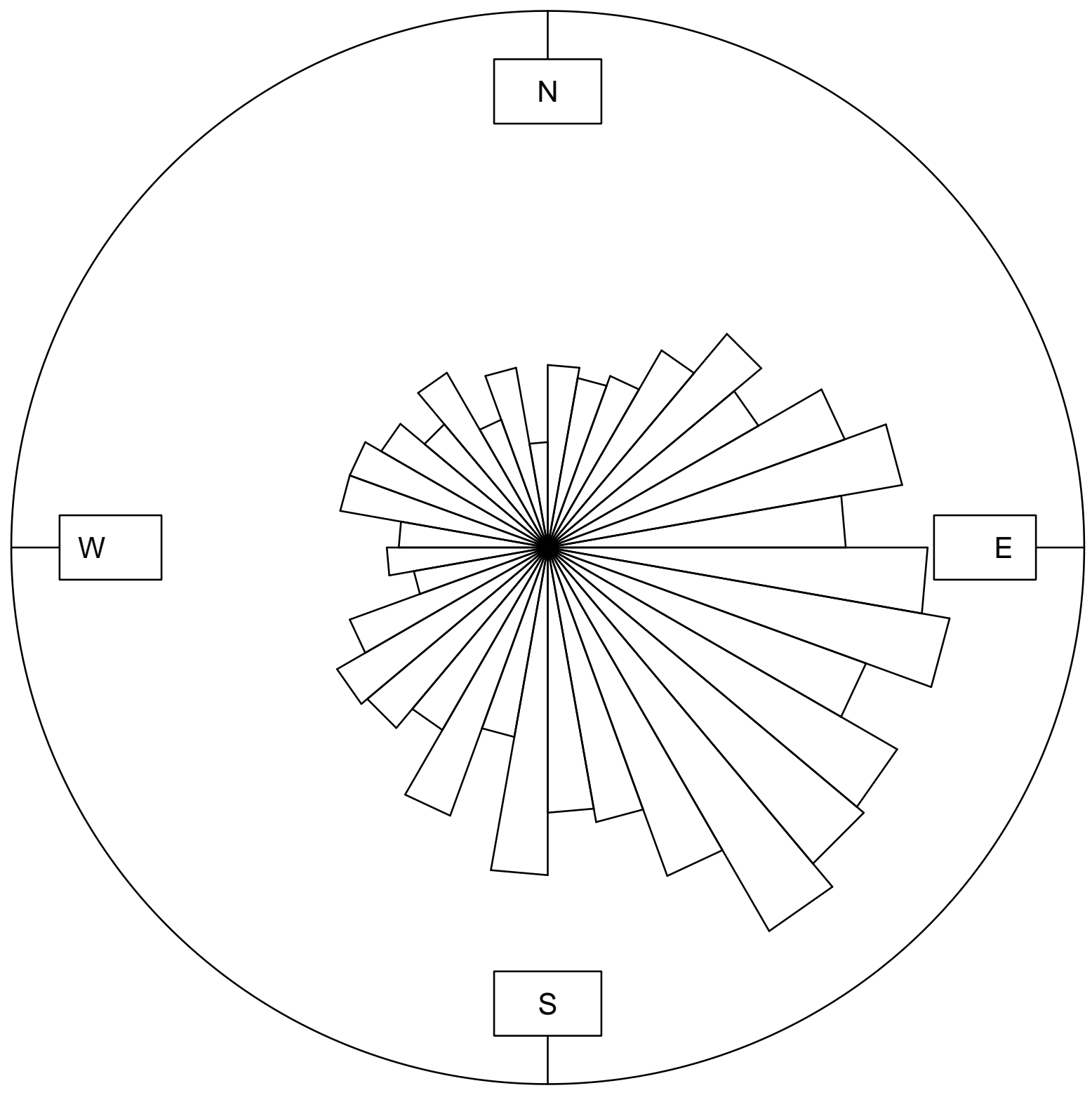




\section{bootstrap 526}

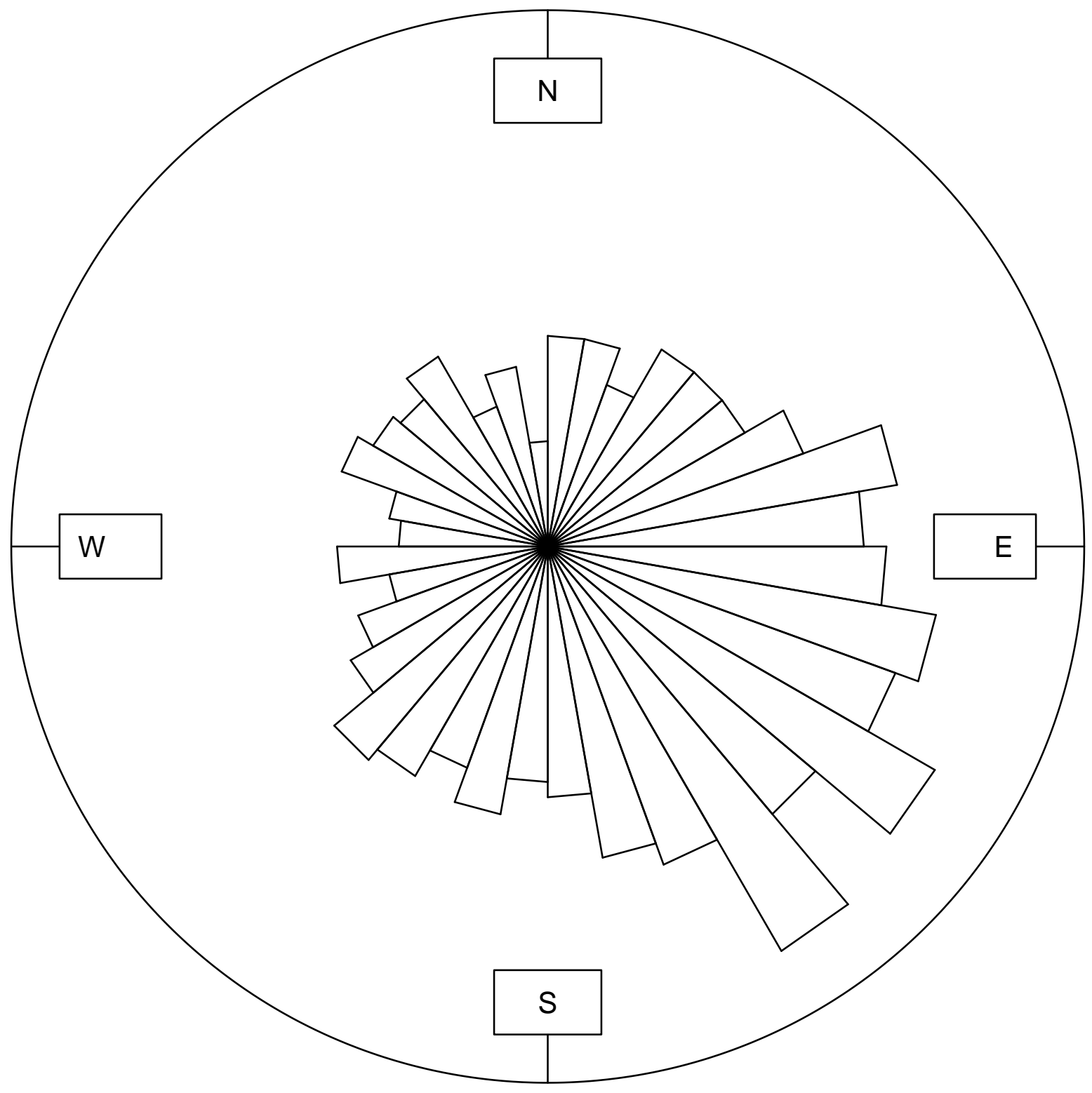


bootstrap 527

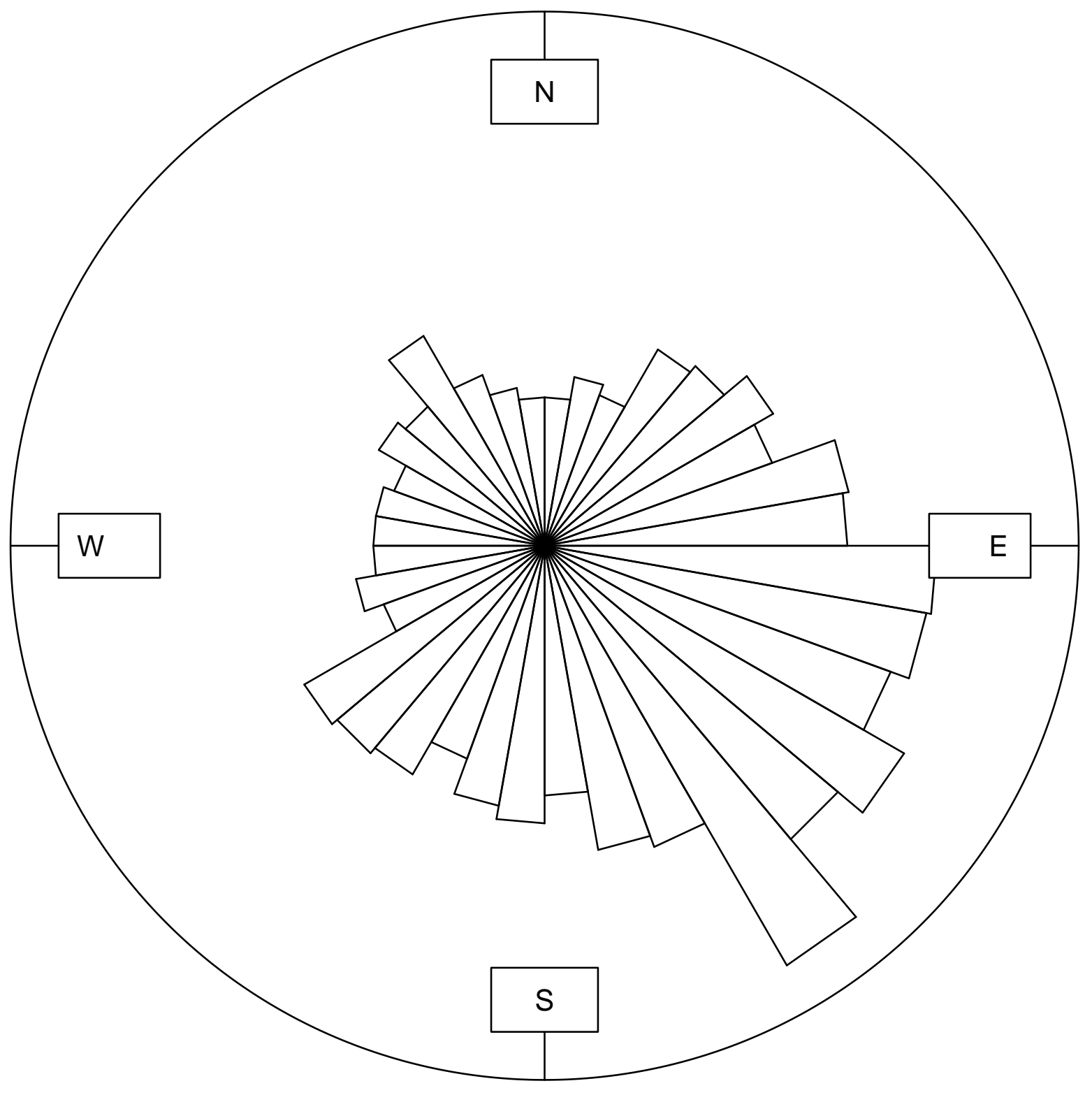


bootstrap 528

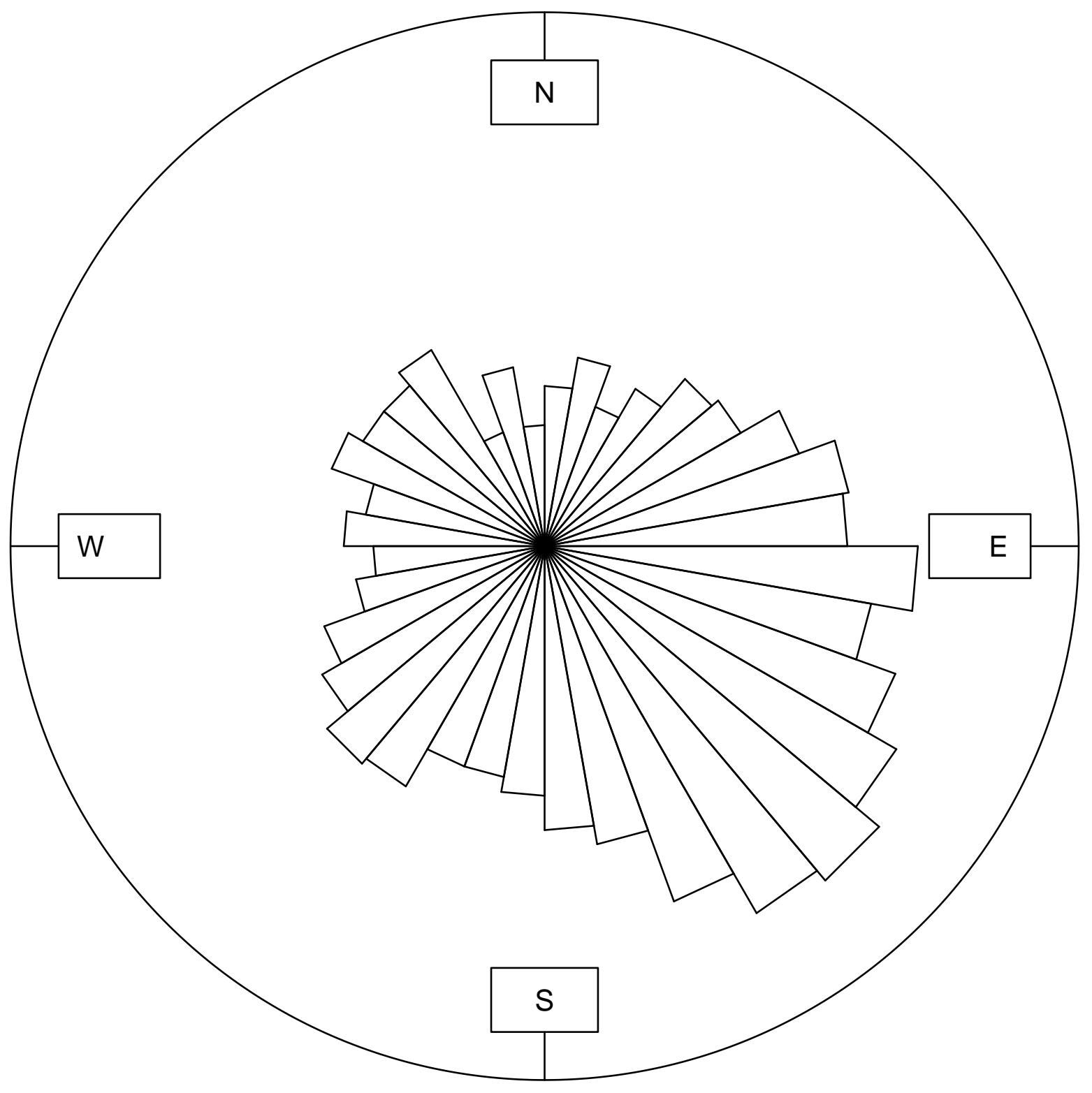


bootstrap 529

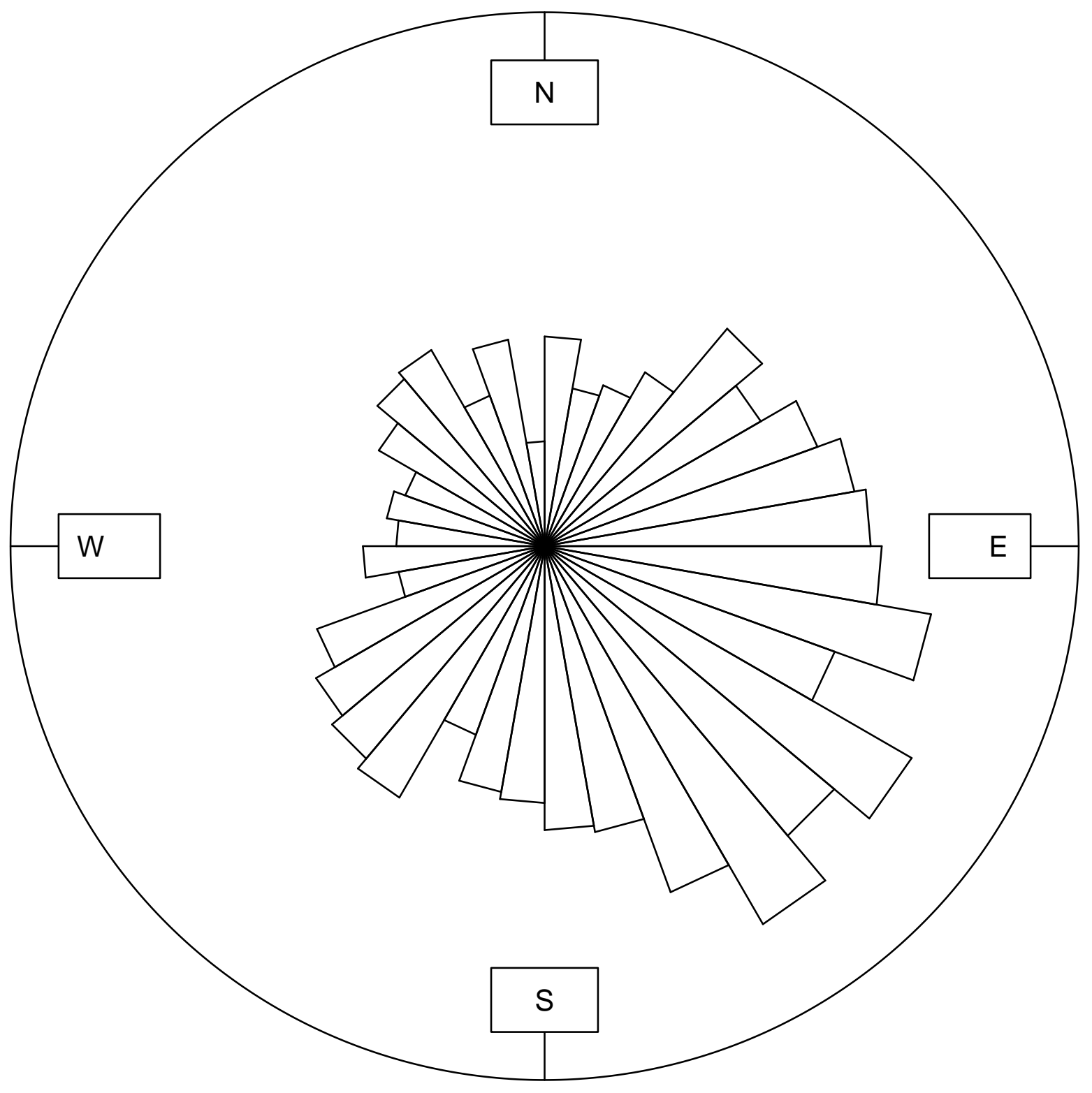


bootstrap 530

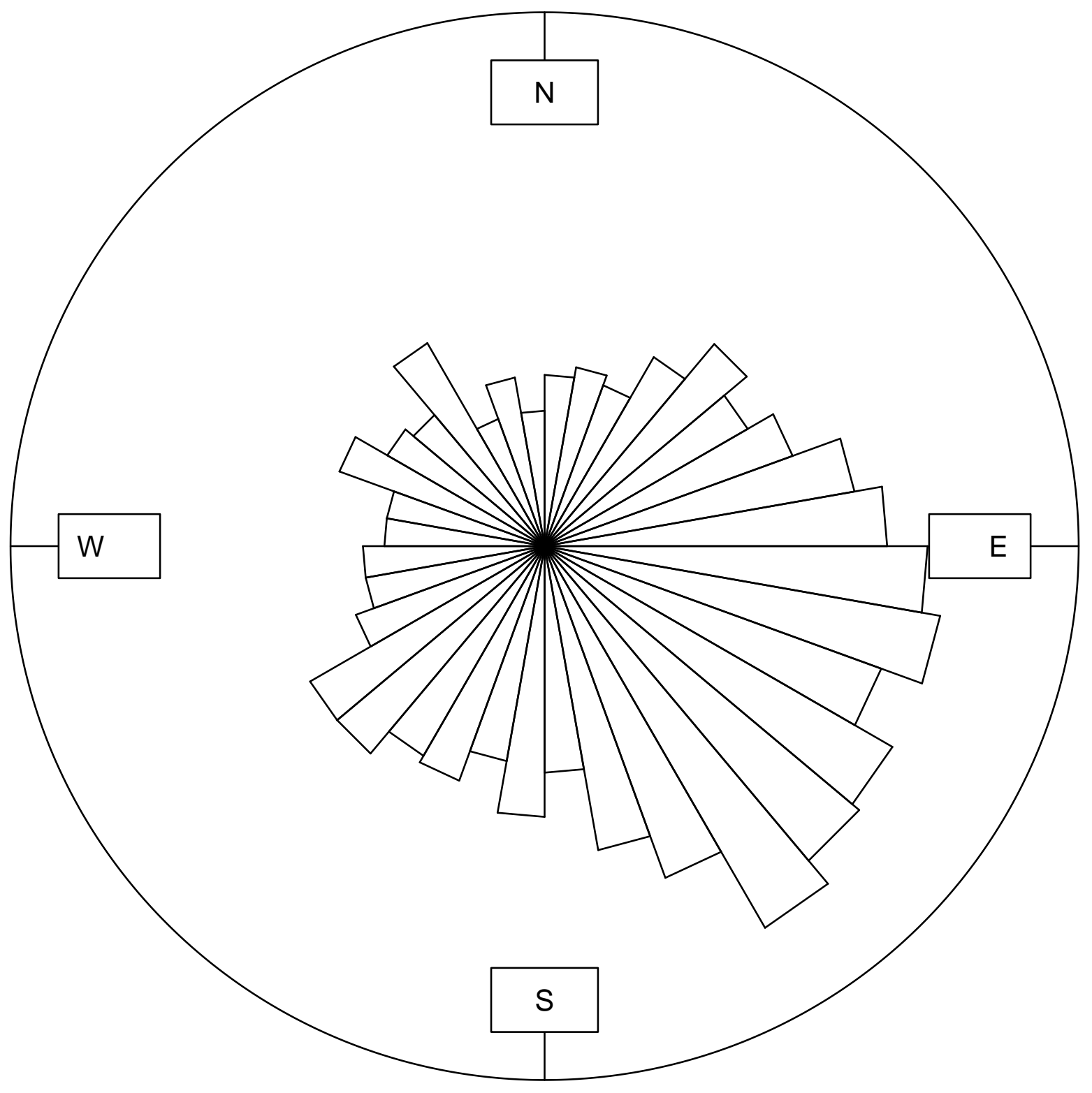


bootstrap 531

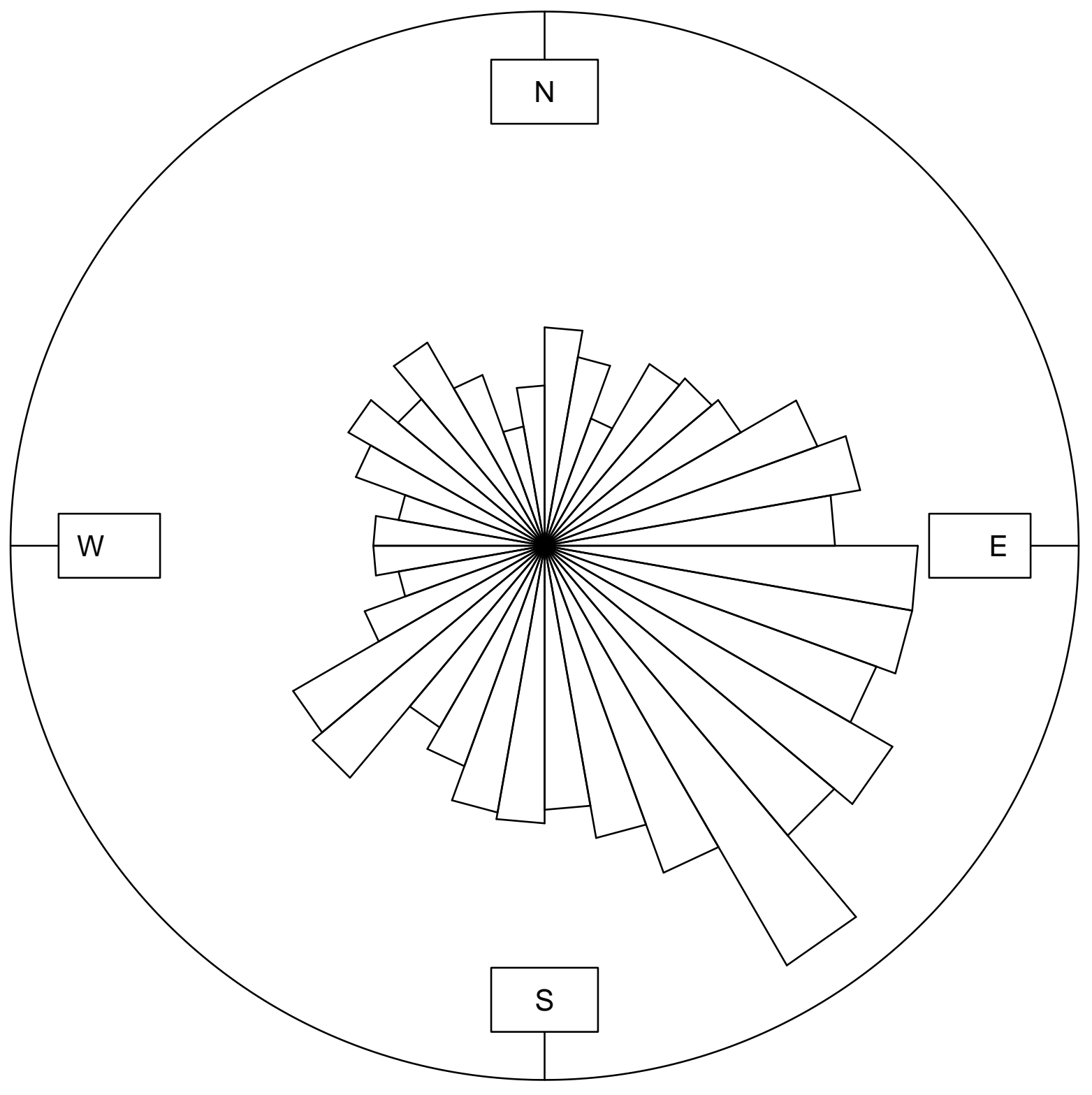


bootstrap 532

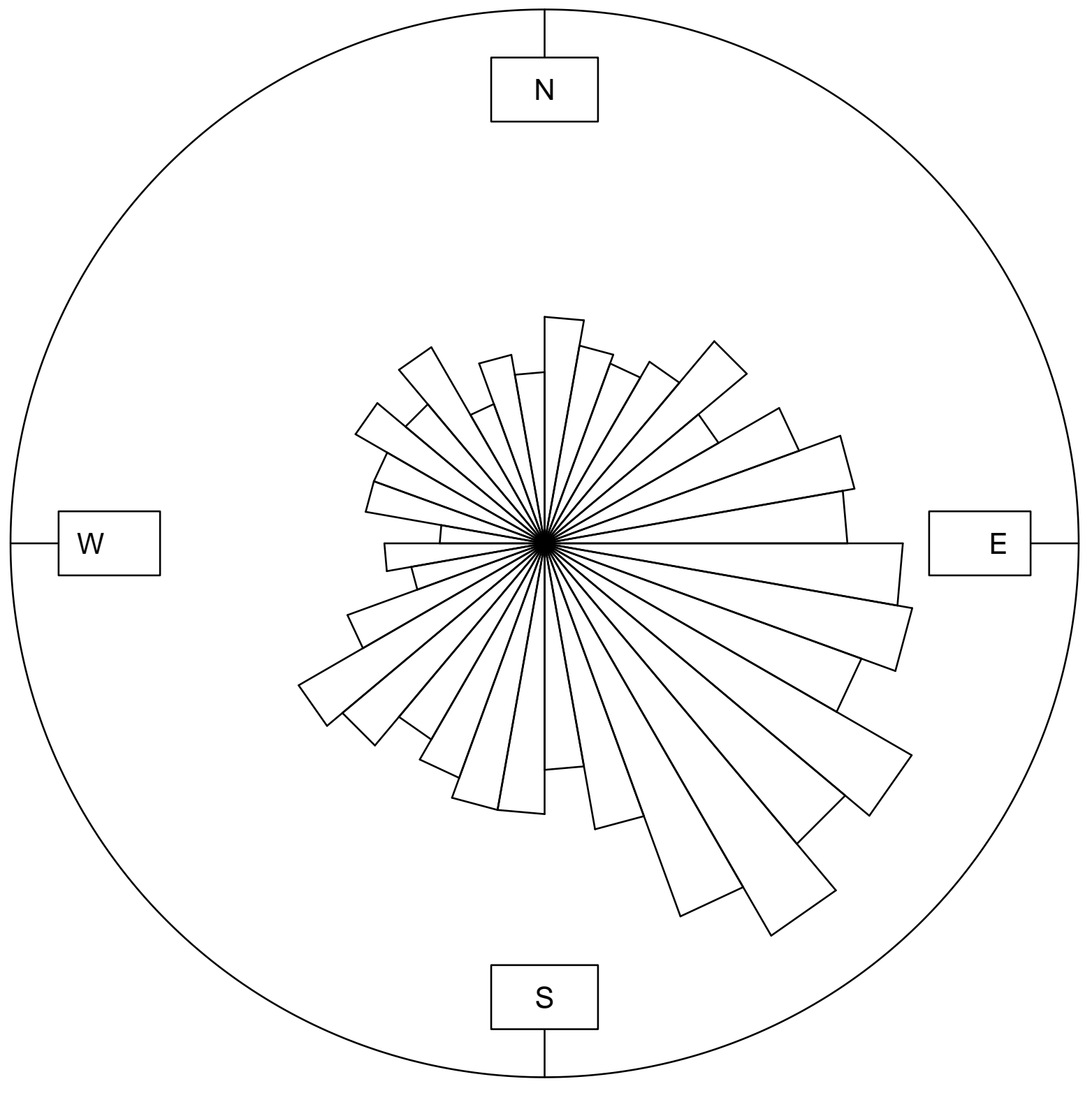




\section{bootstrap 533}

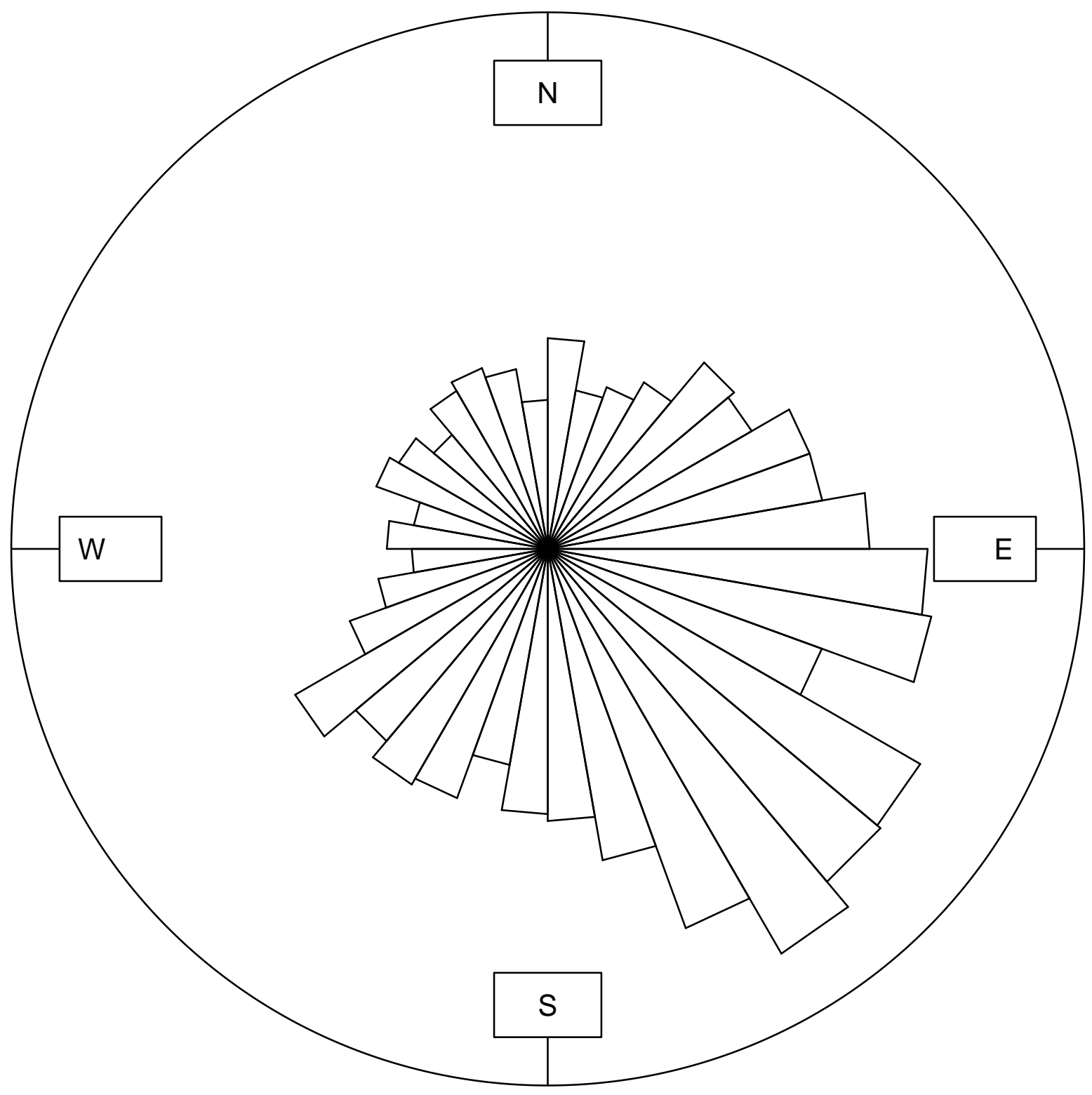




\section{bootstrap 534}

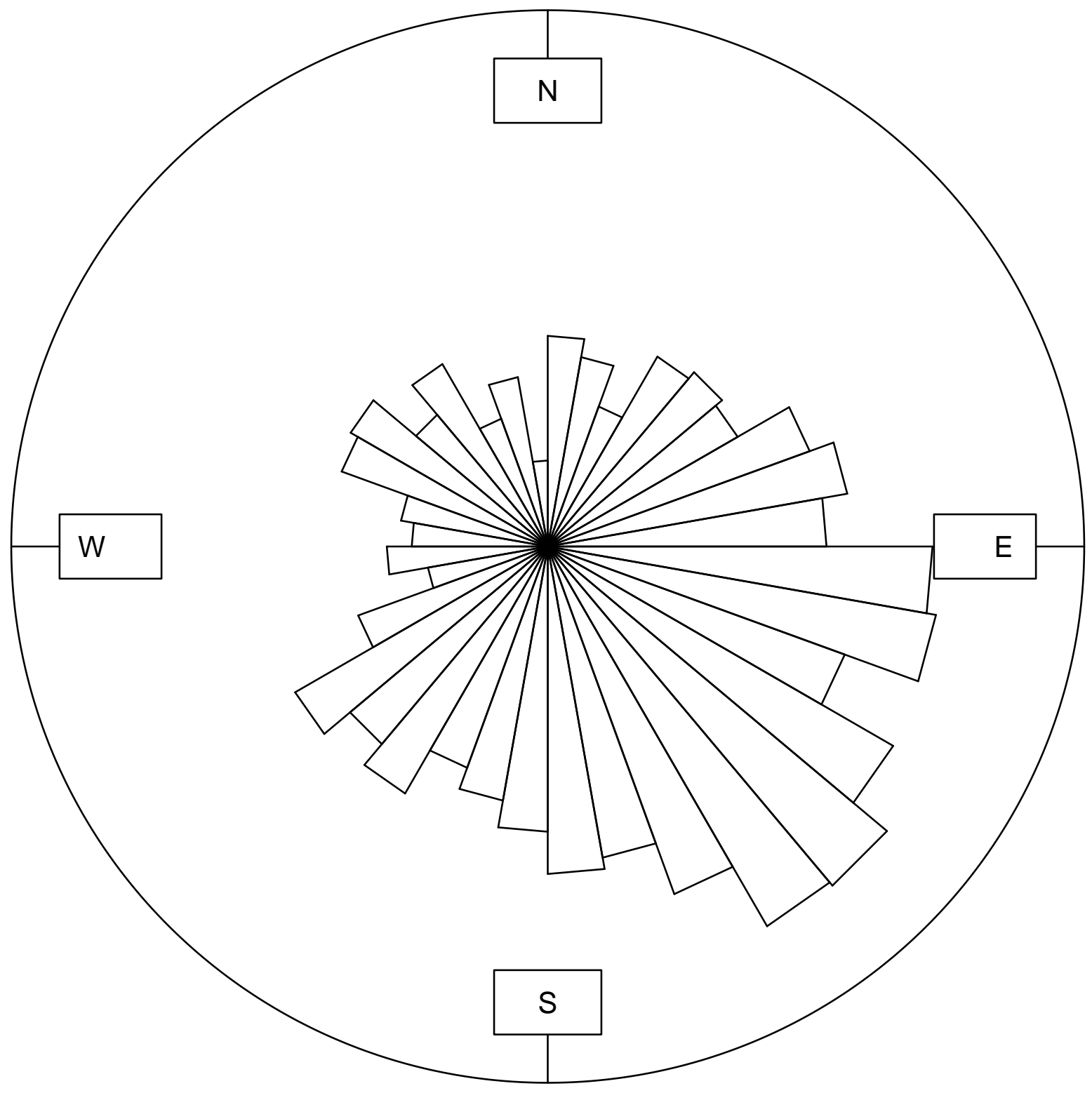




\section{bootstrap 535}

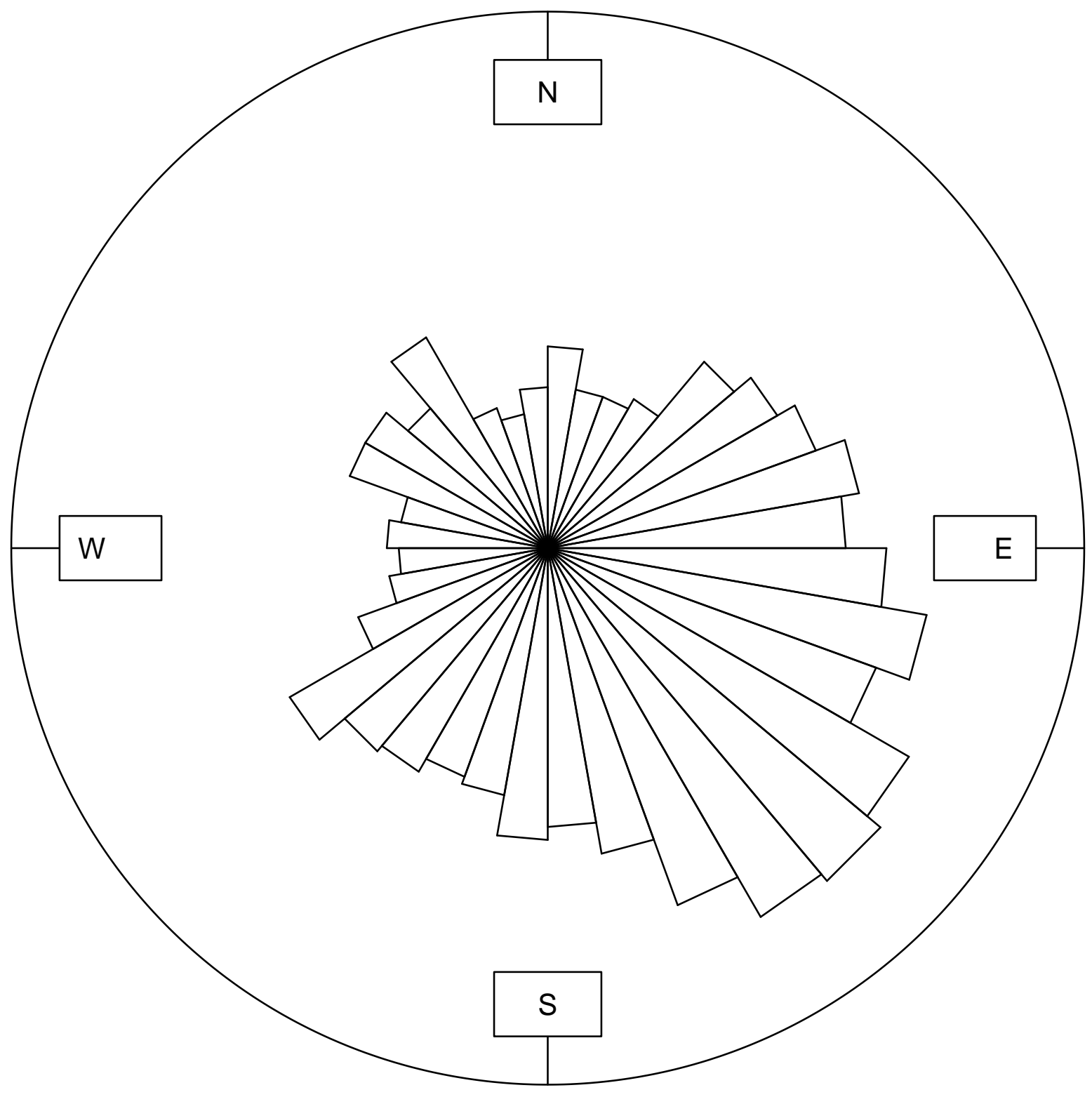




\section{bootstrap 536}

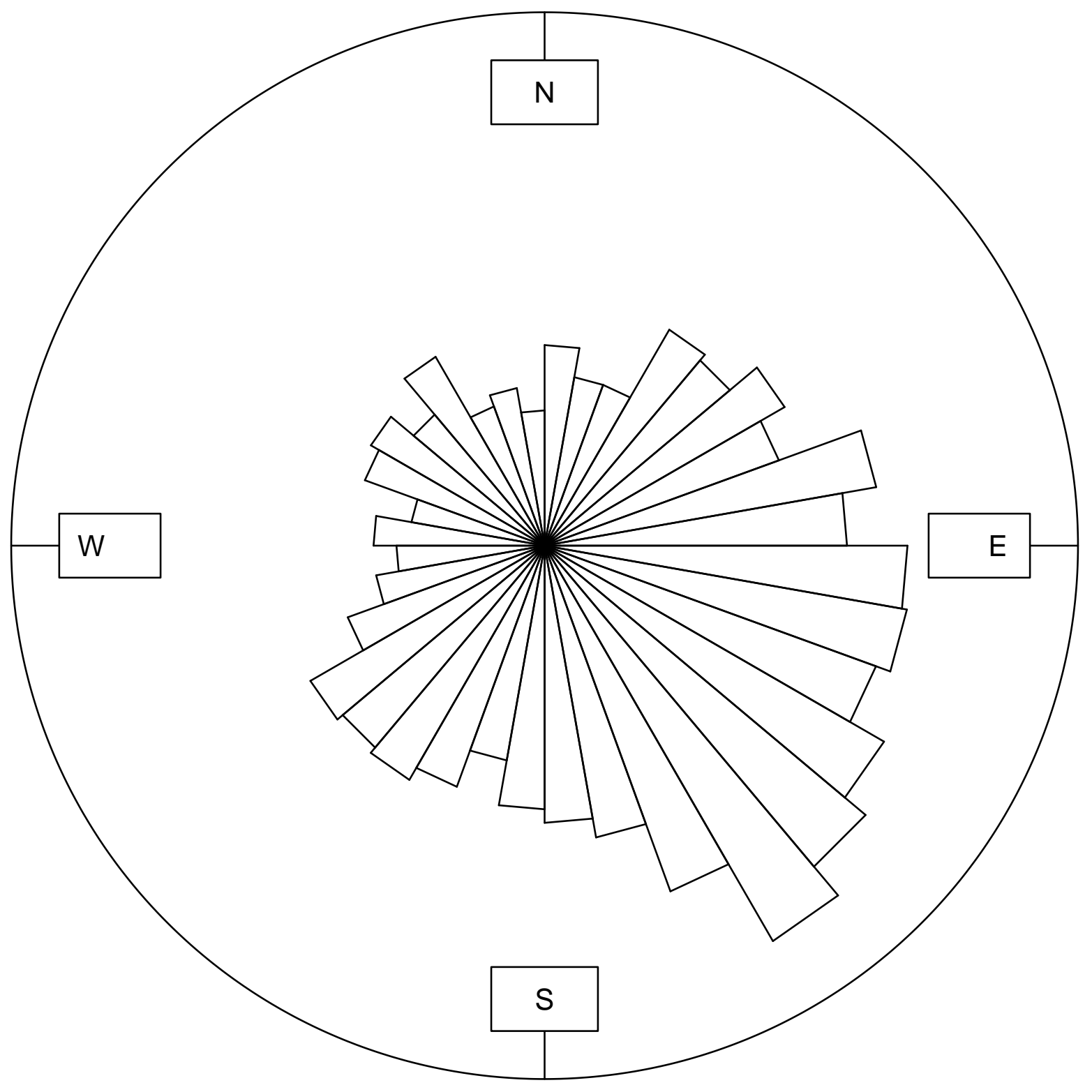


bootstrap 537

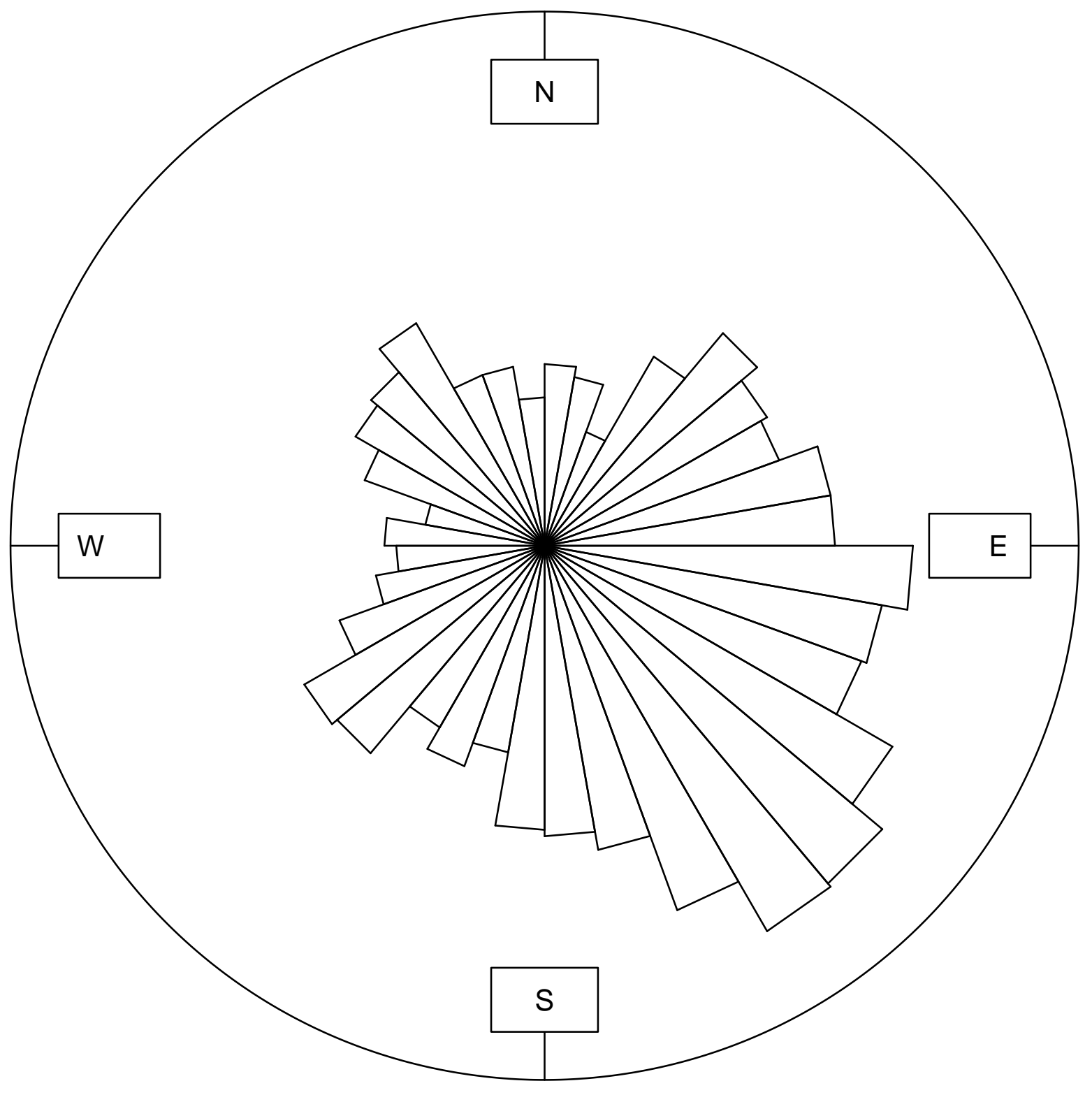


bootstrap 538

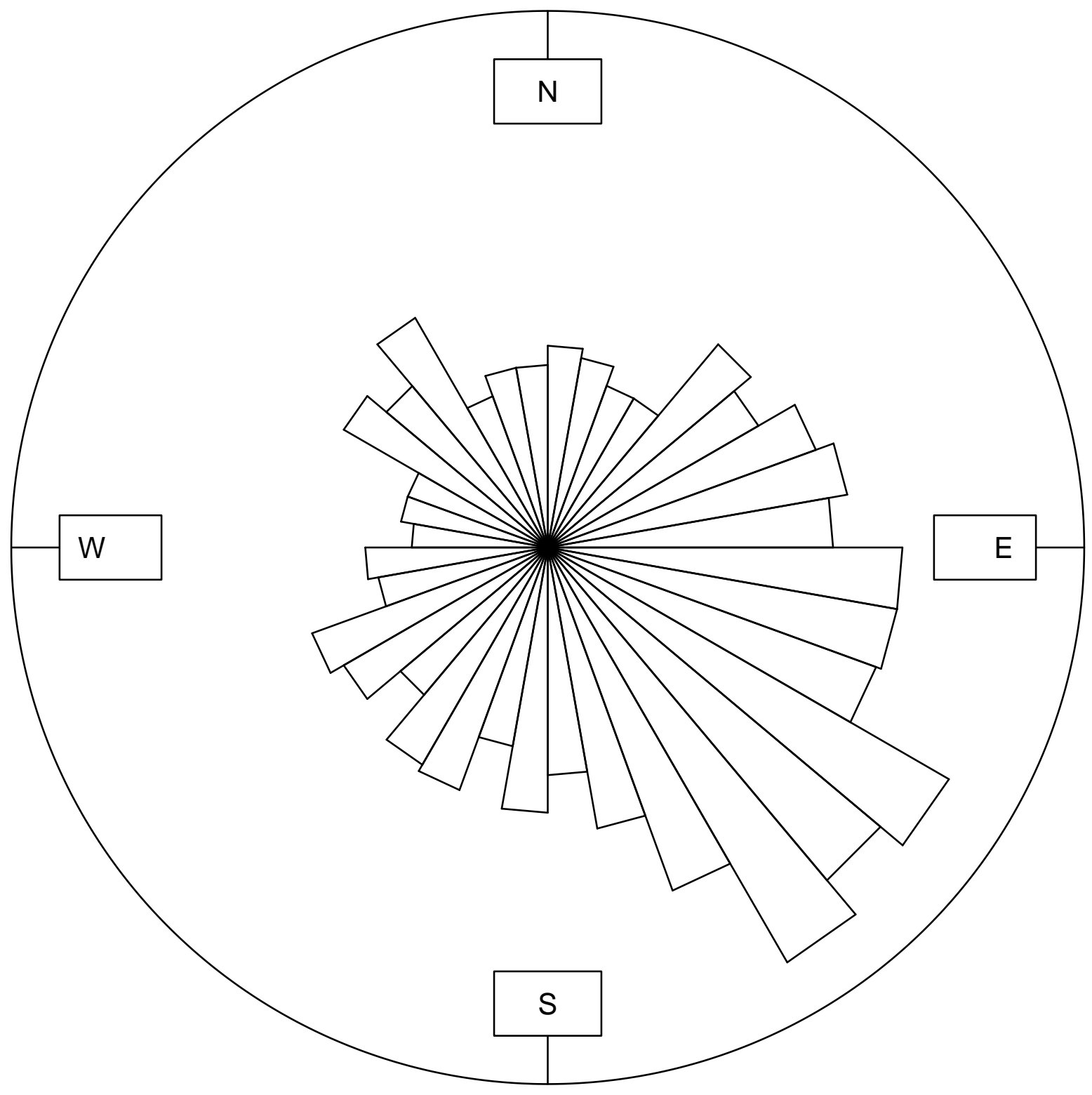


bootstrap 539

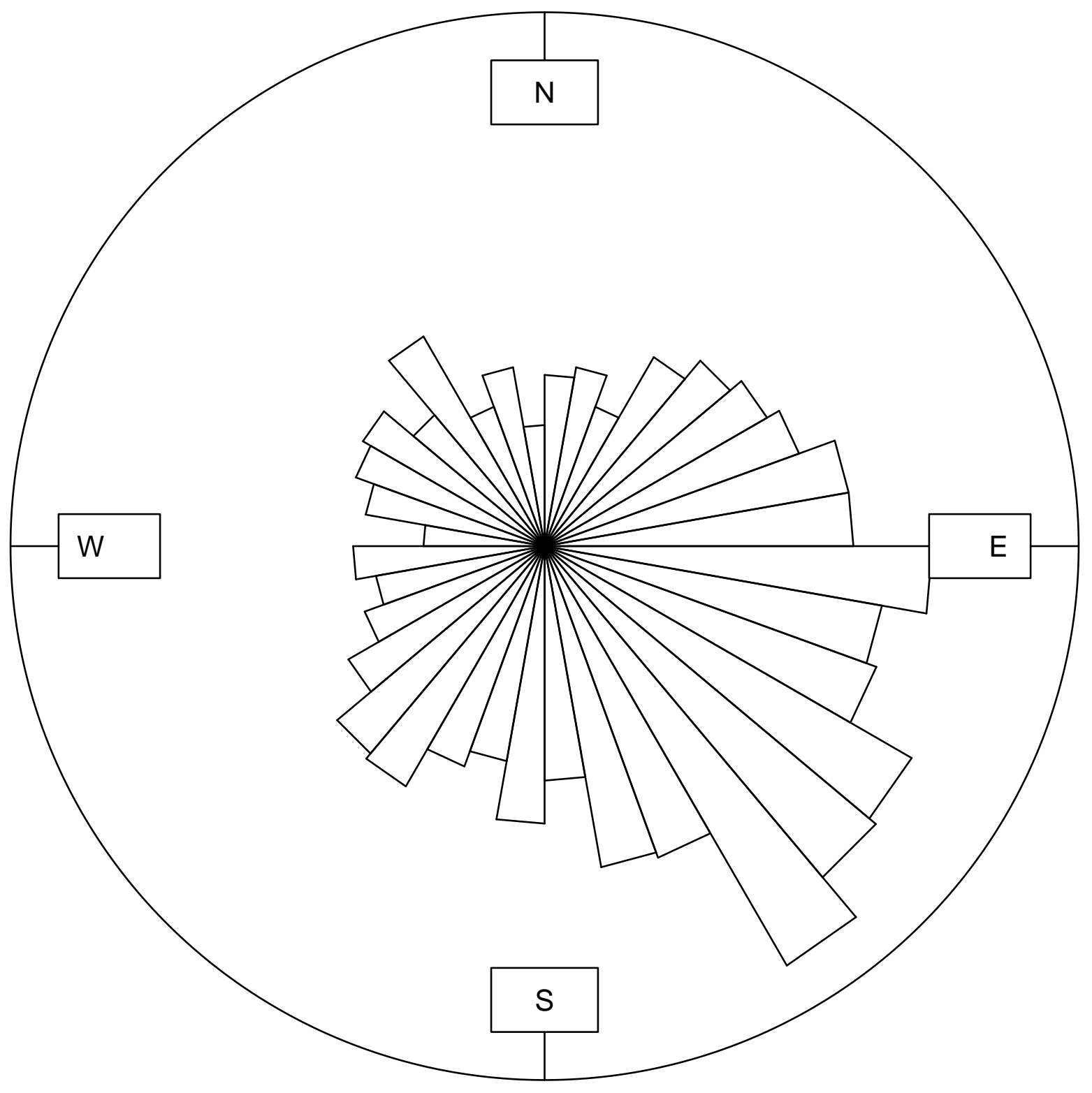


bootstrap 540

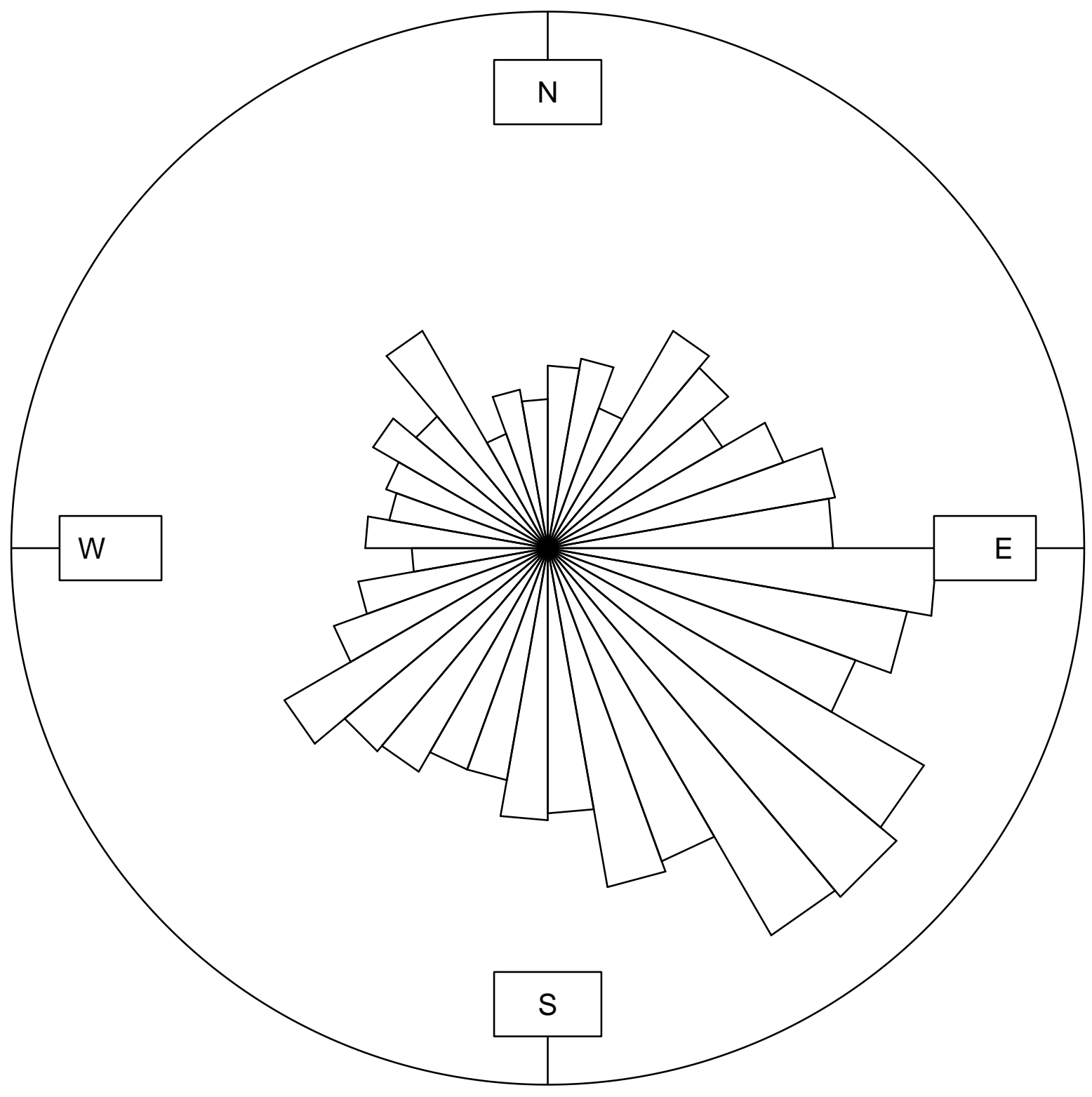




\section{bootstrap 541}

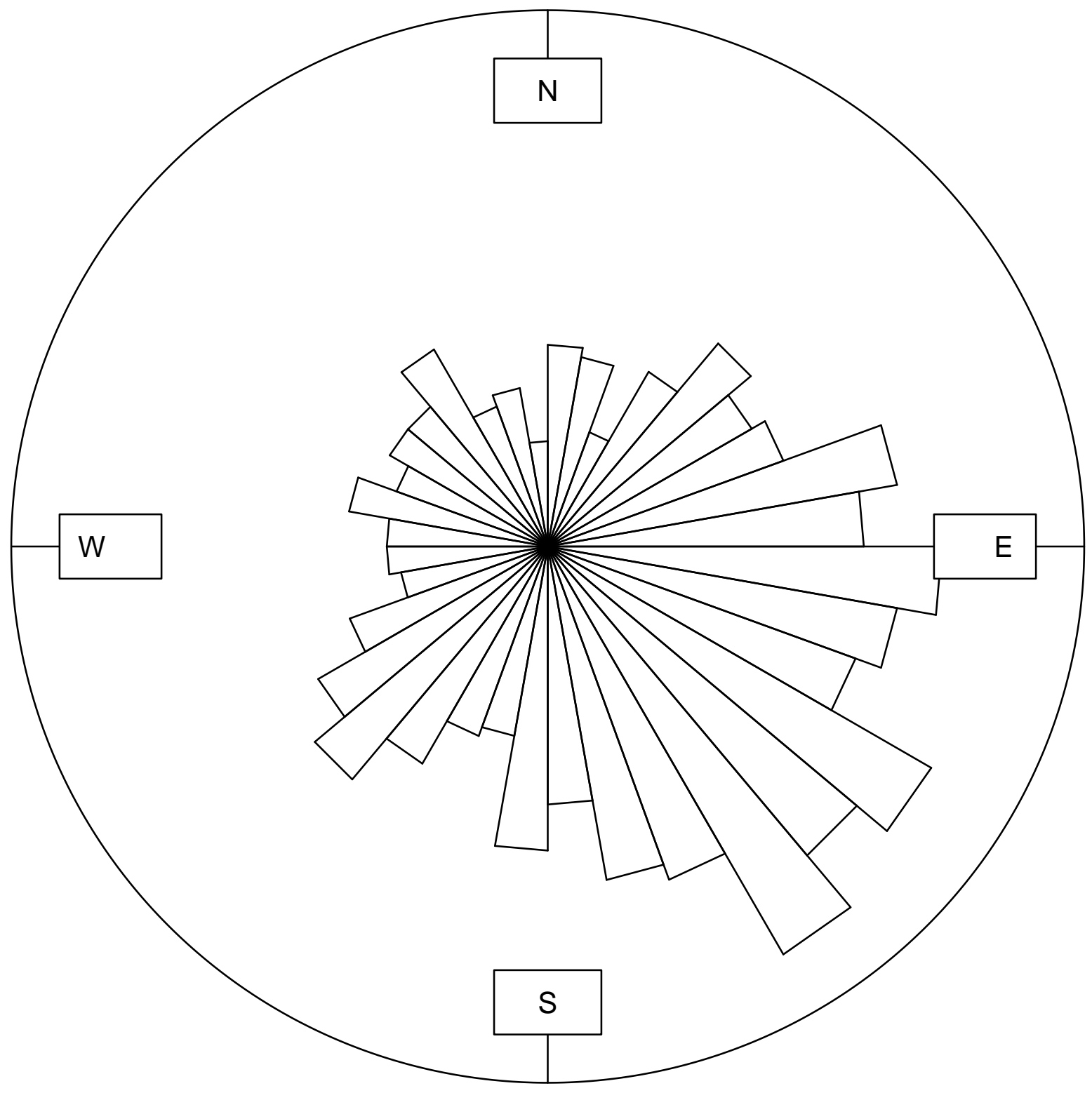




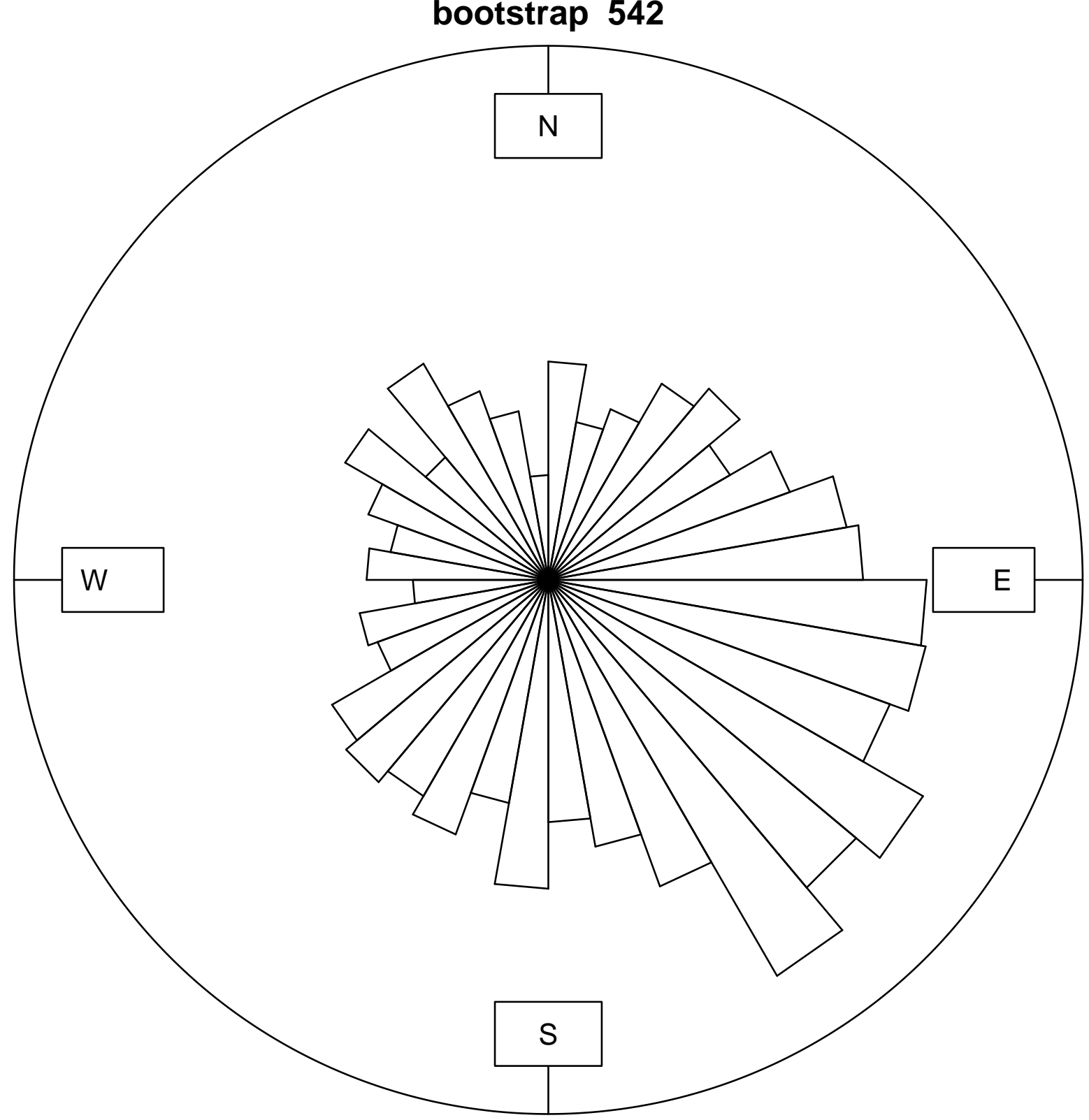




\section{bootstrap 543}

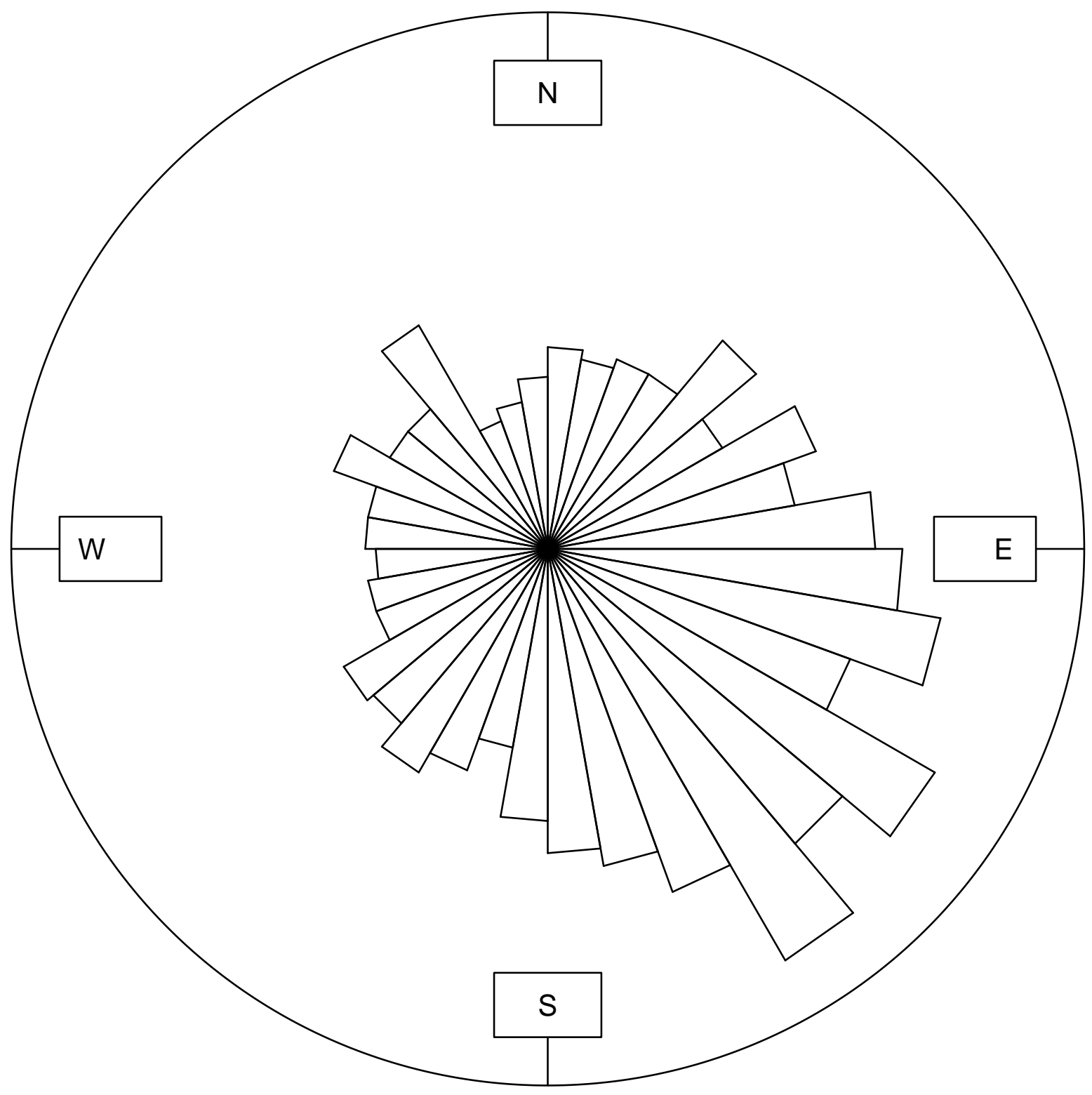




\section{bootstrap 544}

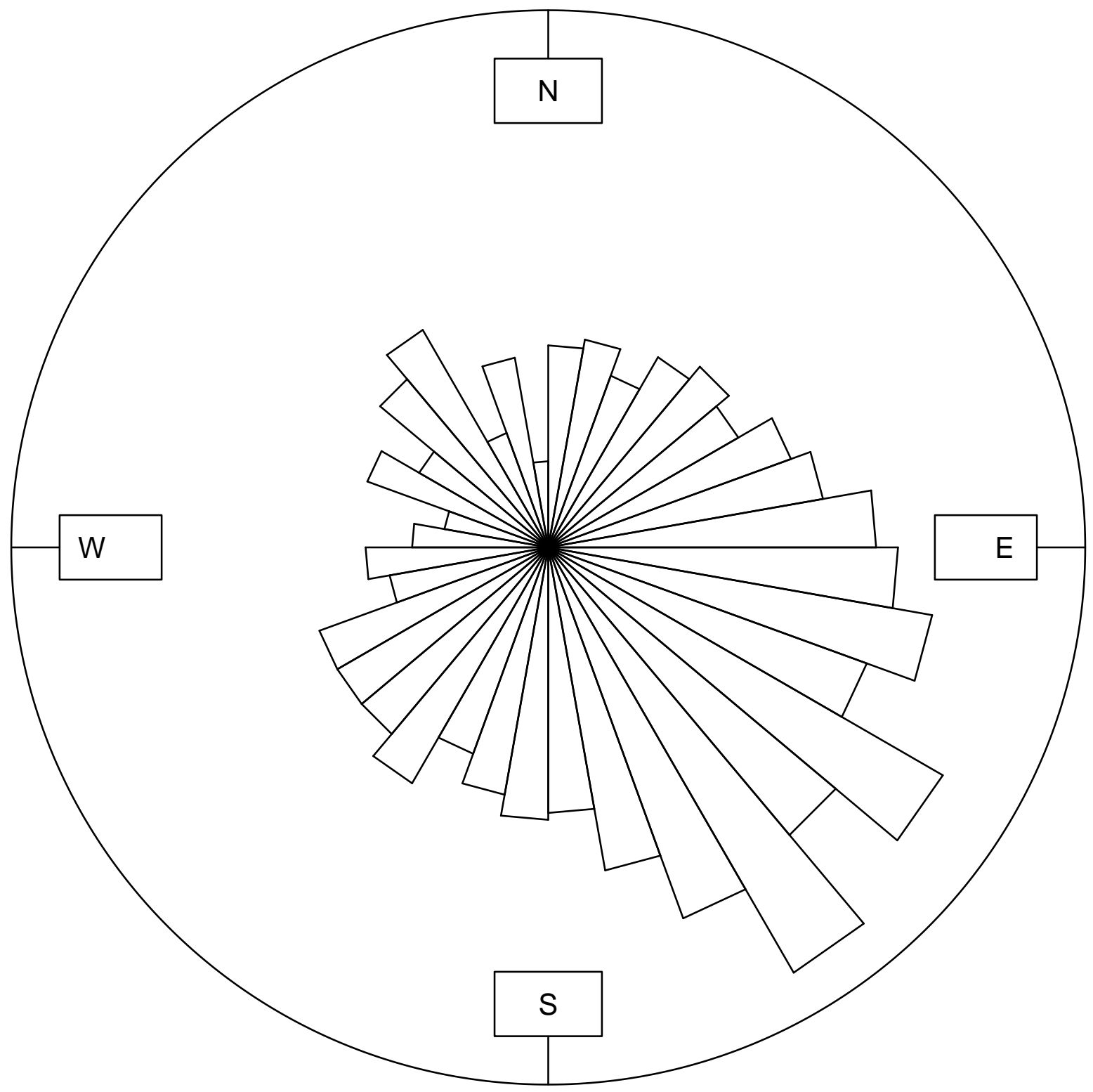




\section{bootstrap 545}

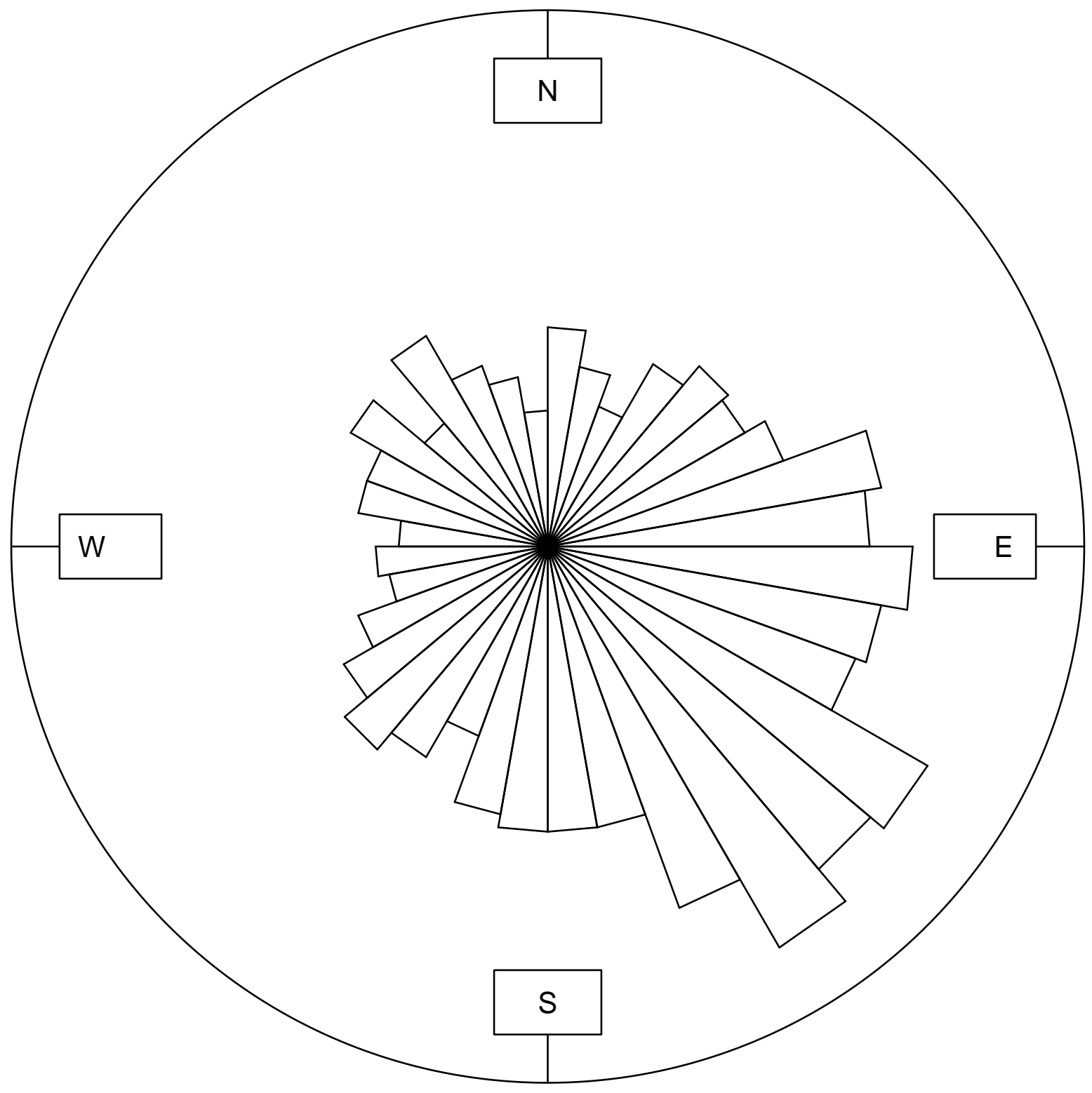


bootstrap 546

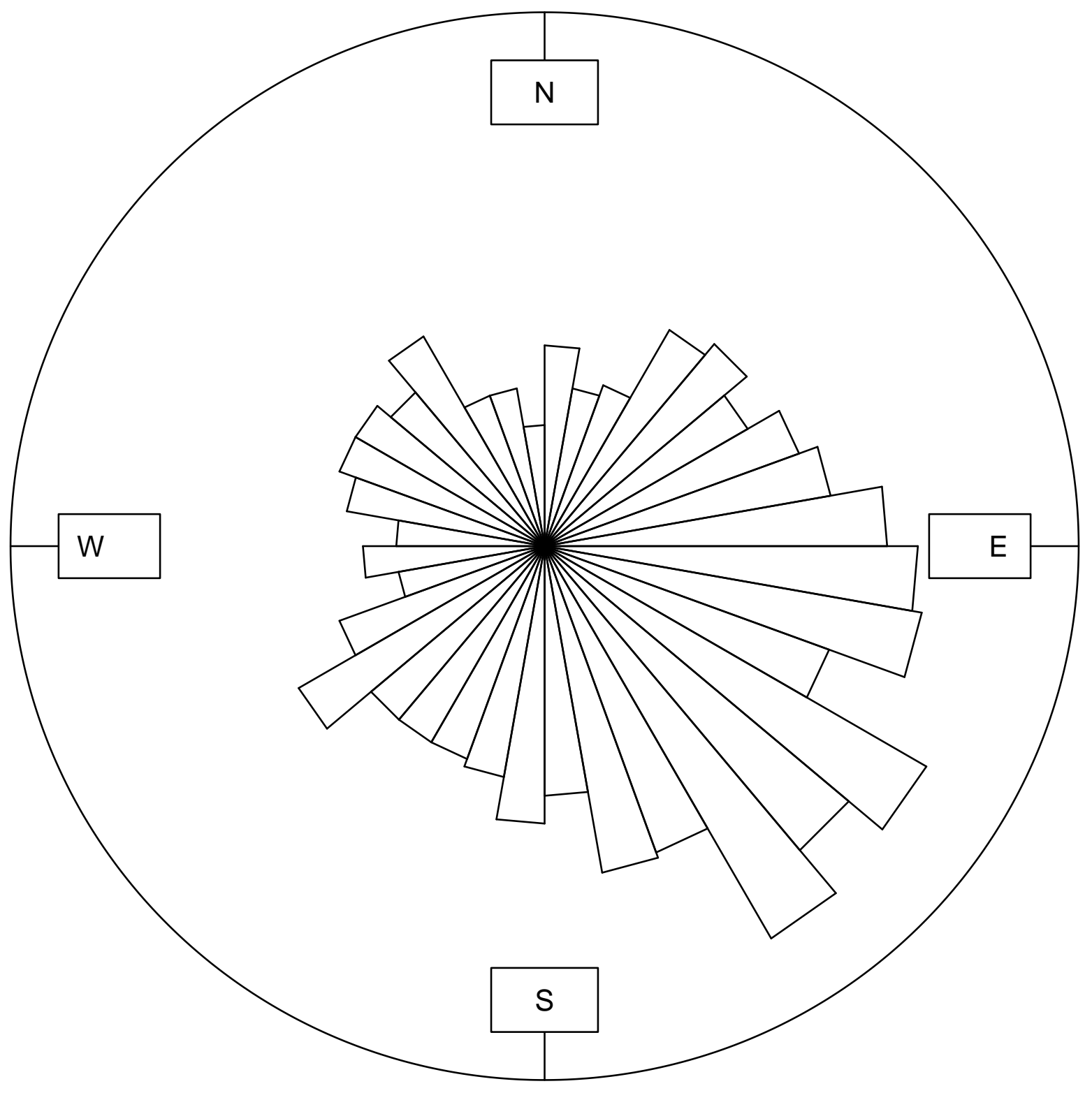


bootstrap 547

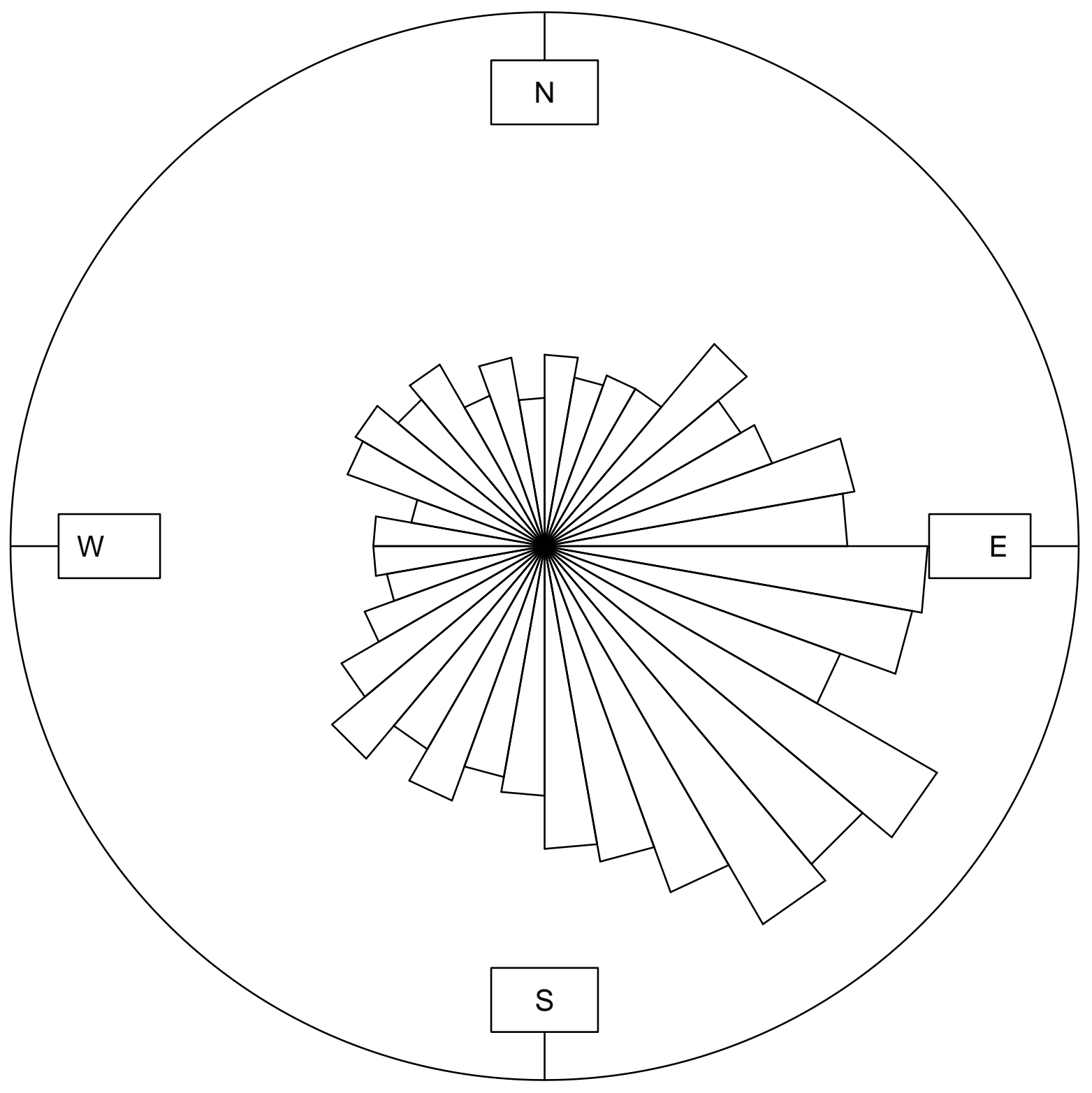


bootstrap 548

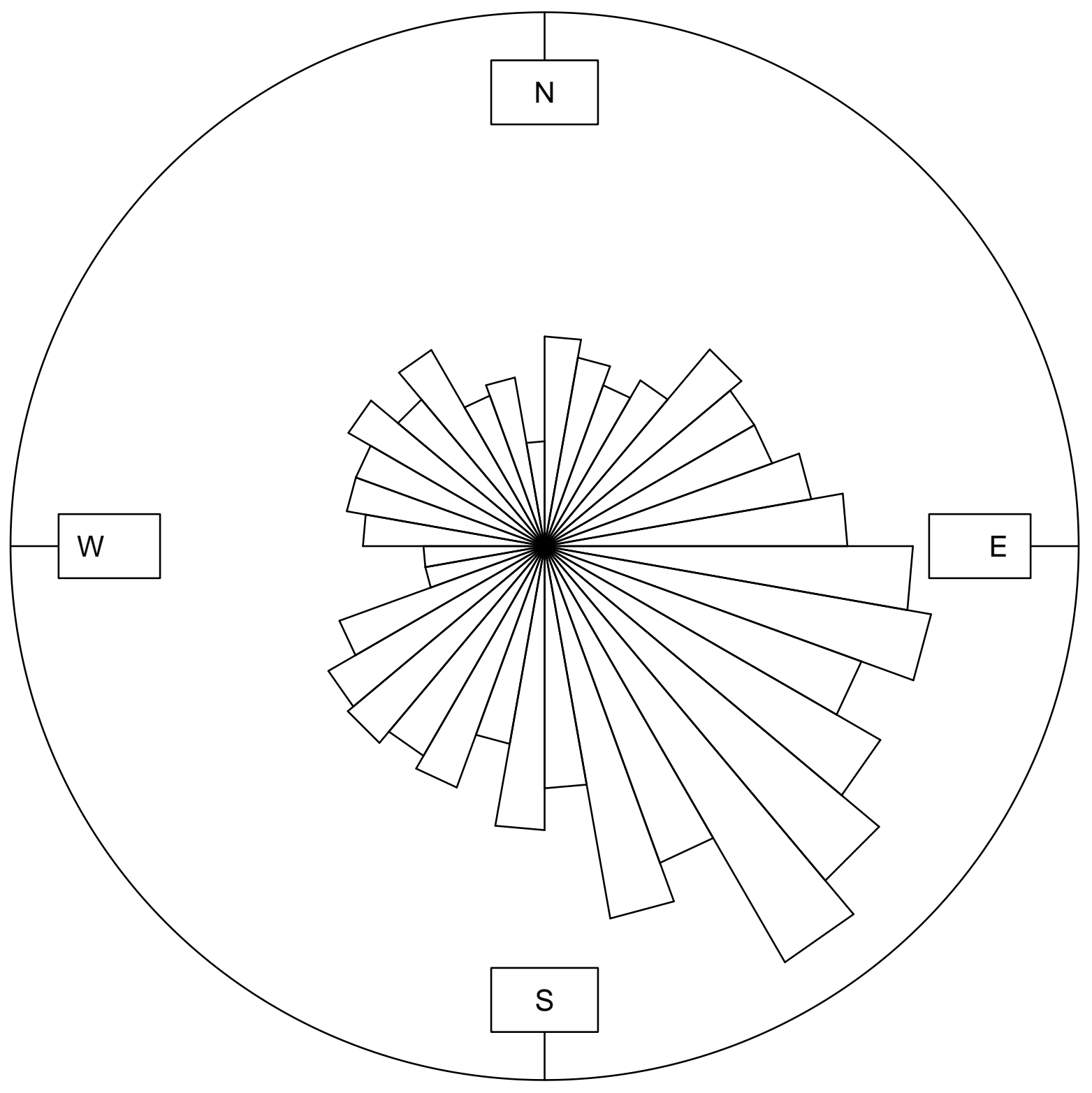


bootstrap 549

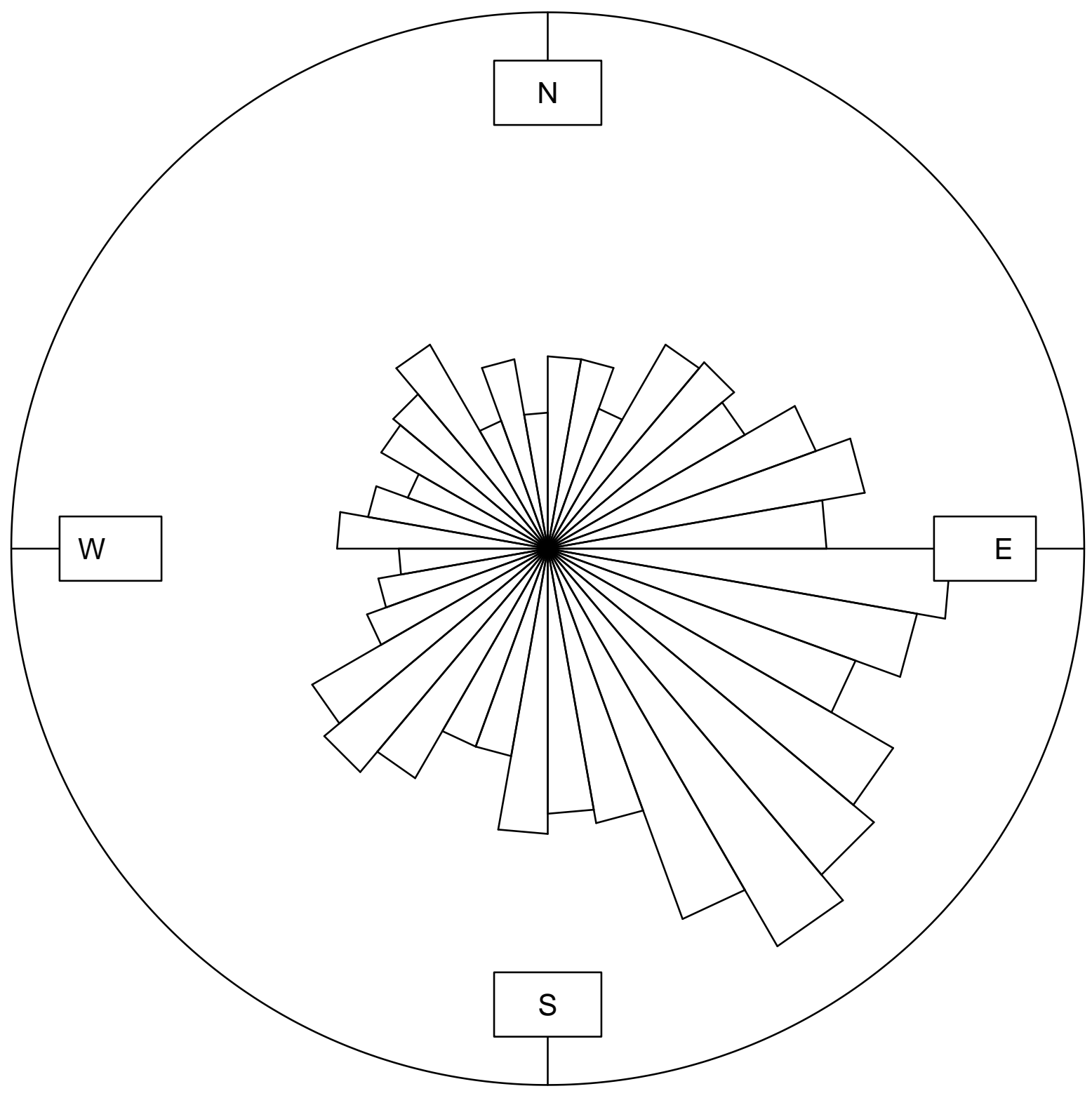


bootstrap 550

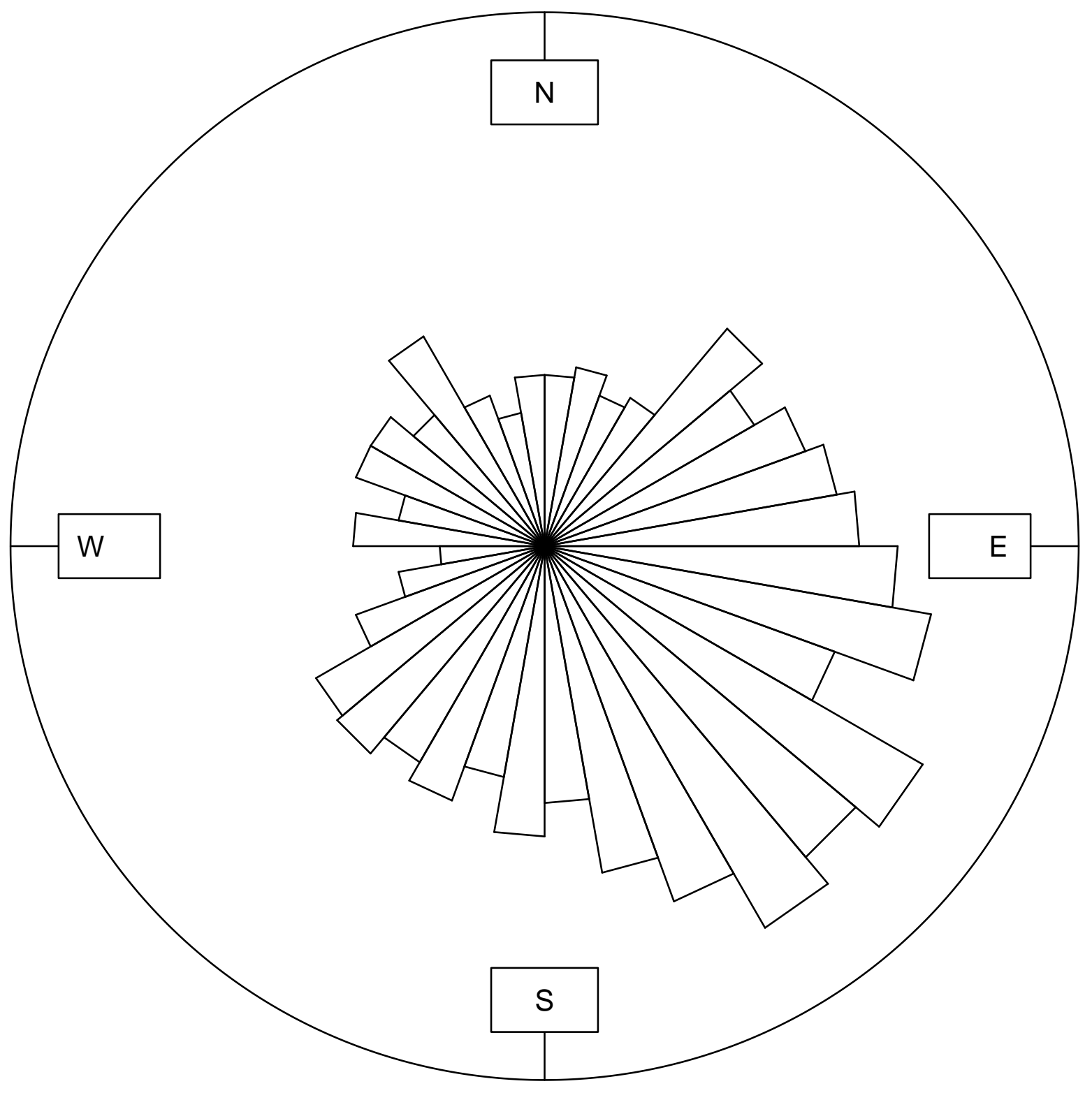


bootstrap 551

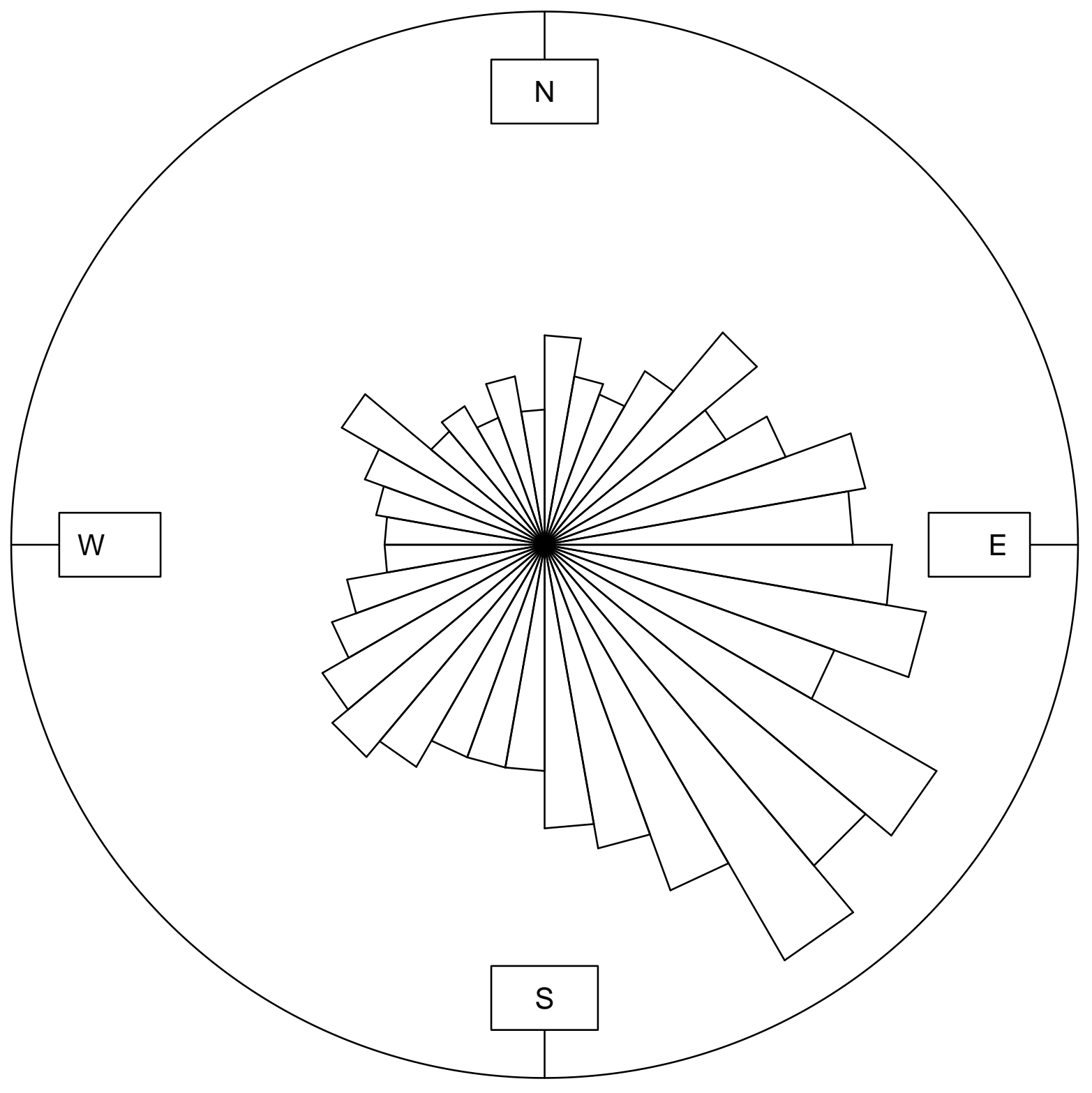


bootstrap 552

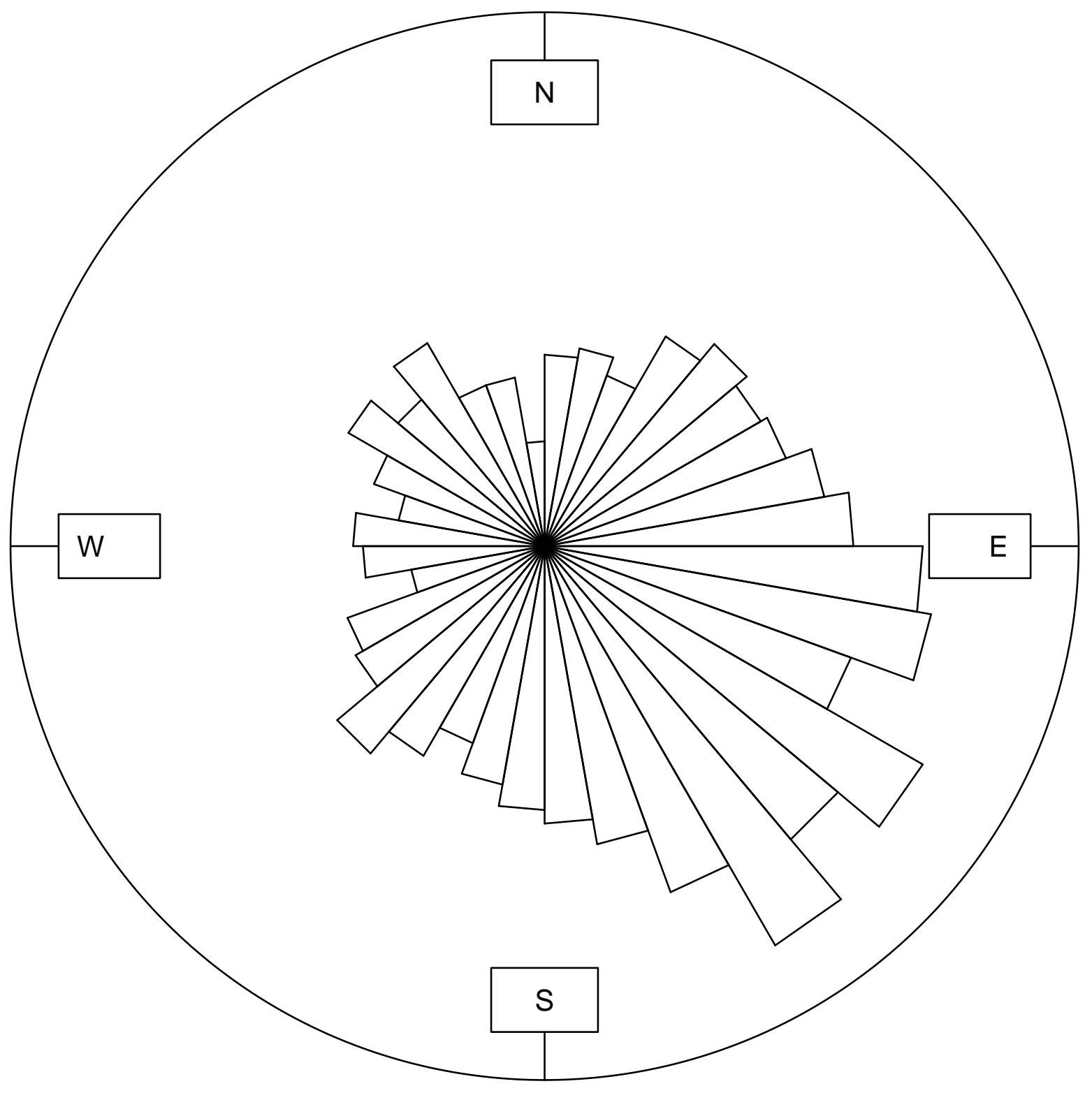




\section{bootstrap 553}

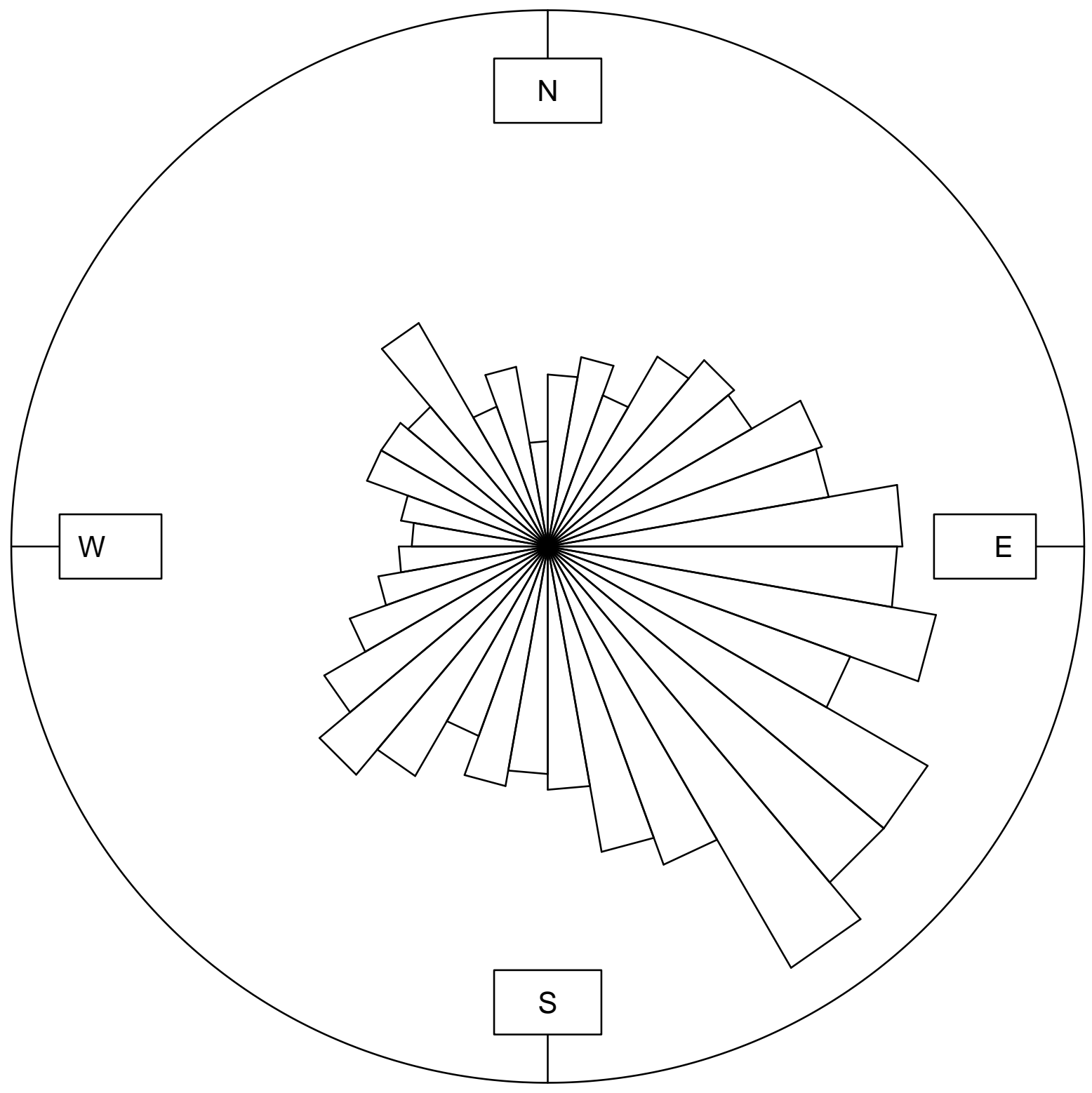




\section{bootstrap 554}

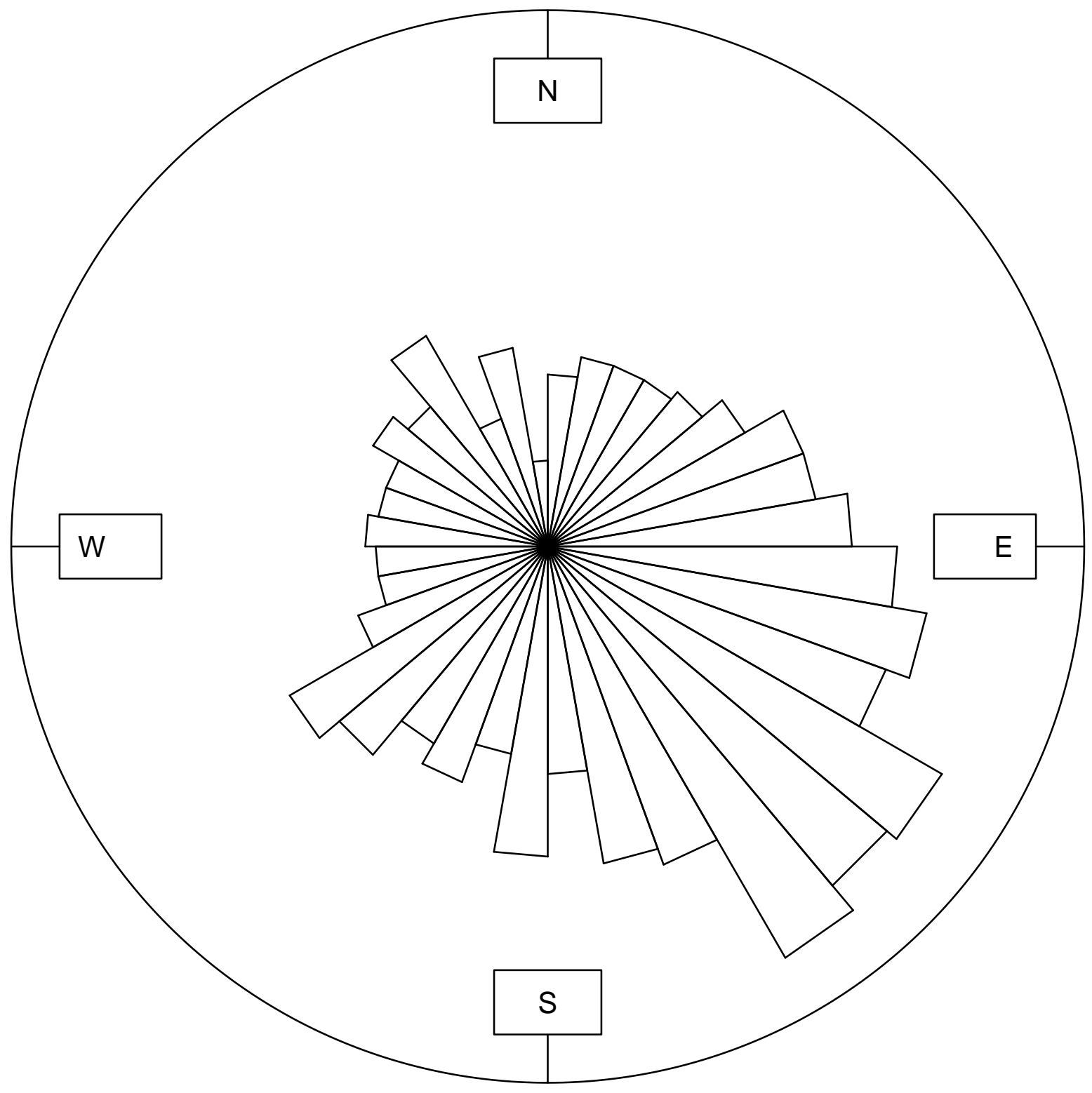




\section{bootstrap 555}

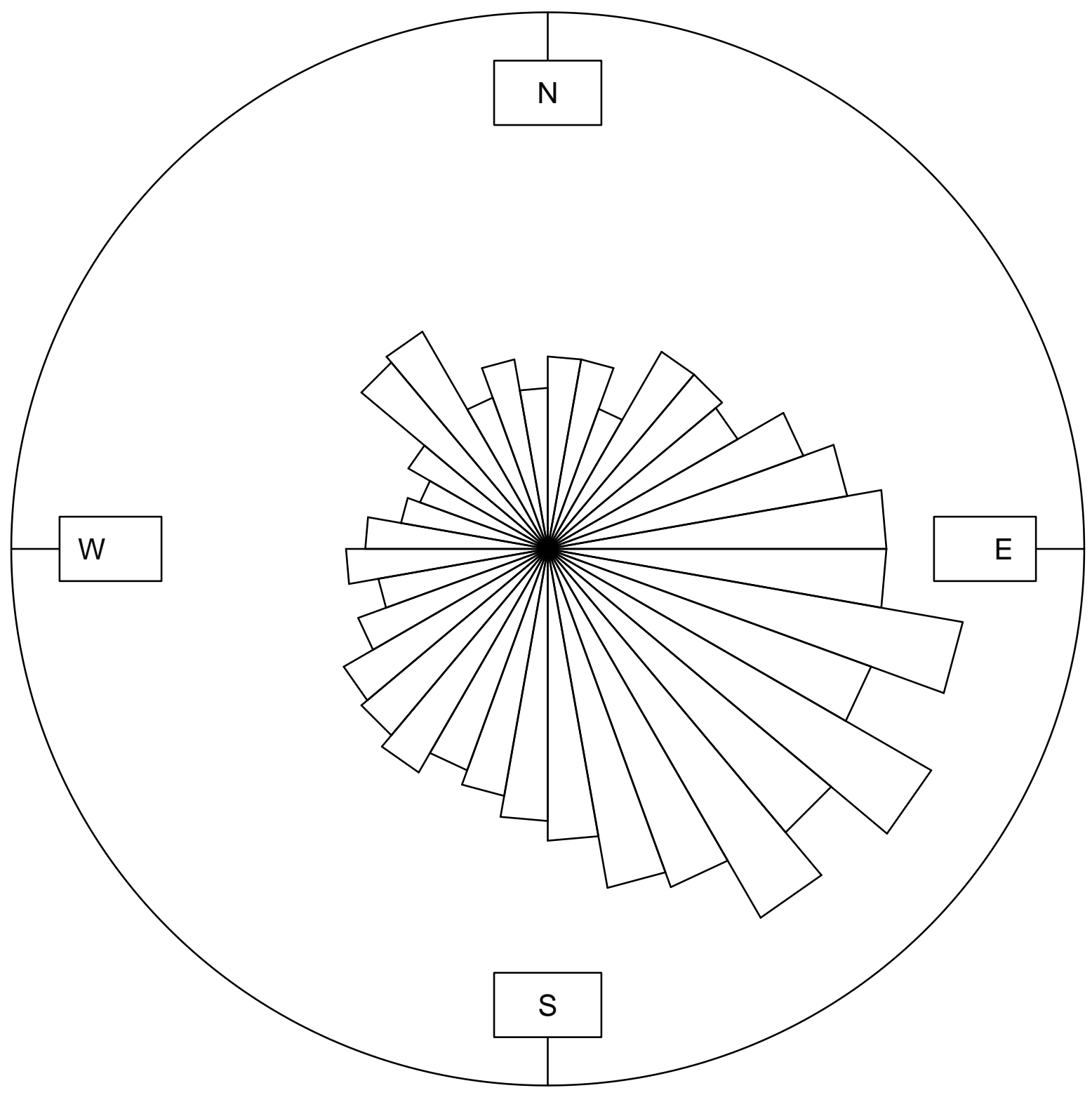




\section{bootstrap 556}

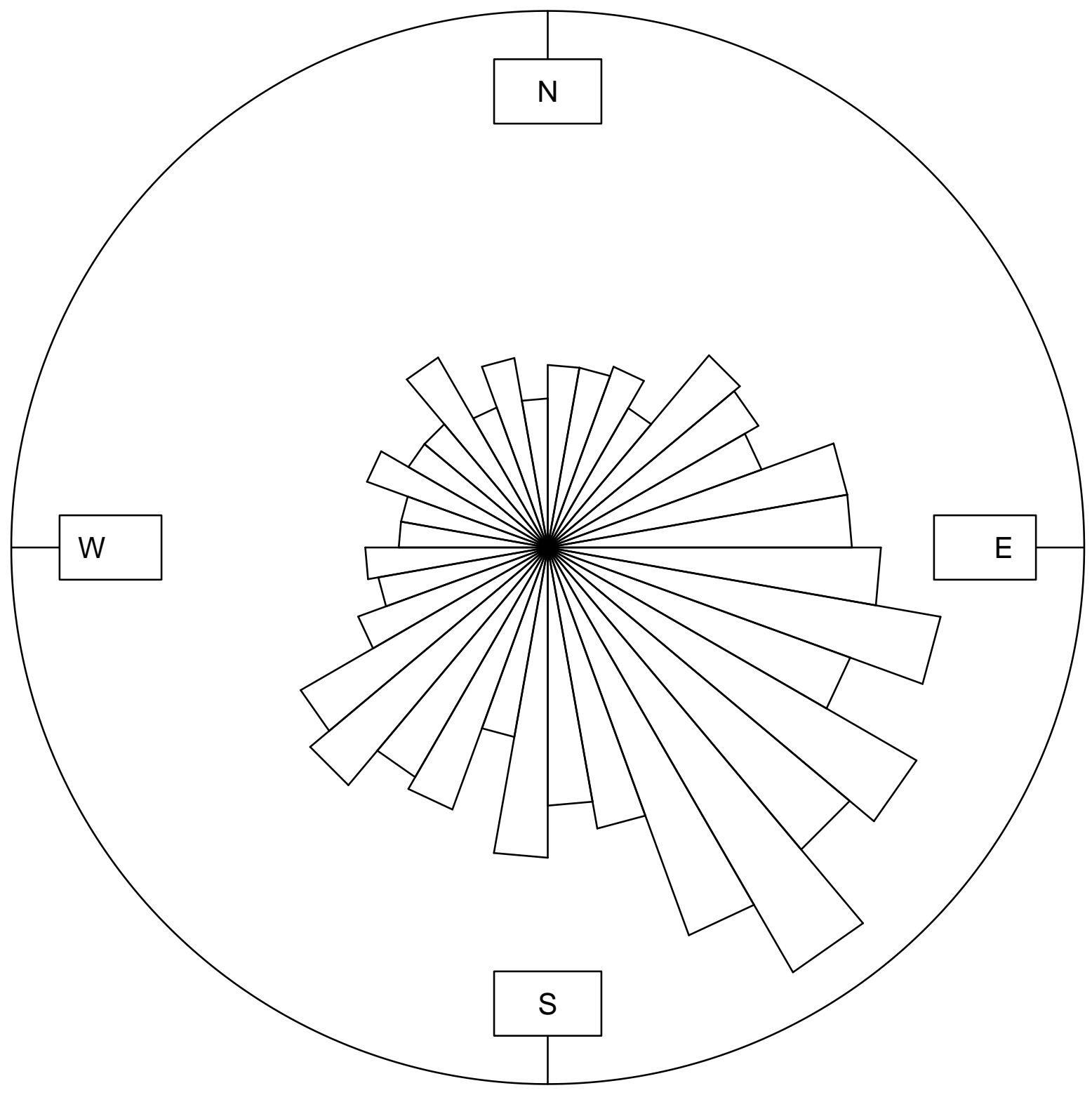


bootstrap 557

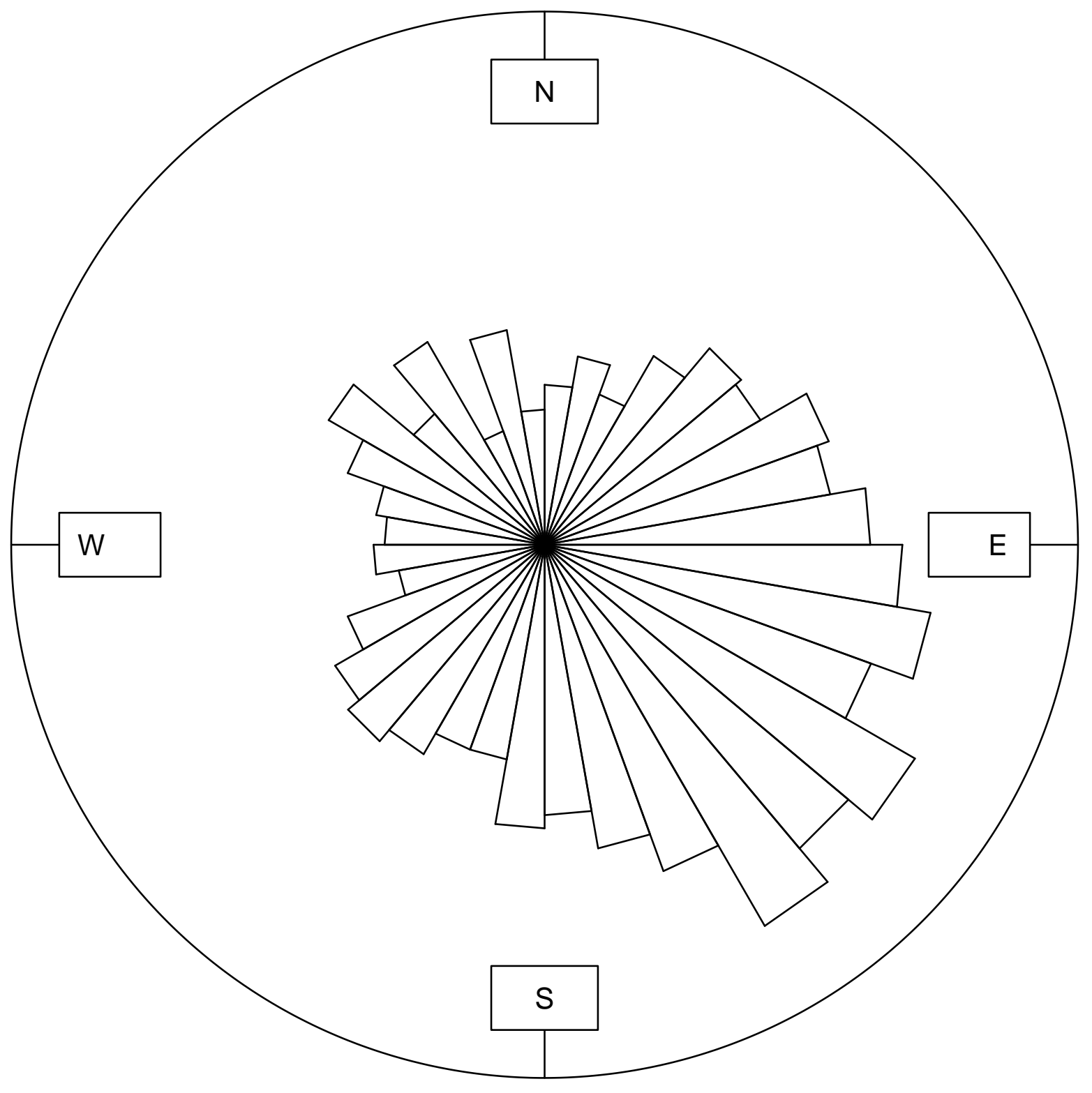


bootstrap 558

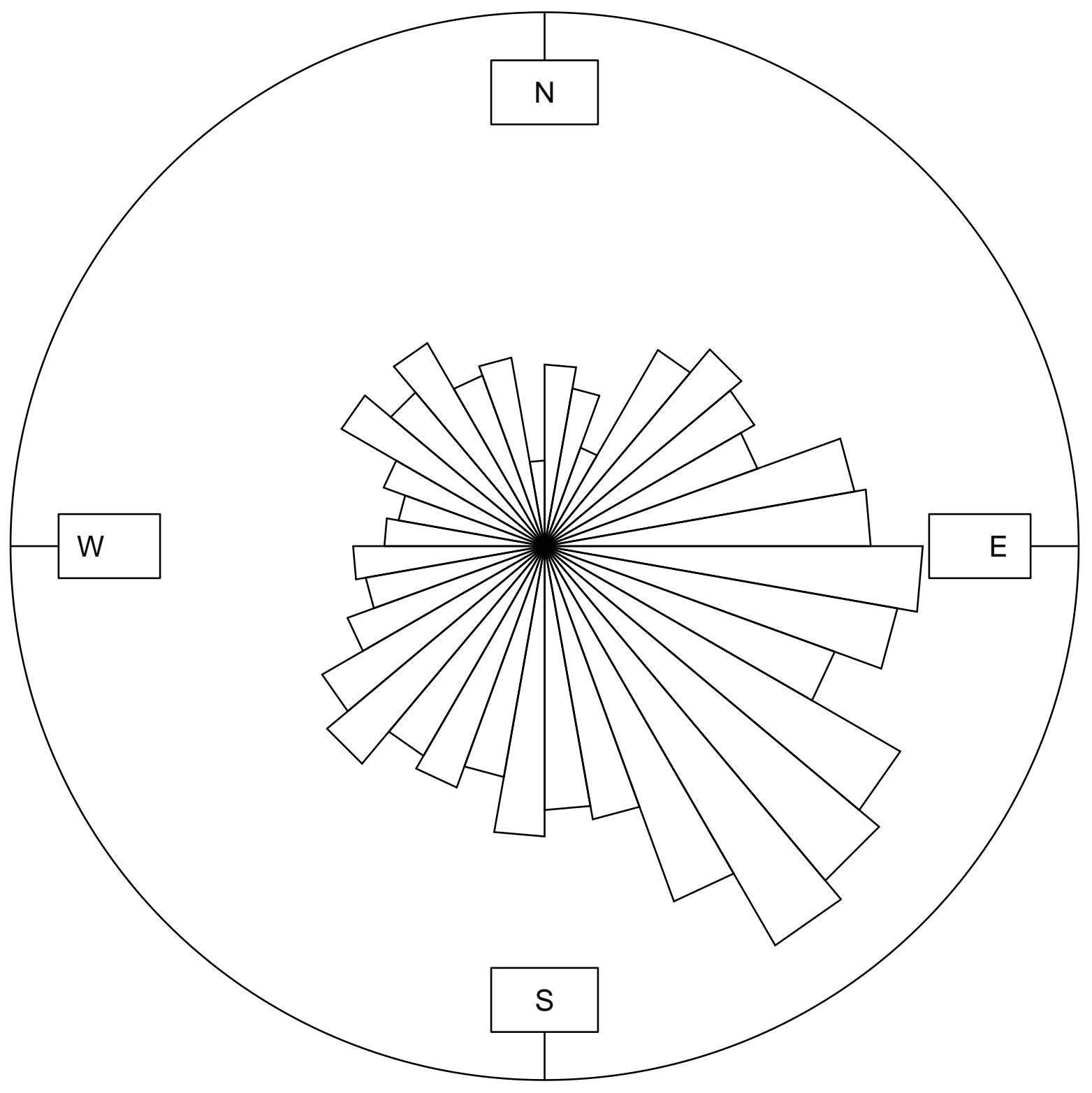


bootstrap 559

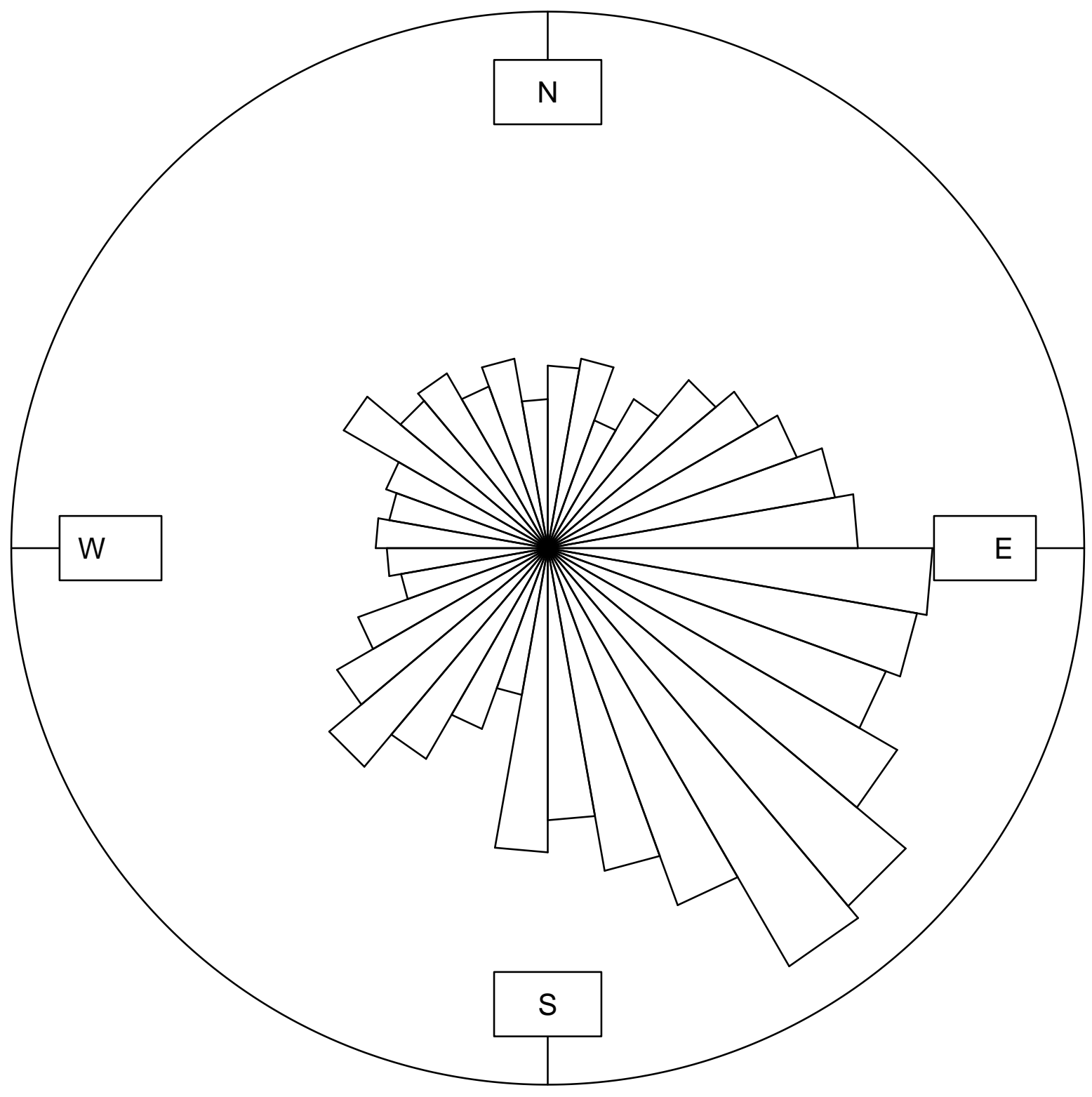


bootstrap 560

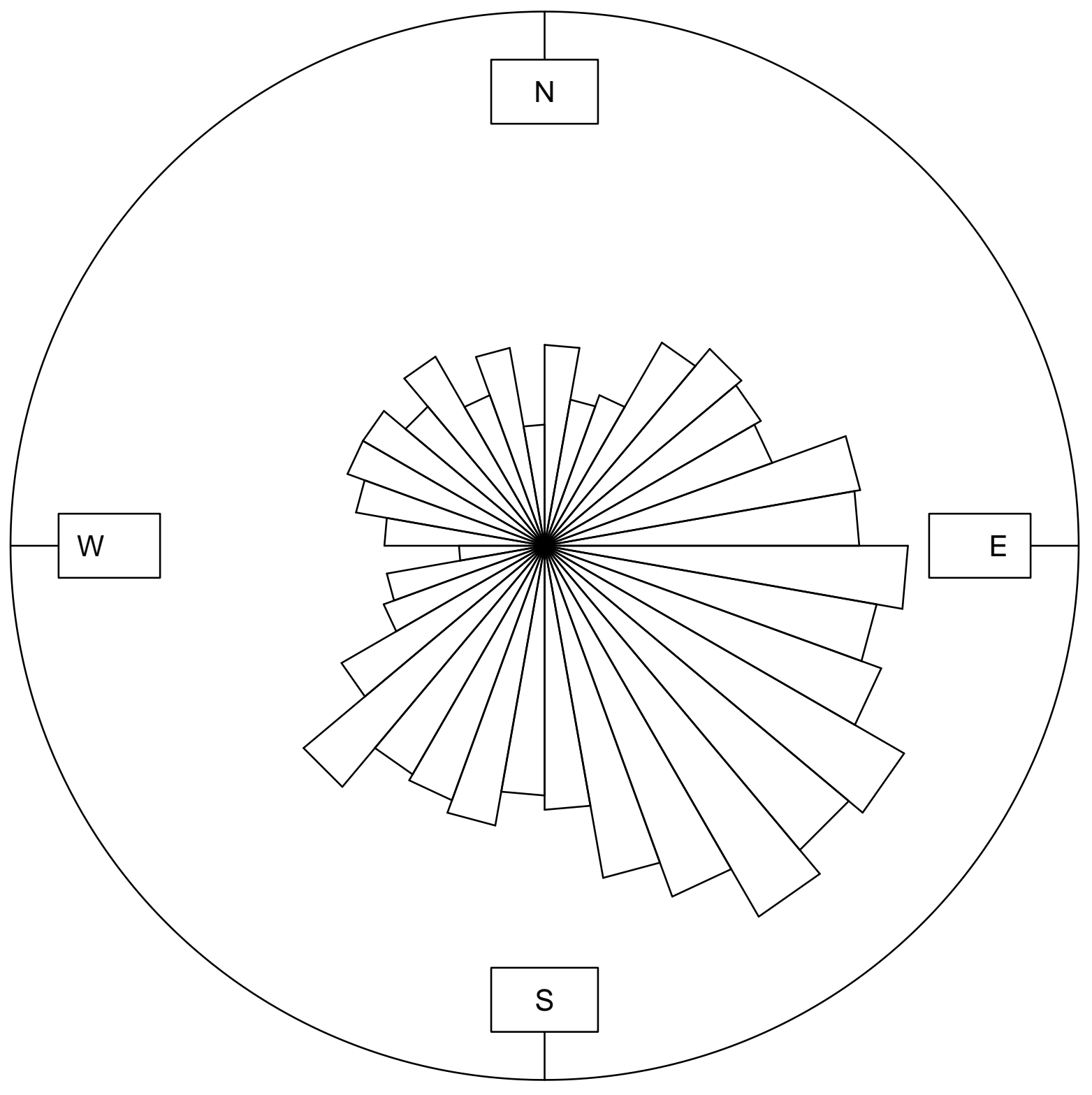


bootstrap 561

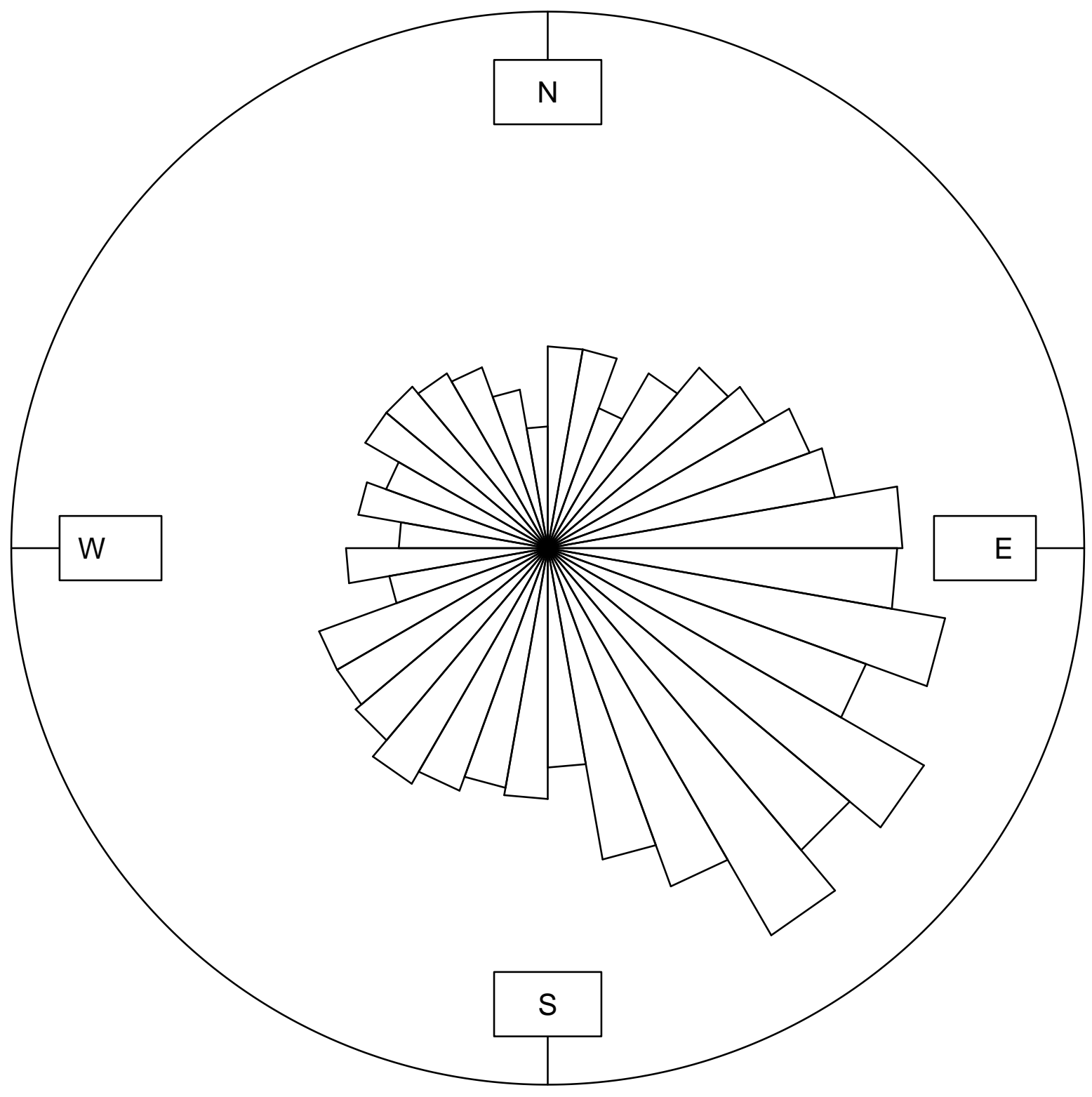


bootstrap 562

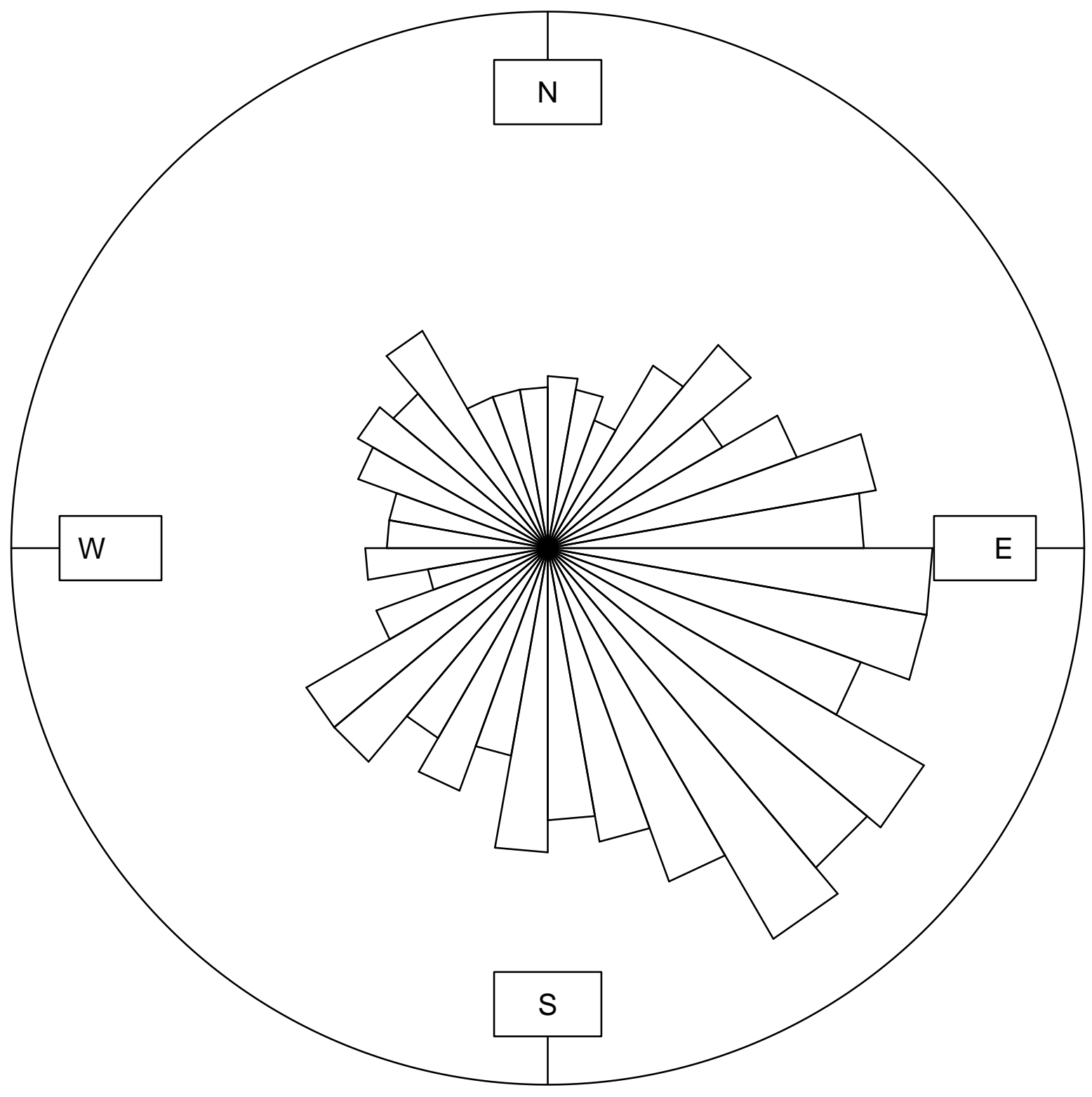


bootstrap 563

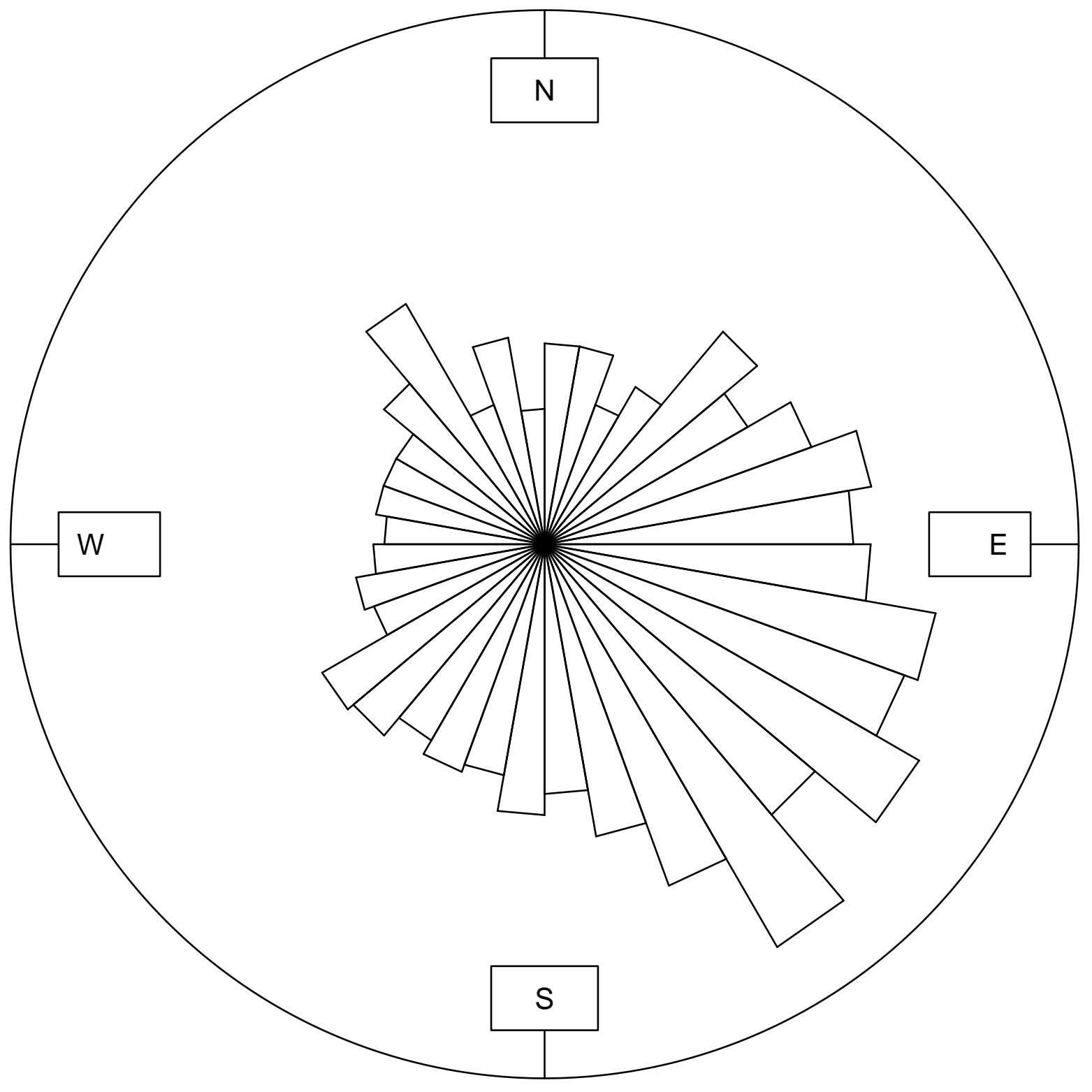




\section{bootstrap 564}

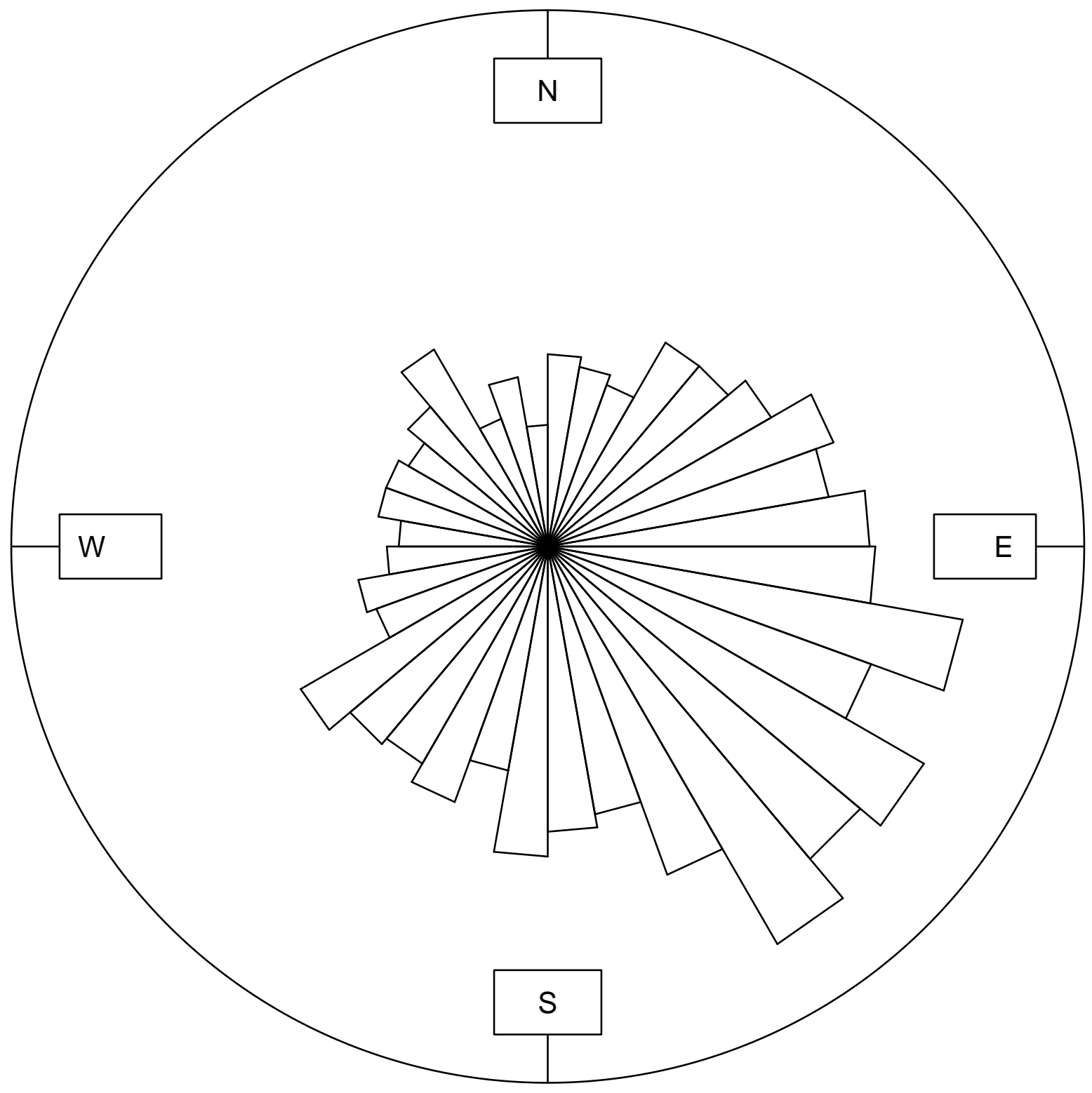


bootstrap 565

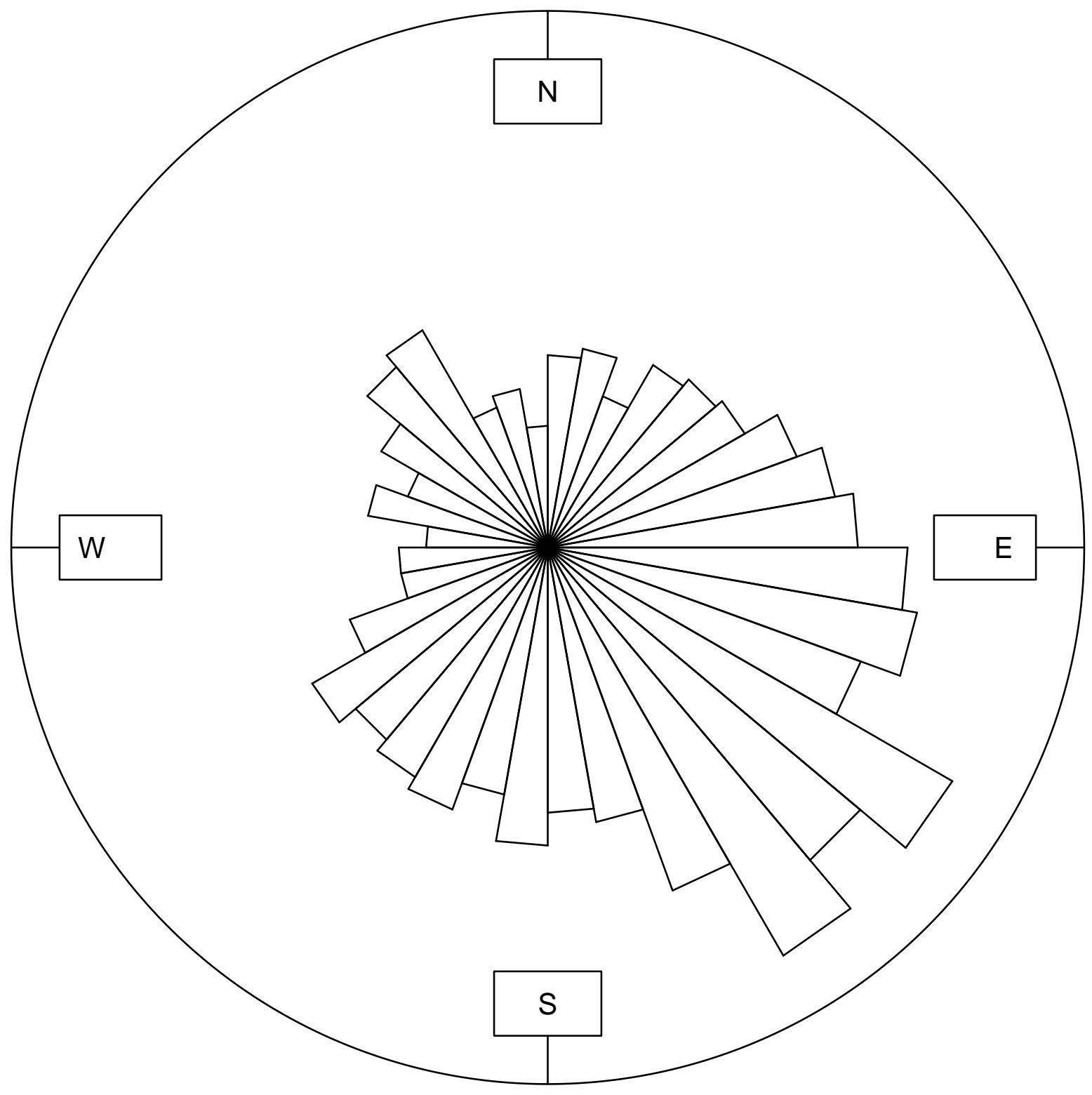


bootstrap 566

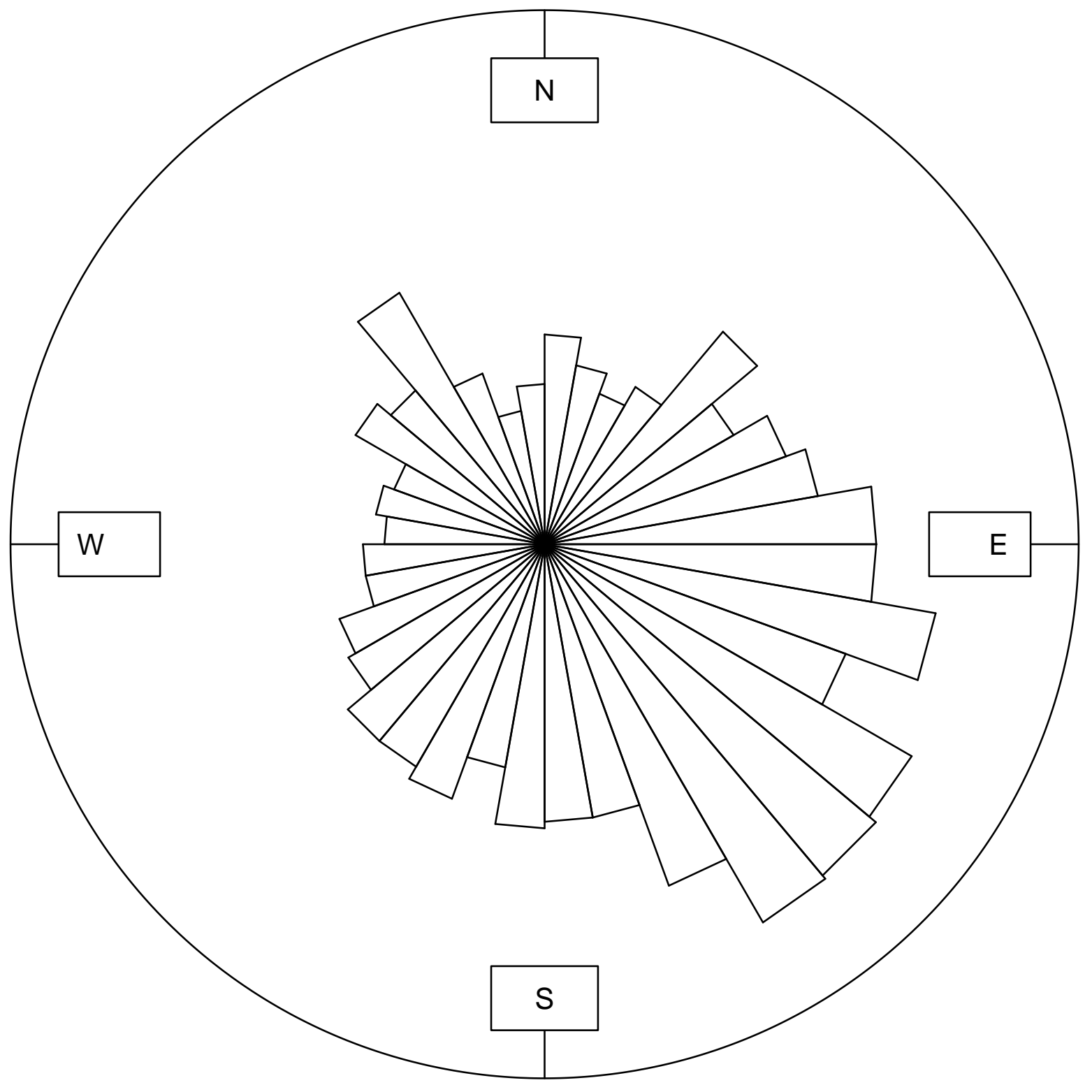


bootstrap 567

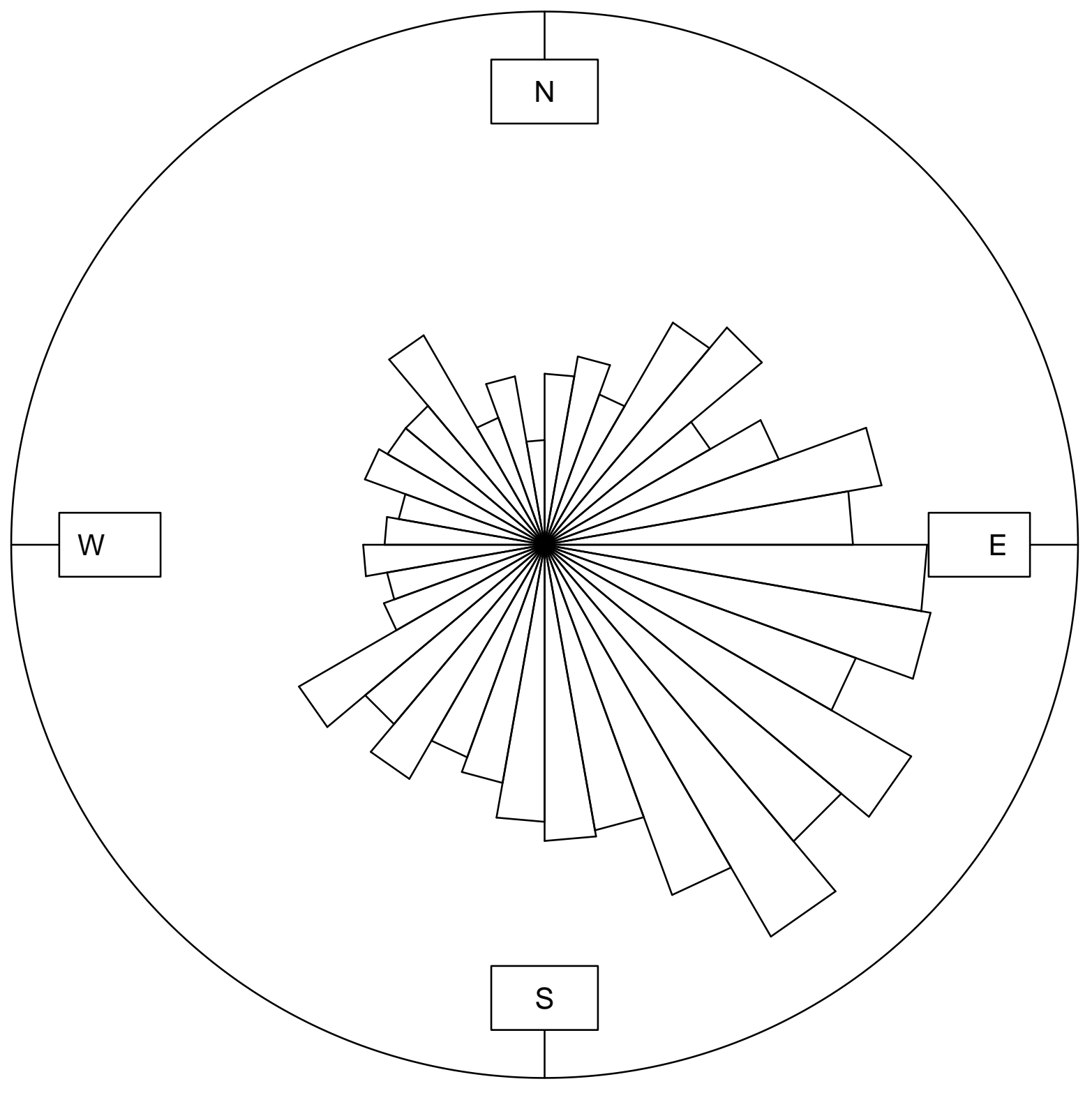


bootstrap 568

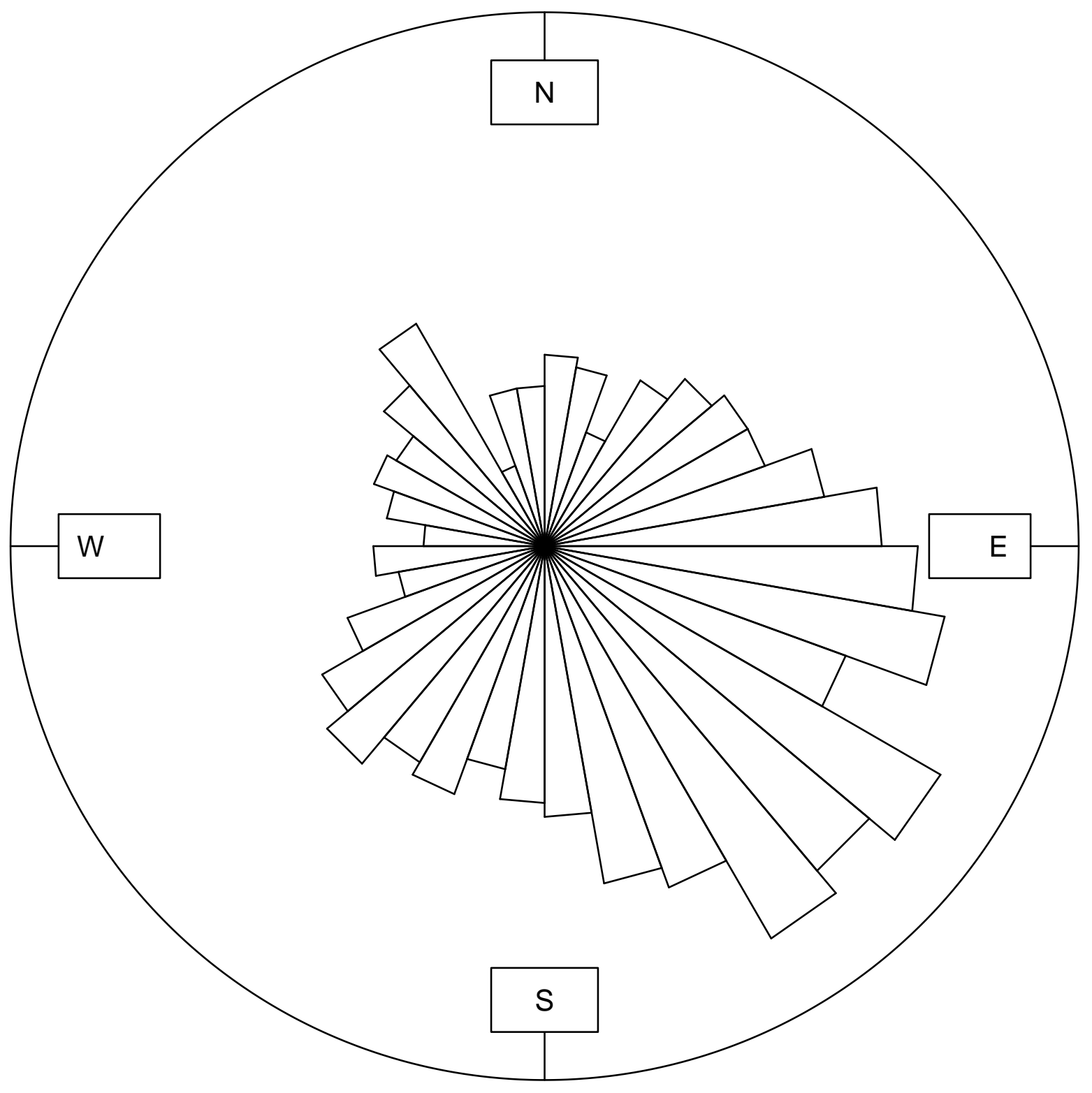


bootstrap 569

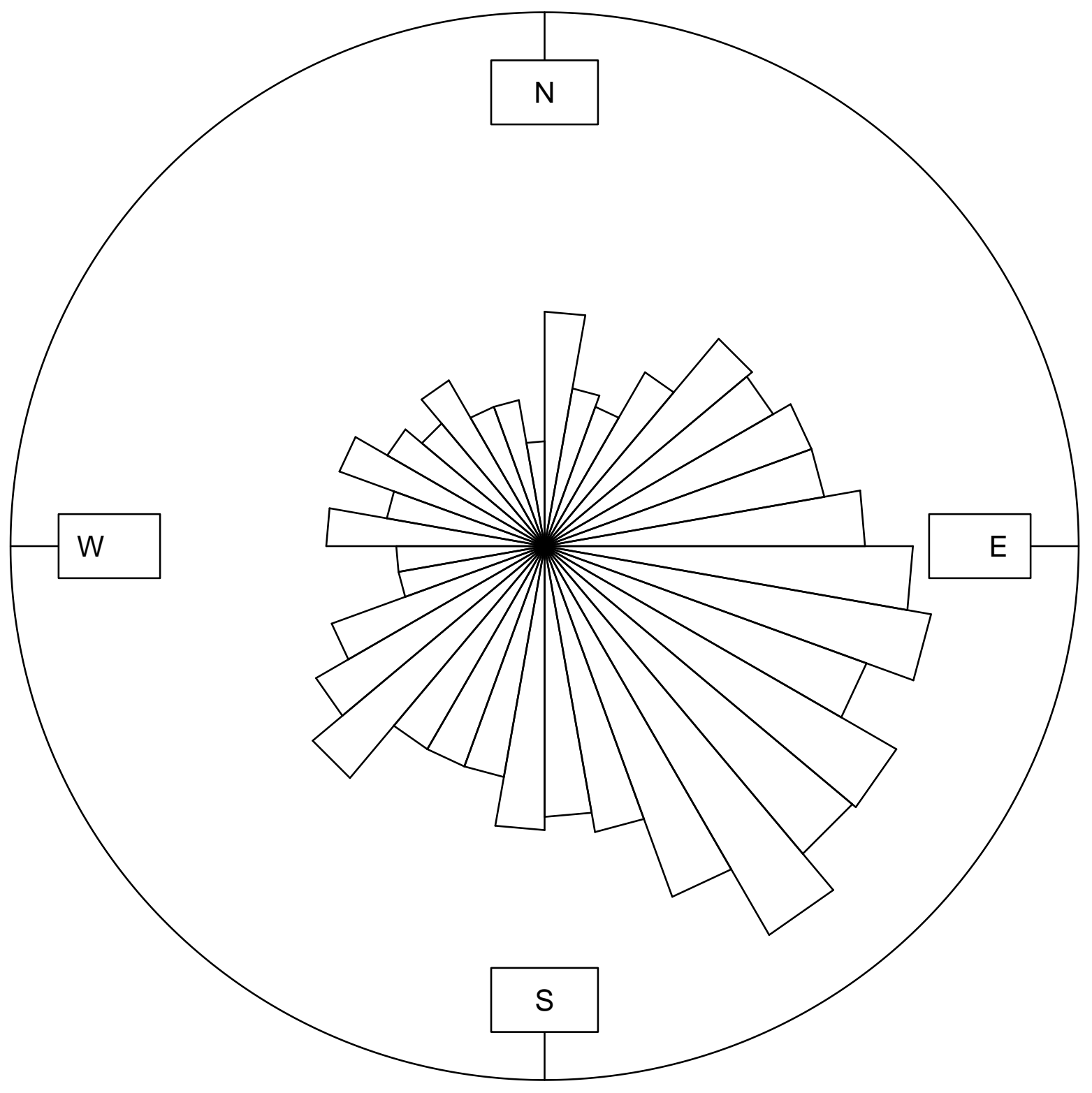


bootstrap 570

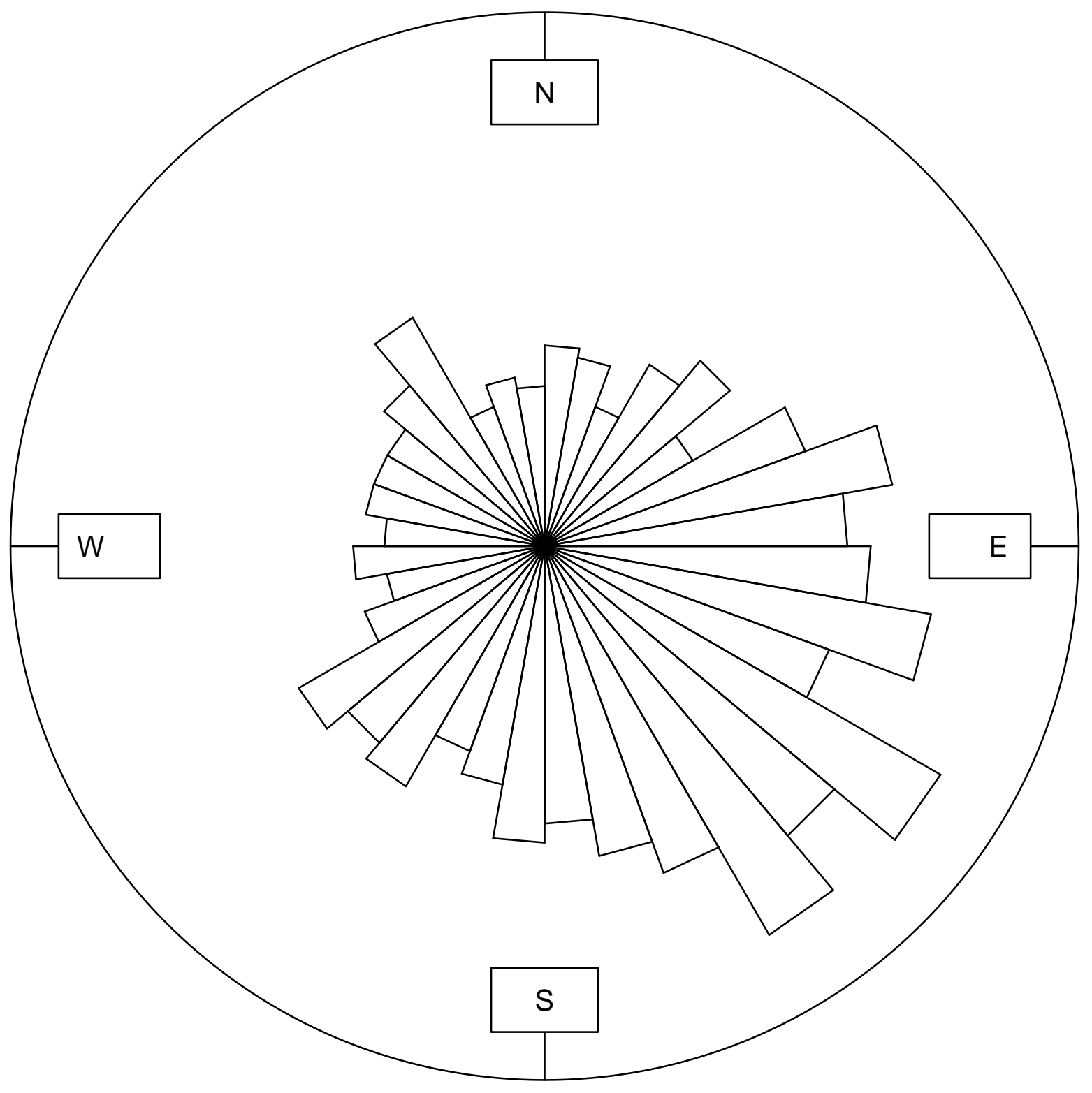


bootstrap 571

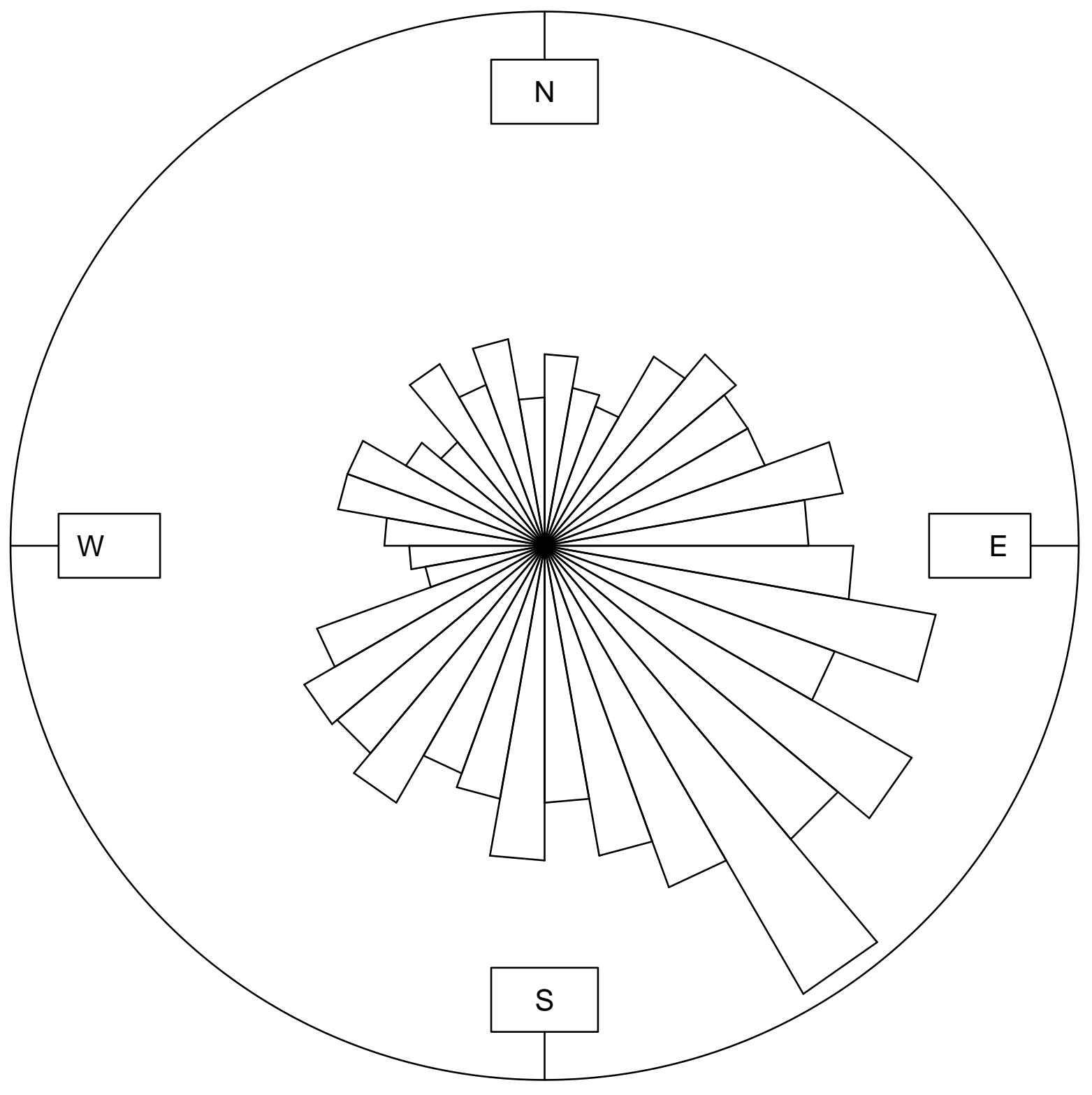


bootstrap 572

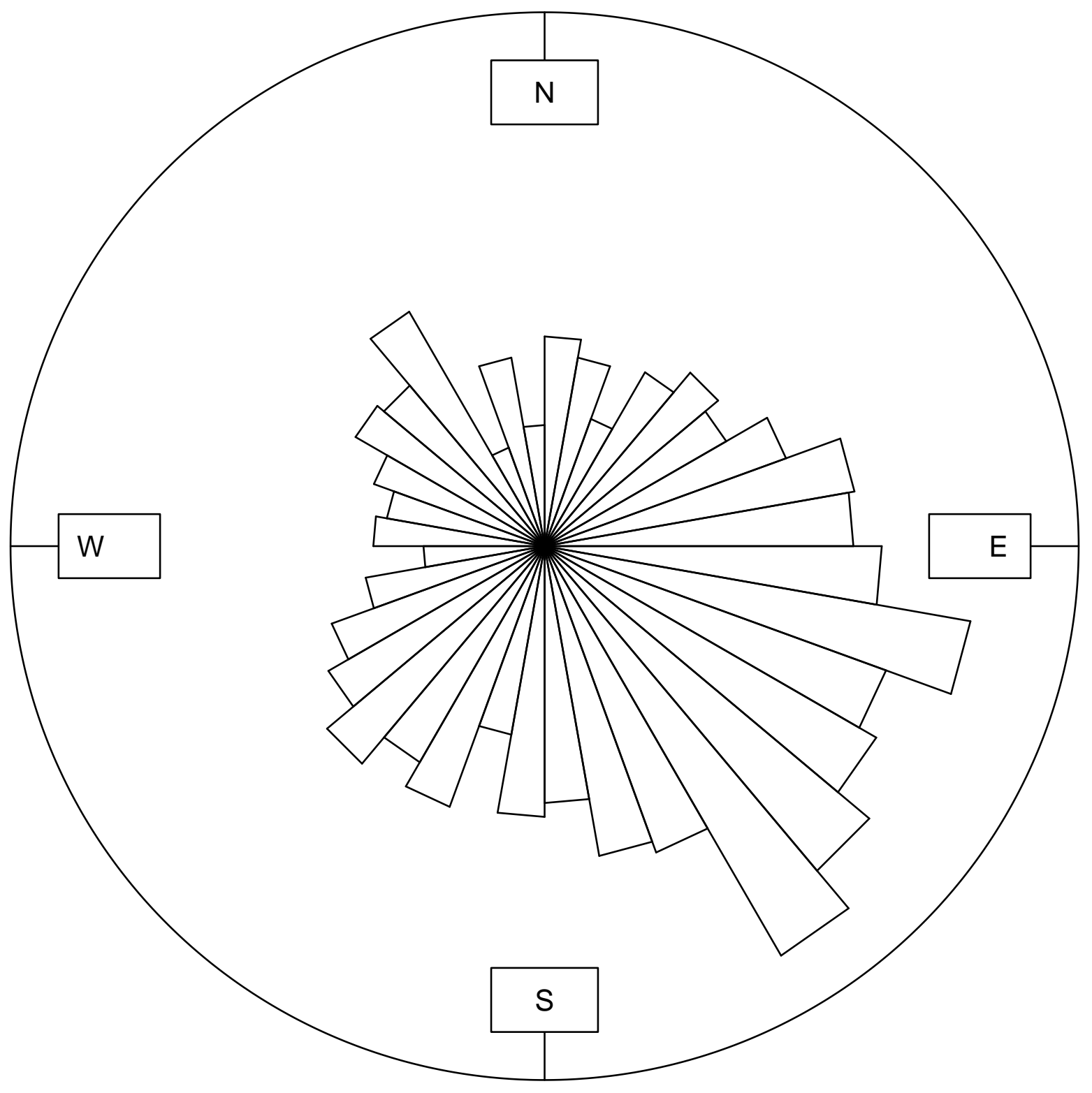


bootstrap 573

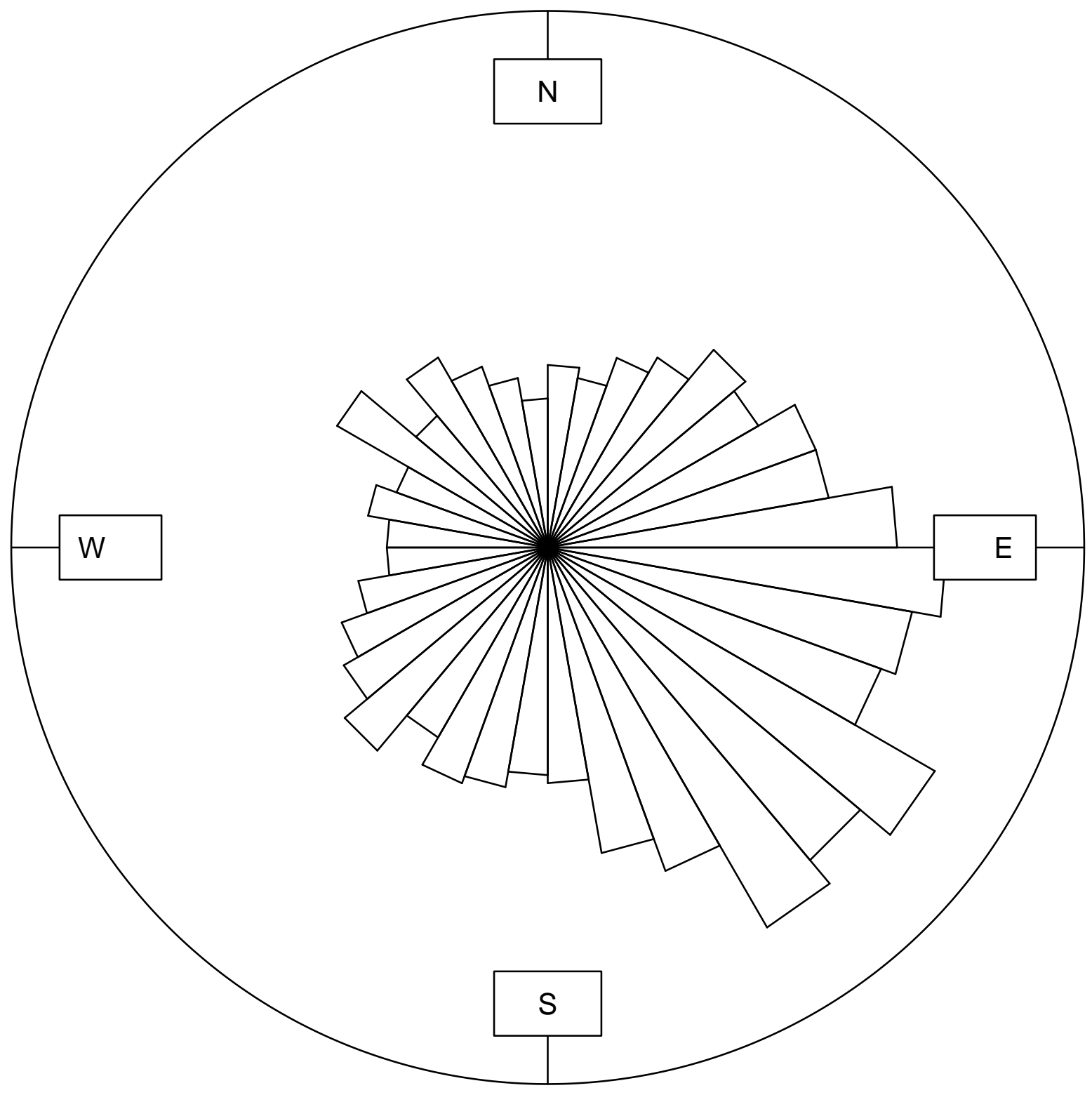


bootstrap 574

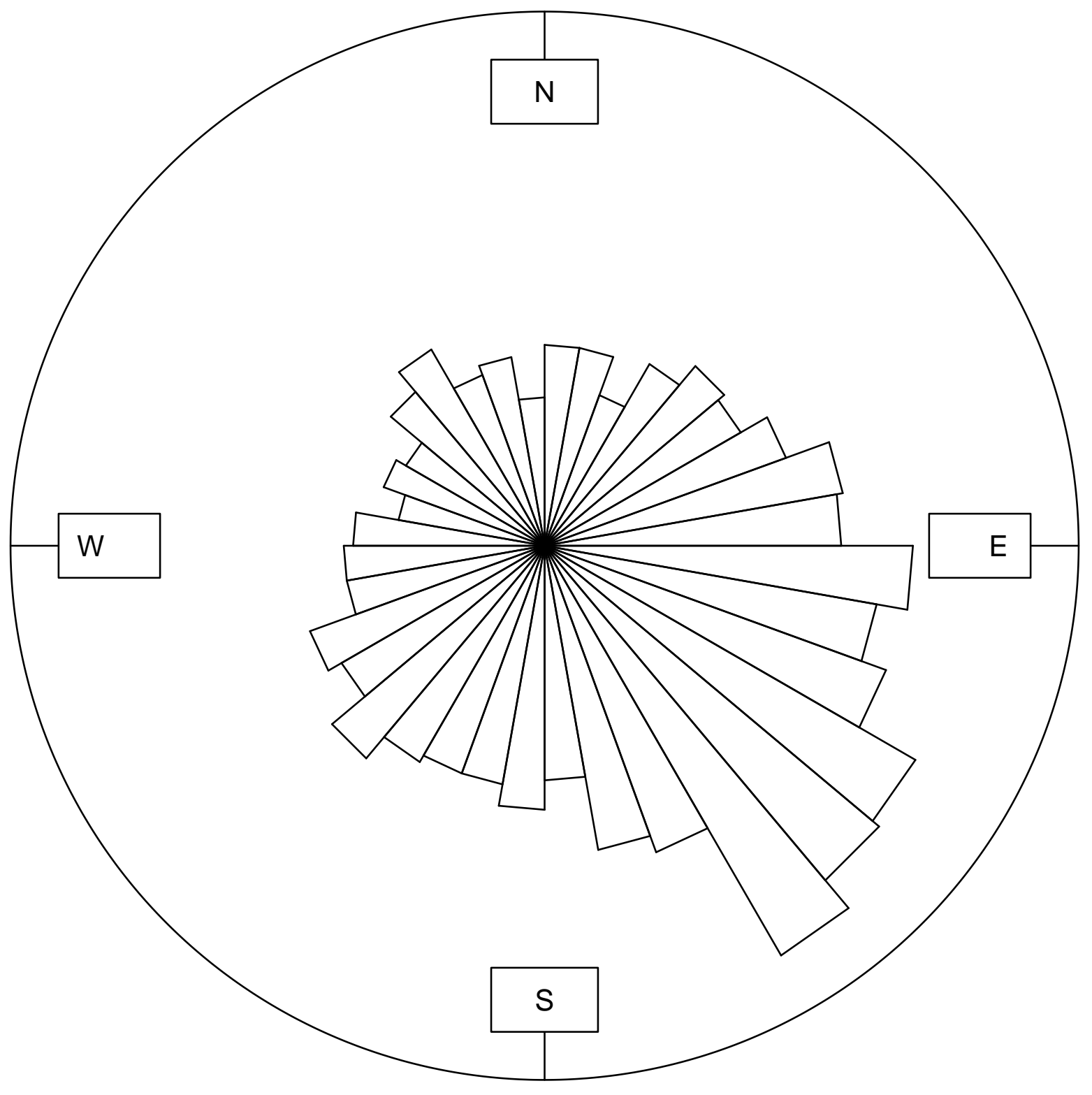




\section{bootstrap 575}

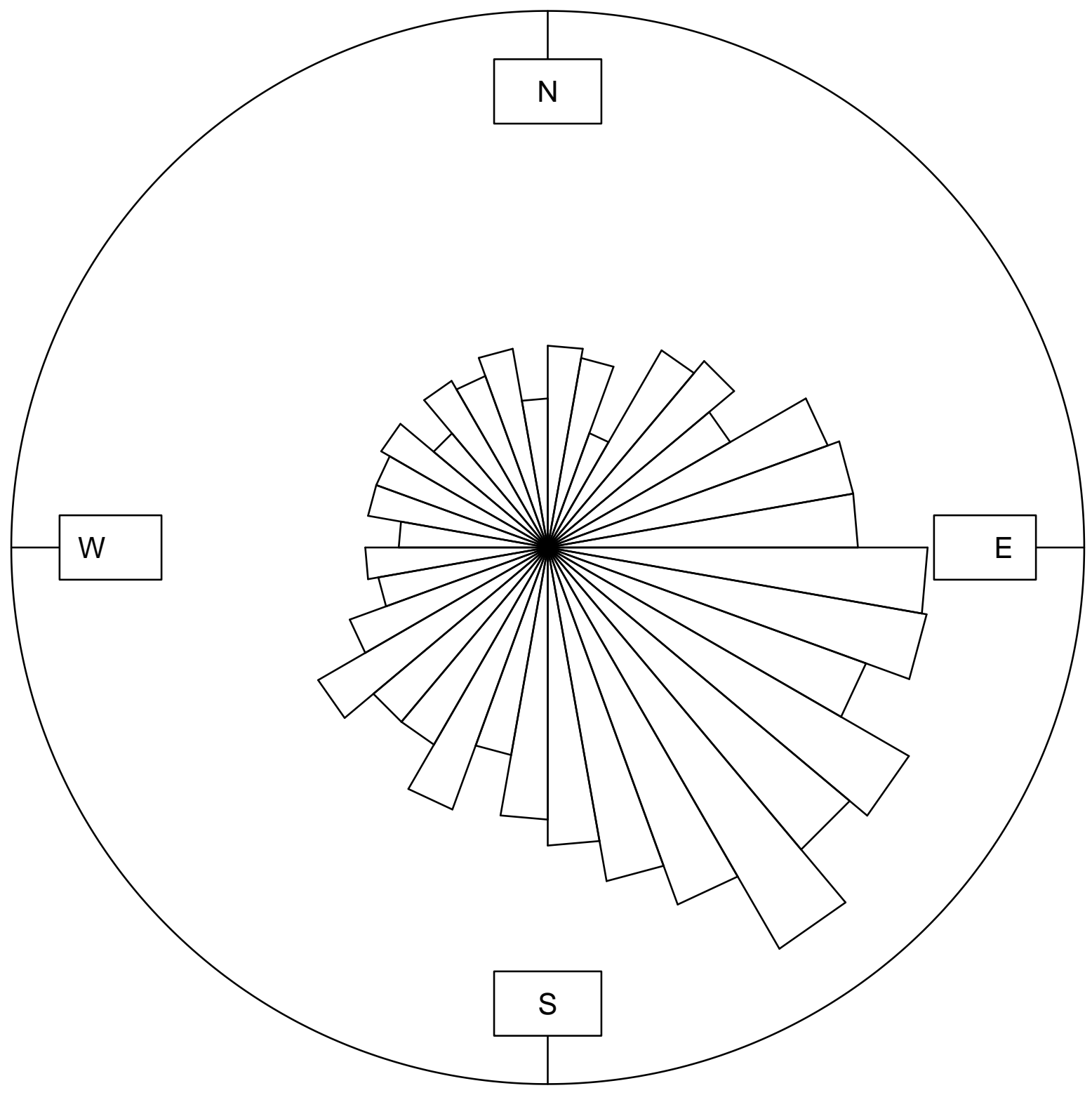


bootstrap 576

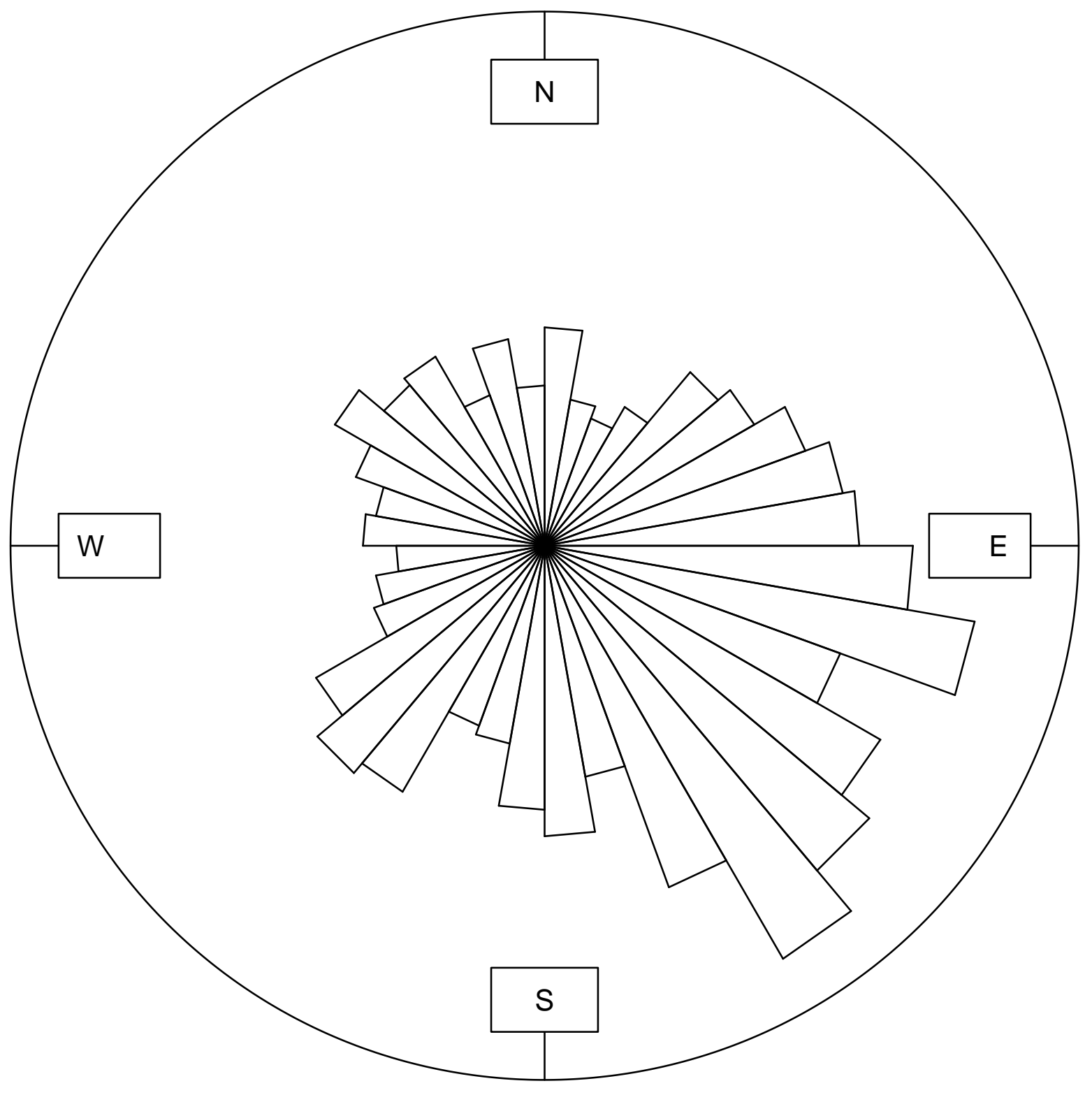


bootstrap 577

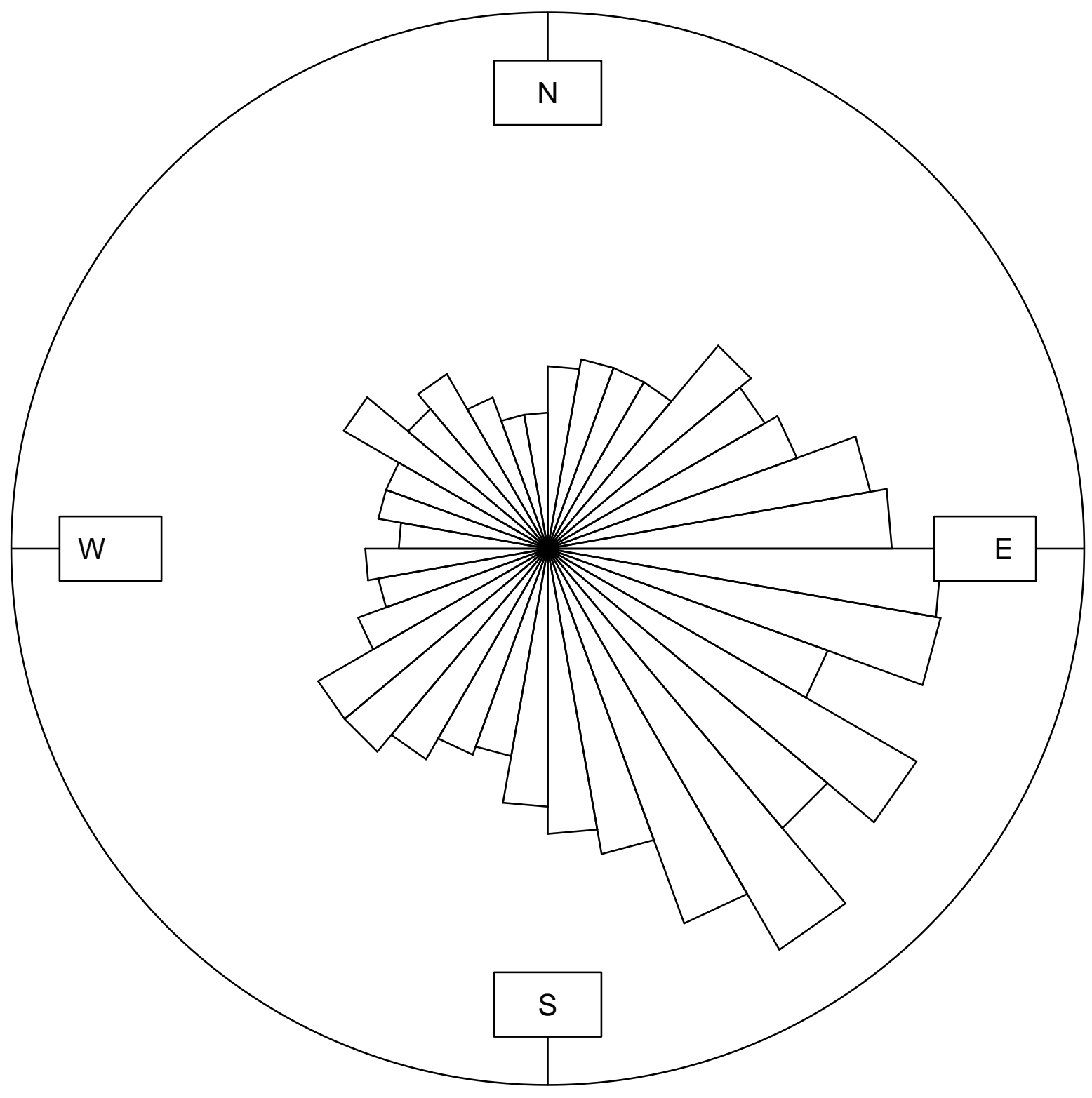


bootstrap 578

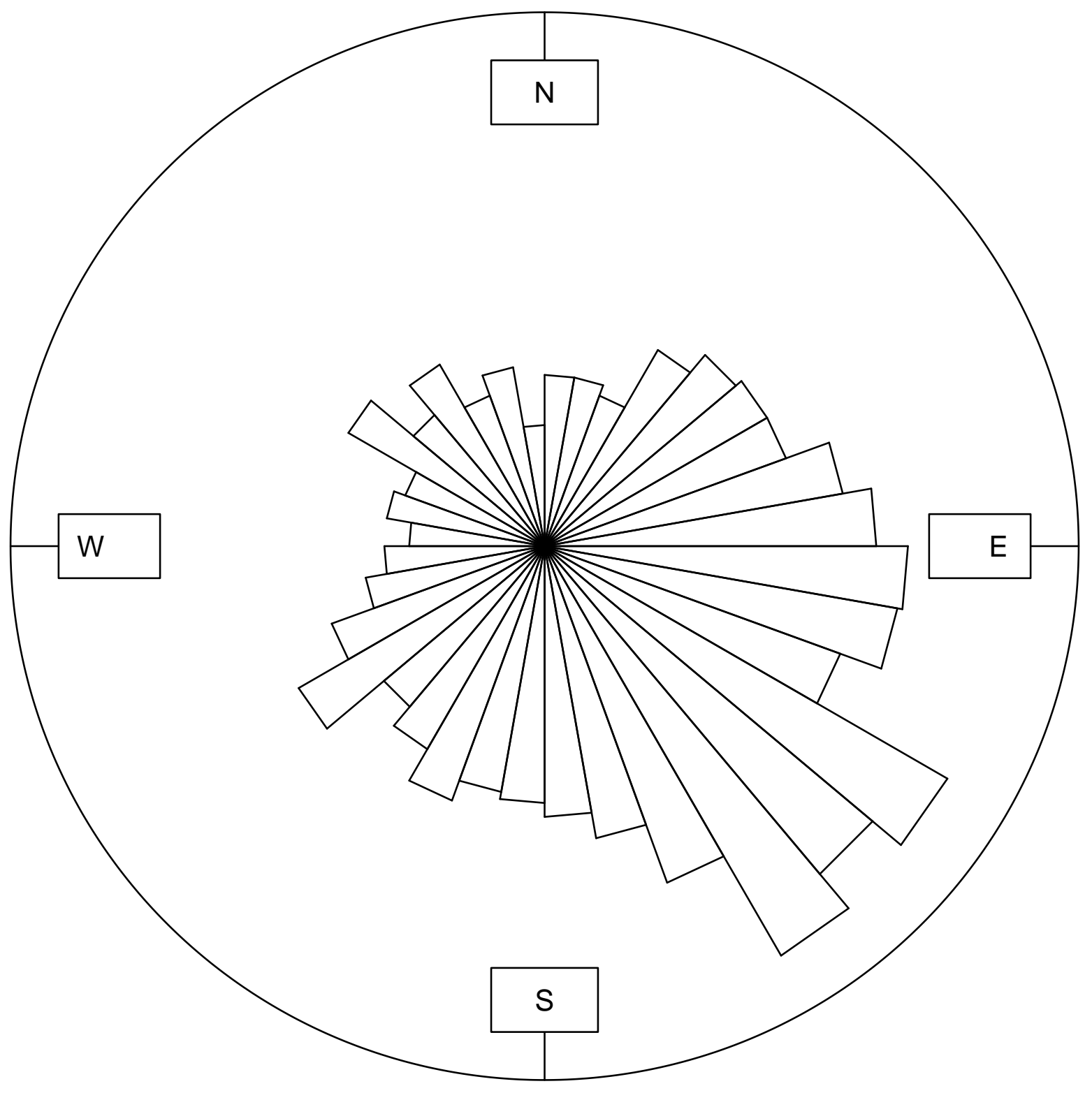


bootstrap 579

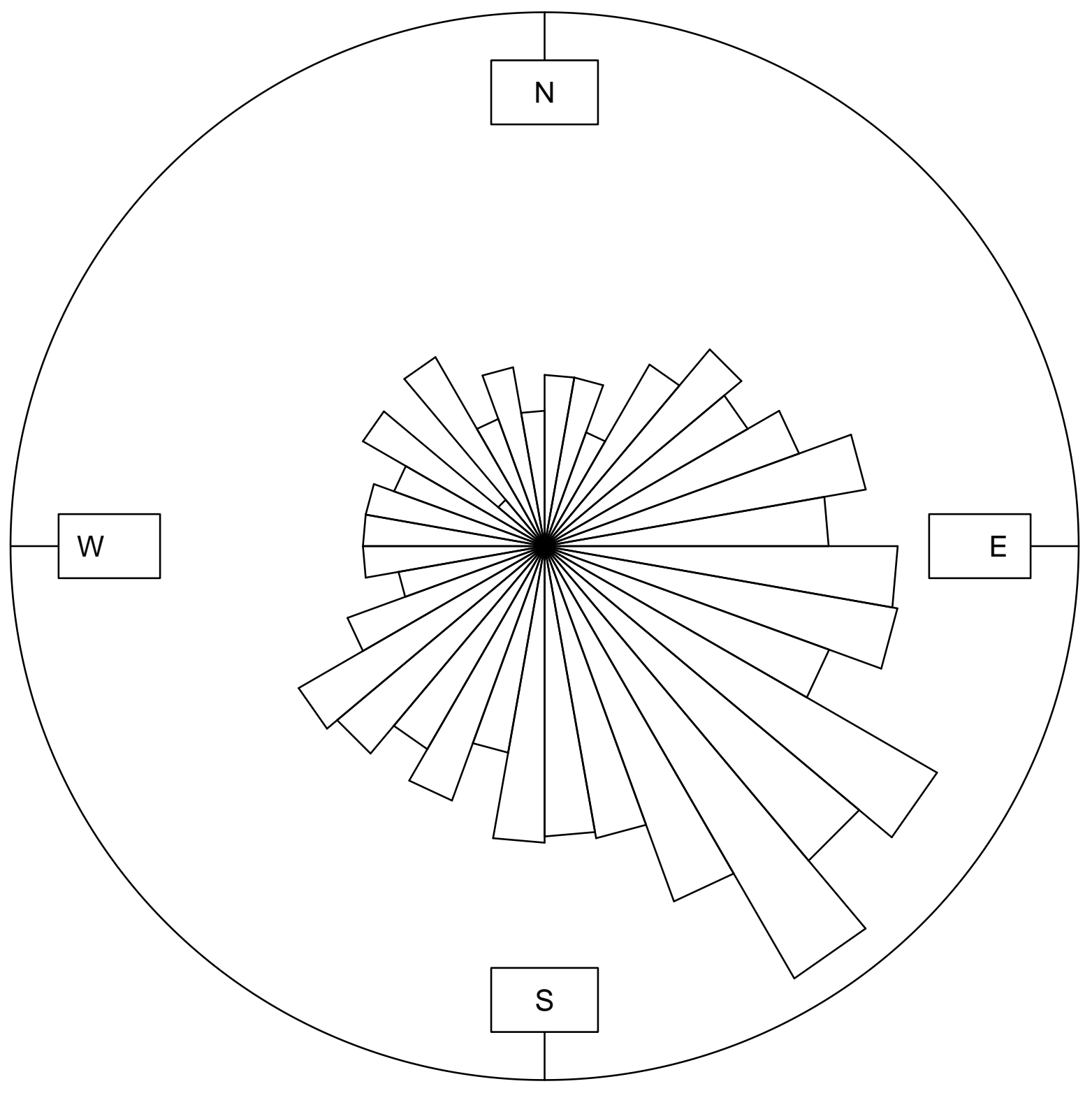


bootstrap 580

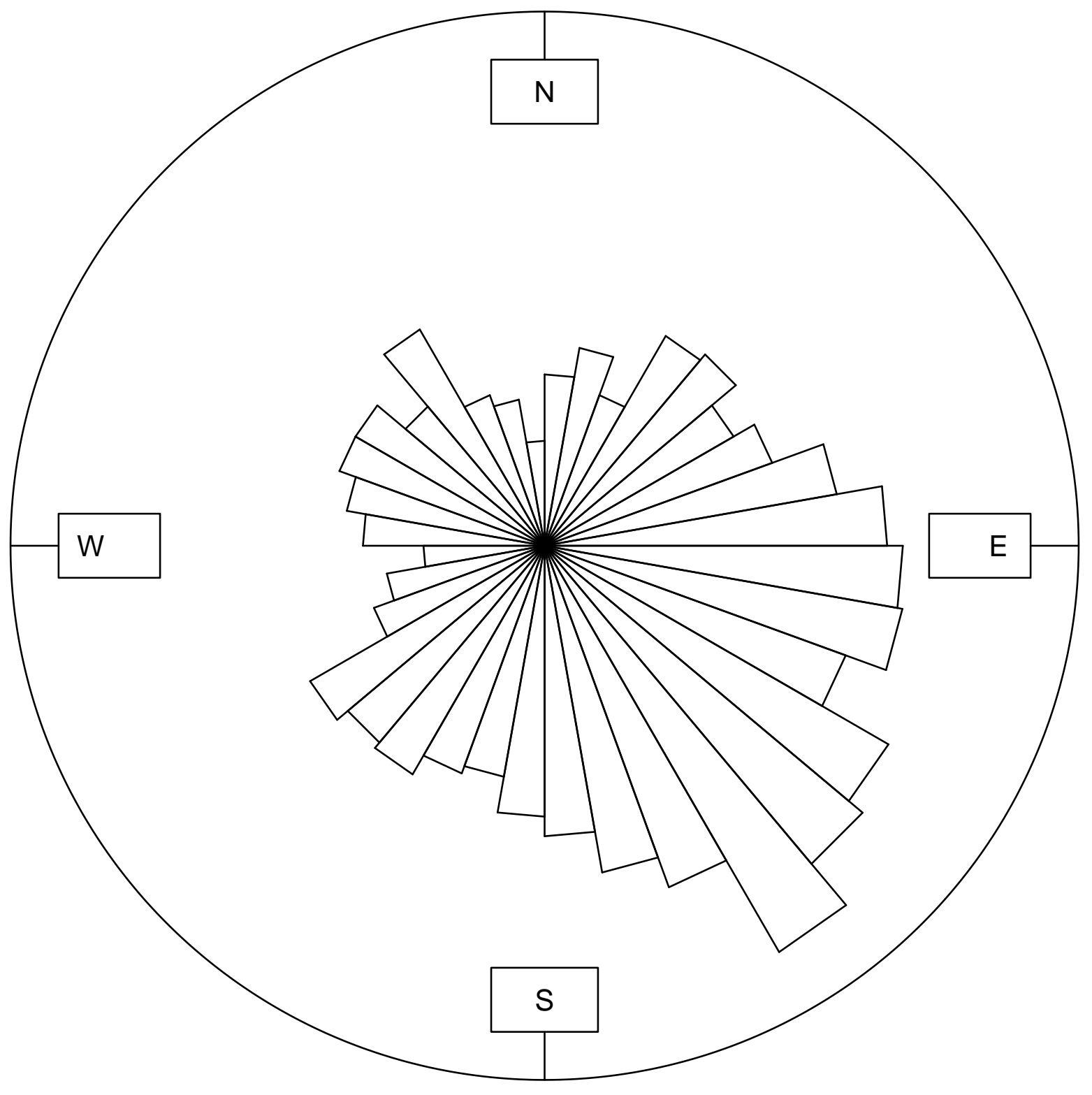




\section{bootstrap 581}

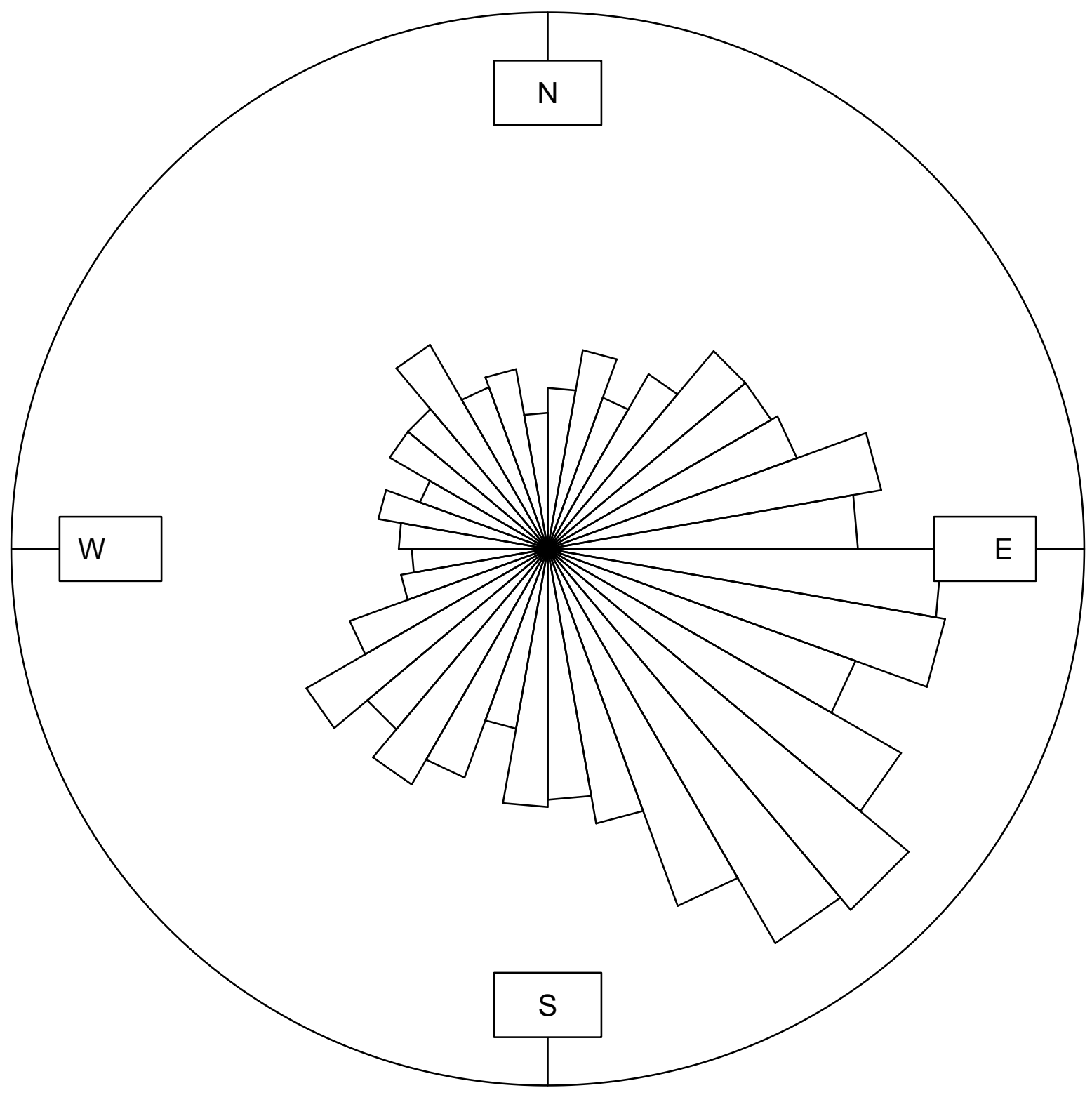


bootstrap 582

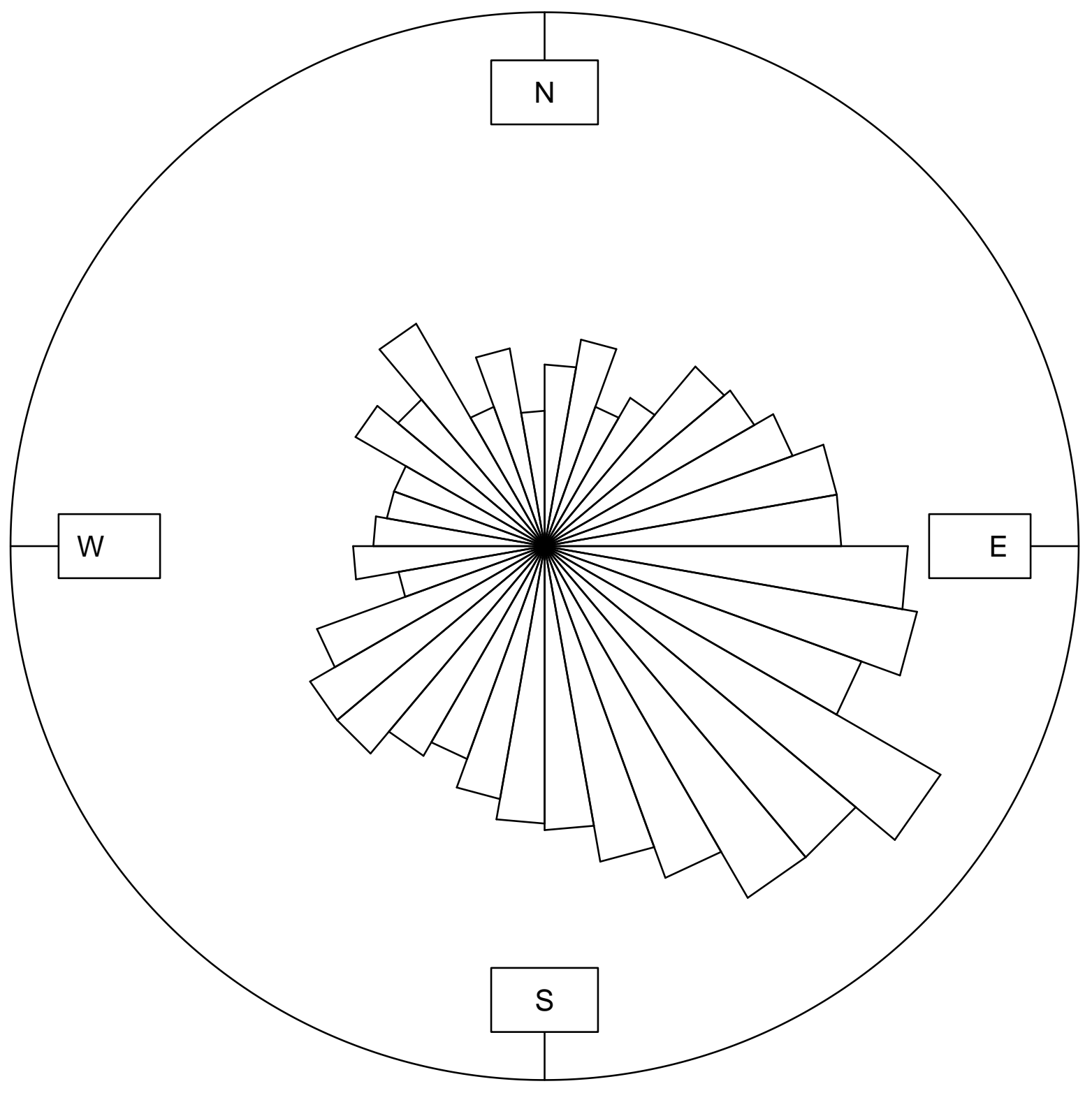




\section{bootstrap 583}

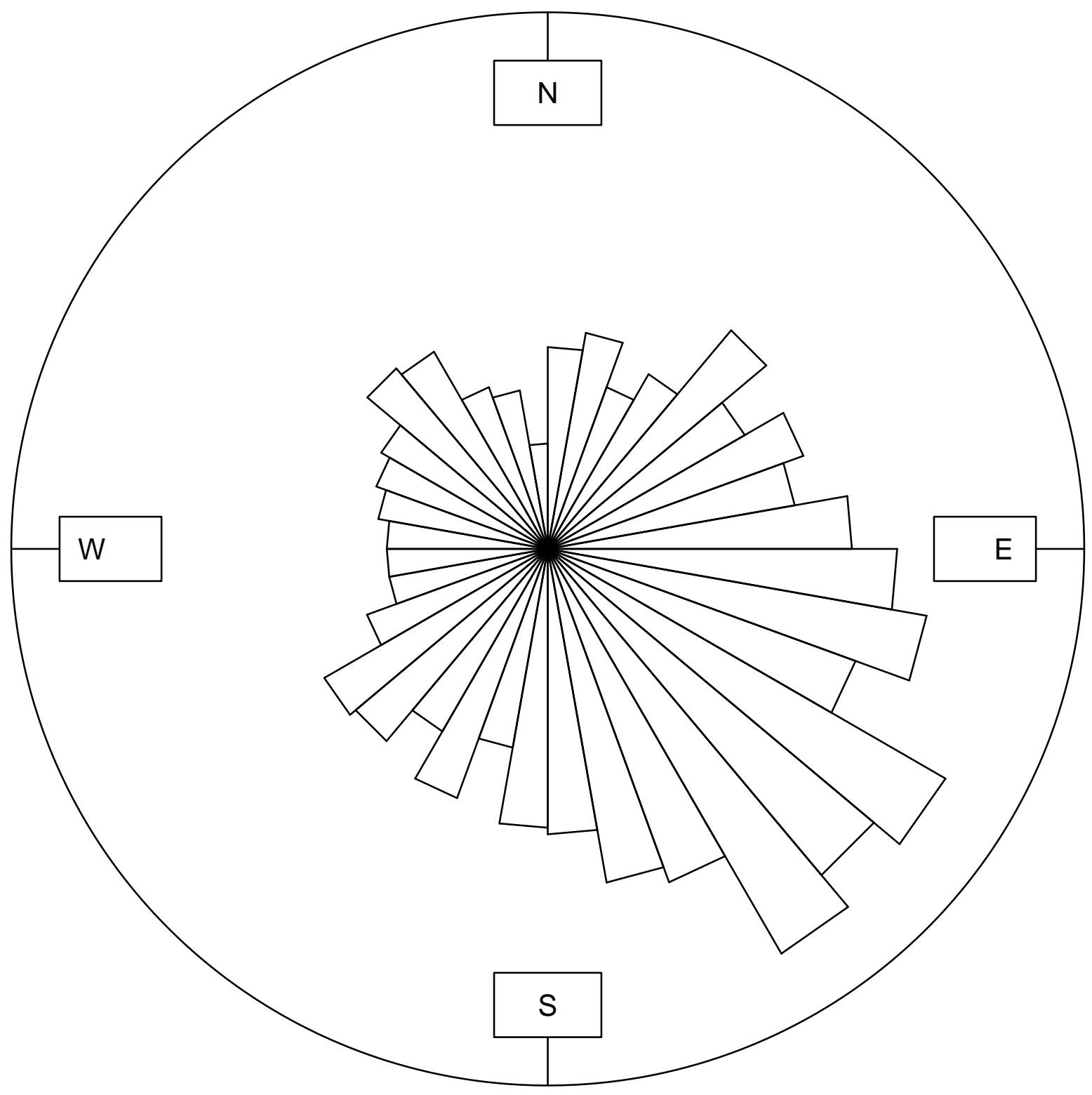




\section{bootstrap 584}

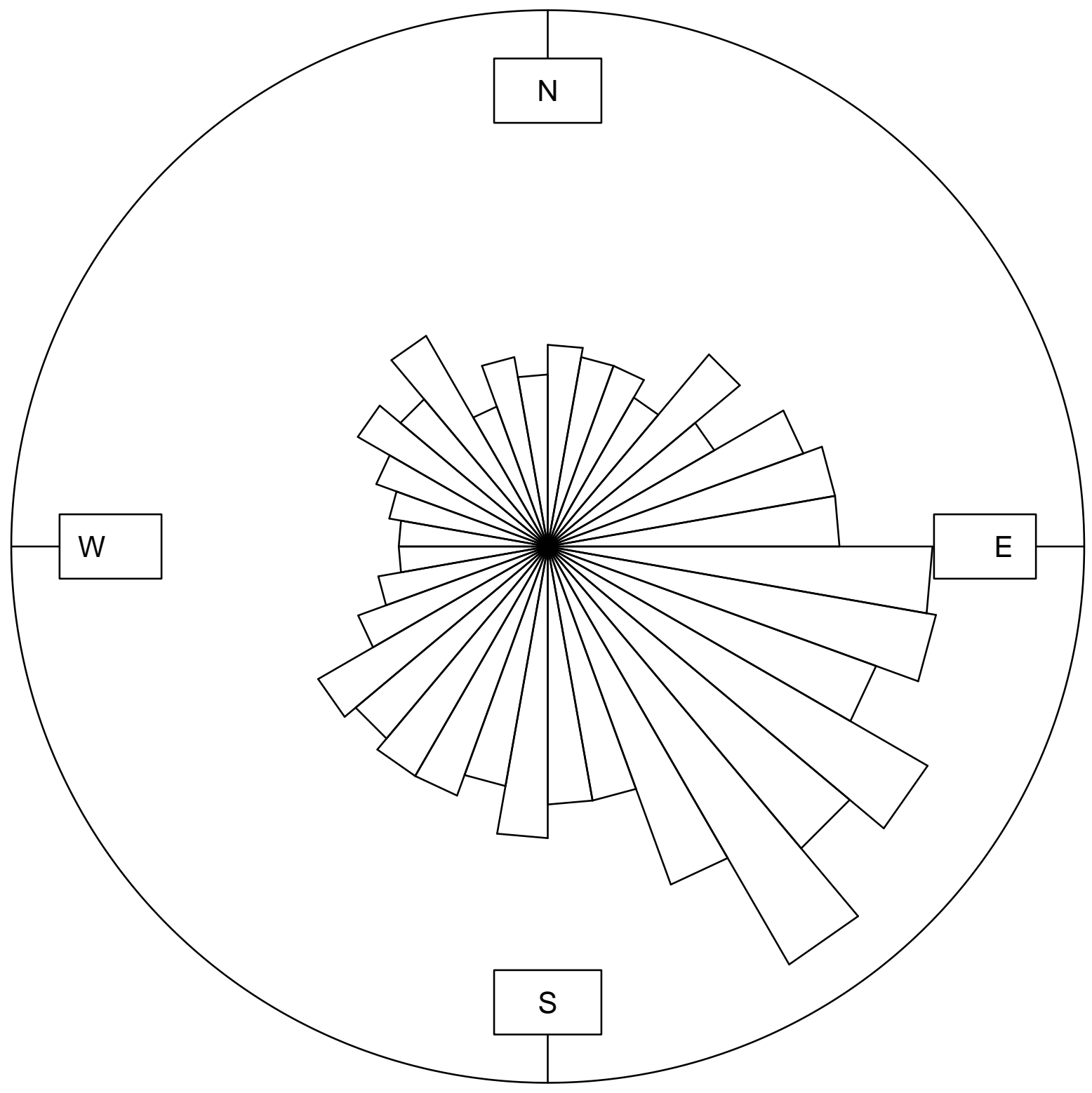


bootstrap 585

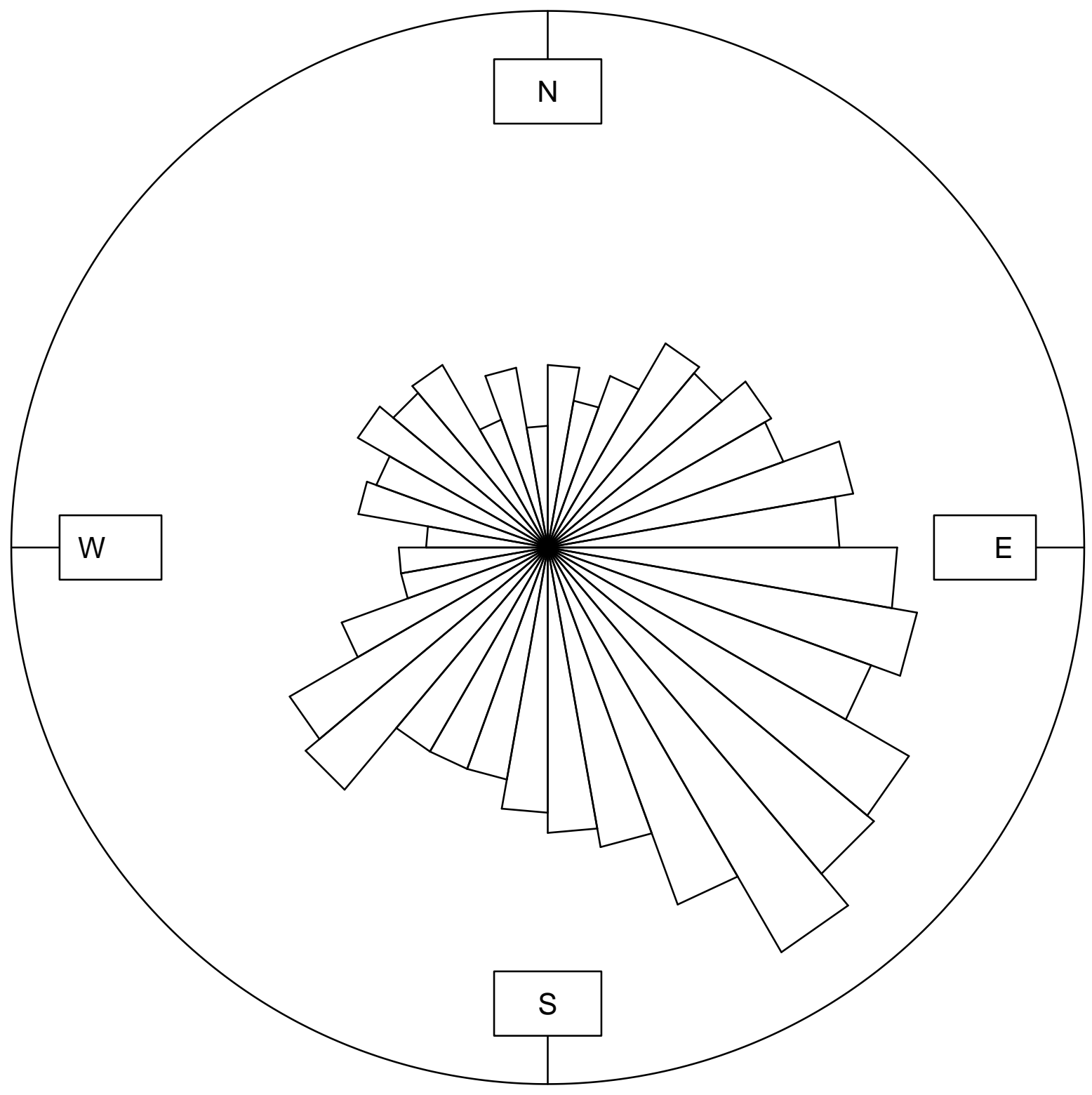


bootstrap 586

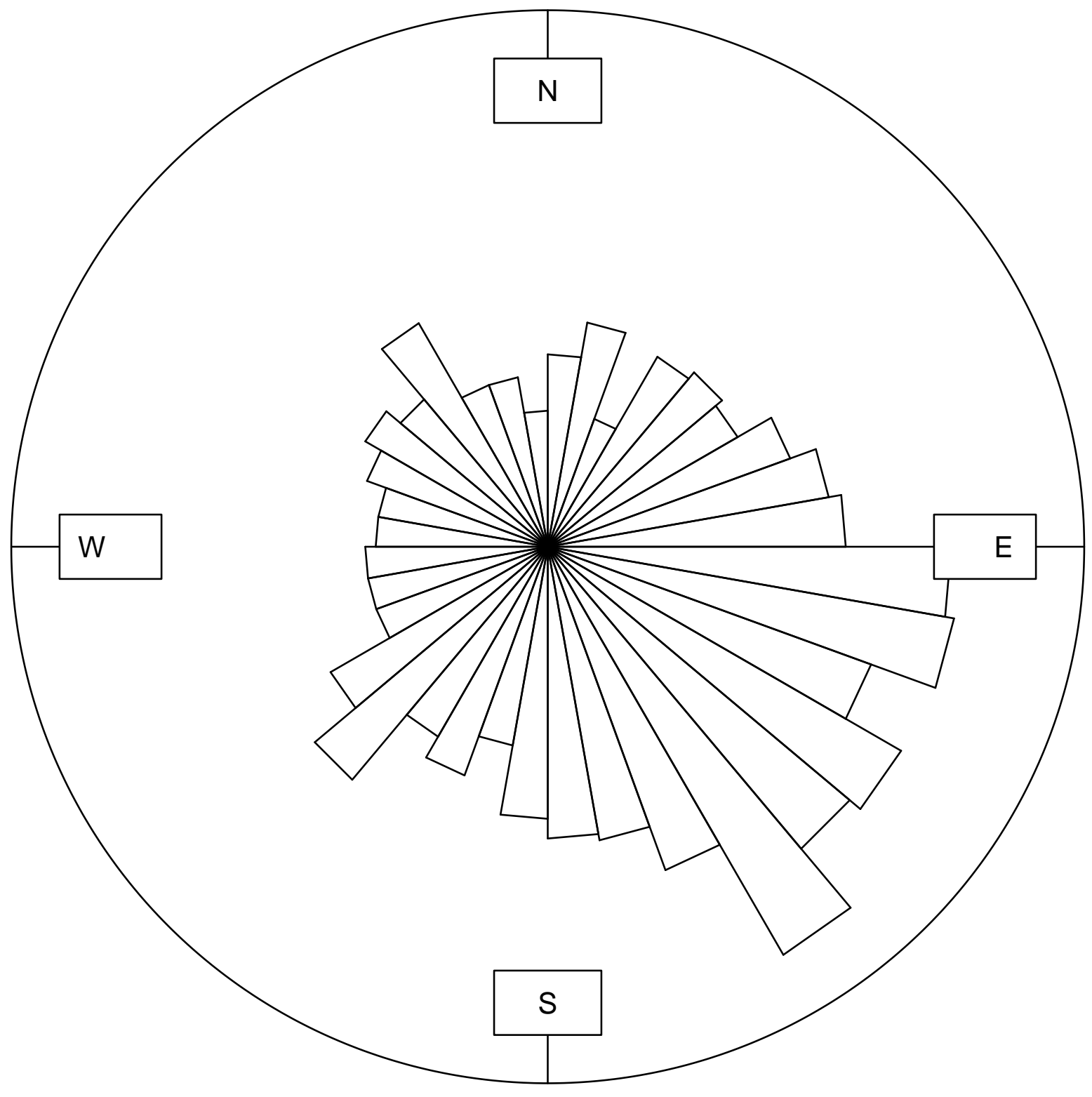


bootstrap 587

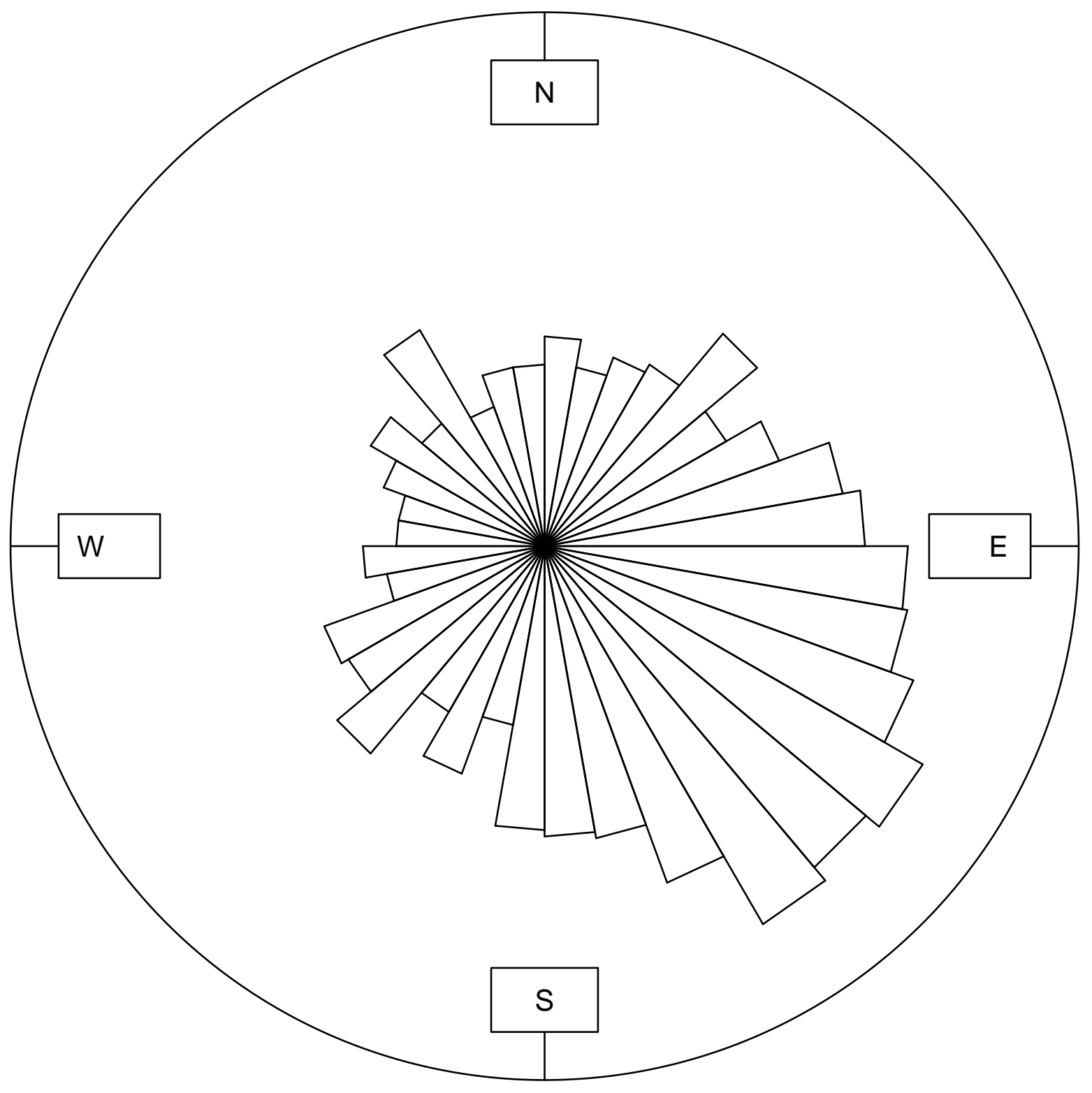


bootstrap 588

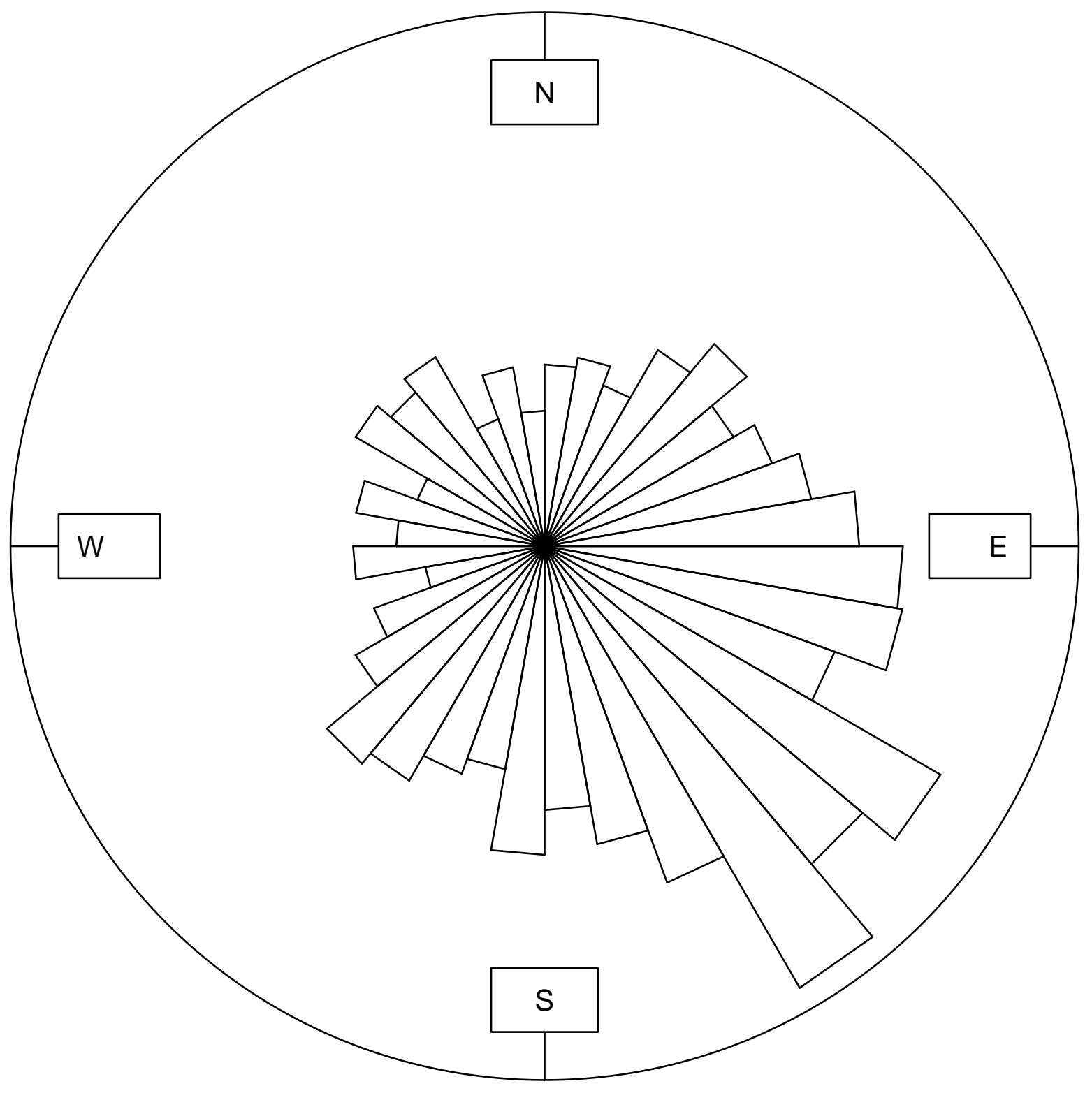


bootstrap 589

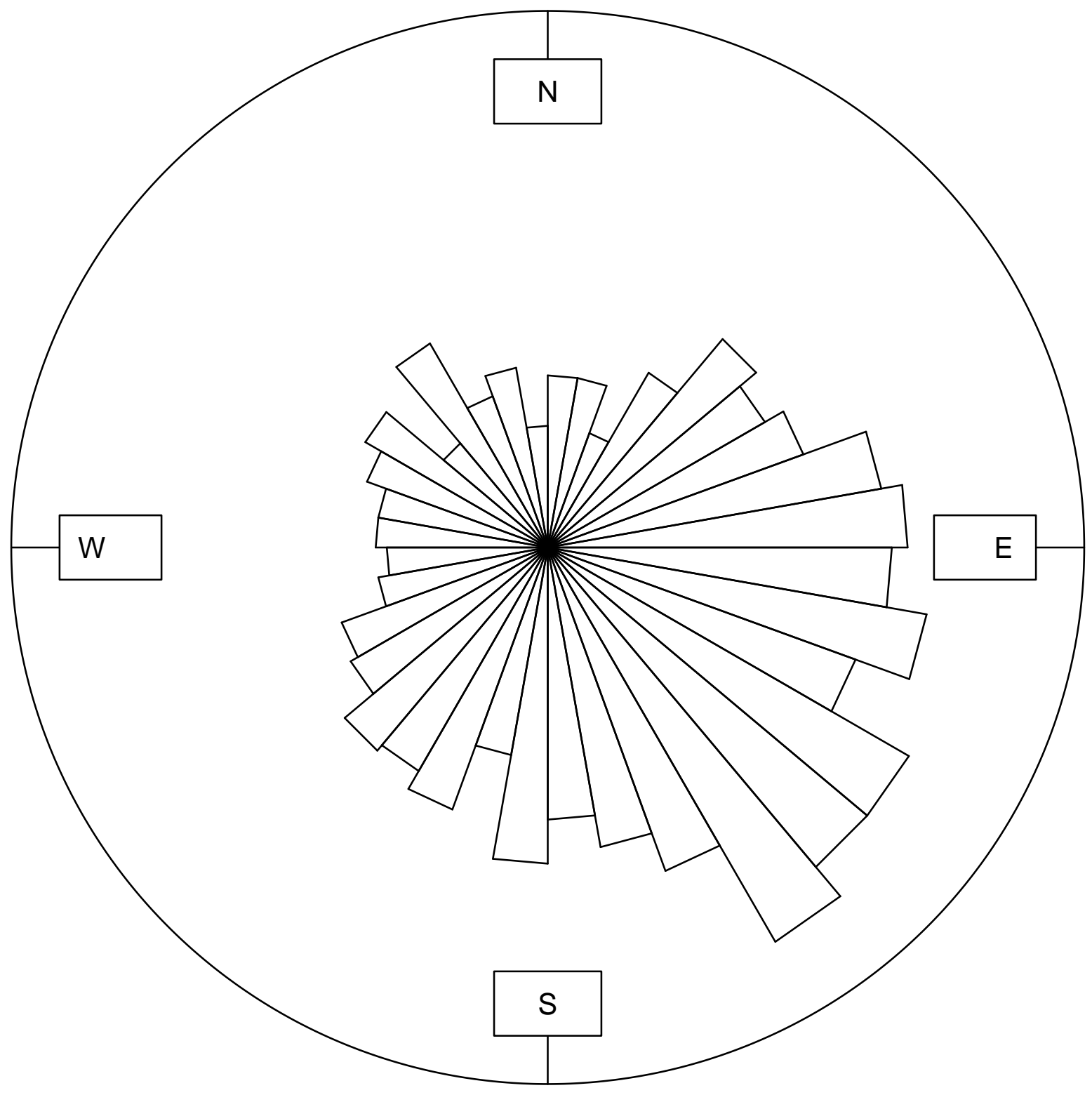


bootstrap 590

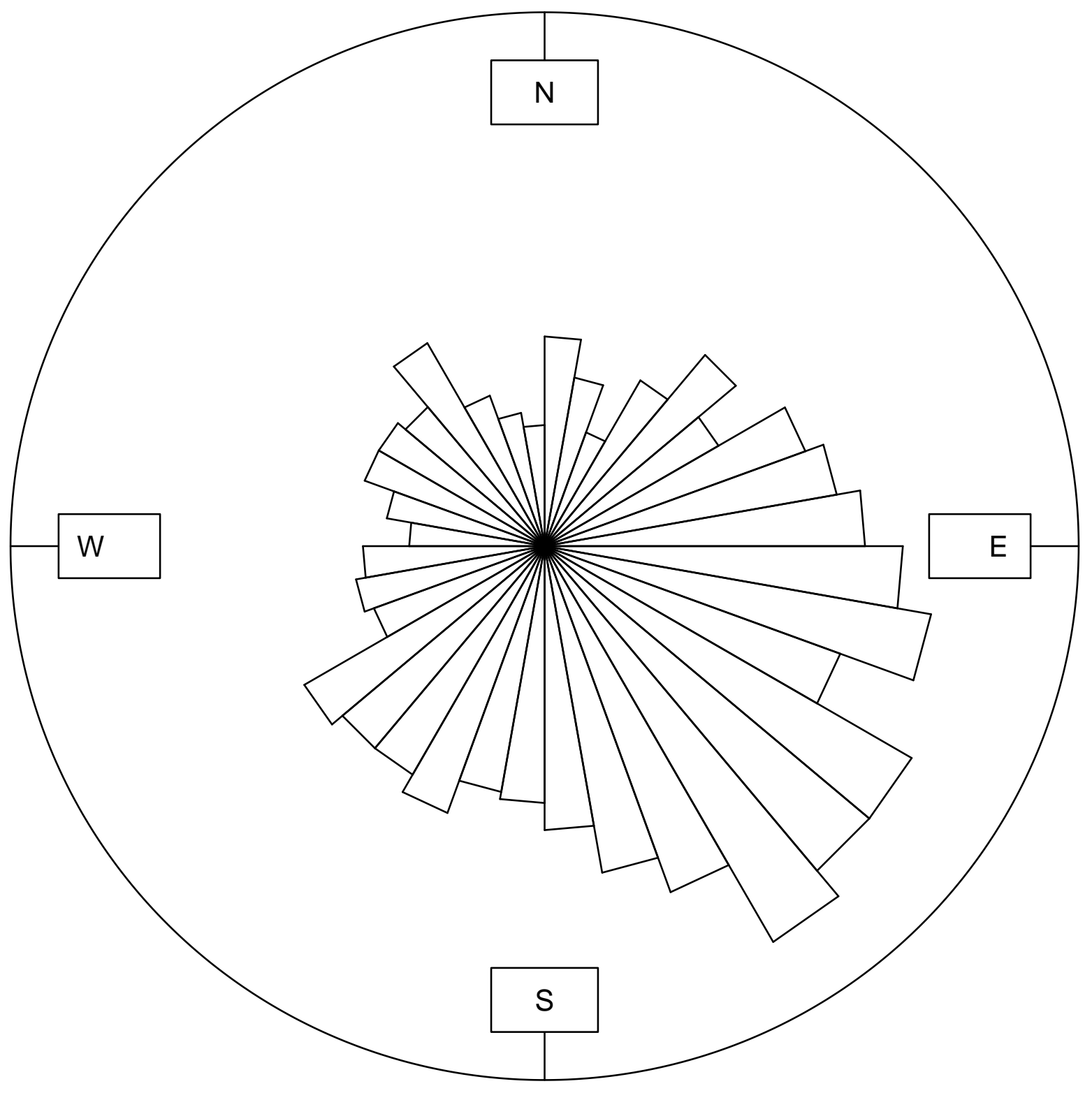


bootstrap 591

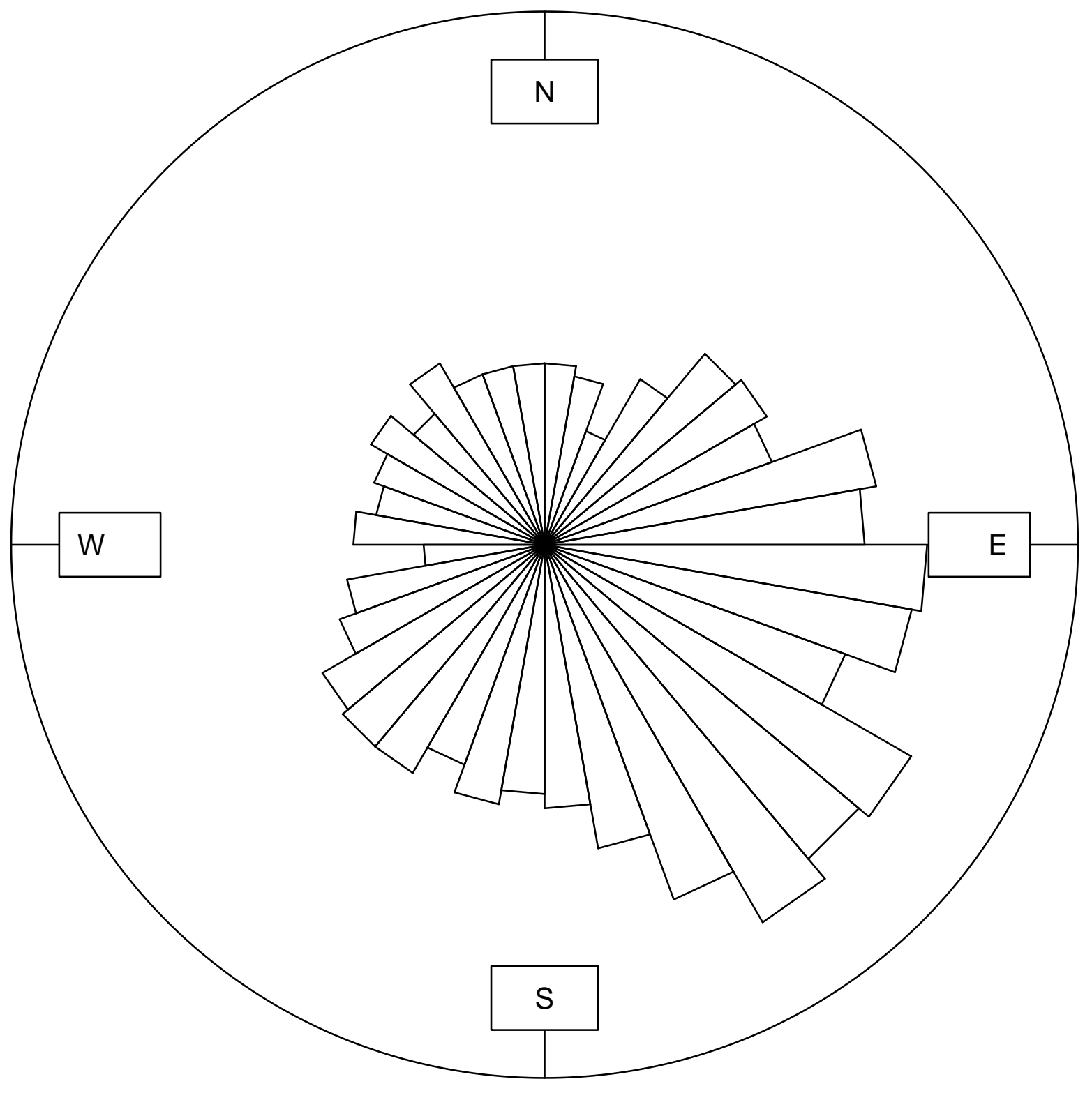


bootstrap 592

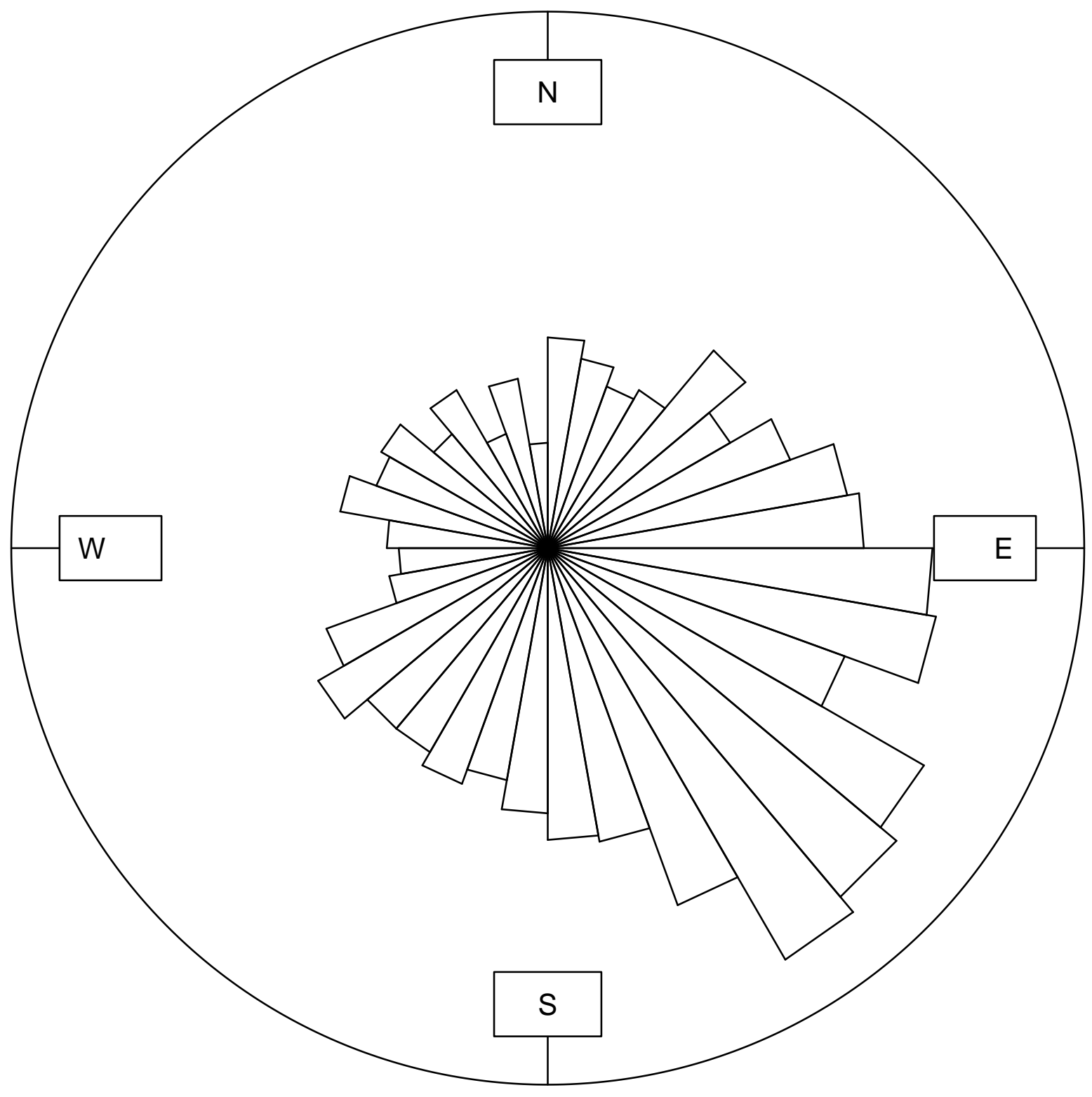




\section{bootstrap 593}

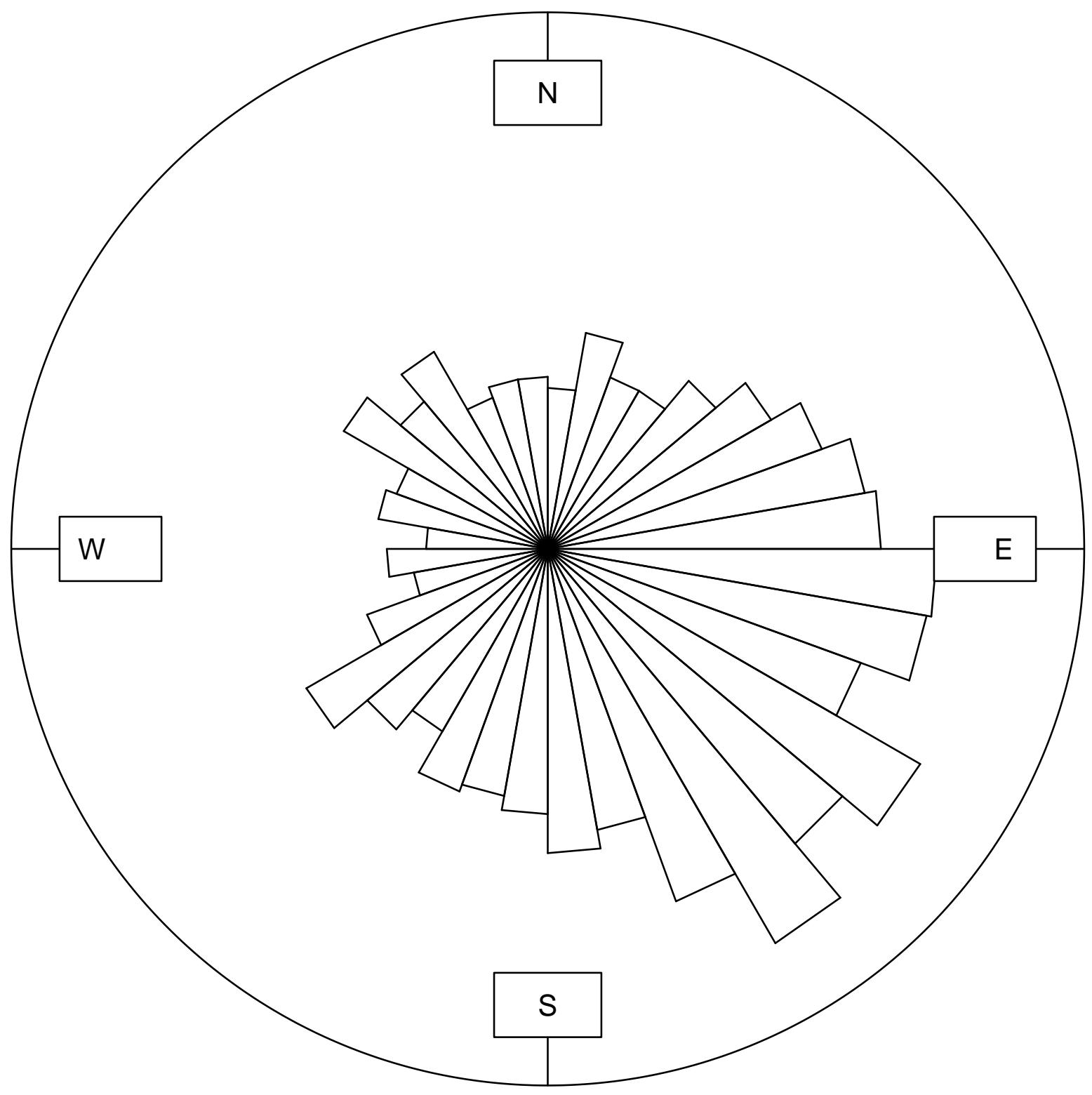


bootstrap 594

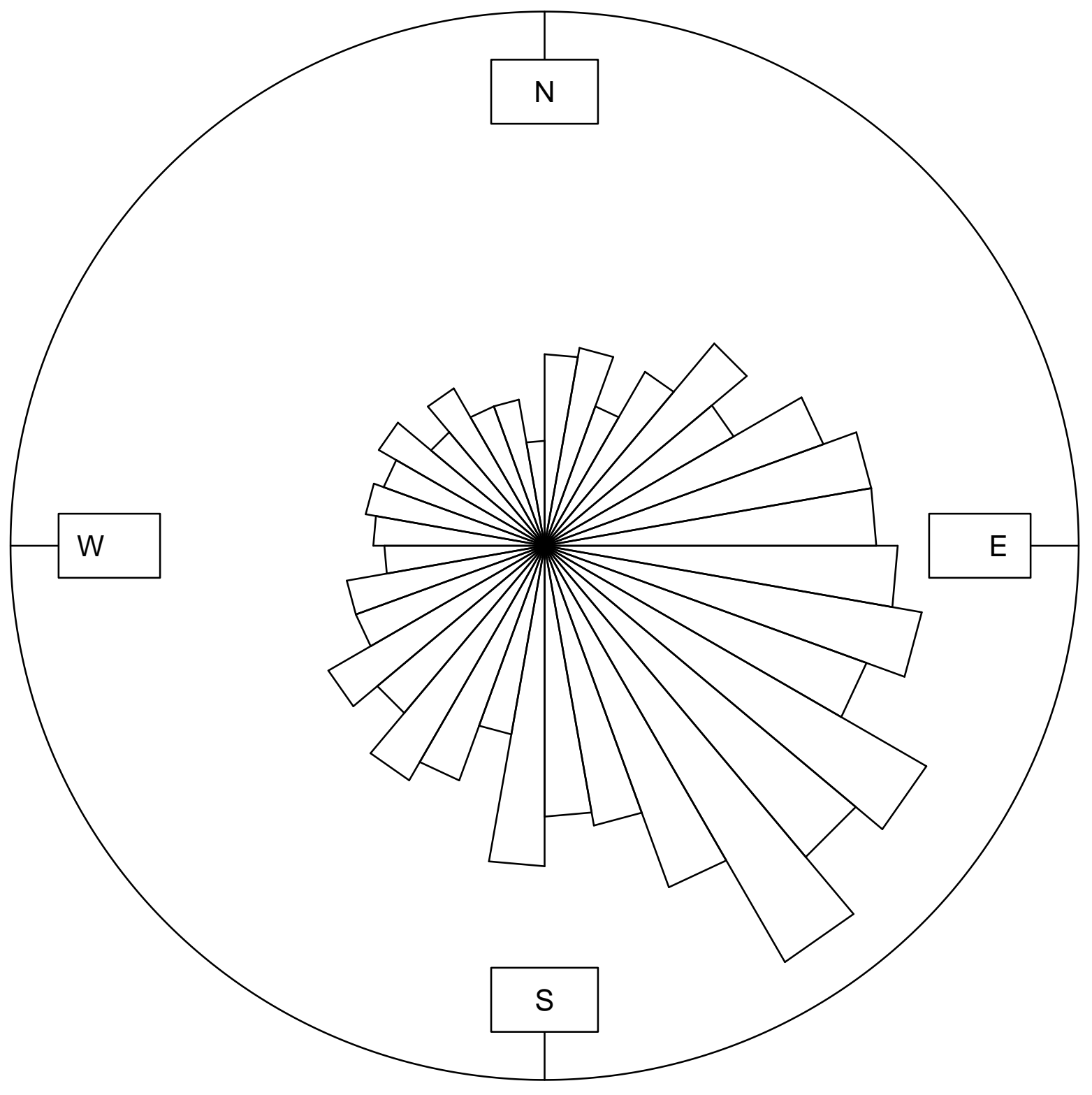


bootstrap 595

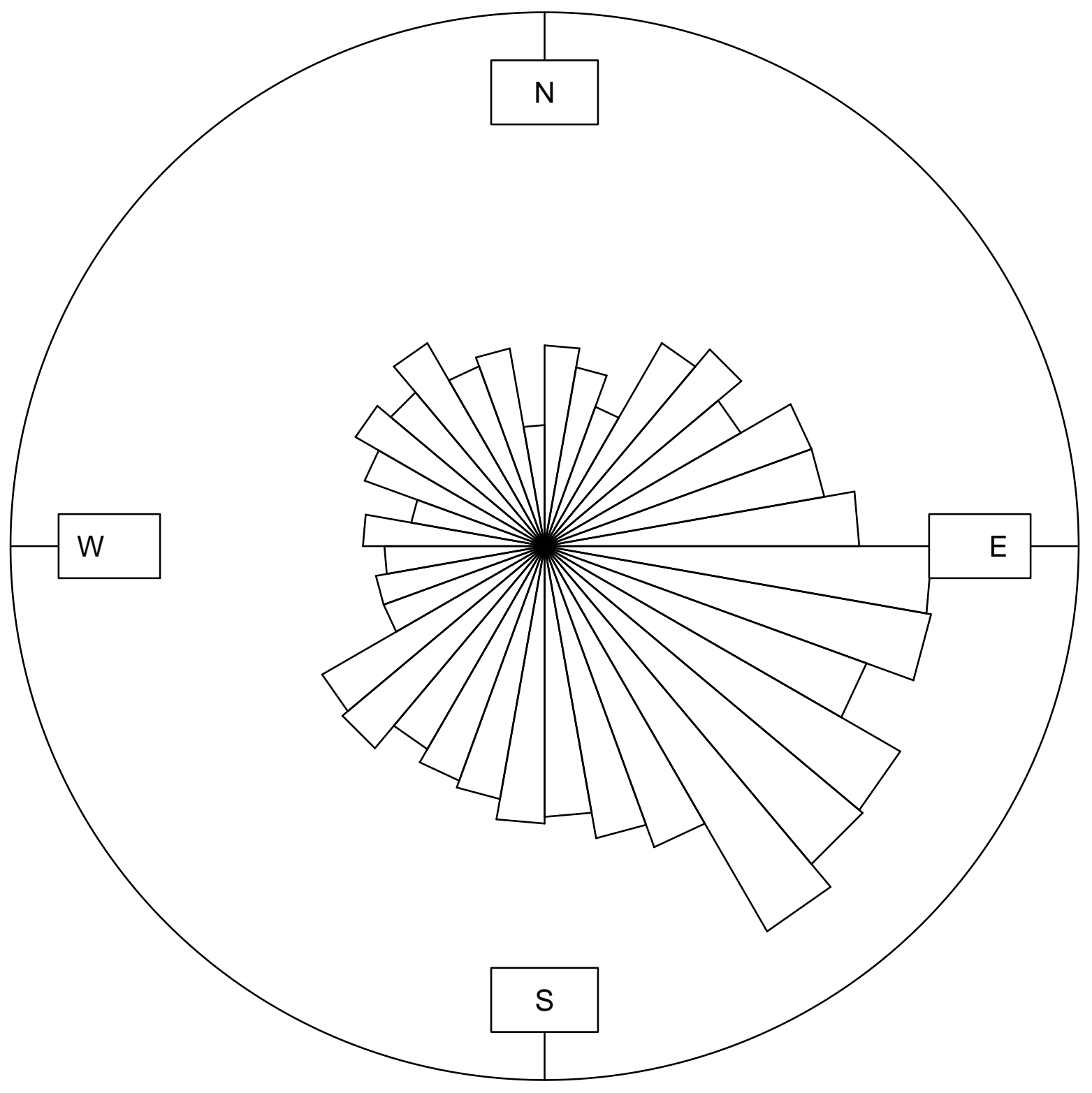


bootstrap 596

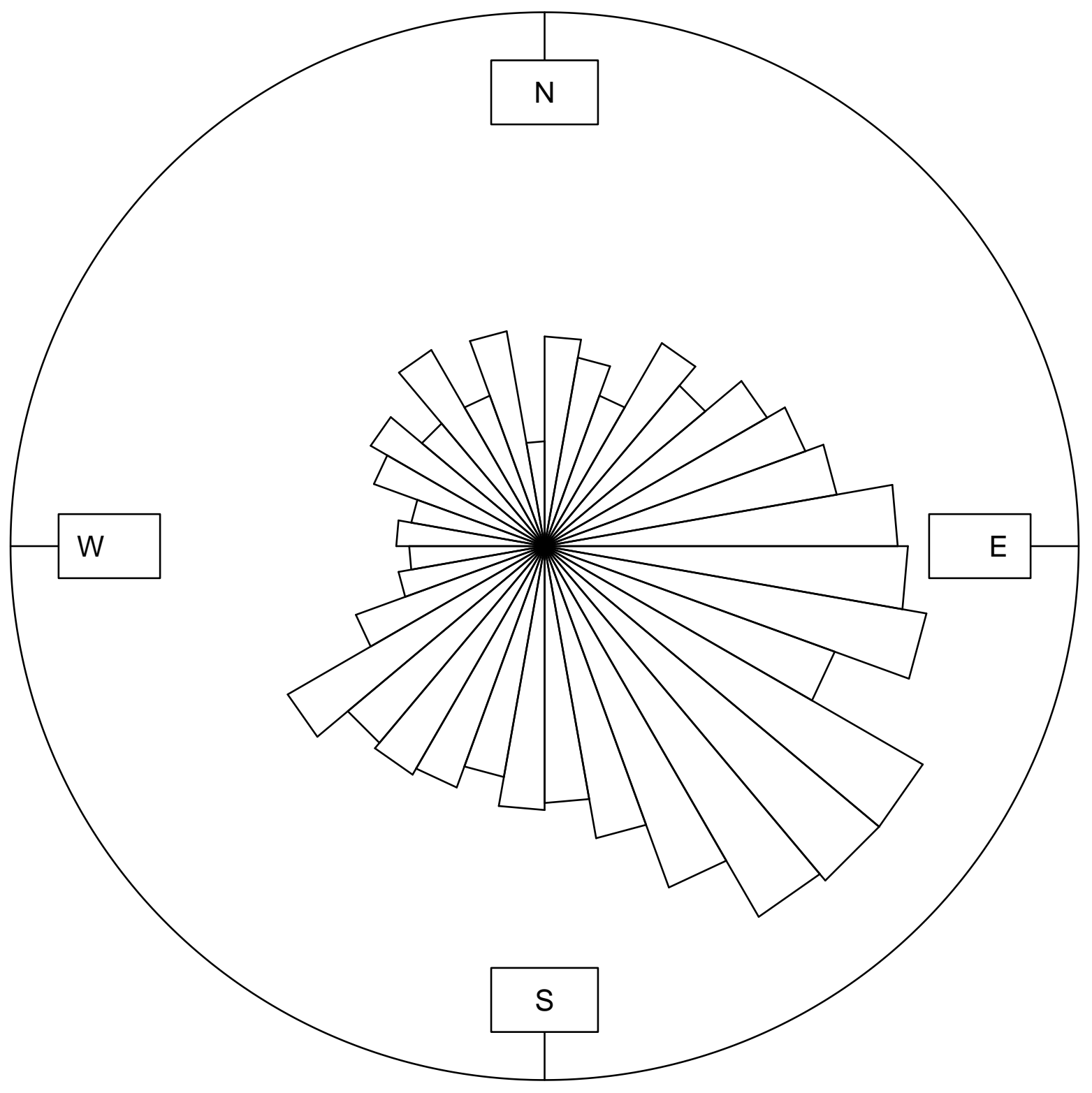


bootstrap 597

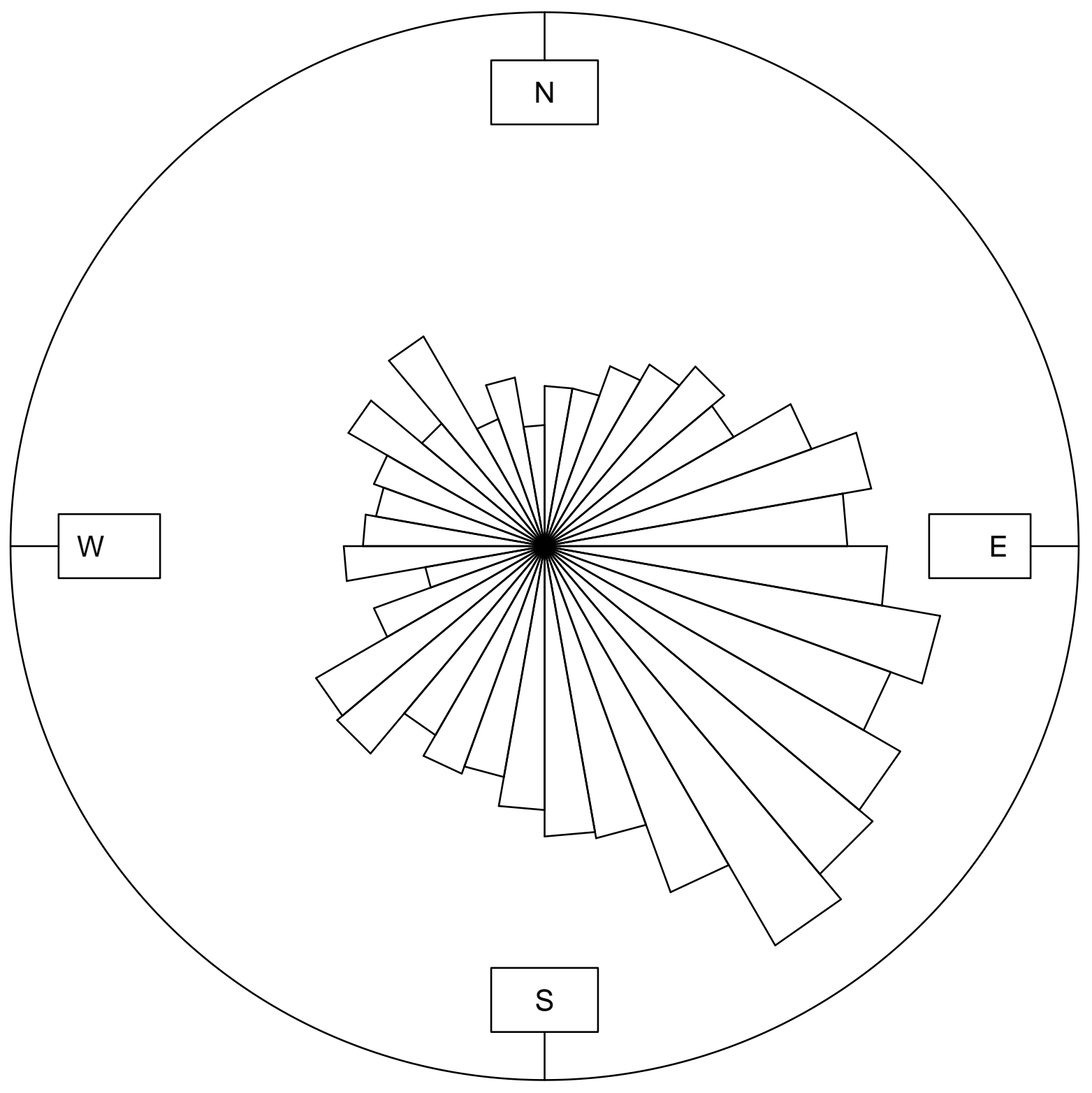


bootstrap 598

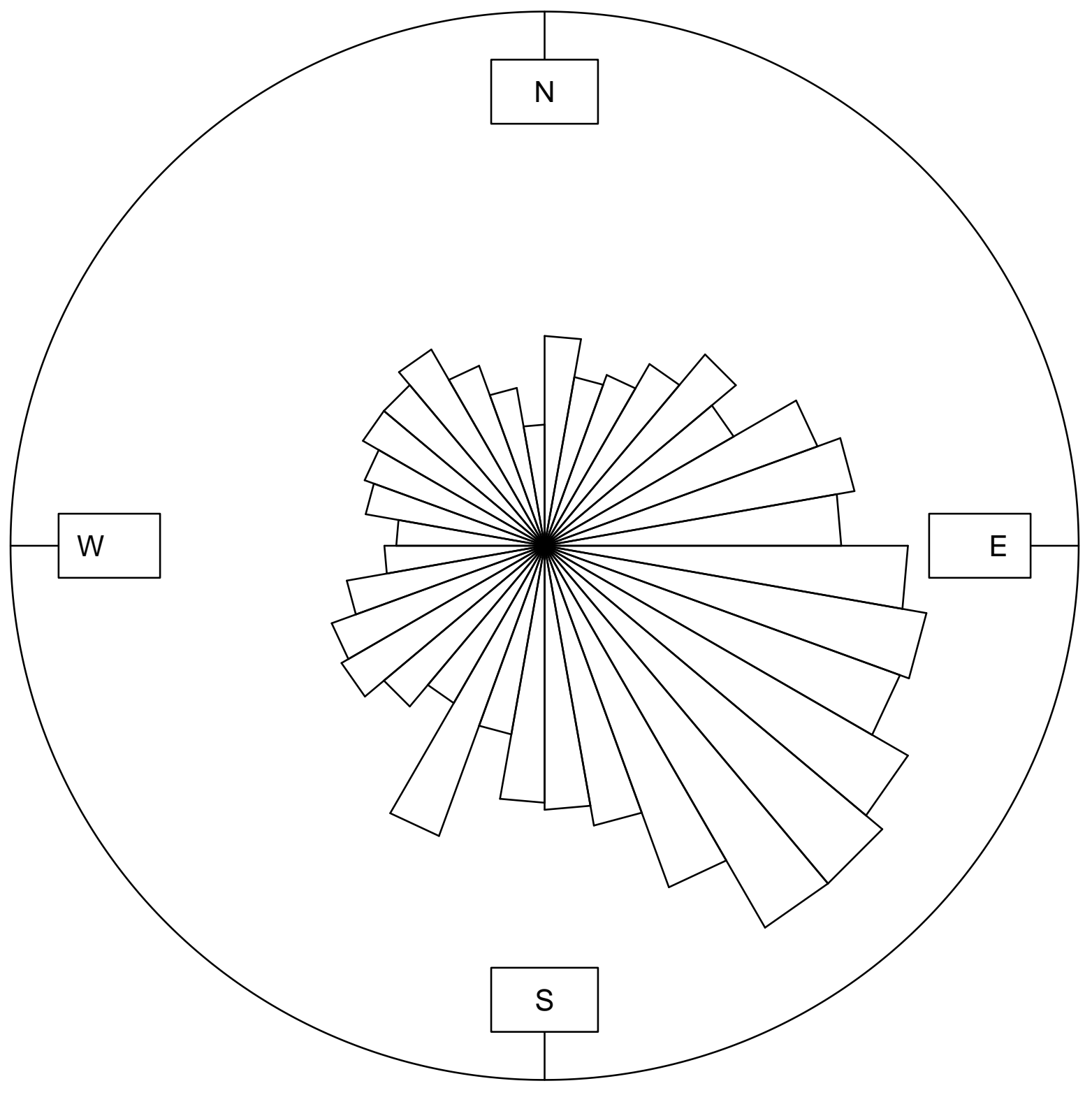


bootstrap 599

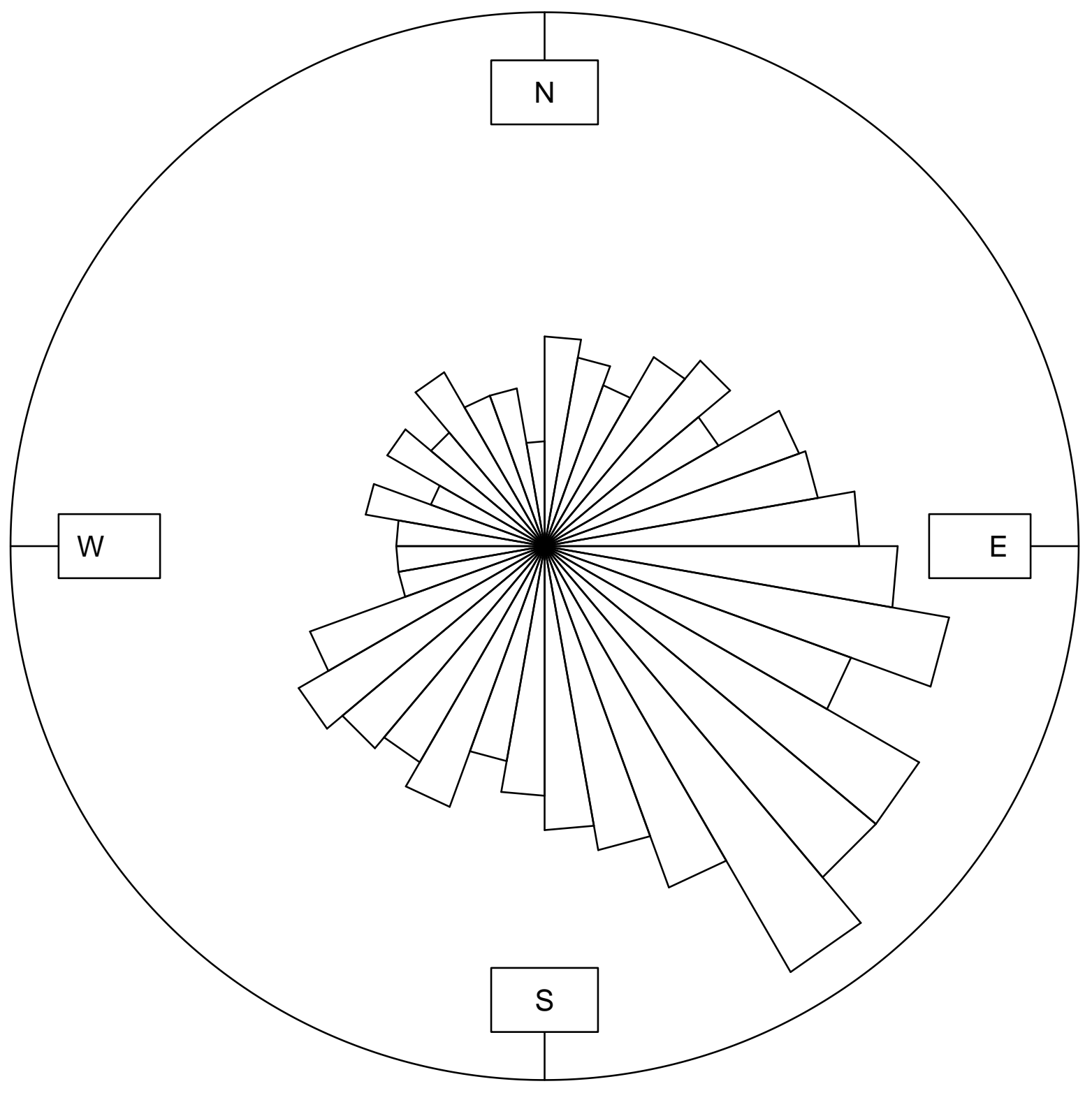


bootstrap 600

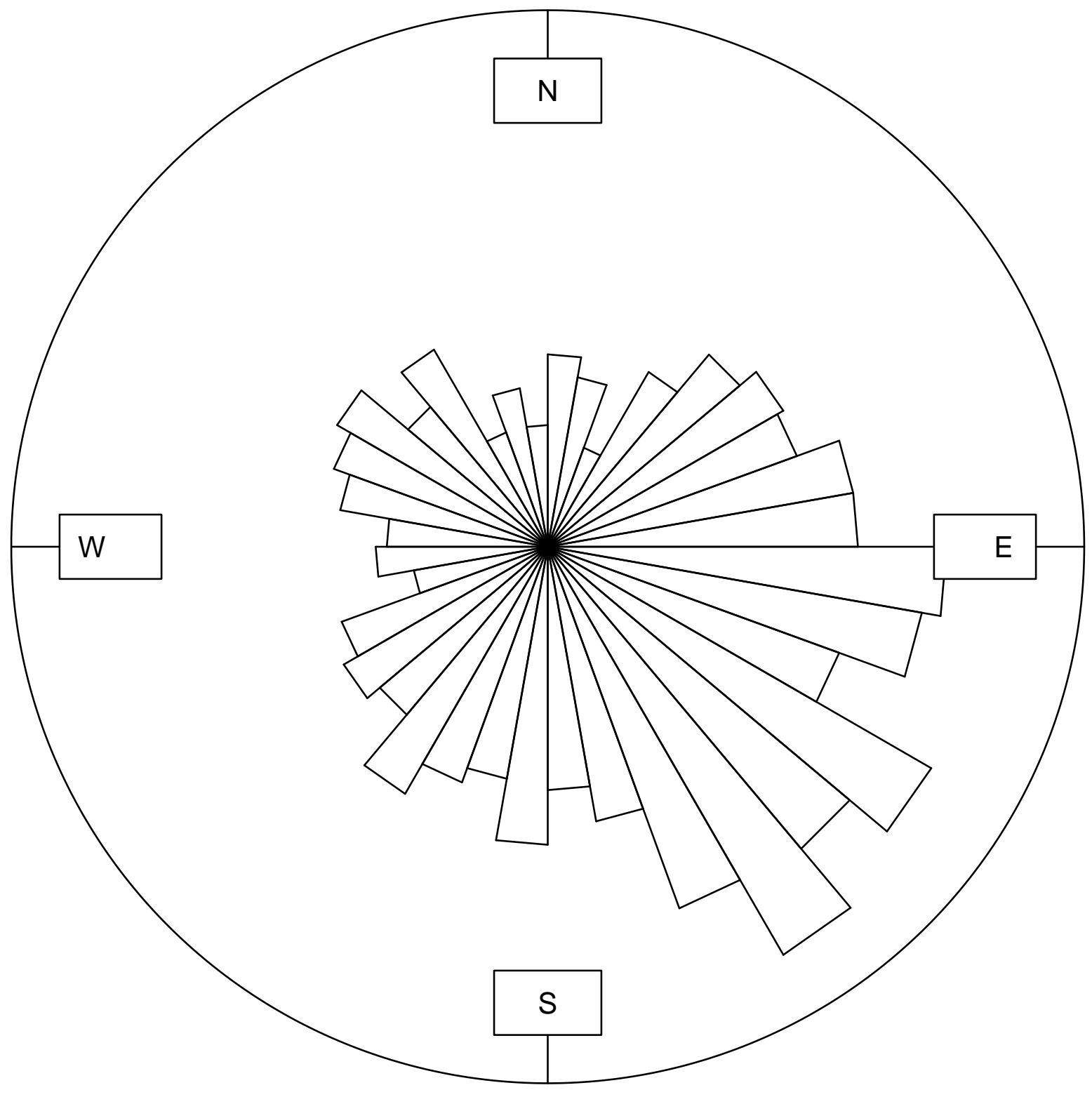


bootstrap 601

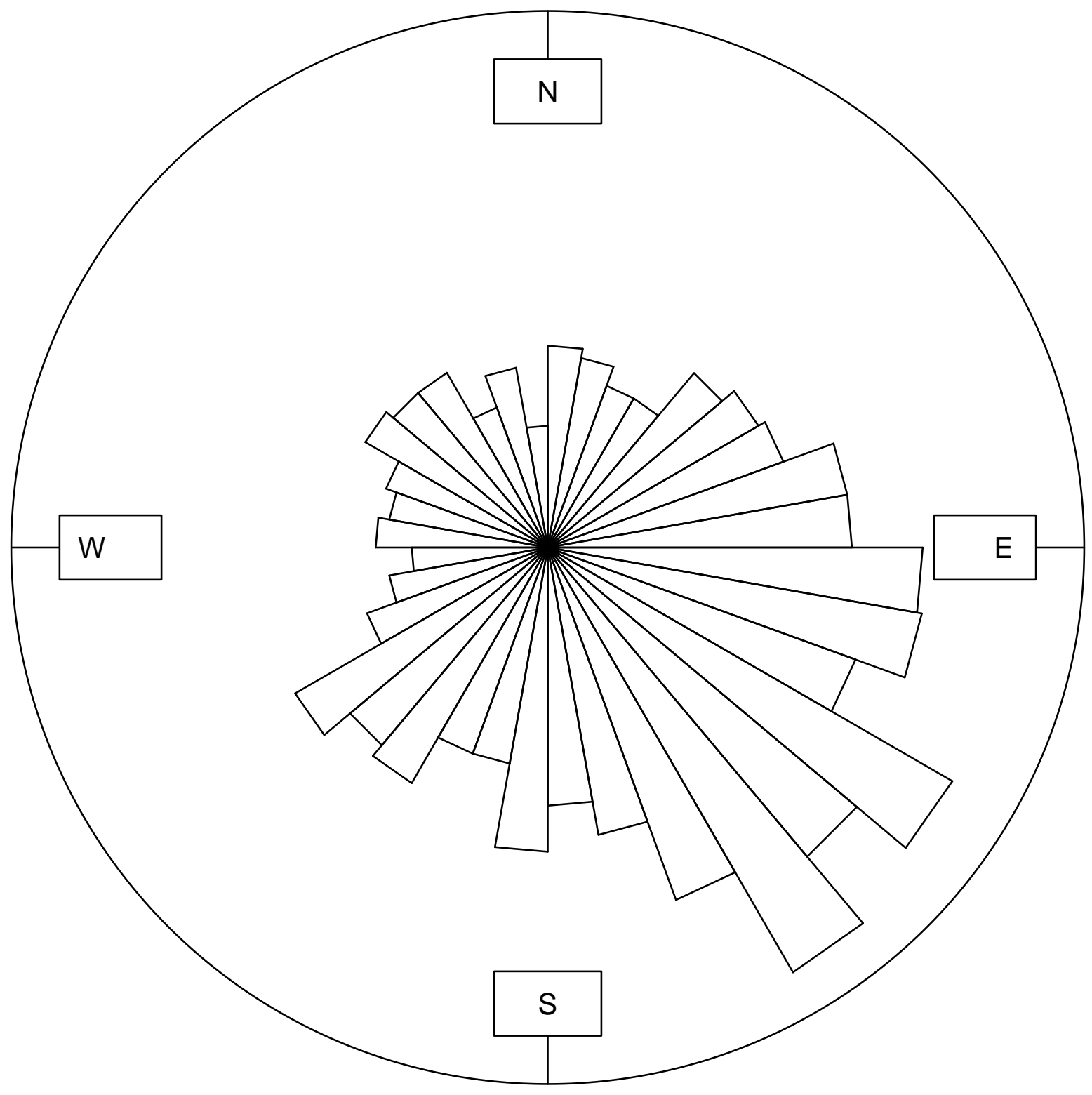


bootstrap 602

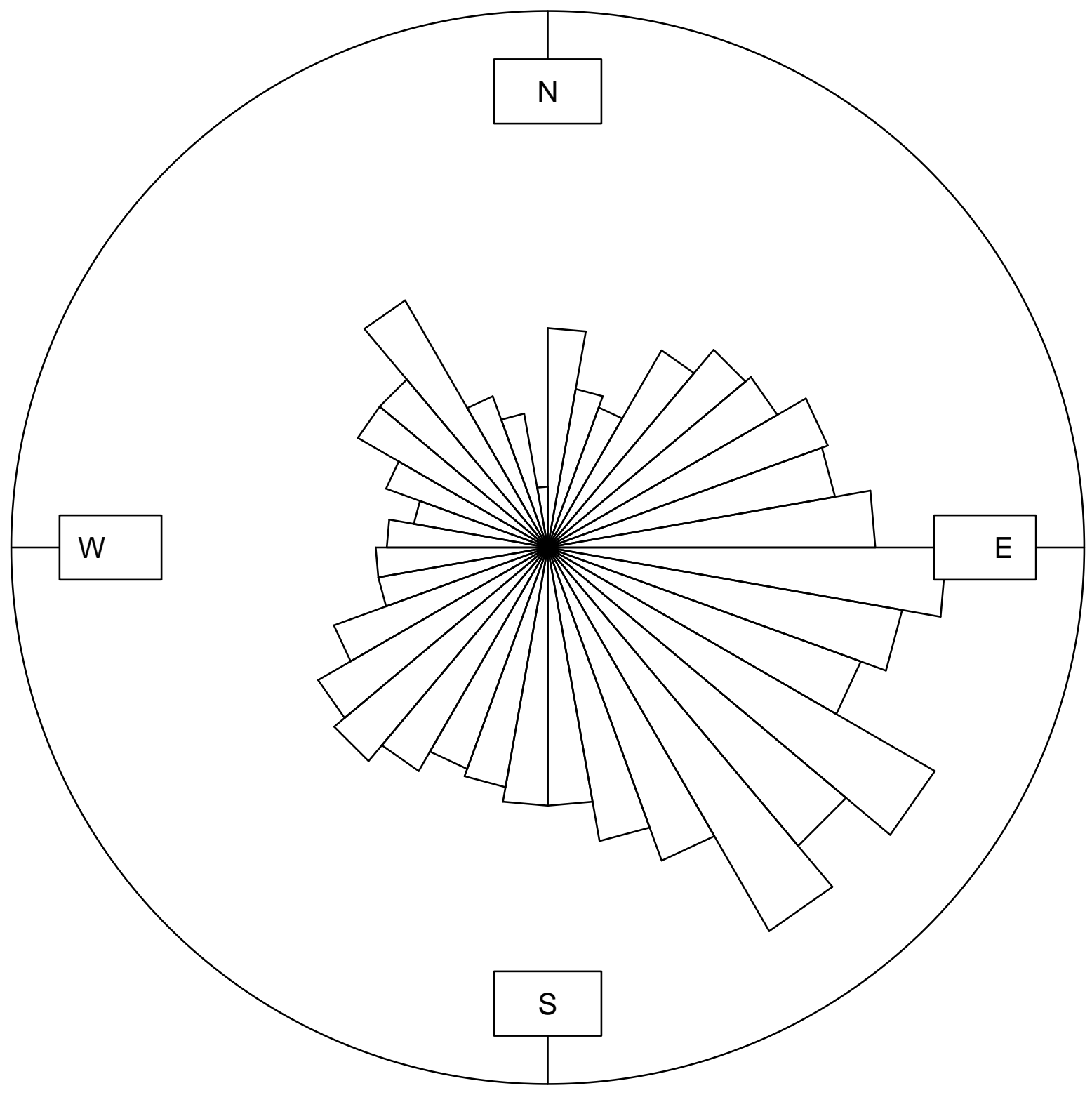


bootstrap 603

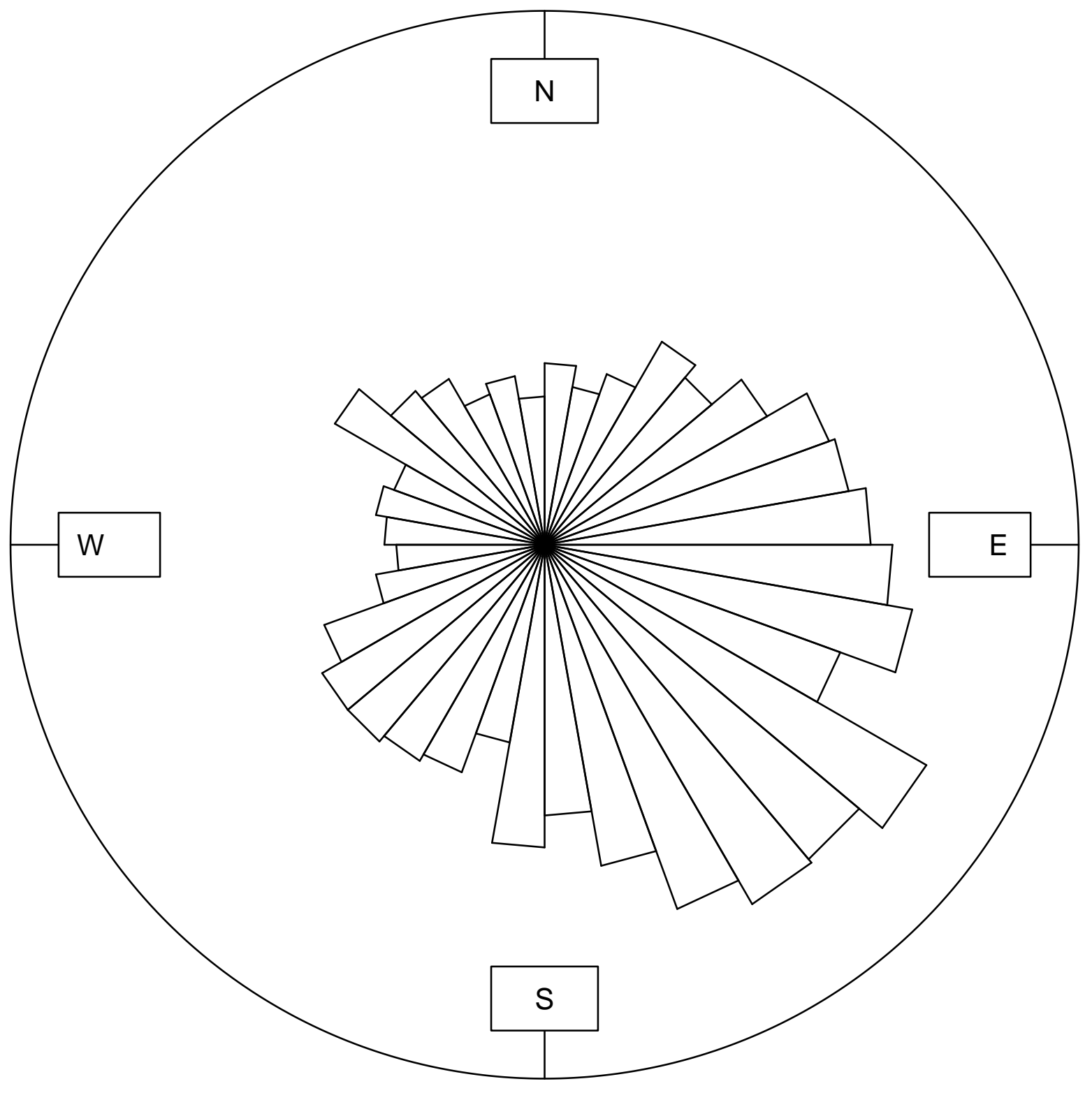




\section{bootstrap 604}

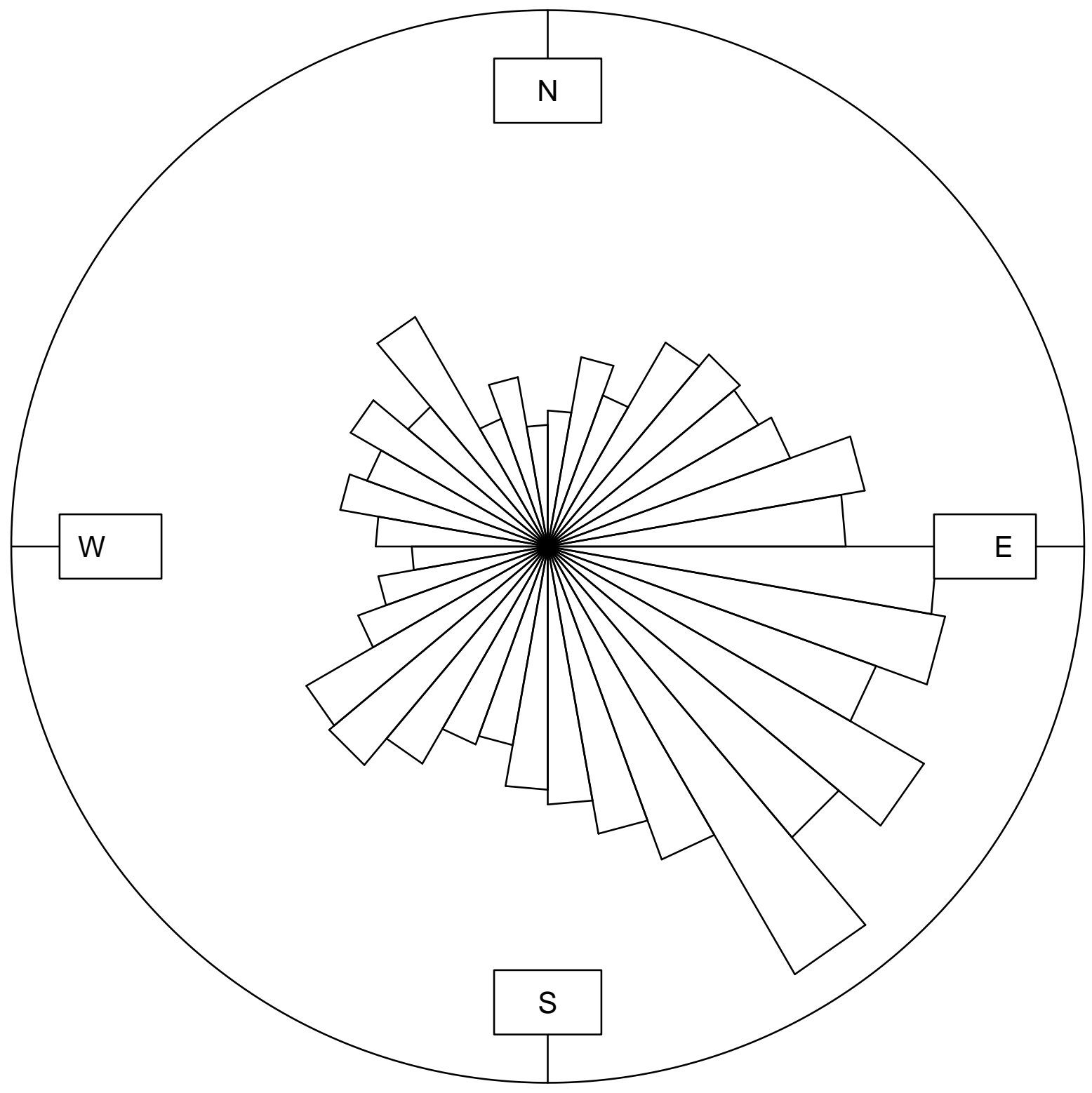




\section{bootstrap 605}

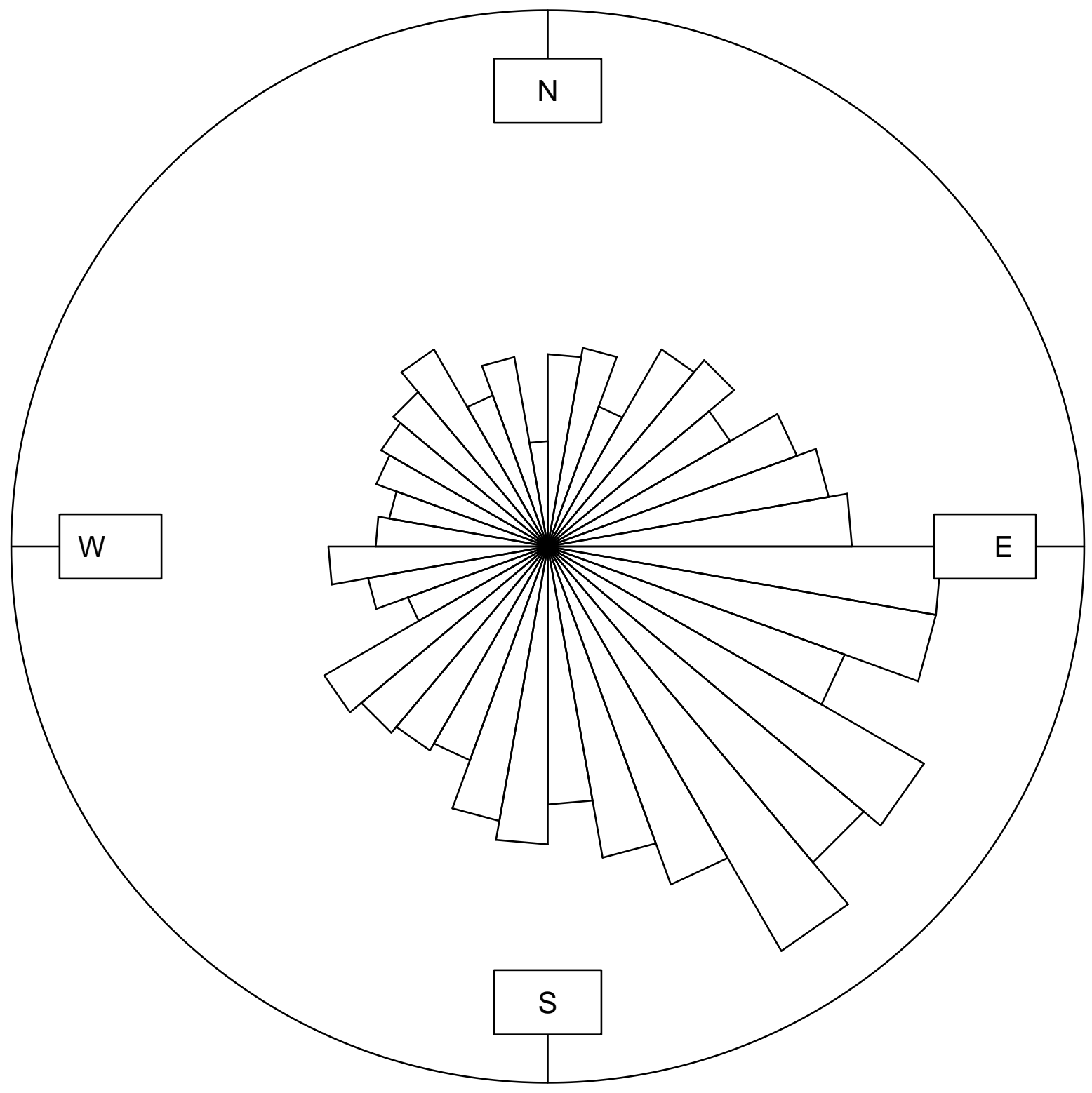


bootstrap 606

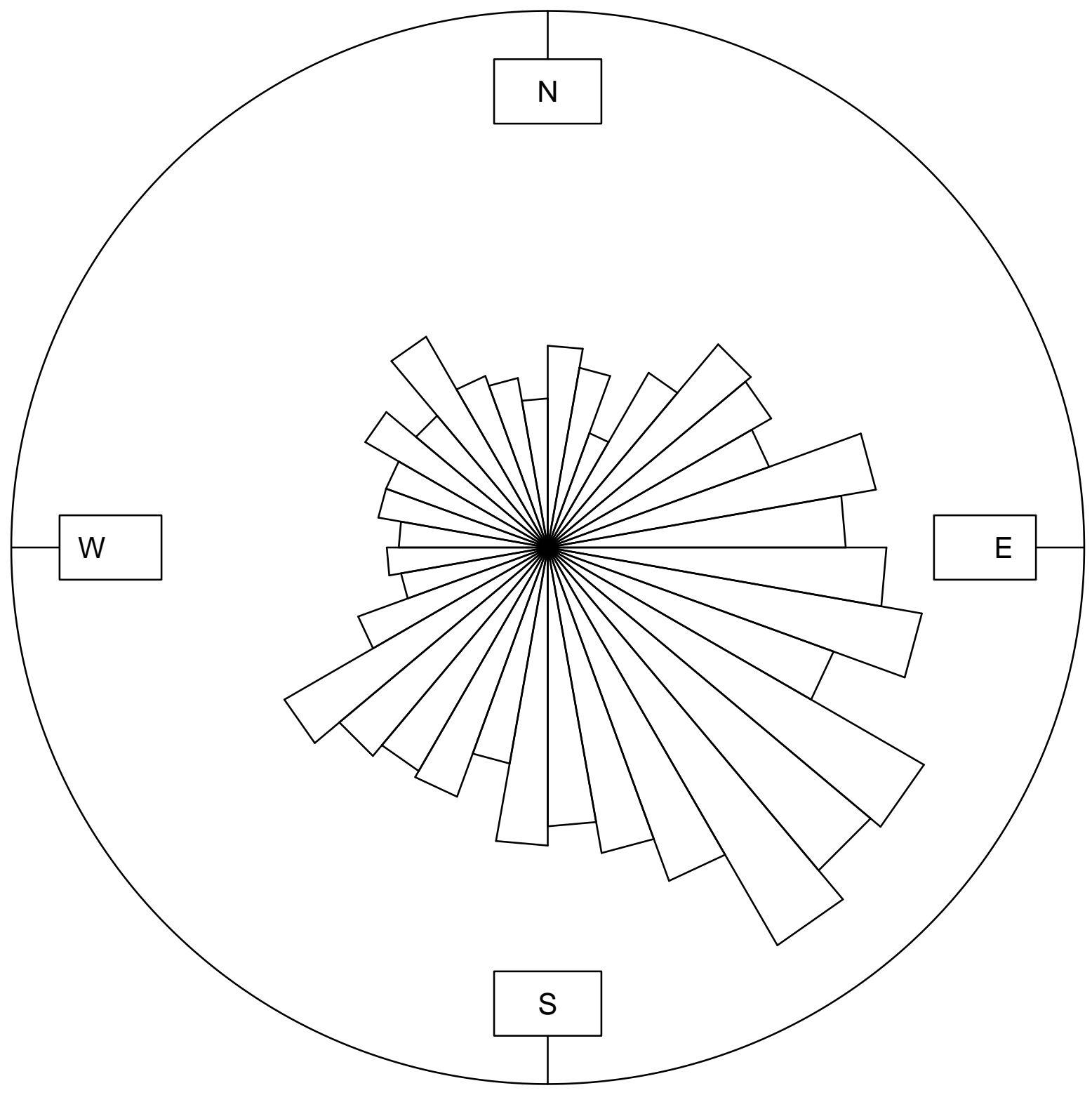


bootstrap 607

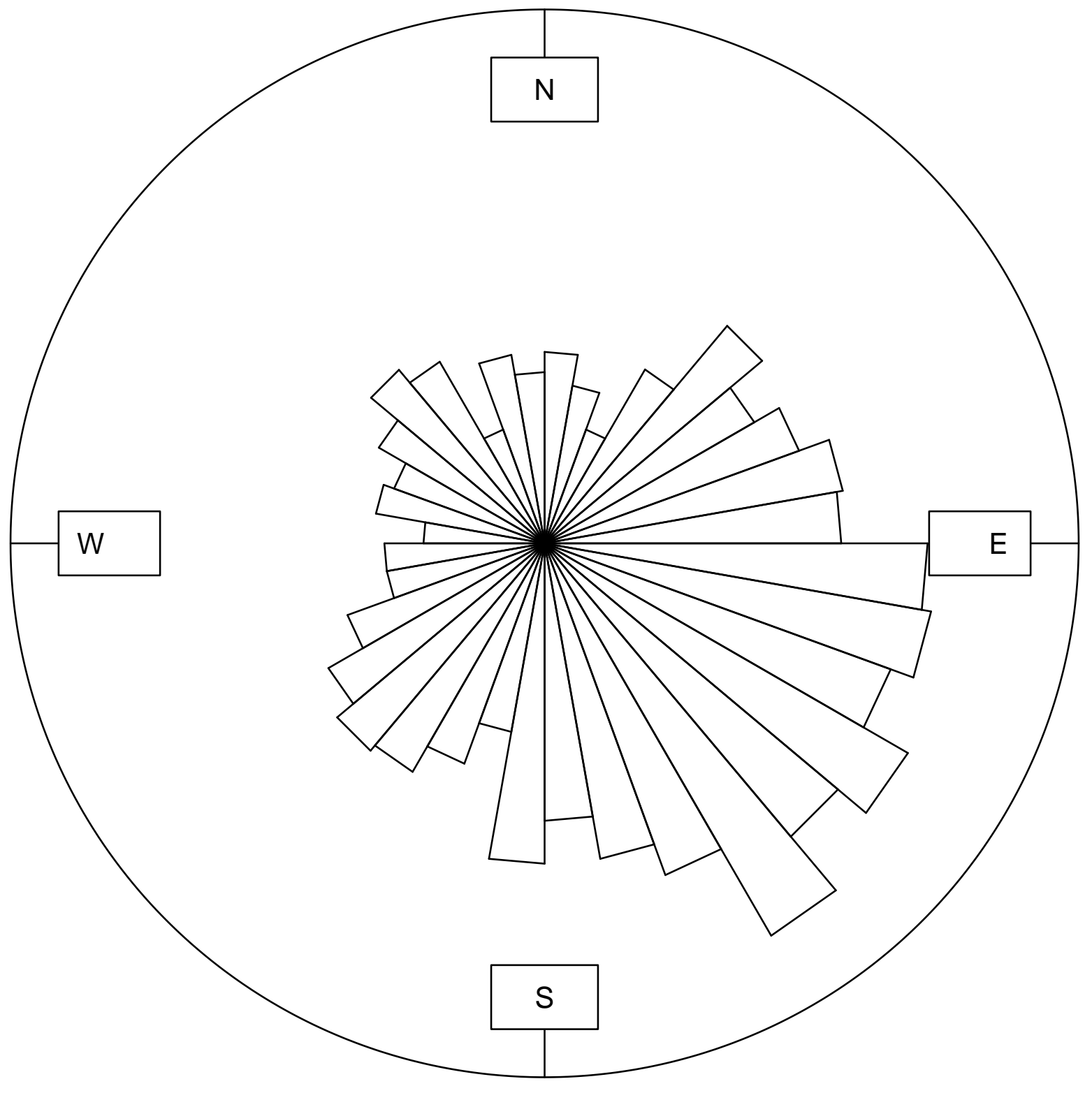


bootstrap 608

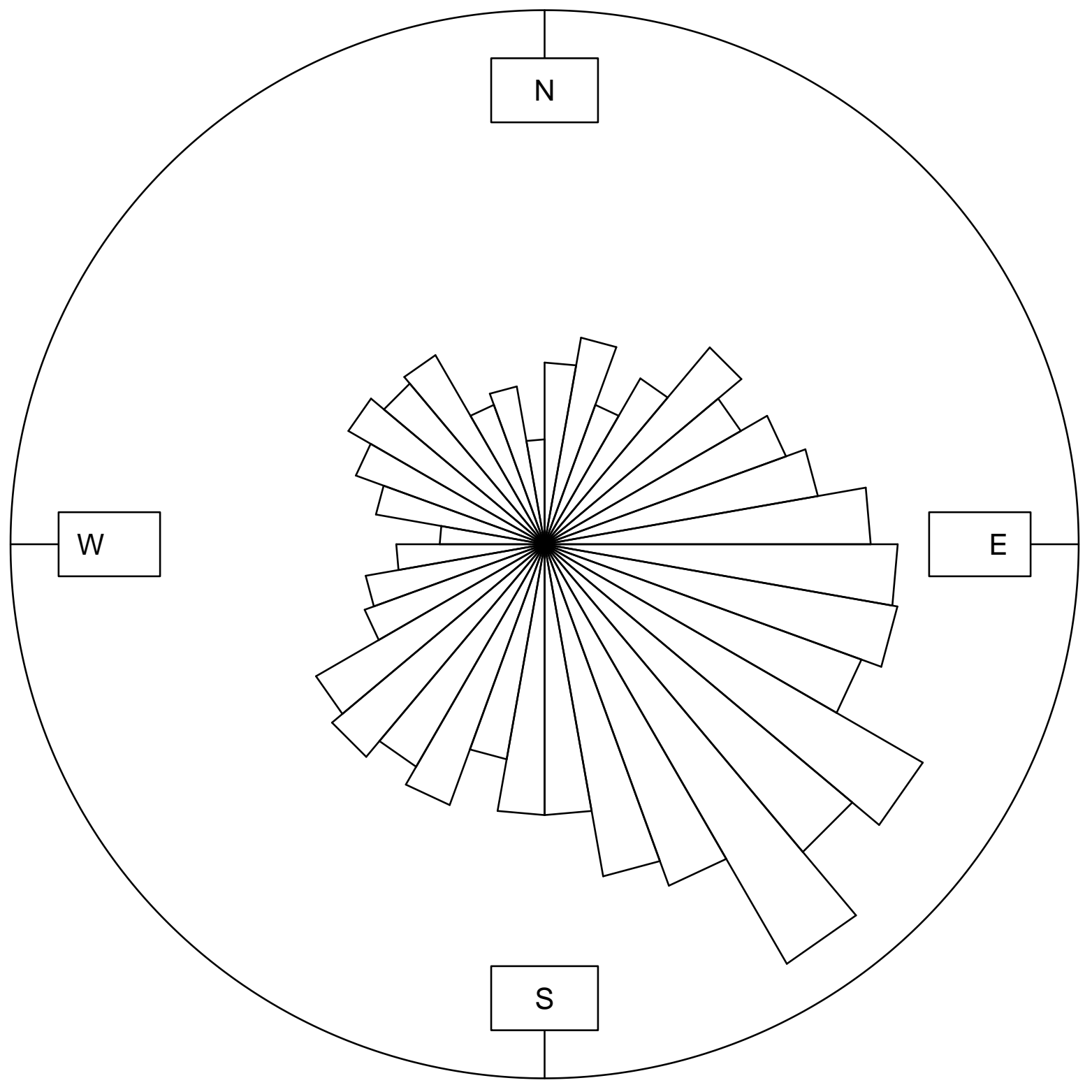


bootstrap 609

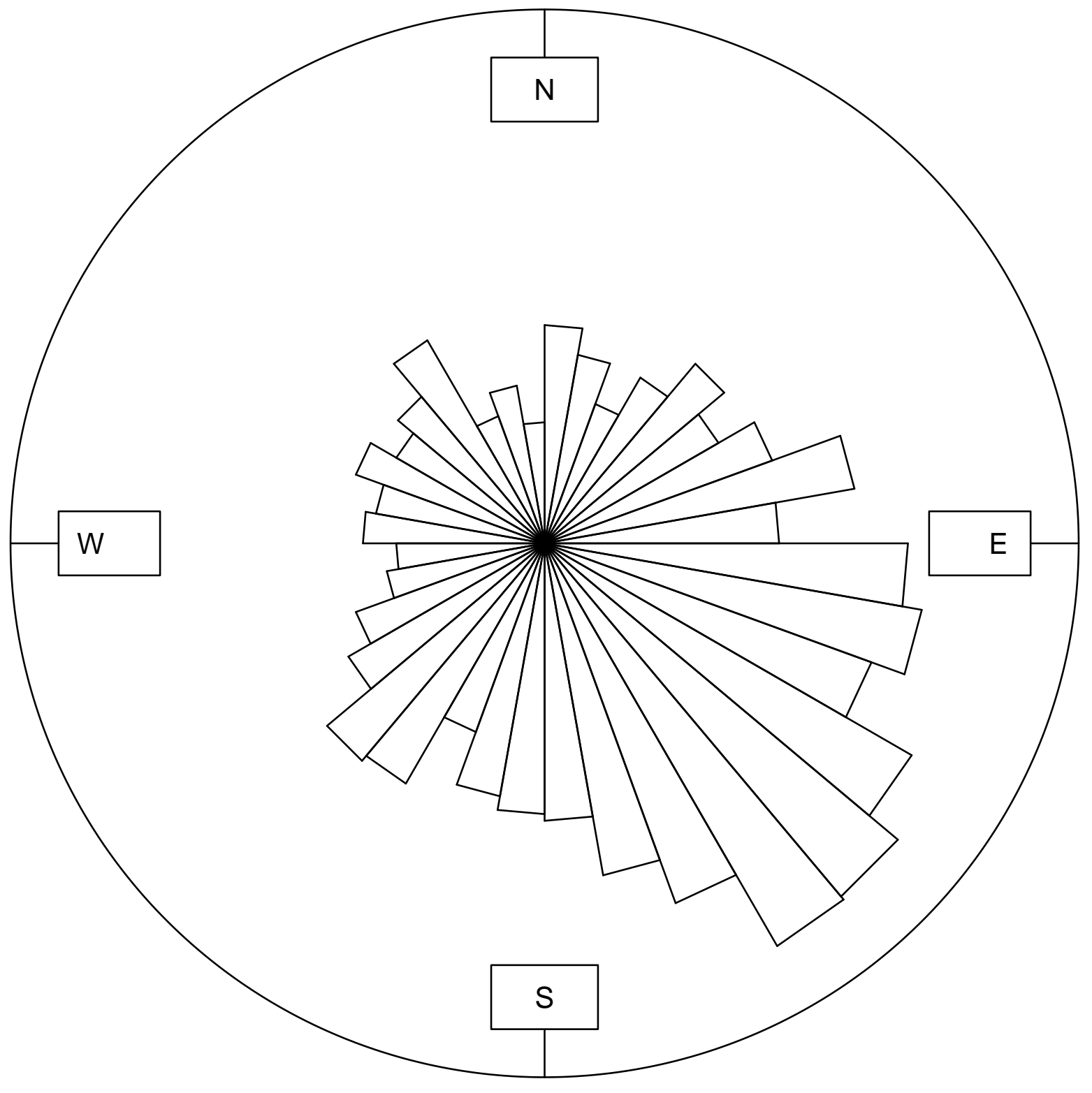


bootstrap 610

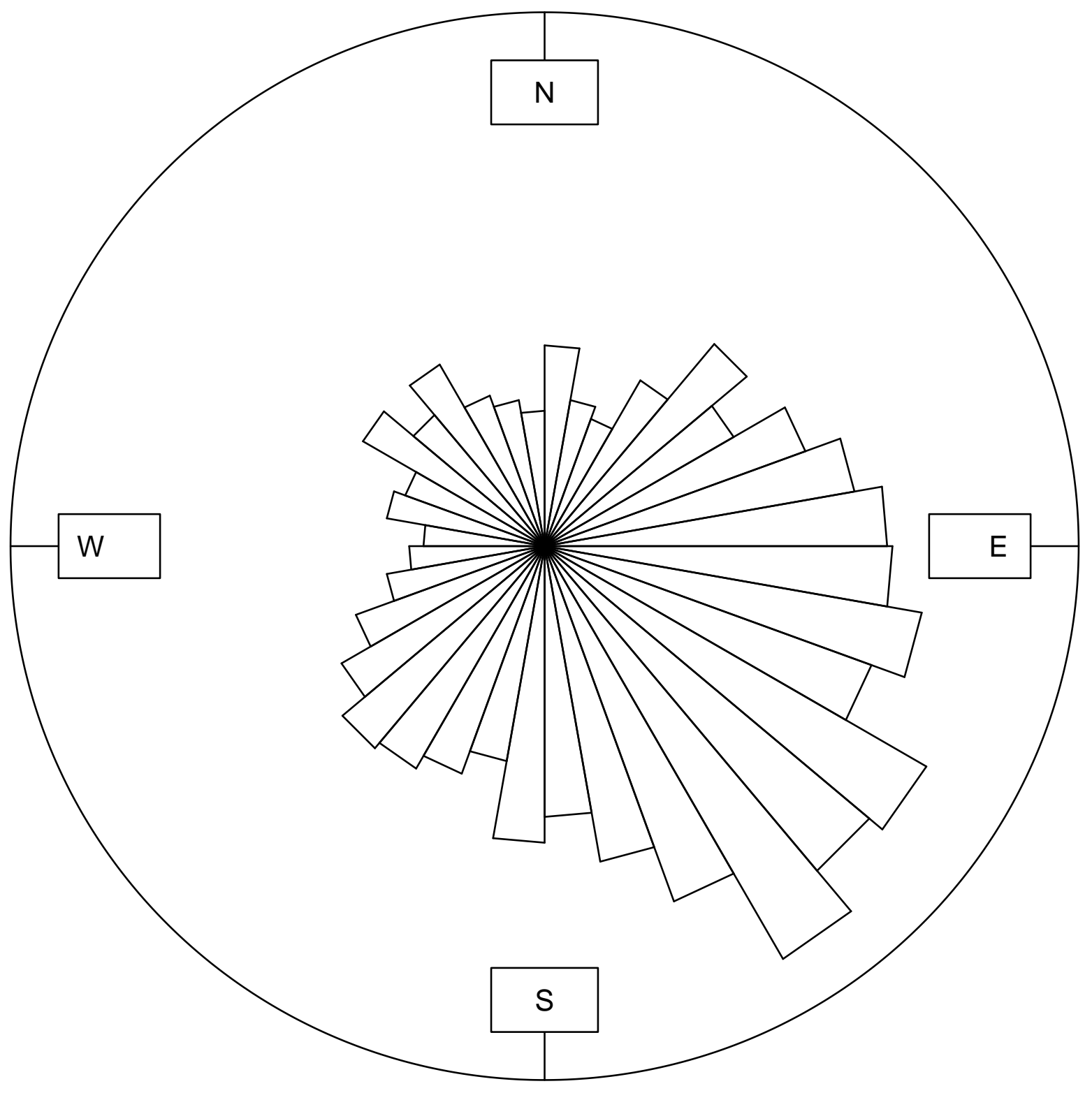


bootstrap 611

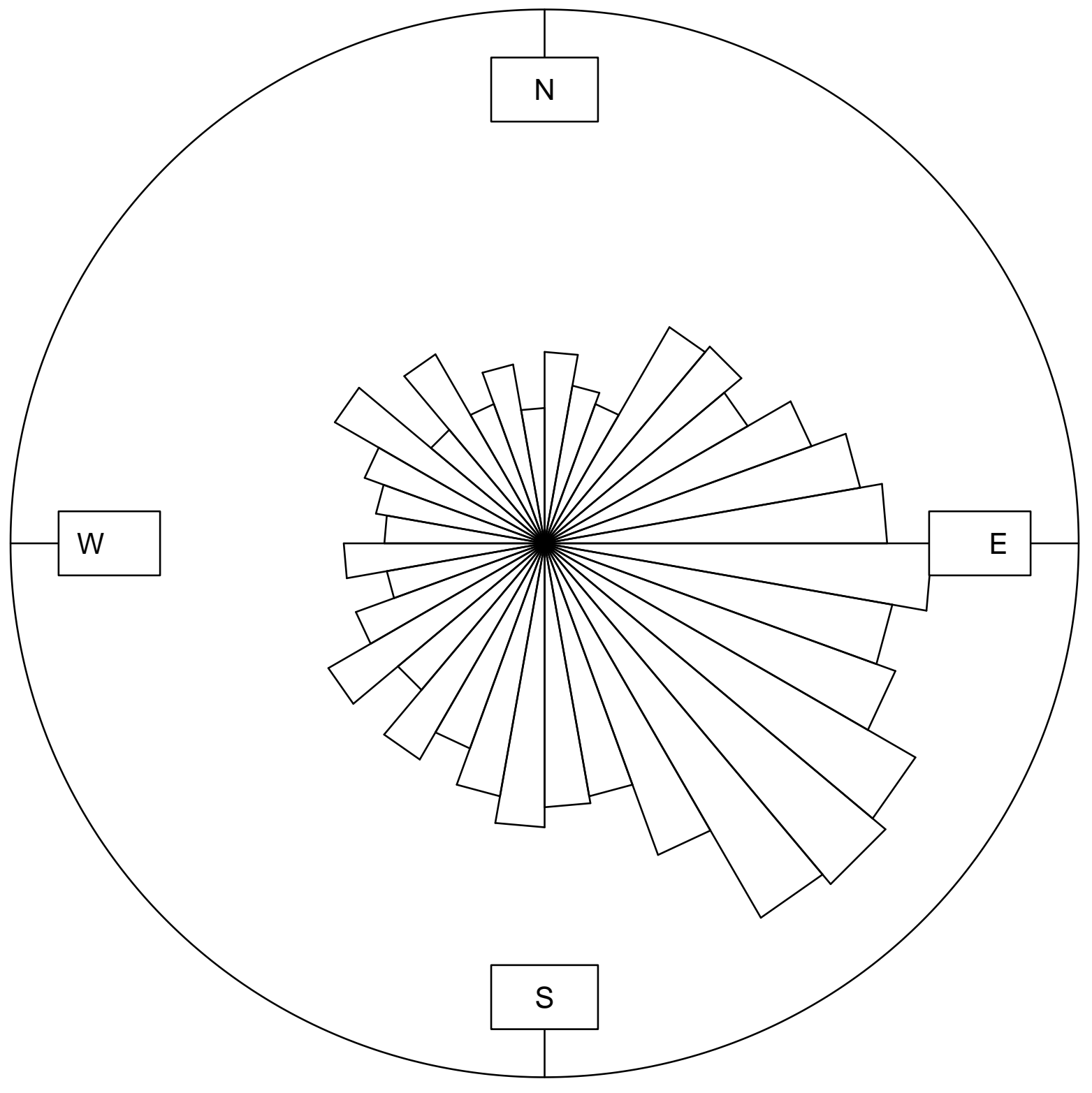


bootstrap 612

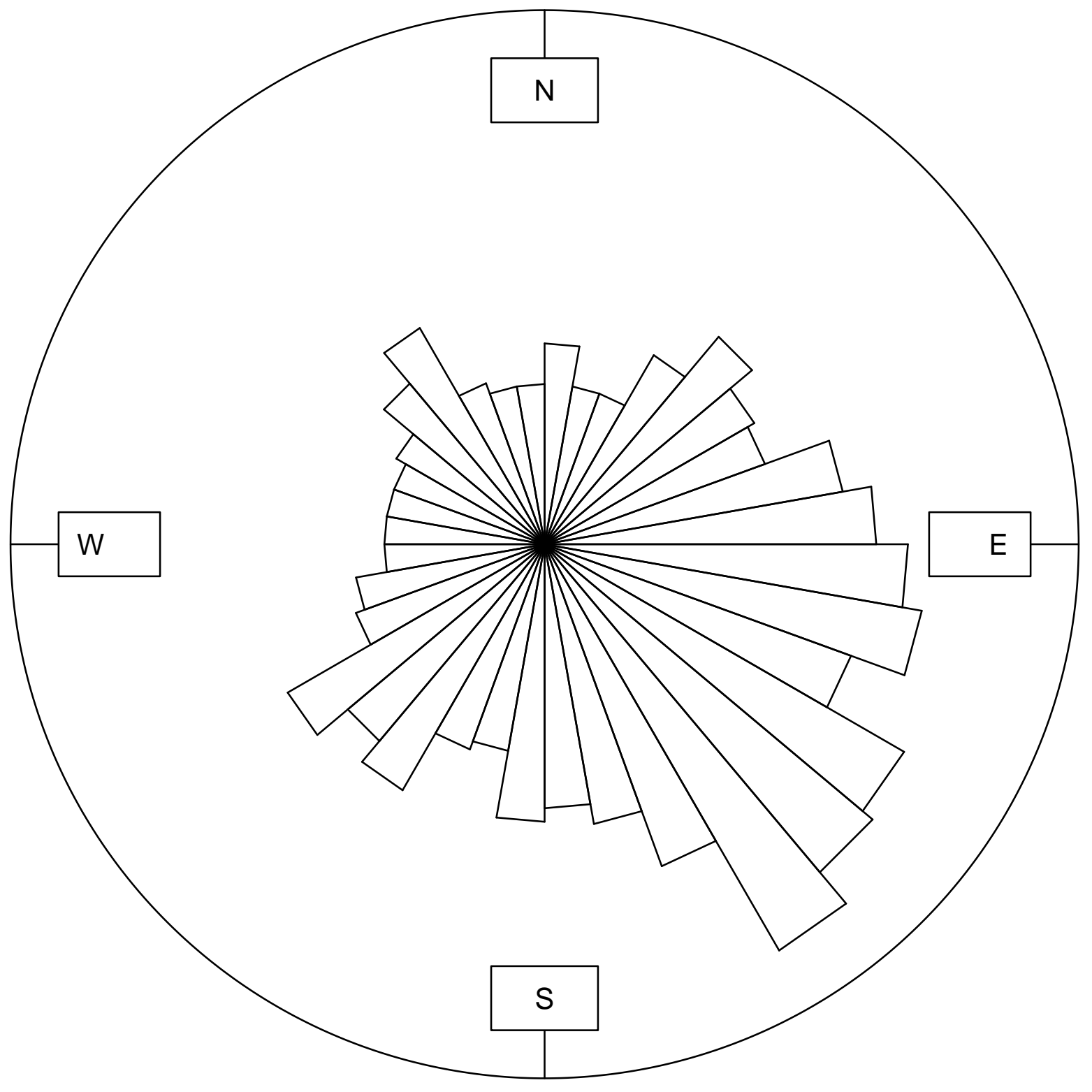


bootstrap 613

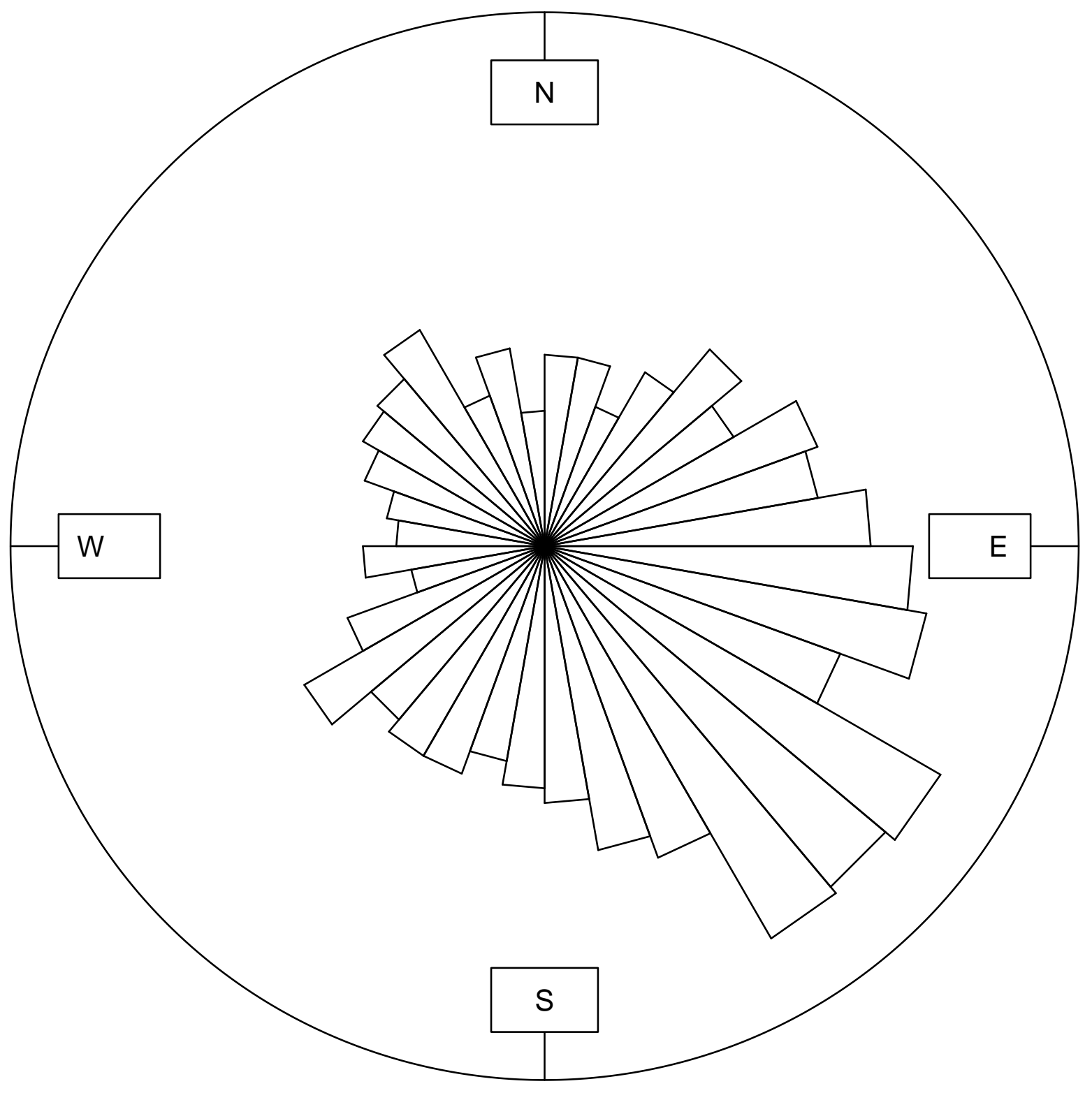


bootstrap 614

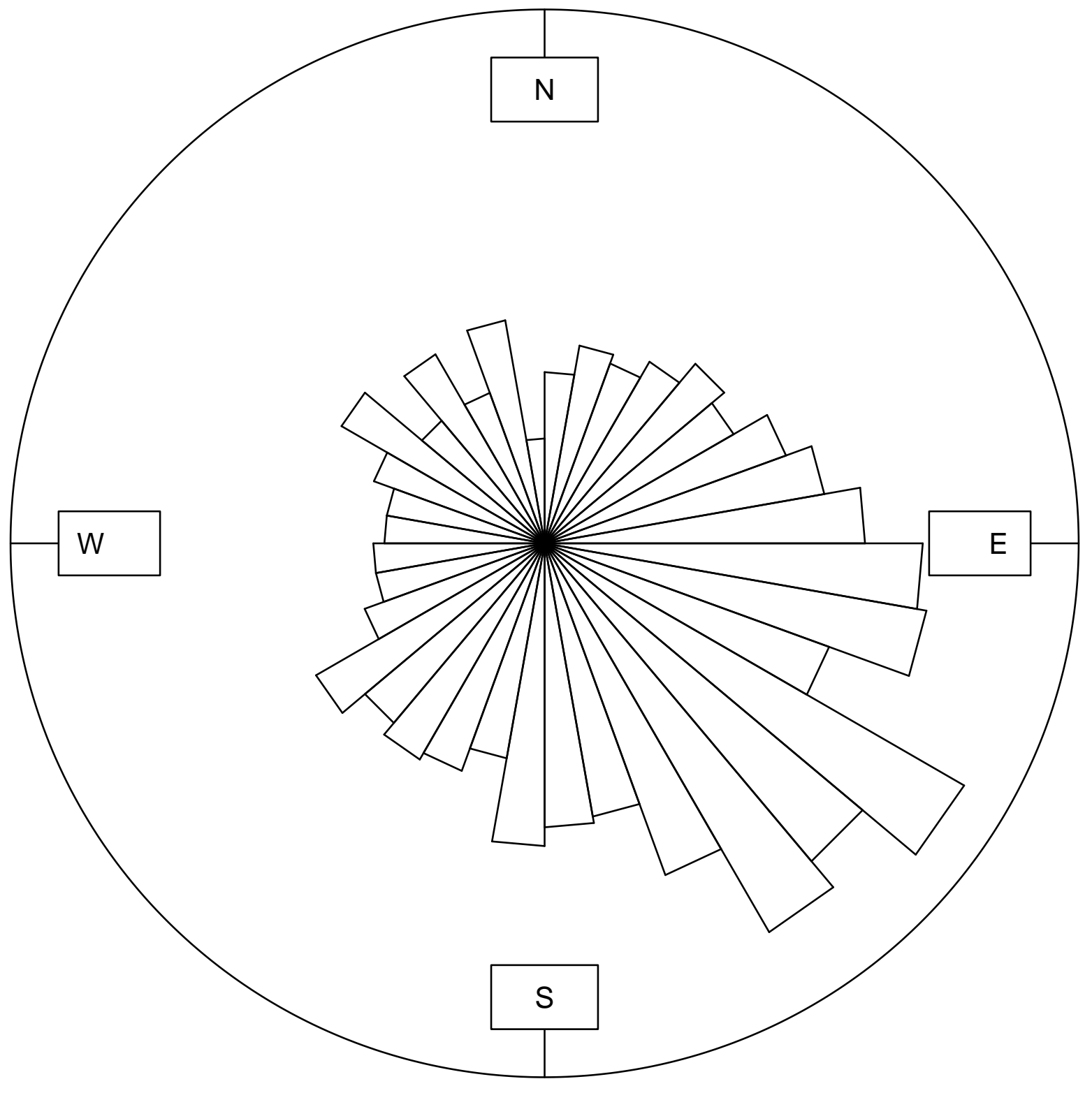


bootstrap 615

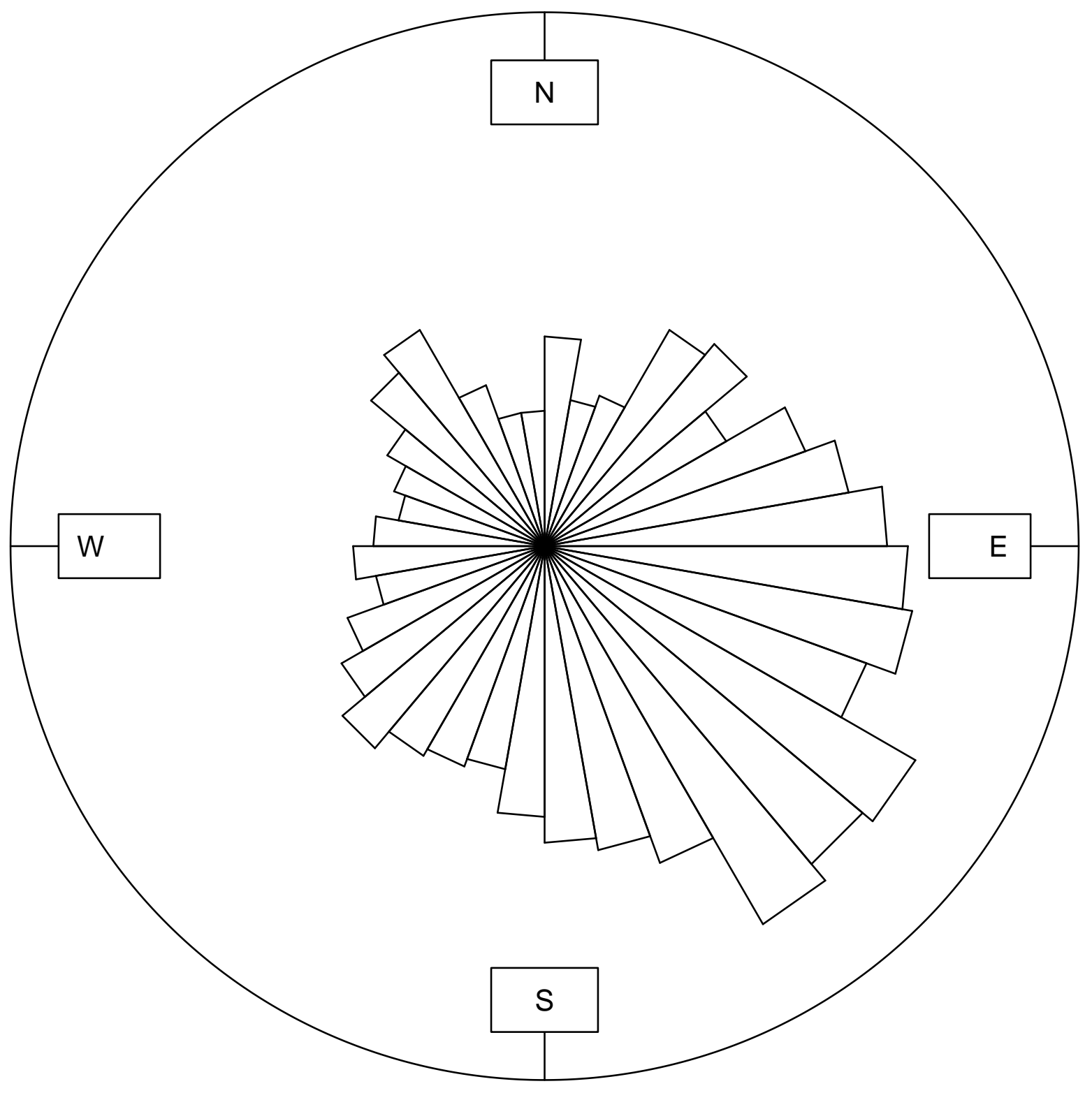


bootstrap 616

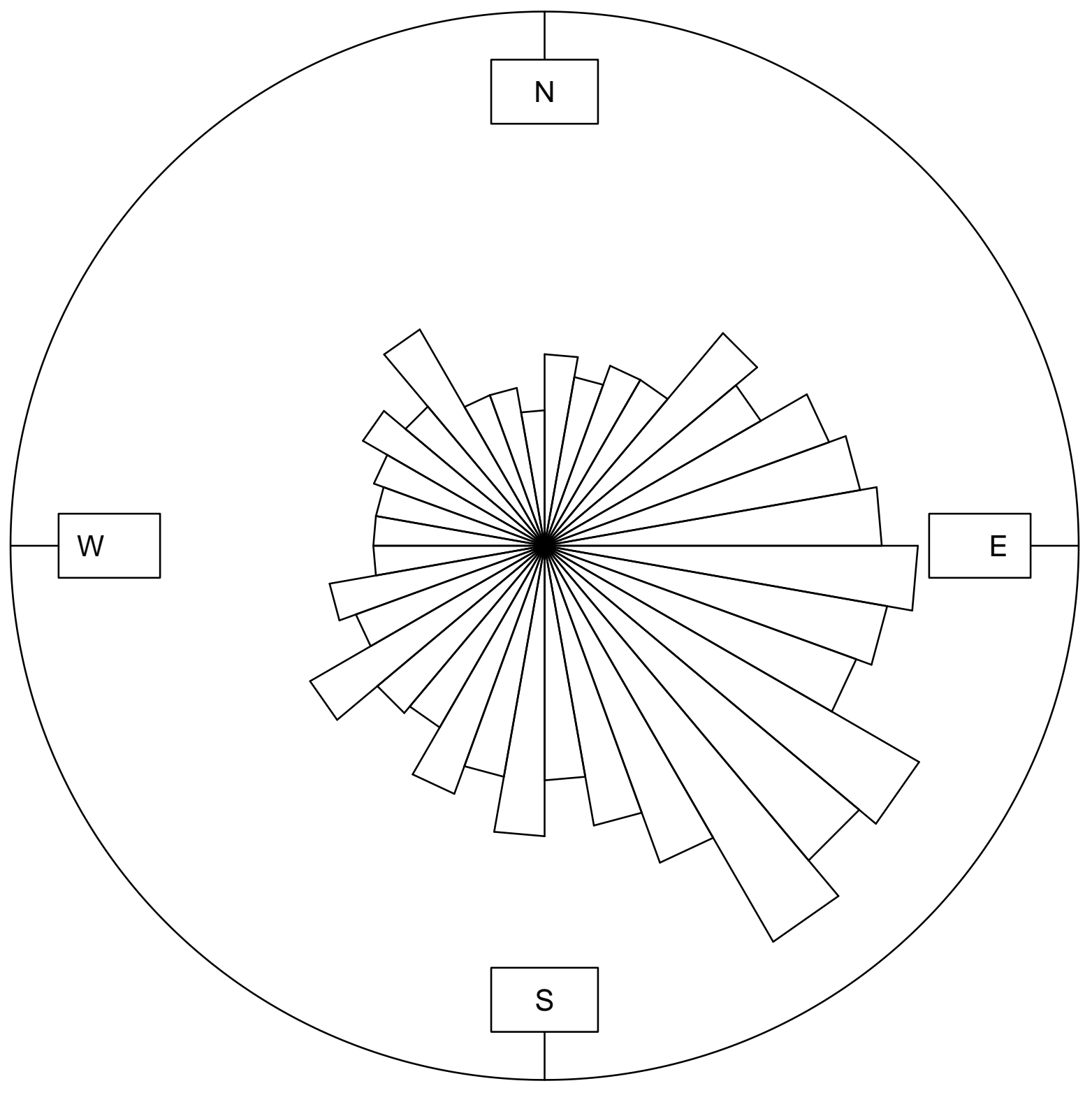


bootstrap 617

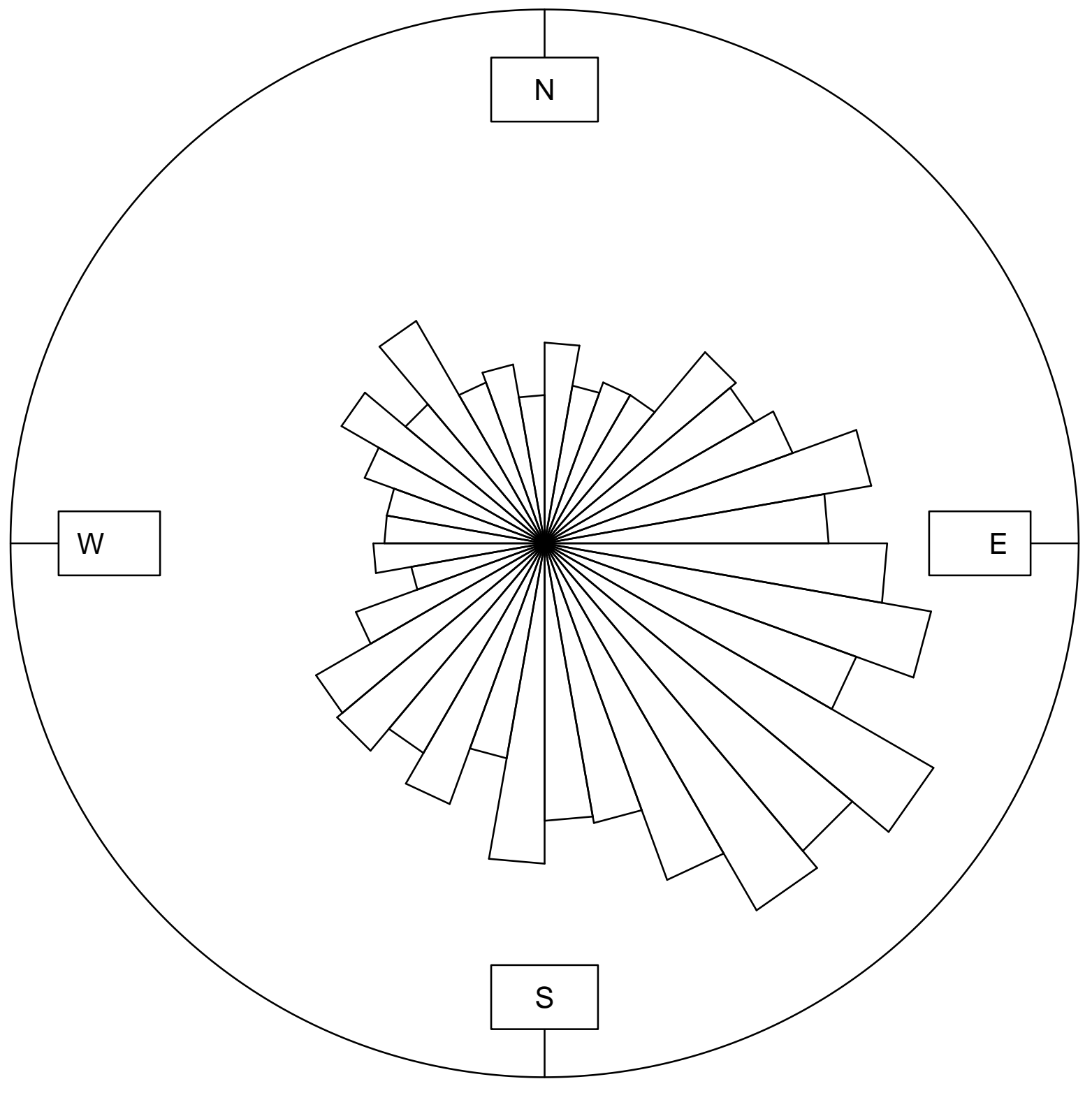


bootstrap 618

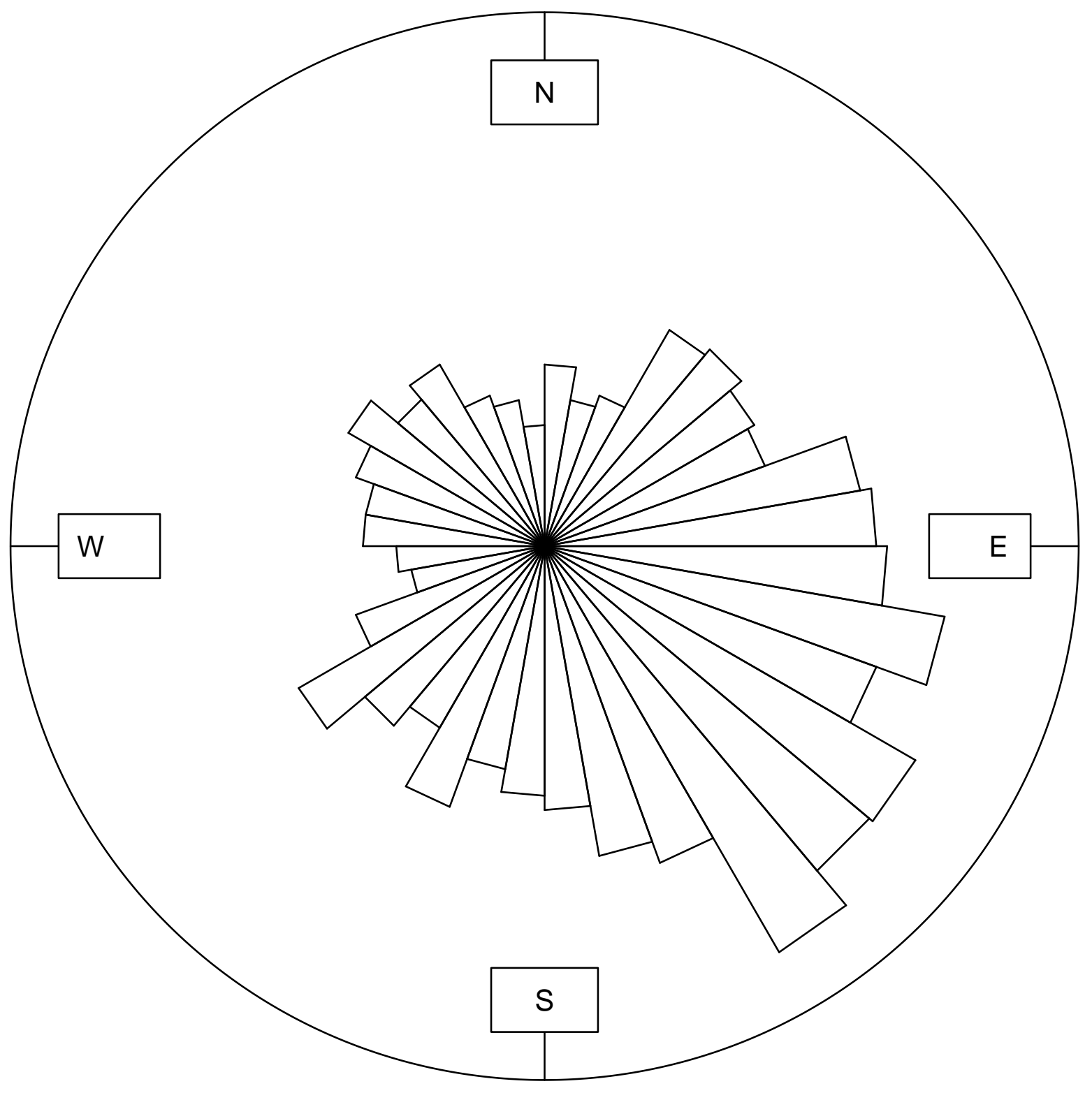


bootstrap 619

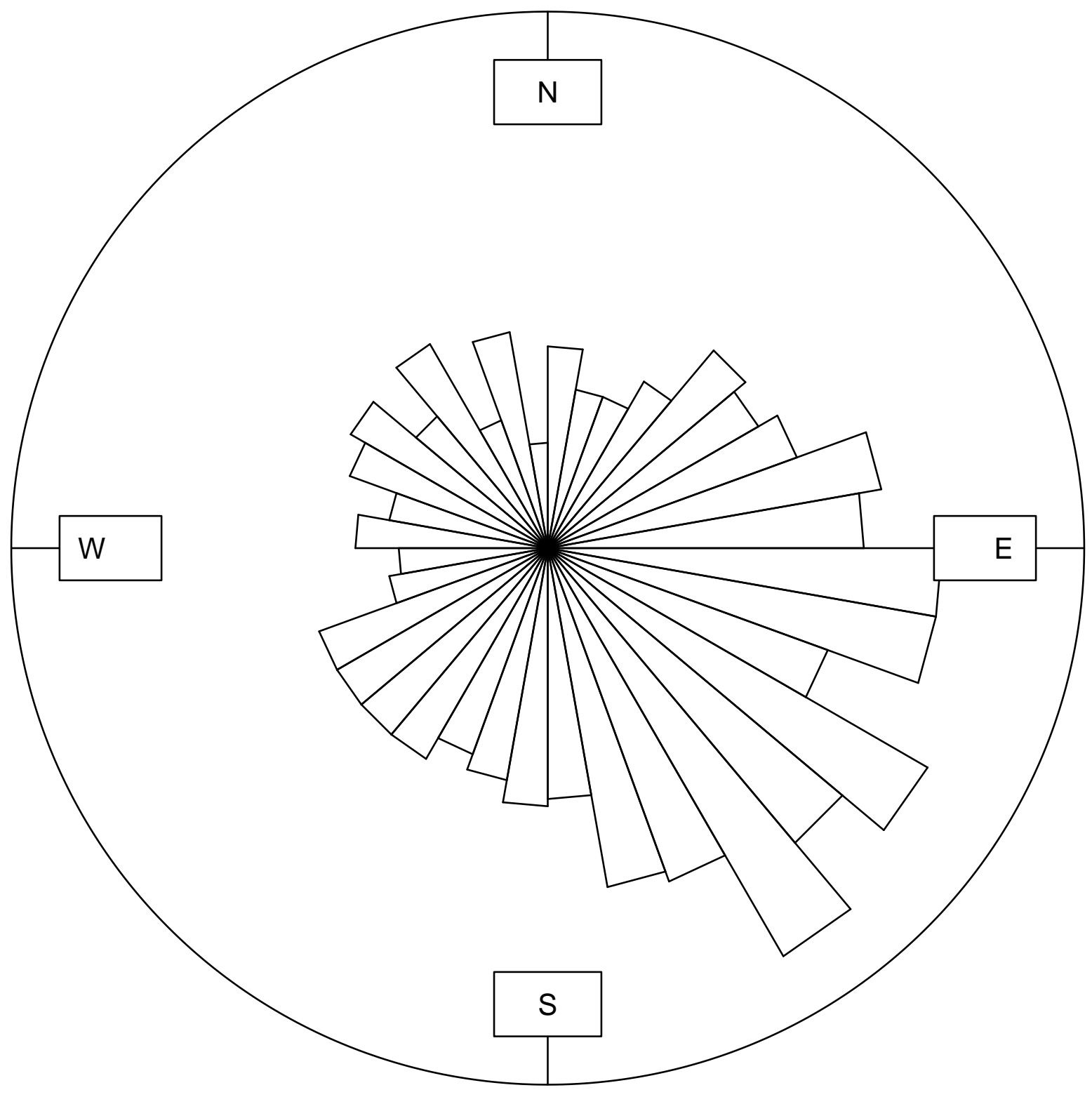


bootstrap 620

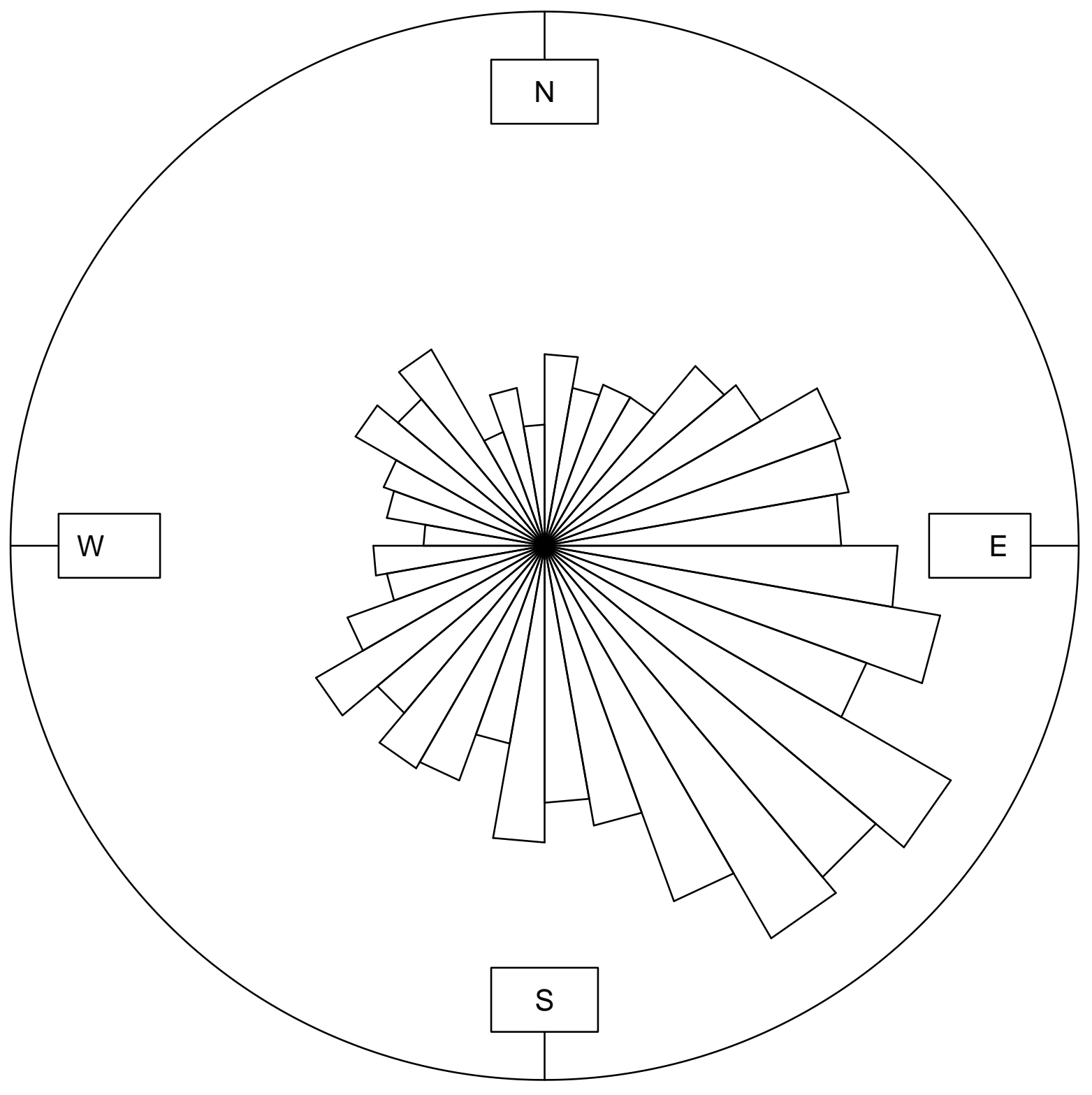


bootstrap 621

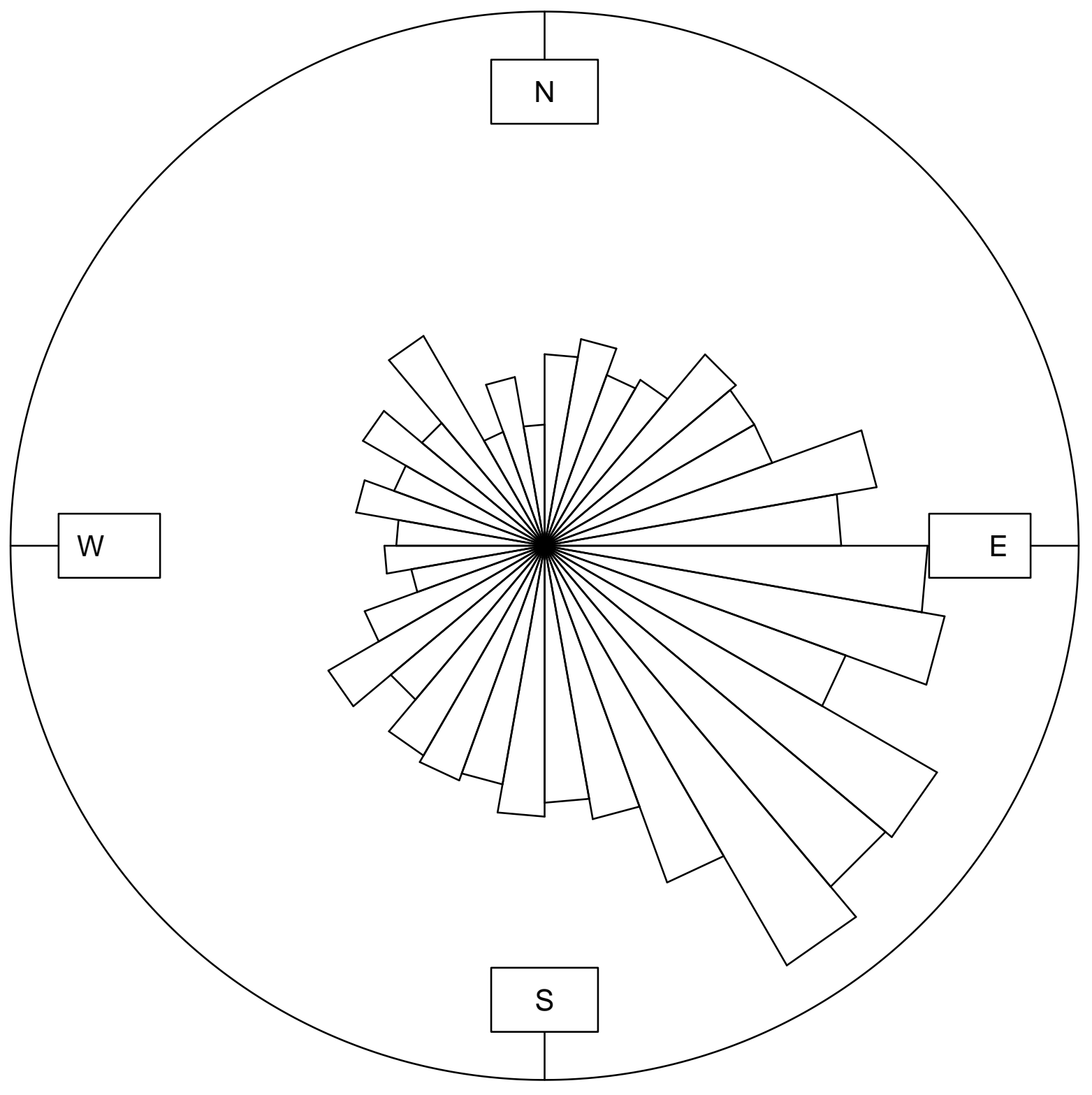


bootstrap 622

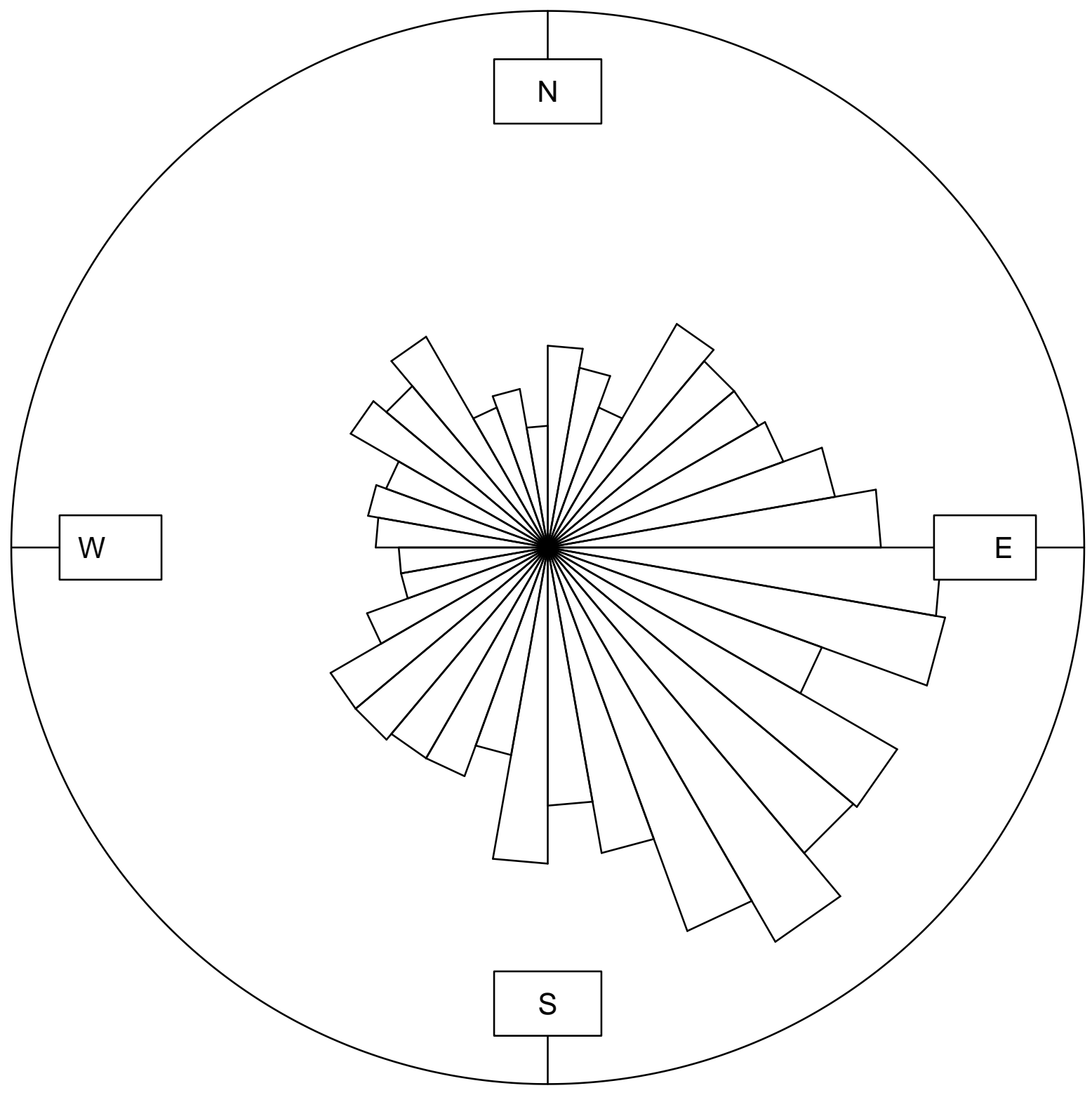




\section{bootstrap 623}

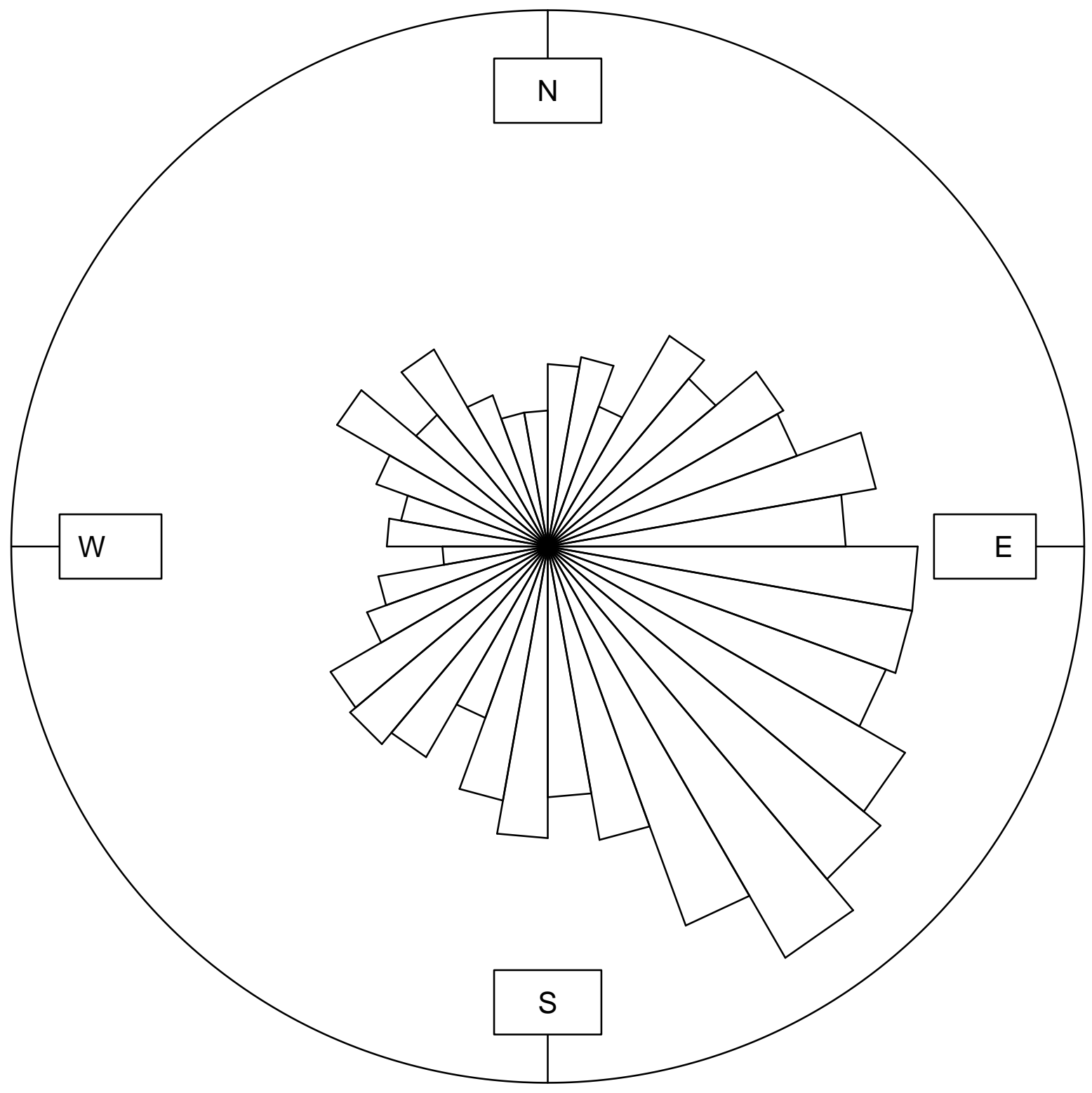




\section{bootstrap 624}

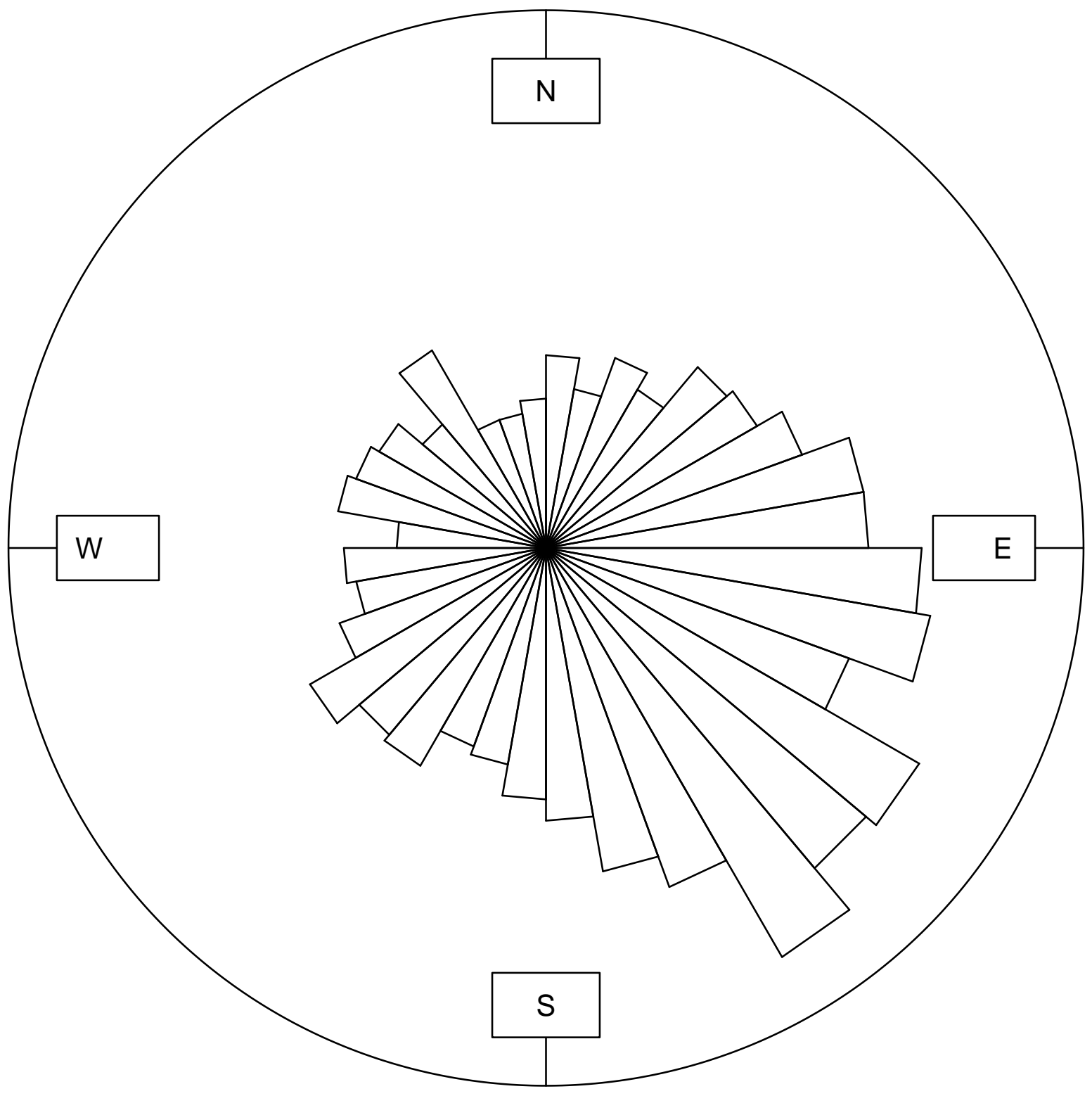


bootstrap 625

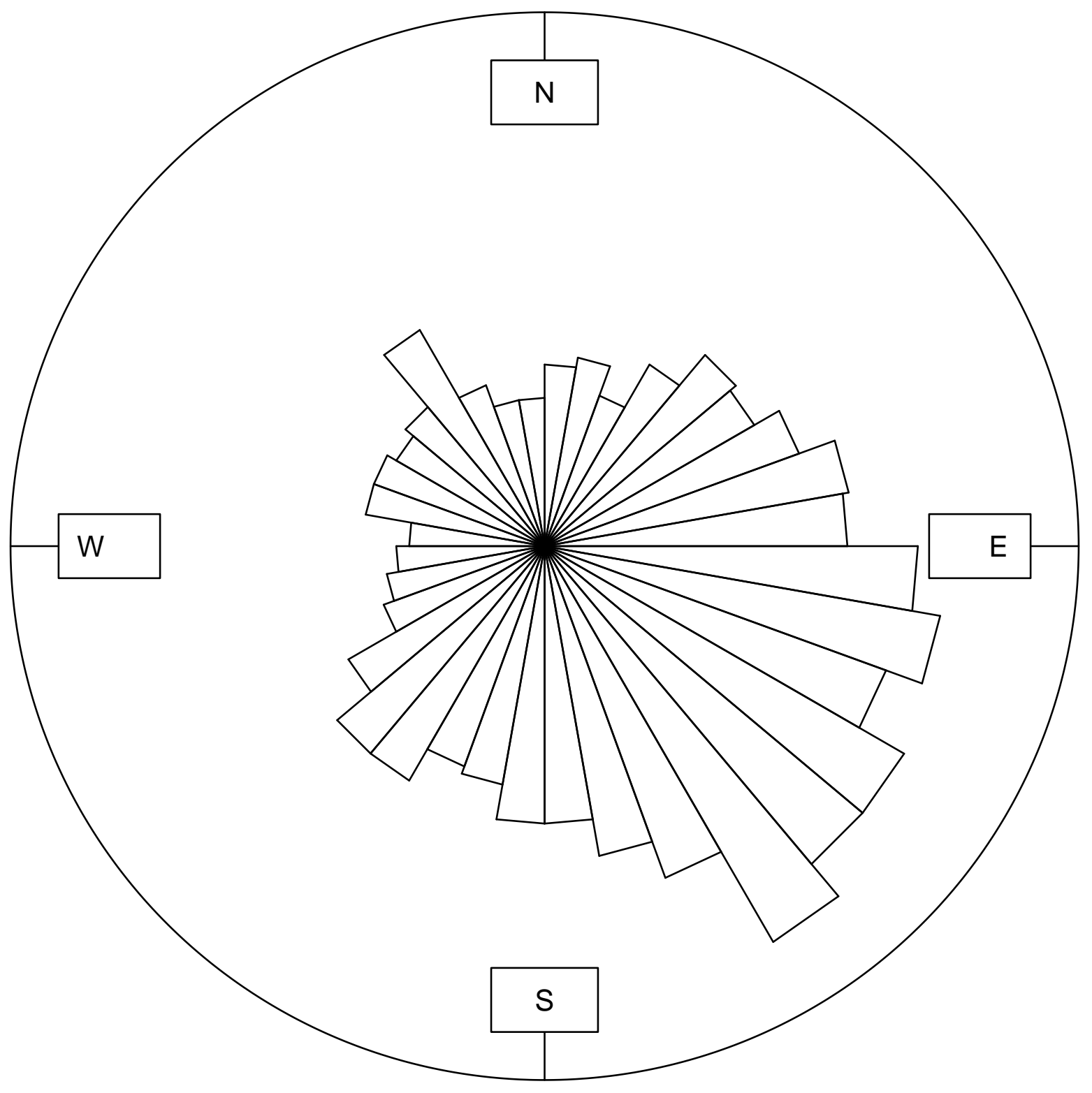




\section{bootstrap 626}

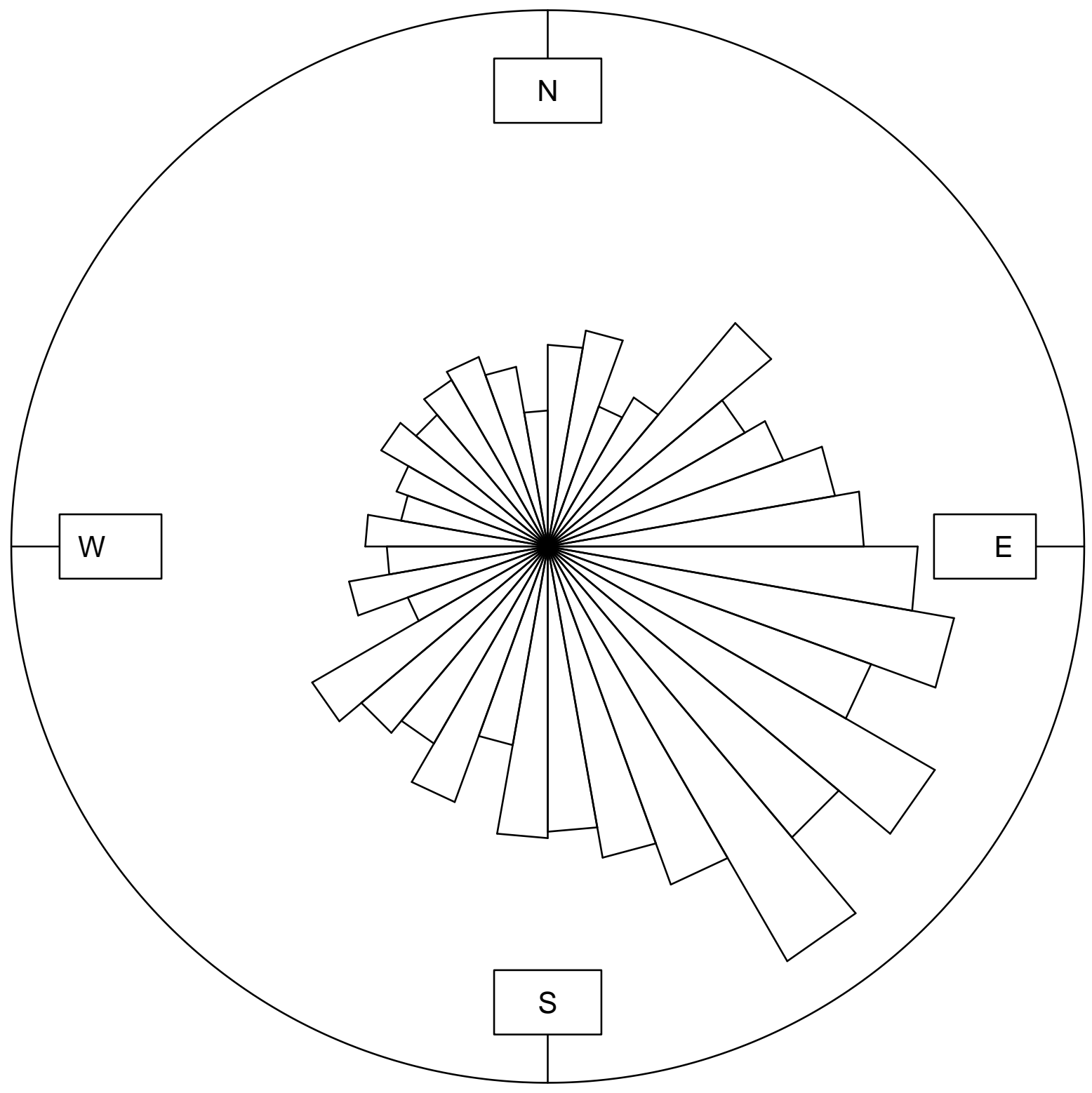


bootstrap 627

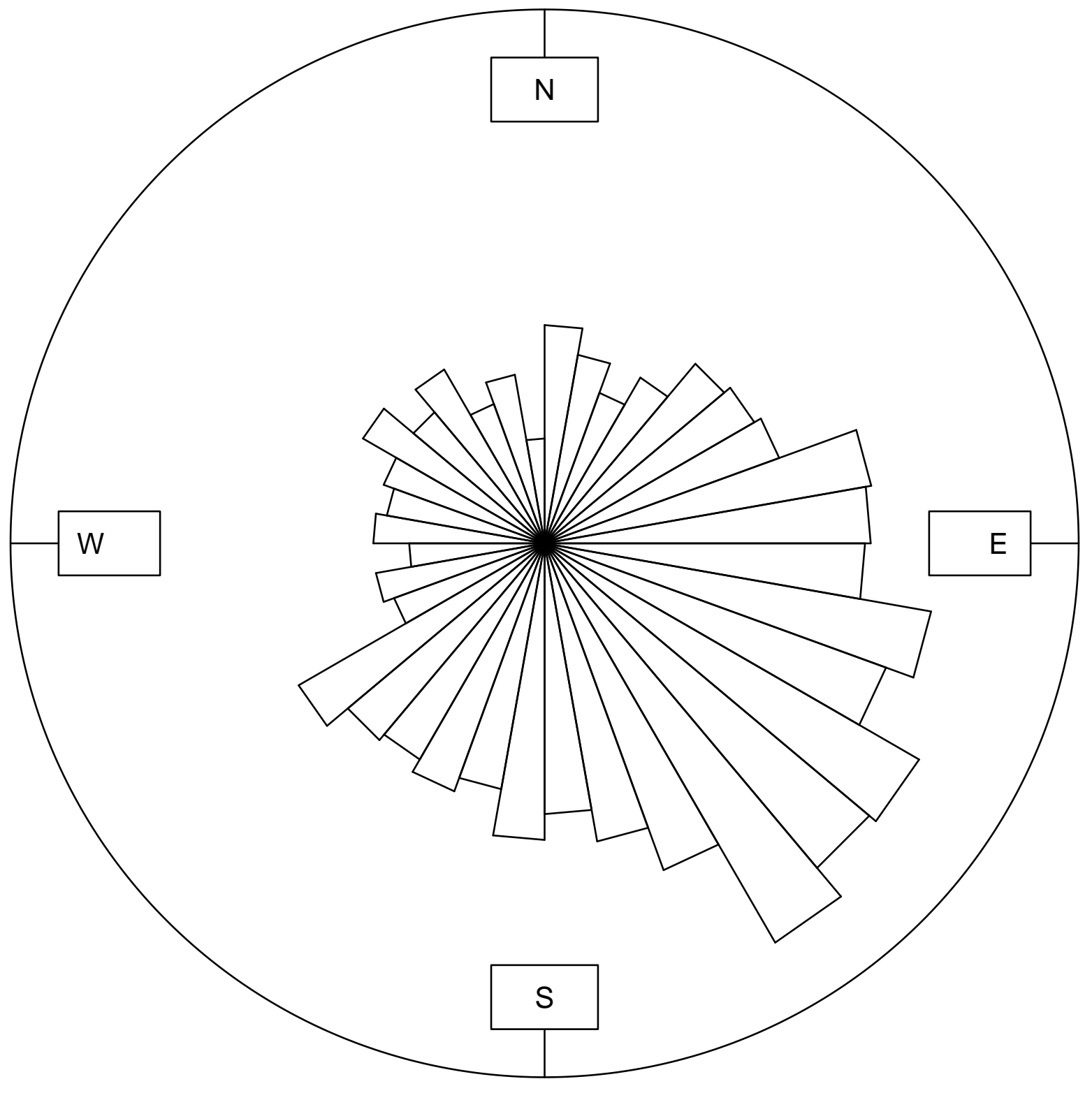


bootstrap 628

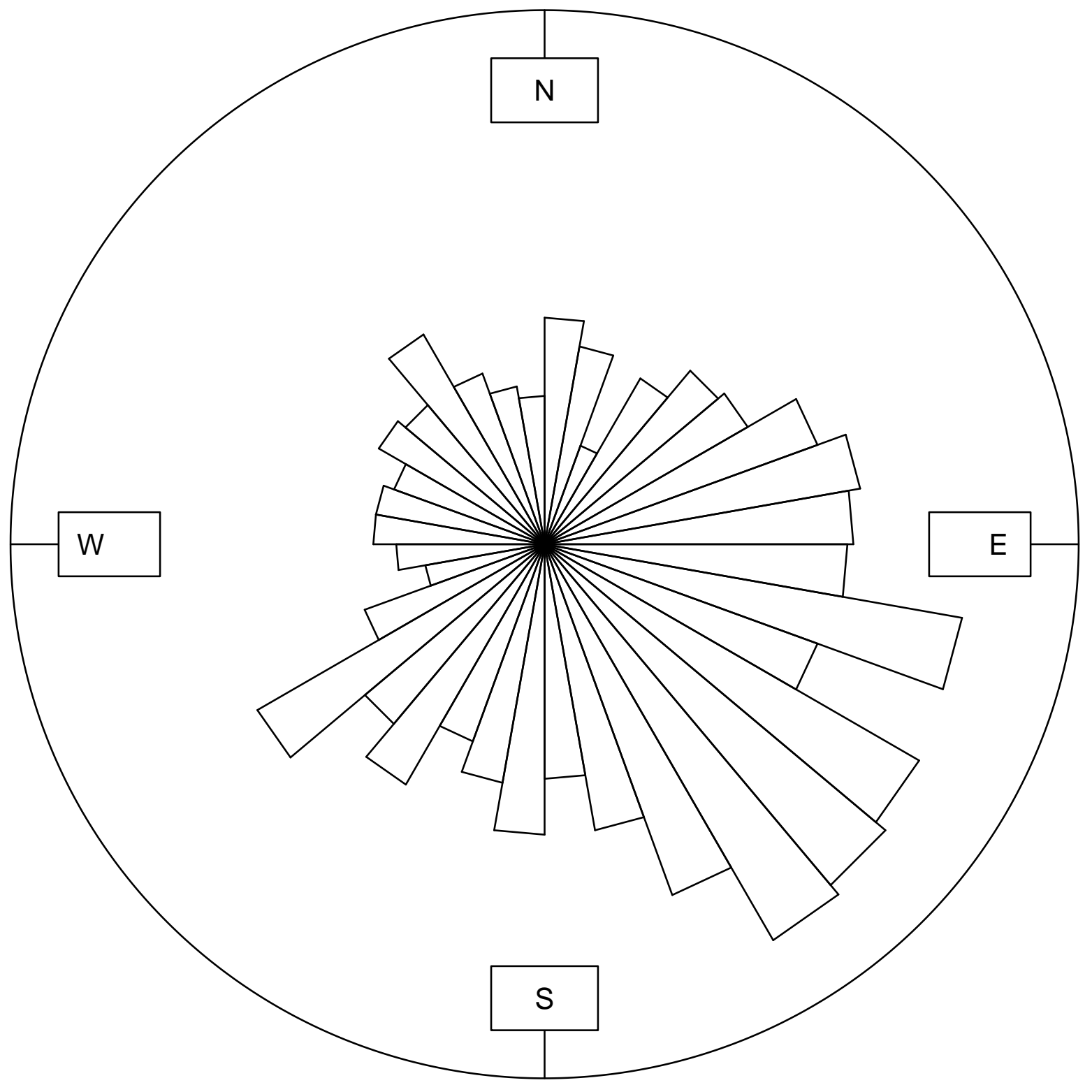


bootstrap 629

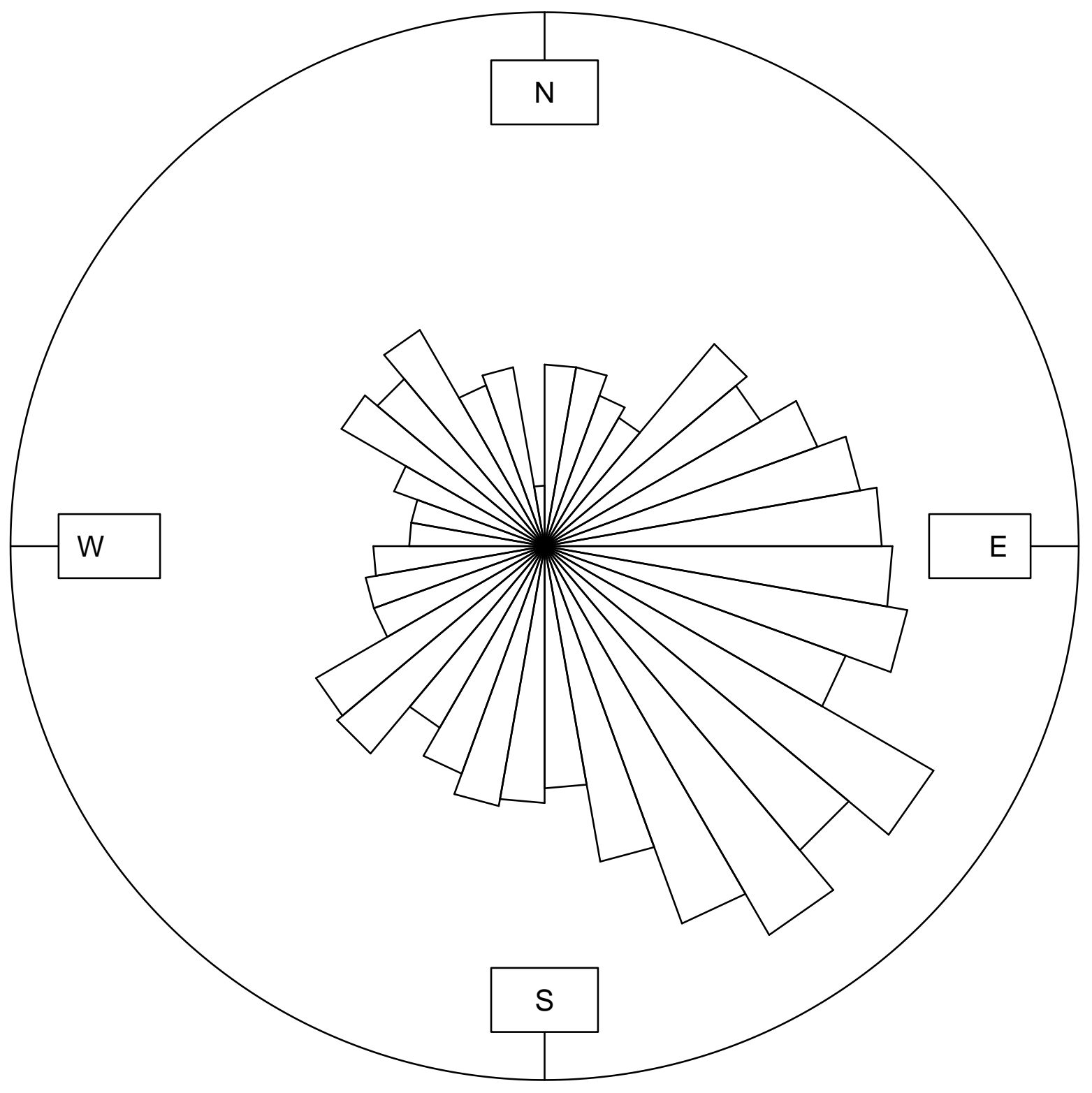


bootstrap 630

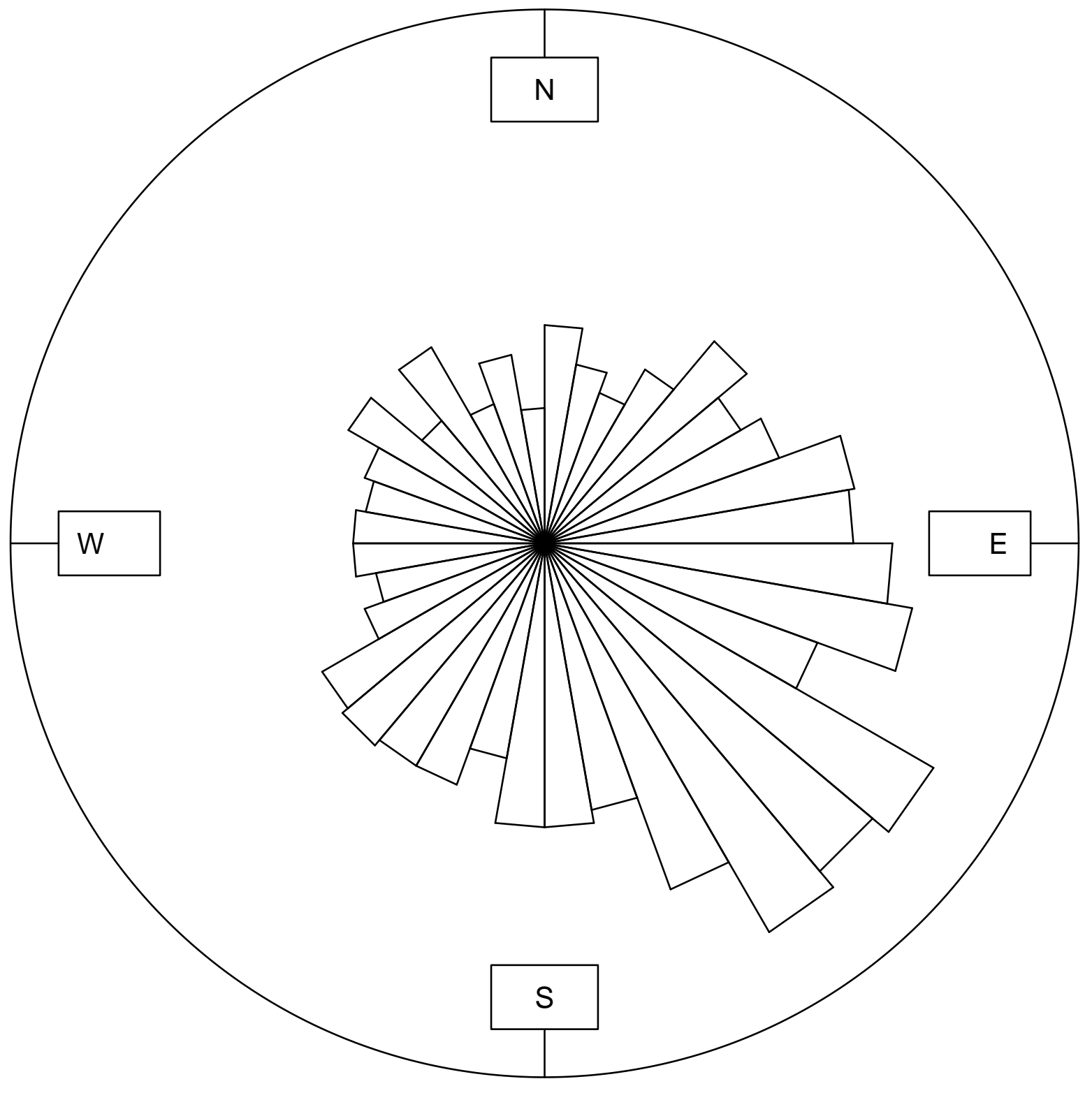


bootstrap 631

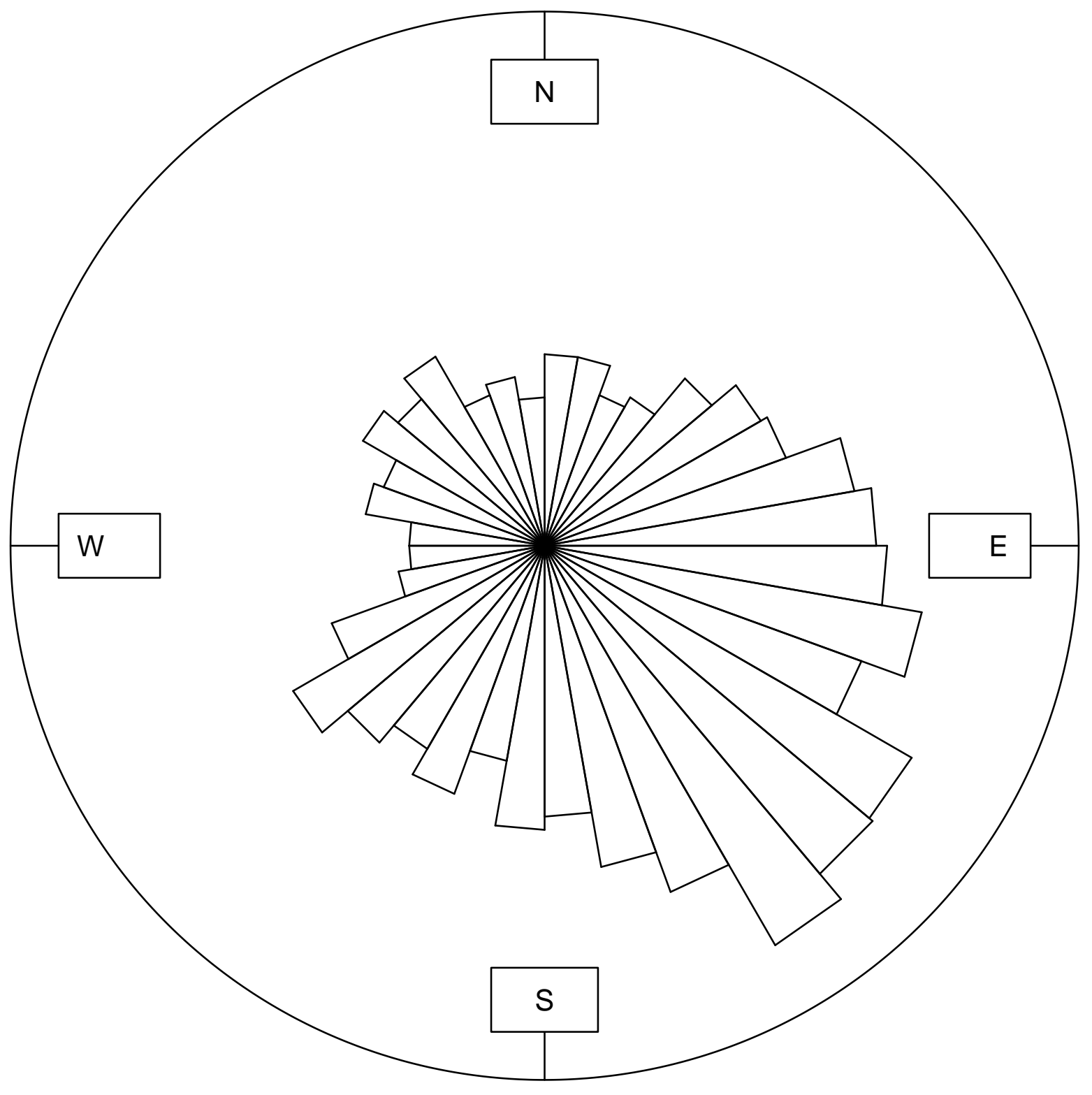


bootstrap 632

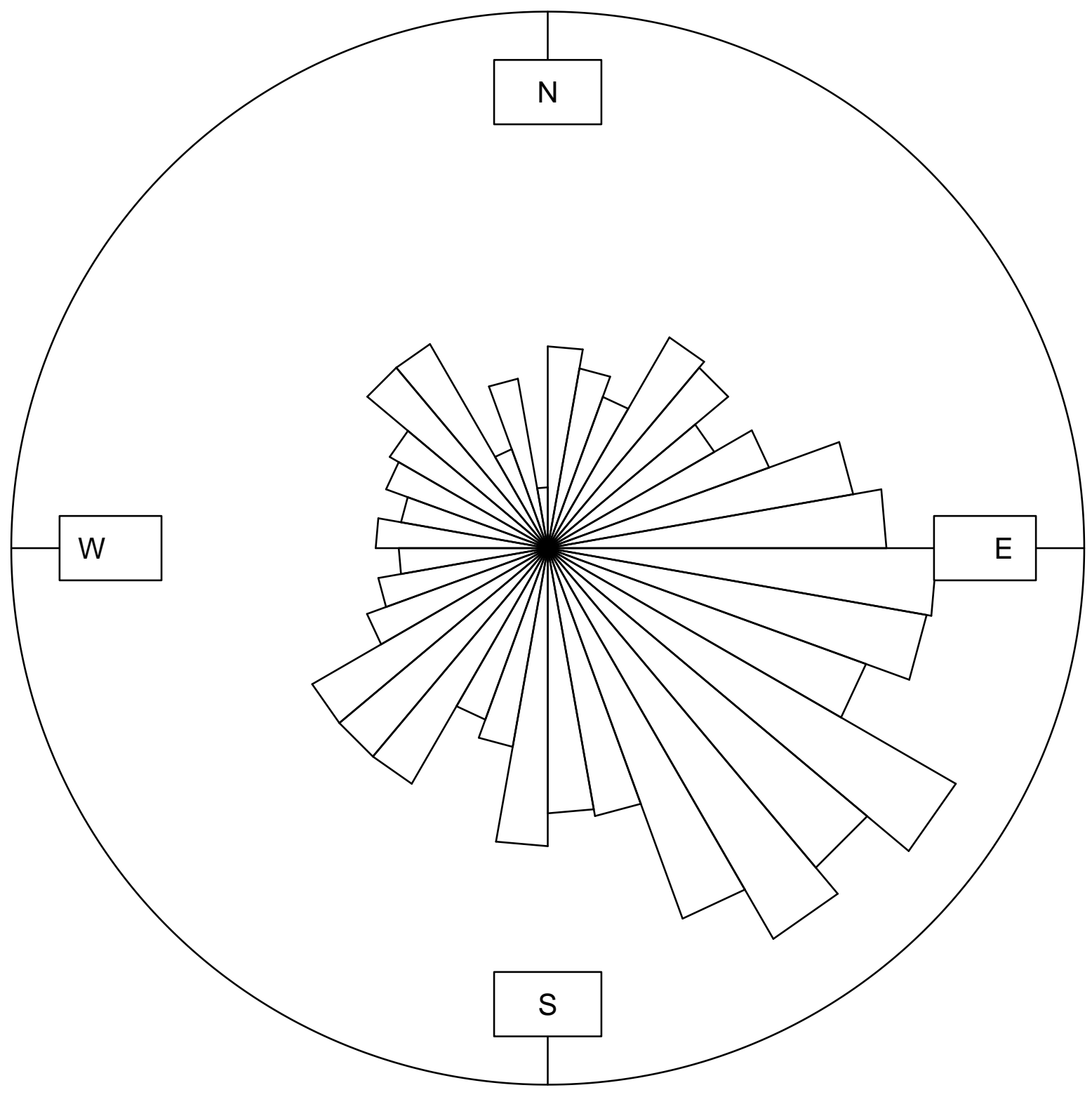




\section{bootstrap 633}

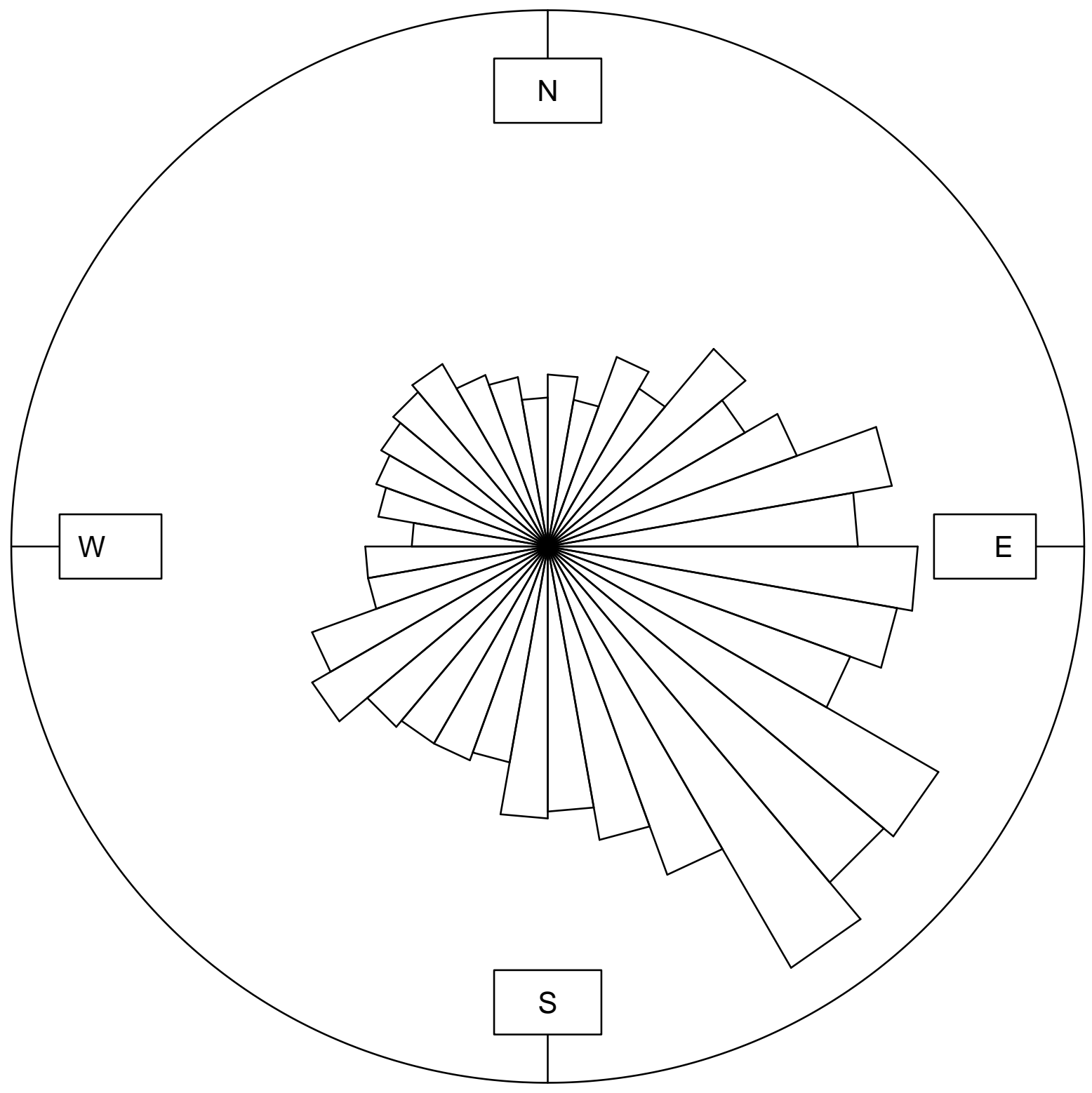




\section{bootstrap 634}

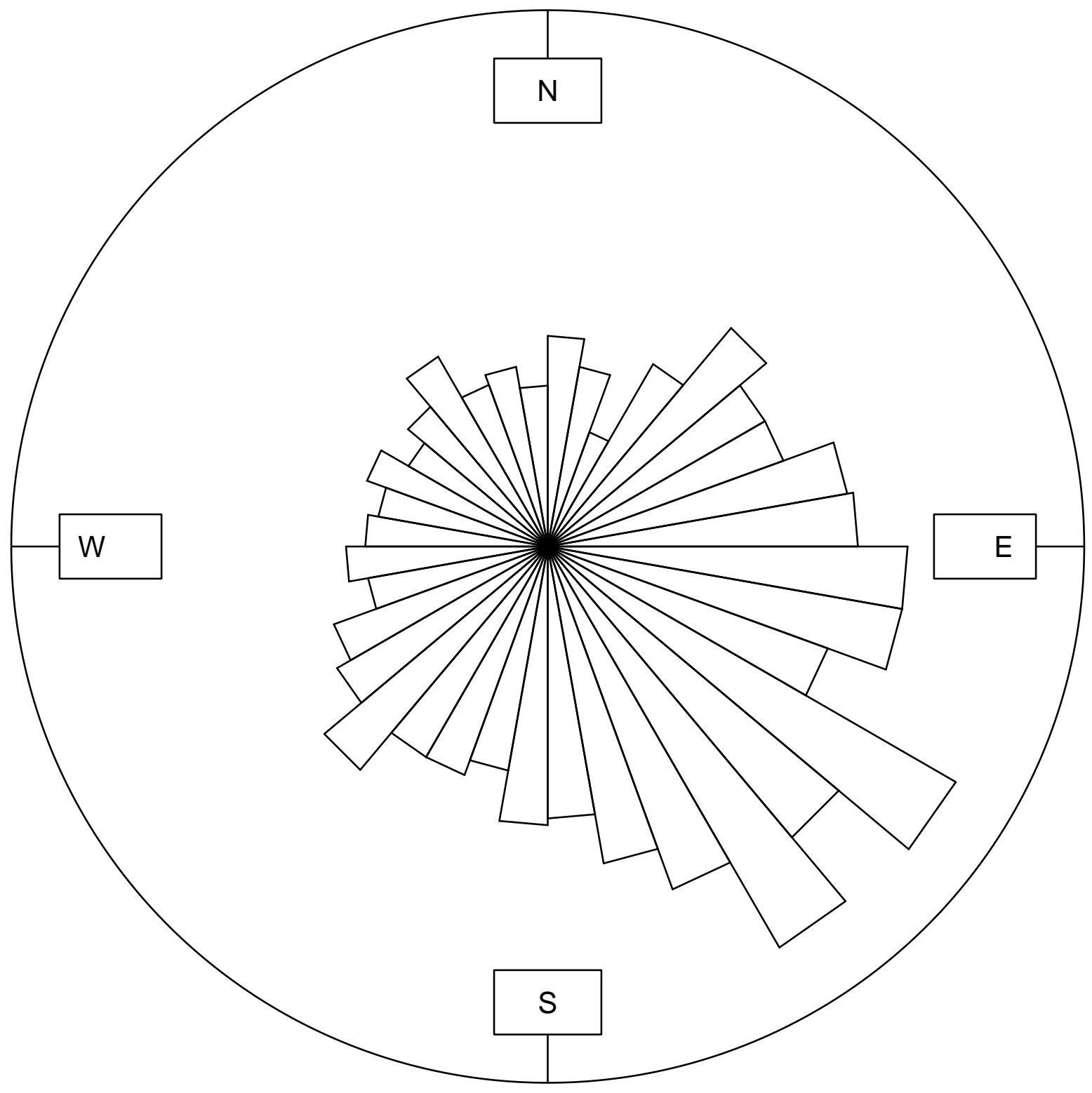




\section{bootstrap 635}

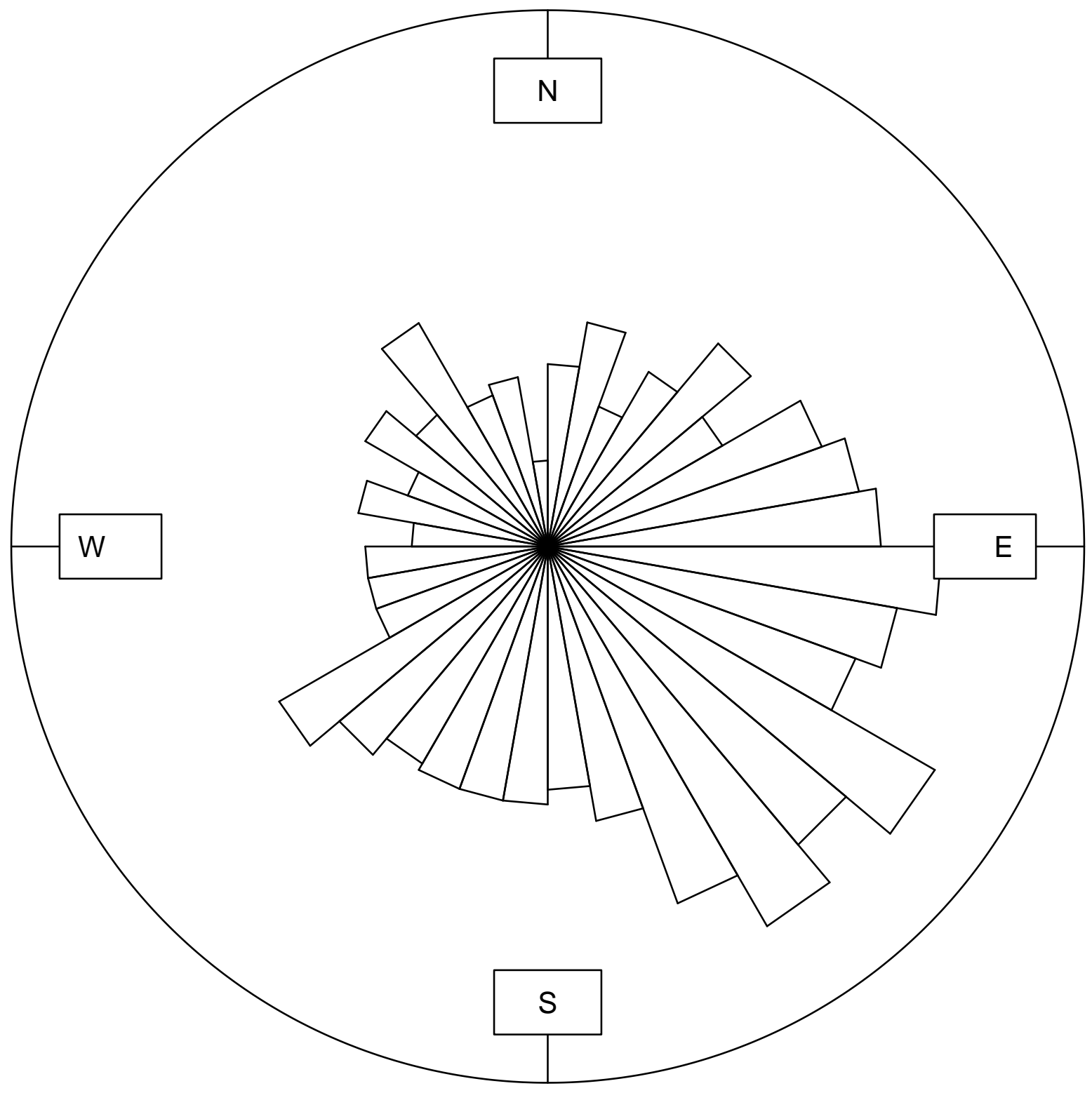




\section{bootstrap 636}

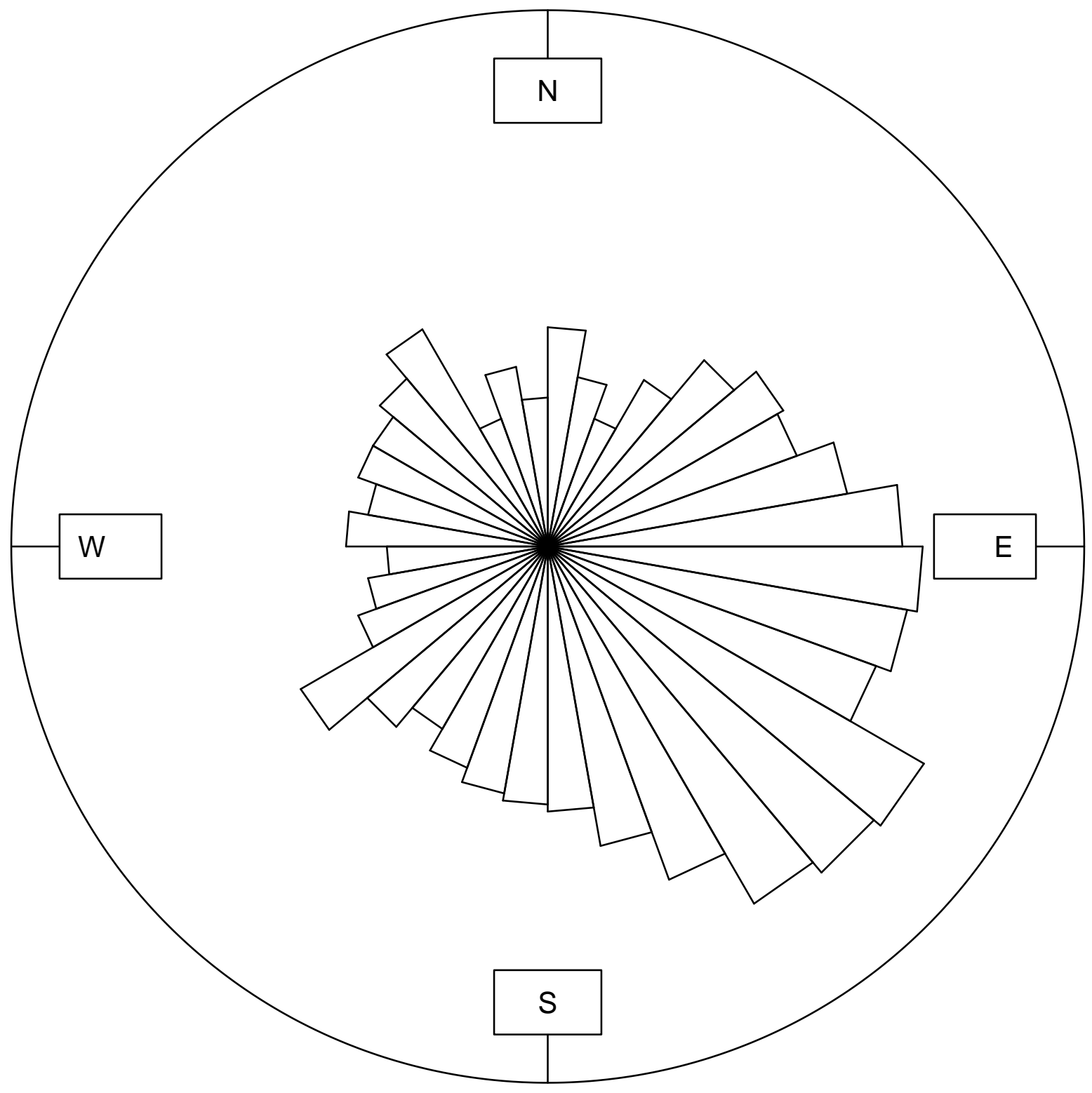


bootstrap 637

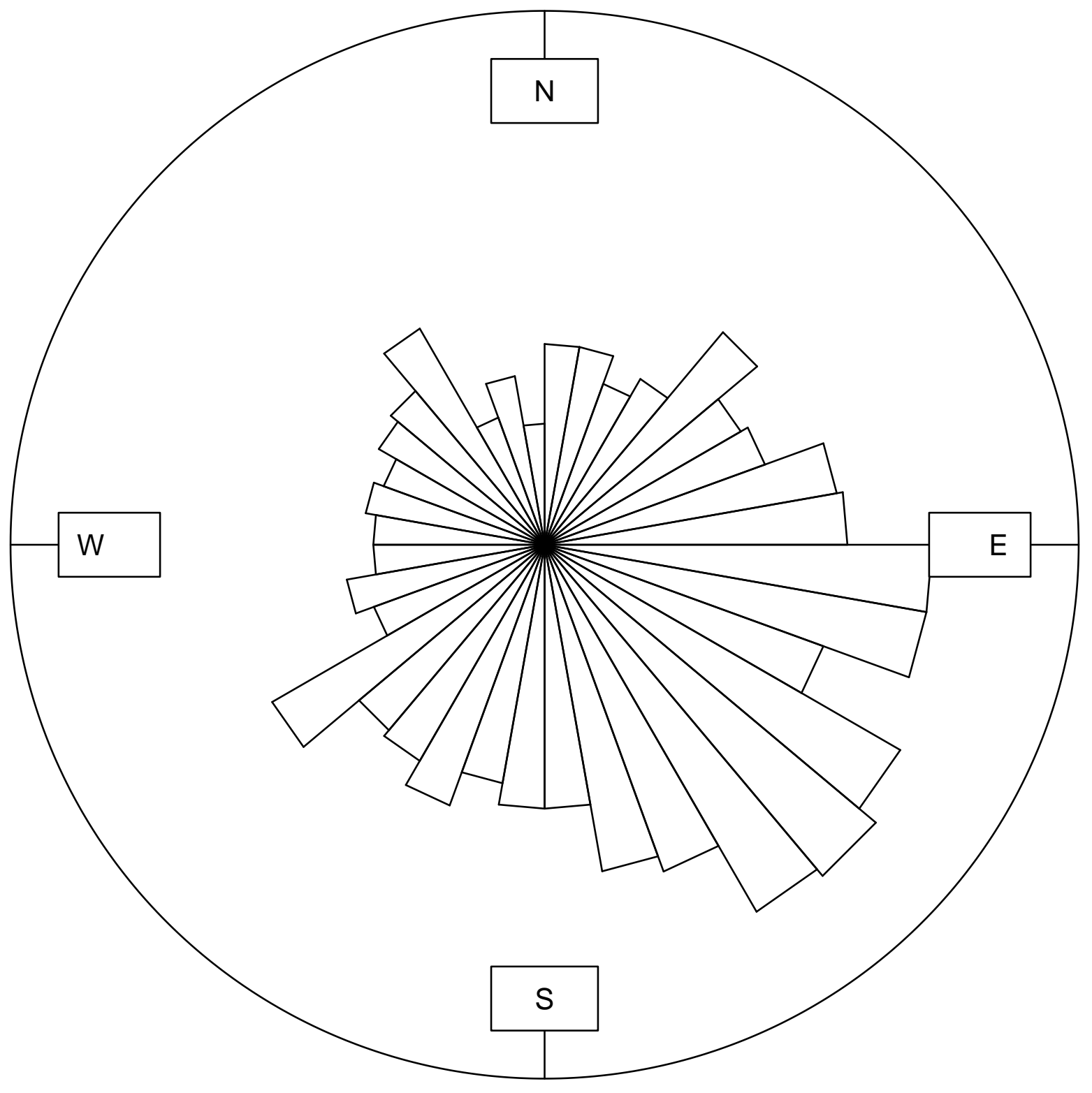


bootstrap 638

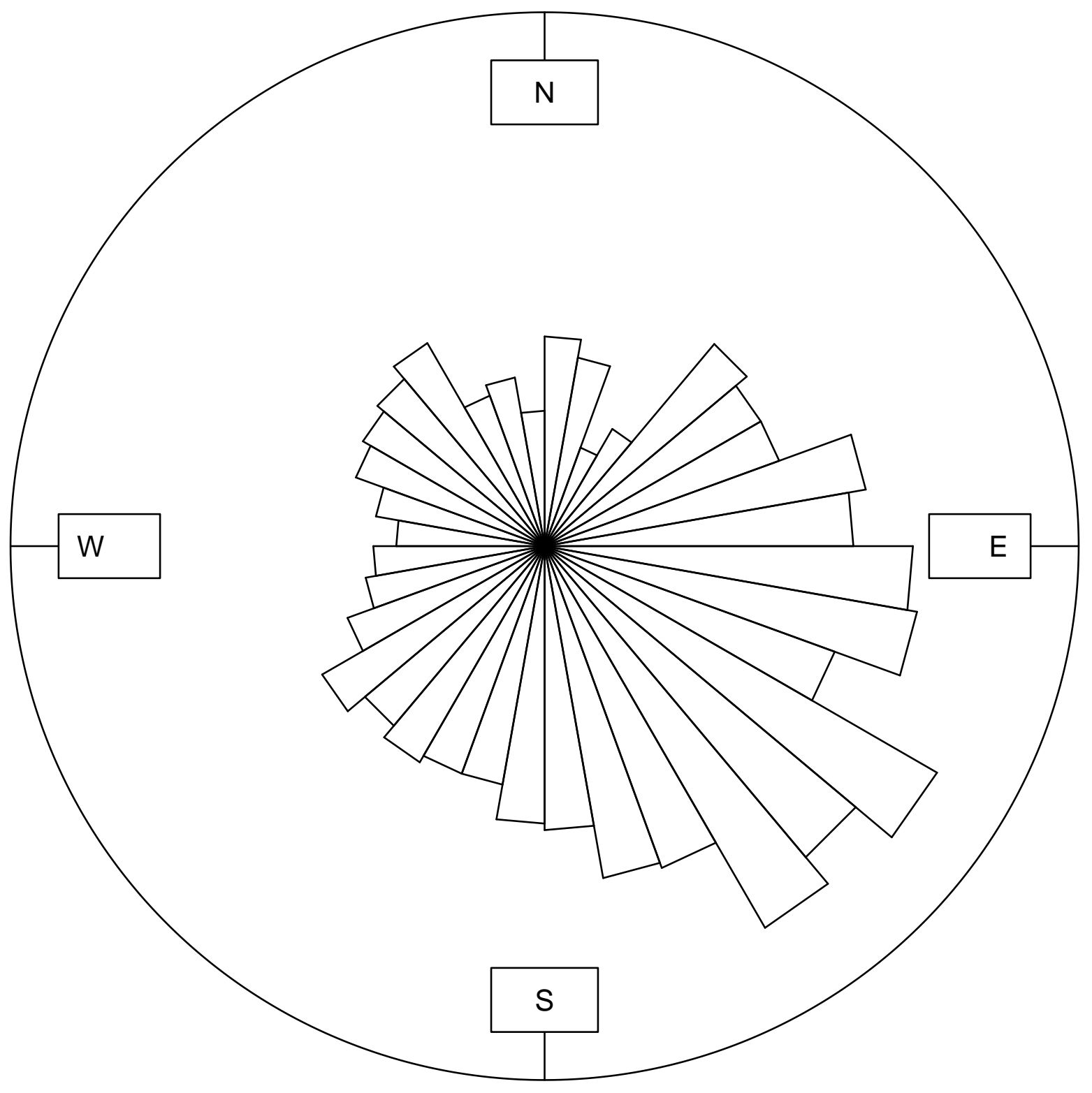


bootstrap 639

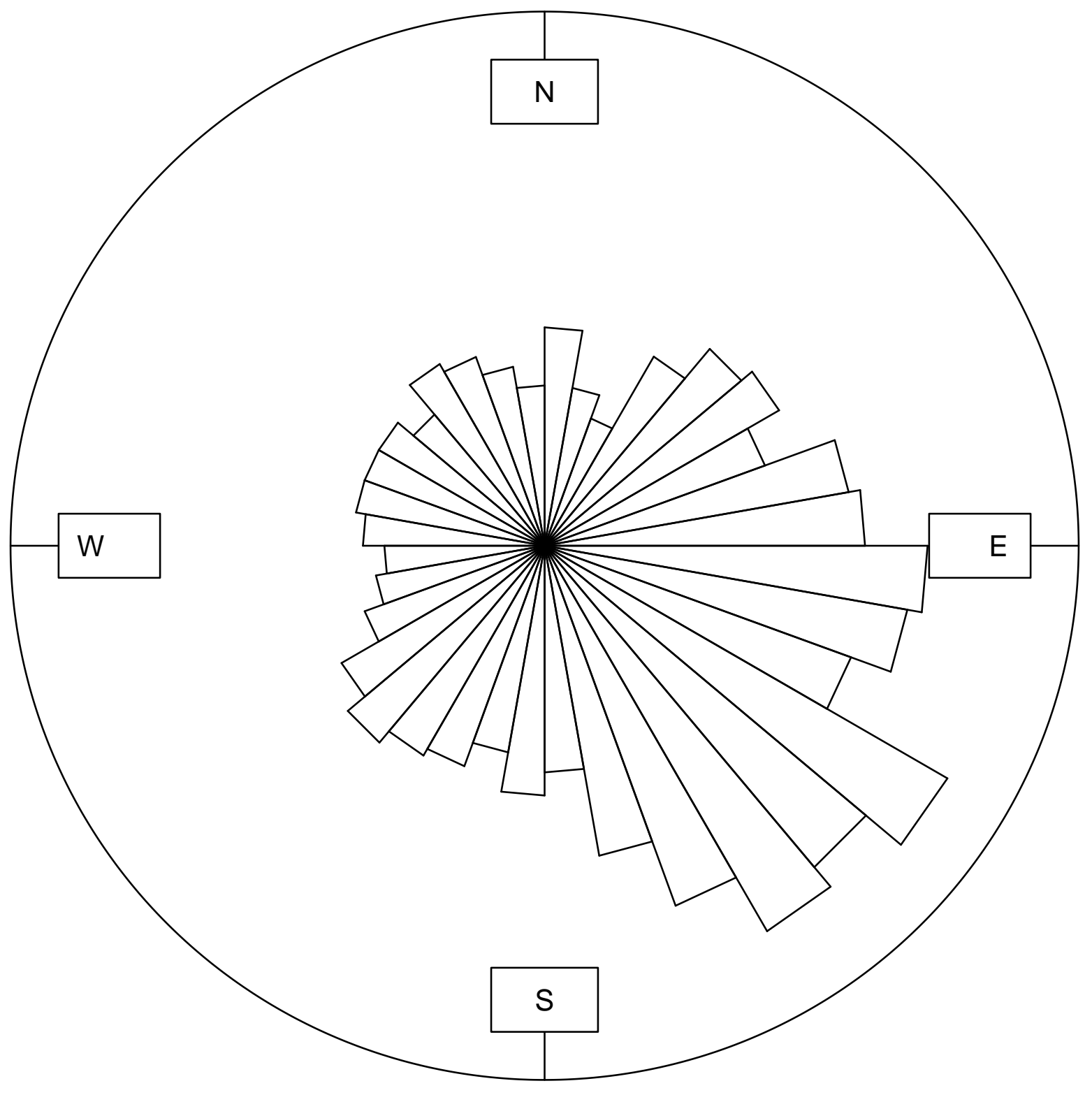


bootstrap 640

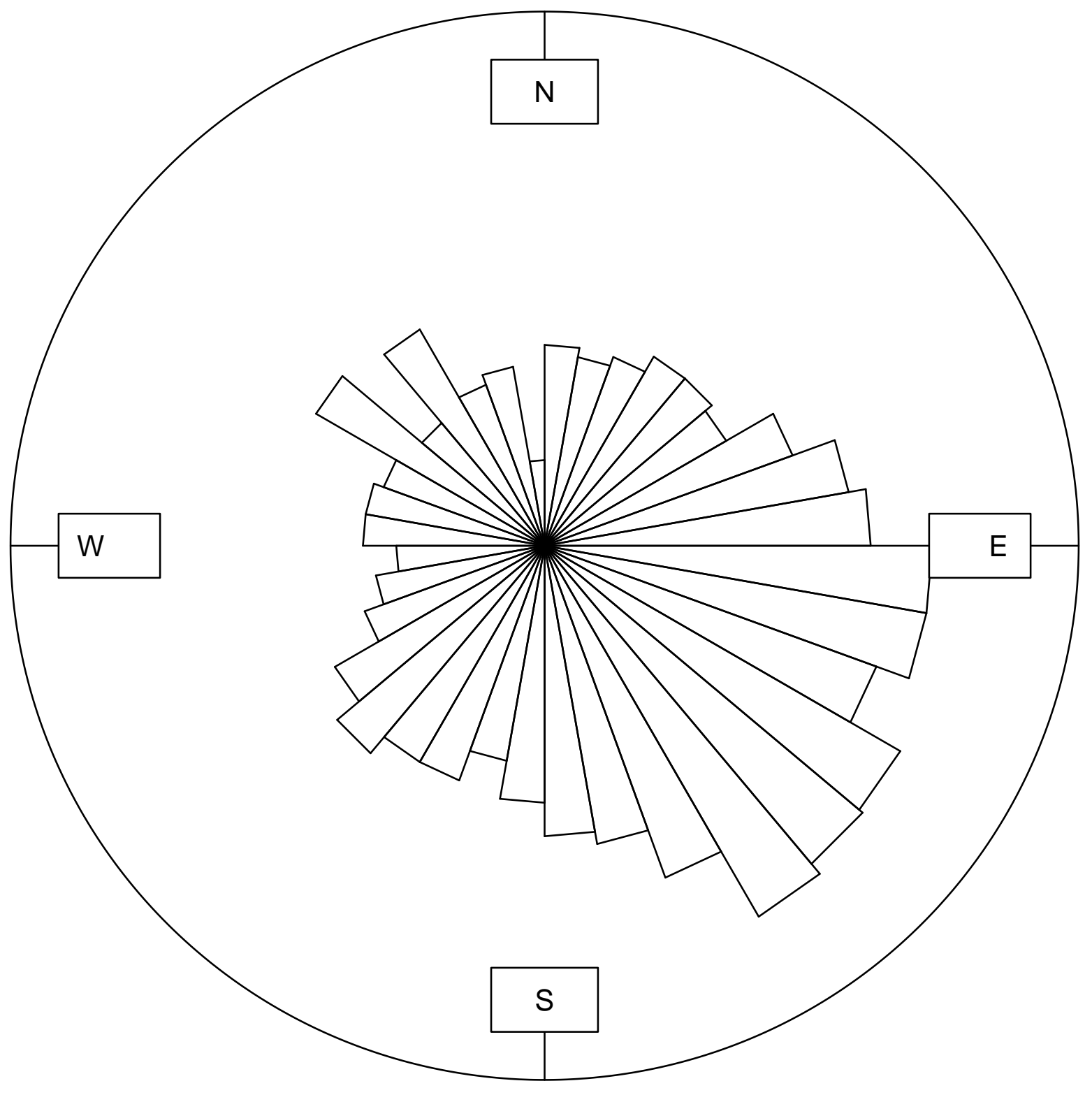




\section{bootstrap 641}

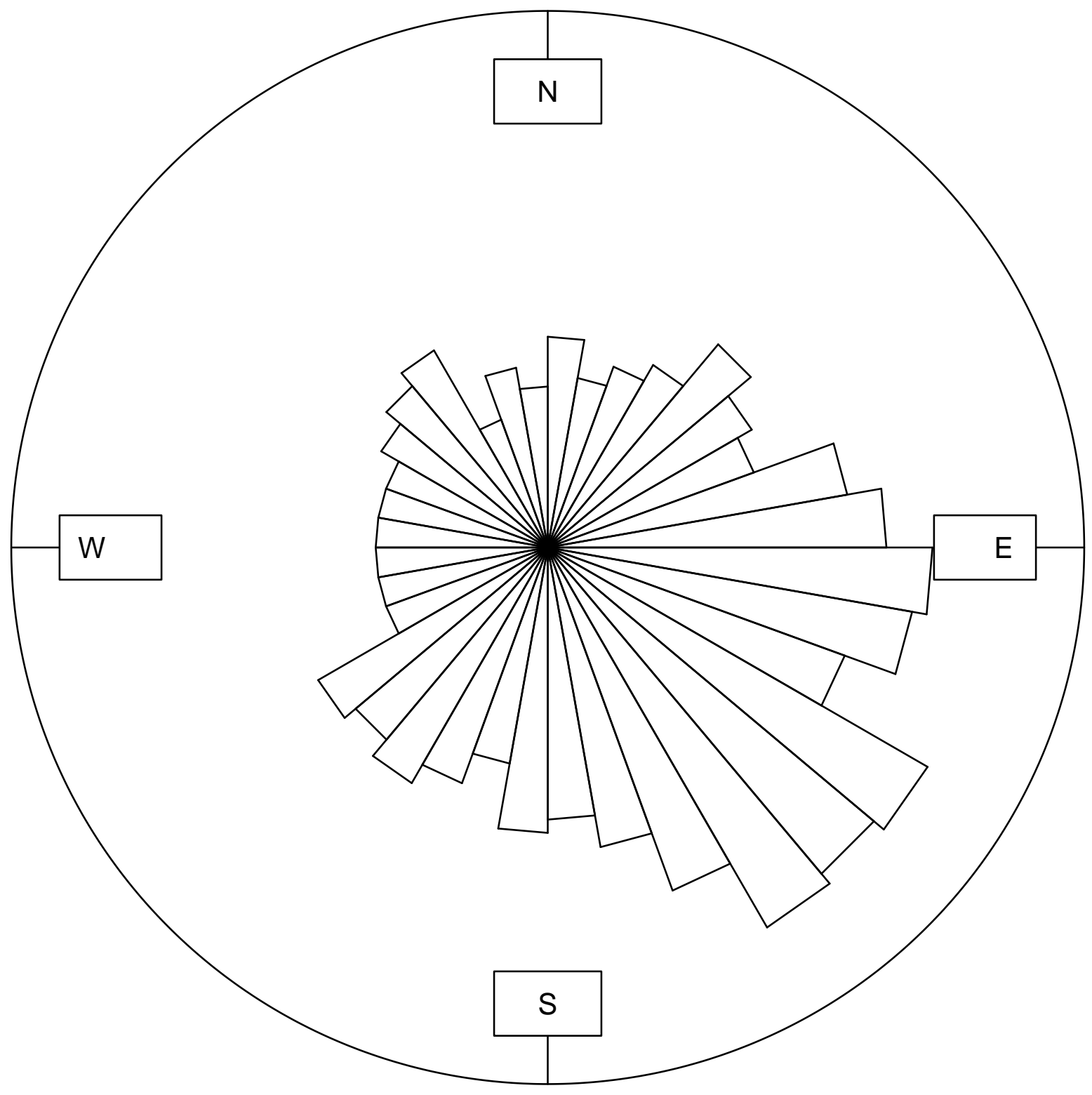


bootstrap 642

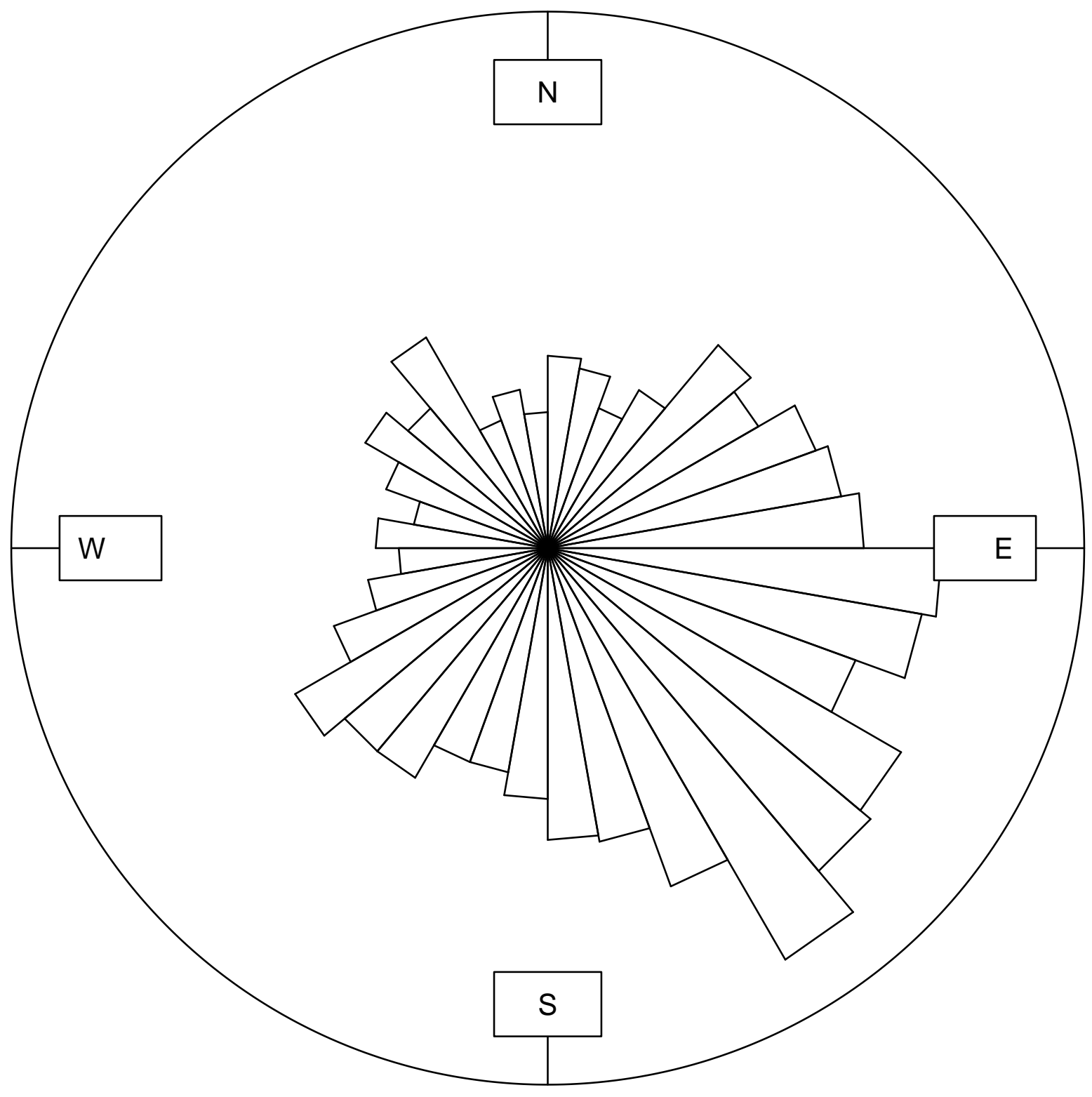


bootstrap 643

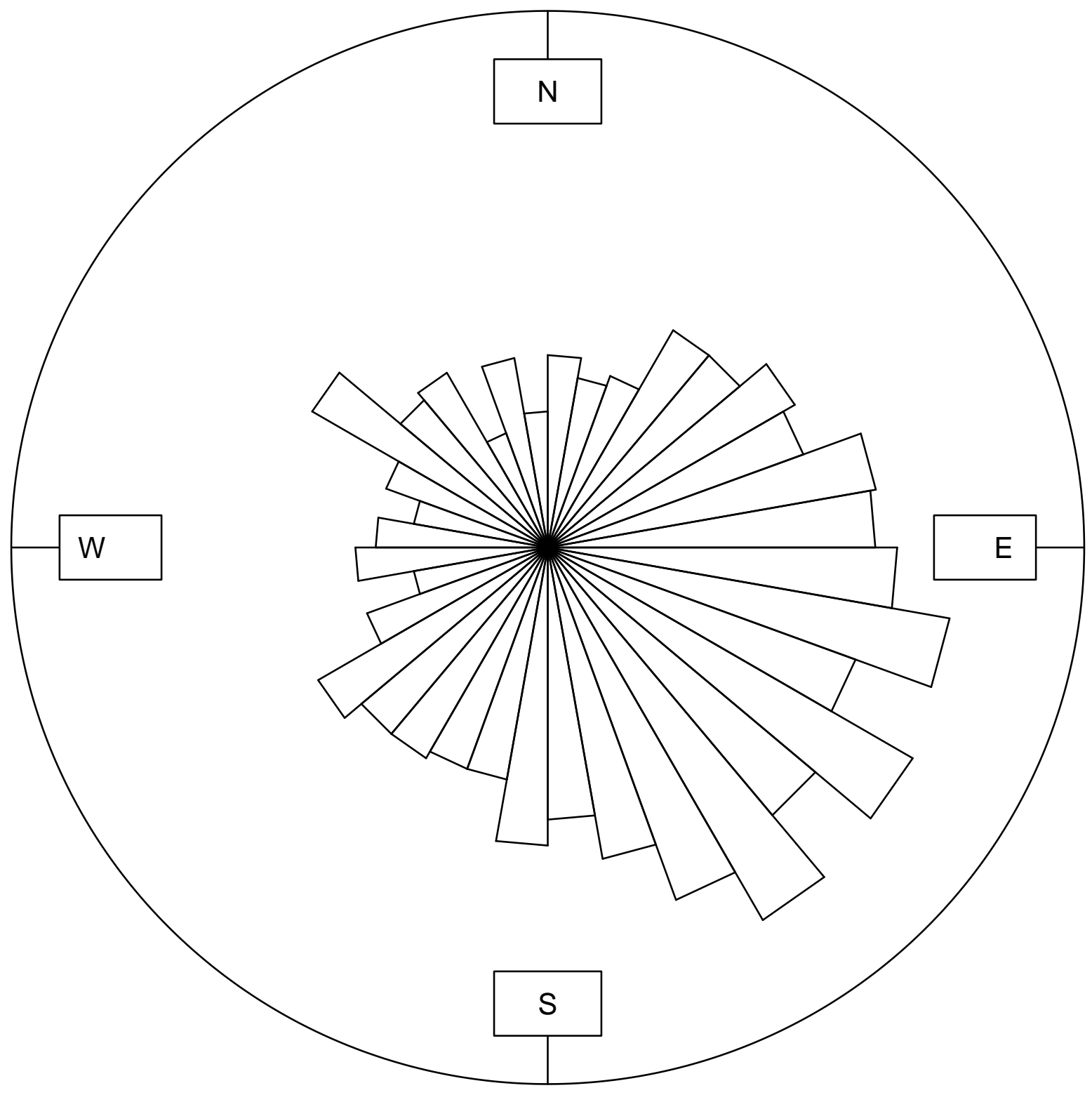




\section{bootstrap 644}

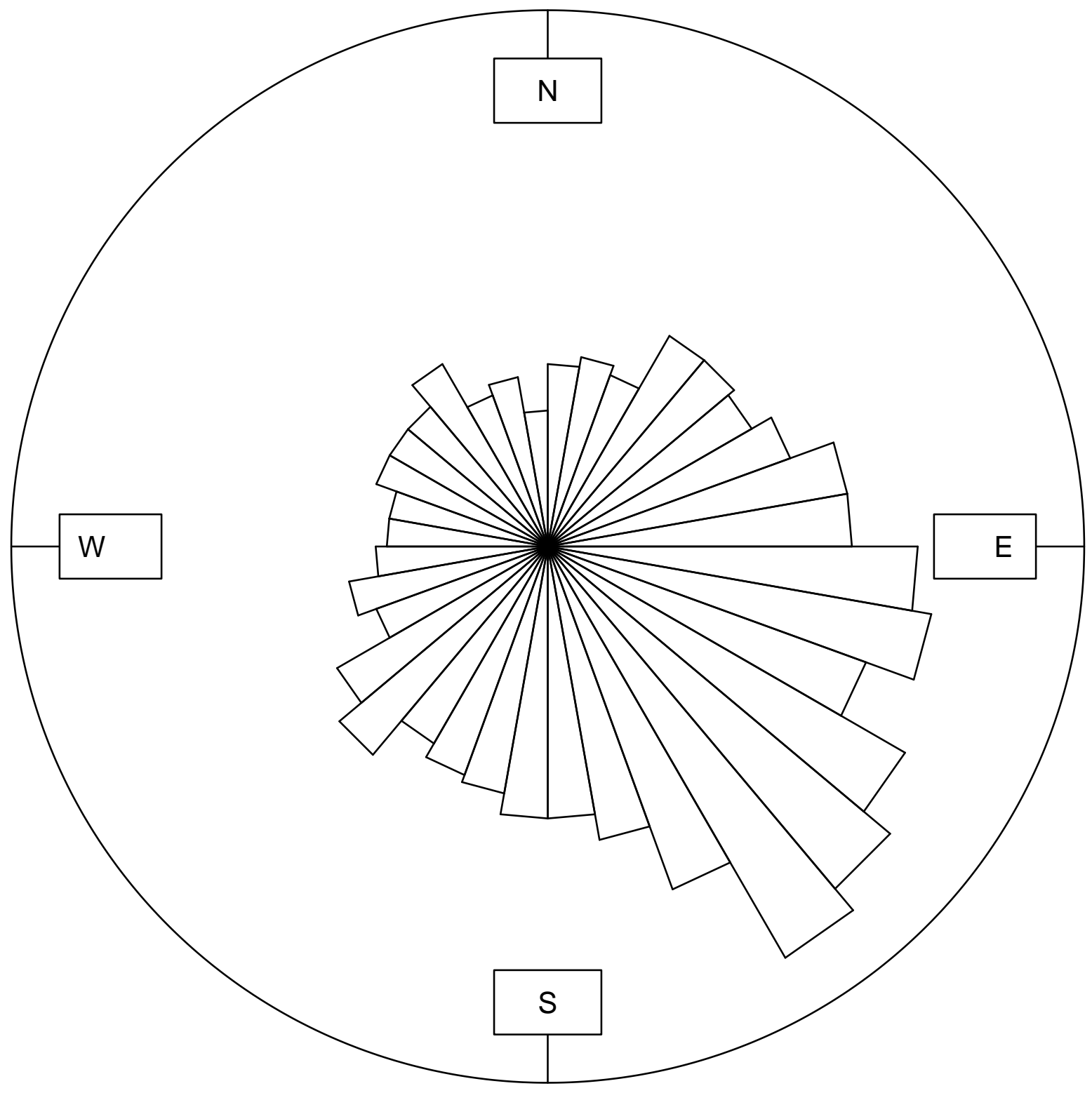




\section{bootstrap 645}

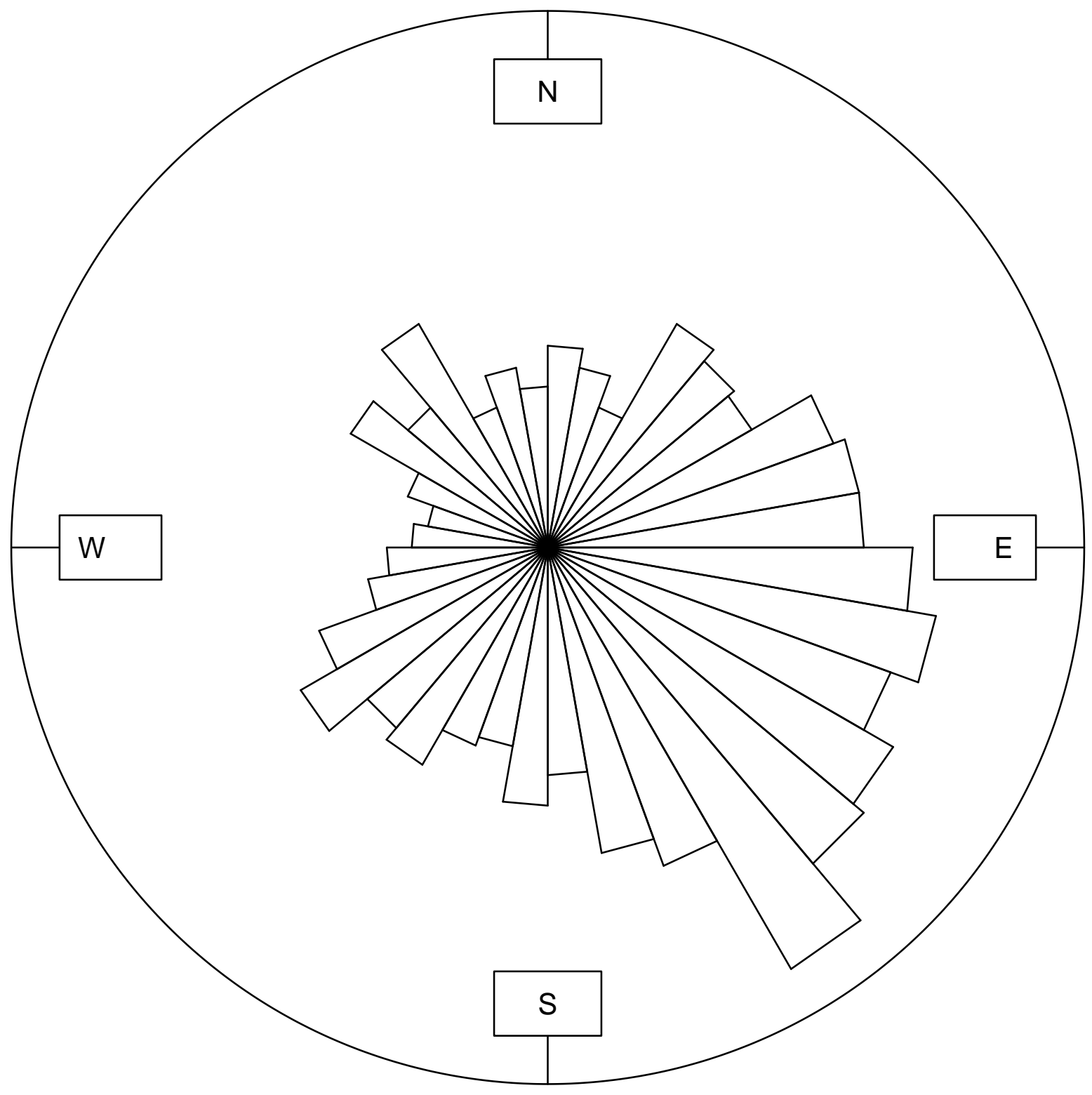


bootstrap 646

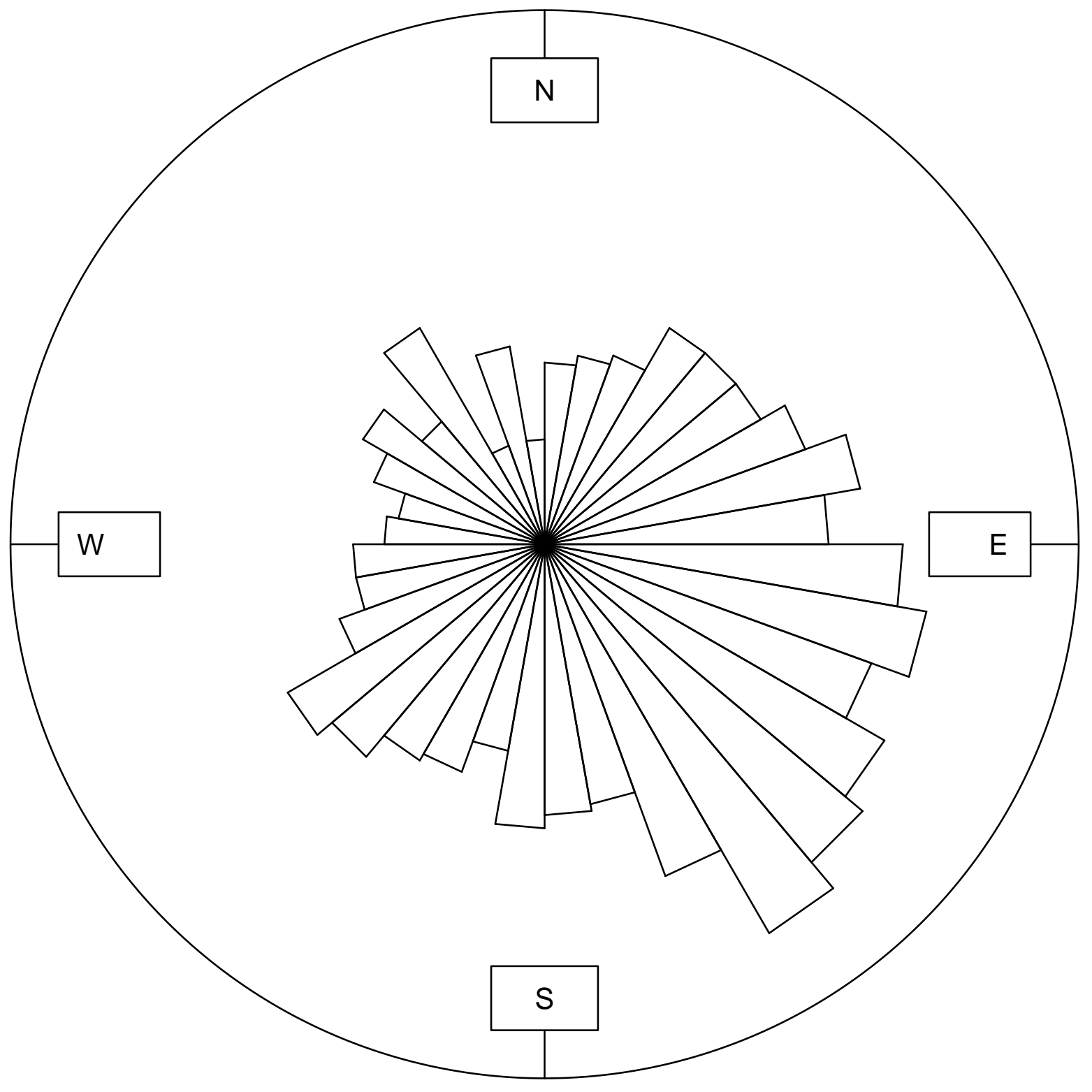


bootstrap 647

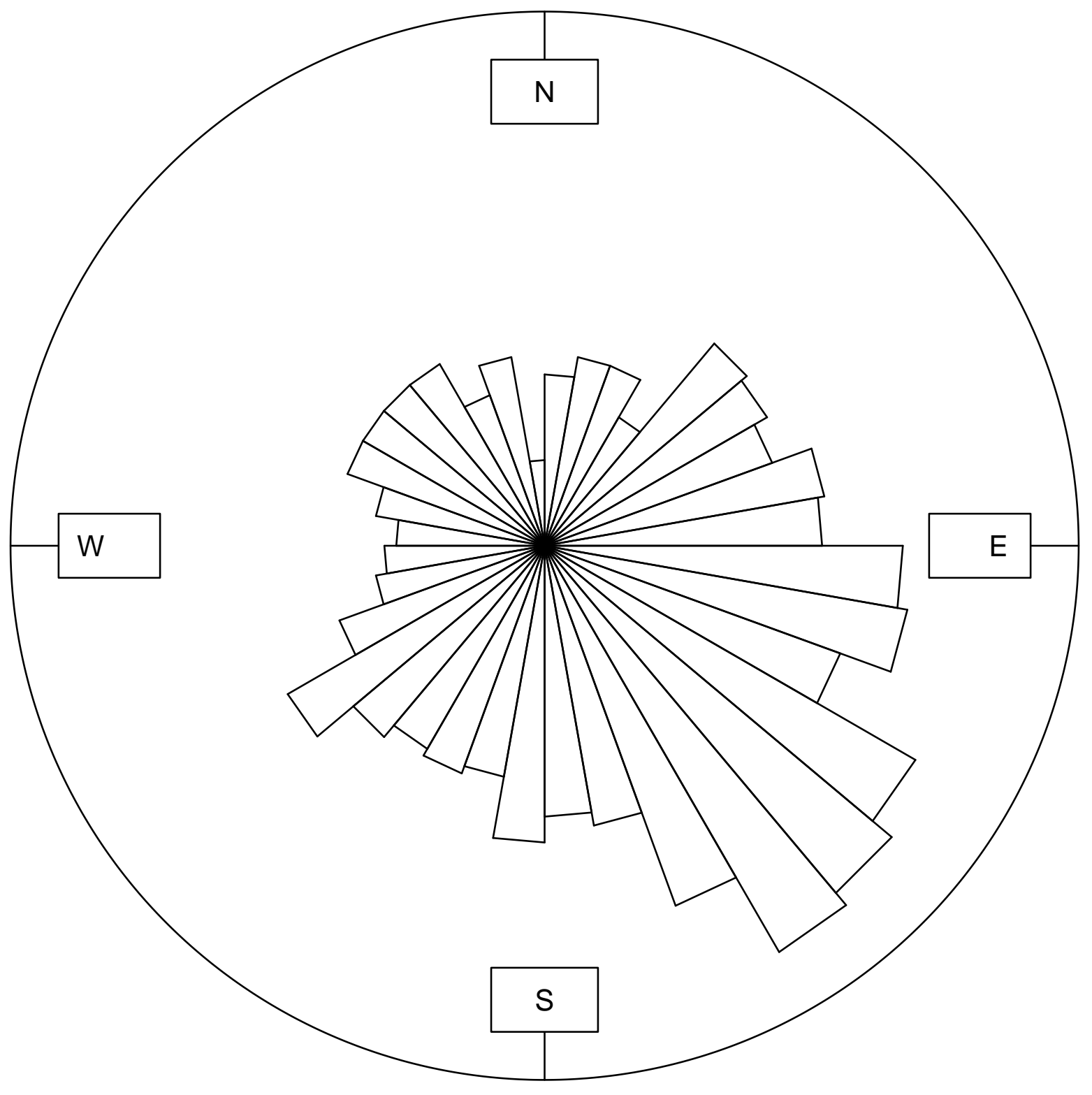


bootstrap 648

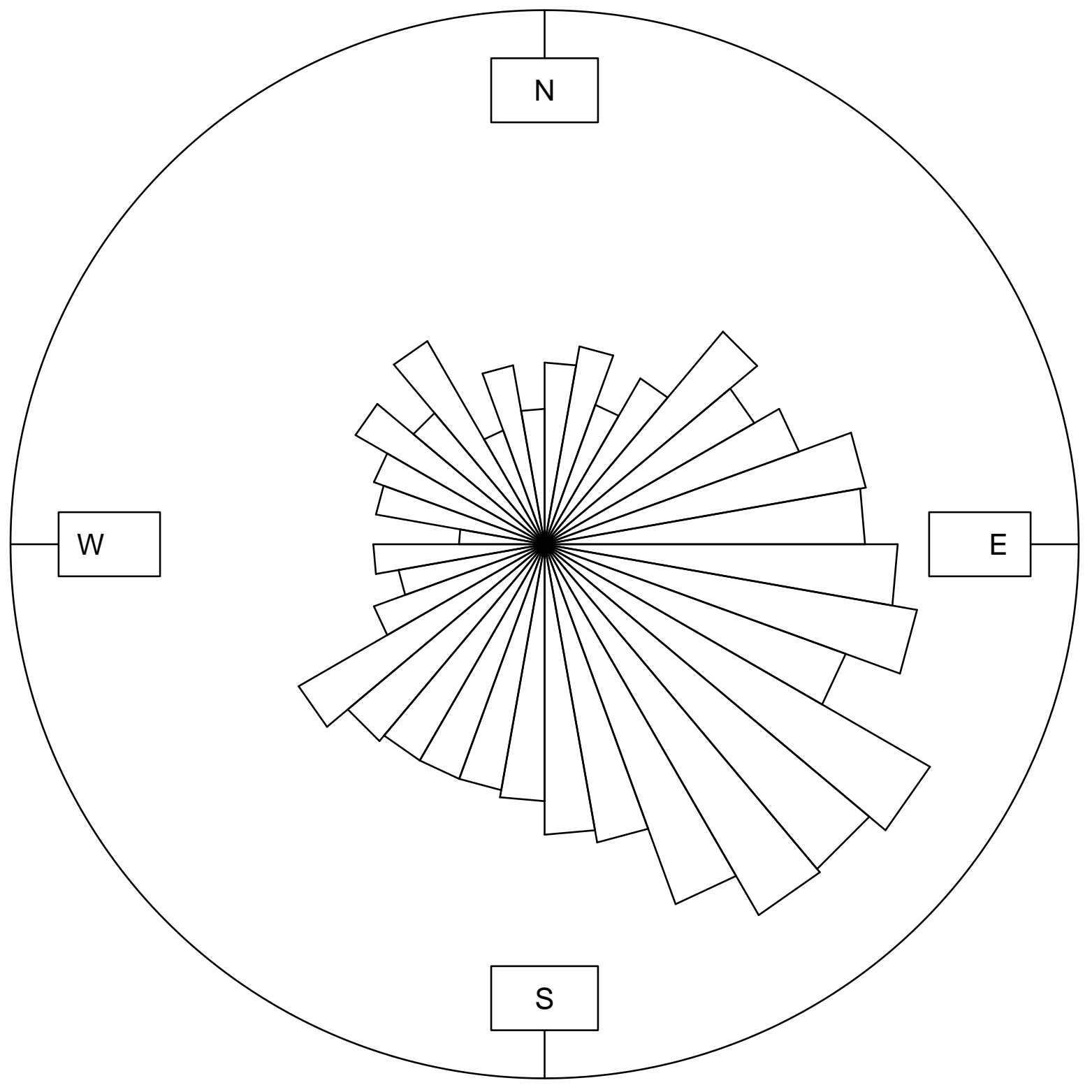


bootstrap 649

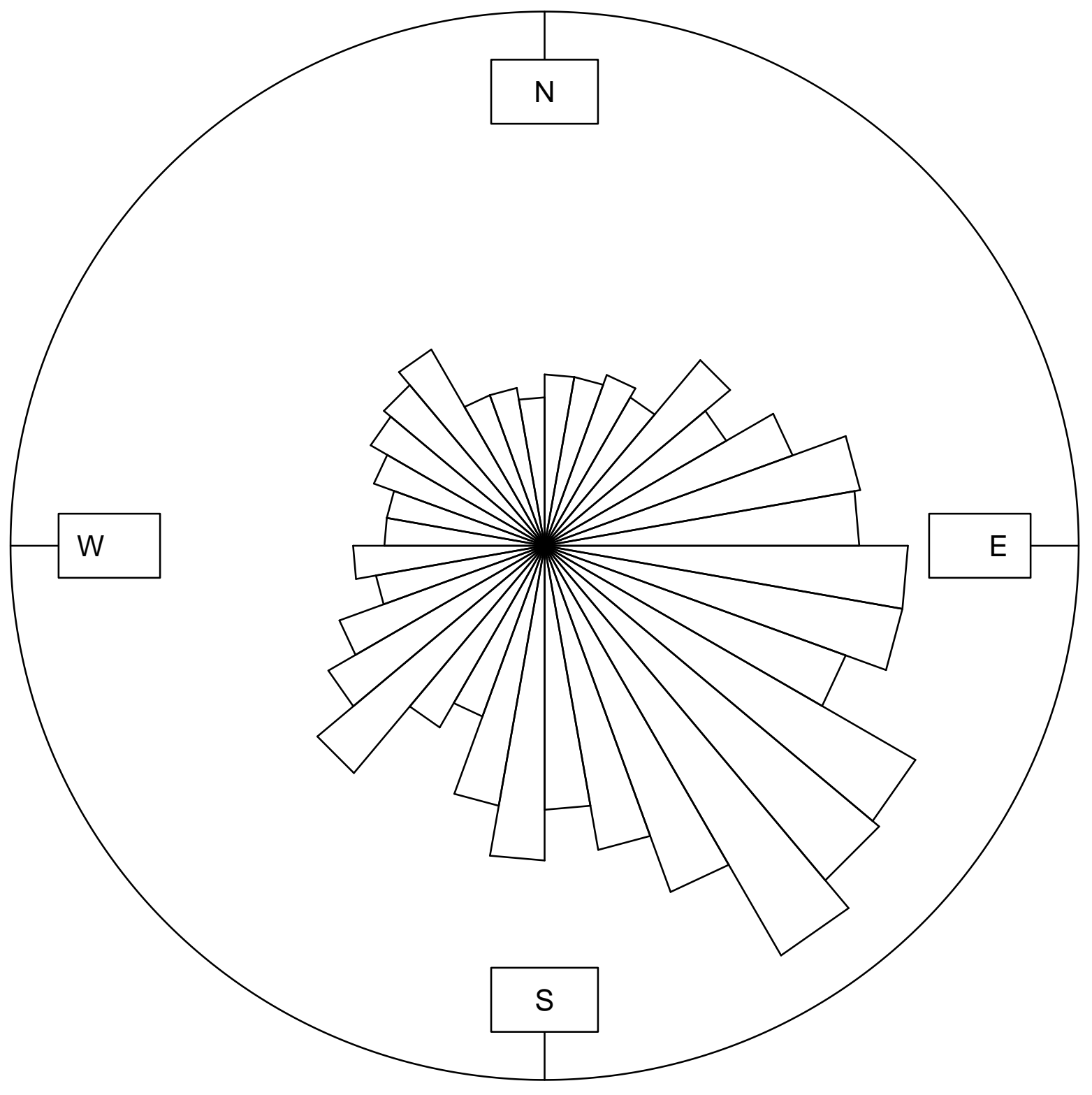


bootstrap 650

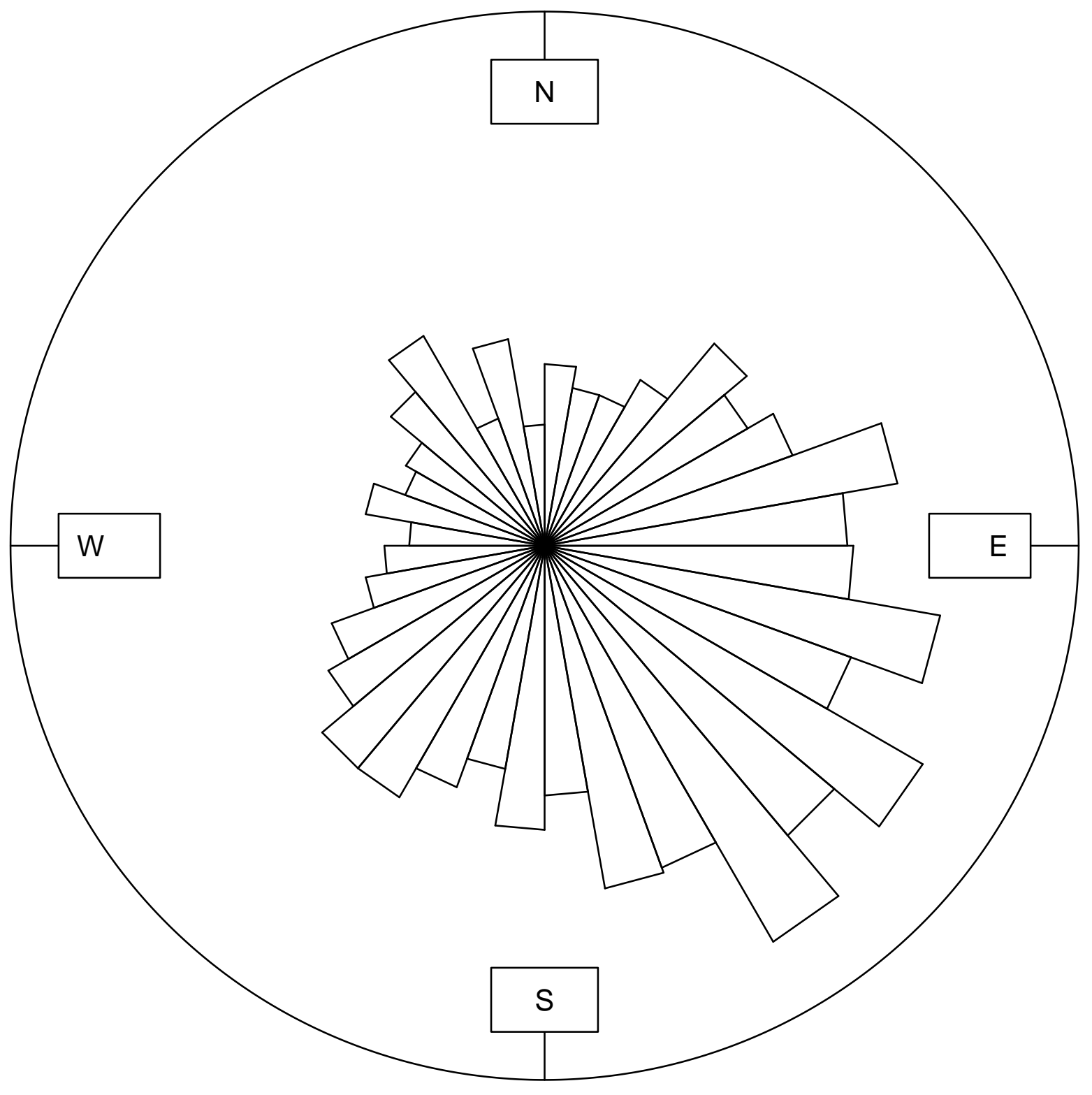




\section{bootstrap 651}

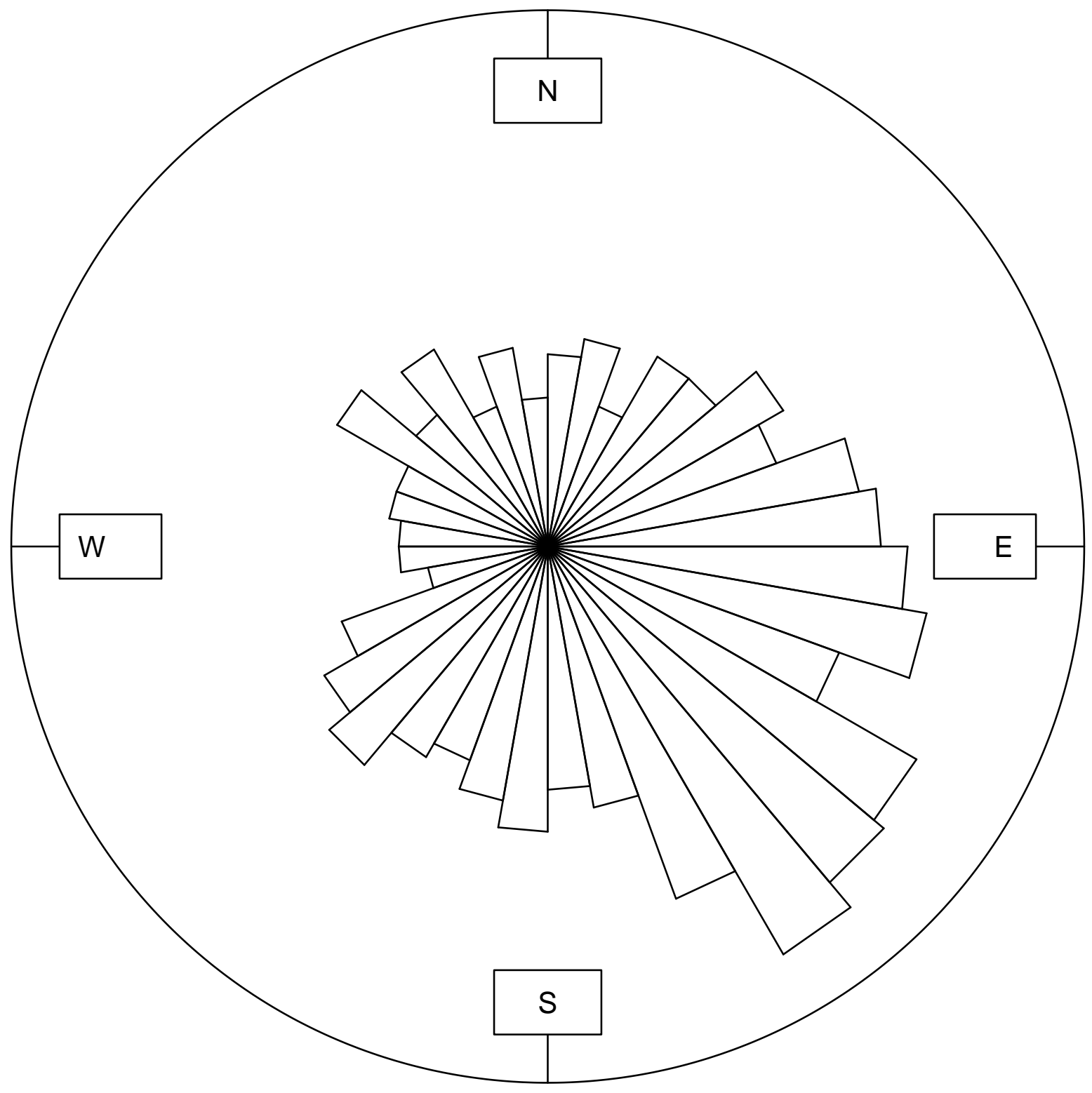


bootstrap 652

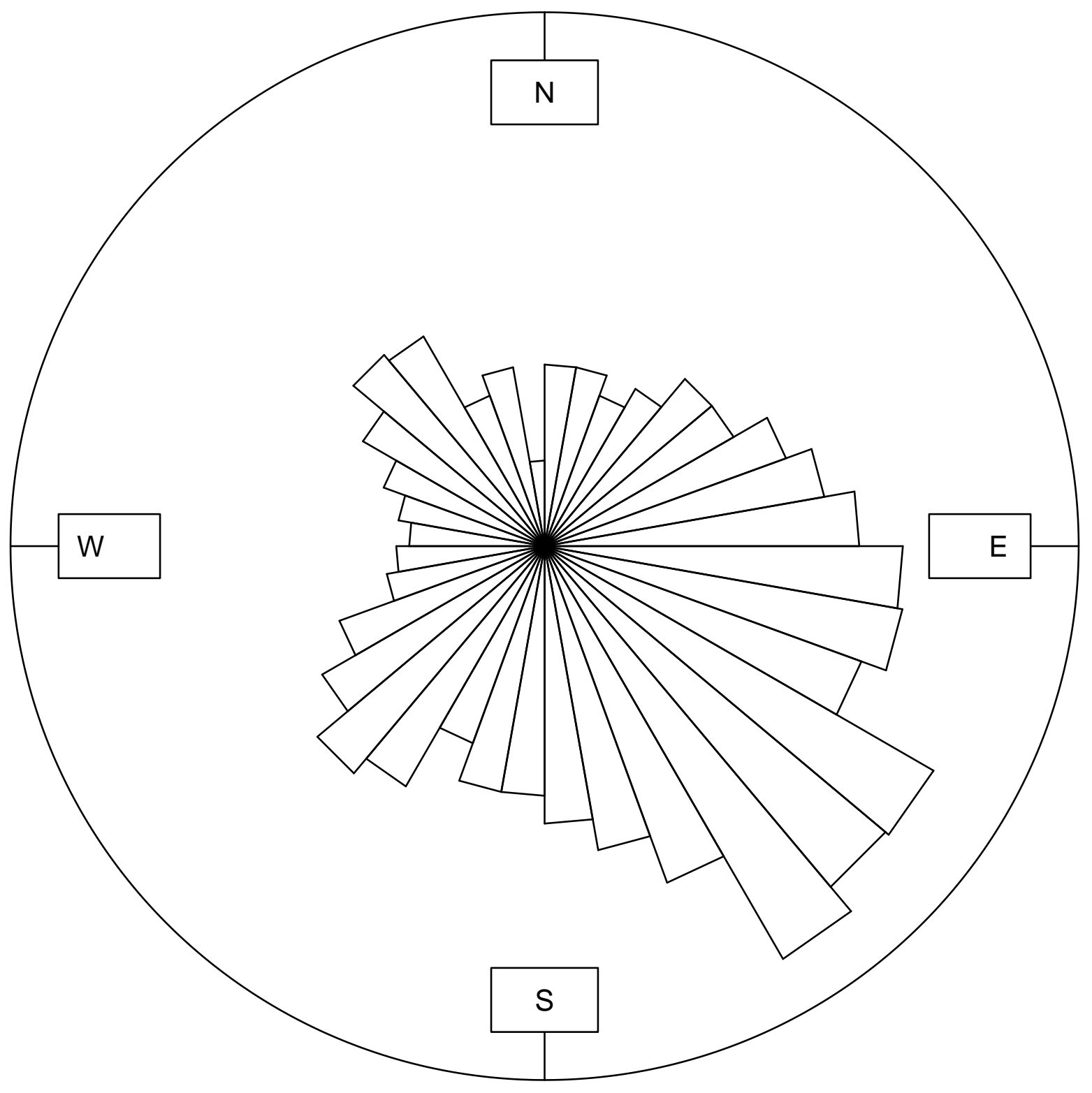




\section{bootstrap 653}

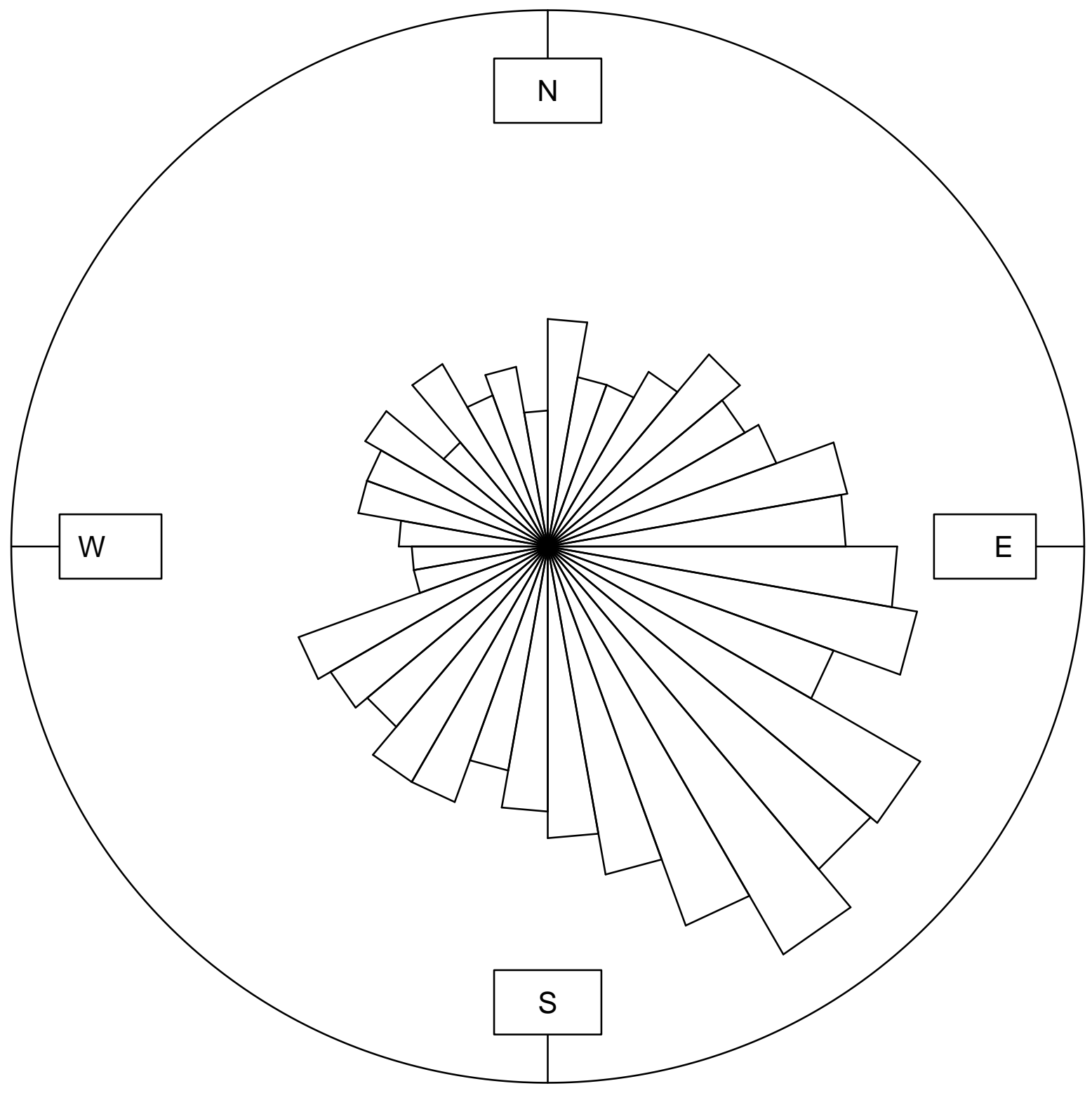




\section{bootstrap 654}

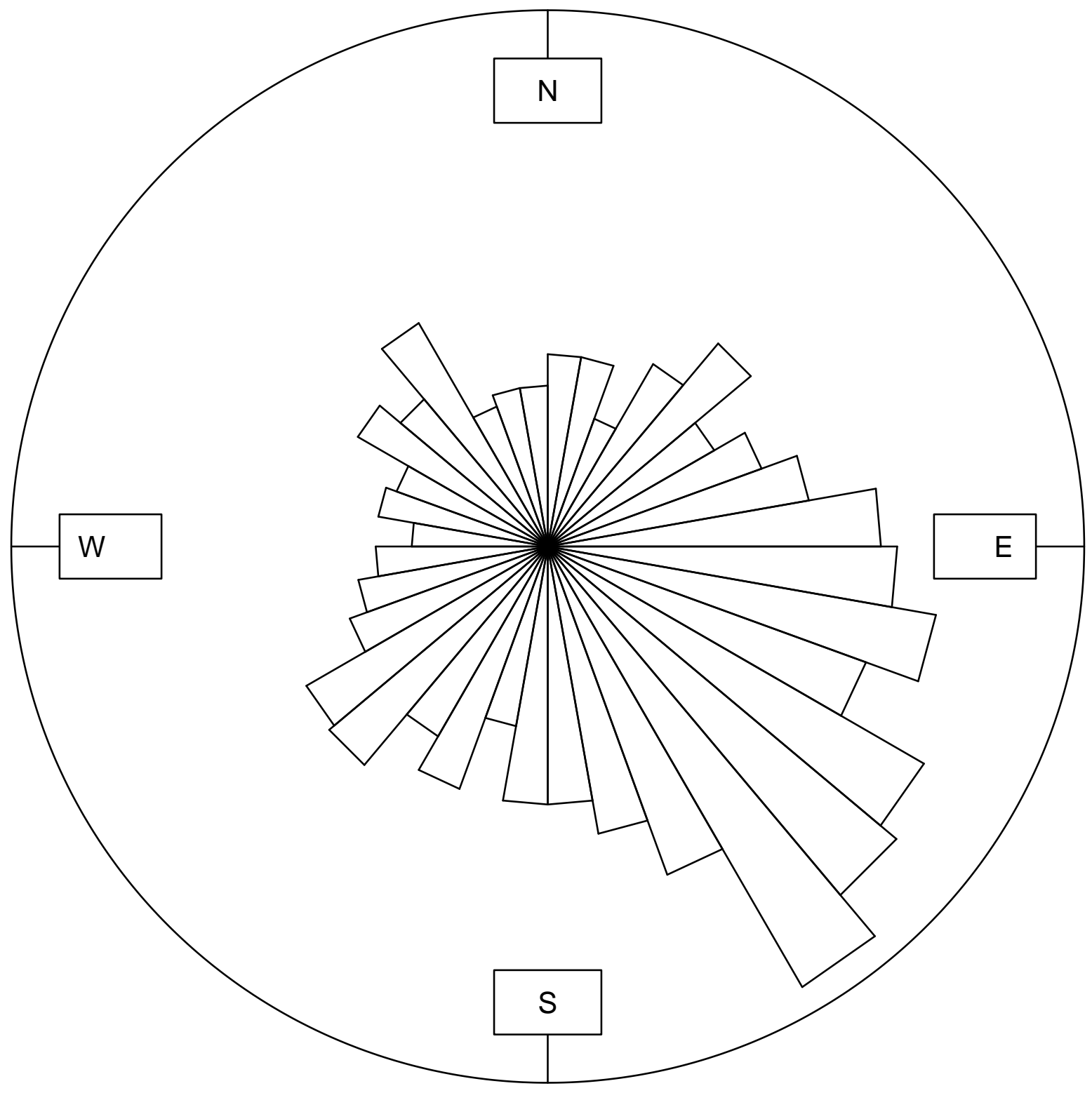




\section{bootstrap 655}

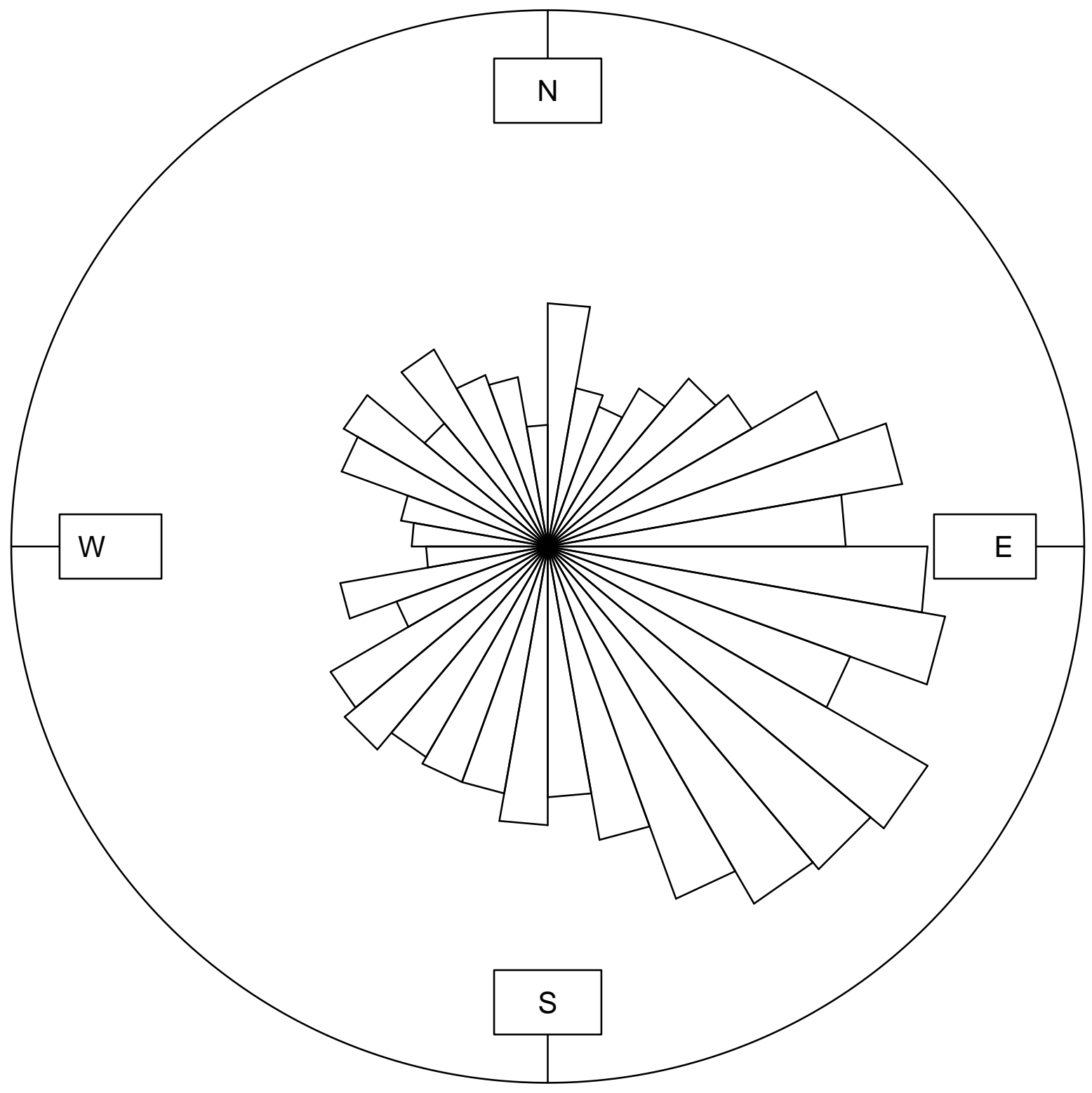




\section{bootstrap 656}

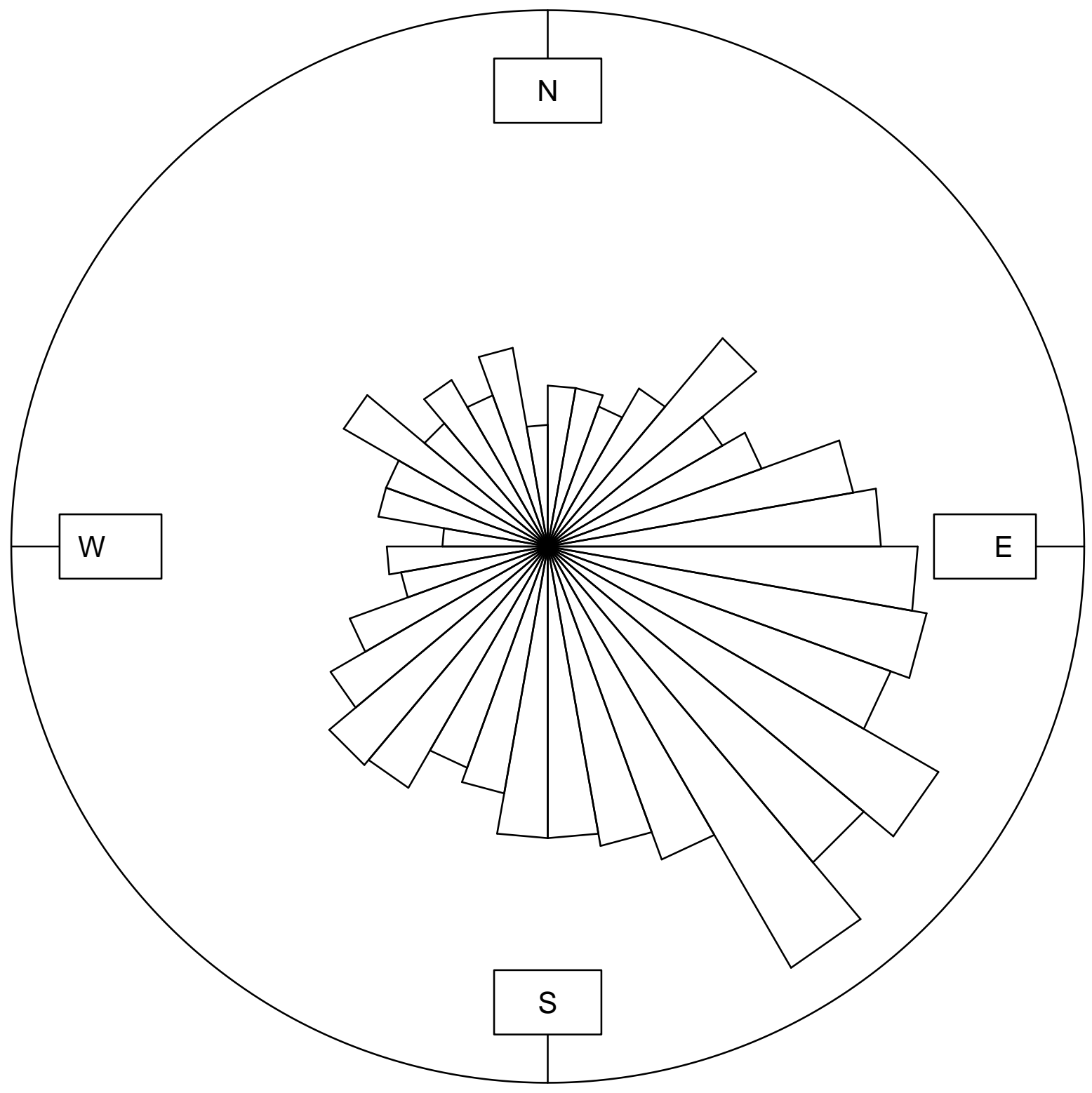


bootstrap 657

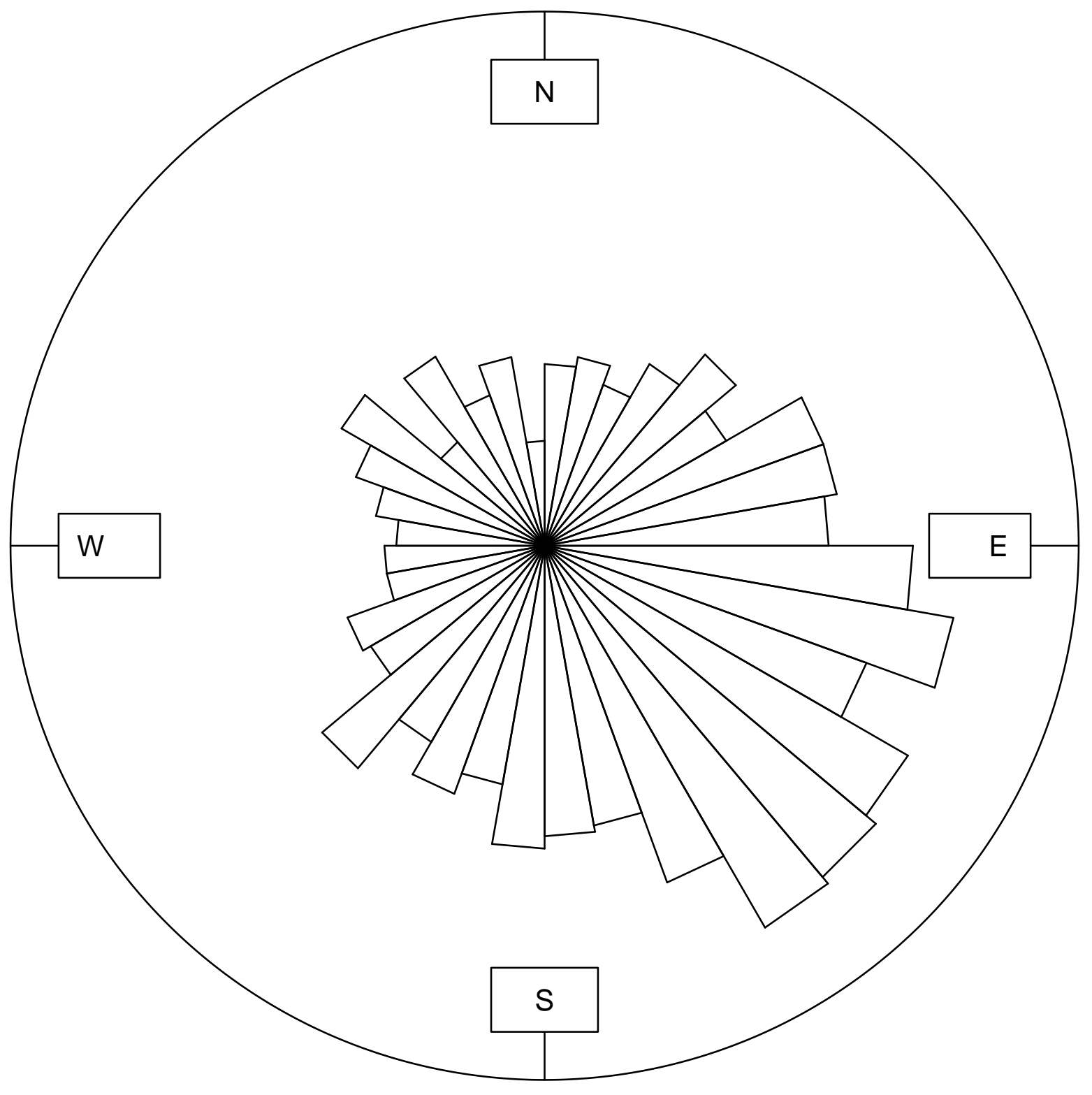


bootstrap 658

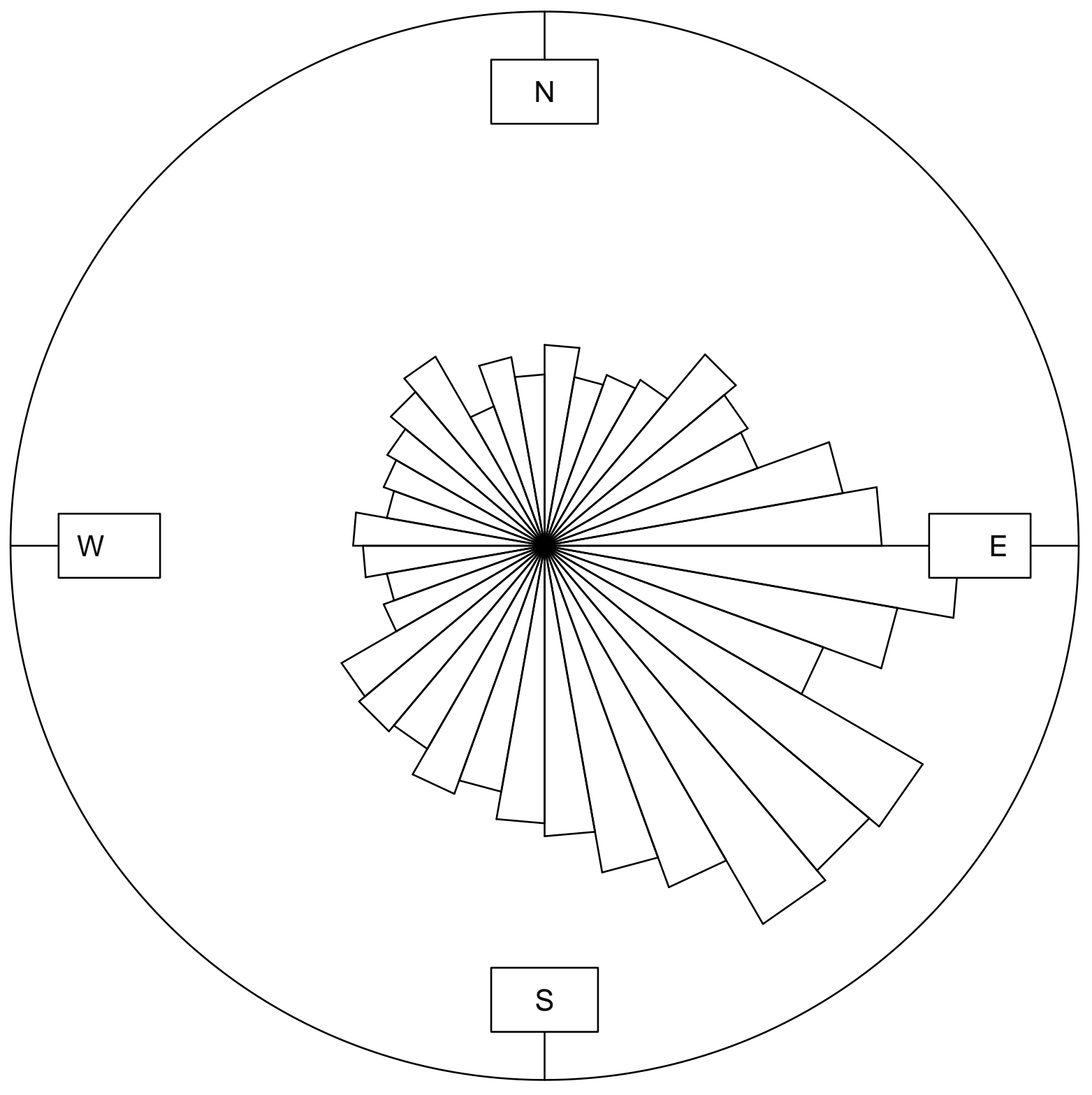


bootstrap 659

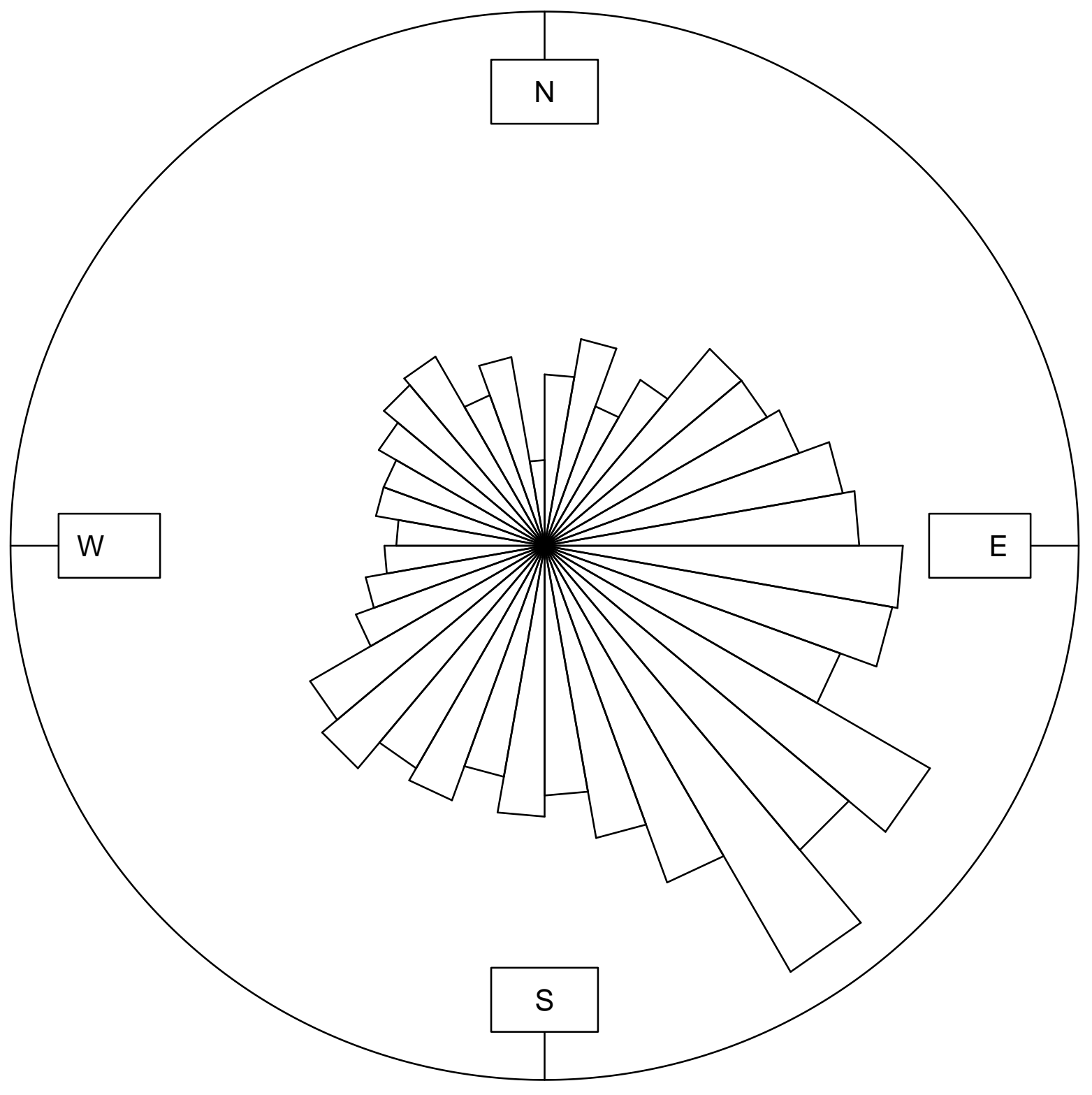


bootstrap 660

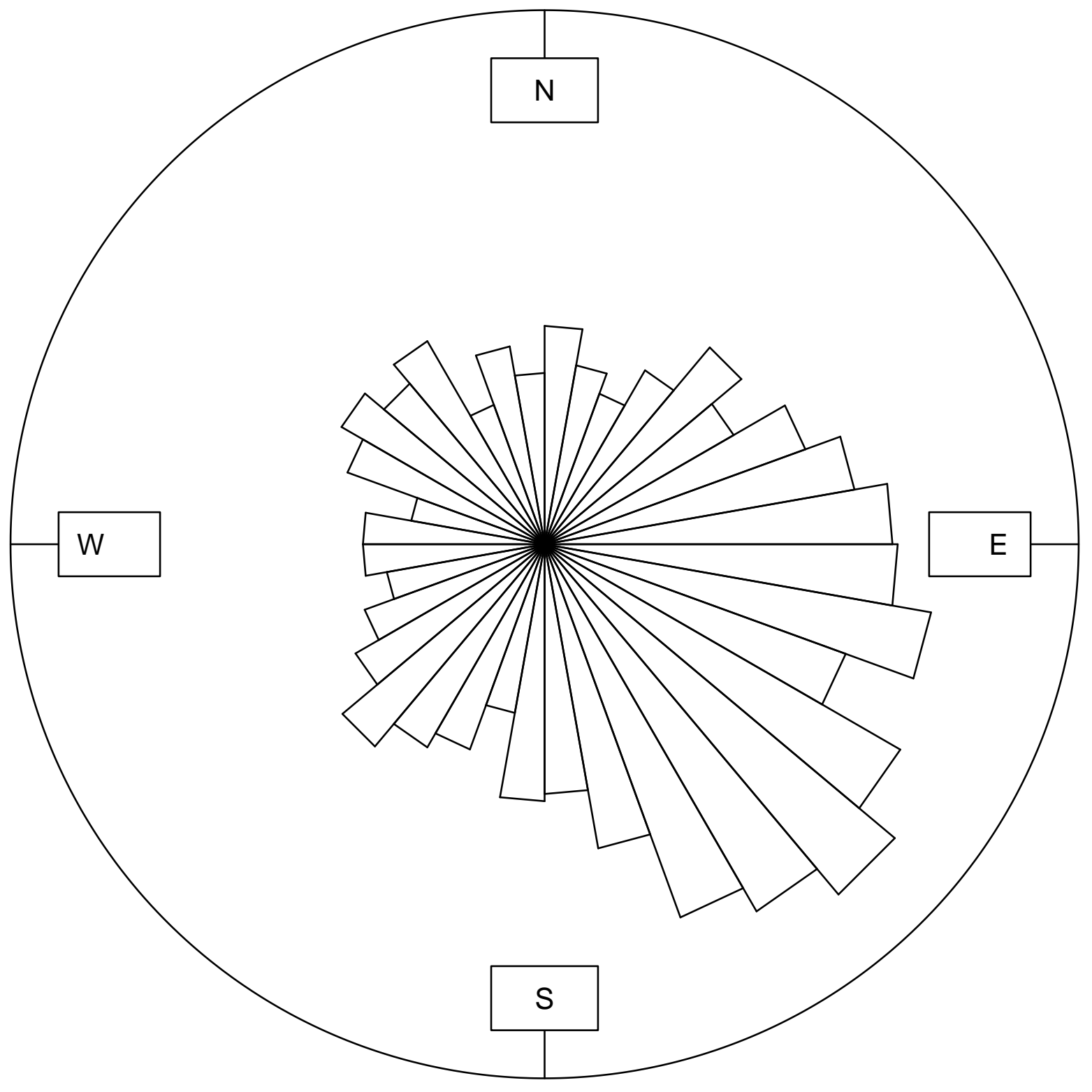




\section{bootstrap 661}

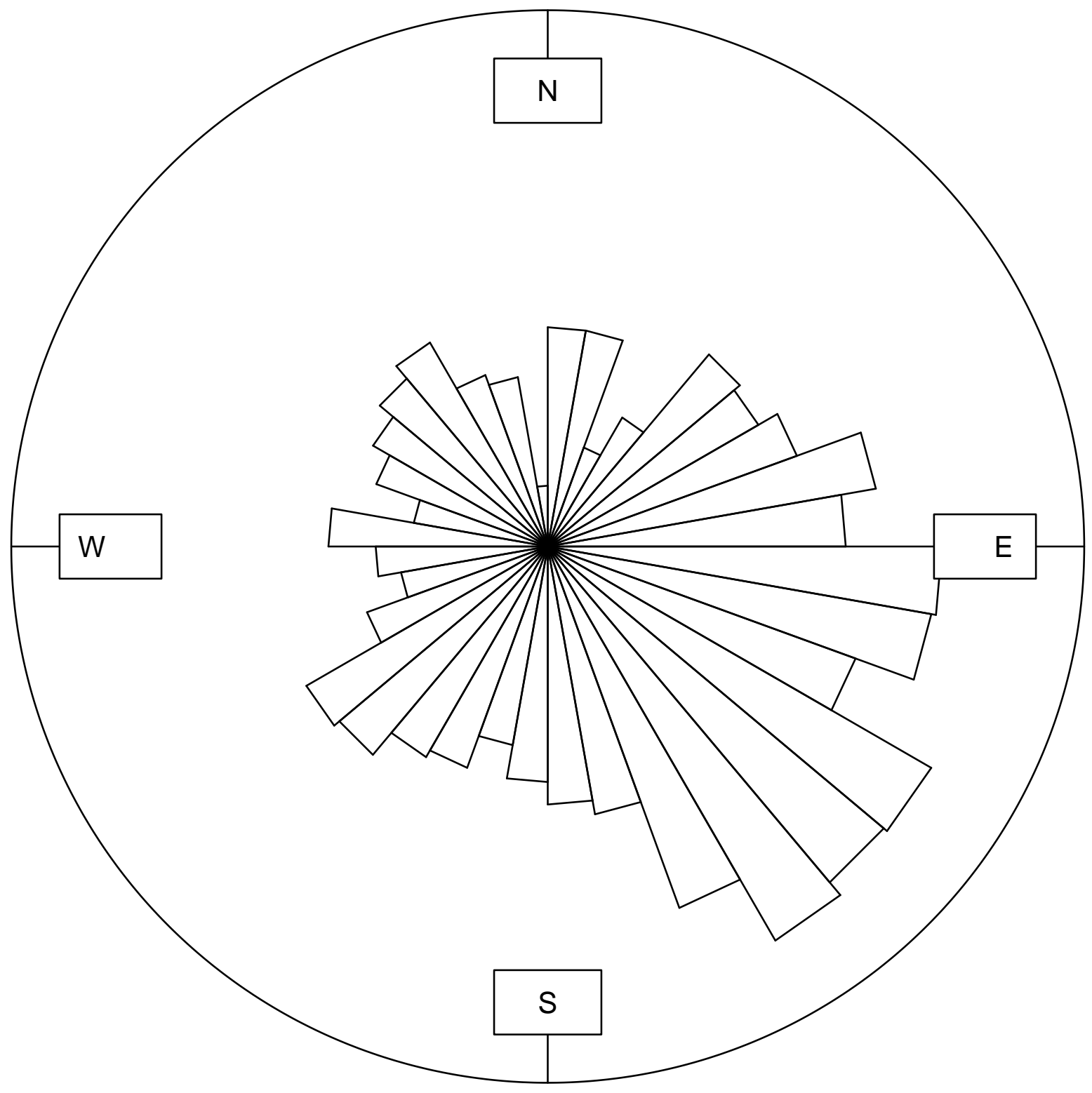


bootstrap 662

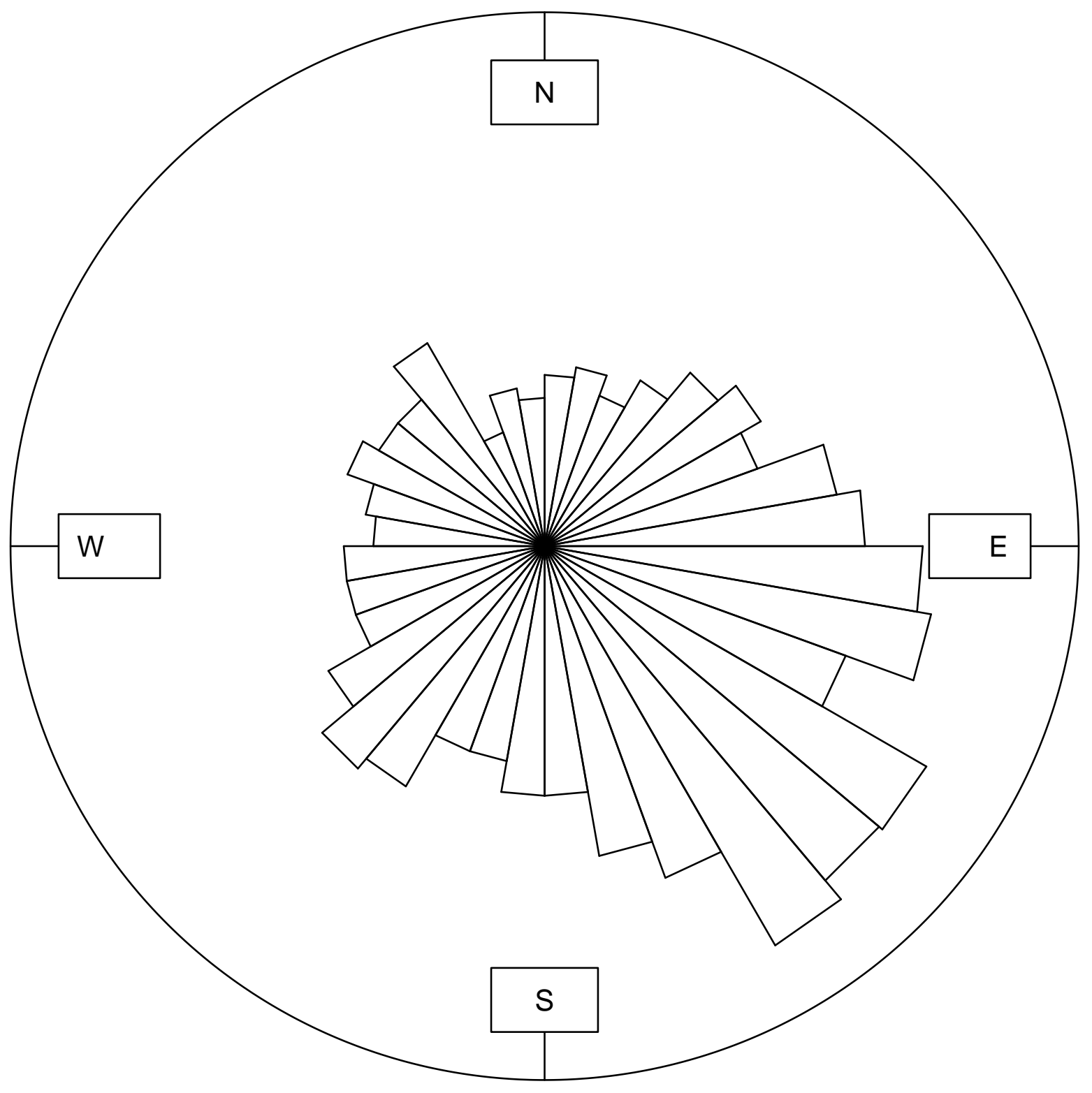




\section{bootstrap 663}

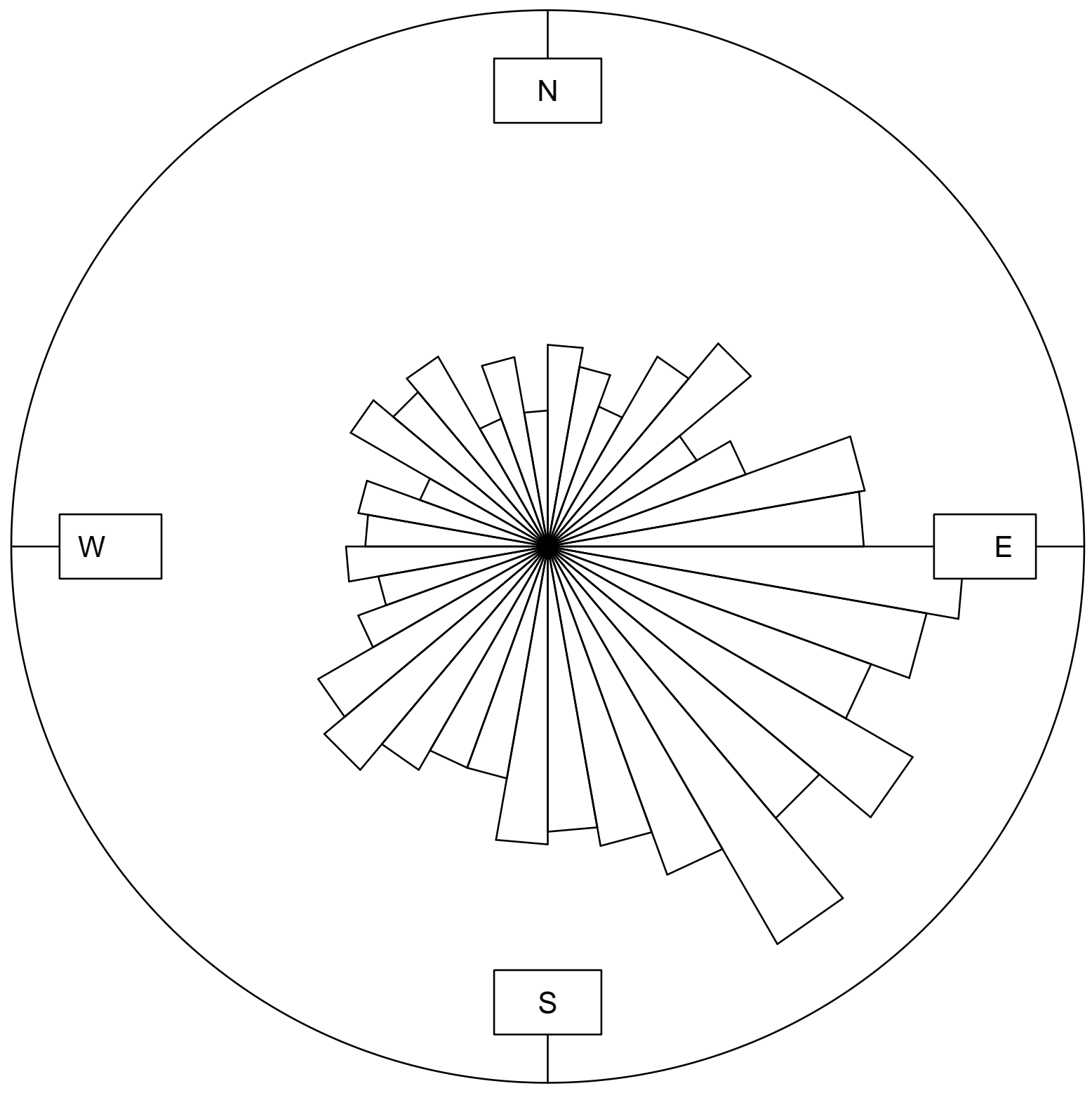




\section{bootstrap 664}

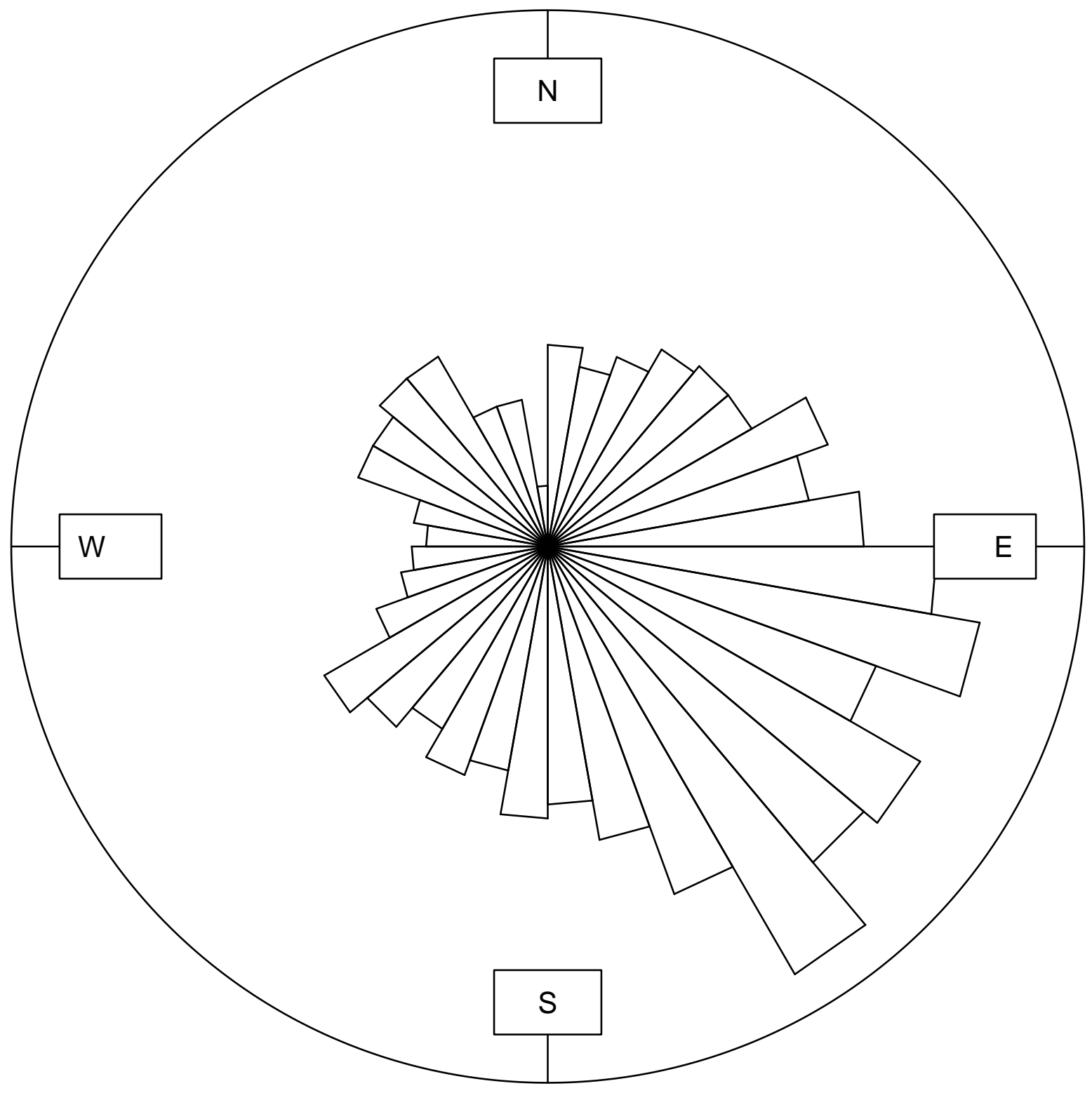




\section{bootstrap 665}

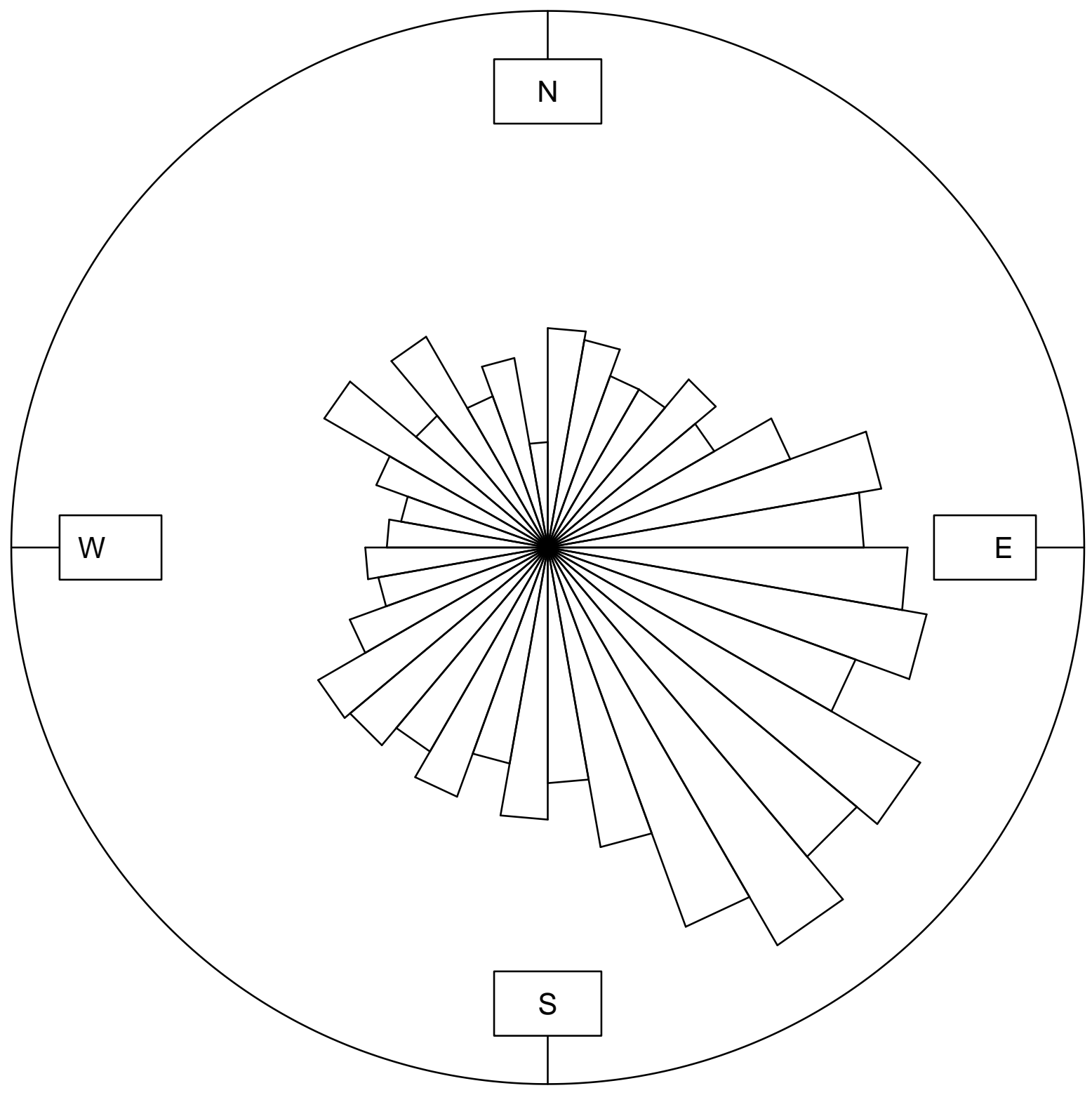




\section{bootstrap 666}

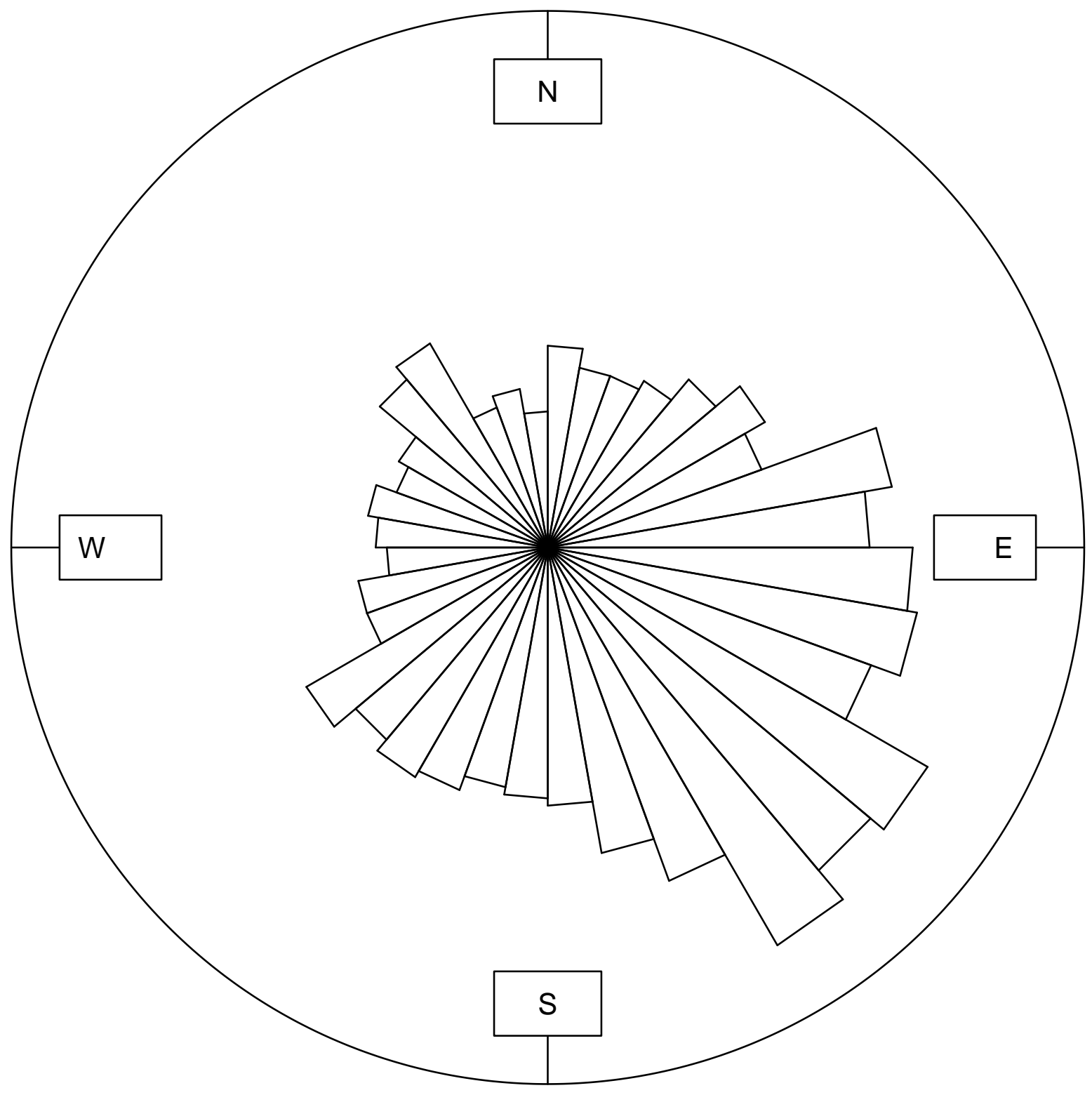


bootstrap 667

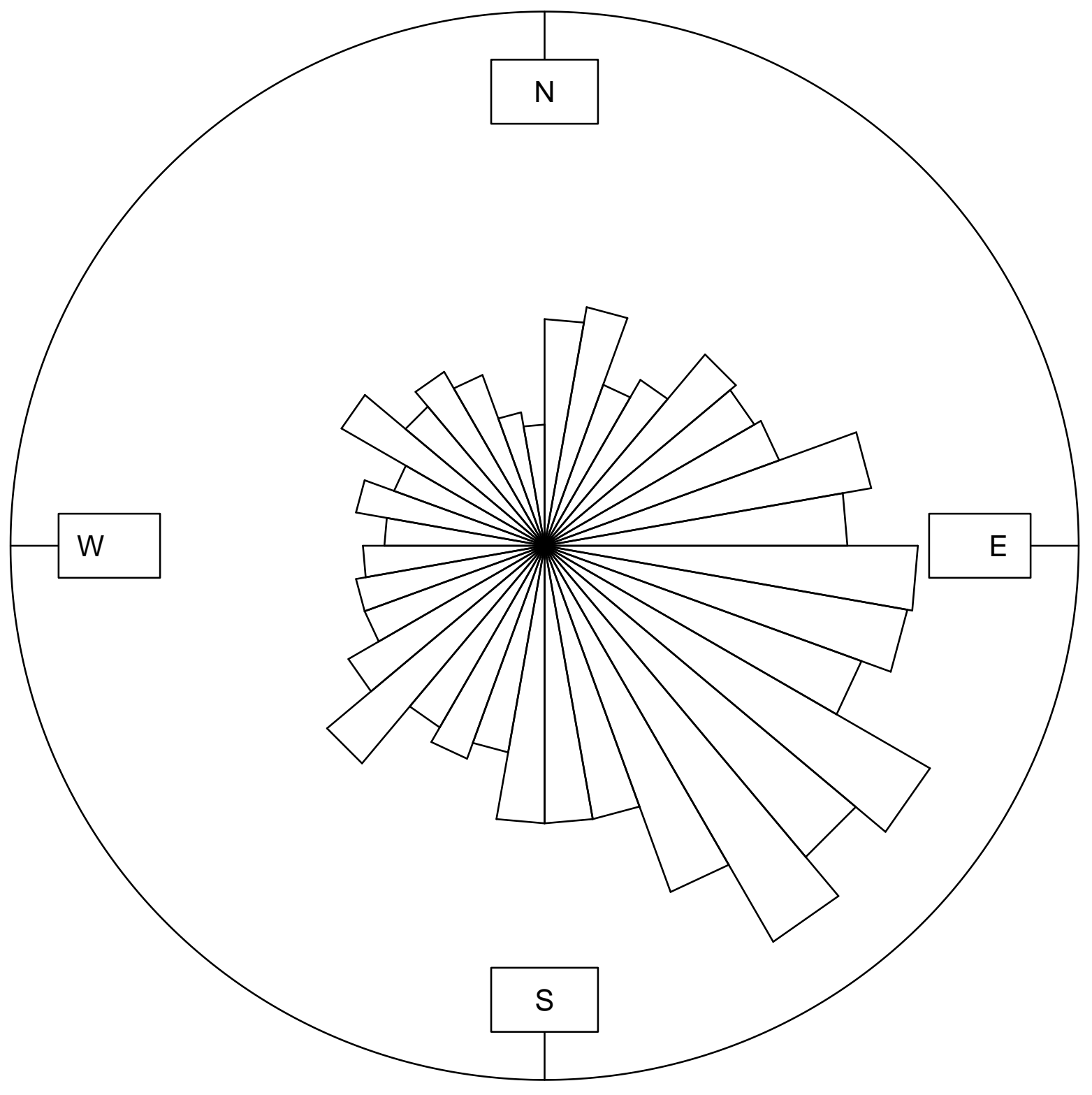


bootstrap 668

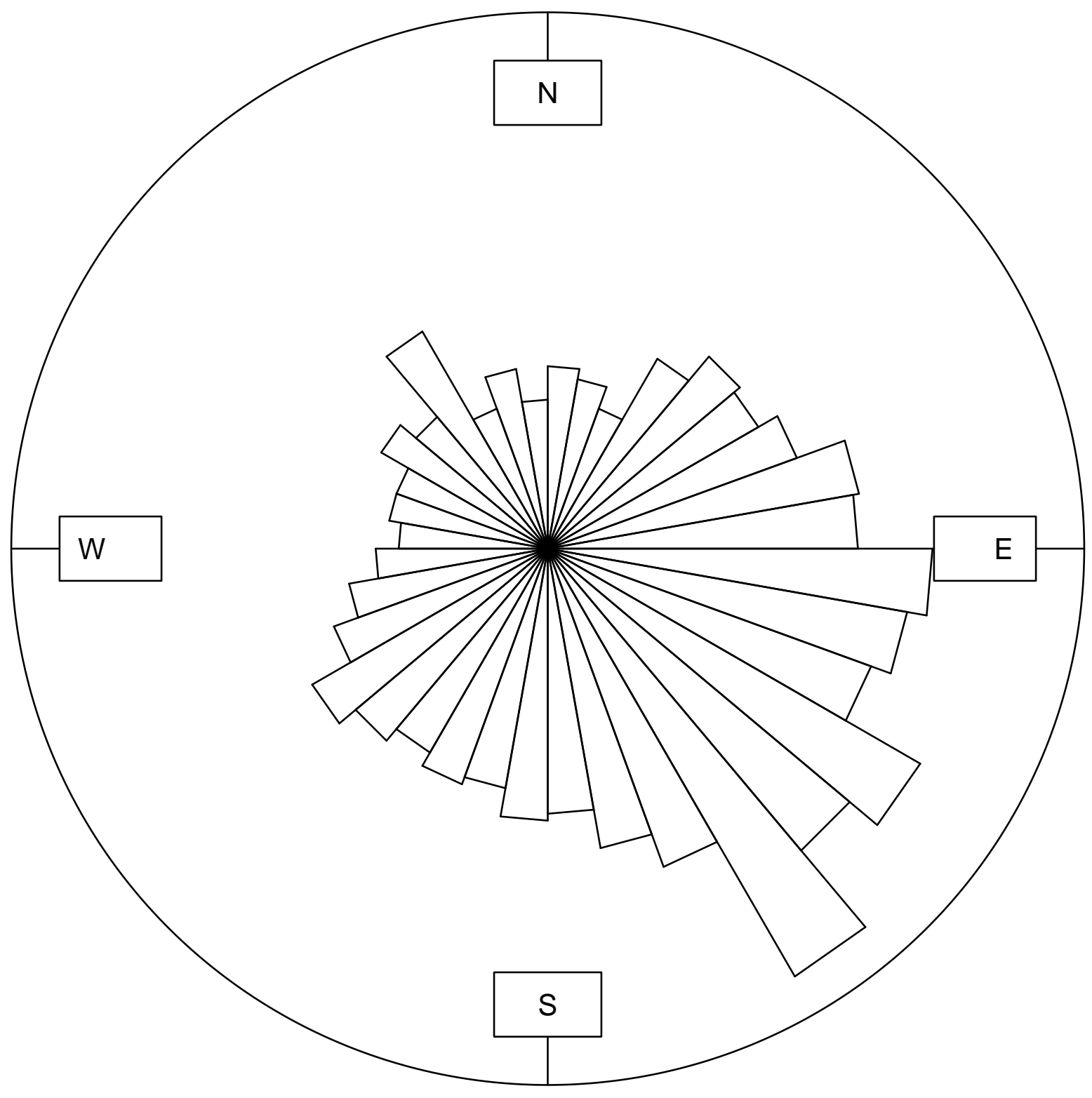


bootstrap 669

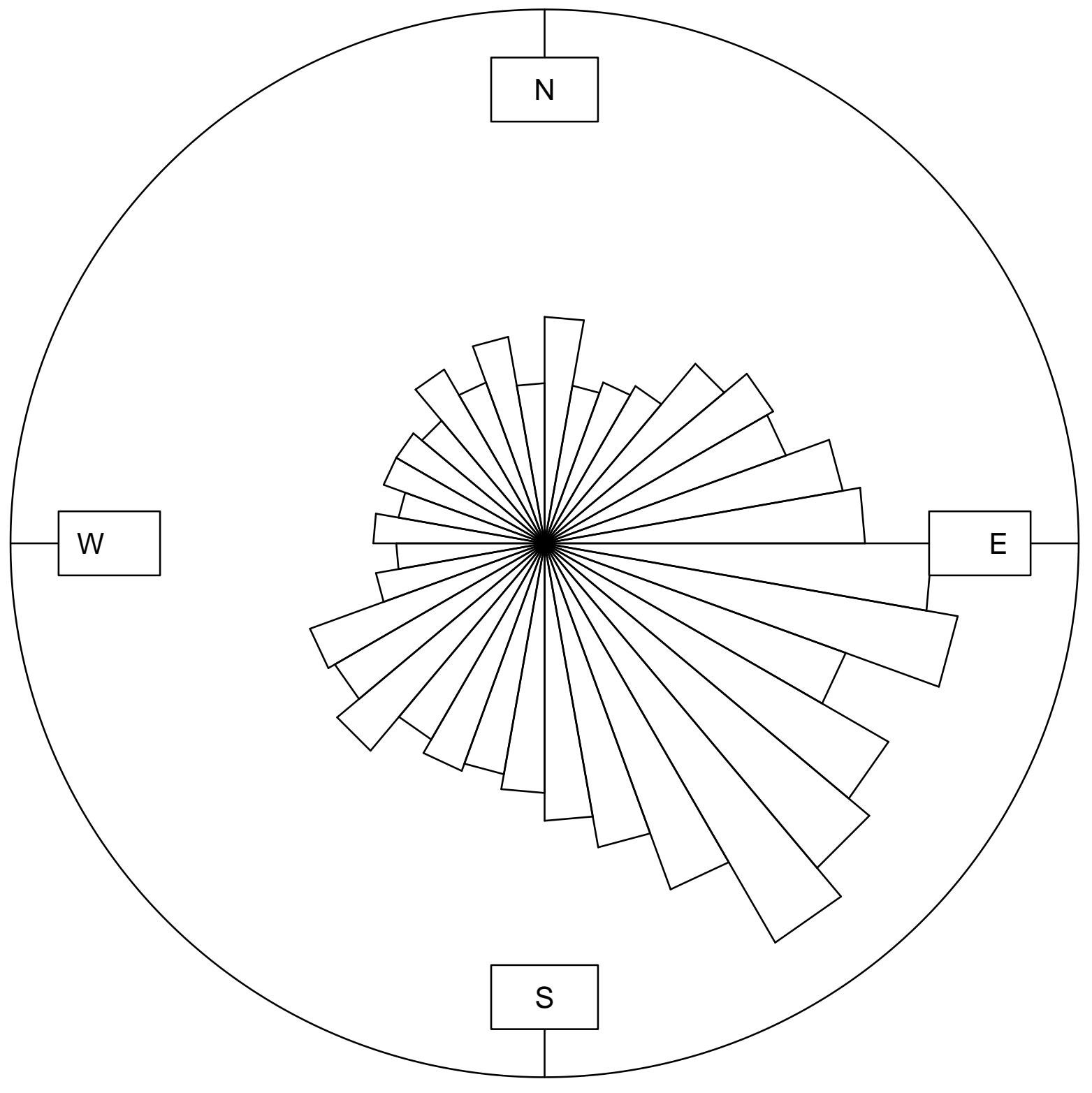


bootstrap 670

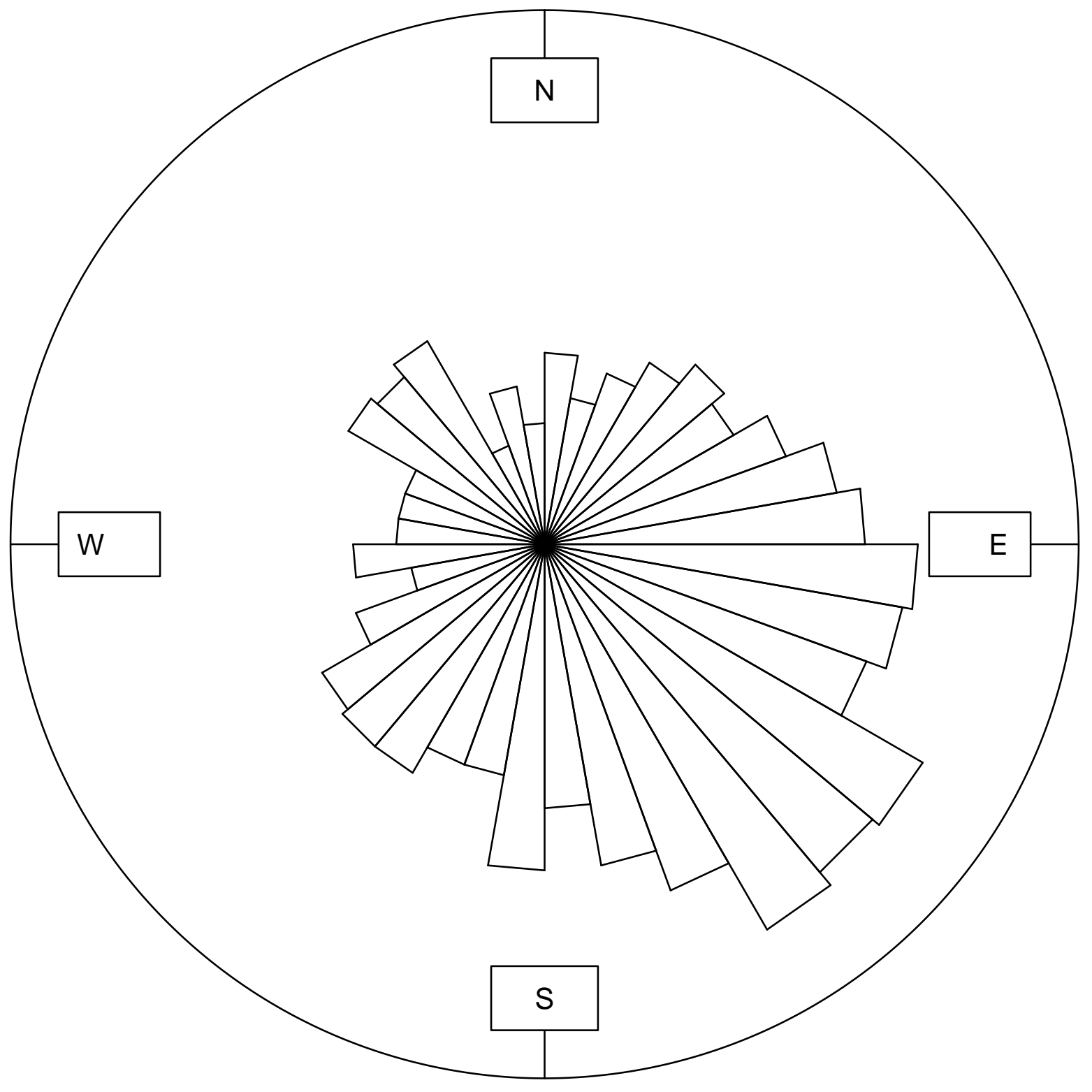


bootstrap 671

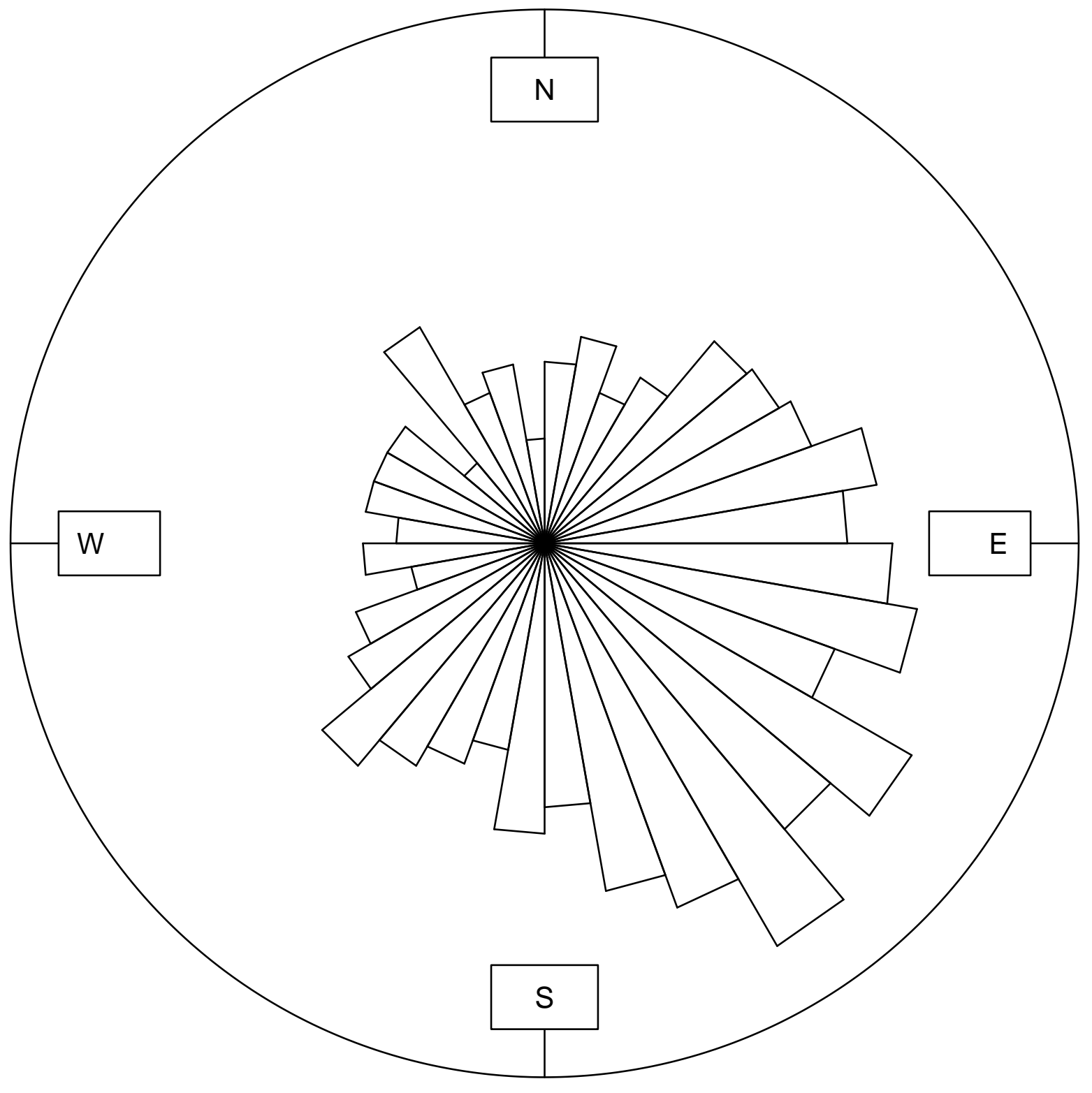


bootstrap 672

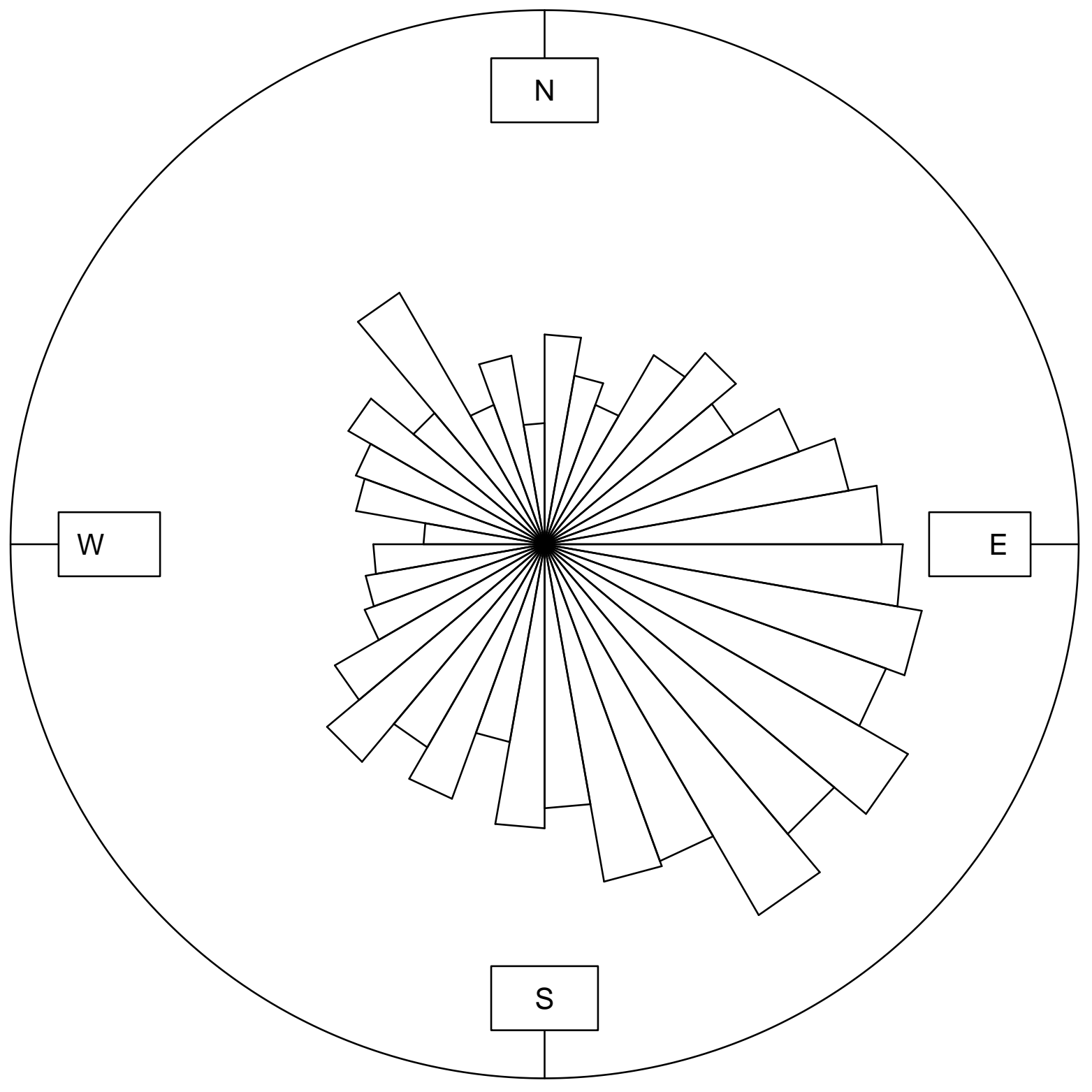


bootstrap 673

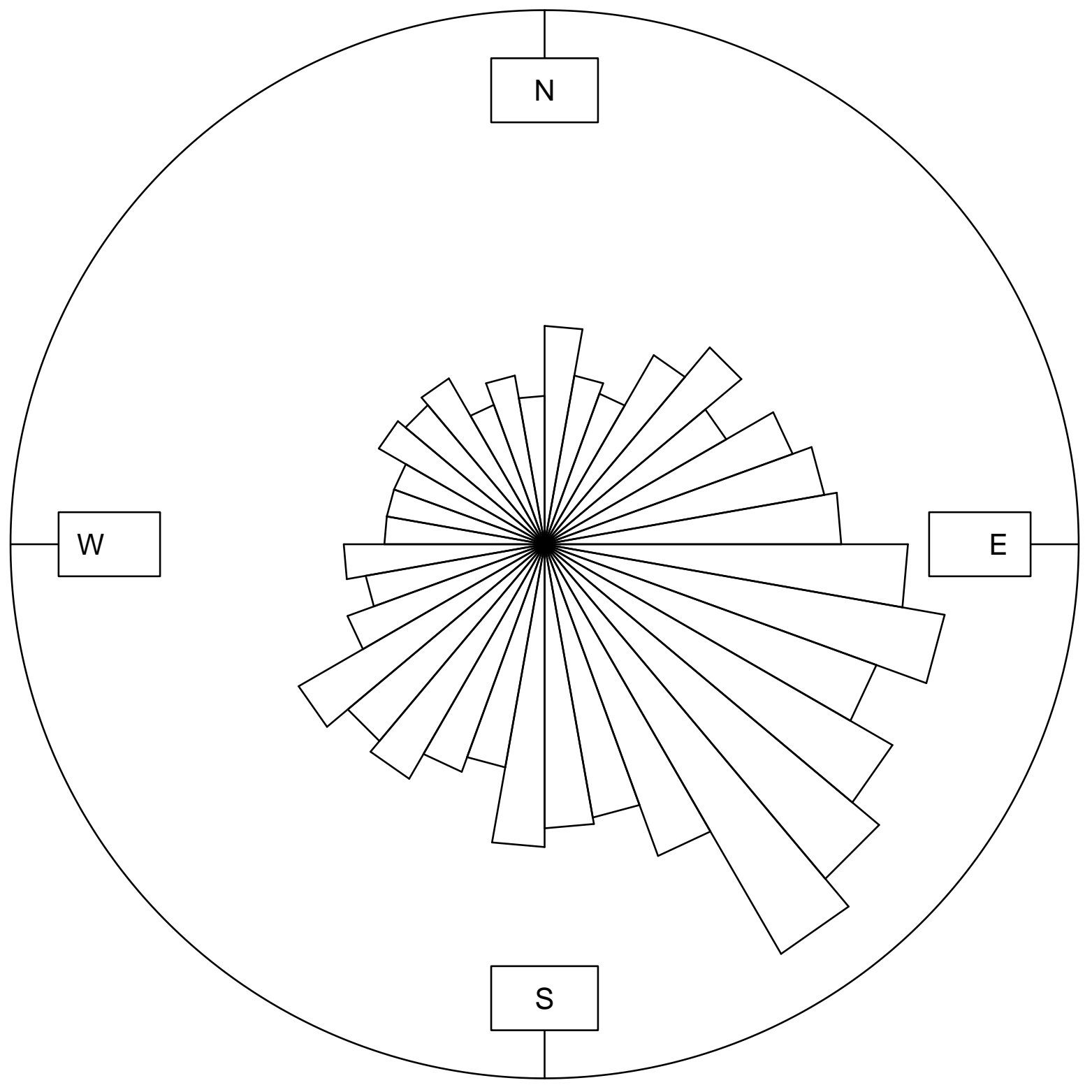


bootstrap 674

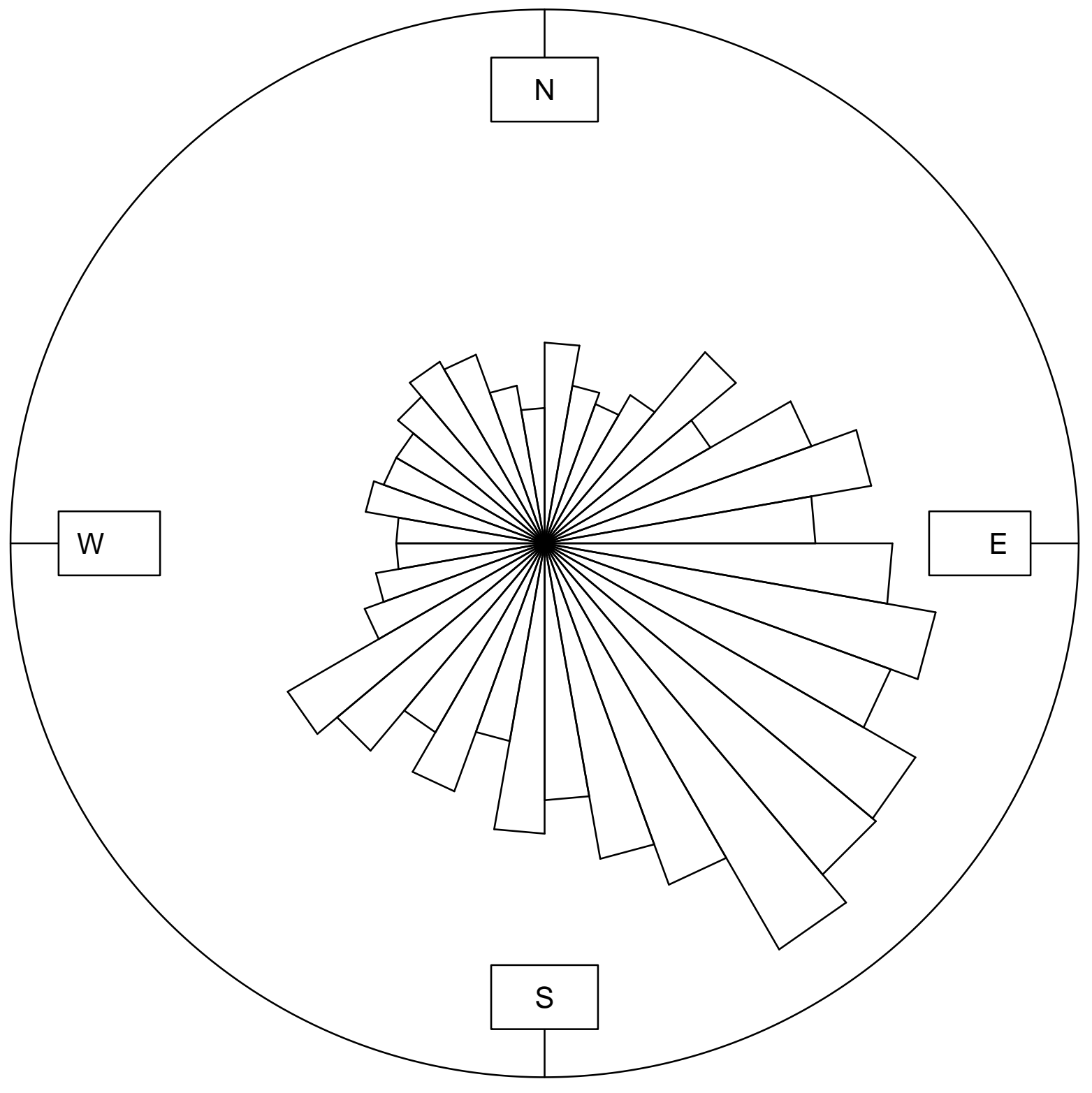


bootstrap 675

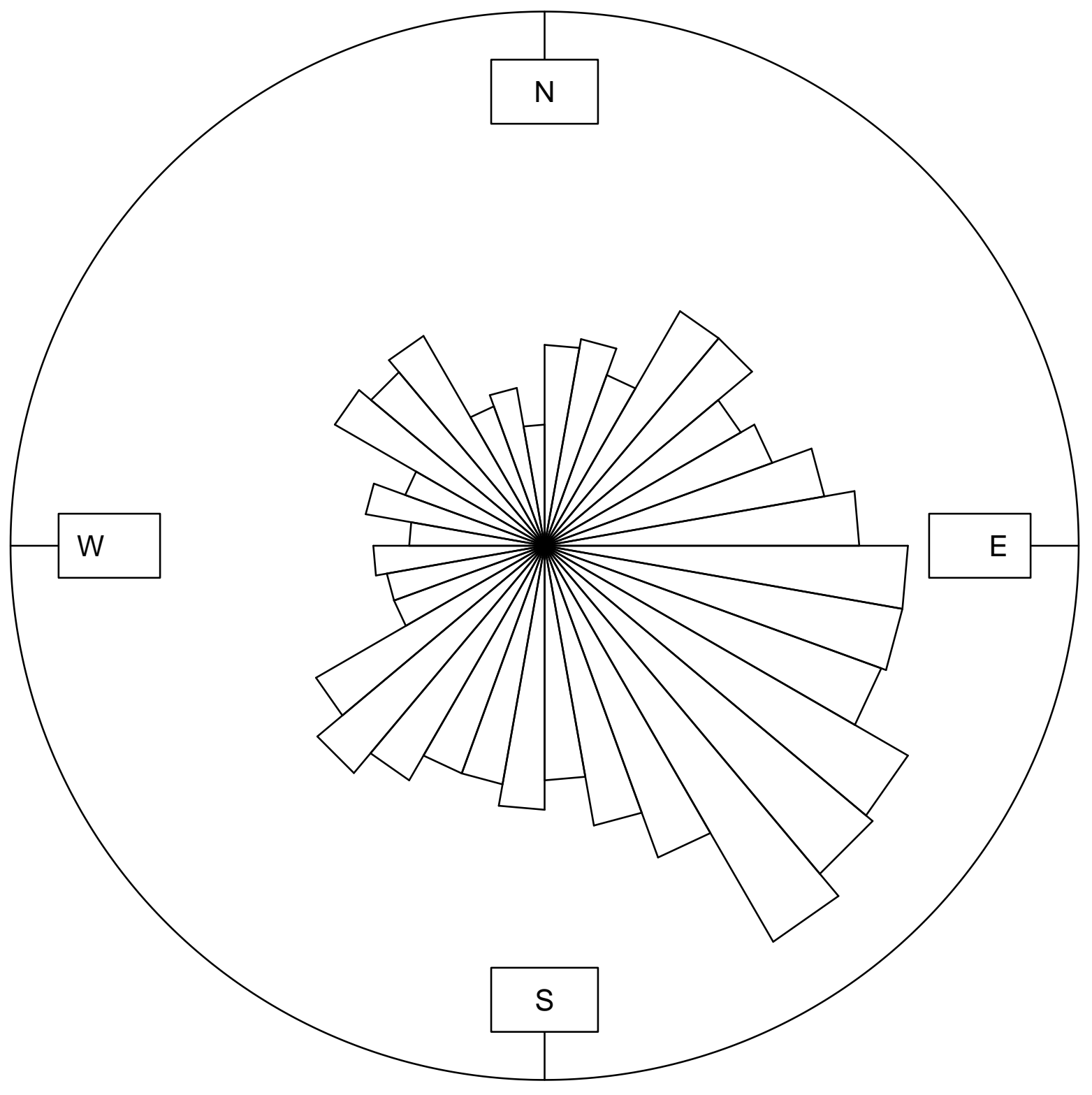


bootstrap 676

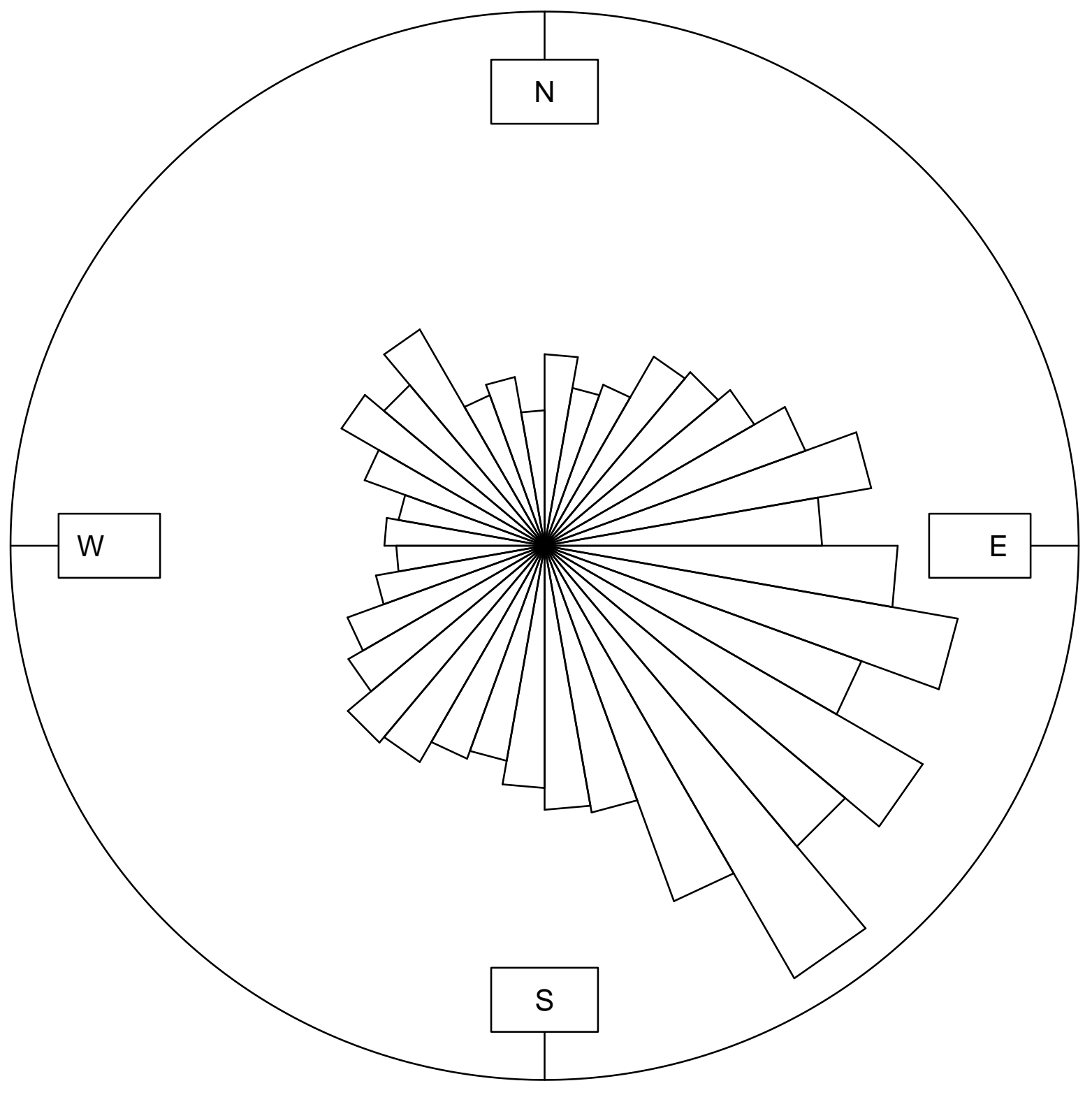


bootstrap 677

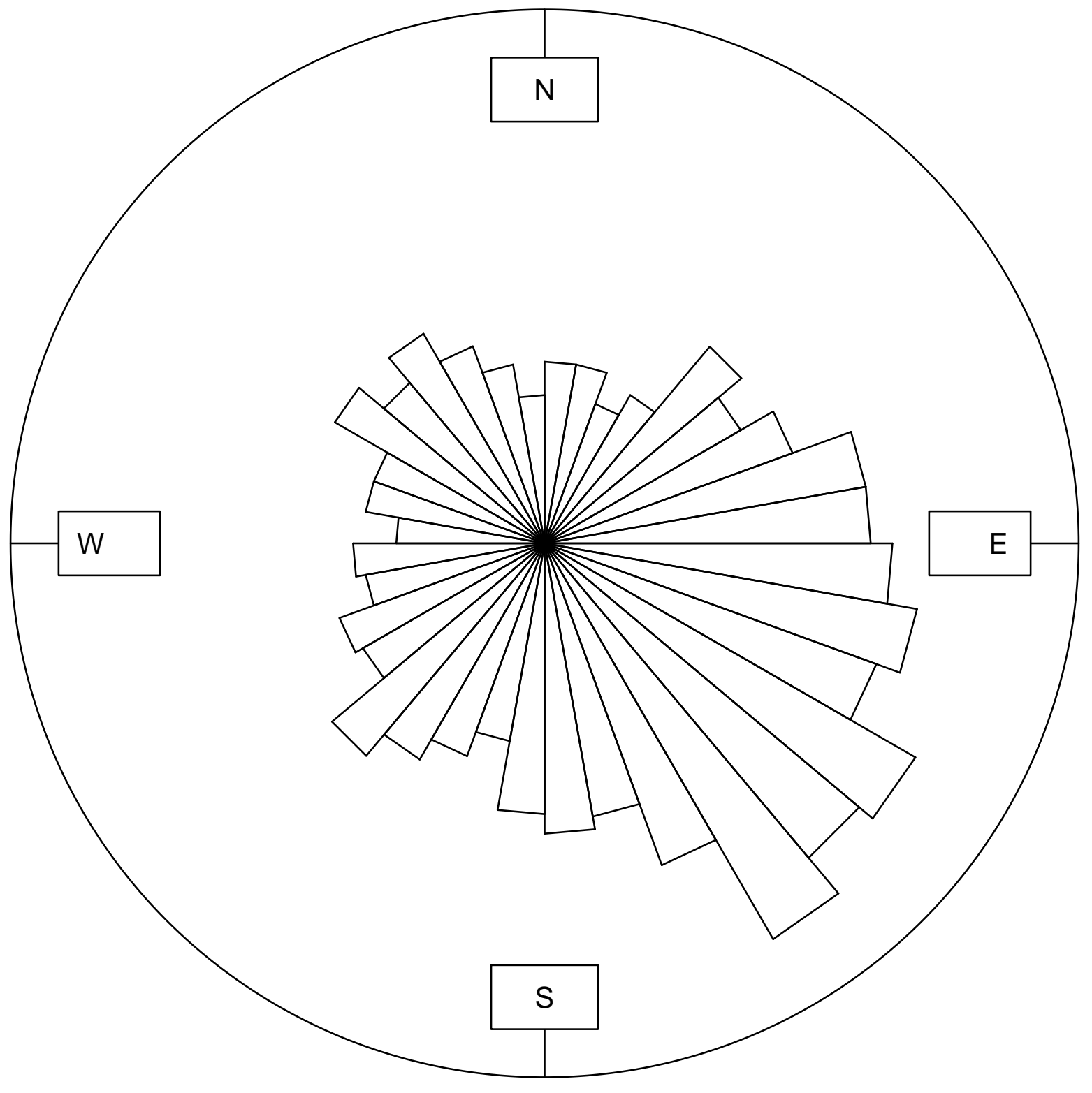


bootstrap 678

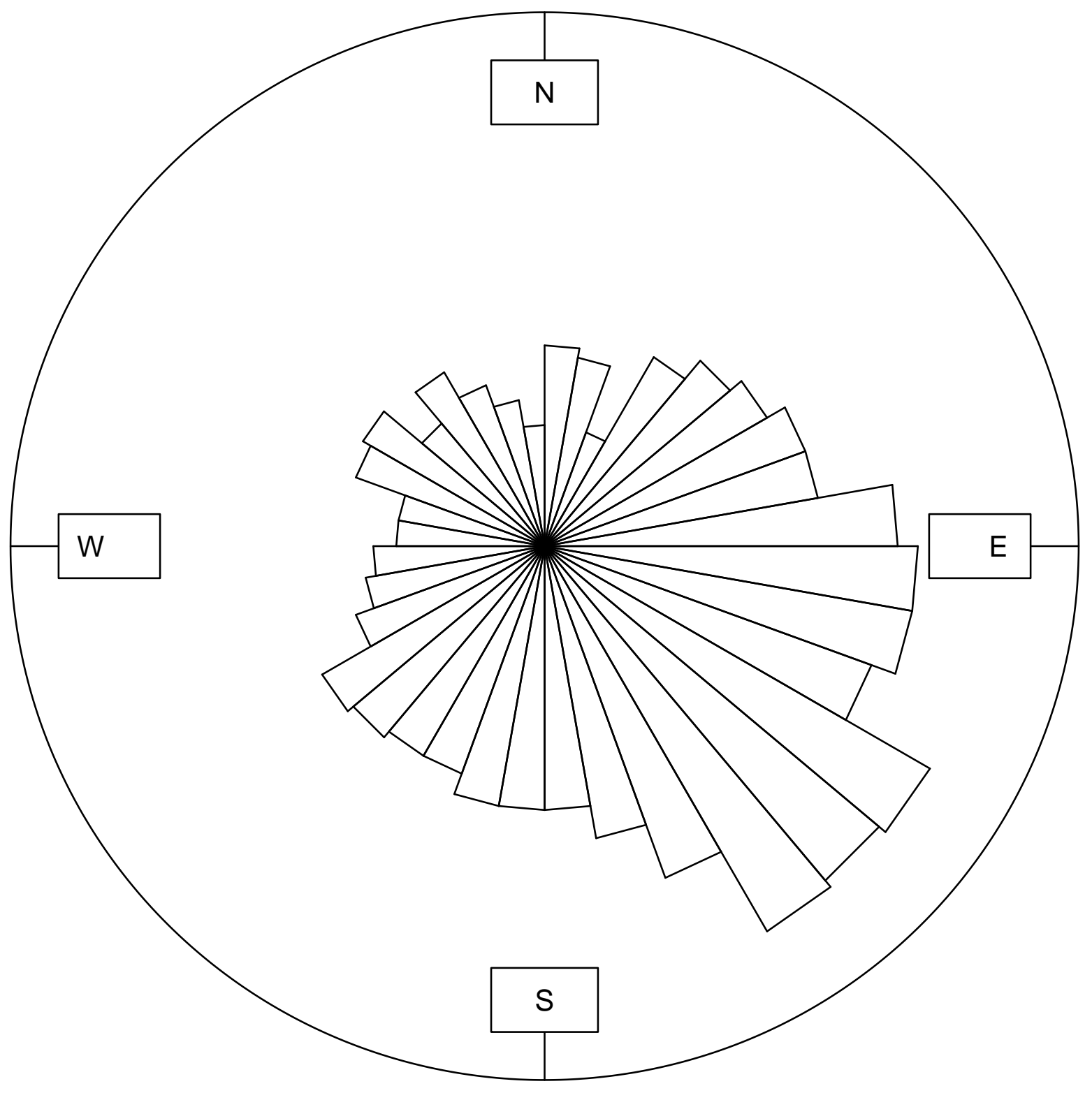


bootstrap 679

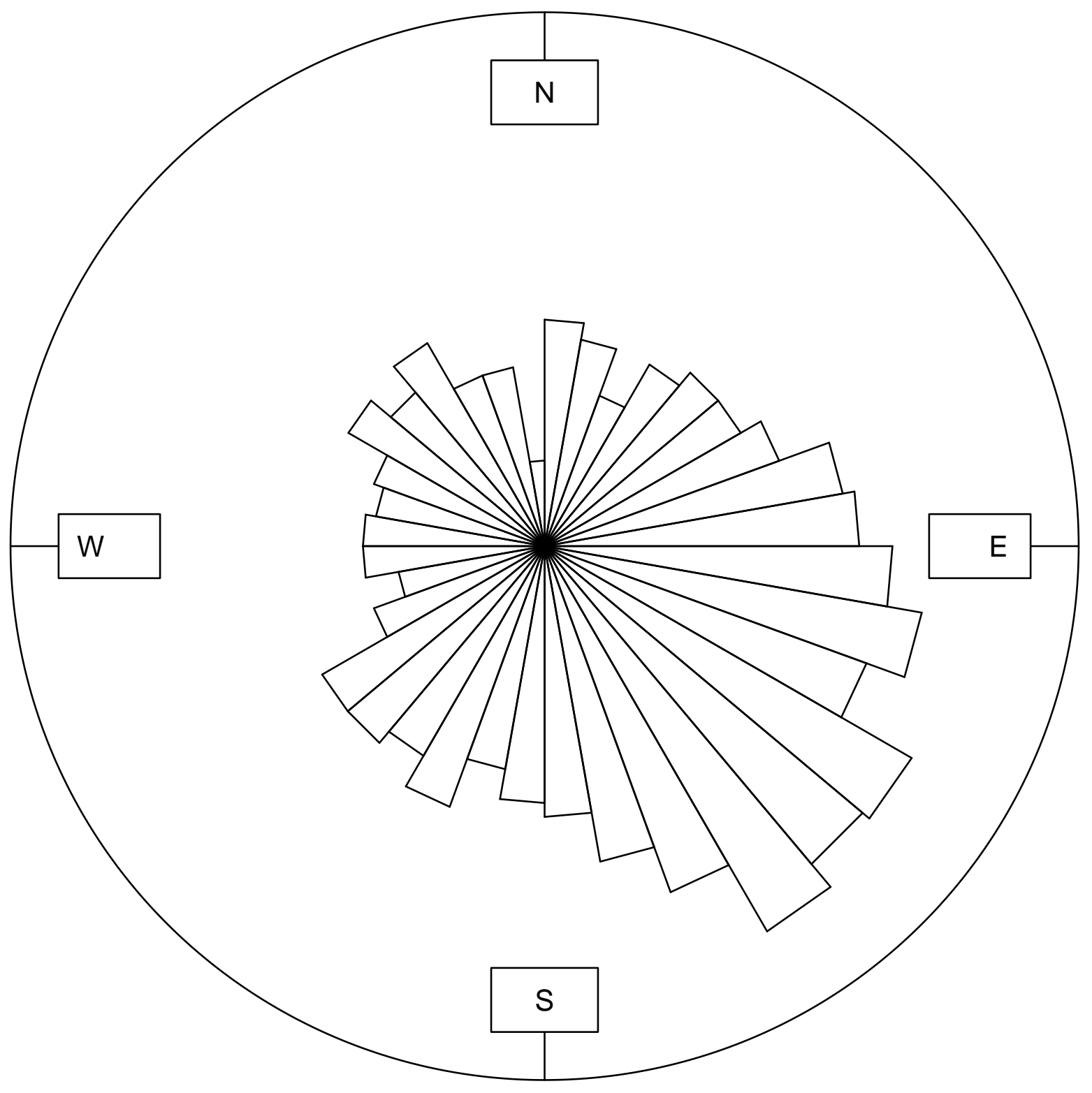


bootstrap 680

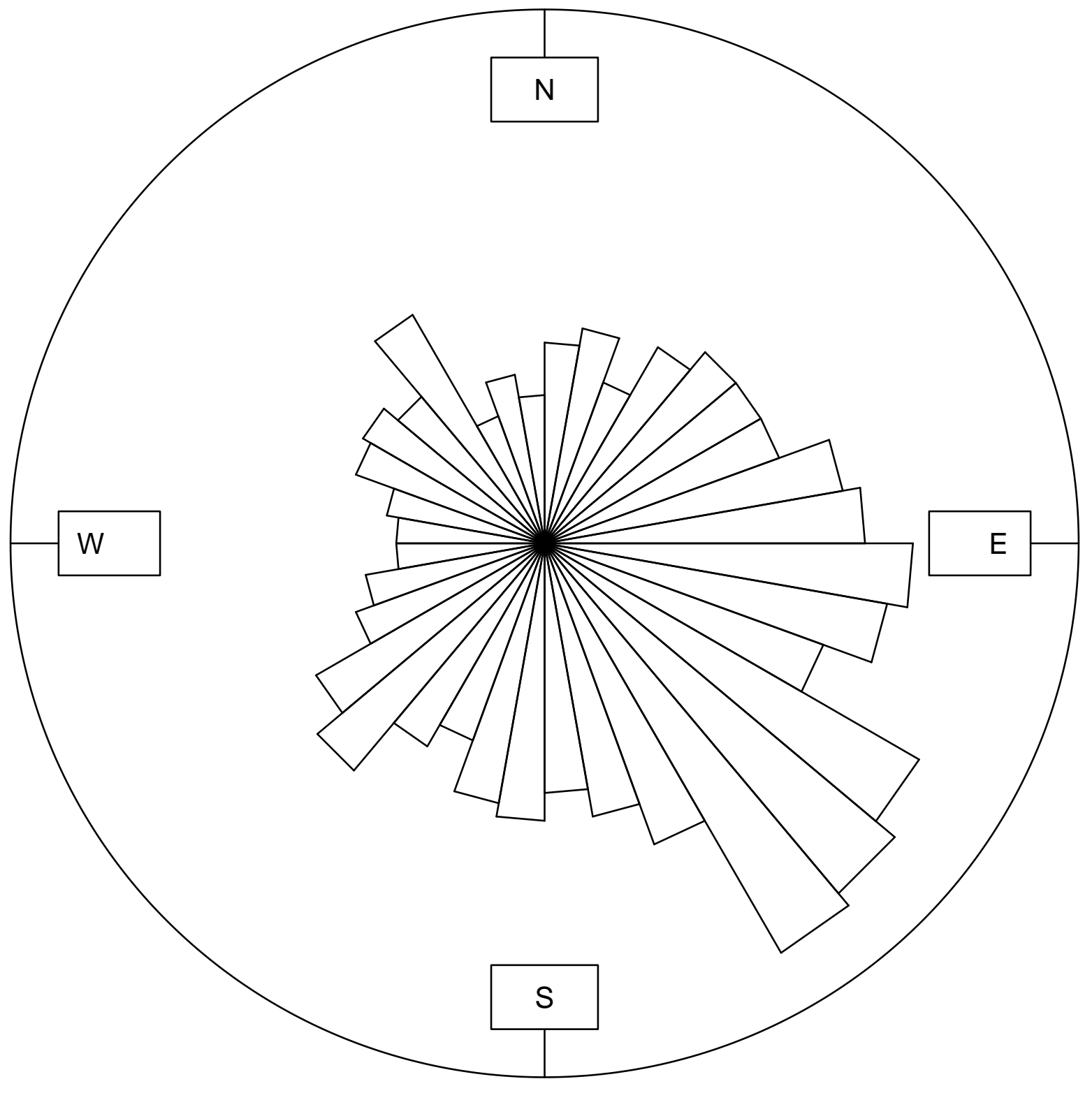




\section{bootstrap 681}

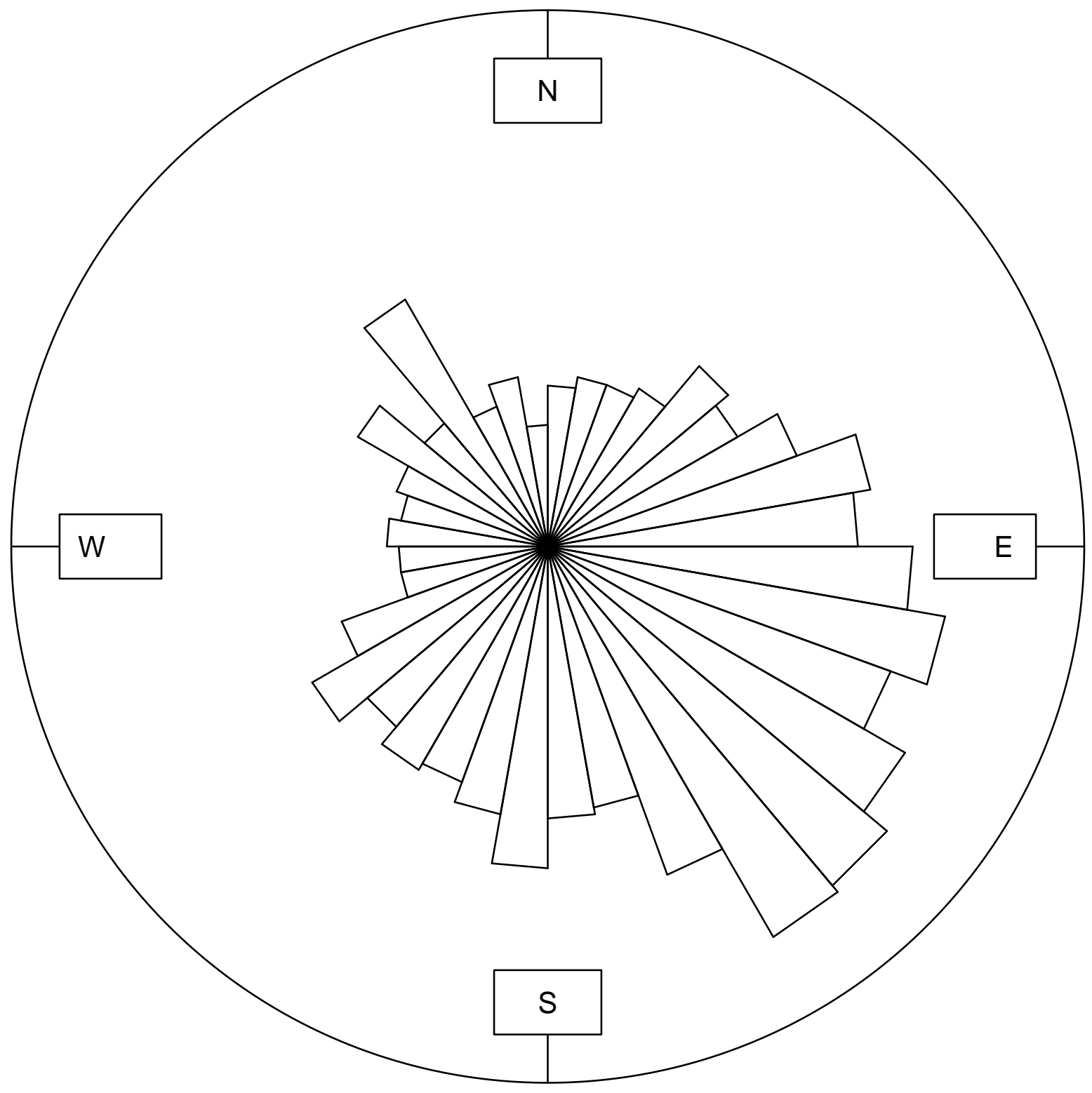


bootstrap 682

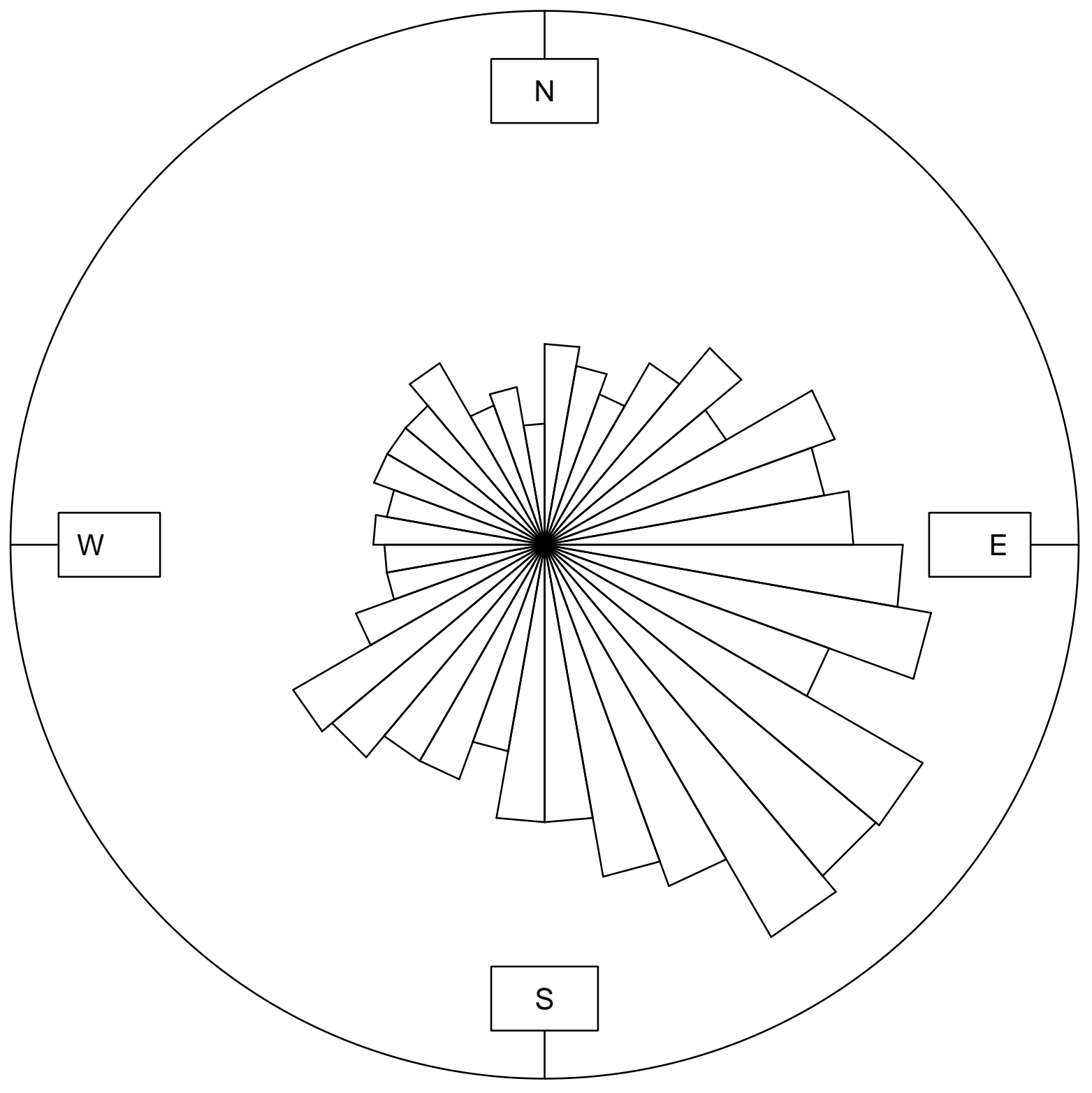




\section{bootstrap 683}

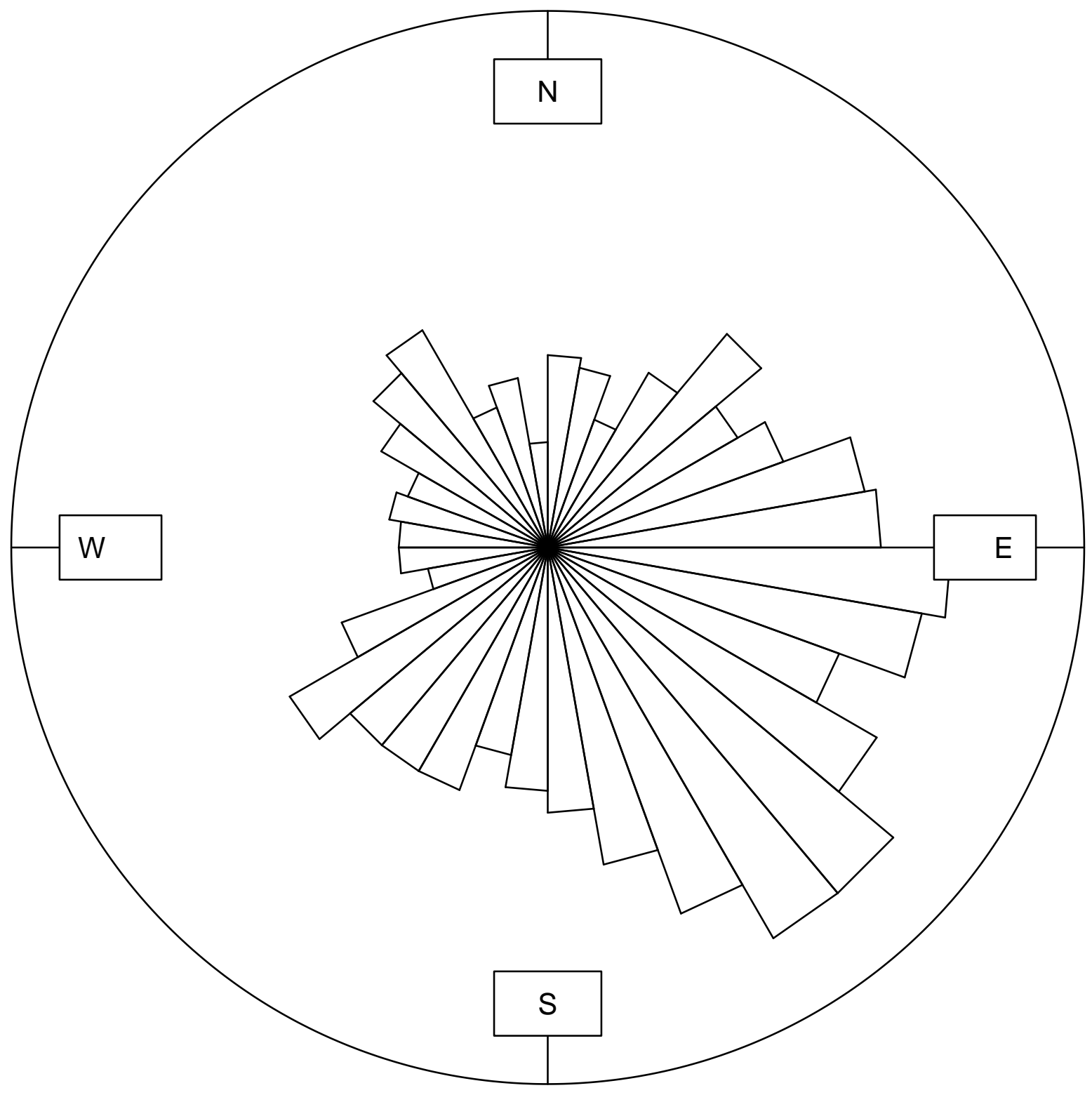




\section{bootstrap 684}

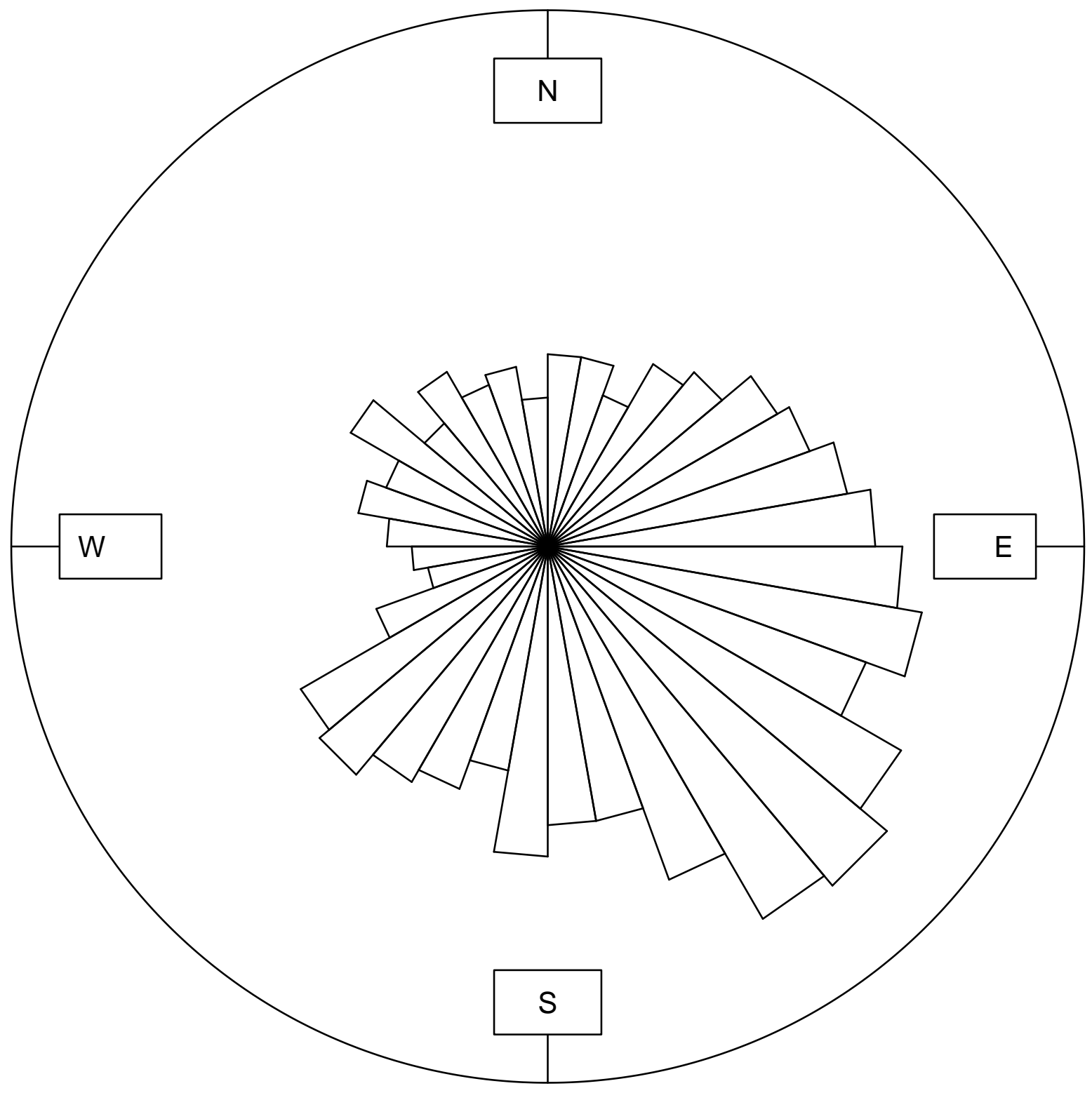




\section{bootstrap 685}

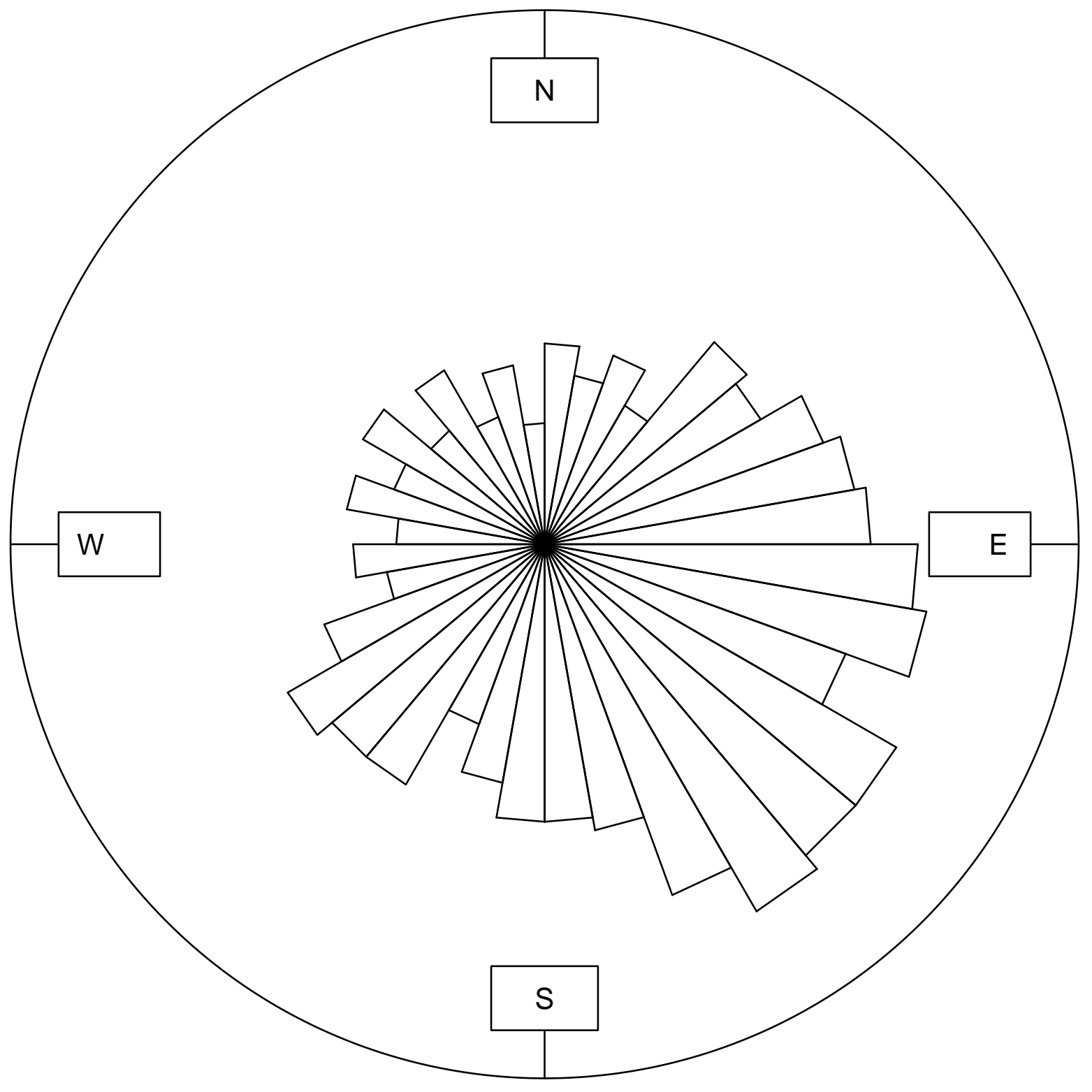




\section{bootstrap 686}

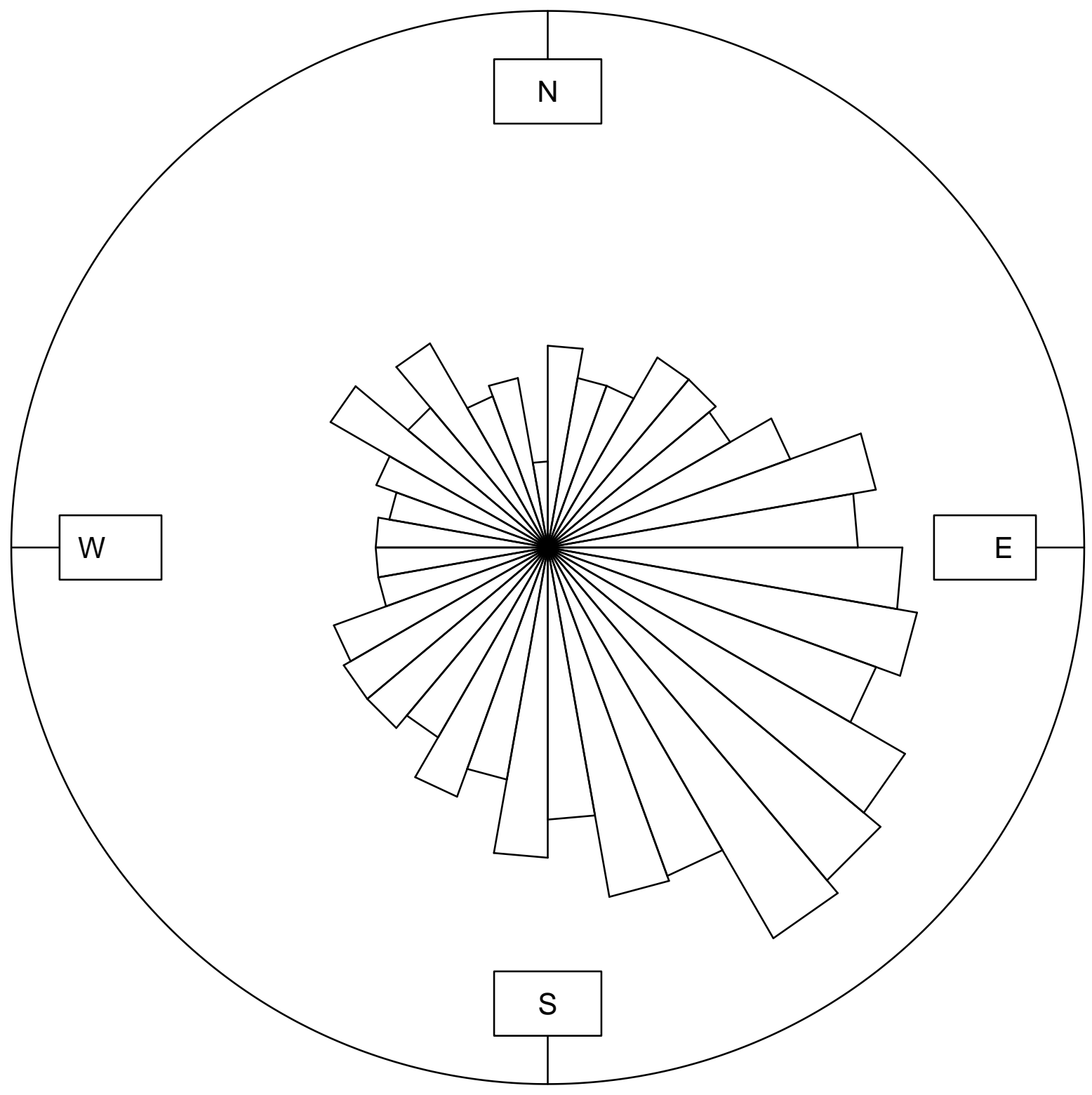


bootstrap 687

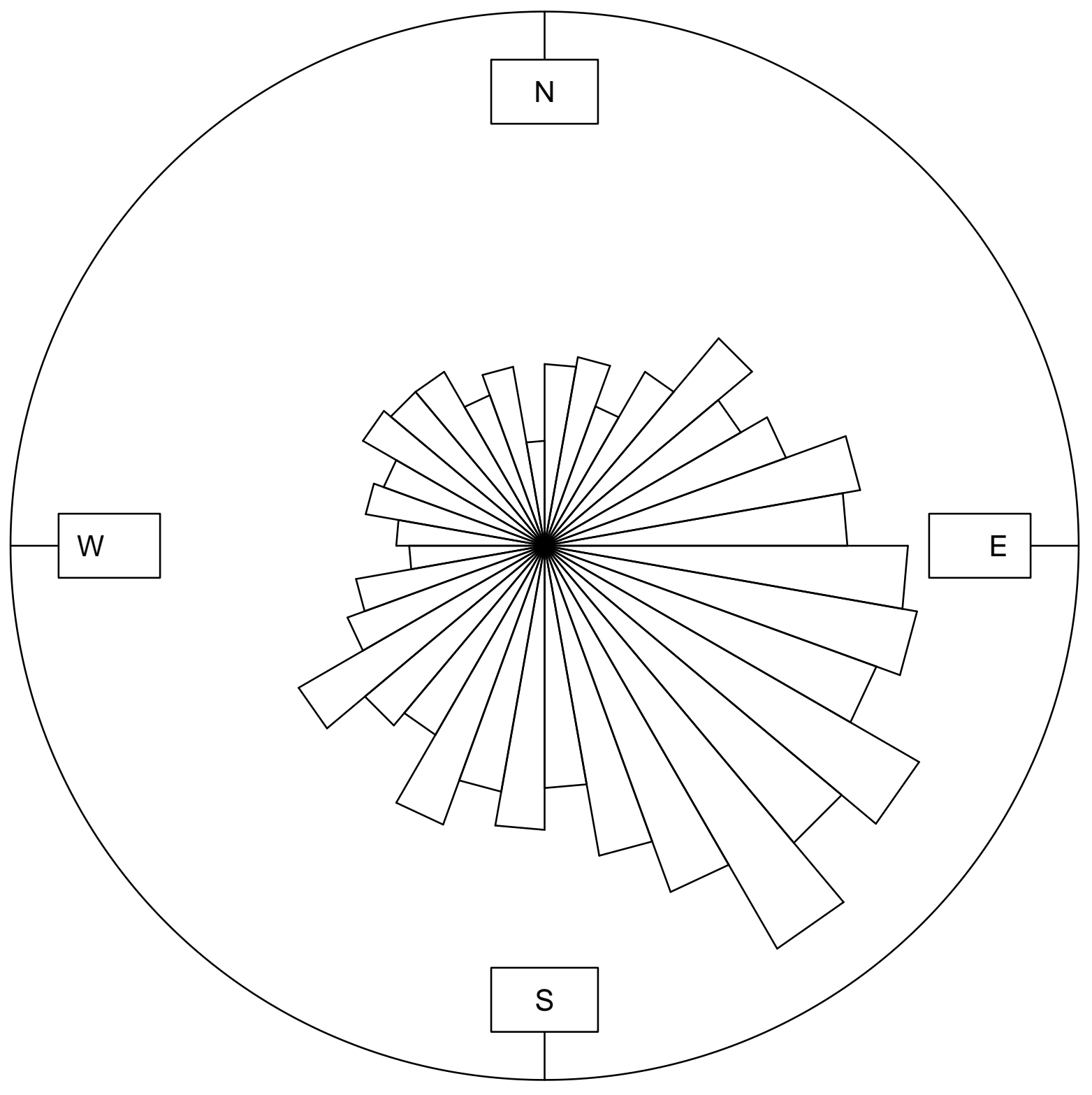


bootstrap 688

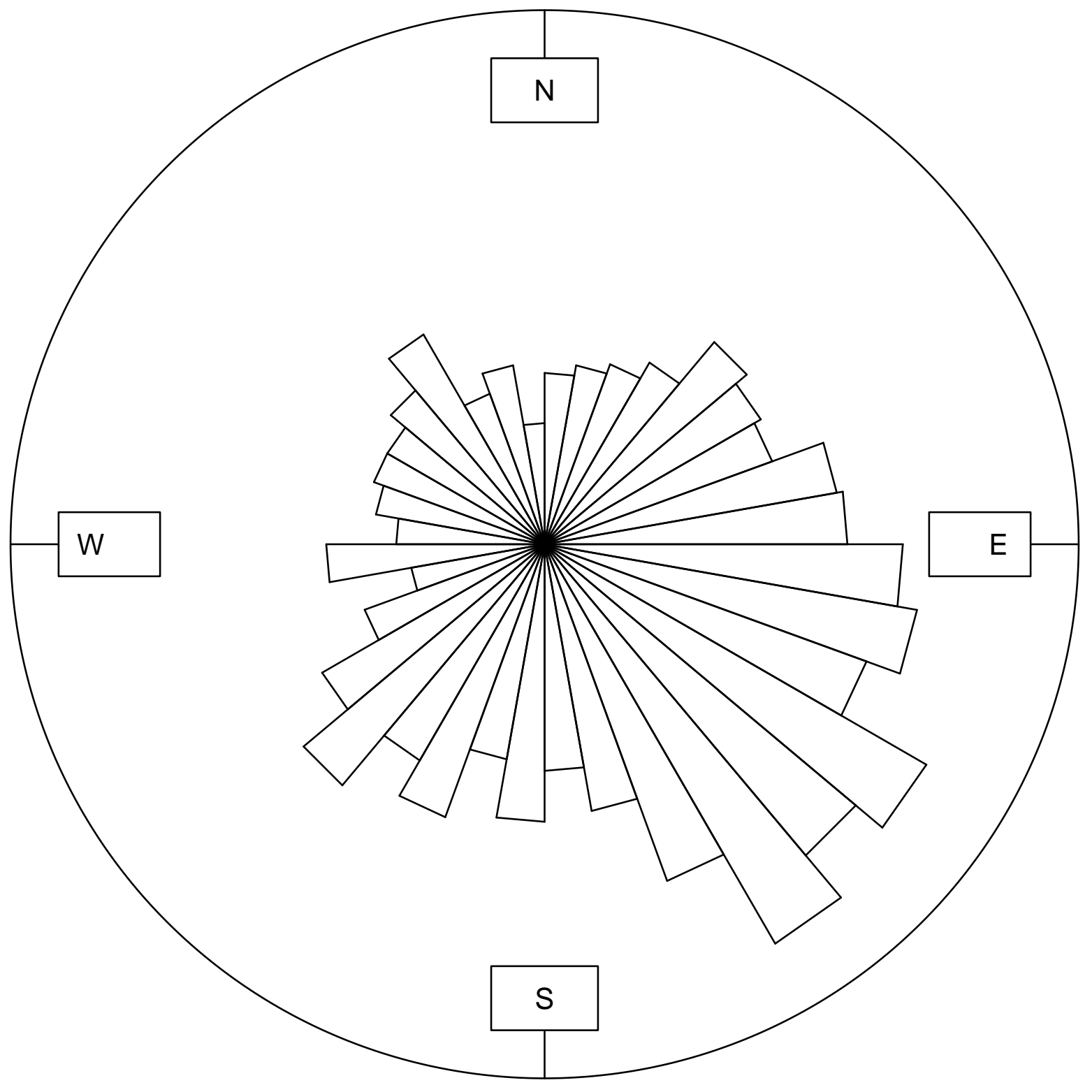


bootstrap 689

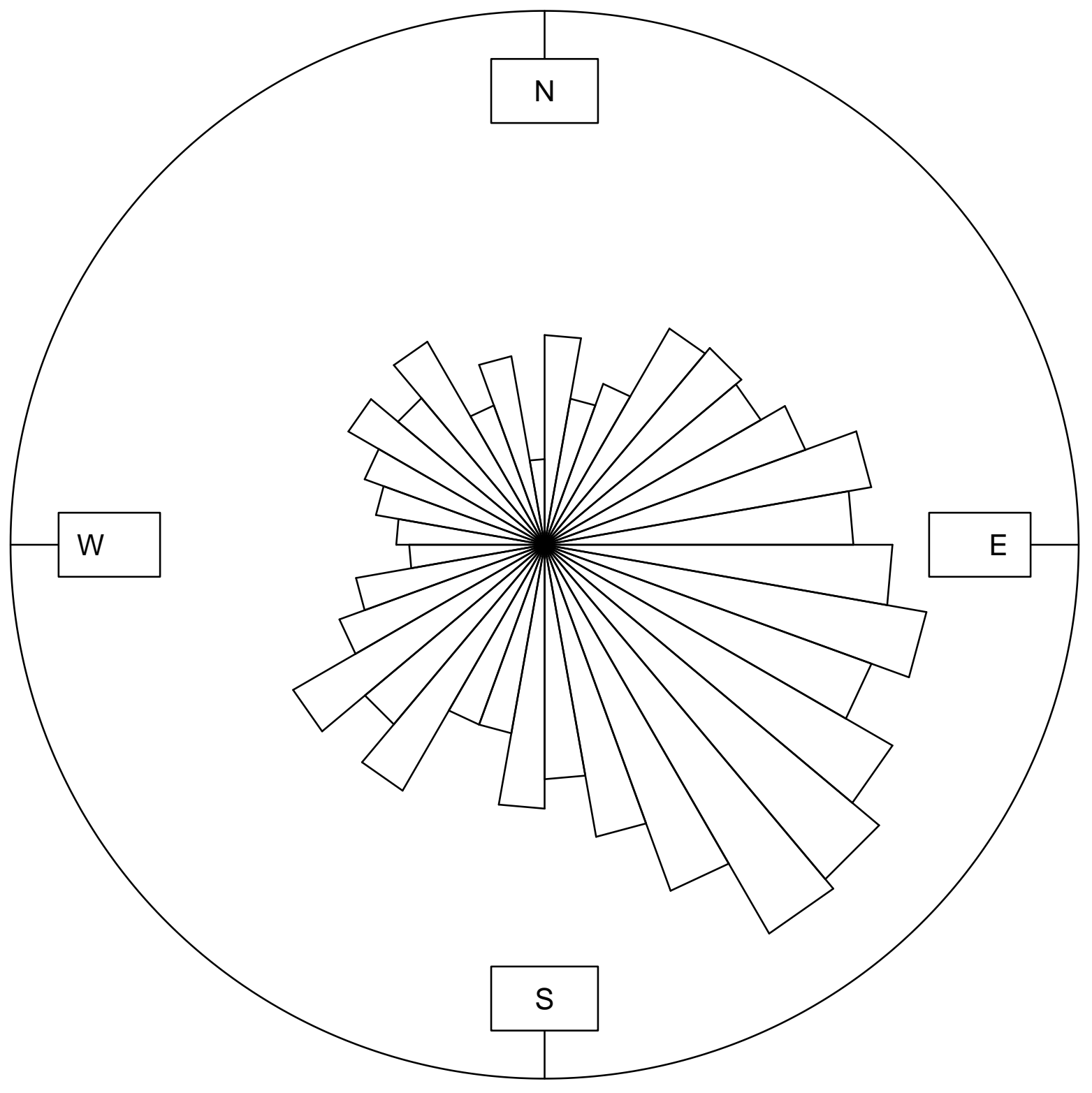


bootstrap 690

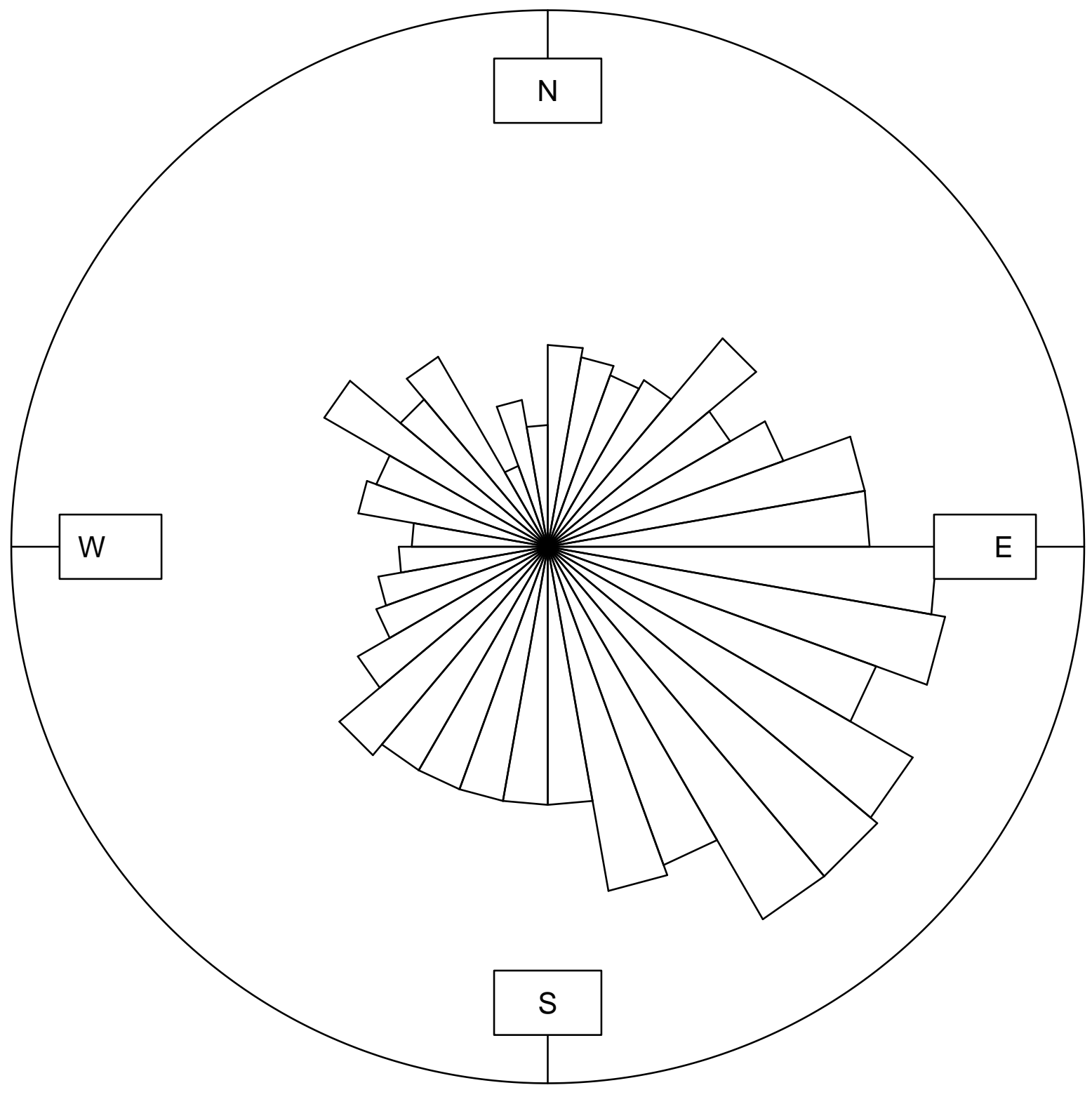


bootstrap 691

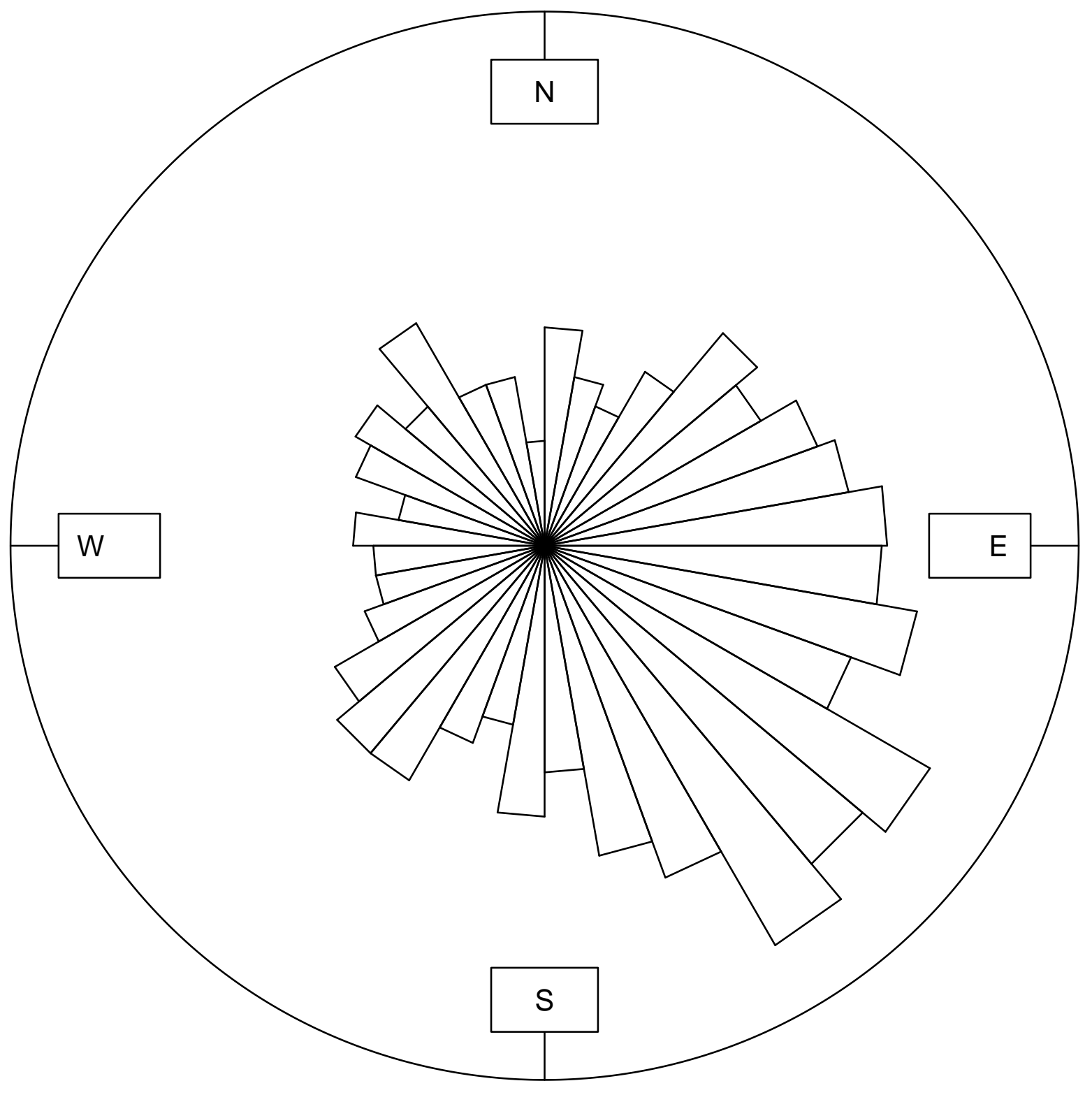


bootstrap 692

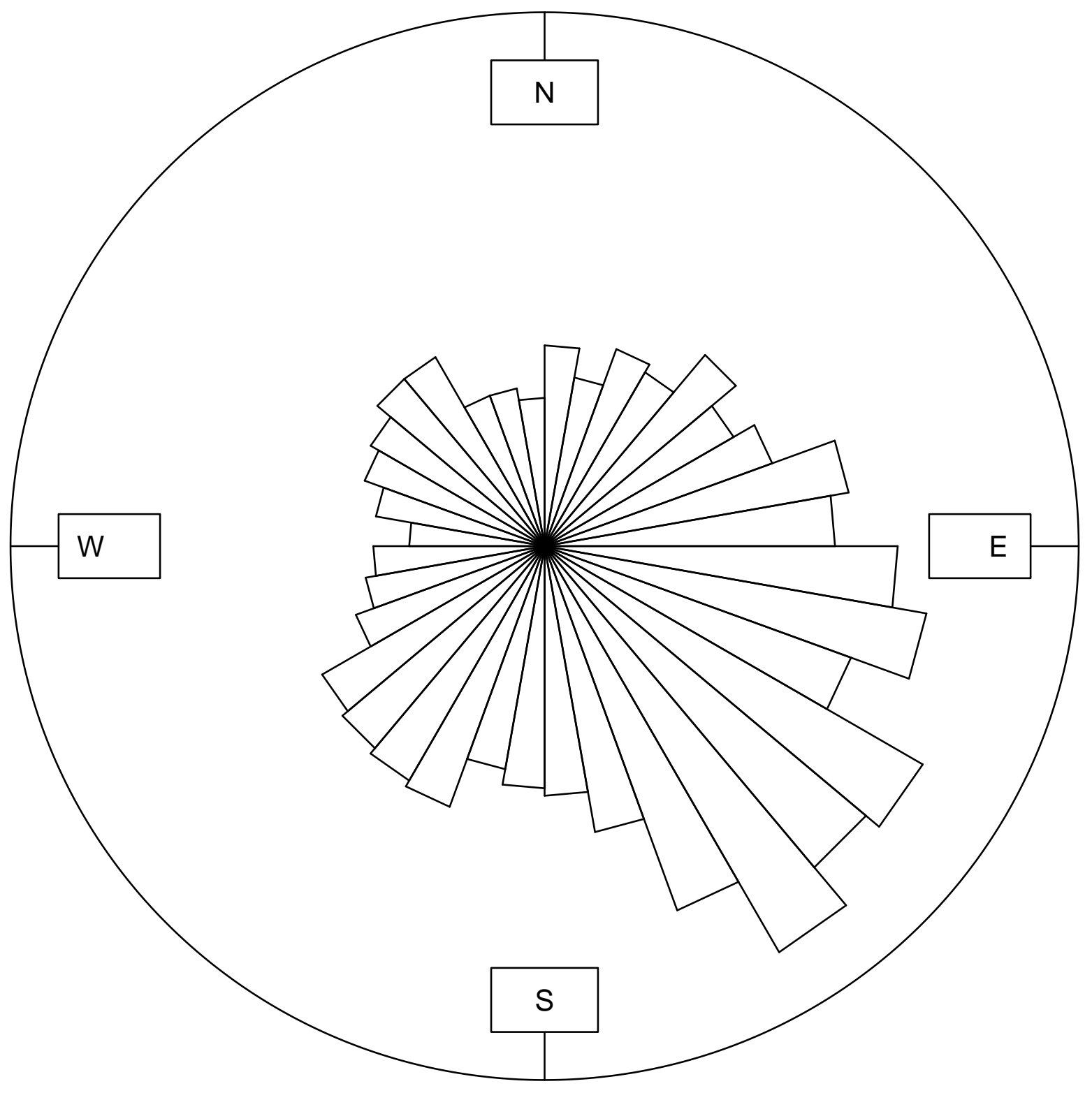




\section{bootstrap 693}

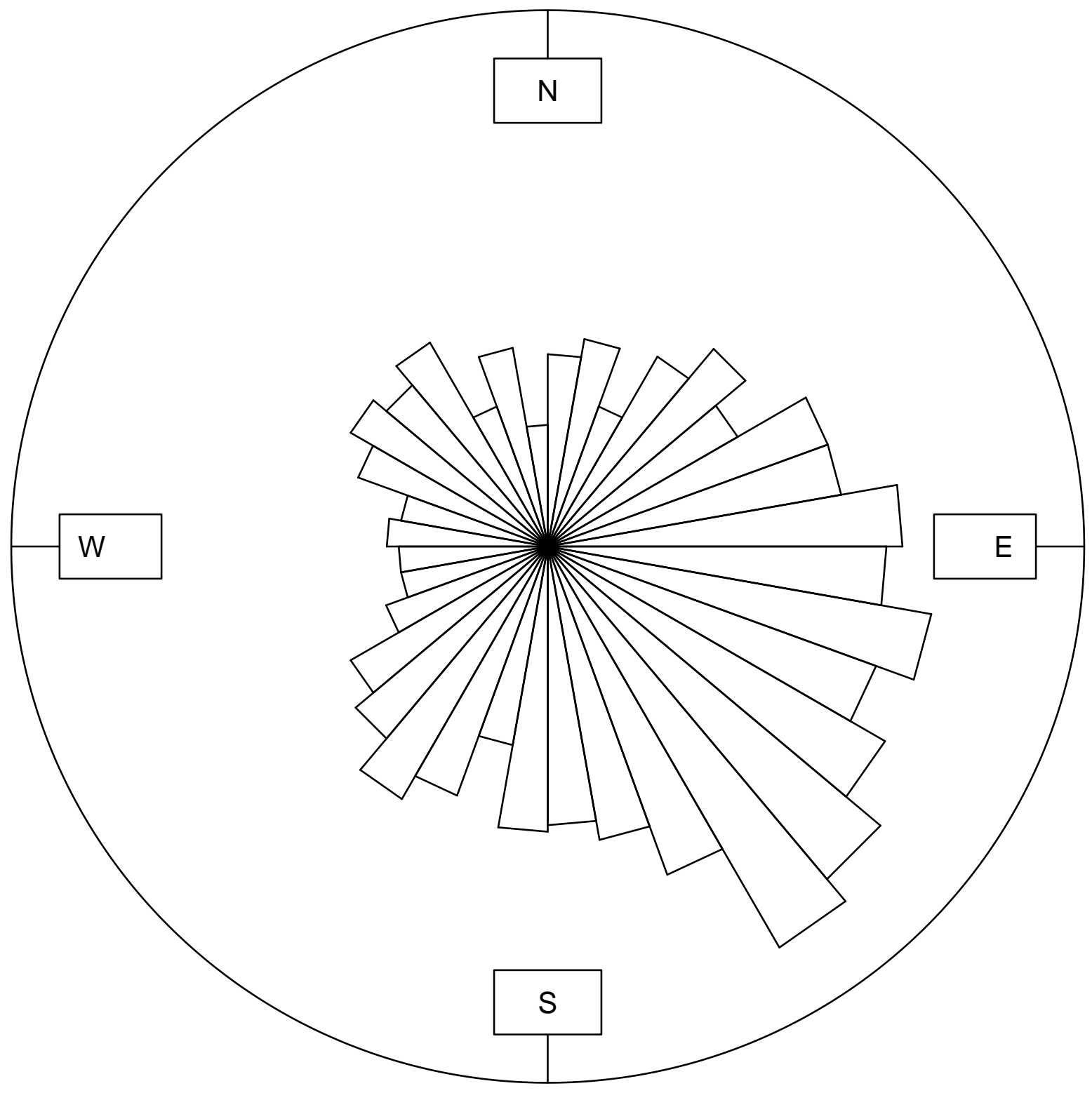




\section{bootstrap 694}

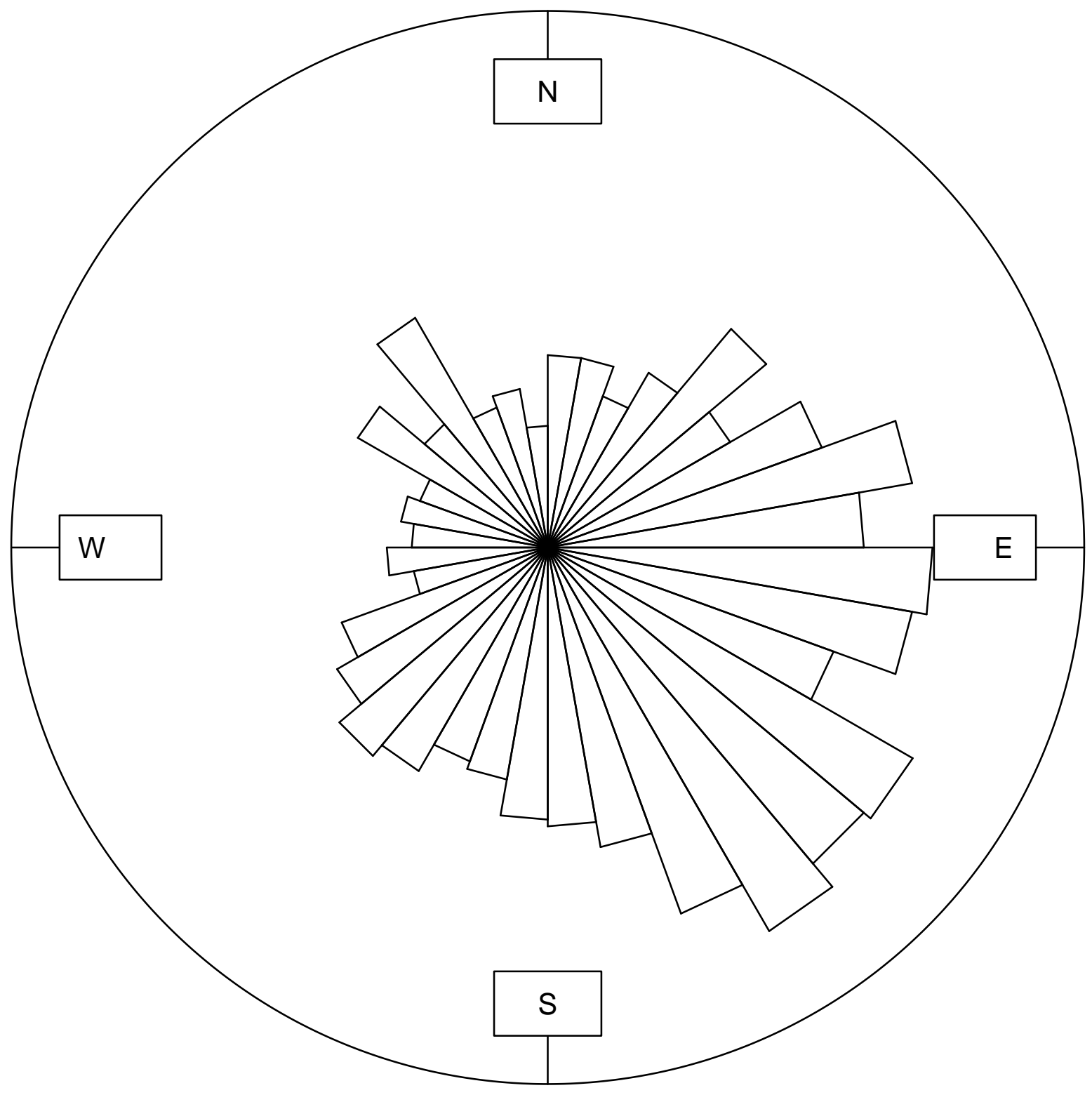




\section{bootstrap 695}

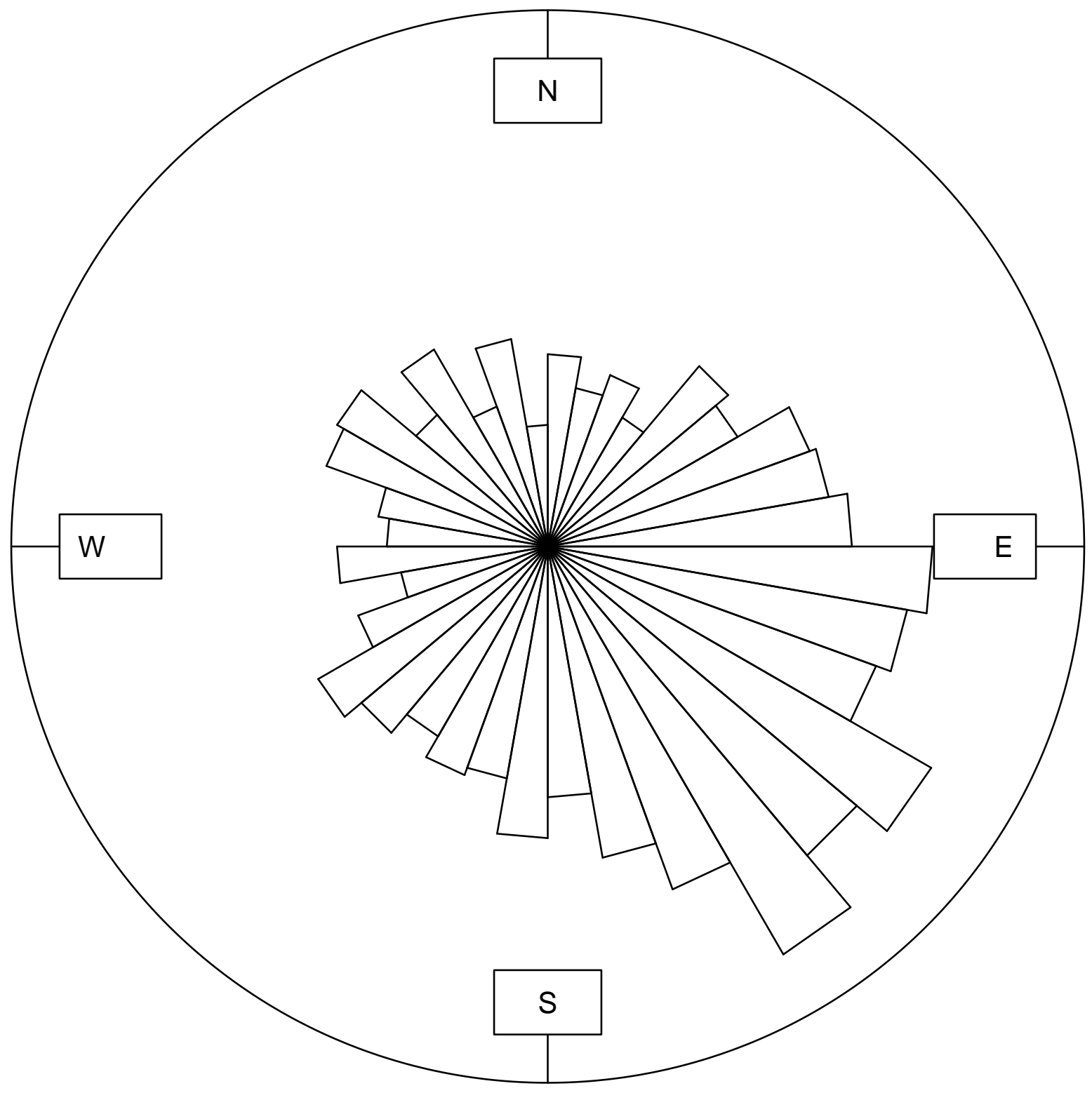


bootstrap 696

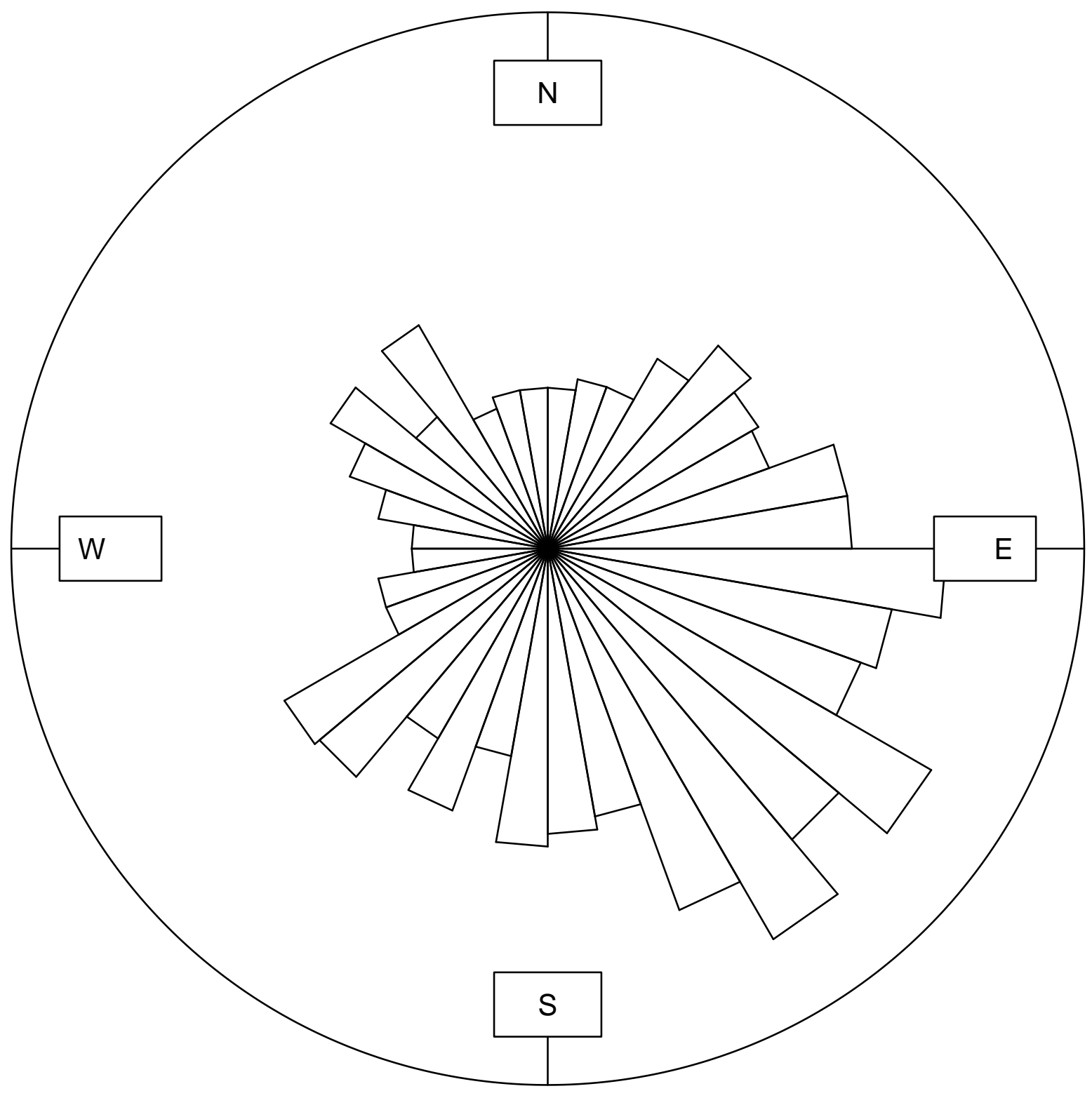


bootstrap 697

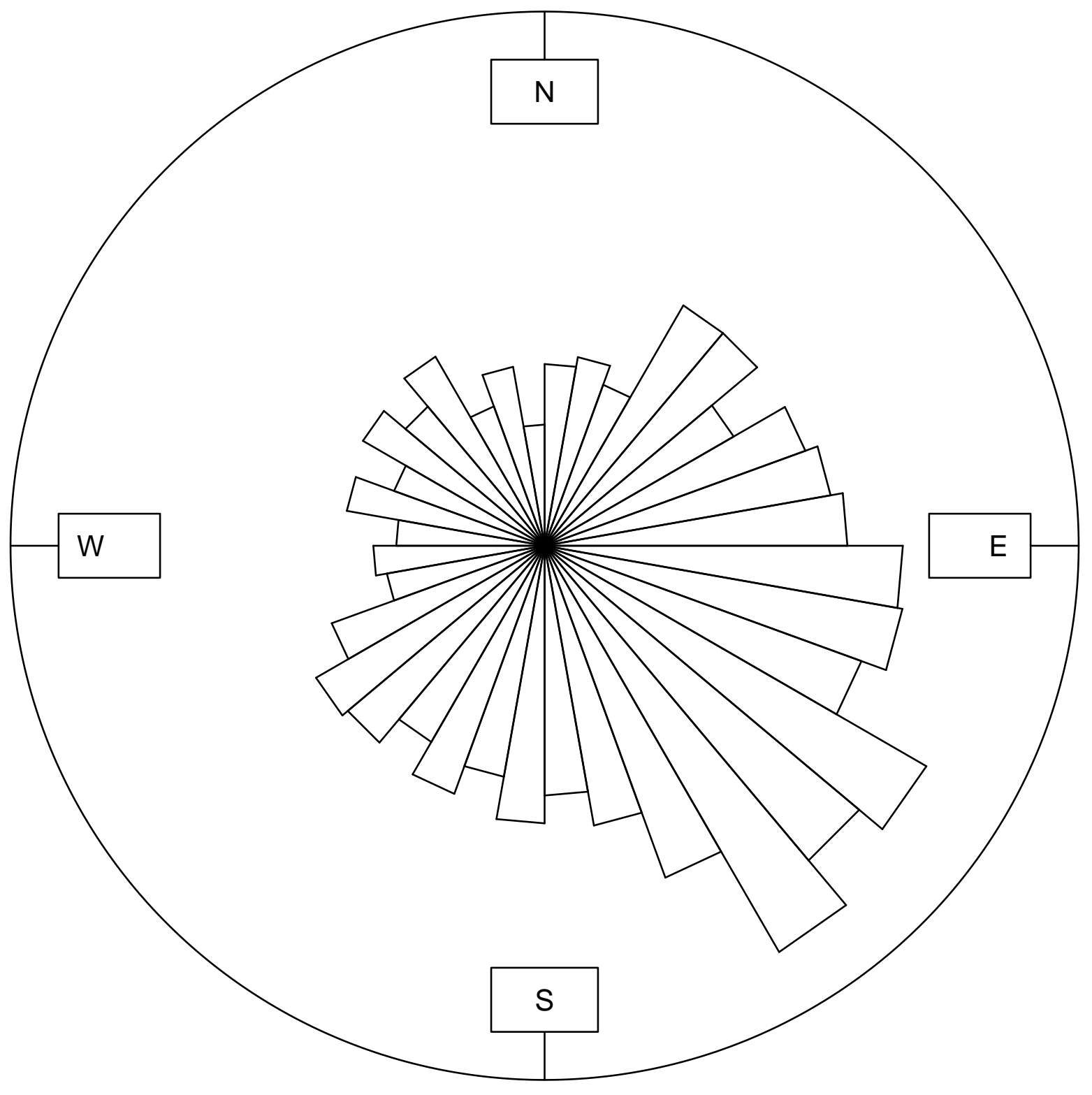


bootstrap 698

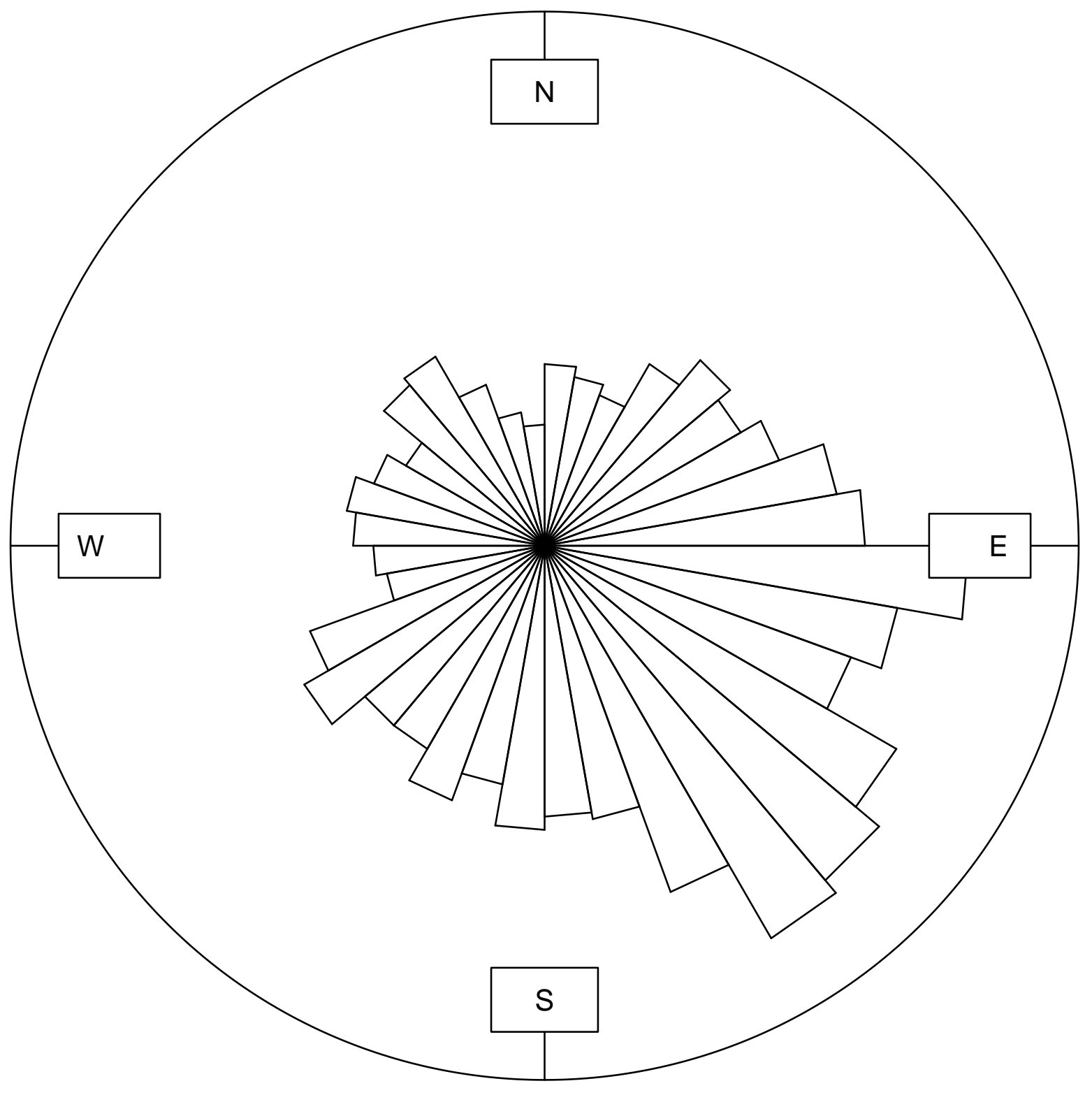


bootstrap 699

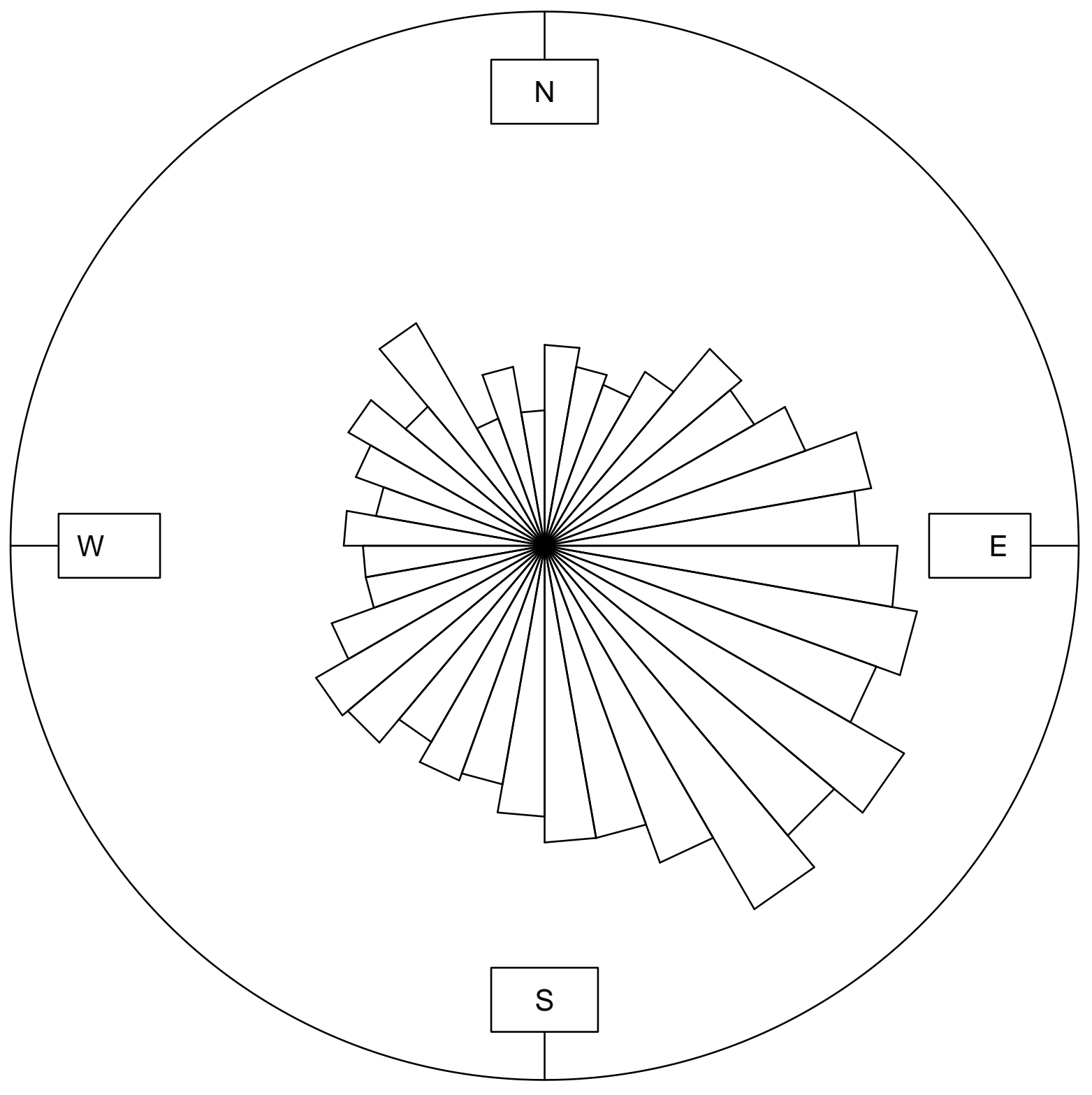


bootstrap 700

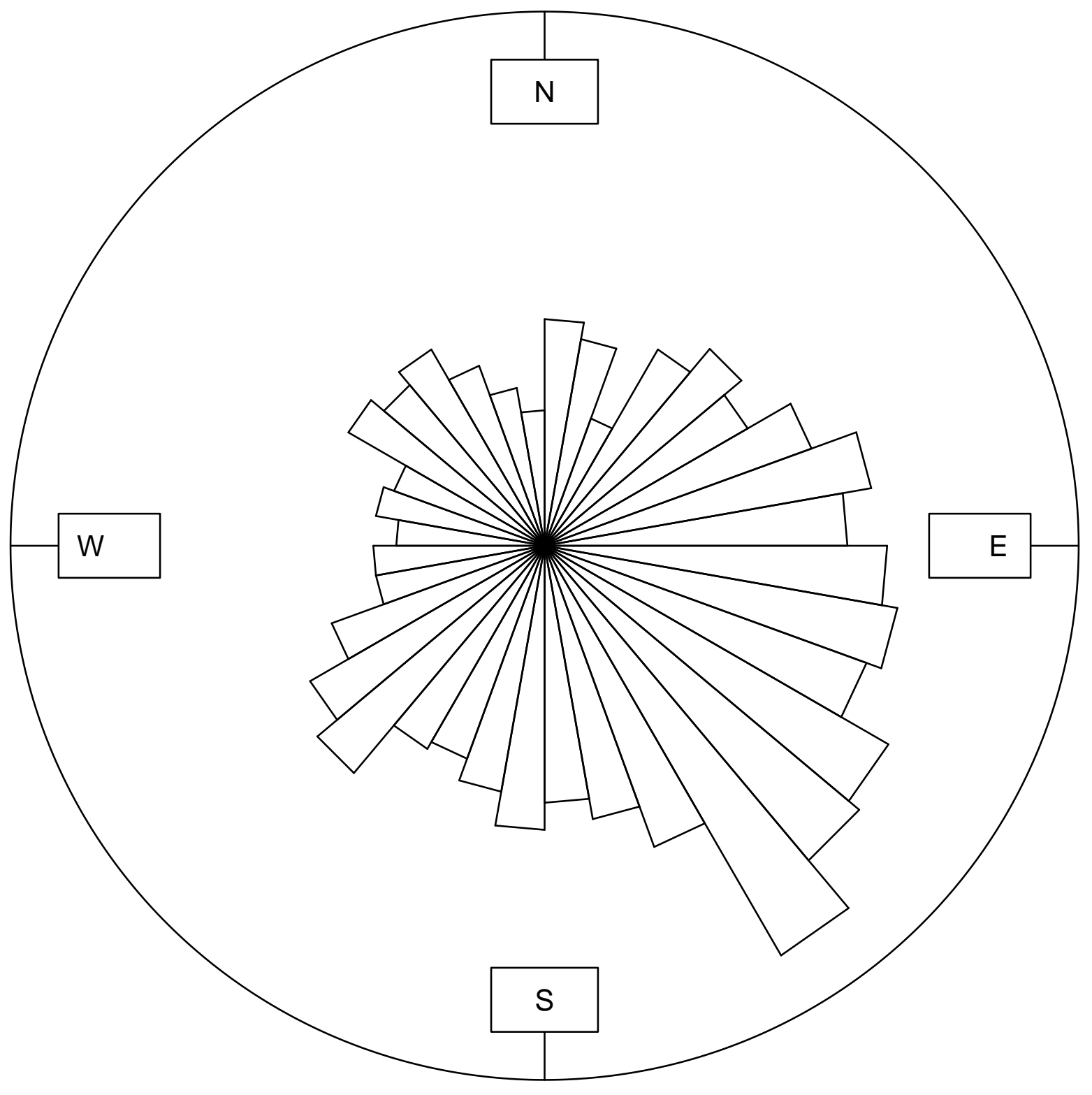


bootstrap 701

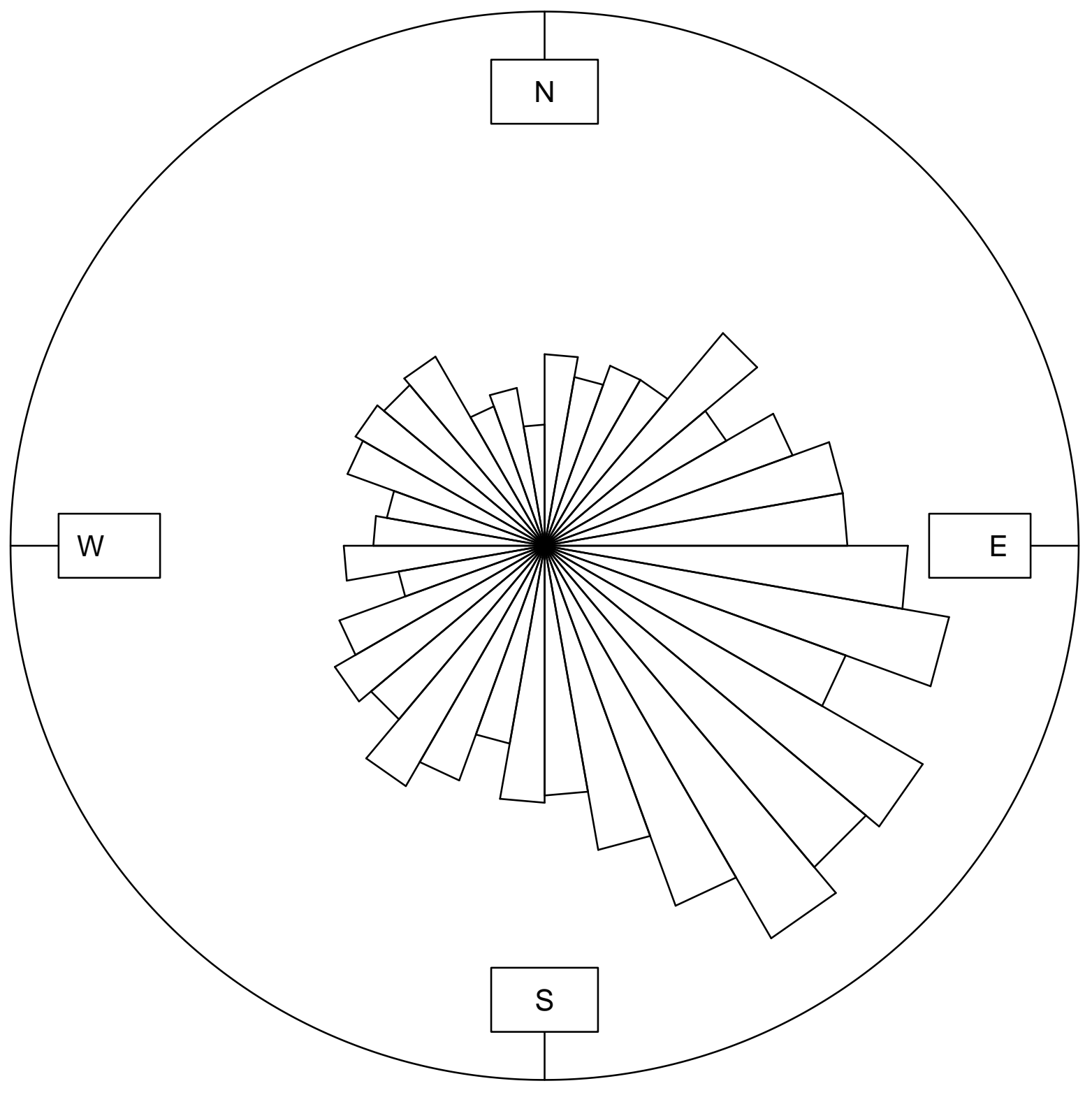


bootstrap 702

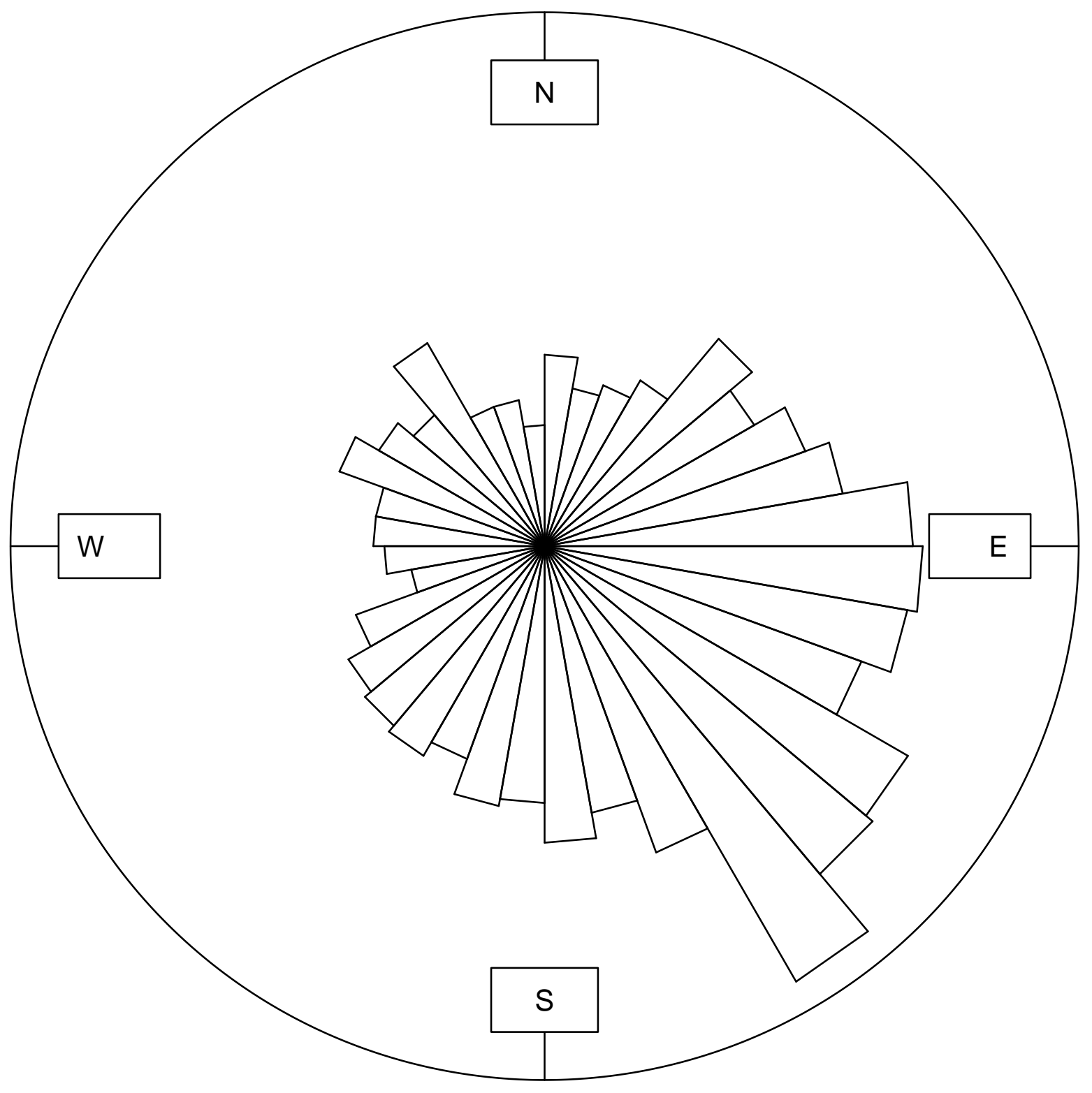




\section{bootstrap 703}

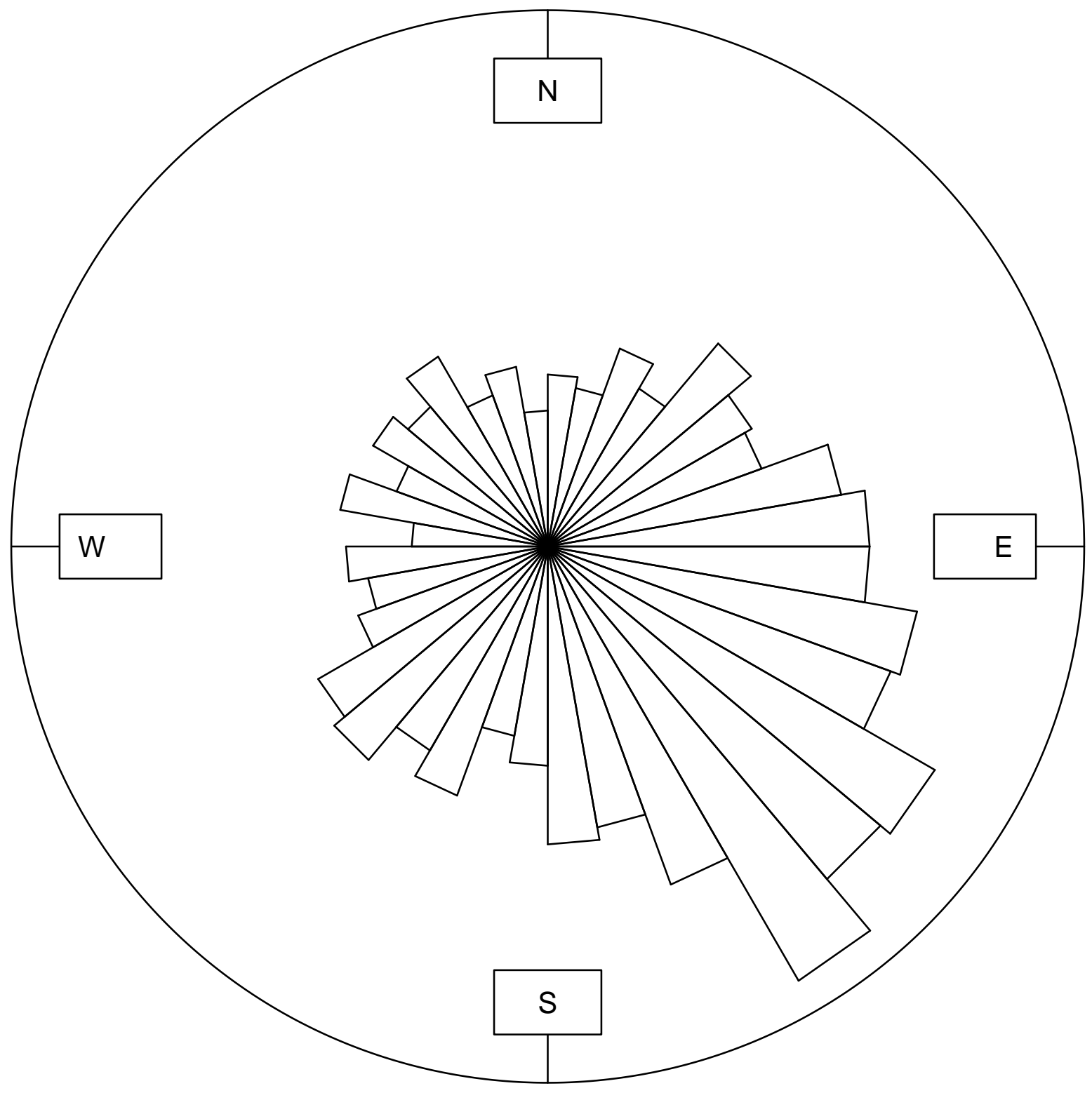


bootstrap 704

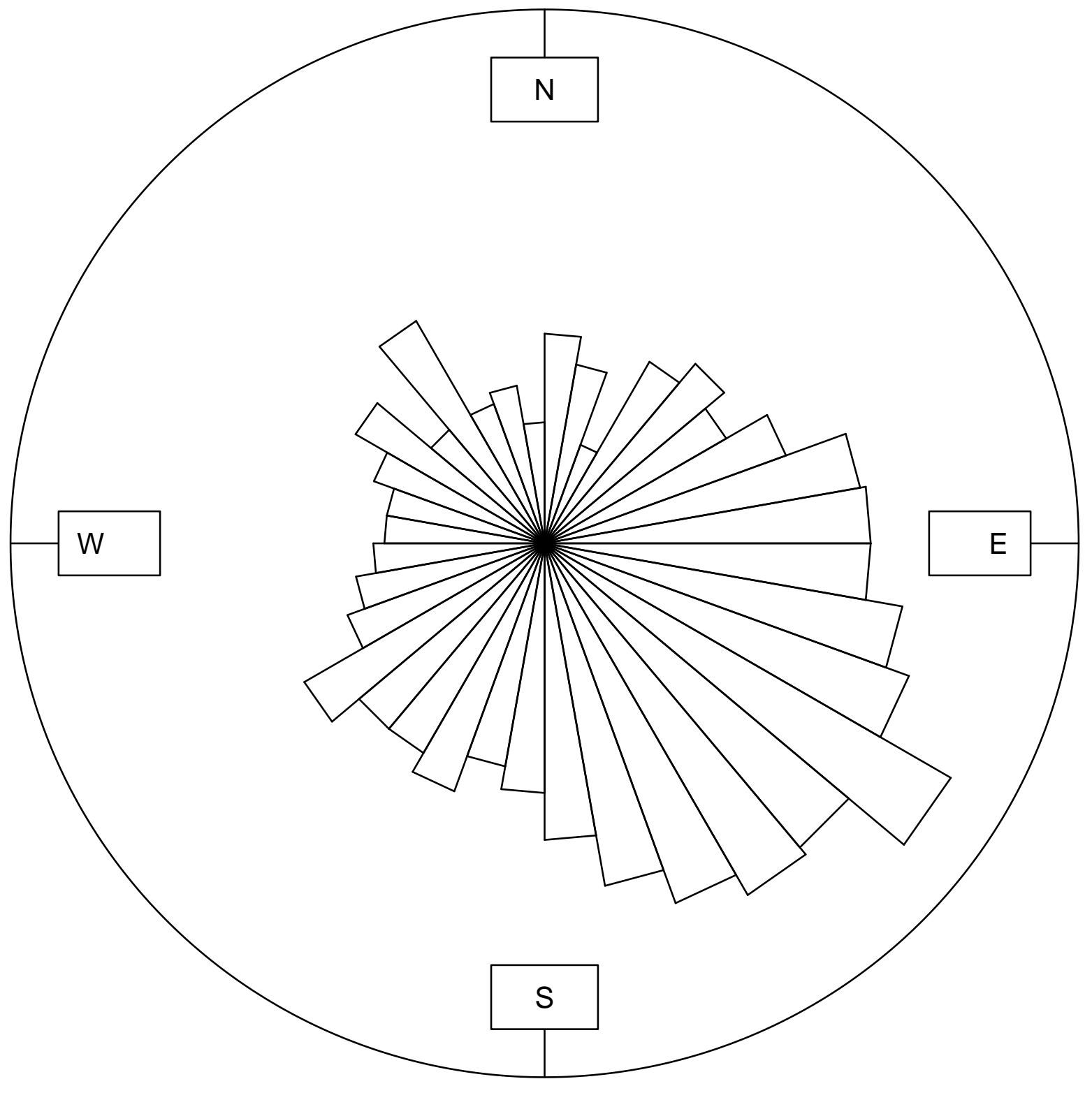


bootstrap 705

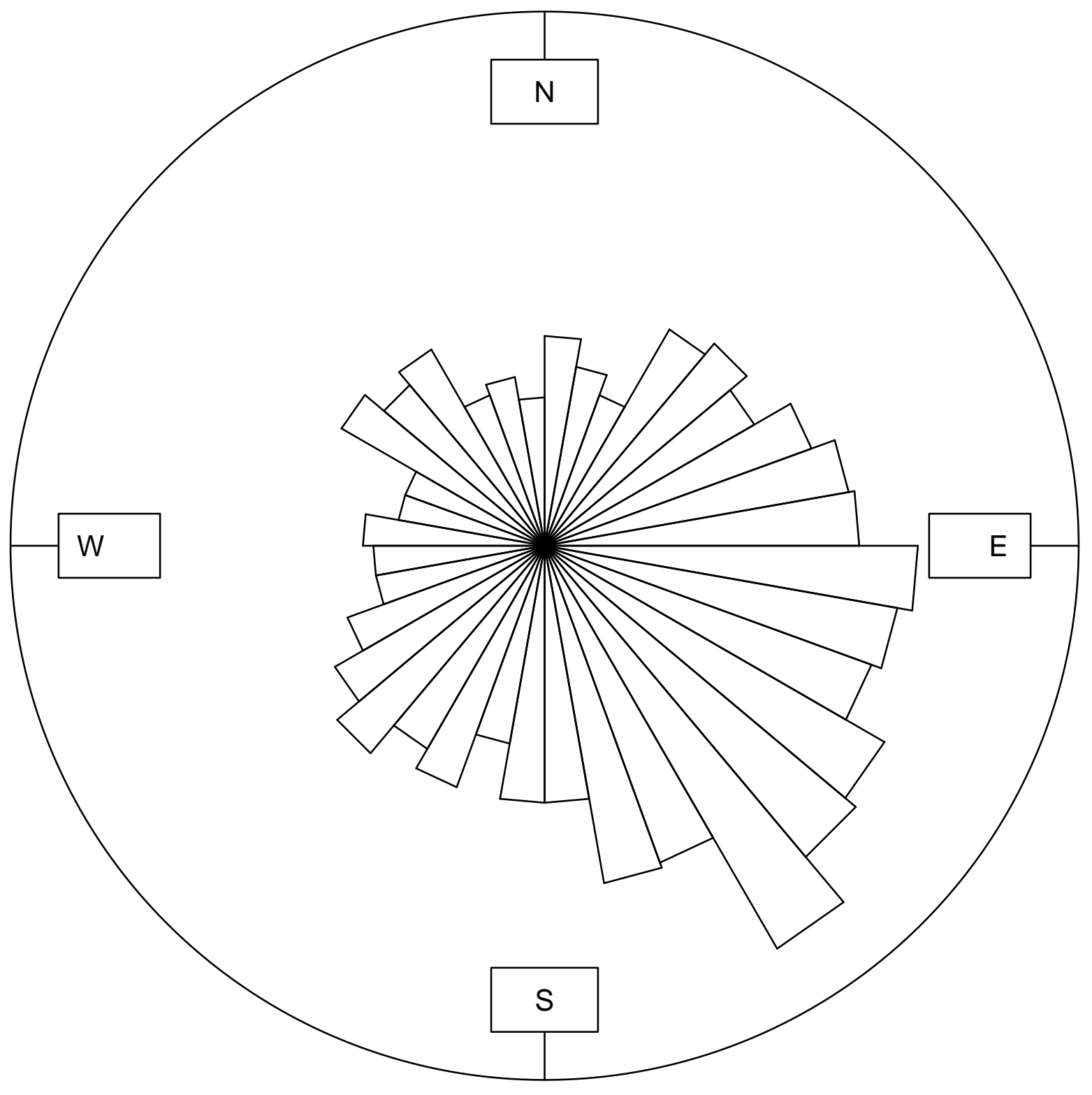


bootstrap 706

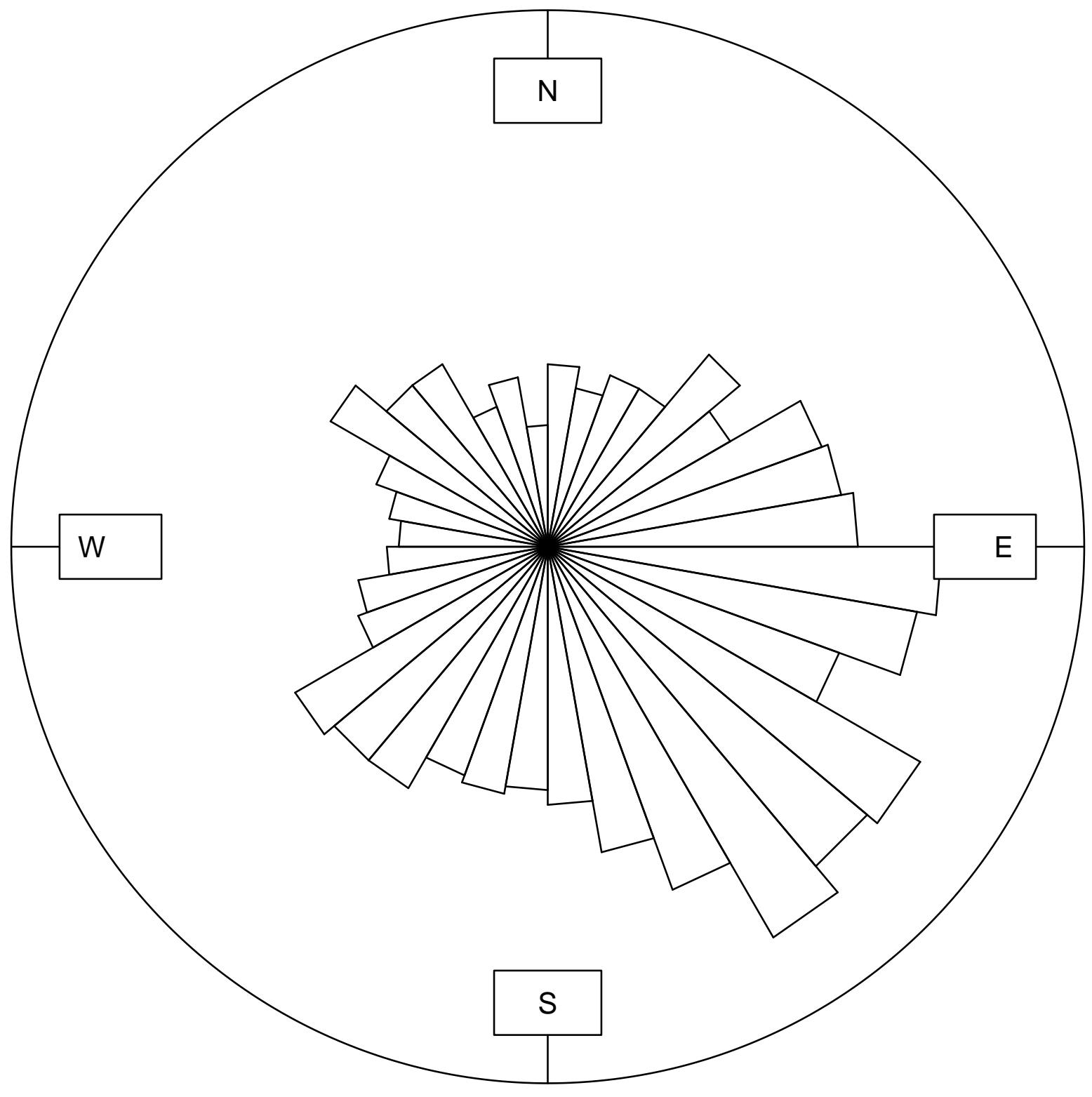


bootstrap 707

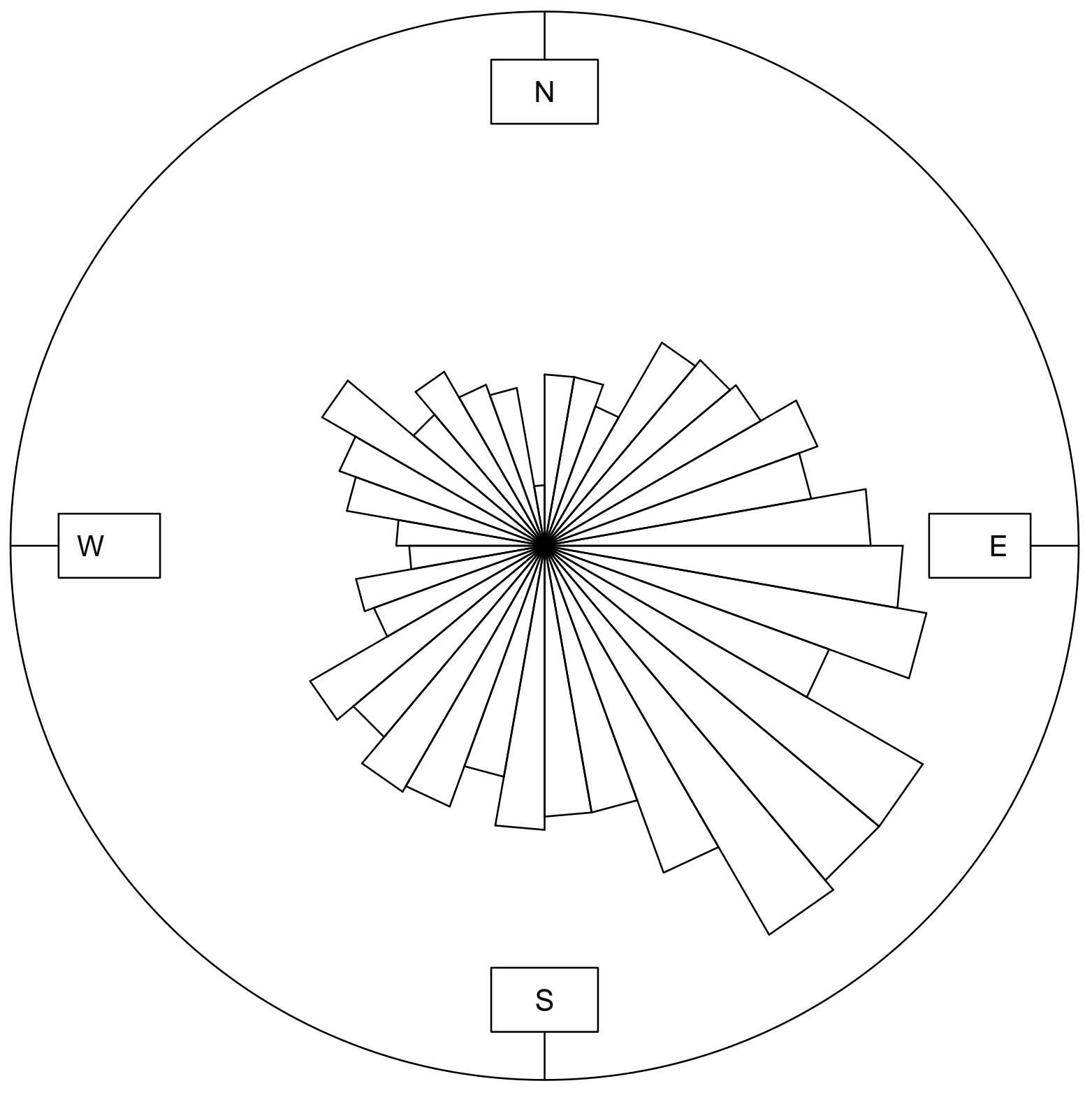


bootstrap 708

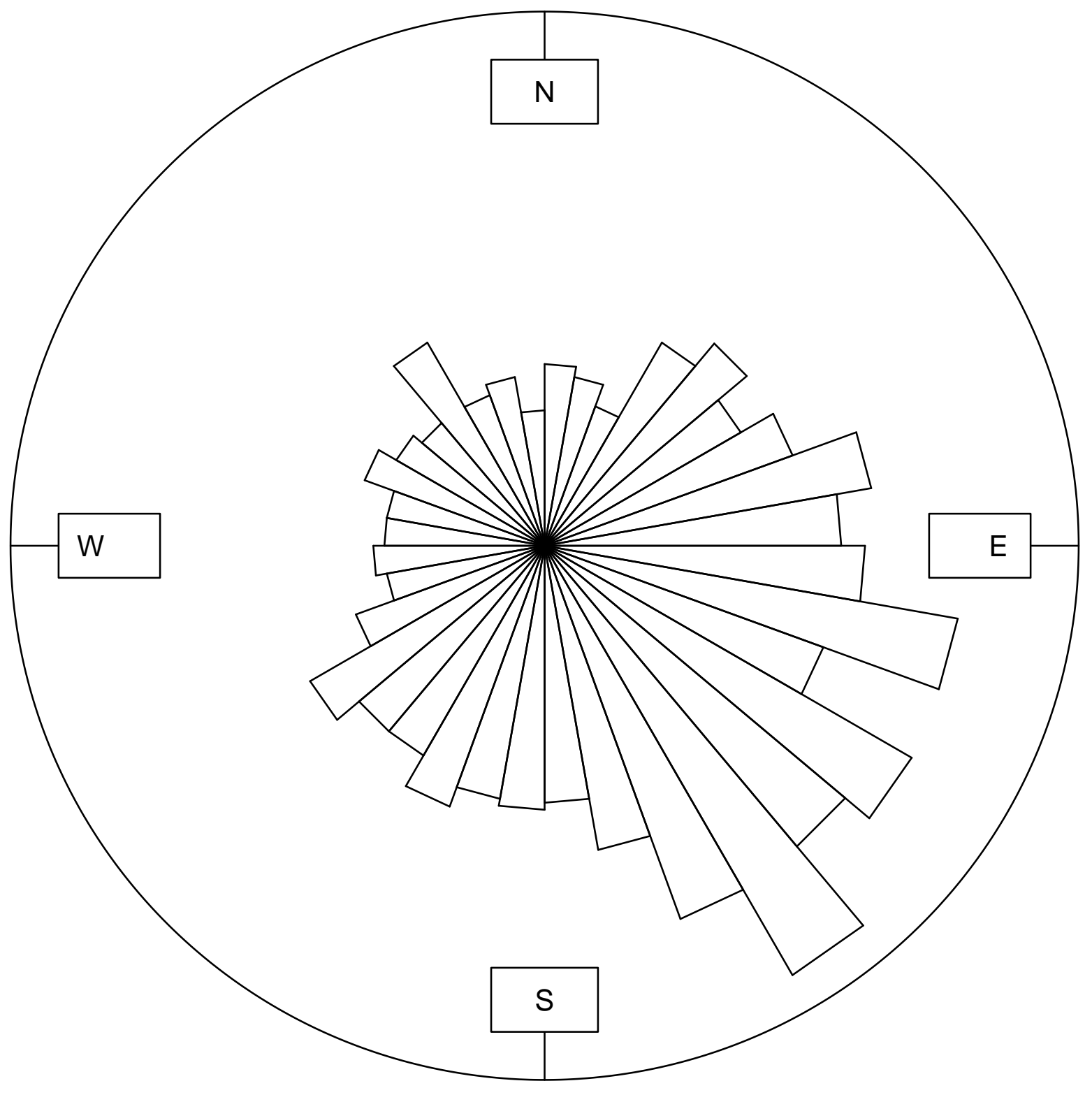


bootstrap 709

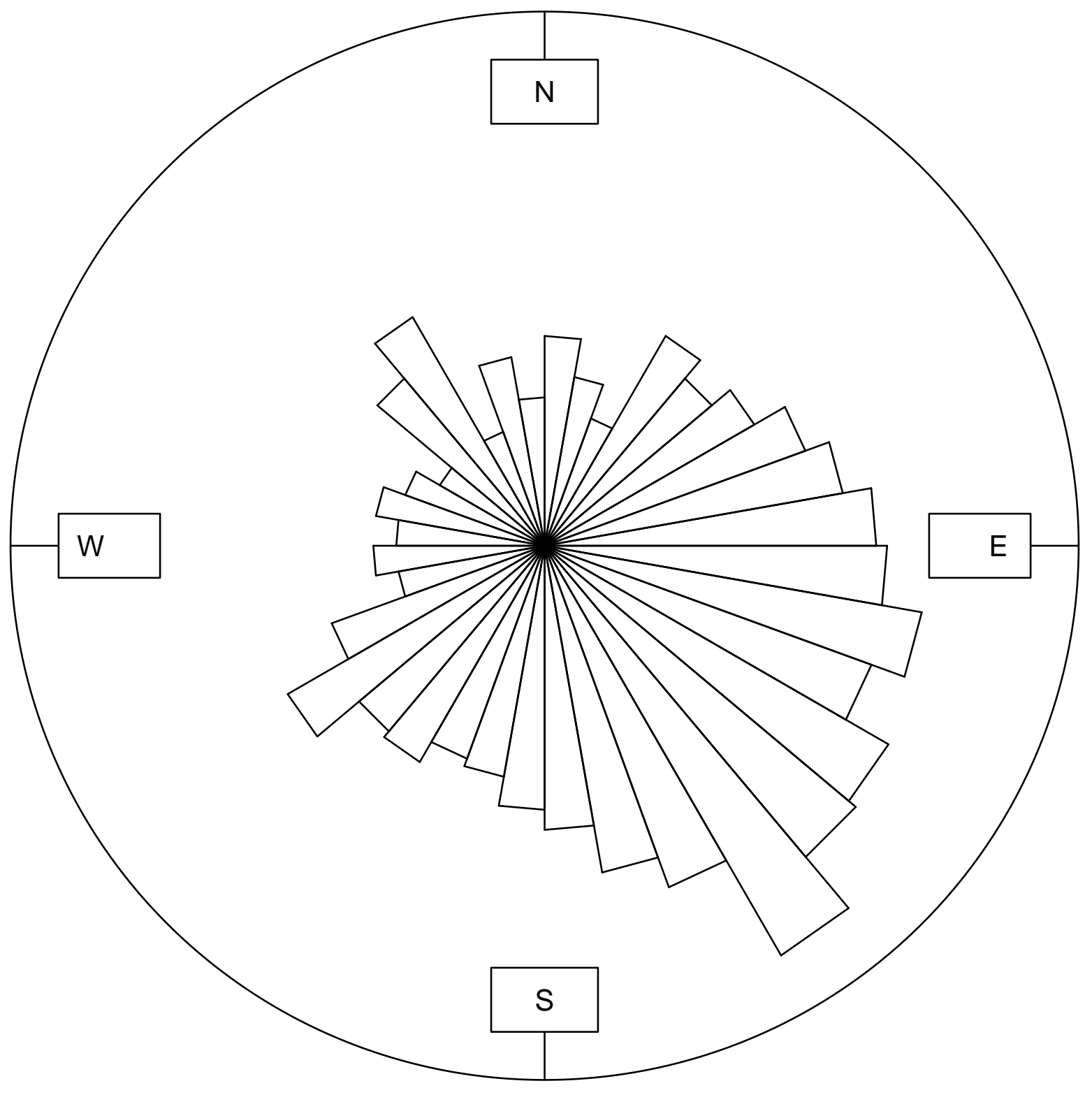


bootstrap 710

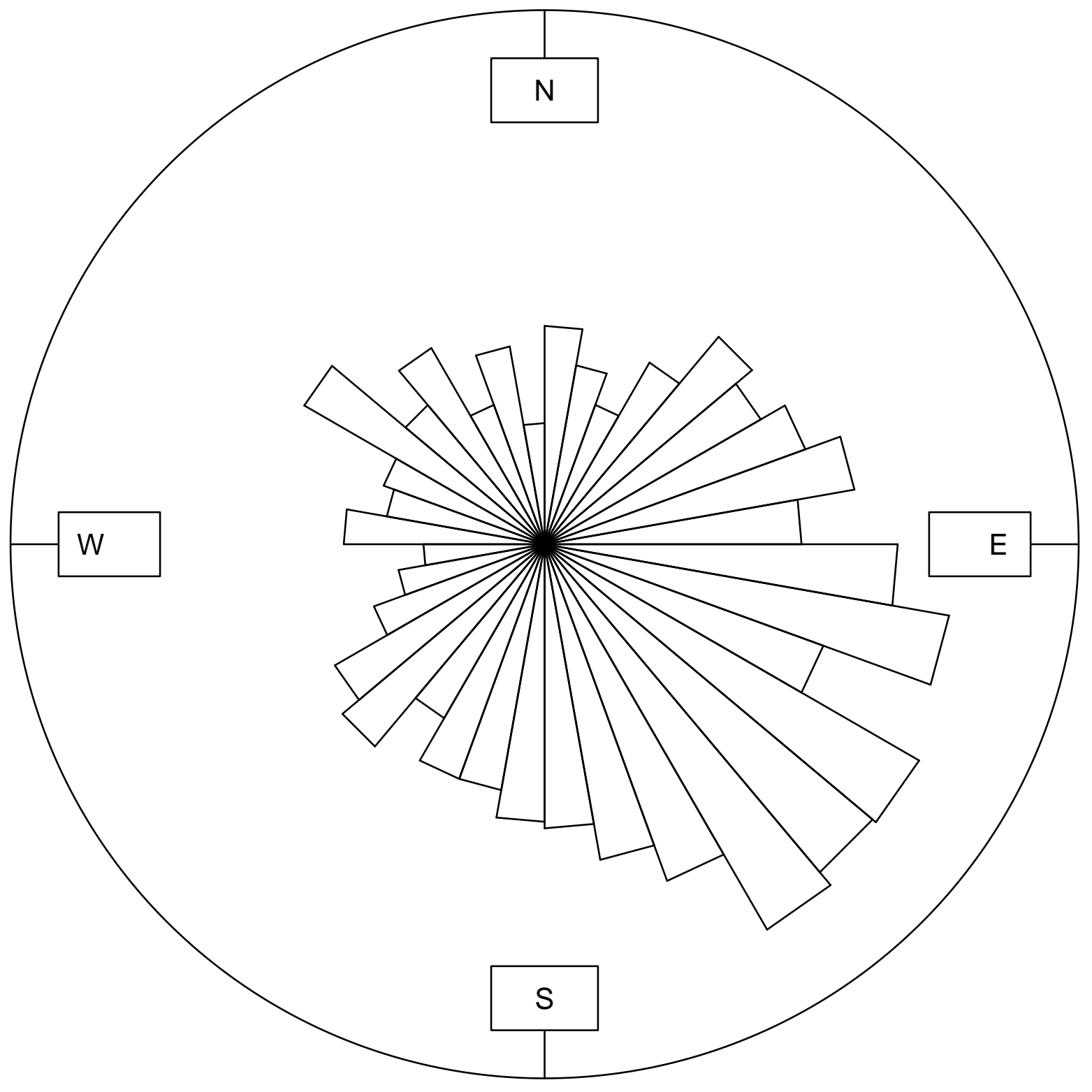


bootstrap 711

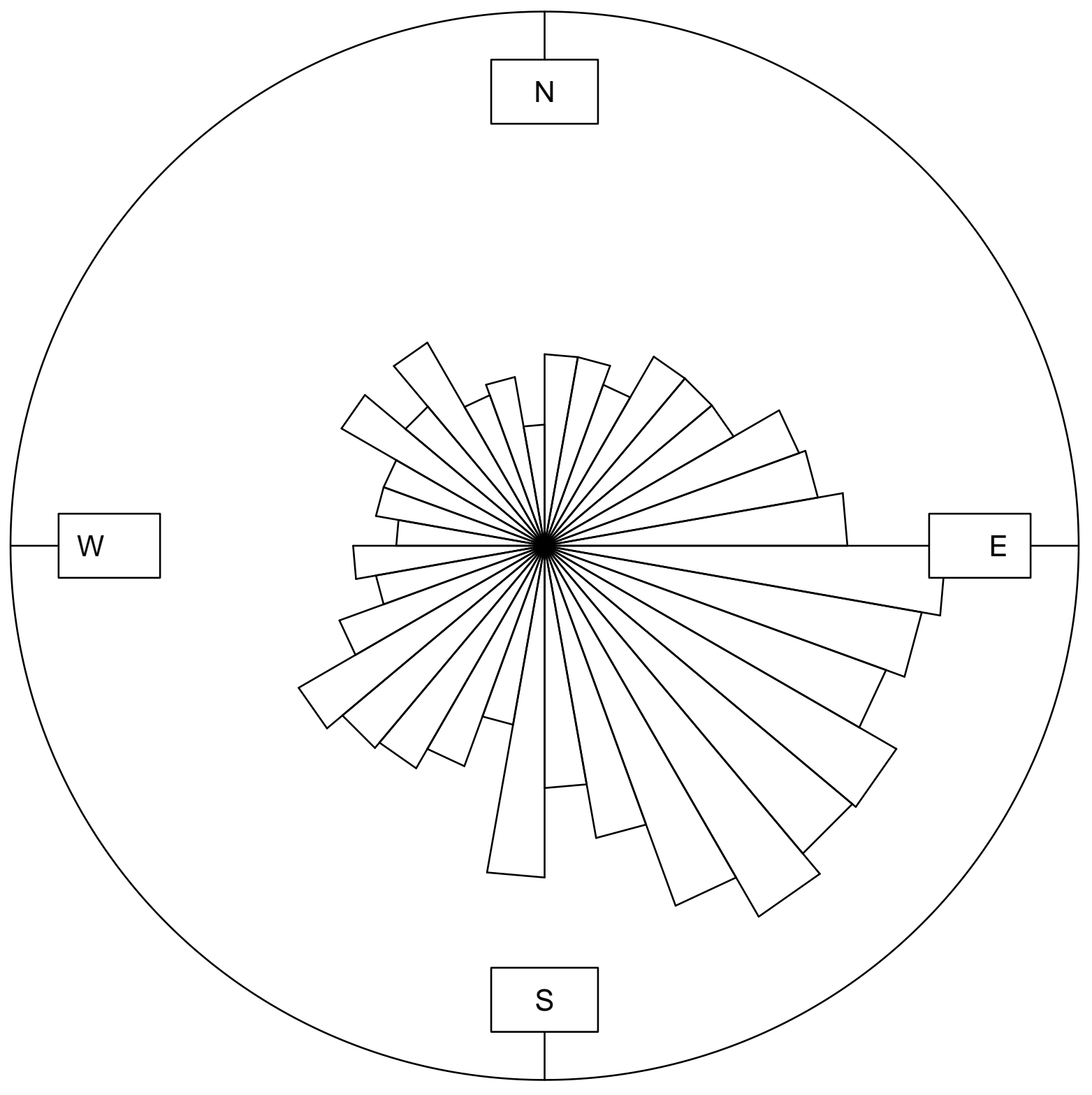


bootstrap 712

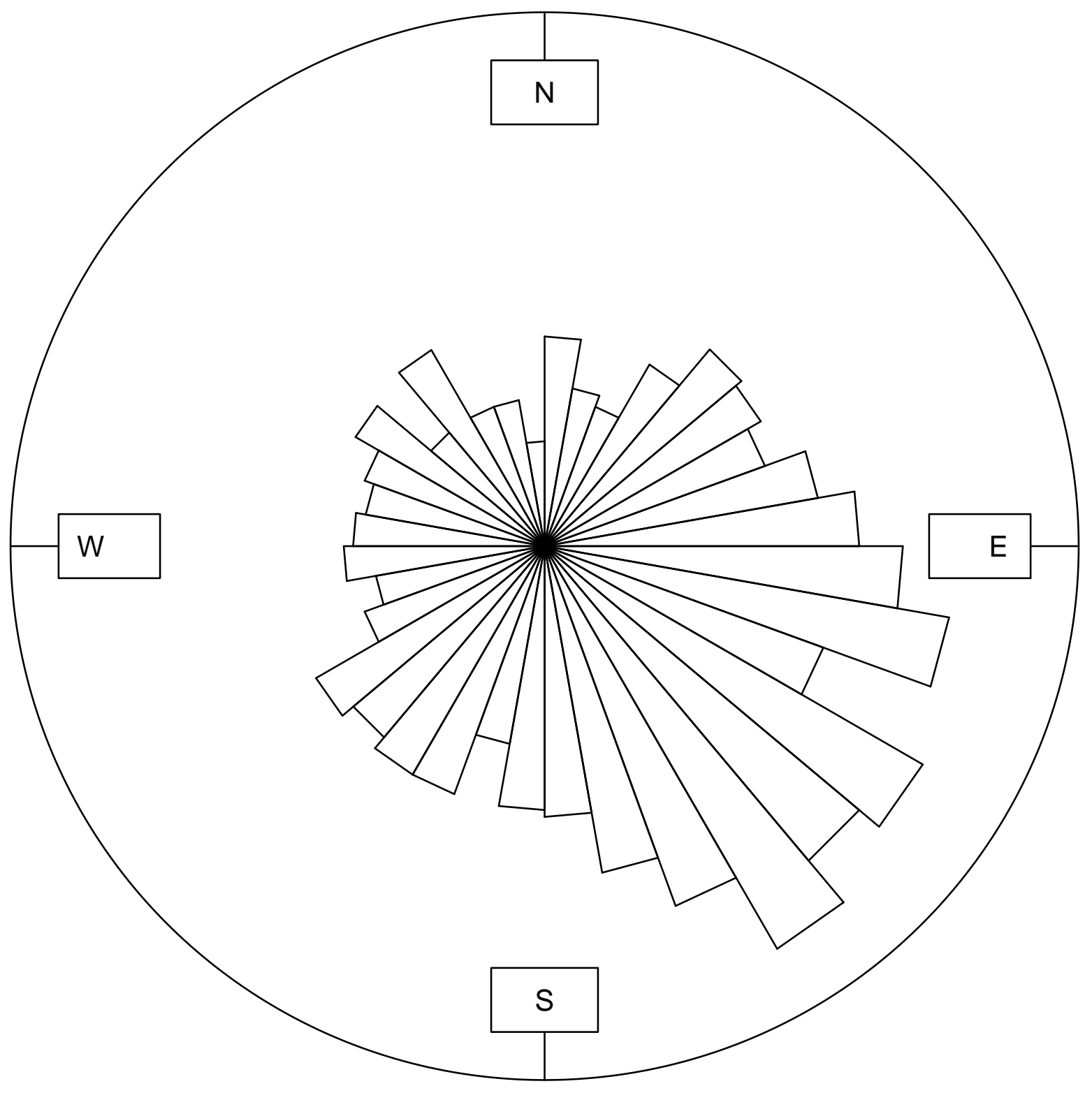


bootstrap 713

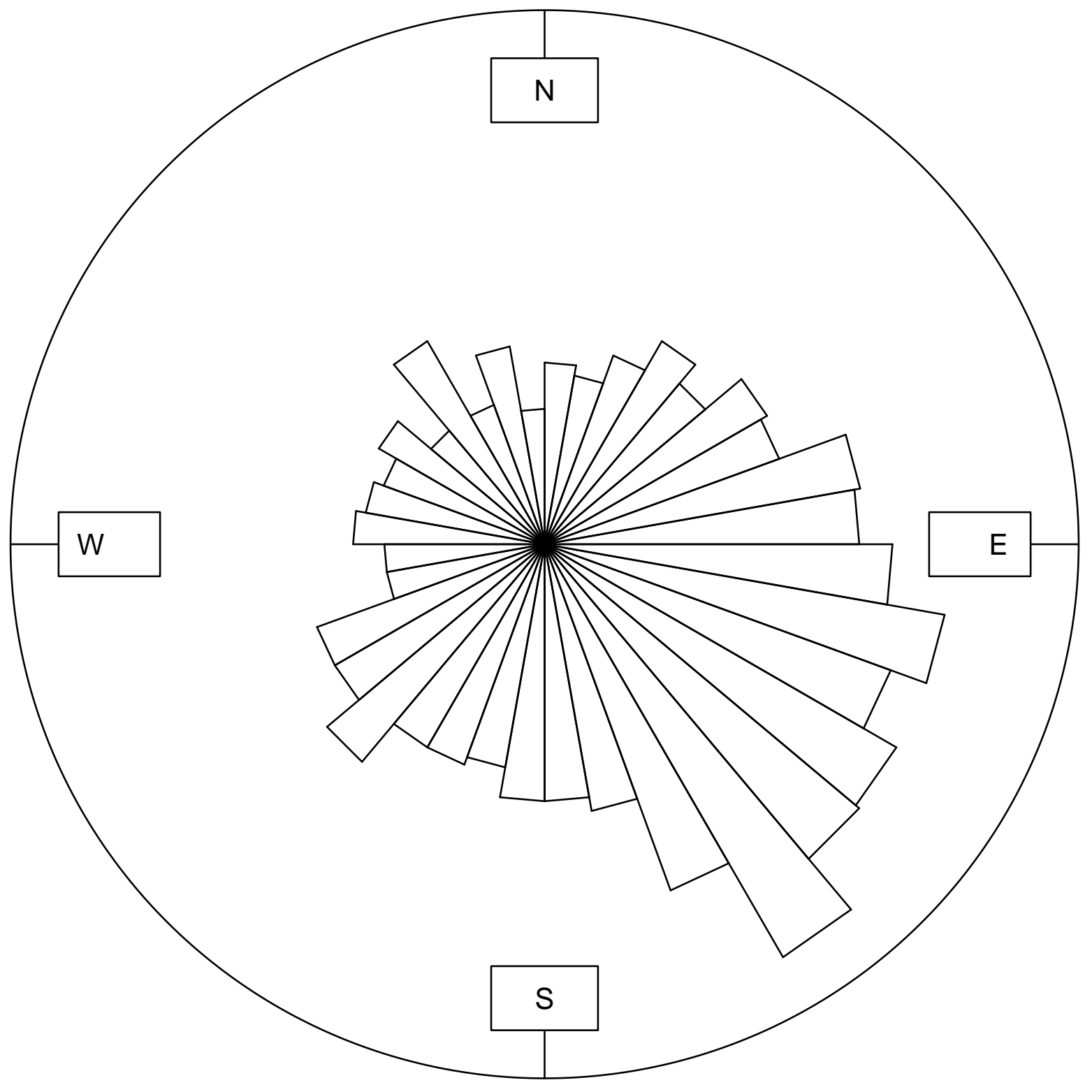


bootstrap 714

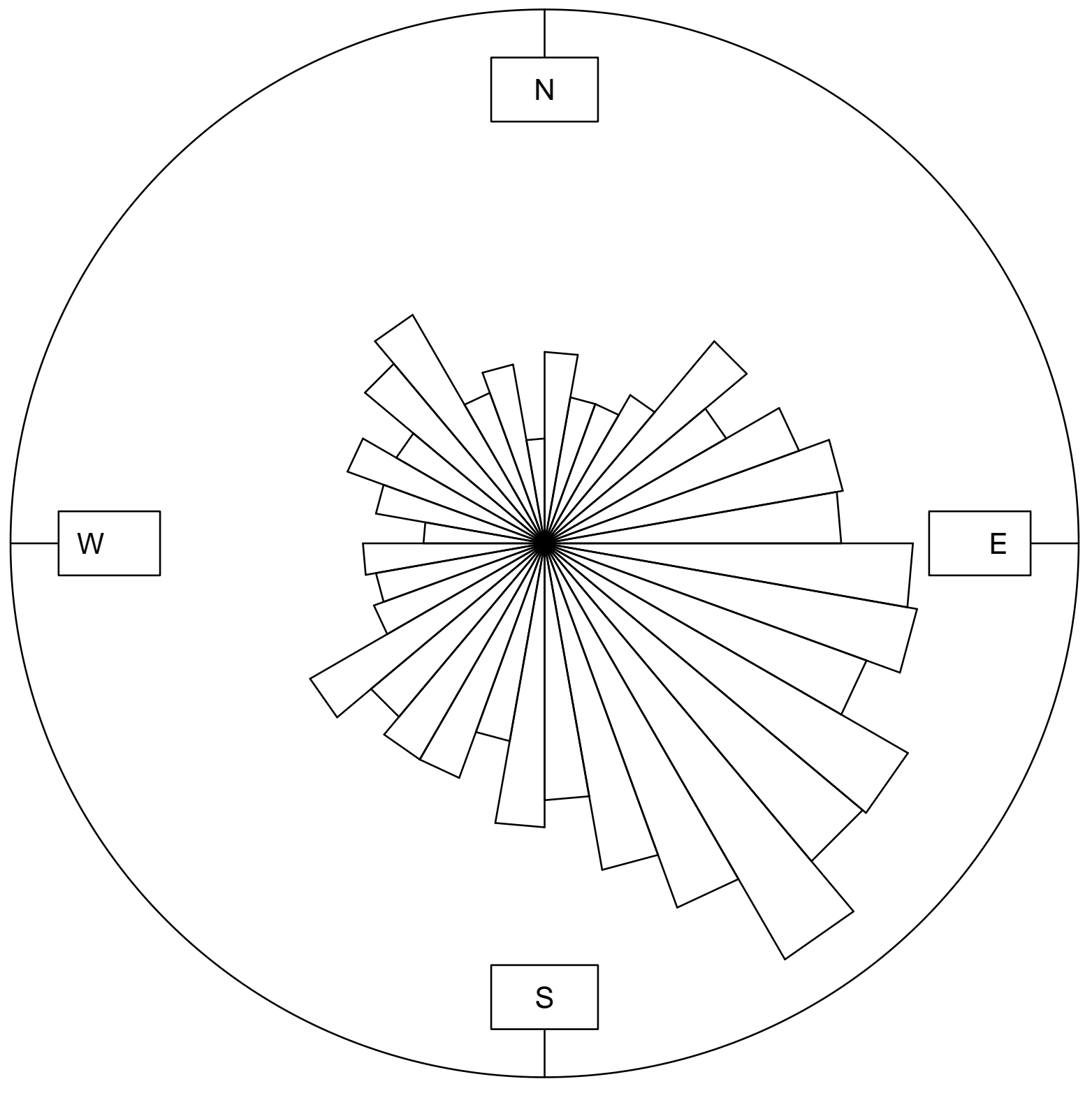




\section{bootstrap 715}

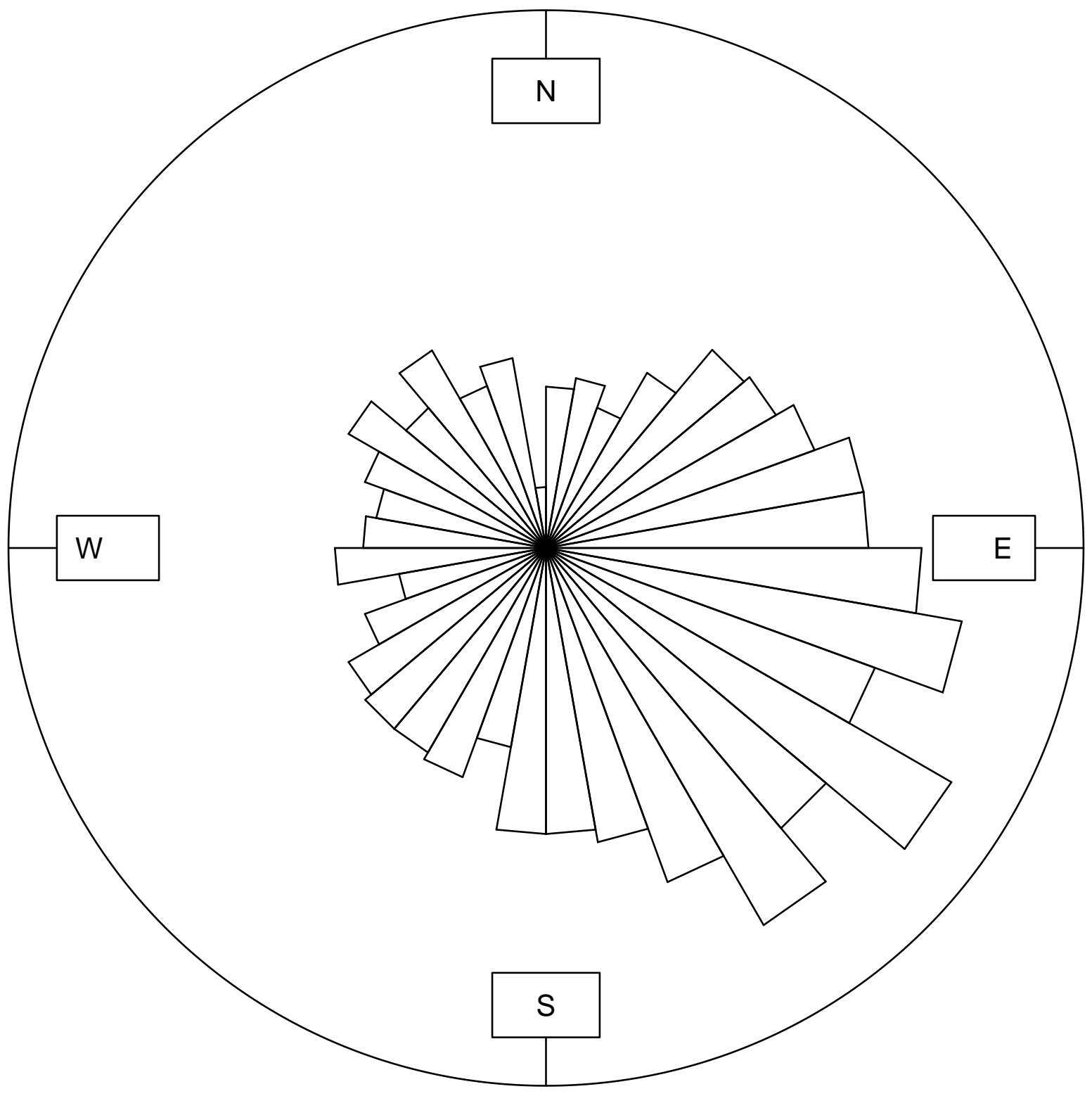


bootstrap 716

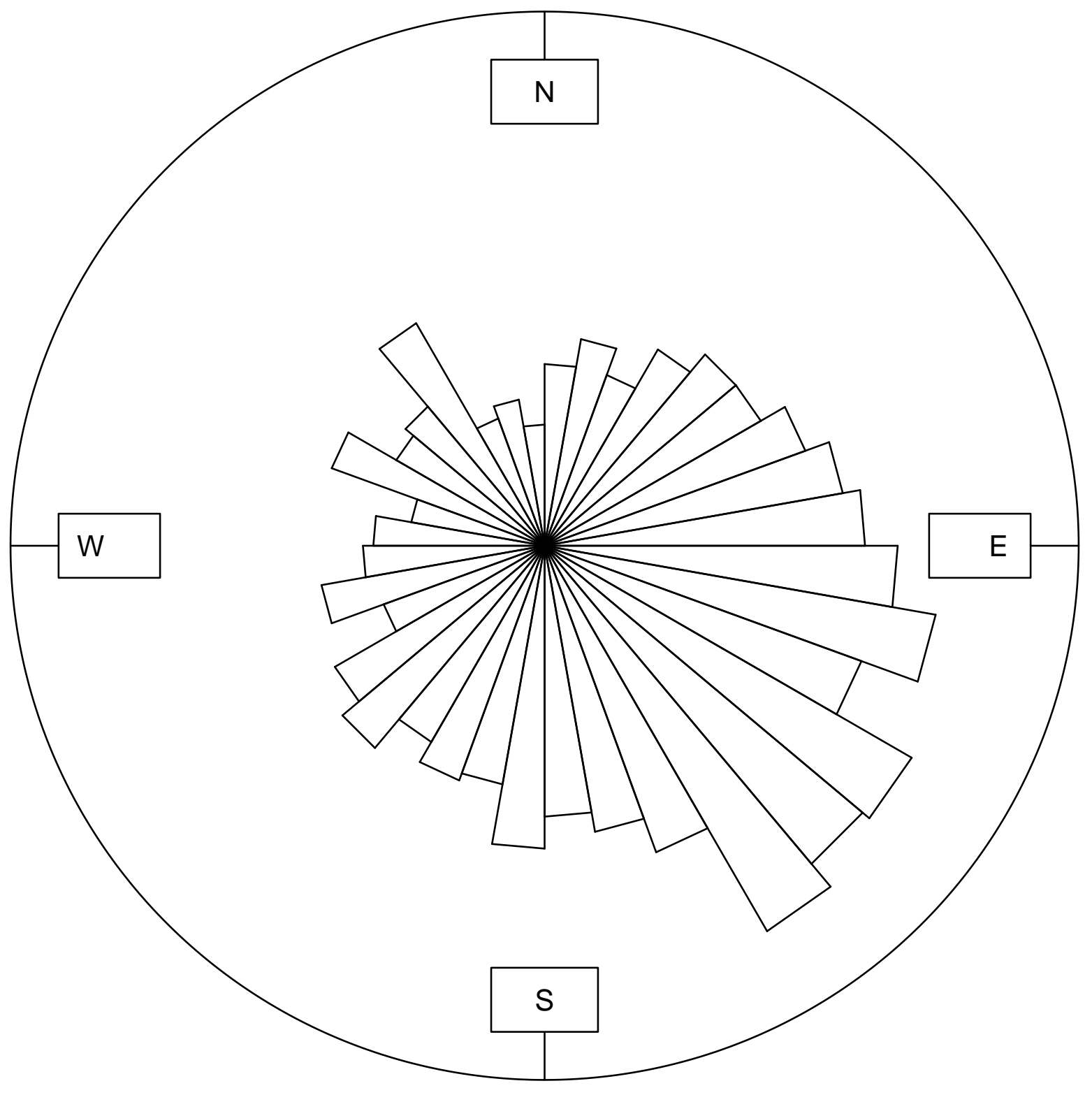


bootstrap 717

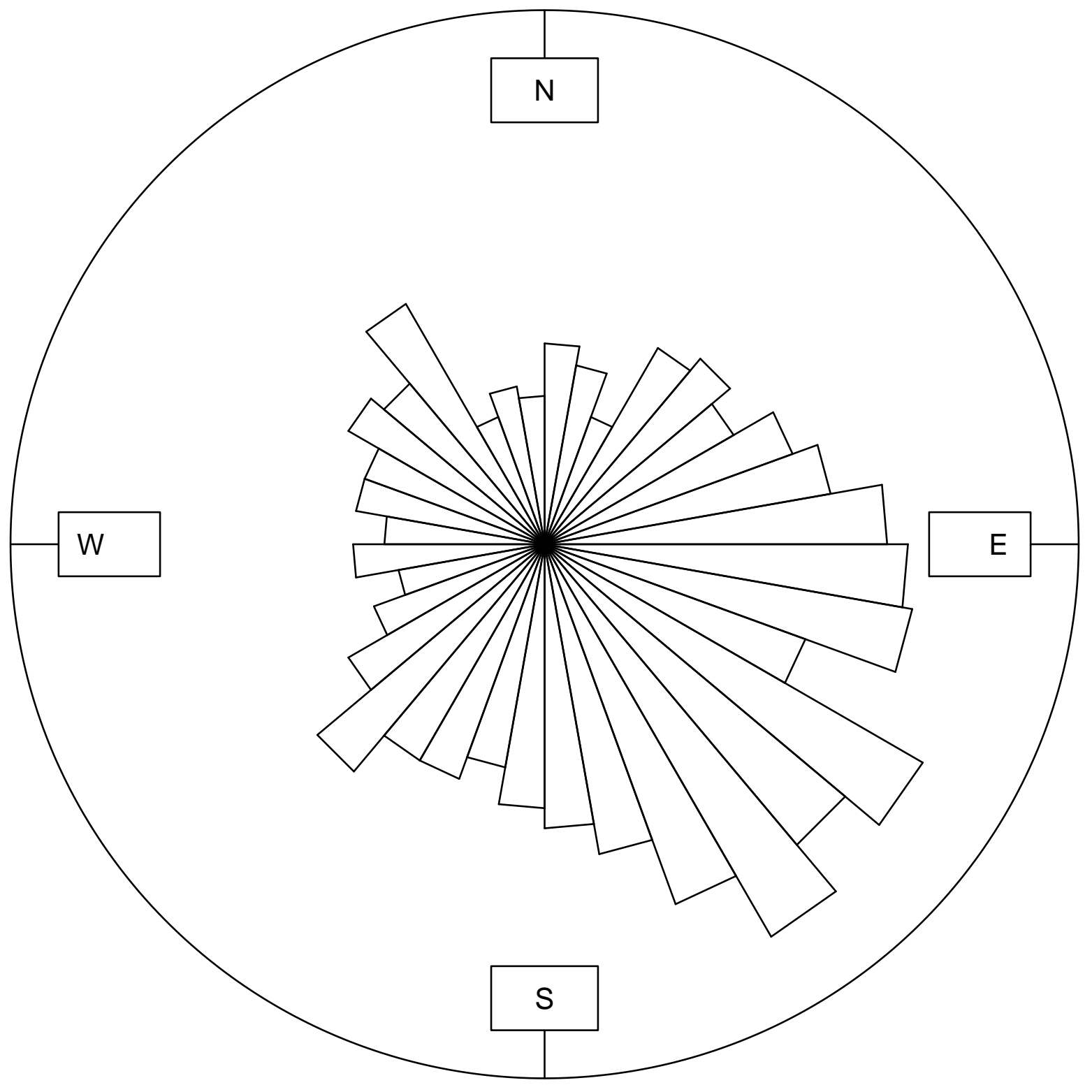


bootstrap 718

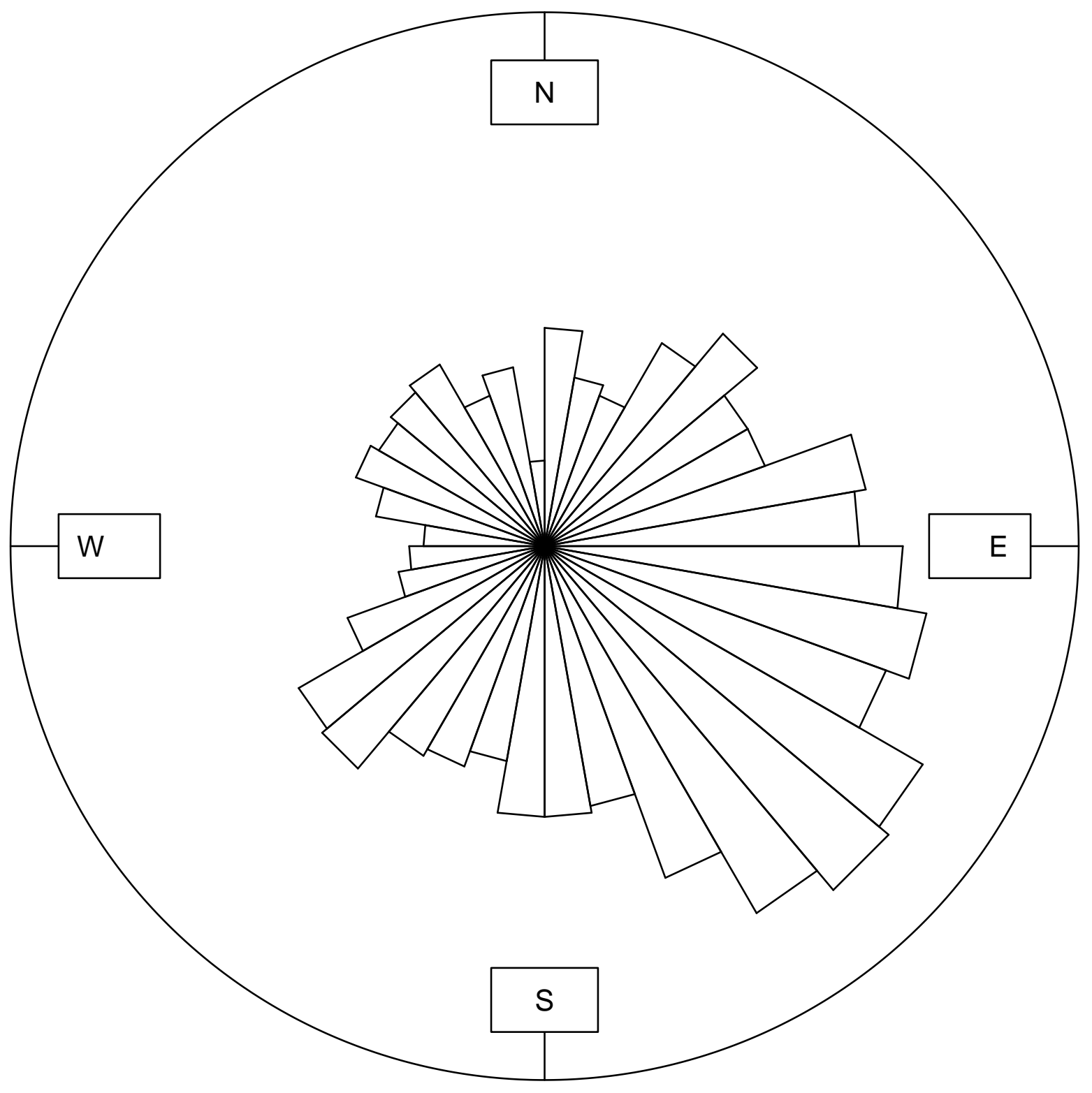


bootstrap 719

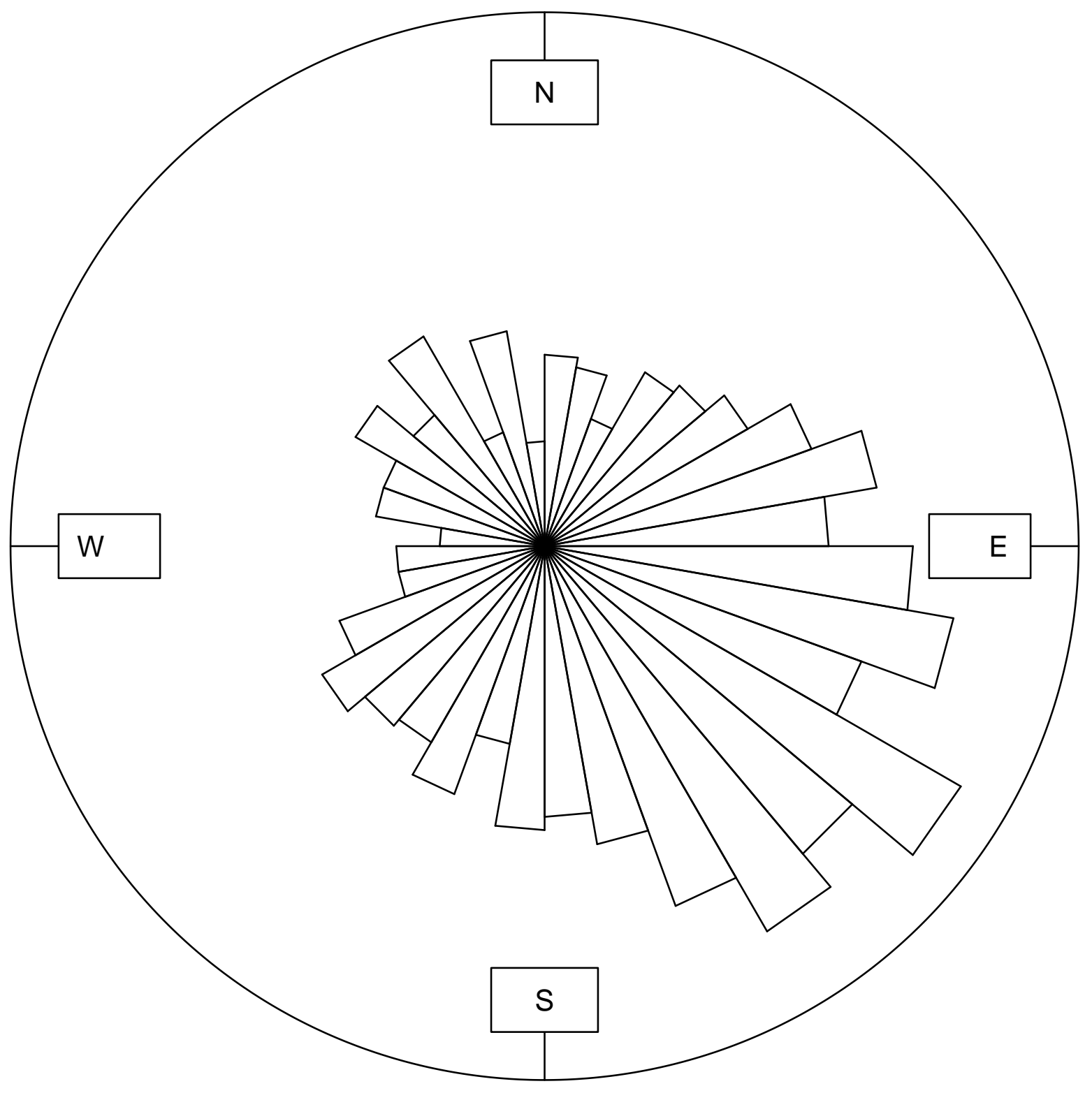


bootstrap 720

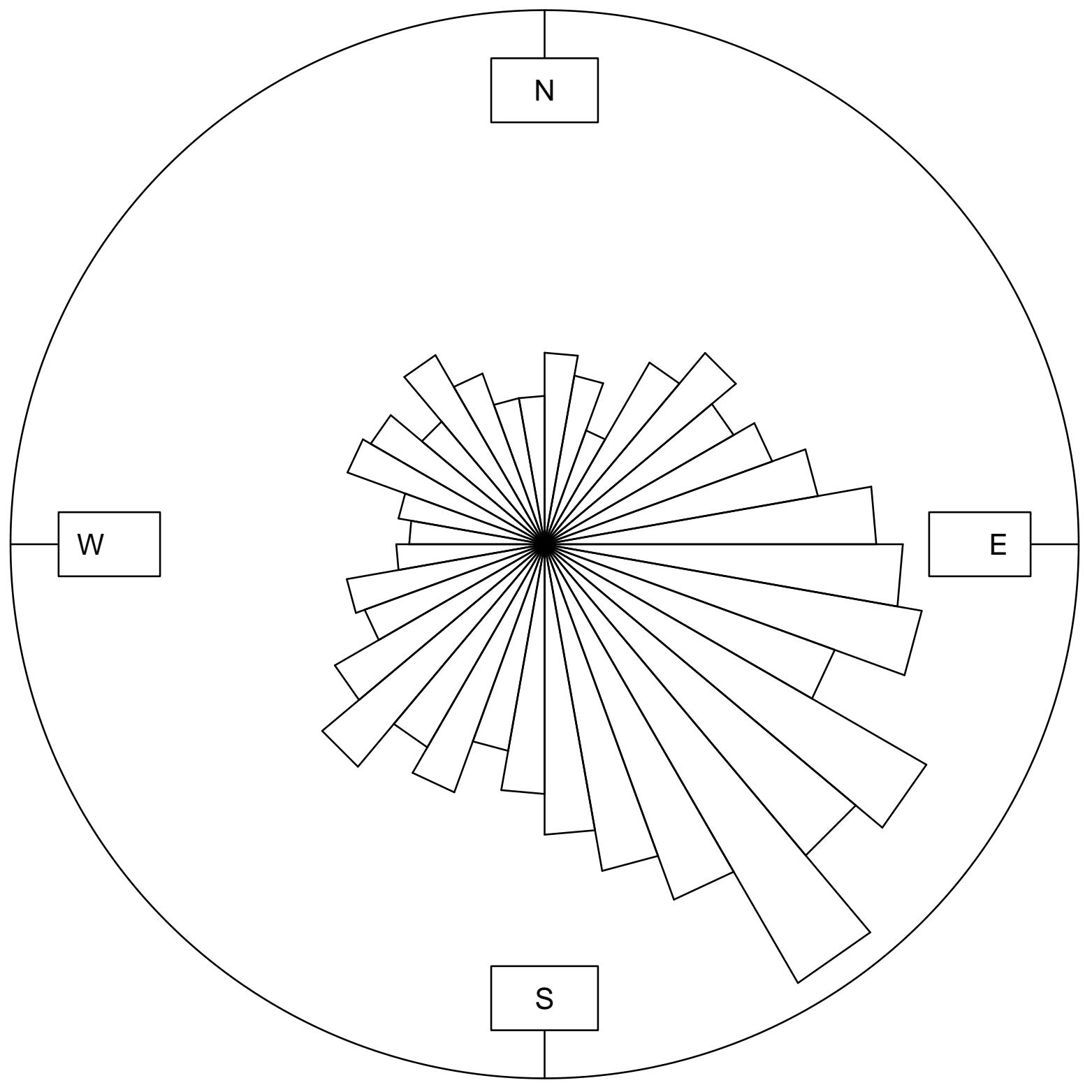


bootstrap 721

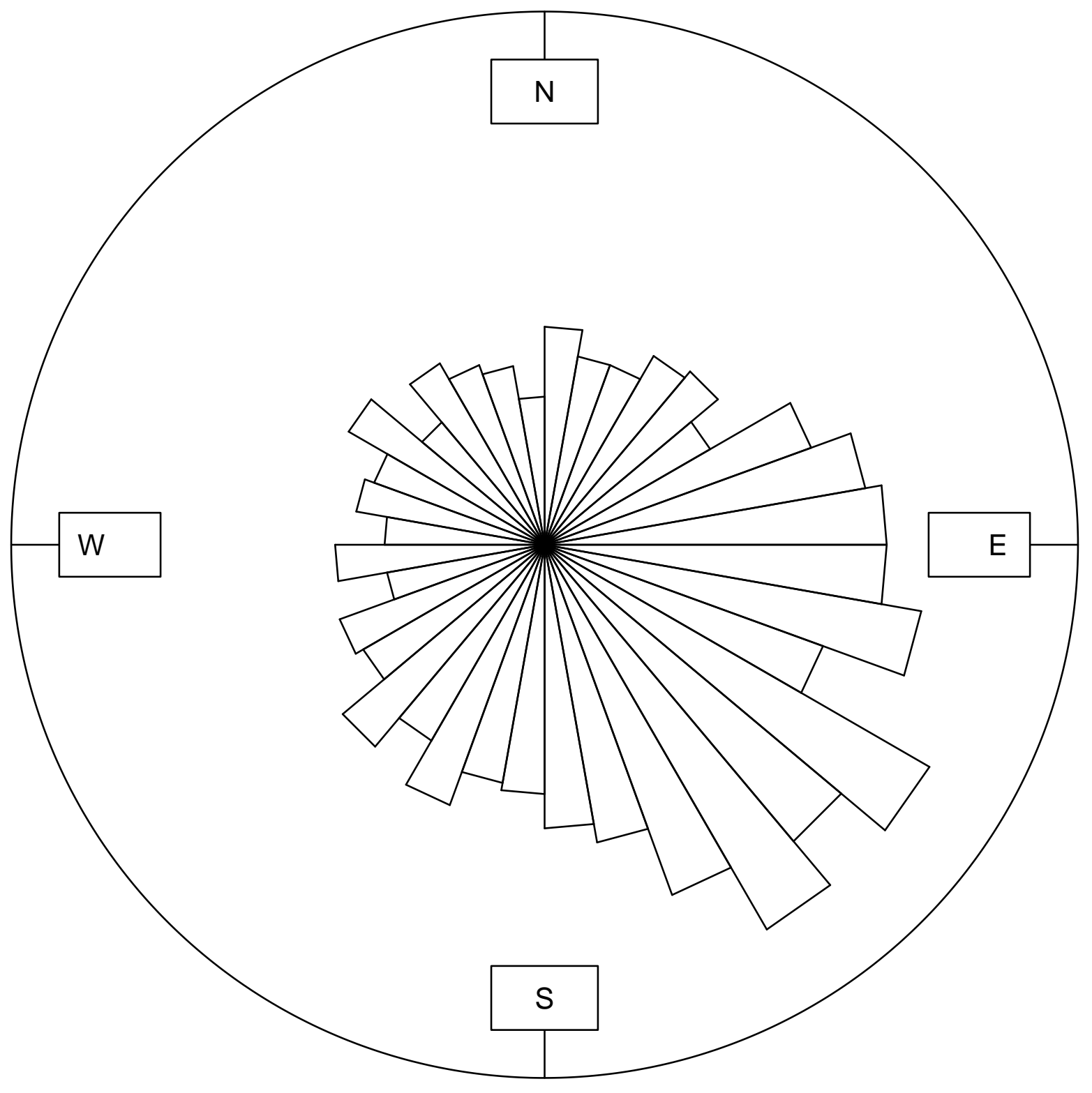


bootstrap 722

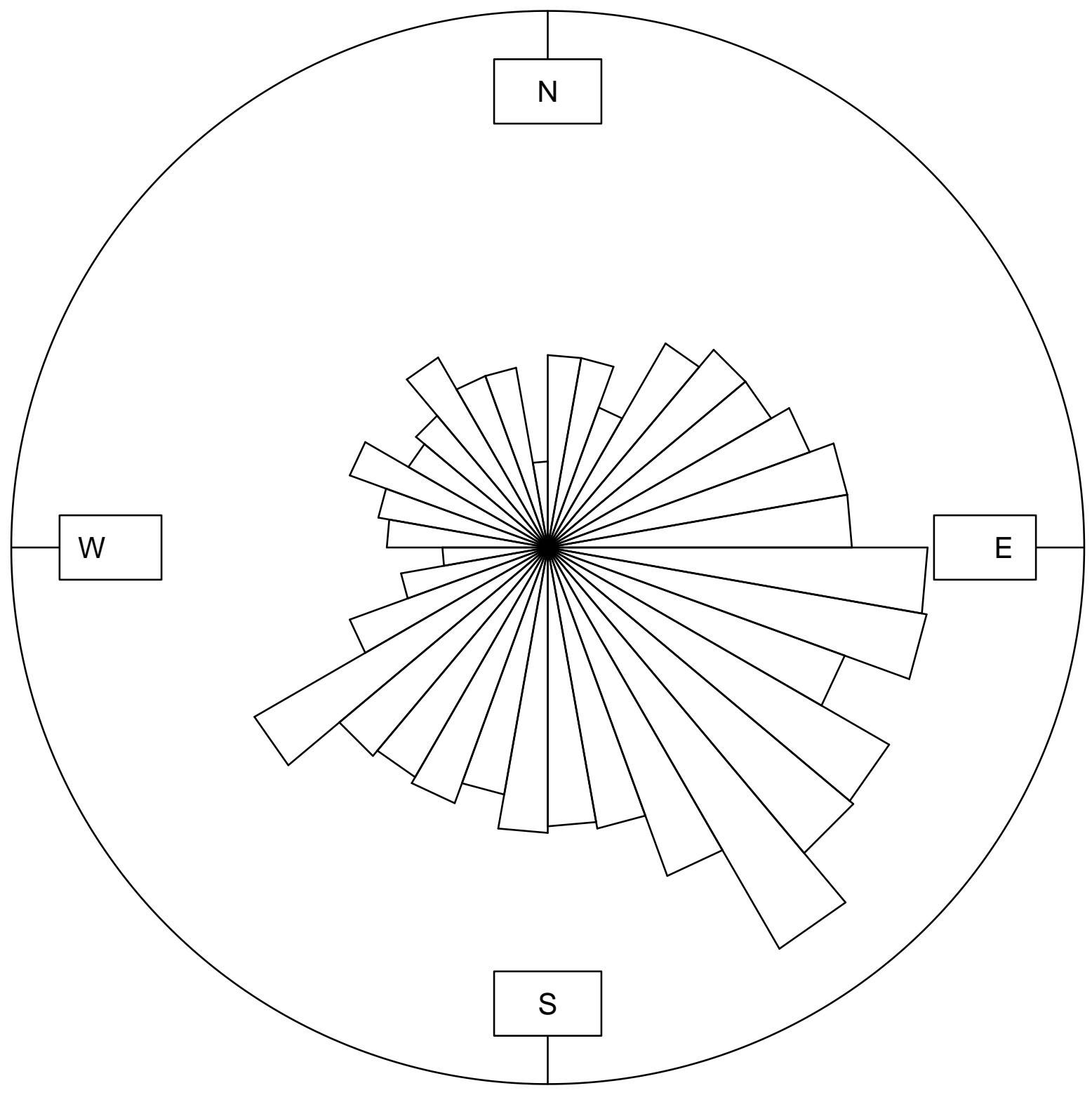


bootstrap 723

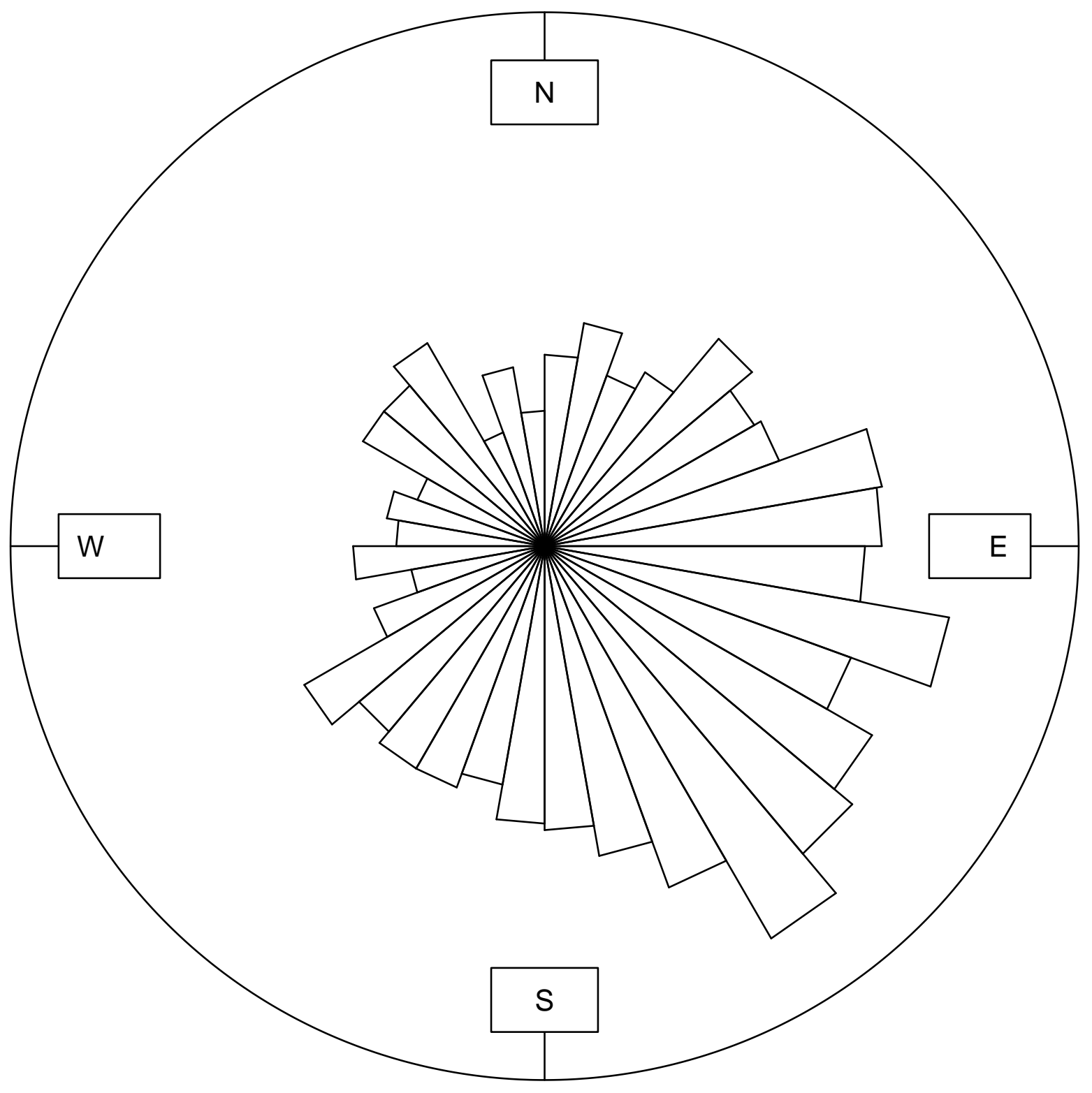


bootstrap 724

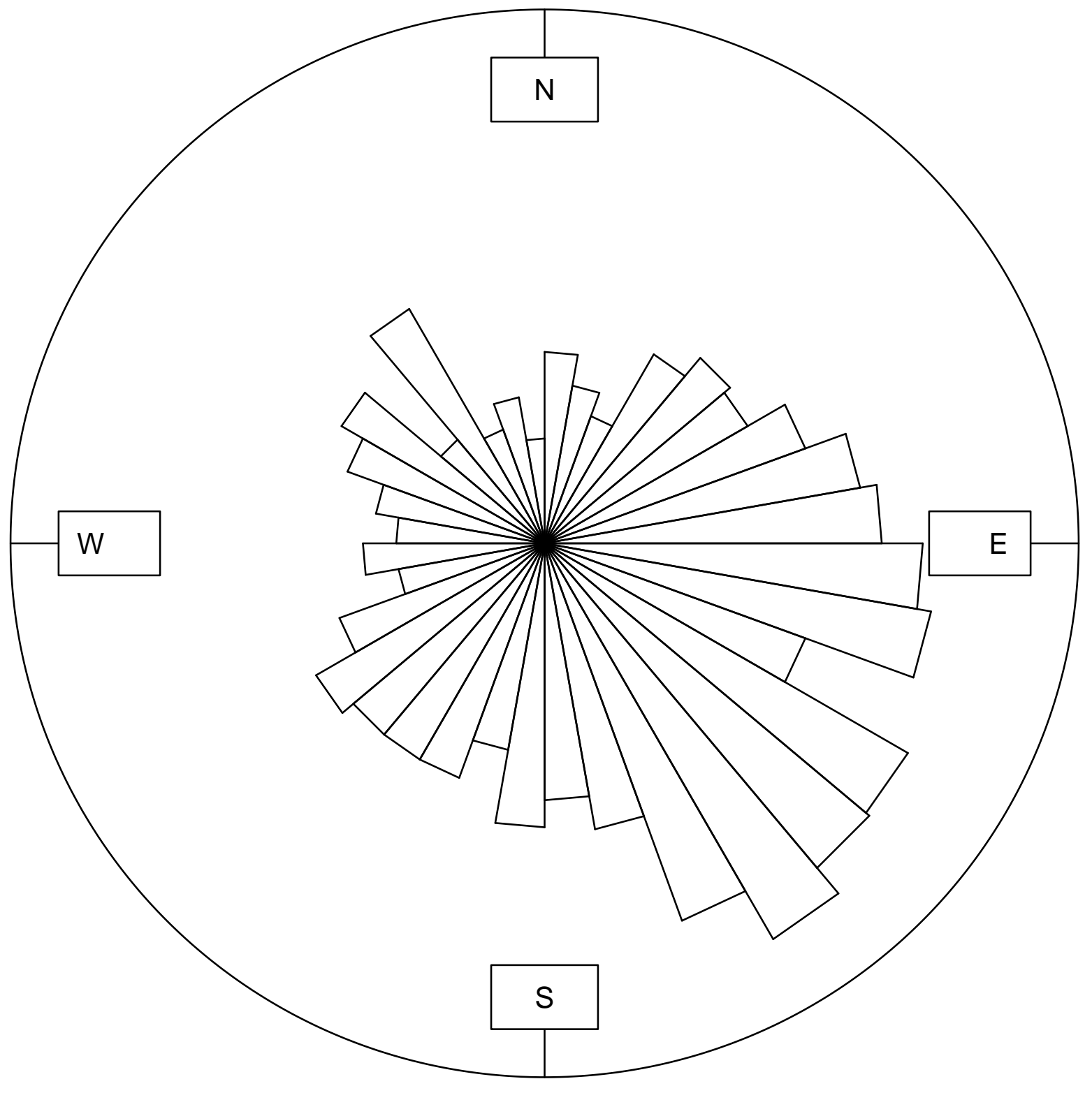


bootstrap 725

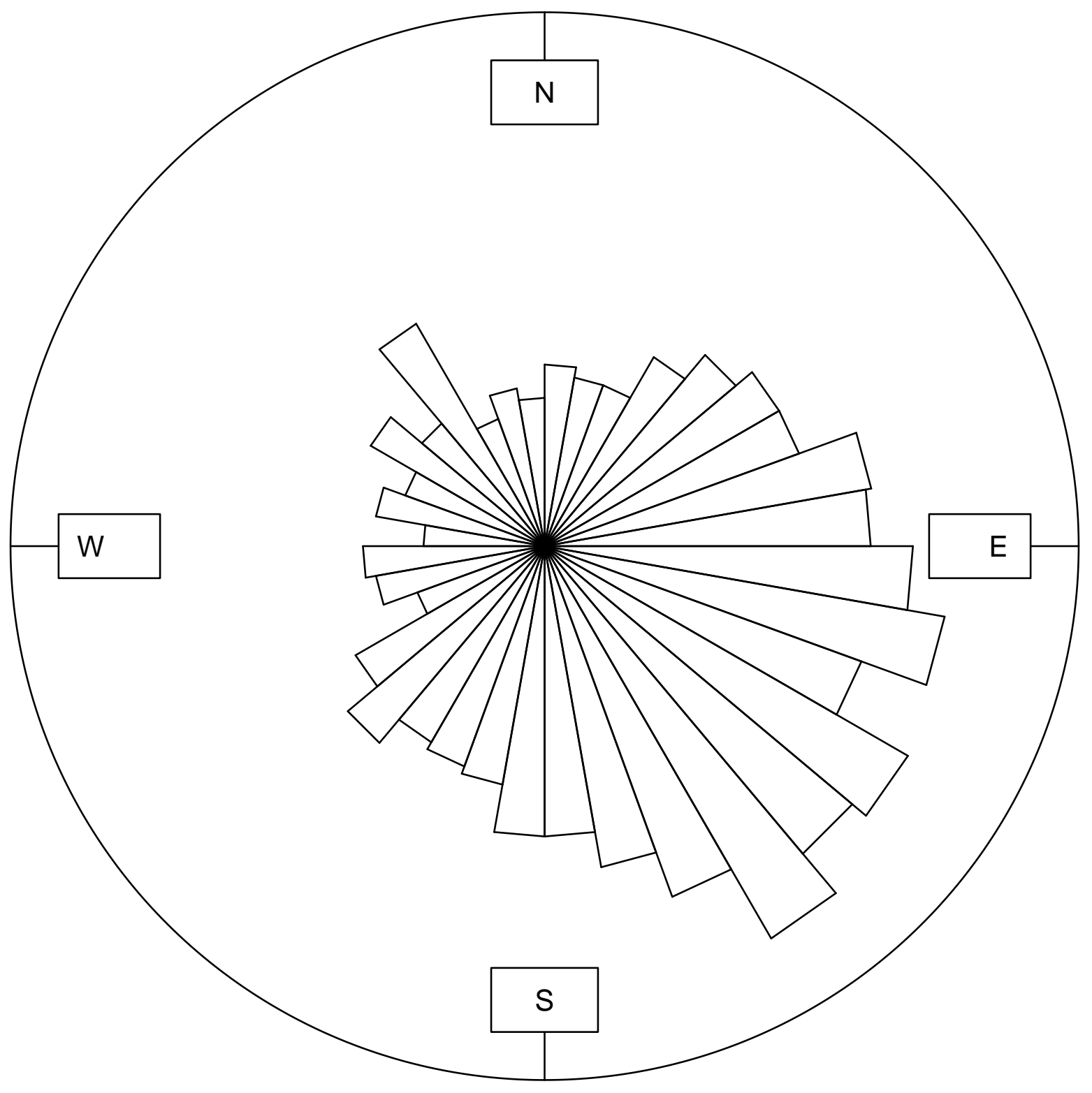




\section{bootstrap 726}

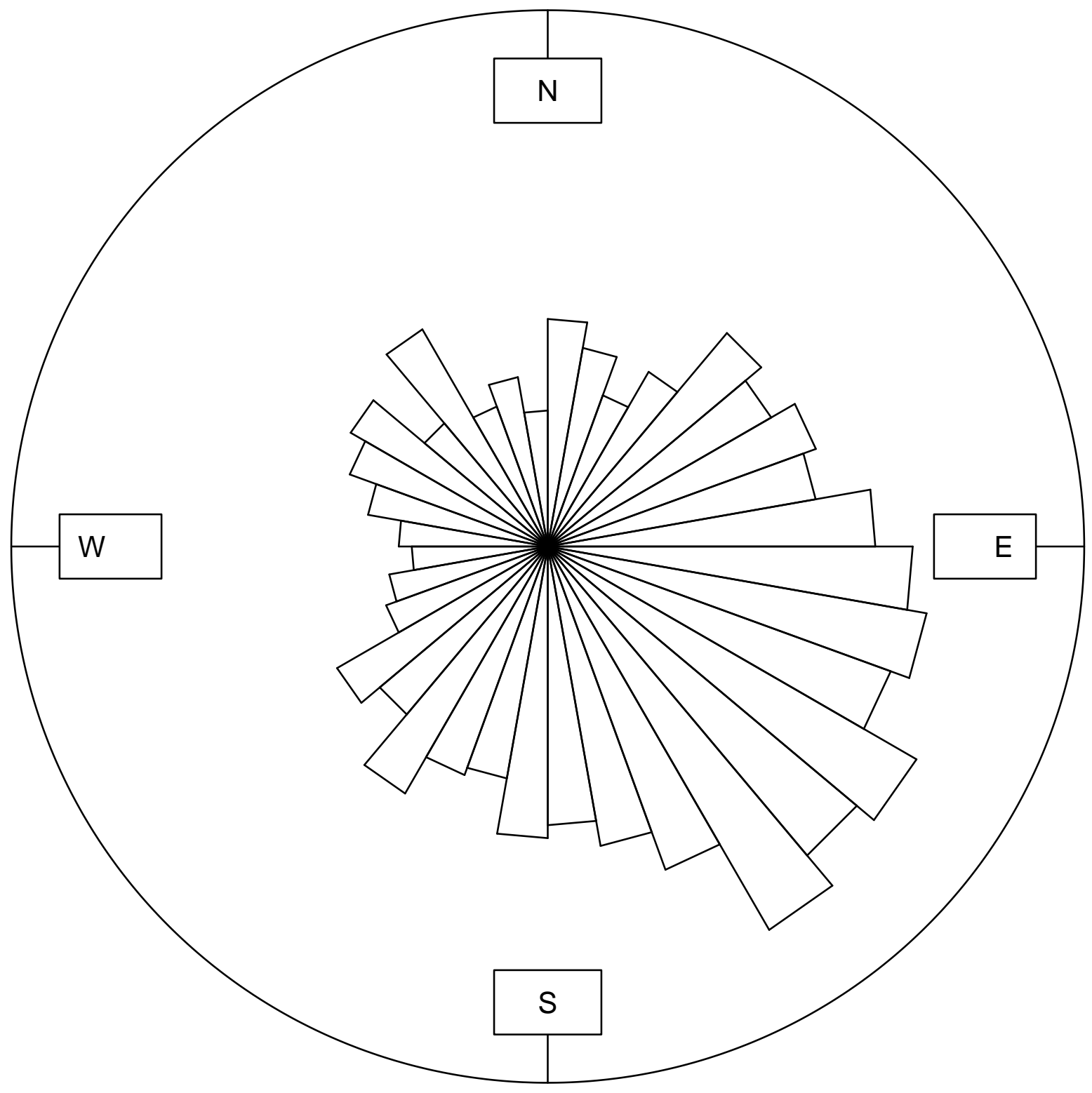




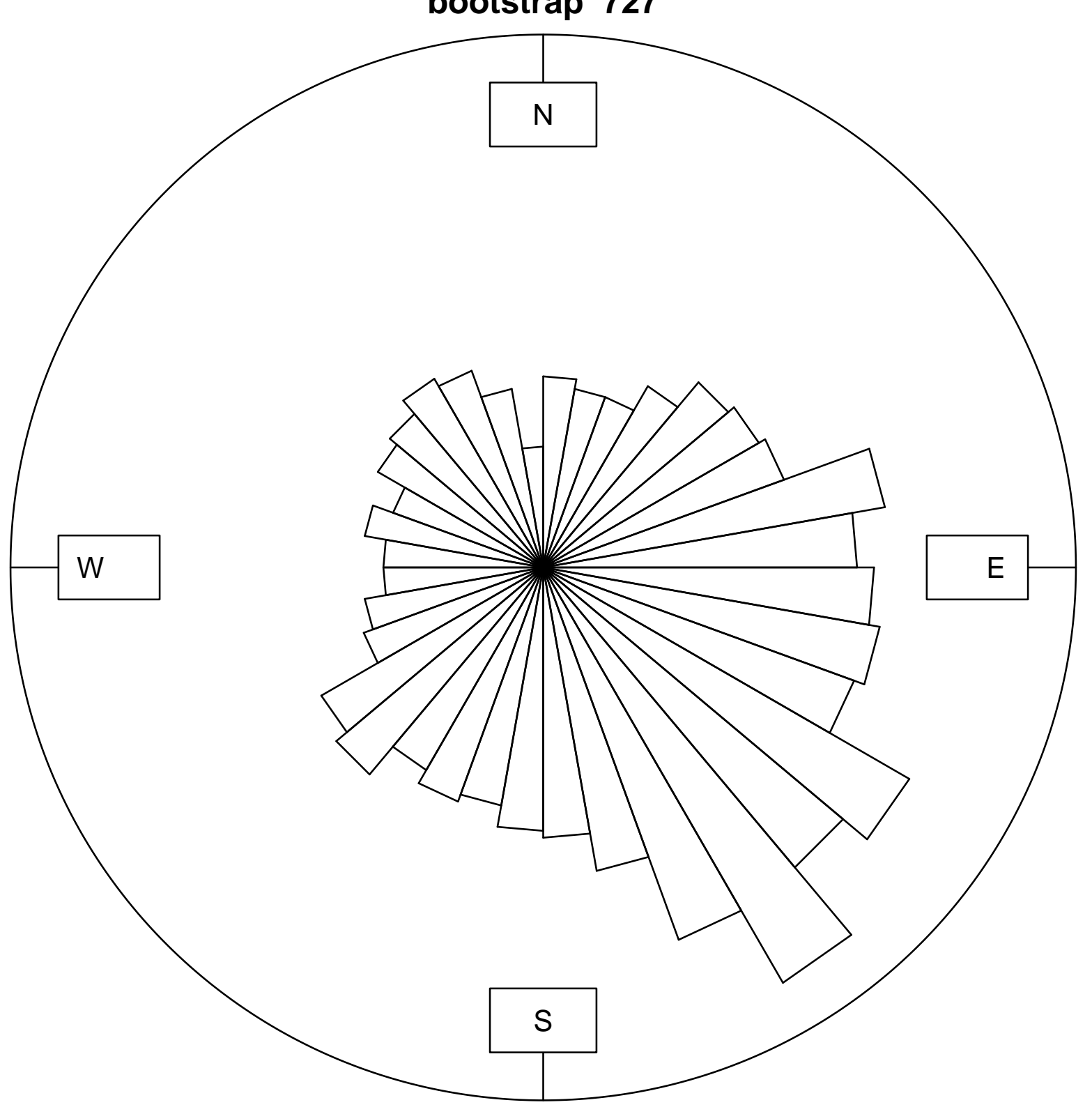


bootstrap 728

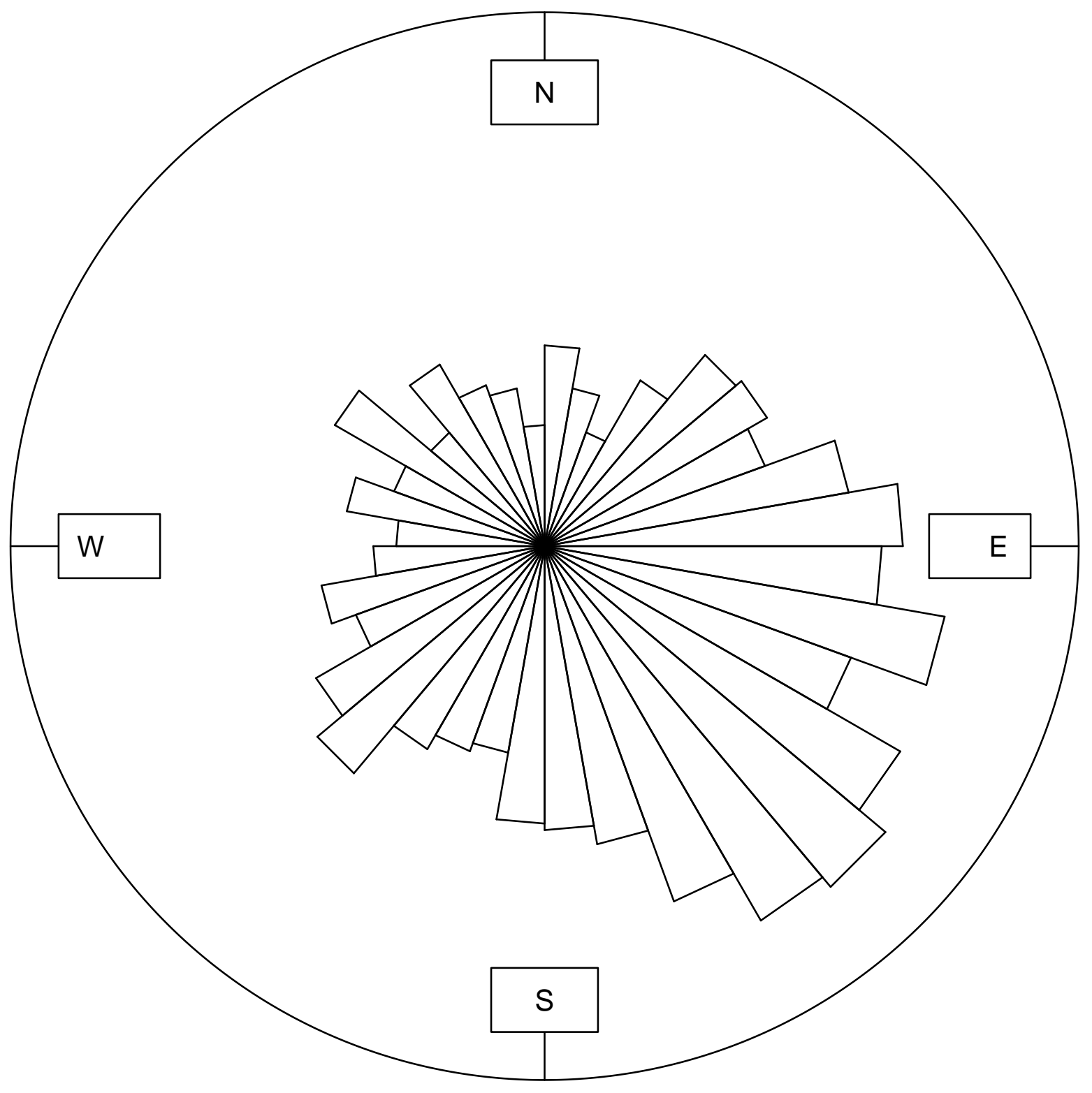


bootstrap 729

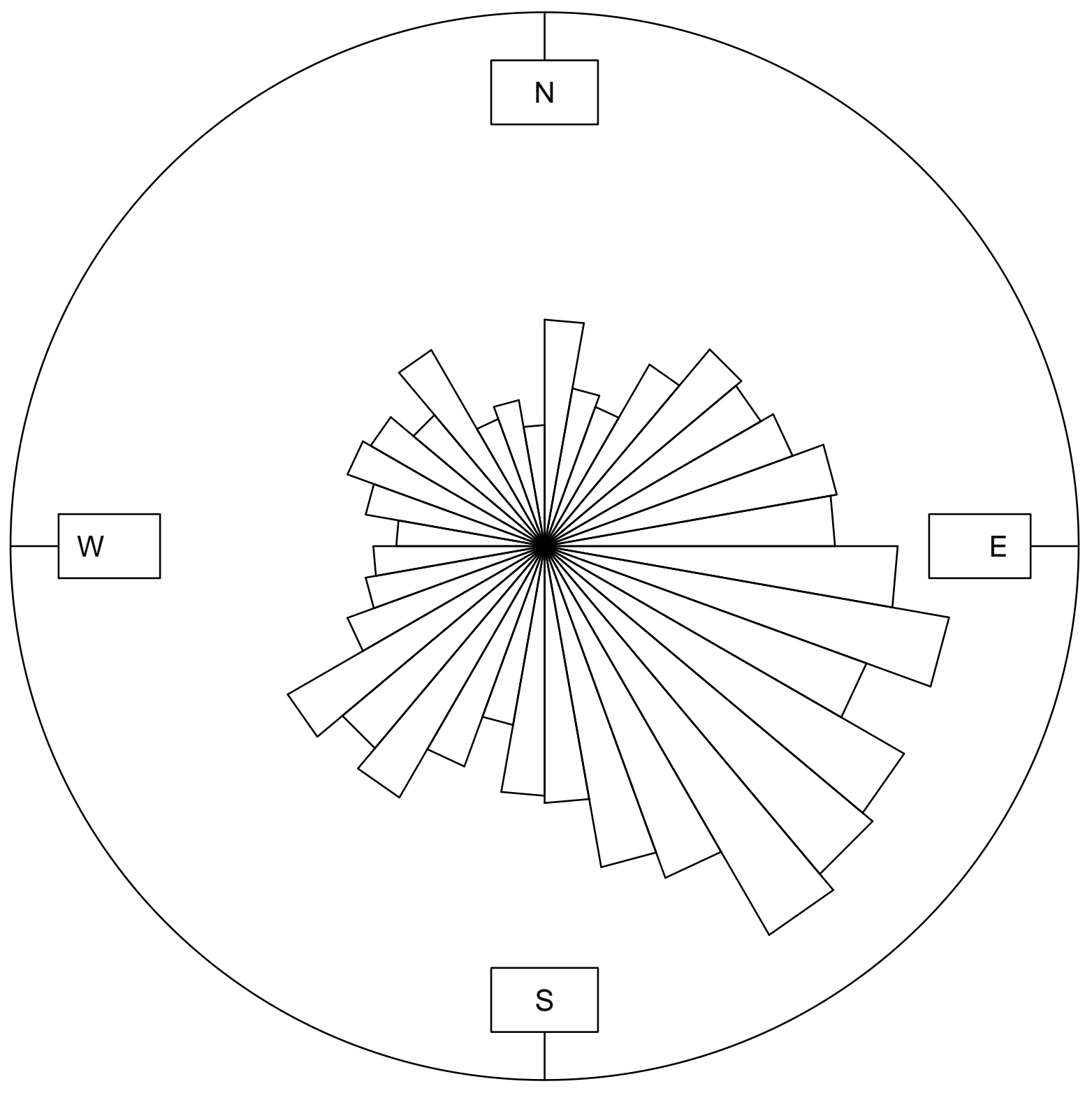


bootstrap 730

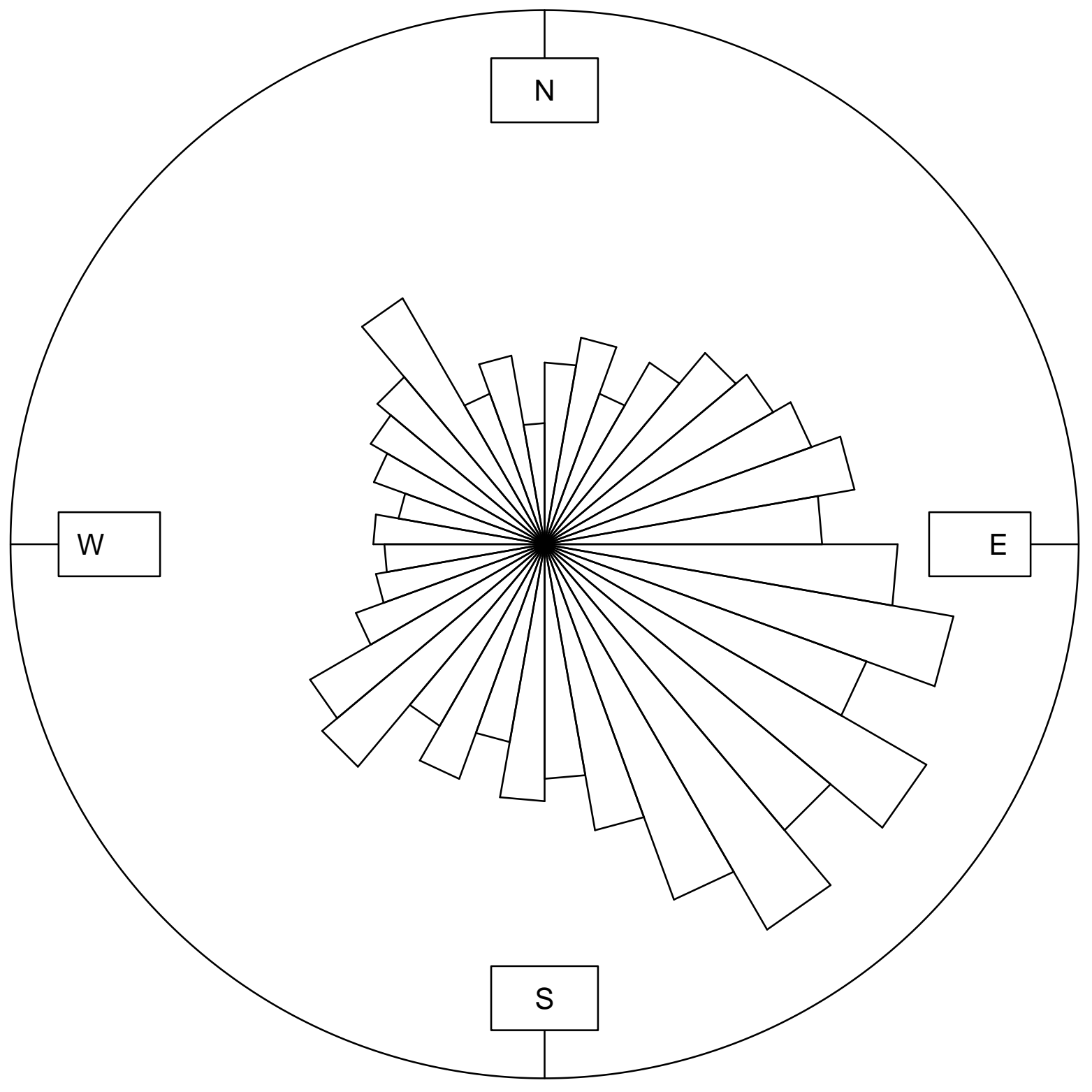


bootstrap 731

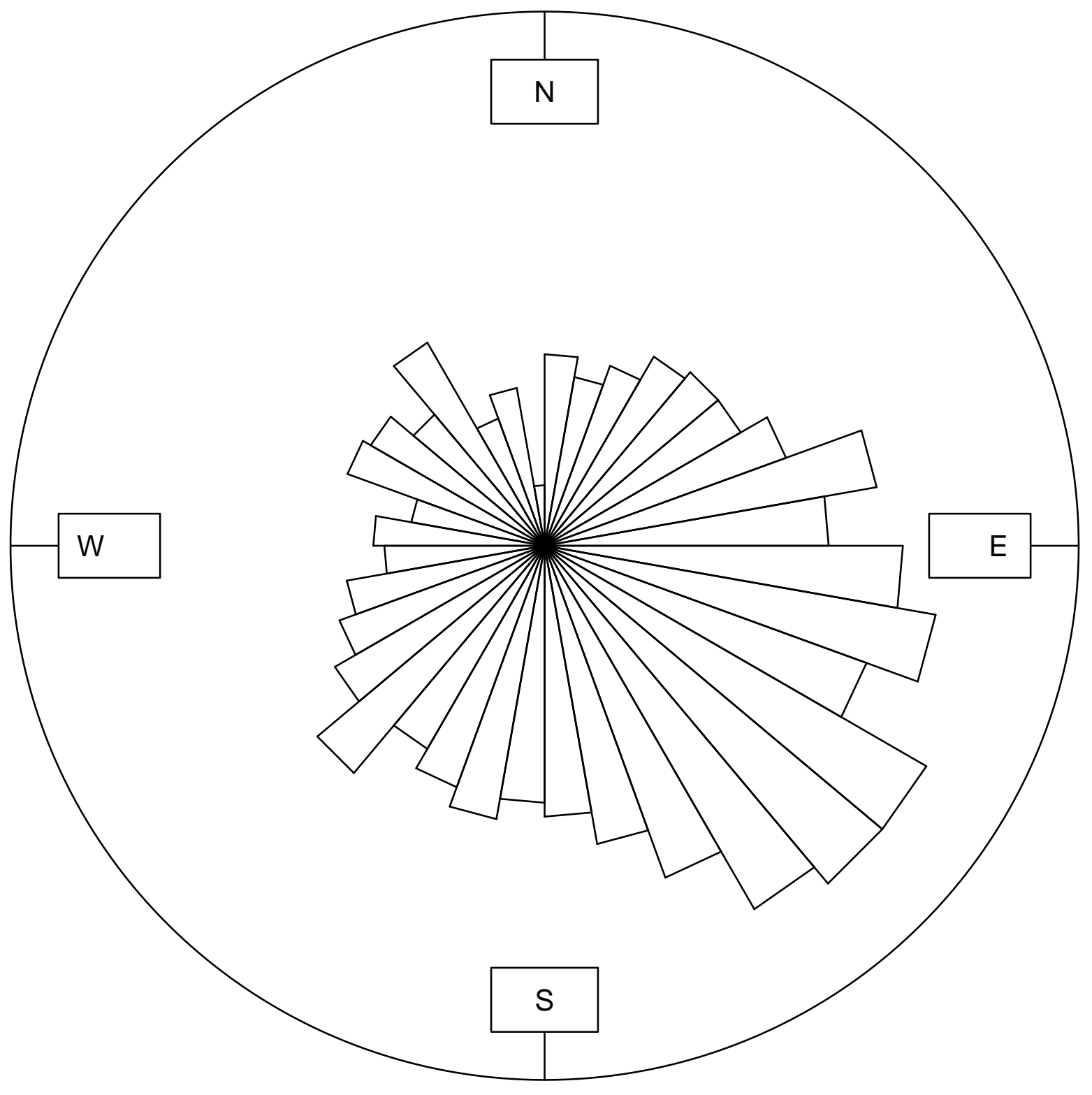


bootstrap 732

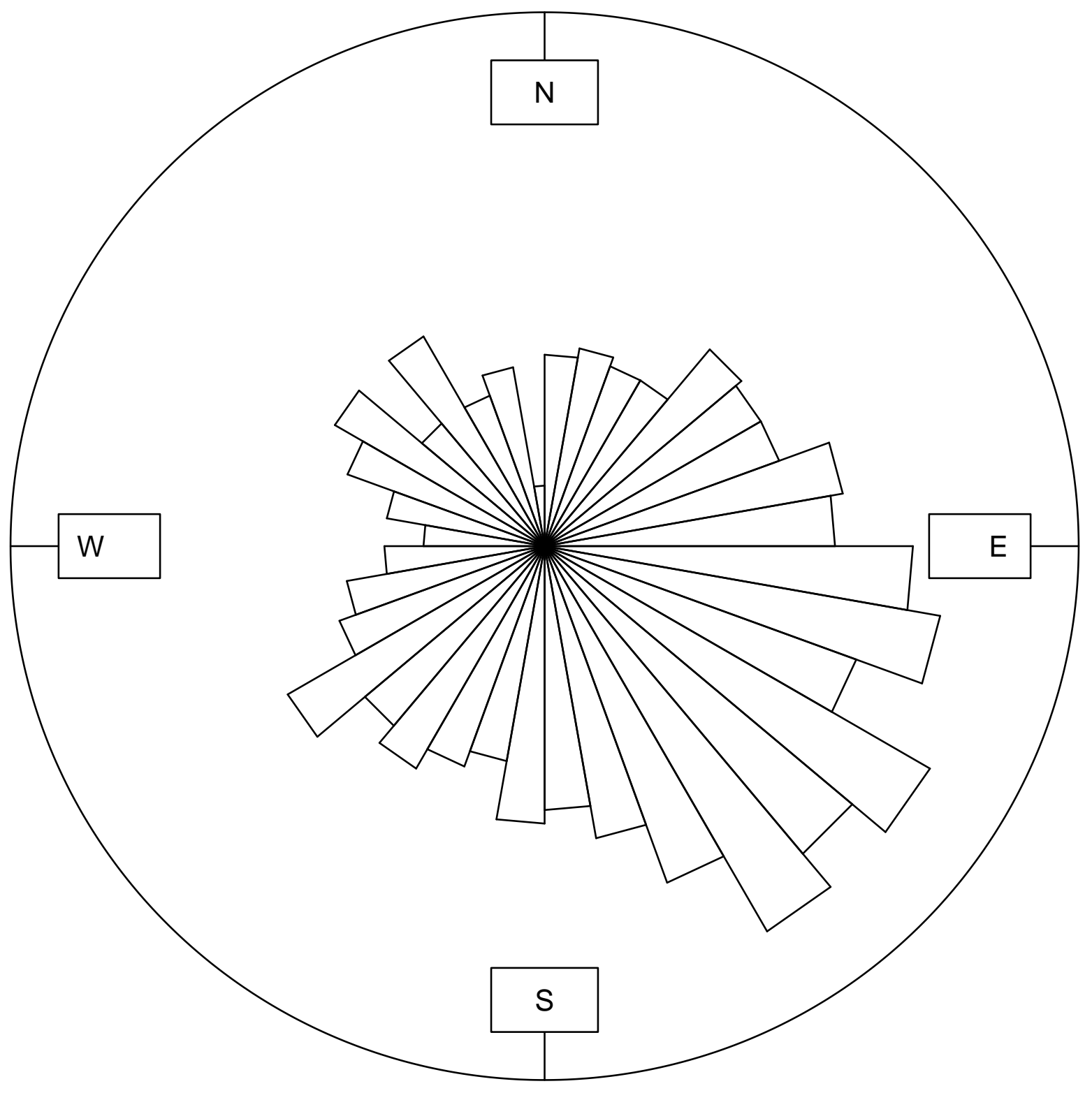


bootstrap 733

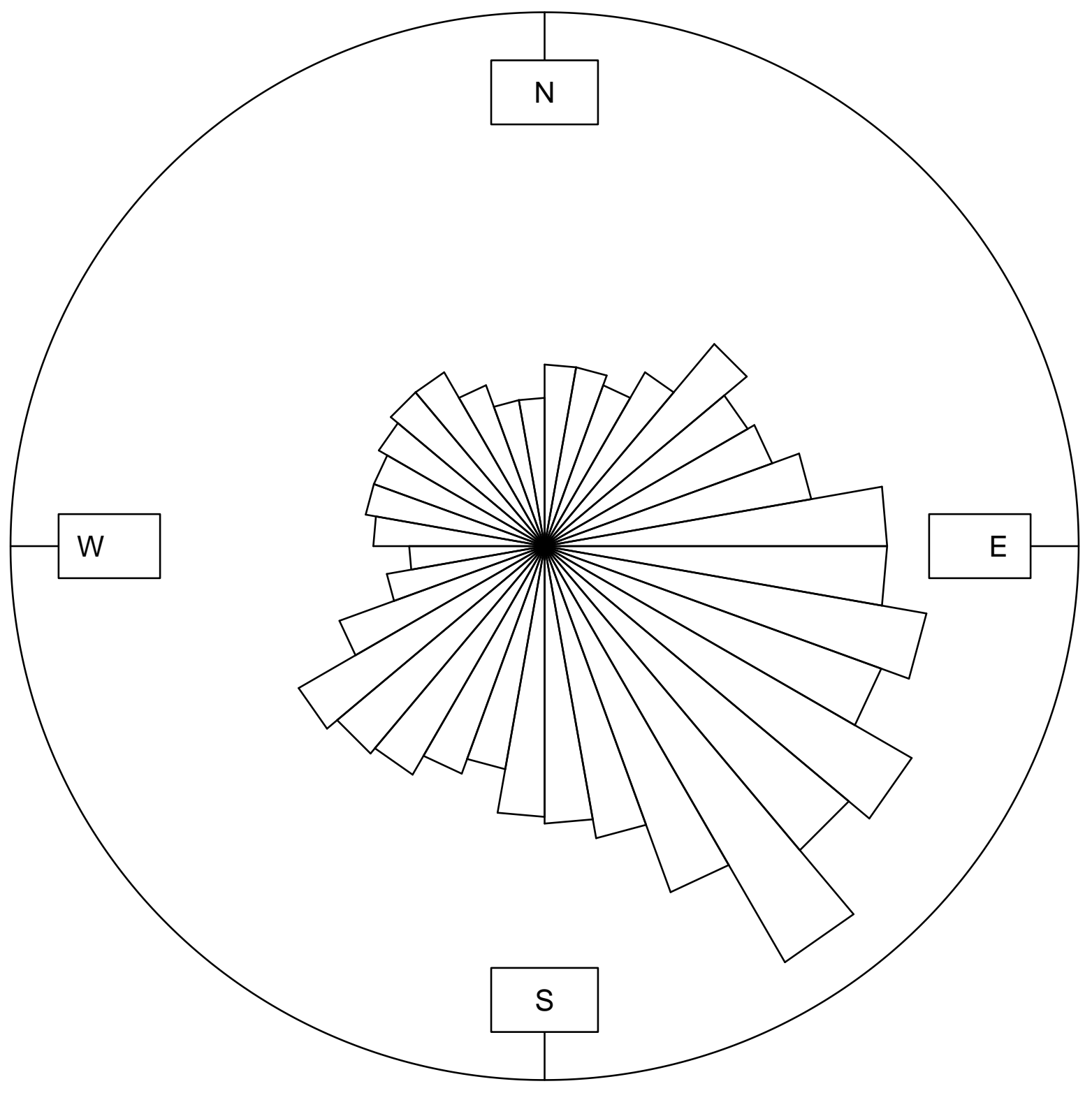


bootstrap 734

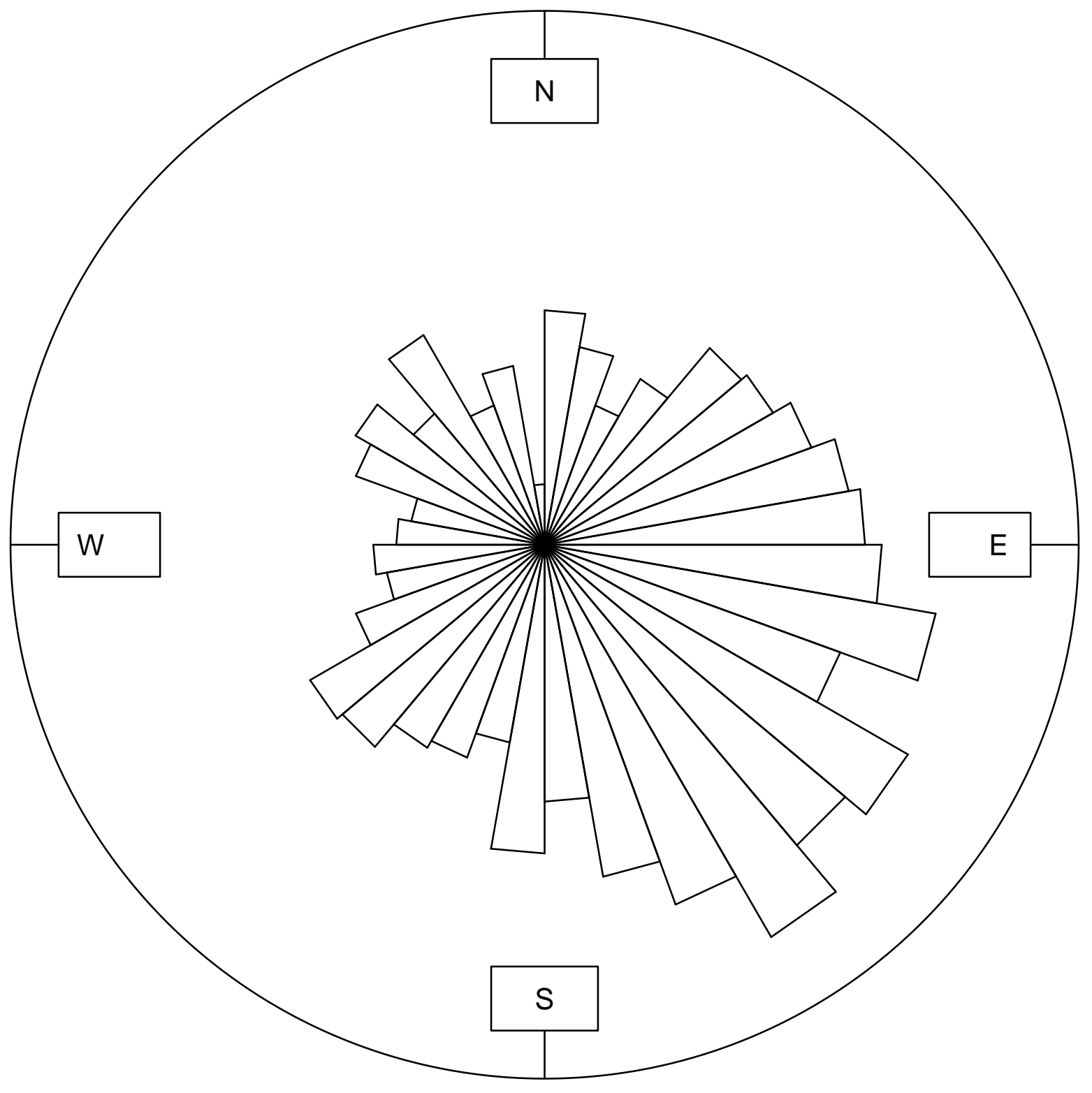




\section{bootstrap 735}

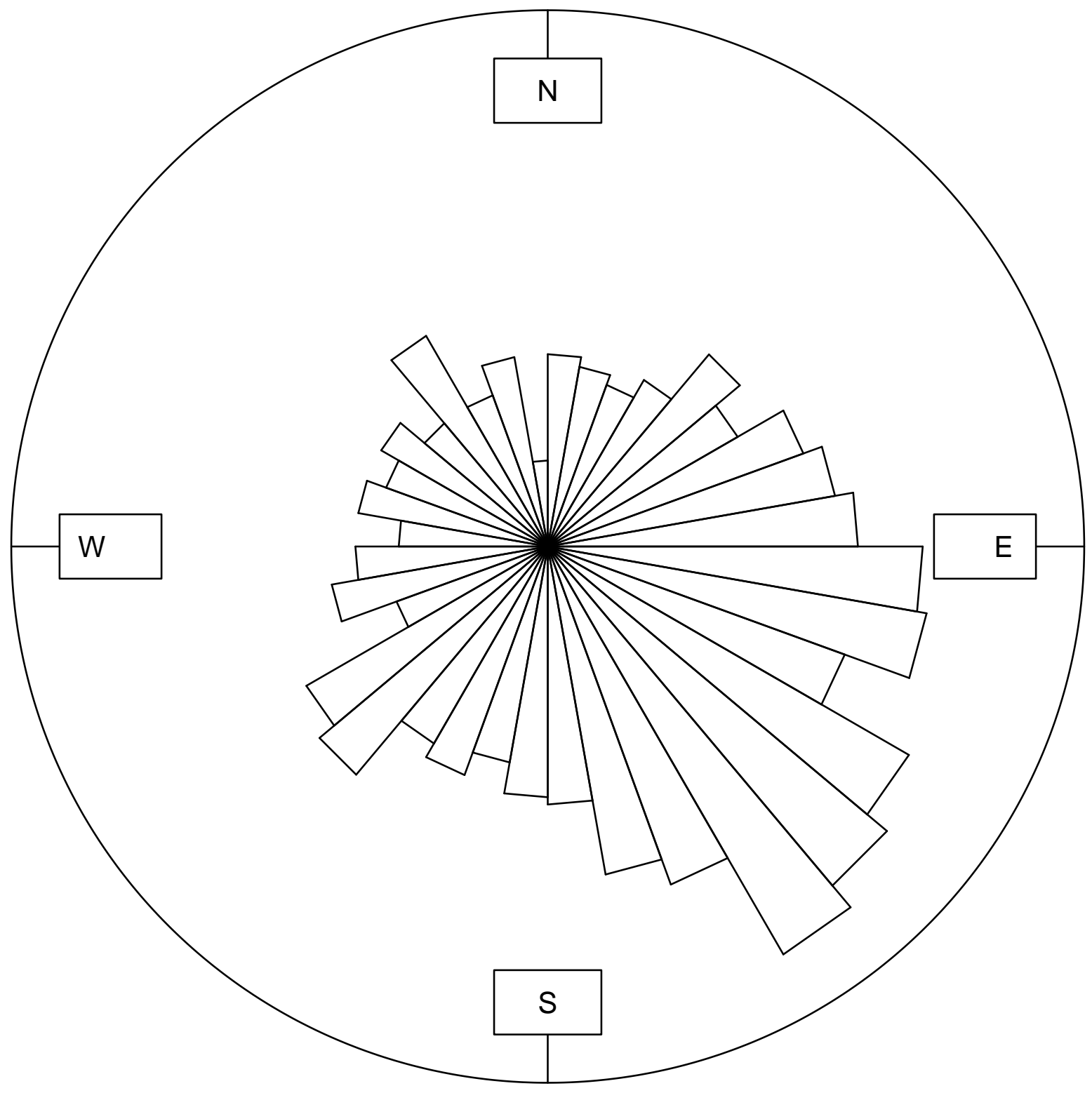




\section{bootstrap 736}

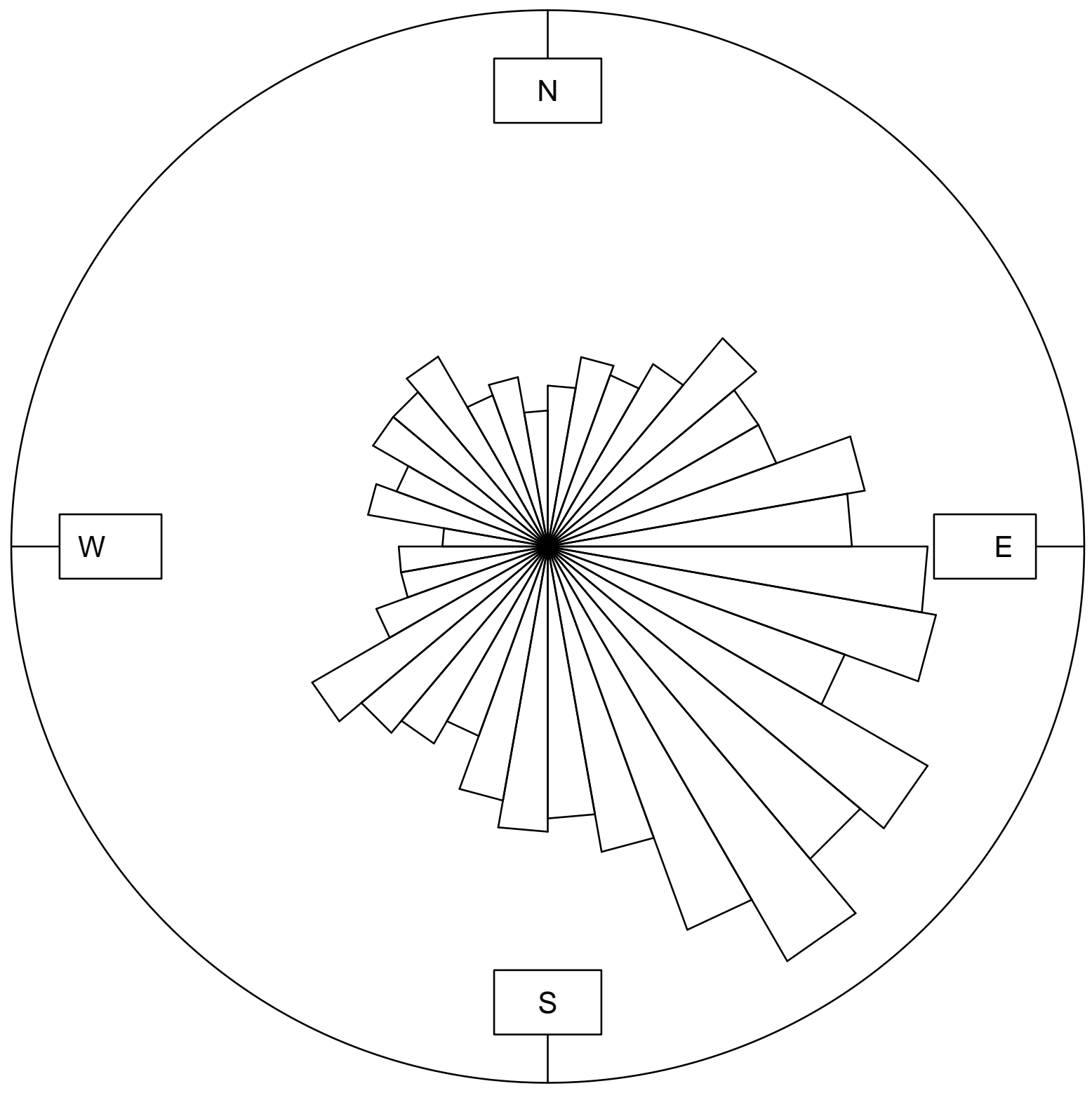


bootstrap 737

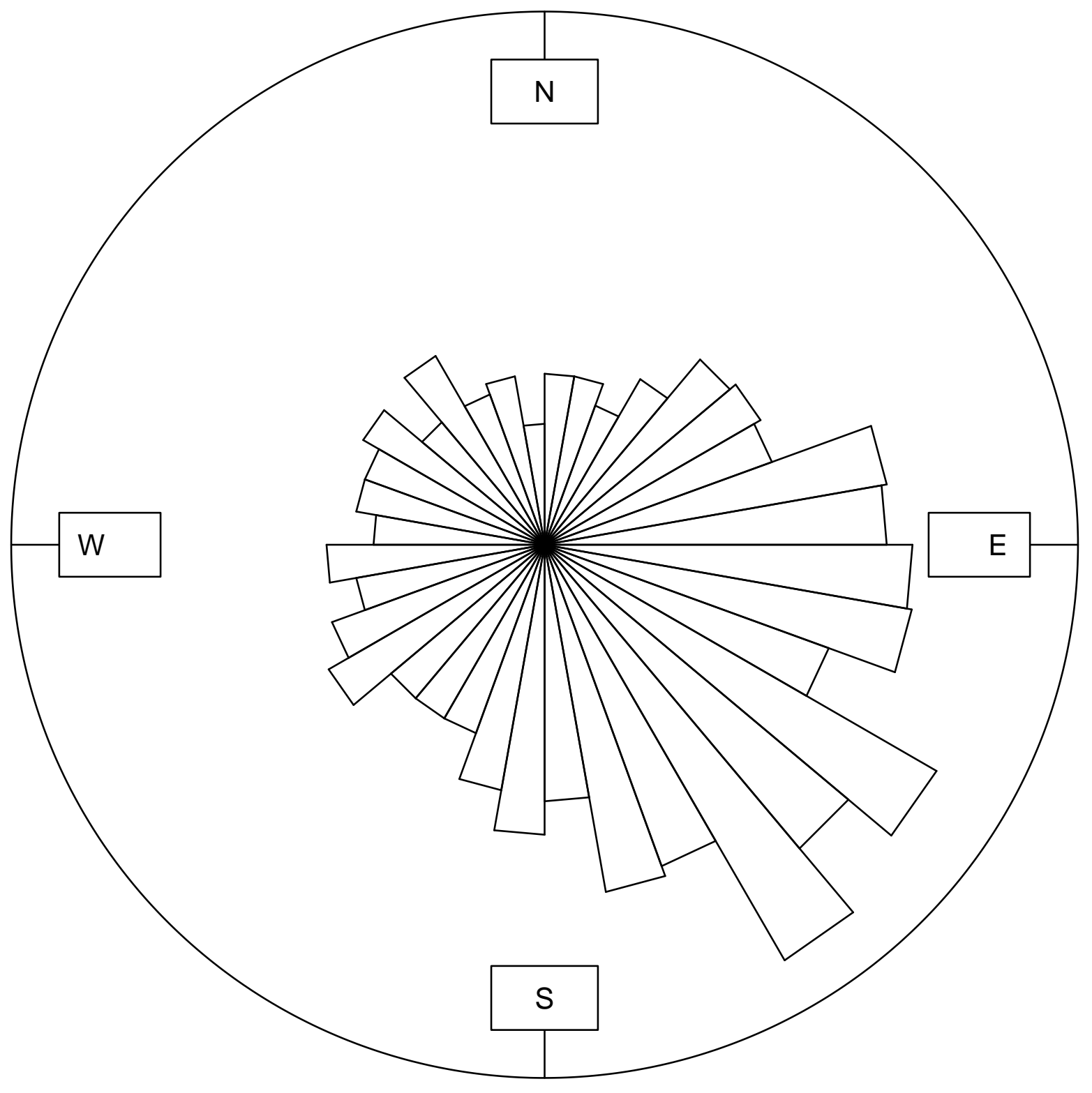


bootstrap 738

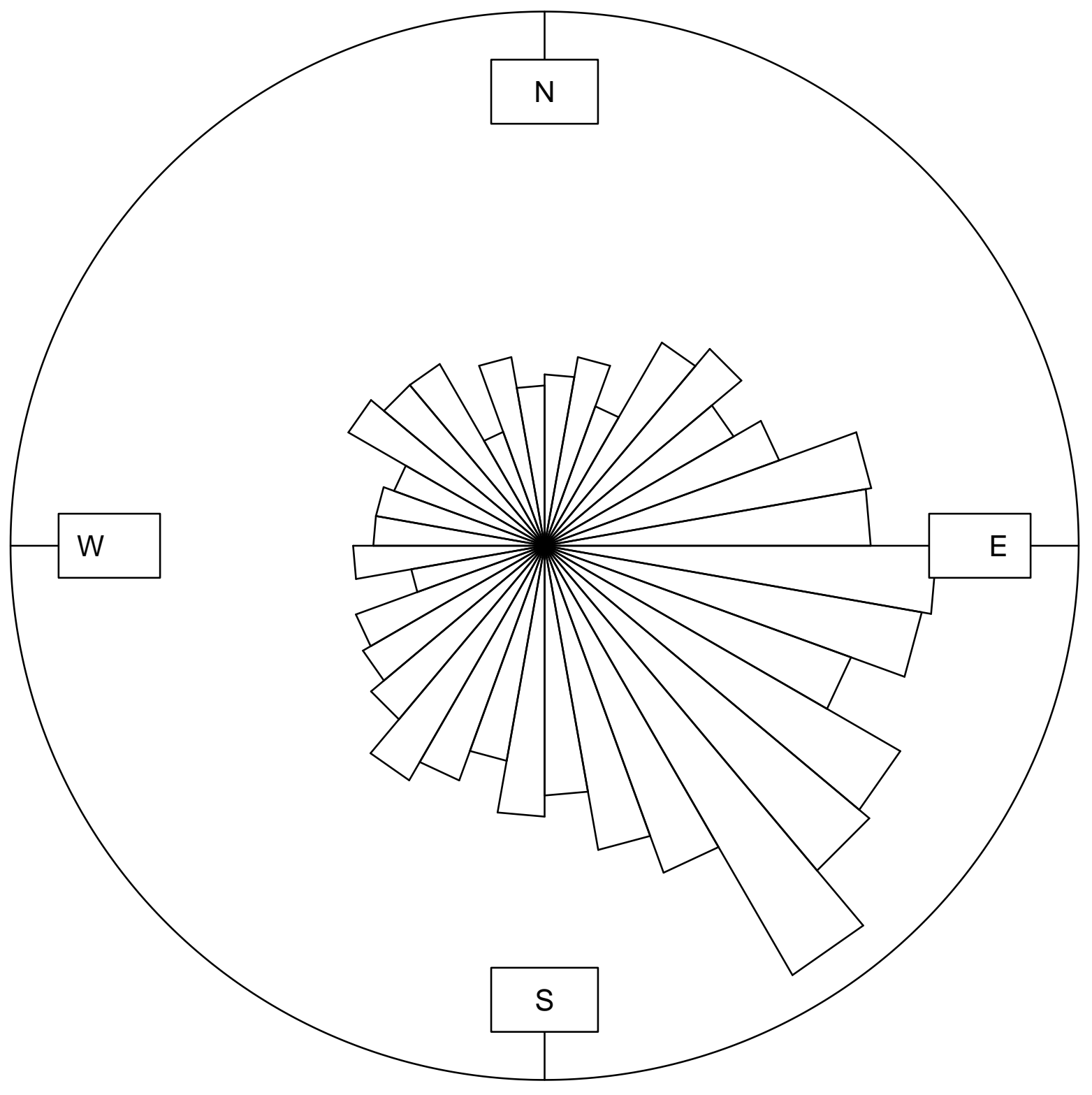


bootstrap 739

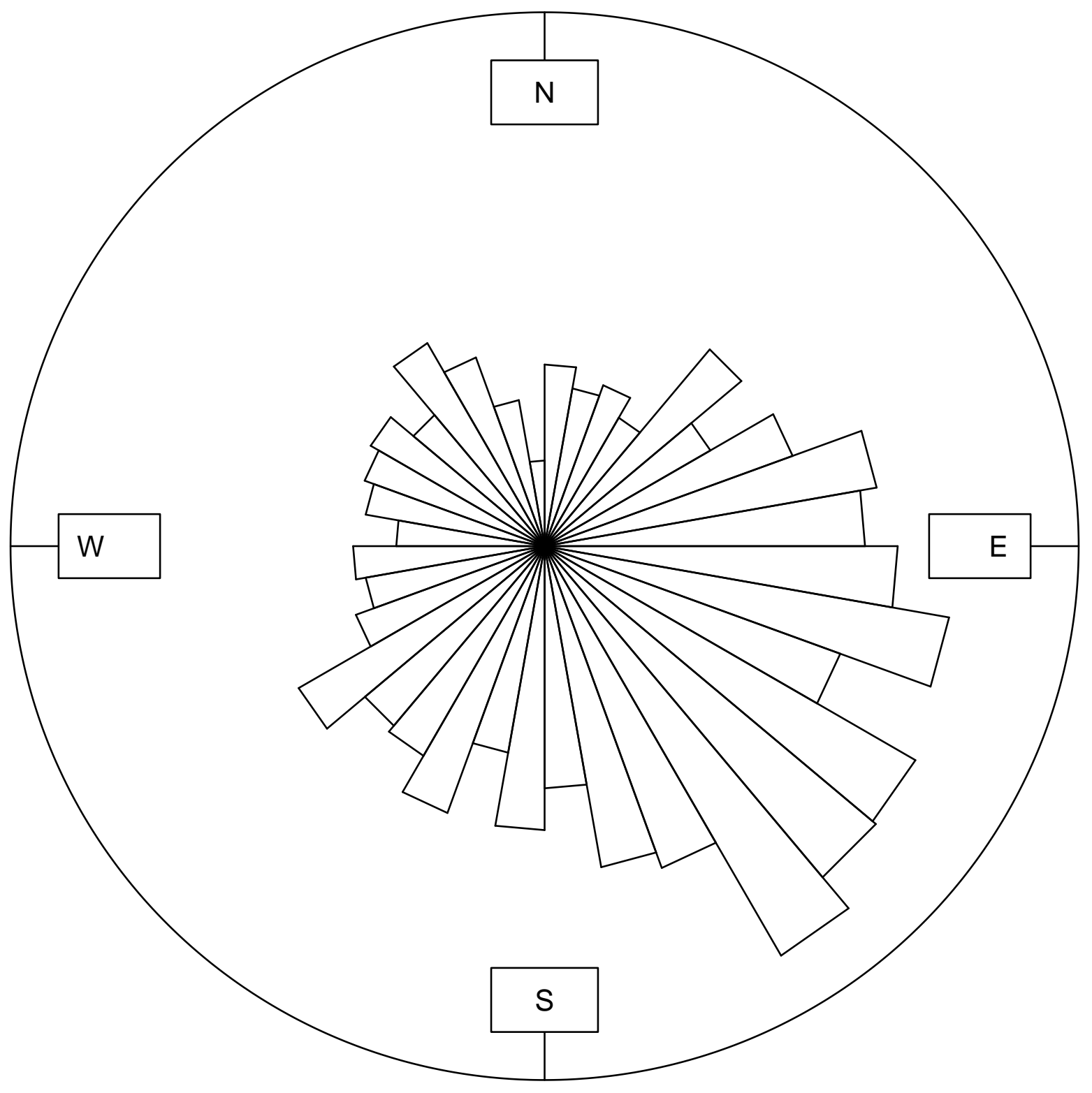


bootstrap 740

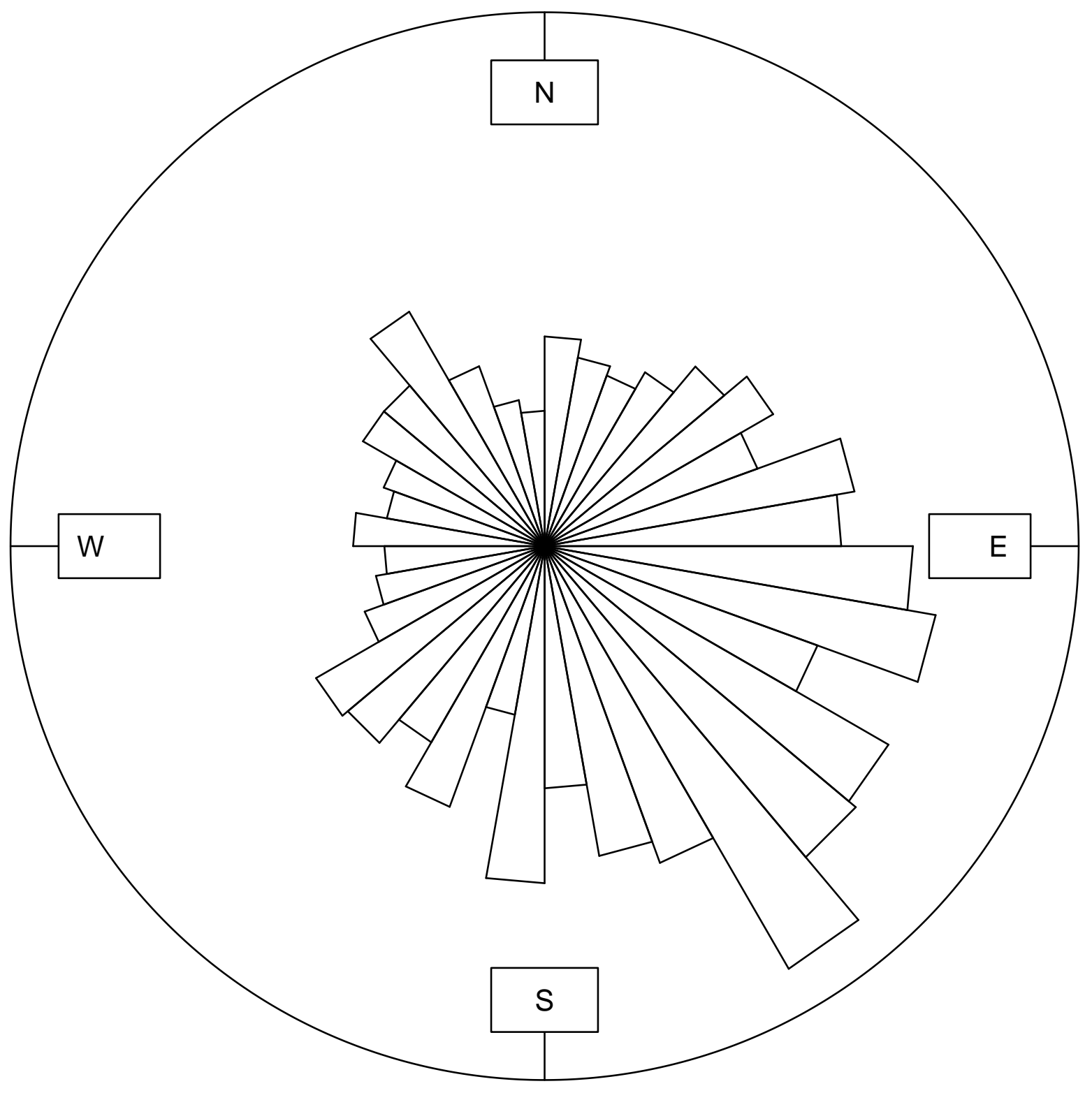




\section{bootstrap 741}

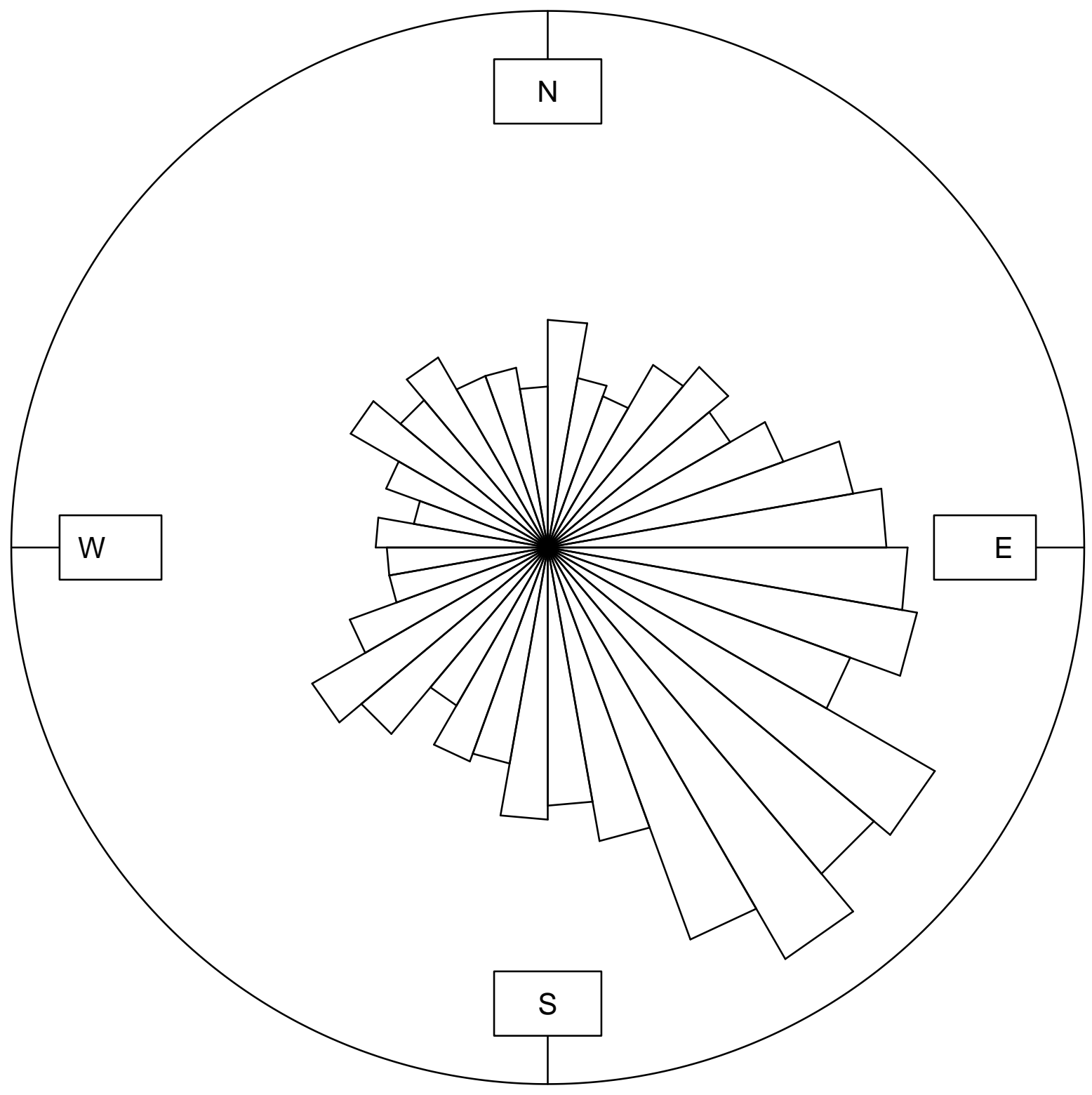


bootstrap 742

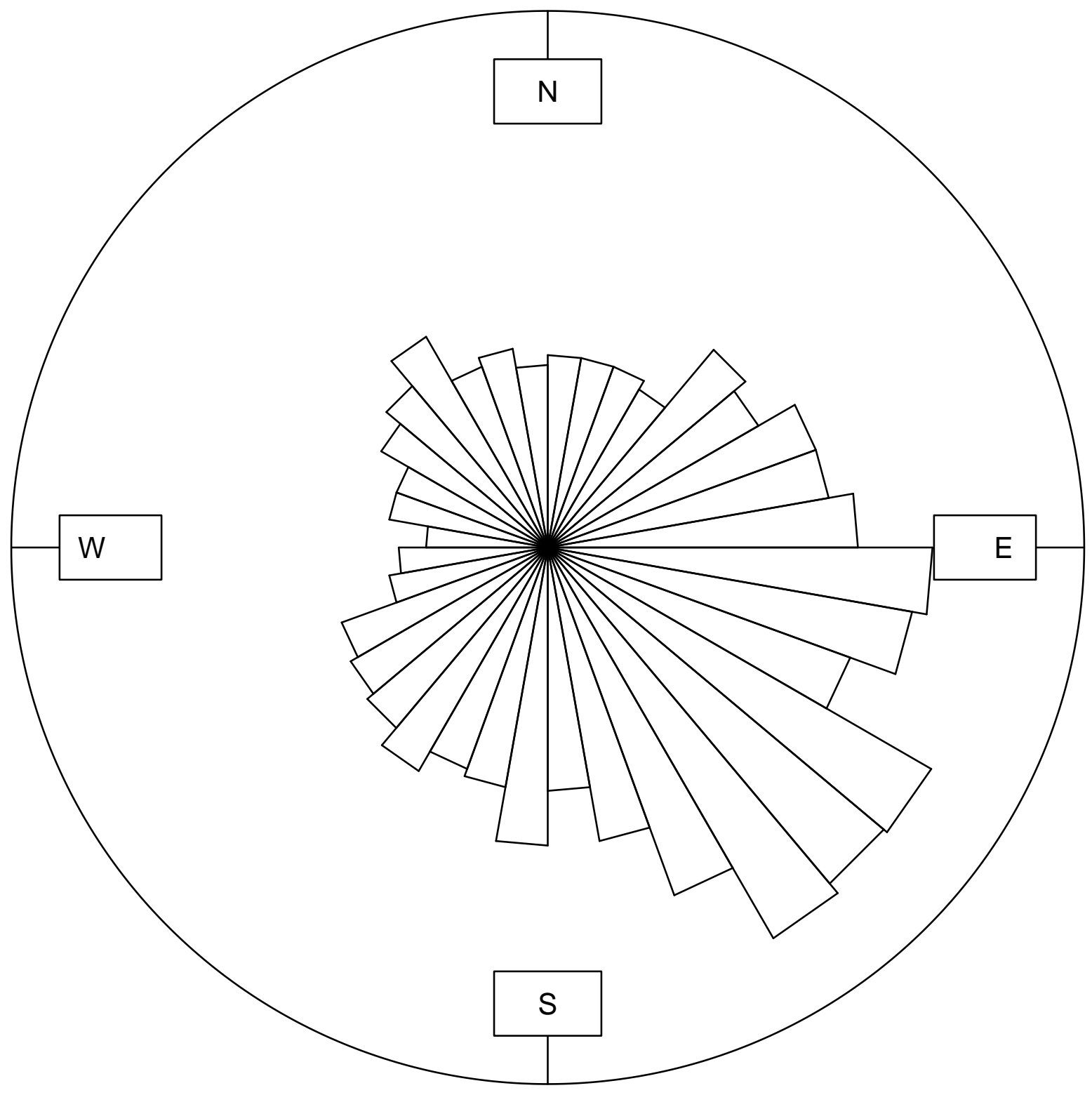




\section{bootstrap 743}

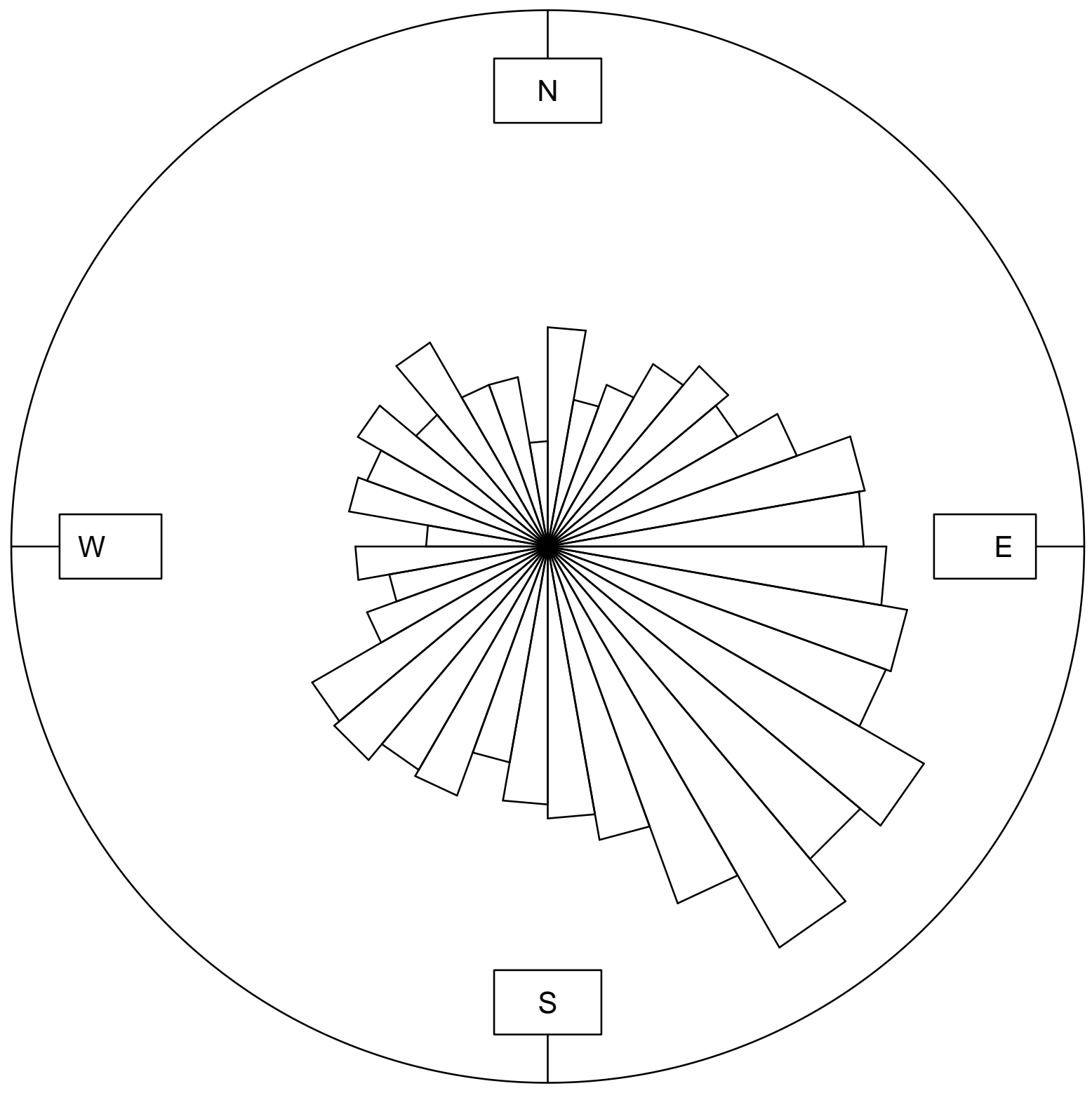




\section{bootstrap 744}

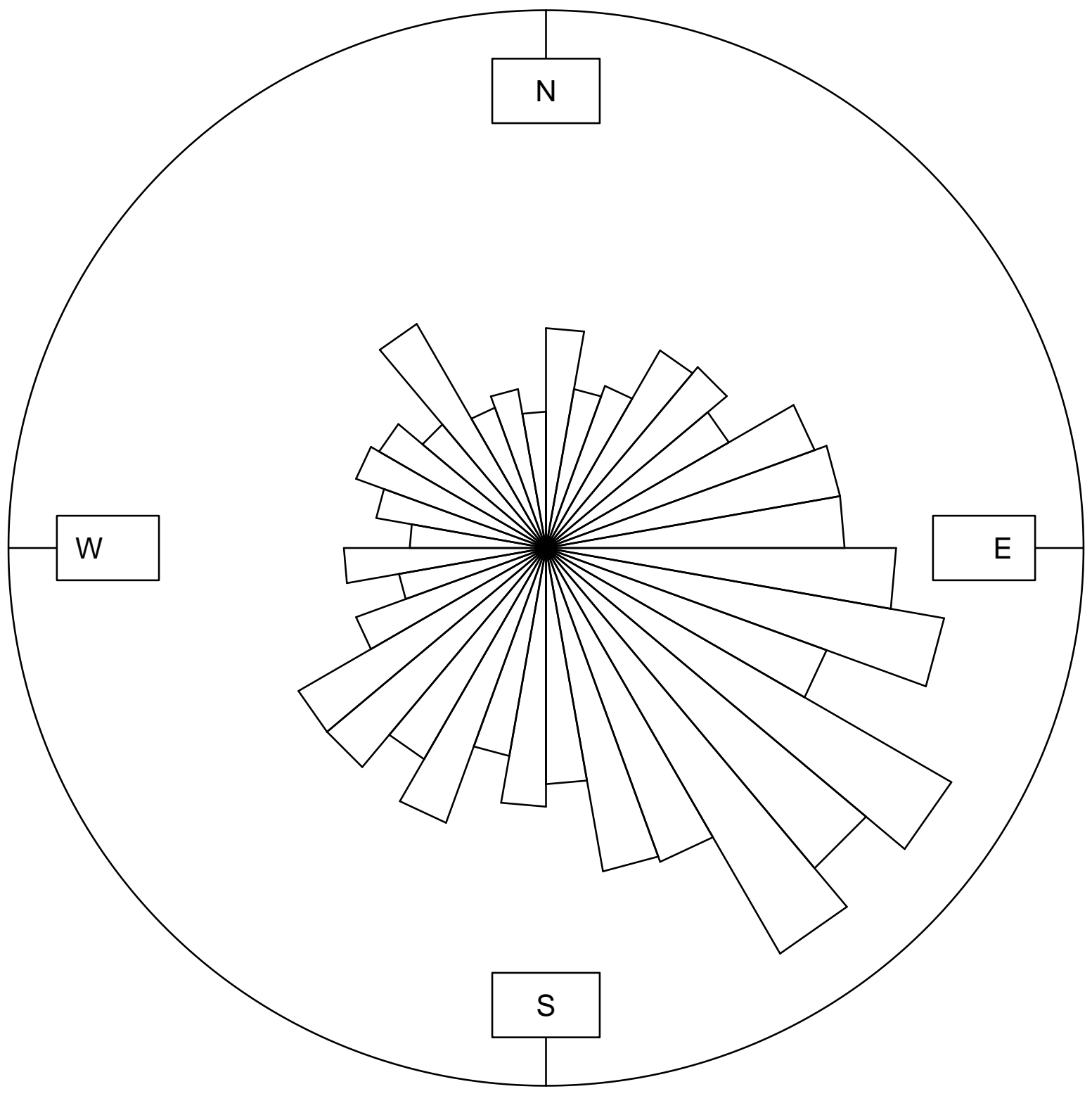


bootstrap 745

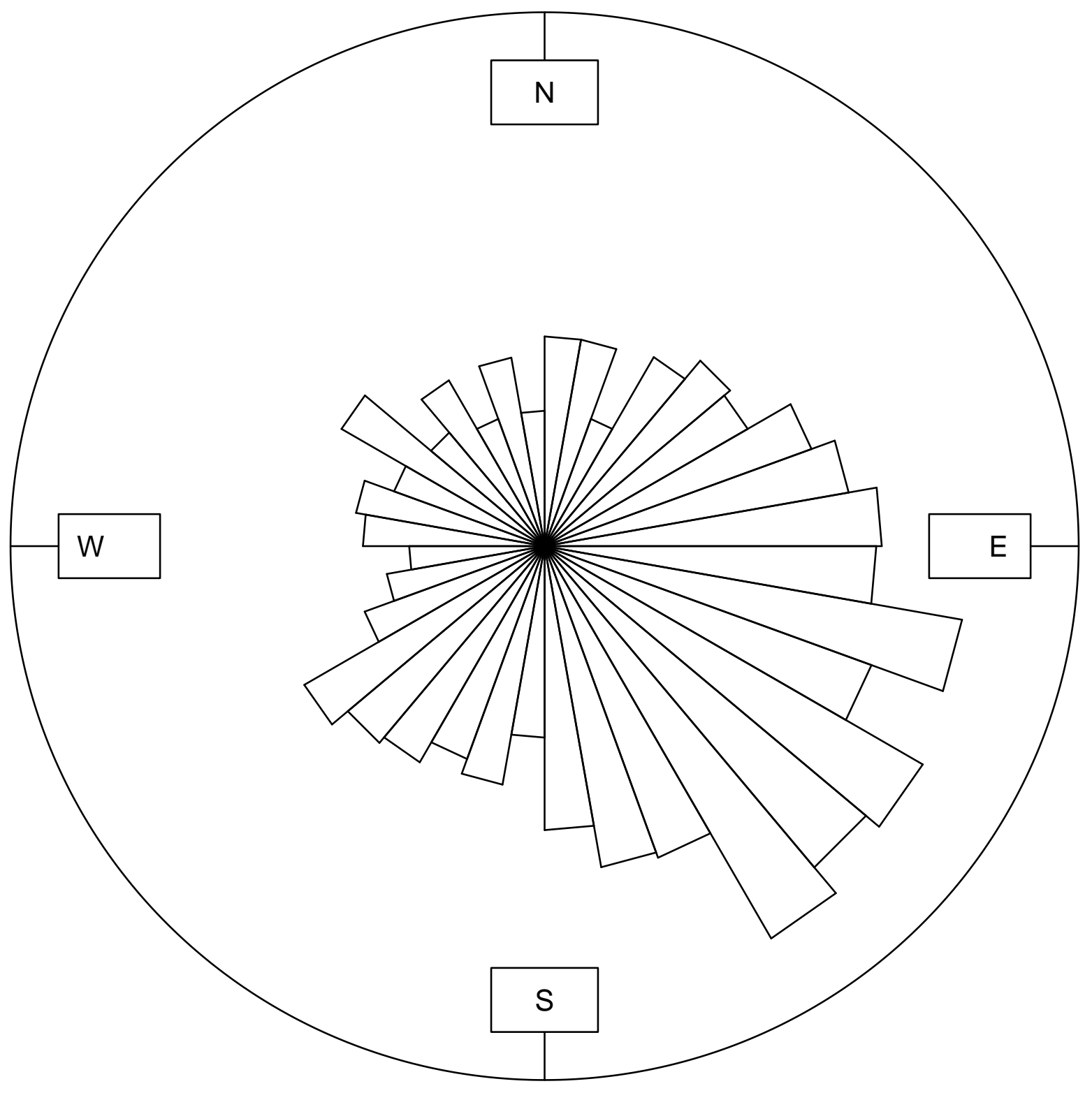


bootstrap 746

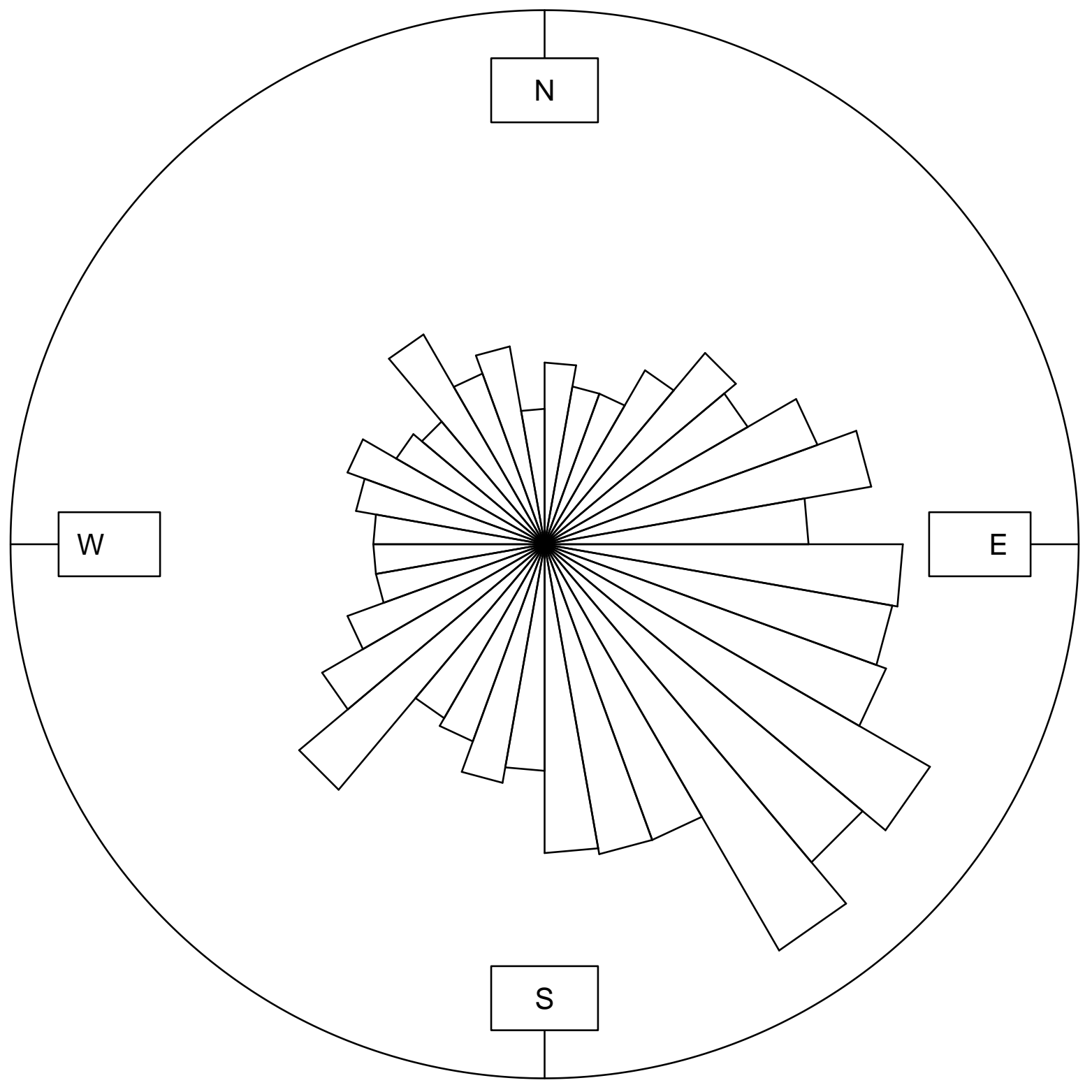


bootstrap 747

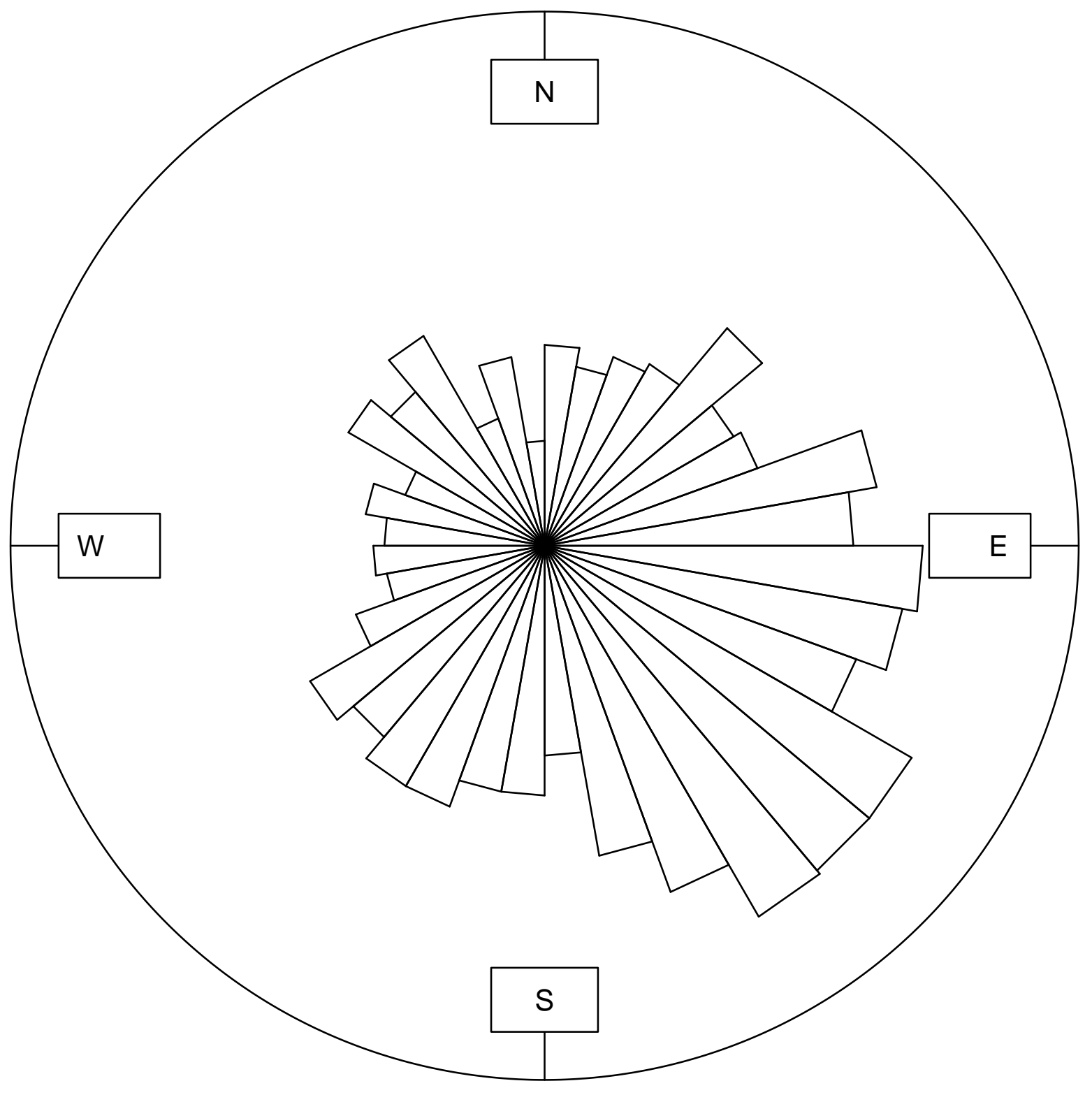


bootstrap 748

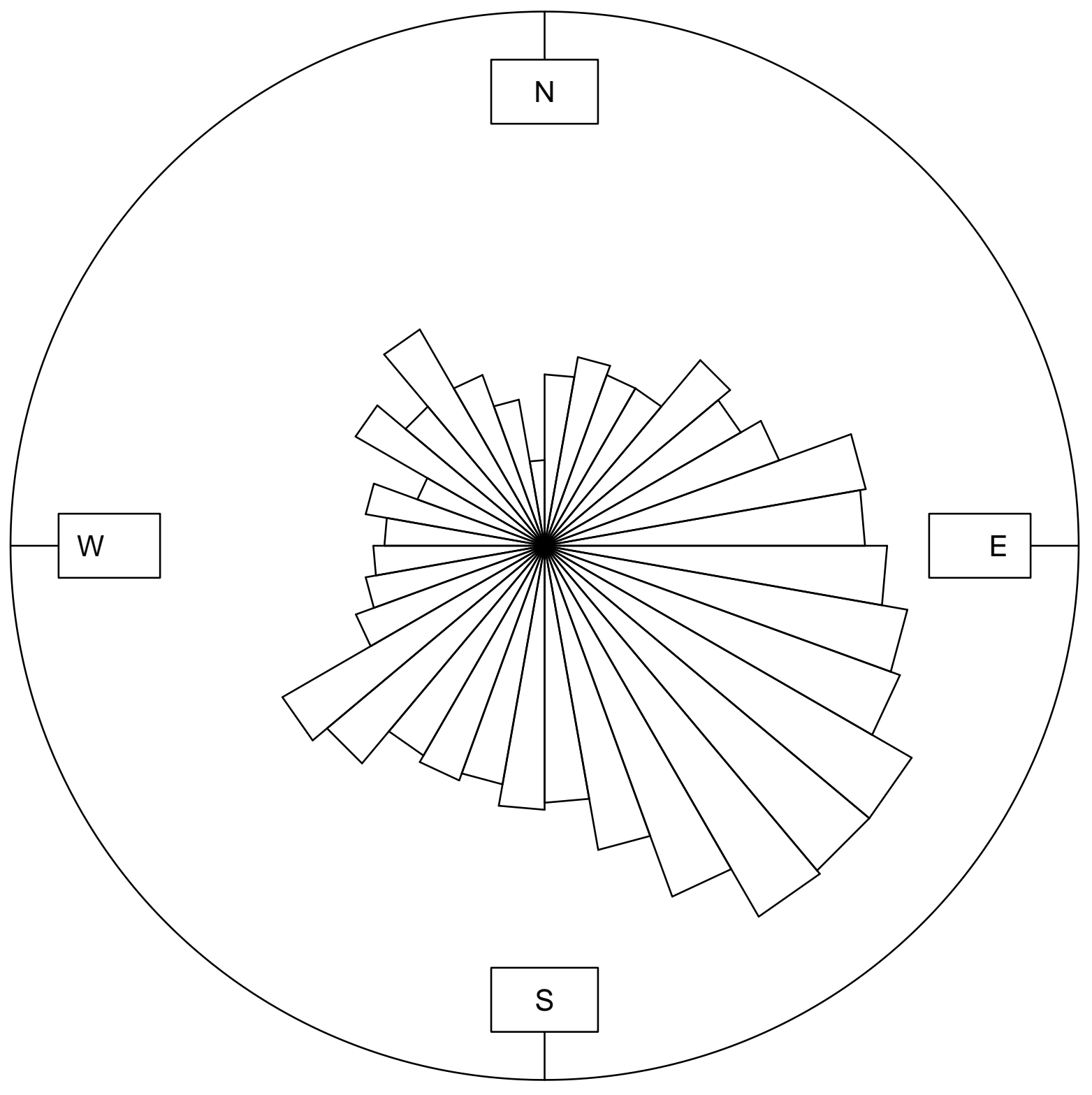


bootstrap 749

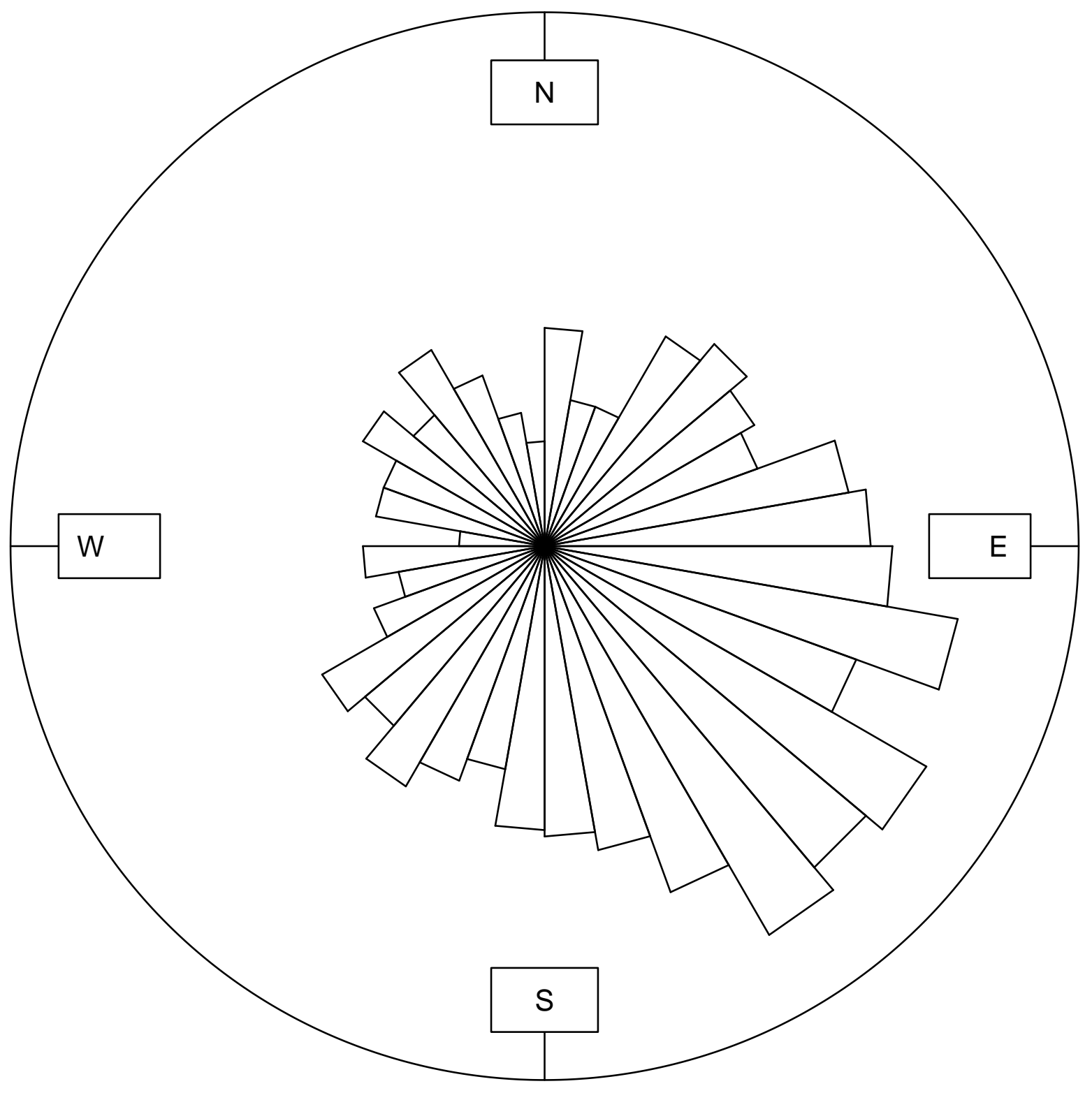


bootstrap 750

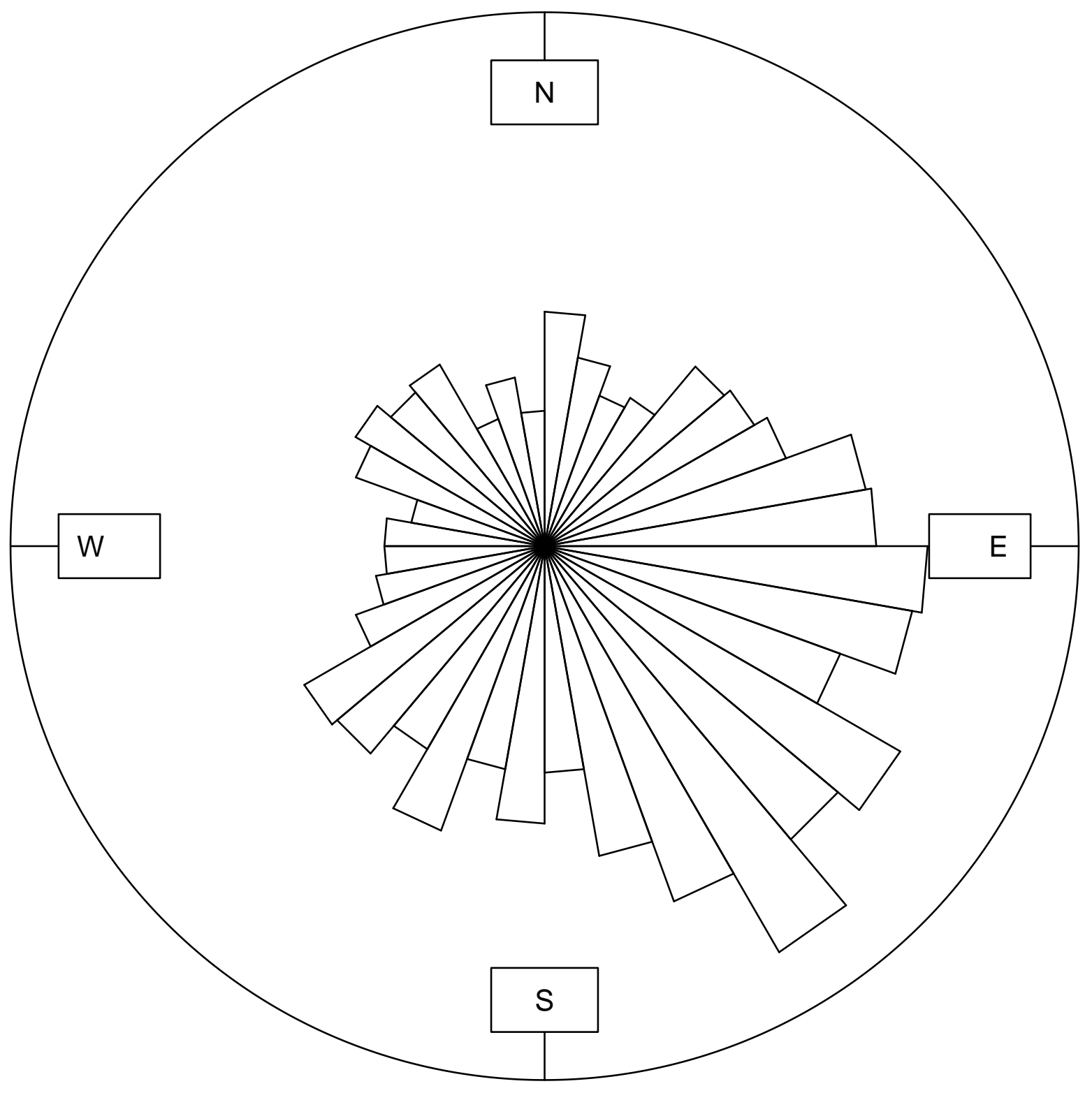


bootstrap 751

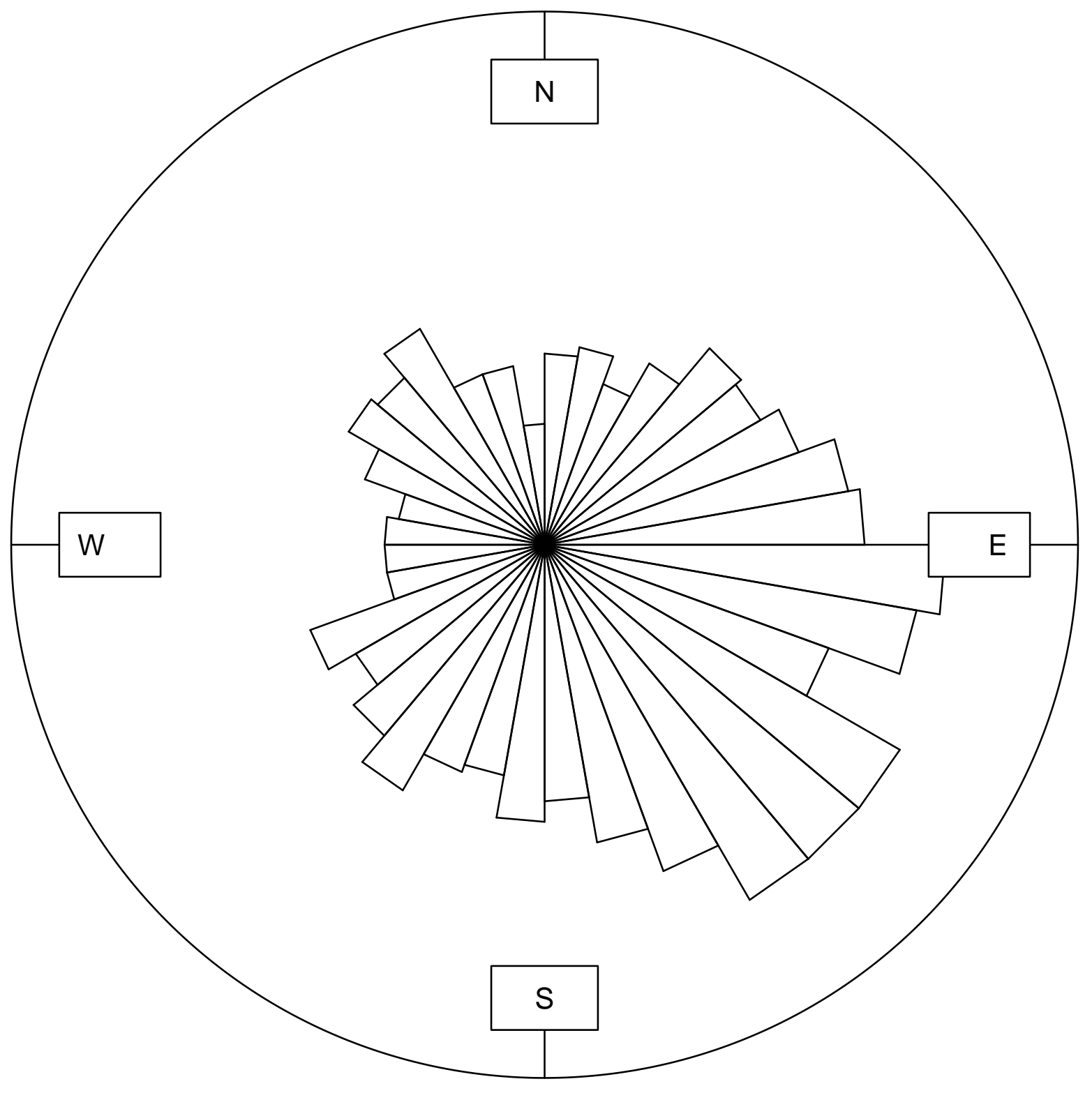


bootstrap 752

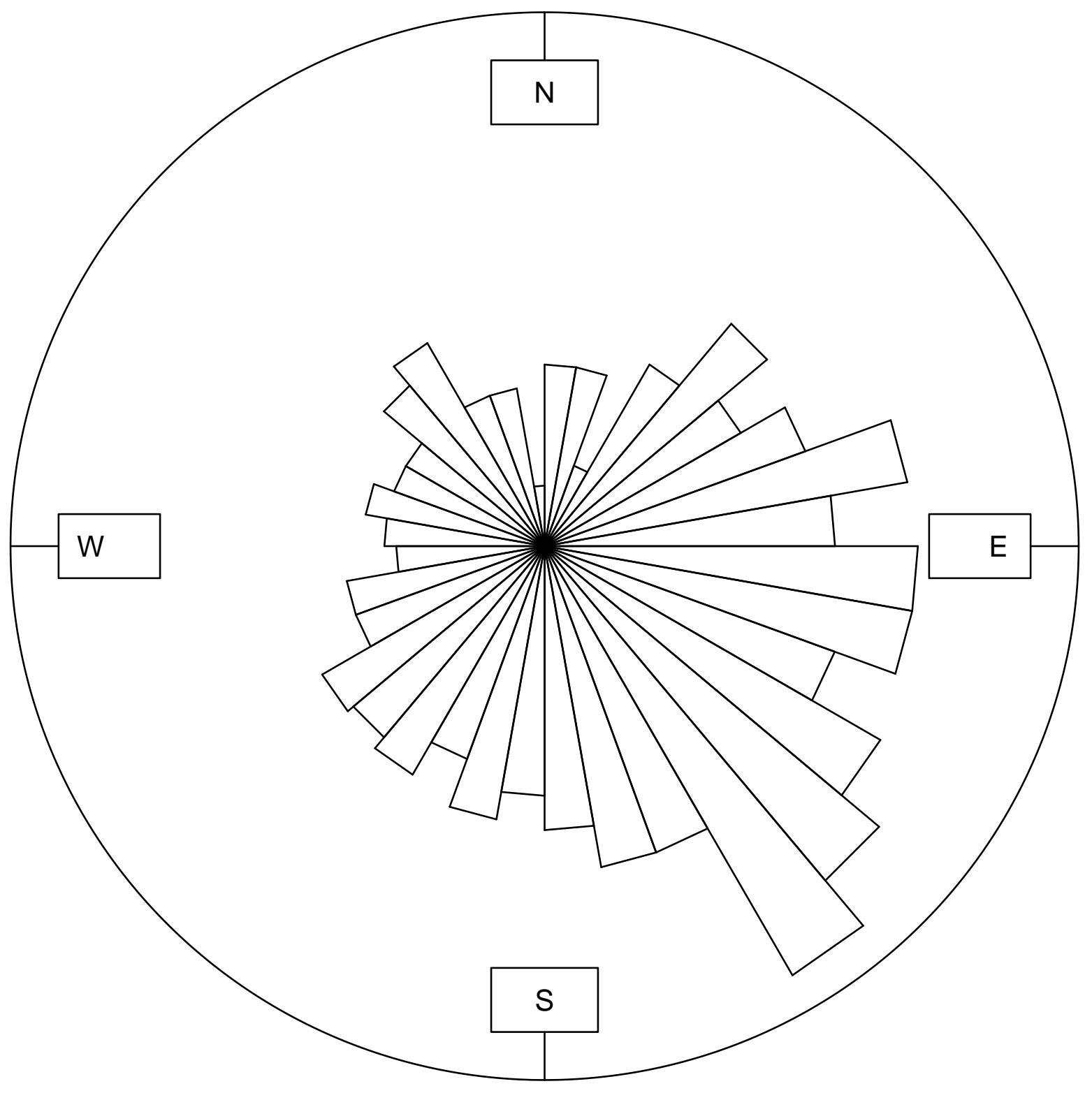




\section{bootstrap 753}

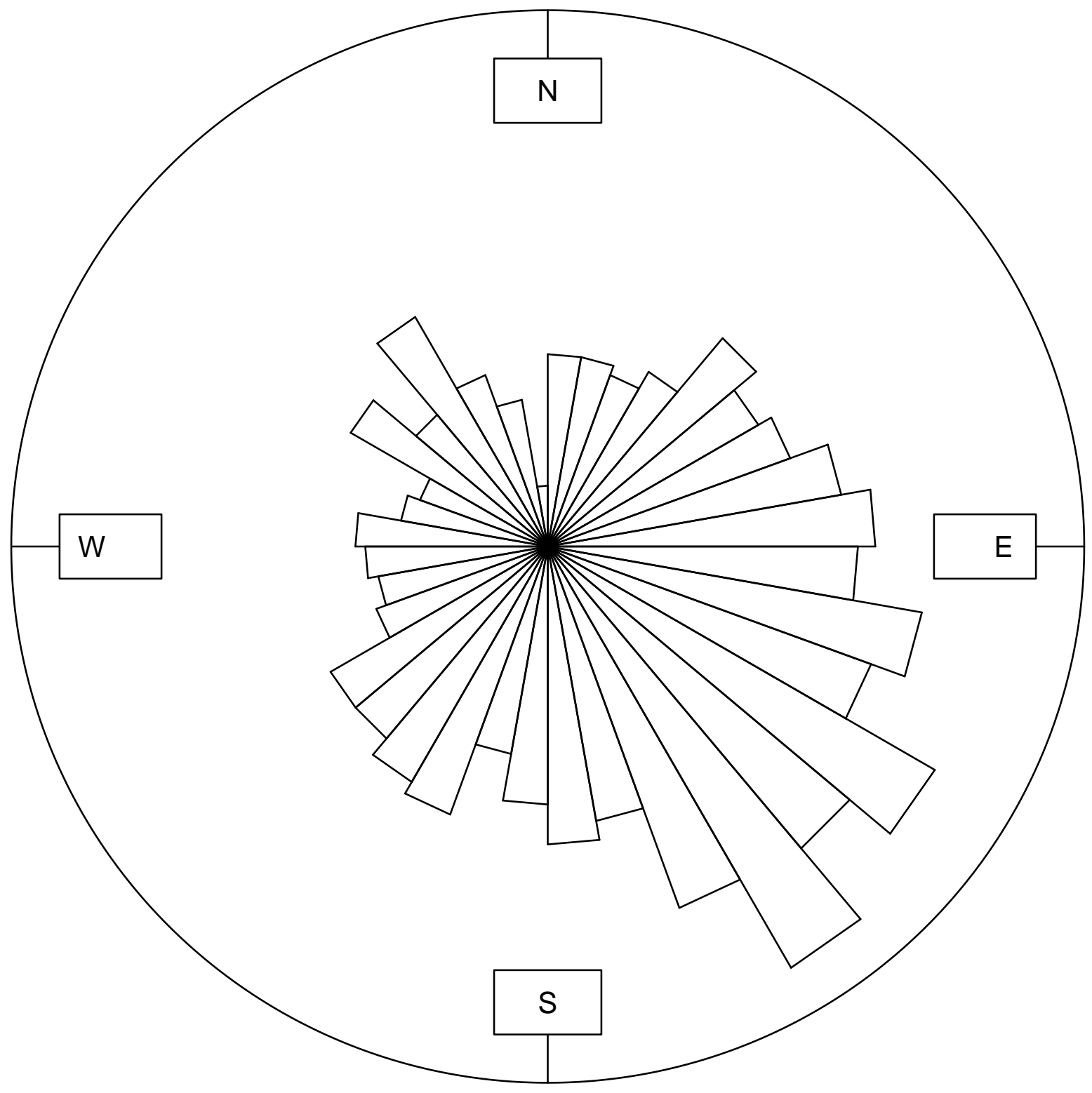


bootstrap 754

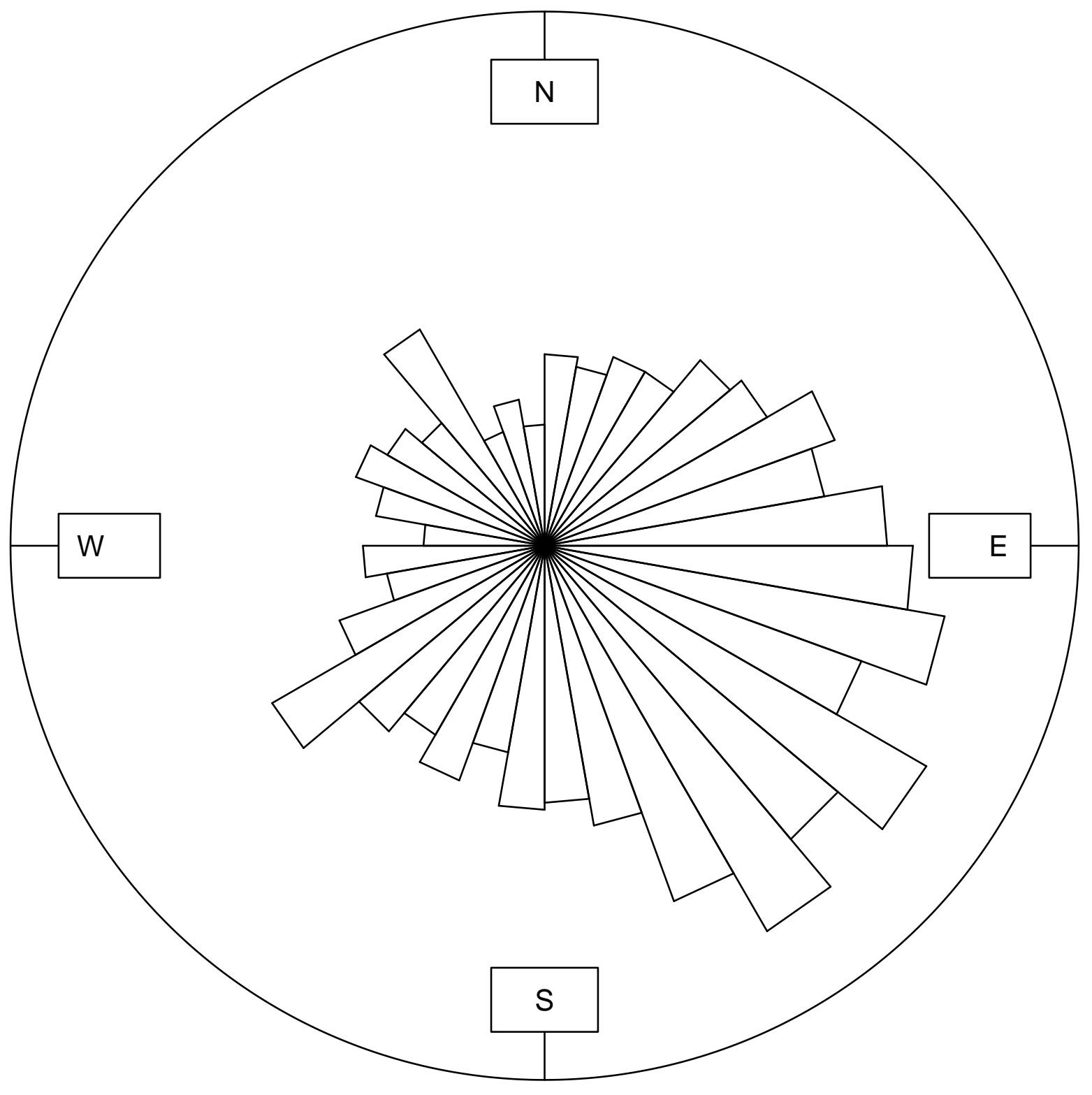




\section{bootstrap 755}

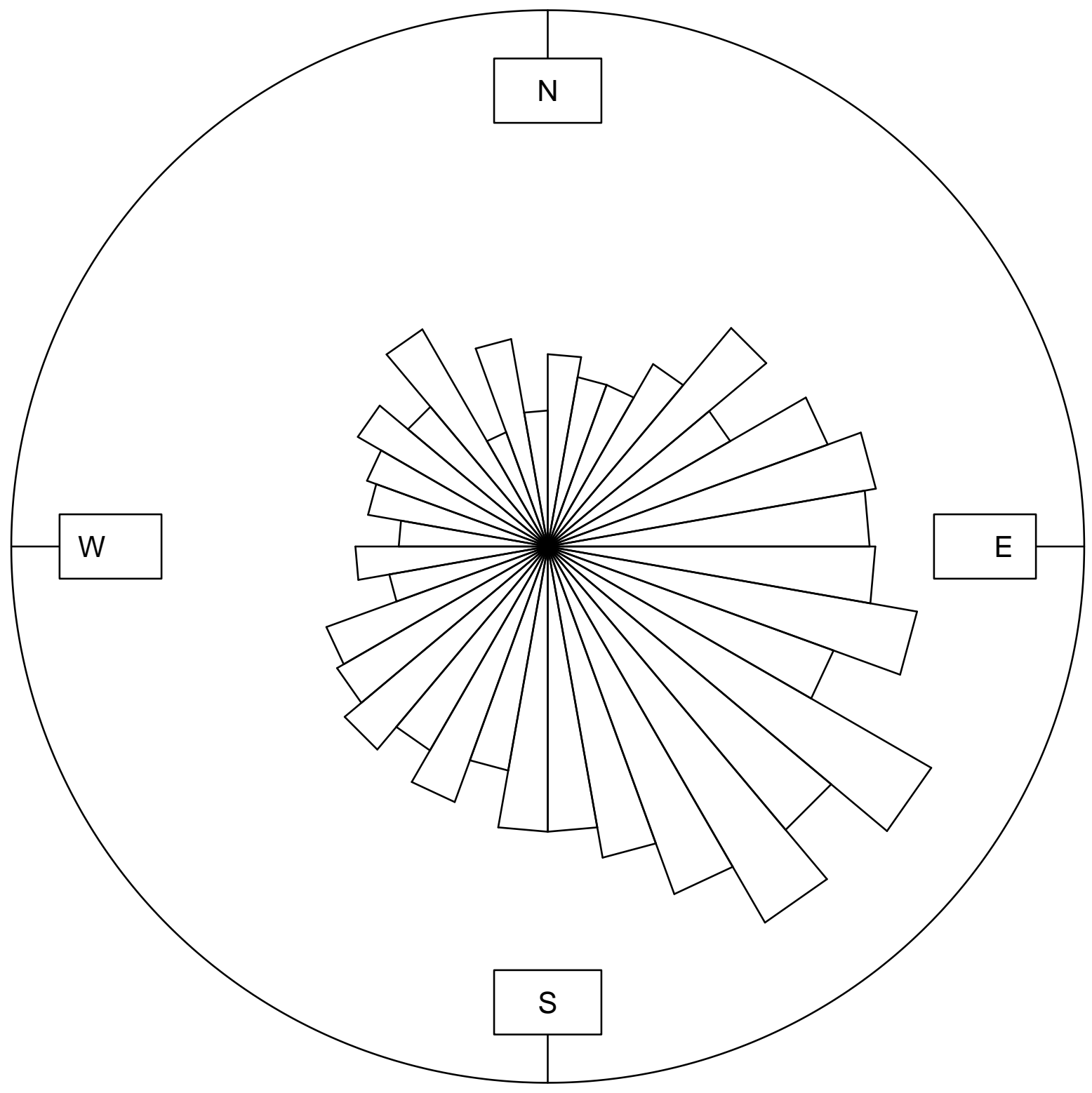


bootstrap 756

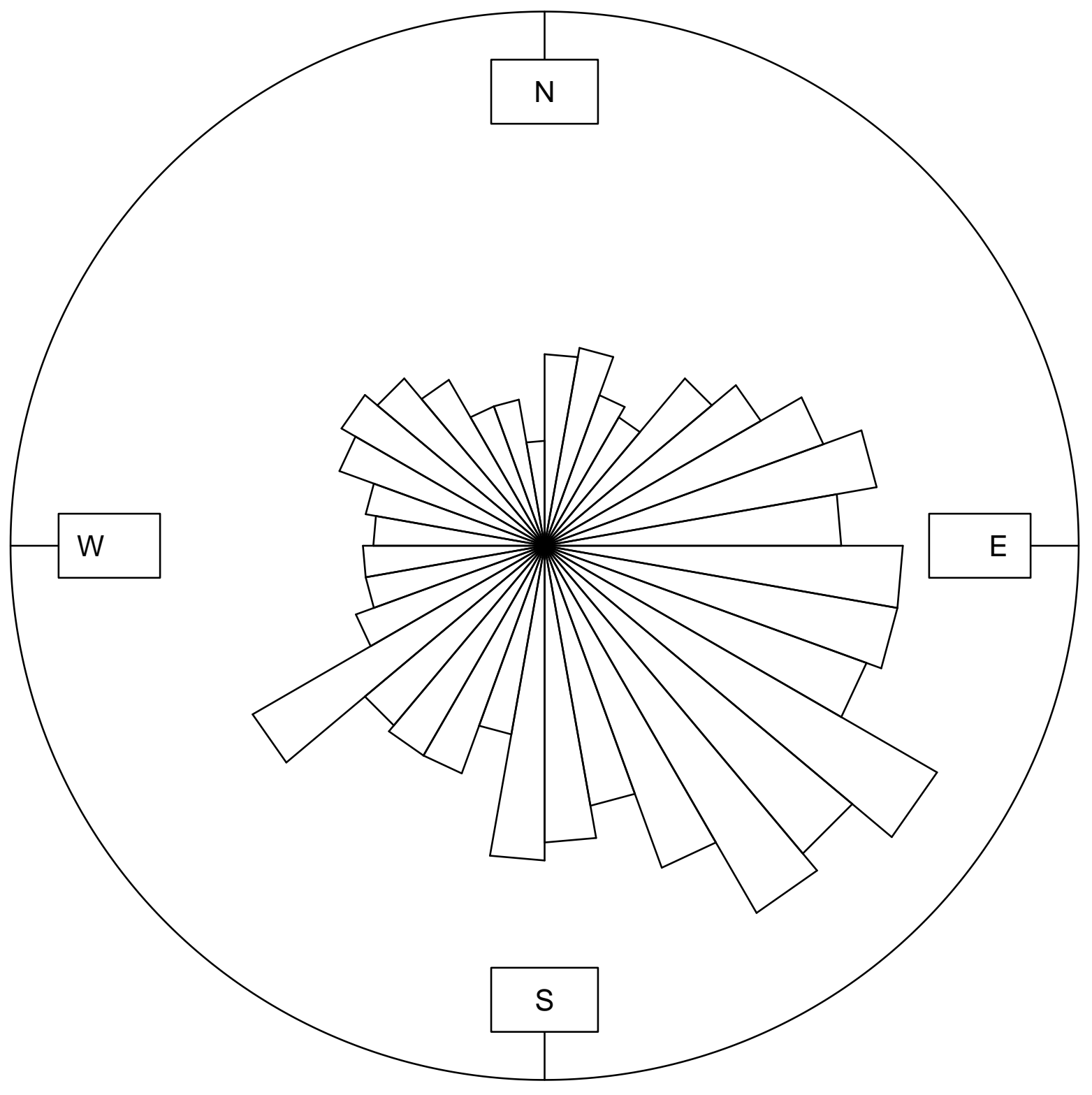


bootstrap 757

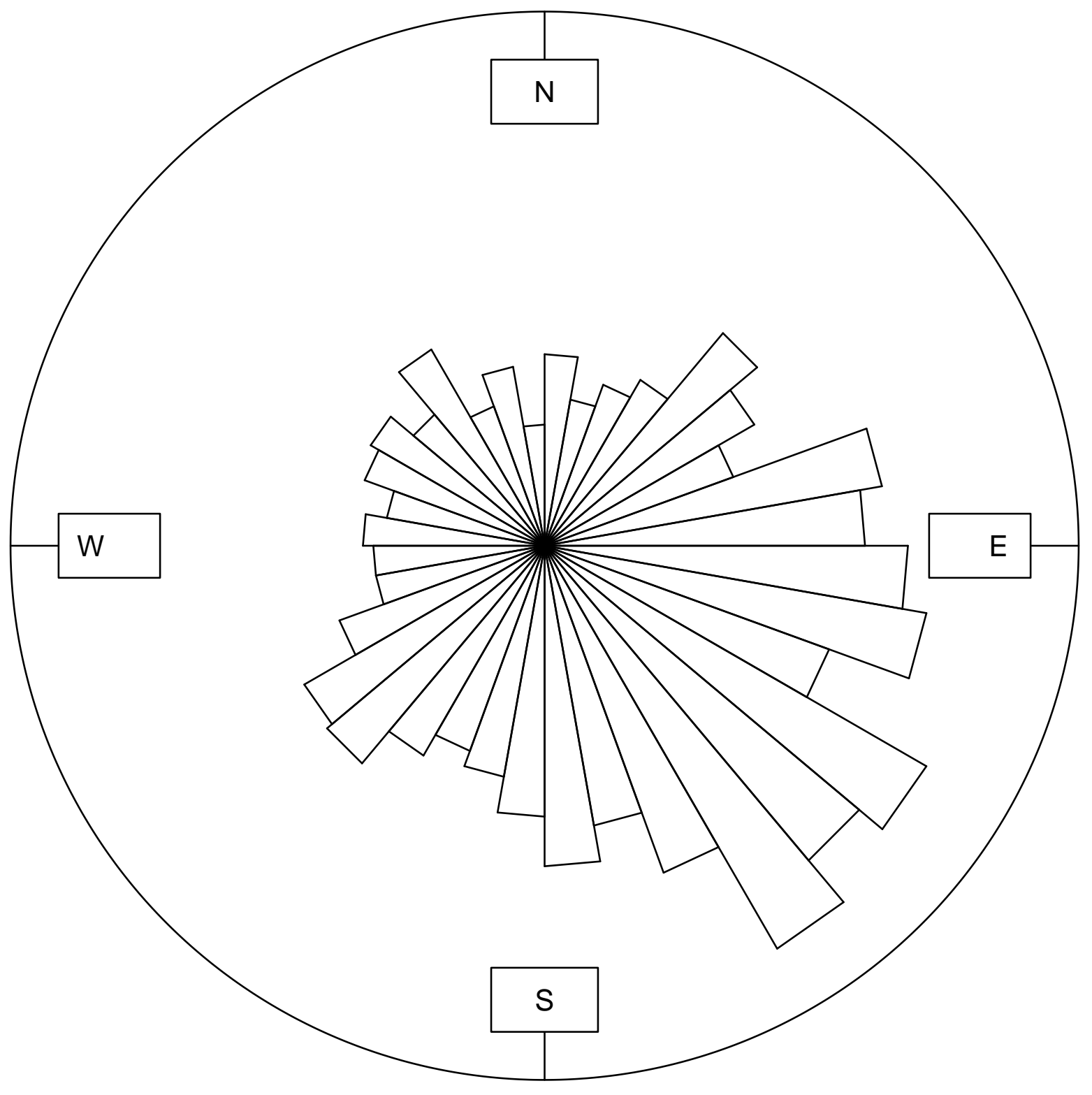


bootstrap 758

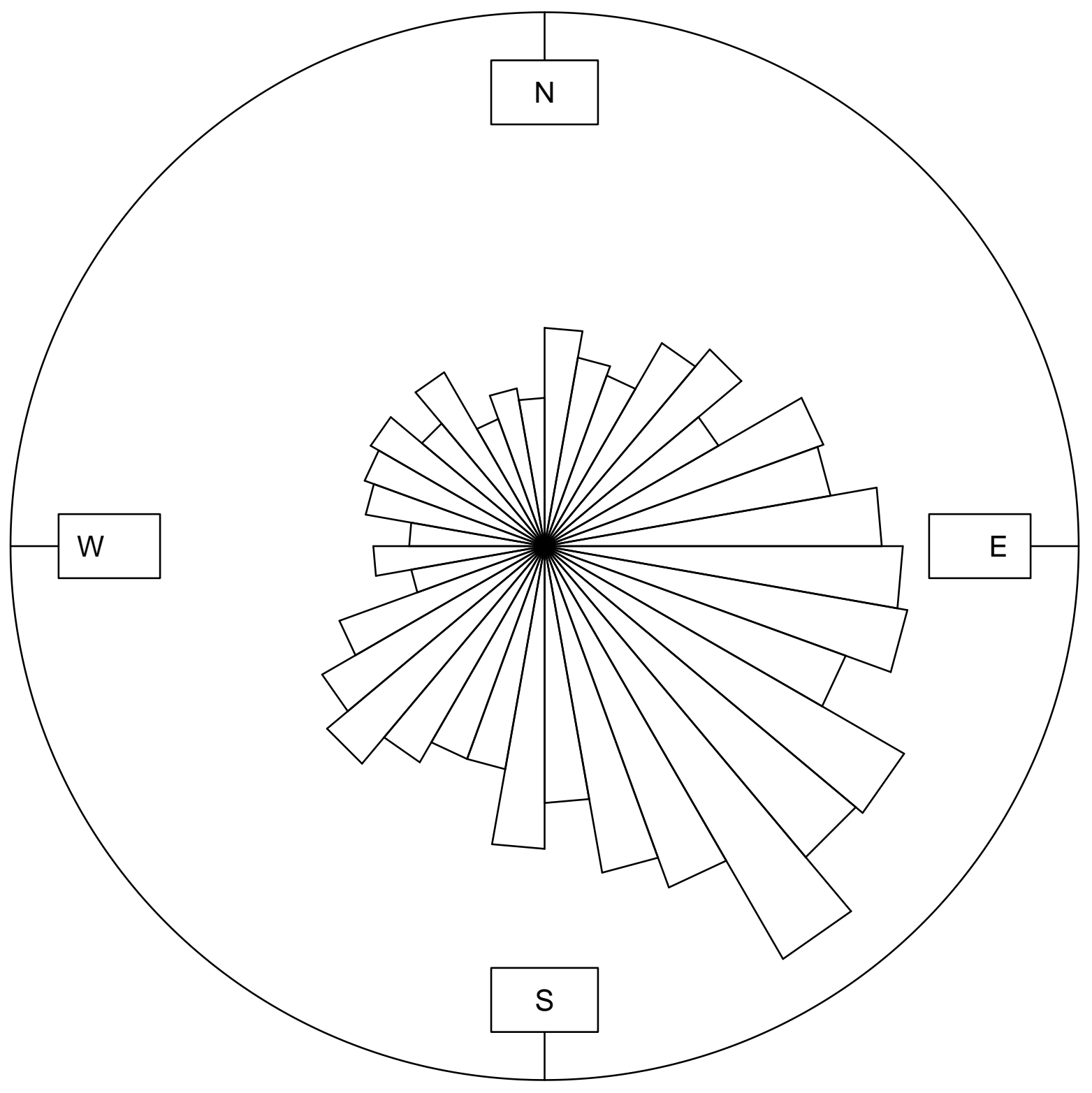


bootstrap 759

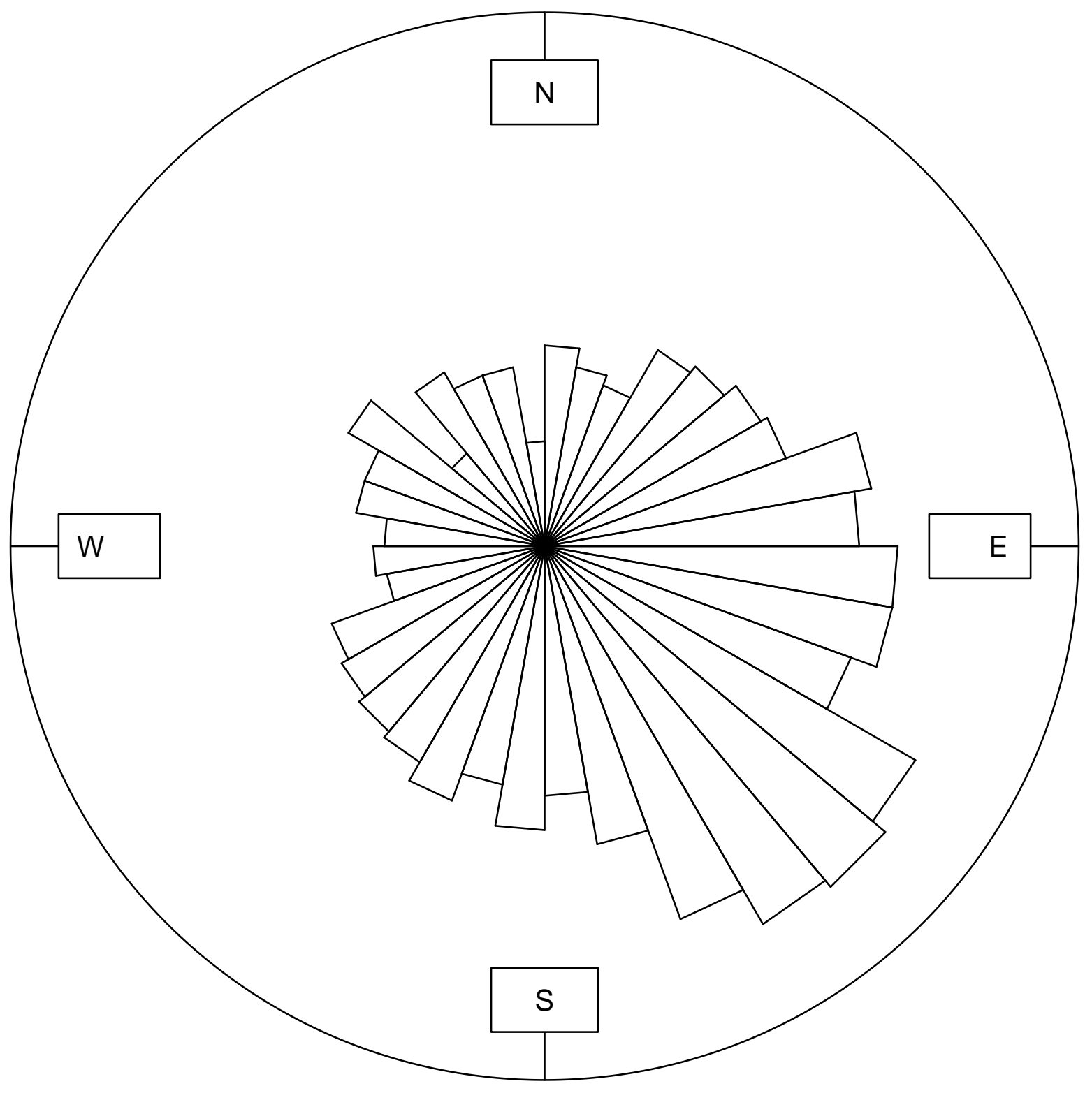


bootstrap 760

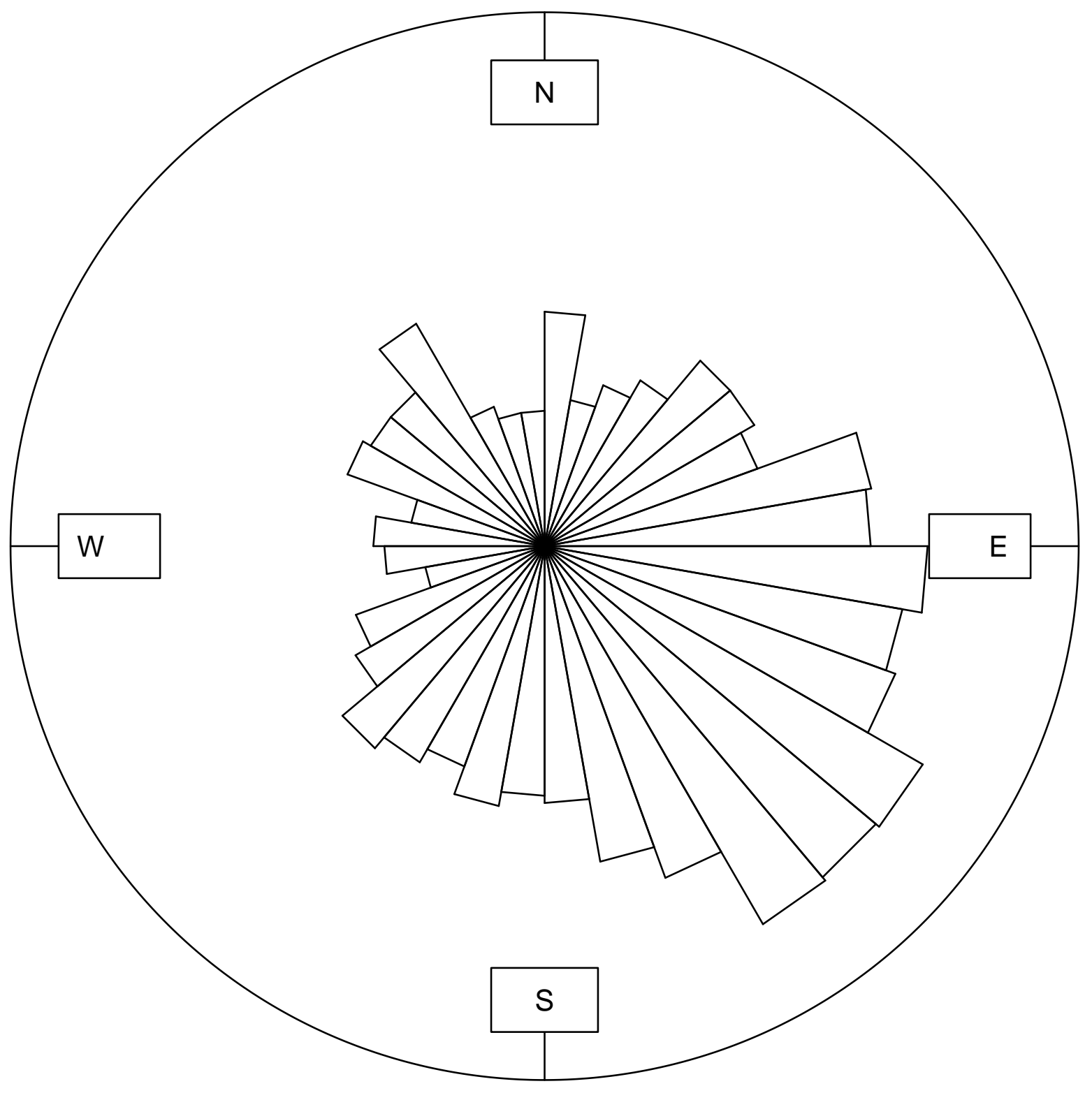


bootstrap 761

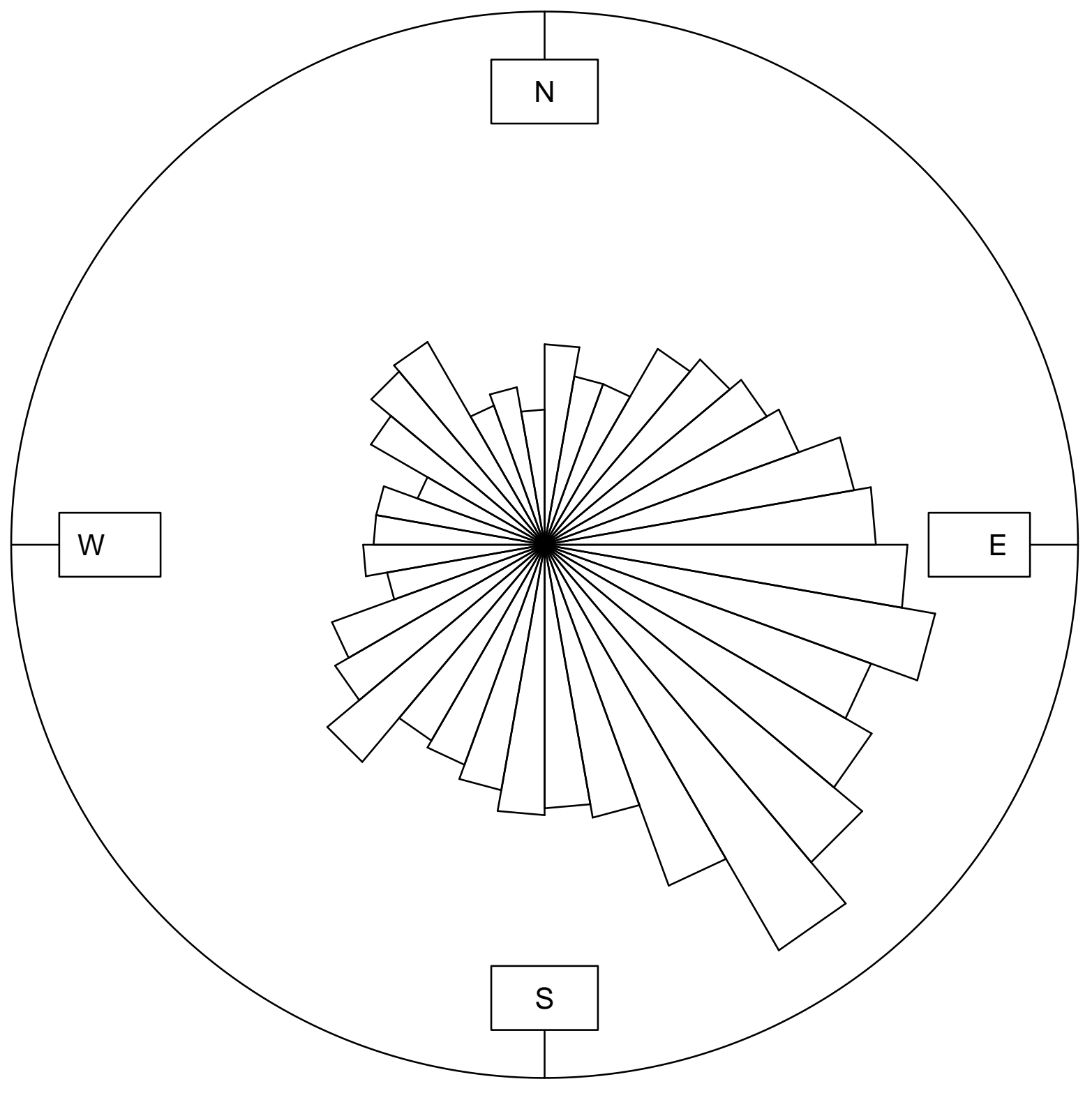


bootstrap 762

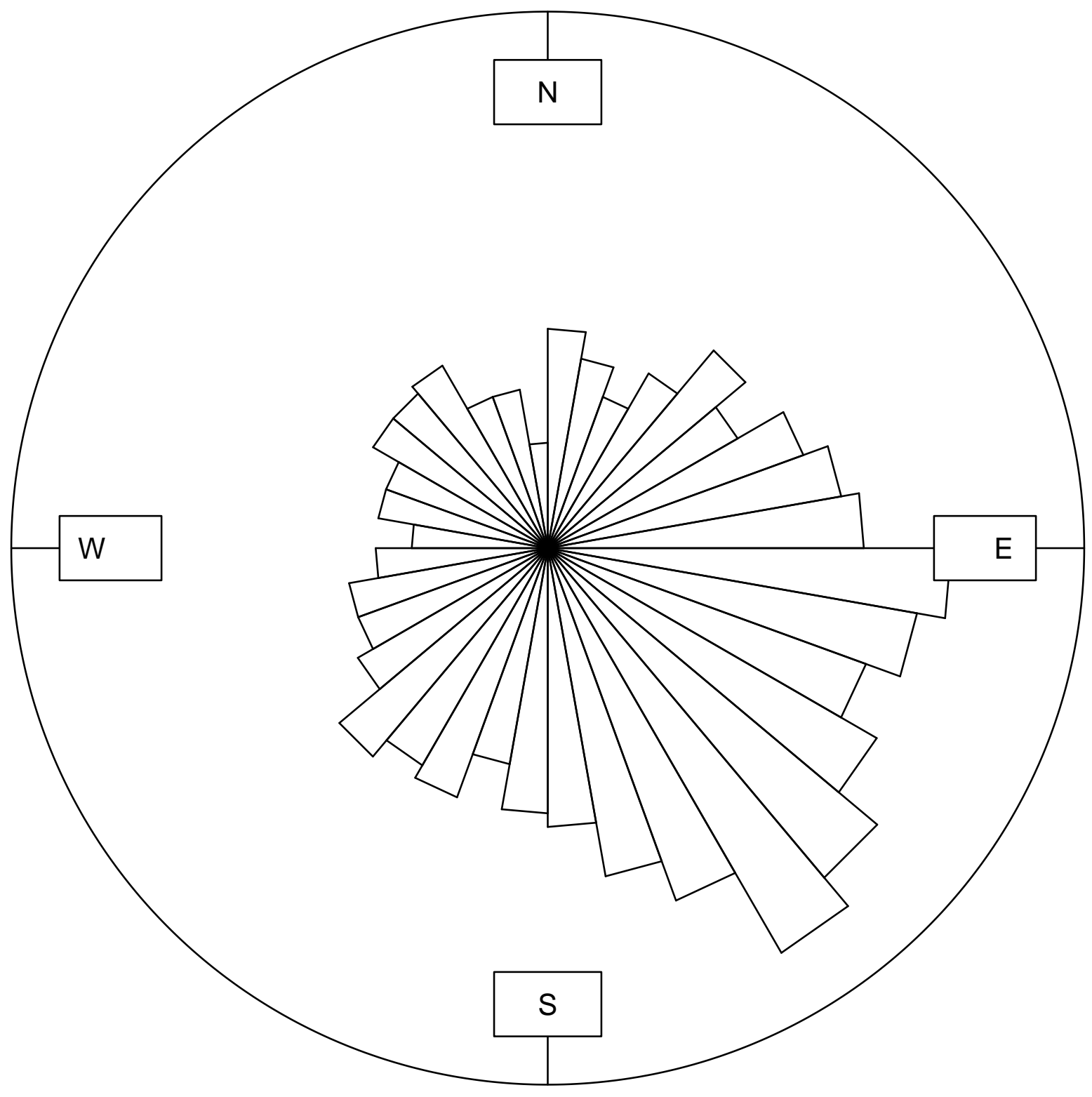


bootstrap 763

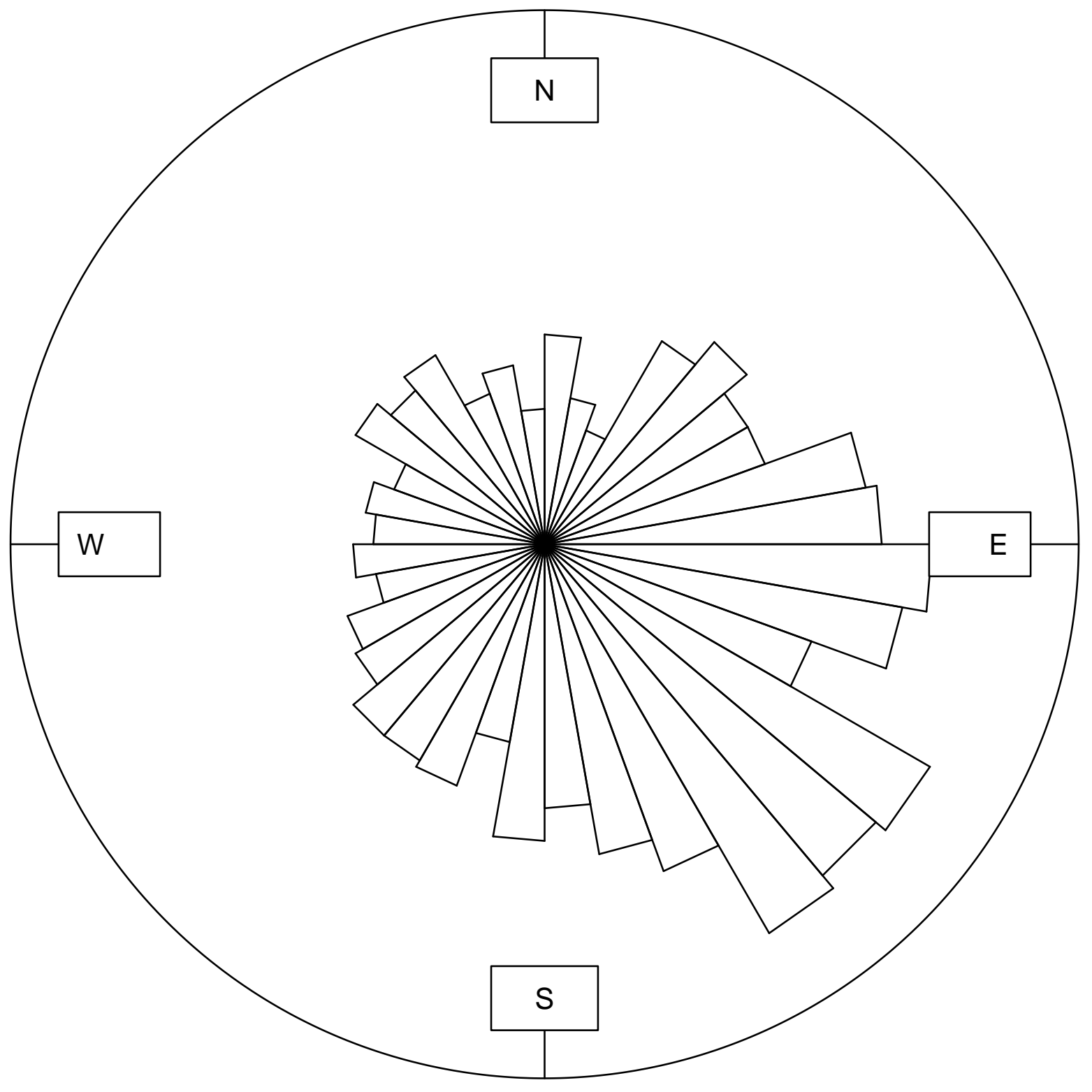




\section{bootstrap 764}

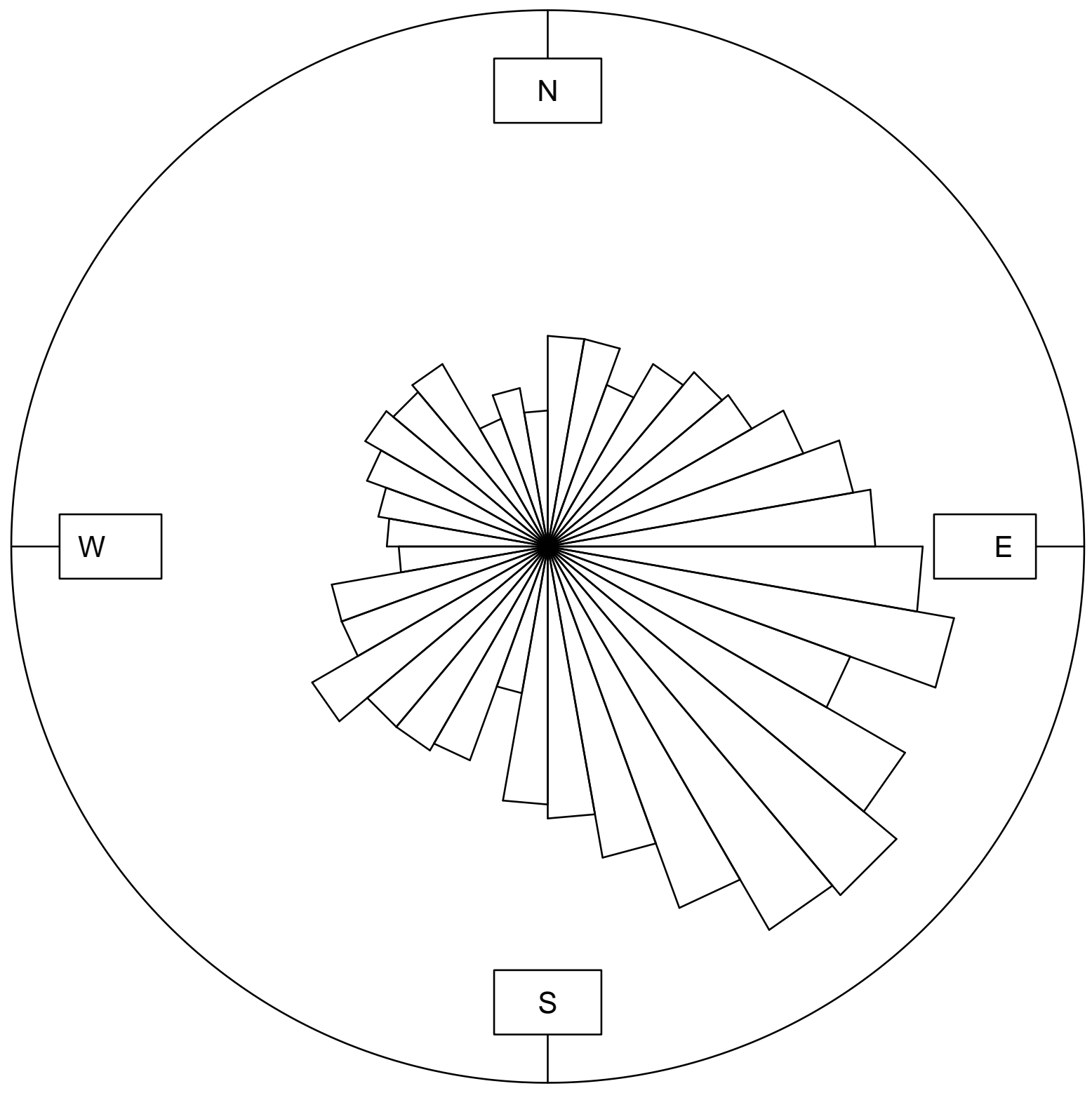


bootstrap 765

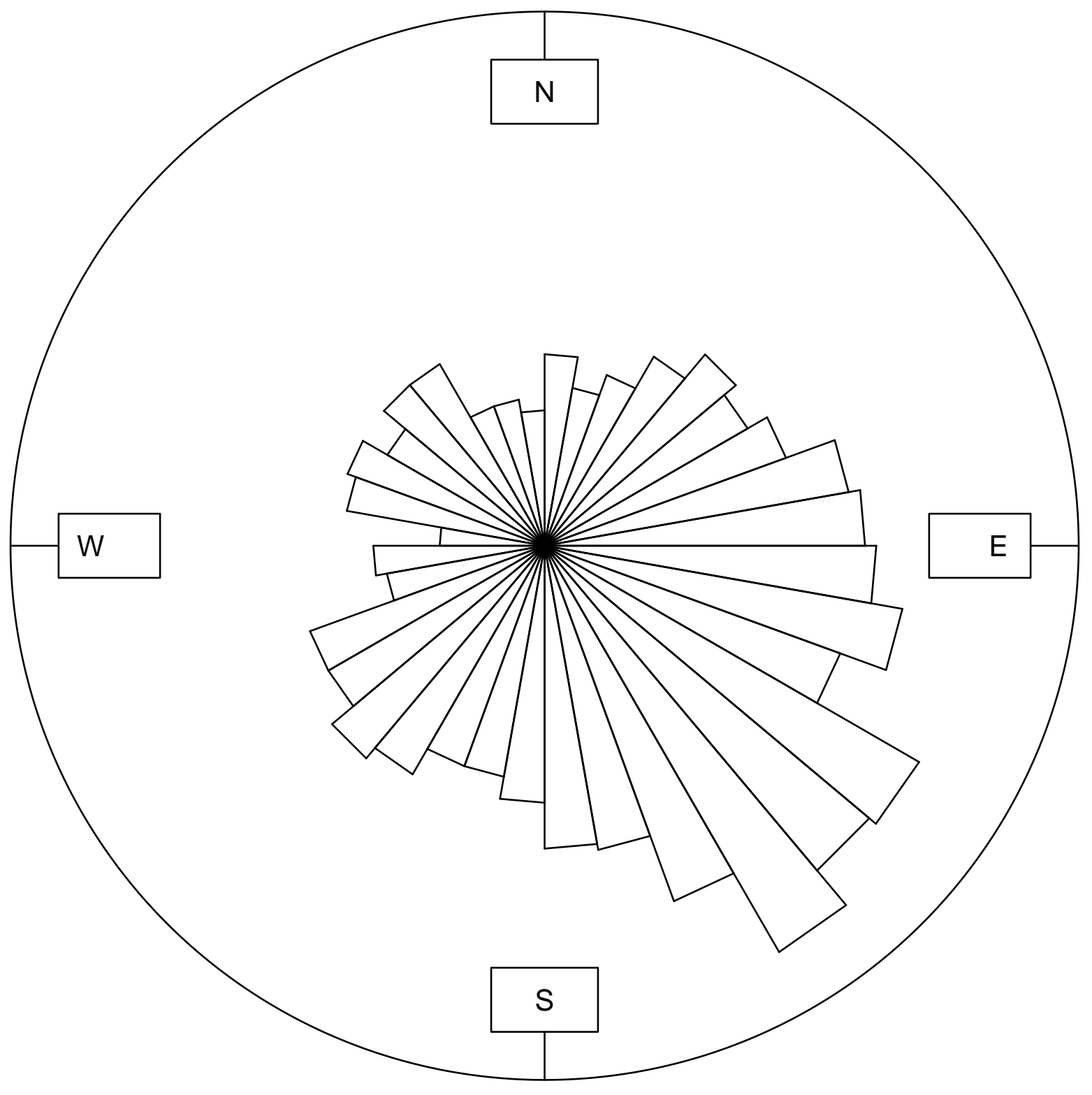


bootstrap 766

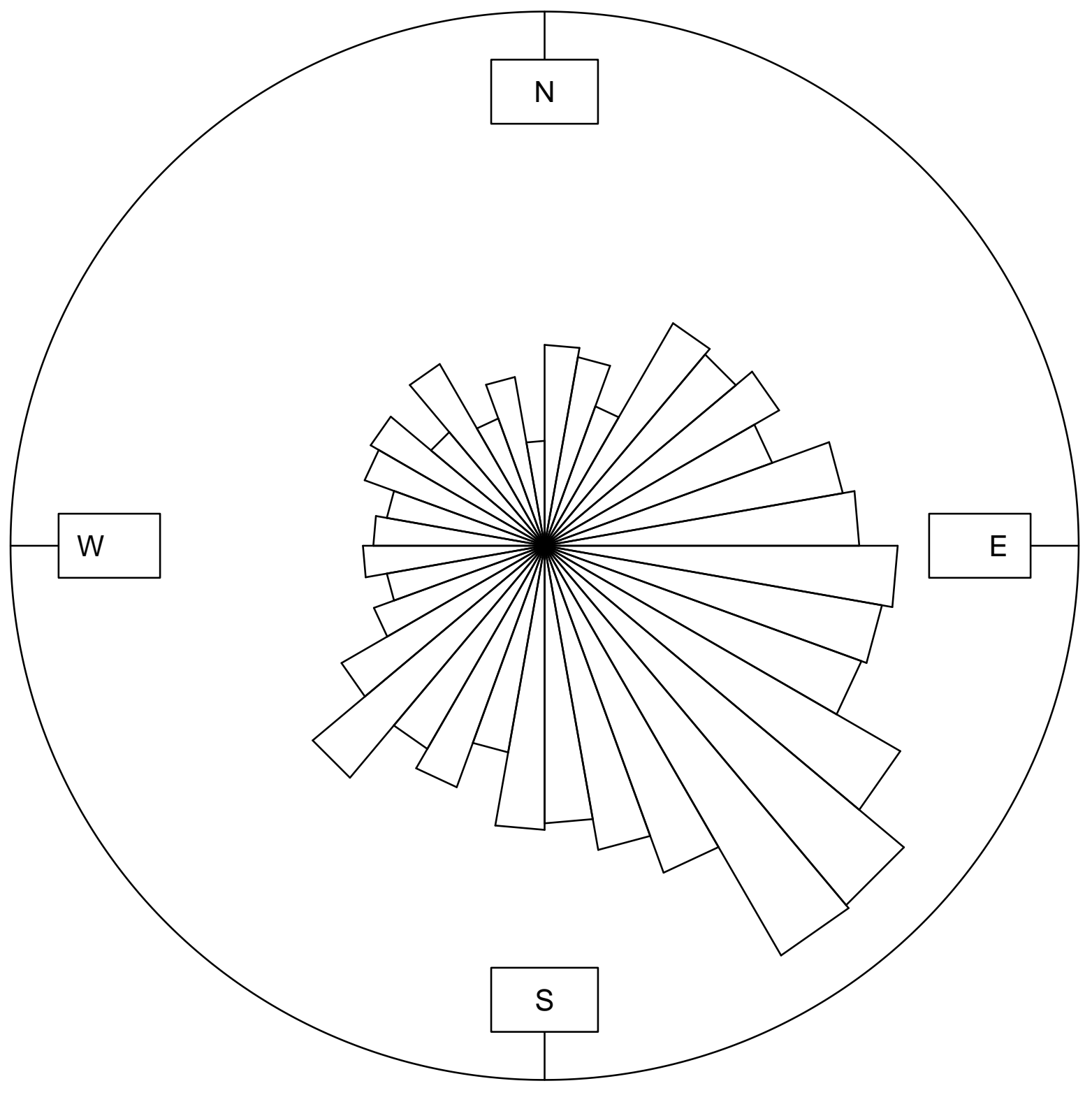


bootstrap 767

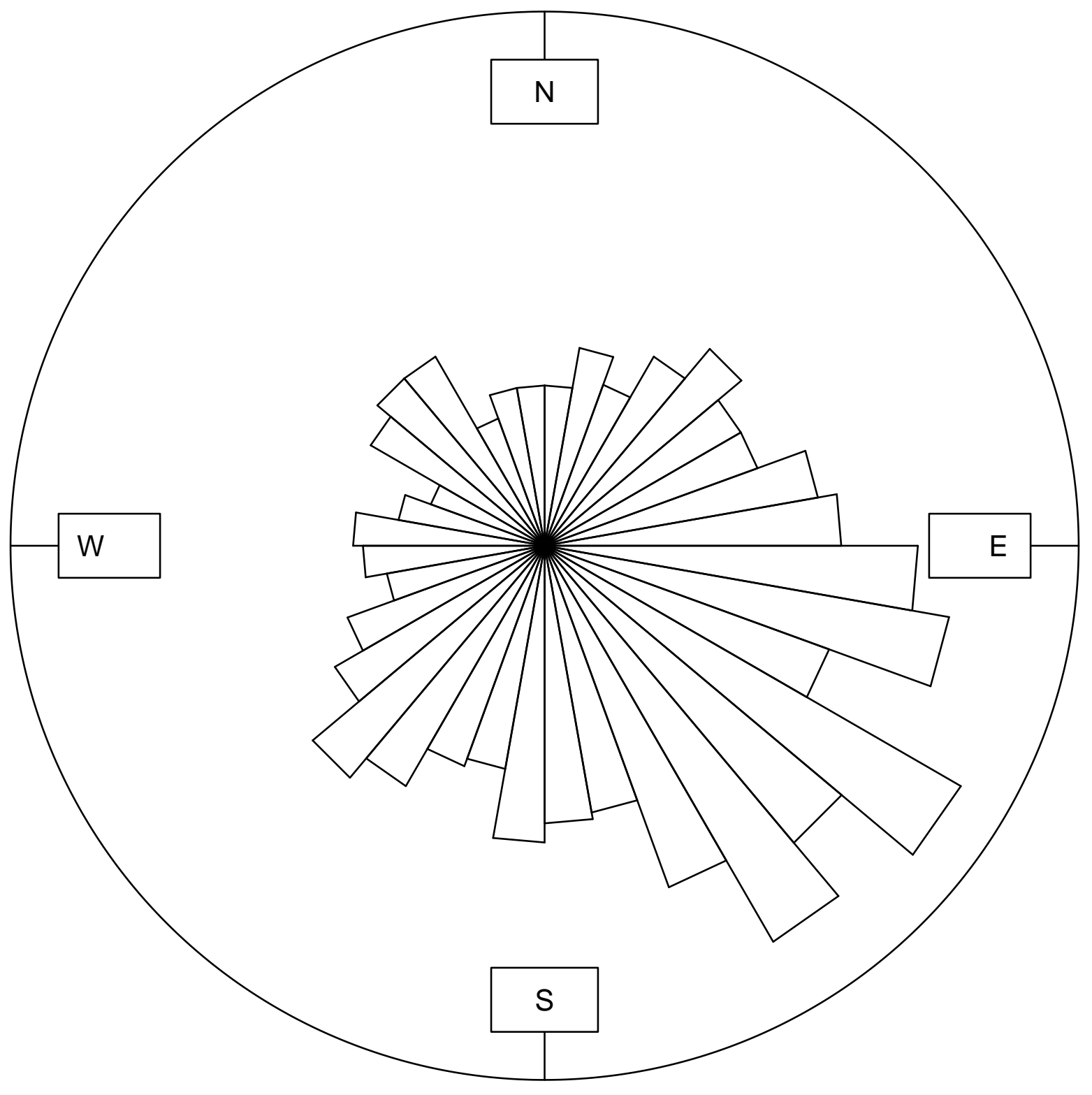


bootstrap 768

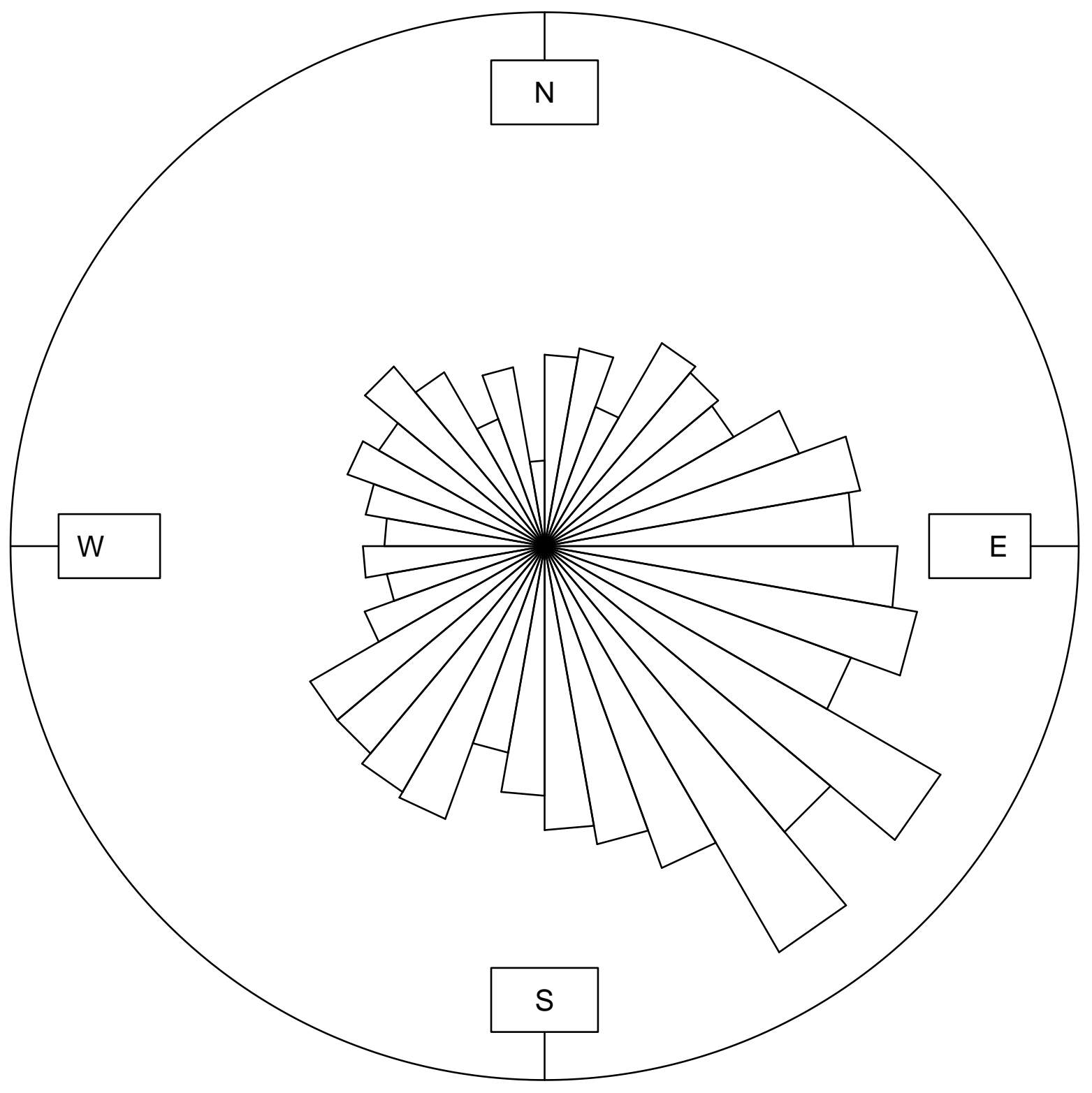


bootstrap 769

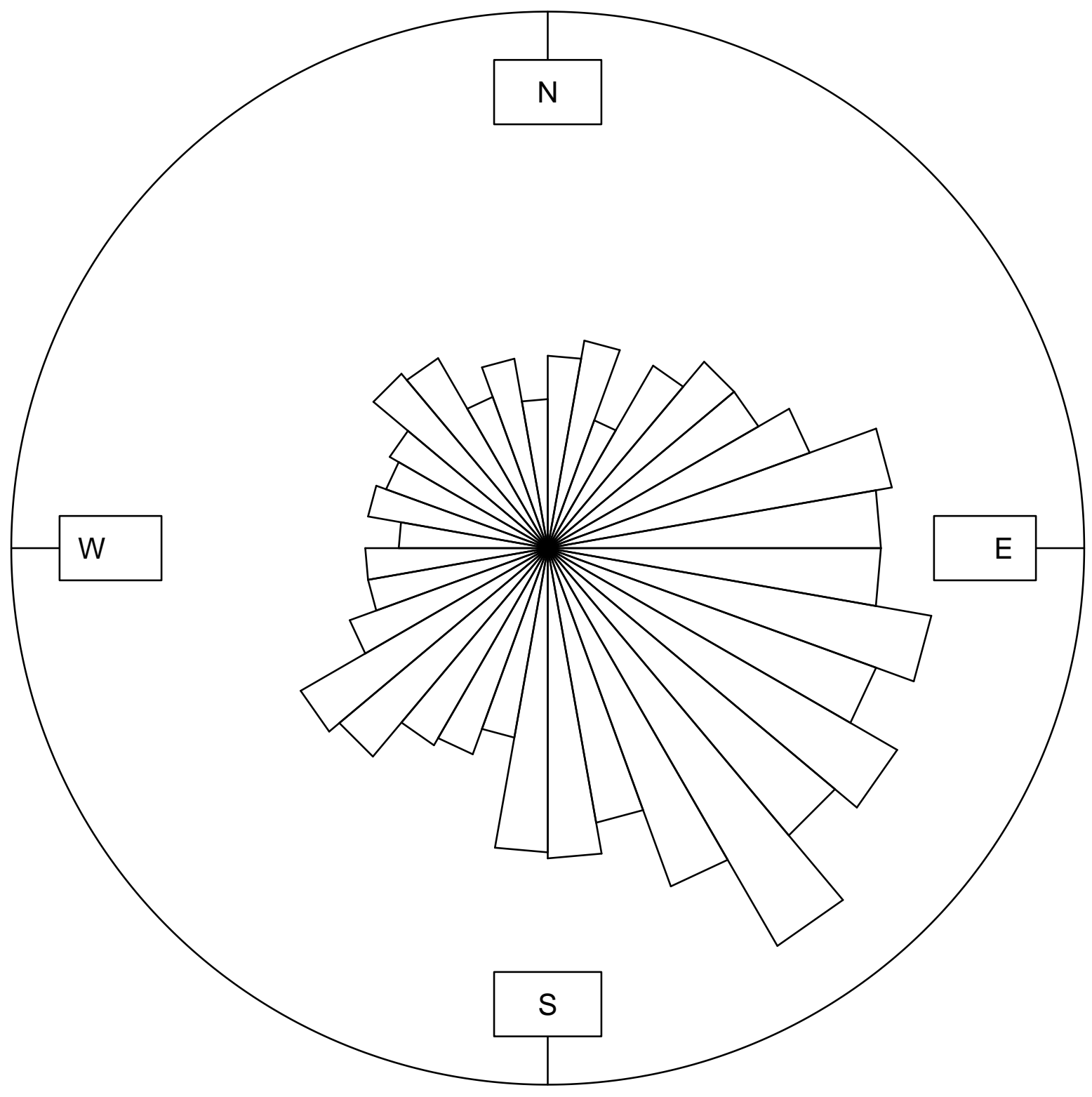


bootstrap 770

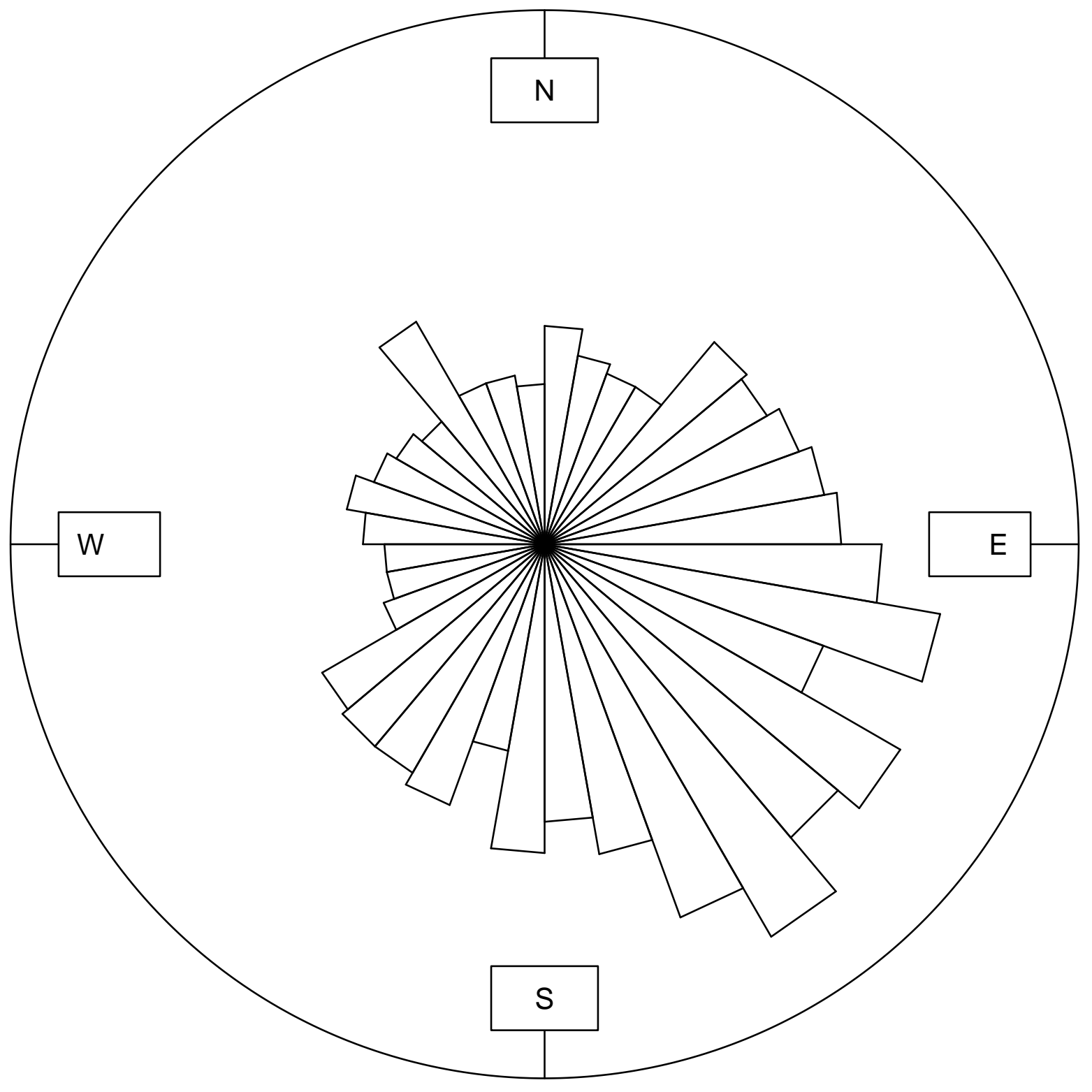


bootstrap 771

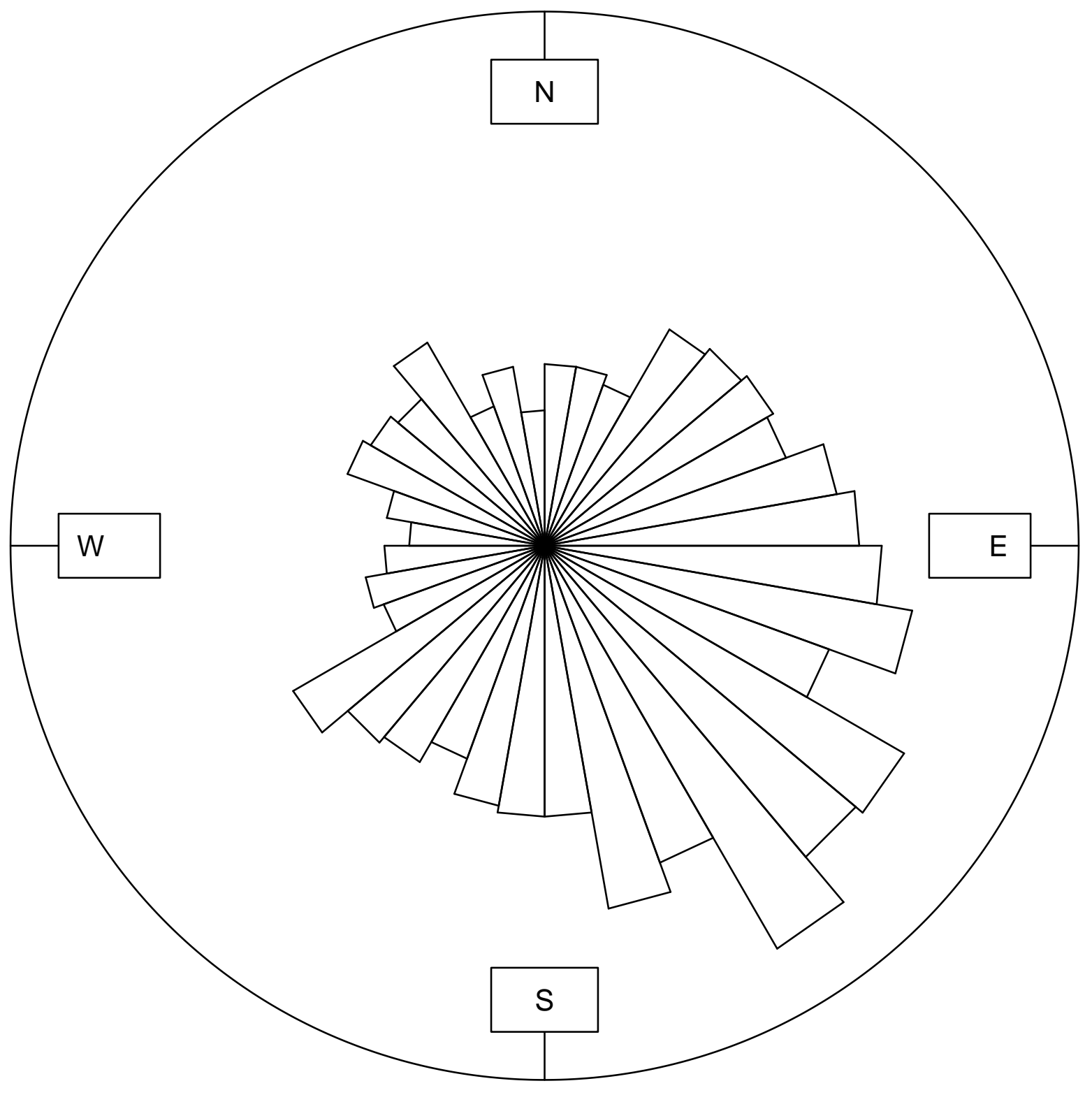


bootstrap 772

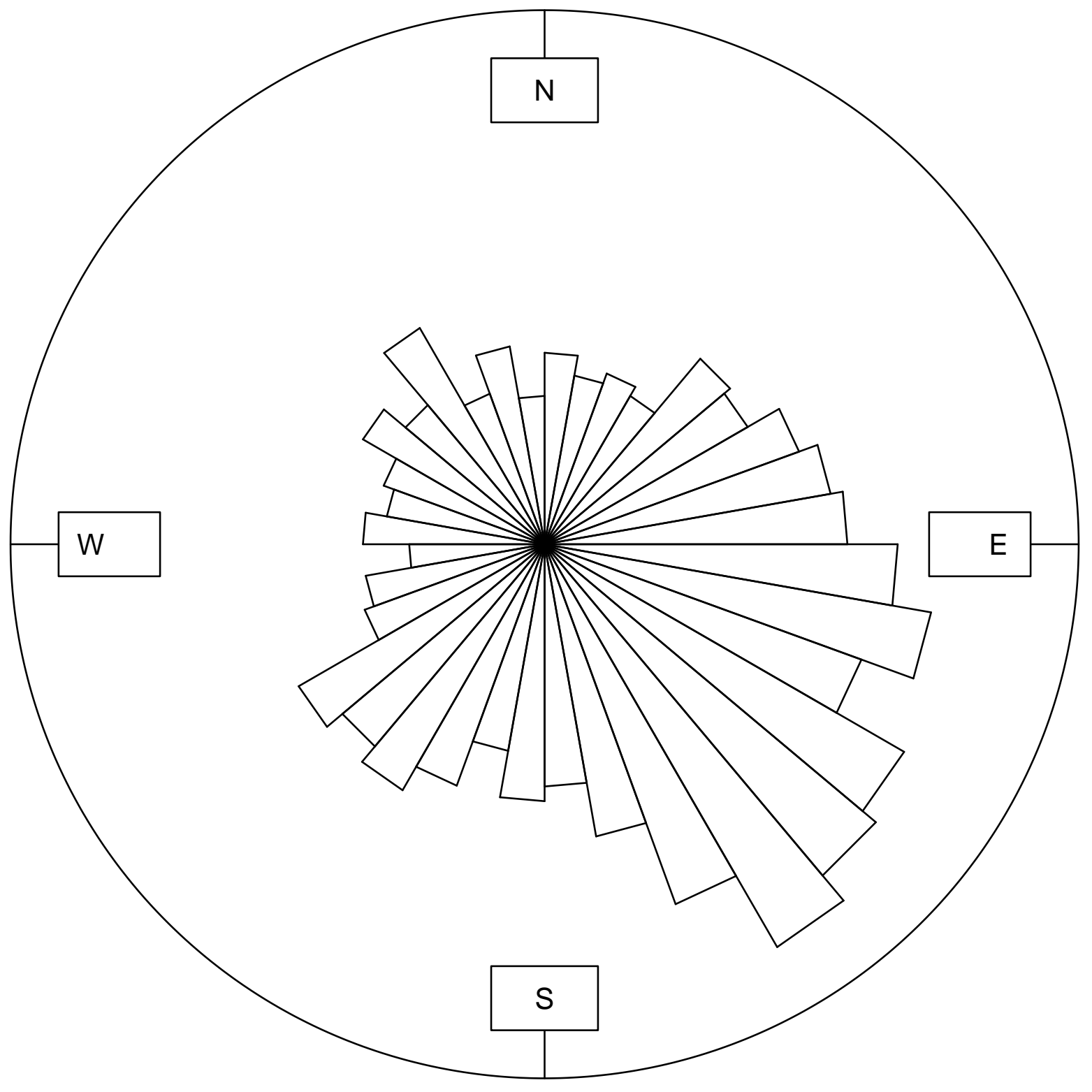


bootstrap 773

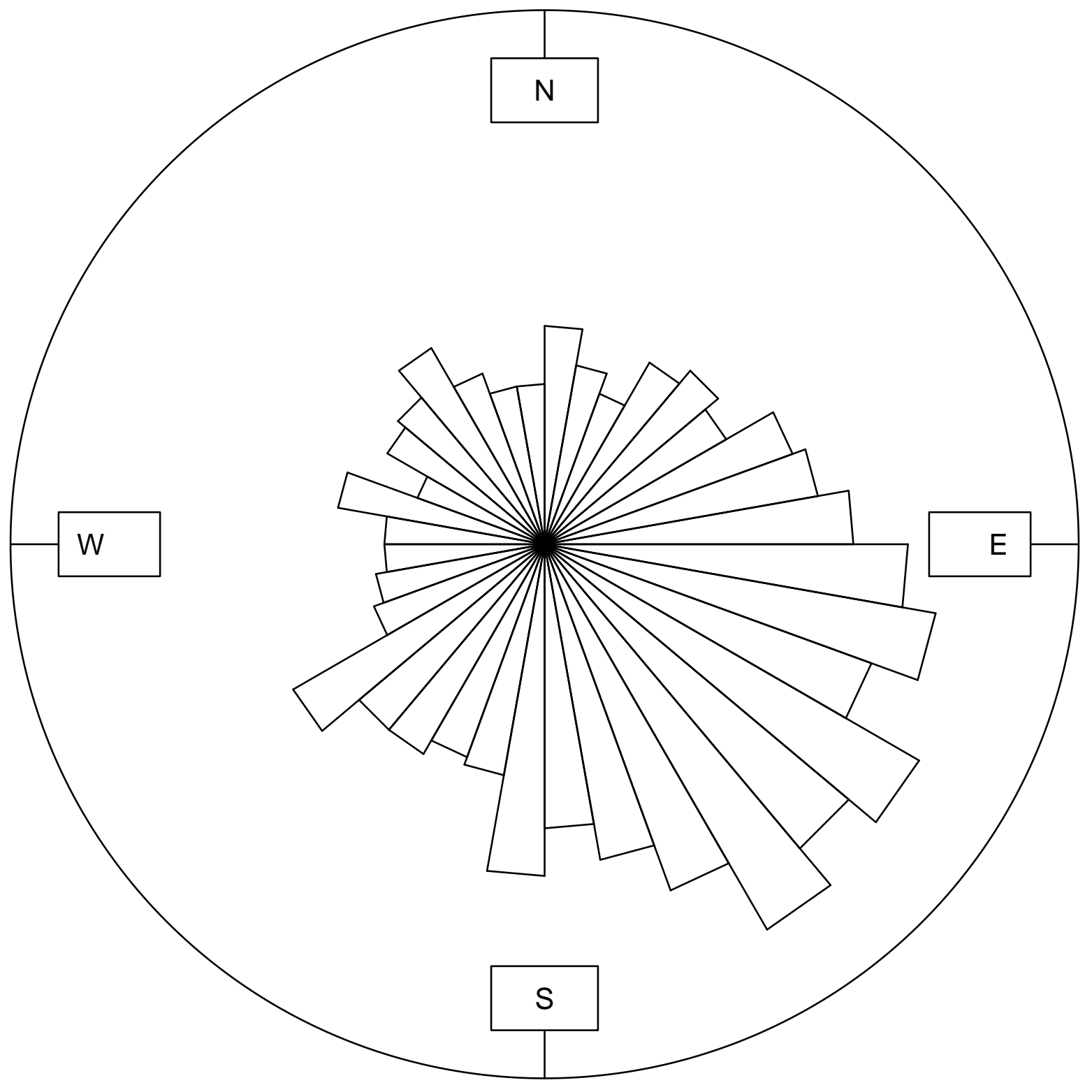


bootstrap 774

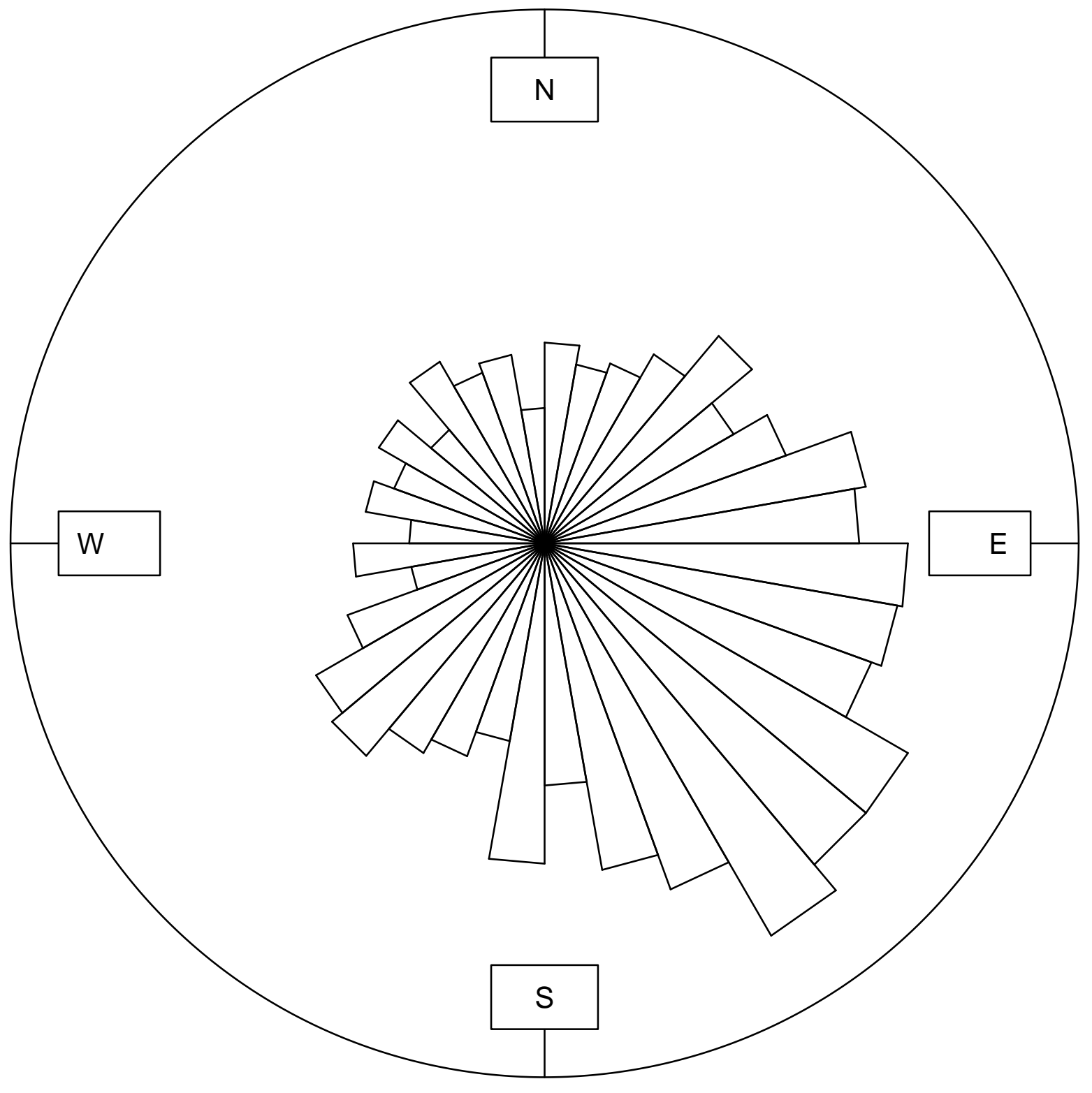


bootstrap 775

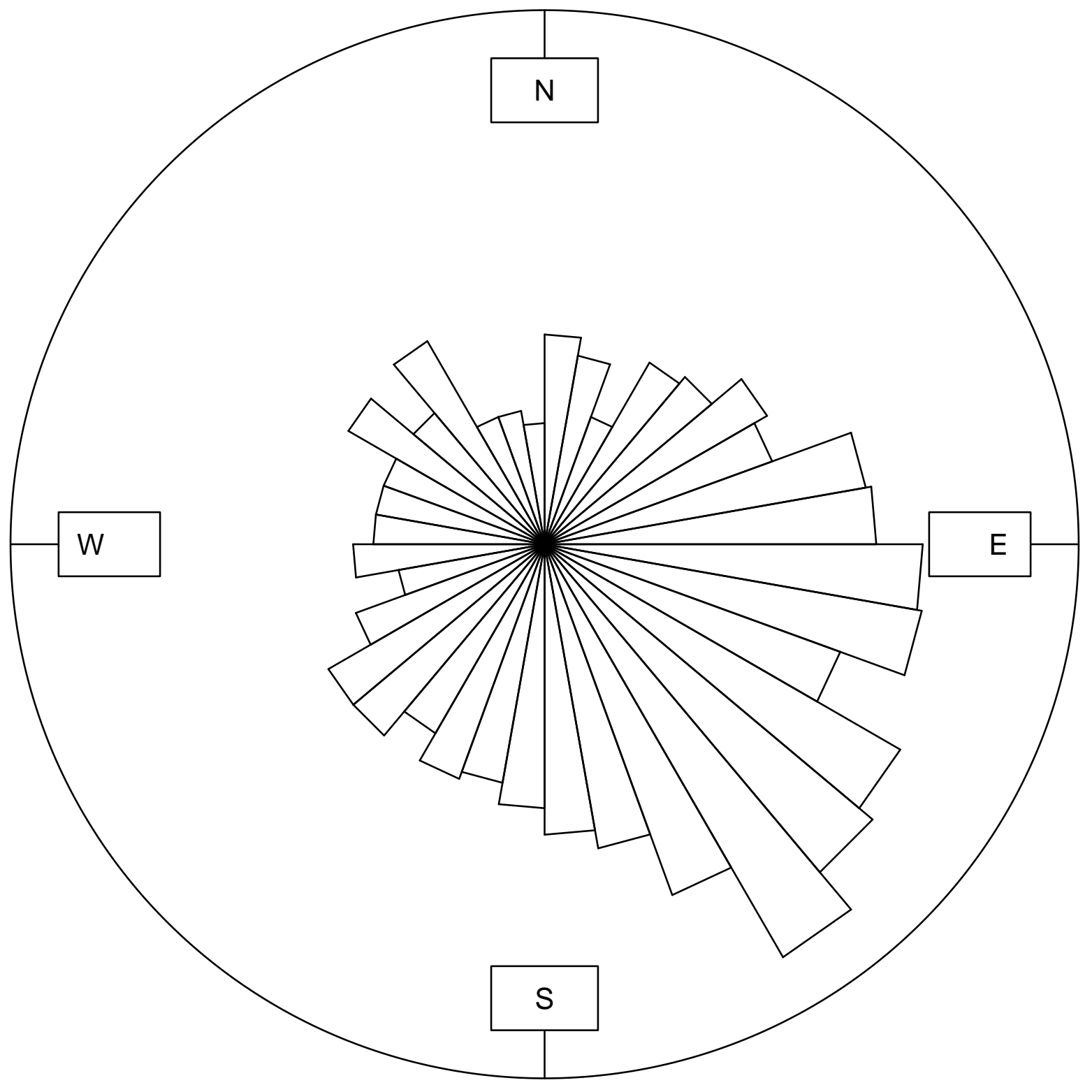


bootstrap 776

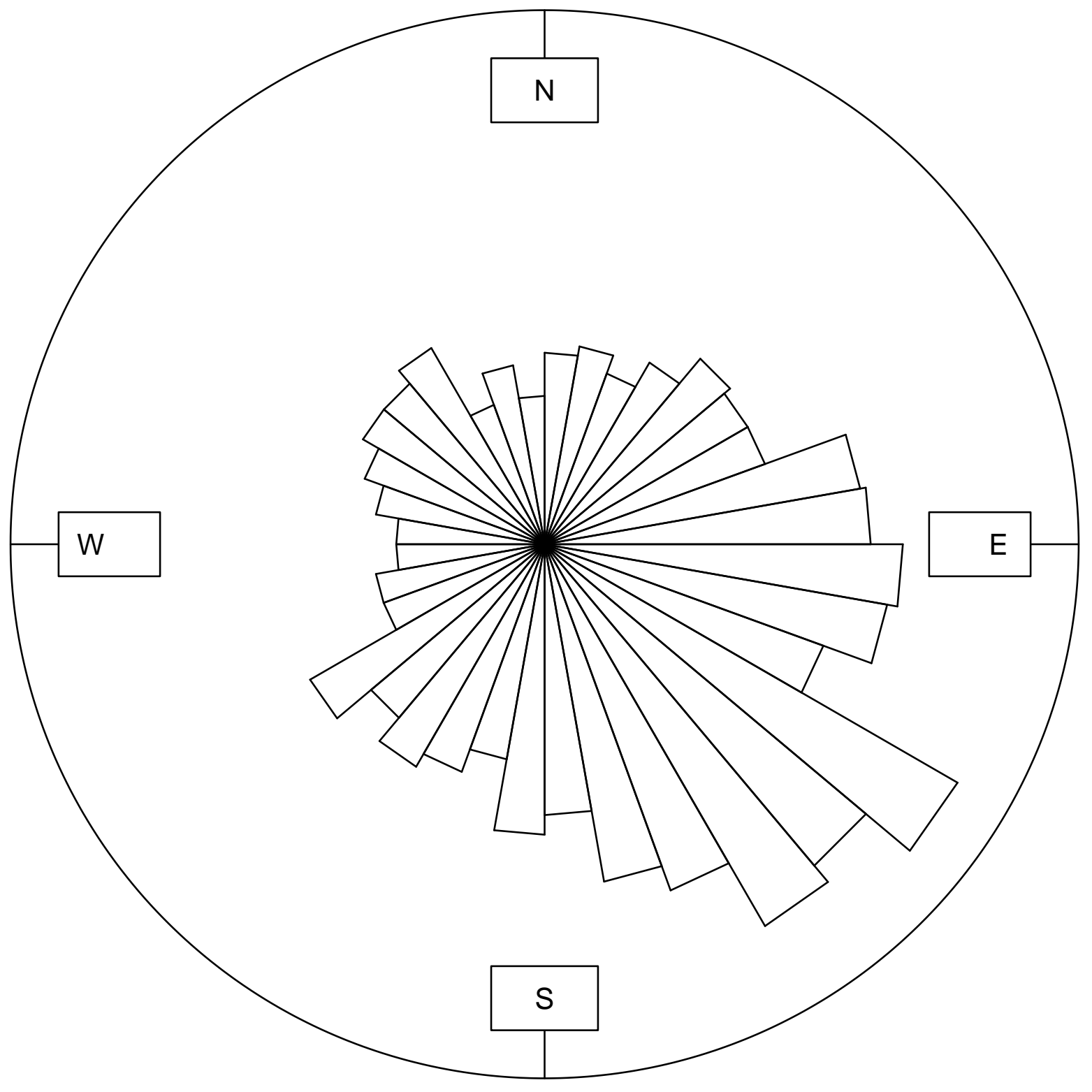


bootstrap 777

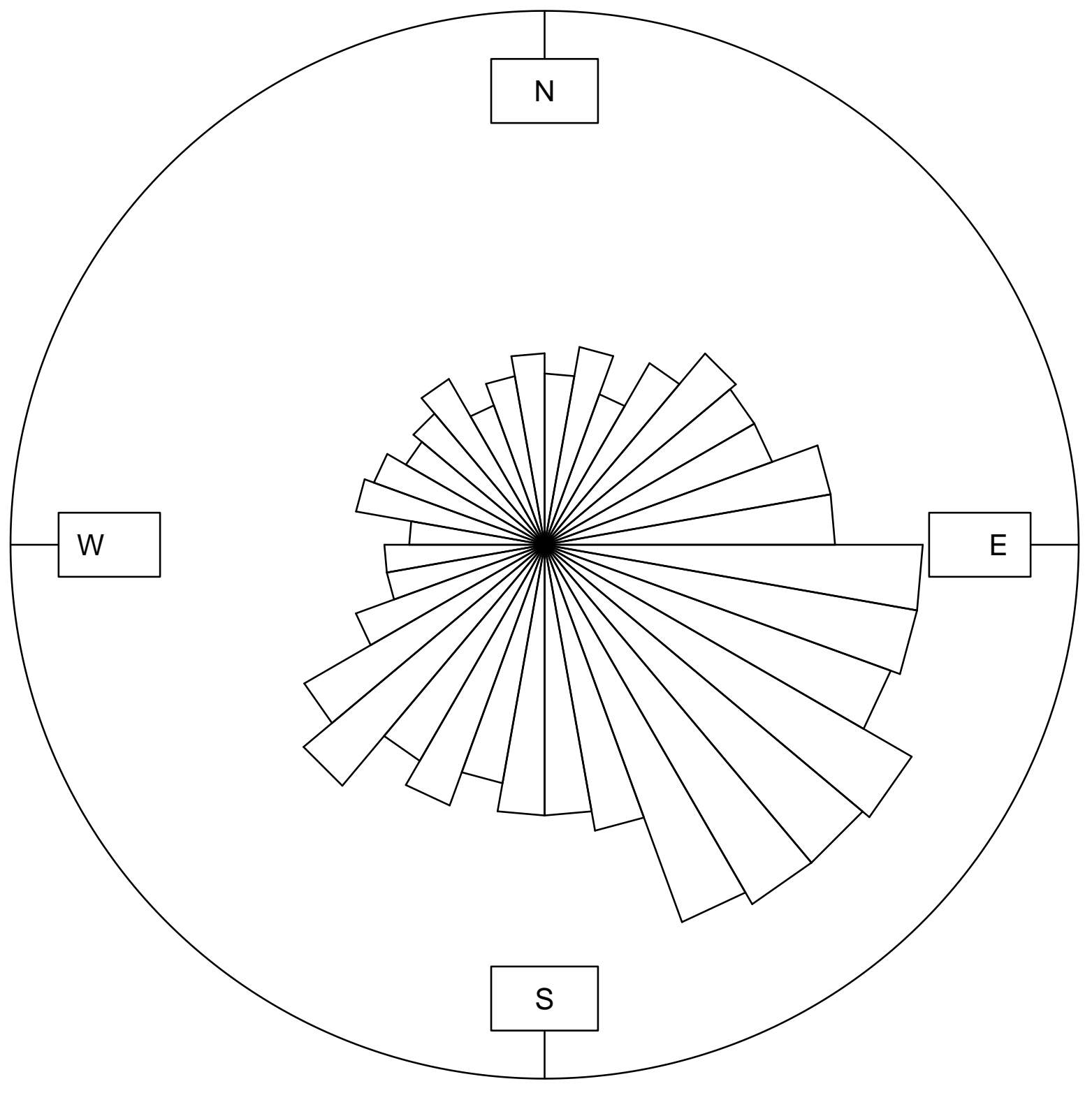


bootstrap 778

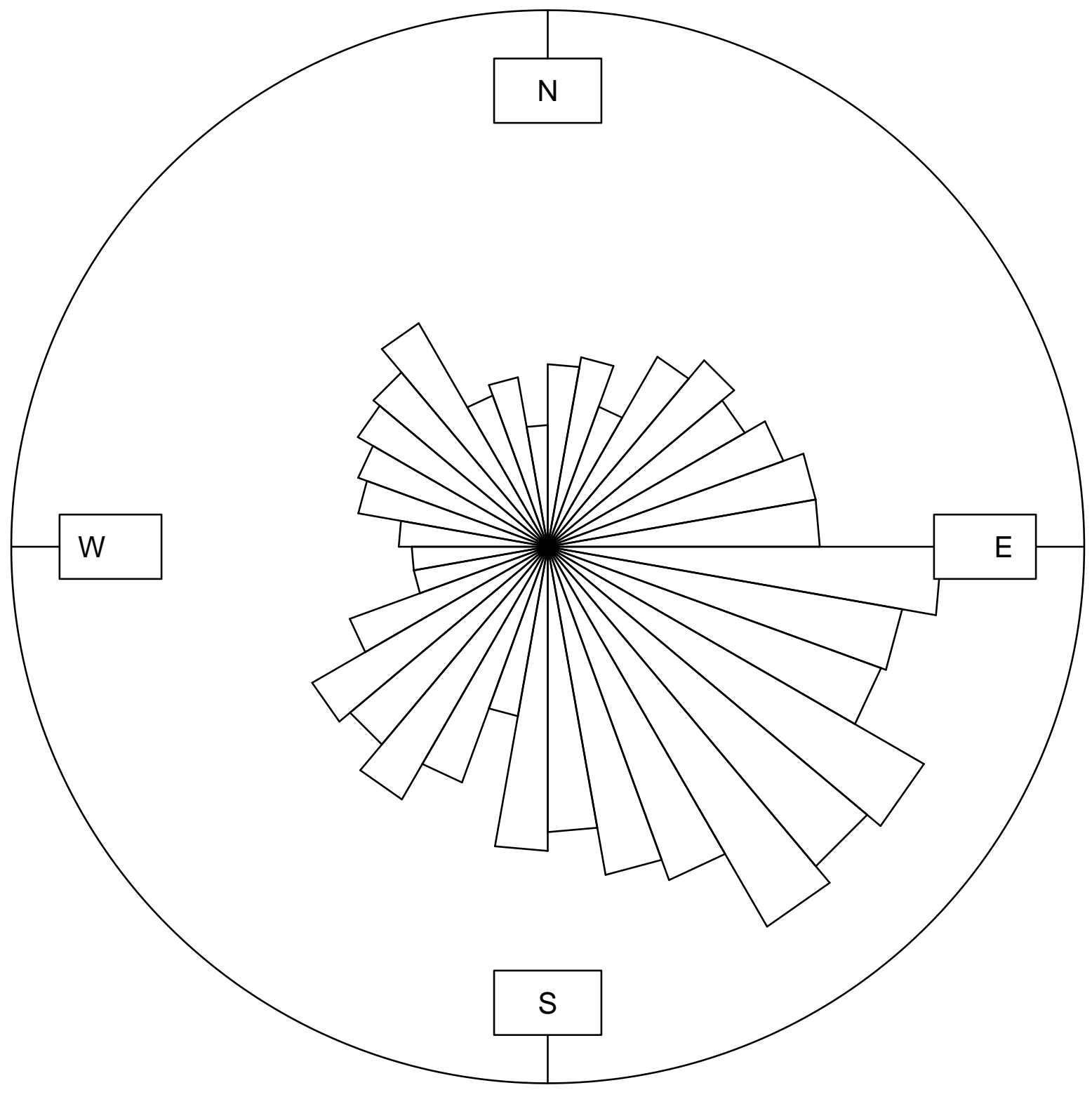


bootstrap 779

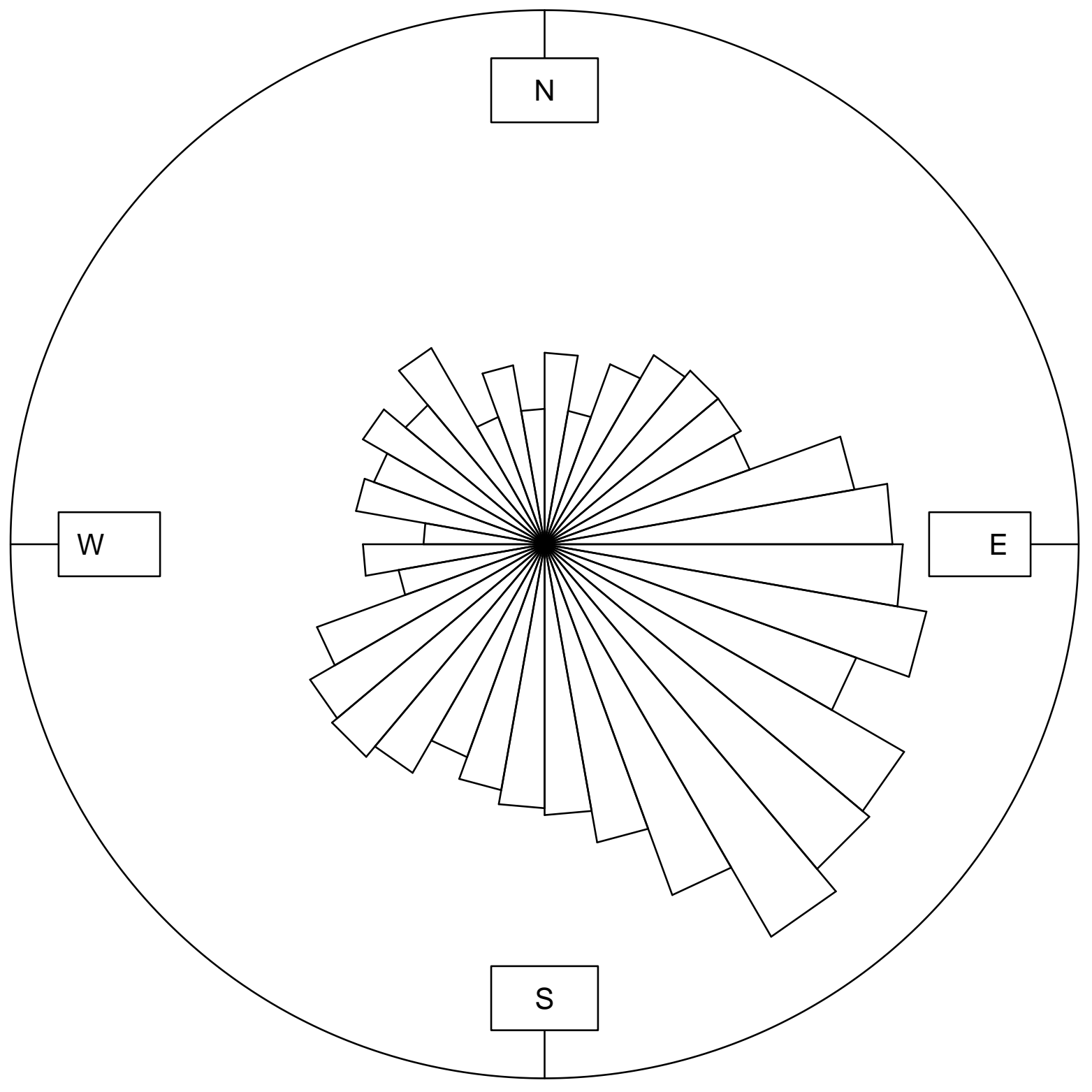


bootstrap 780

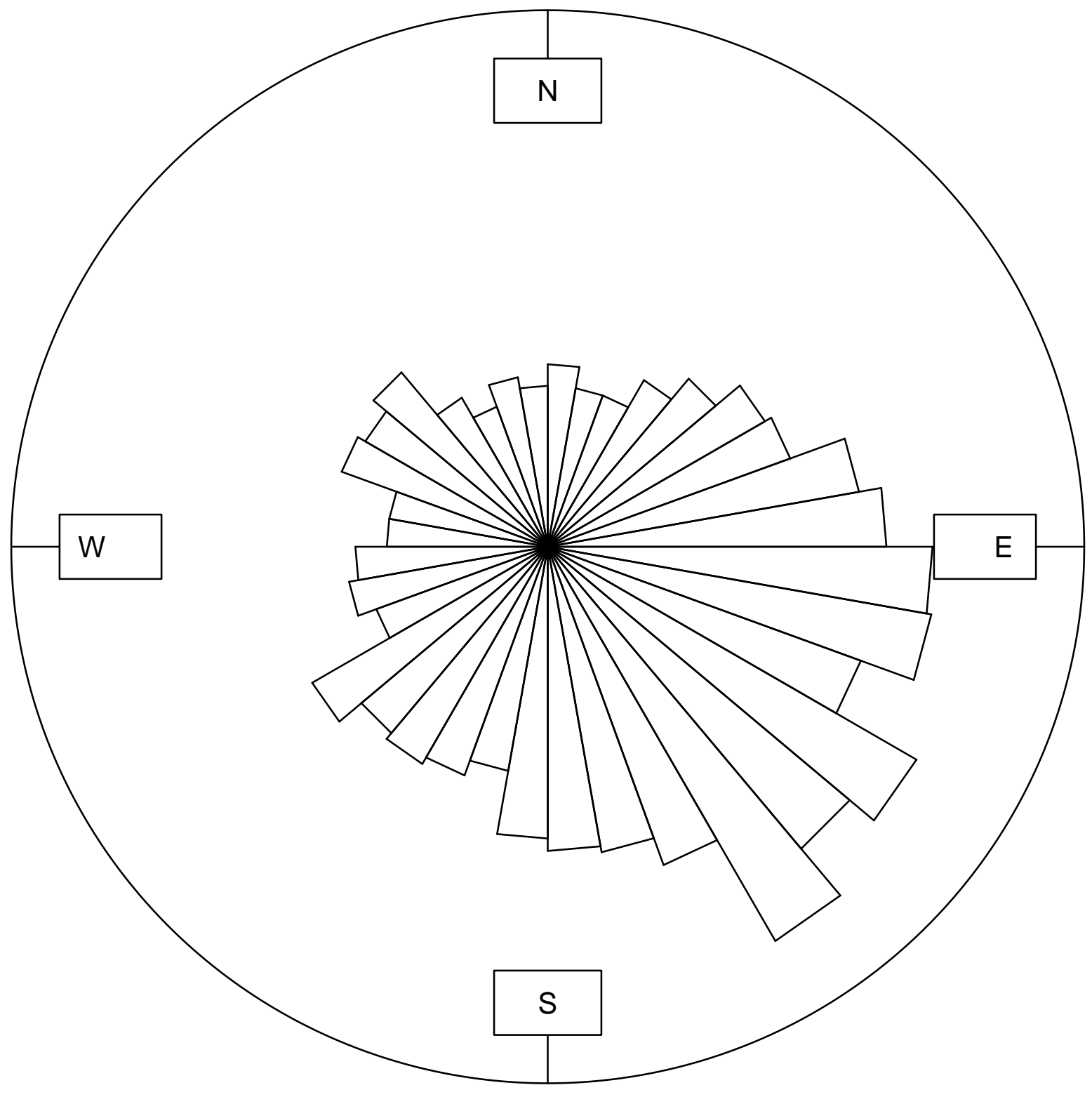




\section{bootstrap 781}

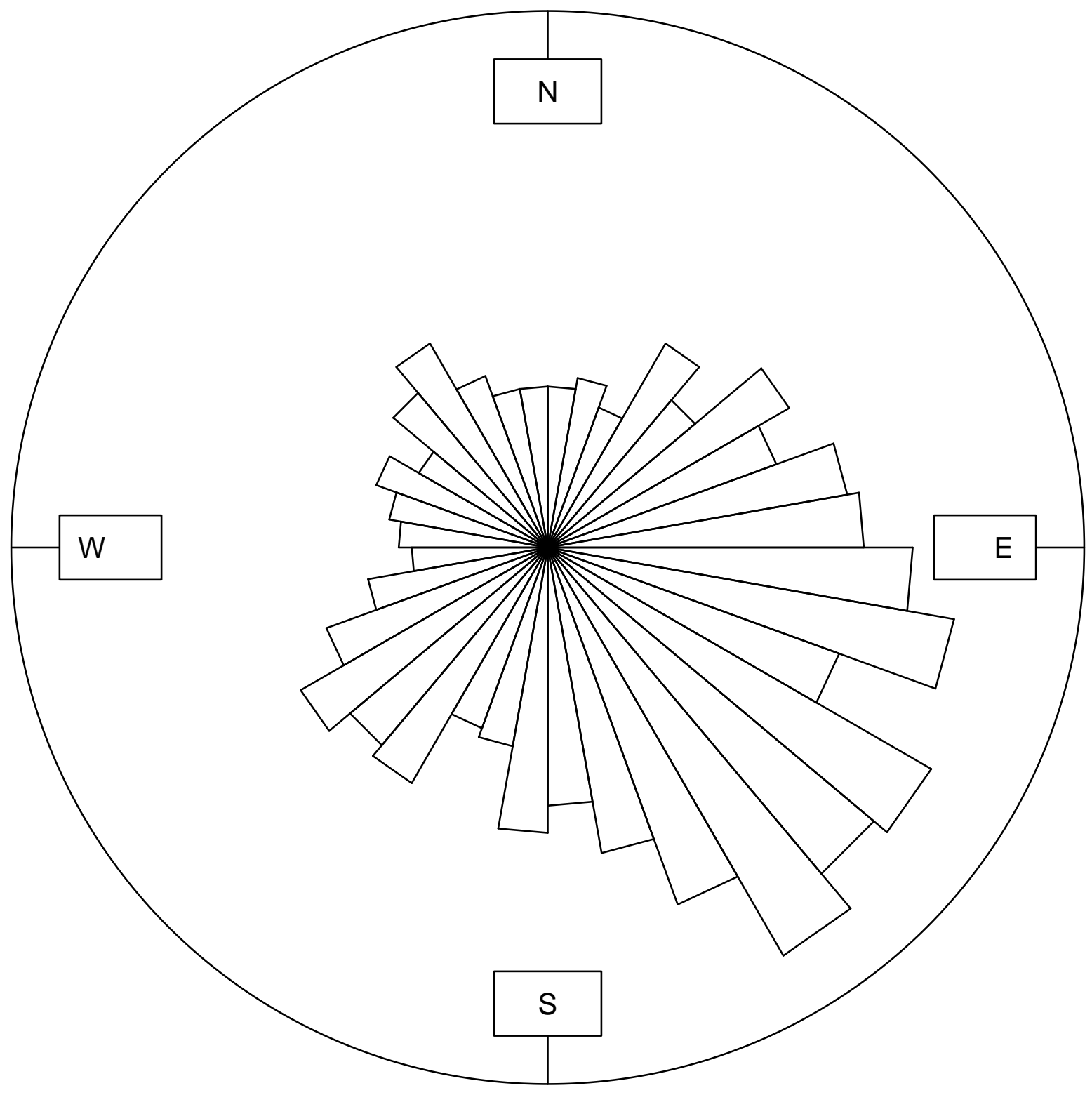


bootstrap 782

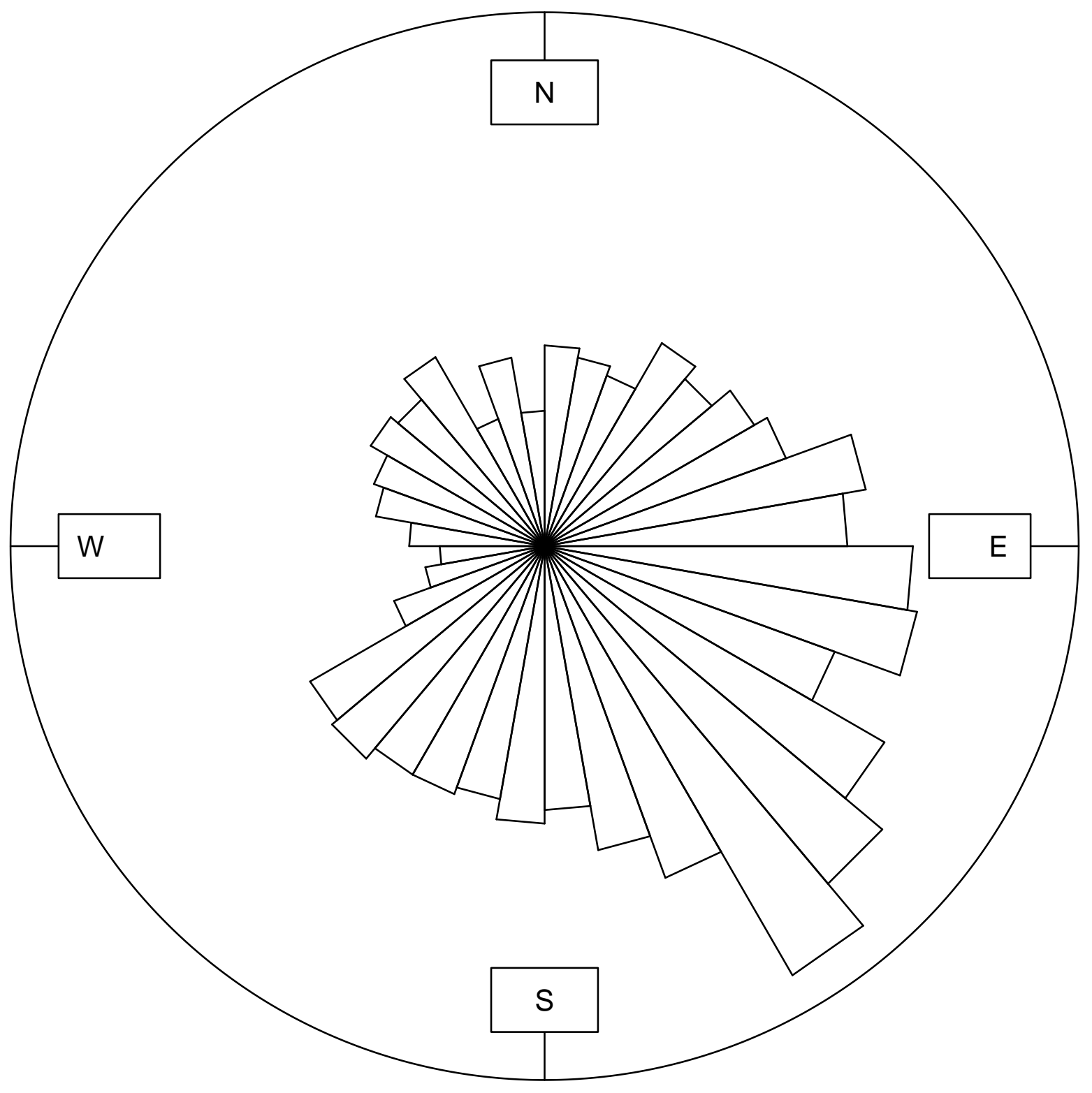




\section{bootstrap 783}

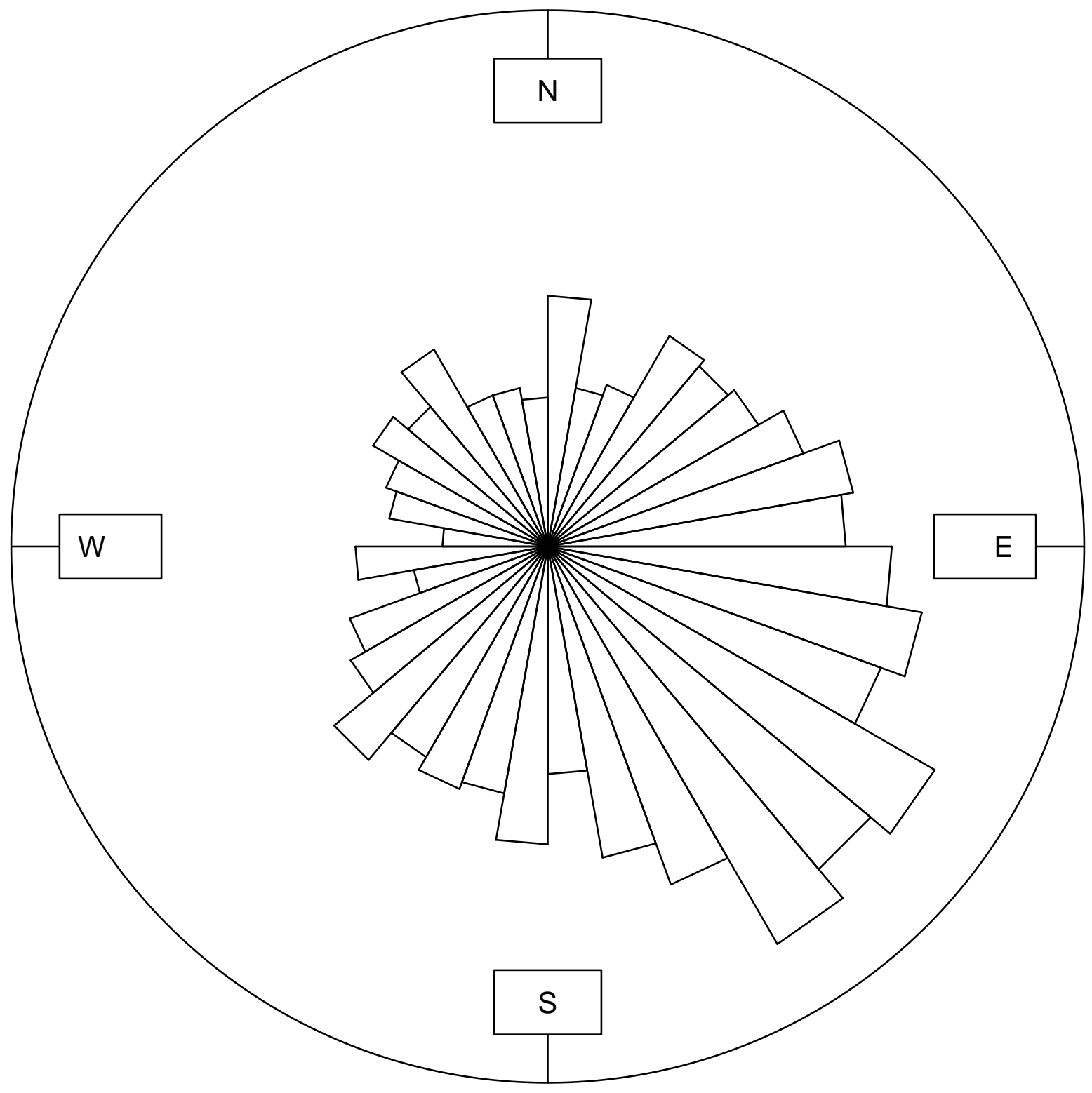


bootstrap 784

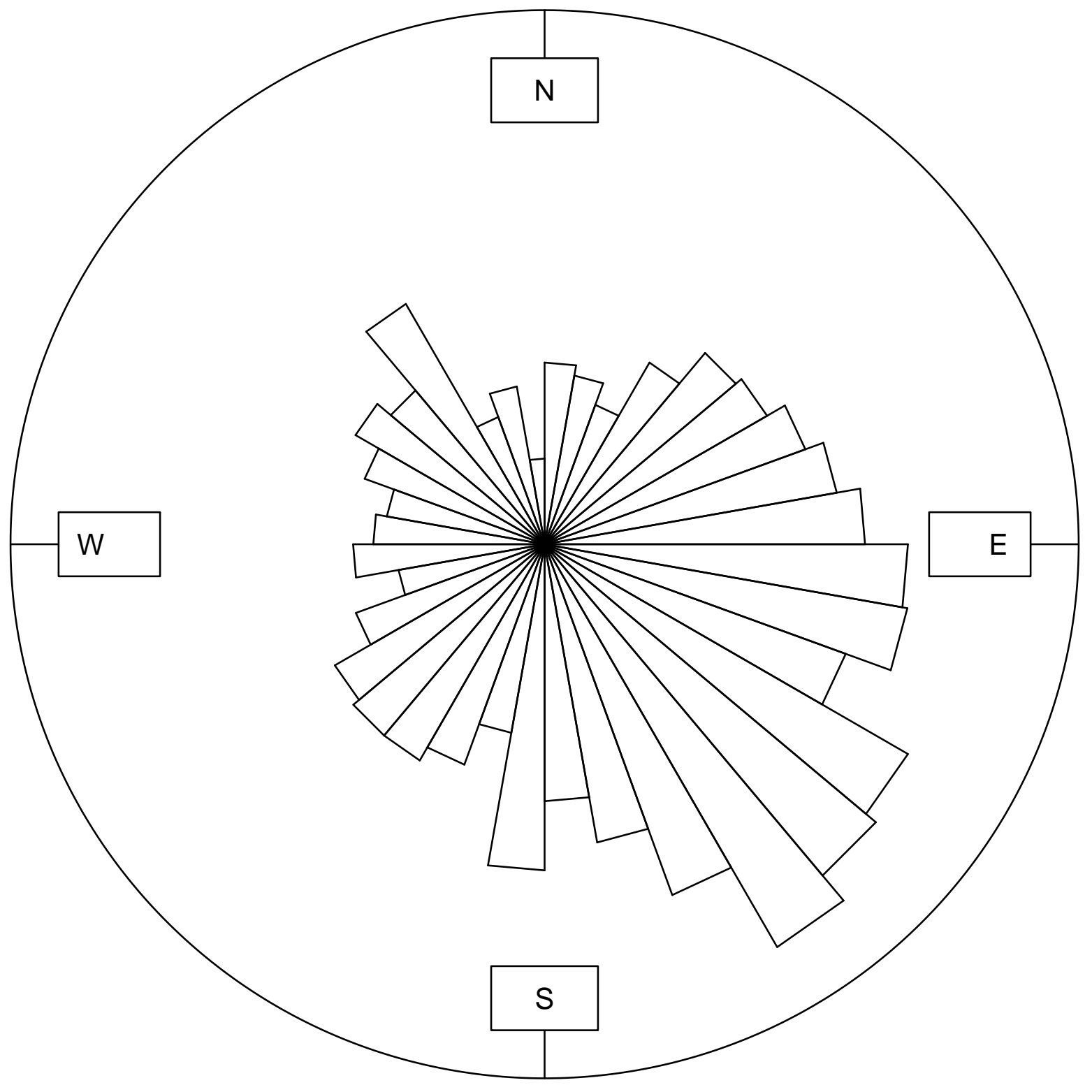


bootstrap 785

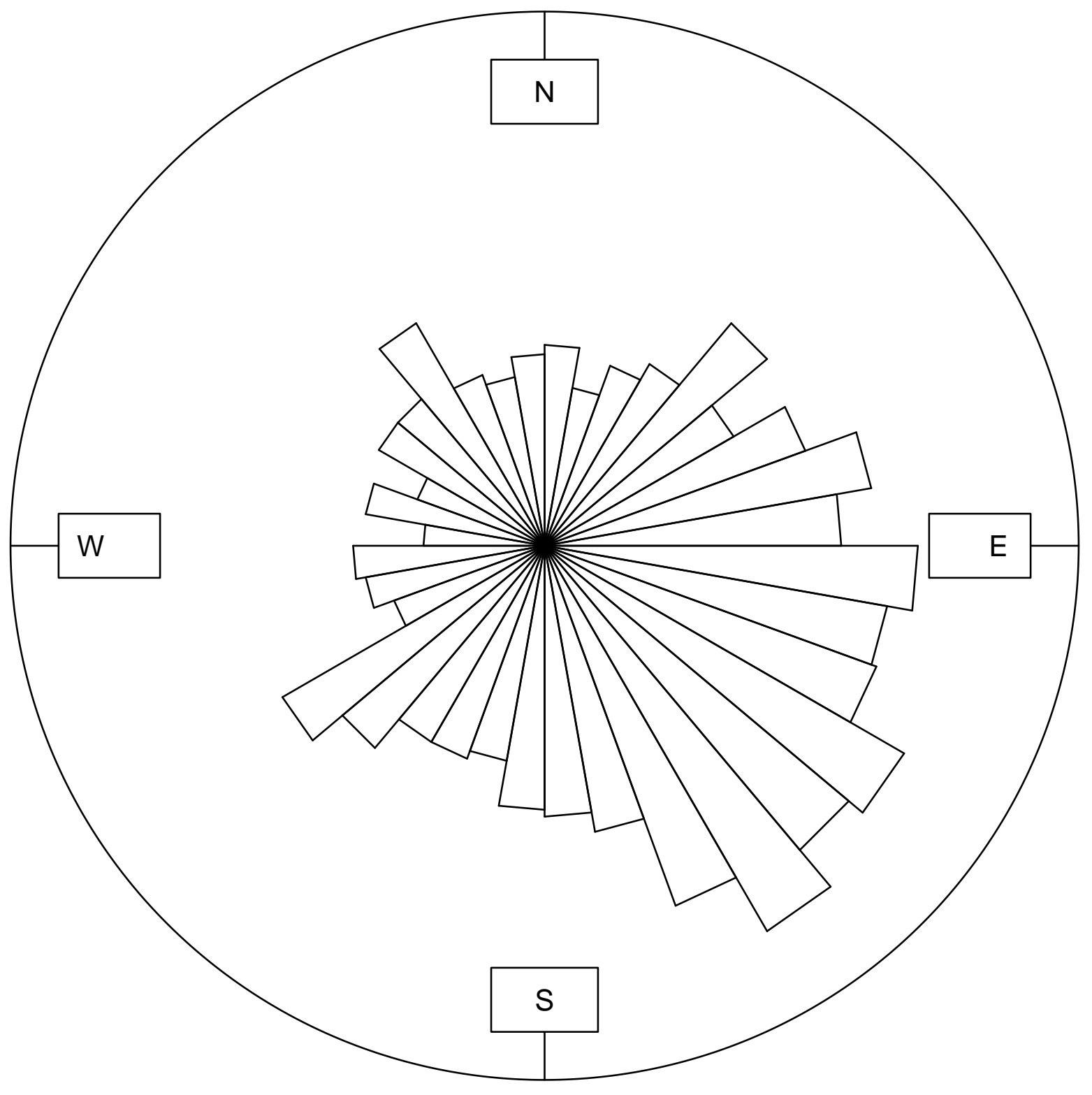


bootstrap 786

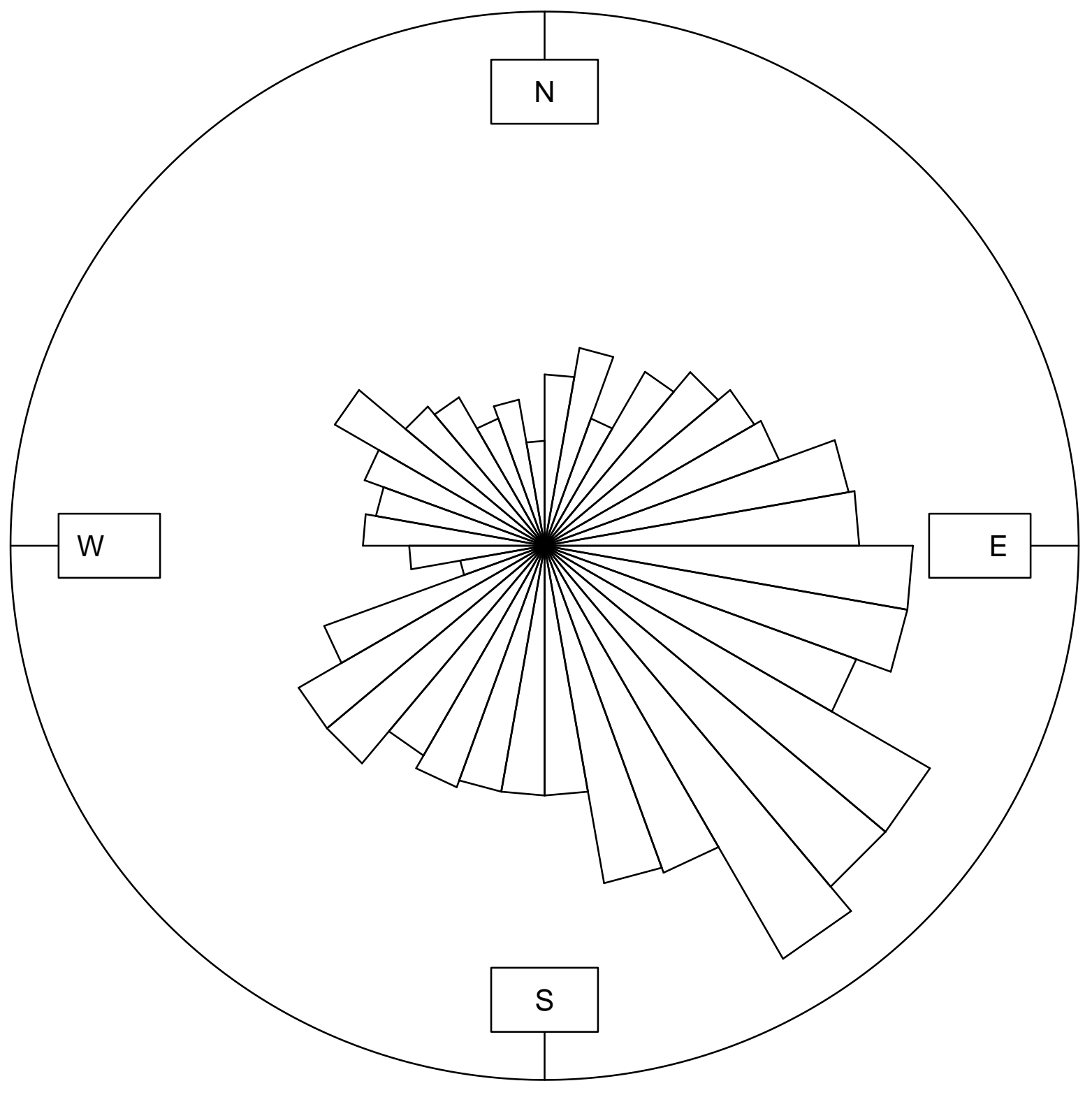


bootstrap 787

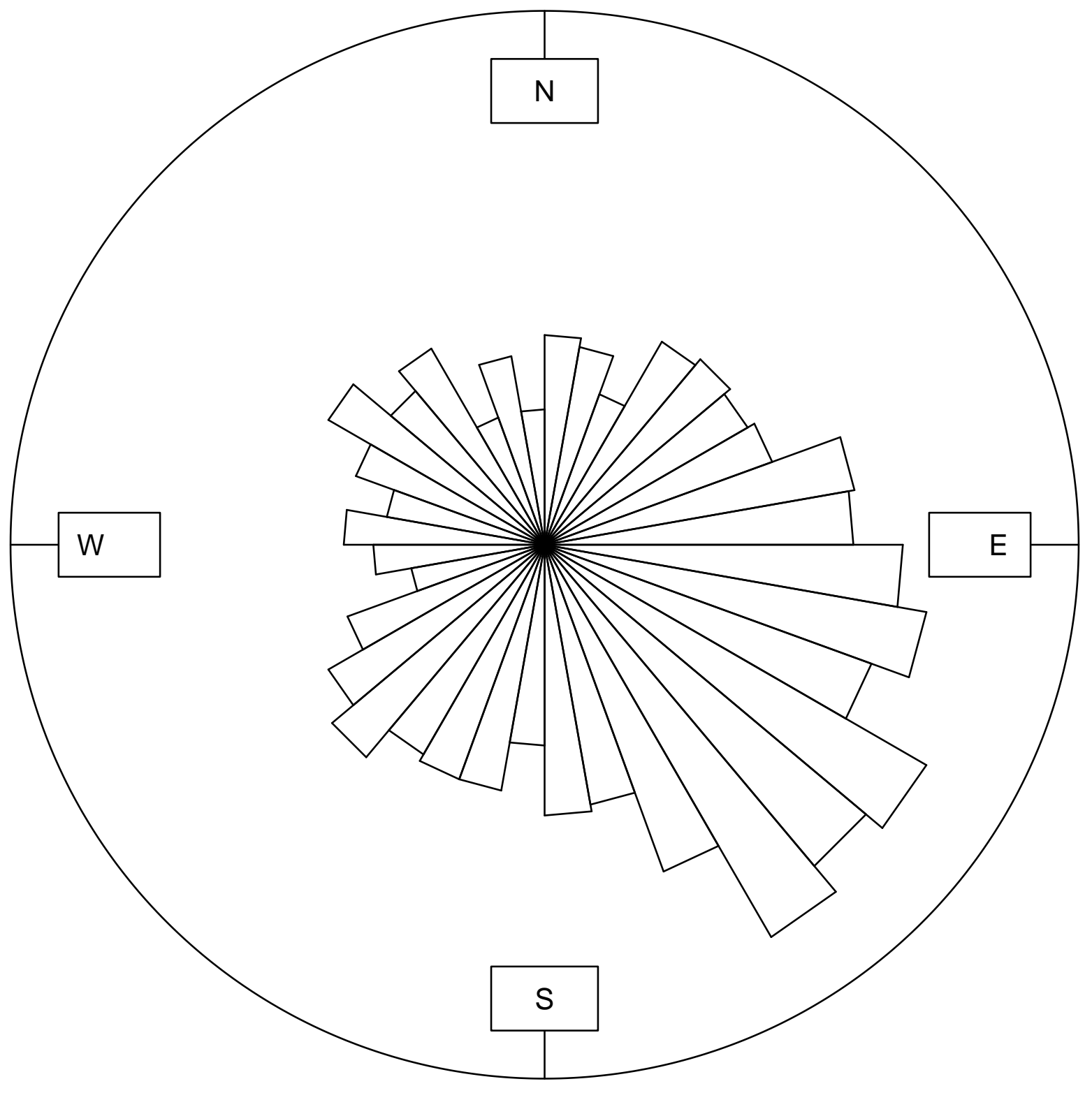


bootstrap 788

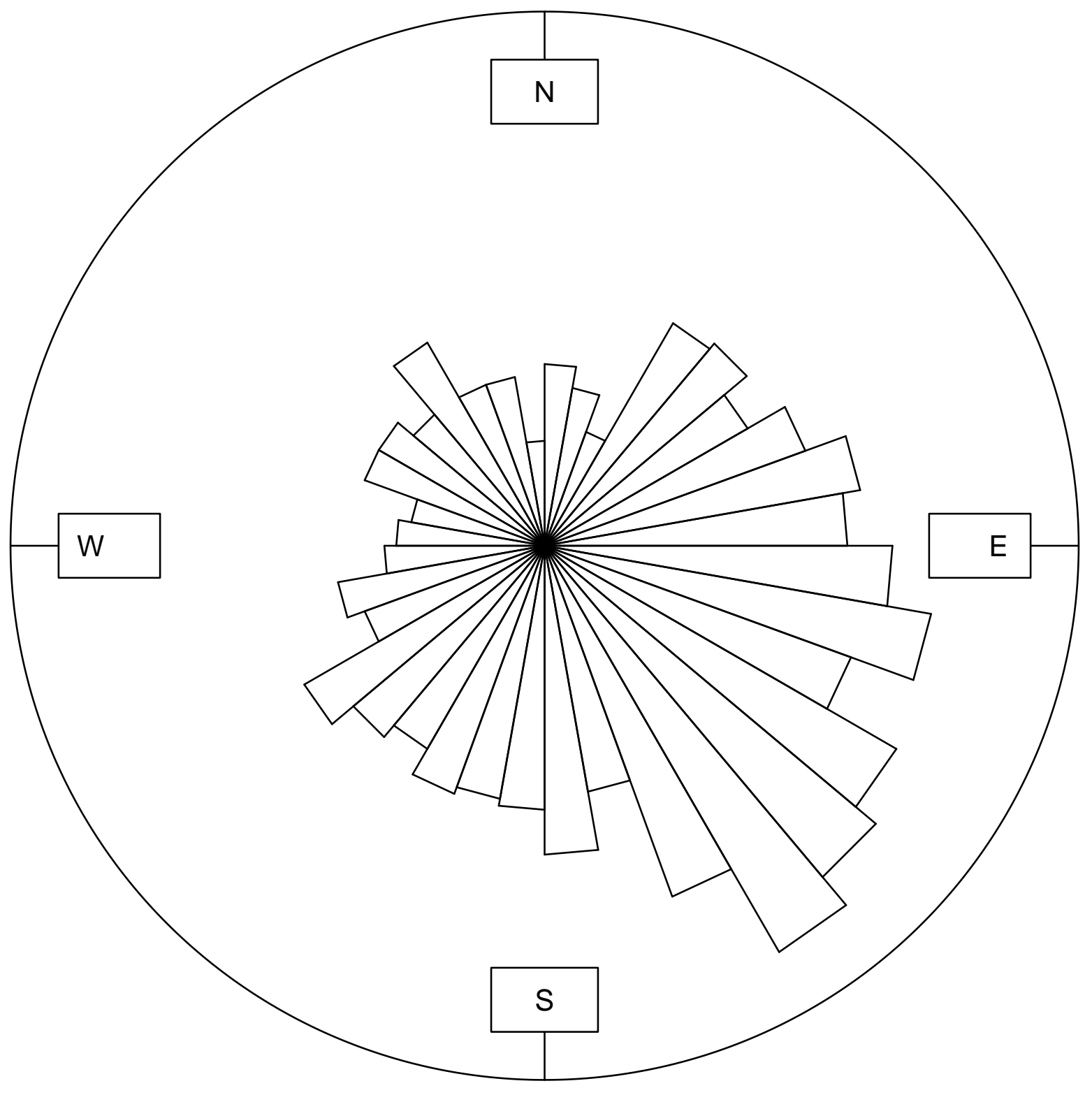


bootstrap 789

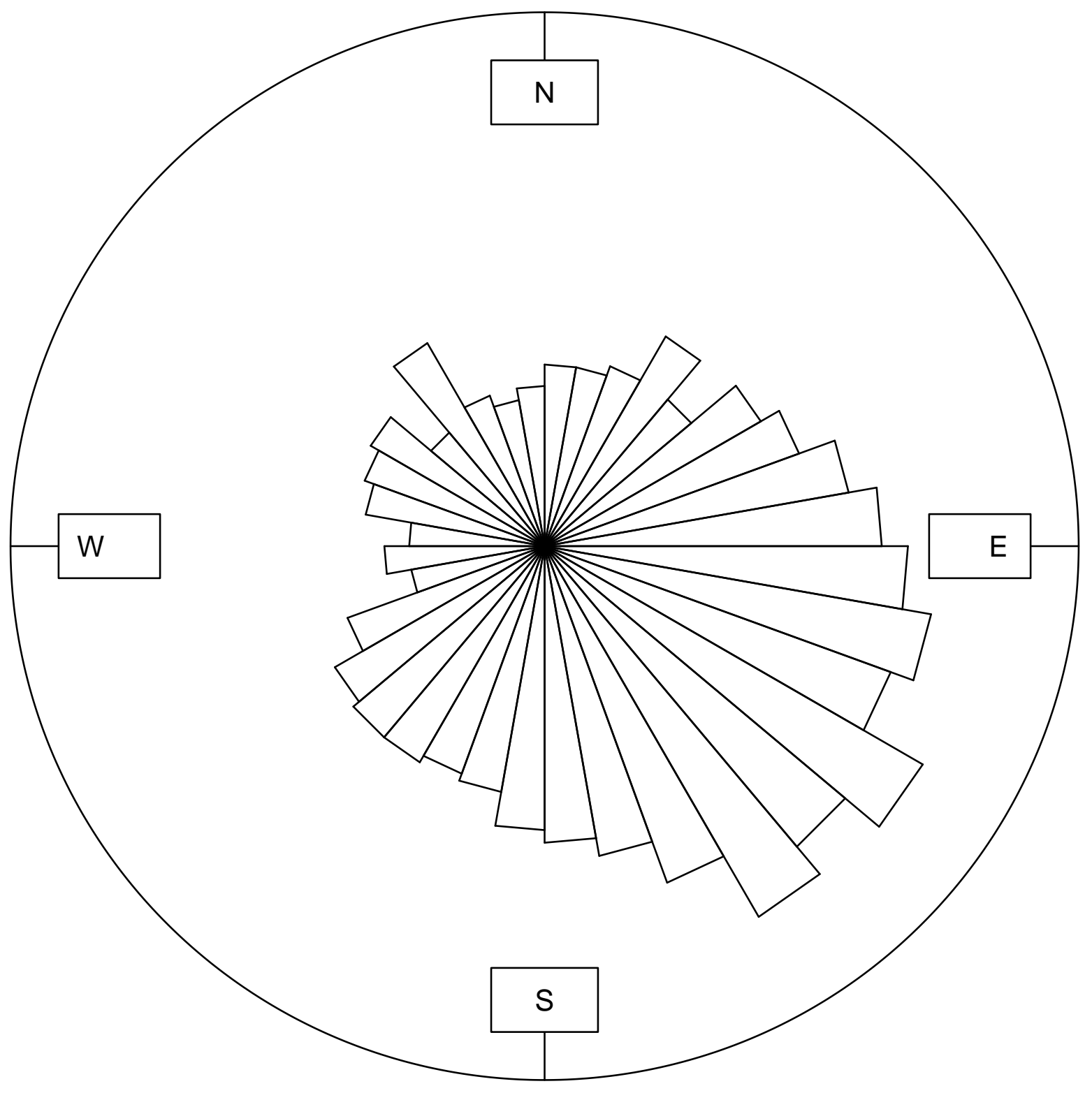


bootstrap 790

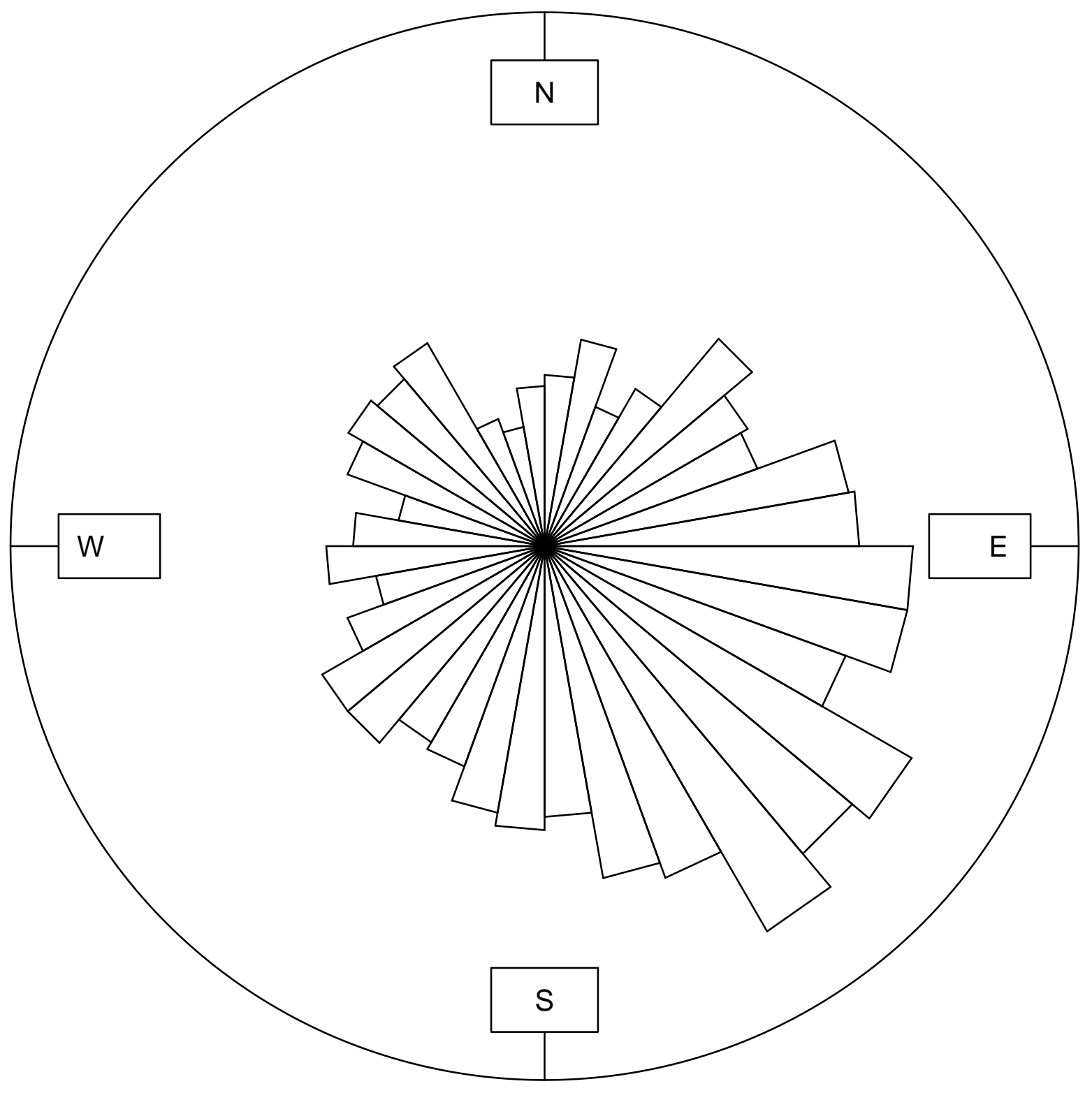




\section{bootstrap 791}

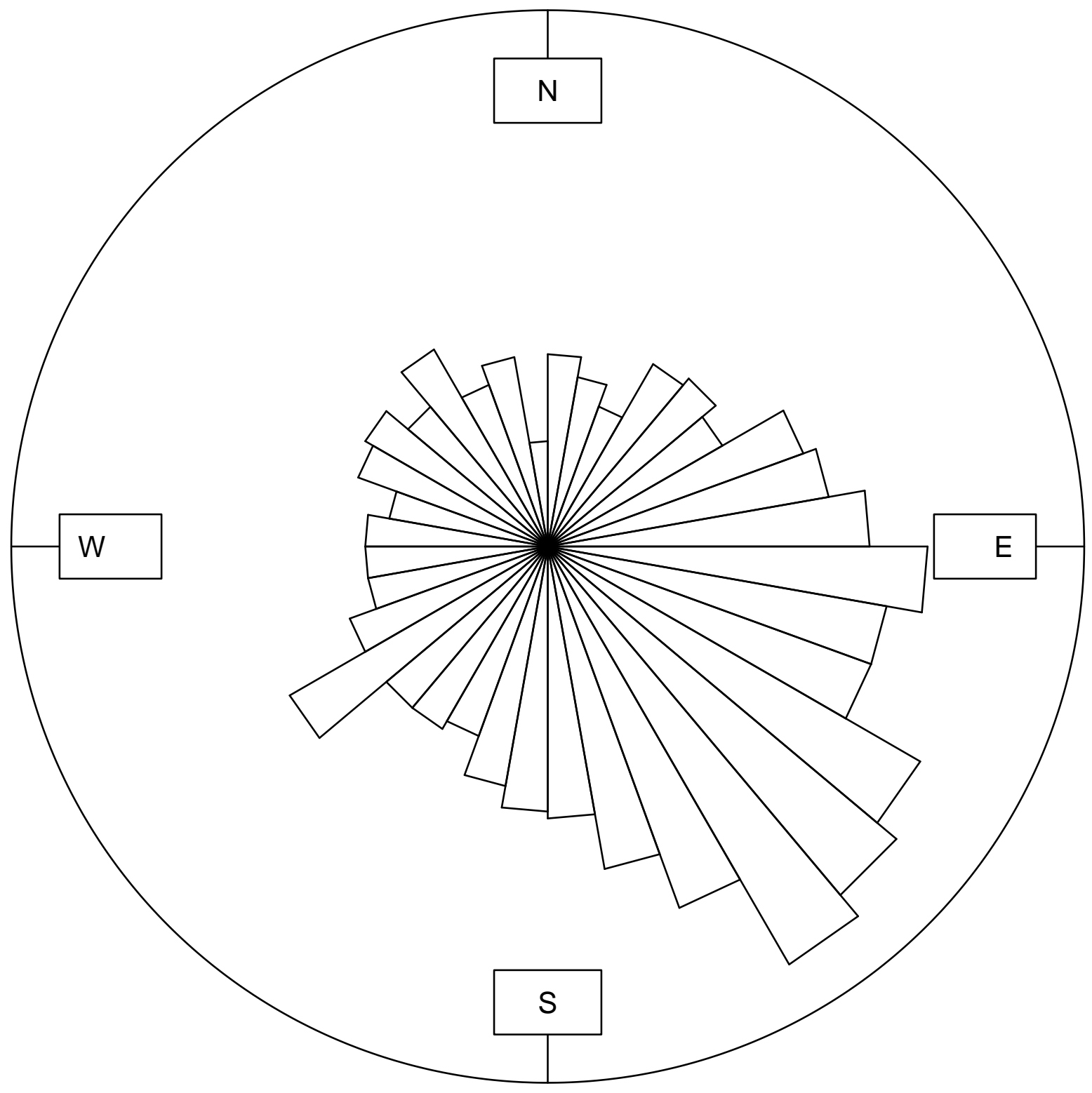


bootstrap 792

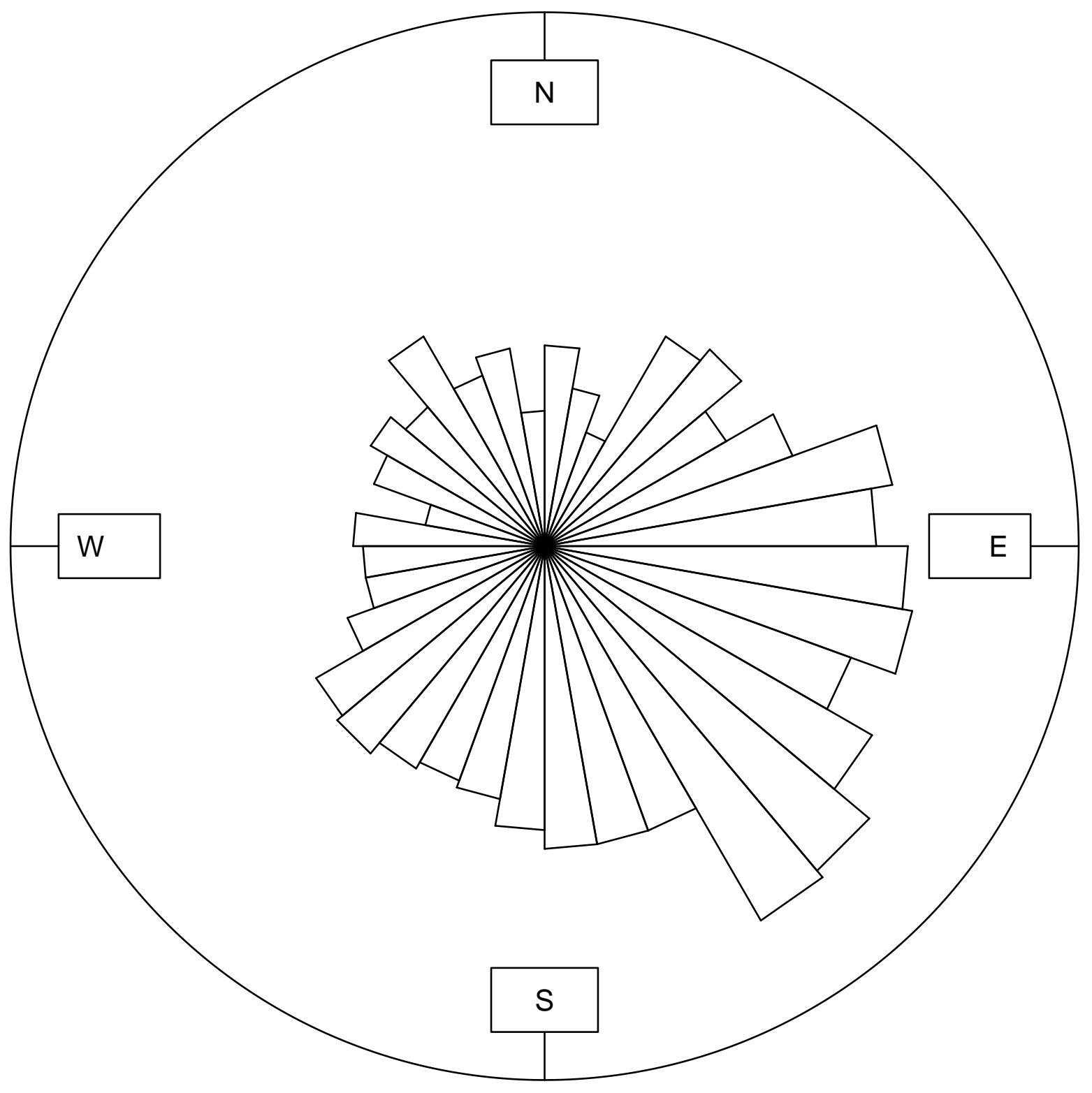




\section{bootstrap 793}

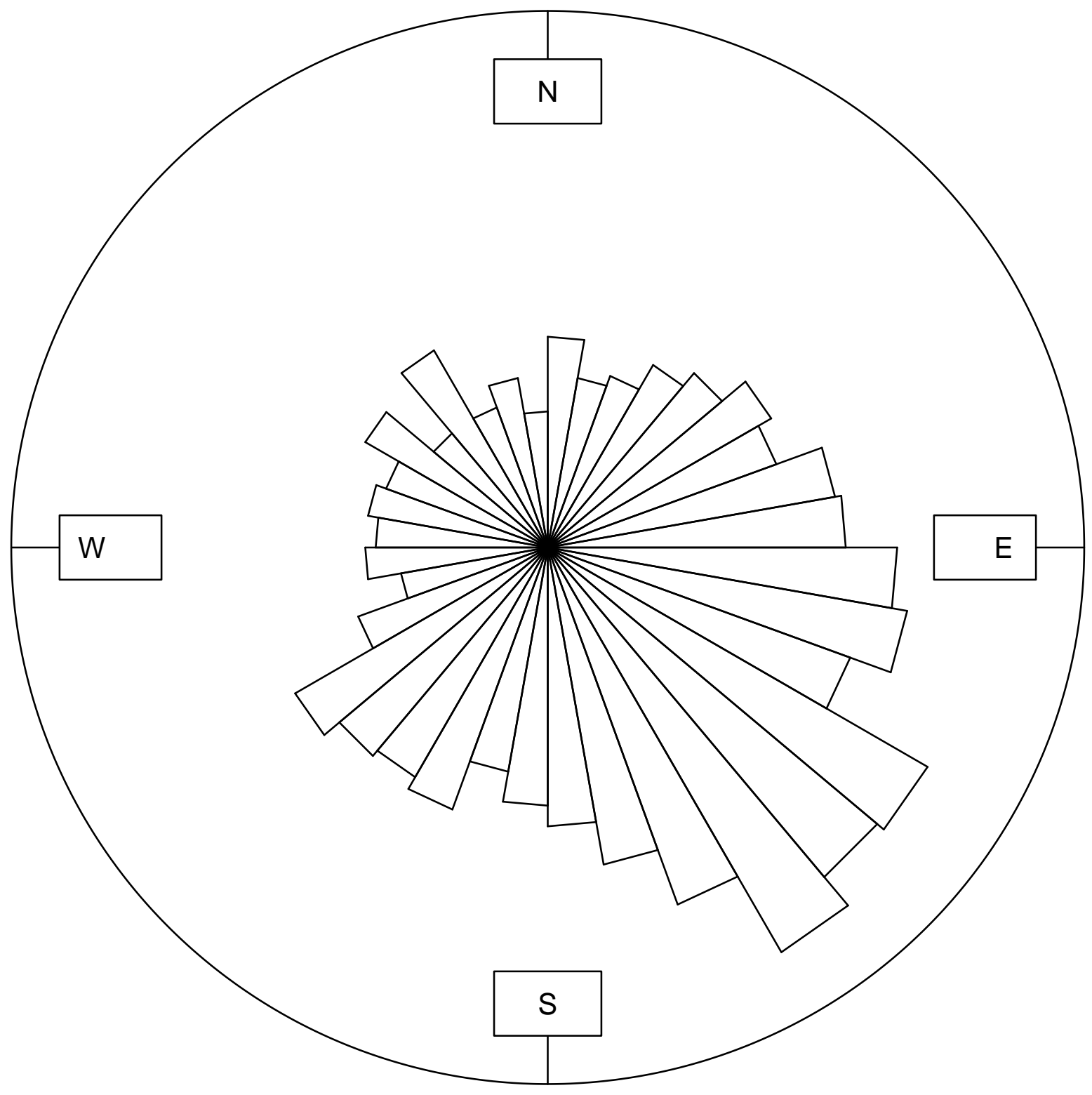


bootstrap 794

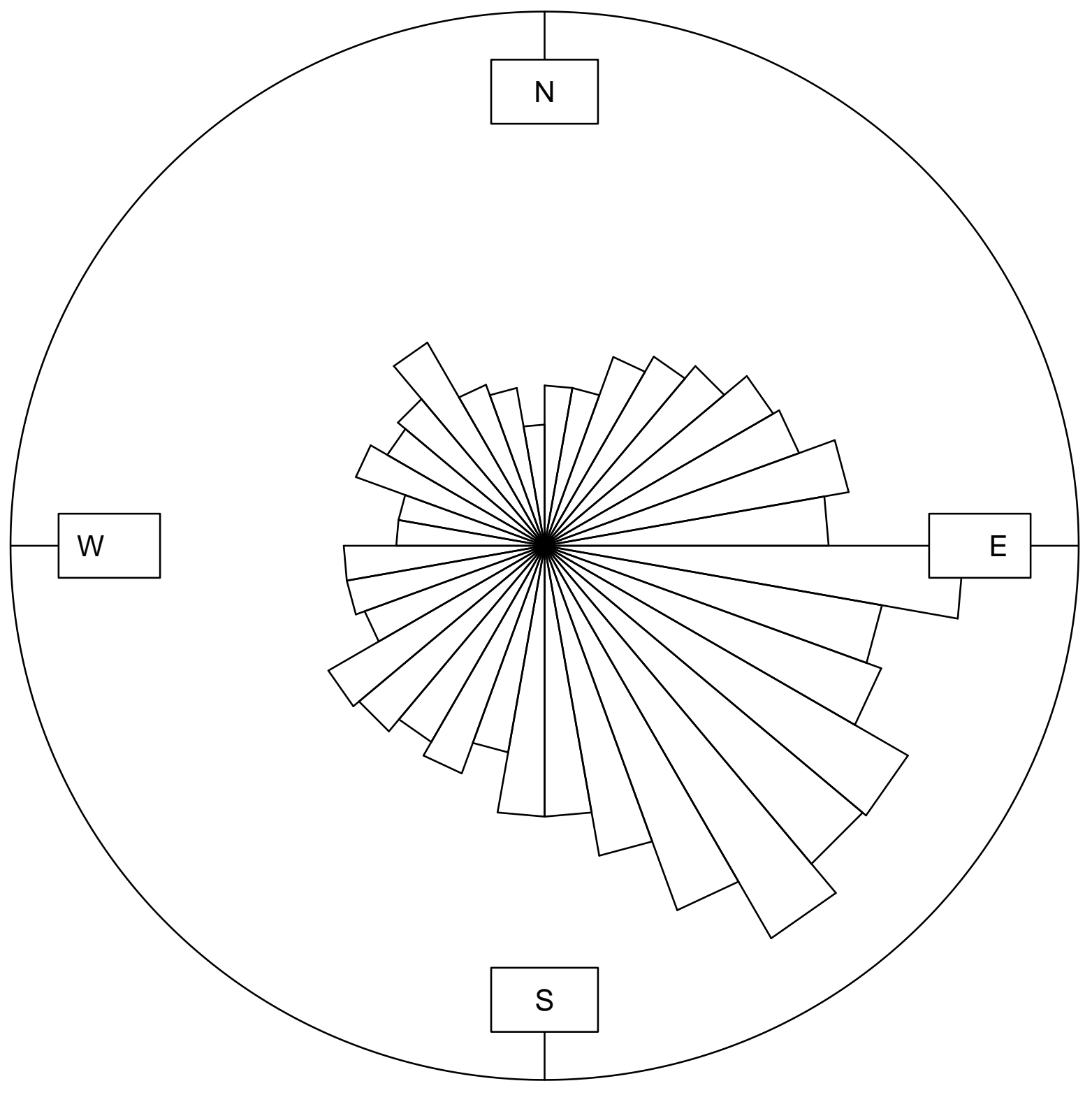


bootstrap 795

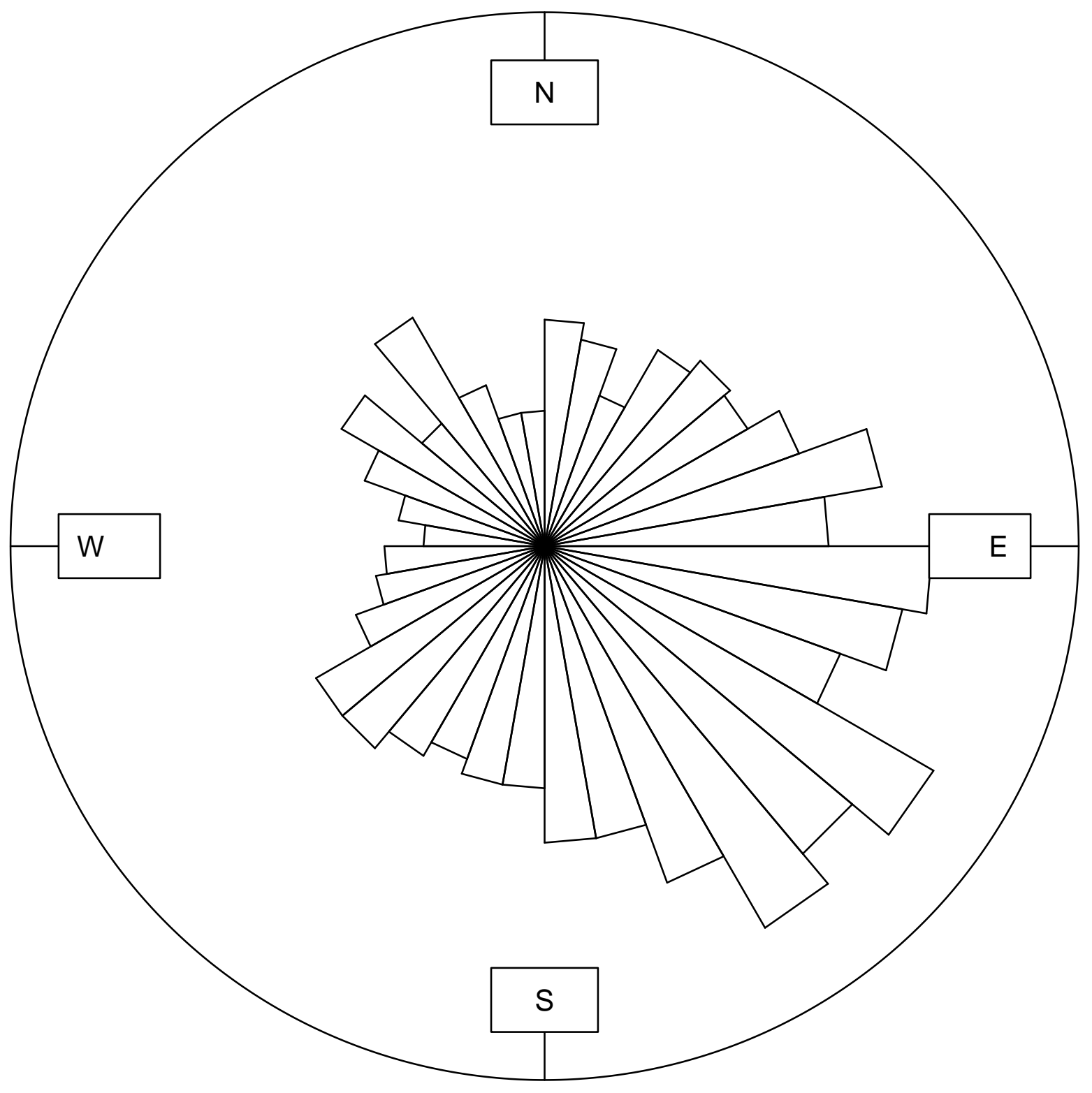




\section{bootstrap 796}

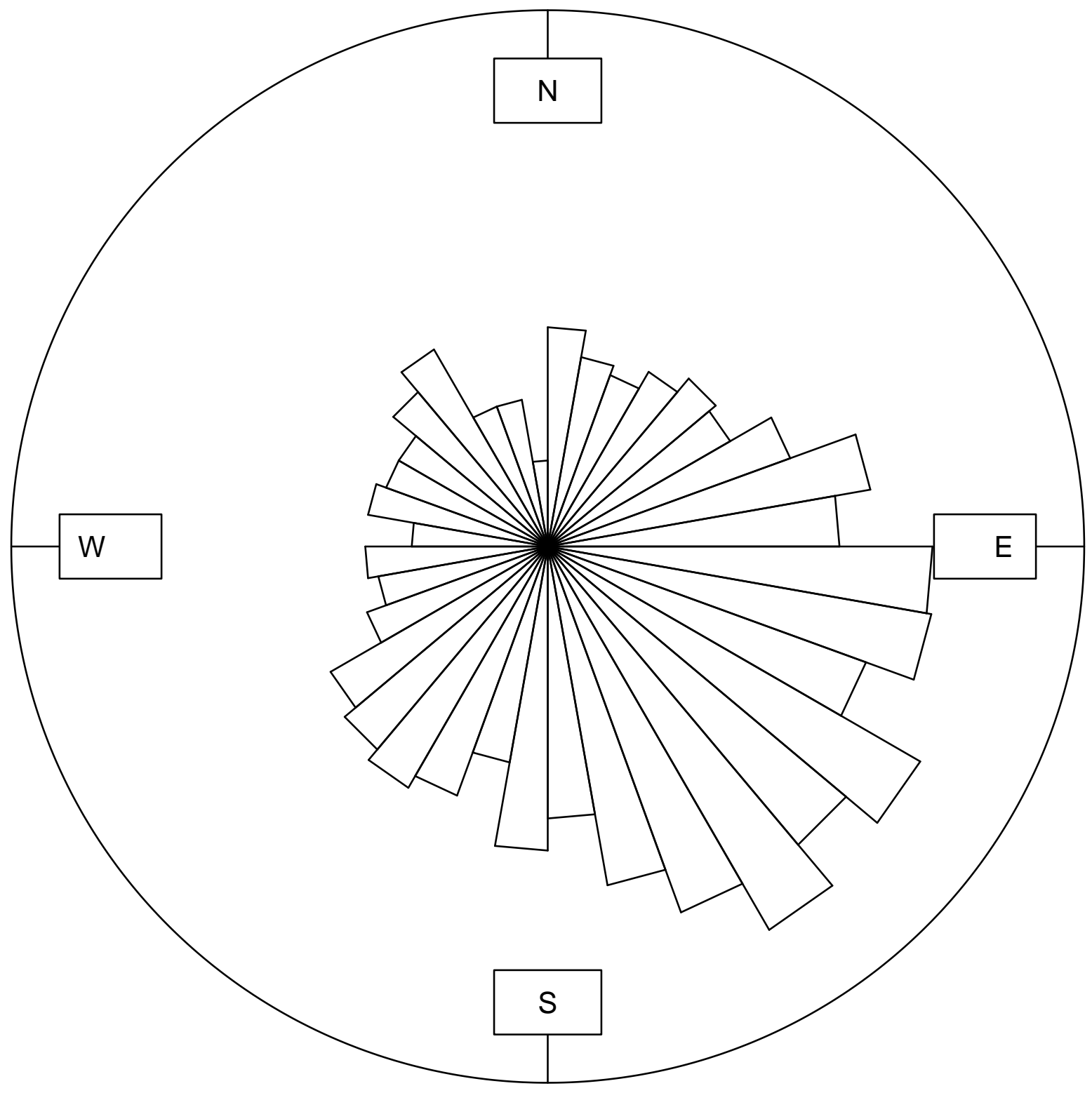


bootstrap 798

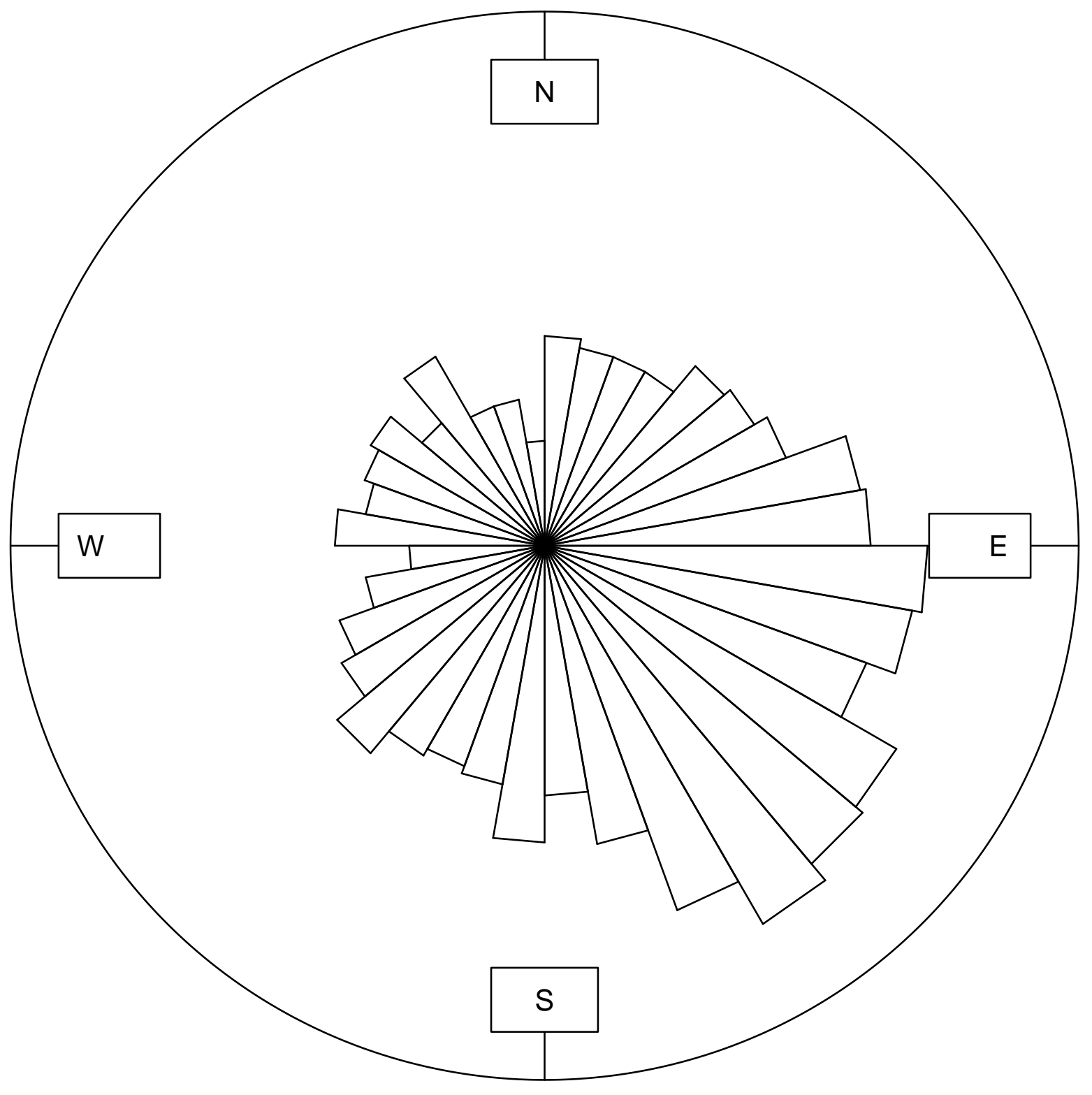


bootstrap 799

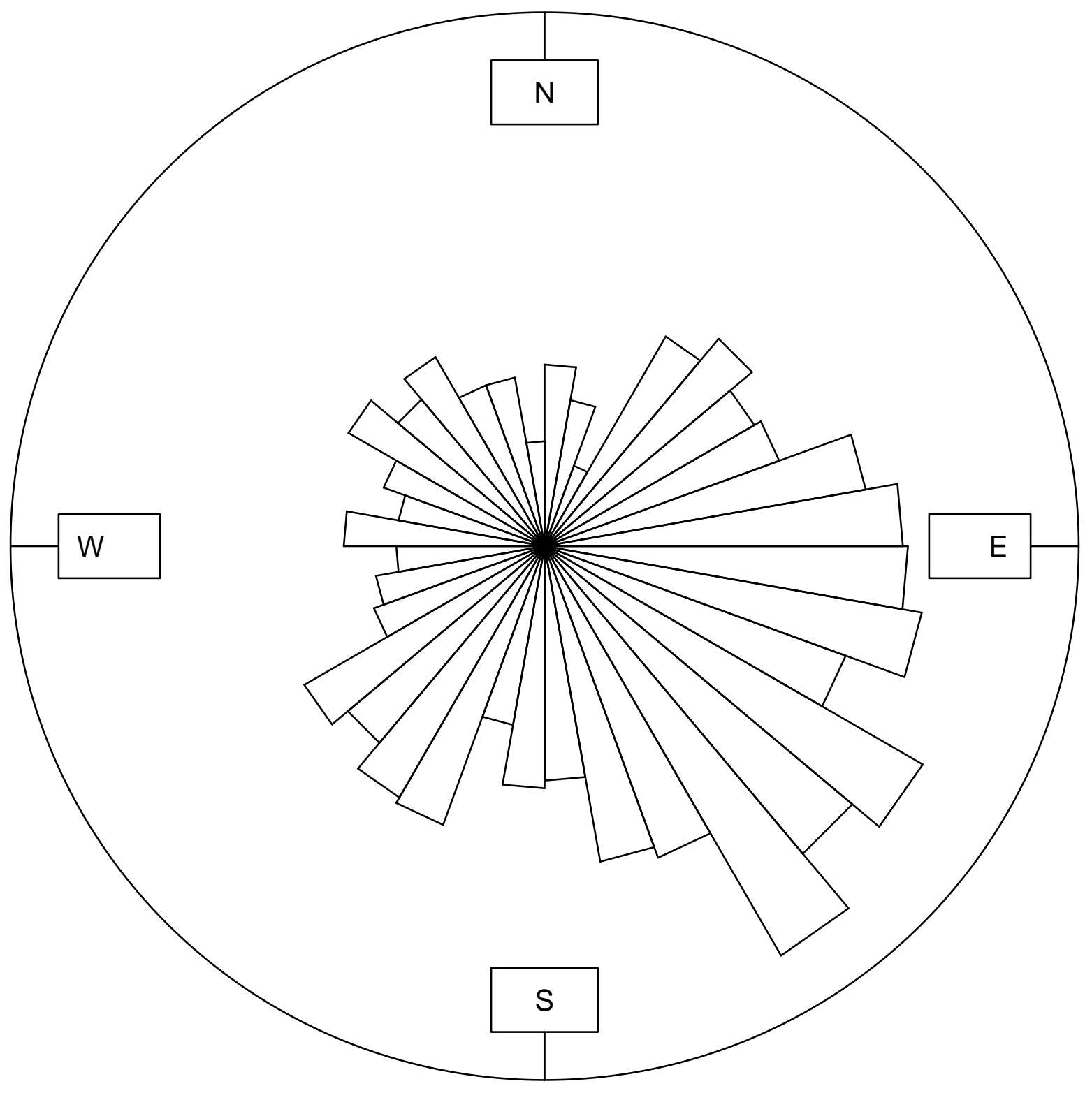


bootstrap 800

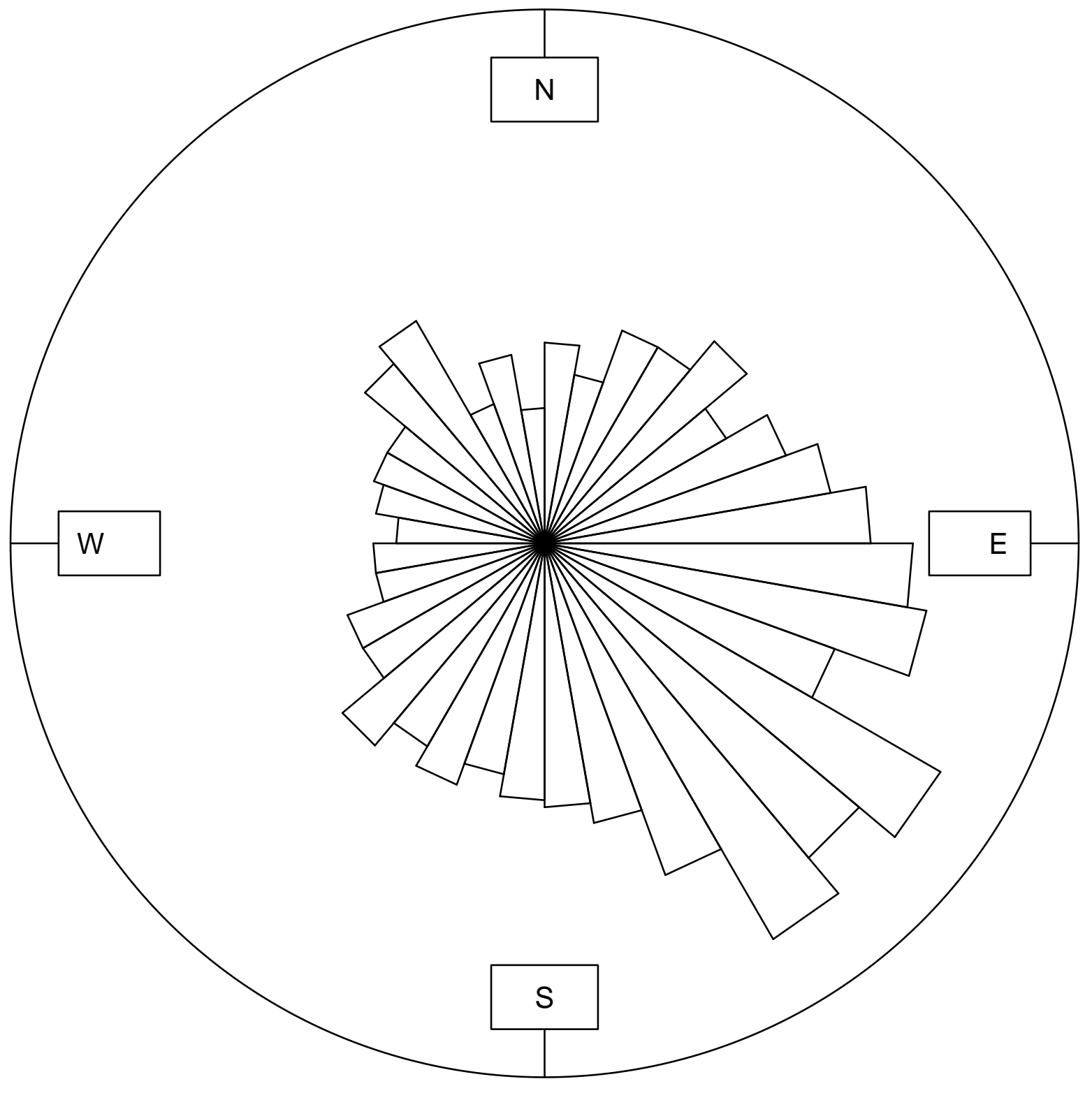




\section{bootstrap 801}

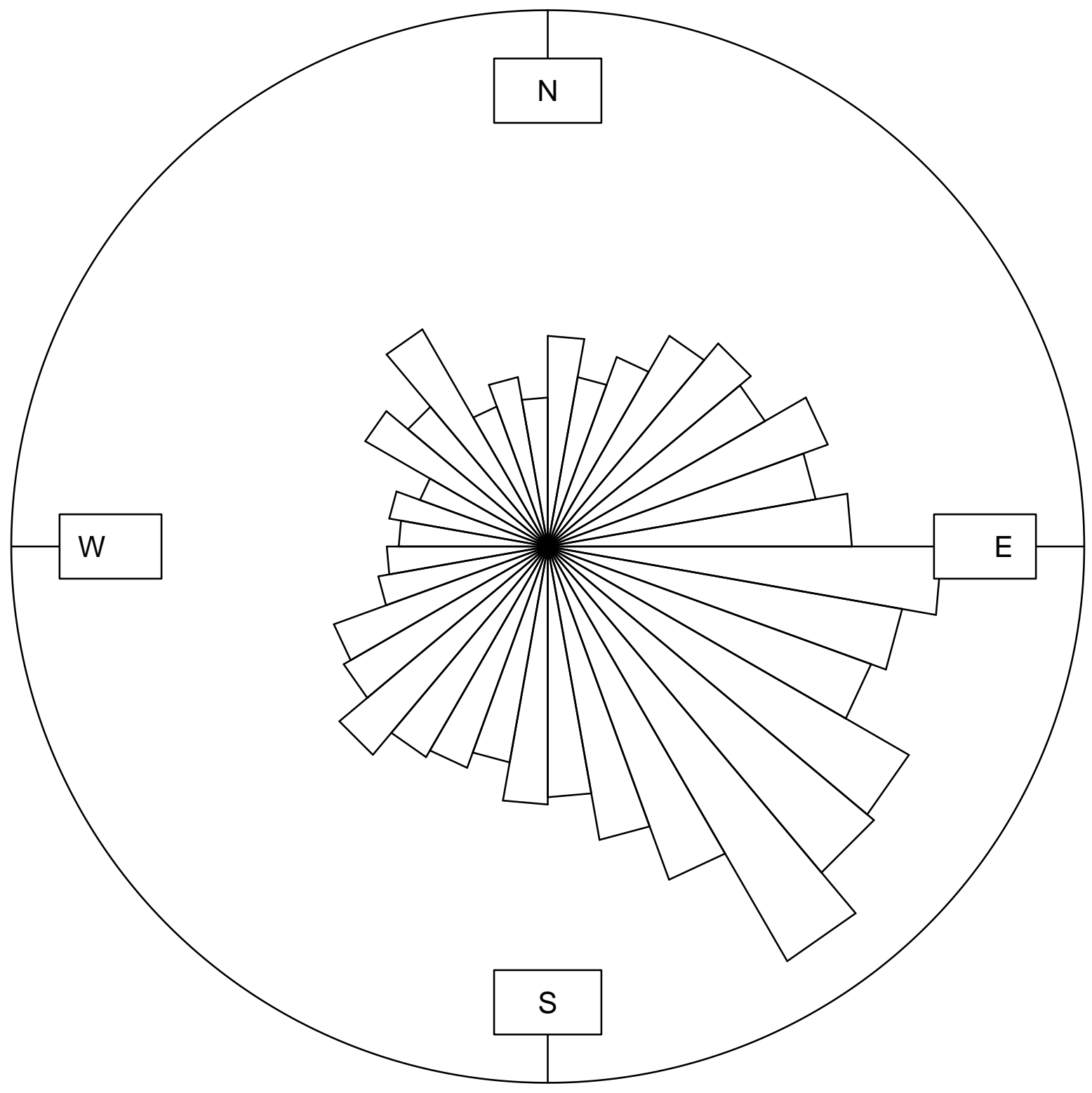


bootstrap 802

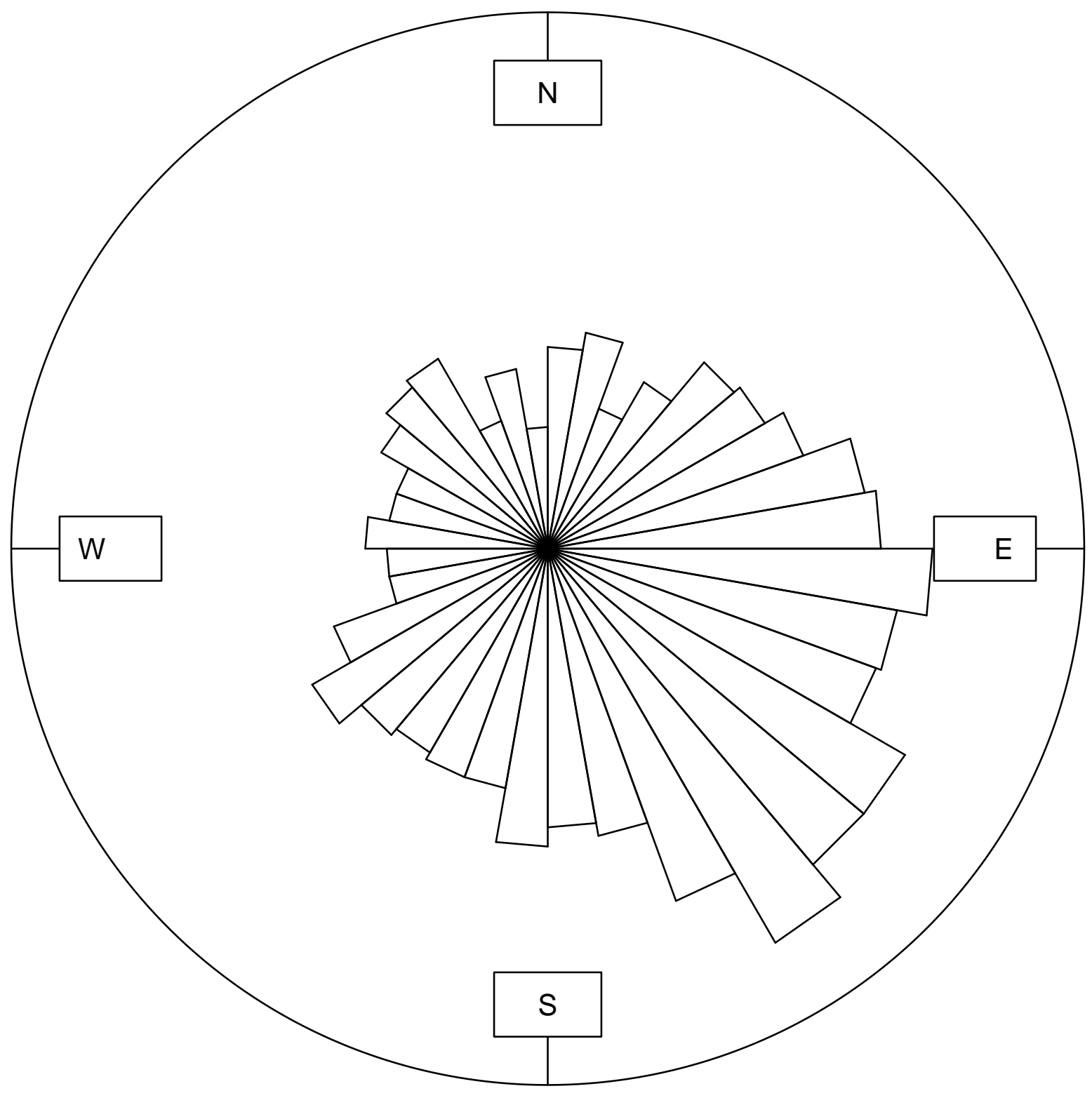


bootstrap 803

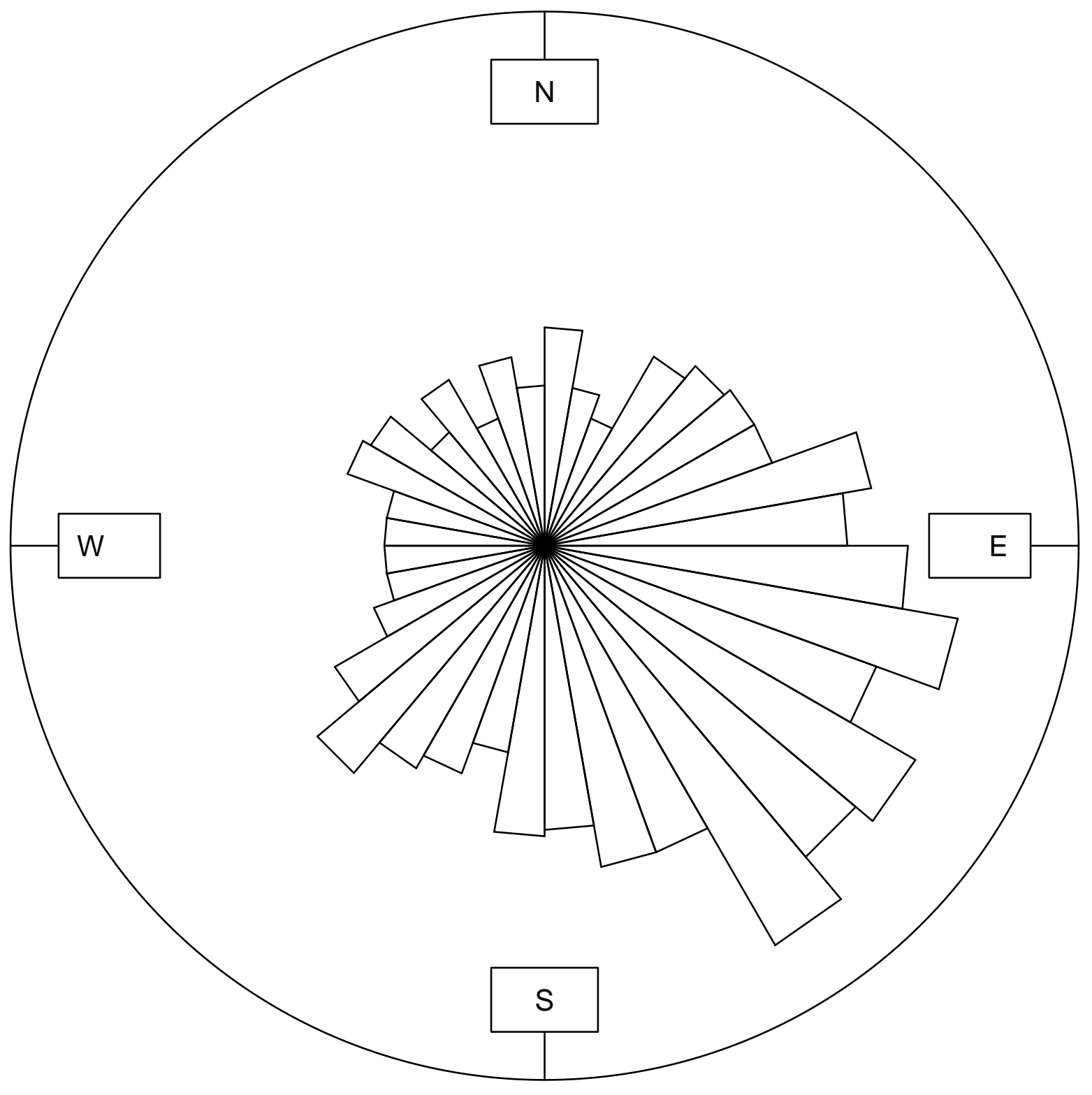




\section{bootstrap 804}

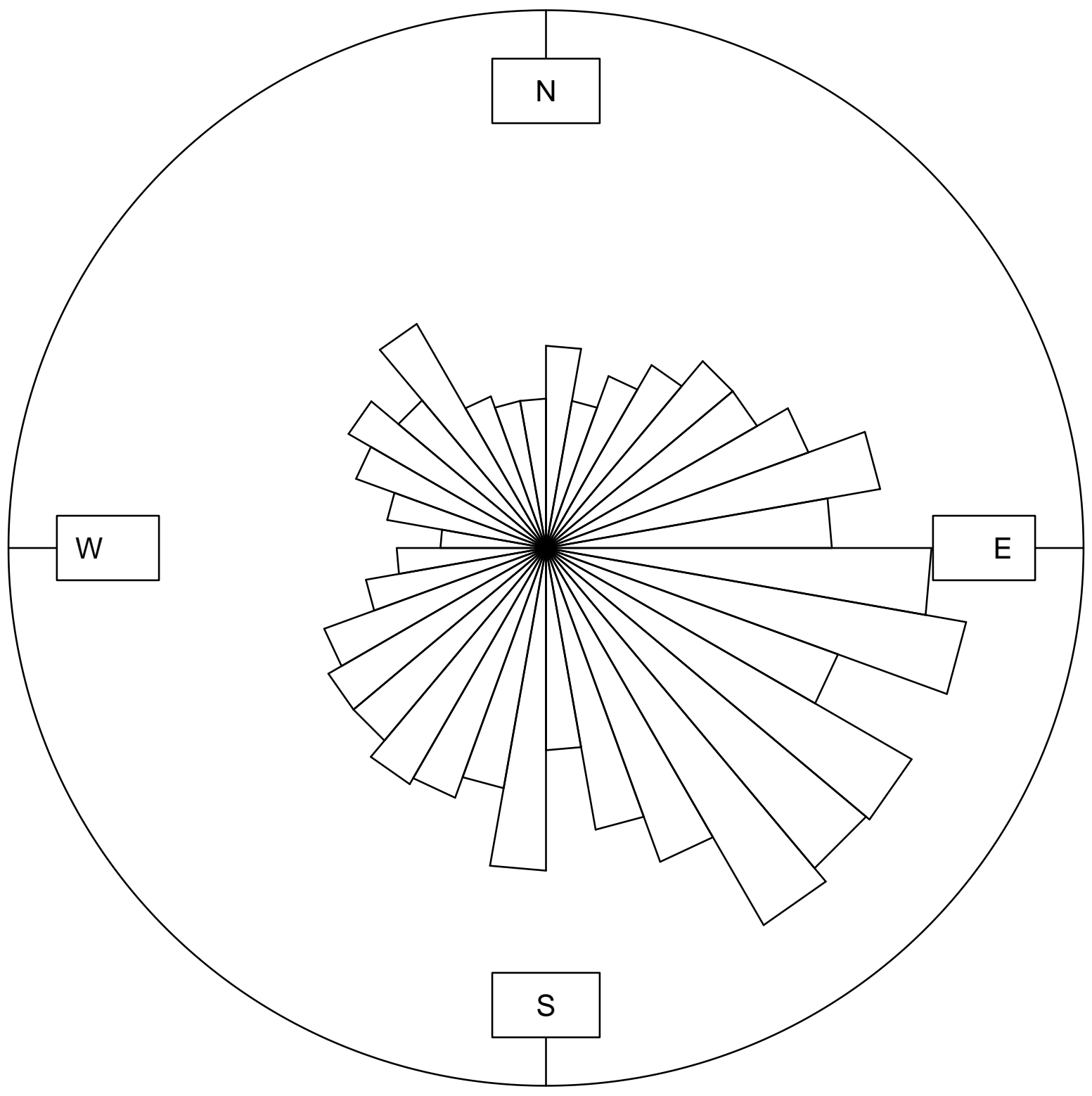




\section{bootstrap 805}

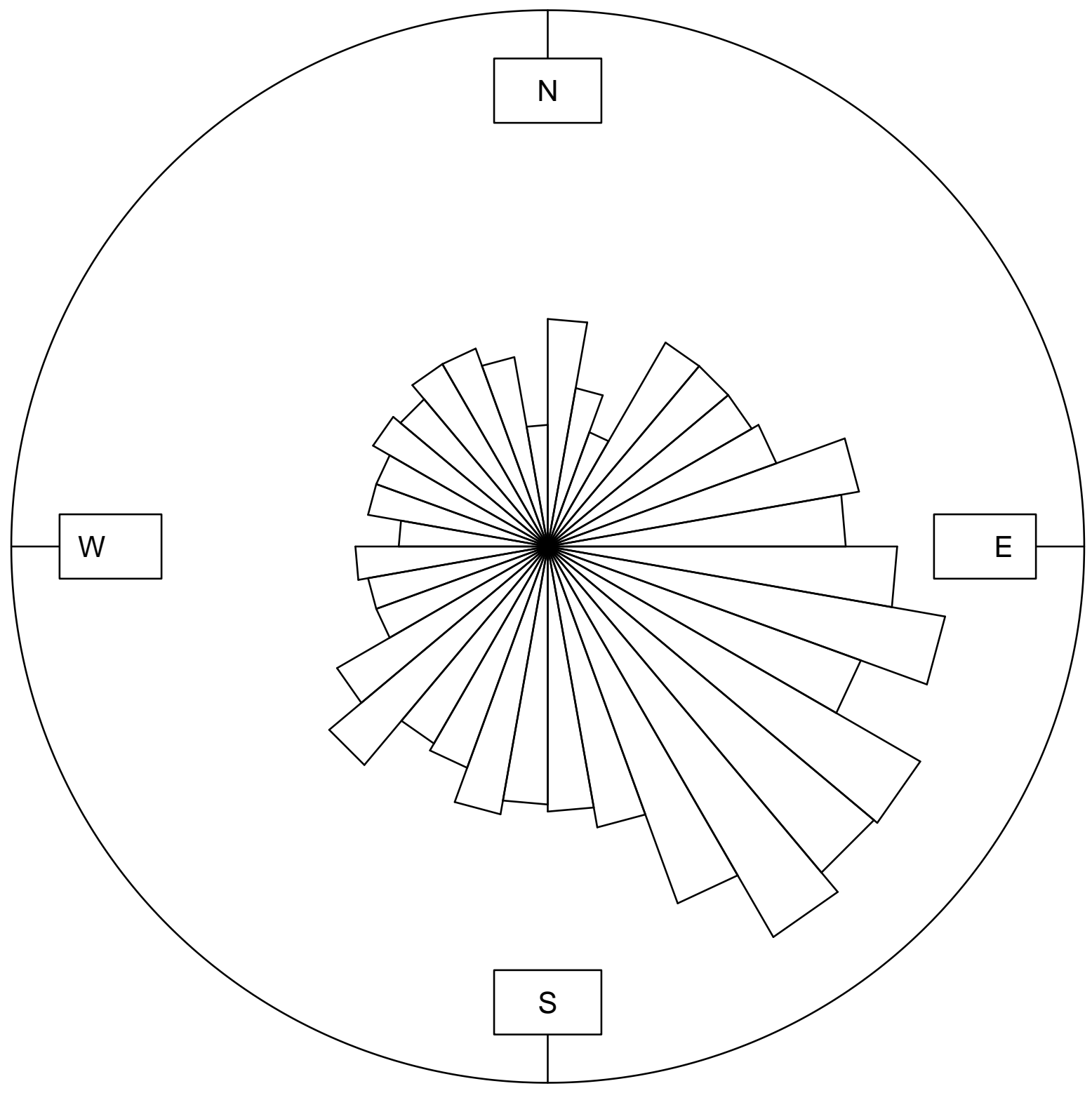


bootstrap 806

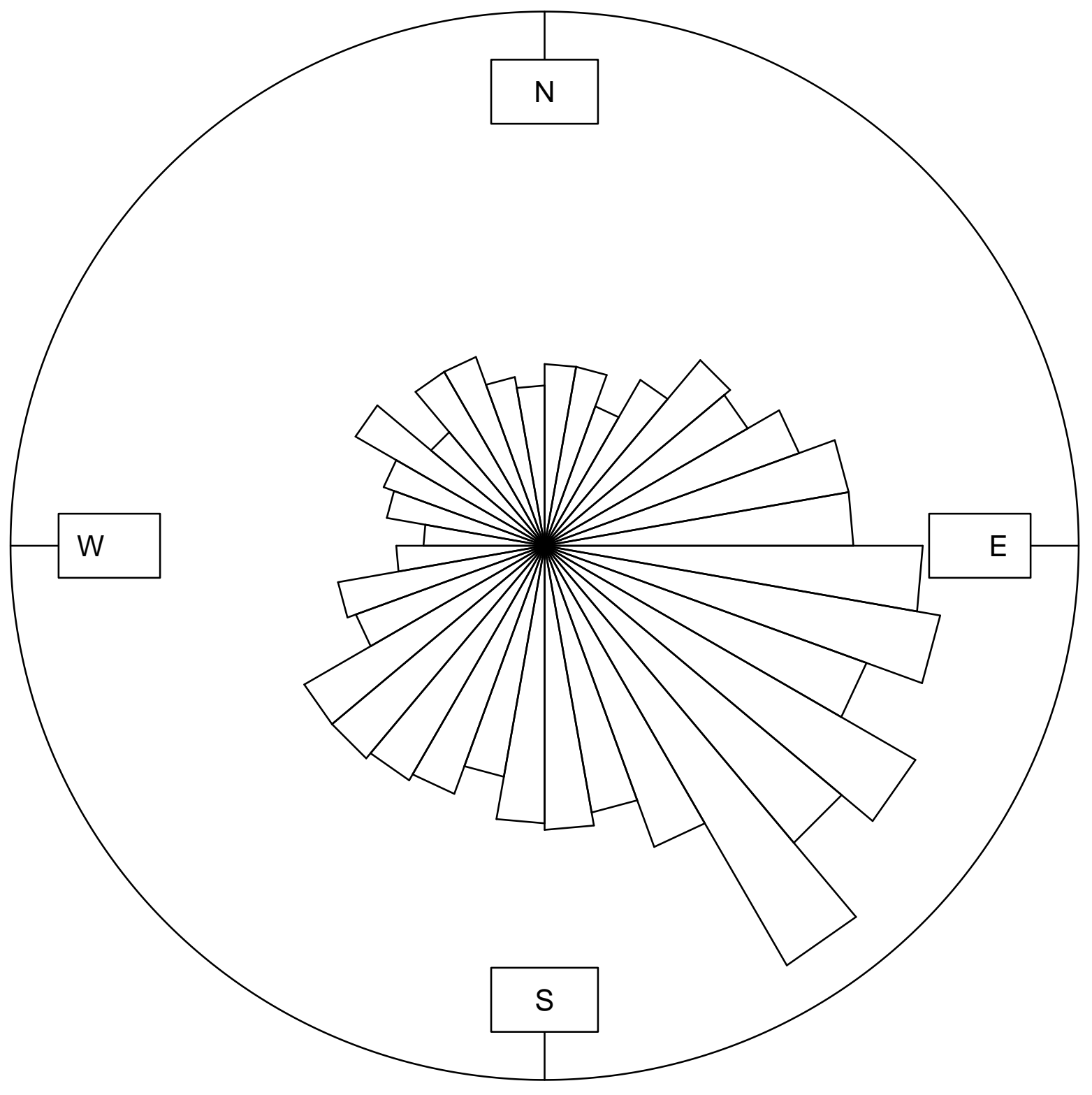


bootstrap 807

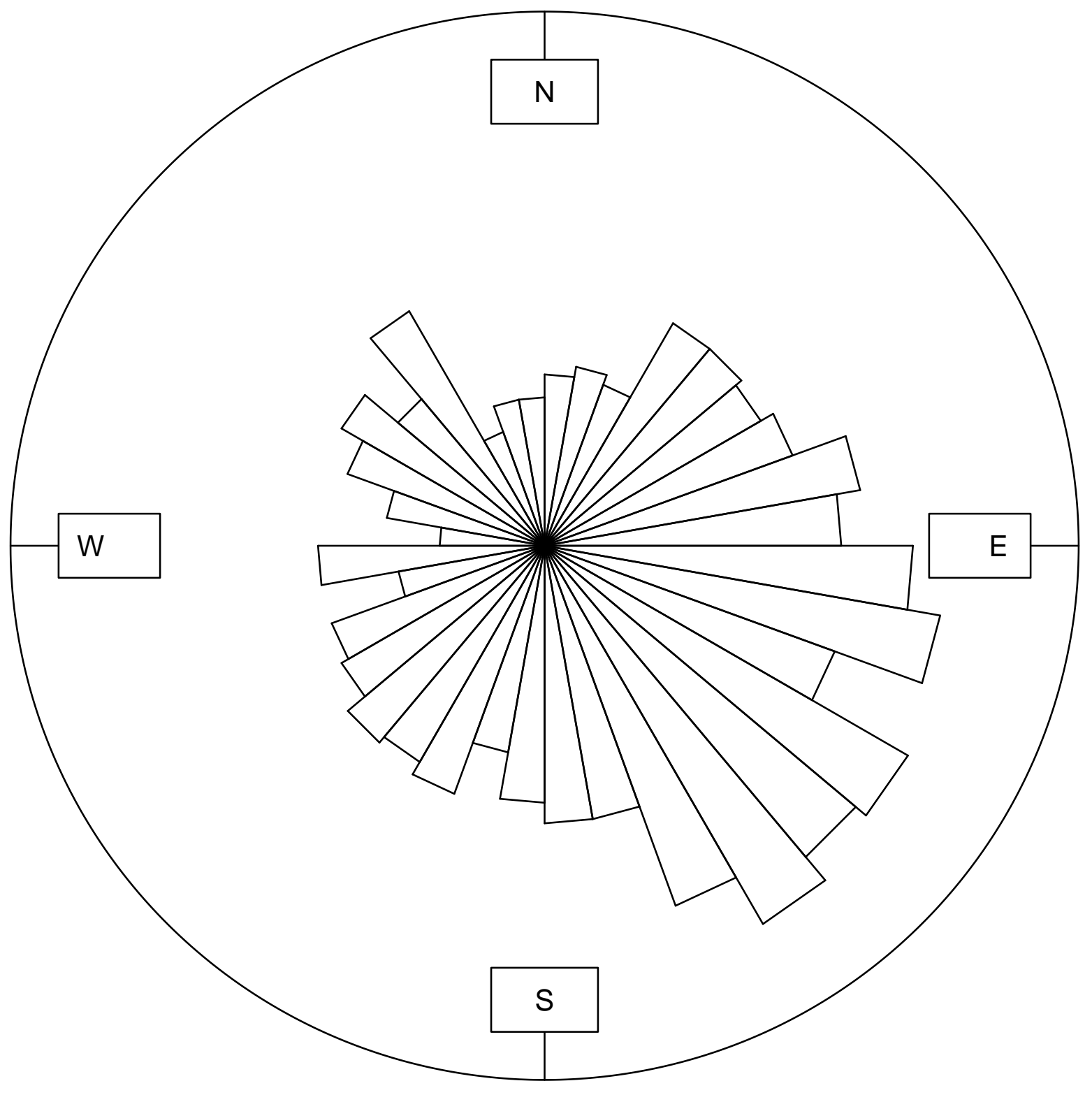


bootstrap 808

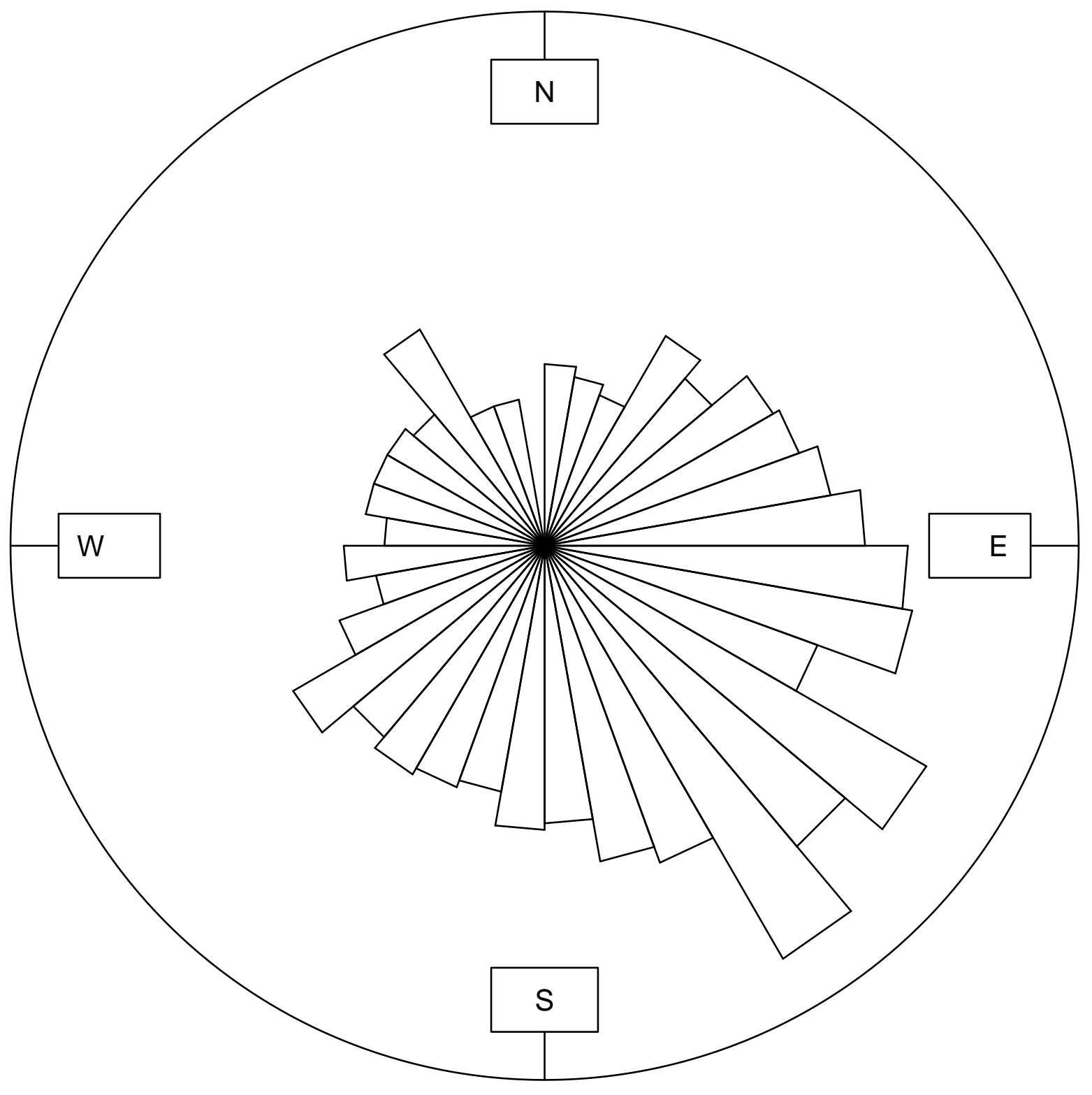


bootstrap 809

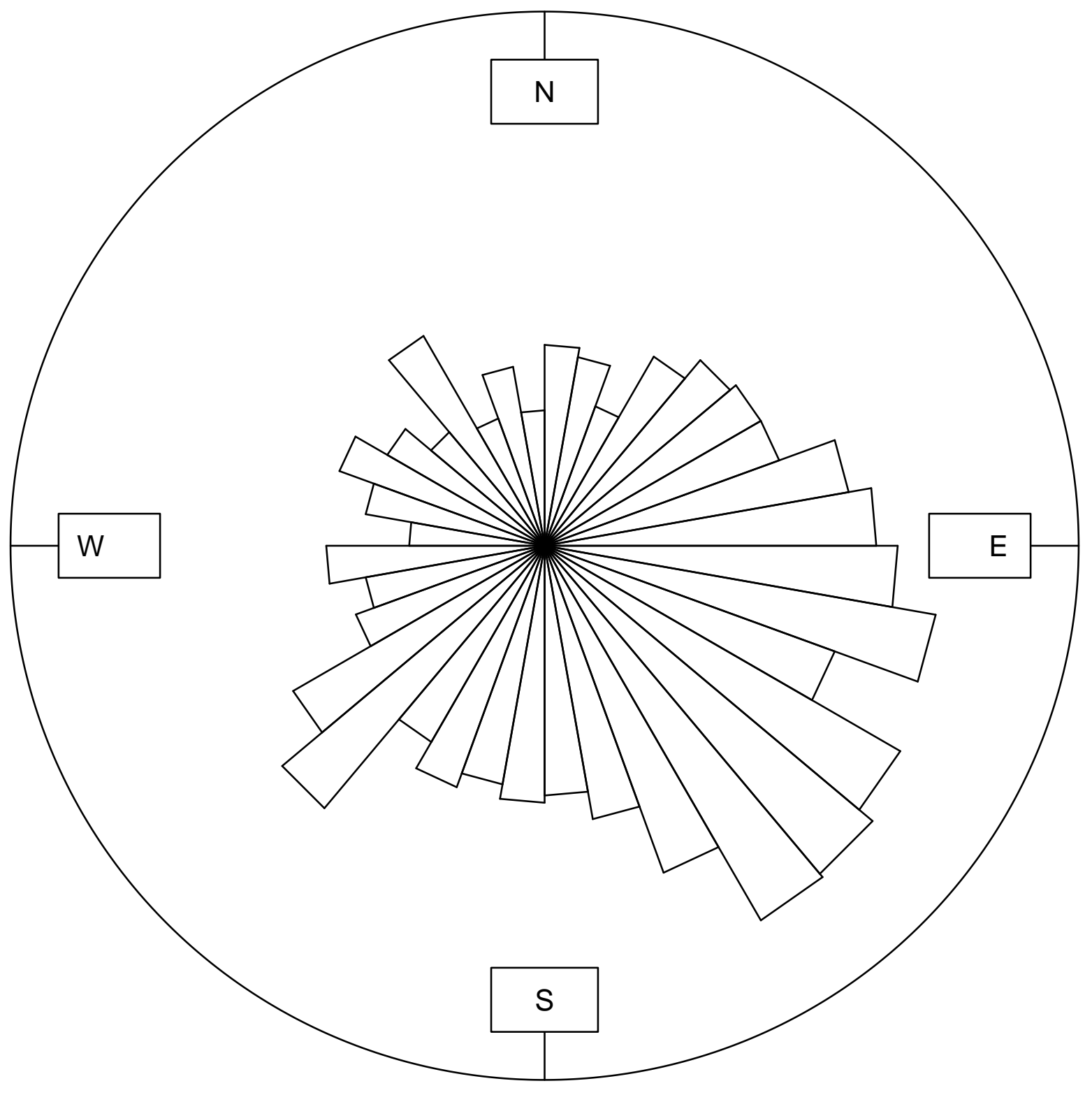


bootstrap 810

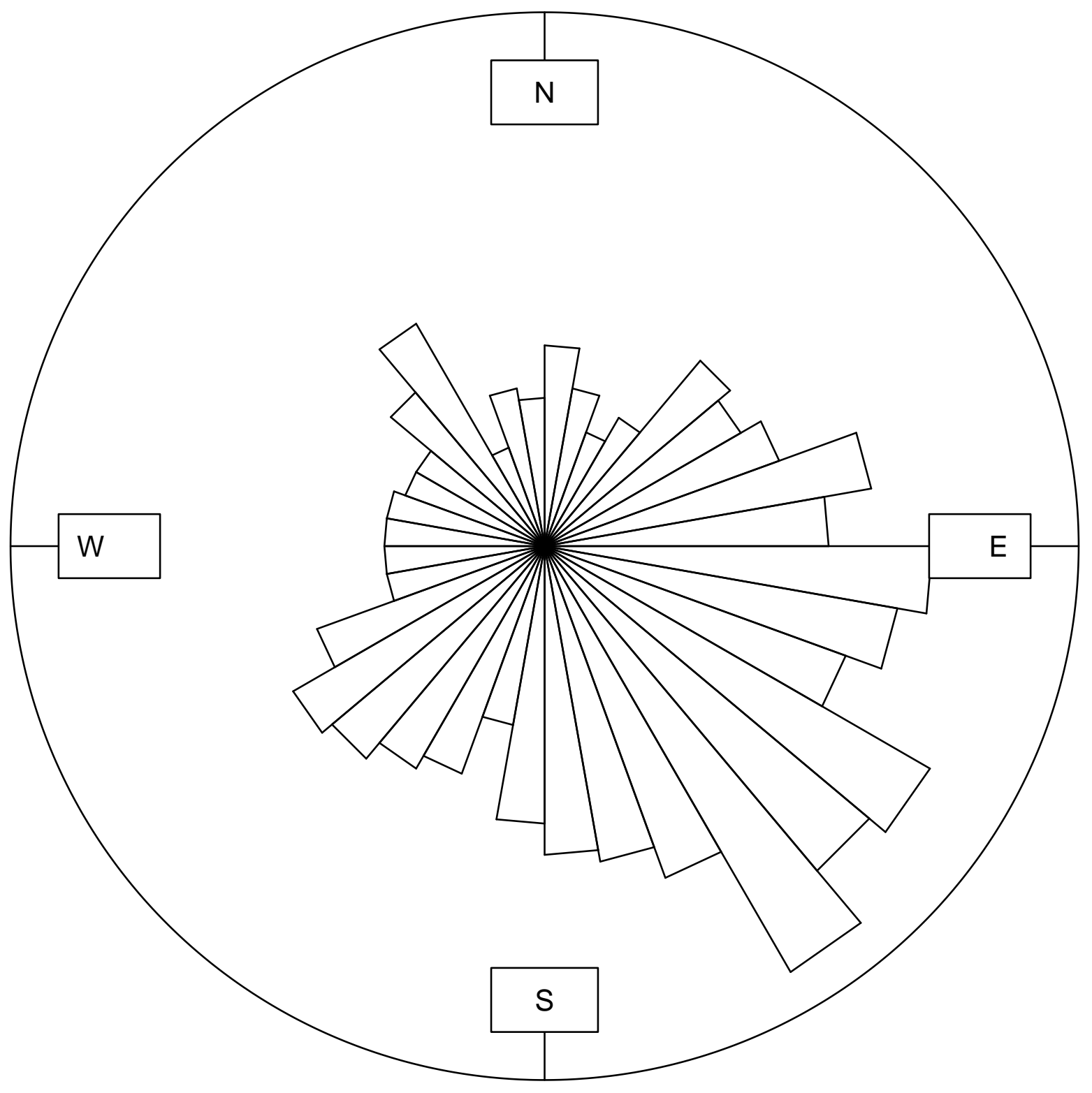


bootstrap 811

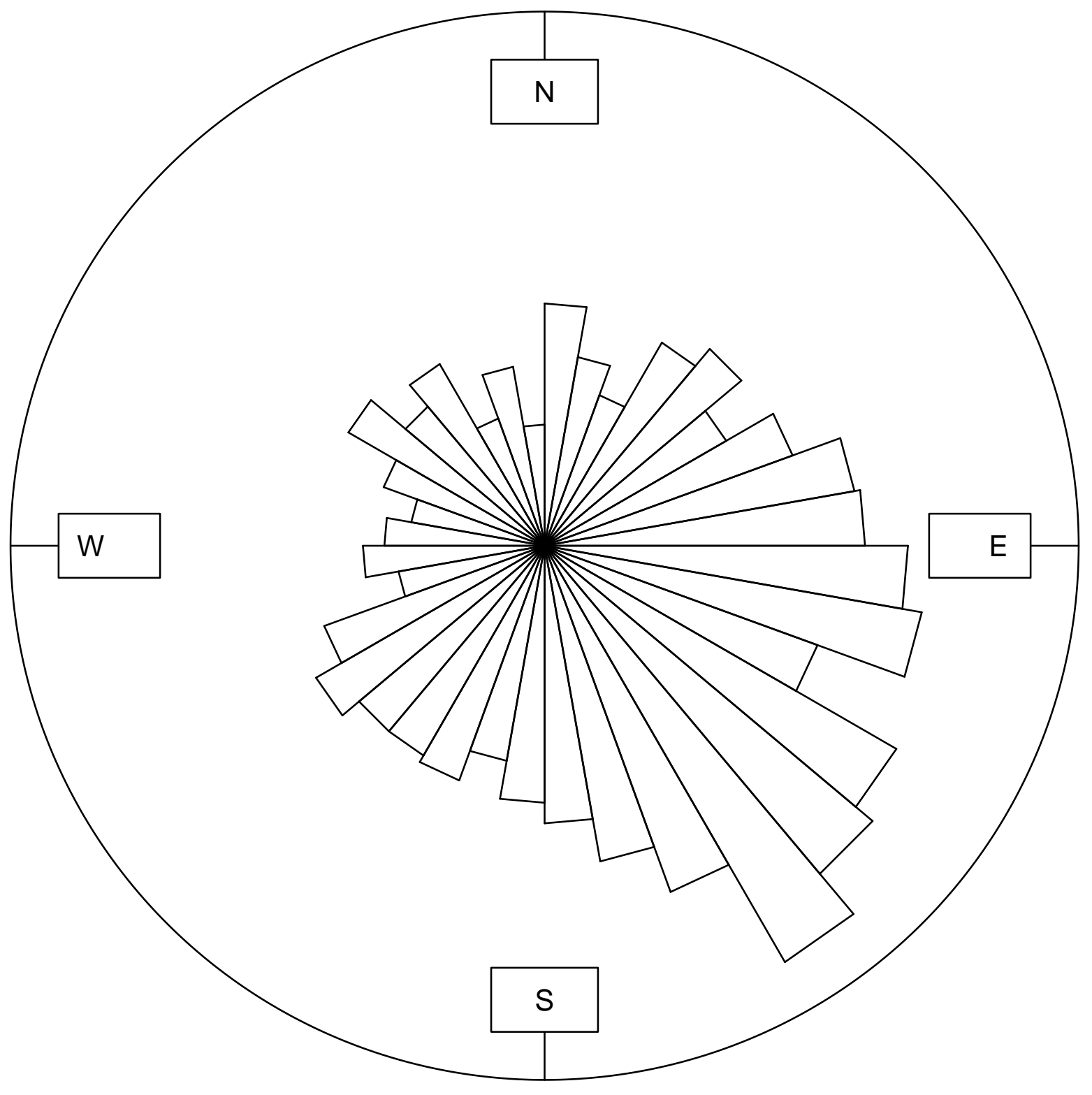


bootstrap 812

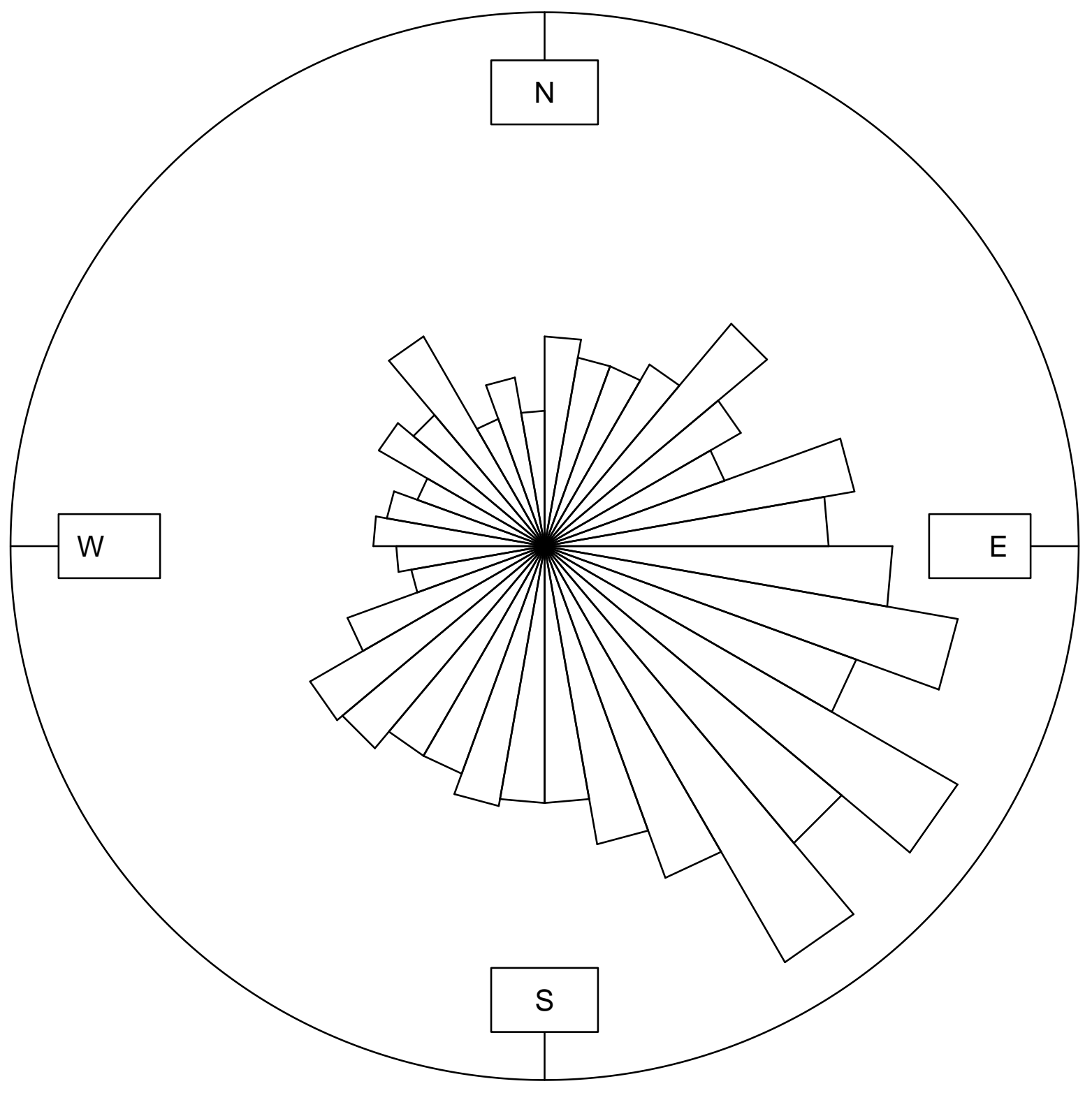


bootstrap 813

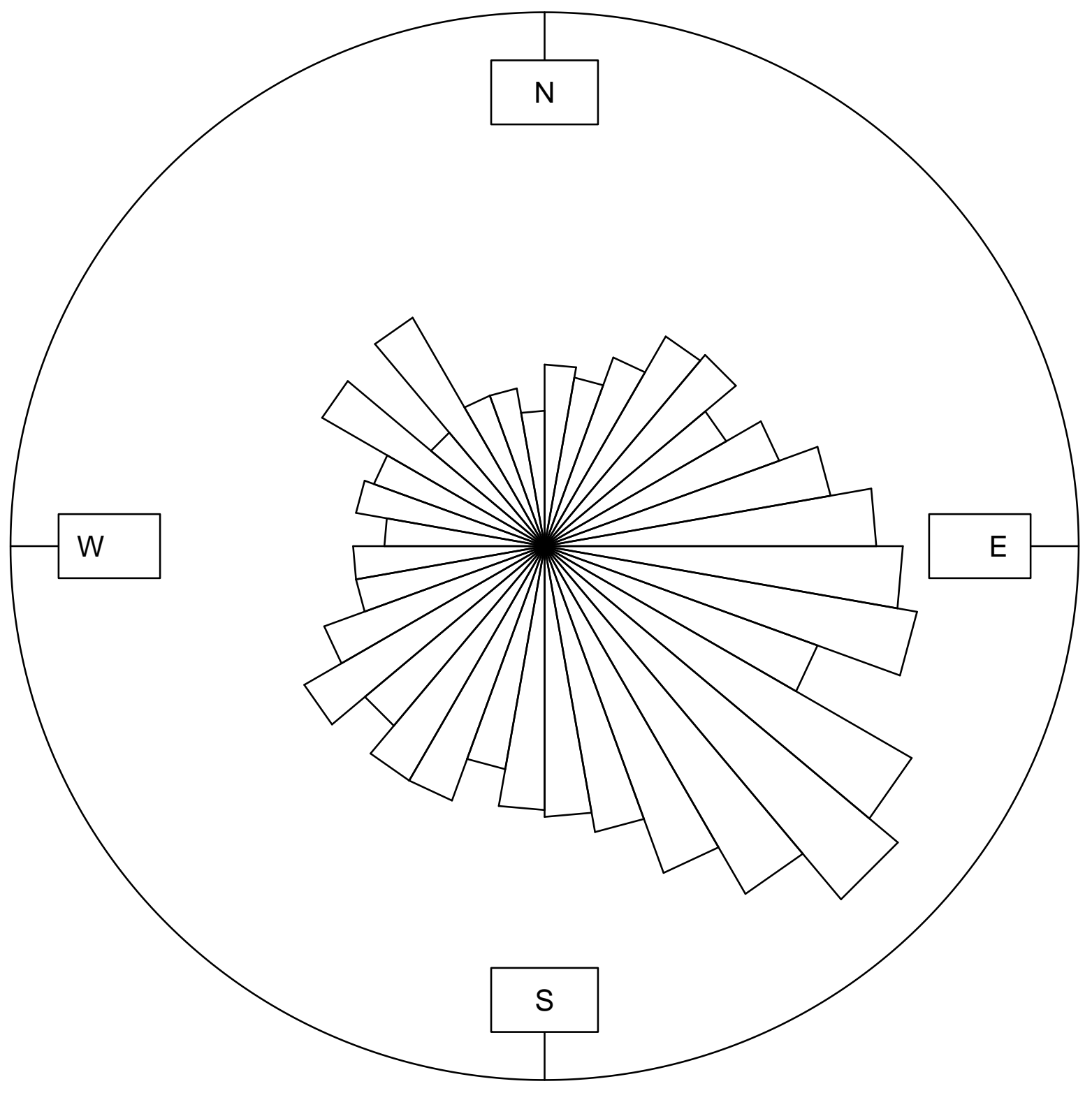


bootstrap 814

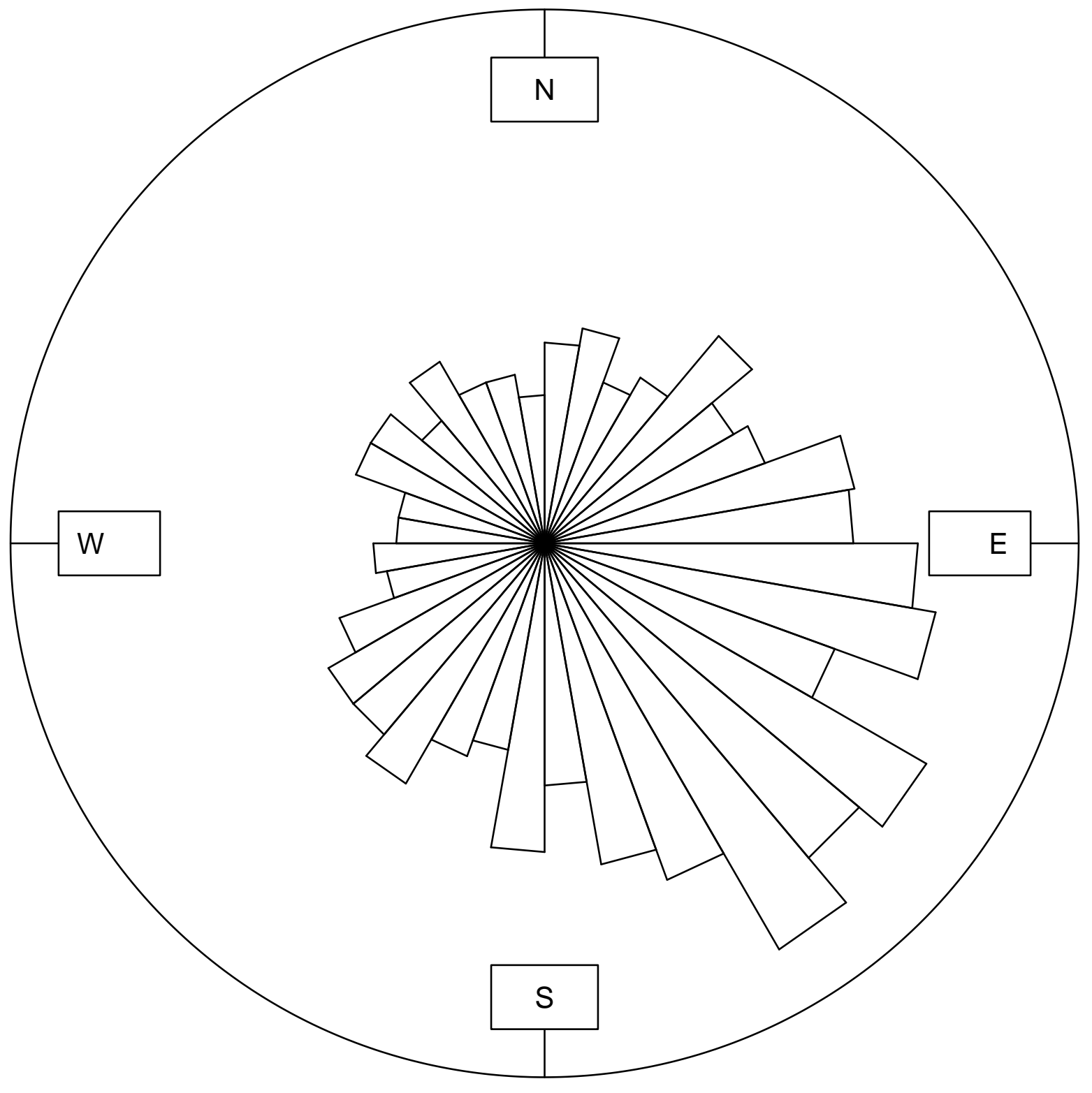




\section{bootstrap 815}

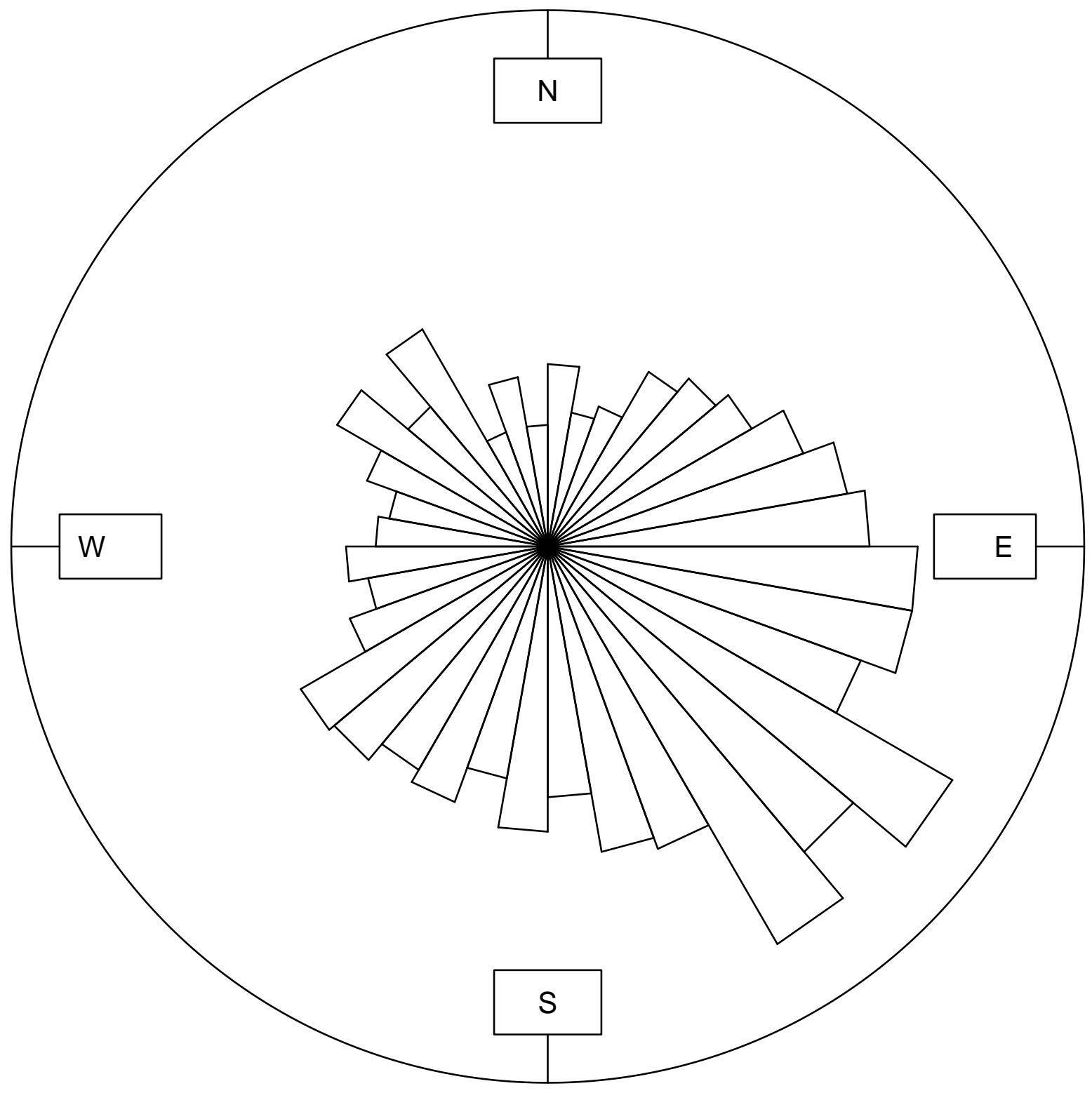


bootstrap 816

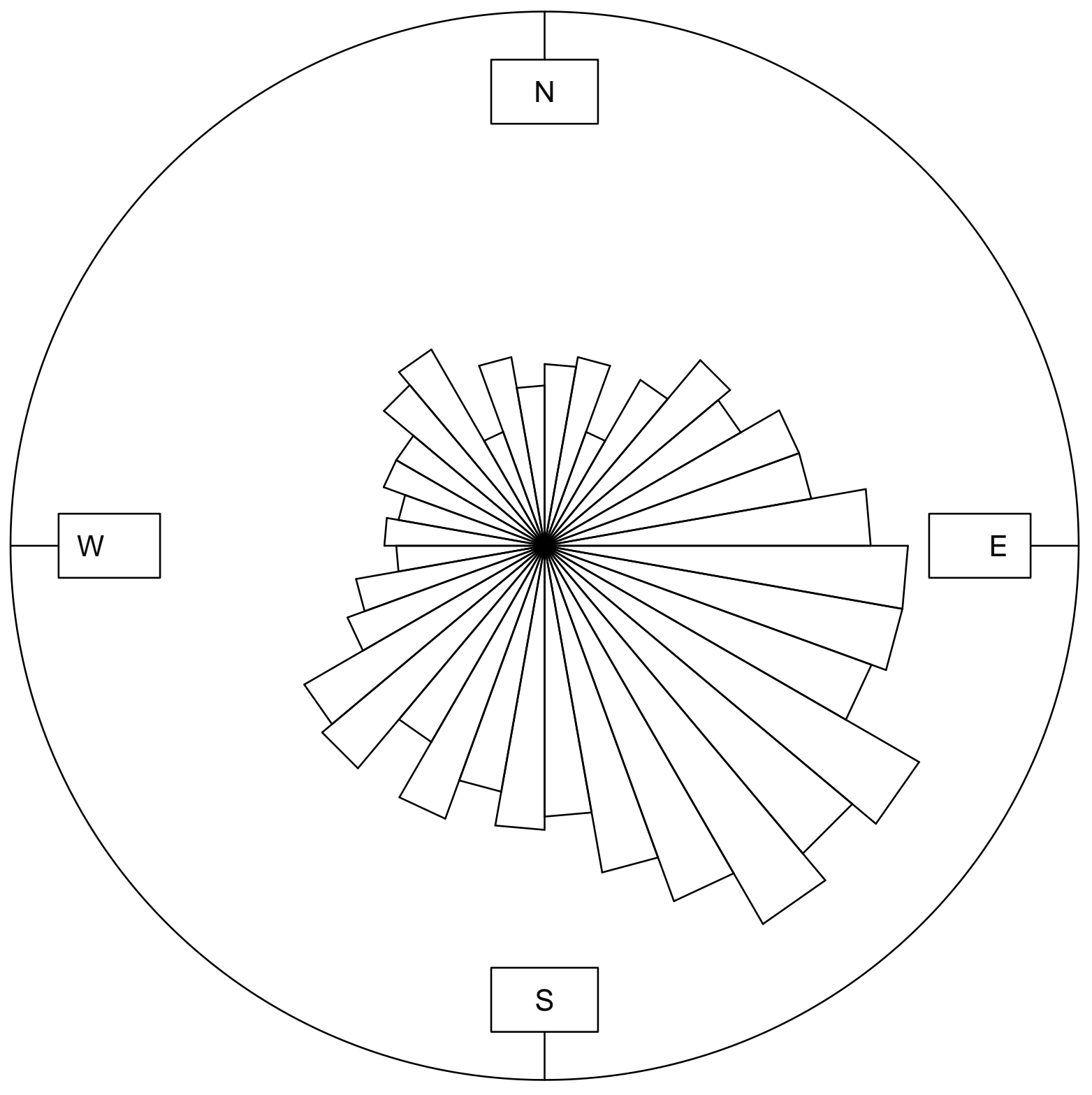


bootstrap 817

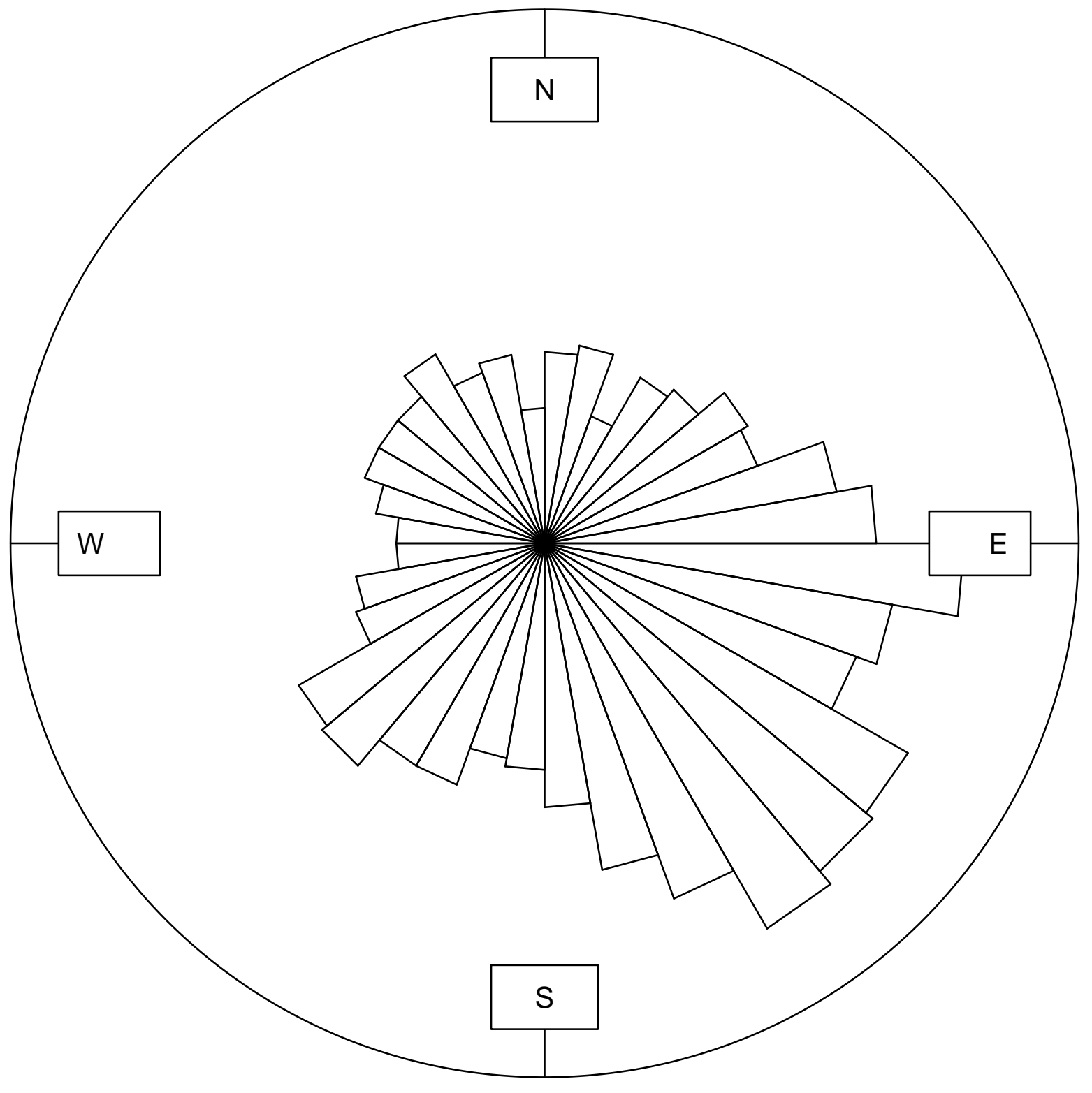


bootstrap 818

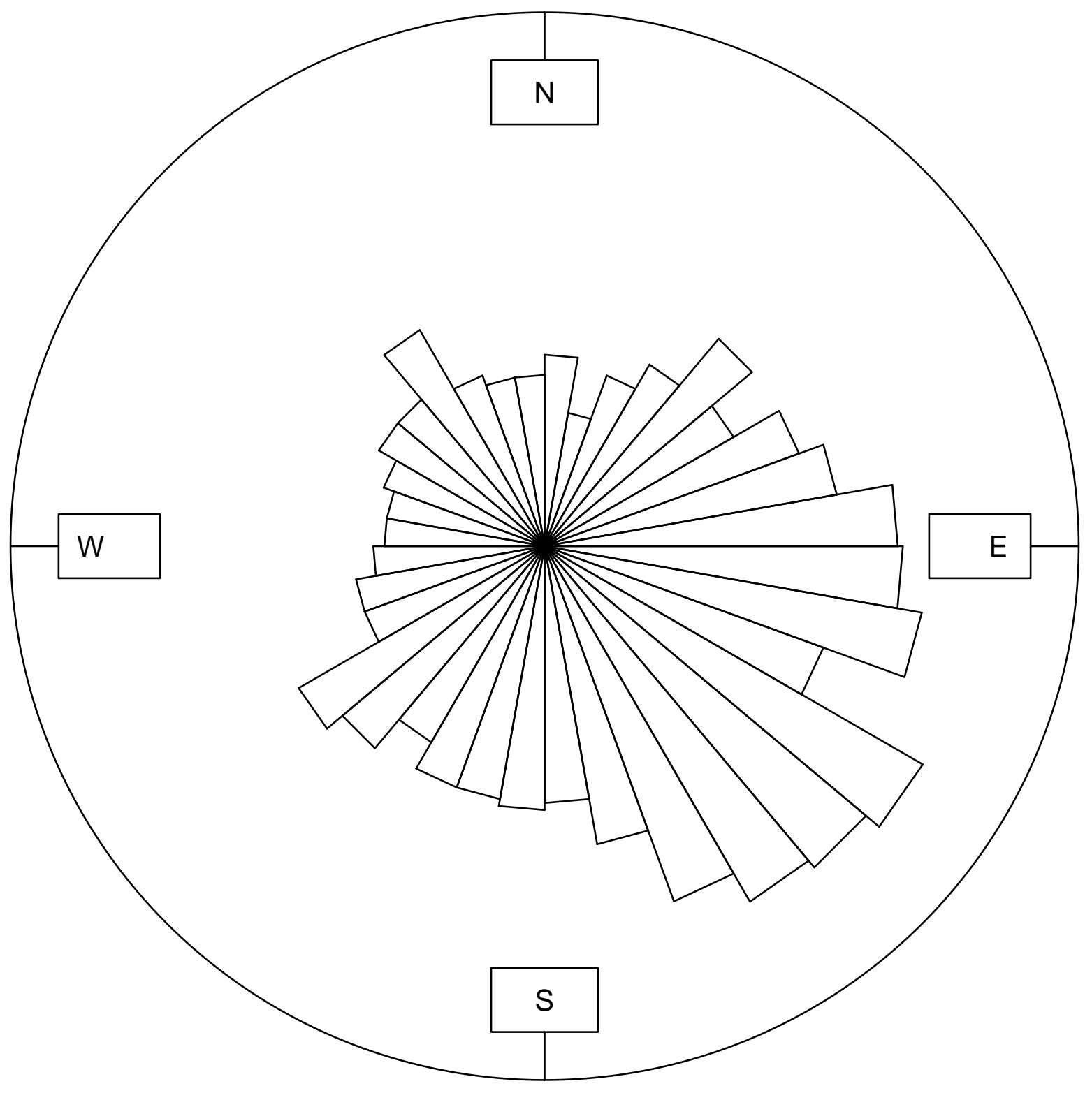


bootstrap 819

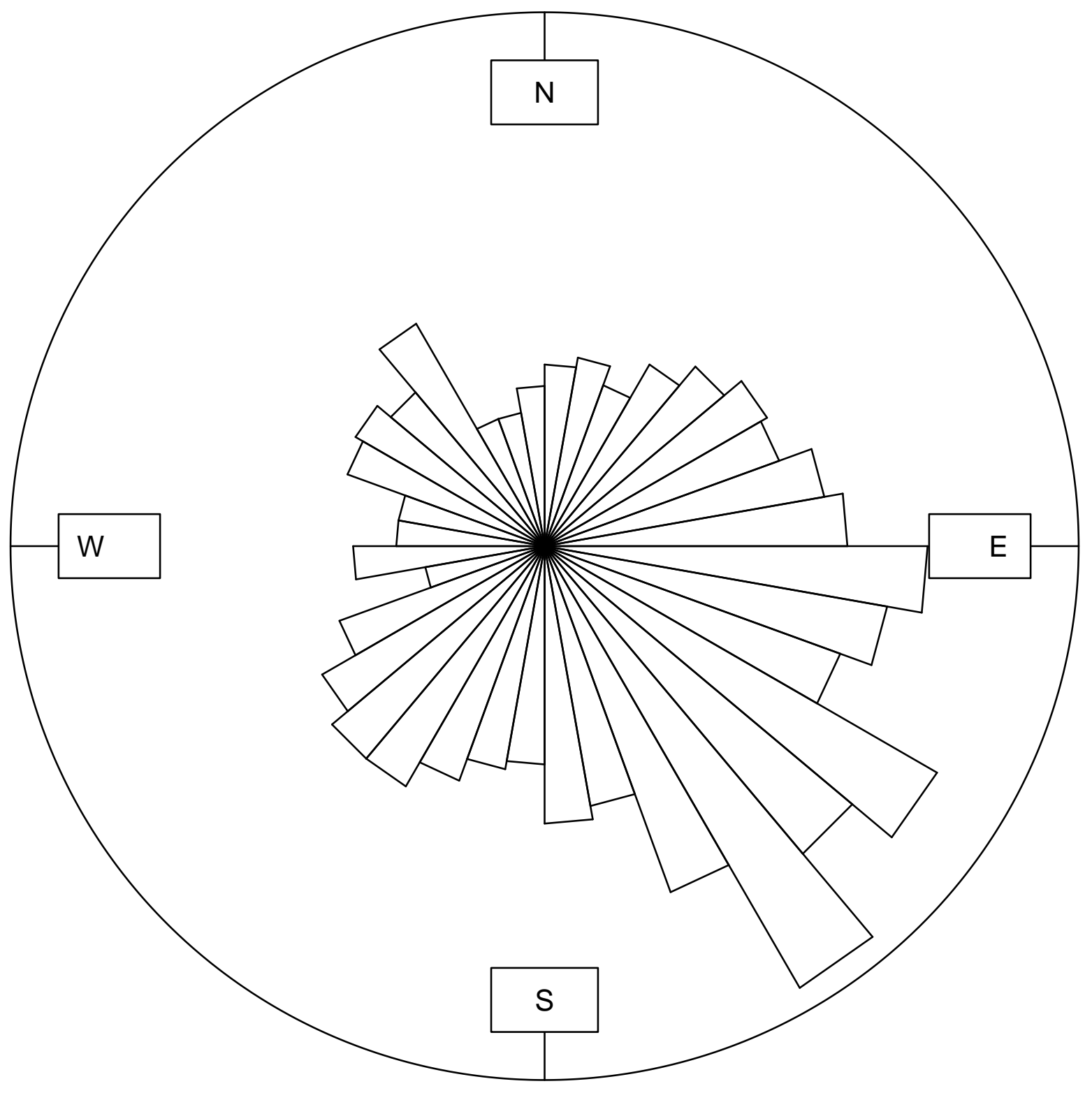




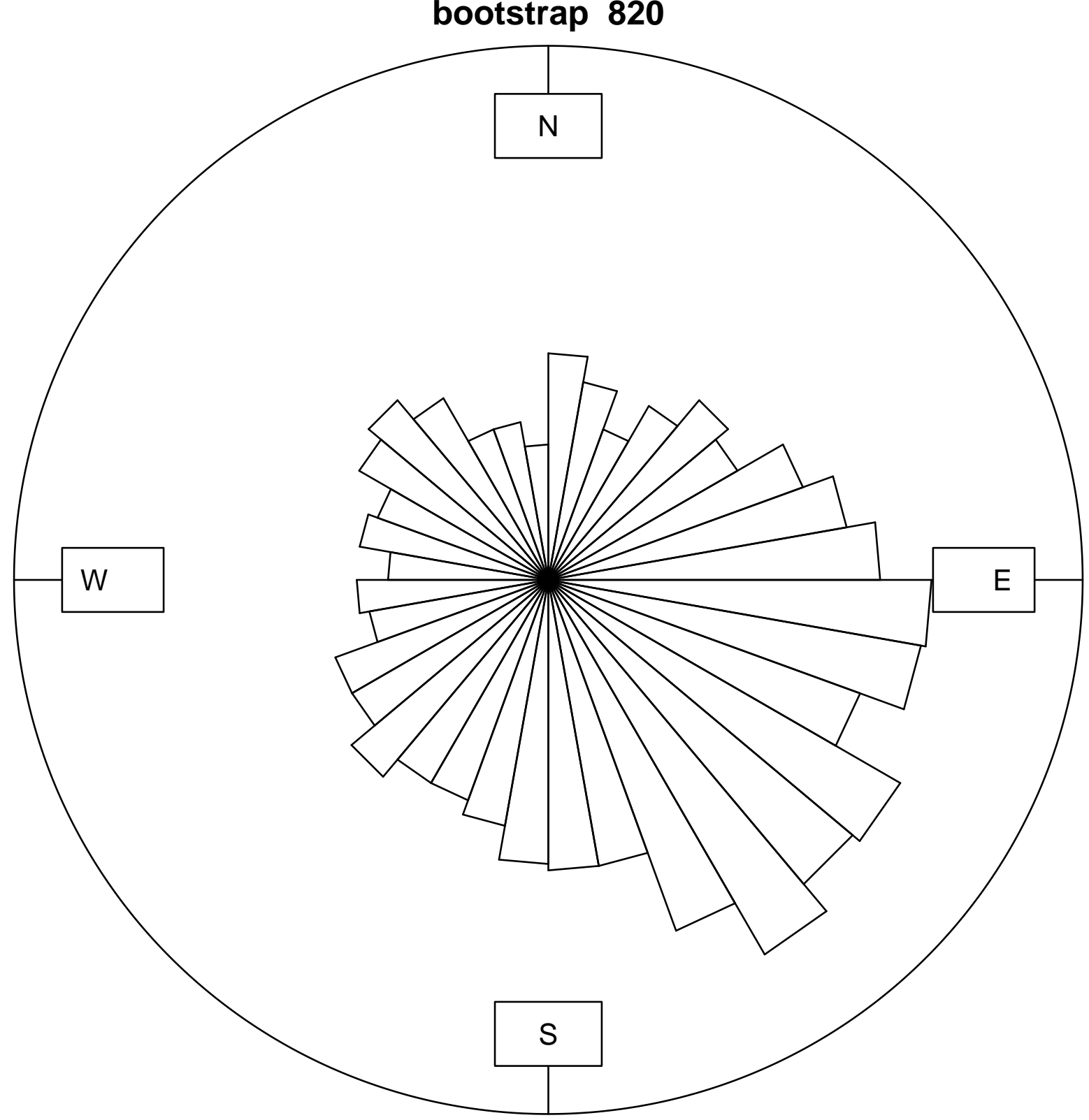


bootstrap 821

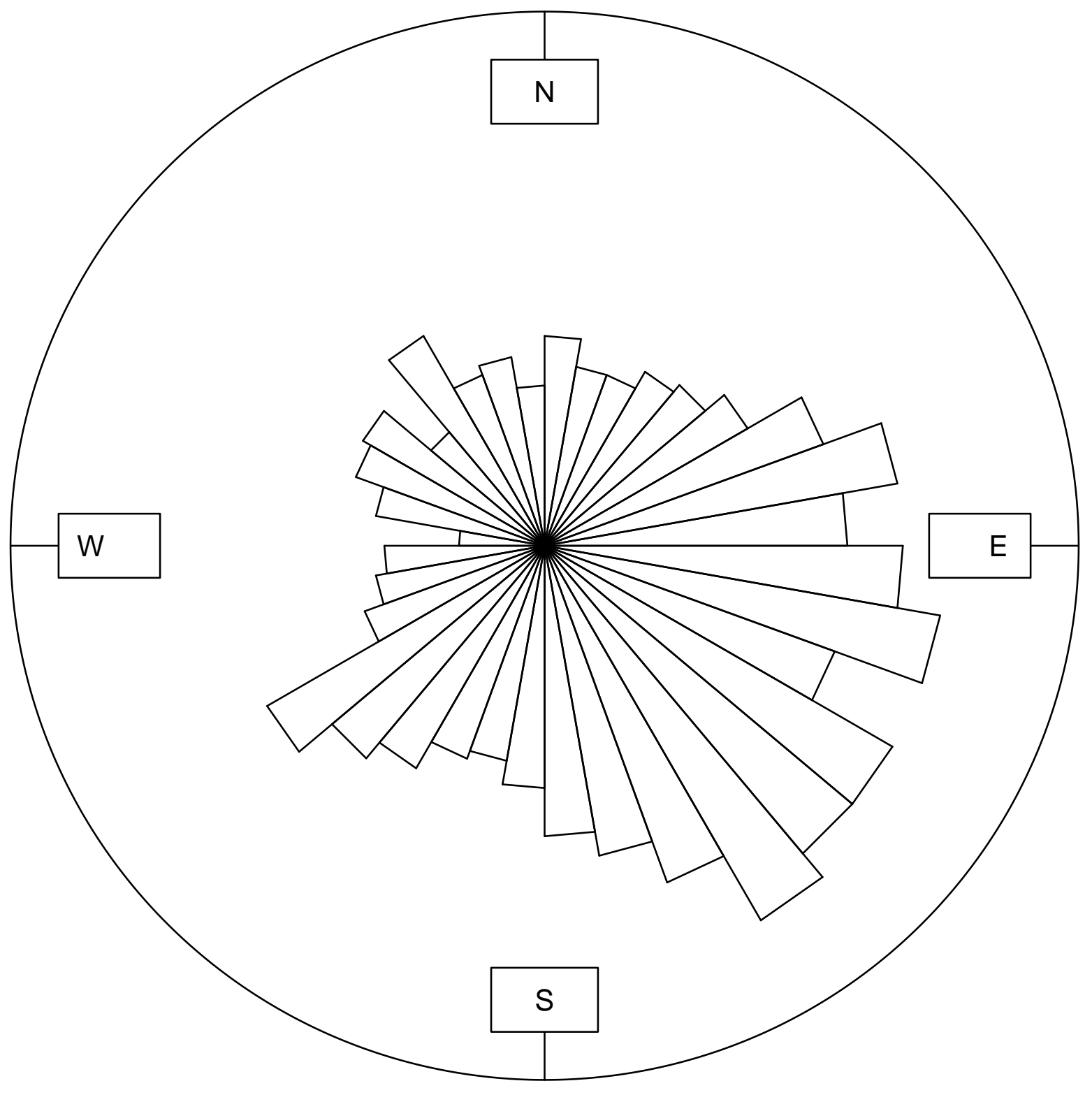


bootstrap 822

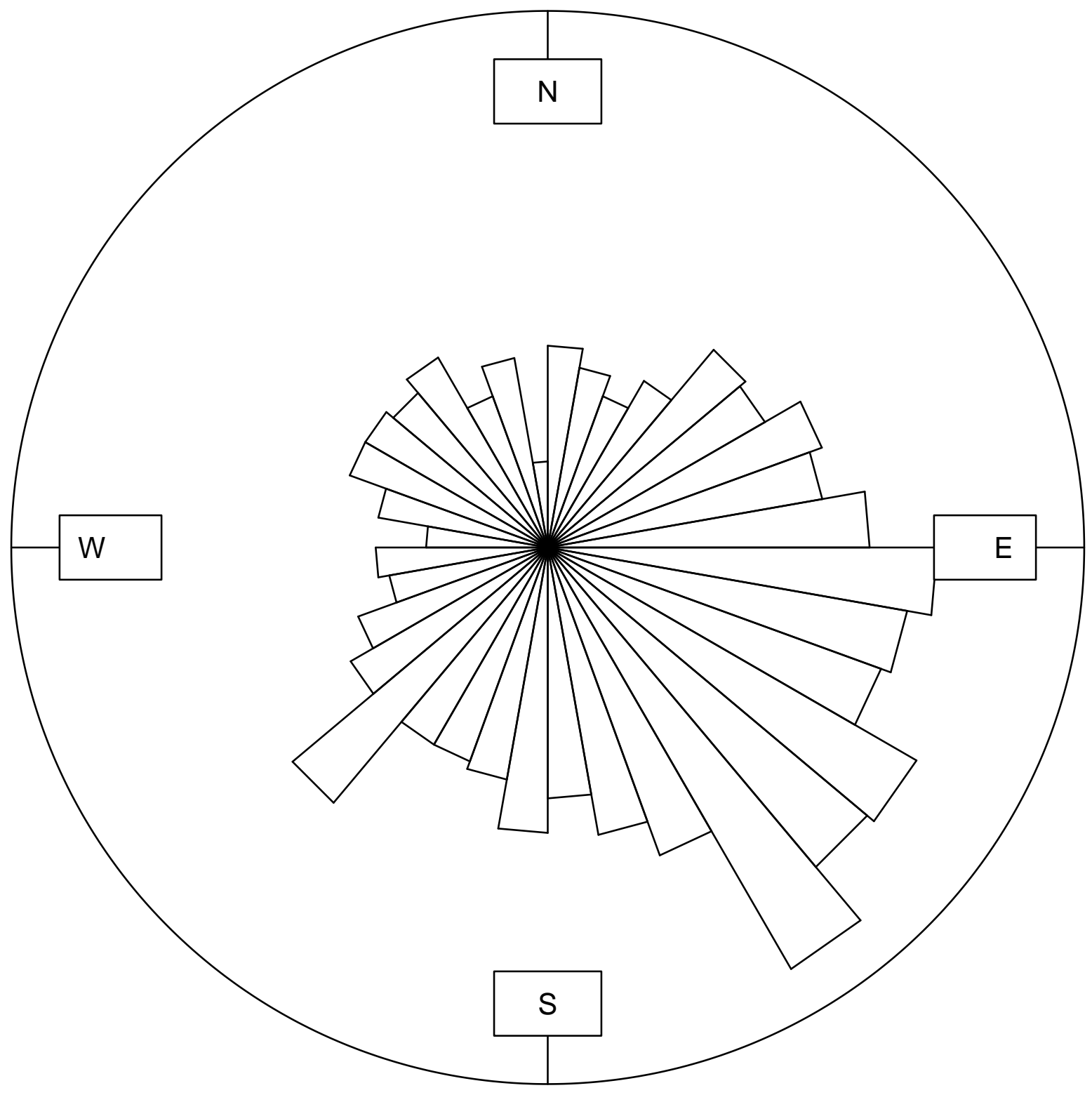


bootstrap 823

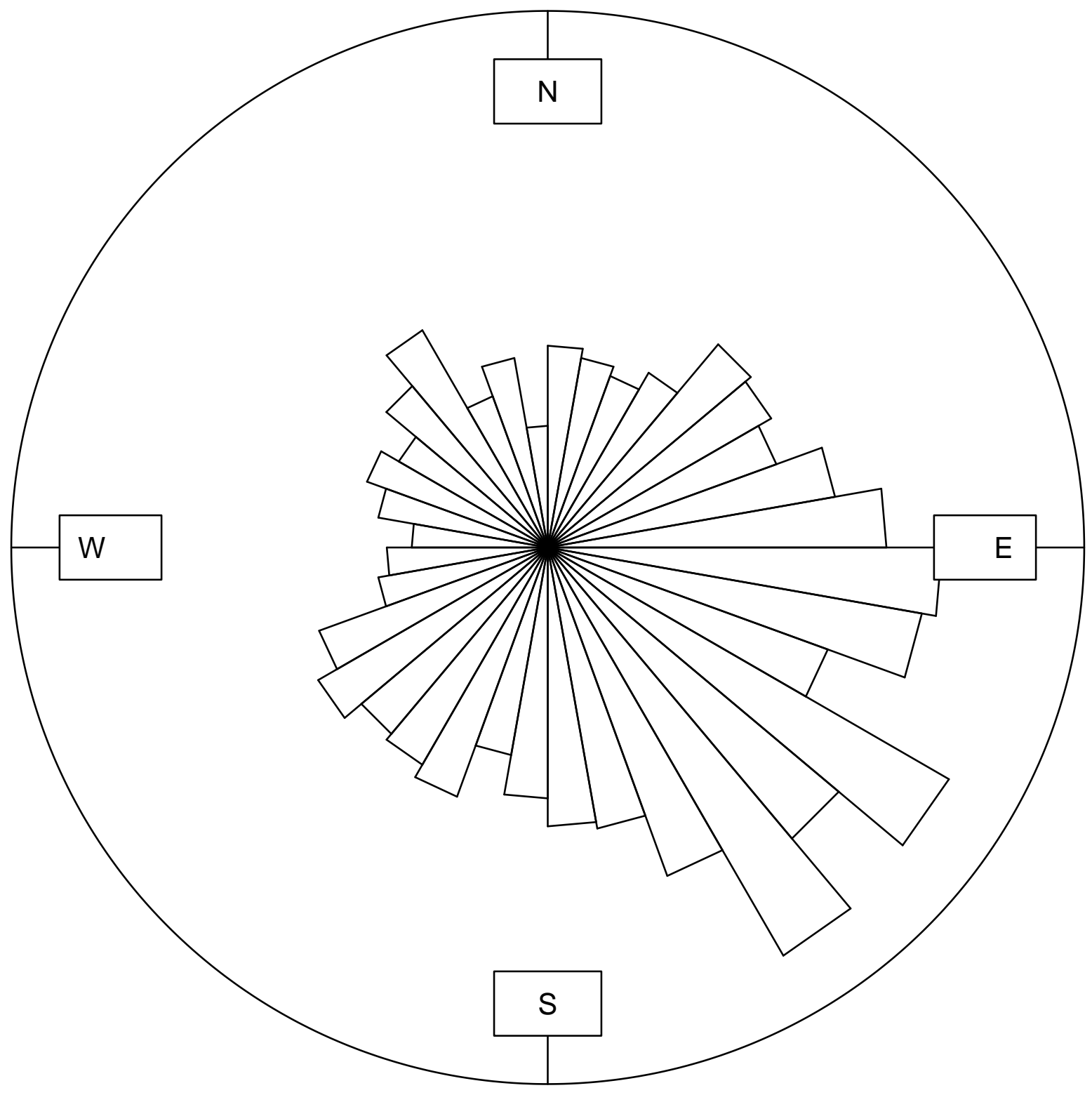




\section{bootstrap 824}

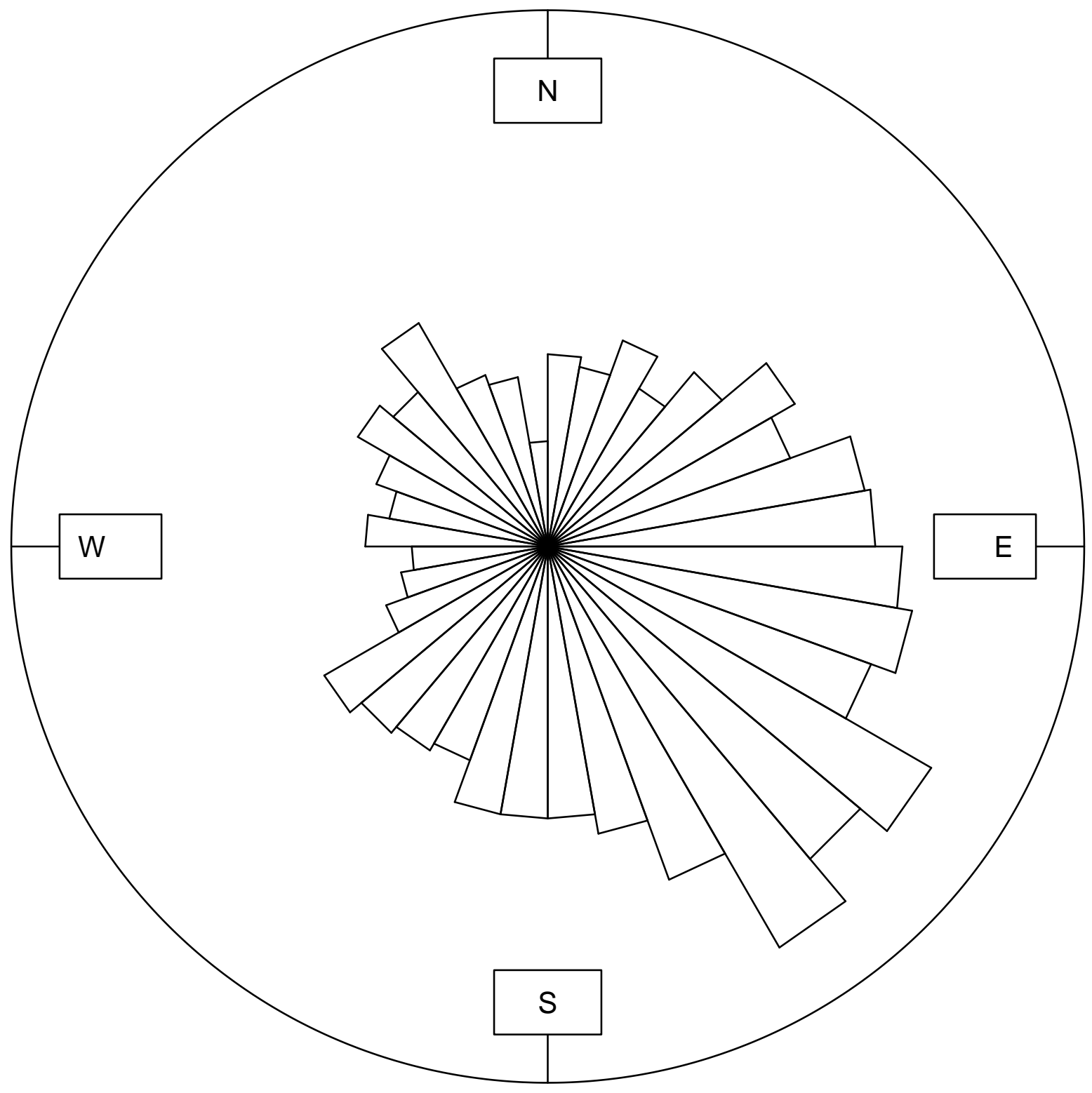


bootstrap 825

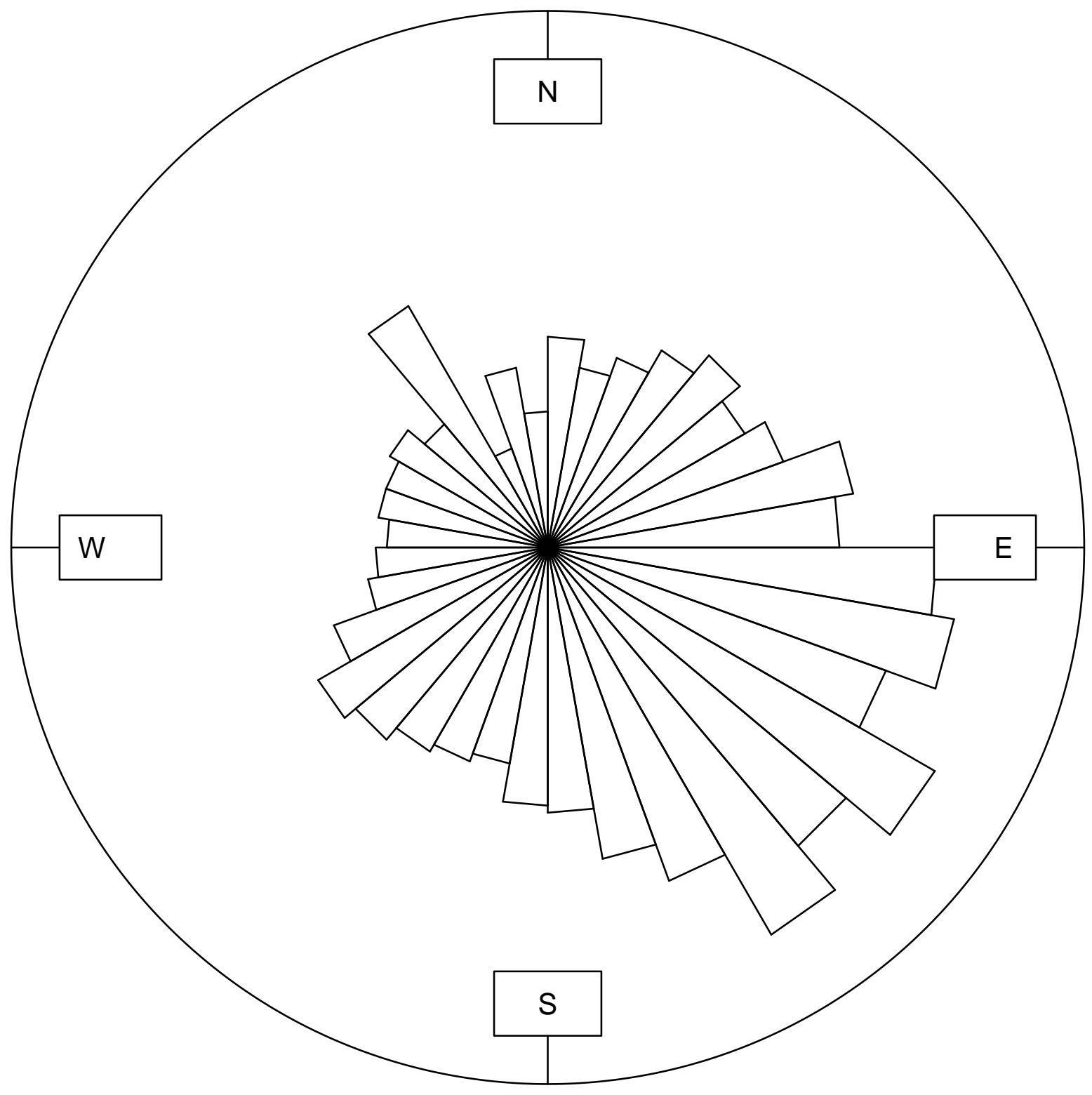


bootstrap 826

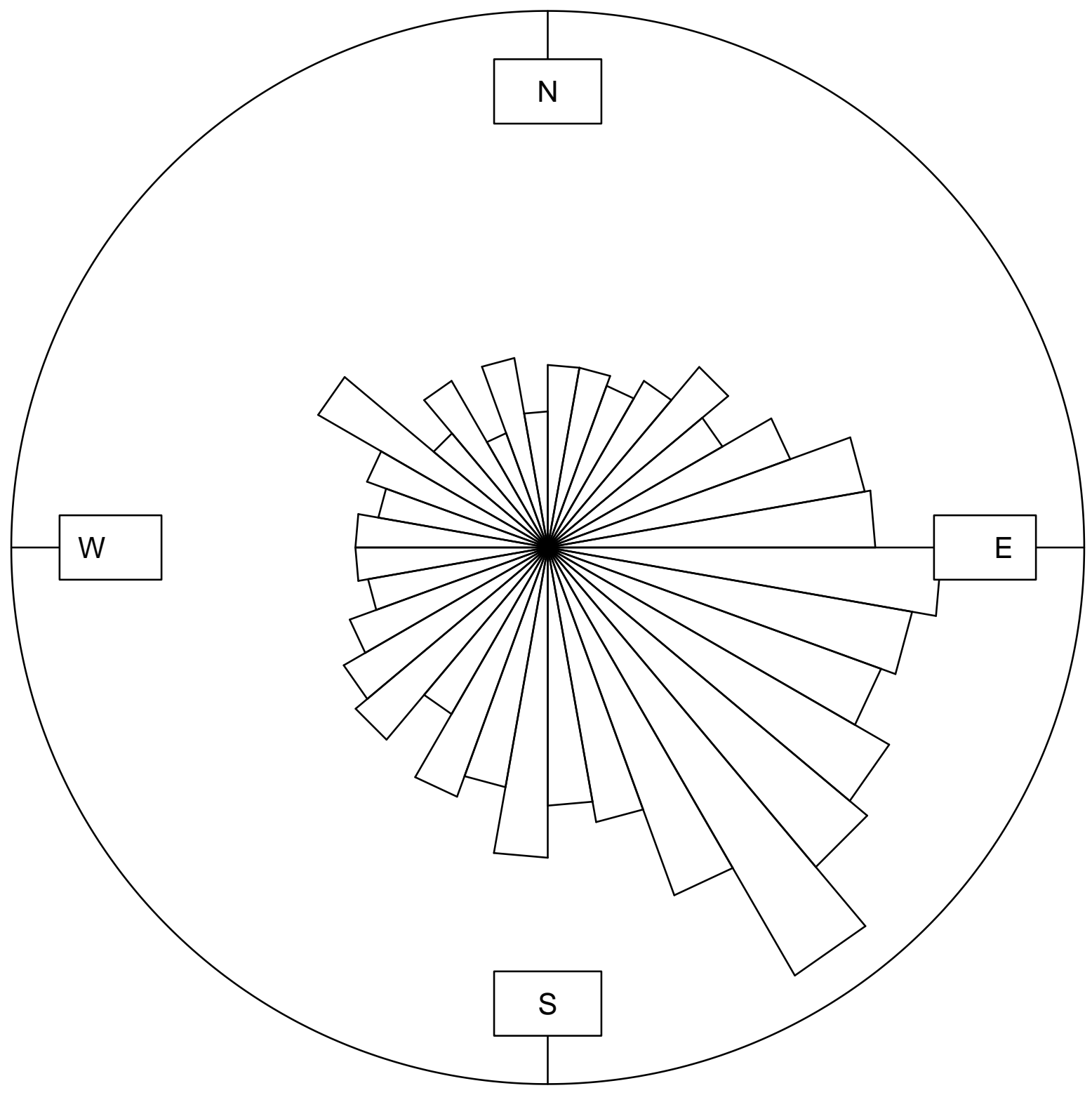


bootstrap 827

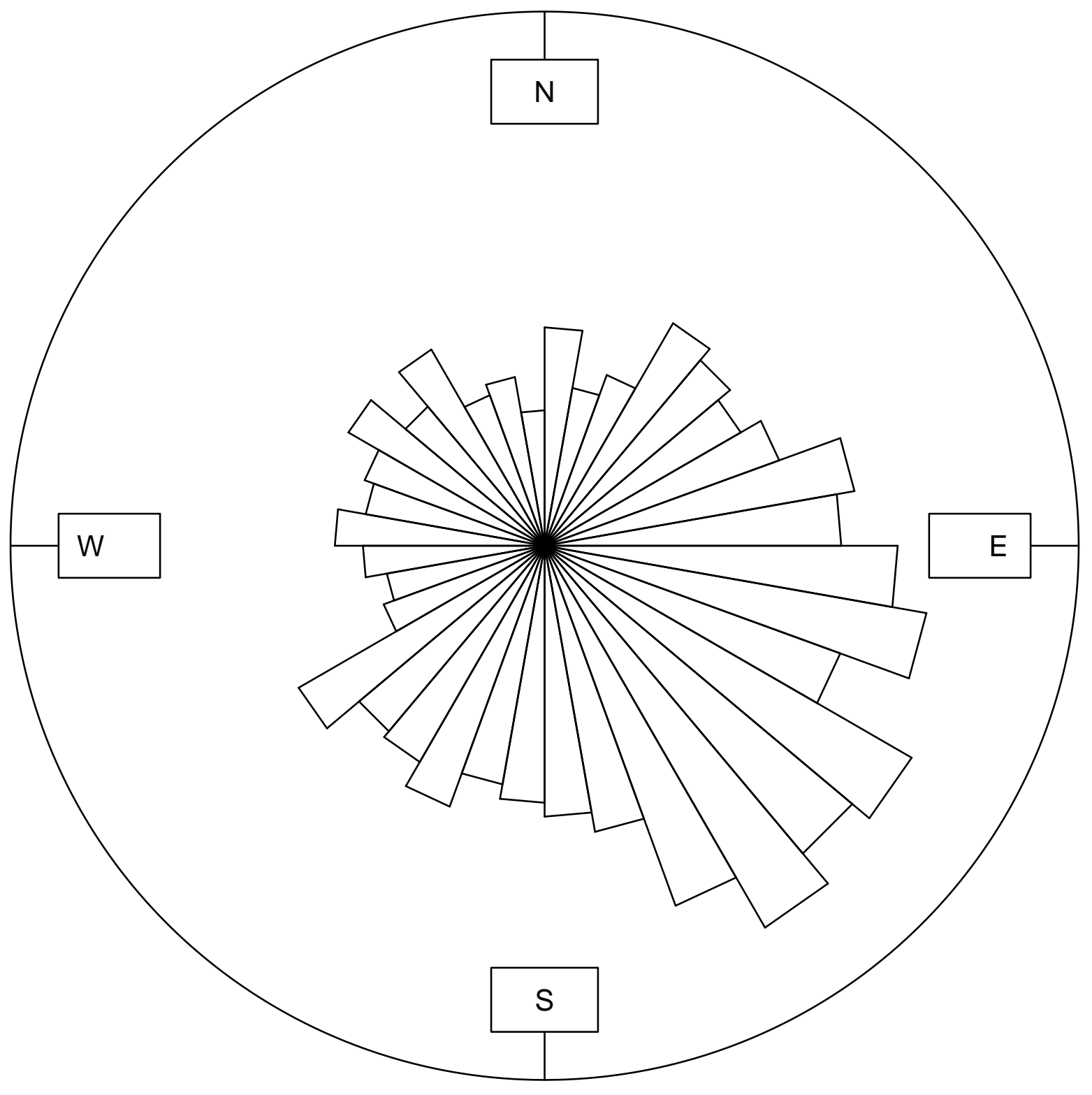


bootstrap 828

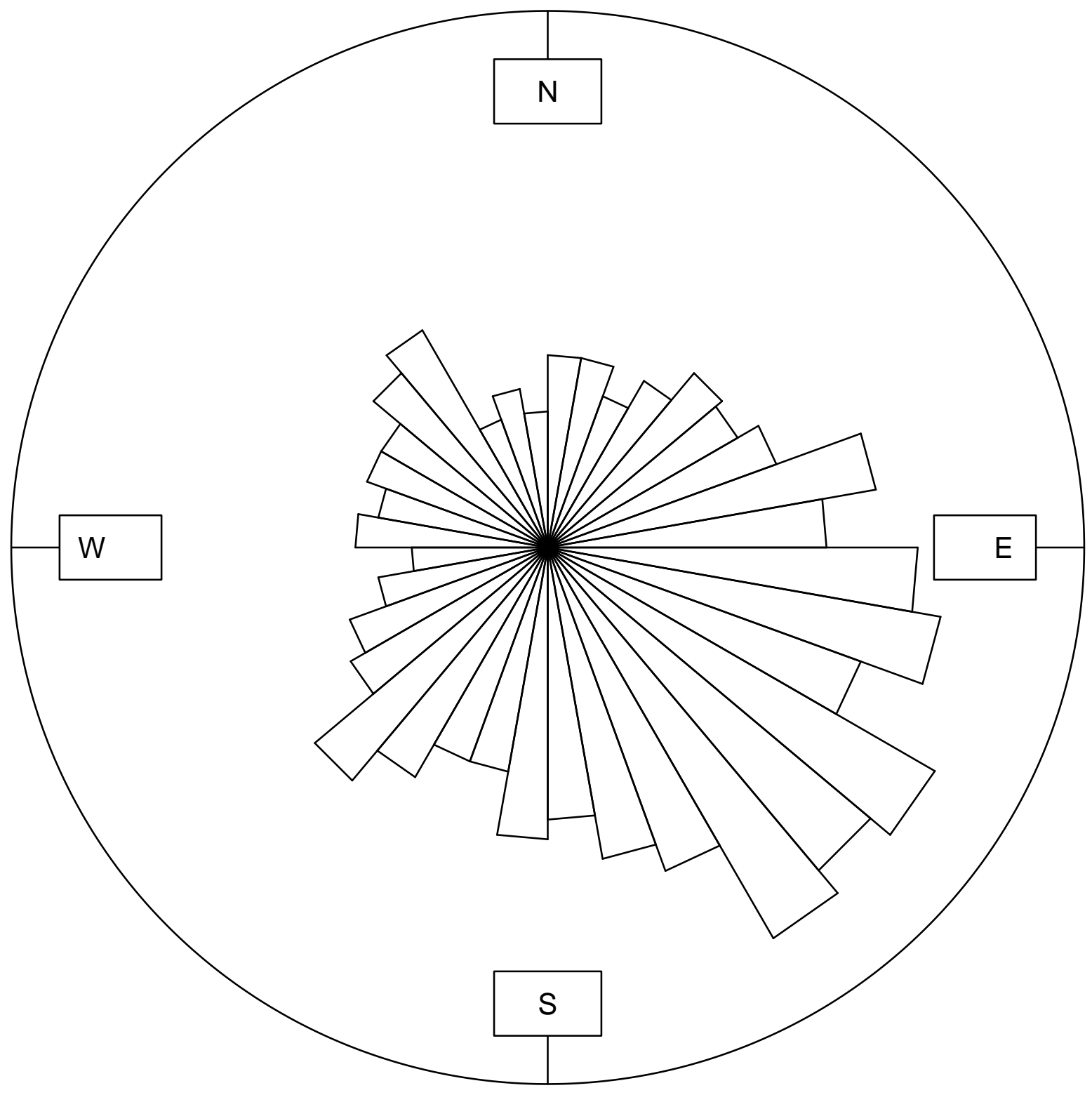


bootstrap 829

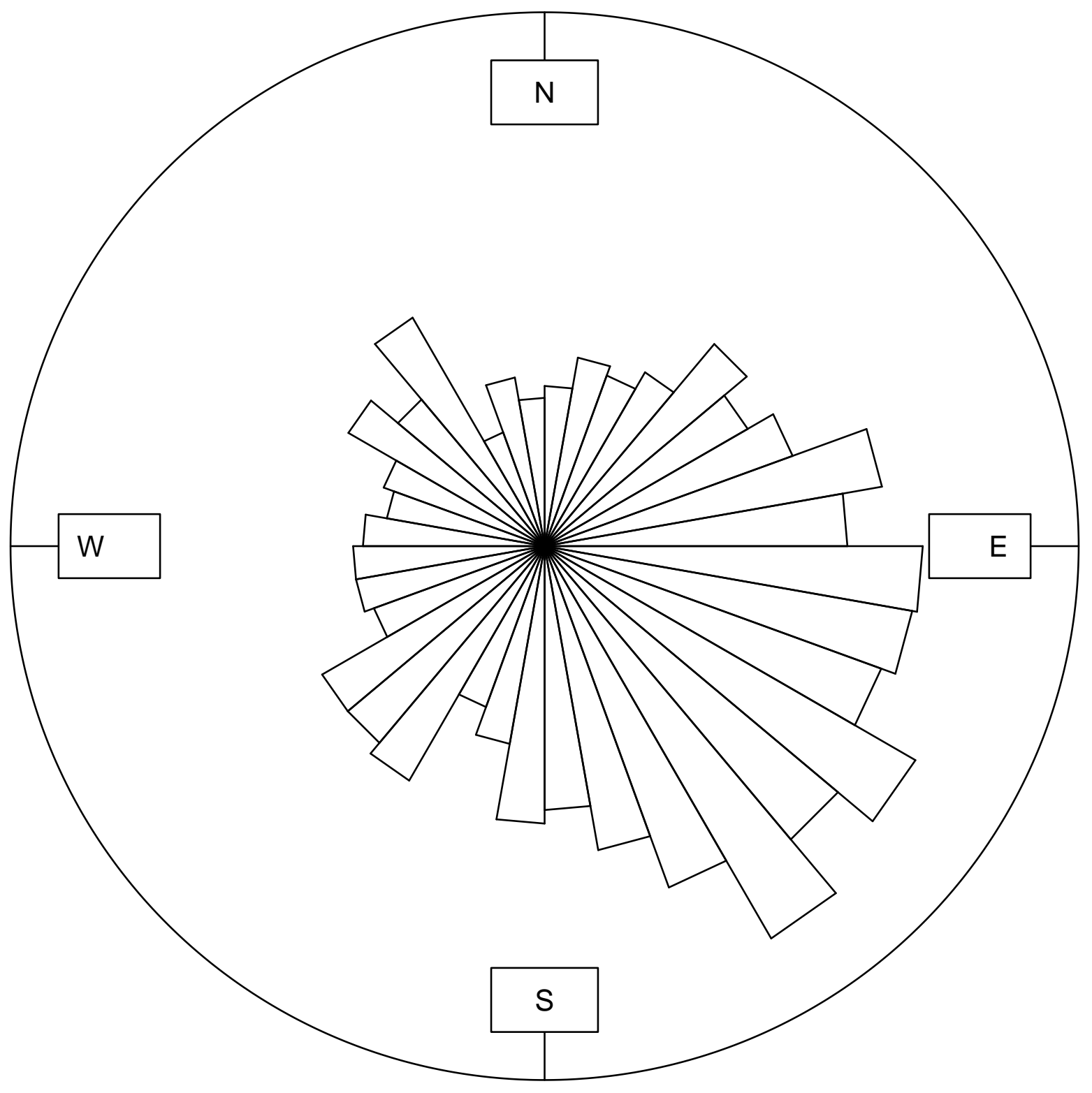


bootstrap 830

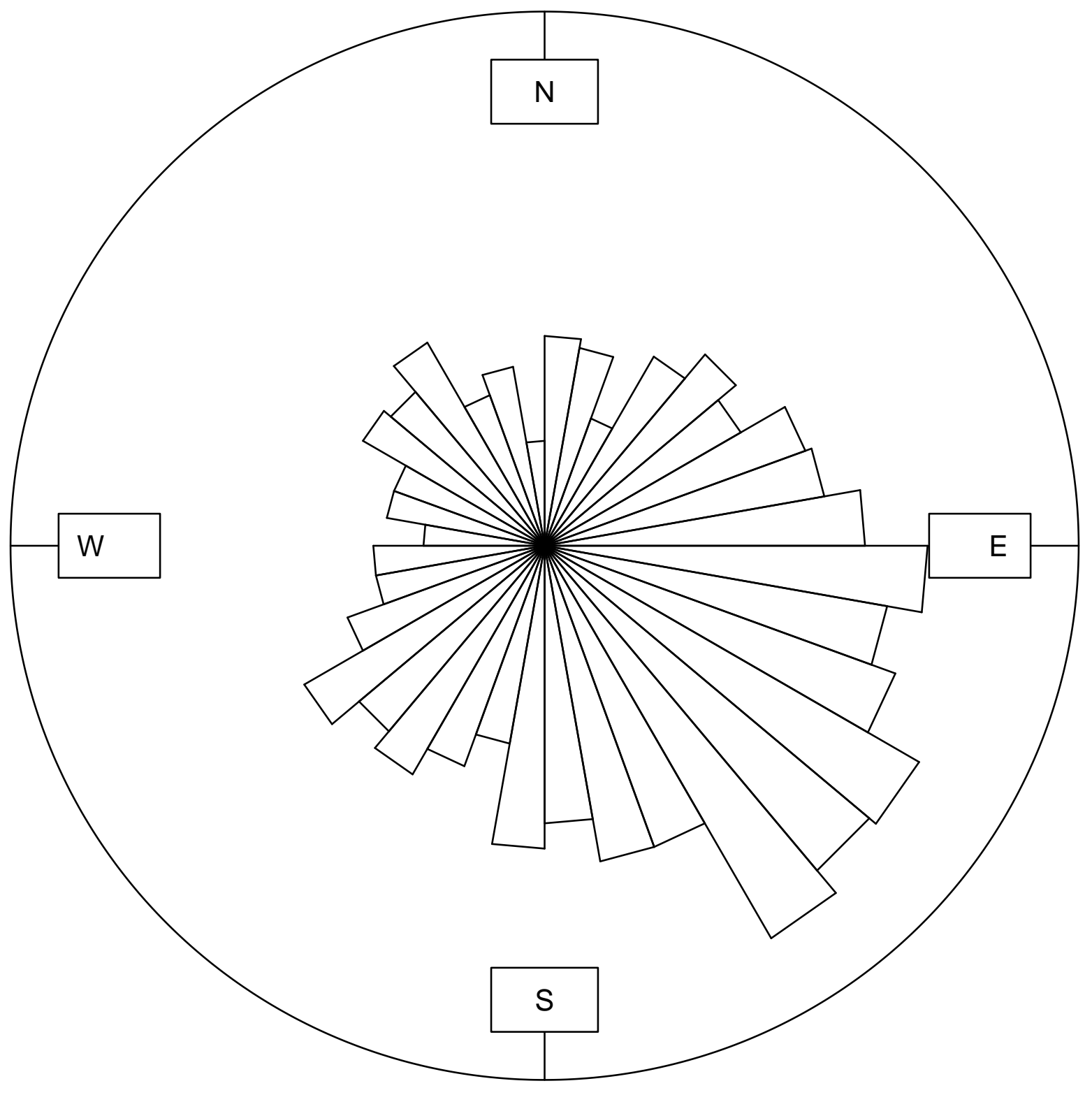


bootstrap 831

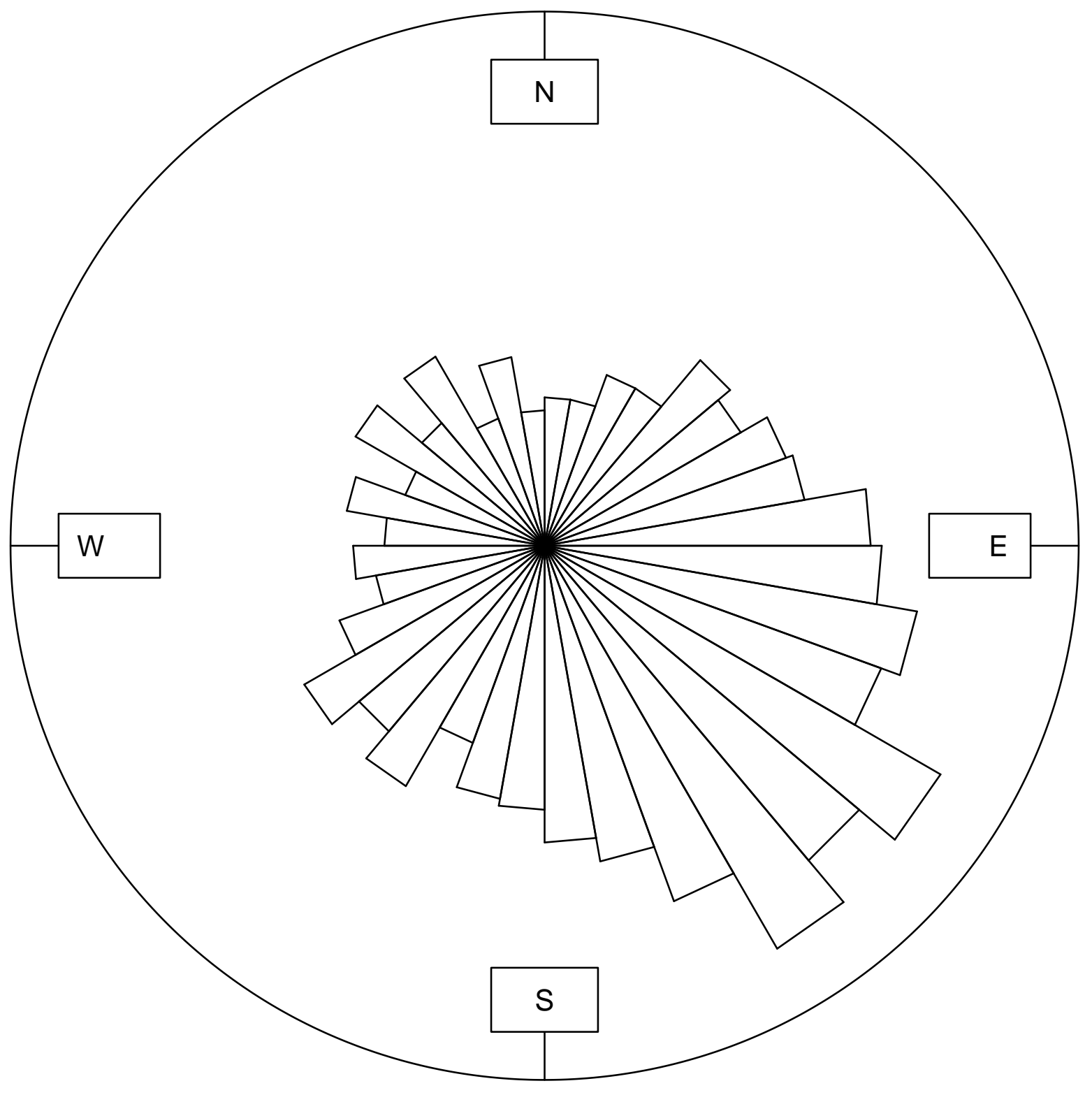


bootstrap 832

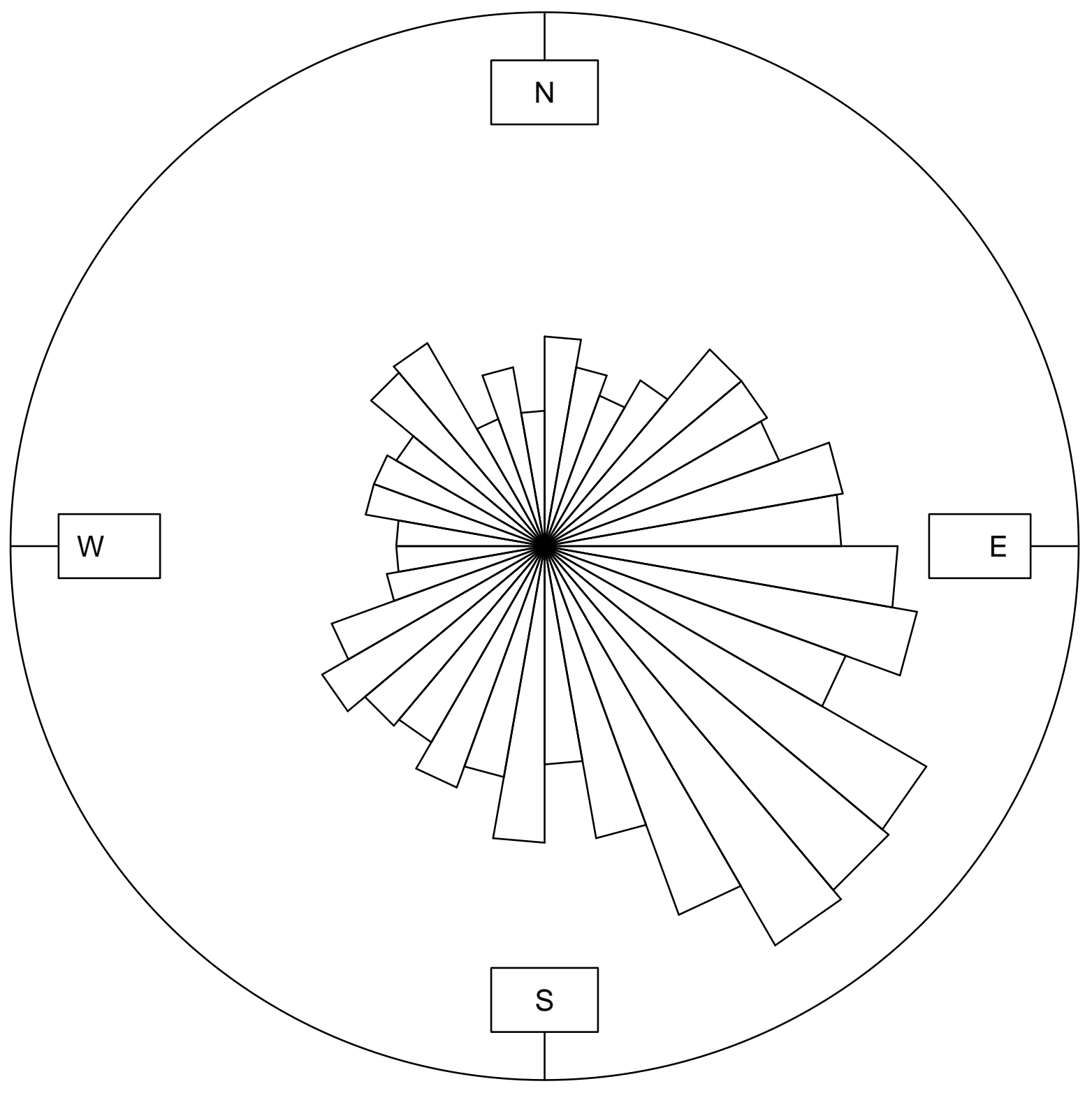




\section{bootstrap 833}

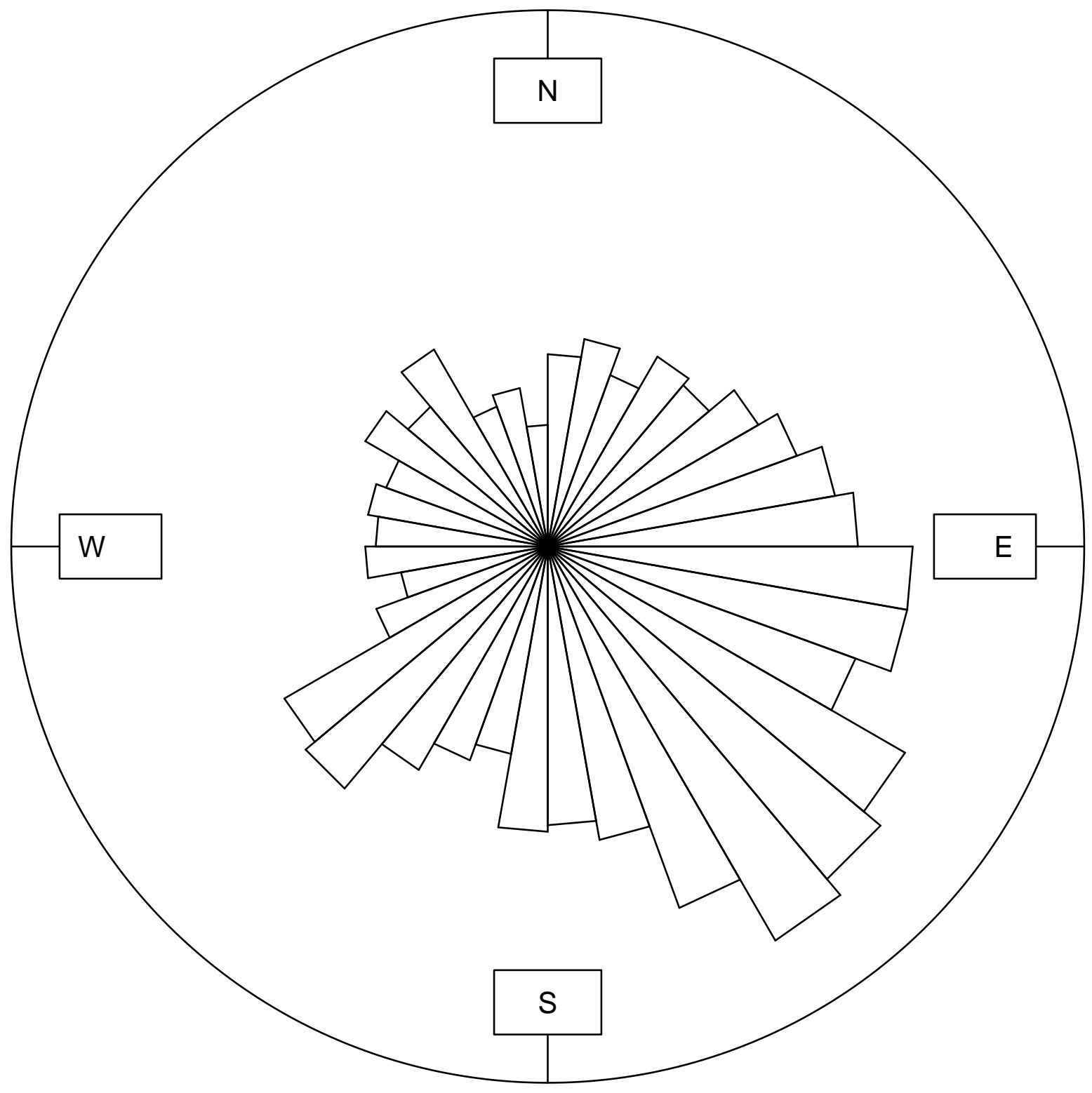




\section{bootstrap 834}

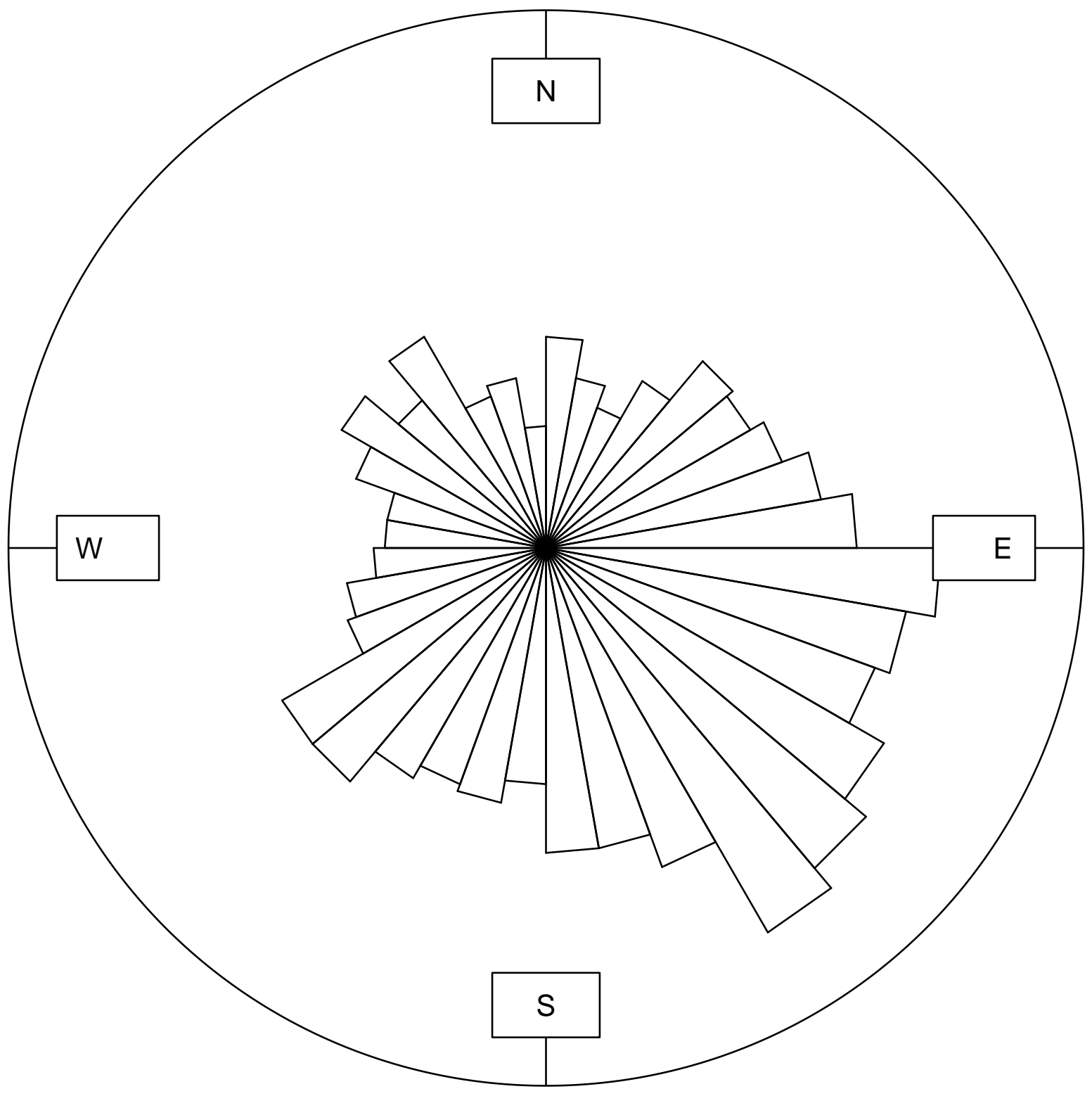




\section{bootstrap 835}

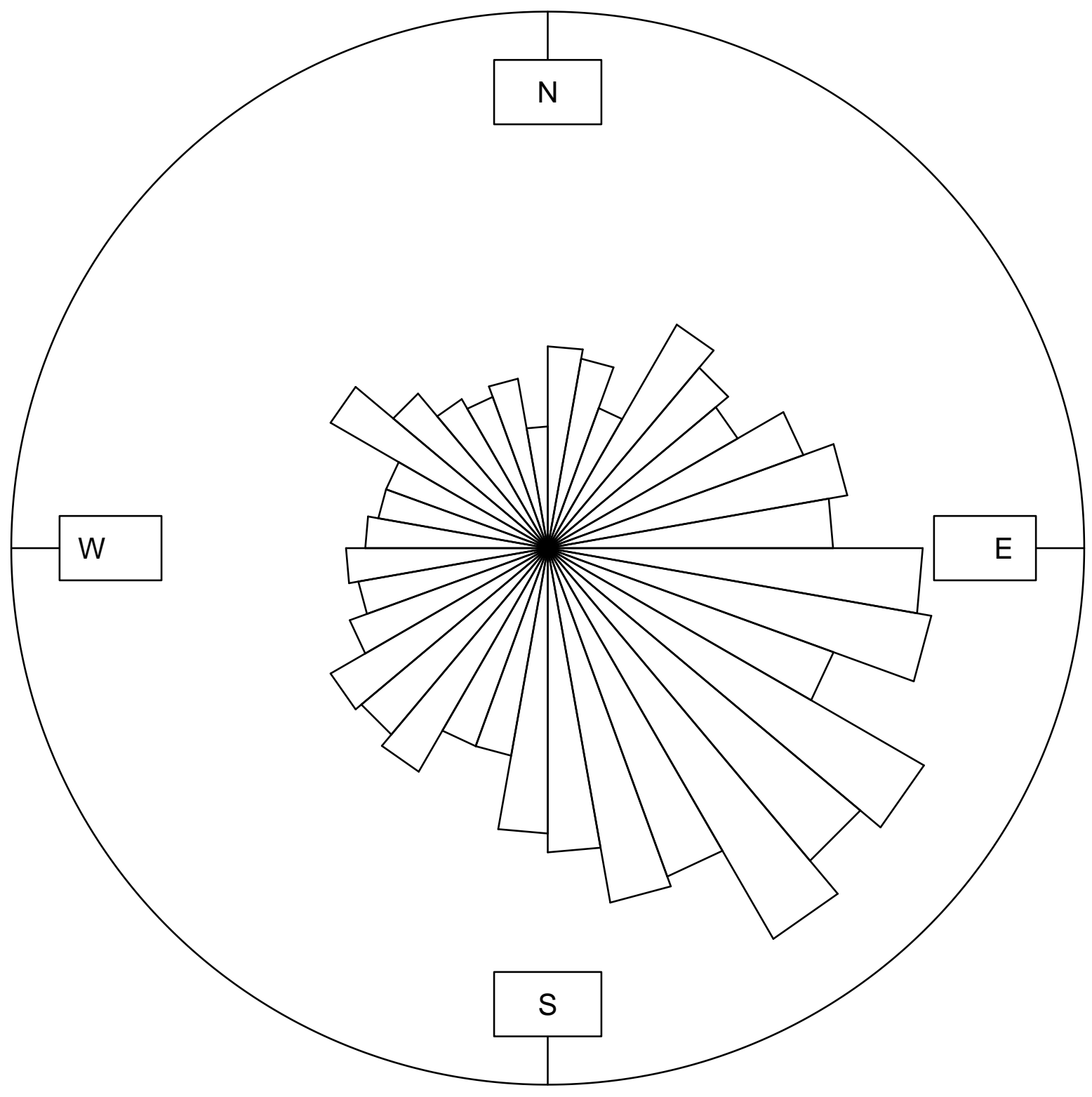




\section{bootstrap 836}

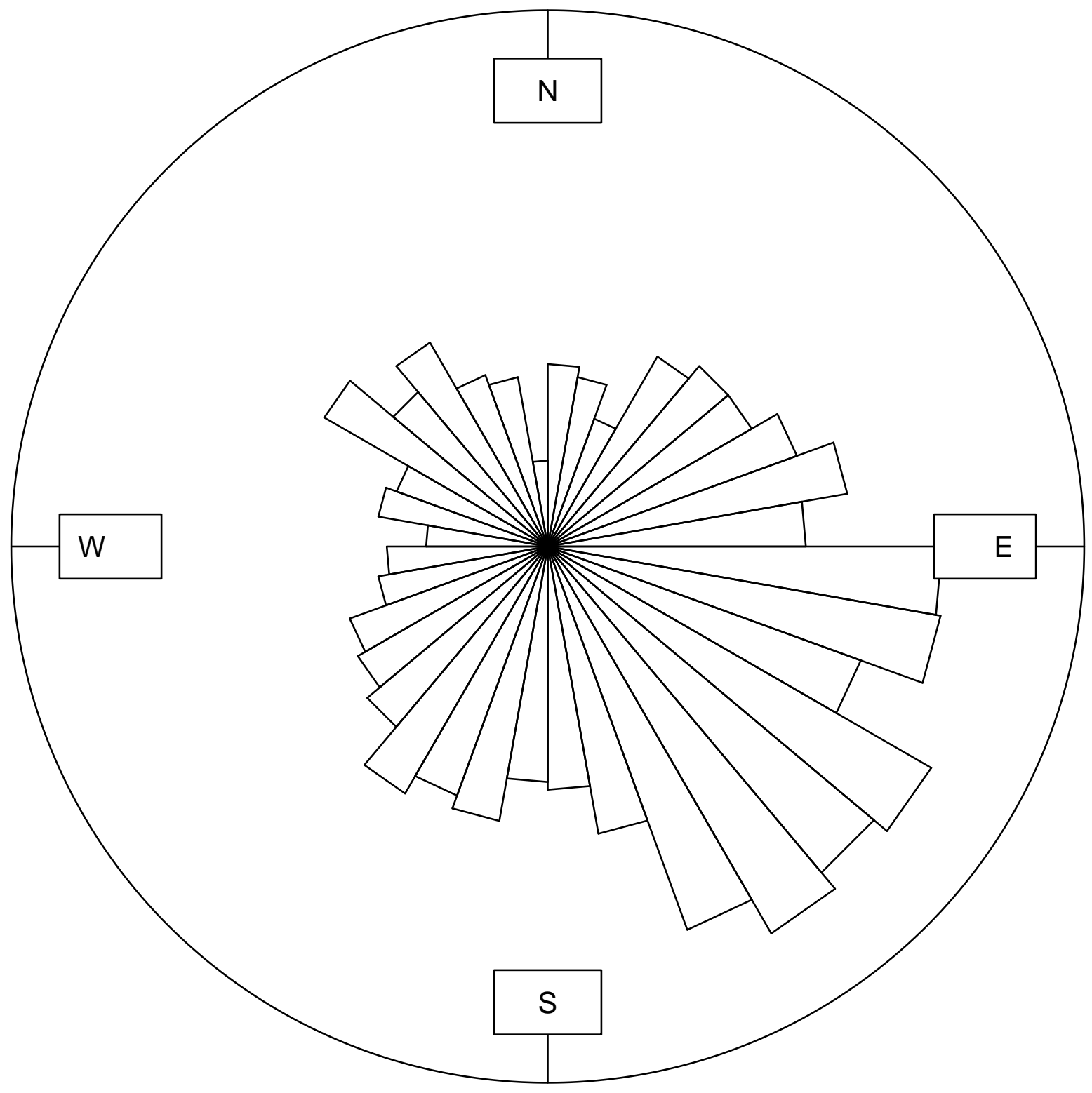


bootstrap 837

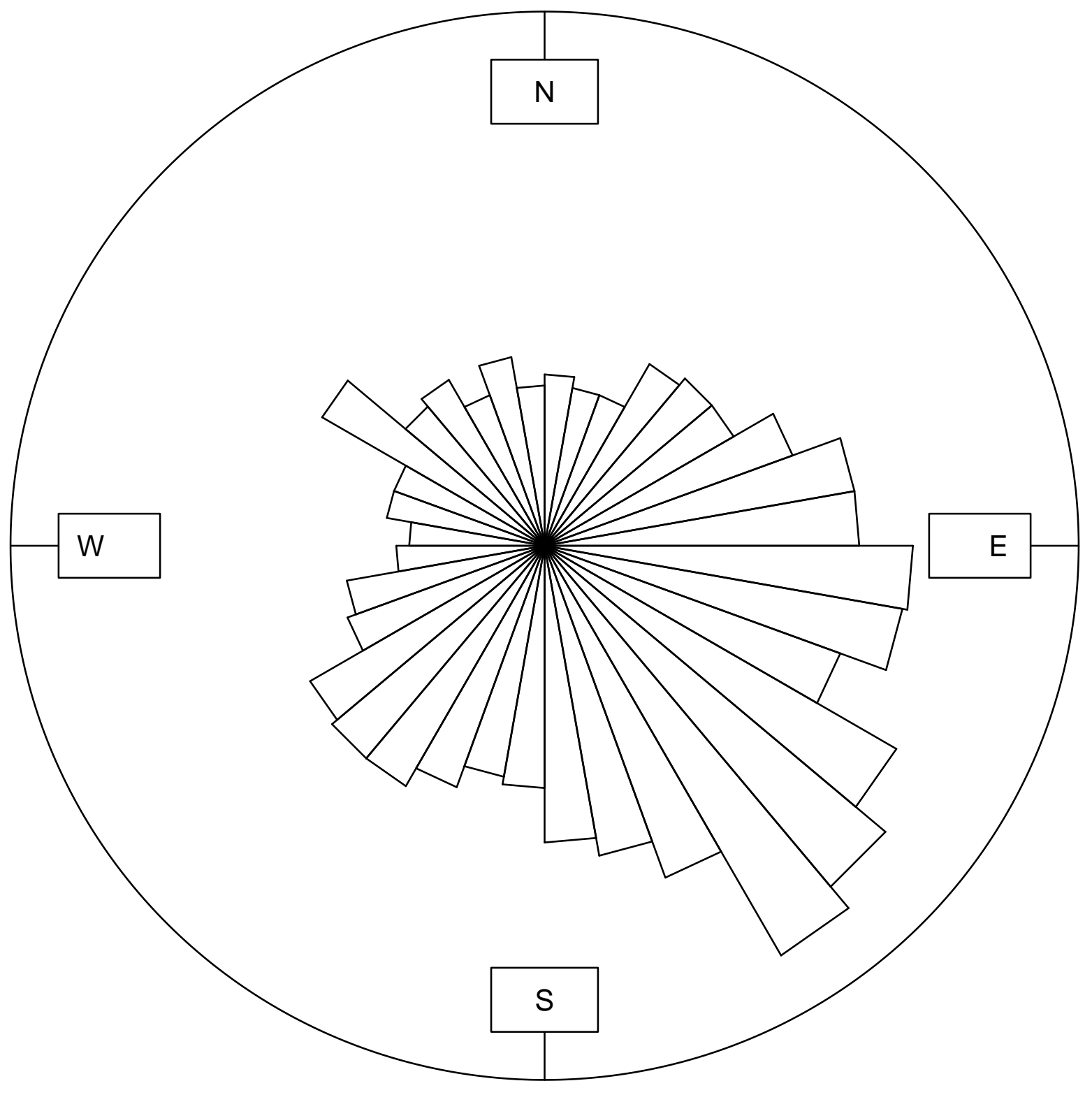


bootstrap 838

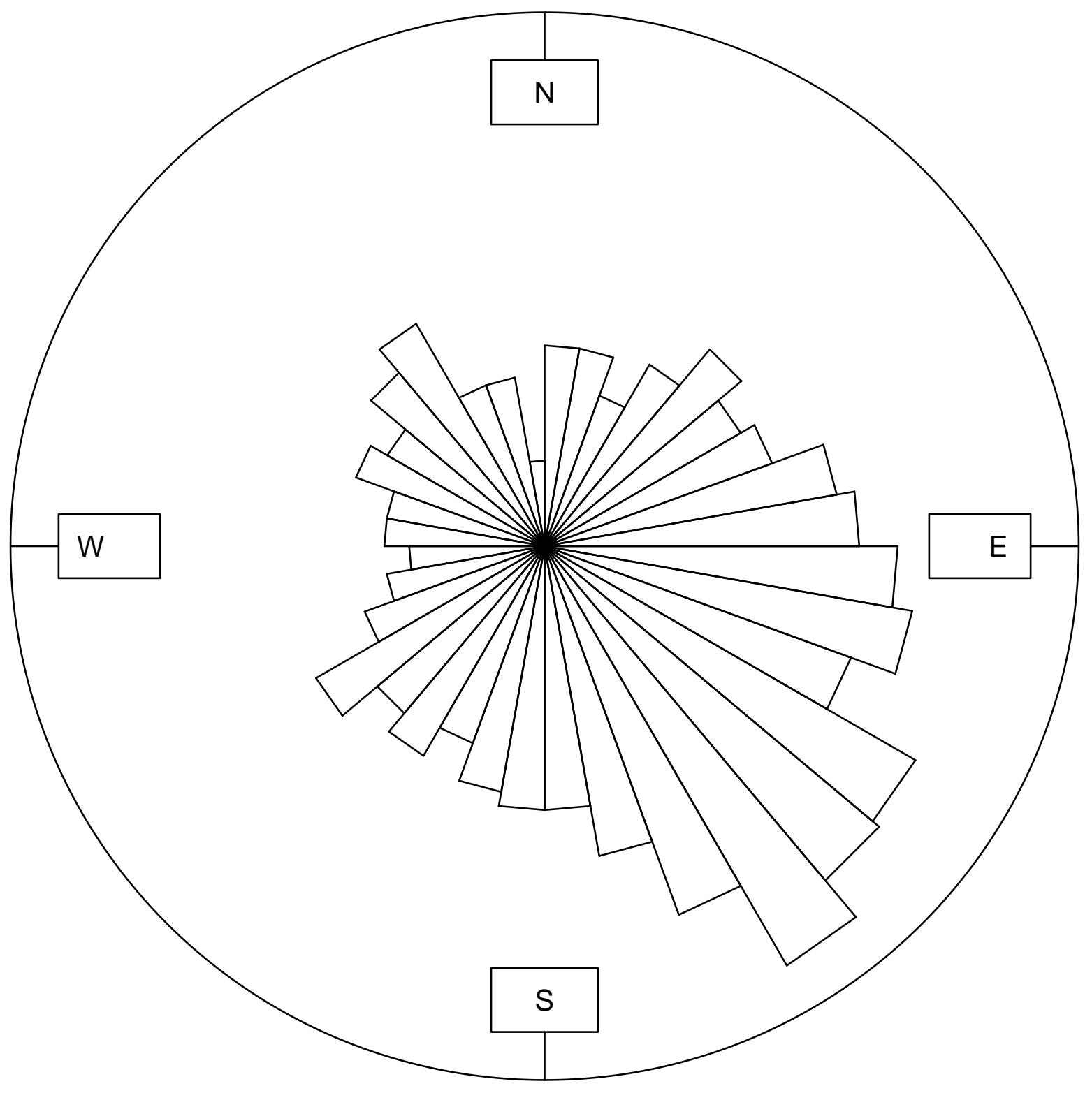


bootstrap 839

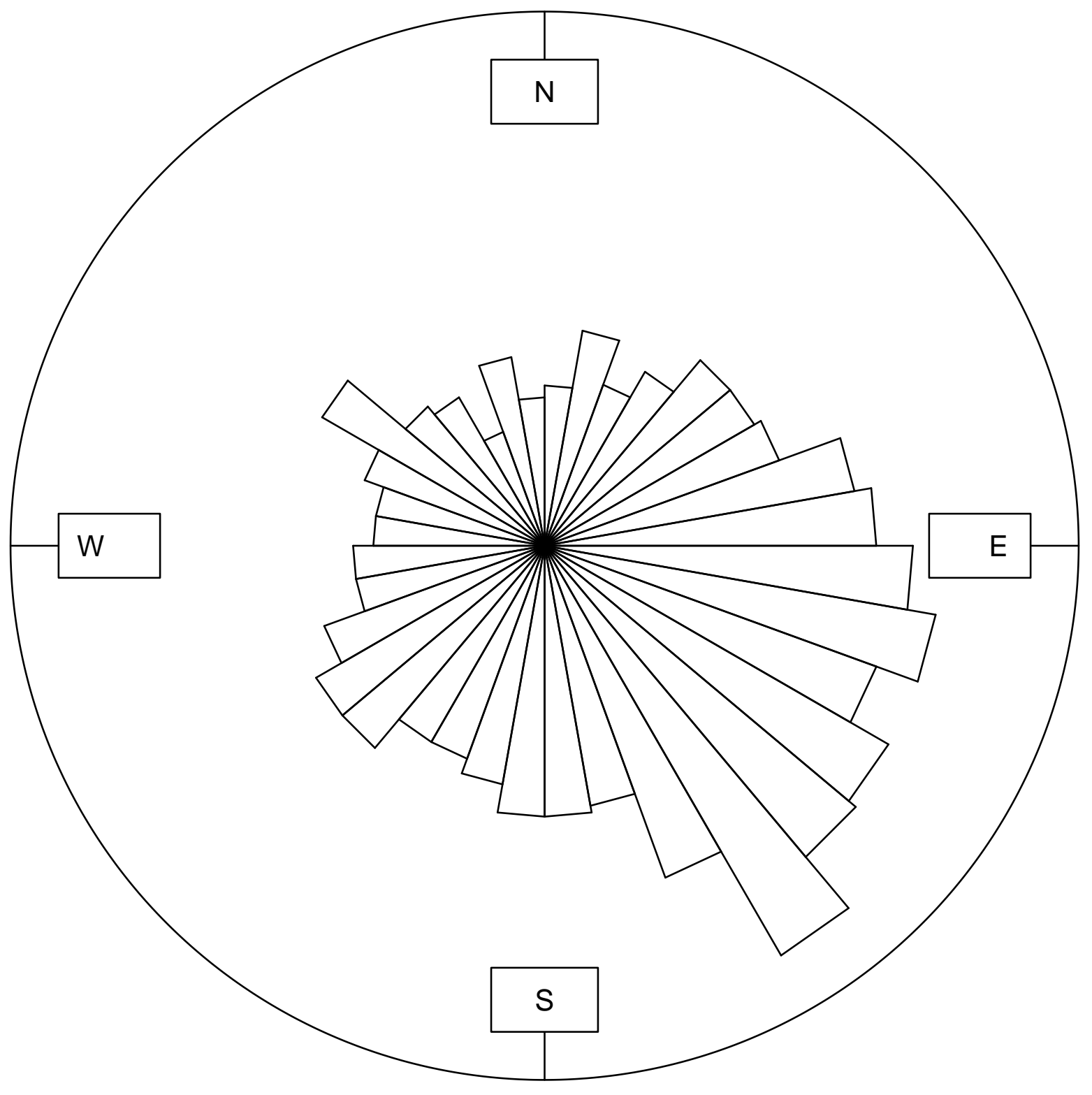


bootstrap 840

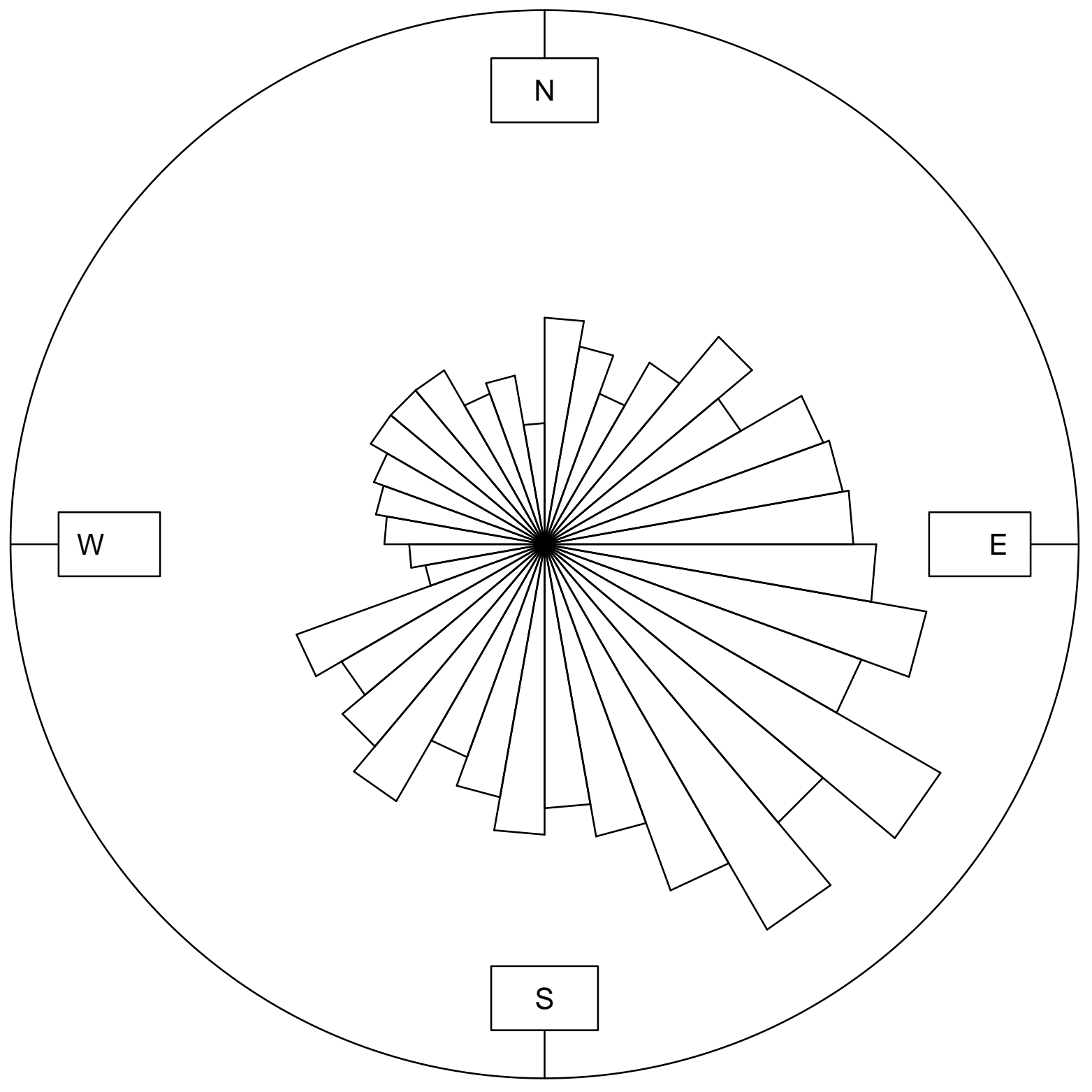




\section{bootstrap 841}

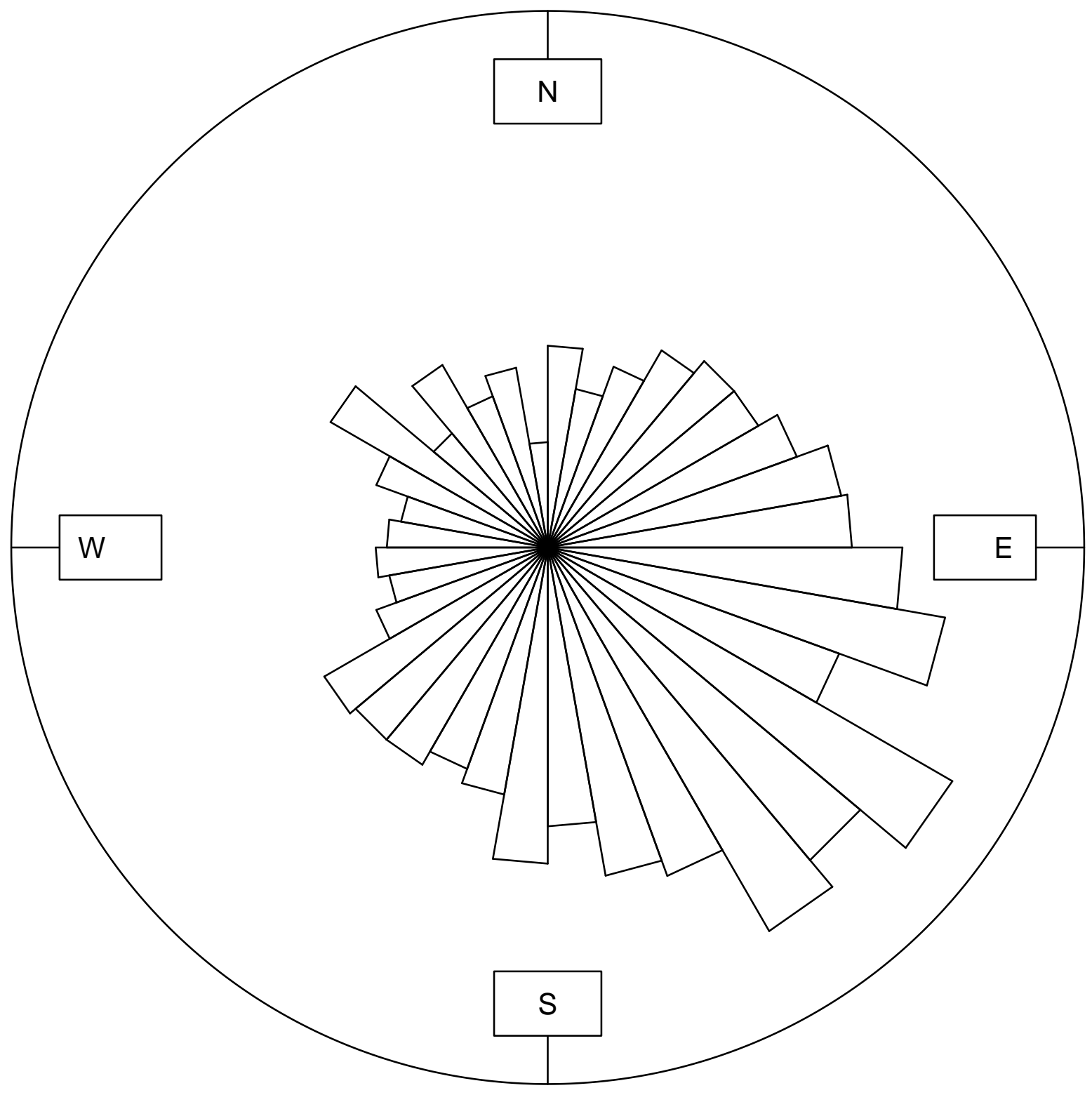


bootstrap 842

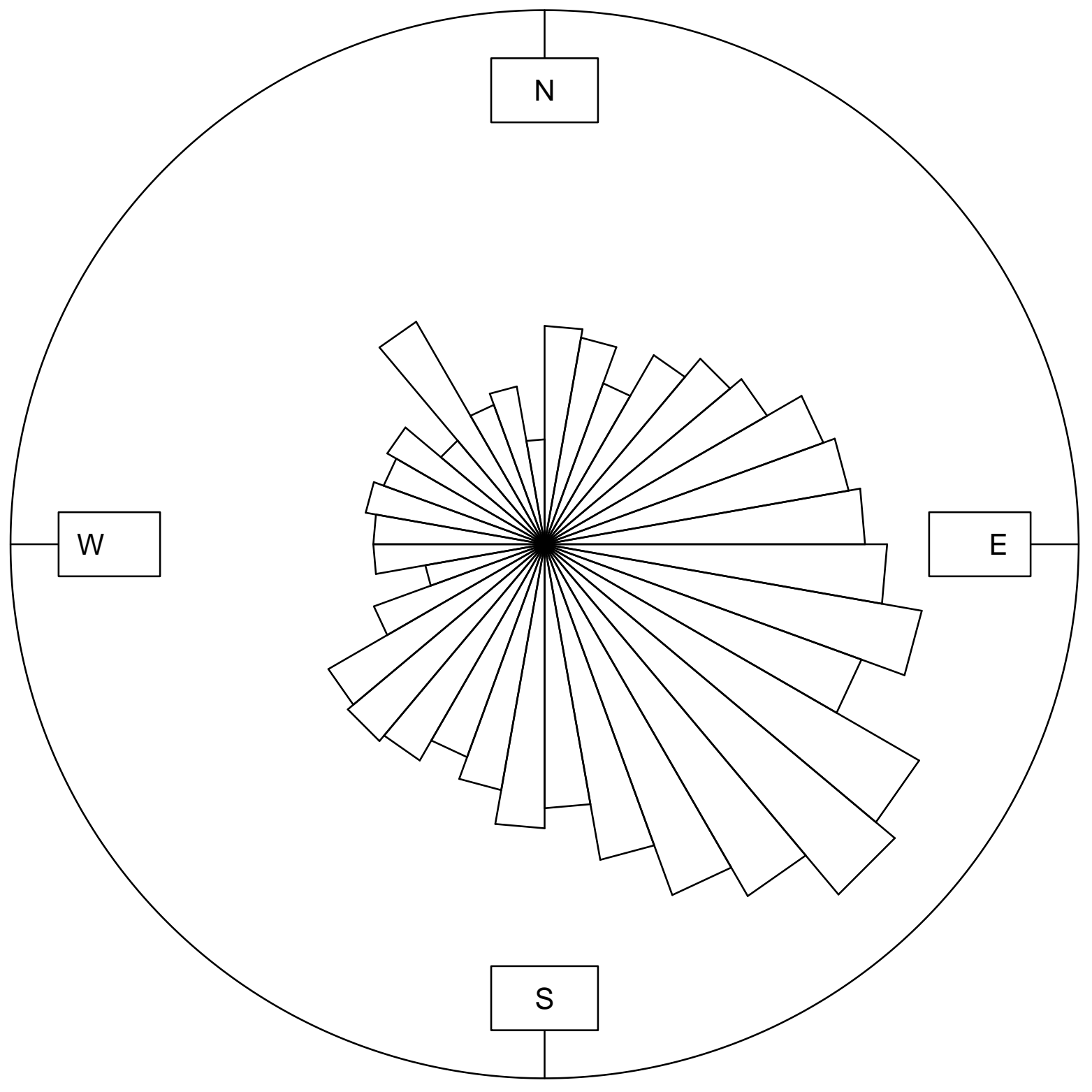




\section{bootstrap 843}

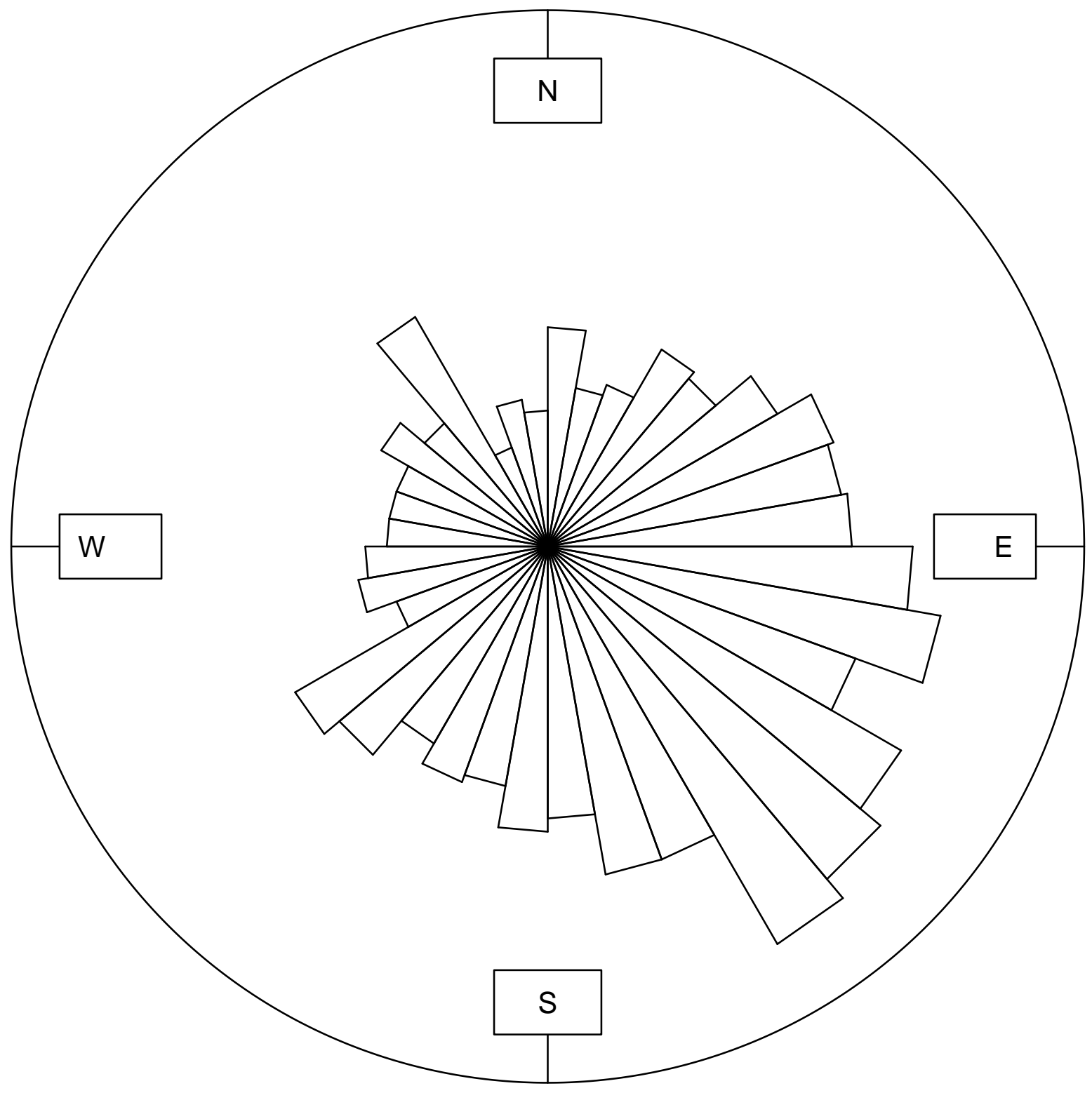




\section{bootstrap 844}

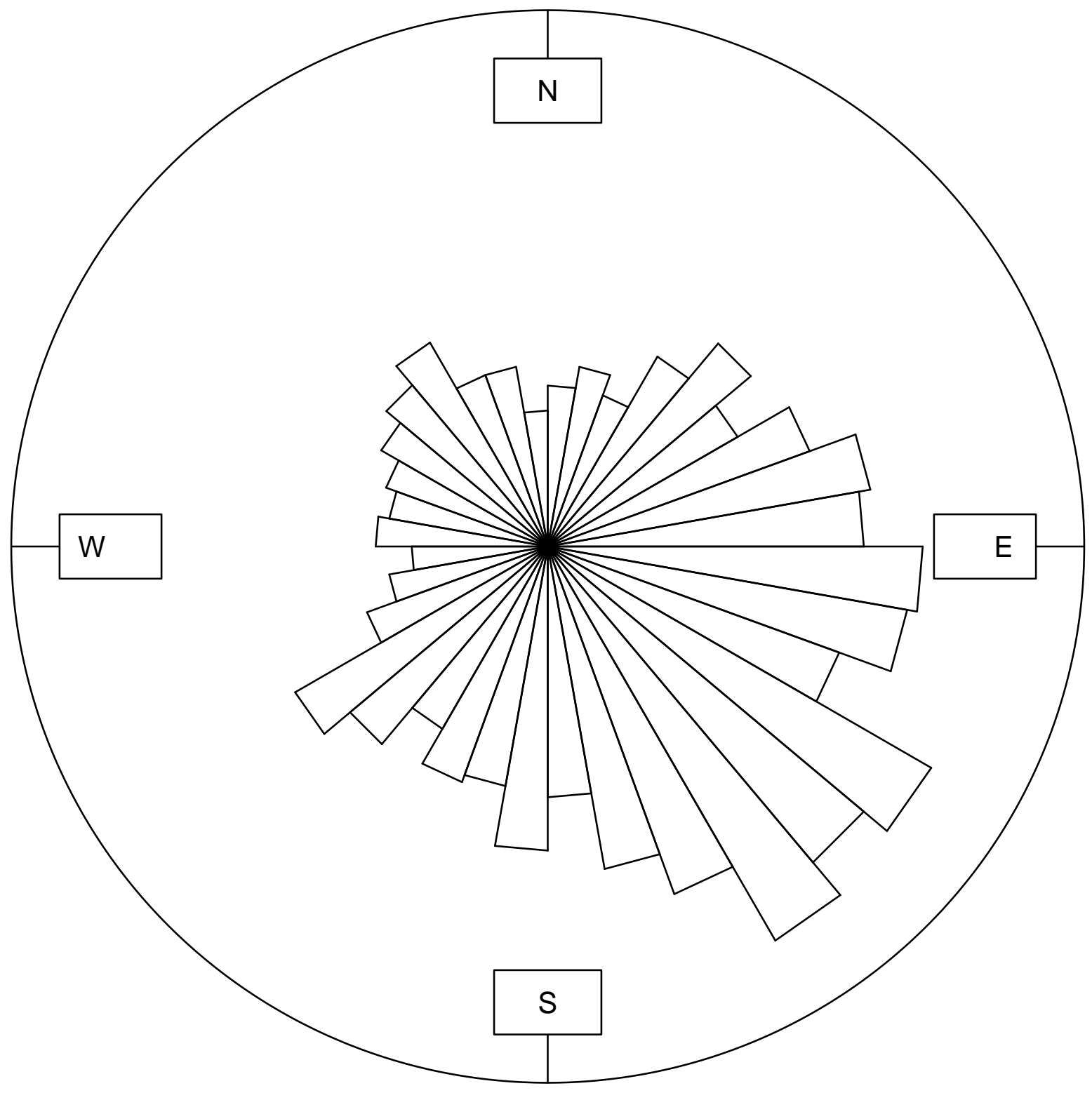




\section{bootstrap 845}

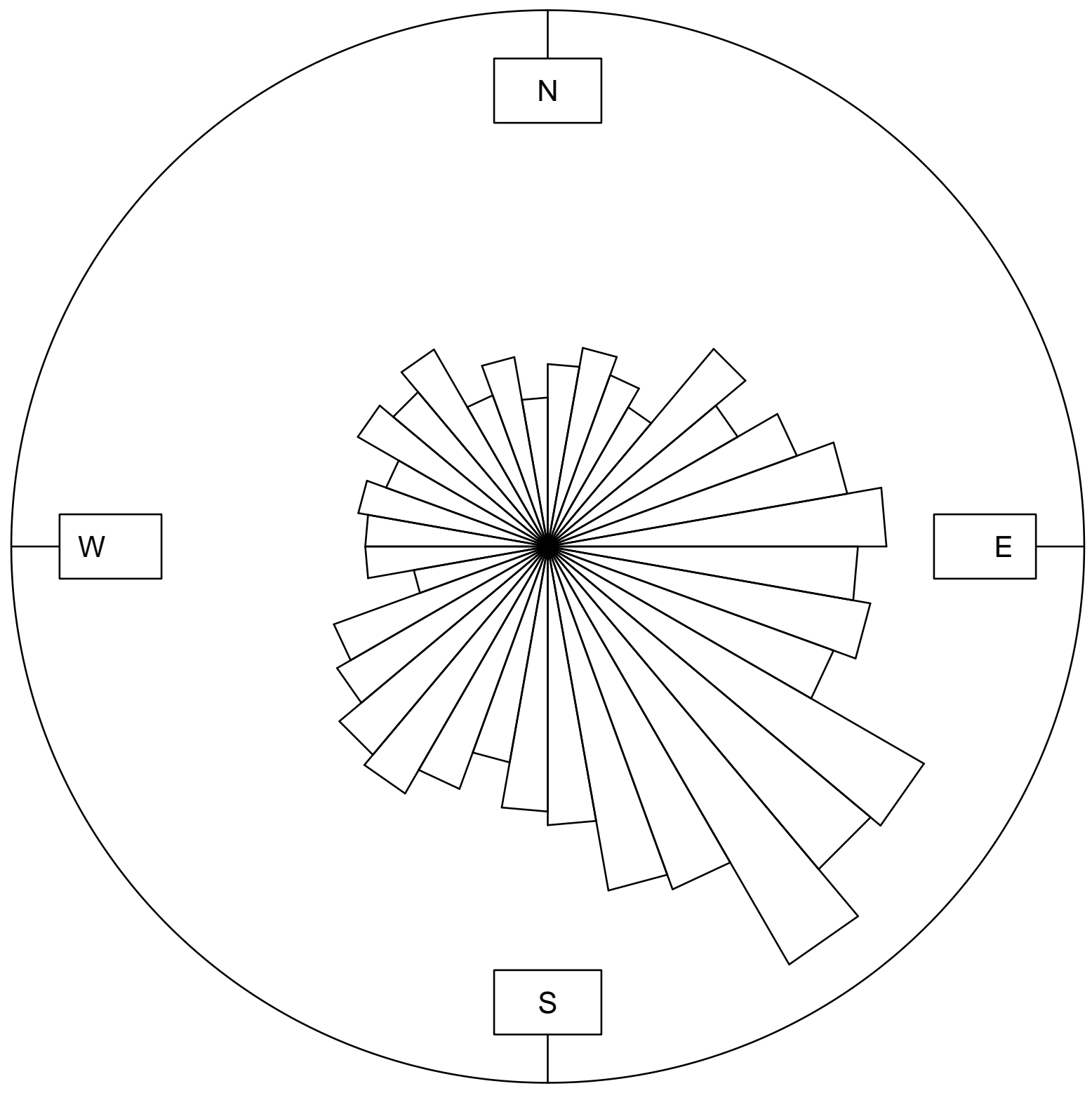


bootstrap 846

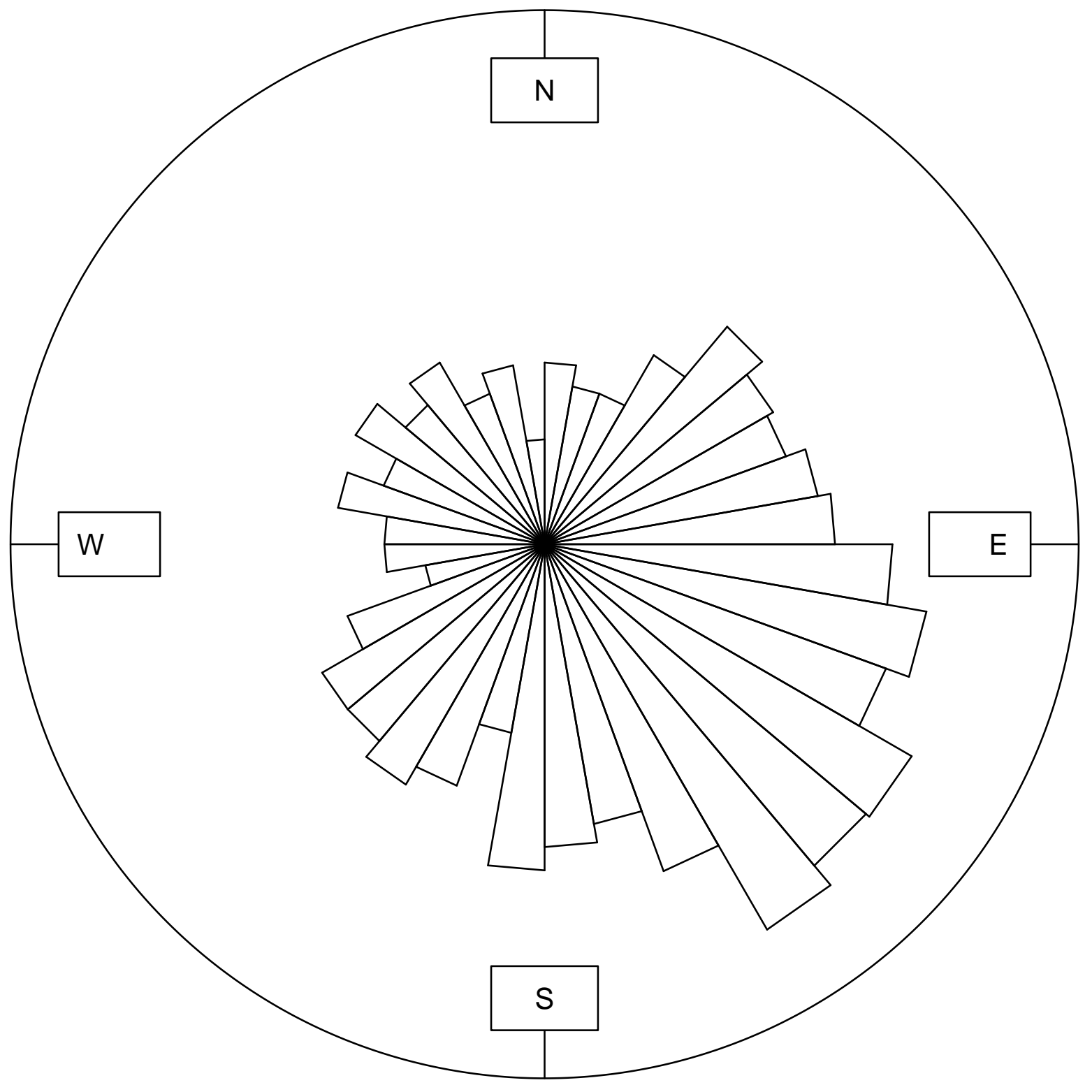


bootstrap 847

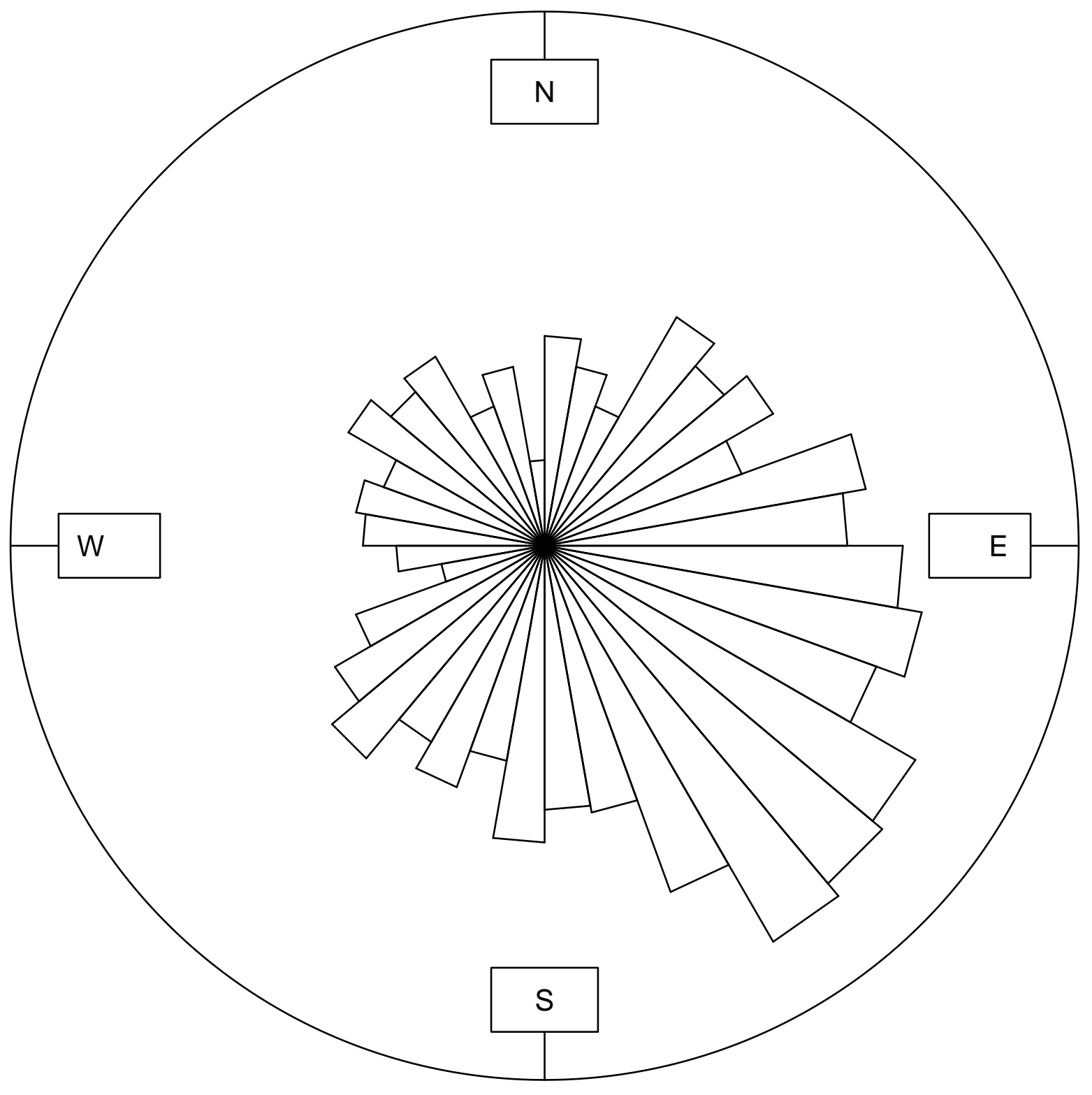


bootstrap 848

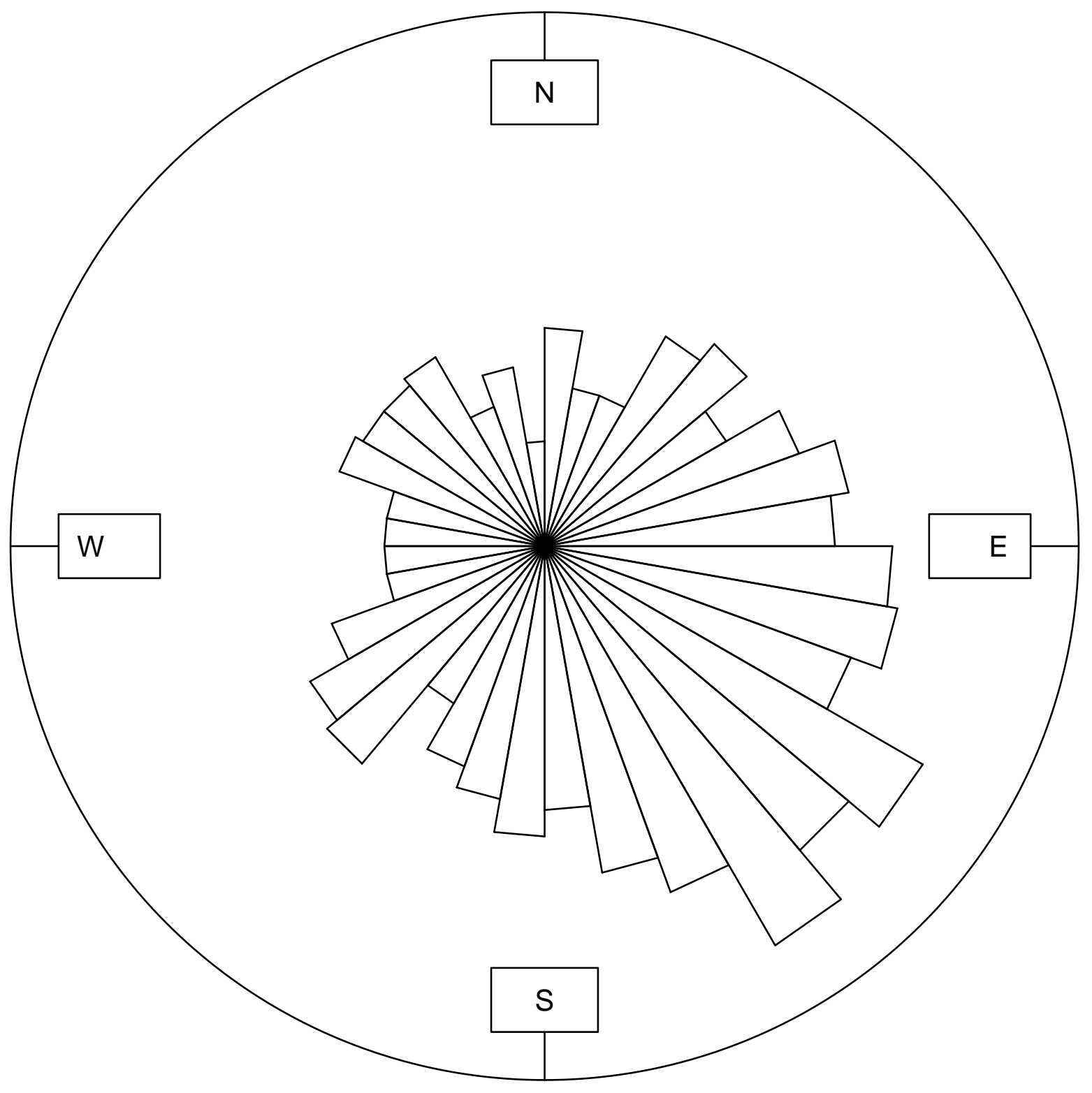


bootstrap 849

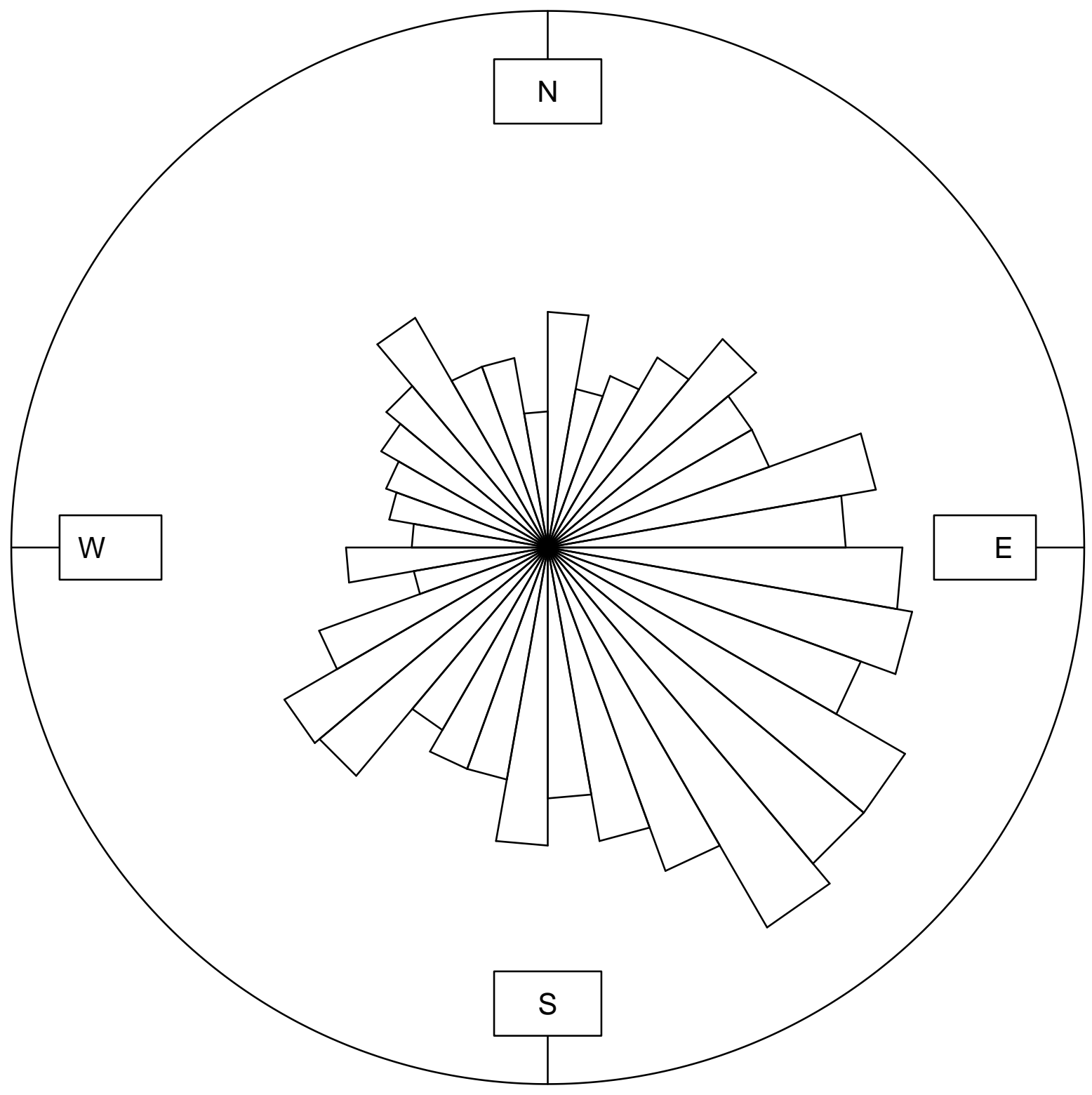


bootstrap 850

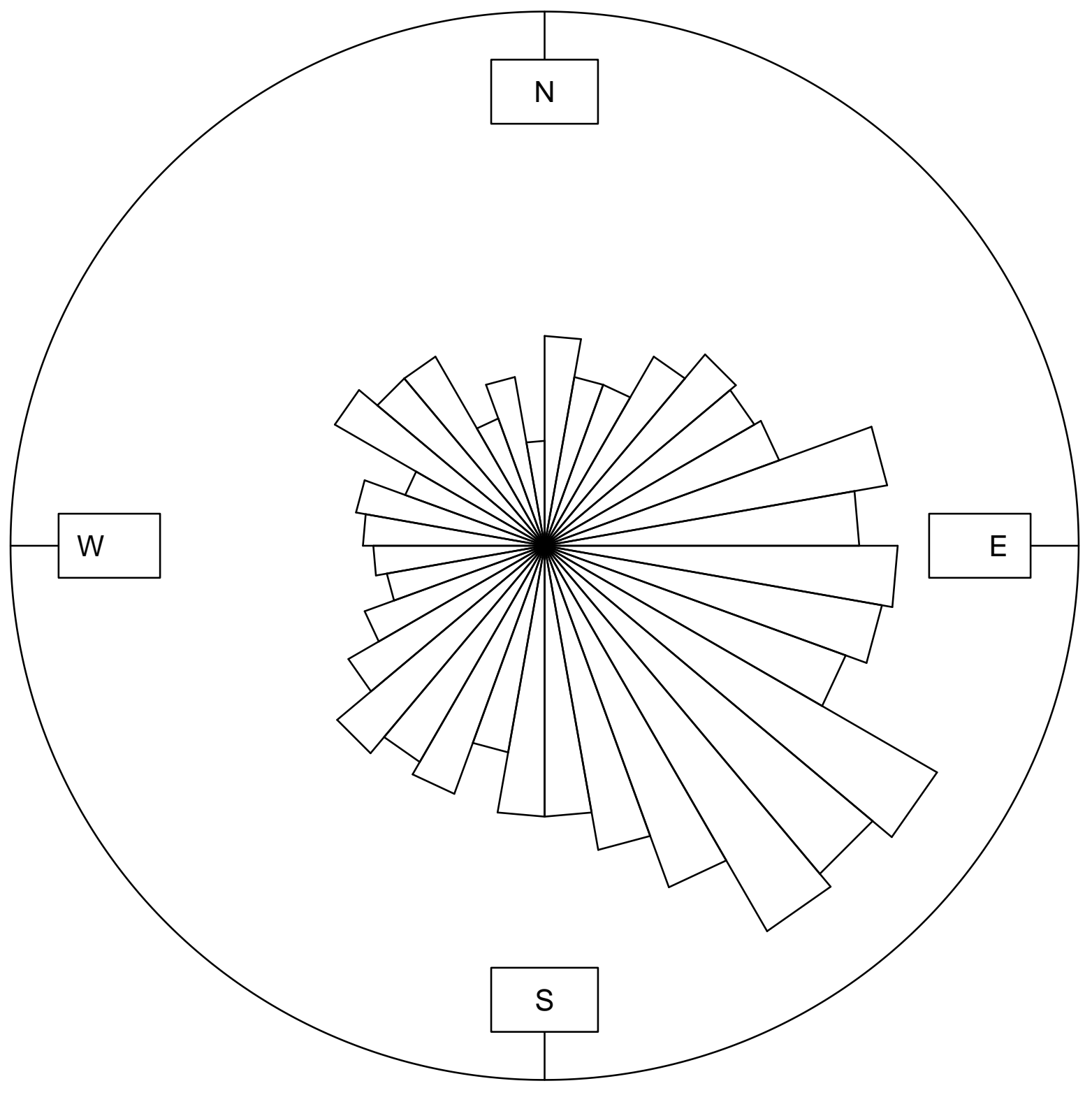


bootstrap 851

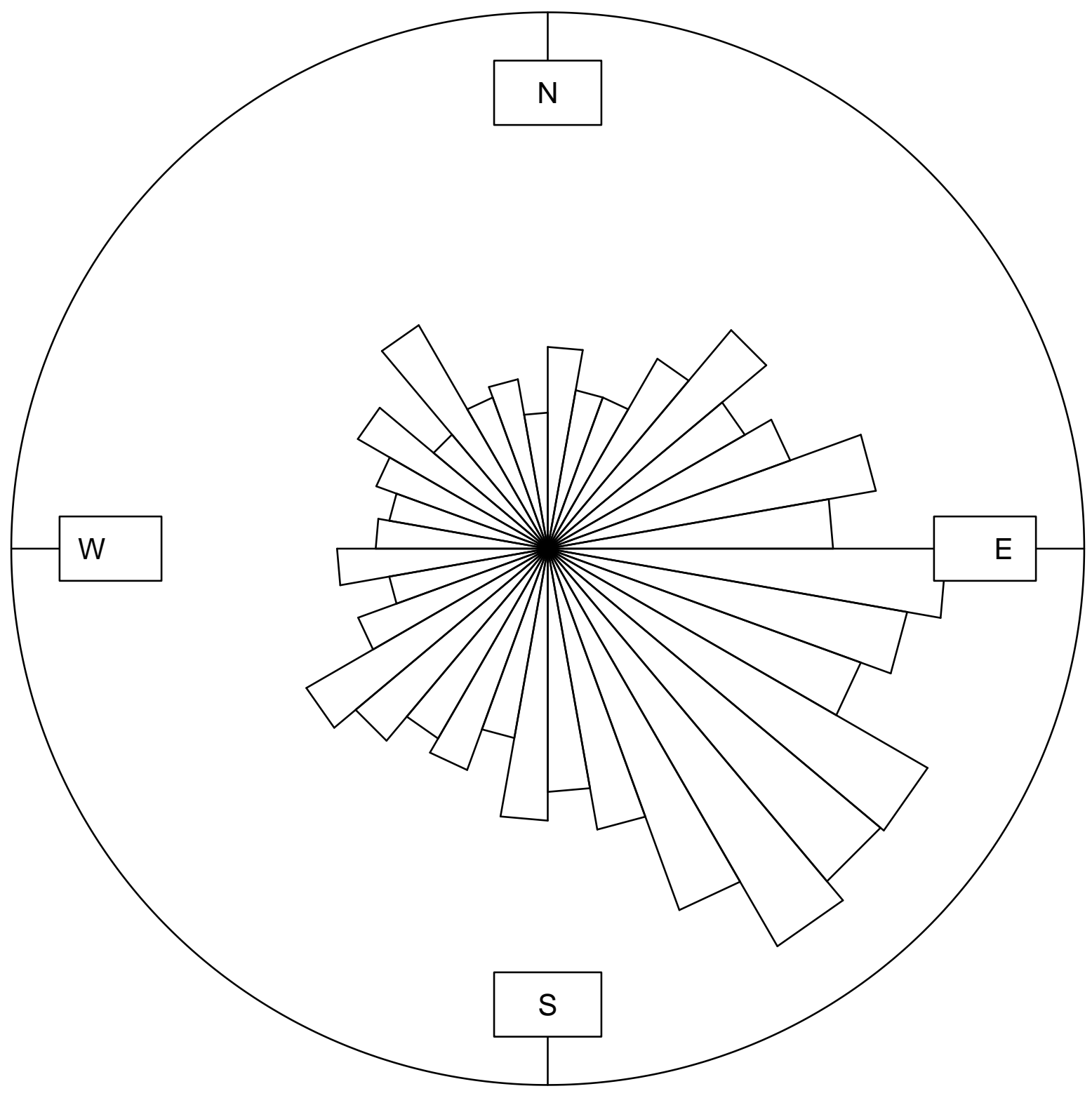


bootstrap 852

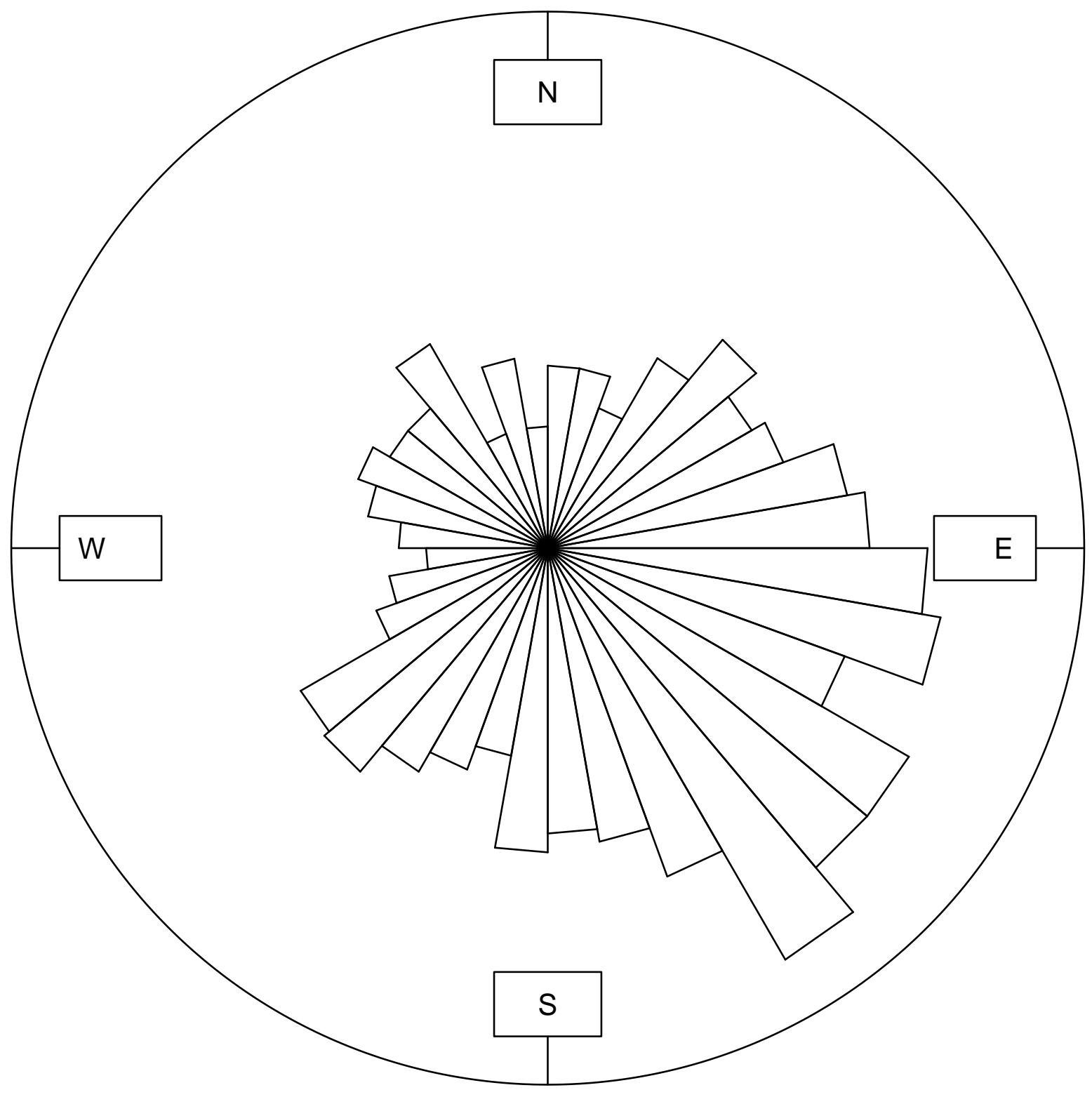




\section{bootstrap 853}

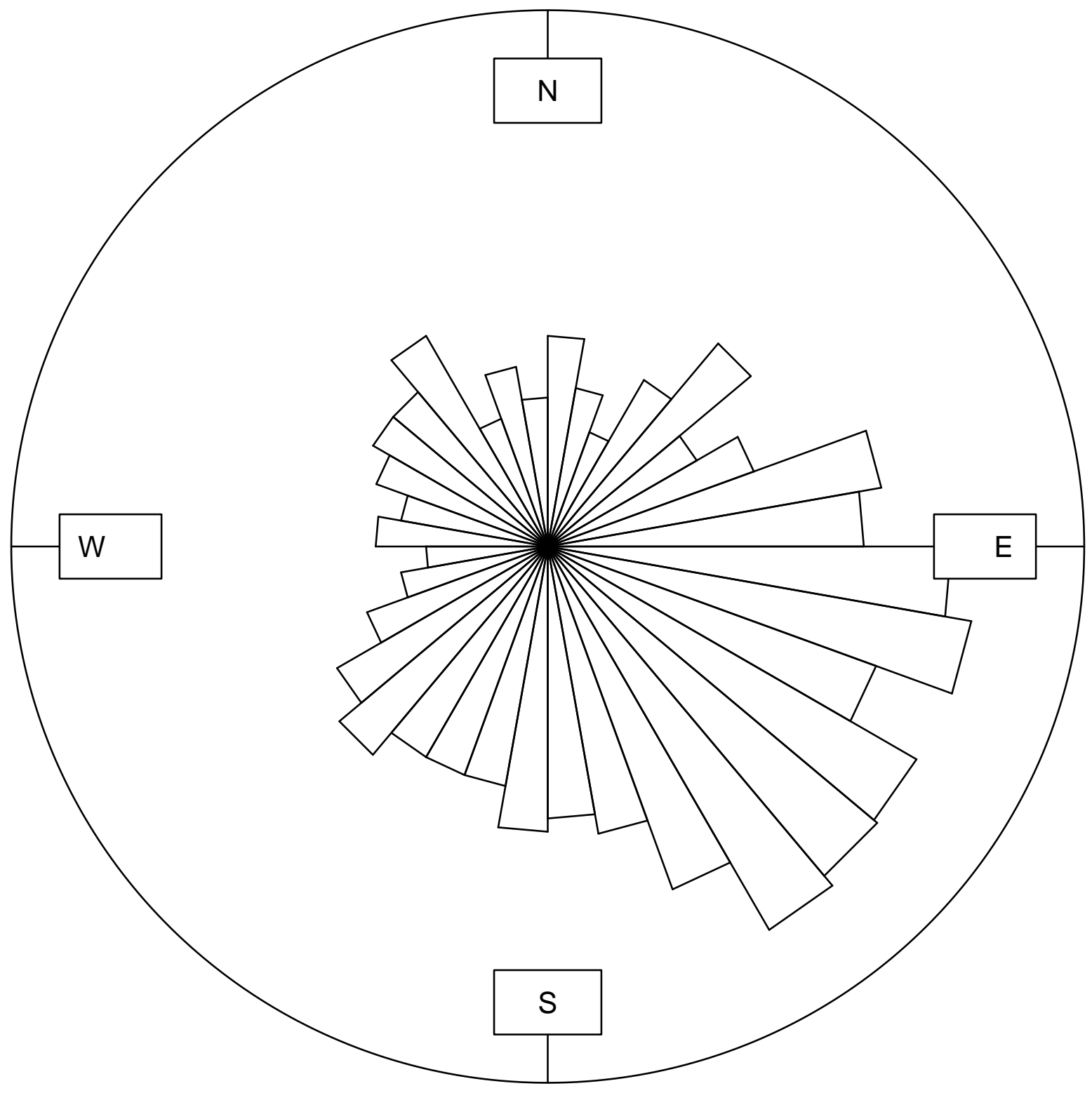




\section{bootstrap 854}

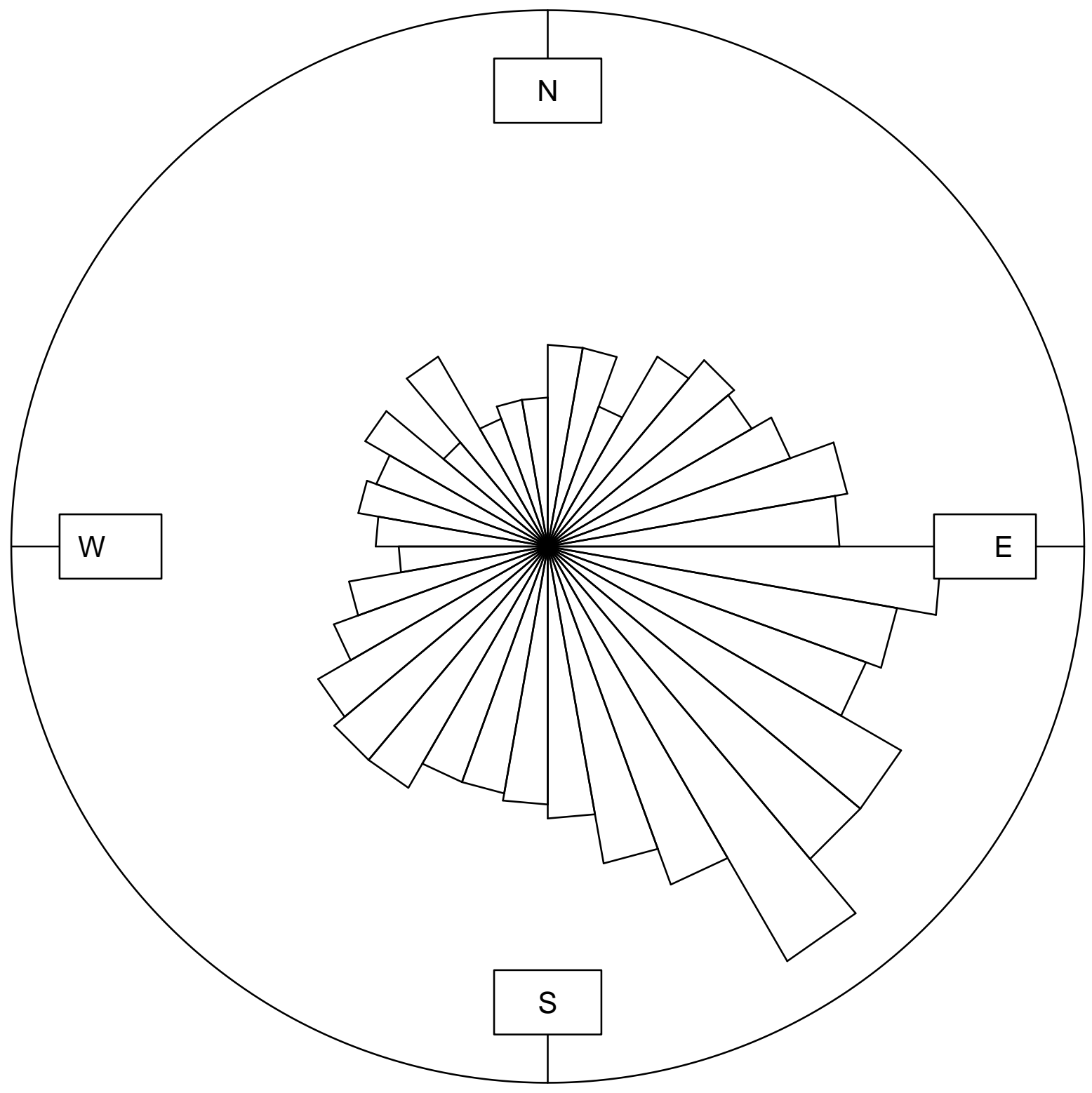




\section{bootstrap 855}

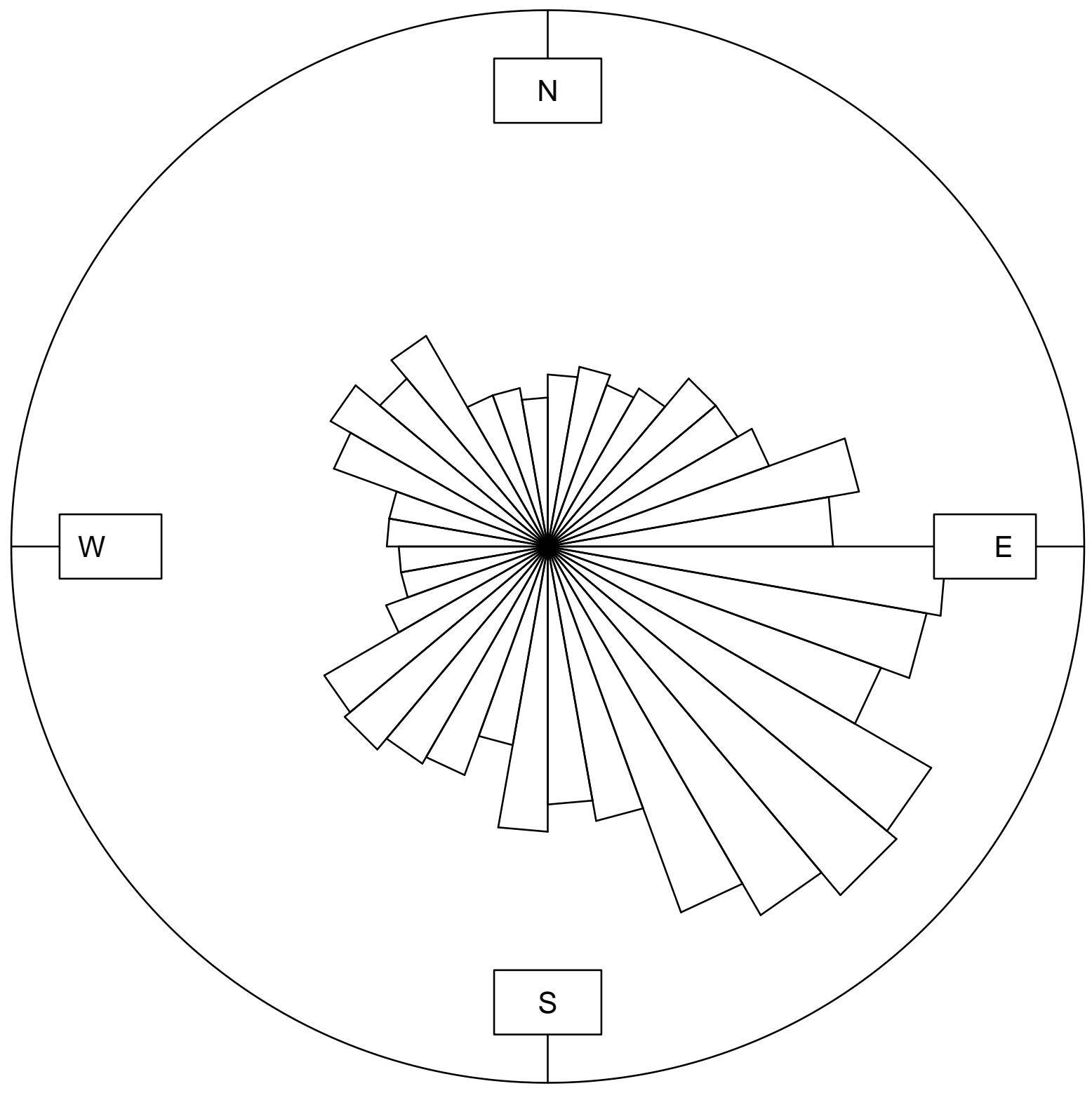


bootstrap 856

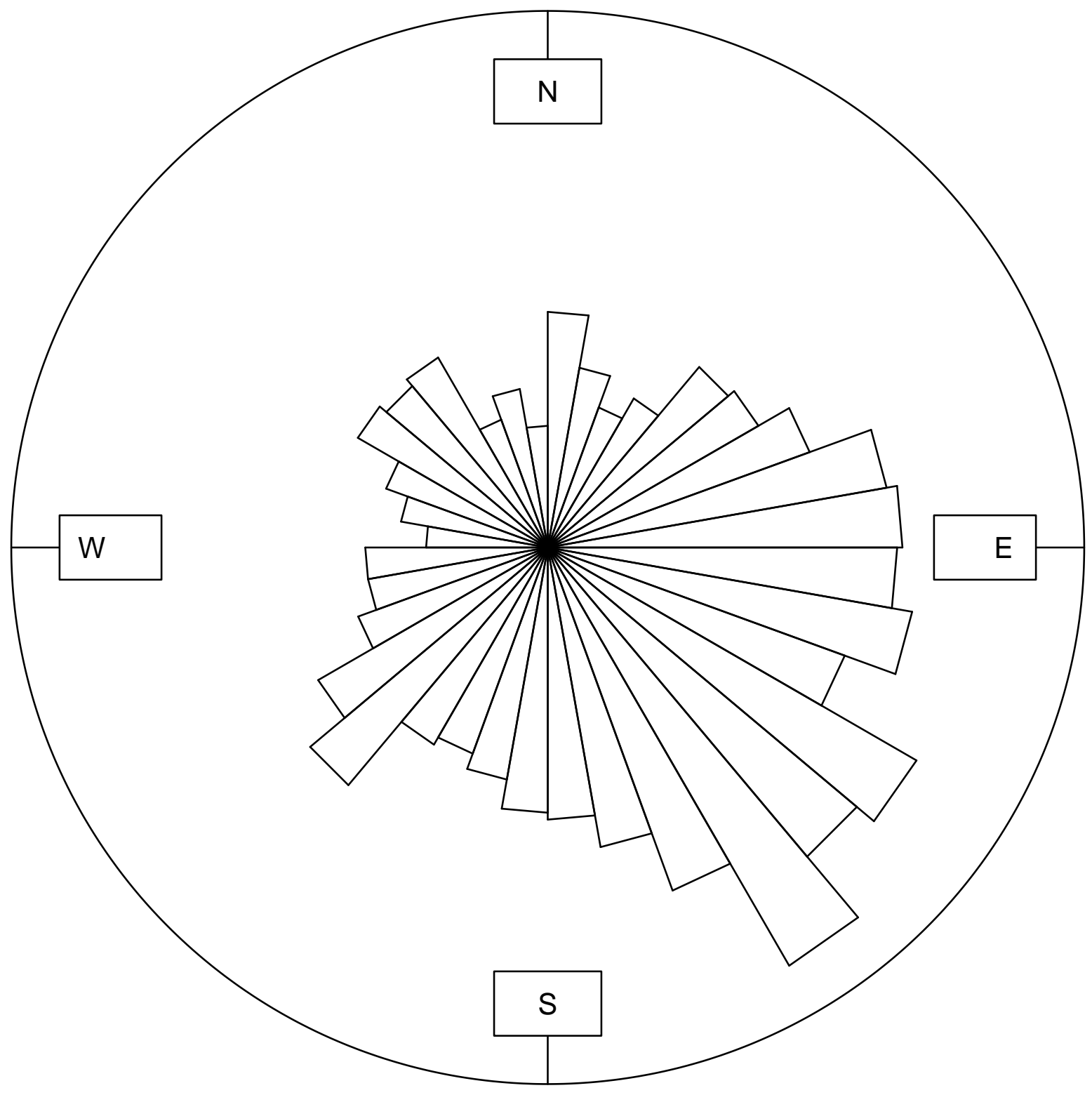


bootstrap 857

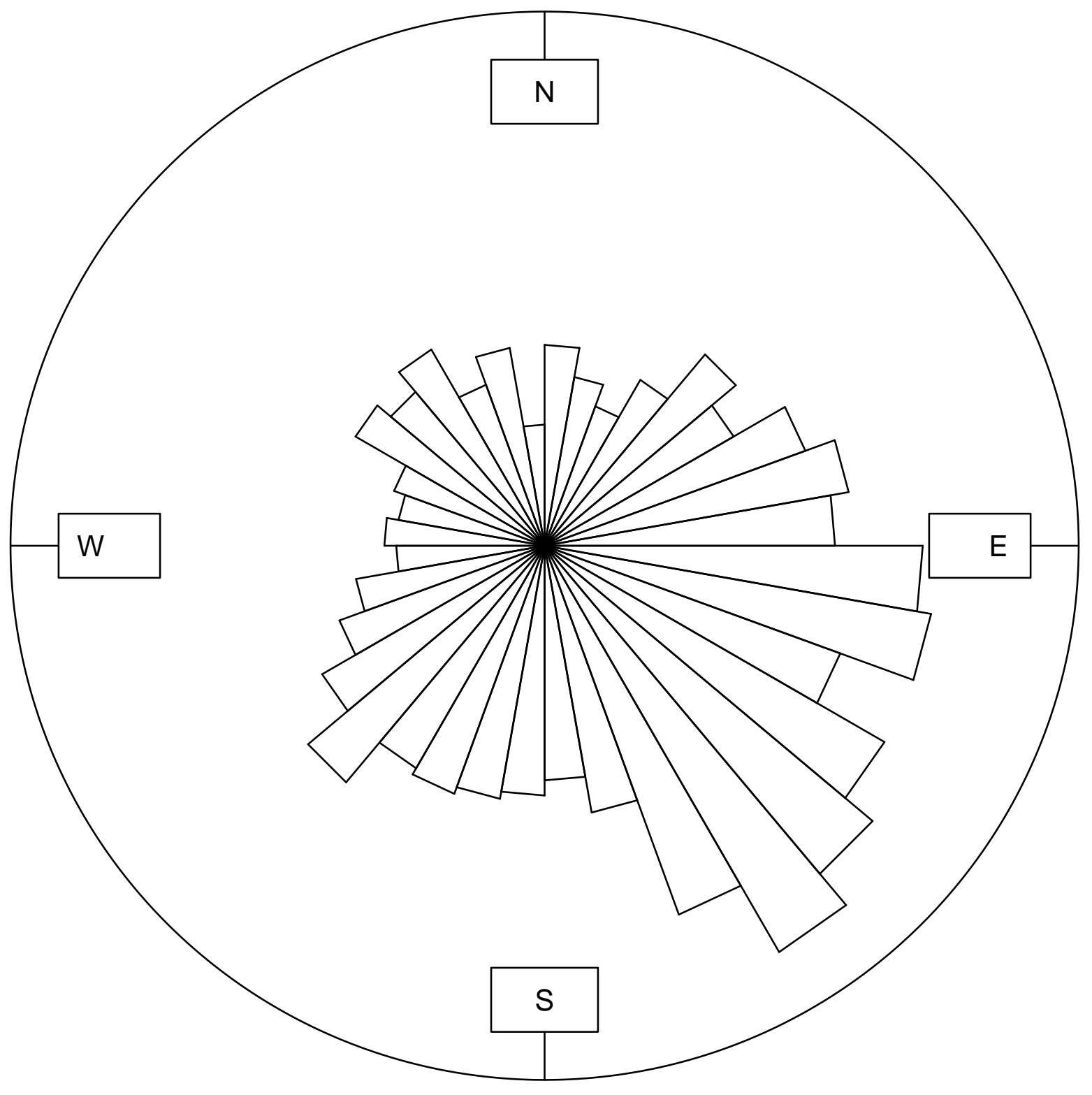


bootstrap 858

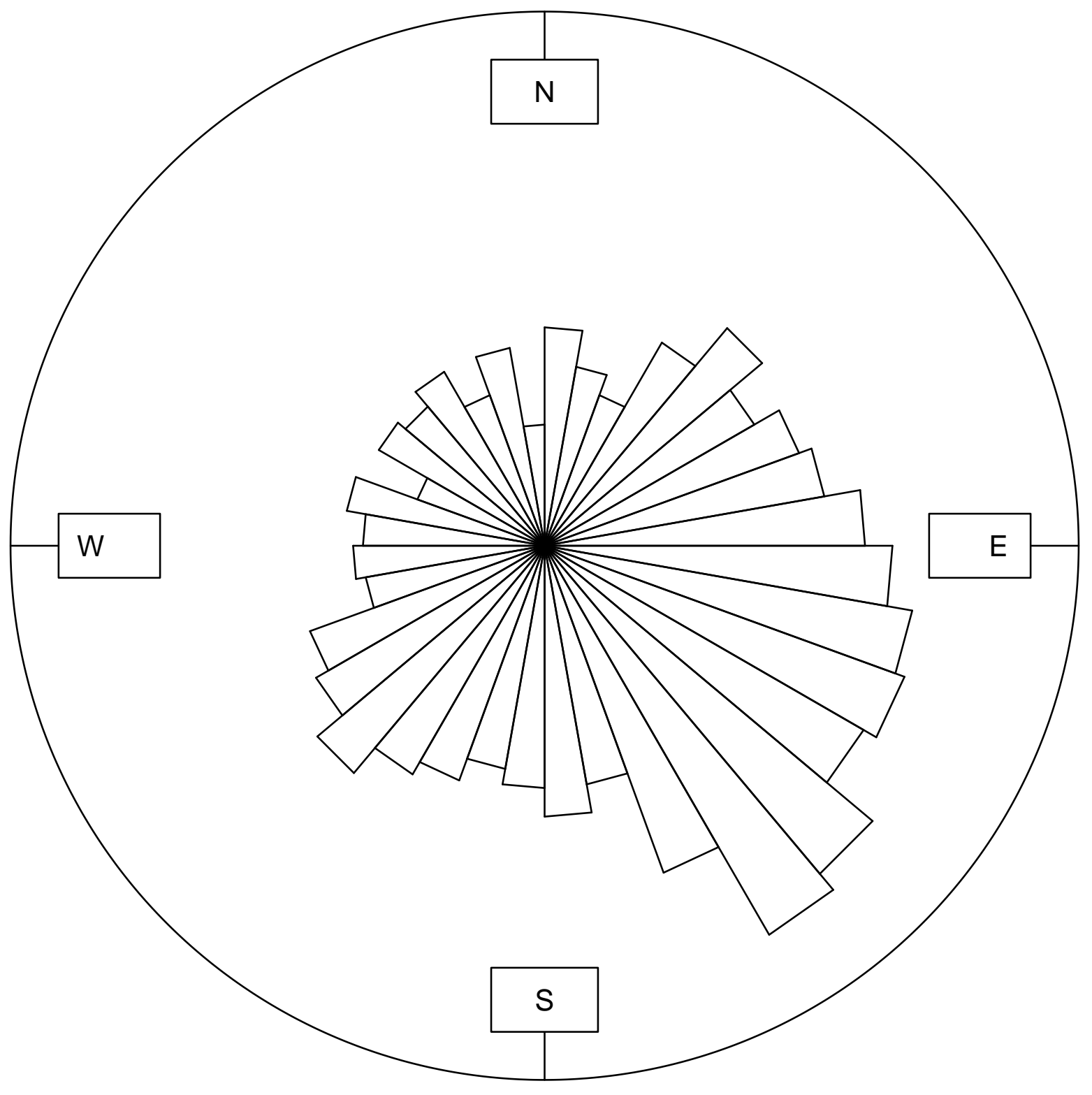


bootstrap 859

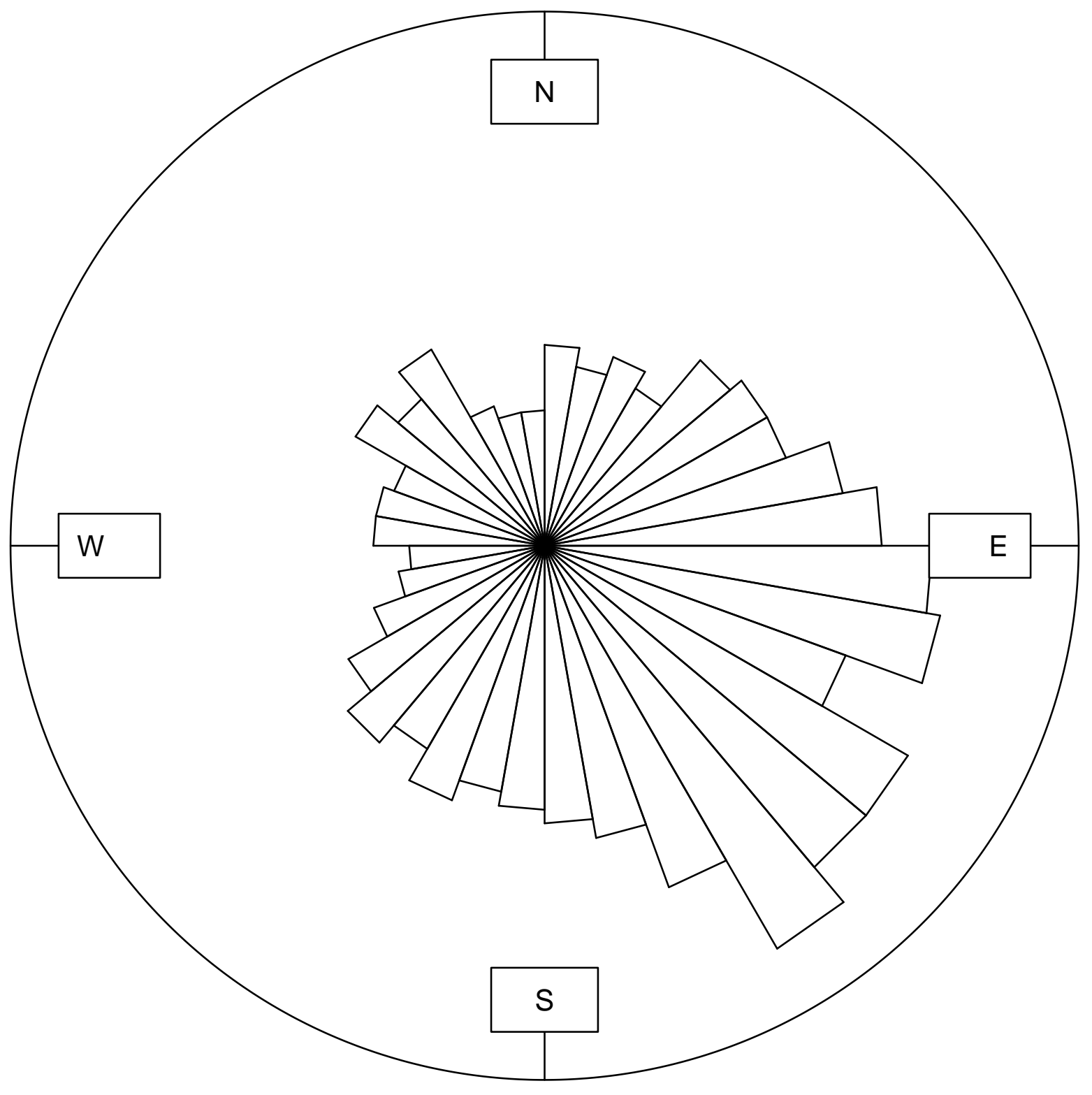


bootstrap 860

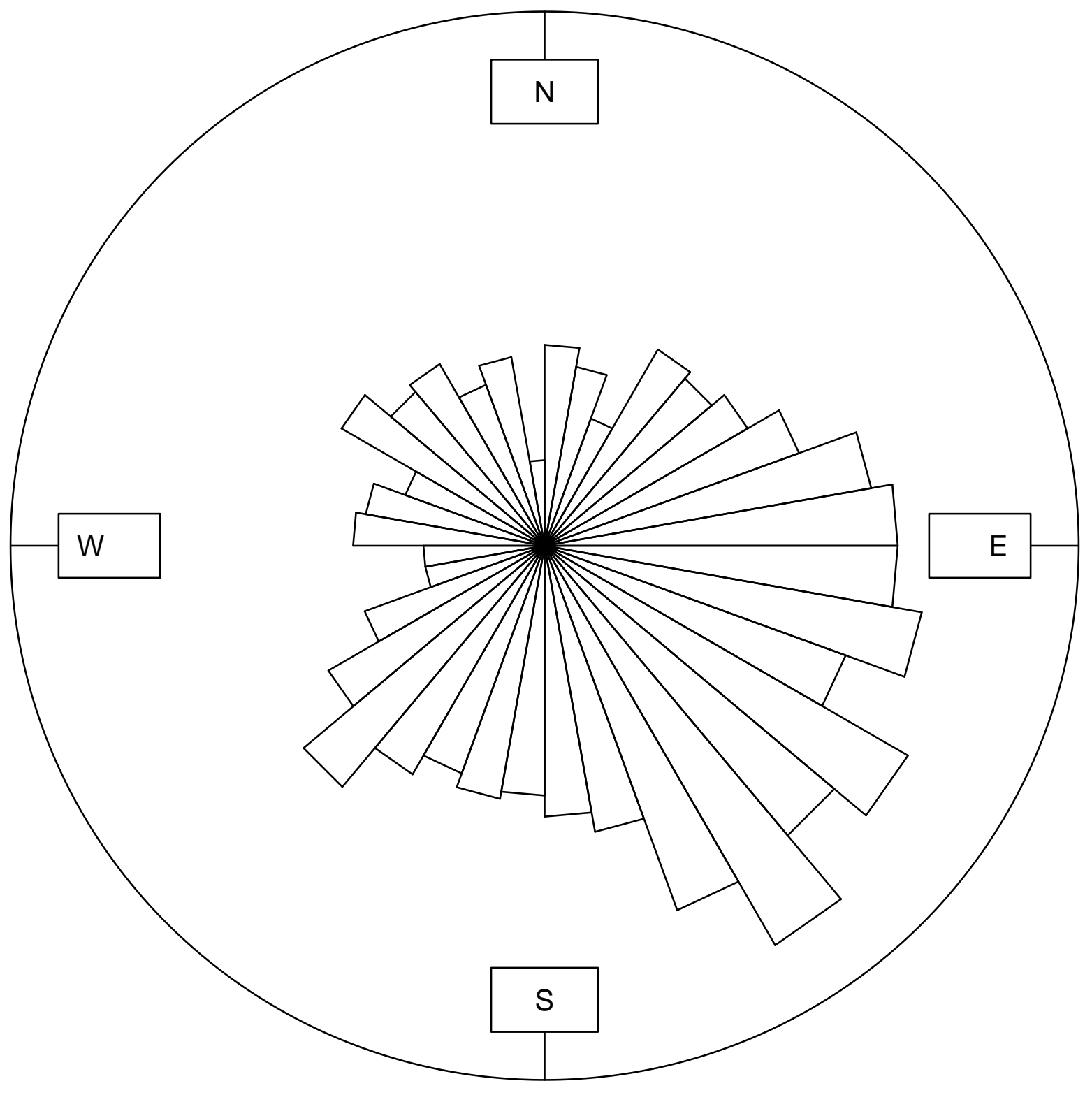


bootstrap 861

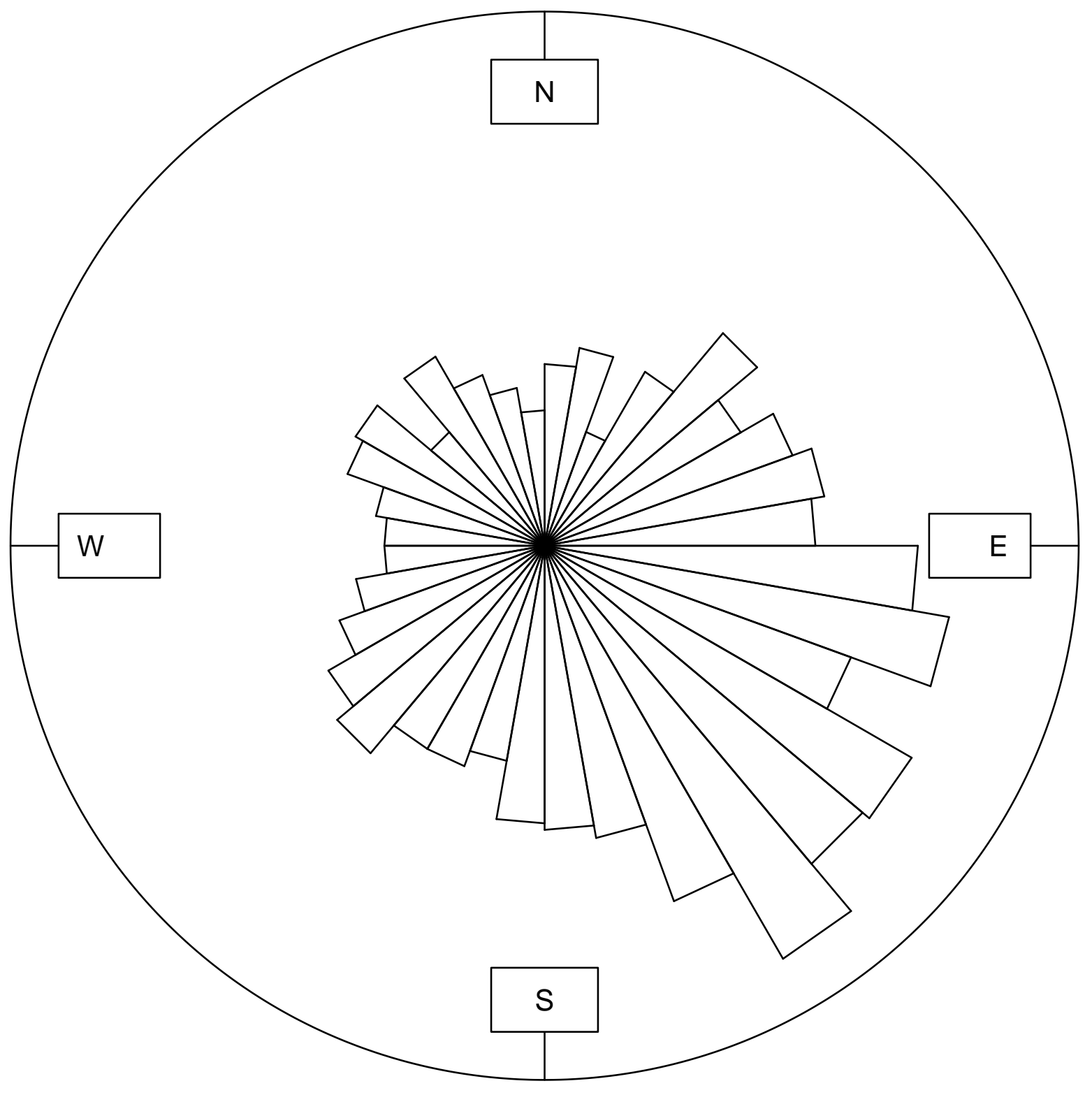


bootstrap 862

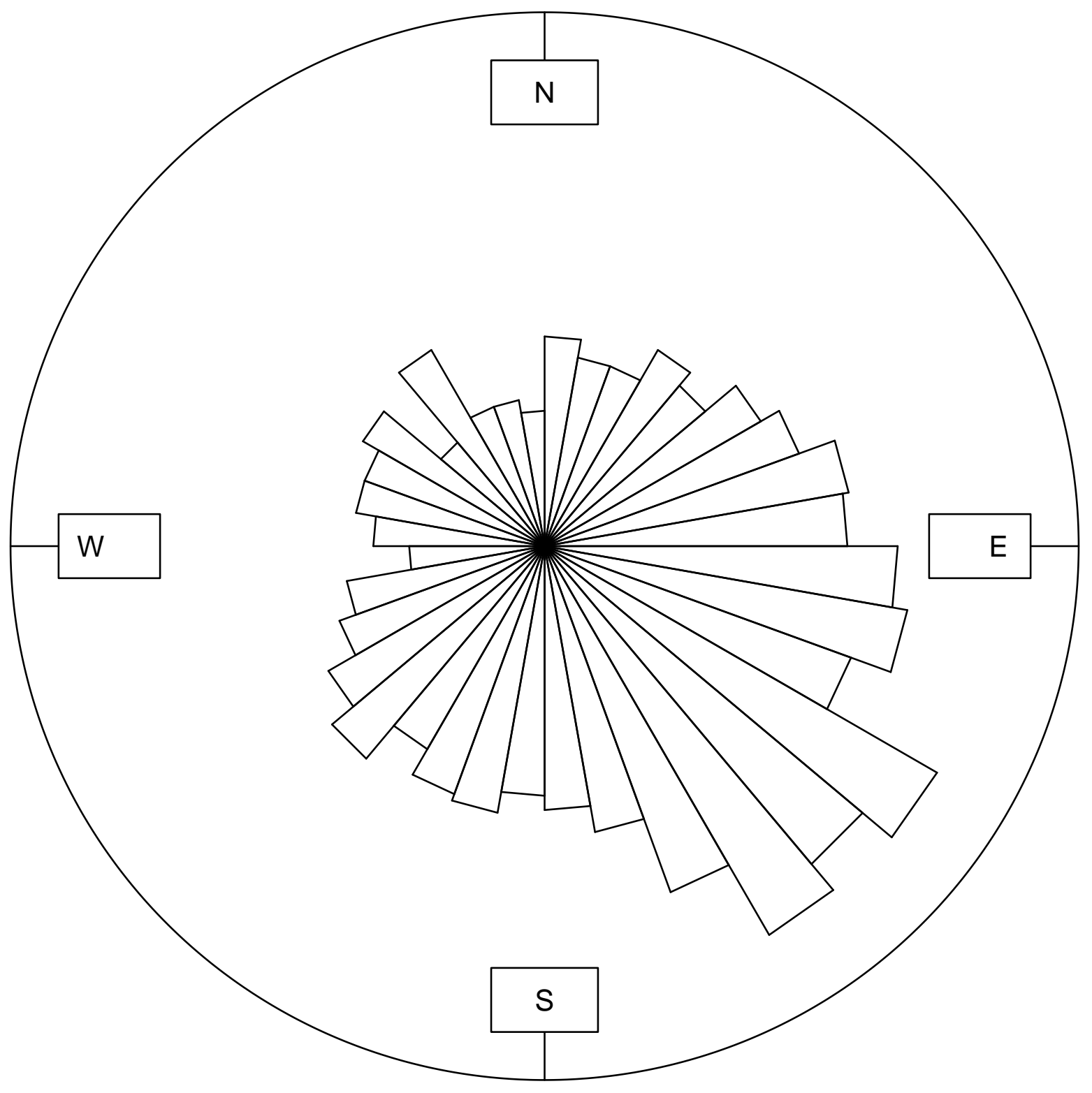




\section{bootstrap 863}

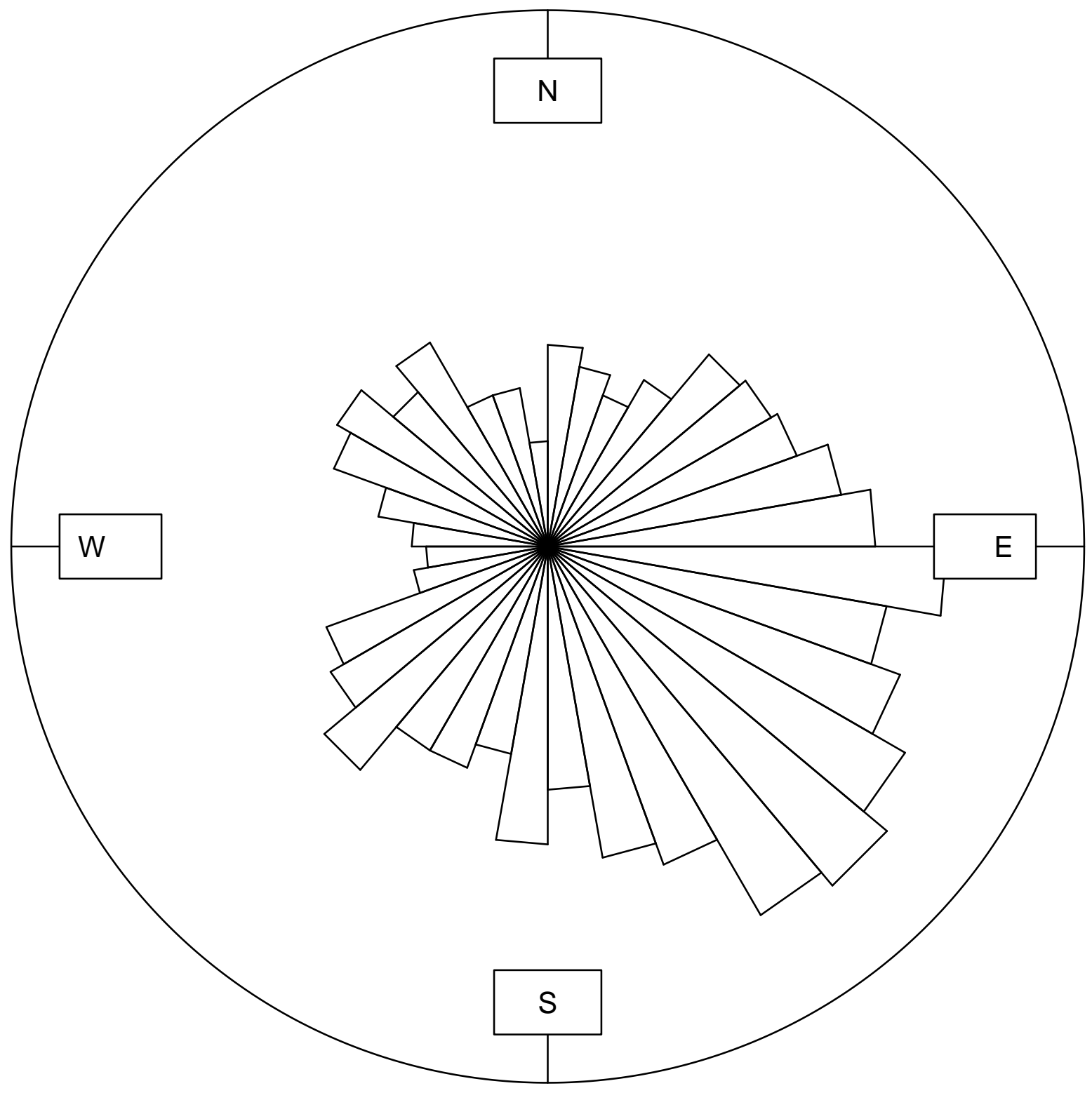




\section{bootstrap 864}

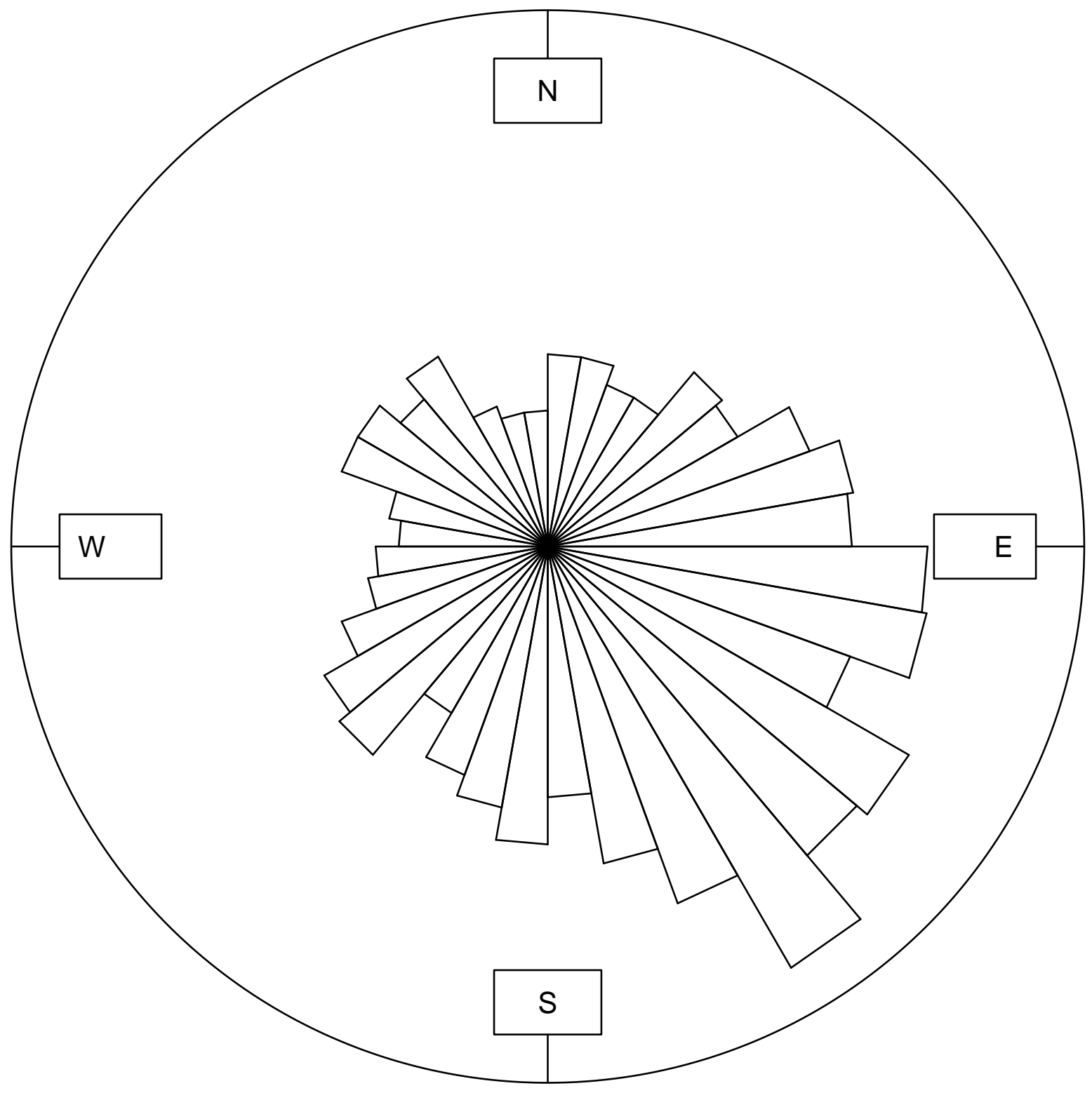




\section{bootstrap 865}

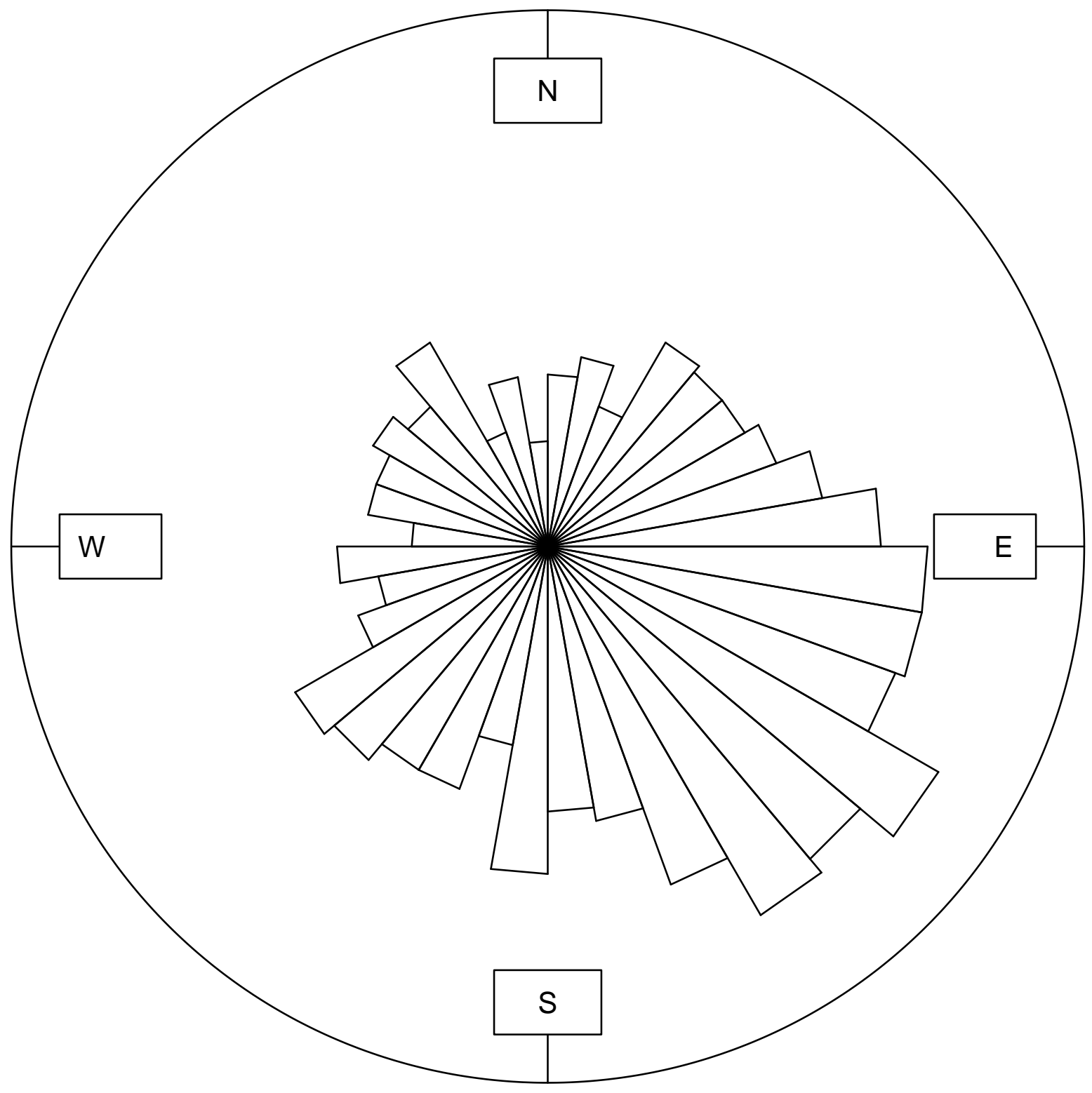


bootstrap 866

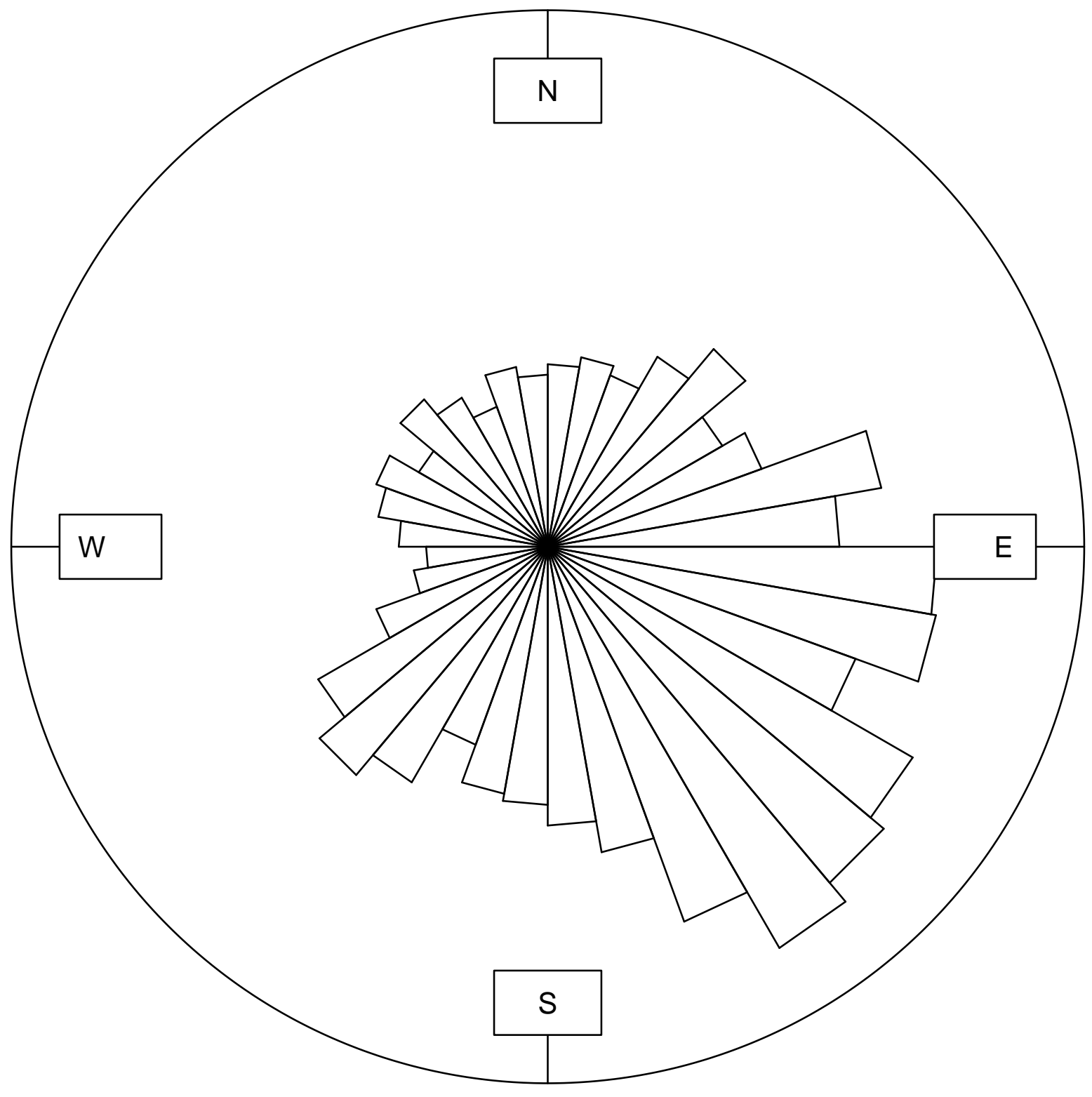


bootstrap 867

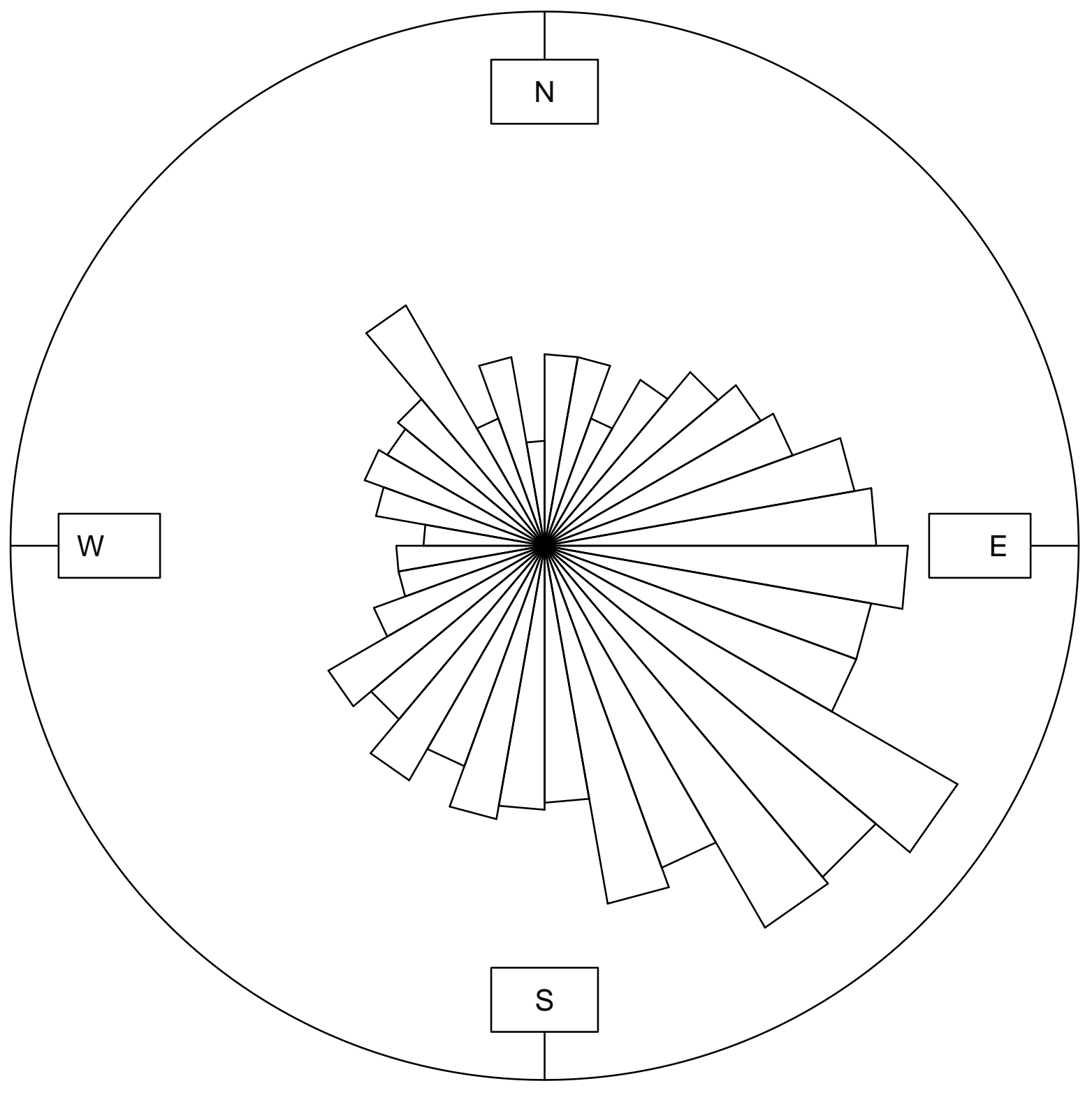


bootstrap 868

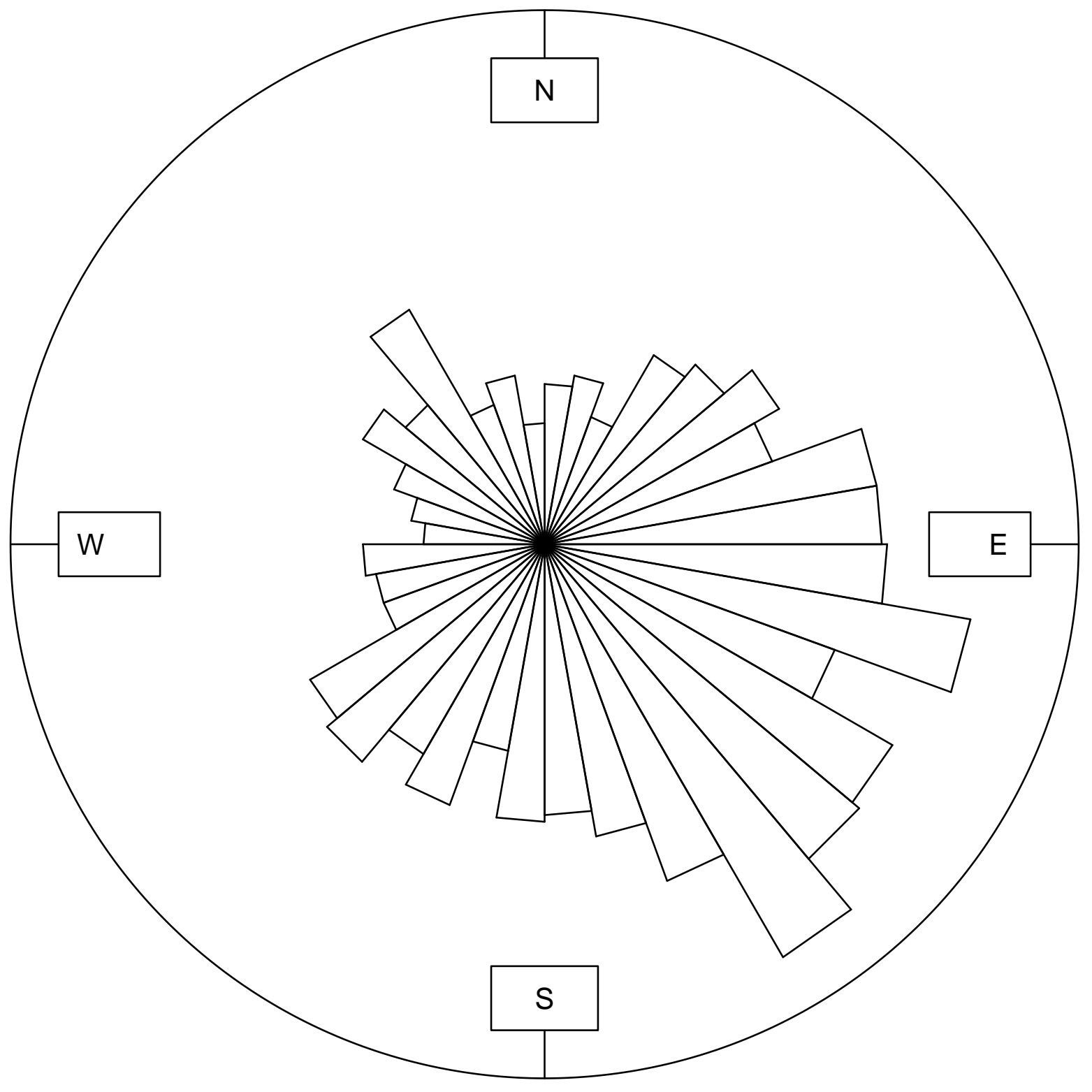


bootstrap 869

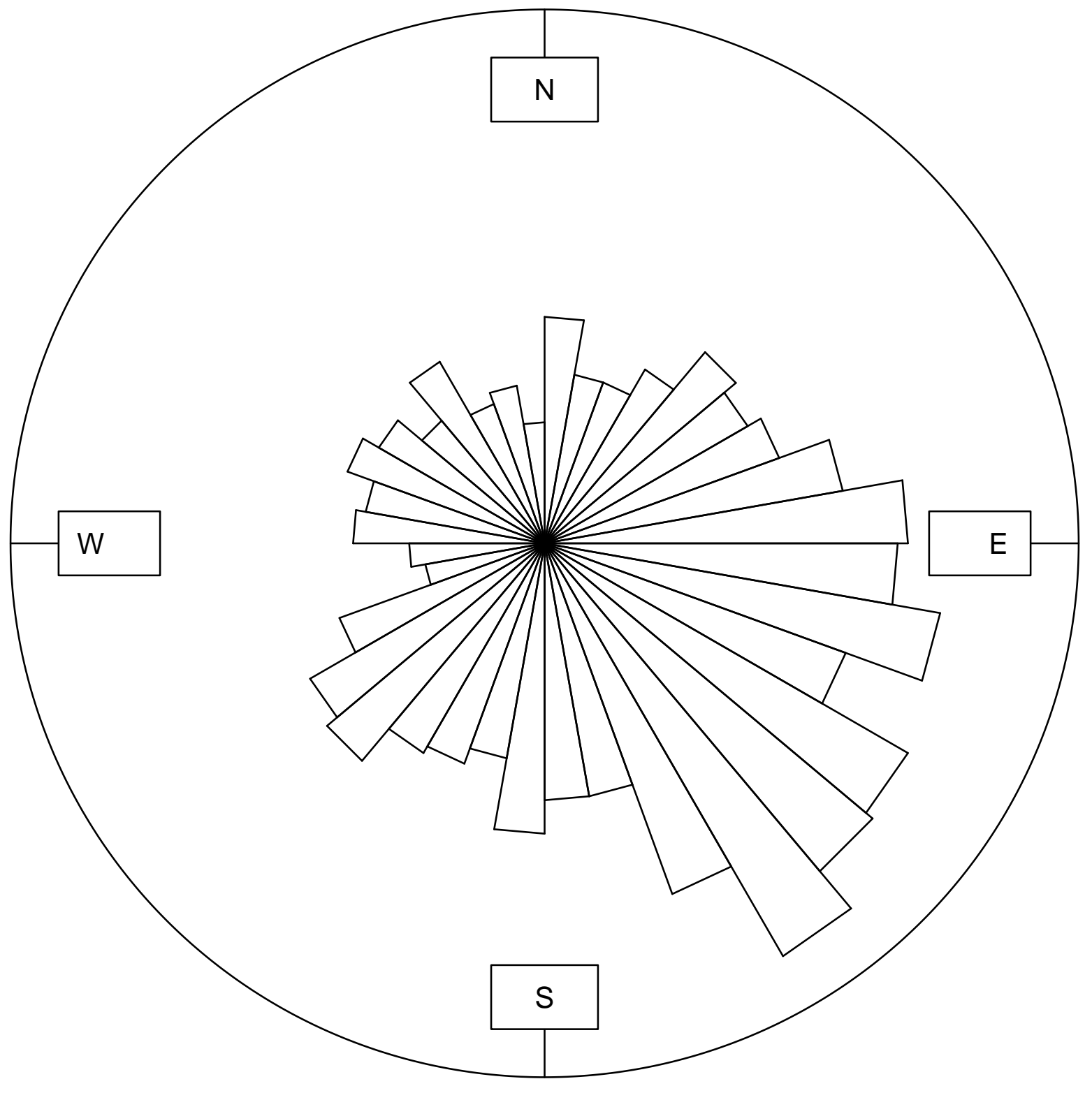


bootstrap 870

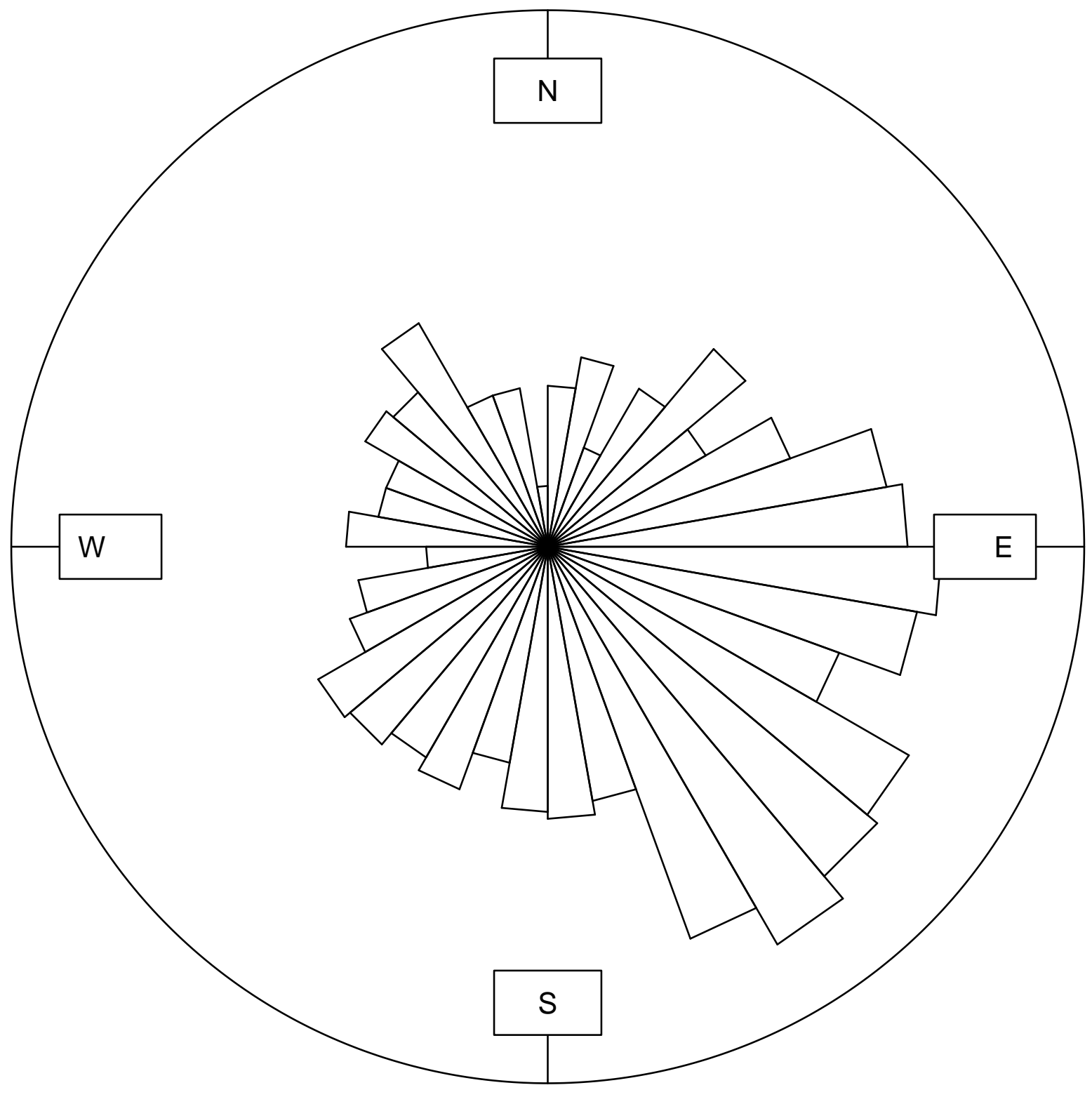


bootstrap 871

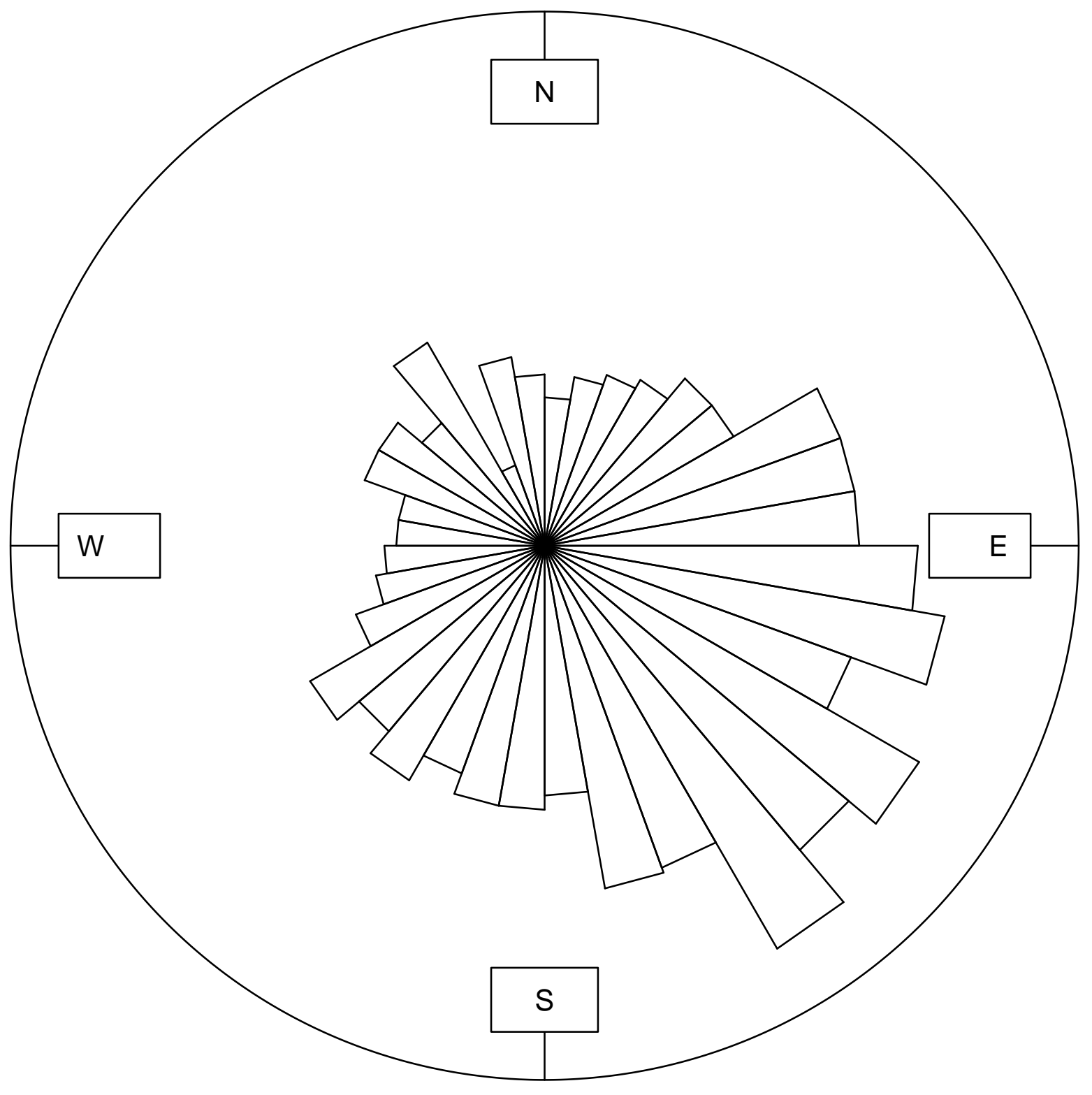


bootstrap 872

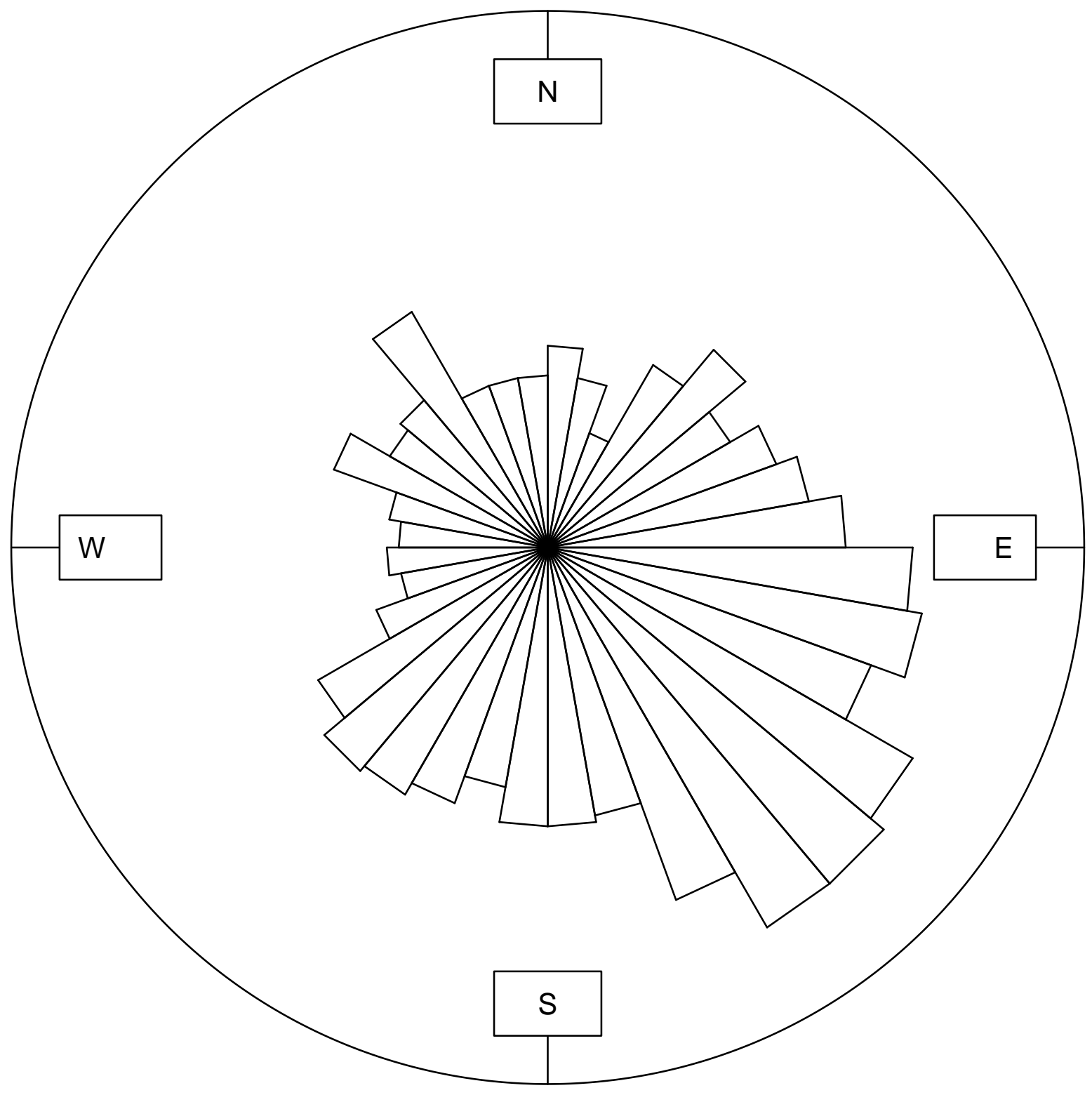


bootstrap 873

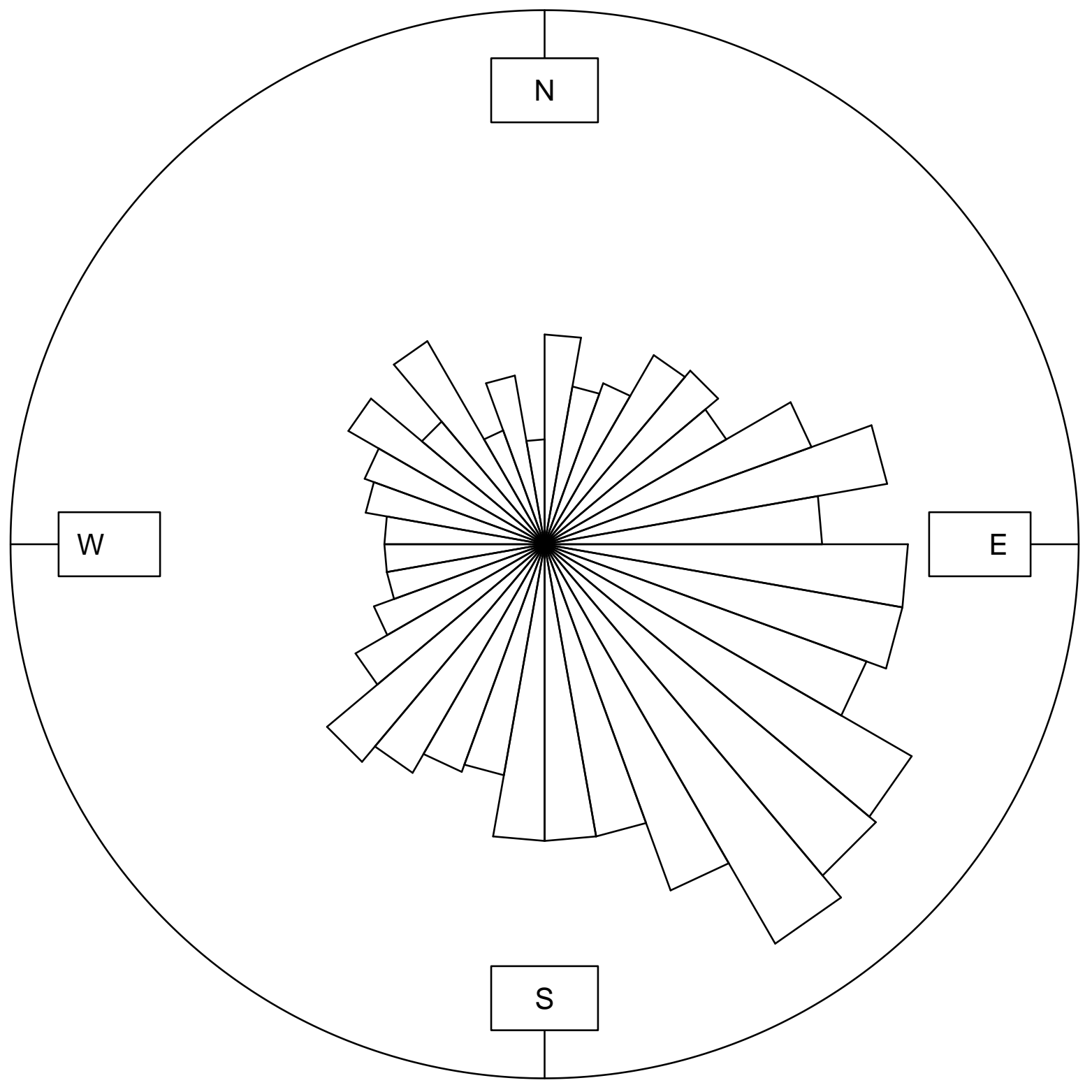


bootstrap 874

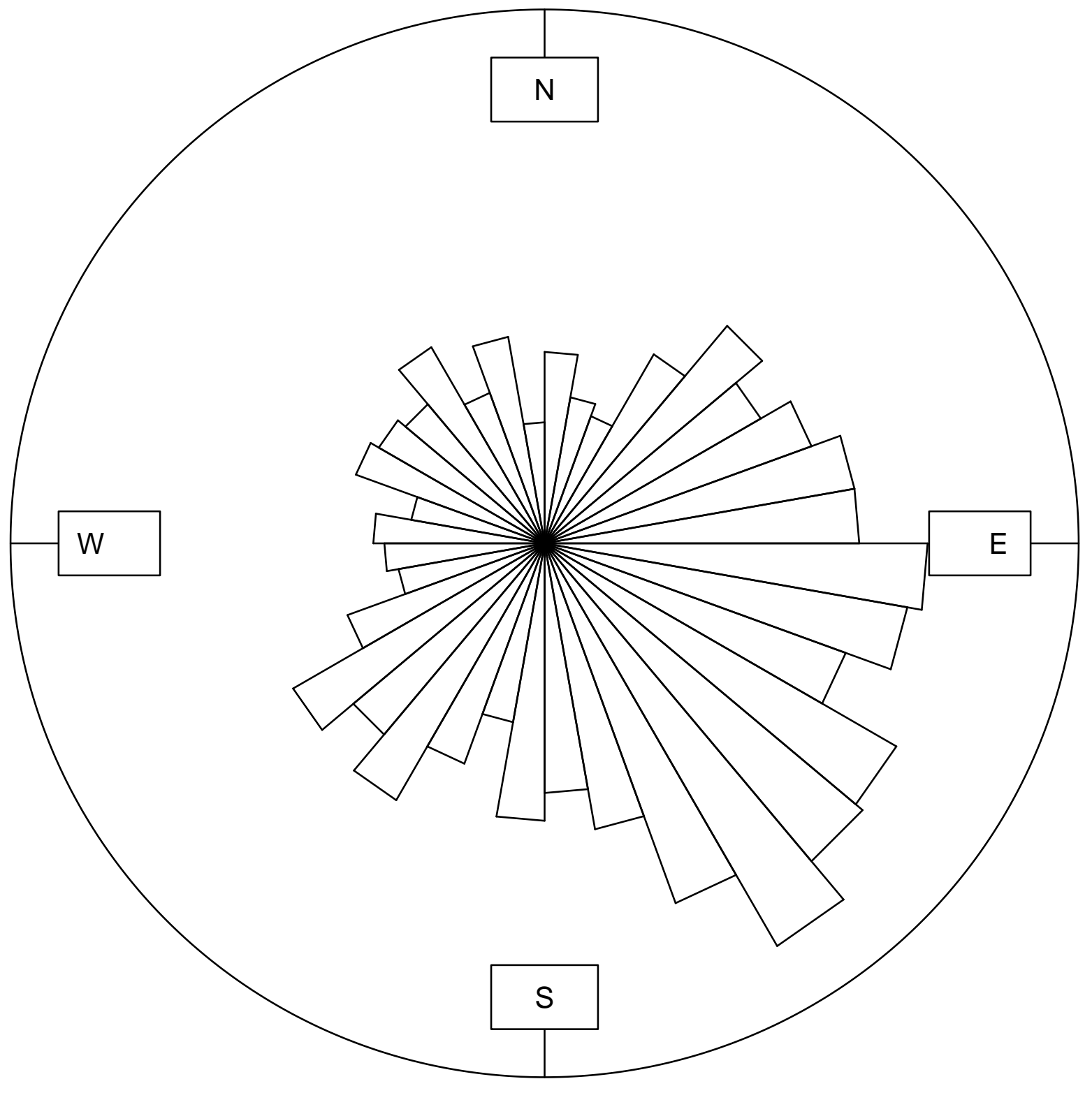


bootstrap 875

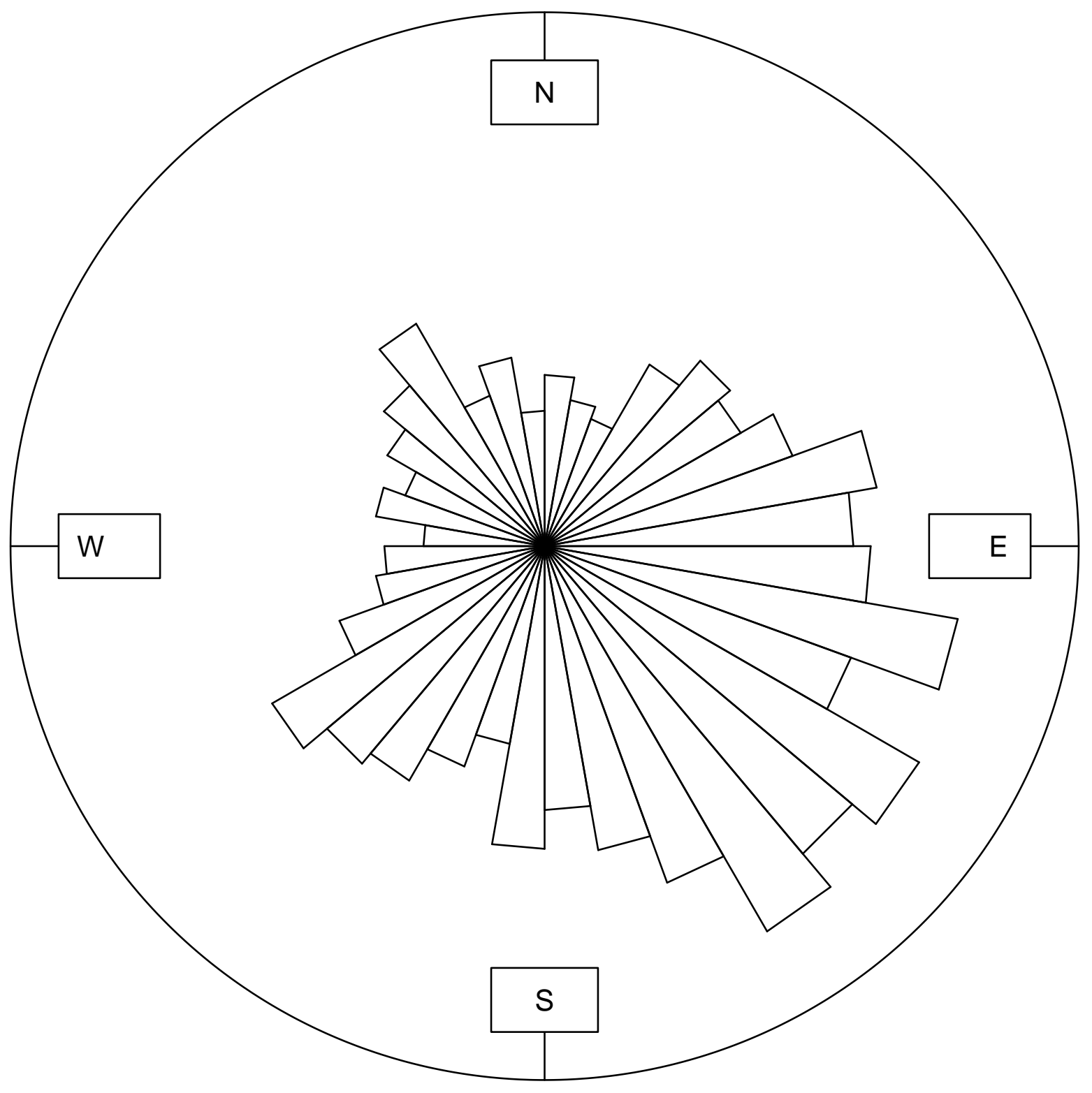


bootstrap 876

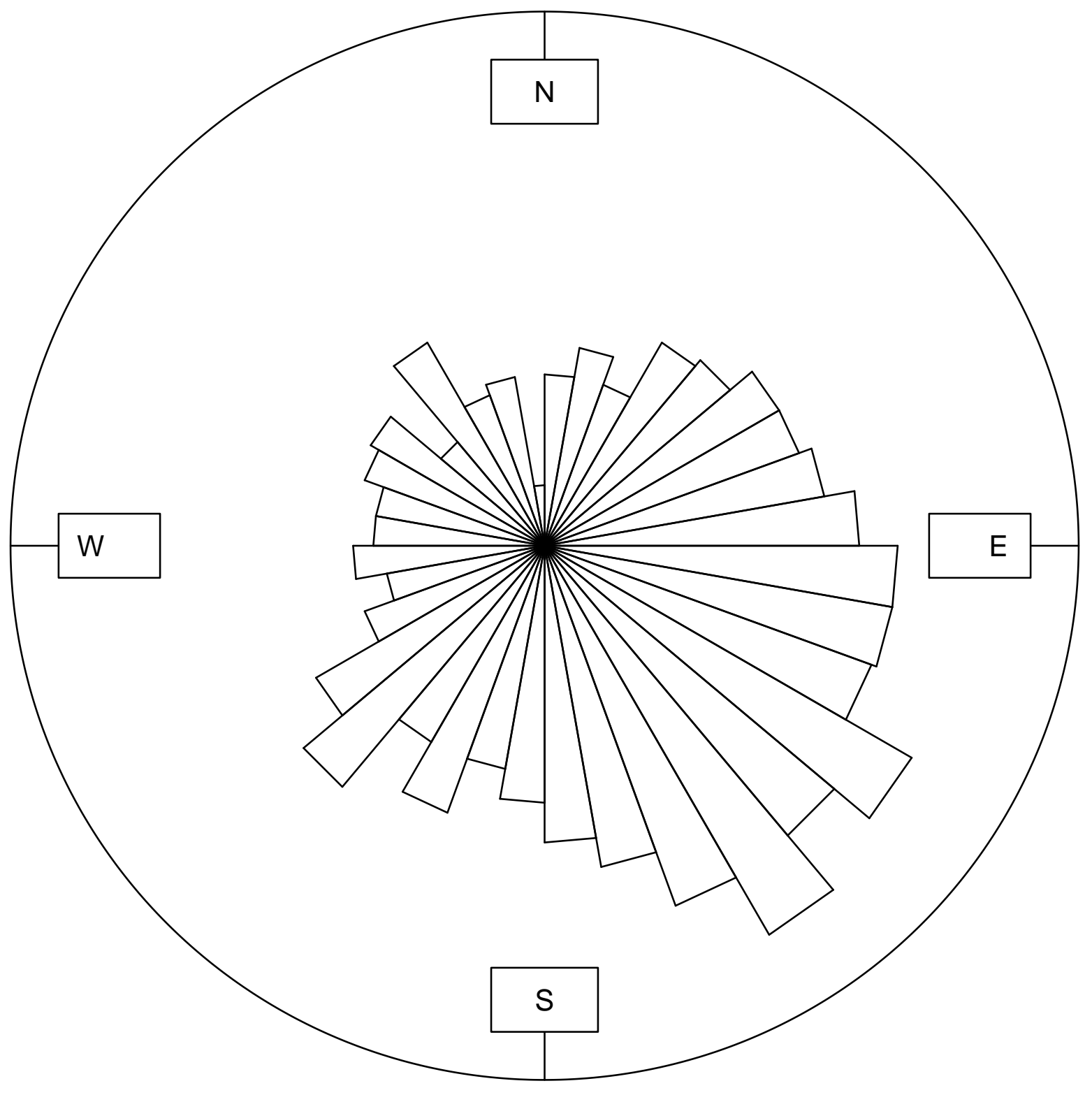


bootstrap 877

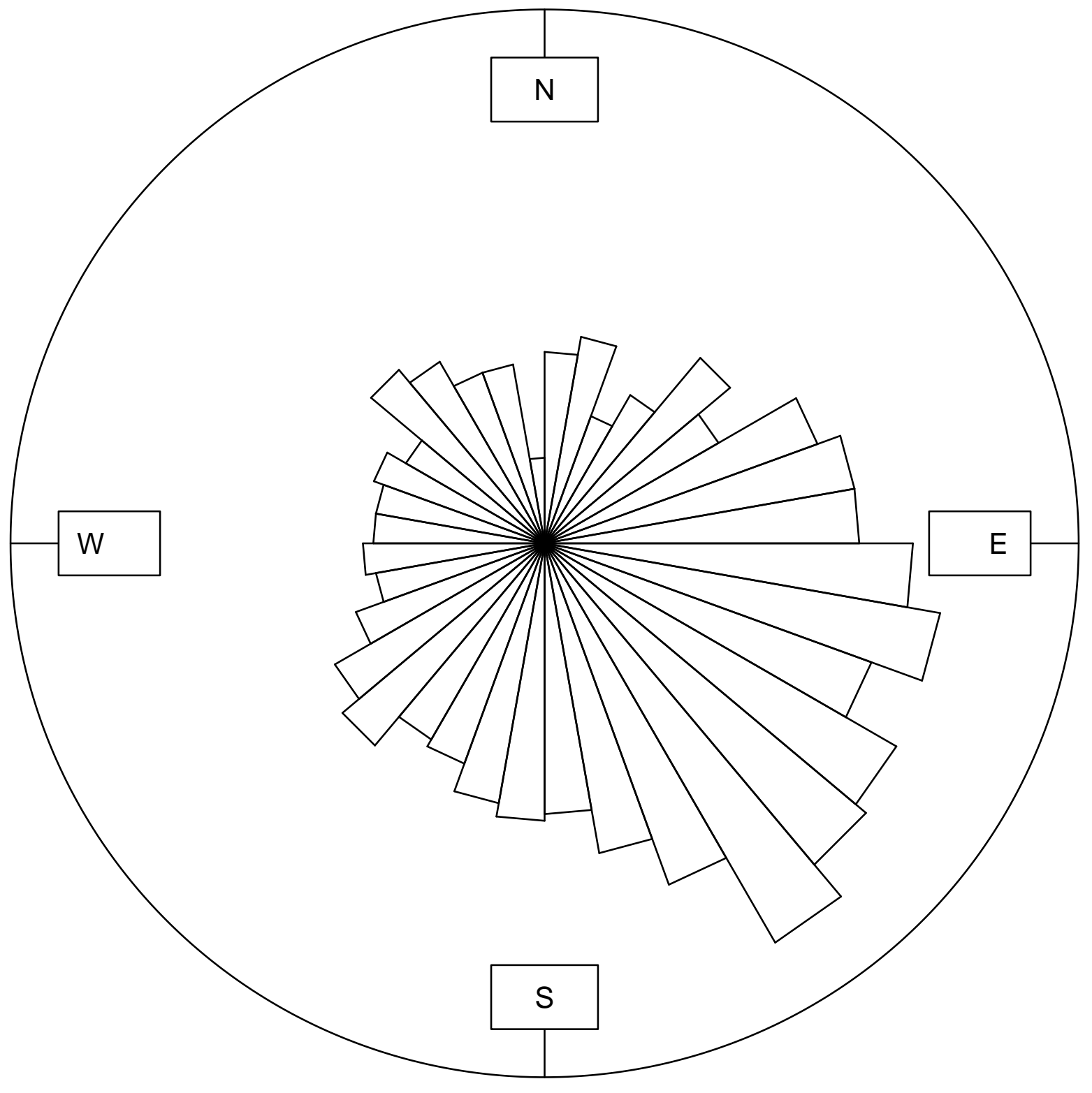


bootstrap 878

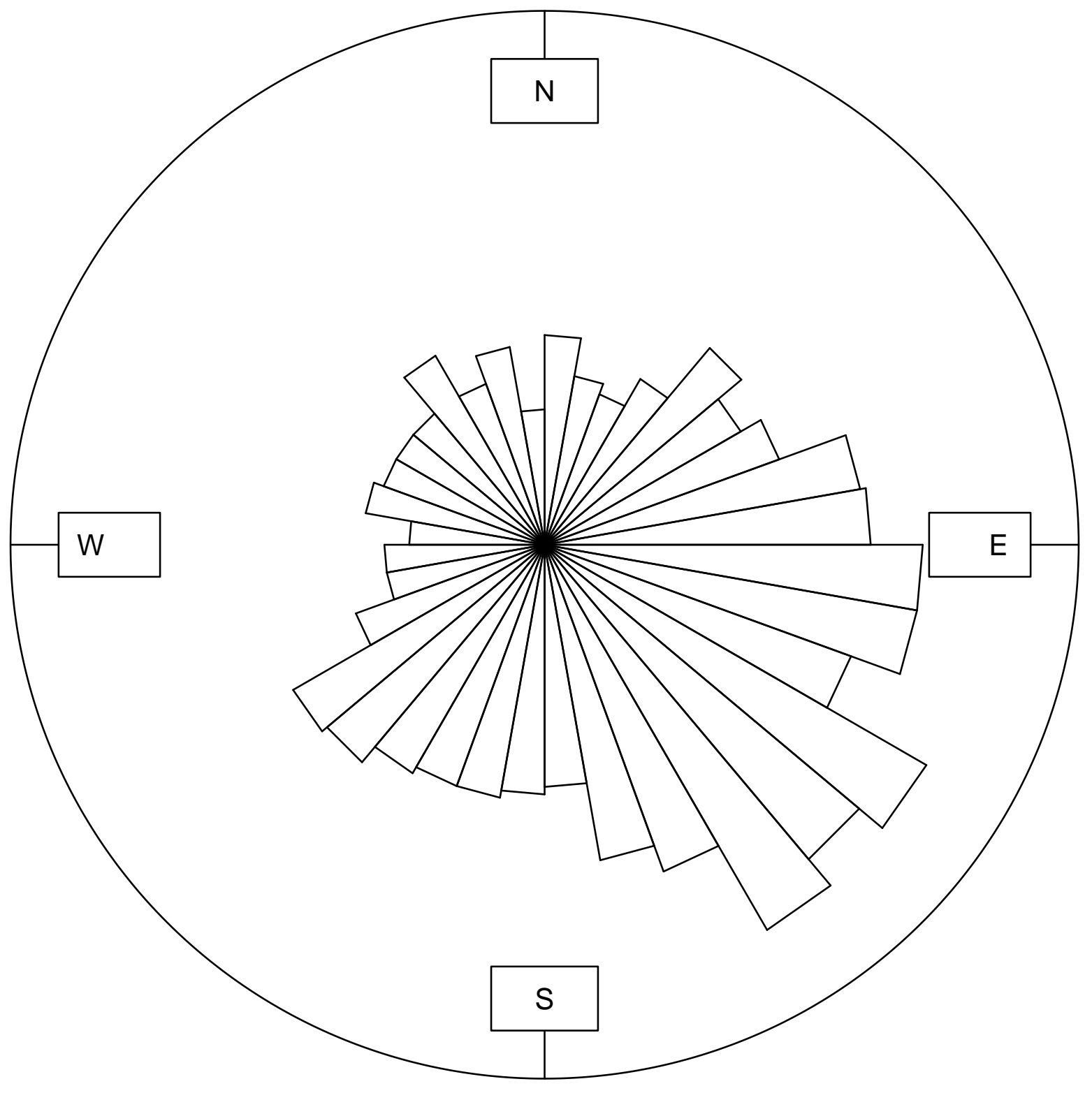


bootstrap 879

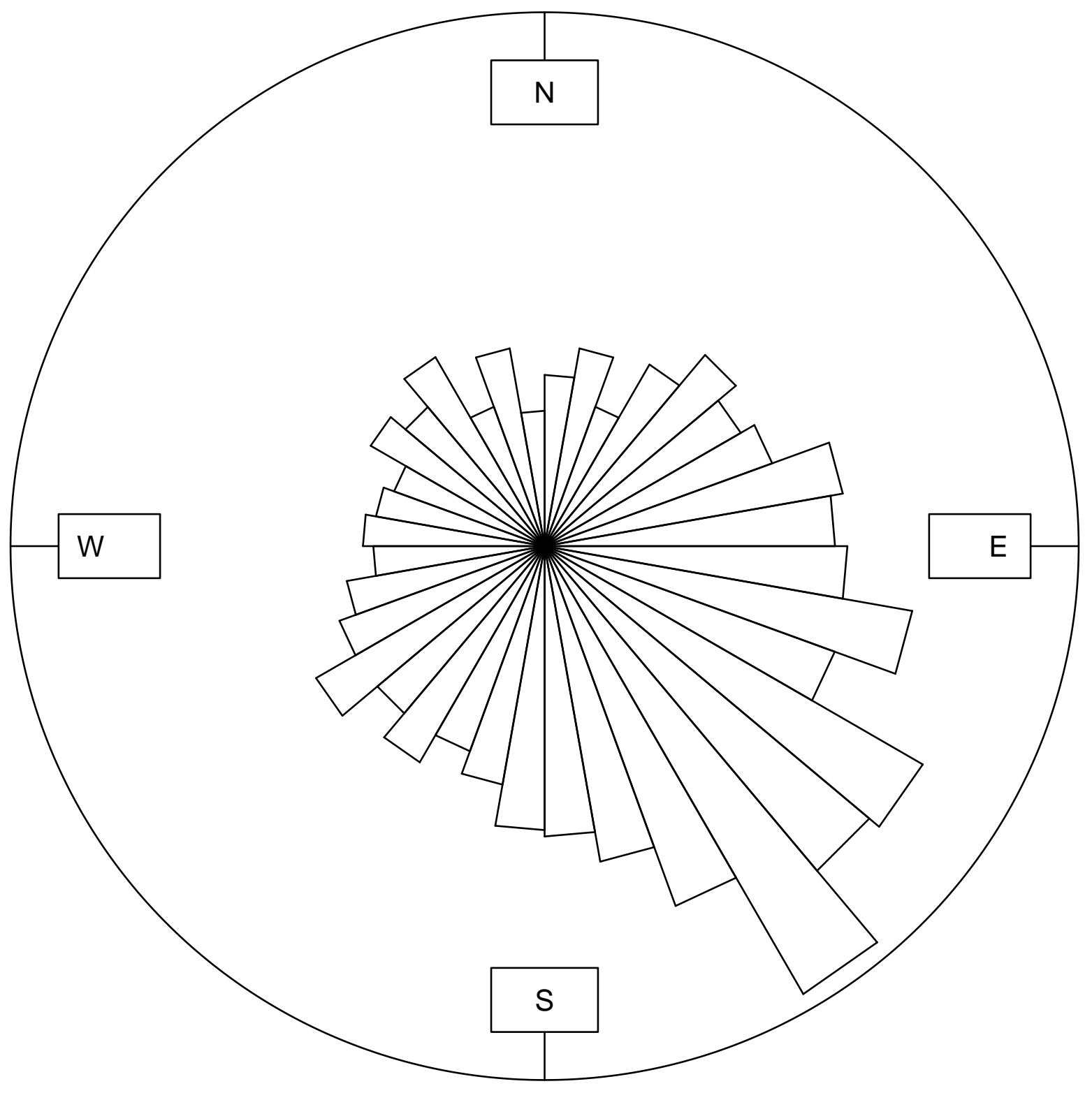


bootstrap 880

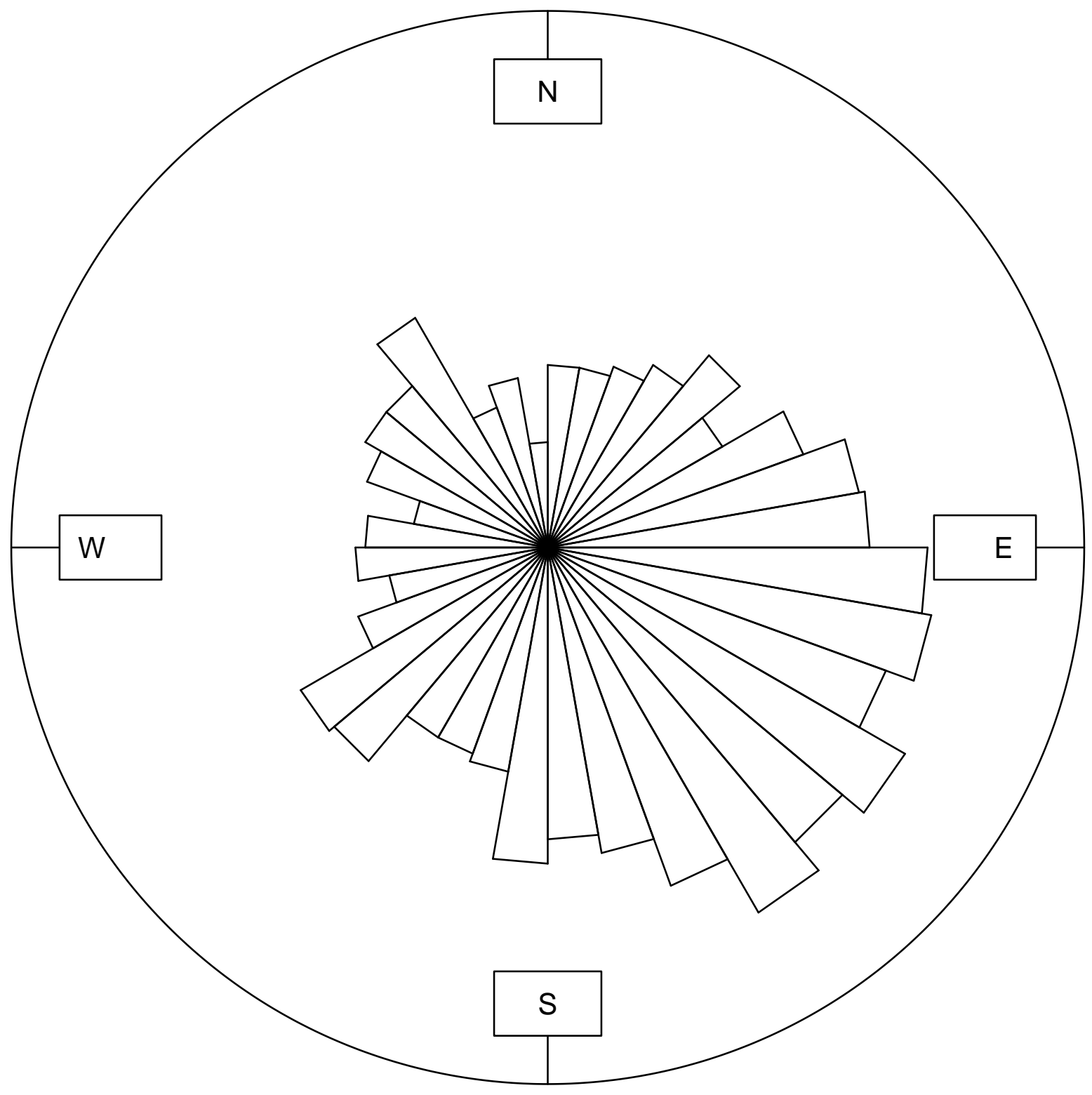


bootstrap 881

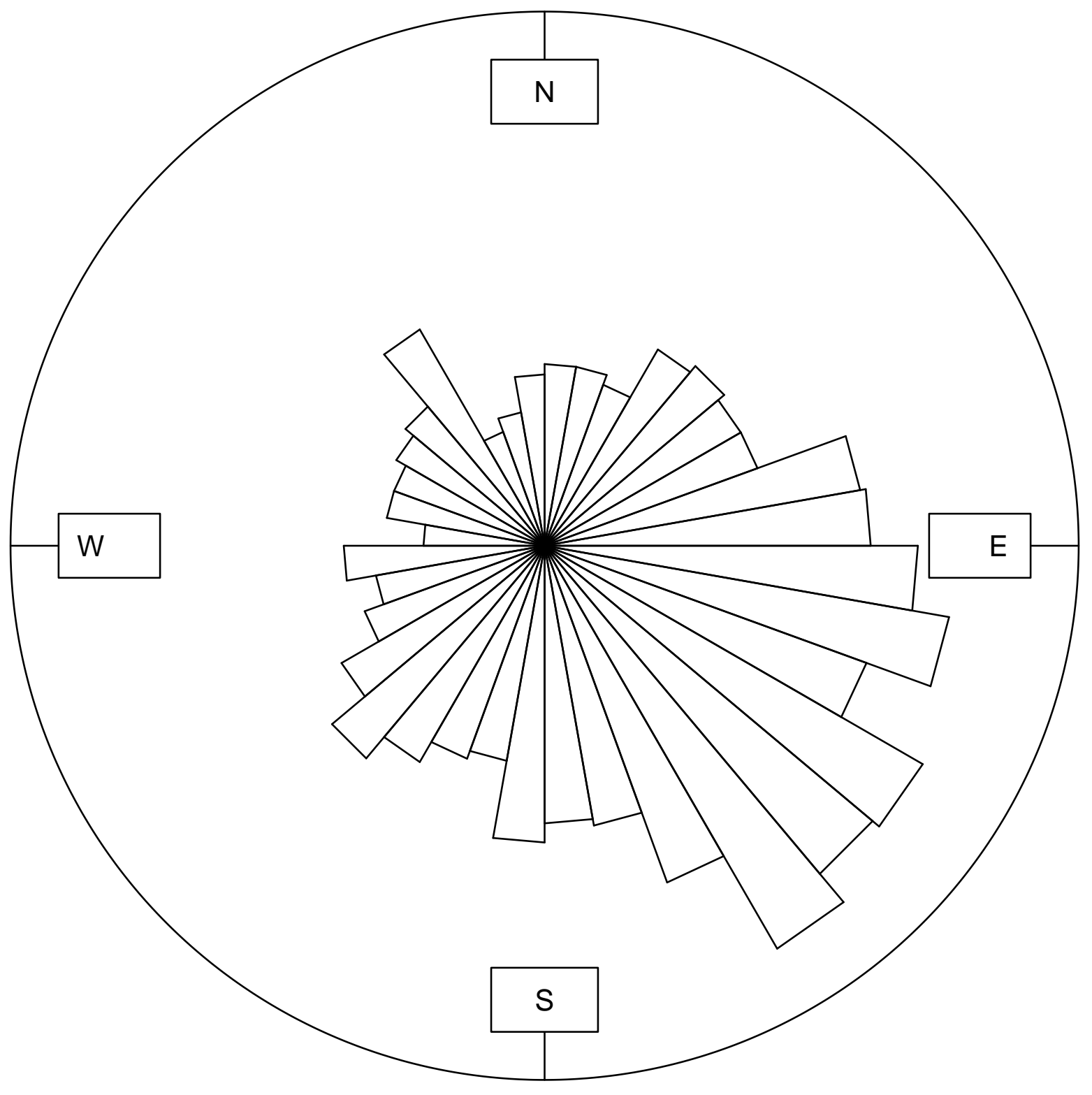


bootstrap 882

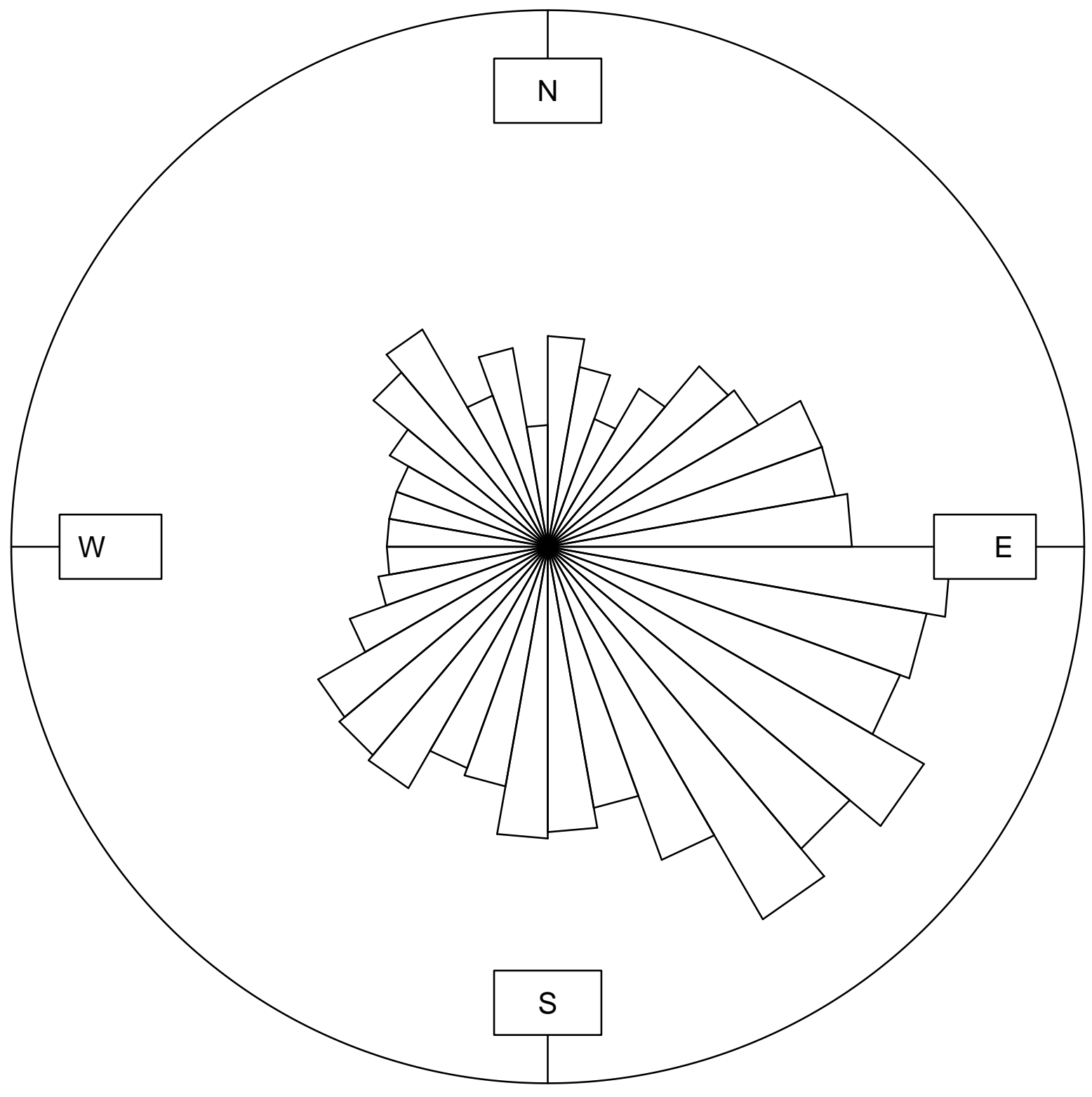


bootstrap 883

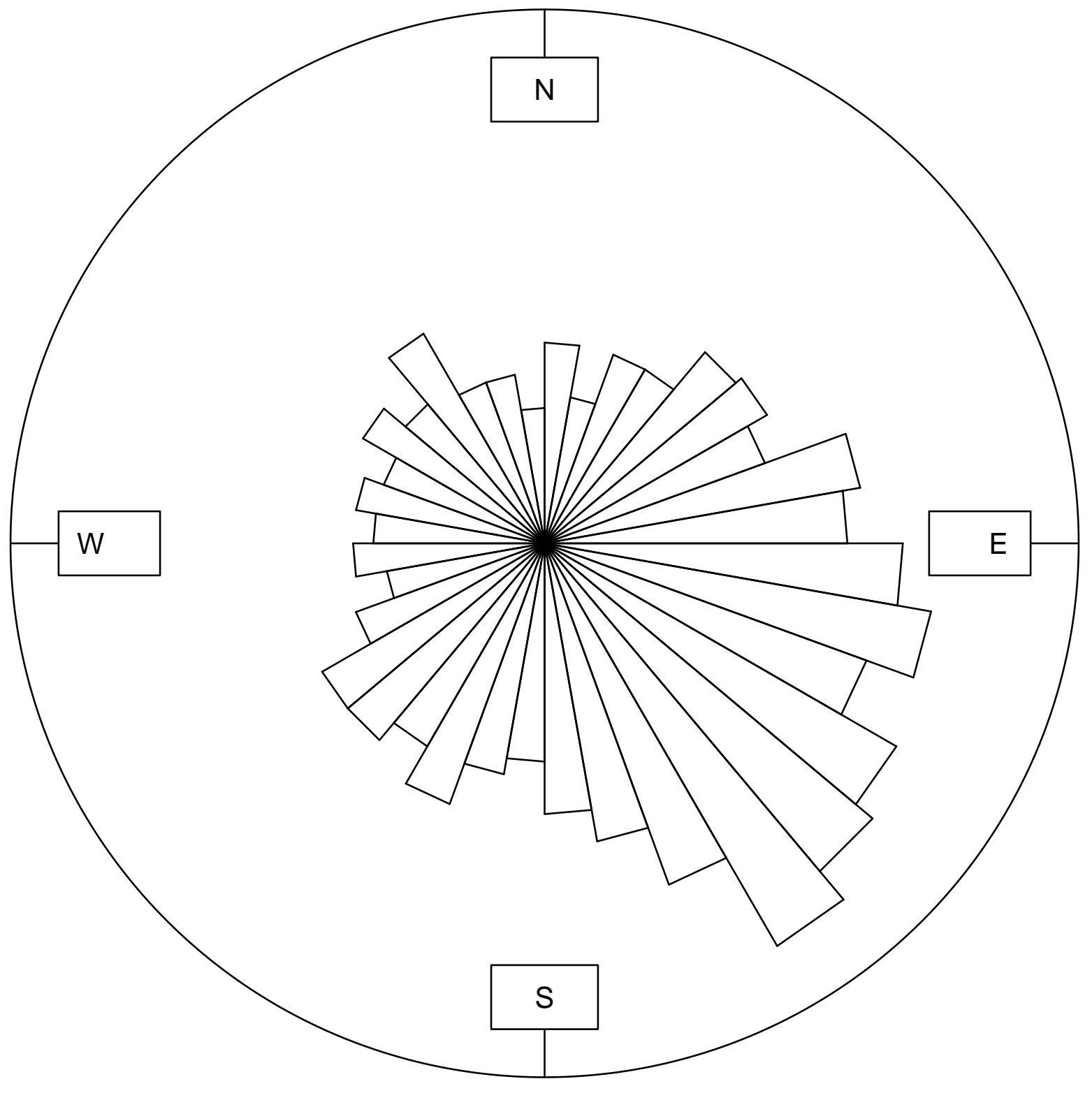




\section{bootstrap 884}

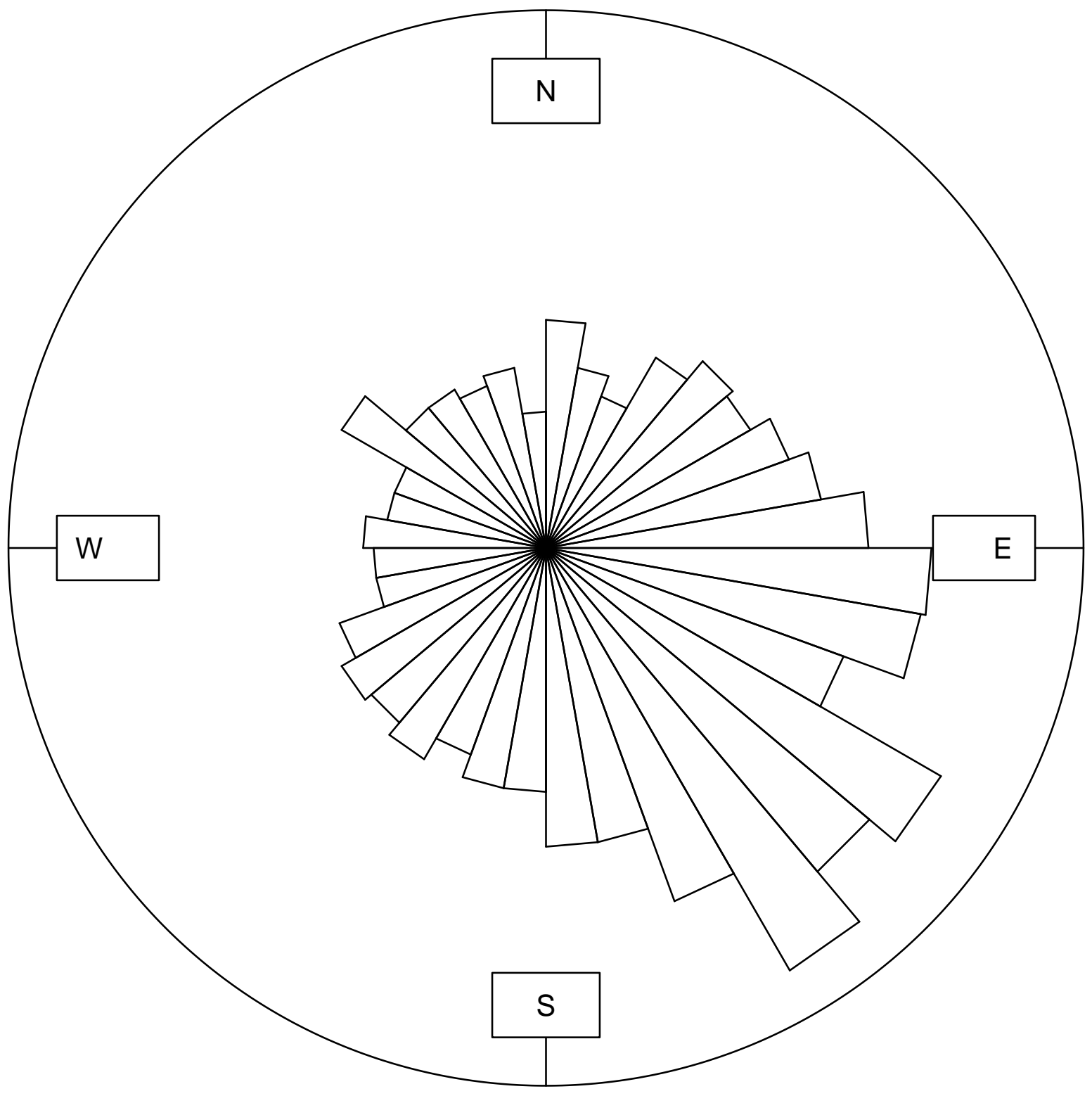




\section{bootstrap 885}

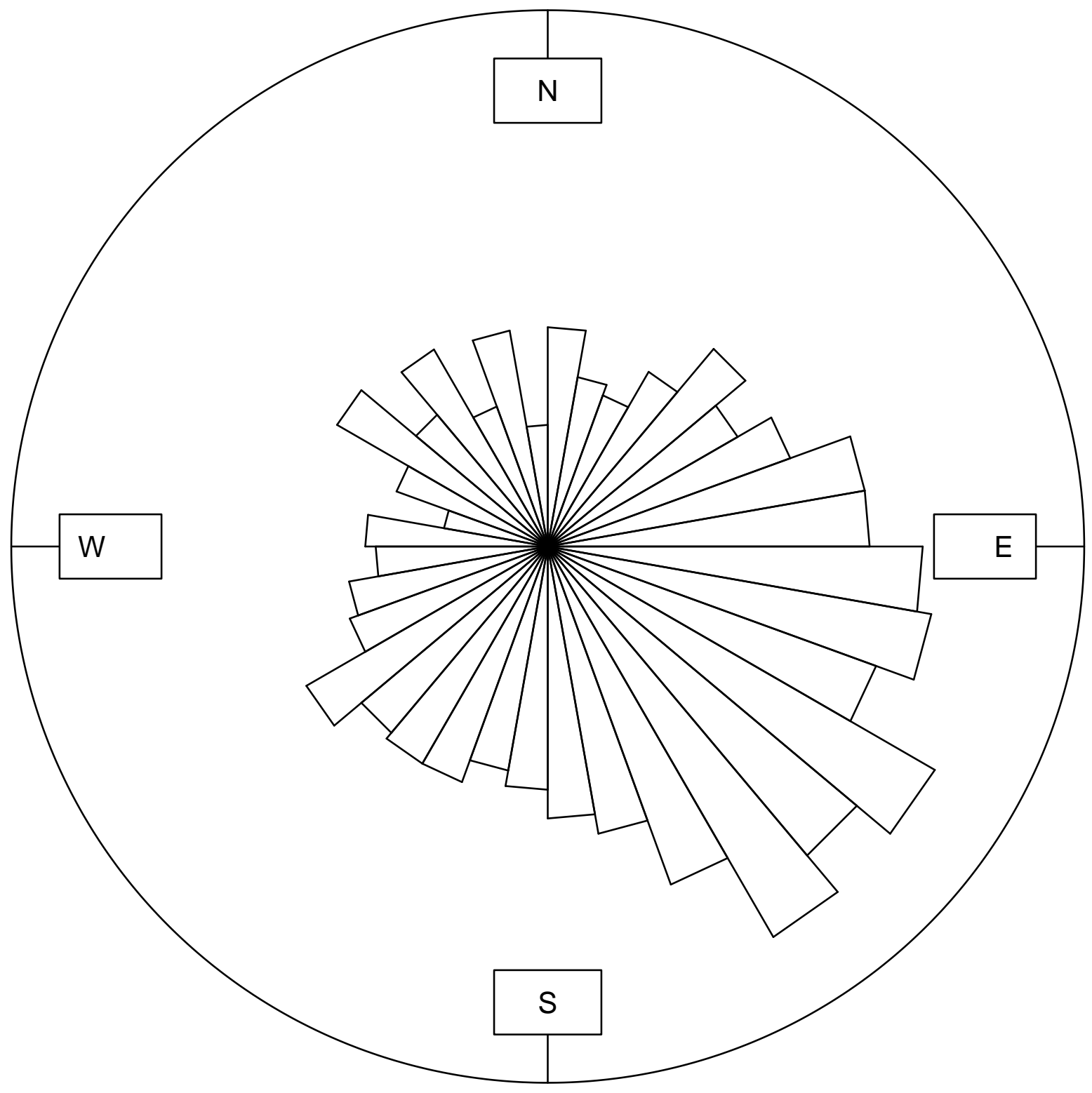


bootstrap 886

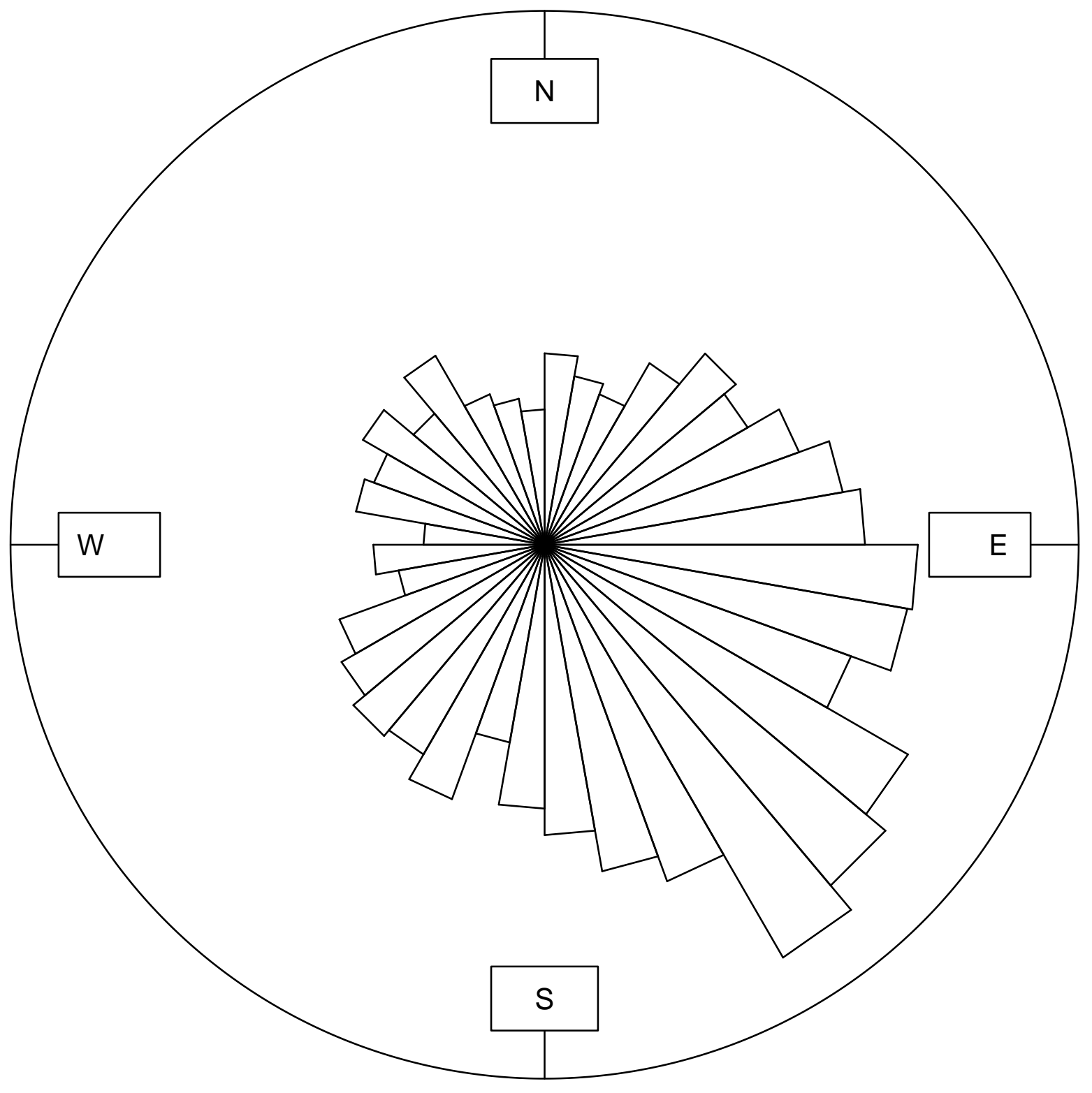


bootstrap 888

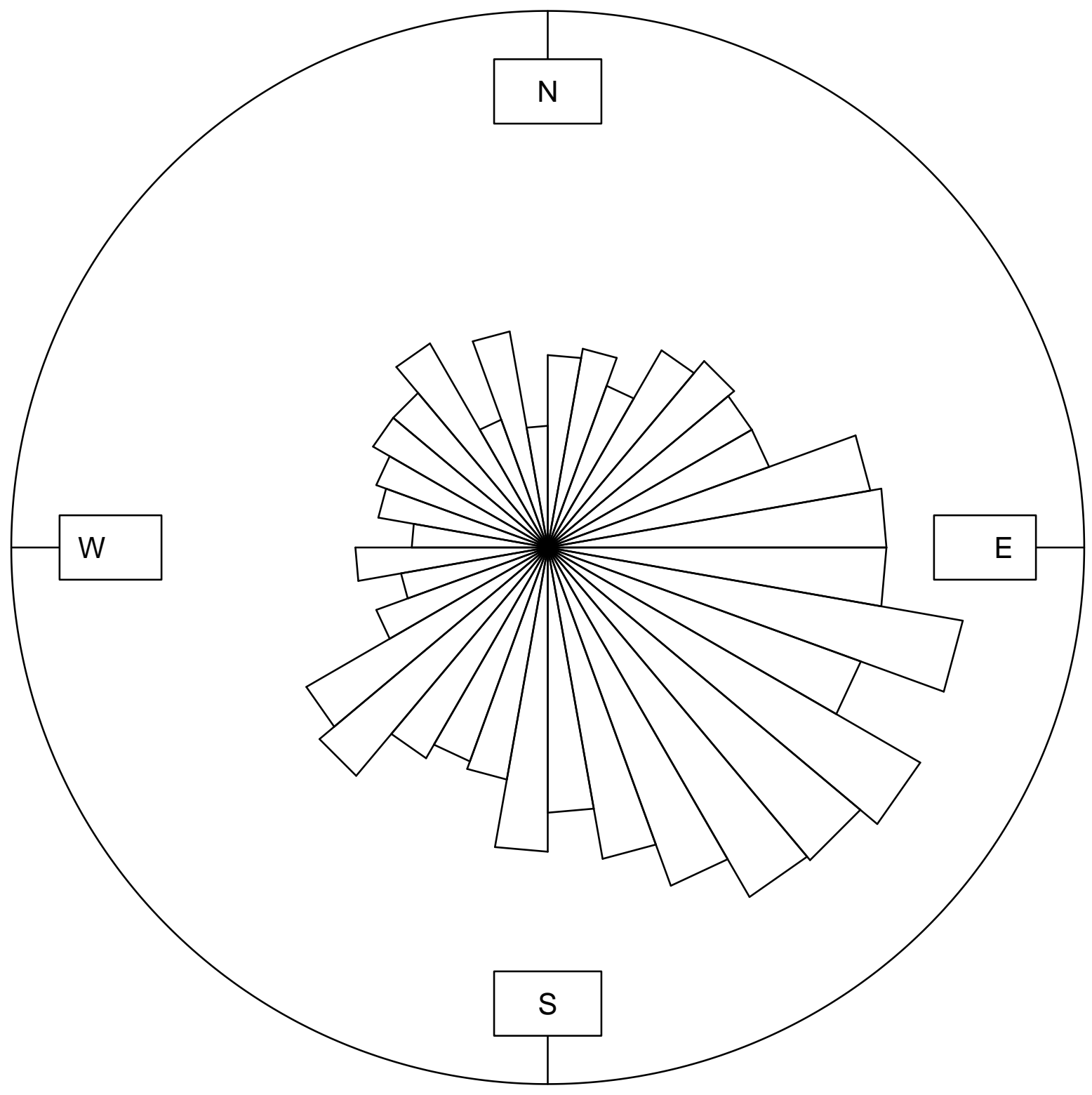


bootstrap 889

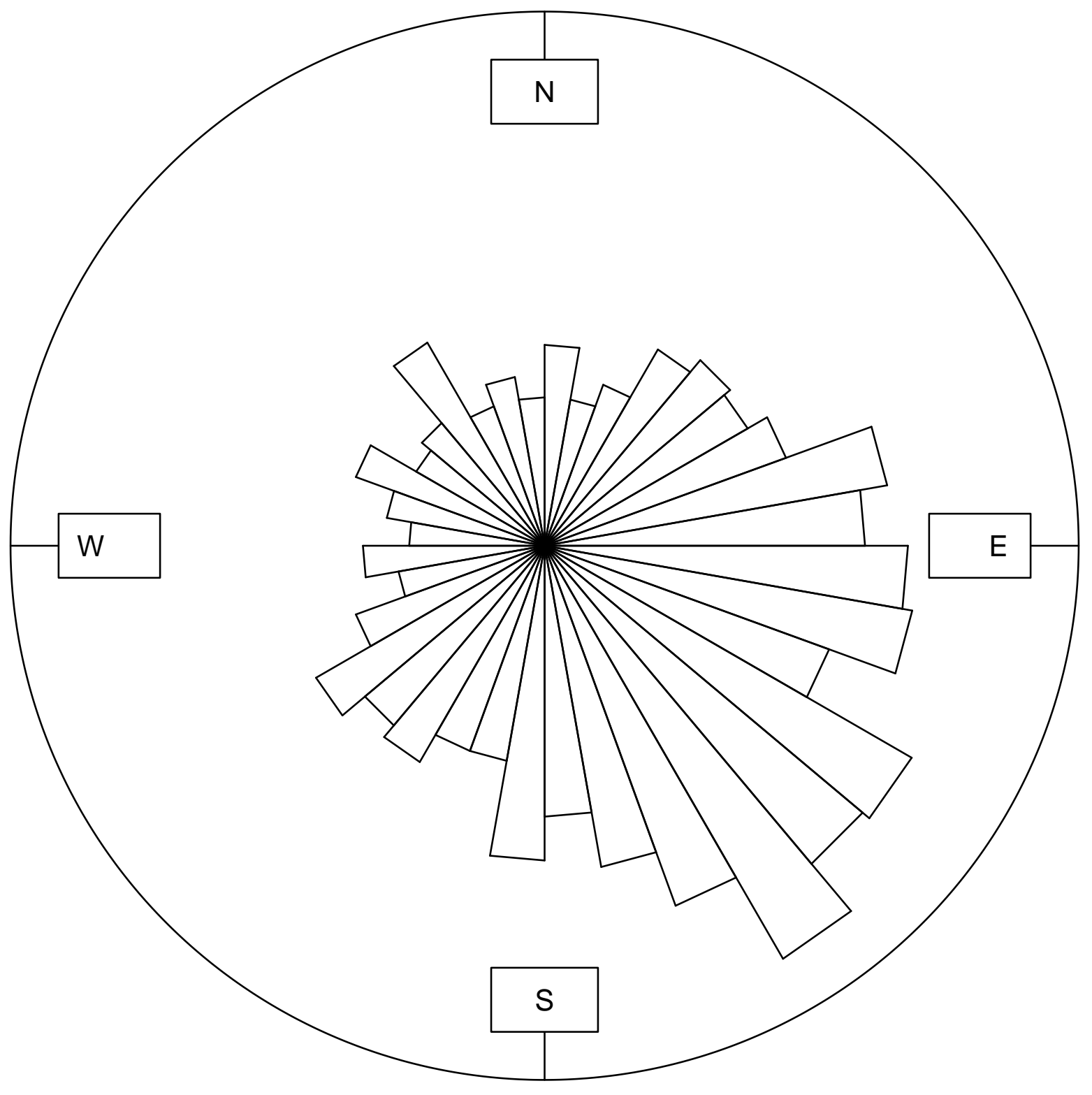


bootstrap 890

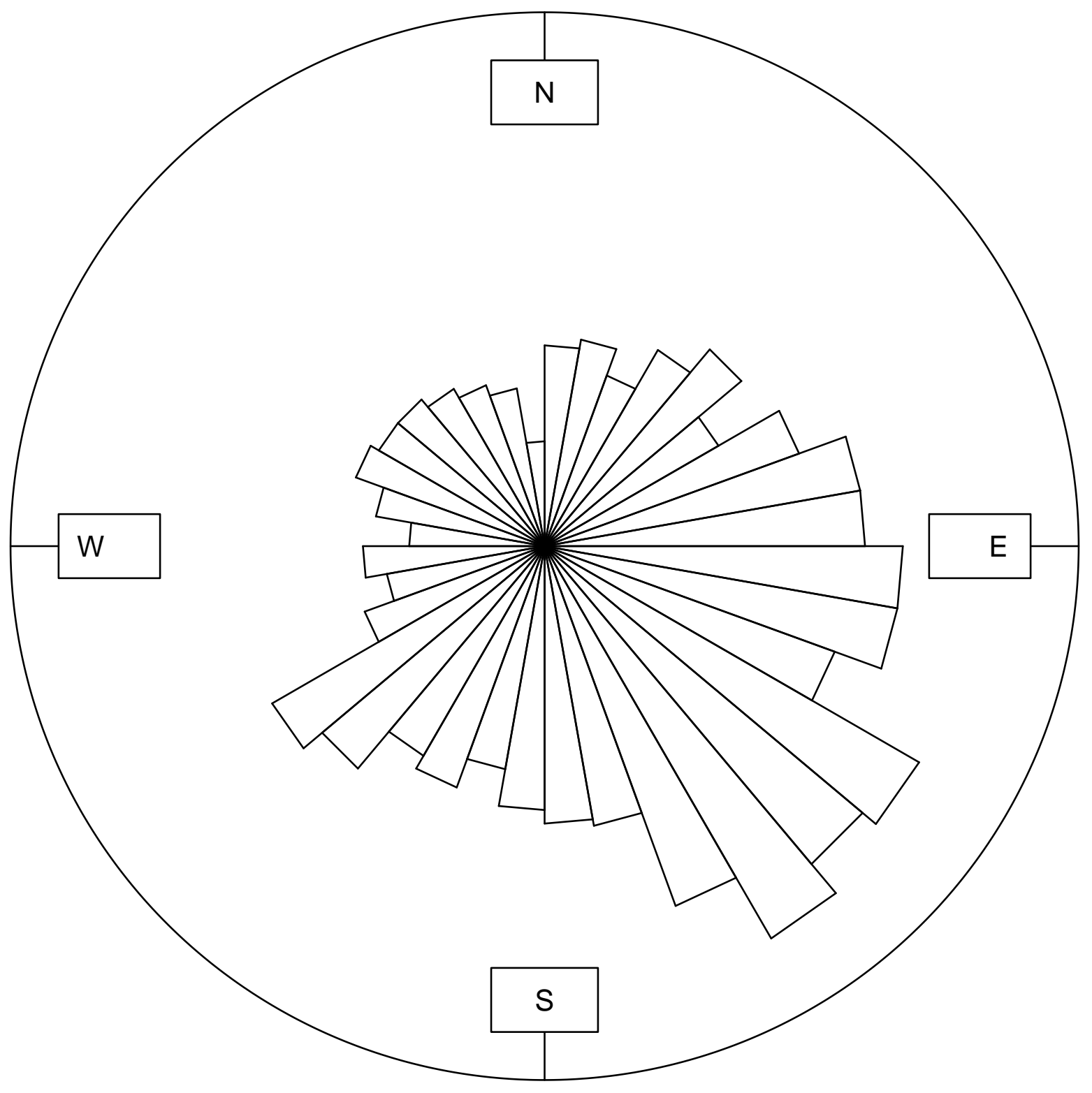


bootstrap 891

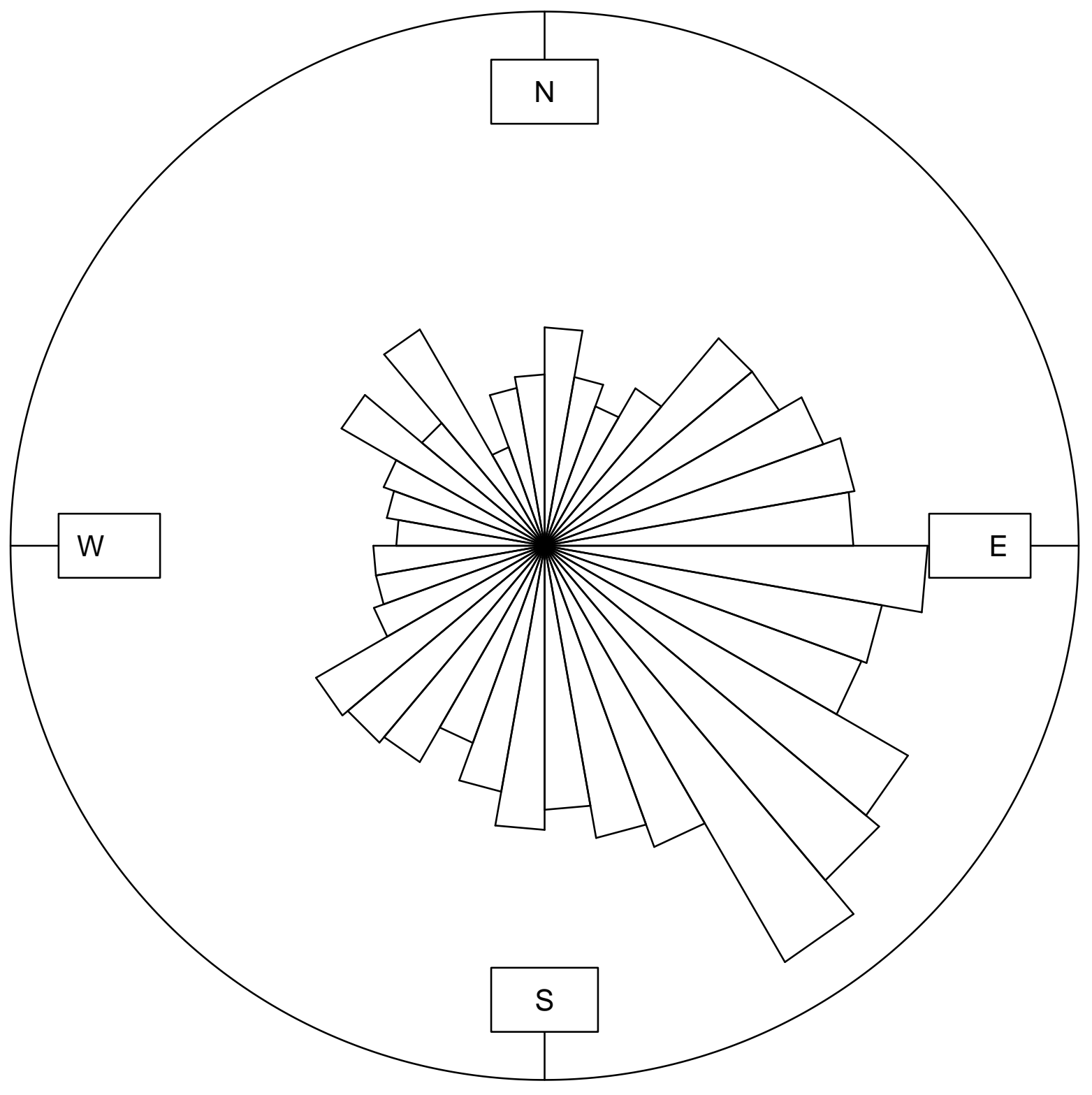


bootstrap 892

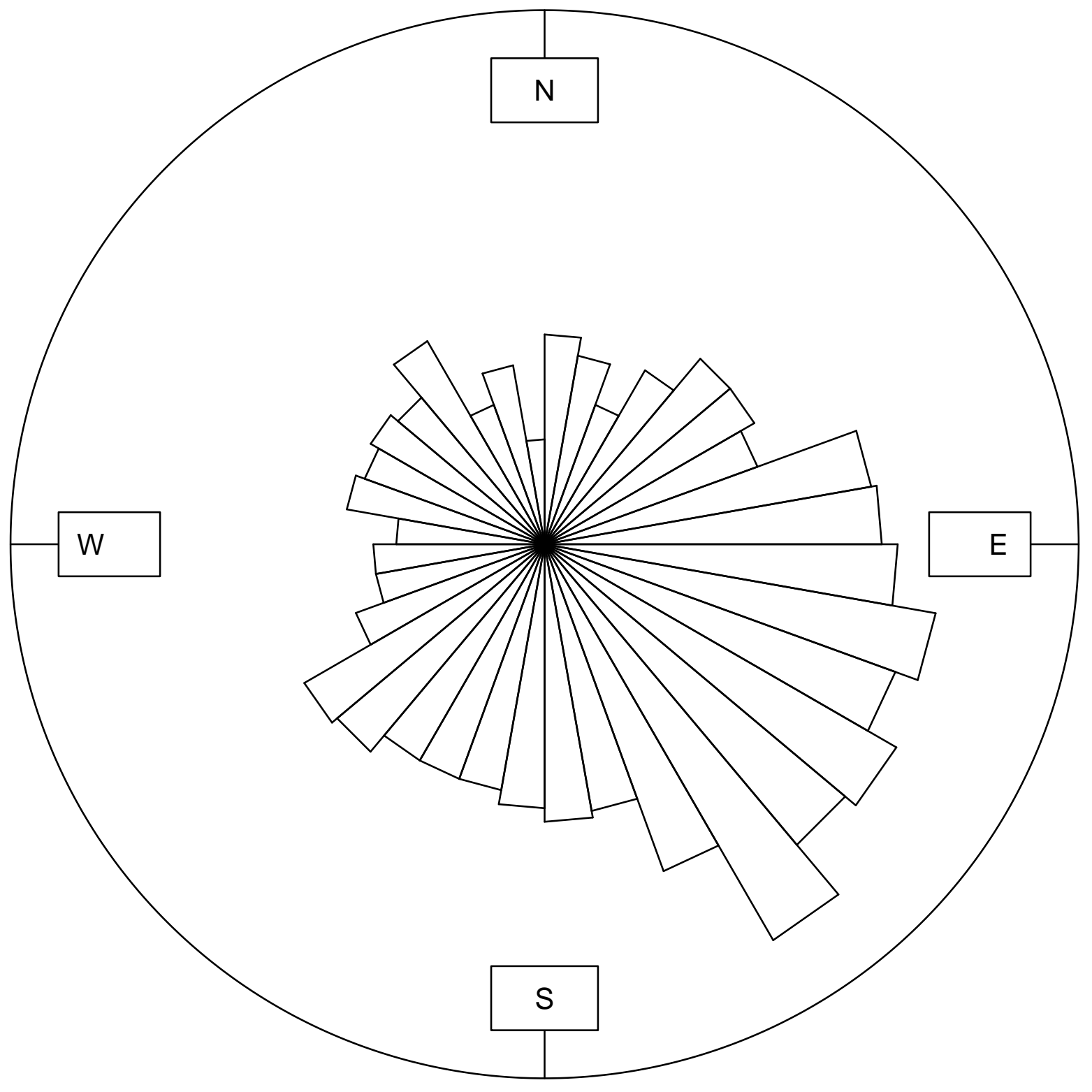




\section{bootstrap 893}

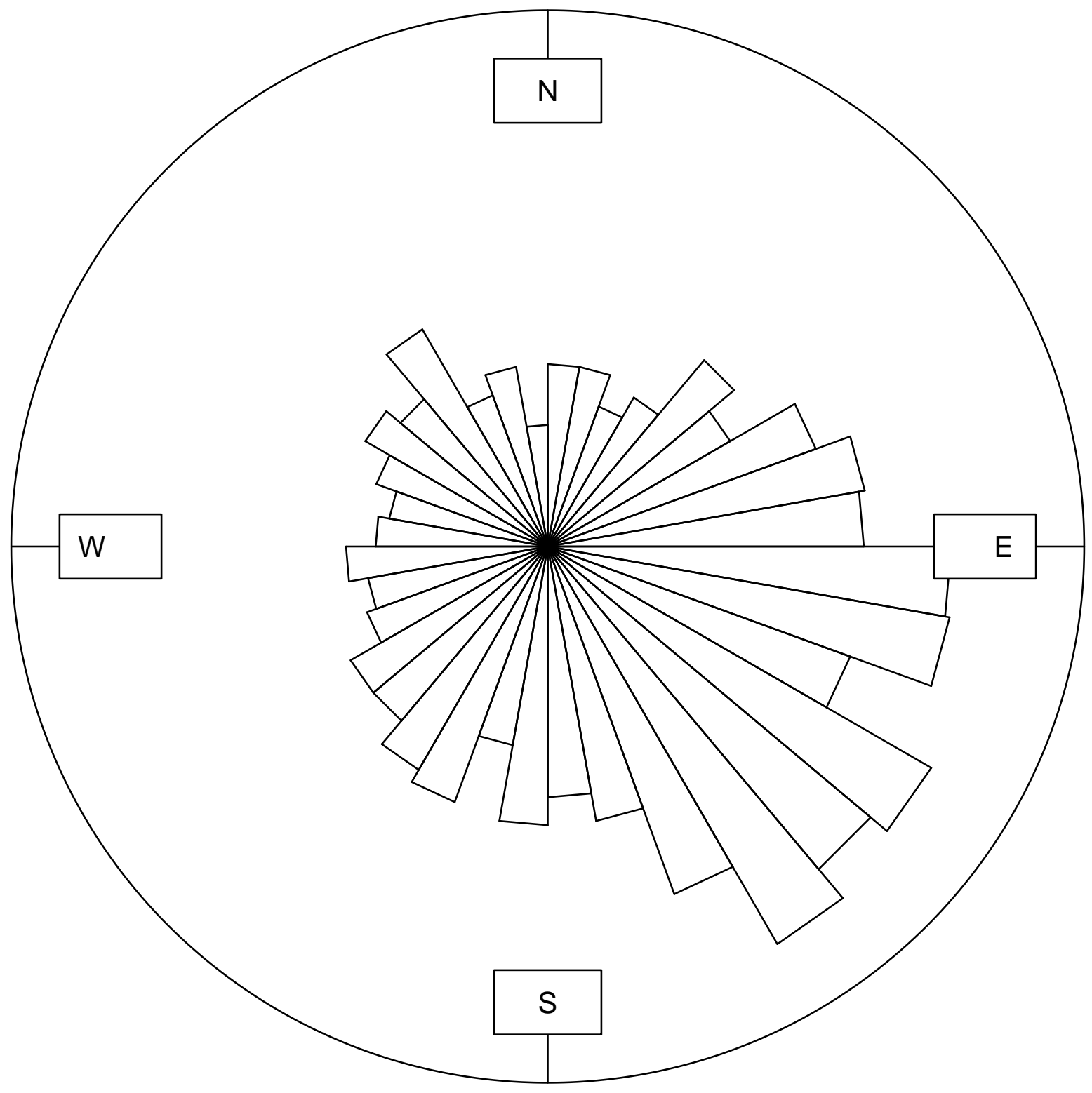




\section{bootstrap 895}

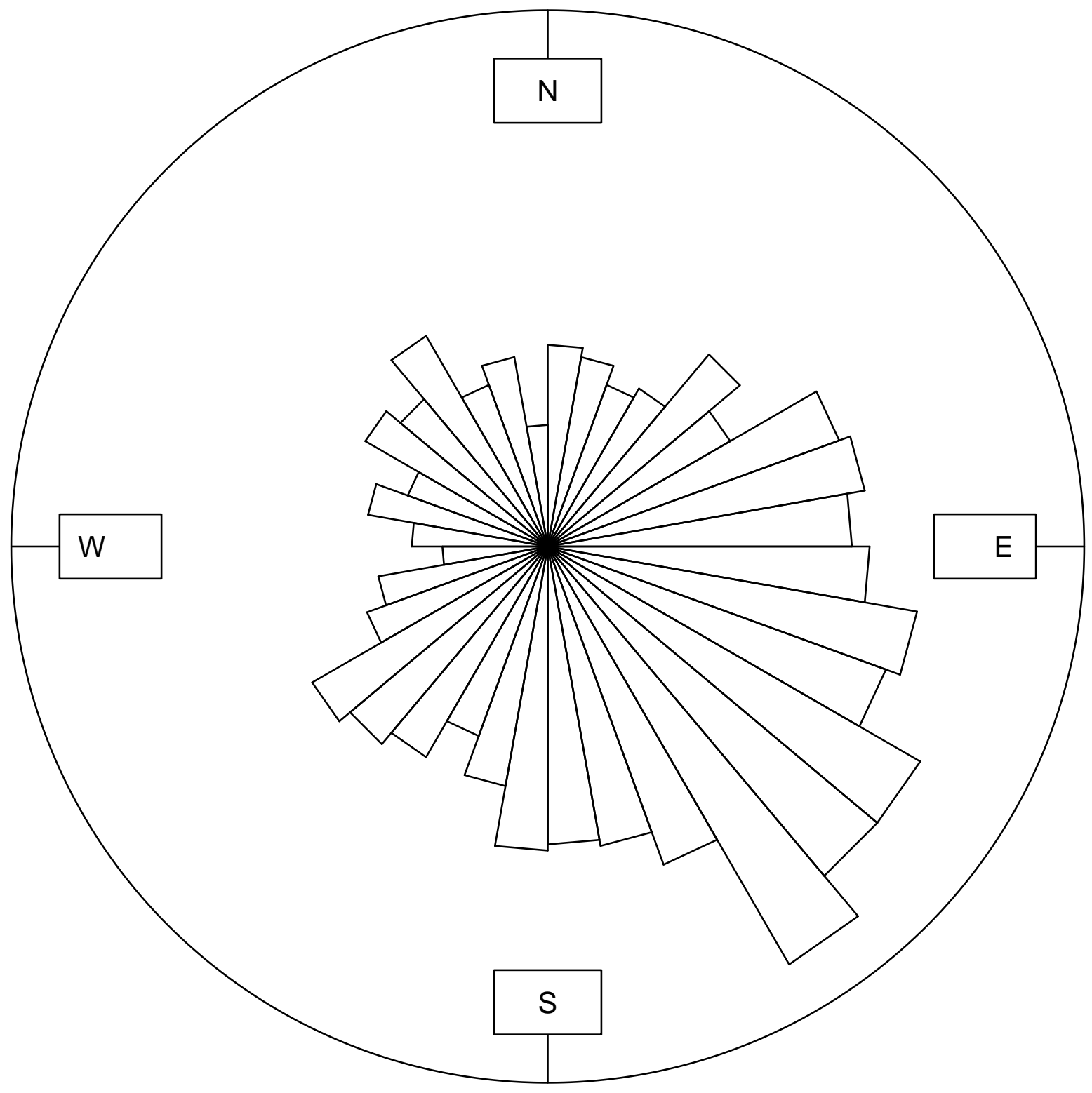


bootstrap 896

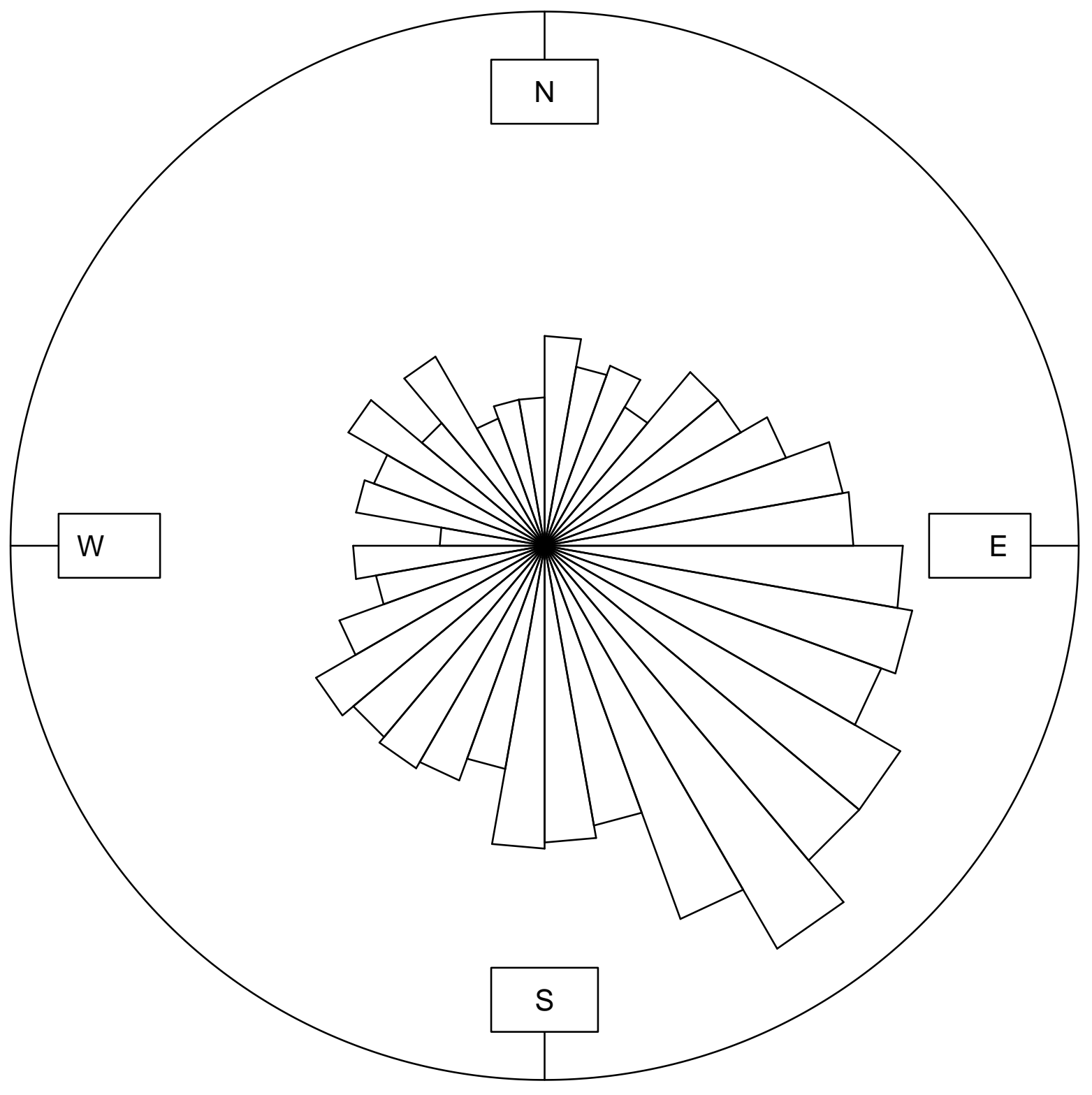


bootstrap 897

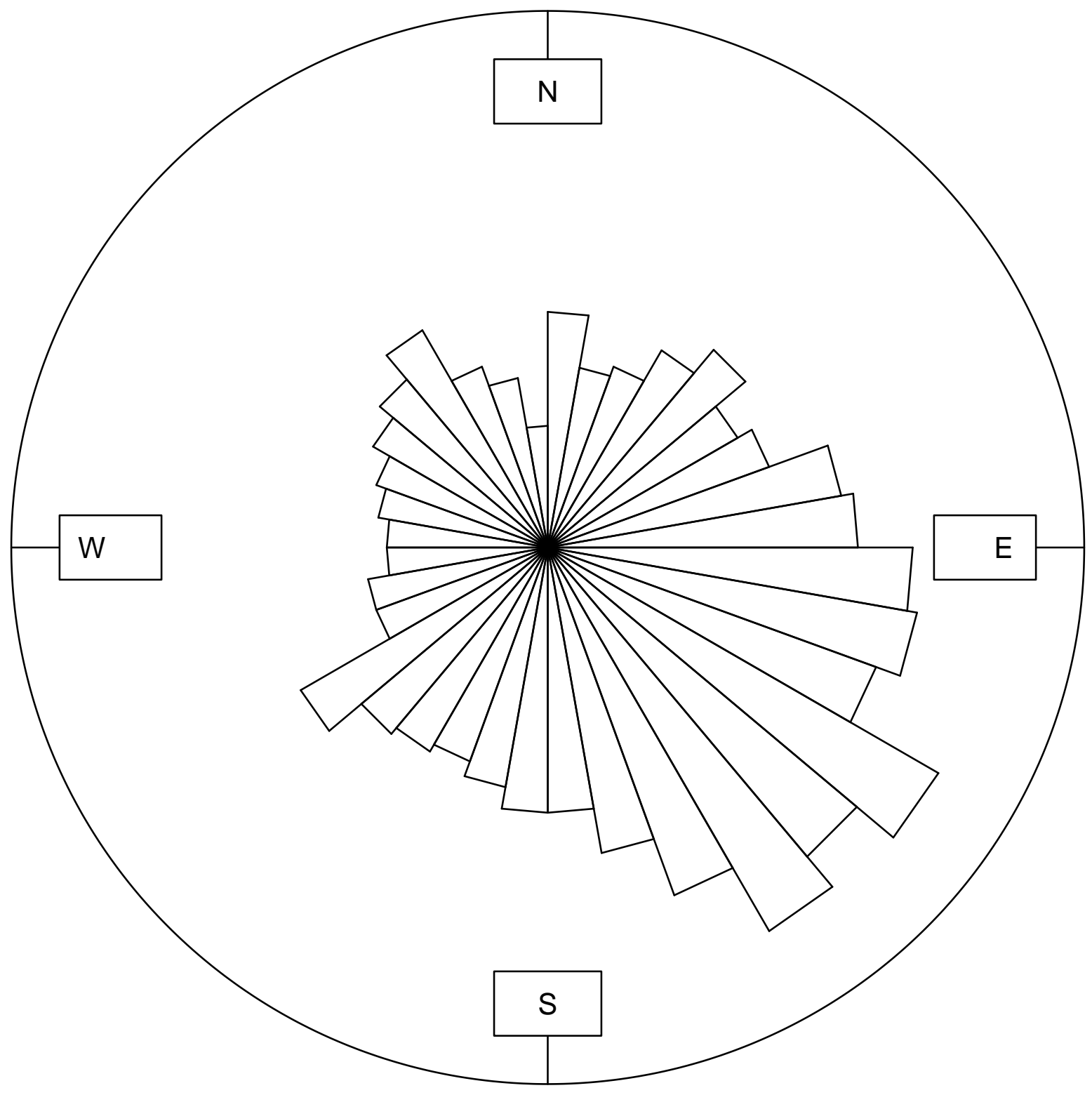


bootstrap 898

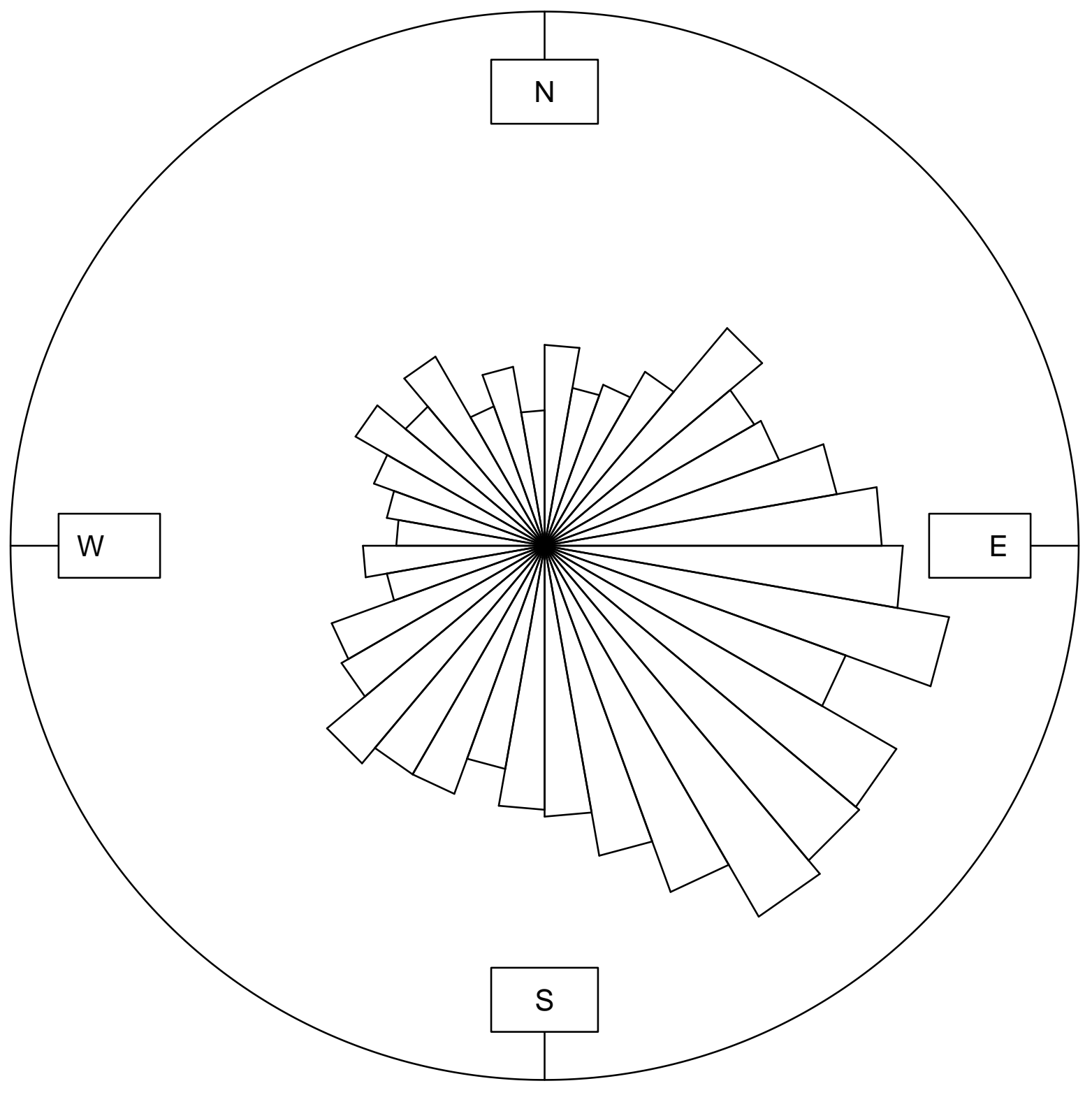


bootstrap 899

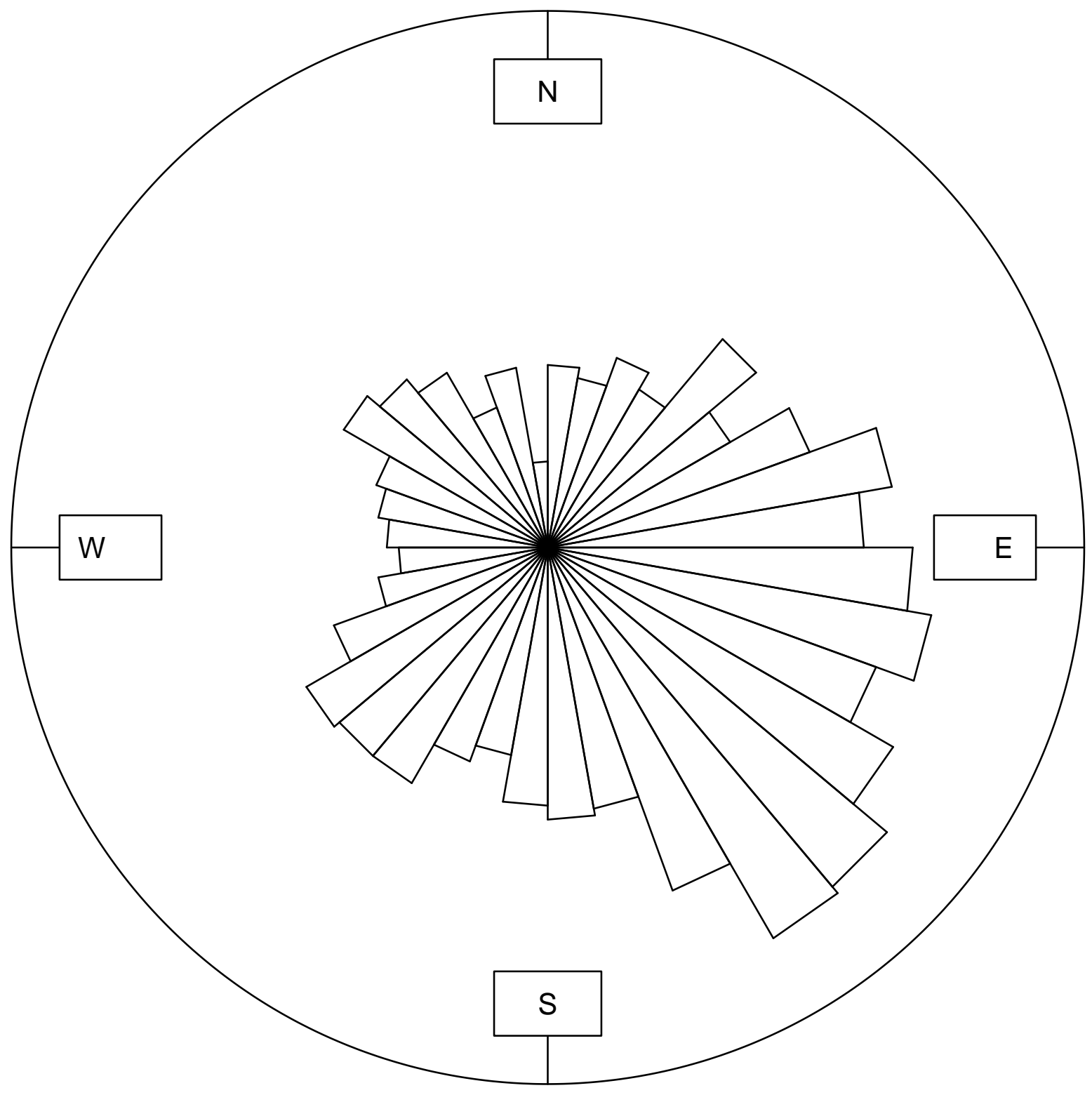


bootstrap 900

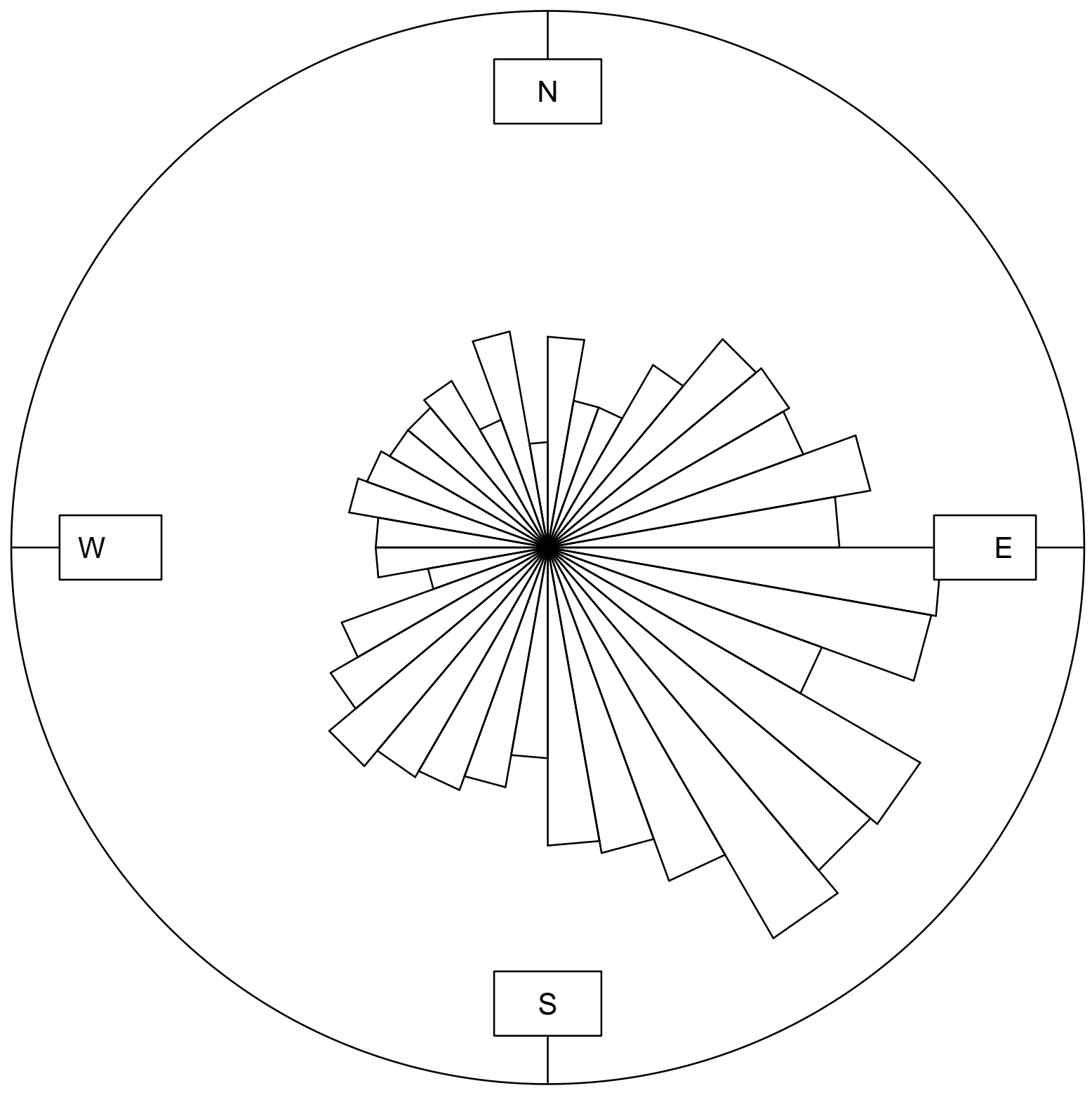


bootstrap 901

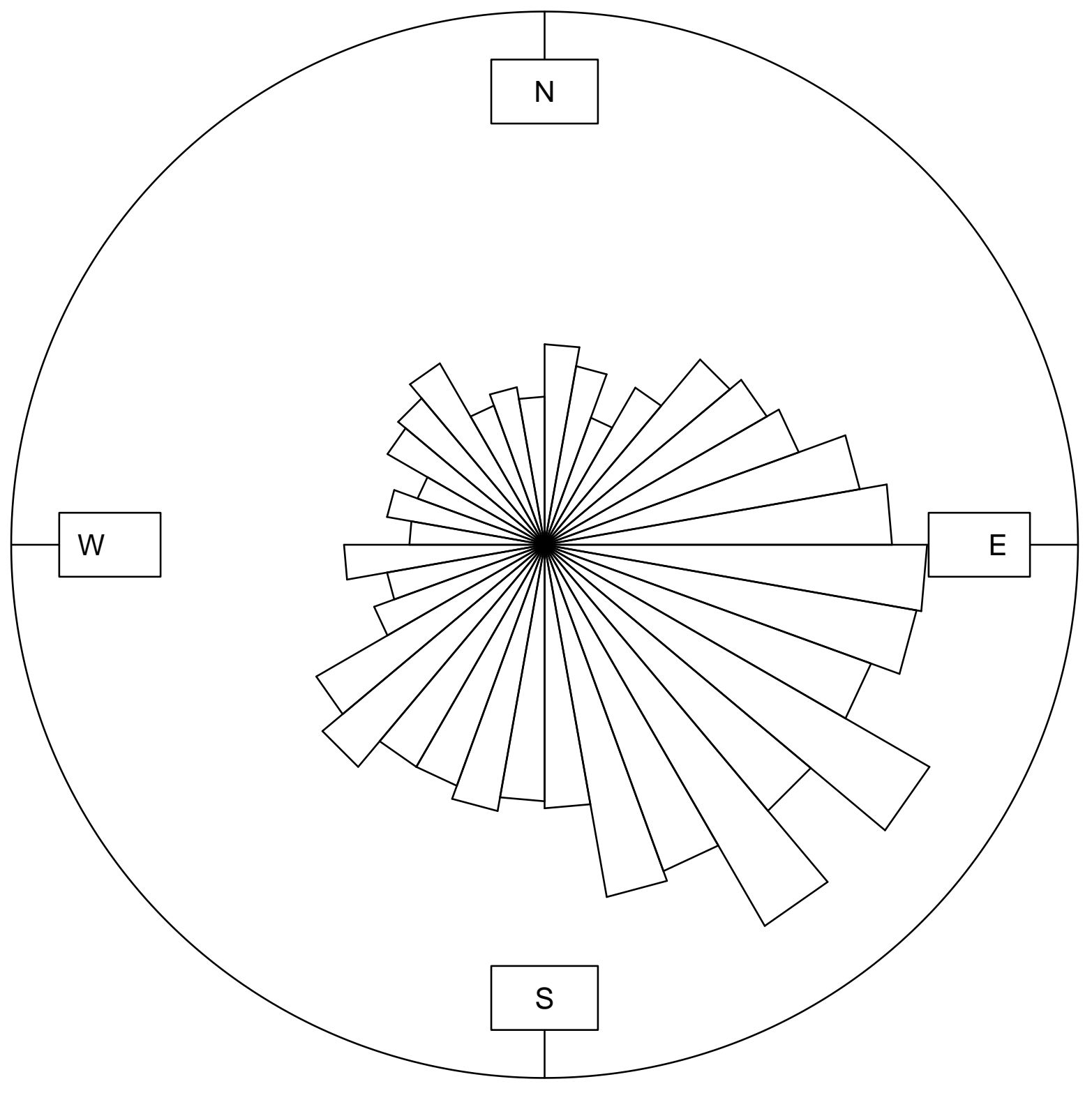


bootstrap 902

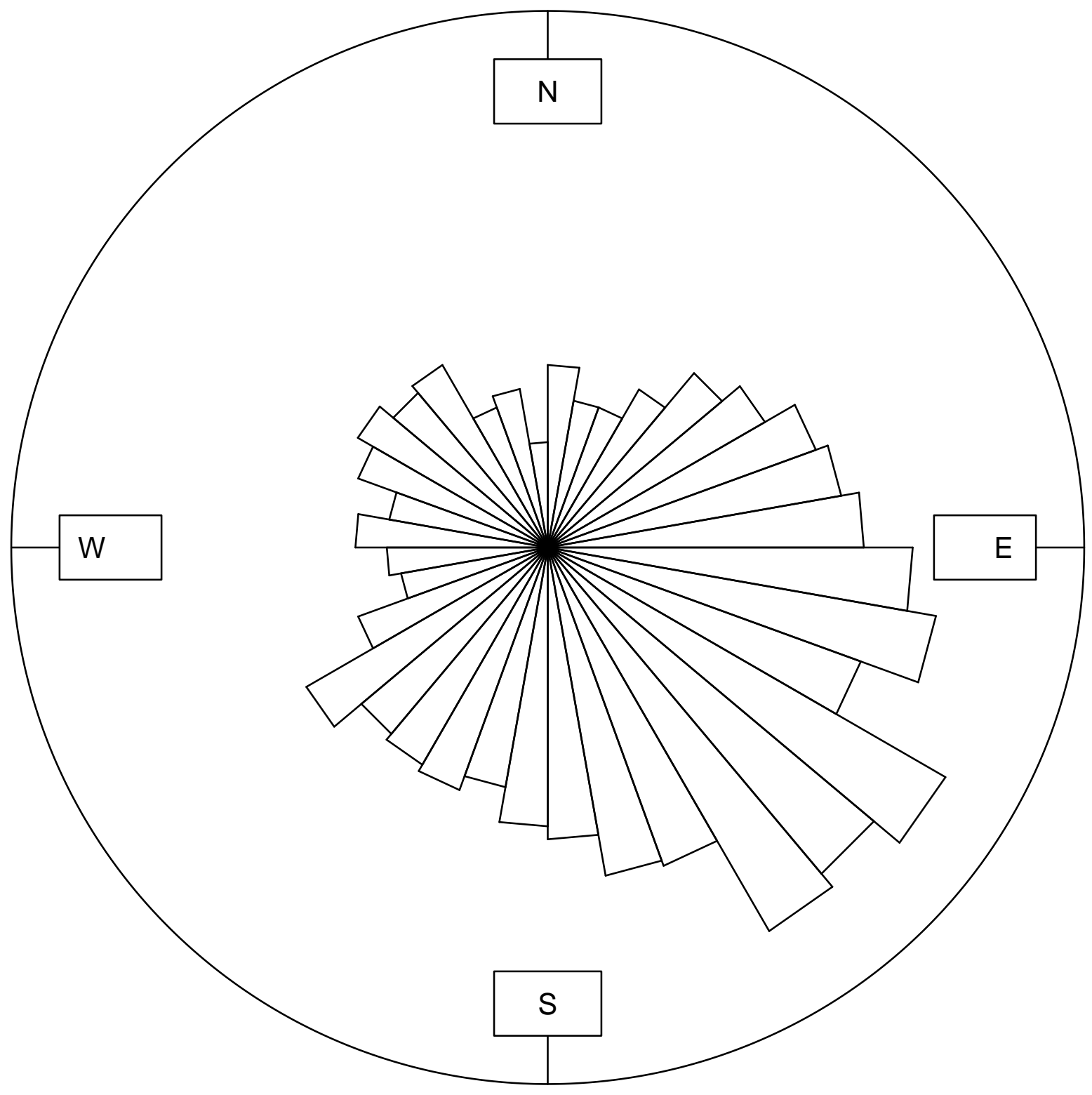


bootstrap 903

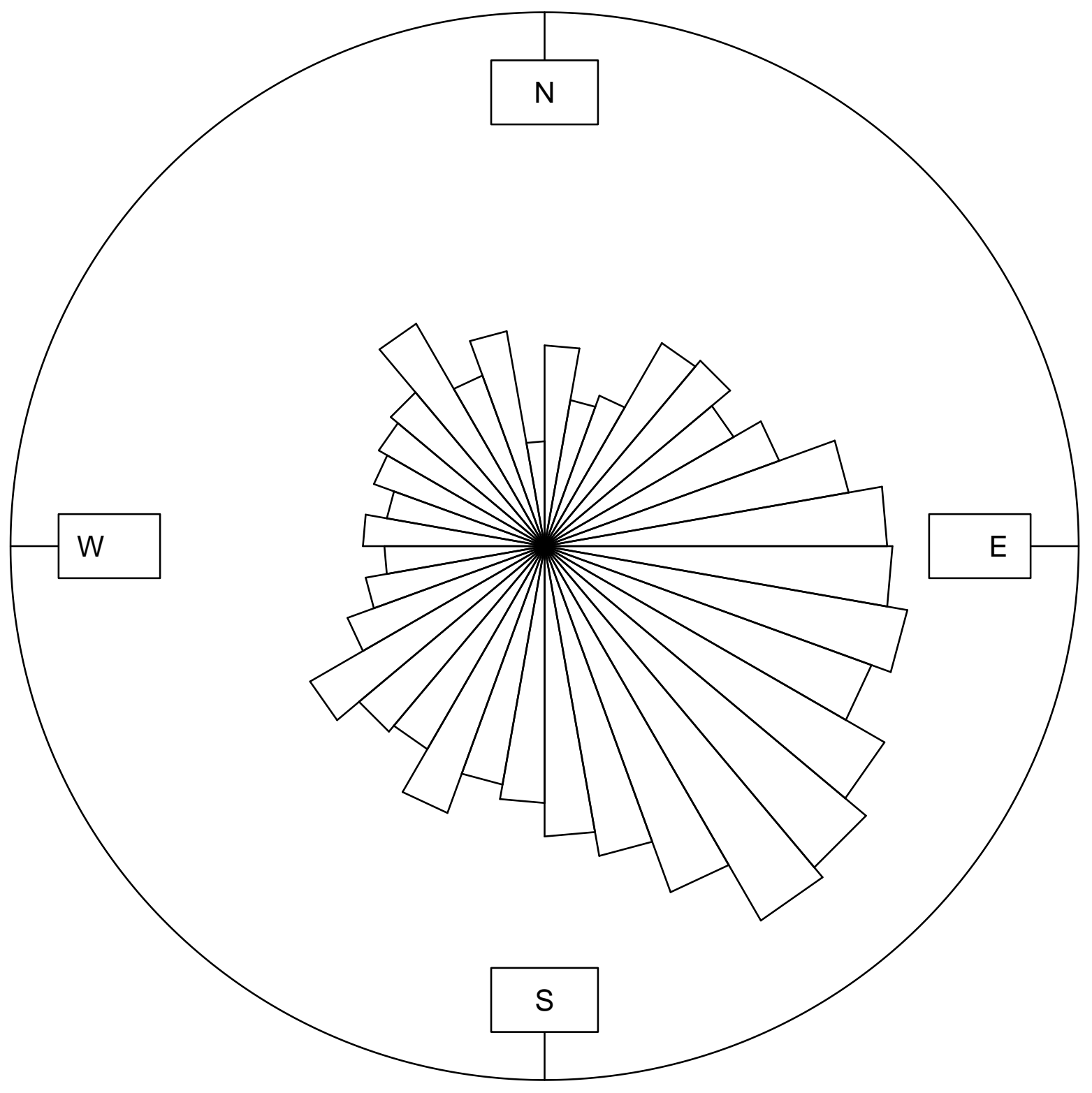




\section{bootstrap 904}

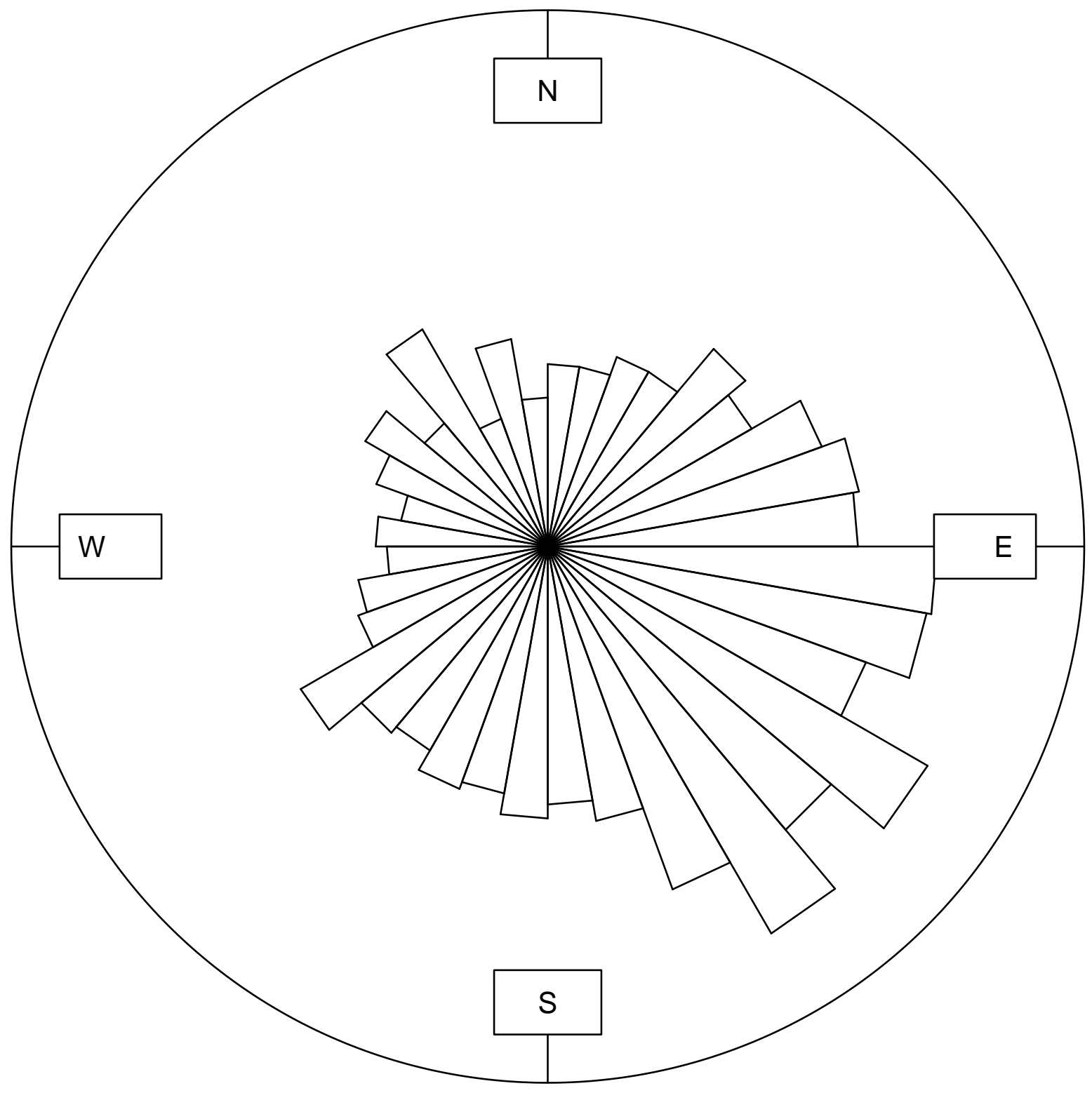




\section{bootstrap 905}

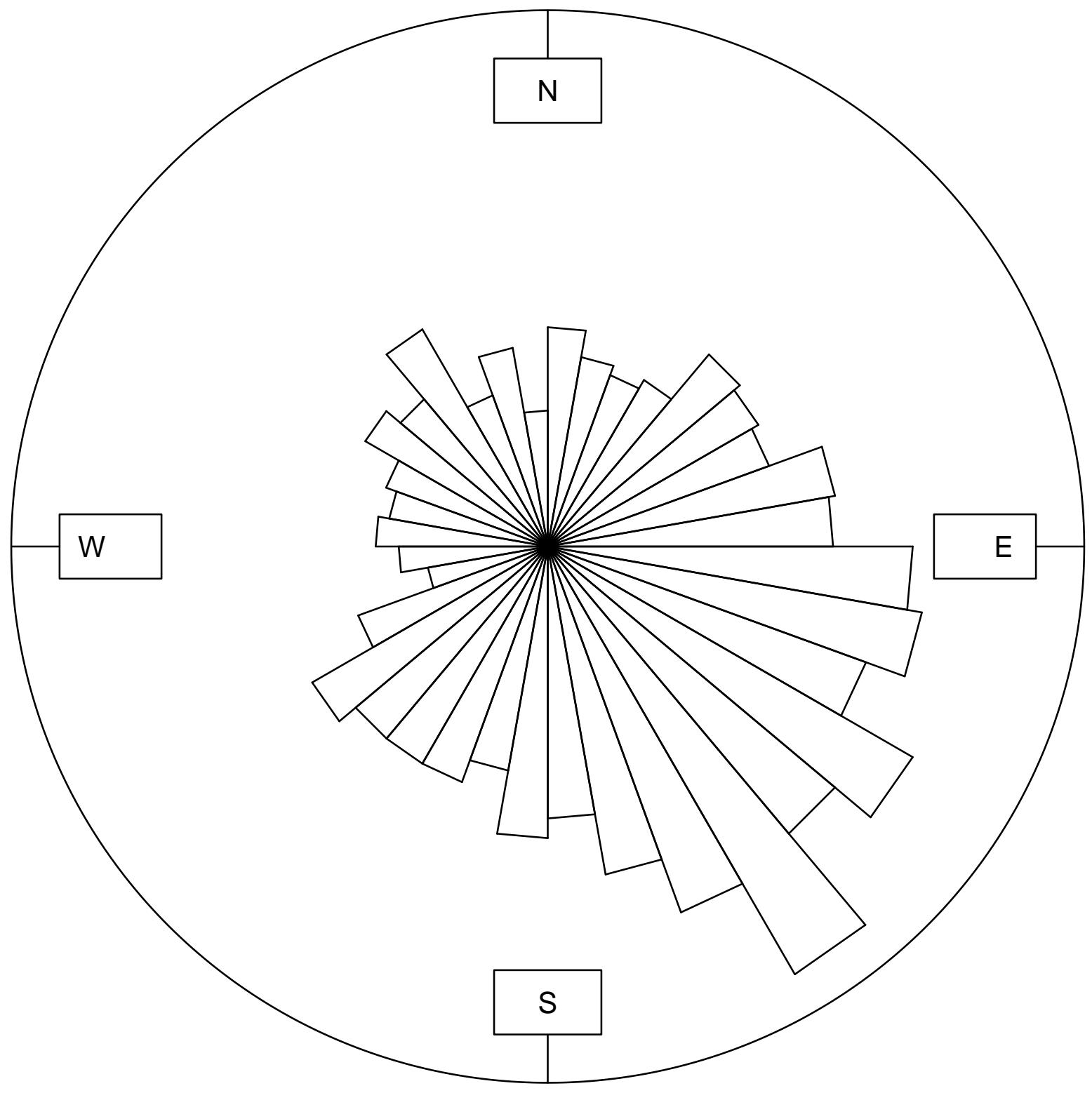


bootstrap 906

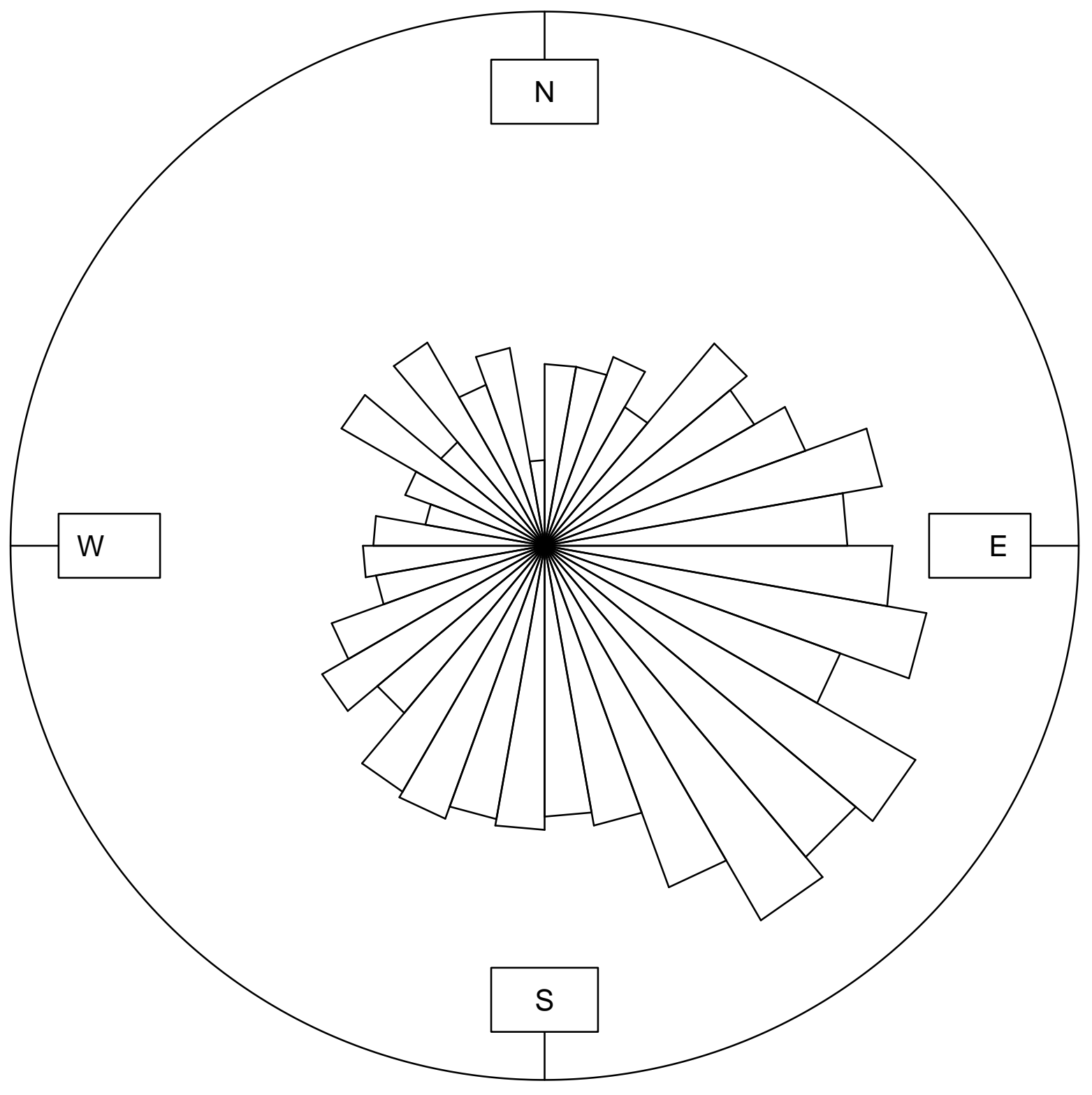


bootstrap 907

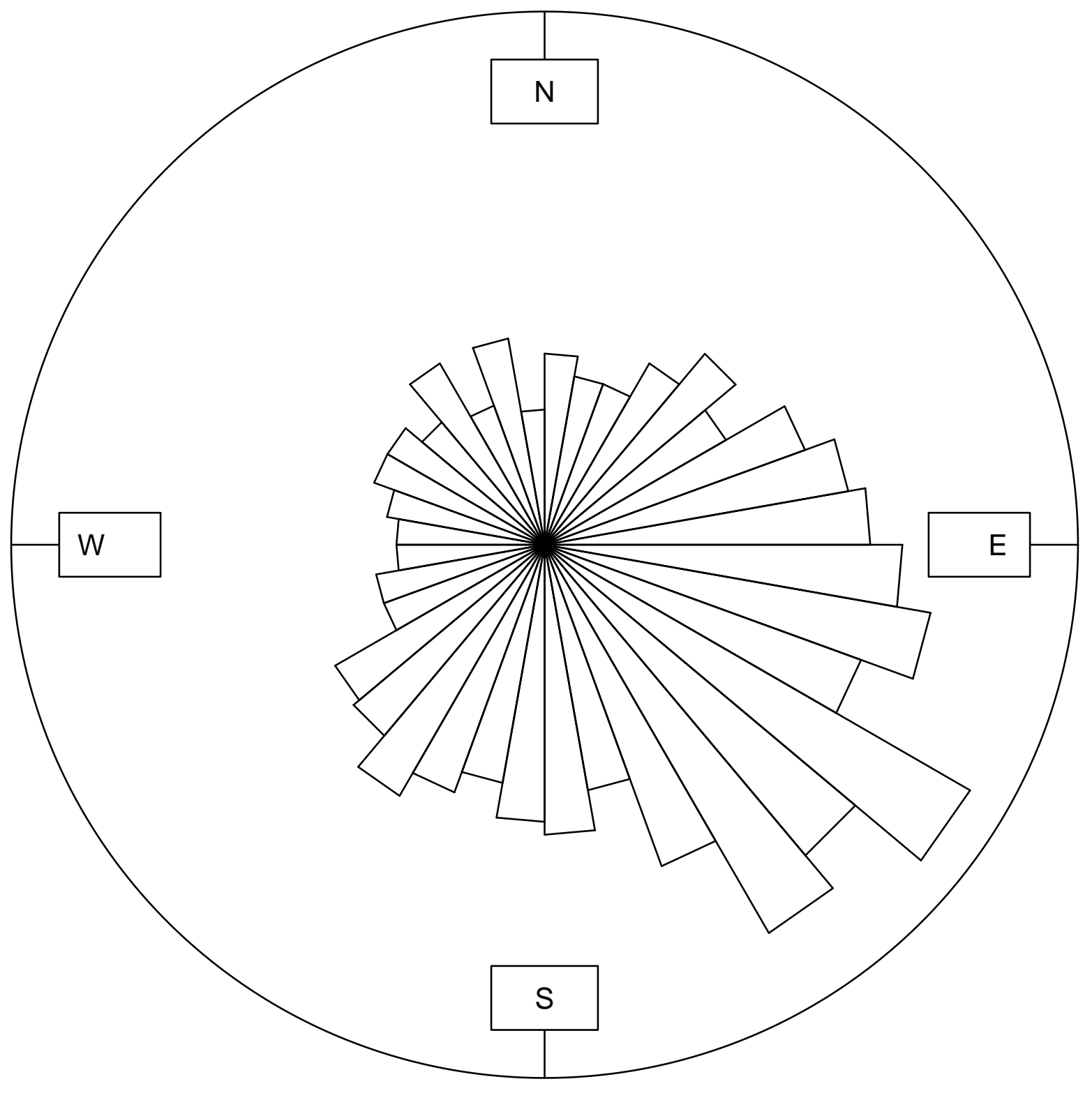


bootstrap 908

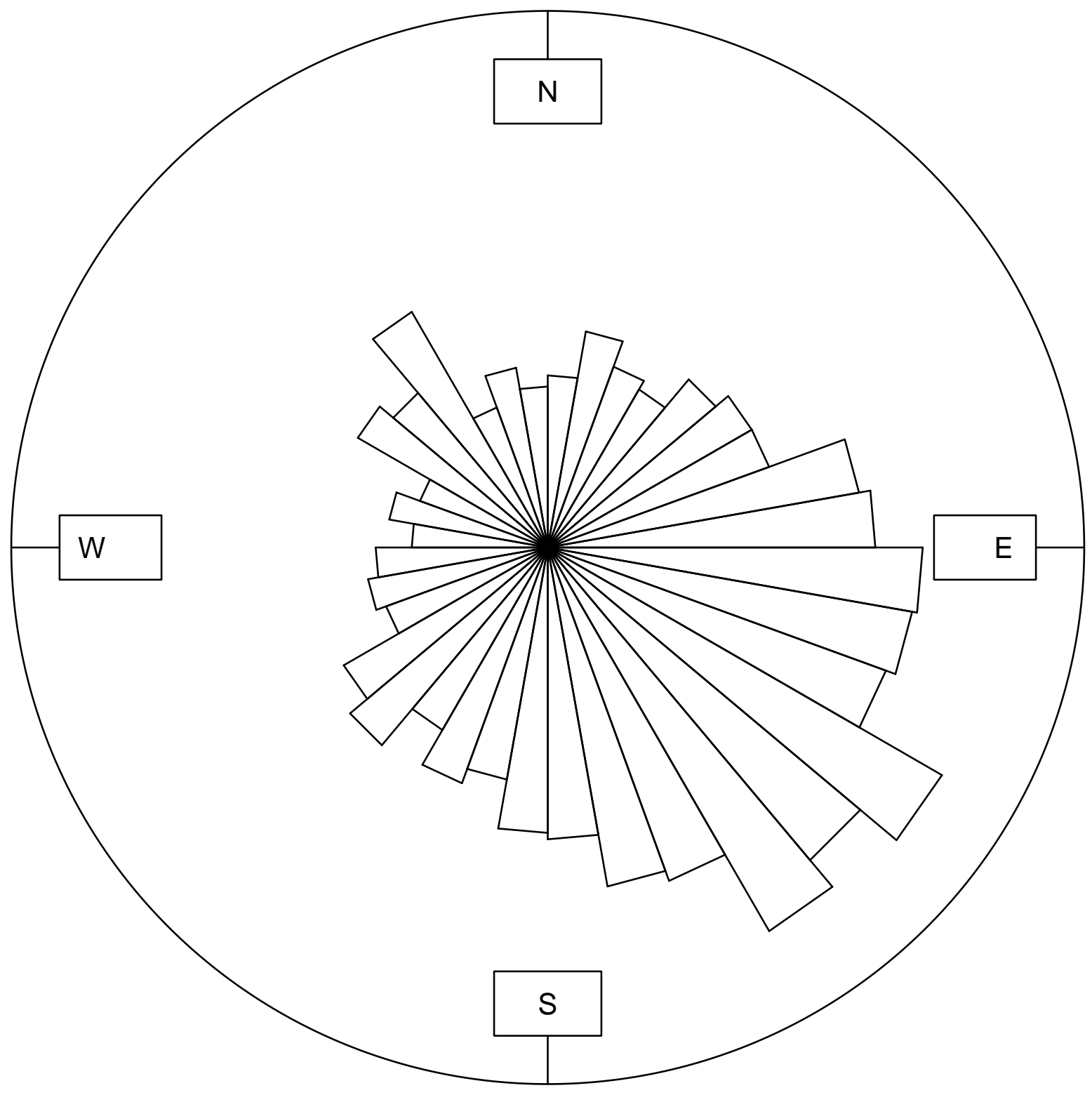


bootstrap 909

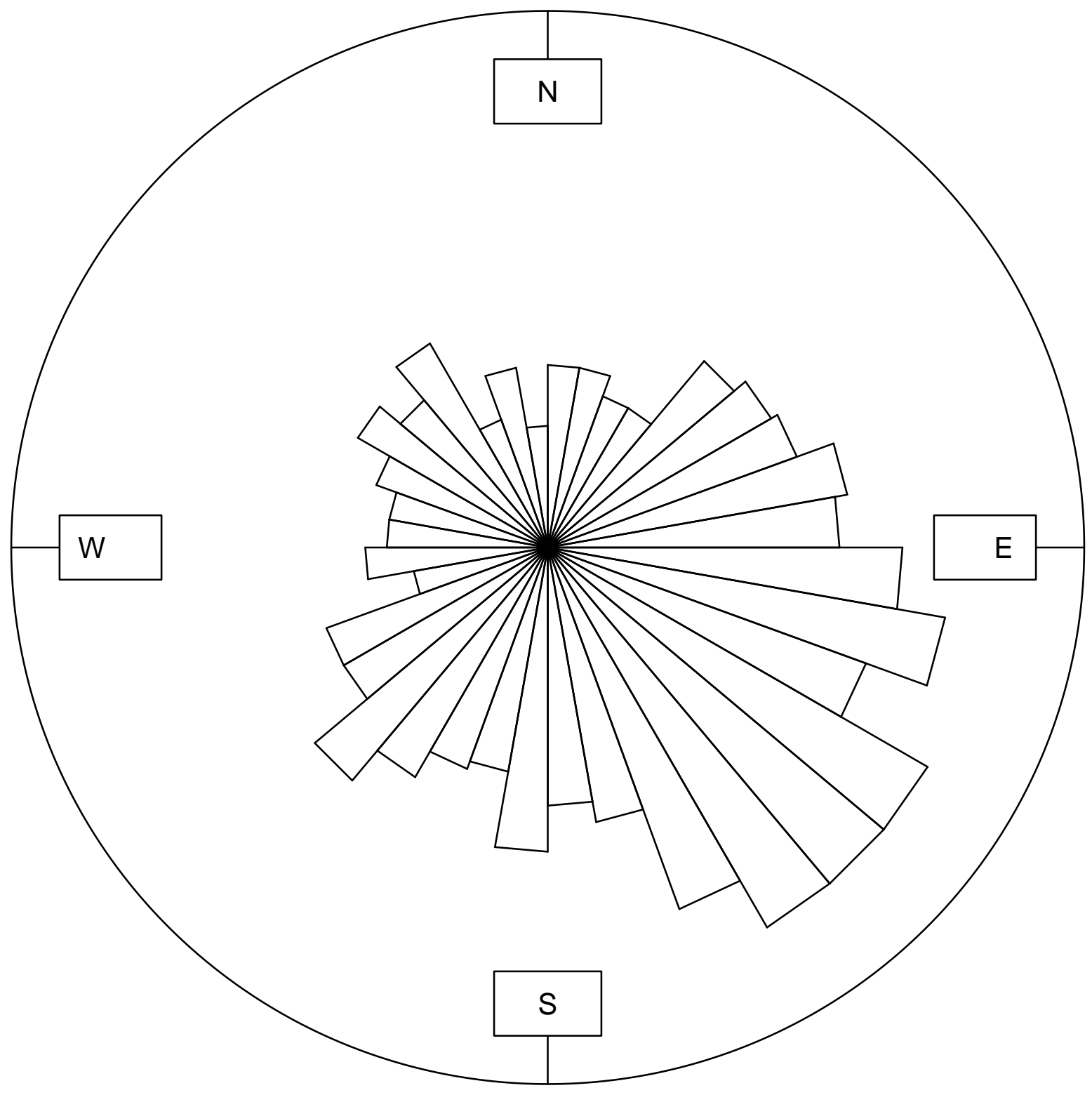


bootstrap 910

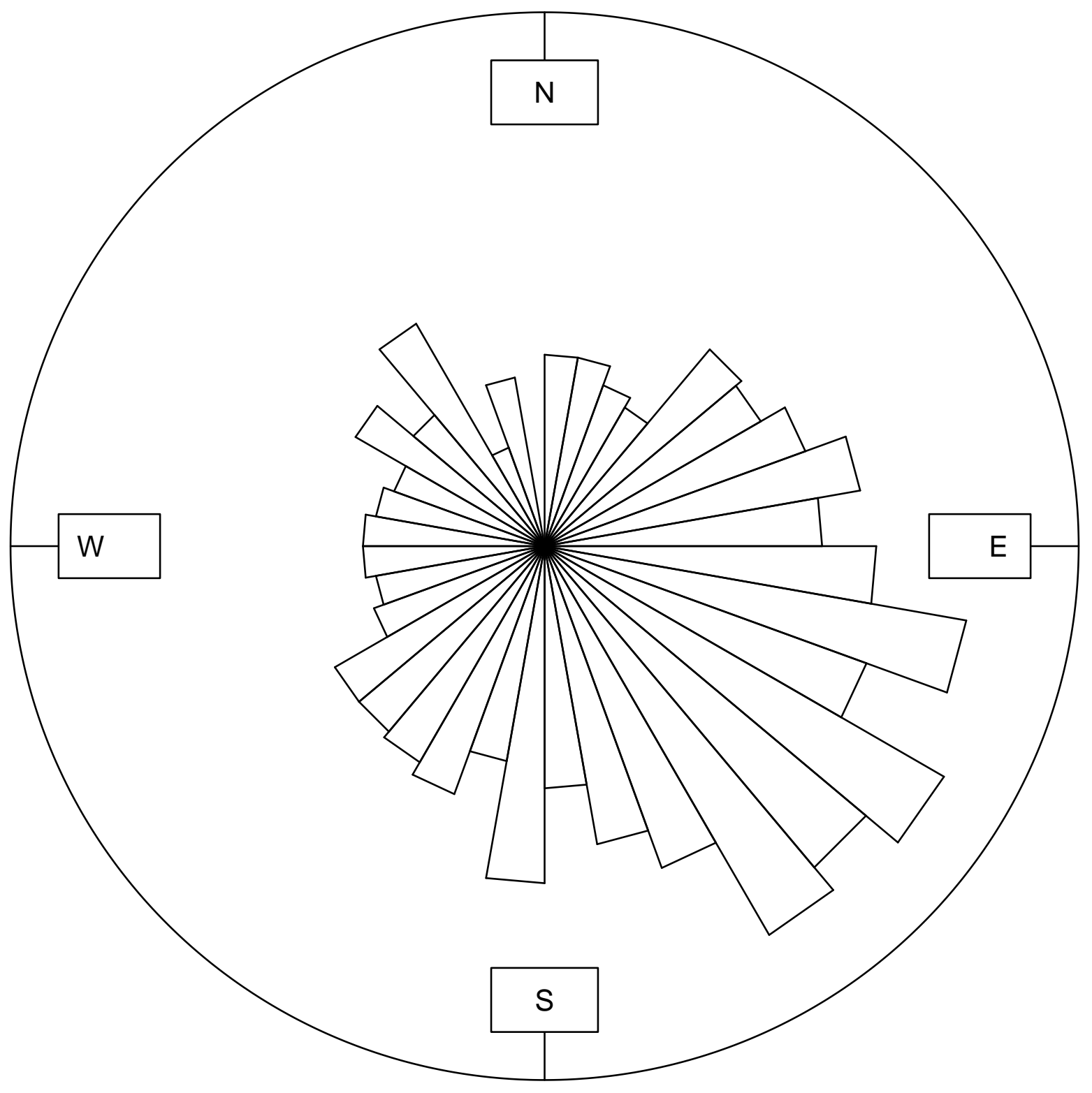


bootstrap 911

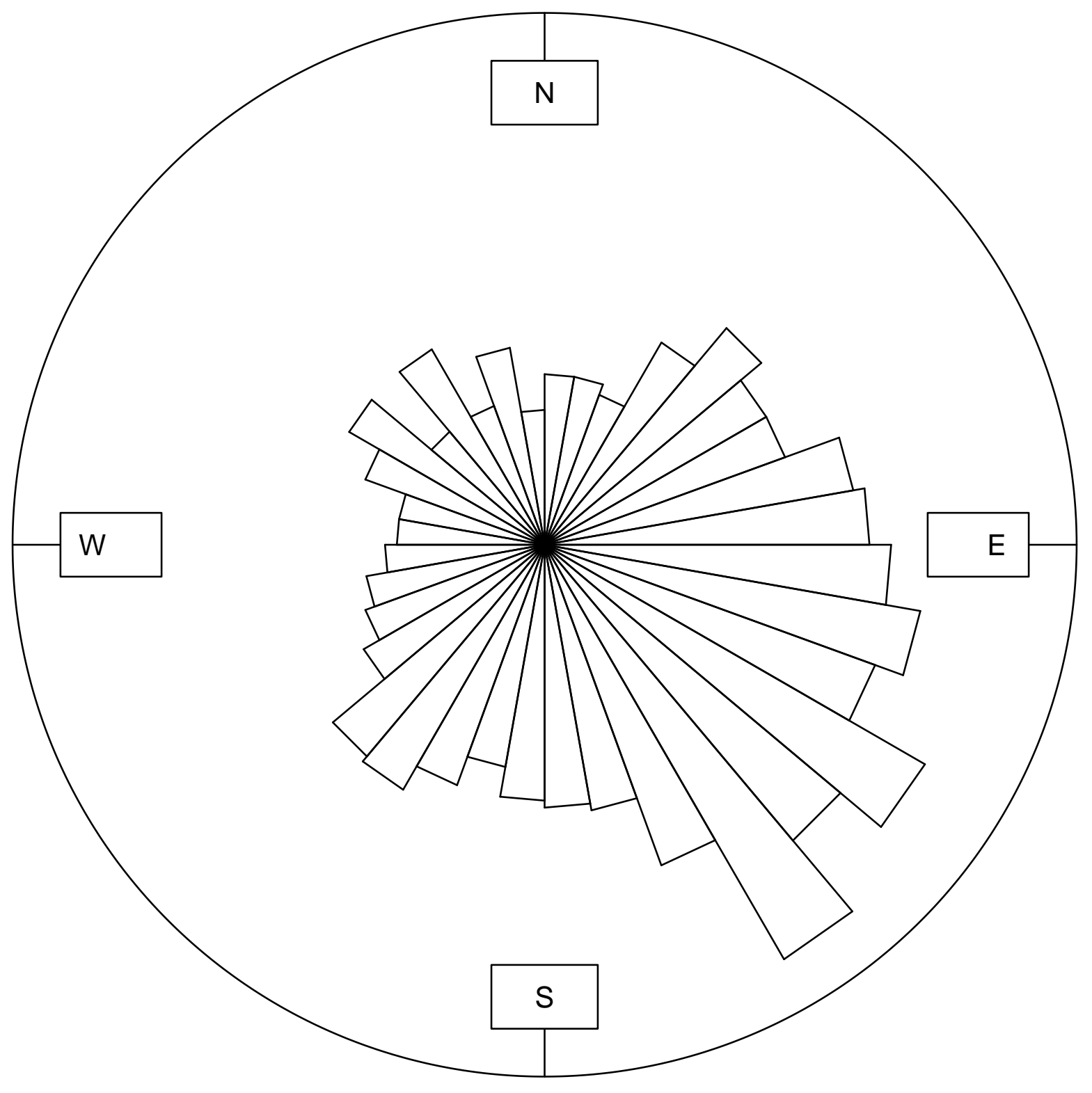


bootstrap 912

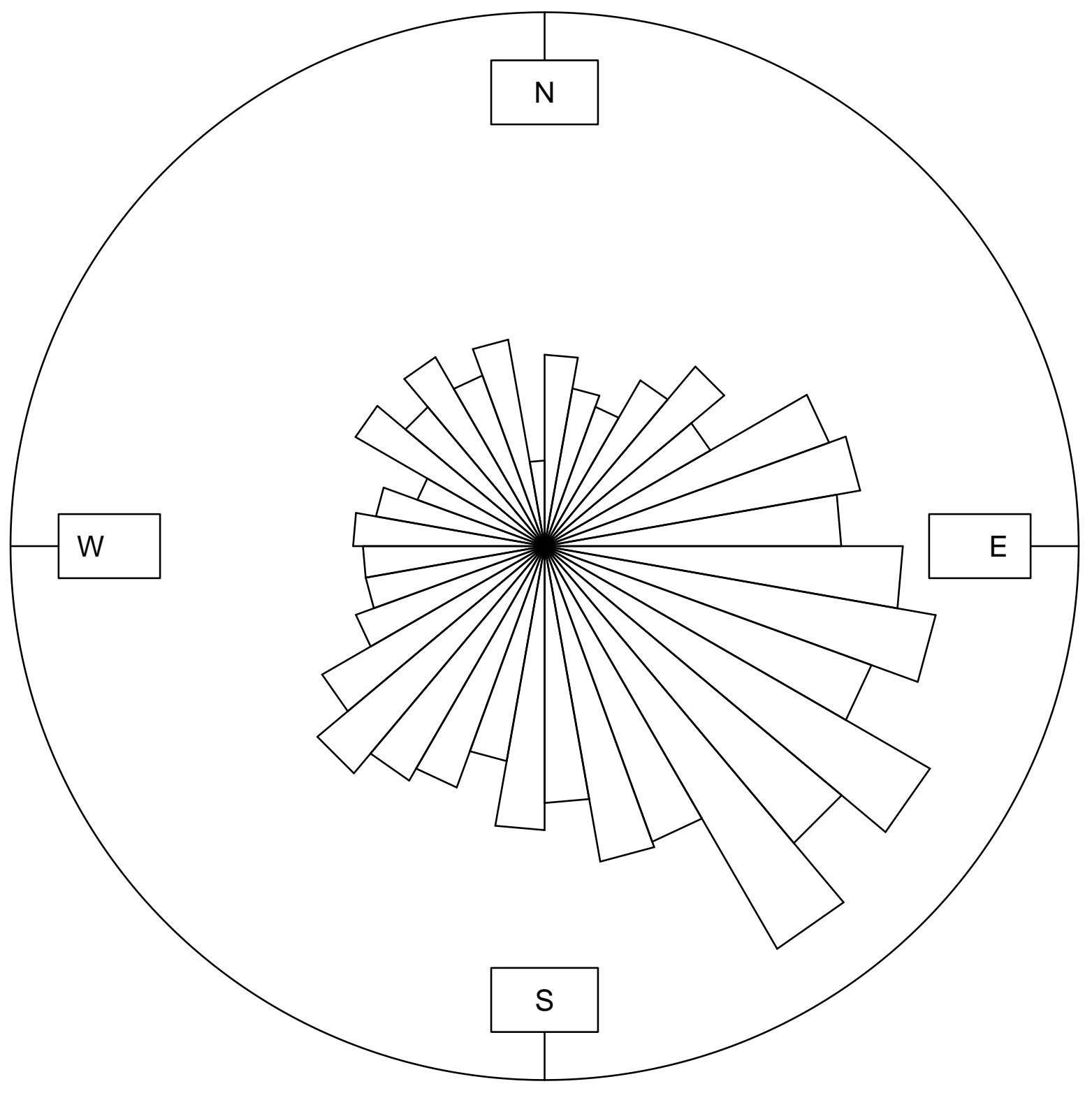


bootstrap 913

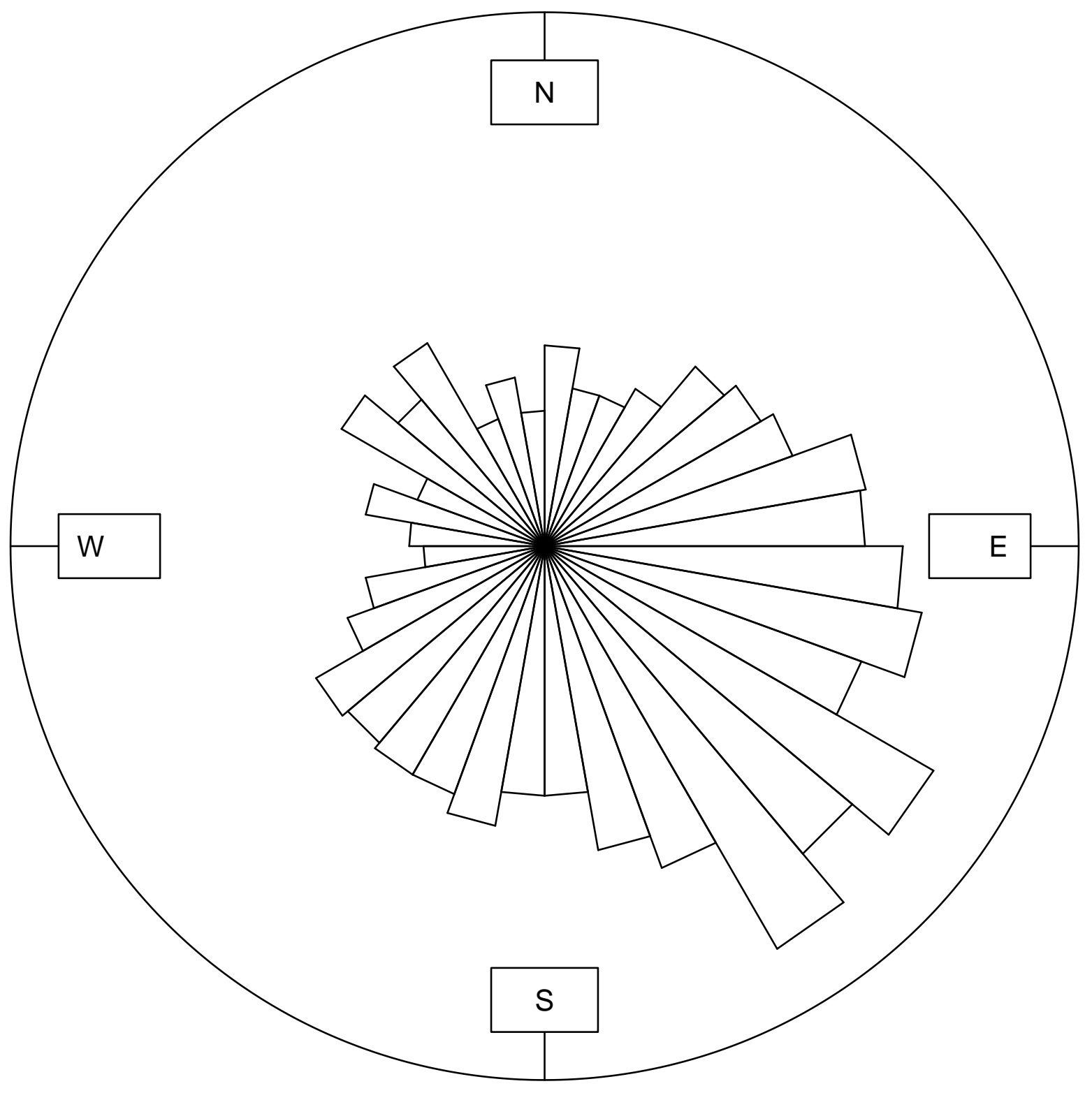


bootstrap 914

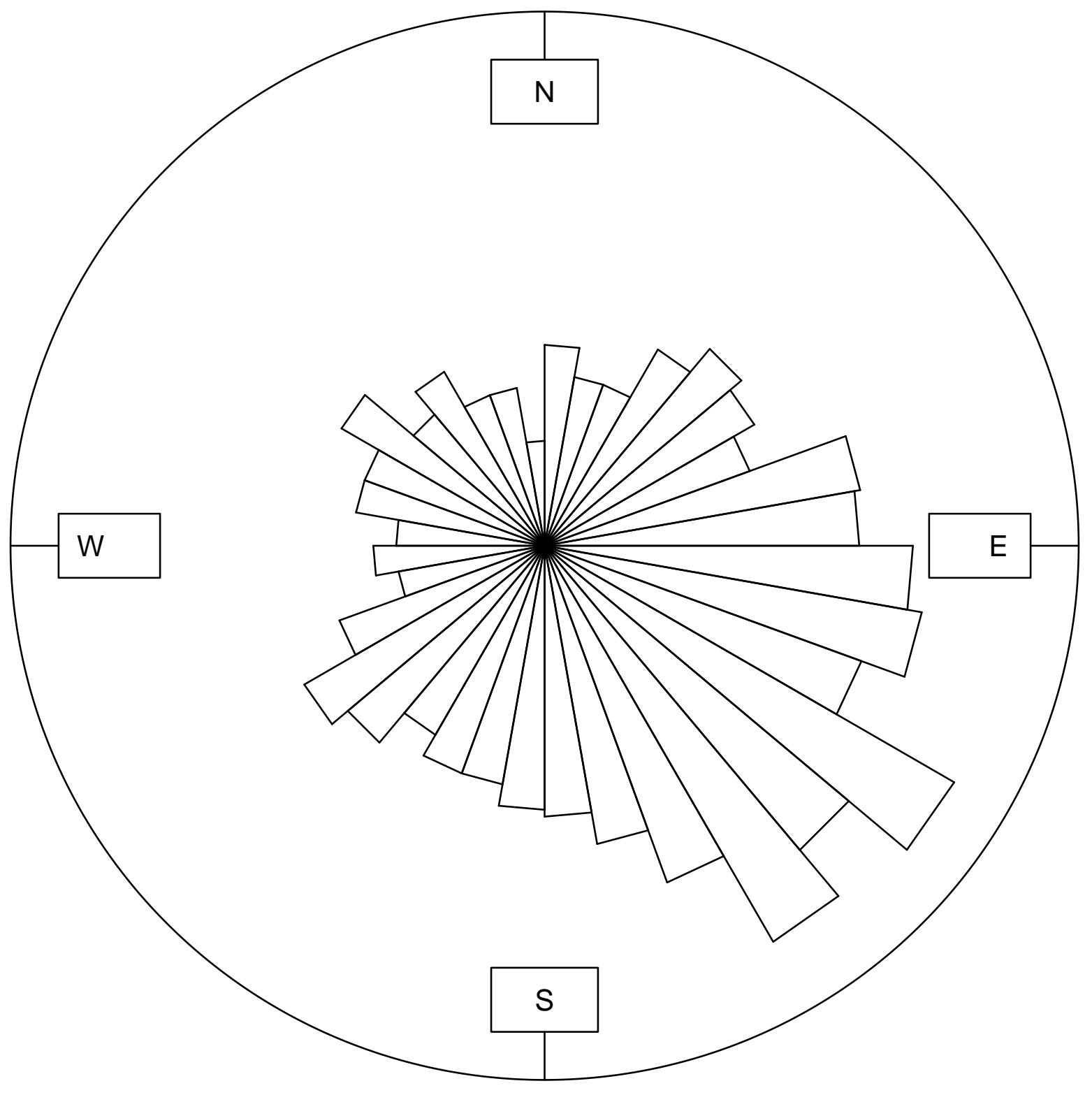




\section{bootstrap 915}

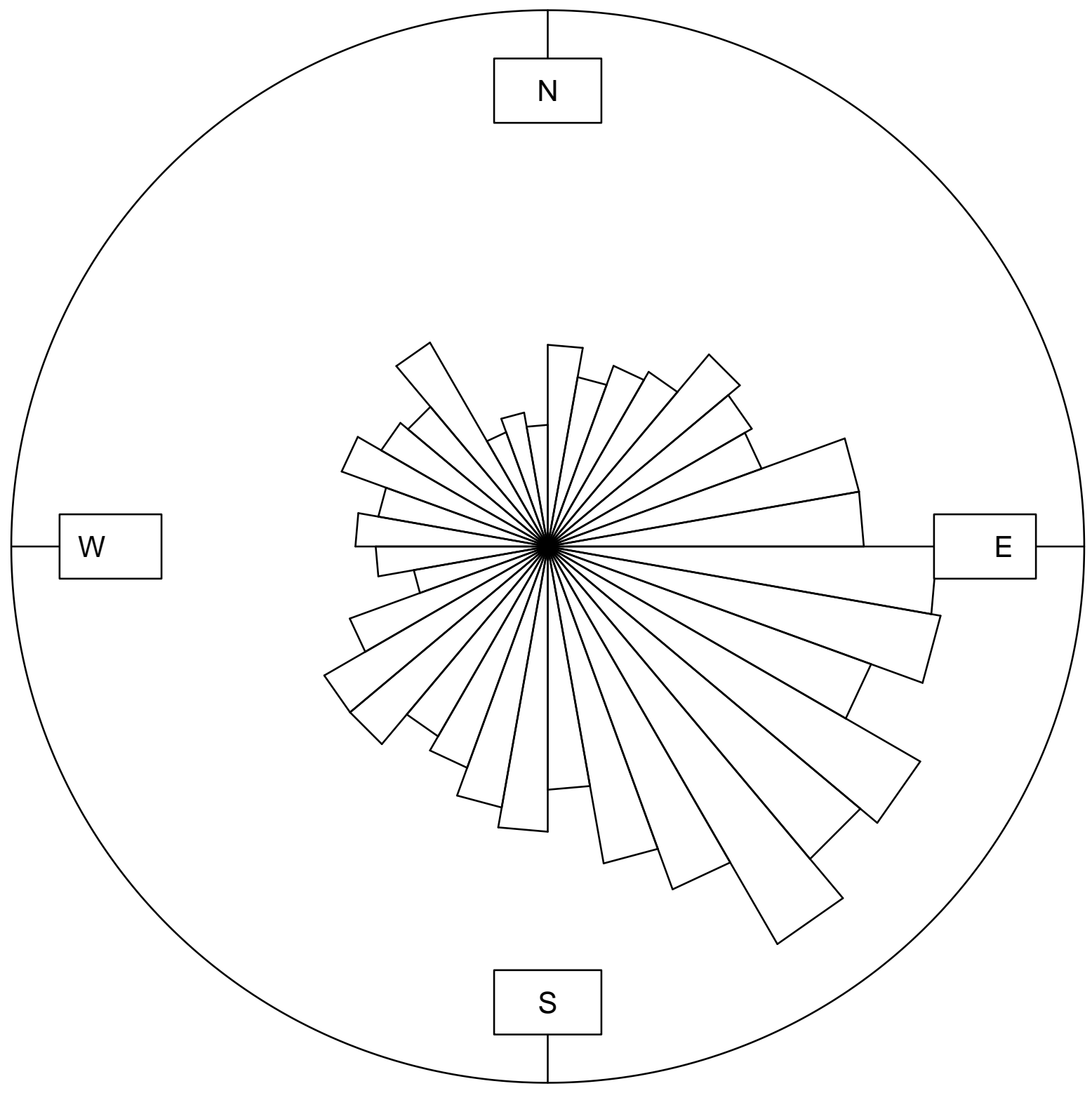


bootstrap 916

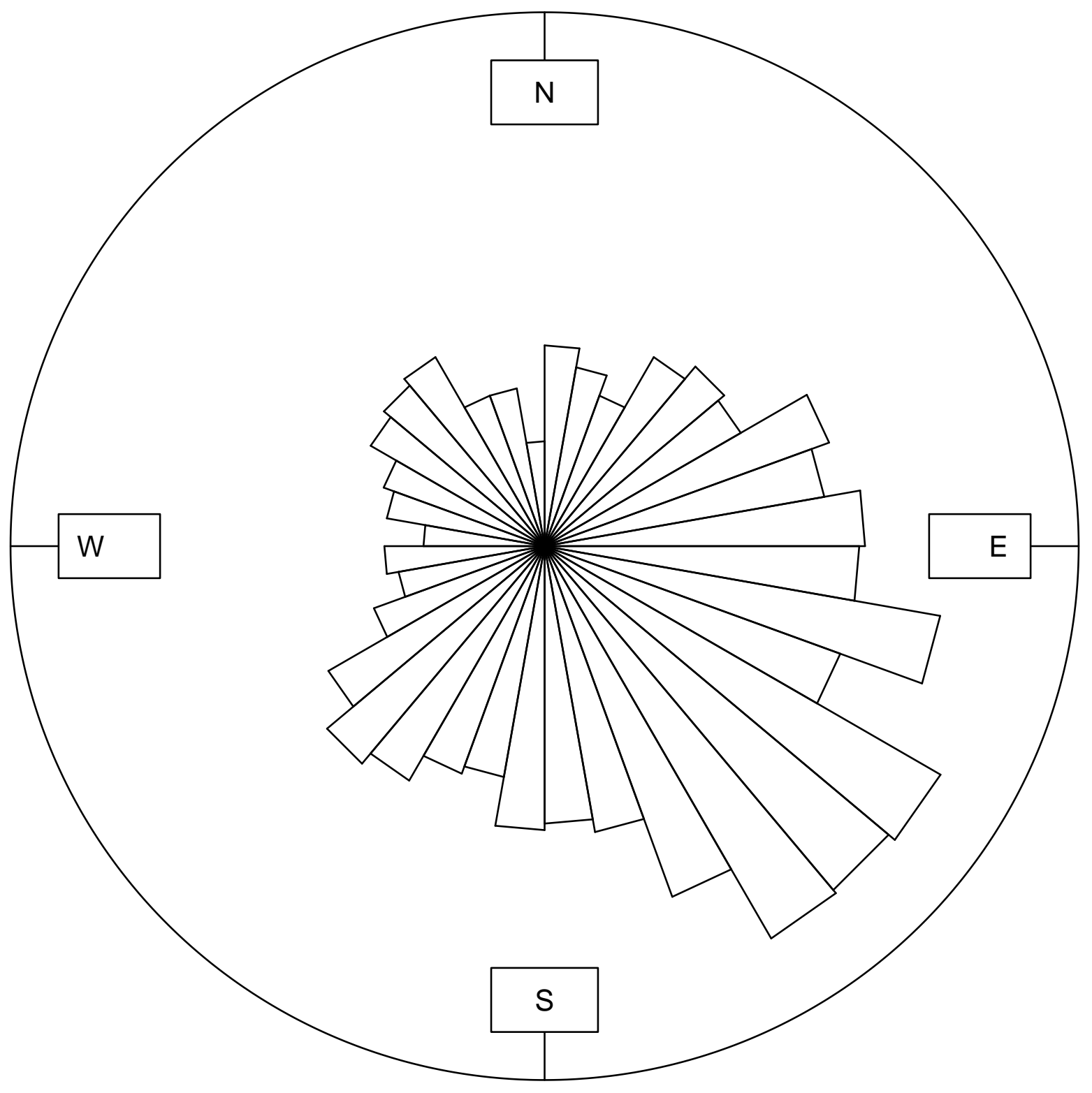


bootstrap 917

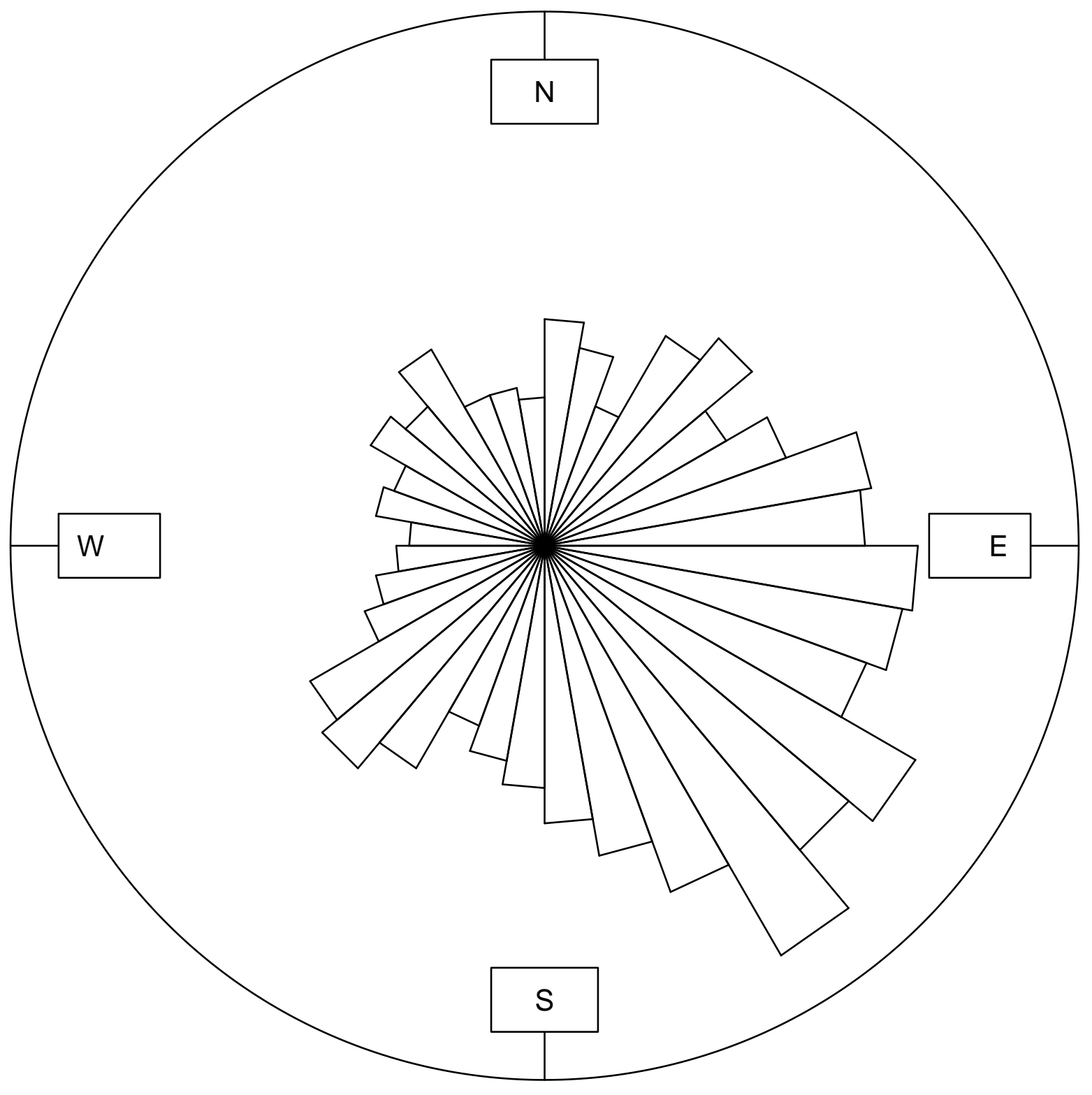


bootstrap 918

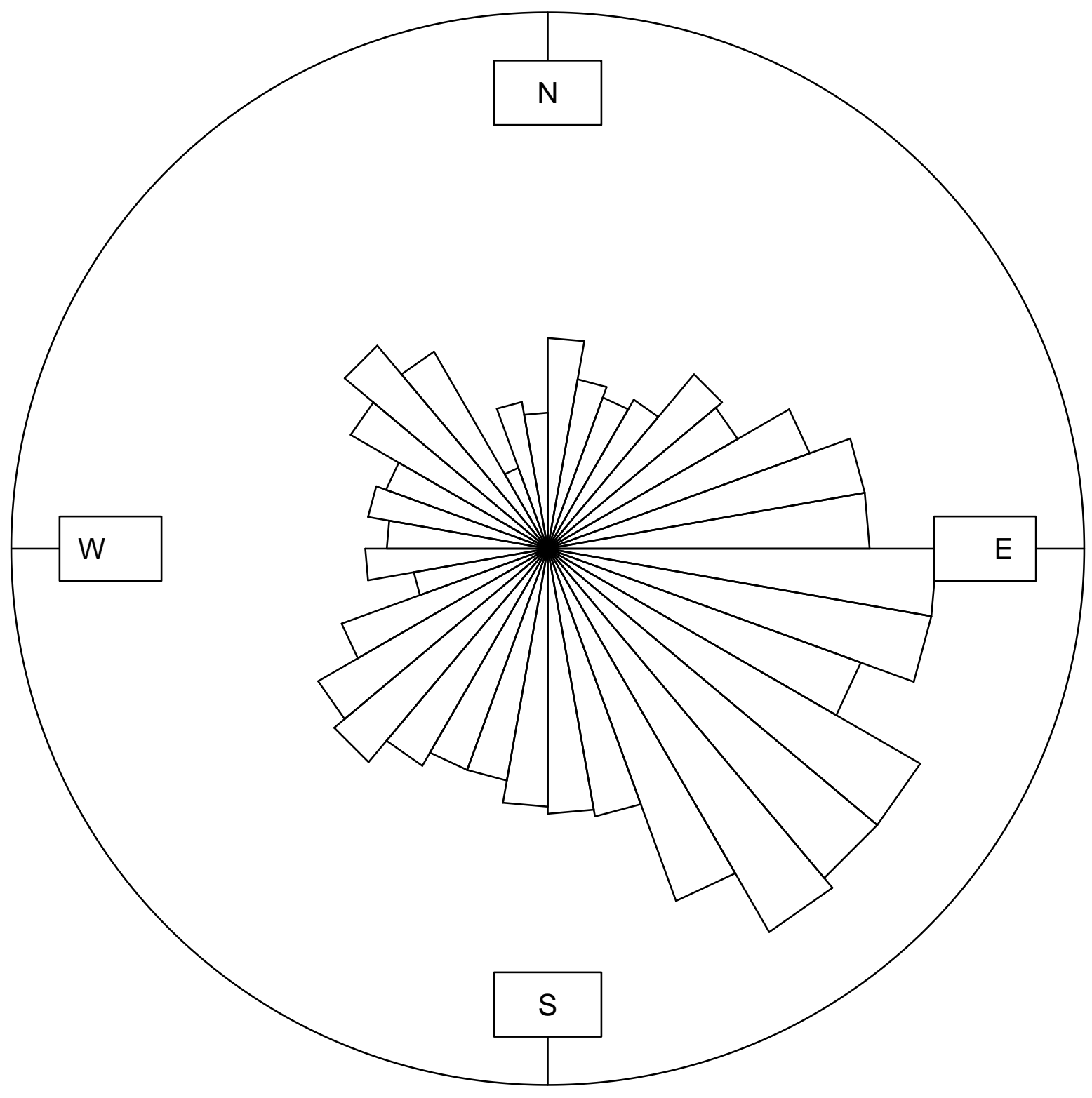


bootstrap 919

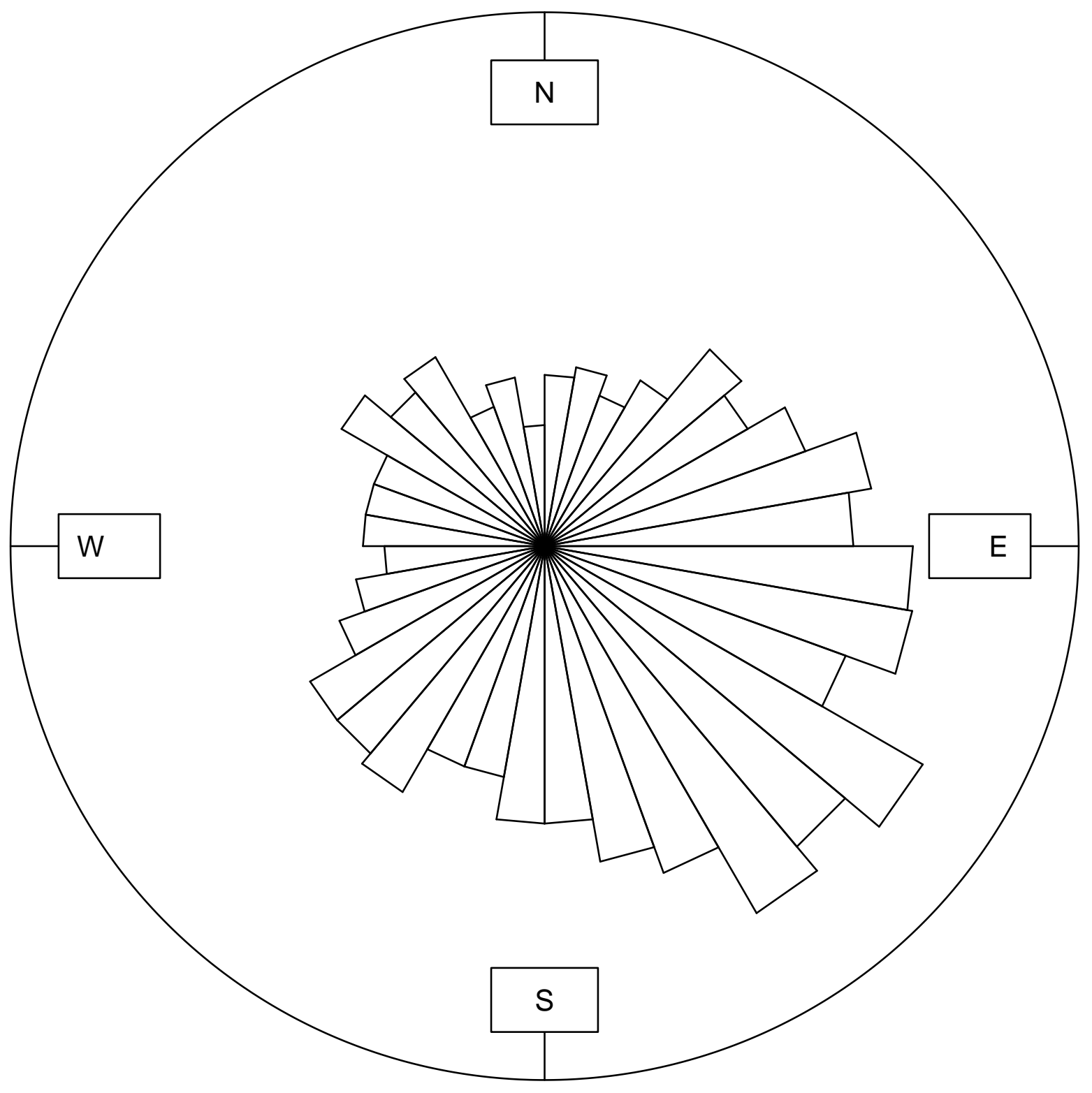


bootstrap 920

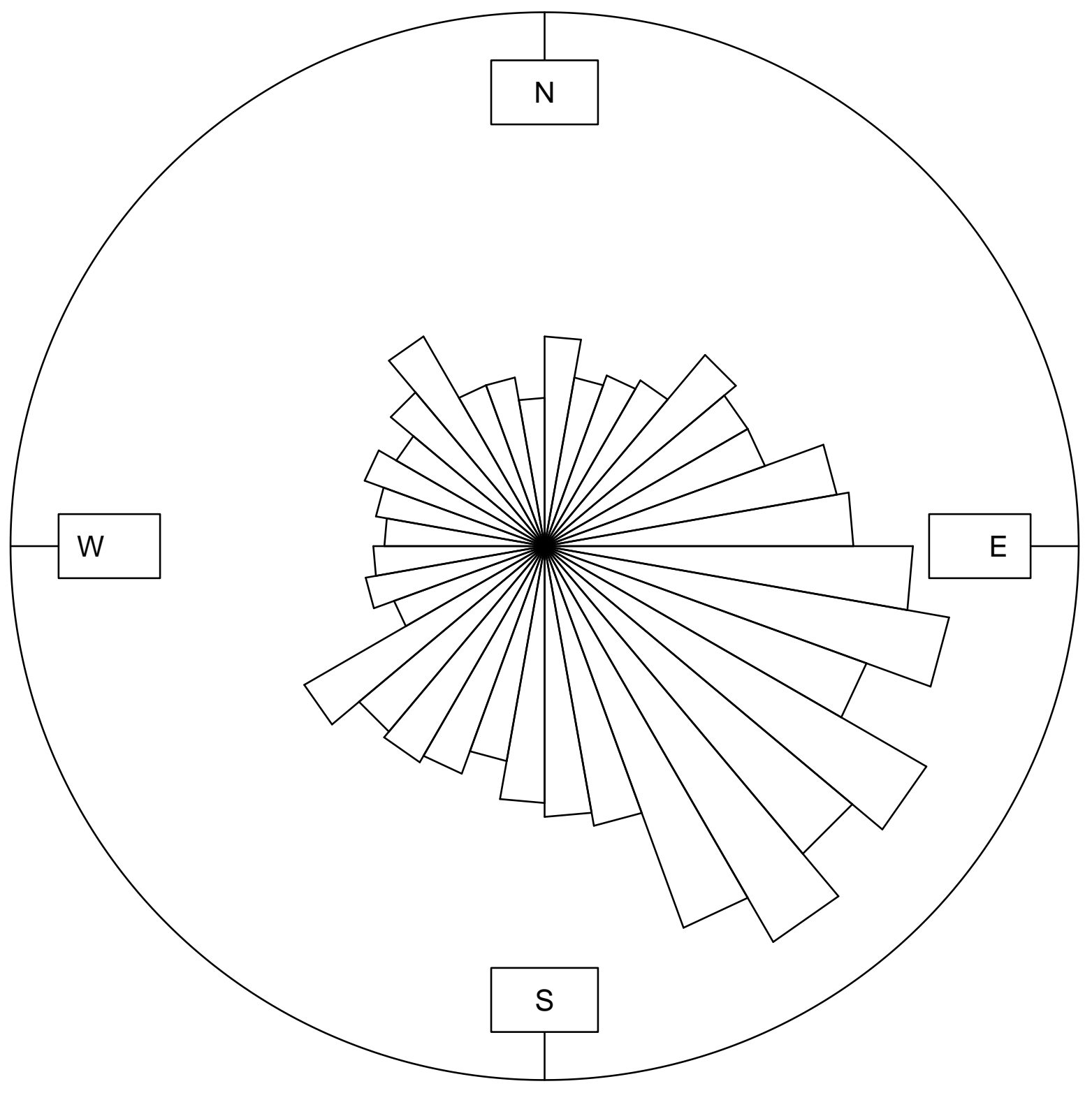


bootstrap 921

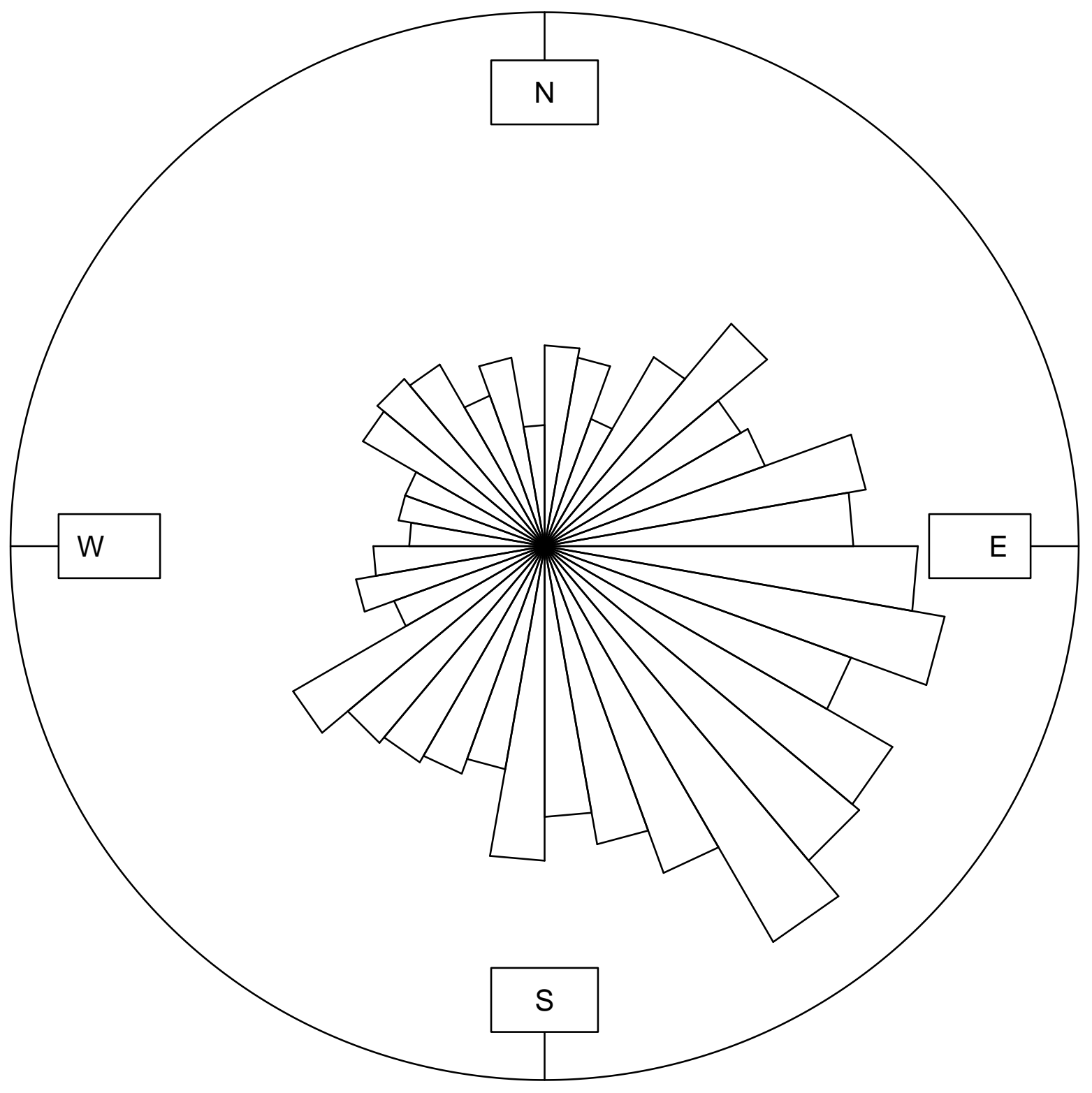


bootstrap 922

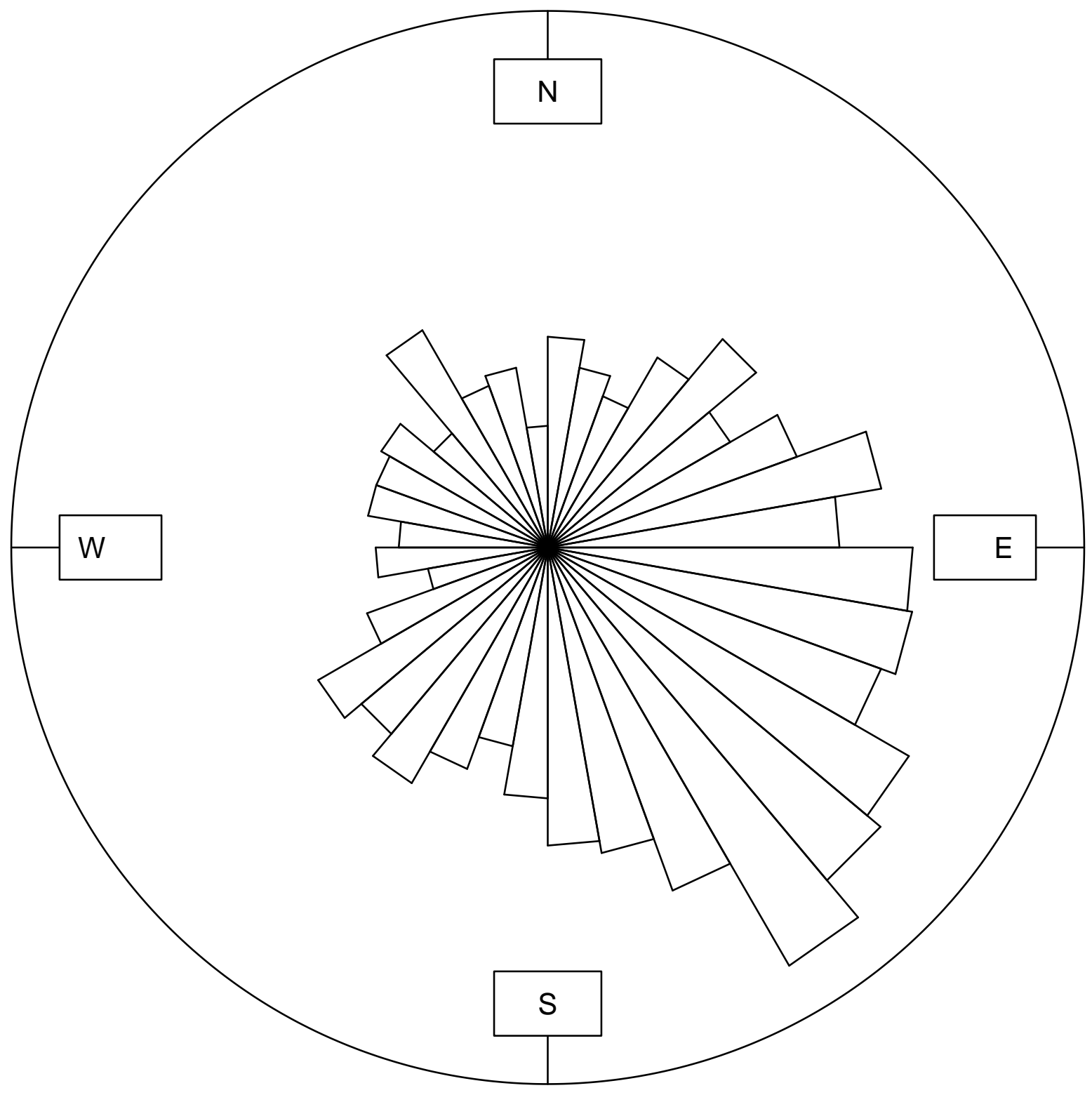


bootstrap 923

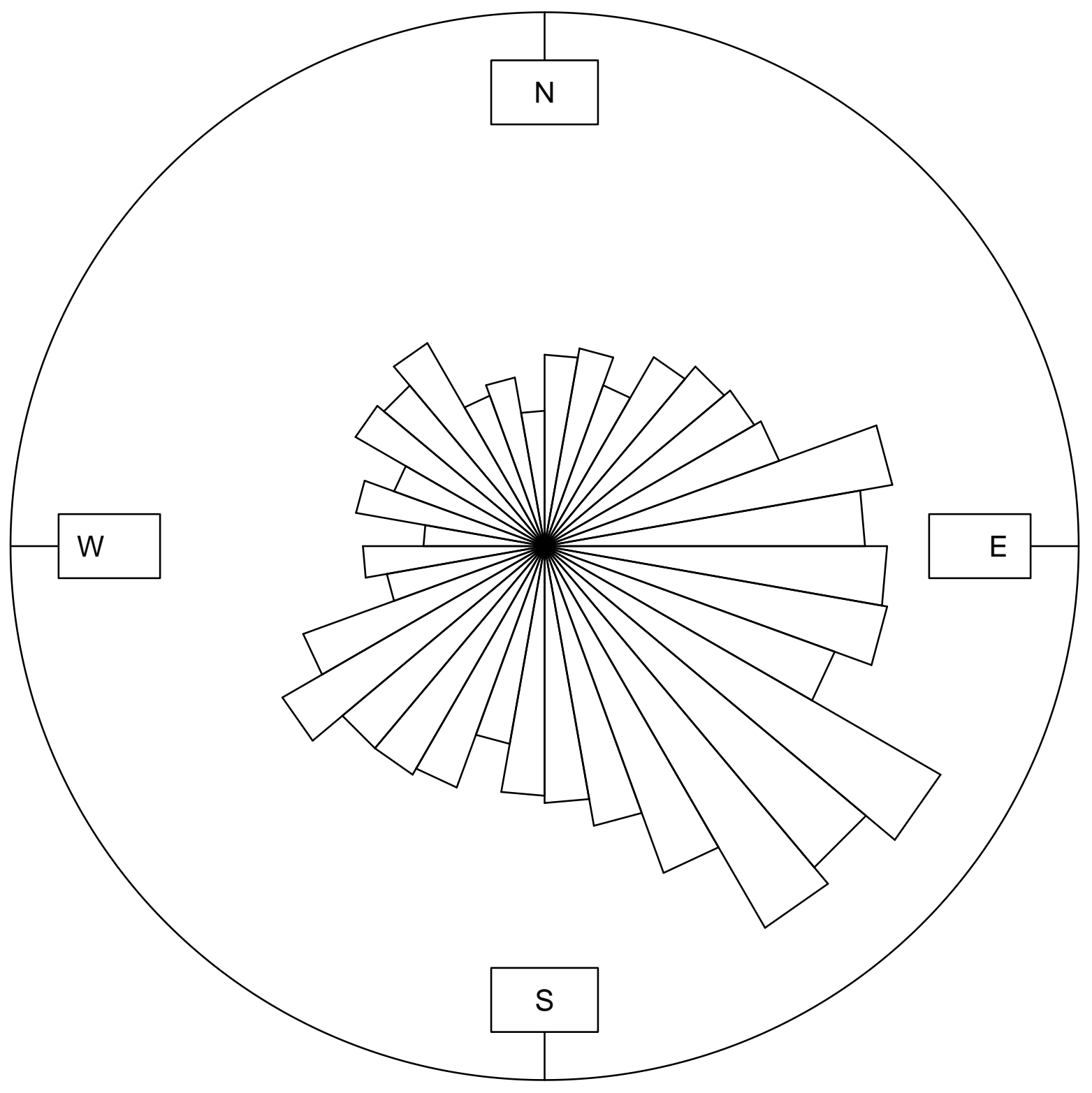


bootstrap 924

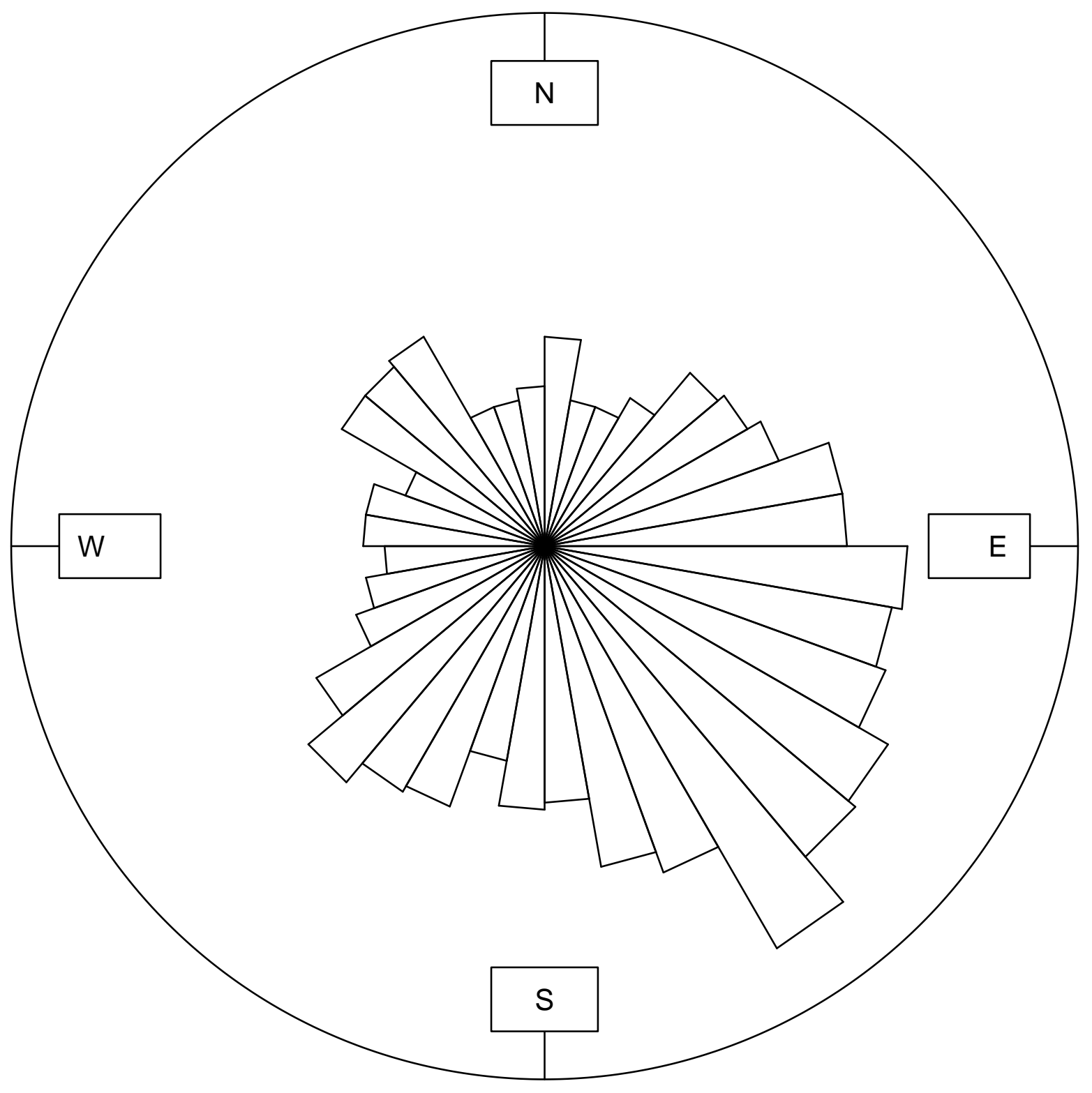




\section{bootstrap 925}

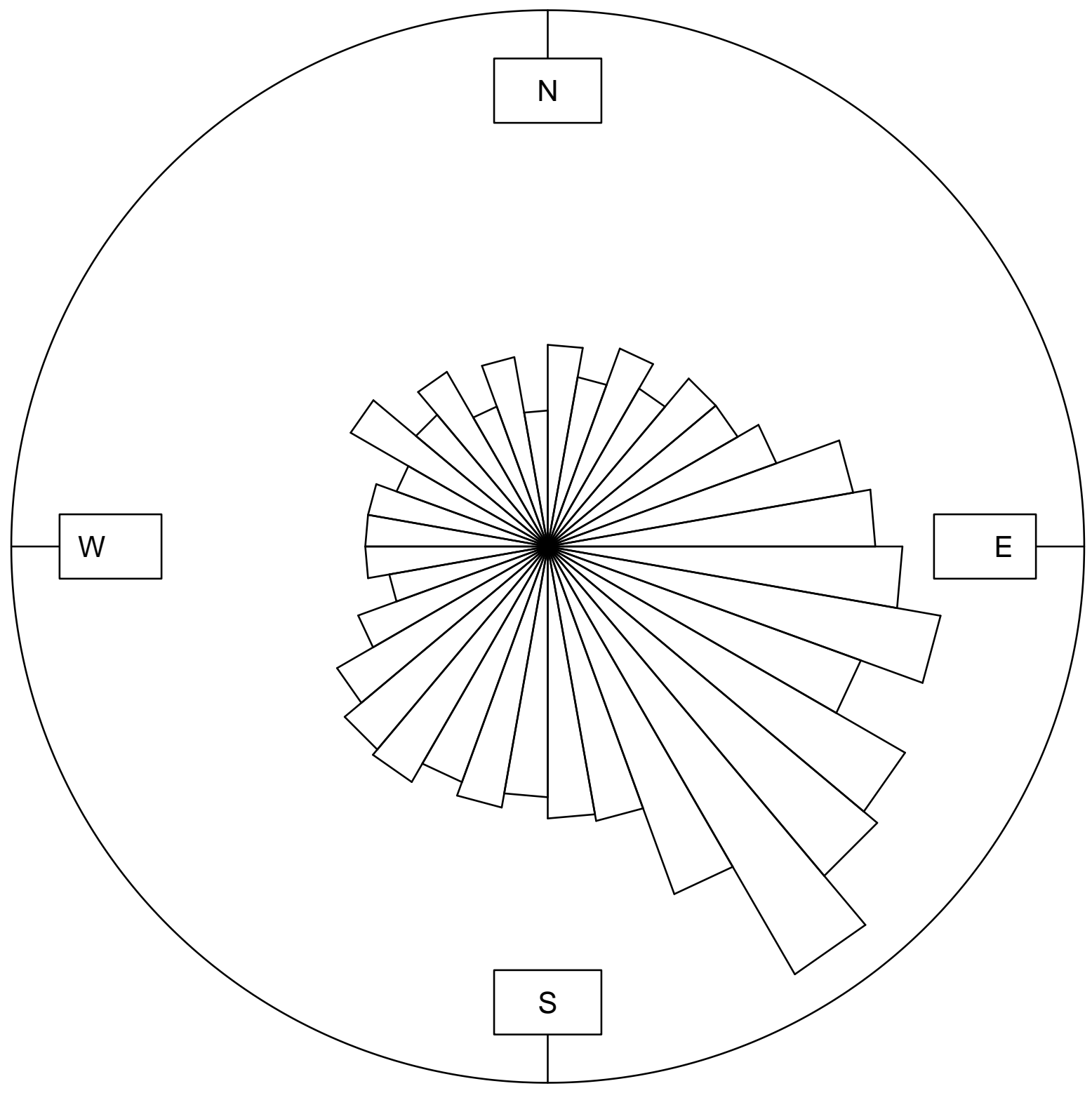




\section{bootstrap 926}

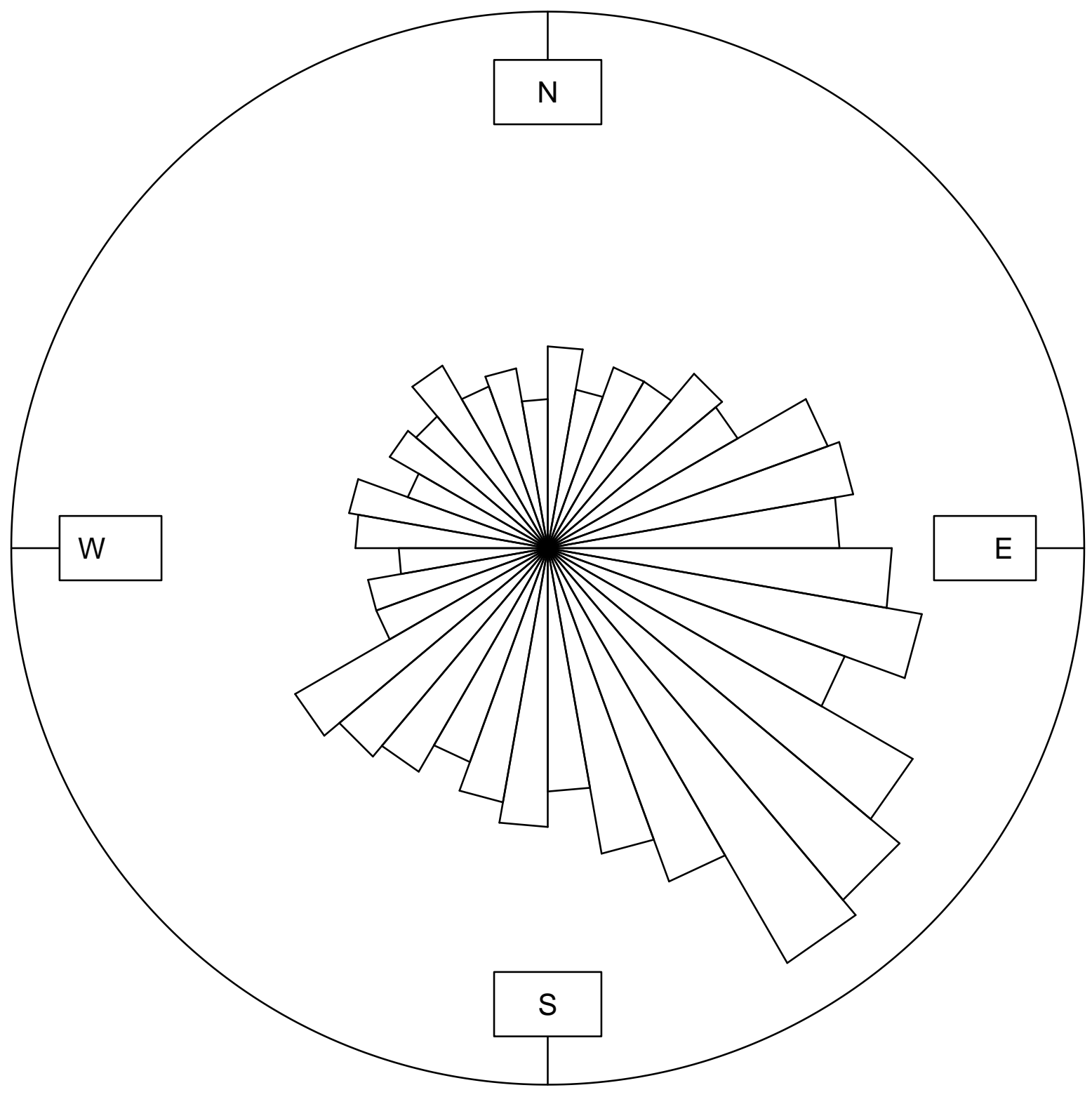


bootstrap 927

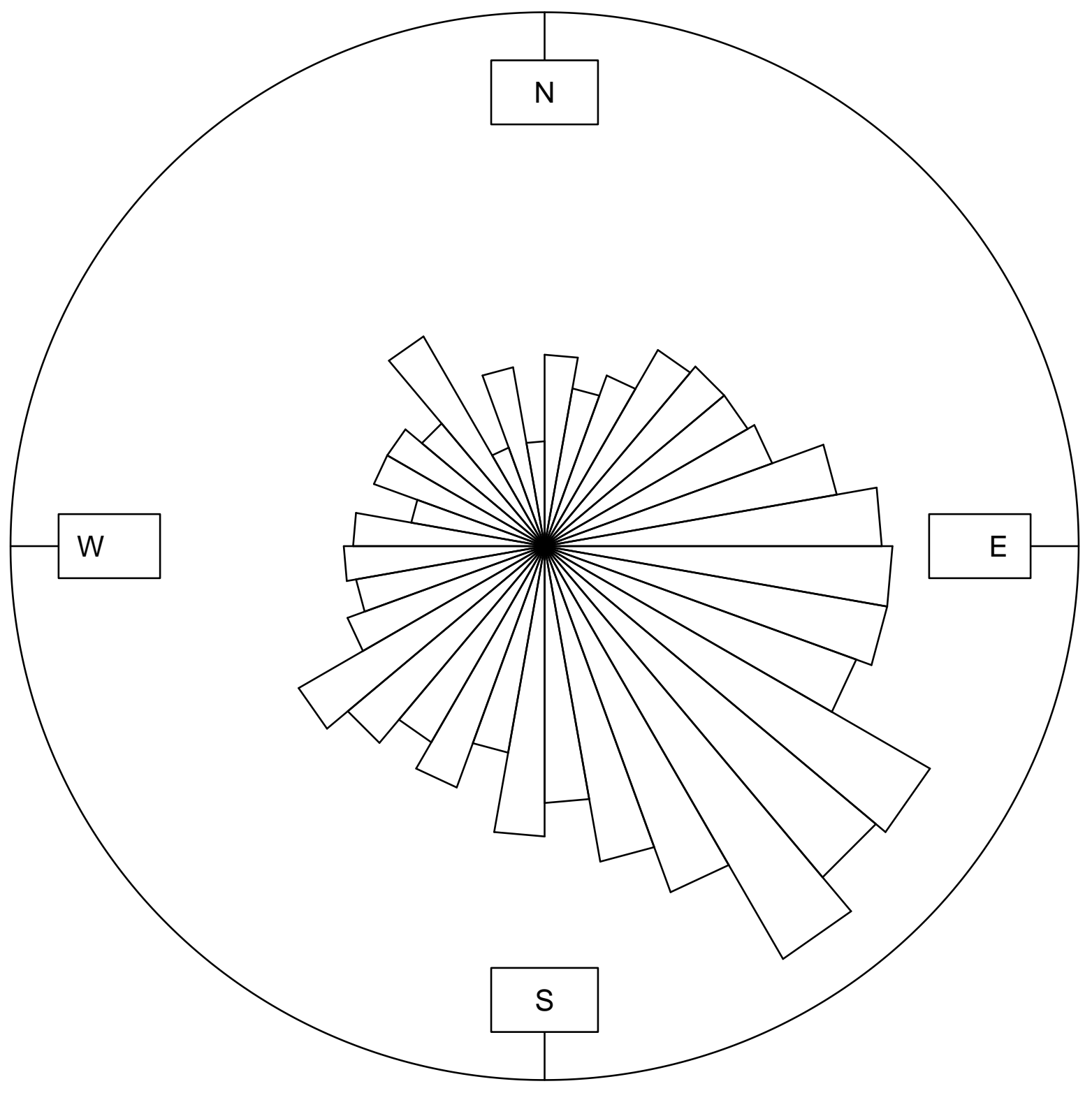


bootstrap 928

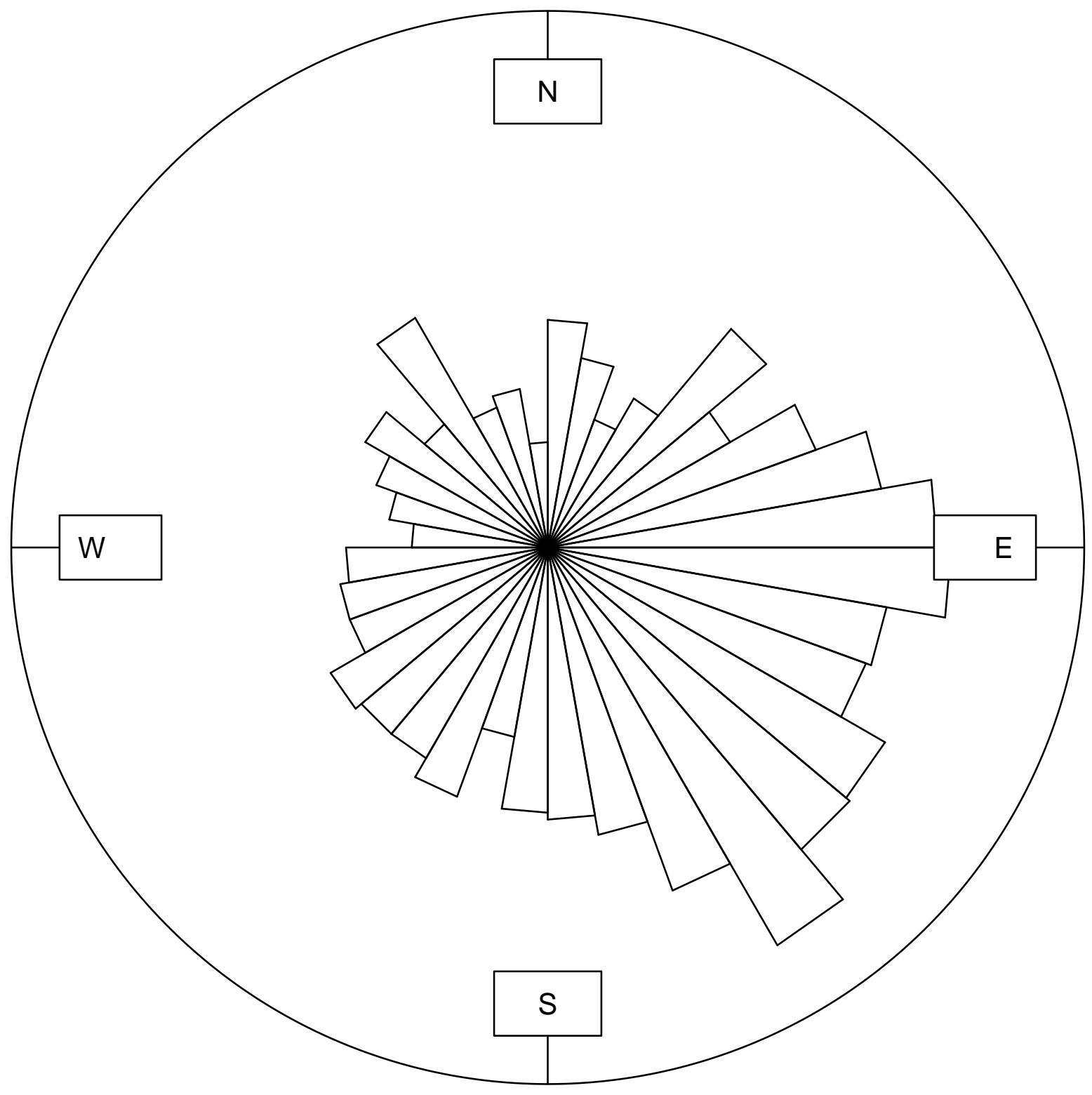


bootstrap 929

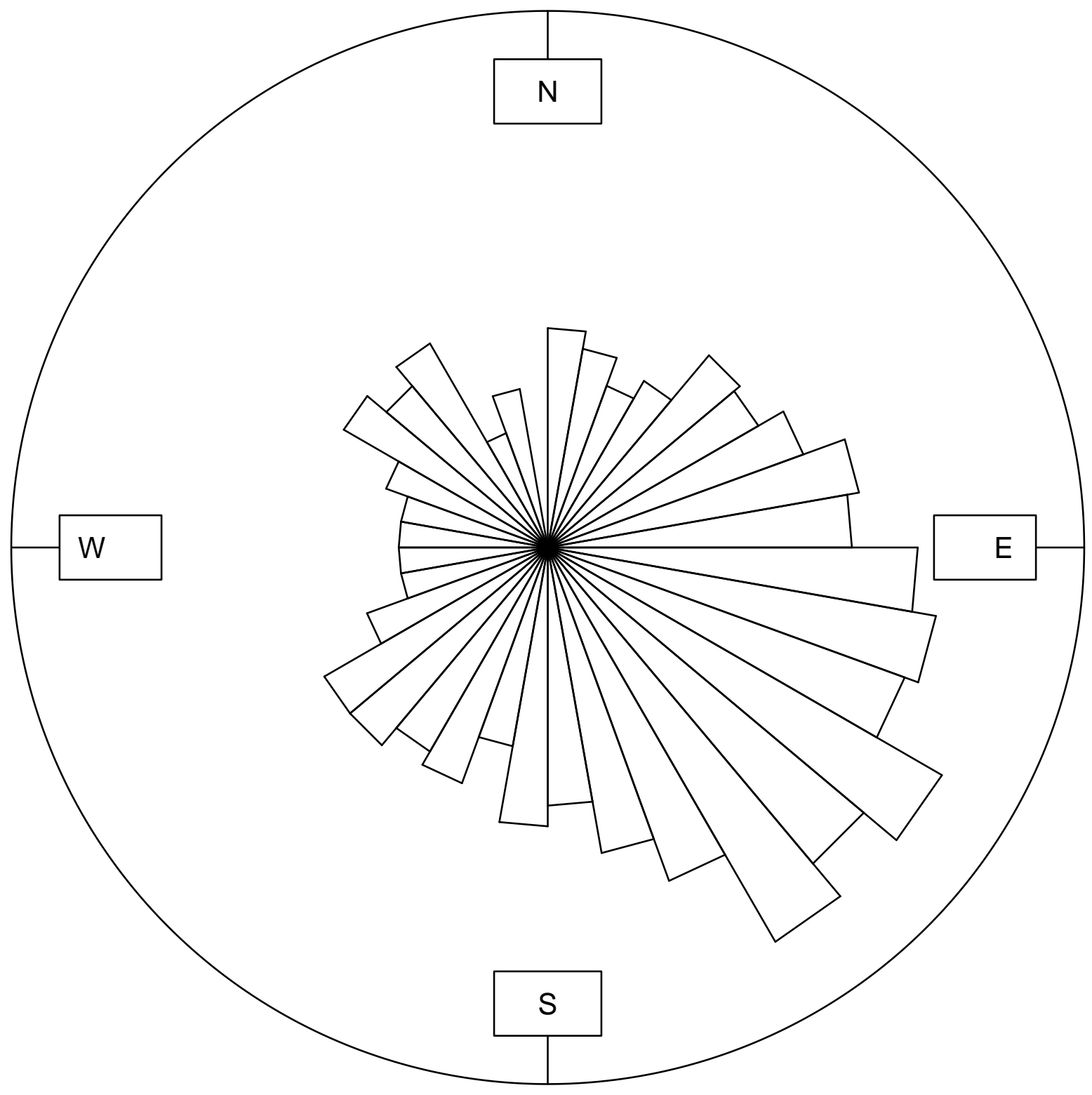


bootstrap 930

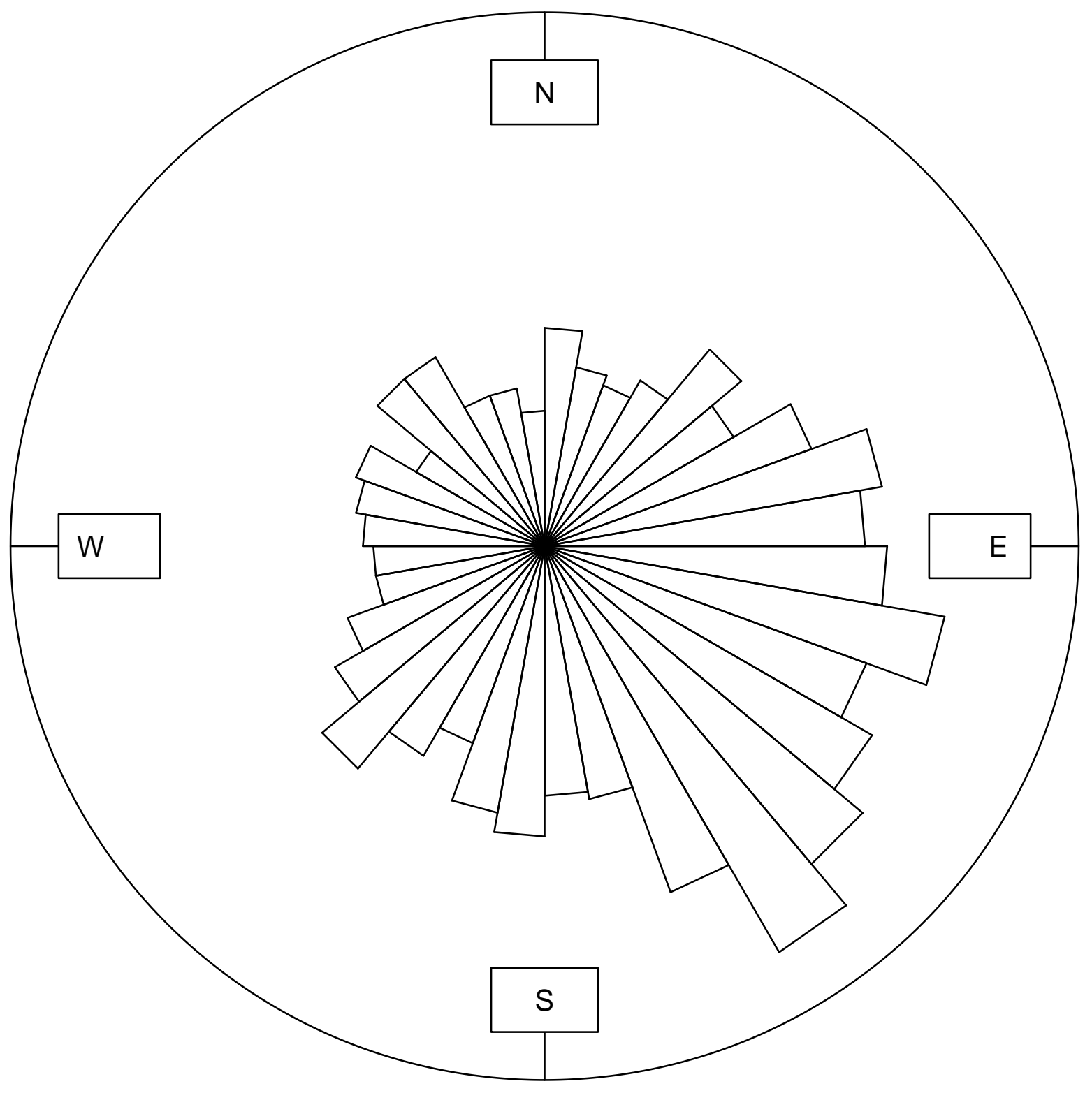


bootstrap 931

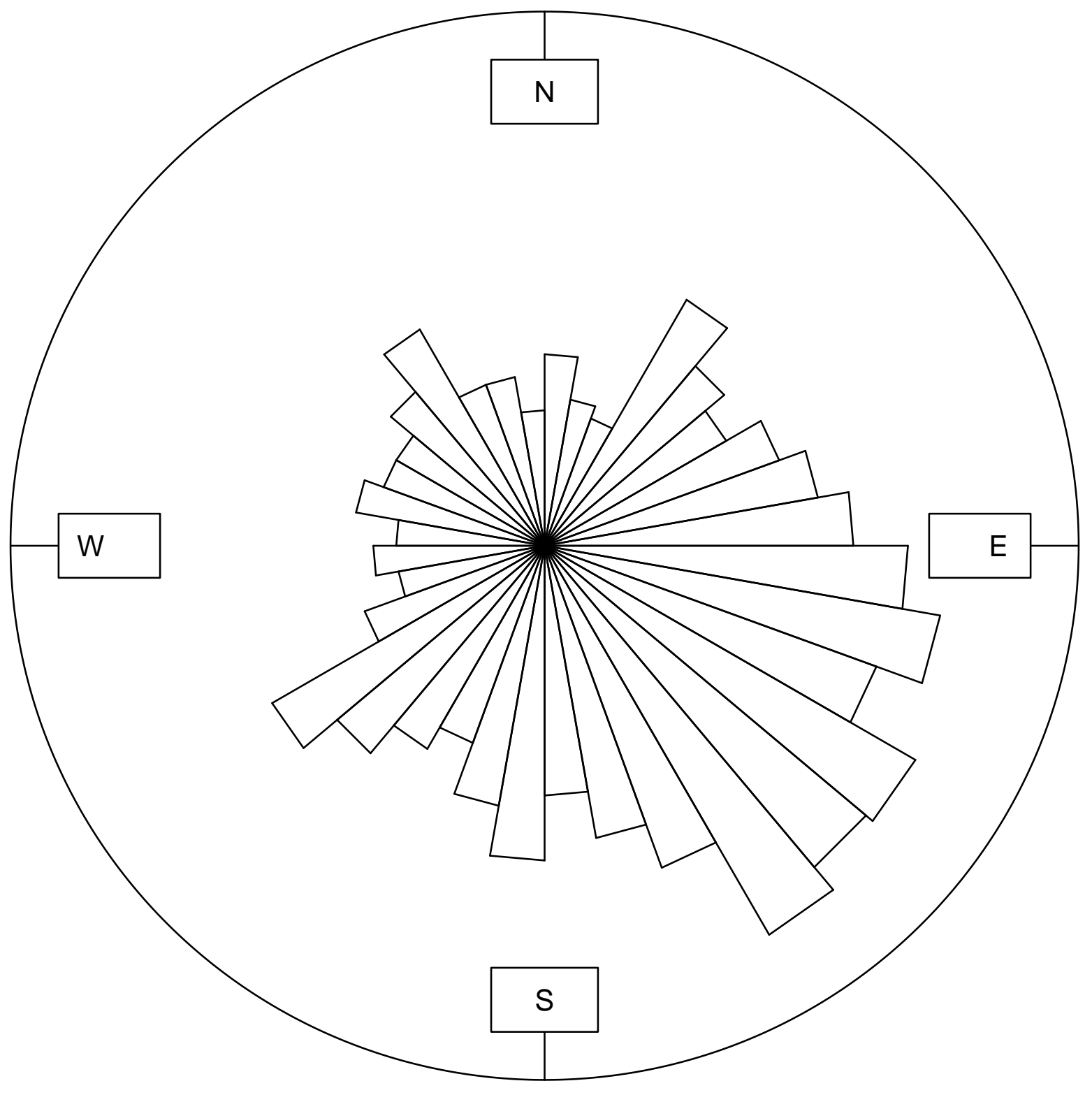


bootstrap 932

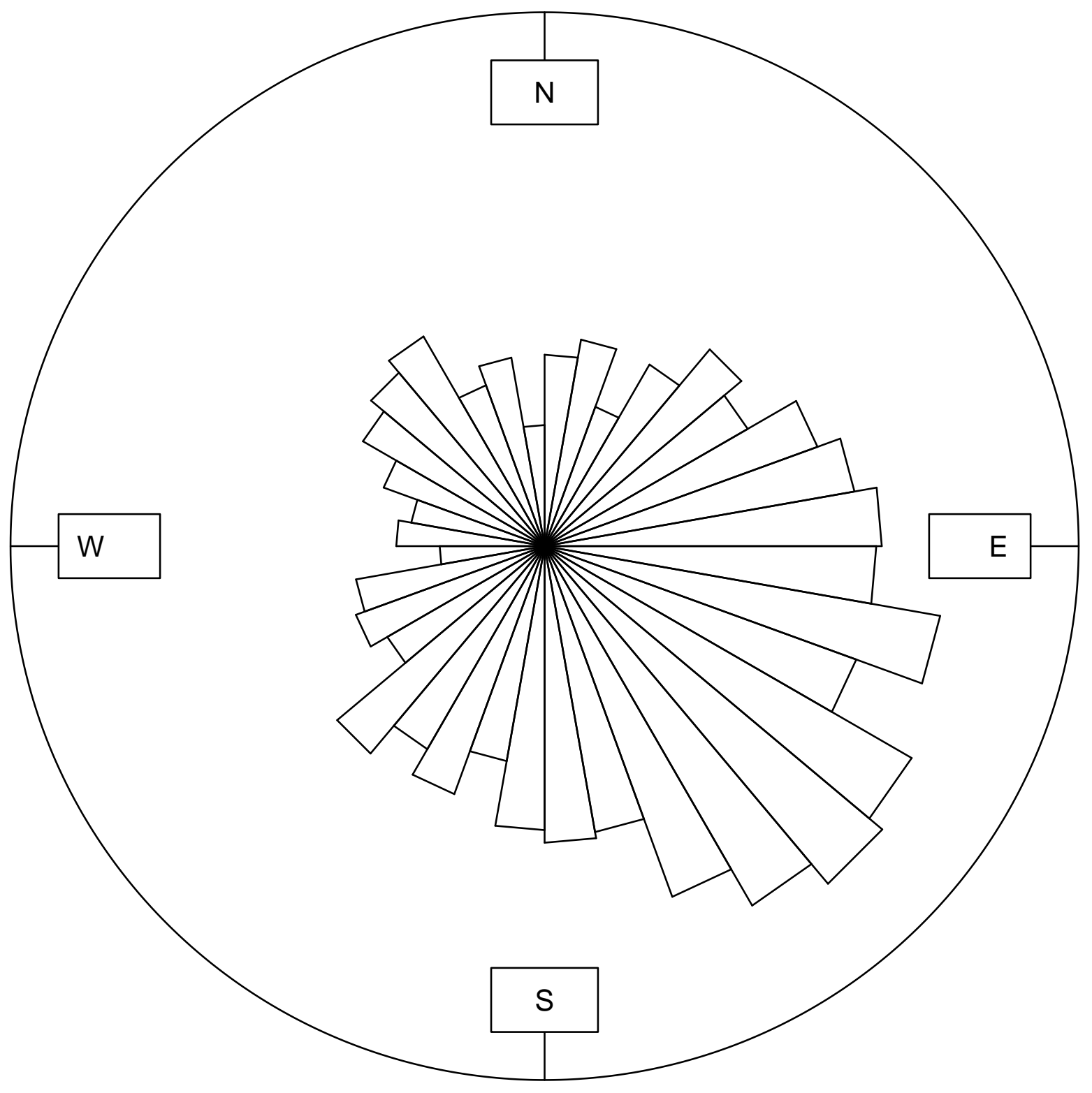




\section{bootstrap 933}

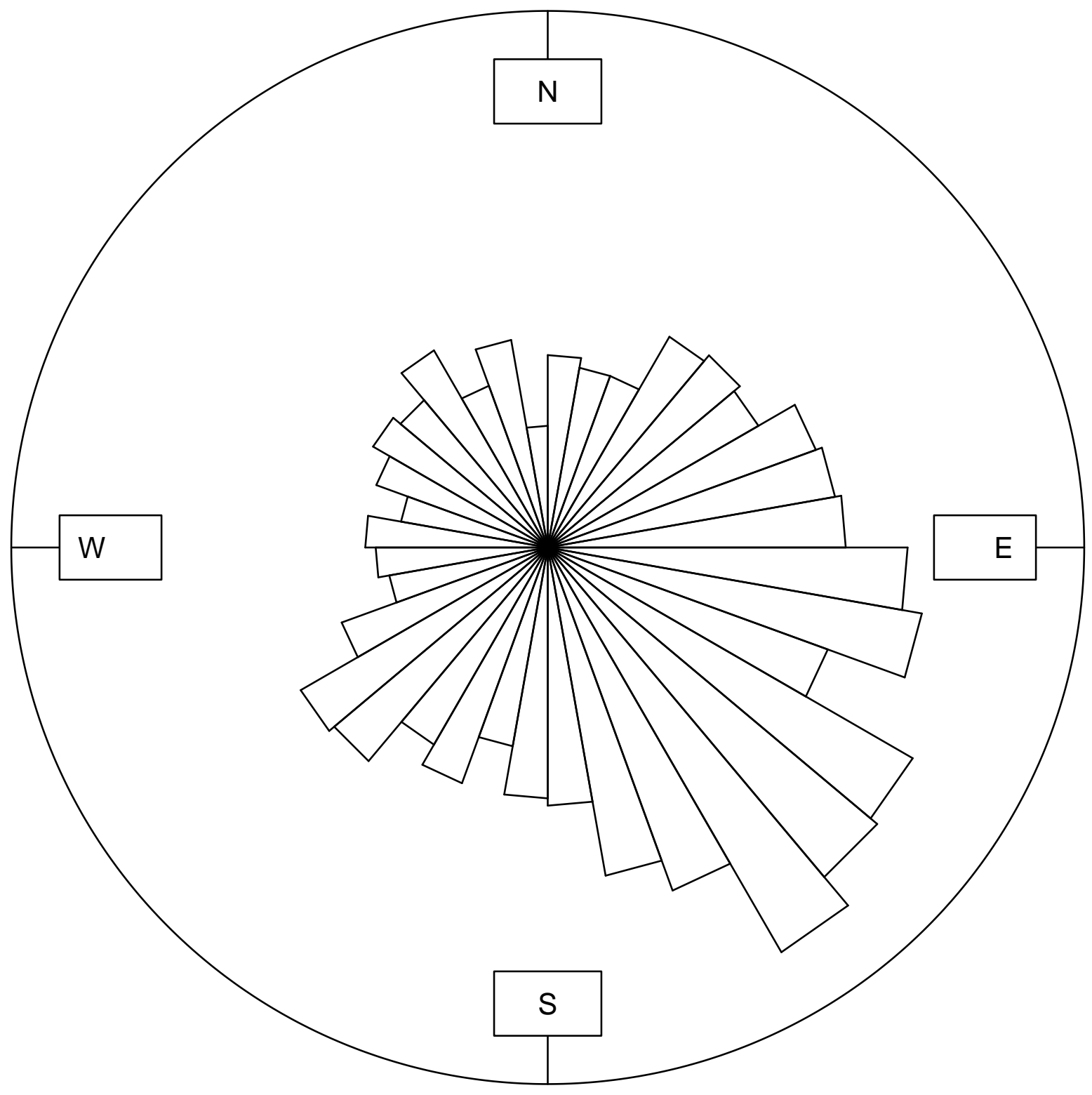




\section{bootstrap 934}

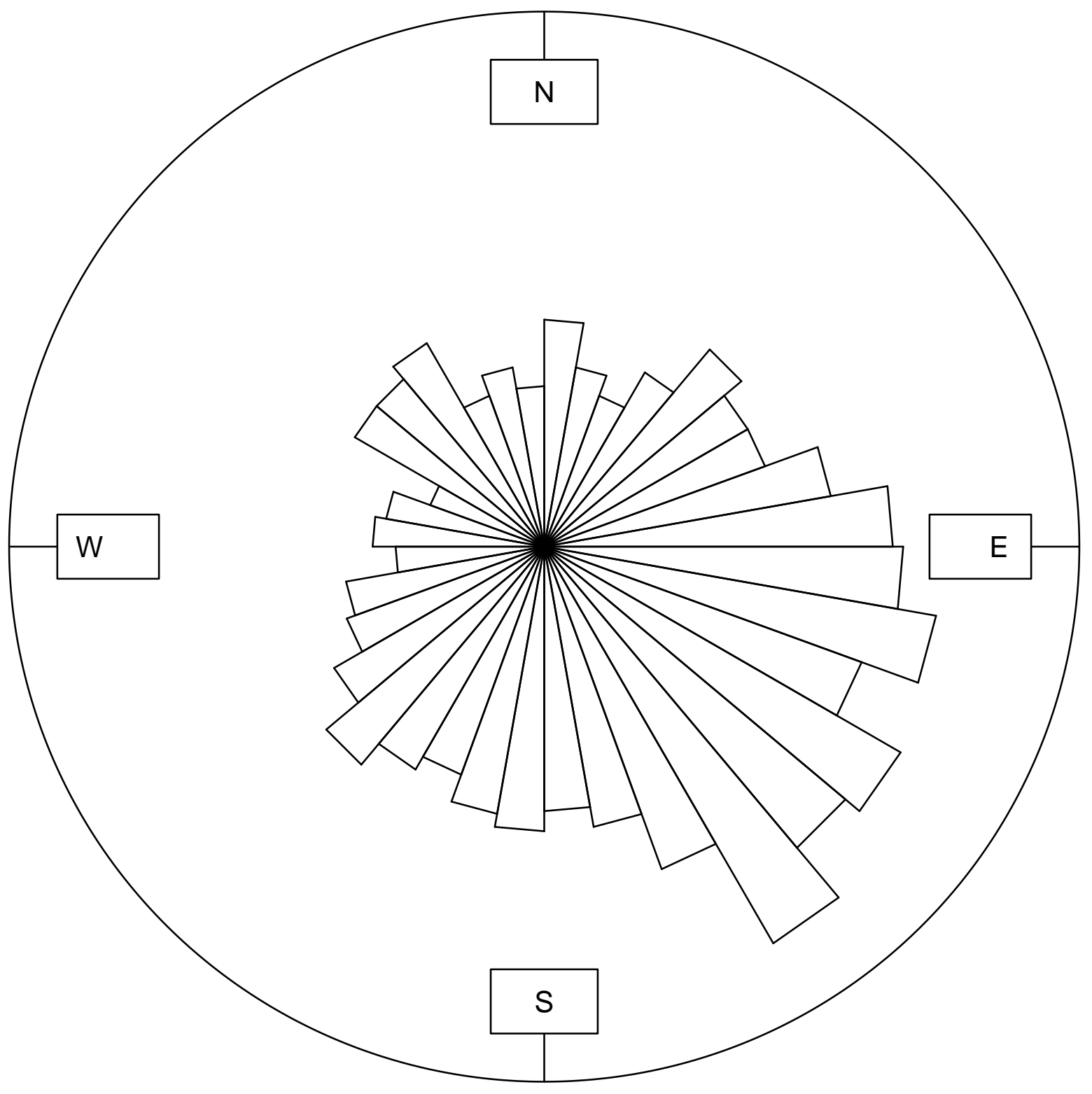




\section{bootstrap 935}

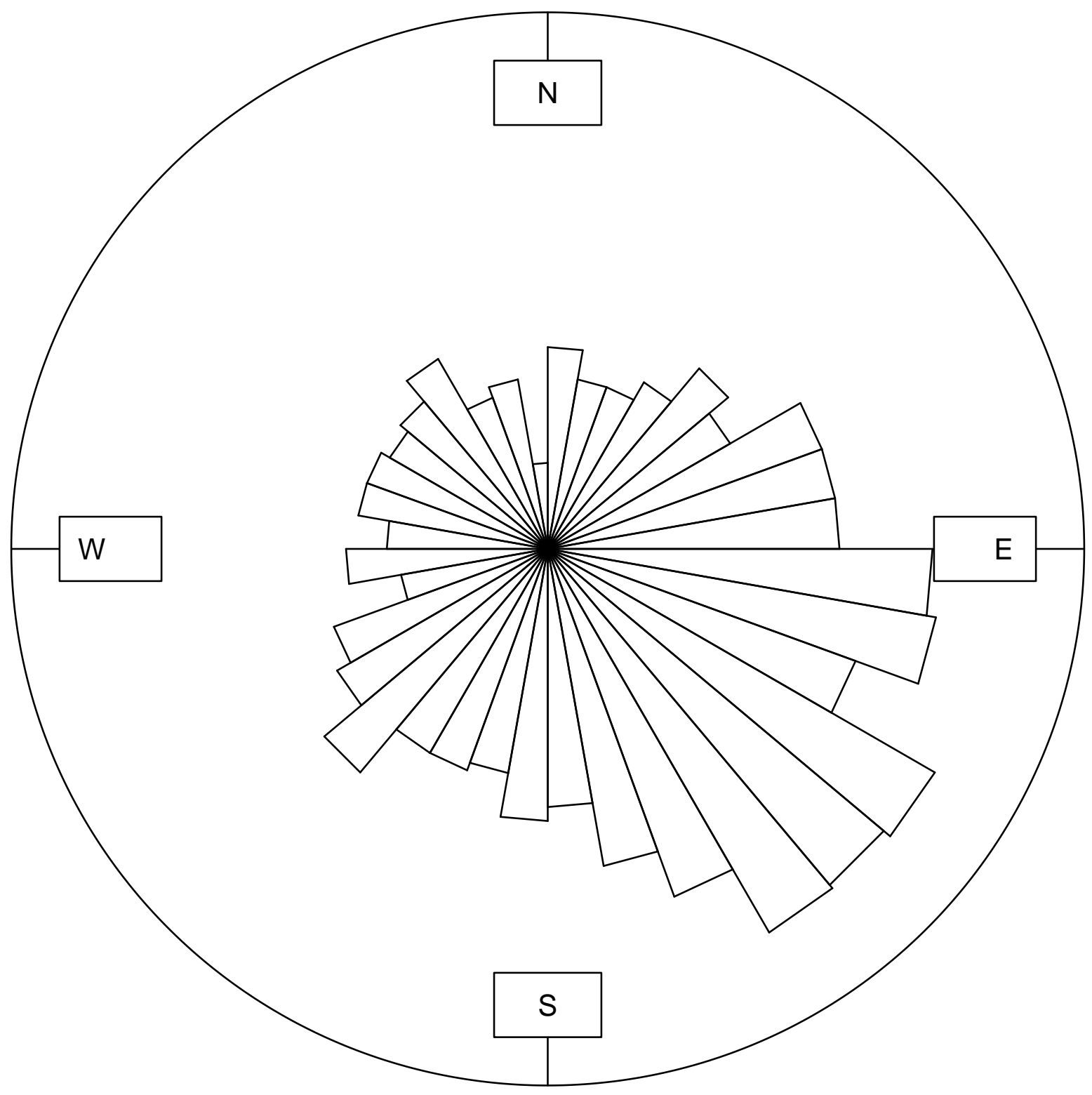




\section{bootstrap 936}

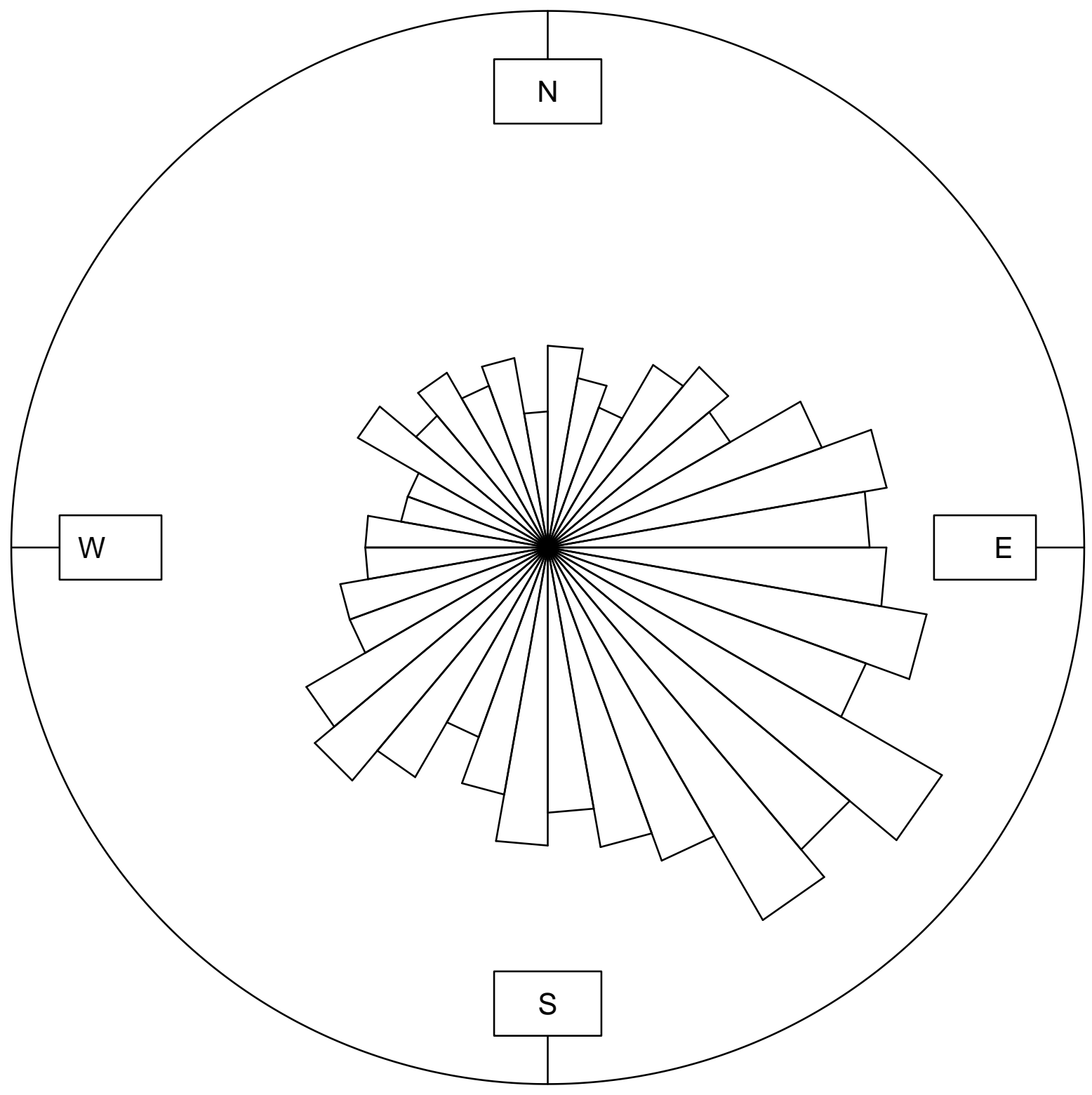


bootstrap 937

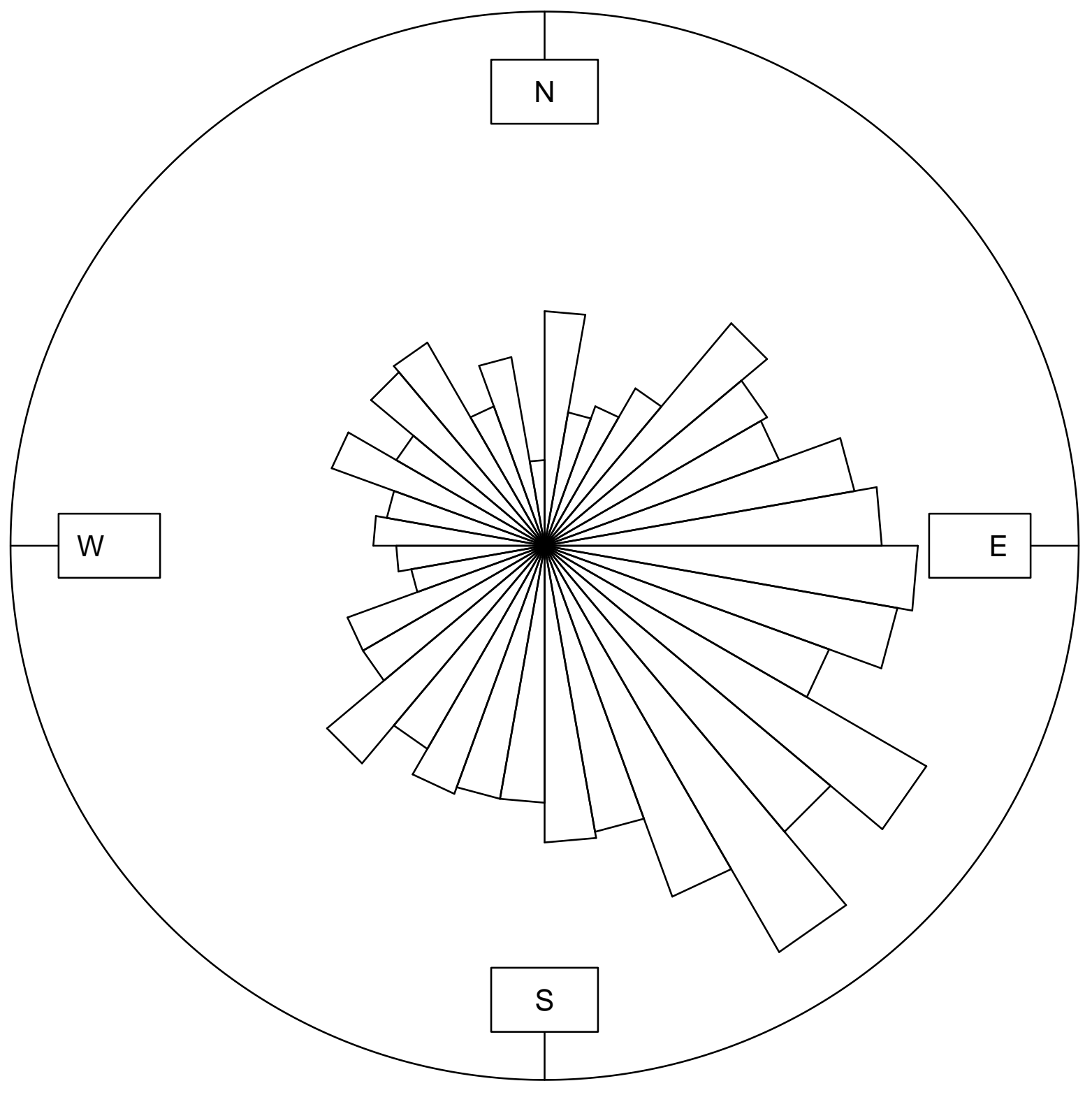


bootstrap 938

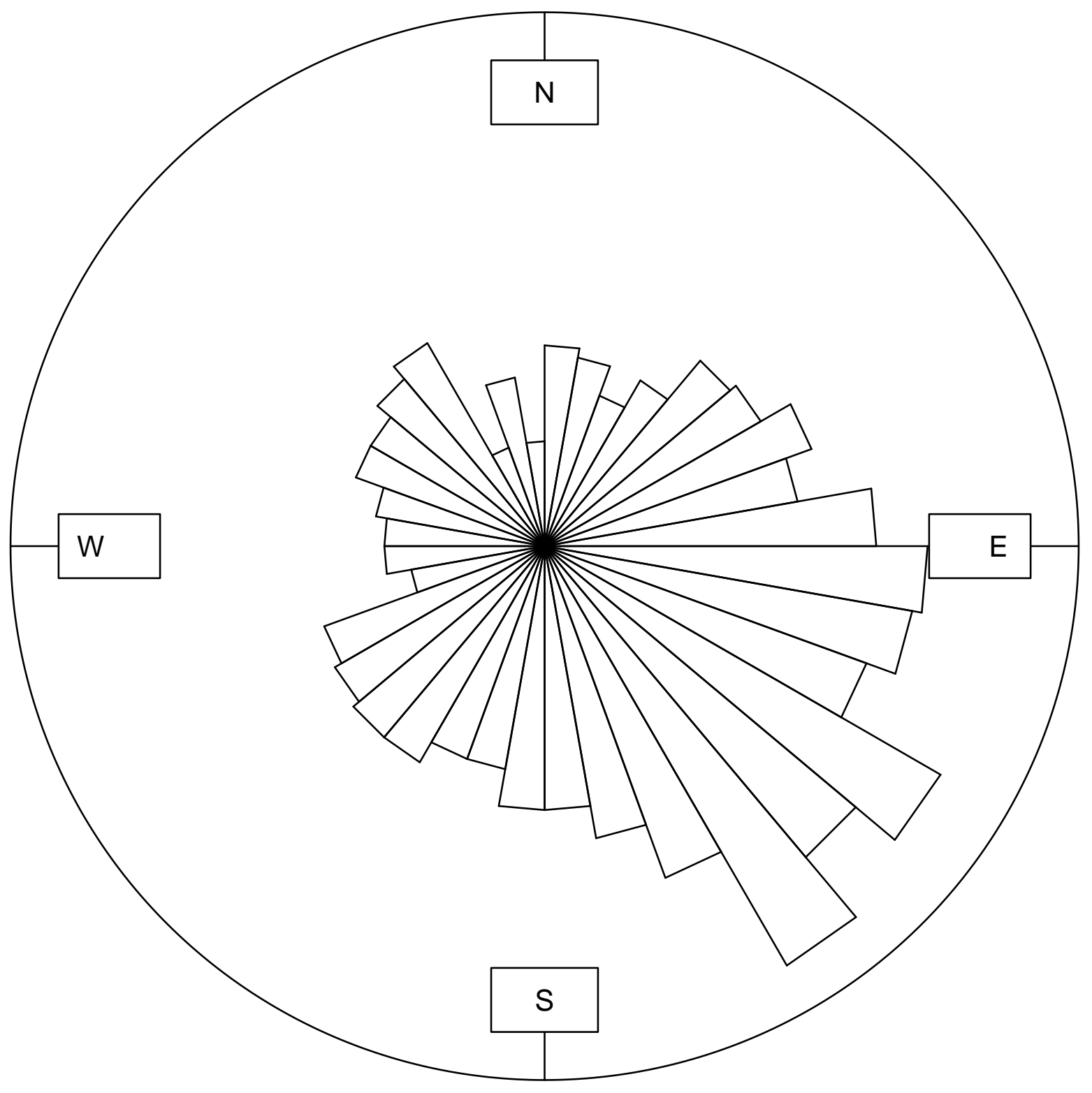


bootstrap 939

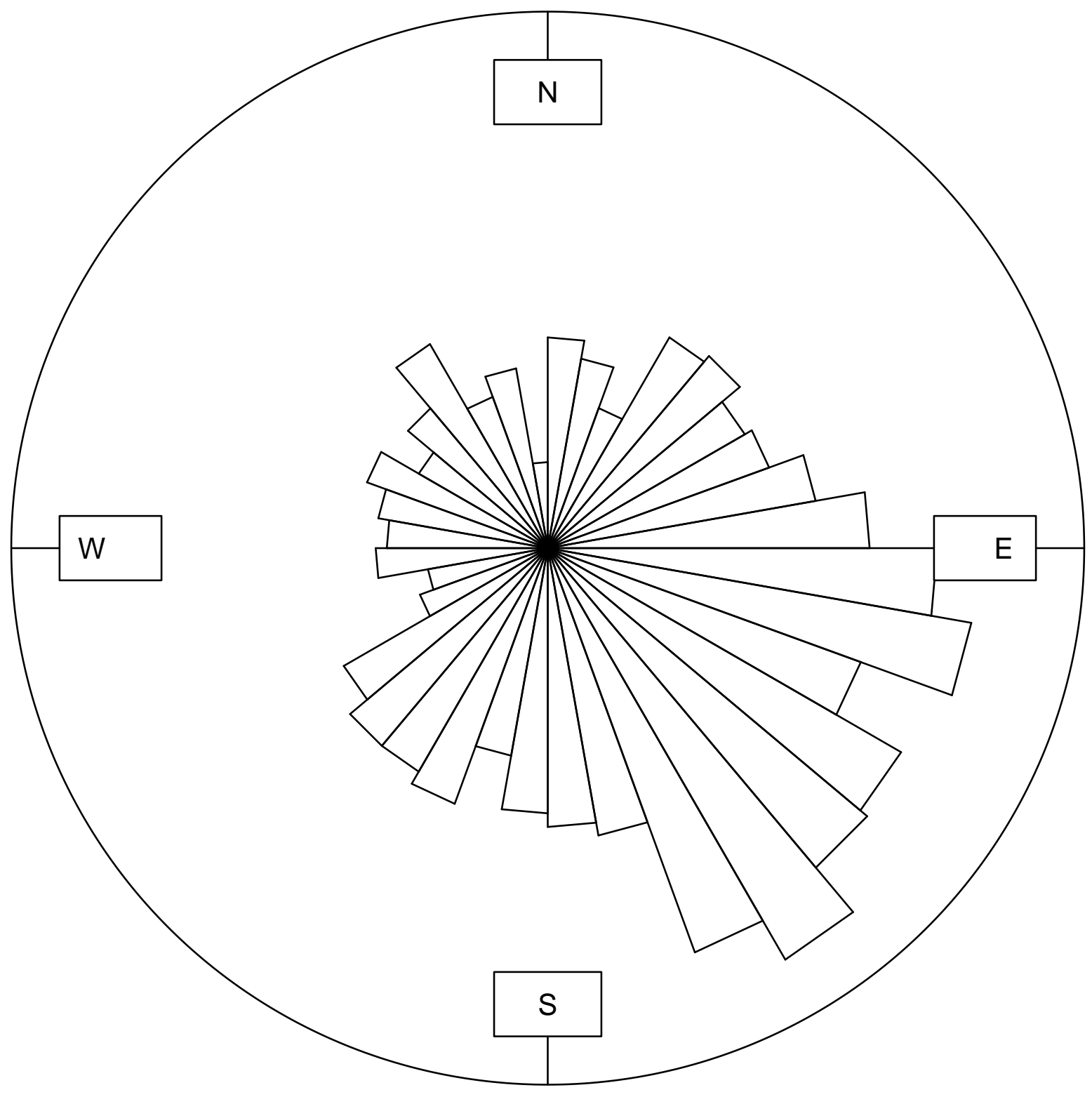


bootstrap 940

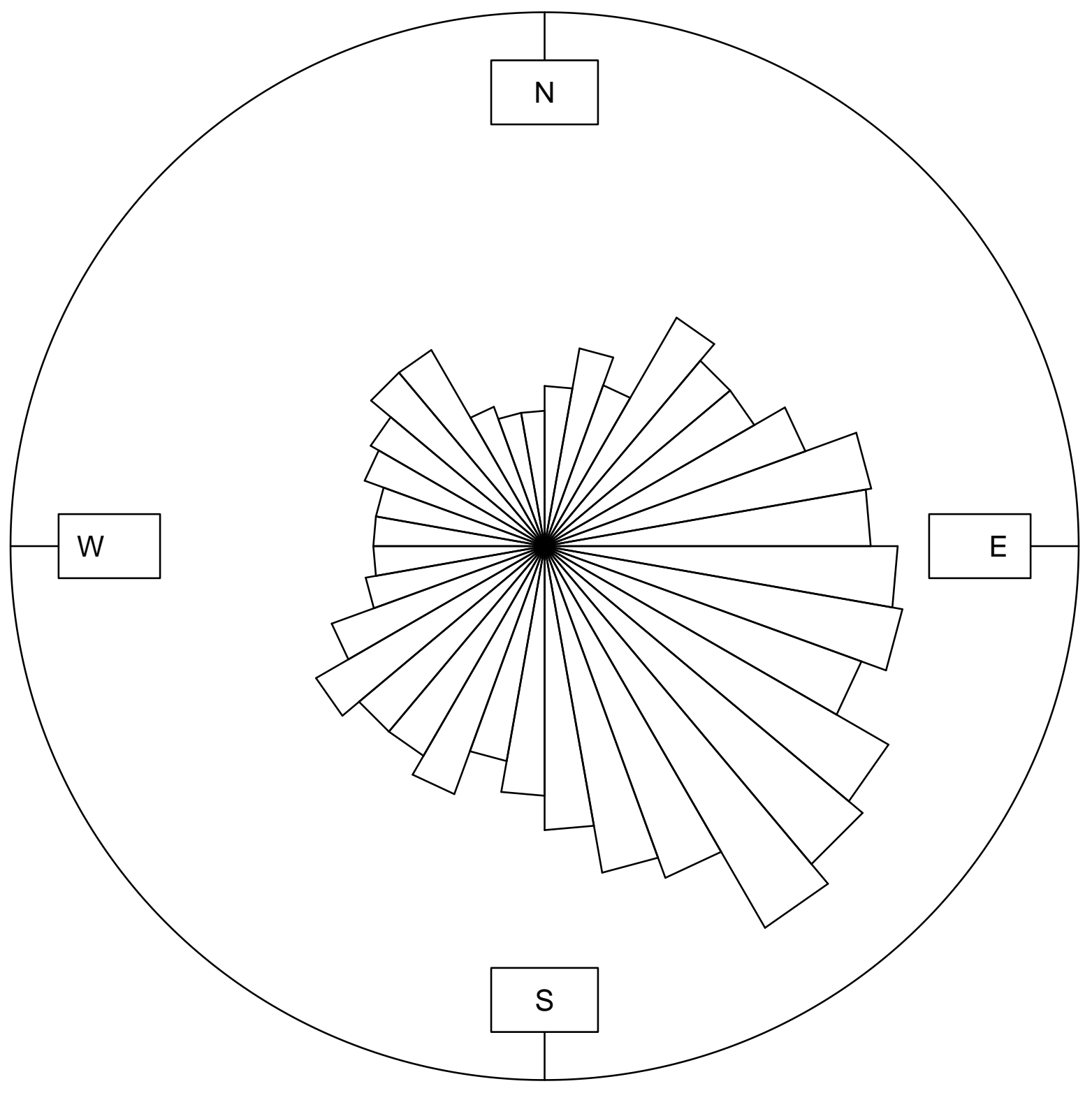


bootstrap 941

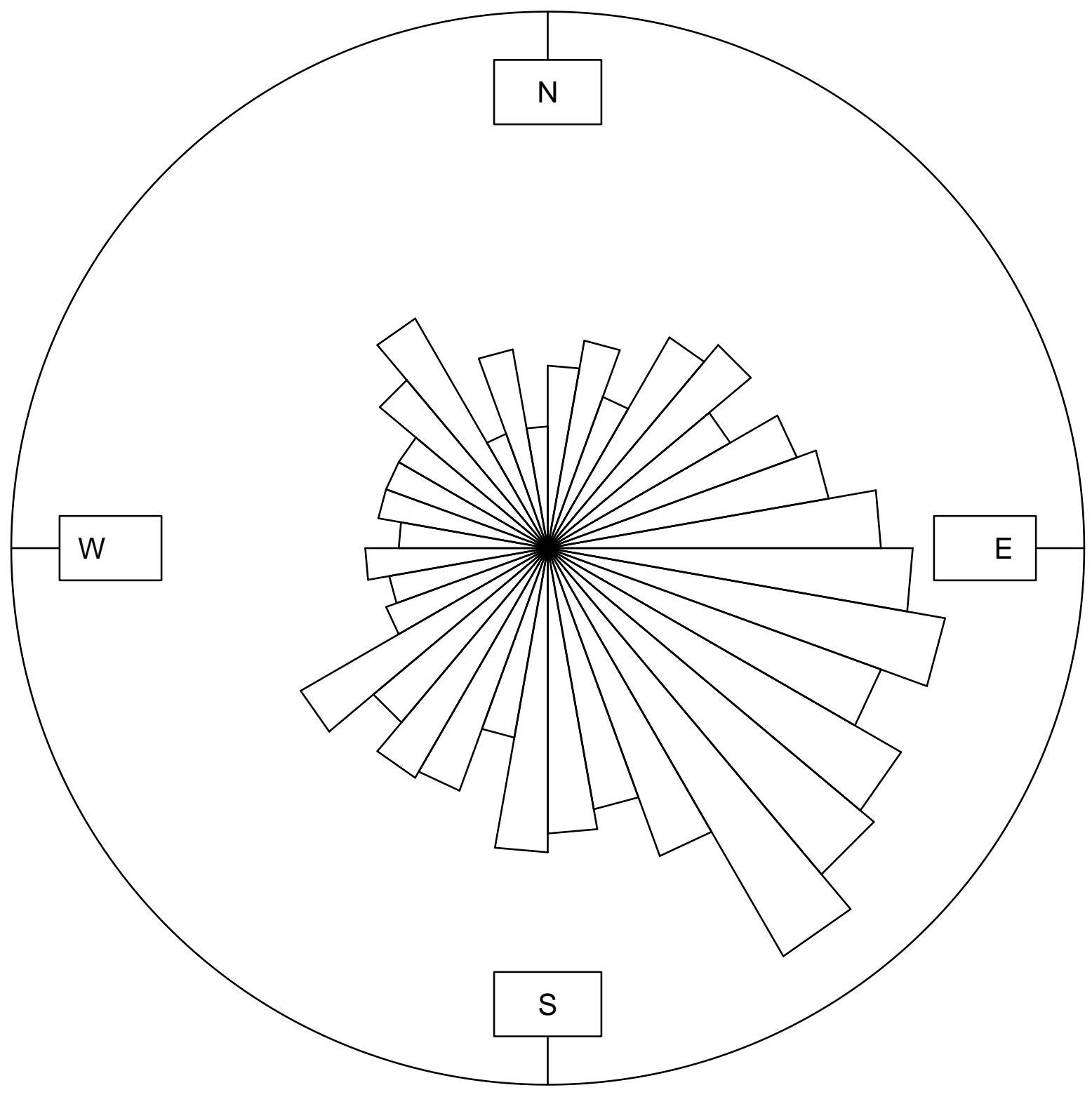


bootstrap 942

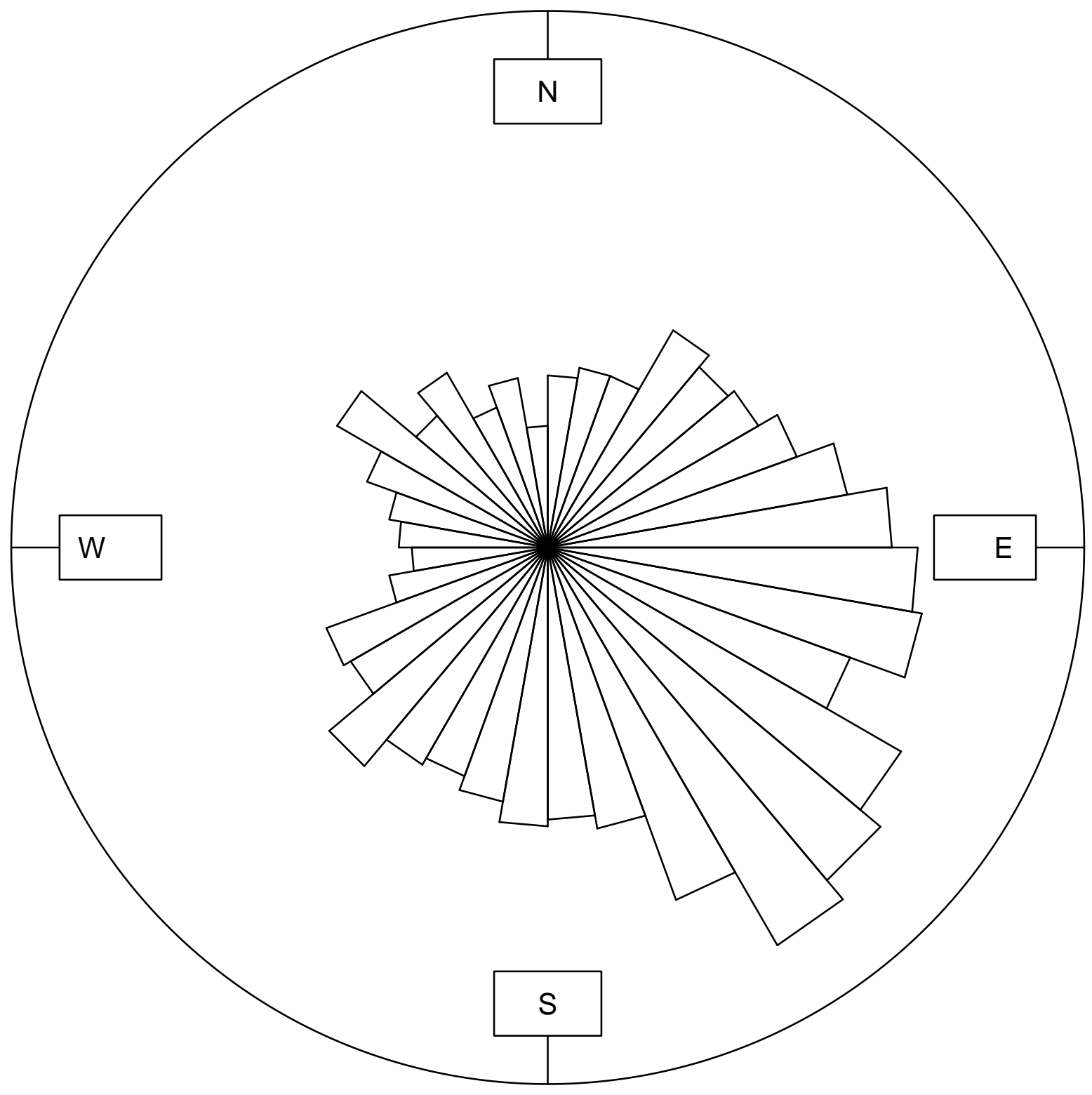




\section{bootstrap 943}

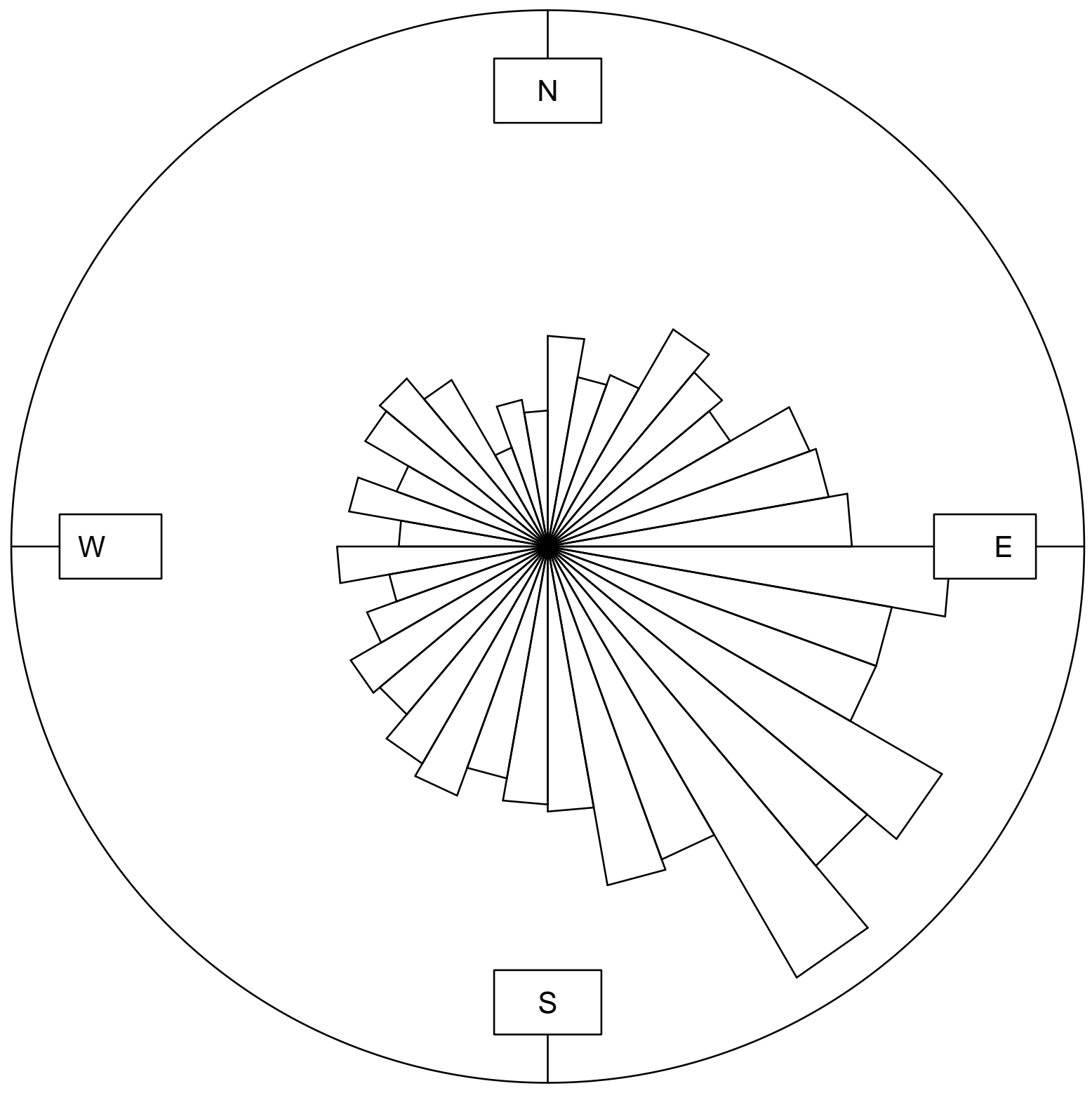


bootstrap 944

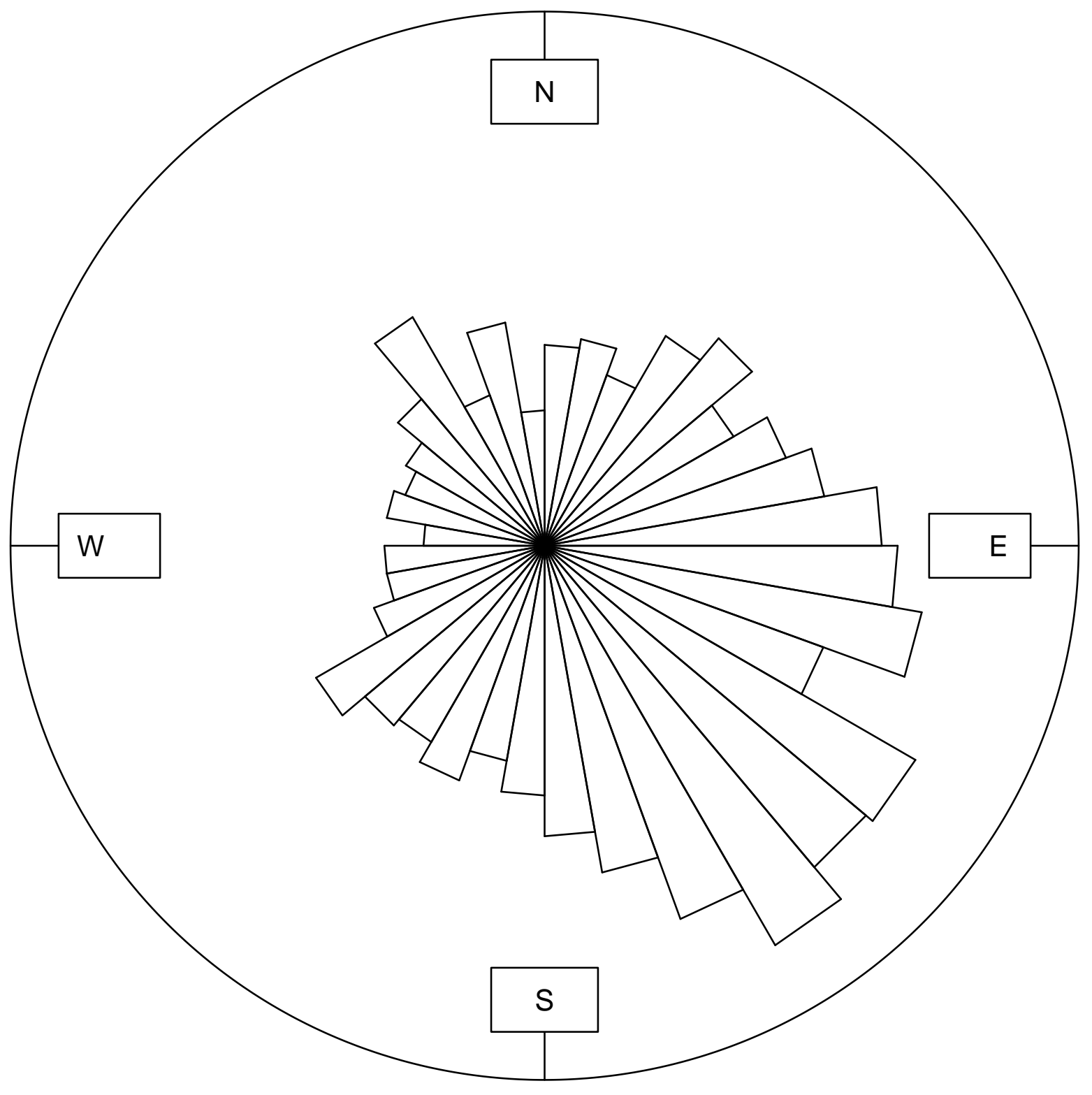


bootstrap 945

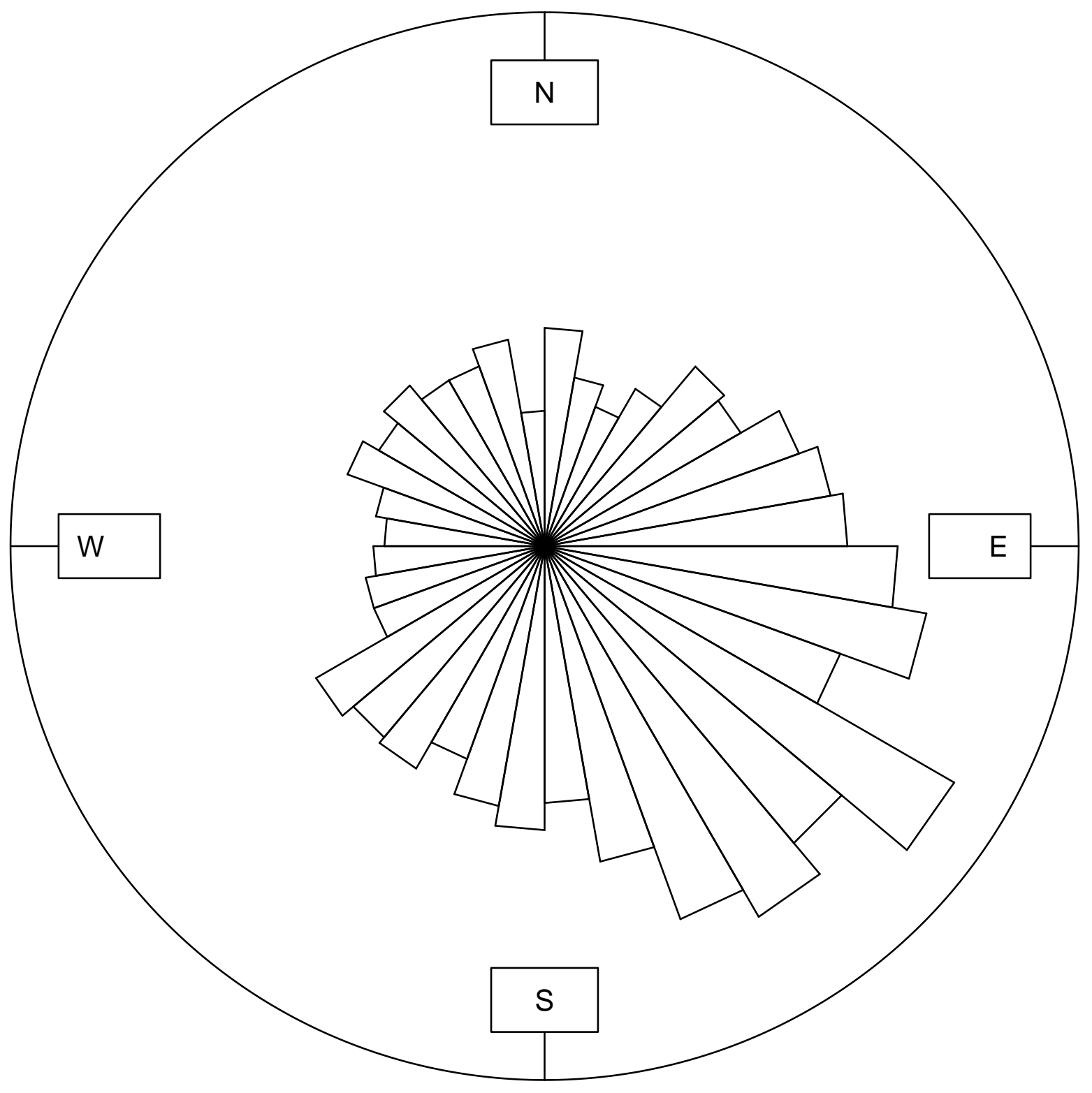


bootstrap 946

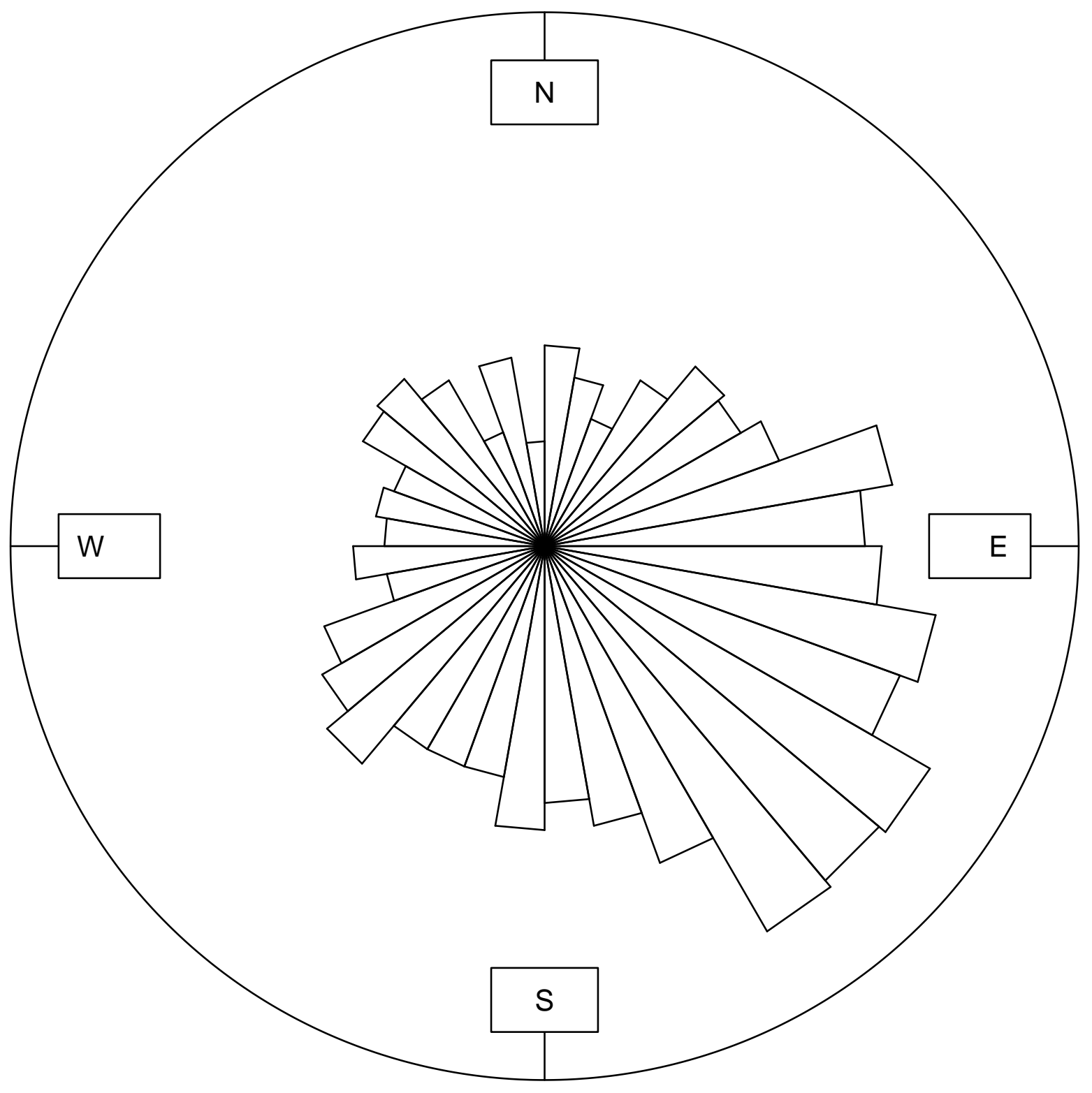


bootstrap 947

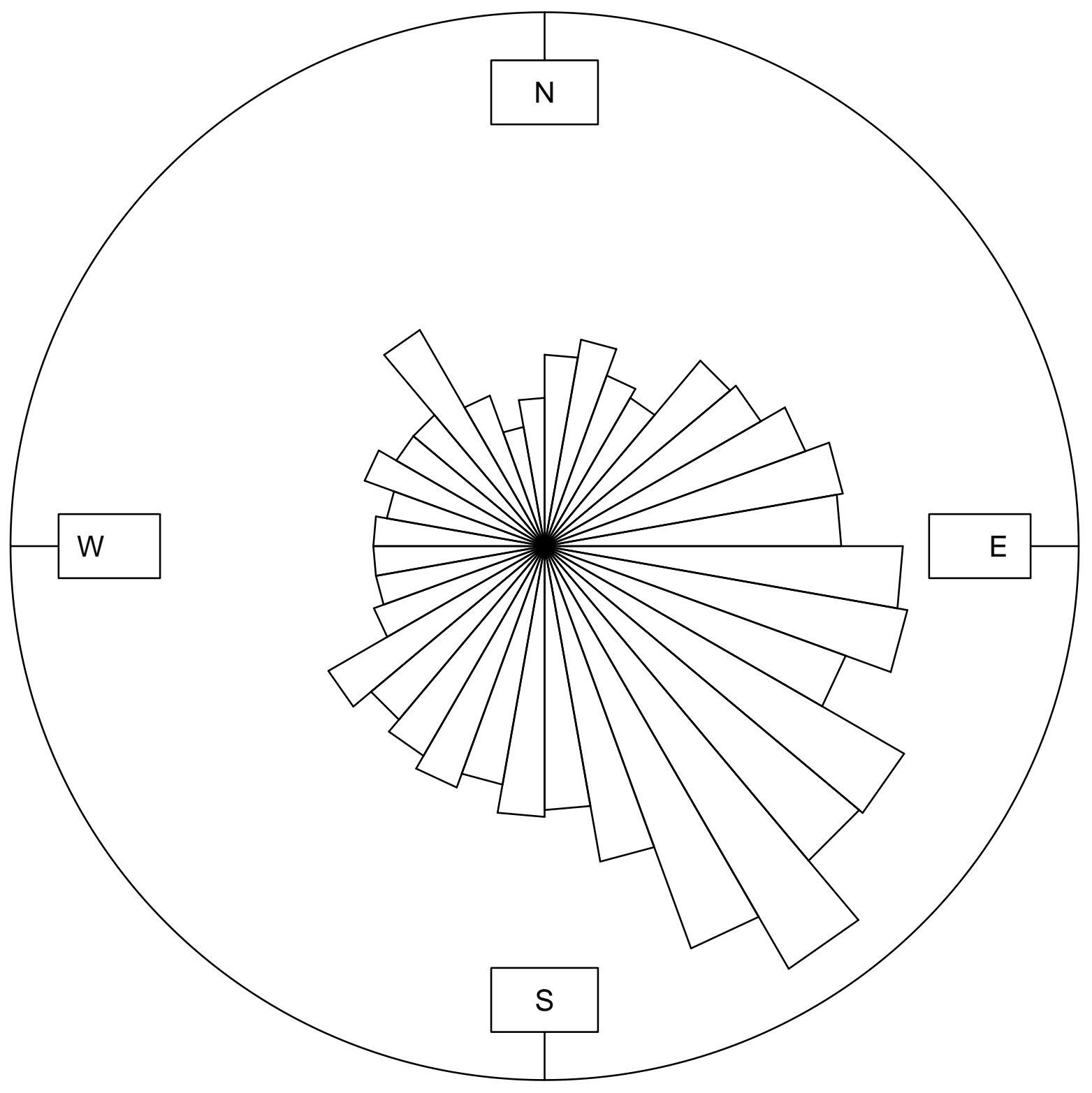


bootstrap 948

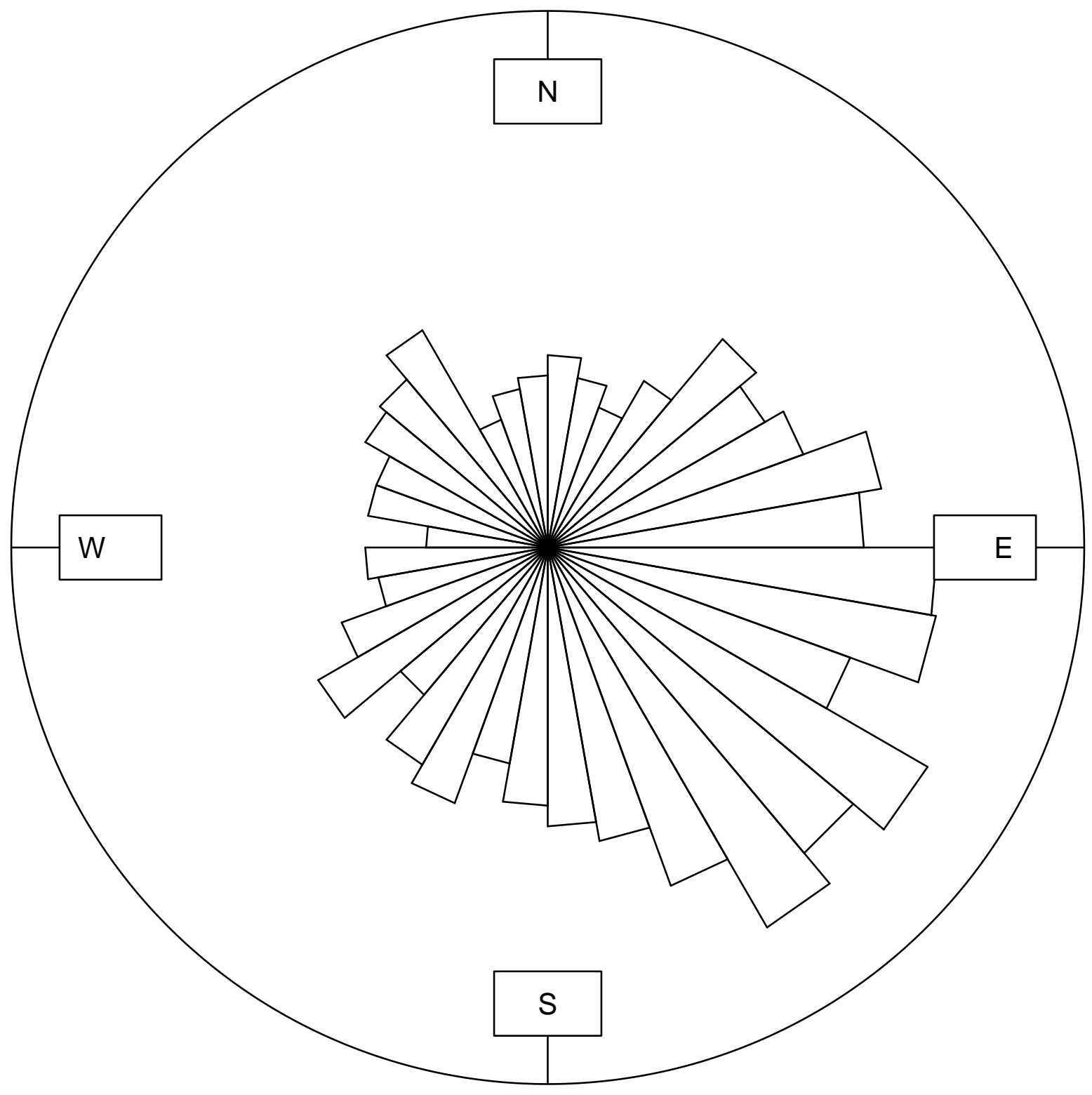


bootstrap 949

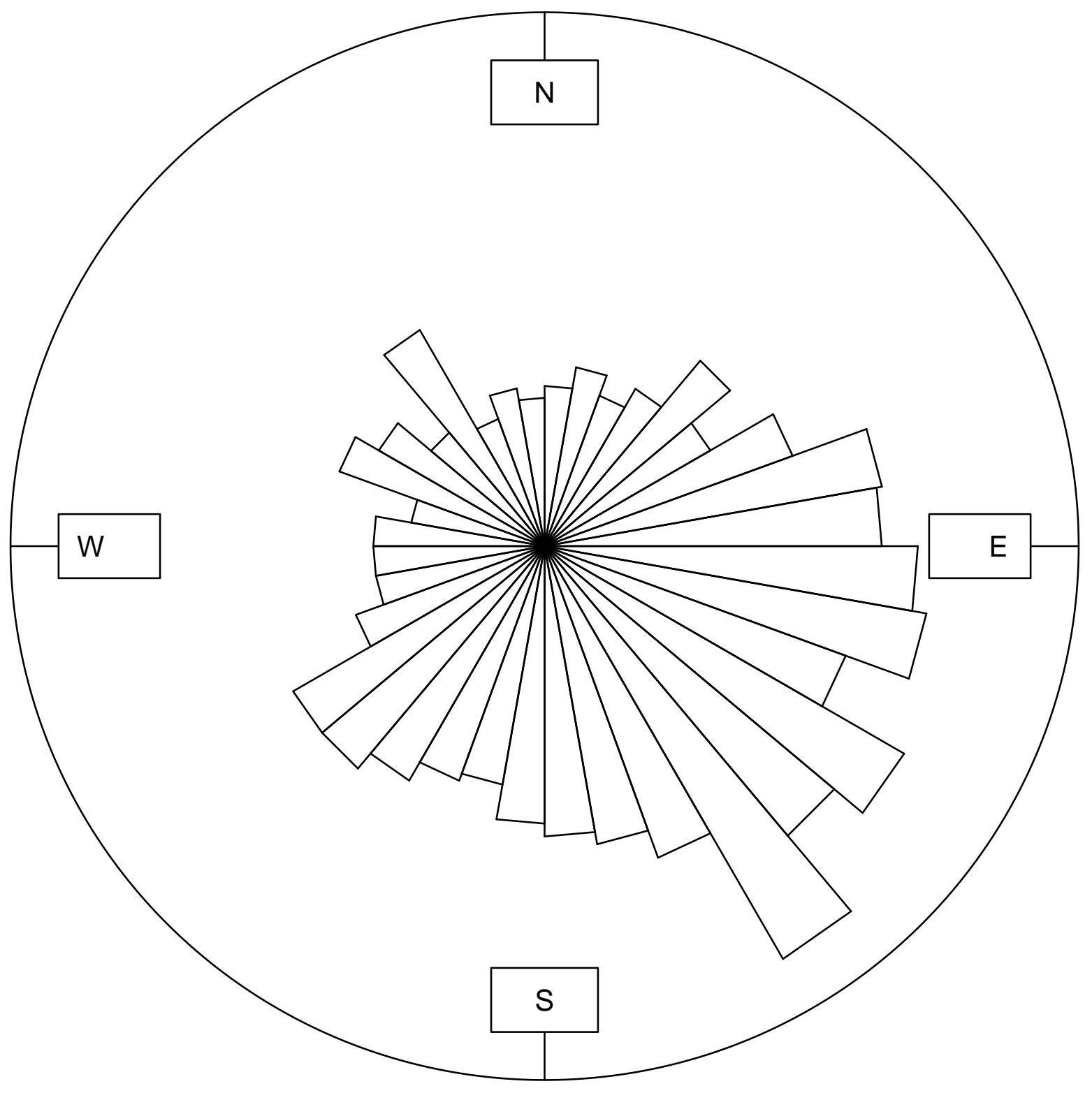


bootstrap 950

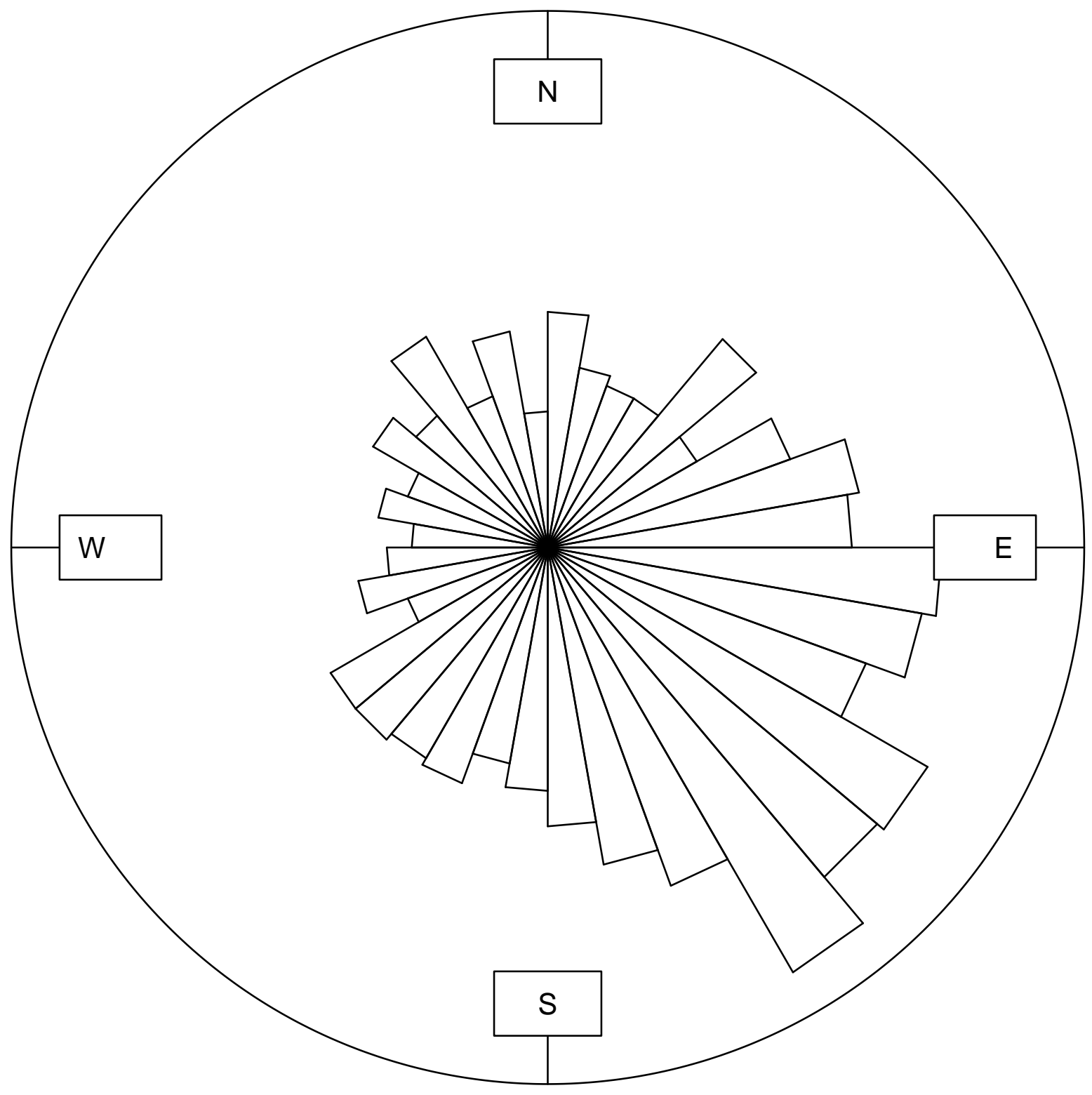


bootstrap 951

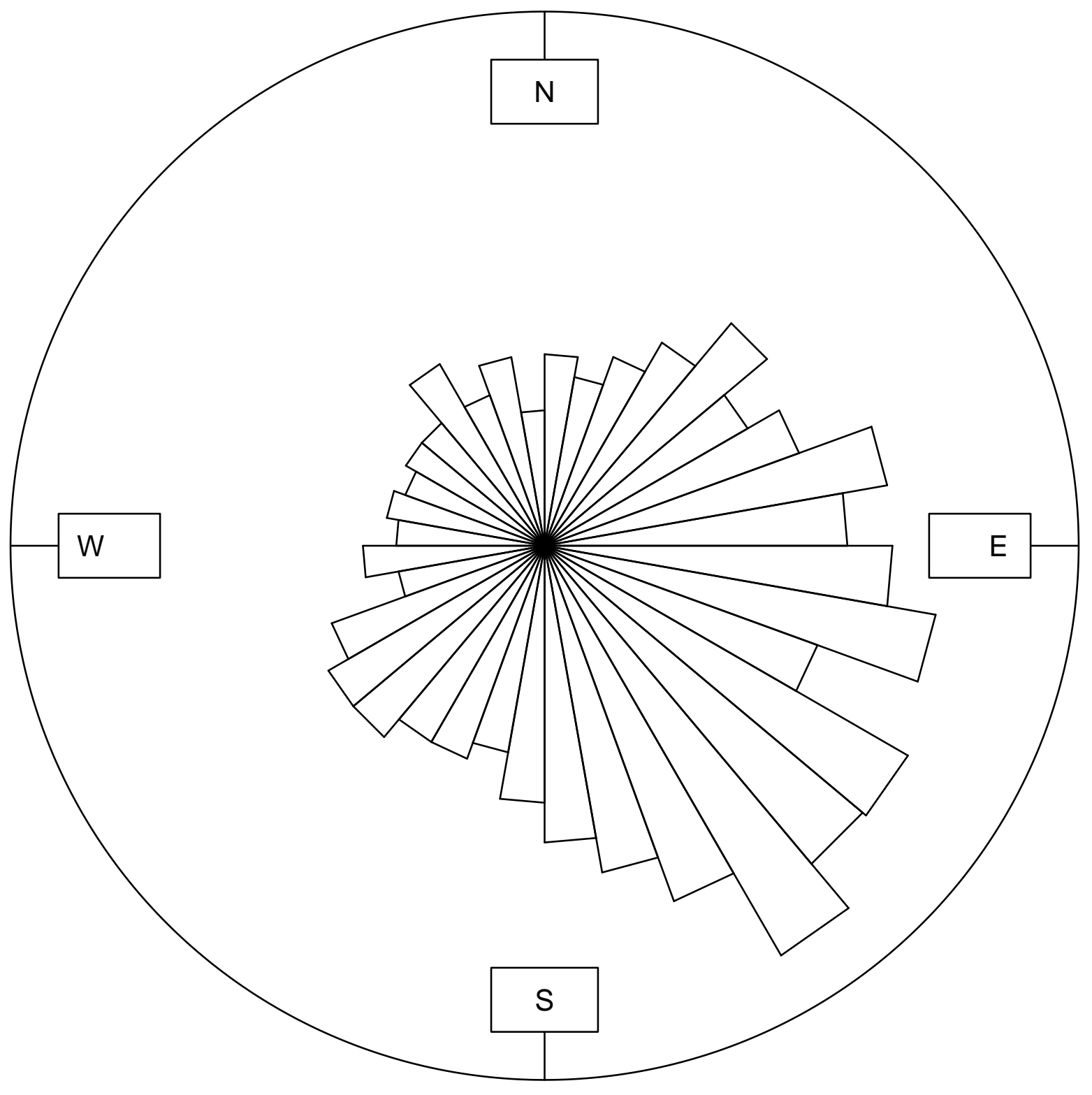


bootstrap 952

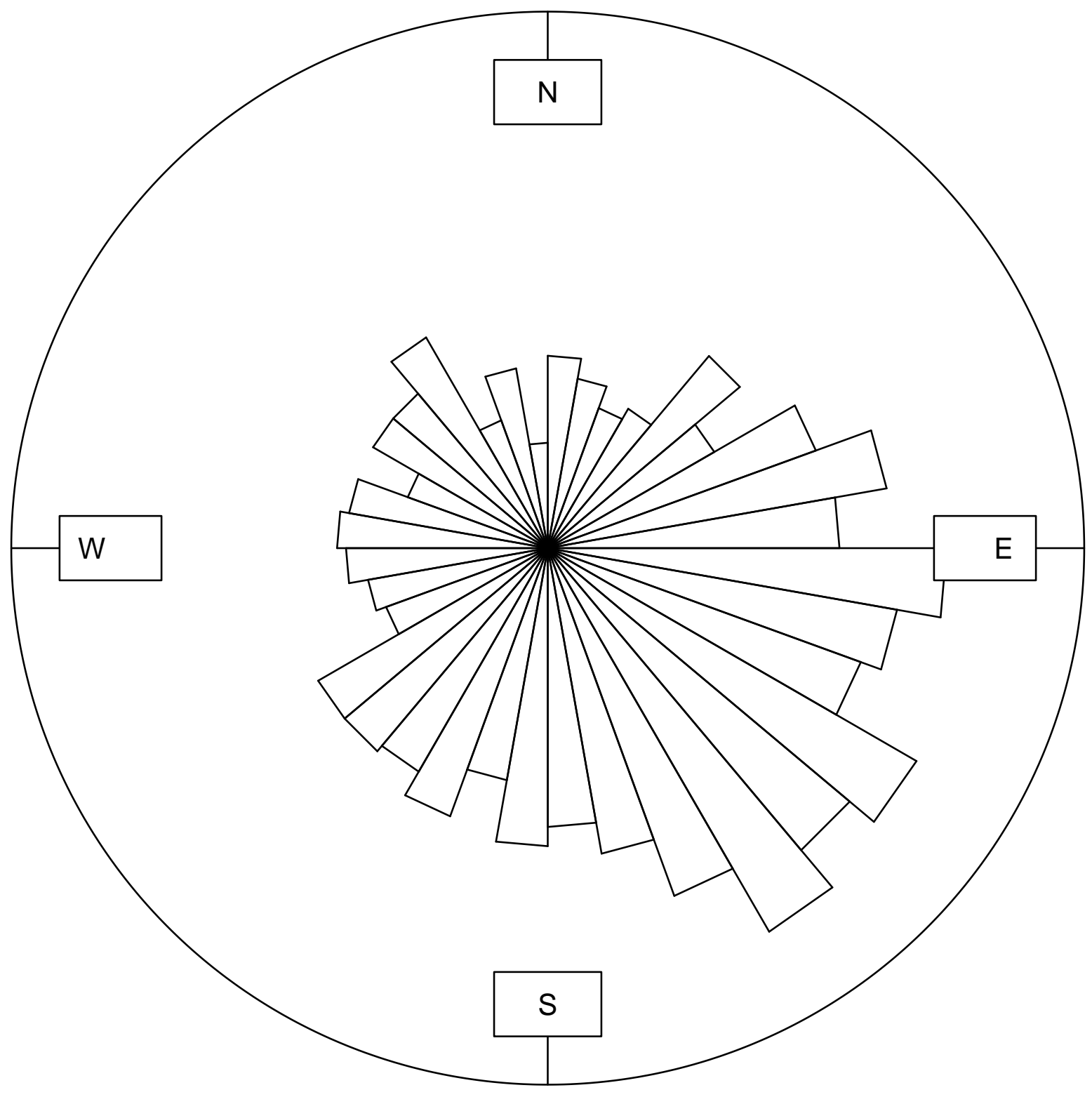




\section{bootstrap 953}

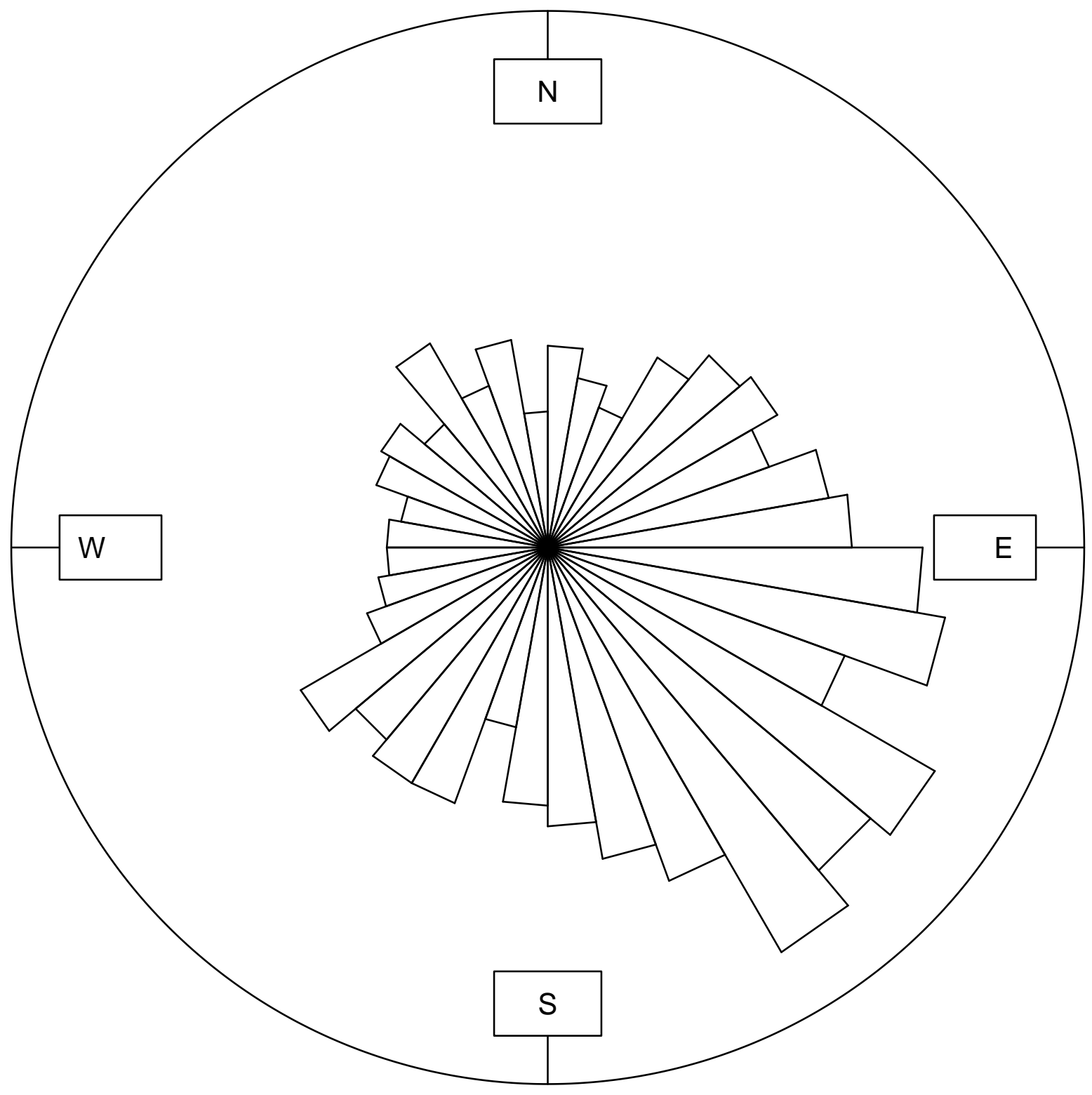




\section{bootstrap 954}

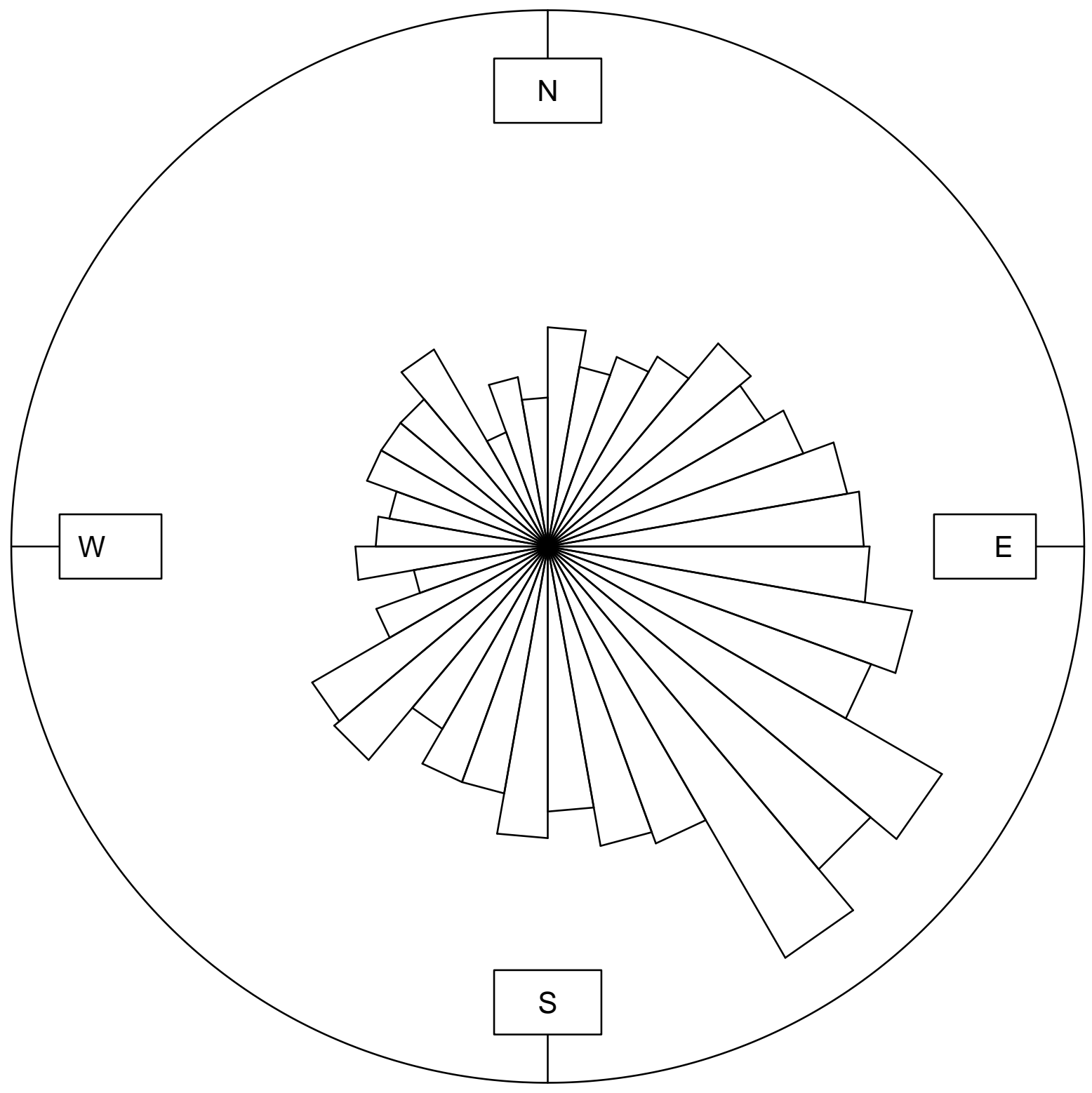




\section{bootstrap 955}

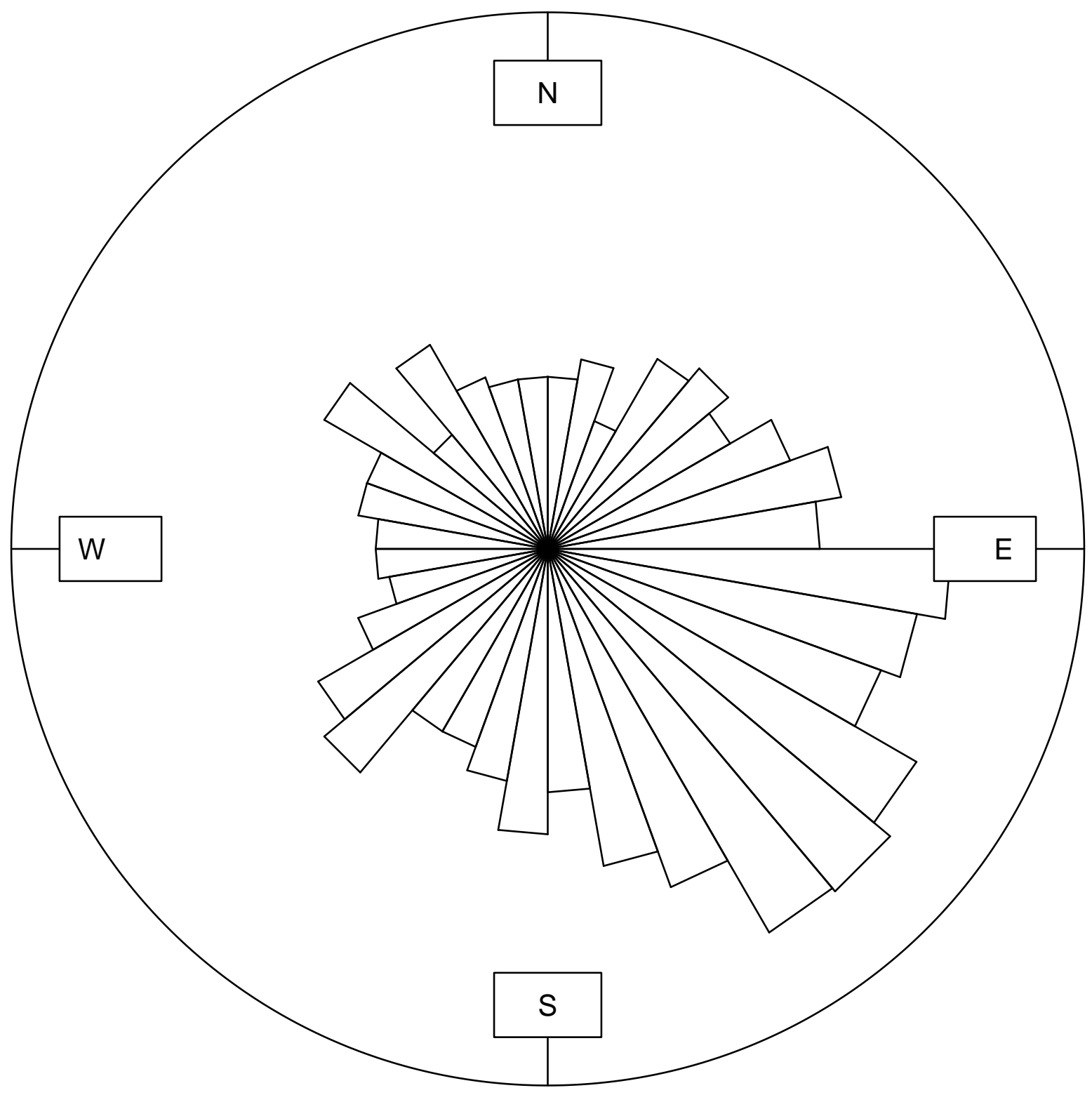


bootstrap 956

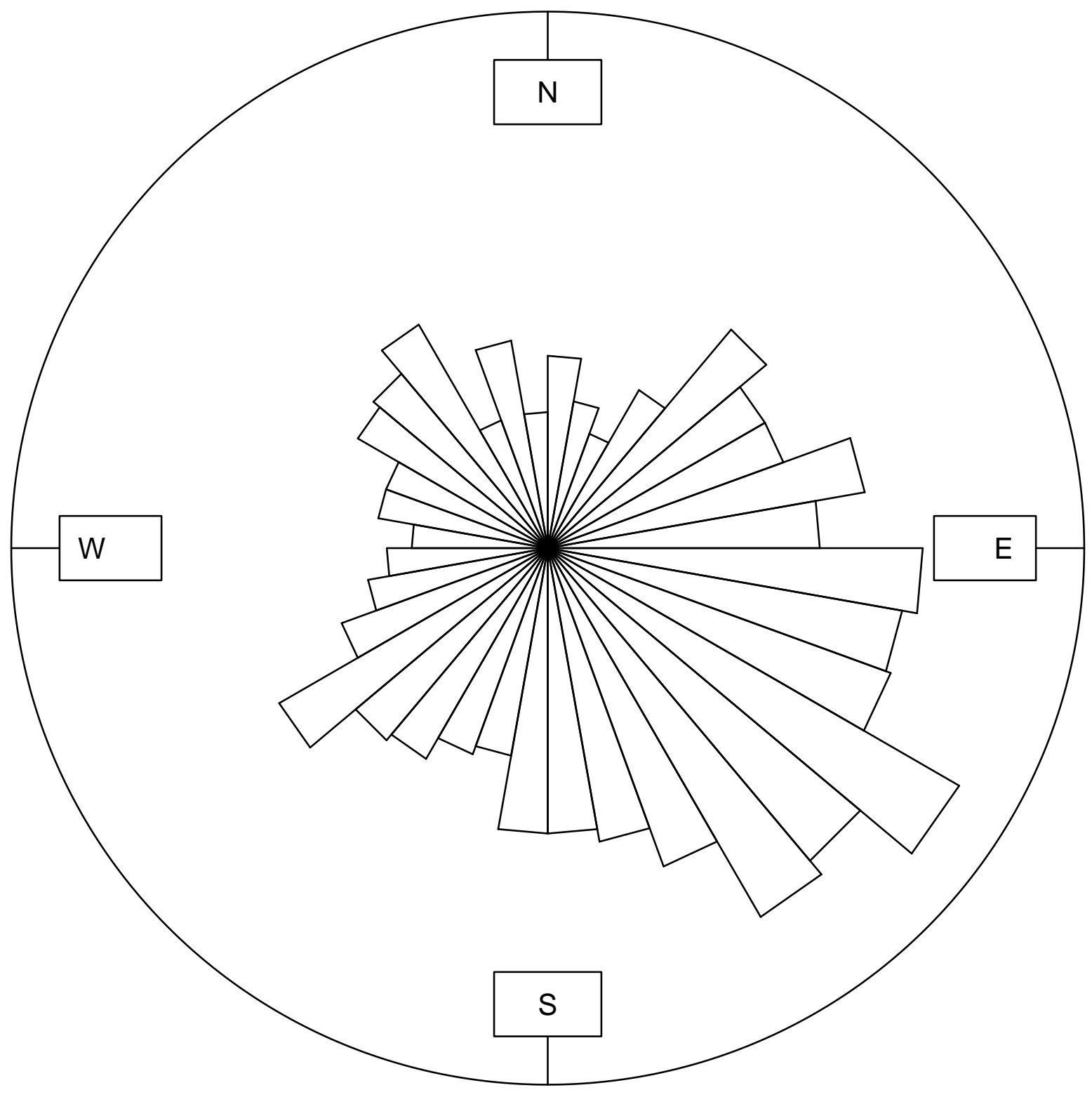


bootstrap 957

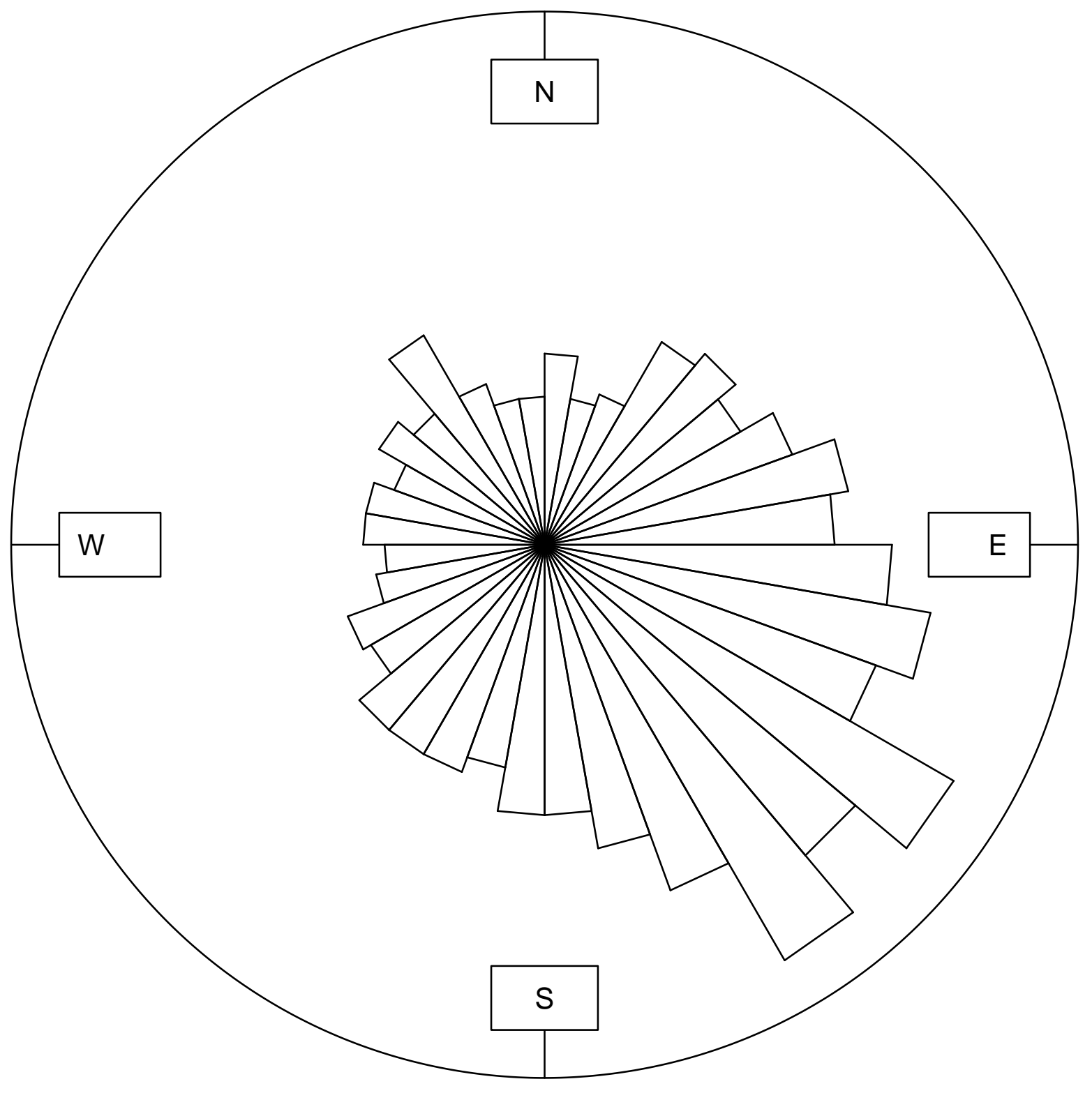


bootstrap 958

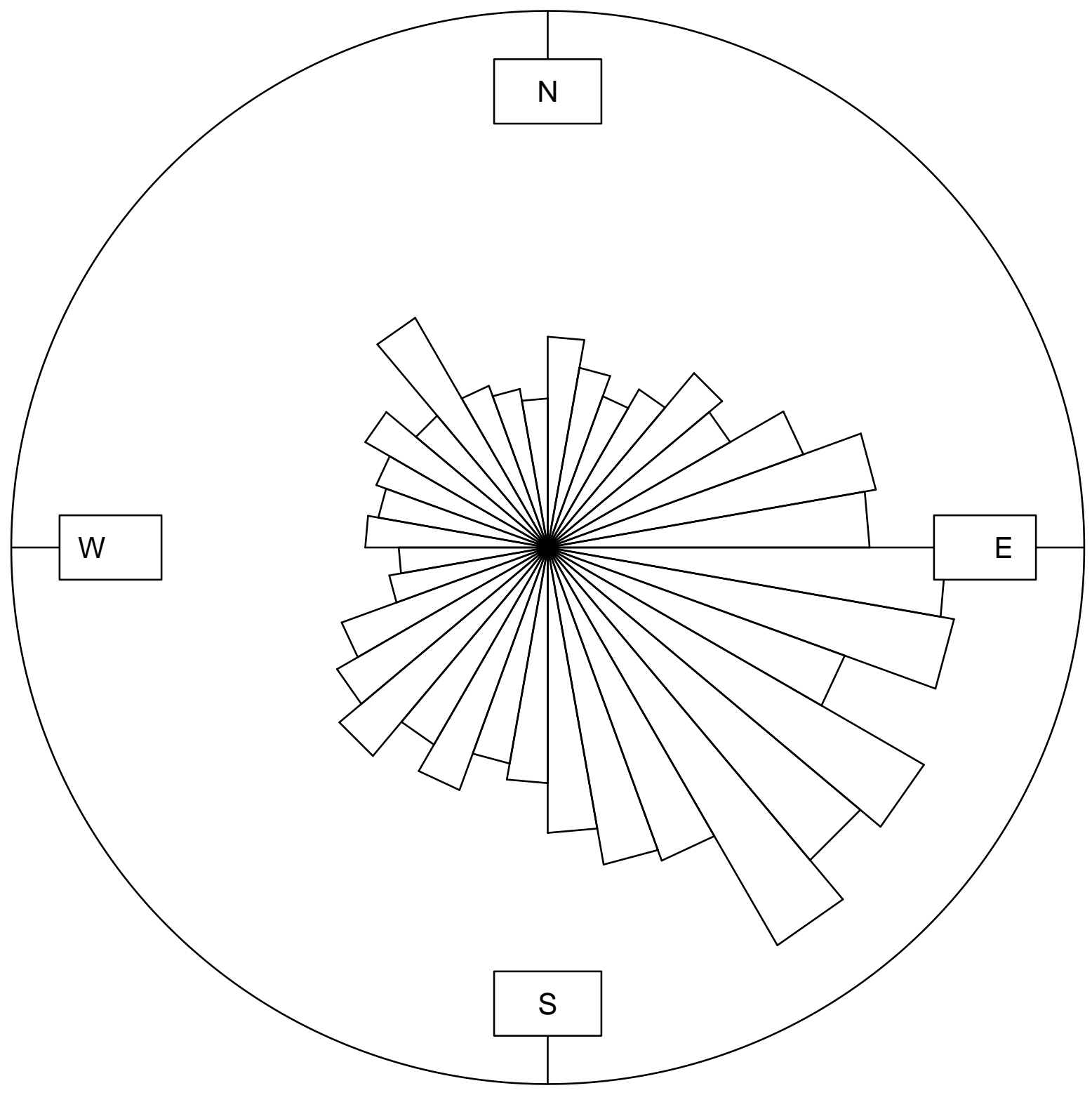


bootstrap 959

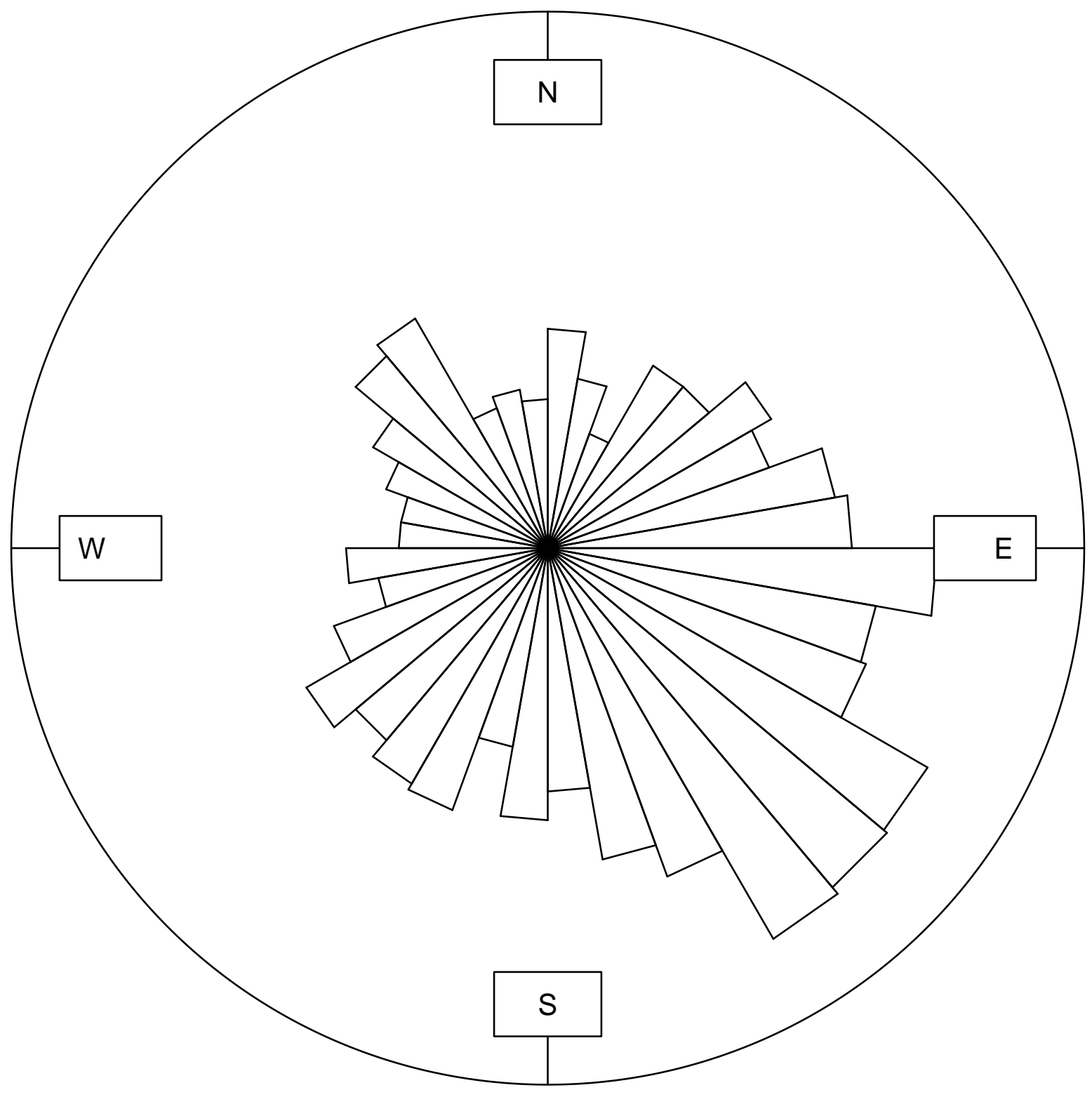


bootstrap 960

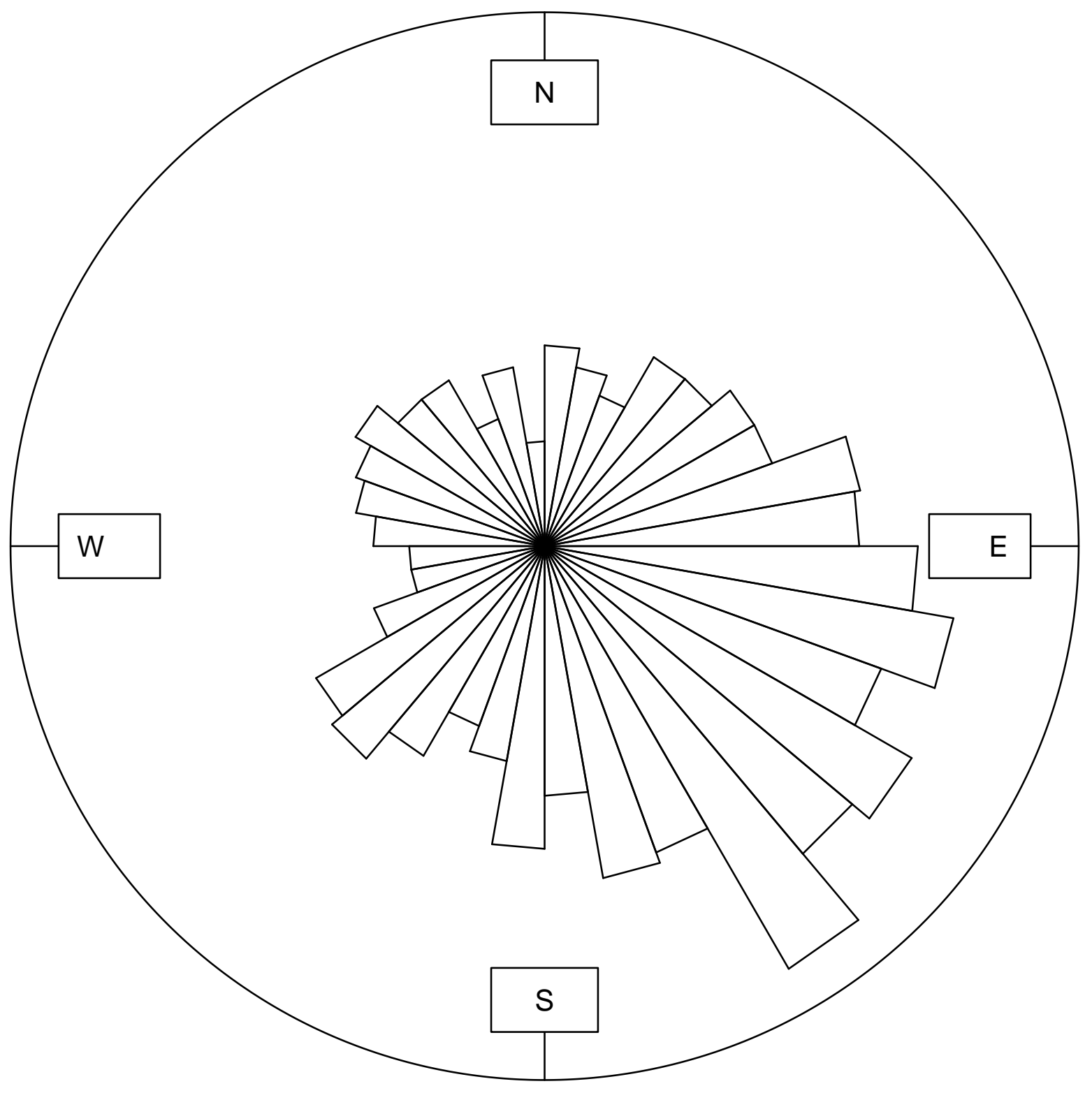




\section{bootstrap 961}

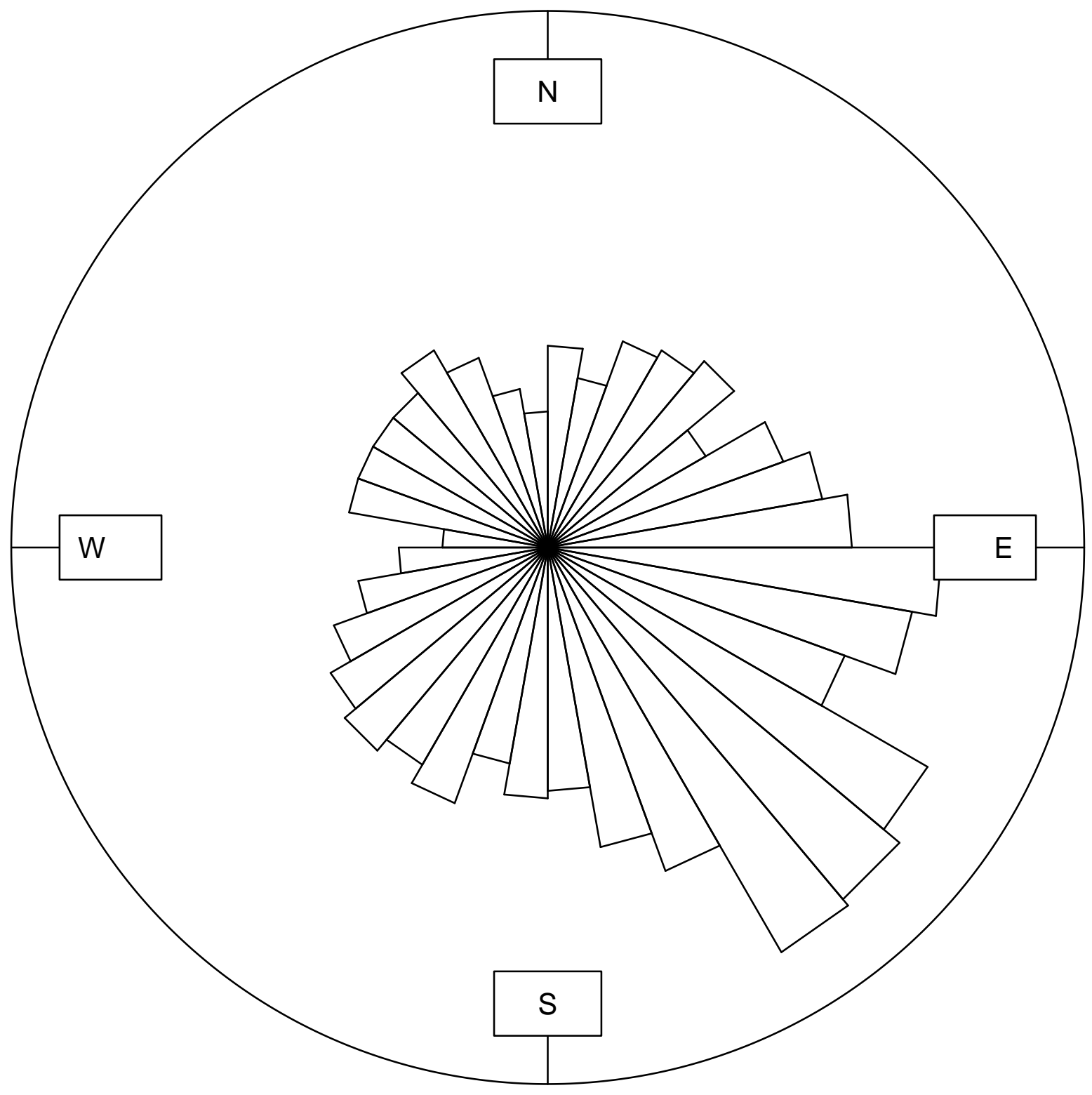


bootstrap 962

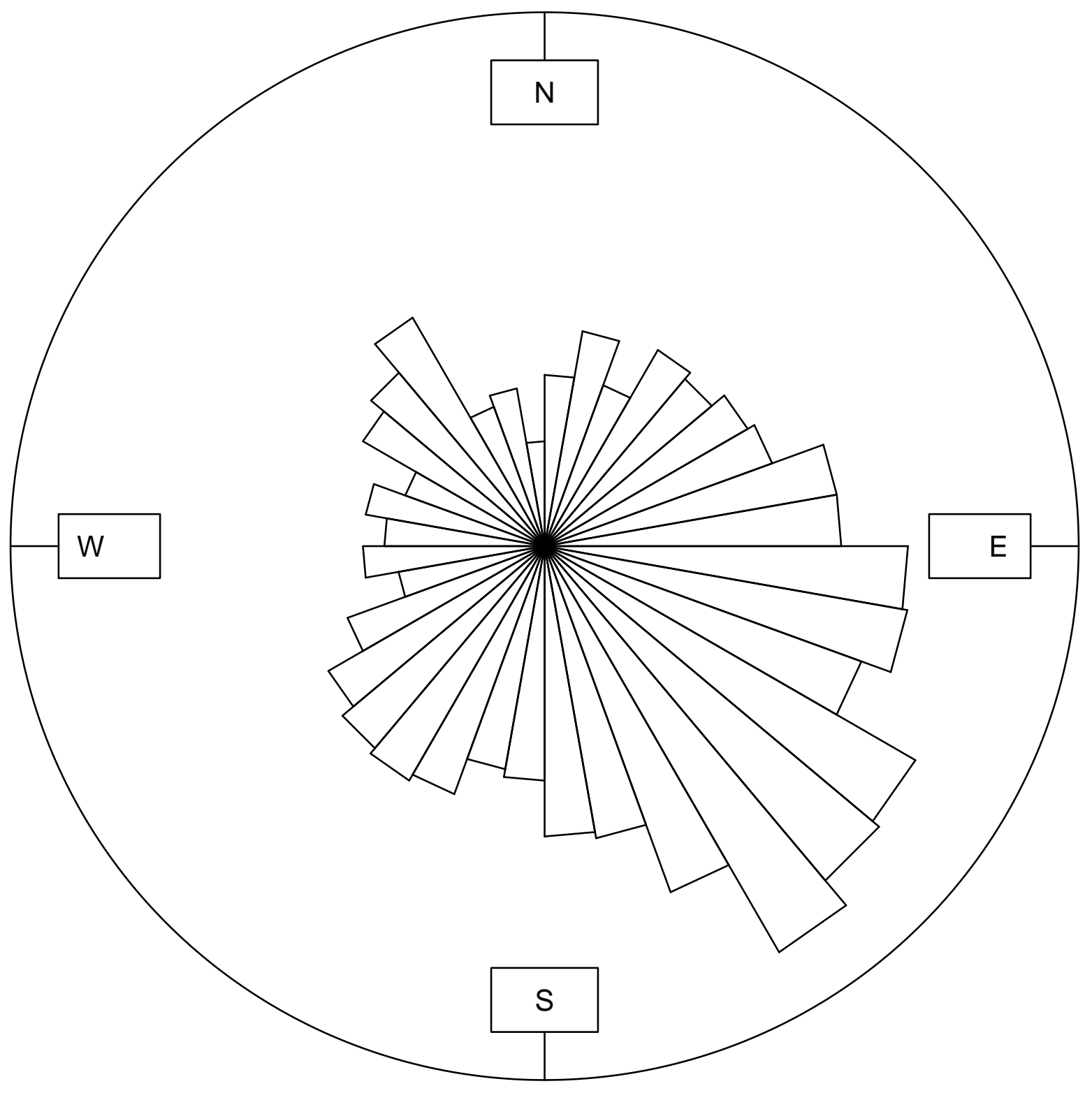




\section{bootstrap 963}

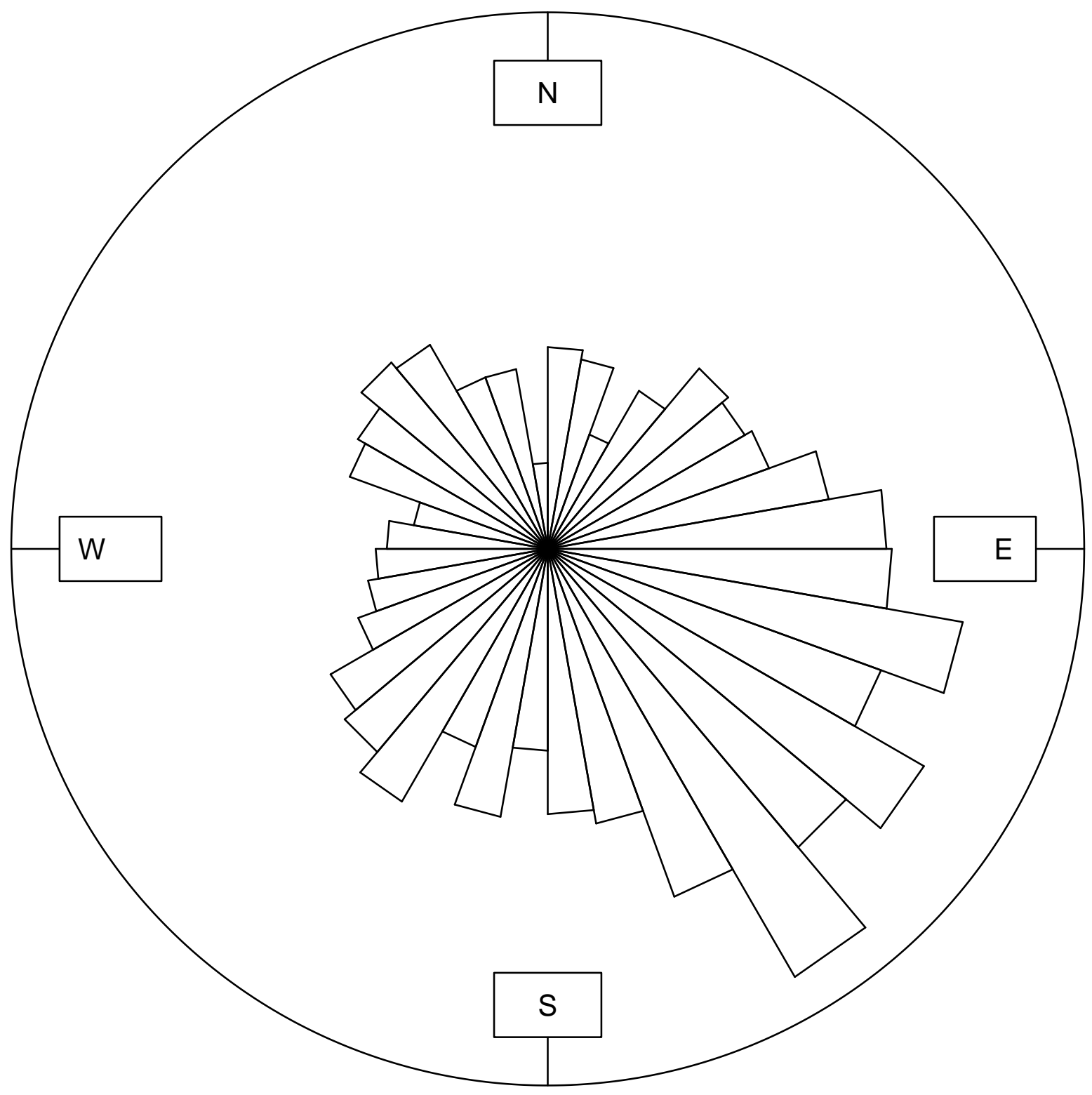




\section{bootstrap 964}

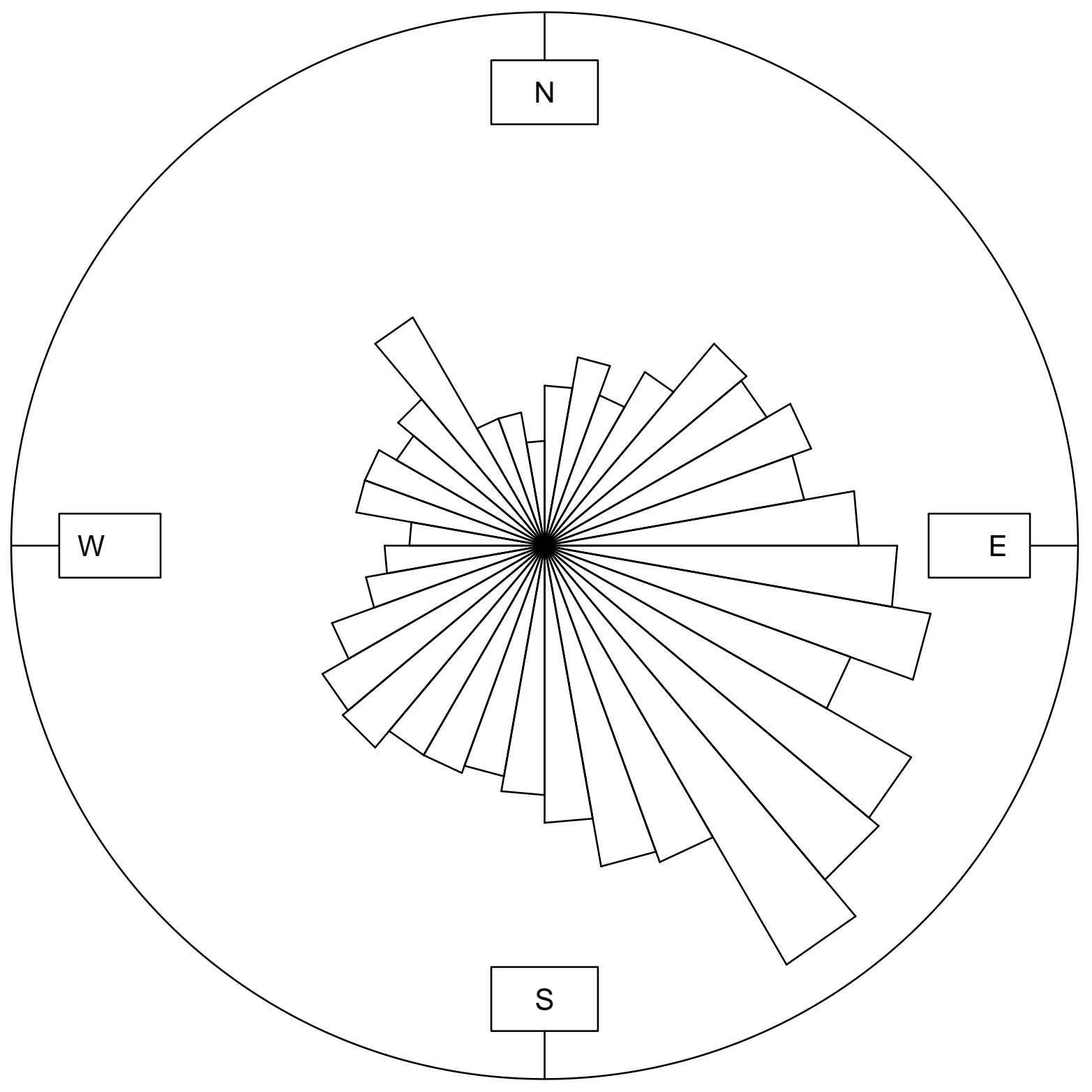


bootstrap 965

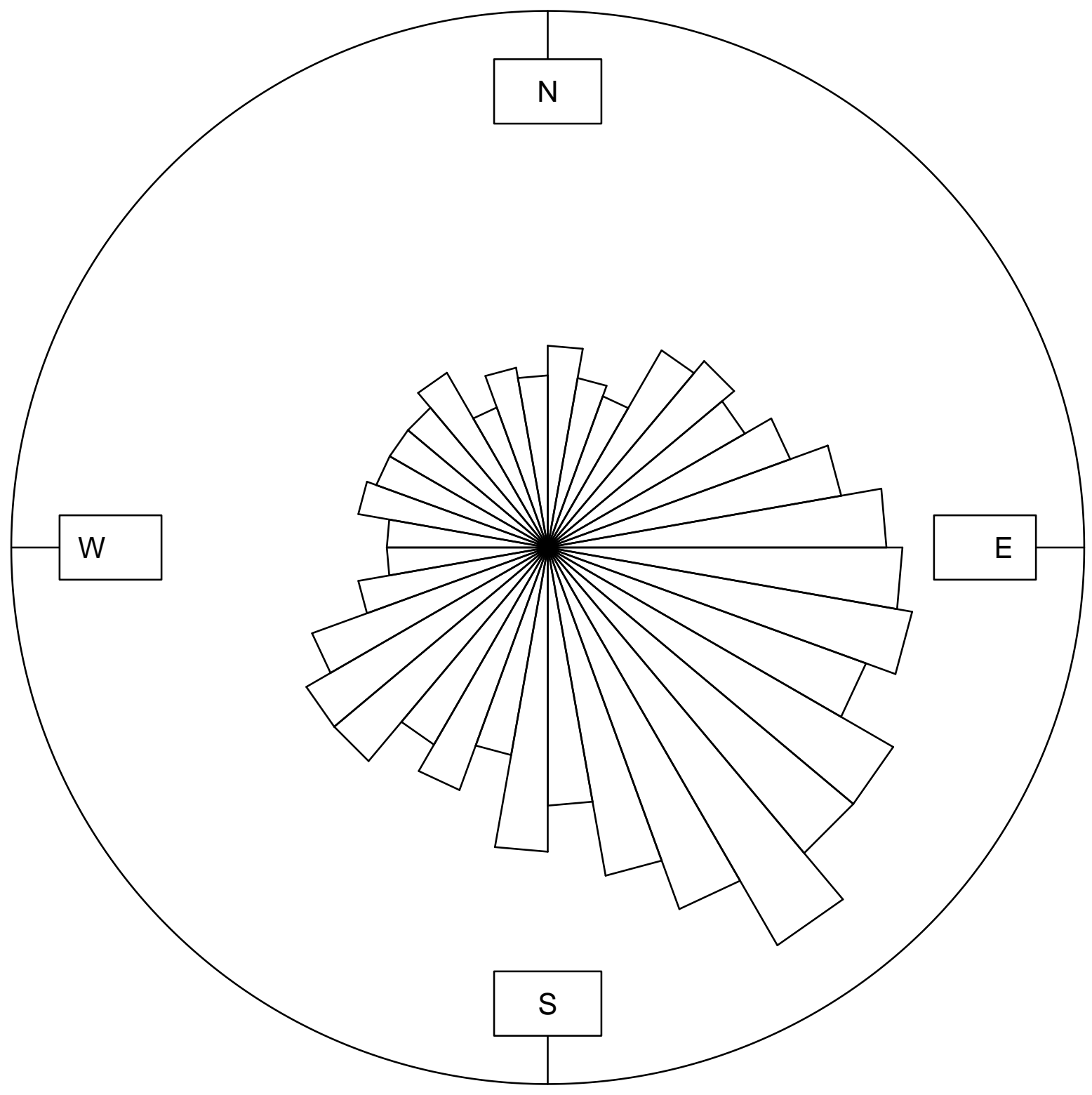


bootstrap 966

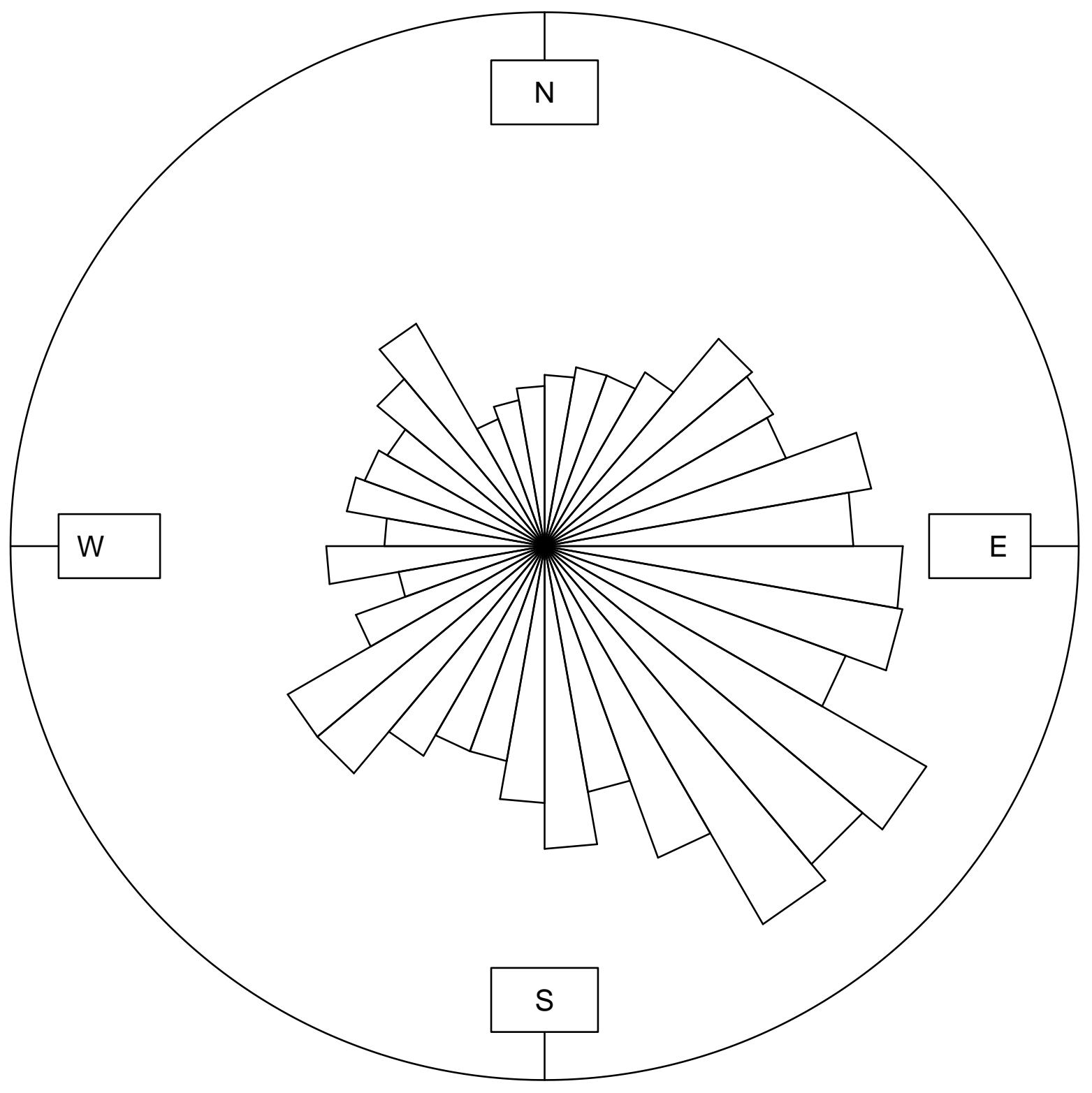


bootstrap 967

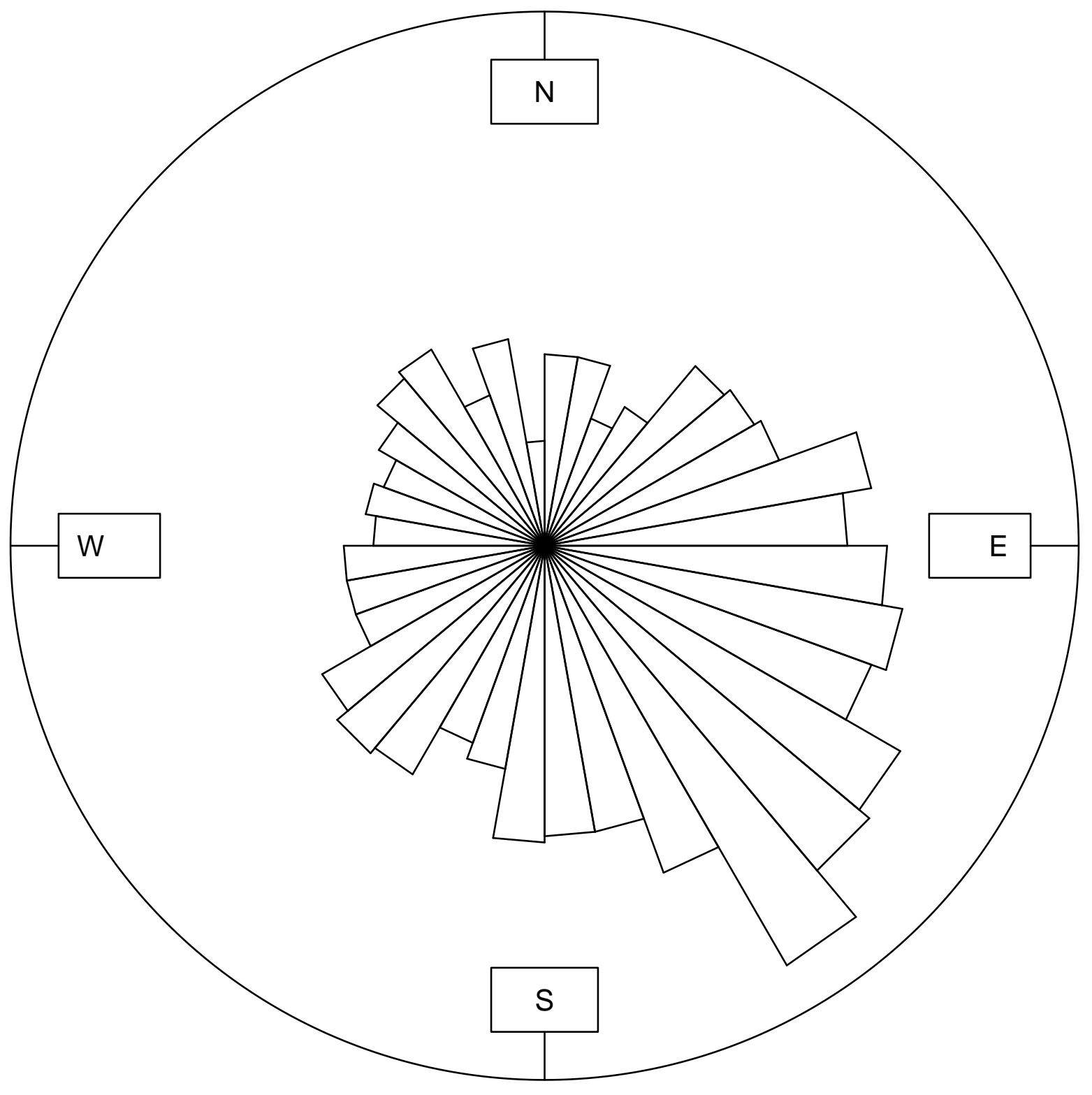


bootstrap 968

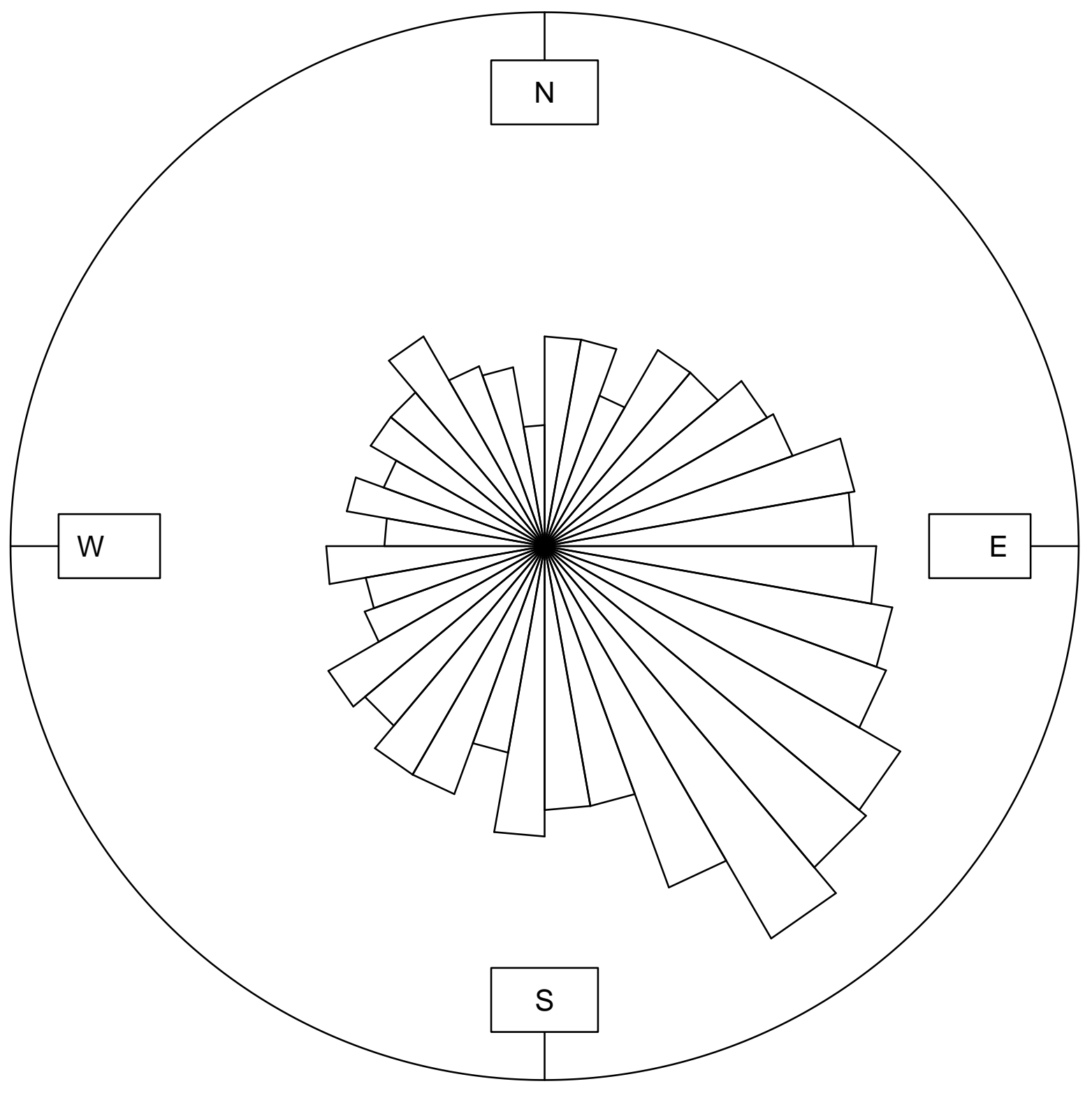


bootstrap 969

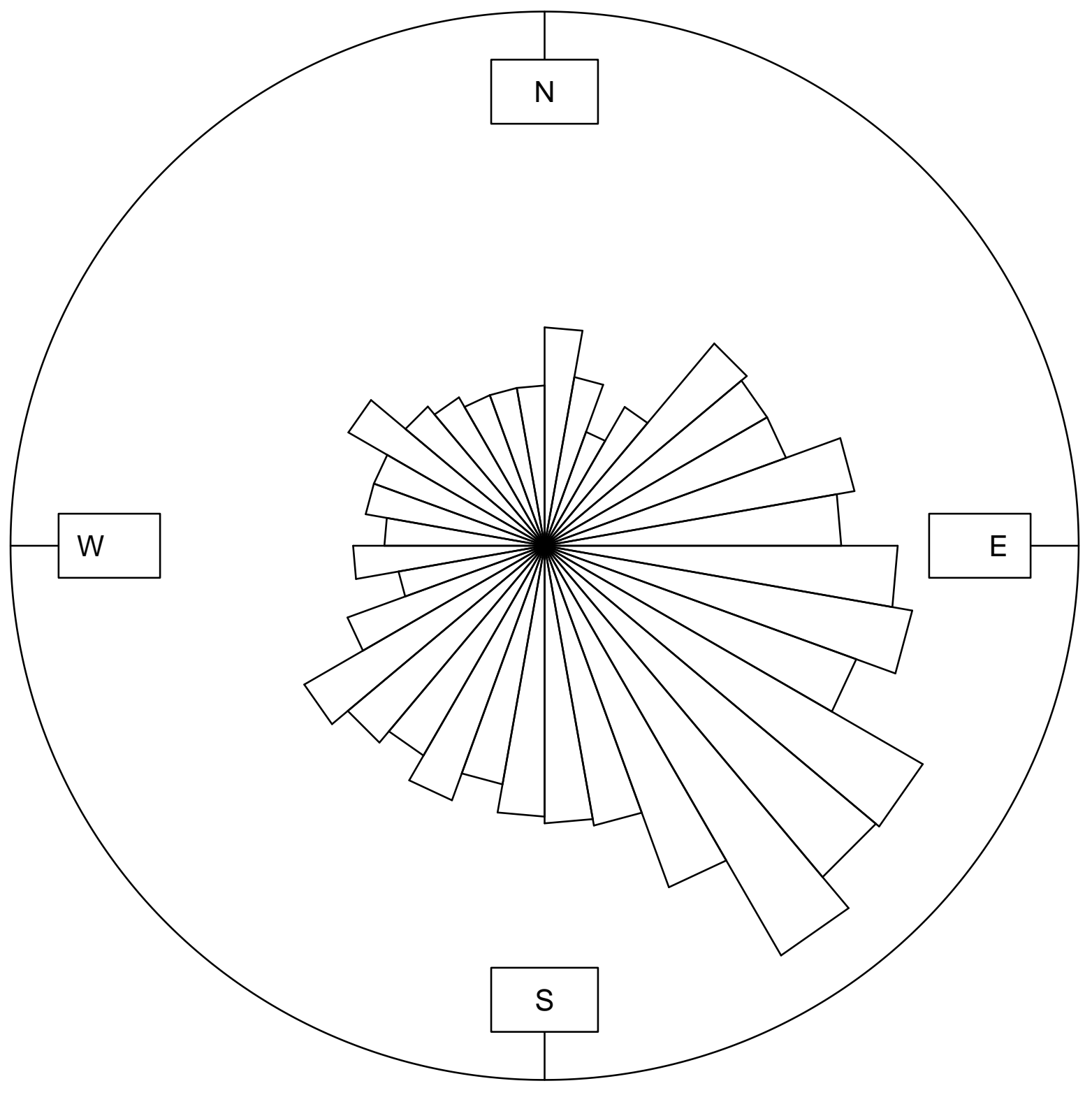


bootstrap 970

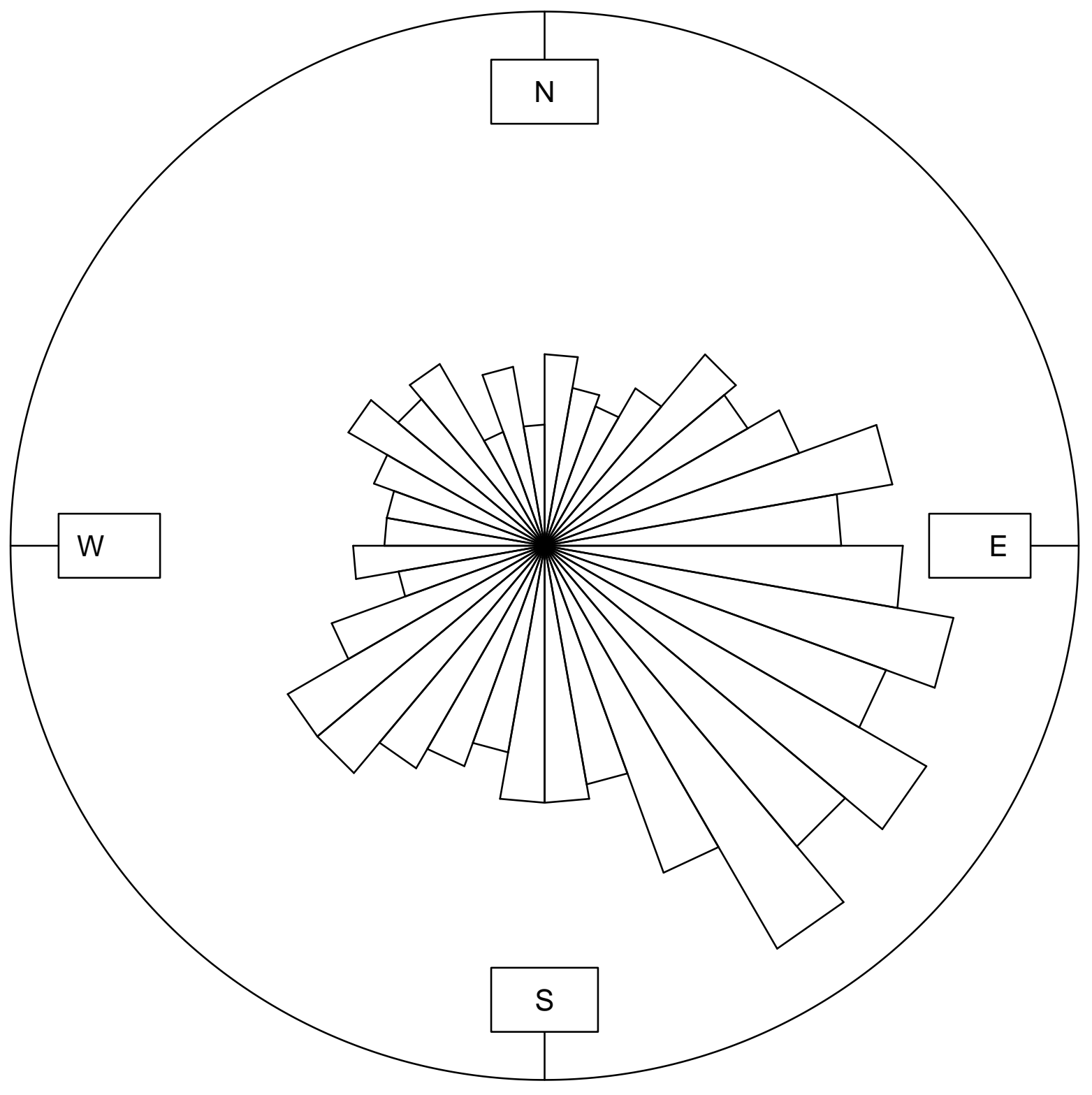


bootstrap 971

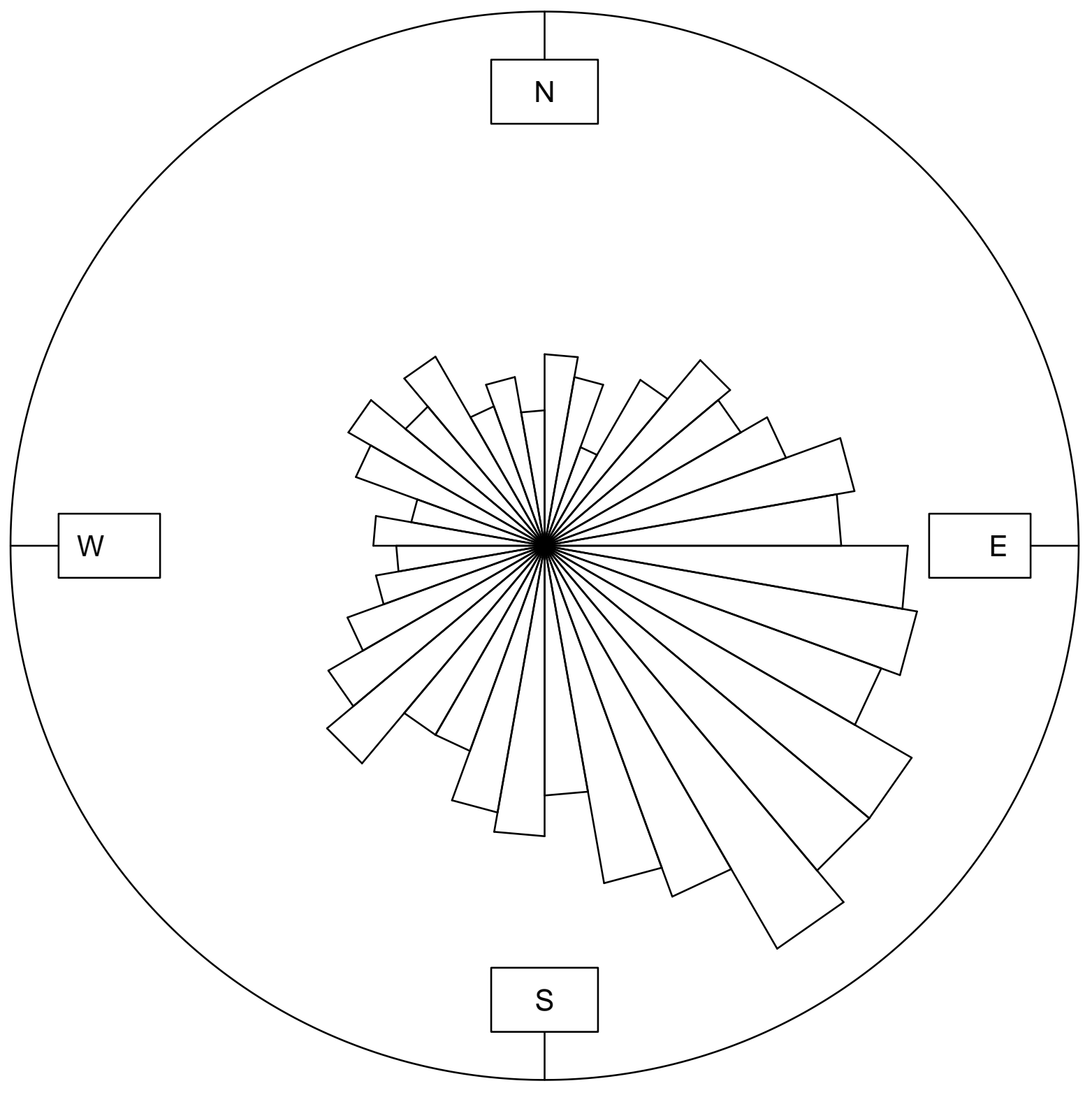


bootstrap 972

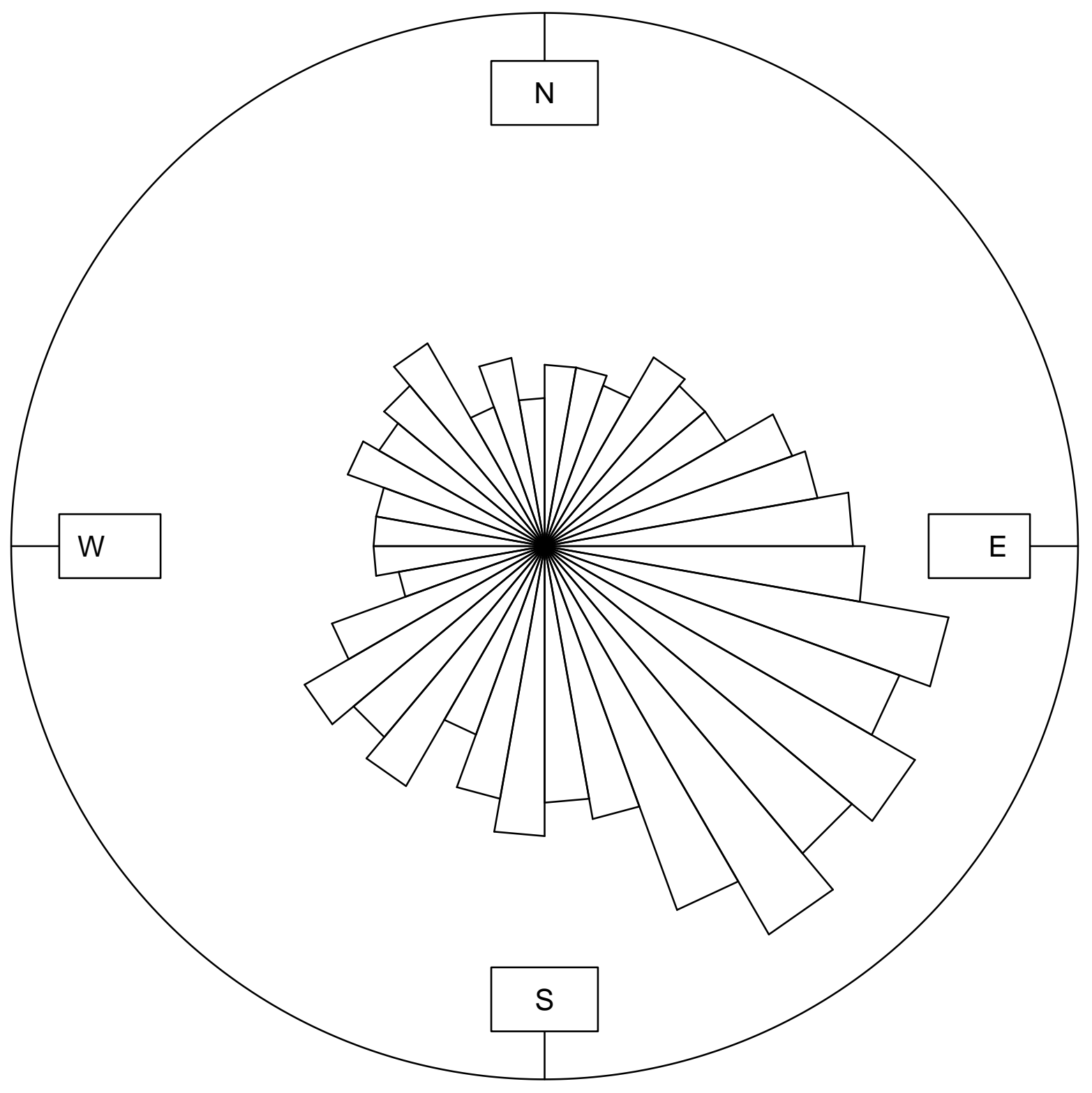


bootstrap 973

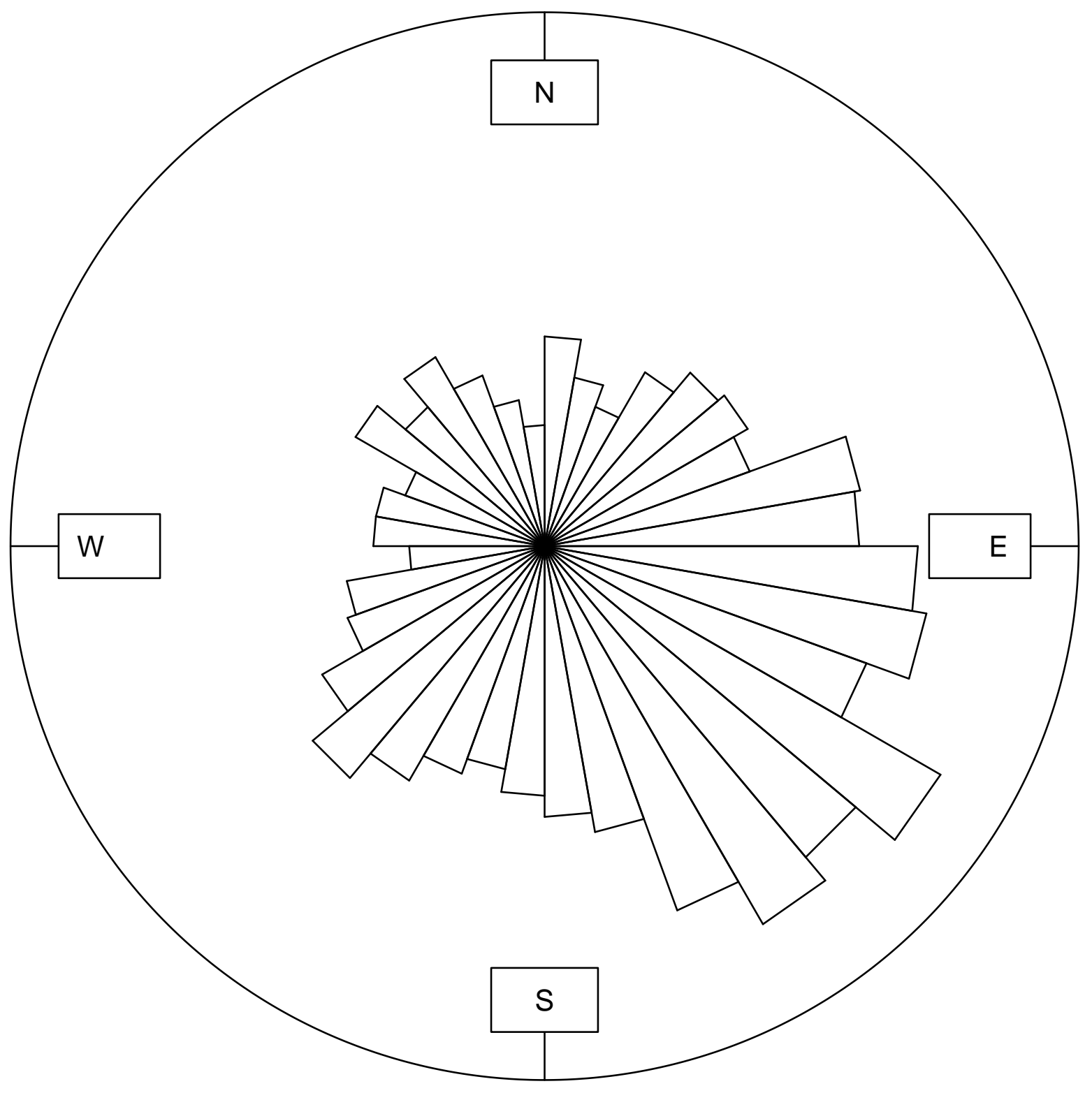




\section{bootstrap 974}

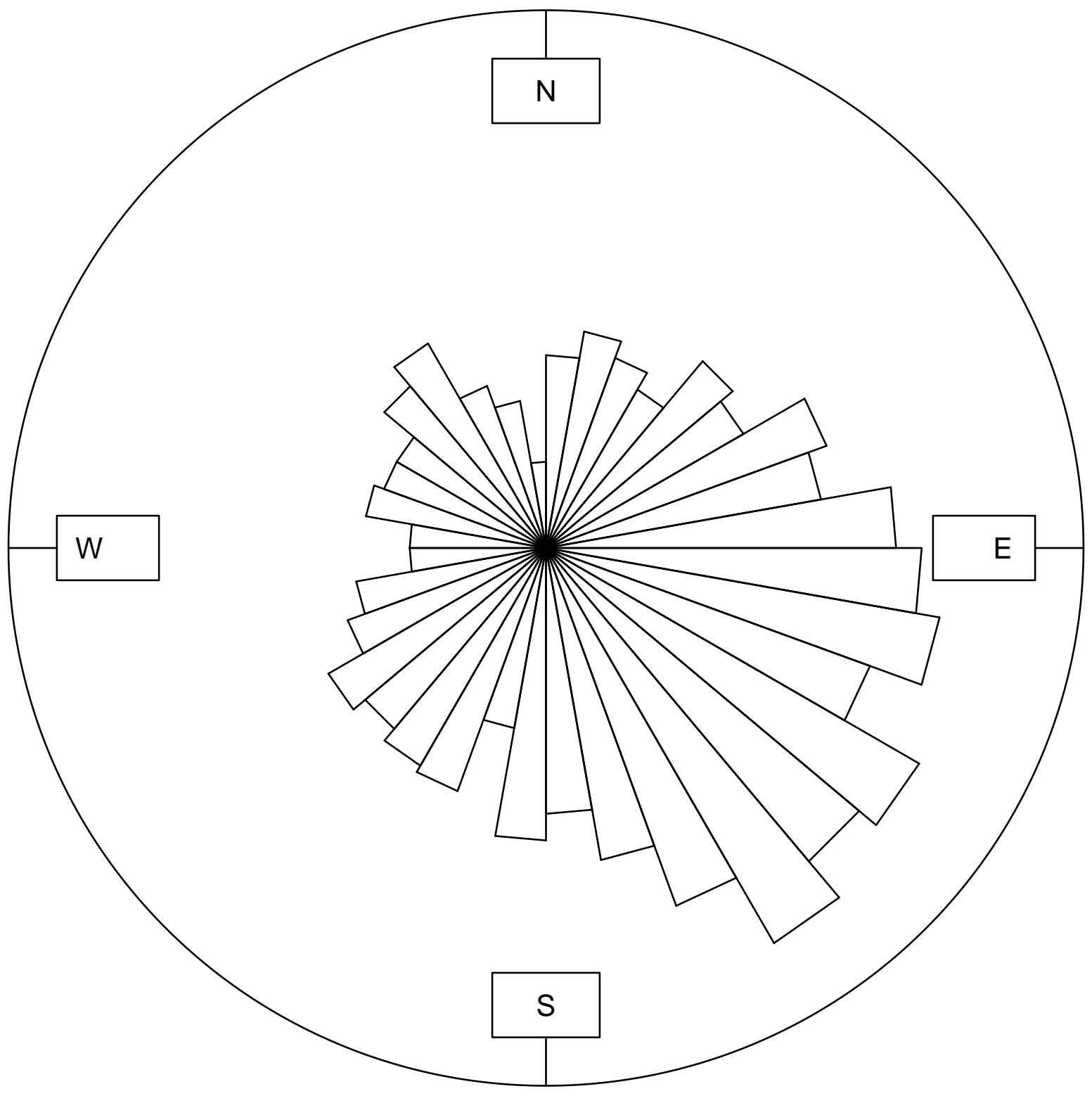


bootstrap 976

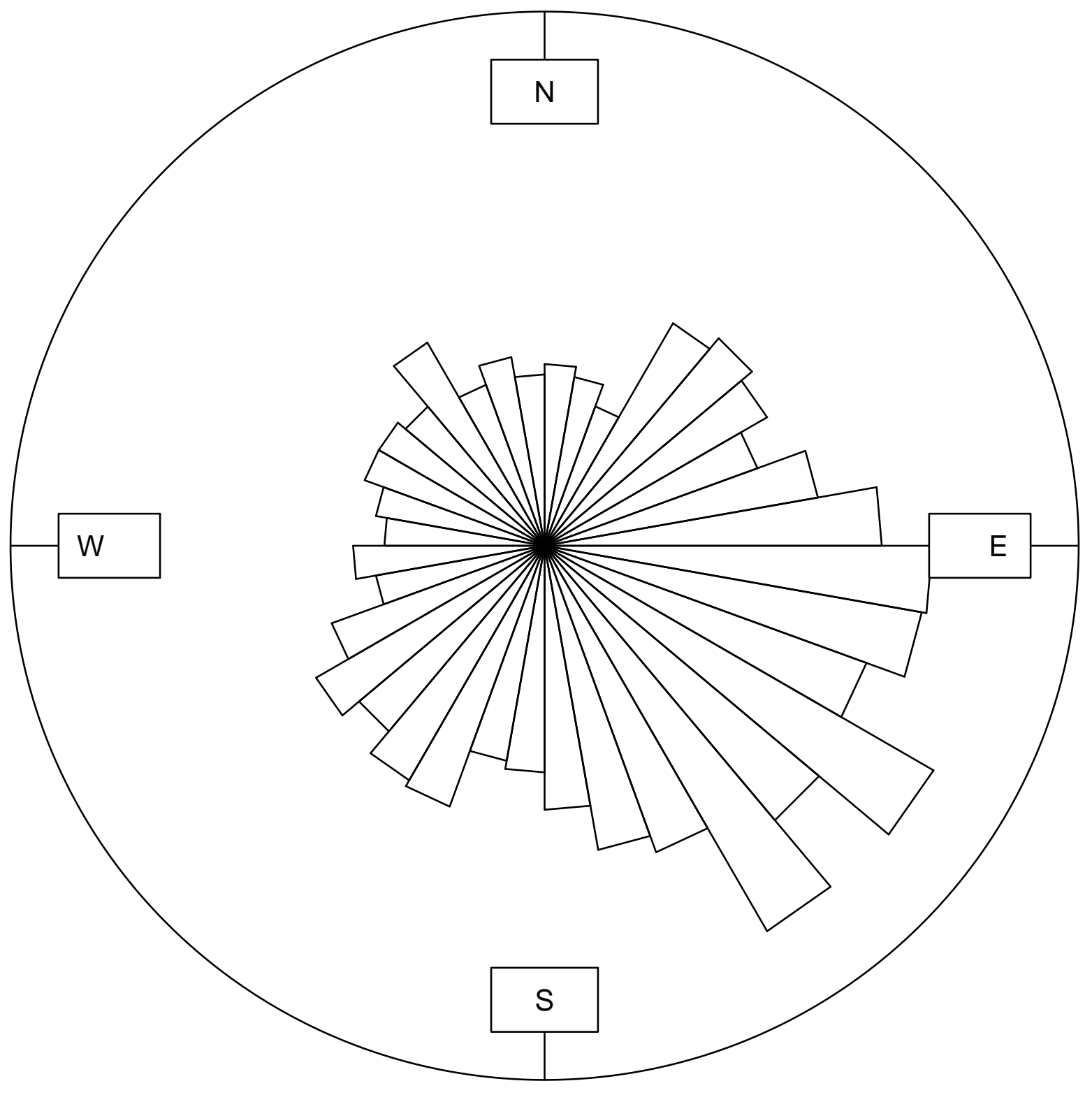


bootstrap 977

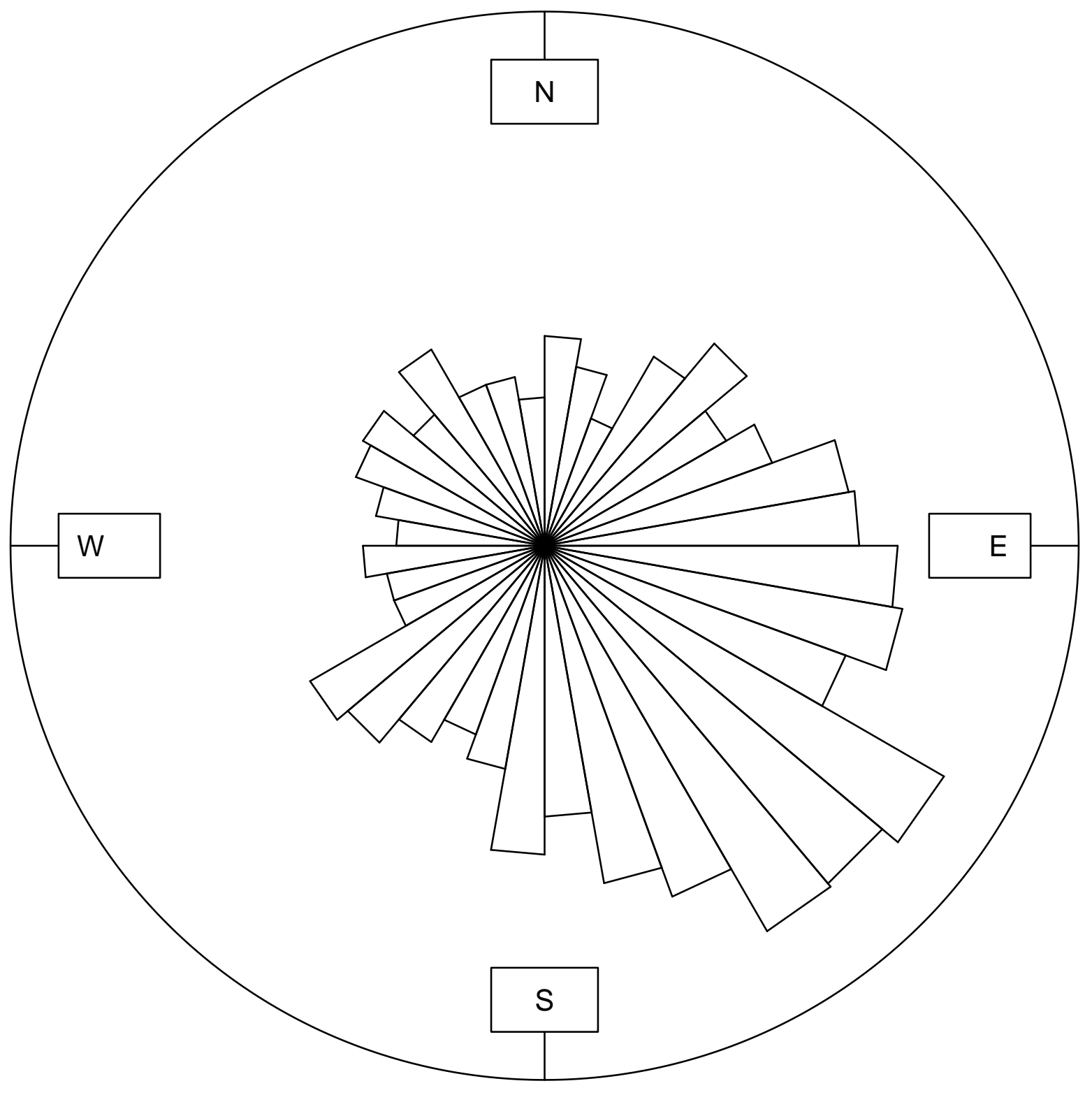


bootstrap 978

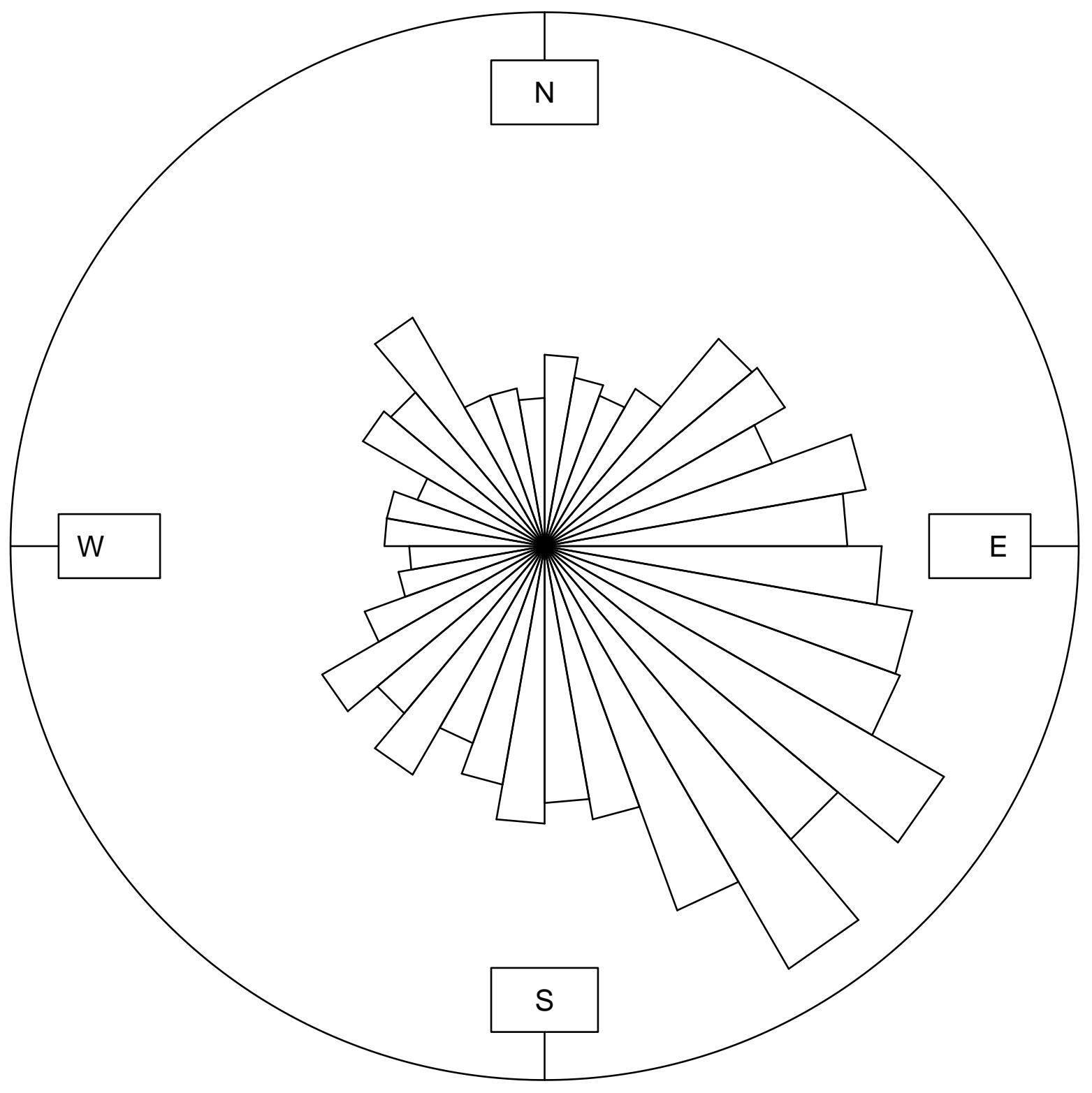


bootstrap 979

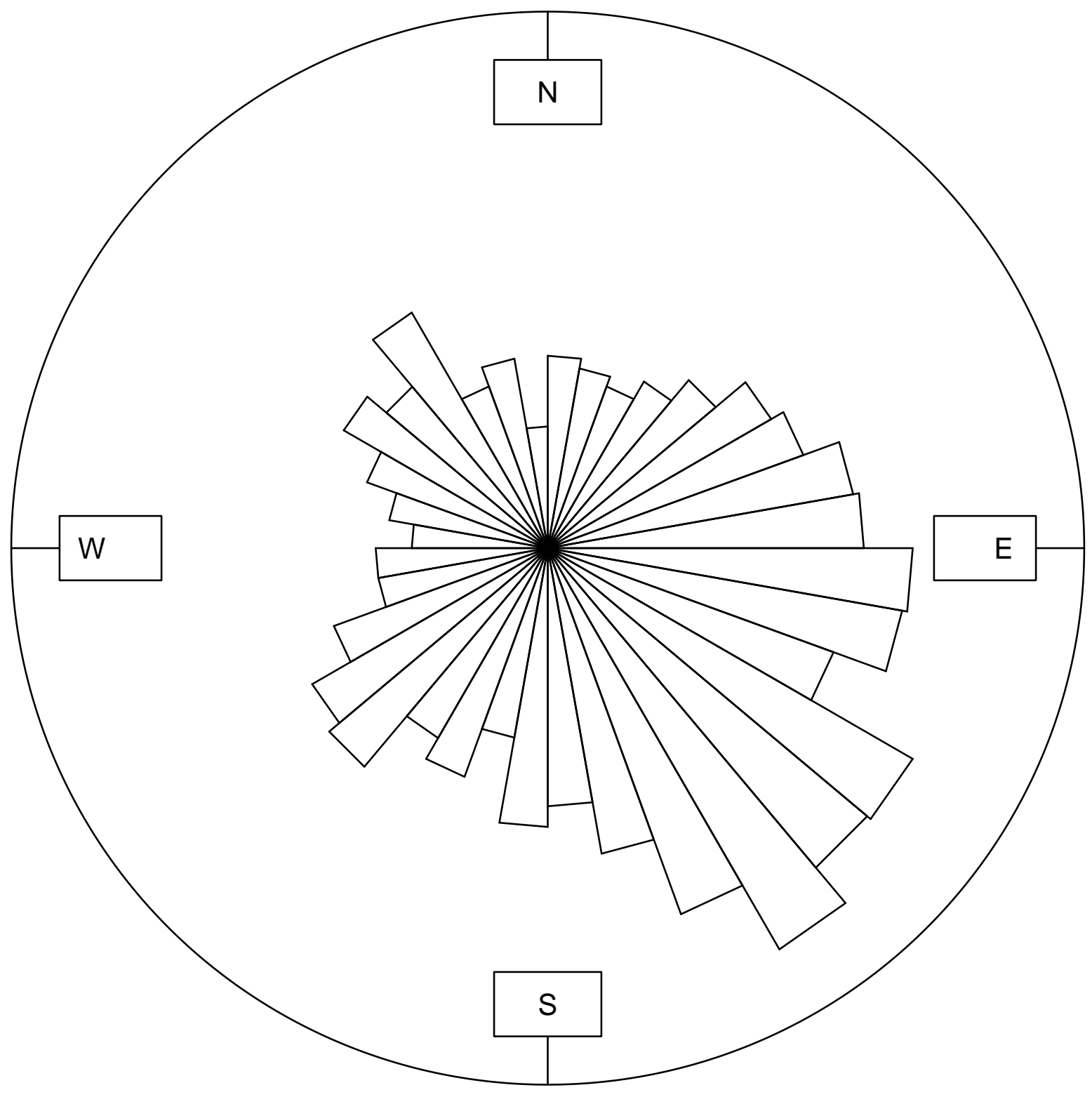


bootstrap 980

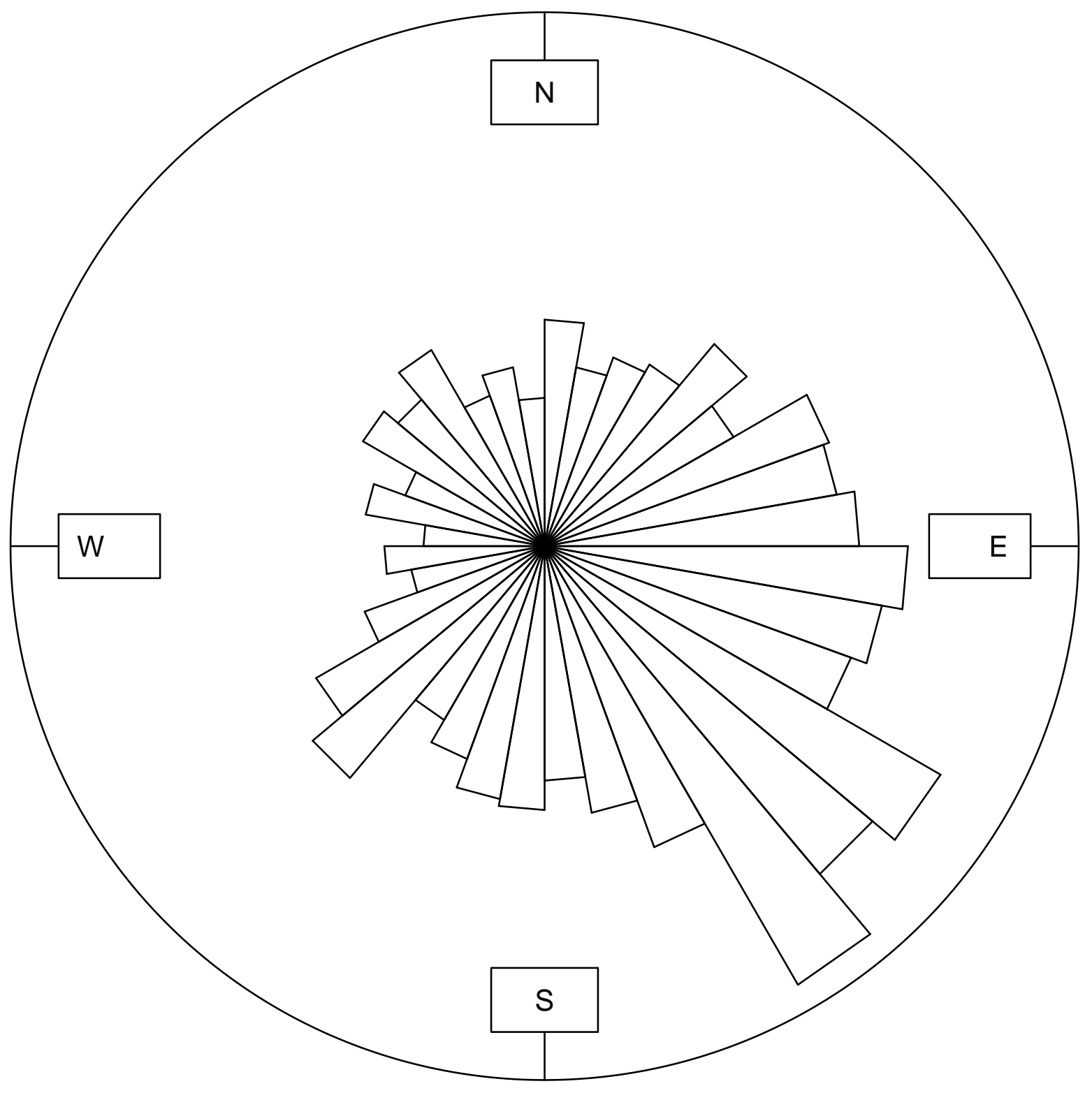


bootstrap 981

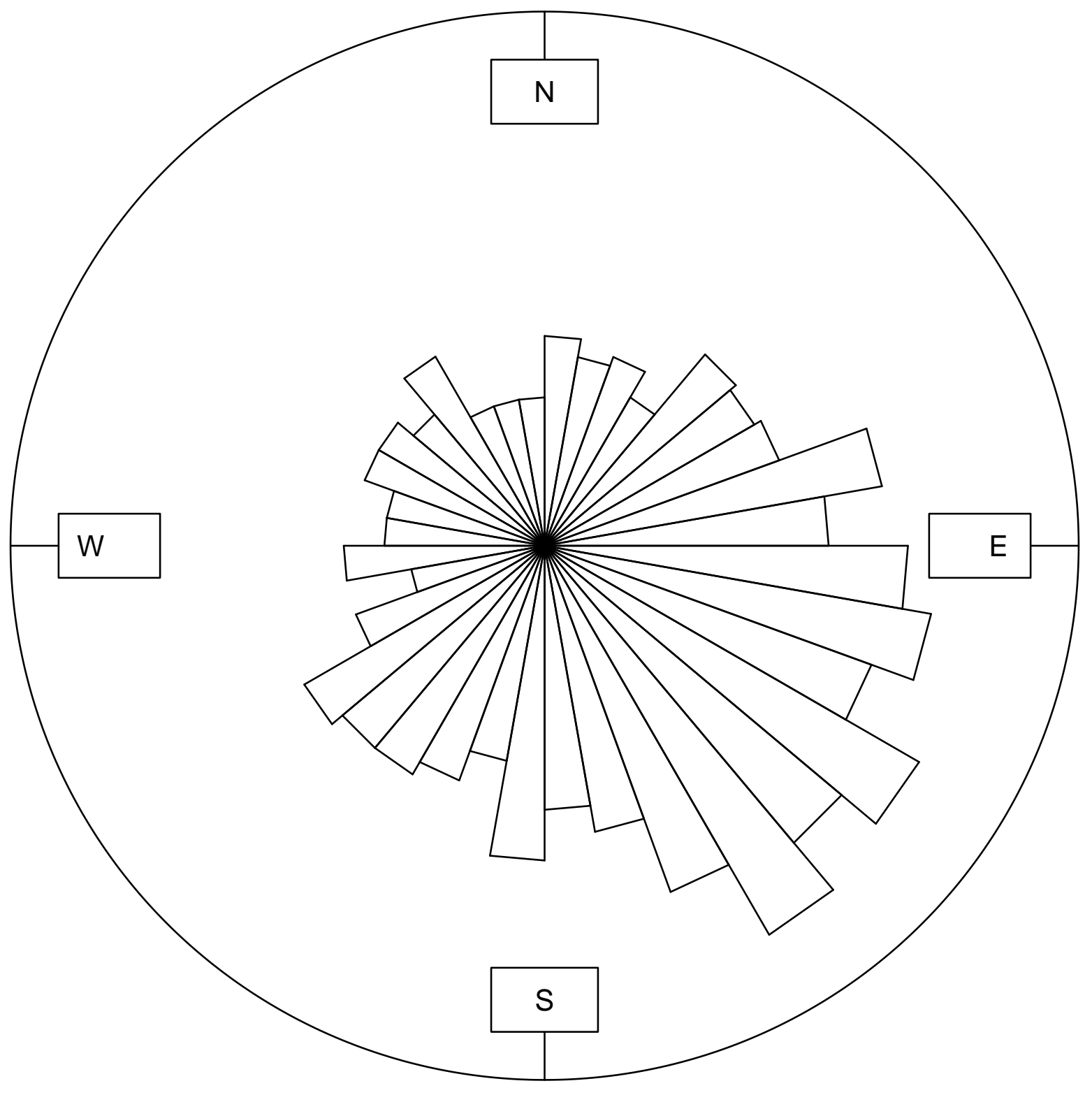


bootstrap 982

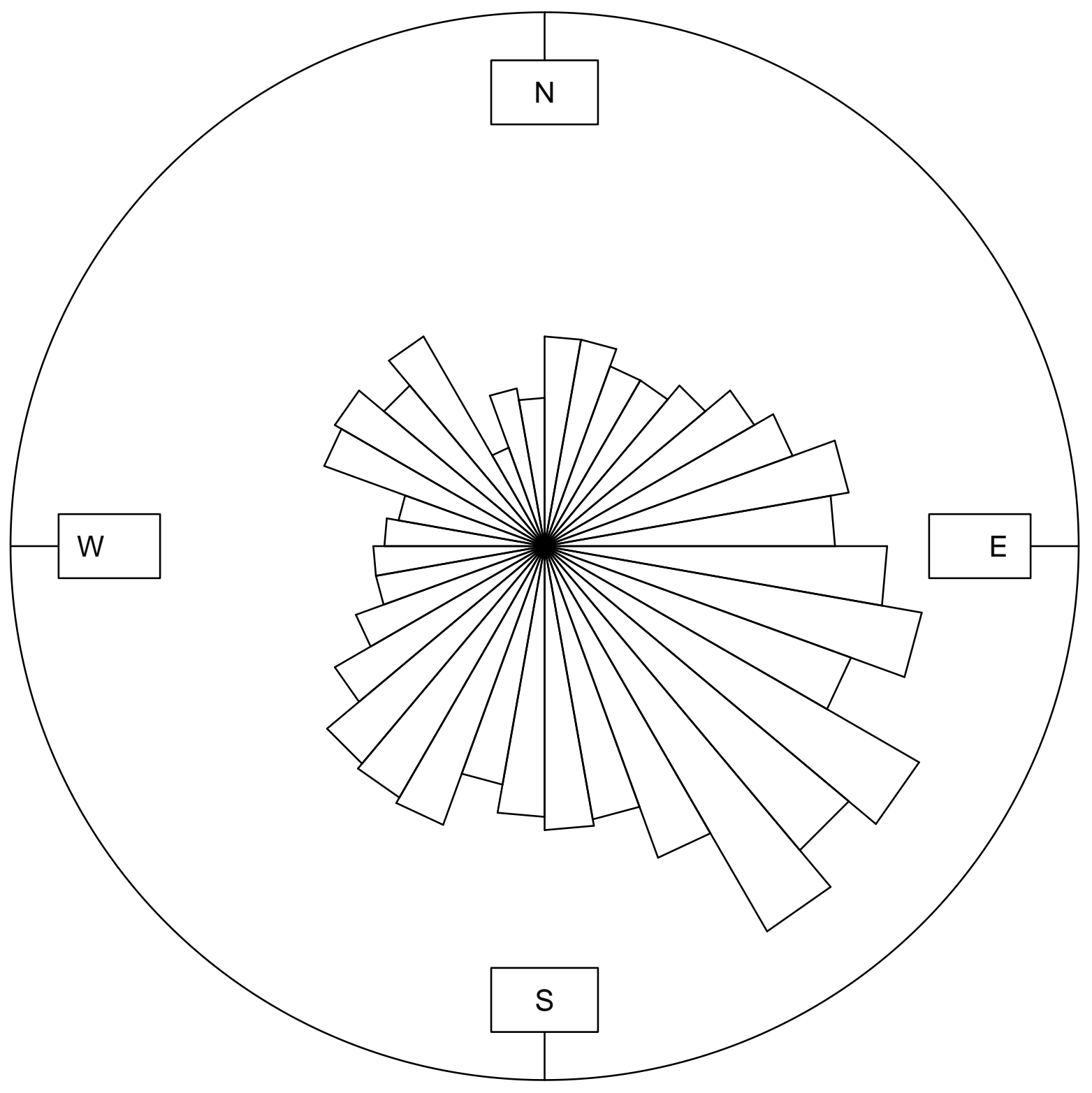


bootstrap 983

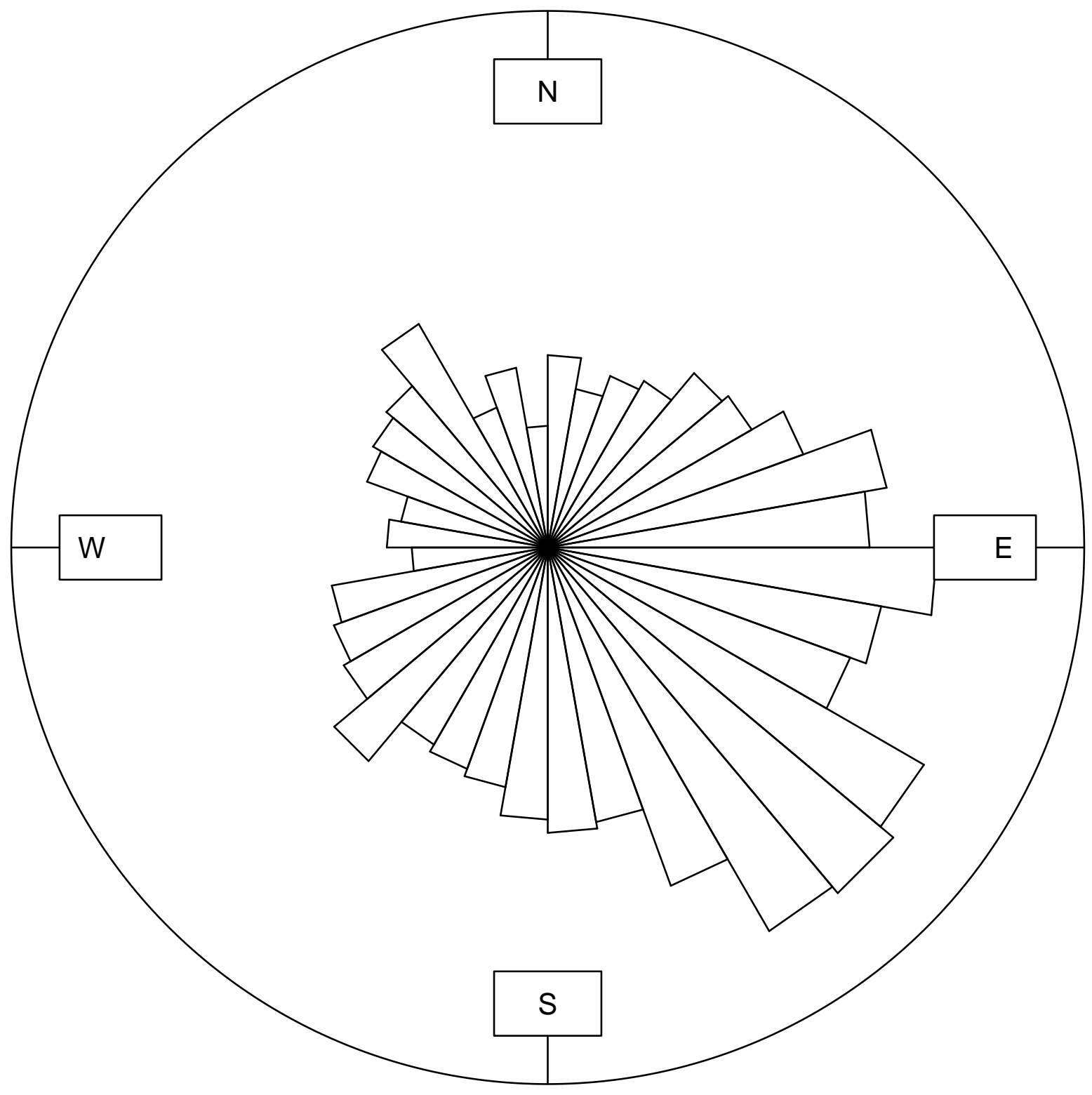




\section{bootstrap 984}

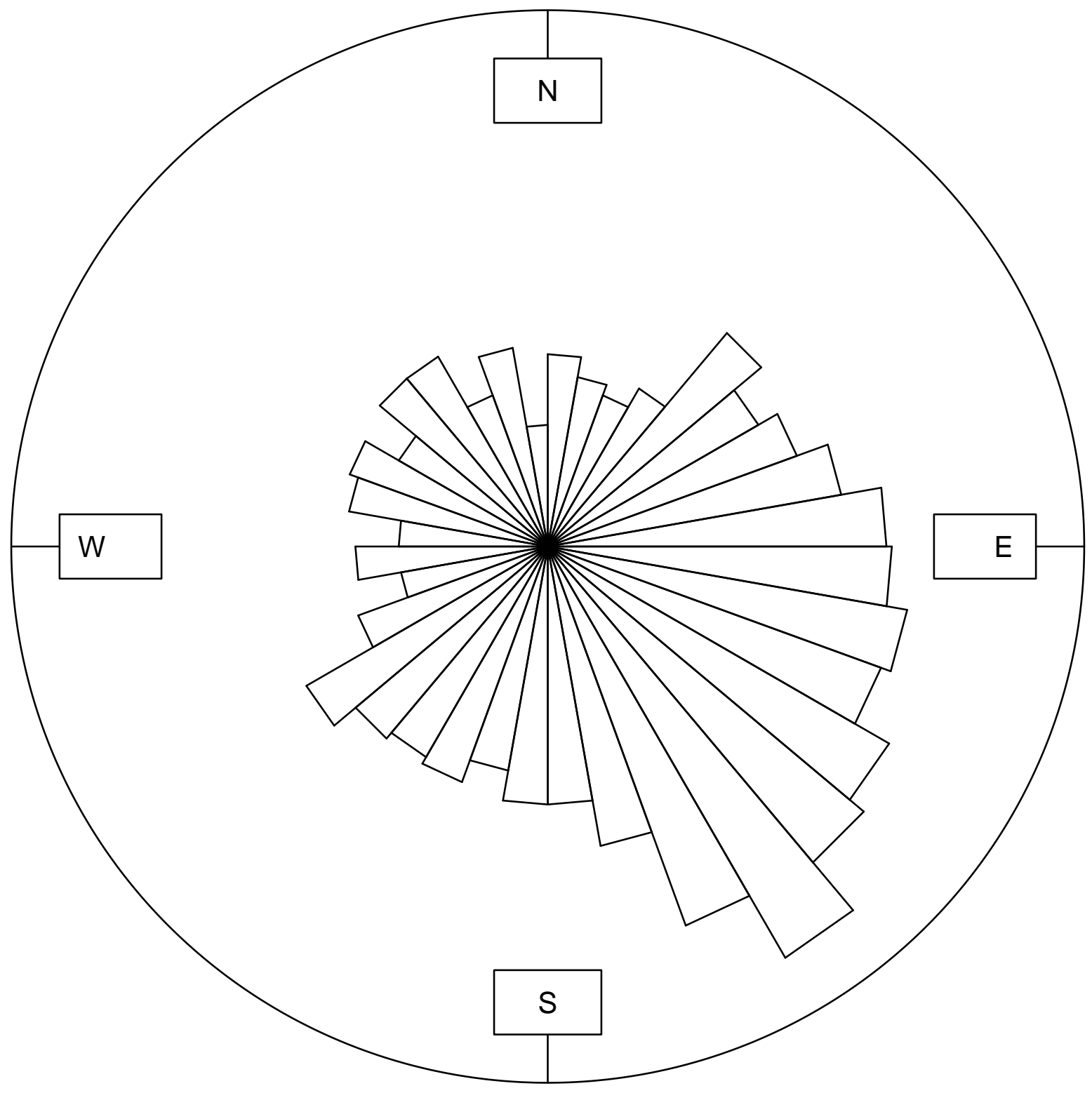




\section{bootstrap 985}

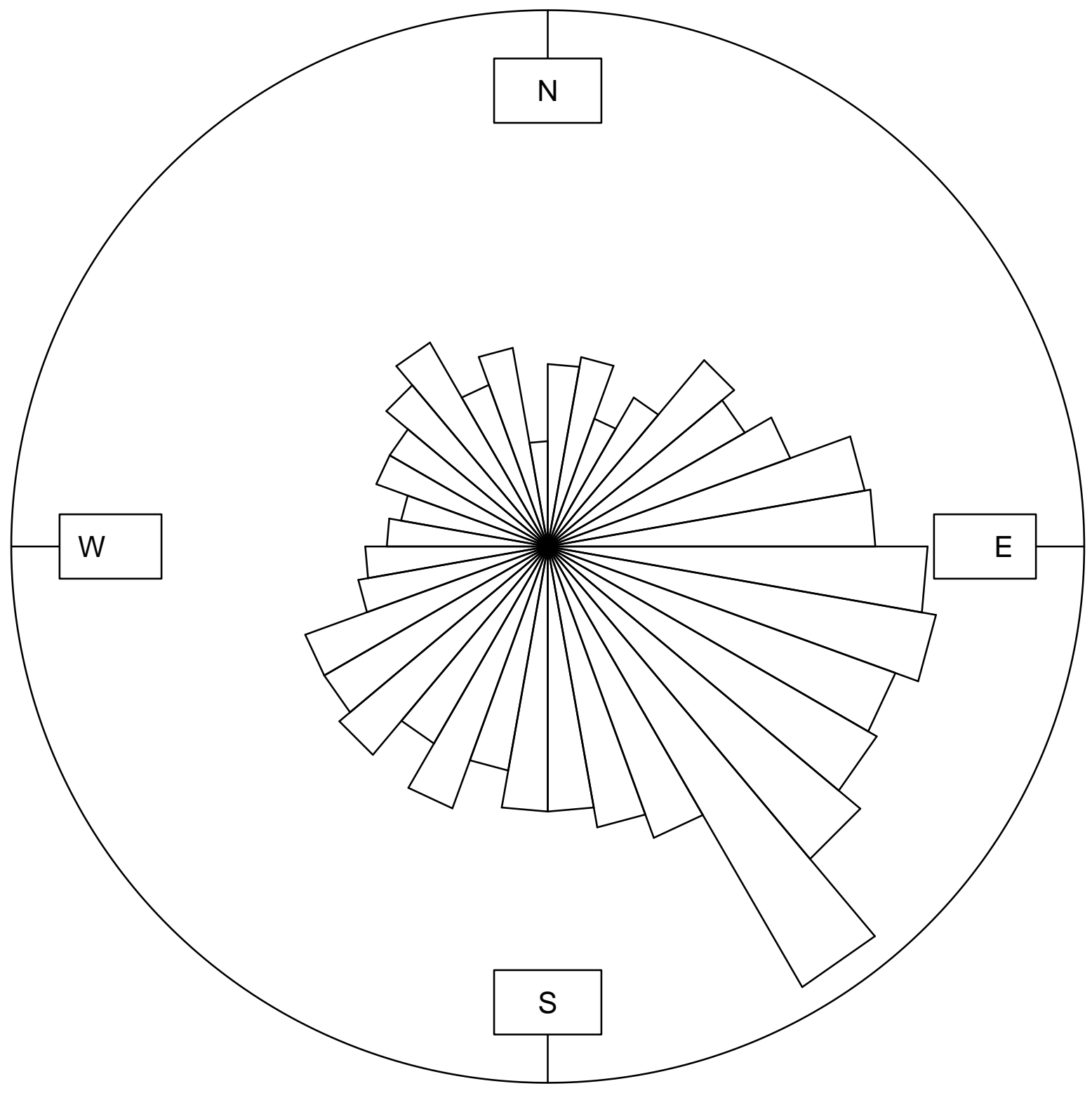


bootstrap 986

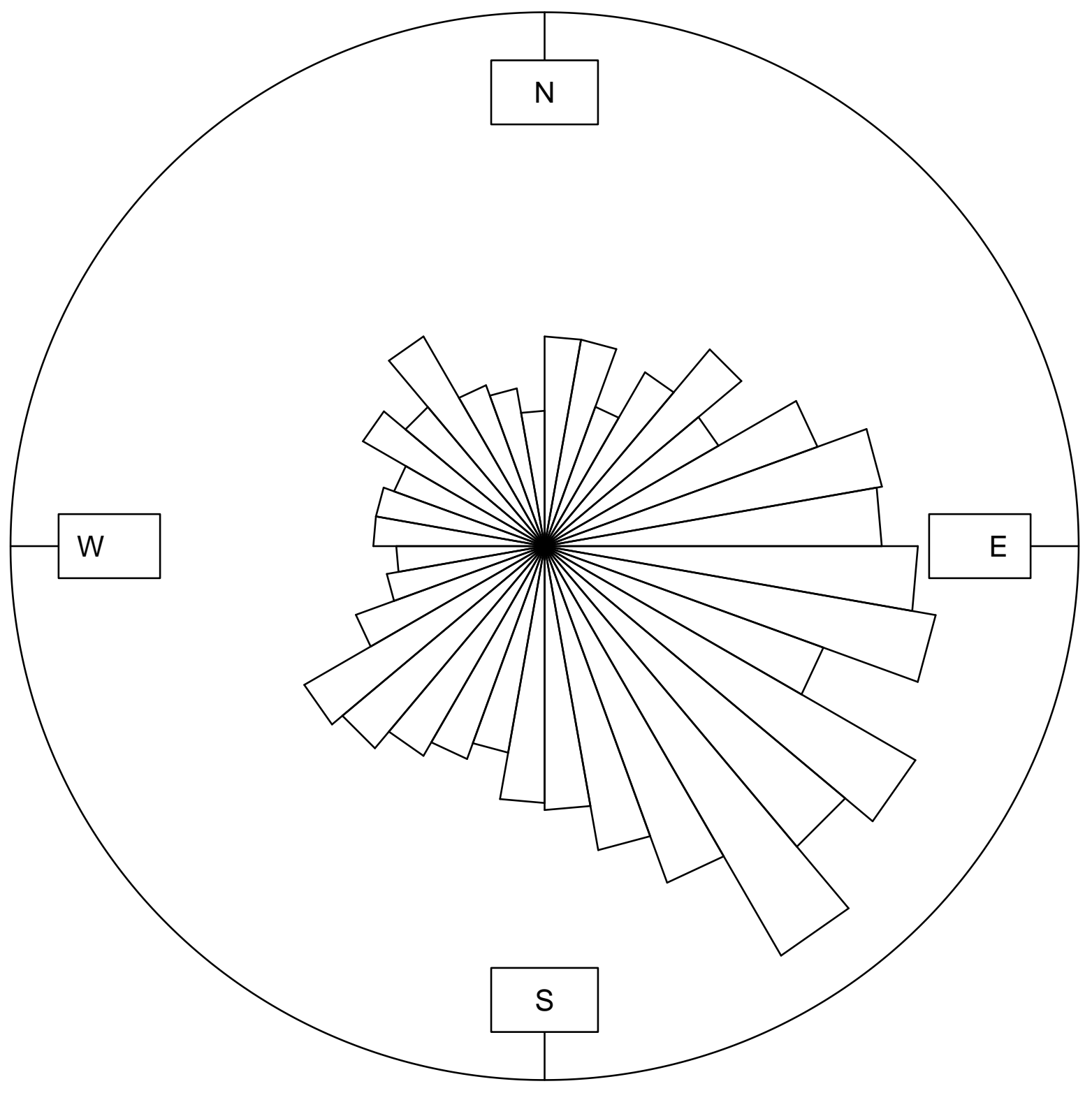


bootstrap 987

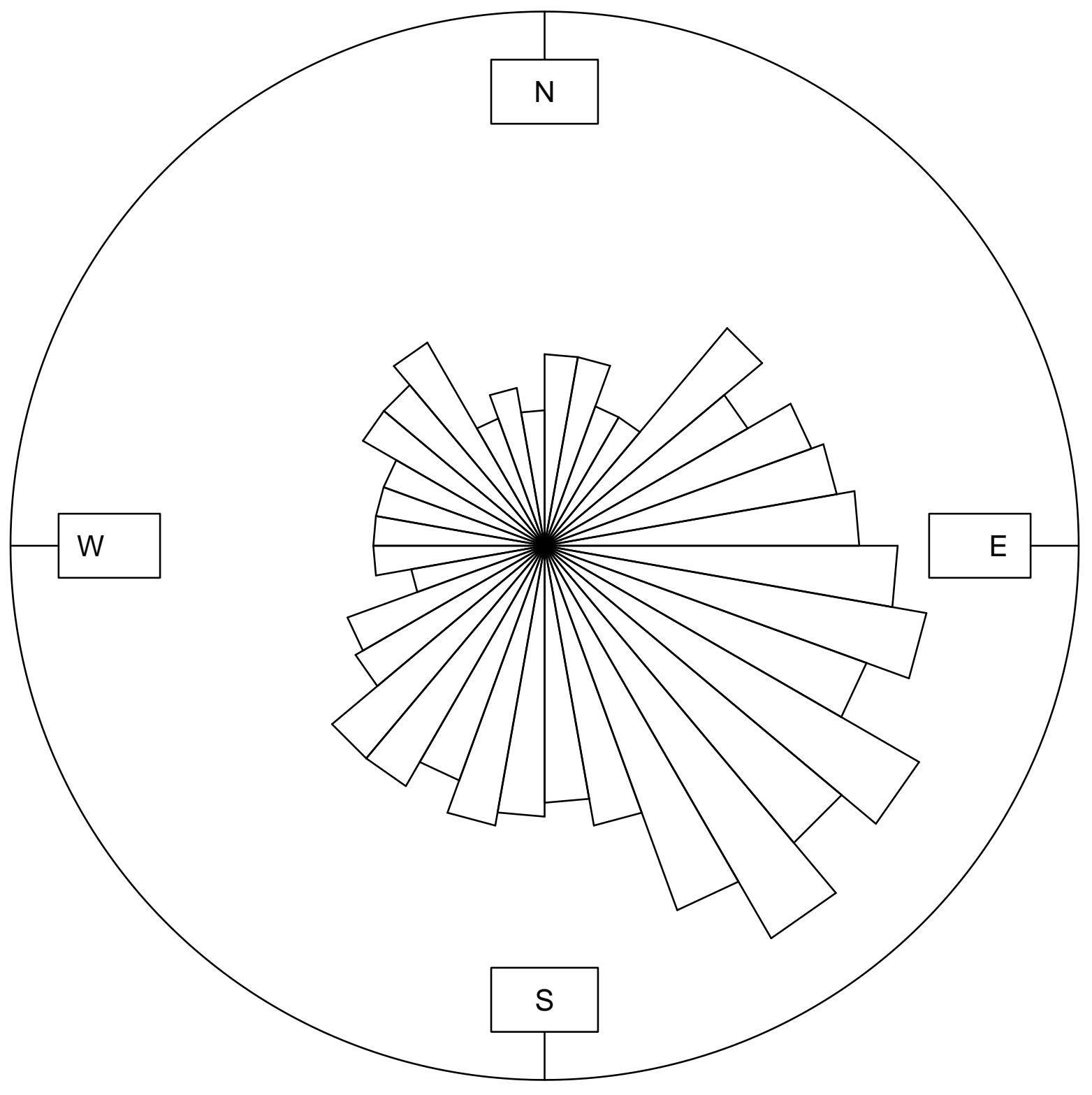


bootstrap 988

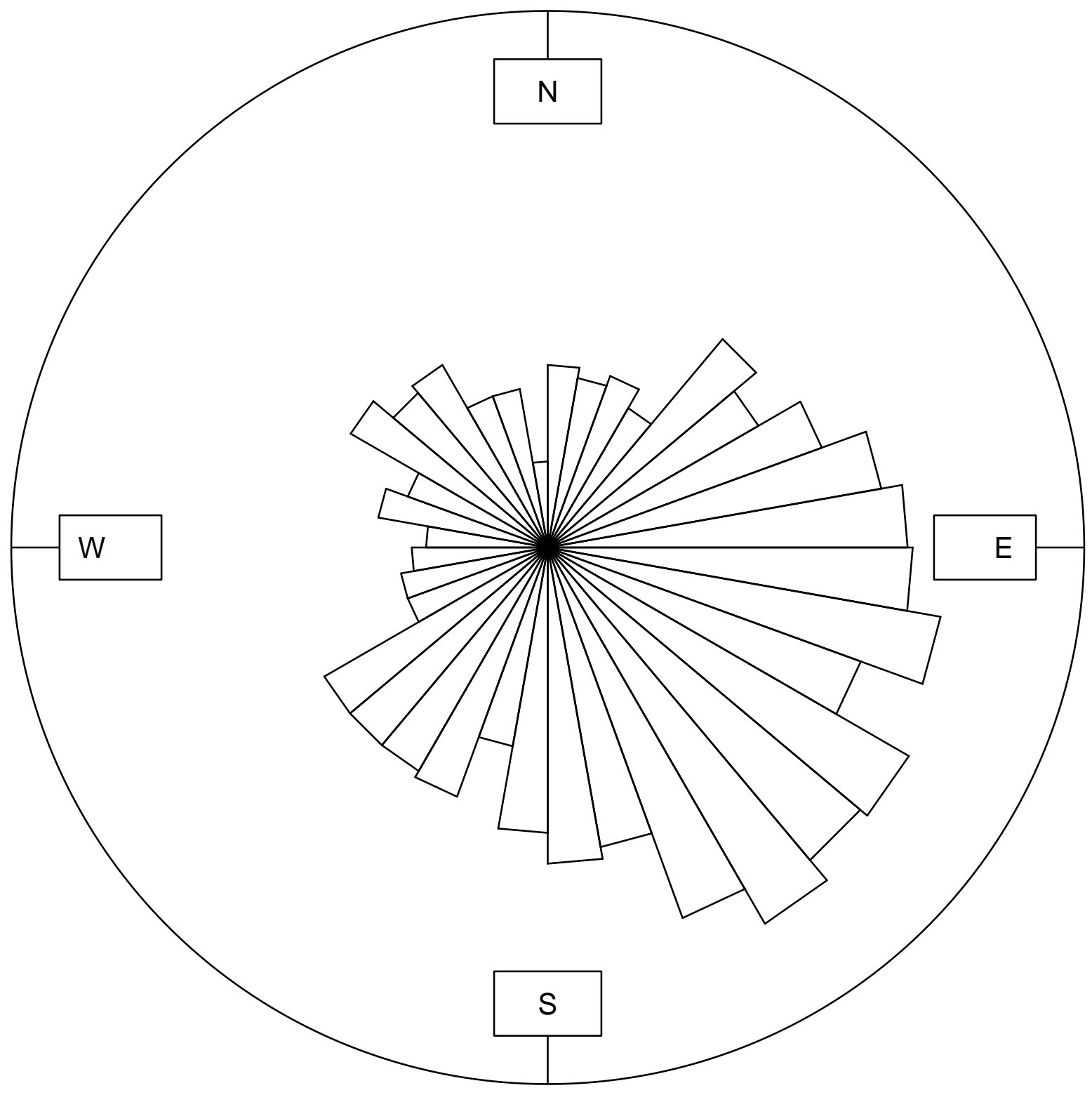


bootstrap 989

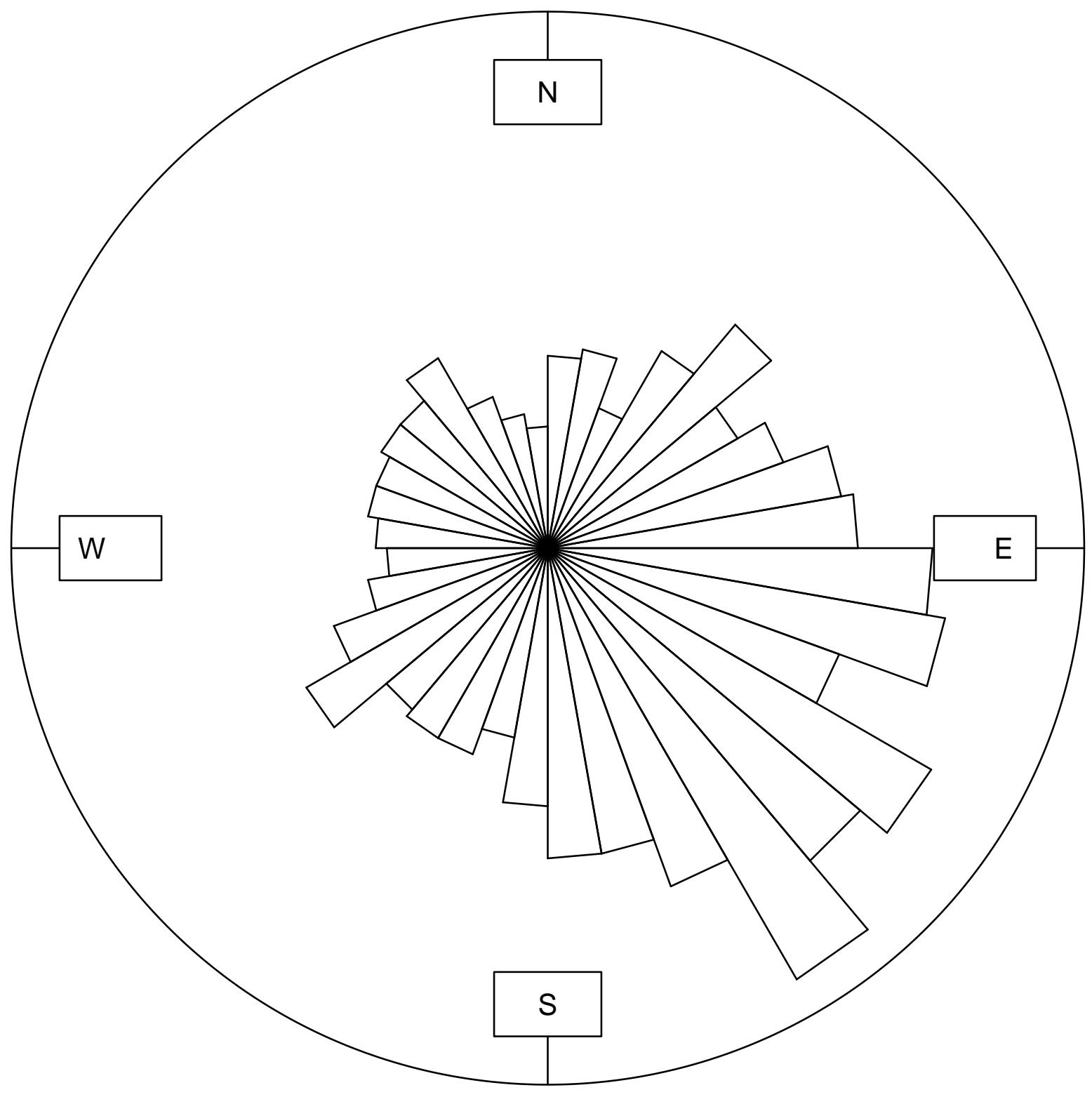


bootstrap 990

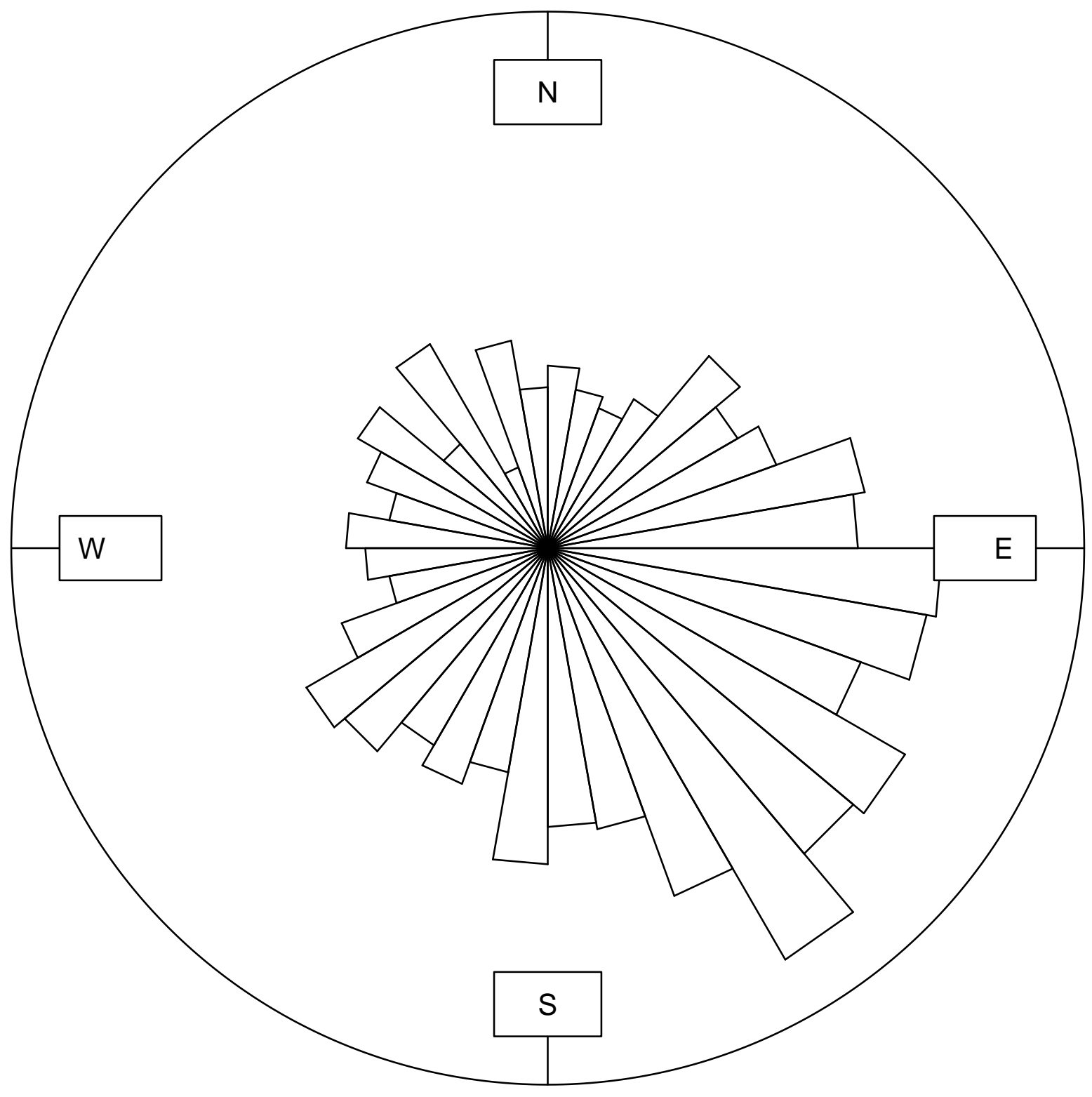


bootstrap 991

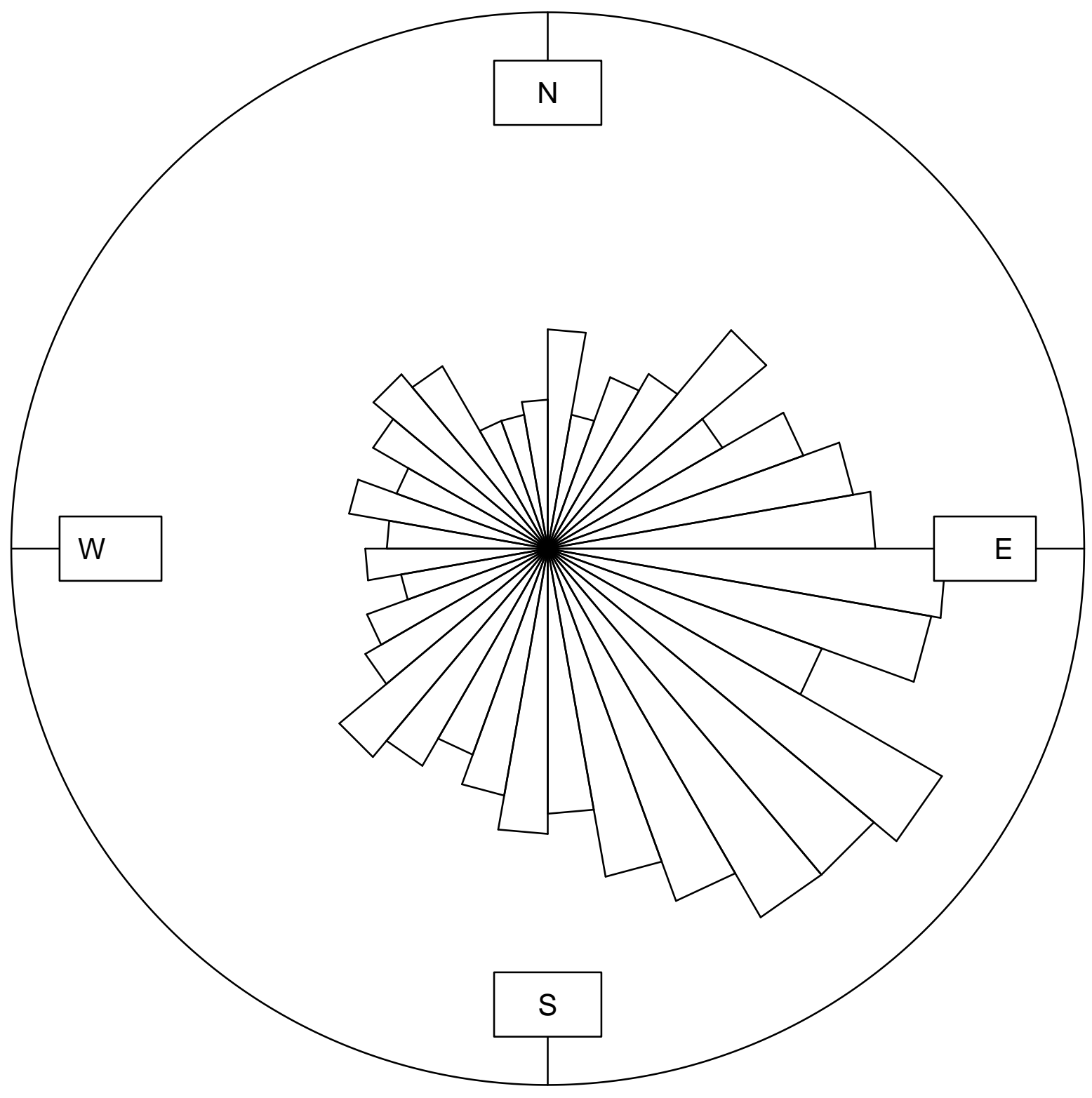


bootstrap 992

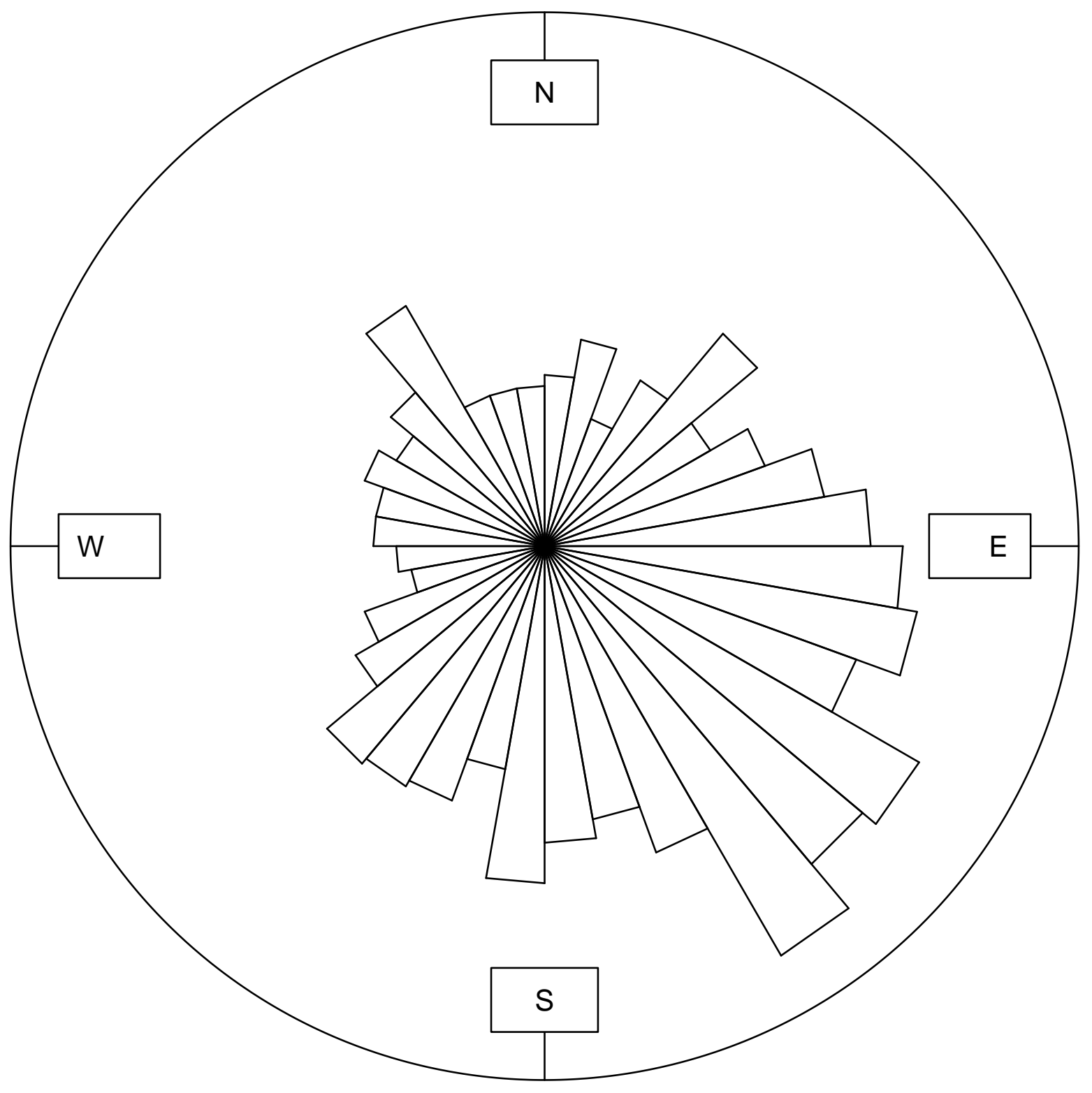




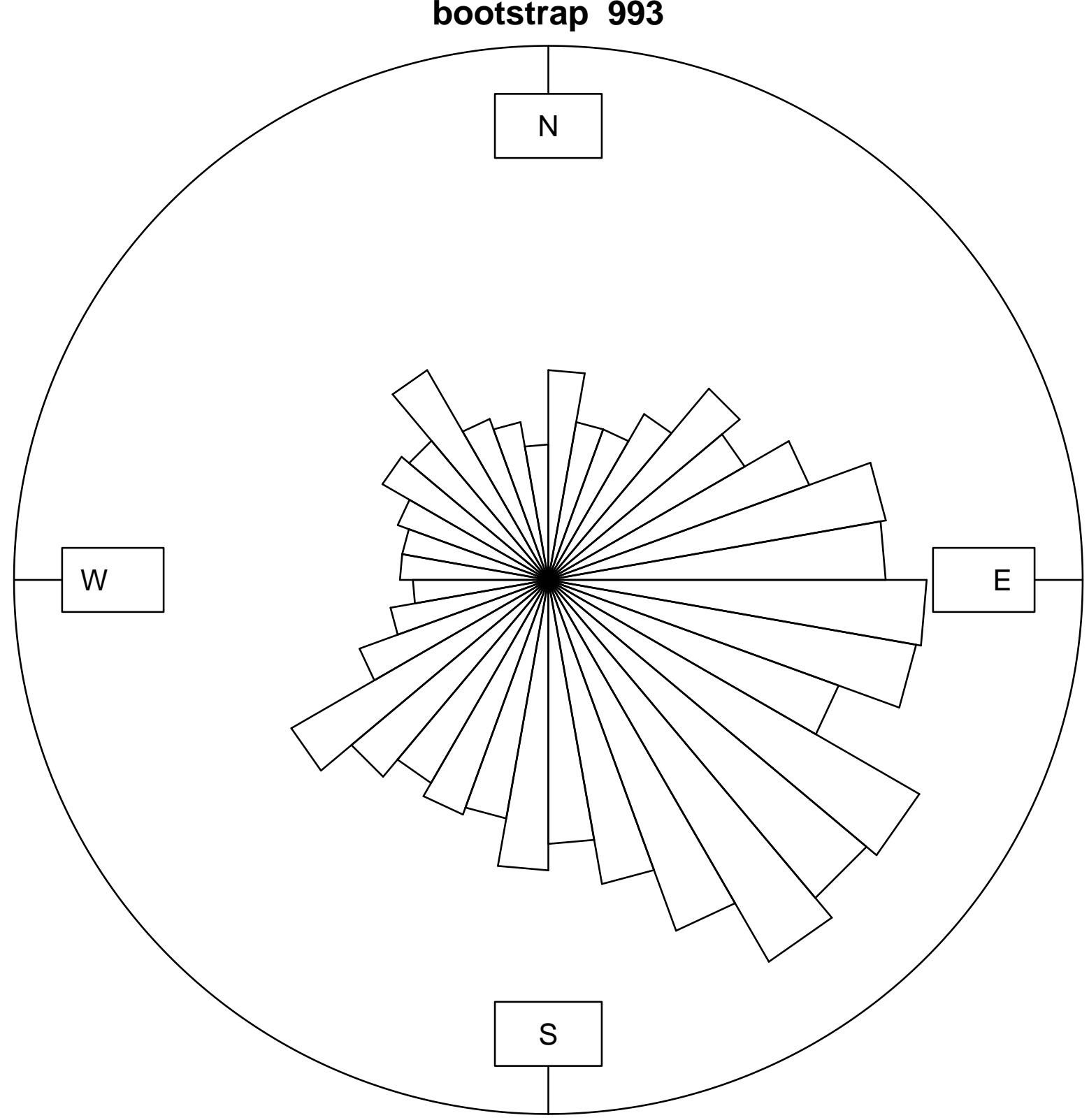




\section{bootstrap 994}

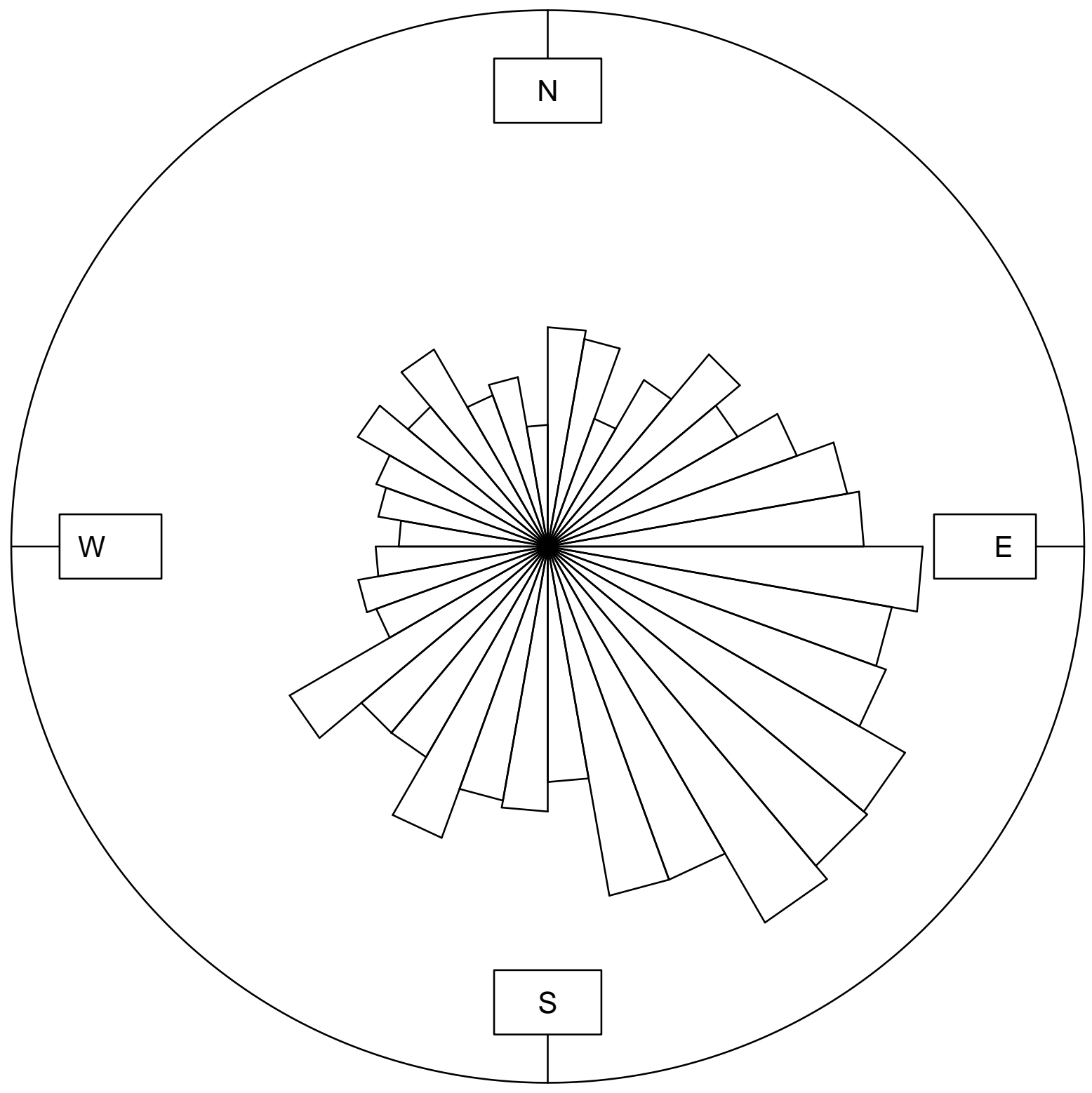


bootstrap 995

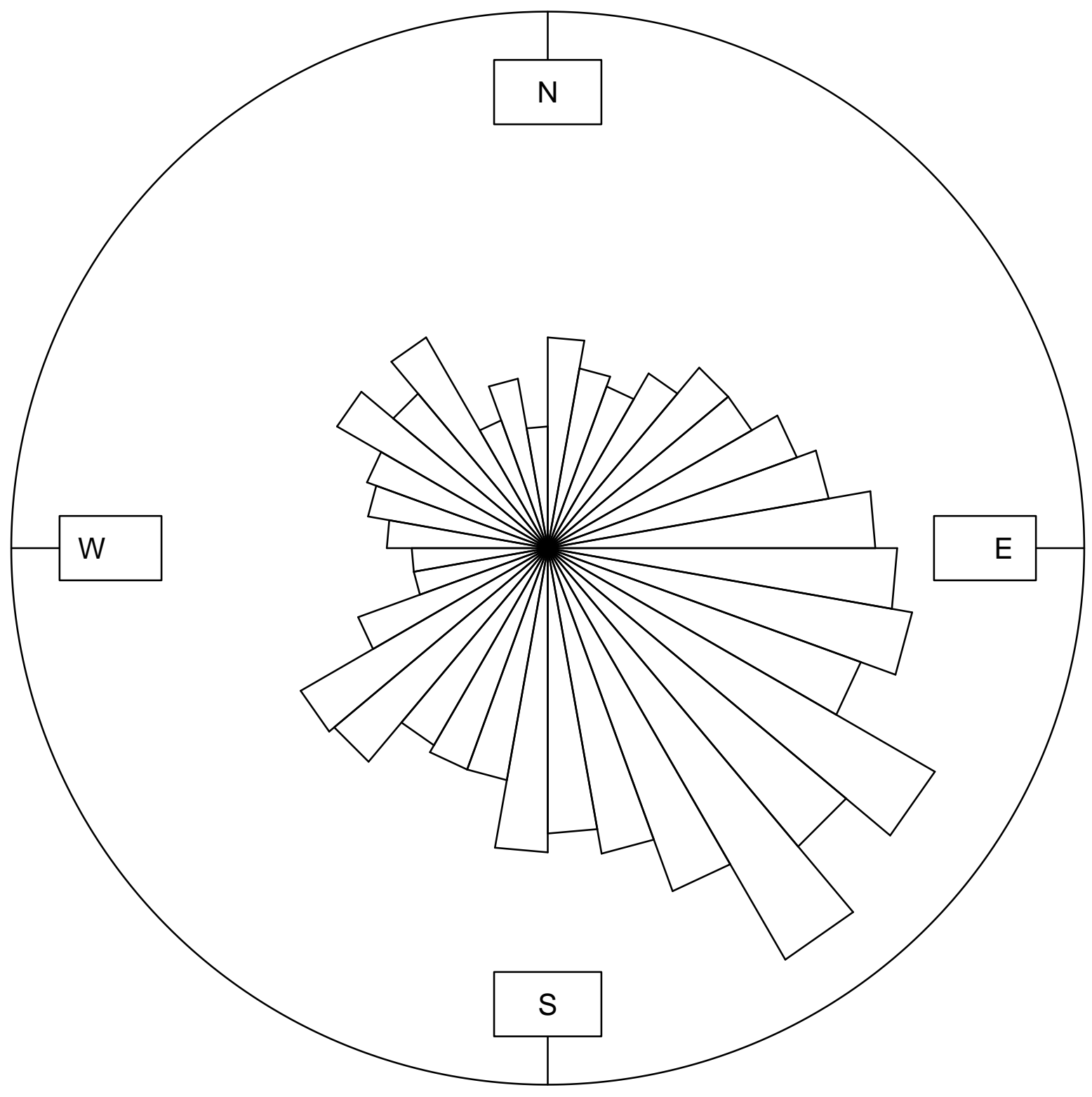


bootstrap 996

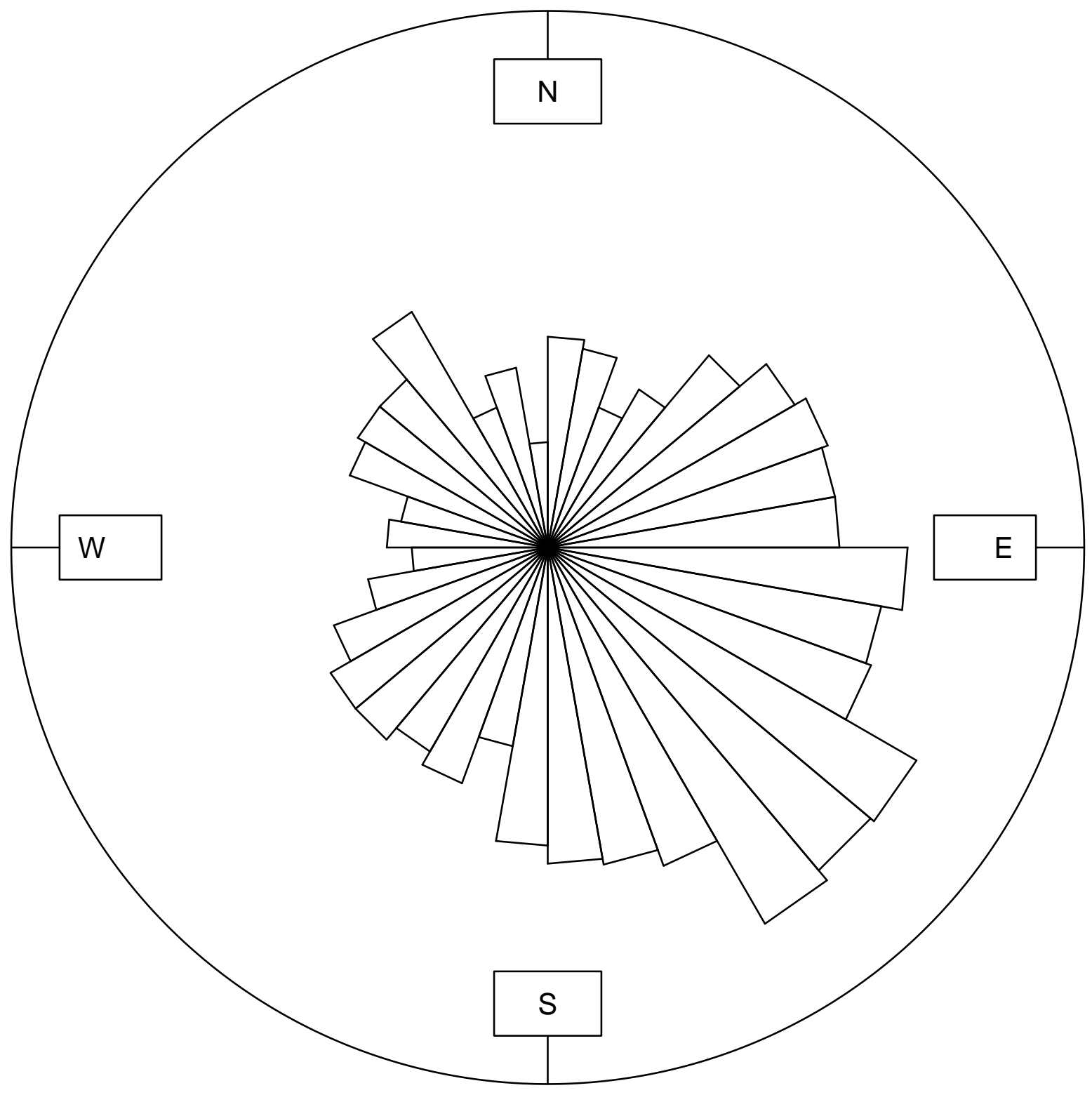


bootstrap 997

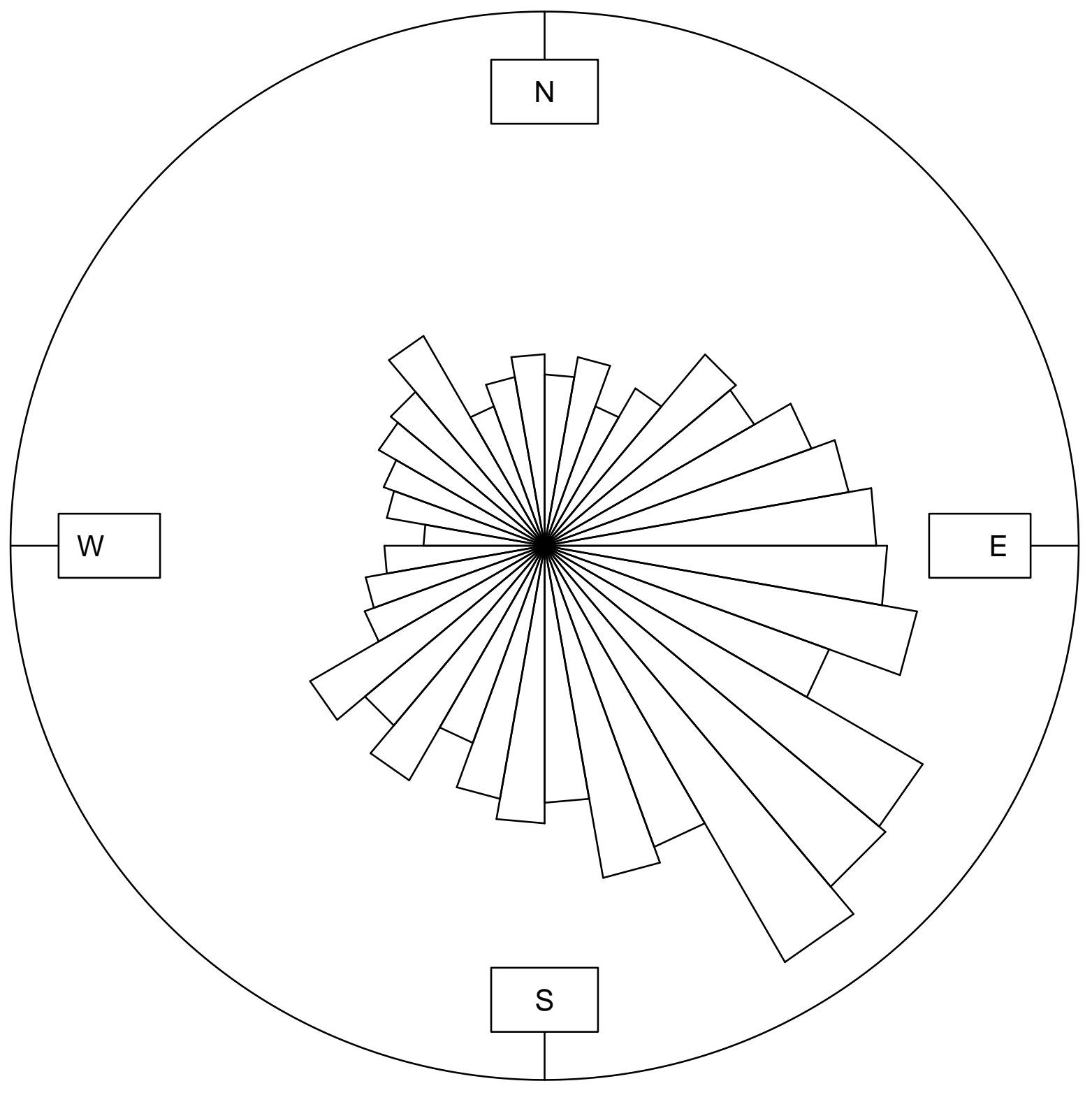


bootstrap 998

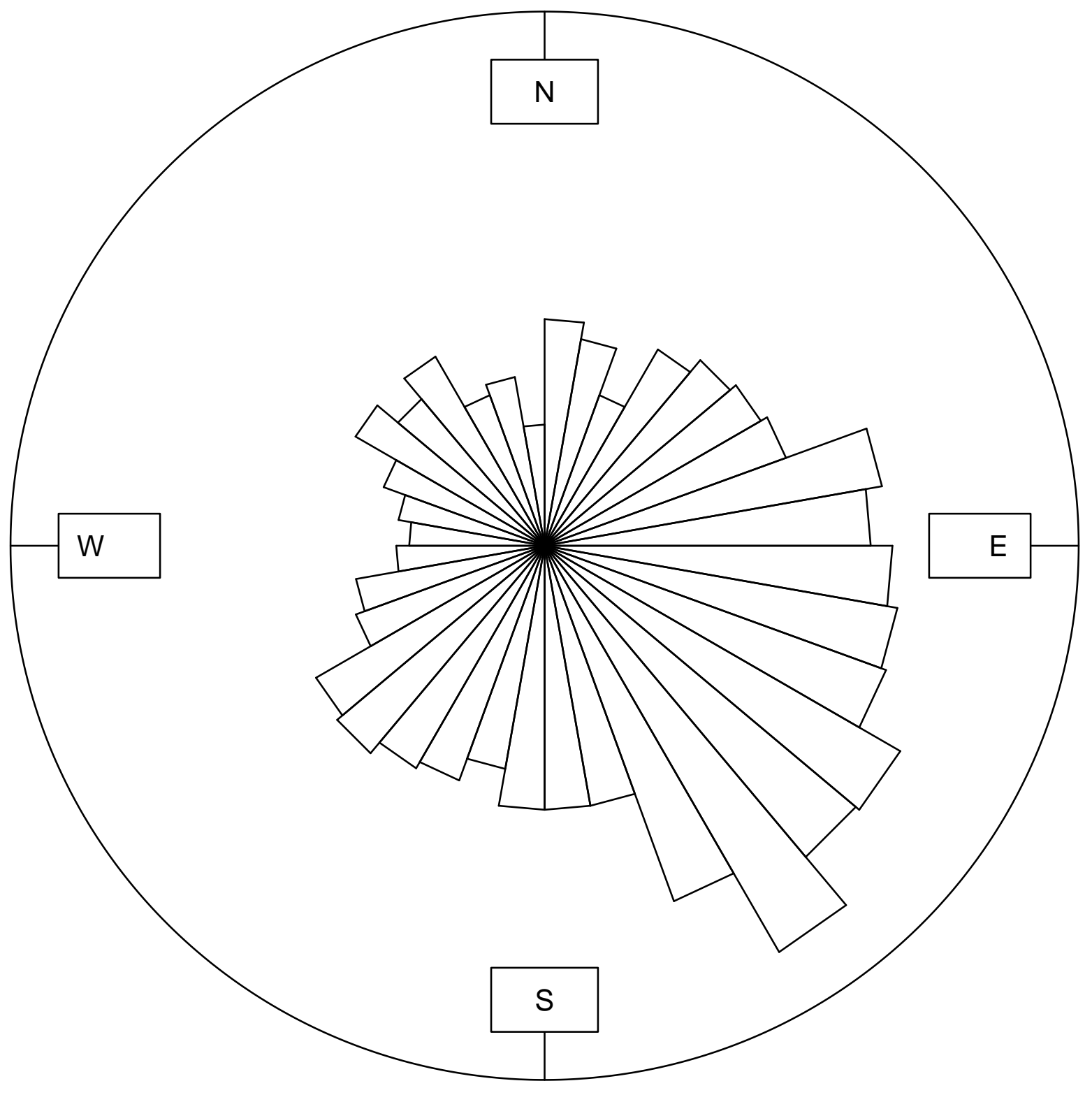


bootstrap 999

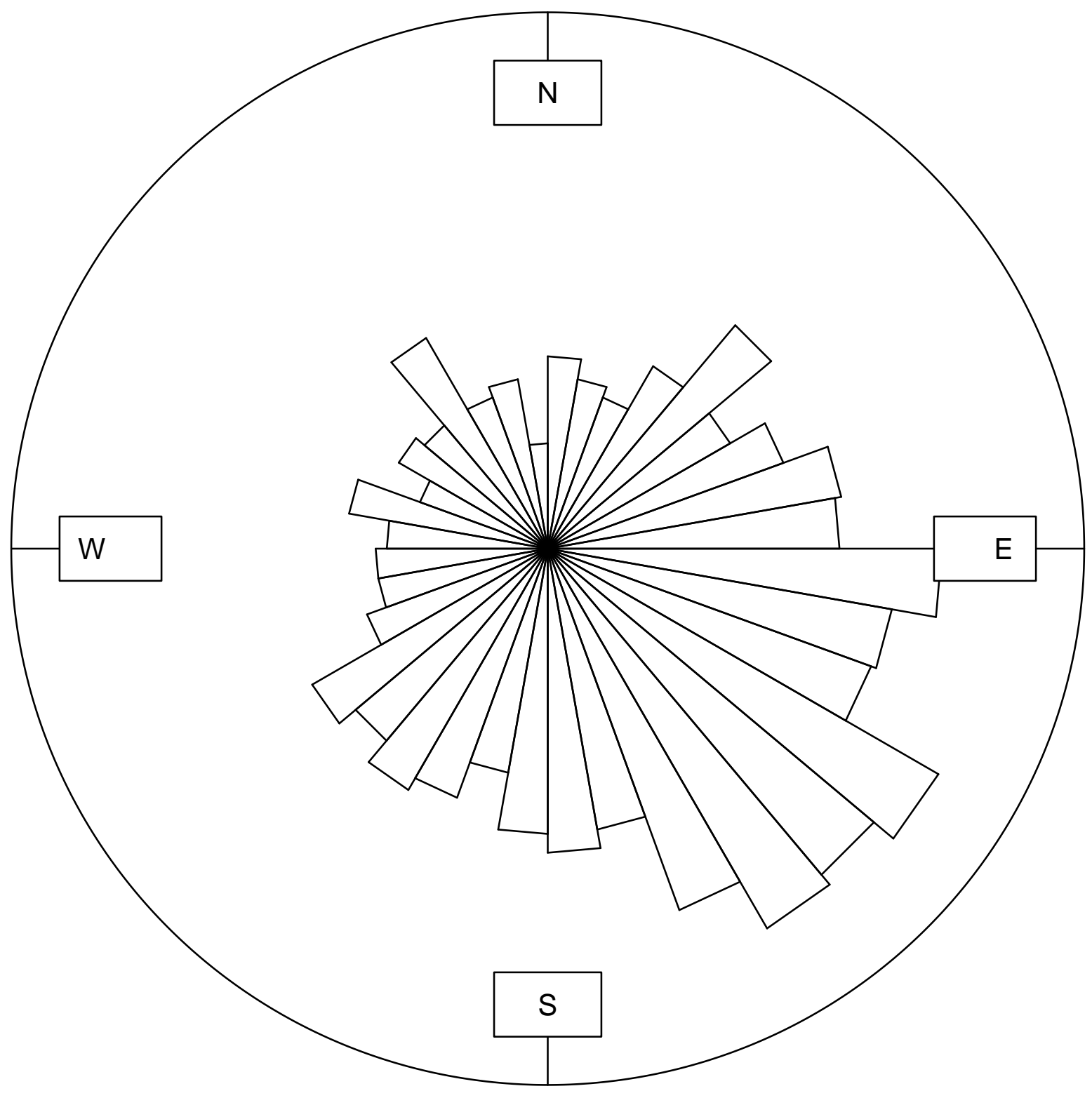


bootstrap 1000

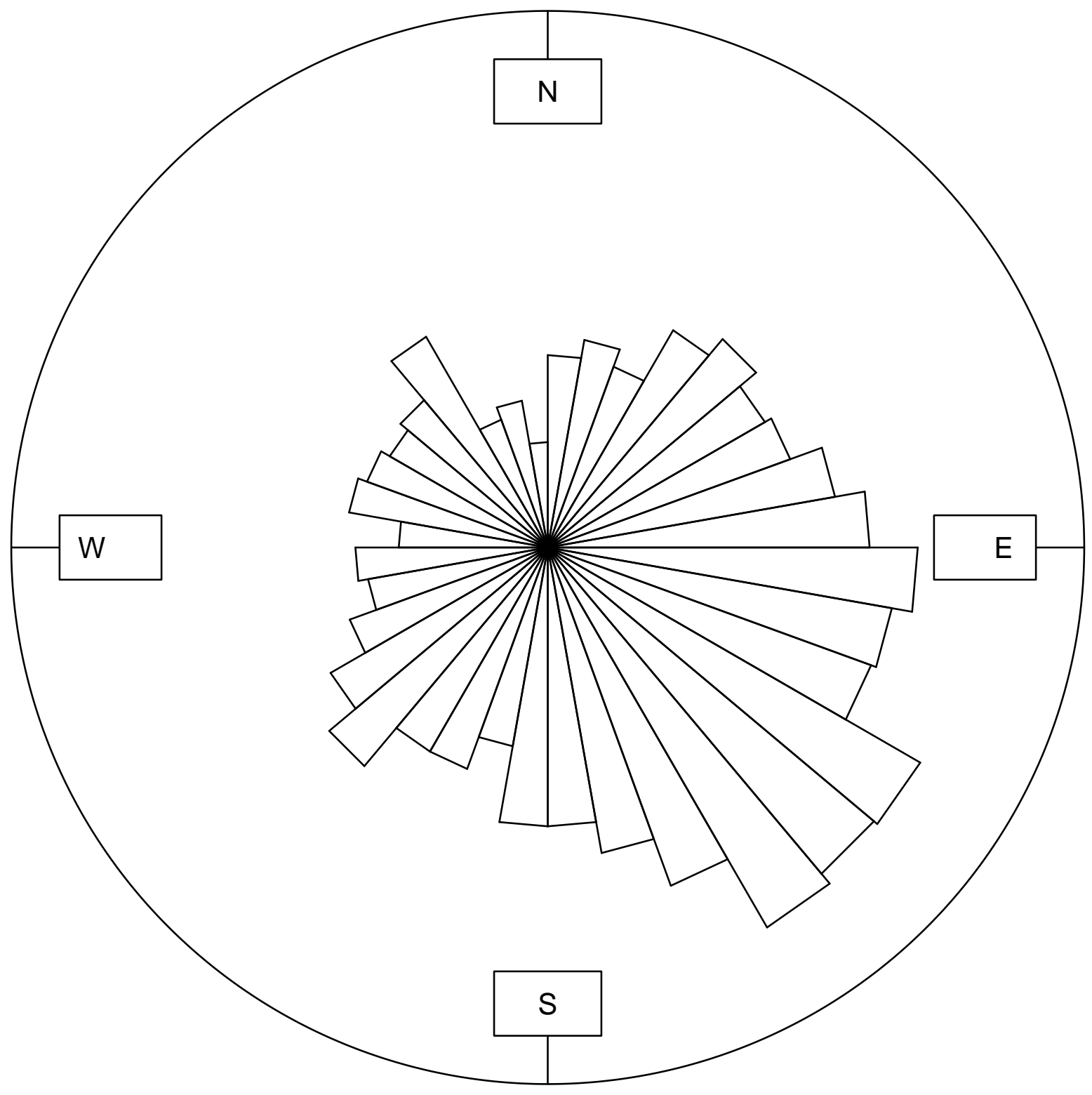

